\title{
Sodium-Bearing Waste Treatment Technology Evaluation Report
}

Charles M. Barnes Arlin L. Olson Dean D. Taylor

February 2004

Idaho National Engineering and Environmental Laboratory Bechtel BWXT Idaho, LLC 
INEEL/EXT-04-01692

\title{
Sodium-Bearing Waste Treatment Technology Evaluation Report
}

\author{
Charles M. Barnes \\ Arlin L. Olson \\ Dean D. Taylor
}

February 2004

Idaho National Engineering and Environmental Laboratory Idaho Completion Project Idaho Falls, Idaho 83415

Prepared for the

U.S. Department of Energy

Assistant Secretary for Environmental Management

Under DOE Idaho Operations Office

Contract DE-AC07-99ID13727 
This page intentionally left blank. 


\section{EXECUTIVE SUMMARY}

A Settlement Agreement between the U.S. Department of Energy (DOE) and the State of Idaho mandates treatment of sodium-bearing waste (SBW) stored at the Idaho Nuclear Technology and Engineering Center located within the Idaho National Engineering and Environmental Laboratory (INEEL). One of the requirements of the Settlement Agreement is to complete treatment of SBW by December 31, 2012. Hence, SBW disposition is one of the Idaho Operation Office's (NE-ID) and State of Idaho's top priorities at the INEEL.

The INEEL has been working over the past several years to identify a treatment technology that meets NE-ID and regulatory treatment requirements, including consideration of stakeholder input. Many studies, including the High-Level Waste and Facilities Disposition Environmental Impact Statement, have resulted in the identification of five treatment alternatives that form a short list of perhaps the most appropriate technologies for the DOE to select from. The alternatives are (a) calcination with maximum achievable control technology (MACT) upgrade, (b) steam reforming, (c) cesium ion exchange (CsIX) with immobilization, (d) direct evaporation, and (e) vitrification. Each alternative has undergone some degree of applied technical development and preliminary process design over the past four years.

Applied technology development and preliminary process design activities have been required to provide the data necessary for informed decision making and evaluation of the treatment options. A technically defensible selection of a SBW treatment process has been a key driver for the collection of needed data. This document presents a summary of the data gathered in recent years and evaluates the technical maturity of each option by listing and discussing the identified higher-risk technical uncertainties. The SBW issue and the five alternatives are described, and their technical performance (throughputs, waste product descriptions and volumes, preliminary facility footprints, etc.) is summarized in the main body. Details of preliminary process design and applied development activities for three of the alternatives (steam reforming, CsIX with immobilization, and direct evaporation) are presented in three appendices; a recent feasibility study provides the details for calcination. There have been no recent activities performed with regard to vitrification; that section summarizes and references previous work.

This report only addresses technical issues that have been identified through examination of waste acceptance criteria (WAC), operability issues, and process design activities. It is assumed in this report that the SBW would be classified as a transuranic waste, suitable for disposal at the Waste Isolation Pilot Plant (WIPP), located in the State of New Mexico, after appropriate treatment to meet transportation requirements and WAC. This report does not address issues that are programmatic in nature. Examples of these include how the SBW will be classified with respect to the Nuclear Waste Policy Act, status of WIPP permits and waste storage availability, available funding for implementation, stakeholder issues, and meeting State of Idaho Settlement Agreement milestones. This report also does not present detailed cost and implementation schedule information for the treatment options. 


\section{CONTENTS}

Executive Summary …………………………………………………………………………ii

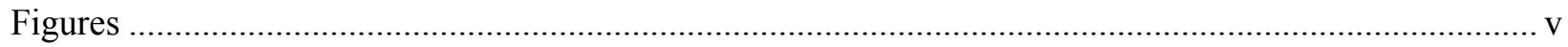

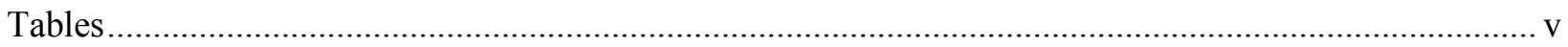

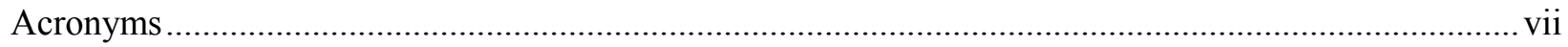

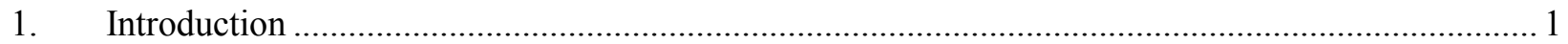

$1.1 \quad$ Purpose and Objectives …………………………………………………………….. 1

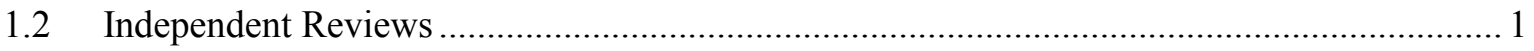

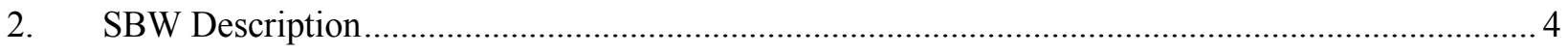

3. Technical Data for Treatment Alternatives ............................................................................... 6

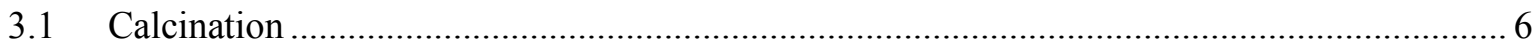

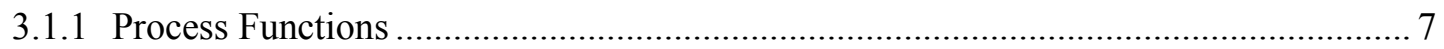

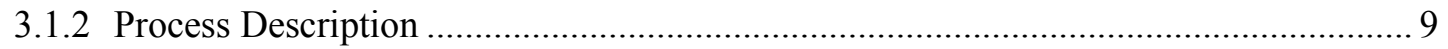

3.1.3 Summary of Technical Performance ...................................................................... 16

3.1.4 Technical Uncertainties and Potential Impacts............................................................. 20

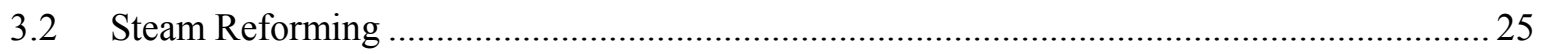

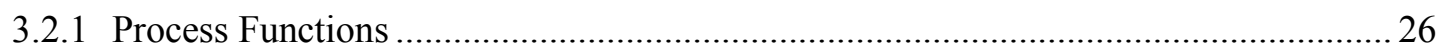

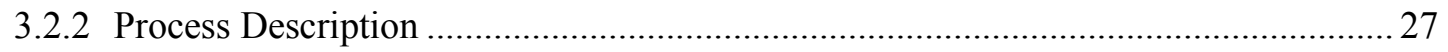

3.2.3 Summary of Technical Performance …………………………………………........ 31

3.2.4 Technical Uncertainties and Potential Impacts............................................................. 35

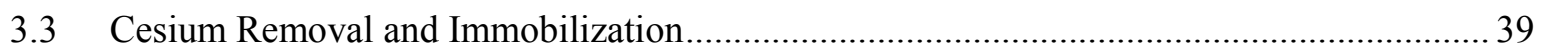

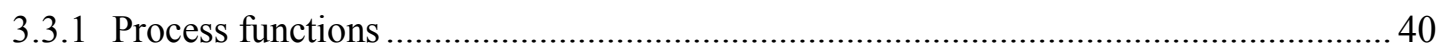

3.3.2 Process Description ...................................................................................... 41

3.3.3 Summary of Technical Performance …………………………………………... 44

3.3.4 Technical Uncertainties and Potential Impacts.......................................................... 48

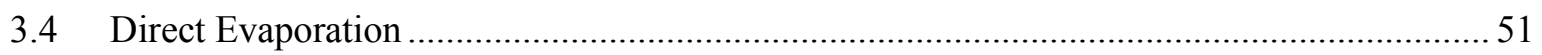

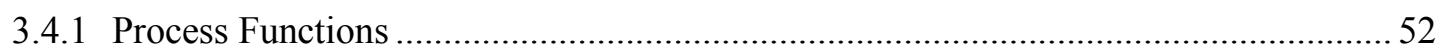

3.4.2 Process Description .................................................................................... 53

3.4.3 Summary of Technical Performance …………………………………………….....59

3.4.4 Technical Uncertainties and Potential Impacts............................................................ 62

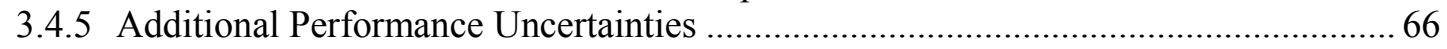




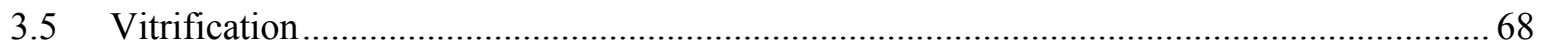

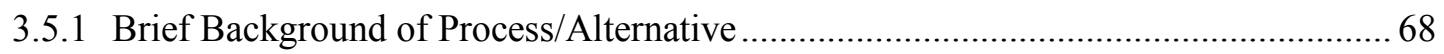

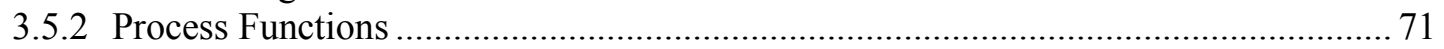

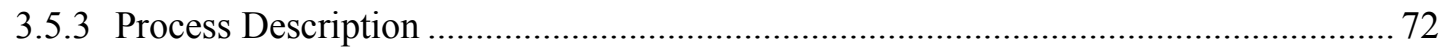

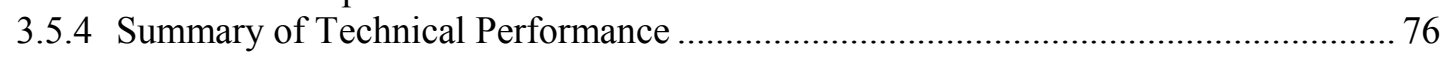

3.5.5 Technical Uncertainties and Potential Impacts........................................................ 81

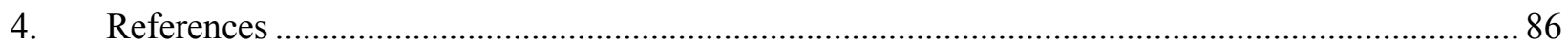

\section{FIGURES}

Figure 1. CMACT block flow diagram: Tank Farm, NWCF, \& CPF. ............................................... 10

Figure 2. CMACT block flow diagram: MACT Compliance Facility................................................ 11

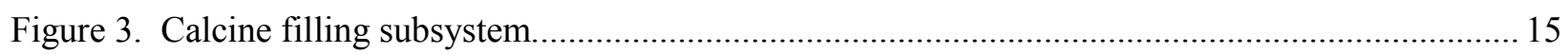

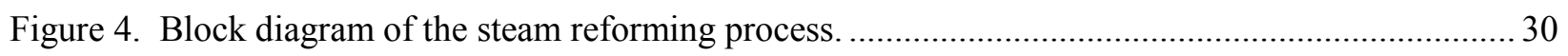

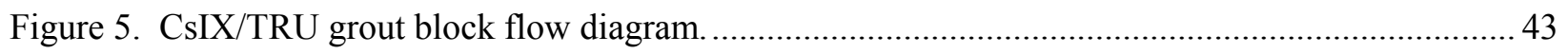

Figure 6. Revised baseline direct evaporation block flow diagram. .................................................. 57

Figure 7. Alkaline scrubber direct evaporation block flow diagram................................................... 58

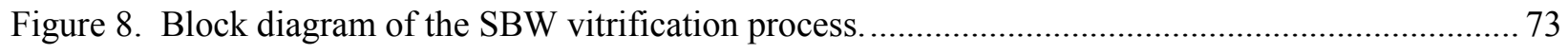

\section{TABLES}

Table 1. Expected feed range for major SBW constituents. ............................................................... 5

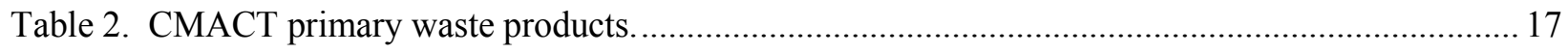

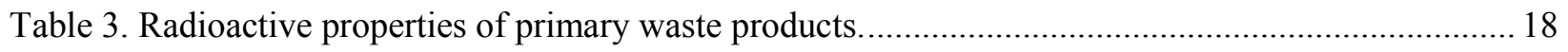

Table 4. Summary of CMACT uncertainties identified in the Applied Technology Plan....................... 20

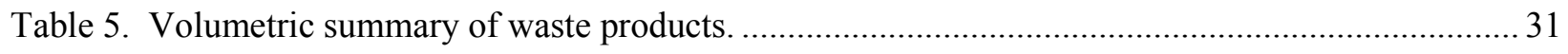

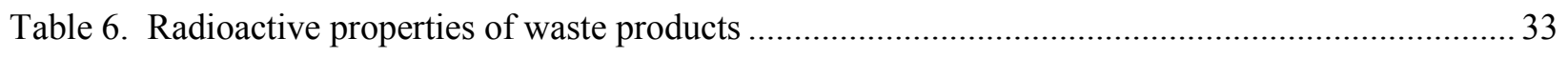

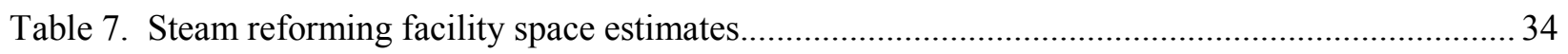

Table 8. Steam reforming facility space estimates for 1 year processing schedule............................... 34

Table 9. Summary of steam reforming uncertainties identified in risk assessment. ............................... 35 


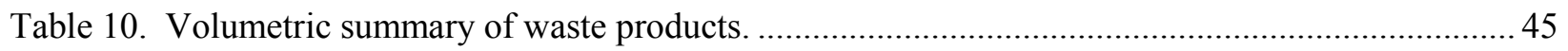

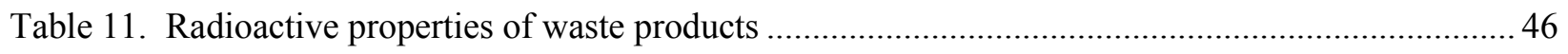

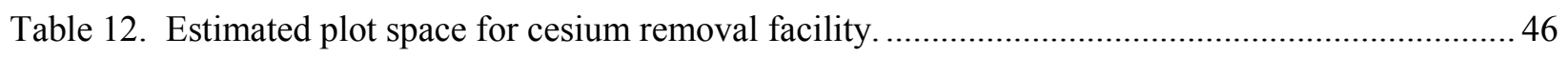

Table 13. Estimated increase of facility plot space for cesium removal facility................................... 48

Table 14. Summary of cesium removal uncertainties identified in risk assessment.............................. 48

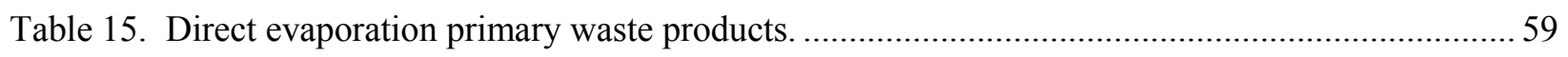

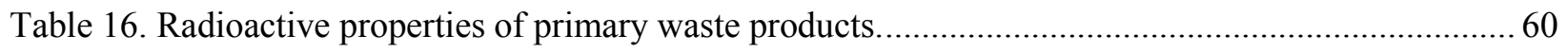

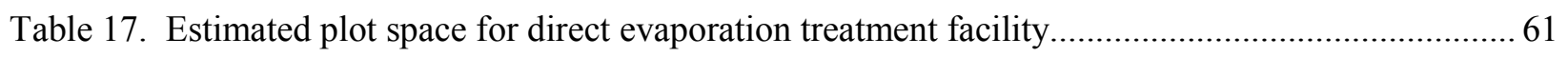

Table 18. Estimated floor space for the direct evaporation treatment facility.................................... 62

Table 19. Summary of direct evaporation uncertainties identified in risk assessment. .......................... 63

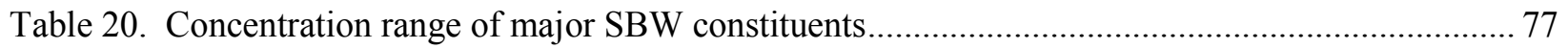

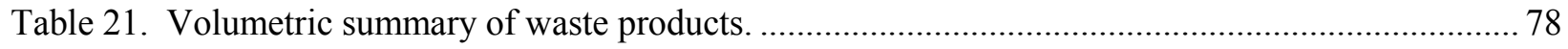

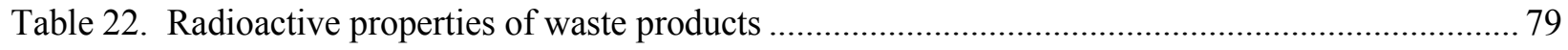

Table 23. Vitrification facility space estimates for 2 -year processing schedule.................................... 80

Table 24. Vitrification facility space estimates for 1 -yr processing schedule. ....................................... 81 


\section{ACRONYMS}

AAR aluminum to alkali mole ratio

APS

Atmospheric Protection System

BBWI Bechtel BWXT Idaho, LLC

BSG borosilicate glass

CCIM Cold Crucible Induction Melter

CEM continuous emission monitor

$\mathrm{CH}$

contact-handled

CMACT Calciner/MACT

$\mathrm{CPF}$

CsIX

Calcine Packaging Facility

CST

cesium ion exchange

crystalline silicotitanate

DBE design basis element

DOE Department of Energy

DWPF Defense Waste Processing Facility

EDF Engineering Design File

EIS

EPA

Environmental Impact Statement

ETS

Environmental Protection Agency

Evaporative Tank System

FY fiscal year

GAC granulated activated carbon

HEME High Efficiency Mist Eliminator

HEPA high efficiency particulate air (filter)

HLLWE High Level Liquid Waste Evaporator

HLW high-level waste

HVAC heating, ventilation and air conditioning

HWC Hazardous Waste Combustion

INEEL Idaho National Engineering and Environmental Laboratory

INTEC Idaho Nuclear Technology and Engineering Center

IRT Independent Review Team

IWVF Idaho Waste Vitrification Facility

LDR Land Disposal Restrictions

LET\&D Liquid Effluent Treatment and Disposal

MACT Maximum Achievable Control Technology

MCF MACT Compliance Facility

NAR nozzle-air-ratio

NE-ID DOE Idaho Operations Office

NESHAP National Emission Standards for Hazardous Air Pollutants

NGLW newly generated liquid waste 


$\begin{array}{ll}\text { NO }_{\mathrm{x}} & \text { gas containing both NO and NO2 } \\ \text { NRC } & \text { Nuclear Regulatory Commission } \\ \text { NWCF } & \text { New Waste Calcining Facility } \\ \text { NWPA } & \text { Nuclear Waste Policy Act } \\ \text { PUREX } & \text { plutonium-uranium extraction } \\ & \\ \text { RAM } & \text { reliability, availability, and maintainability } \\ \text { RCRA } & \text { Resource Conservation and Recovery Act } \\ \text { RH } & \text { remote-handled } \\ & \\ \text { SAIC } & \text { Science Application International Corporation } \\ \text { SBW } & \text { sodium-bearing waste } \\ \text { S-GAC } & \text { sulfur-impregnated, granulated activated carbon } \\ \text { SRS } & \text { Savannah River Site } \\ & \\ \text { T\&FR } & \text { Technical and Functional Requirements } \\ \text { TFF } & \text { Tank Farm Facility } \\ \text { THOR } & \text { Thermal Organic Reduction } \\ \text { THOREX } & \text { thorium extraction } \\ \text { TRU } & \text { transuranic (waste) } \\ \text { TTT } & \text { THOR } \\ \text { TWR } & \text { ThermoCheatment Technologies } \\ & \\ \text { WAC } & \text { Waste Acceptance Criteria } \\ \text { WCF } & \text { Waste Calcining Facility } \\ \text { WFQP } & \text { Waste Form Qualification Plan } \\ \text { WIPP } & \text { Waste Isolation Pilot Plant } \\ \text { WVDP } & \text { West Valley Demonstration Project } \\ \end{array}$




\section{Sodium-Bearing Waste Treatment Technology Evaluation Report}

\section{INTRODUCTION}

Sodium-bearing waste (SBW) disposition is one of the U.S. Department of Energy (DOE) Idaho Operation Office's (NE-ID) and State of Idaho's top priorities at the Idaho National Engineering and Environmental Laboratory (INEEL). The INEEL has been working over the past several years to identify a treatment technology that meets NE-ID and regulatory treatment requirements, including consideration of stakeholder input. Many studies, including the High-Level Waste and Facilities Disposition Environmental Impact Statement (EIS), have resulted in the identification of five treatment alternatives that form a short list of perhaps the most appropriate technologies for the DOE to select from. The alternatives are (a) calcination with maximum achievable control technology (MACT) upgrade, (b) steam reforming, (c) cesium ion exchange (CsIX) with immobilization, (d) direct evaporation, and (e) vitrification. Each alternative has undergone some degree of applied technical development and preliminary process design over the past four years.

This report presents a summary of the applied technology and process design activities performed through February 2004. The SBW issue and the five alternatives are described in Sections 2 and 3, respectively. Details of preliminary process design activities for three of the alternatives (steam reforming, CsIX, and direct evaporation) are presented in three appendices. A recent feasibility study provides the details for calcination. There have been no recent activities performed with regard to vitrification; that section summarizes and references previous work.

\section{$1.1 \quad$ Purpose and Objectives}

The purpose of this document is to summarize technical information that is available to enable the DOE to make an informed decision regarding the selection of a treatment technology for a SWB Treatment Facility. This report does not address issues that are programmatic in nature. Examples of these include how the SBW will be classified with respect to the Nuclear Waste Policy Act (NWPA), status of Waste Isolation Pilot Plant (WIPP) permits and waste storage availability, available funding for implementation, stakeholder issues, and meeting State of Idaho Settlement Agreement milestones. This report also does not present detailed cost and implementation schedule information for the treatment options.

This report only addresses technical issues that have been identified through examination of waste acceptance criteria (WAC), operability issues, and process design activities. It is assumed in this report that the SBW would be classified as a transuranic (TRU) waste, suitable for disposal at WIPP, located in the State of New Mexico, after appropriate treatment to meet transportation requirements and WAC.

Descriptions of anticipated treatment processes, preliminary facility sizes and throughputs, waste product descriptions and volumes, and higher risk technical uncertainties and their potential impacts are presented.

\subsection{Independent Reviews}

To ensure the proper emphasis and staging of decision activities, independent reviews have been conducted of the preliminary process design and applied development activities by a standing ad hoc team of DOE Complex technical experts referred to as the Independent Review Team (IRT). The following 
were identified during a February 2003 review and recommended as areas to accelerate or emphasize, thereby reducing technical risk to the project:

- Identify level of risk associated with uncertainties for each alternative

- Clearly focus on high-risk technology uncertainties first in developing test activities

- Use technology roadmaps as tools to aid DOE management in making informed decisions at key decision points in the project.

A Sodium-Bearing Waste Treatment Applied Technology Plan, INEEL/EXT-03-00477, was developed and published in June 2003 to accommodate these suggestions as follows.

An independent review of the results of steam reforming demonstrations performed in December 2002 and January 2003 was conducted in May 2003. Recommendations were:

- $\quad$ The IRT recommends that INEEL give first priority to continuing the Thermal Organic Reduction $\left(\mathrm{THOR}^{\mathrm{sm}}\right.$ ) and ThermoChem Waste Remediation (TWR) steam reforming tests. The INEEL should (1) optimize the conditions on the reducing fluidized bed, (2) conduct longer tests to approach steady state conditions for product quality and throughput rate evaluations, and (3) if a test of the THOR ${ }^{\mathrm{sm}}$ oxidizing fluidized bed concept is desired, reconfigure the existing bed to operate under oxidizing conditions with feed from (2) above, if possible.

- The IRT recommends that quantitative success criteria be defined, such as the following: nitrogen oxide $\left(\mathrm{NO}_{\mathrm{x}}\right)$ gas concentrations, hydrogen $\left(\mathrm{H}_{2}\right)$ concentration in the off-gas, mercury $(\mathrm{Hg})$ capture/speciation, carbon in product, density of product, and compaction of product.

- The IRT recommends that, to the extent possible, INEEL identify and quantify the solid product characteristics that would be required to satisfy the draft WIPP WAC. The extent to which the steam reforming test products meet these criteria should be determined.

- It would be desirable to have a single product stream from the steam reforming process. To approach this goal, the IRT recommends reconfiguring the test apparatus so that the cyclone product can be recycled back to the fluidized bed.

- The IRT endorses all the modifications to Science Applications International Corporation (SAIC)'s STAR equipment proposed by Bechtel BWXT Idaho, LLC (BBWI) and recommends that they install a bottom feed nozzle.

Two more demonstrations of vendor steam reforming technologies were conducted in November and December of 2003 that incorporated these recommendations. The demonstration results are included in Section 3.2.

A review performed in September 2003, focusing on FY 2003 technical results and FY 2004 plans made the following recommendations:

- $\quad$ SBW Characterization: Consideration should be given to mixing the solids in tank WM-187 prior to or during sampling to ensure representative samples and reduce uncertainties. The liquids in the tanks are adequately characterized, and further liquid sampling and analyses should not be performed at this time. 
- Waste Simulant Development: The extensive planned solid simulant development effort does not appear necessary, and the required chemical and physical property data for these solids should be acquired in the characterization of actual waste solids.

- $\quad$ Calcination with Maximum Achievable Control Technology: Boiling the SBW with glycolic acid as a means of reducing volume, which would decrease the treatment time, should not be continued in FY 2004. Other alternative methods for reducing waste volume (and, consequently, schedule) for calcination should be evaluated and tested. No further testing (including pilot-plant scale tests) of granulated activated carbon (GAC) systems for removal of mercury from off-gas should be conducted at this time. Experimental work should continue in FY 2004 to develop adequate technology for selective and effective removal of hydrogen chloride $(\mathrm{HCl})$ from calciner off-gas based on use of GAC or some other suitable sorbent.

- Direct Evaporation: No further pilot-plant scale tests of the direct evaporation process with SBW simulants, either in a Wiped Film Evaporator or with alternative evaporator equipment, should be performed until the new Idaho Cleanup Project contractor is selected (or a technology down selection has been made by DOE). Appropriate tests should be conducted in FY 2004 to establish the technical feasibility of recycling the off-gas condensate from DE process operation to the evaporator. Bench-scale tests should be conducted in FY 2004 to characterize the off-gas at the optimum evaporation end point.

- $\quad$ Cesium lon Exchange: The IRT agrees that crystalline silicotitanate (CST) is the preferred sorbent and recommends that any additional ion exchange work focus on CST. Further testing should be performed in FY 2004 to resolve the uncertainties concerning the instability of CST sorbent use in plant-scale operation. Silica gel for immobilization of the ion exchange effluent should be discontinued. Development work on grouting has exceeded development needs at this time and should be suspended.

These recommendations were, for the most part, implemented during the period of October 2003 through February 2004 and the results, with the exception of Waste Characterization and Simulant Development, are included in the individual sections and appendices. More definitive characterization and simulant data are due to be published in a separate report in June 2004.

A review of data produced and analyzed for each treatment alternative and presented in this report will be conducted in March 2004 as well. 


\section{SBW DESCRIPTION}

Radioactive liquid waste has been generated over the last five decades at the Idaho Nuclear Technology and Engineering Center (INTEC), formerly called the Idaho Chemical Processing Plant, as a result of nuclear fuel reprocessing activities. From December 1963 until June 2000, the Waste Calcining Facility (WCF; now decommissioned) and the New Waste Calcining Facility (NWCF; presently in a stand-by condition, but undergoing a reversible closure) processed the liquid waste into a granular, solid form. As of February 2004 it is anticipated that approximately 1,000,000 gallons of radioactive liquid and heel solids waste, commonly referred to as SBW, will require treatment for disposal. Additional liquid waste will be generated in the future as a result of filter leach operations, equipment and building decontamination activities, Resource Conservation and Recovery Act (RCRA) closure activities, and other operations at INTEC.

An anticipated SBW treatment facility would treat liquids and solids contained in existing tanks at the INTEC. The treatment facility may also treat additional liquid waste, called Newly Generated Liquid Waste (NGLW), that will be generated after 2005 and stored in separate tanks from the SBW.

A complete description of the feed composition for the SBW requiring treatment can be found in report INEEL/EXT-2000-01378, Revision 3. This report presents the most recent compilation of volumes and compositions of the feed streams to the five proposed alternative treatment processes. These liquid wastes have been stored in eleven 300,000-gallon underground tanks at INTEC. The underground tank facility at INTEC is referred to as the Tank Farm Facility (TFF).

Tanks WM-180 and WM-189 presently contain volumes of waste near their administrative capacities and no additions to these tanks are expected. The waste compositions in the report for waste in Tanks WM-180 and WM-189 are based on sample analyses; the compositions of waste in tank WM-187 and WM-188 are based on analyses of source streams, calculations using plant evaporator operations information, and simulation of expected future evaporation of dilute wastes.

As tanks in the TFF have been flushed and closed, solids have accumulated in Tank WM-187. The treatment process would process the solids in this tank as well as solids from the three SBW tanks (WM-180, WM-188, WM-189). Compositions are defined both for alternatives that process solids separately and together with SBW liquid.

The present estimate of the composition of Tank WM-187 solids is based on analyses of samples of tank heels taken prior to being flushed to WM-187. Not all tanks that have been flushed were sampled and analyzed; in addition there are uncertainties in estimated quantities of solids in each tank. Thus, the present estimate of the WM-187 solids composition contains uncertainty. The tank was recently sampled to obtain a more representative sample of all solids in WM-187. The analyses data from this sample should reduce the uncertainty in the WM-187 solids composition.

Projections have been made of the volumes of NGLW streams generated prior to and during operation of the SBW treatment facility. For some of these NGLW streams, chemical composition data are available and have been used in generating treatment facility feed compositions. However, data for radionuclide concentrations in NGLW are extremely limited. Thus, radionuclide concentrations in treatment facility feeds are based solely on data from existing tank waste. Starting in FY 2006, NGLW will be collected in Tanks WM-100, WM-101, and WM-102. The accumulated NGLW may later be blended with SBW for treatment in the SBW treatment facility.

Each potential treatment option would process approximately 1,000,000 gallons of SBW liquid and heel solids. SBW will initially be contained in four TFF tanks: WM-180, WM-187, WM-188, and 
WM-189. Tanks WM-180, WM-188, and WM-189 will contain liquid SBW with relatively small quantities of undissolved solids, while WM-187 will contain the majority of the solids plus dilute liquid wastes. NGLW after 2005 will be collected in three other tanks, WM-100, WM-101 and WM-102. Table 1 lists the concentration range of the major constituents for the feed to the potential treatment processes described in the later sections of this report.

Table 1. Expected feed range for major SBW constituents.

\begin{tabular}{lccc}
\hline Constituent & $\begin{array}{c}\text { Minimum } \\
\text { (Moles/liter) }\end{array}$ & $\begin{array}{c}\text { Maximum } \\
\text { (Moles/liter })\end{array}$ & Max/Min \\
\hline $\mathrm{H}+$ & $1.07 \mathrm{E}+00$ & $2.82 \mathrm{E}+00$ & 2.6 \\
$\mathrm{Al}+3$ & $3.34 \mathrm{E}-01$ & $6.51 \mathrm{E}-01$ & 1.9 \\
$\mathrm{~B}+3$ & $1.00 \mathrm{E}-02$ & $1.94 \mathrm{E}-02$ & 2.1 \\
$\mathrm{Ca}+2$ & $3.20 \mathrm{E}-02$ & $6.69 \mathrm{E}-02$ & 3.3 \\
$\mathrm{Cs}+$ & $2.57 \mathrm{E}-05$ & $8.42 \mathrm{E}-05$ & 1.8 \\
$\mathrm{Cl}-$ & $1.62 \mathrm{E}-02$ & $2.86 \mathrm{E}-02$ & 1.6 \\
$\mathrm{Cr}+3$ & $3.36 \mathrm{E}-03$ & $5.30 \mathrm{E}-03$ & 4.0 \\
$\mathrm{~F}-$ & $1.41 \mathrm{E}-02$ & $5.62 \mathrm{E}-02$ & 1.7 \\
$\mathrm{Fe}+3$ & $1.73 \mathrm{E}-02$ & $2.97 \mathrm{E}-02$ & 1.9 \\
$\mathrm{Mg}+2$ & $1.10 \mathrm{E}-02$ & $2.09 \mathrm{E}-02$ & 4.5 \\
$\mathrm{Hg}+2$ & $1.32 \mathrm{E}-03$ & $5.97 \mathrm{E}-03$ & 1.5 \\
$\mathrm{NO}_{3}-$ & $4.93 \mathrm{E}+00$ & $7.16 \mathrm{E}+00$ & 11 \\
$\mathrm{PO}_{4}-3$ & $1.53 \mathrm{E}-02$ & $1.75 \mathrm{E}-01$ & 2.0 \\
$\mathrm{~K}+$ & $1.76 \mathrm{E}-01$ & $3.50 \mathrm{E}-01$ & 1.4 \\
$\mathrm{Na}^{-}+$ & $1.35 \mathrm{E}+00$ & $1.94 \mathrm{E}+00$ & 3.7 \\
$\mathrm{SO}_{4}-2$ & $2.61 \mathrm{E}-02$ & $9.79 \mathrm{E}-02$ & 199 \\
$\mathrm{Zr}+4$ & $3.34 \mathrm{E}-04$ & $6.66 \mathrm{E}-02$ &
\end{tabular}

For treatment alternatives that co-process liquid and solids, the solids in WM-187 would be distributed, prior to treatment, among three of the four SBW tanks through a series of waste transfers between tanks. Mixing pumps would be installed in these three tanks to keep the solids in suspension. Four of the potential treatment options, all except the CsIX/Immobilization process, would co-process the solids. Solids would be separated from the SBW and treated separately under the CsIX/Immobilization option. 


\section{TECHNICAL DATA FOR TREATMENT ALTERNATIVES}

The following sections present summary descriptions of the five treatment options for SBW that have been actively pursued through process engineering and applied development work since FY 2000. The most recent preferred process flow configurations and process descriptions are included. A summary of the technical performance of each option is discussed at the end of each option description section. The discussion centers on processing rate, quantity and quality of products produced, footprint of potential facilities for processing, variation of scale with throughput, and status of the technical uncertainties and potential impacts.

\subsection{Calcination}

Fluidized bed calcination has been used at the INTEC since the early 1960's to solidify acidic wastes that were produced in fuel reprocessing operations. WCF was operated from 1961 to 1981, and the NWCF from 1981 to 2000. Newby and O'Brien (2000) have summarized the operation of all the WCF campaigns and all but the last NWCF campaign. The WCF processed a total of 4.1 million gallons of feed, and the NWCF processed 3.9 million gallons (Staiger, 2003).

In the calcination process, liquid wastes are sprayed into a fluidized bed of heated spherical calcine particles. The calciner vessel of the WCF had a 2-ft square fluidization section; the calciner vessel of the NWCF has a 5-ft diameter fluidizing section. Until 1967, the WCF fluidized bed was heated to the calcination temperature of $400^{\circ} \mathrm{C}$ by recirculating liquid eutectic sodium/potassium alloy through heat exchange tubes located in the bed. After 1967, in-bed combustion using kerosene fuel provided the heat to maintain the calcination temperature of $500^{\circ} \mathrm{C}$ in the bed in the WCF, and later in the NWCF calciner. The NWCF calciner was operated at $600^{\circ} \mathrm{C}$ during two short runs in 1999 and 2000 (Law, 2000).

The WCF primarily calcined high-level waste (HLW) from reprocessing aluminum, zirconium and stainless steel-clad fuel. The first processing of SBW occurred during WCF campaign H-7, in which one part SBW was blended with four parts of zirconium waste. Later WCF campaigns included SBW bends with a maximum blend ratio of 1 part SBW to 3.5 parts of zirconium waste. Blending SBW was necessary to avoid agglomeration in the bed due to formation of molten salts of sodium and potassium.

The NWCF also processed HLW and HLW/SBW blends during its first three campaigns. The typical concentration of sodium plus potassium in the NWCF feed blends during these campaigns was about 0.25 molar, considerably less that the 2 molar sodium and potassium concentration of present SBW. The fourth NWCF campaign included processing the last of the HLW stored in the INTEC Tank Farms and also the first long-term processing of SBW, blended not with other wastes but with non-radioactive aluminum nitrate.

In 1995 and 1996, development and engineering studies were initiated to modify the calcination process to treat SBW directly without blending it with lower alkali content HLW liquids (Boardman, 1997; Welland, 1997). The result of this effort recommended increasing the operating temperature of the calciner from $500^{\circ} \mathrm{C}$ to $600^{\circ} \mathrm{C}$. Operation at $600^{\circ} \mathrm{C}$ processing SBW was successfully demonstrated in the NWCF during two separate trials during 1999 and 2000 (Law, 2000; Swenson, 2000; Wood, 2001). The conclusion from these demonstration runs was that operation of the existing NWCF at $600^{\circ} \mathrm{C}$ is a viable long-term method for processing SBW.

The NWCF was operated under a State of Idaho Permit to Construct and under interim RCRA status. In the early and mid-1990's, DOE initiated an effort to better determine the calciner off-gas composition and emission inventory to support a RCRA permitting process for the calciner. At the same general time (1996), the Environmental Protection Agency (EPA) proposed the Hazardous Waste 
Combustion (HWC) National Emission Standards for Hazardous Air Pollutants (NESHAP) based on MACT (40 CFR 63 Subpart EEE).

A multi-year emission inventory and risk assessment effort and several studies that evaluated how to upgrade the calciner facility to meet the HWC MACT standards were performed between 1997 and 2001 (Rawlings, 1997; Boardman, 1999; Boardman, 2000; Young, 2000; Ashworth, 2000; Boardman, 2001). In 2000, after off-gas sampling confirmed that the calciner facility would require modifications to become MACT-compliant, DOE temporarily shut down the calciner pending determination of how the remaining SBW should be treated for final disposal. If restarted, it is expected that the NWCF would be regulated as a miscellaneous thermal treatment unit; thus, compliance with MACT emission limits will be imposed. To meet these standards, an upgrade to the calciner off-gas treatment train will be required. Treatment of SBW in the NWCF upgraded to meet MACT standards and packaging product calcine for disposal at WIPP in a new facility is referred to as the Calciner/MACT (CMACT) alternative for processing SBW.

Three documents provided the source of the most of the rest of the information in this report for the CMACT alternative. In FY 2003, the technical and functional requirements (T\&FR) for the CMACT alternative were defined (Beitel, 2003). Also in 2003, a process design was completed for upgrading the NWCF to meet MACT emission limits and to package the calcine produced in remote-handled (RH) canisters ready for shipment to WIPP (Barnes, 2003). This process design was the starting point for a more detailed feasibility study that was completed in early 2004 (Merrick, 2004).

\subsubsection{Process Functions}

The functions of the CMACT treatment process are as follows:

- Mix and retrieve the SBW liquids and solids from storage tanks WM-180, WM-187, WM188, and WM-189, and tanks storing NGLW

- Prepare the SBW and NGLW feeds with additives prior to calcination

- Calcine the SBW and NGLW

- Treat the off-gas to meet MACT emission standards

- Transfer the calcine to a new Calcine Packaging Facility (CPF)

- Package the calcined waste in canisters

- Grout spent calcine scrub solution and package in canisters

- Certify that the packaged waste meets WIPP WAC

- Load the canisters into shipping casks for transfer to WIPP

- Provide adequate lag storage to allow continuous calcining operations.

- Treat, package and qualify for disposal all other wastes incidental to the treatment of SBW and NGLW. 
Prior to SBW treatment, pumps would be installed in TFF tanks and transfers between tanks made to distribute the SBW solids collected in WM-187 among most of the SBW liquid. Solids need to be distributed and mixed with liquid in the tank farm to (a) avoid the expense of alternative systems that would need to transfer and treat the solids separately with new equipment in a new facility, (b) be able to transfer the solids to the NWCF with existing steam and air jets, (c) provide a homogeneous feed to the CMACT process, and (d) be able to process the solids with no changes to and minimal additives in the calcination process.

Once received in the NWCF "blend and hold" tanks, the waste must be prepared for calcination by the addition of aluminum nitrate and calcium nitrate. The aluminum additive is needed to prevent agglomeration in the calciner bed. Calcium is needed to form nonvolatile halide and phosphate salts to prevent corrosion in the calciner and off-gas equipment.

The waste is calcined to produce a dry product acceptable for disposal at WIPP. Calcination removes water and nitric acid in the waste and results in a granular product. The temperature required for calcination results in partially volatilizing certain constituents in the feed such as mercury, and forming others by chemical reaction, such and $\mathrm{NO}_{\mathrm{x}}$ and unburned hydrocarbons. These impurities must be removed from the off-gas so that the remainder - steam, carbon dioxide $\left(\mathrm{CO}_{2}\right)$, nitrogen $\left(\mathrm{N}_{2}\right)$ and oxygen $\left(\mathrm{O}_{2}\right)$ - can be released to the atmosphere.

Primary functions of the off-gas treatment system include (a) cooling the off-gas to permit other treatment steps that require a lower temperature, (b) removing solids entrained in the gas, (c) removing volatile acids such as hydrogen chloride from the off-gas, (d) converting $\mathrm{NO}_{\mathrm{x}}$ in the off-gas to nitrogen and water $\left(\mathrm{H}_{2} \mathrm{O}\right)$, (e) destroying unburned hydrocarbons and carbon monoxide $(\mathrm{CO})$ in the off-gas by oxidation, and (f) removing mercury from the off-gas. Ultimately, the requirements for off-gas treatment are derived from the Clean Air Act and the State of Idaho air quality standards.

In the past, calcine produced in the NWCF has been pneumatically transferred to storage bins. To package the calcine in canisters for disposal at WIPP, a new facility is required, along with associated lines to transfer the calcine to the new facility. The functions of the CPF include (a) receiving the calcine, (b) separating the solid calcine particles from its transport air, (c) cooling the calcine to avoid exceeding temperature limits on canister seal material, (d) transferring the waste into canisters, and (e) closing and decontaminating the waste canisters when full.

Certification to WIPP WAC requires measurements to determine the weight of the container, the waste composition, certain radionuclide concentrations, radiation fields, surface contamination, and the hydrogen generation rate of the waste in the container. Some of the information required for certification may come from sample analysis of waste in the Tank Farm prior to transfer to the NWCF. Certification also requires labeling the waste containers and preparing the required documentation of the waste to be shipped.

To ship the canisters of calcine to WIPP, they must be stored until transport is available, as waste will be generated as a faster rate than it can be shipped. Canisters would be loaded into shipping casks and onto transporters, as they are available.

Off-gas treatment results in a liquid radioactive waste, referred to as "scrub," that must be purged to avoid excessive buildup of solids. The scrub is mostly purged (recycled) to the calciner, but a portion must be sent elsewhere to avoid excessive mercury buildup. To dispose of the scrub purge waste, it is solidified in a grouting operation and packaged in waste containers. After packaging the solidified scrub waste would be sent to a disposal facility. GAC, used to remove mercury from the off-gas, and spent high efficiency particulate air (HEPA) filters will also need to be packaged and disposed. 


\subsubsection{Process Description}

Treatment of the SBW and NGLW in the CMACT alternative involves three facilities. The treatment processes in each of these facilities is summarized in this section. Additional detail can be found in the Feasibility Study Report (Merrick, 2004). Sketches showing the major unit operations of the CMACT process are shown in Figure 1 (for the Tank Farm, NWCF, and CPF) and Figure 2 (for the MACT Compliance Facility [MCF]).

3.1.2.1 New Waste Calcining Facility (NWCF). The NWCF uses a fluidized-bed calcination process to convert radioactive liquid waste into a granular solid called calcine. The NWCF houses the calciner, the Evaporative Tank System (ETS) evaporator, the filter leach system, associated process equipment, an equipment decontamination area, and eating, ventilation and air conditioning (HVAC) equipment. The NWCF building has one above-grade level and two below-grade levels, divided into a decontamination area and the calciner area.

Sodium-bearing waste is transferred by steam or air jets from the tank farm to the calciner blend and hold tanks, where aluminum nitrate and calcium nitrate are added to the SBW to form the calciner feed solution. Scrub recycle is also added to the blend tank. Calciner feed is then transferred from the blend tanks into a feed tank where it then flows by gravity to the calciner vessel. Heated fluidizing air is added to the fluidizing air distributor at the bottom of the vessel. Additional heat is added to the calciner bed using kerosene and oxygen injected through nozzles.

As feed enters the calciner, water and nitric acid in the feed are evaporated, leaving solid particles that add to the number and size of bed particles. Periodically, the oversized bed calcine particles that sink to near the bottom of the bed are removed through a side withdrawal system as calcine product.

The effluent streams from the NWCF include process off-gas, HVAC exhaust air, liquid waste, and granular calcine solids. The process off-gas consists of calciner off-gas, vessel off-gas, and solids transport return air (calcine transport air returned from the calcine storage bins to the top of the calciner vessel). The calciner vessel process off-gas passes through an extensive cleanup system consisting of a cyclone to remove calcine fines, a quench tower, a venturi scrubber, a knockout drum, a condenser, a mist eliminator, a heater, ruthenium adsorbers, a mist eliminator, a second heater, a HEPA-grade pre-filter, and double HEPA filters. Previously the process off-gas was sent to the atmospheric protection system (APS) before being exhausted to the atmosphere through the INTEC Main Stack. With the MACT upgrade, the process off-gas will be sent to the MCF prior to discharge through the INTEC Main Stack.

Liquid radioactive waste purged from the off-gas scrub system is recycled to the calciner feed stream, with a small amount of blow down to be treated for disposal. Decontamination solutions generated in the NWCF are sent to existing storage tanks and evaporators to be concentrated and then added to the NGLW awaiting treatment. Non-radioactive service waste (steam condensates and cooling water) is monitored for radioactivity before being discharged to existing percolation ponds. If the radiological contamination in the service waste stream exceeds a set of contamination limits, the stream is diverted to a waste storage tank, concentrated, and calcined. 


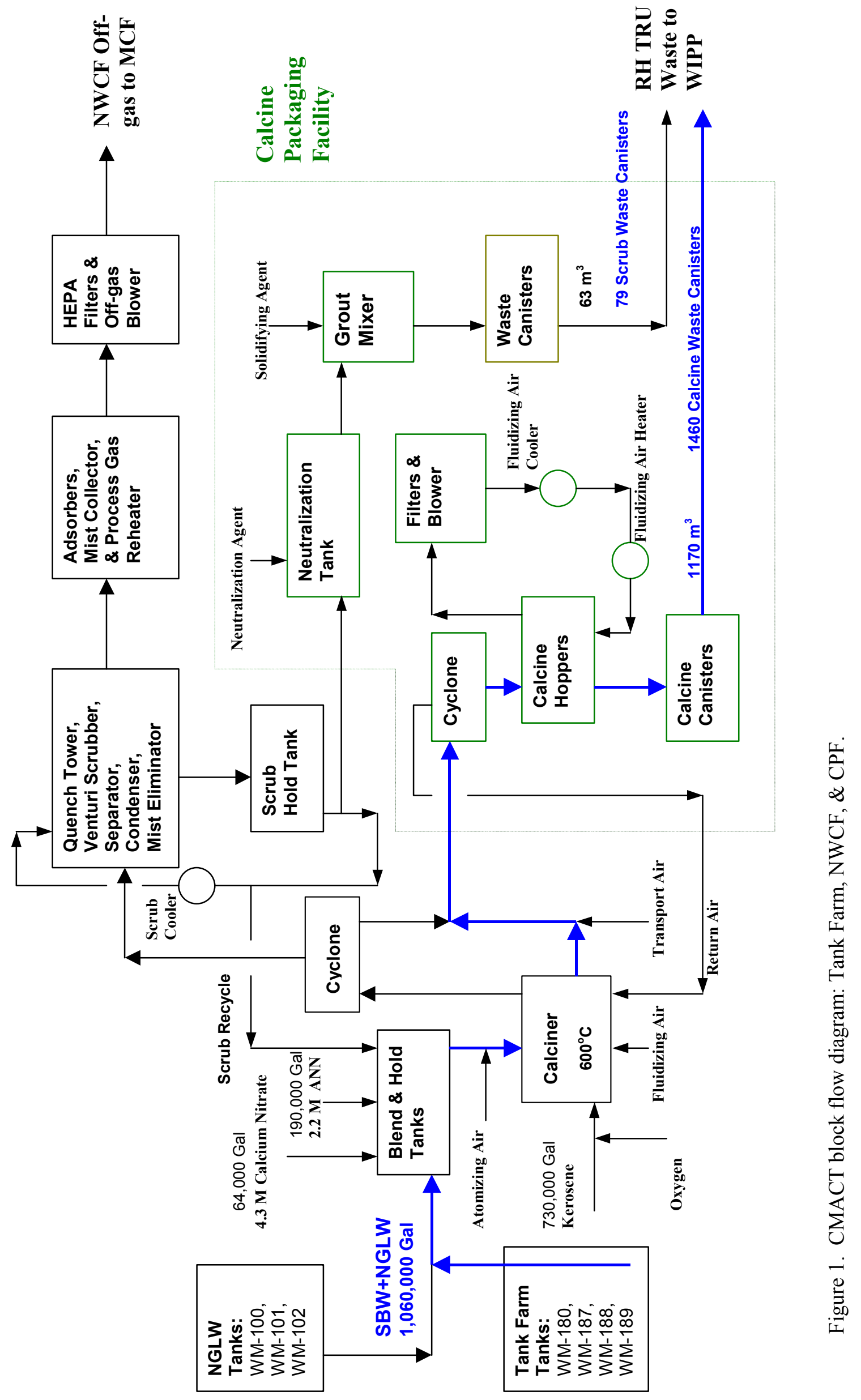



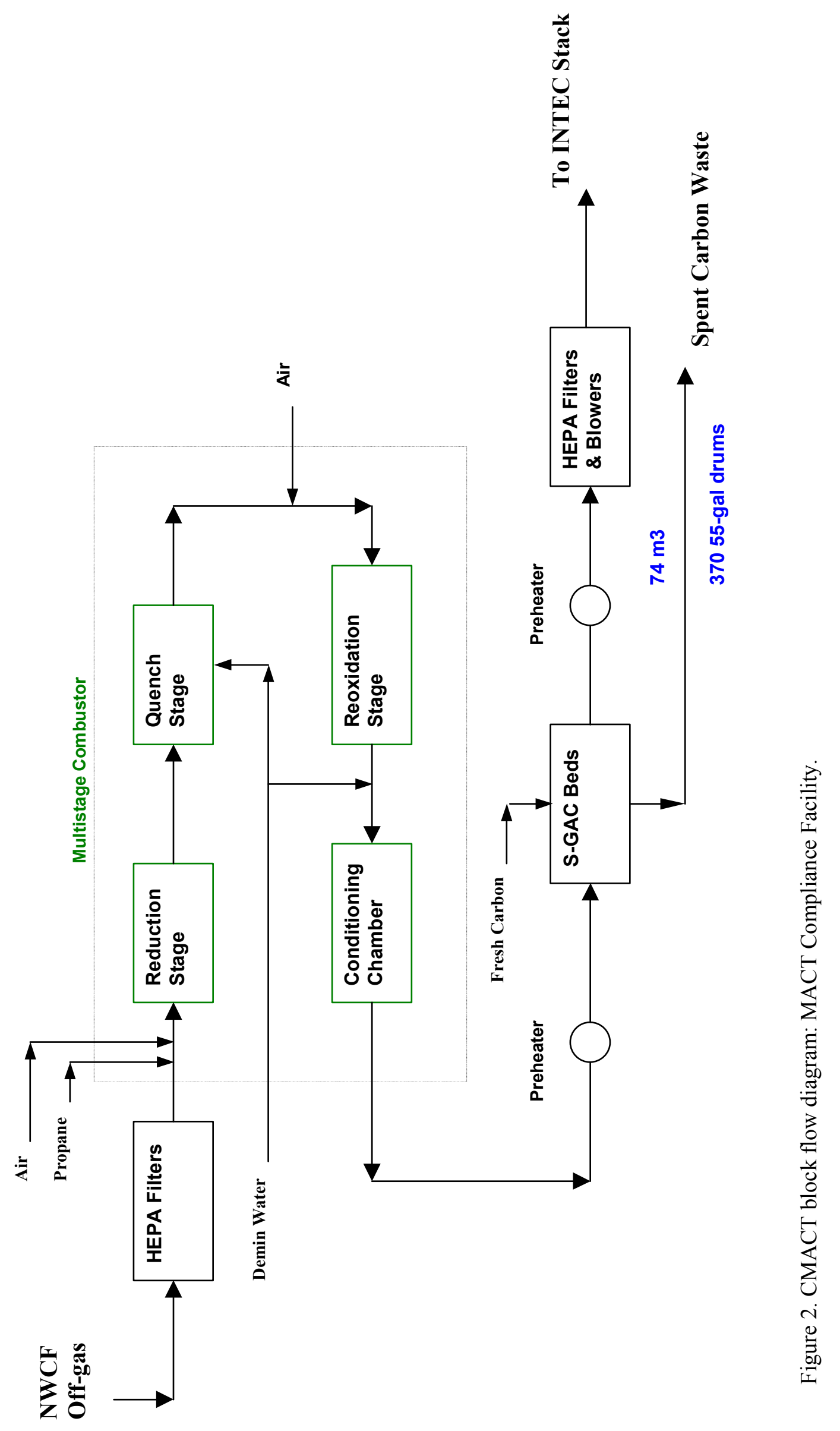
When the calcination process in the NWCF was shut down in 2000, the system was in relatively good condition. However, some of the equipment in the facility needs to be replaced or upgraded since it will be approaching thirty years old by the time the calciner is restarted. NWCF personnel made a list of components and systems they felt should be upgraded or replaced to minimize unplanned shutdowns of the calcination system. They then prepared an engineering design file (EDF) for each of the recommended modifications, complete with a cost estimate, radiation exposure estimate, and schedule. That series of EDFs can be divided into three categories: (1) replacement of equipment or valves due to failure of that system during the last campaign, (2) replacement of equipment because spares are no longer available due to the age of the equipment, and (3) replacement of equipment with new equipment due to advancement in technology and reliability.

Replacement of equipment in the calcination system of the NWCF will expose workers to radiation doses that total an estimated 268.3 man-rem (Bates, 2000). The current administrative limit at the INEEL is $0.700 \mathrm{rem} /$ year while the goal for construction workers is $0.270 \mathrm{rem} /$ year. Depending on the control level, this work could potentially "burn out" from 135 to 1000 workers. Thus, the timing of this work is important. The schedule shows the upgrades to the calcination system of the NWCF occurring simultaneously with the construction of the MCF and CPF. This approach allows workers to rotate to either the MCF or $\mathrm{CPF}$ after having received an allowable radiation dose in the NWCF.

3.1.2.2 MACT Compliance Facility (MCF). Off-gas from the existing NWCF facility must be further treated in a new MCF to meet regulatory standards. That process includes several steps that clean the off-gas. The off-gas coming to the MCF is initially passed through HEPA filters to capture entrained particulates, which is the major transport mode for non-gaseous radioactive isotopes. Once in the MCF, the off-gas is routed in one of two directions, either through the MACT process equipment, where it is treated before exhausting through the INTEC stack, or through a bypass line that routes the off-gas to the existing APS system. This bypass will allow the calciner to continue to operate for brief periods if there should be a problem in the MCF that necessitates its shutdown. The off-gas that enters the MACT facility will be HEPA-filtered again to minimize downstream contamination. Off-gas leaving the HEPA filters will pass through a flow meter and then to the $\mathrm{NO}_{\mathrm{x}}$ reduction furnace.

The reduction furnace is a cylindrical refractory-lined vessel (nominally $8.5 \mathrm{ft}$ diameter by $27 \mathrm{ft}$ long) heated by burning auxiliary fuel (propane) with excess combustion air in a high-intensity burner that is mounted at the front of this furnace. A burner control system is used to sequence operations to safely control and monitor combustion with interlocks to shut off feeds in the event of malfunctions. When the desired operating temperature $\left(\sim 2200^{\circ} \mathrm{F}\right)$ is reached, the system is switched to reducing mode prior to initiating waste gas flow. The reduction furnace is maintained in a stable reducing state by adjusting fuel gas and/or the waste gas such that the amount of oxygen fed is insufficient to fully convert the combustibles in the off-gas to carbon dioxide and water. The waste gas passes through designed spin vanes in the burner to promote turbulent mixing with the fuel gas in the reduction furnace. The exothermic combustion of the fuel gas and residual hydrocarbons in the waste gas rapidly consumes the available oxygen during the typical residence time of 1.5 to 2.0 seconds to yield an off-gas mixture of hydrogen, carbon monoxide, water, carbon dioxide, nitrogen, trace quantities of $\mathrm{NO}_{\mathrm{x}}$, and unburned hydrocarbons. Metered quantities of liquid water are sprayed through quench guns to limit the maximum temperature reached in the reduction furnace to about $2200^{\circ} \mathrm{F}$.

The reduction furnace effluent is then fed to the refractory-lined cylindrical quench chamber $(5.5 \mathrm{ft}$ diameter by $17 \mathrm{ft}$ long) where liquid water is sprayed through quench guns to cool the off-gas to about $1600^{\circ} \mathrm{F}$. This cooling is needed to minimize nitrogen oxide (NO) formation in the subsequent reoxidation stage where excess combustion air is added. 
Excess combustion air is introduced by a blower through a number of injection pipes in a third section to maintain an exiting oxygen level of about $2 \mathrm{vol} \%$ (dry basis) after oxidizing the combustible gases (hydrogen and carbon monoxide) to fully oxidized species (water and carbon dioxide). This reoxidation stage, $6.5 \mathrm{ft}$ diameter by $24.5 \mathrm{ft}$ long, has a residence time of one second and operates at a temperature of about $1800^{\circ} \mathrm{F}$. Higher temperatures would lead to $\mathrm{NO}_{\mathrm{x}}$ formation in this stage.

The effluent from the re-oxidation stage is then cooled to $250^{\circ} \mathrm{F}$ by direct contact with liquid water sprayed from five quench guns into the cylindrical refractory-lined conditioning chamber $(5.5 \mathrm{ft}$ diameter by $15.5 \mathrm{ft}$ long). This exit temperature is the desired inlet temperature to the downstream mercury removal unit.

The quench water used in the staged combustor is provided from a new demineralized water system and storage tank. This vessel provides 30 minutes surge capacity for the staged combustor quench system. The new demineralizer system will supply at least $30 \mathrm{gpm}$ of demineralized water. Additionally, a connection to the existing plant demineralized water system will be made to the storage tank to provide backup demineralized water flow.

After processing the calciner off-gas for $\mathrm{NO}_{\mathrm{x}}$ abatement in a staged combustion unit (which also destroys trace organics and products of incomplete combustion), additional unit operations are needed to achieve compliance with the MACT regulatory standard for mercury emissions of less than $45 \mu \mathrm{g} / \mathrm{dscm}$ (corrected to 7\% oxygen content). Since the off-gas effluent from the staged combustion unit will include a significant fraction of the mercury in the SBW feed as predominately vapor phase elemental mercury plus minor quantities of mercuric halide (Barnes, 2003), a mercury removal efficiency of $99.9 \%$ is needed (Soelberg, 2003). Sulfur-impregnated GAC (S-GAC) pellets are placed in large beds to adsorb this vapor phase mercury.

The effluent off-gas from the conditioning chamber of the staged combustion unit passes through the tubes of a heat exchanger where it can be heated by 150 pound saturated steam in the shell to ensure this off-gas enters the following S-GAC at the desired temperature of $250^{\circ} \mathrm{F}$ with no entrained liquid droplets.

The S-GACs vessels purify the off-gas stream by chemisorption of gaseous mercury compounds plus physical adsorption of residual organic and halogenated compounds using packed beds consisting of S-GAC pellets. The gaseous mercury compounds will react with an equimolar amount of sulfur to form insoluble mercuric sulfide. Residual organic compounds and halogenated compounds will be physically trapped in the tortuous pore structure of the high surface area activated carbon. The spent carbon will thus contain progressively higher quantities of adsorbed mercury plus residual organic and possibly halogenated compounds as processing proceeds. The configuration consists of two parallel beds (one online and one idle) of S-GAC pellets followed by a "guard bed" containing the same material.

The cleaned off-gas from one of the two parallel S-GAC beds then passes through an identical cylindrical vessel ( $16 \mathrm{ft}$ diameter by $10 \mathrm{ft}$ tall tangent to tangent) containing a 5 - $\mathrm{ft}$ guard bed filled with the same $3 \mathrm{~mm}$ S-GAC pellets. Hence, this bed has the same mercury sorption capacity as either of the upstream S-GAC beds.

The purpose of this guard bed is to ensure that the minimum overall desired mercury removal is achieved. If there is a large mercury spike through either of the two upstream S-GAC beds, as shown by the mercury monitor, this guard bed will have sufficient capacity to adsorb mercury over the lifetime of the remaining SBW operation and prevent exceeding emissions limits. 
The off-gas leaving the S-GAC units will be reheated in a steam heat exchanger to above its dew point to avoid condensation in the lines or in the HEPA housings. The heated gas is then filtered through a HEPA filter to capture any particulate carbon fines (containing mercury, halogens, etc.) and remove any residual contamination prior to discharge to the atmosphere through the blowers and stack.

Off-gas will be sampled and the overall off-gas flow rate determined before it is discharged to the atmosphere via the INTEC stack. A new 20-inch line will connect the outlet of the blowers to the stack. The off-gas that has been treated in the MACT facility will not pass through the INTEC APS. However, any off-gas that bypasses the MACT facility, in the case of an emergency shutdown or for brief periods to resolve operating problems, will go to the APS for final filtering before discharge.

3.1.2.3 Calcine Packaging Facility (CPF). The CPF provides three important functions: calcine packaging for shipment to WIPP, immobilization of scrubber liquid, and lag storage of filled calcine and scrubber solution canisters. The calcine packaging portion of the CPF consists of three subsystems. These are the calcine receiving and storage subsystem, the fluidization subsystem, and the canister filling subsystem.

The calcine receiving and storage subsystem consists of a diverter valve, cyclone, two parallel storage hoppers, two parallel sintered metal filters and associated instrumentation. The diverter valve is installed in the solids transport line from the NWCF and allows solids to be bypassed around the CPF to Bin Set 6 (to be used only for short periods of time or in the case of an emergency). This ensures that calciner operations are isolated and independent from the CPF. Transported solids that enter the CPF pass through a cyclone and are discharged into one of two parallel storage/cooling hoppers.

The storage/cooling hoppers are two parallel, fluidized beds. Each bed is sized to hold $45 \mathrm{ft}^{3}$ solids working volume and $60 \mathrm{ft}^{3}$ total solids volume. The nominal solids production rate from the calciner is $45 \mathrm{ft}^{3} /$ day. This additional solids volume allows the hoppers to retain a heel of material during canister filling operations and keep the bed well fluidized. A 4-ft freeboard is provided above the fluidized beds to allow the particles that are elutriated from the bed to disentrain from the fluidizing gas and return to the bed. During normal operation, one hopper will be filled/cooled as the other is emptied. The fluidizing gas cools the bed solids as it flows though the bed.

Two parallel sintered metal or ceramic filters are provided, one on each bed outlet, to remove entrained particles from the fluidizing gas. These filters provide a minimum $2-\mu \mathrm{m}$ absolute filtration capacity for the entrained particles. The filters are equipped with an automatic blowback system to clean the filters as particles build up on the filter elements.

The fluidization subsystem consists of parallel HEPA filters; parallel fluidizing gas recirculation blowers, a cooler, an electrical heater and associated instrumentation and controls. Fluidizing gas exiting the filters on the top of the fluidized beds passes through the HEPA filter to remove any entrained particulate contamination that has passed through the bed-sintered metal blowback filters. The blowers provide the motive force to fluidize the solids in the hoppers and sufficient pressure to recirculate the fluidizing gas. Since a major function of the storage/cooling hoppers is to cool the solids before they are placed in the storage canisters, this heat must be removed from the gas before it is recirculated to the fluidized hoppers. A water-cooled heat exchanger is used for this purpose. Any condensate produced in the heat exchanger is drained to the facility decontamination sump. An electric heater is used to slightly reheat the fluidizing gas and prevent condensation from forming in the fluidizing gas lines or in the bed.

The canister filling subsystem consists of two parallel canister filling stations, one for each of the two storage/cooling hoppers (see Figure 3). Solids will be drained from the hoppers into canisters through a slide valve, a fill spout, a sacrificial seal tube, and through a modified fill-through pintle on the 
shipping canister. The loadout hood will allow manipulator access to the flex hose connector while providing secondary confinement of solids that may become airborne when the fill tube is separated from the filling dock on the bottom of the hood. A two-stage HEPA filter and blower system will be used to control any dust that may escape from the equipment as the canisters are connected to the fill spout and filled. A disposable seal tube will be used for each canister to ensure that the outside of the canister remains clean during the filling operation. Each disposable seal will be replaced with a new one for each canister that is filled. After the canister is filled, the canister is lowered and the plastic bagout bag is sealed using a radio frequency sealing mechanism. The seal is cut, the bag is withdrawn into the top of the canister, and the pintle top blind flange is bolted onto the pintle using remote manipulators. The used seal tube with the other half of the sealed bagout bag is pushed upwards through the hood floor by the subsequent shipping canister's seal tube. This operation assures that no contamination falls onto the shipping containers. The recovered used seal tube is crushed and size reduced to allow the manipulator to drop through the fill port of the subsequent shipping container prior to moving the fill spout from it's idle dock to the in-use dock.

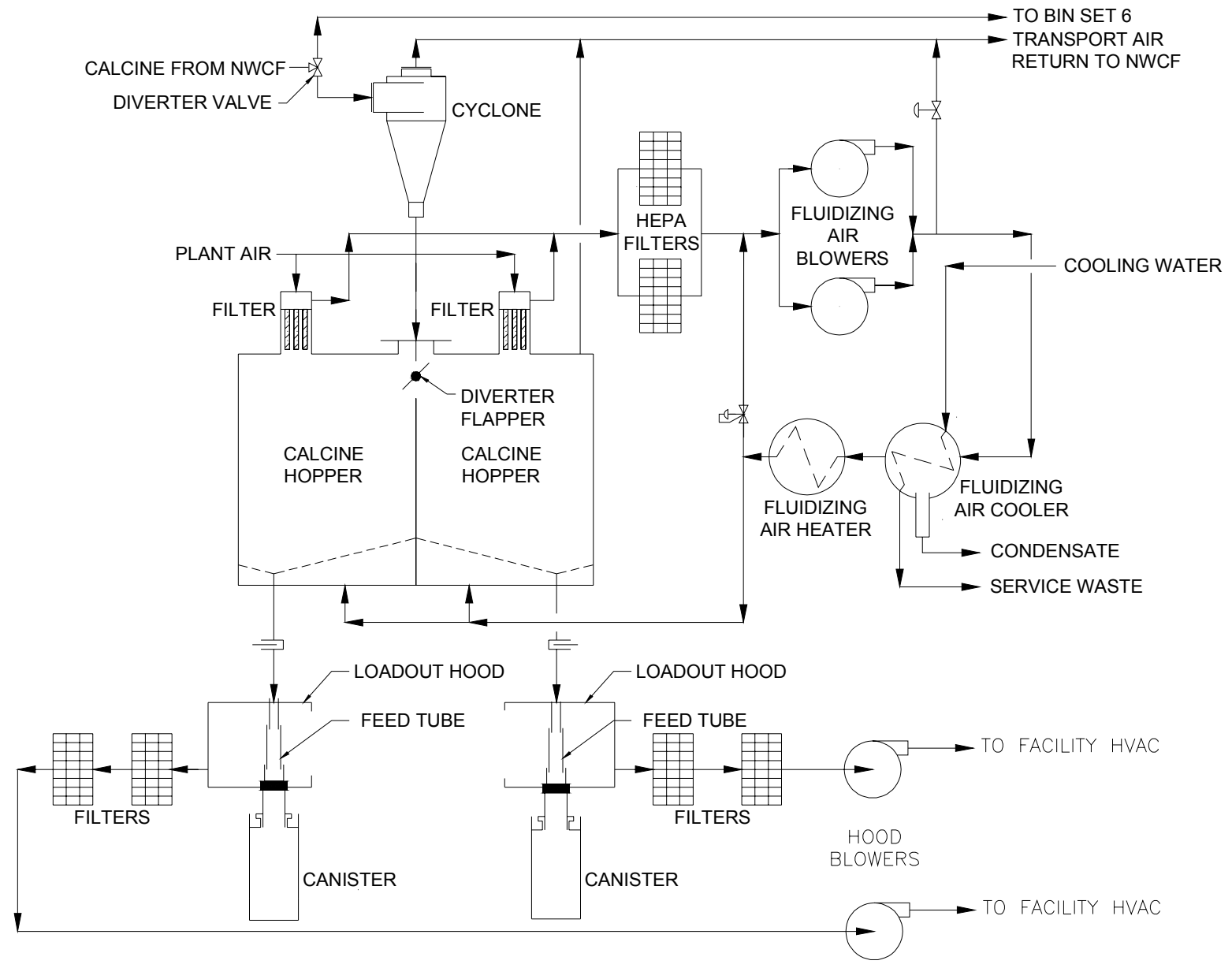

Figure 3. Calcine filling subsystem.

The spent scrub solidification facility consists of four subsystems: the neutralization subsystem, the solidification subsystem, the canister filling subsystem, and the balance of plant subsystem.

The neutralization subsystem consists of a neutralizing agent storage tank, a neutralizing agent metering pump, a spent scrub neutralization and storage tank, and a mixing/recirculation pump. Spent 
scrub from the calciner scrub tank is transferred to the neutralization and storage tank in the CPF. The spent scrub mixes with recirculated scrub and enters the tank through an internal mixing eductor. This helps to mix the tank contents and keep any solids in suspension. A clamp-on cooling jacket is attached to the outside of the neutralization tank and is used to absorb any heat generated by the neutralization reaction. Neutralizing agent is pumped into the neutralization tank as needed to reach the desired $\mathrm{pH}$.

The solidification subsystem consists of a solidification agent storage hopper, a solidification agent loss-in-weight feeder, a continuous in-line mixer, a close-coupled slurry auger, and a mass-flow meter and control valve system. The neutralized scrub is fed to a continuous mixer where it is mixed with a solidifying agent to form a slurry. The slurry is discharged into a vertical auger standpipe where it drops into the canister.

The solidifying agent is fed to the loss-in-weight from a Super Sack ${ }^{\circledR}$ hopper. The loss-in-weight feeder is used to ensure that the proper amount of solidifying agent is fed to the mixer to verify that the product specifications are met. Solids leaving the loss-in-weight feeder pass through a transfer auger (or chute) through the shield wall to the continuous mixer. This allows the Super Sack ${ }^{\circledR}$ hopper and feeder to be outside the remote maintenance area.

The mixed scrub/solidification agent slurry drops into the canister through the vertical standpipe attached to the vertical auger. A weigh cell is used to verify that the canister is not overfilled with slurry. The last few inches of the canister are filled with absorbent clay to ensure that there is no free liquid in the canister. A control valve system is also used to ensure the canister is not overfilled. The canisters are moved under the feed standpipe and lifted into place using a dedicated trolley and lift system. The balance of plant for the spent scrub solidification system consists of a monitored sump, cooling water, and other utilities.

Once the canisters of scrub solution or calcine are filled, lidded, and decontaminated, they are transferred to lag storage. Lag storage provides monitored storage for up to 500 canisters.

3.1.2.4 Tank Mixing System. Three tank-mixing pumps will be installed in each of three tanks VES-WM-187, VES-WM-188, and VES-WM-189 - through existing 12-inch tank risers. These mixing pumps will ensure that solids are homogeneously mixed with tank liquids. The submersible pump motor is located in the tank at the suction head. The submerged pump is installed directly in the waste tank liquid near the bottom of the tank. The unit is supported by a vertical column that, in turn, is supported by a rotational turntable assembly mounted to a riser structure at the top of the waste tank. Each mixing pump weighs in excess of $2000 \mathrm{lbs}$ and cannot be supported from the tank risers. A steel truss will be installed that spans the length of the tank substructure that will support the mixer pumps vertically, laterally, and torsionally.

Power for the Tank Farm area will be provided from existing Substation No. 60. This existing substation is a $13.8 \mathrm{kV}-2400 \mathrm{~V}$ double-ended unit, and the $13.8 \mathrm{kV}$ feeders are from a redundant INEEL $138 \mathrm{kV}-13.8 \mathrm{kV}$ loop.

\subsubsection{Summary of Technical Performance}

3.1.3.1 Processing Rate, Availability. In 2002, Barry O'Brien reviewed operating performance and throughput history of the calciner to select credible SBW feed rates, expected campaign lengths and maintenance turnaround times to be used for the CMACT design (O'Brien, 2002). Based on NWCF operation from 1982 to 2000, O'Brien recommended an average feed rate (SBW plus additives plus scrub recycle) of $182 \mathrm{gph}$, an on-stream factor of $73 \%$, and two turnarounds of 173 days each. Based on a feed aluminum to alkali mole ratio (AAR) of 2.25 , these recommendations would result in a 4-year processing 
schedule for the full inventory of SBW and NGLW. O'Brien noted that aggressive preventative maintenance could improve the on-stream efficiency for at least the first part of the campaign from $73 \%$ to $85 \%$, such as was experienced on Part 1 of the H-4 campaign. O'Brien also noted that if the AAR could be reduced to 1.8, which was later demonstrated in pilot tests performed in January 2004, the total processing time, including turnarounds, could be reduced to 3.5 years (O'Brien, 2002). If the maximum historical sustained feedrate of $223 \mathrm{gph}$ were also achieved, the processing time would be reduced to 3.0 years (O’Brien, 2002).

Two other studies have been performed in attempts to increase throughput or reduce downtime for the CMACT alternative. Doug Stacey developed 14 recommendations that could reduce maintenance turnaround times from 173 to 85 days (Stacey, 2003). Steve Bates reviewed the advantages and feasibility of replacing the cyclone, which currently only removes about $63 \%$ of the carryover calcine fines (Bates, 2003). By replacing the cyclone with one achieving higher efficiency, the fraction of scrub recycle in the calciner feed could be reduced, allowing for an increase in the SBW feed rate and a corresponding reduction in operating schedule. Bates showed that a schedule reduction of 139 days could be achieved by cyclone replacement. The cost to replace the cyclone was later estimated to be $\$ 2.45$ million (Adams, 2003).

The recent CMACT Feasibility Study (Merrick, 2004) included a reliability, availability, and maintainability (RAM) analysis, which concluded that the availability of the MCF taken alone is $91 \%$, the CPF taken alone is $83 \%$, and the scrub solidification system is $93 \%$. Since the availability of each of these new facilities is higher than that of the NWCF $(72 \%)$, it was assumed that maintenance would be performed during NWCF shutdowns; hence, they would not affect the availability of the total processing system.

3.1.3.2 Waste Products Quantity and Quality. The CMACT process would produce an estimated $1168 \mathrm{~m}^{3}$ or 1460 canisters of calcine waste, an RH-TRU waste product. Calcine is a granular solid with a bimodal size distribution of particles. Based on NWCF operation at $600^{\circ} \mathrm{C}$, bed particles average about $0.6 \mathrm{~mm}$ (Swenson, 2000). Based on calcine generated at $600^{\circ} \mathrm{C}$ in $10-\mathrm{cm}$ pilot calciner runs, fines average about $0.05 \mathrm{~mm} \cdot \mathrm{b}$. In the recent pilot calcine tests, the median size of the fines was about $0.01 \mathrm{~mm}$ (Boardman, 2004b). A typical bulk density for SBW calcine, mixed bed and fines, is 1.2 $\mathrm{kg} / \mathrm{liter}$.

Two other waste streams, grouted scrub waste and spent GAC, would be produced intermittently during processing or at the end of processing SBW. Like calcine, the grouted scrub will be an RH-TRU waste. The spent GAC will be a low-level mixed waste. Estimated volumes of these wastes and calcine are shown in Table 2.

Table 2. CMACT primary waste products.

\begin{tabular}{lccccccc}
\hline & WM-180 & WM-187 & WM-188 & WM-189 & D\&D & $\begin{array}{c}\text { Total } \\
\text { containers }\end{array}$ & Total $^{\mathbf{3}}$ \\
\hline Calcine, canisters & 394 & 280 & 377 & 409 & & 1460 & 1168 \\
Scrub waste, canisters & 0 & 16 & 17 & 35 & 20 & 88 & 70 \\
S-GAC, 55-gal drums & 0 & 0 & 0 & 0 & 370 & 370 & 74 \\
\hline
\end{tabular}

b See Figure 1 of Appendix B5 of Welland, 1997 for particle size distribution curves. 
The uncertainty in the above estimate of both scrub and carbon waste is high. Both scrub and carbon wastes are outlets for mercury volatilized in the calciner. The uncertainty in the behavior of mercury downstream of the calciner results in the uncertainty in the waste volumes. The amount of carbon shown in Table 2 assumes two full-sized carbon beds require disposal at the end of processing, and that the third GAC bed is not used during processing. If a bed requires bypass or changeout due to more mercury in the off-gas than presently expected or adsorption of hydrogen chloride $(\mathrm{HCl})$, the amount of carbon waste would increase by $50 \%$.

If more mercury partitions to the off-gas than is presently predicted, the amount of scrub waste will be less. The amount of scrub waste shown in Table 2 is based on purging the scrub when the mercury concentration reaches 55-60 g/liter, a concentration range that was experienced in the NWCF H-4 campaign. The effects on the process of allowing mercury to build up to higher concentrations are not known.

The number of shipments to WIPP of RH waste (i.e., both calcine and scrub waste) is equal to the number of canisters. Carbon beds are sized such that they will not be changed until treatment is complete. However, due to the volume generated, multiple shipping vehicles will be required. A specific shipping schedule of the carbon waste to its disposal site, tentatively identified as Envirocare, has not been determined. Radioactive properties of the CMACT waste streams are shown in Table 3.

Table 3. Radioactive properties of primary waste products.

Calcine $\quad$ Range

All radionuclides, $\mathrm{Ci} /$ canister

$250-840$

Fissile gram equivalent, g/canister

$55-73$

${ }^{239} \mathrm{Pu}$ Equivalent Curies, Ci/canister

$2.7-3.1$

TRU content, $\mathrm{nCi} / \mathrm{g}$

$2,400-3,400$

Surface dose rate, Jan 2008, Rem/hr

$19-66$

Heat generation rate, Watts

$0.8-2.3$

\section{Grouted Scrub Waste}

All radionuclides, $\mathrm{Ci} /$ canister

$30-60$

Fissile gram equivalent, g/canister

$3.2-5.0$

${ }^{239} \mathrm{Pu}$ Equivalent Curies, Ci/canister

$0.15-0.21$

TRU content, $\mathrm{nCi} / \mathrm{g}$

$110-160$

Surface dose rate, Jan 2008

$2.4-3.6$

Heat generation rate, Watts

$0.08-0.13$

\section{Spent GAC (average)}

All radionuclides, $\mu \mathrm{Ci} / \mathrm{drum}$

TRU content, $\mathrm{nCi} / \mathrm{g}$ 
3.1.3.3 Size, Footprint of Processing Facility. The following facility descriptions and estimates are taken from the Calcination with MACT Upgrade Feasibility Study Report (Merrick, 2004). Additional details, including floor plans and elevation drawings, can be found in the referenced report.

The NWCF, an existing facility, is housed in a concrete and steel building that has three main levels, one above grade and two below grade. The building is further divided into two main areas, the decontamination area and the calciner area. The NWCF is approximately 250 feet long and 145 feet wide, and extends 57 feet below grade and 43 feet above grade. The calciner area on the first level is approximately 132 feet wide by 160 feet long and occupies a space about 90 feet wide by 160 feet long on the two below grade levels.

Process control and administrative functions take place on the level above grade. Decontamination area activities are located on Level 1 and on Level 2, which is the first below grade level. Calcination and the evaporation of liquid waste also take place on Level 2 as well as on Level 3 of the NWCF.

The calciner, the High Level Liquid Waste Evaporator (HLLWE), and other associated equipment are located in steel reinforced concrete cells located on Levels 2 and 3. These concrete cells provide the appropriate confinement and shielding of radioactive sources to allow manned entry into adjacent corridors on these levels. All equipment located within these cells is arranged for ease of operation and remote maintenance.

The upper portion of the calciner cells, valve cubicles, and liquid and solid sample stations are located on Level 2. An operating and access corridor borders the cells and cubicles. Shielded viewing windows, tool ports, and master-slave manipulators provide visual and working access from the corridor into the cells. Self-monitoring personnel decontamination stations and double-door passageways are installed at the stairs for personnel protection and contamination control.

A new MCF will be located between the existing NWCF and the existing exhaust stack. The 35-ft tall single story building will house MACT process equipment including the MCF feed filters, staged combustion system, the S-GAC beds, the MCF discharge filters, the off-gas blowers, and associated variable frequency drive units. Adjoining rooms house the analyzers for the S-impregnated Carbon Beds, the continuous emissions monitors for the off-gas stack exhaust, the water treatment system equipment used to provide demineralized water to MACT system, and the atomizing air system, which provides atomizing air to the MACT quench system. The building is approximately 107 feet long by 77 feet wide, plus an additional 33 feet by 37 feet that houses a utility room.

The MCF will be a metal building with flat roof. The rooms housing the staged combustion unit and S-GACs will be lined (floor, walls and ceiling) with a stainless steel liner to facilitate decontamination. The entire building will be protected by a wet pipe sprinkler system supplied from the underground fire water supply at the site. The floor in the process areas will be surrounded with an eightinch curb to provide containment in the event of a sprinkler head discharge.

The CPF is comprised of two buildings. The first is approximately 90 feet tall and houses the process equipment, hot cells, canister fill stations, grout fill station, and lag storage. This building is approximately 240 feet long by 115 feet wide. Lower levels extend about 22 feet below grade. The second is the CPF Administration Building. This 82-ft by 54-ft single-story building will house offices, a radiological laboratory, the control room, the health physics room, restrooms, lockers, showers, personnel decontamination stations, a break/conference room, and a mechanical/electrical equipment room.

The two buildings are connected by a covered walkway. Consideration was given to make the CPF a single building that would be classified as a PC-2, non-reactor nuclear facility. It was found to be more 
cost effective to separate the two buildings, with the CPF meeting the PC-2, non-reactor nuclear facility classification, while allowing the CPF Administration Building to be designed as a non-nuclear facility.

\subsubsection{Technical Uncertainties and Potential Impacts}

3.1.4.1 Uncertainties identified in the SBW Treatment Applied Technology Plan. The Sodium-Bearing Waste Treatment Applied Technology Plan (INEEL/EXT-03-00477, June 2003) identified thirteen uncertainties resulting from technical and operational risk assessments for the CMACT process. These were, for the most part, uncertainties that would have supported the selection of a treatment technology and were not a complete listing of technical issues requiring resolution to support detailed design efforts. The uncertainties were prioritized as High (H), Medium (M), or Low (L) risks. A high risk is categorized as an issue that is likely to occur and would have a significant (\$25 million - \$125 million impact) or critical ( $>\$ 125$ million) consequence. Issues that are unlikely but have significant or critical consequences, or are likely but have marginal (\$5 million - \$25 million) consequences are categorized as medium risks. Issues that have a probability of occurrence of less than $10 \%$, or a higher probability but negligible or marginal consequences are categorized as low risk. Table 4 lists the uncertainties, a short description, and their status as of the writing of this report.

Table 4. Summary of CMACT uncertainties identified in the Applied Technology Plan.

\begin{tabular}{|c|c|c|}
\hline Uncertainty & Description & Status \\
\hline Aluminum Nitrate Ratio (H) & $\begin{array}{l}\text { Based on NWCF-demonstrated AARs, } \\
\text { processing the entire inventory of SBW } \\
\text { will take longer than } 4 \text { years, which will } \\
\text { not meet the required completion date } \\
\text { for SBW treatment. Reducing the AAR } \\
\text { would result in an increased throughput } \\
\text { and reduce processing time. }\end{array}$ & $\begin{array}{l}\text { Pilot tests in January } 2004 \text { demonstrated } \\
\text { calcination of WM-180 simulant at AARs } \\
\text { of } 2.25 \text { and } 1.75 \text {. At an AAR of } 1.75 \text { the } \\
\text { treatment schedule would be reduced by } \\
\text { about } 6 \text { months. It is still uncertain } \\
\text { whether an AAR of } 1.75 \text { is acceptable for } \\
\text { wastes from the other tanks, and whether } \\
\text { pilot results directly correlate with full- } \\
\text { scale calciner operation. }\end{array}$ \\
\hline $\begin{array}{l}\text { Effects of } \mathrm{Zr} \text { and phosphate } \\
\text { on bed agglomeration }(\mathrm{H})\end{array}$ & $\begin{array}{l}\text { Tanks solids are very high in } \mathrm{Zr} \text { and } \\
\text { phosphate. The effect that these solids } \\
\text { will have on bed agglomeration is } \\
\text { uncertain, and whether additional } \\
\text { calcium nitrate will need to be added to } \\
\text { the feed due to the additional phosphate. }\end{array}$ & $\begin{array}{l}\text { No tests have been performed to } \\
\text { determine the effect of tank solids on bed } \\
\text { agglomeration. }\end{array}$ \\
\hline $\begin{array}{l}\text { Scrub recycle minimization } \\
\text { evaluation }(\mathrm{H})\end{array}$ & $\begin{array}{l}\text { Based on operating data, the cyclone } \\
\text { efficiency is about } 63 \% \text {, requiring a } \\
\text { scrub recycle rate of } 23 \% \text { of the total } \\
\text { calciner feed. Replacing the recycle with } \\
\text { a higher efficiency cyclone would allow } \\
\text { a reduction in recycle rate, an increase in } \\
\text { SBW feed rate and a reduction in } \\
\text { required operating time. }\end{array}$ & $\begin{array}{l}\text { EDF-3646 (Bates, 2003) concluded that } \\
\text { replacing the cyclone would reduce } \\
\text { operating time by } 139 \text { days. The cost to } \\
\text { areplace the cyclone was estimated to be } \\
\$ 2.45 \text { million (Adams, 2003). Additional } \\
\text { study is needed to determine what } \\
\text { additional modifications if any are needed } \\
\text { to accommodate the increased pressure } \\
\text { drop of a new cyclone. }\end{array}$ \\
\hline
\end{tabular}


Table 4. Summary of CMACT uncertainties identified in Applied Technology Plan (continued).

\begin{tabular}{|c|c|c|}
\hline Uncertainty & Description & Status \\
\hline $\begin{array}{l}\text { GAC bed chloride removal - } \\
\mathrm{HCl} / \mathrm{Cl} 2 \text { Emissions }(\mathrm{H})\end{array}$ & $\begin{array}{l}\text { Analyses for } \mathrm{HCl} \text { and } \mathrm{Cl}_{2} \text { in NWCF off- } \\
\text { gas samples taken in } 1999 \text { and } 2000 \\
\text { showed concentrations below detection } \\
\text { limits (10-55 ppm total } \mathrm{Cl} \text {, dry basis) } \\
\text { which brackets the } \mathrm{MACT} \text { limit of } 21 \\
\text { ppmv. If the GAC bed does not remove } \\
\mathrm{HCl} \text {, and no other unit operations are } \\
\text { added to the process to remove } \mathrm{Cl} \text {, there } \\
\text { is a risk the MACT } \mathrm{Cl} \text { limit would be } \\
\text { exceeded. }\end{array}$ & $\begin{array}{l}\text { Long-term GAC tests showed negligible } \\
\text { removal of } \mathrm{Cl} \text { (Del Debbio, 2003). Recent } \\
\text { pilot tests (Boardman, 2004b) also show } \\
\text { negligible } \mathrm{Cl} \text { removal by the GAC bed. In } \\
\text { the pilots tests levels of } \mathrm{HCl} \text { in the off-gas } \\
\text { were well below the MACT limit. } \\
\text { However it is still uncertain whether } \mathrm{HCl} \\
\text { concentrations in the off-gas of the full- } \\
\text { scale facility will increase to above the } \\
\text { MACT limit as chlorides build up in the } \\
\text { scrub system. }\end{array}$ \\
\hline $\begin{array}{l}\text { Minimization of } \mathrm{O}_{2} \text { in } \\
\text { calciner off-gas }(\mathrm{M})\end{array}$ & $\begin{array}{l}\text { To destroy } \mathrm{NO}_{\mathrm{x}} \text { in the } \mathrm{MCF} \text {, the initial } \\
\text { stage of the staged combustor must } \\
\text { operate at reducing conditions. The } \mathrm{O}_{2} \\
\text { content of the stage combustor feed is } \\
\text { near } 21 \% \text { on a dry basis, requiring large } \\
\text { quantities of fuel in the combustor to } \\
\text { reach reducing conditions and resulting } \\
\text { in large off-gas flow rates. } \\
\text { Optimization/replacement of calciner air } \\
\text { inflows could result in reductions in } \\
\text { MCF throughput and MCF footprint and } \\
\text { cost. }\end{array}$ & $\begin{array}{l}\text { Discussions with John Zink, (a staged } \\
\text { combustor supplier) during the CMACT } \\
\text { feasibility study resulted in a design } \\
\text { increase in the staged combustor feed } \\
\text { oxygen content (Merrick, 2004). This was } \\
\text { needed in order to ensure proper burner } \\
\text { operation. }\end{array}$ \\
\hline $\begin{array}{l}\text { Combustor operating and } \\
\text { performance parameters }(\mathrm{M})\end{array}$ & $\begin{array}{l}\text { The fuel, water and air additions in the } \\
\text { staged combustor result in more than a } \\
\text { 3-fold increase in volumetric gas rate, } \\
\text { based on data obtained in 1999-2000 } \\
\text { during tests at MSE. Because of } \\
\text { limitations of the MSE test equipment, it } \\
\text { was uncertain whether optimization of } \\
\text { operating parameters could result in a } \\
\text { significant decrease in combustor } \\
\text { throughput. }\end{array}$ & $\begin{array}{l}\text { Engineering of the staged combustor for } \\
\text { the CMACT feasibility study (Merrick, } \\
\text { 2004) and recent kinetic and CFD } \\
\text { modeling of the staged combustor (Wood, } \\
\text { 2004) have shown that no significant } \\
\text { reduction of throughput is possible. }\end{array}$ \\
\hline $\begin{array}{l}\text { GAC bed design and } \\
\text { performance }(\mathrm{M})\end{array}$ & $\begin{array}{l}\text { The GAC beds are large - } 16 \text { feet } \\
\text { diameter by } 10 \text { feet tall (Soelberg, 2003; } \\
\text { Merrick, 2004) and the design calls for } \\
\text { two in series (because of uncertainty in } \\
\text { off-gas Hg content, Hg loading on the } \\
\text { bed, and } \mathrm{Hg} \text { removal by the bed) and a } \\
\text { third in parallel (because of uncertainty } \\
\text { in GAC HCl removal and loading). } \\
\text { Because of these uncertainties, the bed } \\
\text { could be oversized or fewer beds } \\
\text { required. }\end{array}$ & $\begin{array}{l}\text { Long term small scale tests have } \\
\text { demonstrated } 100.0 \pm 2.4 \% \mathrm{Hg} \text { removal } \\
\text { and negligible }(1.6 \pm 7.5 \%) \mathrm{HCl} \text { removal } \\
\text { (Del Debbio, 2003). Recent pilot test data } \\
\text { (Boardman, 2003b) also show adequate } \\
\mathrm{Hg} \text { removal and negligible chloride } \\
\text { removal. These results indicate that a } \\
\text { parallel bed to allow for changeout is } \\
\text { unnecessary. Uncertainties remain } \\
\text { regarding the GAC bed feed } \mathrm{Hg} \\
\text { concentration, the GAC bed carbon } \\
\text { loading and performance at higher } \\
\text { superficial velocities. }\end{array}$ \\
\hline
\end{tabular}


Table 4. Summary of CMACT uncertainties identified in Applied Technology Plan (continued).

\begin{tabular}{|c|c|c|}
\hline Uncertainty & Description & Status \\
\hline $\begin{array}{l}\text { Mercury vapor liquid } \\
\text { equilibrium data (M) }\end{array}$ & $\begin{array}{l}\text { Past attempts have failed to achieve good } \\
\text { closure for Hg in NWCF mass balances. } \\
\text { This uncertainty in partitioning of Hg } \\
\text { between off-gas and scrub impacts the } \\
\text { GAC bed size, the amount of scrub } \\
\text { waste produced, and the timing of scrub } \\
\text { purges. Also the limiting scrub Hg } \\
\text { concentration is unknown, as NWCF } \\
\text { data goes only to about } 60 \mathrm{~g} / \text { liter. }\end{array}$ & $\begin{array}{l}\text { dProgress was made developing models for } \\
\text { predicting Hg VLE (Nichols, 2003, } \\
\text { Taylor, 2003), but management } \\
\text { terminated this work before it was } \\
\text { completed. }\end{array}$ \\
\hline $\begin{array}{l}\text { Off-gas } \mathrm{Cl} \text { concentration and } \\
\text { speciation }(\mathrm{M})\end{array}$ & $\begin{array}{l}\text { Analyses for } \mathrm{HCl} \text { and } \mathrm{Cl}_{2} \text { in NWCF off- } \\
\text { gas samples taken in } 1999 \text { and } 2000 \\
\text { showed concentrations below detection } \\
\text { limits ( } 10-55 \text { ppm total } \mathrm{Cl} \text {, dry basis) } \\
\text { which brackets the MACT limit of } 21 \\
\text { ppmv. The uncertainty in off-gas } \\
\mathrm{HCl} / \mathrm{Cl}_{2} \text { concentrations could lead to } \\
\text { unnecessary off-gas treatment steps or to } \\
\mathrm{Cl} \text { emissions exceeding MACT the limit. }\end{array}$ & $\begin{array}{l}\text { The present process flow diagram shows } \\
\text { no unit operations designed specifically to } \\
\text { remove } \mathrm{HCl} \text {. Recent pilot test data } \\
\text { (Boardman, 2004b) shows off-gas } \\
\text { chloride levels far below the MACT limit. } \\
\text { Uncertainty remains, however, because in } \\
\text { the full-scale plant, chlorides build up in } \\
\text { the scrub system and could result in higher } \\
\text { levels in the off-gas. }\end{array}$ \\
\hline
\end{tabular}

Scrub treatment method and Several treatment methods for highparameters $(\mathrm{M})$

Effects of solids on calcination $(\mathrm{M})$

Feed acidity (L) mercury scrub blow down have been considered, and an initial evaluation favored either grout or absorption on silica gel placed on top of calcine in the packaging process. Subsequent testing provided parameters (loading, formulations) for these two methods and also showed that absorption on silica gel would be feasible. Uncertainties remaining include radiolytic hydrogen generation rates and mixing requirements for the absorbed waste forms.

The effects of undissolved solids on calcination parameters (feed additive quantities and calciner operating parameters) are uncertain.

The waste in three of four SBW tanks has a higher acid molarity than most waste that has been processed in the NWCF. It is uncertain whether this higher acid molarity will cause agglomeration in the bed.
No additional data has been obtained to resolve the uncertainties for the absorbed waste forms. Present designs assume the waste scrub is grouted.

The scale of the pilot plant was inadequate to feed solids; thus the effect of solids remains uncertain.

This issue has not been resolved; the recent pilot tests used WM-180 simulant that has the lowest acid concentration of the SBW waste tanks. 
Table 4. Summary of CMACT uncertainties identified in Applied Technology Plan (continued).

\begin{tabular}{lll}
\hline \multicolumn{1}{c}{ Uncertainty } & \multicolumn{1}{c}{ Description } & \multicolumn{1}{c}{ Status } \\
\hline Calcine densification $(\mathrm{L})$ & $\begin{array}{l}\text { Calcine densification has been suggested Calcine densification is not shown in the } \\
\text { as a means of reducing the volume of } \\
\text { calcine waste in order to reduce the } \\
\text { pumber of waste shipments and the } \\
\text { required lag storage space. However, no flow diagrams and has } \\
\text { undergone neither further evaluation nor } \\
\text { methods of densification have been } \\
\text { tested. }\end{array}$ \\
\hline
\end{tabular}

One of the highest probability risks for the CMACT alternative is not meeting the required treatment deadline of December 31,2012. Because there are process changes that could reduce the treatment schedule, risks associated with these changes are considered technical risks. Two of these are shown in Table 4, "Aluminum nitrate ratio" and "Scrub recycle minimization evaluation."

Reducing the AAR in the blended feed allows for increasing the SBW rate. The SBW high temperature run in 1999 ran with a feed blend having an AAR ratio of 2.0 for two weeks, but the particle size could not be controlled during this time (Wood, 2001). For the 2000 SBW high temperature run, the AAR began at 3.8, was decreased to between 3.5 and 3.0 for most of the run, decreased to 2.8 for seven days, and finally decreased to 2.6 for the last 8 days of the run (Law, 2000). The bed particle size remained constant at about $0.6 \mathrm{~mm}$ for the last month of the run, confirming that it could be controlled at an acceptable size and for the reduced AAR ratio. Guided by this data that showed that an AAR of 2.0 was too low and 2.6 acceptable, O'Brien assumed an AAR of 2.25 in estimating a four-year operating schedule for processing all SBW (O'Brien, 2002).

The first 50-hr calcination pilot test in 2004 used an AAR ratio of 2.25; in the second test the AAR ratio was reduced to 1.75 . No problems with bed growth were seen in either test. Some nozzle plugging was seen with the lower AAR. Also, the feed used for these tests was a surrogate for Tank WM-180 waste, which has less phosphate and zirconium than will the other tank wastes after mixing with tank solids. The effect of the high zirconium phosphate in the tank solids on the bed has not been determined. Thus, while the acceptability of using an AAR ratio of 2.25 was verified, there is still uncertainty whether it can be reduced as low as 1.75 .

A second way to reduce the total operating time is to replace the cyclone separator. The efficiency of the current cyclone separating carryover particulate is only about $63 \%$. With a higher efficiency cyclone, less calcine particles would be carried into the scrub system. Since the recycle rate is determined by the rate the scrub accumulates solids, fewer solids in the cyclone off-gas means a lower rate of scrub recycle to the calciner. Thus, the SBW feed could be increased by the amount that the scrub recycle is reduced. An increase in cyclone efficiency from $63 \%$ to $90 \%$ translates to a reduction in the scrub recycle from $23 \%$ of the total calciner feed to about $6 \%$ and a net increase of $22 \%$ in SBW feedrate.

Bates (2003) estimated the savings in processing time to be 139 days, and Adams (2003) estimated the cost to replace the cyclone would be about $\$ 2.45$ million. A high-efficiency ( $98 \%$ ) cyclone was demonstrated in the 2004 calcination tests, although occasional plugging was observed (Boardman, 2004). Several uncertainties remain in the evaluation of cyclone replacement. A higher efficiency cyclone will have a higher pressure drop; further evaluation is needed to determine how to accommodate this change in pressure profile with the existing off-gas equipment, or whether modifications to the compressor would be required. Modeling the higher efficiency cyclone was based on a scrub recycle rate that kept the aluminum concentration in the scrub below 1.6 molar. Although this constraint is similar to 
historical operation of the NWCF, other constraints, such as chloride buildup or total solids buildup, may affect the actual recycle rate achievable.

There has been uncertainty in the concentrations of hydrogen chloride and chlorine in the off-gas both upstream and downstream of the S-GAC bed. Hydrogen chloride and chlorine in NWCF off-gas sample were not detected, however, detection limits bracket the MACT emission limit. Hydrogen chloride was measured at a concentration of $0.5 \mathrm{ppm}$ in the pilot test off-gas (Boardman, 2004b), much lower than the MACT limit of $21 \mathrm{ppm}$. However, in the full-scale plant, recycle of scrub to the calciner could cause higher hydrogen chloride concentrations in the off-gas as chlorides buildup in the scrub solution. Thus, hydrogen chloride emissions and the need for $\mathrm{HCl} / \mathrm{Cl}_{2}$ removal remain uncertain. Both long-term GAC bed testing (Del Debbio, 2003) and recent pilot tests (Boardman, 2004b) indicate that the S-GAC will not remove a significant amount of hydrogen chloride from the off-gas.

Many of the uncertainties in the CMACT design relate not to performance but to whether a given design is optimized. Some of the questions regarding the MCF have been resolved in design and modeling studies and in the recent calcine pilot tests. The fuel, water, and air feeds to the staged combustor result in a 3-fold increase in throughput in the combustor. Reducing the oxygen content of the calciner off-gas or combustor air rates were suggested as ways to reduce this increase. Kinetic/CFD modeling studies (Wood, 2004), the CMACT Feasibility Study (Merrick, 2004), and the FY 2004 pilot tests (Boardman, 2004b) all showed that combustor throughput reductions are not possible.

Both long-term GAC bed testing (Del Debbio, 2003) and recent pilot testing (Boardman, 2004) have demonstrated that the GAC bed can achieve the required mercury removal efficiency. However, both the small-scale, long-term tests and the pilot tests were performed at lower velocities and with smaller size carbon particles than expected in the design. In addition, uncertainties in the mercury partitioning between off-gas and scrub and inconsistent test results regarding mercury loading on carbon lead to a design that may not be optimum (i.e., the design may have more beds or larger beds than needed).

The CMACT design (Merrick, 2004) has three carbon beds, 16 feet in diameter by 10 feet in height. Two beds are operated in parallel with the third in series. The purpose of the parallel bed was to allow for changeout of a bed while still operating. However, since each bed is sized for capture of all the expected mercury in the off-gas and since the test data shows that hydrogen chloride is not adsorbed in the S-GAC bed, there appears to be no need to have a parallel bed. The need for a second bed in series is uncertain. The pilot data (Boardman, 2004b) shows significantly more capture of mercury in the scrub than assumed in sizing the GAC bed. However, in the full-scale plant, the mercury concentration will increase over time in the scrub and the off-gas. Since nearly all the scrub is recycled, the concentration in the off-gas will increase. Additional GAC bed testing at higher velocities would be needed to evaluate whether the GAC bed diameter can be decreased.

Three methods to solidify the waste scrub have been demonstrated (Herbst, 2003; Scholes, 2003; Raman, 2003). While the option to dispose of the scrub by absorbing it onto calcine is attractive because it eliminates a waste stream, gas generation, foaming, and crusting observed in tests negate this advantage. For the option of absorbing the scrub on silica gel, a higher waste loading could be achieved than if the scrub was grouted. But uncertainties are greater for a silica gel waste form, namely, whether water would desorb over time, whether the higher waste loading and water content would result in excessive radiolytic hydrogen generation, and what mechanical mixing would be needed. Because these questions have not been answered, the present flowsheet shows grouting as the method for treating the scrub. 
3.1.4.2 Risks identified in the Merrick Risk Analysis. The recent CMACT Feasibility Study (Merrick, 2004) included an assessment of program and project risks for the SBW Treatment Projects. Merrick identified risks using the Risk Identification Checklist contained in the Risk Management Plan for the Sodium Bearing Waste Treatment Facility Project (Bonnema, 2002). These risks were then described, categorized, and risk levels determined. Merrick identified no technical or design risks that rated as critical or high. Two risks categorized as technical or design were rated as moderate and are briefly discussed below.

One moderate risk falls under the title "New application of an existing technology". The CMACT alternative includes use of mixing pumps that must fit down 12-in diameter risers in 300,000-gal tanks, a new off-gas treatment system, and remote handling packaging systems. The individual components used with the modified process all involve established technologies. However, they will be applied in a new combination and to some degree in new applications. Off-gas treatment in particular involves many steps that will need to be integrally coordinated. Routine, efficient, and effective performance cannot be guaranteed, particularly during initial operations. Short-term air quality permit violations may occur. Redesign and additional project costs may also occur.

A design risk was identified relating to complex design features and ranked moderate. The remote handling of feed material and product and the treatment of off-gas both involve complex design features. Each component of the off-gas treatment system has certain operating conditions that must be integrated with other unit operations. The off-gas treatment will involve a complex integrated control system to control startup, operation and shutdown, as well as emergency shutdown. Dust control in the CPF is of particular concern. Should the system not integrate properly, air quality objectives and permit limits may not be achieved and there could be contamination issues with packaging calcine.

3.1.4.3 Other Risks. The recent calcination pilot tests confirmed the possibility of another risk involving the GAC bed. Several times during the test, there were temperature excursions in the carbon bed. Additional testing is needed to determine the causes of GAC bed temperature excursions and control measures to avoid these excursions.

\subsection{Steam Reforming}

Steam reforming is a process for producing hydrogen. This is generally done by reacting an organic feed with an oxygen source (e.g., water or carbon dioxide) at elevated temperature (possibly with a catalyst) to form carbon monoxide and hydrogen and various other products (e.g., carbon and/or other hydrocarbons via reactions such as the following:

$$
\mathrm{C}_{\mathrm{x}} \mathrm{H}_{\mathrm{y}}+\mathrm{zH}_{2} \mathrm{O}\left(\text { or } \mathrm{CO}_{2}\right)=\mathrm{mCO}+\mathrm{nH}_{2}+\text { other products }
$$

Steam reforming is considered an alternative to other thermal processes for treating SBW for the following reasons:

- Under favorable conditions, total decomposition of organics can be achieved without openflame combustion and its associated emissions.

- Also under favorable conditions, oxides of nitrogen can be converted to nitrogen. This feature is relevant due to nitrates in SBW that generally produce high levels of $\mathrm{NO}_{\mathrm{x}}$ in off-gas from thermal treatment processes.

Steam reforming processes have long been used commercially to generate hydrogen for ammonia production, for petroleum refining, and to generate synthesis gas. More recently it has been used to 
generate hydrogen for fuel cells, to extract hydrogen from biomass, to treat paper mill black liquor, and to destroy various hazardous wastes. The latter two applications have led to its consideration by DOE for treatment of its radioactive wastes. In 1994, steam reforming was tested to treat Hanford Tank waste in the Synthetica process. In 1996, DOE again funded testing of steam reforming on low-level mixed waste and Hanford tank waste. TWR tested a 42-inch steam reforming fluidized bed reactor at the Portsmouth Gaseous Diffusion Plant for treatment of soil, debris, and aqueous liquids containing PCBs and hazardous organic wastes.

Steam reforming treatment of INEEL SBW was initially considered as a method to convert a low activity liquid waste into a solid that would then be grouted to a final waste form. In tests performed during 2000 a simulant similar to SBW was fed to a bench-scale fluidized bed steam reformer. By operating the test bed with and without added reductants and catalysts these tests also showed that waste denitration could be achieved. In 2001, when DOE was evaluating vitrification of SBW, steam reforming was suggested as a possible alternative treatment. The DOE Idaho Operations Office then sponsored a Tank Focus Area Review Team to review the technology and make a recommendation. The team concluded that although steam reforming by itself is not a feasible alternative to produce an acceptable final waste product it might have merit as a pretreatment prior to vitrification.

In 2002, DOE discontinued funding of SBW vitrification process development and issued guidance to BBWI to assess alternative processes to produce waste forms that would be disposable at WIPP. Pursuant to that decision two analyses were completed (Murphy, et al. 2002 and Perry 2002) which reviewed all processes that had been considered up to that point for treatment of SBW. Also, a feasibility study to treat SBW by steam reforming was prepared in 2002 (Williams 2002) which included a preliminary design, cost estimate, schedule, and risk assessment.

From the 2002 analyses, four processes emerged for final consideration, one of which was stream reforming. Following formulation of the selection process two subcontractors (TWR and THOR ${ }^{\mathrm{sm}}$ Treatment Technologies [TTT]) were asked to provide technical input for fluidized bed treatment of SBW by steam reforming, based on their experience and expertise. (TTT currently uses steam reforming to process commercial radioactive treatment operations at the Studsvik Processing Facility in Erwin, Tennessee, while TWR uses a similar process to treat black liquor from paper/pulp processing.) A benchscale test facility was provided by the INEEL and an SBW surrogate was formulated to use as feed for the tests. The principal test objective was to resolve steam reforming process uncertainties regarding:

- Continuous operability of the process

- Solid product composition and handling/flow characteristics

- Off-gas composition, including $\mathrm{NO}_{\mathrm{x}}$

- Fate of radionuclides and mercury.

In addition to these tests with SBW, similar tests were performed in 2001 on simulated Hanford low activity waste liquid/sludge wastes.

\subsubsection{Process Functions}

The most recent process design anticipates that a Steam Reforming Facility will retrieve waste (including SBW, flushed tank solids, and NGLW from the INTEC TFF) and co-process these wastes through a fluidized-bed, steam-reforming reactor. The functions of this reactor are to:

- Convert the wastes to a solid product that retains most of the radionuclide and hazardous components 
- Convert nitrates in the waste to nitrogen with minimum $\mathrm{NO}_{\mathrm{x}}$ formation.

The process must also treat off-gas to remove and collect product fines, control emissions to comply with MACT requirements, remove radionuclides to applicable regulatory levels (NESHAP), and reduce $\mathrm{NO}_{\mathrm{x}}$ levels to meet project directed emission targets. Additional steam reforming facility functions are to certify, package, and ship the final waste products to disposal sites. The steam reformed product solids will be withdrawn as granular bed particles directly from the reactor vessel and as elutriated fines from the sintered metal filter downstream. Since the reactor will operate at $\sim 700^{\circ} \mathrm{C}$ the products will be cooled prior to packaging. Due to low bulk densities of the fines the product may also require densification before or during packaging in $2-\mathrm{ft} \times 10-\mathrm{ft}$ waste containers.

The solid waste product will have a high radiation field due to its ${ }^{137} \mathrm{Cs} /{ }^{137} \mathrm{Ba}$ content. The steam reformed solids, co processed SBW, and heel solids are expected to contain TRU radionuclides at concentrations greater than $100 \mathrm{nCi} / \mathrm{g}$, the minimum value for acceptance at the WIPP. Therefore, they will be packaged and certified for disposal at WIPP as RH-TRU waste by the following procedures:

- Visual inspection and acceptance

- Canister closure by placement and locking of a lid

- Survey and decontamination as required

- Determination of canister weight, waste composition (including isotope concentrations of interest), and hydrogen generation rate

- Labeling and logging (for recordkeeping)

- Transfer to lag storage or loading into a shipping cask.

- Due to high radiation fields all packaging and certification operations with the canisters will be performed remotely.

Secondary wastes expected from the steam reforming facility include scrub liquor from scrubber blow down and scrub residual after process shutdown, spent GAC, and spent HEPA filters. The classification of the scrub waste is uncertain. The other secondary wastes are expected to be treated as mixed low-level wastes to comply with acceptance criteria at target disposal sites. After waste processing is complete the Steam Reforming Facility and associated subsystems will be closed for decommissioning.

\subsubsection{Process Description}

A process block flow diagram of the steam reforming process is shown in Figure 4. Though either the TTT or the TWR process could be selected the figure is sufficiently generic to represent either process.

The blended waste is retrieved into the processing facility via existing steam jets and mixed with an organic reductant that facilitates reduction of nitrates to nitrogen. This mixture is then pumped into the reactor bed through an atomizing nozzle. The reactor bed is an inert, inorganic granular material fluidized by a flowing gas entering the base of the reactor. Other solids (e.g., a catalyst or a second solid reductant) may also be added to the bed. The SBW and additives undergo various reactions that produce a granular solid mineral product (e.g., alkali carbonates, aluminum oxides, alumino-silicates, etc.) and offgases (mostly nitrogen, $\mathrm{NO}_{\mathrm{x}}$, carbon dioxide, carbon monoxide, hydrogen, steam, and hydrocarbons). 
Solid product emerges from the reactor either as elutriated fines or as granular bed product extracted from the bottom of the reactor. The proportion of bed product-to-fines depends on the choice of operating conditions and additives. Bed product and fines are combined in hoppers, cooled to $56^{\circ} \mathrm{C}$, and transferred to disposal canisters. These are then weighed, inspected, sealed, decontaminated, labeled, and logged prior to interim storage or shipment to WIPP.

Off-gas from the steam reforming vessel is treated prior to release by the following series of unit operations:

- Filtration through a high temperature, pulsed, sintered metal filter to separate out elutriated product fines

- Thermal oxidization to consume hydrogen, carbon monoxide, methane, and unburned hydrocarbons

- Quench cooling

- Wet scrubbing to extract acid gases, soluble mercury, and residual volatile metals (e.g., $\mathrm{Cs}, \mathrm{Pb}$, Cd) that are not extracted by filtration

- HEPA filtration for removing ultra-fine particles

- Activated carbon filtration for polishing residual mercury and iodine.

Compliance with off-gas emission limits is verified by a continuous emission monitor (CEM) system prior to discharge.

Elevated mercury levels may develop in the recirculated scrub solution over time, necessitating periodic replacement of the solution. In addition some residual scrub solution will remain after processing of SBW is completed. The spent scrub solution from these two sources must also be treated for disposal. ${ }^{137} \mathrm{Cs}$ is the major contributor to the gamma radiation dose of the SBW and is expected to partition to the solid products and not to the scrubber liquor. The same behavior is expected for the TRU contaminants. For these reasons the treated scrubber liquor will likely not be disposable at WIPP, but will be disposable as mixed low-level radioactive waste, provided the hazardous metals in the solution are immobilized. The spent scrub is therefore treated to achieve this immobilization and ensure that the acceptance criteria of identified waste disposal sites (e.g., Hanford or Envirocare) are satisfied.

The primary unit operations constituting the scrub liquor treatment process are as follows:

- Partial neutralization

- Mercury precipitation

- Solidification.

Precipitation of mercury from the solution with sulfide will be accomplished by mixing and reaction with calcium sulfide $(\mathrm{CaS})$. The product is mercuric sulfide $(\mathrm{HgS}$, or cinnabar) that is highly insoluble. Precipitation of mercury in this manner immobilizes it in the grouted scrub product and ensures that the waste form meets land disposal restriction (LDR) standards for disposal at a MLLW disposal site. Partial neutralization of the scrub liquor is required to prevent sulfur/acid reactions that can form toxic $\mathrm{H}_{2} \mathrm{~S}$ during the mercury precipitation step. 
The final step in treating the scrub liquor is to solidify the mercury-precipitated slurry by grouting. The three treatment steps (neutralization, precipitation, and grouting) are all represented in the single box labeled "Spent Scrub Tank" in Figure 4. Grouting will be done by mixing $25 \mathrm{wt} \%$ of treated scrub slurry with $75 \mathrm{wt} \%$ of dry additive (Portland cement, blast furnace slag, calcium hydroxide) in a screw mixer and pouring it directly into drums. The final waste form will meet Nuclear Regulatory Commission (NRC) requirements for performance grout. Each filled drum will be decontaminated, as required, and then cured for $\sim 24$ hours prior to being inspected and moved to temporary storage prior to shipment for disposal.

Two additional secondary waste streams, HEPA filters and spent GAC, will also be treated and disposed. HEPA filters may be treated in the INTEC filter leach facility. It is anticipated that GAC will be packaged and disposed of without treatment. However, this is contingent upon the material passing TCLP. Both wastes are expected to be disposed ultimately as mixed low-level wastes.

It should be noted that the preliminary process design configuration, described above, might not be what a steam reforming technology vendor may propose for treating SBW, particularly TTT. There are perhaps other methods of oxidizing the steam reforming reactor off-gas, and minimizing the number of off-gas treatment unit operations to meet emissions requirements and secondary waste quantities. The preliminary process design described above represents INEEL's initial thoughts, and optimization of the design has not been within the work scope performed. 


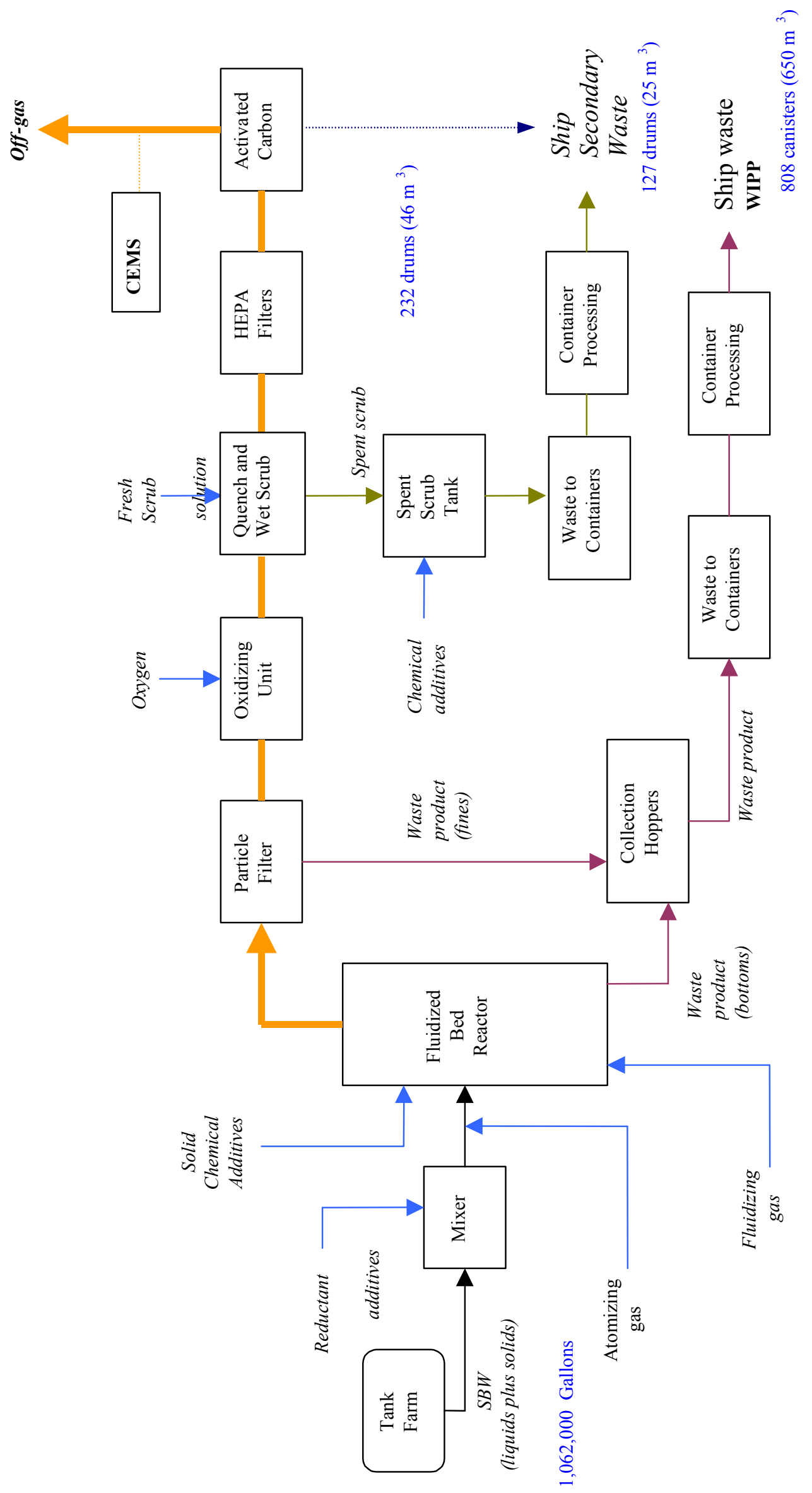

요 


\subsubsection{Summary of Technical Performance}

3.2.3.1 Processing Rate, Availability. A 3-year waste processing period is assumed, which includes six months of transition to processing radioactive waste. Facility availability is assumed to be 200 days per year and 24 hours per day during that period. This translates to an average processing rate of $88.5 \mathrm{gal} / \mathrm{hr}$ or $1.5 \mathrm{gal} / \mathrm{min}$ of combined (SBW liquid + heel solids) feed.

The facility availability is based on many years of operating experience at the INEEL working within DOE regulations. Changes in this operating environment could significantly alter the assumed availability and processing rate. Ideally, the fluidized bed reactor would operate without interruption. Fouling of feed spray nozzles and agglomeration of the bed are two scenarios that could hinder smooth operations; both problems were experienced during bench-scale demonstration tests. Failure to achieve a stable size distribution of the bed particles was also experienced, and could impact the facility availability factor, as could other process uncertainties discussed below in Technical Uncertainties and Potential Impacts.

3.2.3.2 Waste Products Quantity \& Quality. The primary waste product from this option is the steam reformed solid from the SBW feed.

Secondary wastes include the grouted scrub, spent GAC, and contaminated HEPA filters. A quantitative summary of these waste products is shown in Table 5. It has not been determined whether spent GAC will require treatment prior to disposal as mixed low level waste. Volume and mass of the treated HEPA filters have not been estimated.

Table 5. Volumetric summary of waste products.

\begin{tabular}{|c|c|c|c|c|c|c|c|}
\hline Waste Products & WM-180 & WM-187 & WM-188 & WM-189 & Total\# & Total kg & Total $\mathbf{m}^{3}$ \\
\hline Steam Reforming Solids, $\mathrm{kg}$ & 220,000 & 160,000 & 230,000 & 260,000 & & 860,000 & 650 \\
\hline RH canisters, \# (a) & 205 & 149 & 211 & 243 & 808 & & \\
\hline $\mathrm{CH}$ Grout (from scrub), $\mathrm{kg}$ & 14,000 & 9,000 & 15,000 & 14,000 & & 52,000 & $25^{\mathrm{a}}$ \\
\hline $\mathrm{CH}$ or $\mathrm{RH}$ drums, \# & 35 & 22 & 36 & 34 & 127 & & \\
\hline $\mathrm{CH}$ Carbon (spent GAC), kg & 3,300 & 5,000 & 9,200 & 9,500 & & 27,000 & 46 \\
\hline $\mathrm{CH}$ drums, \# (b) & 28 & 43 & 79 & 82 & 232 & & \\
\hline HEPA Filters & & & & & Unknown & & \\
\hline Totals & & & & & 1167 & 939,000 & 721 \\
\hline
\end{tabular}

${ }^{a}$ Assumes aggregated steam reformed solids can be densified to $1.33 \mathrm{~g} / \mathrm{cm}^{3}$ bulk density

${ }^{\mathrm{b}}$ Assumes GAC will pass TCLP test and is direct-disposed after packaging in 55-gal drums

The solid steam reformed product consists of granular bed particles extracted directly from the fluidized bed reactor and elutriated fines that are collected from the off-gas stream by cyclonic action or high-temperature filtration. The bed particles are generally spheroidal in shape and may contain multiple solid phases, depending on processing conditions. In bench-scale tests they ranged between 0.2 and 1.1 $\mathrm{mm}$ in diameter. Bed particle densities were $2.7-3.6 \mathrm{gm} / \mathrm{cm}^{3}$ for individual particles and $1.2-2.2 \mathrm{gm} / \mathrm{cm}^{3}$ in bulk.

The fines product is a freely flowing ash-like powder with limited cohesiveness (forming loose clumps that readily crumble and fluidize when agitated). The portion of the fines collected by cyclonic 
action has higher bulk density than that collected by filtration, and has a broad size spectrum ranging from 10 to as high as $300 \mu \mathrm{m}$. The filter product has a multimodal particle size distribution with a significant fraction in the $0.1-1.0 \mu \mathrm{m}$ range, with bulk densities as low as $0.34 \mathrm{gm} / \mathrm{cm}^{3}$. The fines product bulk density can be increased by a factor of $70-160 \%$ by mechanical agitation or tamping.

Depending on reactor operating conditions (especially stoichiometric proportion of reductant added) the fines product may contain significant amounts of un-oxidized carbon (as high as $50 \mathrm{wt} \%$ in some tests). Data also show that several species ( $\mathrm{Pb}, \mathrm{Cd}, \mathrm{NH}_{3}, \mathrm{Cr}, \mathrm{Ni}, \mathrm{K}, \mathrm{Rh}$ [Tc surrogate], $\mathrm{Na}$, and $\mathrm{SO}_{4}$ ) may be volatile and concentrate in the fine particles.

Radioactive properties of the waste products are shown in Table 6, based on the mass balances in Appendix A. Where a range is specified, the lower limit of the range was determined by using for each radionuclide the minimum concentration from the eight calculated mass balances (four feed cases [tanks WM-180, WM-187, WM-188, WM-189] for each of two flowsheets [TWR and TTT]), and the upper limit was similarly determined. Thus, to the extent that the numbers in the table are sums of minima and maxima (rather than minima and maxima of sums), the ranges are conservative.

Dose rates and heat generation rates have not been calculated for the waste compositions shown in Appendix A. However, these rates are expected to be very similar to those calculated from earlier mass balances (Bohn 2002). For steam reformed product, a canister contact dose rate of $4.5 \times 10^{4} \mathrm{mR} / \mathrm{hr}$ and a heat generation rate of 1.92 watts were estimated previously, based on decay to 2010 .

For the grouted scrub product the corresponding dose rates from an earlier study (Quigley et al 2000) were $460 \mathrm{mR} / \mathrm{hr}$ and 0.0034 watts, respectively, based on an assumed set of radionuclide removal efficiencies in unit operations upstream of the scrubber. For the current mass balance these removal efficiencies were revised based on measurements from the FY 2003 steam reforming tests, and new radionuclide concentrations in the grouted scrub were calculated for the four tank farm feeds. The dose and heating rates for the grouted waste were then re-estimated by scaling the dose rate from the 2000 report by the Cs-137 ratio in the two grouts (Cs-137 is the major contributor to dose). The results indicate dose rates of $145-328 \mathrm{mR} / \mathrm{hr}$ and heating rates of $0.00107-0.00242$ watts. If the four tanks were perfectly blended, the volume-averaged rates would be $225 \mathrm{mR} / \mathrm{hr}$ and 0.00166 watts, respectively. Thus, it remains uncertain whether the grouted scrub product would be contact-handled $(\mathrm{CH})$ or RH. Additional work is needed to determine whether the contact dose rate can be reduced below the $200 \mathrm{mR} / \mathrm{hr}$ limit for $\mathrm{CH}$ waste.

For the drummed, spent GAC the estimated rates from the 2000 feasibility study were $<5 \mathrm{mR} / \mathrm{hr}$ and $<0.001$ watts. Since iodine was not tracked in the FY03 tests these numbers have not been changed and are the best estimates available. 
Table 6. Radioactive properties of waste products

\begin{tabular}{lc}
\hline Steam Reformed Solids & Range \\
All Radionuclides (Ci/canister) & $520-1130$ \\
Fissile Gram Equivalent (g/canister) & $233-630$ \\
${ }^{239}$ Pu Equivalent Curies (Ci/canister) & $4.2-68$ \\
TRU Content (nCi/g) & $7,100-11,800$ \\
\hline Grouted Scrub & $0.04-7.3$ \\
All Radionuclides (Ci/canister) & $1.4 \mathrm{E}-5$ \\
Sum of fractions (Hanford Cat 3) & $1.8 \mathrm{E}-2$ \\
Sum of fractions (NRC Class A) & $0.0-47$ \\
TRU Content (nCi/g) & \\
\hline Spent GAC & $1.0 \mathrm{E}-6-4.3 \mathrm{E}-6$ \\
All Radionuclides (Ci/canister) & $4.2 \mathrm{E}-6$ \\
Sum of fractions (Hanford Cat 1$)$ & $2.0 \mathrm{E}-14$ \\
Sum of fractions (Envirocare) & 0 \\
TRU Content (nCi/g) & \\
\hline
\end{tabular}

3.2.3.3 Size, Footprint of Processing Facility. Preliminary equipment sizing and facility layout were initially performed in 2002 (Williams 2002). This work was revised in late 2003 to reflect process changes (e.g., addition of scrub grouting operations) and updated mass balances. The revised plan drawings are provided in Appendix A. Equipment and room arrangements have not been optimized, as the effort to date has been focused on a preliminary process design only. Also, the latest mass balances, based on FY04 bench-scale test data, are not reflected in the drawings.

Per this layout the steam reforming facility would include two buildings - one for waste treatment, packaging, and certification and one for empty canister storage. These buildings have dimensions of roughly $150 \mathrm{ft} \times 250 \mathrm{ft}$ and $55 \mathrm{ft} \times 65 \mathrm{ft}$, respectively, with footprints of $32,000 \mathrm{ft}^{2}$ and $3600 \mathrm{ft}^{2}$. The processing building has four floors and includes $10,200 \mathrm{ft}^{2}$ of hot cell area. The hot cell consists of 3,600 $\mathrm{ft}^{2}$ of processing area, $1000 \mathrm{ft}^{2}$ for packaging/handling of steam reformed solids, 2,000 $\mathrm{ft}^{2}$ of storage space for filled waste canisters, and the balance $\left(\sim 3,600 \mathrm{ft}^{2}\right)$ for equipment maintenance, decontamination, cell access, and shielding walls. A control room, offices, personnel decontamination room, manipulator repair room, and other support areas are located on the ground floor.

The plot space estimates for the facility are summarized in Table 7. It should be noted that the facility plan was done for an earlier process design that did not include treatment of spent scrub liquor or packaging of spent GAC. Thus, there was no explicit allowance in the facility layout for these operations. The required area can be estimated from a recent study (Merrick, 2004) that included grouting scrub from SBW calcination. The hot cell space for grouting plus calcine packaging in Merrick's study amounted to about $1000 \mathrm{ft}^{2}$. Assuming half of this area for scrub processing would result in an area of $500 \mathrm{ft}^{2}$. 
Table 7. Steam reforming facility space estimates.

\begin{tabular}{|c|c|}
\hline Area Description & Area $\mathrm{ft}^{2}$ \\
\hline Process Equipment & 3,600 \\
\hline GAC Polishing Columns & 2,000 \\
\hline Grouting Equipment/Drum Storage & 500 \\
\hline Waste Packaging and Canister Handling & 1,000 \\
\hline Lag Storage for RH Canister & 2,000 \\
\hline Administrative & 10,400 \\
\hline Mechanical/Electrical & 9,700 \\
\hline
\end{tabular}

3.2.3.4 Effects of Scale, Variation with Throughput. Changing the scale of the process, i.e., designing for an accelerated treatment schedule, would have the most effect on the process operations performed on the lowest floor of the facility. Two areas in particular - the process hot cells and the lag storage area for full waste canisters - would be affected.

Major process equipment in the hot cells includes SBW feed tanks, steam reformer vessel, product cooling bins, and the off-gas treatment train. Decreasing the processing time would increase the floor space required for most of this equipment by the inverse ratio of the processing times to the two-thirds power. Reducing the schedule from 2.5 years to 1 year, the floor space for storage vessels would thus increase by a factor of $\sim(2.5 / 1)^{0.667}=1.84$, and for process equipment by a factor of $\sim(2.5 / 1)^{1.0}=2.5$.

Interim storage for RH waste canisters is driven by (a) the initial lag time between certification of the first canister of waste and actual shipment of it to WIPP, and (b) the excess of the rate at which certified waste canisters are produced over the rate at which WIPP can accept them. For a 2.5 year processing schedule the lag storage requirement is 304 canisters (176 from (a) and 128 from (b), above). For a 1-year schedule the lag storage requirement increases to 728 canisters, necessitating a $140 \%$ increase in the requirement from 2,000 to $4,800 \mathrm{ft}^{2}$. The processing areas for GAC beds to remove mercury from off-gas and for treating/packaging spent scrub liquor may also require scaleup by the same factor as for the process hot cells. The estimated plot space estimates for the facility with a 1-year processing schedule are summarized in Table 8.

Table 8 . Steam reforming facility space estimates for 1 year processing schedule.

\begin{tabular}{lc}
\hline \multicolumn{1}{c}{ Area Description } & $\mathbf{A r e a}_{\mathbf{f t}} \mathbf{2}^{\mathbf{2}}$ \\
\hline Process Equipment & 9,000 \\
GAC Polishing Columns & 5,000 \\
Grouting Equipment/Drum Storage & 500 \\
Waste Packaging and Canister Handling & 1,800 \\
Lag Storage for RH Canister & 4,800 \\
Administrative & 10,400 \\
Mechanical/Electrical & 9,700 \\
\hline
\end{tabular}




\subsubsection{Technical Uncertainties and Potential Impacts}

The Sodium-Bearing Waste Treatment Applied Technology Plan (INEEL/EXT-03-00477, June 2003) identified uncertainties resulting from technical and operational risk assessments. These uncertainties were identified primarily to guide the selection of a treatment technology and were not a complete listing of technical issues requiring resolution during detailed design efforts. The uncertainties were prioritized as High (H), Medium (M), or Low (L) risks. A high risk is categorized as an issue that is likely to occur and would have a significant (\$25 million - \$125 million impact) or critical ( $>\$ 125$ million) consequence. Issues that are unlikely but have significant or critical consequences, or are likely but have marginal ( $\$ 5$ million - \$25 million) consequences are categorized as medium risks. Issues that have a probability of occurrence of less than $10 \%$, or a higher probability but negligible or marginal consequences are categorized as low risk. Table 9 lists and describes these uncertainties as well as their status as of the writing of this report.

Table 9. Summary of steam reforming uncertainties identified in risk assessment.

\begin{tabular}{|c|c|c|}
\hline Uncertainty & Description & Status \\
\hline $\begin{array}{l}\text { Reformer Chemistry and } \\
\text { Operational Mode }(\mathrm{H}) \text { : }\end{array}$ & $\begin{array}{l}\text { Steam reforming chemistry is not } \\
\text { understood sufficiently to define } \\
\text { (a) target product (carbonates or } \\
\text { alumino-silicate), (b) needed } \\
\text { additive types (e.g., reductant) and } \\
\text { blend ratios, (c) ideal processing } \\
\text { temperature, and (d) off-gas } \\
\text { composition. }\end{array}$ & $\begin{array}{l}\text { Bench-scale tests of two } \\
\text { commercial steam reforming } \\
\text { processes were done in FY03 and } \\
\text { 04. Parametric testing indicated } \\
\text { sensitivities of product and off-gas } \\
\text { composition to additive types, } \\
\text { stoichiometry, and process } \\
\text { temperature. Some questions } \\
\text { remain regarding carbon and } \\
\text { hydrocarbon contents of solids and } \\
\text { off-gas fluidized bed stability, and } \\
\text { control of bed agglomeration. }\end{array}$ \\
\hline Product Characterization (M): & $\begin{array}{l}\text { Handling characteristics and } \\
\text { volume of product produced from } \\
\text { unit feed volume are needed to } \\
\text { design packaging system and plan } \\
\text { for disposal of final waste forms. }\end{array}$ & $\begin{array}{l}\text { Bench-scale tests have provided } \\
\text { measurements of solid product } \\
\text { mass per unit of SBW and density; } \\
\text { also, qualitative data on product } \\
\text { flowability, compactability, and } \\
\text { particle sizes, and process-specific } \\
\text { partitioning between coarse and } \\
\text { fine particles. }\end{array}$ \\
\hline Product Densification (L): & $\begin{array}{l}\text { The level to which bulk density of } \\
\text { steam reformed solids may be } \\
\text { increased is not known, leaving } \\
\text { uncertainty as to the final waste } \\
\text { volumes that must be transported } \\
\text { and disposed. }\end{array}$ & $\begin{array}{l}\text { Fines product was compacted } \\
\text { during FY03-04 steam reformer } \\
\text { tests by hand tamping and } \\
\text { vibration. Overall product bulk } \\
\text { density achievable by remote, } \\
\text { mechanized means has not been } \\
\text { determined. }\end{array}$ \\
\hline
\end{tabular}


Table 9. Summary of steam reforming uncertainties identified in risk assessment. (continued)

\begin{tabular}{|c|c|c|}
\hline Uncertainty & Description & Status \\
\hline Off-gas Characterization $(\mathrm{H})$ : & $\begin{array}{l}\text { Off-gas compositions and } \\
\text { effectiveness of emissions control } \\
\text { technologies must be established by } \\
\text { pilot-scale testing. }\end{array}$ & $\begin{array}{l}\text { Off-gas measurements of } \mathrm{H}_{2}, \mathrm{CO}, \\
\mathrm{CO}_{2}, \mathrm{CH}_{4}, \mathrm{H}_{2} \mathrm{O}, \mathrm{NO}_{2}, \mathrm{NO}, \mathrm{SO}_{2}, \\
\mathrm{HCl}, \mathrm{Hg} \text {, and THC were made in } \\
\text { bench-scale tests at various } \\
\text { locations in the off-gas system. Not } \\
\text { all measurements were done at all } \\
\text { times and locations and some } \\
\text { measurements were unreliable. In } \\
\text { particular, THC measurements were } \\
\text { limited. Some significant questions } \\
\text { were raised (see discussion } \\
\text { following table). }\end{array}$ \\
\hline $\begin{array}{l}\text { Speciation \& Fate of Key } \\
\text { Components }(\mathrm{H}) \text { : }\end{array}$ & $\begin{array}{l}\text { Data are needed on fate and } \\
\text { speciation of } \mathrm{Hg}, \mathrm{Cs}, \mathrm{Tc} \text {, and } \mathrm{I} \text { in } \\
\text { order to design off-gas system to } \\
\text { meet emissions requirements. }\end{array}$ & $\begin{array}{l}\text { Elemental and oxidized } \mathrm{Hg} \\
\text { concentrations were measured at } \\
\text { some off-gas locations in bench- } \\
\text { scale tests. Cs and Rh (Tc } \\
\text { surrogate) concentrations were } \\
\text { measured in solid products and } \\
\text { scrub solutions. Iodine data was } \\
\text { not obtained. }\end{array}$ \\
\hline Thermal Oxidizer Kinetics (M): & $\begin{array}{l}\text { Due to high water content and low } \\
\text { oxygen kinetic limitations could } \\
\text { drive destruction efficiency for } \mathrm{CO} \\
\text { and organics below what is } \\
\text { required. }\end{array}$ & $\begin{array}{l}\text { Bench scale thermal oxidizer was } \\
\text { tested in FY03-04 pilot tests. } \\
\text { Measured output CO and THC } \\
\text { concentrations indicate the oxidizer } \\
\text { was effective. }\end{array}$ \\
\hline $\begin{array}{l}\text { Off-gas Treatment Configuration } \\
(\mathrm{H})\end{array}$ & $\begin{array}{l}\text { Off-gas system design has not been } \\
\text { optimized. Off-gas characterization } \\
\text { data are needed before this can be } \\
\text { done. }\end{array}$ & $\begin{array}{l}\text { Off-gas measurements from FY03- } \\
04 \text { bench-scale tests are now } \\
\text { available. Optimization of the off- } \\
\text { gas system has not been performed. }\end{array}$ \\
\hline
\end{tabular}

Mass balances were developed for the preliminary process design to provide a basis to size equipment, determine feed chemical requirements, determine utility requirements, estimate emissions and determine waste volumes, compositions and properties. In order to prepare the mass balances a set of design basis elements (DBEs) was assembled in lieu of hard data addressing the technical uncertainties. The DBEs are assumptions about uncertainties and represent a level of risk to the successful implementation of a production steam reforming facility. Each DBE will require acceptance of technical risk or Applied Technology/Engineering development/demonstration efforts for validation before the next process design steps are taken. Fifty-five DBEs are identified for the preliminary process design. It is expected that more would be identified if this treatment option were selected for implementation. The individual DBEs are amplified with background information, references, explanation, and, in some cases, a discussion of uncertainties in Appendix A. The following is a summary discussion of major outstanding technical uncertainties.

\subsubsection{Feed.}

- There are uncertainties in the physical properties of the SBW inventories in tanks WM-180, $187,188,189$, in NGLW, and in tank farm heel solids. There are also uncertainties associated 
with mixing and transfer of heel solids for co-processing with liquid SBW. These uncertainties are discussed in Section 2.

- The steam reforming process assumes that heel solids will be co-processed with the SBW liquid. During Phase 2 bench-scale tests in FY04 the initial test feed contained both SBW simulant and simulated heel solids. Severe nozzle plugging problems developed until the solids concentration was reduced. This brought to light an uncertainty regarding co-processing of feed solids. At a minimum it indicates the need for feed nozzle design evaluation at a production scale.

- The target carbon ratio in adding reductant to the feed has been set at 5.3 moles $\mathrm{C}$ per mole $\mathrm{NO}_{3}{ }^{-}$(2.7 moles $\mathrm{C}$ from sucrose and 2.6 from solid carbon in the bed). This ratio represents a compromise between maximizing $\mathrm{NO}_{\mathrm{x}}$ destruction and minimizing unburned carbon (or hydrocarbons) in the steam reformed products. It was determined on the basis of the FY03-04 bench-scale tests where $\mathrm{NO}_{\mathrm{x}}$ destruction efficiencies of $89-95 \%$ were achieved.

- No addition of solid catalyst is needed. This assumes there is already sufficient of the catalytic agent in the SBW to achieve the needed $\mathrm{NO}_{x}$ conversion rate. However, a threshold concentration, below which there is insufficient or no catalytic effect, has not been determined.

\subsubsection{Bed Stability.}

- A major operational requirement implementing the steam reforming technology is to be able to control the dynamics of bed particle growth and attrition so that the growth of particles from the input of solids in the feed is balanced by diminution through friction, collision, fracturing and spallation. In addition, the total volume of the bed must be maintained within specified limits through elutriation of fines and/or withdrawal of bed product from the vessel. Finally, the bed must not be prone to agglomeration. When these criteria are satisfied the bed remains fluidized throughout operation and the bed particle size distribution eventually becomes static or mildly oscillatory within static limits. Under these conditions the bed is described as "stable". Such a stable condition was not demonstrated during the FY03-04 bench-scale testing. Rather, a fairly broad spectrum of particle sizes was observed during most of the tests, and bed agglomeration occurred during most (if not all) of the tests. Thus, a major outstanding uncertainty is whether or not a stable bed can be achieved and maintained through extended operating periods.

- Liquid fed to the steam reformer is atomized by a pressurized gas stream. The intensity of the atomization is characterized by the ratio of the standardized volumetric flow rates of gas and liquid (nozzle-air-ratio [NAR]). This ratio has been arbitrarily set at 800 (average of high and low values that were successfully tested). The NAR value influences the bed dynamics through its impact on particle attrition and will almost certainly require adjustment in the search for a set of conditions that ensure a stable bed.

\subsubsection{Stream Factor.}

- The facility design is based on a 2.5-year operating schedule. This, in turn, assumes a threeyear processing time that allows six months of start up and 200 days/year of productive operations. Bench scale testing clearly indicated the potential for off-normal conditions resulting in frequent shutdown of the process. The assumption of 200 days of operation per year thus remains an uncertainty that (if overly optimistic) could impact the schedule. 


\subsubsection{Reformer Product.}

- The mass balance assumes a 58-42\% split of the reformer product solids between coarse bed particles and fines collected in the filter. Process equipment is sized partly on the basis of this split ratio. However, the bench-scale tests showed a wide variation (94-2\% in the TWR test, with $4 \%$ in the cyclone product) so the assumed split ratio constitutes an outstanding uncertainty. Additionally, it has not been determined whether it is feasible to recycle both filter and cyclone fines to the reformer and eliminate the fines product altogether.

- Fines product is very airy and light with a low bulk density $\left(\sim 0.3 \mathrm{gm} / \mathrm{cm}^{3}\right)$. Handling such a product will likely be problematic due concerns with suspension of respirable fines containing alpha-emitting radionuclides. The extent of these problems and their resolution constitutes another technical uncertainty. Included is the question of feasibility of remote mechanical densification to reduce product volume where roughly half of the product mass (and up to $90 \%$ of the initial product volume) is fines.

\subsubsection{Mass Balance.}

- Mercury mass balances were done for all FY03-04 tests and reasonable closures were obtained. From the mass balances the performance of the GAC bed in removing mercury from the offgas was assessed. Performance during the FY03 tests was poor with only $14-76 \%$ of the offgas mercury removed by the GAC. Resulting stack concentrations of mercury were several times higher than the MACT limit. Performance during the FY04 tests was excellent with mercury removal efficiencies $>99.5 \%$ and stack concentrations less than half the MACT limit. The difference in performance was attributed to saturation of available sorption sites in the GAC by unburned hydrocarbons in the off-gas from the earlier tests and consequent poor sorption of mercury. This points to the fact that unburned organic carbon in the off-gas must be tightly controlled in order to achieve MACT-compliant mercury emissions from the process. Whether this can control can be achieved for extended periods of time will need to be demonstrated, especially in light of the following uncertainty.

- In FY03-04 bench scale tests significant amounts of organic carbon were observed in the fines products. The amount of carbon in the solid product was sensitive to the reductant:SBW feed ratio. In one of the FY04 tests a noteworthy discrepancy was observed in the hydrogen balance (as discussed in Appendix A, Section A-6.1). Analysis of the data suggests that either the hydrogen concentration was higher than measurements indicated and/or a significant amount of unburned hydrocarbons were present in the off-gas from the steam reformer. Either possibility raises issues in regard to safety, hydrogen generation in steam reformed solids, accumulation of organics or carbon in scrub liquor, and the likelihood of rendering GAC columns ineffective in removing mercury from off-gas.

- Radioactive iodine will be present in SBW could be the major dose contributor in the stack gas. The fate of iodine in the steam reforming process has not been demonstrated to date.

\subsubsection{Filtration Temperature.}

- During bench-scale tests the temperature of the sintered metal filter was $>500^{\circ} \mathrm{C}$, though a temperature of $400^{\circ} \mathrm{C}$ was recommended by a commercial vendor to capture semi-volatile metals (especially $\mathrm{Cs}$ and $\mathrm{Tc}$ ) and still prevent mercury from condensing and plugging the filter. In some tests $\mathrm{Cs}$ and $\mathrm{Rh}$ (Tc surrogate) were detected in the scrub solution suggesting some penetration of the filter by these semi-volatile metals. Heat loss is expected to be a 
smaller factor in a production scale system, so a cooler may be required to reduce the off-gas temperature upstream from the sintered metal filters. The upper limit on the filtration temperature, however, is not known; if it were the off-gas cooling requirement could possibly be eliminated.

\subsubsection{Scrubbing System Design.}

- The current process flow diagrams show an acidic scrub to extract mercury and acid gases from the off-gas. In bench-scale tests to date, however the scrub solution was plain water that became slightly acidified $(\mathrm{pH}>4.5)$ by the dissolution of acid gases. Moreover, the measured fraction of mercury scrubbed in these tests was $<0.5 \%$. The assumed fraction in the system design is $\sim 100$ times higher (44\%), presumably due to higher $\mathrm{HNO}_{3}$ concentrations in the scrub. Whether this can actually be achieved has not been demonstrated. Moreover, the benefit of doing so may be marginal, since the GAC columns could be sized to capture all mercury (assuming the above-cited uncertainty is resolved). If the scrub were basic rather than highly acidified with $\mathrm{HNO}_{3}$ neutralization and mercury precipitation may be unnecessary and acid gas scrubbing would be more effective. In addition the need for scrub blow down may vanish and the volume of scrub requiring grouting may be significantly reduced. Total scrub recycle may be possible since mass balances from the tests indicate $>90 \%$ of $\mathrm{Cl}$ and fluoride (F) partitions to the solids (however, mass balance closure for $\mathrm{F}$ was poor $-50 \%$ or less). Thus, there remains considerable uncertainty regarding the design requirements and optimization of the scrubbing system.

\subsubsection{Facilities Interfaces.}

- A comparison of projected utilities requirements of the steam reforming process against INTEC capabilities is needed to ensure that no modifications are needed to these systems. A new SBW steam reforming facility would be interfaced with existing INTEC facilities (INTEC TFF, main stack, and APS) and infrastructure (notably, the low pressure steam system, water systems [de-mineralized, de-ionized, distilled, fire water, treated water], power grid for electricity, and high-pressure air supply for process maintenance). The existing facilities have set capacities that must be accounted for in the process design and new equipment selection.

\subsection{Cesium Removal and Immobilization}

Filtration, ion exchange, and grouting are common practices that have been used to treat various radioactive wastes at West Valley, Savannah River, Hanford, Oak Ridge and other DOE complex sites.

Two types of filters have been tested for solid/liquid separation at the INEEL. Tests in 1997-1998 demonstrated the removal of undissolved calcine solids and undissolved solids in SBW using a cross-flow type filter (Tripp, 1997; Mann, 1998). As part of the FY 2000 feasibility study of the CsIX process (Raytheon, 2000, Appendix F), initial tests were performed to demonstrate a cartridge type filter for removal of solids from SBW. The cartridge filter demonstration used a $0.13-\mathrm{ft}^{2}$ filter and test feeds of $\mathrm{TiO}_{2}$ and $\mathrm{TiO}_{2}-\mathrm{SiO}_{2}-\mathrm{ZrO}_{2}$ mixtures. An improved SBW solids simulant has been developed (Barnes, 2003) based on additional SBW solids characterization in late FY 2003. Reviews of both crossflow and Fundabac filtration have been preformed in light of this characterization (Pao, 2003)

Development of ion exchange technology for the selective removal of cesium from SBW, NGLW, and dissolved calcine began in the mid-1990s (Miller et. al, 1995). Gary Stegen proposed processing SBW by cesium ion exchange followed by grouting in late 1997 (Stegen, 1997). Alan Herbst and others began developing and testing grout formulations for anticipated INEEL liquid wastes in 1996 (Herbst, 
1996), and in later years the grout stabilization development program included testing formulations for the liquid effluent from the CsIX process. The original premise behind the CsIX/TRU grout process was that the SBW would be classified as a TRU waste (either as feed to treatment or certainly sans the cesium and tank solids) and could therefore be disposed at WIPP. Cesium was to be removed in order to reduce the dose rate for the waste product containers to under the limit for contact handled waste containers. This was important because of the limited capacity of WIPP for remote handled waste, and to reduce costs of the treatment facility as well as for shipment to and disposal at the repository. As of this writing, a permit for accepting RH waste at WIPP has not been granted. Engineering evaluations and Feasibility Studies were performed for the treatment of SBW with this process in 1998 (Losinski, et. al 1998), 1999 (Valles, et. al, 1999) and 2000 (Raytheon, 2000). Evaluation of this processing alternative was also performed in the High-Level Waste and Facilities Disposition Environmental Impact Statement. Development of the CsIX/TRU Grout Process for treating SBW continued at the INEEL through CY 2003.

\subsubsection{Process functions}

The most recent process design anticipates that a CsIX TRU Grout Facility will retrieve waste (including SBW, flushed tank solids, and NGLW), treat these wastes, and certify, package, and ship the final waste products to disposal sites. After completion of processing, the CsIX TRU Grout Facility and associated subsystems will be closed for decommissioning (although, as with other proposed SBW treatment systems, it could be left in place to support closure of INTEC and other INEEL facilities).

The CsIX TRU Grout process is intended to treat the SBW so that the majority of the waste products can be disposed of as CH-TRU waste. Liquid waste would be treated in the CsIX TRU Grout process by the following primary unit operations:

- Partial neutralization

- Solid-liquid separation

- Cesium ion exchange

- Solidification.

Partial neutralization of the SBW is required, prior to being fed to the ion exchange columns, to prevent excessive chemical degradation of the baseline ion exchange media by acid.

Solid-liquid separation is required for two reasons: (1) to remove high activity solids that would contaminate the grout product (primarily with ${ }^{137} \mathrm{Cs}$ ), and (2) to prevent the solids from collecting in and interfering with operation of the ion exchange columns.

${ }^{137} \mathrm{Cs}$ in the SBW is the major contributor to the gamma radiation dose of the SBW. Removal of the cesium from the bulk liquid is necessary to reduce the dose of the treated liquid to less than 200 $\mathrm{mrem} / \mathrm{hr}$ to meet WIPP WAC for $\mathrm{CH}$ waste.

The Cs-free liquid is solidified in order to meet the "no free liquid" criterion for disposal of waste at WIPP.

The spent CsIX sorbent will have a high radiation field due to its ${ }^{137} \mathrm{Cs} /{ }^{137} \mathrm{Ba}$ content and is also expected to be contaminated with TRU radionuclides to greater than $100 \mathrm{nCi} / \mathrm{g}$, the minimum value for acceptance at the WIPP. Therefore, it would be packaged and disposed of at WIPP as RH-TRU waste.

The solids from the INTEC TFF tanks, as well as the solids removed from the liquid SBW (essentially the same material with an expected smaller particle size distribution), contain sufficient 
activity to be classified as RH-TRU. These solids would be separated from the slurry feed stream, dried and packaged to meet the WIPP RH-WAC, and disposed of at WIPP. The function of the solids treatment steps of decanting, filtration and drying is to reduce the volume of the waste product and produce a waste meeting the WIPP WAC.

\subsubsection{Process Description}

SBW and NGLW would be transferred from INTEC storage tanks by currently existing steam jets to the treatment facility. One tank (WM-187) contains the bulk of the SBW solids, while three other 300,000-gal tanks (WM-180, WM-188 and WM-189) and three 14,000-gal tanks (WM-100, WM-101, WM-102) contain the remainder of the waste to be processed.

A process block flow diagram of the anticipated CsIX/TRU Grout process is shown in Figure 5. The treatment facility would have both liquid and solids processing sections. SBW from Tanks WM-180, WM-188, WM-189 and NGLW would be processed in the liquid processing section, while the inventory of Tank WM-187, containing the bulk of the undissolved heel solids from the other tanks, would be processed in the solids processing section.

The first step in treating SBW liquid is to partially neutralize the waste. Neutralization is performed to reduce chemical degradation of the ion exchange media by acid and to enhance grout formulation and curing. Neutralization is performed by mixing the SBW with a $50 \%$ caustic solution. A jet mixer at the discharge of the recirculation/feed pump enhances mixing and acid adjustment. There will be two tanks, one for acid adjustment and the other for process feed. The feed pump just performs recirculation during acid adjustment. Cooling water in the cooling coils or jacket removes the heat of solution and neutralization. A control valve is opened to a set point value while some liquid recirculates when the tank is feeding the process.

The second step in treating SBW liquid would be to remove trace amounts of undissolved solids by filtration. The undissolved solids are small particulate ( 1-100 micron) and are quite radioactive. If the solids are not separated and removed from the liquid they could contaminate the grout waste product such that the $\mathrm{CH}$ dose limit would be exceeded. The solids would be removed using a cross-flow filter. The cross-flow filtration system uses a large recirculation flow to ensure a high axial velocity to minimize particle buildup on the walls of the sintered metal filter elements. The particle concentration in the slurry to be filtered is controlled via a turbiditometer to ensure that the total suspended solids are less than 30 $\mathrm{g} / \mathrm{L}$. The turbiditometer controls a steam jet orifice that transfers slurry to the clarifier upon reaching the set point.

The third step in treating SBW liquid is to remove cesium by ion exchange. The baseline ion exchange media is IONSIV IE-911, a commercially available CST granular product with high selectivity for cesium. Columns would be preloaded with fresh CST. It is envisioned that the waste will pass through three columns in series. When one column becomes loaded with cesium, it would be taken offline and the media would be in-place rinsed and dried. Then the entire column, with spent media, would be loaded into a waste canister. A new column would be rinsed to remove virgin sorbent fines and placed in the removed column's position. The control sequence will continually switch the $1^{\text {st }}$ column feed to the next column such that a typical sequence will be $1-2-3,2-3-4,3-4-1$, etc. There will be four column positions but only three columns used at any particular time, the other being replaced with a new one.

The final step in treating SBW liquid is to solidify the ion exchange effluent by mixing it with Portland cement, blast furnace slag and slaked lime (calcium hydroxide) in a mixing vessel. The ${ }^{137} \mathrm{Cs}$ depleted SBW should be partially neutralized prior to final waste forming to avoid negative interaction / 
degradation of the cement. The partial neutralization step in front of CsIX is performs this activity. The mixture would be discharged into 55-gal drum. The drum would then be moved into a decontamination area where the drum exterior would be checked for contamination and decontaminated if needed. The drum would then be held for about 24-hours to complete curing. After additional inspection, the drum would be moved to temporary storage to await shipment to WIPP.

Tank solids would be treated separately from the liquid SBW. Undissolved solids from WM-187 would be received in the treatment facility as a slurry of varying solids concentration. The receiving tanks are used as solids settling and decant tanks to increase and maintain the solids concentration in the feed to a filter. Sodium hydroxide is added to the settling tanks to promote flocculation that reduces settling time. After concentration by settling, the slurry is mixed, sampled, and then pumped to a filter that contains multiple filter elements or "candles." The envisioned filter would be of the type manufactured by Fundabac. Solids collect on a filter cloth, while filtrate passes through the cloth and discharges at the top of the unit from a central collection tube. When the solids cake has built up to a given thickness, feed flow is stopped, and steam is passed through the filter to dry the solids in place. Then compressed air is used to expand the filter cloth, causing the solids to fall by gravity into a waste canister.

Waste water decanted from the solids receiving tank, filtrate from the Fundabac filter and wash waters from other plant operations is collected in a waste water tank. The wastewater is treated by evaporation in the existing ETS. The concentrate from the ETS is returned for treatment through the SBW liquid treatment portion of the facility. Overhead from the ETS is condensed and then fractionated in the existing Liquid Effluent Treatment and Disposal (LET\&D) system, another facility at the INTEC. Overheads (water vapor) from the LET\&D are released to the atmosphere through the INTEC stack. LET\&D bottoms, which consist of about 12 molar nitric acid with mercury and chloride impurities, would be sent to WM-100, WM-101 or WM-102 for future processing in the SBW treatment facility. 


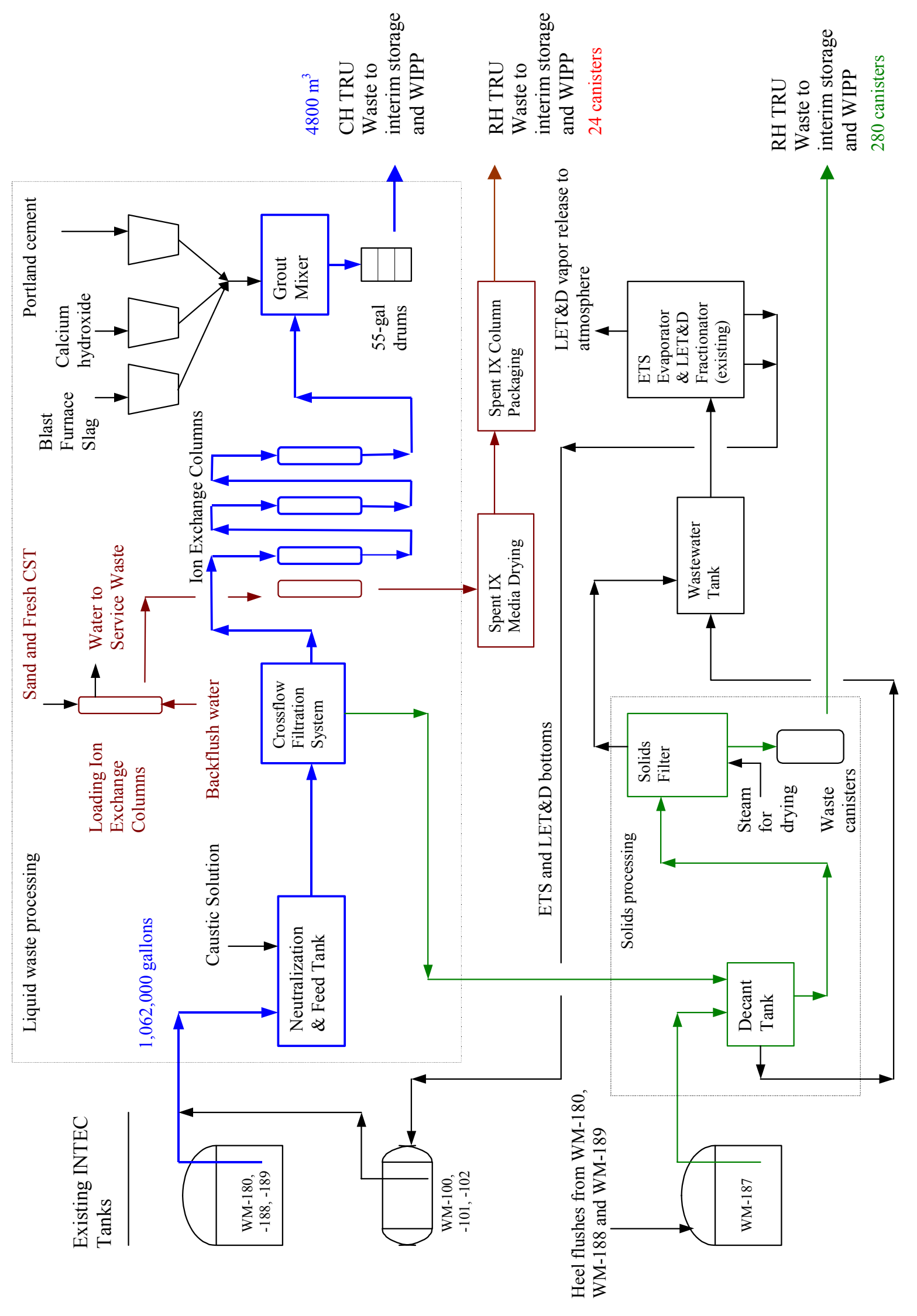




\subsubsection{Summary of Technical Performance}

3.3.3.1 Processing Rate, Availability. A 3-year waste processing period is assumed, which includes six months of transition to processing radioactive waste. Facility availability is assumed to be 200 days per year and 24 hours per day during that period. It is also assumed that a throughput rate of 5 vol\% in excess of the feed volume should be allowed for. This translates to an average liquid processing rate of $85 \mathrm{gal} / \mathrm{hr}$ or $1.4 \mathrm{gal} / \mathrm{min}$ and a feed rate of slurry to the solids processing equipment of $65.9 \mathrm{gal} / \mathrm{hr}$ or $1.1 \mathrm{gal} / \mathrm{min}$. This processing period can be decreased, but at the cost of increased facility size. This is discussed later.

The facility availability, and thus processing rate, is based on many years of operating experience at the INEEL working within DOE regulations. Changes in this operating environment could significantly alter the assumed availability and processing rate either positively or negatively.

A formal RAM analysis has not been performed for this preliminary process design. However, some qualitative statements can be made about the operability and maintainability of the envisioned process and facility. The proposed unit operations are, for the most part, standard industrial practices routinely performed. Mean time to failure data would exist to perform formal analyses for the process. Some experience factors may need to be applied to account for remote operation required by a relatively high radiation environment.

There at least two issues that may affect operating time, but it is believed they can be resolved by detailed engineering efforts. The first is generation of out-of-specification product; appropriate decoupling of unit operations and interim storage will resolve this, but a drawback is perhaps capital cost. The second is restriction of the crossflow filter pores by particles that cannot be dissolved away; this can be resolved by designing the filter for remote removal and replacement. Other issues may be discovered during detailed design, but major ones are not anticipated.

As previously stated, new facilities would be interfaced with existing INTEC facilities to achieve the SBW treatment objectives with this option. The existing facilities have set throughputs and waste composition acceptance criteria that must be accounted for. These must be accounted for in the process design, and have been in the data presented in this report, or new equipment, systems, and/or facilities provided for.

3.3.3.2 Waste Products Quantity \& Quality. Three primary waste products would be produced for disposal with this option. A volumetric and waste package summary of products is shown in Table 10. Tank heel solids and spent ion exchange media are packaged in 2-ft diameter by $10-\mathrm{ft} \mathrm{RH}$ canisters. The ion exchange effluent is grouted and packaged in 55-gal drums as $\mathrm{CH}$ waste. 
Table 10. Volumetric summary of waste products.

\begin{tabular}{lccccccc}
\hline Waste Products & WM-180 & WM-188 & WM-189 & NGLW & Decommissioning & Total & Total $\mathbf{~ m}^{\mathbf{3}}$ \\
\hline Tank Solids & & & & & & 279 & \\
$\quad$ RH canisters & 87 & 90 & 89 & 13 & & & $10 \mathrm{a}$ \\
$\begin{array}{l}\text { Spent CST } \\
\quad \text { RH canisters }\end{array}$ & 5 & 8 & 7 & 1 & 3 & 24 & \\
CH Grout & & & & & & 24,800 \\
$\quad$ CH drums & 6,820 & 8,030 & 7,950 & 1,200 & & 24,000 & \\
\hline
\end{tabular}

a Volume of spent CST based on volume of IX columns and includes sand filter

Tank solids would be dried with steam to a moisture content of about $25 \mathrm{wt} \%$ and will be friable with a bulk density of about $0.68 \mathrm{~g} / \mathrm{cm}^{3}$. This density is based on a measured density of a sample of solids, washed and dried, taken from WM-187 in FY 2004. It is not known whether the dried filter cake density will differ from this value. They would then be packaged in WIPP RH TRU canisters, which have a capacity of $0.89 \mathrm{~m}^{3}$. On average, the solids waste will fill $90 \%$ of the canister volume, or $0.8 \mathrm{~m}^{3}$. Approximately 2-inches of silica gel, equivalent to $0.015 \mathrm{~m}^{3}$ per canister will be added on top of the waste.

Spent ion exchange columns would be disposed in RH-TRU waste canisters, one column per canister. Each column contains about $8 \mathrm{ft}^{3}$ of CST sorbent (bulk density of $60 \mathrm{lbs} / \mathrm{ft}^{3}$, particle density of $2.44 \mathrm{~g} / \mathrm{cm}^{3}$ ) and a sand volume of $0.9 \mathrm{ft}^{3}$. A spent column will be washed with water to reduce the acidity of the spent ion exchange media and then dried to a moisture content of $25 \mathrm{wt} \%$ or less to meet WIPP WAC. Twenty-one spent columns are calculated to be produced during treatment, and three additional columns would be packaged for disposal during decommissioning.

The $\mathrm{CH}$ monolithic grout is $75 \mathrm{wt} \%$ waste partially neutralized with $50 \% \mathrm{NaOH}$, and contains 7 $\mathrm{wt} \%$ calcium hydroxide, $6 \mathrm{wt} \%$ blast furnace slag and $12 \mathrm{wt} \%$ Portland cement. The density of this waste form is $1.5 \mathrm{~kg} / \mathrm{m}^{3}$. Grout will be packaged in 55-gal drums. A drum fill volume of $0.2 \mathrm{~m}^{3}$ is assumed, equivalent to approximately $94 \%$ of the capacity of standard 55 -gal drums.

Radioactive properties of the waste products are shown in Table 11. Dose rates and heat generation rates have not been calculated for waste compositions shown in the present mass balances. However, the grout dose rate is expected to be very similar to that calculated previously. Estimated surface dose rates of 11-21 mR/hr for grout from WM-180 waste and 74-84 mR/hr for grout from WM188 waste, based on decay to 2010 . It was concluded that one-third of the waste drums from the CsIX process would have a surface dose rate less than $50 \mathrm{mR} / \mathrm{hr}$ and two-thirds between 50 and $100 \mathrm{MR} / \mathrm{hr}$ if shipped in the 2010-2012 time period. The estimated heat generation rate for the grouted waste is 0.04 Watts.

Shielding calculations for the spent ion exchange media waste indicate the RH canister, with 0.5inch of steel shielding, would have a surface dose rate of $758 \mathrm{R} / \mathrm{hr}$. A normal shipping cask, again with 0.5 -inch of steel shielding on the canister, would have a 1-meter dose rate of $5.8 \mathrm{mR} / \mathrm{hr}$.

The estimated average surface dose rate for the waste solids canisters in 2010 is $86 \mathrm{R} / \mathrm{hr}$ and heat generation rate 3.2 watts/canister. 
Table 11. Radioactive properties of waste products

\begin{tabular}{lc} 
Grouted Waste & Range \\
All Radionuclides (Ci/drum) & $7.15-14.0$ \\
Fissile Gram Equivalent, g/drum & $3.3-6.4$ \\
${ }^{239} \mathrm{Pu}$ Equivalent Curies, Ci/drum & $0.082-0.11$ \\
TRU Content (nCi/g) & $289-389$ \\
\hline Spent Ion Exchange Media & \\
All Radionuclides (Ci/drum) & $1,030-12,200$ \\
Fissile Gram Equivalent, g/drum & $1.1-2.3$ \\
${ }^{239} \mathrm{Pu}$ Equivalent Curies, Ci/drum & $0.03-0.038$ \\
TRU Content (nCi/g) & $104-135$ \\
\hline Dried Tank Solids & $316-317$ \\
All Radionuclides (Ci/drum) & $47.3-47.7$ \\
Fissile Gram Equivalent, g/drum & 5.2 \\
${ }^{239} \mathrm{Pu}$ Equivalent Curies, Ci/drum & $10,200-10,300$ \\
TRU Content (nCi/g) & \\
\hline
\end{tabular}

3.3.3.3 Size, Footprint of Processing Facility. Preliminary equipment sizing, based on the throughput noted above, has been performed. The sizing information provided the basis for generating layout (plan) drawings for a treatment facility that would accommodate the preliminary process design as described in previous sections. Details and the plan drawings are located in Appendix B. Equipment and room arrangements have not been optimized. Sufficient engineering design has not been performed to provide a strong basis for all of the areas of the facility, as this effort has only been focused on a preliminary process design.

The envisioned facility for the preliminary process design would encompass an area approximately $145 \mathrm{ft}$ wide by $364 \mathrm{ft}$ long at the ground level. It includes waste loading and canister handling operations in the lower level; hot cell process equipment for both liquid and solids processing on the ground floor and non-radioactive activities on the second floor. Equipment in the process hot cell includes the ion exchange columns, seven tanks and both the cross flow and Fundabac filters. The control room, offices, a decontamination room, a manipulator repair room and other support activities are also located on the ground floor. A summary of plot space estimates is given in Table 12.

Table 12. Estimated plot space for cesium removal facility.

\begin{tabular}{lc}
\hline \multicolumn{1}{c}{ Area Description } & Area, $\mathbf{f t}^{\mathbf{2}}$ \\
\hline Process equipment & 2,000 \\
Grouting equipment and drum storage & 13,000 \\
Waste packaging \& canister handling & 5,800 \\
Lag storage for RH canisters & 800 \\
\hline
\end{tabular}


3.3.3.4 Effects of Scale, Variation with Throughput. Changing the scale of the process, i.e., designing for an accelerated treatment schedule, would primarily affect two areas of the facility - the process hot cell and the grouting operation floor space. Minimal effects are expected in the basement with waste packaging, canister handling, and canister storage, based on this preliminary process design effort.

Equipment in the process hot cell includes the ion exchange columns, seven tanks and both the cross-flow and Fundabac filtration equipment. A maximum of about a $40 \%$ increase in capacity of the ion exchange columns (in length and diameter) could be achieved without exceeding the dimension that would fit a RH 2 -ft by 10 -ft canister. Thus, reducing the operating schedule up to about 1.8 years would have no effect on the ion exchange columns. Reducing the schedule further would mean more frequent change-out of columns.

Keeping all other factors equal, decreasing the processing schedule would increase the floor space required for process tanks by the ratio of the decrease to the two-thirds power. For example if the schedule was decreased from 2.5 years to 1 year, the floor space would increase by $(2.5 / 1)^{0.667}=1.84$ or an $84 \%$ increase. Approximately the same increase in space requirements could be expected for the filters, although a trade off study would be needed to better evaluate whether to increase the filter size or install multiple smaller filters.

For the 2.5-year, 200 operating days per year operating schedule, 24,000 drums of grout are produced, equivalent to an average of 2 drums per hour. If in-drum mixing (not the baseline) were used, multiple grouting lines would be required. The FY 2000 feasibility study for the CsIX TRU grout process (Raytheon, 2000) included three grouting lines. However, by using an out-of-drum mixer, a single line could meet the production rate in 2.5 years or a shorter schedule as well. The area required for grouting equipment would increase by the ratio of the schedule decrease to the two-thirds power. However, the area required for storage of grouted waste drums depends only on the lag storage requirement. The lag storage in turn depends of the rate at which WIPP can receive shipments and the initial lag between start of production and sending the first shipment. The projected/assumed shipping rate of $\mathrm{CH}$ handled grout to WIPP is 17 shipments ( 3 HalfPACTs per shipment, 7 drums per HalfPACT) per week for about 68 weeks of the 2.5-year operating schedule (NE-ID, 2003). If the treatment schedule is shortened, it is likely WIPP could receive additional shipments per week. Thus additional storage is not required because of shipping rate limitations. Also, the initial lag time for sending shipments to WIPP could likely be shortened for a shorter schedule, resulting in no dependency of drum storage area on processing schedule.

Insufficient design work has been performed on the RH packaging systems for the CsIX process to evaluate the effect of reducing schedule on basement floor space. The number of ion exchange columns would decrease for a shorter schedule because each would hold more ion exchange media, hence it is unlikely there would be any significant change in floor space for spent ion exchange column packaging. For the same 1-year processing schedule, the rate of production of solids canisters would increase from one per 43 hours to one per 17 hours. Whether this higher rate would require multiple packaging lines has yet to be determined.

Finally, the interim storage for packaged waste is dependent on the initial lag period between producing the first container of waste, having approval to ship that container to WIPP, and the rate at which WIPP can accept waste. While the process will generate, for a 2.5 year schedule, an average of 800 drums of grout and 9 canisters of solids per month, it will take about 3 months to generate the second spent IX column. Perhaps this period could be shortened by starting up on waste with the highest cesium content. However; it is not certain what WIPP would require before qualifying the spend ion exchange media waste canisters. 
Based on discussions with WIPP, it is expected that the disposal facility can receive at least $6 \mathrm{RH}$ canisters per week from the INEEL, or about 300 per year. The total number of RH canisters generated for a 2.5-year production schedule is about 300,3 would be disposed during decommissioning. A shorter schedule would generate less than 300, but more than 280. Hence, WIPP could receive waste without any increase in storage at the INEEL for any treatment schedule of a year or longer.

The facility plot space estimated increase, due to an increased throughput/reduction in processing period to one year, is given in Table 13.

Table 13. Estimated increase of facility plot space for cesium removal facility.

\begin{tabular}{lc}
\hline \multicolumn{1}{c}{ Area Description } & $\mathbf{A r e a}_{\mathbf{f}} \mathbf{f t}^{\mathbf{2}}$ \\
\hline Process equipment & 3,400 \\
Grouting equipment and drum storage & 21,000 \\
Waste packaging \& canister handling & 5,800 \\
Lag storage for RH canisters & 800 \\
\hline
\end{tabular}

\subsubsection{Technical Uncertainties and Potential Impacts}

The Sodium-Bearing Waste Treatment Applied Technology Plan (INEEL/EXT-03-00477, June 2003 ) identified uncertainties resulting from technical and operational risk assessments. These were, for the most part, uncertainties that would have supported the selection of a treatment technology and was not a complete listing of technical issues requiring resolution to support detailed design efforts. The uncertainties were prioritized as High (H), Medium (M), or Low (L) risks. A high risk is categorized as an issue that is likely to occur and would have a significant (\$25 million - \$125 million impact) or critical ( $>\$ 125$ million) consequence. Issues that are unlikely but have significant or critical consequences, or are likely but have marginal (\$5 million - \$25 million) consequences are categorized as medium risks. Issues that have a probability of occurrence of less than $10 \%$, or a higher probability but negligible or marginal consequences are categorized as low risk. Table 14 lists the uncertainties, a short description, and uncertainty status as of the writing of this report.

Mass balances were developed for the preliminary process design to provide a basis for sizing equipment, determining feed chemical requirements, determining utility requirements, estimating emissions and determining waste volumes, compositions and properties. A set of DBEs provides the technical background for preparation of the mass balances. These DBEs are assumptions or uncertainties that represent some level of risk to the successful implementation of a production facility that incorporates this technology; and will require a decision, acceptance of risk, or Applied Technology/Engineering effort to resolve them. Twenty-three DBEs are identified for this preliminary process design. It is expected that several more would be identified as detailed design progresses if this treatment option were to be selected for implementation. The individual DBEs are amplified with background information, references, explanation, and, in some cases, a discussion of uncertainties in Appendix B. The following is a summary discussion. 
Table 14. Summary of cesium removal uncertainties identified in risk assessment.

\begin{tabular}{|c|c|c|}
\hline Uncertainty & Description & Status \\
\hline $\begin{array}{l}\text { Solid/Liquid Separation } \\
\text { Technology }(\mathrm{H}) \text { : }\end{array}$ & $\begin{array}{l}\text { Inappropriately selected/applied solid-liquid } \\
\text { separation equipment can lead to waste } \\
\text { product contamination and/or filter } \\
\text { restrictions leading to downtime. }\end{array}$ & $\begin{array}{l}\text { Lack of heel solids } \\
\text { physical/rheological } \\
\text { characterization data has delayed } \\
\text { resolution of this issue. An } \\
\text { appropriate simulant is required } \\
\text { for validation experiments. A } \\
\text { simulant preparation procedure is } \\
\text { awaiting heel solids data that is } \\
\text { due on June } 30,2004 \text {. }\end{array}$ \\
\hline $\begin{array}{l}\text { Solids Processing and } \\
\text { Disposal }(\mathrm{H}) \text { : }\end{array}$ & $\begin{array}{l}\text { Collection of filtered solids, drying, and } \\
\text { packaging to meet WIPP WAC has not been } \\
\text { demonstrated. }\end{array}$ & $\begin{array}{l}\text { Lack of heel solids } \\
\text { characterization data has delayed } \\
\text { resolution of this issue. An } \\
\text { appropriate simulant is required } \\
\text { for validation experiments. A } \\
\text { simulant preparation procedure is } \\
\text { awaiting heel solids data that is } \\
\text { due on June } 30,2004 \text {. }\end{array}$ \\
\hline $\begin{array}{l}\text { Sorbent } \\
\text { Performance/Selection and } \\
\text { Disposal }(\mathrm{H}) \text { : }\end{array}$ & $\begin{array}{l}\text { The sorbent must selectively remove enough } \\
\text { Cs to render the effluent waste } \mathrm{CH} \text {, not } \\
\text { deteriorate during processing, and contain } \\
\text { enough TRU constituents to be disposable at } \\
\text { WIPP after loading. }\end{array}$ & $\begin{array}{l}\text { A baseline sorbent has been } \\
\text { selected. Latest experimental } \\
\text { results indicate satisfactory Cs } \\
\text { removal and sorbent stability. } \\
\text { Another disposal location for a } \\
\text { non-TRU, high-rad package } \\
\text { could be used. }\end{array}$ \\
\hline Grout Formulation (L): & $\begin{array}{l}\text { Immobilized product physical and chemical } \\
\text { properties are needed to validate design } \\
\text { parameters and assure product acceptance at } \\
\text { WIPP. }\end{array}$ & $\begin{array}{l}\text { Baseline formulation selected, } \\
\text { with properties measured. Long- } \\
\text { term performance not validated } \\
\text { if }>90 \text { days storage is required. }\end{array}$ \\
\hline Silica Gel (L): & $\begin{array}{l}\text { Immobilization of IX effluent is an alternate } \\
\text { waste form to grouting. Physical and } \\
\text { chemical properties are needed to validate } \\
\text { design parameters and assure product } \\
\text { acceptance at WIPP. }\end{array}$ & $\begin{array}{l}\text { Experiments performed and data } \\
\text { obtained indicate that this waste } \\
\text { form is not desirable. }\end{array}$ \\
\hline Grout Mixing (L): & $\begin{array}{l}\text { Data are required with regard to in-drum or } \\
\text { out-of-drum mixing to determine the waste } \\
\text { immobilization mixing method. This has } \\
\text { implications on the system design, amount of } \\
\text { waste produced, and number of } \\
\text { immobilization lines. }\end{array}$ & $\begin{array}{l}\text { Data gathered with regard to out- } \\
\text { of-drum mixing experiments and } \\
\text { process design led to selection of } \\
\text { out-of-drum mixing as baseline. }\end{array}$ \\
\hline
\end{tabular}

3.3.4.1 Feed. The characteristics of the actual waste to be processed are not entirely certain at this juncture. The liquid in two full tanks (WM-180 and WM189) has been sampled, plus solids which where acquired during the sampling event, and the physical, chemical, and radiochemical properties have been documented. Reports are due to be published in June 2004 that detail the results of characterization activities for two other tanks (WM-187 and WM-188) and a plan for synthesizing a representative simulant. The June 2004 characterization report will have the best available solids characterization data. The major issues associated with feed uncertainties are: 
- The quantity of dispersed and heel solids is an estimate based on observations during tank cleaning. The properties of the solids may not be representative of the entire mass. Samples were obtained with existing systems and equipment that only come from one spot in the $50-\mathrm{ft}$ diameter tanks.

- The composition of the NGLW is estimated, and is only a prediction based on past operating experience and sampling events. Future operations at INTEC that involve more decontamination and decommissioning activities than recently experienced could have a significant affect on the volume and characteristics of the NGLW.

These uncertainties affect the following processing activities:

- Solid/liquid separation performance could be negatively affected if the solids quantity and properties differ significantly from simulants used in filter demonstration tests. The quantity of solids can affect the size and processing rate of the solids processing equipment.

- Tanks WM-100, WM-101 and WM-102 are projected to hold all of the NGLW generated from 2006 through 2011. However, the NGLW volume is projected to be a small fraction of the total SBW and is expected to be similar to the SBW composition. The effect of this uncertainty on the process design should be negligible, but devising an appropriate schedule or strategy for processing NGLW in the SBW treatment facility may be challenging.

- This solids concentration affects the operation and schedule of solids treatment. The concentration of solids at which settling would occur in pipes during transfer is not well known, and thought to be much lower than the initial solids content of the tank waste. The amount of water required to transfer the solids is also not known with precision.

3.3.4.2 Solids Separation. The major issue with solids separation is that design parameters have not been determined experimentally with either actual waste or a suitably representative simulant. Lack of characterization data has caused this delay, but the data are expected to be available in July 2004 . The following uncertainties merit the most attention:

- The waste recirculation rate and behavior of very small solids ( $<0.5$ micron) for cross flow filter operation needs to be determined experimentally.

- Solids from different TFF tanks have shown significant differences in settling rates. Based on this data the process flow diagram shows a flocculating agent added to the solids decant tank to decrease settling time. Experiments should be performed to validate the selection of a flocculating agent and the quantity required.

- Small-scale demonstration testing of the Fundabac filtration equipment has been successful. However, the performance of the Fundabac filter with regard to whether the solids can be dried to the required moisture level and then completely removed from the filter into waste disposal containers needs to be validated with a suitably representative simulant or actual waste.

3.3.4.3 Ion Exchange. The major issue with ion exchange is the selection of suitably performing sorbent media. Several sorbents have been experimentally investigated since the mid 1990's. Primary criteria evaluated have been selectivity, capacity, stability with regard to waste composition, and commercial availability. Evaluation of the available data has led to the selection of CST, available commercially in engineered form as IONSIV ${ }^{\mathrm{TM}}$ IE-911, as a baseline sorbent. Uncertainties meriting the most attention with ion exchange are: 
- The Zr binder in CST dissolves to some degree in the acidic waste environment. Partial neutralization of the feed will decrease the degree of dissolution. The quantity of the fines produced by the dissolution and then migrating out of the column could compromise disposal of the grouted waste. A sand layer added to the ion exchange columns to trap sorbent fines is a design feature to reduce the impact of this uncertainty. The most recent experiments (Tranter, 2004a and 2004b) indicate that approximately $2 \mathrm{wt} \%$ of the mass of the sorbent will dissolve during column loading. Cs uptake does not appear to be compromised and is acceptable. However, further radioactive experiments to validate surrogate testing is recommended if this processing option is selected.

- Estimates of TRU activity trapped on/within the spent sorbent, based on tests with a solution spiked with ${ }^{239} \mathrm{Pu}$, indicate that a residual concentration of about 3.7 times the minimum 100 $\mathrm{nCi} / \mathrm{g}$ needed for WIPP disposal will remain. However, this was a scoping experiment and additional experiments should be performed to verify that the sorbent waste would exceed 100 $\mathrm{nCi} / \mathrm{g}$ TRU for all feed cases. Disposal of spent sorbent at a different location would be required if the residual TRU content is below the minimum required at the WIPP, but is not necessarily an issue.

- The method for determining ${ }^{137} \mathrm{Cs}$ breakthrough on an individual column is undetermined. A gamma-measuring device may not be effective, since premature breakthrough may be triggered by ${ }^{137} \mathrm{Ba}$.

3.3.4.4 Immobilized Waste Performance. The major issue with the grout waste form is its performance over time. The potential for, and quantity of, gas generation, water release, and drum corrosion have not been validated. The waste form will be interim stored for an unknown period prior to shipment for disposal at the WIPP. Actual waste immobilized according to the appropriate formula should be tested according to the criteria noted above to validate performance between generation and disposal.

The strategy for Waste Certification has not been investigated in detail. The planning basis has been to "qualify" the process operation such that waste produced under certain operating parameters, and with appropriate analytical data from sampling events, would be "certified" for transportation and disposal (NE-ID, 2003). Detailed design efforts, negotiation with WIPP personnel, and perhaps pilot scale demonstrations will be required to satisfy waste certification requirements.

3.3.4.5 Waste Packaging \& Handling. Equipment and systems associated with the canister filling and handling system for the spent sorbent waste and the tank solids waste have not been addressed in detail. Detailed design studies should be performed to more appropriately define these systems. The systems would undoubtedly require remote application test mockups to validate operability.

3.3.4.6 Facilities Interfaces. The major issue with interfaces is the projected utilities requirement of the process design compared against existing capabilities/capacity at INTEC. An analysis is needed to identify additions and modifications that might be needed for these systems.

\subsection{Direct Evaporation}

Evaporators have a long history of processing radioactive waste at the INEEL and other DOE sites. Development of evaporation specifically for processing SBW into a disposable waste dates back to 1997. At that time, an initial laboratory-scoping test showed that the process was feasible (Kirkham, 1998). Additional development and testing of the process was performed in 2002 using simulated waste from Tank WM-180 (McCray, 2002). In one series of tests, evaporation was carried out in an open one-liter 
stainless steel beaker to represent an "in-can" approach. In a second series, evaporation was carried out in a one-liter flask under vacuum conditions. Results from these tests provided the basis for initial estimates of mass balances (Barnes, 2002a) for the direct evaporation process. Additional testing was performed in 2003 to obtain data at larger scale, to test a second SBW tank waste stimulant, to confirm acceptable performance of a feed containing undissolved tank solids and to test an alternative direct evaporation scheme (Griffith, 2003a, Griffith, 2003b, Griffith 2004, Kirkham, 2003, Kirkham, 2004).

A feasibility study for the direct evaporation process was performed in FY 2002 (Kimmitt, 2003). In this study, the process and facility were defined, equipment sized, mass balances prepared, architectural drawings prepared, development needs defined and project costs estimated.

The direct evaporation process as described in FY 2002 documents has two primary waste products - concentrated, solidified SBW and grouted nitric acid recovered from the SBW evaporator condensate. An engineering evaluation of direct evaporation process variations (Barnes, 2003a) recommended an alternative process scheme that produced a single primary waste product. Both of these process schemes are described in this report. The scheme that grouts recovered acid is referred to as the "Revised Baseline," while the scheme producing a single waste is referred to as the "Alkaline Scrub" process.

\subsubsection{Process Functions}

The Direct Evaporation facility will retrieve waste, including SBW liquids and solids and NGLW, treat the waste so that it can be disposed offsite, and certify, package, and prepare final waste products for shipment to disposal sites. After completion of the processing, the Direct Evaporation facility and associated subsystems will be closed until decommissioning.

The Direct Evaporation process produces a solidified waste from the SBW feed by evaporation to the point that upon cooling the concentrated waste solidifies. The process functions, including both existing and new equipment, are as follows:

\section{$\underline{\text { Revised Baseline Process Functions }}$}

- Mix TFF solids and liquids

- Transfer waste from the TFF and receive SBW and NGLW in the Direct Evaporation Facility

- Evaporate the waste

- Package, cool, store and prepare the concentrated waste for shipment to WIPP

- Condense evaporator overheads, fractionate condensate to reduce the volume, and grout fractionator's bottoms to solidify the recovered nitric acid to permit disposal

- Treat evaporator non-condensable gases for release to the atmosphere

- Treat vent gases for release to the atmosphere.

\section{Alkaline Scrubber Process Functions}

- Mix TFF solids and liquids

- Transfer waste from the TFF and receive SBW and NGLW in the Direct Evaporation Facility 
- Evaporate treatment facility feed

- Package, cool, store and ship the concentrated waste

- Treat evaporator overhead for release to the atmosphere by removing particulate, nitric and hydrochloric acid vapors, and mercury

- Treat vent gases for release to the atmosphere.

Prior to SBW treatment, pumps would be installed in TFF tanks and transfers between tanks made to distribute the SBW solids collected in WM-187 within the SBW liquid. Solids would be distributed and mixed with liquid in the tank farm to (a) avoid the expense of alternative systems that would need to transfer and treat the solids separately in the direct evaporation facility, (b) be able to transfer the solids to the facility with existing steam and air jets, and (c) provide a homogeneous feed to the direct evaporation process.

The treatment facility feed, mixed SBW solids and liquids, as well as NGLW, is evaporated so that when the concentrate cools, it solidifies. Sufficient water and nitric acid in the feed are volatilized during evaporation such that upon cooling solids crystallize and incorporate the remaining water into the waste product as water of hydration.

The two process schemes treat the evaporator overheads differently. The revised baseline process condenses the overheads in order to be able to process it in the existing LET\&D facility. A second function of the condenser is to remove or reduce contaminants in the non-condensable gases, thereby avoiding additional downstream treatment steps for the gases.

It is expected that demister pads, both internal and external to the evaporator, will be required to remove solids, containing both hazardous and radioactive compounds, to levels meeting the LET\&D WAC. The function of the LET\&D is to remove the bulk of the water from the condensate and release it to the atmosphere. The concentrated acid recovered by the LET\&D must then be solidified in order to meet disposal site WAC. Also, the concentrated acid contains hazardous impurities such as mercury that must be rendered non-leachable in the solidified waste.

The final function of the revised baseline process is to treat the non-condensable gases, both process gases and vent gases, to allow release to the atmosphere. These two gas stream are kept separate upstream of their respective blowers because their pressure levels are different.

The alkaline scrubber process also has a demister on the evaporator overheads. The purpose of the demister in this scheme is to reduce the radioactivity in the feed to the scrubber by removal of particulate, thereby allowing easier access to downstream equipment for maintenance.

The purpose of the alkaline scrubber is to remove acid vapors (nitric acid and hydrochloric acid) by reaction with an alkaline compound. The scrubber is expected to also remove additional particulate and some of the mercury. However, an additional unit operation to remove mercury from the gas will also likely be needed. A carbon bed is included in the process for this purpose.

\subsubsection{Process Description}

Prior to treatment in the Direct Evaporation Treatment Facility, mixing pumps would be installed in Tanks WM-187, WM-188 and WM-189. Transfers would be made between tanks to distribute solids 
somewhat evenly between these three tanks. After mixing, SBW and NGLW would be transferred from these tanks by existing steam jets to the new treatment facility feed tank.

Block flow diagrams of both schemes for the direct evaporation process are shown in Figures 1 and 2. SBW would be received from the TFF by the SBW Feed Tank, equipped with an air sparge or other mixing device. Fluid is also mixed in the tank by use of the discharge pump. The SBW liquid/solids mixture would be continuously pumped at a rate of $1.5 \mathrm{gpm}$ to the SBW Evaporator, an agitated thin film type evaporator that is expected to operate at about $120^{\circ} \mathrm{C}$ and about 8.6 psia pressure. Evaporator concentrate will fall from the evaporator discharge into a waste container, also maintained at vacuum pressure. The evaporator is heated with high-pressure steam that is generated within the new facility.

Evaporator vapor would first pass through an internal mist eliminator and then an external demister. The demister provides separation of entrained liquid and solids from the evaporator vapor. Liquid collected in the demister would be periodically pumped to the SBW Feed Tank. The mesh pads in the demister could also be washed with water or condensate.

In the revised baseline scheme, vapor from the demister is condensed by heat exchange with cooling water. Non-condensable gases are superheated and HEPA-filtered before being boosted in pressure and released to the atmosphere through the INTEC stack. Condensate is collected in a tank and periodically transferred to the feed tank of the LET\&D.

Potential corrosion in the Condensate Tank by hydrofluoric acid is controlled by the addition of aluminum nitrate solution, which will form aluminum fluoride complexes. Aluminum nitrate solution is received from a supplier into a small storage tank or drums and pumped as needed to the Condensate Tank.

The existing LET\&D Fractionator would be used to concentrate the condensate, thereby reducing the amount of waste that would require disposal. The condensate is fed by gravity from the LET\&D Feed Tank to one of two identical fractionators, each containing 15 sieve trays. Each fractionator typically recovers at least $99 \%$ of the nitric acid from the feed into the bottoms product. The bottoms product is typically 12 molar nitric acid and contains a high fraction of the other impurities in the feed condensate (hydrogen chloride, fluoride, $\mathrm{HgCl}_{2}$ ). The fractionator overhead is steam with trace impurities. A partial condenser provides reflux to the column. The effluent from the condenser goes to a vapor/liquid separator, with the vapor from the separator superheated, filtered and discharged via a blower to the INTEC-708 stack. When the desired density of the fractionator bottoms is reached, a portion of the solution is drained out of the column and stored in the Bottoms Tank. This recovered acid is cooled by heat exchange with cooling water prior to entering the Bottoms Tank.

LET\&D bottoms would be transferred in batches to a Neutralization Tank. Once a batch was received, solid calcium hydroxide would be added to the tank to neutralize the recovered nitric acid. The Neutralization Tank is equipped with a mixer to ensure adequate mixing of the solid calcium hydroxide with the acid waste, and with a cooling jacket or cooling coils to remove heat generated by the neutralization reaction. The acid waste is expected to contain very low levels of radioactivity, and have a dose rate of $<<1 \mathrm{mR} / \mathrm{hr}$.

After the addition and mixing of calcium hydroxide is complete, the neutralized acid would be pumped through a continuous grout mixer. Blended Portland cement and blast furnace slag in a weight ratio of 3:1 cement:slag would be mixed with the neutralized waste in the grout mixer, which would discharge directly into 55-gal drums, the final waste container. Blast furnace slag is used to bind the mercury in the waste. Filled drums would be conveyed to a swipe/decontamination area where the drum exterior surface would be swiped to test for radioactive contamination. If contamination were found, the 
drum would be decontaminated and re-swiped. Drums that pass the surface swipe would be transferred to a curing area and held for 24-72 hours to complete curing.

Following curing, the drum would be visually inspected for free water. If water were found, silica gel would be added. Following inspection, the drum would move by conveyor to a pallet loading area. A gantry crane would place four drums on a pallet, which when full would be moved to temporary storage until a full load of drums (approximately 60) is available for shipment to the disposal site. An average of 14 drums would be produced per day, although, depending on the feed, as few as 7 or as many as 17 drums would be produced in a day.

The high activity concentrate from the evaporator would be discharged at a rate of about $26 \mathrm{gph}$ into a waste canister, and allowed to cool in the canister. Cooling would be aided by clamp-on cooling jackets. During filling and cooling the canisters would be under vacuum and vented to the process off-gas header.

Empty canisters would be lowered into the Treatment Facility using an overhead crane and placed in a temporary storage area. As needed, the canister would be moved along the canister alley. After being weighed, the canister would be moved into an airlock and then out of the airlock into the fill position. The canister sealing surfaces and seals would be inspected. If the inspection finds any defects, the canister would be moved back to the airlock. The connection of the canister to the evaporator would result in a vacuum in the canister. Gas initially in the canister, and moisture and acid released by the waste during filling would flow up through the evaporator into the process off-gas header. After filling, the canister would be moved to a cooling station to allow the waste additional time to cool and solidify. A cooling jacket could be attached to the canister to reduce the cooling time. When cooling is complete, the cooling jacket and vacuum connection would be detached and a plug inserted into the lid fill hole. A surface swipe of the canister would be performed. If analysis shows no contamination, the canister would be weighed, the dose rate of the canister measured, and the canister moved out of the airlock. If surface contamination were found on the swipe, the canister would be decontaminated, dried, and re-swiped. The canister would then be moved to lag storage area to await loading into a cast for transport to WIPP.

In separate operations, a cask would be loaded onto a transporter and the transporter would be positioned over the canister load-out port. The filled canister would be lifted out of the treatment facility and into the cask. An inner lid would be attached to the cask. The transporter would then be repositioned and the outer lid attached to the cask. The cask would then be rotated into the transport position; the transporter inspected and then would depart from the facility.

In the alkaline scrub scheme, overhead from the SBW evaporator would pass through both an internal mist eliminator and then an external demister vessel as in the revised baseline scheme. However, the vapor from the demister would then enter a packed bed scrubber to contact the overhead vapors with magnesium oxide pellets. The $\mathrm{MgO}$ would react with nitric and halogen acids in the overheads, forming salts that would be transferred to the liquid phase as dissolved solids. Some mercury would also be removed from the vapors. The scrubber temperature would be controlled by a cooler on the scrub circulation stream. Magnesium oxide pellets would be continually added to the bed as a slurry to make up for pellets that dissolve in the scrub liquid and are carried out of the scrubber as dissolved salts.

Vapors exiting the scrubber would be superheated and pass through a GAC bed to remove additional mercury. The mercury-free gas would then be HEPA-filtered, and boosted in pressure to be exhausted through the INTEC stack. Liquid exiting the scrubber would be recycled to the top of the scrubber, with a small portion bled to the evaporator feed. 
A third alternative scheme for direct evaporation is a hybrid of that shown in Figures 6 and 7. Like the revised baseline scheme, the evaporator overhead would be condensed and processed by the existing LET\&D. The LET\&D bottoms would be neutralized with magnesium oxide or aluminum hydroxide and recycled to the evaporator. This scheme would require use of the LET\&D facility, and produce a single final waste product equivalent in volume to that of the alkaline scrub scheme. 

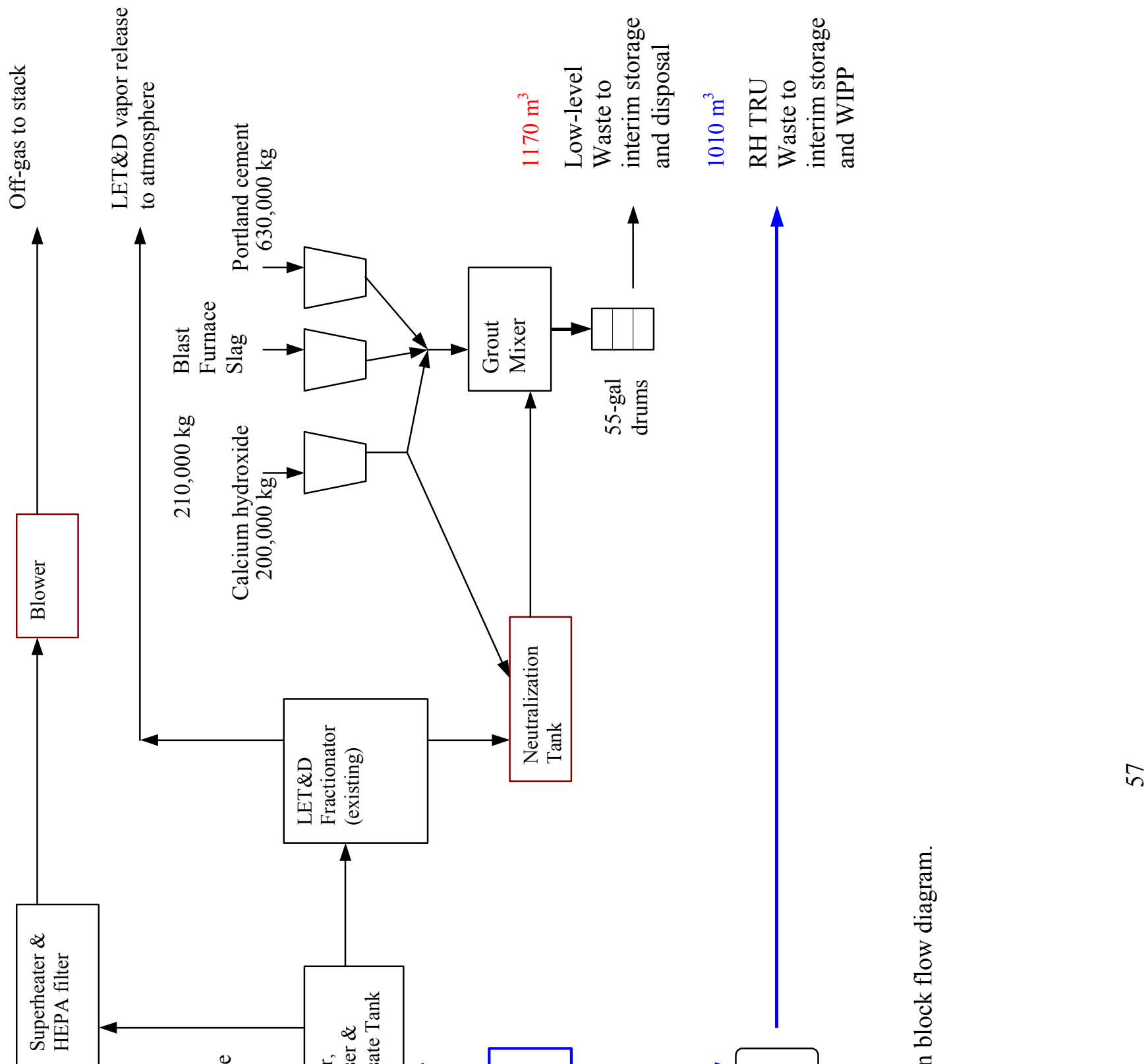

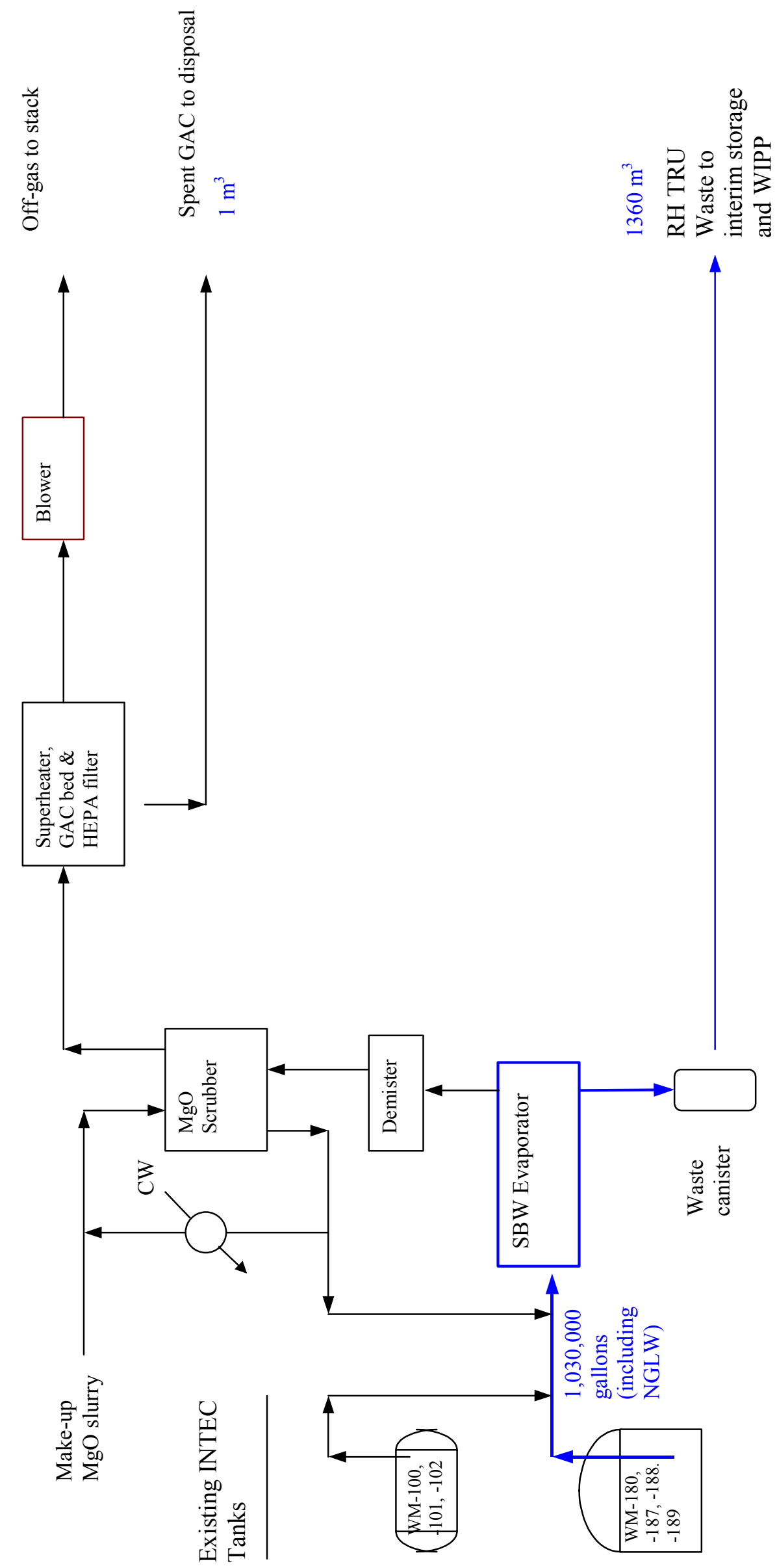


\subsubsection{Summary of Technical Performance}

3.4.3.1 Processing Rate, Availability. Mass balances contained in Appendix C assume a feed rate of about 86 gph based on 12,000 operating hours. The operating time is consistent with the Direct Evaporation T\&FR documents, (Barden, 2003) and is based on 24-hr per day operation, 200 operating days per year and 2.5 years of operation. However, the mass balances are equally applicable to any 500-day operating, such as 2 years at 250 days/year or 20 months at 300 days per year.

The facility availability has not been well established for this type of process. The 200-days/year assumption is based on past operating experience at the INEEL working within DOE regulations. Changes in the operating environment could significantly alter the assumed availability and processing rate.

An analysis of calciner operation 1982-2000 showed an average radioactive on-stream efficiency of $73 \%$ (O'Brien, 2002), equivalent to 266 days per year. Evaporators typically have very high operating efficiencies. For example, 1.3 million gallons of HLW at Hanford was evaporated with an operational efficiency of $99.7 \%$ (Eckard, 2000).

Based on the short test runs to date the availability for an agitated thin film evaporator processing SBW is expected to be high, but cannot be quantified. A major factor in the on-stream time for the direct evaporation process is expected to be the down time required to change-out a canister from under the evaporator. A rough estimate of canister change-out time results in an operating on-stream factor of the process of $70-80 \%$. When combined with downtime for other equipment in the process, the overall onstream time is expected to be $200-250$ days per year.

3.4.3.2 Waste Products Quantity and Quality. The revised baseline version of the direct evaporation process produces two primary waste products - the solidified evaporator bottoms and a grouted LET\&D bottoms waste. The alkaline scrubber scheme or the neutralized and recycled LET\&D bottoms scheme would produce a single primary waste, the solidified evaporator bottoms. For the alkaline scrubber scheme, at the completion of processing, a small amount of spent activated carbon would also need to be disposed. The volumes of waste generated by the revised baseline and alkaline scrubber schemes are shown in Table 15. The volume of waste generated for the neutralized, recycled LET\&D bottoms scheme is equivalent to the alkaline scrubber, except that no spent GAC would be produced.

Table 15. Direct evaporation primary waste products.

\begin{tabular}{|c|c|c|c|c|c|c|}
\hline & WM-180 & $\begin{array}{l}\text { WM-187 } \\
\text { \& NGLW }\end{array}$ & WM-188 & WM-189 & $\begin{array}{c}\text { Total } \\
\text { containers }\end{array}$ & Total $\mathbf{m}^{3}$ \\
\hline \multicolumn{7}{|l|}{ Revised Baseline Scheme } \\
\hline Canisters of SBW evaporator waste & 340 & 182 & 354 & 382 & 1,259 & 1,007 \\
\hline Drums of grouted LET\&D bottoms & 971 & 712 & 1,997 & 2,161 & 5,841 & 1,168 \\
\hline \multicolumn{7}{|l|}{ Alkaline Scrub Scheme } \\
\hline Canisters of SBW evaporator waste & 425 & 224 & 499 & 557 & 1,705 & 1,364 \\
\hline Drums of spent GAC & & & & & 6 & 1 \\
\hline
\end{tabular}

The number of shipments of SBW evaporator waste is equal to the number of canisters produced, i.e., 1007 for the revised baseline scenario or 1364 for the alkaline scrubber scheme. WIPP is expected to 
be able to receive about 300 shipments of RH waste per year, thus shipping will require 4-4.5 years. Based on shipments of sixty drums of grout, 20 shipments of grouted waste would be made. The preferred disposal site for grout is Envirocare; an alternative is Hanford.

The SBW evaporator waste is a solid monolith with an expected bulk density of about $1.75 \mathrm{~kg} / \mathrm{liter}$. In the pilot tests that included solids or magnesium oxide in the test feeds, the range of product bulk density was $1.57-1.86 \mathrm{~kg} /$ liter (Griffith, 2004). Photos of the product produced in various tests can be found in Griffith (2004), Griffith (2003a), Griffith (2003b) and Kirkham (2003).

The grouted LET\&D bottoms waste is also a solid monolith, with a density of about $2.06 \mathrm{~kg} / \mathrm{liter}$ (Herbst, 2002). The spent carbon is in granular form, have a particle size of $3 \mathrm{~mm}$ and a bulk density of $0.48 \mathrm{~kg} / \mathrm{liter}$.

Radioactive properties of the waste products are shown in Table 16. Dose rates and heat generation rates have not been calculated for waste compositions shown in the present mass balances (Appendix C). However, the evaporator waste dose rate is expected to be very similar to that calculated for previous material balances. Based on a date of 2010, the evaporator product canisters are expected to have an average dose rate of $28 \mathrm{R} / \mathrm{hr}$ and generate $1.4 \mathrm{Watts}$ of heat per canister (Bohn, 2002). The drums of grouted acid are estimated to have a dose rate of $0.015 \mathrm{mR} / \mathrm{hr}$ and a heat generation rate of $1.3 \mathrm{x}$ $10^{-7}$ Watts per drum (Bohn, 2002).

Table 16. Radioactive properties of primary waste products.

\section{Revised Baseline Case}

SBW Evaporator Bottoms

All radionuclides, $\mathrm{Ci} /$ canister

$290-500$

Fissile gram equivalent, $\mathrm{g} /$ canister

$60-130$

${ }^{239} \mathrm{Pu}$ Equivalent Curies, Ci/canister

$2.7-4$

TRU content, $\mathrm{nCi} / \mathrm{g}$

$2,100-3,100$

Grouted LET\&D Bottoms

All radionuclides, $\mathrm{Ci} / \mathrm{drum}$

7.8E-04 - 1.3E-03

TRU content, $\mathrm{nCi} / \mathrm{g}$

$0.001-0.004$

Sum of fractions compared to Hanford Cat 1 WAC

$0.02-0.05$

Sum of fractions compared to Envirocare WAC

$0.004-0.006$

Alkaline Scrub Case

SBW Evaporator Bottoms

All radionuclides, $\mathrm{Ci} /$ canister

$240-340$

Fissile gram equivalent, g/canister

$50-90$

${ }^{239} \mathrm{Pu}$ Equivalent Curies, Ci/canister

$1.8-3.2$

TRU content, $\mathrm{nCi} / \mathrm{g}$

$1,400-2,500$

3.4.3.3 Size, Footprint of Processing Facility. Preliminary equipment sizing, based on the throughput noted above, has been performed. The equipment sizing information, along with previous work performed for the Direct Evaporation Feasibility Study (Kimmitt, 2003), provided the basis for 
generating layout (plan) drawings for a treatment facility that would accommodate the preliminary process design as described in previous sections. Details and the plan drawings are located in Appendix C. Equipment and room arrangements have not been optimized. Insufficient engineering design has been performed to provide a strong basis for all of the areas of the facility, as this effort has only been focused on a preliminary process design. The discussion of facility footprint and scale below applies to the revised baseline scheme. For the alkaline scrubber scheme, no grouting equipment is required, which would result in a smaller footprint.

The Direct Evaporation Treatment Facility includes waste loading and canister handling operations in the lower level; hot cell process equipment on the ground floor and cold (non-radioactive) activities plus process and vent gas HEPA filtration on the second floor. The overall dimensions of the facility for the revised baseline scheme are about 310 feet by 140 feet. The control room, offices, a decontamination room, a manipulator repair room and other support activities are located on the ground floor. A summary of plot space estimates is given in Table 17.

Table 17. Estimated plot space for direct evaporation treatment facility.

\begin{tabular}{lc}
\hline \multicolumn{1}{c}{ Area Description } & Area, $\mathbf{f t}$ \\
\hline Process equipment (hot cell space, ground floor) & $5,200 \mathrm{ft}^{2}$ \\
Grouting equipment and drum storage & $18,000 \mathrm{ft}^{2}$ \\
Waste packaging \& canister handling & $1,200 \mathrm{ft}^{2}$ \\
Lag storage for RH canisters & $3,300 \mathrm{ft}^{2}$ \\
\hline
\end{tabular}

3.4.3.4 Effects of Scale, Variation with Throughput. Changing the scale of the process, i.e., designing for an accelerated treatment schedule, would primarily affect four areas of the facility - the process hot cells, the grouting operation floor space, waste packaging and canister handling in the basement and lag storage for waste canisters.

The major equipment in the process hot cell includes the evaporator and four tanks. Other equipment is small and affects of scale on floor space for this equipment is minimal. The evaporator as sized for 2.5 years operation has an outside diameter of $191 / 4$ inches, a length of $10-\mathrm{ft} 8$-in, and heat transfer area of $21.6 \mathrm{ft}^{2}$. For a shorter schedule, the heat transfer area would need to be increased by the schedule reduction ratio. Thus for a schedule of 1-year, the heat transfer area would be $21.6 \times 2.5=54$ $\mathrm{ft}^{2}$. This area could be achieved in an evaporator 1.5 -ft inside diameter by about $17 \mathrm{feet}$ or 2-ft inside diameter by 14-ft. The effect on floor space due would be small, but the hot cell and building height would need to increase to accommodate clearance for evaporator shaft and rotor removal. Alternatively, two evaporators could be used.

Keeping all other factors equal, decreasing the processing schedule would increase the floor space required for process tanks by the inverse ratio of the decrease to the two-thirds power. For example if the schedule was decreased from 2.5 years to 1 year, the floor space for tanks would increase by $(2.5 / 1)^{0.667}=$ 1.84 or an $84 \%$ increase.

For the 2.5-year operating schedule, 5,840 drums of grout are produced, equivalent to an average of 12 drums per operating day. At this production rate, a single grouting line is adequate and would be adequate for shorter treatment schedules as well. Additional space would likely be included in a facility designed for a shorter schedule for empty 55-gal drums fed to the grouting line, to maintain the same schedule for receiving drums. 
The area required for storage of grouted waste drums depends only on the lag storage requirement. Lag storage space in turn depends on the rate at which the disposal site can receive shipments and the initial lag between start of production and sending the first shipment. The intended disposal site for the grouted waste from the direct evaporator process has been Hanford or Envirocare. It is expected that either site could receive this waste at a shipment rate equal to the rate generated for either a 2.5 -yr or a shorter schedule. However, this assumption has not been confirmed.

The alkaline scrub variation of the direct evaporation process, as well as the variation that neutralizes LET\&D bottoms, does not produce a grouted waste product. Besides eliminating the grouting equipment and floor space, these schemes eliminate two of the four major tanks of the process.

The rate at which RH canisters of evaporator product are produced is inversely proportional to any change in processing schedule. For a 2.5-year schedule, on average, one canister is filled in about 10 hours. A single fill station could accommodate shorter processing schedules, but more floor space would be required in the cooling area. The cooling time has yet to be adequately determined, but is expected to be several days. Assuming three days for cooling, space for eight canisters would be needed for a 2.5year schedule or twenty for a 1-year schedule.

Finally, the interim storage for RH waste is dependent upon the initial lag period between producing the first container of waste and having approval to ship that container to WIPP and the rate at which WIPP can accept waste. Based on discussions with WIPP personnel, it is expected that the disposal facility can receive at least $6 \mathrm{RH}$ canisters per week from the INEEL, or about 300 per year. Assuming an initial continuous 40-day operating period prior to waste shipment, storage for 80 canisters would be needed. However, since 1260 total RH canisters of waste are produced and WIPP can receive only about 300 per year, storage for 590 canisters would be required for a 2.5 -year schedule, or 1,040 for a 1 -year schedule. This equates to a $76 \%$ increase in storage space. Estimates of floor space for a treatment facility designed for one-year operation are given in Table 18.

Table 18. Estimated floor space for the direct evaporation treatment facility.

\begin{tabular}{ll}
\hline \multicolumn{1}{c}{ Area Description } & Area, ft \\
\hline Process equipment (hot cell space, ground floor) & $6,200 \mathrm{ft}^{2}$ \\
Grouting equipment and drum storage & $20,000 \mathrm{ft}^{2}$ \\
Waste packaging \& canister handling & $1,300 \mathrm{ft}^{2}$ \\
Lag storage for RH canisters & $5,800 \mathrm{ft}^{2}$ \\
\hline
\end{tabular}

\subsubsection{Technical Uncertainties and Potential Impacts}

\subsubsection{Uncertainties Identified in the SBW Treatment Applied Technology Plan.}

The Sodium-Bearing Waste Treatment Applied Technology Plan (INEEL/EXT-03-00477, June 2003) identified five uncertainties resulting from technical and operational risk assessments for the direct evaporation process. These were, for the most part, uncertainties that would have supported the selection of a treatment technology and was not a complete listing of technical issues requiring resolution to support detailed design efforts. The uncertainties were prioritized as High (H), Medium (M), or Low (L) risks. A high risk is categorized as an issue that is likely to occur and would have a significant ( $\$ 25$ million - \$125 million impact) or critical ( $>\$ 125$ million) consequence. Issues that are unlikely but have significant or critical consequences, or are likely but have marginal (\$5 million - \$25 million) consequences are categorized as medium risks. Issues that have a probability of occurrence of less than 
$10 \%$, or a higher probability but negligible or marginal consequences are categorized as low risk. Table 19 lists the uncertainties, a short description, and their status as of the writing of this report.

Table 19. Summary of direct evaporation uncertainties identified in risk assessment.

\begin{tabular}{ll}
\hline \multicolumn{1}{c}{ Uncertainty } & \multicolumn{1}{c}{ Description } \\
\hline $\begin{array}{l}\text { Product Characterization } \\
\text { (H): }\end{array}$ & The product from processing all of the SBW \\
& wastes may not always meet WIPP WAC and \\
& DOT requirements.
\end{tabular}

Liquid and Heel Solids Co-processing (M):

Evaporator Type (M):

Materials Evaluation (L):

Condensate Treatment

(L):
Co-processing the solids with the SBW liquid could potentially cause fouling and deposition in the evaporator, leading to downtime for maintenance. Also, a plan is needed to process the final tank heel solids.

Different evaporator types have potential advantages and disadvantages for processing SBW. Evaporator demonstrations are needed to verify performance, establish design parameters, and reduce the risk of operation problems for any evaporator type selected.

The high acid and chloride concentrations in the evaporator bottoms will be corrosive.

The initial conception of the direct evaporator process (as defined in Barnes, 2002a) was not optimized. Evaluations supported by test data are needed to optimize the process.

\section{Status}

Data from tests with WM-180 and

WM-189 simulants indicate that if 55-

$60 \%$ of the feed mass is evaporated, the product will be a monolith with no free liquid. Completion of tank solids characterization and solids simulant development, along with testing the other tank waste compositions, is needed to resolve this risk relative to WIPP WAC. The issues of radiolytic hydrogen generation and $\mathrm{NO}_{\mathrm{x}}$ release in the waste canisters also have not been resolved. Production and testing of product from actual SBW is needed to confirm product stability and determine hydrogen generation under radiation fields.

Testing has demonstrated that several types of solids, when added to SBW surrogate feed, present no challenges to the operation of a pilot-scale agitated thin film evaporator. Completion of solids characterization and solids simulant development, followed by additional evaporator tests, would resolve this uncertainty. Planned installation of mixing pumps in three of the SBW tanks eliminates the need to process solids after completion of all liquid SBW processing. Demonstration of mixing solids in the TFF tanks is needed to resolve risks of solids co-processing.

Three test series have been performed using an agitated thin film evaporator. The success of these tests has led to foregoing plans for testing other evaporator types.

No materials tests have been performed.

An evaluation of condensate treatment methods was completed (Barnes, 2003a), and resulted in several improvements to the process. Most of the recommended improvements have been verified by test data and/or cost estimates. 
3.4.4.2 Product Characterization. "Product characterization" was the only high-risk uncertainty identified for the direct evaporation process. The uncertainty encompasses several potential concerns that could lead to waste failing either WIPP WAC or transportation requirements. These concerns included the possibility of (1) free liquids present in the waste due to inadequate control of the process or later phase change of the waste product in its disposal container (2) excessive hydrogen gas generation in the waste canister, (3) excessive $\mathrm{NO}_{\mathrm{x}}$ generation by the waste, (4) corrosion in the canister due to the formation of acidic liquids, (5) waste deliquescence and (6) the possibility of classification of the waste as an oxidation hazard because of its nitrate content.

The second and third series of pilot tests (Griffith, 2003b; Griffith, 2004) demonstrated that the fraction of feed evaporated, and hence the bottoms composition, correlated with the bottoms temperature. The bottoms temperature, and hence the product composition, can thus be controlled by controlling the steam pressure. The tests have thus demonstrated a means of controlling the water content of the bottoms product and ensuring that all water will be incorporated into the solid product. A collection and review of all direct evaporation test data concluded that if 55-60 wt\% of SBW is removed by evaporation, the product will be an acceptable form for disposal (Kirkham, 2004). Continued observations of product up to 2 months after being generated have shown that the solid shows no tendency to reliquify or release water or acid (Griffith, 2004).

Given the radionuclide content of the SBW, the rate of hydrogen generation in the waste product canister depends primarily on the water content of the product waste. Mass balances show a water content of 22-33 wt $\%$, and pilot-scale test data shows 27-33 wt $\%$, based on the taking the difference between feed and condensate. Wendt (2003) modeled hydrogen generation from SBW dried to different levels and recommended removal of water to less than $20 \mathrm{wt} \%$. However Wendt also reported that nitrates present in the waste could inhibit hydrogen generation and allow water in excess of $35 \%$ to be present. The issue of hydrogen generation in the direct evaporation waste product is hence not resolved and requires tests with actual SBW.

Sampling of the head space of cooled waste drums has shown that the head space gas contains levels of $\mathrm{NO}_{\mathrm{x}}$ of up to $100 \mathrm{ppm}$, and if the drum is purged with air and resealed, the $\mathrm{NO}_{\mathrm{x}}$ will return to approximately the same level within days (Griffith, 2004). A specific limit for $\mathrm{NO}_{\mathrm{x}}$ in the headspace has not been determined but the issue has been raised because of the possible effect it would have on the orings used in the $72 \mathrm{~B}$ shipping cask. This issue of the presence and acceptability of $\mathrm{NO}_{\mathrm{x}}$ in the waste containers is not resolved.

After two months of storage of product produced in the pilot tests, lids were removed to verify the absence of liquids. No liquid was found in any of the drums (Griffith, 2004). Thus the risk of corrosion of canisters due to acidic liquids is very small. However, because the waste is deliquescent, any storage of unsealed waste containers will need to have humidity control. Also, based on observations of these waste drums, the evaporator waste contains no particulate and is not friable. Thus it will not be classified as a DOT oxidizer.

3.4.4.3 Solids Co-processing. Prior to testing, there was a concern that co-processing solids with SBW liquid could cause fouling of heating surfaces or plugging of the product outlet. In 2003, tests were performed with a pilot-scale agitated thin film evaporator (Griffith, 2003b) and several types of solids added to the feed, including $2 \mu \mathrm{m}$ silica particles, $5 \mu \mathrm{m}$ silica particles, kaolin clay, zirconia phosphate particles and a composite of the four types of solids. Results from the tests showed that there was no fouling of heating surfaces. No plugging of the outlet was observed in any of the solids tests, even though one run of each feed was made at the expected maximum extent of evaporation. A later test series (Griffith, 2004), confirmed the results regarding no fouling, although bottom plugging was seen in one 
experiment. The plug in this one experiment was attributed to solidified material that remained in the evaporator from the previous experiment.

While the test results seem to allay concerns regarding fouling and plugging, tests with a better solids simulant, i.e., solids that have been demonstrated to behave as actual solids, and tests for longer time periods are still needed to fully resolve this risk. Also, tests of a full-scale evaporator, for which the discharge configuration and method may differ from the pilot-scale unit, are needed to fully resolve this uncertainty.

Another concern raised relative to co-processing was the ability of the evaporator to process solids flushed from the last tank. The presumption was that these solids would be in a very dilute aqueous solution rather that the high salt SBW solution that produces a monolithic waste product. Current plans for co-processing involve the installation of mixing pumps in INTEC Tank Farm tanks. These pumps would deliver a homogeneous SBW liquid/solids feed until heel level is reached. At that time about $99 \%$ of the solids will have been removed from the Tank Farm tank. The heel, if flushed with water, would be concentrated to its original salt concentration by existing INTEC evaporators prior to being sent to treatment, for the last tank as well as the previous ones. Other engineering options have also been suggested for treating the final heel, including putting additional pumps in TFF tanks to directly remove the concentrated SBW heel, or adding cold chemicals to the evaporator feed to maintain evaporator bottoms chemistry if processing solids in a more dilute liquid.

All co-processing scenarios under consideration involve installation of mixing pumps in at least one Tank Farm tank. Mock up tests are needed to verify the performance of these mix pumps.

3.4.4.4 Evaporator Type. Several types of evaporators could potentially be used for SBW evaporation (Packer, 2003). Prior to any testing, there were concerns about maintenance requirements for thin film type evaporators in SBW service. Other types of evaporators had other concerns. Since then three series of tests have been performed with an agitated thin film evaporator (Griffith, 2003a; Griffith, 2003b; Griffith, 2004). While these tests have been too short in duration to quantify maintenance requirements, the smooth operation seen during all of these tests has alleviated the initial concerns about this type of evaporator. The success of the agitated thin film tests also led to cancellation of plans to test alternative evaporator types.

3.4.4.5 Materials Evaluation. High acid and chloride concentrations in the evaporator bottoms will be corrosive. Materials testing has been recommended to identify a material of construction for the evaporator and connecting line to the waste canister that will have an acceptable corrosion rate during the lifetime of the processing facility. These tests have not been performed.

Observations of wastes two months after being generated in pilot tests have shown no liquids, indicating corrosion would not occur once the waste solidified.

3.4.4.6 Condensate Treatment and Process Optimization. Several improvements to the direct evaporation process have been made since it was defined in 2002 (see Kimmitt, 2003 or Barnes, 2002 for the original process configuration). Barnes (2003a) evaluated various options relative to condensate treatment, based on engineering evaluations, new test data and cost estimates, and made several recommendations that have been incorporated into the present design. Appendix $\mathrm{C}$ discusses variations of the direct evaporation process and also contains process flow diagrams, mass balances and equipment lists for two different process schemes. One of the recommended improvements is the use of $\mathrm{MgO}$ to neutralize acid in the evaporator condensate or vapor and recycle the magnesium salt back to the SBW evaporator. This concept was demonstrated in the most recent pilot-scale tests (Griffith, 2004), and eliminates the grouting equipment and grout waste form of the "baseline" process. However, while the 
alkaline scheme process shows savings in equipment cost and facility space, it also produces $35 \%$ more $\mathrm{RH}$ waste. The elusiveness of a definitive WIPP disposal cost precludes making an easy selection between these two options.

Relative to other treatment alternatives the direct evaporation process involves few unit operations. However, additional optimization could still be performed. No economic evaluation has been performed to compare the alkaline scrubber scheme to the LET\&D bottoms neutralization and recycle scheme. Also, significant engineering work needs to be performed relative to canister handling operations.

\subsubsection{Additional Performance Uncertainties}

Mass balances for the direct evaporation process, contained in Appendix C, were developed in order to provide a basis for sizing process equipment, determine utility requirements, determine waste volumes and properties and estimate emissions. A set of DBEs provides the technical background and basis for the mass balances. These DBEs include many assumptions or uncertainties that represent some level of risk to the successful implementation of a production facility that incorporates direct evaporation technology. A summary of the DBEs having the highest uncertainty or impact to plant performance is provided in the following paragraphs. Also included in the discussion below are issues that don't directly affect the mass balance but could significantly impact the cost, schedule or operability of the process.

\subsubsection{Feed Characterization.}

- Analysis of tank solids is incomplete, and past sampling of tank solids may not have resulted in representative samples. Also there is uncertainty in the total amount of tank solids that will be present at the time of treatment. Changes in the amount or composition of the tank solids from present estimates would likely require changes in plant operating parameters. For example, if fewer solids were present than current estimates, the degree of evaporation could be increased and less volume of waste product would be produced. Impacts of this uncertainty are thought to be minimal because of the successful tests using a range of solids in the pilot-scale evaporator (Griffith, 2003b).

- There is uncertainty that Tanks WM-100, WM-101 and WM-102 have sufficient capacity to hold all of the NGLW expected to generated from 2006 through 2011. The Tank Farm Management Plan needs updating with new projections of NGLW volumes and a workable strategy of NGLW storage and treatment. Changes of the Tank Farm Management Plan will result in changes of the feed composition envelop to the SBW treatment facility. While the impact of these changes on plant performance is expected to be small because concentrated NGLW is similar in composition to SBW, the treatment schedule could be impacted, particularly if use of another TFF tank was required.

\subsubsection{Tank Mixing Pump Performance.}

- There is uncertainty in the design and performance of mixing pumps for the Tank Farm tanks. Mock-up testing of Tank Farm Tank mixing pumps is needed to better predict performance. If the mix pumps cannot adequately mix the solids in the tanks, additional equipment would be required in the treatment facility to ensure that the swings in feed solids content can be sufficiently dampened to maintain production of a product with acceptable properties. 


\subsubsection{Evaporator Performance and Product Characterization.}

- Surrogates for WM-180 and WM-189 have been tested and from these tests the extent of evaporation has been determined and product wastes characterized. However the feed composition envelope will include WM-180, three TFF tanks contained mixed SBW liquids and solids of different compositions, and NGLW. After validation of solids simulants is complete, tests with these validated solids and the other tank compositions are needed to confirm and complete the envelope of evaporator operational parameters that will ensure an acceptable product.

- Until testing is performed with actual waste, there will be uncertainty in the fate of certain radionuclides and the effect of the radiation field on the product waste. Evaporation tests with actual waste are needed to evaluate product stability under a radiation field and to determine hydrogen generation in the waste product.

- There are uncertainties in the evaporator performance at full scale. Demonstration tests to date have been at lab and pilot scale. Full-scale tests are needed to confirm operating parameters determined in pilot tests and in particular to validate a design for discharging waste into product canisters. Remote handling of the waste canisters present requirements not present in the pilot tests. Testing is needed to ensure all the components of the packaging system function reliably under the challenging conditions of the highly viscous, corrosive, and rapidly changing waste form as it exits the evaporator and begins to cool.

\subsubsection{Product Cooling.}

- There is uncertainty in the time requirement for product cooling and the processing requirements of gases released during cooling. Modeling of product cooling could supply some of the missing information. If these data are not obtained, the product cooling area could be under designed, resulting in a bottleneck that impacts the treatment schedule; over designed, resulting in a larger area than needed; or inadequately designed, requiring delays for modifications.

\subsubsection{LET\&D Modifications.}

- There are uncertainties in modifications needed to the LET\&D to process the evaporator condensate. An initial review suggests that the only change needed is to change the feed tray. But a more thorough evaluation of processing the evaporator condensate, which has a much higher acid concentration than previous feedstock to the LET\&D, i.e., the Process Effluent Waste Evaporator (PEWE) overhead is needed to determine if other modifications in equipment or operating procedures would be required.

\subsubsection{Off-gas Treatment.}

- There is uncertainty in what emissions standards will be applied to the direct evaporation facility. MACT requirements are not expected to be imposed, but additional off-gas treatment could be required if they are.

- There is uncertainty in both the rate and composition of the evaporator non-condensable offgas. These data are needed to adequately design treatment equipment for the off-gas prior to release to the atmosphere and/or incorporation into secondary waste streams. Of particular interest are mercury, cesium, and $\mathrm{NO}_{\mathrm{x}}$ and hydrogen chloride. While this off-gas treatment 
equipment is expected to amount to a very small part of the capital expenditure of the treatment facility, not including treatment steps because of lack of data could result in unexpected emissions that would cause shutdown and schedule delays for modifications.

- An investigation of blower and compressor capabilities and requirements to better select this equipment, determine the vacuum air rate, and determine whether multiple compression stages are needed.

\subsubsection{Alkaline Scrubber Uncertainties.}

- A cost/benefit analysis is needed to select between the revised baseline scheme, the alkaline scrub scheme and the scheme in which the evaporator overhead is condensed, processed in the LET\&D, and the LET\&D bottoms neutralized and returned to the SBW evaporator.

- No testing of an alkaline scrubber has been performed. Thus the chemistry and mass flow performance of the scrubber contain uncertainty. Removal of nitric acid and hydrogen chloride vapors is expected to be complete but has not been demonstrated. Also, if a packed bed scrubber is used, dissolution of nitrate and chloride salt products is expected to be complete, but has not been confirmed. The extent of mercury removal by the scrubber is unknown. Testing is needed to verify the liquid to gas ratio needed to achieve complete acid gas removal, and to determine the extent of mercury removal. Experimental data is also needed to determine the behavior of the alkaline bed solids (confirmation of negligible pellet degradation or swelling), the build up, if any, of solids in the scrub liquid, and the buildup of soluble species in the scrub purge. Testing is needed to determine the optimum purge rate of scrub solution to the evaporator.

\subsection{Vitrification}

\subsubsection{Brief Background of Process/Alternative}

3.5.1.1 What Is Vitrification? Vitrification is the process of converting materials into glass or a glass-like substance typically through a thermal process. Glass is an amorphous, rigid, noncrystalline structure of low porosity composed primarily of silica, alumina, and oxides of alkali and alkaline earth elements, distinguished from crystalline structures by lack of a definite melting point. When heated it gradually deforms and forms a viscous liquid. Glass has been investigated as a radioactive waste form because of its chemical, thermal, and mechanical stability. Other reasons include the following:

- Organic contaminants are destroyed during vitrification,

- Inorganic contaminants and radionuclides can be stabilized by chemical bonding or by encapsulation in the glass matrix,

- Glass accepts a wide range of waste compositions,

- Glass is less susceptible to radiation damage than crystalline materials,

- Industrial-scale vitrification of radioactive waste has been demonstrated in a number of countries,

- EPA has declared vitrification a treatment standard for mixed HLW. 
Glass has been viewed from a performance perspective as filling three possible roles in radioactive waste disposal:

- As the sole barrier between the radioactive wastes and the biosphere,

- As one barrier of a multi-barrier system (i.e., glass, canister, backfill, and geology) which adequately inhibits radionuclide migration,

- As a convenient container for transport and temporary surface storage.

The single most important characteristic of glass as a waste form is chemical immobilization--the ability to resist leaching of contaminants from the matrix when exposed to liquids--without which the costs of vitrification would not be justified compared with other alternative treatments.

3.5.1.2 Glass Types and Characteristics. Borosilicate glass (BSG), based on the $\mathrm{SiO}_{2}-\mathrm{B}_{2} \mathrm{O}_{3}$ $\mathrm{Na}_{2} \mathrm{O}$ matrix with $\mathrm{SiO}_{2}$ as the main constituent, is a preferred waste form in most countries. Such glasses have been examined for their suitability since the fifties. They are characterized by a high solubility of oxides, and by low melting temperatures $\left(850-1200^{\circ} \mathrm{C}\right)$ and they are now being used in the USA, France, the United Kingdom, Japan and Germany. In the former Soviet Union a phosphate glass was developed for its two vitrification plants. However, Russia is also developing vitrification processes that also will use a BSG.

BSG was the first glass formulated for use in radionuclide waste disposal. Boron is used to reduce the melt viscosity and temperature below $1150^{\circ} \mathrm{C}$. The lower temperature is desirable to reduce the corrosiveness of the melt and to increase the retention of volatile radionuclides such as cesium and technetium. Aluminosilicate glasses have excellent chemical, thermal, and mechanical durability (better than BSG) but also have higher melting temperatures and are thus more corrosive and difficult to process. Other glass types that have been considered include pyrex, glass ceramic (which has a crystalline structure), Synroc (a synthetic three-phase crystalline titanate), iron-enriched basalt (or IEB--an analog to natural basaltic rock), and IEB4 (similar to IEB but with zirconium and titanium added to increase the solubility of uranium oxide and TRU radionuclides (notably, Plutonium).

3.5.1.3 Glass Processing. The combination of high temperature and high-level radioactivity in vitrifying HLW requires sophisticated technologies to meet safety requirements and to permit remote processing. Glass formulations must be designed keeping in mind those species present in target waste streams, which can change the properties of the glass. Sodium, for example, can increase the electric conductivity of the glass melt while decreasing its viscosity. The sodium fraction in the glass must be adapted accordingly to achieve the desired processing characteristics.

Many materials contain adequate quantities of the essential ingredients for glass. However, not all waste materials contain the proper ratios of ingredients so custom formulated additives (known collectively as "frit") are added to tailor the glass to meet performance requirements. Frit components have specific functions for various waste types, one of which is to maximize glass waste loading (typically 15-25 wt\% in BSG). However, the maximum achievable loading is determined by elements with the lowest solubilities in the melt. Low BSG solubility is characteristic of molybdenum, chromium, and sulfur. When solubility limits are exceeded the melt becomes supersaturated and a separate immiscible phase emerges. Accumulation of separate melt phases can have negative impacts on glass quality and on the vitrification process. Limited sulfate solubility, for example, can produce a molten sulfate phase, which can introduce a steam explosion hazard into the process. In technical operation, such accumulations are excluded by the process flowsheet and process control. 
3.5.1.4 Historical Evolution of Radioactive Waste Vitrification. Glass technology has a long history traceable to ancient times, and BSGs have been used since the early 1900s. As early as the 1950s glass was considered an important potential waste form for radioactive materials. Process development for vitrification purposes began in the early 1960s and the basis for current vitrification technology has been created in research facilities worldwide over the last 25-30 years. From these efforts two major production scale processes have emerged. One, developed in France, is the AVM process, which is based on a two-step procedure. The first step is a thermal pretreatment in a rotary tube calciner. The product from this step is melted in an induction-heated metallic furnace. The AVM plant in Marcoule, France was the first vitrification plant worldwide and started radioactive operations in 1978. This technology was adopted by the commercial vitrification plants of La Hague, France, and with minor modifications, in Sellafield, UK, in the 1980s. There is now a long history of industrial scale processing, particularly at La Hague, where over 6,000 canisters have been filled with vitrified HLW.

The second process, developed especially in the United States from the late 1970s, is based on a liquid-fed ceramic melter. Ceramic melters originated from conventional glassmaking technology and were adapted to meet the requirements of nuclear materials. Their major advantage lies in their potential for high throughput and service life. In Germany development of this melter type has been conducted at the INE of FZK (Institut fur Nukleare Entsorgungstechnik of Forschungszentrum Karlsruhe) for over twenty years. Under various national and international programs long-term tests of various types of melters were done on simulated waste. Process technology for the PAMELA vitrification plant in Mol, Belgium was supplied through these activities. Radioactive processing of liquid waste at this facility was begun in 1985 and by the end of operation in 1991, the plant had vitrified $900 \mathrm{~m}^{3}$ of waste.

In the meantime, three more nuclear vitrification plants were equipped with ceramic melting furnaces. The first of these was the Defense Waste Processing Facility (DWPF) at DOE's Savannah River Site (SRS), which began radioactive operations in March 1996. Its purpose is to vitrify into BSG 37 million gallons of high-level radioactive waste in underground tanks. As of October 2001 DWPF had produced 1,178 canisters of HLW glass, representing $20 \%$ of the total required for all SRS high-level tank waste.

The second plant was the West Valley Demonstration Project (WVDP), 30 miles south of Buffalo, New York. Under congressional authorization given in 1980, DOE directed vitrification of 600,000 gal of high-level liquid waste from reprocessing of 700 tons of spent commercial nuclear fuel. The waste was generated by Nuclear Fuel Services, a subsidiary of the W. R. Grace Company. The waste was primarily from the plutonium-uranium extraction (PUREX) raffinates together with about 8,000 gal of thorium extraction (THOREX) process waste. In 1983, DOE selected BSG waste form for the WVDP. From July 1996 until June 1998 the WVDP, operating with an availability factor of $71 \%$, produced 211 canisters of HLW glass.

The third of the three plants mentioned above was at Japan (Tokai Mura). Other facilities were also planned, including the Karlsruhe vitrification plant to manage the HLW from operation of the decommissioned WAK (Wiederaufarbeitungsanlage Karlsruhe) reprocessing plant.

Under the framework of the 1993 Hanford Federal Facility Agreement and Consent Order, signed by the State of Washington, the EPA, and DOE, a Tank Waste Remediation System program was established by DOE to process Hanford Site tank waste for permanent disposal. Under this program the waste will be separated into low- and high-activity streams and vitrified. The inventory to be processed includes 11 million $\mathrm{m}^{3}$ of radioactive fluids, of which, $216,000 \mathrm{~m}^{3}$ (57 million gal) are HLW fluids and sludge, and 6,900 metric tons are nuclear material (including 4,100 metric tons of uranium and 15 metric tons of cesium and strontium capsules). The Hanford vitrification plant is currently under construction. 
3.5.1.5 Vitrification of SBW. In December, 1999 NE-ID requested that BBWI recommend a process to treat SBW stored in the INEEL's INTEC TFF. A broad-based team reviewed work performed over the prior 5-7 years and recommended that an A\&E firm be contracted to provide a preliminary process design to treat SBW by extracting ${ }^{137} \mathrm{Cs}$, grouting the residual liquid as $\mathrm{CH}$ TRU waste, and disposing the grouted waste at WIPP. However, during the summer of 2000, NE-ID requested that the Tanks Focus Area convene a team of nationally recognized experts to independently evaluate all SBW treatment alternatives encompassed by the Draft EIS. This team recommended direct vitrification of SBW as the preferred treatment option, with the CsIX process as a backup.

Pursuant to this recommendation a feasibility study (Quigley 2000) was performed which defined a vitrification process and designed a facility to treat SBW. The study included schedules and cost estimates for construction and operation of the facility, a facility layout with drawings, a process description and flow diagrams, and preliminary equipment requirements and layouts. In late summer of 2000 BBWI prepared a technology roadmap to define the needed development work to resolve technical uncertainties for three possible treatment processes: Direct Vitrification, CsIX, and Solvent Extraction.

At the direction of NE-ID BBWI focused FY01 development work toward direct vitrification of SBW. Under this mandate development activities for FY01 were revised to implement activities identified in the SBW technology roadmap. In April, 2001 a decision analysis meeting was convened to review candidate flowsheets for vitrification of SBW (Seward 2001). This meeting satisfied DOE PEMP 2.1.7.4 and resulted in the selection of a process baseline for vitrification. Later in FY01 a follow up feasibility study (Bates 2001) was performed to study the impacts to the baseline facility if it were designed to treat calcined HLW solids as well as SBW. In addition, an evaluation was performed (McDannel 2001) to assess feasibility of retrofitting the INTEC NWCF to process SBW (and possibly calcine) by installing a series of cold crucible induction melters (CCIMs). Finally, a technical baseline for SBW vitrification was established (Taylor 2001), based on the development work completed in FY01.

In FY02 NE-ID directed BBWI to discontinue development of the SBW vitrification process and issued guidance to assess alternative processes to produce waste forms that would be disposable at WIPP. A summary report was generated by BBWI (Barnes 2002) documenting vitrification development work that was completed during FY01, and documenting outstanding uncertainties that had not been resolved.

[The above summary information was extracted from (NAS 2000), (Roth et al 2000), (Fullmer et al 2000), (Valenti et al 1999), (Norton et al 2002), and (Weber et al 1995).]

\subsubsection{Process Functions}

The primary function of a proposed Idaho Waste Vitrification Facility (IWVF) is to process SBW into a containerized glass waste form that can be transported to a final disposal site. To achieve this, the facility would perform a number of ancillary and subordinate functions. These functions are listed below, based on the processing assumptions current at the time the SBW vitrification baseline was established [see (Taylor et al 2001)]. The facility would:

- Retrieve SBW from the INTEC TFF into holding tanks in the IWVF ("SBW" includes concentrated SBW liquids, NGLW, and suspended solids. Heel solids and solid calcines may eventually also be retrieved and treated; however, the 2001 technical baseline addressed only liquid and suspended solids),

- Blend SBW feed with frit and sugar solution and homogenize the mixture to provide a qualified feed to produce a BSG waste product in a joule-heated melter, 
- Vitrify the blended waste feed and cast the RH waste glass product into 2'x15' disposal canisters,

- Sample and analyze melter feeds and control the vitrification process according to an established quality assurance protocol to ensure that the vitrified waste product will meet WAC of the proposed disposal facility including packaging and shipping requirements,

- Filter and scrub process off-gases to extract solid particles, radionuclides, and gaseous pollutants (mercury, $\mathrm{Cl}$, carbon monoxide, volatile/semi-volatile metals, and hydrocarbons) to comply with MACT and NESHAP requirements,

- Reduce off-gas $\mathrm{NO}_{\mathrm{x}}$ concentration to meet project directed emission targets,

- Extract ${ }^{137} \mathrm{Cs}$ from scrub purge liquid to reduce radioactive content,

- Grout scrub purge and package it in suitable containers for disposal,

- Vitrify spent ion exchange resin used to extract ${ }^{137} \mathrm{Cs}$ from scrub purge liquid (or store for future vitrification with solid calcine),

- Treat and package other low-activity waste streams from the vitrification process (granular activated carbon from off-gas mercury removal, spent HEPA filters),

- Prepare packaged wastes for storage/disposal by performing (a) canister/drum closure, (b) decontamination, (c) certification by visual inspection and acceptance, (d) weighing, (e) determination of waste composition (including isotope concentrations of interest), and hydrogen generation rates, (f) labeling and logging (for recordkeeping), (g) transfer to lag storage or loading into shipping casks.

- Store all packaged wastes awaiting shipment for disposal,

- Receive, store, and blend required process additives (frit and sugar solution),

- Shield and decontaminate personnel to minimize radiation exposure.

\subsubsection{Process Description}

3.5.3.1 Feed Preparation. A process block flow diagram of the SBW vitrification process is shown in Figure 8. One INTEC TFF tank contains SBW tank heel solids (WM-187), and three other 300,000-gal tanks (WM-180, WM-188 and WM-189) together with three 14,000-gal tanks (WM-100, WM-101, and WM-102) contain concentrated SBW (liquid and suspended solids) and NGLW to be processed. These wastes, and possibly solid calcine stored at INTEC, are considered candidate wastes to be processed through the IWVF. However, the 2001 baseline SBW vitrification process addresses only processing of concentrated SBW liquids with suspended solids. With this in mind, the first processing step (performed over several years) is a series of tank transfers, evaporations, and consolidations to homogenize all concentrated liquid waste in the TFF. This is presumed necessary to the degree required by the Waste Form Qualification Plan (WFQP) in order to ensure that the variation in melter feed composition is within acceptable limits for the pre-qualified glass recipe used. 


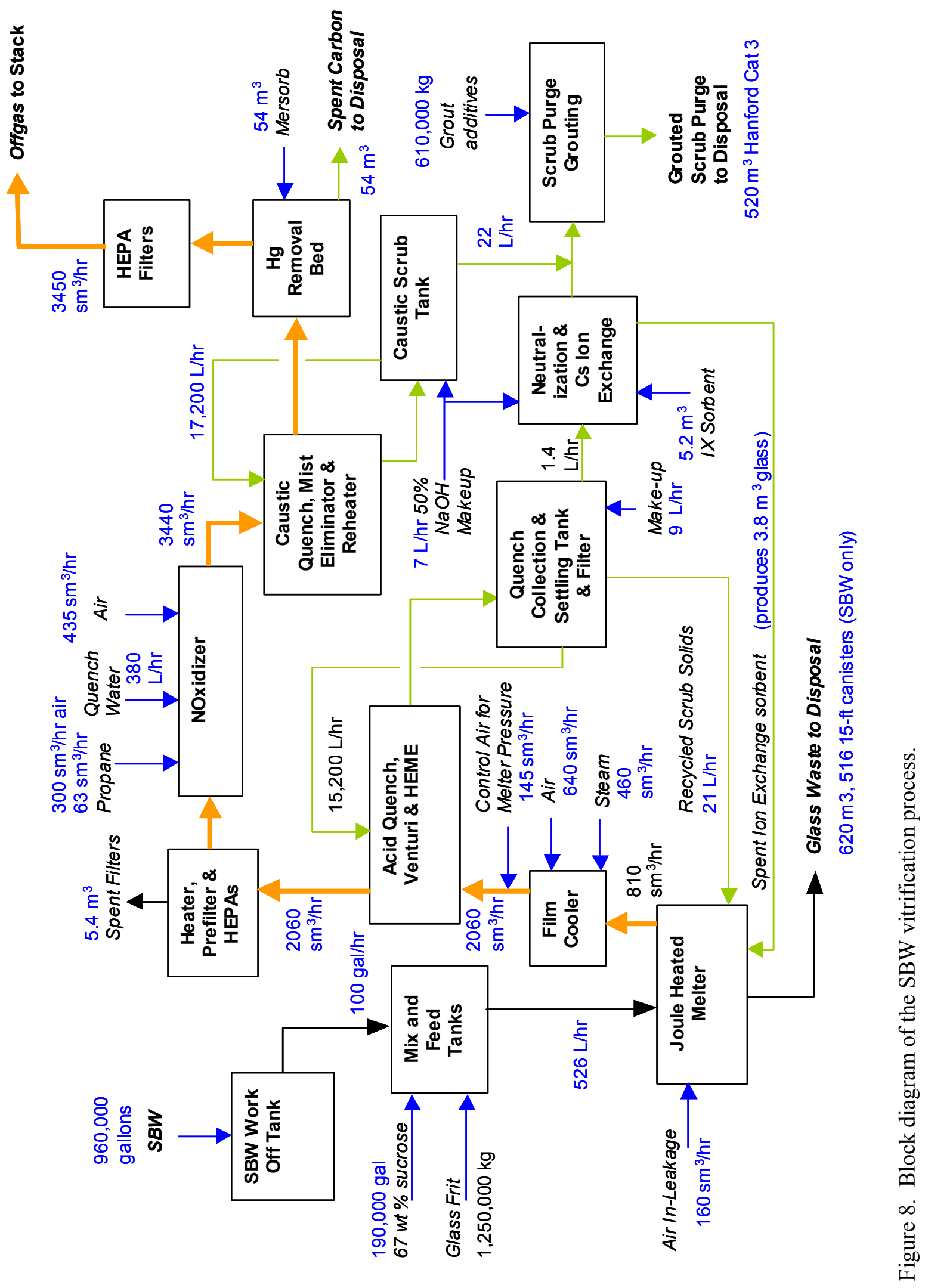


Quantities in Figure 8 apply only to the waste stream "Total SBW". This stream represents the July, 2001 projection (Barnes 2001) of the blended compositions of all liquid waste in the tank farm (excluding heel solids), including the contents of WM-180, WM-188, and WM-189, plus all partially full tanks (as of the time of the projection), plus all concentrated waste from NGLW expected through FY2011.

3.5.3.2 SBW Vitrification. When SBW vitrification begins the liquid waste is transferred from the INTEC Tank Farm into the IWVF SBW Work Off Tank. Feed additives, consisting of glass frit and 67\% sucrose solution are received from offsite and stored until they are metered to a Mix Tank and blended with SBW and with recycled scrub purge liquor from the acid scrub system. The Mix Tanks are cooled to minimize reaction of sucrose with nitric acid. The tanks are also agitated to keep frit and undissolved waste solids in suspension and maintain a uniform composition. When one of the tanks is filled, the tank is sampled to verify that the feed composition is within the limits of the WFQP. After any required adjustments the slurry is transferred to a Feed Tank. The Mix and Feed Tanks are sized to allow time for sample analysis and feed adjustment in the Mix Tanks while maintaining the design melter feed rate from the Feed Tanks.

The Feed Tanks are heated with steam to evaporate water and promote reaction between sugar and nitric acid. $\mathrm{NO}_{\mathrm{x}}$, carbon monoxide, water, and heat are evolved and the remaining solid/liquid slurry and evolved gases are routed to the melter through separate lines. The joule-heated melter is similar to those used at the DWPF and the WVDP. Resistance heating in the molten glass maintains glass temperature at $1150^{\circ} \mathrm{C}$ and evaporates water from the liquid feed. Refractory lining protects the melter vessel from the corrosive melt and power is supplied to embedded Inconel 690 electrodes. Heaters in the melter plenum are used to start the melter and to increase evaporation of water from the feed.

Inside the melter all water evaporates. Sugar and nitrates decompose to form $\mathrm{NO}_{\mathrm{x}}$, nitrogen, carbon monoxide, carbon dioxide, hydrogen, and additional steam. Portions of the mercury, halides, and other volatile and semi-volatile components in the feed leave in the melter off-gas, together with steam and a fraction of the input solids and molten glass entrained by convective processes in the melter.

Empty waste glass canisters brought from offsite are quality checked, cleaned (if necessary), and moved to temporary storage in the IWVF by forklift. Cranes and specially fitted forklifts are used to move the canisters through the facility. When an empty canister is required, it is moved from storage to the melter cell onto a turntable with which it is positioned to receive molten glass. Heated taps on the side of the melter transfer molten glass into a canister. The glass level in the canister is monitored and the tap is closed for canister changeout when the $80 \%$ fill level is reached. Once a canister is filled it is cooled, and then transferred to other cells where it is closed by remote welding, decontaminated, and smeared. It is then moved to interim storage to await shipment for disposal.

3.5.3.3 Off-gas Treatment. As melter off-gas exits the melter it first enters a film cooler, a double-walled duct designed to introduce a film of cool gas (steam and/or air) between the inner duct wall and the melter off-gas. The injected gas cools particles in the off-gas below their softening point to minimize deposits in the duct. Additional air is injected downstream of the film cooler to provide pressure control in the melter. The temperature of the off-gas after the second air addition is about $330^{\circ} \mathrm{C}$. It is cooled further to its dew point temperature (about $80^{\circ} \mathrm{C}$ ) in the quench tower where scrubbing of large-diameter $(>10 \mathrm{~mm})$ solids in the off-gas occurs. The quench liquid is initially plain water but it becomes acidic by scrubbing of $\mathrm{NO}_{2}$, hydrogen chloride, and $\mathrm{HF}$ from the off-gas.

The off-gas next enters a venturi scrubber where extraction of smaller particles and soluble gases continues through intimate gas/liquid contact from spray-atomization of scrub liquor. Most of the atomized liquid droplets are subsequently de-entrained by passage through a knockout drum and high- 
efficiency mist eliminator (HEME). Both water droplets and fine (submicron) particles are collected on the HEME mesh. Water coalesced from the demisting process and from continuous spraying onto the inner face of the mesh provide a washing action, which flushes accumulated solids into the scrub liquid, which exits the HEME.

Removal of ultra-fine solid particles from the off-gas is completed in HEPA filters. The off-gas is heated just prior to entering the HEPA filters to prevent condensation and wetting of the filter fabric. The HEPAs are designed for $>99.97 \%$ removal of particles larger than $0.3 \mu \mathrm{m}$. The filters are replaced when the pressure drop across them reaches a predetermined limit.

After filtration the off-gas is free of most radionuclides and can leave the hot cell. It next enters a three-stage combustion process, which destroys $\mathrm{NO}_{\mathrm{x}}$. In the first stage propane is burned in a fuel-rich, high-temperature $\left(1200-1400^{\circ} \mathrm{C}\right)$ environment to reduce $\mathrm{NO}_{\mathrm{x}}$ to nitrogen. In the second stage the gas is quenched by water injection to a temperature of $760-870^{\circ} \mathrm{C}$. Sufficient air is then bled into the third stage to oxidize the residual hydrocarbons, carbon monoxide, and hydrogen. Reformation of $\mathrm{NO}_{\mathrm{x}}$ is minimal due to kinetic limitations at the reduced temperature.

Following the De- $\mathrm{NO}_{\mathrm{x}}$ process, the off-gas is cooled and scrubbed in a caustic quench scrubber to remove remaining hydrogen chloride, sulfur dioxide $\left(\mathrm{SO}_{2}\right)$, carbon dioxide, and $\mathrm{NO}_{2}$, and also to remove as much HI as possible. Scrubbing of $\mathrm{HI}$ reduces the amount of ${ }^{129} \mathrm{I}$ collected downstream in the GAC beds. The scrub solution is refreshed using 4 molar makeup caustic. Following the caustic scrubber, the off-gas is again demisted before passing through GAC beds and a final bank of HEPA filters. Highefficiency removal of mercury from the off-gas is achieved in the GAC beds with S-GAC. The second bank of HEPA filters removes any carbon from the GAC beds and provides backup to remove any radioactive solids that may have penetrated the upstream unit operations.

3.5.3.4 Spent Scrub Liquor Treatment. Spent scrub liquor from the initial quench, venturi scrubber, and HEME are collected in the acid scrub tank. There the solids separate from the liquid and the supernate is recycled to the quench tower and venturi scrubber. Concentrated solids are recycled to the melter and a portion of the scrub solution is purged. The purge stream is treated by filtering the solids, neutralizing with caustic, removing ${ }^{137} \mathrm{Cs}$ by ion exchange, and grouting the residual liquid. The filtered solids are recycled to the melter with the concentrated solids stream from the scrub tank. Inorganic, spent ion exchange resin is stored, accumulated, and later vitrified.

Grouting the cesium-free scrub purge requires preconditioning the liquid. Caustic is added to achieve $\mathrm{pH}$ 11-12 and calcium hydroxide is added to complex free fluoride. The preconditioned liquid is then blended with blast furnace slag and Portland cement and cast into 55- or 71-gal drums. The grouted drums are decontaminated, surveyed, labeled, and stored until shipment to a disposal site.

3.5.3.5 Treatment of Other Secondary Wastes. Spent GAC is assumed to pass TCLP and will be directly disposed as mixed low-level waste at either Hanford or the Nevada test site. The only treatment assumed is packaging in 55-gal drums and storage until offsite shipment. The process will also generate two classes of spent HEPA filters: High-activity filters (upstream of the $\mathrm{NO}_{x}$ abatement system) and low-activity filters (downstream of $\mathrm{NO}_{\mathrm{x}}$ abatement, upstream of the stack). Both classes will be packaged without treatment and disposed, the former as RH waste at Hanford, and the latter as low-level waste at the Nevada Test Site or Envirocare.

Spent refractory from the $\mathrm{NO}_{\mathrm{x}}$ abatement system will also be generated. However, the operating life of the refractory is expected to extend beyond the processing schedule. It is therefore considered a D\&D item and its treatment and disposal are outside the scope of the SBW vitrification process. 


\subsubsection{Summary of Technical Performance}

3.5.4.1 Quantity and Quality of Feed. A number of processing options and schedules were considered in establishing the FY01 processing baseline. These options included processing of SBW liquids (with suspended solids), concentrated NGLW, tank farm heels solids, and existing calcine solids at INTEC. Two candidate processing schedules emerged, both of which assumed construction of a new tank farm to allow emptying of all current tanks by 2012 in compliance with the 1995 Settlement Agreement with the State of Idaho. The first schedule (or "2012 Schedule") assumed at least two vitrification campaigns. The first campaign, from October 2011 through November 2012, would vitrify 931,000 gal of the concentrated liquid waste inventory (mostly SBW with a small fraction of NGLW). The second campaign, from April 2016 through July 2016 would process the remaining 2012 inventory (44,000 gal) plus 75,000 gal of new liquids (mostly NGLW with a small fraction of SBW). This would leave 75,000 gal of slurry containing about $15 \mathrm{wt} \%$ of tank farm heel solids in dilute liquid waste (from sluicing of solids from emptied tanks). The slurry would be vitrified in a third campaign or stored for later processing with calcine.

The second candidate schedule (the "2016 Schedule") would store all wastes in the new tank farm until June 2016 at which time waste vitrification would begin. The projected waste inventory at that time would consist of 1,025,000 gal of concentrated liquid plus 75,000 gal of heel solids slurry. These wastes, together with 36,000 gal of new waste liquid (generated during vitrification) would be processed between June 2016 and July 2018 (Barnes 2001).

Either schedule would require that the vitrification facility be designed to accommodate multiple feed types (concentrated liquid with a small concentration of suspended solids, slurry with up to $15 \mathrm{wt} \%$ solids, and possibly solid calcine). However, the 2001 vitrification process baseline addresses only concentrated SBW liquid and suspended solids. It should be borne in mind that the process information for SBW vitrification presented here deals only with this baseline.

Table 20 gives the calculated blended composition of the major constituents in the composite SBW waste. Concentrations in the table include contributions from the concentrated SBW liquids plus $2.73 \mathrm{~g} / \mathrm{L}$ of suspended solids. The solids concentration is the weighted average of individual tank contributions listed in (Barnes 2001). Solids composition was assumed to be that of WM-180 solids as given in (Christian 2001). The variability in feed composition indicated in the last column represents the range of concentrations in tanks WM-180, WM-188, and WM-189 as listed in (Taylor et al 2001). A more detailed feed composition can be found in the last reference. 
Table 20. Concentration range of major SBW constituents

\begin{tabular}{|c|c|c|}
\hline Constituent Units & $\begin{array}{c}\text { Nominal } \\
\text { (mol/liter) }\end{array}$ & Max/Min Ratio \\
\hline $\mathrm{H}+$ & 2.4 & 3.4 \\
\hline $\mathrm{Al}+3$ & 0.56 & 1.2 \\
\hline $\mathrm{B}+3$ & 0.019 & 1.82 \\
\hline $\mathrm{Ca}+2$ & 0.052 & 1.5 \\
\hline $\mathrm{Cs}+$ & 0.000022 & 2.7 \\
\hline $\mathrm{Cl}-$ & 0.024 & 1.4 \\
\hline $\mathrm{Cr}+3$ & 0.0046 & 2.0 \\
\hline F- & 0.082 & 2.4 \\
\hline $\mathrm{Fe}+3$ & 0.022 & 1.2 \\
\hline $\mathrm{Mg}+2$ & 0.0088 & 1.6 \\
\hline $\mathrm{Hg}+2$ & 0.0038 & 3.3 \\
\hline NO3- & 5.9 & 1.3 \\
\hline PO4-3 & 0.011 & 3.5 \\
\hline $\mathrm{K}+$ & 0.16 & 1.4 \\
\hline $\mathrm{Na}+$ & 1.5 & 1.7 \\
\hline $\mathrm{SO} 4-2$ & 0.051 & 1.6 \\
\hline $\mathrm{Zr}+4$ & 0.012 & 310. \\
\hline
\end{tabular}

3.5.4.2 Processing Rate, Availability. Processing rates for the IWVF are based on the 2012 Schedule. A 2-year processing period is thus assumed for vitrification operations. Facility availability is assumed to be 200 days per year, 24 hours per day during that period. This translates to an average processing rate of $100 \mathrm{gal} / \mathrm{hr}$ or $1.7 \mathrm{gal} / \mathrm{min}$ of SBW liquid feed.

The facility availability is based on operating experience at the INEEL working within DOE regulations. Changes in this operating environment could significantly alter the assumed availability and processing rate. Ideally, the glass melter would operate without interruption. Off-spec blends of SBW and frit and feed system plugging are two scenarios that could hinder smooth operations. Corrosion failure of melter electrodes could also impact the facility availability factor, as could other process uncertainties discussed below in Technical Uncertainties and Potential Impacts.

3.5.4.3 Waste Products Quantity \& Quality. The primary waste product from this option is glass vitrified from the SBW feed. This product could be disposed at either WIPP or the Federal HLW Repository (Yucca Mountain).

Secondary wastes include glass from vitrification of spent ion exchange resin, the grouted scrub, spent GAC, and contaminated HEPA filters. Spent resin glass, grouted scrub, and untreated HEPA filters could be disposed at Hanford (as Cat 3 waste) or the Nevada Test Site (as NRC Class C waste). Spent GAC could be disposed at Hanford (as Cat 1 waste) or Envirocare. Note that glass from spent resin would not qualify to disposal at WIPP because its TRU content is below $100 \mathrm{nCi} / \mathrm{g}$. 
A quantitative summary of these waste products is shown in Table 21. It is assumed that spent GAC will not require treatment prior to packaging in 55-gal drums and disposal as mixed low level waste.

Table 21. Volumetric summary of waste products.

\begin{tabular}{|c|c|c|c|}
\hline Waste Products & Total\# & Total kg & Total $\mathbf{m}^{3}$ \\
\hline Vitrified SBW & & $1,600,000$ & 618 \\
\hline RH canisters (a) & 515 & & \\
\hline Vitrified Ion Exchange Resin & & 9,900 & 3.8 \\
\hline RH canisters (a) & 3 & & \\
\hline CH Grout (from scrub) & & 940,000 & 521 \\
\hline $\mathrm{CH}$ drums (b) & 2,500 & & \\
\hline CH Carbon (spent GAC) & & 30,000 & 54 \\
\hline $\mathrm{CH}$ drums (b) & 260 & & \\
\hline HEPA Filters & & & 6.3 \\
\hline Totals & 3,300 & $2,600,000$ & 1200 \\
\hline
\end{tabular}

${ }^{a}$ Vitrified wastes are cast in 2'x15' canisters which each hold $1.2 \mathrm{~m}^{3}$ of glass.

${ }^{\mathrm{b}}$ Mixed, low-level wastes are assumed to be packaged for disposal in 55-gal drums.

Radioactive properties of the waste products are shown in Table 22, based on the nominal mass balance in (Taylor et al 2001) for the "Total SBW" stream. Dose rates and heat generation rates were not calculated for this mass balance. However, these rates are expected to be approximately equal to those calculated from the earlier mass balance for the vitrification feasibility study (Quigley et al 2000). The values from this earlier study were apparently calculated based on radionuclide concentrations in SBW in the year 2000, without accounting for radioactive decay up to the time at which vitrification begins and are therefore quite conservative. For vitrified SBW the calculated canister contact dose rate was $65 \mathrm{R} / \mathrm{hr}$ and the heat generation rate was 9.5 watts $^{1}$. For the grouted scrub product the corresponding values were $480 \mathrm{mR} / \mathrm{hr}$ and $3.4 \times 10^{-3}$ watts for scrub liquor without Cs removal, and $2.6 \mathrm{mR} / \mathrm{hr}$ and $1.9 \times 10^{-4}$ watts for the baseline case (with Cs removal).

Radiological dose and heating rates were not estimated for the other secondary wastes from the baseline process.

\footnotetext{
${ }^{1}$ The 2000 feasibility study assumed 2'x10' canisters for the heat generation rate was calculated at 5.7 watts/canister. The number here has been corrected for the larger 2'x15' canisters using the glass volume ratio $(1.2 / 0.72)$.
} 
Table 22. Radioactive properties of waste products

\begin{tabular}{lc}
\hline Virtified SBW & Nominal \\
All Radionuclides (Ci/canister) & 960 \\
Fissile Gram Equivalent (g/canister) & 690 \\
${ }^{239}$ PU Equivalent Curies (Ci/canister) & 6.7 \\
TRU Content (nCi/g) & 2,700 \\
\hline Grouted Scrub & \\
Sum of Fractions (Hanford Cat 3) & $3.9 \mathrm{E}-05$ \\
Sum of Fractions (NRC Class C) & $7.9 \mathrm{E}-04$ \\
\hline Spent GAC & \\
Sum of Fractions (Hanford Cat 1) & $9.5 \mathrm{E}-06$ \\
Sum of Fractions (Envirocare) & $4.7 \mathrm{E}-14$ \\
\hline Vitrified IX Sorbent & \\
Sum of Fractions (Hanford Cat 3) & 0.047 \\
Sum of Fractions (NRC Class C) & 0.12 \\
\hline
\end{tabular}

3.5.4.4 Size, Footprint of Processing Facility. Equipment sizing and facility layout were performed as part of the 2000 feasibility study (Quigley et al 2000). The plan drawings are provided in the referenced external report. The facility was not updated for the 2001 baseline. However, the processes are sufficiently similar that the earlier layouts are considered representative.

Per the feasibility study layout the vitrification facility would consist of two buildings. The first is a multilevel structure, which would house the process building with a footprint of roughly $380 \times 169 \mathrm{ft}$. The structure would extend $32 \mathrm{ft}$ below and $70 \mathrm{ft}$ above grade. The second building is the administrative/support building, a single-level structure with a footprint of $190 \times 70 \mathrm{ft}$, built at grade immediately adjacent to the processing building.

The processing building has five floors and includes $19,400 \mathrm{ft}^{2}$ of hot cell area on the main floor with an additional 17,000 $\mathrm{ft}^{2}$ of hot cell area on the basement level. The main floor hot cell includes roughly 9,200 $\mathrm{ft}^{2}$ of processing area, $1,100 \mathrm{ft}^{2}$ for canister closure, and the balance $\left(9,100 \mathrm{ft}^{2}\right)$ for remote equipment maintenance, access corridors, and air locks. The control room is part of the adjacent administrative area on the ground floor. Post-casting canister closure activities (cooling, decontamination, transfer to interim storage) are done in the basement hot cells. The basement level also includes a storage area for clean canisters. The facility provides no interim storage area for filled, $\mathrm{RH}$ canisters.

Plot space estimates for the IWVF are summarized in Table 23. 
Table 23. Vitrification facility space estimates for 2-year processing schedule.

\begin{tabular}{lc}
\hline \multicolumn{1}{c}{ Area Description } & Area, $\mathbf{f t}^{\mathbf{2}}$ \\
\hline Main Floor & 3,200 \\
Tankage & 1,400 \\
Vitrification & 1,100 \\
Canister Closure/Smear & 1,200 \\
$\mathrm{NO}_{\mathrm{x}}$ Abatement & 1,100 \\
Off-gas Treatment & 1,400 \\
Scrub Purge Grouting & 940 \\
Equipment Decontamination & 14,000 \\
Mechanical/Electrical & \\
\hline Basement & 4,100 \\
Clean Canister Storage (basement) & 1,600 \\
Can Cooling (basement) & 420 \\
Canister Decontamination (basement) & 2,100 \\
Tankage (basement) & \\
\hline Admin Area & 13,000 \\
Administrative & 1,100 \\
Mechanical/Electrical & \\
\hline
\end{tabular}

3.5.4.5 Effects of Scale, Variation with Throughput. Changing the scale of the process, i.e., designing for an accelerated treatment schedule, would have the most effect on the process operations performed on the main floor of the facility. Two areas in particular - the hot processing cells and the cooling area for filled waste canisters - would be affected.

Major process equipment in the hot cells includes SBW work off tank, mix/feed tanks, melter, offgas treatment train, GAC beds, spent scrub ion exchange columns, and grout mixer. Decreasing the processing time would increase the floor space required for most of this equipment by the inverse ratio of the processing times raised to either the two-thirds power (storage vessels) or to the first power (process equipment). Reducing the schedule from 2 years to 1 year, the floor space for storage vessels would thus increase by a factor of $\sim(2 / 1)^{0.667}=1.59$, and for process equipment by a factor of $\sim(2 / 1)^{1.0}=2$.

Provision for interim storage, though not included in the 2000 facility plan, would also be needed. The requirement is driven by (a) the initial lag time between certification of the first canister of glass and actual shipment to the disposal site, and (b) the excess of the rate at which certified waste canisters are produced over the rate at which the disposal site can accept them. The key parameters to determine the actual area requirement are thus (a) the hot operation starting date for the melter, (b) the date at which the target disposal site (e.g., Yucca Mountain) begins accepting INEEL waste canisters, and (c) the rate at which the disposal site can receive, transport, and position the canisters in the facility. Due to large uncertainties in all these parameters, the interim storage facility requirement has not been defined. 
The plot space estimates for the facility with a 1-year processing schedule, obtained from the above scaling considerations, are summarized in Table 24. (No scaleup factor has been applied to equipment items that are assumed to be underutilized in the baseline facility.)

Table 24. Vitrification facility space estimates for 1-yr processing schedule.

\begin{tabular}{lc}
\hline \multicolumn{1}{c}{ Area Description } & Area, $\mathbf{f t}^{\mathbf{2}}$ \\
\hline Main Floor & \\
Tankage & 5,080 \\
Vitrification & 2,800 \\
Canister Closure/Smear & 1,100 \\
$\mathrm{NO}_{\mathrm{x}}$ Abatement & 2,400 \\
Off-gas Treatment & 2,200 \\
Scrub Purge Grouting & 1,400 \\
Equipment Decontamination & 940 \\
Mechanical/Electrical & 14,000 \\
\hline Basement & \\
Clean Canister Storage (basement) & 6,508 \\
Can Cooling (basement) & 2,540 \\
Canister Decontamination (basement) & 420 \\
Tankage (basement) & 3,334 \\
\hline Admin Area & \\
Administrative & 13,000 \\
Mechanical/Electrical & 1,100 \\
\hline
\end{tabular}

\subsubsection{Technical Uncertainties and Potential Impacts}

In 2000 and 2002, prior to the creation of the SBW Treatment Facility Project, alternatives analyses were performed (Murphy et al 2002, Perry 2002 ) that reviewed processing technologies considered for treating SBW up to that point. The 2002 analysis resulted in selection of four technologies considered to be preferred options, based on the screening criteria that were applied. The subsequent applied technology plan (Lauerhass et al 2003) identified uncertainties resulting from technical and operational risk assessments associated with the four selected alternatives. Since vitrification was not among those selected it was not included in the risk assessments and the applied technology plan did not include prioritization and description of its associated technical uncertainties. For this reason there is no tabular summary status of uncertainties from the applied technology plan as there is for the other technologies discussed in this report.

Mass balances were developed for the vitrification technical baseline to provide a basis to size equipment, determine feed chemical requirements, determine utility requirements, estimate emissions, and determine waste volumes, compositions and properties. To prepare the mass balances a set of DBEs was assembled in lieu of hard data addressing technical uncertainties. The DBEs are assumptions about uncertainties and represent a level of risk to the successful implementation of an SBW vitrification facility. Each DBE will require acceptance of technical risk or Applied Technology/Engineering 
development/demonstration efforts for validation before the next process design steps are taken. As of September 2001, 152 DBEs had been identified for the process. It is likely that more would be identified if this treatment option were selected. The individual DBEs are amplified with background information, references, explanation, and, in some cases, a discussion of uncertainties in (Taylor et al 2001).

At the end of FY01 a review of FY01 development work was commissioned (Barnes et al 2002) to update the status of the uncertainties and DBEs. Also, in early December 2001 an EM-50 review of the FY01 SBW vitrification work was conducted at the INEEL. The meeting provided additional insight into high-priority uncertainties that were outstanding.

The following is a summary of some of the major uncertainties that were the subject of these reports and discussions. Underlined items are DBE titles from the 2001 technical baseline. Those that were the focus of discussion by DWPF personnel at the December EM-50 review meeting are so indicated.

\subsubsection{Melter Chemistry.}

- Speciation and partitioning of sulfate in melter. Sulfur has a finite solubility limit in any glass recipe. Once the limit is reached in a melter, excess sulfur will form a separate molten salt phase. Such phases were observed in some subscale and pilot tests during FY01. The salt phase is undesirable for several reasons. First, it introduces the possibility of a steam explosion if mixed with a small amount of water. Second, a salt phase can accelerate corrosion to melter materials. An acceptable feed composition envelope and glass recipe for SBW was not firmly established in FY01. Due to the sulfate content of the SBW waste (both liquid and solid) it will be necessary to both establish and demonstrate a glass recipe which will accommodate all sulfur in the full range of expected feed compositions, or develop and demonstrate a viable means to detect and remove any molten sulfate salt layer that may develop during processing.

- Noble metals concentration in SBW feed. Noble metals are problematic in joule-heated melters due to their propensity to separate and form a separate molten metal phase that can cause electrical shorting between the melter electrodes. Noble metals known to be present in SBW include $\mathrm{Ru}, \mathrm{Rh}, \mathrm{Pd}$, and $\mathrm{Ag}$ and estimates of their concentrations have been made. It has also been reported that selenium and tellurium influence the behavior of noble metals in a melter. However, the amount of molten metal present at steady state under varying conditions with SBW feed has not been quantified, nor has a threshold amount above which problems occur. Reliable measurements of total noble metal concentrations in SBW and of problem thresholds are needed. It may also be required to demonstrate a means to extract any molten, noble metal phase from the melter, should one develop.

- Reductant selection and concentration in melter feed. Sugar is the preferred candidate reductant due to its history of use for various nuclear waste treatment applications and its success in SBW vitrification tests to date. However, other organic reductants (e.g., activated carbon, glycolic acid, corn starch) have been considered for various reasons. Choice and concentration of a reductant was singled out by DWPF personnel as a high-risk uncertainty that impacts several parameters (among them nitrate destruction rates and glass redox ratio, which in turn impacts glass foaming and possibly glass durability).

- $\quad$ Frit vs. glass forming chemicals in melter feed. WAC for waste disposal at Yucca Mountain. Acceptance of INEEL's vitrified waste at Yucca Mountain will most likely be based on demonstrable conformity to a WFQP. The WFQP will document the development of a glass recipe (consisting of prescribed envelopes of feed composition and melter operating 
conditions), which ensures that the resulting glass meets the Yucca Mountain acceptance criteria. The development of this recipe is heavily dependent on the choice of additives that are blended with the waste. The nature of these additives (both the composition and the physical form--as frit or as glass forming chemicals, or GFCs) influences the quality of the glass and the processing behavior. Choice of frit or GFCs will therefore dictate the course of development of the glass recipe and of the WFQP. DWPF personnel flagged this choice as a high-priority uncertainty requiring attention. In addition, it is noted that the WFQP for DWPF is a manyvolume document reflecting a sizable investment of time and money. Development of a WFQP for SBW was barely started when development work for SBW vitrification was terminated at the end of FY01. The WFQP is a long-lead item that influences the system design and would require prompt attention to implement SBW vitrification in a timely fashion. A WFQP would also be required for disposal of a glass waste form at the WIPP. However, the level of effort required to achieve waste certification for WIPP disposal is not anticipated to be nearly as high as for Yucca Mountain.

\subsubsection{Off-gas Scrubbing.}

- $\quad$ Partitioning of acid gases and Mercury during quench/scrubbing operations. Stack concentrations of hydrogen chloride, $\mathrm{Cl}_{2}$, mercury, and ${ }^{129}$ I must be controlled for compliance with MACT and NESHAP standards. In addition, experience in the NWCF indicates the potential for buildup of mercury in acid scrub solutions. The fate of $\mathrm{SO}_{2} / \mathrm{SO}_{3} / \mathrm{H}_{2} \mathrm{SO}_{4}$ is needed in the prediction of sulfate concentrations in recycle streams to the melter to control formation of molten sulfate salts. The fate of carbon dioxide is needed to design the caustic quench column. The vapor/liquid partitioning of these species in acid and caustic scrubbing is needed in system models used to design and optimize unit operations to ensure compliance with stack emission limits.

- $\quad$ Species removal efficiencies in HEME / Soluble species in off-gas / Selection of quench and scrub components in off-gas treatment system. The baseline process scheme includes a film cooler, acid quench, venturi scrubber, HEME, and HEPA filters together with an oxidizer, caustic quench, mist eliminator, GAC bed, and a second HEPA filter bank. Performance of many of these systems depends on both the species solubilities and removal efficiencies in scrubbers and HEMEs. Because of the serial nature of off-gas treatment unit operations and the use of recycle streams, performance of each unit operation may be strongly influenced by (a) species behavior in other unit operations, (b) choice of system components, and (c) configuration of unit operations relative to one another. Underscored uncertainties will thus require resolution in order to design and demonstrate an off-gas system that will achieve the required performance. In particular, DWPF personnel have pointed out the need for judicious choice of quench and scrub components in light of operational problems experienced with plugging/blinding of HEMEs due to submicron particle capture, and penetration of contaminants through HEPA filters that have been wetted by condensation. It was pointed out that alternative unit operations (e.g., customized HEME mesh washing, high-energy scrubbers like the steam-atomized scrubbers used at DWPF, or wet electrostatic precipitators) may be required to preclude major operational problems after the facility is in operation.

\subsubsection{Material Behavior.}

- Corrosion of melter components. This uncertainty was among those flagged by DWPF personnel as high-risk/high-priority. SBW includes high acid, high nitrate materials. Also, the presence of sulfate and chloride provides the potential to form one or more highly destructive acids and/or molten salts in a high temperature environment. Unacceptable electrode loss was 
observed in some tests that may have been due to a low redox ratio or the choice of material for the electrodes. Inadequate understanding of corrosion behavior of melter components could result in a melter design and/or operating parameters that lead to premature system failure and negative cost and schedule impacts.

\subsubsection{Processing Alternatives.}

- $\quad$ Canister closure method. The preferred closure method is welding and the uncertainty associated with the choice was initially assigned a low priority. However, again based on input from experienced DWPF personnel, its priority (together with the issue of selection and demonstration of a method to decontaminate filled glass canisters) was raised.

- Melter selection. An early programmatic decision was made to utilize a joule-heated melter. However, there are compelling reasons to consider other alternatives such as a cold-crucible induction melter (CCIM). A short feasibility study was performed during FY01 addressing the possibility of retrofitting the NWCF with two or more CCIMs. Though the scope of the study was limited, and some measures of feasibility were treated in insufficient detail for definitive conclusions, it was concluded that potential benefits (e.g., capital cost savings) may likely be realized and no insurmountable obstacles were discovered. The study points up the need for a systematic analysis and comparison of alternative melters before proceeding further in detailed process design.

- Effects of recycled scrub on melter and glass. The baseline process assumes that recycled scrub solution will be accumulated and fed back to the melter. The impacts of recycled scrub solution on melter performance are assumed to be (a) water dilution, (b) altered relative concentrations of major species in the glass formulation, and (c) changed mixing/pumping characteristics of the mixing tank solution. The magnitude of these impacts (e.g., the reduction in net SBW throughput resulting from dilution, and the conformity of melter feed with the qualified glass recipe in the WFQP) has not been demonstrated.

\subsubsection{Process Control.}

- Off-gas flow control system / Pressure drops through off-gas system components. The baseline flow control in the vitrification off-gas system is patterned after a system of throttling valves, air bleeds, and compressors similar to that in the NWCF. However, it was pointed out by DWPF personnel that the melter system is far less tolerant of pressure instability and fluctuations than is the NWCF due to the use of hydrostatic pressure to control the flow of molten glass through the pour spout into the canisters. No detailed design or testing has been performed to compare system dynamic behavior with that of (say) DWPF, where a considerable effort was expended before a workable system was developed and demonstrated.

- Flammability limits on hydrogen, carbon monoxide, and unburned HCs in off-gas. The total flammability of a gas mixture is the sum of the percent flammabilities of the separate flammable gases and should be less than $50 \%$ to ensure system safety. The true flammability of the melter off-gas is affected by the melter outlet gas temperature and the presence of other gas species (e.g., water) that can dilute any oxygen that is present. The flammabilities of melter off-gas upstream of the film cooler must be defined (and probably demonstrated) under all expected conditions to preclude any possibility of explosion in the melter or off-gas system. This uncertainty was flagged by DWPF personnel for elevated priority. The need was suggested to evaluate the off-gas composition envelope using the qualified flammability model developed for this purpose at SRS. 


\subsubsection{Stream Factor.}

- The facility design is based on a 2-year operating schedule with 200 days of operation per year. This availability factor is based on NWCF operating experience that may be overly optimistic for a melter.

\subsubsection{Facilities Interfaces.}

- A new SBW vitrification facility would be interfaced with existing INTEC facilities (INTEC TFF, main stack, and APS) and infrastructure (notably, the low pressure steam system, water systems [de-mineralized, de-ionized, distilled, fire water, treated water, potable water], sanitary waste, service waste, power grid for electricity, and high-pressure air supply for process maintenance), and communications systems (alarm, phone, voice, and data). The existing facilities have set capacities that must be accounted for in the process design and new equipment selection.

- A comparison of projected utilities requirements of the SBW vitrification process against INTEC capabilities is needed to ensure that no modifications are needed to these systems. 


\section{REFERENCES}

\section{Section 3.1 - Calcination}

Adams, R. D., (2003), "NWCF Upgrades - Cyclone Replacement," INEEL Interoffice Memorandum (to Steve Bates), RDA-21-03, June 3, 2003.

Ashworth, S. C., D. Harrell, V. Balls, D. Tyson, N. Hertlendy, L. Stuffle, J. Banaee, H. Peterson, D. Rawley, M. Plum, T. Langenwalter, C. Barnes, and W. E. May, (2000), NWCF Mercury Removal Feasibility Study," (2000), INEEL/INT-2000-00539, July 2000.

Barnes, C. M., R. A. Wood, and B. H. O’Brien, (2003), "Calcination with MACT Upgrade Process Design,” Engineering Design File EDF-3387, April 22, 2003.

Barnes, C. M., S. K. Janikowski, and C. B. Millet, (2003b), Feed Composition for the Sodium-Bearing Waste Treatment Process," INEEL/EXT-2000-01378, Rev. 3, September 2003.

Bates, S. O., (2003), "NWCF Cyclone Replacement Options For Calcination With MACT Upgrade SBW Treatment Alternative," Engineering Design File EDF-3646, May 8, 2003.

Boardman, R. D., (1997), “Alternative Calcination Development Status Report,” LMITCO Interdepartmental Communication BDMN-01-97, April 30, 1997.

Boardman, R. D., B. H. O’Brien, C. St. Michael, and G. Anderson, (2004a), SBW Test Plan: High Temperature Calcination with MACT Upgrade Tests (FY04), January 15, 2004.

Boardman, R. D., B. H. O’Brien, N. R. Soelberg, S. O. Bates, R. A. Wood, and C. St. Michael, (2004b), High Temperature Calcination-MACT Upgrade Pilot Plant Tests, INEEL/EXT-04-01625, February, 2004.

Boardman, R. D., L. J. Young, N. R. Soelberg, and L. A. Matejka, (2001), "NWCF Calciner Emissions Inventory - Final Report for Phase IV Testing,” INEEL/EXT/01-00260, February 2001.

Boardman, R. D., L. J. Young, N. R. Soelberg, M. L. Abbott, and D.V. Croson, (1999), Summary of NWCF Calciner Emissions Inventory Data, INEEL/EXT-99-1190, November 1999.

Boardman, R. D., N. R. Soelberg, and L. J. Young, (2000), "Off-gas Emissions for the New Waste Calcining Facility (Calciner)," Nineteenth International Conference on Incineration and Thermal Treatment Technologies, Portland, Oregon, May 8-12, 2000.

Bonnema, B. E., (2002), Risk Management Plan for the Sodium Bearing Waste Treatment Facility Project, PLN-1073, September, 2002.

Del Debbio, J. A., T. L. Watson, and J. B. Heintzelman, (2003), Long-Term Performance of SulfurImpregnated Granulated Activated Carbon $(G A C)$ for Mercury Removal from NWCF Off-Gas," INEEL/EXT-03-01102, September, 2003.

Herbst, A. K., (2003), "Preliminary Results for Solidification of Simulated Liquid Effluents from Sodium-Bearing Waste Treatment Options," Engineering Design File EDF-3788, May 15, 2003. 
Law, J., (2000), Operation of the New Waste Calciner at $600^{\circ} \mathrm{C}$, INEEL/EXT-2000-01263, September 2000.

Merrick \& Company, (2004), Calcination with MACT Upgrade Feasibility Study Report, Project No. 10014501-01, January 23, 2004.

Newby, B. J., and B. H. O’Brien, (2000), Summary of Waste Calcination At the Idaho Nuclear Technology and Engineering Center, INEEL/EXT-2000-01206, October, 2000.

Nichols, T. T., and D. D. Taylor, (2003), Thermodynamic Phase and Chemical Equilibrium at $0-110^{\circ} \mathrm{C}$ for the $\mathrm{H}^{+}-\mathrm{K}^{+}-\mathrm{Na}^{+}-\mathrm{Cl}^{-}-\mathrm{H}_{2} \mathrm{O}$ System up to 16 Molal and the $\mathrm{HNO}_{3}-\mathrm{H}_{2} \mathrm{O}$ System up to 20 Molal Using an Association-Based Pitzer Model Compatible With ASPEN-Plus, INEEL/EXT-03-01167, Rev. 0, September, 2003

O’Brien, B. H., (2002) “Calciner Throughput Evaluation for Calcination with MACT Upgrade SBW Treatment Alternative,” Engineering Design File EDF-312, December 5, 2002.

Raman, S. V., A. K. Herbst, B. A. Scholes, S. H. Hinckley, and R. D. Colby, (2003) Solidification of Simulated Liquid Effluents Originating form Sodium-Bearing Waste at the Idaho Nuclear Technology and Engineering Center, INEEL/Ext-03-01096, September, 2003.

Rawlins, J. K., (1997), Feasibility Study Report for NWCF MACT Compliance Facility, INEL/INT-9700992, November 1997, Volumes 1 \& 2.

Scholes, B. A., (2003), “Adsorption of NWCF Scrubber Solution onto Calcine," Engineering Design File $E D F-3794$, May 19, 2003.

Soelberg, N. R., (2003), Off-gas Mercury Control for the Idaho Tank Farm Project-Calcination with MACT Upgrade SBW Treatment Alternative, INEEL EDF-3292, Feb. 28, 2003.

Stacey, D. E., (2003), "Study \#3b Calciner Throughput Evaluation - Idaho Tank Farm Project Calcination with MACT Upgrade SBW Treatment Alternative," Engineering Design File 3291, April 28, 2003.

Staiger, M. D., (2003), Calcined Waste Storage at the Idaho Nuclear Technology and Engineering Center, INEEL/EXT-98-00455 Rev. 1, June 2003.

Swenson, M. C., (2000), New Waste Calciner High Temperature Operation, INEEL/EXT-2000-01004, September 2000.

Taylor, D. D., and T. T. Nichols, (2003), Revised Computer Program to Regress Parameters for Pitzer's Model, INEEL/EXT-03-01146, Rev. 0, September, 2003.

Welland, H., (1997), NWCF Process Modification for SBW Project Conceptual Design, INEL/INT-9700075 , April 1997.

Wood, R. A., (2001), NWCF High-Temperature Calcination Trial Summary, INEEL/EXT-01-00851, July 2001.

Wood, R. A., (2004), "Modeling of a Multi-Stage Combustor for Off-gas $\mathrm{NO}_{\mathrm{x}}$ and PIC Destruction in Support of the NWCF MACT Upgrade," Engineering Design File EDF-4451, January 30, 2004. 
Young, L. J., N. R. Soelberg, B. H. O’Brien, R. D. Boardman, L. A. Matejka, M. L. Abbott, and G. R. Hassel, (2000), NWCF Calciner Emissions Inventory - Final Report for Test Series 1, 2, and 3, INEEL/EXT-2000-00114, January 2000.

\section{Section 3.2 - Steam Reforming}

Bohn, T. S., (2002), Radiological Shielding Calculations for Screening of Sodium Bearing Waste (SBW) Treatment Alternatives, Revision 1, Interoffice Memo CCN35341, August 29, 2002.

Haefner, D., B. O’Brien, and S. O. Bates, (2003), Steam Reforming Process Design, INEEL EDF-3827, $1 / 30 / 03$.

Merrick \& Company, (2004), Calcination with MACT Upgrade Feasibility Study Report, Project No. 10014501-01, January 23, 2004.

Murphy, J., et al., (2002), Assessment of the SBW Treatment and Disposition Alternatives Decoupled from Calcine Treatment, INEEL/EXT-02-00265, February 2002.

Perry, K. J., (2002), Sodium-Bearing Waste Treatment Facility Project Alternatives Analysis Process and Results, INEEL/EXT-02-00994, September 2002.

Williams, C. L., R. A. Wood, B. L. Blakely, and W. H. Landman, (2002), Feasibility Study Report for the Treatment of Sodium-Bearing Waste by Steam Reforming (Draft), INEEL/EXT-02-01211.

Williams, C. L., R. A. Wood, B. L. Blakely, and W. H. Landman, Feasibility Study Report for Treatment of Sodium-Bearing Waste by Steam Reforming (Draft), INEEL/EXT-02-1211, Rev. A.

\section{Section 3.3 - Cesium Removal and Immobilization}

Barnes, C. M., S. K. Janikowski, and C. B. Millet, (2003), Feed Composition for the Sodium-Bearing Waste Treatment Process, INEEL/EXT-2000-01378, Revision 3, September 2003.

Herbst, A. K. to J. H. Valentine, (1996), FY-96 Status Report on the Low-Level Waste Grout Stabilization Development Program, Lockheed Martin Idaho Technologies Company Interdepartmental Communication AKH-07-96, September 18, 1996.

Losinski, S. J., C. M. Barnes, and B. K. Grover, (1998), CsIX/TRU Grout Feasibility Study, INEEL/EXT99-00034, November 1998.

Mann, N. R., and T. A. Todd, (1998), Evaluation and Testing of the Cells Unit Crossflow Filter on INEEL Dissolved Calcine Slurries, INEEL/EXT-98-00749, August 1998.

Miller, C. J., A. L. Olson, and C. K. Johnson, (1995), Cesium Absorption from Acidic Solutions Using Ammonium Molybdophosphate on a Polyacrylonitrile Support (AMP-PAN), INEL-95/00516, November 17, 1995.

Pao, Jenn-Hai, and R. Lewis, (2003), Experimental Study of Filtration and Solids' Post Treatment from Simulated Sodium-Bearing Waste Derived Slurry, INEEL/EXT-03-01106, September 2003.

Raytheon Engineers and Constructors, (2000), Idaho National Technology \& Engineering Center Sodium Bearing Waste (SBW) Treatment Facility Project CsIX/TRU Grout Process Feasibility Study Report, Volumes 1-4, September 2000. 
Stegen, G. E., to W. B. Palmer, (1997),“Additional SBW Separation and Backup Options,” DSI97-GES051, November 11, 1997.

Tranter, T. J., R.D. Tillotson, M.D. Argyle, and T.A. Batcheller, (2004a), "Evaluation of IONSIV ${ }^{\mathrm{TM}}$ IE911 as a Cesium Removal Option for INEEL Acidic Tank Waste: Phase II Semi-Scale Column Tests," Engineering Design File EDF-4536, January 30, 2004.

Tranter, T. J., (2004b), "Dynamic Stability Testing of IONSIV® IE911 for Cesium Removal from INEEL Acidic Tank Waste," Engineering Design File EDF-4612, February 24, 2004.

Tripp, J. L., and E. L. Wade, (1997), FY-97 Experimental Results of the Cells Unit Cross-Flow Filter Tests at the INEEL, INEEL/EXT-97-01232, November, 1997.

U. S. Department of Energy, Idaho Operations Office, (2003), WIPP Waste Certification Strategy for the Sodium-Bearing Waste Treatment Project, DOE/ID-11057, Rev. 1, August, 2003.

Valles, N. O., C. M. Barnes, C. R. Edinborough, K. H. Liebelt, B. D. Raivo, and D. S. Wendt, (1999), Sodium-Bearing Waste to WIPP Waste $\left(S B W^{3}\right)$ Optimization Study, INEEL/EXT-99-00975, November, 1999.

\section{Section 3.4 - Direct Evaporation}

Barden, R. R., (2003), "Technical and Functional Requirements for the Direct Evaporation SBW Treatment Alternative," TFR-186, draft, May 2003.

Barnes, C. M., R. A. Wood, and B. H. O'Brien, (2002a), "Process Design of SBW Treatment Alternatives," Engineering Design File EDF-2373, September 10, 2002.

Barnes, C. M., and C. B. Millet, (2002b), "INTEC Tank Farm Facility Management Plan," PLN-1112, September 30, 2002.

Barnes, C. M., R. A. Wood, E. B. Packer, and N. A. Chipman, (2003a), "Direct Evaporator Process Condensate Treatment Alternatives Evaluation," Engineering Design File EDF-3356, July 22, 2003.

Bohn, T. S., (2002), Radiological Shielding Calculations for Screening of Sodium Bearing Waste (SBW) Treatment Alternatives, Rev. 1, INEEL Interoffice Memorandum TSB-20, August 26, 2002.

Griffith, D. L., S. J. Losinski, B. E. Olaveson, and L. G. Olson, (2003a), Testing an Agitated Thin Film Evaporator for Solidifying Simulated Sodium-Bearing Waste (Test Series 1), INEEL/EXT-03-00680, June 2003.

Griffith D. L., (2003b), Testing an Agitated Thin Film Evaporator for Solidifying a Mixture of Simulated Sodium-Bearing Waste and Undissolved Solids (Test Series 2), INEEL/EXT-03-01189, November 2003.

Griffith, D. L., R. J. Kirkham, L. G. Olson, W. D. St. Michel, and S. J. Losinski, (2004), Converting Simulated Sodium-Bearing Waste into a Single Solid Waste Form By Evaporation: Laboratory-and Pilot-Scale Test Results on Recycling Evaporator Overheads, INEEL/EXT-04-00172, January, 2004.

Eckard, C., (2000), "Campaign Cascades 1.3 Million Gallons Through Evaporator," The Hanford Reach, May 15, 2000. 
Herbst, A. K., J. A. DelDebbio, R. J. Kirkham, B. A. Scholes, and T. L. Watson, (2002), Idaho Nuclear Technology and Engineering Center Sodium-Bearing Waste Treatment Research and Development FY-2002 Status Report, INEEL/EXT-02-00985, September, 2002.

Kimmitt, R. R., (2003), "Feasibility Study for Direct Evaporation of Sodium Bearing Waste," Engineering Design File EDF-2524, Rev. 0, May 20, 2003.

Kirkham, R. J. to W. H. Landman, (1998) "Sodium Bearing Waste Solidification by Evaporation", Lockheed Martin Idaho Technologies Company Internal Correspondence RJK-6-98, September 29, 1998.

Kirkham, R. J., S. J. Losinski, B. E. Olaveson, and L. G. Olson, (2003), Direct Evaporation of SodiumBearing Waste: Flask-Scale Testing of WM-189 Simulant, INEEL/EXT-03-0657, July 2003.

Kirkham, R. J., B. Olaveson, S. Losinski, , and L. Olson, (2004), "Laboratory-Scale Direct Evaporation Development Report FY-03; Bottoms Product Quality and its Relation to Overheads Concentration and Volume," Engineering Design File EDF-4538 Rev. 1, February 2, 2004.

McCray, J. A., L. G. Olson, and S. J. Losinski, (2002), Investigation of Direct Evaporation and Fractional Crystallization Technologies for Sodium-Bearing Waste Treatment, INEEL/EXT-0201043, September 2002.

O’Brien, B. H., (2002), "Calciner Throughput Evaluation for Calcination with MACT Upgrade SBW Treatment Alternative," Engineering Design File EDF-312, December 5, 2002.

Packer, E. B., (2003), "Preliminary Assessment of Evaporator Technologies for Direct Evaporation of Sodium Bearing Waste," Engineering Design File EDF-3443, April 8, 2003.

Wendt, D. S., (2003), "Radiolytic Hydrogen Gas Generation in Dried SBW UDS and Tank Solids," Engineering Design File EDF-3392, January 27, 2003.

\section{Section 3.5 - Vitrification}

Barnes, C. M., (2001), Feed Composition for the Sodium-Bearing Waste Treatment Process, INEEL/EXT-2000-01378, July 2001.

Barnes, C. M., and D. D. Taylor, (2002), Review of FY2001 Development Work for Vitrification of Sodium Bearing Waste, INEEL/EXT-02-00194, September 2002.

Bates, S. O., B. D. Raivo, J. J. Quigley, S. M. Berry, W. H. Landman, S. L. Palmer, and T. M. Hipp, (2001), Feasibility Study for Vitrification of Calcine in the Waste Vitrification Facility, INEEL/EXT01-00978, September 2001.

Christian, J. D., (2001), Composition and Simulation of Tank WM-180 Sodium-Bearing Waste at the Idaho Nuclear Technology and Engineering Center, INEEL/EXT-2001-00600, May 2001.

Fullmer, K. S., L. W. Fish, and D. K. Fischer, (1994), Vitrification Treatment Options for Disposal of Greater-Than-Class-C Low-Level Waste in a Deep Geologic Repository, National Low-Level Waste Management Program, DOE/LLW-210, November 1994. 
McDannel G. E., and B. D. Preussner, (2001), NWCF Retrofit Viability Evaluation, INEEL/EXT-0101300, September 2001.

Murphy, J. M., et al., (2002), Assessment of the SBW Treatment and Disposition Alternatives Decoupled from Calcine Treatment, INEEL/EXT-02-00265, February 2002.

Murphy, J. M., W. B. Palmer, and K. J. Perry, (2000), Sodium Bearing Waste Processing Alternatives Analysis, INEEL/EXT-2000-00361, May 2000.

NAS, (2000), Glass as a Waste Form and Vitrification Technology: Summary of an International Workshop (1997), http://www/nap.edu/openbook/0309056829/html/3.html copyright 1997. 2000 The National Academy of Sciences.

Norton, M. R., H. B. Shah, M. E. Stone, L. E. Johnson, and R. O’Driscoll, (2002), Overview - Defense Waste Processing Facility Operating Experience, WSRC-MS-2002-00145.

Quigley, J. J., B. D. Raivo, S. O. Bates, S. M. Berry, D. N. Nishioka, and P. J. Bunnell, (2000), Feasibility Study for Vitrification of Sodium-Bearing Waste, INEEL/EXT-2000-00952, September 2000.

Perry, K. J., (2002), Sodium-Bearing Waste Treatment Facility Project Alternatives Analysis Process and Results, INEEL/EXT-02-00994, September 2002.

Lauerhass, L, V. C. Maio, S. K. Merrill, A. L. Olson, and K. J. Perry, (2003), Sodium-Bearing Waste Treatment Applied Technology Plan, INEEL/EXT-03-00477, June 2003.

Roth, G., S. Weisenburger, (2000), Vitrification of High-level Liquid Waste: Glass Chemistry, Process Chemistry and Process Technology, Nuclear Engineering and Design, 202 (2000), pp. 197-207.

Seward, L.C., (2001), Sodium Bearing Waste Vitrification Process Flowsheet - Meeting Summary, Interoffice Memorandum to B. E. Bonema, S. J. Losinski, and L. Lauerhass, LCS-08-01, June 11, 2001.

Taylor, D. D., C. M. Barnes, and L. Lauerhass, (2001), INEEL SBW Vitrification Process, INEEL/EXT01-01139, September 2001.

Valenti, P. J., and D. I. Elliot, (1999), Operating Experience During High-Level Waste Vitrification at the West Valley Demonstration Project, DOE/NE/44139-87, January 1999.

Weber, E. T., R. B. Calmus, and C. N. Wilson, (1995), Vitrification Technology for Hanford Site Tank Waste, presented at the 97th Annual Meeting and Exposition of the American Ceramic Society, April 30-May 3, 1995, WHC-SA-2862-PP, April 1995. 


\section{Appendix A Steam Reforming Process}

Charles M. Barnes

Daryl R. Haefner

Richard A. Wood

Dean D. Taylor

February 2004 
This page intentionally left blank. 


\section{CONTENTS}

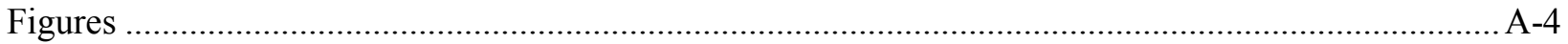

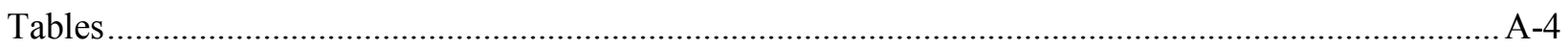

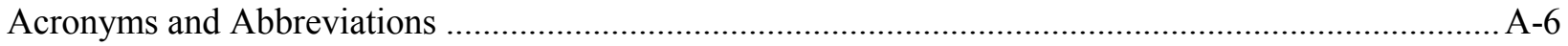

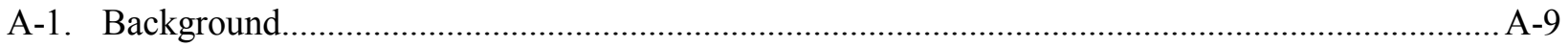

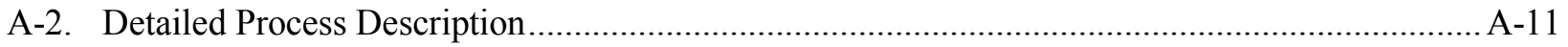

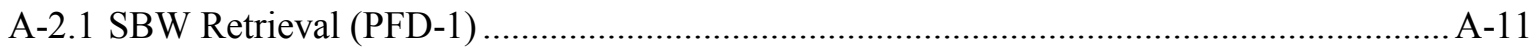

A-2.2 Waste Preparation and Feed (PFD-2A and 2B) ..........................................................

A-2.3 Chemical Additives for the Reformer (PFD-2A and 2B) ............................................A-12

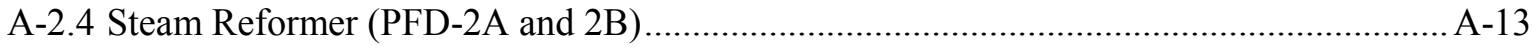

A-2.5 Product Collection and Processing (PFD-2A, PFD-2B, and PFD-4) .............................. A-14

A-2.6 Off-Gas System (PFD-2A, PFD-2B, PFD-3A, PFD-3B) .............................................. A-15

A-2.7 Spent Scrub Solution (PFD-3A, PFD-3B) …….............................................................. A-16

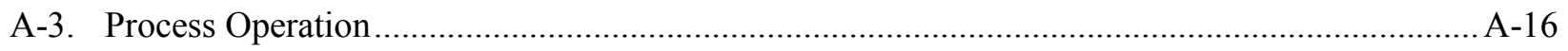

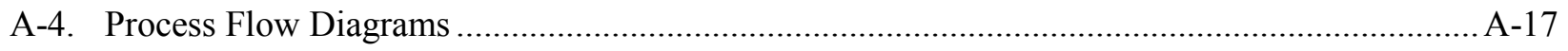

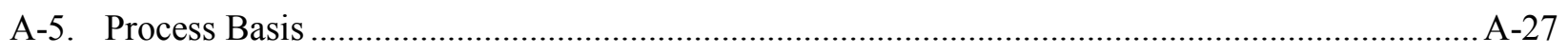

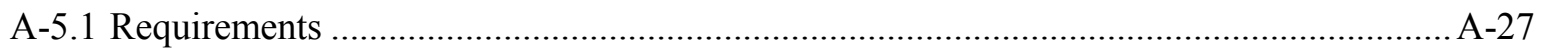

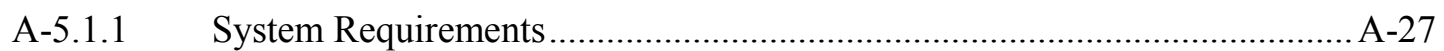

A-5.1.2 Systems, subsystem, and major components....................................... A-30

A-5.1.3 Boundaries and interfaces ………................................................ A-30

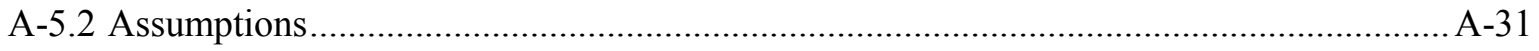

A-5.3 Process Variations ................................................................................................ A-32

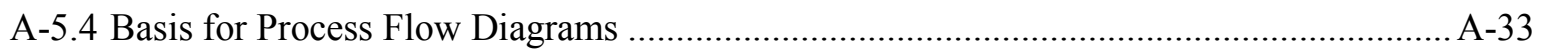

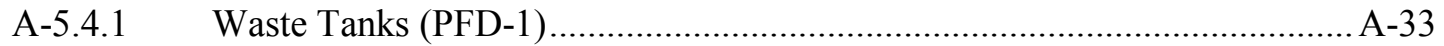

A-5.4.2 Feed Preparation and Steam Reforming (TTT) (PFD-2A) ........................... A-34

A-5.4.3 Steam Reforming (TTT) Off-Gas and Scrub Treatment (PFD-3A)............... A-34

A-5.4.4 Steam Reformer Product Waste Packaging (PFD-4) ................................... A-34

A-5.4.5 Basis for TWR PFDs (PFD-2B \& PFD-3B) ................................................ A-35 


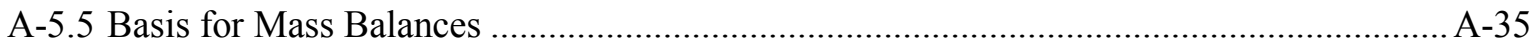

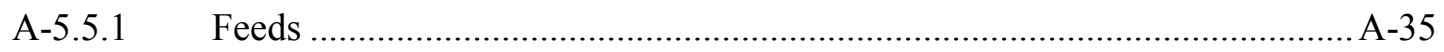

A-5.5.2 Stream Factor, Operating Schedule and Feed Rate.................................... A-39

A-5.5.3 Tank Mixing and Feed Transfer from Tank Farm ..................................... A-39

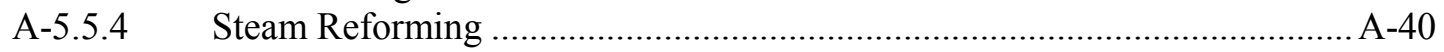

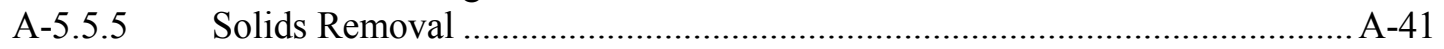

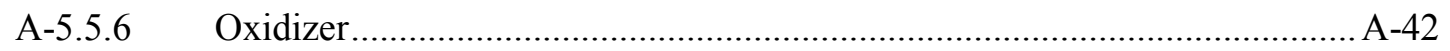

A-5.5.7 Downstream Off-gas Treatment .......................................................... A-42

A-5.5.8 Scrub Purge Treatment .......................................................................... A-45

A-5.5.9 Steam Reformer Product Packaging ...................................................... A-46

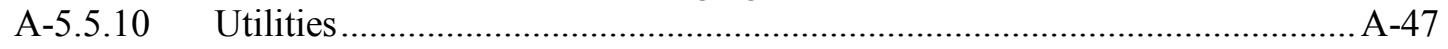

A-5.5.11 Basis for TWR Mass Balance …......................................................... A-48

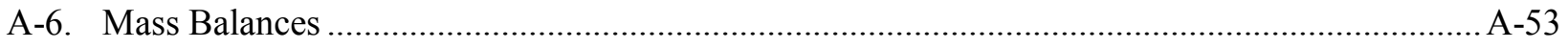

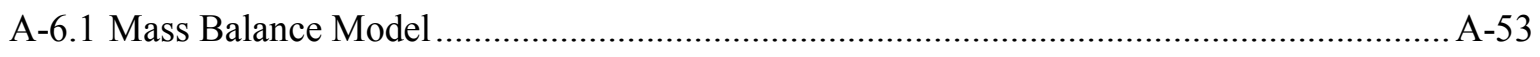

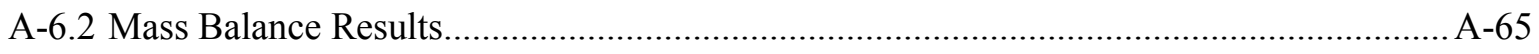

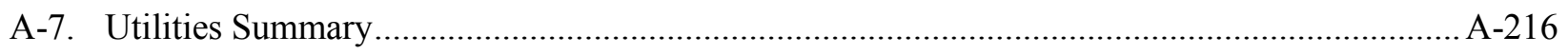

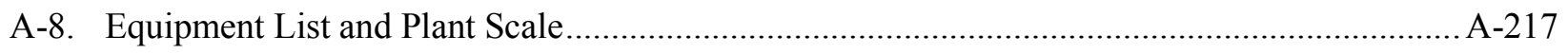

A-9. Layout Drawings ........................................................................................................ A-226

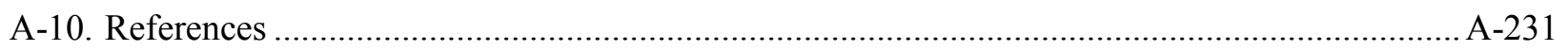

\section{FIGURES}

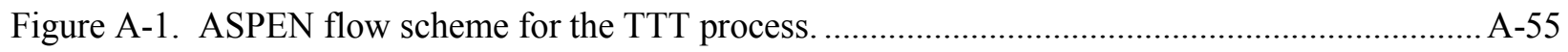

Figure A-2. ASPEN flow scheme for the TWR process................................................................. A-63

\section{TABLES}

Table A-1. Off-gas emission and rate limits.

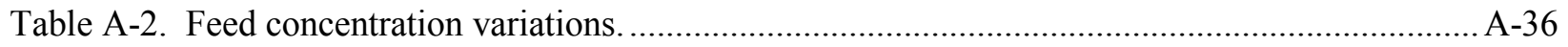

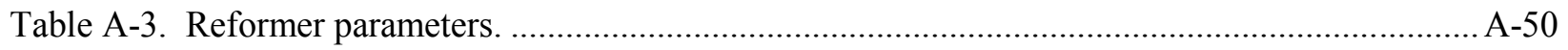

Table A-4. Bed, cyclone, and sintered metal filter separation factors. ............................................. A-51

Table A-5. ASPEN model chemical specie inputs........................................................................... A-54

Table A-6. Carbon composition used for ASPEN model of the TTT flowsheet. .................................. A-57 
Table A-7. Fraction of reformer feeds bypassing the reformer.

Table A-8. Additional reactions in the mass balance model.

Table A-9. Solids capture for the TTT material balance model (fraction of species into each unit captured by that unit)

Table A-10. Consumable chemicals estimate for TTT processing of SBW.

Table A-11. Summary of estimated waste product quantities and properties for TTT processing of SBW.

Table A-12. Consumable chemicals estimate for spent scrub treatment.

Table A-13. Mass balance, Tank WM-180 waste.

Table A-14. Mass balance, Tank WM-187 waste.

Table A-15. Mass balance, Tank WM-188 waste.

Table A-16. Mass balance, Tank WM-189 waste.

Table A-17. Consumable chemicals estimate for TWR processing of SBW.

Table A-18. Summary of estimated waste product quantities and properties for TWR processing of SBW.

Table A-19. Mass balance, Tank WM-180 waste.

Table A-20. Mass balance, Tank WM-187 waste.

Table A-21. Mass balance, Tank WM-188 waste.

Table A-22. Mass balance, Tank WM-189 waste.

Table A-22. Mass balance, Tank WM-189 waste.

Table A-23. Summary of the anticipated major utility uses for the TTT process............................. A-216

Table A-24. Summary of the anticipated major utility uses for the TRW process.

Table A-25. Preliminary list of TTT steam reforming equipment with a description and approximate size. 


\section{ACRONYMS AND ABBREVIATIONS}

\begin{tabular}{|c|c|}
\hline $\begin{array}{l}\text { CEM } \\
\text { CERCLA } \\
\text { cf }\end{array}$ & $\begin{array}{l}\text { continuous emission monitor } \\
\text { Comprehensive Environmental Response, Compensation and Liability Act } \\
\text { cubic foot }\end{array}$ \\
\hline $\begin{array}{l}\text { DBE } \\
\text { DOE } \\
\text { DRE } \\
\text { dscm }\end{array}$ & $\begin{array}{l}\text { design basis element } \\
\text { Department of Energy } \\
\text { destruction removal efficiency } \\
\text { dry standard cubic meter }\end{array}$ \\
\hline EDF & Engineering Design File \\
\hline FGE & fissile gram equivalent \\
\hline $\begin{array}{l}\text { GAC } \\
\text { gph }\end{array}$ & $\begin{array}{l}\text { granulated activated carbon } \\
\text { gallons per hour }\end{array}$ \\
\hline $\begin{array}{l}\text { HEPA } \\
\text { HLW }\end{array}$ & $\begin{array}{l}\text { high efficiency particulate air (filter) } \\
\text { high-level waste }\end{array}$ \\
\hline $\begin{array}{l}\text { INEEL } \\
\text { INTEC }\end{array}$ & $\begin{array}{l}\text { Idaho National Engineering and Environmental Laboratory } \\
\text { Idaho Nuclear Technology and Engineering Center }\end{array}$ \\
\hline $\begin{array}{l}\text { LDR } \\
\text { LET\&D }\end{array}$ & $\begin{array}{l}\text { land disposal restrictions } \\
\text { Liquid Effluent Treatment and Disposal (Facility) }\end{array}$ \\
\hline $\begin{array}{l}\text { MACT } \\
\text { MLLW }\end{array}$ & $\begin{array}{l}\text { Maximum Achievable Control Technology } \\
\text { mixed low level waste }\end{array}$ \\
\hline $\begin{array}{l}\text { NAR } \\
\text { NGLW } \\
\text { NO }_{x} \\
\text { NRC } \\
\text { NSNFP } \\
\text { NWCF }\end{array}$ & $\begin{array}{l}\text { nozzle atomizing air to feed ratio } \\
\text { newly generated liquid waste } \\
\text { an abbreviation for an unspecified mixture of NO and NO2 } \\
\text { Nuclear Regulatory Commission } \\
\text { National Spent Nuclear Fuel Program } \\
\text { New Waste Calcining Facility }\end{array}$ \\
\hline $\begin{array}{l}\text { PCBs } \\
\text { PE-Ci } \\
\text { PFD } \\
\text { POHC } \\
\text { ppm }\end{array}$ & $\begin{array}{l}\text { polychlorinated biphenyl compounds } \\
{ }^{239} \mathrm{Pu} \text {-equivalent curies } \\
\text { process flow diagram } \\
\text { principal organic hazardous constituents } \\
\text { parts per million }\end{array}$ \\
\hline RH & remote handled \\
\hline $\begin{array}{l}\text { SBW } \\
\text { scf } \\
\text { SNF }\end{array}$ & $\begin{array}{l}\text { sodium-bearing waste } \\
\text { standard cubic foot } \\
\text { Spent Nuclear Fuel (Program) }\end{array}$ \\
\hline
\end{tabular}


TEQ toxicity equivalent quotient

TFF Tank Farm Facility

TFRs Technical and functional requirements

THOR THermal Organic Reduction

TRU transuranic

TTT THOR Treatment Technology

TWR Thermochem Waste Remediation

$\mu \mathrm{g} \quad$ microgram

VOCs volatile organic compounds

WAC Waste Acceptance Criteria

w.c. water column

WIPP Waste Isolation Pilot Plant

wscfh wet standard cubic feet per hour 
This page intentionally left blank. 


\section{Appendix A \\ Details of the Steam Reforming Process}

\section{A-1. BACKGROUND}

Steam reforming is the standard industrial process used to produce hydrogen. Steam reforming processes have long been used to generate hydrogen as a feedstock for ammonia production, for petroleum refining steps, for other chemical processes and to generate synthesis gas. More recent uses of steam reforming have been to generate hydrogen for fuel cells, to generate hydrogen from biomass, to treat paper mill black liquor and to destroy various hazardous wastes. These latter applications have led to its development for the treatment of radioactive waste streams.

In the mid-1990's the Department of Energy (DOE)-funded development and demonstrations of steam reforming of both low-level mixed waste and Hanford tank waste (Miller 1995, Voelker 1996; ThermoChem 1998). ThermoChem Waste Remediation (TWR) designed, built and operated a $90 \mathrm{lb} / \mathrm{hr}$ steam reformer test unit at the Portsmouth Gaseous Diffusion Plant that was used to demonstrate treatment of wastes containing polychlorinated biphenyl compounds (PCBs) and other hazardous organics (ThermoChem 1998). The test unit included a 42-inch diameter fluidized bed and a thermal oxidizer to treat off-gas. A diverse set of seven surrogate wastes, from soil to heterogeneous debris to aqueous liquids, were tested during several test periods of up to 20 days (ThermoChem 1998). The test program of the TWR system also included a conceptual design of a full-scale unit.

As early as 1994, steam reforming was proposed and tested for treating Hanford Tank waste (Miller 1995). Testing was performed of the Synthetica steam reforming concept. The Synthetica process first contacts the waste with ceramic spheres in a moving bed evaporator operated at $550^{\circ} \mathrm{C}$. Waste is fed at the top of the bed, coats the spheres and as they flow downward water and volatile species evaporate and nitrates decompose. Steam and carbon dioxide flow up through the bed, converting sodium hydroxide to sodium carbonate. Waste particles separate from the spheres as they are recycled from the bottom to the top of the bed. A unit at Lawrence Livermore National Laboratory was used to demonstrate the concept at up to about $14 \mathrm{lbs} / \mathrm{hr}$ feed. Results of demonstrate tests were inconsistent in the fraction of nitrates destroyed and the composition of off-gas, although high levels of $\mathrm{NO}$ and $\mathrm{NO}_{2}$ were seen in some tests. More recent tests have been performed to demonstrate the use of steam reforming to process Hanford low activity waste (Jantzen 2002, Jantzen 2003).

The first concept for the use of steam reforming in a sodium-bearing waste (SBW) treatment process was as part of a separation process, in which steam reforming would be used to generate an ash from the low activities waste stream (Marshall 2000). The ash would have then been grouted into a final waste form. A bench-scale fluidized bed was used with a simulant similar to SBW composition, and tests with and without reductants and catalysts were performed (Marshall 2000). While the tests demonstrated the concept of waste denitration, data gaps indicated the need for further testing.

In 2001, steam reforming was considered as an alternative to, or complementary process with vitrification for treatment of SBW. The DOE Idaho Operations Office chartered a Tank Focus Area Review Team to conduct an "in-depth evaluation of the technical feasibility and cost incentives, if any, of steam reforming for treating SBW." The conclusions of the Review Team must be viewed in light of the baseline SBW treatment process of that time, namely vitrification. The conclusions of the Review Team were (Gentilucci 2001): 
- Steam reforming of SBW will not generate a waste form qualified for direct disposal in a highlevel waste (HLW) repository.

- There are marginal technical incentives for steam reforming of SBW to generate a waste form for direct disposal in the Waste Isolation Pilot Plant (WIPP) as a remote-handled (RH) transuranic (TRU) waste. However, uncertainties related to waste type (HLW or TRU), the status of Resource Conservation and Recovery Act requirements at WIPP, the impacts of longterm storage of the steam-reformed product, and cost of a facility that satisfies safety requirements at Idaho National Engineering and Environmental Laboratory (INEEL) appear to outweigh the technical incentives.

- Steam reforming of SBW to produce a solid feed for subsequent vitrification appears to be a technically viable option if the presence of chemicals added or created during reforming is shown to not adversely impact melter reliability and if there is an incentive for partitioning sulfate to the off-gas stream.

Based on DOE guidance in 2002 to develop SBW treatment processes that would dispose of the final waste products at WIPP, testing of steam reforming was resumed. In 2003, two subcontractors, TWR and Thermal Organic Reduction (THOR) ${ }^{\mathrm{sm}}$ Treatment Technologies (TTT), each supplied technical recommendations regarding chemical additives and process conditions for treating the SBW in a fluidized bed configuration. The TTT process is in commercial radioactive operations at the Studsvik Processing Facility in Erwin, Tennessee. Additional TTT demonstrations have been implemented specifically for processing DOE nitrate wastes, including tests for treating Hanford waste surrogates as well as those for the INEEL (THOR 2003, Jantzen 2002). The TWR process is being applied commercially to "black liquor," a caustic liquid containing resin and lignin hydrocarbons plus sodium carbonate. Two series of tests were performed for each of the two steam reforming processes.

A bench-scale reactor and support facility was provided by the INEEL for each contractor to demonstrate their technology. Surrogate SBW (containing non-radioactive or naturally occurring isotopes in their natural isotopic distributions) was used for the tests and each trial lasted for about 100-hours. Specific details of the tests are available in the referenced reports (Marshall 2003a, Marshall 2003b, Soelberg 2004a, Soelberg, 2004b). The objectives of the tests were to:

- Verify the operability of treating SBW in a fluidized bed reactor without shutdown due to bed agglomeration,

- Characterize the waste product for composition, density, flow characteristics, handling characteristics, etc.,

- Determine the composition of the off-gas after particle filtration,

- Determine the fate of volatile radionuclides such as cesium and technetium,

- Determine the fate and speciation of mercury,

- Determine the concentration of $\mathrm{NO}_{\mathrm{x}}$ after the reformer.

Both subcontractors successfully completed 100-hour bench-scale tests. Nitrate was destroyed in the steam reformer, with the level of $\mathrm{NO}_{\mathrm{x}}$ in the off-gas ranging from about $1000 \mathrm{ppm}$ (for TWR) to 330 ppm (for TTT). The majority of the solid product was captured from the off-gas as fines. When 
accumulated, the fines formed a free-flowing powder having a low bulk density. Cesium and rhenium (surrogate for technetium) were retained in the solid product and on the bed material.

Results of the bench-scale tests were used to generate a baseline steam reforming process to provide a basis for evaluating steam reforming against other candidate treatments. Material and energy balances were performed using the test results in conjunction with an ASPEN simulation model. It should be pointed out that the equipment provided for the subcontractors was not optimized for either process - a full-scale facility designed by the TTT or TWR may have equipment different than that proposed herein.

The second series of tests were performed in late 2003. Results from the tests were incorporated into the basis for the mass balances shown in later sections of this report.

A feasibility study for treatment of SBW by steam reforming was prepared in 2002 (Williams 2002). The feasibility study included a process design, facility description and drawings, a project cost estimate, project schedule and list of risks and uncertainties. The estimated total project cost shown in the study was \$256 million, at an 85\% confidence level. Testing since 2002 (described above) has led to updates in the process design, which are contained in this Appendix, and expansion of the list of uncertainties. However, no update of the facility design has been performed since the 2002 Feasibility Study.

\section{A-2. DETAILED PROCESS DESCRIPTION}

\section{A-2.1 SBW Retrieval (PFD-1)}

Sodium-bearing waste will be stored in the seven existing tanks shown in process flow diagram (PFD)-1. Tanks VES-WM-180 and VES-WM-189 currently contain waste near their capacity limits, while VES-WM-188 is at about $80 \%$ capacity. VES-WM-187 has been used as a collection tank for liquids and solids flushed from other tanks that are being permanently closed. VES-WM-187 will receive the contents from one more tank and its collection duties will be completed. After 2005, newly generated liquid waste (NGLW) will be stored in the three tanks VES-WM-101, VES-WM-101, and VES-WM-102.

An engineering study concluded that existing Tank Farm equipment is adequate for transferring waste from the Tank Farm to the New Waste Calcining Facility (NWCF) feed tanks (Wood 2002). Liquids and solids can be transferred from tanks VES-WM-180, VES-WM-187, VES-WM-188, VESWM-189, VES-WM-100, VES-WM-101, and VES-WM-102 using existing steam jets, and in the case of VES-WM-189, also an existing airlift. It is assumed that the Tank Farm equipment will also be capable of feeding waste to tanks associated with the steam reforming facility.

The engineering study also evaluated several options for processing tank solids including: separate processing of the solids, batch co-processing, and homogeneous mixture co-processing. These options were evaluated against eight criteria and resulted in recommending co-processing the liquids and solids as a homogeneous mix. This option requires installing new mixing pumps in VES-WM-187, VES-WM-188 and VES-WM-189 to promote uniform distribution of heel solids between the four tanks (Wood 2002).

\section{A-2.2 Waste Preparation and Feed (PFD-2A and 2B)}

Existing steam jets in tanks VES-WM-187, VES-WM-188 and VES-WM-189 will deliver SBW (a near-uniform suspension of solids dispersed in liquid SBW) to the feed preparation tanks VES-100-1, 2. These tanks will be used to combine chemical additives with the SBW in controlled batches. The tanks 
are equipped with mixers to maintain well-stirred conditions, plus cooling coils to remove heat. PFD-2A is based on the TTT process and shows a solid additive, sucrose, being combined with the SBW. The sucrose is stored in a hopper, HOP-800, which supplies an auger/feeder, FEED-400, which in turn provides sucrose to the mixer. TWR uses a liquid chemical additive instead of a solid, and therefore, PFD-2B shows a storage tank VES-115 and associated pump P-201 to supply the SBW preparation feed tank (VES-100-1, 2).

Sucrose and SBW surrogates have been observed undergoing reactions after sitting for several hours. The solutions have effervesced and formed some particulate matter. The induction period is believed to be a function of temperature and acid concentration (Marshall 2003c). If the sucrose/SBW reaction proves problematic, an alternate mixing strategy, for example, an in-line mixer or a stirred tank with a short residence time, could be used.

Once prepared, the stream of combined SBW and chemical additives (stream 102) is pumped to the reforming reactor. The feed is injected and dispersed in the reactor through atomizing nozzles. The TTT process uses nitrogen gas to atomize the feed; the TWR fluid is carbon dioxide. The nitrogen is stored in VES-101 as a low temperature liquid and heated prior to its use as the atomizing fluid.

Based on Phase 2 demonstration tests (Soelberg 2004b), the TWR process uses a single liquid chemical, isopropyl alcohol (IPA), as a reductant. The alcohol is mixed with SBW and recycled scrub solution, prior to injecting this combined feed into the reactor. PFD-2B shows the IPA stored in tank VES-115 and charged through pump P-201 to feed preparation tanks (VES-100-1, 2). As noted above, the preparation tanks could be replaced with in-line mixers if deemed desirable by the project.

TWR uses carbon dioxide $\left(\mathrm{CO}_{2}\right)$ as the primary gas throughout the system and thereby requires no steam generator (to produce steam for fluidization) or nitrogen source tank (for feed atomization). In the TWR process, the feed is injected into the reactor through spray nozzles using carbon dioxide as the atomization fluid. The carbon dioxide is shown on the PFD as being stored (VES-101) as a low temperature liquid, which must be heated and vaporized prior to use. The TWR process also uses carbon dioxide as the bed fluidization media, which requires heating the carbon dioxide (in HE-301) to near the reactor's operating temperature.

\section{A-2.3 Chemical Additives for the Reformer (PFD-2A and 2B)}

In addition to the chemicals added to SBW in the feed preparation tank, solid materials are added directly to the reforming vessel to promote and control the reactions taking place. Hoppers and/or auger feeders are used for storing and transporting these materials. Solid reductant, composed of activated carbon in the TTT process, is stored in HOP-801 and fed via FEED-401 to the reformer. An iron oxide catalyst may also be used in the TTT process, however, if so it will be a relatively small quantity, therefore, no separate storage hopper is required for the catalyst beyond that available as a standard feature on the auger.

Prior to actual waste treatment, starting bed material composed of inert solid particles must be charged to the reactor. Alumina is used in the TTT process and is shown in PFD-2A as being stored in HOP-803. Alumina is only used intermittently: during start-up and possibly on shutdown.

In the bench-scale demonstration, the activated carbon reductant required feeding via a pneumatic injection system due to the low bulk density of the activated carbon. A similar problem was noticed with the reductant used in the FY03 TWR demonstration; however, the solid reductant was eliminated in the FY04 TWR tests and is not present in the current TWR flowsheet. The injection system resulted in a somewhat uneven feed rate (Marshall 2003b). Nonetheless, the PFDs show an injection vessel (VES- 
104) for reductant feed, although an alternative feed system warrants study. The alumina and catalyst are both sufficiently dense to allow these to be fed by gravity to the reformer. For TWR, the contingency to add solid reductant (polypropylene beads) has been maintained in the proposed design, even though the FY 2004 pilot-scale tests did not require this feature. The solid reductant is stored in HOP-801 and fed through FEED-401. The polypropylene beads have a low bulk density and would require use of the pressurized injection vessel VES-104. The injection vessel would use carbon dioxide as the transfer gas.

\section{A-2.4 Steam Reformer (PFD-2A and 2B)}

The steam reformer VES-103 is a fluidized bed reactor that converts the liquid SBW to a freeflowing particulate solid, plus a portion emitted in the off-gas as nitrogen and steam. The reaction vessel consists of three major sections: (1) the distributor, (2) the fluidized bed, and (3) the disengaging or freeboard section (Perry 1973).

The distributor is located at the bottom of the reactor and is where the fluidization gas enters and is dispersed over the cross-section of the reactor. The TTT process uses a combination of steam and oxygen for fluidization. The distributor section also contains a drain to remove heavy or agglomerated particles (bottoms product).

The fluidized bed section contains the bed material, consisting of inert solid particles suspended in the flowing gas. The waste enters in this section and is injected into the bed through atomizing nozzles. Solid chemical reductant (carbon-bearing material) is also introduced in this section. The TTT process uses nitrogen for atomizing the feed and injecting reductant into the reformer. Numerous potential reactions and combinations of reactions occur in this section of the reactor. The following simplified set of reactions is believed significant in the operation of the reformer.

The solid phase carbon (reductant) reactions include the following:

$$
\begin{array}{cc}
\mathrm{C}(\mathrm{s})+\mathrm{H}_{2} \mathrm{O} & \rightarrow \mathrm{CO}+\mathrm{H}_{2} \\
\mathrm{C}(\mathrm{s})+\mathrm{O}_{2} \rightarrow \mathrm{CO}_{2}+\text { heat } & \text { Carbon oxidation } \\
2 \mathrm{C}(\mathrm{s})+\mathrm{NO}_{\mathrm{x}} \rightarrow \mathrm{N}_{2}+\mathrm{CO}+\mathrm{CO}_{2} & \text { Solid phase } \mathrm{NO}_{\mathrm{x}} \text { reduction }
\end{array}
$$

The hydrogen reduces $\mathrm{NO}_{\mathrm{x}}$ to nitrogen via the following reactions:

$$
\begin{gathered}
\mathrm{H}_{2}+\mathrm{NO}_{2} \rightarrow \mathrm{H}_{2} \mathrm{O}+\mathrm{NO} \\
2 \mathrm{H}_{2}+2 \mathrm{NO} \rightarrow 2 \mathrm{H}_{2} \mathrm{O}+\mathrm{N}_{2}
\end{gathered}
$$

Carbon monoxide may react with numerous other species as shown below. Reaction (6) is desirable since it results in increased hydrogen levels, and hydrogen is believed to be more effective in reducing $\mathrm{NO}_{\mathrm{x}}$ than carbon monoxide. Suppression of the methanation reaction (7) is desirable since hydrogen is consumed in this reaction. Reactions (8) and (9) show a potential path for carbon monoxide to reduce $\mathrm{NO}_{\mathrm{x}}$ to nitrogen gas.

$$
\begin{array}{lc}
\mathrm{CO}+\mathrm{H}_{2} \mathrm{O} \rightarrow \mathrm{CO}_{2}+\mathrm{H}_{2} & \text { Water-gas shift } \\
\mathrm{CO}+3 \mathrm{H}_{2} \rightarrow \mathrm{CH}_{4}+\mathrm{H}_{2} \mathrm{O} & \text { Methanation reaction } \\
\mathrm{CO}+\mathrm{NO}_{2} \rightarrow \mathrm{CO}_{2}+\mathrm{NO} &
\end{array}
$$




$$
2 \mathrm{CO}+2 \mathrm{NO} \rightarrow 2 \mathrm{CO}_{2}+\mathrm{N}_{2}
$$

The primary mineral forming constituents in SBW are sodium and aluminum. These may undergo the following denitration reactions and reform the corresponding solid products:

$$
\begin{aligned}
& 4 \mathrm{Al}\left(\mathrm{NO}_{3}\right)_{3} \rightarrow 2 \mathrm{Al}_{2} \mathrm{O}_{3}(\mathrm{~s})+12 \mathrm{NO}_{2}+3 \mathrm{O}_{2} \quad \text { Aluminum nitrate decomp. } \\
& 4 \mathrm{NaNO}_{3} \rightarrow 2 \mathrm{Na}_{2} \mathrm{O}(\mathrm{s})+4 \mathrm{NO}_{2}+\mathrm{O}_{2} \quad \text { Sodium nitrate decomp. } \\
& \mathrm{Na}_{2} \mathrm{O}+\mathrm{CO}_{2} \rightarrow \mathrm{Na}_{2} \mathrm{CO}_{3}(\mathrm{~s}) \quad \text { Sodium carbonate formation }
\end{aligned}
$$

It is desirable to maintain the fluidizing bed section within a defined operating temperature range (about $700^{\circ} \mathrm{C}$ for the TTT process) to minimize detrimental side reactions and prevent bed agglomeration. Similarly, it is desirable to control key gas phase constituents within desired limits to optimize reactor performance (minimize $\mathrm{NO}_{\mathrm{x}}$ emissions, solid product character, etc.).

The upper section of the reformer is the disengaging or freeboard section. This section is wider in diameter than the fluidizing bed section and results in a reduced gas velocity. The reduced velocity causes larger entrained particles to disengage from the gas stream and return to the fluidizing section. The disengaging section also contains an internal cyclone, which captures fine entrained particles and also returns these to the fluidized bed. This results in increasing the average residence time of the particles and should minimize carbon content in the fines product.

The TWR process operates at about $600^{\circ} \mathrm{C}$ and uses carbon dioxide as the fluidization gas and to inject/atomize the feed. Isopropyl alcohol (IPA) is the carbon-bearing reductant used by TWR. Although different chemical amendments are used by TWR and results in a somewhat different reactor chemistry, the desired products from the reaction vessel are similar to those listed above for TTT - nitrates and $\mathrm{NO}_{\mathrm{x}}$ are reduced to nitrogen, and mineral-forming constituents (sodium, potassium, aluminum, etc.) converted to carbonates and oxides. Numerous combinations of reactions are possible between IPA and metalnitrates, however the simplified overall reactions may proceed as follows (using $\mathrm{Na}+$ as a typical cation):

$$
\begin{gathered}
2 \mathrm{C}_{3} \mathrm{H}_{8} \mathrm{O}+2 \mathrm{NaNO}_{3}+\mathrm{CO}_{2} \rightarrow \mathrm{Na}_{2} \mathrm{CO}_{3}+\mathrm{N}_{2}+6 \mathrm{CO}+7 \mathrm{H}_{2}+\mathrm{H}_{2} \mathrm{O} \\
\mathrm{C}_{3} \mathrm{H}_{8} \mathrm{O}+2 \mathrm{NaNO}_{3}+\mathrm{CO}_{2} \rightarrow \mathrm{Na}_{2} \mathrm{CO}_{3}+\mathrm{N}_{2}+2 \mathrm{CO}+4 \mathrm{H}_{2} \mathrm{O}+\mathrm{C}(\mathrm{s}) \\
\mathrm{C}_{3} \mathrm{H}_{8} \mathrm{O}+2 \mathrm{NaNO}_{3}+\mathrm{CO}_{2} \rightarrow \mathrm{Na}_{2} \mathrm{CO}_{3}+\mathrm{N}_{2}+3 \mathrm{CO}+3 \mathrm{H}_{2} \mathrm{O}+\mathrm{H}_{2} \\
\mathrm{C}_{3} \mathrm{H}_{8} \mathrm{O}+2 \mathrm{NaNO}_{3}+\mathrm{CO}_{2} \rightarrow \mathrm{Na}_{2} \mathrm{CO}_{3}+\mathrm{N}_{2}+3 \mathrm{CO}_{2}+4 \mathrm{H}_{2} \\
\mathrm{C}_{3} \mathrm{H}_{8} \mathrm{O}+6 \mathrm{NaNO}_{3}+3 \mathrm{CO}_{2} \rightarrow 3 \mathrm{Na}_{2} \mathrm{CO}_{3}+3 \mathrm{~N}_{2}+6 \mathrm{CO}_{2}+4 \mathrm{H}_{2} \mathrm{O}+4 \mathrm{H}_{2}
\end{gathered}
$$

\section{A-2.5 Product Collection and Processing (PFD-2A, PFD-2B, and PFD-4)}

Solid product may be drawn from the reactor bottom or from the overhead fines collection filter (F100-1, 2). Flow sheets PFD-2A and 2B show bottom product and fines being transported by gravity to a common set of collection hoppers (HOP-804-1, 2, 3). Fresh product will be hot $\left(400\right.$ to $\left.650^{\circ} \mathrm{C}\right)$ and requires cooling prior to being processed into canisters. The proposed design uses cooling gas (nitrogen for the TTT process) being routed directly through the collection hoppers. Three hoppers are used, thereby allowing two to cool while the third is being used for product processing. The cooling gas 
emerging from the collection hoppers may carry some fines; therefore, the gas is routed through a small cyclone (CYC-100-1, 2, 3) to remove this carry-over.

The bench-scale demonstration tests produced overhead product that was free flowing, but having a very low bulk density of 0.15 to $0.35 \mathrm{~g} / \mathrm{cm}^{3}$ (Marshall 2003a, Marshall 2003b). Therefore, once the product is sufficiently cooled, it is sent to an auger/densification device FEED-404, prior to being discharged to canisters. The densification device may be a compactor, pelletizer, or even a simple shaker table. Further study is required to determine the type of densification device recommended and to determine if the added complexity and cost of this equipment and processing is justified.

Canister filling and subsequent processing is shown in PFD-4. Each canister is filled with about $0.8 \mathrm{~m}^{3}$ of product, equivalent to about 85 to $90 \%$ of the canister volume. The filled canister is then visually inspected followed by the lid being placed in position and locked. The sealed canister is then surveyed, decontaminated, and placed in lag storage or a transport cask.

\section{A-2.6 Off-Gas System (PFD-2A, PFD-2B, PFD-3A, PFD-3B)}

The off-gas system consists of a series of unit operations to control radionuclide, hazardous, and particulate emissions. Maximum Achievable Control Technology (MACT) standards are assumed to apply to the off-gas and the proposed design addresses contaminants that will likely be problematic, most notably mercury.

Immediately after leaving the reaction vessel, the off-gas is cooled by introducing relatively cool gases in mixing chamber VES-113. Additional cooling is provided by heat exchanger HE-305, located downstream from the mixing vessel. The off-gas then enters the sintered metal filter vessel $(\mathrm{F}-100-1,2)$, where most of the fines are removed. The filter elements are sintered metal "candles" that retain fine particles. These are periodically pulsed with jets of gas to remove the particles, which are collected and removed from the bottom of the filter vessel. Cooling of the off-gas to about $400^{\circ} \mathrm{C}$ prior to entering the filter is believed to promote removal of volatile metals such as cesium, cadmium, chromium, and lead.

The off-gas emerging from the reformer vessel will contain significant levels of flammable gases, primarily hydrogen, carbon monoxide, and methane, along with trace quantities of organics. An off-gas oxidizer, VES-105, is used to destroy these and is shown immediately downstream of the sintered metal filter. Because of the flammable gas levels and their associated heating values, a flameless thermal oxidation unit is recommended. These units function by passing off-gas fumes through a refractory-lined reactor filled with inert ceramic packing. Air or oxygen is added to the reaction chamber along with any supplementary fuel, if necessary. This type of oxidizer does not use a burner and thereby avoids extreme temperature gradients in the reactor. This leads to improved control of the process chemistry and minimizes generation of undesirable by-products such as nitrogen oxides, un-reacted organics, and products of incomplete combustion. Units ranging in size up to $30,000 \mathrm{scfm}$ are available (Freeman 1998).

After leaving the oxidizer, the gas is routed to the quench and wet scrub system. This system consists of a quench tower (VES-106), the scrubber/absorber (VES-107), a mist eliminator (VES-108), and a solution hold tank (VES-110) plus cooler (HE-303). Provisions are also available to recycle the scrub back to the feed tank. Water is introduced in the quench tower to lower the off-gas temperature.

The gas is then routed to a wet scrubber to remove mercury and chlorine. It is currently proposed to operate the scrubber in a manner similar to that used for the calciner. A recirculating nitric acid solution is used to solubilize mercury until the solution reaches a pre-determined mercury concentration this value was set at $60 \mathrm{~g} / \mathrm{L}$ mercury. The $\mathrm{pH}$ of the scrub solution tank (VES-110) is maintained within 
the desired range by a control loop with a feed make-up of nitric acid (VES-112 and P-204). A second control loop (VES-114 and P-205) is included that uses an aluminum nitrate solution to minimize corrosion due to fluoride and chlorine, however, this loop may be eliminated if the anticipated corrosion is deemed acceptable. The spent scrub solution is discharged to a blow down hold tank (VES-111), where the solution is treated, solidified with cement, and disposed of.

A packed tower is currently proposed, although an alternate device may be specified during detailed design. A packed tower is a departure from the venturi scrubber used in the calcine system. The venturi is more commonly used for removing particulate matter from gases. It is anticipated that the sintered metal filter proposed for the reformer will remove over $98 \%$ of the fines generated; therefore the need for particulate removal by the absorber is reduced. A packed tower typically provides more efficient gas/liquid contact and improved mass transfer compared to a venturi (Treybal 1968, Kohl 1979).

After leaving the packed bed scrubber, the gas is routed through a mist eliminator (VES-108), a heater (HE-304) to raise the gas temperature above its dew point, and then onto a bank of high-efficiency particulate air (HEPA) filters (F-101-1, 2, 3). The filtered gas then passes to a sulfur-impregnated activated carbon bed (VES-109, plus a shallow auxiliary bed), which removes residual mercury and chlorine. Compliance with MACT emission standards is verified by a continuous emission monitoring system on the effluent. Pressure control in the off-gas system is set by adjusting the make-up air to the off-gas blower (BLO-200). From the blower, the off-gas is finally vented to the atmosphere through the stack.

\section{A-2.7 Spent Scrub Solution (PFD-3A, PFD-3B)}

Scrub solution is periodically purged from the scrub hold tank (VES-110) when solubilized mercury accumulates to about $60 \mathrm{~g} / \mathrm{L}$. Scrub purge treatment would not be a continuous operation; batch treatment could be performed during reforming operations or during scheduled shutdowns.

Spent scrub solution is discharged to the blow down hold tank (VES-111). This solution will be acidic and contain high levels of dissolved mercury. Information from the demonstration tests suggests that transuranic nuclide levels will be low; therefore, the solution will presumably be classified as a mixed low-level waste. The proposed treatment includes $\mathrm{pH}$ adjustment to a value near 3 by hydrated lime addition, followed by calcium sulfide to precipitate mercury. The resulting solution/sludge is then combined (in mixer MIX-100) with Portland cement, or similar blended cement, to form a solidified product suitable for disposal as low-level waste.

\section{A-3. PROCESS OPERATION}

This section describes a possible operation scenario.

Prior to processing, mixing pumps would have been installed in Tanks WM-187, WM-188 and WM-189, and the solids initially in Tank WM-187 would be distributed between these three tanks by a series of waste transfers between these tanks. After these transfers, all concentrated NGLW in tanks WM-100, WM-101 and WM-102 would be transferred to WM-187. This NGLW transfer is needed to be able to continue using these smaller tanks to receive and collect NGLW. The four waste tanks - WM180, WM-187, WM-188 and WM-189 - would then be sampled and the analytical results used to support qualification of the wastes from the treatment process.

After plant start-up activities are complete, waste from Tank WM-187 would be processed. Processing this tank first would free it up to receive flush water from the other tanks after they are emptied. Following the completion of processing Tank WM-187 waste, waste from one of the other 
tanks (WM-180, WM-188 or WM-189) would be processed. When waste has been withdrawn to heel level, estimated to be about 3000 gallons, processing of waste from a second of these three tanks would begin. Simultaneously, heel from the emptied tank would be flushed with water to Tank WM-187. After this sequence is repeated for another tank, the treatment process will be processing waste from the last tank, and heels from two tanks will have been collected in WM-187.

The contents of Tank WM-187, mostly flush water, would then be evaporated, with the concentrate sent to WM-100, WM-101 or WM-102 to be held for treatment. After completing treatment of waste in the final waste tank, its heel would be flushed to WM-187, which initially contains a dilute heel. The final evaporation of WM-187 would produce about 3000-4000 gallons of concentrate, which would either be treated in the SBW treatment facility or dispositioned with other NGLW collected during 2012. The heel in WM-187, dilute to begin with, would be flushed using multiple washes to the NGLW tanks. This waste, if concentrated separately from other wastes, would produce only an estimated 300 gallons of concentrate. Thus, flush from WM-187 would more likely be evaporated with other NGLW collected in late 2012, and the concentrate dispositioned with this NGLW.

Transfers of about 3,750 gallons from the Tank Farm to the Steam Reforming Facility would be made about once a day. At a rate of $50 \mathrm{gpm}$, the transfer would take about 1.5 hours. Waste qualification would be based on analysis of samples taken from the Tank Farm tanks, hence no holdup is designed into the treatment facility feed tank for sample turnaround.

Waste processing in the treatment facility would be continuous, 24-hours per day. Waste from each Tank Farm tank would be processed in "campaigns," with no planned shutdown until the tank was empty. Assuming no unscheduled shutdowns, the campaigns for each of the full Tank Farm tanks would take about 125 days (based on a feed rate of 96 gph SBW).

Consumable chemicals will be stored on-site in quantities that an adequate inventory (typically 7 day supply) will be available to maintain constant operation. Similarly, the packaging facility has been designed with enough storage capacity ( 3 storage bins - each with a 2 day accumulation capacity) so that it will not constrain operations. Redundant HEPAs and product-sintered metal filtering systems are currently being recommended. Finally, no change out of refractory from the thermal oxidizer or carbon from the granulated activated carbon (GAC) bed is anticipated during operation.

The spent scrub solution will require periodic treatment. However, the blow down hold tank has been sized to accumulate spent scrub from an entire tank campaign; therefore, treatment can be undertaken during scheduled shutdowns.

\section{A-4. PROCESS FLOW DIAGRAMS}

Sets of PFDs have been prepared based on the bench-scale tests of TTT and TWR. Single diagrams appear for SBW retrieval (PFD-1) and product packaging (PFD-4) since these activities are independent of the reforming process. Although the TTT and TWR use many of the same unit operations and associated equipment, there are enough differences that separate flow diagrams are warranted. The TTT-based process appears on PFD-2A and -3A and the TWR process appears on PFD-2B and $-3 \mathrm{~B}$. The PFDs shown on the following pages thus include:

T-1 Steam Reforming Title Set and Legend

PFD-1 Waste Feed Sources for Steam Reformer

PFD-2A Sodium-Bearing Waste Feed Preparation and Steam Reformer (TTT) 
PFD-3A Steam Reformer (TTT) Off-Gas and Scrub Treatment Systems

PFD-2B Sodium-Bearing Waste Feed Preparation and Steam Reformer (TWR)

PFD-3B Steam Reformer (TWR) Off-gas and Scrub Treatment Systems

PFD-4 Steam Reformer Product Waste Packaging 


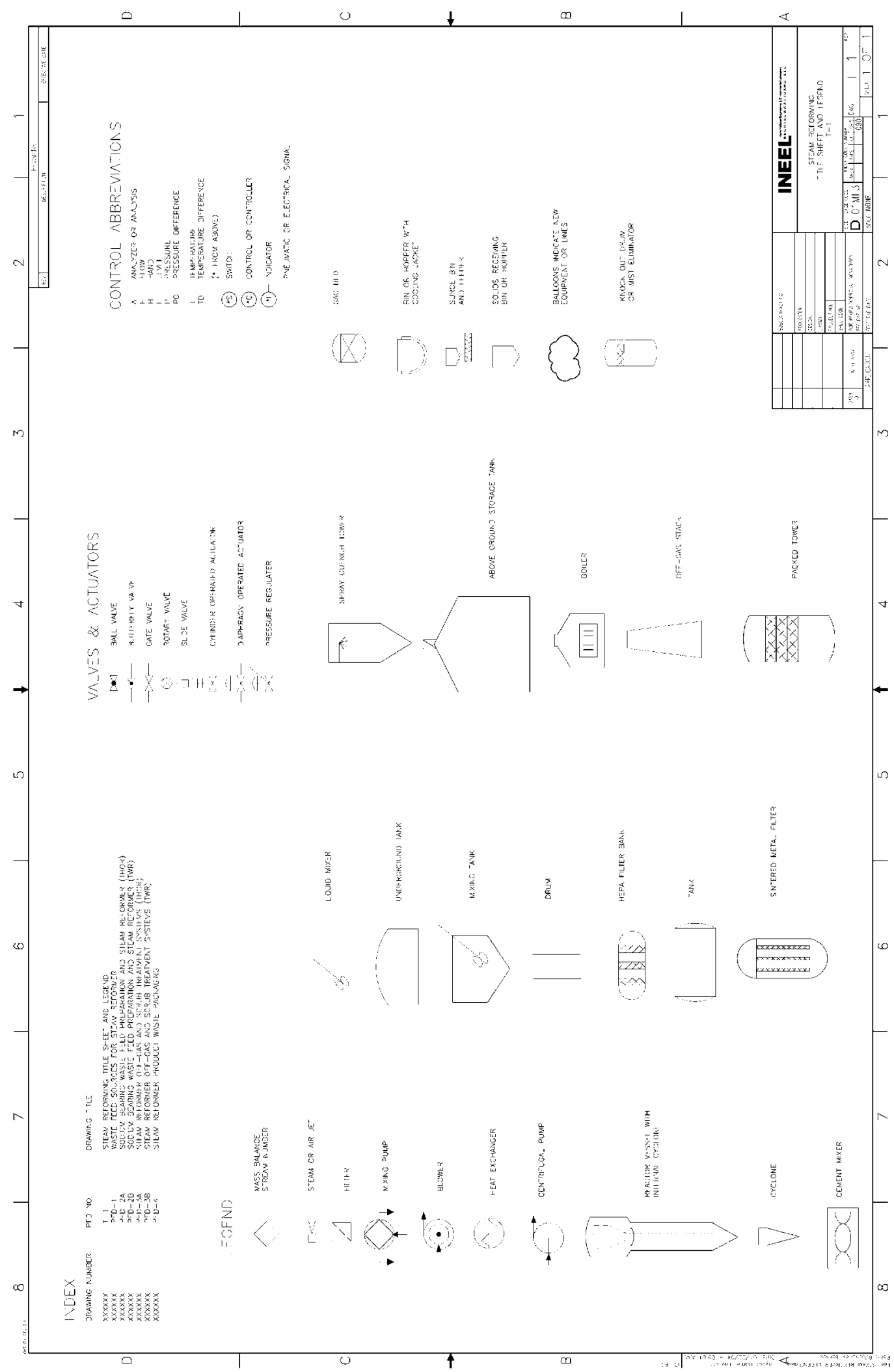




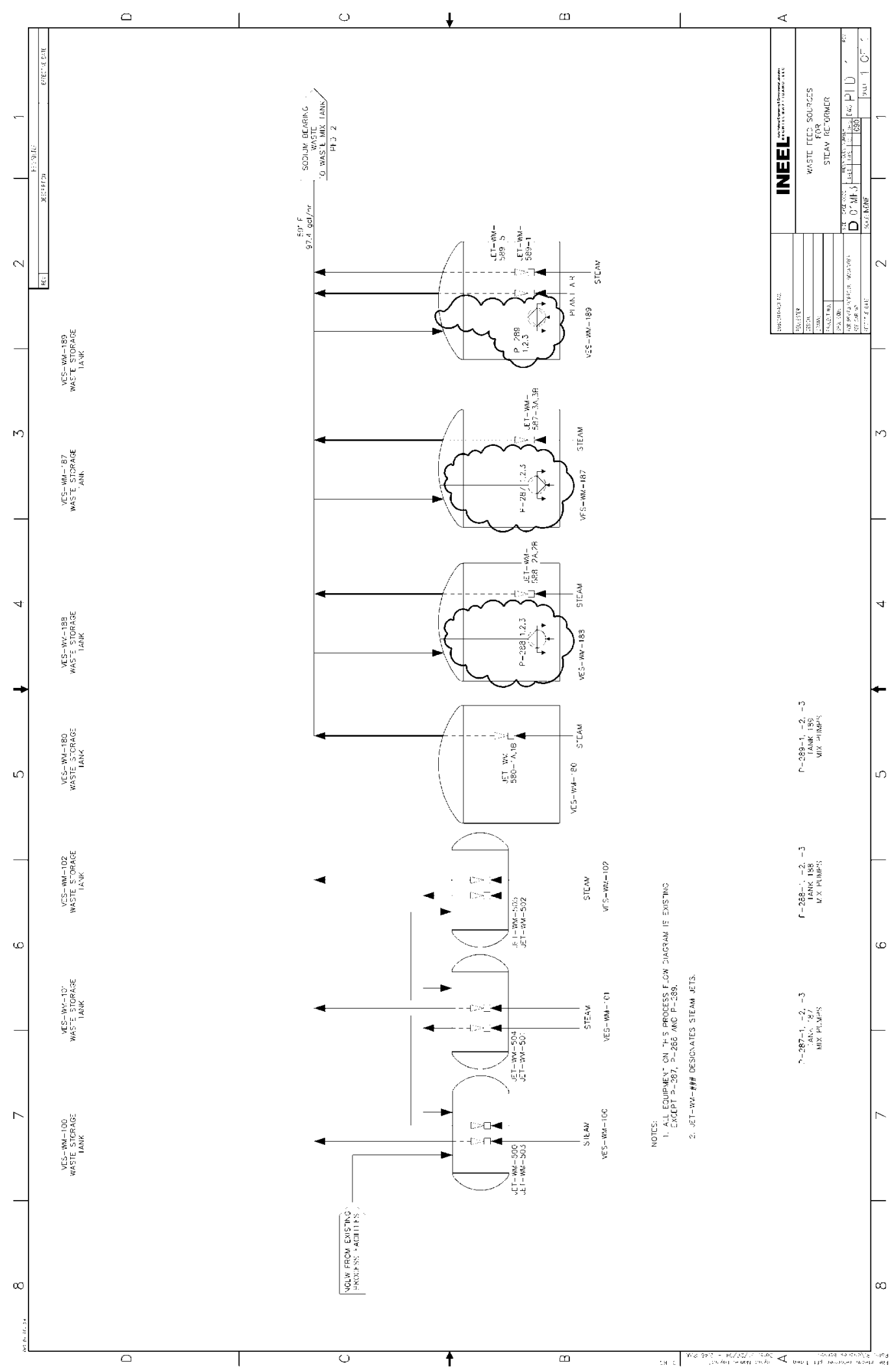




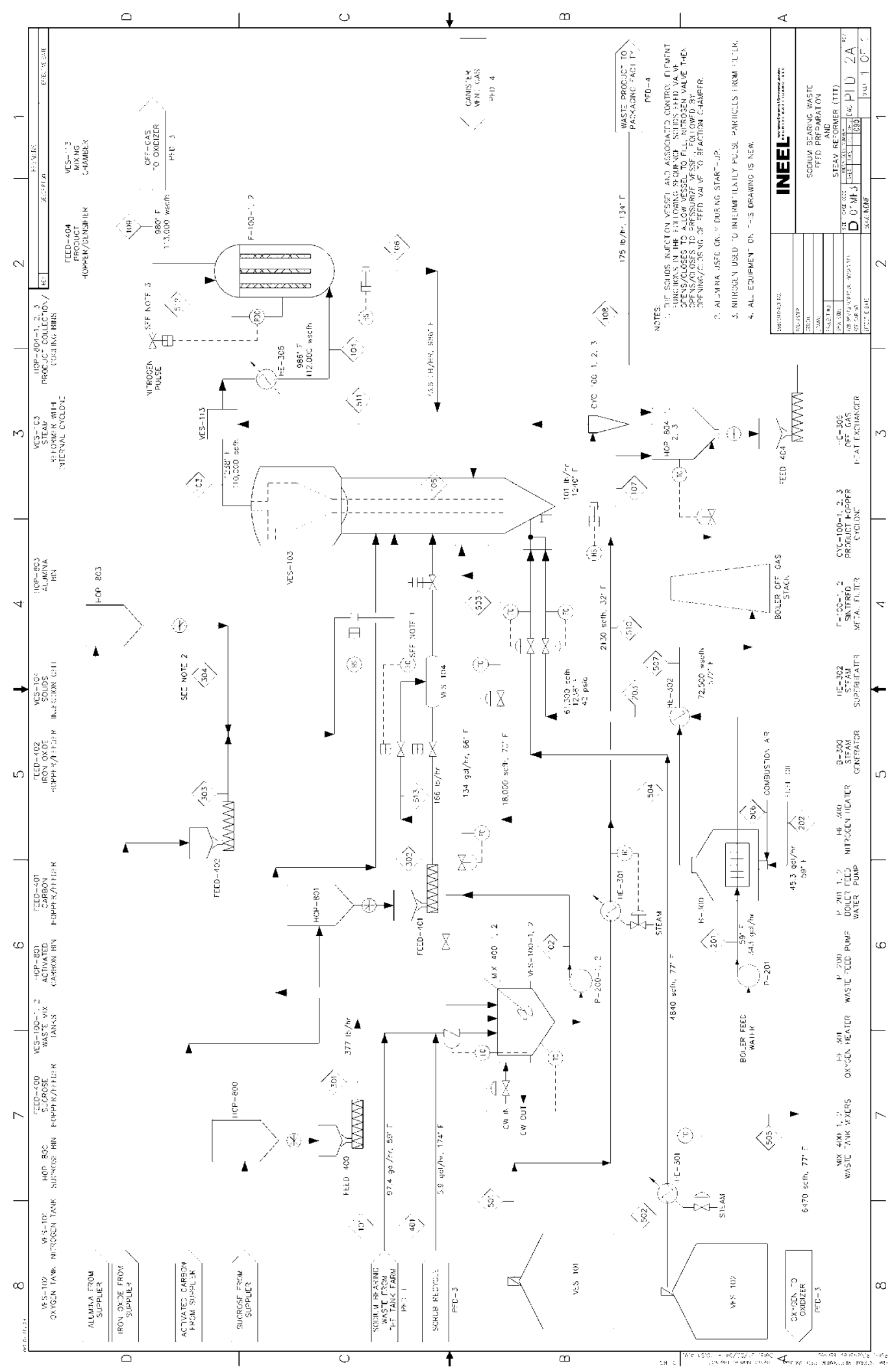




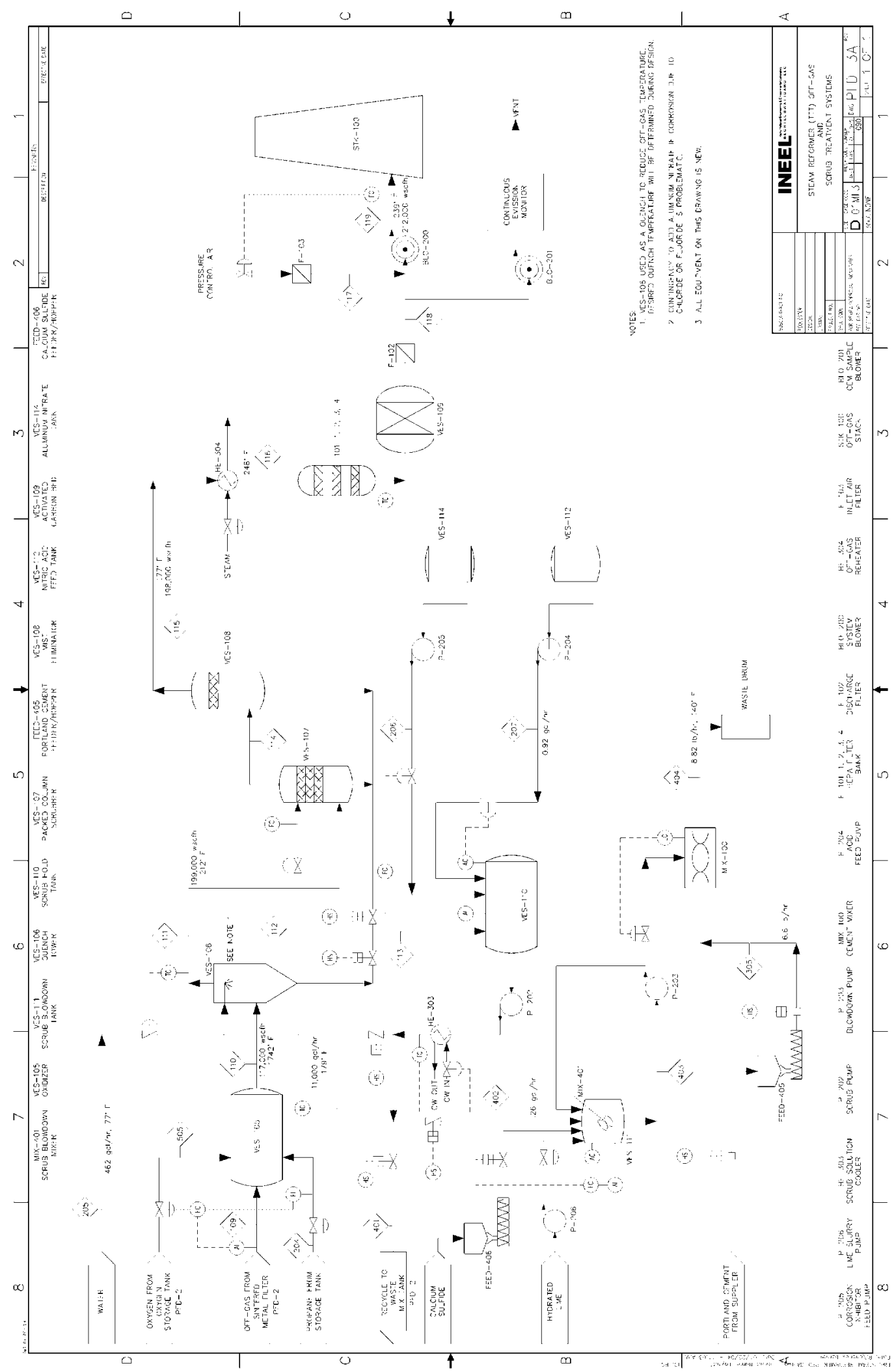




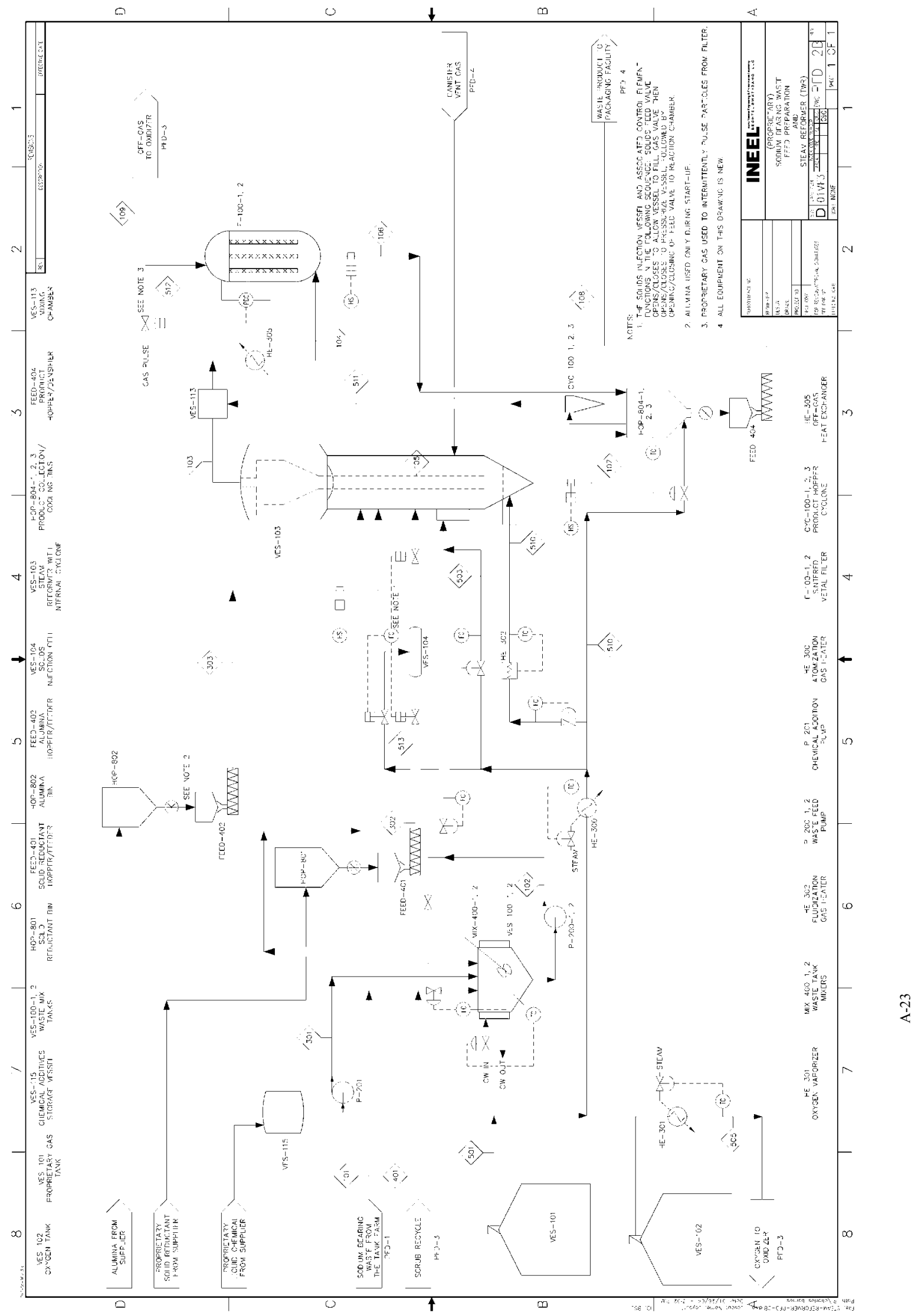




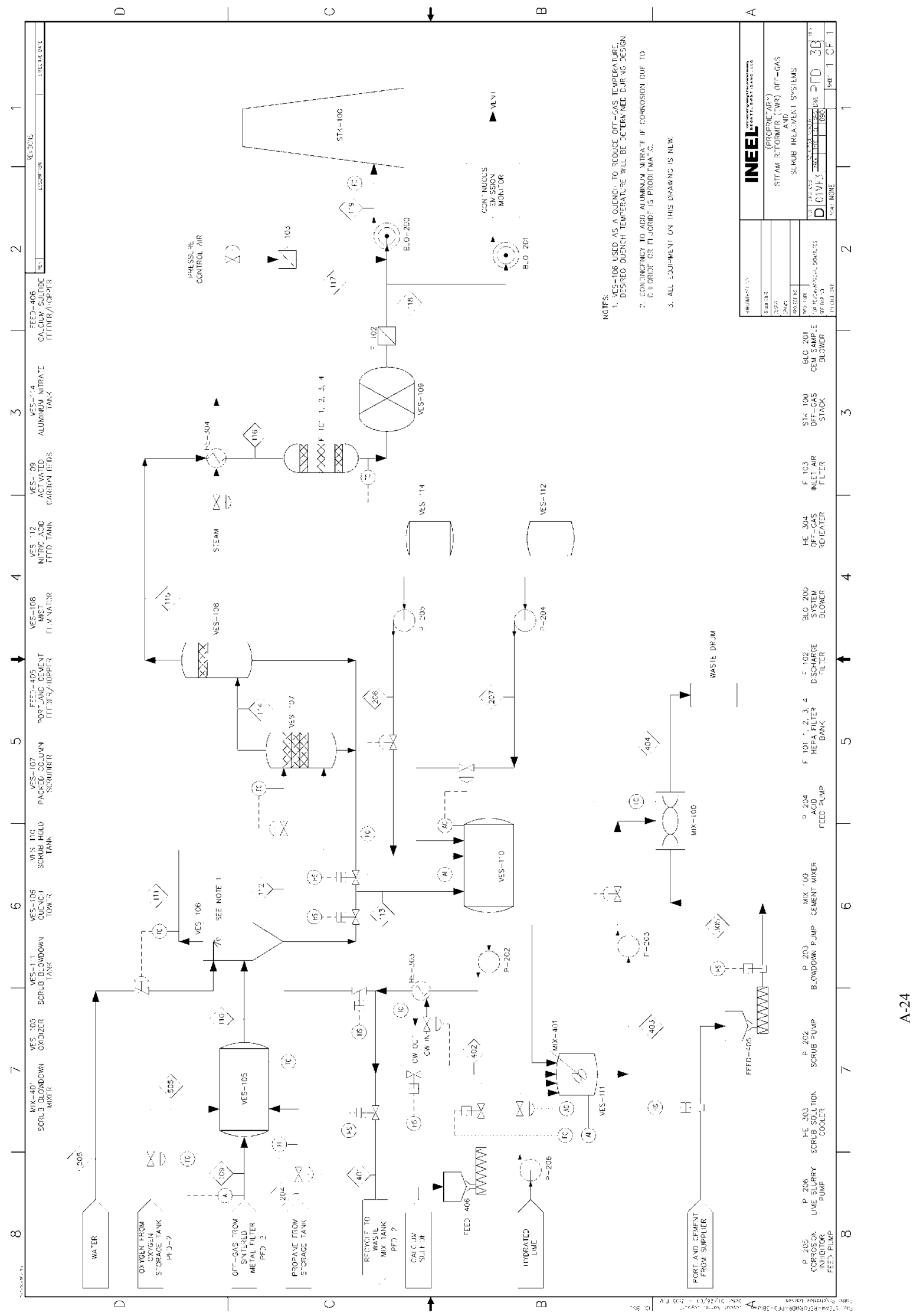




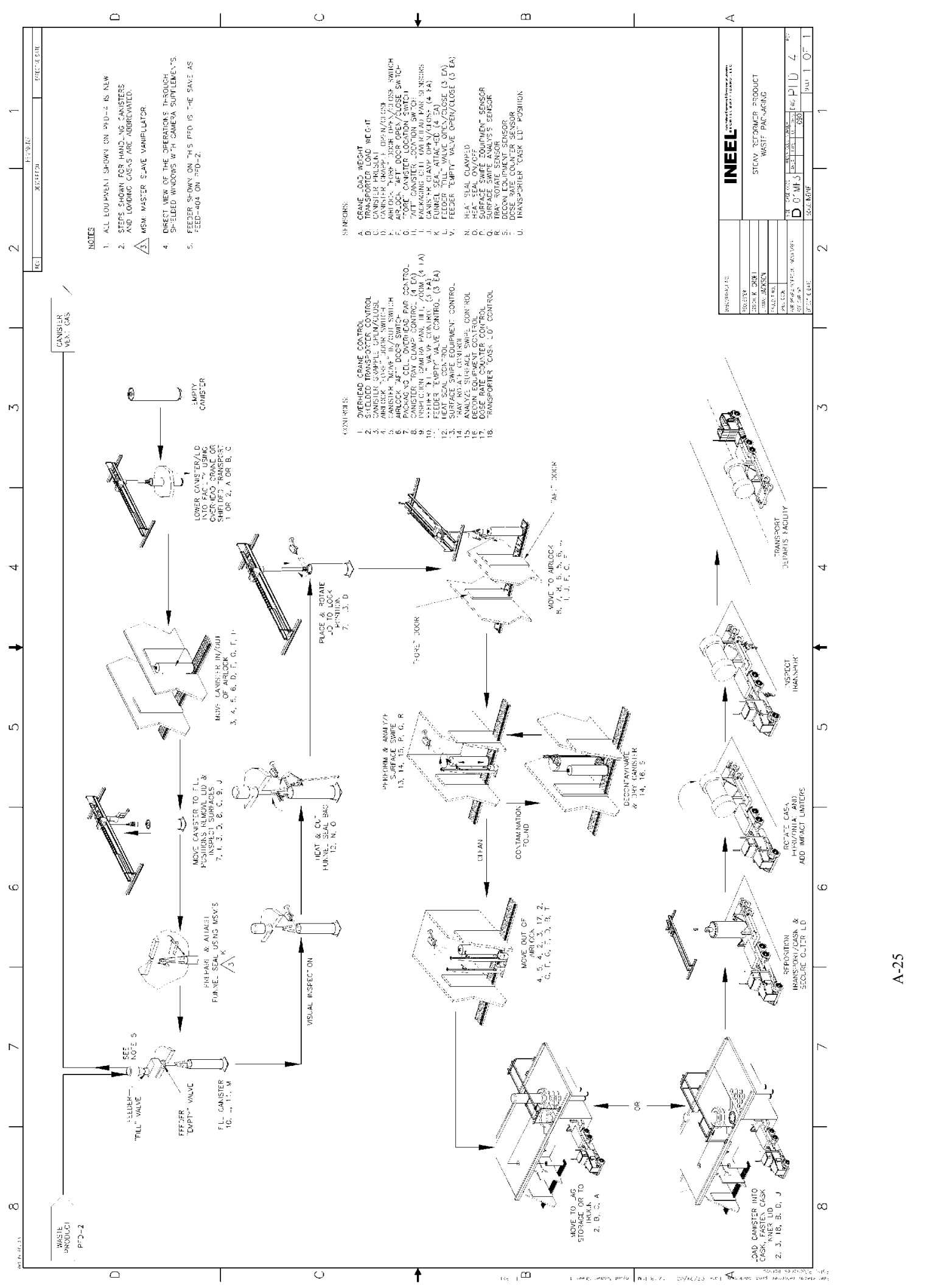




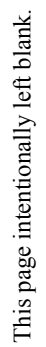




\section{A-5. PROCESS BASIS}

\section{A-5.1 Requirements}

A draft Technical and Functional Requirements (TFRs) document has been prepared for the steam reforming option (Beitel 2003). The requirements pertinent to the process design are restated here; other requirements, justifications, and the bases for requirements are given in the TFRs document. That document should be consulted for additional details.

\section{A-5.1.1 System Requirements}

A-5.1.1.1 Retrieve Waste. The system shall have the capability to retrieve SBW from WM-180, WM-187, WM-188, and WM-189 and NGLW from WM-100, WM-101 and WM-102.

A-5.1.1.2 Prepare Feed for Treatment. The system shall provide for the addition and mixing additives needed to support production of acceptable waste products and maintaining a uniform suspension of SBW solids in the liquids feed.

A-5.1.1.3 Treat Waste. The top-level requirements for waste treatment are given below.

- Volume: The facility shall be capable of treating at least 1.2 million gallons of waste.

- $\quad$ Throughput: The facility shall be capable of processing all SBW (including solids, liquid and designated NGLW) within 3 years. This 3 years includes 6-months of hot start-up and 2.5 years of SBW/NGLW treatment.

- Location: The waste shall be treated and packaged at the INEEL, within the Idaho Nuclear Technology and Engineering Center (INTEC) security enclosure.

- Reformer product: The solid waste product from the reformer shall comply with the remotehandled transuranic waste acceptance criteria (WAC) for WIPP.

1. The activity and mass of each of the following radionuclides shall be established on a payload container Basis: Am-241, Pu-238, Pu-239, Pu-240, Pu-242, U-233, U-234, U238, Sr-90, and Cs-137. If these radionuclides do not account for at least $95 \%$ of the total waste product radioactivity, the mass and activity of additional radionuclides shall be determined until $95 \%$ of the waste product activity is accounted for.

2. Any RH-TRU waste product payload container shall have a dose rate at the surface of the container greater than $200 \mathrm{mrem} / \mathrm{h}$ and less than $1000 \mathrm{rem} / \mathrm{h}$.

3. The ${ }^{239} \mathrm{Pu}$ fissile gram equivalent (FGE) in each payload container, plus its associated uncertainty expressed in terms of one standard deviation, shall be less than $325{ }^{239} \mathrm{Pu}$ FGE.

4. The TRU radionuclide alpha activity concentration, exclusive of the packaging, shall be greater than $100 \mathrm{nCi} / \mathrm{g}$.

5. The ${ }^{239} \mathrm{Pu}$ equivalent curie (PE-Ci) quantities in a payload container shall not exceed 80 PE-Ci for direct loaded RH-TRU waste canisters and shall not exceed $240 \mathrm{PE}-\mathrm{Ci}$ for RH-TRU canisters loaded with three 30 gallon or 55 gallon drums. 
6. The loaded payload container shall contain no detectable liquid.

7. The hydrogen gas generation rate within each payload container shall not exceed the limit specified in the applicable content code.

8. PCB concentrations in wastes destined for WIPP shall not exceed $50 \mathrm{ppm}$.

9. Concentrations of flammable volatile organic compounds (VOCs) in the payload container headspace shall not exceed $500 \mathrm{ppm}$. If calculations cannot show that the VOC concentration in the headspace is less than $500 \mathrm{ppm}$ if all potentially flammable VOCs vaporized into the headspace, sampling of the containers shall be required.

10. The RH-TRU waste shall be placed in a WIPP approved RH-TRU waste canister.

11. The gross weight of a direct loaded RH-TRU canister shall not exceed $5250 \mathrm{lb}$.

12. Surface contamination on loaded RH-TRU payload containers, payload assemblies, and packages shall not exceed $20 \mathrm{dpm} / 100 \mathrm{~cm}^{2}$ alpha and $200 \mathrm{dpm} / 100 \mathrm{~cm}^{2}$ betagamma.

- Off-gas emissions: The off-gas must be treated to meet MACT emission standards and Prevention of Significant Deterioration (for $\mathrm{NO}_{\mathrm{x}}$ emission) levels as shown in Table A-1. Monitoring to demonstrate compliance with the MACT standards shall be performed. $\mathrm{NO}_{2}$ must be controlled to prevent a visible plume when the off-gas exits the facility stack. Radionuclides in the stack release must also meet provisions of 40 CFR 61 Subpart H, "National Emission Standards for Emissions of Radionuclides other than Radon from DOE Facilities."

- Grouted scrub product: The grouted scrub waste product shall comply with the WAC of its disposal site.

- Other secondary wastes: All secondary wastes shall have an acceptable disposal option. Liquid secondary wastes will be either combined with the feed and incorporated into the primary waste product, or stabilized and disposed of as a solid waste.

- Package waste product: The treated waste shall be transferred to a remotely operated packaging station. The waste product packaging station shall have adequate bulk waste product storage capacity to accept the maximum steam reformer product output in the event of a loadout problem to avoid shutting down the steam reformer. All transuranic wastes will be sent to WIPP for disposal. Facilities will be provided to transfer certified payload containers to WIPP-supplied transport vehicles. This project will be responsible to load the waste into approved transport casks and prepare those casks for shipment. WIPP will be responsible for providing the casks, trucks, drivers, and receipt of the waste at WIPP in Carlsbad, Mew Mexico.

1. RH-TRU waste shall be shipped in a RH-TRU 72-B cask.

2. The maximum number of payload containers in a RH-TRU 72-B cask is one.

3. The maximum weight of a loaded $72-\mathrm{B}$ cask is 45,000 pounds. 
4. Sufficient lag storage of RH-TRU waste shall be provided in order to meet WIPP requirements for waste qualification and shipping/receiving rates.

5. A cask loading station compatible with loading WIPP supplied RH-TRU 72-B casks delivered on WIPP supplied transport vehicles shall be provided. The facility shall provide the capability to receive RH-TRU 72-B cask transporters in an enclosed area, raise the cask for loading, load the waste payload assemblies into RH-TRU 72-B cask, and close and lower the RH-TRU 72-B cask onto the transporter.

6. One of the cask loading facilities shall have the capability to wash road grime from the transporters and shipping casks prior to moving into the loading area.

Table A-1. Off-gas emission and rate limits.

\begin{tabular}{|c|c|c|}
\hline Hazardous Air Pollutant & $\begin{array}{l}\text { MACT Emission Standard For New Sources } \\
\text { Corrected to } 7 \text { percent oxygen }\end{array}$ & $\begin{array}{l}\text { PSD Emission Rate } \\
\text { (ton/yr) }\end{array}$ \\
\hline Dioxin/furan & $\begin{array}{l}0.20 \mathrm{ng} \mathrm{TEQ} / \mathrm{dscm} \text {; TEQ is the toxicity } \\
\text { equivalent quotient that relates the toxicity of } \\
\text { various dioxin/furan congeners to the toxicity of } \\
2,3,7,8 \text {-TCDD. }\end{array}$ & \\
\hline Mercury & $45 \mu \mathrm{g} / \mathrm{dscm}$ & 0.1 \\
\hline Particulate matter & $34 \mathrm{mg} / \mathrm{dscm}(0.015 \mathrm{gr} / \mathrm{dscf})$ & 25 \\
\hline $\begin{array}{l}\text { Semi volatile metals (cadmium } \\
\text { and lead) }\end{array}$ & $120 \mu \mathrm{g} / \mathrm{dscm}$ & $0.6(\mathrm{~Pb})$ \\
\hline $\begin{array}{l}\text { Low volatile metals (arsenic, } \\
\text { beryllium and chromium) }\end{array}$ & $97 \mu \mathrm{g} / \mathrm{dscm}$ & $0.0004(\mathrm{Be})$ \\
\hline $\begin{array}{l}\text { Hydrochloric acid and chlorine } \\
\text { gas }\end{array}$ & $21 \mathrm{ppmv}$ & \\
\hline Hydrocarbons or $\mathrm{CO}$ & $\begin{array}{l}10 \text { ppmv reported as propane }- \text { facilities that elect } \\
\text { to continuously comply with the carbon } \\
\text { monoxide standard must also demonstrate } \\
\text { compliance with the hydrocarbon standard during } \\
\text { comprehensive performance testing }\end{array}$ & 100 \\
\hline DRE & $\begin{array}{l}99.99 \% \text { for each principal organic hazardous } \\
\text { constituent (POHC) designated }\end{array}$ & \\
\hline $\mathrm{NO}_{\mathrm{x}}$ & Not Applicable & 40 \\
\hline Fluorides & Not Applicable & 3 \\
\hline
\end{tabular}

Notes: $\mu \mathrm{g}=$ microgram; DRE = destruction removal efficiency; ppmv = parts per million by volume; $\mathrm{ng}=\mathrm{nanogram}$; dscm $=\mathrm{dry}$ standard cubic meter; dscf $=$ dry standard cubic foot; gr = grains.

A-5.1.1.4 Close Facility. The following requirements apply to facility closure:

- All connections between the steam reformer and the tank farm, and any reliance on the tank farm, shall be terminated prior to December 31, 2012. 
- Prior to December 31, 2012, the steam reformer and the off-gas treatment subsystem shall be placed in safe shutdown and have all hazardous waste and hazardous waste residues removed and prepared for acceptable disposal.

- The Packaging, Certification, and Transfer subsystems shall be placed in standby following treating all SBW and NGLW wastes.

\section{A-5.1.2 Systems, subsystem, and major components}

The steam reforming treatment facility shall utilize major support systems and utilities that are available at the INEEL. These include:

- Analytical services.

- Fire protection. This includes a manned fire station, central alarm system, and fire water lines in the vicinity of the waste treatment facility; it does not include fire protection within the physical structures designed and built or used by this project.

- Electrical power. Both normal and standby electrical power are available for use near the INTEC Tank Farm from the INTEC electrical distribution system.

- Steam

- Telephone

- Optical cable

- Security

- Medical

- Radiation records

- Central Facilities maintenance

- Document control

- Configuration management

- Office buildings. The project shall provide adequate office space only for any operations staff, engineers, technicians, supervisors, and managers whose presence is required in the facility full-time during operations, plus limited turn-around office space.

- Roads

- Bus transportation.

\section{A-5.1.3 Boundaries and interfaces}

This system may be constructed using some existing structures, systems, and components within INTEC. When completed and operable it will reside wholly within the INTEC security fence. 
A-5.1.3.1 Liquid Waste Retrieval Feed System. The liquid waste retrieval system interfaces with Tank Farm Operations. All operations within the tank farm will be the responsibility of Tank Farm Operations. Prior to operation of the SBW Treatment Facility, all SBW shall be contained in Tanks WM180, WM-187, WM-188, and WM-189, and NGLW in WM-100, WM-101 and WM-102. Any modifications necessary to retrieve the SBW liquids and solids from these tanks will be the responsibility of this project.

A-5.1.3.2 Utility Interfaces. Interfaces include the following:

- Electric power will be obtained from existing sources at INTEC. The project shall provide the necessary equipment for distribution of power within the facility. The use of existing standby, emergency, or uninterruptible power supplies at INTEC shall be evaluated as the first choice during conceptual design.

- The project shall tie in to the existing INTEC steam distribution piping at an appropriate location adjacent to the facility. If supplemental steam is required, electrical steam generation is preferred to avoid air permitting issues related to direct fired steam boilers.

- The project shall tie in to the existing INTEC water systems piping (de-mineralized, deionized, distilled, fire water, etc.) at an appropriate location adjacent to the facility.

A-5.1.3.3 Tank Farm Closure Project. The system shall interface with the Tank Farm Closure Project. The Tank Farm Closure Project will sequentially remove all liquids and waste solids from the eleven 300,000-gallon Tank Farm tanks WM-180 through 190. The Tank Farm Closure Project will also clean the tanks to performance parameters, grout the tanks, piping and vaults, and close the tanks by December 2012 in accordance with the INTEC Tank Farm Facility (TFF) Management Plan. The SBW Treatment project retrieves the wastes from tanks with the support of the TFC Project and treats it in the Direct Evaporation Facility.

A-5.1.3.4 Tank Farm Soils Project. The system shall interface with the Tank Farm Soils Project, which is responsible to remediate, as required, and close the Tank Farm under the Comprehensive Environmental Response, Compensation and Liability Act (CERCLA) program. The Tank Closure Project shall coordinate all work scope with the INTEC CERCLA Environmental Restoration Program (WAG 3) to ensure no conflicts.

A-5.1.3.5 SNF, NSNFP, and Calcine Disposition. The Steam Reformer SBW Treatment Project shall interface with the Spent Nuclear Fuel (SNF), National Spent Nuclear Fuel Program (NSNFP), and Calcine Disposition Project to ensure that waste product handling systems either interface with, or do not interfere with, calcine handling systems planned or required by the SNF, NSNFP, \& Calcine Disposition Project.

\section{A-5.2 Assumptions}

Process designs considered for the SBW treatments have been based on the following major assumptions. Additional assumptions used in the mass balance are identified in Section A-5.4.

- The SBW, both liquids and solids, and NGLW to be treated are not HLW.

- The reformer waste can be qualified for disposal at the WIPP. 
- The existing INTEC evaporators (Process Equipment Waste Evaporator and Evaporator Tank System) and the Liquid Effluent Treatment and Disposal (LET\&D) acid fractionator will be available for use if needed to process decontamination solutions and other dilute aqueous wastes from the steam reformer process.

- The grouted scrub waste can be disposed of at a mixed low-level waste disposal site such as Hanford or Envirocare provided that the waste meets the site's WAC.

- Toxic Substance Control Act regulations do not apply.

- MACT or equivalent emission standards will apply.

- The facility will not require Nuclear Regulatory Commission (NRC) licensing.

\section{A-5.3 Process Variations}

Numerous variations of the steam reforming process or unit operations within the process are possible, including processes with different:

- Types or modes of operation of the reformer,

- Feed and reformer additives,

- Off-gas treatment unit operations

- Methods of processing secondary waste, including any off-gas scrub wastes

- Methods of collecting, handling and packaging the reformer product.

Both the TTT and TWR processes use fluidized bed reformers. Other types of reformers could be used, such as a moving bed or a gas-phase reactor. The TTT and TWR processes use different fluidization gases, operate at different temperatures, and utilize different methods of achieving the reformer operating temperature. Other differences in operation of a given reformer are possible as well.

Much of the testing of the TTT and TWR processes with SBW simulants has concerned determining and optimizing combinations of feed ingredients and reformer process conditions suitable for treatment of SBW. This optimization is not complete and would need to be done for any steam reforming process. TTT has alternate feed formulations wherein various mineral-forming materials are used in addition to the reductants, with the result that the product characteristics are modified. Tests of steam reforming at Hanford used various reductants such as methane, hydrogen, and ammonia, in addition to sucrose, for reducing nitrates in a fluidized bed reactor configuration (Stegen 1996).

Pretreatment or multiple stage reactors form another set of treatment variations of the steam reforming process. For example, D. Siemer has proposed evaporation of the SBW with an organic such as glycolic acid, followed by a fluidized bed reactor (Siemer 2003). In Siemer's proposed process, the bed is fluidized in part with recycled off-gas, which is primarily steam, but also contains $\mathrm{CO}_{2}$.

The off-gas treatment system shown on PFDs-3A and -3B is largely based on that used at the NWCF - a wet scrub solution is used to remove a large portion of the off-gas mercury. A dry off-gas system may be possible where only enough water is sprayed into the off-gas to reduce its temperature sufficiently to allow downstream treatment operation. Thus all introduced water is vaporized in the off- 
gas stream. To effect mercury removal, the gas would then be passed directly to GAC beds. The attraction of this system is that no spent scrub solution would be generated and therefore, a waste stream would be eliminated and the treatment system simplified. However, there have been no demonstrations or published information that indicates that the GAC system can achieve the required mercury removal to meet the MACT emission limits in this manner (Soelberg 2003).

Another variation in off-gas treatment could be the location and type of oxidation unit. Placing the oxidation unit near the reformer eliminates or reduces the need to reheat the off-gas and favors a thermal oxidizer. If placed downstream of reformer off-gas cleanup, the oxidizer is in a much lower radioactive environment, processes a cleaner stream, and could possibly operate at a lower temperature and with a catalyst.

Testing to date of steam reforming for SBW has focused mostly on the reformer and off-gas treatment sections of the process. Once these are well defined, the requirements for treating secondary wastes can be better determined. Depending on how mercury partitions in the off-gas scrub operations, it may be possible to recycle scrub blow down to the reformer and eliminate the scrub waste. Or alternatively mercury could be removed from the blow down by one of several methods and the scrub recycled to the scrubber with a blow down to the reformer. Whether this is economical depends in part on the radionuclides that get carried into the scrub, which ultimately affect the cost of disposal of a grouted scrub and the cost of additional scrub treatment steps.

Because all product handling and packaging operations are remote, they contribute significantly to the cost and complexity of the facility. The low density of the product suggests an economic benefit to densifying it to reduce the total volume of waste. Various methods of densifying the product are possible, but none have been tested to date. Likewise different concepts for packaging the waste have not yet been adequately defined and evaluated to determine an optimum system.

\section{A-5.4 Basis for Process Flow Diagrams}

The following documents and data formed the basis upon which the PFDs were generated:

- The Technical and Functional Requirements, which provided the overarching goals and identified the high level requirements of the design (Beitel 2003).

- The bench-scale demonstration tests of TTT and TWR, which identified the major unit operations, feed constituents, and process flows.

- Process experience and data from calcine operations at NWCF, which in particular, provided information on the off-gas scrubbing system, and design studies for an upgraded NWCF MACT-compliant SBW treatment facility.

- Results of process simulations using ASPEN Plus.

\section{A-5.4.1 Waste Tanks (PFD-1)}

PFD-1 shows the SBW feed tanks VES-WM-180, VES-WM-187, VES-WM-188 and VES-WM189, and the NGLW tanks VES-WM-100, VES-WM-101 and VES-WM-102. These tanks are all existing tanks and currently in use. New mixing pumps would be installed in VES-WM-187, VES-WM-188 and VES-WM-189 to distribute solids evenly between these three tanks. The basis for the number of pumps (3 per tank) and the blending scenario is documented in a tank heels processing report (Wood 2002). 
A follow-on study evaluated different mixing pumps and recommended submersible pumps over long shaft pump technology (Morrell 2003). Both studies recommend mockup testing of the mixing pumps. Mockup testing is needed to confirm and further define the basis for the mixing pumps and to verify performance. Based on present estimates of total tank solids, it is anticipated that new pumps will be required in three tanks. If future tank samples indicate the total solids are significantly less than current estimates, pumps would only be installed in two tanks.

\section{A-5.4.2 Feed Preparation and Steam Reforming (TTT) (PFD-2A)}

PFD-2A shows the feeds to the reformer, along with the reformer itself and the sintered metal filter on the reformer off-gas. The basis for this section of the process is technology developed by TTT, and demonstration tests in 2003 applying this technology to the treatment of SBW (Marshall 2003b, Soelberg 2004a). One unit operation shown on PFD-2A has not been tested relative to SBW waste treatment - the product densifier. FY 2003 tests, performed on SBW surrogate, showed that the product from the cyclone, contributing $53 \mathrm{wt} \%$ to the total product, had a loose density of $0.24 \mathrm{~g} / \mathrm{cm}^{3}$ and the product from the filter, contributing $20 \mathrm{wt} \%$ of the total, had a loose density of $0.14 \mathrm{~g} / \mathrm{cm}^{3}$ (Marshall 2003b). Tamping increased the density for both of these products to $0.39 \mathrm{~g} / \mathrm{cm}^{3}$ (Marshall 2003b). Without densification the number of product canisters would increase by a factor greater than 4 .

\section{A-5.4.3 Steam Reforming (TTT) Off-Gas and Scrub Treatment (PFD-3A)}

FY 2003 SBW steam reforming demonstration tests (Marshall 2003b, Soelberg, 2004a) showed reformer off-gas contained about 1-2\% CO. A thermal oxidizer is shown on PFD-3A to reduce the CO concentration, as well as methane and other hydrocarbons, to acceptable levels for release to the atmosphere. The FY 2004 SBW steam reforming demonstration tests included a thermal oxidizer as shown on the PFD. Oxygen rather than air is added to the oxidizer unit to minimize the total volumetric rate of off-gas.

The quench tower and subsequent off-gas treatment unit operations are largely based on the NWCF off-gas treatment system. However a packed bed scrubber is shown on the PFD rather than the venturi scrubber of the NWCF system, and a GAC bed is included for additional mercury removal. As discussed in Section 2.2.6, the venturi scrubber on the NWCF system is primarily a particulate removal device, whereas the steam reforming off-gas is expected to have a low particulate loading. A packed bed scrubber is expected to be more efficient removing the acid gas species expected in the steam reformer off-gas. The GAC bed is required to remove mercury to below MACT emission limits. The design of the GAC bed is based on the design of a GAC bed for an upgraded NWCF MACT-compliant SBW treatment facility (Soelberg 2003).

The scrub blow down treatment equipment shown on PFD-3A is based in part on data from the FY 2003 and FY 2004 demonstration tests (Marshall 2003b, Marshall 2004) and in part on the design of similar equipment for an upgraded NWCF MACT-compliant SBW treatment facility (Barnes 2003a).

\section{A-5.4.4 Steam Reformer Product Waste Packaging (PFD-4)}

PFD-4 shows steps to package reformer product into waste canisters, load canisters into casks, and casks onto the transport truck. These steps are a summary of a preliminary concept developed for packaging calcine (Clark 2003). 


\section{A-5.4.5 Basis for TWR PFDs (PFD-2B and PFD-3B)}

The TWR PFDs are primarily based on demonstration tests performed in FY 2003 and FY 2004. These tests sought to optimize feed additives and reformer operating parameters. The TWR process has a liquid organic feed rather than the solid used in the TTT process, so it requires a feed additive tank rather than a hopper. The TWR process has at most one solid additive, polyethylene, rather than the two solids (reductant and possibly catalyst) added in the TTT process, so it has one less solid hopper than TTT. Although polypropylene was not used in the latest demonstration test, the contingency to add it has been maintained in the flow diagram.

The TWR flowsheet uses carbon dioxide gas as the primary fluid as it provides fluidization of the bed, atomization of the feed, pneumatic fluid for the injection cell, and pulsing gas for the sintered metal filter. As for the TTT flowsheet, oxygen and propane are used as feeds to the off-gas oxidizing unit.

Carbon dioxide, oxygen and propane are available as low temperature liquids stored under pressure at near their boiling points. As liquids they are transported in cryogenic tank trailers or railroad cars. Onsite storage tanks are designed to minimize boil-off of the liquid and typically consist of a storage contained within another tank. The annular space between tanks is evacuated and filled with an insulating material. Properly designed, the boil-off rates can be very low.

An alternative to receiving liquefied carbon dioxide from a vendor is to produce it on-site. Carbon dioxide can be derived from directly combusting a fuel such as from the INTEC boiler off-gas; however, the most economical way to produce carbon dioxide is usually to recover it as a byproduct from other companies' manufacturing processes or from natural wells. An engineering study to evaluate on-site production versus purchasing/storing carbon dioxide should be performed to determine the most economical $\mathrm{CO}_{2}$ source.

\section{A-5.5 Basis for Mass Balances}

Mass balances were developed for the Steam Reforming alternative to provide a basis for sizing equipment, determining feed chemical requirements, determining utility requirements, estimating emissions and determining waste volumes, compositions and properties. The sections below document the basis for the mass balance. The basis is capsulated in succinct statements, called "design basis elements" (DBEs), shown in italics in the following paragraphs. The DBEs are amplified with background information, references, explanation, and, in some cases, a discussion of uncertainties. Sections A-5.5.1 through A-5.5.10 apply to the TTT process mass balances and to elements that are common to both the TTT and TWR processes. Unique aspects of the TWR process are discussed in Section 5.5.11

Results from the Phase 2 SBW treatment demonstration tests of the TTT and TWR processes (Soelberg, 2004a; Soelberg, 2004b) were used extensively in defining input to the mass balance models. The Phase 2 TTT tests included two major series - those producing a carbonate product and those producing a mineralized product. For SBW treatment, the carbonate product is assumed and hence results from only the carbonate tests were used as a basis for the TTT mass balances in this Appendix.

\section{A-5.5.1 Feeds}

Feeds to the Steam Reforming process include NGLW and SBW from the INTEC TFF.

A-5.5.1.1 Tank Farm Waste Feeds. According to Tank Farm management plans (Barnes 2002), waste presently in the TFF tanks will be consolidated into four tanks, WM-180, WM-187, WM-188 and WM-189 by the end of 2005 . Tanks WM-180, WM-188 and WM-189 will contain liquid SBW with relatively small quantities of undissolved solids, while WM-187 will be used as a collection tank for 
solids and dilute liquid wastes. Waste generated after 2005 will be collected in WM-100, WM-101 and WM-102. Shortly before treatment, transfers will be made between tanks to distribute solids that have been collected in WM-187 between the three tanks WM-187, WM-188 and WM-189.

\section{DBE \#1: Solids that have been collected in Tank WM-187 will be distributed between Tanks WM- 188, WM-189 and WM-187 by waste transfers between these tanks prior to feeding to the treatment process.}

Pumps will be installed in these three tanks to homogeneously mix the solids with the tank liquid. Approximately equal amounts of waste from WM-188 and WM-189 will first be transferred to Tank WM-187, and then similar volumes of the mixed solids/liquid slurry in WM-187 will be returned to Tanks WM-188 and WM-189 to restore these tanks to their full capacity. The referenced reports describe the tank-blending scenario in more detail (Wood 2002, Barnes 2003b). Mass balances are shown in Section 2.6 for four feed cases. The feed cases correspond to present estimates of waste quantities and compositions that would be transferred from Tanks WM-180, WM-187, WM-188 and WM-189 after waste in these tanks is blended. The WM-187 mass balance case represents a blend of the waste in WM187, after transfers to Tanks WM-188 and WM-189, with NGLW. Whether the NGLW is added to WM187 or sent directly from WM-100, WM-101 or WM-102 to the SBW Treatment Facility feed tanks is of no consequence to the mass balance.

\section{DBE \#2: $\quad$ Four waste compositions, corresponding to waste in Tanks WM-180, WM-188, WM-189 and a combined residual in WM-187 plus $N G L W$, are assumed to adequately envelope the waste fed to the SBW Treatment Facility for conceptual design purposes.}

Table A-2 shows the variability of feed composition between the tanks for major species. Additional variation in feed composition is possible due to: (1) uncertainties in sample analyses, (2) uncertainties in the amount of waste solids in the feed, (3) uncertainties in solids composition, (4) uncertainties in NGLW quantity and composition, and (5) potential changes in the tank mixing or management scenario. The magnitude of some of these uncertainties is known or can be estimated, and is generally within the range shown in Table A-2. Feed composition uncertainties and expected composition ranges are discussed in more detail in the referenced report (Barnes 2003b).

Table A-2. Feed concentration variations.

\begin{tabular}{lccc}
\hline & Minimum & Maximum & Max/Min \\
\hline $\mathrm{H}+$ & $1.07 \mathrm{E}+00$ & $2.42 \mathrm{E}+00$ & 2.27 \\
$\mathrm{Al}+3$ & $3.34 \mathrm{E}-01$ & $6.07 \mathrm{E}-01$ & 1.82 \\
$\mathrm{~B}+3$ & $1.00 \mathrm{E}-02$ & $1.83 \mathrm{E}-02$ & 1.82 \\
$\mathrm{Ca}+2$ & $3.20 \mathrm{E}-02$ & $6.13 \mathrm{E}-02$ & 1.92 \\
$\mathrm{Cs}+$ & $2.57 \mathrm{E}-05$ & $7.37 \mathrm{E}-05$ & 2.86 \\
$\mathrm{Cl}-$ & $1.62 \mathrm{E}-02$ & $2.86 \mathrm{E}-02$ & 1.76 \\
$\mathrm{Cr}+3$ & $3.36 \mathrm{E}-03$ & $5.14 \mathrm{E}-03$ & 1.53 \\
$\mathrm{~F}-$ & $3.32 \mathrm{E}-02$ & $4.98 \mathrm{E}-02$ & 1.50 \\
$\mathrm{Fe}+3$ & $1.92 \mathrm{E}-02$ & $2.97 \mathrm{E}-02$ & 1.54 \\
$\mathrm{Mg}+2$ & $1.10 \mathrm{E}-02$ & $1.94 \mathrm{E}-02$ & 1.76 \\
$\mathrm{Hg}+2$ & $1.46 \mathrm{E}-03$ & $5.44 \mathrm{E}-03$ & 3.73 \\
$\mathrm{NO}_{3-}-$ & $6.63 \mathrm{E}+00$ & 1.34 \\
$\mathrm{PO}_{4}-3$ & $1.93 \mathrm{E}+00$ & $1.46 \mathrm{E}-01$ & 7.50
\end{tabular}




\begin{tabular}{lccc}
\hline & Minimum & Maximum & Max/Min \\
\hline $\mathrm{K}+$ & $1.76 \mathrm{E}-01$ & $2.84 \mathrm{E}-01$ & 1.61 \\
$\mathrm{Na}+$ & $1.36 \mathrm{E}+00$ & $1.89 \mathrm{E}+00$ & 1.39 \\
$\mathrm{SO}_{4}-2$ & $4.04 \mathrm{E}-02$ & $9.08 \mathrm{E}-02$ & 2.25 \\
$\mathrm{Zr}+4$ & $1.32 \mathrm{E}-03$ & $5.58 \mathrm{E}-02$ & 42.17 \\
\hline
\end{tabular}

A-5.5.1.2 Chemical Feeds. In the TTT process, sucrose is dissolved and blended with the SBW prior to injecting this feed into the reactor. Additional carbon, in the form of activated carbon, is added directly to the reactor. The carbonaceous materials promote reduction of the nitrates to nitrogen, and convert mineral-forming species, such as sodium, aluminum, and potassium, to non-agglomerating solids. It is expected that in the full-scale treatment facility alumina would be added at the beginning of operations and during shutdowns or for off-normal conditions, and function as an inert bed material.

$D B E$ \# 3: Sufficient sucrose is added to the feed to result in a carbon to nitrate mole ratio of 2.7.

The sucrose carbon-to-nitrate mole ratio of 2.7 is based on late 2003 bench-scale testing of the TTT flowsheet, specifically tests 3.3 and 3.4 (Soelberg, 2004). Other tests used sucrose to nitrate ratios of 0 to 4.0. The tests showed that sugar ratios lower than 2.7 are feasible with at least partial compensation with increased carbon; however, the low sugar tests were of comparatively short duration.

\section{$D B E$ \#4: Sufficient carbon is be added to the bed to result in a carbon to nitrate in the feed mole ratio of 2.6.}

The solid carbon addition rate is based on that used in tests 3.3 and 3.4 of Phase 2 testing of the TTT process (Soelberg, 2004).

\section{DBE \#5: Alumina will be used as the starting bed media, with bed media addition assumed to be equal to 0.01 wt fraction of the solid product during operation.}

For the TTT mass process, the starting bed media is alumina. During the SBW steam reforming demonstration tests in January 2003, additional bed was added at a rate of about $0.04 \mathrm{~kg}$ alumina per $\mathrm{kg}$ of product collected during the test (Marshall 2003b). Typically, bed media will only be added at startup and for process upsets, so less bed media will be required for full-scale operation and a value of 0.01 is assumed for the model. Future testing may provide a different value for bed media usage.

\section{DBE \#6: No addition of solid catalyst is needed for the TTT process.}

The first series of tests of SBW treatment using the TTT process utilized a solid catalyst to promote $\mathrm{NO}_{\mathrm{x}}$ destruction (Marshall 2003b). Tests were successful in the second series (Soelberg, 2004) with no catalyst. Based on the results of the second test series, the mass balances assume no addition of solid catalyst.

A-5.5.1.3 Fuels. Two fuels are required in the TTT process. The steam boiler will use fuel oil to generate high temperature steam for the process and the oxidizer will use propane to boost the heating value of the steam reformer off-gas. Addition of propane to the oxidizer will allow operation at a temperature of about $1000 \mathrm{oC}$ to ensure destruction of organics and oxidation of $\mathrm{CO}$ in the reformer offgas stream. 


\section{DBE \#7: Fuel oil will be fed to the steam boiler at 42-45 gph based on a required steam flow rate of 2640-2860 lb/hr.}

The fuel oil consumption was calculated assuming combustion with air in a boiler to produce 30psig steam for fluidizing gas for the steam reformer. It was assumed that a recuperator in the boiler offgas would be used to superheat the steam to the bed temperature of $670^{\circ} \mathrm{C}$.

\section{DBE \#8: Propane is fed to the oxidizer at a rate of 34-35 lb/hr to boost the heating value of the reformer off-gas to obtain an oxidizer temperature of $1000^{\circ} \mathrm{C}$ and ensure $99.9 \%$ destruction of organics and $\mathrm{CO}$ in the off-gas.}

The reformer off-gas has some heating value due to its methane, hydrogen and CO content; however, additional fuel will be needed to boost the oxidizer temperature to $1000^{\circ} \mathrm{C}$. The oxidizer will use propane to boost the heating value of the steam reformer off-gas such that a temperature of $1000^{\circ} \mathrm{C}$ can be reached in the oxidizer. This temperature is recommended for destruction of organic species, while minimizing formation of thermal $\mathrm{NO}_{\mathrm{x}}$. In addition, a residence time of 1 to 2 seconds in the oxidizer is needed to ensure adequate organic destruction. Propane was selected as the multi-stage combustor fuel because of: (1) availability, (2) ease of storage and handling, (3) purity, i.e., lack of sulfur present in alternate fuels, and (4) lack of higher hydrocarbons to ensure that the fuel does not add new organic compounds to the off-gas.

A-5.5.1.4 Oxygen and Nitrogen. Oxygen is used rather than air as feed to the oxidizer to minimize the increase in volume of off-gas and to minimize total mass flow of gas to heat up to the oxidizer temperature of $1000^{\circ} \mathrm{C}$.

Nitrogen is used to atomize the feed in the reformer, and also to purge process lines upstream of the oxidizer as this piping may contain combustible levels of $\mathrm{H}_{2}$ and $\mathrm{CO}$. Nitrogen will also be used for direct cooling of the steam reformer product in the collection hopper.

\section{DBE \#9: Oxygen will be fed to the steam reformer with the fluidizing steam at a rate of about 320- $400 \mathrm{lb} / \mathrm{hr}$.}

Oxygen is fed to the steam reformer with the fluidizing steam to partially oxidize the organic reductant and carbon feeds. This provides the heat to evaporate the water in the feed and provide energy for the endothermic steam reforming reactions. For the bench-scale tests, the oxygen was adjusted to maintain temperature. The oxygen input for the ASPEN model was adjusted to match the off-gas $\mathrm{CO} / \mathrm{CO}_{2}$ ratio of $0.164 \mathrm{~mol} / \mathrm{mol}$ measured during the Phase 2 bench scale tests (Soelberg, 2004). When this was done, the predicted hydrogen concentration in the reformer off-gas was close to the value of about $4 \%$ hydrogen measured during the tests. This value and the carbon input will have to be varied to balance heat loss from the vessel with the desired hydrogen concentration in the reformer off-gas.

\section{DBE \#10: The mass balance assumes a rate of 278-300 scfm of nitrogen for feed atomization in the feed nozzles.}

During the Phase 2 tests, the nozzle atomizing air to feed ratio (NAR) was increased in stages from 500 in early tests to 1100 in later tests. The higher ratios were used in the tests to help maintain fluidization of the bed. For the mass balance, the average of these two values was assumed. It is expected that during production the buildup of bed material can be controlled, although this has not yet been demonstrated. 
A-5.5.1.5 Fluidizing Gas. Steam preheated to $670^{\circ} \mathrm{C}$ is used to fluidize the bed media in the steam reformer. Based on the Phase 2 SBW demonstration tests (Soelberg, 2004), the mass balance assumes a fluidizing gas to SBW mass ratio of 2.75. During the Phase 2 tests, the steam rate was increased in stages because of the growth of bed particles. For production, it is expected this growth can be controlled. The rate used for the mass balance is an average of the initial and final steam rates used in the Phase 2 tests.

\section{$D B E$ \# 11: The mass balance assumes a rate of fluidizing steam to the steam reformer of 2.75 times the mass rate of $S B W$, or $2640-2860 \mathrm{lb} / \mathrm{hr}$.}

\section{A-5.5.2 Stream Factor, Operating Schedule and Feed Rate}

The Technical and Functional Requirements (Beitel, 2003) specifies a 3-year processing time that includes six months of hot start up. A stream factor of 200 days per year is assumed. The average feed rate is thus:

$$
1,062,000 \mathrm{gal} /(2.5 \mathrm{yrs} * 200 \mathrm{~d} / \mathrm{yr} * 24 \mathrm{hr} / \mathrm{day})=88.5 \mathrm{gal} / \mathrm{hr} \text { or } 1.5 \mathrm{gal} / \mathrm{min}
$$

$D B E \# 12: \quad$ The total SBW waste inventory will be processed over 2.5 years, during 200 24-hr operating days per year and an average feed rate of $1.5 \mathrm{gpm}$.

\section{A-5.5.3 Tank Mixing and Feed Transfer from Tank Farm}

In an evaluation of options for processing solids in the calciner, it was recommended to mix the solids in the INTEC TFF tanks (Wood 2002, Morrell 2003). This recommendation is equally valid for steam reforming. The concept of mix pumps in the tanks was taken from an earlier conceptual design for tank heel removal (ICF Kaiser 1995). While mock-up tests are recommended to validate the effectiveness of this mix scheme, the studies by Wood and Morrell included an assessment of mixing pump performance and issues (see Section 3 of Wood).

Transfers of waste to the treatment facility will be made using existing steam jets. The mass balance assumed that the volume of waste increases by $5 \%$ due to steam used in the jets. This increase is based on past transfers of waste to the NWCF.

\section{DBE \#13: Mix pumps installed in TFF tanks are assumed to keep solids uniformly distributed throughout the tank waste. Waste will be transferred to the treatment facility using existing steam jets, diluting the waste by $5 \%$.}

SBW from the TFF will contain solids up to about $50 \mathrm{~g}$ solids per liter. The SBW is received and blended with scrub recycle and feed additive solutions in the feed tanks, and then transferred to the reformer.

\section{DBE \#14: Scrub recycle will be blended with SBW and feed makeup chemical solutions in the feed tanks such that the scrub solution portion of the steam reformer feed is equivalent to $5 \%$ of the $S B W$ volumetric feed rate.}

Solids buildup in the acid scrub is slow due to the efficient capture of non-volatiles by the sintered metal filters (also called candle filters). A small amount of acid scrub is purged to the reformer in order to minimize the about of scrub blow down that needs to be treated for disposal. The acidity in the scrub tank continuously decreases due to dissolution of solids. Fresh $13 \mathrm{M} \mathrm{HNO}_{3}$ is added to maintain an acidity of at least 1 molar to ensure partial dissolution of mercury in the scrub. Scrub solution is recycled 
to the steam reformer at a rate equivalent to $5 \mathrm{vol} \%$ of the SBW feed. A nominal recycle of $5 \mathrm{vol} \%$ of the feed was chosen to purge dissolved solids from the scrub system. This flow rate is small $(\sim 0.05 \mathrm{wt} \%)$ compared to the scrub recirculation flow.

\section{DBE \#15: Air sparging is used to keep solids in feed tanks uniformly suspended in the reformer feed.}

Air sparging has been used effectively at INTEC to keep solids suspended in scrub at the NWCF, and based on this experience, this should be adequate for keeping solids in suspension in the steam reformer scrub tank.

\section{A-5.5.4 Steam Reforming}

A-5.5.4.1 Normal Operating Conditions. All Phase 2 carbonate tests were conducted at a nominal reformer bed temperature of $670^{\circ} \mathrm{C}$, and hence this temperature was assumed for the mass balances.

DBE \# 16: The normal operating temperature of the TTT steam reformer will be $670^{\circ} \mathrm{C}$.

Based on a 2.5-yr operating schedule, an average 200 operating days per year, and 24- hrs/day operation, the required SBW feed rate is $88.5 \mathrm{gph}$. Including scrub recycle (see DBE-14) and sugar (DBE-3), the rate of total feed to the reformer is 132-137 gph.

\section{$D B E$ \# 17: The blended feed rate to the reformer is $130 \mathrm{gph}$.}

The steam reformer will be operated under vacuum to control contamination, and the reformer will be at local atmospheric pressure at the distributor. For a bed depth of $3.5 \mathrm{ft}$ (equal to the bed diameter) and a fluidized density of 1.0 to 1.8 (density of $\mathrm{Na}_{2} \mathrm{CO}_{3}$ vs. startup bed material), the pressure drop through the bed will be 42 inches water column (w.c.). Thus, 42 inches w.c. vacuum will be required in the SR vessel above the bed. Vacuum will be increased briefly during nozzle change-outs or bed addition.

\section{DBE \# 18: The Steam reformer vessel will be operated at a vacuum of 42 inches w.c.. Atmospheric pressure at INTEC is 12.27 psia.}

A-5.5.4.2 Steam Reforming Chemistry. The steam reformer operates at conditions that vaporize the feed and provide reducing conditions favorable for decomposition of sodium nitrate and other metal nitrates and for reduction of $\mathrm{NO}_{\mathrm{x}}$ in the off-gas. Metal nitrates in the SBW decompose at the high temperature in the steam reformer to produce metal oxide and/or carbonates and $\mathrm{NO}_{\mathrm{x}}$. The gaseous $\mathrm{NO}_{\mathrm{x}}$ then reacts with $\mathrm{H}_{2}, \mathrm{CO}$, and organics to produce nitrogen, $\mathrm{H}_{2} \mathrm{O}$, and $\mathrm{CO}_{2}$.

Partial oxidation of the sucrose and solid carbon additive generates $\mathrm{CO}, \mathrm{CO}_{2}$, and $\mathrm{H}_{2} \mathrm{O}$ while providing heat to drive the endothermic reforming reactions. The partial oxidation also produces small amounts of products of incomplete combustion.

Steam reforming of the organic feeds in the reformer produces $\mathrm{H}_{2}, \mathrm{CO}$, and $\mathrm{CO}_{2}$. The carbonaceous process additives (sucrose and carbon) facilitate decomposition of nitrates in the SBW feed, and reduction of $\mathrm{NO}_{\mathrm{x}}$ to elemental nitrogen. The pyrolized sucrose and carbon react with the process steam to produce carbon monoxide and hydrogen gas via the water-gas reaction:

$$
\mathrm{C}_{\mathrm{S}}+\mathrm{H}_{2} \mathrm{O} \rightarrow \mathrm{CO}+\mathrm{H}_{2}
$$


A significant portion of the carbon monoxide subsequently reacts with other gaseous species. Examples of this are the water-gas shift reaction that forms hydrogen, the methanation reaction, and reactions with $\mathrm{NO}_{\mathrm{x}}$ to form nitrogen gas.

$$
\begin{aligned}
& \mathrm{CO}+\mathrm{H}_{2} \mathrm{O} \rightarrow \mathrm{CO}_{2}+\mathrm{H}_{2} \\
& \mathrm{CO}+3 \mathrm{H}_{2} \rightarrow \mathrm{CH}_{4}+\mathrm{H}_{2} \mathrm{O} \\
& \mathrm{CO}+\mathrm{NO}_{2} \rightarrow \mathrm{CO}_{2}+\mathrm{NO} \\
& \mathrm{CO}+\mathrm{NO} \rightarrow \mathrm{CO}_{2}+1 / 2 \mathrm{~N}_{2}
\end{aligned}
$$

Hydrogen is believed to be more effective in reducing $\mathrm{NO}_{\mathrm{x}}$ to elemental nitrogen than $\mathrm{CO}$ although reactions with intermediate sugar pyrolysis products may also contribute significantly to $\mathrm{NO}_{\mathrm{x}}$ destruction. Examples of the hydrogen reactions are as follows:

$$
\begin{aligned}
& \mathrm{H}_{2}+\mathrm{NO}_{2} \rightarrow \mathrm{H}_{2} \mathrm{O}+\mathrm{NO} \\
& \mathrm{H}_{2}+\mathrm{NO} \rightarrow \mathrm{H}_{2} \mathrm{O}+1 / 2 \mathrm{~N}_{2}
\end{aligned}
$$

A-5.5.4.3 Reformer Product. In the Phase 2 SBW demonstration tests, bed product, as well as fines from the cyclone and sintered metal filter were collected and analyzed. The results of these analyses were used to define separation factors by chemical specie for input into the mass balance model. These separation factors are shown in Table A-9 (see Section 2.6.2). Based on these factors about $58 \%$ of the total product will be from the bed and the remainder from the filter. The mass balance shows a total product rate of $146-175 \mathrm{lb} / \mathrm{hr}$.

DBE \# 19: Separation factors input in the mass balance model result in a product rate of 146-175 lb/hr.

$D B E$ \# 20: The mass balance model shows that about $58 \%$ of the total reformer product is from the bed and the remainder from the filter.

\section{A-5.5.5 Solids Removal}

A-5.5.5.1 Cyclone. Solids separated by the cyclone were recycled to the reformer bed. This achieves more complete utilization of carbon than if the cyclone solids were collected with the product material. Based on individual specie separation factors shown in Table A-9, the overall cyclone solids removal efficiency is about $62 \%$.

DBE \# 21: The mass balance shows an efficiency of 61-62\% for the cyclone.

DBE \# 22: The cyclone fines will be continuously recycled to the bed at a rate of about $100 \mathrm{lb} / \mathrm{hr}$.

DBE \# 23: The mass balances assume a 5 inches w.c. pressure drop across the cyclone.

A-5.5.5.2 Sintered Metal Filter. The temperature of the sintered metal filter in the Phase 2 TTT demonstration tests (Soelberg, 2004) was $986^{\circ} \mathrm{F}\left(530^{\circ} \mathrm{C}\right)$, and this temperature was assumed for the mass balances. A temperature of $747^{\circ} \mathrm{F}\left(400^{\circ} \mathrm{C}\right)$ at the sintered metal filters is specified by the vendor to capture semi-volatile metals while preventing mercury from condensing and plugging the filters. During the demonstration tests, the temperature drop between the reformer, cyclone and sintered metal filter did not reduce the temperature as much as planned (actual heat loss was less than predicted). Heat loss is 
expected to be less for a larger scale system, so it is expected that a cooler will be required to reduce the temperature prior to the sintered metal filters.

$D B E$ \# 24: An off-gas cooler will be necessary to drop the off-gas temperature to $747^{\circ} \mathrm{F}\left(400^{\circ} \mathrm{C}\right)$ before the filter vessel. However, the mass balance, consistent with Phase 2 demonstration test results, assumes a filter temperature of $530^{\circ} \mathrm{C}$.

Capture of solids and chemical species by the sintered metal filter is based on the Phase 2 demonstration test results as shown in Table A-9.

DBE \# 25: The mass balance assumes non-volatile solids removal by the sintered metal filters of $\mathbf{9 9 . 8 5 \%}$ and a cesium removal of $99.9 \%$ for the sintered metal filters.

DBE \# 26: The filter fines will all be continuously drained at a rate of 62-74 lb/hr.

Pressure drop across the sintered metal filters is estimated at 20 inches w.c. The pressure drop during the short demonstration tests was less, but it was assumed the filter pressure drop would increase with on-line time.

DBE \# 27: The mass balances assume a 20 inches w.c. pressure drop across the sintered metal filters.

\section{A-5.5.6 Oxidizer}

Demonstration tests of staged combustion using NWCF simulated off-gas were performed in 20002001 (Wood 2000, MSE 2000, MSE 2001) and form much of the basis for the mass balance around the combustor. The Phase 2 TTT demonstration test scheme also included an oxidizer. Based on the Phase 2 tests, the mass balance assumed an oxidizer temperature of $1742^{\circ} \mathrm{F}\left(950^{\circ} \mathrm{C}\right)$. This temperature is sufficient for the destruction of residual $\mathrm{H}_{2}, \mathrm{CO}$ and hydrocarbons to the required emission limits while minimizing thermal $\mathrm{NO}_{\mathrm{x}}$ formation from oxidization of $\mathrm{N}_{2}$. Lower temperatures may not result in complete oxidation, while higher temperatures can lead to thermal $\mathrm{NO}_{\mathrm{x}}$ formation. The oxidizer is modeled by the Gibbs routine in ASPEN. As such, the model calculates equilibrium for $\mathrm{CO}, \mathrm{NO}$ and $\mathrm{NO}_{2}$ in the reactor. Based on test data the model results for $\mathrm{NO}_{\mathrm{x}}$ destruction should be viewed as approximate only.

$D B E$ \# 28: The mass balance assumes an oxidizer exit temperature of $1742^{\circ} \mathrm{F}\left(950^{\circ} \mathrm{C}\right)$ in the reduction chamber of the multi-stage combustor.

The pressure drop through the oxidizer vessel was assumed to be low ( 3 inches w.c.) since it is has no internals to restrict flow.

DBE \# 29: The mass balance assumes a pressure drop of 3 inches w.c. through the oxidizer.

The oxidizer effluent oxygen concentration is set at 2 mol \% oxygen (wet basis) based on MSE tests (MSE 2000, MSE 2001).

DBE \#30: The mass balance assumes an oxidizer effluent concentration of 2 mol \% oxygen.

\section{A-5.5.7 Downstream Off-gas Treatment}

A-5.5.7.1 Quench Tower. Water spray injection is used to reduce the temperature of the off-gas out of the oxidizer to a temperature where the gas is still superheated, but low enough to minimize high 
temperature effects on the downstream off-gas piping and scrubber. A temperature above the dew point is desirable to prevent condensation into the scrub solution such that there is no net increase in the scrub volume. A temperature of $212^{\circ} \mathrm{F}\left(100^{\circ} \mathrm{C}\right)$ was chosen for the outlet of the quench tower.

\section{DBE \# 31: The quench water spray flow is $316 \mathrm{gal} / \mathrm{hr}$ for the TTT process and a quench vessel outlet temperature of $100^{\circ} \mathrm{C}$.}

A pressure drop of 5 inches w.c. was estimated for the quench vessel.

\section{DBE \# 32: The mass balance assumes a quench vessel pressure drop of 5 inches w.c.}

A-5.5.7.2 Scrubber. The oxidizer will convert nearly all the mercury in the off-gas to elemental mercury. Therefore, a nitric acid scrubber is included in the process to oxidize and remove a portion of the mercury prior to the GAC beds to ensure that the MACT emission limit can be met. The Phase 2 demonstration test scheme included a venturi scrubber that used water as the scrubbing agent. Based on analysis of the scrub solution, very little ( $2 \%)$ removal of mercury was observed (Soelberg 2004$)$.

A packed-bed scrubber was chosen for good gas-liquid contact for removal of elemental mercury vapor. For the mass balance, it was assumed that the same fraction of mercury (0.437) would be removed by the nitric acid scrub as assumed for the CMACT Upgrade treatment system (Soelberg 2003). This low capture of mercury in the scrub system is reasonable given that the kinetics for dissolution of elemental mercury in nitric acid is slow.

\section{DBE \# 33: The mass balance assumes a packed bed scrubber (VES-107) with two theoretical stages will be required to remove a portion of the elemental mercury from the off-gas.}

\section{DBE \# 34: The mass balance assumes that $43.7 \%$ of the mercury in the off-gas is removed by the scrub system.}

The process flowsheet includes the capability to add 13 molar nitric acid to maintain acidity of the scrub solution at whatever level is desired. The scrub solution composition and temperature are similar to the scrub system at the NWCF, which satisfactorily collects mercury from off-gas. A high scrub acid concentration would be desirable for mercury capture and dissolution, but would require addition of more carbon to destroy the added nitrate and more lime for neutralization of the scrub blow-down stream.

\section{DBE \# 35: The mass balance assumes that 13 molar nitric acid is added to maintain acidity in the scrub at 1 molar.}

Neither modeling nor test data are sufficient to adequately establish the scrub blow down rate. In the NWCF, scrub analyses have shown that mercury has accumulated in the scrub system to concentrations as high as 50-60 g/L. The effect on the process of higher levels is not known. The steam reforming demonstration tests have not recycled scrub or run for long enough periods to predict mercury build up in the scrub. Development of models to accurately predict the chemistry and vapor liquid equilibrium of the scrub system was started in 2003 (Nichols, 2003; Taylor, 2003) but relative to mercury is not complete. For these reasons, the mass balances assume a nominal blow down of $0.26 \mathrm{gph}(1 \mathrm{~kg} / \mathrm{hr})$.

\section{DBE \# 36: The mass balance assumes that scrub tank is purged to control mercury concentration as a blow down rate of $0.26 \mathrm{gph}$.}

A pressure drop of 20 inches w.c. was estimated for the packed-bed scrubber. 


\section{DBE \# 37: The mass balance assumes a pressure drop of 20 inches w.c. across the packed-bed scrubber.}

The required gas-to-liquid ratio for the scrubber was estimated to be similar to that for the quench tower in a vitrification off-gas treatment facility design (Wood 2001). The value of $135 \mathrm{scf} / \mathrm{cf}$ is within the typical range for packed bed designs.

\section{DBE \# 38: The mass balance assumes a gas to liquid ratio of 135 standard cubic feet of gas per cubic foot of liquid, for a scrub solution flow of 7,030 gph.}

A demister is placed downstream of the scrubber to reduce liquid entrainment from the packed-bed scrubber. Mist carryover from the packed-bed scrubber will be minimal as compared to the venturi scrubber used at the NWCF.

\section{A-5.5.7.3 Demister.}

DBE \# 39: The mass balance assumes no liquid entrainment to the off-gas from the scrubber or demister.

The demister pressure drop was estimated to be 5 inches w.c..

DBE \# 40: The mass balance assumes a pressure drop of 5 inches w.c. across the demister.

The demister (VES-102) is modeled as adiabatic, where any condensed liquid is recirculated to the scrub tank.

DBE \# 41: The mass balance assumes a heat duty of 0 BTU/hr for the demister (VES-108).

A-5.5.7.4 Reheater. The reheater temperature is based on maintaining a temperature of $120^{\circ} \mathrm{C}$ at the GAC bed inlet.

$D B E$ \# 42: The mass balance assumes reheater (HE-304) will raise the temperature of the off-gas from to $120^{\circ} \mathrm{C}$ prior to the $\mathrm{GAC}$ beds.

The reheater pressure drop is estimated at 20 inches w.c. A similar reheater for the NWCF has a pressure drop of 40 inches w.c.; however, it is assumed that a new reheater can be designed with a lower pressure drop.

DBE \# 43: The mass balance assumes a reheater pressure drop of 20 inches w.c.

A-5.5.7.5 GAC Beds. The mercury removal efficiency in the GAC bed is based on Phase 2 demonstration tests (Soelberg, 2004), laboratory testing (Del Debbio 2003, Herbst 2002) and discussions with carbon vendors. A total $\mathrm{Hg}$ removal efficiency of $99.9 \%$ would lower the total $\mathrm{Hg}$ concentration to about $30 \mathrm{ug} / \mathrm{dscm}$ (dry, corrected to $7 \% \mathrm{O}_{2}$ ). This outlet total mercury concentration is about $67 \%$ of the MACT limit of $45 \mathrm{ug} / \mathrm{dscm}\left(\mathrm{dry}, 7 \% \mathrm{O}_{2}\right)$. This level should provide assurance of MACT compliance during performance tests and incidental process variations in the event that steam reformer system operation varies from the design parameters. In the Phase 2 demonstration tests, the total mercury removal efficiency of the carbon bed exceeded $99.9 \%$ (Soelberg, 2004). 
DBE \# 44: The mass balance assumes a mercury removal efficiency of $99.9 \%$ in the GAC beds. The mass balance also assumes the $90 \%$ removal of particulate from the off-gas in the GAC beds.

A loading of $95.75 \mathrm{~g}$ mercury per $\mathrm{kg}$ of virgin GAC was achieved in the first bed during Phase I TTT demonstration tests and this loading was assumed for the mass balance. The portion of GAC that is not fully loaded will be dependent on the GAC bed configuration, e.g., parallel versus sequential.

\section{DBE \# 45: The mass balance assumes a mercury loading of to $95.75 \mathrm{~g} \mathrm{per} \mathrm{kg}$ of virgin $\mathrm{GAC}$.}

The total amount of mercury to be absorbed should be very similar to that for the CMACT upgrade alternative, so the GAC bed sizing is assumed to be similar, and the pressure drop is assumed to be 15 inches w.c.. This pressure drop is based on $3 \mathrm{~mm}$ carbon particles and other design parameters discussed in EDF-3292 (Soelberg 2003).

The requirements, basis, performance and design of a GAC bed are discussed in EDF-3292 (Soelberg 2003b). The GAC bed operating conditions are based on operation of two beds in series. The operating temperature is set at $120^{\circ} \mathrm{C}$ to ensure that there is no condensation in the beds. This temperature also provides an adequate margin compared within the vendor-specified temperature limit $\left(150^{\circ} \mathrm{C}\right)$ for effective mercury capture and retention of sulfur on the carbon.

\section{DBE \# 46: The mass balance assumes a GAC bed pressure drop of 15 in water per bed and an operating temperature of $120^{\circ} \mathrm{C}$.}

The final HEPA filter particulate removal efficiency is based on design requirements for new filtration systems in the DOE-ID AE standards. These are to take credit for $99.9 \%$ removal for the first stage HEPA, and $99.8 \%$ removal for the second stage. No credit is taken for the prefilter.

\section{A-5.5.7.6 HEPA Filter and Blower.}

\section{DBE \# 47: The final HEPA filter is assumed to have a DF for particulate of 500,000 (or removal efficiency of $99.9998 \%$}

The blower outlet temperature is calculated in the ASPEN model assuming isentropic compression of the off-gas. The blower outlet pressure is assumed to be 12.4 psia (atmospheric pressure at INTEC is 12.3 psia) and based on a stack sized for negligible back-pressure on the blower.

\section{DBE \# 48: The mass balance assumes a blower (BLO-200) outlet pressure of 12.4 psia.}

The mass balance assumes that pressure control air equal to $10 \%$ of the total flow rate is bled into the off-gas to allow adjustment to maintain constant vacuum on the off-gas system.

\section{DBE \# 49: The blower (BLO-200) pressure control bleed air rate is specified at 10\% of the total flow} rate.

\section{A-5.5.8 Scrub Purge Treatment}

The mass balances in Section 2.6 show scrub purge rates assuming continuous production. Because the mercury concentration in WM-180 will be much lower than in the other tanks, no scrub would be purged when treating Tank WM-180 waste. The mass balance shows purge rates for the tanks of $0.26 \mathrm{gph}$. Because of this low rate, scrub purge treatment would not be a continuous operation. 
Several scenarios are possible and should be further evaluated in conjunction with sizing the treatment equipment. One treatment scenario is to collect the scrub as each SBW tank is depleted and then process it in a short campaign, producing about 32 drums of grouted mixed low level waste (MLLW) per SBW tank. Scrub treatment could be performed either during steam reformer operation, or during scheduled turnarounds.

The mass balance estimates for the scrub purge contain large uncertainties. While the mass balance model is largely based on mercury capture in NWCF scrub, the data does not provide a tight closure for mercury material balances. Because of this uncertainty, the scrub treatment design and schedule will need to be sufficiently robust to process as much as 2-3 times more scrub than estimates in present mass balances. Alternatively, proper operation of the scrub system to maximize mercury concentration could reduce the scrub volume.

The scrub purge will be collected and neutralized in the Spent Scrub Neutralization Tank. The purge will be neutralized with lime slurry to a $\mathrm{pH}$ of 7 . The use of lime slurry is based upon: (1) commercial availability of lime, (2) minimization of water addition, and (3) compatibility with the grout. Sulfide will be added in a stoichiometric amount to precipitate the mercury as insoluble mercury sulfide to ensure that the waste will meet RCRA waste treatment requirements after grouting. The scrub must be neutralized prior to sulfide addition to prevent evolution of $\mathrm{H}_{2} \mathrm{~S}$ gas.

$$
\begin{array}{ll}
2 \mathrm{HNO}_{3}+\mathrm{Ca}(\mathrm{OH})_{2} \rightarrow \mathrm{Ca}\left(\mathrm{NO}_{3}\right)_{2}+2 \mathrm{H}_{2} \mathrm{O} & \text { Acid neutralization } \\
\mathrm{Hg}\left(\mathrm{NO}_{3}\right)_{2}+\mathrm{CaS} \rightarrow \mathrm{Ca}\left(\mathrm{NO}_{3}\right)_{2}+\mathrm{HgS} \downarrow & \text { Sulfide precipitation }
\end{array}
$$

\section{DBE \# 50: The mass balance assumes neutralization of the scrub purge with lime slurry to a pH of 7 and treatment with calcium sulfide to precipitate all the mercury as mercury sulfide.}

The scrub grout is expected to contain very low concentrations of transuranic radionuclides, and thus will likely be classified as mixed-low-level waste. As such it will need to be treated to meet land disposal restriction (LDR) standards for disposal at a MLLW disposal site such as Hanford. It will also need to meet NRC requirements for performance grout. A grout formulation of $25 \mathrm{wt} \%$ neutralized scrub and $75 \mathrm{wt} \%$ grout mix (Portland cement, blast furnace slag, and calcium hydroxide) was assumed in the mass balance. This low waste load will be necessary to ensure that the grout meets compression strength and mercury leach-ability.

\section{DBE \# 51: The mass balance assumes a waste loading of 25 wt \% neutralized scrub with the balance a mixture of Portland cement, blast furnace slag and calcium hydroxide.}

The neutralized and treated scrub blow down stream will be mixed with dry grout mix in a screw mixer and poured directly into drums. The waste will then be allowed to cure prior to shipment to an offsite MLLW disposal site. It was assumed that each waste drum would be filled with $0.2 \mathrm{~m}^{3}$ of grouted waste scrub.

DBE \# 52: Each scrub waste drum will be filled with $0.2 \mathrm{~m}^{3}$ of grouted waste scrub.

\section{A-5.5.9 Steam Reformer Product Packaging}

The reformer bed product and filter fines will be mixed in a hopper and product auger/mixer prior to filling the canister. After densification and cooling, the product will be loaded into canisters. Each 
canister will be filled with $0.8 \mathrm{~m}^{3}$ of reformer waste, equivalent to between $85 \%$ and $90 \%$ of the canister volume.

The maximum temperature of the reformer product is limited by a seal at the top of the waste canisters. The material used for this seal cannot withstand a waste temperature greater than $56^{\circ} \mathrm{C}$. The RH-TRU 72-B Cask SAR lists the maximum temperatures for components of the 72-B cask (WIPP 2003). The maximum waste centerline is $102.7^{\circ} \mathrm{C}\left(217^{\circ} \mathrm{F}\right)$. The maximum canister shell temperature during transport (the surface of the $2 \times 10$ canister) is $56^{\circ} \mathrm{C}\left(132^{\circ} \mathrm{F}\right)$. This means the SR product will need to be cooled before it can be loaded into the 72 -B cask.

\section{$D B E$ \# 53: The mass balance assumes the reformer product will be cooled to a temperature of $56^{\circ} C$ in the reformer product load-out bins prior to loading into canisters.}

The product and fines are cooled in a hopper by direct contact with oxygen-free gas and then transferred using a screw auger that compresses the fluffy material as it is fed into waste canisters. It was assumed that the filter fines bulk density can be increased by a factor of three in the screw auger as it mixes with the bed product. No increase in bulk density is assumed for the bed product. For the TTT process, the reformer bed product has a bulk density of $1.46 \mathrm{~g} / \mathrm{cc}$, and the filter fines were assumed to be compressed from $0.38 \mathrm{~g} / \mathrm{cc}$ up to $80 \%$ of the particle density of $1.44 \mathrm{~g} / \mathrm{cc}$. The mixture density is estimated to be $1.33 \mathrm{~g} / \mathrm{cc}$ from a graph for mixtures of calcine fines and product in ENICO-1100 (Childs 1982).

DBE \# 54: The mass balance assumes the reformer product will have a bulk density of $1.33 \mathrm{~g} / \mathrm{cc}$.

Following cooling, the SR product will be placed in a WIPP-approved RH TRU waste canister, Type A or equivalent. The RH TRU waste canisters must meet the requirements of the 72-B Cask SAR (WIPP 2003). The canister is ten feet tall and two feet in diameter. Filling each canister with $0.8 \mathrm{~m}^{3}$ of SR product utilizes $85-90 \%$ of the canister volume. The amount or SR product then must be determined as part of waste form qualification requirements. The weight will be obtained by subtraction of the tare weight of the canister from the final weight of the filled canister. The canister will be smeared to detect the level of external contamination and decontaminated if needed. Removable surface contamination on RH TRU waste payload containers, payload assemblies, and packaging must not exceed 20 disintegrations per minute (dpm) per 100 square centimeters $\left(\mathrm{cm}^{2}\right)$ alpha and $200 \mathrm{dpm} / 100 \mathrm{~cm}^{2}$ betagamma. The radiation dose from the canister will be measured. The external radiation dose equivalent rate of individual payload containers must be $>200$ milliroentgen equivalent man per hour $(\mathrm{mrem} / \mathrm{h})$, and $<1,000$ roentgen equivalent man per hour $(\mathrm{rem} / \mathrm{h})$ at the surface of the payload container.

DBE \# 55: Each canister will be filled with $0.8 \mathrm{~m} 3$ of steam reformer product.

\section{A-5.5.10 Utilities}

A-5.5.10.1 Cooling Water. Cooling water is assumed to be available from the INTEC raw water supply at $20^{\circ} \mathrm{C}$ and 100 psig.

A-5.5.10.2 Steam. Steam is assumed available from the INTEC steam headers at $35 \mathrm{psig}$ and 150 psig for general building heating.

Process steam at $30 \mathrm{psig}$ and $670^{\circ} \mathrm{C}$ is provided by a new high-temperature low-pressure fuel-oilfired boiler. 
A-5.5.10.3 Air. Air for tank sparging and air purges was assumed to be available from the INTEC plant air supply, at $100 \mathrm{psig}$ and $50 \mathrm{psig}$ with a $-40^{\circ} \mathrm{F}$ dew point. This air will be filtered and regulated to $20 \mathrm{psig}$ for instrument air. Ambient air temperature is assumed to be $77^{\circ} \mathrm{F}\left(25^{\circ} \mathrm{C}\right)$.

A-5.5.10.4 Electricity. The existing electrical supply system is assumed to have adequate capacity for process equipment such as pumps and the off-gas compressor, general equipment, lighting, and building use.

\section{A-5.5.11 Basis for TWR Mass Balance}

Mass balances were developed for the TWR process to provide a basis for sizing equipment, determine feed chemical requirements, determine utility requirements, estimate emissions and determine waste volumes, compositions and properties. The sections below document the basis for the mass balances for the TWR flowsheet where it differs from the TTT flowsheet. The basis is capsulated in succinct statements, called DBEs, shown in italics in the following paragraphs. The DBEs are amplified with background information, references, explanation, and, in some cases, a discussion of uncertainties.

A-5.5.11.1 Feeds. Feeds to the calcination process include waste feeds from the TFF, isopropanol as a chemical reductant, carbon dioxide in various uses, and propane for combustion in the oxidation unit. Nitrogen is assumed in the mass balance as the coolant for reformer product, although alternatively $\mathrm{CO}_{2}$ could be used. $\mathrm{CO}_{2}$ is shown as the coolant on the current PFD.

The amount of liquid reductant was based on Phase 2 bench-scale demonstration tests using simulated WM-180 feed performed in October, 2003 (Soelberg, 2004b).

\section{DBE \#56: Isopropyl alcohol (IPA) is added to the SBW feed in the ratio $0.7 \mathrm{lb} I P A$ per Ib SBW.}

In Phase 1 of the TWR demonstration tests, performed in December 2002 (Marshall, 2003a; Marshall 2003c), a solid reductant, polypropylene, was also fed to the reformer. However, successful tests were completed during phase 2 without the solid reductant (Soelberg, 2004b), and hence the mass balances in this addendum assume no solid reductant.

\section{DBE \#57: No solid organic additive is needed in the TWR flowsheet to obtain the desired $N O_{x}$ destruction.}

For the TWR process, the starting bed media is $300 \mu \mathrm{m}$ AGSCO white alumina grit. During the bench-scale tests, no bed media was drained and the bed mass was allowed to build during the run. As an estimate, total bed media addition of 0.01 fraction of the solids produced was assumed for the mass balance.

\section{DBE \# 58: Starting bed media for the TWR flowsheet will be white AGSCO Alumina grit, with the bed media amount estimated at 0.01 wt fraction of the solid product during operation.}

A-5.5.11.2 Steam Reformer Heating and Process Fuels. The mass balance shows that external heating supplying total input of 1.4-1.8 MM Btu/hr will be required to maintain the process temperature of $600^{\circ} \mathrm{C}$ in the TWR reformer. This is based on the energy balance in the ASPEN model. This heat could be supplied by external heaters, or by adding sufficient oxygen or air to combust with excess IPA in the reformer feed.

The oxidizer will use propane to boost the heating value of the reformer off-gas. Addition of propane to the oxidizer will allow operation at a temperature of $1000^{\circ} \mathrm{C}$ to ensure destruction of organics and oxidation of $\mathrm{CO}$ in the reformate stream. Initial runs of the ASPEN model indicate that because of the 
products of incomplete combustion present in the reformer off-gas, no fuel is required in the oxidizer. Since the Phase 2 demonstration tests had a positive oxidizer fuel rate, the mass balance model was revised with the fuel rate based on the test data. The fuel rate for a relevant portion of the Phase 2 tests averaged $2.9 \mathrm{~kg} / \mathrm{hr}$. Scaling this rate up to a full-scale facility and adjusting for a lower heat loss for a full-scale oxidizer, the expected propane rate would be about $133 \mathrm{~kg} / \mathrm{hr}$. Further "tuning" of the model and analysis of the Phase 2 test data could result in optimization of this rate, as well as reducing or eliminating the reformer duty. Because of specifying both the oxidizer temperature and the feed fuel rate, the ASPEN model necessarily resulted in a required cooling duty for the oxidizer. Further development of the model and/or additional test data is needed to eliminate this cooling duty (see additional discussion at end of Section A-6.1).

DBE \# 59: The mass balance assumes a fuel rate of $133 \mathrm{~kg} / \mathrm{hr}$ propane to the oxidizer based on Phase 2 demonstration test data.

$D B E$ \# 60: The oxidizer will operate at a temperature of $1000^{\circ} \mathrm{C}$ to ensure adequate destruction of organics and $\mathrm{CO}$ in the reformer off-gas.

A-5.5.11.3 Carbon Dioxide and Oxygen. Carbon dioxide is used to atomize the feed in the TWR flowsheet, and will be used for purges in the steam reformer process lines up until the oxidizer, as these points in the piping may contain combustible levels of $\mathrm{H}_{2}$ and $\mathrm{CO}$. Carbon dioxide could also be used for direct cooling of the SR product in the collection hopper, although the mass balance assumes nitrogen is used.

The hydrogen and carbon monoxide concentrations in the off-gas from the reformer were observed to be quite low during both Phase 1 and Phase 2 demonstration tests (Marshall, 2003a, Soelberg, 2004b). The mass balance model predicts large amounts of hydrogen, carbon monoxide, unreacted or partially reacted IPA, and amorphous carbon in the reformer off-gas, somewhat contrary to the results of the tests. Some air in-leakage was suspected during the testing, either into the vessel or, more likely, into the offgas sampling system lines prior to the analyzers. However, the observed low concentration of nitrogen in the off-gas shows that the air in-leakage was small. In an effort to match the test data as close as possible, the concentration of organics in the off-gas was allowed to increase to match more closely observed concentrations of hydrogen, $\mathrm{CO}$ and methane. (See discussion at end of Section A-6.1).

DBE \# 61: The mass balances assume no air in-leakage.

A NAR of 470 was used during the Phase 2 bench-scale tests.

$D B E$ \# 62: Carbon dioxide for feed atomization will be fed to the feed nozzles at a ratio of 470 standard cubic feet per cubic foot of blended feed.

Carbon dioxide gas preheated to the reformer temperature is used to fluidize the bed media in the steam reformer. Based on the Phase 2 demonstration tests, the fluidization gas mass flow rate will need to be about 1.3 times the SBW feed rate.

DBE \#63: The mass balance assumes a fluidizing gas mass flow rate of 1.3 times the $S B W$ feed rate.

A-5.5.11.4 Feed Rate. The feed to the reformer includes SBW plus heels, the organic feed additive, and scrub recycle. The scrub recycle rate is assumed to be 5 vol $\%$ of the SBW rate.

$D B E$ \# 64: The required $S B W$ minimum throughput to meet a 2.5-yr processing schedule is 88.5 gph $(334.8 \mathrm{lph})$. The scrub recycle rate is assumed to be 5 vol \% of the $S B W$ rate. 


\section{A-5.5.11.5 Steam Reforming.}

A-5.5.11.5.1 Normal Operating Conditions-The operating temperature of the steam reformer during Phase 2 bench-scale tests of the TWR flowsheet was $600^{\circ} \mathrm{C}$.

DBE \# 65: The normal operating temperature of the TWR Steam reformer will be $600^{\circ} \mathrm{C}$.

A-5.5.11.5.2 Steam Reforming Chemistry-In the TWR reformer, nitrates are directly reduced to carbonates by the organic additives. Some of the possible reactions are shown in Section A-2.4 (Reactions 1-5). Equilibrium concentrations of the reactants and products are dependant upon the reformer temperature, and were determined by a routine in ASPEN that finds the equilibrium composition by minimizing Gibbs free energy of formation for the products. In reality, some reactions in the reformer do not reach equilibrium, with some of the hydrocarbons, carbon, and $\mathrm{NO}_{\mathrm{x}}$ remaining in the off-gas and solids. To approximate $\mathrm{H}_{2}, \mathrm{CO}, \mathrm{NO}_{\mathrm{x}}$ and $\mathrm{CH}_{4}$ observed in the off-gas during the Phase 2 tests, a portion of the IPA was bypassed in the mass balance model around the Gibbs reactor step, and also certain reactions with set conversions were specified a second reactor step. These are shown in Table A-3.

A-5.5.11.6 SR Product Carryover, Separation and Rate. The fraction of reformer product carried into the cyclone, sintered metal filter vessel, and scrub system was calculated from the Phase 2 bench-scale tests material balance data. Separation factors based on these calculations are shown in Table A-4. Using these separation factors, the mass balance model shows that $93.7 \mathrm{wt} \%$ of the product comes from the bed and the remainder from the filter.

Table A-3. Reformer parameters.

\begin{tabular}{lcc}
\hline \multicolumn{1}{c}{ Bypass of Gibbs reactor } & Fraction Bypassed & \\
\hline \multicolumn{1}{c}{ Reactions } & 0.207 & \\
\hline Nitric acid & Fractional Conversion & Conversion Based on \\
\hline $2 \mathrm{NAF}+\mathrm{H}_{2} \mathrm{O}+\mathrm{CO}_{2}-->2 \mathrm{HF}+\mathrm{NA}_{2} \mathrm{CO}_{3}$ & 0.0626 & $\mathrm{NaF}$ \\
$2 \mathrm{HNO}_{3}+3 \mathrm{H}_{2}-->2 \mathrm{NO}+4 \mathrm{H}_{2} \mathrm{O}$ & 0.9906 & $\mathrm{HNO}_{3}$ \\
$2 \mathrm{NAOH}+\mathrm{CO}_{2}-->\mathrm{NA}_{2} \mathrm{CO}_{3}+\mathrm{H}_{2} \mathrm{O}$ & 1.0000 & $\mathrm{NaOH}$ \\
$2 \mathrm{NAOH}(\mathrm{S})+\mathrm{CO}_{2}-->\mathrm{NA}_{2} \mathrm{CO}_{3}+\mathrm{H}_{2} \mathrm{O}$ & 1.0000 & $\mathrm{NAOH}(\mathrm{S})$ \\
$2 \mathrm{HNO}++\mathrm{H} 2-->2 \mathrm{NO}_{2}+2 \mathrm{H}_{2} \mathrm{O}$ & 1.0000 & $\mathrm{HNO}_{3}$ \\
$\mathrm{CO}+\mathrm{H}_{2} \mathrm{O}-->\mathrm{CO}_{2}+\mathrm{H}_{2}$ & 0.9500 & $\mathrm{CO}$ \\
$\mathrm{Carbon}+2 \mathrm{H} 2-->\mathrm{CH}_{4}$ & 0.0600 & $\mathrm{H}_{2}$ \\
$16 \mathrm{Carbon}+17 \mathrm{H}_{2}-->\mathrm{N}-\mathrm{HEX}-01$ & 1.0000 & $\mathrm{Carbon}$ \\
$2 \mathrm{H}+\mathrm{O}_{2}-->2 \mathrm{H}_{2} \mathrm{O}$ & 1.0000 & $\mathrm{O}_{2}$ \\
$2 \mathrm{NACL}+\mathrm{H}_{2} \mathrm{O}+\mathrm{CO}_{2}-->2 \mathrm{HCL}+\mathrm{NA}_{2} \mathrm{CO}_{3}$ & 0.0037 & $\mathrm{NACL}$ \\
$2 \mathrm{KCL}+\mathrm{H}_{2} \mathrm{O}+\mathrm{CO}_{2}-->2 \mathrm{HCL}+\mathrm{K}_{2} \mathrm{CO}_{3}$ & 0.0037 & $\mathrm{KCL}$ \\
\hline
\end{tabular}

A-5.5.11.7 Sintered Metal Filters. Solids and chemical species capture by the sintered metal filter is based on results from the Phase 2 TWR demonstration tests. The separation factors used in the mass balance are shown in Table A-4. 
A-5.5.11.8 Oxidizer Temperature and Outlet Oxygen Concentration. Based on the Phase 2 TWR demonstration test, the oxidizer temperature is $1000^{\circ} \mathrm{C}$. The mass balance assumed an outlet oxygen concentration of 3 mole $\%$.

DBE \# 66: The oxidizer effluent gas temperature is $1832^{\circ} \mathrm{F}\left(1000^{\circ} \mathrm{C}\right)$. The mass balance assumed an outlet oxygen concentration of about 3 mole $\%$.

Table A-4. Bed, cyclone, and sintered metal filter separation factors.

\begin{tabular}{|c|c|c|c|c|c|}
\hline Component & Product $^{a}$ & Filter $^{\mathrm{a}}$ & Component & Product $^{a}$ & Filter $^{\mathrm{a}}$ \\
\hline $\mathrm{HG}$ & 0.001 & 0.001 & $\mathrm{NA}_{2} \mathrm{SO}_{3}$ & 0.945 & 1.000 \\
\hline $\mathrm{HGCL}_{2}$ & 0.001 & 0.001 & $\mathrm{NAHCO}_{3}$ & 0.945 & 1.000 \\
\hline $\mathrm{NA}_{2} \mathrm{CO}_{3}$ & 0.945 & 1.000 & NAF & 0.945 & 1.000 \\
\hline $\mathrm{K}_{2} \mathrm{CO}_{3}$ & 0.915 & 1.000 & $\mathrm{~K}_{2} \mathrm{SO}_{4}$ & 0.915 & 1.000 \\
\hline $\mathrm{CACO}_{3}$ & 0.948 & 1.000 & $\mathrm{KHSO}_{4}$ & 0.915 & 1.000 \\
\hline $\mathrm{NA}_{2} \mathrm{O}$ & 0.945 & 1.000 & $\mathrm{KHCO}_{3}$ & 0.915 & 1.000 \\
\hline $\mathrm{K}_{2} \mathrm{O}$ & 0.915 & 1.000 & N-HEX-01 & 0.000 & 0.001 \\
\hline $\mathrm{CAO}$ & 0.948 & 1.000 & $\mathrm{CH} 4$ & 0.000 & 0.000 \\
\hline $\mathrm{NAALO}_{2}$ & 0.945 & 1.000 & TRIT-OXD & 0.000 & 0.000 \\
\hline $\mathrm{AL}_{2} \mathrm{O}_{3}$ & 0.942 & 1.000 & $\mathrm{C}_{3} \mathrm{H} 8$ & 0.000 & 0.000 \\
\hline $\mathrm{SIO}_{2}$ & 0.781 & 1.000 & $\mathrm{CS}_{2} \mathrm{CO}_{3}$ & 0.611 & 1.000 \\
\hline $\mathrm{FE}_{2} \mathrm{O}_{3}$ & 0.915 & 0.997 & $\mathrm{NA}_{3} \mathrm{PO}_{4}(\mathrm{~S})$ & 0.945 & 1.000 \\
\hline NACL & 0.945 & 1.000 & $\mathrm{~K}_{3} \mathrm{PO}_{4}$ & 0.915 & 1.000 \\
\hline $\mathrm{KCL}$ & 0.915 & 1.000 & $\mathrm{ALPO}_{4}$ & 0.942 & 1.000 \\
\hline $\mathrm{CAF}_{2}$ & 0.948 & 1.000 & PLUS1 & 0.945 & 1.000 \\
\hline $\mathrm{NANO}_{3}$ & 0.945 & 1.000 & PBO & 0.766 & 1.000 \\
\hline $\mathrm{KNO}_{3}$ & 0.915 & 1.000 & $\mathrm{CR}_{2} \mathrm{O}_{3}$ & 0.920 & 1.000 \\
\hline $\mathrm{CA}\left(\mathrm{NO}_{3}\right)_{2}$ & 0.948 & 1.000 & PLUS4 & 0.942 & 1.000 \\
\hline $\mathrm{TCO}_{3}$ & 0.475 & 0.998 & PLUS5 & 0.942 & 1.000 \\
\hline $\mathrm{AL}\left(\mathrm{NO}_{3}\right)_{3}$ & 0.942 & 1.000 & PLUS6 & 0.942 & 1.000 \\
\hline $\mathrm{CDO}$ & 0.766 & 1.000 & MINUS1 & 0.712 & 0.981 \\
\hline $\mathrm{NAHSO}_{4}$ & 0.945 & 1.000 & $\mathrm{CAOH}+$ & 1.000 & 0.000 \\
\hline $\mathrm{NA}_{2} \mathrm{SO}_{4}$ & 0.945 & 1.000 & $\mathrm{HGOH}+$ & 1.000 & 0.000 \\
\hline NAHS & 0.945 & 1.000 & $\mathrm{HG}(\mathrm{OH})_{2}$ & 0.001 & 0.002 \\
\hline $\mathrm{NA}_{2} \mathrm{~S}$ & 0.945 & 1.000 & $\mathrm{CASO}_{4}(\mathrm{~S})$ & 0.948 & 1.000 \\
\hline $\mathrm{NAOH}$ & 0.945 & 1.000 & $\mathrm{CACL}_{2}(\mathrm{~S})$ & 0.948 & 1.000 \\
\hline $\mathrm{C}_{3} \mathrm{H}_{8} \mathrm{O}-2$ & 0.000 & 0.000 & $\mathrm{KOH}(\mathrm{S})$ & 0.915 & 1.000 \\
\hline CARBON & 0.000 & 0.000 & $\mathrm{NAOH}(\mathrm{S})$ & 0.945 & 1.000 \\
\hline
\end{tabular}

${ }^{\mathrm{a}}$ Factors shown are fractions of specie removed from the bed or by the cyclone or filter. 


\section{A-5.5.11.9 Remainder of Off-gas and Scrub Treatment and Reformer Product}

Packaging. Model input parameters for the remainder of the off-gas treatment system and the scrub recycle and treatment system were assumed the same for the TWR process as for the TTT process. Refer to the forgoing discussion of the TTT process for these parameters, including:

DBE \#29: Oxidizer pressure drop

DBE \#31: Quench tower temperature

DBE \#32: Quench tower pressure drop

DBE \#33: Packed bed scrubber stages

DBE \#34: Fraction of mercury removed in the scrubber

DBE \#35: Control of scrub acidity

DBE \#36: Scrub purge rate

DBE \#37: Scrubber pressure drop

DBE \#38: Scrubber gas to liquid ratio

DBE \#39: Demister entrainment

DBE \#40: Demister pressure drop

DBE \#41: Demister duty

DBE \#42: Reheater temperature

DBE \#43: Reheater pressure drop

DBE \#44: GAC bed $\mathrm{Hg}$ and particulate removal efficiency

DBE \#45: GAC bed Hg loading

DBE \#46: GAC bed pressure drop and temperature

DBE \#47: HEPA filter efficiency

DBE \#48: Blower outlet pressure

DBE \#49: Compressor control air flow rate

DBE \#50: Scrub purge neutralization

DBE \#51: Scrub grout formulation

DBE \#52: Grout waste drum volume

DBE \#53: Product cooler temperature 
DBE \#54: Product density after densification

DBE \#55: Waste canister volume.

The utilities available for the TWR process were assumed the same as for the TTT process.

\section{A-6. MASS BALANCES}

\section{A-6.1 Mass Balance Model}

The material balance for chemical species was performed using ASPEN Plus, a computer program that is part of the ASPEN Engineering Suite 11.1 (ASPEN 2002). ASPEN provides a broad framework for process modeling, including extensive thermodynamic databases, electrolyte solution chemistry models, numerous equation-of-state models, and a comprehensive unit operation library. To simplify the model, many minor species were grouped together based on valence, as shown in Table A-5. Results from the ASPEN Plus model were transferred to a Microsoft Excel spreadsheet, and the grouped species were subsequently separated back into individual components. The ASPEN flow scheme developed for the material balances for the TTT process is shown in Figure A-1. Except where otherwise indicated, the comments below apply to this process (TTT). The model initially developed for steam reforming calculations for EDF-2273 (Barnes 2002a) and EDF-3827 (Haefner, 2003) was modified to reflect updates to the PFDs, and to incorporate the data from Phase 2 bench-scale testing with simulated SBW.

Radionuclides for streams other than the SBW feed stream were calculated from the ratios of radionuclide to non-radioactive element in the SBW feed. Ditritium oxide $\left(\mathrm{T}_{2} \mathrm{O}\right)$ was simulated in ASPEN as a separate compound having the same properties as water. This allowed tritium to be tracked throughout the process. 
Table A-5. ASPEN model chemical specie inputs.

\section{Aspen Model Compound}

Actual Species Represented

\begin{tabular}{|c|c|}
\hline $\mathrm{HgCl}_{2}$ & $\mathrm{Hg}, \mathrm{Cl}$ \\
\hline $\mathrm{CaF}_{2}$ & $\mathrm{~F}, \mathrm{Ca}$ \\
\hline $\mathrm{NaHSO}_{4}$ & $\mathrm{SO}_{4}^{2-}$ \\
\hline $\mathrm{NaCl}$ & $\mathrm{Cl}, \mathrm{Na}$ \\
\hline $\mathrm{Na}_{3} \mathrm{PO}_{4}$ & $\mathrm{Na}, \mathrm{PO}_{4}^{3-}$ \\
\hline $\mathrm{NaNO}_{3}$ & $\mathrm{Na}, \mathrm{NO}_{3}^{-}$, remaining +1 cations \\
\hline $\mathrm{KNO}_{3}$ & $\mathrm{~K}, \mathrm{NO}_{3}^{-}$ \\
\hline $\mathrm{Ca}\left(\mathrm{NO}_{3}\right)_{2}$ & $\mathrm{Ca}, \mathrm{NO}_{3}^{-}$, remaining +2 cations \\
\hline $\mathrm{Al}\left(\mathrm{NO}_{3}\right)_{3} \bullet 9 \mathrm{H}_{2} \mathrm{O}$ & $\mathrm{Al}^{+3}, \mathrm{NO}_{3}^{-}, \mathrm{H}_{2} \mathrm{O}$, remaining +3 cations \\
\hline $\mathrm{ZrO}_{2}$ & $\mathrm{Zr}, \mathrm{O}$, remaining +4 cations \\
\hline $\mathrm{V}_{2} \mathrm{O}_{5}$ & $\mathrm{~V}$, remaining +5 cations, $\mathrm{O}$ \\
\hline $\mathrm{MoO}_{3}$ & Mo, O \\
\hline $\mathrm{HCl}$ & remaining $\mathrm{Cl}$, remaining -1 anions, $\mathrm{H}^{+}$ \\
\hline $\mathrm{HNO}_{3}$ & remaining $\mathrm{H}^{+}, \mathrm{NO}_{3}^{-}$ \\
\hline $\mathrm{CdO}$ & $\mathrm{Cd}, \mathrm{O}$ \\
\hline $\mathrm{Cs}_{2} \mathrm{CO}_{3}$ & $\mathrm{Cs}, \mathrm{C}, \mathrm{O}$ \\
\hline $\mathrm{PbO}$ & $\mathrm{Pb}, \mathrm{O}$ \\
\hline $\mathrm{Cr}_{2} \mathrm{O}_{3}$ & $\mathrm{Cr}, \mathrm{O}$ \\
\hline $\mathrm{TcO}_{3}$ & $\mathrm{Tc}, \mathrm{O}$ \\
\hline Ditritium oxide & Tritium, $\mathrm{O}$ \\
\hline Carbon & $\mathrm{C}$ \\
\hline Sucrose & Sucrose \\
\hline $\mathrm{Fe}_{2} \mathrm{O}_{3}$ & $\mathrm{Fe}, \mathrm{O}$ \\
\hline $\mathrm{SiO}_{2}$ & $\mathrm{Si}, \mathrm{O}$ \\
\hline Radionuclides & $\begin{array}{l}\text { Ratioed from original element composition in the } \\
\text { SBW to chemical element compositions in the SBW }\end{array}$ \\
\hline $\mathrm{H}_{2} \mathrm{O}$ & Remaining $\mathrm{H}_{2} \mathrm{O}$ \\
\hline $\mathrm{NO}_{3}^{-}$ & Remaining $\mathrm{NO}_{3}^{-}$ \\
\hline
\end{tabular}




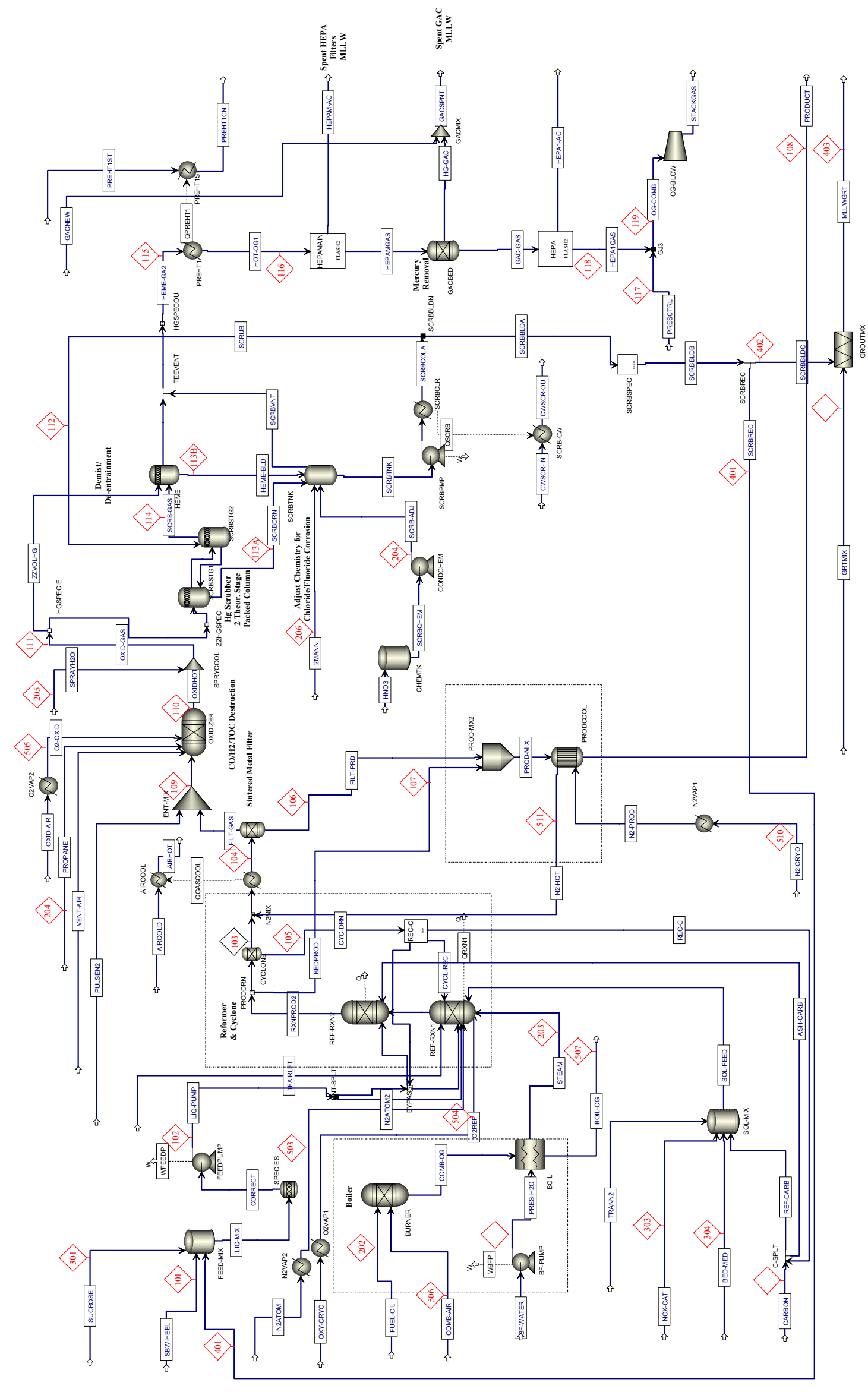

$n$ 
In order to increase confidence in the ASPEN model results, the model was tuned using data from steam reforming demonstration tests (Marshall 2003a, Marshall 2003b, Soelberg 2004a). The Phase 2 demonstration test was similar to the current flowsheet and data from this test was used to the maximum extent. The bench-scale tests used a simulated (non-radioactive) WM-180 feed which included toxic metals, non-radioactive cesium, and non-radioactive rhenium (as a technetium surrogate). Product, and filter fines were collected separately and cyclone fines (which were recycled to the reformer) were sampled. The feed, product, and scrub streams were analyzed. A continuous emission monitoring system was used to analyze the off-gas for $\mathrm{O}_{2}, \mathrm{CO}_{2}, \mathrm{H}_{2}, \mathrm{CO}$ and $\mathrm{CH}_{4}$.

Impurities in the carbon from analyses for the demonstration tests were included in the mass balance. The composition of the carbon as analyzed and input to the ASPEN model is provided in Table A-6.

Table A-6. Carbon composition used for ASPEN model of the TTT flowsheet.

\begin{tabular}{lcccc}
\hline & $\begin{array}{c}\text { Concentration } \\
(\mathbf{m g} / \mathbf{K g})\end{array}$ & $\begin{array}{c}\text { Input Specie for } \\
\mathbf{A S P E N}\end{array}$ & $\begin{array}{c}\text { Concentration of } \\
\text { Specie (mol/g) }\end{array}$ & $\begin{array}{c}\text { Concentration of } \\
\text { Specie (wt\%) }\end{array}$ \\
\hline $\mathrm{Al}$ & 1527 & $\mathrm{Al}_{2} \mathrm{O}_{3}$ & $5.66 \mathrm{E}-05$ & $0.289 \%$ \\
$\mathrm{Ca}$ & 18431 & $\mathrm{CaCO}_{3}$ & $4.60 \mathrm{E}-04$ & $4.60 \%$ \\
$\mathrm{Fe}$ & 867 & $\mathrm{Fe}_{2} \mathrm{O}_{3}$ & $1.55 \mathrm{E}-05$ & $0.124 \%$ \\
$\mathrm{Hg}$ & 1.91 & $\mathrm{Hg}$ & $9.52 \mathrm{E}-09$ & $0.000191 \%$ \\
$\mathrm{P}$ & 733 & $\mathrm{~K}_{3} \mathrm{PO}_{4}$ & $2.37 \mathrm{E}-05$ & $0.502 \%$ \\
$\mathrm{~K}$ & 7107 & $\mathrm{~K}_{2} \mathrm{CO}_{3}$ & $1.82 \mathrm{E}-04$ & $0.712 \%$ \\
$\mathrm{Si}$ & 6233 & $\mathrm{SiO}_{2}$ & $2.22 \mathrm{E}-04$ & $1.33 \%$ \\
$\mathrm{Na}$ & 739 & $\mathrm{Na}_{2} \mathrm{~S}$ & $3.21 \mathrm{E}-05$ & $0.125 \%$ \\
$\mathrm{~S}$ & 598 & $\mathrm{H}_{2} \mathrm{~S}$ & $1.86 \mathrm{E}-05$ & $0.00881 \%$ \\
$\mathrm{Sum}$ of Impurities & 39107 & & $1.09 \mathrm{E}-3$ & $7.70 \%$ \\
$\mathrm{C}$ (balance) & 960893 & $\mathrm{C}$ & $8.00 \mathrm{E}-02$ & $92.3 \%$ \\
$\mathrm{O}$ & & & $2.48 \mathrm{E}-03$ & $3.96 \%$ \\
\hline
\end{tabular}

When the feed, additives, and feed gas $\left(\mathrm{O}_{2}\right.$, steam) flows for the tests were initially tried in the ASPEN model, the resulting $\mathrm{H}_{2}$ and $\mathrm{CO}$ concentrations in the off-gas differed from those observed during the tests. Therefore the feed oxygen rate adjusted in the model to match the hydrogen concentrations measured in the Phase 2 demonstration tests and the $\mathrm{CO}$ yield was adjusted by inputting a reaction that converted a small amount of $\mathrm{CO}_{2}$ to $\mathrm{CO}$.

Equilibrium concentrations of the reactants and products are dependent upon the reformer temperature, and were determined by a routine in ASPEN that determines the final mixture that minimizes Gibbs free energy of formation. The reactions in the reformer do not reach equilibrium, with some of the hydrocarbons, and $\mathrm{NO}_{\mathrm{x}}$ remaining in the off-gas and solids. These fractions of unreacted hydrocarbon and $\mathrm{NO}_{\mathrm{x}}$ are modeled by bypassing equivalent fractions around the reformer. Based on the organic carbon and $\mathrm{NO}_{\mathrm{x}}$ in the products and off-gas, the fractions of hydrocarbon and nitric acid in the feed that are not fully reacted are presented in Table A-7. 
Table A-7. Fraction of reformer feeds bypassing the reformer.

\section{Component}

Sugar-HC

Nitrates
Fraction Not Reacted in Reformer

0.113

0.084-0.21 (varied with feed case)

The predicted concentrations were adjusted to match the volatile species and products found downstream of the reformer during the FY 2003 demonstration tests. This was done by specifying the following additional reactions in the model:

Table A-8. Additional reactions in the mass balance model.

\begin{tabular}{|c|c|c|c|}
\hline Stoichiometry & $\begin{array}{l}\text { Fractional } \\
\text { Conversion }\end{array}$ & of Specie & Basis for Conversion \\
\hline $\begin{array}{r}2 \mathrm{NaCl}+\mathrm{H}_{2} \mathrm{O}+\mathrm{CO}_{2} \rightarrow \\
2 \mathrm{HCL}+\mathrm{Na}_{2} \mathrm{CO}_{3}\end{array}$ & 0.018 & $\mathrm{NaCl}$ & $\begin{array}{l}\text { Based on volatility observed in } \\
\text { demonstration tests. }\end{array}$ \\
\hline $\begin{array}{r}2 \mathrm{NaF}+\mathrm{H}_{2} \mathrm{O}+\mathrm{CO}_{2} \rightarrow \\
2 \mathrm{HF}+\mathrm{Na}_{2} \mathrm{CO}_{3}\end{array}$ & 0.018 & $\mathrm{NaF}$ & Estimate. \\
\hline $2 \mathrm{HNO}_{3}+3 \mathrm{H}_{2} \rightarrow 2 \mathrm{NO}+4 \mathrm{H}_{2} \mathrm{O}$ & 0.9375 & $\mathrm{HNO}_{3}$ & $\begin{array}{l}\text { From } \mathrm{NO} / \mathrm{NO}_{2} \text { ratio observed in } \\
\text { demonstration tests }\end{array}$ \\
\hline $2 \mathrm{HNO}_{3}+\mathrm{H}_{2} \rightarrow 2 \mathrm{NO}_{2}+2 \mathrm{H}_{2} \mathrm{O}$ & 0.059659 & $\mathrm{HNO}_{3}$ & $\begin{array}{l}\text { From } \mathrm{NO} / \mathrm{NO}_{2} \text { ratio observed in } \\
\text { demonstration tests }\end{array}$ \\
\hline $2 \mathrm{NaOH}+\mathrm{CO}_{2} \rightarrow \mathrm{Na}_{2} \mathrm{CO}_{3}+\mathrm{H}_{2} \mathrm{O}$ & 0.96 & $\mathrm{NaOH}$ & $\begin{array}{l}\text { Estimate based on } \\
\text { Carbonate/Hydroxide Equilibrium } \\
\text { predicted by HSC }\end{array}$ \\
\hline $2 \mathrm{NaOH}_{(\mathrm{S})}+\mathrm{CO}_{2} \rightarrow \mathrm{Na}_{2} \mathrm{CO}_{3}+\mathrm{H}_{2} \mathrm{O}$ & 0.96 & $\mathrm{NaOH}_{(\mathrm{S})}$ & $\begin{array}{l}\text { Estimate based on } \\
\text { Carbonate/Hydroxide Equilibrium } \\
\text { predicted by HSC }\end{array}$ \\
\hline $\mathrm{CO}_{2}+2 \mathrm{H}_{2} \mathrm{O} \rightarrow \mathrm{CH}_{4}+\mathrm{O}_{2}$ & 0.00855 & $\mathrm{CO}_{2}$ & From $\mathrm{CH}_{4}$ observed in tests \\
\hline $2 \mathrm{CO}_{2} \rightarrow 2 \mathrm{CO}+\mathrm{O}_{2}$ & 0.107 & $\mathrm{CO}_{2}$ & From $\mathrm{CO}$ observed in tests \\
\hline
\end{tabular}

The fraction of reformer product carried over into to the cyclone, sintered metal filter vessel, and scrub system was calculated from the material balance data from the demonstration tests. Mass fractions of reformer product collected in the bed drain product, cyclone fines, and filter fines for bulk TOCs, Al, $\mathrm{Ca}, \mathrm{Cr}, \mathrm{Cs}, \mathrm{Cr}, \mathrm{Fe}, \mathrm{Pb}, \mathrm{K}, \mathrm{Re}$ (as a surrogate for Tc), $\mathrm{Si}, \mathrm{Na}, \mathrm{Zr}, \mathrm{Cl}, \mathrm{F}$, nitrate, phosphate and sulfate were matched to the those obtained during the tests. The mass fraction of each species collected in the reformer bed drain product was split from the overall amount coming out of the reformer. The fractions of inputs to the reformer, cyclone, filter, and scrub that were retained by each unit for the TTT process are presented in Table A-9. 
Table A-9. Solids capture for the TTT material balance model (fraction of species into each unit captured by that unit)

\begin{tabular}{|c|c|c|c|c|c|c|c|}
\hline Component & Bed & Cyclone Fines & Filter Fines & Component & Bed & Cyclone Fines & Filter Fines \\
\hline $\mathrm{Hg}$ & 0 & 0.00081 & 0.00119 & $\mathrm{Na}_{2} \mathrm{~S}$ & 0.532742 & 0.4483 & 0.9985 \\
\hline $\mathrm{HgCl}_{2}$ & 0 & 0.00081 & 0.00119 & $\mathrm{NaOH}$ & 0.532742 & 0.4483 & 0.9985 \\
\hline $\mathrm{Na}_{2} \mathrm{CO}_{3}$ & 0.532742 & 0.4483 & 0.9985 & Sucrose & 0.0488 & 0.4855 & 0.9985 \\
\hline $\mathrm{K}_{2} \mathrm{CO}_{3}$ & 0.296577 & 0.55 & 0.9985 & Carbon & 0 & 0.95 & 0.9985 \\
\hline $\mathrm{CaCO}_{3}$ & 0.55104 & 0.4615 & 0.9985 & $\mathrm{Na}_{2} \mathrm{SO}_{3}$ & 0.532742 & 0.4483 & 0.9985 \\
\hline $\mathrm{Na}_{2} \mathrm{O}$ & 0.532742 & 0.4483 & 0.9985 & $\mathrm{NaHCO}_{3}$ & 0.532742 & 0.4483 & 0.9985 \\
\hline $\mathrm{K} 2 \mathrm{O}$ & 0.296577 & 0.55 & 0.9985 & $\mathrm{NaF}$ & 0.0703 & 0.362 & 0.9315 \\
\hline $\mathrm{CaO}$ & 0.55104 & 0.4615 & 0.9985 & $\mathrm{~K}_{2} \mathrm{SO}_{4}$ & 0.4078 & 0.335 & 0.9985 \\
\hline $\mathrm{NaAlO}_{2}$ & 0.567821 & 0.484 & 0.9985 & $\mathrm{KHSO}_{4}$ & 0.4078 & 0.4483 & 0.9985 \\
\hline $\mathrm{Al}_{2} \mathrm{O}_{3}$ & 0.567821 & 0.484 & 0.9985 & $\mathrm{KHCO}_{3}$ & 0.296577 & 0.55 & 0.9985 \\
\hline $\mathrm{SiO}_{2}$ & 0.488108 & 0.75 & 0.9985 & $\mathrm{Cs}_{2} \mathrm{CO}_{3}$ & 0.03 & 0.59 & 0.999 \\
\hline $\mathrm{Fe}_{2} \mathrm{O}_{3}$ & 0.706408 & 0.435 & 0.9985 & $\mathrm{Na}_{3} \mathrm{PO}_{4}$ & 0.396 & 0.645 & 0.9985 \\
\hline $\mathrm{NaCl}$ & 0.158205 & 0.483 & 0.9985 & $\mathrm{~K}_{3} \mathrm{PO}_{4}$ & 0.396 & 0.645 & 0.9985 \\
\hline $\mathrm{KCl}$ & 0.158205 & 0.483 & 0.9985 & $\mathrm{AlPO}_{4}$ & 0.396 & 0.645 & 0.9985 \\
\hline $\mathrm{CaF}_{2}$ & 0.0703 & 0.362 & 0.9985 & Other +1 cations & 0.296577 & 0.55 & 0.9985 \\
\hline $\mathrm{NaNO}_{3}$ & 0.2695 & 0.351 & 0.9985 & $\mathrm{PbO}$ & 0.0342 & 0.233 & 0.9985 \\
\hline $\mathrm{KNO}_{3}$ & 0.2695 & 0.351 & 0.9985 & $\mathrm{Cr}_{2} \mathrm{O}_{3}$ & 0.5488 & 0.4145 & 0.9985 \\
\hline $\mathrm{Ca}\left(\mathrm{NO}_{3}\right)_{2}$ & 0.2695 & 0.351 & 0.9985 & +4 cations & 0.548 & 0.5176 & 0.9985 \\
\hline $\mathrm{TcO}_{3}$ & 0.039 & 0.62539 & 0.9985 & +5 cations & 0.548 & 0.5176 & 0.9985 \\
\hline $\mathrm{Al}\left(\mathrm{NO}_{3}\right)_{3}$ & 0.567821 & 0.484 & 0.9985 & +6 cations & 0.548 & 0.5176 & 0.9985 \\
\hline $\mathrm{CdO}$ & 0.5 & 0.5 & 0.9985 & -1 anions & 0.1582 & 0.483 & \\
\hline $\mathrm{NaHSO}_{4}$ & 0.4078 & 0.335 & 0.9985 & $\mathrm{CaSO}_{4}$ & 0.4078 & 0.335 & 0.9985 \\
\hline $\mathrm{Na}_{2} \mathrm{SO}_{4}$ & 0.4078 & 0.335 & 0.9985 & $\mathrm{CaCl}_{2}$ & 0.1582 & 0.483 & 0.9985 \\
\hline $\mathrm{NaHS}$ & 0.532742 & 0.4483 & 0.9985 & $\mathrm{KOH}$ & 0.5 & 0.4483 & 0.9985 \\
\hline $\mathrm{Hg}$ & 0 & 0.00081 & 0.00119 & $\mathrm{Na}_{2} \mathrm{~S}$ & 0.532742 & 0.4483 & 0.9985 \\
\hline
\end{tabular}

Finally it should be noted that while all attempts were made to utilize results from the Phase 2 TTT demonstration test, the test data was still being analyzed and reviewed when the mass balance runs were made. Thus the final Phase 2 test report (Soelberg 2004a) may contain some discrepancies from what was used in the mass balance model.

As for the TTT process, material balances were generated for TWR for chemical species using ASPEN Plus. Much of the discussion above regarding the TTT ASPEN model applies to the TWR process as well. Several organic chemical components (species) such as IPA and propane were added to the TWR model. Results from the Aspen Plus model were transferred to MS Excel, and the grouped species were subsequently separated back into individual components. The ASPEN flow scheme used to 
generate the TWR material balances is shown in Figure A-2. Most input parameters to the model were discussed in Sections A-5.4.5 and A-5.5.11.

Haefner ${ }^{1}$ provides documentation of the ASPEN model as tuned after Phase 1 of the TWR demonstration tests. Data from the tests was used to set "design specifications" in the model, i.e., allowing the program one parameter, such as a feed flow rate, to achieve another, such as a effluent composition or reactor temperature. The following is a list of design specifications in the model based on Phase 2 tests:

1. Airlift: varied the feed rate of air used to transfer SBW to the treatment facility; specification: mass flow rate of air rate $=0.0032 \times$ SBW mass rate.

2. Bed-Med: varied the feed rate of alumina bed material to the reformer; specification: bed media make-up flow rate $=1 \mathrm{wt} \%$ of the total reformer mass effluent rate (solid plus gas).

3. C-Bed: varied the flow of carbon distributed to the bed product to achieve a mass fraction of $0.1 \%$ in the bed product.

4. C-Fines: varied the flow of carbon distributed to the filter product to achieve a mass fraction of $6.5 \%$ in the filter product.

5. Dilu-Air: varied the feed rate of compressor pressure control air; specification: air rate $=10 \mathrm{wt}$ $\%$ of off-gas flow rate.

6. Gacuse: varied the rate of make up GAC; specification: spent GAC mercury loading of $95.75 \mathrm{~g}$ $\mathrm{Hg} / \mathrm{kg} \mathrm{GAC}$.

7. Grtmix: varied the rate of grout additives; specification: grout waste loading of $25 \mathrm{wt} \%$.

8. NAR: varied the rate of atomizing gas; specification: $\mathrm{NAR}=470$.

9. NOX-1: varied the fraction of $\mathrm{HNO}_{3}$ bypassed around the Gibbs reformer reactor to achieve $1800 \mathrm{ppm} \mathrm{NO}$ in the reformer off-gas.

10. NOX-2: varied the fraction of NO in the oxidizer that was treated as inert to achieve an NO concentration of $250 \mathrm{ppm}$ at the oxidizer outlet.

11. Ogcool: varied the rate of air to the off-gas air cooler; specification: effluent air temperature of $80^{\circ} \mathrm{C}$.

12. Prht1st: varied the rate of steam to the off-gas preheater; specification: off-gas exchanger effluent temperature $=120^{\circ} \mathrm{C}$.

13. Prodcool: varied the rate of gas to the product cooler; specification: product temperature of $56^{\circ} \mathrm{C}$.

1 Haefner, D., B. O’Brien, S. O. Bates, 2003, “Steam Reforming Process Design,” Engineering Design File EDF-3827, September 30, 2003. 
14. Propane: varied the flow of oxygen to the thermal oxidizer to achieve an outlet $\mathrm{O}_{2}$ concentration of $3 \mathrm{~mol} . \%$ (wet basis).

15. PulseN2: varied the rate of pulse gas to the sintered metal filter; specification: pulse gas rate $=$ $1 \mathrm{wt} \%$ of off-gas rate.

16. Recvol: varied the rate of scrub recycle to the reformer; specification: recycle scrub rate $=5 \mathrm{vol}$ 5 of SBW rate.

17. Scrbcw: varied the rate of cooling water to the scrub cooler; specification: outlet $\mathrm{CW}$ temperature of $60^{\circ} \mathrm{C}$.

18. Scrbflo: varied the scrub rate to the scrubber; specification: gas to liquid flow in scrubber $=$ 135.

19. Spryqch: varied the rate of water to the quench tower; specification: quench temperature of $100^{\circ} \mathrm{C}$.

20. Tranair1: varied the flow of nitrogen used for transporting bed media to the reformer to equal $10 \mathrm{wt} . \%$ of the solids being transferred.

The model used to simulate the TWR process after Phase 1 testing adjusted air in-leakage to meet a $\mathrm{CO}$ to $\mathrm{CO}_{2}$ off-gas ratio. This resulted in a feed air rate greater than the total $\mathrm{CO}_{2}$ fluidization and atomization rate. This specification was deleted and the air in-leakage set to a small value. However, this resulted in large quantities of organics - unreacted IPA and incomplete products of IPA combustion - in the reformer effluent. This result motivated a more in-depth analysis of the reported data.

The first step in the analysis was to perform mass balance calculations for oxygen for the Phase 2 testing. Using measured quantities of $\mathrm{CO}, \mathrm{CO}_{2}$, and $\mathrm{CO}_{3}$ in the reformer products together with measured flow rates of feed and products, the amount of $\mathrm{O}_{2}$ leaving the system was found to exceed the amount input by a significant amount. In the absence of air in-leakage the only other apparent source for $\mathrm{O}_{2}$ is decomposition of $\mathrm{H}_{2} \mathrm{O}$. Allowing for this possibility, a hydrogen balance was next performed, accounting for the $\mathrm{H}_{2}$ that would be generated in decomposing $\mathrm{H}_{2} \mathrm{O}$ to satisfy $\mathrm{O}_{2}$ balance. The results indicated the presence in the off-gas of either 20 times the amount of $\mathrm{H}_{2}$ that was measured, or of a hydrocarbon (HC) with an $\mathrm{H}: \mathrm{C}$ ratio of about 16 . The validity of this result was examined by considering the heating value that would be represented by the unaccounted $\mathrm{H}_{2}$ or $\mathrm{HC}$. Performing a heat balance on the downstream oxidizer it was found that the measured fuel (propane) input was insufficient to provide the measured temperature rise in the oxidizer unless the unaccounted $\mathrm{H}_{2}$ (or $\mathrm{HC}$ ) was assumed present in the steam reformer off-gas that enters the oxidizer.

This result seemed to confirm the above speculation that the true $\mathrm{H}_{2}$ and/or $\mathrm{HC}$ content of the reformer off-gas was not measured. However, both high postulated $\mathrm{H}_{2}$ concentration $(\sim 30 \%)$ and the high $\mathrm{H}: \mathrm{C}$ ratio in the postulated $\mathrm{HC}$ appeared outside the realm of experience. Nonetheless, the data on which the analysis was based appears valid. Thus, there is a clear need for additional consideration of the TWR test data in order to better model the process, as the model currently has two significant deficiencies. First, the excess organics in the reformer product would result in no propane required in a full-scale oxidizer. This, however, is contrary to the test results and intuition. Second, an excessive organic concentration is calculated in the reformer product. To attempt to address these deficiencies, the Aspen model was adjusted to force the product composition to be comparable with that measured in the Phase 2 tests. Also, in the model the propane rate to the oxidizer was scaled from the test data. As expected from the above analysis, this resulted in an unreasonable cooling duty required in the oxidizer to limit the 
temperature to the target value. These adjustments clearly do not resolve the problem but were done in order to provide a reasonable mass balance for comparison with other candidate processes. The need for additional development is, however, clearly indicated. 


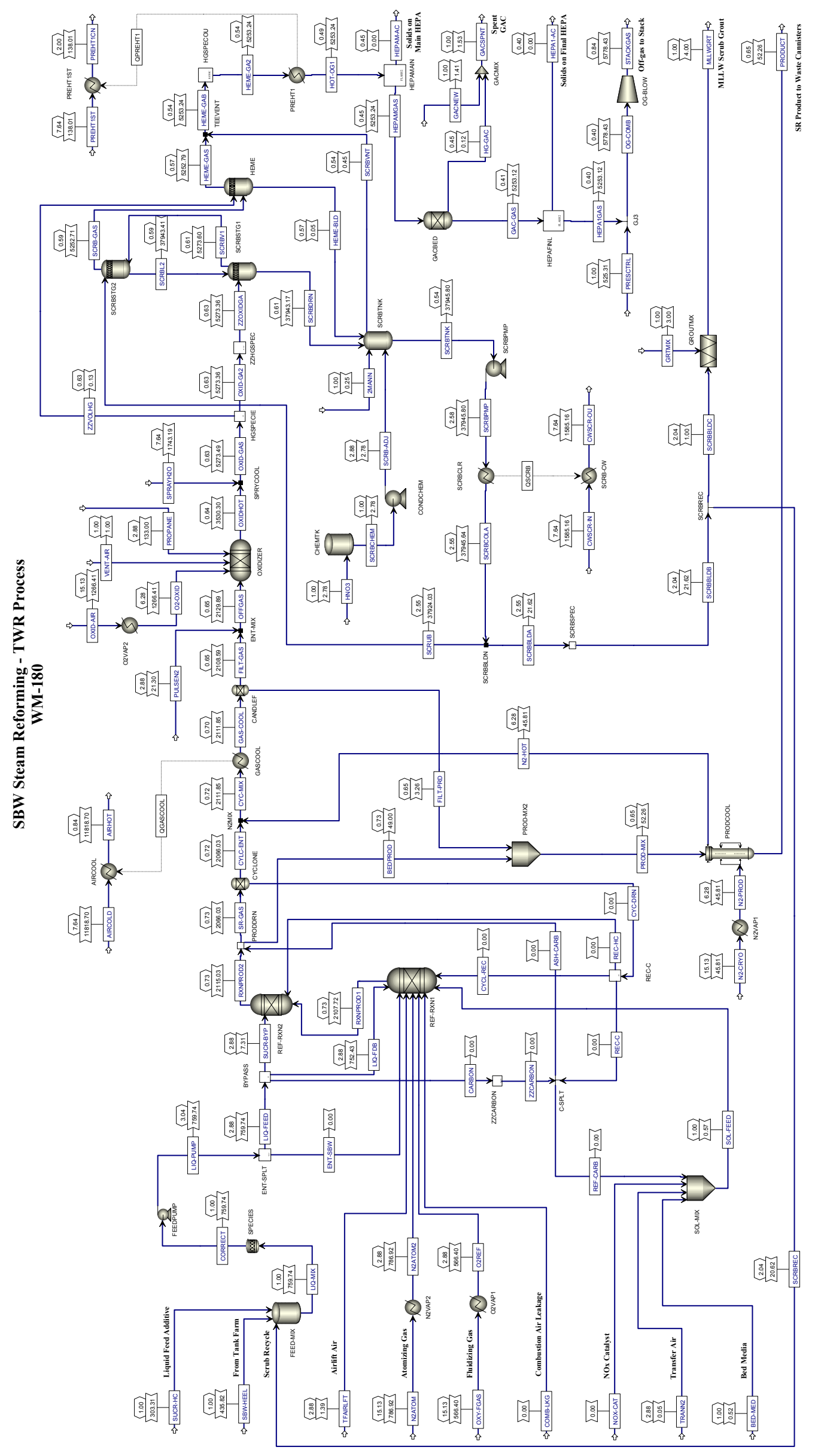

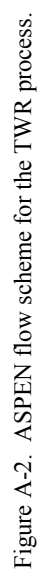




\section{A-6.2 Mass Balance Results}

Table A-10 lists the major chemicals consumed for reforming SBW with the TTT process. Entries in the body of the table are consumption rates for processing individual tanks, while the final column shows the total accumulated material used for complete processing. An iron or iron oxide catalyst may be recommended, however, the quantity called for would be small (no more than several pounds) and would not be costly. Finally, alumina is used as starting bed material and a total of 4 bed change-outs would require about 10,500 pounds.

Table A-10. Consumable chemicals estimate for TTT processing of SBW.

\begin{tabular}{lccccc}
\hline \multicolumn{1}{c}{ Stream } & WM-180 & WM-187 & WM-188 & WM-189 & Total \\
\hline SBW (gal/hr) & 96.0 & 95.8 & 96.6 & 97.4 & $1.16 \times 10^{6}$ gal \\
Gases: & & & & & \\
Nitrogen (lb/hr) & 1410 & 1360 & 1340 & 1460 & $16.7 \times 10^{6} \mathrm{lbs}$ \\
Oxygen (lb/hr) & 840 & 825 & 843 & 937 & $10.4 \times 10^{6} \mathrm{lbs}$ \\
Fuels: & & & & \\
Propane (lb/hr) & 34.5 & 33.9 & 35.0 & 34.5 & $0.41 \times 10^{6} \mathrm{lbs}$ \\
Fuel oil (lb/hr) & 268 & 268 & 275 & 291 & $3.31 \times 10^{6} \mathrm{lbs}$ \\
Reductants: & & & & \\
Sucrose (lb/hr) & 314 & 314 & 325 & 377 & $4.01 \times 10^{6} \mathrm{lbs}$ \\
Carbon (lb/hr) & 138 & 138 & 143 & 166 & $1.76 \times 10^{6} \mathrm{lbs}$ \\
Water: & & & & \\
Boiler feed (lb/hr) & 2640 & 2640 & 2700 & 2860 & $32.6 \times 10^{6} \mathrm{lbs}$ \\
Quench (lb/hr) & 3570 & 3530 & 3620 & 3850 & $43.8 \times 10^{6} \mathrm{lbs}$ \\
Nitric acid (lb/hr) & 4.02 & 8.65 & 8.63 & 9.82 & $9.2 \times 10^{4} \mathrm{lbs}$ \\
Process time (hours) & 3280 & 2100 & 3380 & 3240 & $1.2 \times 10^{4}$ hours \\
\hline
\end{tabular}

Table A-11 summarizes the production rate of reformer product from the TTT process and the associated packaging rate. The values in the table assume a densification operation is used to increase the product's bulk density from about 0.15 to $1.33 \mathrm{~g} / \mathrm{ml}\left(83 \mathrm{lb} / \mathrm{ft}^{3}\right)$. The table also summarizes the estimated decay heat and TRU activity of the final waste. 
Table A-11. Summary of estimated waste product quantities and properties for TTT processing of SBW.

\begin{tabular}{lccccc}
\hline & WM-189 & WM-187 & WM-188 & WM-189 & Total \\
\hline Product mass rate (lb/hr) & 146 & 165 & 147 & 175 & \\
Processing time (hr) & 3280 & 2100 & 3380 & 3240 & 12,000 \\
Canister filling rate (can/day) $^{\mathrm{a}}$ & 1.5 & 1.7 & 1.5 & 1.8 & \\
Number of canisters $^{\mathrm{a}, \mathrm{b}}$ & 205 & 149 & 211 & 243 & 808 \\
Heat generation $\left(\mathrm{W} / \mathrm{m}^{3}\right)$ & 1.99 & 2.62 & 2.70 & 2.92 & \\
Heat per canister $(\mathrm{W} / \mathrm{can})$ & 1.59 & 2.09 & 2.16 & 2.34 & \\
TRU specific activity (nCi/g) & 6040 & 5500 & 5010 & 4360 & \\
\hline
\end{tabular}

a - Waste specific gravity assumed as 1.33 .

$\mathrm{b}-\mathrm{A}$ canister contains $0.8 \mathrm{~m}^{3}$ of waste.

Table A-12 summarizes the chemicals used to treat spent scrub solution to the TTT process. The values in the table are mass amounts (as opposed to rates) since the spent scrub will be blown down in batches and will presumably be treated in batch mode. The lime additions are used to neutralize the acid associated with the waste and are based on a scrub containing 1.02 molar free acid. The lime is assumed to be added as a $50 \mathrm{wt} \%$ slurry. Calcium sulfide is used as a precipitant for mercury, which is assumed present at $60 \mathrm{~g} \mathrm{Hg} / \mathrm{l}$. The final treatment step is to add Portland cement (enough to achieve $75 \mathrm{wt} \%$ cement) for stabilization/solidification of the waste.

Table A-12. Consumable chemicals estimate for spent scrub treatment.

\begin{tabular}{lccccc}
\hline \multicolumn{1}{c}{ Stream } & $\begin{array}{c}\text { WM-180 } \\
\text { (lbs) }\end{array}$ & $\begin{array}{c}\text { WM-187 } \\
\text { (lbs) }\end{array}$ & $\begin{array}{c}\text { WM-188 } \\
(\mathbf{l b s})\end{array}$ & $\begin{array}{c}\text { WM-189 } \\
\text { (lbs) }\end{array}$ & $\begin{array}{c}\text { Total } \\
\text { (lbs) }\end{array}$ \\
\hline Spent scrub & 7180 & 4610 & 7420 & 7090 & 26,300 \\
Lime-50wt\% $\mathrm{Ca}(\mathrm{OH})_{2}$ & 537 & 345 & 555 & 531 & 1970 \\
Calcium sulfide & 153 & 98.5 & 158 & 151 & 561 \\
Portland cement & 23,600 & 15,200 & 24,400 & 23300 & 86,500 \\
$\begin{array}{l}\text { Total mass of stabilized } \\
\text { scrub }\end{array}$ & 31,500 & 20,200 & 32,500 & 31,100 & 115,000 \\
\hline
\end{tabular}

Tables A-13 to A-16 constitute the complete mass balances for the TTT carbonate flowsheet. 
Table A-13. Mass balance, Tank WM-180 waste.

\begin{tabular}{|c|c|c|c|c|c|c|c|c|c|}
\hline PFD \# & PFD-2 & PFD-2 & PFD-2 & PFD-2 & PFD-2 & PFD-2 & PFD-2 & PFD-2 & PFD-2 \\
\hline WM-180, Stream \# & 101 & 102 & 103 & 104 & 105 & 106 & 107 & 108 & 108 \\
\hline Stream Name & SBW & $\begin{array}{l}\text { Reformer } \\
\text { Feed }\end{array}$ & $\begin{array}{l}\text { Reformer } \\
\text { Off-gas }\end{array}$ & $\begin{array}{l}\text { Reformer } \\
\text { Off-gas } \\
\text { Cooled }\end{array}$ & $\begin{array}{c}\text { Cyclone } \\
\text { Drain }\end{array}$ & $\begin{array}{l}\text { Filter } \\
\text { Drain }\end{array}$ & Bed Drain & $\begin{array}{l}\text { Cooled } \\
\text { Product }\end{array}$ & $\begin{array}{l}\text { Product } \\
\text { Shipping } \\
\text { Canisters }\end{array}$ \\
\hline Rate or Volume & $9.60 \mathrm{E}+01$ & $1.32 \mathrm{E}+02$ & $4.56 \mathrm{E}+05$ & $4.11 \mathrm{E}+05$ & $9.84 \mathrm{E}+01$ & $6.22 \mathrm{E}+01$ & $8.38 \mathrm{E}+01$ & $1.46 \mathrm{E}+02$ & $1.49 \mathrm{E}+00$ \\
\hline Volume Flow (standard, we & & & $1.02 \mathrm{E}+05$ & $1.04 \mathrm{E}+05$ & & & & & \\
\hline Volume Flow (standard, dry & & & $3.09 \mathrm{E}+04$ & $3.29 \mathrm{E}+04$ & & & & & \\
\hline Rate Units & $\mathrm{gal} / \mathrm{hr}$ & $\mathrm{gal} / \mathrm{hr}$ & $\mathrm{ft} 3 / \mathrm{hr}$ & $\mathrm{ft} 3 / \mathrm{hr}$ & $\mathrm{lb} / \mathrm{hr}$ & $\mathrm{lb} / \mathrm{hr}$ & $\mathrm{lb} / \mathrm{hr}$ & $\mathrm{lb} / \mathrm{hr}$ & can's/day \\
\hline Rate or Volume, metric & 363 & 501 & $2.90 \mathrm{E}+03$ & $2.95 \mathrm{E}+03$ & 45 & 28.2 & 38.0 & 66.2 & 66.2 \\
\hline Rate Units & $\mathrm{L} / \mathrm{hr}$ & $\mathrm{L} / \mathrm{hr}$ & wscm/hr & wscm/hr & $\mathrm{kg} / \mathrm{hr}$ & $\mathrm{kg} / \mathrm{hr}$ & $\mathrm{kg} / \mathrm{hr}$ & $\mathrm{kg} / \mathrm{hr}$ & $\mathrm{kg} / \mathrm{hr}$ \\
\hline Temperature, ${ }^{\circ} \mathrm{C}$ & 15 & 19 & 670 & 530 & 670 & 530 & 670 & 56 & 56 \\
\hline Temperature, ${ }^{\circ} \mathrm{F}$ & 59 & 67 & 1238 & 986 & 1238 & 986 & 1238 & 133 & 133 \\
\hline Pressure, psia & 14.7 & 44.7 & 10.6 & 10.2 & 10.6 & 9.5 & 10.8 & 9.5 & 9.5 \\
\hline Specific Gravity & 1.20 & 1.20 & $1.98 \mathrm{E}-04$ & $2.26 \mathrm{E}-04$ & 0.24 & 1.15 & 1.46 & 1.33 & 1.33 \\
\hline Chemical Composition & Mol/liter & Mol/liter & $\mathrm{lb} / \mathrm{wscf}$ & $\mathrm{lb} / \mathrm{wscf}$ & Wt frac & Wt frac & Wt frac & Wt frac & Wt frac \\
\hline $\mathrm{H}+$ & $1.02 \mathrm{E}+00$ & $7.89 \mathrm{E}-01$ & $8.63 \mathrm{E}-07$ & $8.47 \mathrm{E}-07$ & $4.55 \mathrm{E}-06$ & $8.84 \mathrm{E}-06$ & $1.36 \mathrm{E}-05$ & $1.16 \mathrm{E}-05$ & $1.16 \mathrm{E}-05$ \\
\hline $\mathrm{Al}+3$ & $5.37 \mathrm{E}-01$ & $3.90 \mathrm{E}-01$ & $3.50 \mathrm{E}-05$ & $3.44 \mathrm{E}-05$ & $3.55 \mathrm{E}-02$ & $5.75 \mathrm{E}-02$ & $1.08 \mathrm{E}-01$ & $8.63 \mathrm{E}-02$ & $8.63 \mathrm{E}-02$ \\
\hline $\mathrm{Sb}+5$ & $1.28 \mathrm{E}-06$ & $9.31 \mathrm{E}-07$ & $3.48 \mathrm{E}-10$ & $3.41 \mathrm{E}-10$ & $3.53 \mathrm{E}-07$ & $5.71 \mathrm{E}-07$ & $1.07 \mathrm{E}-06$ & $8.57 \mathrm{E}-07$ & $8.57 \mathrm{E}-07$ \\
\hline $\mathrm{As}+3$ & $4.12 \mathrm{E}-04$ & $2.99 \mathrm{E}-04$ & $6.87 \mathrm{E}-08$ & $6.75 \mathrm{E}-08$ & $6.98 \mathrm{E}-05$ & $1.13 \mathrm{E}-04$ & $2.11 \mathrm{E}-04$ & $1.69 \mathrm{E}-04$ & $1.69 \mathrm{E}-04$ \\
\hline $\mathrm{Ba}+2$ & $4.61 \mathrm{E}-05$ & $3.35 \mathrm{E}-05$ & $1.51 \mathrm{E}-08$ & $1.48 \mathrm{E}-08$ & $1.35 \mathrm{E}-05$ & $2.48 \mathrm{E}-05$ & $4.21 \mathrm{E}-05$ & $3.47 \mathrm{E}-05$ & $3.47 \mathrm{E}-05$ \\
\hline $\mathrm{Be}+2$ & $7.21 \mathrm{E}-06$ & $5.23 \mathrm{E}-06$ & $1.55 \mathrm{E}-10$ & $1.52 \mathrm{E}-10$ & $1.38 \mathrm{E}-07$ & $2.55 \mathrm{E}-07$ & $4.32 \mathrm{E}-07$ & $3.56 \mathrm{E}-07$ & $3.56 \mathrm{E}-07$ \\
\hline $\mathrm{B}+3$ & $9.59 \mathrm{E}-03$ & $6.96 \mathrm{E}-03$ & $2.31 \mathrm{E}-07$ & $2.26 \mathrm{E}-07$ & $2.34 \mathrm{E}-04$ & $3.79 \mathrm{E}-04$ & $7.09 \mathrm{E}-04$ & $5.68 \mathrm{E}-04$ & $5.68 \mathrm{E}-04$ \\
\hline $\mathrm{Cd}+2$ & $6.60 \mathrm{E}-04$ & $4.79 \mathrm{E}-04$ & $1.94 \mathrm{E}-07$ & $1.90 \mathrm{E}-07$ & $2.01 \mathrm{E}-04$ & $3.18 \mathrm{E}-04$ & $4.73 \mathrm{E}-04$ & $4.07 \mathrm{E}-04$ & $4.07 \mathrm{E}-04$ \\
\hline $\mathrm{Ca}+2$ & $3.41 \mathrm{E}-02$ & $2.47 \mathrm{E}-02$ & $1.09 \mathrm{E}-05$ & $1.07 \mathrm{E}-05$ & $9.70 \mathrm{E}-03$ & $1.79 \mathrm{E}-02$ & $3.03 \mathrm{E}-02$ & $2.50 \mathrm{E}-02$ & $2.50 \mathrm{E}-02$ \\
\hline $\mathrm{Cr}+3$ & $3.10 \mathrm{E}-03$ & $2.25 \mathrm{E}-03$ & $4.10 \mathrm{E}-07$ & $4.02 \mathrm{E}-07$ & $3.02 \mathrm{E}-04$ & $6.74 \mathrm{E}-04$ & $1.04 \mathrm{E}-03$ & $8.84 \mathrm{E}-04$ & $8.84 \mathrm{E}-04$ \\
\hline $\mathrm{Co}+2$ & $1.69 \mathrm{E}-05$ & $1.22 \mathrm{E}-05$ & $2.21 \mathrm{E}-09$ & $2.17 \mathrm{E}-09$ & $2.24 \mathrm{E}-06$ & $3.63 \mathrm{E}-06$ & $6.80 \mathrm{E}-06$ & $5.45 \mathrm{E}-06$ & $5.45 \mathrm{E}-06$ \\
\hline $\mathrm{Cs}+$ & $2.37 \mathrm{E}-05$ & $2.02 \mathrm{E}-05$ & $2.69 \mathrm{E}-08$ & $2.64 \mathrm{E}-08$ & $4.03 \mathrm{E}-05$ & $4.42 \mathrm{E}-05$ & $2.48 \mathrm{E}-06$ & $2.09 \mathrm{E}-05$ & $2.09 \mathrm{E}-05$ \\
\hline $\mathrm{Cu}+2$ & $5.83 \mathrm{E}-04$ & $4.23 \mathrm{E}-04$ & $8.84 \mathrm{E}-08$ & $8.67 \mathrm{E}-08$ & $7.88 \mathrm{E}-05$ & $1.45 \mathrm{E}-04$ & $2.46 \mathrm{E}-04$ & $2.03 \mathrm{E}-04$ & $2.03 \mathrm{E}-04$ \\
\hline $\mathrm{Fe}+3$ & $1.77 \mathrm{E}-02$ & $1.29 \mathrm{E}-02$ & $1.70 \mathrm{E}-06$ & 1.67E-06 & $1.36 \mathrm{E}-03$ & $2.79 \mathrm{E}-03$ & $8.83 \mathrm{E}-03$ & $6.26 \mathrm{E}-03$ & $6.26 \mathrm{E}-03$ \\
\hline $\mathrm{Pb}+2$ & $1.16 \mathrm{E}-03$ & $8.44 \mathrm{E}-04$ & $1.80 \mathrm{E}-06$ & 1.77E-06 & $5.70 \mathrm{E}-04$ & $2.96 \mathrm{E}-03$ & $1.02 \mathrm{E}-04$ & $1.32 \mathrm{E}-03$ & $1.32 \mathrm{E}-03$ \\
\hline $\mathrm{Hg}+2$ & $1.35 \mathrm{E}-03$ & $1.39 \mathrm{E}-03$ & $3.00 \mathrm{E}-06$ & $2.95 \mathrm{E}-06$ & $2.53 \mathrm{E}-06$ & $5.88 \mathrm{E}-06$ & & \begin{tabular}{|l|}
$2.51 \mathrm{E}-06$ \\
\end{tabular} & $2.51 \mathrm{E}-06$ \\
\hline $\mathrm{Mn}+4$ & 1.17E-02 & $8.52 \mathrm{E}-03$ & $1.43 \mathrm{E}-06$ & $1.41 \mathrm{E}-06$ & $1.46 \mathrm{E}-03$ & $2.36 \mathrm{E}-03$ & $4.41 \mathrm{E}-03$ & \begin{tabular}{|l|}
$3.54 \mathrm{E}-03$ \\
\end{tabular} & $3.54 \mathrm{E}-03$ \\
\hline $\mathrm{Ni}+2$ & $1.28 \mathrm{E}-03$ & $9.29 \mathrm{E}-04$ & $1.79 \mathrm{E}-07$ & 1.76E-07 & $1.60 \mathrm{E}-04$ & $2.95 \mathrm{E}-04$ & $4.99 \mathrm{E}-04$ & \begin{tabular}{|l|}
$4.12 \mathrm{E}-04$ \\
\end{tabular} & $4.12 \mathrm{E}-04$ \\
\hline $\mathrm{K}+$ & $1.62 \mathrm{E}-01$ & $1.18 \mathrm{E}-01$ & $3.10 \mathrm{E}-05$ & $3.04 \mathrm{E}-05$ & $3.91 \mathrm{E}-02$ & $5.09 \mathrm{E}-02$ & $3.46 \mathrm{E}-02$ & \begin{tabular}{|l|}
$4.16 \mathrm{E}-02$ \\
\end{tabular} & $4.16 \mathrm{E}-02$ \\
\hline $\mathrm{Se}+4$ & $7.08 \mathrm{E}-05$ & $5.17 \mathrm{E}-05$ & $1.25 \mathrm{E}-08$ & $1.23 \mathrm{E}-08$ & $1.40 \mathrm{E}-05$ & $2.06 \mathrm{E}-05$ & $3.85 \mathrm{E}-05$ & $3.09 \mathrm{E}-05$ & $3.09 \mathrm{E}-05$ \\
\hline $\mathrm{Ag}^{+}$ & $5.60 \mathrm{E}-06$ & $4.07 \mathrm{E}-06$ & $1.62 \mathrm{E}-09$ & $1.59 \mathrm{E}-09$ & $1.35 \mathrm{E}-06$ & $2.66 \mathrm{E}-06$ & $3.81 \mathrm{E}-06$ & $3.32 \mathrm{E}-06$ & $3.32 \mathrm{E}-06$ \\
\hline $\mathrm{Na}+$ & $1.74 \mathrm{E}+00$ & $1.27 \mathrm{E}+00$ & $1.08 \mathrm{E}-04$ & $1.06 \mathrm{E}-04$ & $9.00 \mathrm{E}-02$ & 1.77E-01 & $2.53 \mathrm{E}-01$ & $2.20 \mathrm{E}-01$ & $2.20 \mathrm{E}-01$ \\
\hline $\mathrm{Tl}+3$ & $2.53 \mathrm{E}-05$ & $1.84 \mathrm{E}-05$ & $1.15 \mathrm{E}-08$ & $1.13 \mathrm{E}-08$ & $1.17 \mathrm{E}-05$ & 1.89E-05 & $3.54 \mathrm{E}-05$ & $2.84 \mathrm{E}-05$ & $2.84 \mathrm{E}-05$ \\
\hline $\mathrm{U}+4$ & $3.33 \mathrm{E}-04$ & $2.43 \mathrm{E}-04$ & $1.78 \mathrm{E}-07$ & $1.75 \mathrm{E}-07$ & $1.99 \mathrm{E}-04$ & $2.92 \mathrm{E}-04$ & $5.46 \mathrm{E}-04$ & \begin{tabular}{|l|}
$4.38 \mathrm{E}-04$ \\
\end{tabular} & $4.38 \mathrm{E}-04$ \\
\hline$V+3$ & $8.08 \mathrm{E}-04$ & $5.86 \mathrm{E}-04$ & $9.16 \mathrm{E}-08$ & $8.99 \mathrm{E}-08$ & $1.02 \mathrm{E}-04$ & $1.51 \mathrm{E}-04$ & $2.81 \mathrm{E}-04$ & \begin{tabular}{|l|}
$2.26 \mathrm{E}-04$ \\
\end{tabular} & $2.26 \mathrm{E}-04$ \\
\hline$Z n+2$ & $8.77 \mathrm{E}-04$ & $6.37 \mathrm{E}-04$ & $1.37 \mathrm{E}-07$ & $1.34 \mathrm{E}-07$ & $1.22 \mathrm{E}-04$ & $2.25 \mathrm{E}-04$ & $3.81 \mathrm{E}-04$ & \begin{tabular}{|l|}
$3.14 \mathrm{E}-04$ \\
\end{tabular} & $3.14 \mathrm{E}-04$ \\
\hline $\mathrm{Zr}+4$ & $1.22 \mathrm{E}-03$ & $8.92 \mathrm{E}-04$ & $2.50 \mathrm{E}-07$ & $2.45 \mathrm{E}-07$ & $2.79 \mathrm{E}-04$ & $4.10 \mathrm{E}-04$ & $7.67 \mathrm{E}-04$ & \begin{tabular}{|l|}
$6.15 \mathrm{E}-04$ \\
\end{tabular} & $6.15 \mathrm{E}-04$ \\
\hline $\mathrm{Cl}-$ & $2.64 \mathrm{E}-02$ & $1.92 \mathrm{E}-02$ & $5.44 \mathrm{E}-06$ & $5.34 \mathrm{E}-06$ & $5.13 \mathrm{E}-03$ & $8.67 \mathrm{E}-03$ & $2.34 \mathrm{E}-03$ & $5.04 \mathrm{E}-03$ & $5.04 \mathrm{E}-03$ \\
\hline F- & $3.71 \mathrm{E}-02$ & $2.76 \mathrm{E}-02$ & $5.08 \mathrm{E}-06$ & $4.98 \mathrm{E}-06$ & $2.90 \mathrm{E}-03$ & $7.53 \mathrm{E}-03$ & $7.11 \mathrm{E}-04$ & \begin{tabular}{|c|}
$3.61 \mathrm{E}-03$ \\
\end{tabular} & $3.61 \mathrm{E}-03$ \\
\hline SO4-2 & $4.58 \mathrm{E}-02$ & $3.41 \mathrm{E}-02$ & $2.00 \mathrm{E}-08$ & $1.96 \mathrm{E}-08$ & $1.05 \mathrm{E}-05$ & $3.28 \mathrm{E}-05$ & $2.53 \mathrm{E}-05$ & \begin{tabular}{|l|}
$2.85 \mathrm{E}-05$ \\
\end{tabular} & $2.85 \mathrm{E}-05$ \\
\hline NO3- & $4.48 \mathrm{E}+00$ & $3.29 \mathrm{E}+00$ & $8.33 \mathrm{E}-06$ & $8.17 \mathrm{E}-06$ & $1.22 \mathrm{E}-03$ & $3.51 \mathrm{E}-03$ & $2.70 \mathrm{E}-03$ & \begin{tabular}{|l|}
$3.05 \mathrm{E}-03$ \\
\end{tabular} & $3.05 \mathrm{E}-03$ \\
\hline PO4-3 & $1.80 \mathrm{E}-02$ & $1.31 \mathrm{E}-02$ & $5.78 \mathrm{E}-06$ & $5.67 \mathrm{E}-06$ & $1.09 \mathrm{E}-02$ & $9.50 \mathrm{E}-03$ & $1.30 \mathrm{E}-02$ & \begin{tabular}{|c|}
$1.15 \mathrm{E}-02$ \\
\end{tabular} & $1.15 \mathrm{E}-02$ \\
\hline$A m+4$ & $2.91 \mathrm{E}-08$ & $2.11 \mathrm{E}-08$ & $1.58 \mathrm{E}-11$ & $1.55 \mathrm{E}-11$ & $1.60 \mathrm{E}-08$ & $2.59 \mathrm{E}-08$ & $4.84 \mathrm{E}-08$ & $3.88 \mathrm{E}-08$ & $3.88 \mathrm{E}-08$ \\
\hline $\mathrm{Br}-\mathrm{-}$ & $1.43 \mathrm{E}-07$ & $1.05 \mathrm{E}-07$ & $6.66 \mathrm{E}-11$ & $6.54 \mathrm{E}-11$ & $6.29 \mathrm{E}-08$ & $1.06 \mathrm{E}-07$ & $2.87 \mathrm{E}-08$ & \begin{tabular}{|l|}
$6.18 \mathrm{E}-08$ \\
\end{tabular} & $6.18 \mathrm{E}-08$ \\
\hline $\mathrm{Ce}+4$ & $4.02 \mathrm{E}-05$ & $2.92 \mathrm{E}-05$ & $1.25 \mathrm{E}-08$ & $1.23 \mathrm{E}-08$ & $1.27 \mathrm{E}-05$ & $2.06 \mathrm{E}-05$ & $3.85 \mathrm{E}-05$ & \begin{tabular}{|l|}
$3.09 \mathrm{E}-05$ \\
\end{tabular} & $3.09 \mathrm{E}-05$ \\
\hline $\mathrm{Eu}+3$ & $2.38 \mathrm{E}-07$ & $1.72 \mathrm{E}-07$ & $8.04 \mathrm{E}-11$ & $7.89 \mathrm{E}-11$ & $8.16 \mathrm{E}-08$ & $1.32 \mathrm{E}-07$ & $2.47 \mathrm{E}-07$ & \begin{tabular}{|c|}
$1.98 \mathrm{E}-07$ \\
\end{tabular} & $1.98 \mathrm{E}-07$ \\
\hline $\mathrm{Gd}+3$ & $1.48 \mathrm{E}-04$ & $1.07 \mathrm{E}-04$ & $5.18 \mathrm{E}-08$ & $5.09 \mathrm{E}-08$ & $5.26 \mathrm{E}-05$ & $8.52 \mathrm{E}-05$ & $1.59 \mathrm{E}-04$ & \begin{tabular}{|c|}
$1.28 \mathrm{E}-04$ \\
\end{tabular} & $1.28 \mathrm{E}-04$ \\
\hline $\mathrm{Ge}+4$ & $4.13 \mathrm{E}-09$ & $3.02 \mathrm{E}-09$ & $6.73 \mathrm{E}-13$ & $6.61 \mathrm{E}-13$ & $7.52 \mathrm{E}-10$ & $1.11 \mathrm{E}-09$ & $2.07 \mathrm{E}-09$ & \begin{tabular}{|c|}
$1.66 \mathrm{E}-09$ \\
\end{tabular} & $1.66 \mathrm{E}-09$ \\
\hline $\ln +3$ & $6.50 \mathrm{E}-07$ & $4.72 \mathrm{E}-07$ & $1.66 \mathrm{E}-10$ & $1.63 \mathrm{E}-10$ & $1.69 \mathrm{E}-07$ & $2.73 \mathrm{E}-07$ & $5.11 \mathrm{E}-07$ & \begin{tabular}{|l|}
$4.10 \mathrm{E}-07$ \\
\end{tabular} & $4.10 \mathrm{E}-07$ \\
\hline I- & $1.24 \mathrm{E}-06$ & $9.07 \mathrm{E}-07$ & $9.18 \mathrm{E}-10$ & $9.01 \mathrm{E}-10$ & $8.66 \mathrm{E}-07$ & 1.46E-06 & $3.95 \mathrm{E}-07$ & \begin{tabular}{|l|}
$8.50 \mathrm{E}-07$ \\
\end{tabular} & $8.50 \mathrm{E}-07$ \\
\hline $\mathrm{La}+3$ & $4.32 \mathrm{E}-06$ & $3.13 \mathrm{E}-06$ & $1.33 \mathrm{E}-09$ & $1.31 \mathrm{E}-09$ & $1.35 \mathrm{E}-06$ & $2.19 \mathrm{E}-06$ & $4.10 \mathrm{E}-06$ & $3.29 \mathrm{E}-06$ & $3.29 \mathrm{E}-06$ \\
\hline
\end{tabular}


Table A-13. Mass balance, Tank WM-180 waste (continued).

\begin{tabular}{|c|c|c|c|c|c|c|c|c|c|}
\hline Stream \# & 101 & 102 & 103 & 104 & 105 & 106 & 107 & 108 & 108 \\
\hline Chemical Composition & Mol/liter & Mol/liter & $\mathrm{lb} / \mathrm{wscf}$ & $\mathrm{Ib} /$ wscf & Wt frac & Wt frac & Wt frac & Wt frac & Wt frac \\
\hline Li+ & $4.10 \mathrm{E}-04$ & $2.97 \mathrm{E}-04$ & 7.63E-09 & 7.49E-09 & $6.37 \mathrm{E}-06$ & $1.25 \mathrm{E}-05$ & 1.79E-05 & 1.56E-05 & $1.56 \mathrm{E}-05$ \\
\hline $\mathrm{Mg}+2$ & $1.01 \mathrm{E}-02$ & $7.36 \mathrm{E}-03$ & $5.88 \mathrm{E}-07$ & $5.77 \mathrm{E}-07$ & $5.24 \mathrm{E}-04$ & 9.66E-04 & 1.64E-03 & $1.35 \mathrm{E}-03$ & $1.35 \mathrm{E}-03$ \\
\hline $\mathrm{Mo}+6$ & $1.51 \mathrm{E}-03$ & $1.11 \mathrm{E}-03$ & $3.27 \mathrm{E}-07$ & $3.21 \mathrm{E}-07$ & $3.65 \mathrm{E}-04$ & 5.37E-04 & $1.00 \mathrm{E}-03$ & $8.05 E-04$ & 8.05E-04 \\
\hline $\mathrm{Nd}+3$ & 1.39E-05 & $1.01 \mathrm{E}-05$ & 4.47E-09 & 4.39E-09 & $4.54 \mathrm{E}-06$ & 7.34E-06 & 1.37E-05 & 1.10E-05 & $1.10 \mathrm{E}-05$ \\
\hline $\mathrm{Np}+4$ & 8.05E-06 & $5.84 \mathrm{E}-06$ & $4.25 E-09$ & 4.17E-09 & $4.31 \mathrm{E}-06$ & $6.98 \mathrm{E}-06$ & $1.30 \mathrm{E}-05$ & $1.05 E-05$ & $1.05 \mathrm{E}-05$ \\
\hline $\mathrm{Nb}+5$ & 4.12E-04 & 2.99E-04 & $8.52 \mathrm{E}-08$ & 8.36E-08 & 9.51E-05 & $1.40 \mathrm{E}-04$ & $2.62 \mathrm{E}-04$ & $2.10 \mathrm{E}-04$ & $2.10 \mathrm{E}-04$ \\
\hline $\mathrm{Pd}+4$ & $2.89 \mathrm{E}-05$ & $2.10 \mathrm{E}-05$ & 7.33E-09 & 7.19E-09 & $6.53 \mathrm{E}-06$ & 1.20E-05 & 2.04E-05 & $1.68 \mathrm{E}-05$ & 1.68E-05 \\
\hline $\mathrm{Pu}+4$ & 9.29E-06 & $6.79 \mathrm{E}-06$ & 5.09E-09 & 4.99E-09 & 5.68E-06 & 8.36E-06 & $1.56 \mathrm{E}-05$ & $1.25 \mathrm{E}-05$ & $1.25 \mathrm{E}-05$ \\
\hline $\mathrm{Pr}+4$ & $3.93 \mathrm{E}-06$ & $2.85 \mathrm{E}-06$ & 1.23E-09 & 1.21E-09 & $1.25 \mathrm{E}-06$ & $2.02 \mathrm{E}-06$ & 3.78E-06 & 3.03E-06 & $3.03 E-06$ \\
\hline $\mathrm{Pm}+3$ & $5.95 \mathrm{E}-10$ & $4.31 \mathrm{E}-10$ & 1.92E-13 & $1.88 \mathrm{E}-13$ & $1.95 \mathrm{E}-10$ & $3.15 \mathrm{E}-10$ & $5.90 \mathrm{E}-10$ & $4.73 \mathrm{E}-10$ & $4.73 \mathrm{E}-10$ \\
\hline $\mathrm{Rh}+4$ & 1.69E-06 & 1.23E-06 & $3.88 \mathrm{E}-10$ & $3.81 \mathrm{E}-10$ & 3.94E-07 & $6.37 \mathrm{E}-07$ & 1.19E-06 & 9.56E-07 & 9.56E-07 \\
\hline $\mathrm{Rb}+$ & $2.61 \mathrm{E}-06$ & $1.89 \mathrm{E}-06$ & $5.98 \mathrm{E}-10$ & $5.87 \mathrm{E}-10$ & 4.99E-07 & $9.80 \mathrm{E}-07$ & $1.40 \mathrm{E}-06$ & $1.22 \mathrm{E}-06$ & 1.22E-06 \\
\hline Ru+3 & 1.16E-04 & 8.44E-05 & $2.62 \mathrm{E}-08$ & $2.57 \mathrm{E}-08$ & $2.66 \mathrm{E}-05$ & 4.30E-05 & 8.04E-05 & $6.45 \mathrm{E}-05$ & $6.45 \mathrm{E}-05$ \\
\hline $\mathrm{Sm}+3$ & $2.58 \mathrm{E}-06$ & 1.87E-06 & $8.64 \mathrm{E}-10$ & $8.48 \mathrm{E}-10$ & 8.77E-07 & $1.42 \mathrm{E}-06$ & $2.66 \mathrm{E}-06$ & $2.13 \mathrm{E}-06$ & $2.13 \mathrm{E}-06$ \\
\hline $\mathrm{Si}+4$ & $2.84 \mathrm{E}-03$ & $2.09 \mathrm{E}-03$ & 1.93E-06 & $1.90 \mathrm{E}-06$ & $5.68 \mathrm{E}-03$ & $3.18 \mathrm{E}-03$ & 8.72E-03 & $6.36 \mathrm{E}-03$ & $6.36 \mathrm{E}-03$ \\
\hline $\mathrm{Sr}+2$ & 9.92E-05 & $7.21 \mathrm{E}-05$ & $2.08 \mathrm{E}-08$ & $2.04 \mathrm{E}-08$ & $1.85 \mathrm{E}-05$ & $3.41 \mathrm{E}-05$ & 5.78E-05 & 4.77E-05 & 4.77E-05 \\
\hline $\mathrm{Tc}+7$ & $4.88 \mathrm{E}-06$ & $3.55 \mathrm{E}-06$ & 3.38E-09 & 3.32E-09 & 5.87E-06 & 5.56E-06 & 4.47E-07 & $2.62 \mathrm{E}-06$ & $2.62 \mathrm{E}-06$ \\
\hline $\mathrm{Te}+4$ & $1.36 \mathrm{E}-06$ & 9.91E-07 & $3.88 \mathrm{E}-10$ & $3.81 \mathrm{E}-10$ & 4.34E-07 & $6.38 \mathrm{E}-07$ & 1.19E-06 & 9.56E-07 & $9.56 \mathrm{E}-07$ \\
\hline $\mathrm{Tb}+4$ & 9.94E-10 & $7.21 \mathrm{E}-10$ & $3.51 \mathrm{E}-13$ & $3.45 \mathrm{E}-13$ & $3.57 \mathrm{E}-10$ & $5.78 \mathrm{E}-10$ & 1.08E-09 & $8.66 \mathrm{E}-10$ & $8.66 \mathrm{E}-10$ \\
\hline $\mathrm{Th}+4$ & $9.69 \mathrm{E}-11$ & $7.08 \mathrm{E}-11$ & $5.04 \mathrm{E}-14$ & $4.95 \mathrm{E}-14$ & $5.63 \mathrm{E}-11$ & $8.29 \mathrm{E}-11$ & $1.55 \mathrm{E}-10$ & $1.24 \mathrm{E}-10$ & $1.24 \mathrm{E}-10$ \\
\hline$S n+4$ & 6.81E-05 & 4.98E-05 & 1.81E-08 & 1.78E-08 & 2.02E-05 & 2.98E-05 & 5.57E-05 & 4.46E-05 & 4.46E-05 \\
\hline$T \mathrm{Ti}+4$ & $1.24 \mathrm{E}-04$ & 9.06E-05 & 1.33E-08 & $1.31 \mathrm{E}-08$ & 1.49E-05 & $2.19 \mathrm{E}-05$ & 4.09E-05 & $3.28 \mathrm{E}-05$ & $3.28 \mathrm{E}-05$ \\
\hline $\mathrm{Y}+3$ & $3.22 \mathrm{E}-06$ & 2.34E-06 & $6.37 \mathrm{E}-10$ & $6.25 \mathrm{E}-10$ & $6.46 \mathrm{E}-07$ & 1.05E-06 & 1.96E-06 & 1.57E-06 & 1.57E-06 \\
\hline $\mathrm{OH}-$ & & $3.57 \mathrm{E}-04$ & $2.93 \mathrm{E}-06$ & $2.88 \mathrm{E}-06$ & $2.48 \mathrm{E}-03$ & $4.82 \mathrm{E}-03$ & 7.37E-03 & $6.28 \mathrm{E}-03$ & $6.28 \mathrm{E}-03$ \\
\hline $\mathrm{H} 2 \mathrm{O}$ & $4.68 \mathrm{E}+01$ & $3.63 \mathrm{E}+01$ & $3.27 \mathrm{E}-02$ & $3.21 \mathrm{E}-02$ & $7.61 \mathrm{E}-04$ & $1.51 \mathrm{E}-03$ & $2.30 \mathrm{E}-03$ & 1.97E-03 & 1.97E-03 \\
\hline $\mathrm{SO} 2$ & & 4.34E-07 & 2.85E-09 & 2.80E-09 & & & & & \\
\hline $\mathrm{H} 2 \mathrm{~S}$ & & & $1.26 \mathrm{E}-05$ & $1.23 E-05$ & & & & & \\
\hline $\mathrm{CO}$ & & $8.38 \mathrm{E}-12$ & $7.65 \mathrm{E}-04$ & $7.51 \mathrm{E}-04$ & & & & & \\
\hline $\mathrm{CO} 2$ & & 1.34E-05 & $7.33 \mathrm{E}-03$ & $7.20 \mathrm{E}-03$ & & & & & \\
\hline $\mathrm{H} 2$ & & $1.43 \mathrm{E}-11$ & $2.10 \mathrm{E}-04$ & $2.06 \mathrm{E}-04$ & & & & & \\
\hline $\mathrm{N} 2$ & & $5.88 \mathrm{E}-07$ & $1.30 \mathrm{E}-02$ & $1.41 \mathrm{E}-02$ & & & & & \\
\hline $\mathrm{NO}$ & & 9.83E-09 & 4.49E-05 & $4.41 \mathrm{E}-05$ & & & & & \\
\hline $\mathrm{NO} 2$ & & $1.23 \mathrm{E}-07$ & $4.66 \mathrm{E}-06$ & 4.57E-06 & & & & & \\
\hline $\mathrm{O} 2$ & & $1.42 \mathrm{E}-07$ & $4.27 \mathrm{E}-04$ & 4.19E-04 & & & & & \\
\hline S (other) & & 5.79E-09 & $2.18 \mathrm{E}-07$ & 2.14E-07 & 1.84E-04 & $3.58 \mathrm{E}-04$ & $5.50 \mathrm{E}-04$ & $4.68 \mathrm{E}-04$ & $4.68 \mathrm{E}-04$ \\
\hline $\mathrm{CO} 3$ & 1.19E-05 & $1.01 \mathrm{E}-05$ & 1.60E-04 & 1.57E-04 & $1.46 \mathrm{E}-01$ & $2.64 \mathrm{E}-01$ & $3.78 \mathrm{E}-01$ & $3.29 \mathrm{E}-01$ & $3.29 \mathrm{E}-01$ \\
\hline C (reductant) & & & 2.19E-05 & $2.15 \mathrm{E}-05$ & 4.33E-01 & $3.60 \mathrm{E}-02$ & & $1.53 \mathrm{E}-02$ & 1.53E-02 \\
\hline $\mathrm{O}$ (oxides) & $5.00 \mathrm{E}-02$ & $3.65 \mathrm{E}-02$ & $3.67 \mathrm{E}-05$ & $3.61 \mathrm{E}-05$ & $3.99 \mathrm{E}-02$ & $6.04 \mathrm{E}-02$ & $1.18 \mathrm{E}-01$ & $9.34 \mathrm{E}-02$ & $9.34 \mathrm{E}-02$ \\
\hline C (organic) & 1.64E-02 & $9.98 \mathrm{E}+00$ & 9.19E-05 & 9.02E-05 & $7.25 \mathrm{E}-02$ & 1.21E-01 & 8.99E-03 & 5.69E-02 & 5.69E-02 \\
\hline $\mathrm{H}$ (organic) & $3.01 \mathrm{E}-02$ & $1.83 \mathrm{E}+01$ & $1.74 \mathrm{E}-05$ & $1.71 \mathrm{E}-05$ & $1.12 \mathrm{E}-02$ & $1.87 \mathrm{E}-02$ & $1.38 \mathrm{E}-03$ & $8.75 \mathrm{E}-03$ & $8.75 \mathrm{E}-03$ \\
\hline O (organic) & $1.51 \mathrm{E}-02$ & $9.15 \mathrm{E}+00$ & 9.02E-05 & $8.85 \mathrm{E}-05$ & $8.85 \mathrm{E}-02$ & $1.48 \mathrm{E}-01$ & 1.10E-02 & 6.94E-02 & 6.94E-02 \\
\hline \multirow[t]{2}{*}{ Mass Flow (kg/hr): } & $4.36 \mathrm{E}+02$ & $6.01 \mathrm{E}+02$ & $2.56 \mathrm{E}+03$ & $2.62 \mathrm{E}+03$ & $4.46 \mathrm{E}+01$ & $2.82 \mathrm{E}+01$ & $3.80 \mathrm{E}+01$ & $6.62 \mathrm{E}+01$ & $6.62 \mathrm{E}+01$ \\
\hline & & & & & & & & & \\
\hline \multirow[t]{3}{*}{ Total Canisters Generated } & & & & & & & & 204 & \\
\hline & & & & & & & & & \\
\hline & $\mathrm{w} / \mathrm{m} 3$ & $\mathrm{w} / \mathrm{m} 3$ & $\mathrm{w} / \mathrm{m} 3$ & $\mathrm{w} / \mathrm{m} 3$ & $\mathrm{w} / \mathrm{m} 3$ & $\mathrm{w} / \mathrm{m} 3$ & $\mathrm{w} / \mathrm{m} 3$ & $\mathrm{w} / \mathrm{m} 3$ & w/Canistel \\
\hline Heat Generation, w/m3 & $2.68 \mathrm{E}-01$ & $1.98 \mathrm{E}-01$ & & & $2.05 \mathrm{E}-01$ & $1.45 \mathrm{E}+00$ & $2.44 \mathrm{E}+00$ & $1.99 \mathrm{E}+00$ & $1.59 \mathrm{E}+00$ \\
\hline
\end{tabular}


Table A-13. Mass balance, Tank WM-180 waste (continued).

\begin{tabular}{|c|c|c|c|c|c|c|c|c|c|}
\hline Stream \# & 101 & 102 & 103 & 104 & 105 & 106 & 107 & 108 & 108 \\
\hline Radiological Composition & $\mathrm{Ci} / \mathrm{L}$ & $\mathrm{Ci} / \mathrm{L}$ & Ci/wscm & $\mathrm{Ci} / \mathrm{wscm}$ & $\mathrm{Ci} / \mathrm{kg}$ & $\mathrm{Ci} / \mathrm{kg}$ & $\mathrm{Ci} / \mathrm{kg}$ & $\mathrm{Ci} / \mathrm{kg}$ & Ci/canister \\
\hline $\mathrm{H}-3$ & $1.59 \mathrm{E}-05$ & $1.15 \mathrm{E}-05$ & $1.99 \mathrm{E}-06$ & $1.95 \mathrm{E}-06$ & & & & & \\
\hline C-14 & $5.71 \mathrm{E}-11$ & $4.14 \mathrm{E}-11$ & $6.96 \mathrm{E}-12$ & $6.83 \mathrm{E}-12$ & $9.39 \mathrm{E}-11$ & $3.69 \mathrm{E}-11$ & $1.48 \mathrm{E}-11$ & $2.42 \mathrm{E}-11$ & $2.58 \mathrm{E}-08$ \\
\hline Co-60 & $3.66 \mathrm{E}-06$ & $2.65 \mathrm{E}-06$ & $1.30 \mathrm{E}-07$ & $1.28 \mathrm{E}-07$ & $8.26 \mathrm{E}-06$ & $1.34 \mathrm{E}-05$ & $2.50 \mathrm{E}-05$ & $2.01 \mathrm{E}-05$ & $2.13 \mathrm{E}-02$ \\
\hline Ni-59 & $8.42 \mathrm{E}-08$ & $6.12 \mathrm{E}-08$ & $3.22 \mathrm{E}-09$ & $3.16 \mathrm{E}-09$ & $1.79 \mathrm{E}-07$ & $3.30 \mathrm{E}-07$ & $5.60 \mathrm{E}-07$ & $4.62 \mathrm{E}-07$ & $4.91 \mathrm{E}-04$ \\
\hline $\mathrm{Ni}-63$ & $2.22 \mathrm{E}-05$ & $1.61 \mathrm{E}-05$ & $8.49 \mathrm{E}-07$ & $8.34 \mathrm{E}-07$ & $4.73 \mathrm{E}-05$ & $8.71 \mathrm{E}-05$ & $1.48 \mathrm{E}-04$ & & \\
\hline Se-79 & $2.08 \mathrm{E}-07$ & $1.52 \mathrm{E}-07$ & $7.47 \mathrm{E}-09$ & $7.33 \mathrm{E}-09$ & $5.20 \mathrm{E}-07$ & $7.66 \mathrm{E}-07$ & $1.43 \mathrm{E}-06$ & $1.15 \mathrm{E}-06$ & $1.22 \mathrm{E}-03$ \\
\hline Sr-90 & $1.79 \mathrm{E}-02$ & $1.30 \mathrm{E}-02$ & $6.86 \mathrm{E}-04$ & $6.74 \mathrm{E}-04$ & $3.82 \mathrm{E}-02$ & $7.04 \mathrm{E}-02$ & 1.19E-01 & $9.85 \mathrm{E}-02$ & $1.05 \mathrm{E}+02$ \\
\hline$Y-90$ & $1.79 \mathrm{E}-02$ & $1.30 \mathrm{E}-02$ & $6.40 \mathrm{E}-04$ & $6.28 \mathrm{E}-04$ & $4.05 \mathrm{E}-02$ & $6.56 \mathrm{E}-02$ & $1.23 \mathrm{E}-01$ & $9.84 \mathrm{E}-02$ & $1.05 \mathrm{E}+02$ \\
\hline Zr-93 & $1.00 \mathrm{E}-06$ & $7.35 \mathrm{E}-07$ & $3.61 \mathrm{E}-08$ & $3.55 \mathrm{E}-08$ & $2.52 \mathrm{E}-06$ & $3.71 \mathrm{E}-06$ & $6.92 \mathrm{E}-06$ & $5.55 \mathrm{E}-06$ & $5.90 \mathrm{E}-03$ \\
\hline Nb-93m & $7.74 \mathrm{E}-07$ & $5.62 \mathrm{E}-07$ & $2.76 \mathrm{E}-08$ & $2.71 \mathrm{E}-08$ & $1.93 \mathrm{E}-06$ & $2.83 \mathrm{E}-06$ & $5.29 \mathrm{E}-06$ & $4.25 \mathrm{E}-06$ & $4.51 \mathrm{E}-03$ \\
\hline Nb-94 & $5.42 \mathrm{E}-07$ & $3.93 \mathrm{E}-07$ & $1.93 \mathrm{E}-08$ & $1.90 \mathrm{E}-08$ & $1.35 \mathrm{E}-06$ & $1.98 \mathrm{E}-06$ & $3.70 \mathrm{E}-06$ & $2.97 \mathrm{E}-06$ & $3.16 \mathrm{E}-03$ \\
\hline Tc-99 & $8.30 \mathrm{E}-06$ & $6.03 \mathrm{E}-06$ & $9.40 \mathrm{E}-07$ & $9.22 \mathrm{E}-07$ & $1.02 \mathrm{E}-04$ & $9.64 \mathrm{E}-05$ & $7.76 \mathrm{E}-06$ & $4.55 \mathrm{E}-05$ & $4.84 \mathrm{E}-02$ \\
\hline Ru-106 & $4.43 \mathrm{E}-07$ & $3.21 \mathrm{E}-07$ & $1.58 \mathrm{E}-08$ & $1.55 \mathrm{E}-08$ & $1.00 \mathrm{E}-06$ & $1.62 \mathrm{E}-06$ & $3.03 \mathrm{E}-06$ & $2.43 \mathrm{E}-06$ & $2.58 \mathrm{E}-03$ \\
\hline Rh-102 & $3.91 \mathrm{E}-10$ & $2.84 \mathrm{E}-10$ & $1.39 \mathrm{E}-11$ & $1.37 \mathrm{E}-11$ & $8.83 \mathrm{E}-10$ & $1.43 \mathrm{E}-09$ & $2.67 \mathrm{E}-09$ & $2.14 \mathrm{E}-09$ & $2.28 \mathrm{E}-06$ \\
\hline Rh-106 & $4.43 \mathrm{E}-07$ & $3.21 \mathrm{E}-07$ & $1.58 \mathrm{E}-08$ & $1.55 \mathrm{E}-08$ & $1.00 \mathrm{E}-06$ & $1.62 \mathrm{E}-06$ & $3.03 \mathrm{E}-06$ & $2.43 \mathrm{E}-06$ & $2.58 \mathrm{E}-03$ \\
\hline Pd-107 & $7.49 \mathrm{E}-09$ & $5.44 \mathrm{E}-09$ & $2.87 \mathrm{E}-10$ & $2.81 \mathrm{E}-10$ & $1.59 \mathrm{E}-08$ & $2.94 \mathrm{E}-08$ & $4.98 \mathrm{E}-08$ & $4.11 \mathrm{E}-08$ & $4.37 \mathrm{E}-05$ \\
\hline Cd-113m & $1.51 \mathrm{E}-06$ & $1.09 \mathrm{E}-06$ & $6.29 \mathrm{E}-08$ & $6.18 \mathrm{E}-08$ & $4.09 \mathrm{E}-06$ & $6.46 \mathrm{E}-06$ & $9.60 \mathrm{E}-06$ & $8.26 \mathrm{E}-06$ & $8.78 \mathrm{E}-03$ \\
\hline Sn-121m & $3.03 \mathrm{E}-08$ & $2.22 \mathrm{E}-08$ & $1.09 \mathrm{E}-09$ & $1.07 \mathrm{E}-09$ & $7.60 \mathrm{E}-08$ & $1.12 \mathrm{E}-07$ & $2.09 \mathrm{E}-07$ & $1.68 \mathrm{E}-07$ & $1.78 \mathrm{E}-04$ \\
\hline Sn-126 & $1.95 \mathrm{E}-07$ & $1.43 \mathrm{E}-07$ & $7.02 \mathrm{E}-09$ & $6.89 \mathrm{E}-09$ & $4.90 \mathrm{E}-07$ & $7.21 \mathrm{E}-07$ & $1.35 \mathrm{E}-06$ & $1.08 \mathrm{E}-06$ & $1.15 \mathrm{E}-03$ \\
\hline Sb-125 & $7.28 \mathrm{E}-06$ & $5.28 \mathrm{E}-06$ & $2.60 \mathrm{E}-07$ & $2.55 \mathrm{E}-07$ & $1.64 \mathrm{E}-05$ & $2.66 \mathrm{E}-05$ & $4.98 \mathrm{E}-05$ & $3.99 \mathrm{E}-05$ & $4.24 \mathrm{E}-02$ \\
\hline Sb-126 & $2.61 \mathrm{E}-08$ & $1.89 \mathrm{E}-08$ & $9.30 \mathrm{E}-10$ & $9.13 \mathrm{E}-10$ & $5.89 \mathrm{E}-08$ & $9.54 \mathrm{E}-08$ & $1.78 \mathrm{E}-07$ & 1.43E-07 & $1.52 \mathrm{E}-04$ \\
\hline Te-125m & 1.43E-06 & $1.04 \mathrm{E}-06$ & $5.14 \mathrm{E}-08$ & $5.04 \mathrm{E}-08$ & $3.58 \mathrm{E}-06$ & $5.27 \mathrm{E}-06$ & $9.84 \mathrm{E}-06$ & $7.89 \mathrm{E}-06$ & $8.39 \mathrm{E}-03$ \\
\hline I-129 & $2.21 \mathrm{E}-08$ & $1.61 \mathrm{E}-08$ & $2.06 \mathrm{E}-09$ & $2.02 \mathrm{E}-09$ & $1.21 \mathrm{E}-07$ & $2.05 \mathrm{E}-07$ & $5.54 \mathrm{E}-08$ & $1.19 \mathrm{E}-07$ & $1.27 \mathrm{E}-04$ \\
\hline Cs-134 & $4.72 \mathrm{E}-06$ & $4.02 \mathrm{E}-06$ & $6.45 \mathrm{E}-07$ & $6.33 \mathrm{E}-07$ & $6.03 \mathrm{E}-05$ & $6.62 \mathrm{E}-05$ & $3.71 \mathrm{E}-06$ & $3.13 \mathrm{E}-05$ & $3.32 \mathrm{E}-02$ \\
\hline Cs-135 & 4.07E-07 & $3.46 \mathrm{E}-07$ & $5.56 \mathrm{E}-08$ & $5.45 \mathrm{E}-08$ & $5.19 \mathrm{E}-06$ & $5.70 \mathrm{E}-06$ & $3.20 \mathrm{E}-07$ & $2.69 \mathrm{E}-06$ & $2.86 \mathrm{E}-03$ \\
\hline \begin{tabular}{|l|} 
Cs-137 \\
\end{tabular} & $2.38 \mathrm{E}-02$ & $2.03 \mathrm{E}-02$ & $3.26 \mathrm{E}$ & $3.20 \mathrm{E}$ & $3.04 \mathrm{E}-01$ & $3.34 \mathrm{E}-01$ & $1.87 \mathrm{E}-02$ & $1.58 \mathrm{E}-01$ & $1.68 \mathrm{E}+02$ \\
\hline Ba-137m & $2.25 \mathrm{E}-02$ & $1.64 \mathrm{E}-02$ & $8.62 \mathrm{E}-04$ & $8.46 \mathrm{E}-04$ & $4.80 \mathrm{E}-02$ & $8.84 \mathrm{E}-02$ & $1.50 \mathrm{E}-01$ & $1.24 \mathrm{E}-01$ & $1.31 \mathrm{E}+02$ \\
\hline $\mathrm{Ce}-144$ & $2.98 \mathrm{E}-07$ & $2.17 \mathrm{E}-07$ & $1.06 \mathrm{E}-08$ & $1.04 \mathrm{E}-08$ & $6.74 \mathrm{E}-07$ & $1.09 \mathrm{E}-06$ & $2.04 \mathrm{E}-06$ & $1.64 \mathrm{E}-06$ & $1.74 \mathrm{E}-03$ \\
\hline Pr-144 & $2.98 \mathrm{E}-07$ & $2.17 \mathrm{E}-07$ & $1.06 \mathrm{E}-08$ & $1.04 \mathrm{E}-08$ & $6.74 \mathrm{E}-07$ & $1.09 E-06$ & $2.04 \mathrm{E}-06$ & $1.64 \mathrm{E}-06$ & $1.74 \mathrm{E}-03$ \\
\hline Pm-146 & $2.31 \mathrm{E}-08$ & $1.67 \mathrm{E}-08$ & $8.23 \mathrm{E}-10$ & $8.08 \mathrm{E}-10$ & $5.21 \mathrm{E}-08$ & $8.44 \mathrm{E}-08$ & $1.58 \mathrm{E}-07$ & $1.27 \mathrm{E}-07$ & $1.35 \mathrm{E}-04$ \\
\hline Pm-147 & $8.10 \mathrm{E}-05$ & $5.88 \mathrm{E}-05$ & $2.89 \mathrm{E}-06$ & $2.83 \mathrm{E}-06$ & $1.83 \mathrm{E}-04$ & $2.96 \mathrm{E}-04$ & $5.54 \mathrm{E}-04$ & $4.44 \mathrm{E}-04$ & $4.72 \mathrm{E}-01$ \\
\hline Sm-151 & $1.60 \mathrm{E}-04$ & $1.16 \mathrm{E}-04$ & $5.69 \mathrm{E}-06$ & $5.59 \mathrm{E}-06$ & $3.61 \mathrm{E}-04$ & $5.84 \mathrm{E}-04$ & $1.09 \mathrm{E}-03$ & $8.76 \mathrm{E}-04$ & $9.31 \mathrm{E}-01$ \\
\hline Eu-152 & $1.16 \mathrm{E}-06$ & $8.39 \mathrm{E}-07$ & $4.12 \mathrm{E}-08$ & & $E-06$ & $4.23 \mathrm{E}-06$ & $7.91 \mathrm{E}-06$ & $6.34 \mathrm{E}-06$ & $6.74 \mathrm{E}-03$ \\
\hline \begin{tabular}{|l} 
Eu-154 \\
\end{tabular} & $4.15 \mathrm{E}-05$ & $3.01 \mathrm{E}-05$ & $1.48 \mathrm{E}-06$ & 1.45E-06 & $9.37 \mathrm{E}-05$ & $1.52 \mathrm{E}-04$ & $2.84 \mathrm{E}-04$ & $2.27 \mathrm{E}-04$ & $2.42 \mathrm{E}-01$ \\
\hline Eu-155 & $7.54 \mathrm{E}-05$ & $5.47 \mathrm{E}-05$ & \begin{tabular}{|l|}
$2.69 \mathrm{E}-06$ \\
\end{tabular} & \begin{tabular}{|l|}
$2.64 \mathrm{E}-06$ \\
\end{tabular} & \begin{tabular}{|l|}
$1.70 \mathrm{E}-04$ \\
\end{tabular} & \begin{tabular}{|l|}
$2.76 \mathrm{E}-04$ \\
\end{tabular} & \begin{tabular}{|l|}
$5.16 \mathrm{E}-04$ \\
\end{tabular} & \begin{tabular}{|l|}
$4.14 \mathrm{E}-04$ \\
\end{tabular} & $4.40 \mathrm{E}-01$ \\
\hline & & & & & & & & & \\
\hline Th-230 & $3.96 \mathrm{E}-10$ & $2.89 \mathrm{E}-10$ & & & & & & $2.19 \mathrm{E}-09$ & $2.32 \mathrm{E}-06$ \\
\hline $\mathrm{Pa}-233$ & $1.33 \mathrm{E}-06$ & $9.72 \mathrm{E}-07$ & $4.78 \mathrm{~B}$ & 4.69 & & $4.90 \mathrm{E}-06$ & $9.16 \mathrm{E}-06$ & $7.35 \mathrm{E}-06$ & $7.81 \mathrm{E}-03$ \\
\hline $\mathrm{U}-232$ & $9.54 \mathrm{E}-10$ & $6.97 \mathrm{E}-10$ & $3.43 \mathrm{E}-11$ & $3.36 \mathrm{E}-11$ & $2.39 \mathrm{E}-09$ & $3.52 \mathrm{E}-09$ & $6.57 \mathrm{E}-09$ & $5.27 \mathrm{E}-09$ & $5.60 \mathrm{E}-06$ \\
\hline $\mathrm{U}-233$ & $72 \mathrm{E}-11$ & $2.72 \mathrm{E}-11$ & 1.34 & 1.31 & & & $2.56 \mathrm{E}-10$ & $2.05 \mathrm{E}-10$ & $2.18 \mathrm{E}-07$ \\
\hline U-234 & $9.55 \mathrm{E}-07$ & $6.98 \mathrm{E}-07$ & $3.43 \mathrm{E}-08$ & $3.37 \mathrm{E}-08$ & $2.39 \mathrm{E}-06$ & $3.52 \mathrm{E}-06$ & $6.58 \mathrm{E}-06$ & $5.28 \mathrm{E}-06$ & $5.61 \mathrm{E}-03$ \\
\hline U-235 & $3.49 \mathrm{E}-08$ & $2.55 \mathrm{E}-08$ & 1.25 & 1.23 & & & $2.40 \mathrm{E}-07$ & $1.93 \mathrm{E}-07$ & $2.05 \mathrm{E}-04$ \\
\hline U-236 & $5.18 \mathrm{E}-08$ & $3.79 \mathrm{E}-08$ & $1.86 \mathrm{E}-09$ & $1.83 \mathrm{E}-09$ & $1.30 \mathrm{E}-07$ & $1.91 \mathrm{E}-07$ & $3.57 \mathrm{E}-07$ & $2.86 \mathrm{E}-07$ & $3.04 \mathrm{E}-04$ \\
\hline U-237 & $2.92 \mathrm{E}-09$ & $2.13 \mathrm{E}-09$ & $1.05 \mathrm{E}$ & 1.03 & 7.31 & 1.08 & $2.01 \mathrm{E}-08$ & $1.61 \mathrm{E}-08$ & $1.71 \mathrm{E}-05$ \\
\hline U-238 & $2.06 \mathrm{E}-08$ & $1.51 \mathrm{E}-08$ & $7.42 \mathrm{E}$ & 7.28 & 5.17 & 7.61 & $1.42 \mathrm{E}-07$ & $1.14 \mathrm{E}-07$ & $1.21 \mathrm{E}-04$ \\
\hline Np-237 & $1.34 \mathrm{E}-06$ & $9.76 \mathrm{E}-07$ & $4.79 \mathrm{E}-08$ & $4.70 \mathrm{E}-08$ & $3.04 \mathrm{E}-06$ & $4.92 \mathrm{E}-06$ & $9.20 \mathrm{E}-06$ & $7.37 \mathrm{E}-06$ & $7.84 \mathrm{E}-03$ \\
\hline Pu-236 & $1.91 \mathrm{E}-09$ & 1.40E-09 & $6.88 \mathrm{E}-11$ & $6.75 \mathrm{E}-11$ & $4.80 \mathrm{E}-09$ & $7.06 \mathrm{E}-09$ & $1.32 \mathrm{E}-08$ & 1.06E-08 & $1.12 \mathrm{E}-05$ \\
\hline Pu-238 & $8.73 \mathrm{E}-04$ & $6.38 \mathrm{E}-04$ & $3.14 \mathrm{E}-05$ & $3.08 \mathrm{E}-05$ & $2.19 \mathrm{E}-03$ & $3.22 \mathrm{E}-03$ & $6.01 \mathrm{E}-03$ & $4.82 \mathrm{E}-03$ & $5.13 \mathrm{E}+00$ \\
\hline $\begin{array}{l}\mathrm{Pu}-239 \\
\end{array}$ & $1.32 \mathrm{E}-04$ & $9.68 \mathrm{E}-05$ & $4.76 \mathrm{E}-06$ & $4.67 \mathrm{E}-06$ & $3.32 \mathrm{E}-04$ & $4.88 \mathrm{E}-04$ & $9.12 \mathrm{E}-04$ & $7.32 \mathrm{E}-04$ & $7.78 \mathrm{E}-01$ \\
\hline Pu-240 & $7.19 \mathrm{E}-06$ & $5.25 \mathrm{E}-06$ & $2.58 \mathrm{E}-07$ & $2.54 \mathrm{E}$ & $1.80 \mathrm{E}-05$ & $2.65 \mathrm{E}-05$ & $4.95 \mathrm{E}-05$ & $3.97 \mathrm{E}-05$ & $4.22 \mathrm{E}-02$ \\
\hline Pu-241 & $5.26 \mathrm{E}-04$ & $3.84 \mathrm{E}-04$ & $1.89 \mathrm{E}-05$ & $1.86 \mathrm{E}-05$ & $1.32 \mathrm{E}-03$ & $1.94 \mathrm{E}-03$ & $3.62 \mathrm{E}-03$ & $2.91 \mathrm{E}-03$ & $3.09 \mathrm{E}+00$ \\
\hline Pu-242 & $5.54 \mathrm{E}-09$ & $4.05 \mathrm{E}-09$ & $1.99 \mathrm{E}-10$ & $1.95 \mathrm{E}-10$ & $1.39 \mathrm{E}-08$ & $2.04 \mathrm{E}-08$ & $3.82 \mathrm{E}-08$ & $3.06 \mathrm{E}-08$ & $3.25 \mathrm{E}-05$ \\
\hline Pu-244 & $4.75 \mathrm{E}-16$ & $3.47 \mathrm{E}-16$ & $1.71 \mathrm{E}-17$ & $1.67 \mathrm{E}-17$ & $1.19 \mathrm{E}-15$ & $1.75 \mathrm{E}-15$ & $3.27 \mathrm{E}-15$ & $2.62 \mathrm{E}-15$ & $2.79 \mathrm{E}-12$ \\
\hline Am-241 & $6.32 \mathrm{E}-05$ & $4.59 \mathrm{E}-05$ & $2.25 \mathrm{E}-06$ & $2.21 \mathrm{E}-06$ & $1.43 \mathrm{E}-04$ & $2.31 \mathrm{E}-04$ & $4.32 \mathrm{E}-04$ & 3.47E-04 & $3.69 \mathrm{E}-01$ \\
\hline Am-242m & 6.90E-09 & $5.01 \mathrm{E}-09$ & $2.46 \mathrm{E}-10$ & $2.41 \mathrm{E}-10$ & $1.56 \mathrm{E}-08$ & $2.52 \mathrm{E}-08$ & $4.72 \mathrm{E}-08$ & $3.78 \mathrm{E}-08$ & $4.02 \mathrm{E}-05$ \\
\hline
\end{tabular}


Table A-13. Mass balance, Tank WM-180 waste (continued).

\begin{tabular}{|c|c|c|c|c|c|c|c|c|c|}
\hline Stream \# & 101 & 102 & 103 & 104 & 105 & 106 & 107 & 108 & 108 \\
\hline Radiological Composition & $\mathrm{Ci} / \mathrm{L}$ & $\mathrm{Ci} / \mathrm{L}$ & Ci/wscm & $\mathrm{Ci} / \mathrm{wscm}$ & $\mathrm{Ci} / \mathrm{kg}$ & $\mathrm{Ci} / \mathrm{kg}$ & $\mathrm{Ci} / \mathrm{kg}$ & $\mathrm{Ci} / \mathrm{kg}$ & Ci/Canister \\
\hline Am-243 & $1.00 \mathrm{E}-08$ & 7.27E-09 & $3.57 \mathrm{E}-10$ & $3.50 \mathrm{E}-10$ & $2.26 \mathrm{E}-08$ & $3.66 \mathrm{E}-08$ & 6.85E-08 & 5.49E-08 & $5.84 \mathrm{E}-05$ \\
\hline $\mathrm{Cm}-242$ & 5.69E-09 & 4.16E-09 & $2.05 \mathrm{E}-10$ & $2.01 \mathrm{E}-10$ & $1.43 \mathrm{E}-08$ & $2.10 \mathrm{E}-08$ & 3.92E-08 & $3.14 \mathrm{E}-08$ & 3.34E-05 \\
\hline $\mathrm{Cm}-243$ & $1.64 \mathrm{E}-08$ & $1.20 \mathrm{E}-08$ & $5.88 \mathrm{E}-10$ & $5.77 \mathrm{E}-10$ & $4.10 \mathrm{E}-08$ & $6.04 \mathrm{E}-08$ & $1.13 \mathrm{E}-07$ & 9.04E-08 & 9.61E-05 \\
\hline $\mathrm{Cm}-244$ & 1.02E-06 & $7.44 \mathrm{E}-07$ & $3.66 \mathrm{E}-08$ & $3.59 \mathrm{E}-08$ & $2.55 \mathrm{E}-06$ & $3.75 \mathrm{E}-06$ & $7.01 \mathrm{E}-06$ & $5.62 \mathrm{E}-06$ & 5.97E-03 \\
\hline $\mathrm{Cm}-245$ & $1.72 \mathrm{E}-10$ & $1.26 \mathrm{E}-10$ & $6.19 \mathrm{E}-12$ & $6.08 \mathrm{E}-12$ & $4.32 \mathrm{E}-10$ & $6.35 \mathrm{E}-10$ & 1.19E-09 & $9.52 \mathrm{E}-10$ & $1.01 \mathrm{E}-06$ \\
\hline $\mathrm{Cm}-246$ & $1.13 \mathrm{E}-11$ & $8.27 \mathrm{E}-12$ & $4.07 \mathrm{E}-13$ & $3.99 \mathrm{E}-13$ & $2.83 \mathrm{E}-11$ & $4.17 \mathrm{E}-11$ & $7.79 \mathrm{E}-11$ & $6.25 \mathrm{E}-11$ & $6.64 \mathrm{E}-08$ \\
\hline TRU & 1.09E-03 & $7.99 \mathrm{E}-04$ & 3.93E-05 & 3.86E-05 & $2.72 \mathrm{E}-03$ & 4.03E-03 & $7.53 \mathrm{E}-03$ & $6.04 \mathrm{E}-03$ & $6.42 \mathrm{E}+00$ \\
\hline TRU, nCi/gm & $9.11 \mathrm{E}+02$ & & & & & & & $6.04 \mathrm{E}+03$ & \\
\hline & & & & & & & & & \\
\hline $\begin{array}{l}\text { Gas Stream Bulk } \\
\text { Composition (Wet Basis) }\end{array}$ & $\begin{array}{l}\text { mol\% or } \\
\text { ppmv }\end{array}$ & $\begin{array}{c}\text { mol\% or } \\
\text { ppmv }\end{array}$ & $\begin{array}{l}\text { mol\% or } \\
\text { ppmv }\end{array}$ & $\begin{array}{l}\text { mol\% or } \\
\text { ppmv }\end{array}$ & $\begin{array}{l}\text { mol\% or } \\
\text { ppmv }\end{array}$ & $\begin{array}{c}\mathrm{mol} \% \text { or } \\
\mathrm{ppmv}\end{array}$ & $\begin{array}{c}\mathrm{mol} \% \text { or } \\
\text { ppmv }\end{array}$ & $\begin{array}{c}\text { mol\% or } \\
\text { ppmv }\end{array}$ & $\begin{array}{c}\mathrm{mol} \% \text { or } \\
\mathrm{ppmv}\end{array}$ \\
\hline $\mathrm{H} 2 \mathrm{O}, \mathrm{mol} \%$ & & & $69.76 \%$ & $68.47 \%$ & & & & & \\
\hline $\mathrm{O} 2, \mathrm{~mol} \%$ & & & $0.51 \%$ & $0.50 \%$ & & & & & \\
\hline $\mathrm{N} 2, \mathrm{~mol} \%$ & & & $17.88 \%$ & $19.40 \%$ & & & & & \\
\hline $\mathrm{H} 2, \mathrm{~mol} \%$ & & & $4.01 \%$ & $3.93 \%$ & & & & & \\
\hline $\mathrm{CO} 2, \mathrm{~mol} \%$ & & & $6.41 \%$ & $6.29 \%$ & & & & & \\
\hline $\mathrm{CO}, \mathrm{ppmv}$ & & & $1.05 \mathrm{E}+04$ & $1.03 E+04$ & & & & & \\
\hline $\mathrm{NO}, \mathrm{ppmv}$ & & & $5.76 \mathrm{E}+02$ & $5.65 \mathrm{E}+02$ & & & & & \\
\hline NO2, ppmv & & & $3.90 \mathrm{E}+01$ & $3.82 \mathrm{E}+01$ & & & & & \\
\hline SO2, ppmv & & & $1.71 \mathrm{E}-02$ & 1.68E-02 & & & & & \\
\hline $\mathrm{Cl}, \mathrm{ppmv}$ & & & $5.90 \mathrm{E}+01$ & $5.79 \mathrm{E}+01$ & & & & & \\
\hline $\mathrm{F}, \mathrm{ppmv}$ & & & $1.03 \mathrm{E}+02$ & $1.01 \mathrm{E}+02$ & & & & & \\
\hline C (organic), ppmv & & & $2.94 \mathrm{E}+03$ & $2.89 \mathrm{E}+03$ & & & & & \\
\hline $\mathrm{H}$ (organic), ppmv & & & $6.65 \mathrm{E}+03$ & $6.52 \mathrm{E}+03$ & & & & & \\
\hline $\mathrm{Hg}, \mathrm{ug} / \mathrm{wscm}$ & & & $4.81 \mathrm{E}+04$ & $4.72 \mathrm{E}+04$ & & & & & \\
\hline $\mathrm{PM}, \mathrm{mg} / \mathrm{wscm}$ & & & $9.89 \mathrm{E}+03$ & $9.71 \mathrm{E}+03$ & & & & & \\
\hline SVM, ug/wscm & & & $3.20 \mathrm{E}+04$ & $3.14 \mathrm{E}+04$ & & & & & \\
\hline LVM, ug/wscm & & & $7.68 \mathrm{E}+03$ & $7.53 \mathrm{E}+03$ & & & & & \\
\hline $\begin{array}{l}\text { Gas Stream Bulk } \\
\text { Composition (Dry Basis) }\end{array}$ & $\mathrm{mol} \%$ & $\mathrm{~mol} \%$ & $\mathrm{~mol} \%$ & $\mathrm{~mol} \%$ & $\mathrm{~mol} \%$ & $\mathrm{~mol} \%$ & $\mathrm{~mol} \%$ & $\mathrm{~mol} \%$ & $\mathrm{~mol} \%$ \\
\hline $\mathrm{O} 2, \mathrm{~mol} \%$, dry basis & & & $1.6962 \%$ & $1.5963 \%$ & & & & & \\
\hline $\mathrm{N} 2, \mathrm{~mol} \%$, dry basis & & & $59.12 \%$ & $61.53 \%$ & & & & & \\
\hline $\mathrm{H} 2, \mathrm{~mol} \%$, dry basis & & & $13.25 \%$ & $12.47 \%$ & & & & & \\
\hline $\mathrm{CO} 2, \mathrm{~mol} \%$, dry basis & & & $21.18 \%$ & $19.94 \%$ & & & & & \\
\hline $\begin{array}{l}\text { Gas Stream Bulk } \\
\text { Composition (Dry Basis, } \\
\text { Corrected to } 7 \% \text { O2 with } \\
100 \% \text { O2 Combustion Air) }\end{array}$ & $\begin{array}{l}\text { ppmv, or } \\
\text { ug/dscm } \\
\text { or } \\
\mathrm{mg} / \mathrm{dscm}\end{array}$ & $\begin{array}{l}\mathrm{ppmv}, \text { or } \\
\mathrm{ug} / \mathrm{dscm} \\
\text { or } \\
\mathrm{mg} / \mathrm{dscm}\end{array}$ & $\begin{array}{l}\text { ppmv, or } \\
\text { ug/dscm } \\
\text { or } \\
\mathrm{mg} / \mathrm{dscm}\end{array}$ & $\begin{array}{l}\mathrm{ppmv}, \text { or } \\
\mathrm{ug} / \mathrm{dscm} \\
\text { or } \\
\mathrm{mg} / \mathrm{dscm}\end{array}$ & $\begin{array}{l}\mathrm{ppmv}, \text { or } \\
\text { ug/dscm } \\
\text { or } \\
\mathrm{mg} / \mathrm{dscm}\end{array}$ & $\begin{array}{l}\text { ppmv, or } \\
\text { ug/dscm } \\
\text { or } \\
\mathrm{mg} / \mathrm{dscm}\end{array}$ & $\begin{array}{l}\text { ppmv, or } \\
\text { ug/dscm } \\
\text { or } \\
\mathrm{mg} / \mathrm{dscm}\end{array}$ & $\begin{array}{l}\mathrm{ppmv}, \text { or } \\
\mathrm{ug} / \mathrm{dscm} \\
\text { or } \\
\mathrm{mg} / \mathrm{dscm}\end{array}$ & $\begin{array}{l}\text { ppmv, or } \\
\text { ug/dscm } \\
\text { or } \\
\mathrm{mg} / \mathrm{dscm}\end{array}$ \\
\hline COgas, ppmv, dry basis & & & $4.86 \mathrm{E}+03$ & $4.58 \mathrm{E}+03$ & & & & & \\
\hline $\mathrm{NO}$, ppmv, dry basis & & & $2.67 \mathrm{E}+02$ & $2.51 \mathrm{E}+02$ & & & & & \\
\hline NO2, ppmv, dry basis & & & $1.80 \mathrm{E}+01$ & $1.70 \mathrm{E}+01$ & & & & & \\
\hline SO2, ppmv, dry basis & & & 7.93E-03 & $7.46 \mathrm{E}-03$ & & & & & \\
\hline $\mathrm{Cl}, \mathrm{ppmv}$, dry basis & & & $2.73 \mathrm{E}+01$ & $2.57 \mathrm{E}+01$ & & & & & \\
\hline $\mathrm{F}$, ppmv, dry basis & & & $4.76 \mathrm{E}+01$ & $4.48 \mathrm{E}+01$ & & & & & \\
\hline C (organic), ppmv, dry basis & & & $1.36 \mathrm{E}+03$ & $1.28 \mathrm{E}+03$ & & & & & \\
\hline $\mathrm{H}$ (organic), ppmv, dry basis & & & $3.08 \mathrm{E}+03$ & $2.90 \mathrm{E}+03$ & & & & & \\
\hline $\mathrm{Hg}, \mathrm{ug} / \mathrm{dscm}$ & & & $2.23 \mathrm{E}+04$ & $2.10 \mathrm{E}+04$ & & & & & \\
\hline $\mathrm{PM}, \mathrm{mg} / \mathrm{dscm}$ & & & $4.58 \mathrm{E}+03$ & $4.31 \mathrm{E}+03$ & & & & & \\
\hline SVM, ug/dscm & & & $1.06 \mathrm{E}+05$ & $1.04 \mathrm{E}+05$ & & & & & \\
\hline LVM, ug/dscm & & & $2.54 \mathrm{E}+04$ & $2.49 \mathrm{E}+04$ & & & & & \\
\hline
\end{tabular}


Table A-13. Mass balance, Tank WM-180 waste (continued).

\begin{tabular}{|c|c|c|c|c|c|c|c|c|c|}
\hline PFD \# & PFD-2 & PFD-3 & PFD-3 & PFD-3 & PFD-3 & PFD-3 & PFD-3 & PFD-3 & PFD-3 \\
\hline WM-180, Stream \# & 109 & 110 & 111 & 112 & $113 \mathrm{~A}$ & $113 \mathrm{~B}$ & 114 & 115 & 116 \\
\hline Stream Name & $\begin{array}{c}\text { Off-Gas } \\
\text { from } \\
\text { Filter to } \\
\text { Oxidizer }\end{array}$ & $\begin{array}{l}\text { Oxidizer } \\
\text { Effluent }\end{array}$ & $\begin{array}{c}\text { Quenched } \\
\text { Oxidizer } \\
\text { Off-gas }\end{array}$ & Scrub & $\begin{array}{c}\text { Packed } \\
\text { Scrubber } \\
\text { Drain }\end{array}$ & $\begin{array}{c}\text { Demister } \\
\text { Drain }\end{array}$ & $\begin{array}{c}\text { Scrubber } \\
\text { Effluent } \\
\text { Gas }\end{array}$ & $\begin{array}{c}\text { Demister } \\
\text { Effluent } \\
\text { Gas }\end{array}$ & $\begin{array}{c}\text { GAC Bed } \\
\text { Feed }\end{array}$ \\
\hline Rate or Volume & $4.44 \mathrm{E}+05$ & $7.13 E+05$ & $3.72 \mathrm{E}+05$ & $1.02 \mathrm{E}+04$ & $1.02 \mathrm{E}+04$ & $0.00 \mathrm{E}+00$ & $3.80 \mathrm{E}+05$ & $3.89 \mathrm{E}+05$ & $4.72 \mathrm{E}+05$ \\
\hline Volume Flow (standard, wet) & $1.05 E+05$ & $1.09 \mathrm{E}+05$ & $1.85 \mathrm{E}+05$ & & & & $1.84 \mathrm{E}+05$ & $1.85 E+05$ & $1.85 E+05$ \\
\hline Volume Flow (standard, dry) & $3.35 \mathrm{E}+04$ & $3.22 \mathrm{E}+04$ & $3.21 \mathrm{E}+04$ & & & & $3.21 \mathrm{E}+04$ & $3.22 \mathrm{E}+04$ & $3.22 \mathrm{E}+04$ \\
\hline Rate Units & $\mathrm{ft} 3 / \mathrm{hr}$ & $\mathrm{ft} 3 / \mathrm{hr}$ & $\mathrm{ft} 3 / \mathrm{hr}$ & $\mathrm{gal} / \mathrm{hr}$ & $\mathrm{gal} / \mathrm{hr}$ & $\mathrm{gal} / \mathrm{hr}$ & $\mathrm{ft} 3 / \mathrm{hr}$ & $\mathrm{ft} 3 / \mathrm{hr}$ & $\mathrm{ft} 3 / \mathrm{hr}$ \\
\hline Rate or Volume, metric & $2.97 \mathrm{E}+03$ & $3.09 \mathrm{E}+03$ & $5.23 \mathrm{E}+03$ & $3.87 E+04$ & $3.88 \mathrm{E}+04$ & 0.00 & $5.20 \mathrm{E}+03$ & $5.22 \mathrm{E}+03$ & $5.22 \mathrm{E}+03$ \\
\hline Rate Units & wscm/hr & wscm/hr & wscm/hr & $\mathrm{L} / \mathrm{hr}$ & $\mathrm{L} / \mathrm{hr}$ & $\mathrm{L} / \mathrm{hr}$ & wscm/hr & wscm/hr & wscm/hr \\
\hline Temperature, ${ }^{\circ} \mathrm{C}$ & 527 & 950 & 100 & 82 & 83 & 0 & 82 & 81 & 120 \\
\hline Temperature, ${ }^{\circ} \mathrm{F}$ & 980 & 1742 & 212 & 179 & 181 & 32 & 179 & 178 & 248 \\
\hline Pressure, psia & 9.5 & 9.4 & 9.3 & 38.1 & 9.0 & 8.4 & 8.6 & 8.4 & 7.7 \\
\hline Specific Gravity & $2.09 \mathrm{E}-04$ & $1.42 \mathrm{E}-04$ & $4.25 \mathrm{E}-04$ & 1.01 & 1.01 & 1.01 & $4.15 \mathrm{E}-04$ & 4.06E-04 & 3.34E-04 \\
\hline Chemical Composition & $\mathrm{lb} / \mathrm{wscf}$ & $\mathrm{lb} / \mathrm{wscf}$ & $\mathrm{lb} / \mathrm{wscf}$ & Mol/liter & Mol/liter & Mol/liter & $\mathrm{lb} /$ wscf & $\mathrm{lb} / \mathrm{wscf}$ & lb/wscf \\
\hline $\mathrm{H}+$ & $8.35 \mathrm{E}-07$ & $2.46 \mathrm{E}-08$ & $2.51 \mathrm{E}-08$ & $1.02 \mathrm{E}+00$ & $1.02 \mathrm{E}+00$ & $1.02 \mathrm{E}+00$ & $2.22 \mathrm{E}-07$ & $2.21 \mathrm{E}-07$ & $2.21 \mathrm{E}-07$ \\
\hline $\mathrm{Al}+3$ & $5.12 \mathrm{E}-08$ & 4.92E-08 & 2.91E-08 & $1.78 \mathrm{E}-03$ & \begin{tabular}{|l|}
$1.78 \mathrm{E}-03$ \\
\end{tabular} & 1.78E-03 & & & \\
\hline $\mathrm{Sb}+5$ & $5.08 \mathrm{E}-13$ & $4.89 \mathrm{E}-13$ & $2.89 \mathrm{E}-13$ & 3.93E-09 & 3.93E-09 & 3.93E-09 & & & \\
\hline As+3 & $1.00 \mathrm{E}-10$ & $9.66 \mathrm{E}-11$ & $5.71 \mathrm{E}-11$ & $1.26 \mathrm{E}-06$ & $1.26 \mathrm{E}-06$ & $1.26 \mathrm{E}-06$ & & & \\
\hline $\mathrm{Ba}+2$ & $2.21 \mathrm{E}-11$ & $2.12 \mathrm{E}-11$ & $4.05 \mathrm{E}-13$ & $2.52 \mathrm{E}-07$ & $2.51 \mathrm{E}-07$ & $2.51 \mathrm{E}-07$ & & & \\
\hline $\mathrm{Be}+2$ & $2.27 \mathrm{E}-13$ & $2.18 \mathrm{E}-13$ & $4.16 \mathrm{E}-15$ & 3.93E-08 & 3.93E-08 & 3.93E-08 & & & \\
\hline $\mathrm{B}+3$ & $3.37 \mathrm{E}-10$ & $3.24 \mathrm{E}-10$ & $1.92 \mathrm{E}-10$ & 2.93E-05 & 2.93E-05 & 2.93E-05 & & & \\
\hline $\mathrm{Cd}+2$ & $2.83 \mathrm{E}-10$ & $2.72 \mathrm{E}-10$ & $1.61 \mathrm{E}-10$ & 1.97E-06 & 1.97E-06 & 1.97E-06 & & & \\
\hline $\mathrm{Ca}+2$ & 1.59E-08 & 1.53E-08 & $2.92 \mathrm{E}-10$ & $6.21 \mathrm{E}-04$ & 6.20E-04 & 6.20E-04 & & & \\
\hline $\mathrm{Cr}+3$ & $5.99 \mathrm{E}-10$ & $5.76 \mathrm{E}-10$ & $3.41 \mathrm{E}-10$ & 7.94E-06 & 7.94E-06 & 7.94E-06 & & & \\
\hline $\mathrm{Co}+2$ & $3.23 \mathrm{E}-12$ & $3.11 \mathrm{E}-12$ & $1.84 \mathrm{E}-12$ & 5.16E-08 & 5.16E-08 & $5.16 \mathrm{E}-08$ & & & \\
\hline $\mathrm{Cs}+$ & $2.62 \mathrm{E}-11$ & $2.52 \mathrm{E}-11$ & $1.49 \mathrm{E}-11$ & 6.66E-05 & 6.65E-05 & 6.65E-05 & & & \\
\hline $\mathrm{Cu}+2$ & $1.29 \mathrm{E}-10$ & $1.24 \mathrm{E}-10$ & $2.37 \mathrm{E}-12$ & $3.18 \mathrm{E}-06$ & 3.18E-06 & $3.18 \mathrm{E}-06$ & & & \\
\hline $\mathrm{Fe}+3$ & 2.48E-09 & 2.39E-09 & 1.41E-09 & 9.87E-05 & 9.86E-05 & 9.86E-05 & & & \\
\hline $\mathrm{Pb}+2$ & 2.64E-09 & 2.54E-09 & 1.50E-09 & 6.90E-06 & 6.90E-06 & 6.90E-06 & & & \\
\hline $\mathrm{Hg}+2$ & 2.92E-06 & $2.81 \mathrm{E}-06$ & $1.66 \mathrm{E}-06$ & $9.22 \mathrm{E}-03$ & 9.21E-03 & 9.21E-03 & & $9.36 \mathrm{E}-07$ & $9.36 \mathrm{E}-07$ \\
\hline $\mathrm{Mn}+4$ & $2.10 \mathrm{E}-09$ & 2.02E-09 & 1.19E-09 & 3.59E-05 & 3.59E-05 & 3.59E-05 & & & \\
\hline $\mathrm{Ni}+2$ & $2.62 \mathrm{E}-10$ & $2.52 \mathrm{E}-10$ & $4.81 \mathrm{E}-12$ & 6.99E-06 & 6.98E-06 & 6.98E-06 & & & \\
\hline $\mathrm{K}+$ & $4.53 \mathrm{E}-08$ & $4.36 \mathrm{E}-08$ & $2.57 \mathrm{E}-08$ & 1.53E-03 & 1.53E-03 & 1.53E-03 & & & \\
\hline $\mathrm{Se}+4$ & $1.83 \mathrm{E}-11$ & $1.76 \mathrm{E}-11$ & $1.04 \mathrm{E}-11$ & $8.75 \mathrm{E}-06$ & $8.74 \mathrm{E}-06$ & $8.74 \mathrm{E}-06$ & & & \\
\hline $\mathrm{Ag}+$ & $8.21 \mathrm{E}-12$ & $1.24 \mathrm{E}-11$ & $7.31 \mathrm{E}-12$ & $5.16 \mathrm{E}-08$ & \begin{tabular}{|l|l|}
$5.17 \mathrm{E}-08$ \\
\end{tabular} & 5.17E-08 & & & \\
\hline $\mathrm{Na}+$ & 5.45E-07 & $5.24 \mathrm{E}-07$ & $3.10 \mathrm{E}-07$ & 5.95E-02 & 5.95E-02 & 5.95E-02 & & & \\
\hline $\mathrm{TI}+3$ & $1.68 \mathrm{E}-11$ & $1.62 \mathrm{E}-11$ & $9.57 \mathrm{E}-12$ & 7.75E-08 & 7.75E-08 & $7.75 \mathrm{E}-08$ & & & \\
\hline$U+4$ & $2.60 \mathrm{E}-10$ & $2.50 \mathrm{E}-10$ & $1.48 \mathrm{E}-10$ & $4.12 \mathrm{E}-05$ & 4.11E-05 & 4.11E-05 & & & \\
\hline$V+3$ & $1.34 \mathrm{E}-10$ & $1.29 \mathrm{E}-10$ & $7.61 \mathrm{E}-11$ & 3.83E-06 & 3.82E-06 & 3.82E-06 & & & \\
\hline$Z n+2$ & $2.00 \mathrm{E}-10$ & $1.92 \mathrm{E}-10$ & $3.67 \mathrm{E}-12$ & 4.79E-06 & 4.78E-06 & $4.78 \mathrm{E}-06$ & & & \\
\hline $\mathrm{Zr}+4$ & $3.65 \mathrm{E}-10$ & $3.51 \mathrm{E}-10$ & $2.07 \mathrm{E}-10$ & $1.51 \mathrm{E}-04$ & 1.51E-04 & $1.51 \mathrm{E}-04$ & & & \\
\hline $\mathrm{Cl}-$ & 1.64E-07 & $1.58 \mathrm{E}-07$ & $9.34 \mathrm{E}-08$ & $2.67 \mathrm{E}-03$ & 2.67E-03 & $2.67 \mathrm{E}-03$ & $1.34 \mathrm{E}-08$ & $1.34 \mathrm{E}-08$ & $1.34 \mathrm{E}-08$ \\
\hline $\mathrm{F}-$ & $4.91 \mathrm{E}-07$ & $4.73 \mathrm{E}-07$ & $2.79 \mathrm{E}-07$ & $1.61 \mathrm{E}-02$ & \begin{tabular}{|l}
$1.61 \mathrm{E}-02$ \\
\end{tabular} & $1.61 \mathrm{E}-02$ & $6.72 \mathrm{E}-07$ & $\begin{array}{l}6.69 \mathrm{E}-07 \\
\end{array}$ & 6.69E-07 \\
\hline SO4-2 & $2.92 \mathrm{E}-11$ & $6.15 \mathrm{E}-07$ & 3.63E-07 & $2.12 \mathrm{E}-02$ & $2.11 \mathrm{E}-02$ & $2.11 \mathrm{E}-02$ & & & \\
\hline NO3- & 6.03E-06 & 4.34E-09 & $2.56 \mathrm{E}-09$ & $1.04 \mathrm{E}+00$ & $1.04 \mathrm{E}+00$ & $1.04 \mathrm{E}+00$ & $1.14 \mathrm{E}-05$ & 1.14E-05 & 1.14E-05 \\
\hline PO4-3 & 8.44E-09 & 8.12E-09 & 4.80E-09 & 5.54E-04 & 5.53E-04 & 5.53E-04 & & & \\
\hline$A m+4$ & $2.30 \mathrm{E}-14$ & $2.22 \mathrm{E}-14$ & $1.31 \mathrm{E}-14$ & $8.92 \mathrm{E}-11$ & \begin{tabular}{|l|}
$8.92 \mathrm{E}-11$ \\
\end{tabular} & $8.92 \mathrm{E}-11$ & & & \\
\hline $\mathrm{Br}-$ & $2.02 \mathrm{E}-12$ & $1.94 \mathrm{E}-12$ & $1.15 \mathrm{E}-12$ & $1.45 \mathrm{E}-08$ & $1.45 \mathrm{E}-08$ & 1.45E-08 & $1.64 \mathrm{E}-13$ & $1.64 \mathrm{E}-13$ & $1.64 \mathrm{E}-13$ \\
\hline $\mathrm{Ce}+4$ & $1.83 \mathrm{E}-11$ & $1.76 \mathrm{E}-11$ & $1.04 \mathrm{E}-11$ & $1.23 \mathrm{E}-07$ & $1.23 \mathrm{E}-07$ & $1.23 \mathrm{E}-07$ & & & \\
\hline Eu+3 & $1.17 \mathrm{E}-13$ & $1.13 \mathrm{E}-13$ & $6.68 \mathrm{E}-14$ & $7.27 \mathrm{E}-10$ & $7.27 \mathrm{E}-10$ & $7.27 \mathrm{E}-10$ & & & \\
\hline $\mathrm{Gd}+3$ & $7.57 \mathrm{E}-11$ & $7.28 \mathrm{E}-11$ & $4.30 \mathrm{E}-11$ & $4.53 \mathrm{E}-07$ & $4.53 \mathrm{E}-07$ & $4.53 \mathrm{E}-07$ & & & \\
\hline $\mathrm{Ge}+4$ & $9.84 \mathrm{E}-16$ & $9.46 \mathrm{E}-16$ & 5.59E-16 & $5.11 \mathrm{E}-10$ & $5.10 \mathrm{E}-10$ & $5.10 \mathrm{E}-10$ & & & \\
\hline $\ln +3$ & $2.43 \mathrm{E}-13$ & $2.34 \mathrm{E}-13$ & $1.38 \mathrm{E}-13$ & 1.99E-09 & 1.99E-09 & 1.99E-09 & & & \\
\hline I- & $2.77 \mathrm{E}-11$ & $2.67 \mathrm{E}-11$ & $1.58 \mathrm{E}-11$ & $1.26 \mathrm{E}-07$ & \begin{tabular}{|l}
$1.26 \mathrm{E}-07$ \\
\end{tabular} & $1.26 \mathrm{E}-07$ & $2.26 \mathrm{E}-12$ & $2.26 \mathrm{E}-12$ & $2.26 \mathrm{E}-12$ \\
\hline $\mathrm{La}+3$ & $1.95 \mathrm{E}-12$ & $1.88 \mathrm{E}-12$ & $1.11 \mathrm{E}-12$ & $1.32 \mathrm{E}-08$ & 1.32E-08 & 1.32E-08 & & & \\
\hline
\end{tabular}


Table A-13. Mass balance, Tank WM-180 waste (continued).

\begin{tabular}{|c|c|c|c|c|c|c|c|c|c|}
\hline Stream \# & 109 & 110 & 111 & 112 & $113 \mathrm{~A}$ & $113 \mathrm{~B}$ & 114 & 115 & 116 \\
\hline Chemical Composition & $\mathrm{lb} / \mathrm{wscf}$ & $\mathrm{lb} / \mathrm{wscf}$ & $\mathrm{lb} / \mathrm{wscf}$ & Mol/liter & Mol/liter & Mol/liter & $\mathrm{lb} / \mathrm{wscf}$ & $\mathrm{lb} /$ wscf & lb/wscf \\
\hline $\mathrm{Li}+$ & $3.86 \mathrm{E}-11$ & $5.82 \mathrm{E}-11$ & $3.44 \mathrm{E}-11$ & $3.77 \mathrm{E}-06$ & $3.78 \mathrm{E}-06$ & $3.78 \mathrm{E}-06$ & & & \\
\hline$M g+2$ & $8.59 \mathrm{E}-10$ & $8.26 \mathrm{E}-10$ & $1.58 \mathrm{E}-11$ & $5.53 \mathrm{E}-05$ & $5.53 \mathrm{E}-05$ & $5.53 \mathrm{E}-05$ & & & \\
\hline $\mathrm{Mo}+6$ & $4.78 \mathrm{E}-10$ & $4.60 \mathrm{E}-10$ & $2.72 \mathrm{E}-10$ & $2.54 \mathrm{E}-04$ & $2.54 \mathrm{E}-04$ & $2.54 \mathrm{E}-04$ & & & \\
\hline $\mathrm{Nd}+3$ & $6.53 \mathrm{E}-12$ & $6.28 \mathrm{E}-12$ & $3.71 \mathrm{E}-12$ & $4.26 \mathrm{E}-08$ & $4.26 \mathrm{E}-08$ & $4.26 \mathrm{E}-08$ & & & \\
\hline $\mathrm{Np}+4$ & $6.20 \mathrm{E}-12$ & 5.97E-12 & $3.53 \mathrm{E}-12$ & $2.46 \mathrm{E}-08$ & $2.46 \mathrm{E}-08$ & $2.46 \mathrm{E}-08$ & & & \\
\hline $\mathrm{Nb}+5$ & $1.24 \mathrm{E}-10$ & $1.20 \mathrm{E}-10$ & $7.08 \mathrm{E}-11$ & $1.95 \mathrm{E}-06$ & $1.95 \mathrm{E}-06$ & 1.95E-06 & & & \\
\hline $\mathrm{Pd}+4$ & 1.07E-11 & $1.03 \mathrm{E}-11$ & $1.96 \mathrm{E}-13$ & $1.58 \mathrm{E}-07$ & 1.57E-07 & $1.57 \mathrm{E}-07$ & & & \\
\hline $\mathrm{Pu}+4$ & $7.43 \mathrm{E}-12$ & $7.15 \mathrm{E}-12$ & $4.22 \mathrm{E}-12$ & $1.15 \mathrm{E}-06$ & $1.15 \mathrm{E}-06$ & $1.15 \mathrm{E}-06$ & & & \\
\hline $\mathrm{Pr}+4$ & $1.80 \mathrm{E}-12$ & $1.73 \mathrm{E}-12$ & $1.02 \mathrm{E}-12$ & $1.20 \mathrm{E}-08$ & $1.20 \mathrm{E}-08$ & $1.20 \mathrm{E}-08$ & & & \\
\hline $\mathrm{Pm}+3$ & $2.80 \mathrm{E}-16$ & $2.70 \mathrm{E}-16$ & $1.59 \mathrm{E}-16$ & $1.82 \mathrm{E}-12$ & $1.82 \mathrm{E}-12$ & $1.82 \mathrm{E}-12$ & & & \\
\hline $\mathrm{Rh}+4$ & $5.67 \mathrm{E}-13$ & $5.45 \mathrm{E}-13$ & $3.22 \mathrm{E}-13$ & 5.18E-09 & 5.18E-09 & $5.18 \mathrm{E}-09$ & & & \\
\hline $\mathrm{Rb}+$ & $3.03 \mathrm{E}-12$ & $4.56 \mathrm{E}-12$ & $2.69 \mathrm{E}-12$ & $2.40 \mathrm{E}-08$ & $2.40 \mathrm{E}-08$ & $2.40 \mathrm{E}-08$ & & & \\
\hline $\mathrm{Ru}+3$ & $3.82 \mathrm{E}-11$ & $3.68 \mathrm{E}-11$ & $2.17 \mathrm{E}-11$ & $3.56 \mathrm{E}-07$ & $3.56 \mathrm{E}-07$ & $3.56 \mathrm{E}-07$ & & & \\
\hline $\mathrm{Sm}+3$ & $1.26 \mathrm{E}-12$ & $1.21 \mathrm{E}-12$ & $7.18 \mathrm{E}-13$ & 7.90E-09 & 7.90E-09 & 7.90E-09 & & & \\
\hline $\mathrm{Si+4}$ & $2.83 \mathrm{E}-09$ & $2.72 \mathrm{E}-09$ & 1.61E-09 & $6.65 \mathrm{E}-04$ & $6.64 \mathrm{E}-04$ & $6.64 \mathrm{E}-04$ & & & \\
\hline $\mathrm{Sr}+2$ & $3.03 \mathrm{E}-11$ & $2.92 \mathrm{E}-11$ & $5.56 \mathrm{E}-13$ & 5.42E-07 & 5.41E-07 & $5.41 \mathrm{E}-07$ & & & \\
\hline $\mathrm{Tc}+7$ & $4.94 \mathrm{E}-12$ & $4.75 \mathrm{E}-12$ & $2.81 \mathrm{E}-12$ & 5.04E-08 & 5.04E-08 & 5.04E-08 & & & \\
\hline $\mathrm{Te}+4$ & $5.67 \mathrm{E}-13$ & $5.46 \mathrm{E}-13$ & $3.23 \mathrm{E}-13$ & $1.68 \mathrm{E}-07$ & 1.67E-07 & $1.67 \mathrm{E}-07$ & & & \\
\hline $\mathrm{Tb}+4$ & $5.14 \mathrm{E}-16$ & $4.94 \mathrm{E}-16$ & $2.92 \mathrm{E}-16$ & $3.04 \mathrm{E}-12$ & $3.04 \mathrm{E}-12$ & $3.04 \mathrm{E}-12$ & & & \\
\hline $\mathrm{Th}+4$ & $7.37 \mathrm{E}-17$ & $7.09 \mathrm{E}-17$ & $4.19 \mathrm{E}-17$ & $1.20 \mathrm{E}-11$ & $1.20 \mathrm{E}-11$ & $1.20 \mathrm{E}-11$ & & & \\
\hline Sn+4 & $2.65 \mathrm{E}-11$ & $2.55 \mathrm{E}-11$ & $1.51 \mathrm{E}-11$ & $8.42 \mathrm{E}-06$ & $8.41 \mathrm{E}-06$ & $8.41 \mathrm{E}-06$ & & & \\
\hline $\mathrm{Ti}+4$ & $1.95 \mathrm{E}-11$ & $1.87 \mathrm{E}-11$ & $1.11 \mathrm{E}-11$ & 1.53E-05 & 1.53E-05 & 1.53E-05 & & & \\
\hline $\mathrm{Y}+3$ & $9.30 \mathrm{E}-13$ & $8.95 \mathrm{E}-13$ & $5.29 \mathrm{E}-13$ & 9.85E-09 & 9.85E-09 & 9.85E-09 & & & \\
\hline $\mathrm{OH}-$ & $4.28 \mathrm{E}-09$ & 1.19E-07 & $2.49 \mathrm{E}-07$ & $4.06 \mathrm{E}-07$ & 4.07E-07 & 4.07E-07 & & & \\
\hline $\mathrm{H} 2 \mathrm{O}$ & $3.18 \mathrm{E}-02$ & $3.30 \mathrm{E}-02$ & 3.88E-02 & $5.24 \mathrm{E}+01$ & $5.23 \mathrm{E}+01$ & $5.23 \mathrm{E}+01$ & 3.87E-02 & $3.86 \mathrm{E}-02$ & $3.86 \mathrm{E}-02$ \\
\hline $\mathrm{SO} 2$ & $2.78 \mathrm{E}-09$ & $2.17 \mathrm{E}-05$ & $1.28 \mathrm{E}-05$ & 9.75E-06 & 9.78E-06 & $9.78 \mathrm{E}-06$ & 1.29E-05 & $1.29 \mathrm{E}-05$ & 1.29E-05 \\
\hline $\mathrm{H} 2 \mathrm{~S}$ & 1.22E-05 & $2.71 \mathrm{E}-21$ & & & & & & & \\
\hline $\mathrm{CO}$ & $7.45 \mathrm{E}-04$ & $1.48 \mathrm{E}-09$ & $8.75 \mathrm{E}-10$ & $1.88 \mathrm{E}-10$ & $1.90 \mathrm{E}-10$ & $1.90 \mathrm{E}-10$ & $8.80 \mathrm{E}-10$ & $8.77 \mathrm{E}-10$ & $8.77 \mathrm{E}-10$ \\
\hline $\mathrm{CO} 2$ & $7.14 \mathrm{E}-03$ & $9.01 \mathrm{E}-03$ & $5.32 \mathrm{E}-03$ & $3.00 \mathrm{E}-04$ & 3.09E-04 & $3.09 \mathrm{E}-04$ & $5.35 \mathrm{E}-03$ & $5.33 \mathrm{E}-03$ & $5.33 \mathrm{E}-03$ \\
\hline $\mathrm{H} 2$ & $2.05 E-04$ & $6.16 \mathrm{E}-10$ & $3.64 \mathrm{E}-10$ & $3.21 \mathrm{E}-10$ & $5.06 \mathrm{E}-10$ & $5.06 \mathrm{E}-10$ & 7.70E-09 & & \\
\hline N2 & $1.46 \mathrm{E}-02$ & $1.40 \mathrm{E}-02$ & 8.30E-03 & 1.32E-05 & $2.52 \mathrm{E}-05$ & $2.52 \mathrm{E}-05$ & 8.34E-03 & $8.31 \mathrm{E}-03$ & $8.31 \mathrm{E}-03$ \\
\hline NO & $4.38 \mathrm{E}-05$ & 4.76E-05 & $2.81 \mathrm{E}-05$ & $2.21 \mathrm{E}-07$ & $2.77 \mathrm{E}-07$ & $2.77 \mathrm{E}-07$ & $2.83 E-05$ & $2.82 \mathrm{E}-05$ & $2.82 \mathrm{E}-05$ \\
\hline $\mathrm{NO} 2$ & $4.54 \mathrm{E}-06$ & $2.60 \mathrm{E}-07$ & $1.53 \mathrm{E}-07$ & $2.77 \mathrm{E}-06$ & $2.76 \mathrm{E}-06$ & $2.76 \mathrm{E}-06$ & 1.54E-07 & $1.54 \mathrm{E}-07$ & 1.54E-07 \\
\hline $\mathrm{O} 2$ & 4.16E-04 & 1.83E-03 & $1.08 \mathrm{E}-03$ & $3.19 \mathrm{E}-06$ & $4.88 \mathrm{E}-06$ & $4.88 \mathrm{E}-06$ & $1.08 \mathrm{E}-03$ & $1.08 \mathrm{E}-03$ & $1.08 \mathrm{E}-03$ \\
\hline S (other) & $3.18 \mathrm{E}-10$ & $1.60 \mathrm{E}-25$ & & $1.30 \mathrm{E}-07$ & \begin{tabular}{|l|}
$1.31 \mathrm{E}-07$ \\
\end{tabular} & 1.31E-07 & & & \\
\hline $\mathrm{CO} 3$ & $2.34 \mathrm{E}-07$ & $1.46 \mathrm{E}-11$ & 3.36E-12 & 3.33E-05 & 3.32E-05 & 3.32E-05 & & & \\
\hline C (reductant) & $3.20 \mathrm{E}-08$ & $1.30 \mathrm{E}-18$ & & & & & & & \\
\hline $\mathrm{O}$ (oxides) & 5.37E-08 & $5.01 \mathrm{E}-08$ & $2.96 \mathrm{E}-08$ & $4.92 \mathrm{E}-03$ & 4.91E-03 & 4.91E-03 & & & \\
\hline $\mathrm{C}$ (organic) & $1.77 \mathrm{E}-05$ & $1.66 \mathrm{E}-34$ & & & & & & & \\
\hline $\mathrm{H}$ (organic) & 5.93E-06 & $5.59 \mathrm{E}-35$ & & & & & & & \\
\hline $\mathrm{O}$ (organic) & 1.32E-07 & & & & & & & & \\
\hline \multirow[t]{5}{*}{ Mass Flow (kg/hr): } & $2.62 \mathrm{E}+03$ & $2.87 \mathrm{E}+03$ & $4.49 \mathrm{E}+03$ & $3.92 \mathrm{E}+04$ & $3.93 \mathrm{E}+04$ & & $4.47 \mathrm{E}+03$ & $4.47 E+03$ & $4.47 \mathrm{E}+03$ \\
\hline & & & & & & & & & \\
\hline & & & & & & & & & \\
\hline & & & & & & & & & \\
\hline & $\mathrm{w} / \mathrm{m} 3$ & $\mathrm{w} / \mathrm{m} 3$ & $\mathrm{w} / \mathrm{m} 3$ & $\mathrm{w} / \mathrm{m} 3$ & $\mathrm{w} / \mathrm{m} 3$ & $\mathrm{w} / \mathrm{m} 3$ & $\mathrm{w} / \mathrm{m} 3$ & $\mathrm{w} / \mathrm{m} 3$ & $\mathrm{w} / \mathrm{m} 3$ \\
\hline Heat Generation, w/m3 & & & & $7.26 \mathrm{E}-02$ & $7.25 \mathrm{E}-02$ & & & & \\
\hline
\end{tabular}


Table A-13. Mass balance, Tank WM-180 waste (continued).

\begin{tabular}{|c|c|c|c|c|c|c|c|c|c|}
\hline Stream \# & 109 & 110 & 111 & 112 & $113 \mathrm{~A}$ & 113B & 114 & 115 & 116 \\
\hline Radiological Composition & $\mathrm{Ci} / \mathrm{wscm}$ & $\mathrm{Ci} / \mathrm{wscm}$ & $\mathrm{Ci} / \mathrm{wscm}$ & $\mathrm{Ci} / \mathrm{L}$ & $\mathrm{Ci} / \mathrm{L}$ & $\mathrm{Ci} / \mathrm{L}$ & $\mathrm{Ci} / \mathrm{wscm}$ & $\mathrm{Ci} / \mathrm{wscm}$ & $\mathrm{Ci} / \mathrm{wscm}$ \\
\hline $\mathrm{H}-3$ & 1.94E-06 & $1.87 \mathrm{E}-06$ & 1.10E-06 & 1.64E-06 & 1.63E-06 & $1.63 \mathrm{E}-06$ & $1.10 \mathrm{E}-06$ & $1.10 \mathrm{E}-06$ & 1.10E-06 \\
\hline C-14 & $6.43 \mathrm{E}-12$ & $6.24 \mathrm{E}-12$ & $3.69 \mathrm{E}-12$ & $6.34 \mathrm{E}-16$ & $6.51 \mathrm{E}-16$ & $6.51 \mathrm{E}-16$ & $3.71 \mathrm{E}-12$ & $3.69 \mathrm{E}-12$ & $3.69 \mathrm{E}-12$ \\
\hline Co-60 & $1.91 \mathrm{E}-10$ & $1.83 \mathrm{E}-10$ & $1.08 \mathrm{E}-10$ & 1.12E-08 & 1.12E-08 & 1.12E-08 & & & \\
\hline $\mathrm{Ni}-59$ & $4.71 \mathrm{E}-12$ & $4.53 \mathrm{E}-12$ & $8.63 \mathrm{E}-14$ & $4.60 \mathrm{E}-10$ & $4.59 \mathrm{E}-10$ & $4.59 \mathrm{E}-10$ & & & \\
\hline $\mathrm{Ni}-63$ & $1.24 \mathrm{E}-09$ & $1.19 \mathrm{E}-09$ & $2.28 \mathrm{E}-11$ & $1.21 \mathrm{E}-07$ & $1.21 \mathrm{E}-07$ & $1.21 \mathrm{E}-07$ & & & \\
\hline Se-79 & $1.09 \mathrm{E}-11$ & $1.05 \mathrm{E}-11$ & $6.20 \mathrm{E}-12$ & $2.57 \mathrm{E}-08$ & $2.56 \mathrm{E}-08$ & $2.56 \mathrm{E}-08$ & & & \\
\hline Sr-90 & $1.00 \mathrm{E}-06$ & $9.65 \mathrm{E}-07$ & $1.84 \mathrm{E}-08$ & $9.80 \mathrm{E}-05$ & $9.79 \mathrm{E}-05$ & 9.79E-05 & & & \\
\hline Y-90 & 9.35E-07 & $9.00 \mathrm{E}-07$ & 5.32E-07 & 5.49E-05 & 5.49E-05 & 5.49E-05 & & & \\
\hline Zr-93 & $5.28 \mathrm{E}-11$ & $5.08 \mathrm{E}-11$ & $3.00 \mathrm{E}-11$ & $1.24 \mathrm{E}-07$ & $1.24 \mathrm{E}-07$ & $1.24 \mathrm{E}-07$ & & & \\
\hline $\mathrm{Nb}-93 \mathrm{~m}$ & $4.04 \mathrm{E}-11$ & $3.88 \mathrm{E}-11$ & $2.30 \mathrm{E}-11$ & $3.67 \mathrm{E}-09$ & $3.67 \mathrm{E}-09$ & 3.67E-09 & & & \\
\hline $\mathrm{Nb}-94$ & $2.82 \mathrm{E}-11$ & $2.72 \mathrm{E}-11$ & $1.61 \mathrm{E}-11$ & 2.57E-09 & 2.56E-09 & 2.56E-09 & & & \\
\hline Tc-99 & $1.37 \mathrm{E}-09$ & $1.32 \mathrm{E}-09$ & $7.81 \mathrm{E}-10$ & $8.57 \mathrm{E}-08$ & 8.57E-08 & $8.57 \mathrm{E}-08$ & & & \\
\hline Ru-106 & $2.31 \mathrm{E}-11$ & $2.22 \mathrm{E}-11$ & 1.31E-11 & 1.35E-09 & 1.35E-09 & 1.35E-09 & & & \\
\hline Rh-102 & $2.04 \mathrm{E}-14$ & $1.96 \mathrm{E}-14$ & 1.16E-14 & $1.20 \mathrm{E}-12$ & $1.20 \mathrm{E}-12$ & $1.20 \mathrm{E}-12$ & & & \\
\hline Rh-106 & $2.31 \mathrm{E}-11$ & $2.22 \mathrm{E}-11$ & $1.31 \mathrm{E}-11$ & $1.35 \mathrm{E}-09$ & $1.35 \mathrm{E}-09$ & $1.35 \mathrm{E}-09$ & & & \\
\hline Pd-107 & $4.19 \mathrm{E}-13$ & $4.03 \mathrm{E}-13$ & $7.68 \mathrm{E}-15$ & $4.09 \mathrm{E}-11$ & $4.09 \mathrm{E}-11$ & $4.09 \mathrm{E}-11$ & & & \\
\hline $\mathrm{Cd}-113 \mathrm{~m}$ & $9.20 \mathrm{E}-11$ & $8.85 \mathrm{E}-11$ & $5.23 \mathrm{E}-11$ & $4.50 \mathrm{E}-09$ & $4.50 \mathrm{E}-09$ & 4.50E-09 & & & \\
\hline Sn-121m & $1.59 \mathrm{E}-12$ & $1.53 \mathrm{E}-12$ & $9.06 \mathrm{E}-13$ & $3.75 \mathrm{E}-09$ & $3.74 \mathrm{E}-09$ & $3.74 \mathrm{E}-09$ & & & \\
\hline Sn-126 & $1.03 E-11$ & $9.87 \mathrm{E}-12$ & $5.83 E-12$ & $2.42 \mathrm{E}-08$ & $2.41 \mathrm{E}-08$ & $2.41 \mathrm{E}-08$ & & & \\
\hline Sb-125 & $3.79 \mathrm{E}-10$ & $3.65 \mathrm{E}-10$ & $2.16 \mathrm{E}-10$ & 2.23E-08 & 2.23E-08 & 2.23E-08 & & & \\
\hline Sb-126 & $1.36 \mathrm{E}-12$ & $1.31 \mathrm{E}-12$ & $7.72 \mathrm{E}-13$ & $7.98 \mathrm{E}-11$ & $7.98 \mathrm{E}-11$ & $7.98 \mathrm{E}-11$ & & & \\
\hline Te-125m & $7.50 \mathrm{E}-11$ & $7.22 \mathrm{E}-11$ & $4.27 \mathrm{E}-11$ & 1.77E-07 & 1.76E-07 & 1.76E-07 & & & \\
\hline I-129 & $6.23 \mathrm{E}-11$ & $5.99 \mathrm{E}-11$ & $3.54 \mathrm{E}-11$ & $2.24 \mathrm{E}-09$ & \begin{tabular}{|l}
$2.24 \mathrm{E}-09$ \\
\end{tabular} & 2.24E-09 & $5.08 \mathrm{E}-12$ & $5.07 \mathrm{E}-12$ & $5.07 \mathrm{E}-12$ \\
\hline Cs-134 & $6.29 \mathrm{E}-10$ & $6.05 \mathrm{E}-10$ & $3.57 \mathrm{E}-10$ & 1.33E-05 & 1.32E-05 & $1.32 \mathrm{E}-05$ & & & \\
\hline Cs-135 & $5.41 \mathrm{E}-11$ & $5.21 \mathrm{E}-11$ & $3.08 \mathrm{E}-11$ & 1.14E-06 & 1.14E-06 & 1.14E-06 & & & \\
\hline Cs-137 & $3.17 \mathrm{E}-06$ & $3.05 \mathrm{E}-06$ & $1.80 \mathrm{E}-06$ & 6.69E-02 & 6.68E-02 & $6.68 \mathrm{E}-02$ & & & \\
\hline Ba-137m & $1.26 \mathrm{E}-06$ & $1.21 \mathrm{E}-06$ & $2.31 \mathrm{E}-08$ & 1.23E-04 & $1.23 \mathrm{E}-04$ & $1.23 \mathrm{E}-04$ & & & \\
\hline $\mathrm{Ce}-144$ & $1.55 \mathrm{E}-11$ & $1.50 \mathrm{E}-11$ & $8.83 \mathrm{E}-12$ & $9.13 \mathrm{E}-10$ & $9.13 \mathrm{E}-10$ & $9.13 \mathrm{E}-10$ & & & \\
\hline \begin{tabular}{|l|} 
Pr-144 \\
\end{tabular} & $1.55 \mathrm{E}-11$ & $1.50 \mathrm{E}-11$ & 8.83E-12 & $9.13 \mathrm{E}-10$ & $9.13 \mathrm{E}-10$ & $9.13 \mathrm{E}-10$ & & & \\
\hline $\mathrm{Pm}-146$ & $1.20 \mathrm{E}-12$ & $1.16 \mathrm{E}-12$ & $6.83 \mathrm{E}-13$ & $7.06 \mathrm{E}-11$ & $7.06 \mathrm{E}-11$ & $7.06 \mathrm{E}-11$ & & & \\
\hline Pm-147 & $4.22 \mathrm{E}-09$ & 4.06E-09 & $2.40 \mathrm{E}-09$ & $2.48 \mathrm{E}-07$ & \begin{tabular}{|l|}
$2.48 \mathrm{E}-07$ \\
\end{tabular} & $2.48 \mathrm{E}-07$ & & & \\
\hline Sm-151 & $8.32 \mathrm{E}-09$ & 8.00E-09 & 4.73E-09 & 4.89E-07 & 4.89E-07 & 4.89E-07 & & & \\
\hline Eu-152 & $6.02 \mathrm{E}-11$ & $5.79 \mathrm{E}-11$ & $3.42 \mathrm{E}-11$ & 3.54E-09 & 3.54E-09 & 3.54E-09 & & & \\
\hline Eu-154 & $2.16 \mathrm{E}-09$ & $2.08 \mathrm{E}-09$ & 1.23E-09 & $1.27 \mathrm{E}-07$ & $1.27 \mathrm{E}-07$ & $1.27 \mathrm{E}-07$ & & & \\
\hline Eu-155 & 3.93E-09 & 3.78E-09 & $2.23 \mathrm{E}-09$ & $2.31 \mathrm{E}-07$ & $2.31 \mathrm{E}-07$ & $2.31 \mathrm{E}-07$ & & & \\
\hline Th-230 & $2.08 \mathrm{E}-14$ & $2.00 \mathrm{E}-14$ & $1.18 \mathrm{E}-14$ & $4.89 \mathrm{E}-11$ & $4.89 \mathrm{E}-11$ & $4.89 \mathrm{E}-11$ & & & \\
\hline $\mathrm{Pa}-233$ & $6.98 \mathrm{E}-11$ & $6.72 \mathrm{E}-11$ & $3.97 \mathrm{E}-11$ & $1.64 \mathrm{E}-07$ & $1.64 \mathrm{E}-07$ & \begin{tabular}{|l|}
$1.64 \mathrm{E}-07$ \\
\end{tabular} & & & \\
\hline U-232 & 5.01E-14 & $4.82 \mathrm{E}-14$ & $2.85 \mathrm{E}-14$ & $1.18 \mathrm{E}-10$ & $1.18 \mathrm{E}-10$ & $1.18 \mathrm{E}-10$ & & & \\
\hline U-233 & $1.95 \mathrm{E}-15$ & $1.88 \mathrm{E}-15$ & $1.11 \mathrm{E}-15$ & $4.60 \mathrm{E}-12$ & $4.59 \mathrm{E}-12$ & $4.59 \mathrm{E}-12$ & & & \\
\hline U-234 & 5.02E-11 & $4.83 \mathrm{E}-11$ & $2.85 \mathrm{E}-11$ & $1.18 \mathrm{E}-07$ & 1.18E-07 & 1.18E-07 & & & \\
\hline U-235 & $1.83 \mathrm{E}-12$ & $1.76 \mathrm{E}-12$ & $1.04 \mathrm{E}-12$ & $4.31 \mathrm{E}-09$ & \begin{tabular}{|l}
$4.31 \mathrm{E}-09$ \\
\end{tabular} & 4.31E-09 & & & \\
\hline U-236 & $2.72 \mathrm{E}-12$ & $2.62 \mathrm{E}-12$ & $1.55 \mathrm{E}-12$ & $6.40 \mathrm{E}-09$ & 6.39E-09 & 6.39E-09 & & & \\
\hline U-237 & $1.53 \mathrm{E}-13$ & $1.48 \mathrm{E}-13$ & $8.72 \mathrm{E}-14$ & $3.61 \mathrm{E}-10$ & $3.60 \mathrm{E}-10$ & $3.60 \mathrm{E}-10$ & & & \\
\hline U-238 & $1.08 \mathrm{E}-12$ & $1.04 \mathrm{E}-12$ & $6.16 \mathrm{E}-13$ & $2.55 \mathrm{E}-09$ & $2.55 \mathrm{E}-09$ & 2.55E-09 & & & \\
\hline Np-237 & $7.00 \mathrm{E}-11$ & $6.74 \mathrm{E}-11$ & $3.98 \mathrm{E}-11$ & $4.11 \mathrm{E}-09$ & 4.11E-09 & $4.11 \mathrm{E}-09$ & & & \\
\hline Pu-236 & $1.01 \mathrm{E}-13$ & $9.67 \mathrm{E}-14$ & $5.72 \mathrm{E}-14$ & $2.37 \mathrm{E}-10$ & $2.36 \mathrm{E}-10$ & $2.36 \mathrm{E}-10$ & & & \\
\hline Pu-238 & $4.59 \mathrm{E}-08$ & 4.41E-08 & $2.61 \mathrm{E}-08$ & $1.08 \mathrm{E}-04$ & 1.08E-04 & 1.08E-04 & & & \\
\hline Pu-239 & 6.96E-09 & 6.69E-09 & $3.96 \mathrm{E}-09$ & 1.64E-05 & $1.64 \mathrm{E}-05$ & 1.64E-05 & & & \\
\hline Pu-240 & $3.78 \mathrm{E}-10$ & $3.63 \mathrm{E}-10$ & $2.15 \mathrm{E}-10$ & 8.89E-07 & 8.88E-07 & 8.88E-07 & & & \\
\hline Pu-241 & $2.76 \mathrm{E}-08$ & $2.66 \mathrm{E}-08$ & $1.57 \mathrm{E}-08$ & 6.50E-05 & 6.49E-05 & 6.49E-05 & & & \\
\hline Pu-242 & $2.91 \mathrm{E}-13$ & $2.80 \mathrm{E}-13$ & $1.65 \mathrm{E}-13$ & $6.85 \mathrm{E}-10$ & $6.84 \mathrm{E}-10$ & $6.84 \mathrm{E}-10$ & & & \\
\hline Pu-244 & $2.49 \mathrm{E}-20$ & $2.40 \mathrm{E}-20$ & $1.42 \mathrm{E}-20$ & $5.87 \mathrm{E}-17$ & $5.86 \mathrm{E}-17$ & $5.86 \mathrm{E}-17$ & & & \\
\hline Am-241 & $3.29 E-09$ & $3.17 \mathrm{E}-09$ & 1.87E-09 & $1.93 \mathrm{E}-07$ & 1.93E-07 & 1.93E-07 & & & \\
\hline$A m-242 m$ & $3.59 \mathrm{E}-13$ & $3.46 \mathrm{E}-13$ & $2.04 \mathrm{E}-13$ & $2.11 \mathrm{E}-11$ & $2.11 \mathrm{E}-11$ & $2.11 \mathrm{E}-11$ & & & \\
\hline
\end{tabular}


Table A-13. Mass balance, Tank WM-180 waste (continued).

\begin{tabular}{|c|c|c|c|c|c|c|c|c|c|}
\hline Stream \# & 109 & 110 & 111 & 112 & $113 \mathrm{~A}$ & 113B & 114 & 115 & 116 \\
\hline & $\mathrm{Ci} / \mathrm{wscm}$ & $\mathrm{Ci} / \mathrm{wscm}$ & $\mathrm{Ci} / \mathrm{wscm}$ & $\mathrm{Ci} / \mathrm{L}$ & $\mathrm{Ci} / \mathrm{L}$ & $\mathrm{Ci} / \mathrm{L}$ & $\mathrm{Ci} / \mathrm{wscm}$ & $\mathrm{Ci} / \mathrm{wscm}$ & $\mathrm{Ci} / \mathrm{wscm}$ \\
\hline Am-243 & $5.22 \mathrm{E}-13$ & $5.02 \mathrm{E}-13$ & $2.97 \mathrm{E}-13$ & $3.06 \mathrm{E}-11$ & \begin{tabular}{|c|}
$3.06 \mathrm{E}-11$ \\
\end{tabular} & $3.06 \mathrm{E}-11$ & & & \\
\hline $\mathrm{Cm}-242$ & $2.99 \mathrm{E}-13$ & $2.88 \mathrm{E}-13$ & $1.70 \mathrm{E}-13$ & 7.04E-10 & $7.03 \mathrm{E}-10$ & $7.03 E-10$ & & & \\
\hline $\mathrm{Cm}-243$ & $8.60 \mathrm{E}-13$ & $8.27 \mathrm{E}-13$ & $4.89 \mathrm{E}-13$ & $2.02 \mathrm{E}-09$ & $2.02 \mathrm{E}-09$ & $2.02 \mathrm{E}-09$ & & & \\
\hline $\mathrm{Cm}-244$ & $5.34 \mathrm{E}-11$ & $5.14 \mathrm{E}-11$ & $3.04 \mathrm{E}-11$ & $1.26 \mathrm{E}-07$ & 1.26E-07 & $1.26 \mathrm{E}-07$ & & & \\
\hline $\mathrm{Cm}-245$ & $9.05 \mathrm{E}-15$ & $8.70 \mathrm{E}-15$ & $5.14 \mathrm{E}-15$ & $2.13 \mathrm{E}-11$ & $2.13 \mathrm{E}-11$ & $2.13 \mathrm{E}-11$ & & & \\
\hline $\mathrm{Cm}-246$ & $5.94 \mathrm{E}-16$ & $5.72 \mathrm{E}-16$ & $3.38 \mathrm{E}-16$ & $1.40 \mathrm{E}-12$ & $1.40 \mathrm{E}-12$ & $1.40 \mathrm{E}-12$ & & & \\
\hline TRU & $5.74 \mathrm{E}-08$ & $5.52 \mathrm{E}-08$ & $3.26 \mathrm{E}-08$ & 1.27E-04 & 1.27E-04 & 1.27E-04 & & & \\
\hline \multicolumn{10}{|l|}{ TRU, nCi/gm } \\
\hline & & & & & & & & & \\
\hline $\begin{array}{l}\text { Gas Stream Bulk } \\
\text { Composition (Wet Basis) }\end{array}$ & $\begin{array}{l}\mathrm{mol} \% \text { or } \\
\mathrm{ppmv}\end{array}$ & $\begin{array}{l}\mathrm{mol} \% \text { or } \\
\mathrm{ppmv}\end{array}$ & $\begin{array}{c}\text { mol\% or } \\
\text { ppmv }\end{array}$ & $\begin{array}{c}\mathrm{mol} \% \text { or } \\
\mathrm{ppmv}\end{array}$ & $\begin{array}{c}\mathrm{mol} \% \text { or } \\
\mathrm{ppmv}\end{array}$ & $\begin{array}{c}\mathrm{mol} \% \text { or } \\
\mathrm{ppmv}\end{array}$ & $\begin{array}{c}\mathrm{mol} \% \text { or } \\
\mathrm{ppmv}\end{array}$ & $\begin{array}{c}\text { mol\% or } \\
\text { ppmv }\end{array}$ & $\begin{array}{c}\text { mol\% or } \\
\text { ppmv }\end{array}$ \\
\hline $\mathrm{H} 2 \mathrm{O}, \mathrm{mol} \%$ & $68.11 \%$ & $70.52 \%$ & $82.64 \%$ & & & & $82.54 \%$ & $82.54 \%$ & $82.54 \%$ \\
\hline $\mathrm{O} 2, \mathrm{~mol} \%$ & $0.50 \%$ & $2.20 \%$ & $1.29 \%$ & & & & $1.30 \%$ & $1.30 \%$ & $1.30 \%$ \\
\hline $\mathrm{N} 2, \mathrm{~mol} \%$ & $20.06 \%$ & $19.32 \%$ & $11.37 \%$ & & & & $11.43 \%$ & $11.43 \%$ & $11.43 \%$ \\
\hline $\mathrm{H} 2, \mathrm{~mol} \%$ & $3.91 \%$ & $0.00001 \%$ & $0.00001 \%$ & & & & $0.0001 \%$ & & $0.00 \%$ \\
\hline $\mathrm{CO} 2, \mathrm{~mol} \%$ & $6.25 \%$ & $7.89 \%$ & $4.64 \%$ & & & & $4.67 \%$ & $4.67 \%$ & $4.67 \%$ \\
\hline $\mathrm{CO}, \mathrm{ppmv}$ & $1.03 E+04$ & $2.04 \mathrm{E}-02$ & $1.20 \mathrm{E}-02$ & & & & $1.21 \mathrm{E}-02$ & $1.21 \mathrm{E}-02$ & 1.21E-02 \\
\hline $\mathrm{NO}, \mathrm{ppmv}$ & $5.62 \mathrm{E}+02$ & $6.11 \mathrm{E}+02$ & $3.60 \mathrm{E}+02$ & & & & $3.62 \mathrm{E}+02$ & $3.62 \mathrm{E}+02$ & $3.62 \mathrm{E}+02$ \\
\hline NO2, ppmv & $3.80 \mathrm{E}+01$ & $2.17 \mathrm{E}+00$ & $1.28 \mathrm{E}+00$ & & & & $1.29 \mathrm{E}+00$ & $1.29 \mathrm{E}+00$ & $1.29 E+00$ \\
\hline SO2, ppmv & 1.67E-02 & $1.31 \mathrm{E}+02$ & $7.70 \mathrm{E}+01$ & & & & $7.73 \mathrm{E}+01$ & $7.73 \mathrm{E}+01$ & $7.73 E+01$ \\
\hline $\mathrm{Cl}, \mathrm{ppmv}$ & $1.79 \mathrm{E}+00$ & $1.72 \mathrm{E}+00$ & $1.01 \mathrm{E}+00$ & & & & $1.45 \mathrm{E}-01$ & 1.45E-01 & 1.45E-01 \\
\hline $\mathrm{F}, \mathrm{ppmv}$ & $9.97 \mathrm{E}+00$ & $9.59 \mathrm{E}+00$ & $5.65 \mathrm{E}+00$ & & & & $1.36 \mathrm{E}+01$ & $1.36 \mathrm{E}+01$ & $1.36 \mathrm{E}+01$ \\
\hline C (organic), ppmv & $5.68 \mathrm{E}+02$ & $5.34 \mathrm{E}-27$ & & & & & & & \\
\hline $\mathrm{H}$ (organic), ppmv & $2.27 E+03$ & $2.14 \mathrm{E}-26$ & & & & & & & \\
\hline $\mathrm{Hg}, \mathrm{ug} / \mathrm{wscm}$ & $4.68 \mathrm{E}+04$ & $4.50 \mathrm{E}+04$ & $2.66 \mathrm{E}+04$ & & & & & $1.50 \mathrm{E}+04$ & $1.50 \mathrm{E}+04$ \\
\hline $\mathrm{PM}, \mathrm{mg} / \mathrm{wscm}$ & $4.44 \mathrm{E}+02$ & $7.00 \mathrm{E}+01$ & $4.90 \mathrm{E}+01$ & & & & $1.19 \mathrm{E}+01$ & $2.68 \mathrm{E}+01$ & $2.68 \mathrm{E}+01$ \\
\hline SVM, ug/wscm & $4.68 \mathrm{E}+01$ & $4.50 \mathrm{E}+01$ & $2.66 \mathrm{E}+01$ & & & & & & \\
\hline LVM, ug/wscm & $1.12 \mathrm{E}+01$ & $1.08 \mathrm{E}+01$ & $6.37 \mathrm{E}+00$ & & & & & & \\
\hline $\begin{array}{l}\text { Gas Stream Bulk } \\
\text { Composition (Dry Basis) }\end{array}$ & $\mathrm{mol} \%$ & $\mathrm{~mol} \%$ & $\mathrm{~mol} \%$ & $\mathrm{~mol} \%$ & $\mathrm{~mol} \%$ & $\mathrm{~mol} \%$ & $\mathrm{~mol} \%$ & $\mathrm{~mol} \%$ & $\mathrm{~mol} \%$ \\
\hline $\mathrm{O} 2, \mathrm{~mol} \%$, dry basis & $1.5705 \%$ & $7.4569 \%$ & $7.4567 \%$ & & & & $7.4538 \%$ & $7.4534 \%$ & $7.4534 \%$ \\
\hline $\mathrm{N} 2, \mathrm{~mol} \%$, dry basis & $62.91 \%$ & $65.53 \%$ & $65.53 \%$ & & & & $65.50 \%$ & $65.50 \%$ & $65.50 \%$ \\
\hline $\mathrm{H} 2, \mathrm{~mol} \%$, dry basis & $12.27 \%$ & $0.00004 \%$ & $0.00004 \%$ & & & & $0.00084 \%$ & & $0.00 \%$ \\
\hline $\mathrm{CO} 2, \mathrm{~mol} \%$, dry basis & $19.61 \%$ & $26.76 \%$ & $26.76 \%$ & & & & $26.74 \%$ & $26.74 \%$ & $26.74 \%$ \\
\hline $\begin{array}{l}\text { Gas Stream Bulk } \\
\text { Composition (Dry Basis, } \\
\text { Corrected to } 7 \% \text { O2 with } \\
100 \% \text { O2 Combustion Air) }\end{array}$ & $\begin{array}{l}\text { ppmv, or } \\
\text { ug/dscm } \\
\text { or } \\
\mathrm{mg} / \mathrm{dscm}\end{array}$ & $\begin{array}{l}\text { ppmv, or } \\
\text { ug/dscm } \\
\text { or } \\
\mathrm{mg} / \mathrm{dscm}\end{array}$ & $\begin{array}{l}\mathrm{ppmv}, \text { or } \\
\mathrm{ug} / \mathrm{dscm} \\
\text { or } \\
\mathrm{mg} / \mathrm{dscm}\end{array}$ & $\begin{array}{l}\text { ppmv, or } \\
\text { ug/dscm } \\
\text { or } \\
\mathrm{mg} / \mathrm{dscm}\end{array}$ & $\begin{array}{l}\text { ppmv, or } \\
\text { ug/dscm } \\
\text { or } \\
\mathrm{mg} / \mathrm{dscm}\end{array}$ & $\begin{array}{l}\text { ppmv, or } \\
\text { ug/dscm } \\
\text { or } \\
\mathrm{mg} / \mathrm{dscm}\end{array}$ & $\begin{array}{l}\text { ppmv, or } \\
\text { ug/dscm } \\
\text { or } \\
\mathrm{mg} / \mathrm{dscm}\end{array}$ & $\begin{array}{l}\text { ppmv, or } \\
\text { ug/dscm } \\
\text { or } \\
\mathrm{mg} / \mathrm{dscm}\end{array}$ & $\begin{array}{l}\text { ppmv, or } \\
\text { ug/dscm } \\
\text { or } \\
\mathrm{mg} / \mathrm{dscm}\end{array}$ \\
\hline $\mathrm{CO}, \mathrm{ppmv}$, dry basis & $4.50 \mathrm{E}+03$ & $9.68 \mathrm{E}-03$ & $9.68 \mathrm{E}-03$ & & & & $9.68 \mathrm{E}-03$ & $9.68 \mathrm{E}-03$ & \begin{tabular}{|l|}
$9.68 \mathrm{E}-03$ \\
\end{tabular} \\
\hline $\mathrm{NO}, \mathrm{ppmv}$, dry basis & $2.47 \mathrm{E}+02$ & $2.90 \mathrm{E}+02$ & $2.90 \mathrm{E}+02$ & & & & $2.90 \mathrm{E}+02$ & $2.90 \mathrm{E}+02$ & $2.90 \mathrm{E}+02$ \\
\hline NO2, ppmv, dry basis & $1.67 E+01$ & $1.03 \mathrm{E}+00$ & $1.03 E+00$ & & & & $1.03 \mathrm{E}+00$ & $1.03 E+00$ & $1.03 E+00$ \\
\hline SO2, ppmv, dry basis & $7.34 \mathrm{E}-03$ & $6.21 \mathrm{E}+01$ & $6.21 \mathrm{E}+01$ & & & & $6.21 \mathrm{E}+01$ & $6.21 \mathrm{E}+01$ & $6.21 \mathrm{E}+01$ \\
\hline $\mathrm{Cl}, \mathrm{ppmv}$, dry basis & $7.85 \mathrm{E}-01$ & $8.17 \mathrm{E}-01$ & $8.17 \mathrm{E}-01$ & & & & 1.17E-01 & 1.17E-01 & 1.17E-01 \\
\hline $\mathrm{F}$, ppmv, dry basis & $4.38 \mathrm{E}+00$ & $4.56 \mathrm{E}+00$ & $4.56 \mathrm{E}+00$ & & & & $1.09 \mathrm{E}+01$ & $1.09 \mathrm{E}+01$ & $1.09 \mathrm{E}+01$ \\
\hline C (organic), ppmv, dry basis & $2.50 \mathrm{E}+02$ & & & & & & & & \\
\hline $\mathrm{H}$ (organic), ppmv, dry basis & $9.95 \mathrm{E}+02$ & & & & & & & & \\
\hline $\mathrm{Hg}, \mathrm{ug} / \mathrm{dscm}$ & $2.06 \mathrm{E}+04$ & $2.14 \mathrm{E}+04$ & $2.15 \mathrm{E}+04$ & & & & & $1.20 \mathrm{E}+04$ & $1.20 \mathrm{E}+04$ \\
\hline $\mathrm{PM}, \mathrm{mg} / \mathrm{dscm}$ & $1.95 \mathrm{E}+02$ & $3.33 \mathrm{E}+01$ & $3.95 \mathrm{E}+01$ & & & & $9.56 \mathrm{E}+00$ & $2.15 \mathrm{E}+01$ & $2.15 \mathrm{E}+01$ \\
\hline SVM, ug/dscm & $1.55 \mathrm{E}+02$ & $1.49 \mathrm{E}+02$ & $8.80 \mathrm{E}+01$ & & & & & & \\
\hline LVM, ug/dscm & $3.71 \mathrm{E}+01$ & $3.57 \mathrm{E}+01$ & $2.11 \mathrm{E}+01$ & & & & & & \\
\hline
\end{tabular}


Table A-13. Mass balance, Tank WM-180 waste (continued).

\begin{tabular}{|c|c|c|c|c|c|c|c|c|}
\hline PFD \# & PFD-3 & PFD-3 & PFD-3 & PFD-3 & PFD-2 & PFD-2 & PFD-2 & PFD-3 \\
\hline WM-180, Stream \# & 117 & 118 & 119 & 120 & 201 & 202 & 203 & 204 \\
\hline Stream Name & $\begin{array}{c}\text { Pressure } \\
\text { Control } \\
\text { Bleed Air }\end{array}$ & $\begin{array}{c}\text { Final } \\
\text { HEPA Off- } \\
\text { Gas Outlet }\end{array}$ & $\begin{array}{c}\text { Off-Gas to } \\
\text { Blower }\end{array}$ & $\begin{array}{l}\text { Spent } \\
\text { GAC }\end{array}$ & $\begin{array}{l}\text { Boiler } \\
\text { Feed } \\
\text { Water }\end{array}$ & $\begin{array}{c}\text { Fuel Oil to } \\
\text { Boiler }\end{array}$ & $\begin{array}{l}\text { Steam To } \\
\text { Reformer }\end{array}$ & $\begin{array}{l}\text { Propane } \\
\text { to Oxidizer }\end{array}$ \\
\hline Rate or Volume & $1.34 \mathrm{E}+04$ & $6.10 \mathrm{E}+05$ & $6.43 E+05$ & $2.18 E+00$ & $3.16 \mathrm{E}+02$ & $4.18 \mathrm{E}+01$ & $6.32 \mathrm{E}+04$ & $1.07 \mathrm{E}+02$ \\
\hline Volume Flow (standard, wet) & $1.31 \mathrm{E}+04$ & $1.85 \mathrm{E}+05$ & $\begin{array}{l}1.97 \mathrm{E}+05 \\
\end{array}$ & & & & $5.66 \mathrm{E}+04$ & $3.02 \mathrm{E}+02$ \\
\hline Volume Flow (standard, dry) & $1.31 \mathrm{E}+04$ & $3.22 \mathrm{E}+04$ & $4.53 \mathrm{E}+04$ & & & & & $3.02 \mathrm{E}+02$ \\
\hline Rate Units & $\mathrm{ft} 3 / \mathrm{hr}$ & $\mathrm{ft} 3 / \mathrm{hr}$ & $\mathrm{ft} 3 / \mathrm{hr}$ & $\mathrm{lb} / \mathrm{hr}$ & gal/hr & gal/hr & $\mathrm{ft} 3 / \mathrm{hr}$ & $\mathrm{ft} 3 / \mathrm{hr}$ \\
\hline Rate or Volume, metric & $3.72 \mathrm{E}+02$ & $5.22 \mathrm{E}+03$ & $5.59 \mathrm{E}+03$ & $9.88 \mathrm{E}-01$ & $1.20 \mathrm{E}+03$ & $1.58 \mathrm{E}+02$ & $1.60 \mathrm{E}+03$ & $8.56 \mathrm{E}+00$ \\
\hline Rate Units & $\mathrm{wscm} / \mathrm{hr}$ & wscm $/ \mathrm{hr}$ & $\mathrm{wscm} / \mathrm{hr}$ & $\mathrm{kg} / \mathrm{hr}$ & $\mathrm{L} / \mathrm{hr}$ & $\mathrm{L} / \mathrm{hr}$ & wscm $/ \mathrm{hr}$ & $\mathrm{wscm} / \mathrm{hr}$ \\
\hline Temperature, ${ }^{\circ} \mathrm{C}$ & 25 & 120 & 115 & 28 & 15 & 15 & 670 & 25 \\
\hline Temperature, ${ }^{\circ} \mathrm{F}$ & 77 & 248 & 239 & 82 & 59 & 59 & 1238 & 77 \\
\hline Pressure, psia & 14.7 & 6.0 & 6.0 & 14.7 & 42.3 & 14.7 & 42.3 & 42.3 \\
\hline Specific Gravity & $1.18 \mathrm{E}-03$ & $2.59 \mathrm{E}-04$ & $2.70 \mathrm{E}-04$ & $5.86 \mathrm{E}-01$ & $1.00 \mathrm{E}+00$ & $7.70 \mathrm{E}-01$ & $6.70 \mathrm{E}-04$ & $5.19 \mathrm{E}-03$ \\
\hline Chemical Composition & $\mathrm{lb} / \mathrm{wscf}$ & $\mathrm{lb} / \mathrm{wscf}$ & lb/wscf & Wt frac & Mol/liter & Mol/liter & $\mathrm{lb} / \mathrm{wscf}$ & $\mathrm{lb} / \mathrm{wscf}$ \\
\hline $\mathrm{H}+$ & & $2.21 \mathrm{E}-07$ & $2.07 \mathrm{E}-07$ & $2.90 \mathrm{E}-05$ & & $2.40 \mathrm{E}-01$ & & \\
\hline \multicolumn{9}{|l|}{\begin{tabular}{|l|}
$\mathrm{Al}+3$ \\
\end{tabular}} \\
\hline \multirow{2}{*}{\multicolumn{9}{|c|}{$\mathrm{Sb}+5$}} \\
\hline \multirow{2}{*}{\multicolumn{9}{|c|}{$\frac{\mathrm{As}+3}{\mathrm{Ba}+2}$}} \\
\hline & & & & & & & & \\
\hline \multicolumn{9}{|l|}{\begin{tabular}{|l|}
$\mathrm{Ba}+2$ \\
$\mathrm{Be}+2$
\end{tabular}} \\
\hline \multicolumn{9}{|l|}{$\mathrm{B}+3$} \\
\hline \multicolumn{9}{|l|}{$\mathrm{Cd}+2$} \\
\hline \multirow{2}{*}{\multicolumn{9}{|c|}{\begin{tabular}{|l|}
$\mathrm{Ca}+2$ \\
$\mathrm{Cr}+3$
\end{tabular}}} \\
\hline & & & & & & & & \\
\hline \multicolumn{9}{|l|}{$\mathrm{Co}+2$} \\
\hline \multicolumn{9}{|l|}{ Cs+ } \\
\hline \multicolumn{9}{|l|}{$\mathrm{Cu}+2$} \\
\hline \multirow{2}{*}{\multicolumn{9}{|c|}{$\frac{\mathrm{Fe}+3}{\mathrm{~Pb}+2}$}} \\
\hline & & & & & & \multicolumn{3}{|c|}{$\mathrm{Pb}+2$} \\
\hline $\mathrm{Hg}+2$ & & $9.36 \mathrm{E}-10$ & $8.76 \mathrm{E}-10$ & $7.93 \mathrm{E}-02$ & & & & \\
\hline \multirow{2}{*}{\multicolumn{9}{|c|}{\begin{tabular}{|l|}
$\mathrm{Mn}+4$ \\
$\mathrm{Ni}+2$
\end{tabular}}} \\
\hline \multirow{2}{*}{\multicolumn{9}{|c|}{\begin{tabular}{|l|}
$\mathrm{Ni}+2$ \\
$\mathrm{~K}+$
\end{tabular}}} \\
\hline & & & & & & & & \\
\hline \multicolumn{9}{|l|}{$\mathrm{Se}+4$} \\
\hline \multicolumn{9}{|l|}{$\mathrm{Ag}+$} \\
\hline \multirow{2}{*}{\multicolumn{9}{|c|}{\begin{tabular}{|l}
$\mathrm{Na}+$ \\
$\mathrm{Tl}+3$
\end{tabular}}} \\
\hline & & & & & & & & \\
\hline $\mathrm{U}+4$ & & & & & & & & \\
\hline $\mathrm{V}+3$ & & & & & & & & \\
\hline$Z n+2$ & & & & & & & & \\
\hline $\mathrm{Zr}+4$ & & & & & & & & \\
\hline $\mathrm{Cl}-$ & & $1.34 \mathrm{E}-09$ & $1.25 \mathrm{E}-09$ & $1.02 \mathrm{E}-03$ & & & & \\
\hline F- & & $6.69 \mathrm{E}-07$ & $6.26 \mathrm{E}-07$ & & & & & \\
\hline SO4-2 & & & & & & & & \\
\hline NO3- & & $1.14 \mathrm{E}-05$ & $1.07 \mathrm{E}-05$ & & & & & \\
\hline PO4-3 & & & & & & & & \\
\hline$A m+4$ & & & & & & & & \\
\hline $\mathrm{Br}-$ & & $1.64 \mathrm{E}-14$ & $1.53 \mathrm{E}-14$ & $1.25 \mathrm{E}-08$ & & & & \\
\hline $\mathrm{Ce}+4$ & & & & & & & & \\
\hline $\mathrm{Eu}+3$ & & & & & & & & \\
\hline$G d+3$ & & & & & & & & \\
\hline $\mathrm{Ge}+4$ & & & & & & & & \\
\hline $\ln +3$ & & & & & & & & \\
\hline$|-|$ & & $2.26 \mathrm{E}-13$ & $2.11 \mathrm{E}-13$ & $1.72 \mathrm{E}-07$ & & & & \\
\hline $\mathrm{La}+3$ & & & & & & & & \\
\hline
\end{tabular}


Table A-13. Mass balance, Tank WM-180 waste (continued).

\begin{tabular}{|c|c|c|c|c|c|c|c|c|}
\hline Stream \# & 117 & 118 & 119 & 120 & 201 & 202 & 203 & 204 \\
\hline Chemical Composition & $\mathrm{lb} / \mathrm{wscf}$ & $\mathrm{lb} /$ wscf & $\mathrm{lb} /$ wscf & Wt frac & Mol/liter & Mol/liter & $\mathrm{lb} / \mathrm{wscf}$ & $\mathrm{lb} / \mathrm{wscf}$ \\
\hline \multicolumn{9}{|l|}{$\mathrm{Li}+$} \\
\hline \multicolumn{9}{|l|}{$\mathrm{Mg}+2$} \\
\hline \multirow{2}{*}{\multicolumn{9}{|c|}{\begin{tabular}{|l|}
$\mathrm{Mo}+6$ \\
$\mathrm{Nd}+3$
\end{tabular}}} \\
\hline & & & & & & & & \\
\hline \multicolumn{9}{|l|}{$\frac{\mathrm{Nd}+3}{\mathrm{~Np}+4}$} \\
\hline \multicolumn{9}{|l|}{$\mathrm{Nb}+5$} \\
\hline \multicolumn{9}{|l|}{$\mathrm{Pd}+4$} \\
\hline \multirow{2}{*}{\multicolumn{9}{|c|}{\begin{tabular}{|l|}
$\mathrm{Pu}+4$ \\
$\mathrm{Pr}+4$
\end{tabular}}} \\
\hline & & & & & & & & \\
\hline \multicolumn{9}{|l|}{\begin{tabular}{|l}
$\mathrm{Pr}+4$ \\
$\mathrm{Pm}+3$ \\
\end{tabular}} \\
\hline \multicolumn{9}{|l|}{$\mathrm{Rh}+4$} \\
\hline \multicolumn{9}{|l|}{$\mathrm{Rb}+$} \\
\hline \multirow{2}{*}{\multicolumn{9}{|c|}{$\mathrm{Ru+3}$}} \\
\hline \multirow{2}{*}{\multicolumn{9}{|c|}{$\frac{S m+3}{S i+4}$}} \\
\hline & & & & & & & & \\
\hline \multicolumn{9}{|l|}{$\mathrm{Sr}+2$} \\
\hline \multicolumn{9}{|l|}{$\mathrm{Tc}+7$} \\
\hline \multicolumn{9}{|l|}{$\mathrm{Te}+4$} \\
\hline \multirow{2}{*}{\multicolumn{9}{|c|}{$\mathrm{Tb}+4$}} \\
\hline \multirow{2}{*}{\multicolumn{9}{|c|}{$\begin{array}{l}\mathrm{Th}+4 \\
\mathrm{Sn}+4\end{array}$}} \\
\hline & & & & & & & & \\
\hline \multicolumn{9}{|l|}{$T i+4$} \\
\hline \multicolumn{9}{|l|}{$\mathrm{Y}+3$} \\
\hline \multicolumn{9}{|l|}{$\mathrm{OH}-$} \\
\hline $\mathrm{H} 2 \mathrm{O}$ & $4.68 \mathrm{E}-06$ & $3.86 \mathrm{E}-02$ & $3.61 \mathrm{E}-02$ & & $5.56 \mathrm{E}+01$ & & $4.68 \mathrm{E}-02$ & \\
\hline $\mathrm{SO} 2$ & & $1.29 \mathrm{E}-05$ & $1.20 \mathrm{E}-05$ & & & & & \\
\hline $\mathrm{H} 2 \mathrm{~S}$ & & & & & & $1.20 \mathrm{E}-01$ & & \\
\hline $\mathrm{CO}$ & & $8.77 \mathrm{E}-10$ & $8.20 \mathrm{E}-10$ & & & & & \\
\hline $\mathrm{CO} 2$ & & $5.33 \mathrm{E}-03$ & $4.99 \mathrm{E}-03$ & & & & & \\
\hline \multicolumn{9}{|l|}{$\mathrm{H} 2$} \\
\hline $\mathrm{N} 2$ & $5.75 \mathrm{E}-02$ & $8.31 \mathrm{E}-03$ & $1.16 \mathrm{E}-02$ & & & & & \\
\hline $\mathrm{NO}$ & & $2.82 \mathrm{E}-05$ & $2.63 \mathrm{E}-05$ & & & & & \\
\hline NO2 & & $1.54 \mathrm{E}-07$ & $1.44 \mathrm{E}-07$ & & & & & \\
\hline $\mathrm{O} 2$ & $1.74 \mathrm{E}-02$ & $1.08 \mathrm{E}-03$ & $2.17 \mathrm{E}-03$ & & & & & \\
\hline S (other) & & & & & & & & \\
\hline $\mathrm{CO} 3$ & & & & & & & & \\
\hline C (reductant) & & & & $9.20 \mathrm{E}-01$ & & & & \\
\hline $\mathrm{O}$ (oxides) & & & & & & & & \\
\hline C (organic) & & & & & & $5.41 \mathrm{E}+01$ & & $9.35 \mathrm{E}-02$ \\
\hline $\mathrm{H}$ (organic) & & & & & & $1.15 \mathrm{E}+02$ & & $2.09 \mathrm{E}-02$ \\
\hline $\mathrm{O}$ (organic) & & & & & & & & \\
\hline Mass Flow (kg/hr): & $4.47 \mathrm{E}+02$ & $4.47 \mathrm{E}+03$ & $4.91 \mathrm{E}+03$ & $9.88 \mathrm{E}-01$ & $1.20 \mathrm{E}+03$ & $1.22 \mathrm{E}+02$ & $1.20 \mathrm{E}+03$ & $1.57 \mathrm{E}+01$ \\
\hline & & & & & & & & \\
\hline & & & & & & & & \\
\hline & 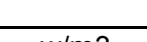 & ? & 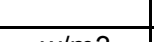 & 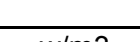 & 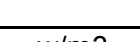 & 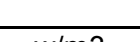 & 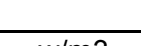 & 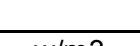 \\
\hline Heat Generation, w/m3 & womins & Winits & Wuims & $2.50 \mathrm{E}-11$ & Wwims & & Wwimis & \\
\hline
\end{tabular}


Table A-13. Mass balance, Tank WM-180 waste (continued).

\begin{tabular}{|c|c|c|c|c|c|c|c|c|}
\hline Stream \# & 117 & 118 & 119 & 120 & 201 & 202 & 203 & 204 \\
\hline Radiological Composition & $\mathrm{Ci} / \mathrm{wscm}$ & $\mathrm{Ci} / \mathrm{wscm}$ & $\mathrm{Ci} / \mathrm{wscm}$ & $\mathrm{Ci} / \mathrm{kg}$ & $\mathrm{Ci} / \mathrm{L}$ & $\mathrm{Ci} / \mathrm{L}$ & $\mathrm{Ci} / \mathrm{wscm}$ & Ci/wscm \\
\hline $\mathrm{H}-3$ & & $1.10 \mathrm{E}-06$ & $1.03 \mathrm{E}-06$ & & & & & \\
\hline C-14 & & $3.69 \mathrm{E}-12$ & $3.45 \mathrm{E}-12$ & $1.46 \mathrm{E}-10$ & & & & \\
\hline \multicolumn{9}{|l|}{ Co-60 } \\
\hline \multicolumn{9}{|l|}{$\mathrm{Ni}-59$} \\
\hline \multicolumn{9}{|l|}{$\mathrm{Ni}-63$} \\
\hline \multicolumn{9}{|l|}{ Se-79 } \\
\hline \multicolumn{9}{|l|}{ Sr-90 } \\
\hline \multicolumn{9}{|l|}{$\mathrm{Y}-90$} \\
\hline \multicolumn{9}{|l|}{ Zr-93 } \\
\hline \multicolumn{9}{|l|}{$\mathrm{Nb}-93 \mathrm{~m}$} \\
\hline \multicolumn{9}{|l|}{$\mathrm{Nb}-94$} \\
\hline \multicolumn{9}{|l|}{ Tc-99 } \\
\hline \multicolumn{9}{|l|}{ Ru-106 } \\
\hline \multicolumn{9}{|l|}{ Rh-102 } \\
\hline \multicolumn{9}{|l|}{ Rh-106 } \\
\hline \multicolumn{9}{|l|}{\begin{tabular}{|l}
$\mathrm{Pd}-107$ \\
\end{tabular}} \\
\hline \multicolumn{9}{|l|}{ Cd-113m } \\
\hline \multicolumn{9}{|l|}{ Sn-121m } \\
\hline Sn-126 & & & & & & & & \\
\hline $\mathrm{Sb}-125$ & & & & & & & & \\
\hline Sb-126 & & & & & & & & \\
\hline Te-125m & & & & & & & & \\
\hline $\mid-129$ & & $5.07 \mathrm{E}-13$ & $4.74 \mathrm{E}-13$ & $2.41 \mathrm{E}-08$ & & & & \\
\hline Cs-134 & & & & & & & & \\
\hline Cs-135 & & & & & & & & \\
\hline Cs-137 & & & & & & & & \\
\hline $\mathrm{Ba}-137 \mathrm{~m}$ & & & & & & & & \\
\hline Ce-144 & & & & & & & & \\
\hline Pr-144 & & & & & & & & \\
\hline $\mathrm{Pm}-146$ & & & & & & & & \\
\hline $\mathrm{Pm}-147$ & & & & & & & & \\
\hline Sm-151 & & & & & & & & \\
\hline Eu-152 & & & & & & & & \\
\hline Eu-154 & & & & & & & & \\
\hline Eu-155 & & & & & & & & \\
\hline & & & & & & & & \\
\hline Th-230 & & & & & & & & \\
\hline Pa-233 & & & & & & & & \\
\hline $\mathrm{U}-232$ & & & & & & & & \\
\hline U-233 & & & & & & & & \\
\hline U-234 & & & & & & & & \\
\hline U-235 & & & & & & & & \\
\hline U-236 & & & & & & & & \\
\hline U-237 & & & & & & & & \\
\hline U-238 & & & & & & & & \\
\hline Np-237 & & & & & & & & \\
\hline Pu-236 & & & & & & & & \\
\hline Pu-238 & & & & & & & & \\
\hline Pu-239 & & & & & & & & \\
\hline Pu-240 & & & & & & & & \\
\hline Pu-241 & & & & & & & & \\
\hline Pu-242 & & & & & & & & \\
\hline Pu-244 & & & & & & & & \\
\hline Am-241 & & & & & & & & \\
\hline$A m-242 m$ & & & & & & & & \\
\hline
\end{tabular}


Table A-13. Mass balance, Tank WM-180 waste (continued).

\begin{tabular}{|c|c|c|c|c|c|c|c|c|}
\hline Stream \# & 117 & 118 & 119 & 120 & 201 & 202 & 203 & 204 \\
\hline & $\mathrm{Ci} / \mathrm{wscm}$ & $\mathrm{Ci} / \mathrm{wscm}$ & $\mathrm{Ci} / \mathrm{wscm}$ & $\mathrm{Ci} / \mathrm{kg}$ & $\mathrm{Ci} / \mathrm{L}$ & $\mathrm{Ci} / \mathrm{L}$ & Ci/wscm & Ci/wscm \\
\hline \multicolumn{9}{|l|}{ Am-243 } \\
\hline \multicolumn{9}{|l|}{$\mathrm{Cm}-242$} \\
\hline \multicolumn{9}{|l|}{$\mathrm{Cm}-243$} \\
\hline \multicolumn{9}{|l|}{$\mathrm{Cm}-244$} \\
\hline \multicolumn{9}{|l|}{$\mathrm{Cm}-245$} \\
\hline \multicolumn{9}{|l|}{$\mathrm{Cm}-246$} \\
\hline \multicolumn{9}{|l|}{ TRU } \\
\hline & & & & & & & & \\
\hline & & & & & & & & \\
\hline $\begin{array}{l}\text { Gas Stream Bulk } \\
\text { Composition (Wet Basis) }\end{array}$ & $\begin{array}{l}\text { mol\% or } \\
\text { ppmv }\end{array}$ & $\begin{array}{l}\text { mol\% or } \\
\text { ppmv }\end{array}$ & $\begin{array}{l}\text { mol\% or } \\
\text { ppmv }\end{array}$ & $\begin{array}{l}\text { mol\% or } \\
\text { ppmv }\end{array}$ & $\begin{array}{l}\text { mol\% or } \\
\text { ppmv }\end{array}$ & $\begin{array}{l}\text { mol\% or } \\
\text { ppmv }\end{array}$ & $\begin{array}{c}\text { mol\% or } \\
\text { ppmv }\end{array}$ & $\begin{array}{c}\mathrm{mol} \% \text { or } \\
\text { ppmv }\end{array}$ \\
\hline $\mathrm{H} 2 \mathrm{O}, \mathrm{mol} \%$ & $0.01 \%$ & $82.55 \%$ & $77.05 \%$ & & & & $100.00 \%$ & \\
\hline $\mathrm{O} 2, \mathrm{~mol} \%$ & $20.99 \%$ & $1.30 \%$ & $2.61 \%$ & & & & & \\
\hline $\mathrm{N} 2, \mathrm{~mol} \%$ & $79.00 \%$ & $11.43 \%$ & $15.93 \%$ & & & & & \\
\hline \multicolumn{9}{|l|}{$\mathrm{H} 2, \mathrm{~mol} \%$} \\
\hline $\mathrm{CO} 2, \mathrm{~mol} \%$ & & $4.67 \%$ & $4.36 \%$ & & & & & \\
\hline $\mathrm{CO}, \mathrm{ppmv}$ & & $1.21 \mathrm{E}-02$ & $1.13 \mathrm{E}-02$ & & & & & \\
\hline $\mathrm{NO}, \mathrm{ppmv}$ & & $3.62 \mathrm{E}+02$ & $3.38 \mathrm{E}+02$ & & & & & \\
\hline NO2, ppmv & & $1.29 \mathrm{E}+00$ & $1.20 \mathrm{E}+00$ & & & & & \\
\hline SO2, ppmv & & $7.73 E+01$ & $7.22 \mathrm{E}+01$ & & & & & \\
\hline $\mathrm{Cl}, \mathrm{ppmv}$ & & $1.45 \mathrm{E}-02$ & $1.36 \mathrm{E}-02$ & & & & & \\
\hline $\mathrm{F}, \mathrm{ppmv}$ & & $1.36 \mathrm{E}+01$ & $1.27 \mathrm{E}+01$ & & & & & \\
\hline C (organic), ppmv & & & & & & & & $3.00 \mathrm{E}+06$ \\
\hline $\mathrm{H}$ (organic), ppmv & & & & & & & & $8.00 \mathrm{E}+06$ \\
\hline $\mathrm{Hg}, \mathrm{ug} / \mathrm{wscm}$ & & $1.50 \mathrm{E}+01$ & $1.40 \mathrm{E}+01$ & & & & & \\
\hline $\mathrm{PM}, \mathrm{mg} / \mathrm{wscm}$ & $1.60 \mathrm{E}+01$ & $1.19 \mathrm{E}+01$ & $1.22 \mathrm{E}+01$ & & & & $1.16 \mathrm{E}+01$ & $1.83 \mathrm{E}+06$ \\
\hline \multicolumn{9}{|l|}{ SVM, ug/wscm } \\
\hline \multicolumn{9}{|l|}{ LVM, ug/wscm } \\
\hline $\begin{array}{l}\text { Gas Stream Bulk } \\
\text { Composition (Dry Basis) }\end{array}$ & $\mathrm{mol} \%$ & $\mathrm{~mol} \%$ & $\mathrm{~mol} \%$ & $\mathrm{~mol} \%$ & $\mathrm{~mol} \%$ & $\mathrm{~mol} \%$ & $\mathrm{~mol} \%$ & $\mathrm{~mol} \%$ \\
\hline $02, \mathrm{~mol} \%$, dry basis & $20.9921 \%$ & $1.0443 \%$ & $11.3790 \%$ & & & & & \\
\hline $\mathrm{N} 2, \mathrm{~mol} \%$, dry basis & $79.01 \%$ & $65.50 \%$ & $69.42 \%$ & & & & & \\
\hline \multicolumn{9}{|l|}{$\mathrm{H} 2, \mathrm{~mol} \%$, dry basis } \\
\hline $\mathrm{CO} 2, \mathrm{~mol} \%$, dry basis & & $26.74 \%$ & $18.99 \%$ & & & & & \\
\hline $\begin{array}{l}\text { Gas Stream Bulk } \\
\text { Composition (Dry Basis, } \\
\text { Corrected to } 7 \% \text { O2 with } \\
100 \% \text { O2 Combustion Air) }\end{array}$ & $\begin{array}{l}\text { ppmv, or } \\
\text { ug/dscm or } \\
\text { mg/dscm }\end{array}$ & $\begin{array}{l}\text { ppmv, or } \\
\text { ug/dscm } \\
\text { or } \\
\mathrm{mg} / \mathrm{dscm}\end{array}$ & $\begin{array}{l}\text { ppmv, or } \\
\text { ug/dscm } \\
\text { or } \\
\mathrm{mg} / \mathrm{dscm}\end{array}$ & $\begin{array}{l}\text { ppmv, or } \\
\text { ug/dscm } \\
\text { or } \\
\mathrm{mg} / \mathrm{dscm}\end{array}$ & $\begin{array}{l}\mathrm{ppmv}, \text { or } \\
\mathrm{ug} / \mathrm{dscm} \\
\text { or } \\
\mathrm{mg} / \mathrm{dscm}\end{array}$ & $\begin{array}{l}\text { ppmv, or } \\
\text { ug/dscm } \\
\text { or } \\
\mathrm{mg} / \mathrm{dscm}\end{array}$ & $\begin{array}{l}\mathrm{ppmv}, \text { or } \\
\mathrm{ug} / \mathrm{dscm} \\
\text { or } \\
\mathrm{mg} / \mathrm{dscm}\end{array}$ & $\begin{array}{l}\mathrm{ppmv} \text {, or } \\
\mathrm{ug} / \mathrm{dscm} \\
\text { or } \\
\mathrm{mg} / \mathrm{dscm}\end{array}$ \\
\hline $\mathrm{CO}, \mathrm{ppmv}$, dry basis & & $9.68 \mathrm{E}-03$ & $6.88 \mathrm{E}-03$ & & & & & \\
\hline $\mathrm{NO}$, ppmv, dry basis & & $2.90 \mathrm{E}+02$ & $2.06 \mathrm{E}+02$ & & & & & \\
\hline NO2, ppmv, dry basis & & $1.03 \mathrm{E}+00$ & 7.34E-01 & & & & & \\
\hline SO2, ppmv, dry basis & & $6.21 \mathrm{E}+01$ & $4.41 \mathrm{E}+01$ & & & & & \\
\hline $\mathrm{Cl}, \mathrm{ppmv}$, dry basis & & $1.17 \mathrm{E}-02$ & $8.28 \mathrm{E}-03$ & & & & & \\
\hline $\mathrm{F}, \mathrm{ppmv}$, dry basis & & $1.09 \mathrm{E}+01$ & $7.74 \mathrm{E}+00$ & & & & & \\
\hline \multicolumn{9}{|l|}{ C (organic), ppmv, dry basis } \\
\hline \multicolumn{9}{|l|}{$\mathrm{H}$ (organic), ppmv, dry basis } \\
\hline $\mathrm{Hg}, \mathrm{ug} / \mathrm{dscm}$ & & $1.20 \mathrm{E}+01$ & $8.57 E+00$ & & & & & \\
\hline $\mathrm{PM}, \mathrm{mg} / \mathrm{dscm}$ & $2.25 \mathrm{E}+00$ & $9.55 \mathrm{E}+00$ & $7.45 \mathrm{E}+00$ & & & & & \\
\hline \multicolumn{9}{|l|}{ SVM, ug/dscm } \\
\hline LVM, ug/dscm & & & & & & & & \\
\hline
\end{tabular}


Table A-13. Mass balance, Tank WM-180 waste (continued).

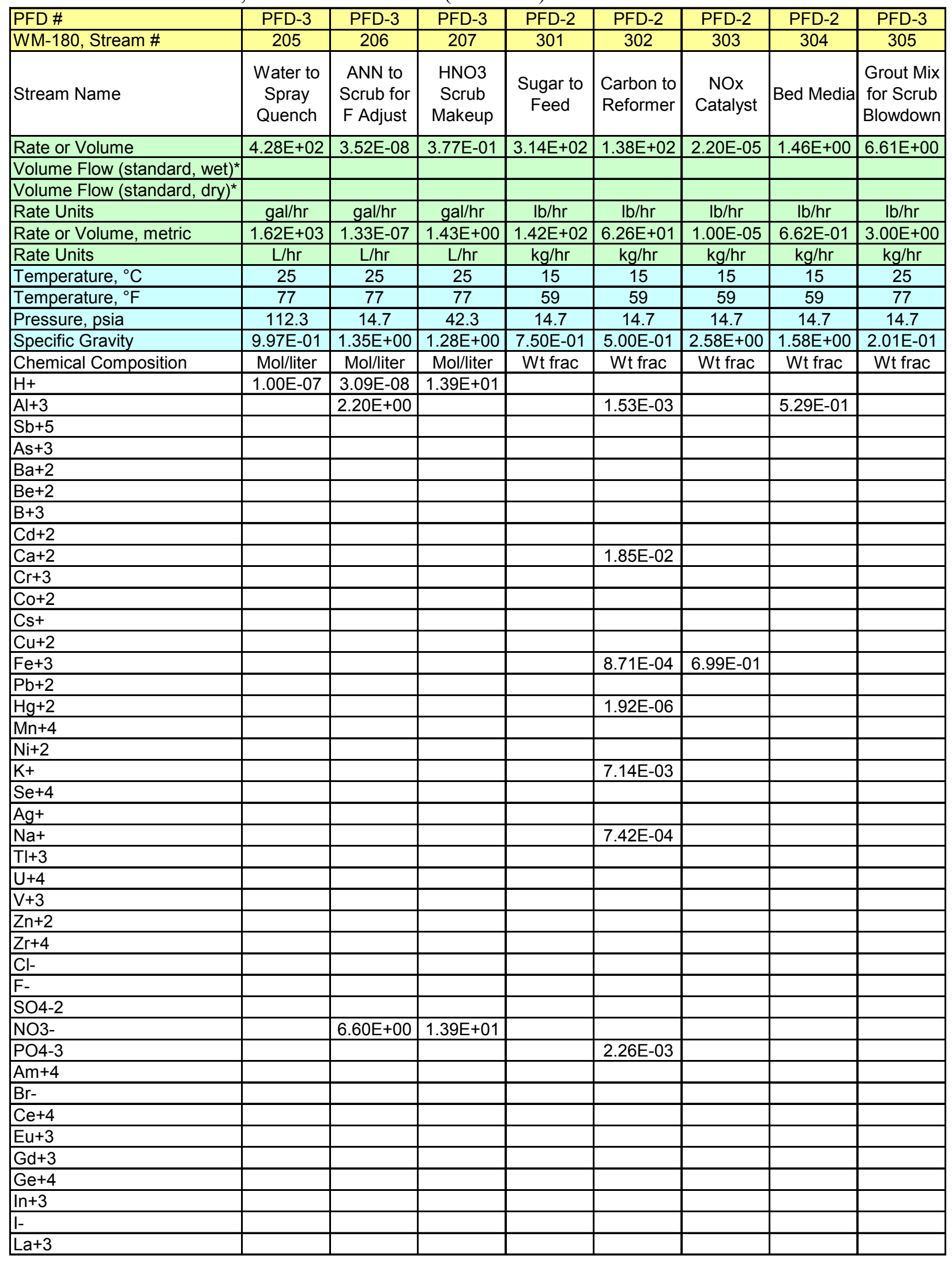


Table A-13. Mass balance, Tank WM-180 waste (continued).

\begin{tabular}{|c|c|c|c|c|c|c|c|c|}
\hline Stream \# & 205 & 206 & 207 & 301 & 302 & 303 & 304 & 305 \\
\hline Chemical Composition & Mol/liter & Mol/liter & Mol/liter & Wt frac & Wt frac & Wt frac & Wt frac & Wt frac \\
\hline \multicolumn{9}{|l|}{$\mathrm{Li}+$} \\
\hline \multicolumn{9}{|l|}{$\mathrm{Mg}+2$} \\
\hline \multicolumn{9}{|l|}{$\mathrm{Mo}+6$} \\
\hline \multicolumn{9}{|l|}{$\mathrm{Nd}+3$} \\
\hline \multicolumn{9}{|l|}{$\mathrm{Np}+4$} \\
\hline \multicolumn{9}{|l|}{$\mathrm{Nb}+5$} \\
\hline \multicolumn{9}{|l|}{$\mathrm{Pd}+4$} \\
\hline \multicolumn{9}{|l|}{$\mathrm{Pu}+4$} \\
\hline \multicolumn{9}{|l|}{$\mathrm{Pr}+4$} \\
\hline \multicolumn{9}{|l|}{$\mathrm{Pm}+3$} \\
\hline \multicolumn{9}{|l|}{$\mathrm{Rh}+4$} \\
\hline \multicolumn{9}{|l|}{$\mathrm{Rb}+$} \\
\hline \multicolumn{9}{|l|}{$\mathrm{Ru}+3$} \\
\hline \multicolumn{9}{|l|}{$\mathrm{Sm}+3$} \\
\hline $\mathrm{Si+4}$ & & & & & $6.26 \mathrm{E}-03$ & & & 4.67E-01 \\
\hline \multicolumn{9}{|l|}{$\mathrm{Sr}+2$} \\
\hline \multicolumn{9}{|l|}{$\mathrm{Tc}+7$} \\
\hline \multicolumn{9}{|l|}{$\mathrm{Te}+4$} \\
\hline \multicolumn{9}{|l|}{$\mathrm{Tb}+4$} \\
\hline \multicolumn{9}{|l|}{$\mathrm{Th}+4$} \\
\hline \multicolumn{9}{|l|}{$\mathrm{Sn}+4$} \\
\hline \multicolumn{9}{|l|}{$\mathrm{Ti}+4$} \\
\hline \multicolumn{9}{|l|}{$\mathrm{Y}+3$} \\
\hline $\mathrm{OH}-$ & $1.00 \mathrm{E}-07$ & 3.09E-08 & & & & & & \\
\hline $\mathrm{H} 2 \mathrm{O}$ & $5.54 \mathrm{E}+01$ & $4.89 \mathrm{E}+01$ & $2.27 \mathrm{E}+01$ & & & & & \\
\hline \multicolumn{9}{|l|}{$\mathrm{SO} 2$} \\
\hline $\mathrm{H} 2 \mathrm{~S}$ & & & & & & & & \\
\hline $\mathrm{CO}$ & & & & & & & & \\
\hline $\mathrm{CO} 2$ & & & & & & & & \\
\hline $\mathrm{H} 2$ & & & & & & & & \\
\hline $\mathrm{N} 2$ & & & & & & & & \\
\hline NO & & & & & & & & \\
\hline $\mathrm{NO} 2$ & & & & & & & & \\
\hline $\mathrm{O} 2$ & & & & & & & & \\
\hline S (other) & & & & & $5.18 \mathrm{E}-04$ & & & \\
\hline $\mathrm{CO} 3$ & & & & & $3.11 \mathrm{E}-02$ & & & \\
\hline C (reductant) & & & & & 9.22E-01 & & & \\
\hline $\mathrm{O}$ (oxides) & & & & & $8.88 \mathrm{E}-03$ & 3.01E-01 & 4.71E-01 & $5.33 \mathrm{E}-01$ \\
\hline C (organic) & & & & $4.21 \mathrm{E}-01$ & & & & \\
\hline $\mathrm{H}$ (organic) & & & & $6.48 \mathrm{E}-02$ & & & & \\
\hline $\mathrm{O}$ (organic) & & & & 5.14E-01 & & & & \\
\hline Mass Flow (kg/hr): & $1.62 \mathrm{E}+03$ & $1.80 \mathrm{E}-07$ & $1.83 E+00$ & $1.42 \mathrm{E}+02$ & $6.26 \mathrm{E}+01$ & 1.00E-05 & $6.62 \mathrm{E}-01$ & $3.00 \mathrm{E}+00$ \\
\hline
\end{tabular}


Table A-13. Mass balance, Tank WM-180 waste (continued).

\begin{tabular}{|c|c|c|c|c|c|c|c|c|}
\hline PFD \# & PFD-3 & PFD-3 & PFD-3 & PFD-2 & PFD-2 & PFD-2 & PFD-3 & PFD-2 \\
\hline WM-180, Stream \# & 401 & 402 & 404 & 404 & 503 & 504 & 505 & 506 \\
\hline Stream Name & $\begin{array}{c}\text { Scrub } \\
\text { Recycled } \\
\text { to Feed }\end{array}$ & $\begin{array}{c}\text { Scrub } \\
\text { Blowdown } \\
\text { to Grout } \\
\text { Mixer }\end{array}$ & $\begin{array}{l}\text { MLLW } \\
\text { Grout }\end{array}$ & $\begin{array}{l}\text { MLLW } \\
\text { Grout } \\
\text { Drums }\end{array}$ & $\begin{array}{c}\text { Feed } \\
\text { Atomizing } \\
\text { Gas }\end{array}$ & $\begin{array}{l}\text { Oxygen to } \\
\text { Reformer }\end{array}$ & $\begin{array}{c}\text { Oxygen to } \\
\text { Oxidizer }\end{array}$ & $\begin{array}{l}\text { Air to } \\
\text { Boiler }\end{array}$ \\
\hline Rate or Volume & $5.89 \mathrm{E}+00$ & \begin{tabular}{|l|}
$2.60 \mathrm{E}-01$ \\
\end{tabular} & $8.82 \mathrm{E}+00$ & $2.29 \mathrm{E}-01$ & $2.79 \mathrm{E}+03$ & $6.52 \mathrm{E}+02$ & $9.89 \mathrm{E}+02$ & $6.19 \mathrm{E}+04$ \\
\hline Volume Flow (standard, wet) & & & & & $1.74 \mathrm{E}+04$ & $4.02 \mathrm{E}+03$ & $6.11 \mathrm{E}+03$ & $6.30 E+04$ \\
\hline Volume Flow (standard, dry) $)^{*}$ & & & & & $1.74 \mathrm{E}+04$ & $4.02 \mathrm{E}+03$ & $6.11 \mathrm{E}+03$ & $6.30 \mathrm{E}+04$ \\
\hline Rate Units & $\mathrm{gal} / \mathrm{hr}$ & gal/hr & $\mathrm{lb} / \mathrm{hr}$ & Drums/day & $\mathrm{ft} 3 / \mathrm{hr}$ & $\mathrm{ft} 3 / \mathrm{hr}$ & $\mathrm{ft} 3 / \mathrm{hr}$ & $\mathrm{ft} 3 / \mathrm{hr}$ \\
\hline Rate or Volume, metric & $2.23 \mathrm{E}+01$ & $9.86 \mathrm{E}-01$ & $4.00 \mathrm{E}+00$ & $4.00 \mathrm{E}+00$ & $4.94 \mathrm{E}+02$ & $1.14 \mathrm{E}+02$ & $1.73 \mathrm{E}+02$ & $1.78 \mathrm{E}+03$ \\
\hline Rate Units & $\mathrm{L} / \mathrm{hr}$ & $\mathrm{L} / \mathrm{hr}$ & $\mathrm{kg} / \mathrm{hr}$ & $\mathrm{kg} / \mathrm{hr}$ & wscm/hr & wscm $/ \mathrm{hr}$ & wscm $/ \mathrm{hr}$ & wscm $/ \mathrm{hr}$ \\
\hline Temperature, ${ }^{\circ} \mathrm{C}$ & 79 & 79 & 60 & 60 & 21 & 25 & 25 & 15 \\
\hline Temperature, ${ }^{\circ} \mathrm{F}$ & 174 & 174 & 140 & 140 & 70 & 77 & 77 & 59 \\
\hline Pressure, psia & 12.3 & 12.3 & 12.3 & 12.3 & 92.3 & 92.3 & 92.3 & 14.7 \\
\hline Specific Gravity & $1.01 \mathrm{E}+00$ & $1.01 \mathrm{E}+00$ & $2.10 \mathrm{E}+00$ & $2.10 \mathrm{E}+00$ & $7.29 \mathrm{E}-03$ & $8.21 \mathrm{E}-03$ & $8.21 \mathrm{E}-03$ & $1.22 \mathrm{E}-03$ \\
\hline Chemical Composition & Mol/liter & Mol/liter & Wt frac & Wt frac & $\mathrm{lb} /$ wscf & $\mathrm{lb} /$ wscf & $\mathrm{lb} /$ wscf & Ib/wscf \\
\hline $\mathrm{H}+$ & $1.03 \mathrm{E}+00$ & $1.03 \mathrm{E}+00$ & $2.56 \mathrm{E}-04$ & $2.56 \mathrm{E}-04$ & & & & \\
\hline$A \mathrm{Al}+3$ & $1.78 \mathrm{E}-03$ & $1.78 \mathrm{E}-03$ & $1.19 \mathrm{E}-05$ & $1.19 \mathrm{E}-05$ & & & & \\
\hline $\mathrm{Sb}+5$ & $3.93 \mathrm{E}-09$ & $3.93 \mathrm{E}-09$ & $1.18 \mathrm{E}-10$ & $1.18 \mathrm{E}-10$ & & & & \\
\hline As+3 & $1.26 \mathrm{E}-06$ & $1.26 \mathrm{E}-06$ & $2.33 \mathrm{E}-08$ & $2.33 \mathrm{E}-08$ & & & & \\
\hline $\mathrm{Ba}+2$ & $2.52 \mathrm{E}-07$ & $2.52 \mathrm{E}-07$ & $8.52 \mathrm{E}-09$ & $8.52 \mathrm{E}-09$ & & & & \\
\hline $\mathrm{Be}+2$ & $3.93 \mathrm{E}-08$ & $3.93 \mathrm{E}-08$ & $8.74 \mathrm{E}-11$ & $8.74 \mathrm{E}-11$ & & & & \\
\hline$B+3$ & $2.93 \mathrm{E}-05$ & $2.93 \mathrm{E}-05$ & $7.82 \mathrm{E}-08$ & $7.82 \mathrm{E}-08$ & & & & \\
\hline $\mathrm{Cd}+2$ & $1.97 \mathrm{E}-06$ & $1.97 \mathrm{E}-06$ & $5.46 \mathrm{E}-08$ & $5.46 \mathrm{E}-08$ & & & & \\
\hline $\mathrm{Ca}+2$ & $6.21 \mathrm{E}-04$ & $6.21 \mathrm{E}-04$ & $6.13 \mathrm{E}-06$ & $6.13 \mathrm{E}-06$ & & & & \\
\hline $\mathrm{Cr}+3$ & $7.94 \mathrm{E}-06$ & $7.94 \mathrm{E}-06$ & $1.02 \mathrm{E}-07$ & $1.02 \mathrm{E}-07$ & & & & \\
\hline $\mathrm{Co}+2$ & $5.16 \mathrm{E}-08$ & $5.16 \mathrm{E}-08$ & $7.50 \mathrm{E}-10$ & $7.50 \mathrm{E}-10$ & & & & \\
\hline Cs+ & $6.66 \mathrm{E}-05$ & $6.66 \mathrm{E}-05$ & $2.25 \mathrm{E}-06$ & $2.25 \mathrm{E}-06$ & & & & \\
\hline $\mathrm{Cu}+2$ & $3.18 \mathrm{E}-06$ & $3.18 \mathrm{E}-06$ & $4.98 \mathrm{E}-08$ & $4.98 \mathrm{E}-08$ & & & & \\
\hline $\mathrm{Fe}+3$ & $9.87 \mathrm{E}-05$ & $9.87 \mathrm{E}-05$ & $1.36 \mathrm{E}-06$ & $1.36 \mathrm{E}-06$ & & & & \\
\hline $\mathrm{Pb}+2$ & $6.90 \mathrm{E}-06$ & $6.90 \mathrm{E}-06$ & $3.52 \mathrm{E}-07$ & $3.52 \mathrm{E}-07$ & & & & \\
\hline $\mathrm{Hg}+2$ & $9.22 \mathrm{E}-03$ & $9.22 \mathrm{E}-03$ & $4.56 \mathrm{E}-04$ & $4.56 \mathrm{E}-04$ & & & & \\
\hline $\mathrm{Mn}+4$ & $3.59 \mathrm{E}-05$ & $3.59 \mathrm{E}-05$ & $4.86 \mathrm{E}-07$ & $4.86 \mathrm{E}-07$ & & & & \\
\hline $\mathrm{Ni}+2$ & $6.99 \mathrm{E}-06$ & $6.99 \mathrm{E}-06$ & $1.01 \mathrm{E}-07$ & $1.01 \mathrm{E}-07$ & & & & \\
\hline $\mathrm{K}+$ & $1.53 \mathrm{E}-03$ & $1.53 \mathrm{E}-03$ & $1.48 \mathrm{E}-05$ & $1.48 \mathrm{E}-05$ & & & & \\
\hline $\mathrm{Se}+4$ & $8.75 \mathrm{E}-06$ & $8.75 \mathrm{E}-06$ & $1.70 \mathrm{E}-07$ & $1.70 \mathrm{E}-07$ & & & & \\
\hline $\mathrm{Ag}+$ & $5.16 \mathrm{E}-08$ & $5.16 \mathrm{E}-08$ & $1.37 \mathrm{E}-09$ & $1.37 \mathrm{E}-09$ & & & & \\
\hline $\mathrm{Na}+$ & $5.95 \mathrm{E}-02$ & $5.95 \mathrm{E}-02$ & 3.37E-04 & $3.37 \mathrm{E}-04$ & & & & \\
\hline $\mathrm{Tl}+3$ & $7.75 \mathrm{E}-08$ & $7.75 \mathrm{E}-08$ & $3.90 \mathrm{E}-09$ & $3.90 \mathrm{E}-09$ & & & & \\
\hline $\mathrm{U}+4$ & $4.12 \mathrm{E}-05$ & $4.12 \mathrm{E}-05$ & $2.42 \mathrm{E}-06$ & $2.42 \mathrm{E}-06$ & & & & \\
\hline $\mathrm{V}+3$ & $3.83 \mathrm{E}-06$ & $3.83 \mathrm{E}-06$ & $4.80 \mathrm{E}-08$ & $4.80 \mathrm{E}-08$ & & & & \\
\hline$Z n+2$ & $4.79 \mathrm{E}-06$ & $4.79 \mathrm{E}-06$ & $7.72 \mathrm{E}-08$ & $7.72 \mathrm{E}-08$ & & & & \\
\hline $\mathrm{Zr}+4$ & $1.51 \mathrm{E}-04$ & $1.51 \mathrm{E}-04$ & $3.39 \mathrm{E}-06$ & $3.39 \mathrm{E}-06$ & & & & \\
\hline $\mathrm{Cl}-$ & $2.67 \mathrm{E}-03$ & $2.67 \mathrm{E}-03$ & $2.33 \mathrm{E}-05$ & $2.33 \mathrm{E}-05$ & & & & \\
\hline F- & $1.61 \mathrm{E}-02$ & $1.61 \mathrm{E}-02$ & $7.56 \mathrm{E}-05$ & $7.56 \mathrm{E}-05$ & & & & \\
\hline SO4-2 & $2.12 \mathrm{E}-02$ & $2.12 \mathrm{E}-02$ & $5.01 \mathrm{E}-04$ & $5.01 \mathrm{E}-04$ & & & & \\
\hline $\mid \mathrm{NO3}^{-}$ & $1.04 \mathrm{E}+00$ & $1.04 \mathrm{E}+00$ & $1.58 \mathrm{E}-02$ & $1.58 \mathrm{E}-02$ & & & & \\
\hline PO4-3 & $5.54 \mathrm{E}-04$ & $5.54 \mathrm{E}-04$ & $1.30 \mathrm{E}-05$ & $1.30 \mathrm{E}-05$ & & & & \\
\hline$A m+4$ & $8.92 \mathrm{E}-11$ & $8.92 \mathrm{E}-11$ & $5.34 \mathrm{E}-12$ & $5.34 \mathrm{E}-12$ & & & & \\
\hline $\mathrm{Br}-$ & $1.45 \mathrm{E}-08$ & $1.45 \mathrm{E}-08$ & $2.86 \mathrm{E}-10$ & $2.86 \mathrm{E}-10$ & & & & \\
\hline $\mathrm{Ce}+4$ & $1.23 \mathrm{E}-07$ & $1.23 \mathrm{E}-07$ & $4.25 \mathrm{E}-09$ & $4.25 \mathrm{E}-09$ & & & & \\
\hline $\mathrm{Eu}+3$ & $7.27 \mathrm{E}-10$ & $7.27 \mathrm{E}-10$ & $2.72 \mathrm{E}-11$ & $2.72 \mathrm{E}-11$ & & & & \\
\hline $\mathrm{Gd}+3$ & $4.53 \mathrm{E}-07$ & $4.53 \mathrm{E}-07$ & $1.76 \mathrm{E}-08$ & $1.76 \mathrm{E}-08$ & & & & \\
\hline $\mathrm{Ge}+4$ & $5.11 \mathrm{E}-10$ & $5.11 \mathrm{E}-10$ & $9.14 \mathrm{E}-12$ & $9.14 \mathrm{E}-12$ & & & & \\
\hline $\ln +3$ & $1.99 \mathrm{E}-09$ & $1.99 \mathrm{E}-09$ & $5.63 \mathrm{E}-11$ & $5.63 \mathrm{E}-11$ & & & & \\
\hline $\mid-$ & $1.26 \mathrm{E}-07$ & $1.26 \mathrm{E}-07$ & $3.94 \mathrm{E}-09$ & $3.94 \mathrm{E}-09$ & & & & \\
\hline $\mathrm{La}+3$ & $1.32 \mathrm{E}-08$ & $1.32 \mathrm{E}-08$ & $4.52 \mathrm{E}-10$ & $4.52 \mathrm{E}-10$ & & & & \\
\hline
\end{tabular}


Table A-13. Mass balance, Tank WM-180 waste (continued).

\begin{tabular}{|c|c|c|c|c|c|c|c|c|}
\hline Stream \# & 401 & 402 & 404 & 404 & 503 & 504 & 505 & 506 \\
\hline Chemical Composition & Mol/liter & Mol/liter & Wt frac & Wt frac & $\mathrm{lb} / \mathrm{wscf}$ & $\mathrm{lb} / \mathrm{wscf}$ & $\mathrm{lb} / \mathrm{wscf}$ & $\mathrm{lb} / \mathrm{wscf}$ \\
\hline $\mathrm{Li+}$ & $3.77 \mathrm{E}-06$ & $3.77 \mathrm{E}-06$ & $6.45 \mathrm{E}-09$ & $6.45 \mathrm{E}-09$ & & & & \\
\hline $\mathrm{Mg}+2$ & $5.53 \mathrm{E}-05$ & $5.53 \mathrm{E}-05$ & $3.31 \mathrm{E}-07$ & $3.31 \mathrm{E}-07$ & & & & \\
\hline $\mathrm{Mo}+6$ & $2.54 \mathrm{E}-04$ & $2.54 \mathrm{E}-04$ & $6.02 \mathrm{E}-06$ & $6.02 \mathrm{E}-06$ & & & & \\
\hline $\mathrm{Nd}+3$ & $4.26 \mathrm{E}-08$ & $4.26 \mathrm{E}-08$ & $1.51 \mathrm{E}-09$ & $1.51 \mathrm{E}-09$ & & & & \\
\hline $\mathrm{Np}+4$ & $2.46 \mathrm{E}-08$ & $2.46 \mathrm{E}-08$ & 1.44E-09 & 1.44E-09 & & & & \\
\hline $\mathrm{Nb}+5$ & $1.95 \mathrm{E}-06$ & $1.95 \mathrm{E}-06$ & $4.47 \mathrm{E}-08$ & 4.47E-08 & & & & \\
\hline $\mathrm{Pd}+4$ & $1.58 \mathrm{E}-07$ & $1.58 \mathrm{E}-07$ & $4.13 \mathrm{E}-09$ & $4.13 \mathrm{E}-09$ & & & & \\
\hline $\mathrm{Pu}+4$ & $1.15 \mathrm{E}-06$ & $1.15 \mathrm{E}-06$ & $6.91 \mathrm{E}-08$ & $6.91 \mathrm{E}-08$ & & & & \\
\hline $\mathrm{Pr}+4$ & $1.20 \mathrm{E}-08$ & $1.20 \mathrm{E}-08$ & $4.17 \mathrm{E}-10$ & $4.17 \mathrm{E}-10$ & & & & \\
\hline $\mathrm{Pm}+3$ & $1.82 \mathrm{E}-12$ & $1.82 \mathrm{E}-12$ & $6.50 \mathrm{E}-14$ & $6.50 \mathrm{E}-14$ & & & & \\
\hline $\mathrm{Rh}+4$ & $5.18 \mathrm{E}-09$ & $5.18 \mathrm{E}-09$ & $1.31 \mathrm{E}-10$ & $1.31 \mathrm{E}-10$ & & & & \\
\hline $\mathrm{Rb}+$ & $2.40 \mathrm{E}-08$ & $2.40 \mathrm{E}-08$ & $5.06 \mathrm{E}-10$ & $5.06 \mathrm{E}-10$ & & & & \\
\hline $\mathrm{Ru+3}$ & $3.56 \mathrm{E}-07$ & $3.56 \mathrm{E}-07$ & $8.87 \mathrm{E}-09$ & $8.87 \mathrm{E}-09$ & & & & \\
\hline $\mathrm{Sm}+3$ & $7.90 \mathrm{E}-09$ & $7.90 \mathrm{E}-09$ & $2.93 \mathrm{E}-10$ & $2.93 \mathrm{E}-10$ & & & & \\
\hline $\mathrm{Si+4}$ & $6.65 \mathrm{E}-04$ & $6.65 \mathrm{E}-04$ & $3.51 \mathrm{E}-01$ & $3.51 \mathrm{E}-01$ & & & & \\
\hline$\overline{\mathrm{Sr}+2}$ & $5.42 \mathrm{E}-07$ & $5.42 \mathrm{E}-07$ & $1.17 \mathrm{E}-08$ & $1.17 \mathrm{E}-08$ & & & & \\
\hline $\mathrm{Tc}+7$ & $5.04 \mathrm{E}-08$ & $5.04 \mathrm{E}-08$ & $1.22 \mathrm{E}-09$ & $1.22 \mathrm{E}-09$ & & & & \\
\hline $\mathrm{Te}+4$ & $1.68 \mathrm{E}-07$ & $1.68 \mathrm{E}-07$ & $5.27 \mathrm{E}-09$ & $5.27 \mathrm{E}-09$ & & & & \\
\hline $\mathrm{Tb}+4$ & $3.04 \mathrm{E}-12$ & $3.04 \mathrm{E}-12$ & $1.19 \mathrm{E}-13$ & $1.19 \mathrm{E}-13$ & & & & \\
\hline $\mathrm{Th}+4$ & $1.20 \mathrm{E}-11$ & $1.20 \mathrm{E}-11$ & $6.85 \mathrm{E}-13$ & $6.85 \mathrm{E}-13$ & & & & \\
\hline $\mathrm{Sn+4}$ & $8.42 \mathrm{E}-06$ & $8.42 \mathrm{E}-06$ & $2.46 \mathrm{E}-07$ & $2.46 \mathrm{E}-07$ & & & & \\
\hline $\mathrm{Ti}+4$ & $1.53 \mathrm{E}-05$ & $1.53 \mathrm{E}-05$ & $1.81 \mathrm{E}-07$ & $1.81 \mathrm{E}-07$ & & & & \\
\hline $\mathrm{Y}+3$ & $9.85 \mathrm{E}-09$ & $9.85 \mathrm{E}-09$ & $2.16 \mathrm{E}-10$ & $2.16 \mathrm{E}-10$ & & & & \\
\hline $\mathrm{OH}-$ & $8.01 \mathrm{E}-03$ & $8.01 \mathrm{E}-03$ & $3.36 \mathrm{E}-05$ & $3.36 \mathrm{E}-05$ & & & & \\
\hline $\mathrm{H} 2 \mathrm{O}$ & $5.23 \mathrm{E}+01$ & $5.23 \mathrm{E}+01$ & $2.32 \mathrm{E}-01$ & $2.32 \mathrm{E}-01$ & & & & $4.68 \mathrm{E}-06$ \\
\hline $\mathrm{SO} 2$ & $9.75 \mathrm{E}-06$ & $9.75 \mathrm{E}-06$ & $1.54 \mathrm{E}-07$ & 1.54E-07 & & & & \\
\hline \multicolumn{9}{|l|}{$\mathrm{H} 2 \mathrm{~S}$} \\
\hline $\mathrm{CO}$ & $1.88 \mathrm{E}-10$ & $1.88 \mathrm{E}-10$ & $1.30 \mathrm{E}-12$ & $1.30 \mathrm{E}-12$ & & & & \\
\hline $\mathrm{CO} 2$ & $3.00 \mathrm{E}-04$ & $3.00 \mathrm{E}-04$ & $3.25 \mathrm{E}-06$ & $3.25 \mathrm{E}-06$ & & & & \\
\hline $\mathrm{H} 2$ & $3.21 \mathrm{E}-10$ & $3.21 \mathrm{E}-10$ & $1.59 \mathrm{E}-13$ & $1.59 \mathrm{E}-13$ & & & & \\
\hline N2 & $1.32 \mathrm{E}-05$ & $1.32 \mathrm{E}-05$ & $9.11 \mathrm{E}-08$ & $9.11 \mathrm{E}-08$ & $7.27 \mathrm{E}-02$ & & & $5.74 \mathrm{E}-02$ \\
\hline NO & $2.21 \mathrm{E}-07$ & $2.21 \mathrm{E}-07$ & $1.63 \mathrm{E}-09$ & $1.63 \mathrm{E}-09$ & & & & \\
\hline NO2 & $2.77 \mathrm{E}-06$ & $2.77 \mathrm{E}-06$ & $3.14 \mathrm{E}-08$ & $3.14 \mathrm{E}-08$ & & & & \\
\hline $\mathrm{O} 2$ & $3.19 \mathrm{E}-06$ & $3.19 \mathrm{E}-06$ & $2.51 \mathrm{E}-08$ & $2.51 \mathrm{E}-08$ & & $8.30 \mathrm{E}-02$ & $8.30 \mathrm{E}-02$ & $1.74 \mathrm{E}-02$ \\
\hline S (other) & $1.30 \mathrm{E}-07$ & $1.30 \mathrm{E}-07$ & $1.03 \mathrm{E}-09$ & $1.03 \mathrm{E}-09$ & & & & \\
\hline $\mathrm{CO} 3$ & $3.33 \mathrm{E}-05$ & $3.33 \mathrm{E}-05$ & $4.92 \mathrm{E}-07$ & $4.92 \mathrm{E}-07$ & & & & \\
\hline \multicolumn{9}{|l|}{$\mathrm{C}$ (reductant) } \\
\hline $\mathrm{O}$ (oxides) & $4.92 \mathrm{E}-03$ & $4.92 \mathrm{E}-03$ & $3.99 \mathrm{E}-01$ & $3.99 \mathrm{E}-01$ & & & & \\
\hline \multicolumn{9}{|l|}{ C (organic) } \\
\hline \multirow{2}{*}{\multicolumn{9}{|c|}{$\frac{\mathrm{H} \text { (organic) }}{\mathrm{O} \text { (organic) }}$}} \\
\hline & & & & & & & & \\
\hline \multirow{2}{*}{ Mass Flow $(\mathrm{kg} / \mathrm{hr})$ : } & $2.26 \mathrm{E}+01$ & $1.00 \mathrm{E}+00$ & $4.00 \mathrm{E}+00$ & $4.00 E+00$ & $5.75 E+02$ & $1.52 E+02$ & $2.30 E+02$ & $2.14 \mathrm{E}+03$ \\
\hline & & & & & & & & \\
\hline \multirow[t]{2}{*}{ Total Drums Generated } & & & & 31 & & & & \\
\hline & $\mathrm{w} / \mathrm{m} 3$ & $\mathrm{w} / \mathrm{m} 3$ & $\mathrm{w} / \mathrm{m} 3$ & w/Drum & $\mathrm{w} / \mathrm{m} 3$ & $\mathrm{w} / \mathrm{m} 3$ & $\mathrm{w} / \mathrm{m} 3$ & $\mathrm{w} / \mathrm{m} 3$ \\
\hline Heat Generation, w/m3 & $7.26 \mathrm{E}-02$ & $7.26 \mathrm{E}-02$ & $3.86 \mathrm{E}-02$ & $7.73 \mathrm{E}-03$ & & & & \\
\hline
\end{tabular}


Table A-13. Mass balance, Tank WM-180 waste (continued).

\begin{tabular}{|c|c|c|c|c|c|c|c|c|}
\hline Stream \# & 401 & 402 & 404 & 404 & 503 & 504 & 505 & 506 \\
\hline Radiological Composition & $\mathrm{Ci} / \mathrm{L}$ & $\mathrm{Ci} / \mathrm{L}$ & $\mathrm{Ci} / \mathrm{kg}$ & Ci/Drum & Ci/wscm & $\mathrm{Ci} / \mathrm{wscm}$ & $\mathrm{Ci} / \mathrm{wscm}$ & Ci/wscm \\
\hline \multicolumn{9}{|l|}{$\mathrm{H}-3$} \\
\hline $\mathrm{C}-14$ & $6.34 \mathrm{E}-16$ & $6.34 \mathrm{E}-16$ & $1.56 \mathrm{E}-16$ & $6.56 \mathrm{E}-14$ & & & & \\
\hline Co-60 & $1.12 \mathrm{E}-08$ & $1.12 \mathrm{E}-08$ & $2.76 \mathrm{E}-09$ & $1.16 \mathrm{E}-06$ & & & & \\
\hline $\mathrm{Ni}-59$ & $4.60 \mathrm{E}-10$ & $4.60 \mathrm{E}-10$ & $1.13 \mathrm{E}-10$ & $4.76 \mathrm{E}-08$ & & & & \\
\hline $\mathrm{Ni}-63$ & $1.21 \mathrm{E}-07$ & $1.21 \mathrm{E}-07$ & $2.99 \mathrm{E}-08$ & $1.26 \mathrm{E}-05$ & & & & \\
\hline Se-79 & $2.57 \mathrm{E}-08$ & $2.57 \mathrm{E}-08$ & $6.33 \mathrm{E}-09$ & $2.66 \mathrm{E}-06$ & & & & \\
\hline Sr-90 & $9.80 \mathrm{E}-05$ & $9.80 \mathrm{E}-05$ & $2.42 \mathrm{E}-05$ & $1.01 \mathrm{E}-02$ & & & & \\
\hline Y-90 & $5.49 \mathrm{E}-05$ & $5.49 \mathrm{E}-05$ & $1.35 \mathrm{E}-05$ & $5.69 \mathrm{E}-03$ & & & & \\
\hline Zr-93 & $1.24 \mathrm{E}-07$ & $1.24 \mathrm{E}-07$ & $3.06 \mathrm{E}-08$ & $1.29 \mathrm{E}-05$ & & & & \\
\hline $\mathrm{Nb}-93 \mathrm{~m}$ & $3.67 \mathrm{E}-09$ & $3.67 \mathrm{E}-09$ & $9.04 \mathrm{E}-10$ & $3.80 \mathrm{E}-07$ & & & & \\
\hline $\mathrm{Nb}-94$ & $2.57 \mathrm{E}-09$ & $2.57 \mathrm{E}-09$ & $6.32 \mathrm{E}-10$ & $2.66 \mathrm{E}-07$ & & & & \\
\hline Tc-99 & $8.57 \mathrm{E}-08$ & $8.57 \mathrm{E}-08$ & $2.11 \mathrm{E}-08$ & $8.87 \mathrm{E}-06$ & & & & \\
\hline Ru-106 & $1.35 \mathrm{E}-09$ & $1.35 \mathrm{E}-09$ & $3.34 \mathrm{E}-10$ & $1.40 \mathrm{E}-07$ & & & & \\
\hline Rh-102 & $1.20 \mathrm{E}-12$ & $1.20 \mathrm{E}-12$ & $2.95 \mathrm{E}-13$ & $1.24 \mathrm{E}-10$ & & & & \\
\hline Rh-106 & $1.35 \mathrm{E}-09$ & $1.35 \mathrm{E}-09$ & $3.34 \mathrm{E}-10$ & $1.40 \mathrm{E}-07$ & & & & \\
\hline $\mathrm{Pd}-107$ & $4.09 \mathrm{E}-11$ & $4.09 \mathrm{E}-11$ & $1.01 \mathrm{E}-11$ & $4.24 \mathrm{E}-09$ & & & & \\
\hline Cd-113m & $4.50 \mathrm{E}-09$ & $4.50 \mathrm{E}-09$ & $1.11 \mathrm{E}-09$ & $4.66 \mathrm{E}-07$ & & & & \\
\hline Sn-121m & $3.75 \mathrm{E}-09$ & $3.75 \mathrm{E}-09$ & $9.24 \mathrm{E}-10$ & $3.88 \mathrm{E}-07$ & & & & \\
\hline Sn-126 & $2.42 \mathrm{E}-08$ & $2.42 \mathrm{E}-08$ & $5.95 \mathrm{E}-09$ & $2.50 \mathrm{E}-06$ & & & & \\
\hline Sb-125 & $2.23 \mathrm{E}-08$ & $2.23 \mathrm{E}-08$ & $5.49 \mathrm{E}-09$ & $2.31 \mathrm{E}-06$ & & & & \\
\hline Sb-126 & $7.98 \mathrm{E}-11$ & $7.98 \mathrm{E}-11$ & $1.97 \mathrm{E}-11$ & $8.26 \mathrm{E}-09$ & & & & \\
\hline Te-125m & $1.77 \mathrm{E}-07$ & $1.77 \mathrm{E}-07$ & $4.35 \mathrm{E}-08$ & $1.83 \mathrm{E}-05$ & & & & \\
\hline I-129 & $2.24 \mathrm{E}-09$ & $2.24 \mathrm{E}-09$ & $5.52 \mathrm{E}-10$ & $2.32 \mathrm{E}-07$ & & & & \\
\hline Cs-134 & $1.33 \mathrm{E}-05$ & $1.33 \mathrm{E}-05$ & $3.36 \mathrm{E}-06$ & $1.41 \mathrm{E}-03$ & & & & \\
\hline Cs-135 & $1.14 \mathrm{E}-06$ & $1.14 \mathrm{E}-06$ & $2.90 \mathrm{E}-07$ & $1.22 \mathrm{E}-04$ & & & & \\
\hline \begin{tabular}{|l|} 
Cs-137 \\
\end{tabular} & $6.69 \mathrm{E}-02$ & $6.69 \mathrm{E}-02$ & $1.70 \mathrm{E}-02$ & $7.13 \mathrm{E}+00$ & & & & \\
\hline Ba-137m & $1.23 \mathrm{E}-04$ & $1.23 \mathrm{E}-04$ & $3.03 \mathrm{E}-05$ & $1.27 \mathrm{E}-02$ & & & & \\
\hline \begin{tabular}{|l|}
$\mathrm{Ce}-144$ \\
\end{tabular} & $9.13 \mathrm{E}-10$ & $9.13 \mathrm{E}-10$ & $2.25 \mathrm{E}-10$ & $9.45 \mathrm{E}-08$ & & & & \\
\hline Pr-144 & $9.13 \mathrm{E}-10$ & $9.13 \mathrm{E}-10$ & $2.25 \mathrm{E}-10$ & $9.45 \mathrm{E}-08$ & & & & \\
\hline Pm-146 & $7.06 \mathrm{E}-11$ & $7.06 \mathrm{E}-11$ & $1.74 \mathrm{E}-11$ & $7.31 \mathrm{E}-09$ & & & & \\
\hline Pm-147 & $2.48 \mathrm{E}-07$ & $2.48 \mathrm{E}-07$ & $6.11 \mathrm{E}-08$ & $2.57 \mathrm{E}-05$ & & & & \\
\hline Sm-151 & $4.89 \mathrm{E}-07$ & $4.89 \mathrm{E}-07$ & $1.20 \mathrm{E}-07$ & $5.06 \mathrm{E}-05$ & & & & \\
\hline Eu-152 & $3.54 \mathrm{E}-09$ & $3.54 \mathrm{E}-09$ & $8.72 \mathrm{E}-10$ & $3.66 \mathrm{E}-07$ & & & & \\
\hline Eu-154 & $1.27 \mathrm{E}-07$ & $1.27 \mathrm{E}-07$ & $3.13 \mathrm{E}-08$ & $1.31 \mathrm{E}-05$ & & & & \\
\hline \multirow[t]{2}{*}{ Eu-155 } & $2.31 \mathrm{E}-07$ & $2.31 \mathrm{E}-07$ & $5.69 \mathrm{E}-08$ & $2.39 \mathrm{E}-05$ & & & & \\
\hline & & & & & & & & \\
\hline Th-230 & $4.89 \mathrm{E}-11$ & $4.89 \mathrm{E}-11$ & $1.21 \mathrm{E}-11$ & $5.07 \mathrm{E}-09$ & & & & \\
\hline Pa-233 & $1.64 \mathrm{E}-07$ & 1.64E-07 & $4.05 \mathrm{E}-08$ & $1.70 \mathrm{E}-05$ & & & & \\
\hline U-232 & $1.18 \mathrm{E}-10$ & $1.18 \mathrm{E}-10$ & $2.91 \mathrm{E}-11$ & $1.22 \mathrm{E}-08$ & & & & \\
\hline $\mathrm{U}-233$ & $4.60 \mathrm{E}-12$ & $4.60 \mathrm{E}-12$ & $1.13 \mathrm{E}-12$ & $4.76 \mathrm{E}-10$ & & & & \\
\hline U-234 & $1.18 \mathrm{E}-07$ & $1.18 \mathrm{E}-07$ & $2.91 \mathrm{E}-08$ & $1.22 \mathrm{E}-05$ & & & & \\
\hline U-235 & $4.31 \mathrm{E}-09$ & $4.31 \mathrm{E}-09$ & 1.06E-09 & $4.47 \mathrm{E}-07$ & & & & \\
\hline U-236 & $6.40 \mathrm{E}-09$ & $6.40 \mathrm{E}-09$ & $1.58 \mathrm{E}-09$ & $6.63 \mathrm{E}-07$ & & & & \\
\hline U-237 & $3.61 \mathrm{E}-10$ & $3.61 \mathrm{E}-10$ & $8.90 \mathrm{E}-11$ & $3.74 \mathrm{E}-08$ & & & & \\
\hline U-238 & $2.55 \mathrm{E}-09$ & $2.55 \mathrm{E}-09$ & $6.29 \mathrm{E}-10$ & $2.64 \mathrm{E}-07$ & & & & \\
\hline Np-237 & $4.11 \mathrm{E}-09$ & $4.11 \mathrm{E}-09$ & $1.01 \mathrm{E}-09$ & $4.26 \mathrm{E}-07$ & & & & \\
\hline Pu-236 & $2.37 \mathrm{E}-10$ & $2.37 \mathrm{E}-10$ & $5.83 \mathrm{E}-11$ & $2.45 \mathrm{E}-08$ & & & & \\
\hline Pu-238 & $1.08 \mathrm{E}-04$ & $1.08 \mathrm{E}-04$ & $2.66 \mathrm{E}-05$ & $1.12 \mathrm{E}-02$ & & & & \\
\hline Pu-239 & $1.64 \mathrm{E}-05$ & $1.64 \mathrm{E}-05$ & $4.04 \mathrm{E}-06$ & $1.70 \mathrm{E}-03$ & & & & \\
\hline Pu-240 & $8.89 \mathrm{E}-07$ & $8.89 \mathrm{E}-07$ & $2.19 \mathrm{E}-07$ & $9.20 \mathrm{E}-05$ & & & & \\
\hline Pu-241 & $6.50 \mathrm{E}-05$ & $6.50 \mathrm{E}-05$ & $1.60 \mathrm{E}-05$ & $6.73 \mathrm{E}-03$ & & & & \\
\hline Pu-242 & $6.85 \mathrm{E}-10$ & $6.85 \mathrm{E}-10$ & $1.69 \mathrm{E}-10$ & $7.09 \mathrm{E}-08$ & & & & \\
\hline Pu-244 & $5.87 \mathrm{E}-17$ & $5.87 \mathrm{E}-17$ & 1.45E-17 & $6.08 \mathrm{E}-15$ & & & & \\
\hline Am-241 & $1.93 \mathrm{E}-07$ & $1.93 \mathrm{E}-07$ & $4.77 \mathrm{E}-08$ & $2.00 \mathrm{E}-05$ & & & & \\
\hline$A m-242 m$ & $2.11 \mathrm{E}-11$ & $2.11 \mathrm{E}-11$ & $5.20 \mathrm{E}-12$ & $2.19 \mathrm{E}-09$ & & & & \\
\hline
\end{tabular}


Table A-13. Mass balance, Tank WM-180 waste (continued).

\begin{tabular}{|c|c|c|c|c|c|c|c|c|}
\hline Stream \# & 401 & 402 & 404 & 404 & 503 & 504 & 505 & 506 \\
\hline & $\mathrm{Ci} / \mathrm{L}$ & $\mathrm{Ci} / \mathrm{L}$ & $\mathrm{Ci} / \mathrm{kg}$ & Ci/Drum & $\mathrm{Ci} / \mathrm{wscm}$ & $\mathrm{Ci} / \mathrm{wscm}$ & $\mathrm{Ci} / \mathrm{wscm}$ & $\mathrm{Ci} / \mathrm{wscm}$ \\
\hline Am-243 & $3.06 \mathrm{E}-11$ & $3.06 \mathrm{E}-11$ & $7.55 \mathrm{E}-12$ & 3.17E-09 & & & & \\
\hline $\mathrm{Cm}-242$ & 7.04E-10 & $7.04 \mathrm{E}-10$ & 1.73E-10 & $7.28 \mathrm{E}-08$ & & & & \\
\hline $\mathrm{Cm}-243$ & 2.02E-09 & 2.02E-09 & 4.99E-10 & $2.09 \mathrm{E}-07$ & & & & \\
\hline $\mathrm{Cm}-244$ & $1.26 \mathrm{E}-07$ & $1.26 \mathrm{E}-07$ & $3.10 \mathrm{E}-08$ & $1.30 \mathrm{E}-05$ & & & & \\
\hline $\mathrm{Cm}-245$ & $2.13 \mathrm{E}-11$ & $2.13 \mathrm{E}-11$ & $5.25 \mathrm{E}-12$ & $2.20 \mathrm{E}-09$ & & & & \\
\hline $\mathrm{Cm}-246$ & $1.40 \mathrm{E}-12$ & $1.40 \mathrm{E}-12$ & $3.45 \mathrm{E}-13$ & $1.45 \mathrm{E}-10$ & & & & \\
\hline TRU & $1.27 \mathrm{E}-04$ & 1.27E-04 & $3.14 \mathrm{E}-05$ & $1.32 \mathrm{E}-02$ & & & & \\
\hline \multicolumn{9}{|l|}{ TRU, nCi/gm } \\
\hline & & & & & & & & \\
\hline $\begin{array}{l}\text { Gas Stream Bulk } \\
\text { Composition (Wet Basis) }\end{array}$ & $\begin{array}{c}\text { mol\% or } \\
\text { ppmv }\end{array}$ & $\begin{array}{l}\text { mol\% or } \\
\text { ppmv }\end{array}$ & $\begin{array}{c}\text { mol\% or } \\
\text { ppmv }\end{array}$ & $\begin{array}{c}\text { mol\% or } \\
\text { ppmv }\end{array}$ & $\begin{array}{c}\text { mol\% or } \\
\text { ppmv }\end{array}$ & $\begin{array}{c}\text { mol\% or } \\
\text { ppmv }\end{array}$ & $\begin{array}{c}\text { mol\% or } \\
\text { ppmv }\end{array}$ & $\begin{array}{c}\text { mol\% or } \\
\text { ppmv }\end{array}$ \\
\hline $\mathrm{H} 2 \mathrm{O}, \mathrm{mol} \%$ & & & & & & & & $0.01 \%$ \\
\hline $\mathrm{O} 2, \mathrm{~mol} \%$ & & & & & & $100.00 \%$ & $100.00 \%$ & $20.99 \%$ \\
\hline $\mathrm{N} 2, \mathrm{~mol} \%$ & & & & & $100.00 \%$ & & & $79.00 \%$ \\
\hline \multicolumn{9}{|l|}{$\mathrm{H} 2, \mathrm{~mol} \%$} \\
\hline \multicolumn{9}{|l|}{$\mathrm{CO} 2, \mathrm{~mol} \%$} \\
\hline \multicolumn{9}{|l|}{$\mathrm{CO}, \mathrm{ppmv}$} \\
\hline \multicolumn{9}{|l|}{$\mathrm{NO}, \mathrm{ppmv}$} \\
\hline \multicolumn{9}{|l|}{ NO2, ppmv } \\
\hline \multicolumn{9}{|l|}{ SO2, ppmv } \\
\hline \multicolumn{9}{|l|}{$\mathrm{Cl}, \mathrm{ppmv}$} \\
\hline \multicolumn{9}{|l|}{$\mathrm{F}, \mathrm{ppmv}$} \\
\hline \multicolumn{9}{|l|}{$\mathrm{C}$ (organic), ppmv } \\
\hline \multicolumn{9}{|l|}{$\mathrm{H}$ (organic), ppmv } \\
\hline \multicolumn{9}{|l|}{$\mathrm{Hg}, \mathrm{ug} / \mathrm{wscm}$} \\
\hline $\mathrm{PM}, \mathrm{mg} / \mathrm{wscm}$ & & & & & $2.00 \mathrm{E}+01$ & $1.48 \mathrm{E}-01$ & & $1.60 \mathrm{E}+01$ \\
\hline \multicolumn{9}{|l|}{ SVM, ug/wscm } \\
\hline \multicolumn{9}{|l|}{ LVM, ug/wscm } \\
\hline $\begin{array}{l}\text { Gas Stream Bulk } \\
\text { Composition (Dry Basis) }\end{array}$ & $\mathrm{mol} \%$ & $\mathrm{~mol} \%$ & $\mathrm{~mol} \%$ & $\mathrm{~mol} \%$ & $\mathrm{~mol} \%$ & $\mathrm{~mol} \%$ & $\mathrm{~mol} \%$ & $\mathrm{~mol} \%$ \\
\hline $\mathrm{O} 2, \mathrm{~mol} \%$, dry basis & & & & & & & & $20.99 \%$ \\
\hline $\mathrm{N} 2, \mathrm{~mol} \%$, dry basis & & & & & & & & $79.01 \%$ \\
\hline \multicolumn{9}{|l|}{$\mathrm{H} 2, \mathrm{~mol} \%$, dry basis } \\
\hline \multicolumn{9}{|l|}{$\mathrm{CO} 2, \mathrm{~mol} \%$, dry basis } \\
\hline $\begin{array}{l}\text { Gas Stream Bulk } \\
\text { Composition (Dry Basis, } \\
\text { Corrected to } 7 \% \text { O2 with } \\
100 \% \text { O2 Combustion Air) }\end{array}$ & $\begin{array}{l}\mathrm{ppmv}, \text { or } \\
\text { ug/dscm } \\
\text { or } \\
\mathrm{mg} / \mathrm{dscm}\end{array}$ & $\begin{array}{l}\mathrm{ppmv}, \text { or } \\
\mathrm{ug} / \mathrm{dscm} \\
\text { or } \\
\mathrm{mg} / \mathrm{dscm}\end{array}$ & $\begin{array}{l}\mathrm{ppmv}, \text { or } \\
\mathrm{ug} / \mathrm{dscm} \\
\text { or } \\
\mathrm{mg} / \mathrm{dscm}\end{array}$ & $\begin{array}{l}\mathrm{ppmv}, \text { or } \\
\mathrm{ug} / \mathrm{dscm} \\
\text { or } \\
\mathrm{mg} / \mathrm{dscm}\end{array}$ & $\begin{array}{l}\mathrm{ppmv}, \text { or } \\
\mathrm{ug} / \mathrm{dscm} \\
\text { or } \\
\mathrm{mg} / \mathrm{dscm}\end{array}$ & $\begin{array}{l}\mathrm{ppmv}, \text { or } \\
\mathrm{ug} / \mathrm{dscm} \\
\text { or } \\
\mathrm{mg} / \mathrm{dscm}\end{array}$ & $\begin{array}{l}\mathrm{ppmv}, \text { or } \\
\mathrm{ug} / \mathrm{dscm} \\
\text { or } \\
\mathrm{mg} / \mathrm{dscm}\end{array}$ & $\begin{array}{l}\text { ppmv, or } \\
\text { ug/dscm } \\
\text { or } \\
\mathrm{mg} / \mathrm{dscm}\end{array}$ \\
\hline \multicolumn{9}{|l|}{$\mathrm{CO}, \mathrm{ppmv}$, dry basis } \\
\hline \multicolumn{9}{|l|}{$\mathrm{NO}$, ppmv, dry basis } \\
\hline \multicolumn{9}{|l|}{ NO2, ppmv, dry basis } \\
\hline \multicolumn{9}{|l|}{ SO2, ppmv, dry basis } \\
\hline \multicolumn{9}{|l|}{$\mathrm{Cl}, \mathrm{ppmv}$, dry basis } \\
\hline $\mathrm{F}, \mathrm{ppmv}$, dry basis & & & & & & & & \\
\hline C (organic), ppmv, dry basis & & & & & & & & \\
\hline $\mathrm{H}$ (organic), ppmv, dry basis & & & & & & & & \\
\hline $\mathrm{Hg}, \mathrm{ug} / \mathrm{dscm}$ & & & & & & & & \\
\hline $\mathrm{PM}, \mathrm{mg} / \mathrm{dscm}$ & & & & & & & & $2.25 \mathrm{E}+00$ \\
\hline SVM, ug/dscm & & & & & & & & \\
\hline LVM, ug/dscm & & & & & & & & \\
\hline
\end{tabular}


Table A-13. Mass balance, Tank WM-180 waste (continued).

\begin{tabular}{|c|c|c|c|c|c|c|c|c|}
\hline \begin{tabular}{|l|l} 
PFD \# \\
\end{tabular} & PFD-2 & PFD-3 & PFD-3 & PFD-3 & PFD-3 & PFD-3 & PFD-3 & $\frac{\text { PFD-3 }}{516}$ \\
\hline WM-180, Stream \# & 507 & 510 & 511 & 512 & 513 & 514 & 515 & 516 \\
\hline Stream Name & $\begin{array}{l}\text { Off-Gas } \\
\text { from Boiler }\end{array}$ & $\begin{array}{c}\text { Nitrogen to } \\
\text { Cool } \\
\text { Product }\end{array}$ & $\begin{array}{l}\text { N2 from } \\
\text { Product } \\
\text { Cooler }\end{array}$ & $\begin{array}{c}\text { Filter } \\
\text { Backpulse } \\
\text { N2 }\end{array}$ & $\begin{array}{l}\text { Solids } \\
\text { Transport } \\
\text { Nitrogen }\end{array}$ & $\begin{array}{c}\text { Tank Farm } \\
\text { Transfer } \\
\text { Airlift Air }\end{array}$ & $\begin{array}{c}\text { Ventilation } \\
\text { Air }\end{array}$ & Fresh GAC \\
\hline Rate or Volume & $1.31 \mathrm{E}+05$ & $2.88 \mathrm{E}+02$ & $9.15 \mathrm{E}+02$ & $2.72 \mathrm{E}+02$ & $6.55 \mathrm{E}+01$ & $1.40 \mathrm{E}+01$ & $2.89 \mathrm{E}+01$ & $2.00 \mathrm{E}+00$ \\
\hline Volume Flow (standard, wet) & $6.70 \mathrm{E}+04$ & $1.94 \mathrm{E}+03$ & $1.94 \mathrm{E}+03$ & $7.95 \mathrm{E}+02$ & $1.92 \mathrm{E}+02$ & $4.11 \mathrm{E}+01$ & $2.94 \mathrm{E}+01$ & \\
\hline Volume Flow (standard, dry) & $5.92 \mathrm{E}+04$ & $1.94 \mathrm{E}+03$ & $1.94 \mathrm{E}+03$ & $7.95 \mathrm{E}+02$ & $1.92 \mathrm{E}+02$ & $4.11 \mathrm{E}+01$ & $2.94 \mathrm{E}+01$ & \\
\hline Rate Units & $\mathrm{ft} 3 / \mathrm{hr}$ & $\mathrm{ft} 3 / \mathrm{hr}$ & $\mathrm{ft} 3 / \mathrm{hr}$ & $\mathrm{ft} 3 / \mathrm{hr}$ & $\mathrm{ft} 3 / \mathrm{hr}$ & $\mathrm{ft} 3 / \mathrm{hr}$ & $\mathrm{ft} 3 / \mathrm{hr}$ & $\mathrm{Ib} / \mathrm{hr}$ \\
\hline Rate or Volume, metric & $1.90 \mathrm{E}+03$ & $5.50 \mathrm{E}+01$ & $5.50 \mathrm{E}+01$ & $2.25 \mathrm{E}+01$ & $5.43 \mathrm{E}+00$ & $1.16 \mathrm{E}+00$ & $8.34 \mathrm{E}-01$ & $9.09 \mathrm{E}-01$ \\
\hline Rate Units & wscm $/ \mathrm{hr}$ & wscm $/ \mathrm{hr}$ & wscm/hr & wscm/hr & $\mathrm{wscm} / \mathrm{hr}$ & wscm $/ \mathrm{hr}$ & wscm/hr & $\mathrm{kg} / \mathrm{hr}$ \\
\hline Temperature, ${ }^{\circ} \mathrm{C}$ & 300 & 0 & 594 & 15 & 15 & 15 & 15 & 25 \\
\hline Temperature, ${ }^{\circ} \mathrm{F}$ & 572 & 32 & 1102 & 59 & 59 & 59 & 59 & 77 \\
\hline Pressure, psia & 14.7 & 92.3 & 92.3 & 42.3 & 42.3 & 42.3 & 14.7 & 14.7 \\
\hline Specific Gravity & $6.10 \mathrm{E}-04$ & $7.85 \mathrm{E}-03$ & $2.47 \mathrm{E}-03$ & $3.41 \mathrm{E}-03$ & $3.41 \mathrm{E}-03$ & $3.51 \mathrm{E}-03$ & $1.22 \mathrm{E}-03$ & $4.81 \mathrm{E}-01$ \\
\hline \multicolumn{9}{|c|}{ Gas Stream Bulk Composition (Wet Basis) } \\
\hline & Mol \% & Mol \% & Mol \% & Mol \% & Mol \% & Mol \% & Mol \% & Wt \% \\
\hline $\mathrm{H} 2 \mathrm{O}$ & $11.52 \%$ & & & & & $0.01 \%$ & $0.01 \%$ & \\
\hline $\mathrm{O} 2$ & $3.00 \%$ & & & & & $20.99 \%$ & $20.99 \%$ & \\
\hline $\mathrm{N} 2$ & $74.11 \%$ & $100.00 \%$ & $100.00 \%$ & $100.00 \%$ & $100.00 \%$ & $79.00 \%$ & $79.00 \%$ & \\
\hline $\mathrm{H} 2$ & $0.05 \%$ & & & & & & & \\
\hline \multirow[t]{2}{*}{$\mathrm{CO} 2$} & $10.64 \%$ & & & & & & & \\
\hline & $\mathrm{ppmv}$ & & & & & & & \\
\hline $\mathrm{CO}$ & $2.21 \mathrm{E}+03$ & & & & & & & \\
\hline NO & $4.30 \mathrm{E}+03$ & & & & & & & \\
\hline NO2 & $1.97 \mathrm{E}+00$ & & & & & & & \\
\hline $\mathrm{SO} 2$ & $2.40 \mathrm{E}+02$ & & & & & & & \\
\hline \multicolumn{9}{|l|}{\begin{tabular}{|l} 
C(organic) \\
\end{tabular}} \\
\hline \multicolumn{9}{|l|}{$\mathrm{H}$ (organic) } \\
\hline & & & & & & & & \\
\hline \multicolumn{9}{|c|}{ Gas Stream Bulk Composition (Dry Basis) } \\
\hline & Mol \% & Mol \% & Mol \% & Mol \% & Mol \% & Mol \% & Mol \% & \\
\hline $\mathrm{O} 2$ & $3.39 \%$ & & & & & $20.99 \%$ & $20.99 \%$ & \\
\hline N2 & $83.77 \%$ & & & & & $79.01 \%$ & $79.01 \%$ & \\
\hline $\mathrm{H} 2$ & $0.05 \%$ & & & & & & & \\
\hline $\mathrm{CO} 2$ & $12.03 \%$ & & & & & & & \\
\hline \multicolumn{9}{|c|}{ Gas Stream Bulk Composition (Dry Basis, Corrected to $7 \%$ O2 with $100 \%$ O2 Combustion Air) } \\
\hline & ppmv & & & & & & & \\
\hline $\mathrm{CO}$ & $3.49 \mathrm{E}+02$ & & & & & & & \\
\hline NO & $6.80 \mathrm{E}+02$ & & & & & & & \\
\hline $\mathrm{NO} 2$ & $3.12 \mathrm{E}-01$ & & & & & & & \\
\hline SO2 & $3.80 \mathrm{E}+01$ & & & & & & & \\
\hline \multicolumn{9}{|l|}{ C(organic) } \\
\hline \multicolumn{9}{|l|}{$\mathrm{H}$ (organic) } \\
\hline PM, mg/dscm & $2.62 \mathrm{E}+00$ & & & & & $2.20 \mathrm{E}+00$ & $2.20 \mathrm{E}+00$ & \\
\hline \multirow{2}{*}{\multicolumn{9}{|c|}{$\mathrm{Hg}, \mathrm{ug} / \mathrm{dscm}$}} \\
\hline & & & & & & & & \\
\hline & & & & & & & & \\
\hline GAC & & & & & & & & $100 \%$ \\
\hline
\end{tabular}


Table A-14. Mass balance, Tank WM-187 waste.

\begin{tabular}{|c|c|c|c|c|c|c|c|c|c|}
\hline PFD\# & PFD-2 & PFD-2 & PFD-2 & PFD-2 & PFD-2 & PFD-2 & PFD-2 & PFD-2 & PFD-2 \\
\hline WM-187, Stream \# & 101 & 102 & 103 & 104 & 105 & 106 & 107 & 108 & 108 \\
\hline Stream Name & SBW & $\begin{array}{l}\text { Reformer } \\
\text { Feed }\end{array}$ & $\begin{array}{c}\text { Reformer } \\
\text { Off-gas }\end{array}$ & $\begin{array}{c}\text { Reformer } \\
\text { Off-gas } \\
\text { Cooled }\end{array}$ & $\begin{array}{l}\text { Cyclone } \\
\text { Drain }\end{array}$ & $\begin{array}{l}\text { Filter } \\
\text { Drain }\end{array}$ & Bed Drain & $\begin{array}{l}\text { Cooled } \\
\text { Product }\end{array}$ & $\begin{array}{l}\text { Product } \\
\text { Shipping } \\
\text { Canisters }\end{array}$ \\
\hline Rate or Volume & $9.58 \mathrm{E}+01$ & $1.32 \mathrm{E}+02$ & $4.49 \mathrm{E}+05$ & $4.04 \mathrm{E}+05$ & $1.11 \mathrm{E}+02$ & $6.93 \mathrm{E}+01$ & $9.60 \mathrm{E}+01$ & $1.65 \mathrm{E}+02$ & $1.69 \mathrm{E}+00$ \\
\hline Volume Flow (standard, we & & & $1.01 \mathrm{E}+05$ & $1.03 \mathrm{E}+05$ & & & & & \\
\hline Volume Flow (standard, dry & & & $3.03 \mathrm{E}+04$ & $3.23 \mathrm{E}+04$ & & & & & \\
\hline Rate Units & $\mathrm{gal} / \mathrm{hr}$ & $\mathrm{gal} / \mathrm{hr}$ & $\mathrm{ft} 3 / \mathrm{hr}$ & $\mathrm{ft} 3 / \mathrm{hr}$ & $\mathrm{lb} / \mathrm{hr}$ & $\mathrm{lb} / \mathrm{hr}$ & $\mathrm{lb} / \mathrm{hr}$ & $\mathrm{lb} / \mathrm{hr}$ & can's/day \\
\hline Rate or Volume, metric & 363 & 499 & $2.85 \mathrm{E}+03$ & $2.91 \mathrm{E}+03$ & 50 & 31.4 & 43.5 & 75.0 & 75.0 \\
\hline Rate Units & $\mathrm{L} / \mathrm{hr}$ & $\mathrm{L} / \mathrm{hr}$ & wscm/hr & wscm/hr & $\mathrm{kg} / \mathrm{hr}$ & $\mathrm{kg} / \mathrm{hr}$ & $\mathrm{kg} / \mathrm{hr}$ & $\mathrm{kg} / \mathrm{hr}$ & $\mathrm{kg} / \mathrm{hr}$ \\
\hline Temperature, ${ }^{\circ} \mathrm{C}$ & 15 & 19 & 670 & 530 & 670 & 530 & 670 & 56 & 56 \\
\hline Temperature, ${ }^{\circ} \mathrm{F}$ & 59 & 66 & 1238 & 986 & 1238 & 986 & 1238 & 134 & 134 \\
\hline Pressure, psia & 14.7 & 44.7 & 10.6 & 10.2 & 10.6 & 9.5 & 10.8 & 9.5 & 9.5 \\
\hline Specific Gravity & 1.20 & 1.20 & $1.99 \mathrm{E}-04$ & $2.26 \mathrm{E}-04$ & 0.24 & 1.15 & 1.46 & 1.33 & 1.33 \\
\hline Chemical Composition & Mol/liter & Mol/liter & $\mathrm{lb} / \mathrm{wscf}$ & $\mathrm{lb} / \mathrm{wscf}$ & Wt frac & Wt frac & Wt frac & Wt frac & Wt frac \\
\hline $\mathrm{H}+$ & $2.71 \mathrm{E}+00$ & $2.02 \mathrm{E}+00$ & $8.27 \mathrm{E}-07$ & $8.11 \mathrm{E}-07$ & $3.25 \mathrm{E}-06$ & $6.38 \mathrm{E}-06$ & $9.54 \mathrm{E}-06$ & $8.22 \mathrm{E}-06$ & $8.22 \mathrm{E}-06$ \\
\hline $\mathrm{Al}+3$ & $3.09 \mathrm{E}-01$ & \begin{tabular}{|l|}
$2.24 \mathrm{E}-01$ \\
\end{tabular} & $2.35 \mathrm{E}-05$ & $2.31 \mathrm{E}-05$ & $2.74 \mathrm{E}-02$ & $3.41 \mathrm{E}-02$ & $5.61 \mathrm{E}-02$ & $4.69 \mathrm{E}-02$ & $4.69 \mathrm{E}-02$ \\
\hline $\mathrm{Sb}+5$ & $2.45 \mathrm{E}-05$ & $1.78 \mathrm{E}-05$ & $7.25 \mathrm{E}-09$ & $7.11 \mathrm{E}-09$ & $8.44 \mathrm{E}-06$ & $1.05 \mathrm{E}-05$ & $1.73 \mathrm{E}-05$ & $1.44 \mathrm{E}-05$ & $1.44 \mathrm{E}-05$ \\
\hline $\mathrm{As}+3$ & $1.43 \mathrm{E}-04$ & \begin{tabular}{|l|}
$1.04 \mathrm{E}-04$ \\
\end{tabular} & \begin{tabular}{|l|}
$2.59 \mathrm{E}-08$ \\
\end{tabular} & $2.55 \mathrm{E}-08$ & $3.02 \mathrm{E}-05$ & $3.76 \mathrm{E}-05$ & $6.19 \mathrm{E}-05$ & $5.17 \mathrm{E}-05$ & $5.17 \mathrm{E}-05$ \\
\hline $\mathrm{Ba}+2$ & $6.00 \mathrm{E}-05$ & \begin{tabular}{|l|}
$4.36 \mathrm{E}-05$ \\
\end{tabular} & $1.99 \mathrm{E}-08$ & $1.96 \mathrm{E}-08$ & $1.55 \mathrm{E}-05$ & $2.89 \mathrm{E}-05$ & $4.77 \mathrm{E}-05$ & $3.98 \mathrm{E}-05$ & $3.98 \mathrm{E}-05$ \\
\hline $\mathrm{Be}+2$ & $1.33 \mathrm{E}-05$ & $9.65 \mathrm{E}-06$ & $2.89 \mathrm{E}-10$ & $2.84 \mathrm{E}-10$ & $2.25 \mathrm{E}-07$ & $4.20 \mathrm{E}-07$ & $6.92 \mathrm{E}-07$ & $5.78 \mathrm{E}-07$ & $5.78 \mathrm{E}-07$ \\
\hline $\mathrm{B}+3$ & $9.26 \mathrm{E}-03$ & \begin{tabular}{|l|}
$6.73 \mathrm{E}-03$ \\
\end{tabular} & $2.43 \mathrm{E}-07$ & $2.38 \mathrm{E}-07$ & $2.83 \mathrm{E}-04$ & $3.52 \mathrm{E}-04$ & $5.79 \mathrm{E}-04$ & $4.84 \mathrm{E}-04$ & $4.84 \mathrm{E}-04$ \\
\hline $\mathrm{Cd}+2$ & $1.48 \mathrm{E}-03$ & \begin{tabular}{|l|}
$1.08 \mathrm{E}-03$ \\
\end{tabular} & \begin{tabular}{|l|}
$4.41 \mathrm{E}-07$ \\
\end{tabular} & $4.33 \mathrm{E}-07$ & $4.01 \mathrm{E}-04$ & $6.40 \mathrm{E}-04$ & $9.26 \mathrm{E}-04$ & $8.06 \mathrm{E}-04$ & $8.06 \mathrm{E}-04$ \\
\hline $\mathrm{Ca}+2$ & $2.96 \mathrm{E}-02$ & \begin{tabular}{|l|}
$2.15 \mathrm{E}-02$ \\
\end{tabular} & $1.06 \mathrm{E}-05$ & $1.04 \mathrm{E}-05$ & $8.26 \mathrm{E}-03$ & $1.54 \mathrm{E}-02$ & $2.54 \mathrm{E}-02$ & $2.12 \mathrm{E}-02$ & $2.12 \mathrm{E}-02$ \\
\hline $\mathrm{Cr}+3$ & $3.83 \mathrm{E}-03$ & \begin{tabular}{|l|}
$2.79 \mathrm{E}-03$ \\
\end{tabular} & 5.14E-07 & $5.05 \mathrm{E}-07$ & $3.31 \mathrm{E}-04$ & $7.46 \mathrm{E}-04$ & $1.12 \mathrm{E}-03$ & $9.64 \mathrm{E}-04$ & $9.64 \mathrm{E}-04$ \\
\hline $\mathrm{Co}+2$ & $1.69 \mathrm{E}-03$ & $1.23 \mathrm{E}-03$ & \begin{tabular}{|l|}
$2.41 \mathrm{E}-07$ \\
\end{tabular} & $2.37 \mathrm{E}-07$ & $2.81 \mathrm{E}-04$ & $3.50 \mathrm{E}-04$ & $5.76 \mathrm{E}-04$ & $4.81 \mathrm{E}-04$ & $4.81 \mathrm{E}-04$ \\
\hline Cs+ & $6.81 \mathrm{E}-05$ & \begin{tabular}{|l|}
$5.24 \mathrm{E}-05$ \\
\end{tabular} & \begin{tabular}{|l|}
$7.08 \mathrm{E}-08$ \\
\end{tabular} & $6.94 \mathrm{E}-08$ & $9.26 \mathrm{E}-05$ & $1.03 \mathrm{E}-04$ & $5.60 \mathrm{E}-06$ & $5.00 \mathrm{E}-05$ & $5.00 \mathrm{E}-05$ \\
\hline $\mathrm{Cu}+2$ & $3.97 \mathrm{E}-04$ & \begin{tabular}{|l|}
$2.89 \mathrm{E}-04$ \\
\end{tabular} & \begin{tabular}{|l|}
$6.11 \mathrm{E}-08$ \\
\end{tabular} & $5.99 \mathrm{E}-08$ & $4.76 \mathrm{E}-05$ & $8.86 \mathrm{E}-05$ & 1.46E-04 & $1.22 \mathrm{E}-04$ & $1.22 \mathrm{E}-04$ \\
\hline $\mathrm{Fe}+3$ & $2.06 \mathrm{E}-02$ & \begin{tabular}{|l|}
$1.49 \mathrm{E}-02$ \\
\end{tabular} & \begin{tabular}{|l|}
$1.96 \mathrm{E}-06$ \\
\end{tabular} & $1.92 \mathrm{E}-06$ & $1.37 \mathrm{E}-03$ & $2.84 \mathrm{E}-03$ & $8.76 \mathrm{E}-03$ & $6.28 \mathrm{E}-03$ & $6.28 \mathrm{E}-03$ \\
\hline $\mathrm{Pb}+2$ & $5.43 \mathrm{E}-04$ & \begin{tabular}{|l|}
$3.95 \mathrm{E}-04$ \\
\end{tabular} & \begin{tabular}{|l|}
$8.54 \mathrm{E}-07$ \\
\end{tabular} & \begin{tabular}{|l|}
$8.38 \mathrm{E}-07$ \\
\end{tabular} & $2.36 \mathrm{E}-04$ & $1.24 \mathrm{E}-03$ & $4.14 \mathrm{E}-05$ & $5.44 \mathrm{E}-04$ & $5.44 \mathrm{E}-04$ \\
\hline $\mathrm{Hg}+2$ & $3.95 \mathrm{E}-03$ & \begin{tabular}{|l|}
$3.33 \mathrm{E}-03$ \\
\end{tabular} & \begin{tabular}{|l|}
$7.29 \mathrm{E}-06$ \\
\end{tabular} & \begin{tabular}{|l|}
$7.16 \mathrm{E}-06$ \\
\end{tabular} & $5.37 \mathrm{E}-06$ & $1.26 \mathrm{E}-05$ & & $5.29 \mathrm{E}-06$ & $5.29 \mathrm{E}-06$ \\
\hline$M n+4$ & $1.52 \mathrm{E}-02$ & $1.11 \mathrm{E}-02$ & $2.03 \mathrm{E}-06$ & $1.99 \mathrm{E}-06$ & $2.36 \mathrm{E}-03$ & $2.95 \mathrm{E}-03$ & $4.84 \mathrm{E}-03$ & $4.05 \mathrm{E}-03$ & $4.05 \mathrm{E}-03$ \\
\hline $\mathrm{Ni}+2$ & $1.15 \mathrm{E}-03$ & $8.33 \mathrm{E}-04$ & 1.63E-07 & $1.60 \mathrm{E}-07$ & $1.27 \mathrm{E}-04$ & $2.36 \mathrm{E}-04$ & $3.89 \mathrm{E}-04$ & $3.25 \mathrm{E}-04$ & $3.25 \mathrm{E}-04$ \\
\hline $\mathrm{K}+$ & $2.62 \mathrm{E}-01$ & $1.91 \mathrm{E}-01$ & $4.73 \mathrm{E}-05$ & $4.64 \mathrm{E}-05$ & $5.24 \mathrm{E}-02$ & $6.86 \mathrm{E}-02$ & $4.60 \mathrm{E}-02$ & $5.55 \mathrm{E}-02$ & $5.55 \mathrm{E}-02$ \\
\hline $\mathrm{Se}+4$ & $3.60 \mathrm{E}-04$ & \begin{tabular}{|l|}
$2.62 \mathrm{E}-04$ \\
\end{tabular} & \begin{tabular}{|l|}
$6.43 \mathrm{E}-08$ \\
\end{tabular} & $6.31 \mathrm{E}-08$ & $6.27 \mathrm{E}-05$ & $9.33 \mathrm{E}-05$ & $1.70 \mathrm{E}-04$ & $1.38 \mathrm{E}-04$ & $1.38 \mathrm{E}-04$ \\
\hline $\mathrm{Ag}^{+}$ & $5.47 \mathrm{E}-04$ & \begin{tabular}{|l|}
$3.98 \mathrm{E}-04$ \\
\end{tabular} & \begin{tabular}{|l|}
$1.63 \mathrm{E}-07$ \\
\end{tabular} & $1.60 \mathrm{E}-07$ & $1.17 \mathrm{E}-04$ & $2.35 \mathrm{E}-04$ & $3.23 \mathrm{E}-04$ & $2.86 \mathrm{E}-04$ & $2.86 \mathrm{E}-04$ \\
\hline $\mathrm{Na}+$ & $1.49 \mathrm{E}+00$ & \begin{tabular}{|l|}
$1.09 \mathrm{E}+00$ \\
\end{tabular} & \begin{tabular}{|c|}
$9.50 \mathrm{E}-05$ \\
\end{tabular} & $9.32 \mathrm{E}-05$ & $6.86 \mathrm{E}-02$ & $1.37 \mathrm{E}-01$ & \begin{tabular}{|l|}
$1.88 \mathrm{E}-01$ \\
\end{tabular} & $1.67 \mathrm{E}-01$ & 1.67E-01 \\
\hline $\mathrm{TI}+3$ & 4.87E-06 & \begin{tabular}{|l|}
$3.54 \mathrm{E}-06$ \\
\end{tabular} & \begin{tabular}{|l|}
$2.42 \mathrm{E}-09$ \\
\end{tabular} & \begin{tabular}{|l|}
$2.37 \mathrm{E}-09$ \\
\end{tabular} & $2.81 \mathrm{E}-06$ & $3.50 \mathrm{E}-06$ & $5.76 \mathrm{E}-06$ & $4.81 \mathrm{E}-06$ & $4.81 \mathrm{E}-06$ \\
\hline $\mathrm{U}+4$ & $2.29 \mathrm{E}-04$ & \begin{tabular}{|l|}
$1.66 \mathrm{E}-04$ \\
\end{tabular} & \begin{tabular}{|l|}
$1.23 \mathrm{E}-07$ \\
\end{tabular} & $1.21 \mathrm{E}-07$ & $1.20 \mathrm{E}-04$ & $1.79 \mathrm{E}-04$ & $3.25 \mathrm{E}-04$ & $2.63 \mathrm{E}-04$ & $2.63 \mathrm{E}-04$ \\
\hline $\mathrm{V}+3$ & $5.19 \mathrm{E}-05$ & \begin{tabular}{|l|}
$3.77 \mathrm{E}-05$ \\
\end{tabular} & \begin{tabular}{|l|}
$5.97 \mathrm{E}-09$ \\
\end{tabular} & $5.86 \mathrm{E}-09$ & $5.82 \mathrm{E}-06$ & $8.66 \mathrm{E}-06$ & 1.57E-05 & $1.28 \mathrm{E}-05$ & $1.28 \mathrm{E}-05$ \\
\hline$Z n+2$ & $6.42 \mathrm{E}-04$ & \begin{tabular}{|l|}
$4.67 \mathrm{E}-04$ \\
\end{tabular} & \begin{tabular}{|l|}
$1.02 \mathrm{E}-07$ \\
\end{tabular} & $9.98 \mathrm{E}-08$ & $7.92 \mathrm{E}-05$ & $1.48 \mathrm{E}-04$ & $2.43 \mathrm{E}-04$ & $2.03 \mathrm{E}-04$ & $2.03 \mathrm{E}-04$ \\
\hline $\mathrm{Zr}+4$ & $5.15 \mathrm{E}-02$ & \begin{tabular}{|l|}
$3.74 \mathrm{E}-02$ \\
\end{tabular} & \begin{tabular}{|l|}
$1.06 \mathrm{E}-05$ \\
\end{tabular} & $1.04 \mathrm{E}-05$ & $1.04 \mathrm{E}-02$ & $1.54 \mathrm{E}-02$ & $2.80 \mathrm{E}-02$ & $2.27 \mathrm{E}-02$ & $2.27 \mathrm{E}-02$ \\
\hline $\mathrm{Cl}-$ & $1.50 \mathrm{E}-02$ & \begin{tabular}{|l|}
$1.10 \mathrm{E}-02$ \\
\end{tabular} & $3.15 \mathrm{E}-06$ & $3.09 \mathrm{E}-06$ & $2.60 \mathrm{E}-03$ & $4.44 \mathrm{E}-03$ & $1.17 \mathrm{E}-03$ & $2.54 \mathrm{E}-03$ & $2.54 \mathrm{E}-03$ \\
\hline F- & $4.60 \mathrm{E}-02$ & \begin{tabular}{|l|}
$3.43 \mathrm{E}-02$ \\
\end{tabular} & \begin{tabular}{|l|}
$6.39 \mathrm{E}-06$ \\
\end{tabular} & $6.27 \mathrm{E}-06$ & $3.18 \mathrm{E}-03$ & $8.36 \mathrm{E}-03$ & $7.68 \mathrm{E}-04$ & $3.95 \mathrm{E}-03$ & $3.95 \mathrm{E}-03$ \\
\hline SO4-2 & $4.21 \mathrm{E}-02$ & \begin{tabular}{|l|}
$3.15 \mathrm{E}-02$ \\
\end{tabular} & \begin{tabular}{|l|}
$1.44 \mathrm{E}-08$ \\
\end{tabular} & $1.42 \mathrm{E}-08$ & $6.61 \mathrm{E}-06$ & $2.10 \mathrm{E}-05$ & $1.57 \mathrm{E}-05$ & $1.79 \mathrm{E}-05$ & $1.79 \mathrm{E}-05$ \\
\hline $\mathrm{NO}^{3}$ & & $3.70 \mathrm{E}+00$ & \begin{tabular}{|l|}
$8.76 \mathrm{E}-06$ \\
\end{tabular} & \begin{tabular}{|l|}
$8.60 \mathrm{E}-06$ \\
\end{tabular} & & $3.14 \mathrm{E}-03$ & $3.56 \mathrm{E}-03$ & $3.39 \mathrm{E}-03$ & $3.39 \mathrm{E}-03$ \\
\hline PO4-3 & $1.35 \mathrm{E}-01$ & \begin{tabular}{|c|}
$9.82 \mathrm{E}-02$ \\
\end{tabular} & \begin{tabular}{|l|}
$3.69 \mathrm{E}-05$ \\
\end{tabular} & \begin{tabular}{|l|}
$3.62 \mathrm{E}-05$ \\
\end{tabular} & $6.09 \mathrm{E}-02$ & $5.35 \mathrm{E}-02$ & $7.14 \mathrm{E}-02$ & $6.39 \mathrm{E}-02$ & $6.39 \mathrm{E}-02$ \\
\hline $\mathrm{Am}+4$ & $5.83 \mathrm{E}-08$ & \begin{tabular}{|l|}
$4.24 \mathrm{E}-08$ \\
\end{tabular} & \begin{tabular}{|l|}
$3.43 \mathrm{E}-11$ \\
\end{tabular} & $3.37 \mathrm{E}-11$ & $4.00 \mathrm{E}-08$ & $4.98 \mathrm{E}-08$ & $8.19 \mathrm{E}-08$ & $6.85 \mathrm{E}-08$ & $6.85 \mathrm{E}-08$ \\
\hline $\mathrm{Br}-$ & $2.10 \mathrm{E}-07$ & \begin{tabular}{|c|}
$1.54 \mathrm{E}-07$ \\
\end{tabular} & \begin{tabular}{|l|}
$9.91 \mathrm{E}-11$ \\
\end{tabular} & $9.72 \mathrm{E}-11$ & $8.19 \mathrm{E}-08$ & $1.40 \mathrm{E}-07$ & $3.68 \mathrm{E}-08$ & $8.01 \mathrm{E}-08$ & $8.01 \mathrm{E}-08$ \\
\hline $\mathrm{Ce}+4$ & $3.64 \mathrm{E}-05$ & \begin{tabular}{|l|}
$2.65 \mathrm{E}-05$ \\
\end{tabular} & \begin{tabular}{|c|}
$1.24 \mathrm{E}-08$ \\
\end{tabular} & $1.21 \mathrm{E}-08$ & 1.44E-05 & $1.80 \mathrm{E}-05$ & $2.95 \mathrm{E}-05$ & $2.47 \mathrm{E}-05$ & $2.47 \mathrm{E}-05$ \\
\hline $\mathrm{Eu}+3$ & $3.49 \mathrm{E}-07$ & \begin{tabular}{|l|}
$2.53 \mathrm{E}-07$ \\
\end{tabular} & \begin{tabular}{|l|}
$1.29 \mathrm{E}-10$ \\
\end{tabular} & $1.26 \mathrm{E}-10$ & $1.50 \mathrm{E}-07$ & $1.86 \mathrm{E}-07$ & $3.07 \mathrm{E}-07$ & $2.56 \mathrm{E}-07$ & $2.56 \mathrm{E}-07$ \\
\hline $\mathrm{Gd}+3$ & $1.27 \mathrm{E}-04$ & \begin{tabular}{|c|}
$9.22 \mathrm{E}-05$ \\
\end{tabular} & $4.84 \mathrm{E}-08$ & $4.75 \mathrm{E}-08$ & $5.64 \mathrm{E}-05$ & $7.02 \mathrm{E}-05$ & $1.15 \mathrm{E}-04$ & $9.64 \mathrm{E}-05$ & $9.64 \mathrm{E}-05$ \\
\hline $\mathrm{Ge}+4$ & $6.05 \mathrm{E}-09$ & \begin{tabular}{|l|}
$4.40 \mathrm{E}-09$ \\
\end{tabular} & \begin{tabular}{|c|}
$9.92 \mathrm{E}-13$ \\
\end{tabular} & $9.74 \mathrm{E}-13$ & $9.68 \mathrm{E}-10$ & $1.44 \mathrm{E}-09$ & \begin{tabular}{|r|}
$2.62 \mathrm{E}-09$ \\
\end{tabular} & $2.12 \mathrm{E}-09$ & $2.12 \mathrm{E}-09$ \\
\hline $\ln +3$ & $1.01 \mathrm{E}-06$ & \begin{tabular}{|l|}
$7.36 \mathrm{E}-07$ \\
\end{tabular} & \begin{tabular}{|l|}
$2.82 \mathrm{E}-10$ \\
\end{tabular} & \begin{tabular}{|l|}
$2.77 \mathrm{E}-10$ \\
\end{tabular} & $3.28 \mathrm{E}-07$ & $4.09 \mathrm{E}-07$ & $6.72 \mathrm{E}-07$ & $5.62 \mathrm{E}-07$ & $5.62 \mathrm{E}-07$ \\
\hline I- & $2.73 \mathrm{E}-06$ & \begin{tabular}{|l|}
$2.00 \mathrm{E}-06$ \\
\end{tabular} & \begin{tabular}{|l|}
$2.05 \mathrm{E}-09$ \\
\end{tabular} & $2.01 \mathrm{E}-09$ & 1.69E-06 & $2.89 \mathrm{E}-06$ & $7.61 \mathrm{E}-07$ & $1.66 \mathrm{E}-06$ & $1.66 \mathrm{E}-06$ \\
\hline $\mathrm{La}+3$ & $6.32 \mathrm{E}-06$ & \begin{tabular}{|l|}
$4.59 \mathrm{E}-06$ \\
\end{tabular} & \begin{tabular}{|l|}
$2.13 \mathrm{E}-09$ \\
\end{tabular} & $2.09 \mathrm{E}-09$ & $2.48 \mathrm{E}-06$ & $3.09 \mathrm{E}-06$ & $5.08 \mathrm{E}-06$ & $4.24 \mathrm{E}-06$ & $4.24 \mathrm{E}-06$ \\
\hline
\end{tabular}


Table A-14. Mass balance, Tank WM-187 waste (continued).

\begin{tabular}{|c|c|c|c|c|c|c|c|c|c|}
\hline Stream \# & 101 & 102 & 103 & 104 & 105 & 106 & 107 & 108 & 108 \\
\hline Chemical Composition & Mol/liter & Mol/liter & $\mathrm{lb} /$ wscf & $\mathrm{lb} /$ wscf & Wt frac & Wt frac & Wt frac & Wt frac & Wt frac \\
\hline $\mathrm{Li+}$ & $5.32 \mathrm{E}-04$ & $3.87 \mathrm{E}-04$ & $1.02 \mathrm{E}-08$ & $9.98 \mathrm{E}-09$ & $7.35 \mathrm{E}-06$ & $1.47 \mathrm{E}-05$ & $2.02 \mathrm{E}-05$ & $1.79 \mathrm{E}-05$ & $1.79 \mathrm{E}-05$ \\
\hline $\mathrm{Mg}+2$ & $1.44 \mathrm{E}-02$ & $1.05 \mathrm{E}-02$ & $8.48 \mathrm{E}-07$ & $8.32 \mathrm{E}-07$ & $6.61 \mathrm{E}-04$ & $1.23 \mathrm{E}-03$ & $2.03 \mathrm{E}-03$ & 1.69E-03 & $1.69 \mathrm{E}-03$ \\
\hline $\mathrm{Mo}+6$ & $7.88 \mathrm{E}-02$ & $5.73 \mathrm{E}-02$ & $1.71 \mathrm{E}-05$ & $1.68 \mathrm{E}-05$ & $1.67 \mathrm{E}-02$ & $2.48 \mathrm{E}-02$ & $4.51 \mathrm{E}-02$ & $3.66 \mathrm{E}-02$ & $3.66 \mathrm{E}-02$ \\
\hline $\mathrm{Nd}+3$ & $2.04 \mathrm{E}-05$ & $1.48 \mathrm{E}-05$ & $7.13 \mathrm{E}-09$ & $7.00 \mathrm{E}-09$ & $8.30 \mathrm{E}-06$ & $1.03 \mathrm{E}-05$ & $1.70 \mathrm{E}-05$ & $1.42 \mathrm{E}-05$ & $1.42 \mathrm{E}-05$ \\
\hline $\mathrm{Np}+4$ & $3.21 \mathrm{E}-06$ & $2.33 \mathrm{E}-06$ & 1.84E-09 & $1.81 \mathrm{E}-09$ & $2.15 \mathrm{E}-06$ & $2.67 \mathrm{E}-06$ & $4.40 \mathrm{E}-06$ & $3.67 \mathrm{E}-06$ & $3.67 \mathrm{E}-06$ \\
\hline $\mathrm{Nb}+5$ & $1.43 \mathrm{E}-03$ & 1.04E-03 & $3.01 \mathrm{E}-07$ & $2.96 \mathrm{E}-07$ & $2.94 \mathrm{E}-04$ & $4.37 E-04$ & 7.94E-04 & $6.44 \mathrm{E}-04$ & $6.44 \mathrm{E}-04$ \\
\hline $\mathrm{Pd}+4$ & $2.34 \mathrm{E}-03$ & $1.70 \mathrm{E}-03$ & $6.03 \mathrm{E}-07$ & $5.92 \mathrm{E}-07$ & $4.70 \mathrm{E}-04$ & $8.75 \mathrm{E}-04$ & $1.44 \mathrm{E}-03$ & $1.20 \mathrm{E}-03$ & $1.20 \mathrm{E}-03$ \\
\hline $\mathrm{Pu}+4$ & $7.51 \mathrm{E}-06$ & $5.46 \mathrm{E}-06$ & $4.14 \mathrm{E}-09$ & $4.06 \mathrm{E}-09$ & $4.04 \mathrm{E}-06$ & $6.01 \mathrm{E}-06$ & $1.09 \mathrm{E}-05$ & $8.86 \mathrm{E}-06$ & $8.86 \mathrm{E}-06$ \\
\hline $\mathrm{Pr}+4$ & $5.75 \mathrm{E}-06$ & $4.18 \mathrm{E}-06$ & 1.96E-09 & 1.93E-09 & $2.29 \mathrm{E}-06$ & $2.85 \mathrm{E}-06$ & $4.68 \mathrm{E}-06$ & $3.91 \mathrm{E}-06$ & $3.91 \mathrm{E}-06$ \\
\hline $\mathrm{Pm}+3$ & $1.40 \mathrm{E}-09$ & 1.02E-09 & $4.92 \mathrm{E}-13$ & $4.83 \mathrm{E}-13$ & $5.73 \mathrm{E}-10$ & $7.14 \mathrm{E}-10$ & 1.17E-09 & $9.81 \mathrm{E}-10$ & $9.81 \mathrm{E}-10$ \\
\hline $\mathrm{Rh}+4$ & $2.48 \mathrm{E}-06$ & 1.80E-06 & $6.19 \mathrm{E}-10$ & $6.07 \mathrm{E}-10$ & $7.21 \mathrm{E}-07$ & 8.98E-07 & 1.48E-06 & $1.23 E-06$ & $1.23 \mathrm{E}-06$ \\
\hline $\mathrm{Rb}+$ & 3.82E-06 & $2.77 \mathrm{E}-06$ & $8.98 \mathrm{E}-10$ & $8.81 \mathrm{E}-10$ & 6.49E-07 & $1.30 \mathrm{E}-06$ & 1.78E-06 & $1.58 \mathrm{E}-06$ & 1.58E-06 \\
\hline$R u+3$ & $1.07 \mathrm{E}-03$ & $7.78 \mathrm{E}-04$ & $2.62 \mathrm{E}-07$ & $2.57 \mathrm{E}-07$ & $3.06 \mathrm{E}-04$ & 3.81E-04 & $6.26 \mathrm{E}-04$ & 5.23E-04 & $5.23 \mathrm{E}-04$ \\
\hline $\mathrm{Sm}+3$ & $3.81 \mathrm{E}-06$ & $2.77 \mathrm{E}-06$ & 1.39E-09 & $1.36 \mathrm{E}-09$ & $1.62 \mathrm{E}-06$ & $2.02 \mathrm{E}-06$ & $3.32 \mathrm{E}-06$ & $2.77 \mathrm{E}-06$ & $2.77 \mathrm{E}-06$ \\
\hline $\mathrm{Si}+4$ & $1.23 \mathrm{E}-01$ & $8.91 \mathrm{E}-02$ & $9.56 \mathrm{E}-06$ & $9.38 \mathrm{E}-06$ & $1.24 \mathrm{E}-02$ & $1.39 \mathrm{E}-02$ & $2.76 \mathrm{E}-02$ & $2.19 \mathrm{E}-02$ & $2.19 \mathrm{E}-02$ \\
\hline $\mathrm{Sr}+2$ & $8.35 \mathrm{E}-05$ & $6.08 \mathrm{E}-05$ & $1.77 \mathrm{E}-08$ & $1.74 \mathrm{E}-08$ & $1.38 \mathrm{E}-05$ & $2.57 \mathrm{E}-05$ & $4.24 \mathrm{E}-05$ & $3.54 \mathrm{E}-05$ & $3.54 \mathrm{E}-05$ \\
\hline Tc+7 & $6.12 \mathrm{E}-06$ & $4.45 \mathrm{E}-06$ & $4.30 \mathrm{E}-09$ & $4.22 \mathrm{E}-09$ & $6.52 \mathrm{E}-06$ & $6.24 \mathrm{E}-06$ & $4.89 \mathrm{E}-07$ & $2.90 \mathrm{E}-06$ & $2.90 \mathrm{E}-06$ \\
\hline $\mathrm{Te}+4$ & $3.28 \mathrm{E}-06$ & $2.38 \mathrm{E}-06$ & $9.45 \mathrm{E}-10$ & $9.28 \mathrm{E}-10$ & $9.22 \mathrm{E}-07$ & 1.37E-06 & $2.49 \mathrm{E}-06$ & $2.02 \mathrm{E}-06$ & $2.02 \mathrm{E}-06$ \\
\hline $\mathrm{Tb}+4$ & $1.45 \mathrm{E}-09$ & $1.06 \mathrm{E}-09$ & $5.61 \mathrm{E}-13$ & $5.50 \mathrm{E}-13$ & $6.53 \mathrm{E}-10$ & $8.13 \mathrm{E}-10$ & 1.34E-09 & 1.12E-09 & 1.12E-09 \\
\hline $\mathrm{Th}+4$ & 1.72E-05 & $1.25 \mathrm{E}-05$ & 9.01E-09 & 8.84E-09 & 8.79E-06 & $1.31 \mathrm{E}-05$ & $2.38 \mathrm{E}-05$ & 1.93E-05 & 1.93E-05 \\
\hline$S n+4$ & $2.49 \mathrm{E}-03$ & $1.81 \mathrm{E}-03$ & $6.69 \mathrm{E}-07$ & $6.57 \mathrm{E}-07$ & $6.53 \mathrm{E}-04$ & 9.71E-04 & $1.76 \mathrm{E}-03$ & $1.43 \mathrm{E}-03$ & $1.43 \mathrm{E}-03$ \\
\hline $\mathrm{Ti}+4$ & $1.26 \mathrm{E}-03$ & $9.15 \mathrm{E}-04$ & $1.36 \mathrm{E}-07$ & 1.34E-07 & 1.33E-04 & 1.97E-04 & 3.59E-04 & $2.91 \mathrm{E}-04$ & $2.91 \mathrm{E}-04$ \\
\hline$Y+3$ & $4.71 \mathrm{E}-06$ & $3.42 \mathrm{E}-06$ & $1.02 \mathrm{E}-09$ & $9.97 \mathrm{E}-10$ & $1.18 \mathrm{E}-06$ & $1.47 \mathrm{E}-06$ & $2.42 \mathrm{E}-06$ & $2.02 \mathrm{E}-06$ & $2.02 \mathrm{E}-06$ \\
\hline $\mathrm{OH}-$ & & 5.22E-04 & $2.55 \mathrm{E}-06$ & $2.50 \mathrm{E}-06$ & $1.88 \mathrm{E}-03$ & 3.70E-03 & 5.51E-03 & $4.75 \mathrm{E}-03$ & $4.75 \mathrm{E}-03$ \\
\hline $\mathrm{H} 2 \mathrm{O}$ & $4.26 \mathrm{E}+01$ & $3.32 E+01$ & $3.28 \mathrm{E}-02$ & $3.21 \mathrm{E}-02$ & 1.09E-03 & $2.09 \mathrm{E}-03$ & 3.08E-03 & 2.67E-03 & 2.67E-03 \\
\hline $\mathrm{SO} 2$ & & $3.88 \mathrm{E}-07$ & $2.69 \mathrm{E}-09$ & $2.64 \mathrm{E}-09$ & & & & & \\
\hline $\mathrm{H} 2 \mathrm{~S}$ & & & $1.19 \mathrm{E}-05$ & $1.16 \mathrm{E}-05$ & & & & & \\
\hline $\mathrm{CO}$ & & $8.19 \mathrm{E}-12$ & $7.79 \mathrm{E}-04$ & 7.64E-04 & & & & & \\
\hline $\mathrm{CO} 2$ & & $1.30 \mathrm{E}-05$ & $7.52 \mathrm{E}-03$ & $7.38 \mathrm{E}-03$ & & & & & \\
\hline $\mathrm{H} 2$ & & $3.12 \mathrm{E}-11$ & $2.10 \mathrm{E}-04$ & $2.06 \mathrm{E}-04$ & & & & & \\
\hline $\mathrm{N} 2$ & & $5.46 \mathrm{E}-07$ & $1.28 \mathrm{E}-02$ & $1.39 \mathrm{E}-02$ & & & & & \\
\hline $\mathrm{NO}$ & & 9.86E-09 & 4.79E-05 & $4.70 \mathrm{E}-05$ & & & & & \\
\hline NO2 & & $1.25 \mathrm{E}-07$ & $4.97 \mathrm{E}-06$ & $4.87 \mathrm{E}-06$ & & & & & \\
\hline $\mathrm{O} 2$ & & $1.37 \mathrm{E}-07$ & $4.35 \mathrm{E}-04$ & $4.27 \mathrm{E}-04$ & & & & & \\
\hline S (other) & & $5.21 \mathrm{E}-09$ & 1.87E-07 & $1.84 \mathrm{E}-07$ & 1.38E-04 & 2.72E-04 & $4.06 \mathrm{E}-04$ & $3.50 \mathrm{E}-04$ & $3.50 \mathrm{E}-04$ \\
\hline CO3 & 3.40E-05 & $2.62 \mathrm{E}-05$ & $1.57 \mathrm{E}-04$ & $1.54 \mathrm{E}-04$ & $1.30 \mathrm{E}-01$ & $2.28 \mathrm{E}-01$ & $3.02 \mathrm{E}-01$ & $2.71 \mathrm{E}-01$ & $2.71 \mathrm{E}-01$ \\
\hline C (reductant) & & & 2.23E-05 & $2.18 \mathrm{E}-05$ & 3.84E-01 & $3.23 E-02$ & & $1.35 \mathrm{E}-02$ & $1.35 \mathrm{E}-02$ \\
\hline $\mathrm{O}$ (oxides) & $6.36 \mathrm{E}-01$ & $4.62 \mathrm{E}-01$ & $4.05 \mathrm{E}-05$ & 3.97E-05 & 4.07E-02 & $5.88 \mathrm{E}-02$ & 1.11E-01 & 8.91E-02 & 8.91E-02 \\
\hline C (organic) & $1.71 \mathrm{E}-01$ & $1.01 \mathrm{E}+01$ & $9.43 \mathrm{E}-05$ & $9.25 \mathrm{E}-05$ & $6.51 \mathrm{E}-02$ & $1.10 \mathrm{E}-01$ & $7.94 \mathrm{E}-03$ & $5.08 \mathrm{E}-02$ & $5.08 \mathrm{E}-02$ \\
\hline $\mathrm{H}$ (organic) & $3.14 \mathrm{E}-01$ & $1.86 \mathrm{E}+01$ & $1.79 \mathrm{E}-05$ & $1.75 \mathrm{E}-05$ & $1.00 \mathrm{E}-02$ & $1.69 \mathrm{E}-02$ & $1.22 \mathrm{E}-03$ & $7.81 \mathrm{E}-03$ & $7.81 \mathrm{E}-03$ \\
\hline O (organic) & $1.57 \mathrm{E}-01$ & $9.28 \mathrm{E}+00$ & $9.26 \mathrm{E}-05$ & $9.09 \mathrm{E}-05$ & $7.95 \mathrm{E}-02$ & $1.34 \mathrm{E}-01$ & $9.69 \mathrm{E}-03$ & \begin{tabular}{|l|}
$6.20 \mathrm{E}-02$ \\
\end{tabular} & $6.20 \mathrm{E}-02$ \\
\hline Mass Flow $(\mathrm{kg} / \mathrm{hr})$ : & $4.36 \mathrm{E}+02$ & $6.00 \mathrm{E}+02$ & $2.53 E+03$ & $2.59 \mathrm{E}+03$ & $5.03 E+01$ & $3.14 \mathrm{E}+01$ & $4.35 E+01$ & $7.50 \mathrm{E}+01$ & $7.50 \mathrm{E}+01$ \\
\hline Total Canisters Generated & & & & & & & & 148 & \\
\hline & & & & & & & & & \\
\hline & $w / m 3$ & $w / m 3$ & $w / m 3$ & w/m3 & $\mathrm{w} / \mathrm{m} 3$ & $\mathrm{w} / \mathrm{m} 3$ & $\mathrm{w} / \mathrm{m} 3$ & $\mathrm{w} / \mathrm{m} 3$ & w/Canistel \\
\hline Heat Generation, w/m3 & $4.01 \mathrm{E}-01$ & $2.93 \mathrm{E}-01$ & & & $2.96 \mathrm{E}-01$ & $1.96 \mathrm{E}+00$ & $3.11 \mathrm{E}+00$ & $2.62 \mathrm{E}+00$ & $2.09 \mathrm{E}+00$ \\
\hline
\end{tabular}


Table A-14. Mass balance, Tank WM-187 waste (continued).

\begin{tabular}{|c|c|c|c|c|c|c|c|c|c|}
\hline Stream \# & 101 & 102 & 103 & 104 & 105 & 106 & 107 & 108 & 108 \\
\hline Radiological Composition & $\mathrm{Ci} / \mathrm{L}$ & $\mathrm{Ci} / \mathrm{L}$ & Ci/wscm & Ci/wscm & $\mathrm{Ci} / \mathrm{kg}$ & $\mathrm{Ci} / \mathrm{kg}$ & $\mathrm{Ci} / \mathrm{kg}$ & $\mathrm{Ci} / \mathrm{kg}$ & Ci/canister \\
\hline $\mathrm{H}-3$ & 9.35E-06 & $6.79 \mathrm{E}-06$ & 1.19E-06 & 1.17E-06 & & & & & \\
\hline $\mathrm{C}-14$ & $1.35 \mathrm{E}-10$ & $9.81 \mathrm{E}-11$ & $1.67 \mathrm{E}-11$ & $1.64 \mathrm{E}-11$ & $1.96 \mathrm{E}-10$ & $7.75 \mathrm{E}-11$ & $2.82 \mathrm{E}-11$ & $4.89 \mathrm{E}-11$ & $5.21 \mathrm{E}-08$ \\
\hline Co-60 & 1.45E-05 & 1.05E-05 & $5.63 \mathrm{E}-07$ & $5.52 \mathrm{E}-07$ & 4.09E-05 & 5.09E-05 & 8.38E-05 & $7.00 \mathrm{E}-05$ & $7.45 \mathrm{E}-02$ \\
\hline \begin{tabular}{|l|}
$\mathrm{Ni}-59$ \\
\end{tabular} & $1.59 \mathrm{E}-06$ & $1.16 \mathrm{E}-06$ & $6.16 \mathrm{E}-08$ & $6.05 \mathrm{E}-08$ & $3.00 \mathrm{E}-06$ & $5.58 \mathrm{E}-06$ & $9.20 \mathrm{E}-06$ & $7.68 \mathrm{E}-06$ & $8.18 \mathrm{E}-03$ \\
\hline $\mathrm{Ni}-63$ & $3.70 \mathrm{E}-05$ & $2.69 \mathrm{E}-05$ & $1.43 \mathrm{E}-06$ & $1.41 \mathrm{E}-06$ & 6.97E-05 & $1.30 \mathrm{E}-04$ & $2.14 \mathrm{E}-04$ & $1.79 \mathrm{E}-04$ & $1.90 \mathrm{E}-01$ \\
\hline Se-79 & $4.90 \mathrm{E}-07$ & $3.56 \mathrm{E}-07$ & 1.77E-08 & $1.74 \mathrm{E}-08$ & 1.08E-06 & $1.60 \mathrm{E}-06$ & $2.92 \mathrm{E}-06$ & $2.37 \mathrm{E}-06$ & $2.52 \mathrm{E}-03$ \\
\hline Sr-90 & $2.77 \mathrm{E}-02$ & $2.02 \mathrm{E}-02$ & $1.08 \mathrm{E}-03$ & $1.06 \mathrm{E}-03$ & $5.23 \mathrm{E}-02$ & $9.74 \mathrm{E}-02$ & 1.61E-01 & $1.34 \mathrm{E}-01$ & $1.43 \mathrm{E}+02$ \\
\hline Y-90 & $2.77 \mathrm{E}-02$ & $2.02 \mathrm{E}-02$ & 1.08E-03 & 1.06E-03 & 7.84E-02 & 9.76E-02 & $1.60 \mathrm{E}-01$ & 1.34E-01 & $1.43 \mathrm{E}+02$ \\
\hline Zr-93 & $1.56 \mathrm{E}-06$ & $1.14 \mathrm{E}-06$ & $5.66 \mathrm{E}-08$ & $5.55 \mathrm{E}-08$ & $3.45 \mathrm{E}-06$ & $5.13 \mathrm{E}-06$ & 9.32E-06 & $7.56 \mathrm{E}-06$ & 8.05E-03 \\
\hline $\mathrm{Nb}-93 \mathrm{~m}$ & $1.20 \mathrm{E}-06$ & $8.76 \mathrm{E}-07$ & $4.36 \mathrm{E}-08$ & $4.28 \mathrm{E}-08$ & $2.65 \mathrm{E}-06$ & $3.95 \mathrm{E}-06$ & $7.18 \mathrm{E}-06$ & $5.82 \mathrm{E}-06$ & $6.20 \mathrm{E}-03$ \\
\hline $\mathrm{Nb}-94$ & $1.27 \mathrm{E}-06$ & $9.24 \mathrm{E}-07$ & $4.60 \mathrm{E}-08$ & $4.52 \mathrm{E}-08$ & $2.80 \mathrm{E}-06$ & 4.17E-06 & 7.58E-06 & $6.15 \mathrm{E}-06$ & $6.55 \mathrm{E}-03$ \\
\hline Tc-99 & $1.47 \mathrm{E}-05$ & $1.07 \mathrm{E}-05$ & $1.69 \mathrm{E}-06$ & $1.66 \mathrm{E}-06$ & 1.60E-04 & $1.53 \mathrm{E}-04$ & $1.20 \mathrm{E}-05$ & $7.12 \mathrm{E}-05$ & $7.58 \mathrm{E}-02$ \\
\hline Ru-106 & $1.05 \mathrm{E}-06$ & $7.62 \mathrm{E}-07$ & 4.07E-08 & $4.00 \mathrm{E}-08$ & $2.96 \mathrm{E}-06$ & $3.69 \mathrm{E}-06$ & $6.06 \mathrm{E}-06$ & 5.07E-06 & $5.40 \mathrm{E}-03$ \\
\hline Rh-102 & $6.08 \mathrm{E}-10$ & $4.42 \mathrm{E}-10$ & $2.36 \mathrm{E}-11$ & $2.32 \mathrm{E}-11$ & 1.72E-09 & 2.14E-09 & 3.52E-09 & 2.94E-09 & $3.13 \mathrm{E}-06$ \\
\hline Rh-106 & $1.05 \mathrm{E}-06$ & $7.62 \mathrm{E}-07$ & 4.07E-08 & $4.00 \mathrm{E}-08$ & $2.96 \mathrm{E}-06$ & 3.69E-06 & $6.06 \mathrm{E}-06$ & 5.07E-06 & $5.40 \mathrm{E}-03$ \\
\hline $\mathrm{Pd}-107$ & 1.17E-08 & 8.48E-09 & $4.52 \mathrm{E}-10$ & $4.44 \mathrm{E}-10$ & $2.20 \mathrm{E}-08$ & $4.10 \mathrm{E}-08$ & $6.75 \mathrm{E}-08$ & 5.64E-08 & $6.00 \mathrm{E}-05$ \\
\hline Cd-113m & $2.34 \mathrm{E}-06$ & $1.70 \mathrm{E}-06$ & 9.93E-08 & $9.75 \mathrm{E}-08$ & $5.64 \mathrm{E}-06$ & $9.00 \mathrm{E}-06$ & $1.30 \mathrm{E}-05$ & $1.13 \mathrm{E}-05$ & $1.21 \mathrm{E}-02$ \\
\hline Sn-121m & $4.72 \mathrm{E}-08$ & $3.43 \mathrm{E}-08$ & 1.71E-09 & 1.68E-09 & 1.04E-07 & $1.55 \mathrm{E}-07$ & $2.81 \mathrm{E}-07$ & $2.28 \mathrm{E}-07$ & $2.43 E-04$ \\
\hline Sn-126 & $4.61 \mathrm{E}-07$ & $3.35 \mathrm{E}-07$ & 1.67E-08 & $1.64 \mathrm{E}-08$ & $1.02 \mathrm{E}-06$ & $1.51 \mathrm{E}-06$ & $2.75 \mathrm{E}-06$ & $2.23 \mathrm{E}-06$ & $2.37 \mathrm{E}-03$ \\
\hline Sb-125 & $5.53 \mathrm{E}-04$ & $4.02 \mathrm{E}-04$ & $2.15 \mathrm{E}-05$ & $2.11 \mathrm{E}-05$ & $1.56 \mathrm{E}-03$ & $1.94 \mathrm{E}-03$ & $3.20 \mathrm{E}-03$ & $2.67 \mathrm{E}-03$ & $2.84 \mathrm{E}+00$ \\
\hline Sb-126 & $4.06 \mathrm{E}-08$ & $2.95 \mathrm{E}-08$ & $1.58 \mathrm{E}-09$ & 1.55E-09 & 1.15E-07 & 1.43E-07 & $2.35 \mathrm{E}-07$ & 1.96E-07 & $2.09 \mathrm{E}-04$ \\
\hline Te-125m & $2.22 \mathrm{E}-06$ & 1.62E-06 & $8.05 \mathrm{E}-08$ & 7.89E-08 & 4.90E-06 & $7.29 \mathrm{E}-06$ & 1.32E-05 & 1.07E-05 & 1.14E-02 \\
\hline $\mid-129$ & $5.94 \mathrm{E}-08$ & $4.35 \mathrm{E}-08$ & $5.62 \mathrm{E}-09$ & 5.51E-09 & $2.90 \mathrm{E}-07$ & $4.96 \mathrm{E}-07$ & $1.30 \mathrm{E}-07$ & $2.83 \mathrm{E}-07$ & $3.02 \mathrm{E}-04$ \\
\hline Cs-134 & 5.82E-05 & $4.48 \mathrm{E}-05$ & $7.29 \mathrm{E}-06$ & $7.15 \mathrm{E}-06$ & 5.95E-04 & 6.61E-04 & $3.60 \mathrm{E}-05$ & 3.22E-04 & $3.43 \mathrm{E}-01$ \\
\hline Cs-135 & $9.26 \mathrm{E}-07$ & $7.13 \mathrm{E}-07$ & 1.16E-07 & 1.14E-07 & $9.48 \mathrm{E}-06$ & 1.05E-05 & $5.73 \mathrm{E}-07$ & $5.12 \mathrm{E}-06$ & $5.45 \mathrm{E}-03$ \\
\hline Cs-137 & $3.76 \mathrm{E}-02$ & $2.89 \mathrm{E}-02$ & $4.71 \mathrm{E}-03$ & $4.62 \mathrm{E}-03$ & 3.84E-01 & 4.27E-01 & $2.33 \mathrm{E}-02$ & 2.08E-01 & $2.21 \mathrm{E}+02$ \\
\hline Ba-137m & $3.55 \mathrm{E}-02$ & $2.58 \mathrm{E}-02$ & $1.38 \mathrm{E}-03$ & $1.35 \mathrm{E}-03$ & 6.70E-02 & $1.25 \mathrm{E}-01$ & $2.06 \mathrm{E}-01$ & 1.72E-01 & $1.83 E+02$ \\
\hline $\mathrm{Ce}-144$ & $7.08 \mathrm{E}-07$ & $5.15 \mathrm{E}-07$ & $2.75 \mathrm{E}-08$ & $2.70 \mathrm{E}-08$ & $2.00 \mathrm{E}-06$ & $2.49 \mathrm{E}-06$ & $4.10 \mathrm{E}-06$ & $3.42 \mathrm{E}-06$ & $3.65 \mathrm{E}-03$ \\
\hline Pr-144 & $7.08 \mathrm{E}-07$ & $5.15 \mathrm{E}-07$ & $2.75 \mathrm{E}-08$ & $2.70 \mathrm{E}-08$ & $2.00 \mathrm{E}-06$ & $2.49 \mathrm{E}-06$ & $4.10 \mathrm{E}-06$ & $3.42 \mathrm{E}-06$ & $3.65 \mathrm{E}-03$ \\
\hline $\mathrm{Pm}-146$ & $3.59 \mathrm{E}-08$ & $2.61 \mathrm{E}-08$ & $1.40 \mathrm{E}-09$ & 1.37E-09 & $1.01 \mathrm{E}-07$ & $1.26 \mathrm{E}-07$ & $2.08 \mathrm{E}-07$ & $1.74 \mathrm{E}-07$ & $1.85 \mathrm{E}-04$ \\
\hline $\mathrm{Pm}-147$ & $1.91 \mathrm{E}-04$ & 1.39E-04 & $7.41 \mathrm{E}-06$ & $7.27 \mathrm{E}-06$ & 5.38E-04 & $6.71 \mathrm{E}-04$ & $1.10 \mathrm{E}-03$ & $9.22 \mathrm{E}-04$ & 9.81E-01 \\
\hline Sm-151 & 3.77E-04 & $2.74 \mathrm{E}-04$ & 1.47E-05 & $1.44 \mathrm{E}-05$ & 1.07E-03 & $1.33 \mathrm{E}-03$ & $2.18 \mathrm{E}-03$ & 1.82E-03 & $1.94 \mathrm{E}+00$ \\
\hline Eu-152 & $2.27 \mathrm{E}-06$ & $1.65 \mathrm{E}-06$ & $8.81 \mathrm{E}-08$ & $8.64 \mathrm{E}-08$ & $6.40 \mathrm{E}-06$ & $7.98 \mathrm{E}-06$ & $1.31 \mathrm{E}-05$ & $1.10 \mathrm{E}-05$ & $1.17 \mathrm{E}-02$ \\
\hline Eu-154 & 8.52E-05 & $6.19 \mathrm{E}-05$ & $3.31 \mathrm{E}-06$ & $3.25 \mathrm{E}-06$ & $2.41 \mathrm{E}-04$ & $3.00 \mathrm{E}-04$ & $4.93 E-04$ & $4.12 \mathrm{E}-04$ & 4.39E-01 \\
\hline Eu-155 & 8.97E-05 & 6.52E-05 & $3.49 \mathrm{E}-06$ & $3.42 E-06$ & 2.53E-04 & $3.16 \mathrm{E}-04$ & 5.19E-04 & 4.34E-04 & 4.62E-01 \\
\hline Th-230 & $84 \mathrm{E}-10$ & $6.43 \mathrm{E}-10$ & $3.20 \mathrm{E}-11$ & $3.14 \mathrm{E}-11$ & $1.95 \mathrm{E}-09$ & $2.90 \mathrm{E}-09$ & $5.27 \mathrm{E}-09$ & 4.27E-09 & $4.55 \mathrm{E}-06$ \\
\hline $\mathrm{Pa}-233$ & $2.07 \mathrm{E}-06$ & $1.50 \mathrm{E}-06$ & $7.49 \mathrm{E}-08$ & $7.35 \mathrm{E}-08$ & $4.56 \mathrm{E}-06$ & $6.78 \mathrm{E}-06$ & 1.23E-05 & $1.00 \mathrm{E}-05$ & $1.06 \mathrm{E}-02$ \\
\hline U-232 & 2.03E-09 & 1.48E-09 & $7.36 \mathrm{E}-11$ & $7.22 \mathrm{E}-11$ & 4.48E-09 & 6.67E-09 & $1.21 \mathrm{E}-08$ & 9.83E-09 & $1.05 \mathrm{E}-05$ \\
\hline U-233 & $6.73 \mathrm{E}-11$ & $4.89 \mathrm{E}-11$ & $2.44 \mathrm{E}-12$ & $2.39 \mathrm{E}-12$ & $1.48 \mathrm{E}-10$ & $2.21 \mathrm{E}-10$ & $4.01 \mathrm{E}-10$ & $3.25 \mathrm{E}-10$ & $3.46 \mathrm{E}-07$ \\
\hline U-234 & $1.11 \mathrm{E}-06$ & 8.08E-07 & $4.03 \mathrm{E}-08$ & $3.95 \mathrm{E}-08$ & $2.45 \mathrm{E}-06$ & $3.65 \mathrm{E}-06$ & 6.63E-06 & 5.38E-06 & $5.73 \mathrm{E}-03$ \\
\hline U-235 & 5.79E-08 & $4.21 \mathrm{E}-08$ & $2.10 \mathrm{E}-09$ & 2.06E-09 & $1.28 \mathrm{E}-07$ & $1.90 \mathrm{E}-07$ & $3.45 \mathrm{E}-07$ & 2.80E-07 & $2.98 \mathrm{E}-04$ \\
\hline U-236 & $5.39 \mathrm{E}-08$ & $3.92 \mathrm{E}-08$ & $1.95 \mathrm{E}-09$ & $1.91 \mathrm{E}-09$ & $1.19 \mathrm{E}-07$ & $1.77 \mathrm{E}-07$ & $3.21 \mathrm{E}-07$ & $2.61 \mathrm{E}-07$ & $2.78 \mathrm{E}-04$ \\
\hline U-237 & 4.54E-09 & 3.30E-09 & $1.64 \mathrm{E}-10$ & $1.61 \mathrm{E}-10$ & $1.00 \mathrm{E}-08$ & $1.49 \mathrm{E}-08$ & $2.71 \mathrm{E}-08$ & $2.20 \mathrm{E}-08$ & $2.34 \mathrm{E}-05$ \\
\hline U-238 & $1.96 \mathrm{E}-08$ & $1.42 \mathrm{E}-08$ & $7.09 \mathrm{E}-10$ & $6.95 \mathrm{E}-10$ & $4.32 \mathrm{E}-08$ & 6.42E-08 & 1.17E-07 & 9.47E-08 & $1.01 \mathrm{E}-04$ \\
\hline $\mathrm{Np}-237$ & 5.36E-07 & 3.89E-07 & $2.08 \mathrm{E}-08$ & $2.04 \mathrm{E}-08$ & $1.51 \mathrm{E}-06$ & $1.88 \mathrm{E}-06$ & $3.10 \mathrm{E}-06$ & $2.59 \mathrm{E}-06$ & $2.76 \mathrm{E}-03$ \\
\hline Pu-236 & 3.19E-09 & 2.32E-09 & $1.15 \mathrm{E}-10$ & $1.13 \mathrm{E}-10$ & 7.02E-09 & $1.04 \mathrm{E}-08$ & 1.90E-08 & 1.54E-08 & 1.64E-05 \\
\hline Pu-238 & $40 \mathrm{E}-04$ & $6.83 \mathrm{E}-04$ & $3.40 \mathrm{E}-05$ & 3.34E-05 & 2.07E-03 & $3.08 \mathrm{E}-03$ & $5.60 \mathrm{E}-03$ & 4.54E-03 & $4.84 \mathrm{E}+00$ \\
\hline Pu-239 & $1.04 \mathrm{E}-04$ & $7.59 \mathrm{E}-05$ & $3.78 \mathrm{E}-06$ & $3.71 \mathrm{E}-06$ & $2.30 \mathrm{E}-04$ & $3.42 \mathrm{E}-04$ & $6.22 \mathrm{E}-04$ & 5.05E-04 & 5.37E-01 \\
\hline Pu-240 & 1.19E-05 & 8.67E-06 & $4.32 \mathrm{E}-07$ & $4.23 E-07$ & 2.63E-05 & 3.91E-05 & 7.11E-05 & 5.76E-05 & $6.14 \mathrm{E}-02$ \\
\hline Pu-241 & $7.09 \mathrm{E}-04$ & $5.16 \mathrm{E}-04$ & $2.57 \mathrm{E}-05$ & $2.52 \mathrm{E}-05$ & $1.56 \mathrm{E}-03$ & $2.33 \mathrm{E}-03$ & $4.23 \mathrm{E}-03$ & $3.43 \mathrm{E}-03$ & $3.65 \mathrm{E}+00$ \\
\hline Pu-242 & 9.36E-09 & $6.80 \mathrm{E}-09$ & $3.39 \mathrm{E}-10$ & $3.32 \mathrm{E}-10$ & $2.06 \mathrm{E}-08$ & $3.07 \mathrm{E}-08$ & 5.57E-08 & $4.52 \mathrm{E}-08$ & 4.82E-05 \\
\hline Pu-244 & $5.60 \mathrm{E}-16$ & 4.07E-16 & $2.03 \mathrm{E}-17$ & $1.99 \mathrm{E}-17$ & $1.23 \mathrm{E}-15$ & $1.83 \mathrm{E}-15$ & 3.33E-15 & $2.71 \mathrm{E}-15$ & $2.88 \mathrm{E}-12$ \\
\hline Am-241 & 6.02E-05 & 4.37E-05 & $2.34 \mathrm{E}-06$ & $2.29 \mathrm{E}-06$ & $1.70 \mathrm{E}-04$ & 2.12E-04 & 3.48E-04 & 2.91E-04 & $3.10 \mathrm{E}-01$ \\
\hline Am-242m & 1.09E-08 & 7.92E-09 & $4.23 \mathrm{E}-10$ & $4.15 \mathrm{E}-10$ & 3.08E-08 & 3.83E-08 & $6.30 \mathrm{E}-08$ & 5.27E-08 & 5.61E-05 \\
\hline
\end{tabular}


Table A-14. Mass balance, Tank WM-187 waste (continued).

\begin{tabular}{|c|c|c|c|c|c|c|c|c|c|}
\hline Stream \# & 101 & 102 & 103 & 104 & 105 & 106 & 107 & 108 & 108 \\
\hline Radiological Composition & $\mathrm{Ci} / \mathrm{L}$ & $\mathrm{Ci} / \mathrm{L}$ & Ci/wscm & Ci/wscm & $\mathrm{Ci} / \mathrm{kg}$ & $\mathrm{Ci} / \mathrm{kg}$ & $\mathrm{Ci} / \mathrm{kg}$ & $\mathrm{Ci} / \mathrm{kg}$ & Ci/Canister \\
\hline Am-243 & 2.07E-08 & $1.50 \mathrm{E}-08$ & $8.03 E-10$ & $7.88 \mathrm{E}-10$ & $5.84 \mathrm{E}-08$ & $7.28 \mathrm{E}-08$ & $1.20 \mathrm{E}-07$ & 1.00E-07 & 1.06E-04 \\
\hline $\mathrm{Cm}-242$ & $2.14 \mathrm{E}-08$ & 1.55E-08 & $7.74 \mathrm{E}-10$ & $7.60 \mathrm{E}-10$ & $4.71 \mathrm{E}-08$ & 7.01E-08 & 1.27E-07 & 1.03E-07 & 1.10E-04 \\
\hline $\mathrm{Cm}-243$ & $3.10 \mathrm{E}-08$ & $2.25 \mathrm{E}-08$ & 1.12E-09 & 1.10E-09 & $6.82 \mathrm{E}-08$ & $1.01 \mathrm{E}-07$ & $1.84 \mathrm{E}-07$ & $1.50 \mathrm{E}-07$ & $1.59 \mathrm{E}-04$ \\
\hline $\mathrm{Cm}-244$ & 1.38E-06 & $1.00 \mathrm{E}-06$ & $4.99 \mathrm{E}-08$ & $4.90 \mathrm{E}-08$ & $3.04 \mathrm{E}-06$ & $4.52 \mathrm{E}-06$ & $8.22 \mathrm{E}-06$ & $6.67 \mathrm{E}-06$ & $7.10 \mathrm{E}-03$ \\
\hline $\mathrm{Cm}-245$ & $3.26 \mathrm{E}-10$ & $2.37 \mathrm{E}-10$ & $1.18 \mathrm{E}-11$ & $1.16 \mathrm{E}-11$ & $7.19 \mathrm{E}-10$ & 1.07E-09 & 1.94E-09 & $1.58 \mathrm{E}-09$ & $1.68 \mathrm{E}-06$ \\
\hline $\mathrm{Cm}-246$ & $2.14 \mathrm{E}-11$ & $1.55 \mathrm{E}-11$ & $7.73 \mathrm{E}-13$ & $7.59 \mathrm{E}-13$ & $4.71 \mathrm{E}-11$ & $7.01 \mathrm{E}-11$ & $1.27 \mathrm{E}-10$ & $1.03 \mathrm{E}-10$ & $1.10 \mathrm{E}-07$ \\
\hline TRU & 1.14E-03 & $8.27 \mathrm{E}-04$ & 4.14E-05 & 4.06E-05 & $2.55 \mathrm{E}-03$ & $3.75 \mathrm{E}-03$ & 6.77E-03 & $5.50 \mathrm{E}-03$ & $5.86 \mathrm{E}+00$ \\
\hline TRU, nCi/gm & $9.47 E+02$ & & & & & & & $5.50 \mathrm{E}+03$ & \\
\hline & & & & & & & & & \\
\hline $\begin{array}{l}\text { Gas Stream Bulk } \\
\text { Composition (Wet Basis) }\end{array}$ & $\begin{array}{c}\mathrm{mol} \% \text { or } \\
\mathrm{ppmv}\end{array}$ & $\begin{array}{c}\text { mol\% or } \\
\text { ppmv }\end{array}$ & $\begin{array}{l}\mathrm{mol} \% \text { or } \\
\text { ppmv }\end{array}$ & $\begin{array}{l}\text { mol\% or } \\
\text { ppmv }\end{array}$ & $\begin{array}{c}\mathrm{mol} \% \text { or } \\
\mathrm{ppmv}\end{array}$ & $\begin{array}{c}\mathrm{mol} \% \text { or } \\
\mathrm{ppmv}\end{array}$ & $\begin{array}{c}\mathrm{mol} \% \text { or } \\
\text { ppmv }\end{array}$ & $\begin{array}{c}\mathrm{mol} \% \text { or } \\
\text { ppmv }\end{array}$ & $\begin{array}{c}\mathrm{mol} \% \text { or } \\
\mathrm{ppmv}\end{array}$ \\
\hline $\mathrm{H} 2 \mathrm{O}, \mathrm{mol} \%$ & & & $69.88 \%$ & $68.57 \%$ & & & & & \\
\hline $\mathrm{O} 2, \mathrm{~mol} \%$ & & & $0.52 \%$ & $0.51 \%$ & & & & & \\
\hline $\mathrm{N} 2, \mathrm{~mol} \%$ & & & $17.54 \%$ & $19.09 \%$ & & & & & \\
\hline $\mathrm{H} 2, \mathrm{~mol} \%$ & & & $4.01 \%$ & $3.93 \%$ & & & & & \\
\hline $\mathrm{CO} 2, \mathrm{~mol} \%$ & & & $6.57 \%$ & $6.44 \%$ & & & & & \\
\hline $\mathrm{CO}, \mathrm{ppmv}$ & & & $1.07 \mathrm{E}+04$ & $1.05 \mathrm{E}+04$ & & & & & \\
\hline $\mathrm{NO}, \mathrm{ppmv}$ & & & $6.13 \mathrm{E}+02$ & $6.02 \mathrm{E}+02$ & & & & & \\
\hline NO2, ppmv & & & $4.15 \mathrm{E}+01$ & $4.07 E+01$ & & & & & \\
\hline SO2, ppmv & & & 1.61E-02 & 1.58E-02 & & & & & \\
\hline $\mathrm{Cl}, \mathrm{ppmv}$ & & & $3.41 \mathrm{E}+01$ & $3.35 \mathrm{E}+01$ & & & & & \\
\hline $\mathrm{F}, \mathrm{ppmv}$ & & & $1.29 \mathrm{E}+02$ & $1.27 E+02$ & & & & & \\
\hline C (organic), ppmv & & & $3.02 \mathrm{E}+03$ & $2.96 \mathrm{E}+03$ & & & & & \\
\hline $\mathrm{H}$ (organic), ppmv & & & $6.81 \mathrm{E}+03$ & $6.68 \mathrm{E}+03$ & & & & & \\
\hline $\mathrm{Hg}, \mathrm{ug} / \mathrm{wscm}$ & & & $1.17 \mathrm{E}+05$ & $1.15 \mathrm{E}+05$ & & & & & \\
\hline $\mathrm{PM}, \mathrm{mg} / \mathrm{wscm}$ & & & $1.08 \mathrm{E}+04$ & $1.06 \mathrm{E}+04$ & & & & & \\
\hline SVM, ug/wscm & & & $2.08 \mathrm{E}+04$ & $2.04 \mathrm{E}+04$ & & & & & \\
\hline LVM, ug/wscm & & & $8.67 E+03$ & $8.50 \mathrm{E}+03$ & & & & & \\
\hline $\begin{array}{l}\text { Gas Stream Bulk } \\
\text { Composition (Dry Basis) }\end{array}$ & $\mathrm{mol} \%$ & $\mathrm{~mol} \%$ & $\mathrm{~mol} \%$ & $\mathrm{~mol} \%$ & $\mathrm{~mol} \%$ & $\mathrm{~mol} \%$ & $\mathrm{~mol} \%$ & $\mathrm{~mol} \%$ & $\mathrm{~mol} \%$ \\
\hline $\mathrm{O} 2, \mathrm{~mol} \%$, dry basis & & & $1.73 \%$ & $1.63 \%$ & & & & & \\
\hline $\mathrm{N} 2, \mathrm{~mol} \%$, dry basis & & & $58.25 \%$ & $60.74 \%$ & & & & & \\
\hline $\mathrm{H} 2, \mathrm{~mol} \%$, dry basis & & & $13.30 \%$ & $12.51 \%$ & & & & & \\
\hline $\mathrm{CO} 2, \mathrm{~mol} \%$, dry basis & & & $21.80 \%$ & $20.50 \%$ & & & & & \\
\hline $\begin{array}{l}\text { Gas Stream Bulk } \\
\text { Composition (Dry Basis, } \\
\text { Corrected to } 7 \% \text { O2 with } \\
100 \% \text { O2 Combustion Air) }\end{array}$ & $\begin{array}{l}\text { ppmv, or } \\
\text { ug/dscm } \\
\text { or } \\
\mathrm{mg} / \mathrm{dscm}\end{array}$ & $\begin{array}{l}\mathrm{ppmv}, \text { or } \\
\mathrm{ug} / \mathrm{dscm} \\
\text { or } \\
\mathrm{mg} / \mathrm{dscm}\end{array}$ & $\begin{array}{l}\text { ppmv, or } \\
\text { ug/dscm } \\
\text { or } \\
\mathrm{mg} / \mathrm{dscm}\end{array}$ & $\begin{array}{l}\mathrm{ppmv}, \text { or } \\
\mathrm{ug} / \mathrm{dscm} \\
\text { or } \\
\mathrm{mg} / \mathrm{dscm}\end{array}$ & $\begin{array}{l}\mathrm{ppmv}, \text { or } \\
\mathrm{ug} / \mathrm{dscm} \\
\text { or } \\
\mathrm{mg} / \mathrm{dscm}\end{array}$ & $\begin{array}{l}\text { ppmv, or } \\
\text { ug/dscm } \\
\text { or } \\
\mathrm{mg} / \mathrm{dscm}\end{array}$ & $\begin{array}{l}\text { ppmv, or } \\
\text { ug/dscm } \\
\text { or } \\
\mathrm{mg} / \mathrm{dscm}\end{array}$ & $\begin{array}{l}\mathrm{ppmv}, \text { or } \\
\text { ug/dscm } \\
\text { or } \\
\mathrm{mg} / \mathrm{dscm}\end{array}$ & $\begin{array}{l}\text { ppmv, or } \\
\text { ug/dscm } \\
\text { or } \\
\mathrm{mg} / \mathrm{dscm}\end{array}$ \\
\hline COgas, ppmv, dry basis & & & $4.97 \mathrm{E}+03$ & $4.67 \mathrm{E}+03$ & & & & & \\
\hline $\mathrm{NO}$, ppmv, dry basis & & & $2.85 \mathrm{E}+02$ & $2.68 \mathrm{E}+02$ & & & & & \\
\hline NO2, ppmv, dry basis & & & $1.93 \mathrm{E}+01$ & $1.81 \mathrm{E}+01$ & & & & & \\
\hline SO2, ppmv, dry basis & & & $7.50 \mathrm{E}-03$ & $7.06 \mathrm{E}-03$ & & & & & \\
\hline $\mathrm{Cl}, \mathrm{ppmv}$, dry basis & & & $1.59 \mathrm{E}+01$ & $1.49 \mathrm{E}+01$ & & & & & \\
\hline $\mathrm{F}$, ppmv, dry basis & & & $6.01 \mathrm{E}+01$ & $5.65 \mathrm{E}+01$ & & & & & \\
\hline C (organic), ppmv, dry basis & & & $1.40 \mathrm{E}+03$ & $1.32 \mathrm{E}+03$ & & & & & \\
\hline $\mathrm{H}$ (organic), ppmv, dry basis & & & $3.16 \mathrm{E}+03$ & $2.98 \mathrm{E}+03$ & & & & & \\
\hline $\mathrm{Hg}, \mathrm{ug} / \mathrm{dscm}$ & & & $5.43 \mathrm{E}+04$ & $5.11 \mathrm{E}+04$ & & & & & \\
\hline $\mathrm{PM}, \mathrm{mg} / \mathrm{dscm}$ & & & $5.01 \mathrm{E}+03$ & $4.71 \mathrm{E}+03$ & & & & & \\
\hline SVM, ug/dscm & & & $6.90 \mathrm{E}+04$ & $6.77 \mathrm{E}+04$ & & & & & \\
\hline LVM, ug/dscm & & & $2.88 \mathrm{E}+04$ & $2.82 \mathrm{E}+04$ & & & & & \\
\hline
\end{tabular}


Table A-14. Mass balance, Tank WM-187 waste (continued).

\begin{tabular}{|c|c|c|c|c|c|c|c|c|c|}
\hline PFD \# & PFD-2 & PFD-3 & PFD-3 & PFD-3 & PFD-3 & PFD-3 & PFD-3 & PFD-3 & PFD-3 \\
\hline WM-187, Stream \# & 109 & 110 & 111 & 112 & $113 \mathrm{~A}$ & 113B & 114 & 115 & 116 \\
\hline Stream Name & $\begin{array}{l}\text { Off-Gas } \\
\text { from } \\
\text { Filter to } \\
\text { Oxidizer }\end{array}$ & $\begin{array}{l}\text { Oxidizer } \\
\text { Effluent }\end{array}$ & $\begin{array}{c}\text { Quenched } \\
\text { Oxidizer } \\
\text { Off-gas }\end{array}$ & Scrub & $\begin{array}{c}\text { Packed } \\
\text { Scrubber } \\
\text { Drain }\end{array}$ & $\begin{array}{c}\text { Demister } \\
\text { Drain }\end{array}$ & $\begin{array}{c}\text { Scrubber } \\
\text { Effluent } \\
\text { Gas }\end{array}$ & $\begin{array}{c}\text { Demister } \\
\text { Effluent } \\
\text { Gas }\end{array}$ & $\begin{array}{c}\text { GAC Bed } \\
\text { Feed }\end{array}$ \\
\hline Rate or Volume & $4.37 \mathrm{E}+05$ & $7.03 \mathrm{E}+05$ & $3.67 \mathrm{E}+05$ & $1.01 \mathrm{E}+04$ & $1.01 \mathrm{E}+04$ & $0.00 \mathrm{E}+00$ & $3.76 \mathrm{E}+05$ & $3.85 \mathrm{E}+05$ & $4.67 \mathrm{E}+05$ \\
\hline Volume Flow (standard, wet) ${ }^{*}$ & $1.03 \mathrm{E}+05$ & $1.08 \mathrm{E}+05$ & $1.82 \mathrm{E}+05$ & & & & $1.82 \mathrm{E}+05$ & $1.83 \mathrm{E}+05$ & $1.83 \mathrm{E}+05$ \\
\hline Volume Flow (standard, dry) ${ }^{*}$ & $3.29 \mathrm{E}+04$ & $3.16 \mathrm{E}+04$ & $3.15 \mathrm{E}+04$ & & & & $3.15 \mathrm{E}+04$ & $3.16 \mathrm{E}+04$ & $3.16 \mathrm{E}+04$ \\
\hline \begin{tabular}{|l|} 
Rate Units \\
\end{tabular} & $\mathrm{ft} 3 / \mathrm{hr}$ & $\mathrm{ft} 3 / \mathrm{hr}$ & $\mathrm{ft} 3 / \mathrm{hr}$ & $\mathrm{gal} / \mathrm{hr}$ & $\mathrm{gal} / \mathrm{hr}$ & $\mathrm{gal} / \mathrm{hr}$ & $\mathrm{ft} 3 / \mathrm{hr}$ & $\mathrm{ft} 3 / \mathrm{hr}$ & $\mathrm{ft} 3 / \mathrm{hr}$ \\
\hline Rate or Volume, metric & $2.93 E+03$ & $3.05 \mathrm{E}+03$ & $5.16 \mathrm{E}+03$ & $3.82 \mathrm{E}+04$ & $3.82 \mathrm{E}+04$ & 0.00 & $5.15 \mathrm{E}+03$ & $5.17 \mathrm{E}+03$ & $5.17 \mathrm{E}+03$ \\
\hline Rate Units & wscm/hr & wscm/hr & wscm/hr & $\mathrm{L} / \mathrm{hr}$ & $\mathrm{L} / \mathrm{hr}$ & $\mathrm{L} / \mathrm{hr}$ & wscm/hr & wscm/hr & wscm/hr \\
\hline Temperature, ${ }^{\circ} \mathrm{C}$ & 527 & 951 & 100 & 82 & 83 & 0 & 82 & 81 & 120 \\
\hline Temperature, ${ }^{\circ} \mathrm{F}$ & 980 & 1743 & 212 & 179 & 181 & 32 & 179 & 178 & 248 \\
\hline Pressure, psia & 9.5 & 9.4 & 9.3 & 38.1 & 9.0 & 8.4 & 8.6 & 8.4 & 7.7 \\
\hline Specific Gravity & 2.09E-04 & $1.42 \mathrm{E}-04$ & $4.26 \mathrm{E}-04$ & 1.01 & 1.01 & 1.01 & $4.15 \mathrm{E}-04$ & $4.06 \mathrm{E}-04$ & 3.34E-04 \\
\hline Chemical Composition & $\mathrm{lb} / \mathrm{wscf}$ & lb/wscf & Ib/wscf & Mol/liter & Mol/liter & Mol/liter & $\mathrm{lb} / \mathrm{wscf}$ & lb/wscf & Ib/wscf \\
\hline $\mathrm{H}+$ & 8.01E-07 & 3.09E-08 & $3.03 E-08$ & $1.02 \mathrm{E}+00$ & $1.02 \mathrm{E}+00$ & $1.02 \mathrm{E}+00$ & $2.33 \mathrm{E}-07$ & $2.32 \mathrm{E}-07$ & $2.32 \mathrm{E}-07$ \\
\hline $\mathrm{Al}+3$ & $3.44 \mathrm{E}-08$ & $3.30 \mathrm{E}-08$ & 1.95E-08 & 1.54E-03 & 1.54E-03 & 1.54E-03 & & & \\
\hline $\mathrm{Sb}+5$ & 1.06E-11 & $1.02 \mathrm{E}-11$ & $6.01 \mathrm{E}-12$ & 1.05E-07 & 1.05E-07 & 1.05E-07 & & & \\
\hline As+3 & $3.79 \mathrm{E}-11$ & $3.64 \mathrm{E}-11$ & $2.15 \mathrm{E}-11$ & $6.13 \mathrm{E}-07$ & 6.13E-07 & $6.13 \mathrm{E}-07$ & & & \\
\hline $\mathrm{Ba}+2$ & $2.91 \mathrm{E}-11$ & $2.80 \mathrm{E}-11$ & $5.76 \mathrm{E}-13$ & 3.37E-07 & 3.37E-07 & 3.37E-07 & & & \\
\hline $\mathrm{Be}+2$ & $4.23 \mathrm{E}-13$ & $4.07 E-13$ & $8.36 \mathrm{E}-15$ & $7.47 \mathrm{E}-08$ & $7.46 \mathrm{E}-08$ & $7.46 \mathrm{E}-08$ & & & \\
\hline $\mathrm{B}+3$ & $3.55 \mathrm{E}-10$ & $3.41 \mathrm{E}-10$ & $2.01 \mathrm{E}-10$ & $3.98 \mathrm{E}-05$ & $3.98 \mathrm{E}-05$ & 3.98E-05 & & & \\
\hline $\mathrm{Cd}+2$ & $6.45 \mathrm{E}-10$ & $6.20 \mathrm{E}-10$ & $3.66 \mathrm{E}-10$ & $2.59 \mathrm{E}-06$ & 2.60E-06 & $2.60 \mathrm{E}-06$ & & & \\
\hline $\mathrm{Ca}+2$ & $1.55 \mathrm{E}-08$ & $1.49 \mathrm{E}-08$ & $3.06 \mathrm{E}-10$ & 6.15E-04 & 6.15E-04 & $6.15 \mathrm{E}-04$ & & & \\
\hline $\mathrm{Cr}+3$ & $7.51 \mathrm{E}-10$ & $7.23 \mathrm{E}-10$ & $4.27 \mathrm{E}-10$ & $8.23 \mathrm{E}-06$ & $8.25 \mathrm{E}-06$ & $8.25 \mathrm{E}-06$ & & & \\
\hline $\mathrm{Co}+2$ & $3.53 \mathrm{E}-10$ & $3.39 \mathrm{E}-10$ & $2.00 \mathrm{E}-10$ & $7.26 \mathrm{E}-06$ & 7.26E-06 & $7.26 \mathrm{E}-06$ & & & \\
\hline $\mathrm{Cs}+$ & $6.89 \mathrm{E}-11$ & $6.63 \mathrm{E}-11$ & $3.91 \mathrm{E}-11$ & $6.96 \mathrm{E}-05$ & 6.96E-05 & 6.96E-05 & & & \\
\hline $\mathrm{Cu}+2$ & 8.92E-11 & $8.58 \mathrm{E}-11$ & $1.76 \mathrm{E}-12$ & $2.23 \mathrm{E}-06$ & 2.23E-06 & $2.23 \mathrm{E}-06$ & & & \\
\hline $\mathrm{Fe}+3$ & $2.86 \mathrm{E}-09$ & $2.75 \mathrm{E}-09$ & 1.63E-09 & 1.02E-04 & 1.02E-04 & $1.02 \mathrm{E}-04$ & & & \\
\hline $\mathrm{Pb}+2$ & 1.25E-09 & 1.20E-09 & $7.09 \mathrm{E}-10$ & 5.05E-06 & 5.05E-06 & \begin{tabular}{|l}
$5.05 \mathrm{E}-06$ \\
\end{tabular} & & & \\
\hline $\mathrm{Hg}+2$ & 7.09E-06 & $6.82 \mathrm{E}-06$ & 4.03E-06 & 1.07E-02 & 1.07E-02 & 1.07E-02 & & $2.26 \mathrm{E}-06$ & $2.26 \mathrm{E}-06$ \\
\hline $\mathrm{Mn}+4$ & 2.97E-09 & 2.85E-09 & 1.68E-09 & $6.55 \mathrm{E}-05$ & 6.55E-05 & 6.55E-05 & & & \\
\hline $\mathrm{Ni}+2$ & $2.38 \mathrm{E}-10$ & $2.29 \mathrm{E}-10$ & 4.70E-12 & $6.45 \mathrm{E}-06$ & $6.44 \mathrm{E}-06$ & 6.44E-06 & & & \\
\hline $\mathrm{K}+$ & 6.91E-08 & $6.65 \mathrm{E}-08$ & 3.93E-08 & 1.66E-03 & 1.66E-03 & 1.66E-03 & & & \\
\hline $\mathrm{Se}+4$ & 9.39E-11 & $9.03 E-11$ & $5.33 \mathrm{E}-11$ & 1.46E-06 & 1.46E-06 & $1.46 \mathrm{E}-06$ & & & \\
\hline $\mathrm{Ag}+$ & 1.07E-09 & $5.96 \mathrm{E}-10$ & $3.52 \mathrm{E}-10$ & 2.09E-06 & $2.10 \mathrm{E}-06$ & $2.10 \mathrm{E}-06$ & & & \\
\hline $\mathrm{Na}+$ & $6.26 \mathrm{E}-07$ & $6.02 \mathrm{E}-07$ & $3.55 \mathrm{E}-07$ & \begin{tabular}{|l|}
$6.14 \mathrm{E}-02$ \\
\end{tabular} & \begin{tabular}{|l}
$6.14 \mathrm{E}-02$ \\
\end{tabular} & \begin{tabular}{|l}
$6.14 \mathrm{E}-02$ \\
\end{tabular} & & & \\
\hline $\mathrm{TI}+3$ & $3.53 \mathrm{E}-12$ & $3.39 \mathrm{E}-12$ & $2.00 \mathrm{E}-12$ & $2.09 \mathrm{E}-08$ & $2.09 \mathrm{E}-08$ & $2.09 \mathrm{E}-08$ & & & \\
\hline $\mathrm{U}+4$ & $1.80 \mathrm{E}-10$ & $1.73 \mathrm{E}-10$ & $1.02 \mathrm{E}-10$ & 9.27E-07 & 9.27E-07 & 9.27E-07 & & & \\
\hline $\mathrm{V}+3$ & $8.72 \mathrm{E}-12$ & $8.38 \mathrm{E}-12$ & $4.95 \mathrm{E}-12$ & $2.10 \mathrm{E}-07$ & $2.10 \mathrm{E}-07$ & $2.10 \mathrm{E}-07$ & & & \\
\hline$Z n+2$ & $1.49 \mathrm{E}-10$ & $1.43 \mathrm{E}-10$ & $2.94 \mathrm{E}-12$ & $3.62 \mathrm{E}-06$ & 3.61E-06 & 3.61E-06 & & & \\
\hline $\mathrm{Zr}+4$ & $1.55 \mathrm{E}-08$ & $1.49 \mathrm{E}-08$ & 8.81E-09 & $2.09 \mathrm{E}-04$ & $2.09 \mathrm{E}-04$ & $2.09 \mathrm{E}-04$ & & & \\
\hline $\mathrm{Cl}-$ & $8.56 \mathrm{E}-08$ & $8.23 E-08$ & $4.86 \mathrm{E}-08$ & $2.16 \mathrm{E}-03$ & 2.16E-03 & $2.16 \mathrm{E}-03$ & $1.03 \mathrm{E}-08$ & $1.02 \mathrm{E}-08$ & 1.02E-08 \\
\hline F- & $6.21 \mathrm{E}-07$ & 5.97E-07 & 3.53E-07 & $2.10 \mathrm{E}-02$ & 2.09E-02 & $2.09 \mathrm{E}-02$ & 8.81E-07 & 8.78E-07 & $8.78 \mathrm{E}-07$ \\
\hline SO4-2 & $2.11 \mathrm{E}-11$ & $8.25 E-07$ & 4.87E-07 & $2.22 \mathrm{E}-02$ & $2.22 \mathrm{E}-02$ & $2.22 \mathrm{E}-02$ & & & \\
\hline \begin{tabular}{|l|} 
NO3- \\
\end{tabular} & $6.42 \mathrm{E}-06$ & 5.64E-09 & 3.33E-09 & $1.04 \mathrm{E}+00$ & $1.04 \mathrm{E}+00$ & $1.04 \mathrm{E}+00$ & $1.14 \mathrm{E}-05$ & 1.14E-05 & 1.14E-05 \\
\hline PO4-3 & 5.39E-08 & $5.18 \mathrm{E}-08$ & 3.06E-08 & $6.75 \mathrm{E}-04$ & $6.75 \mathrm{E}-04$ & $6.75 \mathrm{E}-04$ & & & \\
\hline $\mathrm{Am}+4$ & $5.02 \mathrm{E}-14$ & $4.83 \mathrm{E}-14$ & $2.85 \mathrm{E}-14$ & $2.50 \mathrm{E}-10$ & $2.50 \mathrm{E}-10$ & $2.50 \mathrm{E}-10$ & & & \\
\hline $\mathrm{Br}-$ & $2.70 \mathrm{E}-12$ & $2.59 \mathrm{E}-12$ & $1.53 \mathrm{E}-12$ & $3.02 \mathrm{E}-08$ & 3.02E-08 & 3.02E-08 & $3.24 \mathrm{E}-13$ & $3.23 \mathrm{E}-13$ & $3.23 \mathrm{E}-13$ \\
\hline $\mathrm{Ce}+4$ & $1.81 \mathrm{E}-11$ & $1.74 \mathrm{E}-11$ & $1.03 \mathrm{E}-11$ & $1.56 \mathrm{E}-07$ & $1.56 \mathrm{E}-07$ & $1.56 \mathrm{E}-07$ & & & \\
\hline Eu+3 & $1.88 \mathrm{E}-13$ & $1.81 \mathrm{E}-13$ & $1.07 \mathrm{E}-13$ & 1.50E-09 & 1.50E-09 & 1.50E-09 & & & \\
\hline $\mathrm{Gd}+3$ & $7.07 \mathrm{E}-11$ & $6.80 \mathrm{E}-11$ & $4.01 \mathrm{E}-11$ & $5.45 \mathrm{E}-07$ & $5.45 \mathrm{E}-07$ & 5.45E-07 & & & \\
\hline $\mathrm{Ge}+4$ & $1.45 \mathrm{E}-15$ & 1.39E-15 & $8.23 E-16$ & $2.45 \mathrm{E}-11$ & $2.45 \mathrm{E}-11$ & $2.45 \mathrm{E}-11$ & & & \\
\hline $\ln +3$ & $4.12 \mathrm{E}-13$ & $3.96 \mathrm{E}-13$ & $2.34 \mathrm{E}-13$ & 4.35E-09 & 4.35E-09 & 4.35E-09 & & & \\
\hline |- & $5.58 \mathrm{E}-11$ & $5.36 \mathrm{E}-11$ & $3.17 \mathrm{E}-11$ & 3.93E-07 & 3.93E-07 & 3.93E-07 & $6.70 \mathrm{E}-12$ & $6.68 \mathrm{E}-12$ & $6.68 \mathrm{E}-12$ \\
\hline $\mathrm{La}+3$ & $3.11 \mathrm{E}-12$ & $2.99 \mathrm{E}-12$ & $1.77 \mathrm{E}-12$ & $2.71 \mathrm{E}-08$ & $2.72 \mathrm{E}-08$ & $2.72 \mathrm{E}-08$ & & & \\
\hline
\end{tabular}


Table A-14. Mass balance, Tank WM-187 waste (continued).

\begin{tabular}{|c|c|c|c|c|c|c|c|c|c|}
\hline Stream \# & 109 & 110 & 111 & 112 & $113 \mathrm{~A}$ & $113 \mathrm{~B}$ & 114 & 115 & 116 \\
\hline Chemical Composition & $\mathrm{lb} / \mathrm{wscf}$ & $\mathrm{lb} / \mathrm{wscf}$ & $\mathrm{lb} / \mathrm{wscf}$ & Mol/liter & Mol/liter & Mol/liter & $\mathrm{lb} /$ wscf & $\mathrm{lb} /$ wscf & $\mathrm{lb} /$ wscf \\
\hline $\mathrm{Li+}$ & $6.70 \mathrm{E}-11$ & $3.73 \mathrm{E}-11$ & $2.20 \mathrm{E}-11$ & $2.03 \mathrm{E}-06$ & $2.04 \mathrm{E}-06$ & $2.04 \mathrm{E}-06$ & & & \\
\hline $\mathrm{Mg}+2$ & 1.24E-09 & 1.19E-09 & $2.45 \mathrm{E}-11$ & $8.12 \mathrm{E}-05$ & $8.11 \mathrm{E}-05$ & $8.11 \mathrm{E}-05$ & & & \\
\hline Mo+6 & $2.50 \mathrm{E}-08$ & $2.40 \mathrm{E}-08$ & $1.42 \mathrm{E}-08$ & $3.20 \mathrm{E}-04$ & $3.20 \mathrm{E}-04$ & $3.20 \mathrm{E}-04$ & & & \\
\hline $\mathrm{Nd}+3$ & $1.04 \mathrm{E}-11$ & $1.00 \mathrm{E}-11$ & $5.91 \mathrm{E}-12$ & $8.75 \mathrm{E}-08$ & $8.76 \mathrm{E}-08$ & $8.76 \mathrm{E}-08$ & & & \\
\hline $\mathrm{Np}+4$ & $2.69 \mathrm{E}-12$ & $2.59 \mathrm{E}-12$ & $1.53 \mathrm{E}-12$ & $1.38 \mathrm{E}-08$ & $1.38 \mathrm{E}-08$ & $1.38 \mathrm{E}-08$ & & & \\
\hline $\mathrm{Nb}+5$ & $4.40 \mathrm{E}-10$ & $4.23 E-10$ & $2.50 \mathrm{E}-10$ & $5.81 \mathrm{E}-06$ & 5.82E-06 & $5.82 \mathrm{E}-06$ & & & \\
\hline $\mathrm{Pd}+4$ & $8.81 \mathrm{E}-10$ & $8.47 \mathrm{E}-10$ & $1.74 \mathrm{E}-11$ & $1.32 \mathrm{E}-05$ & $1.32 \mathrm{E}-05$ & $1.32 \mathrm{E}-05$ & & & \\
\hline $\mathrm{Pu}+4$ & $6.05 \mathrm{E}-12$ & $5.82 \mathrm{E}-12$ & $3.44 \mathrm{E}-12$ & $3.05 \mathrm{E}-08$ & $3.05 \mathrm{E}-08$ & $3.05 \mathrm{E}-08$ & & & \\
\hline $\mathrm{Pr}+4$ & $2.87 \mathrm{E}-12$ & $2.76 \mathrm{E}-12$ & $1.63 \mathrm{E}-12$ & $2.47 \mathrm{E}-08$ & $2.47 \mathrm{E}-08$ & $2.47 \mathrm{E}-08$ & & & \\
\hline $\mathrm{Pm}+3$ & $7.19 \mathrm{E}-16$ & $6.91 \mathrm{E}-16$ & $4.08 \mathrm{E}-16$ & $6.01 \mathrm{E}-12$ & $6.01 \mathrm{E}-12$ & $6.01 \mathrm{E}-12$ & & & \\
\hline $\mathrm{Rh}+4$ & $9.04 \mathrm{E}-13$ & $8.69 \mathrm{E}-13$ & $5.13 \mathrm{E}-13$ & 1.06E-08 & 1.07E-08 & 1.07E-08 & & & \\
\hline $\mathrm{Rb}+$ & 5.92E-12 & $3.29 \mathrm{E}-12$ & $1.94 \mathrm{E}-12$ & $1.46 \mathrm{E}-08$ & 1.46E-08 & $1.46 \mathrm{E}-08$ & & & \\
\hline Ru+3 & $3.83 \mathrm{E}-10$ & $3.69 \mathrm{E}-10$ & $2.18 \mathrm{E}-10$ & $4.60 \mathrm{E}-06$ & $4.60 \mathrm{E}-06$ & $4.60 \mathrm{E}-06$ & & & \\
\hline $\mathrm{Sm}+3$ & $2.03 \mathrm{E}-12$ & $1.95 \mathrm{E}-12$ & $1.15 \mathrm{E}-12$ & $1.64 \mathrm{E}-08$ & $1.64 \mathrm{E}-08$ & 1.64E-08 & & & \\
\hline $\mathrm{Si}+4$ & $1.40 \mathrm{E}-08$ & 1.34E-08 & 7.93E-09 & $8.21 \mathrm{E}-04$ & $8.21 \mathrm{E}-04$ & $8.21 \mathrm{E}-04$ & & & \\
\hline $\mathrm{Sr}+2$ & $2.59 \mathrm{E}-11$ & $2.49 \mathrm{E}-11$ & $5.12 \mathrm{E}-13$ & $4.70 \mathrm{E}-07$ & $4.70 \mathrm{E}-07$ & $4.70 \mathrm{E}-07$ & & & \\
\hline $\mathrm{Tc}+7$ & $6.28 \mathrm{E}-12$ & $6.04 \mathrm{E}-12$ & $3.57 \mathrm{E}-12$ & $5.26 \mathrm{E}-08$ & $5.27 \mathrm{E}-08$ & $5.27 \mathrm{E}-08$ & & & \\
\hline $\mathrm{Te}+4$ & $1.38 \mathrm{E}-12$ & $1.33 \mathrm{E}-12$ & $7.84 \mathrm{E}-13$ & $1.33 \mathrm{E}-08$ & $1.33 \mathrm{E}-08$ & $1.33 \mathrm{E}-08$ & & & \\
\hline $\mathrm{Tb}+4$ & $8.19 \mathrm{E}-16$ & $7.88 \mathrm{E}-16$ & $4.65 \mathrm{E}-16$ & $6.25 \mathrm{E}-12$ & $6.25 \mathrm{E}-12$ & $6.25 \mathrm{E}-12$ & & & \\
\hline $\mathrm{Th}+4$ & 1.32E-11 & $1.27 \mathrm{E}-11$ & $7.47 \mathrm{E}-12$ & $6.96 \mathrm{E}-08$ & 6.97E-08 & 6.97E-08 & & & \\
\hline$S n+4$ & $9.78 \mathrm{E}-10$ & $9.40 \mathrm{E}-10$ & $5.55 \mathrm{E}-10$ & $1.01 \mathrm{E}-05$ & $1.01 \mathrm{E}-05$ & 1.01E-05 & & & \\
\hline $\mathrm{Ti}+4$ & $1.99 \mathrm{E}-10$ & $1.91 \mathrm{E}-10$ & $1.13 \mathrm{E}-10$ & $5.10 \mathrm{E}-06$ & $5.10 \mathrm{E}-06$ & $5.10 \mathrm{E}-06$ & & & \\
\hline$Y+3$ & $1.48 \mathrm{E}-12$ & $1.43 \mathrm{E}-12$ & $8.43 \mathrm{E}-13$ & $2.02 \mathrm{E}-08$ & $2.02 \mathrm{E}-08$ & $2.02 \mathrm{E}-08$ & & & \\
\hline $\mathrm{OH}-$ & 3.72E-09 & 1.43E-07 & $2.88 \mathrm{E}-07$ & 4.97E-07 & 4.99E-07 & 4.99E-07 & & & \\
\hline $\mathrm{H} 2 \mathrm{O}$ & 3.19E-02 & $3.30 \mathrm{E}-02$ & 3.88E-02 & $5.23 E+01$ & $5.23 E+01$ & $5.23 E+01$ & $3.88 \mathrm{E}-02$ & $3.87 \mathrm{E}-02$ & $3.87 \mathrm{E}-02$ \\
\hline $\mathrm{SO} 2$ & 2.62E-09 & $2.03 E-05$ & $1.20 \mathrm{E}-05$ & $9.10 \mathrm{E}-06$ & $9.11 \mathrm{E}-06$ & $9.11 \mathrm{E}-06$ & $1.20 \mathrm{E}-05$ & $1.20 \mathrm{E}-05$ & 1.20E-05 \\
\hline $\mathrm{H} 2 \mathrm{~S}$ & $1.15 \mathrm{E}-05$ & $2.53 \mathrm{E}-21$ & & & & & & & \\
\hline $\mathrm{CO}$ & $7.59 \mathrm{E}-04$ & $1.52 \mathrm{E}-09$ & $8.96 \mathrm{E}-10$ & $1.92 \mathrm{E}-10$ & $1.94 \mathrm{E}-10$ & $1.94 \mathrm{E}-10$ & $8.96 \mathrm{E}-10$ & $8.93 \mathrm{E}-10$ & $8.93 \mathrm{E}-10$ \\
\hline $\mathrm{CO} 2$ & 7.32E-03 & $9.20 \mathrm{E}-03$ & 5.43E-03 & $3.05 E-04$ & 3.14E-04 & $3.14 \mathrm{E}-04$ & 5.44E-03 & 5.42E-03 & 5.42E-03 \\
\hline $\mathrm{H} 2$ & $2.05 \mathrm{E}-04$ & $6.17 \mathrm{E}-10$ & $3.64 \mathrm{E}-10$ & $7.32 \mathrm{E}-10$ & $1.19 \mathrm{E}-09$ & 1.19E-09 & $1.81 \mathrm{E}-08$ & & \\
\hline N2 & 1.44E-02 & 1.38E-02 & $8.16 \mathrm{E}-03$ & $1.28 \mathrm{E}-05$ & $2.47 \mathrm{E}-05$ & $2.47 \mathrm{E}-05$ & $8.17 \mathrm{E}-03$ & $8.14 \mathrm{E}-03$ & $8.14 \mathrm{E}-03$ \\
\hline $\mathrm{NO}$ & 4.66E-05 & 5.07E-05 & $2.99 \mathrm{E}-05$ & $2.31 \mathrm{E}-07$ & 2.93E-07 & $2.93 \mathrm{E}-07$ & $2.99 \mathrm{E}-05$ & $2.98 \mathrm{E}-05$ & 2.98E-05 \\
\hline $\mathrm{NO} 2$ & 4.84E-06 & $2.78 \mathrm{E}-07$ & 1.64E-07 & $2.94 \mathrm{E}-06$ & $2.94 \mathrm{E}-06$ & 2.94E-06 & 1.64E-07 & 1.64E-07 & 1.64E-07 \\
\hline $\mathrm{O} 2$ & $4.23 \mathrm{E}-04$ & $1.86 \mathrm{E}-03$ & 1.10E-03 & $3.20 \mathrm{E}-06$ & 4.94E-06 & 4.94E-06 & 1.10E-03 & 1.09E-03 & 1.09E-03 \\
\hline S (other) & $2.74 \mathrm{E}-10$ & 1.79E-25 & & 1.22E-07 & $1.22 \mathrm{E}-07$ & 1.22E-07 & & & \\
\hline $\mathrm{CO} 3$ & $2.30 \mathrm{E}-07$ & $4.57 \mathrm{E}-11$ & $8.84 \mathrm{E}-12$ & $3.48 \mathrm{E}-05$ & $3.48 \mathrm{E}-05$ & $3.48 \mathrm{E}-05$ & & & \\
\hline C (reductant) & $3.25 \mathrm{E}-08$ & $1.32 \mathrm{E}-18$ & & & & & & & \\
\hline $\mathrm{O}$ (oxides) & 5.92E-08 & 5.54E-08 & $3.27 \mathrm{E}-08$ & $5.02 \mathrm{E}-03$ & $5.02 \mathrm{E}-03$ & $5.02 \mathrm{E}-03$ & & & \\
\hline C (organic) & 1.80E-05 & $1.70 \mathrm{E}-34$ & & & & & & & \\
\hline $\mathrm{H}$ (organic) & 6.04E-06 & $5.70 \mathrm{E}-35$ & & & & & & & \\
\hline O (organic) & 1.35E-07 & & & & & & & & \\
\hline \multirow[t]{4}{*}{ Mass Flow (kg/hr): } & $2.58 \mathrm{E}+03$ & $2.83 E+03$ & $4.42 \mathrm{E}+03$ & $3.87 E+04$ & $3.87 E+04$ & & $4.42 \mathrm{E}+03$ & $4.42 \mathrm{E}+03$ & $4.42 \mathrm{E}+03$ \\
\hline & & & & & & & & & \\
\hline & & & & & & & & & \\
\hline & $\mathrm{w} / \mathrm{m} 3$ & $\mathrm{w} / \mathrm{m} 3$ & $\mathrm{w} / \mathrm{m} 3$ & $\mathrm{w} / \mathrm{m} 3$ & $\mathrm{w} / \mathrm{m} 3$ & $\mathrm{w} / \mathrm{m} 3$ & $\mathrm{w} / \mathrm{m} 3$ & $\mathrm{w} / \mathrm{m} 3$ & $\mathrm{w} / \mathrm{m} 3$ \\
\hline Heat Generation, w/m3 & & & & $4.12 \mathrm{E}-02$ & $4.12 \mathrm{E}-02$ & & & & \\
\hline
\end{tabular}


Table A-14. Mass balance, Tank WM-187 waste (continued).

\begin{tabular}{|c|c|c|c|c|c|c|c|c|c|}
\hline Stream \# & 109 & 110 & 111 & 112 & $113 \mathrm{~A}$ & $113 \mathrm{~B}$ & 114 & 115 & 116 \\
\hline Radiological Composition & Ci/wscm & Ci/wscm & Ci/wscm & $\mathrm{Ci} / \mathrm{L}$ & $\mathrm{Ci} / \mathrm{L}$ & $\mathrm{Ci} / \mathrm{L}$ & Ci/wscm & Ci/wscm & Ci/wscm \\
\hline $\mathrm{H}-3$ & 1.16E-06 & $1.11 \mathrm{E}-06$ & $6.57 \mathrm{E}-07$ & $9.73 \mathrm{E}-07$ & $9.72 \mathrm{E}-07$ & 9.72E-07 & $6.57 \mathrm{E}-07$ & $6.54 \mathrm{E}-07$ & $6.54 \mathrm{E}-07$ \\
\hline $\mathrm{C}-14$ & $1.55 \mathrm{E}-11$ & $1.50 \mathrm{E}-11$ & $8.85 \mathrm{E}-12$ & $1.52 \mathrm{E}-15$ & $1.56 \mathrm{E}-15$ & $1.56 \mathrm{E}-15$ & $8.86 \mathrm{E}-12$ & $8.83 \mathrm{E}-12$ & $8.83 \mathrm{E}-12$ \\
\hline Co-60 & $8.22 \mathrm{E}-10$ & $7.90 \mathrm{E}-10$ & $4.67 \mathrm{E}-10$ & $6.22 \mathrm{E}-08$ & $6.22 \mathrm{E}-08$ & $6.22 \mathrm{E}-08$ & & & \\
\hline $\mathrm{Ni}-59$ & $9.00 \mathrm{E}-11$ & $8.66 \mathrm{E}-11$ & $1.78 \mathrm{E}-12$ & $8.94 \mathrm{E}-09$ & $8.94 \mathrm{E}-09$ & 8.94E-09 & & & \\
\hline $\mathrm{Ni}-63$ & 2.09E-09 & $2.01 \mathrm{E}-09$ & $4.14 \mathrm{E}-11$ & $2.08 \mathrm{E}-07$ & $2.08 \mathrm{E}-07$ & $2.08 \mathrm{E}-07$ & & & \\
\hline Se-79 & $2.59 \mathrm{E}-11$ & $2.49 \mathrm{E}-11$ & $1.47 \mathrm{E}-11$ & 1.98E-09 & 1.98E-09 & 1.98E-09 & & & \\
\hline Sr-90 & 1.57E-06 & $1.51 \mathrm{E}-06$ & $3.11 \mathrm{E}-08$ & $1.56 \mathrm{E}-04$ & $1.56 \mathrm{E}-04$ & $1.56 \mathrm{E}-04$ & & & \\
\hline $\mathrm{Y}-90$ & 1.57E-06 & $1.51 \mathrm{E}-06$ & $8.94 \mathrm{E}-07$ & 1.19E-04 & 1.19E-04 & 1.19E-04 & & & \\
\hline Zr-93 & $8.27 \mathrm{E}-11$ & $7.95 \mathrm{E}-11$ & $4.70 \mathrm{E}-11$ & $6.34 \mathrm{E}-09$ & $6.34 \mathrm{E}-09$ & 6.34E-09 & & & \\
\hline $\mathrm{Nb}-93 \mathrm{~m}$ & $6.37 \mathrm{E}-11$ & $6.13 \mathrm{E}-11$ & $3.62 \mathrm{E}-11$ & 4.88E-09 & $4.88 \mathrm{E}-09$ & 4.88E-09 & & & \\
\hline $\mathrm{Nb}-94$ & $6.72 \mathrm{E}-11$ & $6.47 \mathrm{E}-11$ & $3.82 \mathrm{E}-11$ & 5.15E-09 & 5.15E-09 & $5.15 \mathrm{E}-09$ & & & \\
\hline Tc-99 & $2.47 \mathrm{E}-09$ & $2.38 \mathrm{E}-09$ & $1.40 \mathrm{E}-09$ & $1.27 \mathrm{E}-07$ & $1.27 \mathrm{E}-07$ & $1.27 \mathrm{E}-07$ & & & \\
\hline Ru-106 & $5.95 \mathrm{E}-11$ & $5.72 \mathrm{E}-11$ & $3.38 \mathrm{E}-11$ & 4.50E-09 & $4.50 \mathrm{E}-09$ & 4.50E-09 & & & \\
\hline Rh-102 & $3.45 \mathrm{E}-14$ & $3.32 \mathrm{E}-14$ & $1.96 \mathrm{E}-14$ & $2.61 \mathrm{E}-12$ & $2.61 \mathrm{E}-12$ & $2.61 \mathrm{E}-12$ & & & \\
\hline Rh-106 & $5.95 \mathrm{E}-11$ & $5.72 \mathrm{E}-11$ & $3.38 \mathrm{E}-11$ & $4.50 \mathrm{E}-09$ & $4.50 \mathrm{E}-09$ & 4.50E-09 & & & \\
\hline Pd-107 & $6.61 \mathrm{E}-13$ & $6.35 \mathrm{E}-13$ & $1.31 \mathrm{E}-14$ & $6.56 \mathrm{E}-11$ & $6.56 \mathrm{E}-11$ & $6.56 \mathrm{E}-11$ & & & \\
\hline $\mathrm{Cd}-113 \mathrm{~m}$ & $1.45 \mathrm{E}-10$ & $1.40 \mathrm{E}-10$ & $8.24 \mathrm{E}-11$ & 4.09E-09 & $4.10 \mathrm{E}-09$ & 4.10E-09 & & & \\
\hline Sn-121m & $2.50 \mathrm{E}-12$ & $2.40 \mathrm{E}-12$ & $1.42 \mathrm{E}-12$ & $1.91 \mathrm{E}-10$ & $1.91 \mathrm{E}-10$ & $1.91 \mathrm{E}-10$ & & & \\
\hline Sn-126 & $2.44 \mathrm{E}-11$ & $2.34 \mathrm{E}-11$ & $1.38 \mathrm{E}-11$ & 1.87E-09 & 1.87E-09 & 1.87E-09 & & & \\
\hline Sb-125 & $3.14 \mathrm{E}-08$ & $3.02 \mathrm{E}-08$ & $1.78 \mathrm{E}-08$ & $2.37 \mathrm{E}-06$ & $2.37 \mathrm{E}-06$ & $2.37 \mathrm{E}-06$ & & & \\
\hline Sb-126 & $2.30 \mathrm{E}-12$ & $2.22 \mathrm{E}-12$ & $1.31 \mathrm{E}-12$ & $1.74 \mathrm{E}-10$ & $1.74 \mathrm{E}-10$ & $1.74 \mathrm{E}-10$ & & & \\
\hline Te-125m & $1.18 \mathrm{E}-10$ & $1.13 \mathrm{E}-10$ & $6.68 \mathrm{E}-11$ & 9.01E-09 & 9.01E-09 & 9.01E-09 & & & \\
\hline |-129 & $1.53 \mathrm{E}-10$ & $1.47 \mathrm{E}-10$ & $8.68 \mathrm{E}-11$ & 8.54E-09 & 8.54E-09 & 8.54E-09 & $1.84 \mathrm{E}-11$ & $1.83 \mathrm{E}-11$ & $1.83 \mathrm{E}-11$ \\
\hline Cs-134 & $7.10 \mathrm{E}-09$ & $6.83 \mathrm{E}-09$ & $4.03 \mathrm{E}-09$ & 5.95E-05 & $5.95 \mathrm{E}-05$ & 5.95E-05 & & & \\
\hline Cs-135 & $1.13 \mathrm{E}-10$ & $1.09 \mathrm{E}-10$ & $6.42 \mathrm{E}-11$ & 9.47E-07 & 9.47E-07 & 9.47E-07 & & & \\
\hline Cs-137 & $4.59 \mathrm{E}-06$ & $4.41 \mathrm{E}-06$ & $2.60 \mathrm{E}-06$ & $3.84 \mathrm{E}-02$ & $3.84 \mathrm{E}-02$ & $3.84 \mathrm{E}-02$ & & & \\
\hline Ba-137m & $2.01 \mathrm{E}-06$ & $1.94 \mathrm{E}-06$ & $3.98 \mathrm{E}-08$ & $2.00 \mathrm{E}-04$ & $2.00 \mathrm{E}-04$ & $2.00 \mathrm{E}-04$ & & & \\
\hline $\mathrm{Ce}-144$ & $4.02 \mathrm{E}-11$ & $3.87 \mathrm{E}-11$ & $2.28 \mathrm{E}-11$ & 3.04E-09 & $3.04 \mathrm{E}-09$ & 3.04E-09 & & & \\
\hline $\operatorname{Pr}-144$ & $4.02 \mathrm{E}-11$ & $3.87 \mathrm{E}-11$ & $2.28 \mathrm{E}-11$ & 3.04E-09 & $3.04 \mathrm{E}-09$ & 3.04E-09 & & & \\
\hline Pm-146 & $2.04 \mathrm{E}-12$ & $1.96 \mathrm{E}-12$ & 1.16E-12 & $1.54 \mathrm{E}-10$ & $1.54 \mathrm{E}-10$ & \begin{tabular}{|c|}
$1.54 \mathrm{E}-10$ \\
\end{tabular} & & & \\
\hline $\mathrm{Pm}-147$ & 1.08E-08 & 1.04E-08 & $6.14 \mathrm{E}-09$ & $8.19 E-07$ & 8.19E-07 & 8.19E-07 & & & \\
\hline Sm-151 & $2.14 \mathrm{E}-08$ & $2.06 \mathrm{E}-08$ & $1.22 \mathrm{E}-08$ & 1.62E-06 & $1.62 \mathrm{E}-06$ & 1.62E-06 & & & \\
\hline Eu-152 & $1.29 \mathrm{E}-10$ & $1.24 \mathrm{E}-10$ & 7.31E-11 & 9.74E-09 & 9.74E-09 & 9.74E-09 & & & \\
\hline Eu-154 & 4.84E-09 & 4.65E-09 & $2.75 \mathrm{E}-09$ & $3.66 \mathrm{E}-07$ & $3.66 \mathrm{E}-07$ & $3.66 \mathrm{E}-07$ & & & \\
\hline Eu-155 & 5.09E-09 & 4.90E-09 & 2.89E-09 & $3.85 \mathrm{E}-07$ & 3.86E-07 & 3.86E-07 & & & \\
\hline Th-230 & $4.67 \mathrm{E}-14$ & $4.50 \mathrm{E}-14$ & $2.65 \mathrm{E}-14$ & $3.58 \mathrm{E}-12$ & $3.58 \mathrm{E}-12$ & $3.58 \mathrm{E}-12$ & & & \\
\hline $\mathrm{Pa}-233$ & $1.09 \mathrm{E}-10$ & $1.05 \mathrm{E}-10$ & $6.21 \mathrm{E}-11$ & 8.38E-09 & 8.39E-09 & 8.39E-09 & & & \\
\hline U-232 & $1.08 \mathrm{E}-13$ & $1.03 \mathrm{E}-13$ & $6.11 \mathrm{E}-14$ & $8.24 \mathrm{E}-12$ & $8.24 \mathrm{E}-12$ & $8.24 \mathrm{E}-12$ & & & \\
\hline U-233 & $3.56 \mathrm{E}-15$ & $3.42 \mathrm{E}-15$ & $2.02 \mathrm{E}-15$ & $2.73 \mathrm{E}-13$ & $2.73 \mathrm{E}-13$ & \begin{tabular}{|l|}
$2.73 \mathrm{E}-13$ \\
\end{tabular} & & & \\
\hline U-234 & $5.88 \mathrm{E}-11$ & $5.66 \mathrm{E}-11$ & $3.34 \mathrm{E}-11$ & $4.51 \mathrm{E}-09$ & $4.51 \mathrm{E}-09$ & 4.51E-09 & & & \\
\hline U-235 & $3.06 \mathrm{E}-12$ & $2.95 \mathrm{E}-12$ & $1.74 \mathrm{E}-12$ & $2.35 \mathrm{E}-10$ & $2.35 \mathrm{E}-10$ & $2.35 \mathrm{E}-10$ & & & \\
\hline U-236 & $2.85 \mathrm{E}-12$ & $2.74 \mathrm{E}-12$ & $1.62 \mathrm{E}-12$ & $2.19 \mathrm{E}-10$ & $2.19 \mathrm{E}-10$ & $2.19 \mathrm{E}-10$ & & & \\
\hline U-237 & $2.40 \mathrm{E}-13$ & $2.31 \mathrm{E}-13$ & $1.36 \mathrm{E}-13$ & $1.84 \mathrm{E}-11$ & $1.84 \mathrm{E}-11$ & 1.84E-11 & & & \\
\hline U-238 & $1.04 \mathrm{E}-12$ & $9.96 \mathrm{E}-13$ & $5.88 \mathrm{E}-13$ & $7.94 \mathrm{E}-11$ & $7.94 \mathrm{E}-11$ & $7.94 \mathrm{E}-11$ & & & \\
\hline Np-237 & $3.04 \mathrm{E}-11$ & $2.92 \mathrm{E}-11$ & 1.73E-11 & 2.30E-09 & 2.30E-09 & 2.30E-09 & & & \\
\hline Pu-236 & $1.68 \mathrm{E}-13$ & $1.62 \mathrm{E}-13$ & $9.57 \mathrm{E}-14$ & $1.29 \mathrm{E}-11$ & $1.29 \mathrm{E}-11$ & $1.29 \mathrm{E}-11$ & & & \\
\hline Pu-238 & $4.97 \mathrm{E}-08$ & $4.78 \mathrm{E}-08$ & $2.82 \mathrm{E}-08$ & $3.81 \mathrm{E}-06$ & $3.81 \mathrm{E}-06$ & $3.81 \mathrm{E}-06$ & & & \\
\hline Pu-239 & 5.52E-09 & 5.31E-09 & $3.13 \mathrm{E}-09$ & $4.23 \mathrm{E}-07$ & $4.23 \mathrm{E}-07$ & $4.23 \mathrm{E}-07$ & & & \\
\hline Pu-240 & $6.30 \mathrm{E}-10$ & $6.06 \mathrm{E}-10$ & $3.58 \mathrm{E}-10$ & $4.83 \mathrm{E}-08$ & $4.83 E-08$ & $4.83 \mathrm{E}-08$ & & & \\
\hline Pu-241 & $3.75 \mathrm{E}-08$ & $3.61 \mathrm{E}-08$ & $2.13 \mathrm{E}-08$ & $2.87 \mathrm{E}-06$ & $2.88 \mathrm{E}-06$ & $2.88 \mathrm{E}-06$ & & & \\
\hline Pu-242 & $4.95 \mathrm{E}-13$ & $4.76 \mathrm{E}-13$ & $2.81 \mathrm{E}-13$ & $3.79 \mathrm{E}-11$ & $3.79 \mathrm{E}-11$ & $3.79 \mathrm{E}-11$ & & & \\
\hline Pu-244 & $2.96 \mathrm{E}-20$ & $2.85 \mathrm{E}-20$ & $1.68 \mathrm{E}-20$ & $2.27 \mathrm{E}-18$ & $2.27 \mathrm{E}-18$ & $2.27 \mathrm{E}-18$ & & & \\
\hline Am-241 & $3.41 \mathrm{E}-09$ & $3.28 \mathrm{E}-09$ & 1.94E-09 & $2.58 \mathrm{E}-07$ & $2.58 \mathrm{E}-07$ & $2.58 \mathrm{E}-07$ & & & \\
\hline Am-242m & $6.18 \mathrm{E}-13$ & $5.94 \mathrm{E}-13$ & $3.51 \mathrm{E}-13$ & $4.68 \mathrm{E}-11$ & $4.68 \mathrm{E}-11$ & $4.68 \mathrm{E}-11$ & & & \\
\hline
\end{tabular}


Table A-14. Mass balance, Tank WM-187 waste (continued).

\begin{tabular}{|c|c|c|c|c|c|c|c|c|c|}
\hline Stream \# & 109 & 110 & 111 & 112 & $113 \mathrm{~A}$ & 113B & 114 & 115 & 116 \\
\hline & Ci/wscm & Ci/wscm & Ci/wscm & $\mathrm{Ci} / \mathrm{L}$ & $\mathrm{Ci} / \mathrm{L}$ & $\mathrm{Ci} / \mathrm{L}$ & Ci/wscm & Ci/wscm & Ci/wscm \\
\hline Am-243 & 1.17E-12 & $1.13 \mathrm{E}-12$ & 6.66E-13 & 8.88E-11 & 8.88E-11 & $8.88 \mathrm{E}-11$ & & & \\
\hline Cm-242 & $1.13 \mathrm{E}-12$ & 1.09E-12 & $6.42 \mathrm{E}-13$ & $8.67 \mathrm{E}-11$ & 8.67E-11 & $8.67 \mathrm{E}-11$ & & & \\
\hline $\mathrm{Cm}-243$ & $1.64 \mathrm{E}-12$ & $1.57 \mathrm{E}-12$ & $9.29 \mathrm{E}-13$ & $1.25 \mathrm{E}-10$ & $1.25 \mathrm{E}-10$ & $1.25 \mathrm{E}-10$ & & & \\
\hline $\mathrm{Cm}-244$ & $7.29 \mathrm{E}-11$ & $7.01 \mathrm{E}-11$ & $4.14 \mathrm{E}-11$ & 5.59E-09 & 5.59E-09 & 5.59E-09 & & & \\
\hline Cm-245 & $1.72 \mathrm{E}-14$ & $1.66 \mathrm{E}-14$ & $9.79 \mathrm{E}-15$ & $1.32 \mathrm{E}-12$ & $1.32 \mathrm{E}-12$ & $1.32 \mathrm{E}-12$ & & & \\
\hline $\mathrm{Cm}-246$ & $1.13 \mathrm{E}-15$ & $1.09 \mathrm{E}-15$ & $6.42 \mathrm{E}-16$ & $8.66 \mathrm{E}-14$ & $8.66 \mathrm{E}-14$ & $8.66 \mathrm{E}-14$ & & & \\
\hline TRU & $6.04 \mathrm{E}-08$ & $5.81 \mathrm{E}-08$ & $3.43 \mathrm{E}-08$ & $4.63 \mathrm{E}-06$ & 4.63E-06 & $4.63 \mathrm{E}-06$ & & & \\
\hline \multicolumn{10}{|l|}{ TRU, nCi/gm } \\
\hline & & & & & & & & & \\
\hline & & & & & & & & & \\
\hline $\begin{array}{l}\text { Gas Stream Bulk } \\
\text { Composition (Wet Basis) }\end{array}$ & $\begin{array}{l}\text { mol\% or } \\
\text { ppmv }\end{array}$ & $\begin{array}{l}\text { mol\% or } \\
\text { ppmv }\end{array}$ & $\begin{array}{c}\mathrm{mol} \% \text { or } \\
\mathrm{ppmv}\end{array}$ & $\begin{array}{c}\text { mol\% or } \\
\text { ppmv }\end{array}$ & $\begin{array}{c}\text { mol\% or } \\
\text { ppmv }\end{array}$ & $\begin{array}{l}\mathrm{mol} \% \text { or } \\
\text { ppmv }\end{array}$ & $\begin{array}{l}\text { mol\% or } \\
\text { ppmv }\end{array}$ & $\begin{array}{c}\mathrm{mol} \% \text { or } \\
\text { ppmv }\end{array}$ & $\begin{array}{c}\mathrm{mol} \% \text { or } \\
\text { ppmv }\end{array}$ \\
\hline $\mathrm{H} 2 \mathrm{O}, \mathrm{mol} \%$ & $68.23 \%$ & $70.61 \%$ & $82.70 \%$ & & & & $82.69 \%$ & $82.69 \%$ & $82.69 \%$ \\
\hline $\mathrm{O} 2, \mathrm{~mol} \%$ & $0.51 \%$ & $2.24 \%$ & $1.32 \%$ & & & & $1.32 \%$ & $1.32 \%$ & $1.32 \%$ \\
\hline $\mathrm{N} 2, \mathrm{~mol} \%$ & $19.75 \%$ & $19.02 \%$ & $11.19 \%$ & & & & $11.20 \%$ & $11.20 \%$ & $11.20 \%$ \\
\hline $\mathrm{H} 2, \mathrm{~mol} \%$ & $3.91 \%$ & $0.00001 \%$ & $0.00001 \%$ & & & & $0.0003 \%$ & & $0.00 \%$ \\
\hline $\mathrm{CO} 2, \mathrm{~mol} \%$ & $6.41 \%$ & $8.06 \%$ & $4.74 \%$ & & & & $4.74 \%$ & $4.74 \%$ & $4.74 \%$ \\
\hline $\mathrm{CO}, \mathrm{ppmv}$ & $1.04 \mathrm{E}+04$ & $2.09 \mathrm{E}-02$ & $1.23 \mathrm{E}-02$ & & & & $1.23 \mathrm{E}-02$ & $1.23 \mathrm{E}-02$ & 1.23E-02 \\
\hline $\mathrm{NO}, \mathrm{ppmv}$ & $5.99 \mathrm{E}+02$ & $6.51 \mathrm{E}+02$ & $3.83 \mathrm{E}+02$ & & & & $3.83 \mathrm{E}+02$ & $3.83 \mathrm{E}+02$ & $3.83 \mathrm{E}+02$ \\
\hline NO2, ppmv & $4.05 E+01$ & $2.33 \mathrm{E}+00$ & $1.37 \mathrm{E}+00$ & & & & $1.37 \mathrm{E}+00$ & $1.37 \mathrm{E}+00$ & $1.37 \mathrm{E}+00$ \\
\hline SO2, ppmv & 1.58E-02 & $1.22 \mathrm{E}+02$ & $7.20 \mathrm{E}+01$ & & & & $7.20 \mathrm{E}+01$ & $7.20 \mathrm{E}+01$ & $7.20 \mathrm{E}+01$ \\
\hline $\mathrm{Cl}, \mathrm{ppmv}$ & \begin{tabular}{|l|}
$9.30 \mathrm{E}-01$ \\
\end{tabular} & $8.95 \mathrm{E}-01$ & $5.26 \mathrm{E}-01$ & & & & $1.11 \mathrm{E}-01$ & 1.11E-01 & 1.11E-01 \\
\hline $\mathrm{F}, \mathrm{ppmv}$ & $1.26 \mathrm{E}+01$ & $1.21 \mathrm{E}+01$ & $7.13 \mathrm{E}+00$ & & & & $1.78 \mathrm{E}+01$ & $1.78 \mathrm{E}+01$ & $1.78 \mathrm{E}+01$ \\
\hline C (organic), ppmv & $5.79 \mathrm{E}+02$ & \begin{tabular}{|l|}
$5.45 \mathrm{E}-27$ \\
\end{tabular} & & & & & & & \\
\hline $\mathrm{H}$ (organic), ppmv & $2.31 \mathrm{E}+03$ & $2.18 \mathrm{E}-26$ & & & & & & & \\
\hline $\mathrm{Hg}, \mathrm{ug} / \mathrm{wscm}$ & $1.14 \mathrm{E}+05$ & $1.09 \mathrm{E}+05$ & $6.46 \mathrm{E}+04$ & & & & & $3.62 \mathrm{E}+04$ & $3.62 \mathrm{E}+04$ \\
\hline $\mathrm{PM}, \mathrm{mg} / \mathrm{wscm}$ & $5.21 \mathrm{E}+02$ & $1.37 \mathrm{E}+02$ & $8.92 \mathrm{E}+01$ & & & & $1.20 \mathrm{E}+01$ & $4.81 \mathrm{E}+01$ & $4.81 \mathrm{E}+01$ \\
\hline SVM, ug/wscm & $3.03 \mathrm{E}+01$ & $2.92 \mathrm{E}+01$ & $1.72 \mathrm{E}+01$ & & & & & & \\
\hline LVM, ug/wscm & 1.27E+01 & \begin{tabular}{|l|}
$1.22 \mathrm{E}+01$ \\
\end{tabular} & $7.19 \mathrm{E}+00$ & & & & & & \\
\hline $\begin{array}{l}\text { Gas Stream Bulk } \\
\text { Composition (Dry Basis) }\end{array}$ & $\mathrm{mol} \%$ & $\mathrm{~mol} \%$ & $\mathrm{~mol} \%$ & $\mathrm{~mol} \%$ & $\mathrm{~mol} \%$ & $\mathrm{~mol} \%$ & $\mathrm{~mol} \%$ & $\mathrm{~mol} \%$ & $\mathrm{~mol} \%$ \\
\hline $\mathrm{O} 2, \mathrm{~mol} \%$, dry basis & $1.60 \%$ & $7.61 \%$ & $7.61 \%$ & & & & $7.61 \%$ & $7.61 \%$ & $7.61 \%$ \\
\hline $\mathrm{N} 2, \mathrm{~mol} \%$, dry basis & $62.17 \%$ & $64.71 \%$ & $64.70 \%$ & & & & $64.68 \%$ & $64.68 \%$ & $64.68 \%$ \\
\hline $\mathrm{H} 2, \mathrm{~mol} \%$, dry basis & $12.31 \%$ & $0.00004 \%$ & $0.00004 \%$ & & & & $0.002 \%$ & & $0.00 \%$ \\
\hline $\mathrm{CO} 2, \mathrm{~mol} \%$, dry basis & $20.18 \%$ & $27.41 \%$ & $27.41 \%$ & & & & $27.40 \%$ & $27.40 \%$ & $27.40 \%$ \\
\hline $\begin{array}{l}\text { Gas Stream Bulk } \\
\text { Composition (Dry Basis, } \\
\text { Corrected to } 7 \% \text { O2 with } \\
100 \% \text { O2 Combustion Air) }\end{array}$ & $\begin{array}{l}\text { ppmv, or } \\
\text { ug/dscm } \\
\text { or } \\
\mathrm{mg} / \mathrm{dscm}\end{array}$ & $\begin{array}{l}\mathrm{ppmv}, \text { or } \\
\text { ug/dscm } \\
\text { or } \\
\mathrm{mg} / \mathrm{dscm}\end{array}$ & $\begin{array}{c}\text { ppmv, or } \\
\text { ug/dscm or } \\
\mathrm{mg} / \mathrm{dscm}\end{array}$ & $\begin{array}{l}\mathrm{ppmv}, \text { or } \\
\mathrm{ug} / \mathrm{dscm} \\
\text { or } \\
\mathrm{mg} / \mathrm{dscm}\end{array}$ & $\begin{array}{l}\mathrm{ppmv}, \text { or } \\
\mathrm{ug} / \mathrm{dscm} \\
\text { or } \\
\mathrm{mg} / \mathrm{dscm}\end{array}$ & $\begin{array}{l}\text { ppmv, or } \\
\text { ug/dscm } \\
\text { or } \\
\mathrm{mg} / \mathrm{dscm}\end{array}$ & $\begin{array}{l}\mathrm{ppmv}, \text { or } \\
\text { ug/dscm } \\
\text { or } \\
\mathrm{mg} / \mathrm{dscm}\end{array}$ & $\begin{array}{l}\text { ppmv, or } \\
\text { ug/dscm } \\
\text { or } \\
\mathrm{mg} / \mathrm{dscm}\end{array}$ & $\begin{array}{l}\text { ppmv, or } \\
\text { ug/dscm } \\
\text { or } \\
\mathrm{mg} / \mathrm{dscm}\end{array}$ \\
\hline $\mathrm{CO}, \mathrm{ppmv}$, dry basis & $4.60 \mathrm{E}+03$ & 9.94E-03 & 9.94E-03 & & & & 9.94E-03 & 9.94E-03 & 9.94E-03 \\
\hline NO, ppmv, dry basis & $2.64 \mathrm{E}+02$ & $3.10 \mathrm{E}+02$ & $3.10 \mathrm{E}+02$ & & & & $3.10 \mathrm{E}+02$ & $3.10 \mathrm{E}+02$ & $3.10 \mathrm{E}+02$ \\
\hline NO2, ppmv, dry basis & $1.79 \mathrm{E}+01$ & $1.11 \mathrm{E}+00$ & $1.11 \mathrm{E}+00$ & & & & $1.11 \mathrm{E}+00$ & $1.11 \mathrm{E}+00$ & $1.11 \mathrm{E}+00$ \\
\hline SO2, ppmv, dry basis & $6.95 \mathrm{E}-03$ & $5.83 \mathrm{E}+01$ & $5.83 \mathrm{E}+01$ & & & & $5.83 \mathrm{E}+01$ & $5.83 \mathrm{E}+01$ & $5.83 \mathrm{E}+01$ \\
\hline $\mathrm{Cl}, \mathrm{ppmv}$, dry basis & 4.10E-01 & 4.26E-01 & $4.26 \mathrm{E}-01$ & & & & 9.01E-02 & 9.01E-02 & 9.01E-02 \\
\hline $\mathrm{F}, \mathrm{ppmv}$, dry basis & $5.55 \mathrm{E}+00$ & $5.78 \mathrm{E}+00$ & $5.78 \mathrm{E}+00$ & & & & $1.44 \mathrm{E}+01$ & $1.44 \mathrm{E}+01$ & $1.44 \mathrm{E}+01$ \\
\hline C (organic), ppmv, dry basis & $2.55 \mathrm{E}+02$ & & & & & & & & \\
\hline $\mathrm{H}$ (organic), ppmv, dry basis & $1.02 \mathrm{E}+03$ & & & & & & & & \\
\hline $\mathrm{Hg}, \mathrm{ug} / \mathrm{dscm}$ & $5.01 \mathrm{E}+04$ & $5.21 \mathrm{E}+04$ & $5.23 E+04$ & & & & & $2.93 E+04$ & $2.93 E+04$ \\
\hline $\mathrm{PM}, \mathrm{mg} / \mathrm{dscm}$ & $2.30 \mathrm{E}+02$ & $6.55 \mathrm{E}+01$ & $7.23 E+01$ & & & & $9.68 \mathrm{E}+00$ & $3.89 \mathrm{E}+01$ & $3.89 \mathrm{E}+01$ \\
\hline SVM, ug/dscm & $1.01 \mathrm{E}+02$ & $9.69 \mathrm{E}+01$ & $5.72 \mathrm{E}+01$ & & & & & & \\
\hline LVM, ug/dscm & $4.20 \mathrm{E}+01$ & $4.04 \mathrm{E}+01$ & $2.39 \mathrm{E}+01$ & & & & & & \\
\hline
\end{tabular}


Table A-14. Mass balance, Tank WM-187 waste (continued).

\begin{tabular}{|c|c|c|c|c|c|c|c|c|}
\hline PFD \# & PFD-3 & PFD-3 & PFD-3 & PFD-3 & PFD-2 & PFD-2 & PFD-2 & PFD-3 \\
\hline WM-187, Stream \# & 117 & 118 & 119 & 120 & 201 & 202 & 203 & 204 \\
\hline Stream Name & $\begin{array}{l}\text { Pressure } \\
\text { Control } \\
\text { Bleed Air }\end{array}$ & $\begin{array}{c}\text { Final } \\
\text { HEPA Off- } \\
\text { Gas Outlet }\end{array}$ & $\begin{array}{c}\text { Off-Gas to } \\
\text { Blower }\end{array}$ & $\begin{array}{l}\text { Spent } \\
\text { GAC }\end{array}$ & $\begin{array}{l}\text { Boiler } \\
\text { Feed } \\
\text { Water }\end{array}$ & $\begin{array}{c}\text { Fuel Oil to } \\
\text { Boiler }\end{array}$ & $\begin{array}{l}\text { Steam To } \\
\text { Reformer }\end{array}$ & $\begin{array}{l}\text { Propane } \\
\text { to Oxidizer }\end{array}$ \\
\hline Rate or Volume & $1.32 \mathrm{E}+04$ & $6.03 E+05$ & $6.37 \mathrm{E}+05$ & $5.20 \mathrm{E}+00$ & $3.16 \mathrm{E}+02$ & $4.18 \mathrm{E}+01$ & $6.32 \mathrm{E}+04$ & $1.05 \mathrm{E}+02$ \\
\hline $\bar{V}_{\text {Volume Flow (stand, wet) }}^{*}$ & $1.30 \mathrm{E}+04$ & $1.83 \mathrm{E}+05$ & $1.95 \mathrm{E}+05$ & & & & $5.66 \mathrm{E}+04$ & $2.97 \mathrm{E}+02$ \\
\hline Volume Flow (standard, dry) ${ }^{*}$ & $1.30 \mathrm{E}+04$ & $3.16 \mathrm{E}+04$ & $4.46 \mathrm{E}+04$ & & & & & $2.97 \mathrm{E}+02$ \\
\hline Rate Units & $\mathrm{ft} 3 / \mathrm{hr}$ & $\mathrm{ft} 3 / \mathrm{hr}$ & $\mathrm{ft} 3 / \mathrm{hr}$ & $\mathrm{lb} / \mathrm{hr}$ & $\mathrm{gal} / \mathrm{hr}$ & gal/hr & $\mathrm{ft} 3 / \mathrm{hr}$ & $\mathrm{ft} 3 / \mathrm{hr}$ \\
\hline Rate or Volume, metric & $3.68 \mathrm{E}+02$ & $5.17 \mathrm{E}+03$ & $5.53 \mathrm{E}+03$ & $2.36 \mathrm{E}+00$ & $1.20 \mathrm{E}+03$ & $1.58 \mathrm{E}+02$ & $1.60 \mathrm{E}+03$ & $8.42 \mathrm{E}+00$ \\
\hline Rate Units & wscm/hr & wscm $/ \mathrm{hr}$ & wscm/hr & $\mathrm{kg} / \mathrm{hr}$ & $\mathrm{L} / \mathrm{hr}$ & $\mathrm{L} / \mathrm{hr}$ & wscm/hr & wscm $/ \mathrm{hr}$ \\
\hline Temperature, ${ }^{\circ} \mathrm{C}$ & 25 & 120 & 115 & 28 & 15 & 15 & 670 & 25 \\
\hline Temperature, ${ }^{\circ} \mathrm{F}$ & 77 & 248 & 239 & 82 & 59 & 59 & 1238 & 77 \\
\hline Pressure, psia & 14.7 & 6.0 & 6.0 & 14.7 & 42.3 & 14.7 & 42.3 & 42.3 \\
\hline Specific Gravity & $1.18 \mathrm{E}-03$ & $2.59 \mathrm{E}-04$ & $2.70 \mathrm{E}-04$ & $5.86 \mathrm{E}-01$ & $1.00 \mathrm{E}+00$ & $7.70 \mathrm{E}-01$ & $6.70 \mathrm{E}-04$ & $5.19 \mathrm{E}-03$ \\
\hline Chemical Composition & Ib/wscf & $\mathrm{lb} / \mathrm{wscf}$ & Ib/wscf & Wt frac & Mol/liter & Mol/liter & Ib/wscf & Ib/wscf \\
\hline $\mathrm{H}+$ & & $2.32 \mathrm{E}-07$ & $2.17 \mathrm{E}-07$ & $9.20 \mathrm{E}-06$ & & $2.40 \mathrm{E}-01$ & & \\
\hline \multicolumn{9}{|l|}{$\mathrm{Al}+3$} \\
\hline \multirow{2}{*}{\multicolumn{9}{|c|}{$\frac{S b+5}{A c+3}$}} \\
\hline \multirow{2}{*}{\multicolumn{9}{|c|}{ As+3 }} \\
\hline & & & & & & & & \\
\hline \multicolumn{9}{|l|}{$\frac{\mathrm{Ba}+2}{\mathrm{Be}+2}$} \\
\hline \multicolumn{9}{|l|}{$\mathrm{B}+3$} \\
\hline \multicolumn{9}{|l|}{$\mathrm{Cd}+2$} \\
\hline \multirow{2}{*}{\multicolumn{9}{|c|}{$\frac{\mathrm{Ca}+2}{\mathrm{Cr}+3}$}} \\
\hline & & & & & & & & \\
\hline \multicolumn{9}{|l|}{$\mathrm{Co}+2$} \\
\hline \multicolumn{9}{|l|}{ Cs+ } \\
\hline \multicolumn{9}{|l|}{$\mathrm{Cu}+2$} \\
\hline \multirow{2}{*}{\multicolumn{9}{|c|}{\begin{tabular}{|l|}
$\mathrm{Fe}+3$ \\
$\mathrm{Ph}+2$
\end{tabular}}} \\
\hline & & & & & \multicolumn{4}{|c|}{$\mathrm{Pb}+2$} \\
\hline $\mathrm{Hg}+2$ & & $2.26 \mathrm{E}-09$ & $2.12 \mathrm{E}-09$ & $7.93 \mathrm{E}-02$ & & & & \\
\hline \multirow{2}{*}{\multicolumn{9}{|c|}{$\begin{array}{l}\mathrm{Mn}+4 \\
\mathrm{Ni}+2\end{array}$}} \\
\hline \multirow{2}{*}{\multicolumn{9}{|c|}{$\frac{\mathrm{Ni}+2}{\mathrm{~K}+}$}} \\
\hline & & & & & & & & \\
\hline \multicolumn{9}{|l|}{$\mathrm{Se}+4$} \\
\hline \multirow{2}{*}{\multicolumn{9}{|c|}{$\frac{\mathrm{Ag}+}{\mathrm{Na}+}$}} \\
\hline & & & & & & & & \\
\hline \multicolumn{6}{|l|}{$\frac{\mathrm{Na}+}{\mathrm{Tl}+3}$} & & & \\
\hline $\mathrm{U}+4$ & & & & & & & & \\
\hline $\mathrm{V+3}$ & & & & & & & & \\
\hline$Z n+2$ & & & & & & & & \\
\hline $\mathrm{Zr}+4$ & & & & & & & & \\
\hline $\mathrm{Cl}-$ & & 1.02E-09 & $9.58 \mathrm{E}-10$ & $3.24 \mathrm{E}-04$ & & & & \\
\hline F- & & $8.78 \mathrm{E}-07$ & $8.21 \mathrm{E}-07$ & & & & & \\
\hline$\overline{\mathrm{SO} 4-2}$ & & & & & & & & \\
\hline NO3- & & $1.14 \mathrm{E}-05$ & $1.07 \mathrm{E}-05$ & & & & & \\
\hline PO4-3 & & & & & & & & \\
\hline $\mathrm{Am}+4$ & & & & & & & & \\
\hline $\mathrm{Br}-$ & & $3.23 \mathrm{E}-14$ & $3.02 \mathrm{E}-14$ & 1.02E-08 & & & & \\
\hline $\mathrm{Ce}+4$ & & & & & & & & \\
\hline $\mathrm{Eu+3}$ & & & & & & & & \\
\hline $\mathrm{Gd+3}$ & & & & & & & & \\
\hline $\mathrm{Ge}+4$ & & & & & & & & \\
\hline $\ln +3$ & & & & & & & & \\
\hline $1-$ & & $6.68 \mathrm{E}-13$ & $6.24 \mathrm{E}-13$ & $2.11 \mathrm{E}-07$ & & & & \\
\hline $\mathrm{La}+3$ & & & & & & & & \\
\hline
\end{tabular}


Table A-14. Mass balance, Tank WM-187 waste (continued).

\begin{tabular}{|c|c|c|c|c|c|c|c|c|}
\hline Stream \# & 117 & 118 & 119 & 120 & 201 & 202 & 203 & 204 \\
\hline Chemical Composition & $\mathrm{lb} /$ wscf & $\mathrm{lb} / \mathrm{wscf}$ & $\mathrm{lb} /$ wscf & Wt frac & Mol/liter & Mol/liter & $\mathrm{lb} /$ wscf & $\mathrm{lb} / \mathrm{wscf}$ \\
\hline \multicolumn{9}{|l|}{ Li+ } \\
\hline \multicolumn{9}{|l|}{$\mathrm{Mg}+2$} \\
\hline \multicolumn{9}{|l|}{$\mathrm{Mo}+6$} \\
\hline \multicolumn{9}{|l|}{$\mathrm{Nd}+3$} \\
\hline \multicolumn{9}{|l|}{$\mathrm{Np}+4$} \\
\hline \multicolumn{9}{|l|}{$\mathrm{Nb}+5$} \\
\hline \multicolumn{9}{|l|}{$\mathrm{Pd}+4$} \\
\hline \multicolumn{9}{|l|}{$\mathrm{Pu}+4$} \\
\hline \multicolumn{9}{|l|}{$\mathrm{Pr}+4$} \\
\hline \multicolumn{9}{|l|}{$\mathrm{Pm}+3$} \\
\hline \multicolumn{9}{|l|}{$\mathrm{Rh}+4$} \\
\hline \multicolumn{9}{|l|}{$\mathrm{Rb}+$} \\
\hline \multicolumn{9}{|l|}{ Ru+3 } \\
\hline \multicolumn{9}{|l|}{$\mathrm{Sm}+3$} \\
\hline \multicolumn{9}{|l|}{$\mathrm{Si+4}$} \\
\hline \multicolumn{9}{|l|}{$\mathrm{Sr}+2$} \\
\hline \multicolumn{9}{|l|}{ Tc+7 } \\
\hline \multicolumn{9}{|l|}{$\mathrm{Te}+4$} \\
\hline \multicolumn{9}{|l|}{$\mathrm{Tb}+4$} \\
\hline \multicolumn{9}{|l|}{$\mathrm{Th}+4$} \\
\hline \multicolumn{9}{|l|}{ Sn+4 } \\
\hline $\mathrm{Ti}+4$ & & & & & & & & \\
\hline $\mathrm{Y}+3$ & & & & & & & & \\
\hline $\mathrm{OH}-$ & & & & & & & & \\
\hline $\mathrm{H} 2 \mathrm{O}$ & $4.68 \mathrm{E}-06$ & 3.87E-02 & $3.62 \mathrm{E}-02$ & & $5.56 \mathrm{E}+01$ & & 4.68E-02 & \\
\hline $\mathrm{SO} 2$ & & $1.20 \mathrm{E}-05$ & $1.12 \mathrm{E}-05$ & & & & & \\
\hline $\mathrm{H} 2 \mathrm{~S}$ & & & & & & $1.20 \mathrm{E}-01$ & & \\
\hline $\mathrm{CO}$ & & $8.93 E-10$ & $8.35 E-10$ & & & & & \\
\hline $\mathrm{CO} 2$ & & $5.42 \mathrm{E}-03$ & 5.07E-03 & & & & & \\
\hline $\mathrm{H} 2$ & & & & & & & & \\
\hline $\mathrm{N} 2$ & $5.75 \mathrm{E}-02$ & $8.14 \mathrm{E}-03$ & $1.14 \mathrm{E}-02$ & & & & & \\
\hline NO & & $2.98 \mathrm{E}-05$ & $2.79 \mathrm{E}-05$ & & & & & \\
\hline NO2 & & 1.64E-07 & $1.53 \mathrm{E}-07$ & & & & & \\
\hline $\mathrm{O} 2$ & $1.74 \mathrm{E}-02$ & $1.09 \mathrm{E}-03$ & $2.18 \mathrm{E}-03$ & & & & & \\
\hline S (other) & & & & & & & & \\
\hline $\mathrm{CO} 3$ & & & & & & & & \\
\hline C (reductant) & & & & $9.20 \mathrm{E}-01$ & & & & \\
\hline $\mathrm{O}$ (oxides) & & & & & & & & \\
\hline C (organic) & & & & & & $5.41 \mathrm{E}+01$ & & $9.35 \mathrm{E}-02$ \\
\hline $\mathrm{H}$ (organic) & & & & & & $1.15 \mathrm{E}+02$ & & $2.09 \mathrm{E}-02$ \\
\hline O (organic) & & & & & & & & \\
\hline Mass Flow (kg/hr): & $4.42 \mathrm{E}+02$ & $4.42 \mathrm{E}+03$ & $4.86 \mathrm{E}+03$ & $2.36 \mathrm{E}+00$ & $1.20 \mathrm{E}+03$ & $1.22 \mathrm{E}+02$ & $1.20 \mathrm{E}+03$ & $1.54 \mathrm{E}+01$ \\
\hline & & & & & & & & \\
\hline & & & & & & & & \\
\hline & $\mathrm{w} / \mathrm{m} 3$ & $\mathrm{w} / \mathrm{m} 3$ & $w / m 3$ & $\mathrm{w} / \mathrm{m} 3$ & $w / m 3$ & $\mathrm{w} / \mathrm{m} 3$ & $w / m 3$ & $\mathrm{w} / \mathrm{m} 3$ \\
\hline Heat Generation, w/m3 & & & & $5.89 \mathrm{E}-11$ & & & & \\
\hline
\end{tabular}


Table A-14. Mass balance, Tank WM-187 waste (continued).

\begin{tabular}{|c|c|c|c|c|c|c|c|}
\hline Stream \# & 117 & 118 & 119 & 120 & 201 & 202 & 203 \\
\hline Radiological Composition & Ci/wscm & $\mathrm{Ci} / \mathrm{wscm}$ & Ci/wscm & $\mathrm{Ci} / \mathrm{kg}$ & $\mathrm{Ci} / \mathrm{L}$ & $\mathrm{Ci} / \mathrm{L}$ & $\mathrm{Ci} / \mathrm{wscm}$ \\
\hline $\mathrm{H}-3$ & & $6.54 \mathrm{E}-07$ & $6.12 \mathrm{E}-07$ & & & & \\
\hline $\mathrm{C}-14$ & & $8.83 \mathrm{E}-12$ & $8.25 \mathrm{E}-12$ & $3.43 \mathrm{E}-10$ & & & \\
\hline \multicolumn{8}{|l|}{ Co-60 } \\
\hline \multicolumn{8}{|l|}{ Ni-59 } \\
\hline \multicolumn{8}{|l|}{$\mathrm{Ni}-63$} \\
\hline \multirow{2}{*}{\multicolumn{8}{|c|}{\begin{tabular}{|l|} 
Se-79 \\
$S r g n$
\end{tabular}}} \\
\hline & & & & & & & $\frac{S r-90}{Y-90}$ \\
\hline $\mathrm{Y}-90$ & & & & & & & \\
\hline \multicolumn{8}{|l|}{ Zr-93 } \\
\hline \multicolumn{8}{|l|}{$\mathrm{Nb}-93 \mathrm{~m}$} \\
\hline \multirow{2}{*}{\multicolumn{8}{|c|}{$\mathrm{Nb}-94$}} \\
\hline \multirow{2}{*}{\multicolumn{8}{|c|}{$\begin{array}{l}\text { Tc-99 } \\
\text { Ru-106 }\end{array}$}} \\
\hline & & & & & & & \\
\hline \multicolumn{8}{|l|}{ Rh-102 } \\
\hline \multicolumn{8}{|l|}{ Rh-106 } \\
\hline \multirow{2}{*}{\multicolumn{8}{|c|}{$\mathrm{Pd}-107$}} \\
\hline & & & & & & & \begin{tabular}{|l|} 
Cd-113m \\
Sn-121m
\end{tabular} \\
\hline Sn-121m & & & & & & & \\
\hline \multicolumn{8}{|l|}{ Sn-126 } \\
\hline \multicolumn{8}{|l|}{ Sb-125 } \\
\hline \multirow{2}{*}{\multicolumn{8}{|c|}{\begin{tabular}{|l|} 
Sb-126 \\
Te-125m
\end{tabular}}} \\
\hline \multicolumn{6}{|l|}{ Te-125m } & & \\
\hline I-129 & & 1.83E-12 & $1.71 \mathrm{E}-12$ & 3.61E-08 & & & \\
\hline Cs-134 & & & & & & & \\
\hline Cs-135 & & & & & & & \\
\hline Cs-137 & & & & & & & \\
\hline Ba-137m & & & & & & & \\
\hline Ce-144 & & & & & & & \\
\hline Pr-144 & & & & & & & \\
\hline $\mathrm{Pm}-146$ & & & & & & & \\
\hline Pm-147 & & & & & & & \\
\hline Sm-151 & & & & & & & \\
\hline Eu-152 & & & & & & & \\
\hline Eu-154 & & & & & & & \\
\hline Eu-155 & & & & & & & \\
\hline & & & & & & & \\
\hline Th-230 & & & & & & & \\
\hline $\mathrm{Pa}-233$ & & & & & & & \\
\hline $\mathrm{U}-232$ & & & & & & & \\
\hline $\mathrm{U}-233$ & & & & & & & \\
\hline U-234 & & & & & & & \\
\hline$U-235$ & & & & & & & \\
\hline $\mathrm{U}-236$ & & & & & & & \\
\hline $\mathrm{U}-237$ & & & & & & & \\
\hline $\mathrm{U}-238$ & & & & & & & \\
\hline Np-237 & & & & & & & \\
\hline $\mathrm{Pu}-236$ & & & & & & & \\
\hline Pu-238 & & & & & & & \\
\hline Pu-239 & & & & & & & \\
\hline Pu-240 & & & & & & & \\
\hline Pu-241 & & & & & & & \\
\hline Pu-242 & & & & & & & \\
\hline $\mathrm{Pu}-244$ & & & & & & & \\
\hline Am-241 & & & & & & & \\
\hline Am-242m & & & & & & & \\
\hline
\end{tabular}


Table A-14. Mass balance, Tank WM-187 waste (continued).

\begin{tabular}{|c|c|c|c|c|c|c|c|c|}
\hline Stream \# & 117 & 118 & 119 & 120 & 201 & 202 & 203 & 204 \\
\hline & $\mathrm{Ci} / \mathrm{wscm}$ & $\mathrm{Ci} / \mathrm{wscm}$ & $\mathrm{Ci} / \mathrm{wscm}$ & $\mathrm{Ci} / \mathrm{kg}$ & $\mathrm{Ci} / \mathrm{L}$ & $\mathrm{Ci} / \mathrm{L}$ & Ci/wscm & Ci/wscm \\
\hline \multicolumn{9}{|l|}{ Am-243 } \\
\hline \multicolumn{9}{|l|}{$\mathrm{Cm}-242$} \\
\hline \multicolumn{9}{|l|}{$\mathrm{Cm}-243$} \\
\hline \multicolumn{9}{|l|}{$\mathrm{Cm}-244$} \\
\hline \multicolumn{9}{|l|}{$\mathrm{Cm}-245$} \\
\hline \multicolumn{9}{|l|}{$\mathrm{Cm}-246$} \\
\hline \multicolumn{9}{|l|}{ TRU } \\
\hline & & & & & & & & \\
\hline & & & & & & & & \\
\hline $\begin{array}{l}\text { Gas Stream Bulk } \\
\text { Composition (Wet Basis) }\end{array}$ & $\begin{array}{l}\text { mol\% or } \\
\text { ppmv }\end{array}$ & $\begin{array}{l}\text { mol\% or } \\
\text { ppmv }\end{array}$ & $\begin{array}{l}\text { mol\% or } \\
\text { ppmv }\end{array}$ & $\begin{array}{l}\text { mol\% or } \\
\text { ppmv }\end{array}$ & $\begin{array}{l}\text { mol\% or } \\
\text { ppmv }\end{array}$ & $\begin{array}{l}\text { mol\% or } \\
\text { ppmv }\end{array}$ & $\begin{array}{c}\text { mol\% or } \\
\text { ppmv }\end{array}$ & $\begin{array}{c}\mathrm{mol} \% \text { or } \\
\text { ppmv }\end{array}$ \\
\hline $\mathrm{H} 2 \mathrm{O}, \mathrm{mol} \%$ & $0.01 \%$ & $82.69 \%$ & $77.18 \%$ & & & & $100.00 \%$ & \\
\hline $\mathrm{O} 2, \mathrm{~mol} \%$ & $20.99 \%$ & $1.32 \%$ & $2.63 \%$ & & & & & \\
\hline $\mathrm{N} 2, \mathrm{~mol} \%$ & $79.00 \%$ & $11.20 \%$ & $15.71 \%$ & & & & & \\
\hline \multicolumn{9}{|l|}{$\mathrm{H} 2, \mathrm{~mol} \%$} \\
\hline $\mathrm{CO} 2, \mathrm{~mol} \%$ & & $4.74 \%$ & $4.43 \%$ & & & & & \\
\hline $\mathrm{CO}, \mathrm{ppmv}$ & & $1.23 \mathrm{E}-02$ & $1.15 \mathrm{E}-02$ & & & & & \\
\hline $\mathrm{NO}, \mathrm{ppmv}$ & & $3.83 E+02$ & $3.58 \mathrm{E}+02$ & & & & & \\
\hline NO2, ppmv & & $1.37 \mathrm{E}+00$ & $1.28 \mathrm{E}+00$ & & & & & \\
\hline SO2, ppmv & & $7.20 \mathrm{E}+01$ & $6.72 \mathrm{E}+01$ & & & & & \\
\hline $\mathrm{Cl}, \mathrm{ppmv}$ & & $1.11 \mathrm{E}-02$ & $1.04 \mathrm{E}-02$ & & & & & \\
\hline $\mathrm{F}, \mathrm{ppmv}$ & & $1.78 \mathrm{E}+01$ & $1.66 \mathrm{E}+01$ & & & & & \\
\hline C (organic), ppmv & & & & & & & & $3.00 \mathrm{E}+06$ \\
\hline $\mathrm{H}$ (organic), ppmv & & & & & & & & $8.00 \mathrm{E}+06$ \\
\hline $\mathrm{Hg}, \mathrm{ug} / \mathrm{wscm}$ & & $3.62 \mathrm{E}+01$ & $3.39 \mathrm{E}+01$ & & & & & \\
\hline $\mathrm{PM}, \mathrm{mg} / \mathrm{wscm}$ & $1.57 \mathrm{E}+01$ & $1.19 \mathrm{E}+01$ & $1.21 \mathrm{E}+01$ & & & & $1.16 \mathrm{E}+01$ & $1.83 \mathrm{E}+06$ \\
\hline \multicolumn{9}{|l|}{ SVM, ug/wscm } \\
\hline \multicolumn{9}{|l|}{ LVM, ug/wscm } \\
\hline $\begin{array}{l}\text { Gas Stream Bulk } \\
\text { Composition (Dry Basis) }\end{array}$ & $\mathrm{mol} \%$ & $\mathrm{~mol} \%$ & $\mathrm{~mol} \%$ & $\mathrm{~mol} \%$ & $\mathrm{~mol} \%$ & $\mathrm{~mol} \%$ & $\mathrm{~mol} \%$ & $\mathrm{~mol} \%$ \\
\hline $02, \mathrm{~mol} \%$, dry basis & $20.9921 \%$ & $1.0658 \%$ & $11.5102 \%$ & & & & & \\
\hline $\mathrm{N} 2, \mathrm{~mol} \%$, dry basis & $79.01 \%$ & $64.68 \%$ & $68.86 \%$ & & & & & \\
\hline \multicolumn{9}{|l|}{$\mathrm{H} 2, \mathrm{~mol} \%$, dry basis } \\
\hline $\mathrm{CO} 2, \mathrm{~mol} \%$, dry basis & & $27.40 \%$ & $19.41 \%$ & & & & & \\
\hline $\begin{array}{l}\text { Gas Stream Bulk } \\
\text { Composition (Dry Basis, } \\
\text { Corrected to } 7 \% \text { O2 with } \\
100 \% \text { O2 Combustion Air) }\end{array}$ & $\begin{array}{l}\text { ppmv, or } \\
\text { ug/dscm or } \\
\text { mg/dscm }\end{array}$ & $\begin{array}{l}\text { ppmv, or } \\
\text { ug/dscm } \\
\text { or } \\
\mathrm{mg} / \mathrm{dscm}\end{array}$ & $\begin{array}{l}\text { ppmv, or } \\
\text { ug/dscm } \\
\text { or } \\
\mathrm{mg} / \mathrm{dscm}\end{array}$ & $\begin{array}{l}\text { ppmv, or } \\
\text { ug/dscm } \\
\text { or } \\
\mathrm{mg} / \mathrm{dscm}\end{array}$ & $\begin{array}{l}\mathrm{ppmv}, \text { or } \\
\mathrm{ug} / \mathrm{dscm} \\
\text { or } \\
\mathrm{mg} / \mathrm{dscm}\end{array}$ & $\begin{array}{l}\text { ppmv, or } \\
\text { ug/dscm } \\
\text { or } \\
\mathrm{mg} / \mathrm{dscm}\end{array}$ & $\begin{array}{l}\mathrm{ppmv}, \text { or } \\
\mathrm{ug} / \mathrm{dscm} \\
\text { or } \\
\mathrm{mg} / \mathrm{dscm}\end{array}$ & $\begin{array}{l}\mathrm{ppmv} \text {, or } \\
\mathrm{ug} / \mathrm{dscm} \\
\text { or } \\
\mathrm{mg} / \mathrm{dscm}\end{array}$ \\
\hline $\mathrm{CO}, \mathrm{ppmv}$, dry basis & & 9.94E-03 & $7.04 \mathrm{E}-03$ & & & & & \\
\hline $\mathrm{NO}$, ppmv, dry basis & & $3.10 \mathrm{E}+02$ & $2.20 \mathrm{E}+02$ & & & & & \\
\hline NO2, ppmv, dry basis & & $1.11 \mathrm{E}+00$ & 7.87E-01 & & & & & \\
\hline SO2, ppmv, dry basis & & $5.83 \mathrm{E}+01$ & $4.13 \mathrm{E}+01$ & & & & & \\
\hline $\mathrm{Cl}, \mathrm{ppmv}$, dry basis & & $9.01 \mathrm{E}-03$ & $6.39 \mathrm{E}-03$ & & & & & \\
\hline $\mathrm{F}, \mathrm{ppmv}$, dry basis & & $1.44 \mathrm{E}+01$ & $1.02 \mathrm{E}+01$ & & & & & \\
\hline \multicolumn{9}{|l|}{ C (organic), ppmv, dry basis } \\
\hline \multicolumn{9}{|l|}{$\mathrm{H}$ (organic), ppmv, dry basis } \\
\hline $\mathrm{Hg}, \mathrm{ug} / \mathrm{dscm}$ & & $2.93 E+01$ & $2.08 \mathrm{E}+01$ & & & & & \\
\hline $\mathrm{PM}, \mathrm{mg} / \mathrm{dscm}$ & $2.20 \mathrm{E}+00$ & $9.65 \mathrm{E}+00$ & $7.45 \mathrm{E}+00$ & & & & & \\
\hline \multicolumn{9}{|l|}{ SVM, ug/dscm } \\
\hline LVM, ug/dscm & & & & & & & & \\
\hline
\end{tabular}


Table A-14. Mass balance, Tank WM-187 waste (continued).

\begin{tabular}{|c|c|c|c|c|c|c|c|c|}
\hline PFD \# & PFD-3 & PFD-3 & PFD-3 & PFD-2 & PFD-2 & PFD-2 & PFD-2 & PFD-3 \\
\hline WM-187, Stream \# & 205 & 206 & 207 & 301 & 302 & 303 & 304 & 305 \\
\hline Stream Name & $\begin{array}{l}\text { Water to } \\
\text { Spray } \\
\text { Quench }\end{array}$ & $\begin{array}{l}\text { ANN to } \\
\text { Scrub for } \\
\text { F Adjust }\end{array}$ & $\begin{array}{l}\text { HNO3 } \\
\text { Scrub } \\
\text { Makeup }\end{array}$ & $\begin{array}{l}\text { Sugar to } \\
\text { Feed }\end{array}$ & $\begin{array}{l}\text { Carbon to } \\
\text { Reformer }\end{array}$ & $\begin{array}{c}\text { NOx } \\
\text { Catalyst }\end{array}$ & Bed Media & $\begin{array}{l}\text { Grout Mix } \\
\text { for Scrub } \\
\text { Blowdown }\end{array}$ \\
\hline Rate or Volume & $4.23 \mathrm{E}+02$ & $3.52 \mathrm{E}-08$ & $8.10 \mathrm{E}-01$ & $3.14 \mathrm{E}+02$ & $1.38 \mathrm{E}+02$ & $2.20 \mathrm{E}-05$ & $1.66 \mathrm{E}+00$ & $6.61 \mathrm{E}+00$ \\
\hline \multirow{2}{*}{\multicolumn{9}{|c|}{$\begin{array}{ll}\text { Volume Flow (standard, wet) } & \\
\text { Volume Flow (standard, drv) } & \end{array}$}} \\
\hline & & & & & & & & \\
\hline Rate Units & $\mathrm{gal} / \mathrm{hr}$ & $\mathrm{gal} / \mathrm{hr}$ & $\mathrm{gal} / \mathrm{hr}$ & $\mathrm{lb} / \mathrm{hr}$ & $\mathrm{lb} / \mathrm{hr}$ & $\mathrm{Ib} / \mathrm{hr}$ & $\mathrm{lb} / \mathrm{hr}$ & $\mathrm{lb} / \mathrm{hr}$ \\
\hline Rate or Volume, metric & $1.60 \mathrm{E}+03$ & \begin{tabular}{|l|}
$1.33 \mathrm{E}-07$ \\
\end{tabular} & $3.07 \mathrm{E}+00$ & $1.42 \mathrm{E}+02$ & $6.26 \mathrm{E}+01$ & $1.00 \mathrm{E}-05$ & $7.53 \mathrm{E}-01$ & $3.00 \mathrm{E}+00$ \\
\hline Rate Units & $\mathrm{L} / \mathrm{hr}$ & $\mathrm{L} / \mathrm{hr}$ & $\mathrm{L} / \mathrm{hr}$ & $\mathrm{kg} / \mathrm{hr}$ & $\mathrm{kg} / \mathrm{hr}$ & $\mathrm{kg} / \mathrm{hr}$ & $\mathrm{kg} / \mathrm{hr}$ & $\mathrm{kg} / \mathrm{hr}$ \\
\hline Temperature, ${ }^{\circ} \mathrm{C}$ & 25 & 25 & 25 & 15 & 15 & 15 & 15 & 25 \\
\hline Temperature, ${ }^{\circ} \mathrm{F}$ & 77 & 77 & 77 & 59 & 59 & 59 & 59 & 77 \\
\hline Pressure, psia & 112.3 & 14.7 & 42.3 & 14.7 & 14.7 & 14.7 & 14.7 & 14.7 \\
\hline Specific Gravity & $9.97 \mathrm{E}-01$ & $1.35 \mathrm{E}+00$ & $1.28 \mathrm{E}+00$ & $7.50 \mathrm{E}-01$ & $5.00 \mathrm{E}-01$ & $2.58 \mathrm{E}+00$ & $1.58 \mathrm{E}+00$ & $2.01 \mathrm{E}-01$ \\
\hline Chemical Composition & Mol/liter & Mol/liter & \begin{tabular}{|l|} 
Mol/liter \\
\end{tabular} & Wt frac & Wt frac & Wt frac & Wt frac & Wt frac \\
\hline $\mathrm{H}+$ & $1.00 \mathrm{E}-07$ & $3.09 \mathrm{E}-08$ & $1.39 \mathrm{E}+01$ & & & & & \\
\hline $\mathrm{Al}+3$ & & $2.20 \mathrm{E}+00$ & & & $1.53 \mathrm{E}-03$ & & $5.29 \mathrm{E}-01$ & \\
\hline \multicolumn{9}{|l|}{$\mathrm{Sb}+5$} \\
\hline \multicolumn{9}{|l|}{ As+3 } \\
\hline \multirow{2}{*}{\multicolumn{9}{|c|}{\begin{tabular}{|l|}
$\mathrm{Ba}+2$ \\
$\mathrm{Be}+2$
\end{tabular}}} \\
\hline & & & & & & & & \\
\hline \multicolumn{9}{|l|}{$\mathrm{B}+3$} \\
\hline \multicolumn{9}{|l|}{$\mathrm{Cd}+2$} \\
\hline $\mathrm{Ca}+2$ & & & & & $1.85 \mathrm{E}-02$ & & & \\
\hline \multicolumn{9}{|l|}{$\mathrm{Cr}+3$} \\
\hline \multirow{2}{*}{\multicolumn{9}{|c|}{\begin{tabular}{|l|}
$\mathrm{Co}+2$ \\
$\mathrm{Cs}+$ \\
\end{tabular}}} \\
\hline & & & & & & & & \\
\hline \multicolumn{9}{|l|}{$\mathrm{Cu}+2$} \\
\hline $\mathrm{Fe}+3$ & & & & & $8.71 \mathrm{E}-04$ & $6.99 \mathrm{E}-01$ & & \\
\hline \multicolumn{9}{|l|}{$\mathrm{Pb}+2$} \\
\hline $\mathrm{Hg}+2$ & & & & & $1.92 \mathrm{E}-06$ & & & \\
\hline \multicolumn{9}{|l|}{$\mathrm{Mn}+4$} \\
\hline \multicolumn{9}{|l|}{$\mathrm{Ni}+2$} \\
\hline $\mathrm{K}+$ & & & & & $7.14 \mathrm{E}-03$ & & & \\
\hline \multicolumn{9}{|l|}{$\mathrm{Se}+4$} \\
\hline \multicolumn{9}{|l|}{$\mathrm{Ag}+$} \\
\hline $\mathrm{Na}+$ & & & & & $7.42 \mathrm{E}-04$ & & & \\
\hline \multicolumn{9}{|l|}{$\mathrm{Tl}+3$} \\
\hline \multirow{2}{*}{\multicolumn{9}{|c|}{\begin{tabular}{|l|}
$U+4$ \\
$V+3$
\end{tabular}}} \\
\hline & & & & & & & & \\
\hline \multicolumn{9}{|l|}{$Z n+2$} \\
\hline $\mathrm{Zr}+4$ & & & & & & & & \\
\hline $\mathrm{Cl}-$ & & & & & & & & \\
\hline F- & & & & & & & & \\
\hline SO4-2 & & & & & & & & \\
\hline NO3- & & $6.60 \mathrm{E}+00$ & $1.39 \mathrm{E}+01$ & & & & & \\
\hline PO4-3 & & & & & $2.26 \mathrm{E}-03$ & & & \\
\hline$A m+4$ & & & & & & & & \\
\hline $\mathrm{Br}-$ & & & & & & & & \\
\hline $\mathrm{Ce}+4$ & & & & & & & & \\
\hline $\mathrm{Eu}+3$ & & & & & & & & \\
\hline$G d+3$ & & & & & & & & \\
\hline $\mathrm{Ge}+4$ & & & & & & & & \\
\hline $\ln +3$ & & & & & & & & \\
\hline 1- & & & & & & & & \\
\hline $\mathrm{La}+3$ & & & & & & & & \\
\hline
\end{tabular}


Table A-14. Mass balance, Tank WM-187 waste (continued).

\begin{tabular}{|c|c|c|c|c|c|c|c|c|}
\hline Stream \# & 205 & 206 & 207 & 301 & 302 & 303 & 304 & 305 \\
\hline Chemical Composition & Mol/liter & Mol/liter & Mol/liter & Wt frac & Wt frac & Wt frac & Wt frac & Wt frac \\
\hline \multicolumn{9}{|l|}{$\mathrm{Li}+$} \\
\hline \multicolumn{9}{|l|}{$\mathrm{Mg}+2$} \\
\hline \multicolumn{9}{|l|}{$\mathrm{Mo}+6$} \\
\hline \multirow{2}{*}{\multicolumn{9}{|c|}{\begin{tabular}{|l|}
$\mathrm{Nd}+3$ \\
$\mathrm{~Np}+4$
\end{tabular}}} \\
\hline \multirow{2}{*}{\multicolumn{9}{|c|}{$\frac{N p+4}{N b+5}$}} \\
\hline & & & & & & & & \\
\hline \multicolumn{9}{|l|}{$\begin{array}{l}\mathrm{Pd}+4 \\
\end{array}$} \\
\hline \multirow{2}{*}{\multicolumn{9}{|c|}{\begin{tabular}{|l|}
$\mathrm{Pu}+4$ \\
$\mathrm{Pr}+4$
\end{tabular}}} \\
\hline & & & & & & & & \\
\hline \multicolumn{9}{|l|}{\begin{tabular}{|l|}
$\mathrm{Pr}+4$ \\
$\mathrm{Pm}+3$
\end{tabular}} \\
\hline \multicolumn{9}{|l|}{$\mathrm{Rh}+4$} \\
\hline \multicolumn{9}{|l|}{$\mathrm{Rb}+$} \\
\hline \multicolumn{9}{|l|}{$\mathrm{Ru}+3$} \\
\hline \multicolumn{9}{|l|}{$\mathrm{Sm}+3$} \\
\hline $\mathrm{Si+4}$ & & & & & $6.26 \mathrm{E}-03$ & & & $4.67 \mathrm{E}-01$ \\
\hline \multicolumn{9}{|l|}{$\mathrm{Sr}+2$} \\
\hline \multicolumn{9}{|l|}{$\mathrm{Tc}+7$} \\
\hline \multirow{2}{*}{\multicolumn{9}{|c|}{$\mathrm{Te}+4$}} \\
\hline & & & & & & & & $\mathrm{Tb}+4$ \\
\hline \multirow{2}{*}{\multicolumn{9}{|c|}{\begin{tabular}{|l|}
$\mathrm{Th}+4$ \\
$\mathrm{Sn}+4$
\end{tabular}}} \\
\hline & & & & & & & & \\
\hline \multicolumn{9}{|l|}{$\mathrm{Ti+4}$} \\
\hline \multicolumn{9}{|l|}{$\mathrm{Y}+3$} \\
\hline $\mathrm{OH}-$ & $1.00 \mathrm{E}-07$ & $3.09 \mathrm{E}-08$ & & & & & & \\
\hline $\mathrm{H} 2 \mathrm{O}$ & $5.54 \mathrm{E}+01$ & $4.89 \mathrm{E}+01$ & $2.27 \mathrm{E}+01$ & & & & & \\
\hline \multicolumn{9}{|c|}{ 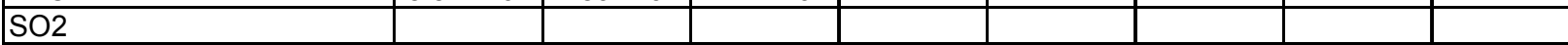 } \\
\hline \multicolumn{9}{|l|}{$\mathrm{H} 2 \mathrm{~S}$} \\
\hline \multicolumn{9}{|l|}{$\mathrm{CO}$} \\
\hline \multirow{2}{*}{\multicolumn{9}{|c|}{\begin{tabular}{|l|}
$\mathrm{CO} 2$ \\
$\mathrm{H} 2$
\end{tabular}}} \\
\hline $\mathrm{H} 2$ & & & & & & & & \\
\hline $\mathrm{N} 2$ & & & & & & & & \\
\hline \begin{tabular}{|l}
$\mathrm{NO}$ \\
\end{tabular} & & & & & & & & \\
\hline $\mathrm{NO} 2$ & & & & & & & & \\
\hline $\mathrm{O} 2$ & & & & & & & & \\
\hline S (other) & & & & & $5.18 \mathrm{E}-04$ & & & \\
\hline $\mathrm{CO} 3$ & & & & & $3.11 \mathrm{E}-02$ & & & \\
\hline C (reductant) & & & & & $9.22 \mathrm{E}-01$ & & & \\
\hline $\mathrm{O}$ (oxides) & & & & & $8.88 \mathrm{E}-03$ & $3.01 \mathrm{E}-01$ & $4.71 \mathrm{E}-01$ & $5.33 \mathrm{E}-01$ \\
\hline C (organic) & & & & $4.21 \mathrm{E}-01$ & & & & \\
\hline $\mathrm{H}$ (organic) & & & & $6.48 \mathrm{E}-02$ & & & & \\
\hline $\mathrm{O}$ (organic) & & & & $5.14 \mathrm{E}-01$ & & & & \\
\hline Mass Flow $(\mathrm{kg} / \mathrm{hr})$ : & $1.60 \mathrm{E}+03$ & $1.80 \mathrm{E}-07$ & $3.94 \mathrm{E}+00$ & $1.42 \mathrm{E}+02$ & $6.26 \mathrm{E}+01$ & $1.00 \mathrm{E}-05$ & $7.53 \mathrm{E}-01$ & $3.00 \mathrm{E}+00$ \\
\hline
\end{tabular}


Table A-14. Mass balance, Tank WM-187 waste (continued).

\begin{tabular}{|c|c|c|c|c|c|c|c|c|}
\hline PFD \# & PFD-3 & PFD-3 & PFD-3 & PFD-2 & PFD-2 & PFD-2 & PFD-3 & PFD-2 \\
\hline WM-187, Stream \# & 401 & 402 & 404 & 404 & 503 & 504 & 505 & 506 \\
\hline Stream Name & $\begin{array}{c}\text { Scrub } \\
\text { Recycled } \\
\text { to Feed }\end{array}$ & \begin{tabular}{|c|} 
Scrub \\
Blowdown \\
to Grout \\
Mixer \\
\end{tabular} & $\begin{array}{l}\text { MLLW } \\
\text { Grout }\end{array}$ & $\begin{array}{l}\text { MLLW } \\
\text { Grout } \\
\text { Drums }\end{array}$ & $\begin{array}{c}\text { Feed } \\
\text { Atomizing } \\
\text { Gas }\end{array}$ & $\begin{array}{l}\text { Oxygen to } \\
\text { Reformer }\end{array}$ & $\begin{array}{c}\text { Oxygen to } \\
\text { Oxidizer }\end{array}$ & $\begin{array}{l}\text { Air to } \\
\text { Boiler }\end{array}$ \\
\hline Rate or Volume & $5.62 \mathrm{E}+00$ & \begin{tabular}{|l|}
$2.60 \mathrm{E}-01$ \\
\end{tabular} & $8.82 \mathrm{E}+00$ & $2.29 \mathrm{E}-01$ & $2.67 \mathrm{E}+03$ & $6.31 \mathrm{E}+02$ & $9.81 \mathrm{E}+02$ & $6.19 \mathrm{E}+04$ \\
\hline Volume Flow (standard, wet)* & & & & & $1.67 \mathrm{E}+04$ & $3.90 \mathrm{E}+03$ & $6.06 \mathrm{E}+03$ & $6.30 \mathrm{E}+04$ \\
\hline Volume Flow (standard, dry) & & & & & $1.67 \mathrm{E}+04$ & $3.90 \mathrm{E}+03$ & $6.06 \mathrm{E}+03$ & $6.30 \mathrm{E}+04$ \\
\hline Rate Units & gal/hr & gal/hr & $\mathrm{lb} / \mathrm{hr}$ & Drums/day & $\mathrm{ft} 3 / \mathrm{hr}$ & $\mathrm{ft} 3 / \mathrm{hr}$ & $\mathrm{ft} 3 / \mathrm{hr}$ & $\mathrm{ft} 3 / \mathrm{hr}$ \\
\hline Rate or Volume, metric & $2.13 \mathrm{E}+01$ & $9.86 \mathrm{E}-01$ & $4.00 \mathrm{E}+00$ & $4.00 \mathrm{E}+00$ & $4.74 \mathrm{E}+02$ & $1.10 \mathrm{E}+02$ & $1.72 \mathrm{E}+02$ & $1.78 \mathrm{E}+03$ \\
\hline Rate Units & $\mathrm{L} / \mathrm{hr}$ & $\mathrm{L} / \mathrm{hr}$ & $\mathrm{kg} / \mathrm{hr}$ & $\mathrm{kg} / \mathrm{hr}$ & wscm/hr & wscm $/ \mathrm{hr}$ & wscm $/ \mathrm{hr}$ & wscm/hr \\
\hline Temperature, ${ }^{\circ} \mathrm{C}$ & 79 & 79 & 60 & 60 & 21 & 25 & 25 & 15 \\
\hline Temperature, ${ }^{\circ} \mathrm{F}$ & 174 & 174 & 140 & 140 & 70 & 77 & 77 & 59 \\
\hline Pressure, psia & 12.3 & 12.3 & 12.3 & 12.3 & 92.3 & 92.3 & 92.3 & 14.7 \\
\hline Specific Gravity & $1.01 \mathrm{E}+00$ & $1.01 \mathrm{E}+00$ & $2.10 \mathrm{E}+00$ & $2.10 \mathrm{E}+00$ & $7.29 \mathrm{E}-03$ & $8.21 \mathrm{E}-03$ & $8.21 \mathrm{E}-03$ & $1.22 \mathrm{E}-03$ \\
\hline Chemical Composition & Mol/liter & Mol/liter & Wt frac & Wt frac & $\mathrm{lb} / \mathrm{wscf}$ & $\mathrm{lb} /$ wscf & Ib/wscf & $\mathrm{Ib} / \mathrm{wscf}$ \\
\hline $\mathrm{H}+$ & $1.03 \mathrm{E}+00$ & $1.03 \mathrm{E}+00$ & $2.57 \mathrm{E}-04$ & $2.57 \mathrm{E}-04$ & & & & \\
\hline $\mathrm{Al}+3$ & $1.54 \mathrm{E}-03$ & $1.54 \mathrm{E}-03$ & $1.03 \mathrm{E}-05$ & $1.03 \mathrm{E}-05$ & & & & \\
\hline $\mathrm{Sb}+5$ & $1.05 \mathrm{E}-07$ & $1.05 \mathrm{E}-07$ & $3.16 \mathrm{E}-09$ & $3.16 \mathrm{E}-09$ & & & & \\
\hline As+3 & $6.13 \mathrm{E}-07$ & $6.13 \mathrm{E}-07$ & $1.13 \mathrm{E}-08$ & $1.13 \mathrm{E}-08$ & & & & \\
\hline $\mathrm{Ba}+2$ & $3.37 \mathrm{E}-07$ & $3.37 \mathrm{E}-07$ & $1.14 \mathrm{E}-08$ & $1.14 \mathrm{E}-08$ & & & & \\
\hline $\mathrm{Be}+2$ & $7.47 \mathrm{E}-08$ & $7.47 \mathrm{E}-08$ & $1.66 \mathrm{E}-10$ & $1.66 \mathrm{E}-10$ & & & & \\
\hline $\mathrm{B}+3$ & $3.98 \mathrm{E}-05$ & $3.98 \mathrm{E}-05$ & $1.06 \mathrm{E}-07$ & $1.06 \mathrm{E}-07$ & & & & \\
\hline $\mathrm{Cd}+2$ & $2.59 \mathrm{E}-06$ & $2.59 \mathrm{E}-06$ & $7.18 \mathrm{E}-08$ & $7.18 \mathrm{E}-08$ & & & & \\
\hline $\mathrm{Ca}+2$ & $6.15 \mathrm{E}-04$ & $6.15 \mathrm{E}-04$ & $6.08 \mathrm{E}-06$ & $6.08 \mathrm{E}-06$ & & & & \\
\hline $\mathrm{Cr}+3$ & $8.23 \mathrm{E}-06$ & $8.23 \mathrm{E}-06$ & $1.06 \mathrm{E}-07$ & $1.06 \mathrm{E}-07$ & & & & \\
\hline $\mathrm{Co}+2$ & $7.26 \mathrm{E}-06$ & $7.26 \mathrm{E}-06$ & 1.05E-07 & $1.05 \mathrm{E}-07$ & & & & \\
\hline Cs+ & $6.96 \mathrm{E}-05$ & $6.96 \mathrm{E}-05$ & $2.46 \mathrm{E}-06$ & $2.46 \mathrm{E}-06$ & & & & \\
\hline $\mathrm{Cu}+2$ & $2.23 \mathrm{E}-06$ & $2.23 \mathrm{E}-06$ & $3.50 \mathrm{E}-08$ & $3.50 \mathrm{E}-08$ & & & & \\
\hline $\mathrm{Fe}+3$ & $1.02 \mathrm{E}-04$ & $1.02 \mathrm{E}-04$ & $1.40 \mathrm{E}-06$ & $1.40 \mathrm{E}-06$ & & & & \\
\hline $\mathrm{Pb}+2$ & $5.05 \mathrm{E}-06$ & $5.05 \mathrm{E}-06$ & $2.58 \mathrm{E}-07$ & $2.58 \mathrm{E}-07$ & & & & \\
\hline $\mathrm{Hg}+2$ & $1.07 \mathrm{E}-02$ & $1.07 \mathrm{E}-02$ & $5.27 \mathrm{E}-04$ & $5.27 \mathrm{E}-04$ & & & & \\
\hline $\mathrm{Mn}+4$ & $6.55 \mathrm{E}-05$ & $6.55 \mathrm{E}-05$ & $8.86 \mathrm{E}-07$ & $8.86 \mathrm{E}-07$ & & & & \\
\hline $\mathrm{Ni}+2$ & $6.45 \mathrm{E}-06$ & $6.45 \mathrm{E}-06$ & $9.33 \mathrm{E}-08$ & $9.33 \mathrm{E}-08$ & & & & \\
\hline $\mathrm{K}+$ & $1.66 \mathrm{E}-03$ & $1.66 \mathrm{E}-03$ & $1.60 \mathrm{E}-05$ & $1.60 \mathrm{E}-05$ & & & & \\
\hline $\mathrm{Se}+4$ & $1.46 \mathrm{E}-06$ & $1.46 \mathrm{E}-06$ & $2.84 \mathrm{E}-08$ & $2.84 \mathrm{E}-08$ & & & & \\
\hline $\mathrm{Ag}+$ & $2.09 \mathrm{E}-06$ & $2.09 \mathrm{E}-06$ & $5.55 \mathrm{E}-08$ & $5.55 \mathrm{E}-08$ & & & & \\
\hline $\mathrm{Na}+$ & $6.14 \mathrm{E}-02$ & $6.14 \mathrm{E}-02$ & $3.48 \mathrm{E}-04$ & $3.48 \mathrm{E}-04$ & & & & \\
\hline $\mathrm{Tl}+3$ & $2.09 \mathrm{E}-08$ & $2.09 \mathrm{E}-08$ & $1.05 \mathrm{E}-09$ & $1.05 \mathrm{E}-09$ & & & & \\
\hline $\mathrm{U}+4$ & $9.27 \mathrm{E}-07$ & $9.27 \mathrm{E}-07$ & $5.44 \mathrm{E}-08$ & $5.44 \mathrm{E}-08$ & & & & \\
\hline $\mathrm{V}+3$ & $2.10 \mathrm{E}-07$ & $2.10 \mathrm{E}-07$ & $2.64 \mathrm{E}-09$ & $2.64 \mathrm{E}-09$ & & & & \\
\hline$Z n+2$ & $3.62 \mathrm{E}-06$ & $3.62 \mathrm{E}-06$ & $5.83 \mathrm{E}-08$ & $5.83 \mathrm{E}-08$ & & & & \\
\hline $2 r+4$ & $2.09 \mathrm{E}-04$ & $2.09 \mathrm{E}-04$ & $4.69 \mathrm{E}-06$ & $4.69 \mathrm{E}-06$ & & & & \\
\hline $\mathrm{Cl}-$ & $2.16 \mathrm{E}-03$ & $2.16 \mathrm{E}-03$ & $1.89 \mathrm{E}-05$ & $1.89 \mathrm{E}-05$ & & & & \\
\hline F- & $2.10 \mathrm{E}-02$ & $2.10 \mathrm{E}-02$ & $9.82 \mathrm{E}-05$ & $9.82 \mathrm{E}-05$ & & & & \\
\hline SO4-2 & $2.22 \mathrm{E}-02$ & $2.22 \mathrm{E}-02$ & $5.25 \mathrm{E}-04$ & $5.25 \mathrm{E}-04$ & & & & \\
\hline $\mid \mathrm{NO3}^{-}$ & $1.04 \mathrm{E}+00$ & $1.04 \mathrm{E}+00$ & $1.58 \mathrm{E}-02$ & $1.58 \mathrm{E}-02$ & & & & \\
\hline PO4-3 & $6.75 \mathrm{E}-04$ & $6.75 \mathrm{E}-04$ & $1.58 \mathrm{E}-05$ & $1.58 \mathrm{E}-05$ & & & & \\
\hline$A m+4$ & $2.50 \mathrm{E}-10$ & $2.50 \mathrm{E}-10$ & $1.50 \mathrm{E}-11$ & $1.50 \mathrm{E}-11$ & & & & \\
\hline $\mathrm{Br}-$ & $3.02 \mathrm{E}-08$ & $3.02 \mathrm{E}-08$ & $5.94 \mathrm{E}-10$ & $5.94 \mathrm{E}-10$ & & & & \\
\hline $\mathrm{Ce}+4$ & $1.56 \mathrm{E}-07$ & $1.56 \mathrm{E}-07$ & $5.40 \mathrm{E}-09$ & $5.40 \mathrm{E}-09$ & & & & \\
\hline $\mathrm{Eu+3}$ & $1.50 \mathrm{E}-09$ & $1.50 \mathrm{E}-09$ & $5.61 \mathrm{E}-11$ & $5.61 \mathrm{E}-11$ & & & & \\
\hline $\mathrm{Gd}+3$ & $5.45 \mathrm{E}-07$ & $5.45 \mathrm{E}-07$ & $2.11 \mathrm{E}-08$ & $2.11 \mathrm{E}-08$ & & & & \\
\hline $\mathrm{Ge}+4$ & $2.45 \mathrm{E}-11$ & $2.45 \mathrm{E}-11$ & $4.39 \mathrm{E}-13$ & $4.39 \mathrm{E}-13$ & & & & \\
\hline $\ln +3$ & $4.35 \mathrm{E}-09$ & $4.35 \mathrm{E}-09$ & $1.23 \mathrm{E}-10$ & $1.23 \mathrm{E}-10$ & & & & \\
\hline $\mid-$ & $3.93 \mathrm{E}-07$ & $3.93 \mathrm{E}-07$ & $1.23 \mathrm{E}-08$ & $1.23 \mathrm{E}-08$ & & & & \\
\hline $\mathrm{La}+3$ & $2.71 \mathrm{E}-08$ & $2.71 \mathrm{E}-08$ & $9.29 \mathrm{E}-10$ & $9.29 \mathrm{E}-10$ & & & & \\
\hline
\end{tabular}


Table A-14. Mass balance, Tank WM-187 waste (continued).

\begin{tabular}{|c|c|c|c|c|c|c|c|c|}
\hline Stream \# & 401 & 402 & 404 & 404 & 503 & 504 & 505 & 506 \\
\hline Chemical Composition & Mol/liter & Mol/liter & Wt frac & Wt frac & $\mathrm{lb} / \mathrm{wscf}$ & $\mathrm{lb} /$ wscf & $\mathrm{lb} / \mathrm{wscf}$ & $\mathrm{lb} / \mathrm{wscf}$ \\
\hline $\mathrm{Li}+$ & $2.03 \mathrm{E}-06$ & 2.03E-06 & $3.47 E-09$ & 3.47E-09 & & & & \\
\hline $\mathrm{Mg}+2$ & $8.12 \mathrm{E}-05$ & $8.12 \mathrm{E}-05$ & 4.86E-07 & 4.86E-07 & & & & \\
\hline $\mathrm{Mo}+6$ & $3.20 \mathrm{E}-04$ & $3.20 \mathrm{E}-04$ & $7.55 \mathrm{E}-06$ & $7.55 \mathrm{E}-06$ & & & & \\
\hline $\mathrm{Nd}+3$ & $8.75 \mathrm{E}-08$ & $8.75 \mathrm{E}-08$ & 3.11E-09 & $3.11 \mathrm{E}-09$ & & & & \\
\hline $\mathrm{Np}+4$ & $1.38 \mathrm{E}-08$ & $1.38 \mathrm{E}-08$ & $8.04 \mathrm{E}-10$ & $8.04 \mathrm{E}-10$ & & & & \\
\hline $\mathrm{Nb}+5$ & $5.81 \mathrm{E}-06$ & $5.81 \mathrm{E}-06$ & 1.33E-07 & $1.33 \mathrm{E}-07$ & & & & \\
\hline $\mathrm{Pd}+4$ & $1.32 \mathrm{E}-05$ & $1.32 \mathrm{E}-05$ & $3.46 \mathrm{E}-07$ & $3.46 \mathrm{E}-07$ & & & & \\
\hline $\mathrm{Pu}+4$ & $3.05 E-08$ & $3.05 E-08$ & 1.83E-09 & 1.83E-09 & & & & \\
\hline $\mathrm{Pr}+4$ & $2.47 E-08$ & $2.47 E-08$ & $8.57 \mathrm{E}-10$ & $8.57 \mathrm{E}-10$ & & & & \\
\hline $\mathrm{Pm}+3$ & $6.01 \mathrm{E}-12$ & $6.01 \mathrm{E}-12$ & $2.15 \mathrm{E}-13$ & $2.15 \mathrm{E}-13$ & & & & \\
\hline $\mathrm{Rh}+4$ & 1.06E-08 & 1.06E-08 & $2.70 \mathrm{E}-10$ & $2.70 \mathrm{E}-10$ & & & & \\
\hline $\mathrm{Rb}+$ & 1.46E-08 & $1.46 \mathrm{E}-08$ & $3.07 E-10$ & $3.07 E-10$ & & & & \\
\hline Ru+3 & 4.60E-06 & 4.60E-06 & 1.15E-07 & $1.15 \mathrm{E}-07$ & & & & \\
\hline $\mathrm{Sm}+3$ & 1.64E-08 & 1.64E-08 & $6.07 \mathrm{E}-10$ & $6.07 \mathrm{E}-10$ & & & & \\
\hline $\mathrm{Si}+4$ & $8.21 \mathrm{E}-04$ & $8.21 \mathrm{E}-04$ & $3.51 \mathrm{E}-01$ & $3.51 \mathrm{E}-01$ & & & & \\
\hline $\mathrm{Sr}+2$ & $4.70 \mathrm{E}-07$ & $4.70 \mathrm{E}-07$ & $1.02 \mathrm{E}-08$ & $1.02 \mathrm{E}-08$ & & & & \\
\hline $\mathrm{Tc}+7$ & $5.26 \mathrm{E}-08$ & $5.26 \mathrm{E}-08$ & 1.27E-09 & 1.27E-09 & & & & \\
\hline $\mathrm{Te}+4$ & 1.33E-08 & 1.33E-08 & $4.18 \mathrm{E}-10$ & $4.18 \mathrm{E}-10$ & & & & \\
\hline $\mathrm{Tb}+4$ & $6.25 \mathrm{E}-12$ & $6.25 \mathrm{E}-12$ & $2.45 \mathrm{E}-13$ & $2.45 \mathrm{E}-13$ & & & & \\
\hline $\mathrm{Th}+4$ & $6.96 \mathrm{E}-08$ & $6.96 \mathrm{E}-08$ & 3.98E-09 & 3.98E-09 & & & & \\
\hline $\mathrm{Sn}+4$ & 1.01E-05 & 1.01E-05 & $2.96 \mathrm{E}-07$ & $2.96 \mathrm{E}-07$ & & & & \\
\hline $\mathrm{Ti}+4$ & $5.10 \mathrm{E}-06$ & $5.10 \mathrm{E}-06$ & $6.02 \mathrm{E}-08$ & $6.02 \mathrm{E}-08$ & & & & \\
\hline $\mathrm{Y}+3$ & $2.02 \mathrm{E}-08$ & $2.02 \mathrm{E}-08$ & $4.43 \mathrm{E}-10$ & $4.43 \mathrm{E}-10$ & & & & \\
\hline $\mathrm{OH}-$ & 1.22E-02 & $1.22 \mathrm{E}-02$ & $5.13 \mathrm{E}-05$ & $5.13 \mathrm{E}-05$ & & & & \\
\hline $\mathrm{H} 2 \mathrm{O}$ & $5.23 E+01$ & $5.23 E+01$ & 2.32E-01 & 2.32E-01 & & & & $4.68 \mathrm{E}-06$ \\
\hline $\mathrm{SO} 2$ & 9.10E-06 & $9.10 \mathrm{E}-06$ & $1.44 \mathrm{E}-07$ & $1.44 \mathrm{E}-07$ & & & & \\
\hline \multicolumn{9}{|l|}{$\mathrm{H} 2 \mathrm{~S}$} \\
\hline $\mathrm{CO}$ & $1.92 \mathrm{E}-10$ & $1.92 \mathrm{E}-10$ & $1.32 \mathrm{E}-12$ & $1.32 \mathrm{E}-12$ & & & & \\
\hline $\mathrm{CO} 2$ & 3.05E-04 & 3.05E-04 & 3.31E-06 & 3.31E-06 & & & & \\
\hline $\mathrm{H} 2$ & $7.32 \mathrm{E}-10$ & $7.32 \mathrm{E}-10$ & $3.64 \mathrm{E}-13$ & $3.64 \mathrm{E}-13$ & & & & \\
\hline $\mathrm{N} 2$ & $1.28 \mathrm{E}-05$ & $1.28 \mathrm{E}-05$ & 8.84E-08 & 8.84E-08 & 7.27E-02 & & & $5.74 \mathrm{E}-02$ \\
\hline $\mathrm{NO}$ & $2.31 \mathrm{E}-07$ & $2.31 \mathrm{E}-07$ & 1.71E-09 & 1.71E-09 & & & & \\
\hline $\mathrm{NO} 2$ & $2.94 \mathrm{E}-06$ & $2.94 \mathrm{E}-06$ & $3.33 \mathrm{E}-08$ & $3.33 \mathrm{E}-08$ & & & & \\
\hline $\mathrm{O} 2$ & $3.20 \mathrm{E}-06$ & $3.20 \mathrm{E}-06$ & $2.52 \mathrm{E}-08$ & $2.52 \mathrm{E}-08$ & & $8.30 \mathrm{E}-02$ & $8.30 \mathrm{E}-02$ & $1.74 \mathrm{E}-02$ \\
\hline S (other) & 1.22E-07 & $1.22 \mathrm{E}-07$ & $9.65 \mathrm{E}-10$ & $9.65 \mathrm{E}-10$ & & & & \\
\hline $\mathrm{CO} 3$ & $3.48 \mathrm{E}-05$ & $3.48 \mathrm{E}-05$ & $5.15 \mathrm{E}-07$ & $5.15 \mathrm{E}-07$ & & & & \\
\hline \multicolumn{9}{|l|}{ C (reductant) } \\
\hline $\mathrm{O}$ (oxides) & $5.02 \mathrm{E}-03$ & $5.02 \mathrm{E}-03$ & 3.99E-01 & $3.99 \mathrm{E}-01$ & & & & \\
\hline \multicolumn{9}{|l|}{ C (organic) } \\
\hline \multicolumn{9}{|l|}{$\mathrm{H}$ (organic) } \\
\hline \multicolumn{9}{|l|}{ O (organic) } \\
\hline \multirow[t]{2}{*}{ Mass Flow (kg/hr): } & $2.16 \mathrm{E}+01$ & $1.00 \mathrm{E}+00$ & $4.00 \mathrm{E}+00$ & $4.00 \mathrm{E}+00$ & $5.52 \mathrm{E}+02$ & $1.47 \mathrm{E}+02$ & $2.28 \mathrm{E}+02$ & $2.14 \mathrm{E}+03$ \\
\hline & & & & & & & & \\
\hline \multirow[t]{2}{*}{ Total Drums Generated } & & & & 20 & & & & \\
\hline & $\mathrm{w} / \mathrm{m} 3$ & $\mathrm{w} / \mathrm{m} 3$ & $\mathrm{w} / \mathrm{m} 3$ & w/Drum & $w / m 3$ & $w / m 3$ & $\mathrm{w} / \mathrm{m} 3$ & $w / m 3$ \\
\hline Heat Generation, w/m3 & $4.12 \mathrm{E}-02$ & $4.12 \mathrm{E}-02$ & $2.30 \mathrm{E}-02$ & $4.59 \mathrm{E}-03$ & & & & \\
\hline
\end{tabular}


Table A-14. Mass balance, Tank WM-187 waste (continued).

\begin{tabular}{|c|c|c|c|c|c|c|c|c|}
\hline Stream \# & 401 & 402 & 404 & 404 & 503 & 504 & 505 & 506 \\
\hline Radiological Composition & $\mathrm{Ci} / \mathrm{L}$ & $\mathrm{Ci} / \mathrm{L}$ & $\mathrm{Ci} / \mathrm{kg}$ & Ci/Drum & Ci/wscm & $\mathrm{Ci} / \mathrm{wscm}$ & $\mathrm{Ci} / \mathrm{wscm}$ & $\mathrm{Ci} / \mathrm{wscm}$ \\
\hline \multicolumn{9}{|l|}{$\mathrm{H}-3$} \\
\hline $\mathrm{C}-14$ & $1.52 \mathrm{E}-15$ & $1.52 \mathrm{E}-15$ & $3.75 \mathrm{E}-16$ & $1.57 \mathrm{E}-13$ & & & & \\
\hline Co-60 & $6.22 \mathrm{E}-08$ & $6.22 \mathrm{E}-08$ & $1.53 \mathrm{E}-08$ & $6.44 \mathrm{E}-06$ & & & & \\
\hline $\mathrm{Ni}-59$ & $8.94 \mathrm{E}-09$ & $8.94 \mathrm{E}-09$ & $2.20 \mathrm{E}-09$ & $9.26 \mathrm{E}-07$ & & & & \\
\hline $\mathrm{Ni}-63$ & $2.08 \mathrm{E}-07$ & $2.08 \mathrm{E}-07$ & $5.13 \mathrm{E}-08$ & $2.15 \mathrm{E}-05$ & & & & \\
\hline Se-79 & $1.98 \mathrm{E}-09$ & $1.98 \mathrm{E}-09$ & $4.89 \mathrm{E}-10$ & $2.05 \mathrm{E}-07$ & & & & \\
\hline Sr-90 & $1.56 \mathrm{E}-04$ & $1.56 \mathrm{E}-04$ & $3.85 \mathrm{E}-05$ & $1.62 \mathrm{E}-02$ & & & & \\
\hline Y-90 & $1.19 \mathrm{E}-04$ & $1.19 \mathrm{E}-04$ & $2.94 \mathrm{E}-05$ & $1.23 \mathrm{E}-02$ & & & & \\
\hline Zr-93 & $6.34 \mathrm{E}-09$ & $6.34 \mathrm{E}-09$ & $1.56 \mathrm{E}-09$ & $6.56 \mathrm{E}-07$ & & & & \\
\hline $\mathrm{Nb}-93 \mathrm{~m}$ & $4.88 \mathrm{E}-09$ & $4.88 \mathrm{E}-09$ & $1.20 \mathrm{E}-09$ & $5.05 \mathrm{E}-07$ & & & & \\
\hline $\mathrm{Nb}-94$ & $5.15 \mathrm{E}-09$ & $5.15 \mathrm{E}-09$ & $1.27 \mathrm{E}-09$ & $5.33 \mathrm{E}-07$ & & & & \\
\hline Tc-99 & $1.27 \mathrm{E}-07$ & $1.27 \mathrm{E}-07$ & $3.12 \mathrm{E}-08$ & $1.31 \mathrm{E}-05$ & & & & \\
\hline Ru-106 & $4.50 \mathrm{E}-09$ & $4.50 \mathrm{E}-09$ & $1.11 \mathrm{E}-09$ & $4.66 \mathrm{E}-07$ & & & & \\
\hline Rh-102 & $2.61 \mathrm{E}-12$ & $2.61 \mathrm{E}-12$ & $6.44 \mathrm{E}-13$ & $2.71 \mathrm{E}-10$ & & & & \\
\hline Rh-106 & $4.50 \mathrm{E}-09$ & $4.50 \mathrm{E}-09$ & $1.11 \mathrm{E}-09$ & $4.66 \mathrm{E}-07$ & & & & \\
\hline $\mathrm{Pd}-107$ & $6.56 \mathrm{E}-11$ & $6.56 \mathrm{E}-11$ & $1.62 \mathrm{E}-11$ & $6.79 \mathrm{E}-09$ & & & & \\
\hline Cd-113m & $4.09 \mathrm{E}-09$ & $4.09 \mathrm{E}-09$ & $1.01 \mathrm{E}-09$ & $4.24 \mathrm{E}-07$ & & & & \\
\hline Sn-121m & $1.91 \mathrm{E}-10$ & $1.91 \mathrm{E}-10$ & $4.71 \mathrm{E}-11$ & $1.98 \mathrm{E}-08$ & & & & \\
\hline Sn-126 & $1.87 \mathrm{E}-09$ & $1.87 \mathrm{E}-09$ & $4.61 \mathrm{E}-10$ & $1.93 \mathrm{E}-07$ & & & & \\
\hline Sb-125 & $2.37 \mathrm{E}-06$ & $2.37 \mathrm{E}-06$ & $5.85 \mathrm{E}-07$ & $2.46 \mathrm{E}-04$ & & & & \\
\hline Sb-126 & $1.74 \mathrm{E}-10$ & $1.74 \mathrm{E}-10$ & $4.30 \mathrm{E}-11$ & $1.80 \mathrm{E}-08$ & & & & \\
\hline Te-125m & $9.01 \mathrm{E}-09$ & $9.01 \mathrm{E}-09$ & $2.22 \mathrm{E}-09$ & $9.32 \mathrm{E}-07$ & & & & \\
\hline I-129 & $8.54 \mathrm{E}-09$ & $8.54 \mathrm{E}-09$ & $2.10 \mathrm{E}-09$ & $8.84 \mathrm{E}-07$ & & & & \\
\hline Cs-134 & $5.95 \mathrm{E}-05$ & $5.95 \mathrm{E}-05$ & $1.58 \mathrm{E}-05$ & $6.65 \mathrm{E}-03$ & & & & \\
\hline Cs-135 & $9.47 \mathrm{E}-07$ & $9.47 \mathrm{E}-07$ & $2.52 \mathrm{E}-07$ & $1.06 \mathrm{E}-04$ & & & & \\
\hline \begin{tabular}{|l|} 
Cs-137 \\
\end{tabular} & $3.84 \mathrm{E}-02$ & $3.84 \mathrm{E}-02$ & $1.02 \mathrm{E}-02$ & $4.30 \mathrm{E}+00$ & & & & \\
\hline Ba-137m & $2.00 \mathrm{E}-04$ & $2.00 \mathrm{E}-04$ & $4.93 \mathrm{E}-05$ & $2.07 \mathrm{E}-02$ & & & & \\
\hline \begin{tabular}{|l|} 
Ce-144 \\
\end{tabular} & $3.04 \mathrm{E}-09$ & $3.04 \mathrm{E}-09$ & $7.50 \mathrm{E}-10$ & $3.15 \mathrm{E}-07$ & & & & \\
\hline Pr-144 & 3.04E-09 & $3.04 \mathrm{E}-09$ & $7.50 \mathrm{E}-10$ & $3.15 \mathrm{E}-07$ & & & & \\
\hline Pm-146 & $1.54 \mathrm{E}-10$ & $1.54 \mathrm{E}-10$ & $3.80 \mathrm{E}-11$ & $1.60 \mathrm{E}-08$ & & & & \\
\hline Pm-147 & $8.19 \mathrm{E}-07$ & $8.19 \mathrm{E}-07$ & $2.02 \mathrm{E}-07$ & $8.47 \mathrm{E}-05$ & & & & \\
\hline Sm-151 & $1.62 \mathrm{E}-06$ & $1.62 \mathrm{E}-06$ & $4.00 \mathrm{E}-07$ & $1.68 \mathrm{E}-04$ & & & & \\
\hline Eu-152 & $9.74 \mathrm{E}-09$ & $9.74 \mathrm{E}-09$ & $2.40 \mathrm{E}-09$ & $1.01 \mathrm{E}-06$ & & & & \\
\hline Eu-154 & $3.66 \mathrm{E}-07$ & $3.66 \mathrm{E}-07$ & $9.02 \mathrm{E}-08$ & $3.79 \mathrm{E}-05$ & & & & \\
\hline \multirow[t]{2}{*}{ Eu-155 } & $3.85 \mathrm{E}-07$ & $3.85 \mathrm{E}-07$ & $9.50 \mathrm{E}-08$ & $3.99 \mathrm{E}-05$ & & & & \\
\hline & & & & & & & & \\
\hline Th-230 & $3.58 \mathrm{E}-12$ & $3.58 \mathrm{E}-12$ & $8.83 \mathrm{E}-13$ & $3.71 \mathrm{E}-10$ & & & & \\
\hline Pa-233 & $8.38 \mathrm{E}-09$ & $8.38 \mathrm{E}-09$ & $2.07 \mathrm{E}-09$ & $8.68 \mathrm{E}-07$ & & & & \\
\hline U-232 & $8.24 \mathrm{E}-12$ & $8.24 \mathrm{E}-12$ & $2.03 \mathrm{E}-12$ & $8.53 \mathrm{E}-10$ & & & & \\
\hline $\mathrm{U}-233$ & $2.73 \mathrm{E}-13$ & $2.73 \mathrm{E}-13$ & $6.72 \mathrm{E}-14$ & $2.82 \mathrm{E}-11$ & & & & \\
\hline U-234 & $4.51 \mathrm{E}-09$ & $4.51 \mathrm{E}-09$ & $1.11 \mathrm{E}-09$ & $4.67 \mathrm{E}-07$ & & & & \\
\hline U-235 & $2.35 \mathrm{E}-10$ & $2.35 \mathrm{E}-10$ & $5.79 \mathrm{E}-11$ & 2.43E-08 & & & & \\
\hline U-236 & $2.19 \mathrm{E}-10$ & $2.19 \mathrm{E}-10$ & $5.38 \mathrm{E}-11$ & $2.26 \mathrm{E}-08$ & & & & \\
\hline U-237 & $1.84 \mathrm{E}-11$ & $1.84 \mathrm{E}-11$ & $4.54 \mathrm{E}-12$ & $1.91 \mathrm{E}-09$ & & & & \\
\hline U-238 & $7.94 \mathrm{E}-11$ & $7.94 \mathrm{E}-11$ & 1.96E-11 & $8.21 \mathrm{E}-09$ & & & & \\
\hline Np-237 & $2.30 \mathrm{E}-09$ & $2.30 \mathrm{E}-09$ & $5.67 \mathrm{E}-10$ & $2.38 \mathrm{E}-07$ & & & & \\
\hline Pu-236 & $1.29 \mathrm{E}-11$ & $1.29 \mathrm{E}-11$ & $3.18 \mathrm{E}-12$ & $1.34 \mathrm{E}-09$ & & & & \\
\hline Pu-238 & $3.81 \mathrm{E}-06$ & $3.81 \mathrm{E}-06$ & $9.38 \mathrm{E}-07$ & $3.94 \mathrm{E}-04$ & & & & \\
\hline Pu-239 & $4.23 \mathrm{E}-07$ & $4.23 \mathrm{E}-07$ & 1.04E-07 & $4.38 \mathrm{E}-05$ & & & & \\
\hline Pu-240 & $4.83 \mathrm{E}-08$ & $4.83 \mathrm{E}-08$ & $1.19 \mathrm{E}-08$ & $5.00 \mathrm{E}-06$ & & & & \\
\hline Pu-241 & $2.87 \mathrm{E}-06$ & $2.87 \mathrm{E}-06$ & $7.08 \mathrm{E}-07$ & $2.98 \mathrm{E}-04$ & & & & \\
\hline Pu-242 & $3.79 \mathrm{E}-11$ & $3.79 \mathrm{E}-11$ & $9.34 \mathrm{E}-12$ & $3.92 \mathrm{E}-09$ & & & & \\
\hline Pu-244 & $2.27 \mathrm{E}-18$ & $2.27 \mathrm{E}-18$ & $5.59 \mathrm{E}-19$ & $2.35 \mathrm{E}-16$ & & & & \\
\hline Am-241 & $2.58 \mathrm{E}-07$ & $2.58 \mathrm{E}-07$ & $6.37 \mathrm{E}-08$ & $2.67 \mathrm{E}-05$ & & & & \\
\hline Am-242m & $4.68 \mathrm{E}-11$ & $4.68 \mathrm{E}-11$ & $1.15 \mathrm{E}-11$ & $4.84 \mathrm{E}-09$ & & & & \\
\hline
\end{tabular}


Table A-14. Mass balance, Tank WM-187 waste (continued).

\begin{tabular}{|c|c|c|c|c|c|c|c|c|}
\hline Stream \# & 401 & 402 & 404 & 404 & 503 & 504 & 505 & 506 \\
\hline & $\mathrm{Ci} / \mathrm{L}$ & $\mathrm{Ci} / \mathrm{L}$ & $\mathrm{Ci} / \mathrm{kg}$ & Ci/Drum & Ci/wscm & $\mathrm{Ci} / \mathrm{wscm}$ & $\mathrm{Ci} / \mathrm{wscm}$ & Ci/wscm \\
\hline Am-243 & $8.88 \mathrm{E}-11$ & $8.88 \mathrm{E}-11$ & $2.19 \mathrm{E}-11$ & 9.19E-09 & & & & \\
\hline $\mathrm{Cm}-242$ & $8.67 \mathrm{E}-11$ & $8.67 \mathrm{E}-11$ & $2.14 \mathrm{E}-11$ & 8.97E-09 & & & & \\
\hline $\mathrm{Cm}-243$ & $1.25 \mathrm{E}-10$ & $1.25 \mathrm{E}-10$ & 3.09E-11 & 1.30E-08 & & & & \\
\hline $\mathrm{Cm}-244$ & 5.59E-09 & 5.59E-09 & 1.38E-09 & $5.78 \mathrm{E}-07$ & & & & \\
\hline $\mathrm{Cm}-245$ & $1.32 \mathrm{E}-12$ & $1.32 \mathrm{E}-12$ & $3.26 \mathrm{E}-13$ & $1.37 \mathrm{E}-10$ & & & & \\
\hline $\mathrm{Cm}-246$ & $8.66 \mathrm{E}-14$ & $8.66 \mathrm{E}-14$ & $2.13 \mathrm{E}-14$ & $8.96 \mathrm{E}-12$ & & & & \\
\hline TRU & $4.63 \mathrm{E}-06$ & 4.63E-06 & 1.14E-06 & 4.79E-04 & & & & \\
\hline \multicolumn{9}{|l|}{ TRU, nCi/gm } \\
\hline & & & & & & & & \\
\hline $\begin{array}{l}\text { Gas Stream Bulk } \\
\text { Composition (Wet Basis) }\end{array}$ & $\begin{array}{l}\text { mol\% or } \\
\text { ppmv }\end{array}$ & $\begin{array}{l}\text { mol\% or } \\
\text { ppmv }\end{array}$ & $\begin{array}{l}\text { mol\% or } \\
\text { ppmv }\end{array}$ & $\begin{array}{l}\text { mol\% or } \\
\text { ppmv }\end{array}$ & $\begin{array}{l}\text { mol\% or } \\
\text { ppmv }\end{array}$ & $\begin{array}{l}\text { mol\% or } \\
\text { ppmv }\end{array}$ & $\begin{array}{l}\text { mol\% or } \\
\text { ppmv }\end{array}$ & $\begin{array}{c}\text { mol\% or } \\
\text { ppmv }\end{array}$ \\
\hline $\mathrm{H} 2 \mathrm{O}, \mathrm{mol} \%$ & & & & & & & & $0.01 \%$ \\
\hline $\mathrm{O} 2, \mathrm{~mol} \%$ & & & & & & $100.00 \%$ & $100.00 \%$ & $20.99 \%$ \\
\hline $\mathrm{N} 2, \mathrm{~mol} \%$ & & & & & $100.00 \%$ & & & $79.00 \%$ \\
\hline \multicolumn{9}{|l|}{$\mathrm{H} 2, \mathrm{~mol} \%$} \\
\hline \multicolumn{9}{|l|}{$\mathrm{CO} 2, \mathrm{~mol} \%$} \\
\hline \multicolumn{9}{|l|}{$\mathrm{CO}, \mathrm{ppmv}$} \\
\hline \multicolumn{9}{|l|}{$\mathrm{NO}, \mathrm{ppmv}$} \\
\hline \multicolumn{9}{|l|}{ NO2, ppmv } \\
\hline \multicolumn{9}{|l|}{ SO2, ppmv } \\
\hline \multicolumn{9}{|l|}{$\mathrm{Cl}, \mathrm{ppmv}$} \\
\hline \multicolumn{9}{|l|}{$\mathrm{F}, \mathrm{ppmv}$} \\
\hline \multicolumn{9}{|l|}{$\mathrm{C}$ (organic), ppmv } \\
\hline \multicolumn{9}{|l|}{$\mathrm{H}$ (organic), ppmv } \\
\hline \multicolumn{9}{|l|}{$\mathrm{Hg}, \mathrm{ug} / \mathrm{wscm}$} \\
\hline $\mathrm{PM}, \mathrm{mg} / \mathrm{wscm}$ & & & & & $2.01 \mathrm{E}+01$ & $1.88 \mathrm{E}-02$ & & $1.60 \mathrm{E}+01$ \\
\hline \multicolumn{9}{|l|}{ SVM, ug/wscm } \\
\hline \multicolumn{9}{|l|}{ LVM, ug/wscm } \\
\hline $\begin{array}{l}\text { Gas Stream Bulk } \\
\text { Composition (Dry Basis) }\end{array}$ & $\mathrm{mol} \%$ & mol\% & $\mathrm{mol} \%$ & $\mathrm{~mol} \%$ & $\mathrm{~mol} \%$ & $\mathrm{~mol} \%$ & $\mathrm{~mol} \%$ & $\mathrm{~mol} \%$ \\
\hline $\mathrm{O} 2, \mathrm{~mol} \%$, dry basis & & & & & & & & $20.9921 \%$ \\
\hline $\mathrm{N} 2, \mathrm{~mol} \%$, dry basis & & & & & & & & $79.01 \%$ \\
\hline \multicolumn{9}{|l|}{$\mathrm{H} 2, \mathrm{~mol} \%$, dry basis } \\
\hline \multicolumn{9}{|l|}{$\mathrm{CO} 2, \mathrm{~mol} \%$, dry basis } \\
\hline $\begin{array}{l}\text { Gas Stream Bulk } \\
\text { Composition (Dry Basis, } \\
\text { Corrected to } 7 \% \text { O2 with } \\
\text { 100\% O2 Combustion Air) }\end{array}$ & $\begin{array}{l}\mathrm{ppmv}, \text { or } \\
\mathrm{ug} / \mathrm{dscm} \\
\text { or } \\
\mathrm{mg} / \mathrm{dscm}\end{array}$ & $\begin{array}{l}\mathrm{ppmv}, \text { or } \\
\mathrm{ug} / \mathrm{dscm} \\
\text { or } \\
\mathrm{mg} / \mathrm{dscm}\end{array}$ & $\begin{array}{l}\text { ppmv, or } \\
\text { ug/dscm } \\
\text { or } \\
\mathrm{mg} / \mathrm{dscm}\end{array}$ & $\begin{array}{l}\mathrm{ppmv}, \text { or } \\
\text { ug/dscm } \\
\text { or } \\
\mathrm{mg} / \mathrm{dscm}\end{array}$ & $\begin{array}{l}\mathrm{ppmv}, \text { or } \\
\mathrm{ug} / \mathrm{dscm} \\
\text { or } \\
\mathrm{mg} / \mathrm{dscm}\end{array}$ & $\begin{array}{l}\mathrm{ppmv}, \text { or } \\
\mathrm{ug} / \mathrm{dscm} \\
\text { or } \\
\mathrm{mg} / \mathrm{dscm}\end{array}$ & $\begin{array}{l}\mathrm{ppmv}, \text { or } \\
\text { ug/dscm } \\
\text { or } \\
\mathrm{mg} / \mathrm{dscm}\end{array}$ & $\begin{array}{l}\mathrm{ppmv}, \text { or } \\
\text { ug/dscm } \\
\text { or } \\
\mathrm{mg} / \mathrm{dscm}\end{array}$ \\
\hline \multicolumn{9}{|l|}{$\mathrm{CO}, \mathrm{ppmv}$, dry basis } \\
\hline \multicolumn{9}{|l|}{$\mathrm{NO}, \mathrm{ppmv}$, dry basis } \\
\hline \multicolumn{9}{|l|}{ NO2, ppmv, dry basis } \\
\hline \multicolumn{9}{|l|}{ SO2, ppmv, dry basis } \\
\hline \multicolumn{9}{|l|}{$\mathrm{Cl}, \mathrm{ppmv}$, dry basis } \\
\hline $\mathrm{F}, \mathrm{ppmv}$, dry basis & & & & & & & & \\
\hline $\mathrm{C}$ (organic), ppmv, dry basis & & & & & & & & \\
\hline $\mathrm{H}$ (organic), ppmv, dry basis & & & & & & & & \\
\hline $\mathrm{Hg}, \mathrm{ug} / \mathrm{dscm}$ & & & & & & & & \\
\hline $\mathrm{PM}, \mathrm{mg} / \mathrm{dscm}$ & & & & & & & & $2.25 \mathrm{E}+00$ \\
\hline SVM, ug/dscm & & & & & & & & \\
\hline LVM, ug/dscm & & & & & & & & \\
\hline
\end{tabular}


Table A-14. Mass balance, Tank WM-187 waste (continued).

\begin{tabular}{|c|c|c|c|c|c|c|c|c|}
\hline PFD \# & PFD-2 & PFD-3 & PFD-3 & PFD-3 & PFD-3 & PFD-3 & PFD-3 & PFD-3 \\
\hline WM-187, Stream \# & 507 & 510 & 511 & 512 & 513 & 514 & 515 & 516 \\
\hline Stream Name & $\begin{array}{c}\text { Off-Gas } \\
\text { from Boiler }\end{array}$ & $\begin{array}{c}\text { Nitrogen to } \\
\text { Cool } \\
\text { Product }\end{array}$ & $\begin{array}{c}\text { N2 from } \\
\text { Product } \\
\text { Cooler }\end{array}$ & $\begin{array}{c}\text { Filter } \\
\text { Backpulse } \\
\text { N2 }\end{array}$ & $\begin{array}{l}\text { Solids } \\
\text { Transport } \\
\text { Nitrogen }\end{array}$ & $\begin{array}{c}\text { Tank Farm } \\
\text { Transfer } \\
\text { Airlift Air }\end{array}$ & $\begin{array}{c}\text { Ventilation } \\
\text { Air }\end{array}$ & Fresh GAC \\
\hline Rate or Volume & $1.31 \mathrm{E}+05$ & $2.86 \mathrm{E}+02$ & $9.08 \mathrm{E}+02$ & $2.68 \mathrm{E}+02$ & $6.56 \mathrm{E}+01$ & $1.40 \mathrm{E}+01$ & $2.89 \mathrm{E}+01$ & $4.79 \mathrm{E}+00$ \\
\hline Volume Flow (standard, wet) & $6.70 \mathrm{E}+04$ & $1.93 \mathrm{E}+03$ & $1.93 \mathrm{E}+03$ & $7.84 \mathrm{E}+02$ & $1.92 \mathrm{E}+02$ & $4.11 \mathrm{E}+01$ & $2.94 \mathrm{E}+01$ & \\
\hline Volume Flow (standard, dry) & $5.92 \mathrm{E}+04$ & $1.93 \mathrm{E}+03$ & $1.93 \mathrm{E}+03$ & $7.84 \mathrm{E}+02$ & $1.92 \mathrm{E}+02$ & $4.11 \mathrm{E}+01$ & $2.94 \mathrm{E}+01$ & \\
\hline Rate Units & $\mathrm{ft} 3 / \mathrm{hr}$ & $\mathrm{ft} 3 / \mathrm{hr}$ & $\mathrm{ft} 3 / \mathrm{hr}$ & $\mathrm{ft} 3 / \mathrm{hr}$ & $\mathrm{ft} 3 / \mathrm{hr}$ & $\mathrm{ft} 3 / \mathrm{hr}$ & $\mathrm{ft} 3 / \mathrm{hr}$ & $\mathrm{lb} / \mathrm{hr}$ \\
\hline Rate or Volume, metric & $1.90 \mathrm{E}+03$ & $5.47 \mathrm{E}+01$ & $5.47 \mathrm{E}+01$ & $2.22 \mathrm{E}+01$ & $5.44 \mathrm{E}+00$ & $1.16 \mathrm{E}+00$ & 8.34E-01 & $2.17 \mathrm{E}+00$ \\
\hline Rate Units & wscm/hr & wscm/hr & wscm/hr & wscm/hr & wscm/hr & wscm/hr & wscm/hr & $\mathrm{kg} / \mathrm{hr}$ \\
\hline Temperature, ${ }^{\circ} \mathrm{C}$ & 300 & 0 & 593 & 15 & 15 & 15 & 15 & 25 \\
\hline Temperature, ${ }^{\circ} \mathrm{F}$ & 572 & 32 & 1099 & 59 & 59 & 59 & 59 & 77 \\
\hline Pressure, psia & 14.7 & 92.3 & 92.3 & 42.3 & 42.3 & 42.3 & 14.7 & 14.7 \\
\hline Specific Gravity & $6.10 \mathrm{E}-04$ & $7.85 \mathrm{E}-03$ & $2.48 \mathrm{E}-03$ & $3.41 \mathrm{E}-03$ & $3.41 \mathrm{E}-03$ & $3.51 \mathrm{E}-03$ & $1.22 \mathrm{E}-03$ & 4.81E-01 \\
\hline \multicolumn{9}{|c|}{ Gas Stream Bulk Composition (Wet Basis) } \\
\hline & Mol \% & Mol \% & Mol \% & Mol \% & Mol \% & Mol \% & Mol \% & Wt \% \\
\hline $\mathrm{H} 2 \mathrm{O}$ & $11.52 \%$ & & & & & $0.01 \%$ & $0.01 \%$ & \\
\hline $\mathrm{O} 2$ & $3.00 \%$ & & & & & $20.99 \%$ & $20.99 \%$ & \\
\hline $\mathrm{N} 2$ & $74.11 \%$ & $100.00 \%$ & $100.00 \%$ & $100.00 \%$ & $100.00 \%$ & $79.00 \%$ & $79.00 \%$ & \\
\hline $\mathrm{H} 2$ & $0.05 \%$ & & & & & & & \\
\hline \multirow[t]{2}{*}{$\mathrm{CO} 2$} & $10.64 \%$ & & & & & & & \\
\hline & ppmv & & & & & & & \\
\hline $\mathrm{CO}$ & $2.21 \mathrm{E}+03$ & & & & & & & \\
\hline NO & $4.30 \mathrm{E}+03$ & & & & & & & \\
\hline $\mathrm{NO} 2$ & $1.97 \mathrm{E}+00$ & & & & & & & \\
\hline $\mathrm{SO} 2$ & $2.40 \mathrm{E}+02$ & & & & & & & \\
\hline \multicolumn{9}{|l|}{$\mathrm{C}$ (organic) } \\
\hline \multicolumn{9}{|l|}{$\mathrm{H}$ (organic) } \\
\hline & & & & & & & & \\
\hline \multicolumn{9}{|c|}{ Gas Stream Bulk Composition (Dry Basis) } \\
\hline & Mol \% & Mol \% & Mol \% & Mol \% & Mol \% & Mol \% & Mol \% & \\
\hline $\mathrm{O} 2$ & $3.39 \%$ & & & & & $20.99 \%$ & $20.99 \%$ & \\
\hline $\mathrm{N} 2$ & $83.77 \%$ & & & & & $79.01 \%$ & $79.01 \%$ & \\
\hline $\mathrm{H} 2$ & $0.05 \%$ & & & & & & & \\
\hline $\mathrm{CO} 2$ & $12.03 \%$ & & & & & & & \\
\hline \multicolumn{9}{|c|}{ Gas Stream Bulk Composition (Dry Basis, Corrected to $7 \%$ O2 with 100\% O2 Combustion Air) } \\
\hline & ppmv & & & & & & & \\
\hline $\mathrm{CO}$ & $3.49 \mathrm{E}+02$ & & & & & & & \\
\hline $\mathrm{NO}$ & $6.80 \mathrm{E}+02$ & & & & & & & \\
\hline $\mathrm{NO} 2$ & $3.12 \mathrm{E}-01$ & & & & & & & \\
\hline $\mathrm{SO} 2$ & $3.80 \mathrm{E}+01$ & & & & & & & \\
\hline \multicolumn{9}{|l|}{ C(organic) } \\
\hline \multicolumn{9}{|l|}{$\mathrm{H}$ (organic) } \\
\hline & & & & & & & & \\
\hline $\mathrm{PM}, \mathrm{mg} / \mathrm{dscm}$ & $2.62 \mathrm{E}+00$ & & & & & $2.22 \mathrm{E}+00$ & $2.20 \mathrm{E}+00$ & \\
\hline \multicolumn{9}{|l|}{$\mathrm{Hg}, \mathrm{ug} / \mathrm{dscm}$} \\
\hline & & & & & & & & \\
\hline & & & & & & & & \\
\hline GAC & & & & & & & & $100 \%$ \\
\hline
\end{tabular}


Table A-15. Mass balance, Tank WM-188 waste.

\begin{tabular}{|c|c|c|c|c|c|c|c|c|c|}
\hline PFD \# & PFD-2 & PFD-2 & PFD-2 & PFD-2 & PFD-2 & PFD-2 & PFD-2 & PFD-2 & PFD-2 \\
\hline WM-188, Stream \# & 101 & 102 & 103 & 104 & 105 & 106 & 107 & 108 & 108 \\
\hline Stream Name & SBW & $\begin{array}{l}\text { Reformer } \\
\text { Feed }\end{array}$ & $\begin{array}{c}\text { Reformer } \\
\text { Off-gas }\end{array}$ & $\begin{array}{c}\text { Reformer } \\
\text { Off-gas } \\
\text { Cooled }\end{array}$ & $\begin{array}{c}\text { Cyclone } \\
\text { Drain }\end{array}$ & $\begin{array}{l}\text { Filter } \\
\text { Drain }\end{array}$ & Bed Drain & $\begin{array}{c}\text { Cooled } \\
\text { Product }\end{array}$ & $\begin{array}{l}\text { Product } \\
\text { Shipping } \\
\text { Canisters }\end{array}$ \\
\hline \begin{tabular}{|l|} 
Rate or Volume \\
\end{tabular} & $9.66 \mathrm{E}+01$ & $1.33 E+02$ & $4.60 \mathrm{E}+05$ & $4.14 \mathrm{E}+05$ & $1.04 \mathrm{E}+02$ & $6.26 \mathrm{E}+01$ & $8.47 \mathrm{E}+01$ & $1.47 \mathrm{E}+02$ & $1.51 \mathrm{E}+00$ \\
\hline Volume Flow (standard, we & & & $1.03 \mathrm{E}+05$ & $1.05 \mathrm{E}+05$ & & & & & \\
\hline Volume Flow (standard, dry & & & $3.07 E+04$ & $3.25 E+04$ & & & & & \\
\hline Rate Units & $\mathrm{gal} / \mathrm{hr}$ & $\mathrm{gal} / \mathrm{hr}$ & $\mathrm{ft} 3 / \mathrm{hr}$ & $\mathrm{ft} 3 / \mathrm{hr}$ & $\mathrm{lb} / \mathrm{hr}$ & $\mathrm{lb} / \mathrm{hr}$ & $\mathrm{lb} / \mathrm{hr}$ & $\mathrm{lb} / \mathrm{hr}$ & can's/day \\
\hline Rate or Volume, metric & 366 & 504 & $2.92 \mathrm{E}+03$ & $2.98 \mathrm{E}+03$ & 47 & 28.4 & 38.4 & 66.8 & 66.8 \\
\hline Rate Units & $\mathrm{L} / \mathrm{hr}$ & $\mathrm{L} / \mathrm{hr}$ & wscm/hr & wscm/hr & $\mathrm{kg} / \mathrm{hr}$ & $\mathrm{kg} / \mathrm{hr}$ & $\mathrm{kg} / \mathrm{hr}$ & $\mathrm{kg} / \mathrm{hr}$ & $\mathrm{kg} / \mathrm{hr}$ \\
\hline Temperature, ${ }^{\circ} \mathrm{C}$ & 15 & 19 & 670 & 530 & 670 & 530 & 670 & 55 & 55 \\
\hline Temperature, ${ }^{\circ} \mathrm{F}$ & 59 & 66 & 1238 & 986 & 1238 & 986 & 1238 & 132 & 132 \\
\hline Pressure, psia & 14.7 & 44.7 & 10.6 & 10.2 & 10.6 & 9.5 & 10.8 & 9.5 & 9.5 \\
\hline Specific Gravity & 1.22 & 1.22 & $1.98 \mathrm{E}-04$ & $2.25 \mathrm{E}-04$ & 0.24 & 1.15 & 1.46 & 1.33 & 1.33 \\
\hline Chemical Composition & Mol/liter & Mol/liter & $\mathrm{lb} / \mathrm{wscf}$ & $\mathrm{lb} / \mathrm{wscf}$ & Wt frac & Wt frac & Wt frac & Wt frac & Wt frac \\
\hline $\mathrm{H}+$ & $2.53 E+00$ & $1.88 \mathrm{E}+00$ & $7.40 \mathrm{E}-07$ & 7.27E-07 & 2.56E-06 & 5.22E-06 & 7.99E-06 & 6.81E-06 & $6.81 \mathrm{E}-06$ \\
\hline $\mathrm{Al}+3$ & 4.63E-01 & 3.36E-01 & 3.14E-05 & 3.09E-05 & $3.47 \mathrm{E}-02$ & 5.17E-02 & $9.23 \mathrm{E}-02$ & $7.51 \mathrm{E}-02$ & $7.51 \mathrm{E}-02$ \\
\hline $\mathrm{Sb}+5$ & $1.45 \mathrm{E}-05$ & 1.05E-05 & 4.05E-09 & 3.98E-09 & 4.46E-06 & 6.66E-06 & 1.19E-05 & 9.67E-06 & 9.67E-06 \\
\hline As+3 & 8.09E-05 & $5.88 \mathrm{E}-05$ & 1.39E-08 & $1.37 \mathrm{E}-08$ & $1.53 \mathrm{E}-05$ & $2.29 \mathrm{E}-05$ & 4.08E-05 & $3.32 \mathrm{E}-05$ & 3.32E-05 \\
\hline $\mathrm{Ba}+2$ & 6.62E-05 & 4.81E-05 & $2.16 \mathrm{E}-08$ & $2.13 \mathrm{E}-08$ & $1.84 \mathrm{E}-05$ & $3.56 \mathrm{E}-05$ & 6.01E-05 & 4.97E-05 & 4.97E-05 \\
\hline $\mathrm{Be}+2$ & 1.67E-05 & $1.22 \mathrm{E}-05$ & $3.59 \mathrm{E}-10$ & $3.53 \mathrm{E}-10$ & $3.06 \mathrm{E}-07$ & 5.91E-07 & 9.98E-07 & $8.25 E-07$ & $8.25 \mathrm{E}-07$ \\
\hline $\mathrm{B}+3$ & $1.45 \mathrm{E}-02$ & $1.05 \mathrm{E}-02$ & $3.59 \mathrm{E}-07$ & $3.53 \mathrm{E}-07$ & 3.96E-04 & 5.91E-04 & 1.05E-03 & 8.58E-04 & 8.58E-04 \\
\hline $\mathrm{Cd}+2$ & $2.21 \mathrm{E}-03$ & $1.61 \mathrm{E}-03$ & $6.46 \mathrm{E}-07$ & $6.35 \mathrm{E}-07$ & $6.42 \mathrm{E}-04$ & $1.06 \mathrm{E}-03$ & $1.58 \mathrm{E}-03$ & 1.36E-03 & $1.36 \mathrm{E}-03$ \\
\hline $\mathrm{Ca}+2$ & 4.41E-02 & $3.21 \mathrm{E}-02$ & $1.20 \mathrm{E}-05$ & 1.18E-05 & 1.02E-02 & 1.98E-02 & 3.34E-02 & $2.76 \mathrm{E}-02$ & $2.76 \mathrm{E}-02$ \\
\hline $\mathrm{Cr}+3$ & $4.13 \mathrm{E}-03$ & $3.00 \mathrm{E}-03$ & $5.45 \mathrm{E}-07$ & 5.36E-07 & 3.83E-04 & 8.97E-04 & 1.38E-03 & 1.18E-03 & 1.18E-03 \\
\hline $\mathrm{Co}+2$ & 3.61E-05 & 2.62E-05 & 4.88E-09 & 4.79E-09 & $5.38 \mathrm{E}-06$ & 8.03E-06 & 1.43E-05 & 1.17E-05 & 1.17E-05 \\
\hline Cs+ & 5.27E-05 & 4.11E-05 & 5.46E-08 & 5.37E-08 & $7.80 \mathrm{E}-05$ & 8.99E-05 & 5.02E-06 & $4.56 \mathrm{E}-05$ & 4.56E-05 \\
\hline $\mathrm{Cu}+2$ & 6.19E-04 & $4.50 \mathrm{E}-04$ & 9.37E-08 & $9.21 \mathrm{E}-08$ & $7.98 \mathrm{E}-05$ & $1.54 \mathrm{E}-04$ & $2.60 \mathrm{E}-04$ & $2.15 \mathrm{E}-04$ & $2.15 \mathrm{E}-04$ \\
\hline $\mathrm{Fe}+3$ & $2.28 \mathrm{E}-02$ & $1.66 \mathrm{E}-02$ & $2.12 \mathrm{E}-06$ & $2.08 \mathrm{E}-06$ & 1.62E-03 & $3.49 \mathrm{E}-03$ & 1.10E-02 & $7.81 \mathrm{E}-03$ & $7.81 \mathrm{E}-03$ \\
\hline $\mathrm{Pb}+2$ & $7.26 \mathrm{E}-04$ & $5.28 \mathrm{E}-04$ & $1.12 \mathrm{E}-06$ & $1.10 \mathrm{E}-06$ & 3.39E-04 & $1.85 \mathrm{E}-03$ & 6.32E-05 & $8.22 \mathrm{E}-04$ & $8.22 \mathrm{E}-04$ \\
\hline $\mathrm{Hg}+2$ & 4.64E-03 & 3.84E-03 & $8.28 \mathrm{E}-06$ & $8.14 \mathrm{E}-06$ & 6.67E-06 & 1.62E-05 & & $6.91 \mathrm{E}-06$ & $6.91 \mathrm{E}-06$ \\
\hline $\mathrm{Mn}+4$ & 1.32E-02 & 9.61E-03 & $1.66 \mathrm{E}-06$ & $1.64 \mathrm{E}-06$ & $1.84 \mathrm{E}-03$ & $2.74 \mathrm{E}-03$ & $4.89 \mathrm{E}-03$ & $3.98 \mathrm{E}-03$ & $3.98 \mathrm{E}-03$ \\
\hline $\mathrm{Ni}+2$ & $1.62 \mathrm{E}-03$ & 1.18E-03 & $2.26 \mathrm{E}-07$ & $2.23 \mathrm{E}-07$ & 1.93E-04 & 3.73E-04 & $6.29 \mathrm{E}-04$ & 5.20E-04 & 5.20E-04 \\
\hline $\mathrm{K}+$ & 1.69E-01 & $1.23 \mathrm{E}-01$ & $3.22 \mathrm{E}-05$ & $3.16 \mathrm{E}-05$ & $3.87 \mathrm{E}-02$ & $5.30 \mathrm{E}-02$ & $3.58 \mathrm{E}-02$ & $4.31 \mathrm{E}-02$ & $4.31 \mathrm{E}-02$ \\
\hline $\mathrm{Se}+4$ & $2.14 \mathrm{E}-04$ & $1.56 \mathrm{E}-04$ & $3.76 \mathrm{E}-08$ & $3.69 \mathrm{E}-08$ & 4.01E-05 & $6.19 \mathrm{E}-05$ & 1.15E-04 & $9.25 \mathrm{E}-05$ & $9.25 \mathrm{E}-05$ \\
\hline $\mathrm{Ag}+$ & 3.33E-04 & 2.42E-04 & 9.71E-08 & $9.55 \mathrm{E}-08$ & 7.71E-05 & 1.59E-04 & $2.25 \mathrm{E}-04$ & 1.97E-04 & 1.97E-04 \\
\hline $\mathrm{Na}+$ & $1.25 \mathrm{E}+00$ & $9.08 \mathrm{E}-01$ & 7.78E-05 & 7.65E-05 & $6.18 \mathrm{E}-02$ & $1.28 \mathrm{E}-01$ & $1.80 \mathrm{E}-01$ & $1.58 \mathrm{E}-01$ & $1.58 \mathrm{E}-01$ \\
\hline $\mathrm{TI}+3$ & 4.05E-06 & 2.94E-06 & 1.90E-09 & 1.86E-09 & 2.09E-06 & 3.12E-06 & 5.57E-06 & 4.53E-06 & 4.53E-06 \\
\hline$U+4$ & $3.20 \mathrm{E}-04$ & $2.33 \mathrm{E}-04$ & $1.70 \mathrm{E}-07$ & $1.67 \mathrm{E}-07$ & $1.81 \mathrm{E}-04$ & $2.79 \mathrm{E}-04$ & $5.20 \mathrm{E}-04$ & 4.17E-04 & 4.17E-04 \\
\hline $\mathrm{V}+3$ & $3.24 \mathrm{E}-05$ & $2.36 \mathrm{E}-05$ & 3.67E-09 & 3.61E-09 & $3.91 \mathrm{E}-06$ & $6.04 \mathrm{E}-06$ & 1.13E-05 & 9.04E-06 & 9.04E-06 \\
\hline$Z n+2$ & 7.17E-04 & $5.21 \mathrm{E}-04$ & $1.12 \mathrm{E}-07$ & $1.10 \mathrm{E}-07$ & 9.51E-05 & 1.84E-04 & $3.10 \mathrm{E}-04$ & $2.57 \mathrm{E}-04$ & $2.57 \mathrm{E}-04$ \\
\hline $\mathrm{Zr}+4$ & 3.34E-02 & $2.42 \mathrm{E}-02$ & $6.76 \mathrm{E}-06$ & $6.65 \mathrm{E}-06$ & $7.21 \mathrm{E}-03$ & $1.11 \mathrm{E}-02$ & $2.07 \mathrm{E}-02$ & $1.67 \mathrm{E}-02$ & $1.67 \mathrm{E}-02$ \\
\hline $\mathrm{Cl}-$ & $2.18 \mathrm{E}-02$ & 1.59E-02 & 4.49E-06 & 4.41E-06 & $4.05 \mathrm{E}-03$ & 7.19E-03 & 1.94E-03 & 4.17E-03 & 4.17E-03 \\
\hline F- & $3.15 \mathrm{E}-02$ & $2.37 \mathrm{E}-02$ & 4.34E-06 & $4.27 \mathrm{E}-06$ & $2.36 \mathrm{E}-03$ & 6.44E-03 & 6.06E-04 & $3.08 \mathrm{E}-03$ & $3.08 \mathrm{E}-03$ \\
\hline SO4-2 & 3.70E-02 & $2.78 \mathrm{E}-02$ & 8.99E-09 & 8.83E-09 & $4.50 \mathrm{E}-06$ & $1.48 \mathrm{E}-05$ & 1.13E-05 & 1.28E-05 & $1.28 \mathrm{E}-05$ \\
\hline NO3- & $5.17 E+00$ & $3.80 \mathrm{E}+00$ & 9.35E-06 & 9.19E-06 & 1.96E-03 & 4.49E-03 & 5.12E-03 & $4.85 \mathrm{E}-03$ & $4.85 \mathrm{E}-03$ \\
\hline PO4-3 & $8.80 \mathrm{E}-02$ & $6.40 \mathrm{E}-02$ & $2.40 \mathrm{E}-05$ & $2.36 \mathrm{E}-05$ & $4.34 \mathrm{E}-02$ & $3.96 \mathrm{E}-02$ & 5.41E-02 & 4.79E-02 & 4.79E-02 \\
\hline $\mathrm{Am}+4$ & $6.13 \mathrm{E}-08$ & $4.45 \mathrm{E}-08$ & $3.41 \mathrm{E}-11$ & $3.35 \mathrm{E}-11$ & $3.76 \mathrm{E}-08$ & 5.62E-08 & $1.00 \mathrm{E}-07$ & $8.15 \mathrm{E}-08$ & $8.15 \mathrm{E}-08$ \\
\hline $\mathrm{Br}-$ & $2.63 \mathrm{E}-07$ & 1.92E-07 & $1.22 \mathrm{E}-10$ & $1.20 \mathrm{E}-10$ & $1.10 \mathrm{E}-07$ & 1.96E-07 & $5.27 \mathrm{E}-08$ & 1.13E-07 & $1.13 \mathrm{E}-07$ \\
\hline $\mathrm{Ce}+4$ & 3.03E-05 & $2.20 \mathrm{E}-05$ & 9.73E-09 & 9.56E-09 & 1.07E-05 & 1.60E-05 & 2.86E-05 & $2.32 \mathrm{E}-05$ & $2.32 \mathrm{E}-05$ \\
\hline Eu+3 & 4.37E-07 & 3.17E-07 & 1.52E-10 & $1.49 \mathrm{E}-10$ & 1.68E-07 & $2.50 \mathrm{E}-07$ & 4.47E-07 & 3.63E-07 & 3.63E-07 \\
\hline $\mathrm{Gd}+3$ & 1.29E-04 & 9.36E-05 & 4.64E-08 & $4.56 \mathrm{E}-08$ & $5.12 \mathrm{E}-05$ & 7.64E-05 & 1.36E-04 & $1.11 \mathrm{E}-04$ & $1.11 \mathrm{E}-04$ \\
\hline $\mathrm{Ge}+4$ & 7.59E-09 & 5.51E-09 & $1.22 \mathrm{E}-12$ & $1.20 \mathrm{E}-12$ & 1.31E-09 & 2.02E-09 & 3.75E-09 & $3.02 \mathrm{E}-09$ & 3.02E-09 \\
\hline $\ln +3$ & 1.33E-06 & 9.70E-07 & $3.51 \mathrm{E}-10$ & $3.45 \mathrm{E}-10$ & 3.87E-07 & 5.78E-07 & 1.03E-06 & 8.39E-07 & 8.39E-07 \\
\hline I- & $2.81 \mathrm{E}-06$ & $2.06 \mathrm{E}-06$ & 2.07E-09 & 2.04E-09 & $1.87 \mathrm{E}-06$ & $3.32 \mathrm{E}-06$ & 8.94E-07 & 1.93E-06 & 1.93E-06 \\
\hline $\mathrm{La}+3$ & 7.93E-06 & $5.76 \mathrm{E}-06$ & 2.52E-09 & 2.48E-09 & $2.78 \mathrm{E}-06$ & $4.15 \mathrm{E}-06$ & 7.41E-06 & 6.03E-06 & $6.03 \mathrm{E}-06$ \\
\hline
\end{tabular}


Table A-15. Mass balance, Tank WM-188 waste (continued).

\begin{tabular}{|c|c|c|c|c|c|c|c|c|c|}
\hline Stream \# & 101 & 102 & 103 & 104 & 105 & 106 & 107 & 108 & 108 \\
\hline Chemical Composition & Mol/liter & Mol/liter & $\mathrm{lb} /$ wscf & $\mathrm{lb} /$ wscf & Wt frac & Wt frac & Wt frac & Wt frac & Wt frac \\
\hline $\mathrm{Li+}$ & $4.14 \mathrm{E}-04$ & $3.01 \mathrm{E}-04$ & $7.77 \mathrm{E}-09$ & $7.63 \mathrm{E}-09$ & $6.17 \mathrm{E}-06$ & $1.27 \mathrm{E}-05$ & $1.80 \mathrm{E}-05$ & $1.58 \mathrm{E}-05$ & $1.58 \mathrm{E}-05$ \\
\hline $\mathrm{Mg}+2$ & $1.63 \mathrm{E}-02$ & $1.18 \mathrm{E}-02$ & $9.41 \mathrm{E}-07$ & $9.25 \mathrm{E}-07$ & 8.01E-04 & $1.55 \mathrm{E}-03$ & $2.62 \mathrm{E}-03$ & $2.16 \mathrm{E}-03$ & $2.16 \mathrm{E}-03$ \\
\hline $\mathrm{Mo}+6$ & $4.78 \mathrm{E}-02$ & $3.47 \mathrm{E}-02$ & $1.02 \mathrm{E}-05$ & $1.00 \mathrm{E}-05$ & $1.09 \mathrm{E}-02$ & $1.68 \mathrm{E}-02$ & $3.12 \mathrm{E}-02$ & $2.51 \mathrm{E}-02$ & $2.51 \mathrm{E}-02$ \\
\hline $\mathrm{Nd}+3$ & $2.56 \mathrm{E}-05$ & $1.86 \mathrm{E}-05$ & $8.45 \mathrm{E}-09$ & $8.30 \mathrm{E}-09$ & $9.32 \mathrm{E}-06$ & $1.39 \mathrm{E}-05$ & $2.48 \mathrm{E}-05$ & $2.02 \mathrm{E}-05$ & $2.02 \mathrm{E}-05$ \\
\hline $\mathrm{Np}+4$ & $2.34 \mathrm{E}-06$ & $1.70 \mathrm{E}-06$ & 1.27E-09 & $1.25 \mathrm{E}-09$ & $1.40 \mathrm{E}-06$ & $2.09 E-06$ & $3.73 \mathrm{E}-06$ & $3.03 E-06$ & $3.03 E-06$ \\
\hline $\mathrm{Nb}+5$ & $9.58 \mathrm{E}-04$ & $6.96 \mathrm{E}-04$ & 1.98E-07 & 1.94E-07 & $2.11 \mathrm{E}-04$ & $3.26 \mathrm{E}-04$ & 6.06E-04 & $4.87 \mathrm{E}-04$ & $4.87 \mathrm{E}-04$ \\
\hline $\mathrm{Pd}+4$ & $1.55 \mathrm{E}-03$ & $1.13 \mathrm{E}-03$ & $3.94 \mathrm{E}-07$ & $3.87 \mathrm{E}-07$ & $3.35 \mathrm{E}-04$ & $6.49 \mathrm{E}-04$ & $1.10 \mathrm{E}-03$ & 9.05E-04 & $9.05 \mathrm{E}-04$ \\
\hline $\mathrm{Pu}+4$ & $6.09 \mathrm{E}-06$ & $4.42 \mathrm{E}-06$ & 3.30E-09 & $3.25 \mathrm{E}-09$ & $3.52 \mathrm{E}-06$ & $5.44 \mathrm{E}-06$ & $1.01 \mathrm{E}-05$ & $8.13 \mathrm{E}-06$ & $8.13 \mathrm{E}-06$ \\
\hline $\mathrm{Pr}+4$ & $7.21 \mathrm{E}-06$ & $5.24 \mathrm{E}-06$ & $2.33 \mathrm{E}-09$ & $2.29 \mathrm{E}-09$ & $2.57 \mathrm{E}-06$ & $3.83 \mathrm{E}-06$ & 6.84E-06 & $5.56 \mathrm{E}-06$ & $5.56 \mathrm{E}-06$ \\
\hline $\mathrm{Pm}+3$ & 1.49E-09 & $1.08 \mathrm{E}-09$ & $4.96 \mathrm{E}-13$ & $4.87 \mathrm{E}-13$ & $5.46 \mathrm{E}-10$ & $8.16 \mathrm{E}-10$ & $1.46 \mathrm{E}-09$ & $1.18 \mathrm{E}-09$ & $1.18 \mathrm{E}-09$ \\
\hline $\mathrm{Rh}+4$ & $3.11 \mathrm{E}-06$ & $2.26 \mathrm{E}-06$ & $7.33 \mathrm{E}-10$ & $7.21 \mathrm{E}-10$ & 8.08E-07 & $1.21 \mathrm{E}-06$ & $2.15 \mathrm{E}-06$ & $1.75 \mathrm{E}-06$ & $1.75 \mathrm{E}-06$ \\
\hline $\mathrm{Rb}+$ & 4.79E-06 & $3.48 \mathrm{E}-06$ & 1.11E-09 & 1.09E-09 & 8.78E-07 & $1.81 \mathrm{E}-06$ & $2.56 \mathrm{E}-06$ & $2.24 \mathrm{E}-06$ & $2.24 \mathrm{E}-06$ \\
\hline$R u+3$ & $7.20 \mathrm{E}-04$ & $5.23 E-04$ & 1.67E-07 & $1.64 \mathrm{E}-07$ & $1.84 \mathrm{E}-04$ & $2.74 \mathrm{E}-04$ & $4.90 \mathrm{E}-04$ & $3.98 \mathrm{E}-04$ & $3.98 \mathrm{E}-04$ \\
\hline $\mathrm{Sm}+3$ & $4.76 \mathrm{E}-06$ & $3.46 \mathrm{E}-06$ & $1.64 \mathrm{E}-09$ & $1.61 \mathrm{E}-09$ & $1.81 \mathrm{E}-06$ & $2.70 \mathrm{E}-06$ & $4.82 \mathrm{E}-06$ & $3.92 \mathrm{E}-06$ & $3.92 \mathrm{E}-06$ \\
\hline $\mathrm{Si}+4$ & $8.17 \mathrm{E}-02$ & $5.94 \mathrm{E}-02$ & $6.90 \mathrm{E}-06$ & $6.78 \mathrm{E}-06$ & $1.08 \mathrm{E}-02$ & $1.14 \mathrm{E}-02$ & $2.40 \mathrm{E}-02$ & $1.86 \mathrm{E}-02$ & $1.86 \mathrm{E}-02$ \\
\hline $\mathrm{Sr}+2$ & $7.11 \mathrm{E}-05$ & $5.17 \mathrm{E}-05$ & $1.48 \mathrm{E}-08$ & $1.46 \mathrm{E}-08$ & $1.26 \mathrm{E}-05$ & $2.44 \mathrm{E}-05$ & $4.12 \mathrm{E}-05$ & $3.41 \mathrm{E}-05$ & $3.41 \mathrm{E}-05$ \\
\hline Tc+7 & $9.28 \mathrm{E}-06$ & $6.74 \mathrm{E}-06$ & $6.41 \mathrm{E}-09$ & $6.30 \mathrm{E}-09$ & $1.06 \mathrm{E}-05$ & $1.05 \mathrm{E}-05$ & $8.46 \mathrm{E}-07$ & $4.97 \mathrm{E}-06$ & $4.97 \mathrm{E}-06$ \\
\hline $\mathrm{Te}+4$ & $3.17 \mathrm{E}-06$ & $2.30 \mathrm{E}-06$ & $8.98 \mathrm{E}-10$ & $8.83 \mathrm{E}-10$ & 9.57E-07 & $1.48 \mathrm{E}-06$ & $2.75 \mathrm{E}-06$ & $2.21 \mathrm{E}-06$ & $2.21 \mathrm{E}-06$ \\
\hline $\mathrm{Tb}+4$ & 1.83E-09 & 1.33E-09 & $6.64 \mathrm{E}-13$ & $6.53 \mathrm{E}-13$ & $7.33 \mathrm{E}-10$ & 1.09E-09 & 1.95E-09 & 1.59E-09 & 1.59E-09 \\
\hline $\mathrm{Th}+4$ & $2.10 \mathrm{E}-05$ & 1.53E-05 & $1.08 \mathrm{E}-08$ & $1.07 \mathrm{E}-08$ & 1.16E-05 & $1.78 \mathrm{E}-05$ & $3.32 \mathrm{E}-05$ & 2.67E-05 & $2.67 \mathrm{E}-05$ \\
\hline$S n+4$ & $1.58 \mathrm{E}-03$ & $1.15 \mathrm{E}-03$ & $4.17 \mathrm{E}-07$ & $4.10 \mathrm{E}-07$ & $4.45 \mathrm{E}-04$ & $6.87 \mathrm{E}-04$ & $1.28 \mathrm{E}-03$ & $1.03 E-03$ & $1.03 \mathrm{E}-03$ \\
\hline $\mathrm{Ti}+4$ & 8.09E-04 & $5.88 \mathrm{E}-04$ & $8.61 \mathrm{E}-08$ & $8.46 \mathrm{E}-08$ & $9.18 \mathrm{E}-05$ & $1.42 \mathrm{E}-04$ & 2.64E-04 & $2.12 \mathrm{E}-04$ & $2.12 \mathrm{E}-04$ \\
\hline$Y+3$ & $5.91 \mathrm{E}-06$ & $4.29 \mathrm{E}-06$ & $1.20 \mathrm{E}-09$ & $1.18 \mathrm{E}-09$ & $1.33 \mathrm{E}-06$ & $1.98 \mathrm{E}-06$ & $3.54 \mathrm{E}-06$ & $2.88 \mathrm{E}-06$ & $2.88 \mathrm{E}-06$ \\
\hline $\mathrm{OH}-$ & & 5.62E-04 & $2.10 \mathrm{E}-06$ & 2.06E-06 & 1.69E-03 & $3.45 \mathrm{E}-03$ & 5.27E-03 & 4.49E-03 & 4.49E-03 \\
\hline $\mathrm{H} 2 \mathrm{O}$ & $4.47 E+01$ & $3.47 E+01$ & $3.29 E-02$ & $3.24 \mathrm{E}-02$ & 1.52E-03 & 2.93E-03 & 4.43E-03 & 3.79E-03 & 3.79E-03 \\
\hline $\mathrm{SO} 2$ & & $3.37 \mathrm{E}-07$ & $2.43 \mathrm{E}-09$ & $2.39 \mathrm{E}-09$ & & & & & \\
\hline $\mathrm{H} 2 \mathrm{~S}$ & & & $1.04 \mathrm{E}-05$ & $1.03 \mathrm{E}-05$ & & & & & \\
\hline $\mathrm{CO}$ & & $8.44 \mathrm{E}-12$ & 7.94E-04 & $7.80 \mathrm{E}-04$ & & & & & \\
\hline $\mathrm{CO} 2$ & & $1.29 \mathrm{E}-05$ & $7.61 \mathrm{E}-03$ & $7.48 \mathrm{E}-03$ & & & & & \\
\hline $\mathrm{H} 2$ & & $3.47 \mathrm{E}-11$ & $2.09 \mathrm{E}-04$ & $2.06 \mathrm{E}-04$ & & & & & \\
\hline $\mathrm{N} 2$ & & $5.06 \mathrm{E}-07$ & $1.24 \mathrm{E}-02$ & $1.35 \mathrm{E}-02$ & & & & & \\
\hline $\mathrm{NO}$ & & 9.58E-09 & $4.81 \mathrm{E}-05$ & $4.73 \mathrm{E}-05$ & & & & & \\
\hline NO2 & & $1.21 \mathrm{E}-07$ & $4.99 \mathrm{E}-06$ & $4.91 \mathrm{E}-06$ & & & & & \\
\hline $\mathrm{O} 2$ & & $1.29 \mathrm{E}-07$ & $4.44 \mathrm{E}-04$ & $4.36 \mathrm{E}-04$ & & & & & \\
\hline S (other) & & $4.50 \mathrm{E}-09$ & $1.49 \mathrm{E}-07$ & $1.46 \mathrm{E}-07$ & $1.20 \mathrm{E}-04$ & $2.45 \mathrm{E}-04$ & 3.75E-04 & $3.20 \mathrm{E}-04$ & $3.20 \mathrm{E}-04$ \\
\hline $\mathrm{CO} 3$ & 2.63E-05 & $2.05 \mathrm{E}-05$ & $1.28 \mathrm{E}-04$ & $1.26 \mathrm{E}-04$ & $1.13 \mathrm{E}-01$ & $2.10 \mathrm{E}-01$ & $2.94 \mathrm{E}-01$ & $2.59 \mathrm{E}-01$ & $2.59 \mathrm{E}-01$ \\
\hline C (reductant) & & & $2.25 \mathrm{E}-05$ & $2.21 \mathrm{E}-05$ & 4.24E-01 & $3.70 \mathrm{E}-02$ & & $1.57 \mathrm{E}-02$ & $1.57 \mathrm{E}-02$ \\
\hline $\mathrm{O}$ (oxides) & $4.25 \mathrm{E}-01$ & 3.09E-01 & $4.31 \mathrm{E}-05$ & $4.24 \mathrm{E}-05$ & $4.58 \mathrm{E}-02$ & $7.10 \mathrm{E}-02$ & 1.37E-01 & $1.09 \mathrm{E}-01$ & $1.09 \mathrm{E}-01$ \\
\hline C (organic) & $6.56 \mathrm{E}-02$ & $1.03 \mathrm{E}+01$ & $9.45 \mathrm{E}-05$ & $9.29 \mathrm{E}-05$ & $7.13 \mathrm{E}-02$ & $1.25 \mathrm{E}-01$ & $9.25 \mathrm{E}-03$ & $5.85 \mathrm{E}-02$ & $5.85 \mathrm{E}-02$ \\
\hline $\mathrm{H}$ (organic) & $1.20 \mathrm{E}-01$ & $1.89 \mathrm{E}+01$ & $1.79 \mathrm{E}-05$ & $1.76 \mathrm{E}-05$ & $1.10 \mathrm{E}-02$ & $1.93 \mathrm{E}-02$ & $1.42 \mathrm{E}-03$ & 9.01E-03 & $9.01 \mathrm{E}-03$ \\
\hline O (organic) & $6.01 \mathrm{E}-02$ & $9.45 \mathrm{E}+00$ & $9.29 \mathrm{E}-05$ & $9.13 \mathrm{E}-05$ & $8.71 \mathrm{E}-02$ & $1.53 \mathrm{E}-01$ & $1.13 \mathrm{E}-02$ & $7.15 \mathrm{E}-02$ & $7.15 \mathrm{E}-02$ \\
\hline Mass Flow $(\mathrm{kg} / \mathrm{hr})$ : & $4.48 \mathrm{E}+02$ & $6.16 \mathrm{E}+02$ & $2.58 \mathrm{E}+03$ & $2.64 \mathrm{E}+03$ & $4.72 \mathrm{E}+01$ & $2.84 \mathrm{E}+01$ & $3.84 \mathrm{E}+01$ & $6.68 \mathrm{E}+01$ & $6.68 \mathrm{E}+01$ \\
\hline Total Canisters Generated & & & & & & & & 213 & \\
\hline & & & & & & & & & \\
\hline & $w / m 3$ & $\mathrm{w} / \mathrm{m} 3$ & $w / m 3$ & w/m3 & $\mathrm{w} / \mathrm{m} 3$ & $\mathrm{w} / \mathrm{m} 3$ & $\mathrm{w} / \mathrm{m} 3$ & $\mathrm{w} / \mathrm{m} 3$ & w/Canistel \\
\hline Heat Generation, w/m3 & $3.68 \mathrm{E}-01$ & $2.68 \mathrm{E}-01$ & & & $2.52 \mathrm{E}-01$ & $1.84 \mathrm{E}+00$ & $3.41 \mathrm{E}+00$ & $2.70 \mathrm{E}+00$ & $2.16 \mathrm{E}+00$ \\
\hline
\end{tabular}


Table A-15. Mass balance, Tank WM-188 waste (continued).

\begin{tabular}{|c|c|c|c|c|c|c|c|c|c|}
\hline Stream \# & 101 & 102 & 103 & 104 & 105 & 106 & 107 & 108 & 108 \\
\hline Radiological Composition & $\overline{\mathrm{Ci} / \mathrm{L}}$ & $\mathrm{Ci} / \mathrm{L}$ & Ci/wscm & $\mathrm{Ci} / \mathrm{wscm}$ & $\mathrm{Ci} / \mathrm{kg}$ & $\mathrm{Ci} / \mathrm{kg}$ & $\mathrm{Ci} / \mathrm{kg}$ & $\mathrm{Ci} / \mathrm{kg}$ & Ci/canister \\
\hline $\mathrm{H}-3$ & $1.06 \mathrm{E}-05$ & $7.71 \mathrm{E}-06$ & $1.33 \mathrm{E}-06$ & $1.30 \mathrm{E}-06$ & & & & & \\
\hline$\overline{C-14}$ & 1.44E-10 & $1.04 \mathrm{E}-10$ & $1.76 \mathrm{E}-11$ & $1.73 \mathrm{E}-11$ & $2.22 \mathrm{E}-10$ & $8.76 \mathrm{E}-11$ & $2.92 \mathrm{E}-11$ & $5.40 \mathrm{E}-11$ & $5.75 \mathrm{E}-08$ \\
\hline Co-60 & $3.09 \mathrm{E}-06$ & 5.88E-06 & 2.97E-07 & 2.92E-07 & 2.04E-05 & 3.05E-05 & 5.45E-05 & $4.43 E-05$ & $4.71 \mathrm{E}-02$ \\
\hline $\mathrm{Ni}-59$ & $1.01 \mathrm{E}-06$ & $7.33 \mathrm{E}-07$ & $3.85 \mathrm{E}-08$ & $3.78 \mathrm{E}-08$ & $2.05 \mathrm{E}-06$ & $3.95 \mathrm{E}-06$ & $6.68 \mathrm{E}-06$ & $5.52 \mathrm{E}-06$ & 5.87E-03 \\
\hline $\mathrm{Ni}-63$ & 3.81E-05 & $2.77 \mathrm{E}-05$ & 1.45E-06 & 1.43E-06 & $7.73 \mathrm{E}-05$ & 1.49E-04 & $2.52 \mathrm{E}-04$ & $2.09 E-04$ & $2.22 \mathrm{E}-01$ \\
\hline Se-79 & $5.22 \mathrm{E}-07$ & $3.79 \mathrm{E}-07$ & $1.86 \mathrm{E}-08$ & 1.83E-08 & $1.24 \mathrm{E}-06$ & $1.91 \mathrm{E}-06$ & $3.55 \mathrm{E}-06$ & $2.85 \mathrm{E}-06$ & $3.03 E-03$ \\
\hline Sr-90 & $3.58 \mathrm{E}-02$ & $2.60 \mathrm{E}-02$ & $1.37 \mathrm{E}-03$ & 1.34E-03 & $7.27 \mathrm{E}-02$ & $1.41 \mathrm{E}-01$ & $2.37 \mathrm{E}-01$ & $1.96 \mathrm{E}-01$ & $2.09 \mathrm{E}+02$ \\
\hline$\overline{Y-90}$ & $3.58 \mathrm{E}-02$ & $2.60 \mathrm{E}-02$ & 1.31E-03 & 1.29E-03 & $9.05 \mathrm{E}-02$ & $1.35 \mathrm{E}-01$ & $2.41 \mathrm{E}-01$ & 1.96E-01 & $2.09 \mathrm{E}+02$ \\
\hline$\overline{Z r-93}$ & $2.06 \mathrm{E}-06$ & $1.50 \mathrm{E}-06$ & 7.34E-08 & $7.22 \mathrm{E}-08$ & $4.89 \mathrm{E}-06$ & $7.55 \mathrm{E}-06$ & $1.41 \mathrm{E}-05$ & $1.13 \mathrm{E}-05$ & $1.20 \mathrm{E}-02$ \\
\hline $\mathrm{Nb}-93 \mathrm{~m}$ & 1.59E-06 & $1.15 \mathrm{E}-06$ & $5.66 \mathrm{E}-08$ & 5.56E-08 & $3.77 \mathrm{E}-06$ & 5.82E-06 & 1.08E-05 & 8.70E-06 & $9.25 \mathrm{E}-03$ \\
\hline $\mathrm{Nb}-94$ & $1.36 \mathrm{E}-06$ & $9.86 \mathrm{E}-07$ & $4.83 \mathrm{E}-08$ & $4.75 \mathrm{E}-08$ & $3.22 \mathrm{E}-06$ & 4.97E-06 & $9.25 \mathrm{E}-06$ & 7.43E-06 & 7.90E-03 \\
\hline Tc-99 & 1.89E-05 & 1.37E-05 & $2.13 \mathrm{E}-06$ & $2.09 \mathrm{E}-06$ & $2.21 \mathrm{E}-04$ & $2.19 \mathrm{E}-04$ & $1.76 \mathrm{E}-05$ & $1.03 \mathrm{E}-04$ & $1.10 \mathrm{E}-01$ \\
\hline Ru-106 & $1.11 \mathrm{E}-06$ & $8.10 \mathrm{E}-07$ & 4.09E-08 & $4.02 \mathrm{E}-08$ & $2.81 \mathrm{E}-06$ & $4.20 \mathrm{E}-06$ & $7.50 \mathrm{E}-06$ & $6.10 \mathrm{E}-06$ & 6.48E-03 \\
\hline Rh-102 & $8.02 \mathrm{E}-10$ & $5.83 \mathrm{E}-10$ & $2.94 \mathrm{E}-11$ & $2.89 \mathrm{E}-11$ & $2.03 \mathrm{E}-09$ & $3.03 E-09$ & $5.40 \mathrm{E}-09$ & $4.39 \mathrm{E}-09$ & $4.67 \mathrm{E}-06$ \\
\hline Rh-106 & 1.11E-06 & $8.10 \mathrm{E}-07$ & 4.09E-08 & $4.02 \mathrm{E}-08$ & $2.81 \mathrm{E}-06$ & $4.20 \mathrm{E}-06$ & $7.50 \mathrm{E}-06$ & $6.10 \mathrm{E}-06$ & 6.48E-03 \\
\hline $\mathrm{Pd}-107$ & 1.54E-08 & 1.12E-08 & $5.87 \mathrm{E}-10$ & 5.77E-10 & $3.12 \mathrm{E}-08$ & $6.03 E-08$ & 1.02E-07 & $8.41 \mathrm{E}-08$ & 8.95E-05 \\
\hline Cd-113m & $3.09 \mathrm{E}-06$ & $2.25 \mathrm{E}-06$ & 1.29E-07 & 1.27E-07 & $7.99 \mathrm{E}-06$ & 1.32E-05 & $1.96 \mathrm{E}-05$ & 1.69E-05 & $1.80 \mathrm{E}-02$ \\
\hline Sn-121m & $6.22 \mathrm{E}-08$ & $4.52 \mathrm{E}-08$ & $2.22 \mathrm{E}-09$ & $2.18 \mathrm{E}-09$ & 1.47E-07 & $2.28 \mathrm{E}-07$ & $4.24 \mathrm{E}-07$ & $3.41 \mathrm{E}-07$ & 3.62E-04 \\
\hline Sn-126 & $4.91 \mathrm{E}-07$ & $3.57 \mathrm{E}-07$ & $1.75 \mathrm{E}-08$ & $1.72 \mathrm{E}-08$ & $1.16 \mathrm{E}-06$ & $1.80 \mathrm{E}-06$ & $3.35 \mathrm{E}-06$ & $2.69 \mathrm{E}-06$ & $2.86 \mathrm{E}-03$ \\
\hline Sb-125 & 3.37E-04 & $2.45 \mathrm{E}-04$ & 1.24E-05 & 1.22E-05 & $8.52 \mathrm{E}-04$ & 1.27E-03 & $2.27 \mathrm{E}-03$ & $1.85 \mathrm{E}-03$ & $1.96 \mathrm{E}+00$ \\
\hline Sb-126 & 5.35E-08 & 3.89E-08 & 1.96E-09 & 1.93E-09 & $1.35 \mathrm{E}-07$ & $2.02 \mathrm{E}-07$ & $3.60 \mathrm{E}-07$ & 2.93E-07 & $3.11 \mathrm{E}-04$ \\
\hline Te-125m & $2.93 \mathrm{E}-06$ & $2.13 \mathrm{E}-06$ & $1.04 \mathrm{E}-07$ & 1.03E-07 & $6.95 \mathrm{E}-06$ & 1.07E-05 & $2.00 \mathrm{E}-05$ & 1.60E-05 & $1.71 \mathrm{E}-02$ \\
\hline $1-129$ & $6.37 \mathrm{E}-08$ & $4.66 \mathrm{E}-08$ & 5.92E-09 & 5.82E-09 & $3.34 \mathrm{E}-07$ & 5.93E-07 & 1.60E-07 & $3.44 \mathrm{E}-07$ & $3.65 \mathrm{E}-04$ \\
\hline Cs-134 & $3.36 \mathrm{E}-05$ & $2.62 \mathrm{E}-05$ & $4.20 \mathrm{E}-06$ & $4.13 \mathrm{E}-06$ & $3.75 \mathrm{E}-04$ & $4.32 \mathrm{E}-04$ & $2.41 \mathrm{E}-05$ & $2.19 \mathrm{E}-04$ & $2.33 \mathrm{E}-01$ \\
\hline Cs-135 & $1.00 \mathrm{E}-06$ & $7.82 \mathrm{E}-07$ & $1.25 \mathrm{E}-07$ & 1.23E-07 & $1.12 \mathrm{E}-05$ & 1.29E-05 & $7.19 \mathrm{E}-07$ & $6.54 \mathrm{E}-06$ & $6.95 \mathrm{E}-03$ \\
\hline Cs-137 & $2.07 \mathrm{E}-02$ & $1.61 \mathrm{E}-02$ & $2.59 \mathrm{E}-03$ & $2.54 \mathrm{E}-03$ & $2.31 \mathrm{E}-01$ & $2.66 \mathrm{E}-01$ & $1.48 \mathrm{E}-02$ & 1.35E-01 & $1.43 E+02$ \\
\hline Ba-137m & 1.96E-02 & 1.42E-02 & 7.47E-04 & 7.34E-04 & 3.97E-02 & 7.68E-02 & $1.30 \mathrm{E}-01$ & 1.07E-01 & $1.14 \mathrm{E}+02$ \\
\hline Ce-144 & $7.52 \mathrm{E}-07$ & 5.46E-07 & $2.76 \mathrm{E}-08$ & $2.71 \mathrm{E}-08$ & 1.90E-06 & $2.84 \mathrm{E}-06$ & $5.06 \mathrm{E}-06$ & $4.12 \mathrm{E}-06$ & $4.38 \mathrm{E}-03$ \\
\hline Pr-144 & $7.52 \mathrm{E}-07$ & $5.46 \mathrm{E}-07$ & $2.76 \mathrm{E}-08$ & $2.71 \mathrm{E}-08$ & 1.90E-06 & $2.84 \mathrm{E}-06$ & $5.06 \mathrm{E}-06$ & $4.12 \mathrm{E}-06$ & $4.38 \mathrm{E}-03$ \\
\hline Pm-146 & $4.74 \mathrm{E}-08$ & $3.44 \mathrm{E}-08$ & 1.74E-09 & 1.71E-09 & $1.20 \mathrm{E}-07$ & 1.79E-07 & $3.19 \mathrm{E}-07$ & $2.59 \mathrm{E}-07$ & $2.76 \mathrm{E}-04$ \\
\hline $\mathrm{Pm}-147$ & $2.03 E-04$ & $1.48 \mathrm{E}-04$ & $7.46 \mathrm{E}-06$ & 7.33E-06 & $5.13 \mathrm{E}-04$ & $7.66 \mathrm{E}-04$ & 1.37E-03 & $1.11 \mathrm{E}-03$ & $1.18 \mathrm{E}+00$ \\
\hline Sm-151 & $4.02 \mathrm{E}-04$ & $2.92 \mathrm{E}-04$ & 1.47E-05 & 1.45E-05 & 1.01E-03 & $1.51 \mathrm{E}-03$ & $2.70 \mathrm{E}-03$ & $2.20 \mathrm{E}-03$ & $2.34 \mathrm{E}+00$ \\
\hline Eu-152 & $2.65 \mathrm{E}-06$ & 1.93E-06 & $9.74 \mathrm{E}-08$ & 9.57E-08 & $6.70 \mathrm{E}-06$ & 1.00E-05 & 1.79E-05 & 1.45E-05 & 1.54E-02 \\
\hline Eu-154 & $52 \mathrm{E}-05$ & $3.28 \mathrm{E}-05$ & $1.66 \mathrm{E}-06$ & 1.63E-06 & 1.14E-04 & 1.70E-04 & 3.04E-04 & $2.47 \mathrm{E}-04$ & $2.63 \mathrm{E}-01$ \\
\hline Eu-155 & $3.92 \mathrm{E}-05$ & $2.85 \mathrm{E}-05$ & $1.44 \mathrm{E}-06$ & $1.41 \mathrm{E}-06$ & 9.89E-05 & $1.48 \mathrm{E}-04$ & $2.64 \mathrm{E}-04$ & $2.14 \mathrm{E}-04$ & $2.28 \mathrm{E}-01$ \\
\hline Th-230 & $53 \mathrm{E}-10$ & $6.93 \mathrm{E}-10$ & $3.39 \mathrm{E}-11$ & $3.34 \mathrm{E}-11$ & $2.26 \mathrm{E}-09$ & 3.49E-09 & $6.50 \mathrm{E}-09$ & $5.22 \mathrm{E}-09$ & $5.55 \mathrm{E}-06$ \\
\hline $\mathrm{Pa}-233$ & $2.73 \mathrm{E}-06$ & $1.98 \mathrm{E}-06$ & $9.72 \mathrm{E}-08$ & 9.55E-08 & $6.47 \mathrm{E}-06$ & 9.99E-06 & $1.86 \mathrm{E}-05$ & 1.49E-05 & $1.59 \mathrm{E}-02$ \\
\hline U-232 & $24 \mathrm{E}-09$ & 1.63E-09 & $7.99 \mathrm{E}-11$ & $7.86 \mathrm{E}-11$ & 5.32E-09 & $8.22 \mathrm{E}-09$ & $1.53 \mathrm{E}-08$ & $1.23 \mathrm{E}-08$ & $1.31 \mathrm{E}-05$ \\
\hline U-233 & 12E-11 & $5.90 \mathrm{E}-11$ & $2.89 \mathrm{E}-12$ & $2.84 \mathrm{E}-12$ & 1.93E-10 & $2.97 \mathrm{E}-10$ & 5.53E-10 & $4.45 \mathrm{E}-10$ & $4.73 \mathrm{E}-07$ \\
\hline U-234 & $1.00 \mathrm{E}-06$ & $7.28 \mathrm{E}-07$ & $3.57 \mathrm{E}-08$ & $3.51 \mathrm{E}-08$ & $2.37 \mathrm{E}-06$ & $3.66 \mathrm{E}-06$ & $6.82 \mathrm{E}-06$ & $5.48 \mathrm{E}-06$ & $5.83 \mathrm{E}-03$ \\
\hline U-235 & $7.36 \mathrm{E}-08$ & $5.35 \mathrm{E}-08$ & $2.62 \mathrm{E}-09$ & $2.58 \mathrm{E}-09$ & $1.74 \mathrm{E}-07$ & $2.69 \mathrm{E}-07$ & $5.01 \mathrm{E}-07$ & 4.03E-07 & $4.28 \mathrm{E}-04$ \\
\hline U-236 & $4.33 \mathrm{E}-08$ & $3.14 \mathrm{E}-08$ & 1.54E-09 & $1.51 \mathrm{E}-09$ & $1.03 \mathrm{E}-07$ & $1.58 \mathrm{E}-07$ & $2.95 \mathrm{E}-07$ & $2.37 \mathrm{E}-07$ & $2.52 \mathrm{E}-04$ \\
\hline U-237 & 5.99E-09 & 4.35E-09 & $2.13 \mathrm{E}-10$ & $2.10 \mathrm{E}-10$ & 1.42E-08 & $2.19 \mathrm{E}-08$ & $4.08 \mathrm{E}-08$ & $3.28 \mathrm{E}-08$ & 3.49E-05 \\
\hline $\mathrm{U}-238$ & $1.31 \mathrm{E}-08$ & 9.52E-09 & $4.67 \mathrm{E}-10$ & $4.59 \mathrm{E}-10$ & $3.11 \mathrm{E}-08$ & $4.80 \mathrm{E}-08$ & 8.93E-08 & $7.17 \mathrm{E}-08$ & 7.62E-05 \\
\hline $\mathrm{Np}-237$ & $90 \mathrm{E}-07$ & $2.84 \mathrm{E}-07$ & 1.43E-08 & $1.41 \mathrm{E}-08$ & 9.86E-07 & 1.47E-06 & $2.63 \mathrm{E}-06$ & $2.14 \mathrm{E}-06$ & $2.27 \mathrm{E}-03$ \\
\hline Pu-236 & 3.28E-09 & $2.38 \mathrm{E}-09$ & $1.17 \mathrm{E}-10$ & $1.15 \mathrm{E}-10$ & 7.77E-09 & 1.20E-08 & $2.23 E-08$ & 1.79E-08 & 1.91E-05 \\
\hline Pu-238 & 7.50E-04 & $5.45 E-04$ & 2.67E-05 & $2.63 \mathrm{E}-05$ & 1.78E-03 & $2.74 \mathrm{E}-03$ & $5.11 \mathrm{E}-03$ & $4.10 \mathrm{E}-03$ & $4.36 \mathrm{E}+00$ \\
\hline Pu-239 & 8.39E-05 & $6.10 \mathrm{E}-05$ & $2.99 \mathrm{E}-06$ & $2.94 \mathrm{E}-06$ & $1.99 \mathrm{E}-04$ & $3.07 \mathrm{E}-04$ & $5.72 \mathrm{E}-04$ & $4.59 \mathrm{E}-04$ & $4.88 \mathrm{E}-01$ \\
\hline Pu-240 & 1.22E-05 & 8.87E-06 & 4.35E-07 & 4.27E-07 & 2.89E-05 & 4.47E-05 & 8.32E-05 & 6.68E-05 & $7.10 \mathrm{E}-02$ \\
\hline $\mathrm{Pu}-241$ & 5.46E-04 & $3.96 \mathrm{E}-04$ & 1.94E-05 & 1.91E-05 & $1.29 \mathrm{E}-03$ & $2.00 \mathrm{E}-03$ & $3.72 \mathrm{E}-03$ & $2.99 \mathrm{E}-03$ & $3.17 \mathrm{E}+00$ \\
\hline Pu-242 & $9.56 \mathrm{E}-09$ & 6.95E-09 & $3.41 \mathrm{E}-10$ & $3.35 \mathrm{E}-10$ & $2.27 \mathrm{E}-08$ & $3.50 \mathrm{E}-08$ & $6.52 \mathrm{E}-08$ & $5.23 E-08$ & $5.56 \mathrm{E}-05$ \\
\hline Pu-244 & $7.30 \mathrm{E}-16$ & 5.30E-16 & $2.60 \mathrm{E}-17$ & $2.55 \mathrm{E}-17$ & 1.73E-15 & $2.67 \mathrm{E}-15$ & 4.97E-15 & $3.99 \mathrm{E}-15$ & $4.25 \mathrm{E}-12$ \\
\hline $\mathrm{Am}-241$ & $5.26 \mathrm{E}-05$ & $3.82 \mathrm{E}-05$ & $1.93 \mathrm{E}-06$ & $1.90 \mathrm{E}-06$ & $1.33 \mathrm{E}-04$ & $1.98 \mathrm{E}-04$ & $3.54 \mathrm{E}-04$ & $2.88 \mathrm{E}-04$ & $3.06 \mathrm{E}-01$ \\
\hline Am-242m & $1.45 \mathrm{E}-08$ & $1.06 \mathrm{E}-08$ & $5.33 \mathrm{E}-10$ & $5.24 \mathrm{E}-10$ & $3.67 \mathrm{E}-08$ & $5.48 \mathrm{E}-08$ & 9.77E-08 & 7.95E-08 & 8.45E-05 \\
\hline
\end{tabular}


Table A-15. Mass balance, Tank WM-188 waste (continued).

\begin{tabular}{|c|c|c|c|c|c|c|c|c|c|}
\hline Stream \# & 101 & 102 & 103 & 104 & 105 & 106 & 107 & 108 & 108 \\
\hline Radiological Composition & $\mathrm{Ci} / \mathrm{L}$ & $\mathrm{Ci} / \mathrm{L}$ & Ci/wscm & Ci/wscm & $\mathrm{Ci} / \mathrm{kg}$ & $\mathrm{Ci} / \mathrm{kg}$ & $\mathrm{Ci} / \mathrm{kg}$ & $\mathrm{Ci} / \mathrm{kg}$ & Ci/Canister \\
\hline Am-243 & $2.37 \mathrm{E}-08$ & $1.72 \mathrm{E}-08$ & $8.70 \mathrm{E}-10$ & $8.55 \mathrm{E}-10$ & 5.99E-08 & $8.94 \mathrm{E}-08$ & 1.59E-07 & 1.30E-07 & $1.38 \mathrm{E}-04$ \\
\hline $\mathrm{Cm}-242$ & $3.15 \mathrm{E}-08$ & $2.29 E-08$ & 1.12E-09 & 1.10E-09 & $7.45 \mathrm{E}-08$ & 1.15E-07 & 2.14E-07 & $1.72 \mathrm{E}-07$ & 1.83E-04 \\
\hline $\mathrm{Cm}-243$ & $3.30 \mathrm{E}-08$ & $2.40 \mathrm{E}-08$ & 1.17E-09 & $1.15 \mathrm{E}-09$ & $7.82 \mathrm{E}-08$ & $1.21 \mathrm{E}-07$ & $2.25 \mathrm{E}-07$ & $1.80 \mathrm{E}-07$ & $1.92 \mathrm{E}-04$ \\
\hline $\mathrm{Cm}-244$ & $1.15 \mathrm{E}-06$ & $8.38 \mathrm{E}-07$ & $4.11 \mathrm{E}-08$ & 4.04E-08 & $2.73 \mathrm{E}-06$ & $4.22 \mathrm{E}-06$ & 7.86E-06 & $6.31 \mathrm{E}-06$ & $6.71 \mathrm{E}-03$ \\
\hline $\mathrm{Cm}-245$ & $3.47 \mathrm{E}-10$ & $2.52 \mathrm{E}-10$ & $1.24 \mathrm{E}-11$ & $1.21 \mathrm{E}-11$ & $8.22 \mathrm{E}-10$ & 1.27E-09 & 2.36E-09 & 1.90E-09 & $2.02 \mathrm{E}-06$ \\
\hline $\mathrm{Cm}-246$ & $2.28 \mathrm{E}-11$ & $1.66 \mathrm{E}-11$ & $8.12 \mathrm{E}-13$ & $7.98 \mathrm{E}-13$ & $5.40 \mathrm{E}-11$ & $8.34 \mathrm{E}-11$ & $1.55 \mathrm{E}-10$ & $1.25 \mathrm{E}-10$ & $1.33 \mathrm{E}-07$ \\
\hline TRU & $9.16 \mathrm{E}-04$ & $6.65 \mathrm{E}-04$ & $3.27 \mathrm{E}-05$ & $3.21 \mathrm{E}-05$ & 2.18E-03 & 3.36E-03 & 6.24E-03 & 5.01E-03 & $5.33 \mathrm{E}+00$ \\
\hline TRU, nCi/gm & $7.48 \mathrm{E}+02$ & & & & & & & $5.01 \mathrm{E}+03$ & \\
\hline & & & & & & & & & \\
\hline $\begin{array}{l}\text { Gas Stream Bulk } \\
\text { Composition (Wet Basis) }\end{array}$ & $\begin{array}{c}\mathrm{mol} \% \text { or } \\
\mathrm{ppmv}\end{array}$ & $\begin{array}{c}\text { mol\% or } \\
\text { ppmv }\end{array}$ & $\begin{array}{l}\mathrm{mol} \% \text { or } \\
\text { ppmv }\end{array}$ & $\begin{array}{l}\text { mol\% or } \\
\text { ppmv }\end{array}$ & $\begin{array}{c}\mathrm{mol} \% \text { or } \\
\mathrm{ppmv}\end{array}$ & $\begin{array}{c}\mathrm{mol} \% \text { or } \\
\mathrm{ppmv}\end{array}$ & $\begin{array}{c}\mathrm{mol} \% \text { or } \\
\text { ppmv }\end{array}$ & $\begin{array}{c}\mathrm{mol} \% \text { or } \\
\text { ppmv }\end{array}$ & $\begin{array}{c}\mathrm{mol} \% \text { or } \\
\text { ppmv }\end{array}$ \\
\hline $\mathrm{H} 2 \mathrm{O}, \mathrm{mol} \%$ & & & $70.27 \%$ & $69.08 \%$ & & & & & \\
\hline $\mathrm{O} 2, \mathrm{~mol} \%$ & & & $0.53 \%$ & $0.52 \%$ & & & & & \\
\hline $\mathrm{N} 2, \mathrm{~mol} \%$ & & & $17.08 \%$ & $18.49 \%$ & & & & & \\
\hline $\mathrm{H} 2, \mathrm{~mol} \%$ & & & $3.99 \%$ & $3.92 \%$ & & & & & \\
\hline $\mathrm{CO} 2, \mathrm{~mol} \%$ & & & $6.65 \%$ & $6.53 \%$ & & & & & \\
\hline $\mathrm{CO}, \mathrm{ppmv}$ & & & $1.09 \mathrm{E}+04$ & $1.07 \mathrm{E}+04$ & & & & & \\
\hline $\mathrm{NO}, \mathrm{ppmv}$ & & & $6.16 \mathrm{E}+02$ & $6.06 \mathrm{E}+02$ & & & & & \\
\hline NO2, ppmv & & & $4.17 E+01$ & $4.10 \mathrm{E}+01$ & & & & & \\
\hline SO2, ppmv & & & 1.46E-02 & 1.43E-02 & & & & & \\
\hline $\mathrm{Cl}, \mathrm{ppmv}$ & & & $4.87 E+01$ & $4.78 \mathrm{E}+01$ & & & & & \\
\hline $\mathrm{F}, \mathrm{ppmv}$ & & & $8.78 \mathrm{E}+01$ & $8.63 \mathrm{E}+01$ & & & & & \\
\hline C (organic), ppmv & & & $3.03 E+03$ & $2.97 E+03$ & & & & & \\
\hline $\mathrm{H}$ (organic), ppmv & & & $6.83 \mathrm{E}+03$ & $6.72 \mathrm{E}+03$ & & & & & \\
\hline $\mathrm{Hg}, \mathrm{ug} / \mathrm{wscm}$ & & & $1.33 \mathrm{E}+05$ & $1.30 \mathrm{E}+05$ & & & & & \\
\hline $\mathrm{PM}, \mathrm{mg} / \mathrm{wscm}$ & & & $9.68 \mathrm{E}+03$ & $9.51 \mathrm{E}+03$ & & & & & \\
\hline SVM, ug/wscm & & & $2.84 \mathrm{E}+04$ & $2.79 \mathrm{E}+04$ & & & & & \\
\hline LVM, ug/wscm & & & $8.97 E+03$ & $8.82 \mathrm{E}+03$ & & & & & \\
\hline $\begin{array}{l}\text { Gas Stream Bulk } \\
\text { Composition (Dry Basis) }\end{array}$ & $\mathrm{mol} \%$ & $\mathrm{~mol} \%$ & $\mathrm{~mol} \%$ & $\mathrm{~mol} \%$ & $\mathrm{~mol} \%$ & $\mathrm{~mol} \%$ & $\mathrm{~mol} \%$ & $\mathrm{~mol} \%$ & $\mathrm{~mol} \%$ \\
\hline $\mathrm{O} 2, \mathrm{~mol} \%$, dry basis & & & $1.79 \%$ & $1.70 \%$ & & & & & \\
\hline $\mathrm{N} 2, \mathrm{~mol} \%$, dry basis & & & $57.47 \%$ & $59.81 \%$ & & & & & \\
\hline $\mathrm{H} 2, \mathrm{~mol} \%$, dry basis & & & $13.42 \%$ & $12.68 \%$ & & & & & \\
\hline $\mathrm{CO} 2, \mathrm{~mol} \%$, dry basis & & & $22.36 \%$ & $21.13 \%$ & & & & & \\
\hline $\begin{array}{l}\text { Gas Stream Bulk } \\
\text { Composition (Dry Basis, } \\
\text { Corrected to } 7 \% \text { O2 with } \\
100 \% \text { O2 Combustion Air) }\end{array}$ & $\begin{array}{l}\text { ppmv, or } \\
\text { ug/dscm } \\
\text { or } \\
\mathrm{mg} / \mathrm{dscm}\end{array}$ & $\begin{array}{l}\mathrm{ppmv}, \text { or } \\
\mathrm{ug} / \mathrm{dscm} \\
\text { or } \\
\mathrm{mg} / \mathrm{dscm}\end{array}$ & $\begin{array}{l}\text { ppmv, or } \\
\text { ug/dscm } \\
\text { or } \\
\mathrm{mg} / \mathrm{dscm}\end{array}$ & $\begin{array}{l}\mathrm{ppmv}, \text { or } \\
\mathrm{ug} / \mathrm{dscm} \\
\text { or } \\
\mathrm{mg} / \mathrm{dscm}\end{array}$ & $\begin{array}{l}\mathrm{ppmv}, \text { or } \\
\text { ug/dscm } \\
\text { or } \\
\mathrm{mg} / \mathrm{dscm}\end{array}$ & $\begin{array}{l}\text { ppmv, or } \\
\text { ug/dscm } \\
\text { or } \\
\mathrm{mg} / \mathrm{dscm}\end{array}$ & $\begin{array}{l}\text { ppmv, or } \\
\text { ug/dscm } \\
\text { or } \\
\mathrm{mg} / \mathrm{dscm}\end{array}$ & $\begin{array}{l}\mathrm{ppmv}, \text { or } \\
\text { ug/dscm } \\
\text { or } \\
\mathrm{mg} / \mathrm{dscm}\end{array}$ & $\begin{array}{l}\text { ppmv, or } \\
\text { ug/dscm } \\
\text { or } \\
\mathrm{mg} / \mathrm{dscm}\end{array}$ \\
\hline COgas, ppmv, dry basis & & & $5.13 \mathrm{E}+03$ & $4.85 \mathrm{E}+03$ & & & & & \\
\hline $\mathrm{NO}$, ppmv, dry basis & & & $2.90 \mathrm{E}+02$ & $2.74 \mathrm{E}+02$ & & & & & \\
\hline NO2, ppmv, dry basis & & & $1.96 \mathrm{E}+01$ & $1.86 \mathrm{E}+01$ & & & & & \\
\hline SO2, ppmv, dry basis & & & $6.86 \mathrm{E}-03$ & $6.48 \mathrm{E}-03$ & & & & & \\
\hline $\mathrm{Cl}, \mathrm{ppmv}$, dry basis & & & $2.29 \mathrm{E}+01$ & $2.17 \mathrm{E}+01$ & & & & & \\
\hline $\mathrm{F}$, ppmv, dry basis & & & $4.14 \mathrm{E}+01$ & $3.91 \mathrm{E}+01$ & & & & & \\
\hline C (organic), ppmv, dry basis & & & $1.43 \mathrm{E}+03$ & $1.35 \mathrm{E}+03$ & & & & & \\
\hline $\mathrm{H}$ (organic), ppmv, dry basis & & & $3.22 \mathrm{E}+03$ & $3.04 \mathrm{E}+03$ & & & & & \\
\hline $\mathrm{Hg}, \mathrm{ug} / \mathrm{dscm}$ & & & $6.25 \mathrm{E}+04$ & $5.91 \mathrm{E}+04$ & & & & & \\
\hline $\mathrm{PM}, \mathrm{mg} / \mathrm{dscm}$ & & & $4.56 \mathrm{E}+03$ & $4.31 \mathrm{E}+03$ & & & & & \\
\hline SVM, ug/dscm & & & $9.54 \mathrm{E}+04$ & $9.38 \mathrm{E}+04$ & & & & & \\
\hline LVM, ug/dscm & & & $3.02 E+04$ & $2.97 \mathrm{E}+04$ & & & & & \\
\hline
\end{tabular}


Table A-15. Mass balance, Tank WM-188 waste (continued).

\begin{tabular}{|c|c|c|c|c|c|c|c|c|c|}
\hline PFD \# & PFD-2 & PFD-3 & PFD-3 & PFD-3 & PFD-3 & PFD-3 & PFD-3 & PFD-3 & PFD-3 \\
\hline WM-188, Stream \# & 109 & 110 & 111 & 112 & $113 \mathrm{~A}$ & 113B & 114 & 115 & 116 \\
\hline Stream Name & $\begin{array}{l}\text { Off-Gas } \\
\text { from } \\
\text { Filter to } \\
\text { Oxidizer }\end{array}$ & $\begin{array}{l}\text { Oxidizer } \\
\text { Effluent }\end{array}$ & $\begin{array}{l}\text { Quenched } \\
\text { Oxidizer Off } \\
\text { gas }\end{array}$ & Scrub & $\begin{array}{c}\text { Packed } \\
\text { Scrubber } \\
\text { Drain }\end{array}$ & $\begin{array}{c}\text { Demister } \\
\text { Drain }\end{array}$ & $\begin{array}{c}\text { Scrubber } \\
\text { Effluent } \\
\text { Gas }\end{array}$ & $\begin{array}{c}\text { Demister } \\
\text { Effluent } \\
\text { Gas }\end{array}$ & $\begin{array}{c}\text { GAC Bed } \\
\text { Feed }\end{array}$ \\
\hline Rate or Volume & $4.47 \mathrm{E}+05$ & $7.20 \mathrm{E}+05$ & $3.76 \mathrm{E}+05$ & $1.03 E+04$ & $1.03 \mathrm{E}+04$ & $2.08 \mathrm{E}-03$ & $3.86 \mathrm{E}+05$ & $3.95 \mathrm{E}+05$ & $4.79 \mathrm{E}+05$ \\
\hline Volume Flow (standard, wet) & $1.06 \mathrm{E}+05$ & $1.10 \mathrm{E}+05$ & $1.87 \mathrm{E}+05$ & & & & $1.86 \mathrm{E}+05$ & $1.87 E+05$ & 1.87E+05 \\
\hline Volume Flow (standard, dry) & $3.31 \mathrm{E}+04$ & $3.18 \mathrm{E}+04$ & $3.17 \mathrm{E}+04$ & & & & $3.17 E+04$ & $3.18 \mathrm{E}+04$ & $3.18 \mathrm{E}+04$ \\
\hline Rate Units & $\mathrm{ft} 3 / \mathrm{hr}$ & $\mathrm{ft} 3 / \mathrm{hr}$ & $\mathrm{ft} 3 / \mathrm{hr}$ & $\mathrm{gal} / \mathrm{hr}$ & $\mathrm{gal} / \mathrm{hr}$ & $\mathrm{gal} / \mathrm{hr}$ & $\mathrm{ft} 3 / \mathrm{hr}$ & $\mathrm{ft} 3 / \mathrm{hr}$ & $\mathrm{ft} 3 / \mathrm{hr}$ \\
\hline Rate or Volume, metric & $3.00 \mathrm{E}+03$ & $3.12 E+03$ & $5.28 \mathrm{E}+03$ & $3.91 \mathrm{E}+04$ & $3.91 \mathrm{E}+04$ & 0.01 & $5.28 E+03$ & $5.30 \mathrm{E}+03$ & $5.30 \mathrm{E}+03$ \\
\hline Rate Units & wscm/hr & wscm/hr & wscm/hr & $\mathrm{L} / \mathrm{hr}$ & $\mathrm{L} / \mathrm{hr}$ & $\mathrm{L} / \mathrm{hr}$ & wscm/hr & wscm/hr & wscm/hr \\
\hline Temperature, ${ }^{\circ} \mathrm{C}$ & 527 & 952 & 100 & 82 & 83 & 81 & 82 & 81 & 120 \\
\hline Temperature, ${ }^{\circ} \mathrm{F}$ & 980 & 1746 & 212 & 180 & 181 & 178 & 179 & 178 & 248 \\
\hline Pressure, psia & 9.5 & 9.4 & 9.3 & 38.1 & 9.0 & 8.4 & 8.6 & 8.4 & 7.7 \\
\hline Specific Gravity & $2.09 \mathrm{E}-04$ & $1.42 \mathrm{E}-04$ & $4.25 \mathrm{E}-04$ & 1.01 & 1.01 & 1.01 & $4.14 \mathrm{E}-04$ & $4.05 \mathrm{E}-04$ & $3.34 \mathrm{E}-04$ \\
\hline Chemical Composition & $\mathrm{lb} / \mathrm{wscf}$ & $\mathrm{lb} / \mathrm{wscf}$ & $\mathrm{lb} /$ wscf & Mol/liter & Mol/liter & Mol/liter & $\mathrm{lb} / \mathrm{wscf}$ & $\mathrm{lb} /$ wscf & $\mathrm{lb} /$ wscf \\
\hline $\mathrm{H}+$ & $7.19 \mathrm{E}-07$ & $2.13 \mathrm{E}-08$ & $2.08 \mathrm{E}-08$ & $1.02 \mathrm{E}+00$ & $1.02 E+00$ & $1.02 \mathrm{E}+00$ & $2.30 \mathrm{E}-07$ & $2.29 \mathrm{E}-07$ & $2.29 \mathrm{E}-07$ \\
\hline $\mathrm{Al}+3$ & $4.60 \mathrm{E}-08$ & $4.42 \mathrm{E}-08$ & $2.61 \mathrm{E}-08$ & $1.65 \mathrm{E}-03$ & $1.65 \mathrm{E}-03$ & $1.65 \mathrm{E}-03$ & & & \\
\hline $\mathrm{Sb}+5$ & $5.92 \mathrm{E}-12$ & $5.70 \mathrm{E}-12$ & $3.36 \mathrm{E}-12$ & $4.71 \mathrm{E}-08$ & $4.72 \mathrm{E}-08$ & $4.72 \mathrm{E}-08$ & & & \\
\hline As+3 & $2.03 \mathrm{E}-11$ & $1.96 \mathrm{E}-11$ & $1.15 \mathrm{E}-11$ & $2.63 \mathrm{E}-07$ & $2.63 \mathrm{E}-07$ & $2.63 \mathrm{E}-07$ & & & \\
\hline $\mathrm{Ba}+2$ & $3.17 \mathrm{E}-11$ & $3.05 \mathrm{E}-11$ & $7.44 \mathrm{E}-13$ & $3.18 \mathrm{E}-07$ & $3.18 \mathrm{E}-07$ & $3.18 \mathrm{E}-07$ & & & \\
\hline $\mathrm{Be}+2$ & $5.25 \mathrm{E}-13$ & $5.06 \mathrm{E}-13$ & $1.23 \mathrm{E}-14$ & $8.04 \mathrm{E}-08$ & $8.04 \mathrm{E}-08$ & $8.04 \mathrm{E}-08$ & & & \\
\hline $\mathrm{B}+3$ & $5.25 \mathrm{E}-10$ & $5.05 \mathrm{E}-10$ & $2.98 \mathrm{E}-10$ & $4.71 \mathrm{E}-05$ & $4.71 \mathrm{E}-05$ & $4.71 \mathrm{E}-05$ & & & \\
\hline $\mathrm{Cd}+2$ & $9.46 \mathrm{E}-10$ & $9.10 \mathrm{E}-10$ & $5.37 \mathrm{E}-10$ & $3.03 \mathrm{E}-06$ & $3.04 \mathrm{E}-06$ & $3.04 \mathrm{E}-06$ & & & \\
\hline $\mathrm{Ca}+2$ & $1.76 \mathrm{E}-08$ & $1.69 \mathrm{E}-08$ & $4.14 \mathrm{E}-10$ & $6.06 \mathrm{E}-04$ & $6.05 \mathrm{E}-04$ & $6.05 \mathrm{E}-04$ & & & \\
\hline $\mathrm{Cr}+3$ & $7.98 \mathrm{E}-10$ & $7.68 \mathrm{E}-10$ & $4.53 \mathrm{E}-10$ & $8.30 \mathrm{E}-06$ & $8.31 \mathrm{E}-06$ & $8.31 \mathrm{E}-06$ & & & \\
\hline $\mathrm{Co}+2$ & $7.14 \mathrm{E}-12$ & $6.87 \mathrm{E}-12$ & $4.05 \mathrm{E}-12$ & $1.17 \mathrm{E}-07$ & 1.17E-07 & $1.17 \mathrm{E}-07$ & & & \\
\hline Cs+ & $5.33 \mathrm{E}-11$ & $5.13 \mathrm{E}-11$ & $3.02 \mathrm{E}-11$ & $6.79 \mathrm{E}-05$ & $6.78 \mathrm{E}-05$ & $6.78 \mathrm{E}-05$ & & & \\
\hline $\mathrm{Cu}+2$ & 1.37E-10 & $1.32 \mathrm{E}-10$ & $3.22 \mathrm{E}-12$ & $2.98 \mathrm{E}-06$ & 2.97E-06 & $2.97 \mathrm{E}-06$ & & & \\
\hline $\mathrm{Fe}+3$ & $3.10 \mathrm{E}-09$ & 2.98E-09 & $1.76 \mathrm{E}-09$ & $1.00 \mathrm{E}-04$ & $1.00 \mathrm{E}-04$ & $1.00 \mathrm{E}-04$ & & & \\
\hline $\mathrm{Pb}+2$ & $1.64 \mathrm{E}-09$ & $1.58 \mathrm{E}-09$ & $9.33 \mathrm{E}-10$ & $5.52 \mathrm{E}-06$ & $5.52 \mathrm{E}-06$ & $5.52 \mathrm{E}-06$ & & & \\
\hline $\mathrm{Hg}+2$ & $8.07 \mathrm{E}-06$ & $7.76 \mathrm{E}-06$ & $4.58 \mathrm{E}-06$ & $1.12 \mathrm{E}-02$ & $1.12 \mathrm{E}-02$ & $1.12 \mathrm{E}-02$ & & $2.55 \mathrm{E}-06$ & $2.55 \mathrm{E}-06$ \\
\hline$M n+4$ & $2.44 \mathrm{E}-09$ & $2.34 \mathrm{E}-09$ & $1.38 \mathrm{E}-09$ & $4.30 \mathrm{E}-05$ & 4.30E-05 & \begin{tabular}{|l|}
$4.30 \mathrm{E}-05$ \\
\end{tabular} & & & \\
\hline $\mathrm{Ni}+2$ & $3.31 \mathrm{E}-10$ & $3.19 \mathrm{E}-10$ & $7.79 \mathrm{E}-12$ & $7.79 \mathrm{E}-06$ & $7.78 \mathrm{E}-06$ & $7.78 \mathrm{E}-06$ & & & \\
\hline $\mathrm{K}+$ & $4.71 \mathrm{E}-08$ & $4.53 \mathrm{E}-08$ & $2.67 \mathrm{E}-08$ & $1.52 \mathrm{E}-03$ & $1.52 \mathrm{E}-03$ & $1.52 \mathrm{E}-03$ & & & \\
\hline $\mathrm{Se}+4$ & $5.50 \mathrm{E}-11$ & $5.29 \mathrm{E}-11$ & $3.12 \mathrm{E}-11$ & $1.19 \mathrm{E}-06$ & $1.19 \mathrm{E}-06$ & 1.19E-06 & & & \\
\hline $\mathrm{Ag}+$ & $5.56 \mathrm{E}-10$ & $4.16 \mathrm{E}-10$ & $2.46 \mathrm{E}-10$ & $1.57 \mathrm{E}-06$ & 1.57E-06 & 1.57E-06 & & & \\
\hline $\mathrm{Na}+$ & $4.45 \mathrm{E}-07$ & $4.28 \mathrm{E}-07$ & $2.52 \mathrm{E}-07$ & $5.84 \mathrm{E}-02$ & 5.84E-02 & 5.84E-02 & & & \\
\hline $\mathrm{TI}+3$ & $2.78 \mathrm{E}-12$ & $2.67 \mathrm{E}-12$ & $1.58 \mathrm{E}-12$ & $1.32 \mathrm{E}-08$ & $1.32 \mathrm{E}-08$ & 1.32E-08 & & & \\
\hline$U+4$ & $2.48 \mathrm{E}-10$ & $2.39 \mathrm{E}-10$ & $1.41 \mathrm{E}-10$ & $1.78 \mathrm{E}-06$ & 1.78E-06 & \begin{tabular}{|l|}
$1.78 \mathrm{E}-06$ \\
\end{tabular} & & & \\
\hline $\mathrm{V}+3$ & $5.37 \mathrm{E}-12$ & $5.17 \mathrm{E}-12$ & $3.05 \mathrm{E}-12$ & $1.82 \mathrm{E}-07$ & $1.82 \mathrm{E}-07$ & 1.82E-07 & & & \\
\hline$Z n+2$ & $1.63 \mathrm{E}-10$ & $1.57 \mathrm{E}-10$ & $3.84 \mathrm{E}-12$ & $3.45 \mathrm{E}-06$ & $3.44 \mathrm{E}-06$ & $3.44 \mathrm{E}-06$ & & & \\
\hline $\mathrm{Zr}+4$ & $9.90 \mathrm{E}-09$ & $9.53 \mathrm{E}-09$ & $5.62 \mathrm{E}-09$ & $1.86 \mathrm{E}-04$ & 1.86E-04 & \begin{tabular}{|l|}
$1.86 \mathrm{E}-04$ \\
\end{tabular} & & & \\
\hline $\mathrm{Cl}-$ & $1.23 \mathrm{E}-07$ & $1.18 \mathrm{E}-07$ & $6.98 \mathrm{E}-08$ & $2.31 \mathrm{E}-03$ & $2.31 \mathrm{E}-03$ & $2.31 \mathrm{E}-03$ & $1.15 \mathrm{E}-08$ & $1.15 \mathrm{E}-08$ & $1.15 \mathrm{E}-08$ \\
\hline F- & $4.26 \mathrm{E}-07$ & $4.10 \mathrm{E}-07$ & $2.42 \mathrm{E}-07$ & $1.86 \mathrm{E}-02$ & 1.86E-02 & 1.86E-02 & $7.82 \mathrm{E}-07$ & \begin{tabular}{|l|}
$7.79 E-07$ \\
\end{tabular} & 7.79E-07 \\
\hline SO4-2 & $1.32 \mathrm{E}-11$ & 4.84E-07 & $2.85 \mathrm{E}-07$ & $2.10 \mathrm{E}-02$ & $2.10 \mathrm{E}-02$ & $2.10 \mathrm{E}-02$ & & & \\
\hline NO3- & $6.47 \mathrm{E}-06$ & $5.35 \mathrm{E}-09$ & $3.16 \mathrm{E}-09$ & $1.04 \mathrm{E}+00$ & $1.04 \mathrm{E}+00$ & $1.04 \mathrm{E}+00$ & $1.16 \mathrm{E}-05$ & $1.15 \mathrm{E}-05$ & $1.15 \mathrm{E}-05$ \\
\hline PO4-3 & $3.52 \mathrm{E}-08$ & $3.39 \mathrm{E}-08$ & $2.00 \mathrm{E}-08$ & $6.13 \mathrm{E}-04$ & $6.13 \mathrm{E}-04$ & $6.13 \mathrm{E}-04$ & & & \\
\hline $\mathrm{Am}+4$ & $4.99 \mathrm{E}-14$ & $4.80 \mathrm{E}-14$ & $2.83 \mathrm{E}-14$ & $1.99 \mathrm{E}-10$ & $1.99 \mathrm{E}-10$ & $1.99 \mathrm{E}-10$ & & & \\
\hline $\mathrm{Br}-$ & $3.35 \mathrm{E}-12$ & $3.22 \mathrm{E}-12$ & $1.90 \mathrm{E}-12$ & $2.78 \mathrm{E}-08$ & $2.78 \mathrm{E}-08$ & $2.78 \mathrm{E}-08$ & $3.13 \mathrm{E}-13$ & $3.12 \mathrm{E}-13$ & $3.12 \mathrm{E}-13$ \\
\hline $\mathrm{Ce}+4$ & $1.42 \mathrm{E}-11$ & $1.37 \mathrm{E}-11$ & $8.08 \mathrm{E}-12$ & 9.84E-08 & 9.85E-08 & 9.85E-08 & & & \\
\hline $\mathrm{Eu}+3$ & $2.23 \mathrm{E}-13$ & $2.14 \mathrm{E}-13$ & $1.26 \mathrm{E}-13$ & $1.42 \mathrm{E}-09$ & 1.42E-09 & 1.42E-09 & & & \\
\hline $\mathrm{Gd}+3$ & $6.80 \mathrm{E}-11$ & $6.54 \mathrm{E}-11$ & $3.86 \mathrm{E}-11$ & 4.19E-07 & $4.19 \mathrm{E}-07$ & 4.19E-07 & & & \\
\hline $\mathrm{Ge}+4$ & $1.79 \mathrm{E}-15$ & $1.72 \mathrm{E}-15$ & $1.02 \mathrm{E}-15$ & $4.22 \mathrm{E}-11$ & $4.22 \mathrm{E}-11$ & $4.22 \mathrm{E}-11$ & & & \\
\hline $\ln +3$ & $5.14 \mathrm{E}-13$ & $4.94 \mathrm{E}-13$ & $2.92 \mathrm{E}-13$ & 4.34E-09 & 4.34E-09 & 4.34E-09 & & & \\
\hline I- & $5.68 \mathrm{E}-11$ & $5.46 \mathrm{E}-11$ & $3.22 \mathrm{E}-11$ & $2.97 \mathrm{E}-07$ & $2.97 \mathrm{E}-07$ & $2.97 \mathrm{E}-07$ & $5.31 \mathrm{E}-12$ & $5.29 \mathrm{E}-12$ & $5.29 \mathrm{E}-12$ \\
\hline$L a+3$ & $3.69 \mathrm{E}-12$ & $3.55 \mathrm{E}-12$ & $2.09 \mathrm{E}-12$ & $2.58 \mathrm{E}-08$ & $2.58 \mathrm{E}-08$ & $2.58 \mathrm{E}-08$ & & & \\
\hline
\end{tabular}


Table A-15. Mass balance, Tank WM-188 waste (continued).

\begin{tabular}{|c|c|c|c|c|c|c|c|c|c|}
\hline Stream \# & 109 & 110 & 111 & 112 & $113 \mathrm{~A}$ & $113 \mathrm{~B}$ & 114 & 115 & 116 \\
\hline Chemical Composition & $\mathrm{lb} /$ wscf & $\mathrm{lb} /$ wscf & $\mathrm{lb} /$ wscf & Mol/liter & Mol/liter & Mol/liter & $\mathrm{lb} / \mathrm{wscf}$ & $\mathrm{lb} /$ wscf & lb/wscf \\
\hline $\mathrm{Li}+$ & $4.44 \mathrm{E}-11$ & $3.33 \mathrm{E}-11$ & $1.96 \mathrm{E}-11$ & 1.95E-06 & 1.95E-06 & $1.95 \mathrm{E}-06$ & & & \\
\hline $\mathrm{Mg}+2$ & $1.38 \mathrm{E}-09$ & $1.32 \mathrm{E}-09$ & $3.23 \mathrm{E}-11$ & $7.81 \mathrm{E}-05$ & $7.81 \mathrm{E}-05$ & $7.81 \mathrm{E}-05$ & & & \\
\hline $\mathrm{Mo}+6$ & $1.49 \mathrm{E}-08$ & $1.43 \mathrm{E}-08$ & $8.46 \mathrm{E}-09$ & $2.88 \mathrm{E}-04$ & $2.88 \mathrm{E}-04$ & $2.88 \mathrm{E}-04$ & & & \\
\hline $\mathrm{Nd}+3$ & $1.24 \mathrm{E}-11$ & $1.19 \mathrm{E}-11$ & $7.02 \mathrm{E}-12$ & $8.30 \mathrm{E}-08$ & $8.31 \mathrm{E}-08$ & $8.31 \mathrm{E}-08$ & & & \\
\hline $\mathrm{Np}+4$ & $1.86 \mathrm{E}-12$ & $1.79 \mathrm{E}-12$ & $1.05 \mathrm{E}-12$ & $7.59 \mathrm{E}-09$ & \begin{tabular}{|l|}
$7.60 \mathrm{E}-09$ \\
\end{tabular} & $7.60 \mathrm{E}-09$ & & & \\
\hline $\mathrm{Nb}+5$ & $2.90 \mathrm{E}-10$ & $2.79 \mathrm{E}-10$ & $1.64 \mathrm{E}-10$ & $5.38 \mathrm{E}-06$ & 5.38E-06 & $5.38 \mathrm{E}-06$ & & & \\
\hline $\mathrm{Pd}+4$ & $5.77 \mathrm{E}-10$ & $5.55 \mathrm{E}-10$ & $1.35 \mathrm{E}-11$ & $7.47 E-06$ & $7.47 \mathrm{E}-06$ & $7.47 \mathrm{E}-06$ & & & \\
\hline $\mathrm{Pu}+4$ & $4.83 \mathrm{E}-12$ & $4.65 \mathrm{E}-12$ & $2.74 \mathrm{E}-12$ & $3.39 \mathrm{E}-08$ & 3.39E-08 & 3.39E-08 & & & \\
\hline $\mathrm{Pr}+4$ & $3.41 \mathrm{E}-12$ & $3.28 \mathrm{E}-12$ & $1.93 \mathrm{E}-12$ & $2.34 \mathrm{E}-08$ & $2.34 \mathrm{E}-08$ & $2.34 \mathrm{E}-08$ & & & \\
\hline $\mathrm{Pm}+3$ & $7.25 \mathrm{E}-16$ & $6.98 \mathrm{E}-16$ & $4.12 \mathrm{E}-16$ & $4.85 \mathrm{E}-12$ & $4.85 \mathrm{E}-12$ & $4.85 \mathrm{E}-12$ & & & \\
\hline $\mathrm{Rh}+4$ & $1.07 \mathrm{E}-12$ & $1.03 \mathrm{E}-12$ & $6.09 \mathrm{E}-13$ & $1.01 \mathrm{E}-08$ & 1.01E-08 & $1.01 \mathrm{E}-08$ & & & \\
\hline $\mathrm{Rb}+$ & $6.32 \mathrm{E}-12$ & $4.74 \mathrm{E}-12$ & $2.80 \mathrm{E}-12$ & $2.25 \mathrm{E}-08$ & $2.26 \mathrm{E}-08$ & $2.26 \mathrm{E}-08$ & & & \\
\hline $\mathrm{Ru}+3$ & $2.44 \mathrm{E}-10$ & $2.35 \mathrm{E}-10$ & $1.38 \mathrm{E}-10$ & $2.34 \mathrm{E}-06$ & $2.34 \mathrm{E}-06$ & $2.34 \mathrm{E}-06$ & & & \\
\hline $\mathrm{Sm}+3$ & $2.40 \mathrm{E}-12$ & $2.31 \mathrm{E}-12$ & $1.36 \mathrm{E}-12$ & $1.55 \mathrm{E}-08$ & \begin{tabular}{|c|}
$1.55 \mathrm{E}-08$ \\
\end{tabular} & $1.55 \mathrm{E}-08$ & & & \\
\hline $\mathrm{Si}+4$ & $1.01 \mathrm{E}-08$ & 9.72E-09 & 5.73E-09 & $7.72 \mathrm{E}-04$ & $7.72 \mathrm{E}-04$ & $7.72 \mathrm{E}-04$ & & & \\
\hline $\mathrm{Sr}+2$ & $2.17 \mathrm{E}-11$ & $2.09 \mathrm{E}-11$ & $5.10 \mathrm{E}-13$ & $3.42 \mathrm{E}-07$ & 3.42E-07 & $3.42 \mathrm{E}-07$ & & & \\
\hline $\mathrm{Tc}+7$ & $9.38 \mathrm{E}-12$ & $9.02 \mathrm{E}-12$ & $5.32 \mathrm{E}-12$ & $5.78 \mathrm{E}-08$ & $5.78 \mathrm{E}-08$ & $5.78 \mathrm{E}-08$ & & & \\
\hline $\mathrm{Te}+4$ & $1.31 \mathrm{E}-12$ & $1.26 \mathrm{E}-12$ & $7.46 \mathrm{E}-13$ & $1.76 \mathrm{E}-08$ & 1.76E-08 & $1.76 \mathrm{E}-08$ & & & \\
\hline $\mathrm{Tb}+4$ & $9.72 \mathrm{E}-16$ & $9.36 \mathrm{E}-16$ & $5.52 \mathrm{E}-16$ & $5.93 \mathrm{E}-12$ & 5.93E-12 & $5.93 \mathrm{E}-12$ & & & \\
\hline $\mathrm{Th}+4$ & $1.59 \mathrm{E}-11$ & $1.53 \mathrm{E}-11$ & $9.00 \mathrm{E}-12$ & 1.17E-07 & 1.17E-07 & 1.17E-07 & & & \\
\hline $\mathrm{Sn}+4$ & $6.11 \mathrm{E}-10$ & $5.87 \mathrm{E}-10$ & $3.46 \mathrm{E}-10$ & $8.80 \mathrm{E}-06$ & $8.80 \mathrm{E}-06$ & $8.80 \mathrm{E}-06$ & & & \\
\hline $\mathrm{Ti}+4$ & $1.26 \mathrm{E}-10$ & $1.21 \mathrm{E}-10$ & $7.15 \mathrm{E}-11$ & $4.50 \mathrm{E}-06$ & $4.50 \mathrm{E}-06$ & $4.50 \mathrm{E}-06$ & & & \\
\hline$\overline{Y+3}$ & $1.76 \mathrm{E}-12$ & 1.69E-12 & $1.00 \mathrm{E}-12$ & 1.92E-08 & 1.92E-08 & 1.92E-08 & & & \\
\hline $\mathrm{OH}-$ & 3.07E-09 & 1.17E-07 & $2.08 \mathrm{E}-07$ & 5.29E-07 & $5.30 \mathrm{E}-07$ & $5.30 \mathrm{E}-07$ & & & \\
\hline $\mathrm{H} 2 \mathrm{O}$ & $3.21 \mathrm{E}-02$ & 3.32E-02 & $3.90 \mathrm{E}-02$ & $5.23 E+01$ & $5.23 E+01$ & $5.23 E+01$ & $3.90 \mathrm{E}-02$ & $3.88 \mathrm{E}-02$ & 3.88E-02 \\
\hline $\mathrm{SO} 2$ & 2.37E-09 & $1.81 \mathrm{E}-05$ & 1.07E-05 & $8.09 \mathrm{E}-06$ & \begin{tabular}{|l}
$8.10 \mathrm{E}-06$ \\
\end{tabular} & $8.10 \mathrm{E}-06$ & $1.07 \mathrm{E}-05$ & $1.07 \mathrm{E}-05$ & 1.07E-05 \\
\hline $\mathrm{H} 2 \mathrm{~S}$ & $1.02 \mathrm{E}-05$ & $2.47 \mathrm{E}-21$ & & & & & & & \\
\hline $\mathrm{CO}$ & 7.74E-04 & 1.60E-09 & $9.45 \mathrm{E}-10$ & $2.02 \mathrm{E}-10$ & $2.05 \mathrm{E}-10$ & $2.05 \mathrm{E}-10$ & $9.45 \mathrm{E}-10$ & $9.42 \mathrm{E}-10$ & $9.42 \mathrm{E}-10$ \\
\hline $\mathrm{CO} 2$ & $7.43 E-03$ & 9.33E-03 & $5.50 \mathrm{E}-03$ & 3.09E-04 & $3.18 \mathrm{E}-04$ & $3.18 \mathrm{E}-04$ & $5.51 \mathrm{E}-03$ & $5.49 \mathrm{E}-03$ & $5.49 \mathrm{E}-03$ \\
\hline $\mathrm{H} 2$ & $2.04 \mathrm{E}-04$ & $6.45 \mathrm{E}-10$ & $3.80 \mathrm{E}-10$ & 8.32E-10 & 1.35E-09 & 1.35E-09 & 2.05E-08 & & \\
\hline N2 & $1.39 \mathrm{E}-02$ & 1.34E-02 & 7.91E-03 & $1.21 \mathrm{E}-05$ & 2.39E-05 & $2.39 \mathrm{E}-05$ & 7.91E-03 & $7.88 \mathrm{E}-03$ & $7.88 \mathrm{E}-03$ \\
\hline NO & 4.69E-05 & $5.10 \mathrm{E}-05$ & 3.01E-05 & $2.30 \mathrm{E}-07$ & $2.93 \mathrm{E}-07$ & $2.93 \mathrm{E}-07$ & $3.01 \mathrm{E}-05$ & $3.00 \mathrm{E}-05$ & $3.00 \mathrm{E}-05$ \\
\hline $\mathrm{NO} 2$ & $4.87 \mathrm{E}-06$ & $2.75 \mathrm{E}-07$ & 1.62E-07 & $2.91 \mathrm{E}-06$ & $2.89 \mathrm{E}-06$ & $2.89 \mathrm{E}-06$ & 1.62E-07 & 1.62E-07 & 1.62E-07 \\
\hline $\mathrm{O} 2$ & $4.33 \mathrm{E}-04$ & $1.82 \mathrm{E}-03$ & 1.07E-03 & $3.08 E-06$ & 4.83E-06 & $4.83 E-06$ & 1.07E-03 & 1.07E-03 & $1.07 \mathrm{E}-03$ \\
\hline S (other) & $2.18 \mathrm{E}-10$ & $1.43 \mathrm{E}-25$ & & $1.08 \mathrm{E}-07$ & $1.08 \mathrm{E}-07$ & 1.08E-07 & & & \\
\hline $\mathrm{CO} 3$ & $1.87 \mathrm{E}-07$ & $2.60 \mathrm{E}-11$ & $6.82 \mathrm{E}-12$ & $3.39 E-05$ & 3.39E-05 & $3.39 \mathrm{E}-05$ & & & \\
\hline C (reductant) & $3.29 \mathrm{E}-08$ & $1.43 \mathrm{E}-18$ & & & & & & & \\
\hline $\mathrm{O}$ (oxides) & $6.31 \mathrm{E}-08$ & $5.90 \mathrm{E}-08$ & $3.48 \mathrm{E}-08$ & $4.97 \mathrm{E}-03$ & 4.97E-03 & 4.97E-03 & & & \\
\hline C (organic) & 1.82E-05 & 1.99E-34 & & & & & & & \\
\hline $\mathrm{H}$ (organic) & 6.07E-06 & $6.67 \mathrm{E}-35$ & & & & & & & \\
\hline O (organic) & $1.36 \mathrm{E}-07$ & & & & & & & & \\
\hline \multirow{5}{*}{ Mass Flow $(\mathrm{kg} / \mathrm{hr})$ : } & & & & & & & & & \\
\hline & $2.64 \mathrm{E}+03$ & $2.89 \mathrm{E}+03$ & $4.53 E+03$ & $3.97 \mathrm{E}+04$ & $3.97 \mathrm{E}+04$ & 8.00E-03 & $4.53 \mathrm{E}+03$ & $4.53 \mathrm{E}+03$ & $4.53 E+03$ \\
\hline & & & & & & & & & \\
\hline & & & & & & & & & \\
\hline & $\mathrm{w} / \mathrm{m} 3$ & $\mathrm{w} / \mathrm{m} 3$ & $\mathrm{w} / \mathrm{m} 3$ & $\mathrm{w} / \mathrm{m} 3$ & $\mathrm{w} / \mathrm{m} 3$ & $\mathrm{w} / \mathrm{m} 3$ & $\mathrm{w} / \mathrm{m} 3$ & $\mathrm{w} / \mathrm{m} 3$ & $\mathrm{w} / \mathrm{m} 3$ \\
\hline Heat Generation, w/m3 & & & & $2.88 \mathrm{E}-02$ & $2.87 \mathrm{E}-02$ & & & & \\
\hline
\end{tabular}


Table A-15. Mass balance, Tank WM-188 waste (continued).

\begin{tabular}{|c|c|c|c|c|c|c|c|c|c|}
\hline Stream \# & 109 & 110 & 111 & 112 & $113 \mathrm{~A}$ & $113 \mathrm{~B}$ & 114 & 115 & 116 \\
\hline Radiological Composition & $\mathrm{Ci} / \mathrm{wscm}$ & Ci/wscm & Ci/wscm & $\mathrm{Ci} / \mathrm{L}$ & $\mathrm{Ci} / \mathrm{L}$ & $\mathrm{Ci} / \mathrm{L}$ & $\mathrm{Ci} / \mathrm{wscm}$ & Ci/wscm & Ci/wscm \\
\hline $\mathrm{H}-3$ & 1.30E-06 & $1.25 \mathrm{E}-06$ & $7.35 \mathrm{E}-07$ & $1.08 \mathrm{E}-06$ & $1.08 \mathrm{E}-06$ & $1.08 \mathrm{E}-06$ & $7.35 \mathrm{E}-07$ & $7.32 \mathrm{E}-07$ & $7.32 \mathrm{E}-07$ \\
\hline $\mathrm{C}-14$ & $1.63 \mathrm{E}-11$ & $1.58 \mathrm{E}-11$ & $9.33 \mathrm{E}-12$ & $1.60 \mathrm{E}-15$ & $1.64 \mathrm{E}-15$ & 1.64E-15 & $9.33 \mathrm{E}-12$ & $9.30 \mathrm{E}-12$ & $9.30 \mathrm{E}-12$ \\
\hline Co-60 & $4.35 \mathrm{E}-10$ & $4.18 \mathrm{E}-10$ & $2.47 \mathrm{E}-10$ & $2.63 \mathrm{E}-08$ & $2.63 \mathrm{E}-08$ & $2.63 \mathrm{E}-08$ & & & \\
\hline $\mathrm{Ni}-59$ & $5.63 \mathrm{E}-11$ & $5.42 \mathrm{E}-11$ & $1.32 \mathrm{E}-12$ & $4.85 \mathrm{E}-09$ & $4.85 \mathrm{E}-09$ & $4.85 \mathrm{E}-09$ & & & \\
\hline $\mathrm{Ni}-63$ & $2.13 E-09$ & $2.05 E-09$ & $5.00 \mathrm{E}-11$ & 1.83E-07 & 1.83E-07 & $1.83 \mathrm{E}-07$ & & & \\
\hline Se-79 & $2.72 \mathrm{E}-11$ & $2.62 \mathrm{E}-11$ & $1.54 \mathrm{E}-11$ & $2.90 \mathrm{E}-09$ & $2.90 \mathrm{E}-09$ & $2.90 \mathrm{E}-09$ & & & \\
\hline Sr-90 & $2.00 \mathrm{E}-06$ & $1.93 \mathrm{E}-06$ & $4.70 \mathrm{E}-08$ & $1.72 \mathrm{E}-04$ & $1.72 \mathrm{E}-04$ & $1.72 \mathrm{E}-04$ & & & \\
\hline$\overline{Y-90}$ & 1.92E-06 & $1.85 \mathrm{E}-06$ & 1.09E-06 & 1.16E-04 & $1.16 \mathrm{E}-04$ & 1.16E-04 & & & \\
\hline Zr-93 & $1.07 \mathrm{E}-10$ & $1.03 \mathrm{E}-10$ & $6.10 \mathrm{E}-11$ & $1.15 \mathrm{E}-08$ & $1.15 \mathrm{E}-08$ & 1.15E-08 & & & \\
\hline $\mathrm{Nb}-93 \mathrm{~m}$ & $8.28 \mathrm{E}-11$ & $7.97 \mathrm{E}-11$ & $4.70 \mathrm{E}-11$ & 8.93E-09 & 8.93E-09 & 8.93E-09 & & & \\
\hline $\mathrm{Nb}-94$ & $7.07 \mathrm{E}-11$ & $6.81 \mathrm{E}-11$ & $4.01 \mathrm{E}-11$ & 7.63E-09 & $7.63 \mathrm{E}-09$ & 7.63E-09 & & & \\
\hline Tc-99 & $3.12 \mathrm{E}-09$ & 3.00E-09 & 1.77E-09 & 1.17E-07 & $1.18 \mathrm{E}-07$ & 1.18E-07 & & & \\
\hline Ru-106 & $5.98 \mathrm{E}-11$ & $5.76 \mathrm{E}-11$ & $3.40 \mathrm{E}-11$ & 3.62E-09 & $3.62 \mathrm{E}-09$ & 3.62E-09 & & & \\
\hline Rh-102 & $4.31 \mathrm{E}-14$ & $4.15 \mathrm{E}-14$ & $2.44 \mathrm{E}-14$ & $2.61 \mathrm{E}-12$ & $2.61 \mathrm{E}-12$ & $2.61 \mathrm{E}-12$ & & & \\
\hline Rh-106 & 5.98E-11 & $5.76 \mathrm{E}-11$ & $3.40 \mathrm{E}-11$ & 3.62E-09 & 3.62E-09 & 3.62E-09 & & & \\
\hline $\mathrm{Pd}-107$ & $8.59 \mathrm{E}-13$ & $8.26 \mathrm{E}-13$ & $2.02 \mathrm{E}-14$ & $7.39 \mathrm{E}-11$ & $7.39 \mathrm{E}-11$ & $7.39 \mathrm{E}-11$ & & & \\
\hline $\mathrm{Cd}-113 \mathrm{~m}$ & $1.89 \mathrm{E}-10$ & $1.81 \mathrm{E}-10$ & $1.07 \mathrm{E}-10$ & $4.24 \mathrm{E}-09$ & $4.25 \mathrm{E}-09$ & $4.25 \mathrm{E}-09$ & & & \\
\hline Sn-121m & $3.24 \mathrm{E}-12$ & $3.12 \mathrm{E}-12$ & 1.84E-12 & $3.46 \mathrm{E}-10$ & $3.46 \mathrm{E}-10$ & $3.46 \mathrm{E}-10$ & & & \\
\hline Sn-126 & $2.56 \mathrm{E}-11$ & $2.46 \mathrm{E}-11$ & $1.45 \mathrm{E}-11$ & $2.73 \mathrm{E}-09$ & $2.73 \mathrm{E}-09$ & 2.73E-09 & & & \\
\hline Sb-125 & $1.81 \mathrm{E}-08$ & 1.74E-08 & 1.03E-08 & 1.10E-06 & 1.10E-06 & 1.10E-06 & & & \\
\hline Sb-126 & $2.87 \mathrm{E}-12$ & $2.77 \mathrm{E}-12$ & $1.63 \mathrm{E}-12$ & $1.74 \mathrm{E}-10$ & $1.74 \mathrm{E}-10$ & $1.74 \mathrm{E}-10$ & & & \\
\hline $\mathrm{Te}-125 \mathrm{~m}$ & $1.53 \mathrm{E}-10$ & $1.47 \mathrm{E}-10$ & $8.67 \mathrm{E}-11$ & 1.63E-08 & 1.63E-08 & 1.63E-08 & & & \\
\hline $1-129$ & $1.62 \mathrm{E}-10$ & $1.56 \mathrm{E}-10$ & $9.21 \mathrm{E}-11$ & 6.73E-09 & $6.74 \mathrm{E}-09$ & $6.74 \mathrm{E}-09$ & $1.52 \mathrm{E}-11$ & $1.51 \mathrm{E}-11$ & $1.51 \mathrm{E}-11$ \\
\hline Cs-134 & $4.10 \mathrm{E}-09$ & 3.94E-09 & 2.33E-09 & 4.33E-05 & 4.33E-05 & 4.33E-05 & & & \\
\hline Cs-135 & $1.22 \mathrm{E}-10$ & $1.18 \mathrm{E}-10$ & $6.93 \mathrm{E}-11$ & $1.29 \mathrm{E}-06$ & 1.29E-06 & 1.29E-06 & & & \\
\hline Cs-137 & $2.52 \mathrm{E}-06$ & $2.43 \mathrm{E}-06$ & $1.43 \mathrm{E}-06$ & $2.67 \mathrm{E}-02$ & $2.67 \mathrm{E}-02$ & $2.67 \mathrm{E}-02$ & & & \\
\hline Ba-137m & 1.09E-06 & $1.05 \mathrm{E}-06$ & $2.57 \mathrm{E}-08$ & 9.41E-05 & 9.41E-05 & 9.41E-05 & & & \\
\hline $\mathrm{Ce}-144$ & $4.04 \mathrm{E}-11$ & $3.89 \mathrm{E}-11$ & $2.29 \mathrm{E}-11$ & $2.44 \mathrm{E}-09$ & $2.44 \mathrm{E}-09$ & $2.44 \mathrm{E}-09$ & & & \\
\hline Pr-144 & $4.04 \mathrm{E}-11$ & $3.89 \mathrm{E}-11$ & $2.29 \mathrm{E}-11$ & $2.44 \mathrm{E}-09$ & 2.44E-09 & $2.44 \mathrm{E}-09$ & & & \\
\hline $\mathrm{Pm}-146$ & $2.54 \mathrm{E}-12$ & $2.45 \mathrm{E}-12$ & $1.44 \mathrm{E}-12$ & $1.54 \mathrm{E}-10$ & $1.54 \mathrm{E}-10$ & $1.54 \mathrm{E}-10$ & & & \\
\hline Pm-147 & $1.09 \mathrm{E}-08$ & 1.05E-08 & $6.19 \mathrm{E}-09$ & $6.60 \mathrm{E}-07$ & $6.60 \mathrm{E}-07$ & $6.60 \mathrm{E}-07$ & & & \\
\hline Sm-151 & $2.16 \mathrm{E}-08$ & $2.08 \mathrm{E}-08$ & $1.22 \mathrm{E}-08$ & $1.30 \mathrm{E}-06$ & 1.31E-06 & $1.31 \mathrm{E}-06$ & & & \\
\hline Eu-152 & $1.43 \mathrm{E}-10$ & $1.37 \mathrm{E}-10$ & $8.09 \mathrm{E}-11$ & 8.62E-09 & 8.62E-09 & 8.62E-09 & & & \\
\hline Eu-154 & $2.43 E-09$ & $2.33 \mathrm{E}-09$ & 1.38E-09 & 1.47E-07 & 1.47E-07 & $1.47 \mathrm{E}-07$ & & & \\
\hline Eu-155 & $2.10 \mathrm{E}-09$ & $2.02 \mathrm{E}-09$ & 1.19E-09 & $1.27 \mathrm{E}-07$ & $1.27 \mathrm{E}-07$ & $1.27 \mathrm{E}-07$ & & & \\
\hline & & & & & & & & & \\
\hline Th-230 & 4.97E-14 & $4.78 \mathrm{E}-14$ & $2.82 \mathrm{E}-14$ & $5.31 \mathrm{E}-12$ & 5.31E-12 & $5.31 \mathrm{E}-12$ & & & \\
\hline $\mathrm{Pa}-233$ & $1.42 \mathrm{E}-10$ & $1.37 \mathrm{E}-10$ & $8.07 \mathrm{E}-11$ & 1.52E-08 & $1.52 \mathrm{E}-08$ & 1.52E-08 & & & \\
\hline U-232 & $1.17 \mathrm{E}-13$ & 1.13E-13 & $6.64 \mathrm{E}-14$ & $1.25 \mathrm{E}-11$ & $1.25 \mathrm{E}-11$ & $1.25 \mathrm{E}-11$ & & & \\
\hline U-233 & $4.23 E-15$ & $4.07 \mathrm{E}-15$ & $2.40 \mathrm{E}-15$ & $4.52 \mathrm{E}-13$ & $4.52 \mathrm{E}-13$ & $4.52 \mathrm{E}-13$ & & & \\
\hline $\mathrm{U}-234$ & $5.22 \mathrm{E}-11$ & $5.02 \mathrm{E}-11$ & $2.96 \mathrm{E}-11$ & 5.57E-09 & 5.57E-09 & 5.57E-09 & & & \\
\hline U-235 & $3.83 \mathrm{E}-12$ & $3.69 \mathrm{E}-12$ & $2.18 \mathrm{E}-12$ & $4.10 \mathrm{E}-10$ & $4.10 \mathrm{E}-10$ & $4.10 \mathrm{E}-10$ & & & \\
\hline U-236 & $2.25 \mathrm{E}-12$ & $2.17 \mathrm{E}-12$ & $1.28 \mathrm{E}-12$ & $2.41 \mathrm{E}-10$ & $2.41 \mathrm{E}-10$ & $2.41 \mathrm{E}-10$ & & & \\
\hline $\mathrm{U}-237$ & $3.12 \mathrm{E}-13$ & $3.00 \mathrm{E}-13$ & $1.77 \mathrm{E}-13$ & $3.33 \mathrm{E}-11$ & 3.33E-11 & 3.33E-11 & & & \\
\hline $\mathrm{U}-238$ & $6.83 \mathrm{E}-13$ & $6.57 \mathrm{E}-13$ & $3.88 \mathrm{E}-13$ & $7.29 \mathrm{E}-11$ & $7.29 \mathrm{E}-11$ & $7.29 \mathrm{E}-11$ & & & \\
\hline $\mathrm{Np}-237$ & $2.10 \mathrm{E}-11$ & $2.02 \mathrm{E}-11$ & 1.19E-11 & 1.27E-09 & 1.27E-09 & 1.27E-09 & & & \\
\hline Pu-236 & $1.71 \mathrm{E}-13$ & $1.64 \mathrm{E}-13$ & $9.69 \mathrm{E}-14$ & $1.82 \mathrm{E}-11$ & $1.82 \mathrm{E}-11$ & $1.82 \mathrm{E}-11$ & & & \\
\hline $\mathrm{Pu}-238$ & $3.91 \mathrm{E}-08$ & $3.76 \mathrm{E}-08$ & $2.22 \mathrm{E}-08$ & $4.17 \mathrm{E}-06$ & 4.17E-06 & 4.17E-06 & & & \\
\hline Pu-239 & 4.37E-09 & 4.21E-09 & 2.48E-09 & 4.67E-07 & 4.67E-07 & 4.67E-07 & & & \\
\hline Pu-240 & $6.36 \mathrm{E}-10$ & $6.12 \mathrm{E}-10$ & $3.61 \mathrm{E}-10$ & $6.80 \mathrm{E}-08$ & $6.80 \mathrm{E}-08$ & $6.80 \mathrm{E}-08$ & & & \\
\hline $\mathrm{Pu}-241$ & $2.84 \mathrm{E}-08$ & $2.74 \mathrm{E}-08$ & 1.61E-08 & 3.04E-06 & $3.04 \mathrm{E}-06$ & 3.04E-06 & & & \\
\hline $\mathrm{Pu}-242$ & $4.98 \mathrm{E}-13$ & $4.80 \mathrm{E}-13$ & $2.83 \mathrm{E}-13$ & $5.32 \mathrm{E}-11$ & $5.32 \mathrm{E}-11$ & $5.32 \mathrm{E}-11$ & & & \\
\hline Pu-244 & $3.80 \mathrm{E}-20$ & $3.66 \mathrm{E}-20$ & $2.16 \mathrm{E}-20$ & $4.06 \mathrm{E}-18$ & $4.06 \mathrm{E}-18$ & $4.06 \mathrm{E}-18$ & & & \\
\hline Am-241 & $2.82 \mathrm{E}-09$ & 2.72E-09 & 1.60E-09 & 1.71E-07 & $1.71 \mathrm{E}-07$ & $1.71 \mathrm{E}-07$ & & & \\
\hline Am-242m & $7.80 \mathrm{E}-13$ & $7.50 \mathrm{E}-13$ & $4.43 \mathrm{E}-13$ & $4.72 \mathrm{E}-11$ & $4.72 \mathrm{E}-11$ & $4.72 \mathrm{E}-11$ & & & \\
\hline
\end{tabular}


Table A-15. Mass balance, Tank WM-188 waste (continued).

\begin{tabular}{|c|c|c|c|c|c|c|c|c|c|}
\hline Stream \# & 109 & 110 & 111 & 112 & $113 \mathrm{~A}$ & $113 \mathrm{~B}$ & 114 & 115 & 116 \\
\hline & Ci/wscm & Ci/wscm & $\mathrm{Ci} / \mathrm{wscm}$ & $\mathrm{Ci} / \mathrm{L}$ & $\mathrm{Ci} / \mathrm{L}$ & $\mathrm{Ci} / \mathrm{L}$ & $\mathrm{Ci} / \mathrm{wscm}$ & $\mathrm{Ci} / \mathrm{wscm}$ & Ci/wscm \\
\hline Am-243 & 1.27E-12 & $1.22 \mathrm{E}-12$ & 7.22E-13 & $7.70 \mathrm{E}-11$ & $7.70 \mathrm{E}-11$ & 7.70E-11 & & & \\
\hline $\mathrm{Cm}-242$ & $1.64 \mathrm{E}-12$ & $1.58 \mathrm{E}-12$ & $9.30 \mathrm{E}-13$ & $1.75 \mathrm{E}-10$ & $1.75 \mathrm{E}-10$ & $1.75 \mathrm{E}-10$ & & & \\
\hline Cm-243 & $1.72 \mathrm{E}-12$ & $1.65 \mathrm{E}-12$ & $9.75 \mathrm{E}-13$ & $1.84 \mathrm{E}-10$ & $1.84 \mathrm{E}-10$ & $1.84 \mathrm{E}-10$ & & & \\
\hline Cm-244 & $6.01 \mathrm{E}-11$ & $5.78 \mathrm{E}-11$ & $3.41 \mathrm{E}-11$ & 6.42E-09 & 6.42E-09 & 6.42E-09 & & & \\
\hline $\mathrm{Cm}-245$ & $1.81 \mathrm{E}-14$ & $1.74 \mathrm{E}-14$ & $1.03 \mathrm{E}-14$ & $1.93 \mathrm{E}-12$ & 1.93E-12 & 1.93E-12 & & & \\
\hline $\mathrm{Cm}-246$ & 1.19E-15 & $1.14 \mathrm{E}-15$ & $6.74 \mathrm{E}-16$ & $1.27 \mathrm{E}-13$ & 1.27E-13 & 1.27E-13 & & & \\
\hline TRU & $4.78 \mathrm{E}-08$ & $4.60 \mathrm{E}-08$ & $2.71 \mathrm{E}-08$ & 4.98E-06 & 4.98E-06 & $4.98 \mathrm{E}-06$ & & & \\
\hline \multicolumn{10}{|l|}{ TRU, nCi/gm } \\
\hline & & & & & & & & & \\
\hline $\begin{array}{l}\text { Gas Stream Bulk } \\
\text { Composition (Wet Basis) }\end{array}$ & $\begin{array}{c}\text { mol\% or } \\
\text { ppmv }\end{array}$ & $\begin{array}{c}\mathrm{mol} \% \text { or } \\
\text { ppmv }\end{array}$ & $\begin{array}{l}\text { mol\% or } \\
\text { ppmv }\end{array}$ & $\begin{array}{c}\text { mol\% or } \\
\text { ppmv }\end{array}$ & $\begin{array}{c}\mathrm{mol} \% \text { or } \\
\text { ppmv }\end{array}$ & $\begin{array}{c}\text { mol\% or } \\
\text { ppmv }\end{array}$ & $\begin{array}{l}\text { mol\% or } \\
\text { ppmv }\end{array}$ & $\begin{array}{c}\text { mol\% or } \\
\text { ppmv }\end{array}$ & $\begin{array}{c}\mathrm{mol} \% \text { or } \\
\mathrm{ppmv}\end{array}$ \\
\hline $\mathrm{H} 2 \mathrm{O}, \mathrm{mol} \%$ & $68.72 \%$ & $71.11 \%$ & $83.02 \%$ & & & & $83.01 \%$ & $83.01 \%$ & $83.01 \%$ \\
\hline $\mathrm{O} 2, \mathrm{~mol} \%$ & $0.52 \%$ & $2.19 \%$ & $1.29 \%$ & & & & $1.29 \%$ & $1.29 \%$ & $1.29 \%$ \\
\hline $\mathrm{N} 2, \mathrm{~mol} \%$ & $19.15 \%$ & $18.45 \%$ & $10.84 \%$ & & & & $10.84 \%$ & $10.84 \%$ & $10.84 \%$ \\
\hline $\mathrm{H} 2, \mathrm{~mol} \%$ & $3.90 \%$ & $0.00001 \%$ & $0.00001 \%$ & & & & $0.0004 \%$ & & $0.00 \%$ \\
\hline $\mathrm{CO} 2, \mathrm{~mol} \%$ & $6.50 \%$ & $8.17 \%$ & $4.80 \%$ & & & & $4.80 \%$ & $4.80 \%$ & $4.80 \%$ \\
\hline $\mathrm{CO}, \mathrm{ppmv}$ & $1.07 \mathrm{E}+04$ & $2.20 \mathrm{E}-02$ & 1.30E-02 & & & & $1.30 \mathrm{E}-02$ & \begin{tabular}{|l|}
$1.30 \mathrm{E}-02$ \\
\end{tabular} & $1.30 \mathrm{E}-02$ \\
\hline $\mathrm{NO}, \mathrm{ppmv}$ & $6.03 \mathrm{E}+02$ & $6.55 \mathrm{E}+02$ & $3.85 \mathrm{E}+02$ & & & & $3.85 \mathrm{E}+02$ & $3.85 \mathrm{E}+02$ & $3.85 \mathrm{E}+02$ \\
\hline NO2, ppmv & $4.08 \mathrm{E}+01$ & $2.31 \mathrm{E}+00$ & $1.36 \mathrm{E}+00$ & & & & $1.35 \mathrm{E}+00$ & $1.36 \mathrm{E}+00$ & $1.36 \mathrm{E}+00$ \\
\hline SO2, ppmv & \begin{tabular}{|l|}
$1.42 \mathrm{E}-02$ \\
\end{tabular} & $1.09 \mathrm{E}+02$ & $6.40 \mathrm{E}+01$ & & & & $6.41 \mathrm{E}+01$ & $6.41 \mathrm{E}+01$ & $6.41 \mathrm{E}+01$ \\
\hline $\mathrm{Cl}, \mathrm{ppmv}$ & $1.34 \mathrm{E}+00$ & $1.29 \mathrm{E}+00$ & $7.56 \mathrm{E}-01$ & & & & $1.25 \mathrm{E}-01$ & $1.25 \mathrm{E}-01$ & $1.25 \mathrm{E}-01$ \\
\hline $\mathrm{F}, \mathrm{ppmv}$ & $8.64 \mathrm{E}+00$ & $8.31 \mathrm{E}+00$ & $4.88 \mathrm{E}+00$ & & & & $1.58 \mathrm{E}+01$ & $1.58 \mathrm{E}+01$ & $1.58 \mathrm{E}+01$ \\
\hline C (organic), ppmv & $5.83 \mathrm{E}+02$ & $6.38 \mathrm{E}-27$ & & & & & & & \\
\hline $\mathrm{H}$ (organic), ppmv & $2.32 \mathrm{E}+03$ & $2.55 \mathrm{E}-26$ & & & & & & & \\
\hline $\mathrm{Hg}, \mathrm{ug} / \mathrm{wscm}$ & $1.29 \mathrm{E}+05$ & $1.24 \mathrm{E}+05$ & $7.33 \mathrm{E}+04$ & & & & & $4.09 \mathrm{E}+04$ & $4.09 \mathrm{E}+04$ \\
\hline $\mathrm{PM}, \mathrm{mg} / \mathrm{wscm}$ & $5.36 \mathrm{E}+02$ & $1.49 \mathrm{E}+02$ & $9.48 \mathrm{E}+01$ & & & & $1.20 \mathrm{E}+01$ & $5.28 \mathrm{E}+01$ & $5.28 \mathrm{E}+01$ \\
\hline SVM, ug/wscm & $4.15 \mathrm{E}+01$ & $4.00 \mathrm{E}+01$ & $2.36 \mathrm{E}+01$ & & & & & & \\
\hline LVM, ug/wscm & $1.31 \mathrm{E}+01$ & $1.26 \mathrm{E}+01$ & $7.44 \mathrm{E}+00$ & & & & & & \\
\hline $\begin{array}{l}\text { Gas Stream Bulk } \\
\text { Composition (Dry Basis) }\end{array}$ & $\mathrm{mol} \%$ & $\mathrm{~mol} \%$ & $\mathrm{~mol} \%$ & $\mathrm{~mol} \%$ & $\mathrm{~mol} \%$ & $\mathrm{~mol} \%$ & $\mathrm{~mol} \%$ & $\mathrm{~mol} \%$ & $\mathrm{~mol} \%$ \\
\hline $\mathrm{O} 2, \mathrm{~mol} \%$, dry basis & $1.67 \%$ & $7.57 \%$ & $7.57 \%$ & & & & $7.57 \%$ & $7.57 \%$ & $7.57 \%$ \\
\hline $\mathrm{N} 2, \mathrm{~mol} \%$, dry basis & $61.23 \%$ & $63.86 \%$ & $63.86 \%$ & & & & $63.84 \%$ & $63.84 \%$ & $63.84 \%$ \\
\hline $\mathrm{H} 2, \mathrm{~mol} \%$, dry basis & $12.47 \%$ & $0.00004 \%$ & $0.00004 \%$ & & & & $0.00230 \%$ & & $0.00 \%$ \\
\hline $\mathrm{CO} 2, \mathrm{~mol} \%$, dry basis & $20.78 \%$ & $28.29 \%$ & $28.29 \%$ & & & & $28.28 \%$ & $28.28 \%$ & $28.28 \%$ \\
\hline $\begin{array}{l}\text { Gas Stream Bulk } \\
\text { Composition (Dry Basis, } \\
\text { Corrected to } 7 \% \text { O2 with } \\
100 \% \text { O2 Combustion Air) } \\
\end{array}$ & $\begin{array}{l}\mathrm{ppmv}, \text { or } \\
\mathrm{ug} / \mathrm{dscm} \\
\text { or } \\
\mathrm{mg} / \mathrm{dscm}\end{array}$ & $\begin{array}{l}\mathrm{ppmv}, \text { or } \\
\text { ug/dscm } \\
\text { or } \\
\mathrm{mg} / \mathrm{dscm}\end{array}$ & $\begin{array}{l}\text { ppmv, or } \\
\text { ug/dscm or } \\
\mathrm{mg} / \mathrm{dscm}\end{array}$ & $\begin{array}{l}\mathrm{ppmv}, \text { or } \\
\mathrm{ug} / \mathrm{dscm} \\
\text { or } \\
\mathrm{mg} / \mathrm{dscm}\end{array}$ & $\begin{array}{l}\text { ppmv, or } \\
\text { ug/dscm } \\
\text { or } \\
\mathrm{mg} / \mathrm{dscm}\end{array}$ & $\begin{array}{l}\text { ppmv, or } \\
\text { ug/dscm } \\
\text { or } \\
\text { mg/dscm }\end{array}$ & $\begin{array}{l}\mathrm{ppmv}, \text { or } \\
\mathrm{ug} / \mathrm{dscm} \\
\text { or } \\
\mathrm{mg} / \mathrm{dscm}\end{array}$ & $\begin{array}{l}\text { ppmv, or } \\
\text { ug/dscm } \\
\text { or } \\
\mathrm{mg} / \mathrm{dscm}\end{array}$ & $\begin{array}{l}\text { ppmv, or } \\
\text { ug/dscm } \\
\text { or } \\
\mathrm{mg} / \mathrm{dscm}\end{array}$ \\
\hline $\mathrm{CO}, \mathrm{ppmv}$, dry basis & $4.77 \mathrm{E}+03$ & 1.07E-02 & 1.07E-02 & & & & 1.07E-02 & 1.07E-02 & 1.07E-02 \\
\hline NO, ppmv, dry basis & $2.70 \mathrm{E}+02$ & $3.18 \mathrm{E}+02$ & $3.18 \mathrm{E}+02$ & & & & $3.18 \mathrm{E}+02$ & $3.18 \mathrm{E}+02$ & $3.18 \mathrm{E}+02$ \\
\hline NO2, ppmv, dry basis & $1.83 \mathrm{E}+01$ & $1.12 \mathrm{E}+00$ & $1.12 \mathrm{E}+00$ & & & & $1.12 \mathrm{E}+00$ & $1.12 \mathrm{E}+00$ & $1.12 \mathrm{E}+00$ \\
\hline SO2, ppmv, dry basis & 6.37E-03 & $5.28 \mathrm{E}+01$ & $5.28 \mathrm{E}+01$ & & & & $5.28 \mathrm{E}+01$ & $5.28 \mathrm{E}+01$ & $5.28 \mathrm{E}+01$ \\
\hline $\mathrm{Cl}, \mathrm{ppmv}$, dry basis & 5.99E-01 & $6.24 \mathrm{E}-01$ & $6.24 \mathrm{E}-01$ & & & & 1.03E-01 & $1.03 \mathrm{E}-01$ & $1.03 \mathrm{E}-01$ \\
\hline $\mathrm{F}, \mathrm{ppmv}$, dry basis & $3.87 \mathrm{E}+00$ & $4.03 E+00$ & $4.03 \mathrm{E}+00$ & & & & $1.30 \mathrm{E}+01$ & $1.30 \mathrm{E}+01$ & $1.30 \mathrm{E}+01$ \\
\hline C (organic), ppmv, dry basis & $2.61 \mathrm{E}+02$ & & & & & & & & \\
\hline $\mathrm{H}$ (organic), ppmv, dry basis & $1.04 \mathrm{E}+03$ & & & & & & & & \\
\hline $\mathrm{Hg}, \mathrm{ug} / \mathrm{dscm}$ & $5.79 \mathrm{E}+04$ & $6.03 \mathrm{E}+04$ & $6.05 \mathrm{E}+04$ & & & & & $3.37 \mathrm{E}+04$ & $3.37 E+04$ \\
\hline $\mathrm{PM}, \mathrm{mg} / \mathrm{dscm}$ & $2.40 \mathrm{E}+02$ & $7.22 \mathrm{E}+01$ & $7.83 \mathrm{E}+01$ & & & & $9.89 \mathrm{E}+00$ & $4.35 \mathrm{E}+01$ & $4.35 \mathrm{E}+01$ \\
\hline SVM, ug/dscm & $1.40 \mathrm{E}+02$ & $1.34 \mathrm{E}+02$ & $7.93 \mathrm{E}+01$ & & & & & & \\
\hline LVM, ug/dscm & $4.42 \mathrm{E}+01$ & $4.25 \mathrm{E}+01$ & $2.50 \mathrm{E}+01$ & & & & & & \\
\hline
\end{tabular}


Table A-15. Mass balance, Tank WM-188 waste (continued).

\begin{tabular}{|c|c|c|c|c|c|c|c|c|}
\hline PFD \# & PFD-3 & PFD-3 & PFD-3 & PFD-3 & PFD-2 & PFD-2 & PFD-2 & $\frac{\text { PFD-3 }}{204}$ \\
\hline WM-188, Stream \# & 117 & 118 & 119 & 120 & 201 & 202 & 203 & 204 \\
\hline Stream Name & $\begin{array}{c}\text { Pressure } \\
\text { Control } \\
\text { Bleed Air }\end{array}$ & $\begin{array}{c}\text { Final } \\
\text { HEPA Off- } \\
\text { Gas Outlet }\end{array}$ & $\begin{array}{c}\text { Off-Gas to } \\
\text { Blower }\end{array}$ & $\begin{array}{l}\text { Spent } \\
\text { GAC }\end{array}$ & $\begin{array}{l}\text { Boiler } \\
\text { Feed } \\
\text { Water }\end{array}$ & $\begin{array}{c}\text { Fuel Oil to } \\
\text { Boiler }\end{array}$ & $\begin{array}{l}\text { Steam To } \\
\text { Reformer }\end{array}$ & $\begin{array}{c}\text { Propane } \\
\text { to Oxidizer }\end{array}$ \\
\hline Rate or Volume & $1.35 \mathrm{E}+04$ & $6.18 \mathrm{E}+05$ & $6.53 \mathrm{E}+05$ & $6.01 \mathrm{E}+00$ & $3.24 \mathrm{E}+02$ & $4.28 \mathrm{E}+01$ & $6.48 \mathrm{E}+04$ & $1.08 \mathrm{E}+02$ \\
\hline Volume Flow (standard, wet) & $1.33 \mathrm{E}+04$ & $1.87 \mathrm{E}+05$ & \begin{tabular}{|l|}
$2.00 \mathrm{E}+05$ \\
\end{tabular} & & & & $5.80 \mathrm{E}+04$ & $3.07 \mathrm{E}+02$ \\
\hline Volume Flow (standard, dry)* & $1.33 \mathrm{E}+04$ & $3.18 \mathrm{E}+04$ & $4.50 \mathrm{E}+04$ & & & & & $3.07 \mathrm{E}+02$ \\
\hline Rate Units & $\mathrm{ft} 3 / \mathrm{hr}$ & $\mathrm{ft} 3 / \mathrm{hr}$ & $\mathrm{ft} 3 / \mathrm{hr}$ & $\mathrm{lb} / \mathrm{hr}$ & $\mathrm{gal} / \mathrm{hr}$ & gal/hr & $\mathrm{ft} 3 / \mathrm{hr}$ & $\mathrm{ft} 3 / \mathrm{hr}$ \\
\hline Rate or Volume, metric & $3.77 \mathrm{E}+02$ & $5.30 \mathrm{E}+03$ & $5.67 \mathrm{E}+03$ & $2.73 \mathrm{E}+00$ & $1.23 \mathrm{E}+03$ & $1.62 \mathrm{E}+02$ & $1.64 \mathrm{E}+03$ & $8.68 \mathrm{E}+00$ \\
\hline Rate Units & wscm $/ \mathrm{hr}$ & $\mathrm{wscm} / \mathrm{hr}$ & wscm $/ \mathrm{hr}$ & $\mathrm{kg} / \mathrm{hr}$ & \begin{tabular}{|l|}
$\mathrm{L} / \mathrm{hr}$ \\
\end{tabular} & $\mathrm{L} / \mathrm{hr}$ & wscm $/ \mathrm{hr}$ & wscm $/ \mathrm{hr}$ \\
\hline Temperature, ${ }^{\circ} \mathrm{C}$ & 25 & 120 & 115 & 28 & 15 & 15 & 670 & 25 \\
\hline Temperature, ${ }^{\circ} \mathrm{F}$ & 77 & 248 & 239 & 82 & 59 & 59 & 1238 & 77 \\
\hline Pressure, psia & 14.7 & 6.0 & 6.0 & 14.7 & 42.3 & 14.7 & 42.3 & 42.3 \\
\hline Specific Gravity & $1.18 \mathrm{E}-03$ & $2.58 \mathrm{E}-04$ & $2.69 \mathrm{E}-04$ & $5.86 \mathrm{E}-01$ & $1.00 \mathrm{E}+00$ & $7.70 \mathrm{E}-01$ & $6.70 \mathrm{E}-04$ & $5.19 \mathrm{E}-03$ \\
\hline Chemical Composition & $\mathrm{lb} / \mathrm{wscf}$ & $\mathrm{lb} / \mathrm{wscf}$ & lb/wscf & Wt frac & Mol/liter & Mol/liter & lb/wscf & $\mathrm{lb} / \mathrm{wscf}$ \\
\hline $\mathrm{H}+$ & & $2.28 \mathrm{E}-07$ & $2.14 \mathrm{E}-07$ & $9.14 \mathrm{E}-06$ & & $2.40 \mathrm{E}-01$ & & \\
\hline \multicolumn{9}{|l|}{\begin{tabular}{|l|l}
$\mathrm{Al}+3$ \\
\end{tabular}} \\
\hline \multirow{2}{*}{\multicolumn{9}{|c|}{$\mathrm{Sb}+5$}} \\
\hline \multirow{2}{*}{\multicolumn{9}{|c|}{\begin{tabular}{|l|}
$\mathrm{As}+3$ \\
$\mathrm{Ba}+2$
\end{tabular}}} \\
\hline & & & & & & & & \\
\hline \multicolumn{9}{|l|}{$\frac{\mathrm{Ba}+2}{\mathrm{Be}+2}$} \\
\hline \multicolumn{9}{|l|}{$B+3$} \\
\hline \multicolumn{9}{|l|}{$\mathrm{Cd}+2$} \\
\hline \multirow{2}{*}{\multicolumn{9}{|c|}{\begin{tabular}{|l|}
$\mathrm{Ca}+2$ \\
$\mathrm{Cr}+3$
\end{tabular}}} \\
\hline & & & & & & & & \\
\hline \multicolumn{9}{|l|}{$\mathrm{Co}+2$} \\
\hline \multicolumn{9}{|l|}{ Cs+ } \\
\hline \multicolumn{9}{|l|}{$\mathrm{Cu}+2$} \\
\hline \multirow{2}{*}{\multicolumn{9}{|c|}{\begin{tabular}{|l|}
$\mathrm{Fe}+3$ \\
$\mathrm{~Pb}+2$
\end{tabular}}} \\
\hline & & & & & & & \multicolumn{2}{|c|}{$\mathrm{Pb}+2$} \\
\hline $\mathrm{Hg}+2$ & & $2.55 \mathrm{E}-09$ & $2.38 \mathrm{E}-09$ & $7.93 \mathrm{E}-02$ & & & & \\
\hline \multirow{2}{*}{\multicolumn{9}{|c|}{\begin{tabular}{|l|}
$\mathrm{Mn}+4$ \\
$\mathrm{Ni}+2$
\end{tabular}}} \\
\hline \multirow{2}{*}{\multicolumn{9}{|c|}{\begin{tabular}{|l|}
$\mathrm{Ni}+2$ \\
$\mathrm{~K}+$ \\
\end{tabular}}} \\
\hline & & & & & & & & \\
\hline \multicolumn{9}{|l|}{$\mathrm{Se}+4$} \\
\hline \multirow{2}{*}{\multicolumn{9}{|c|}{\begin{tabular}{|l|}
$\mathrm{Ag}+$ \\
$\mathrm{Na}+$
\end{tabular}}} \\
\hline & & & & & & & & \\
\hline \multicolumn{9}{|l|}{$\frac{\mathrm{Na}+}{\mathrm{T} \mid+3}$} \\
\hline $\mathrm{U}+4$ & & & & & & & & \\
\hline $\mathrm{V}+3$ & & & & & & & & \\
\hline$Z n+2$ & & & & & & & & \\
\hline $\mathrm{Zr}+4$ & & & & & & & & \\
\hline $\mathrm{Cl}-$ & & $1.15 \mathrm{E}-09$ & $1.07 \mathrm{E}-09$ & $3.21 \mathrm{E}-04$ & & & & \\
\hline F- & & $7.79 \mathrm{E}-07$ & $7.28 \mathrm{E}-07$ & & & & & \\
\hline SO4-2 & & & & & & & & \\
\hline NO3- & & $1.15 \mathrm{E}-05$ & $1.08 \mathrm{E}-05$ & & & & & \\
\hline PO4-3 & & & & & & & & \\
\hline$A m+4$ & & & & & & & & \\
\hline $\mathrm{Br}-\mathrm{B}$ & & $3.12 \mathrm{E}-14$ & $2.92 \mathrm{E}-14$ & $8.74 \mathrm{E}-09$ & & & & \\
\hline $\mathrm{Ce}+4$ & & & & & & & & \\
\hline $\mathrm{Eu+3}$ & & & & & & & & \\
\hline $\mathrm{Gd}+3$ & & & & & & & & \\
\hline $\mathrm{Ge}+4$ & & & & & & & & \\
\hline $\ln +3$ & & & & & & & & \\
\hline$-1-$ & & $5.29 \mathrm{E}-13$ & $4.95 \mathrm{E}-13$ & $1.48 \mathrm{E}-07$ & & & & \\
\hline $\mathrm{La+3}$ & & & & & & & & \\
\hline
\end{tabular}


Table A-15. Mass balance, Tank WM-188 waste (continued).

\begin{tabular}{|c|c|c|c|c|c|c|c|c|}
\hline Stream \# & 117 & 118 & 119 & 120 & 201 & 202 & 203 & 204 \\
\hline Chemical Composition & $\mathrm{lb} /$ wscf & $\mathrm{lb} / \mathrm{wscf}$ & $\mathrm{lb} / \mathrm{wscf}$ & Wt frac & Mol/liter & Mol/liter & $\mathrm{lb} /$ wscf & $\mathrm{lb} / \mathrm{wscf}$ \\
\hline \multicolumn{9}{|l|}{ Li+ } \\
\hline \multicolumn{9}{|l|}{$\mathrm{Mg}+2$} \\
\hline \multicolumn{9}{|l|}{$\mathrm{Mo}+6$} \\
\hline \multicolumn{9}{|l|}{$\mathrm{Nd}+3$} \\
\hline \multicolumn{9}{|l|}{$\mathrm{Np}+4$} \\
\hline \multicolumn{9}{|l|}{$\mathrm{Nb}+5$} \\
\hline \multicolumn{9}{|l|}{$\mathrm{Pd}+4$} \\
\hline \multicolumn{9}{|l|}{$\mathrm{Pu}+4$} \\
\hline \multicolumn{9}{|l|}{$\mathrm{Pr}+4$} \\
\hline \multicolumn{9}{|l|}{$\mathrm{Pm}+3$} \\
\hline \multicolumn{9}{|l|}{$\mathrm{Rh}+4$} \\
\hline \multicolumn{9}{|l|}{$\mathrm{Rb}+$} \\
\hline \multicolumn{9}{|l|}{ Ru+3 } \\
\hline \multicolumn{9}{|l|}{$\mathrm{Sm}+3$} \\
\hline \multicolumn{9}{|l|}{$\mathrm{Si+4}$} \\
\hline \multicolumn{9}{|l|}{$\mathrm{Sr}+2$} \\
\hline \multicolumn{9}{|l|}{$\mathrm{Tc}+7$} \\
\hline \multicolumn{9}{|l|}{$\mathrm{Te}+4$} \\
\hline \multicolumn{9}{|l|}{$\mathrm{Tb}+4$} \\
\hline \multicolumn{9}{|l|}{$\mathrm{Th}+4$} \\
\hline \multicolumn{9}{|l|}{ Sn+4 } \\
\hline $\mathrm{Ti}+4$ & & & & & & & & \\
\hline$Y+3$ & & & & & & & & \\
\hline $\mathrm{OH}-$ & & & & & & & & \\
\hline $\mathrm{H} 2 \mathrm{O}$ & $4.68 \mathrm{E}-06$ & $3.88 \mathrm{E}-02$ & $3.63 E-02$ & & $5.56 \mathrm{E}+01$ & & $4.68 \mathrm{E}-02$ & \\
\hline $\mathrm{SO} 2$ & & 1.07E-05 & $9.96 \mathrm{E}-06$ & & & & & \\
\hline $\mathrm{H} 2 \mathrm{~S}$ & & & & & & $1.20 \mathrm{E}-01$ & & \\
\hline $\mathrm{CO}$ & & $9.42 \mathrm{E}-10$ & $8.81 \mathrm{E}-10$ & & & & & \\
\hline $\mathrm{CO} 2$ & & 5.49E-03 & $5.13 \mathrm{E}-03$ & & & & & \\
\hline $\mathrm{H} 2$ & & & & & & & & \\
\hline $\mathrm{N} 2$ & $5.75 \mathrm{E}-02$ & $7.88 \mathrm{E}-03$ & $1.12 \mathrm{E}-02$ & & & & & \\
\hline NO & & $3.00 \mathrm{E}-05$ & $2.81 \mathrm{E}-05$ & & & & & \\
\hline NO2 & & 1.62E-07 & $1.51 \mathrm{E}-07$ & & & & & \\
\hline $\mathrm{O} 2$ & $1.74 \mathrm{E}-02$ & $1.07 \mathrm{E}-03$ & $2.16 \mathrm{E}-03$ & & & & & \\
\hline S (other) & & & & & & & & \\
\hline $\mathrm{CO} 3$ & & & & & & & & \\
\hline C (reductant) & & & & $9.20 \mathrm{E}-01$ & & & & \\
\hline O (oxides) & & & & & & & & \\
\hline C (organic) & & & & & & $5.41 \mathrm{E}+01$ & & $9.35 \mathrm{E}-02$ \\
\hline $\mathrm{H}$ (organic) & & & & & & $1.15 \mathrm{E}+02$ & & $2.09 \mathrm{E}-02$ \\
\hline O (organic) & & & & & & & & \\
\hline Mass Flow $(\mathrm{kg} / \mathrm{hr})$ : & $4.53 \mathrm{E}+02$ & $4.53 \mathrm{E}+03$ & $4.98 \mathrm{E}+03$ & $2.73 \mathrm{E}+00$ & $1.23 \mathrm{E}+03$ & $1.25 \mathrm{E}+02$ & $1.23 \mathrm{E}+03$ & $1.59 \mathrm{E}+01$ \\
\hline & & & & & & & & \\
\hline & & & & & & & & \\
\hline & $\mathrm{w} / \mathrm{m} 3$ & $\mathrm{w} / \mathrm{m} 3$ & $\mathrm{w} / \mathrm{m} 3$ & $\mathrm{w} / \mathrm{m} 3$ & $w / m 3$ & $\mathrm{w} / \mathrm{m} 3$ & $w / m 3$ & $\mathrm{w} / \mathrm{m} 3$ \\
\hline Heat Generation, w/m3 & & & & $6.13 \mathrm{E}-11$ & & & & \\
\hline
\end{tabular}


Table A-15. Mass balance, Tank WM-188 waste (continued).

\begin{tabular}{|c|c|c|c|c|c|c|c|c|}
\hline Stream \# & 117 & 118 & 119 & 120 & 201 & 202 & 203 & 204 \\
\hline Radiological Composition & $\mathrm{Ci} / \mathrm{wscm}$ & Ci/wscm & Ci/wscm & $\mathrm{Ci} / \mathrm{kg}$ & $\mathrm{Ci} / \mathrm{L}$ & $\mathrm{Ci} / \mathrm{L}$ & Ci/wscm & $\mathrm{Ci} / \mathrm{wscm}$ \\
\hline $\mathrm{H}-3$ & & $7.32 \mathrm{E}-07$ & $6.85 \mathrm{E}-07$ & & & & & \\
\hline C-14 & & $9.30 \mathrm{E}-12$ & $8.70 \mathrm{E}-12$ & $3.57 \mathrm{E}-10$ & & & & \\
\hline \multicolumn{9}{|l|}{ Co-60 } \\
\hline \multicolumn{9}{|l|}{ Ni-59 } \\
\hline \multicolumn{9}{|l|}{$\mathrm{Ni}-63$} \\
\hline \multicolumn{9}{|l|}{ Se-79 } \\
\hline \multicolumn{9}{|l|}{ Sr-90 } \\
\hline \multicolumn{9}{|l|}{$\mathrm{Y}-90$} \\
\hline \multicolumn{9}{|l|}{ Zr-93 } \\
\hline \multicolumn{9}{|l|}{$\mathrm{Nb}-93 \mathrm{~m}$} \\
\hline \multicolumn{9}{|l|}{$\mathrm{Nb}-94$} \\
\hline \multicolumn{9}{|l|}{ Tc-99 } \\
\hline \multicolumn{9}{|l|}{ Ru-106 } \\
\hline \multicolumn{9}{|l|}{\begin{tabular}{|l|} 
Rh-102 \\
\end{tabular}} \\
\hline \multicolumn{9}{|l|}{ Rh-106 } \\
\hline \multicolumn{9}{|l|}{ Pd-107 } \\
\hline \multicolumn{9}{|l|}{ Cd-113m } \\
\hline \multicolumn{9}{|l|}{ Sn-121m } \\
\hline Sn-126 & & & & & & & & \\
\hline Sb-125 & & & & & & & & \\
\hline Sb-126 & & & & & & & & \\
\hline Te-125m & & & & & & & & \\
\hline $\mid$ & & $1.51 \mathrm{E}-12$ & $1.42 \mathrm{E}-12$ & $2.65 \mathrm{E}-08$ & & & & \\
\hline Cs-134 & & & & & & & & \\
\hline Cs-135 & & & & & & & & \\
\hline Cs-137 & & & & & & & & \\
\hline Ba-137m & & & & & & & & \\
\hline Ce-144 & & & & & & & & \\
\hline Pr-144 & & & & & & & & \\
\hline $\mathrm{Pm}-146$ & & & & & & & & \\
\hline $\mathrm{Pm}-147$ & & & & & & & & \\
\hline Sm-151 & & & & & & & & \\
\hline Eu-152 & & & & & & & & \\
\hline Eu-154 & & & & & & & & \\
\hline Eu-155 & & & & & & & & \\
\hline & & & & & & & & \\
\hline Th-230 & & & & & & & & \\
\hline Pa-233 & & & & & & & & \\
\hline U-232 & & & & & & & & \\
\hline U-233 & & & & & & & & \\
\hline U-234 & & & & & & & & \\
\hline U-235 & & & & & & & & \\
\hline U-236 & & & & & & & & \\
\hline U-237 & & & & & & & & \\
\hline U-238 & & & & & & & & \\
\hline Np-237 & & & & & & & & \\
\hline Pu-236 & & & & & & & & \\
\hline Pu-238 & & & & & & & & \\
\hline Pu-239 & & & & & & & & \\
\hline Pu-240 & & & & & & & & \\
\hline Pu-241 & & & & & & & & \\
\hline Pu-242 & & & & & & & & \\
\hline Pu-244 & & & & & & & & \\
\hline Am-241 & & & & & & & & \\
\hline Am-242m & & & & & & & & \\
\hline
\end{tabular}


Table A-15. Mass balance, Tank WM-188 waste (continued).

\begin{tabular}{|c|c|c|c|c|c|c|c|c|}
\hline Stream \# & 117 & 118 & 119 & 120 & 201 & 202 & 203 & 204 \\
\hline & $\mathrm{Ci} / \mathrm{wscm}$ & $\mathrm{Ci} / \mathrm{wscm}$ & $\mathrm{Ci} / \mathrm{wscm}$ & $\mathrm{Ci} / \mathrm{kg}$ & $\mathrm{Ci} / \mathrm{L}$ & $\mathrm{Ci} / \mathrm{L}$ & Ci/wscm & Ci/wscm \\
\hline \multicolumn{9}{|l|}{ Am-243 } \\
\hline \multicolumn{9}{|l|}{$\mathrm{Cm}-242$} \\
\hline \multicolumn{9}{|l|}{$\mathrm{Cm}-243$} \\
\hline \multicolumn{9}{|l|}{$\mathrm{Cm}-244$} \\
\hline \multicolumn{9}{|l|}{$\mathrm{Cm}-245$} \\
\hline \multicolumn{9}{|l|}{$\mathrm{Cm}-246$} \\
\hline \multicolumn{9}{|l|}{ TRU } \\
\hline & & & & & & & & \\
\hline & & & & & & & & \\
\hline $\begin{array}{l}\text { Gas Stream Bulk } \\
\text { Composition (Wet Basis) }\end{array}$ & $\begin{array}{l}\text { mol\% or } \\
\text { ppmv }\end{array}$ & $\begin{array}{l}\text { mol\% or } \\
\text { ppmv }\end{array}$ & $\begin{array}{l}\text { mol\% or } \\
\text { ppmv }\end{array}$ & $\begin{array}{l}\text { mol\% or } \\
\text { ppmv }\end{array}$ & $\begin{array}{l}\text { mol\% or } \\
\text { ppmv }\end{array}$ & $\begin{array}{l}\text { mol\% or } \\
\text { ppmv }\end{array}$ & $\begin{array}{c}\text { mol\% or } \\
\text { ppmv }\end{array}$ & $\begin{array}{c}\mathrm{mol} \% \text { or } \\
\text { ppmv }\end{array}$ \\
\hline $\mathrm{H} 2 \mathrm{O}, \mathrm{mol} \%$ & $0.01 \%$ & $83.01 \%$ & $77.50 \%$ & & & & $100.00 \%$ & \\
\hline $\mathrm{O} 2, \mathrm{~mol} \%$ & $20.99 \%$ & $1.29 \%$ & $2.60 \%$ & & & & & \\
\hline $\mathrm{N} 2, \mathrm{~mol} \%$ & $79.00 \%$ & $10.84 \%$ & $15.37 \%$ & & & & & \\
\hline \multicolumn{9}{|l|}{$\mathrm{H} 2, \mathrm{~mol} \%$} \\
\hline $\mathrm{CO} 2, \mathrm{~mol} \%$ & & $4.80 \%$ & $4.48 \%$ & & & & & \\
\hline $\mathrm{CO}, \mathrm{ppmv}$ & & $1.30 \mathrm{E}-02$ & $1.21 \mathrm{E}-02$ & & & & & \\
\hline $\mathrm{NO}, \mathrm{ppmv}$ & & $3.85 E+02$ & $3.60 \mathrm{E}+02$ & & & & & \\
\hline NO2, ppmv & & $1.36 \mathrm{E}+00$ & $1.26 \mathrm{E}+00$ & & & & & \\
\hline SO2, ppmv & & $6.41 \mathrm{E}+01$ & $5.98 \mathrm{E}+01$ & & & & & \\
\hline $\mathrm{Cl}, \mathrm{ppmv}$ & & $1.25 \mathrm{E}-02$ & 1.16E-02 & & & & & \\
\hline $\mathrm{F}, \mathrm{ppmv}$ & & $1.58 \mathrm{E}+01$ & $1.47 \mathrm{E}+01$ & & & & & \\
\hline C (organic), ppmv & & & & & & & & $3.00 \mathrm{E}+06$ \\
\hline $\mathrm{H}$ (organic), ppmv & & & & & & & & $8.00 \mathrm{E}+06$ \\
\hline $\mathrm{Hg}, \mathrm{ug} / \mathrm{wscm}$ & & $4.09 \mathrm{E}+01$ & $3.82 \mathrm{E}+01$ & & & & & \\
\hline $\mathrm{PM}, \mathrm{mg} / \mathrm{wscm}$ & $1.60 \mathrm{E}+01$ & $1.20 \mathrm{E}+01$ & $1.21 \mathrm{E}+01$ & & & & $1.16 \mathrm{E}+01$ & $1.83 \mathrm{E}+06$ \\
\hline \multicolumn{9}{|l|}{ SVM, ug/wscm } \\
\hline \multicolumn{9}{|l|}{ LVM, ug/wscm } \\
\hline $\begin{array}{l}\text { Gas Stream Bulk } \\
\text { Composition (Dry Basis) }\end{array}$ & $\mathrm{mol} \%$ & $\mathrm{~mol} \%$ & $\mathrm{~mol} \%$ & $\mathrm{~mol} \%$ & $\mathrm{~mol} \%$ & $\mathrm{~mol} \%$ & $\mathrm{~mol} \%$ & $\mathrm{~mol} \%$ \\
\hline $02, \mathrm{~mol} \%$, dry basis & $20.9921 \%$ & $1.0604 \%$ & $11.5328 \%$ & & & & & \\
\hline $\mathrm{N} 2, \mathrm{~mol} \%$, dry basis & $79.01 \%$ & $63.84 \%$ & $68.32 \%$ & & & & & \\
\hline \multicolumn{9}{|l|}{$\mathrm{H} 2, \mathrm{~mol} \%$, dry basis } \\
\hline $\mathrm{CO} 2, \mathrm{~mol} \%$, dry basis & & $28.28 \%$ & $19.93 \%$ & & & & & \\
\hline $\begin{array}{l}\text { Gas Stream Bulk } \\
\text { Composition (Dry Basis, } \\
\text { Corrected to } 7 \% \text { O2 with } \\
100 \% \text { O2 Combustion Air) }\end{array}$ & $\begin{array}{l}\text { ppmv, or } \\
\text { ug/dscm or } \\
\text { mg/dscm }\end{array}$ & $\begin{array}{l}\text { ppmv, or } \\
\text { ug/dscm } \\
\text { or } \\
\mathrm{mg} / \mathrm{dscm}\end{array}$ & $\begin{array}{l}\text { ppmv, or } \\
\text { ug/dscm } \\
\text { or } \\
\mathrm{mg} / \mathrm{dscm}\end{array}$ & $\begin{array}{l}\text { ppmv, or } \\
\text { ug/dscm } \\
\text { or } \\
\mathrm{mg} / \mathrm{dscm}\end{array}$ & $\begin{array}{l}\mathrm{ppmv}, \text { or } \\
\mathrm{ug} / \mathrm{dscm} \\
\text { or } \\
\mathrm{mg} / \mathrm{dscm}\end{array}$ & $\begin{array}{l}\text { ppmv, or } \\
\text { ug/dscm } \\
\text { or } \\
\mathrm{mg} / \mathrm{dscm}\end{array}$ & $\begin{array}{l}\mathrm{ppmv}, \text { or } \\
\mathrm{ug} / \mathrm{dscm} \\
\text { or } \\
\mathrm{mg} / \mathrm{dscm}\end{array}$ & $\begin{array}{l}\mathrm{ppmv} \text {, or } \\
\mathrm{ug} / \mathrm{dscm} \\
\text { or } \\
\mathrm{mg} / \mathrm{dscm}\end{array}$ \\
\hline $\mathrm{CO}, \mathrm{ppmv}$, dry basis & & 1.07E-02 & $7.53 \mathrm{E}-03$ & & & & & \\
\hline $\mathrm{NO}$, ppmv, dry basis & & $3.18 \mathrm{E}+02$ & $2.24 \mathrm{E}+02$ & & & & & \\
\hline NO2, ppmv, dry basis & & $1.12 \mathrm{E}+00$ & $7.88 \mathrm{E}-01$ & & & & & \\
\hline SO2, ppmv, dry basis & & $5.28 \mathrm{E}+01$ & $3.73 \mathrm{E}+01$ & & & & & \\
\hline $\mathrm{Cl}, \mathrm{ppmv}$, dry basis & & 1.03E-02 & $7.25 \mathrm{E}-03$ & & & & & \\
\hline $\mathrm{F}, \mathrm{ppmv}$, dry basis & & $1.30 \mathrm{E}+01$ & $9.18 \mathrm{E}+00$ & & & & & \\
\hline \multicolumn{9}{|l|}{ C (organic), ppmv, dry basis } \\
\hline \multicolumn{9}{|l|}{$\mathrm{H}$ (organic), ppmv, dry basis } \\
\hline $\mathrm{Hg}, \mathrm{ug} / \mathrm{dscm}$ & & $3.37 E+01$ & $2.38 \mathrm{E}+01$ & & & & & \\
\hline $\mathrm{PM}, \mathrm{mg} / \mathrm{dscm}$ & $2.24 \mathrm{E}+00$ & $9.88 \mathrm{E}+00$ & $7.54 \mathrm{E}+00$ & & & & & \\
\hline \multicolumn{9}{|l|}{ SVM, ug/dscm } \\
\hline LVM, ug/dscm & & & & & & & & \\
\hline
\end{tabular}


Table A-15. Mass balance, Tank WM-188 waste (continued).

\begin{tabular}{|c|c|c|c|c|c|c|c|c|}
\hline PFD \# & PFD-3 & PFD-3 & PFD-3 & PFD-2 & PFD-2 & PFD-2 & PFD-2 & PFD-3 \\
\hline WM-188, Stream \# & 205 & 206 & 207 & 301 & 302 & 303 & 304 & 305 \\
\hline Stream Name & $\begin{array}{l}\text { Water to } \\
\text { Spray } \\
\text { Quench }\end{array}$ & $\begin{array}{l}\text { ANN to } \\
\text { Scrub for } \\
\text { F Adjust }\end{array}$ & $\begin{array}{l}\text { HNO3 } \\
\text { Scrub } \\
\text { Makeup }\end{array}$ & $\begin{array}{l}\text { Sugar to } \\
\text { Feed }\end{array}$ & $\begin{array}{l}\text { Carbon to } \\
\text { Reformer }\end{array}$ & $\begin{array}{c}\text { NOx } \\
\text { Catalyst }\end{array}$ & Bed Media & $\begin{array}{l}\text { Grout Mix } \\
\text { for Scrub } \\
\text { Blowdown }\end{array}$ \\
\hline Rate or Volume & $4.34 \mathrm{E}+02$ & $3.52 \mathrm{E}-08$ & $8.08 \mathrm{E}-01$ & $3.25 \mathrm{E}+02$ & $1.43 \mathrm{E}+02$ & $2.20 \mathrm{E}-05$ & $1.47 \mathrm{E}+00$ & $6.61 \mathrm{E}+00$ \\
\hline \multicolumn{9}{|c|}{ Volume Flow (standard, wet) ${ }^{*}$} \\
\hline Volume Flow (standard, dry) & & & & & & & & \\
\hline Rate Units & $\mathrm{gal} / \mathrm{hr}$ & $\mathrm{gal} / \mathrm{hr}$ & $\mathrm{gal} / \mathrm{hr}$ & $\mathrm{lb} / \mathrm{hr}$ & $\mathrm{lb} / \mathrm{hr}$ & $\mathrm{lb} / \mathrm{hr}$ & $\mathrm{lb} / \mathrm{hr}$ & $\mathrm{lb} / \mathrm{hr}$ \\
\hline Rate or Volume, metric & $1.64 \mathrm{E}+03$ & $1.33 \mathrm{E}-07$ & $3.06 \mathrm{E}+00$ & $1.47 \mathrm{E}+02$ & $6.48 \mathrm{E}+01$ & $1.00 \mathrm{E}-05$ & $6.68 \mathrm{E}-01$ & $3.00 \mathrm{E}+00$ \\
\hline Rate Units & $\mathrm{L} / \mathrm{hr}$ & $\mathrm{L} / \mathrm{hr}$ & $\mathrm{L} / \mathrm{hr}$ & $\mathrm{kg} / \mathrm{hr}$ & $\mathrm{kg} / \mathrm{hr}$ & $\mathrm{kg} / \mathrm{hr}$ & $\mathrm{kg} / \mathrm{hr}$ & $\mathrm{kg} / \mathrm{hr}$ \\
\hline Temperature, ${ }^{\circ} \mathrm{C}$ & 25 & 25 & 25 & 15 & 15 & 15 & 15 & 25 \\
\hline Temperature, ${ }^{\circ} \mathrm{F}$ & 77 & 77 & 77 & 59 & 59 & 59 & 59 & 77 \\
\hline Pressure, psia & 112.3 & 14.7 & 42.3 & 14.7 & 14.7 & 14.7 & 14.7 & 14.7 \\
\hline Specific Gravity & 9.97E-01 & $1.35 \mathrm{E}+00$ & $1.28 \mathrm{E}+00$ & $7.50 \mathrm{E}-01$ & $5.00 \mathrm{E}-01$ & $2.58 \mathrm{E}+00$ & $1.58 \mathrm{E}+00$ & $2.01 \mathrm{E}-01$ \\
\hline Chemical Composition & Mol/liter & Mol/liter & \begin{tabular}{|l|} 
Mol/liter \\
\end{tabular} & Wt frac & Wt frac & Wt frac & Wt frac & Wt frac \\
\hline $\mathrm{H}+$ & $1.00 \mathrm{E}-07$ & $3.09 \mathrm{E}-08$ & $1.39 \mathrm{E}+01$ & & & & & \\
\hline $\mathrm{Al}+3$ & & $2.20 \mathrm{E}+00$ & & & $1.53 \mathrm{E}-03$ & & $5.29 \mathrm{E}-01$ & \\
\hline \multicolumn{9}{|l|}{$\mathrm{Sb}+5$} \\
\hline \multicolumn{9}{|l|}{$\mathrm{As}+3$} \\
\hline \multirow{2}{*}{\multicolumn{9}{|c|}{$\frac{\mathrm{Ba}+2}{\mathrm{Be}+2}$}} \\
\hline & & & & & & & & \\
\hline \multicolumn{9}{|l|}{$\mathrm{B}+3$} \\
\hline \multicolumn{9}{|l|}{$\mathrm{Cd}+2$} \\
\hline $\mathrm{Ca}+2$ & & & & & 1.85E-02 & & & \\
\hline \multicolumn{9}{|l|}{$\mathrm{Cr}+3$} \\
\hline \multirow{2}{*}{\multicolumn{9}{|c|}{$\frac{\mathrm{Co}+2}{\mathrm{Cs}+}$}} \\
\hline & & & & & & & & \\
\hline \multicolumn{9}{|l|}{$\mathrm{Cu}+2$} \\
\hline $\mathrm{Fe}+3$ & & & & & $8.71 \mathrm{E}-04$ & $6.99 \mathrm{E}-01$ & & \\
\hline \multicolumn{9}{|l|}{$\mathrm{Pb}+2$} \\
\hline $\mathrm{Hg}+2$ & & & & & $1.92 \mathrm{E}-06$ & & & \\
\hline \multicolumn{9}{|l|}{$\mathrm{Mn}+4$} \\
\hline \multicolumn{9}{|l|}{$\mathrm{Ni}+2$} \\
\hline $\mathrm{K}+$ & & & & & 7.14E-03 & & & \\
\hline \multicolumn{9}{|l|}{$\mathrm{Se}+4$} \\
\hline \multicolumn{9}{|l|}{$\mathrm{Ag}+$} \\
\hline $\mathrm{Na}+$ & & & & & $7.42 \mathrm{E}-04$ & & & \\
\hline \multicolumn{9}{|l|}{$\mathrm{Tl}+3$} \\
\hline \multicolumn{9}{|l|}{$\mathrm{U}+4$} \\
\hline \multicolumn{9}{|l|}{$\mathrm{V}+3$} \\
\hline$Z n+2$ & & & & & & & & \\
\hline$Z r+4$ & & & & & & & & \\
\hline $\mathrm{Cl}-$ & & & & & & & & \\
\hline F- & & & & & & & & \\
\hline SO4-2 & & & & & & & & \\
\hline NO3- & & $6.60 \mathrm{E}+00$ & $1.39 \mathrm{E}+01$ & & & & & \\
\hline PO4-3 & & & & & $2.26 \mathrm{E}-03$ & & & \\
\hline $\mathrm{Am}+4$ & & & & & & & & \\
\hline $\mathrm{Br}-\mathrm{-}$ & & & & & & & & \\
\hline $\mathrm{Ce}+4$ & & & & & & & & \\
\hline $\mathrm{Eu}+3$ & & & & & & & & \\
\hline$G d+3$ & & & & & & & & \\
\hline $\mathrm{Ge}+4$ & & & & & & & & \\
\hline $\ln +3$ & & & & & & & & \\
\hline $1-$ & & & & & & & & \\
\hline $\mathrm{La}+3$ & & & & & & & & \\
\hline
\end{tabular}


Table A-15. Mass balance, Tank WM-188 waste (continued).

\begin{tabular}{|c|c|c|c|c|c|c|c|c|}
\hline Stream \# & 205 & 206 & 207 & 301 & 302 & 303 & 304 & 305 \\
\hline Chemical Composition & Mol/liter & Mol/liter & Mol/liter & Wt frac & Wt frac & Wt frac & Wt frac & Wt frac \\
\hline \multicolumn{9}{|l|}{$\mathrm{Li+}$} \\
\hline \multicolumn{9}{|l|}{$\mathrm{Mg}+2$} \\
\hline \multicolumn{9}{|l|}{$\mathrm{Mo}+6$} \\
\hline \multicolumn{9}{|l|}{$\mathrm{Nd}+3$} \\
\hline \multicolumn{9}{|l|}{$\mathrm{Np}+4$} \\
\hline \multicolumn{9}{|l|}{$\mathrm{Nb}+5$} \\
\hline \multicolumn{9}{|l|}{$\mathrm{Pd}+4$} \\
\hline \multicolumn{9}{|l|}{$\mathrm{Pu}+4$} \\
\hline \multicolumn{9}{|l|}{$\mathrm{Pr}+4$} \\
\hline \multicolumn{9}{|l|}{$\mathrm{Pm}+3$} \\
\hline \multicolumn{9}{|l|}{$\mathrm{Rh}+4$} \\
\hline \multicolumn{9}{|l|}{$\mathrm{Rb}+$} \\
\hline \multicolumn{9}{|l|}{ Ru+3 } \\
\hline \multicolumn{9}{|l|}{$\mathrm{Sm}+3$} \\
\hline $\mathrm{Si}+4$ & & & & & $6.26 \mathrm{E}-03$ & & & 4.67E-01 \\
\hline \multicolumn{9}{|l|}{$\mathrm{Sr}+2$} \\
\hline \multicolumn{9}{|l|}{$\mathrm{Tc}+7$} \\
\hline \multicolumn{9}{|l|}{$\mathrm{Te}+4$} \\
\hline \multicolumn{9}{|l|}{$\mathrm{Tb}+4$} \\
\hline \multicolumn{9}{|l|}{$\mathrm{Th}+4$} \\
\hline \multicolumn{9}{|l|}{$\mathrm{Sn}+4$} \\
\hline \multicolumn{9}{|l|}{$\mathrm{Ti}+4$} \\
\hline \multicolumn{9}{|l|}{$Y+3$} \\
\hline $\mathrm{OH}-$ & $1.00 \mathrm{E}-07$ & $3.09 \mathrm{E}-08$ & & & & & & \\
\hline $\mathrm{H} 2 \mathrm{O}$ & $5.54 \mathrm{E}+01$ & $4.89 \mathrm{E}+01$ & $2.27 \mathrm{E}+01$ & & & & & \\
\hline \multicolumn{9}{|l|}{$\mathrm{SO} 2$} \\
\hline $\mathrm{H} 2 \mathrm{~S}$ & & & & & & & & \\
\hline $\mathrm{CO}$ & & & & & & & & \\
\hline $\mathrm{CO} 2$ & & & & & & & & \\
\hline $\mathrm{H} 2$ & & & & & & & & \\
\hline $\mathrm{N} 2$ & & & & & & & & \\
\hline NO & & & & & & & & \\
\hline $\mathrm{NO} 2$ & & & & & & & & \\
\hline $\mathrm{O} 2$ & & & & & & & & \\
\hline S (other) & & & & & $5.18 \mathrm{E}-04$ & & & \\
\hline $\mathrm{CO} 3$ & & & & & $3.11 \mathrm{E}-02$ & & & \\
\hline C (reductant) & & & & & 9.22E-01 & & & \\
\hline $\mathrm{O}$ (oxides) & & & & & $8.88 \mathrm{E}-03$ & 3.01E-01 & 4.71E-01 & 5.33E-01 \\
\hline C (organic) & & & & $4.21 \mathrm{E}-01$ & & & & \\
\hline $\mathrm{H}$ (organic) & & & & $6.48 \mathrm{E}-02$ & & & & \\
\hline $\mathrm{O}$ (organic) & & & & 5.14E-01 & & & & \\
\hline Mass Flow $(\mathrm{kg} / \mathrm{hr})$ : & $1.64 \mathrm{E}+03$ & 1.80E-07 & $3.93 E+00$ & $1.47 \mathrm{E}+02$ & $6.48 \mathrm{E}+01$ & $1.00 \mathrm{E}-05$ & $6.68 \mathrm{E}-01$ & $3.00 \mathrm{E}+00$ \\
\hline
\end{tabular}


Table A-15. Mass balance, Tank WM-188 waste (continued).

\begin{tabular}{|c|c|c|c|c|c|c|c|c|}
\hline PFD \# & PFD-3 & PFD-3 & PFD-3 & PFD-2 & PFD-2 & PFD-2 & PFD-3 & PFD-2 \\
\hline WM-188, Stream \# & 401 & 402 & 404 & 404 & 503 & 504 & 505 & 506 \\
\hline Stream Name & $\begin{array}{c}\text { Scrub } \\
\text { Recycled } \\
\text { to Feed }\end{array}$ & \begin{tabular}{|c|} 
Scrub \\
Blowdown \\
to Grout \\
Mixer \\
\end{tabular} & $\begin{array}{l}\text { MLLW } \\
\text { Grout }\end{array}$ & $\begin{array}{l}\text { MLLW } \\
\text { Grout } \\
\text { Drums }\end{array}$ & $\begin{array}{c}\text { Feed } \\
\text { Atomizing } \\
\text { Gas }\end{array}$ & $\begin{array}{l}\text { Oxygen to } \\
\text { Reformer }\end{array}$ & $\begin{array}{c}\text { Oxygen to } \\
\text { Oxidizer }\end{array}$ & $\begin{array}{l}\text { Air to } \\
\text { Boiler }\end{array}$ \\
\hline Rate or Volume & $5.55 \mathrm{E}+00$ & \begin{tabular}{|l|}
$2.60 \mathrm{E}-01$ \\
\end{tabular} & $8.82 \mathrm{E}+00$ & $2.29 \mathrm{E}-01$ & $2.66 \mathrm{E}+03$ & $6.54 \mathrm{E}+02$ & $9.95 \mathrm{E}+02$ & $6.35 \mathrm{E}+04$ \\
\hline Volume Flow (standard, wet) & & & & & $1.67 E+04$ & $4.04 \mathrm{E}+03$ & $6.15 E+03$ & $6.46 \mathrm{E}+04$ \\
\hline Volume Flow (standard, dry) & & & & & $1.67 \mathrm{E}+04$ & $4.04 \mathrm{E}+03$ & $6.15 \mathrm{E}+03$ & $6.46 \mathrm{E}+04$ \\
\hline Rate Units & gal/hr & gal/hr & $\mathrm{lb} / \mathrm{hr}$ & Drums/day & $\mathrm{ft} 3 / \mathrm{hr}$ & $\mathrm{ft} 3 / \mathrm{hr}$ & $\mathrm{ft} 3 / \mathrm{hr}$ & $\mathrm{ft} 3 / \mathrm{hr}$ \\
\hline Rate or Volume, metric & $2.10 \mathrm{E}+01$ & $9.86 \mathrm{E}-01$ & $4.00 \mathrm{E}+00$ & $4.00 \mathrm{E}+00$ & $4.72 \mathrm{E}+02$ & $1.14 \mathrm{E}+02$ & $1.74 \mathrm{E}+02$ & $1.83 \mathrm{E}+03$ \\
\hline Rate Units & $\mathrm{L} / \mathrm{hr}$ & $\mathrm{L} / \mathrm{hr}$ & $\mathrm{kg} / \mathrm{hr}$ & $\mathrm{kg} / \mathrm{hr}$ & wscm/hr & wscm $/ \mathrm{hr}$ & wscm $/ \mathrm{hr}$ & wscm/hr \\
\hline Temperature, ${ }^{\circ} \mathrm{C}$ & 79 & 79 & 60 & 60 & 21 & 25 & 25 & 15 \\
\hline Temperature, ${ }^{\circ} \mathrm{F}$ & 174 & 174 & 140 & 140 & 70 & 77 & 77 & 59 \\
\hline Pressure, psia & 12.3 & 12.3 & 12.3 & 12.3 & 92.3 & 92.3 & 92.3 & 14.7 \\
\hline Specific Gravity & $1.01 \mathrm{E}+00$ & $1.01 \mathrm{E}+00$ & $2.10 \mathrm{E}+00$ & $2.10 \mathrm{E}+00$ & $7.29 \mathrm{E}-03$ & $8.21 \mathrm{E}-03$ & $8.21 \mathrm{E}-03$ & $1.22 \mathrm{E}-03$ \\
\hline Chemical Composition & Mol/liter & Mol/liter & Wt frac & Wt frac & $\mathrm{lb} / \mathrm{wscf}$ & $\mathrm{lb} / \mathrm{wscf}$ & Ib/wscf & $\mathrm{lb} / \mathrm{wscf}$ \\
\hline $\mathrm{H}+$ & $1.03 \mathrm{E}+00$ & $1.03 \mathrm{E}+00$ & $2.57 \mathrm{E}-04$ & $2.57 \mathrm{E}-04$ & & & & \\
\hline $\mathrm{Al}+3$ & $1.65 \mathrm{E}-03$ & $1.65 \mathrm{E}-03$ & $1.10 \mathrm{E}-05$ & $1.10 \mathrm{E}-05$ & & & & \\
\hline $\mathrm{Sb}+5$ & $4.71 \mathrm{E}-08$ & $4.71 \mathrm{E}-08$ & $1.41 \mathrm{E}-09$ & $1.41 \mathrm{E}-09$ & & & & \\
\hline As+3 & $2.63 \mathrm{E}-07$ & $2.63 \mathrm{E}-07$ & $4.85 \mathrm{E}-09$ & $4.85 \mathrm{E}-09$ & & & & \\
\hline $\mathrm{Ba}+2$ & $3.18 \mathrm{E}-07$ & $3.18 \mathrm{E}-07$ & $1.08 \mathrm{E}-08$ & $1.08 \mathrm{E}-08$ & & & & \\
\hline $\mathrm{Be}+2$ & $8.04 \mathrm{E}-08$ & $8.04 \mathrm{E}-08$ & $1.79 \mathrm{E}-10$ & $1.79 \mathrm{E}-10$ & & & & \\
\hline $\mathrm{B}+3$ & $4.71 \mathrm{E}-05$ & $4.71 \mathrm{E}-05$ & $1.25 \mathrm{E}-07$ & $1.25 \mathrm{E}-07$ & & & & \\
\hline $\mathrm{Cd}+2$ & $3.03 \mathrm{E}-06$ & $3.03 \mathrm{E}-06$ & $8.39 \mathrm{E}-08$ & $8.39 \mathrm{E}-08$ & & & & \\
\hline $\mathrm{Ca}+2$ & $6.06 \mathrm{E}-04$ & $6.06 \mathrm{E}-04$ & $5.98 \mathrm{E}-06$ & $5.98 \mathrm{E}-06$ & & & & \\
\hline $\mathrm{Cr}+3$ & $8.30 \mathrm{E}-06$ & $8.30 \mathrm{E}-06$ & $1.06 \mathrm{E}-07$ & $1.06 \mathrm{E}-07$ & & & & \\
\hline $\mathrm{Co}+2$ & $1.17 \mathrm{E}-07$ & 1.17E-07 & $1.70 \mathrm{E}-09$ & $1.70 \mathrm{E}-09$ & & & & \\
\hline Cs+ & $6.79 \mathrm{E}-05$ & $6.79 \mathrm{E}-05$ & $2.47 \mathrm{E}-06$ & $2.47 \mathrm{E}-06$ & & & & \\
\hline $\mathrm{Cu}+2$ & $2.98 \mathrm{E}-06$ & $2.98 \mathrm{E}-06$ & $4.66 \mathrm{E}-08$ & $4.66 \mathrm{E}-08$ & & & & \\
\hline $\mathrm{Fe}+3$ & $1.00 \mathrm{E}-04$ & $1.00 \mathrm{E}-04$ & $1.38 \mathrm{E}-06$ & $1.38 \mathrm{E}-06$ & & & & \\
\hline $\mathrm{Pb}+2$ & $5.52 \mathrm{E}-06$ & $5.52 \mathrm{E}-06$ & $2.82 \mathrm{E}-07$ & $2.82 \mathrm{E}-07$ & & & & \\
\hline $\mathrm{Hg}+2$ & $1.12 \mathrm{E}-02$ & $1.12 \mathrm{E}-02$ & $5.53 \mathrm{E}-04$ & $5.53 \mathrm{E}-04$ & & & & \\
\hline $\mathrm{Mn}+4$ & $4.30 \mathrm{E}-05$ & $4.30 \mathrm{E}-05$ & $5.82 \mathrm{E}-07$ & $5.82 \mathrm{E}-07$ & & & & \\
\hline $\mathrm{Ni}+2$ & $7.79 \mathrm{E}-06$ & $7.79 \mathrm{E}-06$ & $1.13 \mathrm{E}-07$ & $1.13 \mathrm{E}-07$ & & & & \\
\hline $\mathrm{K}+$ & $1.52 \mathrm{E}-03$ & $1.52 \mathrm{E}-03$ & $1.46 \mathrm{E}-05$ & $1.46 \mathrm{E}-05$ & & & & \\
\hline $\mathrm{Se}+4$ & $1.19 \mathrm{E}-06$ & $1.19 \mathrm{E}-06$ & $2.32 \mathrm{E}-08$ & $2.32 \mathrm{E}-08$ & & & & \\
\hline $\mathrm{Ag}+$ & $1.57 \mathrm{E}-06$ & $1.57 \mathrm{E}-06$ & $4.17 \mathrm{E}-08$ & $4.17 \mathrm{E}-08$ & & & & \\
\hline $\mathrm{Na}+$ & $5.84 \mathrm{E}-02$ & $5.84 \mathrm{E}-02$ & $3.31 \mathrm{E}-04$ & $3.31 \mathrm{E}-04$ & & & & \\
\hline $\mathrm{Tl}+3$ & $1.32 \mathrm{E}-08$ & $1.32 \mathrm{E}-08$ & $6.63 \mathrm{E}-10$ & $6.63 \mathrm{E}-10$ & & & & \\
\hline $\mathrm{U}+4$ & $1.78 \mathrm{E}-06$ & $1.78 \mathrm{E}-06$ & $1.05 \mathrm{E}-07$ & $1.05 \mathrm{E}-07$ & & & & \\
\hline $\mathrm{V}+3$ & $1.82 \mathrm{E}-07$ & $1.82 \mathrm{E}-07$ & $2.29 \mathrm{E}-09$ & $2.29 \mathrm{E}-09$ & & & & \\
\hline$Z n+2$ & $3.45 \mathrm{E}-06$ & $3.45 \mathrm{E}-06$ & $5.56 \mathrm{E}-08$ & $5.56 \mathrm{E}-08$ & & & & \\
\hline $\mathrm{Zr}+4$ & $1.86 \mathrm{E}-04$ & $1.86 \mathrm{E}-04$ & $4.17 \mathrm{E}-06$ & $4.17 \mathrm{E}-06$ & & & & \\
\hline $\mathrm{Cl}-$ & $2.31 \mathrm{E}-03$ & $2.31 \mathrm{E}-03$ & $2.01 \mathrm{E}-05$ & $2.01 \mathrm{E}-05$ & & & & \\
\hline F- & $1.86 \mathrm{E}-02$ & $1.86 \mathrm{E}-02$ & $8.72 \mathrm{E}-05$ & $8.72 \mathrm{E}-05$ & & & & \\
\hline SO4-2 & $2.10 \mathrm{E}-02$ & $2.10 \mathrm{E}-02$ & $4.96 \mathrm{E}-04$ & $4.96 \mathrm{E}-04$ & & & & \\
\hline \begin{tabular}{|l|}
$\mathrm{NO3}^{-}$ \\
\end{tabular} & $1.04 \mathrm{E}+00$ & $1.04 \mathrm{E}+00$ & $1.58 \mathrm{E}-02$ & $1.58 \mathrm{E}-02$ & & & & \\
\hline PO4-3 & $6.13 \mathrm{E}-04$ & $6.13 \mathrm{E}-04$ & 1.44E-05 & $1.44 \mathrm{E}-05$ & & & & \\
\hline $\mathrm{Am}+4$ & $1.99 \mathrm{E}-10$ & $1.99 \mathrm{E}-10$ & $1.19 \mathrm{E}-11$ & $1.19 \mathrm{E}-11$ & & & & \\
\hline $\mathrm{Br}-$ & $2.78 \mathrm{E}-08$ & $2.78 \mathrm{E}-08$ & $5.48 \mathrm{E}-10$ & $5.48 \mathrm{E}-10$ & & & & \\
\hline $\mathrm{Ce}+4$ & $9.84 \mathrm{E}-08$ & $9.84 \mathrm{E}-08$ & $3.40 \mathrm{E}-09$ & $3.40 \mathrm{E}-09$ & & & & \\
\hline $\mathrm{Eu}+3$ & $1.42 \mathrm{E}-09$ & $1.42 \mathrm{E}-09$ & $5.31 \mathrm{E}-11$ & $5.31 \mathrm{E}-11$ & & & & \\
\hline $\mathrm{Gd}+3$ & $4.19 \mathrm{E}-07$ & $4.19 \mathrm{E}-07$ & $1.62 \mathrm{E}-08$ & $1.62 \mathrm{E}-08$ & & & & \\
\hline $\mathrm{Ge}+4$ & $4.22 \mathrm{E}-11$ & $4.22 \mathrm{E}-11$ & $7.56 \mathrm{E}-13$ & $7.56 \mathrm{E}-13$ & & & & \\
\hline $\ln +3$ & $4.34 \mathrm{E}-09$ & $4.34 \mathrm{E}-09$ & $1.23 \mathrm{E}-10$ & $1.23 \mathrm{E}-10$ & & & & \\
\hline $\mid-$ & $2.97 \mathrm{E}-07$ & $2.97 \mathrm{E}-07$ & $9.30 \mathrm{E}-09$ & $9.30 \mathrm{E}-09$ & & & & \\
\hline $\mathrm{La}+3$ & $2.58 \mathrm{E}-08$ & $2.58 \mathrm{E}-08$ & $8.82 \mathrm{E}-10$ & $8.82 \mathrm{E}-10$ & & & & \\
\hline
\end{tabular}


Table A-15. Mass balance, Tank WM-188 waste (continued).

\begin{tabular}{|c|c|c|c|c|c|c|c|c|}
\hline Stream \# & 401 & 402 & 404 & 404 & 503 & 504 & 505 & 506 \\
\hline Chemical Composition & Mol/liter & Mol/liter & Wt frac & Wt frac & $\mathrm{lb} / \mathrm{wscf}$ & $\mathrm{lb} / \mathrm{wscf}$ & $\mathrm{lb} / \mathrm{wscf}$ & $\mathrm{lb} / \mathrm{wscf}$ \\
\hline Li+ & $1.95 \mathrm{E}-06$ & 1.95E-06 & 3.33E-09 & 3.33E-09 & & & & \\
\hline $\mathrm{Mg}+2$ & $7.81 \mathrm{E}-05$ & $7.81 \mathrm{E}-05$ & $4.68 \mathrm{E}-07$ & $4.68 \mathrm{E}-07$ & & & & \\
\hline $\mathrm{Mo}+6$ & $2.88 \mathrm{E}-04$ & $2.88 \mathrm{E}-04$ & $6.80 \mathrm{E}-06$ & $6.80 \mathrm{E}-06$ & & & & \\
\hline $\mathrm{Nd}+3$ & $8.30 \mathrm{E}-08$ & 8.30E-08 & 2.95E-09 & $2.95 \mathrm{E}-09$ & & & & \\
\hline $\mathrm{Np}+4$ & $7.59 \mathrm{E}-09$ & $7.59 \mathrm{E}-09$ & $4.43 \mathrm{E}-10$ & $4.43 \mathrm{E}-10$ & & & & \\
\hline $\mathrm{Nb}+5$ & $5.38 \mathrm{E}-06$ & $5.38 \mathrm{E}-06$ & $1.23 \mathrm{E}-07$ & $1.23 \mathrm{E}-07$ & & & & \\
\hline $\mathrm{Pd}+4$ & $7.47 \mathrm{E}-06$ & 7.47E-06 & $1.96 \mathrm{E}-07$ & $1.96 \mathrm{E}-07$ & & & & \\
\hline $\mathrm{Pu}+4$ & $3.39 \mathrm{E}-08$ & $3.39 E-08$ & 2.04E-09 & $2.04 \mathrm{E}-09$ & & & & \\
\hline $\mathrm{Pr}+4$ & $2.34 \mathrm{E}-08$ & $2.34 \mathrm{E}-08$ & $8.13 \mathrm{E}-10$ & $8.13 \mathrm{E}-10$ & & & & \\
\hline $\mathrm{Pm}+3$ & $4.85 \mathrm{E}-12$ & $4.85 \mathrm{E}-12$ & $1.73 \mathrm{E}-13$ & $1.73 \mathrm{E}-13$ & & & & \\
\hline $\mathrm{Rh}+4$ & $1.01 \mathrm{E}-08$ & $1.01 \mathrm{E}-08$ & $2.56 \mathrm{E}-10$ & $2.56 \mathrm{E}-10$ & & & & \\
\hline $\mathrm{Rb}+$ & $2.25 \mathrm{E}-08$ & $2.25 \mathrm{E}-08$ & $4.74 \mathrm{E}-10$ & $4.74 \mathrm{E}-10$ & & & & \\
\hline Ru+3 & $2.34 \mathrm{E}-06$ & $2.34 \mathrm{E}-06$ & $5.82 \mathrm{E}-08$ & $5.82 \mathrm{E}-08$ & & & & \\
\hline $\mathrm{Sm}+3$ & $1.55 \mathrm{E}-08$ & 1.55E-08 & $5.73 \mathrm{E}-10$ & $5.73 \mathrm{E}-10$ & & & & \\
\hline $\mathrm{Si}+4$ & $7.72 \mathrm{E}-04$ & $7.72 \mathrm{E}-04$ & $3.51 \mathrm{E}-01$ & $3.51 \mathrm{E}-01$ & & & & \\
\hline $\mathrm{Sr}+2$ & $3.42 \mathrm{E}-07$ & $3.42 \mathrm{E}-07$ & $7.38 \mathrm{E}-09$ & $7.38 \mathrm{E}-09$ & & & & \\
\hline $\mathrm{Tc}+7$ & $5.78 \mathrm{E}-08$ & $5.78 \mathrm{E}-08$ & 1.39E-09 & 1.39E-09 & & & & \\
\hline $\mathrm{Te}+4$ & $1.76 \mathrm{E}-08$ & $1.76 \mathrm{E}-08$ & $5.54 \mathrm{E}-10$ & $5.54 \mathrm{E}-10$ & & & & \\
\hline$T b+4$ & $5.93 E-12$ & $5.93 \mathrm{E}-12$ & $2.32 \mathrm{E}-13$ & $2.32 \mathrm{E}-13$ & & & & \\
\hline $\mathrm{Th}+4$ & 1.17E-07 & $1.17 \mathrm{E}-07$ & 6.69E-09 & $6.69 \mathrm{E}-09$ & & & & \\
\hline $\mathrm{Sn}+4$ & $8.80 \mathrm{E}-06$ & $8.80 \mathrm{E}-06$ & $2.57 \mathrm{E}-07$ & $2.57 \mathrm{E}-07$ & & & & \\
\hline $\mathrm{Ti}+4$ & $4.50 \mathrm{E}-06$ & $4.50 \mathrm{E}-06$ & $5.31 \mathrm{E}-08$ & $5.31 \mathrm{E}-08$ & & & & \\
\hline $\mathrm{Y}+3$ & 1.92E-08 & $1.92 \mathrm{E}-08$ & $4.21 \mathrm{E}-10$ & $4.21 \mathrm{E}-10$ & & & & \\
\hline $\mathrm{OH}-$ & $1.35 E-02$ & $1.35 \mathrm{E}-02$ & $5.65 \mathrm{E}-05$ & $5.65 \mathrm{E}-05$ & & & & \\
\hline $\mathrm{H} 2 \mathrm{O}$ & $5.23 E+01$ & $5.23 \mathrm{E}+01$ & $2.32 \mathrm{E}-01$ & $2.32 \mathrm{E}-01$ & & & & $4.68 \mathrm{E}-06$ \\
\hline $\mathrm{SO} 2$ & 8.09E-06 & 8.09E-06 & $1.28 \mathrm{E}-07$ & $1.28 \mathrm{E}-07$ & & & & \\
\hline \multicolumn{9}{|l|}{$\mathrm{H} 2 \mathrm{~S}$} \\
\hline $\mathrm{CO}$ & $2.02 \mathrm{E}-10$ & $2.02 \mathrm{E}-10$ & $1.40 \mathrm{E}-12$ & $1.40 \mathrm{E}-12$ & & & & \\
\hline $\mathrm{CO} 2$ & $3.09 \mathrm{E}-04$ & $3.09 \mathrm{E}-04$ & $3.35 E-06$ & $3.35 \mathrm{E}-06$ & & & & \\
\hline $\mathrm{H} 2$ & $8.32 \mathrm{E}-10$ & $8.32 \mathrm{E}-10$ & $4.13 \mathrm{E}-13$ & $4.13 \mathrm{E}-13$ & & & & \\
\hline N2 & $1.21 \mathrm{E}-05$ & $1.21 \mathrm{E}-05$ & $8.38 \mathrm{E}-08$ & $8.38 \mathrm{E}-08$ & $7.27 \mathrm{E}-02$ & & & $5.74 \mathrm{E}-02$ \\
\hline $\mathrm{NO}$ & $2.30 \mathrm{E}-07$ & $2.30 \mathrm{E}-07$ & 1.70E-09 & 1.70E-09 & & & & \\
\hline NO2 & $2.91 \mathrm{E}-06$ & $2.91 \mathrm{E}-06$ & $3.30 \mathrm{E}-08$ & $3.30 \mathrm{E}-08$ & & & & \\
\hline $\mathrm{O} 2$ & $3.08 \mathrm{E}-06$ & $3.08 \mathrm{E}-06$ & $2.43 \mathrm{E}-08$ & $2.43 \mathrm{E}-08$ & & $8.30 \mathrm{E}-02$ & $8.30 \mathrm{E}-02$ & 1.74E-02 \\
\hline S (other) & $1.08 \mathrm{E}-07$ & $1.08 \mathrm{E}-07$ & $8.52 \mathrm{E}-10$ & $8.52 \mathrm{E}-10$ & & & & \\
\hline $\mathrm{CO} 3$ & $3.39 \mathrm{E}-05$ & 3.39E-05 & 5.02E-07 & $5.02 \mathrm{E}-07$ & & & & \\
\hline \multicolumn{9}{|l|}{ C (reductant) } \\
\hline $\mathrm{O}$ (oxides) & 4.97E-03 & 4.97E-03 & 3.99E-01 & 3.99E-01 & & & & \\
\hline \multicolumn{9}{|l|}{ C (organic) } \\
\hline \multicolumn{9}{|l|}{$\mathrm{H}$ (organic) } \\
\hline \multicolumn{9}{|l|}{ O (organic) } \\
\hline \multirow[t]{2}{*}{ Mass Flow $(\mathrm{kg} / \mathrm{hr})$ : } & $2.13 \mathrm{E}+01$ & $1.00 \mathrm{E}+00$ & $4.00 \mathrm{E}+00$ & $4.00 \mathrm{E}+00$ & $5.50 \mathrm{E}+02$ & $1.52 \mathrm{E}+02$ & $2.31 \mathrm{E}+02$ & $2.19 \mathrm{E}+03$ \\
\hline & & & & & & & & \\
\hline \multirow[t]{3}{*}{ Total Drums Generated } & & & & 32 & & & & \\
\hline & & & & & & & & \\
\hline & $\mathrm{w} / \mathrm{m} 3$ & $\mathrm{w} / \mathrm{m} 3$ & $\mathrm{w} / \mathrm{m} 3$ & w/Drum & $w / m 3$ & $\mathrm{w} / \mathrm{m} 3$ & $\mathrm{w} / \mathrm{m} 3$ & $\mathrm{w} / \mathrm{m} 3$ \\
\hline Heat Generation, w/m3 & $2.88 \mathrm{E}-02$ & $2.88 \mathrm{E}-02$ & $1.64 \mathrm{E}-02$ & $3.29 \mathrm{E}-03$ & & & & \\
\hline
\end{tabular}


Table A-15. Mass balance, Tank WM-188 waste (continued).

\begin{tabular}{|c|c|c|c|c|c|c|c|c|}
\hline Stream \# & 401 & 402 & 404 & 404 & 503 & 504 & 505 & 506 \\
\hline Radiological Composition & $\mathrm{Ci} / \mathrm{L}$ & $\mathrm{Ci} / \mathrm{L}$ & $\mathrm{Ci} / \mathrm{kg}$ & Ci/Drum & Ci/wscm & Ci/wscm & $\mathrm{Ci} / \mathrm{wscm}$ & $\mathrm{Ci} / \mathrm{wscm}$ \\
\hline \multicolumn{9}{|l|}{$\mathrm{H}-3$} \\
\hline $\mathrm{C}-14$ & $1.60 \mathrm{E}-15$ & $1.60 \mathrm{E}-15$ & $3.94 \mathrm{E}-16$ & $1.65 \mathrm{E}-13$ & & & & \\
\hline Co-60 & $2.63 \mathrm{E}-08$ & $2.63 \mathrm{E}-08$ & $6.48 \mathrm{E}-09$ & $2.72 \mathrm{E}-06$ & & & & \\
\hline Ni-59 & $4.85 \mathrm{E}-09$ & $4.85 \mathrm{E}-09$ & $1.19 \mathrm{E}-09$ & $5.02 \mathrm{E}-07$ & & & & \\
\hline $\mathrm{Ni}-63$ & $1.83 \mathrm{E}-07$ & $1.83 \mathrm{E}-07$ & $4.51 \mathrm{E}-08$ & $1.90 \mathrm{E}-05$ & & & & \\
\hline Se-79 & $2.90 \mathrm{E}-09$ & $2.90 \mathrm{E}-09$ & $7.16 \mathrm{E}-10$ & $3.01 \mathrm{E}-07$ & & & & \\
\hline Sr-90 & $1.72 \mathrm{E}-04$ & $1.72 \mathrm{E}-04$ & $4.25 \mathrm{E}-05$ & $1.78 \mathrm{E}-02$ & & & & \\
\hline Y-90 & $1.16 \mathrm{E}-04$ & $1.16 \mathrm{E}-04$ & $2.87 \mathrm{E}-05$ & $1.20 \mathrm{E}-02$ & & & & \\
\hline Zr-93 & $1.15 \mathrm{E}-08$ & $1.15 \mathrm{E}-08$ & $2.83 \mathrm{E}-09$ & $1.19 \mathrm{E}-06$ & & & & \\
\hline $\mathrm{Nb}-93 \mathrm{~m}$ & $8.93 \mathrm{E}-09$ & $8.93 \mathrm{E}-09$ & $2.20 \mathrm{E}-09$ & $9.24 \mathrm{E}-07$ & & & & \\
\hline $\mathrm{Nb}-94$ & $7.63 \mathrm{E}-09$ & $7.63 \mathrm{E}-09$ & $1.88 \mathrm{E}-09$ & $7.89 \mathrm{E}-07$ & & & & \\
\hline Tc-99 & $1.17 \mathrm{E}-07$ & 1.17E-07 & $2.89 \mathrm{E}-08$ & $1.22 \mathrm{E}-05$ & & & & \\
\hline Ru-106 & $3.62 \mathrm{E}-09$ & $3.62 \mathrm{E}-09$ & $8.92 \mathrm{E}-10$ & $3.75 \mathrm{E}-07$ & & & & \\
\hline Rh-102 & $2.61 \mathrm{E}-12$ & $2.61 \mathrm{E}-12$ & $6.42 \mathrm{E}-13$ & $2.70 \mathrm{E}-10$ & & & & \\
\hline Rh-106 & $3.62 \mathrm{E}-09$ & $3.62 \mathrm{E}-09$ & $8.92 \mathrm{E}-10$ & $3.75 \mathrm{E}-07$ & & & & \\
\hline $\mathrm{Pd}-107$ & $7.39 \mathrm{E}-11$ & $7.39 \mathrm{E}-11$ & $1.82 \mathrm{E}-11$ & $7.65 \mathrm{E}-09$ & & & & \\
\hline Cd-113m & $4.24 \mathrm{E}-09$ & $4.24 \mathrm{E}-09$ & $1.04 \mathrm{E}-09$ & $4.39 \mathrm{E}-07$ & & & & \\
\hline Sn-121m & $3.46 \mathrm{E}-10$ & $3.46 \mathrm{E}-10$ & $8.54 \mathrm{E}-11$ & $3.59 \mathrm{E}-08$ & & & & \\
\hline Sn-126 & $2.73 \mathrm{E}-09$ & $2.73 \mathrm{E}-09$ & $6.74 \mathrm{E}-10$ & $2.83 \mathrm{E}-07$ & & & & \\
\hline Sb-125 & $1.10 \mathrm{E}-06$ & $1.10 \mathrm{E}-06$ & $2.70 \mathrm{E}-07$ & $1.13 \mathrm{E}-04$ & & & & \\
\hline Sb-126 & $1.74 \mathrm{E}-10$ & $1.74 \mathrm{E}-10$ & $4.28 \mathrm{E}-11$ & $1.80 \mathrm{E}-08$ & & & & \\
\hline Te-125m & $1.63 \mathrm{E}-08$ & $1.63 \mathrm{E}-08$ & $4.02 \mathrm{E}-09$ & $1.69 \mathrm{E}-06$ & & & & \\
\hline I-129 & $6.73 \mathrm{E}-09$ & $6.73 \mathrm{E}-09$ & $1.66 \mathrm{E}-09$ & $6.97 \mathrm{E}-07$ & & & & \\
\hline Cs-134 & $4.33 \mathrm{E}-05$ & $4.33 \mathrm{E}-05$ & $1.19 \mathrm{E}-05$ & $4.98 \mathrm{E}-03$ & & & & \\
\hline Cs-135 & $1.29 \mathrm{E}-06$ & $1.29 \mathrm{E}-06$ & $3.53 \mathrm{E}-07$ & $1.48 \mathrm{E}-04$ & & & & \\
\hline Cs-137 & $2.67 \mathrm{E}-02$ & $2.67 \mathrm{E}-02$ & $7.30 \mathrm{E}-03$ & $3.06 \mathrm{E}+00$ & & & & \\
\hline Ba-137m & $9.41 \mathrm{E}-05$ & $9.41 \mathrm{E}-05$ & $2.32 \mathrm{E}-05$ & $9.74 \mathrm{E}-03$ & & & & \\
\hline \begin{tabular}{|l|} 
Ce-144 \\
\end{tabular} & $2.44 \mathrm{E}-09$ & $2.44 \mathrm{E}-09$ & $6.02 \mathrm{E}-10$ & $2.53 \mathrm{E}-07$ & & & & \\
\hline Pr-144 & $2.44 \mathrm{E}-09$ & $2.44 \mathrm{E}-09$ & $6.02 \mathrm{E}-10$ & $2.53 \mathrm{E}-07$ & & & & \\
\hline Pm-146 & $1.54 \mathrm{E}-10$ & $1.54 \mathrm{E}-10$ & $3.79 \mathrm{E}-11$ & $1.59 \mathrm{E}-08$ & & & & \\
\hline Pm-147 & $6.60 \mathrm{E}-07$ & $6.60 \mathrm{E}-07$ & $1.63 \mathrm{E}-07$ & $6.83 \mathrm{E}-05$ & & & & \\
\hline Sm-151 & $1.30 \mathrm{E}-06$ & $1.30 \mathrm{E}-06$ & $3.21 \mathrm{E}-07$ & $1.35 \mathrm{E}-04$ & & & & \\
\hline Eu-152 & $8.62 \mathrm{E}-09$ & $8.62 \mathrm{E}-09$ & $2.12 \mathrm{E}-09$ & $8.92 \mathrm{E}-07$ & & & & \\
\hline Eu-154 & $1.47 \mathrm{E}-07$ & 1.47E-07 & $3.62 \mathrm{E}-08$ & $1.52 \mathrm{E}-05$ & & & & \\
\hline \multirow[t]{2}{*}{ Eu-155 } & $1.27 \mathrm{E}-07$ & $1.27 \mathrm{E}-07$ & $3.13 \mathrm{E}-08$ & $1.32 \mathrm{E}-05$ & & & & \\
\hline & & & & & & & & \\
\hline Th-230 & $5.31 \mathrm{E}-12$ & $5.31 \mathrm{E}-12$ & $1.31 \mathrm{E}-12$ & $5.49 \mathrm{E}-10$ & & & & \\
\hline Pa-233 & $1.52 \mathrm{E}-08$ & $1.52 \mathrm{E}-08$ & $3.74 \mathrm{E}-09$ & $1.57 \mathrm{E}-06$ & & & & \\
\hline U-232 & $1.25 \mathrm{E}-11$ & $1.25 \mathrm{E}-11$ & $3.08 \mathrm{E}-12$ & $1.29 \mathrm{E}-09$ & & & & \\
\hline $\mathrm{U}-233$ & $4.52 \mathrm{E}-13$ & $4.52 \mathrm{E}-13$ & $1.11 \mathrm{E}-13$ & $4.68 \mathrm{E}-11$ & & & & \\
\hline U-234 & $5.57 \mathrm{E}-09$ & $5.57 \mathrm{E}-09$ & $1.37 \mathrm{E}-09$ & $5.77 \mathrm{E}-07$ & & & & \\
\hline U-235 & $4.10 \mathrm{E}-10$ & $4.10 \mathrm{E}-10$ & $1.01 \mathrm{E}-10$ & $4.24 \mathrm{E}-08$ & & & & \\
\hline U-236 & $2.41 \mathrm{E}-10$ & $2.41 \mathrm{E}-10$ & $5.93 \mathrm{E}-11$ & $2.49 \mathrm{E}-08$ & & & & \\
\hline U-237 & $3.33 \mathrm{E}-11$ & $3.33 \mathrm{E}-11$ & $8.22 \mathrm{E}-12$ & $3.45 \mathrm{E}-09$ & & & & \\
\hline U-238 & $7.29 \mathrm{E}-11$ & $7.29 \mathrm{E}-11$ & $1.80 \mathrm{E}-11$ & $7.55 \mathrm{E}-09$ & & & & \\
\hline Np-237 & $1.27 \mathrm{E}-09$ & $1.27 \mathrm{E}-09$ & $3.13 \mathrm{E}-10$ & $1.31 \mathrm{E}-07$ & & & & \\
\hline Pu-236 & $1.82 \mathrm{E}-11$ & $1.82 \mathrm{E}-11$ & $4.50 \mathrm{E}-12$ & $1.89 \mathrm{E}-09$ & & & & \\
\hline Pu-238 & $4.17 \mathrm{E}-06$ & 4.17E-06 & $1.03 \mathrm{E}-06$ & $4.32 \mathrm{E}-04$ & & & & \\
\hline Pu-239 & $4.67 \mathrm{E}-07$ & $4.67 \mathrm{E}-07$ & 1.15E-07 & $4.83 \mathrm{E}-05$ & & & & \\
\hline Pu-240 & $6.80 \mathrm{E}-08$ & $6.80 \mathrm{E}-08$ & 1.67E-08 & $7.03 \mathrm{E}-06$ & & & & \\
\hline Pu-241 & $3.04 \mathrm{E}-06$ & $3.04 \mathrm{E}-06$ & $7.48 \mathrm{E}-07$ & $3.14 \mathrm{E}-04$ & & & & \\
\hline Pu-242 & $5.32 \mathrm{E}-11$ & $5.32 \mathrm{E}-11$ & $1.31 \mathrm{E}-11$ & $5.51 \mathrm{E}-09$ & & & & \\
\hline Pu-244 & $4.06 \mathrm{E}-18$ & $4.06 \mathrm{E}-18$ & 1.00E-18 & $4.20 \mathrm{E}-16$ & & & & \\
\hline Am-241 & $1.71 \mathrm{E}-07$ & $1.71 \mathrm{E}-07$ & $4.21 \mathrm{E}-08$ & 1.77E-05 & & & & \\
\hline Am-242m & $4.72 \mathrm{E}-11$ & $4.72 \mathrm{E}-11$ & 1.16E-11 & $4.88 \mathrm{E}-09$ & & & & \\
\hline
\end{tabular}


Table A-15. Mass balance, Tank WM-188 waste (continued).

\begin{tabular}{|c|c|c|c|c|c|c|c|c|}
\hline Stream \# & 401 & 402 & 404 & 404 & 503 & 504 & 505 & 506 \\
\hline & $\mathrm{Ci} / \mathrm{L}$ & $\mathrm{Ci} / \mathrm{L}$ & $\mathrm{Ci} / \mathrm{kg}$ & Ci/Drum & $\mathrm{Ci} / \mathrm{wscm}$ & $\mathrm{Ci} / \mathrm{wscm}$ & $\mathrm{Ci} / \mathrm{wscm}$ & $\mathrm{Ci} / \mathrm{wscm}$ \\
\hline $\mathrm{Am}-243$ & $7.70 \mathrm{E}-11$ & $7.70 \mathrm{E}-11$ & 1.90E-11 & 7.97E-09 & & & & \\
\hline $\mathrm{Cm}-242$ & $1.75 \mathrm{E}-10$ & $1.75 \mathrm{E}-10$ & $4.31 \mathrm{E}-11$ & $1.81 \mathrm{E}-08$ & & & & \\
\hline $\mathrm{Cm}-243$ & $1.84 \mathrm{E}-10$ & $1.84 \mathrm{E}-10$ & $4.52 \mathrm{E}-11$ & 1.90E-08 & & & & \\
\hline $\mathrm{Cm}-244$ & 6.42E-09 & 6.42E-09 & 1.58E-09 & 6.64E-07 & & & & \\
\hline $\mathrm{Cm}-245$ & $1.93 \mathrm{E}-12$ & 1.93E-12 & $4.76 \mathrm{E}-13$ & $2.00 \mathrm{E}-10$ & & & & \\
\hline $\mathrm{Cm}-246$ & $1.27 \mathrm{E}-13$ & 1.27E-13 & $3.13 \mathrm{E}-14$ & $1.31 \mathrm{E}-11$ & & & & \\
\hline TRU & 4.98E-06 & $4.98 \mathrm{E}-06$ & 1.23E-06 & $5.15 E-04$ & & & & \\
\hline \multicolumn{9}{|l|}{ TRU, nCi/gm } \\
\hline & & & & & & & & \\
\hline $\begin{array}{l}\text { Gas Stream Bulk } \\
\text { Composition (Wet Basis) }\end{array}$ & $\begin{array}{c}\text { mol\% or } \\
\text { ppmv }\end{array}$ & $\begin{array}{l}\text { mol\% or } \\
\text { ppmv }\end{array}$ & $\begin{array}{c}\text { mol\% or } \\
\text { ppmv }\end{array}$ & $\begin{array}{c}\text { mol\% or } \\
\text { ppmv }\end{array}$ & $\begin{array}{l}\text { mol\% or } \\
\text { ppmv }\end{array}$ & $\begin{array}{c}\text { mol\% or } \\
\text { ppmv }\end{array}$ & $\begin{array}{c}\text { mol\% or } \\
\text { ppmv }\end{array}$ & $\begin{array}{c}\text { mol\% or } \\
\text { ppmv }\end{array}$ \\
\hline $\mathrm{H} 2 \mathrm{O}, \mathrm{mol} \%$ & & & & & & & & $0.01 \%$ \\
\hline $\mathrm{O} 2, \mathrm{~mol} \%$ & & & & & & $100.00 \%$ & $100.00 \%$ & $20.99 \%$ \\
\hline $\mathrm{N} 2, \mathrm{~mol} \%$ & & & & & $100.00 \%$ & & & $79.00 \%$ \\
\hline \multicolumn{9}{|l|}{$\mathrm{H} 2, \mathrm{~mol} \%$} \\
\hline \multicolumn{9}{|l|}{$\mathrm{CO} 2, \mathrm{~mol} \%$} \\
\hline \multicolumn{9}{|l|}{$\mathrm{CO}, \mathrm{ppmv}$} \\
\hline \multicolumn{9}{|l|}{$\mathrm{NO}, \mathrm{ppmv}$} \\
\hline \multicolumn{9}{|l|}{ NO2, ppmv } \\
\hline \multicolumn{9}{|l|}{ SO2, ppmv } \\
\hline \multicolumn{9}{|l|}{$\mathrm{Cl}, \mathrm{ppmv}$} \\
\hline \multicolumn{9}{|l|}{$\mathrm{F}, \mathrm{ppmv}$} \\
\hline \multicolumn{9}{|l|}{ C (organic), ppmv } \\
\hline \multicolumn{9}{|l|}{$\mathrm{H}$ (organic), ppmv } \\
\hline \multicolumn{9}{|l|}{$\mathrm{Hg}, \mathrm{ug} / \mathrm{wscm}$} \\
\hline $\mathrm{PM}, \mathrm{mg} / \mathrm{wscm}$ & & & & & $1.99 \mathrm{E}+01$ & $3.46 \mathrm{E}-01$ & $2.76 \mathrm{E}-01$ & $1.60 \mathrm{E}+01$ \\
\hline \multicolumn{9}{|l|}{ SVM, ug/wscm } \\
\hline \multicolumn{9}{|l|}{ LVM, ug/wscm } \\
\hline $\begin{array}{l}\text { Gas Stream Bulk } \\
\text { Composition (Dry Basis) }\end{array}$ & $\mathrm{mol} \%$ & $\mathrm{~mol} \%$ & $\mathrm{~mol} \%$ & $\mathrm{~mol} \%$ & $\mathrm{~mol} \%$ & $\mathrm{~mol} \%$ & $\mathrm{~mol} \%$ & $\mathrm{~mol} \%$ \\
\hline $\mathrm{O} 2, \mathrm{~mol} \%$, dry basis & & & & & & & & $20.99 \%$ \\
\hline $\mathrm{N} 2, \mathrm{~mol} \%$, dry basis & & & & & & & & $79.01 \%$ \\
\hline \multicolumn{9}{|l|}{$\mathrm{H} 2, \mathrm{~mol} \%$, dry basis } \\
\hline \multicolumn{9}{|l|}{$\mathrm{CO} 2, \mathrm{~mol} \%$, dry basis } \\
\hline $\begin{array}{l}\text { Gas Stream Bulk } \\
\text { Composition (Dry Basis, } \\
\text { Corrected to } 7 \% \text { O2 with } \\
100 \% \text { O2 Combustion Air) }\end{array}$ & $\begin{array}{l}\mathrm{ppmv}, \text { or } \\
\mathrm{ug} / \mathrm{dscm} \\
\text { or } \\
\mathrm{mg} / \mathrm{dscm}\end{array}$ & $\begin{array}{l}\mathrm{ppmv}, \text { or } \\
\mathrm{ug} / \mathrm{dscm} \\
\text { or } \\
\mathrm{mg} / \mathrm{dscm}\end{array}$ & $\begin{array}{l}\mathrm{ppmv}, \text { or } \\
\text { ug/dscm } \\
\text { or } \\
\mathrm{mg} / \mathrm{dscm}\end{array}$ & $\begin{array}{l}\mathrm{ppmv}, \text { or } \\
\text { ug/dscm } \\
\text { or } \\
\mathrm{mg} / \mathrm{dscm}\end{array}$ & $\begin{array}{l}\mathrm{ppmv}, \text { or } \\
\mathrm{ug} / \mathrm{dscm} \\
\text { or } \\
\mathrm{mg} / \mathrm{dscm}\end{array}$ & $\begin{array}{l}\mathrm{ppmv}, \text { or } \\
\mathrm{ug} / \mathrm{dscm} \\
\text { or } \\
\mathrm{mg} / \mathrm{dscm}\end{array}$ & $\begin{array}{l}\mathrm{ppmv}, \text { or } \\
\mathrm{ug} / \mathrm{dscm} \\
\text { or } \\
\mathrm{mg} / \mathrm{dscm}\end{array}$ & $\begin{array}{l}\text { ppmv, or } \\
\text { ug/dscm } \\
\text { or } \\
\mathrm{mg} / \mathrm{dscm}\end{array}$ \\
\hline \multicolumn{9}{|l|}{$\mathrm{CO}, \mathrm{ppmv}$, dry basis } \\
\hline \multicolumn{9}{|l|}{$\mathrm{NO}$, ppmv, dry basis } \\
\hline \multicolumn{9}{|l|}{ NO2, ppmv, dry basis } \\
\hline \multicolumn{9}{|l|}{ SO2, ppmv, dry basis } \\
\hline \multicolumn{9}{|l|}{$\mathrm{Cl}, \mathrm{ppmv}$, dry basis } \\
\hline $\mathrm{F}, \mathrm{ppmv}$, dry basis & & & & & & & & \\
\hline C (organic), ppmv, dry basis & & & & & & & & \\
\hline $\mathrm{H}$ (organic), ppmv, dry basis & & & & & & & & \\
\hline $\mathrm{Hg}, \mathrm{ug} / \mathrm{dscm}$ & & & & & & & & \\
\hline $\mathrm{PM}, \mathrm{mg} / \mathrm{dscm}$ & & & & & & & & $2.24 \mathrm{E}+00$ \\
\hline SVM, ug/dscm & & & & & & & & \\
\hline LVM, ug/dscm & & & & & & & & \\
\hline
\end{tabular}


Table A-15. Mass balance, Tank WM-188 waste (continued).

\begin{tabular}{|c|c|c|c|c|c|c|c|c|}
\hline PFD \# & PFD-2 & PFD-3 & PFD-3 & PFD-3 & PFD-3 & PFD-3 & PFD-3 & PFD-3 \\
\hline WM-188, Stream \# & 507 & 510 & 511 & 512 & 513 & 514 & 515 & 516 \\
\hline Stream Name & $\begin{array}{c}\text { Off-Gas } \\
\text { from Boiler }\end{array}$ & $\begin{array}{c}\text { Nitrogen to } \\
\text { Cool } \\
\text { Product }\end{array}$ & $\begin{array}{l}\text { N2 from } \\
\text { Product } \\
\text { Cooler }\end{array}$ & $\begin{array}{c}\text { Filter } \\
\text { Backpulse } \\
\text { N2 }\end{array}$ & $\begin{array}{l}\text { Solids } \\
\text { Transport } \\
\text { Nitrogen }\end{array}$ & $\begin{array}{c}\text { Tank Farm } \\
\text { Transfer } \\
\text { Airlift Air }\end{array}$ & $\begin{array}{c}\text { Ventilation } \\
\text { Air }\end{array}$ & Fresh GAC \\
\hline Rate or Volume & $1.34 \mathrm{E}+05$ & $2.65 \mathrm{E}+02$ & $8.40 \mathrm{E}+02$ & $2.73 E+02$ & $6.78 \mathrm{E}+01$ & $1.44 \mathrm{E}+01$ & $2.89 \mathrm{E}+01$ & $5.53 \mathrm{E}+00$ \\
\hline Volume Flow (standard, wet) & $6.86 \mathrm{E}+04$ & $1.79 \mathrm{E}+03$ & $1.79 \mathrm{E}+03$ & $8.01 \mathrm{E}+02$ & $1.99 \mathrm{E}+02$ & $4.22 \mathrm{E}+01$ & $2.94 \mathrm{E}+01$ & \\
\hline Volume Flow (standard, dry)* & $6.07 \mathrm{E}+04$ & $1.79 \mathrm{E}+03$ & $1.79 \mathrm{E}+03$ & $8.01 \mathrm{E}+02$ & $1.99 \mathrm{E}+02$ & $4.22 \mathrm{E}+01$ & $2.94 \mathrm{E}+01$ & \\
\hline Rate Units & $\mathrm{ft} 3 / \mathrm{hr}$ & $\mathrm{ft} 3 / \mathrm{hr}$ & $\mathrm{ft} 3 / \mathrm{hr}$ & $\mathrm{ft} 3 / \mathrm{hr}$ & $\mathrm{ft} 3 / \mathrm{hr}$ & $\mathrm{ft} 3 / \mathrm{hr}$ & $\mathrm{ft} 3 / \mathrm{hr}$ & $\mathrm{lb} / \mathrm{hr}$ \\
\hline Rate or Volume, metric & $1.94 \mathrm{E}+03$ & $5.06 \mathrm{E}+01$ & $5.06 \mathrm{E}+01$ & $2.27 \mathrm{E}+01$ & $5.62 \mathrm{E}+00$ & $1.19 \mathrm{E}+00$ & $8.34 \mathrm{E}-01$ & $2.51 \mathrm{E}+00$ \\
\hline Rate Units & wscm $/ \mathrm{hr}$ & $\mathrm{wscm} / \mathrm{hr}$ & $\mathrm{wscm} / \mathrm{hr}$ & wscm/hr & $\mathrm{wscm} / \mathrm{hr}$ & wscm $/ \mathrm{hr}$ & wscm/hr & $\mathrm{kg} / \mathrm{hr}$ \\
\hline Temperature, ${ }^{\circ} \mathrm{C}$ & 300 & 0 & 591 & 15 & 15 & 15 & 15 & 25 \\
\hline Temperature, ${ }^{\circ} \mathrm{F}$ & 572 & 32 & 1096 & 59 & 59 & 59 & 59 & 77 \\
\hline Pressure, psia & 14.7 & 92.3 & 92.3 & 42.3 & 42.3 & 42.3 & 14.7 & 14.7 \\
\hline Specific Gravity & \begin{tabular}{|l|}
$6.10 \mathrm{E}-04$ \\
\end{tabular} & $7.85 \mathrm{E}-03$ & $2.48 \mathrm{E}-03$ & $3.41 \mathrm{E}-03$ & $3.41 \mathrm{E}-03$ & $3.51 \mathrm{E}-03$ & $1.22 \mathrm{E}-03$ & $4.81 \mathrm{E}-01$ \\
\hline \multicolumn{9}{|c|}{ Gas Stream Bulk Composition (Wet Basis) } \\
\hline & Mol \% & Mol \% & Mol \% & Mol \% & Mol \% & Mol \% & Mol \% & Wt \% \\
\hline $\mathrm{H} 2 \mathrm{O}$ & $11.53 \%$ & & & & & $0.01 \%$ & $0.01 \%$ & \\
\hline $\mathrm{O} 2$ & $3.00 \%$ & & & & & $20.99 \%$ & $20.99 \%$ & \\
\hline N2 & $74.11 \%$ & $100.00 \%$ & $100.00 \%$ & $100.00 \%$ & $100.00 \%$ & $79.00 \%$ & $79.00 \%$ & \\
\hline $\mathrm{H} 2$ & $0.05 \%$ & & & & & & & \\
\hline \multirow[t]{2}{*}{$\mathrm{CO} 2$} & $10.64 \%$ & & & & & & & \\
\hline & ppmv & & & & & & & \\
\hline $\mathrm{CO}$ & $2.21 \mathrm{E}+03$ & & & & & & & \\
\hline NO & $4.30 \mathrm{E}+03$ & & & & & & & \\
\hline $\mathrm{NO} 2$ & $1.97 \mathrm{E}+00$ & & & & & & & \\
\hline $\mathrm{SO} 2$ & $2.40 \mathrm{E}+02$ & & & & & & & \\
\hline C(organic) & $4.39 \mathrm{E}-14$ & & & & & & & \\
\hline $\mathrm{H}$ (organic) & $1.76 \mathrm{E}-13$ & & & & & & & \\
\hline \multicolumn{9}{|c|}{ Gas Stream Bulk Composition (Dry Basis) } \\
\hline & Mol \% & Mol \% & Mol \% & Mol \% & Mol \% & Mol \% & Mol \% & \\
\hline $\mathrm{O} 2$ & $3.39 \%$ & & & & & $20.99 \%$ & $20.99 \%$ & \\
\hline N2 & $83.77 \%$ & & & & & $79.01 \%$ & $79.01 \%$ & \\
\hline $\mathrm{H} 2$ & $0.05 \%$ & & & & & & & \\
\hline $\mathrm{CO} 2$ & $12.03 \%$ & & & & & & & \\
\hline \multicolumn{9}{|c|}{ Gas Stream Bulk Composition (Dry Basis, Corrected to $7 \%$ O2 with $100 \%$ O2 Combustion Air) } \\
\hline & ppmv & & & & & & & \\
\hline $\mathrm{CO}$ & $3.51 \mathrm{E}+02$ & & & & & & & \\
\hline NO & $6.80 \mathrm{E}+02$ & & & & & & & \\
\hline NO2 & $3.12 \mathrm{E}-01$ & & & & & & & \\
\hline \begin{tabular}{|l|}
$\mathrm{SO} 2$ \\
\end{tabular} & $3.80 \mathrm{E}+01$ & & & & & & & \\
\hline \begin{tabular}{|l} 
C(organic) \\
\end{tabular} & $6.96 \mathrm{E}-15$ & & & & & & & \\
\hline $\mathrm{H}$ (organic) & $2.78 \mathrm{E}-14$ & & & & & & & \\
\hline PM ma/dscm & $251 \mathrm{~F}+00$ & & & & & $2105+00$ & 2005000 & \\
\hline \multirow{2}{*}{\multicolumn{9}{|c|}{$\mathrm{Hg}, \mathrm{ug} / \mathrm{dscm}$}} \\
\hline & & & & & & & & \\
\hline & & & & & & & & \\
\hline GAC & & & & & & & & $100 \%$ \\
\hline
\end{tabular}


Table A-16. Mass balance, Tank WM-189 waste.

\begin{tabular}{|c|c|c|c|c|c|c|c|c|c|}
\hline DFD \# & PFD-2 & PFD-2 & PFD-2 & PFD-2 & PFD-2 & PFD-2 & PFD-2 & PFD-2 & PFD-2 \\
\hline WM-189, Stream \# & 101 & 102 & 103 & 104 & 105 & 106 & 107 & 108 & 108 \\
\hline Stream Name & SBW & $\begin{array}{c}\text { Reformer } \\
\text { Feed }\end{array}$ & $\begin{array}{c}\text { Reformer } \\
\text { Off-gas }\end{array}$ & $\begin{array}{l}\text { Reformer } \\
\text { Off-gas } \\
\text { Cooled }\end{array}$ & $\begin{array}{l}\text { Cyclone } \\
\text { Drain }\end{array}$ & $\begin{array}{l}\text { Filter } \\
\text { Drain }\end{array}$ & Bed Drain & $\begin{array}{l}\text { Cooled } \\
\text { Product }\end{array}$ & $\begin{array}{l}\text { Product } \\
\text { Shipping } \\
\text { Canisters }\end{array}$ \\
\hline Rate or Volume & $9.74 \mathrm{E}+01$ & $1.37 \mathrm{E}+02$ & $4.90 \mathrm{E}+05$ & $4.42 \mathrm{E}+05$ & $1.21 \mathrm{E}+02$ & $7.36 \mathrm{E}+01$ & $1.01 \mathrm{E}+02$ & $1.75 \mathrm{E}+02$ & $1.79 \mathrm{E}+00$ \\
\hline Volume Flow (standard, wet & & & $1.10 \mathrm{E}+05$ & $1.12 \mathrm{E}+05$ & & & & & \\
\hline Volume Flow (standard, dry & & & $3.39 \mathrm{E}+04$ & $3.60 \mathrm{E}+04$ & & & & & \\
\hline \begin{tabular}{|l|} 
Rate Units \\
\end{tabular} & $\mathrm{gal} / \mathrm{hr}$ & $\mathrm{gal} / \mathrm{hr}$ & $\mathrm{ft} 3 / \mathrm{hr}$ & $\mathrm{ft} 3 / \mathrm{hr}$ & $\mathrm{lb} / \mathrm{hr}$ & $\mathrm{Ib} / \mathrm{hr}$ & $\mathrm{lb} / \mathrm{hr}$ & $\mathrm{lb} / \mathrm{hr}$ & can's/day \\
\hline Rate or Volume, metric & 369 & 520 & $3.12 \mathrm{E}+03$ & $3.18 \mathrm{E}+03$ & 55 & 33.4 & 46.0 & 79.4 & 79.4 \\
\hline Rate Units & $\mathrm{L} / \mathrm{hr}$ & $\mathrm{L} / \mathrm{hr}$ & wscm/hr & wscm/hr & $\mathrm{kg} / \mathrm{hr}$ & $\mathrm{kg} / \mathrm{hr}$ & $\mathrm{kg} / \mathrm{hr}$ & $\mathrm{kg} / \mathrm{hr}$ & $\mathrm{kg} / \mathrm{hr}$ \\
\hline Temperature, ${ }^{\circ} \mathrm{C}$ & 15 & 19 & 670 & 530 & 670 & 530 & 670 & 57 & 57 \\
\hline Temperature, ${ }^{\circ} \mathrm{F}$ & 59 & 66 & 1238 & 986 & 1238 & 986 & 1238 & 134 & 134 \\
\hline Pressure, psia & 14.7 & 44.7 & 10.6 & 10.2 & 10.6 & 9.5 & 10.8 & 9.5 & 9.5 \\
\hline Specific Gravity & 1.28 & 1.28 & $2.00 \mathrm{E}-04$ & $2.28 \mathrm{E}-04$ & 0.24 & 1.15 & 1.46 & 1.33 & 1.33 \\
\hline Chemical Composition & Mol/liter & Mol/liter & Ib/wscf & $\mathrm{lb} / \mathrm{wscf}$ & Wt frac & Wt frac & Wt frac & Wt frac & Wt frac \\
\hline $\mathrm{H}+$ & $2.80 \mathrm{E}+00$ & $2.03 \mathrm{E}+00$ & $1.35 \mathrm{E}-06$ & $1.33 \mathrm{E}-06$ & $5.60 \mathrm{E}-06$ & $1.13 \mathrm{E}-05$ & $1.70 \mathrm{E}-05$ & $1.46 \mathrm{E}-05$ & $1.46 \mathrm{E}-05$ \\
\hline $\mathrm{Al}+3$ & $5.52 \mathrm{E}-01$ & $3.92 \mathrm{E}-01$ & $3.54 \mathrm{E}-05$ & $3.47 \mathrm{E}-05$ & $3.53 \mathrm{E}-02$ & $5.28 \mathrm{E}-02$ & $9.28 \mathrm{E}-02$ & $7.60 \mathrm{E}-02$ & $7.60 \mathrm{E}-02$ \\
\hline$S b+5$ & $1.64 \mathrm{E}-05$ & $1.17 \mathrm{E}-05$ & $4.32 \mathrm{E}-09$ & $4.24 \mathrm{E}-09$ & $4.31 \mathrm{E}-06$ & $6.45 \mathrm{E}-06$ & $1.13 \mathrm{E}-05$ & $9.28 \mathrm{E}-06$ & $9.28 \mathrm{E}-06$ \\
\hline $\mathrm{As}+3$ & $8.19 \mathrm{E}-05$ & $5.81 \mathrm{E}-05$ & $1.33 \mathrm{E}-08$ & $1.30 \mathrm{E}-08$ & $1.32 \mathrm{E}-05$ & $1.98 \mathrm{E}-05$ & $3.48 \mathrm{E}-05$ & $2.85 \mathrm{E}-05$ & $2.85 \mathrm{E}-05$ \\
\hline $\mathrm{Ba}+2$ & $6.23 \mathrm{E}-05$ & $4.42 \mathrm{E}-05$ & $1.93 \mathrm{E}-08$ & $1.89 \mathrm{E}-08$ & $1.50 \mathrm{E}-05$ & $2.87 \mathrm{E}-05$ & $4.76 \mathrm{E}-05$ & $3.97 \mathrm{E}-05$ & $3.97 \mathrm{E}-05$ \\
\hline $\mathrm{Be}+2$ & $2.02 \mathrm{E}-05$ & $1.44 \mathrm{E}-05$ & $4.11 \mathrm{E}-10$ & $4.03 \mathrm{E}-10$ & $3.20 \mathrm{E}-07$ & $6.13 \mathrm{E}-07$ & $1.02 \mathrm{E}-06$ & 8.47E-07 & $8.47 \mathrm{E}-07$ \\
\hline $\mathrm{B}+3$ & $1.66 \mathrm{E}-02$ & $1.18 \mathrm{E}-02$ & $3.88 \mathrm{E}-07$ & $3.81 \mathrm{E}-07$ & $3.88 \mathrm{E}-04$ & $5.79 \mathrm{E}-04$ & $1.02 \mathrm{E}-03$ & $8.33 \mathrm{E}-04$ & $8.33 \mathrm{E}-04$ \\
\hline $\mathrm{Cd}+2$ & $2.96 \mathrm{E}-03$ & $2.10 \mathrm{E}-03$ & $8.20 \mathrm{E}-07$ & $8.05 \mathrm{E}-07$ & $7.46 \mathrm{E}-04$ & $1.22 \mathrm{E}-03$ & $1.78 \mathrm{E}-03$ & \begin{tabular}{|l|}
$1.55 \mathrm{E}-03$ \\
\end{tabular} & $1.55 \mathrm{E}-03$ \\
\hline $\mathrm{Ca}+2$ & $5.57 \mathrm{E}-02$ & $3.95 \mathrm{E}-02$ & $1.35 \mathrm{E}-05$ & $1.33 \mathrm{E}-05$ & $1.05 \mathrm{E}-02$ & $2.02 \mathrm{E}-02$ & $3.35 \mathrm{E}-02$ & $2.79 \mathrm{E}-02$ & $2.79 \mathrm{E}-02$ \\
\hline $\mathrm{Cr}+3$ & $4.67 \mathrm{E}-03$ & $3.31 \mathrm{E}-03$ & $5.82 \mathrm{E}-07$ & $5.71 \mathrm{E}-07$ & $3.75 \mathrm{E}-04$ & $8.69 \mathrm{E}-04$ & $1.31 \mathrm{E}-03$ & $1.13 \mathrm{E}-03$ & $1.13 \mathrm{E}-03$ \\
\hline $\mathrm{Co}+2$ & $3.88 \mathrm{E}-05$ & $2.76 \mathrm{E}-05$ & $4.95 \mathrm{E}-09$ & $4.85 \mathrm{E}-09$ & $4.94 \mathrm{E}-06$ & $7.38 \mathrm{E}-06$ & $1.30 \mathrm{E}-05$ & $1.06 \mathrm{E}-05$ & $1.06 \mathrm{E}-05$ \\
\hline Cs+ & $5.54 \mathrm{E}-05$ & $4.17 \mathrm{E}-05$ & $5.37 \mathrm{E}-08$ & $5.27 \mathrm{E}-08$ & $7.03 \mathrm{E}-05$ & $8.02 \mathrm{E}-05$ & $4.39 \mathrm{E}-06$ & $4.06 \mathrm{E}-05$ & $4.06 \mathrm{E}-05$ \\
\hline $\mathrm{Cu}+2$ & $7.99 \mathrm{E}-04$ & $5.67 \mathrm{E}-04$ & $1.14 \mathrm{E}-07$ & $\begin{array}{l}1.12 \mathrm{E}-07 \\
\end{array}$ & $8.91 \mathrm{E}-05$ & $1.71 \mathrm{E}-04$ & $2.83 \mathrm{E}-04$ & $2.36 \mathrm{E}-04$ & $2.36 \mathrm{E}-04$ \\
\hline $\mathrm{Fe}+3$ & $2.70 \mathrm{E}-02$ & $1.91 \mathrm{E}-02$ & $2.36 \mathrm{E}-06$ & $2.32 \mathrm{E}-06$ & $1.65 \mathrm{E}-03$ & $3.53 \mathrm{E}-03$ & $1.09 \mathrm{E}-02$ & $7.81 \mathrm{E}-03$ & $7.81 \mathrm{E}-03$ \\
\hline $\mathrm{Pb}+2$ & $9.05 \mathrm{E}-04$ & $6.42 \mathrm{E}-04$ & $1.32 \mathrm{E}-06$ & \begin{tabular}{|l|}
$1.30 \mathrm{E}-06$ \\
\end{tabular} & $3.65 \mathrm{E}-04$ & $1.98 \mathrm{E}-03$ & $6.63 \mathrm{E}-05$ & $8.69 \mathrm{E}-04$ & $8.69 \mathrm{E}-04$ \\
\hline $\mathrm{Ha}+2$ & $4.94 \mathrm{E}-03$ & $4.14 \mathrm{E}-03$ & $8.65 \mathrm{E}-06$ & $8.49 \mathrm{E}-06$ & $6.37 \mathrm{E}-06$ & $1.54 \mathrm{E}-05$ & & $6.47 \mathrm{E}-06$ & $6.47 \mathrm{E}-06$ \\
\hline$M n+4$ & $1.50 \mathrm{E}-02$ & $1.06 \mathrm{E}-02$ & $1.78 \mathrm{E}-06$ & $\begin{array}{l}1.74 \mathrm{E}-06 \\
\end{array}$ & $1.77 \mathrm{E}-03$ & $2.65 \mathrm{E}-03$ & $4.66 \mathrm{E}-03$ & $3.81 \mathrm{E}-03$ & $3.81 \mathrm{E}-03$ \\
\hline $\mathrm{Ni}+2$ & $1.89 \mathrm{E}-03$ & $1.34 \mathrm{E}-03$ & $2.50 \mathrm{E}-07$ & \begin{tabular}{|l|}
$2.46 \mathrm{E}-07$ \\
\end{tabular} & $1.95 \mathrm{E}-04$ & $3.74 \mathrm{E}-04$ & $6.19 \mathrm{E}-04$ & \begin{tabular}{|l|}
$5.16 \mathrm{E}-04$ \\
\end{tabular} & $5.16 \mathrm{E}-04$ \\
\hline $\mathrm{K}+$ & $1.84 \mathrm{E}-01$ & $1.30 \mathrm{E}-01$ & $3.32 \mathrm{E}-05$ & $3.25 \mathrm{E}-05$ & $3.66 \mathrm{E}-02$ & $4.95 \mathrm{E}-02$ & $3.32 \mathrm{E}-02$ & \begin{tabular}{|l|}
$4.01 \mathrm{E}-02$ \\
\end{tabular} & $4.01 \mathrm{E}-02$ \\
\hline $\mathrm{Se}+4$ & $2.35 \mathrm{E}-04$ & $1.67 \mathrm{E}-04$ & $3.90 \mathrm{E}-08$ & \begin{tabular}{|l|}
$3.83 \mathrm{E}-08$ \\
\end{tabular} & $3.80 \mathrm{E}-05$ & $5.82 \mathrm{E}-05$ & $1.06 \mathrm{E}-04$ & \begin{tabular}{|l|}
$8.61 \mathrm{E}-05$ \\
\end{tabular} & $8.61 \mathrm{E}-05$ \\
\hline $\mathrm{Ag}+$ & $3.68 \mathrm{E}-04$ & $2.61 \mathrm{E}-04$ & $9.99 \mathrm{E}-08$ & $9.80 \mathrm{E}-08$ & $7.28 \mathrm{E}-05$ & $1.49 \mathrm{E}-04$ & $2.10 \mathrm{E}-04$ & \begin{tabular}{|c|}
$1.84 \mathrm{E}-04$ \\
\end{tabular} & $1.84 \mathrm{E}-04$ \\
\hline $\mathrm{Na}+$ & $1.55 \mathrm{E}+00$ & $1.10 \mathrm{E}+00$ & $8.99 \mathrm{E}-05$ & \begin{tabular}{|l|}
$8.82 \mathrm{E}-05$ \\
\end{tabular} & $6.56 \mathrm{E}-02$ & $1.34 \mathrm{E}-01$ & $1.89 \mathrm{E}-01$ & \begin{tabular}{|l|}
$1.66 \mathrm{E}-01$ \\
\end{tabular} & $1.66 \mathrm{E}-01$ \\
\hline $\mathrm{TI}+3$ & $4.32 \mathrm{E}-06$ & $3.07 \mathrm{E}-06$ & $1.91 \mathrm{E}-09$ & $1.87 \mathrm{E}-09$ & $1.91 \mathrm{E}-06$ & $2.85 \mathrm{E}-06$ & $5.01 \mathrm{E}-06$ & \begin{tabular}{|l|}
$4.10 \mathrm{E}-06$ \\
\end{tabular} & $4.10 \mathrm{E}-06$ \\
\hline $\mathrm{U}+4$ & $5.11 \mathrm{E}-04$ & $3.63 \mathrm{E}-04$ & $2.56 \mathrm{E}-07$ & $2.51 \mathrm{E}-07$ & $2.49 \mathrm{E}-04$ & $3.82 \mathrm{E}-04$ & $6.97 \mathrm{E}-04$ & $5.65 \mathrm{E}-04$ & $5.65 \mathrm{E}-04$ \\
\hline $\mathrm{V}+3$ & $2.87 \mathrm{E}-05$ & $2.04 \mathrm{E}-05$ & $3.08 \mathrm{E}-09$ & $3.02 \mathrm{E}-09$ & $3.00 \mathrm{E}-06$ & $4.59 \mathrm{E}-06$ & $8.38 \mathrm{E}-06$ & \begin{tabular}{|l|}
$6.79 \mathrm{E}-06$ \\
\end{tabular} & $6.79 \mathrm{E}-06$ \\
\hline $\mathrm{Zn+2}$ & $8.56 \mathrm{E}-04$ & $6.08 \mathrm{E}-04$ & $1.26 \mathrm{E}-07$ & $1.24 \mathrm{E}-07$ & $9.82 \mathrm{E}-05$ & $1.88 \mathrm{E}-04$ & $3.12 \mathrm{E}-04$ & $2.60 \mathrm{E}-04$ & $2.60 \mathrm{E}-04$ \\
\hline $\mathrm{Zr}+4$ & $3.53 \mathrm{E}-02$ & $2.50 \mathrm{E}-02$ & $6.77 \mathrm{E}-06$ & \begin{tabular}{|l|}
$6.64 \mathrm{E}-06$ \\
\end{tabular} & $6.60 \mathrm{E}-03$ & $1.01 \mathrm{E}-02$ & $1.85 \mathrm{E}-02$ & $1.49 \mathrm{E}-02$ & $1.49 \mathrm{E}-02$ \\
\hline $\mathrm{Cl}-$ & $1.86 \mathrm{E}-02$ & $1.33 \mathrm{E}-02$ & $3.63 \mathrm{E}-06$ & \begin{tabular}{|c|}
$3.56 \mathrm{E}-06$ \\
\end{tabular} & $2.99 \mathrm{E}-03$ & $5.26 \mathrm{E}-03$ & $1.39 \mathrm{E}-03$ & \begin{tabular}{|l|}
$3.02 \mathrm{E}-03$ \\
\end{tabular} & $3.02 \mathrm{E}-03$ \\
\hline F- & $3.02 \mathrm{E}-02$ & $2.18 \mathrm{E}-02$ & $3.86 \mathrm{E}-06$ & \begin{tabular}{|l|}
$3.79 \mathrm{E}-06$ \\
\end{tabular} & $1.92 \mathrm{E}-03$ & $5.19 \mathrm{E}-03$ & $4.79 \mathrm{E}-04$ & \begin{tabular}{|l|}
$2.46 \mathrm{E}-03$ \\
\end{tabular} & $2.46 \mathrm{E}-03$ \\
\hline SO4-2 & $8.25 \mathrm{E}-02$ & $5.93 \mathrm{E}-02$ & $2.40 \mathrm{E}-08$ & \begin{tabular}{|l|}
$2.36 \mathrm{E}-08$ \\
\end{tabular} & $1.10 \mathrm{E}-05$ & $3.59 \mathrm{E}-05$ & $2.70 \mathrm{E}-05$ & \begin{tabular}{|l|}
$3.07 \mathrm{E}-05$ \\
\end{tabular} & 3.07E-05 \\
\hline NO3- & $5.96 \mathrm{E}+00$ & $4.27 \mathrm{E}+00$ & $9.43 \mathrm{E}-06$ & \begin{tabular}{|l|}
$9.25 \mathrm{E}-06$ \\
\end{tabular} & $2.13 \mathrm{E}-03$ & $4.77 \mathrm{E}-03$ & $5.33 \mathrm{E}-03$ & \begin{tabular}{|l|}
$5.10 \mathrm{E}-03$ \\
\end{tabular} & $5.10 \mathrm{E}-03$ \\
\hline PO4-3 & $9.76 \mathrm{E}-02$ & $6.93 \mathrm{E}-02$ & $2.53 \mathrm{E}-05$ & $2.48 \mathrm{E}-05$ & 4.17E-02 & $3.77 \mathrm{E}-02$ & $5.06 \mathrm{E}-02$ & $4.52 \mathrm{E}-02$ & $4.52 \mathrm{E}-02$ \\
\hline $\mathrm{Am}+4$ & $5.93 \mathrm{E}-08$ & $4.21 \mathrm{E}-08$ & $3.11 \mathrm{E}-11$ & $3.05 E-11$ & $3.11 \mathrm{E}-08$ & $4.65 \mathrm{E}-08$ & $8.17 \mathrm{E}-08$ & \begin{tabular}{|l|}
$6.69 \mathrm{E}-08$ \\
\end{tabular} & $6.69 \mathrm{E}-08$ \\
\hline $\mathrm{Br}-$ & $2.46 \mathrm{E}-07$ & $1.76 \mathrm{E}-07$ & $1.08 \mathrm{E}-10$ & $1.06 \mathrm{E}-10$ & $8.94 \mathrm{E}-08$ & $1.57 \mathrm{E}-07$ & $4.15 \mathrm{E}-08$ & \begin{tabular}{|l|}
$9.01 \mathrm{E}-08$ \\
\end{tabular} & $9.01 \mathrm{E}-08$ \\
\hline $\mathrm{Ce}+4$ & $3.60 \mathrm{E}-05$ & $2.55 \mathrm{E}-05$ & \begin{tabular}{|l|}
$1.09 \mathrm{E}-08$ \\
\end{tabular} & $1.07 \mathrm{E}-08$ & $1.09 \mathrm{E}-05$ & $1.62 \mathrm{E}-05$ & $2.85 \mathrm{E}-05$ & \begin{tabular}{|l|}
$2.34 \mathrm{E}-05$ \\
\end{tabular} & $2.34 \mathrm{E}-05$ \\
\hline $\mathrm{Eu}+3$ & $4.09 \mathrm{E}-07$ & $2.90 \mathrm{E}-07$ & \begin{tabular}{|c|}
$1.34 \mathrm{E}-10$ \\
\end{tabular} & $1.32 \mathrm{E}-10$ & $1.34 \mathrm{E}-07$ & $2.01 \mathrm{E}-07$ & $3.52 \mathrm{E}-07$ & $2.89 \mathrm{E}-07$ & $2.89 \mathrm{E}-07$ \\
\hline $\mathrm{Gd}+3$ & $1.23 \mathrm{E}-04$ & $8.72 \mathrm{E}-05$ & \begin{tabular}{|l|}
$4.17 \mathrm{E}-08$ \\
\end{tabular} & \begin{tabular}{|l|}
$4.09 \mathrm{E}-08$ \\
\end{tabular} & 4.17E-05 & $6.23 \mathrm{E}-05$ & $1.09 \mathrm{E}-04$ & $8.96 \mathrm{E}-05$ & $8.96 \mathrm{E}-05$ \\
\hline $\mathrm{Ge}+4$ & $7.11 \mathrm{E}-09$ & $5.04 \mathrm{E}-09$ & $1.08 \mathrm{E}-12$ & $1.06 \mathrm{E}-12$ & $1.06 \mathrm{E}-09$ & $1.62 \mathrm{E}-09$ & $2.96 \mathrm{E}-09$ & $2.39 \mathrm{E}-09$ & $2.39 \mathrm{E}-09$ \\
\hline $\ln +3$ & $1.14 \mathrm{E}-06$ & $8.09 \mathrm{E}-07$ & $2.83 \mathrm{E}-10$ & $2.77 \mathrm{E}-10$ & $2.82 \mathrm{E}-07$ & $4.22 \mathrm{E}-07$ & $7.42 \mathrm{E}-07$ & $6.07 \mathrm{E}-07$ & $6.07 \mathrm{E}-07$ \\
\hline I- & $2.74 \mathrm{E}-06$ & $1.96 \mathrm{E}-06$ & \begin{tabular}{|c|}
$1.91 \mathrm{E}-09$ \\
\end{tabular} & \begin{tabular}{|c|}
$1.88 \mathrm{E}-09$ \\
\end{tabular} & $1.58 \mathrm{E}-06$ & $2.77 \mathrm{E}-06$ & $7.33 \mathrm{E}-07$ & \begin{tabular}{|l|}
$1.59 \mathrm{E}-06$ \\
\end{tabular} & $1.59 \mathrm{E}-06$ \\
\hline $\mathrm{La}+3$ & $7.42 \mathrm{E}-06$ & \begin{tabular}{|l|}
$5.27 \mathrm{E}-06$ \\
\end{tabular} & \begin{tabular}{|l|}
$2.23 \mathrm{E}-09$ \\
\end{tabular} & \begin{tabular}{|l|}
$2.19 \mathrm{E}-09$ \\
\end{tabular} & \begin{tabular}{|l|}
$2.23 \mathrm{E}-06$ \\
\end{tabular} & $3.33 \mathrm{E}-06$ & \begin{tabular}{|l|}
$5.84 \mathrm{E}-06$ \\
\end{tabular} & \begin{tabular}{|l|}
$4.79 \mathrm{E}-06$ \\
\end{tabular} & $4.79 \mathrm{E}-06$ \\
\hline
\end{tabular}


Table A-16. Mass balance, Tank WM-189 waste (continued).

\begin{tabular}{|c|c|c|c|c|c|c|c|c|c|}
\hline Stream \# & 101 & 102 & 103 & 104 & 105 & 106 & 107 & 108 & 108 \\
\hline Chemical Composition & Mol/liter & Mol/liter & $\mathrm{lb} /$ wscf & $\mathrm{lb} /$ wscf & Wt frac & Wt frac & Wt frac & Wt frac & Wt frac \\
\hline $\mathrm{Li+}$ & $4.92 \mathrm{E}-04$ & $3.49 \mathrm{E}-04$ & $8.60 \mathrm{E}-09$ & $8.44 \mathrm{E}-09$ & $6.27 \mathrm{E}-06$ & $1.28 \mathrm{E}-05$ & $1.81 \mathrm{E}-05$ & $1.59 \mathrm{E}-05$ & $1.59 \mathrm{E}-05$ \\
\hline $\mathrm{Mg}+2$ & $1.76 \mathrm{E}-02$ & $1.25 \mathrm{E}-02$ & $9.63 \mathrm{E}-07$ & $9.45 \mathrm{E}-07$ & $7.50 \mathrm{E}-04$ & $1.44 \mathrm{E}-03$ & $2.38 \mathrm{E}-03$ & $1.98 \mathrm{E}-03$ & $1.98 \mathrm{E}-03$ \\
\hline $\mathrm{Mo}+6$ & $5.31 \mathrm{E}-02$ & $3.77 \mathrm{E}-02$ & $1.07 \mathrm{E}-05$ & $1.05 \mathrm{E}-05$ & $1.04 \mathrm{E}-02$ & $1.60 \mathrm{E}-02$ & $2.92 \mathrm{E}-02$ & $2.36 \mathrm{E}-02$ & $2.36 \mathrm{E}-02$ \\
\hline $\mathrm{Nd}+3$ & $2.39 \mathrm{E}-05$ & $1.70 \mathrm{E}-05$ & $7.46 \mathrm{E}-09$ & $7.32 \mathrm{E}-09$ & $7.45 \mathrm{E}-06$ & $1.11 \mathrm{E}-05$ & $1.96 \mathrm{E}-05$ & $1.60 \mathrm{E}-05$ & $1.60 \mathrm{E}-05$ \\
\hline $\mathrm{Np}+4$ & $2.49 \mathrm{E}-06$ & $1.76 \mathrm{E}-06$ & 1.27E-09 & $1.25 \mathrm{E}-09$ & $1.27 \mathrm{E}-06$ & $1.90 \mathrm{E}-06$ & 3.34E-06 & $2.73 \mathrm{E}-06$ & $2.73 \mathrm{E}-06$ \\
\hline $\mathrm{Nb}+5$ & $1.04 \mathrm{E}-03$ & $7.36 \mathrm{E}-04$ & $2.02 \mathrm{E}-07$ & $1.99 \mathrm{E}-07$ & 1.97E-04 & $3.02 E-04$ & 5.52E-04 & $4.47 \mathrm{E}-04$ & $4.47 \mathrm{E}-04$ \\
\hline $\mathrm{Pd}+4$ & $1.53 \mathrm{E}-03$ & $1.09 \mathrm{E}-03$ & $3.68 \mathrm{E}-07$ & $3.61 \mathrm{E}-07$ & $2.87 \mathrm{E}-04$ & $5.49 \mathrm{E}-04$ & $9.09 \mathrm{E}-04$ & $7.58 \mathrm{E}-04$ & $7.58 \mathrm{E}-04$ \\
\hline $\mathrm{Pu}+4$ & $6.03 E-06$ & $4.28 \mathrm{E}-06$ & $3.09 \mathrm{E}-09$ & $3.03 E-09$ & $3.02 E-06$ & $4.62 \mathrm{E}-06$ & $8.43 \mathrm{E}-06$ & $6.83 E-06$ & $6.83 \mathrm{E}-06$ \\
\hline $\mathrm{Pr}+4$ & $6.75 \mathrm{E}-06$ & $4.79 \mathrm{E}-06$ & $2.06 \mathrm{E}-09$ & $2.02 \mathrm{E}-09$ & $2.05 \mathrm{E}-06$ & $3.07 \mathrm{E}-06$ & $5.39 \mathrm{E}-06$ & $4.41 \mathrm{E}-06$ & $4.41 \mathrm{E}-06$ \\
\hline $\mathrm{Pm}+3$ & 1.36E-09 & $9.62 \mathrm{E}-10$ & $4.25 \mathrm{E}-13$ & $4.17 \mathrm{E}-13$ & $4.24 \mathrm{E}-10$ & $6.34 \mathrm{E}-10$ & $1.11 \mathrm{E}-09$ & $9.12 \mathrm{E}-10$ & $9.12 \mathrm{E}-10$ \\
\hline $\mathrm{Rh}+4$ & 2.91E-06 & $2.07 E-06$ & $6.47 \mathrm{E}-10$ & $6.35 \mathrm{E}-10$ & $6.47 \mathrm{E}-07$ & 9.66E-07 & $1.70 \mathrm{E}-06$ & 1.39E-06 & 1.39E-06 \\
\hline $\mathrm{Rb}+$ & 4.48E-06 & $3.18 \mathrm{E}-06$ & $9.65 \mathrm{E}-10$ & $9.47 \mathrm{E}-10$ & 7.04E-07 & 1.44E-06 & $2.03 \mathrm{E}-06$ & $1.78 \mathrm{E}-06$ & 1.78E-06 \\
\hline$R u+3$ & 8.04E-04 & $5.71 \mathrm{E}-04$ & $1.76 \mathrm{E}-07$ & $1.72 \mathrm{E}-07$ & $1.75 \mathrm{E}-04$ & $2.62 \mathrm{E}-04$ & $4.60 \mathrm{E}-04$ & $3.77 \mathrm{E}-04$ & $3.77 \mathrm{E}-04$ \\
\hline $\mathrm{Sm}+3$ & $4.46 \mathrm{E}-06$ & $3.17 \mathrm{E}-06$ & $1.45 \mathrm{E}-09$ & $1.42 \mathrm{E}-09$ & $1.45 \mathrm{E}-06$ & $2.16 \mathrm{E}-06$ & $3.80 \mathrm{E}-06$ & $3.11 \mathrm{E}-06$ & $3.11 \mathrm{E}-06$ \\
\hline $\mathrm{Si}+4$ & 8.94E-02 & $6.35 \mathrm{E}-02$ & $7.24 \mathrm{E}-06$ & $7.10 \mathrm{E}-06$ & $1.05 \mathrm{E}-02$ & $1.08 \mathrm{E}-02$ & $2.25 \mathrm{E}-02$ & $1.76 \mathrm{E}-02$ & $1.76 \mathrm{E}-02$ \\
\hline $\mathrm{Sr}+2$ & $1.08 \mathrm{E}-04$ & 7.67E-05 & $2.13 \mathrm{E}-08$ & $2.09 \mathrm{E}-08$ & $1.66 \mathrm{E}-05$ & $3.18 \mathrm{E}-05$ & $5.27 \mathrm{E}-05$ & 4.39E-05 & $4.39 \mathrm{E}-05$ \\
\hline Tc+7 & $5.33 \mathrm{E}-06$ & $3.78 \mathrm{E}-06$ & $3.48 \mathrm{E}-09$ & $3.41 \mathrm{E}-09$ & $5.28 \mathrm{E}-06$ & $5.19 \mathrm{E}-06$ & $4.09 \mathrm{E}-07$ & $2.42 \mathrm{E}-06$ & $2.42 \mathrm{E}-06$ \\
\hline $\mathrm{Te}+4$ & $5.22 \mathrm{E}-06$ & $3.70 \mathrm{E}-06$ & $1.40 \mathrm{E}-09$ & 1.37E-09 & $1.36 \mathrm{E}-06$ & $2.09 \mathrm{E}-06$ & $3.81 \mathrm{E}-06$ & $3.09 \mathrm{E}-06$ & $3.09 \mathrm{E}-06$ \\
\hline $\mathrm{Tb}+4$ & 1.71E-09 & $1.21 \mathrm{E}-09$ & $5.87 \mathrm{E}-13$ & $5.76 \mathrm{E}-13$ & $5.86 \mathrm{E}-10$ & $8.76 \mathrm{E}-10$ & 1.54E-09 & 1.26E-09 & 1.26E-09 \\
\hline $\mathrm{Th}+4$ & $2.59 \mathrm{E}-05$ & $1.84 \mathrm{E}-05$ & $1.26 \mathrm{E}-08$ & $1.24 \mathrm{E}-08$ & 1.23E-05 & $1.88 \mathrm{E}-05$ & $3.44 \mathrm{E}-05$ & $2.79 \mathrm{E}-05$ & $2.79 \mathrm{E}-05$ \\
\hline$S n+4$ & $1.75 \mathrm{E}-03$ & $1.24 \mathrm{E}-03$ & $4.36 \mathrm{E}-07$ & $4.28 \mathrm{E}-07$ & $4.25 \mathrm{E}-04$ & $6.50 \mathrm{E}-04$ & $1.19 \mathrm{E}-03$ & $9.62 \mathrm{E}-04$ & $9.62 \mathrm{E}-04$ \\
\hline $\mathrm{Ti}+4$ & 9.02E-04 & $6.40 \mathrm{E}-04$ & $9.08 \mathrm{E}-08$ & $8.91 \mathrm{E}-08$ & $8.85 \mathrm{E}-05$ & 1.35E-04 & $2.47 \mathrm{E}-04$ & $2.00 \mathrm{E}-04$ & $2.00 \mathrm{E}-04$ \\
\hline$Y+3$ & $5.53 \mathrm{E}-06$ & $3.93 \mathrm{E}-06$ & $1.06 \mathrm{E}-09$ & $1.04 \mathrm{E}-09$ & $1.06 \mathrm{E}-06$ & $1.59 \mathrm{E}-06$ & $2.79 \mathrm{E}-06$ & $2.28 \mathrm{E}-06$ & $2.28 \mathrm{E}-06$ \\
\hline $\mathrm{OH}-$ & & 8.50E-04 & 2.47E-06 & $2.42 \mathrm{E}-06$ & 1.83E-03 & $3.69 \mathrm{E}-03$ & 5.52E-03 & $4.75 \mathrm{E}-03$ & $4.75 \mathrm{E}-03$ \\
\hline $\mathrm{H} 2 \mathrm{O}$ & $4.43 E+01$ & $3.37 E+01$ & $3.24 \mathrm{E}-02$ & $3.18 \mathrm{E}-02$ & $1.66 \mathrm{E}-03$ & $3.12 \mathrm{E}-03$ & 4.61E-03 & 3.99E-03 & 3.99E-03 \\
\hline $\mathrm{SO} 2$ & & $7.08 \mathrm{E}-07$ & 4.74E-09 & $4.65 \mathrm{E}-09$ & & & & & \\
\hline $\mathrm{H} 2 \mathrm{~S}$ & & & $2.09 \mathrm{E}-05$ & $2.05 \mathrm{E}-05$ & & & & & \\
\hline $\mathrm{CO}$ & & 8.99E-12 & $8.65 \mathrm{E}-04$ & $8.49 \mathrm{E}-04$ & & & & & \\
\hline $\mathrm{CO} 2$ & & $1.43 \mathrm{E}-05$ & $8.25 \mathrm{E}-03$ & $8.09 \mathrm{E}-03$ & & & & & \\
\hline $\mathrm{H} 2$ & & $3.77 \mathrm{E}-11$ & $2.09 \mathrm{E}-04$ & $2.05 \mathrm{E}-04$ & & & & & \\
\hline N2 & & 5.49E-07 & $1.27 \mathrm{E}-02$ & $1.38 \mathrm{E}-02$ & & & & & \\
\hline $\mathrm{NO}$ & & 9.53E-09 & $4.53 \mathrm{E}-05$ & 4.44E-05 & & & & & \\
\hline NO2 & & $1.20 \mathrm{E}-07$ & $4.70 \mathrm{E}-06$ & $4.61 \mathrm{E}-06$ & & & & & \\
\hline $\mathrm{O} 2$ & & $1.36 \mathrm{E}-07$ & $4.83 \mathrm{E}-04$ & $4.73 \mathrm{E}-04$ & & & & & \\
\hline S (other) & & $9.40 \mathrm{E}-09$ & $2.94 \mathrm{E}-07$ & $2.88 \mathrm{E}-07$ & $2.17 \mathrm{E}-04$ & $4.38 \mathrm{E}-04$ & $6.58 \mathrm{E}-04$ & $5.66 \mathrm{E}-04$ & $5.66 \mathrm{E}-04$ \\
\hline $\mathrm{CO} 3$ & 2.77E-05 & $2.08 \mathrm{E}-05$ & $1.47 \mathrm{E}-04$ & $1.44 \mathrm{E}-04$ & $1.18 \mathrm{E}-01$ & $2.19 \mathrm{E}-01$ & 3.04E-01 & $2.68 \mathrm{E}-01$ & $2.68 \mathrm{E}-01$ \\
\hline C (reductant) & & & $2.45 \mathrm{E}-05$ & $2.40 \mathrm{E}-05$ & $4.22 \mathrm{E}-01$ & $3.65 \mathrm{E}-02$ & & $1.54 \mathrm{E}-02$ & $1.54 \mathrm{E}-02$ \\
\hline $\mathrm{O}$ (oxides) & $4.70 \mathrm{E}-01$ & 3.33E-01 & $4.75 \mathrm{E}-05$ & $4.66 \mathrm{E}-05$ & $4.60 \mathrm{E}-02$ & 7.09E-02 & 1.35E-01 & $1.08 \mathrm{E}-01$ & $1.08 \mathrm{E}-01$ \\
\hline C (organic) & $4.39 \mathrm{E}-02$ & $1.16 \mathrm{E}+01$ & $1.03 \mathrm{E}-04$ & $1.01 \mathrm{E}-04$ & $7.08 \mathrm{E}-02$ & $1.23 \mathrm{E}-01$ & 8.93E-03 & $5.70 \mathrm{E}-02$ & $5.70 \mathrm{E}-02$ \\
\hline $\mathrm{H}$ (organic) & $8.05 \mathrm{E}-02$ & $2.12 \mathrm{E}+01$ & $1.95 \mathrm{E}-05$ & $1.91 \mathrm{E}-05$ & $1.09 \mathrm{E}-02$ & $1.90 \mathrm{E}-02$ & $1.37 \mathrm{E}-03$ & $8.77 \mathrm{E}-03$ & $8.77 \mathrm{E}-03$ \\
\hline O (organic) & $4.02 \mathrm{E}-02$ & $1.06 \mathrm{E}+01$ & $1.01 \mathrm{E}-04$ & $9.89 \mathrm{E}-05$ & $8.65 \mathrm{E}-02$ & $1.51 \mathrm{E}-01$ & $1.09 \mathrm{E}-02$ & 6.96E-02 & 6.96E-02 \\
\hline Mass Flow $(\mathrm{kg} / \mathrm{hr})$ : & $4.73 E+02$ & $6.67 E+02$ & $2.78 \mathrm{E}+03$ & $2.85 E+03$ & $5.49 \mathrm{E}+01$ & $3.34 \mathrm{E}+01$ & $4.60 \mathrm{E}+01$ & $7.94 \mathrm{E}+01$ & $7.94 \mathrm{E}+01$ \\
\hline Total Canisters Generated & & & & & & & & 242 & \\
\hline & & & & & & & & & \\
\hline & $w / m 3$ & $w / m 3$ & $w / m 3$ & w/m3 & $w / m 3$ & $\mathrm{w} / \mathrm{m} 3$ & $\mathrm{w} / \mathrm{m} 3$ & $\mathrm{w} / \mathrm{m} 3$ & w/Canistel \\
\hline Heat Generation, w/m3 & $4.64 \mathrm{E}-01$ & $3.31 \mathrm{E}-01$ & & & $3.04 \mathrm{E}-01$ & $2.18 \mathrm{E}+00$ & $3.46 \mathrm{E}+00$ & $2.92 \mathrm{E}+00$ & $2.34 \mathrm{E}+00$ \\
\hline
\end{tabular}


Table A-16. Mass balance, Tank WM-189 waste (continued).

\begin{tabular}{|c|c|c|c|c|c|c|c|c|c|}
\hline Stream \# & 101 & 102 & 103 & 104 & 105 & 106 & 107 & 108 & 108 \\
\hline Radiological Composition & $\mathrm{Ci} / \mathrm{L}$ & $\mathrm{Ci} / \mathrm{L}$ & Ci/wscm & $\mathrm{Ci} / \mathrm{wscm}$ & $\mathrm{Ci} / \mathrm{kg}$ & Ci $/ \mathrm{kg}$ & Ci $/ \mathrm{kg}$ & Ci $/ \mathrm{kg}$ & Ci/canister \\
\hline $\mathrm{H}-3$ & $8.12 \mathrm{E}-06$ & $5.76 \mathrm{E}-06$ & $9.60 \mathrm{E}-07$ & $9.42 \mathrm{E}-07$ & & & & & \\
\hline C-14 & $1.31 \mathrm{E}-10$ & $9.27 \mathrm{E}-11$ & $1.51 \mathrm{E}-11$ & $1.48 \mathrm{E}-11$ & $1.75 \mathrm{E}-10$ & $6.91 \mathrm{E}-11$ & $2.37 \mathrm{E}-11$ & $4.28 \mathrm{E}-11$ & $4.55 \mathrm{E}-08$ \\
\hline Co-60 & $2.65 \mathrm{E}-05$ & $1.88 \mathrm{E}-05$ & $9.18 \mathrm{E}-07$ & $9.00 \mathrm{E}-07$ & $5.72 \mathrm{E}-05$ & $8.55 \mathrm{E}-05$ & $1.50 \mathrm{E}-04$ & $1.23 \mathrm{E}-04$ & $1.31 \mathrm{E}-01$ \\
\hline Ni-59 & $1.11 \mathrm{E}-06$ & $7.90 \mathrm{E}-07$ & $4.02 \mathrm{E}-08$ & $3.94 \mathrm{E}-08$ & $1.95 \mathrm{E}-06$ & $3.74 \mathrm{E}-06$ & $6.20 \mathrm{E}-06$ & $5.16 \mathrm{E}-06$ & $5.50 \mathrm{E}-03$ \\
\hline $\mathrm{Ni}-63$ & $3.47 \mathrm{E}-05$ & $2.46 \mathrm{E}-05$ & $1.25 \mathrm{E}-06$ & $1.23 \mathrm{E}-06$ & $6.08 \mathrm{E}-05$ & 1.17E-04 & $1.93 \mathrm{E}-04$ & $1.61 \mathrm{E}-04$ & $1.71 \mathrm{E}-01$ \\
\hline Se-79 & $4.74 \mathrm{E}-07$ & $3.37 \mathrm{E}-07$ & $1.60 \mathrm{E}-08$ & $1.57 \mathrm{E}-08$ & $9.72 \mathrm{E}-07$ & $1.49 \mathrm{E}-06$ & $2.72 \mathrm{E}-06$ & $2.20 \mathrm{E}-06$ & $2.34 \mathrm{E}-03$ \\
\hline Sr-90 & $3.15 \mathrm{E}-02$ & $2.23 \mathrm{E}-02$ & $1.13 \mathrm{E}-03$ & $1.11 \mathrm{E}-03$ & $5.52 \mathrm{E}-02$ & $1.06 \mathrm{E}-01$ & $1.75 \mathrm{E}-01$ & & $1.55 \mathrm{E}+02$ \\
\hline $\mathrm{Y}-90$ & $3.15 \mathrm{E}-02$ & $2.23 \mathrm{E}-02$ & $1.09 \mathrm{E}-03$ & $1.07 \mathrm{E}-03$ & $6.79 \mathrm{E}-02$ & $1.01 \mathrm{E}-01$ & $1.78 \mathrm{E}-01$ & $1.46 \mathrm{E}-01$ & $1.55 \mathrm{E}+02$ \\
\hline Zr-93 & $1.76 \mathrm{E}-06$ & $1.25 \mathrm{E}-06$ & $5.93 \mathrm{E}-08$ & $5.82 \mathrm{E}-08$ & $3.61 \mathrm{E}-06$ & $5.53 \mathrm{E}-06$ & $1.01 \mathrm{E}-05$ & $8.17 \mathrm{E}-06$ & $8.70 \mathrm{E}-03$ \\
\hline Nb-93m & $1.36 \mathrm{E}-06$ & $9.63 \mathrm{E}-07$ & $4.57 \mathrm{E}-08$ & \begin{tabular}{|l|}
$4.48 \mathrm{E}-08$ \\
\end{tabular} & $2.78 \mathrm{E}-06$ & $4.26 \mathrm{E}-06$ & $7.78 \mathrm{E}-06$ & $6.30 \mathrm{E}-06$ & $6.70 \mathrm{E}-03$ \\
\hline $\mathrm{Nb}-94$ & $1.23 \mathrm{E}-06$ & $8.75 \mathrm{E}-07$ & $4.15 \mathrm{E}-08$ & \begin{tabular}{|l|}
$4.07 \mathrm{E}-08$ \\
\end{tabular} & $2.53 \mathrm{E}-06$ & $3.87 \mathrm{E}-06$ & $7.07 \mathrm{E}-06$ & $5.72 \mathrm{E}-06$ & $6.09 \mathrm{E}-03$ \\
\hline Tc-99 & $1.25 \mathrm{E}-05$ & $8.88 \mathrm{E}-06$ & $1.34 \mathrm{E}-06$ & \begin{tabular}{|l|}
$1.31 \mathrm{E}-06$ \\
\end{tabular} & $1.27 \mathrm{E}-04$ & $1.24 \mathrm{E}-04$ & $9.80 \mathrm{E}-06$ & $5.80 \mathrm{E}-05$ & $6.17 \mathrm{E}-02$ \\
\hline Ru-106 & $1.01 \mathrm{E}-06$ & $7.19 \mathrm{E}-07$ & $3.51 \mathrm{E}-08$ & \begin{tabular}{|l|}
$3.44 \mathrm{E}-08$ \\
\end{tabular} & $2.19 \mathrm{E}-06$ & $3.27 \mathrm{E}-06$ & $5.75 \mathrm{E}-06$ & $4.70 \mathrm{E}-06$ & $5.01 \mathrm{E}-03$ \\
\hline Rh-102 & $6.85 \mathrm{E}-10$ & $4.86 \mathrm{E}-10$ & $2.37 \mathrm{E}-11$ & \begin{tabular}{|l|}
$2.33 \mathrm{E}-11$ \\
\end{tabular} & $1.48 \mathrm{E}-09$ & $2.21 \mathrm{E}-09$ & $3.88 \mathrm{E}-09$ & $3.18 \mathrm{E}-09$ & $3.38 \mathrm{E}-06$ \\
\hline Rh-106 & $1.01 \mathrm{E}-06$ & $7.19 \mathrm{E}-07$ & $3.51 \mathrm{E}-08$ & $3.44 \mathrm{E}-08$ & $2.19 \mathrm{E}-06$ & $3.27 \mathrm{E}-06$ & $5.75 \mathrm{E}-06$ & $4.70 \mathrm{E}-06$ & $5.01 \mathrm{E}-03$ \\
\hline $\mathrm{Pd}-107$ & $1.31 \mathrm{E}-08$ & $9.32 \mathrm{E}-09$ & $4.74 \mathrm{E}-10$ & $4.65 \mathrm{E}-10$ & $2.30 \mathrm{E}-08$ & $4.42 \mathrm{E}-08$ & $7.31 \mathrm{E}-08$ & $6.09 \mathrm{E}-08$ & $6.49 \mathrm{E}-05$ \\
\hline $\begin{array}{ll}d-113 m \\
\end{array}$ & $2.64 \mathrm{E}-06$ & 1.87E-06 & 1.04E-07 & \begin{tabular}{|l|}
$1.02 \mathrm{E}-07$ \\
\end{tabular} & $5.91 \mathrm{E}-06$ & $9.70 \mathrm{E}-06$ & $1.41 \mathrm{E}-05$ & $1.22 \mathrm{E}-05$ & $1.30 \mathrm{E}-02$ \\
\hline Sn-121m & $5.32 \mathrm{E}-08$ & $3.77 \mathrm{E}-08$ & $1.79 \mathrm{E}-09$ & $1.76 \mathrm{E}-09$ & $1.09 \mathrm{E}-07$ & 1.67E-07 & $3.05 \mathrm{E}-07$ & \begin{tabular}{|l|}
$2.47 \mathrm{E}-07$ \\
\end{tabular} & $2.63 \mathrm{E}-04$ \\
\hline Sn-126 & $4.47 \mathrm{E}-07$ & $3.17 \mathrm{E}-07$ & $1.50 \mathrm{E}-08$ & $1.48 \mathrm{E}-08$ & $9.15 \mathrm{E}-07$ & $1.40 \mathrm{E}-06$ & $2.56 \mathrm{E}-06$ & $2.07 \mathrm{E}-06$ & $2.21 \mathrm{E}-03$ \\
\hline Sb-125 & $3.72 \mathrm{E}-04$ & $2.64 \mathrm{E}-04$ & $1.29 \mathrm{E}-05$ & $1.26 \mathrm{E}-05$ & $8.04 \mathrm{E}-04$ & $1.20 \mathrm{E}-03$ & $2.11 \mathrm{E}-03$ & $1.73 \mathrm{E}-03$ & $1.84 \mathrm{E}+00$ \\
\hline Sb-126 & $4.57 \mathrm{E}-08$ & $3.24 \mathrm{E}-08$ & $1.58 \mathrm{E}-09$ & $1.55 \mathrm{E}-09$ & $9.87 \mathrm{E}-08$ & 1.47E-07 & $2.59 \mathrm{E}-07$ & $2.12 \mathrm{E}-07$ & $2.26 \mathrm{E}-04$ \\
\hline Te-125m & $2.50 \mathrm{E}-06$ & $1.78 \mathrm{E}-06$ & $8.43 \mathrm{E}-08$ & \begin{tabular}{|l|}
$8.27 \mathrm{E}-08$ \\
\end{tabular} & $5.13 \mathrm{E}-06$ & $7.86 \mathrm{E}-06$ & $1.44 \mathrm{E}-05$ & $1.16 \mathrm{E}-05$ & $1.24 \mathrm{E}-02$ \\
\hline I-129 & $5.79 \mathrm{E}-08$ & $4.15 \mathrm{E}-08$ & $5.11 \mathrm{E}-09$ & $5.01 \mathrm{E}-09$ & $2.63 \mathrm{E}-07$ & $4.62 \mathrm{E}-07$ & $1.22 \mathrm{E}-07$ & $2.65 \mathrm{E}-07$ & $2.82 \mathrm{E}-04$ \\
\hline Cs-134 & $5.75 \mathrm{E}-05$ & $4.32 \mathrm{E}-05$ & $6.71 \mathrm{E}-06$ & \begin{tabular}{|l|}
$6.59 \mathrm{E}-06$ \\
\end{tabular} & $5.49 \mathrm{E}-04$ & $6.26 \mathrm{E}-04$ & $3.43 \mathrm{E}-05$ & $3.17 \mathrm{E}-04$ & $3.37 \mathrm{E}-01$ \\
\hline Cs-1 & $9.07 \mathrm{E}-07$ & $6.82 \mathrm{E}-07$ & $1.06 \mathrm{E}-07$ & $1.04 \mathrm{E}-07$ & $8.65 \mathrm{E}-06$ & $9.87 \mathrm{E}-06$ & $5.41 \mathrm{E}-07$ & $5.00 \mathrm{E}-06$ & $5.32 \mathrm{E}-03$ \\
\hline \begin{tabular}{|l|} 
Cs-137 \\
\end{tabular} & $4.70 \mathrm{E}-02$ & $3.53 \mathrm{E}-02$ & $5.48 \mathrm{E}-03$ & $5.38 \mathrm{E}-03$ & $4.48 \mathrm{E}-01$ & $5.11 \mathrm{E}-01$ & $2.80 \mathrm{E}-02$ & $2.59 \mathrm{E}-01$ & $2.76 \mathrm{E}+02$ \\
\hline Ba-137m & $45 \mathrm{E}-02$ & $3.16 \mathrm{E}-02$ & $1.60 \mathrm{E}-03$ & $1.57 \mathrm{E}-03$ & $7.80 \mathrm{E}-02$ & 1.49E-01 & $2.47 \mathrm{E}-01$ & $2.06 \mathrm{E}-01$ & $2.20 \mathrm{E}+02$ \\
\hline Ce-144 & $.85 \mathrm{E}-07$ & $4.86 \mathrm{E}-07$ & $2.37 \mathrm{E}-08$ & $2.32 \mathrm{E}-08$ & $1.48 \mathrm{E}-06$ & $2.21 \mathrm{E}-06$ & $3.88 \mathrm{E}-06$ & $3.18 \mathrm{E}-06$ & $3.38 \mathrm{E}-03$ \\
\hline Pr-144 & $6.85 \mathrm{E}-07$ & $4.86 \mathrm{E}-07$ & $2.37 \mathrm{E}-08$ & \begin{tabular}{|l|}
$2.32 \mathrm{E}-08$ \\
\end{tabular} & $1.48 \mathrm{E}-06$ & $2.21 \mathrm{E}-06$ & $3.88 \mathrm{E}-06$ & $3.18 \mathrm{E}-06$ & $3.38 \mathrm{E}-03$ \\
\hline Pm-146 & $4.05 \mathrm{E}-08$ & $2.87 \mathrm{E}-08$ & $1.40 \mathrm{E}-09$ & \begin{tabular}{|l|}
$1.37 \mathrm{E}-09$ \\
\end{tabular} & $8.73 \mathrm{E}-08$ & $1.30 \mathrm{E}-07$ & $2.29 \mathrm{E}-07$ & $1.88 \mathrm{E}-07$ & $2.00 \mathrm{E}-04$ \\
\hline Pm-147 & $1.85 \mathrm{E}-04$ & $1.31 \mathrm{E}-04$ & $6.39 \mathrm{E}-06$ & \begin{tabular}{|l|}
$6.27 \mathrm{E}-06$ \\
\end{tabular} & $3.99 \mathrm{E}-04$ & $5.96 \mathrm{E}-04$ & $1.05 \mathrm{E}-03$ & $8.57 \mathrm{E}-04$ & $9.12 \mathrm{E}-01$ \\
\hline $\mathrm{Sm-1}$ & $3.65 \mathrm{E}-04$ & $2.59 \mathrm{E}-04$ & $1.26 \mathrm{E}-05$ & \begin{tabular}{|l|}
$1.24 \mathrm{E}-05$ \\
\end{tabular} & $7.88 \mathrm{E}-04$ & $1.18 \mathrm{E}-03$ & $2.07 \mathrm{E}-03$ & $1.69 \mathrm{E}-03$ & $1.80 \mathrm{E}+00$ \\
\hline \begin{tabular}{|l|} 
Eu-152 \\
\end{tabular} & $2.34 \mathrm{E}-06$ & $1.66 \mathrm{E}-06$ & $8.11 \mathrm{E}-08$ & \begin{tabular}{|l|}
$7.96 \mathrm{E}-08$ \\
\end{tabular} & $5.06 \mathrm{E}-06$ & $7.56 \mathrm{E}-06$ & $1.33 \mathrm{E}-05$ & $1.09 \mathrm{E}-05$ & $1.16 \mathrm{E}-02$ \\
\hline Eu-154 & $1.40 \mathrm{E}-04$ & $9.93 \mathrm{E}-05$ & $4.84 \mathrm{E}-06$ & \begin{tabular}{|l|l}
$4.75 \mathrm{E}-06$ \\
\end{tabular} & $3.02 \mathrm{E}-04$ & $4.51 \mathrm{E}-04$ & $7.93 \mathrm{E}-04$ & $6.49 \mathrm{E}-04$ & $6.91 \mathrm{E}-01$ \\
\hline Eu-155 & $1.32 \mathrm{E}-04$ & $9.35 \mathrm{E}-05$ & \begin{tabular}{|l|}
$4.56 \mathrm{E}-06$ \\
\end{tabular} & \begin{tabular}{|l|}
$4.47 \mathrm{E}-06$ \\
\end{tabular} & \begin{tabular}{|l|}
$2.84 \mathrm{E}-04$ \\
\end{tabular} & \begin{tabular}{|l|}
$4.25 \mathrm{E}-04$ \\
\end{tabular} & $7.46 \mathrm{E}-04$ & \begin{tabular}{|c|c|}
$6.11 \mathrm{E}-04$ \\
\end{tabular} & $6.50 \mathrm{E}-01$ \\
\hline & & & & & & & & & \\
\hline Th-2: & $2 \mathrm{E}-10$ & $6.12 \mathrm{E}-10$ & $\mathrm{E}-11$ & $85 \mathrm{E}-11$ & 7E-09 & $.70 \mathrm{E}-09$ & $4.94 \mathrm{E}-09$ & $4.00 \mathrm{E}-09$ & $4.26 \mathrm{E}-06$ \\
\hline $\mathrm{Pa}-233$ & $33 \mathrm{E}-06$ & $1.65 \mathrm{E}-06$ & $7.85 \mathrm{E}-08$ & $7.70 \mathrm{E}-08$ & $4.78 \mathrm{E}-06$ & $7.31 \mathrm{E}-06$ & $1.34 \mathrm{E}-05$ & $1.08 \mathrm{E}-05$ & $1.15 \mathrm{E}-02$ \\
\hline $\mathrm{U}-232$ & $2 \mathrm{E}-09$ & $1.43 \mathrm{E}-09$ & $\begin{array}{l}6.79 \mathrm{E}-11 \\
\end{array}$ & \begin{tabular}{|l|}
$6.66 \mathrm{E}-11$ \\
\end{tabular} & $4.13 \mathrm{E}-09$ & $6.33 \mathrm{E}-09$ & $1.16 \mathrm{E}-08$ & $9.36 \mathrm{E}-09$ & $9.96 \mathrm{E}-06$ \\
\hline U-233 & $1 \mathrm{E}-11$ & $5.05 \mathrm{E}-11$ & $2.39 \mathrm{E}-12$ & \begin{tabular}{|l|}
$2.35 \mathrm{E}-12$ \\
\end{tabular} & $\mathrm{E}-10$ & $2.23 \mathrm{E}-10$ & 4.07E-10 & $3.30 \mathrm{E}-10$ & $3.51 \mathrm{E}-07$ \\
\hline 234 & $40 \mathrm{E}-06$ & $9.90 \mathrm{E}-07$ & $4.70 \mathrm{E}-08$ & \begin{tabular}{|l|}
$4.61 \mathrm{E}-08$ \\
\end{tabular} & $2.86 \mathrm{E}-06$ & $4.38 \mathrm{E}-06$ & $8.00 \mathrm{E}-06$ & $6.47 \mathrm{E}-06$ & $6.89 \mathrm{E}-03$ \\
\hline 35 & 63E-08 & $3.99 \mathrm{E}-08$ & $1.89 \mathrm{E}-09$ & $1.86 \mathrm{E}-09$ & $1.15 \mathrm{E}-07$ & 1.77E-07 & $3.22 \mathrm{E}-07$ & $2.61 \mathrm{E}-07$ & $2.78 \mathrm{E}-04$ \\
\hline 236 & $6.53 \mathrm{E}-08$ & $4.63 \mathrm{E}-08$ & $2.20 \mathrm{E}-09$ & $2.16 \mathrm{E}-09$ & $1.34 \mathrm{E}-07$ & $2.05 \mathrm{E}-07$ & $3.74 \mathrm{E}-07$ & $3.03 \mathrm{E}-07$ & $3.22 \mathrm{E}-04$ \\
\hline 237 & $5.12 \mathrm{E}-09$ & $3.63 \mathrm{E}-09$ & $1.72 \mathrm{E}-10$ & 1.69E-10 & $1.05 \mathrm{E}-08$ & $1.61 \mathrm{E}-08$ & $2.93 \mathrm{E}-08$ & $2.37 \mathrm{E}-08$ & $2.53 \mathrm{E}-05$ \\
\hline U-238 & $3.10 \mathrm{E}-08$ & $2.20 \mathrm{E}-08$ & $1.04 \mathrm{E}-09$ & \begin{tabular}{|l|}
$1.02 \mathrm{E}-09$ \\
\end{tabular} & $6.35 \mathrm{E}-08$ & $9.71 \mathrm{E}-08$ & $1.77 \mathrm{E}-07$ & $1.44 \mathrm{E}-07$ & $1.53 \mathrm{E}-04$ \\
\hline Np-237 & 4.15E-07 & $2.95 \mathrm{E}-07$ & $1.44 \mathrm{E}-08$ & $1.41 \mathrm{E}-08$ & $8.96 \mathrm{E}-07$ & $1.34 \mathrm{E}-06$ & $2.35 \mathrm{E}-06$ & $1.93 \mathrm{E}-06$ & $2.05 \mathrm{E}-03$ \\
\hline Pu-236 & $3.29 \mathrm{E}-09$ & $2.33 \mathrm{E}-09$ & $1.11 \mathrm{E}-10$ & $1.09 \mathrm{E}-10$ & $6.74 \mathrm{E}-09$ & 1.03E-08 & $1.88 \mathrm{E}-08$ & $1.53 \mathrm{E}-08$ & 1.62E-05 \\
\hline Pu-238 & $7.59 \mathrm{E}-04$ & $5.39 \mathrm{E}-04$ & $2.56 \mathrm{E}-05$ & $2.51 \mathrm{E}-05$ & $1.56 \mathrm{E}-03$ & $2.38 \mathrm{E}-03$ & $4.35 \mathrm{E}-03$ & $3.52 \mathrm{E}-03$ & $3.75 \mathrm{E}+00$ \\
\hline Pu-239 & $8.29 \mathrm{E}-05$ & $5.88 \mathrm{E}-05$ & $2.79 \mathrm{E}-06$ & $2.74 \mathrm{E}-06$ & $1.70 \mathrm{E}-04$ & $2.60 \mathrm{E}-04$ & $4.75 \mathrm{E}-04$ & $3.85 \mathrm{E}-04$ & $4.10 \mathrm{E}-01$ \\
\hline Pu-240 & $1.23 \mathrm{E}-05$ & $8.70 \mathrm{E}-06$ & $4.13 \mathrm{E}-07$ & $4.05 \mathrm{E}-07$ & $2.51 \mathrm{E}-05$ & $3.85 \mathrm{E}-05$ & $7.03 \mathrm{E}-05$ & $5.69 \mathrm{E}-05$ & $6.06 \mathrm{E}-02$ \\
\hline Pu-241 & $6.64 \mathrm{E}-04$ & $4.71 \mathrm{E}-04$ & $2.24 \mathrm{E}-05$ & $2.19 \mathrm{E}-05$ & $1.36 \mathrm{E}-03$ & $2.08 \mathrm{E}-03$ & $3.81 \mathrm{E}-03$ & $3.08 \mathrm{E}-03$ & $3.28 \mathrm{E}+00$ \\
\hline Pu-242 & $9.60 \mathrm{E}-09$ & $6.81 \mathrm{E}-09$ & $3.23 \mathrm{E}-10$ & $3.17 \mathrm{E}-10$ & $1.97 \mathrm{E}-08$ & $3.01 \mathrm{E}-08$ & $5.50 \mathrm{E}-08$ & $4.45 \mathrm{E}-08$ & $4.74 \mathrm{E}-05$ \\
\hline Pu-244 & $2.86 \mathrm{E}-16$ & $2.03 \mathrm{E}-16$ & $9.64 \mathrm{E}-18$ & $9.46 \mathrm{E}-18$ & $5.87 \mathrm{E}-16$ & $8.98 \mathrm{E}-16$ & $1.64 \mathrm{E}-15$ & \begin{tabular}{|l|l|}
$1.33 \mathrm{E}-15$ \\
\end{tabular} & $1.41 \mathrm{E}-12$ \\
\hline Am-241 & $6.48 \mathrm{E}-05$ & $4.60 \mathrm{E}-05$ & $2.24 \mathrm{E}-06$ & $2.20 \mathrm{E}-06$ & $1.40 \mathrm{E}-04$ & $2.09 \mathrm{E}-04$ & $3.67 \mathrm{E}-04$ & $3.00 \mathrm{E}-04$ & $3.20 \mathrm{E}-01$ \\
\hline $\mathrm{Am}-242 \mathrm{~m}$ & $1.21 \mathrm{E}-08$ & \begin{tabular}{|l|}
$8.62 \mathrm{E}-09$ \\
\end{tabular} & \begin{tabular}{|l|}
$4.20 \mathrm{E}-10$ \\
\end{tabular} & \begin{tabular}{|l|}
$4.12 \mathrm{E}-10$ \\
\end{tabular} & $2.62 \mathrm{E}-08$ & $3.92 \mathrm{E}-08$ & $6.88 \mathrm{E}-08$ & $5.64 \mathrm{E}-08$ & $6.00 \mathrm{E}-05$ \\
\hline
\end{tabular}


Table A-16. Mass balance, Tank WM-189 waste (continued).

\begin{tabular}{|c|c|c|c|c|c|c|c|c|c|}
\hline Stream \# & 101 & 102 & 103 & 104 & 105 & 106 & 107 & 108 & 108 \\
\hline Radiological Composition & $\mathrm{Ci} / \mathrm{L}$ & $\mathrm{Ci} / \mathrm{L}$ & Ci/wscm & Ci/wscm & $\mathrm{Ci} / \mathrm{kg}$ & $\mathrm{Ci} / \mathrm{kg}$ & $\mathrm{Ci} / \mathrm{kg}$ & $\mathrm{Ci} / \mathrm{kg}$ & Ci/Canister \\
\hline Am-243 & 2.07E-08 & 1.47E-08 & $7.17 \mathrm{E}-10$ & $7.03 \mathrm{E}-10$ & 4.47E-08 & 6.68E-08 & 1.17E-07 & $9.61 \mathrm{E}-08$ & 1.02E-04 \\
\hline $\mathrm{Cm}-242$ & $2.43 \mathrm{E}-08$ & $1.72 \mathrm{E}-08$ & $8.17 \mathrm{E}-10$ & $8.02 E-10$ & 4.97E-08 & 7.61E-08 & 1.39E-07 & 1.13E-07 & 1.20E-04 \\
\hline $\mathrm{Cm}-243$ & 2.99E-08 & $2.12 \mathrm{E}-08$ & $1.01 \mathrm{E}-09$ & $9.86 \mathrm{E}-10$ & $6.12 \mathrm{E}-08$ & 9.37E-08 & $1.71 \mathrm{E}-07$ & 1.39E-07 & 1.47E-04 \\
\hline $\mathrm{Cm}-244$ & $1.26 \mathrm{E}-06$ & $8.97 \mathrm{E}-07$ & $4.26 \mathrm{E}-08$ & $4.18 \mathrm{E}-08$ & $2.59 \mathrm{E}-06$ & $3.97 \mathrm{E}-06$ & $7.25 \mathrm{E}-06$ & 5.87E-06 & $6.25 \mathrm{E}-03$ \\
\hline $\mathrm{Cm}-245$ & $3.14 \mathrm{E}-10$ & $2.23 \mathrm{E}-10$ & $1.06 \mathrm{E}-11$ & $1.04 \mathrm{E}-11$ & $6.44 \mathrm{E}-10$ & $9.86 \mathrm{E}-10$ & 1.80E-09 & 1.46E-09 & $1.55 \mathrm{E}-06$ \\
\hline $\mathrm{Cm}-246$ & $2.06 \mathrm{E}-11$ & $1.46 \mathrm{E}-11$ & $6.95 \mathrm{E}-13$ & $6.82 \mathrm{E}-13$ & $4.23 \mathrm{E}-11$ & $6.47 \mathrm{E}-11$ & $1.18 \mathrm{E}-10$ & 9.57E-11 & $1.02 \mathrm{E}-07$ \\
\hline TRU & $9.40 \mathrm{E}-04$ & $6.67 \mathrm{E}-04$ & $3.17 \mathrm{E}-05$ & $3.11 \mathrm{E}-05$ & 1.93E-03 & $2.96 \mathrm{E}-03$ & 5.38E-03 & $4.36 \mathrm{E}-03$ & $4.64 \mathrm{E}+00$ \\
\hline TRU, nCi/gm & $7.33 \mathrm{E}+02$ & & & & & & & $4.36 \mathrm{E}+03$ & \\
\hline & & & & & & & & & \\
\hline $\begin{array}{l}\text { Gas Stream Bulk } \\
\text { Composition (Wet Basis) }\end{array}$ & $\begin{array}{c}\mathrm{mol} \% \text { or } \\
\mathrm{ppmv}\end{array}$ & $\begin{array}{c}\text { mol\% or } \\
\text { ppmv }\end{array}$ & $\begin{array}{l}\mathrm{mol} \% \text { or } \\
\text { ppmv }\end{array}$ & $\begin{array}{l}\text { mol\% or } \\
\text { ppmv }\end{array}$ & $\begin{array}{c}\text { mol\% or } \\
\text { ppmv }\end{array}$ & $\begin{array}{c}\mathrm{mol} \% \text { or } \\
\mathrm{ppmv}\end{array}$ & $\begin{array}{c}\mathrm{mol} \% \text { or } \\
\text { ppmv }\end{array}$ & $\begin{array}{c}\mathrm{mol} \% \text { or } \\
\text { ppmv }\end{array}$ & $\begin{array}{c}\mathrm{mol} \% \text { or } \\
\text { ppmv }\end{array}$ \\
\hline $\mathrm{H} 2 \mathrm{O}, \mathrm{mol} \%$ & & & $69.24 \%$ & $67.93 \%$ & & & & & \\
\hline $\mathrm{O} 2, \mathrm{~mol} \%$ & & & $0.58 \%$ & $0.57 \%$ & & & & & \\
\hline $\mathrm{N} 2, \mathrm{~mol} \%$ & & & $17.38 \%$ & $18.95 \%$ & & & & & \\
\hline $\mathrm{H} 2, \mathrm{~mol} \%$ & & & $3.99 \%$ & $3.91 \%$ & & & & & \\
\hline $\mathrm{CO} 2, \mathrm{~mol} \%$ & & & $7.20 \%$ & $7.07 \%$ & & & & & \\
\hline $\mathrm{CO}, \mathrm{ppmv}$ & & & $1.19 \mathrm{E}+04$ & $1.17 \mathrm{E}+04$ & & & & & \\
\hline $\mathrm{NO}, \mathrm{ppmv}$ & & & $5.80 \mathrm{E}+02$ & $5.69 \mathrm{E}+02$ & & & & & \\
\hline NO2, ppmv & & & $3.92 \mathrm{E}+01$ & $3.85 \mathrm{E}+01$ & & & & & \\
\hline SO2, ppmv & & & 2.84E-02 & $2.79 \mathrm{E}-02$ & & & & & \\
\hline $\mathrm{Cl}, \mathrm{ppmv}$ & & & $3.93 \mathrm{E}+01$ & $3.86 \mathrm{E}+01$ & & & & & \\
\hline $\mathrm{F}, \mathrm{ppmv}$ & & & $7.81 \mathrm{E}+01$ & $7.66 \mathrm{E}+01$ & & & & & \\
\hline C (organic), ppmv & & & $3.29 \mathrm{E}+03$ & $3.22 \mathrm{E}+03$ & & & & & \\
\hline $\mathrm{H}$ (organic), ppmv & & & $7.42 \mathrm{E}+03$ & $7.28 \mathrm{E}+03$ & & & & & \\
\hline $\mathrm{Hg}, \mathrm{ug} / \mathrm{wscm}$ & & & $1.39 \mathrm{E}+05$ & $1.36 \mathrm{E}+05$ & & & & & \\
\hline $\mathrm{PM}, \mathrm{mg} / \mathrm{wscm}$ & & & $1.07 E+04$ & $1.05 \mathrm{E}+04$ & & & & & \\
\hline SVM, ug/wscm & & & $3.44 \mathrm{E}+04$ & $3.37 \mathrm{E}+04$ & & & & & \\
\hline LVM, ug/wscm & & & $9.56 \mathrm{E}+03$ & $9.38 \mathrm{E}+03$ & & & & & \\
\hline $\begin{array}{l}\text { Gas Stream Bulk } \\
\text { Composition (Dry Basis) }\end{array}$ & $\mathrm{mol} \%$ & $\mathrm{~mol} \%$ & $\mathrm{~mol} \%$ & $\mathrm{~mol} \%$ & $\mathrm{~mol} \%$ & $\mathrm{~mol} \%$ & $\mathrm{~mol} \%$ & $\mathrm{~mol} \%$ & $\mathrm{~mol} \%$ \\
\hline $\mathrm{O} 2, \mathrm{~mol} \%$, dry basis & & & $1.88 \%$ & $1.77 \%$ & & & & & \\
\hline $\mathrm{N} 2, \mathrm{~mol} \%$, dry basis & & & $56.51 \%$ & $59.08 \%$ & & & & & \\
\hline $\mathrm{H} 2, \mathrm{~mol} \%$, dry basis & & & $12.96 \%$ & $12.20 \%$ & & & & & \\
\hline $\mathrm{CO} 2, \mathrm{~mol} \%$, dry basis & & & $23.42 \%$ & $22.04 \%$ & & & & & \\
\hline $\begin{array}{l}\text { Gas Stream Bulk } \\
\text { Composition (Dry Basis, } \\
\text { Corrected to } 7 \% \text { O2 with } \\
100 \% \text { O2 Combustion Air) }\end{array}$ & $\begin{array}{l}\text { ppmv, or } \\
\text { ug/dscm } \\
\text { or } \\
\mathrm{mg} / \mathrm{dscm}\end{array}$ & $\begin{array}{l}\mathrm{ppmv}, \text { or } \\
\mathrm{ug} / \mathrm{dscm} \\
\text { or } \\
\mathrm{mg} / \mathrm{dscm}\end{array}$ & $\begin{array}{l}\text { ppmv, or } \\
\text { ug/dscm } \\
\text { or } \\
\mathrm{mg} / \mathrm{dscm}\end{array}$ & $\begin{array}{l}\mathrm{ppmv}, \text { or } \\
\mathrm{ug} / \mathrm{dscm} \\
\text { or } \\
\mathrm{mg} / \mathrm{dscm}\end{array}$ & $\begin{array}{l}\mathrm{ppmv}, \text { or } \\
\mathrm{ug} / \mathrm{dscm} \\
\text { or } \\
\mathrm{mg} / \mathrm{dscm}\end{array}$ & $\begin{array}{l}\text { ppmv, or } \\
\text { ug/dscm } \\
\text { or } \\
\mathrm{mg} / \mathrm{dscm}\end{array}$ & $\begin{array}{l}\text { ppmv, or } \\
\text { ug/dscm } \\
\text { or } \\
\mathrm{mg} / \mathrm{dscm}\end{array}$ & $\begin{array}{l}\mathrm{ppmv}, \text { or } \\
\text { ug/dscm } \\
\text { or } \\
\mathrm{mg} / \mathrm{dscm}\end{array}$ & $\begin{array}{l}\text { ppmv, or } \\
\text { ug/dscm } \\
\text { or } \\
\mathrm{mg} / \mathrm{dscm}\end{array}$ \\
\hline COgas, ppmv, dry basis & & & $5.41 \mathrm{E}+03$ & $5.09 \mathrm{E}+03$ & & & & & \\
\hline $\mathrm{NO}$, ppmv, dry basis & & & $2.64 \mathrm{E}+02$ & $2.48 \mathrm{E}+02$ & & & & & \\
\hline NO2, ppmv, dry basis & & & $1.79 \mathrm{E}+01$ & $1.68 \mathrm{E}+01$ & & & & & \\
\hline SO2, ppmv, dry basis & & & $1.29 \mathrm{E}-02$ & $1.22 \mathrm{E}-02$ & & & & & \\
\hline $\mathrm{Cl}, \mathrm{ppmv}$, dry basis & & & $1.79 \mathrm{E}+01$ & $1.69 \mathrm{E}+01$ & & & & & \\
\hline $\mathrm{F}$, ppmv, dry basis & & & $3.55 \mathrm{E}+01$ & $3.34 \mathrm{E}+01$ & & & & & \\
\hline C (organic), ppmv, dry basis & & & $1.50 \mathrm{E}+03$ & $1.41 \mathrm{E}+03$ & & & & & \\
\hline $\mathrm{H}$ (organic), ppmv, dry basis & & & $3.38 \mathrm{E}+03$ & $3.18 \mathrm{E}+03$ & & & & & \\
\hline $\mathrm{Hg}, \mathrm{ug} / \mathrm{dscm}$ & & & $6.31 \mathrm{E}+04$ & $5.94 \mathrm{E}+04$ & & & & & \\
\hline $\mathrm{PM}, \mathrm{mg} / \mathrm{dscm}$ & & & $4.87 \mathrm{E}+03$ & $4.58 \mathrm{E}+03$ & & & & & \\
\hline SVM, ug/dscm & & & $1.12 \mathrm{E}+05$ & $1.10 \mathrm{E}+05$ & & & & & \\
\hline LVM, ug/dscm & & & $3.11 \mathrm{E}+04$ & $3.05 \mathrm{E}+04$ & & & & & \\
\hline
\end{tabular}


Table A-16. Mass balance, Tank WM-189 waste (continued).

\begin{tabular}{|c|c|c|c|c|c|c|c|c|c|}
\hline PFD \# & PFD-2 & PFD-3 & PFD-3 & PFD-3 & PFD-3 & PFD-3 & PFD-3 & PFD-3 & PFD-3 \\
\hline WM-189, Stream \# & 109 & 110 & 111 & 112 & $113 \mathrm{~A}$ & 113B & 114 & 115 & 116 \\
\hline Stream Name & $\begin{array}{l}\text { Off-Gas } \\
\text { from } \\
\text { Filter to } \\
\text { Oxidizer }\end{array}$ & $\begin{array}{l}\text { Oxidizer } \\
\text { Effluent }\end{array}$ & $\begin{array}{l}\text { Quenched } \\
\text { Oxidizer Off- } \\
\text { gas }\end{array}$ & Scrub & $\begin{array}{c}\text { Packed } \\
\text { Scrubber } \\
\text { Drain }\end{array}$ & $\begin{array}{c}\text { Demister } \\
\text { Drain }\end{array}$ & $\begin{array}{c}\text { Scrubber } \\
\text { Effluent } \\
\text { Gas }\end{array}$ & $\begin{array}{c}\text { Demister } \\
\text { Effluent } \\
\text { Gas }\end{array}$ & $\begin{array}{c}\text { GAC Bed } \\
\text { Feed }\end{array}$ \\
\hline Rate or Volume & $4.77 \mathrm{E}+05$ & $7.66 \mathrm{E}+05$ & $4.01 \mathrm{E}+05$ & $1.10 \mathrm{E}+04$ & $1.10 \mathrm{E}+04$ & $1.33 \mathrm{E}-02$ & $4.09 \mathrm{E}+05$ & $4.18 \mathrm{E}+05$ & $5.08 \mathrm{E}+05$ \\
\hline Volume Flow (standard, wet)* & $1.13 \mathrm{E}+05$ & $1.17 \mathrm{E}+05$ & $1.99 \mathrm{E}+05$ & & & & $1.98 \mathrm{E}+05$ & $1.98 \mathrm{E}+05$ & $1.98 \mathrm{E}+05$ \\
\hline Volume Flow (standard, dry) & $3.66 \mathrm{E}+04$ & $3.51 \mathrm{E}+04$ & $3.50 \mathrm{E}+04$ & & & & $3.50 \mathrm{E}+04$ & $3.51 \mathrm{E}+04$ & $3.51 \mathrm{E}+04$ \\
\hline Rate Units & $\mathrm{ft} 3 / \mathrm{hr}$ & $\mathrm{ft} 3 / \mathrm{hr}$ & $\mathrm{ft} 3 / \mathrm{hr}$ & $\mathrm{gal} / \mathrm{hr}$ & $\mathrm{gal} / \mathrm{hr}$ & $\mathrm{gal} / \mathrm{hr}$ & $\mathrm{ft} 3 / \mathrm{hr}$ & $\mathrm{ft} 3 / \mathrm{hr}$ & $\mathrm{ft} 3 / \mathrm{hr}$ \\
\hline Rate or Volume, metric & $3.20 \mathrm{E}+03$ & $3.32 E+03$ & $5.63 \mathrm{E}+03$ & $4.16 \mathrm{E}+04$ & $4.17 \mathrm{E}+04$ & 0.05 & $5.60 \mathrm{E}+03$ & $5.62 \mathrm{E}+03$ & $5.62 E+03$ \\
\hline Rate Units & wscm/hr & wscm/hr & wscm/hr & $\mathrm{L} / \mathrm{hr}$ & L/hr & $\mathrm{L} / \mathrm{hr}$ & wscm/hr & wscm/hr & wscm/hr \\
\hline Temperature, ${ }^{\circ} \mathrm{C}$ & 527 & 950 & 100 & 81 & 83 & 81 & 82 & 81 & 120 \\
\hline Temperature, ${ }^{\circ} \mathrm{F}$ & 980 & 1742 & 212 & 179 & 181 & 177 & 179 & 177 & 248 \\
\hline Pressure, psia & 9.5 & 9.4 & 9.3 & 38.1 & 9.0 & 8.4 & 8.6 & 8.4 & 7.7 \\
\hline Specific Gravity & $2.11 \mathrm{E}-04$ & 1.43E-04 & $4.28 \mathrm{E}-04$ & 1.02 & 1.01 & 1.01 & $4.17 \mathrm{E}-04$ & $4.08 \mathrm{E}-04$ & 3.36E-04 \\
\hline Chemical Composition & $\mathrm{lb} /$ wscf & $\mathrm{lb} /$ wscf & $\mathrm{lb} / \mathrm{wscf}$ & Mol/liter & Mol/liter & Mol/liter & $\mathrm{lb} /$ wscf & $\mathrm{lb} / \mathrm{wscf}$ & $\mathrm{lb} /$ wscf \\
\hline $\mathrm{H}+$ & $1.31 \mathrm{E}-06$ & $1.89 \mathrm{E}-08$ & $2.01 \mathrm{E}-08$ & $1.02 \mathrm{E}+00$ & $1.02 \mathrm{E}+00$ & $1.02 \mathrm{E}+00$ & 2.07E-07 & $2.06 \mathrm{E}-07$ & 2.06E-07 \\
\hline $\mathrm{Al}+3$ & $5.17 \mathrm{E}-08$ & $4.98 \mathrm{E}-08$ & $2.94 \mathrm{E}-08$ & 1.90E-03 & $1.90 \mathrm{E}-03$ & 1.90E-03 & & & \\
\hline $\mathrm{Sb}+5$ & $6.31 \mathrm{E}-12$ & $6.08 \mathrm{E}-12$ & $3.59 \mathrm{E}-12$ & $5.15 \mathrm{E}-08$ & $5.15 \mathrm{E}-08$ & $5.15 \mathrm{E}-08$ & & & \\
\hline As+3 & 1.94E-11 & $1.87 \mathrm{E}-11$ & $1.10 \mathrm{E}-11$ & $2.57 \mathrm{E}-07$ & 2.57E-07 & 2.57E-07 & & & \\
\hline $\mathrm{Ba}+2$ & $2.81 \mathrm{E}-11$ & $2.71 \mathrm{E}-11$ & $3.12 \mathrm{E}-13$ & $2.05 \mathrm{E}-07$ & $2.05 \mathrm{E}-07$ & $2.05 \mathrm{E}-07$ & & & \\
\hline $\mathrm{Be}+2$ & $6.00 \mathrm{E}-13$ & $5.78 \mathrm{E}-13$ & $6.66 \mathrm{E}-15$ & $6.68 \mathrm{E}-08$ & $6.67 \mathrm{E}-08$ & $6.67 \mathrm{E}-08$ & & & \\
\hline $\mathrm{B}+3$ & $5.67 \mathrm{E}-10$ & $5.46 \mathrm{E}-10$ & $3.22 \mathrm{E}-10$ & 5.21E-05 & 5.21E-05 & 5.21E-05 & & & \\
\hline $\mathrm{Cd}+2$ & $1.20 \mathrm{E}-09$ & 1.15E-09 & $6.81 \mathrm{E}-10$ & 5.74E-06 & 5.74E-06 & 5.74E-06 & & & \\
\hline $\mathrm{Ca}+2$ & $1.98 \mathrm{E}-08$ & $1.90 \mathrm{E}-08$ & $2.19 \mathrm{E}-10$ & 4.94E-04 & 4.94E-04 & 4.94E-04 & & & \\
\hline $\mathrm{Cr}+3$ & $8.51 \mathrm{E}-10$ & $8.20 \mathrm{E}-10$ & $4.84 \mathrm{E}-10$ & $1.18 \mathrm{E}-05$ & $1.18 \mathrm{E}-05$ & $1.18 \mathrm{E}-05$ & & & \\
\hline $\mathrm{Co}+2$ & $7.22 \mathrm{E}-12$ & $6.96 \mathrm{E}-12$ & $4.11 \mathrm{E}-12$ & $1.22 \mathrm{E}-07$ & 1.22E-07 & $1.22 \mathrm{E}-07$ & & & \\
\hline Cs+ & $5.23 \mathrm{E}-11$ & $5.04 \mathrm{E}-11$ & $2.97 \mathrm{E}-11$ & $5.45 \mathrm{E}-05$ & $5.45 \mathrm{E}-05$ & $5.45 \mathrm{E}-05$ & & & \\
\hline $\mathrm{Cu}+2$ & $1.67 \mathrm{E}-10$ & $1.61 \mathrm{E}-10$ & $1.85 \mathrm{E}-12$ & $2.64 \mathrm{E}-06$ & $2.63 \mathrm{E}-06$ & $2.63 \mathrm{E}-06$ & & & \\
\hline $\mathrm{Fe}+3$ & $3.45 \mathrm{E}-09$ & 3.33E-09 & 1.96E-09 & 9.96E-05 & 9.96E-05 & 9.96E-05 & & & \\
\hline $\mathrm{Pb}+2$ & 1.93E-09 & $1.86 \mathrm{E}-09$ & $1.10 \mathrm{E}-09$ & $7.19 \mathrm{E}-06$ & $7.19 \mathrm{E}-06$ & $7.19 \mathrm{E}-06$ & & & \\
\hline $\mathrm{Hg}+2$ & $8.41 \mathrm{E}-06$ & $8.11 \mathrm{E}-06$ & 4.79E-06 & $1.48 \mathrm{E}-02$ & 1.48E-02 & 1.48E-02 & & $2.58 \mathrm{E}-06$ & $2.58 \mathrm{E}-06$ \\
\hline$M n+4$ & 2.59E-09 & $2.50 \mathrm{E}-09$ & 1.48E-09 & 4.69E-05 & $4.69 \mathrm{E}-05$ & 4.69E-05 & & & \\
\hline $\mathrm{Ni}+2$ & $3.66 \mathrm{E}-10$ & $3.53 \mathrm{E}-10$ & $4.06 \mathrm{E}-12$ & $6.25 \mathrm{E}-06$ & $6.24 \mathrm{E}-06$ & $6.24 \mathrm{E}-06$ & & & \\
\hline $\mathrm{K}+$ & $4.84 \mathrm{E}-08$ & $4.67 \mathrm{E}-08$ & $2.76 \mathrm{E}-08$ & $1.59 \mathrm{E}-03$ & $1.59 \mathrm{E}-03$ & $1.59 \mathrm{E}-03$ & & & \\
\hline $\mathrm{Se}+4$ & $5.70 \mathrm{E}-11$ & $5.49 \mathrm{E}-11$ & $3.24 \mathrm{E}-11$ & 1.19E-06 & 1.19E-06 & $1.19 \mathrm{E}-06$ & & & \\
\hline $\mathrm{Ag}+$ & $4.73 \mathrm{E}-10$ & $2.89 \mathrm{E}-10$ & $1.71 \mathrm{E}-10$ & $2.04 \mathrm{E}-06$ & $2.04 \mathrm{E}-06$ & $2.04 \mathrm{E}-06$ & & & \\
\hline $\mathrm{Na}+$ & $4.26 \mathrm{E}-07$ & $4.10 \mathrm{E}-07$ & $2.42 \mathrm{E}-07$ & $5.28 \mathrm{E}-02$ & $5.28 \mathrm{E}-02$ & $5.28 \mathrm{E}-02$ & & & \\
\hline$T \mid+3$ & $2.79 \mathrm{E}-12$ & $2.69 \mathrm{E}-12$ & $1.59 \mathrm{E}-12$ & $1.36 \mathrm{E}-08$ & $1.36 \mathrm{E}-08$ & $1.36 \mathrm{E}-08$ & & & \\
\hline$U+4$ & $3.74 \mathrm{E}-10$ & $3.60 \mathrm{E}-10$ & $2.12 \mathrm{E}-10$ & $2.59 \mathrm{E}-06$ & $2.58 \mathrm{E}-06$ & $2.58 \mathrm{E}-06$ & & & \\
\hline$V+3$ & $4.49 \mathrm{E}-12$ & $4.33 \mathrm{E}-12$ & $2.55 \mathrm{E}-12$ & $1.48 \mathrm{E}-07$ & 1.48E-07 & 1.48E-07 & & & \\
\hline$Z n+2$ & $1.84 \mathrm{E}-10$ & $1.78 \mathrm{E}-10$ & $2.04 \mathrm{E}-12$ & $2.82 \mathrm{E}-06$ & $2.82 \mathrm{E}-06$ & 2.82E-06 & & & \\
\hline $\mathrm{Zr}+4$ & 9.89E-09 & 9.53E-09 & $5.62 \mathrm{E}-09$ & $1.79 \mathrm{E}-04$ & $1.78 \mathrm{E}-04$ & 1.78E-04 & & & \\
\hline $\mathrm{Cl}-$ & $1.05 \mathrm{E}-07$ & $1.01 \mathrm{E}-07$ & $5.98 \mathrm{E}-08$ & $2.58 \mathrm{E}-03$ & $2.58 \mathrm{E}-03$ & $2.58 \mathrm{E}-03$ & $1.26 \mathrm{E}-08$ & $1.25 \mathrm{E}-08$ & $1.25 \mathrm{E}-08$ \\
\hline F- & 3.75E-07 & $3.62 \mathrm{E}-07$ & $2.13 \mathrm{E}-07$ & $8.06 \mathrm{E}-03$ & 8.03E-03 & 8.03E-03 & 3.34E-07 & 3.33E-07 & 3.33E-07 \\
\hline SO4-2 & $3.51 \mathrm{E}-11$ & 5.71E-07 & 3.37E-07 & 1.89E-02 & $1.89 \mathrm{E}-02$ & 1.89E-02 & & & \\
\hline NO3- & 6.08E-06 & 5.68E-09 & 3.35E-09 & $1.06 \mathrm{E}+00$ & $1.05 \mathrm{E}+00$ & $1.05 \mathrm{E}+00$ & 1.16E-05 & $1.16 \mathrm{E}-05$ & 1.16E-05 \\
\hline PO4-3 & 3.69E-08 & $3.56 \mathrm{E}-08$ & $2.10 \mathrm{E}-08$ & 6.11E-04 & 6.10E-04 & 6.10E-04 & & & \\
\hline $\mathrm{Am}+4$ & $4.55 \mathrm{E}-14$ & $4.38 \mathrm{E}-14$ & $2.59 \mathrm{E}-14$ & $1.86 \mathrm{E}-10$ & $1.86 \mathrm{E}-10$ & $1.86 \mathrm{E}-10$ & & & \\
\hline $\mathrm{Br}-$ & $3.14 \mathrm{E}-12$ & $3.03 \mathrm{E}-12$ & $1.79 \mathrm{E}-12$ & $3.42 \mathrm{E}-08$ & $3.42 \mathrm{E}-08$ & $3.42 \mathrm{E}-08$ & $3.76 \mathrm{E}-13$ & $3.75 \mathrm{E}-13$ & $3.75 \mathrm{E}-13$ \\
\hline $\mathrm{Ce}+4$ & $1.59 \mathrm{E}-11$ & $1.53 \mathrm{E}-11$ & $9.04 \mathrm{E}-12$ & $1.13 \mathrm{E}-07$ & $1.13 \mathrm{E}-07$ & $1.13 \mathrm{E}-07$ & & & \\
\hline $\mathrm{Eu}+3$ & $1.96 \mathrm{E}-13$ & $1.89 \mathrm{E}-13$ & $1.12 \mathrm{E}-13$ & $1.28 \mathrm{E}-09$ & $1.28 \mathrm{E}-09$ & 1.28E-09 & & & \\
\hline$G d+3$ & $6.10 \mathrm{E}-11$ & $5.88 \mathrm{E}-11$ & $3.47 \mathrm{E}-11$ & $3.85 \mathrm{E}-07$ & $3.85 \mathrm{E}-07$ & $3.85 \mathrm{E}-07$ & & & \\
\hline $\mathrm{Ge}+4$ & $1.58 \mathrm{E}-15$ & $1.53 \mathrm{E}-15$ & $9.01 \mathrm{E}-16$ & $3.60 \mathrm{E}-11$ & $3.59 \mathrm{E}-11$ & $3.59 \mathrm{E}-11$ & & & \\
\hline $\ln +3$ & $4.13 \mathrm{E}-13$ & $3.98 \mathrm{E}-13$ & $2.35 \mathrm{E}-13$ & $3.57 \mathrm{E}-09$ & 3.57E-09 & $3.57 \mathrm{E}-09$ & & & \\
\hline $\mid$ & $5.55 \mathrm{E}-11$ & $5.34 \mathrm{E}-11$ & $3.15 \mathrm{E}-11$ & 3.81E-07 & $3.80 \mathrm{E}-07$ & $3.80 \mathrm{E}-07$ & $6.64 \mathrm{E}-12$ & \begin{tabular}{|l|}
$6.61 \mathrm{E}-12$ \\
\end{tabular} & $6.61 \mathrm{E}-12$ \\
\hline $\mathrm{La}+3$ & $3.25 \mathrm{E}-12$ & $3.14 \mathrm{E}-12$ & $1.85 \mathrm{E}-12$ & $2.33 \mathrm{E}-08$ & $2.33 \mathrm{E}-08$ & $2.33 \mathrm{E}-08$ & & & \\
\hline
\end{tabular}


Table A-16. Mass balance, Tank WM-189 waste (continued).

\begin{tabular}{|c|c|c|c|c|c|c|c|c|c|}
\hline Stream \# & 109 & 110 & 111 & 112 & $113 \mathrm{~A}$ & $113 \mathrm{~B}$ & 114 & 115 & 116 \\
\hline Chemical Composition & $\mathrm{lb} /$ wscf & $\mathrm{lb} /$ wscf & $\mathrm{lb} /$ wscf & Mol/liter & Mol/liter & Mol/liter & $\mathrm{lb} / \mathrm{wscf}$ & $\mathrm{lb} /$ wscf & $\mathrm{lb} / \mathrm{wscf}$ \\
\hline $\mathrm{Li+}$ & $4.07 \mathrm{E}-11$ & $2.49 \mathrm{E}-11$ & $1.47 \mathrm{E}-11$ & $2.73 \mathrm{E}-06$ & $2.73 \mathrm{E}-06$ & $2.73 \mathrm{E}-06$ & & & \\
\hline $\mathrm{Mg}+2$ & $1.41 \mathrm{E}-09$ & $1.36 \mathrm{E}-09$ & $1.56 \mathrm{E}-11$ & $5.80 \mathrm{E}-05$ & 5.79E-05 & $5.79 \mathrm{E}-05$ & & & \\
\hline $\mathrm{Mo}+6$ & $1.56 \mathrm{E}-08$ & 1.51E-08 & 8.89E-09 & 2.81E-04 & $2.81 \mathrm{E}-04$ & 2.81E-04 & & & \\
\hline $\mathrm{Nd}+3$ & $1.09 \mathrm{E}-11$ & $1.05 \mathrm{E}-11$ & $6.20 \mathrm{E}-12$ & $7.51 \mathrm{E}-08$ & $7.50 \mathrm{E}-08$ & $7.50 \mathrm{E}-08$ & & & \\
\hline $\mathrm{Np}+4$ & $1.86 \mathrm{E}-12$ & $1.79 \mathrm{E}-12$ & $1.06 \mathrm{E}-12$ & 7.79E-09 & 7.79E-09 & $7.79 \mathrm{E}-09$ & & & \\
\hline $\mathrm{Nb}+5$ & $2.96 \mathrm{E}-10$ & $2.85 \mathrm{E}-10$ & $1.68 \mathrm{E}-10$ & 5.34E-06 & $5.34 \mathrm{E}-06$ & 5.34E-06 & & & \\
\hline $\mathrm{Pd}+4$ & $5.37 \mathrm{E}-10$ & $5.18 \mathrm{E}-10$ & $5.96 \mathrm{E}-12$ & $5.06 \mathrm{E}-06$ & $5.06 \mathrm{E}-06$ & $5.06 \mathrm{E}-06$ & & & \\
\hline $\mathrm{Pu}+4$ & $4.52 \mathrm{E}-12$ & $4.35 \mathrm{E}-12$ & $2.57 \mathrm{E}-12$ & $3.05 \mathrm{E}-08$ & $3.05 \mathrm{E}-08$ & $3.05 \mathrm{E}-08$ & & & \\
\hline $\mathrm{Pr}+4$ & $3.00 \mathrm{E}-12$ & $2.89 \mathrm{E}-12$ & $1.71 \mathrm{E}-12$ & $2.12 \mathrm{E}-08$ & $2.12 \mathrm{E}-08$ & $2.12 \mathrm{E}-08$ & & & \\
\hline $\mathrm{Pm}+3$ & $6.21 \mathrm{E}-16$ & $5.98 \mathrm{E}-16$ & $3.53 \mathrm{E}-16$ & $4.25 \mathrm{E}-12$ & $4.25 \mathrm{E}-12$ & $4.25 \mathrm{E}-12$ & & & \\
\hline $\mathrm{Rh}+4$ & $9.46 \mathrm{E}-13$ & $9.11 \mathrm{E}-13$ & $5.38 \mathrm{E}-13$ & $9.13 \mathrm{E}-09$ & $9.13 \mathrm{E}-09$ & $9.13 \mathrm{E}-09$ & & & \\
\hline $\mathrm{Rb}+$ & $4.57 \mathrm{E}-12$ & $2.79 \mathrm{E}-12$ & $1.65 \mathrm{E}-12$ & $2.49 \mathrm{E}-08$ & $2.49 \mathrm{E}-08$ & 2.49E-08 & & & \\
\hline Ru+3 & $2.56 \mathrm{E}-10$ & $2.47 \mathrm{E}-10$ & $1.46 \mathrm{E}-10$ & $2.52 \mathrm{E}-06$ & $2.52 \mathrm{E}-06$ & 2.52E-06 & & & \\
\hline $\mathrm{Sm}+3$ & $2.12 \mathrm{E}-12$ & $2.04 \mathrm{E}-12$ & $1.20 \mathrm{E}-12$ & $1.40 \mathrm{E}-08$ & \begin{tabular}{|l|}
$1.40 \mathrm{E}-08$ \\
\end{tabular} & $1.40 \mathrm{E}-08$ & & & \\
\hline $\mathrm{Si}+4$ & $1.06 \mathrm{E}-08$ & $1.02 \mathrm{E}-08$ & $6.01 \mathrm{E}-09$ & $7.40 \mathrm{E}-04$ & 7.39E-04 & 7.39E-04 & & & \\
\hline $\mathrm{Sr}+2$ & $3.12 \mathrm{E}-11$ & $3.00 \mathrm{E}-11$ & $3.46 \mathrm{E}-13$ & $3.57 \mathrm{E}-07$ & $3.56 \mathrm{E}-07$ & $3.56 \mathrm{E}-07$ & & & \\
\hline $\mathrm{Tc}+7$ & $5.08 \mathrm{E}-12$ & $4.90 \mathrm{E}-12$ & $2.89 \mathrm{E}-12$ & $6.22 \mathrm{E}-08$ & 6.21E-08 & $6.21 \mathrm{E}-08$ & & & \\
\hline $\mathrm{Te}+4$ & $2.04 \mathrm{E}-12$ & $1.97 \mathrm{E}-12$ & $1.16 \mathrm{E}-12$ & $2.64 \mathrm{E}-08$ & $2.64 \mathrm{E}-08$ & $2.64 \mathrm{E}-08$ & & & \\
\hline $\mathrm{Tb}+4$ & $8.57 \mathrm{E}-16$ & $8.26 \mathrm{E}-16$ & $4.87 \mathrm{E}-16$ & $5.36 \mathrm{E}-12$ & $5.36 \mathrm{E}-12$ & $5.36 \mathrm{E}-12$ & & & \\
\hline $\mathrm{Th}+4$ & $1.84 \mathrm{E}-11$ & $1.78 \mathrm{E}-11$ & $1.05 \mathrm{E}-11$ & $1.31 \mathrm{E}-07$ & 1.31E-07 & $1.31 \mathrm{E}-07$ & & & \\
\hline$S n+4$ & $6.37 \mathrm{E}-10$ & $6.14 \mathrm{E}-10$ & $3.62 \mathrm{E}-10$ & $8.84 \mathrm{E}-06$ & $8.83 \mathrm{E}-06$ & 8.83E-06 & & & \\
\hline $\mathrm{Ti}+4$ & $1.33 \mathrm{E}-10$ & $1.28 \mathrm{E}-10$ & $7.54 \mathrm{E}-11$ & $4.56 \mathrm{E}-06$ & $4.56 \mathrm{E}-06$ & 4.56E-06 & & & \\
\hline $\mathrm{Y}+3$ & $1.55 \mathrm{E}-12$ & $1.50 \mathrm{E}-12$ & $8.83 E-13$ & $1.73 \mathrm{E}-08$ & $1.73 \mathrm{E}-08$ & $1.73 \mathrm{E}-08$ & & & \\
\hline $\mathrm{OH}-$ & $3.61 \mathrm{E}-09$ & 8.69E-08 & $2.02 \mathrm{E}-07$ & 7.39E-07 & 7.46E-07 & 7.46E-07 & & & \\
\hline $\mathrm{H} 2 \mathrm{O}$ & $3.16 \mathrm{E}-02$ & $3.28 \mathrm{E}-02$ & $3.87 E-02$ & $5.23 E+01$ & $5.23 E+01$ & $5.23 E+01$ & $3.86 \mathrm{E}-02$ & $3.85 \mathrm{E}-02$ & $3.85 \mathrm{E}-02$ \\
\hline $\mathrm{SO} 2$ & 4.61E-09 & $3.65 \mathrm{E}-05$ & $2.15 \mathrm{E}-05$ & \begin{tabular}{|l|}
$1.65 \mathrm{E}-05$ \\
\end{tabular} & \begin{tabular}{|l|}
$1.65 \mathrm{E}-05$ \\
\end{tabular} & 1.65E-05 & $2.17 \mathrm{E}-05$ & $\begin{array}{l}2.16 \mathrm{E}-05 \\
\end{array}$ & $2.16 \mathrm{E}-05$ \\
\hline $\mathrm{H} 2 \mathrm{~S}$ & $2.04 \mathrm{E}-05$ & $4.53 \mathrm{E}-21$ & & & & & & & \\
\hline $\mathrm{CO}$ & $8.43 \mathrm{E}-04$ & $1.64 \mathrm{E}-09$ & $9.68 \mathrm{E}-10$ & $2.09 \mathrm{E}-10$ & $2.12 \mathrm{E}-10$ & $2.12 \mathrm{E}-10$ & $9.73 \mathrm{E}-10$ & $9.70 \mathrm{E}-10$ & $9.70 \mathrm{E}-10$ \\
\hline $\mathrm{CO} 2$ & 8.03E-03 & 9.97E-03 & $5.88 \mathrm{E}-03$ & 3.33E-04 & 3.43E-04 & $3.43 E-04$ & 5.92E-03 & 5.89E-03 & 5.89E-03 \\
\hline $\mathrm{H} 2$ & $2.04 \mathrm{E}-04$ & $6.12 \mathrm{E}-10$ & $3.61 \mathrm{E}-10$ & $8.76 \mathrm{E}-10$ & 1.42E-09 & 1.42E-09 & $2.15 \mathrm{E}-08$ & & \\
\hline N2 & $1.43 \mathrm{E}-02$ & $1.38 \mathrm{E}-02$ & $8.12 \mathrm{E}-03$ & $1.28 \mathrm{E}-05$ & $2.47 \mathrm{E}-05$ & $2.47 \mathrm{E}-05$ & $8.17 \mathrm{E}-03$ & $8.13 \mathrm{E}-03$ & $8.13 \mathrm{E}-03$ \\
\hline $\mathrm{NO}$ & $4.41 \mathrm{E}-05$ & $4.80 \mathrm{E}-05$ & $2.83 \mathrm{E}-05$ & $2.22 \mathrm{E}-07$ & $2.81 \mathrm{E}-07$ & $2.81 \mathrm{E}-07$ & $2.85 \mathrm{E}-05$ & $2.84 \mathrm{E}-05$ & 2.84E-05 \\
\hline $\mathrm{NO} 2$ & 4.57E-06 & $2.62 \mathrm{E}-07$ & $1.55 \mathrm{E}-07$ & $2.79 \mathrm{E}-06$ & $2.79 \mathrm{E}-06$ & 2.79E-06 & $1.55 \mathrm{E}-07$ & $1.55 \mathrm{E}-07$ & $1.55 \mathrm{E}-07$ \\
\hline $\mathrm{O} 2$ & $4.70 \mathrm{E}-04$ & 1.83E-03 & $1.08 \mathrm{E}-03$ & $3.16 \mathrm{E}-06$ & 4.89E-06 & 4.89E-06 & $1.08 \mathrm{E}-03$ & $1.08 \mathrm{E}-03$ & 1.08E-03 \\
\hline S (other) & $4.29 \mathrm{E}-10$ & $1.96 \mathrm{E}-25$ & & $2.19 \mathrm{E}-07$ & $2.19 \mathrm{E}-07$ & $2.19 \mathrm{E}-07$ & & & \\
\hline $\mathrm{CO} 3$ & $2.15 \mathrm{E}-07$ & $2.08 \mathrm{E}-11$ & $6.71 \mathrm{E}-12$ & $2.73 \mathrm{E}-05$ & 2.72E-05 & 2.72E-05 & & & \\
\hline C (reductant) & $3.57 \mathrm{E}-08$ & $1.44 \mathrm{E}-18$ & & & & & & & \\
\hline $\mathrm{O}$ (oxides) & $6.93 \mathrm{E}-08$ & $6.49 \mathrm{E}-08$ & $3.83 \mathrm{E}-08$ & 5.26E-03 & $5.26 \mathrm{E}-03$ & 5.26E-03 & & & \\
\hline C (organic) & 1.97E-05 & $1.82 \mathrm{E}-34$ & & & & & & & \\
\hline $\mathrm{H}$ (organic) & 6.59E-06 & $6.11 \mathrm{E}-35$ & & & & & & & \\
\hline O (organic) & 1.47E-07 & & & & & & & & \\
\hline Mass Flow (kg/hr): & $2.85 \mathrm{E}+03$ & $3.11 \mathrm{E}+03$ & $4.85 \mathrm{E}+03$ & $4.23 \mathrm{E}+04$ & $4.23 \mathrm{E}+04$ & $5.11 \mathrm{E}-02$ & $4.83 \mathrm{E}+03$ & $4.83 \mathrm{E}+03$ & $4.83 \mathrm{E}+03$ \\
\hline & & & & & & & & & \\
\hline & & & & & & & & & \\
\hline & $\mathrm{w} / \mathrm{m} 3$ & $\mathrm{w} / \mathrm{m} 3$ & $\mathrm{w} / \mathrm{m} 3$ & $\mathrm{w} / \mathrm{m} 3$ & $\mathrm{w} / \mathrm{m} 3$ & $\mathrm{w} / \mathrm{m} 3$ & $\mathrm{w} / \mathrm{m} 3$ & $\mathrm{w} / \mathrm{m} 3$ & $\mathrm{w} / \mathrm{m} 3$ \\
\hline Heat Generation, w/m3 & & & & 4.87E-02 & $4.86 \mathrm{E}-02$ & & & & \\
\hline
\end{tabular}


Table A-16. Mass balance, Tank WM-189 waste (continued).

\begin{tabular}{|c|c|c|c|c|c|c|c|c|c|}
\hline Stream \# & 109 & 110 & 111 & 112 & $113 \mathrm{~A}$ & 113B & 114 & 115 & 116 \\
\hline Radiological Composition & $\mathrm{Ci} / \mathrm{wscm}$ & $\mathrm{Ci} / \mathrm{wscm}$ & $\mathrm{Ci} / \mathrm{wscm}$ & $\mathrm{Ci} / \mathrm{L}$ & $\mathrm{Ci} / \mathrm{L}$ & $\mathrm{Ci} / \mathrm{L}$ & $\mathrm{Ci} / \mathrm{wscm}$ & $\mathrm{Ci} / \mathrm{wscm}$ & $\mathrm{Ci} / \mathrm{wscm}$ \\
\hline $\mathrm{H}-3$ & 9.35E-07 & 9.01E-07 & 5.32E-07 & $7.89 \mathrm{E}-07$ & $7.88 \mathrm{E}-07$ & $7.88 \mathrm{E}-07$ & $5.31 \mathrm{E}-07$ & $5.29 \mathrm{E}-07$ & $5.29 \mathrm{E}-07$ \\
\hline C-14 & $1.40 \mathrm{E}-11$ & $1.36 \mathrm{E}-11$ & $8.00 \mathrm{E}-12$ & $1.35 \mathrm{E}-15$ & $1.38 \mathrm{E}-15$ & $1.38 \mathrm{E}-15$ & $8.05 \mathrm{E}-12$ & $8.02 \mathrm{E}-12$ & $8.02 \mathrm{E}-12$ \\
\hline Co-60 & 1.34E-09 & 1.29E-09 & $7.62 \mathrm{E}-10$ & $8.31 \mathrm{E}-08$ & $8.31 \mathrm{E}-08$ & $8.31 \mathrm{E}-08$ & & & \\
\hline Ni-59 & $5.87 \mathrm{E}-11$ & $5.65 \mathrm{E}-11$ & $6.51 \mathrm{E}-13$ & 3.67E-09 & 3.67E-09 & 3.67E-09 & & & \\
\hline $\mathrm{Ni}-63$ & $1.83 \mathrm{E}-09$ & $1.76 \mathrm{E}-09$ & $2.03 E-11$ & $1.14 \mathrm{E}-07$ & 1.14E-07 & $1.14 \mathrm{E}-07$ & & & \\
\hline Se-79 & $2.33 \mathrm{E}-11$ & $2.25 \mathrm{E}-11$ & $1.33 \mathrm{E}-11$ & $2.40 \mathrm{E}-09$ & $2.40 \mathrm{E}-09$ & $2.40 \mathrm{E}-09$ & & & \\
\hline Sr-90 & $1.66 \mathrm{E}-06$ & $1.60 \mathrm{E}-06$ & $1.84 \mathrm{E}-08$ & 1.04E-04 & 1.04E-04 & 1.04E-04 & & & \\
\hline Y-90 & $1.59 \mathrm{E}-06$ & $1.53 \mathrm{E}-06$ & 9.04E-07 & $9.86 \mathrm{E}-05$ & $9.86 \mathrm{E}-05$ & $9.86 \mathrm{E}-05$ & & & \\
\hline Zr-93 & $8.66 \mathrm{E}-11$ & $8.35 \mathrm{E}-11$ & $4.93 \mathrm{E}-11$ & $8.91 \mathrm{E}-09$ & $8.91 \mathrm{E}-09$ & $8.91 \mathrm{E}-09$ & & & \\
\hline $\mathrm{Nb}-93 \mathrm{~m}$ & $6.68 \mathrm{E}-11$ & $6.43 \mathrm{E}-11$ & $3.80 \mathrm{E}-11$ & 6.99E-09 & 6.99E-09 & 6.99E-09 & & & \\
\hline $\mathrm{Nb}-94$ & $6.07 \mathrm{E}-11$ & $5.85 \mathrm{E}-11$ & $3.45 \mathrm{E}-11$ & $6.35 \mathrm{E}-09$ & $6.35 \mathrm{E}-09$ & $6.35 E-09$ & & & \\
\hline Tc-99 & 1.95E-09 & $1.88 \mathrm{E}-09$ & 1.11E-09 & $1.46 \mathrm{E}-07$ & $1.46 \mathrm{E}-07$ & $1.46 \mathrm{E}-07$ & & & \\
\hline Ru-106 & $5.13 E-11$ & $4.94 \mathrm{E}-11$ & $2.91 \mathrm{E}-11$ & 3.18E-09 & $3.18 \mathrm{E}-09$ & $3.18 \mathrm{E}-09$ & & & \\
\hline Rh-102 & $3.46 \mathrm{E}-14$ & $3.34 \mathrm{E}-14$ & $1.97 \mathrm{E}-14$ & $2.15 \mathrm{E}-12$ & $2.15 \mathrm{E}-12$ & $2.15 \mathrm{E}-12$ & & & \\
\hline Rh-106 & $5.13 \mathrm{E}-11$ & $4.94 \mathrm{E}-11$ & $2.91 \mathrm{E}-11$ & 3.18E-09 & $3.18 \mathrm{E}-09$ & $3.18 \mathrm{E}-09$ & & & \\
\hline $\mathrm{Pd}-107$ & $6.92 \mathrm{E}-13$ & $6.67 \mathrm{E}-13$ & $7.68 \mathrm{E}-15$ & $4.33 \mathrm{E}-11$ & $4.33 \mathrm{E}-11$ & $4.33 \mathrm{E}-11$ & & & \\
\hline $\mathrm{Cd}-113 \mathrm{~m}$ & $1.52 \mathrm{E}-10$ & $1.47 \mathrm{E}-10$ & $8.65 \mathrm{E}-11$ & $5.11 \mathrm{E}-09$ & 5.12E-09 & 5.12E-09 & & & \\
\hline Sn-121m & $2.61 \mathrm{E}-12$ & $2.52 \mathrm{E}-12$ & $1.49 \mathrm{E}-12$ & $2.69 \mathrm{E}-10$ & $2.69 \mathrm{E}-10$ & $2.69 \mathrm{E}-10$ & & & \\
\hline Sn-126 & $2.20 \mathrm{E}-11$ & $2.12 \mathrm{E}-11$ & $1.25 \mathrm{E}-11$ & $2.26 \mathrm{E}-09$ & $2.26 \mathrm{E}-09$ & $2.26 \mathrm{E}-09$ & & & \\
\hline Sb-125 & $1.88 \mathrm{E}-08$ & $1.81 \mathrm{E}-08$ & 1.07E-08 & $1.17 \mathrm{E}-06$ & 1.17E-06 & 1.17E-06 & & & \\
\hline Sb-126 & $2.31 \mathrm{E}-12$ & $2.23 \mathrm{E}-12$ & $1.31 \mathrm{E}-12$ & $1.43 \mathrm{E}-10$ & $1.43 \mathrm{E}-10$ & $1.43 \mathrm{E}-10$ & & & \\
\hline Te-125m & $1.23 \mathrm{E}-10$ & 1.19E-10 & $7.01 \mathrm{E}-11$ & 1.27E-08 & 1.27E-08 & 1.27E-08 & & & \\
\hline $\mid-129$ & $1.48 \mathrm{E}-10$ & $1.43 \mathrm{E}-10$ & $8.42 \mathrm{E}-11$ & $8.05 \mathrm{E}-09$ & $8.05 E-09$ & $8.05 \mathrm{E}-09$ & $1.77 \mathrm{E}-11$ & $1.77 \mathrm{E}-11$ & $1.77 \mathrm{E}-11$ \\
\hline Cs-134 & 6.54E-09 & 6.30E-09 & 3.72E-09 & $5.66 \mathrm{E}-05$ & \begin{tabular}{|l|}
$5.65 E-05$ \\
\end{tabular} & 5.65E-05 & & & \\
\hline Cs-135 & $1.03 \mathrm{E}-10$ & $9.94 \mathrm{E}-11$ & $5.86 \mathrm{E}-11$ & 8.92E-07 & 8.91E-07 & 8.91E-07 & & & \\
\hline Cs-137 & $5.34 \mathrm{E}-06$ & $5.15 \mathrm{E}-06$ & $3.04 \mathrm{E}-06$ & $4.62 \mathrm{E}-02$ & $4.62 \mathrm{E}-02$ & $4.62 \mathrm{E}-02$ & & & \\
\hline Ba-137m & $2.34 \mathrm{E}-06$ & $2.26 \mathrm{E}-06$ & $2.60 \mathrm{E}-08$ & $1.47 \mathrm{E}-04$ & $1.46 \mathrm{E}-04$ & $1.46 \mathrm{E}-04$ & & & \\
\hline $\mathrm{Ce}-144$ & $3.46 \mathrm{E}-11$ & $3.34 \mathrm{E}-11$ & $1.97 \mathrm{E}-11$ & $2.15 \mathrm{E}-09$ & $2.15 \mathrm{E}-09$ & $2.15 \mathrm{E}-09$ & & & \\
\hline Pr-144 & $3.46 \mathrm{E}-11$ & 3.34E-11 & $1.97 \mathrm{E}-11$ & $2.15 \mathrm{E}-09$ & $2.15 \mathrm{E}-09$ & $2.15 \mathrm{E}-09$ & & & \\
\hline $\mathrm{Pm}-146$ & $2.05 \mathrm{E}-12$ & $1.97 \mathrm{E}-12$ & $1.16 \mathrm{E}-12$ & $1.27 \mathrm{E}-10$ & $1.27 \mathrm{E}-10$ & $1.27 \mathrm{E}-10$ & & & \\
\hline $\mathrm{Pm}-147$ & 9.34E-09 & 9.00E-09 & 5.31E-09 & 5.79E-07 & 5.79E-07 & 5.79E-07 & & & \\
\hline Sm-151 & $1.85 \mathrm{E}-08$ & $1.78 \mathrm{E}-08$ & $1.05 \mathrm{E}-08$ & 1.15E-06 & $1.14 \mathrm{E}-06$ & 1.14E-06 & & & \\
\hline Eu-152 & $1.19 \mathrm{E}-10$ & $1.14 \mathrm{E}-10$ & $6.74 \mathrm{E}-11$ & 7.35E-09 & $7.35 \mathrm{E}-09$ & 7.35E-09 & & & \\
\hline Eu-154 & 7.07E-09 & 6.82E-09 & 4.02E-09 & 4.39E-07 & 4.38E-07 & $4.38 \mathrm{E}-07$ & & & \\
\hline Eu-155 & $6.66 \mathrm{E}-09$ & $6.42 \mathrm{E}-09$ & $3.79 \mathrm{E}-09$ & $4.13 \mathrm{E}-07$ & \begin{tabular}{|l|}
$4.13 \mathrm{E}-07$ \\
\end{tabular} & \begin{tabular}{|l|}
$4.13 \mathrm{E}-07$ \\
\end{tabular} & & & \\
\hline Th-230 & $4.24 \mathrm{E}-14$ & $4.09 \mathrm{E}-14$ & $2.41 \mathrm{E}-14$ & $4.36 \mathrm{E}-12$ & $4.36 \mathrm{E}-12$ & $4.36 \mathrm{E}-12$ & & & \\
\hline $\mathrm{Pa}-233$ & $1.15 \mathrm{E}-10$ & $1.10 \mathrm{E}-10$ & $6.52 \mathrm{E}-11$ & $1.18 \mathrm{E}-08$ & 1.18E-08 & $1.18 \mathrm{E}-08$ & & & \\
\hline $\mathrm{U}-232$ & $9.92 \mathrm{E}-14$ & $9.56 \mathrm{E}-14$ & $5.64 \mathrm{E}-14$ & $1.02 \mathrm{E}-11$ & $1.02 \mathrm{E}-11$ & $1.02 \mathrm{E}-11$ & & & \\
\hline U-233 & $3.50 \mathrm{E}-15$ & $3.37 \mathrm{E}-15$ & $1.99 \mathrm{E}-15$ & $3.60 \mathrm{E}-13$ & $3.59 \mathrm{E}-13$ & $3.59 \mathrm{E}-13$ & & & \\
\hline U-234 & $6.86 \mathrm{E}-11$ & $6.61 \mathrm{E}-11$ & $3.90 \mathrm{E}-11$ & $7.06 \mathrm{E}-09$ & 7.05E-09 & $7.05 E-09$ & & & \\
\hline U-235 & $2.77 \mathrm{E}-12$ & $2.67 \mathrm{E}-12$ & $1.57 \mathrm{E}-12$ & $2.85 \mathrm{E}-10$ & $2.85 \mathrm{E}-10$ & $2.85 \mathrm{E}-10$ & & & \\
\hline U-236 & $3.21 \mathrm{E}-12$ & $3.09 \mathrm{E}-12$ & $1.83 \mathrm{E}-12$ & 3.30E-10 & $3.30 \mathrm{E}-10$ & $3.30 \mathrm{E}-10$ & & & \\
\hline U-237 & $2.52 \mathrm{E}-13$ & $2.43 \mathrm{E}-13$ & $1.43 \mathrm{E}-13$ & $2.59 \mathrm{E}-11$ & $2.59 \mathrm{E}-11$ & $2.59 \mathrm{E}-11$ & & & \\
\hline U-238 & $1.52 \mathrm{E}-12$ & $1.47 \mathrm{E}-12$ & $8.66 \mathrm{E}-13$ & $1.57 \mathrm{E}-10$ & 1.57E-10 & $1.57 \mathrm{E}-10$ & & & \\
\hline $\mathrm{Np}-237$ & $2.10 \mathrm{E}-11$ & $2.02 \mathrm{E}-11$ & 1.19E-11 & 1.30E-09 & 1.30E-09 & 1.30E-09 & & & \\
\hline Pu-236 & $1.62 \mathrm{E}-13$ & $1.56 \mathrm{E}-13$ & $9.20 \mathrm{E}-14$ & $1.66 \mathrm{E}-11$ & $1.66 \mathrm{E}-11$ & $1.66 \mathrm{E}-11$ & & & \\
\hline Pu-238 & $3.73 \mathrm{E}-08$ & $3.60 \mathrm{E}-08$ & $2.12 \mathrm{E}-08$ & $3.84 \mathrm{E}-06$ & 3.84E-06 & $3.84 \mathrm{E}-06$ & & & \\
\hline Pu-239 & $4.08 \mathrm{E}-09$ & 3.93E-09 & $2.32 \mathrm{E}-09$ & $4.20 \mathrm{E}-07$ & 4.19E-07 & 4.19E-07 & & & \\
\hline Pu-240 & $6.03 \mathrm{E}-10$ & $5.81 \mathrm{E}-10$ & $3.43 E-10$ & $6.20 \mathrm{E}-08$ & $6.20 \mathrm{E}-08$ & $6.20 \mathrm{E}-08$ & & & \\
\hline Pu-241 & $3.27 E-08$ & $3.15 \mathrm{E}-08$ & 1.86E-08 & $3.36 \mathrm{E}-06$ & $3.36 \mathrm{E}-06$ & $3.36 \mathrm{E}-06$ & & & \\
\hline Pu-242 & $4.72 \mathrm{E}-13$ & $4.55 \mathrm{E}-13$ & $2.69 \mathrm{E}-13$ & $4.86 \mathrm{E}-11$ & $4.85 \mathrm{E}-11$ & $4.85 \mathrm{E}-11$ & & & \\
\hline Pu-244 & $1.41 \mathrm{E}-20$ & $1.36 \mathrm{E}-20$ & $8.01 \mathrm{E}-21$ & $1.45 \mathrm{E}-18$ & $1.45 \mathrm{E}-18$ & $1.45 \mathrm{E}-18$ & & & \\
\hline $\mathrm{Am}-241$ & $3.27 \mathrm{E}-09$ & $3.16 \mathrm{E}-09$ & $1.86 \mathrm{E}-09$ & $2.03 \mathrm{E}-07$ & $2.03 \mathrm{E}-07$ & $2.03 E-07$ & & & \\
\hline Am-242m & $6.14 \mathrm{E}-13$ & $5.92 \mathrm{E}-13$ & $3.49 \mathrm{E}-13$ & $3.81 \mathrm{E}-11$ & 3.81E-11 & $3.81 \mathrm{E}-11$ & & & \\
\hline
\end{tabular}


Table A-16. Mass balance, Tank WM-189 waste (continued).

\begin{tabular}{|c|c|c|c|c|c|c|c|c|c|}
\hline Stream \# & 109 & 110 & 111 & 112 & $113 \mathrm{~A}$ & $113 \mathrm{~B}$ & 114 & 115 & 116 \\
\hline & Ci/wscm & Ci/wscm & $\mathrm{Ci} / \mathrm{wscm}$ & $\mathrm{Ci} / \mathrm{L}$ & $\mathrm{Ci} / \mathrm{L}$ & \begin{tabular}{|c|}
$\mathrm{Ci} / \mathrm{L}$ \\
\end{tabular} & Ci/wscm & $\mathrm{Ci} / \mathrm{wscm}$ & Ci/wscm \\
\hline Am-243 & $1.05 \mathrm{E}-12$ & $1.01 \mathrm{E}-12$ & $5.95 \mathrm{E}-13$ & $6.49 \mathrm{E}-11$ & $6.49 \mathrm{E}-11$ & $6.49 \mathrm{E}-11$ & & & \\
\hline $\mathrm{Cm}-242$ & $1.19 \mathrm{E}-12$ & $1.15 \mathrm{E}-12$ & $6.79 \mathrm{E}-13$ & $1.23 \mathrm{E}-10$ & $1.23 \mathrm{E}-10$ & $1.23 \mathrm{E}-10$ & & & \\
\hline $\mathrm{Cm}-243$ & $1.47 \mathrm{E}-12$ & $1.42 \mathrm{E}-12$ & $8.35 \mathrm{E}-13$ & $1.51 \mathrm{E}-10$ & $1.51 \mathrm{E}-10$ & $1.51 \mathrm{E}-10$ & & & \\
\hline $\mathrm{Cm}-244$ & $6.22 \mathrm{E}-11$ & $6.00 \mathrm{E}-11$ & $3.54 \mathrm{E}-11$ & $6.40 \mathrm{E}-09$ & 6.39E-09 & $6.39 E-09$ & & & \\
\hline $\mathrm{Cm}-245$ & $1.55 \mathrm{E}-14$ & 1.49E-14 & $8.79 \mathrm{E}-15$ & $1.59 \mathrm{E}-12$ & $1.59 \mathrm{E}-12$ & $1.59 \mathrm{E}-12$ & & & \\
\hline $\mathrm{Cm}-246$ & $1.01 \mathrm{E}-15$ & $9.78 \mathrm{E}-16$ & $5.77 \mathrm{E}-16$ & $1.04 \mathrm{E}-13$ & $1.04 \mathrm{E}-13$ & $1.04 \mathrm{E}-13$ & & & \\
\hline TRU & $4.63 \mathrm{E}-08$ & $4.46 \mathrm{E}-08$ & $2.63 \mathrm{E}-08$ & 4.63E-06 & 4.63E-06 & $4.63 E-06$ & & & \\
\hline \multicolumn{10}{|l|}{ TRU, nCi/gm } \\
\hline & & & & & & & & & \\
\hline $\begin{array}{l}\text { Gas Stream Bulk } \\
\text { Composition (Wet Basis) }\end{array}$ & $\begin{array}{l}\text { mol\% or } \\
\text { ppmv }\end{array}$ & $\begin{array}{c}\mathrm{mol} \% \text { or } \\
\text { ppmv }\end{array}$ & $\begin{array}{l}\text { mol\% or } \\
\text { ppmv }\end{array}$ & $\begin{array}{l}\text { mol\% or } \\
\text { ppmv }\end{array}$ & $\begin{array}{l}\text { mol\% or } \\
\text { ppmv }\end{array}$ & $\begin{array}{l}\text { mol\% or } \\
\text { ppmv }\end{array}$ & $\begin{array}{l}\text { mol\% or } \\
\text { ppmv }\end{array}$ & $\begin{array}{c}\mathrm{mol} \% \text { or } \\
\text { ppmv }\end{array}$ & $\begin{array}{c}\text { mol\% or } \\
\text { ppmv }\end{array}$ \\
\hline $\mathrm{H} 2 \mathrm{O}, \mathrm{mol} \%$ & $67.58 \%$ & $70.06 \%$ & $82.39 \%$ & & & & $82.29 \%$ & $82.29 \%$ & $82.29 \%$ \\
\hline $\mathrm{O} 2, \mathrm{~mol} \%$ & $0.57 \%$ & $2.20 \%$ & $1.29 \%$ & & & & $1.30 \%$ & $1.30 \%$ & $1.30 \%$ \\
\hline $\mathrm{N} 2, \mathrm{~mol} \%$ & $19.62 \%$ & $18.93 \%$ & $11.13 \%$ & & & & $11.19 \%$ & $11.19 \%$ & $11.19 \%$ \\
\hline $\mathrm{H} 2, \mathrm{~mol} \%$ & $3.89 \%$ & $0.00001 \%$ & $0.00001 \%$ & & & & $0.0004 \%$ & & $0.00 \%$ \\
\hline $\mathrm{CO} 2, \mathrm{~mol} \%$ & $7.03 \%$ & $8.73 \%$ & $5.13 \%$ & & & & $5.16 \%$ & $5.16 \%$ & $5.16 \%$ \\
\hline $\mathrm{CO}, \mathrm{ppmv}$ & $1.16 \mathrm{E}+04$ & $2.26 \mathrm{E}-02$ & 1.33E-02 & & & & 1.33E-02 & 1.33E-02 & 1.33E-02 \\
\hline $\mathrm{NO}, \mathrm{ppmv}$ & $5.66 \mathrm{E}+02$ & $6.17 \mathrm{E}+02$ & $3.63 \mathrm{E}+02$ & & & & $3.65 \mathrm{E}+02$ & $3.65 \mathrm{E}+02$ & $3.65 \mathrm{E}+02$ \\
\hline NO2, ppmv & $3.83 \mathrm{E}+01$ & $2.19 \mathrm{E}+00$ & $1.29 \mathrm{E}+00$ & & & & $1.30 \mathrm{E}+00$ & $1.30 \mathrm{E}+00$ & $1.30 \mathrm{E}+00$ \\
\hline SO2, ppmv & $2.77 \mathrm{E}-02$ & $2.19 \mathrm{E}+02$ & $1.29 \mathrm{E}+02$ & & & & $1.30 \mathrm{E}+02$ & $1.30 \mathrm{E}+02$ & $1.30 \mathrm{E}+02$ \\
\hline $\mathrm{Cl}, \mathrm{ppmv}$ & $1.14 \mathrm{E}+00$ & $1.10 \mathrm{E}+00$ & $6.48 \mathrm{E}-01$ & & & & $1.36 \mathrm{E}-01$ & 1.36E-01 & 1.36E-01 \\
\hline $\mathrm{F}, \mathrm{ppmv}$ & $7.61 \mathrm{E}+00$ & $7.34 \mathrm{E}+00$ & $4.31 \mathrm{E}+00$ & & & & $6.75 \mathrm{E}+00$ & $6.75 \mathrm{E}+00$ & $6.75 \mathrm{E}+00$ \\
\hline C (organic), ppmv & $6.32 \mathrm{E}+02$ & $5.84 \mathrm{E}-27$ & & & & & & & \\
\hline $\mathrm{H}$ (organic), ppmv & $2.52 \mathrm{E}+03$ & $2.34 \mathrm{E}-26$ & & & & & & & \\
\hline $\mathrm{Hg}, \mathrm{ug} / \mathrm{wscm}$ & $1.35 \mathrm{E}+05$ & $1.30 \mathrm{E}+05$ & $7.67 \mathrm{E}+04$ & & & & & $4.14 \mathrm{E}+04$ & $4.14 \mathrm{E}+04$ \\
\hline $\mathrm{PM}, \mathrm{mg} / \mathrm{wscm}$ & $5.66 \mathrm{E}+02$ & $1.54 \mathrm{E}+02$ & $9.78 \mathrm{E}+01$ & & & & $1.18 \mathrm{E}+01$ & $5.31 \mathrm{E}+01$ & $5.31 \mathrm{E}+01$ \\
\hline SVM, ug/wscm & $5.02 \mathrm{E}+01$ & $4.84 \mathrm{E}+01$ & $2.86 \mathrm{E}+01$ & & & & & & \\
\hline \multirow[t]{2}{*}{ LVM, ug/wscm } & $1.40 \mathrm{E}+01$ & $1.35 \mathrm{E}+01$ & $7.93 \mathrm{E}+00$ & & & & & & \\
\hline & & & & & & & & & \\
\hline $\begin{array}{l}\text { Gas Stream Bulk } \\
\text { Composition (Dry Basis) }\end{array}$ & $\mathrm{mol} \%$ & $\mathrm{~mol} \%$ & $\mathrm{~mol} \%$ & $\mathrm{~mol} \%$ & $\mathrm{~mol} \%$ & $\mathrm{~mol} \%$ & $\mathrm{~mol} \%$ & $\mathrm{~mol} \%$ & $\mathrm{~mol} \%$ \\
\hline $\mathrm{O} 2, \mathrm{~mol} \%$, dry basis & $1.75 \%$ & $7.35 \%$ & $7.35 \%$ & & & & $7.34 \%$ & $7.34 \%$ & $7.34 \%$ \\
\hline $\mathrm{N} 2, \mathrm{~mol} \%$, dry basis & $60.51 \%$ & $63.21 \%$ & $63.21 \%$ & & & & $63.19 \%$ & $63.19 \%$ & $63.19 \%$ \\
\hline $\mathrm{H} 2, \mathrm{~mol} \%$, dry basis & $12.01 \%$ & $0.00004 \%$ & $0.00004 \%$ & & & & $0.002 \%$ & & $0.00 \%$ \\
\hline $\mathrm{CO} 2, \mathrm{~mol} \%$, dry basis & $21.69 \%$ & $29.15 \%$ & $29.15 \%$ & & & & $29.14 \%$ & $29.14 \%$ & $29.14 \%$ \\
\hline $\begin{array}{l}\text { Gas Stream Bulk } \\
\text { Composition (Dry Basis, } \\
\text { Corrected to } 7 \% \text { O2 with } \\
100 \% \text { O2 Combustion Air) }\end{array}$ & $\begin{array}{l}\text { ppmv, or } \\
\text { ug/dscm } \\
\text { or } \\
\mathrm{mg} / \mathrm{dscm}\end{array}$ & $\begin{array}{l}\mathrm{ppmv}, \text { or } \\
\mathrm{ug} / \mathrm{dscm} \text { or } \\
\mathrm{mg} / \mathrm{dscm}\end{array}$ & $\begin{array}{l}\mathrm{ppmv}, \text { or } \\
\text { ug/dscm or } \\
\mathrm{mg} / \mathrm{dscm}\end{array}$ & $\begin{array}{l}\text { ppmv, or } \\
\text { ug/dscm } \\
\text { or } \\
\mathrm{mg} / \mathrm{dscm}\end{array}$ & $\begin{array}{l}\text { ppmv, or } \\
\text { ug/dscm } \\
\text { or } \\
\mathrm{mg} / \mathrm{dscm}\end{array}$ & $\begin{array}{l}\text { ppmv, or } \\
\text { ug/dscm } \\
\text { or } \\
\mathrm{mg} / \mathrm{dscm}\end{array}$ & $\begin{array}{l}\text { ppmv, or } \\
\text { ug/dscm } \\
\text { or } \\
\mathrm{mg} / \mathrm{dscm}\end{array}$ & $\begin{array}{l}\text { ppmv, or } \\
\text { ug/dscm } \\
\text { or } \\
\mathrm{mg} / \mathrm{dscm}\end{array}$ & $\begin{array}{l}\mathrm{ppmv}, \text { or } \\
\mathrm{ug} / \mathrm{dscm} \\
\text { or } \\
\mathrm{mg} / \mathrm{dscm}\end{array}$ \\
\hline $\mathrm{CO}$, ppmv, dry basis & $5.01 \mathrm{E}+03$ & $1.06 \mathrm{E}-02$ & $1.06 \mathrm{E}-02$ & & & & $1.06 \mathrm{E}-02$ & 1.06E-02 & 1.06E-02 \\
\hline NO, ppmv, dry basis & $2.44 \mathrm{E}+02$ & $2.89 \mathrm{E}+02$ & $2.89 \mathrm{E}+02$ & & & & $2.88 \mathrm{E}+02$ & $2.88 \mathrm{E}+02$ & $2.88 \mathrm{E}+02$ \\
\hline NO2, ppmv, dry basis & $1.65 \mathrm{E}+01$ & $1.03 \mathrm{E}+00$ & $1.03 \mathrm{E}+00$ & & & & $1.03 \mathrm{E}+00$ & $1.03 E+00$ & $1.03 \mathrm{E}+00$ \\
\hline SO2, ppmv, dry basis & $1.20 \mathrm{E}-02$ & $1.03 \mathrm{E}+02$ & $1.03 \mathrm{E}+02$ & & & & $1.03 \mathrm{E}+02$ & $1.03 E+02$ & $1.03 \mathrm{E}+02$ \\
\hline $\mathrm{Cl}, \mathrm{ppmv}$, dry basis & 4.94E-01 & $5.16 \mathrm{E}-01$ & $5.16 \mathrm{E}-01$ & & & & $1.08 \mathrm{E}-01$ & $1.08 \mathrm{E}-01$ & $1.08 \mathrm{E}-01$ \\
\hline $\mathrm{F}, \mathrm{ppmv}$, dry basis & $3.29 \mathrm{E}+00$ & $3.43 \mathrm{E}+00$ & $3.43 \mathrm{E}+00$ & & & & $5.34 \mathrm{E}+00$ & $5.34 \mathrm{E}+00$ & $5.34 \mathrm{E}+00$ \\
\hline C (organic), ppmv, dry basis & $2.73 \mathrm{E}+02$ & & & & & & & & \\
\hline $\mathrm{H}$ (organic), ppmv, dry basis & $1.09 \mathrm{E}+03$ & & & & & & & & \\
\hline $\mathrm{Hg}, \mathrm{ug} / \mathrm{dscm}$ & $5.82 \mathrm{E}+04$ & $6.08 \mathrm{E}+04$ & $6.10 \mathrm{E}+04$ & & & & & $3.27 \mathrm{E}+04$ & $3.27 \mathrm{E}+04$ \\
\hline $\mathrm{PM}, \mathrm{mg} / \mathrm{dscm}$ & $2.44 \mathrm{E}+02$ & $7.20 \mathrm{E}+01$ & $7.79 \mathrm{E}+01$ & & & & $9.31 \mathrm{E}+00$ & $4.20 \mathrm{E}+01$ & $4.20 \mathrm{E}+01$ \\
\hline SVM, ug/dscm & $1.63 \mathrm{E}+02$ & $1.57 \mathrm{E}+02$ & $9.28 \mathrm{E}+01$ & & & & & & \\
\hline LVM, ug/dscm & $4.54 \mathrm{E}+01$ & $4.37 \mathrm{E}+01$ & $2.58 \mathrm{E}+01$ & & & & & & \\
\hline
\end{tabular}


Table A-16. Mass balance, Tank WM-189 waste (continued).

\begin{tabular}{|c|c|c|c|c|c|c|c|c|}
\hline PFD \# & PFD-3 & PFD-3 & PFD-3 & PFD-3 & PFD-2 & PFD-2 & PFD-2 & PFD-3 \\
\hline WM-189, Stream \# & 117 & 118 & 119 & 120 & 201 & 202 & 203 & 204 \\
\hline Stream Name & $\begin{array}{c}\text { Pressure } \\
\text { Control } \\
\text { Bleed Air }\end{array}$ & $\begin{array}{c}\text { Final HEPA } \\
\text { Off-Gas } \\
\text { Outlet }\end{array}$ & $\begin{array}{c}\text { Off-Gas to } \\
\text { Blower }\end{array}$ & Spent GAC & $\left|\begin{array}{c}\text { Boiler Feed } \\
\text { Water }\end{array}\right|$ & $\begin{array}{c}\text { Fuel Oil to } \\
\text { Boiler }\end{array}$ & $\begin{array}{l}\text { Steam To } \\
\text { Reformer }\end{array}$ & $\begin{array}{c}\text { Propane to } \\
\text { Oxidizer }\end{array}$ \\
\hline Rate or Volume & $1.45 \mathrm{E}+04$ & $6.55 \mathrm{E}+05$ & $6.92 \mathrm{E}+05$ & $6.46 \mathrm{E}+00$ & $3.43 \mathrm{E}+02$ & $4.53 \mathrm{E}+01$ & $6.85 \mathrm{E}+04$ & $1.07 \mathrm{E}+02$ \\
\hline Volume Flow (standard, wet)* & $1.42 \mathrm{E}+04$ & $1.98 \mathrm{E}+05$ & $2.12 \mathrm{E}+05$ & & & & $6.13 \mathrm{E}+04$ & $3.02 \mathrm{E}+02$ \\
\hline Volume Flow (standard, dry) & $1.42 \mathrm{E}+04$ & $3.51 \mathrm{E}+04$ & $4.93 \mathrm{E}+04$ & & & & & $3.02 \mathrm{E}+02$ \\
\hline Rate Units & $\mathrm{ft} 3 / \mathrm{hr}$ & $\mathrm{ft} 3 / \mathrm{hr}$ & $\mathrm{ft} 3 / \mathrm{hr}$ & $\mathrm{lb} / \mathrm{hr}$ & gal/hr & gal/hr & $\mathrm{ft} 3 / \mathrm{hr}$ & $\mathrm{ft} 3 / \mathrm{hr}$ \\
\hline Rate or Volume, metric & $4.02 \mathrm{E}+02$ & $5.62 \mathrm{E}+03$ & $6.01 \mathrm{E}+03$ & $2.93 \mathrm{E}+00$ & $1.30 \mathrm{E}+03$ & $1.71 \mathrm{E}+02$ & $1.74 \mathrm{E}+03$ & $8.54 \mathrm{E}+00$ \\
\hline Rate Units & wscm/hr & wscm/hr & wscm $/ \mathrm{hr}$ & $\mathrm{kg} / \mathrm{hr}$ & $\mathrm{L} / \mathrm{hr}$ & $\mathrm{L} / \mathrm{hr}$ & wscm $/ \mathrm{hr}$ & wscm/hr \\
\hline Temperature, ${ }^{\circ} \mathrm{C}$ & 25 & 120 & 115 & 28 & 15 & 15 & 670 & 25 \\
\hline Temperature, ${ }^{\circ} \mathrm{F}$ & 77 & 248 & 239 & 82 & 59 & 59 & 1238 & 77 \\
\hline Pressure, psia & 14.7 & 6.0 & 6.0 & 14.7 & 42.3 & 14.7 & 42.3 & 42.3 \\
\hline Specific Gravity & $1.18 \mathrm{E}-03$ & $2.60 \mathrm{E}-04$ & $2.71 \mathrm{E}-04$ & $5.86 \mathrm{E}-01$ & $1.00 \mathrm{E}+00$ & $7.70 \mathrm{E}-01$ & $6.70 \mathrm{E}-04$ & $5.19 \mathrm{E}-03$ \\
\hline Chemical Composition & $\mathrm{lb} / \mathrm{wscf}$ & $\mathrm{lb} / \mathrm{wscf}$ & $\mathrm{lb} / \mathrm{wscf}$ & Wt frac & Mol/liter & Mol/liter & Ib/wscf & $\mathrm{lb} / \mathrm{wscf}$ \\
\hline $\mathrm{H}+$ & & $2.06 \mathrm{E}-07$ & $1.92 \mathrm{E}-07$ & $9.87 \mathrm{E}-06$ & & $2.40 \mathrm{E}-01$ & & \\
\hline \multicolumn{9}{|l|}{$\mathrm{Al}+3$} \\
\hline \multirow{2}{*}{\multicolumn{9}{|c|}{$\mathrm{Sb}+5$}} \\
\hline \multirow{2}{*}{\multicolumn{9}{|c|}{$\mathrm{As}+3$}} \\
\hline & & & & & & & & \\
\hline \multicolumn{9}{|l|}{$\frac{\mathrm{Bd}+2}{\mathrm{Be}+2}$} \\
\hline \multicolumn{9}{|l|}{$B+3$} \\
\hline \multirow{2}{*}{\multicolumn{9}{|c|}{$\frac{C d+2}{C a+2}$}} \\
\hline & & & & & & & & \\
\hline \multicolumn{9}{|l|}{$\mathrm{Cr}+3$} \\
\hline \multicolumn{9}{|l|}{$\mathrm{Co}+2$} \\
\hline \multirow{2}{*}{\multicolumn{9}{|c|}{\begin{tabular}{|l}
$\mathrm{Cs}+$ \\
$\mathrm{Cu}+2$
\end{tabular}}} \\
\hline & & & & & & & & \\
\hline \multicolumn{9}{|l|}{$\mathrm{Fe}+3$} \\
\hline \multicolumn{9}{|l|}{$\mathrm{Pb}+2$} \\
\hline $\mathrm{Hg}+2$ & & $2.58 \mathrm{E}-09$ & $2.42 \mathrm{E}-09$ & $7.93 \mathrm{E}-02$ & & & & \\
\hline \multicolumn{9}{|l|}{$M n+4$} \\
\hline \multirow{2}{*}{\multicolumn{9}{|c|}{$\begin{array}{ll}\mathrm{Ni}+2 \\
\mathrm{~K}+\end{array}$}} \\
\hline \multirow{2}{*}{\multicolumn{9}{|c|}{\begin{tabular}{|l|}
$\mathrm{K}+$ \\
$\mathrm{Se}+4$
\end{tabular}}} \\
\hline & & & & & & & & \\
\hline \multirow{2}{*}{\multicolumn{9}{|c|}{$\frac{\mathrm{Ag}+}{\mathrm{Na}+}$}} \\
\hline \multirow{2}{*}{\multicolumn{9}{|c|}{$\frac{\mathrm{Na}+}{\mathrm{TI}+3}$}} \\
\hline & & & & & & & & \\
\hline \multicolumn{9}{|l|}{$\mathrm{U}+4$} \\
\hline $\mathrm{V}+3$ & & & & & & & & \\
\hline $\mathrm{Zn+2}$ & & & & & & & & \\
\hline $\mathrm{Zr}+4$ & & & & & & & & \\
\hline$\overline{\mathrm{Cl}-}$ & & $1.25 \mathrm{E}-09$ & $1.17 \mathrm{E}-09$ & $3.47 \mathrm{E}-04$ & & & & \\
\hline F- & & $3.33 \mathrm{E}-07$ & $3.11 \mathrm{E}-07$ & & & & & \\
\hline $\mathrm{SO} 4-2$ & & & & & & & & \\
\hline NO3- & & $1.16 \mathrm{E}-05$ & $1.08 \mathrm{E}-05$ & & & & & \\
\hline$\overline{\mathrm{PO} 4-3}$ & & & & & & & & \\
\hline $\mathrm{Am}+4$ & & & & & & & & \\
\hline $\mathrm{Br}-$ & & $3.75 \mathrm{E}-14$ & $3.50 \mathrm{E}-14$ & $1.04 \mathrm{E}-08$ & & & & \\
\hline $\mathrm{Ce}+4$ & & & & & & & & \\
\hline$E u+3$ & & & & & & & & \\
\hline $\mathrm{Gd}+3$ & & & & & & & & \\
\hline $\mathrm{Ge}+4$ & & & & & & & & \\
\hline $\ln +3$ & & & & & & & & \\
\hline $1-$ & & $6.61 \mathrm{E}-13$ & $6.18 \mathrm{E}-13$ & $1.83 \mathrm{E}-07$ & & & & \\
\hline $\mathrm{La+3}$ & & & & & & & & \\
\hline
\end{tabular}


Table A-16. Mass balance, Tank WM-189 waste (continued).

\begin{tabular}{|c|c|c|c|c|c|c|c|c|}
\hline Stream \# & 117 & 118 & 119 & 120 & 201 & 202 & 203 & 204 \\
\hline Chemical Composition & $\mathrm{lb} /$ wscf & $\mathrm{lb} /$ wscf & $\mathrm{lb} / \mathrm{wscf}$ & Wt frac & Mol/liter & Mol/liter & $\mathrm{lb} /$ wscf & $\mathrm{lb} / \mathrm{wscf}$ \\
\hline \multicolumn{9}{|l|}{$\mathrm{Li}+$} \\
\hline \multicolumn{9}{|l|}{$\mathrm{Mg}+2$} \\
\hline \multicolumn{9}{|l|}{$\mathrm{Mo}+6$} \\
\hline \multicolumn{9}{|l|}{$\mathrm{Nd}+3$} \\
\hline \multicolumn{9}{|l|}{$\mathrm{Np}+4$} \\
\hline \multicolumn{9}{|l|}{$\mathrm{Nb}+5$} \\
\hline \multicolumn{9}{|l|}{$\mathrm{Pd}+4$} \\
\hline \multicolumn{9}{|l|}{$\mathrm{Pu}+4$} \\
\hline \multicolumn{9}{|l|}{$\mathrm{Pr}+4$} \\
\hline \multicolumn{9}{|l|}{$\mathrm{Pm}+3$} \\
\hline \multicolumn{9}{|l|}{$\mathrm{Rh}+4$} \\
\hline \multicolumn{9}{|l|}{$\mathrm{Rb}+$} \\
\hline \multicolumn{9}{|l|}{ Ru+3 } \\
\hline \multicolumn{9}{|l|}{$\mathrm{Sm}+3$} \\
\hline \multicolumn{9}{|l|}{$\mathrm{Si}+4$} \\
\hline \multicolumn{9}{|l|}{$\mathrm{Sr}+2$} \\
\hline \multicolumn{9}{|l|}{$\mathrm{Tc}+7$} \\
\hline \multicolumn{9}{|l|}{$\mathrm{Te}+4$} \\
\hline \multicolumn{9}{|l|}{$\mathrm{Tb}+4$} \\
\hline \multicolumn{9}{|l|}{$\mathrm{Th}+4$} \\
\hline $\mathrm{Sn}+4$ & & & & & & & & \\
\hline $\mathrm{Ti}+4$ & & & & & & & & \\
\hline $\mathrm{Y}+3$ & & & & & & & & \\
\hline $\mathrm{OH}-$ & & & & & & & & \\
\hline $\mathrm{H} 2 \mathrm{O}$ & $4.68 \mathrm{E}-06$ & $3.85 \mathrm{E}-02$ & $3.60 \mathrm{E}-02$ & & $5.56 \mathrm{E}+01$ & & $4.68 \mathrm{E}-02$ & \\
\hline $\mathrm{SO} 2$ & & $2.16 \mathrm{E}-05$ & $2.02 \mathrm{E}-05$ & & & & & \\
\hline $\mathrm{H} 2 \mathrm{~S}$ & & & & & & $1.20 \mathrm{E}-01$ & & \\
\hline $\mathrm{CO}$ & & $9.70 \mathrm{E}-10$ & $9.07 \mathrm{E}-10$ & & & & & \\
\hline $\mathrm{CO} 2$ & & $5.89 \mathrm{E}-03$ & 5.51E-03 & & & & & \\
\hline $\mathrm{H} 2$ & & & & & & & & \\
\hline N2 & $5.75 \mathrm{E}-02$ & $8.13 \mathrm{E}-03$ & $1.15 \mathrm{E}-02$ & & & & & \\
\hline NO & & 2.84E-05 & 2.65E-05 & & & & & \\
\hline $\mathrm{NO} 2$ & & $1.55 \mathrm{E}-07$ & $1.45 \mathrm{E}-07$ & & & & & \\
\hline $\mathrm{O} 2$ & $1.74 \mathrm{E}-02$ & $1.08 \mathrm{E}-03$ & $2.18 \mathrm{E}-03$ & & & & & \\
\hline S (other) & & & & & & & & \\
\hline $\mathrm{CO} 3$ & & & & & & & & \\
\hline C (reductant) & & & & $9.20 \mathrm{E}-01$ & & & & \\
\hline $\mathrm{O}$ (oxides) & & & & & & & & \\
\hline C (organic) & & & & & & $5.41 \mathrm{E}+01$ & & 9.35E-02 \\
\hline $\mathrm{H}$ (organic) & & & & & & $1.15 \mathrm{E}+02$ & & $2.09 \mathrm{E}-02$ \\
\hline $\mathrm{O}$ (organic) & & & & & & & & \\
\hline & & & & & & & & \\
\hline Mass Flow (kg/hr): & $4.83 \mathrm{E}+02$ & $4.83 \mathrm{E}+03$ & $5.31 \mathrm{E}+03$ & $2.93 \mathrm{E}+00$ & $1.30 \mathrm{E}+03$ & $1.32 \mathrm{E}+02$ & $1.30 \mathrm{E}+03$ & $1.57 \mathrm{E}+01$ \\
\hline & & & & & & & & \\
\hline & & & & & & & & \\
\hline & $\mathrm{w} / \mathrm{m} 3$ & $\mathrm{w} / \mathrm{m} 3$ & $\mathrm{w} / \mathrm{m} 3$ & $\mathrm{w} / \mathrm{m} 3$ & $\mathrm{w} / \mathrm{m} 3$ & $\mathrm{w} / \mathrm{m} 3$ & $\mathrm{w} / \mathrm{m} 3$ & $\mathrm{w} / \mathrm{m} 3$ \\
\hline Heat Generation, w/m3 & & & & $4.92 \mathrm{E}-11$ & & & & \\
\hline
\end{tabular}


Table A-16. Mass balance, Tank WM-189 waste (continued).

\begin{tabular}{|c|c|c|c|c|c|c|c|c|}
\hline Stream \# & 117 & 118 & 119 & 120 & 201 & 202 & 203 & 204 \\
\hline Radiological Composition & Ci/wscm & $\mathrm{Ci} / \mathrm{wscm}$ & $\mathrm{Ci} / \mathrm{wscm}$ & $\mathrm{Ci} / \mathrm{kg}$ & $\mathrm{Ci} / \mathrm{L}$ & $\mathrm{Ci} / \mathrm{L}$ & $\mathrm{Ci} / \mathrm{wscm}$ & $\mathrm{Ci} / \mathrm{wscm}$ \\
\hline $\mathrm{H}-3$ & & $5.29 \mathrm{E}-07$ & $4.95 \mathrm{E}-07$ & & & & & \\
\hline C-14 & & $8.02 \mathrm{E}-12$ & $7.50 \mathrm{E}-12$ & $2.86 \mathrm{E}-10$ & & & & \\
\hline Co-60 & & & & & & & & \\
\hline $\mathrm{Ni}-59$ & & & & & & & & \\
\hline \multicolumn{9}{|l|}{$\mathrm{Ni}-63$} \\
\hline \multicolumn{9}{|l|}{ Se-79 } \\
\hline \multirow{2}{*}{\multicolumn{9}{|c|}{\begin{tabular}{|l|} 
Sr-90 \\
$Y-90$
\end{tabular}}} \\
\hline & & & & & & & & \\
\hline \multicolumn{9}{|l|}{ Zr-93 } \\
\hline \multirow{2}{*}{\multicolumn{9}{|c|}{\begin{tabular}{|l|}
$\mathrm{Nb}-93 \mathrm{~m}$ \\
$\mathrm{Nb}-94$
\end{tabular}}} \\
\hline & & & & & & & & \\
\hline \multicolumn{9}{|l|}{ Tc-99 } \\
\hline \multirow{2}{*}{\multicolumn{9}{|c|}{\begin{tabular}{|l|} 
Ru-106 \\
Rh-102
\end{tabular}}} \\
\hline & & & & & & & & \\
\hline \multicolumn{9}{|l|}{ Rh-106 } \\
\hline \multicolumn{9}{|l|}{\begin{tabular}{|l|}
$\mathrm{Pd}-107$ \\
\end{tabular}} \\
\hline \multirow{2}{*}{\multicolumn{9}{|c|}{\begin{tabular}{|l|} 
Cd-113m \\
Sn-121m
\end{tabular}}} \\
\hline & & & & & & & & \\
\hline \multicolumn{9}{|l|}{$\begin{array}{l}\text { Sn-126 } \\
\end{array}$} \\
\hline \multicolumn{9}{|l|}{ Sb-125 } \\
\hline \multicolumn{9}{|l|}{ Sb-126 } \\
\hline \multicolumn{9}{|l|}{ Te-125m } \\
\hline I-129 & & $1.77 \mathrm{E}-12$ & $1.65 \mathrm{E}-12$ & $3.05 \mathrm{E}-08$ & & & & \\
\hline \multicolumn{9}{|l|}{ Cs-134 } \\
\hline Cs-135 & & & & & & & & \\
\hline Cs-137 & & & & & & & & \\
\hline Ba-137m & & & & & & & & \\
\hline Ce-144 & & & & & & & & \\
\hline Pr-144 & & & & & & & & \\
\hline Pm-146 & & & & & & & & \\
\hline $\mathrm{Pm}-147$ & & & & & & & & \\
\hline Sm-151 & & & & & & & & \\
\hline Eu-152 & & & & & & & & \\
\hline Eu-154 & & & & & & & & \\
\hline Eu-155 & & & & & & & & \\
\hline & & & & & & & & \\
\hline Th-230 & & & & & & & & \\
\hline $\mathrm{Pa}-233$ & & & & & & & & \\
\hline $\mathrm{U}-232$ & & & & & & & & \\
\hline $\mathrm{U}-233$ & & & & & & & & \\
\hline $\mathrm{U}-234$ & & & & & & & & \\
\hline $\mathrm{U}-235$ & & & & & & & & \\
\hline $\mathrm{U}-236$ & & & & & & & & \\
\hline $\mathrm{U}-237$ & & & & & & & & \\
\hline U-238 & & & & & & & & \\
\hline $\mathrm{Np}-237$ & & & & & & & & \\
\hline Pu-236 & & & & & & & & \\
\hline $\mathrm{Pu}-238$ & & & & & & & & \\
\hline $\mathrm{Pu}-239$ & & & & & & & & \\
\hline $\mathrm{Pu}-240$ & & & & & & & & \\
\hline Pu-241 & & & & & & & & \\
\hline Pu-242 & & & & & & & & \\
\hline Pu-244 & & & & & & & & \\
\hline $\mathrm{Am-241}$ & & & & & & & & \\
\hline Am-242m & & & & & & & & \\
\hline
\end{tabular}


Table A-16. Mass balance, Tank WM-189 waste (continued).

\begin{tabular}{|c|c|c|c|c|c|c|c|c|}
\hline Stream \# & 117 & 118 & 119 & 120 & 201 & 202 & 203 & 204 \\
\hline & $\mathrm{Ci} / \mathrm{wscm}$ & $\mathrm{Ci} / \mathrm{wscm}$ & $\mathrm{Ci} / \mathrm{wscm}$ & $\mathrm{Ci} / \mathrm{kg}$ & $\mathrm{Ci} / \mathrm{L}$ & $\mathrm{Ci} / \mathrm{L}$ & $\mathrm{Ci} / \mathrm{wscm}$ & $\mathrm{Ci} / \mathrm{wscm}$ \\
\hline \multicolumn{9}{|l|}{ Am-243 } \\
\hline \multicolumn{9}{|l|}{$\mathrm{Cm}-242$} \\
\hline \multicolumn{9}{|l|}{$\mathrm{Cm}-243$} \\
\hline \multicolumn{9}{|l|}{$\mathrm{Cm}-244$} \\
\hline \multicolumn{9}{|l|}{ Cm-245 } \\
\hline \multicolumn{9}{|l|}{$\mathrm{Cm}-246$} \\
\hline \multicolumn{9}{|l|}{ TRU } \\
\hline & & & & & & & & \\
\hline & & & & & & & & \\
\hline & & & & & & & & \\
\hline $\begin{array}{l}\text { Gas Stream Bulk } \\
\text { Composition (Wet Basis) }\end{array}$ & $\begin{array}{c}\text { mol\% or } \\
\text { ppmv }\end{array}$ & $\begin{array}{c}\text { mol\% or } \\
\text { ppmv }\end{array}$ & $\begin{array}{c}\text { mol\% or } \\
\text { ppmv }\end{array}$ & $\begin{array}{c}\text { mol\% or } \\
\text { ppmv }\end{array}$ & $\begin{array}{c}\text { mol\% or } \\
\text { ppmv }\end{array}$ & $\begin{array}{c}\text { mol\% or } \\
\text { ppmv }\end{array}$ & $\begin{array}{c}\text { mol\% or } \\
\text { ppmv }\end{array}$ & $\begin{array}{c}\text { mol\% or } \\
\text { ppmv }\end{array}$ \\
\hline $\mathrm{H} 2 \mathrm{O}, \mathrm{mol} \%$ & $0.01 \%$ & $82.29 \%$ & $76.79 \%$ & & & & $100.00 \%$ & \\
\hline $\mathrm{O} 2, \mathrm{~mol} \%$ & $20.99 \%$ & $1.30 \%$ & $2.62 \%$ & & & & & \\
\hline $\mathrm{N} 2, \mathrm{~mol} \%$ & $79.00 \%$ & $11.19 \%$ & $15.72 \%$ & & & & & \\
\hline \multicolumn{9}{|l|}{$\mathrm{H} 2, \mathrm{~mol} \%$} \\
\hline $\mathrm{CO} 2, \mathrm{~mol} \%$ & & $5.16 \%$ & $4.81 \%$ & & & & & \\
\hline $\mathrm{CO}, \mathrm{ppmv}$ & & 1.33E-02 & 1.24E-02 & & & & & \\
\hline $\mathrm{NO}, \mathrm{ppmv}$ & & $3.65 \mathrm{E}+02$ & $3.40 \mathrm{E}+02$ & & & & & \\
\hline NO2, ppmv & & $1.30 \mathrm{E}+00$ & $1.21 \mathrm{E}+00$ & & & & & \\
\hline SO2, ppmv & & $1.30 \mathrm{E}+02$ & $1.21 \mathrm{E}+02$ & & & & & \\
\hline $\mathrm{Cl}, \mathrm{ppmv}$ & & 1.36E-02 & $1.27 \mathrm{E}-02$ & & & & & \\
\hline $\mathrm{F}, \mathrm{ppmv}$ & & $6.75 \mathrm{E}+00$ & $6.30 \mathrm{E}+00$ & & & & & \\
\hline C (organic), ppmv & & & & & & & & $3.00 \mathrm{E}+06$ \\
\hline $\mathrm{H}$ (organic), ppmv & & & & & & & & $8.00 \mathrm{E}+06$ \\
\hline $\mathrm{Hg}, \mathrm{ug} / \mathrm{wscm}$ & & $4.14 \mathrm{E}+01$ & $3.87 \mathrm{E}+01$ & & & & & \\
\hline $\mathrm{PM}, \mathrm{mg} / \mathrm{wscm}$ & $1.58 \mathrm{E}+01$ & $1.18 \mathrm{E}+01$ & $1.22 \mathrm{E}+01$ & & & & 1.17E+01 & $1.83 \mathrm{E}+06$ \\
\hline \multicolumn{9}{|l|}{ SVM, ug/wscm } \\
\hline \multicolumn{9}{|l|}{ LVM, ug/wscm } \\
\hline & & & & & & & & \\
\hline $\begin{array}{l}\text { Gas Stream Bulk } \\
\text { Composition (Dry Basis) }\end{array}$ & $\mathrm{mol} \%$ & $\mathrm{~mol} \%$ & $\mathrm{~mol} \%$ & $\mathrm{~mol} \%$ & $\mathrm{~mol} \%$ & $\mathrm{~mol} \%$ & $\mathrm{~mol} \%$ & $\mathrm{~mol} \%$ \\
\hline $\mathrm{O} 2, \mathrm{~mol} \%$, dry basis & $20.99 \%$ & $1.03 \%$ & $11.27 \%$ & & & & & \\
\hline $\mathrm{N} 2, \mathrm{~mol} \%$, dry basis & $79.01 \%$ & $63.19 \%$ & $67.75 \%$ & & & & & \\
\hline \multicolumn{9}{|l|}{$\mathrm{H} 2, \mathrm{~mol} \%$, dry basis } \\
\hline $\mathrm{CO} 2, \mathrm{~mol} \%$, dry basis & & $29.14 \%$ & $20.75 \%$ & & & & & \\
\hline $\begin{array}{l}\text { Gas Stream Bulk } \\
\text { Composition (Dry Basis, } \\
\text { Corrected to } 7 \% \text { O2 with } \\
100 \% \text { O2 Combustion Air) }\end{array}$ & $\begin{array}{c}\text { ppmv, or } \\
\text { ug/dscm or } \\
\mathrm{mg} / \mathrm{dscm}\end{array}$ & $\begin{array}{c}\mathrm{ppmv}, \text { or } \\
\mathrm{ug} / \mathrm{dscm} \text { or } \\
\mathrm{mg} / \mathrm{dscm}\end{array}$ & $\begin{array}{c}\mathrm{ppmv}, \text { or } \\
\mathrm{ug} / \mathrm{dscm} \text { or } \\
\mathrm{mg} / \mathrm{dscm}\end{array}$ & $\begin{array}{c}\mathrm{ppmv}, \text { or } \\
\text { ug/dscm or } \\
\mathrm{mg} / \mathrm{dscm}\end{array}$ & $\begin{array}{l}\text { ppmv, or } \\
\text { ug/dscm or } \\
\mathrm{mg} / \mathrm{dscm}\end{array}$ & $\begin{array}{c}\mathrm{ppmv}, \text { or } \\
\mathrm{ug} / \mathrm{dscm} \text { or } \\
\mathrm{mg} / \mathrm{dscm}\end{array}$ & $\begin{array}{c}\mathrm{ppmv}, \text { or } \\
\mathrm{ug} / \mathrm{dscm} \text { or } \\
\mathrm{mg} / \mathrm{dscm}\end{array}$ & $\begin{array}{l}\text { ppmv, or } \\
\text { ug/dscm or } \\
\text { mg/dscm }\end{array}$ \\
\hline $\mathrm{CO}, \mathrm{ppmv}$, dry basis & & $1.06 \mathrm{E}-02$ & $7.52 \mathrm{E}-03$ & & & & & \\
\hline $\mathrm{NO}$, ppmv, dry basis & & $2.88 \mathrm{E}+02$ & $2.05 \mathrm{E}+02$ & & & & & \\
\hline NO2, ppmv, dry basis & & $1.03 \mathrm{E}+00$ & $7.30 \mathrm{E}-01$ & & & & & \\
\hline SO2, ppmv, dry basis & & $1.03 \mathrm{E}+02$ & $7.31 \mathrm{E}+01$ & & & & & \\
\hline $\mathrm{Cl}, \mathrm{ppmv}$, dry basis & & $1.08 \mathrm{E}-02$ & $7.68 \mathrm{E}-03$ & & & & & \\
\hline $\mathrm{F}, \mathrm{ppmv}$, dry basis & & $5.34 \mathrm{E}+00$ & $3.80 \mathrm{E}+00$ & & & & & \\
\hline \multicolumn{9}{|l|}{ C (organic), ppmv, dry basis } \\
\hline \multicolumn{9}{|l|}{$\mathrm{H}$ (organic), ppmv, dry basis } \\
\hline $\mathrm{Hg}, \mathrm{ug} / \mathrm{dscm}$ & & $3.28 \mathrm{E}+01$ & $2.34 \mathrm{E}+01$ & & & & & \\
\hline $\mathrm{PM}, \mathrm{mg} / \mathrm{dscm}$ & $2.21 \mathrm{E}+00$ & $9.37 \mathrm{E}+00$ & $7.36 \mathrm{E}+00$ & & & & & \\
\hline \multicolumn{9}{|l|}{ SVM, ug/dscm } \\
\hline LVM, ug/dscm & & & & & & & & \\
\hline
\end{tabular}


Table A-16. Mass balance, Tank WM-189 waste (continued).

\begin{tabular}{|c|c|c|c|c|c|c|c|c|}
\hline PFD \# & PFD-3 & PFD-3 & PFD-3 & PFD-2 & PFD-2 & PFD-2 & PFD-2 & $\frac{\text { PFD-3 }}{305}$ \\
\hline WM-189, Stream \# & 205 & 206 & 207 & 301 & 302 & 303 & 304 & 305 \\
\hline Stream Name & $\begin{array}{l}\text { Water to } \\
\text { Spray } \\
\text { Quench }\end{array}$ & $\begin{array}{l}\text { ANN to } \\
\text { Scrub for } \\
\text { F Adjust }\end{array}$ & $\begin{array}{l}\text { HNO3 } \\
\text { Scrub } \\
\text { Makeup }\end{array}$ & $\begin{array}{l}\text { Sugar to } \\
\text { Feed }\end{array}$ & $\begin{array}{l}\text { Carbon to } \\
\text { Reformer }\end{array}$ & $\begin{array}{c}\text { NOx } \\
\text { Catalyst }\end{array}$ & Bed Media & $\begin{array}{l}\text { Grout Mix } \\
\text { for Scrub } \\
\text { Blowdown }\end{array}$ \\
\hline Rate or Volume & $4.62 \mathrm{E}+02$ & $3.52 \mathrm{E}-08$ & $9.20 \mathrm{E}-01$ & $3.77 \mathrm{E}+02$ & $1.66 \mathrm{E}+02$ & $2.20 \mathrm{E}-05$ & $1.79 \mathrm{E}+00$ & $6.61 \mathrm{E}+00$ \\
\hline \multicolumn{9}{|c|}{ Volume Flow (standard, wet)* } \\
\hline \multicolumn{9}{|l|}{ Volume Flow (standard, dry)* } \\
\hline Rate Units & $\mathrm{gal} / \mathrm{hr}$ & $\mathrm{gal} / \mathrm{hr}$ & $\mathrm{gal} / \mathrm{hr}$ & $\mathrm{lb} / \mathrm{hr}$ & $\mathrm{lb} / \mathrm{hr}$ & $\mathrm{lb} / \mathrm{hr}$ & $\mathrm{Ib} / \mathrm{hr}$ & $\mathrm{Ib} / \mathrm{hr}$ \\
\hline Rate or Volume, metric & $1.75 \mathrm{E}+03$ & $1.33 \mathrm{E}-07$ & $3.48 \mathrm{E}+00$ & $1.71 \mathrm{E}+02$ & $7.52 \mathrm{E}+01$ & $1.00 \mathrm{E}-05$ & $8.14 \mathrm{E}-01$ & $3.00 \mathrm{E}+00$ \\
\hline Rate Units & $\mathrm{L} / \mathrm{hr}$ & $\mathrm{L} / \mathrm{hr}$ & \begin{tabular}{|l|}
$\mathrm{L} / \mathrm{hr}$ \\
\end{tabular} & $\mathrm{kg} / \mathrm{hr}$ & $\mathrm{kg} / \mathrm{hr}$ & $\mathrm{kg} / \mathrm{hr}$ & $\mathrm{kg} / \mathrm{hr}$ & $\mathrm{kg} / \mathrm{hr}$ \\
\hline Temperature, ${ }^{\circ} \mathrm{C}$ & 25 & 25 & 25 & 15 & 15 & 15 & 15 & 25 \\
\hline Temperature, ${ }^{\circ} \mathrm{F}$ & 77 & 77 & 77 & 59 & 59 & 59 & 59 & 77 \\
\hline Pressure, psia & 112.3 & 14.7 & 42.3 & 14.7 & 14.7 & 14.7 & 14.7 & 14.7 \\
\hline Specific Gravity & $9.97 \mathrm{E}-01$ & $1.35 \mathrm{E}+00$ & $1.28 \mathrm{E}+00$ & $7.50 \mathrm{E}-01$ & $5.00 \mathrm{E}-01$ & $2.58 \mathrm{E}+00$ & $1.58 \mathrm{E}+00$ & $2.01 \mathrm{E}-01$ \\
\hline Chemical Composition & Mol/liter & Mol/liter & Mol/liter & Wt frac & Wt frac & Wt frac & Wt frac & Wt frac \\
\hline $\mathrm{H}+$ & $1.00 \mathrm{E}-07$ & $3.09 \mathrm{E}-08$ & $1.39 \mathrm{E}+01$ & & & & & \\
\hline $\mathrm{Al}+3$ & & $2.20 \mathrm{E}+00$ & & & $1.53 \mathrm{E}-03$ & & $5.29 \mathrm{E}-01$ & \\
\hline \multicolumn{9}{|l|}{$S b+5$} \\
\hline \multicolumn{9}{|l|}{$\mathrm{As}+3$} \\
\hline \multicolumn{9}{|l|}{$\mathrm{Ba}+2$} \\
\hline \multirow{2}{*}{\multicolumn{9}{|c|}{$\frac{\mathrm{Be}+2}{\mathrm{~B}+3}$}} \\
\hline & & & & & & & & \\
\hline \multicolumn{9}{|l|}{$C d+2$} \\
\hline $\mathrm{Ca}+2$ & & & & & $1.85 \mathrm{E}-02$ & & & \\
\hline \multicolumn{9}{|l|}{$\mathrm{Cr}+3$} \\
\hline \multicolumn{9}{|l|}{$\mathrm{Co}+2$} \\
\hline \multirow{2}{*}{\multicolumn{9}{|c|}{$\frac{\mathrm{Cs}+}{\mathrm{Cu}+2}$}} \\
\hline & & & & & & & & $\mathrm{Cu}+2$ \\
\hline $\mathrm{Fe}+3$ & & & & & $8.71 \mathrm{E}-04$ & $6.99 \mathrm{E}-01$ & & \\
\hline \multicolumn{9}{|l|}{$\mathrm{Pb}+2$} \\
\hline $\mathrm{Hg}+2$ & & & & & $1.92 \mathrm{E}-06$ & & & \\
\hline \multicolumn{9}{|l|}{$\mathrm{Mn}+4$} \\
\hline \multicolumn{9}{|l|}{$\mathrm{Ni}+2$} \\
\hline $\mathrm{K}+$ & & & & & $7.14 \mathrm{E}-03$ & & & \\
\hline \multicolumn{9}{|l|}{$\mathrm{Se}+4$} \\
\hline \multicolumn{9}{|l|}{$\mathrm{Ag}+$} \\
\hline $\mathrm{Na}+$ & & & & & $7.42 \mathrm{E}-04$ & & & \\
\hline \multicolumn{9}{|l|}{$\mathrm{TI}+3$} \\
\hline $\mathrm{U}+4$ & & & & & & & & \\
\hline $\mathrm{V+3}$ & & & & & & & & \\
\hline$Z n+2$ & & & & & & & & \\
\hline$Z r+4$ & & & & & & & & \\
\hline $\mathrm{Cl}-$ & & & & & & & & \\
\hline F- & & & & & & & & \\
\hline SO4-2 & & & & & & & & \\
\hline NO3- & & $6.60 \mathrm{E}+00$ & $1.39 \mathrm{E}+01$ & & & & & \\
\hline PO4-3 & & & & & $2.26 \mathrm{E}-03$ & & & \\
\hline $\mathrm{Am}+4$ & & & & & & & & \\
\hline $\mathrm{Br}-$ & & & & & & & & \\
\hline $\mathrm{Ce}+4$ & & & & & & & & \\
\hline $\mathrm{Eu+3}$ & & & & & & & & \\
\hline $\mathrm{Gd}+3$ & & & & & & & & \\
\hline $\mathrm{Ge}+4$ & & & & & & & & \\
\hline $\ln +3$ & & & & & & & & \\
\hline $\mid$ & & & & & & & & \\
\hline La+3 & & & & & & & & \\
\hline
\end{tabular}


Table A-16. Mass balance, Tank WM-189 waste (continued).

\begin{tabular}{|c|c|c|c|c|c|c|c|c|}
\hline Stream \# & 205 & 206 & 207 & 301 & 302 & 303 & 304 & 305 \\
\hline Chemical Composition & Mol/liter & Mol/liter & Mol/liter & Wt frac & Wt frac & Wt frac & Wt frac & Wt frac \\
\hline \multicolumn{9}{|l|}{$\mathrm{Li+}$} \\
\hline \multicolumn{9}{|l|}{$\mathrm{Mg}+2$} \\
\hline \multicolumn{9}{|l|}{$\mathrm{Mo}+6$} \\
\hline \multicolumn{9}{|l|}{$\mathrm{Nd}+3$} \\
\hline \multicolumn{9}{|l|}{$\mathrm{Np}+4$} \\
\hline \multicolumn{9}{|l|}{$\mathrm{Nb}+5$} \\
\hline \multicolumn{9}{|l|}{$\mathrm{Pd}+4$} \\
\hline \multicolumn{9}{|l|}{$\mathrm{Pu}+4$} \\
\hline \multicolumn{9}{|l|}{$\mathrm{Pr}+4$} \\
\hline \multicolumn{9}{|l|}{$\mathrm{Pm}+3$} \\
\hline \multicolumn{9}{|l|}{$\mathrm{Rh}+4$} \\
\hline \multicolumn{9}{|l|}{$\mathrm{Rb}+$} \\
\hline \multicolumn{9}{|l|}{ Ru+3 } \\
\hline \multicolumn{9}{|l|}{$\mathrm{Sm}+3$} \\
\hline $\mathrm{Si}+4$ & & & & & $6.26 \mathrm{E}-03$ & & & 4.67E-01 \\
\hline \multicolumn{9}{|l|}{$\mathrm{Sr}+2$} \\
\hline \multicolumn{9}{|l|}{$\mathrm{Tc}+7$} \\
\hline \multicolumn{9}{|l|}{$\mathrm{Te}+4$} \\
\hline \multicolumn{9}{|l|}{$\mathrm{Tb}+4$} \\
\hline \multicolumn{9}{|l|}{$\mathrm{Th}+4$} \\
\hline \multicolumn{9}{|l|}{$\mathrm{Sn}+4$} \\
\hline \multicolumn{9}{|l|}{$\mathrm{Ti}+4$} \\
\hline \multicolumn{9}{|l|}{$Y+3$} \\
\hline $\mathrm{OH}-$ & $1.00 \mathrm{E}-07$ & $3.09 \mathrm{E}-08$ & & & & & & \\
\hline $\mathrm{H} 2 \mathrm{O}$ & $5.54 \mathrm{E}+01$ & $4.89 \mathrm{E}+01$ & $2.27 \mathrm{E}+01$ & & & & & \\
\hline \multicolumn{9}{|l|}{$\mathrm{SO} 2$} \\
\hline $\mathrm{H} 2 \mathrm{~S}$ & & & & & & & & \\
\hline $\mathrm{CO}$ & & & & & & & & \\
\hline $\mathrm{CO} 2$ & & & & & & & & \\
\hline $\mathrm{H} 2$ & & & & & & & & \\
\hline $\mathrm{N} 2$ & & & & & & & & \\
\hline NO & & & & & & & & \\
\hline $\mathrm{NO} 2$ & & & & & & & & \\
\hline $\mathrm{O} 2$ & & & & & & & & \\
\hline S (other) & & & & & $5.18 \mathrm{E}-04$ & & & \\
\hline $\mathrm{CO} 3$ & & & & & $3.11 \mathrm{E}-02$ & & & \\
\hline C (reductant) & & & & & 9.22E-01 & & & \\
\hline $\mathrm{O}$ (oxides) & & & & & $8.88 \mathrm{E}-03$ & 3.01E-01 & 4.71E-01 & 5.33E-01 \\
\hline C (organic) & & & & $4.21 \mathrm{E}-01$ & & & & \\
\hline $\mathrm{H}$ (organic) & & & & $6.48 \mathrm{E}-02$ & & & & \\
\hline $\mathrm{O}$ (organic) & & & & 5.14E-01 & & & & \\
\hline Mass Flow $(\mathrm{kg} / \mathrm{hr})$ : & $1.74 \mathrm{E}+03$ & 1.80E-07 & $4.47 E+00$ & $1.71 \mathrm{E}+02$ & $7.52 \mathrm{E}+01$ & $1.00 \mathrm{E}-05$ & $8.14 \mathrm{E}-01$ & $3.00 \mathrm{E}+00$ \\
\hline
\end{tabular}


Table A-16. Mass balance, Tank WM-189 waste (continued).

\begin{tabular}{|c|c|c|c|c|c|c|c|c|}
\hline PFD \# & PFD-3 & PFD-3 & PFD-3 & PFD-2 & PFD-2 & PFD-2 & PFD-3 & PFD-2 \\
\hline WM-189, Stream \# & 401 & 402 & 404 & 404 & 503 & 504 & 505 & 506 \\
\hline Stream Name & $\begin{array}{c}\text { Scrub } \\
\text { Recycled } \\
\text { to Feed }\end{array}$ & \begin{tabular}{|c|} 
Scrub \\
Blowdown \\
to Grout \\
Mixer \\
\end{tabular} & $\begin{array}{l}\text { MLLW } \\
\text { Grout }\end{array}$ & $\begin{array}{l}\text { MLLW } \\
\text { Grout } \\
\text { Drums }\end{array}$ & $\begin{array}{c}\text { Feed } \\
\text { Atomizing } \\
\text { Gas }\end{array}$ & $\begin{array}{l}\text { Oxygen to } \\
\text { Reformer }\end{array}$ & $\begin{array}{c}\text { Oxygen to } \\
\text { Oxidizer }\end{array}$ & $\begin{array}{l}\text { Air to } \\
\text { Boiler }\end{array}$ \\
\hline Rate or Volume & $5.90 \mathrm{E}+00$ & \begin{tabular}{|l|}
$2.60 \mathrm{E}-01$ \\
\end{tabular} & $8.82 \mathrm{E}+00$ & $2.29 \mathrm{E}-01$ & $2.88 \mathrm{E}+03$ & $7.84 \mathrm{E}+02$ & $1.05 \mathrm{E}+03$ & $6.71 \mathrm{E}+04$ \\
\hline Volume Flow (standard, wet)* & & & & & $1.80 \mathrm{E}+04$ & $4.84 \mathrm{E}+03$ & $6.47 \mathrm{E}+03$ & $6.82 \mathrm{E}+04$ \\
\hline Volume Flow (standard, dry) & & & & & $1.80 \mathrm{E}+04$ & $4.84 \mathrm{E}+03$ & $6.47 \mathrm{E}+03$ & $6.82 \mathrm{E}+04$ \\
\hline Rate Units & $\mathrm{gal} / \mathrm{hr}$ & $\mathrm{gal} / \mathrm{hr}$ & $\mathrm{lb} / \mathrm{hr}$ & Drums/day & $\mathrm{ft} 3 / \mathrm{hr}$ & $\mathrm{ft} 3 / \mathrm{hr}$ & $\mathrm{ft} 3 / \mathrm{hr}$ & $\mathrm{ft} 3 / \mathrm{hr}$ \\
\hline Rate or Volume, metric & $2.23 \mathrm{E}+01$ & $9.84 \mathrm{E}-01$ & $4.00 \mathrm{E}+00$ & $4.00 \mathrm{E}+00$ & $5.10 \mathrm{E}+02$ & $1.37 \mathrm{E}+02$ & $1.83 \mathrm{E}+02$ & $1.93 \mathrm{E}+03$ \\
\hline Rate Units & $\mathrm{L} / \mathrm{hr}$ & $\mathrm{L} / \mathrm{hr}$ & $\mathrm{kg} / \mathrm{hr}$ & $\mathrm{kg} / \mathrm{hr}$ & wscm/hr & wscm $/ \mathrm{hr}$ & wscm $/ \mathrm{hr}$ & wscm $/ \mathrm{hr}$ \\
\hline Temperature, ${ }^{\circ} \mathrm{C}$ & 79 & 79 & 60 & 60 & 21 & 25 & 25 & 15 \\
\hline Temperature, ${ }^{\circ} \mathrm{F}$ & 174 & 174 & 140 & 140 & 70 & 77 & 77 & 59 \\
\hline Pressure, psia & 12.3 & 12.3 & 12.3 & 12.3 & 92.3 & 92.3 & 92.3 & 14.7 \\
\hline Specific Gravity & $1.02 \mathrm{E}+00$ & $1.02 \mathrm{E}+00$ & $2.10 \mathrm{E}+00$ & $2.10 \mathrm{E}+00$ & $7.29 \mathrm{E}-03$ & $8.21 \mathrm{E}-03$ & $8.21 \mathrm{E}-03$ & $1.22 \mathrm{E}-03$ \\
\hline Chemical Composition & Mol/liter & Mol/liter & Wt frac & Wt frac & $\mathrm{lb} / \mathrm{wscf}$ & $\mathrm{lb} / \mathrm{wscf}$ & Ib/wscf & $\mathrm{lb} / \mathrm{wscf}$ \\
\hline $\mathrm{H}+$ & $1.04 \mathrm{E}+00$ & $1.04 \mathrm{E}+00$ & $2.58 \mathrm{E}-04$ & $2.58 \mathrm{E}-04$ & & & & \\
\hline $\mathrm{Al}+3$ & $1.90 \mathrm{E}-03$ & $1.90 \mathrm{E}-03$ & $1.26 \mathrm{E}-05$ & $1.26 \mathrm{E}-05$ & & & & \\
\hline $\mathrm{Sb}+5$ & $5.15 \mathrm{E}-08$ & $5.15 \mathrm{E}-08$ & $1.54 \mathrm{E}-09$ & $1.54 \mathrm{E}-09$ & & & & \\
\hline As+3 & $2.57 \mathrm{E}-07$ & $2.57 \mathrm{E}-07$ & $4.73 \mathrm{E}-09$ & $4.73 \mathrm{E}-09$ & & & & \\
\hline $\mathrm{Ba}+2$ & $2.05 \mathrm{E}-07$ & $2.05 \mathrm{E}-07$ & $6.94 \mathrm{E}-09$ & $6.94 \mathrm{E}-09$ & & & & \\
\hline $\mathrm{Be}+2$ & $6.68 \mathrm{E}-08$ & $6.68 \mathrm{E}-08$ & $1.48 \mathrm{E}-10$ & $1.48 \mathrm{E}-10$ & & & & \\
\hline $\mathrm{B}+3$ & $5.21 \mathrm{E}-05$ & $5.21 \mathrm{E}-05$ & $1.39 \mathrm{E}-07$ & $1.39 \mathrm{E}-07$ & & & & \\
\hline $\mathrm{Cd}+2$ & $5.74 \mathrm{E}-06$ & $5.74 \mathrm{E}-06$ & $1.59 \mathrm{E}-07$ & $1.59 \mathrm{E}-07$ & & & & \\
\hline $\mathrm{Ca}+2$ & $4.94 \mathrm{E}-04$ & $4.94 \mathrm{E}-04$ & $4.88 \mathrm{E}-06$ & $4.88 \mathrm{E}-06$ & & & & \\
\hline $\mathrm{Cr}+3$ & $1.18 \mathrm{E}-05$ & $1.18 \mathrm{E}-05$ & $1.51 \mathrm{E}-07$ & $1.51 \mathrm{E}-07$ & & & & \\
\hline $\mathrm{Co}+2$ & $1.22 \mathrm{E}-07$ & $1.22 \mathrm{E}-07$ & $1.77 \mathrm{E}-09$ & $1.77 \mathrm{E}-09$ & & & & \\
\hline Cs+ & $5.45 \mathrm{E}-05$ & $5.45 \mathrm{E}-05$ & $2.00 \mathrm{E}-06$ & $2.00 \mathrm{E}-06$ & & & & \\
\hline $\mathrm{Cu}+2$ & $2.64 \mathrm{E}-06$ & $2.64 \mathrm{E}-06$ & $4.12 \mathrm{E}-08$ & $4.12 \mathrm{E}-08$ & & & & \\
\hline $\mathrm{Fe}+3$ & $9.96 \mathrm{E}-05$ & $9.96 \mathrm{E}-05$ & $1.37 \mathrm{E}-06$ & $1.37 \mathrm{E}-06$ & & & & \\
\hline $\mathrm{Pb}+2$ & $7.19 \mathrm{E}-06$ & $7.19 \mathrm{E}-06$ & 3.67E-07 & $3.67 \mathrm{E}-07$ & & & & \\
\hline $\mathrm{Hg}+2$ & $1.48 \mathrm{E}-02$ & $1.48 \mathrm{E}-02$ & $7.32 \mathrm{E}-04$ & $7.32 \mathrm{E}-04$ & & & & \\
\hline $\mathrm{Mn}+4$ & $4.69 \mathrm{E}-05$ & $4.69 \mathrm{E}-05$ & $6.34 \mathrm{E}-07$ & $6.34 \mathrm{E}-07$ & & & & \\
\hline $\mathrm{Ni}+2$ & $6.25 \mathrm{E}-06$ & $6.25 \mathrm{E}-06$ & $9.03 \mathrm{E}-08$ & $9.03 \mathrm{E}-08$ & & & & \\
\hline $\mathrm{K}+$ & $1.59 \mathrm{E}-03$ & $1.59 \mathrm{E}-03$ & $1.53 \mathrm{E}-05$ & $1.53 \mathrm{E}-05$ & & & & \\
\hline $\mathrm{Se}+4$ & $1.19 \mathrm{E}-06$ & $1.19 \mathrm{E}-06$ & $2.31 \mathrm{E}-08$ & $2.31 \mathrm{E}-08$ & & & & \\
\hline $\mathrm{Ag}+$ & $2.04 \mathrm{E}-06$ & $2.04 \mathrm{E}-06$ & $5.42 \mathrm{E}-08$ & $5.42 \mathrm{E}-08$ & & & & \\
\hline $\mathrm{Na}+$ & $5.28 \mathrm{E}-02$ & $5.28 \mathrm{E}-02$ & $2.99 \mathrm{E}-04$ & $2.99 \mathrm{E}-04$ & & & & \\
\hline $\mathrm{Tl}+3$ & $1.36 \mathrm{E}-08$ & $1.36 \mathrm{E}-08$ & $6.82 \mathrm{E}-10$ & $6.82 \mathrm{E}-10$ & & & & \\
\hline $\mathrm{U}+4$ & $2.59 \mathrm{E}-06$ & $2.59 \mathrm{E}-06$ & $1.52 \mathrm{E}-07$ & $1.52 \mathrm{E}-07$ & & & & \\
\hline $\mathrm{V}+3$ & $1.48 \mathrm{E}-07$ & $1.48 \mathrm{E}-07$ & $1.85 \mathrm{E}-09$ & $1.85 \mathrm{E}-09$ & & & & \\
\hline$Z n+2$ & $2.82 \mathrm{E}-06$ & $2.82 \mathrm{E}-06$ & $4.55 \mathrm{E}-08$ & $4.55 \mathrm{E}-08$ & & & & \\
\hline $\mathrm{Zr}+4$ & $1.79 \mathrm{E}-04$ & $1.79 \mathrm{E}-04$ & $4.01 \mathrm{E}-06$ & $4.01 \mathrm{E}-06$ & & & & \\
\hline $\mathrm{Cl}-$ & $2.58 \mathrm{E}-03$ & $2.58 \mathrm{E}-03$ & $2.25 \mathrm{E}-05$ & $2.25 \mathrm{E}-05$ & & & & \\
\hline F- & $8.06 \mathrm{E}-03$ & $8.06 \mathrm{E}-03$ & $\begin{array}{l}3.77 \mathrm{E}-05 \\
\end{array}$ & $3.77 \mathrm{E}-05$ & & & & \\
\hline SO4-2 & $1.89 \mathrm{E}-02$ & $1.89 \mathrm{E}-02$ & $4.48 \mathrm{E}-04$ & $4.48 \mathrm{E}-04$ & & & & \\
\hline \begin{tabular}{|l|}
$\mathrm{NO3}^{-}$ \\
\end{tabular} & $1.06 \mathrm{E}+00$ & $1.06 \mathrm{E}+00$ & $1.60 \mathrm{E}-02$ & $1.60 \mathrm{E}-02$ & & & & \\
\hline PO4-3 & $6.11 \mathrm{E}-04$ & $6.11 \mathrm{E}-04$ & $1.43 \mathrm{E}-05$ & $1.43 \mathrm{E}-05$ & & & & \\
\hline $\mathrm{Am}+4$ & $1.86 \mathrm{E}-10$ & $1.86 \mathrm{E}-10$ & $1.11 \mathrm{E}-11$ & $1.11 \mathrm{E}-11$ & & & & \\
\hline $\mathrm{Br}-$ & $3.42 \mathrm{E}-08$ & $3.42 \mathrm{E}-08$ & $6.73 \mathrm{E}-10$ & $6.73 \mathrm{E}-10$ & & & & \\
\hline $\mathrm{Ce}+4$ & $1.13 \mathrm{E}-07$ & $1.13 \mathrm{E}-07$ & $3.89 \mathrm{E}-09$ & $3.89 \mathrm{E}-09$ & & & & \\
\hline $\mathrm{Eu}+3$ & $1.28 \mathrm{E}-09$ & $1.28 \mathrm{E}-09$ & $4.80 \mathrm{E}-11$ & $4.80 \mathrm{E}-11$ & & & & \\
\hline $\mathrm{Gd}+3$ & $3.85 \mathrm{E}-07$ & $3.85 \mathrm{E}-07$ & 1.49E-08 & $1.49 \mathrm{E}-08$ & & & & \\
\hline $\mathrm{Ge}+4$ & $3.60 \mathrm{E}-11$ & $3.60 \mathrm{E}-11$ & $6.43 \mathrm{E}-13$ & $6.43 \mathrm{E}-13$ & & & & \\
\hline $\ln +3$ & $3.57 \mathrm{E}-09$ & $3.57 \mathrm{E}-09$ & $1.01 \mathrm{E}-10$ & $1.01 \mathrm{E}-10$ & & & & \\
\hline $\mid-$ & $3.81 \mathrm{E}-07$ & $3.81 \mathrm{E}-07$ & 1.19E-08 & $1.19 \mathrm{E}-08$ & & & & \\
\hline $\mathrm{La}+3$ & $2.33 \mathrm{E}-08$ & $2.33 \mathrm{E}-08$ & $7.96 \mathrm{E}-10$ & $7.96 \mathrm{E}-10$ & & & & \\
\hline
\end{tabular}


Table A-16. Mass balance, Tank WM-189 waste (continued).

\begin{tabular}{|c|c|c|c|c|c|c|c|c|}
\hline Stream \# & 401 & 402 & 404 & 404 & 503 & 504 & 505 & 506 \\
\hline Chemical Composition & Mol/liter & Mol/liter & Wt frac & Wt frac & $\mathrm{lb} / \mathrm{wscf}$ & $\mathrm{lb} /$ wscf & $\mathrm{lb} / \mathrm{wscf}$ & $\mathrm{lb} / \mathrm{wscf}$ \\
\hline $\mathrm{Li}+$ & $2.73 \mathrm{E}-06$ & $2.73 \mathrm{E}-06$ & 4.67E-09 & 4.67E-09 & & & & \\
\hline $\mathrm{Mg}+2$ & 5.80E-05 & 5.80E-05 & 3.47E-07 & 3.47E-07 & & & & \\
\hline $\mathrm{Mo}+6$ & $2.81 \mathrm{E}-04$ & $2.81 \mathrm{E}-04$ & 6.64E-06 & $6.64 \mathrm{E}-06$ & & & & \\
\hline $\mathrm{Nd}+3$ & 7.51E-08 & 7.51E-08 & $2.66 \mathrm{E}-09$ & $2.66 \mathrm{E}-09$ & & & & \\
\hline $\mathrm{Np}+4$ & 7.79E-09 & 7.79E-09 & $4.55 \mathrm{E}-10$ & $4.55 \mathrm{E}-10$ & & & & \\
\hline $\mathrm{Nb}+5$ & 5.34E-06 & 5.34E-06 & $1.22 \mathrm{E}-07$ & $1.22 \mathrm{E}-07$ & & & & \\
\hline $\mathrm{Pd}+4$ & $5.06 \mathrm{E}-06$ & $5.06 \mathrm{E}-06$ & 1.33E-07 & $1.33 \mathrm{E}-07$ & & & & \\
\hline $\mathrm{Pu}+4$ & $3.05 E-08$ & $3.05 E-08$ & 1.83E-09 & 1.83E-09 & & & & \\
\hline $\mathrm{Pr}+4$ & $2.12 \mathrm{E}-08$ & $2.12 \mathrm{E}-08$ & $7.34 \mathrm{E}-10$ & $7.34 \mathrm{E}-10$ & & & & \\
\hline $\mathrm{Pm}+3$ & $4.25 \mathrm{E}-12$ & $4.25 \mathrm{E}-12$ & $1.52 \mathrm{E}-13$ & $1.52 \mathrm{E}-13$ & & & & \\
\hline $\mathrm{Rh}+4$ & 9.13E-09 & 9.13E-09 & $2.31 \mathrm{E}-10$ & $2.31 \mathrm{E}-10$ & & & & \\
\hline $\mathrm{Rb}+$ & $2.49 \mathrm{E}-08$ & $2.49 \mathrm{E}-08$ & $5.24 \mathrm{E}-10$ & $5.24 \mathrm{E}-10$ & & & & \\
\hline $\mathrm{Ru}+3$ & $2.52 \mathrm{E}-06$ & $2.52 \mathrm{E}-06$ & $6.27 \mathrm{E}-08$ & $6.27 \mathrm{E}-08$ & & & & \\
\hline $\mathrm{Sm}+3$ & $1.40 \mathrm{E}-08$ & $1.40 \mathrm{E}-08$ & $5.18 \mathrm{E}-10$ & $5.18 \mathrm{E}-10$ & & & & \\
\hline $\mathrm{Si}+4$ & $7.40 \mathrm{E}-04$ & $7.40 \mathrm{E}-04$ & 3.51E-01 & $3.51 \mathrm{E}-01$ & & & & \\
\hline $\mathrm{Sr}+2$ & $3.57 \mathrm{E}-07$ & $3.57 \mathrm{E}-07$ & 7.69E-09 & 7.69E-09 & & & & \\
\hline $\mathrm{Tc}+7$ & $6.22 \mathrm{E}-08$ & $6.22 \mathrm{E}-08$ & 1.50E-09 & 1.50E-09 & & & & \\
\hline $\mathrm{Te}+4$ & $2.64 \mathrm{E}-08$ & $2.64 \mathrm{E}-08$ & $8.29 \mathrm{E}-10$ & $8.29 \mathrm{E}-10$ & & & & \\
\hline $\mathrm{Tb}+4$ & $5.36 \mathrm{E}-12$ & $5.36 \mathrm{E}-12$ & $2.10 \mathrm{E}-13$ & $2.10 \mathrm{E}-13$ & & & & \\
\hline $\mathrm{Th}+4$ & $1.31 \mathrm{E}-07$ & $1.31 \mathrm{E}-07$ & $7.48 \mathrm{E}-09$ & $7.48 \mathrm{E}-09$ & & & & \\
\hline $\mathrm{Sn}+4$ & 8.84E-06 & $8.84 \mathrm{E}-06$ & $2.58 \mathrm{E}-07$ & $2.58 \mathrm{E}-07$ & & & & \\
\hline $\mathrm{Ti}+4$ & 4.56E-06 & 4.56E-06 & $5.38 \mathrm{E}-08$ & $5.38 \mathrm{E}-08$ & & & & \\
\hline $\mathrm{Y}+3$ & 1.73E-08 & 1.73E-08 & $3.80 \mathrm{E}-10$ & $3.80 \mathrm{E}-10$ & & & & \\
\hline $\mathrm{OH}-$ & $1.98 \mathrm{E}-02$ & $1.98 \mathrm{E}-02$ & $8.27 \mathrm{E}-05$ & 8.27E-05 & & & & \\
\hline $\mathrm{H} 2 \mathrm{O}$ & $5.23 \mathrm{E}+01$ & $5.23 E+01$ & 2.32E-01 & 2.32E-01 & & & & $4.68 \mathrm{E}-06$ \\
\hline $\mathrm{SO} 2$ & 1.65E-05 & 1.65E-05 & $2.60 \mathrm{E}-07$ & $2.60 \mathrm{E}-07$ & & & & \\
\hline \multicolumn{9}{|l|}{$\mathrm{H} 2 \mathrm{~S}$} \\
\hline $\mathrm{CO}$ & $2.09 \mathrm{E}-10$ & $2.09 \mathrm{E}-10$ & $1.44 \mathrm{E}-12$ & $1.44 \mathrm{E}-12$ & & & & \\
\hline $\mathrm{CO} 2$ & 3.33E-04 & 3.33E-04 & $3.60 \mathrm{E}-06$ & $3.60 \mathrm{E}-06$ & & & & \\
\hline $\mathrm{H} 2$ & $8.76 \mathrm{E}-10$ & $8.76 \mathrm{E}-10$ & $4.34 \mathrm{E}-13$ & $4.34 \mathrm{E}-13$ & & & & \\
\hline N2 & $1.28 \mathrm{E}-05$ & $1.28 \mathrm{E}-05$ & 8.80E-08 & 8.80E-08 & $7.27 \mathrm{E}-02$ & & & $5.74 \mathrm{E}-02$ \\
\hline $\mathrm{NO}$ & $2.22 \mathrm{E}-07$ & $2.22 \mathrm{E}-07$ & 1.64E-09 & 1.64E-09 & & & & \\
\hline $\mathrm{NO} 2$ & $2.79 \mathrm{E}-06$ & $2.79 \mathrm{E}-06$ & $3.16 \mathrm{E}-08$ & $3.16 \mathrm{E}-08$ & & & & \\
\hline $\mathrm{O} 2$ & $3.16 \mathrm{E}-06$ & $3.16 \mathrm{E}-06$ & $2.49 \mathrm{E}-08$ & $2.49 \mathrm{E}-08$ & & $8.30 \mathrm{E}-02$ & $8.30 \mathrm{E}-02$ & 1.74E-02 \\
\hline S (other) & $2.19 \mathrm{E}-07$ & 2.19E-07 & 1.73E-09 & 1.73E-09 & & & & \\
\hline $\mathrm{CO} 3$ & $2.73 \mathrm{E}-05$ & $2.73 \mathrm{E}-05$ & $4.03 \mathrm{E}-07$ & $4.03 \mathrm{E}-07$ & & & & \\
\hline \multicolumn{9}{|l|}{ C (reductant) } \\
\hline $\mathrm{O}$ (oxides) & $5.26 \mathrm{E}-03$ & $5.26 \mathrm{E}-03$ & 3.99E-01 & $3.99 \mathrm{E}-01$ & & & & \\
\hline \multicolumn{9}{|l|}{ C (organic) } \\
\hline \multicolumn{9}{|l|}{$\mathrm{H}$ (organic) } \\
\hline \multicolumn{9}{|l|}{$\mathrm{O}$ (organic) } \\
\hline Mass Flow (kg/hr): & $2.27 \mathrm{E}+01$ & $1.00 \mathrm{E}+00$ & $4.00 \mathrm{E}+00$ & $4.00 \mathrm{E}+00$ & $5.94 \mathrm{E}+02$ & $1.82 \mathrm{E}+02$ & $2.44 \mathrm{E}+02$ & $2.32 \mathrm{E}+03$ \\
\hline & & & & & & & & \\
\hline \multirow[t]{2}{*}{ Total Drums Generated } & & & & 31 & & & & \\
\hline & $\mathrm{w} / \mathrm{m} 3$ & $w / m 3$ & $w / m 3$ & w/Drum & $\mathrm{w} / \mathrm{m} 3$ & $w / m 3$ & $w / m 3$ & $\mathrm{w} / \mathrm{m} 3$ \\
\hline Heat Generation, w/m3 & $4.87 \mathrm{E}-02$ & $4.87 \mathrm{E}-02$ & $2.81 \mathrm{E}-02$ & $5.62 \mathrm{E}-03$ & & & & \\
\hline
\end{tabular}


Table A-16. Mass balance, Tank WM-189 waste (continued).

\begin{tabular}{|c|c|c|c|c|c|c|c|c|}
\hline Stream \# & 401 & 402 & 404 & 404 & 503 & 504 & 505 & 506 \\
\hline Radiological Composition & $\mathrm{Ci} / \mathrm{L}$ & $\mathrm{Ci} / \mathrm{L}$ & $\mathrm{Ci} / \mathrm{kg}$ & Ci/Drum & Ci/wscm & Ci/wscm & $\mathrm{Ci} / \mathrm{wscm}$ & $\mathrm{Ci} / \mathrm{wscm}$ \\
\hline $\mathrm{H}-3$ & & & & & & & & \\
\hline $\mathrm{C}-14$ & $1.35 \mathrm{E}-15$ & $1.35 \mathrm{E}-15$ & $3.31 \mathrm{E}-16$ & $1.39 \mathrm{E}-13$ & & & & \\
\hline Co-60 & $8.31 \mathrm{E}-08$ & $8.31 \mathrm{E}-08$ & $2.05 \mathrm{E}-08$ & $8.59 \mathrm{E}-06$ & & & & \\
\hline Ni-59 & $3.67 \mathrm{E}-09$ & $3.67 \mathrm{E}-09$ & $9.04 \mathrm{E}-10$ & $3.80 \mathrm{E}-07$ & & & & \\
\hline $\mathrm{Ni}-63$ & $1.14 \mathrm{E}-07$ & $1.14 \mathrm{E}-07$ & $2.82 \mathrm{E}-08$ & $1.18 \mathrm{E}-05$ & & & & \\
\hline Se-79 & $2.40 \mathrm{E}-09$ & $2.40 \mathrm{E}-09$ & $5.91 \mathrm{E}-10$ & $2.48 \mathrm{E}-07$ & & & & \\
\hline Sr-90 & $1.04 \mathrm{E}-04$ & $1.04 \mathrm{E}-04$ & $2.55 \mathrm{E}-05$ & $1.07 \mathrm{E}-02$ & & & & \\
\hline Y-90 & $9.86 \mathrm{E}-05$ & $9.86 \mathrm{E}-05$ & $2.43 \mathrm{E}-05$ & $1.02 \mathrm{E}-02$ & & & & \\
\hline Zr-93 & $8.91 \mathrm{E}-09$ & $8.91 \mathrm{E}-09$ & $2.19 \mathrm{E}-09$ & $9.21 \mathrm{E}-07$ & & & & \\
\hline $\mathrm{Nb}-93 \mathrm{~m}$ & $6.99 \mathrm{E}-09$ & $6.99 \mathrm{E}-09$ & $1.72 \mathrm{E}-09$ & $7.23 \mathrm{E}-07$ & & & & \\
\hline $\mathrm{Nb}-94$ & $6.35 \mathrm{E}-09$ & $6.35 \mathrm{E}-09$ & $1.56 \mathrm{E}-09$ & $6.57 \mathrm{E}-07$ & & & & \\
\hline Tc-99 & $1.46 \mathrm{E}-07$ & $1.46 \mathrm{E}-07$ & $3.59 \mathrm{E}-08$ & $1.51 \mathrm{E}-05$ & & & & \\
\hline Ru-106 & $3.18 \mathrm{E}-09$ & $3.18 \mathrm{E}-09$ & $7.82 \mathrm{E}-10$ & $3.29 \mathrm{E}-07$ & & & & \\
\hline Rh-102 & $2.15 \mathrm{E}-12$ & $2.15 \mathrm{E}-12$ & $5.29 \mathrm{E}-13$ & $2.22 \mathrm{E}-10$ & & & & \\
\hline Rh-106 & $3.18 \mathrm{E}-09$ & $3.18 \mathrm{E}-09$ & $7.82 \mathrm{E}-10$ & $3.29 \mathrm{E}-07$ & & & & \\
\hline $\mathrm{Pd}-107$ & $4.33 \mathrm{E}-11$ & $4.33 \mathrm{E}-11$ & 1.07E-11 & $4.48 \mathrm{E}-09$ & & & & \\
\hline Cd-113m & $5.11 \mathrm{E}-09$ & $5.11 \mathrm{E}-09$ & $1.26 \mathrm{E}-09$ & $5.28 \mathrm{E}-07$ & & & & \\
\hline Sn-121m & $2.69 \mathrm{E}-10$ & $2.69 \mathrm{E}-10$ & $6.62 \mathrm{E}-11$ & $2.78 \mathrm{E}-08$ & & & & \\
\hline Sn-126 & $2.26 \mathrm{E}-09$ & $2.26 \mathrm{E}-09$ & $5.56 \mathrm{E}-10$ & $2.34 \mathrm{E}-07$ & & & & \\
\hline Sb-125 & $1.17 \mathrm{E}-06$ & $1.17 \mathrm{E}-06$ & $2.87 \mathrm{E}-07$ & $1.21 \mathrm{E}-04$ & & & & \\
\hline Sb-126 & $1.43 \mathrm{E}-10$ & $1.43 \mathrm{E}-10$ & $3.53 \mathrm{E}-11$ & $1.48 \mathrm{E}-08$ & & & & \\
\hline Te-125m & $1.27 \mathrm{E}-08$ & $1.27 \mathrm{E}-08$ & $3.12 \mathrm{E}-09$ & $1.31 \mathrm{E}-06$ & & & & \\
\hline I-129 & $8.05 \mathrm{E}-09$ & $8.05 \mathrm{E}-09$ & $1.98 \mathrm{E}-09$ & $8.32 \mathrm{E}-07$ & & & & \\
\hline Cs-134 & $5.66 \mathrm{E}-05$ & $5.66 \mathrm{E}-05$ & $1.56 \mathrm{E}-05$ & $6.55 \mathrm{E}-03$ & & & & \\
\hline Cs-135 & $8.92 \mathrm{E}-07$ & $8.92 \mathrm{E}-07$ & $2.46 \mathrm{E}-07$ & $1.03 \mathrm{E}-04$ & & & & \\
\hline \begin{tabular}{|l|} 
Cs-137 \\
\end{tabular} & $4.62 \mathrm{E}-02$ & $4.62 \mathrm{E}-02$ & $1.27 \mathrm{E}-02$ & $5.35 \mathrm{E}+00$ & & & & \\
\hline Ba-137m & $1.47 \mathrm{E}-04$ & 1.47E-04 & $3.61 \mathrm{E}-05$ & $1.52 \mathrm{E}-02$ & & & & \\
\hline \begin{tabular}{|l|} 
Ce-144 \\
\end{tabular} & $2.15 \mathrm{E}-09$ & $2.15 \mathrm{E}-09$ & $5.28 \mathrm{E}-10$ & $2.22 \mathrm{E}-07$ & & & & \\
\hline Pr-144 & $2.15 \mathrm{E}-09$ & $2.15 \mathrm{E}-09$ & $5.28 \mathrm{E}-10$ & $2.22 \mathrm{E}-07$ & & & & \\
\hline Pm-146 & $1.27 \mathrm{E}-10$ & $1.27 \mathrm{E}-10$ & $3.12 \mathrm{E}-11$ & $1.31 \mathrm{E}-08$ & & & & \\
\hline Pm-147 & $5.79 \mathrm{E}-07$ & $5.79 \mathrm{E}-07$ & $1.43 \mathrm{E}-07$ & $5.99 \mathrm{E}-05$ & & & & \\
\hline Sm-151 & $1.15 \mathrm{E}-06$ & $1.15 \mathrm{E}-06$ & $2.82 \mathrm{E}-07$ & $1.18 \mathrm{E}-04$ & & & & \\
\hline Eu-152 & $7.35 \mathrm{E}-09$ & $7.35 \mathrm{E}-09$ & $1.81 \mathrm{E}-09$ & $7.60 \mathrm{E}-07$ & & & & \\
\hline Eu-154 & $4.39 \mathrm{E}-07$ & $4.39 \mathrm{E}-07$ & $1.08 \mathrm{E}-07$ & $4.53 \mathrm{E}-05$ & & & & \\
\hline Eu-155 & $4.13 \mathrm{E}-07$ & $4.13 \mathrm{E}-07$ & 1.02E-07 & $4.27 \mathrm{E}-05$ & & & & \\
\hline & & & & & & & & \\
\hline Th-230 & 4.36E-12 & $4.36 \mathrm{E}-12$ & $1.07 \mathrm{E}-12$ & $4.51 \mathrm{E}-10$ & & & & \\
\hline Pa-233 & $1.18 \mathrm{E}-08$ & $1.18 \mathrm{E}-08$ & $2.90 \mathrm{E}-09$ & $1.22 \mathrm{E}-06$ & & & & \\
\hline$U-232$ & $1.02 \mathrm{E}-11$ & $1.02 \mathrm{E}-11$ & $2.51 \mathrm{E}-12$ & $1.05 \mathrm{E}-09$ & & & & \\
\hline $\mathrm{U}-233$ & $3.60 \mathrm{E}-13$ & $3.60 \mathrm{E}-13$ & $8.85 \mathrm{E}-14$ & $3.72 \mathrm{E}-11$ & & & & \\
\hline $\mathrm{U}-234$ & $7.06 \mathrm{E}-09$ & $7.06 \mathrm{E}-09$ & $1.74 \mathrm{E}-09$ & $7.30 \mathrm{E}-07$ & & & & \\
\hline U-235 & $2.85 \mathrm{E}-10$ & $2.85 \mathrm{E}-10$ & $7.01 \mathrm{E}-11$ & $2.94 \mathrm{E}-08$ & & & & \\
\hline U-236 & $3.30 \mathrm{E}-10$ & $3.30 \mathrm{E}-10$ & $8.13 \mathrm{E}-11$ & $3.41 \mathrm{E}-08$ & & & & \\
\hline U-237 & $2.59 \mathrm{E}-11$ & $2.59 \mathrm{E}-11$ & $6.37 \mathrm{E}-12$ & $2.68 \mathrm{E}-09$ & & & & \\
\hline U-238 & $1.57 \mathrm{E}-10$ & $1.57 \mathrm{E}-10$ & $3.85 \mathrm{E}-11$ & $1.62 \mathrm{E}-08$ & & & & \\
\hline Np-237 & $1.30 \mathrm{E}-09$ & $1.30 \mathrm{E}-09$ & $3.20 \mathrm{E}-10$ & $1.35 \mathrm{E}-07$ & & & & \\
\hline Pu-236 & $1.66 \mathrm{E}-11$ & $1.66 \mathrm{E}-11$ & $4.09 \mathrm{E}-12$ & $1.72 \mathrm{E}-09$ & & & & \\
\hline Pu-238 & $3.84 \mathrm{E}-06$ & $3.84 \mathrm{E}-06$ & $9.46 \mathrm{E}-07$ & $3.97 \mathrm{E}-04$ & & & & \\
\hline Pu-239 & $4.20 \mathrm{E}-07$ & $4.20 \mathrm{E}-07$ & 1.03E-07 & $4.34 \mathrm{E}-05$ & & & & \\
\hline Pu-240 & $6.20 \mathrm{E}-08$ & $6.20 \mathrm{E}-08$ & $1.53 \mathrm{E}-08$ & $6.41 \mathrm{E}-06$ & & & & \\
\hline Pu-241 & $3.36 \mathrm{E}-06$ & $3.36 \mathrm{E}-06$ & $8.27 \mathrm{E}-07$ & $3.47 \mathrm{E}-04$ & & & & \\
\hline Pu-242 & $4.86 \mathrm{E}-11$ & $4.86 \mathrm{E}-11$ & $1.20 \mathrm{E}-11$ & $5.02 \mathrm{E}-09$ & & & & \\
\hline Pu-244 & $1.45 \mathrm{E}-18$ & 1.45E-18 & $3.56 \mathrm{E}-19$ & $1.50 \mathrm{E}-16$ & & & & \\
\hline Am-241 & $2.03 \mathrm{E}-07$ & $2.03 \mathrm{E}-07$ & $5.00 \mathrm{E}-08$ & $2.10 \mathrm{E}-05$ & & & & \\
\hline Am-242m & $3.81 \mathrm{E}-11$ & $3.81 \mathrm{E}-11$ & $9.37 \mathrm{E}-12$ & $3.94 \mathrm{E}-09$ & & & & \\
\hline
\end{tabular}


Table A-16. Mass balance, Tank WM-189 waste (continued).

\begin{tabular}{|c|c|c|c|c|c|c|c|c|}
\hline Stream \# & 401 & 402 & 404 & 404 & 503 & 504 & 505 & 506 \\
\hline & $\mathrm{Ci} / \mathrm{L}$ & $\mathrm{Ci} / \mathrm{L}$ & $\mathrm{Ci} / \mathrm{kg}$ & Ci/Drum & Ci/wscm & $\mathrm{Ci} / \mathrm{wscm}$ & $\mathrm{Ci} / \mathrm{wscm}$ & Ci/wscm \\
\hline Am-243 & $6.49 \mathrm{E}-11$ & $6.49 \mathrm{E}-11$ & $1.60 \mathrm{E}-11$ & $6.71 \mathrm{E}-09$ & & & & \\
\hline $\mathrm{Cm}-242$ & $1.23 \mathrm{E}-10$ & $1.23 \mathrm{E}-10$ & $3.02 \mathrm{E}-11$ & 1.27E-08 & & & & \\
\hline $\mathrm{Cm}-243$ & $1.51 \mathrm{E}-10$ & $1.51 \mathrm{E}-10$ & $3.72 \mathrm{E}-11$ & $1.56 \mathrm{E}-08$ & & & & \\
\hline $\mathrm{Cm}-244$ & $6.40 \mathrm{E}-09$ & $6.40 \mathrm{E}-09$ & 1.57E-09 & $6.61 \mathrm{E}-07$ & & & & \\
\hline $\mathrm{Cm}-245$ & $1.59 \mathrm{E}-12$ & $1.59 \mathrm{E}-12$ & $3.91 \mathrm{E}-13$ & $1.64 \mathrm{E}-10$ & & & & \\
\hline $\mathrm{Cm}-246$ & $1.04 \mathrm{E}-13$ & $1.04 \mathrm{E}-13$ & $2.57 \mathrm{E}-14$ & $1.08 \mathrm{E}-11$ & & & & \\
\hline TRU & $4.63 \mathrm{E}-06$ & 4.63E-06 & 1.14E-06 & $4.79 \mathrm{E}-04$ & & & & \\
\hline \multicolumn{9}{|l|}{ TRU, nCi/gm } \\
\hline & & & & & & & & \\
\hline $\begin{array}{l}\text { Gas Stream Bulk } \\
\text { Composition (Wet Basis) }\end{array}$ & $\begin{array}{l}\text { mol\% or } \\
\text { ppmv }\end{array}$ & $\begin{array}{l}\text { mol\% or } \\
\text { ppmv }\end{array}$ & $\begin{array}{l}\text { mol\% or } \\
\text { ppmv }\end{array}$ & $\begin{array}{l}\text { mol\% or } \\
\text { ppmv }\end{array}$ & $\begin{array}{l}\text { mol\% or } \\
\text { ppmv }\end{array}$ & $\begin{array}{l}\text { mol\% or } \\
\text { ppmv }\end{array}$ & $\begin{array}{l}\text { mol\% or } \\
\text { ppmv }\end{array}$ & $\begin{array}{c}\text { mol\% or } \\
\text { ppmv }\end{array}$ \\
\hline $\mathrm{H} 2 \mathrm{O}, \mathrm{mol} \%$ & & & & & & & & $0.01 \%$ \\
\hline $\mathrm{O} 2, \mathrm{~mol} \%$ & & & & & & $100.00 \%$ & $100.00 \%$ & $20.99 \%$ \\
\hline $\mathrm{N} 2, \mathrm{~mol} \%$ & & & & & $100.00 \%$ & & & $79.00 \%$ \\
\hline \multicolumn{9}{|l|}{$\mathrm{H} 2, \mathrm{~mol} \%$} \\
\hline \multicolumn{9}{|l|}{$\mathrm{CO} 2, \mathrm{~mol} \%$} \\
\hline \multicolumn{9}{|l|}{$\mathrm{CO}, \mathrm{ppmv}$} \\
\hline \multicolumn{9}{|l|}{$\mathrm{NO}, \mathrm{ppmv}$} \\
\hline \multicolumn{9}{|l|}{ NO2, ppmv } \\
\hline \multicolumn{9}{|l|}{ SO2, ppmv } \\
\hline \multicolumn{9}{|l|}{$\mathrm{Cl}, \mathrm{ppmv}$} \\
\hline \multicolumn{9}{|l|}{$\mathrm{F}, \mathrm{ppmv}$} \\
\hline \multicolumn{9}{|l|}{ C (organic), ppmv } \\
\hline \multicolumn{9}{|l|}{$\mathrm{H}$ (organic), ppmv } \\
\hline \multicolumn{9}{|l|}{$\mathrm{Hg}, \mathrm{ug} / \mathrm{wscm}$} \\
\hline $\mathrm{PM}, \mathrm{mg} / \mathrm{wscm}$ & & & & & $1.98 \mathrm{E}+01$ & $2.60 \mathrm{E}-01$ & & $1.57 E+01$ \\
\hline \multicolumn{9}{|l|}{ SVM, ug/wscm } \\
\hline \multicolumn{9}{|l|}{ LVM, ug/wscm } \\
\hline $\begin{array}{l}\text { Gas Stream Bulk } \\
\text { Composition (Dry Basis) }\end{array}$ & $\mathrm{mol} \%$ & $\mathrm{~mol} \%$ & $\mathrm{~mol} \%$ & $\mathrm{~mol} \%$ & $\mathrm{~mol} \%$ & $\mathrm{~mol} \%$ & $\mathrm{~mol} \%$ & $\mathrm{~mol} \%$ \\
\hline $\mathrm{O} 2, \mathrm{~mol} \%$, dry basis & & & & & & & & $20.99 \%$ \\
\hline $\mathrm{N} 2, \mathrm{~mol} \%$, dry basis & & & & & & & & $79.01 \%$ \\
\hline \multicolumn{9}{|l|}{$\mathrm{H} 2, \mathrm{~mol} \%$, dry basis } \\
\hline \multicolumn{9}{|l|}{$\mathrm{CO} 2, \mathrm{~mol} \%$, dry basis } \\
\hline $\begin{array}{l}\text { Gas Stream Bulk } \\
\text { Composition (Dry Basis, } \\
\text { Corrected to } 7 \% \text { O2 with } \\
100 \% \text { O2 Combustion Air) }\end{array}$ & $\begin{array}{l}\mathrm{ppmv}, \text { or } \\
\text { ug/dscm } \\
\text { or } \\
\mathrm{mg} / \mathrm{dscm}\end{array}$ & $\begin{array}{l}\mathrm{ppmv}, \text { or } \\
\text { ug/dscm } \\
\text { or } \\
\mathrm{mg} / \mathrm{dscm}\end{array}$ & $\begin{array}{l}\mathrm{ppmv}, \text { or } \\
\text { ug/dscm } \\
\text { or } \\
\mathrm{mg} / \mathrm{dscm}\end{array}$ & $\begin{array}{l}\mathrm{ppmv}, \text { or } \\
\text { ug/dscm } \\
\text { or } \\
\mathrm{mg} / \mathrm{dscm}\end{array}$ & $\begin{array}{l}\mathrm{ppmv}, \text { or } \\
\text { ug/dscm } \\
\text { or } \\
\mathrm{mg} / \mathrm{dscm}\end{array}$ & $\begin{array}{l}\mathrm{ppmv}, \text { or } \\
\text { ug/dscm } \\
\text { or } \\
\mathrm{mg} / \mathrm{dscm}\end{array}$ & $\begin{array}{l}\mathrm{ppmv}, \text { or } \\
\text { ug/dscm } \\
\text { or } \\
\mathrm{mg} / \mathrm{dscm}\end{array}$ & $\begin{array}{l}\text { ppmv, or } \\
\text { ug/dscm } \\
\text { or } \\
\mathrm{mg} / \mathrm{dscm}\end{array}$ \\
\hline \multicolumn{9}{|l|}{$\mathrm{CO}, \mathrm{ppmv}$, dry basis } \\
\hline \multicolumn{9}{|l|}{$\mathrm{NO}$, ppmv, dry basis } \\
\hline \multicolumn{9}{|l|}{ NO2, ppmv, dry basis } \\
\hline \multicolumn{9}{|l|}{ SO2, ppmv, dry basis } \\
\hline \multicolumn{9}{|l|}{$\mathrm{Cl}, \mathrm{ppmv}$, dry basis } \\
\hline $\mathrm{F}, \mathrm{ppmv}$, dry basis & & & & & & & & \\
\hline C (organic), ppmv, dry basis & & & & & & & & \\
\hline $\mathrm{H}$ (organic), ppmv, dry basis & & & & & & & & \\
\hline $\mathrm{Hg}, \mathrm{ug} / \mathrm{dscm}$ & & & & & & & & \\
\hline $\mathrm{PM}, \mathrm{mg} / \mathrm{dscm}$ & & & & & & & & $2.20 \mathrm{E}+00$ \\
\hline SVM, ug/dscm & & & & & & & & \\
\hline LVM, ug/dscm & & & & & & & & \\
\hline
\end{tabular}


Table A-16. Mass balance, Tank WM-189 waste (continued).

\begin{tabular}{|c|c|c|c|c|c|c|c|c|}
\hline PFD \# & PFD-2 & PFD-3 & PFD-3 & PFD-3 & PFD-3 & PFD-3 & PFD-3 & PFD-3 \\
\hline WM-189, Stream \# & 507 & 510 & 511 & 512 & 513 & 514 & 515 & 516 \\
\hline Stream Name & $\begin{array}{l}\text { Off-Gas } \\
\text { from Boiler }\end{array}$ & $\begin{array}{c}\text { Nitrogen to } \\
\text { Cool } \\
\text { Product }\end{array}$ & $\begin{array}{l}\text { N2 from } \\
\text { Product } \\
\text { Cooler }\end{array}$ & $\begin{array}{c}\text { Filter } \\
\text { Backpulse } \\
\text { N2 }\end{array}$ & $\begin{array}{l}\text { Solids } \\
\text { Transport } \\
\text { Nitrogen }\end{array}$ & $\begin{array}{c}\text { Tank Farm } \\
\text { Transfer } \\
\text { Airlift Air }\end{array}$ & $\begin{array}{c}\text { Ventilation } \\
\text { Air }\end{array}$ & Fresh GAC \\
\hline Rate or Volume & $1.42 \mathrm{E}+05$ & $3.16 \mathrm{E}+02$ & $1.00 \mathrm{E}+03$ & $2.95 \mathrm{E}+02$ & $7.87 \mathrm{E}+01$ & $1.52 \mathrm{E}+01$ & $2.89 \mathrm{E}+01$ & $5.94 \mathrm{E}+00$ \\
\hline Volume Flow (standard, wet) & $7.25 \mathrm{E}+04$ & $2.13 \mathrm{E}+03$ & $2.13 \mathrm{E}+03$ & $8.63 \mathrm{E}+02$ & $2.30 \mathrm{E}+02$ & $4.46 \mathrm{E}+01$ & $2.94 \mathrm{E}+01$ & \\
\hline Volume Flow (standard, dry) & $6.42 \mathrm{E}+04$ & $2.13 \mathrm{E}+03$ & $2.13 \mathrm{E}+03$ & $8.63 \mathrm{E}+02$ & $2.30 \mathrm{E}+02$ & $4.46 \mathrm{E}+01$ & $2.94 \mathrm{E}+01$ & \\
\hline Rate Units & $\mathrm{ft} 3 / \mathrm{hr}$ & $\mathrm{ft} 3 / \mathrm{hr}$ & $\mathrm{ft} 3 / \mathrm{hr}$ & $\mathrm{ft} 3 / \mathrm{hr}$ & $\mathrm{ft} 3 / \mathrm{hr}$ & $\mathrm{ft} 3 / \mathrm{hr}$ & $\mathrm{ft} 3 / \mathrm{hr}$ & $\mathrm{lb} / \mathrm{hr}$ \\
\hline Rate or Volume, metric & $2.05 \mathrm{E}+03$ & $6.03 \mathrm{E}+01$ & $6.03 \mathrm{E}+01$ & $2.44 \mathrm{E}+01$ & $6.52 \mathrm{E}+00$ & $1.26 \mathrm{E}+00$ & $8.34 \mathrm{E}-01$ & $2.70 \mathrm{E}+00$ \\
\hline Rate Units & wscm/hr & wscm $/ \mathrm{hr}$ & $\mathrm{wscm} / \mathrm{hr}$ & wscm $/ \mathrm{hr}$ & $\mathrm{wscm} / \mathrm{hr}$ & wscm $/ \mathrm{hr}$ & wscm $/ \mathrm{hr}$ & $\mathrm{kg} / \mathrm{hr}$ \\
\hline Temperature, ${ }^{\circ} \mathrm{C}$ & 300 & 0 & 592 & 15 & 15 & 15 & 15 & 25 \\
\hline Temperature, ${ }^{\circ} \mathrm{F}$ & 572 & 32 & 1098 & 59 & 59 & 59 & 59 & 77 \\
\hline Pressure, psia & 14.7 & 92.3 & 92.3 & 42.3 & 42.3 & 42.3 & 14.7 & 14.7 \\
\hline Specific Gravity & $6.10 \mathrm{E}-04$ & $7.85 \mathrm{E}-03$ & $2.48 \mathrm{E}-03$ & $3.41 \mathrm{E}-03$ & $3.41 \mathrm{E}-03$ & $3.51 \mathrm{E}-03$ & $1.22 \mathrm{E}-03$ & $4.81 \mathrm{E}-01$ \\
\hline \multicolumn{9}{|c|}{ Gas Stream Bulk Composition (Wet Basis) } \\
\hline & Mol \% & Mol \% & Mol \% & Mol \% & Mol \% & Mol \% & Mol \% & Wt \% \\
\hline $\mathrm{H} 2 \mathrm{O}$ & $11.53 \%$ & & & & & $0.01 \%$ & $0.01 \%$ & \\
\hline $\mathrm{O} 2$ & $3.00 \%$ & & & & & $20.99 \%$ & $20.99 \%$ & \\
\hline $\mathrm{N} 2$ & $74.11 \%$ & $100.00 \%$ & $100.00 \%$ & $100.00 \%$ & $100.00 \%$ & $79.00 \%$ & $79.00 \%$ & \\
\hline $\mathrm{H} 2$ & $0.05 \%$ & & & & & & & \\
\hline \multirow[t]{2}{*}{$\mathrm{CO} 2$} & $10.64 \%$ & & & & & & & \\
\hline & ppmv & & & & & & & \\
\hline $\mathrm{CO}$ & $2.21 \mathrm{E}+03$ & & & & & & & \\
\hline NO & $4.30 \mathrm{E}+03$ & & & & & & & \\
\hline NO2 & $1.97 \mathrm{E}+00$ & & & & & & & \\
\hline $\mathrm{SO} 2$ & $2.40 \mathrm{E}+02$ & & & & & & & \\
\hline C(organic) & $4.36 \mathrm{E}-14$ & & & & & & & \\
\hline $\mathrm{H}$ (organic) & $1.74 \mathrm{E}-13$ & & & & & & & \\
\hline \multirow{2}{*}{\multicolumn{9}{|c|}{ Gas Stream Bulk Composition (Dry Basis) }} \\
\hline & & & & & & & & \\
\hline & Mol \% & Mol \% & Mol \% & Mol \% & Mol \% & Mol \% & Mol \% & \\
\hline $\mathrm{O} 2$ & $3.39 \%$ & & & & & $20.99 \%$ & $20.99 \%$ & \\
\hline $\mathrm{N} 2$ & $83.77 \%$ & & & & & $79.01 \%$ & $79.01 \%$ & \\
\hline $\mathrm{H} 2$ & $0.05 \%$ & & & & & & & \\
\hline $\mathrm{CO} 2$ & $12.03 \%$ & & & & & & & \\
\hline \multirow{2}{*}{\multicolumn{9}{|c|}{ Gas Stream Bulk Composition (Dry Basis, Corrected to $7 \%$ O2 with $100 \%$ O2 Combustion Air) }} \\
\hline & & & & & & & & \\
\hline $\mathrm{CO}$ & $\frac{\mathrm{ppmv}}{3.50 \mathrm{~F}+02}$ & & & & & & & \\
\hline NO & $6.80 \mathrm{E}+02$ & & & & & & & \\
\hline $\mathrm{NO2}$ & $3.12 \mathrm{E}-01$ & & & & & & & \\
\hline $\mathrm{SO} 2$ & $3.80 \mathrm{E}+01$ & & & & & & & \\
\hline C(organic) & $6.90 \mathrm{E}-15$ & & & & & & & \\
\hline H(organic) & $2.76 \mathrm{E}-14$ & & & & & & & \\
\hline & & & & & & & & \\
\hline $\mathrm{PM}, \mathrm{mg} / \mathrm{dscm}$ & $2.53 \mathrm{E}+00$ & & & & & $2.17 \mathrm{E}+00$ & $2.20 \mathrm{E}+00$ & \\
\hline \multirow{2}{*}{\multicolumn{9}{|c|}{$\mathrm{Hg}, \mathrm{ug} / \mathrm{dscm}$}} \\
\hline & & & & & & & & \\
\hline & & & & & & & & \\
\hline & & & & & & & & \\
\hline GAC & & & & & & & & $100 \%$ \\
\hline
\end{tabular}


Table A-17 lists the major chemicals consumed for reforming SBW with the TWR process. Entries in the body of the table are consumption rates for processing individual tank wastes, while the final column shows the total accumulated material used for complete processing. Alumina is used as starting bed material and a total of 4 bed change-outs would require about 10,500 pounds.

Table A-17. Consumable chemicals estimate for TWR processing of SBW.

\begin{tabular}{lcccccc}
\hline \multicolumn{1}{c}{ Stream } & WM-180 & WM-187 & WM-188 & WM-189 & Totals \\
\hline SBW (gal/hr) & $9.59 \mathrm{E}+01$ & $9.54 \mathrm{E}+01$ & $9.65 \mathrm{E}+01$ & $9.73 \mathrm{E}+01$ & $1.16 \mathrm{E}+06$ & gal \\
Gases: & & & & & & \\
$\quad \mathrm{CO}_{2}(\mathrm{lb} / \mathrm{hr})$ & $2.98 \mathrm{E}+03$ & $2.95 \mathrm{E}+03$ & $2.86 \mathrm{E}+03$ & $2.92 \mathrm{E}+03$ & $3.51 \mathrm{E}+07$ & $\mathrm{lb}$ \\
$\quad \mathrm{N}_{2}(\mathrm{lb} / \mathrm{hr})$ & $1.01 \mathrm{E}+02$ & $1.01 \mathrm{E}+02$ & $8.91 \mathrm{E}+01$ & $1.08 \mathrm{E}+02$ & $1.19 \mathrm{E}+06$ & $\mathrm{lb}$ \\
$\quad \mathrm{O}_{2}(\mathrm{lb} / \mathrm{hr})$ & $2.79 \mathrm{E}+03$ & $2.77 \mathrm{E}+03$ & $2.78 \mathrm{E}+03$ & $2.80 \mathrm{E}+03$ & $3.34 \mathrm{E}+07$ & $\mathrm{lb}$ \\
Propane $(\mathrm{lb} / \mathrm{hr})$ & $2.93 \mathrm{E}+02$ & $2.93 \mathrm{E}+02$ & $2.93 \mathrm{E}+02$ & $2.93 \mathrm{E}+02$ & $3.52 \mathrm{E}+06$ & $\mathrm{lb}$ \\
Isopropyl Alcohol $(\mathrm{lb} / \mathrm{hr})$ & $6.69 \mathrm{E}+02$ & $6.69 \mathrm{E}+02$ & $9.86 \mathrm{E}+02$ & $7.06 \mathrm{E}+02$ & $9.22 \mathrm{E}+06$ & $\mathrm{lb}$ \\
Quench water $(\mathrm{lb} / \mathrm{hr})$ & $3.84 \mathrm{E}+03$ & $3.79 \mathrm{E}+03$ & $3.81 \mathrm{E}+03$ & $3.87 \mathrm{E}+03$ & $4.60 \mathrm{E}+07$ & $\mathrm{lb}$ \\
Nitric acid $(\mathrm{lb} / \mathrm{hr})$ & $6.13 \mathrm{E}+00$ & $6.13 \mathrm{E}+00$ & $6.13 \mathrm{E}+00$ & $6.13 \mathrm{E}+00$ & $7.35 \mathrm{E}+04$ & $\mathrm{lb}$ \\
Processing time $(\mathrm{hrs})$ & $3.28 \mathrm{E}+03$ & $2.10 \mathrm{E}+03$ & $3.38 \mathrm{E}+03$ & $3.24 \mathrm{E}+03$ & $1.20 \mathrm{E}+04$ & $\mathrm{hr}$ \\
\hline
\end{tabular}

Table A-18 summarizes the production rate of reformer product and the associated packaging rate for TWR. The values in the table assume a densification operation is used to increase the product's bulk density from about 0.15 to $1.42 \mathrm{~g} / \mathrm{ml}(89 \mathrm{lb} / \mathrm{ft} 3)$. The table also summarizes the estimated decay heat and TRU activity of the final waste.

Table A-18. Summary of estimated waste product quantities and properties for TWR processing of SBW.

\begin{tabular}{|c|c|c|c|c|c|}
\hline & WM-180 & WM-187 & WM-188 & WM-189 & Total \\
\hline Product mass rate $(\mathrm{lb} / \mathrm{hr})$ & 115 & 134 & 115 & 138 & \\
\hline Processing time $(\mathrm{hr})$ & 3,280 & 2,100 & 3,380 & 3,240 & 12,000 \\
\hline Canister filling rate (can/day) $)^{\mathrm{a}}$ & 1.11 & 1.29 & 1.11 & 1.32 & \\
\hline Number of canisters ${ }^{\mathrm{a}, \mathrm{b}}$ & 152 & 113 & 156 & 178 & 599 \\
\hline Heat generation $\left(\mathrm{W} / \mathrm{m}^{3}\right)$ & 2.64 & 3.38 & 3.65 & 3.88 & \\
\hline Heat per canister (W/can) & 2.12 & 2.71 & 2.92 & 3.10 & \\
\hline TRU specific activity (nCi/g) & 7,600 & 6,770 & 6,410 & 5,540 & \\
\hline
\end{tabular}

${ }^{\mathrm{a}}$ Waste specific gravity assumed as 1.42 .

${ }^{\mathrm{b}} \mathrm{A}$ canister contains $0.8 \mathrm{~m}^{3}$ waste.

The estimated spent scrub quantities and treatment process are the same for the TWR and TTT processes.

Mass balances for Tanks WM-180, WM-187, WM-188 and WM-189 are shown below in Tables A-19, A-20, A-21, and A-22, respectively. 
Table A-19. Mass balance, Tank WM-180 waste.

\begin{tabular}{|c|c|c|c|c|c|c|c|}
\hline PFD Number & PFD-2 & PFD-2 & PFD-2 & PFD-2 & PFD-2 & PFD-2 & PFD-2 \\
\hline Stream Number & 101 & 102 & 103 & 104 & 106 & 107 & 108 \\
\hline Stream Name & SBW & $\begin{array}{l}\text { Reformer } \\
\text { Feed }\end{array}$ & $\begin{array}{c}\text { Reformer } \\
\text { Off-gas }\end{array}$ & $\begin{array}{c}\text { Reformer } \\
\text { Off-gas } \\
\text { Cooled }\end{array}$ & Filter Drain & Bed Drain & $\begin{array}{l}\text { Cooled } \\
\text { Product }\end{array}$ \\
\hline Rate or Volume & $9.59 \mathrm{E}+01$ & $1.67 \mathrm{E}+02$ & $2.20 \mathrm{E}+05$ & $1.81 \mathrm{E}+05$ & $7.19 \mathrm{E}+00$ & $1.08 \mathrm{E}+02$ & $1.15 \mathrm{E}+02$ \\
\hline Volume Flow (standard, wet) ${ }^{*}$ & & & $5.35 \mathrm{E}+04$ & $5.49 \mathrm{E}+04$ & & & \\
\hline Volume Flow (standard, dry) & & & $3.70 \mathrm{E}+04$ & $3.84 \mathrm{E}+04$ & & & \\
\hline Rate Units & $\mathrm{gal} / \mathrm{hr}$ & $\mathrm{gal} / \mathrm{hr}$ & $\mathrm{ft} 3 / \mathrm{hr}$ & $\mathrm{ft} 3 / \mathrm{hr}$ & $\mathrm{lb} / \mathrm{hr}$ & $\mathrm{lb} / \mathrm{hr}$ & $\mathrm{lb} / \mathrm{hr}$ \\
\hline Rate or Volume, metric & $3.63 \mathrm{E}+02$ & $6.33 \mathrm{E}+02$ & $1.51 \mathrm{E}+03$ & $1.55 \mathrm{E}+03$ & $3.26 \mathrm{E}+00$ & $4.90 \mathrm{E}+01$ & $5.23 \mathrm{E}+01$ \\
\hline Rate Units & $\mathrm{L} / \mathrm{hr}$ & $\mathrm{L} / \mathrm{hr}$ & wscm $/ \mathrm{hr}$ & wscm $/ \mathrm{hr}$ & $\mathrm{kg} / \mathrm{hr}$ & $\mathrm{kg} / \mathrm{hr}$ & $\mathrm{kg} / \mathrm{hr}$ \\
\hline Temperature, ${ }^{\circ} \mathrm{C}$ & 15 & 17 & 600 & 400 & 400 & 600 & 56 \\
\hline Temperature, ${ }^{\circ} \mathrm{F}$ & 59 & 63 & 1112 & 752 & 752 & 1112 & 133 \\
\hline Pressure, psia & 14.70 & 44.70 & 10.62 & 10.22 & 9.50 & 10.80 & 9.50 \\
\hline Specific Gravity & 1.20 & 1.20 & $3.31 \mathrm{E}-04$ & $4.12 \mathrm{E}-04$ & 0.79 & 1.46 & 1.42 \\
\hline Chemical Composition & Mol/liter & Mol/liter & $\mathrm{Ib} / \mathrm{wscf}$ & $\mathrm{lb} / \mathrm{wscf}$ & Wt Frac & Wt Frac & Wt frac \\
\hline $\mathrm{H}+$ & $1.03 \mathrm{E}+00$ & $6.19 \mathrm{E}-01$ & $1.31 \mathrm{E}-06$ & $1.28 \mathrm{E}-06$ & $2.22 \mathrm{E}-05$ & $7.75 \mathrm{E}-05$ & $7.41 \mathrm{E}-05$ \\
\hline $\mathrm{Al}+3$ & $5.38 \mathrm{E}-01$ & $3.09 \mathrm{E}-01$ & $1.32 \mathrm{E}-05$ & $1.28 \mathrm{E}-05$ & $9.81 \mathrm{E}-02$ & $1.07 \mathrm{E}-01$ & $1.06 \mathrm{E}-01$ \\
\hline $\mathrm{Sb}+5$ & $1.28 \mathrm{E}-06$ & $7.37 \mathrm{E}-07$ & $1.35 \mathrm{E}-10$ & $1.31 \mathrm{E}-10$ & $1.00 \mathrm{E}-06$ & $1.09 \mathrm{E}-06$ & $1.09 \mathrm{E}-06$ \\
\hline $\mathrm{As}+3$ & $4.12 \mathrm{E}-04$ & $2.37 \mathrm{E}-04$ & 2.67E-08 & $2.60 \mathrm{E}-08$ & $1.98 \mathrm{E}-04$ & $2.16 \mathrm{E}-04$ & $2.15 \mathrm{E}-04$ \\
\hline $\mathrm{Ba}+2$ & $4.61 \mathrm{E}-05$ & $2.64 \mathrm{E}-05$ & $4.96 \mathrm{E}-09$ & $4.83 \mathrm{E}-09$ & $3.69 \mathrm{E}-05$ & $4.45 \mathrm{E}-05$ & $4.40 \mathrm{E}-05$ \\
\hline $\mathrm{Be}+2$ & $7.21 \mathrm{E}-06$ & $4.14 \mathrm{E}-06$ & $5.09 \mathrm{E}-11$ & $4.96 \mathrm{E}-11$ & $3.78 \mathrm{E}-07$ & $4.56 \mathrm{E}-07$ & $4.51 \mathrm{E}-07$ \\
\hline$B+3$ & $9.59 \mathrm{E}-03$ & $5.51 \mathrm{E}-03$ & $8.95 \mathrm{E}-08$ & $8.73 \mathrm{E}-08$ & $6.66 \mathrm{E}-04$ & $7.25 \mathrm{E}-04$ & $7.21 \mathrm{E}-04$ \\
\hline $\mathrm{Cd}+2$ & $6.60 \mathrm{E}-04$ & $3.79 \mathrm{E}-04$ & $2.60 \mathrm{E}-07$ & $2.53 \mathrm{E}-07$ & $1.93 \mathrm{E}-03$ & $4.21 \mathrm{E}-04$ & $5.16 \mathrm{E}-04$ \\
\hline $\mathrm{Ca}+2$ & $3.41 \mathrm{E}-02$ & $1.95 \mathrm{E}-02$ & 1.07E-06 & $1.04 \mathrm{E}-06$ & $7.95 \mathrm{E}-03$ & $9.59 \mathrm{E}-03$ & $9.49 \mathrm{E}-03$ \\
\hline $\mathrm{Cr}+3$ & $3.10 \mathrm{E}-03$ & $1.78 \mathrm{E}-03$ & $1.93 \mathrm{E}-07$ & $1.88 \mathrm{E}-07$ & $1.43 \mathrm{E}-03$ & $1.10 \mathrm{E}-03$ & $1.12 \mathrm{E}-03$ \\
\hline $\mathrm{Co}+2$ & 1.69E-05 & $9.69 \mathrm{E}-06$ & $8.59 \mathrm{E}-10$ & $8.37 \mathrm{E}-10$ & $6.39 \mathrm{E}-06$ & $6.95 \mathrm{E}-06$ & $6.91 \mathrm{E}-06$ \\
\hline $\mathrm{Cs}+$ & $2.37 \mathrm{E}-05$ & $1.36 \mathrm{E}-05$ & $1.84 \mathrm{E}-08$ & $1.79 \mathrm{E}-08$ & $1.37 \mathrm{E}-04$ & $1.43 \mathrm{E}-05$ & $2.19 \mathrm{E}-05$ \\
\hline $\mathrm{Cu}+2$ & $5.83 \mathrm{E}-04$ & $3.34 \mathrm{E}-04$ & $2.90 \mathrm{E}-08$ & $2.83 \mathrm{E}-08$ & $2.16 \mathrm{E}-04$ & $2.60 \mathrm{E}-04$ & $2.57 \mathrm{E}-04$ \\
\hline $\mathrm{Fe}+3$ & $1.77 \mathrm{E}-02$ & $1.02 \mathrm{E}-02$ & $1.26 \mathrm{E}-06$ & $1.22 \mathrm{E}-06$ & $9.31 \mathrm{E}-03$ & $6.72 \mathrm{E}-03$ & $6.88 \mathrm{E}-03$ \\
\hline $\mathrm{Pb}+2$ & $1.16 \mathrm{E}-03$ & $6.68 \mathrm{E}-04$ & $8.44 \mathrm{E}-07$ & $8.23 \mathrm{E}-07$ & $6.28 \mathrm{E}-03$ & $1.37 \mathrm{E}-03$ & $1.68 \mathrm{E}-03$ \\
\hline $\mathrm{Hg}+2$ & 1.35E-03 & $1.83 \mathrm{E}-03$ & $9.55 \mathrm{E}-06$ & $9.31 \mathrm{E}-06$ & $7.10 \mathrm{E}-05$ & $4.73 \mathrm{E}-06$ & $8.87 \mathrm{E}-06$ \\
\hline $\mathrm{Mn}+4$ & 1.17E-02 & $6.74 \mathrm{E}-03$ & $5.57 \mathrm{E}-07$ & $5.43 \mathrm{E}-07$ & $4.14 \mathrm{E}-03$ & $4.51 \mathrm{E}-03$ & $4.49 \mathrm{E}-03$ \\
\hline $\mathrm{Ni}+2$ & $1.28 \mathrm{E}-03$ & $7.34 \mathrm{E}-04$ & $5.88 \mathrm{E}-08$ & $5.73 \mathrm{E}-08$ & $4.37 \mathrm{E}-04$ & $5.28 \mathrm{E}-04$ & $5.22 \mathrm{E}-04$ \\
\hline $\mathrm{K}+$ & 1.62E-01 & $9.31 \mathrm{E}-02$ & $8.08 \mathrm{E}-06$ & $7.88 \mathrm{E}-06$ & $6.01 \mathrm{E}-02$ & $4.30 \mathrm{E}-02$ & $4.41 \mathrm{E}-02$ \\
\hline $\mathrm{Se}+4$ & $7.08 \mathrm{E}-05$ & $4.06 \mathrm{E}-05$ & $4.82 \mathrm{E}-09$ & $4.70 \mathrm{E}-09$ & $3.59 \mathrm{E}-05$ & $3.90 \mathrm{E}-05$ & $3.88 \mathrm{E}-05$ \\
\hline $\mathrm{Ag}+$ & $5.61 \mathrm{E}-06$ & $3.22 \mathrm{E}-06$ & $4.98 \mathrm{E}-10$ & $4.86 \mathrm{E}-10$ & $3.71 \mathrm{E}-06$ & $4.23 \mathrm{E}-06$ & $4.20 \mathrm{E}-06$ \\
\hline $\mathrm{Na}+$ & $1.74 \mathrm{E}+00$ & 9.99E-01 & $3.30 \mathrm{E}-05$ & $3.22 \mathrm{E}-05$ & $2.45 \mathrm{E}-01$ & $2.80 \mathrm{E}-01$ & $2.78 \mathrm{E}-01$ \\
\hline $\mathrm{Tl}+3$ & $2.53 \mathrm{E}-05$ & 1.45E-05 & 4.47E-09 & $4.36 \mathrm{E}-09$ & $3.33 \mathrm{E}-05$ & $3.62 \mathrm{E}-05$ & $3.60 \mathrm{E}-05$ \\
\hline $\mathrm{U}+4$ & $3.33 \mathrm{E}-04$ & $1.91 \mathrm{E}-04$ & $6.84 \mathrm{E}-08$ & $6.67 \mathrm{E}-08$ & $5.09 \mathrm{E}-04$ & $5.54 \mathrm{E}-04$ & $5.51 \mathrm{E}-04$ \\
\hline $\mathrm{V}+3$ & $8.08 \mathrm{E}-04$ & $4.63 \mathrm{E}-04$ & $3.55 \mathrm{E}-08$ & $3.46 \mathrm{E}-08$ & $2.64 \mathrm{E}-04$ & $2.87 \mathrm{E}-04$ & $2.86 \mathrm{E}-04$ \\
\hline $\mathrm{Zn}+2$ & $8.77 \mathrm{E}-04$ & $5.03 \mathrm{E}-04$ & 4.49E-08 & 4.38E-08 & 3.34E-04 & 4.03E-04 & $3.98 \mathrm{E}-04$ \\
\hline $\mathrm{Zr}+4$ & $1.22 \mathrm{E}-03$ & $7.00 \mathrm{E}-04$ & $9.60 \mathrm{E}-08$ & $9.36 \mathrm{E}-08$ & $7.14 \mathrm{E}-04$ & $7.77 \mathrm{E}-04$ & $7.73 \mathrm{E}-04$ \\
\hline $\mathrm{Cl}-$ & $2.64 \mathrm{E}-02$ & $1.51 \mathrm{E}-02$ & $8.47 \mathrm{E}-07$ & $8.26 \mathrm{E}-07$ & $5.92 \mathrm{E}-03$ & $6.51 \mathrm{E}-03$ & $6.48 \mathrm{E}-03$ \\
\hline F- & $3.71 \mathrm{E}-02$ & $2.15 \mathrm{E}-02$ & $1.22 \mathrm{E}-06$ & $1.19 \mathrm{E}-06$ & $4.08 \mathrm{E}-03$ & $4.66 \mathrm{E}-03$ & $4.63 \mathrm{E}-03$ \\
\hline SO4-2 & $4.58 \mathrm{E}-02$ & $2.63 \mathrm{E}-02$ & $1.55 \mathrm{E}-12$ & $1.51 \mathrm{E}-12$ & 1.15E-08 & 1.32E-08 & $1.31 \mathrm{E}-08$ \\
\hline NO3- & & $2.61 \mathrm{E}+00$ & $1.28 \mathrm{E}-06$ & $1.25 \mathrm{E}-06$ & $9.55 \mathrm{E}-03$ & $3.49 \mathrm{E}-03$ & $3.86 \mathrm{E}-03$ \\
\hline PO4-3 & $1.80 \mathrm{E}-02$ & $1.03 \mathrm{E}-02$ & $1.48 \mathrm{E}-06$ & $1.44 \mathrm{E}-06$ & $1.10 \mathrm{E}-02$ & $1.19 \mathrm{E}-02$ & $1.19 \mathrm{E}-02$ \\
\hline $\mathrm{Am}+4$ & $2.91 \mathrm{E}-08$ & $1.67 \mathrm{E}-08$ & $6.12 \mathrm{E}-12$ & $5.96 \mathrm{E}-12$ & $4.55 \mathrm{E}-08$ & $4.95 \mathrm{E}-08$ & $4.93 \mathrm{E}-08$ \\
\hline $\mathrm{Br}-$ & $1.43 \mathrm{E}-07$ & $8.23 \mathrm{E}-08$ & $1.04 \mathrm{E}-11$ & $1.01 \mathrm{E}-11$ & $7.26 \mathrm{E}-08$ & $7.98 \mathrm{E}-08$ & 7.94E-08 \\
\hline $\mathrm{Ce}+4$ & $4.02 \mathrm{E}-05$ & $2.31 \mathrm{E}-05$ & $4.86 \mathrm{E}-09$ & $4.74 \mathrm{E}-09$ & $3.62 \mathrm{E}-05$ & $3.94 \mathrm{E}-05$ & $3.92 \mathrm{E}-05$ \\
\hline $\mathrm{Eu+3}$ & $2.38 \mathrm{E}-07$ & $1.37 \mathrm{E}-07$ & $3.12 \mathrm{E}-11$ & $3.04 \mathrm{E}-11$ & $2.32 \mathrm{E}-07$ & $2.53 \mathrm{E}-07$ & $2.51 \mathrm{E}-07$ \\
\hline $\mathrm{Gd+3}$ & $1.48 \mathrm{E}-04$ & $8.51 \mathrm{E}-05$ & $2.01 \mathrm{E}-08$ & $1.96 \mathrm{E}-08$ & $1.50 \mathrm{E}-04$ & $1.63 \mathrm{E}-04$ & $1.62 \mathrm{E}-04$ \\
\hline $\mathrm{Ge}+4$ & 4.13E-09 & $2.37 \mathrm{E}-09$ & $2.59 \mathrm{E}-13$ & $2.52 \mathrm{E}-13$ & $1.93 \mathrm{E}-09$ & $2.10 \mathrm{E}-09$ & $2.09 \mathrm{E}-09$ \\
\hline $\ln +3$ & $6.51 \mathrm{E}-07$ & $3.74 \mathrm{E}-07$ & $6.45 \mathrm{E}-11$ & $6.29 \mathrm{E}-11$ & $4.80 \mathrm{E}-07$ & $5.22 \mathrm{E}-07$ & $5.20 \mathrm{E}-07$ \\
\hline $1-$ & $1.24 \mathrm{E}-06$ & $7.14 \mathrm{E}-07$ & $1.43 \mathrm{E}-10$ & $1.39 \mathrm{E}-10$ & $1.00 \mathrm{E}-06$ & $1.10 \mathrm{E}-06$ & $1.09 \mathrm{E}-06$ \\
\hline$L a+3$ & $4.32 \mathrm{E}-06$ & $2.48 \mathrm{E}-06$ & $5.18 \mathrm{E}-10$ & $5.05 \mathrm{E}-10$ & $3.85 \mathrm{E}-06$ & $4.19 \mathrm{E}-06$ & $4.17 \mathrm{E}-06$ \\
\hline
\end{tabular}


Table A-19. Mass balance, Tank WM-180 waste (continued).

\begin{tabular}{|c|c|c|c|c|c|c|c|}
\hline Stream \# & 101 & 102 & 103 & 104 & 106 & 107 & 108 \\
\hline Chemical Composition & Mol/liter & Mol/liter & $\mathrm{lb} / \mathrm{wscf}$ & $\mathrm{Ib} / \mathrm{wscf}$ & Wt frac & Wt frac & Wt frac \\
\hline $\mathrm{Li}+$ & $4.10 \mathrm{E}-04$ & $2.35 \mathrm{E}-04$ & $2.34 \mathrm{E}-09$ & $2.28 \mathrm{E}-09$ & $1.74 \mathrm{E}-05$ & $1.99 \mathrm{E}-05$ & $1.98 \mathrm{E}-05$ \\
\hline $\mathrm{Mg}+2$ & $1.01 \mathrm{E}-02$ & $5.82 \mathrm{E}-03$ & $1.93 \mathrm{E}-07$ & $1.88 \mathrm{E}-07$ & $1.43 \mathrm{E}-03$ & $1.73 \mathrm{E}-03$ & $1.71 \mathrm{E}-03$ \\
\hline $\mathrm{Mo}+6$ & $1.52 \mathrm{E}-03$ & $8.69 \mathrm{E}-04$ & $1.25 \mathrm{E}-07$ & $1.22 \mathrm{E}-07$ & $9.33 \mathrm{E}-04$ & $1.02 \mathrm{E}-03$ & $1.01 \mathrm{E}-03$ \\
\hline $\mathrm{Nd}+3$ & $1.39 \mathrm{E}-05$ & $8.00 \mathrm{E}-06$ & $1.73 \mathrm{E}-09$ & $1.69 \mathrm{E}-09$ & $1.29 \mathrm{E}-05$ & $1.40 \mathrm{E}-05$ & $1.40 \mathrm{E}-05$ \\
\hline $\mathrm{Np}+4$ & $8.05 \mathrm{E}-06$ & 4.62E-06 & $1.65 \mathrm{E}-09$ & 1.61E-09 & $1.23 \mathrm{E}-05$ & $1.33 \mathrm{E}-05$ & $1.33 \mathrm{E}-05$ \\
\hline $\mathrm{Nb}+5$ & $4.12 \mathrm{E}-04$ & $2.36 \mathrm{E}-04$ & $3.30 \mathrm{E}-08$ & $3.22 \mathrm{E}-08$ & $2.45 \mathrm{E}-04$ & $2.67 \mathrm{E}-04$ & $2.66 \mathrm{E}-04$ \\
\hline $\mathrm{Pd}+4$ & $2.89 \mathrm{E}-05$ & $1.66 \mathrm{E}-05$ & $2.40 \mathrm{E}-09$ & $2.34 \mathrm{E}-09$ & $1.79 \mathrm{E}-05$ & $2.16 \mathrm{E}-05$ & $2.13 \mathrm{E}-05$ \\
\hline $\mathrm{Pu}+4$ & $9.29 \mathrm{E}-06$ & $5.33 \mathrm{E}-06$ & $1.96 \mathrm{E}-09$ & $1.91 \mathrm{E}-09$ & $1.45 \mathrm{E}-05$ & $1.58 \mathrm{E}-05$ & $1.58 \mathrm{E}-05$ \\
\hline $\mathrm{Pr}+4$ & $3.93 \mathrm{E}-06$ & $2.25 \mathrm{E}-06$ & $4.78 \mathrm{E}-10$ & $4.66 \mathrm{E}-10$ & $3.55 \mathrm{E}-06$ & $3.87 \mathrm{E}-06$ & $3.85 \mathrm{E}-06$ \\
\hline $\mathrm{Pm}+3$ & $5.95 \mathrm{E}-10$ & $3.42 \mathrm{E}-10$ & $7.45 \mathrm{E}-14$ & $7.26 \mathrm{E}-14$ & $5.54 \mathrm{E}-10$ & $6.03 \mathrm{E}-10$ & $6.00 \mathrm{E}-10$ \\
\hline $\mathrm{Rh}+4$ & $1.69 \mathrm{E}-06$ & $9.73 \mathrm{E}-07$ & $1.51 \mathrm{E}-10$ & $1.47 \mathrm{E}-10$ & $1.12 \mathrm{E}-06$ & $1.22 \mathrm{E}-06$ & $1.21 \mathrm{E}-06$ \\
\hline $\mathrm{Rb}+$ & $2.61 \mathrm{E}-06$ & $1.50 \mathrm{E}-06$ & $1.84 \mathrm{E}-10$ & $1.79 \mathrm{E}-10$ & $1.37 \mathrm{E}-06$ & $1.56 \mathrm{E}-06$ & $1.55 \mathrm{E}-06$ \\
\hline $\mathrm{Ru}+3$ & $1.16 \mathrm{E}-04$ & $6.68 \mathrm{E}-05$ & $1.02 \mathrm{E}-08$ & $9.90 \mathrm{E}-09$ & $7.56 \mathrm{E}-05$ & $8.22 \mathrm{E}-05$ & $8.18 \mathrm{E}-05$ \\
\hline $\mathrm{Sm+3}$ & $2.58 \mathrm{E}-06$ & $1.48 \mathrm{E}-06$ & $3.35 \mathrm{E}-10$ & $3.27 \mathrm{E}-10$ & $2.50 \mathrm{E}-06$ & $2.72 \mathrm{E}-06$ & $2.70 \mathrm{E}-06$ \\
\hline $\mathrm{Si+4}$ & $2.84 \mathrm{E}-03$ & $1.63 \mathrm{E}-03$ & $6.88 \mathrm{E}-08$ & $6.70 \mathrm{E}-08$ & $5.11 \mathrm{E}-04$ & $5.57 \mathrm{E}-04$ & $5.54 \mathrm{E}-04$ \\
\hline $\mathrm{Sr}+2$ & 9.93E-05 & $5.70 \mathrm{E}-05$ & $6.81 \mathrm{E}-09$ & $6.64 \mathrm{E}-09$ & $5.06 \mathrm{E}-05$ & $6.11 \mathrm{E}-05$ & $6.04 \mathrm{E}-05$ \\
\hline $\mathrm{Tc}+7$ & $4.89 \mathrm{E}-06$ & $2.81 \mathrm{E}-06$ & $3.77 \mathrm{E}-09$ & $3.67 \mathrm{E}-09$ & $2.80 \mathrm{E}-05$ & 1.69E-06 & $3.33 \mathrm{E}-06$ \\
\hline $\mathrm{Te}+4$ & $1.36 \mathrm{E}-06$ & $7.78 \mathrm{E}-07$ & $1.49 \mathrm{E}-10$ & $1.46 \mathrm{E}-10$ & $1.11 \mathrm{E}-06$ & $1.21 \mathrm{E}-06$ & $1.20 \mathrm{E}-06$ \\
\hline $\mathrm{Tb}+4$ & $9.94 \mathrm{E}-10$ & $5.71 \mathrm{E}-10$ & $1.36 \mathrm{E}-13$ & $1.33 \mathrm{E}-13$ & $1.01 \mathrm{E}-09$ & $1.10 \mathrm{E}-09$ & $1.10 \mathrm{E}-09$ \\
\hline $\mathrm{Th}+4$ & $9.69 \mathrm{E}-11$ & $5.56 \mathrm{E}-11$ & $1.94 \mathrm{E}-14$ & $1.89 \mathrm{E}-14$ & $1.44 \mathrm{E}-10$ & $1.57 \mathrm{E}-10$ & $1.56 \mathrm{E}-10$ \\
\hline$S n+4$ & $6.81 \mathrm{E}-05$ & $3.91 \mathrm{E}-05$ & $6.97 \mathrm{E}-09$ & $6.80 \mathrm{E}-09$ & $5.19 \mathrm{E}-05$ & $5.65 \mathrm{E}-05$ & $5.62 \mathrm{E}-05$ \\
\hline $\mathrm{Ti}+4$ & $1.24 \mathrm{E}-04$ & $7.11 \mathrm{E}-05$ & $5.12 \mathrm{E}-09$ & $4.99 \mathrm{E}-09$ & $3.81 \mathrm{E}-05$ & $4.15 \mathrm{E}-05$ & $4.12 \mathrm{E}-05$ \\
\hline$Y+3$ & $3.22 \mathrm{E}-06$ & $1.85 \mathrm{E}-06$ & $2.47 \mathrm{E}-10$ & $2.41 \mathrm{E}-10$ & $1.84 \mathrm{E}-06$ & $2.00 \mathrm{E}-06$ & $1.99 \mathrm{E}-06$ \\
\hline $\mathrm{OH}-$ & & $3.86 \mathrm{E}-08$ & $3.01 \mathrm{E}-10$ & $2.94 \mathrm{E}-10$ & $2.24 \mathrm{E}-06$ & $1.61 \mathrm{E}-06$ & $1.65 \mathrm{E}-06$ \\
\hline $\mathrm{H} 2 \mathrm{O}$ & $4.68 \mathrm{E}+01$ & $2.85 \mathrm{E}+01$ & $1.44 \mathrm{E}-02$ & $1.40 \mathrm{E}-02$ & $5.02 \mathrm{E}-03$ & $3.25 \mathrm{E}-03$ & $3.36 \mathrm{E}-03$ \\
\hline & & & & & & & \\
\hline $\mathrm{SO} 2$ & & $2.44 \mathrm{E}-08$ & $1.62 \mathrm{E}-12$ & $1.58 \mathrm{E}-12$ & & & \\
\hline $\mathrm{H} 2 \mathrm{~S}$ & & & $2.15 \mathrm{E}-05$ & $2.10 \mathrm{E}-05$ & & & \\
\hline $\mathrm{CO}$ & & $8.72 \mathrm{E}-11$ & 3.53E-04 & $3.44 \mathrm{E}-04$ & & & \\
\hline $\mathrm{CO} 2$ & & $6.64 \mathrm{E}-06$ & $6.21 \mathrm{E}-02$ & $6.06 \mathrm{E}-02$ & & & \\
\hline $\mathrm{H} 2$ & & $5.59 \mathrm{E}-10$ & $5.60 \mathrm{E}-04$ & $5.46 \mathrm{E}-04$ & & & \\
\hline N2 & & $2.04 \mathrm{E}-10$ & $9.44 \mathrm{E}-04$ & $2.76 \mathrm{E}-03$ & & & \\
\hline $\mathrm{NO}$ & & $2.82 \mathrm{E}-09$ & $1.41 \mathrm{E}-04$ & $1.38 \mathrm{E}-04$ & & & \\
\hline $\mathrm{NO2}$ & & $4.27 \mathrm{E}-07$ & $2.06 \mathrm{E}-06$ & $2.01 \mathrm{E}-06$ & & & \\
\hline O2 & & $7.02 \mathrm{E}-10$ & & & & & \\
\hline S (other) & & $3.46 \mathrm{E}-10$ & 9.36E-08 & $9.12 \mathrm{E}-08$ & $6.96 \mathrm{E}-04$ & 7.95E-04 & 7.89E-04 \\
\hline $\mathrm{CO} 3$ & 1.19E-05 & $6.81 \mathrm{E}-06$ & $4.98 \mathrm{E}-05$ & $4.86 \mathrm{E}-05$ & $3.71 \mathrm{E}-01$ & $4.05 \mathrm{E}-01$ & $4.03 \mathrm{E}-01$ \\
\hline \multicolumn{8}{|l|}{ C (reductant) } \\
\hline $\mathrm{O}$ (oxides) & $5.00 \mathrm{E}-02$ & $2.87 \mathrm{E}-02$ & $1.32 \mathrm{E}-05$ & $1.28 \mathrm{E}-05$ & $9.78 \%$ & $10.41 \%$ & $10.37 \%$ \\
\hline C (organic) & $4.11 \mathrm{E}-03$ & $2.39 \mathrm{E}+01$ & $5.44 \mathrm{E}-03$ & $5.30 \mathrm{E}-03$ & $5.52 \mathrm{E}-02$ & $8.48 \mathrm{E}-04$ & $4.24 \mathrm{E}-03$ \\
\hline $\mathrm{H}$ (organic) & $1.10 \mathrm{E}-02$ & $6.38 \mathrm{E}+01$ & $1.02 \mathrm{E}-03$ & $9.94 \mathrm{E}-04$ & $9.84 \mathrm{E}-03$ & $1.51 \mathrm{E}-04$ & $7.56 \mathrm{E}-04$ \\
\hline \multirow[t]{2}{*}{$\mathrm{O}$ (organic) } & $1.37 \mathrm{E}-03$ & $7.97 \mathrm{E}+00$ & & & & & \\
\hline & & & & & & & \\
\hline Mass Flow (kg/hr): & $4.36 \mathrm{E}+02$ & $7.60 \mathrm{E}+02$ & $2.07 \mathrm{E}+03$ & $2.11 \mathrm{E}+03$ & $3.26 \mathrm{E}+00$ & $4.90 \mathrm{E}+01$ & $5.23 \mathrm{E}+01$ \\
\hline Canister Rate, canisters/day & & & & & & & 1.11 \\
\hline \multirow[t]{2}{*}{ Total Canisters Generated } & & & & & & & 151 \\
\hline & $\mathrm{w} / \mathrm{m} 3$ & $\mathrm{w} / \mathrm{m} 3$ & $\mathrm{w} / \mathrm{m} 3$ & $\mathrm{w} / \mathrm{m} 3$ & $\mathrm{w} / \mathrm{m} 3$ & $\mathrm{w} / \mathrm{m} 3$ & $\mathrm{w} / \mathrm{m} 3$ \\
\hline Heat Generation & $2.68 \mathrm{E}-01$ & $1.54 \mathrm{E}-01$ & & & $2.02 \mathrm{E}+00$ & $2.66 \mathrm{E}+00$ & $2.64 \mathrm{E}+00$ \\
\hline
\end{tabular}


Table A-19. Mass balance, Tank WM-180 waste (continued).

\begin{tabular}{|c|c|c|c|c|c|c|c|}
\hline Stream \# & 101 & 102 & 103 & 104 & 106 & 107 & 108 \\
\hline Radiological Composition & $\mathrm{Ci} / \mathrm{L}$ & $\mathrm{Ci} / \mathrm{L}$ & Ci/wscm & Ci/wscm & $\mathrm{Ci} / \mathrm{kg}$ & $\mathrm{Ci} / \mathrm{kg}$ & $\mathrm{Ci} / \mathrm{kg}$ \\
\hline $\mathrm{H}-3$ & $1.59 \mathrm{E}-05$ & $9.12 \mathrm{E}-06$ & $3.81 \mathrm{E}-06$ & $3.71 \mathrm{E}-06$ & & & \\
\hline C-14 & $5.71 \mathrm{E}-11$ & $3.28 \mathrm{E}-11$ & $1.36 \mathrm{E}-11$ & 1.33E-11 & $4.87 \mathrm{E}-12$ & $3.08 \mathrm{E}-12$ & $3.19 \mathrm{E}-12$ \\
\hline Co-60 & $3.66 \mathrm{E}-06$ & $2.10 \mathrm{E}-06$ & $5.06 \mathrm{E}-08$ & $4.93 \mathrm{E}-08$ & $2.35 \mathrm{E}-05$ & $2.56 \mathrm{E}-05$ & $2.55 \mathrm{E}-05$ \\
\hline Ni-59 & $8.43 \mathrm{E}-08$ & $4.83 \mathrm{E}-08$ & $1.06 \mathrm{E}-09$ & $1.03 \mathrm{E}-09$ & $4.91 \mathrm{E}-07$ & $5.92 \mathrm{E}-07$ & $5.85 \mathrm{E}-07$ \\
\hline $\mathrm{Ni}-63$ & $2.22 \mathrm{E}-05$ & $1.27 \mathrm{E}-05$ & $2.79 \mathrm{E}-07$ & $2.72 \mathrm{E}-07$ & $1.29 \mathrm{E}-04$ & $1.56 \mathrm{E}-04$ & $1.54 \mathrm{E}-04$ \\
\hline Se-79 & $2.08 \mathrm{E}-07$ & $1.19 \mathrm{E}-07$ & $2.87 \mathrm{E}-09$ & $2.80 \mathrm{E}-09$ & $1.33 \mathrm{E}-06$ & $1.45 \mathrm{E}-06$ & $1.44 \mathrm{E}-06$ \\
\hline Sr-90 & $1.80 \mathrm{E}-02$ & $1.03 \mathrm{E}-02$ & $2.25 \mathrm{E}-04$ & $2.19 \mathrm{E}-04$ & $1.05 \mathrm{E}-01$ & $1.26 \mathrm{E}-01$ & $1.25 \mathrm{E}-01$ \\
\hline Y-90 & $1.80 \mathrm{E}-02$ & $1.03 \mathrm{E}-02$ & $2.48 \mathrm{E}-04$ & $2.42 \mathrm{E}-04$ & $1.15 \mathrm{E}-01$ & $1.26 \mathrm{E}-01$ & $1.25 \mathrm{E}-01$ \\
\hline Zr-93 & $1.01 \mathrm{E}-06$ & $5.77 \mathrm{E}-07$ & $1.39 \mathrm{E}-08$ & $1.35 \mathrm{E}-08$ & $6.45 \mathrm{E}-06$ & $7.02 \mathrm{E}-06$ & $6.98 \mathrm{E}-06$ \\
\hline $\mathrm{Nb}-93 \mathrm{~m}$ & $7.75 \mathrm{E}-07$ & $4.44 \mathrm{E}-07$ & 1.07E-08 & $1.04 \mathrm{E}-08$ & 4.97E-06 & $5.41 \mathrm{E}-06$ & $5.38 \mathrm{E}-06$ \\
\hline $\mathrm{Nb}-94$ & $5.42 \mathrm{E}-07$ & $3.11 \mathrm{E}-07$ & $7.49 \mathrm{E}-09$ & $7.30 \mathrm{E}-09$ & $3.48 \mathrm{E}-06$ & $3.78 \mathrm{E}-06$ & $3.76 \mathrm{E}-06$ \\
\hline Tc-99 & $8.31 \mathrm{E}-06$ & $4.77 \mathrm{E}-06$ & $1.05 \mathrm{E}-06$ & $1.02 \mathrm{E}-06$ & $4.85 \mathrm{E}-04$ & $2.92 \mathrm{E}-05$ & 5.77E-05 \\
\hline Ru-106 & 4.43E-07 & $2.54 \mathrm{E}-07$ & $6.12 \mathrm{E}-09$ & 5.97E-09 & $2.84 \mathrm{E}-06$ & $3.09 \mathrm{E}-06$ & $3.08 \mathrm{E}-06$ \\
\hline Rh-102 & $3.91 \mathrm{E}-10$ & $2.25 \mathrm{E}-10$ & $5.41 \mathrm{E}-12$ & $5.27 \mathrm{E}-12$ & $2.51 \mathrm{E}-09$ & $2.73 \mathrm{E}-09$ & $2.72 \mathrm{E}-09$ \\
\hline Rh-106 & $4.43 \mathrm{E}-07$ & $2.54 \mathrm{E}-07$ & $6.12 \mathrm{E}-09$ & $5.97 \mathrm{E}-09$ & $2.84 \mathrm{E}-06$ & $3.09 \mathrm{E}-06$ & $3.08 \mathrm{E}-06$ \\
\hline $\mathrm{Pd}-107$ & $7.50 \mathrm{E}-09$ & $4.30 \mathrm{E}-09$ & $9.40 \mathrm{E}-11$ & $9.16 \mathrm{E}-11$ & $4.36 \mathrm{E}-08$ & $5.26 \mathrm{E}-08$ & $5.21 \mathrm{E}-08$ \\
\hline Cd-113m & $1.51 \mathrm{E}-06$ & $8.64 \mathrm{E}-07$ & $8.45 \mathrm{E}-08$ & $8.23 \mathrm{E}-08$ & $3.92 \mathrm{E}-05$ & $8.56 \mathrm{E}-06$ & 1.05E-05 \\
\hline Sn-121m & $3.03 \mathrm{E}-08$ & $1.74 \mathrm{E}-08$ & $4.19 \mathrm{E}-10$ & $4.09 \mathrm{E}-10$ & $1.95 \mathrm{E}-07$ & $2.12 \mathrm{E}-07$ & $2.11 \mathrm{E}-07$ \\
\hline Sn-126 & $1.95 \mathrm{E}-07$ & $1.12 \mathrm{E}-07$ & $2.70 \mathrm{E}-09$ & $2.63 \mathrm{E}-09$ & $1.25 \mathrm{E}-06$ & $1.36 \mathrm{E}-06$ & $1.36 \mathrm{E}-06$ \\
\hline Sb-125 & $7.28 \mathrm{E}-06$ & $4.18 \mathrm{E}-06$ & $1.01 \mathrm{E}-07$ & $9.82 \mathrm{E}-08$ & $4.68 \mathrm{E}-05$ & $5.09 \mathrm{E}-05$ & 5.06E-05 \\
\hline Sb-126 & $2.61 \mathrm{E}-08$ & $1.50 \mathrm{E}-08$ & $3.61 \mathrm{E}-10$ & $3.52 \mathrm{E}-10$ & $1.68 \mathrm{E}-07$ & 1.82E-07 & $1.81 \mathrm{E}-07$ \\
\hline Te-125m & $1.43 \mathrm{E}-06$ & $8.20 \mathrm{E}-07$ & $1.97 \mathrm{E}-08$ & $1.92 \mathrm{E}-08$ & $9.17 \mathrm{E}-06$ & $9.98 \mathrm{E}-06$ & $9.93 \mathrm{E}-06$ \\
\hline \begin{tabular}{|l|l|}
$\mathrm{I}-129$ \\
\end{tabular} & $2.21 \mathrm{E}-08$ & $1.27 \mathrm{E}-08$ & $3.21 \mathrm{E}-10$ & $3.13 \mathrm{E}-10$ & $1.40 \mathrm{E}-07$ & $1.54 \mathrm{E}-07$ & 1.53E-07 \\
\hline Cs-134 & $4.73 \mathrm{E}-06$ & $2.71 \mathrm{E}-06$ & $4.41 \mathrm{E}-07$ & $4.30 \mathrm{E}-07$ & $2.05 \mathrm{E}-04$ & $2.14 \mathrm{E}-05$ & $3.28 \mathrm{E}-05$ \\
\hline Cs-135 & $4.07 \mathrm{E}-07$ & $2.33 \mathrm{E}-07$ & $3.80 \mathrm{E}-08$ & $3.70 \mathrm{E}-08$ & $1.76 \mathrm{E}-05$ & $1.84 \mathrm{E}-06$ & $2.83 \mathrm{E}-06$ \\
\hline Cs-137 & $2.38 \mathrm{E}-02$ & $1.37 \mathrm{E}-02$ & $2.22 \mathrm{E}-03$ & $2.17 \mathrm{E}-03$ & $1.03 E+00$ & $1.08 \mathrm{E}-01$ & $1.66 \mathrm{E}-01$ \\
\hline Ba-137m & $2.26 \mathrm{E}-02$ & $1.29 \mathrm{E}-02$ & $2.83 \mathrm{E}-04$ & $2.76 \mathrm{E}-04$ & $1.31 \mathrm{E}-01$ & $1.58 \mathrm{E}-01$ & 1.57E-01 \\
\hline $\begin{array}{l}\mathrm{Ce}-144 \\
\end{array}$ & $2.98 \mathrm{E}-07$ & $1.71 \mathrm{E}-07$ & $4.13 \mathrm{E}-09$ & $4.02 \mathrm{E}-09$ & $1.92 \mathrm{E}-06$ & $2.09 \mathrm{E}-06$ & $2.08 \mathrm{E}-06$ \\
\hline Pr-144 & $2.98 \mathrm{E}-07$ & $1.71 \mathrm{E}-07$ & $4.13 \mathrm{E}-09$ & $4.02 \mathrm{E}-09$ & $1.92 \mathrm{E}-06$ & $2.09 \mathrm{E}-06$ & $2.08 \mathrm{E}-06$ \\
\hline $\mathrm{Pm}-146$ & $2.31 \mathrm{E}-08$ & $1.33 \mathrm{E}-08$ & $3.19 \mathrm{E}-10$ & $3.11 \mathrm{E}-10$ & $1.48 \mathrm{E}-07$ & $1.61 \mathrm{E}-07$ & 1.61E-07 \\
\hline $\mathrm{Pm}-147$ & $8.10 \mathrm{E}-05$ & $4.65 \mathrm{E}-05$ & $1.12 \mathrm{E}-06$ & $1.09 \mathrm{E}-06$ & $5.20 \mathrm{E}-04$ & $5.66 \mathrm{E}-04$ & $5.63 \mathrm{E}-04$ \\
\hline Sm-151 & 1.60E-04 & 9.17E-05 & $2.21 \mathrm{E}-06$ & $2.15 \mathrm{E}-06$ & $1.03 \mathrm{E}-03$ & $1.12 \mathrm{E}-03$ & $1.11 \mathrm{E}-03$ \\
\hline Eu-152 & $1.16 \mathrm{E}-06$ & $6.64 \mathrm{E}-07$ & $1.60 \mathrm{E}-08$ & $1.56 \mathrm{E}-08$ & $7.43 \mathrm{E}-06$ & $8.08 \mathrm{E}-06$ & $8.04 \mathrm{E}-06$ \\
\hline Eu-154 & $4.15 \mathrm{E}-05$ & $2.38 \mathrm{E}-05$ & $5.74 \mathrm{E}-07$ & $5.59 \mathrm{E}-07$ & $2.66 \mathrm{E}-04$ & $2.90 \mathrm{E}-04$ & $2.89 \mathrm{E}-04$ \\
\hline Eu-155 & $7.55 \mathrm{E}-05$ & 4.33E-05 & $1.04 \mathrm{E}-06$ & $1.02 \mathrm{E}-06$ & $4.85 \mathrm{E}-04$ & $5.28 \mathrm{E}-04$ & $5.25 \mathrm{E}-04$ \\
\hline Th-230 & $3.96 \mathrm{E}-10$ & $2.27 \mathrm{E}-10$ & $5.47 \mathrm{E}-12$ & 5.33E-12 & $2.54 \mathrm{E}-09$ & $2.76 \mathrm{E}-09$ & $2.75 \mathrm{E}-09$ \\
\hline Pa-233 & $1.33 \mathrm{E}-06$ & $7.63 \mathrm{E}-07$ & $1.84 \mathrm{E}-08$ & $1.79 \mathrm{E}-08$ & $8.53 \mathrm{E}-06$ & $9.29 \mathrm{E}-06$ & $9.24 \mathrm{E}-06$ \\
\hline $\mathrm{U}-232$ & $9.54 \mathrm{E}-10$ & $5.47 \mathrm{E}-10$ & $1.32 \mathrm{E}-11$ & $1.28 \mathrm{E}-11$ & $6.12 \mathrm{E}-09$ & $6.66 \mathrm{E}-09$ & $6.63 \mathrm{E}-09$ \\
\hline U-233 & $3.72 \mathrm{E}-11$ & $2.13 \mathrm{E}-11$ & $5.14 \mathrm{E}-13$ & $5.01 \mathrm{E}-13$ & $2.39 \mathrm{E}-10$ & $2.60 \mathrm{E}-10$ & $2.58 \mathrm{E}-10$ \\
\hline U-234 & $9.56 \mathrm{E}-07$ & $5.48 \mathrm{E}-07$ & $1.32 \mathrm{E}-08$ & $1.29 \mathrm{E}-08$ & $6.13 \mathrm{E}-06$ & $6.67 \mathrm{E}-06$ & $6.64 \mathrm{E}-06$ \\
\hline $\mathrm{U}-235$ & $3.49 \mathrm{E}-08$ & $2.00 \mathrm{E}-08$ & $4.82 \mathrm{E}-10$ & $4.70 \mathrm{E}-10$ & $2.24 \mathrm{E}-07$ & $2.44 \mathrm{E}-07$ & 2.43E-07 \\
\hline $\mathrm{U}-236$ & $5.18 \mathrm{E}-08$ & $2.97 \mathrm{E}-08$ & $7.16 \mathrm{E}-10$ & $6.98 \mathrm{E}-10$ & $3.32 \mathrm{E}-07$ & $3.62 \mathrm{E}-07$ & $3.60 \mathrm{E}-07$ \\
\hline U-237 & $2.92 \mathrm{E}-09$ & $1.68 \mathrm{E}-09$ & $4.04 \mathrm{E}-11$ & $3.93 \mathrm{E}-11$ & $1.87 \mathrm{E}-08$ & $2.04 \mathrm{E}-08$ & 2.03E-08 \\
\hline U-238 & $2.06 \mathrm{E}-08$ & $1.18 \mathrm{E}-08$ & $2.85 \mathrm{E}-10$ & $2.78 \mathrm{E}-10$ & $1.32 \mathrm{E}-07$ & 1.44E-07 & 1.43E-07 \\
\hline Np-237 & $1.34 \mathrm{E}-06$ & $7.72 \mathrm{E}-07$ & $1.86 \mathrm{E}-08$ & $1.81 \mathrm{E}-08$ & $8.64 \mathrm{E}-06$ & $9.40 \mathrm{E}-06$ & $9.35 \mathrm{E}-06$ \\
\hline $\mathrm{Pu}-236$ & $1.91 \mathrm{E}-09$ & $1.10 \mathrm{E}-09$ & $2.65 \mathrm{E}-11$ & $2.58 \mathrm{E}-11$ & $1.23 \mathrm{E}-08$ & $1.34 \mathrm{E}-08$ & $1.33 \mathrm{E}-08$ \\
\hline $\mathrm{Pu}-238$ & $8.73 \mathrm{E}-04$ & $5.01 \mathrm{E}-04$ & $1.21 \mathrm{E}-05$ & $1.18 \mathrm{E}-05$ & $5.60 \mathrm{E}-03$ & $6.10 \mathrm{E}-03$ & $6.07 \mathrm{E}-03$ \\
\hline Pu-239 & $1.33 \mathrm{E}-04$ & $7.60 \mathrm{E}-05$ & $1.83 \mathrm{E}-06$ & $1.78 \mathrm{E}-06$ & $8.50 \mathrm{E}-04$ & $9.25 \mathrm{E}-04$ & $9.21 \mathrm{E}-04$ \\
\hline Pu-240 & $7.19 \mathrm{E}-06$ & $4.12 \mathrm{E}-06$ & $9.94 \mathrm{E}-08$ & $9.68 \mathrm{E}-08$ & 4.61E-05 & $5.02 \mathrm{E}-05$ & $5.00 \mathrm{E}-05$ \\
\hline $\mathrm{Pu}-241$ & $5.26 \mathrm{E}-04$ & $3.02 \mathrm{E}-04$ & $7.27 \mathrm{E}-06$ & $7.09 \mathrm{E}-06$ & $3.38 \mathrm{E}-03$ & $3.67 \mathrm{E}-03$ & $3.65 \mathrm{E}-03$ \\
\hline Pu-242 & $5.54 \mathrm{E}-09$ & $3.18 \mathrm{E}-09$ & $7.66 \mathrm{E}-11$ & $7.47 \mathrm{E}-11$ & $3.56 \mathrm{E}-08$ & $3.87 \mathrm{E}-08$ & $3.85 \mathrm{E}-08$ \\
\hline Pu-244 & $4.75 \mathrm{E}-16$ & $2.72 \mathrm{E}-16$ & $6.56 \mathrm{E}-18$ & $6.40 \mathrm{E}-18$ & $3.05 \mathrm{E}-15$ & $3.32 \mathrm{E}-15$ & $3.30 \mathrm{E}-15$ \\
\hline Am-241 & $6.32 \mathrm{E}-05$ & $3.63 \mathrm{E}-05$ & $8.75 \mathrm{E}-07$ & $8.53 \mathrm{E}-07$ & $4.06 \mathrm{E}-04$ & $4.42 \mathrm{E}-04$ & $4.40 \mathrm{E}-04$ \\
\hline Am-242m & $6.90 \mathrm{E}-09$ & $3.96 \mathrm{E}-09$ & $9.55 \mathrm{E}-11$ & $9.31 \mathrm{E}-11$ & $4.43 \mathrm{E}-08$ & $4.82 \mathrm{E}-08$ & $4.80 \mathrm{E}-08$ \\
\hline
\end{tabular}


Table A-19. Mass balance, Tank WM-180 waste (continued).

\begin{tabular}{|c|c|c|c|c|c|c|c|}
\hline Stream \# & 101 & 102 & 103 & 104 & 106 & 107 & 108 \\
\hline Radiological Composition & $\mathrm{Ci} / \mathrm{L}$ & $\mathrm{Ci} / \mathrm{L}$ & Ci/wscm & Ci/wscm & $\mathrm{Ci} / \mathrm{kg}$ & $\mathrm{Ci} / \mathrm{kg}$ & $\mathrm{Ci} / \mathrm{kg}$ \\
\hline $\mathrm{Am}-243$ & $1.00 \mathrm{E}-08$ & $5.75 \mathrm{E}-09$ & $1.39 \mathrm{E}-10$ & $1.35 \mathrm{E}-10$ & $6.43 \mathrm{E}-08$ & $7.00 \mathrm{E}-08$ & $6.97 \mathrm{E}-08$ \\
\hline $\mathrm{Cm}-242$ & $5.69 \mathrm{E}-09$ & $3.27 \mathrm{E}-09$ & $7.87 \mathrm{E}-11$ & $7.67 \mathrm{E}-11$ & $3.65 \mathrm{E}-08$ & $3.98 \mathrm{E}-08$ & $3.96 \mathrm{E}-08$ \\
\hline $\mathrm{Cm}-243$ & $1.64 \mathrm{E}-08$ & $9.39 \mathrm{E}-09$ & $2.26 \mathrm{E}-10$ & $2.21 \mathrm{E}-10$ & $1.05 \mathrm{E}-07$ & $1.14 \mathrm{E}-07$ & $1.14 \mathrm{E}-07$ \\
\hline $\mathrm{Cm}-244$ & $1.02 \mathrm{E}-06$ & $5.84 \mathrm{E}-07$ & $1.41 \mathrm{E}-08$ & $1.37 \mathrm{E}-08$ & $6.53 \mathrm{E}-06$ & $7.10 \mathrm{E}-06$ & $7.07 \mathrm{E}-06$ \\
\hline $\mathrm{Cm}-245$ & $1.72 \mathrm{E}-10$ & $9.88 \mathrm{E}-11$ & $2.38 \mathrm{E}-12$ & $2.32 \mathrm{E}-12$ & $1.11 \mathrm{E}-09$ & $1.20 \mathrm{E}-09$ & $1.20 \mathrm{E}-09$ \\
\hline $\mathrm{Cm}-246$ & $1.13 \mathrm{E}-11$ & $6.49 \mathrm{E}-12$ & $1.56 \mathrm{E}-13$ & $1.52 \mathrm{E}-13$ & $7.26 \mathrm{E}-11$ & $7.90 \mathrm{E}-11$ & $7.86 \mathrm{E}-11$ \\
\hline TRU & $1.09 \mathrm{E}-03$ & $6.27 \mathrm{E}-04$ & $1.51 \mathrm{E}-05$ & $1.47 \mathrm{E}-05$ & $7.02 \mathrm{E}-03$ & $7.64 \mathrm{E}-03$ & $7.60 \mathrm{E}-03$ \\
\hline & & & & & & & \\
\hline & & & & & & & \\
\hline $\begin{array}{l}\text { Gas Stream Bulk Composition } \\
\text { (Wet Basis) }\end{array}$ & $\begin{array}{c}\text { mol\% or } \\
\text { ppmv }\end{array}$ & $\begin{array}{c}\text { mol\% or } \\
\text { ppmv }\end{array}$ & $\begin{array}{c}\text { mol\% or } \\
\text { ppmv }\end{array}$ & $\begin{array}{c}\text { mol\% or } \\
\text { ppmv }\end{array}$ & $\begin{array}{c}\mathrm{mol} \% \text { or } \\
\text { ppmv }\end{array}$ & $\begin{array}{c}\text { mol\% or } \\
\text { ppmv }\end{array}$ & $\begin{array}{c}\text { mol\% or } \\
\text { ppmv }\end{array}$ \\
\hline $\mathrm{H} 2 \mathrm{O}, \mathrm{mol} \%$ & & & $30.81 \%$ & $30.03 \%$ & & & \\
\hline $\mathrm{O} 2, \mathrm{~mol} \%$ & & & & & & & \\
\hline $\mathrm{N} 2, \mathrm{~mol} \%$ & & & $1.30 \%$ & $3.80 \%$ & & & \\
\hline $\mathrm{H} 2, \mathrm{~mol} \%$ & & & $10.70 \%$ & $10.43 \%$ & & & \\
\hline $\mathrm{CO} 2, \mathrm{~mol} \%$ & & & $54.40 \%$ & $53.02 \%$ & & & \\
\hline COgas, ppmv & & & $4.85 \mathrm{E}+03$ & $4.73 \mathrm{E}+03$ & & & \\
\hline NO, ppmv & & & $1.81 \mathrm{E}+03$ & $1.77 \mathrm{E}+03$ & & & \\
\hline NO2, ppmv & & & $1.73 \mathrm{E}+01$ & $1.68 \mathrm{E}+01$ & & & \\
\hline SO2, ppmv & & & 9.76E-06 & 9.52E-06 & & & \\
\hline $\mathrm{Cl}, \mathrm{ppmv}$ & & & $9.21 \mathrm{E}+00$ & $8.97 \mathrm{E}+00$ & & & \\
\hline $\mathrm{F}, \mathrm{ppmv}$ & & & $2.47 \mathrm{E}+01$ & $2.41 \mathrm{E}+01$ & & & \\
\hline $\mathrm{C}$ (organic), ppmv & & & $1.74 \mathrm{E}+05$ & $1.70 \mathrm{E}+05$ & & & \\
\hline $\mathrm{H}$ (organic), ppmv & & & $3.90 \mathrm{E}+05$ & $3.80 \mathrm{E}+05$ & & & \\
\hline & & & & & & & \\
\hline $\mathrm{Hg}, \mathrm{ug} / \mathrm{wscm}$ & & & $1.53 \mathrm{E}+05$ & $1.49 \mathrm{E}+05$ & & & \\
\hline $\mathrm{PM}, \mathrm{mg} / \mathrm{wscm}$ & & & $1.06 \mathrm{E}+05$ & $1.03 \mathrm{E}+05$ & & & \\
\hline SVM, ug/wscm & & & $1.77 \mathrm{E}+04$ & $1.72 \mathrm{E}+04$ & & & \\
\hline LVM, ug/wscm & & & $3.52 \mathrm{E}+03$ & $3.43 \mathrm{E}+03$ & & & \\
\hline $\begin{array}{l}\text { Gas Stream Bulk Composition } \\
\text { (Dry Basis) }\end{array}$ & $\mathrm{mol} \%$ & $\mathrm{~mol} \%$ & $\mathrm{~mol} \%$ & $\mathrm{~mol} \%$ & $\mathrm{~mol} \%$ & $\mathrm{~mol} \%$ & $\mathrm{~mol} \%$ \\
\hline $\mathrm{O} 2, \mathrm{~mol} \%$, dry basis & & & & & & & \\
\hline $\mathrm{N} 2, \mathrm{~mol} \%$, dry basis & & & $1.88 \%$ & $5.43 \%$ & & & \\
\hline $\mathrm{H} 2, \mathrm{~mol} \%$, dry basis & & & $15.46 \%$ & $14.90 \%$ & & & \\
\hline $\mathrm{CO} 2, \mathrm{~mol} \%$, dry basis & & & $78.62 \%$ & $75.77 \%$ & & & \\
\hline $\begin{array}{l}\text { Gas Stream Bulk Composition } \\
\text { (Dry Basis, Corrected to } 7 \% \\
\text { O2 with } 100 \% \text { O2 Combustion } \\
\text { Air) }\end{array}$ & $\begin{array}{l}\mathrm{ppmv} \text {, or } \\
\text { ug/dscm } \\
\text { or } \\
\mathrm{mg} / \mathrm{dscm} \\
\end{array}$ & $\begin{array}{l}\text { ppmv, or } \\
\text { ug/dscm } \\
\text { or } \\
\mathrm{mg} / \mathrm{dscm} \\
\end{array}$ & $\begin{array}{l}\text { ppmv, or } \\
\text { ug/dscm } \\
\text { or } \\
\mathrm{mg} / \mathrm{dscm} \\
\end{array}$ & $\begin{array}{l}\mathrm{ppmv}, \text { or } \\
\mathrm{ug} / \mathrm{dscm} \\
\text { or } \\
\mathrm{mg} / \mathrm{dscm} \\
\end{array}$ & $\begin{array}{l}\mathrm{ppmv}, \text { or } \\
\mathrm{ug} / \mathrm{dscm} \text { or } \\
\mathrm{mg} / \mathrm{dscm}\end{array}$ & $\begin{array}{l}\text { ppmv, or } \\
\text { ug/dscm or } \\
\mathrm{mg} / \mathrm{dscm}\end{array}$ & $\begin{array}{l}\text { ppmv, or } \\
\text { ug/dscm or } \\
\mathrm{mg} / \mathrm{dscm}\end{array}$ \\
\hline COgas, ppmv, dry basis & & & $9.82 \mathrm{E}+02$ & $9.46 \mathrm{E}+02$ & & & \\
\hline NO, ppmv, dry basis & & & $3.67 \mathrm{E}+02$ & $3.54 \mathrm{E}+02$ & & & \\
\hline NO2, ppmv, dry basis & & & $3.50 \mathrm{E}+00$ & $3.37 \mathrm{E}+00$ & & & \\
\hline SO2, ppmv, dry basis & & & $1.98 \mathrm{E}-06$ & $1.90 \mathrm{E}-06$ & & & \\
\hline $\mathrm{Cl}$, ppmv, dry basis & & & $1.86 \mathrm{E}+00$ & $1.80 \mathrm{E}+00$ & & & \\
\hline $\mathrm{F}, \mathrm{ppmv}$, dry basis & & & $5.00 \mathrm{E}+00$ & $4.82 \mathrm{E}+00$ & & & \\
\hline C (organic), ppmv, dry basis & & & $3.53 \mathrm{E}+04$ & $3.40 \mathrm{E}+04$ & & & \\
\hline $\mathrm{H}$ (organic), ppmv, dry basis & & & $7.89 \mathrm{E}+04$ & $7.60 \mathrm{E}+04$ & & & \\
\hline $\mathrm{Hg}, \mathrm{ug} / \mathrm{dscm}$ & & & $3.10 \mathrm{E}+04$ & $2.98 \mathrm{E}+04$ & & & \\
\hline $\mathrm{PM}, \mathrm{mg} / \mathrm{dscm}$ & & & $2.14 \mathrm{E}+04$ & $2.06 \mathrm{E}+04$ & & & \\
\hline SVM, ug/dscm & & & $2.56 \mathrm{E}+04$ & $2.49 \mathrm{E}+04$ & & & \\
\hline LVM, ug/dscm & & & $5.08 \mathrm{E}+03$ & $4.96 \mathrm{E}+03$ & & & \\
\hline
\end{tabular}


Table A-19. Mass balance, Tank WM-180 waste (continued).

\begin{tabular}{|c|c|c|c|c|c|c|c|c|}
\hline PFD Number & PFD-2 & PFD-2 & PFD-3 & PFD-3 & PFD-3 & PFD-3 & PFD-3 & PFD-3 \\
\hline Stream Number & 108 & 109 & 110 & 111 & 112 & $113 \mathrm{~A}$ & 113B & 114 \\
\hline Stream Name & $\begin{array}{l}\text { Product } \\
\text { Shipping } \\
\text { Canisters }\end{array}$ & $\begin{array}{l}\text { Off-Gas } \\
\text { from Filter } \\
\text { to Oxidizer }\end{array}$ & $\begin{array}{l}\text { Outlet of } \\
\text { Oxidizer }\end{array}$ & $\begin{array}{c}\text { Quenched } \\
\text { Oxidizer } \\
\text { Off-gas }\end{array}$ & Scrub & $\begin{array}{l}\text { Packed } \\
\text { Scrubber } \\
\text { Drain }\end{array}$ & $\begin{array}{l}\text { Demister } \\
\text { Drain }\end{array}$ & $\begin{array}{l}\text { Off-Gas to } \\
\text { Demister }\end{array}$ \\
\hline Rate or Volume & $1.11 \mathrm{E}+00$ & $1.96 \mathrm{E}+05$ & $6.48 \mathrm{E}+05$ & $3.58 \mathrm{E}+05$ & $9.82 \mathrm{E}+03$ & $9.84 \mathrm{E}+03$ & 0 & $3.60 \mathrm{E}+05$ \\
\hline Volume Flow (standard, wet) & & $5.55 E+04$ & $9.54 \mathrm{E}+04$ & $1.78 \mathrm{E}+05$ & & & & $1.76 \mathrm{E}+05$ \\
\hline Volume Flow (standard, dry) ${ }^{*}$ & & $3.90 \mathrm{E}+04$ & $5.25 E+04$ & $5.25 \mathrm{E}+04$ & & & & $5.23 \mathrm{E}+04$ \\
\hline Rate Units & cont/day & $\mathrm{ft} 3 / \mathrm{hr}$ & $\mathrm{ft} 3 / \mathrm{hr}$ & $\mathrm{ft} 3 / \mathrm{hr}$ & $\mathrm{gal} / \mathrm{hr}$ & $\mathrm{gal} / \mathrm{hr}$ & $\mathrm{gal} / \mathrm{hr}$ & $\mathrm{ft} 3 / \mathrm{hr}$ \\
\hline Rate or Volume, metric & $5.23 E+01$ & $1.57 \mathrm{E}+03$ & $2.70 \mathrm{E}+03$ & $5.03 E+03$ & $3.72 E+04$ & $3.72 E+04$ & 0 & $4.98 E+03$ \\
\hline Rate Units & $\mathrm{kg} / \mathrm{hr}$ & wscm/hr & wscm/hr & wscm/hr & $\mathrm{L} / \mathrm{hr}$ & $\mathrm{L} / \mathrm{hr}$ & $\mathrm{L} / \mathrm{hr}$ & wscm/hr \\
\hline Temperature, ${ }^{\circ} \mathrm{C}$ & 56 & 397 & 1000 & 100 & 77 & 79 & 338 & 78 \\
\hline Temperature, ${ }^{\circ} \mathrm{F}$ & 133 & 747 & 1832 & 212 & 171 & 174 & 170 & 172 \\
\hline Pressure, psia & 9.50 & 9.50 & 9.39 & 9.28 & 37.52 & 8.96 & 8.42 & 8.60 \\
\hline Specific Gravity & 1.42 & $3.83 E-04$ & 1.92E-04 & $5.20 \mathrm{E}-04$ & 1.02 & 1.02 & 1.02 & $5.16 \mathrm{E}-04$ \\
\hline Chemical Composition & Wt frac & $\mathrm{lb} /$ wscf & $\mathrm{lb} / \mathrm{wscf}$ & $\mathrm{lb} /$ wscf & Mol/liter & Mol/liter & Mol/liter & $\mathrm{lb} /$ wscf \\
\hline $\mathrm{H}+$ & $7.41 \mathrm{E}-05$ & $1.26 \mathrm{E}-06$ & $2.08 \mathrm{E}-08$ & $1.12 \mathrm{E}-08$ & $9.72 \mathrm{E}-01$ & $9.70 \mathrm{E}-01$ & & $1.23 \mathrm{E}-07$ \\
\hline $\mathrm{Al}+3$ & $1.06 \mathrm{E}-01$ & & & & $1.08 \mathrm{E}-02$ & $1.08 \mathrm{E}-02$ & & \\
\hline $\mathrm{Sb}+5$ & $1.09 \mathrm{E}-06$ & & & & $2.46 \mathrm{E}-08$ & $2.45 \mathrm{E}-08$ & & \\
\hline$A s+3$ & $2.15 \mathrm{E}-04$ & & & & $7.90 \mathrm{E}-06$ & 7.89E-06 & & \\
\hline $\mathrm{Ba}+2$ & $4.40 \mathrm{E}-05$ & & & & & & & \\
\hline $\mathrm{Be}+2$ & $4.51 \mathrm{E}-07$ & & & & & & & \\
\hline $\mathrm{B}+3$ & $7.21 \mathrm{E}-04$ & & & & $1.84 \mathrm{E}-04$ & $1.83 \mathrm{E}-04$ & & \\
\hline $\mathrm{Cd}+2$ & $5.16 \mathrm{E}-04$ & & & & & & & \\
\hline $\mathrm{Ca}+2$ & $9.49 \mathrm{E}-03$ & & & & & & & \\
\hline $\mathrm{Cr}+3$ & $1.12 \mathrm{E}-03$ & & & & & & & \\
\hline $\mathrm{Co}+2$ & $6.91 \mathrm{E}-06$ & & & & $3.23 \mathrm{E}-07$ & $3.23 \mathrm{E}-07$ & & \\
\hline Cs+ & $2.19 \mathrm{E}-05$ & & & & & & & \\
\hline $\mathrm{Cu}+2$ & $2.57 \mathrm{E}-04$ & & & & & & & \\
\hline $\mathrm{Fe}+3$ & $6.88 \mathrm{E}-03$ & $3.79 \mathrm{E}-09$ & $2.21 \mathrm{E}-09$ & $1.19 \mathrm{E}-09$ & $7.26 \mathrm{E}-05$ & $7.26 \mathrm{E}-05$ & & \\
\hline $\mathrm{Pb}+2$ & $1.68 \mathrm{E}-03$ & & & & & & & \\
\hline $\mathrm{Hg}+2$ & $8.87 \mathrm{E}-06$ & $9.19 \mathrm{E}-06$ & $5.35 \mathrm{E}-06$ & $2.87 \mathrm{E}-06$ & $3.30 \mathrm{E}-02$ & $3.30 \mathrm{E}-02$ & & $1.14 \mathrm{E}-06$ \\
\hline $\mathrm{Mn}+4$ & 4.49E-03 & & & & $2.25 \mathrm{E}-04$ & $2.24 \mathrm{E}-04$ & & \\
\hline $\mathrm{Ni}+2$ & $5.22 \mathrm{E}-04$ & & & & & & & \\
\hline $\mathrm{K}+$ & $4.41 \mathrm{E}-02$ & & & & & & & \\
\hline $\mathrm{Se}+4$ & $3.88 \mathrm{E}-05$ & & & & & & & \\
\hline $\mathrm{Ag}+$ & $4.20 \mathrm{E}-06$ & & & & & & & \\
\hline $\mathrm{Na}+$ & $2.78 \mathrm{E}-01$ & & & & & & & \\
\hline$T \mathrm{TI}+3$ & $3.60 \mathrm{E}-05$ & & & & $4.85 \mathrm{E}-07$ & $4.84 \mathrm{E}-07$ & & \\
\hline $\mathrm{U}+4$ & $5.51 \mathrm{E}-04$ & & & & & & & \\
\hline $\mathrm{V}+3$ & $2.86 \mathrm{E}-04$ & & & & & & & \\
\hline$Z n+2$ & $3.98 \mathrm{E}-04$ & & & & & & & \\
\hline $\mathrm{Zr}+4$ & $7.73 \mathrm{E}-04$ & & & & & & & \\
\hline $\mathrm{Cl}-$ & $6.48 \mathrm{E}-03$ & 4.92E-08 & $2.87 \mathrm{E}-08$ & $1.54 \mathrm{E}-08$ & $5.43 \mathrm{E}-04$ & $5.43 \mathrm{E}-04$ & & $9.46 \mathrm{E}-09$ \\
\hline F- & $4.63 \mathrm{E}-03$ & $6.46 \mathrm{E}-07$ & $3.76 \mathrm{E}-07$ & $2.02 \mathrm{E}-07$ & 5.99E-03 & $5.98 \mathrm{E}-03$ & & $1.75 \mathrm{E}-07$ \\
\hline SO4-2 & $1.31 \mathrm{E}-08$ & & $1.48 \mathrm{E}-10$ & $7.96 \mathrm{E}-11$ & & & & \\
\hline NO3- & $3.86 \mathrm{E}-03$ & $9.65 \mathrm{E}-12$ & $5.66 \mathrm{E}-12$ & $3.04 \mathrm{E}-12$ & $8.77 \mathrm{E}-01$ & $8.75 \mathrm{E}-01$ & & 6.99E-06 \\
\hline PO4-3 & 1.19E-02 & & & & & & & \\
\hline $\mathrm{Am}+4$ & $4.93 \mathrm{E}-08$ & & & & $5.58 \mathrm{E}-10$ & $5.57 \mathrm{E}-10$ & & \\
\hline $\mathrm{Br}-$ & 7.94E-08 & $6.04 \mathrm{E}-13$ & $3.51 \mathrm{E}-13$ & $1.89 \mathrm{E}-13$ & 2.95E-09 & $2.95 \mathrm{E}-09$ & & $1.16 \mathrm{E}-13$ \\
\hline $\mathrm{Ce}+4$ & $3.92 \mathrm{E}-05$ & & & & $7.70 \mathrm{E}-07$ & $7.68 \mathrm{E}-07$ & & \\
\hline $\mathrm{Eu}+3$ & $2.51 \mathrm{E}-07$ & & & & $4.55 \mathrm{E}-09$ & $4.55 \mathrm{E}-09$ & & \\
\hline $\mathrm{Gd}+3$ & $1.62 \mathrm{E}-04$ & & & & $2.84 \mathrm{E}-06$ & $2.83 \mathrm{E}-06$ & & \\
\hline $\mathrm{Ge}+4$ & 2.09E-09 & & & & & & & \\
\hline $\ln +3$ & $5.20 \mathrm{E}-07$ & & & & $1.25 \mathrm{E}-08$ & $1.24 \mathrm{E}-08$ & & \\
\hline I- & $1.09 \mathrm{E}-06$ & $8.31 \mathrm{E}-12$ & $4.84 \mathrm{E}-12$ & $2.60 \mathrm{E}-12$ & $2.56 \mathrm{E}-08$ & $2.56 \mathrm{E}-08$ & & $1.60 \mathrm{E}-12$ \\
\hline $\mathrm{La}+3$ & 4.17E-06 & & & & 8.27E-08 & $8.26 \mathrm{E}-08$ & & \\
\hline
\end{tabular}


Table A-19. Mass balance, Tank WM-180 waste (continued).

\begin{tabular}{|c|c|c|c|c|c|c|c|c|}
\hline Stream \# & 108 & 109 & 110 & 111 & 112 & $113 \mathrm{~A}$ & $113 \mathrm{~B}$ & 114 \\
\hline Chemical Composition & Wt frac & $\mathrm{Ib} / \mathrm{wscf}$ & $\mathrm{Ib} / \mathrm{wscf}$ & $\mathrm{lb} / \mathrm{wscf}$ & Mol/liter & Mol/liter & Mol/liter & $\mathrm{lb} / \mathrm{wscf}$ \\
\hline $\mathrm{Li}+$ & $1.98 \mathrm{E}-05$ & & & & & & & \\
\hline $\mathrm{Mg}+2$ & $1.71 \mathrm{E}-03$ & & & & & & & \\
\hline $\mathrm{Mo}+6$ & $1.01 \mathrm{E}-03$ & & & & & & & \\
\hline $\mathrm{Nd}+3$ & $1.40 \mathrm{E}-05$ & & & & $2.67 \mathrm{E}-07$ & $2.66 \mathrm{E}-07$ & & \\
\hline $\mathrm{Np}+4$ & 1.33E-05 & & & & $1.54 \mathrm{E}-07$ & $1.54 \mathrm{E}-07$ & & \\
\hline $\mathrm{Nb}+5$ & $2.66 \mathrm{E}-04$ & & & & & & & \\
\hline $\mathrm{Pd}+4$ & $2.13 \mathrm{E}-05$ & & & & & & & \\
\hline $\mathrm{Pu}+4$ & $1.58 \mathrm{E}-05$ & & & & & & & \\
\hline $\mathrm{Pr}+4$ & $3.85 \mathrm{E}-06$ & & & & $7.52 \mathrm{E}-08$ & $7.51 \mathrm{E}-08$ & & \\
\hline $\mathrm{Pm}+3$ & $6.00 \mathrm{E}-10$ & & & & $1.14 \mathrm{E}-11$ & $1.14 \mathrm{E}-11$ & & \\
\hline $\mathrm{Rh}+4$ & $1.21 \mathrm{E}-06$ & & & & $3.24 \mathrm{E}-08$ & $3.24 \mathrm{E}-08$ & & \\
\hline $\mathrm{Rb}+$ & $1.55 \mathrm{E}-06$ & & & & & & & \\
\hline$R u+3$ & $8.18 \mathrm{E}-05$ & & & & $2.23 \mathrm{E}-06$ & $2.23 \mathrm{E}-06$ & & \\
\hline $\mathrm{Sm}+3$ & $2.70 \mathrm{E}-06$ & & & & $4.95 \mathrm{E}-08$ & $4.94 \mathrm{E}-08$ & & \\
\hline $\mathrm{Si}+4$ & $5.54 \mathrm{E}-04$ & & & & & & & \\
\hline $\mathrm{Sr}+2$ & $6.04 \mathrm{E}-05$ & & & & & & & \\
\hline $\mathrm{Tc}+7$ & $3.33 \mathrm{E}-06$ & $7.62 \mathrm{E}-12$ & $4.44 \mathrm{E}-12$ & $2.38 \mathrm{E}-12$ & $8.21 \mathrm{E}-08$ & $8.20 \mathrm{E}-08$ & & \\
\hline $\mathrm{Te}+4$ & $1.20 \mathrm{E}-06$ & & & & & & & \\
\hline $\mathrm{Tb}+4$ & 1.10E-09 & & & & $1.90 \mathrm{E}-11$ & $1.90 \mathrm{E}-11$ & & \\
\hline $\mathrm{Th}+4$ & $1.56 \mathrm{E}-10$ & & & & & & & \\
\hline $\mathrm{Sn+4}$ & $5.62 \mathrm{E}-05$ & & & & & & & \\
\hline $\mathrm{Ti}+4$ & $4.12 \mathrm{E}-05$ & & & & & & & \\
\hline $\mathrm{Y}+3$ & $1.99 \mathrm{E}-06$ & & & & $6.17 \mathrm{E}-08$ & $6.16 \mathrm{E}-08$ & & \\
\hline $\mathrm{OH}-$ & $1.65 \mathrm{E}-06$ & & $6.56 \mathrm{E}-21$ & $3.69 \mathrm{E}-11$ & $1.21 \mathrm{E}-06$ & $1.21 \mathrm{E}-06$ & & \\
\hline $\mathrm{H} 2 \mathrm{O}$ & $3.36 \mathrm{E}-03$ & $1.39 \mathrm{E}-02$ & $2.10 \mathrm{E}-02$ & $3.29 \mathrm{E}-02$ & $5.31 \mathrm{E}+01$ & $5.31 \mathrm{E}+01$ & & $3.30 \mathrm{E}-02$ \\
\hline $\mathrm{SO} 2$ & & $1.56 \mathrm{E}-12$ & $2.27 \mathrm{E}-05$ & $1.22 \mathrm{E}-05$ & $7.64 \mathrm{E}-07$ & $8.04 \mathrm{E}-07$ & & $1.23 \mathrm{E}-05$ \\
\hline $\mathrm{H} 2 \mathrm{~S}$ & & $2.08 \mathrm{E}-05$ & $6.72 \mathrm{E}-21$ & $3.61 \mathrm{E}-21$ & & & & \\
\hline $\mathrm{CO}$ & & $3.40 \mathrm{E}-04$ & $2.14 \mathrm{E}-08$ & $1.15 \mathrm{E}-08$ & $2.73 \mathrm{E}-09$ & $2.82 \mathrm{E}-09$ & & $1.16 \mathrm{E}-08$ \\
\hline $\mathrm{CO} 2$ & & $5.99 \mathrm{E}-02$ & $5.55 \mathrm{E}-02$ & $2.98 \mathrm{E}-02$ & $2.08 \mathrm{E}-04$ & $3.11 \mathrm{E}-04$ & & $3.01 \mathrm{E}-02$ \\
\hline $\mathrm{H} 2$ & & 5.39E-04 & $8.14 \mathrm{E}-10$ & $4.37 \mathrm{E}-10$ & $1.75 \mathrm{E}-08$ & $1.48 \mathrm{E}-08$ & & $1.32 \mathrm{E}-08$ \\
\hline N2 & & $3.57 \mathrm{E}-03$ & $2.13 \mathrm{E}-03$ & $1.14 \mathrm{E}-03$ & $6.38 \mathrm{E}-09$ & $2.47 \mathrm{E}-07$ & & $1.15 \mathrm{E}-03$ \\
\hline $\mathrm{NO}$ & & $1.36 \mathrm{E}-04$ & $1.95 \mathrm{E}-05$ & 1.05E-05 & $8.82 \mathrm{E}-08$ & $1.39 \mathrm{E}-07$ & & $1.05 \mathrm{E}-05$ \\
\hline $\mathrm{NO} 2$ & & 1.99E-06 & 1.16E-06 & $6.22 \mathrm{E}-07$ & 1.34E-05 & 1.34E-05 & & $6.27 \mathrm{E}-07$ \\
\hline $\mathrm{O} 2$ & & & 2.89E-03 & $1.55 \mathrm{E}-03$ & $2.20 \mathrm{E}-08$ & 5.05E-07 & & 1.57E-03 \\
\hline S (other) & $7.89 \mathrm{E}-04$ & & & & $1.08 \mathrm{E}-08$ & 1.12E-08 & & \\
\hline $\mathrm{CO} 3$ & $4.03 \mathrm{E}-01$ & & & & $1.19 \mathrm{E}-10$ & $1.77 \mathrm{E}-10$ & & \\
\hline C (reductant) & & & $2.23 \mathrm{E}-17$ & $1.20 \mathrm{E}-17$ & & & & \\
\hline $\mathrm{O}$ (oxides) & $10.37 \%$ & 1.63E-09 & $9.51 \mathrm{E}-10$ & $5.11 \mathrm{E}-10$ & 1.09E-04 & 1.09E-04 & & \\
\hline C (organic) & & $5.23 E-03$ & & & & & & \\
\hline $\mathrm{H}$ (organic) & & 9.81E-04 & & & & & & \\
\hline \multicolumn{9}{|l|}{ O (organic) } \\
\hline & & & & & & & & \\
\hline Mass Flow (ka/hr). & $523 \mathrm{~F}+01$ & $213 \mathrm{~F}+03$ & $353 F+03$ & $527 \mathrm{~F}+03$ & $379 \mathrm{~F}+04$ & $379 F+04$ & & $525 \mathrm{~F}+03$ \\
\hline \multicolumn{9}{|l|}{ Canister Rate, canisters/day } \\
\hline \multicolumn{9}{|l|}{ Total Canisters Generated } \\
\hline & & & & & & & & \\
\hline & w/Canister & $\mathrm{w} / \mathrm{m} 3$ & $\mathrm{w} / \mathrm{m} 3$ & $\mathrm{w} / \mathrm{m} 3$ & $\mathrm{w} / \mathrm{m} 3$ & $\mathrm{w} / \mathrm{m} 3$ & $\mathrm{w} / \mathrm{m} 3$ & $\mathrm{w} / \mathrm{m} 3$ \\
\hline Heat Generation & $2.12 \mathrm{E}+00$ & & & & $1.95 \mathrm{E}-03$ & 1.94E-03 & & \\
\hline
\end{tabular}


Table A-19. Mass balance, Tank WM-180 waste (continued).

\begin{tabular}{|c|c|c|c|c|c|c|c|c|}
\hline Stream \# & 108 & 109 & 110 & 111 & 112 & $113 \mathrm{~A}$ & $113 \mathrm{~B}$ & 114 \\
\hline Radiological Composition & Ci/Canister & Ci/wscm & Ci/wscm & Ci/wscm & $\mathrm{Ci} / \mathrm{L}$ & $\mathrm{Ci} / \mathrm{L}$ & $\mathrm{Ci} / \mathrm{L}$ & $\mathrm{Ci} / \mathrm{wscm}$ \\
\hline $\mathrm{H}-3$ & & $3.67 E-06$ & $2.14 \mathrm{E}-06$ & 1.15E-06 & $2.02 \mathrm{E}-06$ & $2.01 \mathrm{E}-06$ & & 1.15E-06 \\
\hline $\mathrm{C}-14$ & $3.62 \mathrm{E}-09$ & $1.31 \mathrm{E}-11$ & $7.63 \mathrm{E}-12$ & $4.10 \mathrm{E}-12$ & $7.84 \mathrm{E}-17$ & $1.17 \mathrm{E}-16$ & & $4.13 \mathrm{E}-12$ \\
\hline Co-60 & $2.89 \mathrm{E}-02$ & & & & $7.01 \mathrm{E}-08$ & $7.00 \mathrm{E}-08$ & & \\
\hline $\mathrm{Ni}-59$ & $6.64 \mathrm{E}-04$ & & & & & & & \\
\hline $\mathrm{Ni}-63$ & $1.75 \mathrm{E}-01$ & & & & & & & \\
\hline Se-79 & $1.64 \mathrm{E}-03$ & & & & & & & \\
\hline Sr-90 & $1.42 \mathrm{E}+02$ & & & & & & & \\
\hline Y-90 & $1.42 \mathrm{E}+02$ & & & & $3.44 \mathrm{E}-04$ & $3.43 \mathrm{E}-04$ & & \\
\hline $\mathrm{Zr}-93$ & $7.92 \mathrm{E}-03$ & & & & & & & \\
\hline \begin{tabular}{|l|}
$\mathrm{Nb}-93 m$ \\
\end{tabular} & $6.11 \mathrm{E}-03$ & & & & & & & \\
\hline \begin{tabular}{|l|}
$\mathrm{Nb}-94$ \\
\end{tabular} & $4.27 \mathrm{E}-03$ & & & & & & & \\
\hline Tc-99 & $6.55 \mathrm{E}-02$ & $2.12 \mathrm{E}-09$ & $1.23 \mathrm{E}-09$ & $6.62 \mathrm{E}-10$ & $1.40 \mathrm{E}-07$ & $1.39 \mathrm{E}-07$ & & \\
\hline Ru-106 & $3.49 \mathrm{E}-03$ & & & & $8.48 \mathrm{E}-09$ & $8.47 \mathrm{E}-09$ & & \\
\hline Rh-102 & $3.09 \mathrm{E}-06$ & & & & $7.49 \mathrm{E}-12$ & $7.48 \mathrm{E}-12$ & & \\
\hline Rh-106 & $3.49 \mathrm{E}-03$ & & & & 8.48E-09 & 8.47E-09 & & \\
\hline $\mathrm{Pd}-107$ & 5.91E-05 & & & & & & & \\
\hline $\mathrm{Cd}-113 \mathrm{~m}$ & $1.19 \mathrm{E}-02$ & & & & & & & \\
\hline Sn-121m & $2.39 \mathrm{E}-04$ & & & & & & & \\
\hline Sn-126 & $1.54 \mathrm{E}-03$ & & & & & & & \\
\hline Sb-125 & $5.75 \mathrm{E}-02$ & & & & $1.39 \mathrm{E}-07$ & $1.39 \mathrm{E}-07$ & & \\
\hline Sb-126 & $2.06 \mathrm{E}-04$ & & & & $5.00 \mathrm{E}-10$ & $4.99 \mathrm{E}-10$ & & \\
\hline Te-125m & 1.13E-02 & & & & & & & \\
\hline |-129 & 1.74E-04 & $1.87 \mathrm{E}-11$ & $1.09 \mathrm{E}-11$ & $5.83 \mathrm{E}-12$ & $4.56 \mathrm{E}-10$ & $4.55 \mathrm{E}-10$ & & $3.58 \mathrm{E}-12$ \\
\hline Cs-134 & $3.73 \mathrm{E}-02$ & & & & & & & \\
\hline Cs-135 & $3.21 \mathrm{E}-03$ & & & & & & & \\
\hline Cs-137 & $1.88 \mathrm{E}+02$ & & & & & & & \\
\hline Ba-137m & $1.78 \mathrm{E}+02$ & & & & & & & \\
\hline $\mathrm{Ce}-144$ & $2.36 \mathrm{E}-03$ & & & & $5.72 \mathrm{E}-09$ & $5.71 \mathrm{E}-09$ & & \\
\hline $\operatorname{Pr}-144$ & $2.36 \mathrm{E}-03$ & & & & 5.72E-09 & 5.71E-09 & & \\
\hline $\mathrm{Pm}-146$ & $1.82 \mathrm{E}-04$ & & & & $4.42 \mathrm{E}-10$ & $4.42 \mathrm{E}-10$ & & \\
\hline $\mathrm{Pm}-147$ & 6.39E-01 & & & & $1.55 \mathrm{E}-06$ & $1.55 \mathrm{E}-06$ & & \\
\hline Sm-151 & $1.26 \mathrm{E}+00$ & & & & $3.06 \mathrm{E}-06$ & $3.05 E-06$ & & \\
\hline Eu-152 & $9.12 \mathrm{E}-03$ & & & & $2.21 \mathrm{E}-08$ & $2.21 \mathrm{E}-08$ & & \\
\hline Eu-154 & $3.27 \mathrm{E}-01$ & & & & $7.95 \mathrm{E}-07$ & $7.93 \mathrm{E}-07$ & & \\
\hline Eu-155 & $5.96 \mathrm{E}-01$ & & & & $1.45 \mathrm{E}-06$ & $1.44 \mathrm{E}-06$ & & \\
\hline & & & & & & & & \\
\hline Th-230 & $3.12 \mathrm{E}-06$ & & & & & & & \\
\hline $\mathrm{Pa}-233$ & $1.05 \mathrm{E}-02$ & & & & & & & \\
\hline $\mathrm{U}-232$ & $7.52 \mathrm{E}-06$ & & & & & & & \\
\hline$U-233$ & $2.93 \mathrm{E}-07$ & & & & & & & \\
\hline U-234 & $7.53 \mathrm{E}-03$ & & & & & & & \\
\hline U-235 & $2.75 \mathrm{E}-04$ & & & & & & & \\
\hline U-236 & $4.08 \mathrm{E}-04$ & & & & & & & \\
\hline U-237 & $2.30 \mathrm{E}-05$ & & & & & & & \\
\hline U-238 & 1.63E-04 & & & & & & & \\
\hline Np-237 & $1.06 \mathrm{E}-02$ & & & & $2.58 \mathrm{E}-08$ & $2.57 \mathrm{E}-08$ & & \\
\hline Pu-236 & $1.51 \mathrm{E}-05$ & & & & & & & \\
\hline Pu-238 & $6.88 \mathrm{E}+00$ & & & & & & & \\
\hline Pu-239 & $1.04 \mathrm{E}+00$ & & & & & & & \\
\hline Pu-240 & 5.67E-02 & & & & & & & \\
\hline Pu-241 & $4.15 \mathrm{E}+00$ & & & & & & & \\
\hline Pu-242 & 4.37E-05 & & & & & & & \\
\hline $\mathrm{Pu}-244$ & $3.74 \mathrm{E}-12$ & & & & & & & \\
\hline Am-241 & 4.99E-01 & & & & $1.21 \mathrm{E}-06$ & $1.21 \mathrm{E}-06$ & & \\
\hline Am-242m & $5.45 \mathrm{E}-05$ & & & & $1.32 \mathrm{E}-10$ & $1.32 \mathrm{E}-10$ & & \\
\hline
\end{tabular}


Table A-19. Mass balance, Tank WM-180 waste (continued).

\begin{tabular}{|c|c|c|c|c|c|c|c|c|}
\hline Stream \# & 108 & 109 & 110 & 111 & 112 & $113 \mathrm{~A}$ & $113 \mathrm{~B}$ & 114 \\
\hline Radiological Composition & Ci/Canister & $\mathrm{Ci} / \mathrm{wscm}$ & Ci/wscm & Ci/wscm & $\mathrm{Ci} / \mathrm{L}$ & $\mathrm{Ci} / \mathrm{L}$ & $\mathrm{Ci} / \mathrm{L}$ & Ci/wscm \\
\hline Am-243 & 7.91E-05 & & & & $1.92 \mathrm{E}-10$ & $1.92 \mathrm{E}-10$ & & \\
\hline $\mathrm{Cm}-242$ & $4.49 E-05$ & & & & & & & \\
\hline $\mathrm{Cm}-243$ & $1.29 \mathrm{E}-04$ & & & & & & & \\
\hline $\mathrm{Cm}-244$ & $8.02 E-03$ & & & & & & & \\
\hline $\mathrm{Cm}-245$ & $1.36 \mathrm{E}-06$ & & & & & & & \\
\hline $\mathrm{Cm}-246$ & $8.92 \mathrm{E}-08$ & & & & & & & \\
\hline TRU & $8.62 \mathrm{E}+00$ & & & & $1.24 \mathrm{E}-06$ & $1.24 \mathrm{E}-06$ & & \\
\hline & & & & & & & & \\
\hline & & & & & & & & \\
\hline $\begin{array}{l}\text { Gas Stream Bulk Composition } \\
\text { (Wet Basis) }\end{array}$ & $\begin{array}{l}\text { mol\% or } \\
\text { ppmv }\end{array}$ & $\begin{array}{l}\text { mol\% or } \\
\text { ppmv }\end{array}$ & $\begin{array}{c}\text { mol\% or } \\
\text { ppmv }\end{array}$ & $\begin{array}{c}\text { mol\% or } \\
\text { ppmv }\end{array}$ & $\begin{array}{c}\text { mol\% or } \\
\text { ppmv }\end{array}$ & $\begin{array}{l}\text { mol\% or } \\
\text { ppmv }\end{array}$ & $\begin{array}{l}\text { mol\% or } \\
\text { ppmv }\end{array}$ & $\begin{array}{c}\text { mol\% or } \\
\text { ppmv }\end{array}$ \\
\hline $\mathrm{H} 2 \mathrm{O}, \mathrm{mol} \%$ & & $29.69 \%$ & $44.95 \%$ & $70.43 \%$ & & & & $70.26 \%$ \\
\hline $\mathrm{O} 2, \mathrm{~mol} \%$ & & & $3.4827 \%$ & $1.8707 \%$ & & & & $1.8811 \%$ \\
\hline $\mathrm{N} 2, \mathrm{~mol} \%$ & & $4.92 \%$ & $2.92 \%$ & $1.57 \%$ & & & & $1.58 \%$ \\
\hline $\mathrm{H} 2, \mathrm{~mol} \%$ & & $10.31 \%$ & $0.00002 \%$ & $0.00001 \%$ & & & & $0.0003 \%$ \\
\hline $\mathrm{CO} 2, \mathrm{~mol} \%$ & & $52.43 \%$ & $48.60 \%$ & $26.10 \%$ & & & & $26.25 \%$ \\
\hline COgas, ppmv & & $4.68 \mathrm{E}+03$ & $2.95 \mathrm{E}-01$ & $1.58 \mathrm{E}-01$ & & & & $1.59 \mathrm{E}-01$ \\
\hline $\mathrm{NO}, \mathrm{ppmv}$ & & $1.75 \mathrm{E}+03$ & $2.50 \mathrm{E}+02$ & $1.34 \mathrm{E}+02$ & & & & $1.35 \mathrm{E}+02$ \\
\hline $\mathrm{NO} 2, \mathrm{ppmv}$ & & $1.67 \mathrm{E}+01$ & $9.70 \mathrm{E}+00$ & $5.21 \mathrm{E}+00$ & & & & $5.23 \mathrm{E}+00$ \\
\hline $\mathrm{SO} 2, \mathrm{ppmv}$ & & $9.41 \mathrm{E}-06$ & $1.37 \mathrm{E}+02$ & $7.34 \mathrm{E}+01$ & & & & $7.38 \mathrm{E}+01$ \\
\hline $\mathrm{Cl}, \mathrm{ppmv}$ & & $5.35 \mathrm{E}-01$ & $3.11 \mathrm{E}-01$ & $1.67 \mathrm{E}-01$ & & & & $1.02 \mathrm{E}-01$ \\
\hline $\mathrm{F}, \mathrm{ppmv}$ & & $1.31 \mathrm{E}+01$ & $7.63 \mathrm{E}+00$ & $4.10 \mathrm{E}+00$ & & & & $3.54 \mathrm{E}+00$ \\
\hline C (organic), ppmv & & $1.68 \mathrm{E}+05$ & & & & & & \\
\hline $\mathrm{H}$ (organic), ppmv & & $3.75 \mathrm{E}+05$ & & & & & & \\
\hline & & & & & & & & \\
\hline $\mathrm{Hg}, \mathrm{ug} / \mathrm{wscm}$ & & $1.47 \mathrm{E}+05$ & $8.57 \mathrm{E}+04$ & $4.60 \mathrm{E}+04$ & & & & \\
\hline $\mathrm{PM}, \mathrm{mg} / \mathrm{wscm}$ & & $9.97 \mathrm{E}+04$ & $9.19 \mathrm{E}+01$ & $5.45 \mathrm{E}+01$ & & & & $8.63 E+00$ \\
\hline SVM, ug/wscm & & & & & & & & \\
\hline LVM, ug/wscm & & & & & & & & \\
\hline & & & & & & & & \\
\hline $\begin{array}{l}\text { Gas Stream Bulk Composition } \\
\text { (Dry Basis) }\end{array}$ & $\mathrm{mol} \%$ & $\mathrm{~mol} \%$ & $\mathrm{~mol} \%$ & $\mathrm{~mol} \%$ & $\mathrm{~mol} \%$ & $\mathrm{~mol} \%$ & $\mathrm{~mol} \%$ & $\mathrm{~mol} \%$ \\
\hline $\mathrm{O} 2, \mathrm{~mol} \%$, dry basis & & & $6.3267 \%$ & $6.3267 \%$ & & & & $6.3262 \%$ \\
\hline $\mathrm{N} 2, \mathrm{~mol} \%$, dry basis & & $6.99 \%$ & $5.31 \%$ & $5.31 \%$ & & & & $5.31 \%$ \\
\hline $\mathrm{H} 2, \mathrm{~mol} \%$, dry basis & & $14.66 \%$ & $0.00003 \%$ & $0.00003 \%$ & & & & $0.001 \%$ \\
\hline $\mathrm{CO} 2, \mathrm{~mol} \%$, dry basis & & $74.57 \%$ & $88.29 \%$ & $88.29 \%$ & & & & $88.27 \%$ \\
\hline $\begin{array}{l}\text { Gas Stream Bulk Composition } \\
\text { (Dry Basis, Corrected to } 7 \% \\
\text { O2 with } 100 \% \text { O2 Combustion } \\
\text { Air) }\end{array}$ & $\begin{array}{c}\mathrm{ppmv}, \text { or } \\
\mathrm{ug} / \mathrm{dscm} \text { or } \\
\mathrm{mg} / \mathrm{dscm}\end{array}$ & $\begin{array}{l}\mathrm{ppmv}, \text { or } \\
\mathrm{ug} / \mathrm{dscm} \text { or } \\
\mathrm{mg} / \mathrm{dscm}\end{array}$ & $\begin{array}{l}\text { ppmv, or } \\
\text { ug/dscm or } \\
\mathrm{mg} / \mathrm{dscm}\end{array}$ & $\begin{array}{l}\mathrm{ppmv}, \text { or } \\
\text { ug/dscm or } \\
\mathrm{mg} / \mathrm{dscm}\end{array}$ & $\begin{array}{c}\mathrm{ppmv}, \text { or } \\
\mathrm{ug} / \mathrm{dscm} \text { or } \\
\mathrm{mg} / \mathrm{dscm}\end{array}$ & $\begin{array}{c}\mathrm{ppmv}, \text { or } \\
\mathrm{ug} / \mathrm{dscm} \text { or } \\
\mathrm{mg} / \mathrm{dscm}\end{array}$ & $\begin{array}{l}\mathrm{ppmv}, \text { or } \\
\text { ug/dscm or } \\
\mathrm{mg} / \mathrm{dscm}\end{array}$ & $\begin{array}{l}\text { ppmv, or } \\
\text { ug/dscm or } \\
\mathrm{mg} / \mathrm{dscm}\end{array}$ \\
\hline COgas, ppmv, dry basis & & $9.31 \mathrm{E}+02$ & $7.50 \mathrm{E}-02$ & $7.50 \mathrm{E}-02$ & & & & $7.50 \mathrm{E}-02$ \\
\hline $\mathrm{NO}, \mathrm{ppmv}$, dry basis & & $3.48 \mathrm{E}+02$ & $6.36 \mathrm{E}+01$ & $6.36 \mathrm{E}+01$ & & & & $6.36 \mathrm{E}+01$ \\
\hline NO2, ppmv, dry basis & & $3.32 \mathrm{E}+00$ & $2.47 \mathrm{E}+00$ & $2.47 \mathrm{E}+00$ & & & & $2.47 \mathrm{E}+00$ \\
\hline SO2, ppmv, dry basis & & $1.87 \mathrm{E}-06$ & $3.48 \mathrm{E}+01$ & $3.48 \mathrm{E}+01$ & & & & $3.48 \mathrm{E}+01$ \\
\hline $\mathrm{Cl}, \mathrm{ppmv}$, dry basis & & $1.07 \mathrm{E}-01$ & 7.93E-02 & $7.93 E-02$ & & & & 4.83E-02 \\
\hline $\mathrm{F}, \mathrm{ppmv}$, dry basis & & $2.61 \mathrm{E}+00$ & $1.94 \mathrm{E}+00$ & $1.94 \mathrm{E}+00$ & & & & $1.67 \mathrm{E}+00$ \\
\hline C (organic), ppmv, dry basis & & $3.34 \mathrm{E}+04$ & & & & & & \\
\hline $\mathrm{H}$ (organic), ppmv, dry basis & & $7.47 \mathrm{E}+04$ & & & & & & \\
\hline $\mathrm{Hg}, \mathrm{ug} / \mathrm{dscm}$ & & $2.93 \mathrm{E}+04$ & $2.18 \mathrm{E}+04$ & $2.18 \mathrm{E}+04$ & & & & \\
\hline $\mathrm{PM}, \mathrm{mg} / \mathrm{dscm}$ & & $1.98 \mathrm{E}+04$ & $2.34 \mathrm{E}+01$ & $2.58 \mathrm{E}+01$ & & & & $4.07 \mathrm{E}+00$ \\
\hline SVM, ug/dscm & & & & & & & & \\
\hline LVM, ug/dscm & & & & & & & & \\
\hline
\end{tabular}


Table A-19. Mass balance, Tank WM-180 waste (continued).

\begin{tabular}{|c|c|c|c|c|c|c|}
\hline PFD Number & PFD-3 & PFD-3 & PFD-3 & PFD-3 & PFD-3 & PFD-3 \\
\hline Stream Number & 115 & 116 & 117 & 118 & 119 & 120 \\
\hline Stream Name & $\begin{array}{l}\text { Scrubbed } \\
\text { Off-Gas to } \\
\text { Preheater }\end{array}$ & $\begin{array}{c}\text { Preheated } \\
\text { Off-gas to } \\
\text { GAC }\end{array}$ & $\begin{array}{c}\text { Pressure } \\
\text { Control } \\
\text { Bleed Air }\end{array}$ & $\begin{array}{c}\text { Final HEPA } \\
\text { Off-Gas } \\
\text { Outlet }\end{array}$ & $\begin{array}{l}\text { Off-gas to } \\
\text { Exhaust } \\
\text { Blower }\end{array}$ & Spent GAC \\
\hline Rate or Volume & $3.93 \mathrm{E}+05$ & $4.86 \mathrm{E}+05$ & $1.57 \mathrm{E}+04$ & $5.91 \mathrm{E}+05$ & $6.32 \mathrm{E}+05$ & $3.38 \mathrm{E}+00$ \\
\hline Volume Flow (standard, wet) ${ }^{*}$ & $1.77 \mathrm{E}+05$ & $1.77 \mathrm{E}+05$ & $1.55 \mathrm{E}+04$ & $1.77 \mathrm{E}+05$ & $1.92 \mathrm{E}+05$ & \\
\hline Volume Flow (standard, dry) ${ }^{*}$ & $5.25 \mathrm{E}+04$ & $5.25 \mathrm{E}+04$ & $1.55 \mathrm{E}+04$ & $5.25 \mathrm{E}+04$ & $6.79 \mathrm{E}+04$ & \\
\hline Rate Units & $\mathrm{ft} 3 / \mathrm{hr}$ & $\mathrm{ft} 3 / \mathrm{hr}$ & $\mathrm{ft} 3 / \mathrm{hr}$ & $\mathrm{ft} 3 / \mathrm{hr}$ & $\mathrm{ft} 3 / \mathrm{hr}$ & $\mathrm{lb} / \mathrm{hr}$ \\
\hline Rate or Volume, metric & $5.00 \mathrm{E}+03$ & $5.00 \mathrm{E}+03$ & $4.38 \mathrm{E}+02$ & $5.00 \mathrm{E}+03$ & $5.43 \mathrm{E}+03$ & $1.53 \mathrm{E}+00$ \\
\hline Rate Units & wscm $/ \mathrm{hr}$ & wscm $/ \mathrm{hr}$ & wscm $/ \mathrm{hr}$ & wscm $/ \mathrm{hr}$ & wscm $/ \mathrm{hr}$ & $\mathrm{kg} / \mathrm{hr}$ \\
\hline Temperature, ${ }^{\circ} \mathrm{C}$ & 77 & 120 & 25 & 120 & 114 & 28 \\
\hline Temperature, ${ }^{\circ} \mathrm{F}$ & 170 & 248 & 77 & 248 & 237 & 82 \\
\hline Pressure, psia & 7.88 & 7.16 & 14.70 & 5.89 & 5.89 & 14.70 \\
\hline Specific Gravity & $4.72 \mathrm{E}-04$ & $3.81 \mathrm{E}-04$ & $1.18 \mathrm{E}-03$ & $3.14 \mathrm{E}-04$ & $3.23 \mathrm{E}-04$ & $5.86 \mathrm{E}-01$ \\
\hline Chemical Composition & $\mathrm{lb} /$ wscf & lb/wscf & lb/wscf & $\mathrm{lb} / \mathrm{wscf}$ & $\mathrm{lb} /$ wscf & wt frac \\
\hline $\mathrm{H}+$ & $1.23 \mathrm{E}-07$ & $1.23 \mathrm{E}-07$ & & $1.22 \mathrm{E}-07$ & $1.13 \mathrm{E}-07$ & $1.03 \mathrm{E}-05$ \\
\hline \multicolumn{7}{|l|}{$\mathrm{Al}+3$} \\
\hline \multicolumn{7}{|l|}{$\mathrm{Sb}+5$} \\
\hline \multirow{2}{*}{\multicolumn{7}{|c|}{$\frac{\mathrm{As}+3}{\mathrm{Ba}+2}$}} \\
\hline \multirow{2}{*}{\multicolumn{7}{|c|}{$\frac{\mathrm{Ba}+2}{\mathrm{Be}+2}$}} \\
\hline & & & & & & \\
\hline \multicolumn{7}{|l|}{$\mathrm{B}+3$} \\
\hline \multirow{2}{*}{\multicolumn{7}{|c|}{$\frac{C d+2}{C a+2}$}} \\
\hline & & & & & & \\
\hline \multicolumn{7}{|l|}{$\frac{\mathrm{Ca}+2}{\mathrm{Cr}+3}$} \\
\hline \multicolumn{7}{|l|}{$\mathrm{Co}+2$} \\
\hline \multicolumn{7}{|l|}{ Cs+ } \\
\hline \multicolumn{7}{|l|}{$\mathrm{Cu}+2$} \\
\hline \multirow{2}{*}{\multicolumn{7}{|c|}{$\begin{array}{l}\mathrm{Fe}+3 \\
\mathrm{~Pb}+2\end{array}$}} \\
\hline & & & & & & \\
\hline $\mathrm{Hg}+2$ & $1.14 \mathrm{E}-06$ & $1.14 \mathrm{E}-06$ & & $1.52 \mathrm{E}-09$ & $1.40 \mathrm{E}-09$ & $5.93 \mathrm{E}-02$ \\
\hline \multicolumn{7}{|l|}{$\mathrm{Mn}+4$} \\
\hline \multirow{2}{*}{\multicolumn{7}{|c|}{$\begin{array}{ll}\mathrm{Ni}+2 \\
\mathrm{~K}+\end{array}$}} \\
\hline \multirow{2}{*}{\multicolumn{7}{|c|}{\begin{tabular}{|l}
$\mathrm{K}+$ \\
$\mathrm{Se}+4$
\end{tabular}}} \\
\hline & & & & & & \\
\hline \multicolumn{7}{|l|}{$\frac{\mathrm{Se}+4}{\mathrm{Ag}+}$} \\
\hline \multicolumn{7}{|l|}{$\mathrm{Na}+$} \\
\hline \multicolumn{7}{|l|}{$\mathrm{TI}+3$} \\
\hline \multicolumn{7}{|l|}{$\mathrm{U}+4$} \\
\hline $\mathrm{V}+3$ & & & & & & \\
\hline $\mathrm{Zn}+2$ & & & & & & \\
\hline $\mathrm{Zr}+4$ & & & & & & \\
\hline $\mathrm{Cl}-$ & $9.42 \mathrm{E}-09$ & $9.42 \mathrm{E}-09$ & & $2.46 \mathrm{E}-09$ & $2.27 \mathrm{E}-09$ & $3.64 \mathrm{E}-04$ \\
\hline F- & $1.75 \mathrm{E}-07$ & $1.75 \mathrm{E}-07$ & & $1.75 \mathrm{E}-07$ & $1.61 \mathrm{E}-07$ & \\
\hline SO4-2 & & & & & & \\
\hline NO3- & $6.96 \mathrm{E}-06$ & $6.96 \mathrm{E}-06$ & & $6.96 \mathrm{E}-06$ & $6.41 \mathrm{E}-06$ & \\
\hline PO4-3 & & & & & & \\
\hline $\mathrm{Am}+4$ & & & & & & \\
\hline $\mathrm{Br}-$ & $1.16 \mathrm{E}-13$ & $1.16 \mathrm{E}-13$ & & $3.01 \mathrm{E}-14$ & $2.78 \mathrm{E}-14$ & $4.46 \mathrm{E}-09$ \\
\hline $\mathrm{Ce}+4$ & & & & & & \\
\hline $\mathrm{Eu}+3$ & & & & & & \\
\hline $\mathrm{Gd}+3$ & & & & & & \\
\hline $\mathrm{Ge}+4$ & & & & & & \\
\hline $\ln +3$ & & & & & & \\
\hline $1-$ & $1.59 \mathrm{E}-12$ & $1.59 \mathrm{E}-12$ & & $4.15 \mathrm{E}-13$ & $3.82 \mathrm{E}-13$ & $6.14 \mathrm{E}-08$ \\
\hline $\mathrm{La}+3$ & & & & & & \\
\hline
\end{tabular}


Table A-19. Mass balance, Tank WM-180 waste (continued).

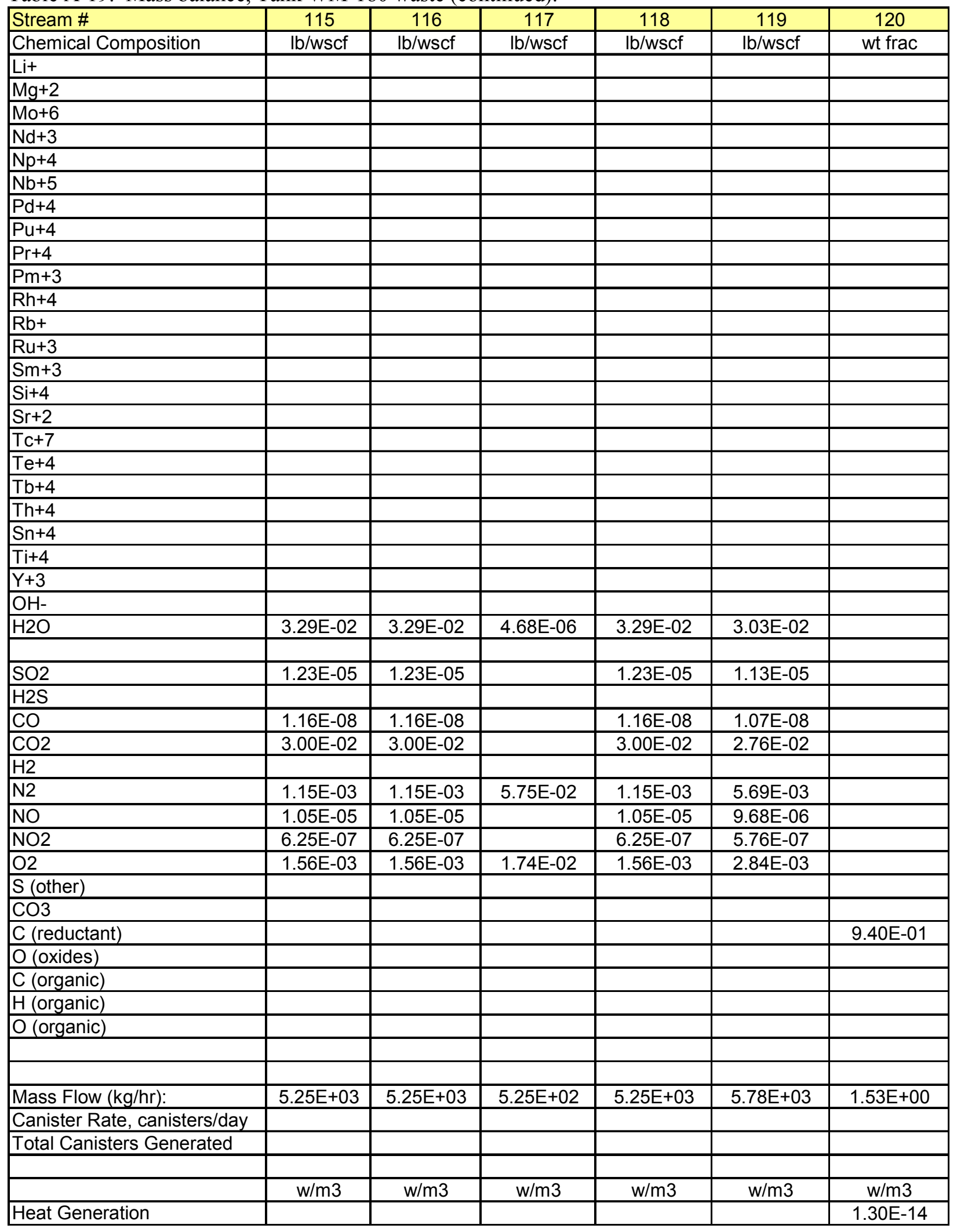


Table A-19. Mass balance, Tank WM-180 waste (continued).

\begin{tabular}{|c|c|c|c|c|c|c|}
\hline Stream \# & 115 & 116 & 117 & 118 & 119 & 120 \\
\hline Radiological Composition & $\mathrm{Ci} / \mathrm{wscm}$ & Ci/wscm & $\mathrm{Ci} / \mathrm{wscm}$ & $\mathrm{Ci} / \mathrm{wscm}$ & $\mathrm{Ci} / \mathrm{wscm}$ & $\mathrm{Ci} / \mathrm{kg}$ \\
\hline $\mathrm{H}-3$ & $1.15 \mathrm{E}-06$ & $1.15 \mathrm{E}-06$ & & $1.15 \mathrm{E}-06$ & $1.05 \mathrm{E}-06$ & \\
\hline C-14 & $4.12 \mathrm{E}-12$ & $4.12 \mathrm{E}-12$ & & $4.12 \mathrm{E}-12$ & $3.79 \mathrm{E}-12$ & $2.89 \mathrm{E}-11$ \\
\hline \multicolumn{7}{|l|}{ Co-60 } \\
\hline \multicolumn{7}{|l|}{ Ni-59 } \\
\hline \multicolumn{7}{|l|}{$\mathrm{Ni}-63$} \\
\hline \multicolumn{7}{|l|}{ Se-79 } \\
\hline \multicolumn{7}{|l|}{ Sr-90 } \\
\hline \multicolumn{7}{|l|}{ Y-90 } \\
\hline \multicolumn{7}{|l|}{ Zr-93 } \\
\hline \multirow{2}{*}{\multicolumn{7}{|c|}{ Nb-93m }} \\
\hline \multirow{2}{*}{\multicolumn{7}{|c|}{$\begin{array}{l}\mathrm{Nb}-94 \\
\mathrm{Tc}-99\end{array}$}} \\
\hline & & & & & & \\
\hline \multicolumn{7}{|l|}{ Ru-106 } \\
\hline \multicolumn{7}{|l|}{ Rh-102 } \\
\hline \multirow{2}{*}{\multicolumn{7}{|c|}{\begin{tabular}{|l|} 
Rh-106 \\
Pd-107 \\
\end{tabular}}} \\
\hline \multirow{2}{*}{\multicolumn{7}{|c|}{\begin{tabular}{|l|}
$\mathrm{Pd}-107$ \\
$\mathrm{Cd}-113 \mathrm{~m}$
\end{tabular}}} \\
\hline & & & & & & \\
\hline \multicolumn{7}{|l|}{ Sn-121m } \\
\hline \multicolumn{7}{|l|}{ Sn-126 } \\
\hline \multirow{2}{*}{\multicolumn{7}{|c|}{$\begin{array}{l}\text { Sb-125 } \\
\text { Sb-126 }\end{array}$}} \\
\hline & & & & & & \\
\hline \multicolumn{7}{|l|}{\begin{tabular}{|l|} 
Sb-126 \\
Te-125m
\end{tabular}} \\
\hline $1-129$ & $3.57 \mathrm{E}-12$ & $3.57 \mathrm{E}-12$ & & $9.32 \mathrm{E}-13$ & $8.59 \mathrm{E}-13$ & $8.61 \mathrm{E}-09$ \\
\hline Cs-134 & & & & & & \\
\hline Cs-135 & & & & & & \\
\hline Cs-137 & & & & & & \\
\hline Ba-137m & & & & & & \\
\hline Ce-144 & & & & & & \\
\hline Pr-144 & & & & & & \\
\hline $\mathrm{Pm}-146$ & & & & & & \\
\hline Pm-147 & & & & & & \\
\hline Sm-151 & & & & & & \\
\hline Eu-152 & & & & & & \\
\hline Eu-154 & & & & & & \\
\hline Eu-155 & & & & & & \\
\hline & & & & & & \\
\hline Th-230 & & & & & & \\
\hline Pa-233 & & & & & & \\
\hline $\mathrm{U}-232$ & & & & & & \\
\hline $\mathrm{U}-233$ & & & & & & \\
\hline U-234 & & & & & & \\
\hline U-235 & & & & & & \\
\hline $\mathrm{U}-236$ & & & & & & \\
\hline $\mathrm{U}-237$ & & & & & & \\
\hline $\mathrm{U}-238$ & & & & & & \\
\hline Np-237 & & & & & & \\
\hline Pu-236 & & & & & & \\
\hline Pu-238 & & & & & & \\
\hline Pu-239 & & & & & & \\
\hline Pu-240 & & & & & & \\
\hline Pu-241 & & & & & & \\
\hline $\mathrm{Pu}-242$ & & & & & & \\
\hline $\mathrm{Pu}-244$ & & & & & & \\
\hline Am-241 & & & & & & \\
\hline $\mathrm{Am}-242 \mathrm{~m}$ & & & & & & \\
\hline
\end{tabular}


Table A-19. Mass balance, Tank WM-180 waste (continued).

\begin{tabular}{|c|c|c|c|c|c|c|}
\hline Stream \# & 115 & 116 & 117 & 118 & 119 & 120 \\
\hline Radiological Composition & Ci/wscm & Ci/wscm & $\mathrm{Ci} / \mathrm{wscm}$ & Ci/wscm & $\mathrm{Ci} / \mathrm{wscm}$ & $\mathrm{Ci} / \mathrm{kg}$ \\
\hline \multicolumn{7}{|l|}{$\mathrm{Am}-243$} \\
\hline \multicolumn{7}{|l|}{$\mathrm{Cm}-242$} \\
\hline \multicolumn{7}{|l|}{$\mathrm{Cm}-243$} \\
\hline \multicolumn{7}{|l|}{$\mathrm{Cm}-244$} \\
\hline \multicolumn{7}{|l|}{ Cm-245 } \\
\hline \multirow{2}{*}{\multicolumn{7}{|c|}{ Cm-246 }} \\
\hline & & & & & & TRU \\
\hline & & & & & & \\
\hline & & & & & & \\
\hline $\begin{array}{l}\text { Gas Stream Bulk } \\
\text { Composition (Wet Basis) }\end{array}$ & $\begin{array}{c}\mathrm{mol} \% \text { or } \\
\mathrm{ppmv}\end{array}$ & $\begin{array}{c}\mathrm{mol} \% \text { or } \\
\mathrm{ppmv}\end{array}$ & $\begin{array}{c}\mathrm{mol} \% \text { or } \\
\mathrm{ppmv}\end{array}$ & $\begin{array}{c}\mathrm{mol} \% \text { or } \\
\mathrm{ppmv}\end{array}$ & $\begin{array}{c}\text { mol\% or } \\
\text { ppmv }\end{array}$ & $\begin{array}{c}\text { mol\% or } \\
\text { ppmv }\end{array}$ \\
\hline $\mathrm{H} 2 \mathrm{O}, \mathrm{mol} \%$ & $70.27 \%$ & $70.27 \%$ & $0.01 \%$ & $70.27 \%$ & $64.61 \%$ & \\
\hline $\mathrm{O} 2, \mathrm{~mol} \%$ & $1.88 \%$ & $1.88 \%$ & $20.99 \%$ & $1.88 \%$ & $3.42 \%$ & \\
\hline $\mathrm{N} 2, \mathrm{~mol} \%$ & $1.58 \%$ & $1.58 \%$ & $79.00 \%$ & $1.58 \%$ & $7.81 \%$ & \\
\hline \multicolumn{7}{|l|}{$\mathrm{H} 2, \mathrm{~mol} \%$} \\
\hline $\mathrm{CO} 2, \mathrm{~mol} \%$ & $26.25 \%$ & $26.25 \%$ & & $26.25 \%$ & $24.13 \%$ & \\
\hline COgas, ppmv & $1.59 \mathrm{E}-01$ & $1.59 \mathrm{E}-01$ & & $1.59 \mathrm{E}-01$ & $1.46 \mathrm{E}-01$ & \\
\hline NO, ppmv & $1.35 \mathrm{E}+02$ & $1.35 \mathrm{E}+02$ & & $1.35 \mathrm{E}+02$ & $1.24 \mathrm{E}+02$ & \\
\hline NO2, ppmv & $5.23 \mathrm{E}+00$ & $5.23 \mathrm{E}+00$ & & $5.23 \mathrm{E}+00$ & $4.81 \mathrm{E}+00$ & \\
\hline SO2, ppmv & $7.38 \mathrm{E}+01$ & $7.38 \mathrm{E}+01$ & & $7.38 \mathrm{E}+01$ & $6.78 \mathrm{E}+01$ & \\
\hline$\overline{\mathrm{Cl}, \mathrm{ppmv}}$ & $1.02 \mathrm{E}-01$ & $1.02 \mathrm{E}-01$ & & $2.67 \mathrm{E}-02$ & $2.46 \mathrm{E}-02$ & \\
\hline$\overline{F, p p m v}$ & $3.54 \mathrm{E}+00$ & $3.54 \mathrm{E}+00$ & & $3.54 \mathrm{E}+00$ & $3.26 \mathrm{E}+00$ & \\
\hline \multicolumn{7}{|l|}{$\mathrm{C}$ (organic), ppmv } \\
\hline \multirow{2}{*}{\multicolumn{7}{|c|}{$\mathrm{H}$ (organic), ppmv }} \\
\hline & & & & & & \\
\hline $\mathrm{Hg}, \mathrm{ug} / \mathrm{wscm}$ & $2.43 \mathrm{E}+04$ & $2.43 \mathrm{E}+04$ & & $2.43 \mathrm{E}+01$ & $2.24 \mathrm{E}+01$ & \\
\hline$\overline{P M}, \mathrm{mg} / \mathrm{wscm}$ & $3.29 \mathrm{E}+01$ & $3.29 \mathrm{E}+01$ & $1.58 \mathrm{E}+01$ & $8.64 \mathrm{E}+00$ & $9.31 \mathrm{E}+00$ & \\
\hline \multicolumn{7}{|l|}{ SVM, ug/wscm } \\
\hline \multicolumn{7}{|l|}{ LVM, ug/wscm } \\
\hline $\begin{array}{l}\text { Gas Stream Bulk } \\
\text { Composition (Dry Basis) }\end{array}$ & $\mathrm{mol} \%$ & $\mathrm{~mol} \%$ & $\mathrm{~mol} \%$ & $\mathrm{~mol} \%$ & $\mathrm{~mol} \%$ & $\mathrm{~mol} \%$ \\
\hline $\mathrm{O} 2, \mathrm{~mol} \%$, dry basis & $6.33 \%$ & $6.33 \%$ & $20.99 \%$ & $0.89 \%$ & $9.66 \%$ & \\
\hline $\mathrm{N} 2, \mathrm{~mol} \%$, dry basis & $5.31 \%$ & $5.31 \%$ & $79.01 \%$ & $5.31 \%$ & $22.08 \%$ & \\
\hline \multicolumn{7}{|l|}{$\mathrm{H} 2, \mathrm{~mol} \%$, dry basis } \\
\hline \multicolumn{7}{|l|}{$\mathrm{CO} 2, \mathrm{~mol} \%$, dry basis } \\
\hline $\begin{array}{l}\text { Gas Stream Bulk } \\
\text { Composition (Dry Basis, } \\
\text { Corrected to } 7 \% \text { O2 with } \\
100 \% \text { O2 Combustion Air) }\end{array}$ & $\begin{array}{c}\mathrm{ppmv} \text {, or } \\
\mathrm{ug} / \mathrm{dscm} \text { or } \\
\mathrm{mg} / \mathrm{dscm}\end{array}$ & $\begin{array}{l}\text { ppmv, or } \\
\text { ug/dscm or } \\
\mathrm{mg} / \mathrm{dscm}\end{array}$ & $\begin{array}{l}\mathrm{ppmv} \text {, or } \\
\mathrm{ug} / \mathrm{dscm} \text { or } \\
\mathrm{mg} / \mathrm{dscm}\end{array}$ & $\begin{array}{l}\text { ppmv, or } \\
\text { ug/dscm or } \\
\mathrm{mg} / \mathrm{dscm}\end{array}$ & $\begin{array}{l}\text { ppmv, or } \\
\text { ug/dscm or } \\
\mathrm{mg} / \mathrm{dscm}\end{array}$ & $\begin{array}{l}\text { ppmv, or } \\
\text { ug/dscm or } \\
\mathrm{mg} / \mathrm{dscm}\end{array}$ \\
\hline COgas, ppmv, dry basis & $7.50 \mathrm{E}-02$ & $7.50 \mathrm{E}-02$ & & $7.50 \mathrm{E}-02$ & $5.79 \mathrm{E}-02$ & \\
\hline NO, ppmv, dry basis & $6.36 \mathrm{E}+01$ & $6.36 \mathrm{E}+01$ & & $6.36 \mathrm{E}+01$ & $4.91 \mathrm{E}+01$ & \\
\hline NO2, ppmv, dry basis & $2.47 \mathrm{E}+00$ & $2.47 \mathrm{E}+00$ & & $2.47 \mathrm{E}+00$ & $1.91 \mathrm{E}+00$ & \\
\hline SO2, ppmv, dry basis & $3.48 \mathrm{E}+01$ & $3.48 \mathrm{E}+01$ & & $3.48 \mathrm{E}+01$ & $2.69 \mathrm{E}+01$ & \\
\hline $\mathrm{Cl}, \mathrm{ppmv}$, dry basis & $4.83 \mathrm{E}-02$ & $4.83 \mathrm{E}-02$ & & $1.26 \mathrm{E}-02$ & $9.73 \mathrm{E}-03$ & \\
\hline F, ppmv, dry basis & $1.67 \mathrm{E}+00$ & $1.67 \mathrm{E}+00$ & & $1.67 \mathrm{E}+00$ & $1.29 \mathrm{E}+00$ & \\
\hline \multicolumn{7}{|l|}{$\mathrm{C}$ (organic), ppmv, dry basis } \\
\hline \multicolumn{7}{|l|}{$\mathrm{H}$ (organic), ppmv, dry basis } \\
\hline $\mathrm{Hg}, \mathrm{ug} / \mathrm{dscm}$ & $1.15 \mathrm{E}+04$ & $1.15 \mathrm{E}+04$ & & $1.15 \mathrm{E}+01$ & $8.88 \mathrm{E}+00$ & \\
\hline $\mathrm{PM}, \mathrm{mg} / \mathrm{dscm}$ & $1.55 \mathrm{E}+01$ & $1.55 \mathrm{E}+01$ & $2.21 \mathrm{E}+00$ & $4.07 \mathrm{E}+00$ & $3.69 \mathrm{E}+00$ & \\
\hline \multicolumn{7}{|l|}{ SVM, ug/dscm } \\
\hline LVM, ug/dscm & & & & & & \\
\hline
\end{tabular}


Table A-19. Mass balance, Tank WM-180 waste (continued).

\begin{tabular}{|c|c|c|c|c|c|c|c|}
\hline \begin{tabular}{|l} 
PFD Number \\
\end{tabular} & PFD-3 & PFD-3 & PFD-3 & PFD-3 & PFD-3 & PFD-3 & PFD-2 \\
\hline Stream Number & 203 & 204 & 205 & 206 & 207 & 301 & 303 \\
\hline Stream Name & $\begin{array}{l}\text { Fluidizing } \\
\text { Gas to } \\
\text { Reformer }\end{array}$ & $\begin{array}{l}\text { Propane to } \\
\text { Oxidizer }\end{array}$ & $\begin{array}{l}\text { Water to } \\
\text { Spray } \\
\text { Quench }\end{array}$ & $\begin{array}{l}\text { ANN to } \\
\text { Scrub for } F \\
\text { Adjust }\end{array}$ & $\begin{array}{l}\text { HNO3 to } \\
\text { Scrub for } \\
\mathrm{H}+\text { Adjust }\end{array}$ & Isopropanol & Bed Media \\
\hline Rate or Volume & $1.11 \mathrm{E}+04$ & $9.05 \mathrm{E}+02$ & $4.64 \mathrm{E}+02$ & $3.66 \mathrm{E}-02$ & $-6.17 \mathrm{E}-03$ & $7.77 \mathrm{E}+01$ & $1.15 \mathrm{E}+00$ \\
\hline Volume Flow (standard, wet) & $1.09 \mathrm{E}+04$ & $2.56 \mathrm{E}+03$ & & & & & \\
\hline Volume Flow (standard, dry) $)^{*}$ & $1.09 \mathrm{E}+04$ & $2.56 \mathrm{E}+03$ & & & & & \\
\hline Rate Units & $\mathrm{ft} 3 / \mathrm{hr}$ & $\mathrm{ft} 3 / \mathrm{hr}$ & gal/hr & gal/hr & $\mathrm{gal} / \mathrm{hr}$ & $\mathrm{gal} / \mathrm{hr}$ & $\mathrm{Ib} / \mathrm{hr}$ \\
\hline Rate or Volume, metric & $3.10 \mathrm{E}+02$ & $7.26 \mathrm{E}+01$ & $1.76 \mathrm{E}+03$ & $1.39 \mathrm{E}-01$ & $-2.34 \mathrm{E}-02$ & $2.94 \mathrm{E}+02$ & $5.22 \mathrm{E}-01$ \\
\hline Rate Units & wscm/hr & wscm/hr & $\mathrm{L} / \mathrm{hr}$ & $\mathrm{L} / \mathrm{hr}$ & $\mathrm{L} / \mathrm{hr}$ & $\mathrm{L} / \mathrm{hr}$ & $\mathrm{kg} / \mathrm{hr}$ \\
\hline Temperature, ${ }^{\circ} \mathrm{C}$ & 580 & 25 & 25 & 25 & 25 & 15 & 15 \\
\hline Temperature, ${ }^{\circ} \mathrm{F}$ & 1076 & 77 & 77 & 77 & 77 & 59 & 59 \\
\hline Pressure, psia & 42.30 & 42.30 & 112.30 & 14.70 & 42.30 & 14.70 & 14.70 \\
\hline Specific Gravity & $1.81 \mathrm{E}-03$ & $5.19 \mathrm{E}-03$ & $9.93 \mathrm{E}-01$ & $1.80 \mathrm{E}+00$ & $1.23 \mathrm{E}+00$ & $1.03 \mathrm{E}+00$ & $1.58 \mathrm{E}+00$ \\
\hline Chemical Composition & Mol \% & lb/wscf & Mol/liter & Mol/liter & Mol/liter & Mol/liter & Wt frac \\
\hline $\mathrm{H}+$ & & & $9.95 \mathrm{E}-08$ & & $1.33 \mathrm{E}+01$ & & \\
\hline $\mathrm{Al}+3$ & & & & $2.20 \mathrm{E}+00$ & & & 0.529 \\
\hline \multicolumn{8}{|l|}{$\mathrm{Sb}+5$} \\
\hline \multirow{2}{*}{\multicolumn{8}{|c|}{$\frac{\text { Ast3 }}{\mathrm{Ba}+2}$}} \\
\hline & & & & & & & \\
\hline \multicolumn{8}{|l|}{$\mathrm{Be}+2$} \\
\hline \multicolumn{8}{|l|}{$B+3$} \\
\hline \multirow{2}{*}{\multicolumn{8}{|c|}{\begin{tabular}{|l}
$\mathrm{Cd}+2$ \\
$\mathrm{Ca}+2$
\end{tabular}}} \\
\hline & & & & & & & \\
\hline \multicolumn{8}{|l|}{$\frac{\mathrm{Ca}+2}{\mathrm{Cr}+3}$} \\
\hline \multicolumn{8}{|l|}{$\mathrm{Co}+2$} \\
\hline \multirow{2}{*}{\multicolumn{8}{|c|}{$\begin{array}{l}\mathrm{Cs}+ \\
\mathrm{Cu}+2\end{array}$}} \\
\hline & & & & & & & \\
\hline \multicolumn{8}{|l|}{$\frac{\mathrm{Cu}+2}{\mathrm{Fe}+3}$} \\
\hline \multicolumn{8}{|l|}{$\mathrm{Pb}+2$} \\
\hline \multirow{2}{*}{\multicolumn{8}{|c|}{$\frac{\mathrm{Hg}+2}{\mathrm{Mn}+4}$}} \\
\hline & & & & & & & \\
\hline \multicolumn{8}{|l|}{\begin{tabular}{|l|}
$\mathrm{Mn}+4$ \\
$\mathrm{Ni}+2$ \\
\end{tabular}} \\
\hline \multicolumn{8}{|l|}{$\mathrm{K}+$} \\
\hline \multirow{2}{*}{\multicolumn{8}{|c|}{$\mathrm{Se}+4$}} \\
\hline \multirow{2}{*}{\multicolumn{8}{|c|}{\begin{tabular}{|l|}
$\mathrm{Ag}+$ \\
$\mathrm{Na}+$
\end{tabular}}} \\
\hline & & & & & & & \\
\hline \multicolumn{8}{|l|}{$\frac{\mid l v a r}{T+1+3}$} \\
\hline \multicolumn{8}{|l|}{$\mathrm{U}+4$} \\
\hline \multirow{2}{*}{\multicolumn{8}{|c|}{\begin{tabular}{|l|}
$V+3$ \\
$7 n+2$
\end{tabular}}} \\
\hline \multirow{2}{*}{\multicolumn{8}{|c|}{$\frac{Z n+2}{Z r+4}$}} \\
\hline & & & & & & & \\
\hline $\mathrm{Cl}-$ & & & & & & & \\
\hline F- & & & & & & & \\
\hline SO4-2 & & & & & & & \\
\hline \begin{tabular}{|l|} 
NO3- \\
\end{tabular} & & & & $6.60 \mathrm{E}+00$ & $1.33 \mathrm{E}+01$ & & \\
\hline $\mathrm{CO} 2$ & 100 & & & & & & \\
\hline $\mathrm{OH}-$ & & & $9.95 \mathrm{E}-08$ & $7.31 \mathrm{E}-08$ & & & \\
\hline $\mathrm{H} 2 \mathrm{O}$ & & & $5.51 \mathrm{E}+01$ & $7.41 \mathrm{E}+01$ & $2.17 \mathrm{E}+01$ & & \\
\hline $\mathrm{O}$ (oxides) & & & & & & & 0.471 \\
\hline C (organic) & & $9.35 \mathrm{E}-02$ & & & & $5.15 \mathrm{E}+01$ & \\
\hline $\mathrm{H}$ (organic) & & $2.09 \mathrm{E}-02$ & & & & $1.37 \mathrm{E}+02$ & \\
\hline $\mathrm{O}$ (organic) & & & & & & $1.72 \mathrm{E}+01$ & \\
\hline Mass Flow (kg/hr): & $5.66 \mathrm{E}+02$ & $1.33 \mathrm{E}+02$ & $1.74 \mathrm{E}+03$ & $2.50 \mathrm{E}-01$ & & $3.03 \mathrm{E}+02$ & $5.22 \mathrm{E}-01$ \\
\hline
\end{tabular}


Table A-19. Mass balance, Tank WM-180 waste (continued).

\begin{tabular}{|c|c|c|c|c|c|c|c|}
\hline Stream Number & 305 & 401 & 402 & 404 & 404 & 503 & 505 \\
\hline Stream Name & $\begin{array}{l}\text { Grout Mix } \\
\text { for Scrub } \\
\text { Blowdown }\end{array}$ & $\begin{array}{c}\text { Scrub } \\
\text { Recycled to } \\
\text { Feed }\end{array}$ & $\begin{array}{c}\text { Scrub } \\
\text { Blowdown } \\
\text { to Grout } \\
\text { Mixer }\end{array}$ & $\begin{array}{c}\text { MLLW } \\
\text { Grout from } \\
\text { Scrub }\end{array}$ & $\begin{array}{l}\text { MLLW } \\
\text { Grout } \\
\text { Drums }\end{array}$ & $\begin{array}{c}\text { Feed } \\
\text { Atomizing } \\
\text { Gas }\end{array}$ & $\begin{array}{l}\text { Oxygen to } \\
\text { Oxidizer }\end{array}$ \\
\hline Rate or Volume & $6.61 \mathrm{E}+00$ & $5.34 \mathrm{E}+00$ & $2.59 \mathrm{E}-01$ & $8.82 \mathrm{E}+00$ & $2.29 \mathrm{E}-01$ & $5.30 \mathrm{E}+03$ & $5.44 \mathrm{E}+03$ \\
\hline Volume Flow (standard, wet) ${ }^{*}$ & & & & & & $1.52 \mathrm{E}+04$ & $3.36 \mathrm{E}+04$ \\
\hline Volume Flow (standard, dry) & & & & & & $1.52 \mathrm{E}+04$ & $3.36 \mathrm{E}+04$ \\
\hline Rate Units & $\mathrm{lb} / \mathrm{hr}$ & $\mathrm{gal} / \mathrm{hr}$ & $\mathrm{gal} / \mathrm{hr}$ & $\mathrm{lb} / \mathrm{hr}$ & Drums/day & $\mathrm{ft} 3 / \mathrm{hr}$ & $\mathrm{ft} 3 / \mathrm{hr}$ \\
\hline Rate or Volume, metric & $3.00 \mathrm{E}+00$ & $2.02 \mathrm{E}+01$ & $9.81 \mathrm{E}-01$ & $4.00 \mathrm{E}+00$ & $4.00 \mathrm{E}+00$ & $4.30 \mathrm{E}+02$ & $9.52 \mathrm{E}+02$ \\
\hline Rate Units & $\mathrm{kg} / \mathrm{hr}$ & $\mathrm{L} / \mathrm{hr}$ & $\mathrm{L} / \mathrm{hr}$ & $\mathrm{kg} / \mathrm{hr}$ & $\mathrm{kg} / \mathrm{hr}$ & wscm $/ \mathrm{hr}$ & wscm/hr \\
\hline Temperature, ${ }^{\circ} \mathrm{C}$ & 25 & 70 & 70 & 54 & 54 & 21 & 25 \\
\hline Temperature, ${ }^{\circ} \mathrm{F}$ & 77 & 158 & 158 & 129 & 129 & 70 & 77 \\
\hline Pressure, psia & 14.70 & 29.98 & 29.98 & 14.70 & 14.70 & 42.30 & 92.30 \\
\hline Specific Gravity & $2.01 \mathrm{E}-01$ & $1.02 \mathrm{E}+00$ & $1.02 \mathrm{E}+00$ & $2.10 \mathrm{E}+00$ & $2.10 \mathrm{E}+00$ & $5.25 \mathrm{E}-03$ & $8.21 \mathrm{E}-03$ \\
\hline Chemical Composition & Wt frac & Mol/liter & Mol/liter & Wt frac & Wt frac & $\mathrm{lb} / \mathrm{wscf}$ & Ib/wscf \\
\hline $\mathrm{H}+$ & & $9.72 \mathrm{E}-01$ & $9.72 \mathrm{E}-01$ & $2.40 \mathrm{E}-04$ & $2.40 \mathrm{E}-04$ & & \\
\hline $\mathrm{Al}+3$ & & $1.08 \mathrm{E}-02$ & $1.08 \mathrm{E}-02$ & $0.01 \%$ & $0.01 \%$ & & \\
\hline$\overline{S b+5}$ & & $2.46 \mathrm{E}-08$ & $2.46 \mathrm{E}-08$ & $7.34 \mathrm{E}-10$ & $7.34 \mathrm{E}-10$ & & \\
\hline $\mathrm{As}+3$ & & $7.90 \mathrm{E}-06$ & $7.90 \mathrm{E}-06$ & $1.45 \mathrm{E}-07$ & $1.45 \mathrm{E}-07$ & & \\
\hline \multicolumn{8}{|l|}{$\mathrm{Ba}+2$} \\
\hline \multicolumn{8}{|l|}{$\mathrm{Be}+2$} \\
\hline $\mathrm{B}+3$ & & 1.84E-04 & 1.84E-04 & $4.87 \mathrm{E}-07$ & $4.87 \mathrm{E}-07$ & & \\
\hline \multicolumn{8}{|l|}{$C d+2$} \\
\hline \multicolumn{8}{|l|}{$\mathrm{Ca}+2$} \\
\hline \multicolumn{8}{|l|}{$\mathrm{Cr}+3$} \\
\hline $\mathrm{Co}+2$ & & $3.23 \mathrm{E}-07$ & $3.23 \mathrm{E}-07$ & $4.67 \mathrm{E}-09$ & $4.67 \mathrm{E}-09$ & & \\
\hline \multicolumn{8}{|l|}{$\mathrm{Cs}^{+}$} \\
\hline \multicolumn{8}{|l|}{$\mathrm{Cu}+2$} \\
\hline $\mathrm{Fe}+3$ & & $7.26 \mathrm{E}-05$ & $7.26 \mathrm{E}-05$ & $9.95 \mathrm{E}-07$ & $9.95 \mathrm{E}-07$ & & \\
\hline \multicolumn{8}{|l|}{$\mathrm{Pb}+2$} \\
\hline $\mathrm{Hg}+2$ & & $3.30 \mathrm{E}-02$ & $3.30 \mathrm{E}-02$ & $1.62 \mathrm{E}-03$ & $1.62 \mathrm{E}-03$ & & \\
\hline $\mathrm{Mn}+4$ & & $2.25 \mathrm{E}-04$ & $2.25 \mathrm{E}-04$ & $3.03 \mathrm{E}-06$ & $3.03 \mathrm{E}-06$ & & \\
\hline \multirow{2}{*}{\multicolumn{8}{|c|}{$\mathrm{Ni}+2$}} \\
\hline \multirow{2}{*}{\multicolumn{8}{|c|}{\begin{tabular}{|l|}
$\mathrm{K}+$ \\
$\mathrm{Se}+4$
\end{tabular}}} \\
\hline & & & & & & & \\
\hline \multirow{2}{*}{\multicolumn{8}{|c|}{\begin{tabular}{|l}
$\mathrm{Ag}+$ \\
$\mathrm{Na}+$
\end{tabular}}} \\
\hline & & & & & & & \\
\hline \multicolumn{8}{|c|}{$2.43 \mathrm{E}-08$} \\
\hline \multicolumn{8}{|l|}{$\frac{U+4}{V+3}$} \\
\hline $\mathrm{V+3}$ & & & & & & & \\
\hline \multicolumn{8}{|l|}{$\mathrm{Zn+2}$} \\
\hline \multicolumn{8}{|l|}{$\mathrm{Zr}+4$} \\
\hline Cl- & & $5.43 \mathrm{E}-04$ & $5.43 \mathrm{E}-04$ & $4.72 \mathrm{E}-06$ & $4.72 \mathrm{E}-06$ & & \\
\hline $\mathrm{F}-$ & & $5.99 \mathrm{E}-03$ & $5.99 \mathrm{E}-03$ & $2.79 \mathrm{E}-05$ & $2.79 \mathrm{E}-05$ & & \\
\hline \multicolumn{8}{|l|}{ SO4-2 } \\
\hline NO3- & & $8.77 \mathrm{E}-01$ & $8.77 \mathrm{E}-01$ & $1.33 \mathrm{E}-02$ & $1.33 \mathrm{E}-02$ & & \\
\hline \multicolumn{8}{|l|}{ PO4-3 } \\
\hline$\overline{A m+4}$ & & $5.58 \mathrm{E}-10$ & $5.58 \mathrm{E}-10$ & $3.33 \mathrm{E}-11$ & $3.33 \mathrm{E}-11$ & & \\
\hline $\mathrm{Br}-$ & & $2.95 \mathrm{E}-09$ & $2.95 \mathrm{E}-09$ & $5.79 \mathrm{E}-11$ & $5.79 \mathrm{E}-11$ & & \\
\hline $\mathrm{Ce}+4$ & & $7.70 \mathrm{E}-07$ & $7.70 \mathrm{E}-07$ & $2.64 \mathrm{E}-08$ & $2.64 \mathrm{E}-08$ & & \\
\hline $\mathrm{Eu+3}$ & & $4.55 \mathrm{E}-09$ & $4.55 \mathrm{E}-09$ & $1.70 \mathrm{E}-10$ & $1.70 \mathrm{E}-10$ & & \\
\hline $\mathrm{Gd+3}$ & & $2.84 \mathrm{E}-06$ & $2.84 \mathrm{E}-06$ & $1.09 \mathrm{E}-07$ & $1.09 \mathrm{E}-07$ & & \\
\hline $\mathrm{Ge}+4$ & & & & & & & \\
\hline $\ln +3$ & & $1.25 \mathrm{E}-08$ & $1.25 \mathrm{E}-08$ & $3.51 \mathrm{E}-10$ & $3.51 \mathrm{E}-10$ & & \\
\hline $1-$ & & $2.56 \mathrm{E}-08$ & $2.56 \mathrm{E}-08$ & $7.97 \mathrm{E}-10$ & $7.97 \mathrm{E}-10$ & & \\
\hline $\mathrm{La}+3$ & & $8.27 \mathrm{E}-08$ & $8.27 \mathrm{E}-08$ & $2.82 \mathrm{E}-09$ & $2.82 \mathrm{E}-09$ & & \\
\hline
\end{tabular}


Table A-19. Mass balance, Tank WM-180 waste (continued).

\begin{tabular}{|c|c|c|c|c|c|c|c|}
\hline Stream \# & 305 & 401 & 402 & 404 & 404 & 503 & 505 \\
\hline Chemical Composition & Wt frac & Mol/liter & Mol/liter & Wt frac & Wt frac & $\mathrm{Ib} / \mathrm{wscf}$ & $\mathrm{lb} / \mathrm{wscf}$ \\
\hline \multicolumn{8}{|l|}{$\mathrm{Li}+$} \\
\hline \multicolumn{8}{|l|}{$\mathrm{Mg}+2$} \\
\hline \multicolumn{8}{|l|}{$\mathrm{Mo}+6$} \\
\hline $\mathrm{Nd}+3$ & & $2.67 \mathrm{E}-07$ & $2.67 \mathrm{E}-07$ & $9.43 \mathrm{E}-09$ & $9.43 \mathrm{E}-09$ & & \\
\hline $\mathrm{Np}+4$ & & $1.54 \mathrm{E}-07$ & $1.54 \mathrm{E}-07$ & $8.96 \mathrm{E}-09$ & $8.96 \mathrm{E}-09$ & & \\
\hline \multicolumn{8}{|l|}{$\mathrm{Nb}+5$} \\
\hline \multicolumn{8}{|l|}{$\mathrm{Pd}+4$} \\
\hline \multicolumn{8}{|l|}{$\mathrm{Pu}+4$} \\
\hline $\mathrm{Pr}+4$ & & $7.52 \mathrm{E}-08$ & $7.52 \mathrm{E}-08$ & $2.60 \mathrm{E}-09$ & $2.60 \mathrm{E}-09$ & & \\
\hline $\mathrm{Pm}+3$ & & $1.14 \mathrm{E}-11$ & $1.14 \mathrm{E}-11$ & $4.05 \mathrm{E}-13$ & $4.05 \mathrm{E}-13$ & & \\
\hline $\mathrm{Rh}+4$ & & $3.24 \mathrm{E}-08$ & $3.24 \mathrm{E}-08$ & $8.19 \mathrm{E}-10$ & $8.19 \mathrm{E}-10$ & & \\
\hline \multicolumn{8}{|l|}{$\mathrm{Rb}+$} \\
\hline $\mathrm{Ru}+3$ & & $2.23 \mathrm{E}-06$ & $2.23 \mathrm{E}-06$ & $5.52 \mathrm{E}-08$ & $5.52 \mathrm{E}-08$ & & \\
\hline $\mathrm{Sm}+3$ & & $4.95 \mathrm{E}-08$ & $4.95 \mathrm{E}-08$ & $1.82 \mathrm{E}-09$ & $1.82 \mathrm{E}-09$ & & \\
\hline$S i+4$ & $4.67 \mathrm{E}-01$ & & & $3.51 \mathrm{E}-01$ & $3.51 \mathrm{E}-01$ & & \\
\hline \multicolumn{8}{|l|}{$\mathrm{Sr}+2$} \\
\hline $\mathrm{Tc}+7$ & & $8.21 \mathrm{E}-08$ & $8.21 \mathrm{E}-08$ & $1.97 \mathrm{E}-09$ & 1.97E-09 & & \\
\hline \multicolumn{8}{|l|}{$\mathrm{Te}+4$} \\
\hline $\mathrm{Tb}+4$ & & $1.90 \mathrm{E}-11$ & $1.90 \mathrm{E}-11$ & $7.42 \mathrm{E}-13$ & $7.42 \mathrm{E}-13$ & & \\
\hline \multicolumn{8}{|l|}{$\mathrm{Th}+4$} \\
\hline \multicolumn{8}{|l|}{$S n+4$} \\
\hline \multicolumn{8}{|l|}{$\mathrm{Ti}+4$} \\
\hline$Y+3$ & & $6.17 \mathrm{E}-08$ & $6.17 \mathrm{E}-08$ & 1.34E-09 & 1.34E-09 & & \\
\hline $\mathrm{OH}-$ & & $1.21 \mathrm{E}-06$ & $1.21 \mathrm{E}-06$ & $5.04 \mathrm{E}-09$ & $5.04 \mathrm{E}-09$ & & \\
\hline \multirow[t]{2}{*}{$\mathrm{H} 2 \mathrm{O}$} & & $5.31 \mathrm{E}+01$ & $5.31 \mathrm{E}+01$ & $2.35 \mathrm{E}-01$ & $2.35 \mathrm{E}-01$ & & \\
\hline & & 761507 & 761507 & $120 \mathrm{~F} 08$ & $120 \mathrm{~F}_{-08}$ & & \\
\hline \\
\hline $\mathrm{CO}$ & & $2.73 \mathrm{E}-09$ & $2.73 \mathrm{E}-09$ & $1.87 \mathrm{E}-11$ & $1.87 \mathrm{E}-11$ & & \\
\hline $\mathrm{CO} 2$ & & $2.08 \mathrm{E}-04$ & $2.08 \mathrm{E}-04$ & $2.24 \mathrm{E}-06$ & $2.24 \mathrm{E}-06$ & $1.14 \mathrm{E}-01$ & \\
\hline $\mathrm{H} 2$ & & $1.75 \mathrm{E}-08$ & $1.75 \mathrm{E}-08$ & $8.65 \mathrm{E}-12$ & $8.65 \mathrm{E}-12$ & & \\
\hline N2 & & $6.38 \mathrm{E}-09$ & $6.38 \mathrm{E}-09$ & $4.38 \mathrm{E}-11$ & $4.38 \mathrm{E}-11$ & & \\
\hline $\mathrm{NO}$ & & $8.82 \mathrm{E}-08$ & $8.82 \mathrm{E}-08$ & $6.49 \mathrm{E}-10$ & $6.49 \mathrm{E}-10$ & & \\
\hline NO2 & & $1.34 \mathrm{E}-05$ & $1.34 \mathrm{E}-05$ & $1.51 \mathrm{E}-07$ & $1.51 \mathrm{E}-07$ & & \\
\hline $\mathrm{O} 2$ & & $2.20 \mathrm{E}-08$ & $2.20 \mathrm{E}-08$ & $1.72 \mathrm{E}-10$ & $1.72 \mathrm{E}-10$ & & $8.30 \mathrm{E}-02$ \\
\hline S (other) & & $1.08 \mathrm{E}-08$ & $1.08 \mathrm{E}-08$ & $8.52 \mathrm{E}-11$ & $8.52 \mathrm{E}-11$ & & \\
\hline \multicolumn{8}{|l|}{$\mathrm{CO} 3$} \\
\hline \multicolumn{8}{|l|}{ C (reductant) } \\
\hline $\mathrm{O}$ (oxides) & $53.26 \%$ & 1.09E-04 & $1.09 \mathrm{E}-04$ & $39.94 \%$ & $39.94 \%$ & & \\
\hline \multicolumn{8}{|l|}{ C (organic) } \\
\hline \multicolumn{8}{|l|}{$\mathrm{H}$ (organic) } \\
\hline \multicolumn{8}{|l|}{$\mathrm{O}$ (organic) } \\
\hline & & & & & & & \\
\hline Mass Flow (kg/hr): & $3.00 \mathrm{E}+00$ & $2.06 \mathrm{E}+01$ & $1.00 \mathrm{E}+00$ & $4.00 \mathrm{E}+00$ & $4.00 \mathrm{E}+00$ & $7.87 \mathrm{E}+02$ & $1.27 \mathrm{E}+03$ \\
\hline Waste Rate, drums/day & & & & & 0.23 & & \\
\hline Total drums Generated & & & & & 31 & & \\
\hline & & & & & & & \\
\hline \begin{tabular}{|l} 
Heat Generation \\
Heat Generation
\end{tabular} & $\mathrm{w} / \mathrm{m} 3$ & $\frac{\mathrm{w} / \mathrm{m} 3}{1.95 \mathrm{E}-03}$ & $\frac{\mathrm{w} / \mathrm{m} 3}{1.95 \mathrm{E}-03}$ & $\frac{\mathrm{w} / \mathrm{m} 3}{1.00 \mathrm{E}-03}$ & $\frac{\text { w/Drum }}{2.01 \mathrm{E}-04}$ & $\mathrm{w} / \mathrm{m} 3$ & $\mathrm{w} / \mathrm{m} 3$ \\
\hline
\end{tabular}


Table A-19. Mass balance, Tank WM-180 waste (continued).

\begin{tabular}{|c|c|c|c|c|c|c|c|}
\hline Stream \# & 305 & 401 & 402 & 404 & 404 & 503 & 505 \\
\hline Radiological Composition & $\mathrm{Ci} / \mathrm{kg}$ & $\mathrm{Ci} / \mathrm{L}$ & $\mathrm{Ci} / \mathrm{L}$ & $\mathrm{Ci} / \mathrm{kg}$ & Ci/Drum & $\mathrm{Ci} / \mathrm{wscm}$ & $\mathrm{Ci} / \mathrm{wscm}$ \\
\hline \multicolumn{8}{|l|}{$\mathrm{H}-3$} \\
\hline C-14 & & $7.84 \mathrm{E}-17$ & $7.84 \mathrm{E}-17$ & $1.92 \mathrm{E}-17$ & $8.08 \mathrm{E}-15$ & & \\
\hline Co-60 & & $7.01 \mathrm{E}-08$ & $7.01 \mathrm{E}-08$ & $1.72 \mathrm{E}-08$ & $7.22 \mathrm{E}-06$ & & \\
\hline \multicolumn{8}{|l|}{ Ni-59 } \\
\hline \multicolumn{8}{|l|}{$\mathrm{Ni}-63$} \\
\hline \multirow{2}{*}{\multicolumn{8}{|c|}{\begin{tabular}{|l|} 
Se-79 \\
Sr-90
\end{tabular}}} \\
\hline & & & & & \multicolumn{3}{|c|}{ Sr-90 } \\
\hline $\mathrm{Y}-90$ & & $3.44 \mathrm{E}-04$ & $3.44 \mathrm{E}-04$ & $8.43 \mathrm{E}-05$ & $3.54 \mathrm{E}-02$ & & \\
\hline \multicolumn{8}{|l|}{ Zr-93 } \\
\hline \multicolumn{8}{|l|}{$\mathrm{Nb}-93 \mathrm{~m}$} \\
\hline \multicolumn{8}{|l|}{$\mathrm{Nb}-94$} \\
\hline Tc-99 & & 1.40E-07 & $1.40 \mathrm{E}-07$ & $3.42 \mathrm{E}-08$ & $1.44 \mathrm{E}-05$ & & \\
\hline Ru-106 & & $8.48 \mathrm{E}-09$ & $8.48 \mathrm{E}-09$ & $2.08 \mathrm{E}-09$ & $8.73 \mathrm{E}-07$ & & \\
\hline Rh-102 & & $7.49 \mathrm{E}-12$ & $7.49 \mathrm{E}-12$ & $1.84 \mathrm{E}-12$ & $7.71 \mathrm{E}-10$ & & \\
\hline Rh-106 & & $8.48 \mathrm{E}-09$ & $8.48 \mathrm{E}-09$ & $2.08 \mathrm{E}-09$ & $8.73 \mathrm{E}-07$ & & \\
\hline \multicolumn{8}{|l|}{$P d-107$} \\
\hline \multicolumn{8}{|l|}{ Cd-113m } \\
\hline \multirow{2}{*}{\multicolumn{8}{|c|}{\begin{tabular}{|l|} 
Sn-121m \\
Sn-126
\end{tabular}}} \\
\hline & & & & & & & \\
\hline Sb-125 & & $1.39 \mathrm{E}-07$ & $1.39 \mathrm{E}-07$ & $3.42 \mathrm{E}-08$ & $1.44 \mathrm{E}-05$ & & \\
\hline Sb-126 & & $5.00 \mathrm{E}-10$ & $5.00 \mathrm{E}-10$ & $1.23 \mathrm{E}-10$ & $5.15 \mathrm{E}-08$ & & \\
\hline \multicolumn{8}{|l|}{ Te-125m } \\
\hline $\mid-129$ & & $4.56 \mathrm{E}-10$ & $4.56 \mathrm{E}-10$ & $1.12 \mathrm{E}-10$ & $4.69 \mathrm{E}-08$ & & \\
\hline \multicolumn{8}{|l|}{ Cs-134 } \\
\hline \multicolumn{8}{|l|}{ Cs-135 } \\
\hline \multirow{2}{*}{\multicolumn{8}{|c|}{\begin{tabular}{|l|} 
Cs-137 \\
Ba-137m
\end{tabular}}} \\
\hline & & & & & & \multicolumn{2}{|c|}{ Ba-137m } \\
\hline \begin{tabular}{|l|l} 
Ce-144 \\
\end{tabular} & & $5.72 \mathrm{E}-09$ & $5.72 \mathrm{E}-09$ & $1.40 \mathrm{E}-09$ & $5.89 \mathrm{E}-07$ & & \\
\hline Pr-144 & & $5.72 \mathrm{E}-09$ & $5.72 \mathrm{E}-09$ & $1.40 \mathrm{E}-09$ & $5.89 \mathrm{E}-07$ & & \\
\hline $\mathrm{Pm}-146$ & & $4.42 \mathrm{E}-10$ & $4.42 \mathrm{E}-10$ & $1.08 \mathrm{E}-10$ & $4.55 \mathrm{E}-08$ & & \\
\hline Pm-147 & & $1.55 \mathrm{E}-06$ & $1.55 \mathrm{E}-06$ & $3.80 \mathrm{E}-07$ & $1.60 \mathrm{E}-04$ & & \\
\hline Sm-151 & & $3.06 \mathrm{E}-06$ & $3.06 \mathrm{E}-06$ & $7.50 \mathrm{E}-07$ & $3.15 \mathrm{E}-04$ & & \\
\hline Eu-152 & & $2.21 \mathrm{E}-08$ & $2.21 \mathrm{E}-08$ & $5.43 \mathrm{E}-09$ & $2.28 \mathrm{E}-06$ & & \\
\hline Eu-154 & & $7.95 \mathrm{E}-07$ & $7.95 \mathrm{E}-07$ & $1.95 \mathrm{E}-07$ & $8.18 \mathrm{E}-05$ & & \\
\hline Eu-155 & & $1.45 \mathrm{E}-06$ & $1.45 \mathrm{E}-06$ & $3.54 \mathrm{E}-07$ & $1.49 \mathrm{E}-04$ & & \\
\hline Th-230 & & & & & & & \\
\hline $\mathrm{Pa}-233$ & & & & & & & \\
\hline $\mathrm{U}-232$ & & & & & & & \\
\hline $\mathrm{U}-233$ & & & & & & & \\
\hline U-234 & & & & & & & \\
\hline U-235 & & & & & & & \\
\hline U-236 & & & & & & & \\
\hline U-237 & & & & & & & \\
\hline U-238 & & & & & & & \\
\hline Np-237 & & $2.58 \mathrm{E}-08$ & $2.58 \mathrm{E}-08$ & $6.32 \mathrm{E}-09$ & $2.65 \mathrm{E}-06$ & & \\
\hline Pu-236 & & & & & & & \\
\hline Pu-238 & & & & & & & \\
\hline Pu-239 & & & & & & & \\
\hline Pu-240 & & & & & & & \\
\hline Pu-241 & & & & & & & \\
\hline Pu-242 & & & & & & & \\
\hline Pu-244 & & & & & & & \\
\hline Am-241 & & $1.21 \mathrm{E}-06$ & $1.21 \mathrm{E}-06$ & $2.97 \mathrm{E}-07$ & $1.25 \mathrm{E}-04$ & & \\
\hline Am-242m & & $1.32 \mathrm{E}-10$ & $1.32 \mathrm{E}-10$ & $3.24 \mathrm{E}-11$ & $1.36 \mathrm{E}-08$ & & \\
\hline
\end{tabular}


Table A-19. Mass balance, Tank WM-180 waste (continued).

\begin{tabular}{|c|c|c|c|c|c|c|c|}
\hline Stream \# & 305 & 401 & 402 & 404 & 404 & 503 & 505 \\
\hline Radiological Composition & $\mathrm{Ci} / \mathrm{kg}$ & $\mathrm{Ci} / \mathrm{L}$ & $\mathrm{Ci} / \mathrm{L}$ & $\mathrm{Ci} / \mathrm{kg}$ & Ci/Drum & $\mathrm{Ci} / \mathrm{wscm}$ & Ci/wscm \\
\hline Am-243 & & $1.92 \mathrm{E}-10$ & $1.92 \mathrm{E}-10$ & $4.70 \mathrm{E}-11$ & $1.98 \mathrm{E}-08$ & & \\
\hline \multicolumn{8}{|l|}{$\mathrm{Cm}-242$} \\
\hline \multicolumn{8}{|l|}{$\mathrm{Cm}-243$} \\
\hline \multicolumn{8}{|l|}{$\mathrm{Cm}-244$} \\
\hline \multicolumn{8}{|l|}{ Cm-245 } \\
\hline \multicolumn{8}{|l|}{$\mathrm{Cm}-246$} \\
\hline TRU & & $1.24 \mathrm{E}-06$ & $1.24 \mathrm{E}-06$ & 3.03E-07 & $1.27 \mathrm{E}-04$ & & \\
\hline & & & & & & & \\
\hline & & & & & & & \\
\hline $\begin{array}{l}\text { Gas Stream Bulk } \\
\text { Composition (Wet Basis) }\end{array}$ & $\begin{array}{l}\text { mol\% or } \\
\text { ppmv }\end{array}$ & $\begin{array}{l}\text { mol\% or } \\
\text { ppmv }\end{array}$ & $\begin{array}{l}\text { mol\% or } \\
\text { ppmv }\end{array}$ & $\begin{array}{l}\text { mol\% or } \\
\text { ppmv }\end{array}$ & $\begin{array}{l}\text { mol\% or } \\
\text { ppmv }\end{array}$ & $\begin{array}{l}\text { mol\% or } \\
\text { ppmv }\end{array}$ & $\begin{array}{l}\text { mol\% or } \\
\text { ppmv }\end{array}$ \\
\hline \multicolumn{8}{|l|}{$\mathrm{H} 2 \mathrm{O}, \mathrm{mol} \%$} \\
\hline $\mathrm{O} 2, \mathrm{~mol} \%$ & & & & & & & $100 \%$ \\
\hline \multicolumn{8}{|l|}{$\mathrm{N} 2, \mathrm{~mol} \%$} \\
\hline \multicolumn{8}{|l|}{$\mathrm{H} 2, \mathrm{~mol} \%$} \\
\hline $\mathrm{CO} 2, \mathrm{~mol} \%$ & & & & & & $100 \%$ & \\
\hline \multicolumn{8}{|l|}{ COgas, ppmv } \\
\hline \multicolumn{8}{|l|}{$\mathrm{NO}, \mathrm{ppmv}$} \\
\hline \multicolumn{8}{|l|}{ NO2, ppmv } \\
\hline \multicolumn{8}{|l|}{ SO2, ppmv } \\
\hline \multicolumn{8}{|l|}{$\mathrm{Cl}, \mathrm{ppmv}$} \\
\hline \multicolumn{8}{|l|}{$\mathrm{F}, \mathrm{ppmv}$} \\
\hline \multicolumn{8}{|l|}{ C (organic), ppmv } \\
\hline \multicolumn{8}{|l|}{$\mathrm{H}$ (organic), ppmv } \\
\hline \multicolumn{8}{|l|}{$\mathrm{Hg}, \mathrm{ug} / \mathrm{wscm}$} \\
\hline $\mathrm{PM}, \mathrm{mg} / \mathrm{wscm}$ & & & & & & & $1.67 \mathrm{E}-01$ \\
\hline \multicolumn{8}{|l|}{ SVM, ug/wscm } \\
\hline \multicolumn{8}{|l|}{ LVM, ug/wscm } \\
\hline $\begin{array}{l}\text { Gas Stream Bulk } \\
\text { Composition (Dry Basis) }\end{array}$ & $\mathrm{mol} \%$ & $\mathrm{~mol} \%$ & $\mathrm{~mol} \%$ & $\mathrm{~mol} \%$ & $\mathrm{~mol} \%$ & $\mathrm{~mol} \%$ & $\mathrm{~mol} \%$ \\
\hline \multicolumn{8}{|l|}{$\mathrm{O} 2, \mathrm{~mol} \%$, dry basis } \\
\hline \multicolumn{8}{|l|}{$\mathrm{N} 2, \mathrm{~mol} \%$, dry basis } \\
\hline \multicolumn{8}{|l|}{$\mathrm{H} 2, \mathrm{~mol} \%$, dry basis } \\
\hline $\mathrm{CO} 2, \mathrm{~mol} \%$, dry basis & & & & & & & \\
\hline $\begin{array}{l}\text { Gas Stream Bulk } \\
\text { Composition (Dry Basis, } \\
\text { Corrected to } 7 \% \text { O2 with } \\
100 \% \text { O2 Combustion Air) }\end{array}$ & $\begin{array}{l}\text { ppmv, or } \\
\text { ug/dscm or } \\
\text { mg/dscm }\end{array}$ & $\begin{array}{l}\text { ppmv, or } \\
\text { ug/dscm or } \\
\text { mg/dscm }\end{array}$ & $\begin{array}{l}\text { ppmv, or } \\
\text { ug/dscm or } \\
\text { mg/dscm }\end{array}$ & $\begin{array}{l}\mathrm{ppmv}, \text { or } \\
\mathrm{ug} / \mathrm{dscm} \text { or } \\
\mathrm{mg} / \mathrm{dscm}\end{array}$ & $\begin{array}{l}\text { ppmv, or } \\
\text { ug/dscm or } \\
\mathrm{mg} / \mathrm{dscm}\end{array}$ & $\begin{array}{c}\text { ppmv, or } \\
\text { ug/dscm or } \\
\text { mg/dscm }\end{array}$ & $\begin{array}{l}\text { ppmv, or } \\
\text { ug/dscm or } \\
\mathrm{mg} / \mathrm{dscm}\end{array}$ \\
\hline COgas, ppmv, dry basis & & & & & & & \\
\hline $\mathrm{NO}$, ppmv, dry basis & & & & & & & \\
\hline NO2, ppmv, dry basis & & & & & & & \\
\hline SO2, ppmv, dry basis & & & & & & & \\
\hline $\mathrm{Cl}, \mathrm{ppmv}$, dry basis & & & & & & & \\
\hline $\mathrm{F}, \mathrm{ppmv}$, dry basis & & & & & & & \\
\hline C (organic), ppmv, dry basis & & & & & & & \\
\hline $\mathrm{H}$ (organic), ppmv, dry basis & & & & & & & \\
\hline $\mathrm{Hg}, \mathrm{ug} / \mathrm{dscm}$ & & & & & & & \\
\hline $\mathrm{PM}, \mathrm{mg} / \mathrm{dscm}$ & & & & & & & \\
\hline SVM, ug/dscm & & & & & & & \\
\hline LVM, ug/dscm & & & & & & & \\
\hline
\end{tabular}


Table A-19. Mass balance, Tank WM-180 waste (continued).

\begin{tabular}{|c|c|c|c|c|c|c|c|c|}
\hline PFD Number & PFD-3 & PFD-3 & PFD-3 & PFD-3 & PFD-3 & PFD-3 & PFD-3 & PFD-3 \\
\hline Stream Number & 510 & 511 & 512 & 513 & 514 & 515 & 516 & 517 \\
\hline Stream Name & $\begin{array}{c}\text { Gas to } \\
\text { Cool } \\
\text { Product }\end{array}$ & $\begin{array}{l}\text { Hot Gas } \\
\text { after } \\
\text { Product } \\
\text { Cooling }\end{array}$ & $\begin{array}{c}\text { Backpulse } \\
\text { Gas for } \\
\text { Candle } \\
\text { Filters }\end{array}$ & $\begin{array}{c}\text { Bed/ Solid } \\
\text { Reductant } \\
\text { Transport } \\
\text { Gas }\end{array}$ & $\begin{array}{c}\text { TF Transfer } \\
\text { \& Tank } \\
\text { Sparge Air }\end{array}$ & $\begin{array}{c}\text { Ventilation } \\
\text { Air }\end{array}$ & New GAC & $\begin{array}{c}\text { Off-gas } \\
\text { Preaheater } \\
\text { Steam }\end{array}$ \\
\hline Rate or Volume & $2.06 \mathrm{E}+02$ & $6.45 \mathrm{E}+02$ & $2.21 \mathrm{E}+02$ & $5.41 \mathrm{E}-01$ & $1.40 \mathrm{E}+01$ & $2.89 \mathrm{E}+01$ & $3.11 \mathrm{E}+00$ & $1.31 \mathrm{E}+03$ \\
\hline Volume Flow (standard, wet) ${ }^{*}$ & $1.39 \mathrm{E}+03$ & $1.39 \mathrm{E}+03$ & $6.46 \mathrm{E}+02$ & $1.58 \mathrm{E}+00$ & $4.11 \mathrm{E}+01$ & $2.94 \mathrm{E}+01$ & & $6.51 \mathrm{E}+03$ \\
\hline Volume Flow (standard, dry) & $1.39 \mathrm{E}+03$ & $1.39 \mathrm{E}+03$ & $6.46 \mathrm{E}+02$ & $1.58 \mathrm{E}+00$ & $4.11 \mathrm{E}+01$ & $2.94 \mathrm{E}+01$ & & $0.00 \mathrm{E}+00$ \\
\hline Rate Units & $\mathrm{ft} 3 / \mathrm{hr}$ & $\mathrm{ft} 3 / \mathrm{hr}$ & $\mathrm{ft} 3 / \mathrm{hr}$ & $\mathrm{ft} 3 / \mathrm{hr}$ & $\mathrm{ft} 3 / \mathrm{hr}$ & $\mathrm{ft} 3 / \mathrm{hr}$ & $\mathrm{lb} / \mathrm{hr}$ & $\mathrm{ft} 3 / \mathrm{hr}$ \\
\hline Rate or Volume, metric & $3.93 E+01$ & $3.93 \mathrm{E}+01$ & $1.83 \mathrm{E}+01$ & 4.49E-02 & $1.16 \mathrm{E}+00$ & $8.34 \mathrm{E}-01$ & $1.41 \mathrm{E}+00$ & $1.84 \mathrm{E}+02$ \\
\hline Rate Units & wscm/hr & wscm/hr & wscm/hr & wscm/hr & wscm/hr & wscm/hr & $\mathrm{kg} / \mathrm{hr}$ & wscm/hr \\
\hline Temperature, ${ }^{\circ} \mathrm{C}$ & 0 & 581 & 15 & 15 & 15 & 15 & 25 & 177 \\
\hline Temperature, ${ }^{\circ} \mathrm{F}$ & 32 & 1078 & 59 & 59 & 59 & 59 & 77 & 350 \\
\hline Pressure, psia & 92.30 & 92.30 & 42.30 & 42.30 & 42.30 & 14.70 & 14.70 & 112.30 \\
\hline Specific Gravity & $7.85 \mathrm{E}-03$ & $2.51 \mathrm{E}-03$ & $3.41 \mathrm{E}-03$ & $3.41 \mathrm{E}-03$ & $3.51 \mathrm{E}-03$ & $1.22 \mathrm{E}-03$ & 4.81E-01 & $3.73 \mathrm{E}-03$ \\
\hline Chemical Composition & $\mathrm{lb} / \mathrm{wscf}$ & $\mathrm{lb} / \mathrm{wscf}$ & $\mathrm{lb} /$ wscf & $\mathrm{lb} /$ wscf & $\mathrm{lb} /$ wscf & $\mathrm{lb} / \mathrm{wscf}$ & Wt. \% & $\mathrm{lb} / \mathrm{wscf}$ \\
\hline C (reductant) & & & & & & & $100 \%$ & \\
\hline & & & & & & & & \\
\hline Gas Composition, wet basis & $\mathrm{mol} \%$ & $\mathrm{~mol} \%$ & $\mathrm{~mol} \%$ & $\mathrm{~mol} \%$ & $\mathrm{~mol} \%$ & $\mathrm{~mol} \%$ & $\mathrm{~mol} \%$ & $\mathrm{~mol} \%$ \\
\hline $\mathrm{H} 2 \mathrm{O}, \mathrm{mol} \%$ & & & & & $0.01 \%$ & $0.01 \%$ & & $100 \%$ \\
\hline $\mathrm{O} 2, \mathrm{~mol} \%$ & & & & & $21 \%$ & $21 \%$ & & \\
\hline $\mathrm{N} 2, \mathrm{~mol} \%$ & $100 \%$ & $100 \%$ & $100 \%$ & $100 \%$ & $79 \%$ & $79 \%$ & & \\
\hline $\mathrm{PM}, \mathrm{mg} / \mathrm{wscm}$ & $1.99 \mathrm{E}+01$ & $1.99 \mathrm{E}+01$ & $2.00 \mathrm{E}+01$ & $2.10 \mathrm{E}+01$ & $1.57 \mathrm{E}+01$ & $1.57 \mathrm{E}+01$ & & $1.19 \mathrm{E}+01$ \\
\hline & & & & & & & & \\
\hline $\mathrm{kg} / \mathrm{hr}$ & $4.58 \mathrm{E}+01$ & $4.58 \mathrm{E}+01$ & $2.13 \mathrm{E}+01$ & $5.22 \mathrm{E}-02$ & $1.39 \mathrm{E}+00$ & $1.00 \mathrm{E}+00$ & $1.41 \mathrm{E}+00$ & $1.38 \mathrm{E}+02$ \\
\hline
\end{tabular}


Table A-20. Mass balance, Tank WM-187 waste.

\begin{tabular}{|c|c|c|c|c|c|c|c|}
\hline PFD Number & PFD-2 & PFD-2 & PFD-2 & PFD-2 & PFD-2 & PFD-2 & PFD-2 \\
\hline Stream Number & 101 & 102 & 103 & 104 & 106 & 107 & 108 \\
\hline Stream Name & SBW & $\begin{array}{l}\text { Reformer } \\
\text { Feed }\end{array}$ & $\begin{array}{c}\text { Reformer } \\
\text { Off-gas }\end{array}$ & $\begin{array}{c}\text { Reformer } \\
\text { Off-gas } \\
\text { Cooled }\end{array}$ & Filter Drain & Bed Drain & $\begin{array}{l}\text { Cooled } \\
\text { Product }\end{array}$ \\
\hline Rate or Volume & $9.54 \mathrm{E}+01$ & $1.67 \mathrm{E}+02$ & $2.16 \mathrm{E}+05$ & $1.77 \mathrm{E}+05$ & $8.56 \mathrm{E}+00$ & $1.26 \mathrm{E}+02$ & $1.34 \mathrm{E}+02$ \\
\hline Volume Flow (standard, wet) $^{*}$ & & & $5.23 E+04$ & $5.37 \mathrm{E}+04$ & & & \\
\hline Volume Flow (standard, dry)* & & & $3.65 E+04$ & $3.79 \mathrm{E}+04$ & & & \\
\hline Rate Units & $\mathrm{gal} / \mathrm{hr}$ & gal/hr & $\mathrm{ft} 3 / \mathrm{hr}$ & $\mathrm{ft} 3 / \mathrm{hr}$ & $\mathrm{lb} / \mathrm{hr}$ & $\mathrm{lb} / \mathrm{hr}$ & $\mathrm{lb} / \mathrm{hr}$ \\
\hline Rate or Volume, metric & $3.61 \mathrm{E}+02$ & $6.30 \mathrm{E}+02$ & $1.48 \mathrm{E}+03$ & $1.52 \mathrm{E}+03$ & $3.88 \mathrm{E}+00$ & $5.71 \mathrm{E}+01$ & $6.09 \mathrm{E}+01$ \\
\hline Rate Units & $\mathrm{L} / \mathrm{hr}$ & $\mathrm{L} / \mathrm{hr}$ & wscm $/ \mathrm{hr}$ & wscm $/ \mathrm{hr}$ & $\mathrm{kg} / \mathrm{hr}$ & $\mathrm{kg} / \mathrm{hr}$ & $\mathrm{kg} / \mathrm{hr}$ \\
\hline Temperature, ${ }^{\circ} \mathrm{C}$ & 15 & 17 & 600 & 400 & 400 & 600 & 56 \\
\hline Temperature, ${ }^{\circ} \mathrm{F}$ & 59 & 63 & 1112 & 752 & 752 & 1112 & 132 \\
\hline Pressure, psia & 14.70 & 44.70 & 10.62 & 10.22 & 9.50 & 10.80 & 9.50 \\
\hline Specific Gravity & 1.20 & 1.20 & $3.35 \mathrm{E}-04$ & $4.16 \mathrm{E}-04$ & 0.79 & 1.46 & 1.42 \\
\hline Chemical Composition & Mol/liter & Mol/liter & lb/wscf & lb/wscf & Wt Frac & Wt Frac & Wt frac \\
\hline $\mathrm{H}+$ & $2.72 \mathrm{E}+00$ & $1.59 \mathrm{E}+00$ & $1.25 \mathrm{E}-06$ & $1.22 \mathrm{E}-06$ & $1.54 \mathrm{E}-05$ & $4.74 \mathrm{E}-05$ & $4.54 \mathrm{E}-05$ \\
\hline $\mathrm{Al}+3$ & $3.10 \mathrm{E}-01$ & $1.78 \mathrm{E}-01$ & $8.14 \mathrm{E}-06$ & $7.93 \mathrm{E}-06$ & $4.97 \mathrm{E}-02$ & $5.53 \mathrm{E}-02$ & $5.50 \mathrm{E}-02$ \\
\hline $\mathrm{Sb}+5$ & $2.46 \mathrm{E}-05$ & $1.41 \mathrm{E}-05$ & $2.64 \mathrm{E}-09$ & $2.57 \mathrm{E}-09$ & $1.61 \mathrm{E}-05$ & $1.79 \mathrm{E}-05$ & $1.78 \mathrm{E}-05$ \\
\hline As+3 & $1.43 \mathrm{E}-04$ & $8.23 \mathrm{E}-05$ & $9.44 \mathrm{E}-09$ & $9.20 \mathrm{E}-09$ & $5.77 \mathrm{E}-05$ & $6.42 \mathrm{E}-05$ & $6.38 \mathrm{E}-05$ \\
\hline $\mathrm{Ba}+2$ & $6.02 \mathrm{E}-05$ & $3.45 \mathrm{E}-05$ & $6.58 \mathrm{E}-09$ & $6.41 \mathrm{E}-09$ & $4.02 \mathrm{E}-05$ & $4.96 \mathrm{E}-05$ & $4.90 \mathrm{E}-05$ \\
\hline $\mathrm{Be}+2$ & $1.33 \mathrm{E}-05$ & $7.63 \mathrm{E}-06$ & $9.56 \mathrm{E}-11$ & $9.31 \mathrm{E}-11$ & $5.84 \mathrm{E}-07$ & $7.20 \mathrm{E}-07$ & $7.11 \mathrm{E}-07$ \\
\hline$B+3$ & $9.30 \mathrm{E}-03$ & $5.34 \mathrm{E}-03$ & $8.83 \mathrm{E}-08$ & $8.61 \mathrm{E}-08$ & $5.40 \mathrm{E}-04$ & $6.01 \mathrm{E}-04$ & $5.97 \mathrm{E}-04$ \\
\hline$C d+2$ & $1.49 \mathrm{E}-03$ & $8.54 \mathrm{E}-04$ & $5.96 \mathrm{E}-07$ & $5.81 \mathrm{E}-07$ & $3.64 \mathrm{E}-03$ & $8.12 \mathrm{E}-04$ & $9.93 \mathrm{E}-04$ \\
\hline $\mathrm{Ca}+2$ & $2.97 \mathrm{E}-02$ & $1.70 \mathrm{E}-02$ & $9.47 \mathrm{E}-07$ & $9.23 \mathrm{E}-07$ & $5.79 \mathrm{E}-03$ & $7.14 \mathrm{E}-03$ & $7.05 \mathrm{E}-03$ \\
\hline $\mathrm{Cr}+3$ & $3.85 \mathrm{E}-03$ & $2.21 \mathrm{E}-03$ & $2.43 \mathrm{E}-07$ & $2.37 \mathrm{E}-07$ & $1.49 \mathrm{E}-03$ & $1.17 \mathrm{E}-03$ & $1.19 \mathrm{E}-03$ \\
\hline $\mathrm{Co}+2$ & $1.70 \mathrm{E}-03$ & $9.74 \mathrm{E}-04$ & $8.79 \mathrm{E}-08$ & $8.56 \mathrm{E}-08$ & $5.37 \mathrm{E}-04$ & $5.97 \mathrm{E}-04$ & 5.94E-04 \\
\hline Cs+ & $6.83 \mathrm{E}-05$ & $3.91 \mathrm{E}-05$ & $5.38 \mathrm{E}-08$ & $5.24 \mathrm{E}-08$ & $3.29 \mathrm{E}-04$ & $3.51 \mathrm{E}-05$ & $5.38 \mathrm{E}-05$ \\
\hline $\mathrm{Cu}+2$ & $3.98 \mathrm{E}-04$ & $2.28 \mathrm{E}-04$ & $2.02 \mathrm{E}-08$ & $1.96 \mathrm{E}-08$ & $1.23 \mathrm{E}-04$ & $1.52 \mathrm{E}-04$ & $1.50 \mathrm{E}-04$ \\
\hline $\mathrm{Fe}+3$ & $2.06 \mathrm{E}-02$ & $1.18 \mathrm{E}-02$ & $1.49 \mathrm{E}-06$ & $1.45 \mathrm{E}-06$ & $9.05 \mathrm{E}-03$ & $6.68 \mathrm{E}-03$ & $6.83 \mathrm{E}-03$ \\
\hline $\mathrm{Pb}+2$ & $5.45 \mathrm{E}-04$ & $3.12 \mathrm{E}-04$ & $4.02 \mathrm{E}-07$ & $3.92 \mathrm{E}-07$ & $2.46 \mathrm{E}-03$ & $5.48 \mathrm{E}-04$ & $6.70 \mathrm{E}-04$ \\
\hline $\mathrm{Hg}+2$ & $3.97 \mathrm{E}-03$ & $4.30 \mathrm{E}-03$ & $2.29 \mathrm{E}-05$ & $2.23 \mathrm{E}-05$ & $1.40 \mathrm{E}-04$ & $9.52 \mathrm{E}-06$ & $1.78 \mathrm{E}-05$ \\
\hline$M n+4$ & $1.53 \mathrm{E}-02$ & $8.78 \mathrm{E}-03$ & $7.39 \mathrm{E}-07$ & $7.20 \mathrm{E}-07$ & $4.52 \mathrm{E}-03$ & $5.02 \mathrm{E}-03$ & $4.99 \mathrm{E}-03$ \\
\hline $\mathrm{Ni}+2$ & $1.15 \mathrm{E}-03$ & $6.59 \mathrm{E}-04$ & $5.38 \mathrm{E}-08$ & $5.24 \mathrm{E}-08$ & $3.28 \mathrm{E}-04$ & $4.05 \mathrm{E}-04$ & $4.00 \mathrm{E}-04$ \\
\hline $\mathrm{K}+$ & $2.63 \mathrm{E}-01$ & $1.51 \mathrm{E}-01$ & $1.33 \mathrm{E}-05$ & $1.30 \mathrm{E}-05$ & $8.15 \mathrm{E}-02$ & $5.96 \mathrm{E}-02$ & $6.10 \mathrm{E}-02$ \\
\hline $\mathrm{Se}+4$ & $3.62 \mathrm{E}-04$ & $2.07 \mathrm{E}-04$ & $2.51 \mathrm{E}-08$ & $2.44 \mathrm{E}-08$ & $1.53 \mathrm{E}-04$ & $1.70 \mathrm{E}-04$ & $1.69 \mathrm{E}-04$ \\
\hline $\mathrm{Ag}^{+}$ & $5.50 \mathrm{E}-04$ & $3.15 \mathrm{E}-04$ & $4.97 \mathrm{E}-08$ & $4.84 \mathrm{E}-08$ & $3.04 \mathrm{E}-04$ & $3.55 \mathrm{E}-04$ & $3.52 \mathrm{E}-04$ \\
\hline $\mathrm{Na}+$ & $1.50 \mathrm{E}+00$ & $8.60 \mathrm{E}-01$ & $2.89 \mathrm{E}-05$ & $2.82 \mathrm{E}-05$ & $1.77 \mathrm{E}-01$ & $2.06 \mathrm{E}-01$ & $2.05 \mathrm{E}-01$ \\
\hline $\mathrm{TI}+3$ & $4.89 \mathrm{E}-06$ & $2.81 \mathrm{E}-06$ & $8.79 \mathrm{E}-10$ & $8.56 \mathrm{E}-10$ & $5.37 \mathrm{E}-06$ & $5.98 \mathrm{E}-06$ & $5.94 \mathrm{E}-06$ \\
\hline$U+4$ & $2.30 \mathrm{E}-04$ & $1.32 \mathrm{E}-04$ & $4.80 \mathrm{E}-08$ & $4.67 \mathrm{E}-08$ & $2.93 \mathrm{E}-04$ & $3.26 \mathrm{E}-04$ & $3.24 \mathrm{E}-04$ \\
\hline$V+3$ & $5.21 \mathrm{E}-05$ & $2.98 \mathrm{E}-05$ & $2.33 \mathrm{E}-09$ & $2.27 \mathrm{E}-09$ & $1.42 \mathrm{E}-05$ & $1.58 \mathrm{E}-05$ & $1.57 \mathrm{E}-05$ \\
\hline$Z n+2$ & $6.45 \mathrm{E}-04$ & $3.69 \mathrm{E}-04$ & $3.36 \mathrm{E}-08$ & $3.27 \mathrm{E}-08$ & $2.05 \mathrm{E}-04$ & $2.53 \mathrm{E}-04$ & $2.50 \mathrm{E}-04$ \\
\hline $\mathrm{Zr}+4$ & $5.17 \mathrm{E}-02$ & $2.96 \mathrm{E}-02$ & $4.14 \mathrm{E}-06$ & $4.03 \mathrm{E}-06$ & $2.53 \mathrm{E}-02$ & $2.82 \mathrm{E}-02$ & $2.80 \mathrm{E}-02$ \\
\hline $\mathrm{Cl}-$ & $1.51 \mathrm{E}-02$ & $8.64 \mathrm{E}-03$ & $4.99 \mathrm{E}-07$ & $4.86 \mathrm{E}-07$ & $2.87 \mathrm{E}-03$ & $3.18 \mathrm{E}-03$ & $3.16 \mathrm{E}-03$ \\
\hline F- & $4.62 \mathrm{E}-02$ & $2.67 \mathrm{E}-02$ & $1.55 \mathrm{E}-06$ & $1.51 \mathrm{E}-06$ & $4.25 \mathrm{E}-03$ & $4.96 \mathrm{E}-03$ & $4.92 \mathrm{E}-03$ \\
\hline \begin{tabular}{|l|}
$\mathrm{SO} 4-2$ \\
\end{tabular} & $4.22 \mathrm{E}-02$ & $2.42 \mathrm{E}-02$ & $1.15 \mathrm{E}-12$ & $1.12 \mathrm{E}-12$ & $7.04 \mathrm{E}-09$ & $8.23 \mathrm{E}-09$ & $8.16 \mathrm{E}-09$ \\
\hline \begin{tabular}{|l|} 
NO3- \\
\end{tabular} & $5.07 \mathrm{E}+00$ & $2.94 \mathrm{E}+00$ & 1.67E-06 & $1.63 \mathrm{E}-06$ & $1.02 \mathrm{E}-02$ & $3.76 \mathrm{E}-03$ & $4.17 \mathrm{E}-03$ \\
\hline PO4-3 & $1.36 \mathrm{E}-01$ & $7.77 \mathrm{E}-02$ & $1.13 \mathrm{E}-05$ & $1.10 \mathrm{E}-05$ & $6.92 \mathrm{E}-02$ & $7.68 \mathrm{E}-02$ & $7.63 \mathrm{E}-02$ \\
\hline$A m+4$ & $5.85 \mathrm{E}-08$ & $3.36 \mathrm{E}-08$ & $1.25 \mathrm{E}-11$ & $1.22 \mathrm{E}-11$ & $7.64 \mathrm{E}-08$ & $8.50 \mathrm{E}-08$ & $8.44 \mathrm{E}-08$ \\
\hline $\mathrm{Br}-$ & $2.11 \mathrm{E}-07$ & $1.21 \mathrm{E}-07$ & $1.57 \mathrm{E}-11$ & $1.53 \mathrm{E}-11$ & $9.04 \mathrm{E}-08$ & $1.00 \mathrm{E}-07$ & $9.95 \mathrm{E}-08$ \\
\hline $\mathrm{Ce}+4$ & $3.66 \mathrm{E}-05$ & $2.10 \mathrm{E}-05$ & $4.50 \mathrm{E}-09$ & $4.39 \mathrm{E}-09$ & $2.75 \mathrm{E}-05$ & $3.06 \mathrm{E}-05$ & $3.04 \mathrm{E}-05$ \\
\hline $\mathrm{Eu}+3$ & $3.50 \mathrm{E}-07$ & $2.01 \mathrm{E}-07$ & $4.68 \mathrm{E}-11$ & $4.56 \mathrm{E}-11$ & $2.86 \mathrm{E}-07$ & $3.18 \mathrm{E}-07$ & $3.16 \mathrm{E}-07$ \\
\hline $\mathrm{Gd}+3$ & $1.27 \mathrm{E}-04$ & $7.31 \mathrm{E}-05$ & $1.76 \mathrm{E}-08$ & $1.72 \mathrm{E}-08$ & $1.08 \mathrm{E}-04$ & $1.20 \mathrm{E}-04$ & $1.19 \mathrm{E}-04$ \\
\hline $\mathrm{Ge}+4$ & $6.07 \mathrm{E}-09$ & $3.48 \mathrm{E}-09$ & $3.87 \mathrm{E}-13$ & $3.77 \mathrm{E}-13$ & $2.36 \mathrm{E}-09$ & $2.63 \mathrm{E}-09$ & $2.61 \mathrm{E}-09$ \\
\hline $\ln +3$ & $1.02 \mathrm{E}-06$ & $5.83 \mathrm{E}-07$ & $1.03 \mathrm{E}-10$ & $9.99 \mathrm{E}-11$ & $6.27 \mathrm{E}-07$ & $6.97 \mathrm{E}-07$ & $6.93 \mathrm{E}-07$ \\
\hline \begin{tabular}{|l|}
- \\
-
\end{tabular} & $2.74 \mathrm{E}-06$ & $1.57 \mathrm{E}-06$ & $3.25 \mathrm{E}-10$ & $3.17 \mathrm{E}-10$ & $1.87 \mathrm{E}-06$ & $2.07 \mathrm{E}-06$ & $2.06 \mathrm{E}-06$ \\
\hline $\mathrm{La+3}$ & $6.35 \mathrm{E}-06$ & $3.64 \mathrm{E}-06$ & $7.75 \mathrm{E}-10$ & $7.55 \mathrm{E}-10$ & $4.74 \mathrm{E}-06$ & $5.27 \mathrm{E}-06$ & $5.23 \mathrm{E}-06$ \\
\hline
\end{tabular}


Table A-20. Mass balance, Tank WM-187 waste (continued).

\begin{tabular}{|c|c|c|c|c|c|c|c|}
\hline Stream \# & 101 & 102 & 103 & 104 & 106 & 107 & 108 \\
\hline Chemical Composition & Mol/liter & Mol/liter & $\mathrm{Ib} / \mathrm{wscf}$ & $\mathrm{lb} / \mathrm{wscf}$ & Wt frac & Wt frac & Wt frac \\
\hline $\mathrm{Li}+$ & $5.34 \mathrm{E}-04$ & $3.06 \mathrm{E}-04$ & $3.11 \mathrm{E}-09$ & $3.03 E-09$ & $1.90 \mathrm{E}-05$ & $2.22 \mathrm{E}-05$ & $2.20 \mathrm{E}-05$ \\
\hline$M g+2$ & $1.45 \mathrm{E}-02$ & $8.29 \mathrm{E}-03$ & $2.80 \mathrm{E}-07$ & $2.73 \mathrm{E}-07$ & $1.71 \mathrm{E}-03$ & $2.11 \mathrm{E}-03$ & $2.09 \mathrm{E}-03$ \\
\hline $\mathrm{Mo}+6$ & $7.92 \mathrm{E}-02$ & $4.53 \mathrm{E}-02$ & $6.66 \mathrm{E}-06$ & $6.49 \mathrm{E}-06$ & $4.07 \mathrm{E}-02$ & $4.53 \mathrm{E}-02$ & $4.50 \mathrm{E}-02$ \\
\hline $\mathrm{Nd}+3$ & $2.05 \mathrm{E}-05$ & $1.17 \mathrm{E}-05$ & $2.59 \mathrm{E}-09$ & $2.53 \mathrm{E}-09$ & $1.59 \mathrm{E}-05$ & $1.76 \mathrm{E}-05$ & $1.75 \mathrm{E}-05$ \\
\hline $\mathrm{Np}+4$ & $3.22 \mathrm{E}-06$ & $1.85 \mathrm{E}-06$ & $6.71 \mathrm{E}-10$ & $6.53 \mathrm{E}-10$ & $4.10 \mathrm{E}-06$ & $4.56 \mathrm{E}-06$ & $4.53 \mathrm{E}-06$ \\
\hline $\mathrm{Nb}+5$ & $1.44 \mathrm{E}-03$ & $8.25 \mathrm{E}-04$ & $1.17 \mathrm{E}-07$ & $1.14 \mathrm{E}-07$ & $7.18 \mathrm{E}-04$ & $7.98 \mathrm{E}-04$ & $7.93 \mathrm{E}-04$ \\
\hline $\mathrm{Pd}+4$ & $2.35 \mathrm{E}-03$ & $1.35 \mathrm{E}-03$ & $1.99 \mathrm{E}-07$ & $1.94 \mathrm{E}-07$ & $1.22 \mathrm{E}-03$ & $1.50 \mathrm{E}-03$ & $1.48 \mathrm{E}-03$ \\
\hline $\mathrm{Pu}+4$ & $7.55 \mathrm{E}-06$ & $4.32 \mathrm{E}-06$ & $1.62 \mathrm{E}-09$ & $1.57 \mathrm{E}-09$ & $9.87 \mathrm{E}-06$ & $1.10 \mathrm{E}-05$ & $1.09 \mathrm{E}-05$ \\
\hline $\mathrm{Pr}+4$ & $5.77 \mathrm{E}-06$ & $3.31 \mathrm{E}-06$ & $7.15 \mathrm{E}-10$ & $6.96 \mathrm{E}-10$ & $4.37 \mathrm{E}-06$ & $4.86 \mathrm{E}-06$ & $4.83 \mathrm{E}-06$ \\
\hline $\mathrm{Pm}+3$ & $1.41 \mathrm{E}-09$ & $8.07 \mathrm{E}-10$ & $1.79 \mathrm{E}-13$ & $1.74 \mathrm{E}-13$ & $1.09 \mathrm{E}-09$ & $1.22 \mathrm{E}-09$ & $1.21 \mathrm{E}-09$ \\
\hline $\mathrm{Rh}+4$ & $2.49 \mathrm{E}-06$ & $1.43 \mathrm{E}-06$ & $2.25 \mathrm{E}-10$ & $2.19 \mathrm{E}-10$ & $1.38 \mathrm{E}-06$ & $1.53 \mathrm{E}-06$ & $1.52 \mathrm{E}-06$ \\
\hline $\mathrm{Rb}+$ & 3.83E-06 & $2.20 \mathrm{E}-06$ & $2.75 \mathrm{E}-10$ & $2.68 \mathrm{E}-10$ & $1.68 \mathrm{E}-06$ & $1.96 \mathrm{E}-06$ & $1.94 \mathrm{E}-06$ \\
\hline $\mathrm{Ru}+3$ & $1.07 \mathrm{E}-03$ & $6.17 \mathrm{E}-04$ & $9.55 \mathrm{E}-08$ & $9.30 \mathrm{E}-08$ & $5.84 \mathrm{E}-04$ & $6.49 \mathrm{E}-04$ & $6.45 \mathrm{E}-04$ \\
\hline $\mathrm{Sm+3}$ & $3.83 \mathrm{E}-06$ & $2.20 \mathrm{E}-06$ & $5.06 \mathrm{E}-10$ & $4.93 \mathrm{E}-10$ & $3.09 \mathrm{E}-06$ & $3.44 \mathrm{E}-06$ & $3.42 \mathrm{E}-06$ \\
\hline $\mathrm{Si+4}$ & $1.23 \mathrm{E}-01$ & $7.05 \mathrm{E}-02$ & $3.03 E-06$ & $2.95 \mathrm{E}-06$ & $1.85 \mathrm{E}-02$ & $2.06 \mathrm{E}-02$ & $2.05 \mathrm{E}-02$ \\
\hline $\mathrm{Sr}+2$ & $8.39 \mathrm{E}-05$ & $4.80 \mathrm{E}-05$ & $5.85 \mathrm{E}-09$ & $5.70 \mathrm{E}-09$ & $3.58 \mathrm{E}-05$ & $4.41 \mathrm{E}-05$ & $4.36 \mathrm{E}-05$ \\
\hline $\mathrm{Tc}+7$ & $6.15 \mathrm{E}-06$ & $3.52 \mathrm{E}-06$ & $4.82 \mathrm{E}-09$ & $4.70 \mathrm{E}-09$ & $2.94 \mathrm{E}-05$ & $1.81 \mathrm{E}-06$ & $3.57 \mathrm{E}-06$ \\
\hline $\mathrm{Te}+4$ & $3.29 \mathrm{E}-06$ & $1.89 \mathrm{E}-06$ & $3.69 \mathrm{E}-10$ & $3.59 \mathrm{E}-10$ & $2.25 \mathrm{E}-06$ & $2.51 \mathrm{E}-06$ & $2.49 \mathrm{E}-06$ \\
\hline $\mathrm{Tb}+4$ & $1.46 \mathrm{E}-09$ & $8.38 \mathrm{E}-10$ & $2.04 \mathrm{E}-13$ & $1.99 \mathrm{E}-13$ & $1.25 \mathrm{E}-09$ & $1.39 \mathrm{E}-09$ & $1.38 \mathrm{E}-09$ \\
\hline $\mathrm{Th}+4$ & $1.73 \mathrm{E}-05$ & $9.88 \mathrm{E}-06$ & $3.51 \mathrm{E}-09$ & $3.42 \mathrm{E}-09$ & $2.15 \mathrm{E}-05$ & $2.39 \mathrm{E}-05$ & $2.37 \mathrm{E}-05$ \\
\hline$S n+4$ & $2.51 \mathrm{E}-03$ & $1.43 \mathrm{E}-03$ & $2.61 \mathrm{E}-07$ & $2.54 \mathrm{E}-07$ & $1.59 \mathrm{E}-03$ & $1.77 \mathrm{E}-03$ & $1.76 \mathrm{E}-03$ \\
\hline Ti+4 & $1.26 \mathrm{E}-03$ & $7.24 \mathrm{E}-04$ & $5.31 \mathrm{E}-08$ & $5.17 \mathrm{E}-08$ & $3.24 \mathrm{E}-04$ & $3.61 \mathrm{E}-04$ & $3.59 \mathrm{E}-04$ \\
\hline $\mathrm{Y}+3$ & $4.73 \mathrm{E}-06$ & $2.72 \mathrm{E}-06$ & $3.70 \mathrm{E}-10$ & $3.60 \mathrm{E}-10$ & $2.26 \mathrm{E}-06$ & $2.51 \mathrm{E}-06$ & $2.50 \mathrm{E}-06$ \\
\hline $\mathrm{OH}-$ & & $4.01 \mathrm{E}-08$ & $3.84 \mathrm{E}-10$ & $3.75 \mathrm{E}-10$ & $2.35 \mathrm{E}-06$ & $1.72 \mathrm{E}-06$ & $1.76 \mathrm{E}-06$ \\
\hline $\mathrm{H} 2 \mathrm{O}$ & $4.28 \mathrm{E}+01$ & $2.62 \mathrm{E}+01$ & $1.41 \mathrm{E}-02$ & 1.37E-02 & $7.57 \mathrm{E}-03$ & $3.52 \mathrm{E}-03$ & $3.77 \mathrm{E}-03$ \\
\hline $\mathrm{SO} 2$ & & $2.24 \mathrm{E}-08$ & $1.55 \mathrm{E}-12$ & $1.51 \mathrm{E}-12$ & & & \\
\hline $\mathrm{H} 2 \mathrm{~S}$ & & & $2.04 \mathrm{E}-05$ & $1.99 \mathrm{E}-05$ & & & \\
\hline $\mathrm{CO}$ & & $8.62 \mathrm{E}-11$ & $3.59 \mathrm{E}-04$ & $3.49 \mathrm{E}-04$ & & & \\
\hline $\mathrm{CO} 2$ & & $6.73 \mathrm{E}-06$ & $6.31 \mathrm{E}-02$ & $6.15 \mathrm{E}-02$ & & & \\
\hline $\mathrm{H} 2$ & & $8.91 \mathrm{E}-10$ & $5.40 \mathrm{E}-04$ & $5.26 \mathrm{E}-04$ & & & \\
\hline N2 & & $2.07 E-10$ & $1.08 \mathrm{E}-03$ & 2.93E-03 & & & \\
\hline NO & & $2.76 \mathrm{E}-09$ & $1.41 \mathrm{E}-04$ & $1.37 \mathrm{E}-04$ & & & \\
\hline NO2 & & $4.14 \mathrm{E}-07$ & $2.06 \mathrm{E}-06$ & $2.01 \mathrm{E}-06$ & & & \\
\hline $\mathrm{O} 2$ & & $6.82 \mathrm{E}-10$ & & & & & \\
\hline $\mathrm{S}$ (other) & & $3.28 \mathrm{E}-10$ & $7.90 \mathrm{E}-08$ & $7.69 \mathrm{E}-08$ & $4.83 \mathrm{E}-04$ & $5.64 \mathrm{E}-04$ & $5.58 \mathrm{E}-04$ \\
\hline $\mathrm{CO} 3$ & $3.42 \mathrm{E}-05$ & $1.96 \mathrm{E}-05$ & $4.88 \mathrm{E}-05$ & $4.75 \mathrm{E}-05$ & $2.98 \mathrm{E}-01$ & $3.22 \mathrm{E}-01$ & $3.20 \mathrm{E}-01$ \\
\hline $\mathrm{C}$ (reductant) & & & & & & & \\
\hline $\mathrm{O}$ (oxides) & $6.38 \mathrm{E}-01$ & $3.66 \mathrm{E}-01$ & $1.47 \mathrm{E}-05$ & $1.43 \mathrm{E}-05$ & $8.98 \%$ & $9.76 \%$ & $9.71 \%$ \\
\hline C (organic) & & $2.40 \mathrm{E}+01$ & $5.54 \mathrm{E}-03$ & $5.40 \mathrm{E}-03$ & $5.52 \mathrm{E}-02$ & $8.56 \mathrm{E}-04$ & $4.32 \mathrm{E}-03$ \\
\hline $\mathrm{H}$ (organic) & & $6.40 \mathrm{E}+01$ & $1.04 \mathrm{E}-03$ & $1.01 \mathrm{E}-03$ & $9.84 \mathrm{E}-03$ & $1.53 \mathrm{E}-04$ & $7.70 \mathrm{E}-04$ \\
\hline $\mathrm{O}$ (organic) & & $8.00 \mathrm{E}+00$ & & & & & \\
\hline & & & & & & & \\
\hline Mass Flow (kg/hr): & $4.34 \mathrm{E}+02$ & $7.58 \mathrm{E}+02$ & $2.04 \mathrm{E}+03$ & $2.09 \mathrm{E}+03$ & $3.88 \mathrm{E}+00$ & $5.71 \mathrm{E}+01$ & $6.09 E+01$ \\
\hline Canister Rate, canisters/day & & & & & & & 1.29 \\
\hline Total Canisters Generated & & & & & & & 113 \\
\hline & $\mathrm{w} / \mathrm{m} 3$ & $\mathrm{w} / \mathrm{m} 3$ & $\mathrm{w} / \mathrm{m} 3$ & $\mathrm{w} / \mathrm{m} 3$ & $\mathrm{w} / \mathrm{m} 3$ & $\mathrm{w} / \mathrm{m} 3$ & $\mathrm{w} / \mathrm{m} 3$ \\
\hline Heat Generation & 4.03E-01 & $2.31 \mathrm{E}-01$ & & & $2.59 \mathrm{E}+00$ & $3.40 \mathrm{E}+00$ & $3.38 \mathrm{E}+00$ \\
\hline
\end{tabular}


Table A-20. Mass balance, Tank WM-187 waste (continued).

\begin{tabular}{|c|c|c|c|c|c|c|c|}
\hline Stream \# & 101 & 102 & 103 & 104 & 106 & 107 & 108 \\
\hline Radiological Composition & $\mathrm{Ci} / \mathrm{L}$ & $\mathrm{Ci} / \mathrm{L}$ & Ci/wscm & Ci/wscm & $\mathrm{Ci} / \mathrm{kg}$ & $\mathrm{Ci} / \mathrm{kg}$ & $\mathrm{Ci} / \mathrm{kg}$ \\
\hline $\mathrm{H}-3$ & $9.39 \mathrm{E}-06$ & $5.38 \mathrm{E}-06$ & $2.29 \mathrm{E}-06$ & $2.23 \mathrm{E}-06$ & & & \\
\hline $\mathrm{C}-14$ & $1.36 \mathrm{E}-10$ & $7.77 \mathrm{E}-11$ & $3.28 \mathrm{E}-11$ & $3.20 \mathrm{E}-11$ & $1.03 \mathrm{E}-11$ & $5.84 \mathrm{E}-12$ & $6.12 \mathrm{E}-12$ \\
\hline Co-60 & $1.45 \mathrm{E}-05$ & $8.35 \mathrm{E}-06$ & $2.05 \mathrm{E}-07$ & 1.99E-07 & $7.81 \mathrm{E}-05$ & $8.69 \mathrm{E}-05$ & $8.63 \mathrm{E}-05$ \\
\hline $\mathrm{Ni}-59$ & $1.60 \mathrm{E}-06$ & $9.14 \mathrm{E}-07$ & $2.03 \mathrm{E}-08$ & $1.98 \mathrm{E}-08$ & $7.76 \mathrm{E}-06$ & $9.57 \mathrm{E}-06$ & $9.46 \mathrm{E}-06$ \\
\hline $\mathrm{Ni}-63$ & $3.71 \mathrm{E}-05$ & $2.13 \mathrm{E}-05$ & $4.73 \mathrm{E}-07$ & $4.61 \mathrm{E}-07$ & $1.81 \mathrm{E}-04$ & $2.23 \mathrm{E}-04$ & $2.20 \mathrm{E}-04$ \\
\hline Se-79 & $4.92 \mathrm{E}-07$ & $2.82 \mathrm{E}-07$ & $6.91 \mathrm{E}-09$ & $6.73 \mathrm{E}-09$ & $2.64 \mathrm{E}-06$ & $2.93 \mathrm{E}-06$ & $2.91 \mathrm{E}-06$ \\
\hline Sr-90 & $2.78 \mathrm{E}-02$ & $1.60 \mathrm{E}-02$ & $3.55 \mathrm{E}-04$ & $3.46 \mathrm{E}-04$ & $1.35 \mathrm{E}-01$ & 1.67E-01 & $1.65 \mathrm{E}-01$ \\
\hline $\mathrm{Y}-90$ & $2.78 \mathrm{E}-02$ & $1.60 \mathrm{E}-02$ & $3.92 \mathrm{E}-04$ & $3.82 \mathrm{E}-04$ & $1.50 \mathrm{E}-01$ & 1.66E-01 & $1.65 \mathrm{E}-01$ \\
\hline Zr-93 & 1.57E-06 & $8.99 \mathrm{E}-07$ & $2.21 \mathrm{E}-08$ & $2.15 \mathrm{E}-08$ & $8.42 \mathrm{E}-06$ & $9.37 \mathrm{E}-06$ & $9.31 \mathrm{E}-06$ \\
\hline $\mathrm{Nb}-93 \mathrm{~m}$ & $1.21 \mathrm{E}-06$ & $6.93 \mathrm{E}-07$ & $1.70 \mathrm{E}-08$ & $1.66 \mathrm{E}-08$ & $6.49 \mathrm{E}-06$ & $7.22 \mathrm{E}-06$ & $7.17 \mathrm{E}-06$ \\
\hline $\mathrm{Nb}-94$ & $1.28 \mathrm{E}-06$ & $7.31 \mathrm{E}-07$ & $1.79 \mathrm{E}-08$ & $1.75 \mathrm{E}-08$ & $6.84 \mathrm{E}-06$ & $7.62 \mathrm{E}-06$ & $7.57 \mathrm{E}-06$ \\
\hline Tc-99 & $1.48 \mathrm{E}-05$ & $8.48 \mathrm{E}-06$ & $1.90 \mathrm{E}-06$ & $1.85 \mathrm{E}-06$ & $7.22 \mathrm{E}-04$ & $4.45 \mathrm{E}-05$ & $8.77 \mathrm{E}-05$ \\
\hline Ru-106 & $1.05 \mathrm{E}-06$ & $6.04 \mathrm{E}-07$ & $1.48 \mathrm{E}-08$ & $1.44 \mathrm{E}-08$ & $5.65 \mathrm{E}-06$ & $6.29 \mathrm{E}-06$ & $6.25 \mathrm{E}-06$ \\
\hline Rh-102 & $6.11 \mathrm{E}-10$ & $3.51 \mathrm{E}-10$ & $8.60 \mathrm{E}-12$ & $8.38 \mathrm{E}-12$ & $3.28 \mathrm{E}-09$ & $3.65 \mathrm{E}-09$ & $3.63 \mathrm{E}-09$ \\
\hline Rh-106 & $1.05 \mathrm{E}-06$ & $6.04 \mathrm{E}-07$ & $1.48 \mathrm{E}-08$ & $1.44 \mathrm{E}-08$ & $5.65 \mathrm{E}-06$ & $6.29 \mathrm{E}-06$ & $6.25 \mathrm{E}-06$ \\
\hline $\mathrm{Pd}-107$ & $1.17 \mathrm{E}-08$ & $6.71 \mathrm{E}-09$ & $1.49 \mathrm{E}-10$ & $1.45 \mathrm{E}-10$ & $5.70 \mathrm{E}-08$ & $7.02 \mathrm{E}-08$ & $6.94 \mathrm{E}-08$ \\
\hline $\mathrm{Cd}-113 \mathrm{~m}$ & $2.35 \mathrm{E}-06$ & $1.35 \mathrm{E}-06$ & $1.34 \mathrm{E}-07$ & $1.31 \mathrm{E}-07$ & $5.12 \mathrm{E}-05$ & $1.14 \mathrm{E}-05$ & $1.39 \mathrm{E}-05$ \\
\hline Sn-121m & $4.74 \mathrm{E}-08$ & $2.71 \mathrm{E}-08$ & $6.66 \mathrm{E}-10$ & $6.49 \mathrm{E}-10$ & $2.54 \mathrm{E}-07$ & $2.83 \mathrm{E}-07$ & $2.81 \mathrm{E}-07$ \\
\hline Sn-126 & $4.63 \mathrm{E}-07$ & $2.65 \mathrm{E}-07$ & $6.51 \mathrm{E}-09$ & $6.34 \mathrm{E}-09$ & $2.48 \mathrm{E}-06$ & $2.76 \mathrm{E}-06$ & $2.74 \mathrm{E}-06$ \\
\hline Sb-125 & $5.55 \mathrm{E}-04$ & $3.19 \mathrm{E}-04$ & $7.81 \mathrm{E}-06$ & $7.61 \mathrm{E}-06$ & $2.98 \mathrm{E}-03$ & $3.32 \mathrm{E}-03$ & $3.30 \mathrm{E}-03$ \\
\hline Sb-126 & $4.08 \mathrm{E}-08$ & $2.34 \mathrm{E}-08$ & $5.74 \mathrm{E}-10$ & $5.59 \mathrm{E}-10$ & $2.19 \mathrm{E}-07$ & $2.44 \mathrm{E}-07$ & $2.42 \mathrm{E}-07$ \\
\hline Te-125m & $2.23 \mathrm{E}-06$ & $1.28 \mathrm{E}-06$ & $3.14 \mathrm{E}-08$ & $3.06 \mathrm{E}-08$ & $1.20 \mathrm{E}-05$ & $1.33 \mathrm{E}-05$ & $1.32 \mathrm{E}-05$ \\
\hline $\mathrm{I}-129$ & $5.96 \mathrm{E}-08$ & $3.42 \mathrm{E}-08$ & $8.92 \mathrm{E}-10$ & $8.69 \mathrm{E}-10$ & $3.20 \mathrm{E}-07$ & $3.55 \mathrm{E}-07$ & $3.52 \mathrm{E}-07$ \\
\hline Cs-134 & $5.84 \mathrm{E}-05$ & $3.35 \mathrm{E}-05$ & $5.54 \mathrm{E}-06$ & $5.40 \mathrm{E}-06$ & $2.11 \mathrm{E}-03$ & $2.26 \mathrm{E}-04$ & $3.46 \mathrm{E}-04$ \\
\hline Cs-135 & $9.30 \mathrm{E}-07$ & $5.33 \mathrm{E}-07$ & $8.82 \mathrm{E}-08$ & $8.59 \mathrm{E}-08$ & $3.37 \mathrm{E}-05$ & $3.60 \mathrm{E}-06$ & $5.51 \mathrm{E}-06$ \\
\hline Cs-137 & $3.77 \mathrm{E}-02$ & $2.16 \mathrm{E}-02$ & $3.58 \mathrm{E}-03$ & $3.49 \mathrm{E}-03$ & $1.37 \mathrm{E}+00$ & $1.46 \mathrm{E}-01$ & $2.24 \mathrm{E}-01$ \\
\hline Ba-137m & $3.57 \mathrm{E}-02$ & $2.04 \mathrm{E}-02$ & $4.55 \mathrm{E}-04$ & $4.43 \mathrm{E}-04$ & $1.74 \mathrm{E}-01$ & $2.14 \mathrm{E}-01$ & $2.11 \mathrm{E}-01$ \\
\hline $\mathrm{Ce}-144$ & $7.11 \mathrm{E}-07$ & $4.08 \mathrm{E}-07$ & $1.00 \mathrm{E}-08$ & $9.76 \mathrm{E}-09$ & $3.82 \mathrm{E}-06$ & $4.25 \mathrm{E}-06$ & $4.22 \mathrm{E}-06$ \\
\hline Pr-144 & $7.11 \mathrm{E}-07$ & $4.08 \mathrm{E}-07$ & $1.00 \mathrm{E}-08$ & $9.76 \mathrm{E}-09$ & $3.82 \mathrm{E}-06$ & $4.25 \mathrm{E}-06$ & $4.22 \mathrm{E}-06$ \\
\hline Pm-146 & $3.61 \mathrm{E}-08$ & $2.07 \mathrm{E}-08$ & $5.08 \mathrm{E}-10$ & $4.95 \mathrm{E}-10$ & $1.94 \mathrm{E}-07$ & $2.16 \mathrm{E}-07$ & $2.14 \mathrm{E}-07$ \\
\hline Pm-147 & 1.91E-04 & $1.10 \mathrm{E}-04$ & $2.70 \mathrm{E}-06$ & $2.63 \mathrm{E}-06$ & $1.03 \mathrm{E}-03$ & $1.14 \mathrm{E}-03$ & $1.14 \mathrm{E}-03$ \\
\hline Sm-151 & $3.79 \mathrm{E}-04$ & $2.18 \mathrm{E}-04$ & $5.34 \mathrm{E}-06$ & $5.20 \mathrm{E}-06$ & $2.04 \mathrm{E}-03$ & $2.26 \mathrm{E}-03$ & $2.25 \mathrm{E}-03$ \\
\hline Eu-152 & $2.28 \mathrm{E}-06$ & $1.31 \mathrm{E}-06$ & $3.21 \mathrm{E}-08$ & $3.12 \mathrm{E}-08$ & $1.22 \mathrm{E}-05$ & $1.36 \mathrm{E}-05$ & $1.35 \mathrm{E}-05$ \\
\hline Eu-154 & $8.56 \mathrm{E}-05$ & $4.91 \mathrm{E}-05$ & $1.20 \mathrm{E}-06$ & $1.17 \mathrm{E}-06$ & $4.60 \mathrm{E}-04$ & $5.11 \mathrm{E}-04$ & $5.08 \mathrm{E}-04$ \\
\hline Eu-155 & $9.01 \mathrm{E}-05$ & $5.17 \mathrm{E}-05$ & $1.27 \mathrm{E}-06$ & $1.24 \mathrm{E}-06$ & $4.84 \mathrm{E}-04$ & $5.39 \mathrm{E}-04$ & $5.35 \mathrm{E}-04$ \\
\hline Th-230 & $8.88 \mathrm{E}-10$ & $5.08 \mathrm{E}-10$ & $1.25 \mathrm{E}-11$ & $1.22 \mathrm{E}-11$ & $4.76 \mathrm{E}-09$ & $5.30 \mathrm{E}-09$ & $5.26 \mathrm{E}-09$ \\
\hline $\mathrm{Pa}-233$ & $2.08 \mathrm{E}-06$ & $1.19 \mathrm{E}-06$ & $2.92 \mathrm{E}-08$ & $2.84 \mathrm{E}-08$ & $1.11 \mathrm{E}-05$ & $1.24 \mathrm{E}-05$ & $1.23 \mathrm{E}-05$ \\
\hline $\mathrm{U}-232$ & $2.04 \mathrm{E}-09$ & $1.17 \mathrm{E}-09$ & $2.87 \mathrm{E}-11$ & $2.80 \mathrm{E}-11$ & $1.09 \mathrm{E}-08$ & $1.22 \mathrm{E}-08$ & $1.21 \mathrm{E}-08$ \\
\hline $\mathrm{U}-233$ & $6.76 \mathrm{E}-11$ & $3.87 \mathrm{E}-11$ & $9.50 \mathrm{E}-13$ & $9.25 \mathrm{E}-13$ & $3.62 \mathrm{E}-10$ & $4.03 \mathrm{E}-10$ & $4.01 \mathrm{E}-10$ \\
\hline $\mathrm{U}-234$ & $1.12 \mathrm{E}-06$ & $6.40 \mathrm{E}-07$ & $1.57 \mathrm{E}-08$ & $1.53 \mathrm{E}-08$ & $5.99 \mathrm{E}-06$ & $6.66 \mathrm{E}-06$ & $6.62 \mathrm{E}-06$ \\
\hline $\mathrm{U}-235$ & $5.82 \mathrm{E}-08$ & $3.33 \mathrm{E}-08$ & $8.18 \mathrm{E}-10$ & $7.97 \mathrm{E}-10$ & $3.12 \mathrm{E}-07$ & $3.47 \mathrm{E}-07$ & $3.45 \mathrm{E}-07$ \\
\hline $\mathrm{U}-236$ & $5.41 \mathrm{E}-08$ & $3.10 \mathrm{E}-08$ & $7.61 \mathrm{E}-10$ & $7.41 \mathrm{E}-10$ & $2.90 \mathrm{E}-07$ & $3.23 \mathrm{E}-07$ & $3.21 \mathrm{E}-07$ \\
\hline $\mathrm{U}-237$ & $4.56 \mathrm{E}-09$ & $2.61 \mathrm{E}-09$ & $6.41 \mathrm{E}-11$ & $6.24 \mathrm{E}-11$ & $2.45 \mathrm{E}-08$ & $2.72 \mathrm{E}-08$ & $2.70 \mathrm{E}-08$ \\
\hline $\mathrm{U}-238$ & $1.97 \mathrm{E}-08$ & $1.13 \mathrm{E}-08$ & $2.76 \mathrm{E}-10$ & $2.69 \mathrm{E}-10$ & $1.05 \mathrm{E}-07$ & $1.17 \mathrm{E}-07$ & $1.17 \mathrm{E}-07$ \\
\hline Np-237 & $5.38 \mathrm{E}-07$ & $3.09 \mathrm{E}-07$ & $7.57 \mathrm{E}-09$ & $7.38 \mathrm{E}-09$ & $2.89 \mathrm{E}-06$ & $3.21 \mathrm{E}-06$ & $3.19 \mathrm{E}-06$ \\
\hline Pu-236 & $3.20 \mathrm{E}-09$ & $1.83 \mathrm{E}-09$ & $4.50 \mathrm{E}-11$ & $4.38 \mathrm{E}-11$ & $1.71 \mathrm{E}-08$ & 1.91E-08 & $1.90 \mathrm{E}-08$ \\
\hline Pu-238 & $9.43 \mathrm{E}-04$ & $5.40 \mathrm{E}-04$ & $1.33 \mathrm{E}-05$ & $1.29 \mathrm{E}-05$ & $5.06 \mathrm{E}-03$ & $5.63 \mathrm{E}-03$ & $5.59 \mathrm{E}-03$ \\
\hline Pu-239 & $1.05 \mathrm{E}-04$ & $6.00 \mathrm{E}-05$ & $1.47 \mathrm{E}-06$ & $1.44 \mathrm{E}-06$ & $5.62 \mathrm{E}-04$ & $6.25 \mathrm{E}-04$ & $6.21 \mathrm{E}-04$ \\
\hline Pu-240 & $1.20 \mathrm{E}-05$ & 6.86E-06 & $1.68 \mathrm{E}-07$ & 1.64E-07 & $6.42 \mathrm{E}-05$ & $7.14 \mathrm{E}-05$ & $7.09 \mathrm{E}-05$ \\
\hline Pu-241 & $7.12 \mathrm{E}-04$ & $4.08 \mathrm{E}-04$ & $1.00 \mathrm{E}-05$ & $9.75 \mathrm{E}-06$ & $3.82 \mathrm{E}-03$ & $4.25 \mathrm{E}-03$ & $4.22 \mathrm{E}-03$ \\
\hline Pu-242 & $9.39 \mathrm{E}-09$ & $5.38 \mathrm{E}-09$ & $1.32 \mathrm{E}-10$ & $1.29 \mathrm{E}-10$ & $5.04 \mathrm{E}-08$ & $5.60 \mathrm{E}-08$ & $5.57 \mathrm{E}-08$ \\
\hline Pu-244 & $5.62 \mathrm{E}-16$ & $3.22 \mathrm{E}-16$ & $7.90 \mathrm{E}-18$ & $7.69 \mathrm{E}-18$ & $3.01 \mathrm{E}-15$ & $3.35 \mathrm{E}-15$ & $3.33 \mathrm{E}-15$ \\
\hline $\mathrm{Am}-241$ & $6.04 \mathrm{E}-05$ & $3.47 \mathrm{E}-05$ & $8.51 \mathrm{E}-07$ & $8.29 \mathrm{E}-07$ & $3.24 \mathrm{E}-04$ & $3.61 \mathrm{E}-04$ & $3.59 \mathrm{E}-04$ \\
\hline$A m-242 m$ & 1.09E-08 & $6.28 \mathrm{E}-09$ & $1.54 \mathrm{E}-10$ & $1.50 \mathrm{E}-10$ & $5.87 \mathrm{E}-08$ & $6.54 \mathrm{E}-08$ & $6.49 \mathrm{E}-08$ \\
\hline
\end{tabular}


Table A-20. Mass balance, Tank WM-187 waste (continued).

\begin{tabular}{|c|c|c|c|c|c|c|c|}
\hline Stream \# & 101 & 102 & 103 & 104 & 106 & 107 & 108 \\
\hline Radiological Composition & $\mathrm{Ci} / \mathrm{L}$ & $\mathrm{Ci} / \mathrm{L}$ & $\mathrm{Ci} / \mathrm{wscm}$ & Ci/wscm & $\mathrm{Ci} / \mathrm{kg}$ & $\mathrm{Ci} / \mathrm{kg}$ & $\mathrm{Ci} / \mathrm{kg}$ \\
\hline $\mathrm{Am}-243$ & $2.08 \mathrm{E}-08$ & $1.19 \mathrm{E}-08$ & $2.92 \mathrm{E}-10$ & $2.85 \mathrm{E}-10$ & $1.12 \mathrm{E}-07$ & $1.24 \mathrm{E}-07$ & $1.23 \mathrm{E}-07$ \\
\hline $\mathrm{Cm}-242$ & $2.15 \mathrm{E}-08$ & $1.23 \mathrm{E}-08$ & $3.02 \mathrm{E}-10$ & $2.94 \mathrm{E}-10$ & $1.15 \mathrm{E}-07$ & $1.28 \mathrm{E}-07$ & $1.27 \mathrm{E}-07$ \\
\hline $\mathrm{Cm}-243$ & $3.11 \mathrm{E}-08$ & $1.78 \mathrm{E}-08$ & $4.37 \mathrm{E}-10$ & $4.25 \mathrm{E}-10$ & $1.67 \mathrm{E}-07$ & $1.85 \mathrm{E}-07$ & $1.84 \mathrm{E}-07$ \\
\hline $\mathrm{Cm}-244$ & $1.38 \mathrm{E}-06$ & $7.93 \mathrm{E}-07$ & $1.95 \mathrm{E}-08$ & $1.90 \mathrm{E}-08$ & $7.42 \mathrm{E}-06$ & $8.26 \mathrm{E}-06$ & $8.20 \mathrm{E}-06$ \\
\hline $\mathrm{Cm}-245$ & $3.27 \mathrm{E}-10$ & $1.88 \mathrm{E}-10$ & $4.60 \mathrm{E}-12$ & $4.48 \mathrm{E}-12$ & $1.76 \mathrm{E}-09$ & 1.95E-09 & $1.94 \mathrm{E}-09$ \\
\hline $\mathrm{Cm}-246$ & $2.15 \mathrm{E}-11$ & $1.23 \mathrm{E}-11$ & $3.02 \mathrm{E}-13$ & $2.94 \mathrm{E}-13$ & $1.15 \mathrm{E}-10$ & $1.28 \mathrm{E}-10$ & $1.27 \mathrm{E}-10$ \\
\hline TRU & $1.14 \mathrm{E}-03$ & $6.55 \mathrm{E}-04$ & $1.61 \mathrm{E}-05$ & $1.56 \mathrm{E}-05$ & $6.13 \mathrm{E}-03$ & $6.82 \mathrm{E}-03$ & $6.77 \mathrm{E}-03$ \\
\hline & & & & & & & \\
\hline & & & & & & & \\
\hline $\begin{array}{l}\text { Gas Stream Bulk } \\
\text { Composition (Wet Basis) }\end{array}$ & $\begin{array}{c}\text { mol\% or } \\
\text { ppmv }\end{array}$ & $\begin{array}{c}\text { mol\% or } \\
\text { ppmv }\end{array}$ & $\begin{array}{c}\mathrm{mol} \% \text { or } \\
\text { ppmv }\end{array}$ & $\begin{array}{c}\mathrm{mol} \% \text { or } \\
\mathrm{ppmv}\end{array}$ & $\begin{array}{c}\text { mol\% or } \\
\text { ppmv }\end{array}$ & $\begin{array}{c}\mathrm{mol} \% \text { or } \\
\mathrm{ppmv}\end{array}$ & $\begin{array}{c}\text { mol\% or } \\
\text { ppmv }\end{array}$ \\
\hline $\mathrm{H} 2 \mathrm{O}, \mathrm{mol} \%$ & & & $30.16 \%$ & $29.38 \%$ & & & \\
\hline $\mathrm{O} 2, \mathrm{~mol} \%$ & & & & & & & \\
\hline $\mathrm{N} 2, \mathrm{~mol} \%$ & & & $1.49 \%$ & $4.03 \%$ & & & \\
\hline $\mathrm{H} 2, \mathrm{~mol} \%$ & & & $10.31 \%$ & $10.05 \%$ & & & \\
\hline $\mathrm{CO} 2, \mathrm{~mol} \%$ & & & $55.21 \%$ & $53.78 \%$ & & & \\
\hline COgas, ppmv & & & $4.93 \mathrm{E}+03$ & $4.80 \mathrm{E}+03$ & & & \\
\hline $\mathrm{NO}, \mathrm{ppmv}$ & & & $1.81 \mathrm{E}+03$ & $1.76 \mathrm{E}+03$ & & & \\
\hline NO2, ppmv & & & $1.73 \mathrm{E}+01$ & $1.68 \mathrm{E}+01$ & & & \\
\hline SO2, ppmv & & & $9.30 \mathrm{E}-06$ & $9.06 \mathrm{E}-06$ & & & \\
\hline $\mathrm{Cl}, \mathrm{ppmv}$ & & & $5.42 \mathrm{E}+00$ & $5.28 \mathrm{E}+00$ & & & \\
\hline F, ppmv & & & $3.13 \mathrm{E}+01$ & $3.05 \mathrm{E}+01$ & & & \\
\hline $\mathrm{C}$ (organic), ppmv & & & $1.78 \mathrm{E}+05$ & $1.73 \mathrm{E}+05$ & & & \\
\hline $\mathrm{H}$ (organic), ppmv & & & $3.97 \mathrm{E}+05$ & $3.87 \mathrm{E}+05$ & & & \\
\hline & & & & & & & \\
\hline $\mathrm{Hg}, \mathrm{ug} / \mathrm{wscm}$ & & & $3.67 \mathrm{E}+05$ & $3.57 \mathrm{E}+05$ & & & \\
\hline $\mathrm{PM}, \mathrm{mg} / \mathrm{wscm}$ & & & $1.08 \mathrm{E}+05$ & $1.05 \mathrm{E}+05$ & & & \\
\hline SVM, ug/wscm & & & $1.60 \mathrm{E}+04$ & $1.56 \mathrm{E}+04$ & & & \\
\hline LVM, ug/wscm & & & $4.05 \mathrm{E}+03$ & $3.95 \mathrm{E}+03$ & & & \\
\hline \begin{tabular}{|l|} 
Gas Stream Bulk \\
Composition (Dry Basis)
\end{tabular} & $\mathrm{mol} \%$ & $\mathrm{~mol} \%$ & $\mathrm{~mol} \%$ & $\mathrm{~mol} \%$ & $\mathrm{~mol} \%$ & $\mathrm{~mol} \%$ & $\mathrm{~mol} \%$ \\
\hline $\mathrm{O} 2, \mathrm{~mol} \%$, dry basis & & & & & & & \\
\hline $\mathrm{N} 2, \mathrm{~mol} \%$, dry basis & & & $2.13 \%$ & $5.71 \%$ & & & \\
\hline $\mathrm{H} 2$, mol \%, dry basis & & & $14.77 \%$ & $14.23 \%$ & & & \\
\hline $\mathrm{CO} 2, \mathrm{~mol} \%$, dry basis & & & $79.04 \%$ & $76.15 \%$ & & & \\
\hline $\begin{array}{l}\text { Gas Stream Bulk } \\
\text { Composition (Dry Basis, } \\
\text { Corrected to } 7 \% \text { O2 with } \\
100 \% \text { O2 Combustion Air) }\end{array}$ & $\begin{array}{l}\mathrm{ppmv}, \text { or } \\
\mathrm{ug} / \mathrm{dscm} \\
\text { or } \\
\mathrm{mg} / \mathrm{dscm}\end{array}$ & $\begin{array}{l}\text { ppmv, or } \\
\text { ug/dscm } \\
\text { or } \\
\mathrm{mg} / \mathrm{dscm}\end{array}$ & $\begin{array}{l}\text { ppmv, or } \\
\text { ug/dscm } \\
\text { or } \\
\mathrm{mg} / \mathrm{dscm}\end{array}$ & $\begin{array}{l}\mathrm{ppmv}, \text { or } \\
\mathrm{ug} / \mathrm{dscm} \\
\text { or } \\
\mathrm{mg} / \mathrm{dscm}\end{array}$ & $\begin{array}{l}\text { ppmv, or } \\
\mathrm{ug} / \mathrm{dscm} \text { or } \\
\mathrm{mg} / \mathrm{dscm}\end{array}$ & $\begin{array}{l}\text { ppmv, or } \\
\mathrm{ug} / \mathrm{dscm} \text { or } \\
\mathrm{mg} / \mathrm{dscm}\end{array}$ & $\begin{array}{l}\text { ppmv, or } \\
\text { ug/dscm or } \\
\mathrm{mg} / \mathrm{dscm}\end{array}$ \\
\hline COgas, ppmv, dry basis & & & $9.89 \mathrm{E}+02$ & $9.53 \mathrm{E}+02$ & & & \\
\hline NO, ppmv, dry basis & & & $3.63 \mathrm{E}+02$ & $3.50 \mathrm{E}+02$ & & & \\
\hline NO2, ppmv, dry basis & & & $3.46 \mathrm{E}+00$ & $3.33 \mathrm{E}+00$ & & & \\
\hline SO2, ppmv, dry basis & & & $1.87 \mathrm{E}-06$ & $1.80 \mathrm{E}-06$ & & & \\
\hline $\mathrm{Cl}, \mathrm{ppmv}$, dry basis & & & $1.09 \mathrm{E}+00$ & $1.05 \mathrm{E}+00$ & & & \\
\hline $\mathrm{F}, \mathrm{ppmv}$, dry basis & & & $6.28 \mathrm{E}+00$ & $6.05 \mathrm{E}+00$ & & & \\
\hline C (organic), ppmv, dry basis & & & $3.56 \mathrm{E}+04$ & $3.43 \mathrm{E}+04$ & & & \\
\hline $\mathrm{H}$ (organic), ppmv, dry basis & & & $7.96 \mathrm{E}+04$ & $7.67 \mathrm{E}+04$ & & & \\
\hline $\mathrm{Hg}, \mathrm{ug} / \mathrm{dscm}$ & & & $7.35 \mathrm{E}+04$ & $7.08 \mathrm{E}+04$ & & & \\
\hline $\mathrm{PM}, \mathrm{mg} / \mathrm{dscm}$ & & & $2.16 \mathrm{E}+04$ & $2.09 \mathrm{E}+04$ & & & \\
\hline SVM, ug/dscm & & & $2.29 \mathrm{E}+04$ & $2.23 \mathrm{E}+04$ & & & \\
\hline LVM, ug/dscm & & & $5.80 \mathrm{E}+03$ & $5.65 \mathrm{E}+03$ & & & \\
\hline
\end{tabular}


Table A-20. Mass balance, Tank WM-187 waste (continued).

\begin{tabular}{|c|c|c|c|c|c|c|c|c|}
\hline PFD Number & PFD-2 & PFD-2 & PFD-3 & PFD-3 & PFD-3 & PFD-3 & PFD-3 & PFD-3 \\
\hline Stream Number & 108 & 109 & 110 & 111 & 112 & $113 \mathrm{~A}$ & $113 \mathrm{~B}$ & 114 \\
\hline Stream Name & $\begin{array}{l}\text { Product } \\
\text { Shipping } \\
\text { Canisters }\end{array}$ & $\begin{array}{l}\text { Off-Gas } \\
\text { from Filter } \\
\text { to Oxidizer }\end{array}$ & $\begin{array}{l}\text { Outlet of } \\
\text { Oxidizer }\end{array}$ & $\begin{array}{l}\text { Quenched } \\
\text { Oxidizer Off } \\
\text { gas }\end{array}$ & Scrub & $\begin{array}{l}\text { Packed } \\
\text { Scrubber } \\
\text { Drain }\end{array}$ & $\begin{array}{c}\text { Demister } \\
\text { Drain }\end{array}$ & $\begin{array}{l}\text { Off-Gas to } \\
\text { Demister }\end{array}$ \\
\hline Rate or Volume & $1.29 \mathrm{E}+00$ & $1.92 \mathrm{E}+05$ & $6.40 \mathrm{E}+05$ & $3.53 \mathrm{E}+05$ & $9.69 \mathrm{E}+03$ & $9.71 E+03$ & 0 & $3.55 \mathrm{E}+05$ \\
\hline Volume Flow (standard, wet) $^{*}$ & & $5.43 \mathrm{E}+04$ & $9.41 \mathrm{E}+04$ & $1.75 \mathrm{E}+05$ & & & & $1.74 \mathrm{E}+05$ \\
\hline Volume Flow (standard, dry) & & $3.85 \mathrm{E}+04$ & $5.23 \mathrm{E}+04$ & $5.23 \mathrm{E}+04$ & & & & $5.21 \mathrm{E}+04$ \\
\hline Rate Units & cont/day & $\mathrm{ft} 3 / \mathrm{hr}$ & $\mathrm{ft} 3 / \mathrm{hr}$ & $\mathrm{ft} 3 / \mathrm{hr}$ & gal/hr & gal/hr & $\mathrm{gal} / \mathrm{hr}$ & $\mathrm{ft} 3 / \mathrm{hr}$ \\
\hline Rate or Volume, metric & $6.09 \mathrm{E}+01$ & $1.54 \mathrm{E}+03$ & $2.66 \mathrm{E}+03$ & $4.96 \mathrm{E}+03$ & $3.67 \mathrm{E}+04$ & $3.67 E+04$ & 1 & $4.92 \mathrm{E}+03$ \\
\hline Rate Units & $\mathrm{kg} / \mathrm{hr}$ & wscm/hr & wscm/hr & wscm/hr & $\mathrm{L} / \mathrm{hr}$ & $\mathrm{L} / \mathrm{hr}$ & $\mathrm{L} / \mathrm{hr}$ & wscm/hr \\
\hline Temperature, ${ }^{\circ} \mathrm{C}$ & 56 & 397 & 1000 & 100 & 77 & 79 & 339 & 78 \\
\hline Temperature, ${ }^{\circ} \mathrm{F}$ & 132 & 747 & 1832 & 212 & 171 & 173 & 170 & 172 \\
\hline \begin{tabular}{|l} 
Pressure, psia \\
\end{tabular} & 9.50 & 9.50 & 9.39 & 9.28 & 37.52 & 8.96 & 8.42 & 8.60 \\
\hline Specific Gravity & 1.42 & $3.87 \mathrm{E}-04$ & $1.93 \mathrm{E}-04$ & $5.22 \mathrm{E}-04$ & 1.03 & 1.03 & 1.03 & $5.17 \mathrm{E}-04$ \\
\hline Chemical Composition & Wt frac & $\mathrm{lb} / \mathrm{wscf}$ & $\mathrm{Ib} / \mathrm{wscf}$ & $\mathrm{lb} / \mathrm{wscf}$ & Mol/liter & Mol/liter & Mol/liter & $\mathrm{lb} / \mathrm{wscf}$ \\
\hline $\mathrm{H}+$ & $4.54 \mathrm{E}-05$ & $1.21 \mathrm{E}-06$ & $2.55 \mathrm{E}-08$ & $1.37 \mathrm{E}-08$ & $9.55 \mathrm{E}-01$ & $9.54 \mathrm{E}-01$ & & $1.25 \mathrm{E}-07$ \\
\hline $\mathrm{Al}+3$ & $5.50 \mathrm{E}-02$ & & & & $1.27 \mathrm{E}-02$ & $1.26 \mathrm{E}-02$ & & \\
\hline $\mathrm{Sb}+5$ & $1.78 \mathrm{E}-05$ & & & & $9.09 \mathrm{E}-07$ & $9.08 \mathrm{E}-07$ & & \\
\hline $\mathrm{As}+3$ & $6.38 \mathrm{E}-05$ & & & & $5.29 \mathrm{E}-06$ & $5.28 \mathrm{E}-06$ & & \\
\hline $\mathrm{Ba}+2$ & $4.90 \mathrm{E}-05$ & & & & & & & \\
\hline $\mathrm{Be}+2$ & $7.11 \mathrm{E}-07$ & & & & & & & \\
\hline$B+3$ & $5.97 \mathrm{E}-04$ & & & & $3.43 \mathrm{E}-04$ & $3.43 \mathrm{E}-04$ & & \\
\hline $\mathrm{Cd}+2$ & $9.93 \mathrm{E}-04$ & & & & & & & \\
\hline $\mathrm{Ca}+2$ & $7.05 \mathrm{E}-03$ & & & & & & & \\
\hline $\mathrm{Cr}+3$ & $1.19 \mathrm{E}-03$ & & & & & & & \\
\hline $\mathrm{Co}+2$ & $5.94 \mathrm{E}-04$ & & & & $6.26 \mathrm{E}-05$ & $6.25 \mathrm{E}-05$ & & \\
\hline $\begin{array}{l}\mathrm{Cs}+ \\
\end{array}$ & $5.38 \mathrm{E}-05$ & & & & & & & \\
\hline $\begin{array}{ll}\mathrm{Cu}+2 \\
\end{array}$ & $1.50 \mathrm{E}-04$ & & & & & & & \\
\hline $\mathrm{Fe}+3$ & $6.83 \mathrm{E}-03$ & $4.48 \mathrm{E}-09$ & $2.59 \mathrm{E}-09$ & $1.39 \mathrm{E}-09$ & $8.43 \mathrm{E}-05$ & $8.42 \mathrm{E}-05$ & & \\
\hline $\mathrm{Pb}+2$ & $6.70 \mathrm{E}-04$ & & & & & & & \\
\hline $\mathrm{Hg}+2$ & $1.78 \mathrm{E}-05$ & $2.20 \mathrm{E}-05$ & $1.27 \mathrm{E}-05$ & $6.82 \mathrm{E}-06$ & $6.43 \mathrm{E}-02$ & $6.42 \mathrm{E}-02$ & & $3.47 \mathrm{E}-06$ \\
\hline $\mathrm{Mn}+4$ & $4.99 \mathrm{E}-03$ & & & & $5.65 \mathrm{E}-04$ & $5.64 \mathrm{E}-04$ & & \\
\hline $\mathrm{Ni+2}$ & $4.00 \mathrm{E}-04$ & & & & & & & \\
\hline $\mathrm{K}+$ & $6.10 \mathrm{E}-02$ & & & & & & & \\
\hline $\mathrm{Se}+4$ & $1.69 \mathrm{E}-04$ & & & & & & & \\
\hline $\mathrm{Ag}^{+}$ & $3.52 \mathrm{E}-04$ & & & & & & & \\
\hline $\mathrm{Na}+$ & $2.05 \mathrm{E}-01$ & & & & & & & \\
\hline $\mathrm{Tl}+3$ & $5.94 \mathrm{E}-06$ & & & & $1.81 \mathrm{E}-07$ & $1.80 \mathrm{E}-07$ & & \\
\hline $\mathrm{U}+4$ & $3.24 \mathrm{E}-04$ & & & & & & & \\
\hline $\mathrm{V}+3$ & $1.57 \mathrm{E}-05$ & & & & & & & \\
\hline$Z n+2$ & $2.50 \mathrm{E}-04$ & & & & & & & \\
\hline $\mathrm{Zr}+4$ & $2.80 \mathrm{E}-02$ & & & & & & & \\
\hline $\mathrm{Cl}-$ & $3.16 \mathrm{E}-03$ & $2.86 \mathrm{E}-08$ & $1.65 \mathrm{E}-08$ & $8.87 \mathrm{E}-09$ & $3.15 \mathrm{E}-04$ & $3.14 \mathrm{E}-04$ & & $5.43 \mathrm{E}-09$ \\
\hline F- & $4.92 \mathrm{E}-03$ & $8.18 \mathrm{E}-07$ & $4.72 \mathrm{E}-07$ & $2.54 \mathrm{E}-07$ & $7.38 \mathrm{E}-03$ & $7.37 \mathrm{E}-03$ & & $2.21 \mathrm{E}-07$ \\
\hline SO4-2 & $8.16 \mathrm{E}-09$ & & $1.37 \mathrm{E}-10$ & $7.36 \mathrm{E}-11$ & & & & \\
\hline NO3- & 4.17E-03 & $1.23 \mathrm{E}-11$ & $7.17 \mathrm{E}-12$ & $3.85 \mathrm{E}-12$ & $9.09 \mathrm{E}-01$ & $9.08 \mathrm{E}-01$ & & $6.97 \mathrm{E}-06$ \\
\hline PO4-3 & $7.63 \mathrm{E}-02$ & & & & & & & \\
\hline $\mathrm{Am}+4$ & $8.44 \mathrm{E}-08$ & & & & $2.16 \mathrm{E}-09$ & $2.16 \mathrm{E}-09$ & & \\
\hline $\mathrm{Br}-$ & $9.95 \mathrm{E}-08$ & $9.02 \mathrm{E}-13$ & $5.21 \mathrm{E}-13$ & $2.80 \mathrm{E}-13$ & $4.40 \mathrm{E}-09$ & $4.40 \mathrm{E}-09$ & & $1.71 \mathrm{E}-13$ \\
\hline $\mathrm{Ce}+4$ & $3.04 \mathrm{E}-05$ & & & & $1.35 \mathrm{E}-06$ & $1.35 \mathrm{E}-06$ & & \\
\hline$E u+3$ & $3.16 \mathrm{E}-07$ & & & & $1.29 \mathrm{E}-08$ & $1.29 \mathrm{E}-08$ & & \\
\hline$G d+3$ & 1.19E-04 & & & & $4.70 \mathrm{E}-06$ & $4.69 \mathrm{E}-06$ & & \\
\hline $\mathrm{Ge}+4$ & $2.61 \mathrm{E}-09$ & & & & & & & \\
\hline $\ln +3$ & $6.93 \mathrm{E}-07$ & & & & 3.75E-08 & $3.74 \mathrm{E}-08$ & & \\
\hline |- & $2.06 \mathrm{E}-06$ & $1.86 \mathrm{E}-11$ & $1.08 \mathrm{E}-11$ & $5.78 \mathrm{E}-12$ & $5.73 \mathrm{E}-08$ & $5.72 \mathrm{E}-08$ & & $3.53 \mathrm{E}-12$ \\
\hline $\mathrm{La}+3$ & $5.23 \mathrm{E}-06$ & & & & $2.34 \mathrm{E}-07$ & $2.34 \mathrm{E}-07$ & & \\
\hline
\end{tabular}


Table A-20. Mass balance, Tank WM-187 waste (continued).

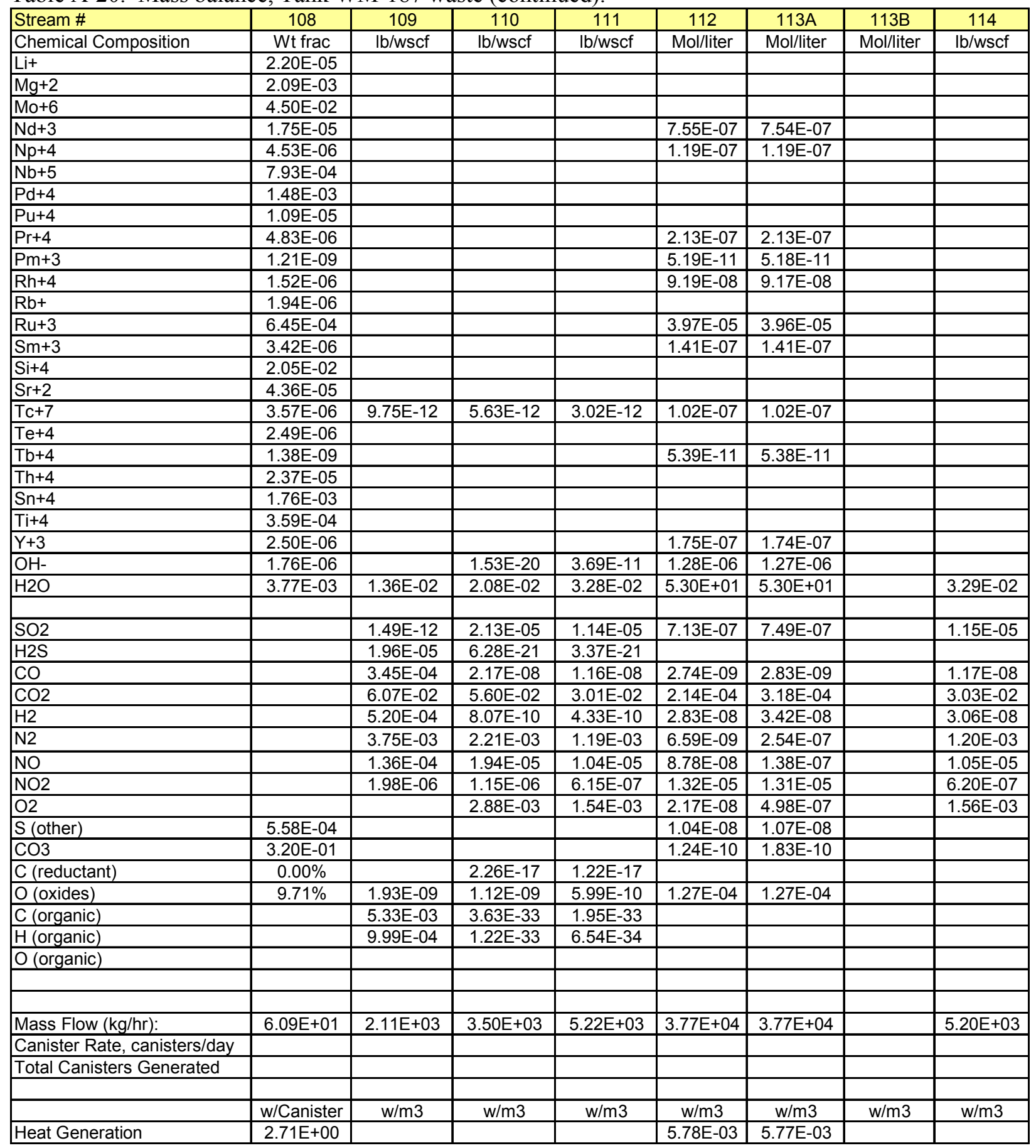


Table A-20. Mass balance, Tank WM-187 waste (continued).

\begin{tabular}{|c|c|c|c|c|c|c|c|c|}
\hline Stream \# & 108 & 109 & 110 & 111 & 112 & $113 \mathrm{~A}$ & 113B & 114 \\
\hline Radiological Composition & $\mathrm{Ci} /$ Canister & Ci/wscm & $\mathrm{Ci} / \mathrm{wscm}$ & Ci/wscm & $\mathrm{Ci} / \mathrm{L}$ & $\mathrm{Ci} / \mathrm{L}$ & $\mathrm{Ci} / \mathrm{L}$ & $\mathrm{Ci} / \mathrm{wscm}$ \\
\hline $\mathrm{H}-3$ & & $2.20 \mathrm{E}-06$ & $1.27 \mathrm{E}-06$ & $6.83 \mathrm{E}-07$ & $1.19 \mathrm{E}-06$ & 1.19E-06 & & $6.85 \mathrm{E}-07$ \\
\hline $\mathrm{C}-14$ & $6.94 \mathrm{E}-09$ & $3.16 \mathrm{E}-11$ & $1.83 \mathrm{E}-11$ & $9.81 \mathrm{E}-12$ & $1.92 \mathrm{E}-16$ & $2.85 \mathrm{E}-16$ & & $9.88 \mathrm{E}-12$ \\
\hline Co-60 & 9.79E-02 & & & & 5.37E-07 & $5.36 \mathrm{E}-07$ & & \\
\hline Ni-59 & 1.07E-02 & & & & & & & \\
\hline $\mathrm{Ni}-63$ & $2.49 \mathrm{E}-01$ & & & & & & & \\
\hline Se-79 & 3.30E-03 & & & & & & & \\
\hline Sr-90 & $1.87 \mathrm{E}+02$ & & & & & & & \\
\hline$\overline{Y-90}$ & $1.88 \mathrm{E}+02$ & & & & $1.03 \mathrm{E}-03$ & $1.03 \mathrm{E}-03$ & & \\
\hline Zr-93 & 1.06E-02 & & & & & & & \\
\hline $\mathrm{Nb}-93 \mathrm{~m}$ & $8.13 \mathrm{E}-03$ & & & & & & & \\
\hline Nb-94 & $8.58 \mathrm{E}-03$ & & & & & & & \\
\hline Tc-99 & 9.94E-02 & $3.84 \mathrm{E}-09$ & $2.21 \mathrm{E}-09$ & $1.19 \mathrm{E}-09$ & $2.47 \mathrm{E}-07$ & $2.46 \mathrm{E}-07$ & & \\
\hline Ru-106 & $7.09 \mathrm{E}-03$ & & & & 3.88E-08 & $3.88 \mathrm{E}-08$ & & \\
\hline Rh-102 & $4.11 \mathrm{E}-06$ & & & & $2.25 \mathrm{E}-11$ & $2.25 \mathrm{E}-11$ & & \\
\hline Rh-106 & $7.09 \mathrm{E}-03$ & & & & $3.88 \mathrm{E}-08$ & $3.88 \mathrm{E}-08$ & & \\
\hline Pd-107 & 7.87E-05 & & & & & & & \\
\hline $\mathrm{Cd}-113 \mathrm{~m}$ & $1.58 \mathrm{E}-02$ & & & & & & & \\
\hline Sn-121m & $3.18 \mathrm{E}-04$ & & & & & & & \\
\hline Sn-126 & $3.11 \mathrm{E}-03$ & & & & & & & \\
\hline Sb-125 & $3.74 \mathrm{E}+00$ & & & & $2.05 \mathrm{E}-05$ & $2.05 \mathrm{E}-05$ & & \\
\hline Sb-126 & $2.74 \mathrm{E}-04$ & & & & $1.50 \mathrm{E}-09$ & 1.50E-09 & & \\
\hline Te-125m & $1.50 \mathrm{E}-02$ & & & & & & & \\
\hline $\mid-129$ & $4.00 \mathrm{E}-04$ & $5.11 \mathrm{E}-11$ & $2.95 \mathrm{E}-11$ & $1.59 \mathrm{E}-11$ & $1.24 \mathrm{E}-09$ & $1.24 \mathrm{E}-09$ & & $9.69 \mathrm{E}-12$ \\
\hline Cs-134 & 3.93E-01 & & & & & & & \\
\hline Cs-135 & $6.25 E-03$ & & & & & & & \\
\hline Cs-137 & $2.53 \mathrm{E}+02$ & & & & & & & \\
\hline Ba-137m & $2.40 \mathrm{E}+02$ & & & & & & & \\
\hline $\mathrm{Ce}-144$ & 4.79E-03 & & & & $2.63 \mathrm{E}-08$ & $2.62 \mathrm{E}-08$ & & \\
\hline Pr-144 & 4.79E-03 & & & & $2.63 \mathrm{E}-08$ & $2.62 \mathrm{E}-08$ & & \\
\hline $\mathrm{Pm}-146$ & $2.43 \mathrm{E}-04$ & & & & 1.33E-09 & 1.33E-09 & & \\
\hline Pm-147 & $1.29 \mathrm{E}+00$ & & & & 7.06E-06 & 7.05E-06 & & \\
\hline Sm-151 & $2.55 \mathrm{E}+00$ & & & & $1.40 \mathrm{E}-05$ & $1.40 \mathrm{E}-05$ & & \\
\hline Eu-152 & $1.53 \mathrm{E}-02$ & & & & $8.40 \mathrm{E}-08$ & $8.39 \mathrm{E}-08$ & & \\
\hline Eu-154 & $5.76 \mathrm{E}-01$ & & & & $3.16 \mathrm{E}-06$ & $3.15 E-06$ & & \\
\hline Eu-155 & $6.07 \mathrm{E}-01$ & & & & 3.33E-06 & 3.32E-06 & & \\
\hline & & & & & & & & \\
\hline Th-230 & 5.97E-06 & & & & & & & \\
\hline $\mathrm{Pa}-233$ & $1.40 \mathrm{E}-02$ & & & & & & & \\
\hline U-232 & $1.37 \mathrm{E}-05$ & & & & & & & \\
\hline U-233 & $4.54 \mathrm{E}-07$ & & & & & & & \\
\hline U-234 & $7.51 \mathrm{E}-03$ & & & & & & & \\
\hline U-235 & $3.91 \mathrm{E}-04$ & & & & & & & \\
\hline U-236 & $3.64 \mathrm{E}-04$ & & & & & & & \\
\hline U-237 & 3.07E-05 & & & & & & & \\
\hline U-238 & $1.32 \mathrm{E}-04$ & & & & & & & \\
\hline Np-237 & $3.62 \mathrm{E}-03$ & & & & $1.99 \mathrm{E}-08$ & $1.98 \mathrm{E}-08$ & & \\
\hline Pu-236 & $2.15 \mathrm{E}-05$ & & & & & & & \\
\hline Pu-238 & $6.34 \mathrm{E}+00$ & & & & & & & \\
\hline Pu-239 & 7.04E-01 & & & & & & & \\
\hline Pu-240 & $8.05 \mathrm{E}-02$ & & & & & & & \\
\hline Pu-241 & $4.79 \mathrm{E}+00$ & & & & & & & \\
\hline Pu-242 & $6.31 \mathrm{E}-05$ & & & & & & & \\
\hline Pu-244 & $3.78 \mathrm{E}-12$ & & & & & & & \\
\hline Am-241 & 4.07E-01 & & & & $2.23 \mathrm{E}-06$ & $2.23 \mathrm{E}-06$ & & \\
\hline Am-242m & $7.36 \mathrm{E}-05$ & & & & $4.04 \mathrm{E}-10$ & $4.03 \mathrm{E}-10$ & & \\
\hline
\end{tabular}


Table A-20. Mass balance, Tank WM-187 waste (continued).

\begin{tabular}{|c|c|c|c|c|c|c|c|c|}
\hline Stream \# & 108 & 109 & 110 & 111 & 112 & $113 \mathrm{~A}$ & $113 \mathrm{~B}$ & 114 \\
\hline Radiological Composition & $\mathrm{Ci} /$ Canister & Ci/wscm & $\mathrm{Ci} / \mathrm{wscm}$ & Ci/wscm & $\mathrm{Ci} / \mathrm{L}$ & $\mathrm{Ci} / \mathrm{L}$ & $\mathrm{Ci} / \mathrm{L}$ & $\mathrm{Ci} / \mathrm{wscm}$ \\
\hline $\mathrm{Am}-243$ & $1.40 \mathrm{E}-04$ & & & & $7.66 \mathrm{E}-10$ & $7.65 \mathrm{E}-10$ & & \\
\hline Cm-242 & $1.44 \mathrm{E}-04$ & & & & & & & \\
\hline $\mathrm{Cm}-243$ & $2.09 \mathrm{E}-04$ & & & & & & & \\
\hline $\mathrm{Cm}-244$ & $9.30 \mathrm{E}-03$ & & & & & & & \\
\hline $\mathrm{Cm}-245$ & $2.20 \mathrm{E}-06$ & & & & & & & \\
\hline $\mathrm{Cm}-246$ & $1.44 \mathrm{E}-07$ & & & & & & & \\
\hline TRU & $7.68 \mathrm{E}+00$ & & & & $2.25 \mathrm{E}-06$ & $2.25 \mathrm{E}-06$ & & \\
\hline & & & & & & & & \\
\hline & & & & & & & & \\
\hline $\begin{array}{l}\text { Gas Stream Bulk } \\
\text { Composition (Wet Basis) }\end{array}$ & $\begin{array}{c}\text { mol\% or } \\
\text { ppmv }\end{array}$ & $\begin{array}{c}\mathrm{mol} \% \text { or } \\
\mathrm{ppmv}\end{array}$ & $\begin{array}{c}\mathrm{mol} \% \text { or } \\
\mathrm{ppmv}\end{array}$ & $\begin{array}{c}\mathrm{mol} \% \text { or } \\
\mathrm{ppmv}\end{array}$ & $\begin{array}{c}\mathrm{mol} \% \text { or } \\
\mathrm{ppmv}\end{array}$ & $\begin{array}{c}\mathrm{mol} \% \text { or } \\
\text { ppmv }\end{array}$ & $\begin{array}{c}\mathrm{mol} \% \text { or } \\
\text { ppmv }\end{array}$ & $\begin{array}{c}\mathrm{mol} \% \text { or } \\
\mathrm{ppmv}\end{array}$ \\
\hline $\mathrm{H} 2 \mathrm{O}, \mathrm{mol} \%$ & & $29.05 \%$ & $44.44 \%$ & $70.17 \%$ & & & & $70.04 \%$ \\
\hline $\mathrm{O} 2, \mathrm{~mol} \%$ & & & $3.46 \%$ & $1.86 \%$ & & & & $1.87 \%$ \\
\hline $\mathrm{N} 2, \mathrm{~mol} \%$ & & $5.16 \%$ & $3.04 \%$ & $1.63 \%$ & & & & $1.64 \%$ \\
\hline $\mathrm{H} 2, \mathrm{~mol} \%$ & & $9.93 \%$ & $0.00002 \%$ & $0.00001 \%$ & & & & $0.001 \%$ \\
\hline $\mathrm{CO} 2, \mathrm{~mol} \%$ & & $53.18 \%$ & $49.02 \%$ & $26.32 \%$ & & & & $26.43 \%$ \\
\hline COgas, ppmv & & $4.75 \mathrm{E}+03$ & $2.98 \mathrm{E}-01$ & $1.60 \mathrm{E}-01$ & & & & $1.61 \mathrm{E}-01$ \\
\hline $\mathrm{NO}, \mathrm{ppmv}$ & & $1.74 \mathrm{E}+03$ & $2.49 \mathrm{E}+02$ & $1.34 \mathrm{E}+02$ & & & & $1.34 \mathrm{E}+02$ \\
\hline NO2, ppmv & & $1.66 \mathrm{E}+01$ & $9.60 \mathrm{E}+00$ & $5.15 \mathrm{E}+00$ & & & & $5.18 \mathrm{E}+00$ \\
\hline SO2, ppmv & & $8.96 \mathrm{E}-06$ & $1.28 \mathrm{E}+02$ & $6.88 \mathrm{E}+01$ & & & & $6.91 \mathrm{E}+01$ \\
\hline $\mathrm{Cl}, \mathrm{ppmv}$ & & $3.11 \mathrm{E}-01$ & $1.80 \mathrm{E}-01$ & 9.64E-02 & & & & $5.87 \mathrm{E}-02$ \\
\hline $\mathrm{F}, \mathrm{ppmv}$ & & $1.66 \mathrm{E}+01$ & $9.58 \mathrm{E}+00$ & $5.14 \mathrm{E}+00$ & & & & $4.47 \mathrm{E}+00$ \\
\hline C (organic), ppmv & & $1.71 \mathrm{E}+05$ & & & & & & \\
\hline $\mathrm{H}$ (organic), ppmv & & $3.82 \mathrm{E}+05$ & & & & & & \\
\hline & & & & & & & & \\
\hline $\mathrm{Hg}, \mathrm{ug} / \mathrm{wscm}$ & & $3.53 \mathrm{E}+05$ & $2.04 \mathrm{E}+05$ & $1.09 \mathrm{E}+05$ & & & & \\
\hline $\mathrm{PM}, \mathrm{mg} / \mathrm{wscm}$ & & $1.02 \mathrm{E}+05$ & $2.09 \mathrm{E}+02$ & $1.18 \mathrm{E}+02$ & & & & $8.51 \mathrm{E}+00$ \\
\hline SVM, ug/wscm & & & & & & & & \\
\hline LVM, ug/wscm & & & & & & & & \\
\hline $\begin{array}{l}\text { Gas Stream Bulk } \\
\text { Composition (Dry Basis) }\end{array}$ & $\mathrm{mol} \%$ & $\mathrm{~mol} \%$ & $\mathrm{~mol} \%$ & $\mathrm{~mol} \%$ & $\mathrm{~mol} \%$ & $\mathrm{~mol} \%$ & $\mathrm{~mol} \%$ & $\mathrm{~mol} \%$ \\
\hline $\mathrm{O} 2, \mathrm{~mol} \%$, dry basis & & & $6.23 \%$ & $6.23 \%$ & & & & $6.23 \%$ \\
\hline $\mathrm{N} 2, \mathrm{~mol} \%$, dry basis & & $7.27 \%$ & $5.48 \%$ & $5.48 \%$ & & & & $5.48 \%$ \\
\hline $\mathrm{H} 2, \mathrm{~mol} \%$, dry basis & & $14.00 \%$ & $0.00003 \%$ & $0.00003 \%$ & & & & $0.002 \%$ \\
\hline $\mathrm{CO} 2, \mathrm{~mol} \%$, dry basis & & $74.95 \%$ & $88.22 \%$ & $88.22 \%$ & & & & $88.20 \%$ \\
\hline $\begin{array}{l}\text { Gas Stream Bulk } \\
\text { Composition (Dry Basis, } \\
\text { Corrected to } 7 \% \text { O2 with } \\
100 \% \text { O2 Combustion Air) } \\
\end{array}$ & $\begin{array}{l}\text { ppmv, or } \\
\text { ug/dscm or } \\
\mathrm{mg} / \mathrm{dscm}\end{array}$ & $\begin{array}{c}\mathrm{ppmv}, \text { or } \\
\text { ug/dscm or } \\
\mathrm{mg} / \mathrm{dscm}\end{array}$ & $\begin{array}{l}\mathrm{ppmv}, \text { or } \\
\mathrm{ug} / \mathrm{dscm} \text { or } \\
\mathrm{mg} / \mathrm{dscm}\end{array}$ & $\begin{array}{l}\mathrm{ppmv}, \text { or } \\
\mathrm{ug} / \mathrm{dscm} \text { or } \\
\mathrm{mg} / \mathrm{dscm}\end{array}$ & $\begin{array}{l}\mathrm{ppmv}, \text { or } \\
\mathrm{ug} / \mathrm{dscm} \\
\text { or } \\
\mathrm{mg} / \mathrm{dscm}\end{array}$ & $\begin{array}{l}\mathrm{ppmv}, \text { or } \\
\mathrm{ug} / \mathrm{dscm} \text { or } \\
\mathrm{mg} / \mathrm{dscm}\end{array}$ & $\begin{array}{l}\mathrm{ppmv}, \text { or } \\
\mathrm{ug} / \mathrm{dscm} \\
\text { or } \\
\mathrm{mg} / \mathrm{dscm}\end{array}$ & $\begin{array}{l}\mathrm{ppmv}, \text { or } \\
\text { ug/dscm or } \\
\mathrm{mg} / \mathrm{dscm}\end{array}$ \\
\hline COgas, ppmv, dry basis & & $9.37 \mathrm{E}+02$ & $7.51 \mathrm{E}-02$ & $7.51 \mathrm{E}-02$ & & & & $7.51 \mathrm{E}-02$ \\
\hline NO, ppmv, dry basis & & $3.44 \mathrm{E}+02$ & $6.28 \mathrm{E}+01$ & $6.28 \mathrm{E}+01$ & & & & $6.28 \mathrm{E}+01$ \\
\hline NO2, ppmv, dry basis & & $3.28 \mathrm{E}+00$ & $2.42 \mathrm{E}+00$ & $2.42 \mathrm{E}+00$ & & & & $2.42 \mathrm{E}+00$ \\
\hline SO2, ppmv, dry basis & & $1.77 \mathrm{E}-06$ & $3.23 \mathrm{E}+01$ & $3.23 \mathrm{E}+01$ & & & & $3.23 \mathrm{E}+01$ \\
\hline $\mathrm{Cl}, \mathrm{ppmv}$, dry basis & & $6.14 \mathrm{E}-02$ & $4.53 \mathrm{E}-02$ & $4.53 \mathrm{E}-02$ & & & & $2.75 \mathrm{E}-02$ \\
\hline $\mathrm{F}, \mathrm{ppmv}$, dry basis & & $3.27 E+00$ & $2.42 \mathrm{E}+00$ & $2.42 \mathrm{E}+00$ & & & & $2.09 \mathrm{E}+00$ \\
\hline C (organic), ppmv, dry basis & & $3.37 \mathrm{E}+04$ & $2.93 E-26$ & $2.93 \mathrm{E}-26$ & & & & \\
\hline $\mathrm{H}$ (organic), ppmv, dry basis & & $7.53 \mathrm{E}+04$ & $1.17 \mathrm{E}-25$ & $1.17 \mathrm{E}-25$ & & & & \\
\hline $\mathrm{Hg}, \mathrm{ug} / \mathrm{dscm}$ & & $6.96 \mathrm{E}+04$ & $5.13 \mathrm{E}+04$ & $5.13 \mathrm{E}+04$ & & & & \\
\hline $\mathrm{PM}, \mathrm{mg} / \mathrm{dscm}$ & & $2.01 \mathrm{E}+04$ & $5.28 \mathrm{E}+01$ & $5.53 \mathrm{E}+01$ & & & & $3.98 \mathrm{E}+00$ \\
\hline SVM, ug/dscm & & & & & & & & \\
\hline LVM, ug/dscm & & & & & & & & \\
\hline
\end{tabular}


Table A-20. Mass balance, Tank WM-187 waste (continued).

\begin{tabular}{|c|c|c|c|c|c|c|}
\hline PFD Number & PFD-3 & PFD-3 & PFD-3 & PFD-3 & PFD-3 & PFD-3 \\
\hline Stream Number & 115 & 116 & 117 & 118 & 119 & 120 \\
\hline Stream Name & $\begin{array}{l}\text { Scrubbed } \\
\text { Off-Gas to } \\
\text { Preheater }\end{array}$ & $\begin{array}{c}\text { Preheated } \\
\text { Off-gas to } \\
\text { GAC }\end{array}$ & $\begin{array}{c}\text { Pressure } \\
\text { Control Bleed } \\
\text { Air }\end{array}$ & $\begin{array}{c}\text { Final HEPA } \\
\text { Off-Gas } \\
\text { Outlet }\end{array}$ & $\begin{array}{l}\text { Off-gas to } \\
\text { Exhaust } \\
\text { Blower }\end{array}$ & Spent GAC \\
\hline Rate or Volume & $3.88 \mathrm{E}+05$ & $4.80 \mathrm{E}+05$ & $1.56 \mathrm{E}+04$ & $5.83 E+05$ & $6.24 \mathrm{E}+05$ & $4.66 \mathrm{E}+00$ \\
\hline Volume Flow (standard, wet) & $1.74 \mathrm{E}+05$ & $1.74 \mathrm{E}+05$ & $1.53 \mathrm{E}+04$ & $1.74 \mathrm{E}+05$ & $1.89 \mathrm{E}+05$ & \\
\hline Volume Flow (standard, dry) ${ }^{*}$ & $5.23 \mathrm{E}+04$ & $5.23 \mathrm{E}+04$ & $1.53 \mathrm{E}+04$ & $5.23 \mathrm{E}+04$ & $6.75 \mathrm{E}+04$ & \\
\hline Rate Units & $\mathrm{ft} 3 / \mathrm{hr}$ & $\mathrm{ft} 3 / \mathrm{hr}$ & $\mathrm{ft} 3 / \mathrm{hr}$ & $\mathrm{ft} 3 / \mathrm{hr}$ & $\mathrm{ft} 3 / \mathrm{hr}$ & $\mathrm{Ib} / \mathrm{hr}$ \\
\hline Rate or Volume, metric & $4.94 \mathrm{E}+03$ & $4.94 \mathrm{E}+03$ & $4.33 \mathrm{E}+02$ & $4.94 \mathrm{E}+03$ & $5.36 \mathrm{E}+03$ & $2.11 \mathrm{E}+00$ \\
\hline Rate Units & $\mathrm{wscm} / \mathrm{hr}$ & $\mathrm{wscm} / \mathrm{hr}$ & $\mathrm{wscm} / \mathrm{hr}$ & wscm $/ \mathrm{hr}$ & $\mathrm{wscm} / \mathrm{hr}$ & $\mathrm{kg} / \mathrm{hr}$ \\
\hline Temperature, ${ }^{\circ} \mathrm{C}$ & 77 & 120 & 25 & 120 & 114 & 27 \\
\hline Temperature, ${ }^{\circ} \mathrm{F}$ & 170 & 248 & 77 & 248 & 237 & 81 \\
\hline Pressure, psia & 7.88 & 7.16 & 14.70 & 5.89 & 5.89 & 14.70 \\
\hline Specific Gravity & $4.73 \mathrm{E}-04$ & $3.82 \mathrm{E}-04$ & $1.18 \mathrm{E}-03$ & $3.15 \mathrm{E}-04$ & $3.24 \mathrm{E}-04$ & $5.86 \mathrm{E}-01$ \\
\hline Chemical Composition & Ib/wscf & $\mathrm{lb} / \mathrm{wscf}$ & lb/wscf & Ib/wscf & $\mathrm{lb} /$ wscf & wt frac \\
\hline $\mathrm{H}+$ & $1.25 \mathrm{E}-07$ & $1.25 \mathrm{E}-07$ & & $1.25 \mathrm{E}-07$ & $1.15 \mathrm{E}-07$ & $4.27 \mathrm{E}-06$ \\
\hline \multicolumn{7}{|l|}{$\mathrm{Al}+3$} \\
\hline \multicolumn{7}{|l|}{$\mathrm{Sb}+5$} \\
\hline \multirow{2}{*}{\multicolumn{7}{|c|}{ As+3 }} \\
\hline \multirow{2}{*}{\multicolumn{7}{|c|}{$\frac{\mathrm{Ba}+2}{\mathrm{Be}+2}$}} \\
\hline & & & & & & $\mathrm{Be}+2$ \\
\hline \multicolumn{7}{|l|}{$\mathrm{B}+3$} \\
\hline \multirow{2}{*}{\multicolumn{7}{|c|}{$\frac{\mathrm{Cd}+2}{\mathrm{Ca}+2}$}} \\
\hline & & & & & & \\
\hline \multicolumn{7}{|l|}{\begin{tabular}{|l}
$\mathrm{Ca}+2$ \\
$\mathrm{Cr}+3$ \\
\end{tabular}} \\
\hline \multicolumn{7}{|l|}{$\mathrm{Co}+2$} \\
\hline \multicolumn{7}{|l|}{ Cs+ } \\
\hline \multicolumn{7}{|l|}{$\mathrm{Cu}+2$} \\
\hline \multirow{2}{*}{\multicolumn{7}{|c|}{$\frac{\mathrm{Fe}+3}{\mathrm{~Pb}+2}$}} \\
\hline & & & & & \multicolumn{2}{|c|}{$\mathrm{Pb}+2$} \\
\hline $\mathrm{Hg}+2$ & $3.46 \mathrm{E}-06$ & $3.46 \mathrm{E}-06$ & & $2.12 \mathrm{E}-09$ & $1.95 \mathrm{E}-09$ & $1.30 \mathrm{E}-01$ \\
\hline \multicolumn{7}{|l|}{$\mathrm{Mn}+4$} \\
\hline \multirow{2}{*}{\multicolumn{7}{|c|}{$\mathrm{Ni}+2$}} \\
\hline \multirow{2}{*}{\multicolumn{5}{|c|}{\begin{tabular}{|l}
$\mathrm{K}+$ \\
$\mathrm{Se}+4$
\end{tabular}}} & & \\
\hline \multirow{2}{*}{\multicolumn{7}{|c|}{$\frac{\mathrm{Se}+4}{\mathrm{Ag}+}$}} \\
\hline & & & & & & \\
\hline $\mathrm{Na}+$ & & & & & & \\
\hline $\mathrm{Tl}+3$ & & & & & & \\
\hline $\mathrm{U}+4$ & & & & & & \\
\hline $\mathrm{V}+3$ & & & & & & \\
\hline$Z n+2$ & & & & & & \\
\hline $\mathrm{Zr}+4$ & & & & & & \\
\hline $\mathrm{Cl}-$ & $5.41 \mathrm{E}-09$ & $5.41 \mathrm{E}-09$ & & 1.40E-09 & $1.29 \mathrm{E}-09$ & $1.50 \mathrm{E}-04$ \\
\hline F- & $2.20 \mathrm{E}-07$ & $2.20 \mathrm{E}-07$ & & $2.20 \mathrm{E}-07$ & $2.03 \mathrm{E}-07$ & \\
\hline SO4-2 & & & & & & \\
\hline NO3- & $6.94 \mathrm{E}-06$ & $6.94 \mathrm{E}-06$ & & $6.94 \mathrm{E}-06$ & $6.39 \mathrm{E}-06$ & \\
\hline PO4-3 & & & & & & \\
\hline$A m+4$ & & & & & & \\
\hline $\mathrm{Br}-$ & $1.70 \mathrm{E}-13$ & $1.70 \mathrm{E}-13$ & & $4.40 \mathrm{E}-14$ & $4.05 \mathrm{E}-14$ & $4.73 \mathrm{E}-09$ \\
\hline $\mathrm{Ce}+4$ & & & & & & \\
\hline $\mathrm{Eu}+3$ & & & & & & \\
\hline $\mathrm{Gd}+3$ & & & & & & \\
\hline $\mathrm{Ge}+4$ & & & & & & \\
\hline $\ln +3$ & & & & & & \\
\hline I- & $3.52 \mathrm{E}-12$ & $3.52 \mathrm{E}-12$ & & $9.10 \mathrm{E}-13$ & $8.38 \mathrm{E}-13$ & $9.79 \mathrm{E}-08$ \\
\hline $\mathrm{La}+3$ & & & & & & \\
\hline
\end{tabular}


Table A-20. Mass balance, Tank WM-187 waste (continued).

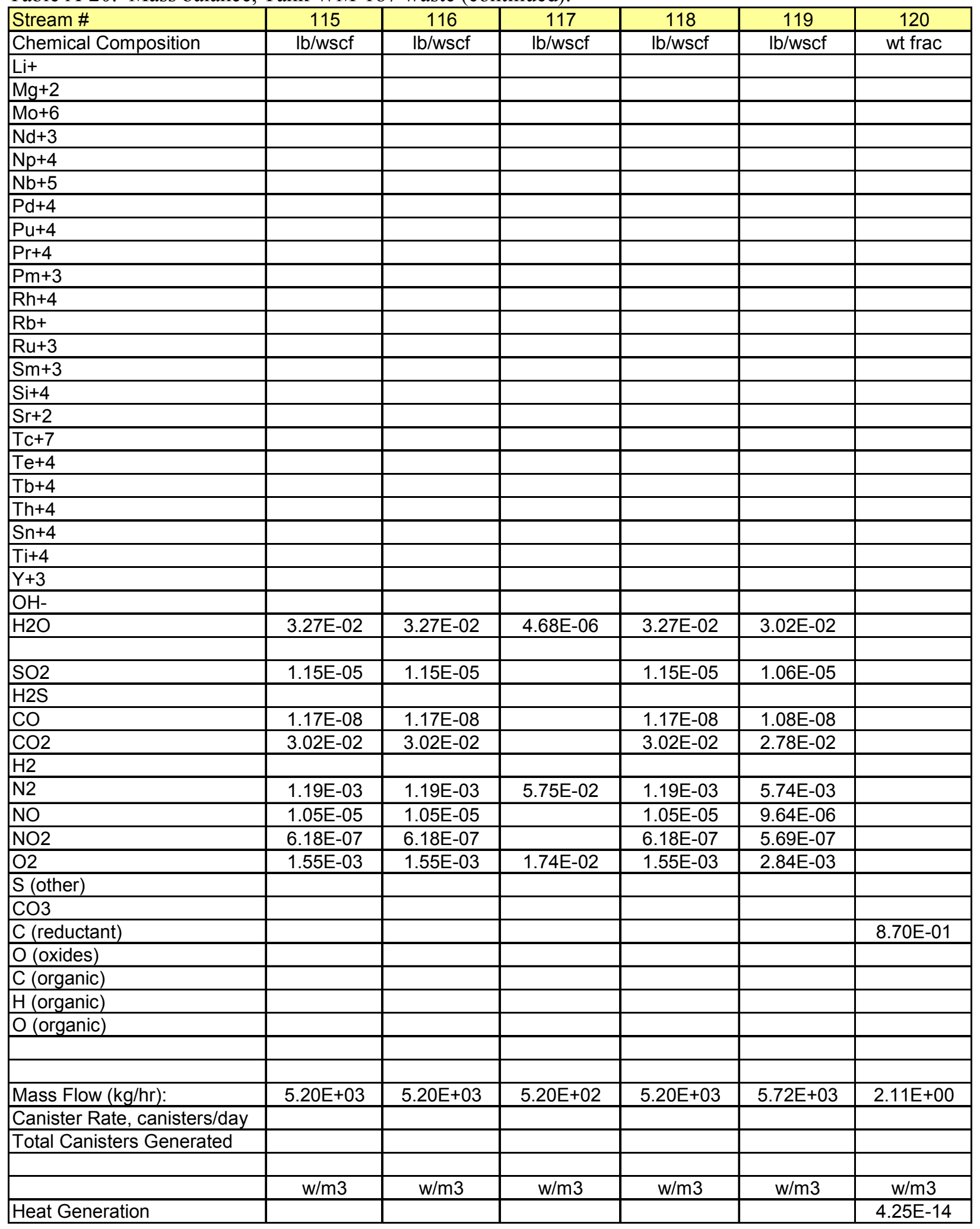


Table A-20. Mass balance, Tank WM-187 waste (continued).

\begin{tabular}{|c|c|c|c|c|c|c|}
\hline Stream \# & 115 & 116 & 117 & 118 & 119 & 120 \\
\hline Radiological Composition & Ci/wscm & Ci/wscm & Ci/wscm & $\mathrm{Ci} / \mathrm{wscm}$ & Ci/wscm & $\mathrm{Ci} / \mathrm{kg}$ \\
\hline $\mathrm{H}-3$ & $6.82 \mathrm{E}-07$ & $6.82 \mathrm{E}-07$ & & $6.82 \mathrm{E}-07$ & $6.28 \mathrm{E}-07$ & \\
\hline C-14 & $9.85 \mathrm{E}-12$ & $9.85 \mathrm{E}-12$ & & $9.85 \mathrm{E}-12$ & $9.07 \mathrm{E}-12$ & $6.87 \mathrm{E}-11$ \\
\hline \multicolumn{7}{|l|}{\begin{tabular}{|l|} 
Co-60 \\
Ni-59
\end{tabular}} \\
\hline $\mathrm{Ni}-59$ & & & & & & \\
\hline \multicolumn{7}{|l|}{$\mathrm{Ni}-63$} \\
\hline \multicolumn{7}{|l|}{ Se-79 } \\
\hline \multicolumn{7}{|l|}{ Sr-90 } \\
\hline \multicolumn{7}{|l|}{ Y-90 } \\
\hline \multicolumn{7}{|l|}{ Zr-93 } \\
\hline \multirow{2}{*}{\multicolumn{7}{|c|}{$\begin{array}{l}\mathrm{Nb}-93 \mathrm{~m} \\
\mathrm{Nb}-94\end{array}$}} \\
\hline & & & & & & \\
\hline \multicolumn{7}{|l|}{$\begin{array}{l}\mathrm{Nb}-94 \\
\mathrm{Tc}-99\end{array}$} \\
\hline \multicolumn{7}{|l|}{ Ru-106 } \\
\hline \multicolumn{7}{|l|}{ Rh-102 } \\
\hline \multicolumn{7}{|l|}{ Rh-106 } \\
\hline \multicolumn{7}{|l|}{ Pd-107 } \\
\hline \multicolumn{7}{|l|}{ Cd-113m } \\
\hline \multicolumn{7}{|l|}{ Sn-121m } \\
\hline \multirow{2}{*}{\multicolumn{7}{|c|}{\begin{tabular}{|l|} 
Sn-126 \\
Sb-125
\end{tabular}}} \\
\hline & & & & & & \\
\hline \multicolumn{7}{|l|}{\begin{tabular}{|l|}
$S b-125$ \\
$S b-126$
\end{tabular}} \\
\hline \multicolumn{7}{|l|}{ Te-125m } \\
\hline $\mid$ & $9.66 \mathrm{E}-12$ & $9.66 \mathrm{E}-12$ & & $2.50 \mathrm{E}-12$ & $2.30 \mathrm{E}-12$ & $1.68 \mathrm{E}-08$ \\
\hline \multicolumn{7}{|l|}{ Cs-134 } \\
\hline Cs-135 & & & & & & \\
\hline Cs-137 & & & & & & \\
\hline Ba-137m & & & & & & \\
\hline Ce-144 & & & & & & \\
\hline Pr-144 & & & & & & \\
\hline $\mathrm{Pm}-146$ & & & & & & \\
\hline $\mathrm{Pm}-147$ & & & & & & \\
\hline Sm-151 & & & & & & \\
\hline Eu-152 & & & & & & \\
\hline Eu-154 & & & & & & \\
\hline Eu-155 & & & & & & \\
\hline Th-230 & & & & & & \\
\hline $\mathrm{Pa}-233$ & & & & & & \\
\hline $\mathrm{U}-232$ & & & & & & \\
\hline $\mathrm{U}-233$ & & & & & & \\
\hline $\mathrm{U}-234$ & & & & & & \\
\hline $\mathrm{U}-235$ & & & & & & \\
\hline $\mathrm{U}-236$ & & & & & & \\
\hline U-237 & & & & & & \\
\hline U-238 & & & & & & \\
\hline $\mathrm{Np}-237$ & & & & & & \\
\hline Pu-236 & & & & & & \\
\hline Pu-238 & & & & & & \\
\hline Pu-239 & & & & & & \\
\hline Pu-240 & & & & & & \\
\hline Pu-241 & & & & & & \\
\hline Pu-242 & & & & & & \\
\hline Pu-244 & & & & & & \\
\hline Am-241 & & & & & & \\
\hline$A m-242 m$ & & & & & & \\
\hline
\end{tabular}


Table A-20. Mass balance, Tank WM-187 waste (continued).

\begin{tabular}{|c|c|c|c|c|c|c|}
\hline Stream \# & 115 & 116 & 117 & 118 & 119 & 120 \\
\hline Radiological Composition & Ci/wscm & Ci/wscm & Ci/wscm & Ci/wscm & Ci/wscm & $\mathrm{Ci} / \mathrm{kg}$ \\
\hline \multicolumn{7}{|l|}{ Am-243 } \\
\hline \multicolumn{7}{|l|}{$\mathrm{Cm}-242$} \\
\hline \multicolumn{7}{|l|}{$\mathrm{Cm}-243$} \\
\hline \multicolumn{7}{|l|}{$\frac{\mathrm{Cm}-244}{\mathrm{Cm}-245}$} \\
\hline \multirow{2}{*}{\multicolumn{7}{|c|}{$\frac{\mathrm{Cm}-245}{\mathrm{Cm}-246}$}} \\
\hline & & & & & & \\
\hline \multicolumn{7}{|l|}{ TRU } \\
\hline & & & & & & \\
\hline & & & & & & \\
\hline $\begin{array}{l}\text { Gas Stream Bulk } \\
\text { Composition (Wet Basis) }\end{array}$ & $\begin{array}{c}\text { mol\% or } \\
\text { ppmv }\end{array}$ & $\begin{array}{c}\text { mol\% or } \\
\text { ppmv }\end{array}$ & $\begin{array}{c}\text { mol\% or } \\
\text { ppmv }\end{array}$ & $\begin{array}{c}\mathrm{mol} \% \text { or } \\
\text { ppmv }\end{array}$ & $\begin{array}{c}\text { mol\% or } \\
\text { ppmv }\end{array}$ & $\begin{array}{c}\text { mol\% or } \\
\text { ppmv }\end{array}$ \\
\hline $\mathrm{H} 2 \mathrm{O}, \mathrm{mol} \%$ & $70.03 \%$ & $70.03 \%$ & $0.01 \%$ & $70.03 \%$ & $64.38 \%$ & \\
\hline $\mathrm{O} 2, \mathrm{~mol} \%$ & $1.87 \%$ & $1.87 \%$ & $20.99 \%$ & $1.87 \%$ & $3.41 \%$ & \\
\hline $\mathrm{N} 2, \mathrm{~mol} \%$ & $1.64 \%$ & $1.64 \%$ & $79.00 \%$ & $1.64 \%$ & $7.88 \%$ & \\
\hline \multicolumn{7}{|l|}{$\mathrm{H} 2, \mathrm{~mol} \%$} \\
\hline $\mathrm{CO} 2, \mathrm{~mol} \%$ & $26.43 \%$ & $26.43 \%$ & & $26.43 \%$ & $24.30 \%$ & \\
\hline COgas, ppmv & $1.61 \mathrm{E}-01$ & $1.61 \mathrm{E}-01$ & & $1.61 \mathrm{E}-01$ & $1.48 \mathrm{E}-01$ & \\
\hline NO, ppmv & $1.34 \mathrm{E}+02$ & $1.34 \mathrm{E}+02$ & & $1.34 \mathrm{E}+02$ & $1.24 \mathrm{E}+02$ & \\
\hline $\mathrm{NO} 2, \mathrm{ppmv}$ & $5.18 \mathrm{E}+00$ & $5.18 \mathrm{E}+00$ & & $5.18 \mathrm{E}+00$ & $4.76 \mathrm{E}+00$ & \\
\hline SO2, ppmv & $6.91 \mathrm{E}+01$ & $6.91 \mathrm{E}+01$ & & $6.91 \mathrm{E}+01$ & $6.35 \mathrm{E}+01$ & \\
\hline $\mathrm{Cl}, \mathrm{ppmv}$ & $5.88 \mathrm{E}-02$ & $5.88 \mathrm{E}-02$ & & $1.52 \mathrm{E}-02$ & $1.40 \mathrm{E}-02$ & \\
\hline $\mathrm{F}, \mathrm{ppmv}$ & $4.47 \mathrm{E}+00$ & $4.47 \mathrm{E}+00$ & & $4.47 \mathrm{E}+00$ & $4.11 \mathrm{E}+00$ & \\
\hline \multicolumn{7}{|l|}{$\mathrm{C}$ (organic), ppmv } \\
\hline \multicolumn{7}{|l|}{$\mathrm{H}$ (organic), ppmv } \\
\hline $\mathrm{Hg}, \mathrm{ug} / \mathrm{wscm}$ & $3.40 \mathrm{E}+04$ & $3.40 \mathrm{E}+04$ & & $3.40 \mathrm{E}+01$ & $3.13 \mathrm{E}+01$ & \\
\hline $\mathrm{PM}, \mathrm{mg} / \mathrm{wscm}$ & $4.26 \mathrm{E}+01$ & $4.26 \mathrm{E}+01$ & $1.58 \mathrm{E}+01$ & $8.61 \mathrm{E}+00$ & $9.27 \mathrm{E}+00$ & \\
\hline \multicolumn{7}{|l|}{ SVM, ug/wscm } \\
\hline \multicolumn{7}{|l|}{ LVM, ug/wscm } \\
\hline $\begin{array}{l}\text { Gas Stream Bulk } \\
\text { Composition (Dry Basis) }\end{array}$ & $\mathrm{mol} \%$ & $\mathrm{~mol} \%$ & $\mathrm{~mol} \%$ & $\mathrm{~mol} \%$ & $\mathrm{~mol} \%$ & $\mathrm{~mol} \%$ \\
\hline $\mathrm{O} 2, \mathrm{~mol} \%$, dry basis & $6.23 \%$ & $6.23 \%$ & $20.99 \%$ & $0.87 \%$ & $9.57 \%$ & \\
\hline $\mathrm{N} 2, \mathrm{~mol} \%$, dry basis & $5.48 \%$ & $5.48 \%$ & $79.01 \%$ & $5.48 \%$ & $22.13 \%$ & \\
\hline \multicolumn{7}{|l|}{$\mathrm{H} 2, \mathrm{~mol} \%$, dry basis } \\
\hline $\mathrm{CO} 2, \mathrm{~mol} \%$, dry basis & $88.21 \%$ & $88.21 \%$ & & $88.21 \%$ & $68.23 \%$ & \\
\hline $\begin{array}{l}\text { Gas Stream Bulk } \\
\text { Composition (Dry Basis, } \\
\text { Corrected to } 7 \% \text { O2 with } \\
100 \% \text { O2 Combustion Air) }\end{array}$ & $\begin{array}{l}\mathrm{ppmv}, \text { or } \\
\mathrm{ug} / \mathrm{dscm} \text { or } \\
\mathrm{mg} / \mathrm{dscm}\end{array}$ & $\begin{array}{l}\mathrm{ppmv}, \text { or } \\
\text { ug/dscm or } \\
\mathrm{mg} / \mathrm{dscm}\end{array}$ & $\begin{array}{l}\mathrm{ppmv}, \text { or } \\
\mathrm{ug} / \mathrm{dscm} \text { or } \\
\mathrm{mg} / \mathrm{dscm}\end{array}$ & $\begin{array}{l}\mathrm{ppmv}, \text { or } \\
\mathrm{ug} / \mathrm{dscm} \text { or } \\
\mathrm{mg} / \mathrm{dscm}\end{array}$ & $\begin{array}{l}\text { ppmv, or } \\
\text { ug/dscm or } \\
\mathrm{mg} / \mathrm{dscm}\end{array}$ & $\begin{array}{l}\text { ppmv, or } \\
\mathrm{ug} / \mathrm{dscm} \text { or } \\
\mathrm{mg} / \mathrm{dscm}\end{array}$ \\
\hline COgas, ppmv, dry basis & $7.51 \mathrm{E}-02$ & $7.51 \mathrm{E}-02$ & & $7.51 \mathrm{E}-02$ & $5.81 \mathrm{E}-02$ & \\
\hline NO, ppmv, dry basis & $6.28 \mathrm{E}+01$ & $6.28 \mathrm{E}+01$ & & $6.28 \mathrm{E}+01$ & $4.86 \mathrm{E}+01$ & \\
\hline NO2, ppmv, dry basis & $2.42 \mathrm{E}+00$ & $2.42 \mathrm{E}+00$ & & $2.42 \mathrm{E}+00$ & $1.87 \mathrm{E}+00$ & \\
\hline SO2, ppmv, dry basis & $3.23 \mathrm{E}+01$ & $3.23 \mathrm{E}+01$ & & $3.23 \mathrm{E}+01$ & $2.50 \mathrm{E}+01$ & \\
\hline $\mathrm{Cl}, \mathrm{ppmv}$, dry basis & $2.75 \mathrm{E}-02$ & $2.75 \mathrm{E}-02$ & & $7.10 \mathrm{E}-03$ & $5.49 \mathrm{E}-03$ & \\
\hline F, ppmv, dry basis & $2.09 \mathrm{E}+00$ & $2.09 \mathrm{E}+00$ & & $2.09 \mathrm{E}+00$ & $1.62 \mathrm{E}+00$ & \\
\hline \multicolumn{7}{|l|}{$\mathrm{C}$ (organic), ppmv, dry basis } \\
\hline \multicolumn{7}{|l|}{$\mathrm{H}$ (organic), ppmv, dry basis } \\
\hline $\mathrm{Hg}, \mathrm{ug} / \mathrm{dscm}$ & $1.59 \mathrm{E}+04$ & $1.59 \mathrm{E}+04$ & & $1.59 \mathrm{E}+01$ & $1.23 \mathrm{E}+01$ & \\
\hline $\mathrm{PM}, \mathrm{mg} / \mathrm{dscm}$ & $1.99 \mathrm{E}+01$ & $1.99 \mathrm{E}+01$ & $2.21 \mathrm{E}+00$ & $4.02 \mathrm{E}+00$ & $3.65 \mathrm{E}+00$ & \\
\hline \multicolumn{7}{|l|}{ SVM, ug/dscm } \\
\hline LVM, ug/dscm & & & & & & \\
\hline
\end{tabular}


Table A-20. Mass balance, Tank WM-187 waste (continued).

\begin{tabular}{|c|c|c|c|c|c|c|c|}
\hline \begin{tabular}{|l|} 
PFD Number \\
\end{tabular} & PFD-3 & PFD-3 & PFD-3 & PFD-3 & PFD-3 & PFD-3 & PFD-2 \\
\hline Stream Number & 203 & 204 & 205 & 206 & 207 & 301 & 303 \\
\hline Stream Name & $\begin{array}{l}\text { Fluidizing } \\
\text { Gas to } \\
\text { Reformer }\end{array}$ & $\begin{array}{c}\text { Propane to } \\
\text { Oxidizer }\end{array}$ & $\begin{array}{l}\text { Water to } \\
\text { Spray } \\
\text { Quench }\end{array}$ & $\begin{array}{l}\text { ANN to } \\
\text { Scrub for F } \\
\text { Adjust }\end{array}$ & $\begin{array}{l}\text { HNO3 to } \\
\text { Scrub for } \\
\mathrm{H}+\text { Adjust }\end{array}$ & Isopropanol & Bed Media \\
\hline Rate or Volume & $1.11 \mathrm{E}+04$ & $9.05 \mathrm{E}+02$ & $4.58 \mathrm{E}+02$ & $4.48 \mathrm{E}-02$ & $1.22 \mathrm{E}+00$ & $7.77 \mathrm{E}+01$ & $1.34 \mathrm{E}+00$ \\
\hline Volume Flow (standard, wet) ${ }^{*}$ & $1.09 \mathrm{E}+04$ & $2.56 \mathrm{E}+03$ & & & & & \\
\hline Volume Flow (standard, dry) ${ }^{*}$ & $1.09 \mathrm{E}+04$ & $2.56 \mathrm{E}+03$ & & & & & \\
\hline Rate Units & $\mathrm{ft} 3 / \mathrm{hr}$ & $\mathrm{ft} 3 / \mathrm{hr}$ & $\mathrm{gal} / \mathrm{hr}$ & $\mathrm{gal} / \mathrm{hr}$ & $\mathrm{gal} / \mathrm{hr}$ & $\mathrm{gal} / \mathrm{hr}$ & $\mathrm{lb} / \mathrm{hr}$ \\
\hline Rate or Volume, metric & $3.10 \mathrm{E}+02$ & $7.26 \mathrm{E}+01$ & $1.73 \mathrm{E}+03$ & $1.70 \mathrm{E}-01$ & $4.60 \mathrm{E}+00$ & $2.94 \mathrm{E}+02$ & $6.09 \mathrm{E}-01$ \\
\hline Rate Units & wscm $/ \mathrm{hr}$ & wscm $/ \mathrm{hr}$ & $\mathrm{L} / \mathrm{hr}$ & $\mathrm{L} / \mathrm{hr}$ & $\mathrm{L} / \mathrm{hr}$ & $\mathrm{L} / \mathrm{hr}$ & $\mathrm{kg} / \mathrm{hr}$ \\
\hline Temperature, ${ }^{\circ} \mathrm{C}$ & 580 & 25 & 25 & 25 & 25 & 15 & 15 \\
\hline Temperature, ${ }^{\circ} \mathrm{F}$ & 1076 & 77 & 77 & 77 & 77 & 59 & 59 \\
\hline Pressure, psia & 42.30 & 42.30 & 112.30 & 14.70 & 42.30 & 14.70 & 14.70 \\
\hline Specific Gravity & $1.81 \mathrm{E}-03$ & $5.19 \mathrm{E}-03$ & $9.93 \mathrm{E}-01$ & $1.80 \mathrm{E}+00$ & $1.23 \mathrm{E}+00$ & $1.03 \mathrm{E}+00$ & $1.58 \mathrm{E}+00$ \\
\hline Chemical Composition & Mol \% & $\mathrm{lb} /$ wscf & Mol/liter & Mol/liter & Mol/liter & Mol/liter & Wt frac \\
\hline $\mathrm{H}+$ & & & $9.95 \mathrm{E}-08$ & & $1.33 \mathrm{E}+01$ & & \\
\hline $\mathrm{Al}+3$ & & & & $2.20 \mathrm{E}+00$ & & & 0.529 \\
\hline \multicolumn{8}{|l|}{$\mathrm{Sb}+5$} \\
\hline \multirow{2}{*}{\multicolumn{8}{|c|}{$\frac{\mathrm{As}+3}{\mathrm{Ba}+2}$}} \\
\hline & & & & & & & \\
\hline \multicolumn{8}{|l|}{$\mathrm{Be}+2$} \\
\hline \multicolumn{8}{|l|}{$B+3$} \\
\hline \multirow{2}{*}{\multicolumn{8}{|c|}{\begin{tabular}{|l|}
$\mathrm{Cd}+2$ \\
$\mathrm{Ca}+2$
\end{tabular}}} \\
\hline & & & & & & & \\
\hline \multicolumn{8}{|l|}{$\mathrm{Cr}+3$} \\
\hline \multirow{2}{*}{\multicolumn{8}{|c|}{\begin{tabular}{|l|}
$\mathrm{Co}+2$ \\
$\mathrm{Cs}+$ \\
\end{tabular}}} \\
\hline & & & & & & & \\
\hline \multicolumn{8}{|l|}{$\mathrm{Cu}+2$} \\
\hline \multicolumn{8}{|l|}{$\mathrm{Fe}+3$} \\
\hline \multirow{2}{*}{\multicolumn{8}{|c|}{$\frac{\mathrm{Pb}+2}{\mathrm{Hg}+2}$}} \\
\hline & & & & & & & \\
\hline \multicolumn{8}{|l|}{$M n+4$} \\
\hline \multirow{2}{*}{\multicolumn{8}{|c|}{$\begin{array}{ll}\mathrm{Ni}+2 \\
\mathrm{~K}+\end{array}$}} \\
\hline & & & & & & & \\
\hline \multicolumn{8}{|l|}{$\mathrm{Se}+4$} \\
\hline \multirow{2}{*}{\multicolumn{8}{|c|}{$\mathrm{Ag}+$}} \\
\hline \multirow{2}{*}{\multicolumn{8}{|c|}{\begin{tabular}{|l|}
$\mathrm{Na}+$ \\
$\mathrm{Tl}+3$ \\
\end{tabular}}} \\
\hline \multirow{2}{*}{\multicolumn{8}{|c|}{\begin{tabular}{|l|}
$\mathrm{T} \mid+3$ \\
$\mathrm{U}+4$
\end{tabular}}} \\
\hline & & & & & & & \\
\hline \multicolumn{8}{|l|}{$\mathrm{V}+3$} \\
\hline \multicolumn{8}{|l|}{$Z n+2$} \\
\hline \multicolumn{8}{|l|}{$\mathrm{Zr}+4$} \\
\hline $\mathrm{Cl}-$ & & & & & & & \\
\hline F- & & & & & & & \\
\hline SO4-2 & & & & & & & \\
\hline NO3- & & & & $6.60 \mathrm{E}+00$ & $1.33 \mathrm{E}+01$ & & \\
\hline$\overline{\mathrm{CO} 2}$ & 100 & & & & & & \\
\hline $\mathrm{OH}-$ & & & $9.95 \mathrm{E}-08$ & 7.31E-08 & & & \\
\hline $\mathrm{H} 2 \mathrm{O}$ & & & $5.51 \mathrm{E}+01$ & $7.41 \mathrm{E}+01$ & $2.17 \mathrm{E}+01$ & & \\
\hline $\mathrm{O}$ (oxides) & & & & & & & 0.471 \\
\hline C (organic) & & $9.35 \mathrm{E}-02$ & & & & $5.15 \mathrm{E}+01$ & \\
\hline $\mathrm{H}$ (organic) & & $2.09 \mathrm{E}-02$ & & & & $1.37 \mathrm{E}+02$ & \\
\hline $\mathrm{O}$ (organic) & & & & & & $1.72 \mathrm{E}+01$ & \\
\hline Mass Flow $(\mathrm{kg} / \mathrm{hr})$ : & $5.66 \mathrm{E}+02$ & $1.33 \mathrm{E}+02$ & $1.72 \mathrm{E}+03$ & $3.06 \mathrm{E}-01$ & $5.66 \mathrm{E}+00$ & $3.03 \mathrm{E}+02$ & $6.09 \mathrm{E}-01$ \\
\hline
\end{tabular}


Table A-20. Mass balance, Tank WM-187 waste (continued).

\begin{tabular}{|c|c|c|c|c|c|c|c|}
\hline \begin{tabular}{|l|} 
PFD Number \\
\end{tabular} & PFD-3 & PFD-3 & PFD-3 & PFD-3 & PFD-2 & PFD-2 & $\frac{\text { PFD-3 }}{505}$ \\
\hline Stream Number & 305 & 401 & 402 & 404 & 404 & 503 & 505 \\
\hline Stream Name & $\begin{array}{l}\text { Grout Mix } \\
\text { for Scrub } \\
\text { Blowdown }\end{array}$ & $\begin{array}{c}\text { Scrub } \\
\text { Recycled to } \\
\text { Feed }\end{array}$ & $\begin{array}{c}\text { Scrub } \\
\text { Blowdown } \\
\text { to Grout } \\
\text { Mixer }\end{array}$ & $\begin{array}{l}\text { MLLW } \\
\text { Grout from } \\
\text { Scrub }\end{array}$ & $\begin{array}{c}\text { MLLW } \\
\text { Grout } \\
\text { Drums }\end{array}$ & $\begin{array}{c}\text { Feed } \\
\text { Atomizing } \\
\text { Gas }\end{array}$ & $\begin{array}{c}\text { Oxygen to } \\
\text { Oxidizer }\end{array}$ \\
\hline Rate or Volume & $6.61 \mathrm{E}+00$ & $5.24 \mathrm{E}+00$ & $2.57 \mathrm{E}-01$ & $8.82 \mathrm{E}+00$ & $2.29 \mathrm{E}-01$ & $5.20 \mathrm{E}+03$ & $5.40 \mathrm{E}+03$ \\
\hline Volume Flow (standard, wet) & & & & & & $1.49 \mathrm{E}+04$ & $3.33 \mathrm{E}+04$ \\
\hline Volume Flow (standard, dry) ${ }^{*}$ & & & & & & $1.49 \mathrm{E}+04$ & $3.33 \mathrm{E}+04$ \\
\hline Rate Units & $\mathrm{Ib} / \mathrm{hr}$ & $\mathrm{gal} / \mathrm{hr}$ & $\mathrm{gal} / \mathrm{hr}$ & $\mathrm{lb} / \mathrm{hr}$ & Drums/day & $\mathrm{ft} 3 / \mathrm{hr}$ & $\mathrm{ft} 3 / \mathrm{hr}$ \\
\hline Rate or Volume, metric & $3.00 \mathrm{E}+00$ & $1.98 \mathrm{E}+01$ & $9.74 \mathrm{E}-01$ & $4.00 \mathrm{E}+00$ & $4.00 \mathrm{E}+00$ & $4.23 \mathrm{E}+02$ & $9.44 \mathrm{E}+02$ \\
\hline Rate Units & $\mathrm{kg} / \mathrm{hr}$ & $\mathrm{L} / \mathrm{hr}$ & $\mathrm{L} / \mathrm{hr}$ & $\mathrm{kg} / \mathrm{hr}$ & $\mathrm{kg} / \mathrm{hr}$ & wscm $/ \mathrm{hr}$ & wscm/hr \\
\hline Temperature, ${ }^{\circ} \mathrm{C}$ & 25 & 70 & 70 & 54 & 54 & 21 & 25 \\
\hline Temperature, ${ }^{\circ} \mathrm{F}$ & 77 & 158 & 158 & 129 & 129 & 70 & 77 \\
\hline Pressure, psia & 14.70 & 29.98 & 29.98 & 14.70 & 14.70 & 42.30 & 92.30 \\
\hline Specific Gravity & $2.01 \mathrm{E}-01$ & $1.03 \mathrm{E}+00$ & $1.03 \mathrm{E}+00$ & $2.10 \mathrm{E}+00$ & $2.10 \mathrm{E}+00$ & $5.25 \mathrm{E}-03$ & $8.21 \mathrm{E}-03$ \\
\hline Chemical Composition & Wt frac & Mol/liter & Mol/liter & Wt frac & Wt frac & $\mathrm{lb} / \mathrm{wscf}$ & $\mathrm{lb} /$ wscf \\
\hline $\mathrm{H}+$ & & $9.55 \mathrm{E}-01$ & $9.55 \mathrm{E}-01$ & $2.35 \mathrm{E}-04$ & $2.35 \mathrm{E}-04$ & & \\
\hline$A \mathrm{Al}+3$ & & $1.27 \mathrm{E}-02$ & $1.27 \mathrm{E}-02$ & $0.01 \%$ & $0.01 \%$ & & \\
\hline $\mathrm{Sb}+5$ & & $9.09 \mathrm{E}-07$ & $9.09 \mathrm{E}-07$ & $2.70 \mathrm{E}-08$ & $2.70 \mathrm{E}-08$ & & \\
\hline$A s+3$ & & $5.29 \mathrm{E}-06$ & $5.29 \mathrm{E}-06$ & $9.66 \mathrm{E}-08$ & $9.66 \mathrm{E}-08$ & & \\
\hline \multicolumn{8}{|l|}{$\mathrm{Ba}+2$} \\
\hline \multicolumn{8}{|l|}{$\mathrm{Be}+2$} \\
\hline $\mathrm{B}+3$ & & $3.43 \mathrm{E}-04$ & $3.43 \mathrm{E}-04$ & $9.04 \mathrm{E}-07$ & $9.04 \mathrm{E}-07$ & & \\
\hline \multicolumn{8}{|l|}{$C d+2$} \\
\hline \multicolumn{8}{|l|}{$\mathrm{Ca}+2$} \\
\hline \multicolumn{8}{|l|}{$\mathrm{Cr}+3$} \\
\hline $\mathrm{Co}+2$ & & $6.26 \mathrm{E}-05$ & $6.26 \mathrm{E}-05$ & $8.99 \mathrm{E}-07$ & $8.99 \mathrm{E}-07$ & & \\
\hline \multicolumn{8}{|l|}{ Cs+ } \\
\hline \multicolumn{8}{|l|}{$\mathrm{Cu}+2$} \\
\hline $\mathrm{Fe}+3$ & & $8.43 \mathrm{E}-05$ & $8.43 \mathrm{E}-05$ & $1.15 \mathrm{E}-06$ & $1.15 \mathrm{E}-06$ & & \\
\hline \multicolumn{8}{|l|}{$\mathrm{Pb}+2$} \\
\hline $\mathrm{Hg}+2$ & & $6.43 \mathrm{E}-02$ & $6.43 \mathrm{E}-02$ & $3.14 \mathrm{E}-03$ & $3.14 \mathrm{E}-03$ & & \\
\hline$M n+4$ & & $5.65 \mathrm{E}-04$ & $5.65 \mathrm{E}-04$ & $7.56 \mathrm{E}-06$ & $7.56 \mathrm{E}-06$ & & \\
\hline \multicolumn{8}{|l|}{$\mathrm{Ni}+2$} \\
\hline \multicolumn{8}{|l|}{$\mathrm{K}+$} \\
\hline \multicolumn{8}{|l|}{$\mathrm{Se}+4$} \\
\hline \multirow{2}{*}{\multicolumn{8}{|c|}{\begin{tabular}{|l|}
$\mathrm{Ag}+$ \\
$\mathrm{Na}+$
\end{tabular}}} \\
\hline & & & & & & & \\
\hline$T \mid+3$ & & $1.81 \mathrm{E}-07$ & $1.81 \mathrm{E}-07$ & $8.99 \mathrm{E}-09$ & $8.99 \mathrm{E}-09$ & & \\
\hline \multicolumn{8}{|l|}{$\mathrm{U}+4$} \\
\hline \multirow{2}{*}{\multicolumn{8}{|c|}{\begin{tabular}{|l|}
$V+3$ \\
$7 n+2$
\end{tabular}}} \\
\hline \multirow{2}{*}{\multicolumn{8}{|c|}{$Z n+2$}} \\
\hline & & & & & & & $\mathrm{Zr}+4$ \\
\hline $\mathrm{Cl}-$ & & $3.15 \mathrm{E}-04$ & $3.15 \mathrm{E}-04$ & $2.72 \mathrm{E}-06$ & $2.72 \mathrm{E}-06$ & & \\
\hline F- & & $7.38 \mathrm{E}-03$ & $7.38 \mathrm{E}-03$ & $3.41 \mathrm{E}-05$ & $3.41 \mathrm{E}-05$ & & \\
\hline \multicolumn{8}{|l|}{\begin{tabular}{|l|}
$\mathrm{SO} 4-2$ \\
\end{tabular}} \\
\hline NO3- & & $9.09 \mathrm{E}-01$ & $9.09 \mathrm{E}-01$ & $1.37 \mathrm{E}-02$ & $1.37 \mathrm{E}-02$ & & \\
\hline \multicolumn{8}{|l|}{ PO4-3 } \\
\hline$A m+4$ & & $2.16 \mathrm{E}-09$ & $2.16 \mathrm{E}-09$ & $1.28 \mathrm{E}-10$ & $1.28 \mathrm{E}-10$ & & \\
\hline $\mathrm{Br}-$ & & $4.40 \mathrm{E}-09$ & $4.40 \mathrm{E}-09$ & $8.56 \mathrm{E}-11$ & $8.56 \mathrm{E}-11$ & & \\
\hline $\mathrm{Ce}+4$ & & $1.35 \mathrm{E}-06$ & $1.35 \mathrm{E}-06$ & $4.61 \mathrm{E}-08$ & $4.61 \mathrm{E}-08$ & & \\
\hline $\mathrm{Eu}+3$ & & $1.29 \mathrm{E}-08$ & $1.29 \mathrm{E}-08$ & $4.78 \mathrm{E}-10$ & $4.78 \mathrm{E}-10$ & & \\
\hline $\mathrm{Gd}+3$ & & $4.70 \mathrm{E}-06$ & $4.70 \mathrm{E}-06$ & $1.80 \mathrm{E}-07$ & $1.80 \mathrm{E}-07$ & & \\
\hline $\mathrm{Ge}+4$ & & & & & & & \\
\hline $\ln +3$ & & $3.75 \mathrm{E}-08$ & $3.75 \mathrm{E}-08$ & $1.05 \mathrm{E}-09$ & $1.05 \mathrm{E}-09$ & & \\
\hline $\mid-$ & & $5.73 \mathrm{E}-08$ & $5.73 \mathrm{E}-08$ & $1.77 \mathrm{E}-09$ & 1.77E-09 & & \\
\hline La+3 & & $2.34 \mathrm{E}-07$ & $2.34 \mathrm{E}-07$ & $7.93 \mathrm{E}-09$ & $7.93 \mathrm{E}-09$ & & \\
\hline
\end{tabular}


Table A-20. Mass balance, Tank WM-187 waste (continued).

\begin{tabular}{|c|c|c|c|c|c|c|c|}
\hline Stream \# & 305 & 401 & 402 & 404 & 404 & 503 & 505 \\
\hline Chemical Composition & Wt frac & Mol/liter & Mol/liter & Wt frac & Wt frac & Ib/wscf & $\mathrm{Ib} / \mathrm{wscf}$ \\
\hline \multicolumn{8}{|l|}{$\mathrm{Li}+$} \\
\hline \multicolumn{8}{|l|}{$\mathrm{Mg}+2$} \\
\hline \multicolumn{8}{|l|}{$\mathrm{Mo}+6$} \\
\hline $\mathrm{Nd}+3$ & & $7.55 \mathrm{E}-07$ & $7.55 \mathrm{E}-07$ & $2.65 \mathrm{E}-08$ & $2.65 \mathrm{E}-08$ & & \\
\hline $\mathrm{Np}+4$ & & $1.19 \mathrm{E}-07$ & $1.19 \mathrm{E}-07$ & $6.86 \mathrm{E}-09$ & $6.86 \mathrm{E}-09$ & & \\
\hline \multicolumn{8}{|l|}{$\mathrm{Nb}+5$} \\
\hline \multicolumn{8}{|l|}{$\mathrm{Pd}+4$} \\
\hline \multicolumn{8}{|l|}{$\mathrm{Pu}+4$} \\
\hline $\mathrm{Pr}+4$ & & $2.13 \mathrm{E}-07$ & $2.13 \mathrm{E}-07$ & $7.31 \mathrm{E}-09$ & $7.31 \mathrm{E}-09$ & & \\
\hline $\mathrm{Pm}+3$ & & $5.19 \mathrm{E}-11$ & $5.19 \mathrm{E}-11$ & $1.83 \mathrm{E}-12$ & $1.83 \mathrm{E}-12$ & & \\
\hline $\mathrm{Rh}+4$ & & $9.19 \mathrm{E}-08$ & $9.19 \mathrm{E}-08$ & $2.30 \mathrm{E}-09$ & $2.30 \mathrm{E}-09$ & & \\
\hline \multicolumn{8}{|l|}{$\mathrm{Rb}+$} \\
\hline $\mathrm{Ru}+3$ & & $3.97 \mathrm{E}-05$ & $3.97 \mathrm{E}-05$ & $9.77 \mathrm{E}-07$ & $9.77 \mathrm{E}-07$ & & \\
\hline $\mathrm{Sm+3}$ & & $1.41 \mathrm{E}-07$ & $1.41 \mathrm{E}-07$ & $5.18 \mathrm{E}-09$ & $5.18 \mathrm{E}-09$ & & \\
\hline$S i+4$ & $4.67 \mathrm{E}-01$ & & & $3.51 \mathrm{E}-01$ & $3.51 \mathrm{E}-01$ & & \\
\hline \multicolumn{8}{|l|}{$\mathrm{Sr}+2$} \\
\hline$T \mathrm{Tc}+7$ & & $1.02 \mathrm{E}-07$ & $1.02 \mathrm{E}-07$ & $2.44 \mathrm{E}-09$ & $2.44 \mathrm{E}-09$ & & \\
\hline \multicolumn{8}{|l|}{$\mathrm{Te}+4$} \\
\hline $\mathrm{Tb}+4$ & & $5.39 \mathrm{E}-11$ & $5.39 \mathrm{E}-11$ & $2.09 \mathrm{E}-12$ & $2.09 \mathrm{E}-12$ & & \\
\hline \multicolumn{8}{|l|}{$\mathrm{Th}+4$} \\
\hline \multirow{2}{*}{\multicolumn{8}{|c|}{$\begin{array}{l}\mathrm{Sn}+4 \\
\mathrm{Ti}+4\end{array}$}} \\
\hline & & & & & & & \\
\hline $\mathrm{Y}+3$ & & $1.75 \mathrm{E}-07$ & $1.75 \mathrm{E}-07$ & $3.78 \mathrm{E}-09$ & $3.78 \mathrm{E}-09$ & & \\
\hline $\mathrm{OH}-$ & & $1.28 \mathrm{E}-06$ & $1.28 \mathrm{E}-06$ & $5.28 \mathrm{E}-09$ & $5.28 \mathrm{E}-09$ & & \\
\hline \multirow[t]{2}{*}{$\mathrm{H} 2 \mathrm{O}$} & & $5.30 \mathrm{E}+01$ & $5.30 \mathrm{E}+01$ & $2.33 \mathrm{E}-01$ & $2.33 \mathrm{E}-01$ & & \\
\hline & & & & & & & \\
\hline $\mathrm{SO} 2$ & & $7.13 \mathrm{E}-07$ & $7.13 \mathrm{E}-07$ & $1.11 \mathrm{E}-08$ & $1.11 \mathrm{E}-08$ & & \\
\hline \multicolumn{8}{|l|}{$\mathrm{H} 2 \mathrm{~S}$} \\
\hline $\mathrm{CO}$ & & $2.74 \mathrm{E}-09$ & $2.74 \mathrm{E}-09$ & $1.87 \mathrm{E}-11$ & $1.87 \mathrm{E}-11$ & & \\
\hline $\mathrm{CO} 2$ & & $2.14 \mathrm{E}-04$ & $2.14 \mathrm{E}-04$ & $2.29 \mathrm{E}-06$ & $2.29 \mathrm{E}-06$ & $1.14 \mathrm{E}-01$ & \\
\hline $\mathrm{H} 2$ & & $2.83 \mathrm{E}-08$ & $2.83 \mathrm{E}-08$ & $1.39 \mathrm{E}-11$ & $1.39 \mathrm{E}-11$ & & \\
\hline N2 & & $6.59 \mathrm{E}-09$ & $6.59 \mathrm{E}-09$ & $4.50 \mathrm{E}-11$ & $4.50 \mathrm{E}-11$ & & \\
\hline $\mathrm{NO}$ & & $8.78 \mathrm{E}-08$ & $8.78 \mathrm{E}-08$ & $6.42 \mathrm{E}-10$ & $6.42 \mathrm{E}-10$ & & \\
\hline $\mathrm{NO} 2$ & & $1.32 \mathrm{E}-05$ & 1.32E-05 & $1.48 \mathrm{E}-07$ & $1.48 \mathrm{E}-07$ & & \\
\hline $\mathrm{O} 2$ & & $2.17 \mathrm{E}-08$ & $2.17 \mathrm{E}-08$ & $1.69 \mathrm{E}-10$ & $1.69 \mathrm{E}-10$ & & $8.30 \mathrm{E}-02$ \\
\hline $\mathrm{S}$ (other) & & $1.04 \mathrm{E}-08$ & $1.04 \mathrm{E}-08$ & $8.16 \mathrm{E}-11$ & $8.16 \mathrm{E}-11$ & & \\
\hline \multicolumn{8}{|l|}{$\mathrm{CO} 3$} \\
\hline \multicolumn{8}{|l|}{ C (reductant) } \\
\hline $\mathrm{O}$ (oxides) & $53.26 \%$ & $1.27 \mathrm{E}-04$ & $1.27 \mathrm{E}-04$ & $39.94 \%$ & $39.94 \%$ & & \\
\hline \multicolumn{8}{|l|}{ C (organic) } \\
\hline \multicolumn{8}{|l|}{$\mathrm{H}$ (organic) } \\
\hline \multicolumn{8}{|l|}{$\mathrm{O}$ (organic) } \\
\hline & & & & & & & \\
\hline Mass Flow (kg/hr): & $3.00 \mathrm{E}+00$ & $2.03 \mathrm{E}+01$ & $1.00 \mathrm{E}+00$ & $4.00 \mathrm{E}+00$ & $4.00 \mathrm{E}+00$ & $7.73 \mathrm{E}+02$ & $1.26 \mathrm{E}+03$ \\
\hline Canister Rate, canisters/day & & & & & 0.23 & & \\
\hline Total Canisters Generated & & & & & 20 & & \\
\hline Heat Generation & $\mathrm{w} / \mathrm{m} 3$ & $\mathrm{w} / \mathrm{m} 3$ & $\mathrm{w} / \mathrm{m} 3$ & $\mathrm{w} / \mathrm{m} 3$ & w/Drum & $\mathrm{w} / \mathrm{m} 3$ & $\mathrm{w} / \mathrm{m} 3$ \\
\hline Heat Generation & & $5.78 \mathrm{E}-03$ & $5.78 \mathrm{E}-03$ & $2.96 \mathrm{E}-03$ & $5.91 \mathrm{E}-04$ & & \\
\hline
\end{tabular}


Table A-20. Mass balance, Tank WM-187 waste (continued).

\begin{tabular}{|c|c|c|c|c|c|c|c|}
\hline Stream \# & 305 & 401 & 402 & 404 & 404 & 503 & 505 \\
\hline Radiological Composition & $\mathrm{Ci} / \mathrm{kg}$ & $\mathrm{Ci} / \mathrm{L}$ & $\mathrm{Ci} / \mathrm{L}$ & $\mathrm{Ci} / \mathrm{kg}$ & Ci/Drum & Ci/wscm & Ci/wscm \\
\hline \multicolumn{8}{|l|}{$\mathrm{H}-3$} \\
\hline C-14 & & 1.92E-16 & $1.92 \mathrm{E}-16$ & $4.67 \mathrm{E}-17$ & $1.96 \mathrm{E}-14$ & & \\
\hline Co-60 & & $5.37 \mathrm{E}-07$ & $5.37 \mathrm{E}-07$ & $1.31 \mathrm{E}-07$ & $5.49 \mathrm{E}-05$ & & \\
\hline \multicolumn{8}{|l|}{ Ni-59 } \\
\hline \multicolumn{8}{|l|}{$\mathrm{Ni}-63$} \\
\hline \multicolumn{8}{|l|}{ Se-79 } \\
\hline \multicolumn{8}{|l|}{ Sr-90 } \\
\hline Y-90 & & $1.03 \mathrm{E}-03$ & $1.03 \mathrm{E}-03$ & $2.50 \mathrm{E}-04$ & $1.05 \mathrm{E}-01$ & & \\
\hline \multicolumn{8}{|l|}{ Zr-93 } \\
\hline \multicolumn{8}{|l|}{$\mathrm{Nb}-93 \mathrm{~m}$} \\
\hline \multicolumn{8}{|l|}{$\mathrm{Nb}-94$} \\
\hline Tc-99 & & $2.47 \mathrm{E}-07$ & $2.47 \mathrm{E}-07$ & $6.00 \mathrm{E}-08$ & $2.52 \mathrm{E}-05$ & & \\
\hline Ru-106 & & $3.88 \mathrm{E}-08$ & $3.88 \mathrm{E}-08$ & $9.46 \mathrm{E}-09$ & $3.97 \mathrm{E}-06$ & & \\
\hline $\mathrm{Rh}-102$ & & $2.25 \mathrm{E}-11$ & $2.25 \mathrm{E}-11$ & $5.49 \mathrm{E}-12$ & $2.31 \mathrm{E}-09$ & & \\
\hline Rh-106 & & $3.88 \mathrm{E}-08$ & $3.88 \mathrm{E}-08$ & $9.46 \mathrm{E}-09$ & $3.97 \mathrm{E}-06$ & & \\
\hline \multicolumn{8}{|l|}{$\mathrm{Pd}-107$} \\
\hline \multicolumn{8}{|l|}{ Cd-113m } \\
\hline \multicolumn{8}{|l|}{ Sn-121m } \\
\hline \multicolumn{8}{|l|}{ Sn-126 } \\
\hline Sb-125 & & $2.05 \mathrm{E}-05$ & $2.05 \mathrm{E}-05$ & $4.99 \mathrm{E}-06$ & $2.10 \mathrm{E}-03$ & & \\
\hline Sb-126 & & $1.50 \mathrm{E}-09$ & $1.50 \mathrm{E}-09$ & $3.66 \mathrm{E}-10$ & $1.54 \mathrm{E}-07$ & & \\
\hline \multicolumn{8}{|l|}{ Te-125m } \\
\hline $\mathrm{I}-129$ & & $1.24 \mathrm{E}-09$ & $1.24 \mathrm{E}-09$ & $3.03 \mathrm{E}-10$ & $1.27 \mathrm{E}-07$ & & \\
\hline \multicolumn{8}{|l|}{ Cs-134 } \\
\hline \multicolumn{8}{|l|}{ Cs-135 } \\
\hline \multirow{2}{*}{\multicolumn{8}{|c|}{$\frac{\text { Cs-137 }}{\text { Ba-137m }}$}} \\
\hline \multirow{2}{*}{\multicolumn{5}{|c|}{$\frac{\mathrm{Ba}-137 \mathrm{~m}}{\mathrm{Ce}-144}$}} & & & \\
\hline & & $2.63 \mathrm{E}-08$ & $2.63 \mathrm{E}-08$ & $6.39 \mathrm{E}-09$ & & & \\
\hline Pr-144 & & $2.63 \mathrm{E}-08$ & $2.63 \mathrm{E}-08$ & $6.39 \mathrm{E}-09$ & $2.69 \mathrm{E}-06$ & & \\
\hline $\mathrm{Pm}-146$ & & $1.33 \mathrm{E}-09$ & $1.33 \mathrm{E}-09$ & $3.24 \mathrm{E}-10$ & $1.36 \mathrm{E}-07$ & & \\
\hline Pm-147 & & $7.06 \mathrm{E}-06$ & $7.06 \mathrm{E}-06$ & $1.72 \mathrm{E}-06$ & $7.23 \mathrm{E}-04$ & & \\
\hline Sm-151 & & $1.40 \mathrm{E}-05$ & $1.40 \mathrm{E}-05$ & $3.41 \mathrm{E}-06$ & $1.43 \mathrm{E}-03$ & & \\
\hline Eu-152 & & $8.40 \mathrm{E}-08$ & $8.40 \mathrm{E}-08$ & $2.05 \mathrm{E}-08$ & $8.60 \mathrm{E}-06$ & & \\
\hline Eu-154 & & $3.16 \mathrm{E}-06$ & $3.16 \mathrm{E}-06$ & $7.69 \mathrm{E}-07$ & $3.23 \mathrm{E}-04$ & & \\
\hline Eu-155 & & 3.33E-06 & 3.33E-06 & $8.10 \mathrm{E}-07$ & $3.40 \mathrm{E}-04$ & & \\
\hline & & & & & & & \\
\hline Th-230 & & & & & & & \\
\hline $\mathrm{Pa}-233$ & & & & & & & \\
\hline $\mathrm{U}-232$ & & & & & & & \\
\hline $\mathrm{U}-233$ & & & & & & & \\
\hline $\mathrm{U}-234$ & & & & & & & \\
\hline $\mathrm{U}-235$ & & & & & & & \\
\hline $\mathrm{U}-236$ & & & & & & & \\
\hline $\mathrm{U}-237$ & & & & & & & \\
\hline $\mathrm{U}-238$ & & & & & & & \\
\hline Np-237 & & 1.99E-08 & 1.99E-08 & 4.84E-09 & $2.03 \mathrm{E}-06$ & & \\
\hline Pu-236 & & & & & & & \\
\hline $\mathrm{Pu}-238$ & & & & & & & \\
\hline Pu-239 & & & & & & & \\
\hline Pu-240 & & & & & & & \\
\hline Pu-241 & & & & & & & \\
\hline Pu-242 & & & & & & & \\
\hline Pu-244 & & & & & & & \\
\hline Am-241 & & $2.23 \mathrm{E}-06$ & $2.23 \mathrm{E}-06$ & $5.43 \mathrm{E}-07$ & $2.28 \mathrm{E}-04$ & & \\
\hline Am-242m & & $4.04 \mathrm{E}-10$ & $4.04 \mathrm{E}-10$ & $9.83 \mathrm{E}-11$ & $4.13 \mathrm{E}-08$ & & \\
\hline
\end{tabular}


Table A-20. Mass balance, Tank WM-187 waste (continued).

\begin{tabular}{|c|c|c|c|c|c|c|c|}
\hline Stream \# & 305 & 401 & 402 & 404 & 404 & 503 & 505 \\
\hline Radiological Composition & $\mathrm{Ci} / \mathrm{kg}$ & $\mathrm{Ci} / \mathrm{L}$ & $\mathrm{Ci} / \mathrm{L}$ & $\mathrm{Ci} / \mathrm{kg}$ & Ci/Drum & $\mathrm{Ci} / \mathrm{wscm}$ & $\mathrm{Ci} / \mathrm{wscm}$ \\
\hline Am-243 & & $7.66 \mathrm{E}-10$ & $7.66 \mathrm{E}-10$ & $1.87 \mathrm{E}-10$ & 7.84E-08 & & \\
\hline \multicolumn{8}{|l|}{$\mathrm{Cm}-242$} \\
\hline \multicolumn{8}{|l|}{$\mathrm{Cm}-243$} \\
\hline \multicolumn{8}{|l|}{$\mathrm{Cm}-244$} \\
\hline \multicolumn{8}{|l|}{$\mathrm{Cm}-245$} \\
\hline \multicolumn{8}{|l|}{$\mathrm{Cm}-246$} \\
\hline TRU & & $2.25 \mathrm{E}-06$ & $2.25 \mathrm{E}-06$ & $5.48 \mathrm{E}-07$ & $2.30 \mathrm{E}-04$ & & \\
\hline & & & & & & & \\
\hline & & & & & & & \\
\hline $\begin{array}{l}\text { Gas Stream Bulk Composition } \\
\text { (Wet Basis) }\end{array}$ & $\begin{array}{l}\text { mol\% or } \\
\text { ppmv }\end{array}$ & $\begin{array}{l}\mathrm{mol} \% \text { or } \\
\text { ppmv }\end{array}$ & $\begin{array}{l}\text { mol\% or } \\
\text { ppmv }\end{array}$ & $\begin{array}{l}\mathrm{mol} \% \text { or } \\
\text { ppmv }\end{array}$ & $\begin{array}{l}\text { mol\% or } \\
\text { ppmv }\end{array}$ & $\begin{array}{l}\text { mol\% or } \\
\text { ppmv }\end{array}$ & $\begin{array}{l}\text { mol\% or } \\
\text { ppmv }\end{array}$ \\
\hline \multicolumn{8}{|l|}{$\mathrm{H} 2 \mathrm{O}, \mathrm{mol} \%$} \\
\hline $\mathrm{O} 2, \mathrm{~mol} \%$ & & & & & & & $100 \%$ \\
\hline \multicolumn{8}{|l|}{$\mathrm{N} 2, \mathrm{~mol} \%$} \\
\hline \multicolumn{8}{|l|}{$\mathrm{H} 2, \mathrm{~mol} \%$} \\
\hline $\mathrm{CO} 2, \mathrm{~mol} \%$ & & & & & & $100 \%$ & \\
\hline \multicolumn{8}{|l|}{ COgas, ppmv } \\
\hline \multicolumn{8}{|l|}{$\mathrm{NO}, \mathrm{ppmv}$} \\
\hline \multicolumn{8}{|l|}{ NO2, ppmv } \\
\hline \multicolumn{8}{|l|}{ SO2, ppmv } \\
\hline \multicolumn{8}{|l|}{$\mathrm{Cl}, \mathrm{ppmv}$} \\
\hline \multicolumn{8}{|l|}{$\mathrm{F}, \mathrm{ppmv}$} \\
\hline \multicolumn{8}{|l|}{$\mathrm{C}$ (organic), ppmv } \\
\hline \multicolumn{8}{|l|}{$\mathrm{H}$ (organic), ppmv } \\
\hline & & & & & & & \\
\hline \multicolumn{8}{|l|}{$\mathrm{Hg}, \mathrm{ug} / \mathrm{wscm}$} \\
\hline \multicolumn{8}{|l|}{$\mathrm{PM}, \mathrm{mg} / \mathrm{wscm}$} \\
\hline \multicolumn{8}{|l|}{ SVM, ug/wscm } \\
\hline \multicolumn{8}{|l|}{ LVM, ug/wscm } \\
\hline $\begin{array}{l}\text { Gas Stream Bulk Composition } \\
\text { (Dry Basis) }\end{array}$ & $\mathrm{mol} \%$ & $\mathrm{~mol} \%$ & $\mathrm{~mol} \%$ & $\mathrm{~mol} \%$ & $\mathrm{~mol} \%$ & $\mathrm{~mol} \%$ & $\mathrm{~mol} \%$ \\
\hline \multicolumn{8}{|l|}{$\mathrm{O} 2, \mathrm{~mol} \%$, dry basis } \\
\hline $\mathrm{N} 2, \mathrm{~mol} \%$, dry basis & & & & & & & \\
\hline $\mathrm{H} 2, \mathrm{~mol} \%$, dry basis & & & & & & & \\
\hline $\mathrm{CO} 2, \mathrm{~mol} \%$, dry basis & & & & & & & \\
\hline $\begin{array}{l}\text { Gas Stream Bulk Composition } \\
\text { (Dry Basis, Corrected to } 7 \% \\
\text { O2 with } 100 \% \text { O2 Combustion } \\
\text { Air) }\end{array}$ & $\begin{array}{c}\text { ppmv, or } \\
\text { ug/dscm or } \\
\text { mg/dscm }\end{array}$ & $\begin{array}{c}\text { ppmv, or } \\
\text { ug/dscm or } \\
\text { mg/dscm }\end{array}$ & $\begin{array}{c}\text { ppmv, or } \\
\text { ug/dscm or } \\
\text { mg/dscm }\end{array}$ & $\begin{array}{c}\text { ppmv, or } \\
\text { ug/dscm or } \\
\text { mg/dscm }\end{array}$ & $\begin{array}{l}\text { ppmv, or } \\
\text { ug/dscm or } \\
\text { mg/dscm }\end{array}$ & $\begin{array}{c}\text { ppmv, or } \\
\text { ug/dscm or } \\
\mathrm{mg} / \mathrm{dscm}\end{array}$ & $\begin{array}{l}\text { ppmv, or } \\
\text { ug/dscm or } \\
\text { mg/dscm }\end{array}$ \\
\hline COgas, ppmv, dry basis & & & & & & & \\
\hline $\mathrm{NO}$, ppmv, dry basis & & & & & & & \\
\hline NO2, ppmv, dry basis & & & & & & & \\
\hline SO2, ppmv, dry basis & & & & & & & \\
\hline $\mathrm{Cl}, \mathrm{ppmv}$, dry basis & & & & & & & \\
\hline $\mathrm{F}, \mathrm{ppmv}$, dry basis & & & & & & & \\
\hline C (organic), ppmv, dry basis & & & & & & & \\
\hline $\mathrm{H}$ (organic), ppmv, dry basis & & & & & & & \\
\hline $\mathrm{Hg}, \mathrm{ug} / \mathrm{dscm}$ & & & & & & & \\
\hline $\mathrm{PM}, \mathrm{mg} / \mathrm{dscm}$ & & & & & & & \\
\hline SVM, ug/dscm & & & & & & & \\
\hline LVM, ug/dscm & & & & & & & \\
\hline
\end{tabular}


Table A-20. Mass balance, Tank WM-187 waste (continued).

\begin{tabular}{|c|c|c|c|c|c|c|c|c|}
\hline PFD Number & PFD-3 & PFD-3 & PFD-3 & PFD-3 & PFD-3 & PFD-3 & PFD-3 & PFD-3 \\
\hline Stream Number & 510 & 511 & 512 & 513 & 514 & 515 & 516 & 517 \\
\hline Stream Name & $\begin{array}{c}\text { Gas to } \\
\text { Cool } \\
\text { Product }\end{array}$ & $\begin{array}{c}\text { Hot Gas } \\
\text { after } \\
\text { Product } \\
\text { Cooling }\end{array}$ & $\begin{array}{c}\text { Backpulse } \\
\text { Gas for } \\
\text { Candle } \\
\text { Filters }\end{array}$ & $\begin{array}{c}\text { Bed/ Solid } \\
\text { Reductant } \\
\text { Transport } \\
\text { Gas }\end{array}$ & \begin{tabular}{|c|} 
TF \\
Transfer \& \\
Tank \\
Sparge Air
\end{tabular} & $\begin{array}{c}\text { Ventilation } \\
\text { Air }\end{array}$ & New GAC & $\begin{array}{c}\text { Off-gas } \\
\text { Preaheater } \\
\text { Steam }\end{array}$ \\
\hline Rate or Volume & $2.06 \mathrm{E}+02$ & $6.42 E+02$ & $2.18 \mathrm{E}+02$ & $6.31 \mathrm{E}-01$ & $1.40 \mathrm{E}+01$ & $2.89 \mathrm{E}+01$ & $4.29 \mathrm{E}+00$ & $1.29 \mathrm{E}+03$ \\
\hline Volume Flow (standard, wet) ${ }^{*}$ & $1.39 \mathrm{E}+03$ & $1.39 \mathrm{E}+03$ & $6.38 \mathrm{E}+02$ & $1.85 \mathrm{E}+00$ & $4.09 \mathrm{E}+01$ & $2.94 \mathrm{E}+01$ & & $6.42 \mathrm{E}+03$ \\
\hline Volume Flow (standard, dry) & $1.39 \mathrm{E}+03$ & $1.39 \mathrm{E}+03$ & $6.38 \mathrm{E}+02$ & $1.85 \mathrm{E}+00$ & $4.09 \mathrm{E}+01$ & $2.94 \mathrm{E}+01$ & & $0.00 \mathrm{E}+00$ \\
\hline Rate Units & $\mathrm{ft} 3 / \mathrm{hr}$ & $\mathrm{ft} 3 / \mathrm{hr}$ & $\mathrm{ft} 3 / \mathrm{hr}$ & $\mathrm{ft} 3 / \mathrm{hr}$ & $\mathrm{ft} 3 / \mathrm{hr}$ & $\mathrm{ft} 3 / \mathrm{hr}$ & $\mathrm{lb} / \mathrm{hr}$ & $\mathrm{ft} 3 / \mathrm{hr}$ \\
\hline Rate or Volume, metric & $3.93 \mathrm{E}+01$ & $3.93 \mathrm{E}+01$ & $1.81 \mathrm{E}+01$ & $5.23 E-02$ & $1.16 \mathrm{E}+00$ & 8.34E-01 & $1.94 \mathrm{E}+00$ & $1.82 \mathrm{E}+02$ \\
\hline Rate Units & wscm/hr & wscm/hr & wscm/hr & wscm/hr & wscm/hr & wscm/hr & $\mathrm{kg} / \mathrm{hr}$ & wscm/hr \\
\hline Temperature, ${ }^{\circ} \mathrm{C}$ & 0 & 580 & 15 & 15 & 15 & 15 & 25 & 177 \\
\hline Temperature, ${ }^{\circ} \mathrm{F}$ & 32 & 1076 & 59 & 59 & 59 & 59 & 77 & 350 \\
\hline Pressure, psia & 92.30 & 92.30 & 42.30 & 42.30 & 42.30 & 14.70 & 14.70 & 112.30 \\
\hline Specific Gravity & 7.85E-03 & $2.51 \mathrm{E}-03$ & $3.41 \mathrm{E}-03$ & $3.41 \mathrm{E}-03$ & $3.51 \mathrm{E}-03$ & $1.22 \mathrm{E}-03$ & 4.81E-01 & 3.73E-03 \\
\hline Chemical Composition & lb/wscf & $\mathrm{lb} / \mathrm{wscf}$ & $\mathrm{lb} /$ wscf & $\mathrm{lb} / \mathrm{wscf}$ & lb/wscf & $\mathrm{lb} / \mathrm{wscf}$ & Wt. \% & $\mathrm{lb} /$ wscf \\
\hline C (reductant) & & & & & & & $100 \%$ & \\
\hline Gas Composition, wet basis & $\mathrm{mol} \%$ & $\mathrm{~mol} \%$ & $\mathrm{~mol} \%$ & $\mathrm{~mol} \%$ & $\mathrm{~mol} \%$ & $\mathrm{~mol} \%$ & $\mathrm{~mol} \%$ & $\mathrm{~mol} \%$ \\
\hline $\mathrm{H} 2 \mathrm{O}, \mathrm{mol} \%$ & & & & & $0.01 \%$ & $0.01 \%$ & & $100 \%$ \\
\hline $\mathrm{O} 2, \mathrm{~mol} \%$ & & & & & $21 \%$ & $21 \%$ & & \\
\hline $\mathrm{N} 2, \mathrm{~mol} \%$ & $100 \%$ & $100 \%$ & $100 \%$ & $100 \%$ & $79 \%$ & $79 \%$ & & \\
\hline $\mathrm{PM}, \mathrm{mg} / \mathrm{wscm}$ & $2.01 \mathrm{E}+01$ & $2.01 \mathrm{E}+01$ & 1.97E+01 & $1.99 \mathrm{E}+01$ & $1.54 \mathrm{E}+01$ & $1.57 \mathrm{E}+01$ & & $1.16 \mathrm{E}+01$ \\
\hline & & & & & & & & \\
\hline $\mathrm{kg} / \mathrm{hr}$ & $4.57 \mathrm{E}+01$ & $4.57 \mathrm{E}+01$ & $2.11 \mathrm{E}+01$ & $6.09 \mathrm{E}-02$ & $1.39 \mathrm{E}+00$ & $1.00 \mathrm{E}+00$ & $1.94 \mathrm{E}+00$ & $1.36 \mathrm{E}+02$ \\
\hline
\end{tabular}


Table A-21. Mass balance, Tank WM-188 waste.

\begin{tabular}{|c|c|c|c|c|c|c|c|}
\hline PFD Number & PFD-2 & PFD-2 & PFD-2 & PFD-2 & PFD-2 & PFD-2 & PFD-2 \\
\hline Stream Number & 101 & 102 & 103 & 104 & 106 & 107 & 108 \\
\hline Stream Name & SBW & $\begin{array}{l}\text { Reformer } \\
\text { Feed }\end{array}$ & $\begin{array}{c}\text { Reformer } \\
\text { Off-gas }\end{array}$ & $\begin{array}{l}\text { Reformer } \\
\text { Off-gas } \\
\text { Cooled }\end{array}$ & Filter Drain & Bed Drain & $\begin{array}{l}\text { Cooled } \\
\text { Product }\end{array}$ \\
\hline Rate or Volume & $9.65 \mathrm{E}+01$ & $1.68 \mathrm{E}+02$ & $2.18 \mathrm{E}+05$ & $1.78 \mathrm{E}+05$ & $7.29 \mathrm{E}+00$ & $1.08 \mathrm{E}+02$ & $1.15 \mathrm{E}+02$ \\
\hline $\bar{V}_{\text {Volume Flow (stand, wet }}^{*}$ & & & $5.28 \mathrm{E}+04$ & $5.40 \mathrm{E}+04$ & & & \\
\hline Volume Flow (standard, dry) $)^{*}$ & & & $3.64 \mathrm{E}+04$ & $3.76 \mathrm{E}+04$ & & & \\
\hline Rate Units & $\mathrm{gal} / \mathrm{hr}$ & $\mathrm{gal} / \mathrm{hr}$ & $\mathrm{ft} 3 / \mathrm{hr}$ & $\mathrm{ft} 3 / \mathrm{hr}$ & $\mathrm{lb} / \mathrm{hr}$ & $\mathrm{lb} / \mathrm{hr}$ & $\mathrm{lb} / \mathrm{hr}$ \\
\hline Rate or Volume, metric & $3.65 \mathrm{E}+02$ & $6.37 \mathrm{E}+02$ & $1.50 \mathrm{E}+03$ & $1.53 \mathrm{E}+03$ & $3.30 \mathrm{E}+00$ & $4.90 \mathrm{E}+01$ & $5.23 \mathrm{E}+01$ \\
\hline Rate Units & $\mathrm{L} / \mathrm{hr}$ & $\mathrm{L} / \mathrm{hr}$ & wscm $/ \mathrm{hr}$ & wscm/hr & $\mathrm{kg} / \mathrm{hr}$ & $\mathrm{kg} / \mathrm{hr}$ & $\mathrm{kg} / \mathrm{hr}$ \\
\hline Temperature, ${ }^{\circ} \mathrm{C}$ & 15 & 17 & 600 & 400 & 400 & 600 & 55 \\
\hline Temperature, ${ }^{\circ} \mathrm{F}$ & 59 & 63 & 1112 & 752 & 752 & 1112 & 132 \\
\hline Pressure, psia & 14.70 & 44.70 & 10.62 & 10.22 & 9.50 & 10.80 & 9.50 \\
\hline Specific Gravity & 1.22 & 1.22 & $3.30 \mathrm{E}-04$ & $4.10 \mathrm{E}-04$ & 0.79 & 1.46 & 1.42 \\
\hline Chemical Composition & Mol/liter & Mol/liter & $\mathrm{lb} / \mathrm{wscf}$ & $\mathrm{lb} / \mathrm{wscf}$ & Wt Frac & Wt Frac & Wt frac \\
\hline $\mathrm{H}+$ & $2.53 \mathrm{E}+00$ & $1.48 \mathrm{E}+00$ & $1.11 \mathrm{E}-06$ & $1.08 \mathrm{E}-06$ & $1.35 \mathrm{E}-05$ & $4.23 \mathrm{E}-05$ & $4.05 \mathrm{E}-05$ \\
\hline $\mathrm{Al}+3$ & $4.63 \mathrm{E}-01$ & $2.66 \mathrm{E}-01$ & $1.17 \mathrm{E}-05$ & $1.14 \mathrm{E}-05$ & $8.45 \mathrm{E}-02$ & $9.33 \mathrm{E}-02$ & $9.27 \mathrm{E}-02$ \\
\hline $\mathrm{Sb}+5$ & $1.45 \mathrm{E}-05$ & $8.34 \mathrm{E}-06$ & $1.56 \mathrm{E}-09$ & $1.52 \mathrm{E}-09$ & $1.13 \mathrm{E}-05$ & $1.24 \mathrm{E}-05$ & $1.24 \mathrm{E}-05$ \\
\hline $\mathrm{As}+3$ & $8.10 \mathrm{E}-05$ & $4.65 \mathrm{E}-05$ & 5.34E-09 & $5.22 \mathrm{E}-09$ & $3.87 \mathrm{E}-05$ & $4.27 \mathrm{E}-05$ & $4.25 \mathrm{E}-05$ \\
\hline $\mathrm{Ba}+2$ & $6.63 \mathrm{E}-05$ & $3.80 \mathrm{E}-05$ & $7.26 \mathrm{E}-09$ & $7.09 \mathrm{E}-09$ & $5.26 \mathrm{E}-05$ & $6.43 \mathrm{E}-05$ & $6.36 \mathrm{E}-05$ \\
\hline $\mathrm{Be}+2$ & $1.67 \mathrm{E}-05$ & $9.61 \mathrm{E}-06$ & $1.20 \mathrm{E}-10$ & $1.18 \mathrm{E}-10$ & $8.73 \mathrm{E}-07$ & 1.07E-06 & 1.05E-06 \\
\hline$B+3$ & $1.45 \mathrm{E}-02$ & $8.33 \mathrm{E}-03$ & $1.38 \mathrm{E}-07$ & $1.35 \mathrm{E}-07$ & $1.00 \mathrm{E}-03$ & $1.10 \mathrm{E}-03$ & $1.10 \mathrm{E}-03$ \\
\hline $\mathrm{Cd}+2$ & $2.21 \mathrm{E}-03$ & $1.27 \mathrm{E}-03$ & $8.87 \mathrm{E}-07$ & $8.67 \mathrm{E}-07$ & $6.43 \mathrm{E}-03$ & $1.42 \mathrm{E}-03$ & $1.74 \mathrm{E}-03$ \\
\hline $\mathrm{Ca}+2$ & $4.42 \mathrm{E}-02$ & $2.53 \mathrm{E}-02$ & $1.41 \mathrm{E}-06$ & $1.38 \mathrm{E}-06$ & $1.02 \mathrm{E}-02$ & $1.25 \mathrm{E}-02$ & $1.24 \mathrm{E}-02$ \\
\hline $\mathrm{Cr}+3$ & $4.14 \mathrm{E}-03$ & $2.37 \mathrm{E}-03$ & $2.62 \mathrm{E}-07$ & $2.56 \mathrm{E}-07$ & $1.90 \mathrm{E}-03$ & $1.48 \mathrm{E}-03$ & $1.50 \mathrm{E}-03$ \\
\hline $\mathrm{Co}+2$ & $3.62 \mathrm{E}-05$ & $2.08 \mathrm{E}-05$ & $1.88 \mathrm{E}-09$ & $1.83 \mathrm{E}-09$ & $1.36 \mathrm{E}-05$ & $1.50 \mathrm{E}-05$ & $1.49 \mathrm{E}-05$ \\
\hline Cs+ & $5.27 \mathrm{E}-05$ & $3.03 \mathrm{E}-05$ & $4.16 \mathrm{E}-08$ & $4.07 \mathrm{E}-08$ & $3.01 \mathrm{E}-04$ & $3.19 \mathrm{E}-05$ & $4.90 \mathrm{E}-05$ \\
\hline $\mathrm{Cu}+2$ & $6.20 \mathrm{E}-04$ & $3.56 \mathrm{E}-04$ & $3.14 \mathrm{E}-08$ & $3.07 \mathrm{E}-08$ & $2.28 \mathrm{E}-04$ & $2.79 \mathrm{E}-04$ & $2.75 \mathrm{E}-04$ \\
\hline $\mathrm{Fe}+3$ & $2.28 \mathrm{E}-02$ & $1.31 \mathrm{E}-02$ & $1.65 \mathrm{E}-06$ & $1.61 \mathrm{E}-06$ & $1.19 \mathrm{E}-02$ & $8.70 \mathrm{E}-03$ & $8.91 \mathrm{E}-03$ \\
\hline $\mathrm{Pb}+2$ & $7.27 \mathrm{E}-04$ & $4.17 \mathrm{E}-04$ & $5.37 \mathrm{E}-07$ & $5.25 \mathrm{E}-07$ & $3.89 \mathrm{E}-03$ & $8.61 \mathrm{E}-04$ & $1.05 \mathrm{E}-03$ \\
\hline $\mathrm{Hg}+2$ & $4.65 \mathrm{E}-03$ & $4.90 \mathrm{E}-03$ & $2.61 \mathrm{E}-05$ & $2.55 \mathrm{E}-05$ & $1.89 \mathrm{E}-04$ & $1.28 \mathrm{E}-05$ & $2.39 \mathrm{E}-05$ \\
\hline $\mathrm{Mn}+4$ & $1.32 \mathrm{E}-02$ & $7.60 \mathrm{E}-03$ & $6.40 \mathrm{E}-07$ & $6.26 \mathrm{E}-07$ & $4.64 \mathrm{E}-03$ & $5.12 \mathrm{E}-03$ & $5.09 \mathrm{E}-03$ \\
\hline $\mathrm{Ni}+2$ & 1.62E-03 & $9.30 \mathrm{E}-04$ & $7.59 \mathrm{E}-08$ & $7.42 \mathrm{E}-08$ & $5.50 \mathrm{E}-04$ & $6.73 \mathrm{E}-04$ & $6.65 \mathrm{E}-04$ \\
\hline $\mathrm{K}+$ & $1.69 \mathrm{E}-01$ & $9.72 \mathrm{E}-02$ & $8.59 \mathrm{E}-06$ & $8.40 \mathrm{E}-06$ & $6.23 \mathrm{E}-02$ & $4.52 \mathrm{E}-02$ & $4.63 \mathrm{E}-02$ \\
\hline $\mathrm{Se}+4$ & $2.14 \mathrm{E}-04$ & $1.23 \mathrm{E}-04$ & $1.49 \mathrm{E}-08$ & $1.45 \mathrm{E}-08$ & $1.08 \mathrm{E}-04$ & $1.19 \mathrm{E}-04$ & $1.18 \mathrm{E}-04$ \\
\hline $\mathrm{Ag}+$ & $3.34 \mathrm{E}-04$ & $1.91 \mathrm{E}-04$ & $3.02 \mathrm{E}-08$ & $2.96 \mathrm{E}-08$ & $2.19 \mathrm{E}-04$ & $2.54 \mathrm{E}-04$ & $2.52 \mathrm{E}-04$ \\
\hline $\mathrm{Na}+$ & $1.25 \mathrm{E}+00$ & $7.17 \mathrm{E}-01$ & $2.41 \mathrm{E}-05$ & $2.36 \mathrm{E}-05$ & $1.75 \mathrm{E}-01$ & $2.02 \mathrm{E}-01$ & $2.01 \mathrm{E}-01$ \\
\hline $\mathrm{TI}+3$ & $4.06 \mathrm{E}-06$ & $2.33 \mathrm{E}-06$ & $7.30 \mathrm{E}-10$ & $7.13 \mathrm{E}-10$ & $5.29 \mathrm{E}-06$ & $5.83 \mathrm{E}-06$ & $5.80 \mathrm{E}-06$ \\
\hline $\mathrm{U}+4$ & $3.21 \mathrm{E}-04$ & $1.84 \mathrm{E}-04$ & $6.71 \mathrm{E}-08$ & $6.56 \mathrm{E}-08$ & $4.86 \mathrm{E}-04$ & $5.37 \mathrm{E}-04$ & $5.33 \mathrm{E}-04$ \\
\hline $\mathrm{V}+3$ & $3.25 \mathrm{E}-05$ & $1.86 \mathrm{E}-05$ & $1.45 \mathrm{E}-09$ & 1.42E-09 & $1.05 \mathrm{E}-05$ & $1.16 \mathrm{E}-05$ & $1.16 \mathrm{E}-05$ \\
\hline$Z n+2$ & $7.18 \mathrm{E}-04$ & $4.12 \mathrm{E}-04$ & $3.75 \mathrm{E}-08$ & $3.66 \mathrm{E}-08$ & $2.71 \mathrm{E}-04$ & $3.32 \mathrm{E}-04$ & $3.28 \mathrm{E}-04$ \\
\hline $\mathrm{Zr}+4$ & $3.34 \mathrm{E}-02$ & $1.92 \mathrm{E}-02$ & $2.68 \mathrm{E}-06$ & $2.62 \mathrm{E}-06$ & $1.94 \mathrm{E}-02$ & $2.14 \mathrm{E}-02$ & $2.13 \mathrm{E}-02$ \\
\hline$\overline{\mathrm{Cl}-}$ & $2.18 \mathrm{E}-02$ & $1.25 \mathrm{E}-02$ & $7.24 \mathrm{E}-07$ & $7.08 \mathrm{E}-07$ & $4.94 \mathrm{E}-03$ & $5.43 \mathrm{E}-03$ & $5.40 \mathrm{E}-03$ \\
\hline F- & $3.16 \mathrm{E}-02$ & $1.83 \mathrm{E}-02$ & $1.06 \mathrm{E}-06$ & $1.03 \mathrm{E}-06$ & $3.45 \mathrm{E}-03$ & $3.99 \mathrm{E}-03$ & $3.96 \mathrm{E}-03$ \\
\hline SO4-2 & $3.71 \mathrm{E}-02$ & $2.13 \mathrm{E}-02$ & $6.40 \mathrm{E}-13$ & $6.26 \mathrm{E}-13$ & $4.64 \mathrm{E}-09$ & $5.39 \mathrm{E}-09$ & $5.34 \mathrm{E}-09$ \\
\hline NO3- & $5.19 \mathrm{E}+00$ & $3.00 \mathrm{E}+00$ & $2.27 \mathrm{E}-06$ & $2.22 \mathrm{E}-06$ & $1.64 \mathrm{E}-02$ & $5.51 \mathrm{E}-03$ & $6.20 \mathrm{E}-03$ \\
\hline PO4-3 & $8.81 \mathrm{E}-02$ & $5.05 \mathrm{E}-02$ & $7.37 \mathrm{E}-06$ & $7.20 \mathrm{E}-06$ & $5.34 \mathrm{E}-02$ & $5.88 \mathrm{E}-02$ & $5.85 \mathrm{E}-02$ \\
\hline $\mathrm{Am}+4$ & $6.14 \mathrm{E}-08$ & $3.52 \mathrm{E}-08$ & $1.31 \mathrm{E}-11$ & $1.28 \mathrm{E}-11$ & $9.51 \mathrm{E}-08$ & $1.05 \mathrm{E}-07$ & $1.04 \mathrm{E}-07$ \\
\hline $\mathrm{Br}-$ & $2.64 \mathrm{E}-07$ & $1.51 \mathrm{E}-07$ & $1.97 \mathrm{E}-11$ & $1.92 \mathrm{E}-11$ & $1.34 \mathrm{E}-07$ & $1.48 \mathrm{E}-07$ & $1.47 \mathrm{E}-07$ \\
\hline $\mathrm{Ce}+4$ & $3.03 \mathrm{E}-05$ & $1.74 \mathrm{E}-05$ & $3.74 \mathrm{E}-09$ & $3.66 \mathrm{E}-09$ & $2.71 \mathrm{E}-05$ & $2.99 \mathrm{E}-05$ & $2.97 \mathrm{E}-05$ \\
\hline $\mathrm{Eu+3}$ & $4.37 \mathrm{E}-07$ & $2.51 \mathrm{E}-07$ & $5.85 \mathrm{E}-11$ & $5.72 \mathrm{E}-11$ & $4.24 \mathrm{E}-07$ & $4.68 \mathrm{E}-07$ & $4.65 \mathrm{E}-07$ \\
\hline $\mathrm{Gd}+3$ & $1.29 \mathrm{E}-04$ & $7.41 \mathrm{E}-05$ & $1.79 \mathrm{E}-08$ & $1.75 \mathrm{E}-08$ & $1.29 \mathrm{E}-04$ & $1.43 \mathrm{E}-04$ & $1.42 \mathrm{E}-04$ \\
\hline $\mathrm{Ge}+4$ & $7.60 \mathrm{E}-09$ & $4.36 \mathrm{E}-09$ & $4.85 \mathrm{E}-13$ & $4.74 \mathrm{E}-13$ & $3.51 \mathrm{E}-09$ & $3.88 \mathrm{E}-09$ & $3.85 \mathrm{E}-09$ \\
\hline $\ln +3$ & 1.34E-06 & $7.67 \mathrm{E}-07$ & $1.35 \mathrm{E}-10$ & $1.32 \mathrm{E}-10$ & $9.78 \mathrm{E}-07$ & $1.08 \mathrm{E}-06$ & 1.07E-06 \\
\hline |- & $2.82 \mathrm{E}-06$ & $1.62 \mathrm{E}-06$ & $3.34 \mathrm{E}-10$ & $3.27 \mathrm{E}-10$ & $2.28 \mathrm{E}-06$ & $2.51 \mathrm{E}-06$ & $2.49 \mathrm{E}-06$ \\
\hline$L a+3$ & $7.94 \mathrm{E}-06$ & $4.56 \mathrm{E}-06$ & $9.70 \mathrm{E}-10$ & $9.48 \mathrm{E}-10$ & $7.03 \mathrm{E}-06$ & $7.76 \mathrm{E}-06$ & $7.71 \mathrm{E}-06$ \\
\hline
\end{tabular}


Table A-21. Mass balance, Tank WM-188 waste (continued).

\begin{tabular}{|c|c|c|c|c|c|c|c|}
\hline Stream \# & 101 & 102 & 103 & 104 & 106 & 107 & 108 \\
\hline Chemical Composition & Mol/liter & Mol/liter & $\mathrm{Ib} / \mathrm{wscf}$ & $\mathrm{Ib} / \mathrm{wscf}$ & Wt frac & Wt frac & Wt frac \\
\hline Li+ & $4.15 \mathrm{E}-04$ & $2.38 \mathrm{E}-04$ & $2.42 \mathrm{E}-09$ & $2.36 \mathrm{E}-09$ & $1.75 \mathrm{E}-05$ & $2.03 \mathrm{E}-05$ & $2.01 \mathrm{E}-05$ \\
\hline$M g+2$ & $1.63 \mathrm{E}-02$ & $9.33 \mathrm{E}-03$ & $3.16 \mathrm{E}-07$ & $3.08 \mathrm{E}-07$ & $2.29 \mathrm{E}-03$ & $2.80 \mathrm{E}-03$ & $2.76 \mathrm{E}-03$ \\
\hline $\mathrm{Mo}+6$ & $4.78 \mathrm{E}-02$ & $2.74 \mathrm{E}-02$ & $4.03 \mathrm{E}-06$ & $3.94 \mathrm{E}-06$ & $2.92 \mathrm{E}-02$ & $3.22 \mathrm{E}-02$ & $3.21 \mathrm{E}-02$ \\
\hline $\mathrm{Nd}+3$ & $2.56 \mathrm{E}-05$ & $1.47 \mathrm{E}-05$ & $3.25 \mathrm{E}-09$ & $3.18 \mathrm{E}-09$ & $2.35 \mathrm{E}-05$ & $2.60 \mathrm{E}-05$ & $2.58 \mathrm{E}-05$ \\
\hline $\mathrm{Np}+4$ & $2.34 \mathrm{E}-06$ & $1.34 \mathrm{E}-06$ & $4.88 \mathrm{E}-10$ & $4.77 \mathrm{E}-10$ & $3.54 \mathrm{E}-06$ & $3.90 \mathrm{E}-06$ & $3.88 \mathrm{E}-06$ \\
\hline $\mathrm{Nb}+5$ & $9.59 \mathrm{E}-04$ & $5.50 \mathrm{E}-04$ & $7.83 \mathrm{E}-08$ & $7.66 \mathrm{E}-08$ & $5.68 \mathrm{E}-04$ & $6.26 \mathrm{E}-04$ & $6.23 \mathrm{E}-04$ \\
\hline $\mathrm{Pd}+4$ & $1.56 \mathrm{E}-03$ & $8.93 \mathrm{E}-04$ & $1.32 \mathrm{E}-07$ & $1.29 \mathrm{E}-07$ & $9.57 \mathrm{E}-04$ & $1.17 \mathrm{E}-03$ & $1.16 \mathrm{E}-03$ \\
\hline $\mathrm{Pu}+4$ & $6.09 \mathrm{E}-06$ & $3.50 \mathrm{E}-06$ & $1.31 \mathrm{E}-09$ & $1.28 \mathrm{E}-09$ & $9.47 \mathrm{E}-06$ & $1.05 \mathrm{E}-05$ & $1.04 \mathrm{E}-05$ \\
\hline $\mathrm{Pr}+4$ & $7.22 \mathrm{E}-06$ & $4.14 \mathrm{E}-06$ & $8.95 \mathrm{E}-10$ & $8.75 \mathrm{E}-10$ & $6.49 \mathrm{E}-06$ & $7.16 \mathrm{E}-06$ & $7.11 \mathrm{E}-06$ \\
\hline $\mathrm{Pm}+3$ & $1.49 \mathrm{E}-09$ & $8.58 \mathrm{E}-10$ & $1.91 \mathrm{E}-13$ & $1.86 \mathrm{E}-13$ & $1.38 \mathrm{E}-09$ & $1.52 \mathrm{E}-09$ & $1.52 \mathrm{E}-09$ \\
\hline $\mathrm{Rh}+4$ & $3.11 \mathrm{E}-06$ & $1.79 \mathrm{E}-06$ & $2.82 \mathrm{E}-10$ & $2.76 \mathrm{E}-10$ & $2.04 \mathrm{E}-06$ & $2.25 \mathrm{E}-06$ & $2.24 \mathrm{E}-06$ \\
\hline $\mathrm{Rb}+$ & $4.80 \mathrm{E}-06$ & $2.75 \mathrm{E}-06$ & $3.44 \mathrm{E}-10$ & $3.36 \mathrm{E}-10$ & $2.49 \mathrm{E}-06$ & $2.89 \mathrm{E}-06$ & $2.86 \mathrm{E}-06$ \\
\hline $\mathrm{Ru}+3$ & $7.21 \mathrm{E}-04$ & $4.14 \mathrm{E}-04$ & $6.41 \mathrm{E}-08$ & $6.26 \mathrm{E}-08$ & $4.64 \mathrm{E}-04$ & $5.12 \mathrm{E}-04$ & $5.09 \mathrm{E}-04$ \\
\hline$S m+3$ & $4.77 \mathrm{E}-06$ & $2.74 \mathrm{E}-06$ & $6.31 \mathrm{E}-10$ & $6.17 \mathrm{E}-10$ & $4.57 \mathrm{E}-06$ & $5.04 \mathrm{E}-06$ & $5.01 \mathrm{E}-06$ \\
\hline Si+4 & $8.18 \mathrm{E}-02$ & $4.69 \mathrm{E}-02$ & $2.02 \mathrm{E}-06$ & 1.97E-06 & $1.46 \mathrm{E}-02$ & $1.62 \mathrm{E}-02$ & $1.61 \mathrm{E}-02$ \\
\hline $\mathrm{Sr}+2$ & $7.12 \mathrm{E}-05$ & $4.08 \mathrm{E}-05$ & $4.98 \mathrm{E}-09$ & $4.86 \mathrm{E}-09$ & $3.61 \mathrm{E}-05$ & $4.41 \mathrm{E}-05$ & $4.36 \mathrm{E}-05$ \\
\hline $\mathrm{Tc}+7$ & $9.29 \mathrm{E}-06$ & $5.33 \mathrm{E}-06$ & $7.30 \mathrm{E}-09$ & $7.13 \mathrm{E}-09$ & $5.28 \mathrm{E}-05$ & $3.23 \mathrm{E}-06$ & $6.36 \mathrm{E}-06$ \\
\hline $\mathrm{Te}+4$ & $3.17 \mathrm{E}-06$ & $1.82 \mathrm{E}-06$ & $3.56 \mathrm{E}-10$ & $3.47 \mathrm{E}-10$ & $2.58 \mathrm{E}-06$ & $2.84 \mathrm{E}-06$ & $2.83 \mathrm{E}-06$ \\
\hline $\mathrm{Tb}+4$ & $1.83 \mathrm{E}-09$ & 1.05E-09 & $2.56 \mathrm{E}-13$ & $2.50 \mathrm{E}-13$ & $1.85 \mathrm{E}-09$ & $2.04 \mathrm{E}-09$ & $2.03 \mathrm{E}-09$ \\
\hline $\mathrm{Th}+4$ & $2.10 \mathrm{E}-05$ & $1.21 \mathrm{E}-05$ & $4.29 \mathrm{E}-09$ & $4.19 \mathrm{E}-09$ & $3.11 \mathrm{E}-05$ & $3.43 \mathrm{E}-05$ & $3.41 \mathrm{E}-05$ \\
\hline $\mathrm{Sn}+4$ & $1.58 \mathrm{E}-03$ & $9.08 \mathrm{E}-04$ & $1.65 \mathrm{E}-07$ & $1.61 \mathrm{E}-07$ & $1.20 \mathrm{E}-03$ & $1.32 \mathrm{E}-03$ & $1.31 \mathrm{E}-03$ \\
\hline $\mathrm{Ti}+4$ & $8.10 \mathrm{E}-04$ & 4.64E-04 & $3.41 \mathrm{E}-08$ & $3.33 \mathrm{E}-08$ & $2.47 \mathrm{E}-04$ & $2.73 \mathrm{E}-04$ & $2.71 \mathrm{E}-04$ \\
\hline$\overline{\mathrm{Y}+3}$ & $5.92 \mathrm{E}-06$ & $3.40 \mathrm{E}-06$ & $4.63 \mathrm{E}-10$ & $4.52 \mathrm{E}-10$ & $3.35 \mathrm{E}-06$ & $3.70 \mathrm{E}-06$ & $3.68 \mathrm{E}-06$ \\
\hline $\mathrm{OH}-$ & & $3.90 \mathrm{E}-08$ & $3.17 \mathrm{E}-10$ & $3.10 \mathrm{E}-10$ & $2.30 \mathrm{E}-06$ & $1.67 \mathrm{E}-06$ & $1.71 \mathrm{E}-06$ \\
\hline $\mathrm{H} 2 \mathrm{O}$ & $4.48 \mathrm{E}+01$ & $2.73 \mathrm{E}+01$ & $1.45 \mathrm{E}-02$ & $1.42 \mathrm{E}-02$ & $1.22 \mathrm{E}-02$ & $4.83 \mathrm{E}-03$ & $5.30 \mathrm{E}-03$ \\
\hline & & 190 & & 120 & & & \\
\hline $\mathrm{SO} 2$ & & 1.90E-08 & $1.26 \mathrm{E}-12$ & 1.23E-12 & & & \\
\hline $\mathrm{H} 2 \mathrm{~S}$ & & & $1.81 \mathrm{E}-05$ & 1.77E-05 & & & \\
\hline $\mathrm{CO}$ & & $8.65 \mathrm{E}-11$ & $3.55 \mathrm{E}-04$ & $3.47 \mathrm{E}-04$ & & & \\
\hline $\mathrm{CO} 2$ & & $6.29 \mathrm{E}-06$ & $6.13 \mathrm{E}-02$ & $5.99 \mathrm{E}-02$ & & & \\
\hline $\mathrm{H} 2$ & & $9.21 \mathrm{E}-10$ & $5.71 \mathrm{E}-04$ & $5.58 \mathrm{E}-04$ & & & \\
\hline N2 & & $1.73 \mathrm{E}-10$ & $1.11 \mathrm{E}-03$ & $2.73 \mathrm{E}-03$ & & & \\
\hline $\mathrm{NO}$ & & $2.51 \mathrm{E}-09$ & $1.41 \mathrm{E}-04$ & $1.38 \mathrm{E}-04$ & & & \\
\hline $\mathrm{NO} 2$ & & $3.94 \mathrm{E}-07$ & $2.06 \mathrm{E}-06$ & $2.01 \mathrm{E}-06$ & & & \\
\hline $\mathrm{O} 2$ & & $5.22 \mathrm{E}-10$ & & & & & \\
\hline$S$ (other) & & $2.78 \mathrm{E}-10$ & $5.84 \mathrm{E}-08$ & $5.70 \mathrm{E}-08$ & $4.23 \mathrm{E}-04$ & $4.90 \mathrm{E}-04$ & $4.86 \mathrm{E}-04$ \\
\hline $\mathrm{CO} 3$ & $2.64 \mathrm{E}-05$ & $1.51 \mathrm{E}-05$ & $3.98 \mathrm{E}-05$ & $3.89 \mathrm{E}-05$ & $2.88 \mathrm{E}-01$ & $3.15 \mathrm{E}-01$ & $3.13 \mathrm{E}-01$ \\
\hline C (reductant) & & & & & & & \\
\hline $\mathrm{O}$ (oxides) & $4.26 \mathrm{E}-01$ & $2.44 \mathrm{E}-01$ & $1.60 \mathrm{E}-05$ & $1.56 \mathrm{E}-05$ & $11.59 \%$ & $12.46 \%$ & $12.40 \%$ \\
\hline C (organic) & $1.64 \mathrm{E}-02$ & $2.45 \mathrm{E}+01$ & $5.63 \mathrm{E}-03$ & $5.50 \mathrm{E}-03$ & $5.52 \mathrm{E}-02$ & $8.49 \mathrm{E}-04$ & $4.28 \mathrm{E}-03$ \\
\hline $\mathrm{H}$ (organic) & $4.38 \mathrm{E}-02$ & $6.54 \mathrm{E}+01$ & 1.06E-03 & 1.03E-03 & $9.84 \mathrm{E}-03$ & $1.51 \mathrm{E}-04$ & 7.64E-04 \\
\hline $\mathrm{O}$ (organic) & $5.47 \mathrm{E}-03$ & $8.18 \mathrm{E}+00$ & & & & & \\
\hline & & & & & & & \\
\hline Mass Flow (kg/hr): & $4.47 \mathrm{E}+02$ & $7.80 \mathrm{E}+02$ & $2.03 \mathrm{E}+03$ & $2.07 \mathrm{E}+03$ & $3.30 \mathrm{E}+00$ & $4.90 \mathrm{E}+01$ & $5.23 E+01$ \\
\hline Canister Rate, canisters/day & & & & & & & 1.11 \\
\hline Total Canisters Generated & & & & & & & 156 \\
\hline & $\mathrm{w} / \mathrm{m} 3$ & $\mathrm{w} / \mathrm{m} 3$ & $\mathrm{w} / \mathrm{m} 3$ & $\mathrm{w} / \mathrm{m} 3$ & $\mathrm{w} / \mathrm{m} 3$ & $\mathrm{w} / \mathrm{m} 3$ & $\mathrm{w} / \mathrm{m} 3$ \\
\hline Heat Generation & $3.68 \mathrm{E}-01$ & $2.11 \mathrm{E}-01$ & & & $2.42 \mathrm{E}+00$ & $3.71 \mathrm{E}+00$ & $3.65 \mathrm{E}+00$ \\
\hline
\end{tabular}


Table A-21. Mass balance, Tank WM-188 waste (continued).

\begin{tabular}{|c|c|c|c|c|c|c|c|}
\hline Stream \# & 101 & 102 & 103 & 104 & 106 & 107 & 108 \\
\hline Radiological Composition & $\mathrm{Ci} / \mathrm{L}$ & $\mathrm{Ci} / \mathrm{L}$ & Ci/wscm & Ci/wscm & $\mathrm{Ci} / \mathrm{kg}$ & $\mathrm{Ci} / \mathrm{kg}$ & $\mathrm{Ci} / \mathrm{kg}$ \\
\hline $\mathrm{H}-3$ & $1.06 \mathrm{E}-05$ & $6.10 \mathrm{E}-06$ & $2.60 \mathrm{E}-06$ & $2.54 \mathrm{E}-06$ & & & \\
\hline$\overline{C-14}$ & $1.44 \mathrm{E}-10$ & $8.25 \mathrm{E}-11$ & $3.49 \mathrm{E}-11$ & $3.42 \mathrm{E}-11$ & $1.09 \mathrm{E}-11$ & $6.19 \mathrm{E}-12$ & $6.49 \mathrm{E}-12$ \\
\hline Co-60 & $8.10 \mathrm{E}-06$ & $4.65 \mathrm{E}-06$ & $1.14 \mathrm{E}-07$ & $1.12 \mathrm{E}-07$ & $5.17 \mathrm{E}-05$ & $5.70 \mathrm{E}-05$ & $5.67 \mathrm{E}-05$ \\
\hline Ni-59 & $1.01 \mathrm{E}-06$ & $5.79 \mathrm{E}-07$ & $1.29 \mathrm{E}-08$ & $1.26 \mathrm{E}-08$ & $5.84 \mathrm{E}-06$ & $7.14 \mathrm{E}-06$ & $7.06 \mathrm{E}-06$ \\
\hline $\mathrm{Ni}-63$ & $3.82 \mathrm{E}-05$ & $2.19 \mathrm{E}-05$ & $4.88 \mathrm{E}-07$ & $4.76 \mathrm{E}-07$ & $2.21 \mathrm{E}-04$ & $2.70 \mathrm{E}-04$ & $2.67 \mathrm{E}-04$ \\
\hline Se-79 & $5.22 \mathrm{E}-07$ & $3.00 \mathrm{E}-07$ & $7.35 \mathrm{E}-09$ & $7.19 \mathrm{E}-09$ & $3.33 \mathrm{E}-06$ & $3.67 \mathrm{E}-06$ & $3.65 \mathrm{E}-06$ \\
\hline Sr-90 & $3.59 \mathrm{E}-02$ & $2.06 \mathrm{E}-02$ & $4.59 \mathrm{E}-04$ & $4.48 \mathrm{E}-04$ & $2.07 \mathrm{E}-01$ & $2.54 \mathrm{E}-01$ & $2.51 \mathrm{E}-01$ \\
\hline $\mathrm{Y}-90$ & $3.59 \mathrm{E}-02$ & $2.06 \mathrm{E}-02$ & $5.06 \mathrm{E}-04$ & 4.94E-04 & $2.29 \mathrm{E}-01$ & $2.52 \mathrm{E}-01$ & $2.51 \mathrm{E}-01$ \\
\hline $\mathrm{Zr}-93$ & $2.06 \mathrm{E}-06$ & $1.18 \mathrm{E}-06$ & $2.91 \mathrm{E}-08$ & $2.84 \mathrm{E}-08$ & $1.32 \mathrm{E}-05$ & $1.45 \mathrm{E}-05$ & $1.44 \mathrm{E}-05$ \\
\hline $\mathrm{Nb}-93 \mathrm{~m}$ & 1.59E-06 & $9.12 \mathrm{E}-07$ & $2.24 \mathrm{E}-08$ & $2.19 \mathrm{E}-08$ & $1.01 \mathrm{E}-05$ & $1.12 \mathrm{E}-05$ & $1.11 \mathrm{E}-05$ \\
\hline $\mathrm{Nb}-94$ & $1.36 \mathrm{E}-06$ & $7.79 \mathrm{E}-07$ & $1.91 \mathrm{E}-08$ & $1.87 \mathrm{E}-08$ & $8.66 \mathrm{E}-06$ & $9.55 \mathrm{E}-06$ & $9.50 \mathrm{E}-06$ \\
\hline Tc-99 & $1.89 \mathrm{E}-05$ & $1.08 \mathrm{E}-05$ & $2.43 \mathrm{E}-06$ & $2.37 \mathrm{E}-06$ & $1.10 \mathrm{E}-03$ & $6.69 \mathrm{E}-05$ & $1.32 \mathrm{E}-04$ \\
\hline Ru-106 & $1.12 \mathrm{E}-06$ & $6.41 \mathrm{E}-07$ & $1.57 \mathrm{E}-08$ & $1.54 \mathrm{E}-08$ & $7.11 \mathrm{E}-06$ & $7.85 \mathrm{E}-06$ & $7.80 \mathrm{E}-06$ \\
\hline Rh-102 & $8.03 \mathrm{E}-10$ & $4.61 \mathrm{E}-10$ & $1.13 \mathrm{E}-11$ & $1.11 \mathrm{E}-11$ & $5.12 \mathrm{E}-09$ & $5.65 \mathrm{E}-09$ & $5.62 \mathrm{E}-09$ \\
\hline Rh-106 & $1.12 \mathrm{E}-06$ & $6.41 \mathrm{E}-07$ & $1.57 \mathrm{E}-08$ & $1.54 \mathrm{E}-08$ & $7.11 \mathrm{E}-06$ & $7.85 \mathrm{E}-06$ & $7.80 \mathrm{E}-06$ \\
\hline $\mathrm{Pd}-107$ & $1.54 \mathrm{E}-08$ & $8.83 \mathrm{E}-09$ & $1.97 \mathrm{E}-10$ & $1.92 \mathrm{E}-10$ & $8.90 \mathrm{E}-08$ & $1.09 \mathrm{E}-07$ & $1.08 \mathrm{E}-07$ \\
\hline Cd-113m & $3.10 \mathrm{E}-06$ & $1.78 \mathrm{E}-06$ & $1.77 \mathrm{E}-07$ & $1.73 \mathrm{E}-07$ & $8.00 \mathrm{E}-05$ & $1.77 \mathrm{E}-05$ & $2.16 \mathrm{E}-05$ \\
\hline Sn-121m & $6.23 \mathrm{E}-08$ & $3.57 \mathrm{E}-08$ & $8.78 \mathrm{E}-10$ & $8.58 \mathrm{E}-10$ & 3.97E-07 & $4.38 \mathrm{E}-07$ & $4.35 \mathrm{E}-07$ \\
\hline Sn-126 & $4.92 \mathrm{E}-07$ & $2.82 \mathrm{E}-07$ & $6.92 \mathrm{E}-09$ & $6.77 \mathrm{E}-09$ & $3.13 \mathrm{E}-06$ & $3.46 \mathrm{E}-06$ & $3.44 \mathrm{E}-06$ \\
\hline Sb-125 & $3.38 \mathrm{E}-04$ & $1.94 \mathrm{E}-04$ & $4.76 \mathrm{E}-06$ & $4.65 \mathrm{E}-06$ & $2.15 \mathrm{E}-03$ & $2.38 \mathrm{E}-03$ & $2.36 \mathrm{E}-03$ \\
\hline Sb-126 & $5.36 \mathrm{E}-08$ & $3.08 \mathrm{E}-08$ & $7.56 \mathrm{E}-10$ & $7.38 \mathrm{E}-10$ & $3.42 \mathrm{E}-07$ & $3.77 \mathrm{E}-07$ & $3.75 \mathrm{E}-07$ \\
\hline Te-125m & $2.94 \mathrm{E}-06$ & $1.68 \mathrm{E}-06$ & $4.13 \mathrm{E}-08$ & $4.04 \mathrm{E}-08$ & $1.87 \mathrm{E}-05$ & $2.06 \mathrm{E}-05$ & $2.05 \mathrm{E}-05$ \\
\hline $\mathrm{I}-129$ & $6.38 \mathrm{E}-08$ & $3.66 \mathrm{E}-08$ & $9.56 \mathrm{E}-10$ & $9.34 \mathrm{E}-10$ & $4.07 \mathrm{E}-07$ & $4.47 \mathrm{E}-07$ & $4.45 \mathrm{E}-07$ \\
\hline Cs-134 & $3.37 \mathrm{E}-05$ & $1.93 \mathrm{E}-05$ & $3.20 \mathrm{E}-06$ & $3.13 \mathrm{E}-06$ & $1.45 \mathrm{E}-03$ & $1.54 \mathrm{E}-04$ & $2.35 \mathrm{E}-04$ \\
\hline Cs-135 & $1.00 \mathrm{E}-06$ & $5.76 \mathrm{E}-07$ & $9.55 \mathrm{E}-08$ & $9.33 \mathrm{E}-08$ & $4.32 \mathrm{E}-05$ & $4.58 \mathrm{E}-06$ & $7.02 \mathrm{E}-06$ \\
\hline Cs-137 & $2.07 \mathrm{E}-02$ & $1.19 \mathrm{E}-02$ & $1.97 \mathrm{E}-03$ & $1.93 \mathrm{E}-03$ & $8.91 \mathrm{E}-01$ & $9.45 \mathrm{E}-02$ & $1.45 \mathrm{E}-01$ \\
\hline Ba-137m & $1.96 \mathrm{E}-02$ & $1.12 \mathrm{E}-02$ & $2.51 \mathrm{E}-04$ & $2.45 \mathrm{E}-04$ & $1.13 \mathrm{E}-01$ & $1.39 \mathrm{E}-01$ & $1.37 \mathrm{E}-01$ \\
\hline $\mathrm{Ce}-144$ & $7.53 \mathrm{E}-07$ & $4.32 \mathrm{E}-07$ & $1.06 \mathrm{E}-08$ & $1.04 \mathrm{E}-08$ & $4.80 \mathrm{E}-06$ & $5.30 \mathrm{E}-06$ & $5.27 \mathrm{E}-06$ \\
\hline Pr-144 & $7.53 \mathrm{E}-07$ & $4.32 \mathrm{E}-07$ & $1.06 \mathrm{E}-08$ & $1.04 \mathrm{E}-08$ & $4.80 \mathrm{E}-06$ & $5.30 \mathrm{E}-06$ & $5.27 \mathrm{E}-06$ \\
\hline $\mathrm{Pm}-146$ & $4.74 \mathrm{E}-08$ & $2.72 \mathrm{E}-08$ & $6.69 \mathrm{E}-10$ & $6.53 \mathrm{E}-10$ & $3.02 \mathrm{E}-07$ & $3.34 \mathrm{E}-07$ & $3.32 \mathrm{E}-07$ \\
\hline $\mathrm{Pm}-147$ & $2.03 \mathrm{E}-04$ & $1.17 \mathrm{E}-04$ & $2.87 \mathrm{E}-06$ & $2.80 \mathrm{E}-06$ & $1.30 \mathrm{E}-03$ & $1.43 \mathrm{E}-03$ & $1.42 \mathrm{E}-03$ \\
\hline Sm-151 & $4.02 \mathrm{E}-04$ & $2.31 \mathrm{E}-04$ & $5.67 \mathrm{E}-06$ & $5.54 \mathrm{E}-06$ & $2.56 \mathrm{E}-03$ & $2.83 \mathrm{E}-03$ & $2.81 \mathrm{E}-03$ \\
\hline Eu-152 & $2.66 \mathrm{E}-06$ & $1.53 \mathrm{E}-06$ & $3.75 \mathrm{E}-08$ & $3.66 \mathrm{E}-08$ & $1.69 \mathrm{E}-05$ & $1.87 \mathrm{E}-05$ & $1.86 \mathrm{E}-05$ \\
\hline Eu-154 & $4.52 \mathrm{E}-05$ & $2.60 \mathrm{E}-05$ & $6.38 \mathrm{E}-07$ & $6.23 \mathrm{E}-07$ & $2.88 \mathrm{E}-04$ & $3.18 \mathrm{E}-04$ & $3.16 \mathrm{E}-04$ \\
\hline Eu-155 & $3.92 \mathrm{E}-05$ & $2.25 \mathrm{E}-05$ & $5.53 \mathrm{E}-07$ & $5.40 \mathrm{E}-07$ & $2.50 \mathrm{E}-04$ & $2.76 \mathrm{E}-04$ & $2.74 \mathrm{E}-04$ \\
\hline Th-230 & $9.55 \mathrm{E}-10$ & $5.47 \mathrm{E}-10$ & 1.34E-11 & $1.31 \mathrm{E}-11$ & 6.08E-09 & 6.71E-09 & 6.67E-09 \\
\hline $\mathrm{Pa}-233$ & $2.73 \mathrm{E}-06$ & $1.57 \mathrm{E}-06$ & $3.85 \mathrm{E}-08$ & $3.76 \mathrm{E}-08$ & $1.74 \mathrm{E}-05$ & $1.92 \mathrm{E}-05$ & $1.91 \mathrm{E}-05$ \\
\hline $\mathrm{U}-232$ & $2.25 \mathrm{E}-09$ & $1.29 \mathrm{E}-09$ & $3.17 \mathrm{E}-11$ & $3.09 \mathrm{E}-11$ & $1.43 \mathrm{E}-08$ & $1.58 \mathrm{E}-08$ & $1.57 \mathrm{E}-08$ \\
\hline $\mathrm{U}-233$ & $8.13 \mathrm{E}-11$ & $4.66 \mathrm{E}-11$ & $1.15 \mathrm{E}-12$ & $1.12 \mathrm{E}-12$ & $5.18 \mathrm{E}-10$ & $5.72 \mathrm{E}-10$ & $5.68 \mathrm{E}-10$ \\
\hline $\mathrm{U}-234$ & $1.00 \mathrm{E}-06$ & $5.75 \mathrm{E}-07$ & $1.41 \mathrm{E}-08$ & $1.38 \mathrm{E}-08$ & $6.39 \mathrm{E}-06$ & $7.05 \mathrm{E}-06$ & $7.01 \mathrm{E}-06$ \\
\hline $\mathrm{U}-235$ & $7.37 \mathrm{E}-08$ & $4.23 \mathrm{E}-08$ & $1.04 \mathrm{E}-09$ & $1.01 \mathrm{E}-09$ & $4.69 \mathrm{E}-07$ & $5.18 \mathrm{E}-07$ & $5.15 \mathrm{E}-07$ \\
\hline $\mathrm{U}-236$ & $4.33 \mathrm{E}-08$ & $2.48 \mathrm{E}-08$ & $6.10 \mathrm{E}-10$ & $5.96 \mathrm{E}-10$ & $2.76 \mathrm{E}-07$ & $3.04 \mathrm{E}-07$ & $3.03 \mathrm{E}-07$ \\
\hline $\mathrm{U}-237$ & $6.00 \mathrm{E}-09$ & $3.44 \mathrm{E}-09$ & $8.45 \mathrm{E}-11$ & $8.26 \mathrm{E}-11$ & $3.82 \mathrm{E}-08$ & $4.22 \mathrm{E}-08$ & $4.19 \mathrm{E}-08$ \\
\hline $\mathrm{U}-238$ & $1.31 \mathrm{E}-08$ & $7.52 \mathrm{E}-09$ & $1.85 \mathrm{E}-10$ & $1.81 \mathrm{E}-10$ & $8.36 \mathrm{E}-08$ & $9.22 \mathrm{E}-08$ & $9.17 \mathrm{E}-08$ \\
\hline Np-237 & $3.91 \mathrm{E}-07$ & $2.24 \mathrm{E}-07$ & $5.51 \mathrm{E}-09$ & $5.39 \mathrm{E}-09$ & $2.49 \mathrm{E}-06$ & $2.75 \mathrm{E}-06$ & $2.73 \mathrm{E}-06$ \\
\hline Pu-236 & $3.28 \mathrm{E}-09$ & $1.88 \mathrm{E}-09$ & $4.62 \mathrm{E}-11$ & $4.52 \mathrm{E}-11$ & $2.09 \mathrm{E}-08$ & $2.31 \mathrm{E}-08$ & $2.29 \mathrm{E}-08$ \\
\hline Pu-238 & $7.51 \mathrm{E}-04$ & $4.31 \mathrm{E}-04$ & $1.06 \mathrm{E}-05$ & $1.03 \mathrm{E}-05$ & $4.78 \mathrm{E}-03$ & $5.28 \mathrm{E}-03$ & $5.25 \mathrm{E}-03$ \\
\hline Pu-239 & $8.40 \mathrm{E}-05$ & $4.82 \mathrm{E}-05$ & $1.18 \mathrm{E}-06$ & $1.16 \mathrm{E}-06$ & $5.35 \mathrm{E}-04$ & $5.91 \mathrm{E}-04$ & $5.87 \mathrm{E}-04$ \\
\hline Pu-240 & $1.22 \mathrm{E}-05$ & $7.01 \mathrm{E}-06$ & $1.72 \mathrm{E}-07$ & $1.68 \mathrm{E}-07$ & $7.79 \mathrm{E}-05$ & $8.59 \mathrm{E}-05$ & $8.54 \mathrm{E}-05$ \\
\hline Pu-241 & $5.46 \mathrm{E}-04$ & $3.13 \mathrm{E}-04$ & $7.69 \mathrm{E}-06$ & $7.52 \mathrm{E}-06$ & $3.48 \mathrm{E}-03$ & $3.84 \mathrm{E}-03$ & $3.82 \mathrm{E}-03$ \\
\hline Pu-242 & $9.57 \mathrm{E}-09$ & $5.49 \mathrm{E}-09$ & $1.35 \mathrm{E}-10$ & $1.32 \mathrm{E}-10$ & $6.10 \mathrm{E}-08$ & $6.73 \mathrm{E}-08$ & $6.69 \mathrm{E}-08$ \\
\hline $\mathrm{Pu}-244$ & $7.31 \mathrm{E}-16$ & $4.19 \mathrm{E}-16$ & $1.03 \mathrm{E}-17$ & $1.01 \mathrm{E}-17$ & $4.65 \mathrm{E}-15$ & $5.14 \mathrm{E}-15$ & $5.11 \mathrm{E}-15$ \\
\hline Am-241 & $5.26 \mathrm{E}-05$ & $3.02 \mathrm{E}-05$ & $7.42 \mathrm{E}-07$ & $7.25 \mathrm{E}-07$ & $3.36 \mathrm{E}-04$ & $3.70 \mathrm{E}-04$ & $3.68 \mathrm{E}-04$ \\
\hline Am-242m & $1.45 \mathrm{E}-08$ & $8.35 \mathrm{E}-09$ & $2.05 \mathrm{E}-10$ & $2.00 \mathrm{E}-10$ & $9.27 \mathrm{E}-08$ & $1.02 \mathrm{E}-07$ & $1.02 \mathrm{E}-07$ \\
\hline
\end{tabular}


Table A-21. Mass balance, Tank WM-188 waste (continued).

\begin{tabular}{|c|c|c|c|c|c|c|c|}
\hline Stream \# & 101 & 102 & 103 & 104 & 106 & 107 & 108 \\
\hline Radiological Composition & $\mathrm{Ci} / \mathrm{L}$ & $\mathrm{Ci} / \mathrm{L}$ & $\mathrm{Ci} / \mathrm{wscm}$ & $\mathrm{Ci} / \mathrm{wscm}$ & $\mathrm{Ci} / \mathrm{kg}$ & $\mathrm{Ci} / \mathrm{kg}$ & $\mathrm{Ci} / \mathrm{kg}$ \\
\hline $\mathrm{Am}-243$ & $2.37 \mathrm{E}-08$ & $1.36 \mathrm{E}-08$ & $3.34 \mathrm{E}-10$ & $3.27 \mathrm{E}-10$ & $1.51 \mathrm{E}-07$ & 1.67E-07 & $1.66 \mathrm{E}-07$ \\
\hline $\mathrm{Cm}-242$ & $3.15 E-08$ & $1.81 \mathrm{E}-08$ & $4.43 \mathrm{E}-10$ & $4.33 \mathrm{E}-10$ & $2.01 \mathrm{E}-07$ & $2.21 \mathrm{E}-07$ & $2.20 \mathrm{E}-07$ \\
\hline $\mathrm{Cm}-243$ & $3.30 \mathrm{E}-08$ & $1.89 \mathrm{E}-08$ & $4.65 \mathrm{E}-10$ & $4.55 \mathrm{E}-10$ & $2.10 \mathrm{E}-07$ & $2.32 \mathrm{E}-07$ & $2.31 \mathrm{E}-07$ \\
\hline $\mathrm{Cm}-244$ & $1.15 \mathrm{E}-06$ & $6.62 \mathrm{E}-07$ & $1.63 \mathrm{E}-08$ & $1.59 \mathrm{E}-08$ & $7.36 \mathrm{E}-06$ & $8.12 \mathrm{E}-06$ & $8.07 \mathrm{E}-06$ \\
\hline $\mathrm{Cm}-245$ & $3.48 \mathrm{E}-10$ & 1.99E-10 & $4.89 \mathrm{E}-12$ & $4.78 \mathrm{E}-12$ & $2.21 \mathrm{E}-09$ & $2.44 \mathrm{E}-09$ & $2.43 \mathrm{E}-09$ \\
\hline $\mathrm{Cm}-246$ & $2.28 \mathrm{E}-11$ & $1.31 \mathrm{E}-11$ & $3.21 \mathrm{E}-13$ & $3.14 \mathrm{E}-13$ & $1.45 \mathrm{E}-10$ & $1.60 \mathrm{E}-10$ & $1.59 \mathrm{E}-10$ \\
\hline TRU & 9.17E-04 & $5.26 \mathrm{E}-04$ & 1.29E-05 & 1.26E-05 & 5.84E-03 & $6.45 \mathrm{E}-03$ & $6.41 \mathrm{E}-03$ \\
\hline & & & & & & & \\
\hline & & & & & & & \\
\hline $\begin{array}{l}\text { Gas Stream Bulk } \\
\text { Composition (Wet Basis) }\end{array}$ & $\begin{array}{l}\text { mol\% or } \\
\text { ppmv }\end{array}$ & $\begin{array}{l}\text { mol\% or } \\
\text { ppmv }\end{array}$ & $\begin{array}{l}\text { mol\% or } \\
\text { ppmv }\end{array}$ & $\begin{array}{l}\text { mol\% or } \\
\text { ppmv }\end{array}$ & $\begin{array}{l}\text { mol\% or } \\
\text { ppmv }\end{array}$ & $\begin{array}{l}\text { mol\% or } \\
\text { ppmv }\end{array}$ & $\begin{array}{l}\text { mol\% or } \\
\text { ppmv }\end{array}$ \\
\hline $\mathrm{H} 2 \mathrm{O}, \mathrm{mol} \%$ & & & $31.07 \%$ & $30.36 \%$ & & & \\
\hline $\mathrm{O} 2, \mathrm{~mol} \%$ & & & & & & & \\
\hline $\mathrm{N} 2, \mathrm{~mol} \%$ & & & $1.53 \%$ & $3.76 \%$ & & & \\
\hline $\mathrm{H} 2, \mathrm{~mol} \%$ & & & $10.92 \%$ & $10.67 \%$ & & & \\
\hline $\mathrm{CO} 2, \mathrm{~mol} \%$ & & & $53.61 \%$ & $52.40 \%$ & & & \\
\hline COgas, ppmv & & & $4.88 \mathrm{E}+03$ & $4.77 \mathrm{E}+03$ & & & \\
\hline $\mathrm{NO}, \mathrm{ppmv}$ & & & $1.81 \mathrm{E}+03$ & $1.77 \mathrm{E}+03$ & & & \\
\hline NO2, ppmv & & & $1.72 \mathrm{E}+01$ & $1.69 \mathrm{E}+01$ & & & \\
\hline SO2, ppmv & & & $7.55 \mathrm{E}-06$ & $7.38 \mathrm{E}-06$ & & & \\
\hline $\mathrm{Cl}, \mathrm{ppmv}$ & & & $7.87 \mathrm{E}+00$ & $7.69 \mathrm{E}+00$ & & & \\
\hline $\mathrm{F}, \mathrm{ppmv}$ & & & $2.15 \mathrm{E}+01$ & $2.10 \mathrm{E}+01$ & & & \\
\hline C (organic), ppmv & & & $1.80 \mathrm{E}+05$ & $1.76 \mathrm{E}+05$ & & & \\
\hline $\mathrm{H}$ (organic), ppmv & & & $4.04 \mathrm{E}+05$ & $3.94 \mathrm{E}+05$ & & & \\
\hline & & & & & & & \\
\hline $\mathrm{Hg}, \mathrm{ug} / \mathrm{wscm}$ & & & $4.18 \mathrm{E}+05$ & $4.09 \mathrm{E}+05$ & & & \\
\hline $\mathrm{PM}, \mathrm{mg} / \mathrm{wscm}$ & & & $1.09 \mathrm{E}+05$ & $1.07 \mathrm{E}+05$ & & & \\
\hline SVM, ug/wscm & & & $2.28 \mathrm{E}+04$ & $2.23 \mathrm{E}+04$ & & & \\
\hline LVM, ug/wscm & & & $4.29 \mathrm{E}+03$ & $4.19 \mathrm{E}+03$ & & & \\
\hline $\begin{array}{l}\text { Gas Stream Bulk } \\
\text { Composition (Dry Basis) } \\
\end{array}$ & $\mathrm{mol} \%$ & $\mathrm{~mol} \%$ & $\mathrm{~mol} \%$ & $\mathrm{~mol} \%$ & $\mathrm{~mol} \%$ & $\mathrm{~mol} \%$ & $\mathrm{~mol} \%$ \\
\hline $\mathrm{O} 2, \mathrm{~mol} \%$, dry basis & & & & & & & \\
\hline $\mathrm{N} 2, \mathrm{~mol} \%$, dry basis & & & $2.22 \%$ & $5.40 \%$ & & & \\
\hline $\mathrm{H} 2, \mathrm{~mol} \%$, dry basis & & & $15.84 \%$ & $15.32 \%$ & & & \\
\hline $\mathrm{CO} 2, \mathrm{~mol} \%$, dry basis & & & $77.77 \%$ & $75.24 \%$ & & & \\
\hline $\begin{array}{l}\text { Gas Stream Bulk } \\
\text { Composition (Dry Basis, } \\
\text { Corrected to } 7 \% \text { O2 with } \\
100 \% \text { O2 Combustion Air) } \\
\end{array}$ & $\begin{array}{l}\mathrm{ppmv}, \text { or } \\
\mathrm{ug} / \mathrm{dscm} \\
\text { or } \\
\mathrm{mg} / \mathrm{dscm}\end{array}$ & $\begin{array}{l}\mathrm{ppmv}, \text { or } \\
\text { ug/dscm } \\
\text { or } \\
\mathrm{mg} / \mathrm{dscm}\end{array}$ & $\begin{array}{l}\mathrm{ppmv}, \text { or } \\
\mathrm{ug} / \mathrm{dscm} \\
\text { or } \\
\mathrm{mg} / \mathrm{dscm}\end{array}$ & $\begin{array}{c}\text { ppmv, or } \\
\text { ug/dscm or } \\
\text { mg/dscm }\end{array}$ & $\begin{array}{c}\text { ppmv, or } \\
\text { ug/dscm or } \\
\text { mg/dscm }\end{array}$ & $\begin{array}{l}\text { ppmv, or } \\
\text { ug/dscm or } \\
\text { mg/dscm }\end{array}$ & $\begin{array}{l}\text { ppmv, or } \\
\text { ug/dscm or } \\
\text { mg/dscm }\end{array}$ \\
\hline COgas, ppmv, dry basis & & & $9.92 \mathrm{E}+02$ & $9.60 \mathrm{E}+02$ & & & \\
\hline $\mathrm{NO}$, ppmv, dry basis & & & $3.67 \mathrm{E}+02$ & $3.55 \mathrm{E}+02$ & & & \\
\hline NO2, ppmv, dry basis & & & $3.50 \mathrm{E}+00$ & $3.39 \mathrm{E}+00$ & & & \\
\hline SO2, ppmv, dry basis & & & $1.53 \mathrm{E}-06$ & $1.48 \mathrm{E}-06$ & & & \\
\hline $\mathrm{Cl}, \mathrm{ppmv}$, dry basis & & & $1.60 \mathrm{E}+00$ & $1.55 \mathrm{E}+00$ & & & \\
\hline $\mathrm{F}, \mathrm{ppmv}$, dry basis & & & $4.36 \mathrm{E}+00$ & $4.22 \mathrm{E}+00$ & & & \\
\hline C (organic), ppmv, dry basis & & & $3.66 \mathrm{E}+04$ & $3.55 \mathrm{E}+04$ & & & \\
\hline $\mathrm{H}$ (organic), ppmv, dry basis & & & $8.20 \mathrm{E}+04$ & $7.93 E+04$ & & & \\
\hline $\mathrm{Hg}, \mathrm{ug} / \mathrm{dscm}$ & & & $8.50 \mathrm{E}+04$ & $8.22 \mathrm{E}+04$ & & & \\
\hline $\mathrm{PM}, \mathrm{mg} / \mathrm{dscm}$ & & & $2.22 \mathrm{E}+04$ & $2.15 \mathrm{E}+04$ & & & \\
\hline SVM, ug/dscm & & & $3.31 \mathrm{E}+04$ & $3.24 \mathrm{E}+04$ & & & \\
\hline LVM, ug/dscm & & & $6.22 \mathrm{E}+03$ & $6.08 \mathrm{E}+03$ & & & \\
\hline
\end{tabular}


Table A-21. Mass balance, Tank WM-188 waste (continued).

\begin{tabular}{|c|c|c|c|c|c|c|c|c|}
\hline PFD Number & PFD-2 & PFD-2 & PFD-3 & PFD-3 & PFD-3 & PFD-3 & PFD-3 & PFD-3 \\
\hline Stream Number & 108 & 109 & 110 & 111 & 112 & $113 \mathrm{~A}$ & $113 \mathrm{~B}$ & 114 \\
\hline Stream Name & $\begin{array}{l}\text { Product } \\
\text { Shipping } \\
\text { Canisters }\end{array}$ & $\begin{array}{l}\text { Off-Gas } \\
\text { from Filter } \\
\text { to Oxidizer }\end{array}$ & $\begin{array}{l}\text { Outlet of } \\
\text { Oxidizer }\end{array}$ & $\begin{array}{c}\text { Quenched } \\
\text { Oxidizer Off } \\
\text { gas }\end{array}$ & Scrub & $\begin{array}{l}\text { Packed } \\
\text { Scrubber } \\
\text { Drain }\end{array}$ & $\begin{array}{c}\text { Demister } \\
\text { Drain }\end{array}$ & $\begin{array}{l}\text { Off-Gas to } \\
\text { Demister }\end{array}$ \\
\hline Rate or Volume & $1.11 \mathrm{E}+00$ & $1.93 \mathrm{E}+05$ & $6.42 \mathrm{E}+05$ & $3.54 \mathrm{E}+05$ & $9.73 E+03$ & $9.74 \mathrm{E}+03$ & 0 & $3.57 \mathrm{E}+05$ \\
\hline Volume Flow (standard, wet) ${ }^{\star}$ & & $5.46 \mathrm{E}+04$ & $9.45 \mathrm{E}+04$ & $1.76 \mathrm{E}+05$ & & & & $1.74 \mathrm{E}+05$ \\
\hline Volume Flow (standard, dry) ${ }^{*}$ & & $3.82 \mathrm{E}+04$ & $5.14 \mathrm{E}+04$ & $5.14 \mathrm{E}+04$ & & & & $5.12 \mathrm{E}+04$ \\
\hline Rate Units & cont/day & $\mathrm{ft} 3 / \mathrm{hr}$ & $\mathrm{ft} 3 / \mathrm{hr}$ & $\mathrm{ft} 3 / \mathrm{hr}$ & $\mathrm{gal} / \mathrm{hr}$ & $\mathrm{gal} / \mathrm{hr}$ & $\mathrm{gal} / \mathrm{hr}$ & $\mathrm{ft} 3 / \mathrm{hr}$ \\
\hline Rate or Volume, metric & $5.23 E+01$ & $1.55 \mathrm{E}+03$ & $2.67 \mathrm{E}+03$ & $4.98 \mathrm{E}+03$ & $3.68 \mathrm{E}+04$ & $3.69 \mathrm{E}+04$ & 1 & $4.94 \mathrm{E}+03$ \\
\hline Rate Units & $\mathrm{kg} / \mathrm{hr}$ & wscm/hr & $\mathrm{wscm} / \mathrm{hr}$ & wscm/hr & $\mathrm{L} / \mathrm{hr}$ & $\mathrm{L} / \mathrm{hr}$ & $\mathrm{L} / \mathrm{hr}$ & $\mathrm{wscm} / \mathrm{hr}$ \\
\hline Temperature, ${ }^{\circ} \mathrm{C}$ & 55 & 397 & 1000 & 100 & 77 & 79 & 339 & 78 \\
\hline Temperature, ${ }^{\circ} \mathrm{F}$ & 132 & 747 & 1832 & 212 & 171 & 174 & 171 & 172 \\
\hline Pressure, psia & 9.50 & 9.50 & 9.39 & 9.28 & 37.52 & 8.96 & 8.42 & 8.60 \\
\hline Specific Gravity & 1.42 & $3.82 \mathrm{E}-04$ & $1.92 \mathrm{E}-04$ & $5.19 \mathrm{E}-04$ & 1.03 & 1.03 & 1.03 & $5.14 \mathrm{E}-04$ \\
\hline Chemical Composition & Wt frac & Ib/wscf & lb/wscf & $\mathrm{lb} / \mathrm{wscf}$ & Mol/liter & Mol/liter & Mol/liter & $\mathrm{lb} / \mathrm{wscf}$ \\
\hline $\mathrm{H}+$ & $4.05 \mathrm{E}-05$ & $1.07 \mathrm{E}-06$ & $1.80 \mathrm{E}-08$ & $9.65 \mathrm{E}-09$ & $9.56 \mathrm{E}-01$ & $9.55 \mathrm{E}-01$ & & $1.26 \mathrm{E}-07$ \\
\hline $\mathrm{Al}+3$ & $9.27 \mathrm{E}-02$ & & & & $9.25 \mathrm{E}-03$ & $9.24 \mathrm{E}-03$ & & \\
\hline $\mathrm{Sb}+5$ & 1.24E-05 & & & & $2.74 \mathrm{E}-07$ & $2.73 \mathrm{E}-07$ & & \\
\hline As+3 & $4.25 \mathrm{E}-05$ & & & & 1.53E-06 & 1.52E-06 & & \\
\hline $\mathrm{Ba}+2$ & $6.36 \mathrm{E}-05$ & & & & & & & \\
\hline $\mathrm{Be}+2$ & $1.05 \mathrm{E}-06$ & & & & & & & \\
\hline $\mathrm{B}+3$ & $1.10 \mathrm{E}-03$ & & & & $2.73 \mathrm{E}-04$ & $2.73 \mathrm{E}-04$ & & \\
\hline $\mathrm{Cd}+2$ & $1.74 \mathrm{E}-03$ & & & & & & & \\
\hline $\mathrm{Ca}+2$ & $1.24 \mathrm{E}-02$ & & & & & & & \\
\hline $\mathrm{Cr}+3$ & $1.50 \mathrm{E}-03$ & & & & & & & \\
\hline $\mathrm{Co}+2$ & $1.49 \mathrm{E}-05$ & & & & $6.81 \mathrm{E}-07$ & $6.80 \mathrm{E}-07$ & & \\
\hline Cs+ & $4.90 \mathrm{E}-05$ & & & & & & & \\
\hline $\mathrm{Cu}+2$ & $2.75 \mathrm{E}-04$ & & & & & & & \\
\hline $\mathrm{Fe}+3$ & $8.91 \mathrm{E}-03$ & 4.98E-09 & 2.88E-09 & $1.55 \mathrm{E}-09$ & $9.46 \mathrm{E}-05$ & $9.46 \mathrm{E}-05$ & & \\
\hline $\mathrm{Pb}+2$ & $1.05 \mathrm{E}-03$ & & & & & & & \\
\hline $\mathrm{Hg}+2$ & $2.39 \mathrm{E}-05$ & $2.52 \mathrm{E}-05$ & $1.46 \mathrm{E}-05$ & $7.83 \mathrm{E}-06$ & $7.43 \mathrm{E}-02$ & $7.42 \mathrm{E}-02$ & & $4.10 \mathrm{E}-06$ \\
\hline $\mathrm{Mn}+4$ & 5.09E-03 & & & & $2.49 \mathrm{E}-04$ & $2.49 \mathrm{E}-04$ & & \\
\hline $\mathrm{Ni}+2$ & $6.65 \mathrm{E}-04$ & & & & & & & \\
\hline $\mathrm{K}+$ & $4.63 \mathrm{E}-02$ & & & & & & & \\
\hline $\mathrm{Se}+4$ & 1.18E-04 & & & & & & & \\
\hline $\mathrm{Ag}+$ & $2.52 \mathrm{E}-04$ & & & & & & & \\
\hline $\mathrm{Na}+$ & $2.01 \mathrm{E}-01$ & & & & & & & \\
\hline $\mathrm{Tl}+3$ & $5.80 \mathrm{E}-06$ & & & & $7.64 \mathrm{E}-08$ & $7.63 \mathrm{E}-08$ & & \\
\hline $\mathrm{U}+4$ & 5.33E-04 & & & & & & & \\
\hline $\mathrm{V}+3$ & 1.16E-05 & & & & & & & \\
\hline$Z n+2$ & $3.28 \mathrm{E}-04$ & & & & & & & \\
\hline $\mathrm{Zr}+4$ & $2.13 \mathrm{E}-02$ & & & & & & & \\
\hline $\mathrm{Cl}-$ & $5.40 \mathrm{E}-03$ & $4.17 \mathrm{E}-08$ & $2.41 \mathrm{E}-08$ & $1.30 \mathrm{E}-08$ & $4.71 \mathrm{E}-04$ & $4.70 \mathrm{E}-04$ & & $7.98 \mathrm{E}-09$ \\
\hline F- & 3.96E-03 & $5.63 \mathrm{E}-07$ & 3.26E-07 & $1.75 \mathrm{E}-07$ & 5.09E-03 & 5.09E-03 & & $1.55 \mathrm{E}-07$ \\
\hline SO4-2 & 5.34E-09 & & $1.17 \mathrm{E}-10$ & $6.30 \mathrm{E}-11$ & & & & \\
\hline NO3- & $6.20 \mathrm{E}-03$ & $1.87 \mathrm{E}-11$ & $1.09 \mathrm{E}-11$ & $5.84 \mathrm{E}-12$ & $9.16 \mathrm{E}-01$ & $9.15 \mathrm{E}-01$ & & $7.20 \mathrm{E}-06$ \\
\hline $\mathrm{PO} 4-3$ & $5.85 \mathrm{E}-02$ & & & & & & & \\
\hline $\mathrm{Am}+4$ & 1.04E-07 & & & & $1.16 \mathrm{E}-09$ & $1.15 \mathrm{E}-09$ & & \\
\hline $\mathrm{Br}-$ & 1.47E-07 & $1.13 \mathrm{E}-12$ & $6.56 \mathrm{E}-13$ & $3.52 \mathrm{E}-13$ & 5.68E-09 & 5.67E-09 & & $2.17 \mathrm{E}-13$ \\
\hline $\mathrm{Ce}+4$ & 2.97E-05 & & & & 5.71E-07 & $5.71 \mathrm{E}-07$ & & \\
\hline Eu+3 & $4.65 \mathrm{E}-07$ & & & & $8.24 \mathrm{E}-09$ & 8.23E-09 & & \\
\hline $\mathrm{Gd}+3$ & $1.42 \mathrm{E}-04$ & & & & $2.43 \mathrm{E}-06$ & $2.43 \mathrm{E}-06$ & & \\
\hline $\mathrm{Ge}+4$ & 3.85E-09 & & & & & & & \\
\hline $\ln +3$ & 1.07E-06 & & & & $2.52 \mathrm{E}-08$ & $2.51 \mathrm{E}-08$ & & \\
\hline I- & $2.49 \mathrm{E}-06$ & $1.93 \mathrm{E}-11$ & $1.11 \mathrm{E}-11$ & $5.98 \mathrm{E}-12$ & $6.07 \mathrm{E}-08$ & $6.07 \mathrm{E}-08$ & & $3.68 \mathrm{E}-12$ \\
\hline $\mathrm{La+3}$ & $7.71 \mathrm{E}-06$ & & & & $1.50 \mathrm{E}-07$ & $1.49 \mathrm{E}-07$ & & \\
\hline
\end{tabular}


Table A-21. Mass balance, Tank WM-188 waste (continued).

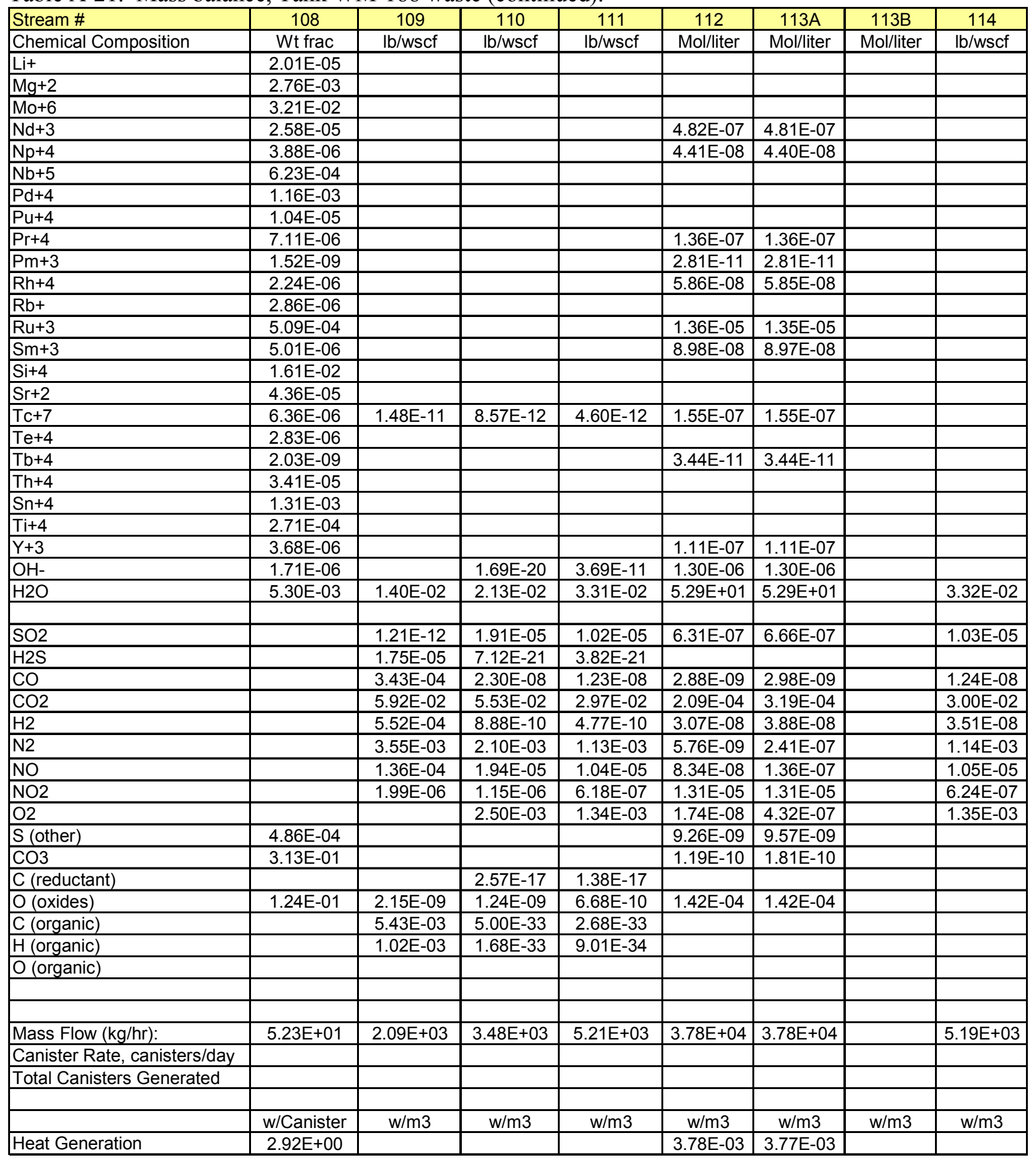


Table A-21. Mass balance, Tank WM-188 waste (continued).

\begin{tabular}{|c|c|c|c|c|c|c|c|c|}
\hline Stream \# & 108 & 109 & 110 & 111 & 112 & $113 \mathrm{~A}$ & $113 \mathrm{~B}$ & 114 \\
\hline Radiological Composition & Ci/Canister & Ci/wscm & Ci/wscm & Ci/wscm & $\mathrm{Ci} / \mathrm{L}$ & $\mathrm{Ci} / \mathrm{L}$ & $\mathrm{Ci} / \mathrm{L}$ & Ci/wscm \\
\hline $\mathrm{H}-3$ & & $2.51 \mathrm{E}-06$ & $1.45 \mathrm{E}-06$ & $7.80 \mathrm{E}-07$ & $1.34 \mathrm{E}-06$ & $1.34 \mathrm{E}-06$ & & $7.81 \mathrm{E}-07$ \\
\hline C-14 & $7.37 \mathrm{E}-09$ & $3.37 \mathrm{E}-11$ & $1.95 \mathrm{E}-11$ & $1.05 \mathrm{E}-11$ & $2.03 E-16$ & $3.09 \mathrm{E}-16$ & & $1.06 \mathrm{E}-11$ \\
\hline Co-60 & $6.43 \mathrm{E}-02$ & & & & $1.53 \mathrm{E}-07$ & $1.52 \mathrm{E}-07$ & & \\
\hline $\mathrm{Ni}-59$ & 8.00E-03 & & & & & & & \\
\hline $\mathrm{Ni}-63$ & 3.02E-01 & & & & & & & \\
\hline Se-79 & 4.14E-03 & & & & & & & \\
\hline Sr-90 & $2.84 \mathrm{E}+02$ & & & & & & & \\
\hline Y-90 & $2.85 \mathrm{E}+02$ & & & & $6.76 \mathrm{E}-04$ & $6.75 \mathrm{E}-04$ & & \\
\hline Zr-93 & $1.64 \mathrm{E}-02$ & & & & & & & \\
\hline $\mathrm{Nb}-93 \mathrm{~m}$ & $1.26 \mathrm{E}-02$ & & & & & & & \\
\hline $\mathrm{Nb}-94$ & $1.08 \mathrm{E}-02$ & & & & & & & \\
\hline Tc-99 & $1.50 \mathrm{E}-01$ & $4.92 \mathrm{E}-09$ & $2.85 \mathrm{E}-09$ & $1.53 \mathrm{E}-09$ & $3.15 \mathrm{E}-07$ & $3.15 \mathrm{E}-07$ & & \\
\hline Ru-106 & $8.85 \mathrm{E}-03$ & & & & $2.10 \mathrm{E}-08$ & $2.10 \mathrm{E}-08$ & & \\
\hline Rh-102 & $6.37 \mathrm{E}-06$ & & & & $1.51 \mathrm{E}-11$ & $1.51 \mathrm{E}-11$ & & \\
\hline Rh-106 & $8.85 \mathrm{E}-03$ & & & & $2.10 \mathrm{E}-08$ & $2.10 \mathrm{E}-08$ & & \\
\hline Pd-107 & $1.22 \mathrm{E}-04$ & & & & & & & \\
\hline Cd-113m & $2.45 \mathrm{E}-02$ & & & & & & & \\
\hline Sn-121m & $4.94 \mathrm{E}-04$ & & & & & & & \\
\hline Sn-126 & $3.90 \mathrm{E}-03$ & & & & & & & \\
\hline Sb-125 & $2.68 \mathrm{E}+00$ & & & & $6.36 \mathrm{E}-06$ & $6.35 \mathrm{E}-06$ & & \\
\hline $\mathrm{Sb}-126$ & $4.25 \mathrm{E}-04$ & & & & $1.01 \mathrm{E}-09$ & $1.01 \mathrm{E}-09$ & & \\
\hline Te-125m & $2.33 \mathrm{E}-02$ & & & & & & & \\
\hline \begin{tabular}{|l|}
$1-129$ \\
\end{tabular} & $5.04 \mathrm{E}-04$ & $5.50 \mathrm{E}-11$ & $3.18 \mathrm{E}-11$ & $1.71 \mathrm{E}-11$ & $1.38 \mathrm{E}-09$ & $1.37 \mathrm{E}-09$ & & $1.05 \mathrm{E}-11$ \\
\hline Cs-134 & 2.67E-01 & & & & & & & \\
\hline Cs-135 & 7.96E-03 & & & & & & & \\
\hline Cs-137 & $1.64 \mathrm{E}+02$ & & & & & & & \\
\hline Ba-137m & $1.55 \mathrm{E}+02$ & & & & & & & \\
\hline $\mathrm{Ce}-144$ & 5.97E-03 & & & & $1.42 \mathrm{E}-08$ & $1.42 \mathrm{E}-08$ & & \\
\hline Pr-144 & 5.97E-03 & & & & $1.42 \mathrm{E}-08$ & $1.42 \mathrm{E}-08$ & & \\
\hline $\mathrm{Pm}-146$ & $3.76 \mathrm{E}-04$ & & & & $8.93 \mathrm{E}-10$ & $8.92 \mathrm{E}-10$ & & \\
\hline $\mathrm{Pm}-147$ & $1.61 \mathrm{E}+00$ & & & & $3.83 \mathrm{E}-06$ & $3.83 E-06$ & & \\
\hline Sm-151 & $3.19 \mathrm{E}+00$ & & & & $7.57 \mathrm{E}-06$ & $7.56 \mathrm{E}-06$ & & \\
\hline Eu-152 & $2.11 \mathrm{E}-02$ & & & & $5.00 \mathrm{E}-08$ & $5.00 \mathrm{E}-08$ & & \\
\hline Eu-154 & $3.59 \mathrm{E}-01$ & & & & $8.52 \mathrm{E}-07$ & $8.51 \mathrm{E}-07$ & & \\
\hline Eu-155 & $3.11 \mathrm{E}-01$ & & & & 7.39E-07 & 7.37E-07 & & \\
\hline & & & & & & & & \\
\hline Th-230 & $7.57 \mathrm{E}-06$ & & & & & & & \\
\hline $\mathrm{Pa}-233$ & $2.17 \mathrm{E}-02$ & & & & & & & \\
\hline U-232 & $1.78 \mathrm{E}-05$ & & & & & & & \\
\hline U-233 & $6.45 \mathrm{E}-07$ & & & & & & & \\
\hline U-234 & $7.95 \mathrm{E}-03$ & & & & & & & \\
\hline U-235 & $5.84 \mathrm{E}-04$ & & & & & & & \\
\hline U-236 & 3.43E-04 & & & & & & & \\
\hline U-237 & 4.75E-05 & & & & & & & \\
\hline U-238 & 1.04E-04 & & & & & & & \\
\hline Np-237 & $3.10 \mathrm{E}-03$ & & & & 7.36E-09 & 7.35E-09 & & \\
\hline Pu-236 & $2.60 \mathrm{E}-05$ & & & & & & & \\
\hline Pu-238 & $5.95 \mathrm{E}+00$ & & & & & & & \\
\hline Pu-239 & $6.66 \mathrm{E}-01$ & & & & & & & \\
\hline Pu-240 & 9.69E-02 & & & & & & & \\
\hline Pu-241 & $4.33 E+00$ & & & & & & & \\
\hline Pu-242 & $7.59 \mathrm{E}-05$ & & & & & & & \\
\hline Pu-244 & $5.79 \mathrm{E}-12$ & & & & & & & \\
\hline Am-241 & $4.18 \mathrm{E}-01$ & & & & 9.91E-07 & $9.90 \mathrm{E}-07$ & & \\
\hline Am-242m & $1.15 \mathrm{E}-04$ & & & & $2.74 \mathrm{E}-10$ & $2.73 \mathrm{E}-10$ & & \\
\hline
\end{tabular}


Table A-21. Mass balance, Tank WM-188 waste (continued).

\begin{tabular}{|c|c|c|c|c|c|c|c|c|}
\hline Stream \# & 108 & 109 & 110 & 111 & 112 & $113 \mathrm{~A}$ & $113 \mathrm{~B}$ & 114 \\
\hline Radiological Composition & Ci/Canister & Ci/wscm & Ci/wscm & Ci/wscm & $\mathrm{Ci} / \mathrm{L}$ & $\mathrm{Ci} / \mathrm{L}$ & $\mathrm{Ci} / \mathrm{L}$ & $\mathrm{Ci} / \mathrm{wscm}$ \\
\hline Am-243 & $1.88 \mathrm{E}-04$ & & & & $4.47 \mathrm{E}-10$ & $4.46 \mathrm{E}-10$ & & \\
\hline $\mathrm{Cm}-242$ & $2.50 \mathrm{E}-04$ & & & & & & & \\
\hline $\mathrm{Cm}-243$ & $2.62 \mathrm{E}-04$ & & & & & & & \\
\hline $\mathrm{Cm}-244$ & $9.15 \mathrm{E}-03$ & & & & & & & \\
\hline $\mathrm{Cm}-245$ & $2.75 \mathrm{E}-06$ & & & & & & & \\
\hline $\mathrm{Cm}-246$ & $1.81 \mathrm{E}-07$ & & & & & & & \\
\hline TRU & $7.27 \mathrm{E}+00$ & & & & $1.00 \mathrm{E}-06$ & $9.98 \mathrm{E}-07$ & & \\
\hline & & & & & & & & \\
\hline & & & & & & & & \\
\hline $\begin{array}{l}\text { Gas Stream Bulk } \\
\text { Composition (Wet Basis) }\end{array}$ & $\begin{array}{l}\text { mol\% or } \\
\text { ppmv }\end{array}$ & $\begin{array}{c}\text { mol\% or } \\
\text { ppmv }\end{array}$ & $\begin{array}{c}\mathrm{mol} \% \text { or } \\
\text { ppmv }\end{array}$ & $\begin{array}{c}\text { mol\% or } \\
\text { ppmv }\end{array}$ & $\begin{array}{l}\mathrm{mol} \% \text { or } \\
\text { ppmv }\end{array}$ & $\begin{array}{l}\text { mol\% or } \\
\text { ppmv }\end{array}$ & $\begin{array}{c}\mathrm{mol} \% \text { or } \\
\text { ppmv }\end{array}$ & $\begin{array}{l}\mathrm{mol} \% \text { or } \\
\text { ppmv }\end{array}$ \\
\hline $\mathrm{H} 2 \mathrm{O}, \mathrm{mol} \%$ & & $30.02 \%$ & $45.60 \%$ & $70.79 \%$ & & & & $70.65 \%$ \\
\hline $\mathrm{O} 2, \mathrm{~mol} \%$ & & & $3.01 \%$ & $1.62 \%$ & & & & $1.62 \%$ \\
\hline $\mathrm{N} 2, \mathrm{~mol} \%$ & & $4.88 \%$ & $2.88 \%$ & $1.55 \%$ & & & & $1.56 \%$ \\
\hline $\mathrm{H} 2, \mathrm{~mol} \%$ & & $10.55 \%$ & $0.00002 \%$ & $0.00001 \%$ & & & & $0.0007 \%$ \\
\hline $\mathrm{CO} 2, \mathrm{~mol} \%$ & & $51.81 \%$ & $48.46 \%$ & $26.02 \%$ & & & & $26.15 \%$ \\
\hline COgas, ppmv & & $4.72 \mathrm{E}+03$ & $3.16 \mathrm{E}-01$ & $1.70 \mathrm{E}-01$ & & & & $1.71 \mathrm{E}-01$ \\
\hline NO, ppmv & & $1.75 \mathrm{E}+03$ & $2.49 \mathrm{E}+02$ & $1.34 \mathrm{E}+02$ & & & & $1.35 \mathrm{E}+02$ \\
\hline NO2, ppmv & & $1.67 \mathrm{E}+01$ & $9.64 \mathrm{E}+00$ & $5.18 \mathrm{E}+00$ & & & & $5.21 \mathrm{E}+00$ \\
\hline SO2, ppmv & & $7.29 \mathrm{E}-06$ & $1.15 \mathrm{E}+02$ & $6.16 \mathrm{E}+01$ & & & & $6.19 \mathrm{E}+01$ \\
\hline $\mathrm{Cl}, \mathrm{ppmv}$ & & $4.53 \mathrm{E}-01$ & $2.62 \mathrm{E}-01$ & $1.41 \mathrm{E}-01$ & & & & $8.64 \mathrm{E}-02$ \\
\hline $\mathrm{F}, \mathrm{ppmv}$ & & $1.14 \mathrm{E}+01$ & $6.61 \mathrm{E}+00$ & $3.55 \mathrm{E}+00$ & & & & $3.13 \mathrm{E}+00$ \\
\hline C (organic), ppmv & & $1.74 \mathrm{E}+05$ & & & & & & \\
\hline $\mathrm{H}$ (organic), ppmv & & $3.90 \mathrm{E}+05$ & & & & & & \\
\hline & & & & & & & & \\
\hline $\mathrm{Hg}, \mathrm{ug} / \mathrm{wscm}$ & & $4.04 \mathrm{E}+05$ & $2.34 \mathrm{E}+05$ & $1.25 \mathrm{E}+05$ & & & & \\
\hline $\mathrm{PM}, \mathrm{mg} / \mathrm{wscm}$ & & $1.04 \mathrm{E}+05$ & $2.40 \mathrm{E}+02$ & $1.34 \mathrm{E}+02$ & & & & $8.72 \mathrm{E}+00$ \\
\hline SVM, ug/wscm & & & & & & & & \\
\hline LVM, ug/wscm & & & & & & & & \\
\hline & & & & & & & & \\
\hline $\begin{array}{l}\text { Gas Stream Bulk } \\
\text { Composition (Dry Basis) }\end{array}$ & $\mathrm{mol} \%$ & $\mathrm{~mol} \%$ & $\mathrm{~mol} \%$ & $\mathrm{~mol} \%$ & $\mathrm{~mol} \%$ & $\mathrm{~mol} \%$ & $\mathrm{~mol} \%$ & $\mathrm{~mol} \%$ \\
\hline $\mathrm{O} 2, \mathrm{~mol} \%$, dry basis & & & $5.54 \%$ & $5.54 \%$ & & & & $5.54 \%$ \\
\hline $\mathrm{N} 2, \mathrm{~mol} \%$, dry basis & & $6.97 \%$ & $5.30 \%$ & $5.30 \%$ & & & & $5.30 \%$ \\
\hline $\mathrm{H} 2, \mathrm{~mol} \%$, dry basis & & $15.08 \%$ & $0.00003 \%$ & $0.00003 \%$ & & & & $0.002 \%$ \\
\hline $\mathrm{CO} 2, \mathrm{~mol} \%$, dry basis & & $74.04 \%$ & $89.09 \%$ & $89.09 \%$ & & & & $89.08 \%$ \\
\hline & & & & & & & & \\
\hline $\begin{array}{l}\text { Gas Stream Bulk } \\
\text { Composition (Dry Basis, } \\
\text { Corrected to } 7 \% \text { O2 with } \\
100 \% \text { O2 Combustion Air) }\end{array}$ & $\begin{array}{l}\text { ppmv, or } \\
\text { ug/dscm or } \\
\mathrm{mg} / \mathrm{dscm}\end{array}$ & $\begin{array}{l}\text { ppmv, or } \\
\text { ug/dscm or } \\
\mathrm{mg} / \mathrm{dscm}\end{array}$ & $\begin{array}{l}\text { ppmv, or } \\
\text { ug/dscm or } \\
\mathrm{mg} / \mathrm{dscm}\end{array}$ & $\begin{array}{l}\text { ppmv, or } \\
\text { ug/dscm or } \\
\mathrm{mg} / \mathrm{dscm}\end{array}$ & $\begin{array}{l}\mathrm{ppmv}, \text { or } \\
\text { ug/dscm } \\
\text { or } \\
\mathrm{mg} / \mathrm{dscm}\end{array}$ & $\begin{array}{l}\mathrm{ppmv} \text {, or } \\
\mathrm{ug} / \mathrm{dscm} \\
\text { or } \\
\mathrm{mg} / \mathrm{dscm}\end{array}$ & $\begin{array}{l}\mathrm{ppmv} \text {, or } \\
\mathrm{ug} / \mathrm{dscm} \\
\text { or } \\
\mathrm{mg} / \mathrm{dscm}\end{array}$ & $\begin{array}{l}\mathrm{ppmv} \text {, or } \\
\mathrm{ug} / \mathrm{dscm} \\
\text { or } \\
\mathrm{mg} / \mathrm{dscm}\end{array}$ \\
\hline COgas, ppmv, dry basis & & $9.44 \mathrm{E}+02$ & $8.14 \mathrm{E}-02$ & $8.14 \mathrm{E}-02$ & & & & $8.14 \mathrm{E}-02$ \\
\hline $\mathrm{NO}$, ppmv, dry basis & & $3.50 \mathrm{E}+02$ & $6.42 \mathrm{E}+01$ & $6.42 \mathrm{E}+01$ & & & & $6.42 \mathrm{E}+01$ \\
\hline NO2, ppmv, dry basis & & $3.34 \mathrm{E}+00$ & $2.48 \mathrm{E}+00$ & $2.48 \mathrm{E}+00$ & & & & $2.49 \mathrm{E}+00$ \\
\hline SO2, ppmv, dry basis & & $1.46 \mathrm{E}-06$ & $2.95 \mathrm{E}+01$ & $2.95 \mathrm{E}+01$ & & & & $2.95 \mathrm{E}+01$ \\
\hline $\mathrm{Cl}, \mathrm{ppmv}$, dry basis & & 9.07E-02 & $6.75 \mathrm{E}-02$ & $6.75 \mathrm{E}-02$ & & & & $4.12 \mathrm{E}-02$ \\
\hline $\mathrm{F}, \mathrm{ppmv}$, dry basis & & $2.28 \mathrm{E}+00$ & $1.70 \mathrm{E}+00$ & $1.70 \mathrm{E}+00$ & & & & $1.49 \mathrm{E}+00$ \\
\hline C (organic), ppmv, dry basis & & $3.48 \mathrm{E}+04$ & $4.13 E-26$ & $4.13 \mathrm{E}-26$ & & & & \\
\hline $\mathrm{H}$ (organic), ppmv, dry basis & & $7.79 \mathrm{E}+04$ & $1.65 \mathrm{E}-25$ & $1.65 \mathrm{E}-25$ & & & & \\
\hline $\mathrm{Hg}, \mathrm{ug} / \mathrm{dscm}$ & & $8.08 \mathrm{E}+04$ & $6.02 \mathrm{E}+04$ & $6.01 \mathrm{E}+04$ & & & & \\
\hline $\mathrm{PM}, \mathrm{mg} / \mathrm{dscm}$ & & $2.07 \mathrm{E}+04$ & $6.18 \mathrm{E}+01$ & $6.43 \mathrm{E}+01$ & & & & $4.16 \mathrm{E}+00$ \\
\hline SVM, ug/dscm & & & & & & & & \\
\hline LVM, ug/dscm & & & & & & & & \\
\hline
\end{tabular}


Table A-21. Mass balance, Tank WM-188 waste (continued).

\begin{tabular}{|c|c|c|c|c|c|c|}
\hline PFD Number & PFD-3 & PFD-3 & PFD-3 & PFD-3 & PFD-3 & PFD-3 \\
\hline Stream Number & 115 & 116 & 117 & 118 & 119 & 120 \\
\hline Stream Name & $\begin{array}{l}\text { Scrubbed } \\
\text { Off-Gas to } \\
\text { Preheater }\end{array}$ & $\begin{array}{l}\text { Preheated } \\
\text { Off-gas to } \\
\text { GAC }\end{array}$ & $\begin{array}{c}\text { Pressure } \\
\text { Control Bleed } \\
\text { Air }\end{array}$ & $\begin{array}{c}\text { Final HEPA } \\
\text { Off-Gas } \\
\text { Outlet }\end{array}$ & $\begin{array}{l}\text { Off-gas to } \\
\text { Exhaust } \\
\text { Blower }\end{array}$ & Spent GAC \\
\hline Rate or Volume & $3.90 \mathrm{E}+05$ & $4.82 \mathrm{E}+05$ & $1.55 \mathrm{E}+04$ & $5.85 \mathrm{E}+05$ & $6.26 \mathrm{E}+05$ & $5.01 \mathrm{E}+00$ \\
\hline Volume Flow (standard, wet) & $1.75 \mathrm{E}+05$ & $1.75 \mathrm{E}+05$ & $1.53 \mathrm{E}+04$ & $1.75 \mathrm{E}+05$ & $1.90 \mathrm{E}+05$ & \\
\hline Volume Flow (standard, dry) & $5.14 \mathrm{E}+04$ & $5.14 \mathrm{E}+04$ & $1.53 \mathrm{E}+04$ & $5.14 \mathrm{E}+04$ & $6.66 \mathrm{E}+04$ & \\
\hline Rate Units & $\mathrm{ft} 3 / \mathrm{hr}$ & $\mathrm{ft} 3 / \mathrm{hr}$ & $\mathrm{ft} 3 / \mathrm{hr}$ & $\mathrm{ft} 3 / \mathrm{hr}$ & $\mathrm{ft} 3 / \mathrm{hr}$ & $\mathrm{lb} / \mathrm{hr}$ \\
\hline Rate or Volume, metric & $4.96 \mathrm{E}+03$ & $4.96 \mathrm{E}+03$ & $4.33 \mathrm{E}+02$ & $4.96 \mathrm{E}+03$ & $5.38 \mathrm{E}+03$ & $2.27 \mathrm{E}+00$ \\
\hline Rate Units & $\mathrm{wscm} / \mathrm{hr}$ & wscm $/ \mathrm{hr}$ & wscm $/ \mathrm{hr}$ & wscm $/ \mathrm{hr}$ & wscm $/ \mathrm{hr}$ & $\mathrm{kg} / \mathrm{hr}$ \\
\hline Temperature, ${ }^{\circ} \mathrm{C}$ & 77 & 120 & 25 & 120 & 114 & 27 \\
\hline Temperature, ${ }^{\circ} \mathrm{F}$ & 171 & 248 & 77 & 248 & 237 & 81 \\
\hline Pressure, psia & 7.88 & 7.16 & 14.70 & 5.89 & 5.89 & 14.70 \\
\hline Specific Gravity & $4.70 \mathrm{E}-04$ & $3.81 \mathrm{E}-04$ & $1.18 \mathrm{E}-03$ & $3.13 \mathrm{E}-04$ & $3.22 \mathrm{E}-04$ & $5.86 \mathrm{E}-01$ \\
\hline Chemical Composition & $\mathrm{lb} /$ wscf & $\mathrm{lb} / \mathrm{wscf}$ & $\mathrm{lb} /$ wscf & $\mathrm{lb} /$ wscf & $\mathrm{lb} / \mathrm{wscf}$ & wt frac \\
\hline $\mathrm{H}+$ & 1.25E-07 & 1.25E-07 & & 1.25E-07 & 1.15E-07 & 5.82E-06 \\
\hline \multicolumn{7}{|l|}{$\mathrm{Al}+3$} \\
\hline \multicolumn{7}{|l|}{$\mathrm{Sb}+5$} \\
\hline \multicolumn{7}{|l|}{$\mathrm{As}+3$} \\
\hline \multirow{2}{*}{\multicolumn{7}{|c|}{$\frac{\mathrm{Ba}+2}{\mathrm{Be}+2}$}} \\
\hline \multirow{2}{*}{\multicolumn{7}{|c|}{$\frac{\mathrm{Be}+2}{\mathrm{~B}+3}$}} \\
\hline & & & & & & \\
\hline \multicolumn{7}{|l|}{$\frac{B+3}{C d+2}$} \\
\hline \multicolumn{7}{|l|}{$\mathrm{Ca}+2$} \\
\hline \multicolumn{7}{|l|}{$\mathrm{Cr}+3$} \\
\hline \multicolumn{7}{|l|}{$\mathrm{Co}+2$} \\
\hline \multirow{2}{*}{\multicolumn{7}{|c|}{$\frac{\mathrm{Cs}+}{\mathrm{Cu}+2}$}} \\
\hline & & & & & & \\
\hline \multicolumn{7}{|l|}{$\mathrm{Fe}+3$} \\
\hline \multicolumn{7}{|l|}{$\mathrm{Pb}+2$} \\
\hline $\mathrm{Hg}+2$ & 4.09E-06 & 4.09E-06 & & $2.27 \mathrm{E}-09$ & $2.09 \mathrm{E}-09$ & 1.43E-01 \\
\hline \multicolumn{7}{|l|}{$\mathrm{Mn}+4$} \\
\hline \multicolumn{7}{|l|}{$\mathrm{Ni}+2$} \\
\hline \multicolumn{7}{|l|}{$\mathrm{K}+$} \\
\hline \multirow{2}{*}{\multicolumn{7}{|c|}{$\frac{\mathrm{Se}+4}{\mathrm{Aq}+}$}} \\
\hline \multirow{2}{*}{\multicolumn{7}{|c|}{$\frac{\mathrm{Ag}+}{\mathrm{Na}+}$}} \\
\hline & & & & & & \\
\hline \multicolumn{7}{|l|}{$\mathrm{TI}+3$} \\
\hline $\mathrm{U}+4$ & & & & & & \\
\hline $\mathrm{V}+3$ & & & & & & \\
\hline$Z n+2$ & & & & & & \\
\hline $\mathrm{Zr}+4$ & & & & & & \\
\hline $\mathrm{Cl}-$ & 7.95E-09 & 7.95E-09 & & $2.09 \mathrm{E}-09$ & 1.93E-09 & 2.05E-04 \\
\hline $\mathrm{F}-$ & $1.54 \mathrm{E}-07$ & $1.54 \mathrm{E}-07$ & & $1.54 \mathrm{E}-07$ & $1.42 \mathrm{E}-07$ & \\
\hline SO4-2 & & & & & & \\
\hline NO3- & 7.17E-06 & 7.17E-06 & & 7.18E-06 & 6.61E-06 & \\
\hline PO4-3 & & & & & & \\
\hline $\mathrm{Am}+4$ & & & & & & \\
\hline $\mathrm{Br}-$ & 2.16E-13 & 2.16E-13 & & $5.69 \mathrm{E}-14$ & $5.25 \mathrm{E}-14$ & 5.57E-09 \\
\hline $\mathrm{Ce}+4$ & & & & & & \\
\hline $\mathrm{Eu}+3$ & & & & & & \\
\hline$G d+3$ & & & & & & \\
\hline $\mathrm{Ge}+4$ & & & & & & \\
\hline $\ln +3$ & & & & & & \\
\hline $1-$ & 3.67E-12 & 3.67E-12 & & 9.67E-13 & $8.91 \mathrm{E}-13$ & $9.46 \mathrm{E}-08$ \\
\hline $\mathrm{La}+3$ & & & & & & \\
\hline
\end{tabular}


Table A-21. Mass balance, Tank WM-188 waste (continued).

\begin{tabular}{|c|c|c|c|c|c|c|}
\hline Stream \# & 115 & 116 & 117 & 118 & 119 & 120 \\
\hline Chemical Composition & $\mathrm{Ib} / \mathrm{wscf}$ & $\mathrm{lb} / \mathrm{wscf}$ & $\mathrm{lb} / \mathrm{wscf}$ & $\mathrm{Ib} / \mathrm{wscf}$ & $\mathrm{lb} / \mathrm{wscf}$ & wt frac \\
\hline \multicolumn{7}{|l|}{$\mathrm{Li}+$} \\
\hline \multicolumn{7}{|l|}{$\mathrm{Mg}+2$} \\
\hline \multicolumn{7}{|l|}{$\mathrm{Mo}+6$} \\
\hline \multicolumn{7}{|l|}{$\mathrm{Nd}+3$} \\
\hline \multicolumn{7}{|l|}{$\mathrm{Np}+4$} \\
\hline \multicolumn{7}{|l|}{$\mathrm{Nb}+5$} \\
\hline \multicolumn{7}{|l|}{$\mathrm{Pd}+4$} \\
\hline \multicolumn{7}{|l|}{$\mathrm{Pu}+4$} \\
\hline \multicolumn{7}{|l|}{$\mathrm{Pr}+4$} \\
\hline \multicolumn{7}{|l|}{$\mathrm{Pm}+3$} \\
\hline \multicolumn{7}{|l|}{$\mathrm{Rh}+4$} \\
\hline \multicolumn{7}{|l|}{$\mathrm{Rb}+$} \\
\hline \multicolumn{7}{|l|}{\begin{tabular}{|l|}
$\mathrm{Ru}+3$ \\
$\mathrm{sm}+3$
\end{tabular}} \\
\hline \multirow{2}{*}{\multicolumn{7}{|c|}{$\frac{\mathrm{Sm}+3}{\mathrm{Si}+4}$}} \\
\hline & & & & & & \\
\hline \multicolumn{7}{|l|}{$\mathrm{Sr}+2$} \\
\hline \multicolumn{7}{|l|}{$\mathrm{Tc}+7$} \\
\hline \multicolumn{7}{|l|}{$\mathrm{Te}+4$} \\
\hline \multicolumn{7}{|l|}{$\mathrm{Tb}+4$} \\
\hline \multirow{2}{*}{\multicolumn{7}{|c|}{$\begin{array}{l}\text { Th+4 } \\
S n+4\end{array}$}} \\
\hline & & & & & & \\
\hline \multicolumn{7}{|l|}{$\frac{S n+4}{T i+4}$} \\
\hline \multicolumn{7}{|l|}{$\frac{11+4}{Y+3}$} \\
\hline $\mathrm{OH}-$ & & & & & & \\
\hline $\mathrm{H} 2 \mathrm{O}$ & $3.30 \mathrm{E}-02$ & $3.30 \mathrm{E}-02$ & $4.68 \mathrm{E}-06$ & $3.30 \mathrm{E}-02$ & $3.04 \mathrm{E}-02$ & \\
\hline$\overline{\mathrm{SO} 2}$ & 1.03E-05 & 1.03E-05 & & $1.03 E-05$ & $9.48 \mathrm{E}-06$ & \\
\hline $\mathrm{H} 2 \mathrm{~S}$ & & & & & & \\
\hline $\mathrm{CO}$ & $1.24 \mathrm{E}-08$ & $1.24 \mathrm{E}-08$ & & $1.24 \mathrm{E}-08$ & $1.14 \mathrm{E}-08$ & \\
\hline $\mathrm{CO} 2$ & 2.99E-02 & $2.99 \mathrm{E}-02$ & & $2.99 \mathrm{E}-02$ & $2.75 \mathrm{E}-02$ & \\
\hline $\mathrm{H} 2$ & & & & & & \\
\hline N2 & $1.13 \mathrm{E}-03$ & 1.13E-03 & $5.75 \mathrm{E}-02$ & 1.13E-03 & $5.66 \mathrm{E}-03$ & \\
\hline $\mathrm{NO}$ & $1.05 \mathrm{E}-05$ & $1.05 \mathrm{E}-05$ & & $1.05 \mathrm{E}-05$ & $9.66 \mathrm{E}-06$ & \\
\hline $\mathrm{NO} 2$ & $6.22 \mathrm{E}-07$ & $6.22 \mathrm{E}-07$ & & $6.22 \mathrm{E}-07$ & $5.73 \mathrm{E}-07$ & \\
\hline $\mathrm{O} 2$ & $1.35 \mathrm{E}-03$ & $1.35 \mathrm{E}-03$ & 1.74E-02 & $1.35 \mathrm{E}-03$ & $2.65 \mathrm{E}-03$ & \\
\hline S (other) & & & & & & \\
\hline $\mathrm{CO} 3$ & & & & & & \\
\hline C (reductant) & & & & & & $8.57 \mathrm{E}-01$ \\
\hline $\mathrm{O}$ (oxides) & & & & & & \\
\hline C (organic) & & & & & & \\
\hline $\mathrm{H}$ (organic) & & & & & & \\
\hline $\mathrm{O}$ (organic) & & & & & & \\
\hline & & & & & & \\
\hline Mass Flow $(\mathrm{kg} / \mathrm{hr})$ : & $5.19 \mathrm{E}+03$ & $5.19 \mathrm{E}+03$ & $5.19 \mathrm{E}+02$ & $5.19 \mathrm{E}+03$ & $5.71 \mathrm{E}+03$ & $2.27 \mathrm{E}+00$ \\
\hline Canister Rate, canister & & & & & & \\
\hline Total Canisters Genera & & & & & & \\
\hline & $\mathrm{w} / \mathrm{m} 3$ & $\mathrm{w} / \mathrm{m} 3$ & $\mathrm{w} / \mathrm{m} 3$ & $\mathrm{w} / \mathrm{m} 3$ & $\mathrm{w} / \mathrm{m} 3$ & $\mathrm{w} / \mathrm{m} 3$ \\
\hline Heat Generation & & & & & & $4.95 \mathrm{E}-14$ \\
\hline
\end{tabular}


Table A-21. Mass balance, Tank WM-188 waste (continued).

\begin{tabular}{|c|c|c|c|c|c|c|}
\hline Stream \# & 115 & 116 & 117 & 118 & 119 & 120 \\
\hline Radiological Composition & $\mathrm{Ci} / \mathrm{wscm}$ & $\mathrm{Ci} / \mathrm{wscm}$ & Ci/wscm & $\mathrm{Ci} / \mathrm{wscm}$ & $\mathrm{Ci} / \mathrm{wscm}$ & $\mathrm{Ci} / \mathrm{kg}$ \\
\hline $\mathrm{H}-3$ & $7.78 \mathrm{E}-07$ & $7.78 \mathrm{E}-07$ & & $7.78 \mathrm{E}-07$ & $7.16 \mathrm{E}-07$ & \\
\hline $\mathrm{C}-14$ & $1.05 \mathrm{E}-11$ & $1.05 \mathrm{E}-11$ & & $1.05 \mathrm{E}-11$ & $9.71 \mathrm{E}-12$ & $7.43 \mathrm{E}-11$ \\
\hline \multicolumn{7}{|l|}{ Co-60 } \\
\hline \multicolumn{7}{|l|}{ Ni-59 } \\
\hline \multirow{2}{*}{\multicolumn{7}{|c|}{$\begin{array}{ll}\mathrm{Ni}-63 \\
70\end{array}$}} \\
\hline \multirow{2}{*}{\multicolumn{5}{|c|}{$\frac{\text { Se-79 }}{\text { Sr-90 }}$}} & & \\
\hline \multirow{2}{*}{\multicolumn{7}{|c|}{$\frac{\text { Sr-90 }}{Y-90}$}} \\
\hline & & & & & & \\
\hline \multicolumn{7}{|l|}{$\mathrm{Zr}-93$} \\
\hline \multicolumn{7}{|l|}{$\mathrm{Nb}-93 \mathrm{~m}$} \\
\hline \multicolumn{7}{|l|}{$\mathrm{Nb}-94$} \\
\hline \multicolumn{7}{|l|}{ Tc-99 } \\
\hline \multicolumn{7}{|l|}{$\frac{\mathrm{Ru}-106}{\mathrm{Rh}-102}$} \\
\hline \multirow{2}{*}{\multicolumn{7}{|c|}{$\frac{\mathrm{Rh}-102}{\mathrm{Rh}-106}$}} \\
\hline \multirow{2}{*}{\multicolumn{7}{|c|}{$\frac{R h-106}{P d-107}$}} \\
\hline & & & & & & \\
\hline \multicolumn{7}{|l|}{$\mathrm{Cd}-113 \mathrm{~m}$} \\
\hline \multicolumn{7}{|l|}{ Sn-121m } \\
\hline \multirow{2}{*}{\multicolumn{7}{|c|}{$\frac{S n-126}{S b-125}$}} \\
\hline \multirow{2}{*}{\multicolumn{7}{|c|}{$\begin{array}{l}\text { Sb-125 } \\
\text { Sb-126 }\end{array}$}} \\
\hline \multirow{2}{*}{\multicolumn{7}{|c|}{$\frac{\mathrm{Sb}-126}{\mathrm{Te}-125 \mathrm{~m}}$}} \\
\hline & & & & & & \\
\hline $\mid-129$ & $1.05 \mathrm{E}-11$ & 1.05E-11 & & $2.76 \mathrm{E}-12$ & $2.55 \mathrm{E}-12$ & 1.69E-08 \\
\hline \multicolumn{7}{|l|}{ Cs-134 } \\
\hline Cs-135 & & & & & & \\
\hline Cs-137 & & & & & & \\
\hline Ba-137m & & & & & & \\
\hline $\mathrm{Ce}-144$ & & & & & & \\
\hline Pr-144 & & & & & & \\
\hline $\mathrm{Pm}-146$ & & & & & & \\
\hline $\mathrm{Pm}-147$ & & & & & & \\
\hline Sm-151 & & & & & & \\
\hline Eu-152 & & & & & & \\
\hline Eu-154 & & & & & & \\
\hline Eu-155 & & & & & & \\
\hline Th-230 & & & & & & \\
\hline $\mathrm{Pa}-233$ & & & & & & \\
\hline $\mathrm{U}-232$ & & & & & & \\
\hline $\mathrm{U}-233$ & & & & & & \\
\hline $\mathrm{U}-234$ & & & & & & \\
\hline $\mathrm{U}-235$ & & & & & & \\
\hline $\mathrm{U}-236$ & & & & & & \\
\hline $\mathrm{U}-237$ & & & & & & \\
\hline $\mathrm{U}-238$ & & & & & & \\
\hline Np-237 & & & & & & \\
\hline Pu-236 & & & & & & \\
\hline Pu-238 & & & & & & \\
\hline Pu-239 & & & & & & \\
\hline Pu-240 & & & & & & \\
\hline $\mathrm{Pu}-241$ & & & & & & \\
\hline Pu-242 & & & & & & \\
\hline Pu-244 & & & & & & \\
\hline Am-241 & & & & & & \\
\hline $\mathrm{Am}-242 \mathrm{~m}$ & & & & & & \\
\hline
\end{tabular}


Table A-21. Mass balance, Tank WM-188 waste (continued).

\begin{tabular}{|c|c|c|c|c|c|c|}
\hline Stream \# & 115 & 116 & 117 & 118 & 119 & 120 \\
\hline Radiological Composition & Ci/wscm & Ci/wscm & $\mathrm{Ci} / \mathrm{wscm}$ & $\mathrm{Ci} / \mathrm{wscm}$ & Ci/wscm & $\mathrm{Ci} / \mathrm{kg}$ \\
\hline \multicolumn{7}{|l|}{$\mathrm{Am}-243$} \\
\hline \multicolumn{7}{|l|}{$\mathrm{Cm}-242$} \\
\hline \multicolumn{7}{|l|}{$\mathrm{Cm}-243$} \\
\hline \multicolumn{7}{|l|}{$\mathrm{Cm}-244$} \\
\hline \multicolumn{7}{|l|}{$\mathrm{Cm}-245$} \\
\hline \multicolumn{7}{|l|}{$\mathrm{Cm}-246$} \\
\hline \multicolumn{7}{|l|}{ TRU } \\
\hline & & & & & & \\
\hline & & & & & & \\
\hline $\begin{array}{l}\text { Gas Stream Bulk } \\
\text { Composition (Wet Basis) }\end{array}$ & $\begin{array}{c}\mathrm{mol} \% \text { or } \\
\text { ppmv }\end{array}$ & $\begin{array}{c}\mathrm{mol} \% \text { or } \\
\text { ppmv }\end{array}$ & $\mathrm{mol} \%$ or $\mathrm{ppmv}$ & $\begin{array}{c}\text { mol\% or } \\
\text { ppmv }\end{array}$ & $\begin{array}{c}\mathrm{mol} \% \text { or } \\
\text { ppmv }\end{array}$ & $\begin{array}{c}\text { mol\% or } \\
\text { ppmv }\end{array}$ \\
\hline $\mathrm{H} 2 \mathrm{O}, \mathrm{mol} \%$ & $70.64 \%$ & $70.64 \%$ & $0.01 \%$ & $70.64 \%$ & $64.97 \%$ & \\
\hline $\mathrm{O} 2, \mathrm{~mol} \%$ & $1.6245 \%$ & $1.6245 \%$ & $20.99 \%$ & $1.6245 \%$ & $3.1804 \%$ & \\
\hline $\mathrm{N} 2, \mathrm{~mol} \%$ & $1.56 \%$ & $1.56 \%$ & $79.00 \%$ & $1.56 \%$ & $7.78 \%$ & \\
\hline \multicolumn{7}{|l|}{$\mathrm{H} 2, \mathrm{~mol} \%$} \\
\hline $\mathrm{CO} 2, \mathrm{~mol} \%$ & $26.15 \%$ & $26.15 \%$ & & $26.15 \%$ & $24.05 \%$ & \\
\hline COgas, ppmv & $1.71 \mathrm{E}-01$ & $1.71 \mathrm{E}-01$ & & $1.71 \mathrm{E}-01$ & $1.57 \mathrm{E}-01$ & \\
\hline $\mathrm{NO}, \mathrm{ppmv}$ & $1.35 \mathrm{E}+02$ & $1.35 \mathrm{E}+02$ & & $1.35 \mathrm{E}+02$ & $1.24 \mathrm{E}+02$ & \\
\hline NO2, ppmv & $5.21 \mathrm{E}+00$ & $5.21 \mathrm{E}+00$ & & $5.21 \mathrm{E}+00$ & $4.79 \mathrm{E}+00$ & \\
\hline SO2, ppmv & $6.19 \mathrm{E}+01$ & $6.19 \mathrm{E}+01$ & & $6.19 \mathrm{E}+01$ & $5.69 \mathrm{E}+01$ & \\
\hline $\mathrm{Cl}, \mathrm{ppmv}$ & $8.64 \mathrm{E}-02$ & $8.64 \mathrm{E}-02$ & & $2.28 \mathrm{E}-02$ & $2.09 \mathrm{E}-02$ & \\
\hline $\mathrm{F}, \mathrm{ppmv}$ & $3.13 \mathrm{E}+00$ & $3.13 \mathrm{E}+00$ & & $3.13 \mathrm{E}+00$ & $2.88 \mathrm{E}+00$ & \\
\hline \multicolumn{7}{|l|}{ C (organic), ppmv } \\
\hline \multirow{2}{*}{\multicolumn{7}{|c|}{$\mathrm{H}$ (organic), ppmv }} \\
\hline & & & & & & \\
\hline $\mathrm{Hg}, \mathrm{ug} / \mathrm{wscm}$ & $3.64 \mathrm{E}+04$ & $3.64 \mathrm{E}+04$ & & $3.64 \mathrm{E}+01$ & $3.35 \mathrm{E}+01$ & \\
\hline $\mathrm{PM}, \mathrm{mg} / \mathrm{wscm}$ & $4.52 \mathrm{E}+01$ & $4.52 \mathrm{E}+01$ & $1.57 \mathrm{E}+01$ & $8.75 \mathrm{E}+00$ & $9.14 \mathrm{E}+00$ & \\
\hline \multicolumn{7}{|l|}{ SVM, ug/wscm } \\
\hline \multicolumn{7}{|l|}{ LVM, ug/wscm } \\
\hline & & & & & & \\
\hline $\begin{array}{l}\text { Gas Stream Bulk } \\
\text { Composition (Dry Basis) }\end{array}$ & $\mathrm{mol} \%$ & $\mathrm{~mol} \%$ & $\mathrm{~mol} \%$ & $\mathrm{~mol} \%$ & $\mathrm{~mol} \%$ & $\mathrm{~mol} \%$ \\
\hline O2, mol \%, dry basis & $5.53 \%$ & $5.53 \%$ & $20.99 \%$ & $0.78 \%$ & $9.08 \%$ & \\
\hline $\mathrm{N} 2, \mathrm{~mol} \%$, dry basis & $5.30 \%$ & $5.30 \%$ & $79.01 \%$ & $5.30 \%$ & $22.20 \%$ & \\
\hline \multicolumn{7}{|l|}{$\mathrm{H} 2, \mathrm{~mol} \%$, dry basis } \\
\hline $\mathrm{CO} 2, \mathrm{~mol} \%$, dry basis & $89.08 \%$ & $89.08 \%$ & & $89.08 \%$ & $68.65 \%$ & \\
\hline $\begin{array}{l}\text { Gas Stream Bulk } \\
\text { Composition (Dry Basis, } \\
\text { Corrected to } 7 \% \text { O2 with } \\
100 \% \text { O2 Combustion Air) }\end{array}$ & $\begin{array}{l}\text { ppmv, or } \\
\text { ug/dscm or } \\
\mathrm{mg} / \mathrm{dscm}\end{array}$ & $\begin{array}{l}\text { ppmv, or } \\
\text { ug/dscm or } \\
\mathrm{mg} / \mathrm{dscm}\end{array}$ & $\begin{array}{l}\mathrm{ppmv}, \text { or } \\
\mathrm{ug} / \mathrm{dscm} \text { or } \\
\mathrm{mg} / \mathrm{dscm}\end{array}$ & $\begin{array}{l}\text { ppmv, or } \\
\text { ug/dscm or } \\
\mathrm{mg} / \mathrm{dscm}\end{array}$ & $\begin{array}{l}\text { ppmv, or } \\
\text { ug/dscm or } \\
\mathrm{mg} / \mathrm{dscm}\end{array}$ & $\begin{array}{l}\mathrm{ppmv}, \text { or } \\
\mathrm{ug} / \mathrm{dscm} \text { or } \\
\mathrm{mg} / \mathrm{dscm}\end{array}$ \\
\hline COgas, ppmv, dry basis & $8.14 \mathrm{E}-02$ & $8.14 \mathrm{E}-02$ & & $8.14 \mathrm{E}-02$ & $6.27 \mathrm{E}-02$ & \\
\hline NO, ppmv, dry basis & $6.42 \mathrm{E}+01$ & $6.42 \mathrm{E}+01$ & & $6.42 \mathrm{E}+01$ & $4.95 \mathrm{E}+01$ & \\
\hline $\mathrm{NO} 2, \mathrm{ppmv}$, dry basis & $2.49 \mathrm{E}+00$ & $2.49 \mathrm{E}+00$ & & $2.49 \mathrm{E}+00$ & $1.92 \mathrm{E}+00$ & \\
\hline SO2, ppmv, dry basis & $2.95 \mathrm{E}+01$ & $2.95 \mathrm{E}+01$ & & $2.95 \mathrm{E}+01$ & $2.28 \mathrm{E}+01$ & \\
\hline Cl, ppmv, dry basis & $4.12 \mathrm{E}-02$ & $4.12 \mathrm{E}-02$ & & $1.09 \mathrm{E}-02$ & $8.37 \mathrm{E}-03$ & \\
\hline F, ppmv, dry basis & $1.49 \mathrm{E}+00$ & $1.49 \mathrm{E}+00$ & & $1.49 \mathrm{E}+00$ & $1.15 \mathrm{E}+00$ & \\
\hline \multicolumn{7}{|l|}{$\mathrm{C}$ (organic), ppmv, dry basis } \\
\hline \multicolumn{7}{|l|}{$\mathrm{H}$ (organic), ppmv, dry basis } \\
\hline $\mathrm{Hg}, \mathrm{ug} / \mathrm{dscm}$ & $1.74 \mathrm{E}+04$ & $1.74 \mathrm{E}+04$ & & $1.74 \mathrm{E}+01$ & $1.34 \mathrm{E}+01$ & \\
\hline $\mathrm{PM}, \mathrm{mg} / \mathrm{dscm}$ & $2.16 \mathrm{E}+01$ & $2.16 \mathrm{E}+01$ & $2.21 \mathrm{E}+00$ & $4.18 \mathrm{E}+00$ & $3.66 \mathrm{E}+00$ & \\
\hline \multicolumn{7}{|l|}{ SVM, ug/dscm } \\
\hline LVM, ug/dscm & & & & & & \\
\hline
\end{tabular}


Table A-21. Mass balance, Tank WM-188 waste (continued).

\begin{tabular}{|c|c|c|c|c|c|c|c|}
\hline PFD Number & PFD-3 & PFD-3 & PFD-3 & PFD-3 & PFD-3 & PFD-3 & PFD-2 \\
\hline Stream Number & 203 & 204 & 205 & 206 & 207 & 301 & 303 \\
\hline Stream Name & $\begin{array}{l}\text { Fluidizing } \\
\text { Gas to } \\
\text { Reformer }\end{array}$ & $\begin{array}{l}\text { Propane to } \\
\text { Oxidizer }\end{array}$ & $\begin{array}{l}\text { Water to } \\
\text { Spray } \\
\text { Quench }\end{array}$ & $\begin{array}{l}\text { ANN to } \\
\text { Scrub for F } \\
\text { Adjust }\end{array}$ & $\begin{array}{l}\text { HNO3 to } \\
\text { Scrub for } \\
\text { H+ Adjust }\end{array}$ & Isopropanol & Bed Media \\
\hline Rate or Volume & $1.11 \mathrm{E}+04$ & $9.05 \mathrm{E}+02$ & $4.59 \mathrm{E}+02$ & $3.12 \mathrm{E}-02$ & $1.21 \mathrm{E}+00$ & $8.02 \mathrm{E}+01$ & $1.15 \mathrm{E}+00$ \\
\hline Volume Flow (standard, wet) & $1.09 \mathrm{E}+04$ & $2.56 \mathrm{E}+03$ & & & & & \\
\hline Volume Flow (standard, dry) & $1.09 \mathrm{E}+04$ & $2.56 \mathrm{E}+03$ & & & & & \\
\hline Rate Units & $\mathrm{ft} 3 / \mathrm{hr}$ & $\mathrm{ft} 3 / \mathrm{hr}$ & gal $/ \mathrm{hr}$ & $\mathrm{gal} / \mathrm{hr}$ & gal/hr & gal/hr & $\mathrm{lb} / \mathrm{hr}$ \\
\hline Rate or Volume, metric & $3.10 \mathrm{E}+02$ & $7.26 \mathrm{E}+01$ & $1.74 \mathrm{E}+03$ & $1.18 \mathrm{E}-01$ & $4.57 \mathrm{E}+00$ & $3.04 \mathrm{E}+02$ & $5.23 \mathrm{E}-01$ \\
\hline Rate Units & wscm $/ \mathrm{hr}$ & wscm $/ \mathrm{hr}$ & $\mathrm{L} / \mathrm{hr}$ & $\mathrm{L} / \mathrm{hr}$ & $\mathrm{L} / \mathrm{hr}$ & $\mathrm{L} / \mathrm{hr}$ & $\mathrm{kg} / \mathrm{hr}$ \\
\hline Temperature, ${ }^{\circ} \mathrm{C}$ & 580 & 25 & 25 & 25 & 25 & 15 & 15 \\
\hline Temperature, ${ }^{\circ} \mathrm{F}$ & 1076 & 77 & 77 & 77 & 77 & 59 & 59 \\
\hline Pressure, psia & 42.30 & 42.30 & 112.30 & 14.70 & 42.30 & 14.70 & 14.70 \\
\hline \begin{tabular}{|l|} 
Specific Gravity \\
\end{tabular} & $1.81 \mathrm{E}-03$ & $5.19 \mathrm{E}-03$ & $9.93 \mathrm{E}-01$ & $1.80 \mathrm{E}+00$ & $1.23 \mathrm{E}+00$ & $1.03 \mathrm{E}+00$ & $1.58 \mathrm{E}+00$ \\
\hline Chemical Composition & Mol \% & $\mathrm{lb} / \mathrm{wscf}$ & Mol/liter & Mol/liter & Mol/liter & Mol/liter & Wt frac \\
\hline $\mathrm{H}+$ & & & $9.95 \mathrm{E}-08$ & & $1.33 \mathrm{E}+01$ & & \\
\hline $\mathrm{Al}+3$ & & & & $2.20 \mathrm{E}+00$ & & & 0.529 \\
\hline \multicolumn{8}{|l|}{$\mathrm{Sb}+5$} \\
\hline \multicolumn{8}{|l|}{ As+3 } \\
\hline \multirow{2}{*}{\multicolumn{8}{|c|}{\begin{tabular}{|l|}
$\mathrm{Ba}+2$ \\
$\mathrm{Be}+2$
\end{tabular}}} \\
\hline \multirow{2}{*}{\multicolumn{8}{|c|}{\begin{tabular}{|l|}
$\mathrm{Be}+2$ \\
$\mathrm{~B}+3$
\end{tabular}}} \\
\hline & & & & & & & \\
\hline \multicolumn{8}{|l|}{$\mathrm{Cd}+2$} \\
\hline \multicolumn{8}{|l|}{$\mathrm{Ca}+2$} \\
\hline \multirow{2}{*}{\multicolumn{8}{|c|}{\begin{tabular}{|l|}
$\mathrm{Cr}+3$ \\
$\mathrm{Co}+2$ \\
\end{tabular}}} \\
\hline & & & & & & & \\
\hline \multicolumn{8}{|l|}{ Cs+ } \\
\hline \multirow{2}{*}{\multicolumn{8}{|c|}{\begin{tabular}{|l|}
$\mathrm{Cu}+2$ \\
$\mathrm{Fe}+3$
\end{tabular}}} \\
\hline & & & & & & & \\
\hline \multicolumn{8}{|l|}{$\mathrm{Pb}+2$} \\
\hline \multicolumn{8}{|l|}{$\mathrm{Hg}+2$} \\
\hline \multicolumn{8}{|l|}{$\mathrm{Mn}+4$} \\
\hline \multirow{2}{*}{\multicolumn{8}{|c|}{$\begin{array}{ll}\mathrm{Ni}+2 \\
\mathrm{~K}+\end{array}$}} \\
\hline & & & & & & & \\
\hline \multirow{2}{*}{\multicolumn{8}{|c|}{\begin{tabular}{|l|}
$\mathrm{Se}+4$ \\
$\mathrm{Ag}+$ \\
\end{tabular}}} \\
\hline \multirow{2}{*}{\multicolumn{8}{|c|}{\begin{tabular}{|l|}
$\mathrm{Ag}+$ \\
$\mathrm{Na}+$ \\
\end{tabular}}} \\
\hline & & & & & & & \\
\hline \multicolumn{8}{|l|}{$\mathrm{TI}+3$} \\
\hline \multicolumn{8}{|l|}{$\mathrm{U}+4$} \\
\hline \multirow{2}{*}{\multicolumn{8}{|c|}{\begin{tabular}{|l|}
$V+3$ \\
$Z n+2$
\end{tabular}}} \\
\hline & & & & & & & \\
\hline $\mathrm{Zr}+4$ & & & & & & & \\
\hline $\mathrm{Cl}-\mathrm{P}$ & & & & & & & \\
\hline F- & & & & & & & \\
\hline SO4-2 & & & & & & & \\
\hline \begin{tabular}{|l|} 
NO3- \\
\end{tabular} & & & & $6.60 \mathrm{E}+00$ & $1.33 \mathrm{E}+01$ & & \\
\hline & & & & & & & \\
\hline $\mathrm{CO} 2$ & 100 & & & & & & \\
\hline $\mathrm{OH}-$ & & & $9.95 \mathrm{E}-08$ & $7.31 \mathrm{E}-08$ & & & \\
\hline $\begin{array}{ll}\mathrm{H} 2 \mathrm{O} \\
\end{array}$ & & & $5.51 \mathrm{E}+01$ & $7.41 \mathrm{E}+01$ & $2.17 \mathrm{E}+01$ & & \\
\hline $\mathrm{O}$ (oxides) & & & & & & & 0.471 \\
\hline C (organic) & & $9.35 \mathrm{E}-02$ & & & & $5.15 \mathrm{E}+01$ & \\
\hline $\mathrm{H}$ (organic) & & $2.09 \mathrm{E}-02$ & & & & $1.37 \mathrm{E}+02$ & \\
\hline $\mathrm{O}$ (organic) & & & & & & $1.72 \mathrm{E}+01$ & \\
\hline Mass Flow (kg/hr): & $5.66 \mathrm{E}+02$ & $1.33 \mathrm{E}+02$ & $1.73 \mathrm{E}+03$ & $2.13 \mathrm{E}-01$ & $5.62 \mathrm{E}+00$ & $3.13 \mathrm{E}+02$ & $5.23 \mathrm{E}-01$ \\
\hline
\end{tabular}


Table A-21. Mass balance, Tank WM-188 waste (continued).

\begin{tabular}{|c|c|c|c|c|c|c|c|}
\hline PFD Number & PFD-3 & PFD-3 & PFD-3 & PFD-3 & PFD-2 & PFD-2 & PFD-3 \\
\hline Stream Number & 305 & 401 & 402 & 404 & 404 & 503 & 505 \\
\hline Stream Name & $\begin{array}{l}\text { Grout Mix } \\
\text { for Scrub } \\
\text { Blowdown }\end{array}$ & $\begin{array}{c}\text { Scrub } \\
\text { Recycled to } \\
\text { Feed }\end{array}$ & $\begin{array}{c}\text { Scrub } \\
\text { Blowdown } \\
\text { to Grout } \\
\text { Mixer }\end{array}$ & $\begin{array}{l}\text { MLLW } \\
\text { Grout from } \\
\text { Scrub }\end{array}$ & $\begin{array}{c}\text { MLLW } \\
\text { Grout } \\
\text { Drums }\end{array}$ & $\begin{array}{c}\text { Feed } \\
\text { Atomizing } \\
\text { Gas }\end{array}$ & $\begin{array}{l}\text { Oxygen to } \\
\text { Oxidizer }\end{array}$ \\
\hline Rate or Volume & $6.61 \mathrm{E}+00$ & $5.06 \mathrm{E}+00$ & $2.57 \mathrm{E}-01$ & $8.82 \mathrm{E}+00$ & $2.29 \mathrm{E}-01$ & $4.93 \mathrm{E}+03$ & $5.42 \mathrm{E}+03$ \\
\hline Volume Flow (standard, wet) & & & & & & $1.41 \mathrm{E}+04$ & $3.35 \mathrm{E}+04$ \\
\hline Volume Flow (standard, dry) $)^{*}$ & & & & & & $1.41 \mathrm{E}+04$ & $3.35 \mathrm{E}+04$ \\
\hline Rate Units & $\mathrm{lb} / \mathrm{hr}$ & gal/hr & gal/hr & $\mathrm{lb} / \mathrm{hr}$ & Drums/day & $\mathrm{ft} 3 / \mathrm{hr}$ & $\mathrm{ft} 3 / \mathrm{hr}$ \\
\hline Rate or Volume, metric & $3.00 \mathrm{E}+00$ & $1.91 \mathrm{E}+01$ & $9.74 \mathrm{E}-01$ & $4.00 \mathrm{E}+00$ & $4.00 \mathrm{E}+00$ & $4.00 \mathrm{E}+02$ & $9.48 \mathrm{E}+02$ \\
\hline Rate Units & $\mathrm{kg} / \mathrm{hr}$ & $\mathrm{L} / \mathrm{hr}$ & $\mathrm{L} / \mathrm{hr}$ & $\mathrm{kg} / \mathrm{hr}$ & $\mathrm{kg} / \mathrm{hr}$ & wscm $/ \mathrm{hr}$ & wscm $/ \mathrm{hr}$ \\
\hline Temperature, ${ }^{\circ} \mathrm{C}$ & 25 & 70 & 70 & 54 & 54 & 21 & 25 \\
\hline Temperature, ${ }^{\circ} \mathrm{F}$ & 77 & 158 & 158 & 129 & 129 & 70 & 77 \\
\hline Pressure, psia & 14.70 & 29.98 & 29.98 & 14.70 & 14.70 & 42.30 & 92.30 \\
\hline Specific Gravity & $2.01 \mathrm{E}-01$ & $1.03 \mathrm{E}+00$ & $1.03 \mathrm{E}+00$ & $2.10 \mathrm{E}+00$ & $2.10 \mathrm{E}+00$ & $5.25 \mathrm{E}-03$ & $8.21 \mathrm{E}-03$ \\
\hline Chemical Composition & Wt frac & Mol/liter & Mol/liter & Wt frac & Wt frac & Ib/wscf & $\mathrm{lb} / \mathrm{wscf}$ \\
\hline $\mathrm{H}+$ & & $9.56 \mathrm{E}-01$ & $9.56 \mathrm{E}-01$ & $2.35 \mathrm{E}-04$ & $2.35 \mathrm{E}-04$ & & \\
\hline$A \mathrm{Al}+3$ & & $9.25 \mathrm{E}-03$ & $9.25 \mathrm{E}-03$ & $0.01 \%$ & $0.01 \%$ & & \\
\hline $\mathrm{Sb}+5$ & & $2.74 \mathrm{E}-07$ & $2.74 \mathrm{E}-07$ & $8.11 \mathrm{E}-09$ & $8.11 \mathrm{E}-09$ & & \\
\hline$A s+3$ & & $1.53 \mathrm{E}-06$ & $1.53 \mathrm{E}-06$ & $2.78 \mathrm{E}-08$ & $2.78 \mathrm{E}-08$ & & \\
\hline \multicolumn{8}{|l|}{$\mathrm{Ba}+2$} \\
\hline \multicolumn{8}{|l|}{$\mathrm{Be}+2$} \\
\hline$B+3$ & & $2.73 \mathrm{E}-04$ & $2.73 \mathrm{E}-04$ & $7.20 \mathrm{E}-07$ & $7.20 \mathrm{E}-07$ & & \\
\hline \multicolumn{8}{|l|}{$\mathrm{Cd}+2$} \\
\hline \multirow{2}{*}{\multicolumn{8}{|c|}{\begin{tabular}{|l|}
$\mathrm{Ca}+2$ \\
$\mathrm{Cr}+3$
\end{tabular}}} \\
\hline & & & \multicolumn{5}{|c|}{$\mathrm{Cr}+3$} \\
\hline $\mathrm{Co}+2$ & & $6.81 \mathrm{E}-07$ & $6.81 \mathrm{E}-07$ & $9.78 \mathrm{E}-09$ & $9.78 \mathrm{E}-09$ & & \\
\hline \multicolumn{8}{|l|}{$\mathrm{Cs}+$} \\
\hline \multicolumn{8}{|l|}{$\mathrm{Cu}+2$} \\
\hline $\mathrm{Fe}+3$ & & $9.46 \mathrm{E}-05$ & $9.46 \mathrm{E}-05$ & $1.29 \mathrm{E}-06$ & $1.29 \mathrm{E}-06$ & & \\
\hline \multicolumn{8}{|l|}{$\mathrm{Pb}+2$} \\
\hline $\mathrm{Hg}+2$ & & $7.43 \mathrm{E}-02$ & $7.43 \mathrm{E}-02$ & $3.63 \mathrm{E}-03$ & $3.63 \mathrm{E}-03$ & & \\
\hline$\overline{M n+4}$ & & $2.49 \mathrm{E}-04$ & $2.49 \mathrm{E}-04$ & $3.34 \mathrm{E}-06$ & $3.34 \mathrm{E}-06$ & & \\
\hline \multirow{2}{*}{\multicolumn{8}{|c|}{$\mathrm{Ni}+2$}} \\
\hline \multirow{2}{*}{\multicolumn{8}{|c|}{\begin{tabular}{|l}
$\mathrm{K}+$ \\
$\mathrm{Se}+4$
\end{tabular}}} \\
\hline & & & & & \multicolumn{3}{|c|}{$\mathrm{Se}+4$} \\
\hline \multirow{2}{*}{\multicolumn{8}{|c|}{\begin{tabular}{|l}
$\mathrm{Ag}+$ \\
$\mathrm{Na}+$
\end{tabular}}} \\
\hline & & & & & & & \\
\hline $\mathrm{TI}+3$ & & $7.64 \mathrm{E}-08$ & $7.64 \mathrm{E}-08$ & $3.80 \mathrm{E}-09$ & $3.80 \mathrm{E}-09$ & & \\
\hline \multicolumn{8}{|l|}{$\begin{array}{l}\mathrm{U}+4 \\
\mathrm{U}\end{array}$} \\
\hline \multicolumn{8}{|l|}{$V+3$} \\
\hline \multicolumn{8}{|l|}{$Z n+2$} \\
\hline \multirow{2}{*}{\multicolumn{8}{|c|}{\begin{tabular}{|l}
$\mathrm{Zr}+4$ \\
$\mathrm{Cl}-$
\end{tabular}}} \\
\hline $\mathrm{Cl}-$ & & $4.71 \mathrm{E}-04$ & $4.71 \mathrm{E}-04$ & $4.06 \mathrm{E}-06$ & $4.06 \mathrm{E}-06$ & & \\
\hline F- & & $5.09 \mathrm{E}-03$ & $5.09 \mathrm{E}-03$ & $2.36 \mathrm{E}-05$ & $2.36 \mathrm{E}-05$ & & \\
\hline \multicolumn{8}{|l|}{ SO4-2 } \\
\hline NO3- & & $9.16 \mathrm{E}-01$ & $9.16 \mathrm{E}-01$ & $1.38 \mathrm{E}-02$ & $1.38 \mathrm{E}-02$ & & \\
\hline \multicolumn{8}{|l|}{\begin{tabular}{|l|} 
PO4-3 \\
\end{tabular}} \\
\hline$A m+4$ & & 1.16E-09 & $1.16 \mathrm{E}-09$ & $6.84 \mathrm{E}-11$ & $6.84 \mathrm{E}-11$ & & \\
\hline $\mathrm{Br}-$ & & $5.68 \mathrm{E}-09$ & $5.68 \mathrm{E}-09$ & $1.11 \mathrm{E}-10$ & $1.11 \mathrm{E}-10$ & & \\
\hline $\mathrm{Ce}+4$ & & $5.71 \mathrm{E}-07$ & $5.71 \mathrm{E}-07$ & $1.95 \mathrm{E}-08$ & $1.95 \mathrm{E}-08$ & & \\
\hline $\mathrm{Eu+3}$ & & $8.24 \mathrm{E}-09$ & $8.24 \mathrm{E}-09$ & $3.05 \mathrm{E}-10$ & $3.05 \mathrm{E}-10$ & & \\
\hline $\mathrm{Gd}+3$ & & $2.43 \mathrm{E}-06$ & $2.43 \mathrm{E}-06$ & $9.31 \mathrm{E}-08$ & $9.31 \mathrm{E}-08$ & & \\
\hline $\mathrm{Ge}+4$ & & & & & & & \\
\hline $\ln +3$ & & $2.52 \mathrm{E}-08$ & $2.52 \mathrm{E}-08$ & $7.04 \mathrm{E}-10$ & $7.04 \mathrm{E}-10$ & & \\
\hline $\mid-$ & & $6.07 \mathrm{E}-08$ & $6.07 \mathrm{E}-08$ & $1.88 \mathrm{E}-09$ & $1.88 \mathrm{E}-09$ & & \\
\hline$L a+3$ & & $1.50 \mathrm{E}-07$ & $1.50 \mathrm{E}-07$ & $5.06 \mathrm{E}-09$ & $5.06 \mathrm{E}-09$ & & \\
\hline
\end{tabular}


Table A-21. Mass balance, Tank WM-188 waste (continued).

\begin{tabular}{|c|c|c|c|c|c|c|c|}
\hline Stream \# & 305 & 401 & 402 & 404 & 404 & 503 & 505 \\
\hline Chemical Composition & Wt frac & Mol/liter & Mol/liter & Wt frac & Wt frac & $\mathrm{Ib} / \mathrm{wscf}$ & $\mathrm{Ib} / \mathrm{wscf}$ \\
\hline \multicolumn{8}{|l|}{$\mathrm{Li}+$} \\
\hline \multicolumn{8}{|l|}{$\mathrm{Mg}+2$} \\
\hline \multicolumn{8}{|l|}{$\mathrm{Mo}+6$} \\
\hline $\mathrm{Nd}+3$ & & $4.82 \mathrm{E}-07$ & $4.82 \mathrm{E}-07$ & $1.69 \mathrm{E}-08$ & 1.69E-08 & & \\
\hline $\mathrm{Np}+4$ & & $4.41 \mathrm{E}-08$ & $4.41 \mathrm{E}-08$ & $2.54 \mathrm{E}-09$ & $2.54 \mathrm{E}-09$ & & \\
\hline \multicolumn{8}{|l|}{$\mathrm{Nb}+5$} \\
\hline \multicolumn{8}{|l|}{$\mathrm{Pd}+4$} \\
\hline \multicolumn{8}{|l|}{$\mathrm{Pu}+4$} \\
\hline $\mathrm{Pr}+4$ & & $1.36 \mathrm{E}-07$ & $1.36 \mathrm{E}-07$ & $4.66 \mathrm{E}-09$ & $4.66 \mathrm{E}-09$ & & \\
\hline $\mathrm{Pm}+3$ & & $2.81 \mathrm{E}-11$ & $2.81 \mathrm{E}-11$ & $9.93 \mathrm{E}-13$ & $9.93 \mathrm{E}-13$ & & \\
\hline $\mathrm{Rh}+4$ & & $5.86 \mathrm{E}-08$ & $5.86 \mathrm{E}-08$ & $1.47 \mathrm{E}-09$ & 1.47E-09 & & \\
\hline \multicolumn{8}{|l|}{$\mathrm{Rb}+$} \\
\hline $\mathrm{Ru}+3$ & & $1.36 \mathrm{E}-05$ & $1.36 \mathrm{E}-05$ & $3.34 \mathrm{E}-07$ & $3.34 \mathrm{E}-07$ & & \\
\hline $\mathrm{Sm+3}$ & & $8.98 \mathrm{E}-08$ & $8.98 \mathrm{E}-08$ & $3.29 \mathrm{E}-09$ & $3.29 \mathrm{E}-09$ & & \\
\hline$S i+4$ & $4.67 \mathrm{E}-01$ & & & $3.51 \mathrm{E}-01$ & $3.51 \mathrm{E}-01$ & & \\
\hline \multicolumn{8}{|l|}{$\mathrm{Sr}+2$} \\
\hline $\mathrm{Tc}+7$ & & $1.55 \mathrm{E}-07$ & $1.55 \mathrm{E}-07$ & $3.70 \mathrm{E}-09$ & $3.70 \mathrm{E}-09$ & & \\
\hline \multicolumn{8}{|l|}{$\mathrm{Te}+4$} \\
\hline $\mathrm{Tb}+4$ & & $3.44 \mathrm{E}-11$ & $3.44 \mathrm{E}-11$ & $1.33 \mathrm{E}-12$ & $1.33 \mathrm{E}-12$ & & \\
\hline \multicolumn{8}{|l|}{$\mathrm{Th}+4$} \\
\hline \multirow{2}{*}{\multicolumn{8}{|c|}{$\frac{S n+4}{T+i+4}$}} \\
\hline & & & & & \multicolumn{3}{|c|}{$\mathrm{Ti}+4$} \\
\hline $\mathrm{Y}+3$ & & $1.11 \mathrm{E}-07$ & $1.11 \mathrm{E}-07$ & $2.41 \mathrm{E}-09$ & $2.41 \mathrm{E}-09$ & & \\
\hline $\mathrm{OH}-$ & & $1.30 \mathrm{E}-06$ & $1.30 \mathrm{E}-06$ & $5.37 \mathrm{E}-09$ & 5.37E-09 & & \\
\hline \multirow[t]{2}{*}{$\mathrm{H} 2 \mathrm{O}$} & & $5.29 \mathrm{E}+01$ & $5.29 \mathrm{E}+01$ & $2.32 \mathrm{E}-01$ & $2.32 \mathrm{E}-01$ & & \\
\hline & & & & & & & \\
\hline $\mathrm{SO} 2$ & & $6.31 \mathrm{E}-07$ & $6.31 \mathrm{E}-07$ & $9.84 \mathrm{E}-09$ & 9.84E-09 & & \\
\hline \multicolumn{8}{|l|}{$\mathrm{H} 2 \mathrm{~S}$} \\
\hline $\mathrm{CO}$ & & $2.88 \mathrm{E}-09$ & $2.88 \mathrm{E}-09$ & $1.96 \mathrm{E}-11$ & $1.96 \mathrm{E}-11$ & & \\
\hline $\mathrm{CO} 2$ & & $2.09 \mathrm{E}-04$ & $2.09 \mathrm{E}-04$ & $2.24 \mathrm{E}-06$ & $2.24 \mathrm{E}-06$ & 1.14E-01 & \\
\hline $\mathrm{H} 2$ & & $3.07 \mathrm{E}-08$ & $3.07 \mathrm{E}-08$ & $1.50 \mathrm{E}-11$ & $1.50 \mathrm{E}-11$ & & \\
\hline $\mathrm{N} 2$ & & $5.76 \mathrm{E}-09$ & $5.76 \mathrm{E}-09$ & $3.93 \mathrm{E}-11$ & $3.93 \mathrm{E}-11$ & & \\
\hline $\mathrm{NO}$ & & $8.34 \mathrm{E}-08$ & $8.34 \mathrm{E}-08$ & $6.09 \mathrm{E}-10$ & $6.09 \mathrm{E}-10$ & & \\
\hline $\mathrm{NO2}$ & & $1.31 \mathrm{E}-05$ & $1.31 \mathrm{E}-05$ & $1.47 \mathrm{E}-07$ & $1.47 \mathrm{E}-07$ & & \\
\hline $\mathrm{O} 2$ & & $1.74 \mathrm{E}-08$ & $1.74 \mathrm{E}-08$ & $1.35 \mathrm{E}-10$ & $1.35 \mathrm{E}-10$ & & $8.30 \mathrm{E}-02$ \\
\hline S (other) & & $9.26 \mathrm{E}-09$ & $9.26 \mathrm{E}-09$ & $7.23 \mathrm{E}-11$ & $7.23 \mathrm{E}-11$ & & \\
\hline \multicolumn{8}{|l|}{$\mathrm{CO} 3$} \\
\hline \multicolumn{8}{|l|}{ C (reductant) } \\
\hline $\mathrm{O}$ (oxides) & $53.26 \%$ & $1.42 \mathrm{E}-04$ & $1.42 \mathrm{E}-04$ & $39.94 \%$ & $39.94 \%$ & & \\
\hline \multicolumn{8}{|l|}{ C (organic) } \\
\hline \multicolumn{8}{|l|}{$\mathrm{H}$ (organic) } \\
\hline \multicolumn{8}{|l|}{$\mathrm{O}$ (organic) } \\
\hline & & & & & & & \\
\hline Mass Flow (kg/hr): & $3.00 \mathrm{E}+00$ & $1.97 \mathrm{E}+01$ & $1.00 \mathrm{E}+00$ & $4.00 \mathrm{E}+00$ & $4.00 \mathrm{E}+00$ & $7.32 \mathrm{E}+02$ & $1.26 \mathrm{E}+03$ \\
\hline Canister Rate, canisters/day & & & & & 0.23 & & \\
\hline Total Canisters Generated & & & & & 32 & & \\
\hline Heat Generation & $\mathrm{w} / \mathrm{m} 3$ & $\mathrm{w} / \mathrm{m} 3$ & $\mathrm{w} / \mathrm{m} 3$ & $\mathrm{w} / \mathrm{m} 3$ & w/Drum & $\mathrm{w} / \mathrm{m} 3$ & $\mathrm{w} / \mathrm{m} 3$ \\
\hline Heat Generation & & $3.78 \mathrm{E}-03$ & $3.78 \mathrm{E}-03$ & $1.93 \mathrm{E}-03$ & $3.87 \mathrm{E}-04$ & & \\
\hline
\end{tabular}


Table A-21. Mass balance, Tank WM-188 waste (continued).

\begin{tabular}{|c|c|c|c|c|c|c|c|}
\hline Stream \# & 305 & 401 & 402 & 404 & 404 & 503 & 505 \\
\hline Radiological Composition & $\mathrm{Ci} / \mathrm{kg}$ & $\mathrm{Ci} / \mathrm{L}$ & $\mathrm{Ci} / \mathrm{L}$ & $\mathrm{Ci} / \mathrm{kg}$ & Ci/Drum & $\mathrm{Ci} / \mathrm{wscm}$ & $\mathrm{Ci} / \mathrm{wscm}$ \\
\hline \multicolumn{8}{|l|}{$\mathrm{H}-3$} \\
\hline C-14 & & $2.03 \mathrm{E}-16$ & $2.03 \mathrm{E}-16$ & $4.94 \mathrm{E}-17$ & $2.07 \mathrm{E}-14$ & & \\
\hline Co-60 & & $1.53 \mathrm{E}-07$ & $1.53 \mathrm{E}-07$ & $3.72 \mathrm{E}-08$ & $1.56 \mathrm{E}-05$ & & \\
\hline \multicolumn{8}{|l|}{$\mathrm{Ni}-59$} \\
\hline \multicolumn{8}{|l|}{$\mathrm{Ni}-63$} \\
\hline \multicolumn{8}{|l|}{ Se-79 } \\
\hline \multicolumn{8}{|l|}{ Sr-90 } \\
\hline $\mathrm{Y}-90$ & & $6.76 \mathrm{E}-04$ & $6.76 \mathrm{E}-04$ & $1.65 \mathrm{E}-04$ & $6.91 \mathrm{E}-02$ & & \\
\hline \multicolumn{8}{|l|}{ Zr-93 } \\
\hline \multicolumn{8}{|l|}{$\mathrm{Nb}-93 \mathrm{~m}$} \\
\hline \multicolumn{8}{|l|}{$\mathrm{Nb}-94$} \\
\hline Tc-99 & & $3.15 \mathrm{E}-07$ & $3.15 \mathrm{E}-07$ & $7.67 \mathrm{E}-08$ & $3.22 \mathrm{E}-05$ & & \\
\hline Ru-106 & & $2.10 \mathrm{E}-08$ & $2.10 \mathrm{E}-08$ & $5.12 \mathrm{E}-09$ & $2.15 \mathrm{E}-06$ & & \\
\hline Rh-102 & & $1.51 \mathrm{E}-11$ & $1.51 \mathrm{E}-11$ & $3.68 \mathrm{E}-12$ & $1.55 \mathrm{E}-09$ & & \\
\hline Rh-106 & & $2.10 \mathrm{E}-08$ & $2.10 \mathrm{E}-08$ & $5.12 \mathrm{E}-09$ & $2.15 \mathrm{E}-06$ & & \\
\hline \multicolumn{8}{|l|}{$\mathrm{Pd}-107$} \\
\hline \multicolumn{8}{|l|}{$\mathrm{Cd}-113 \mathrm{~m}$} \\
\hline \multirow{2}{*}{\multicolumn{8}{|c|}{$\frac{S n-121 m}{S n-126}$}} \\
\hline & & & & \multicolumn{4}{|c|}{$\overline{S n-126}$} \\
\hline Sb-125 & & $6.36 \mathrm{E}-06$ & $6.36 \mathrm{E}-06$ & $1.55 \mathrm{E}-06$ & $6.51 \mathrm{E}-04$ & & \\
\hline Sb-126 & & 1.01E-09 & $1.01 \mathrm{E}-09$ & $2.46 \mathrm{E}-10$ & $1.03 \mathrm{E}-07$ & & \\
\hline \multicolumn{8}{|l|}{$T e-125 m$} \\
\hline |-129 & & $1.38 \mathrm{E}-09$ & $1.38 \mathrm{E}-09$ & $3.35 \mathrm{E}-10$ & $1.41 \mathrm{E}-07$ & & \\
\hline \multicolumn{8}{|l|}{ Cs-134 } \\
\hline \multirow{2}{*}{\multicolumn{8}{|c|}{$\begin{array}{l}\text { Cs-135 } \\
\text { Cs-137 }\end{array}$}} \\
\hline \multirow{2}{*}{\multicolumn{8}{|c|}{$\frac{\mathrm{Cs}-137}{\mathrm{Ba}-137 \mathrm{~m}}$}} \\
\hline & & & & & & & \\
\hline \multicolumn{2}{|l|}{$\frac{\mathrm{Ba}-137 m}{\mathrm{Ce}-144}$} & 1.42E-08 & $1.42 \mathrm{E}-08$ & $3.45 \mathrm{E}-09$ & $1.45 \mathrm{E}-06$ & & \\
\hline Pr-144 & & $1.42 \mathrm{E}-08$ & $1.42 \mathrm{E}-08$ & $3.45 \mathrm{E}-09$ & $1.45 \mathrm{E}-06$ & & \\
\hline$P m-146$ & & $8.93 \mathrm{E}-10$ & $8.93 \mathrm{E}-10$ & $2.17 \mathrm{E}-10$ & $9.13 \mathrm{E}-08$ & & \\
\hline $\mathrm{Pm}-147$ & & $3.83 \mathrm{E}-06$ & $3.83 \mathrm{E}-06$ & $9.33 \mathrm{E}-07$ & $3.92 \mathrm{E}-04$ & & \\
\hline Sm-151 & & $7.57 \mathrm{E}-06$ & $7.57 \mathrm{E}-06$ & $1.84 \mathrm{E}-06$ & 7.74E-04 & & \\
\hline Eu-152 & & $5.00 \mathrm{E}-08$ & $5.00 \mathrm{E}-08$ & $1.22 \mathrm{E}-08$ & $5.12 \mathrm{E}-06$ & & \\
\hline Eu-154 & & $8.52 \mathrm{E}-07$ & $8.52 \mathrm{E}-07$ & $2.07 \mathrm{E}-07$ & $8.71 \mathrm{E}-05$ & & \\
\hline Eu-155 & & $7.39 \mathrm{E}-07$ & $7.39 \mathrm{E}-07$ & $1.80 \mathrm{E}-07$ & $7.55 \mathrm{E}-05$ & & \\
\hline & & & & & & & \\
\hline Th-230 & & & & & & & \\
\hline Pa-233 & & & & & & & \\
\hline $\mathrm{U}-232$ & & & & & & & \\
\hline$U-233$ & & & & & & & \\
\hline U-234 & & & & & & & \\
\hline $\mathrm{U}-235$ & & & & & & & \\
\hline U-236 & & & & & & & \\
\hline U-237 & & & & & & & \\
\hline $\mathrm{U}-238$ & & & & & & & \\
\hline Np-237 & & $7.36 \mathrm{E}-09$ & $7.36 \mathrm{E}-09$ & 1.79E-09 & $7.53 \mathrm{E}-07$ & & \\
\hline Pu-236 & & & & & & & \\
\hline Pu-238 & & & & & & & \\
\hline Pu-239 & & & & & & & \\
\hline Pu-240 & & & & & & & \\
\hline Pu-241 & & & & & & & \\
\hline Pu-242 & & & & & & & \\
\hline Pu-244 & & & & & & & \\
\hline Am-241 & & $9.91 \mathrm{E}-07$ & $9.91 \mathrm{E}-07$ & $2.41 \mathrm{E}-07$ & $1.01 \mathrm{E}-04$ & & \\
\hline Am-242m & & $2.74 \mathrm{E}-10$ & $2.74 \mathrm{E}-10$ & $6.67 \mathrm{E}-11$ & $2.80 \mathrm{E}-08$ & & \\
\hline
\end{tabular}


Table A-21. Mass balance, Tank WM-188 waste (continued).

\begin{tabular}{|c|c|c|c|c|c|c|c|}
\hline Stream \# & 305 & 401 & 402 & 404 & 404 & 503 & 505 \\
\hline Radiological Composition & $\mathrm{Ci} / \mathrm{kg}$ & $\mathrm{Ci} / \mathrm{L}$ & $\mathrm{Ci} / \mathrm{L}$ & $\mathrm{Ci} / \mathrm{kg}$ & Ci/Drum & Ci/wscm & $\mathrm{Ci} / \mathrm{wscm}$ \\
\hline $\mathrm{Am}-243$ & & $4.47 \mathrm{E}-10$ & $4.47 \mathrm{E}-10$ & $1.09 \mathrm{E}-10$ & $4.57 \mathrm{E}-08$ & & \\
\hline \multicolumn{8}{|l|}{$\mathrm{Cm}-242$} \\
\hline \multicolumn{8}{|l|}{$\mathrm{Cm}-243$} \\
\hline \multicolumn{8}{|l|}{$\mathrm{Cm}-244$} \\
\hline \multicolumn{8}{|l|}{$\mathrm{Cm}-245$} \\
\hline \multicolumn{8}{|l|}{$\mathrm{Cm}-246$} \\
\hline \multirow[t]{3}{*}{. } & & $1.00 \mathrm{E}-06$ & $1.00 \mathrm{E}-06$ & $2.43 \mathrm{E}-07$ & $1.02 \mathrm{E}-04$ & & \\
\hline & & & & & & & \\
\hline & & & & & & & \\
\hline $\begin{array}{l}\text { Gas Stream Bulk Composition } \\
\text { (Wet Basis) }\end{array}$ & $\begin{array}{c}\text { mol\% or } \\
\text { ppmv }\end{array}$ & $\begin{array}{c}\mathrm{mol} \% \text { or } \\
\mathrm{ppmv}\end{array}$ & $\begin{array}{c}\mathrm{mol} \% \text { or } \\
\mathrm{ppmv}\end{array}$ & $\begin{array}{c}\text { mol\% or } \\
\text { ppmv }\end{array}$ & $\begin{array}{c}\mathrm{mol} \% \text { or } \\
\mathrm{ppmv}\end{array}$ & $\begin{array}{c}\mathrm{mol} \% \text { or } \\
\text { ppmv }\end{array}$ & $\begin{array}{c}\mathrm{mol} \% \text { or } \\
\mathrm{ppmv}\end{array}$ \\
\hline \multicolumn{8}{|l|}{$\mathrm{H} 2 \mathrm{O}, \mathrm{mol} \%$} \\
\hline $\mathrm{O} 2, \mathrm{~mol} \%$ & & & & & & & $100 \%$ \\
\hline \multicolumn{8}{|l|}{$\mathrm{N} 2, \mathrm{~mol} \%$} \\
\hline \multicolumn{8}{|l|}{$\mathrm{H} 2, \mathrm{~mol} \%$} \\
\hline $\mathrm{CO} 2, \mathrm{~mol} \%$ & & & & & & $100 \%$ & \\
\hline \multicolumn{8}{|l|}{ COgas, ppmv } \\
\hline \multicolumn{8}{|l|}{ NO, ppmv } \\
\hline \multicolumn{8}{|l|}{$\mathrm{NO} 2, \mathrm{ppmv}$} \\
\hline \multirow{2}{*}{\multicolumn{8}{|c|}{$\begin{array}{l}\mathrm{SO} 2, \mathrm{ppmv} \\
\mathrm{Cl}, \mathrm{ppmv}\end{array}$}} \\
\hline \multirow{2}{*}{\multicolumn{8}{|c|}{$\begin{array}{l}\mathrm{Cl}, \mathrm{ppmv} \\
\mathrm{F}, \mathrm{ppmv}\end{array}$}} \\
\hline & & & & & & & \\
\hline \multicolumn{8}{|l|}{ C (organic), ppmv } \\
\hline \multicolumn{8}{|l|}{$\mathrm{H}$ (organic), ppmv } \\
\hline & & & & & & & \\
\hline \multicolumn{8}{|l|}{$\mathrm{Hg}, \mathrm{ug} / \mathrm{wscm}$} \\
\hline $\mathrm{PM}, \mathrm{mg} / \mathrm{wscm}$ & & & & & & $1.89 \mathrm{E}-01$ & $3.05 \mathrm{E}-02$ \\
\hline \multicolumn{8}{|l|}{ SVM, ug/wscm } \\
\hline \multicolumn{8}{|l|}{ LVM, ug/wscm } \\
\hline & & & & & & & \\
\hline $\begin{array}{l}\text { Gas Stream Bulk Composition } \\
\text { (Dry Basis) }\end{array}$ & $\mathrm{mol} \%$ & $\mathrm{~mol} \%$ & $\mathrm{~mol} \%$ & $\mathrm{~mol} \%$ & $\mathrm{~mol} \%$ & $\mathrm{~mol} \%$ & $\mathrm{~mol} \%$ \\
\hline \multicolumn{8}{|l|}{$\mathrm{O} 2, \mathrm{~mol} \%$, dry basis } \\
\hline \multicolumn{8}{|l|}{$\mathrm{N} 2, \mathrm{~mol} \%$, dry basis } \\
\hline $\mathrm{H} 2, \mathrm{~mol} \%$, dry basis & & & & & & & \\
\hline $\mathrm{CO} 2, \mathrm{~mol} \%$, dry basis & & & & & & & \\
\hline $\begin{array}{l}\text { Gas Stream Bulk Composition } \\
\text { (Dry Basis, Corrected to } 7 \% \\
\text { O2 with } 100 \% \text { O2 Combustion } \\
\text { Air) }\end{array}$ & $\begin{array}{l}\mathrm{ppmv} \text {, or } \\
\mathrm{ug} / \mathrm{dscm} \text { or } \\
\mathrm{mg} / \mathrm{dscm}\end{array}$ & $\begin{array}{c}\mathrm{ppmv}, \text { or } \\
\mathrm{ug} / \mathrm{dscm} \text { or } \\
\mathrm{mg} / \mathrm{dscm}\end{array}$ & $\begin{array}{c}\mathrm{ppmv} \text {, or } \\
\mathrm{ug} / \mathrm{dscm} \text { or } \\
\mathrm{mg} / \mathrm{dscm}\end{array}$ & $\begin{array}{l}\text { ppmv, or } \\
\text { ug/dscm or } \\
\mathrm{mg} / \mathrm{dscm}\end{array}$ & $\begin{array}{l}\text { ppmv, or } \\
\text { ug/dscm or } \\
\mathrm{mg} / \mathrm{dscm}\end{array}$ & $\begin{array}{l}\mathrm{ppmv}, \text { or } \\
\mathrm{ug} / \mathrm{dscm} \text { or } \\
\mathrm{mg} / \mathrm{dscm}\end{array}$ & $\begin{array}{l}\mathrm{ppmv}, \text { or } \\
\mathrm{ug} / \mathrm{dscm} \text { or } \\
\mathrm{mg} / \mathrm{dscm}\end{array}$ \\
\hline COgas, ppmv, dry basis & & & & & & & \\
\hline NO, ppmv, dry basis & & & & & & & \\
\hline NO2, ppmv, dry basis & & & & & & & \\
\hline SO2, ppmv, dry basis & & & & & & & \\
\hline $\mathrm{Cl}$, ppmv, dry basis & & & & & & & \\
\hline $\mathrm{F}, \mathrm{ppmv}$, dry basis & & & & & & & \\
\hline $\mathrm{C}$ (organic), ppmv, dry basis & & & & & & & \\
\hline $\mathrm{H}$ (organic), ppmv, dry basis & & & & & & & \\
\hline $\mathrm{Hg}, \mathrm{ug} / \mathrm{dscm}$ & & & & & & & \\
\hline $\mathrm{PM}, \mathrm{mg} / \mathrm{dscm}$ & & & & & & & \\
\hline SVM, ug/dscm & & & & & & & \\
\hline $\mathrm{LVM}, \mathrm{ug} / \mathrm{dscm}$ & & & & & & & \\
\hline
\end{tabular}


Table A-21. Mass balance, Tank WM-188 waste (continued).

\begin{tabular}{|c|c|c|c|c|c|c|c|c|}
\hline PFD Number & PFD-3 & PFD-3 & PFD-3 & PFD-3 & PFD-3 & PFD-3 & PFD-3 & PFD-3 \\
\hline Stream Number & 510 & 511 & 512 & 513 & 514 & 515 & 516 & 517 \\
\hline Stream Name & $\begin{array}{c}\text { Gas to Cool } \\
\text { Product }\end{array}$ & $\begin{array}{l}\text { Hot Gas } \\
\text { after } \\
\text { Product } \\
\text { Cooling }\end{array}$ & $\begin{array}{c}\text { Backpulse } \\
\text { Gas for } \\
\text { Candle } \\
\text { Filters }\end{array}$ & $\begin{array}{c}\text { Bed/ Solid } \\
\text { Reductant } \\
\text { Transport } \\
\text { Gas }\end{array}$ & $\begin{array}{c}\text { TF } \\
\text { Transfer \& } \\
\text { Tank } \\
\text { Sparge Air }\end{array}$ & $\begin{array}{c}\text { Ventilation } \\
\text { Air }\end{array}$ & New GAC & $\begin{array}{c}\text { Off-gas } \\
\text { Preaheater } \\
\text { Steam }\end{array}$ \\
\hline Rate or Volume & $1.82 \mathrm{E}+02$ & $5.68 \mathrm{E}+02$ & $2.16 \mathrm{E}+02$ & $5.41 \mathrm{E}-01$ & $1.44 \mathrm{E}+01$ & $2.89 \mathrm{E}+01$ & $4.61 \mathrm{E}+00$ & $1.29 \mathrm{E}+03$ \\
\hline Volume Flow (standard, wet) $)^{*}$ & $1.22 \mathrm{E}+03$ & $1.22 \mathrm{E}+03$ & $6.34 \mathrm{E}+02$ & $1.59 \mathrm{E}+00$ & $4.21 \mathrm{E}+01$ & $2.94 \mathrm{E}+01$ & & $6.40 \mathrm{E}+03$ \\
\hline Volume Flow (standard, dry) ${ }^{*}$ & $1.22 \mathrm{E}+03$ & $1.22 \mathrm{E}+03$ & $6.34 \mathrm{E}+02$ & $1.59 \mathrm{E}+00$ & $4.21 \mathrm{E}+01$ & $2.94 \mathrm{E}+01$ & & $0.00 \mathrm{E}+00$ \\
\hline Rate Units & $\mathrm{ft} 3 / \mathrm{hr}$ & $\mathrm{ft} 3 / \mathrm{hr}$ & $\mathrm{ft} 3 / \mathrm{hr}$ & $\mathrm{ft} 3 / \mathrm{hr}$ & $\mathrm{ft} 3 / \mathrm{hr}$ & $\mathrm{ft} 3 / \mathrm{hr}$ & $\mathrm{lb} / \mathrm{hr}$ & $\mathrm{ft} 3 / \mathrm{hr}$ \\
\hline Rate or Volume, metric & $3.47 \mathrm{E}+01$ & $3.47 E+01$ & $1.79 \mathrm{E}+01$ & 4.49E-02 & $1.19 \mathrm{E}+00$ & 8.34E-01 & $2.09 \mathrm{E}+00$ & $1.81 \mathrm{E}+02$ \\
\hline Rate Units & wscm/hr & wscm/hr & wscm/hr & wscm/hr & wscm/hr & wscm/hr & $\mathrm{kg} / \mathrm{hr}$ & wscm/hr \\
\hline Temperature, ${ }^{\circ} \mathrm{C}$ & 0 & 580 & 15 & 15 & 15 & 15 & 25 & 177 \\
\hline Temperature, ${ }^{\circ} \mathrm{F}$ & 32 & 1077 & 59 & 59 & 59 & 59 & 77 & 350 \\
\hline Pressure, psia & 92.30 & 92.30 & 42.30 & 42.30 & 42.30 & 14.70 & 14.70 & 112.30 \\
\hline Specific Gravity & $7.85 \mathrm{E}-03$ & $2.51 \mathrm{E}-03$ & $3.41 \mathrm{E}-03$ & $3.41 \mathrm{E}-03$ & $3.51 \mathrm{E}-03$ & $1.22 \mathrm{E}-03$ & $4.81 \mathrm{E}-01$ & $3.73 \mathrm{E}-03$ \\
\hline Chemical Composition & $\mathrm{lb} /$ wscf & lb/wscf & $\mathrm{lb} / \mathrm{wscf}$ & $\mathrm{lb} / \mathrm{wscf}$ & $\mathrm{lb} / \mathrm{wscf}$ & lb/wscf & Wt. \% & $\mathrm{lb} / \mathrm{wscf}$ \\
\hline C (reductant) & & & & & & & $100 \%$ & \\
\hline & & & & & & & & \\
\hline Gas Composition, wet basis & $\mathrm{mol} \%$ & $\mathrm{~mol} \%$ & $\mathrm{~mol} \%$ & $\mathrm{~mol} \%$ & $\mathrm{~mol} \%$ & $\mathrm{~mol} \%$ & $\mathrm{~mol} \%$ & $\mathrm{~mol} \%$ \\
\hline $\mathrm{H} 2 \mathrm{O}, \mathrm{mol} \%$ & & & & & $0.01 \%$ & $0.01 \%$ & & $100 \%$ \\
\hline $\mathrm{O} 2, \mathrm{~mol} \%$ & & & & & $21 \%$ & $21 \%$ & & \\
\hline $\mathrm{N} 2, \mathrm{~mol} \%$ & $100 \%$ & $100 \%$ & $100 \%$ & $100 \%$ & $79 \%$ & $79 \%$ & & \\
\hline $\mathrm{PM}, \mathrm{mg} / \mathrm{wscm}$ & $1.98 \mathrm{E}+01$ & $1.98 \mathrm{E}+01$ & $2.02 \mathrm{E}+01$ & $1.94 \mathrm{E}+01$ & $1.62 \mathrm{E}+01$ & $1.57 \mathrm{E}+01$ & & $1.17 \mathrm{E}+01$ \\
\hline & & & & & & & & \\
\hline $\mathrm{kg} / \mathrm{hr}$ & $4.04 \mathrm{E}+01$ & $4.04 \mathrm{E}+01$ & $2.09 \mathrm{E}+01$ & $5.23 \mathrm{E}-02$ & $1.43 E+00$ & $1.00 \mathrm{E}+00$ & $2.09 \mathrm{E}+00$ & $1.36 \mathrm{E}+02$ \\
\hline
\end{tabular}


Table A-22. Mass balance, Tank WM-189 waste.

\begin{tabular}{|c|c|c|c|c|c|c|c|}
\hline \begin{tabular}{|l|} 
PFD Number \\
\end{tabular} & PFD-2 & PFD-2 & PFD-2 & PFD-2 & PFD-2 & PFD-2 & PFD-2 \\
\hline Stream Number & 101 & 102 & 103 & 104 & 106 & 107 & 108 \\
\hline Stream Name & SBW & $\begin{array}{l}\text { Reformer } \\
\text { Feed }\end{array}$ & $\begin{array}{c}\text { Reformer } \\
\text { Off-gas }\end{array}$ & $\begin{array}{c}\text { Reformer } \\
\text { Off-gas } \\
\text { Cooled }\end{array}$ & Filter Drain & Bed Drain & $\begin{array}{l}\text { Cooled } \\
\text { Product }\end{array}$ \\
\hline Rate or Volume & $9.73 E+01$ & $1.67 \mathrm{E}+02$ & $2.23 E+05$ & $1.83 \mathrm{E}+05$ & $8.69 \mathrm{E}+00$ & $1.29 \mathrm{E}+02$ & $1.38 \mathrm{E}+02$ \\
\hline Volume Flow (standard, wet) & & & $5.40 \mathrm{E}+04$ & $5.55 \mathrm{E}+04$ & & & \\
\hline Volume Flow (standard, dry) ${ }^{*}$ & & & $3.73 \mathrm{E}+04$ & $3.88 \mathrm{E}+04$ & & & \\
\hline Rate Units & gal/hr & $\mathrm{gal} / \mathrm{hr}$ & $\mathrm{ft} 3 / \mathrm{hr}$ & $\mathrm{ft} 3 / \mathrm{hr}$ & $\mathrm{Ib} / \mathrm{hr}$ & $\mathrm{Ib} / \mathrm{hr}$ & $\mathrm{lb} / \mathrm{hr}$ \\
\hline Rate or Volume, metric & $3.68 \mathrm{E}+02$ & $6.34 \mathrm{E}+02$ & $1.53 \mathrm{E}+03$ & $1.57 \mathrm{E}+03$ & $3.94 \mathrm{E}+00$ & $5.86 \mathrm{E}+01$ & $6.26 \mathrm{E}+01$ \\
\hline Rate Units & $\mathrm{L} / \mathrm{hr}$ & $\mathrm{L} / \mathrm{hr}$ & wscm $/ \mathrm{hr}$ & wscm $/ \mathrm{hr}$ & $\mathrm{kg} / \mathrm{hr}$ & $\mathrm{kg} / \mathrm{hr}$ & $\mathrm{kg} / \mathrm{hr}$ \\
\hline Temperature, ${ }^{\circ} \mathrm{C}$ & 15 & 17 & 600 & 400 & 400 & 600 & 56 \\
\hline Temperature, ${ }^{\circ} \mathrm{F}$ & 59 & 63 & 1112 & 752 & 752 & 1112 & 133 \\
\hline Pressure, psia & 14.70 & 44.70 & 10.62 & 10.22 & 9.50 & 10.80 & 9.50 \\
\hline Specific Gravity & 1.28 & 1.28 & $3.30 \mathrm{E}-04$ & $4.10 \mathrm{E}-04$ & 0.79 & 1.46 & 1.42 \\
\hline Chemical Composition & Mol/liter & Mol/liter & Ib/wscf & Ib/wscf & Wt Frac & Wt Frac & Wt frac \\
\hline $\mathrm{H}+$ & $2.80 \mathrm{E}+00$ & $1.65 \mathrm{E}+00$ & $2.36 \mathrm{E}-06$ & $2.30 \mathrm{E}-06$ & $3.04 \mathrm{E}-05$ & $5.74 \mathrm{E}-05$ & $5.57 \mathrm{E}-05$ \\
\hline $\mathrm{Al}+3$ & $5.52 \mathrm{E}-01$ & $3.21 \mathrm{E}-01$ & $1.37 \mathrm{E}-05$ & $1.33 \mathrm{E}-05$ & $8.52 \mathrm{E}-02$ & $9.36 \mathrm{E}-02$ & $9.31 \mathrm{E}-02$ \\
\hline $\mathrm{Sb}+5$ & $1.64 \mathrm{E}-05$ & $9.56 \mathrm{E}-06$ & $1.74 \mathrm{E}-09$ & $1.69 \mathrm{E}-09$ & $1.08 \mathrm{E}-05$ & $1.19 \mathrm{E}-05$ & $1.18 \mathrm{E}-05$ \\
\hline$A s+3$ & $8.20 \mathrm{E}-05$ & $4.77 \mathrm{E}-05$ & $5.33 \mathrm{E}-09$ & $5.18 \mathrm{E}-09$ & $3.31 \mathrm{E}-05$ & $3.64 \mathrm{E}-05$ & $3.62 \mathrm{E}-05$ \\
\hline $\mathrm{Ba}+2$ & $6.23 \mathrm{E}-05$ & $3.62 \mathrm{E}-05$ & $6.73 \mathrm{E}-09$ & $6.55 \mathrm{E}-09$ & $4.18 \mathrm{E}-05$ & $5.09 \mathrm{E}-05$ & $5.04 \mathrm{E}-05$ \\
\hline $\mathrm{Be}+2$ & $2.03 \mathrm{E}-05$ & $1.18 \mathrm{E}-05$ & $1.44 \mathrm{E}-10$ & $1.40 \mathrm{E}-10$ & $8.92 \mathrm{E}-07$ & $1.09 \mathrm{E}-06$ & $1.08 \mathrm{E}-06$ \\
\hline$B+3$ & $1.66 \mathrm{E}-02$ & $9.67 \mathrm{E}-03$ & $1.56 \mathrm{E}-07$ & $1.52 \mathrm{E}-07$ & $9.69 \mathrm{E}-04$ & $1.07 \mathrm{E}-03$ & $1.06 \mathrm{E}-03$ \\
\hline$C d+2$ & $2.97 \mathrm{E}-03$ & $1.72 \mathrm{E}-03$ & $1.17 \mathrm{E}-06$ & $1.14 \mathrm{E}-06$ & $7.29 \mathrm{E}-03$ & $1.61 \mathrm{E}-03$ & $1.96 \mathrm{E}-03$ \\
\hline $\mathrm{Ca}+2$ & $5.57 \mathrm{E}-02$ & $3.24 \mathrm{E}-02$ & $1.76 \mathrm{E}-06$ & $1.71 \mathrm{E}-06$ & $1.09 \mathrm{E}-02$ & $1.33 \mathrm{E}-02$ & $1.32 \mathrm{E}-02$ \\
\hline $\mathrm{Cr}+3$ & $4.67 \mathrm{E}-03$ & $2.71 \mathrm{E}-03$ & $2.92 \mathrm{E}-07$ & $2.84 \mathrm{E}-07$ & $1.81 \mathrm{E}-03$ & $1.40 \mathrm{E}-03$ & $1.43 \mathrm{E}-03$ \\
\hline $\mathrm{Co}+2$ & $3.89 \mathrm{E}-05$ & $2.26 \mathrm{E}-05$ & 1.99E-09 & 1.93E-09 & $1.23 \mathrm{E}-05$ & $1.36 \mathrm{E}-05$ & $1.35 \mathrm{E}-05$ \\
\hline Cs+ & $5.55 \mathrm{E}-05$ & $3.22 \mathrm{E}-05$ & $4.31 \mathrm{E}-08$ & $4.20 \mathrm{E}-08$ & $2.68 \mathrm{E}-04$ & $2.83 \mathrm{E}-05$ & $4.34 \mathrm{E}-05$ \\
\hline $\mathrm{Cu}+2$ & $8.00 \mathrm{E}-04$ & $4.65 \mathrm{E}-04$ & $4.00 \mathrm{E}-08$ & $3.89 \mathrm{E}-08$ & $2.48 \mathrm{E}-04$ & $3.03 \mathrm{E}-04$ & $2.99 \mathrm{E}-04$ \\
\hline $\mathrm{Fe}+3$ & $2.70 \mathrm{E}-02$ & $1.57 \mathrm{E}-02$ & $1.92 \mathrm{E}-06$ & $1.87 \mathrm{E}-06$ & $1.19 \mathrm{E}-02$ & $8.67 \mathrm{E}-03$ & $8.87 \mathrm{E}-03$ \\
\hline $\mathrm{Pb}+2$ & $9.06 \mathrm{E}-04$ & $5.26 \mathrm{E}-04$ & $6.60 \mathrm{E}-07$ & $6.42 \mathrm{E}-07$ & $4.10 \mathrm{E}-03$ & $9.03 \mathrm{E}-04$ & $1.10 \mathrm{E}-03$ \\
\hline $\mathrm{Hg}+2$ & $4.95 \mathrm{E}-03$ & $5.35 \mathrm{E}-03$ & $2.77 \mathrm{E}-05$ & $2.70 \mathrm{E}-05$ & $1.72 \mathrm{E}-04$ & $1.16 \mathrm{E}-05$ & $2.17 \mathrm{E}-05$ \\
\hline $\mathrm{Mn}+4$ & $1.50 \mathrm{E}-02$ & $8.71 \mathrm{E}-03$ & $7.14 \mathrm{E}-07$ & $6.95 \mathrm{E}-07$ & $4.44 \mathrm{E}-03$ & $4.88 \mathrm{E}-03$ & $4.85 \mathrm{E}-03$ \\
\hline $\mathrm{Ni}+2$ & $1.90 \mathrm{E}-03$ & $1.10 \mathrm{E}-03$ & $8.75 \mathrm{E}-08$ & $8.52 \mathrm{E}-08$ & $5.44 \mathrm{E}-04$ & $6.63 \mathrm{E}-04$ & $6.55 \mathrm{E}-04$ \\
\hline $\mathrm{K}+$ & $1.84 \mathrm{E}-01$ & 1.07E-01 & 9.19E-06 & $8.94 \mathrm{E}-06$ & $5.71 \mathrm{E}-02$ & $4.13 \mathrm{E}-02$ & $4.23 \mathrm{E}-02$ \\
\hline $\mathrm{Se}+4$ & $2.35 \mathrm{E}-04$ & $1.37 \mathrm{E}-04$ & $1.61 \mathrm{E}-08$ & $1.57 \mathrm{E}-08$ & $1.00 \mathrm{E}-04$ & $1.10 \mathrm{E}-04$ & $1.09 \mathrm{E}-04$ \\
\hline $\mathrm{Ag}+$ & $3.68 \mathrm{E}-04$ & $2.14 \mathrm{E}-04$ & $3.29 \mathrm{E}-08$ & $3.20 \mathrm{E}-08$ & $2.04 \mathrm{E}-04$ & $2.36 \mathrm{E}-04$ & $2.34 \mathrm{E}-04$ \\
\hline $\mathrm{Na}+$ & $1.55 \mathrm{E}+00$ & $9.00 \mathrm{E}-01$ & $2.95 \mathrm{E}-05$ & $2.87 \mathrm{E}-05$ & $1.83 \mathrm{E}-01$ & $2.11 \mathrm{E}-01$ & $2.10 \mathrm{E}-01$ \\
\hline $\mathrm{TI}+3$ & $4.33 \mathrm{E}-06$ & $2.52 \mathrm{E}-06$ & $7.67 \mathrm{E}-10$ & $7.47 \mathrm{E}-10$ & $4.77 \mathrm{E}-06$ & $5.24 \mathrm{E}-06$ & $5.21 \mathrm{E}-06$ \\
\hline $\mathrm{U}+4$ & $5.12 \mathrm{E}-04$ & $2.97 \mathrm{E}-04$ & $1.06 \mathrm{E}-07$ & $1.03 \mathrm{E}-07$ & $6.56 \mathrm{E}-04$ & $7.21 \mathrm{E}-04$ & 7.17E-04 \\
\hline $\mathrm{V}+3$ & $2.87 \mathrm{E}-05$ & 1.67E-05 & $1.27 \mathrm{E}-09$ & $1.23 \mathrm{E}-09$ & $7.88 \mathrm{E}-06$ & $8.67 \mathrm{E}-06$ & $8.62 \mathrm{E}-06$ \\
\hline$Z n+2$ & $8.57 \mathrm{E}-04$ & $4.98 \mathrm{E}-04$ & $4.41 \mathrm{E}-08$ & $4.29 \mathrm{E}-08$ & $2.74 \mathrm{E}-04$ & $3.34 \mathrm{E}-04$ & $3.30 \mathrm{E}-04$ \\
\hline $\mathrm{Zr}+4$ & $3.53 \mathrm{E}-02$ & $2.05 \mathrm{E}-02$ & $2.79 \mathrm{E}-06$ & $2.72 \mathrm{E}-06$ & $1.74 \mathrm{E}-02$ & $1.91 \mathrm{E}-02$ & $1.90 \mathrm{E}-02$ \\
\hline $\mathrm{Cl}-$ & $1.86 \mathrm{E}-02$ & $1.08 \mathrm{E}-02$ & $6.05 \mathrm{E}-07$ & $5.89 \mathrm{E}-07$ & $3.53 \mathrm{E}-03$ & $3.90 \mathrm{E}-03$ & $3.88 \mathrm{E}-03$ \\
\hline F- & $3.02 \mathrm{E}-02$ & $1.77 \mathrm{E}-02$ & $9.97 \mathrm{E}-07$ & $9.71 \mathrm{E}-07$ & $2.79 \mathrm{E}-03$ & $3.22 \mathrm{E}-03$ & $3.19 \mathrm{E}-03$ \\
\hline SO4-2 & $8.26 \mathrm{E}-02$ & $4.80 \mathrm{E}-02$ & $2.15 \mathrm{E}-12$ & $2.09 \mathrm{E}-12$ & $1.34 \mathrm{E}-08$ & $1.55 \mathrm{E}-08$ & $1.53 \mathrm{E}-08$ \\
\hline NO3- & $5.96 \mathrm{E}+00$ & $3.49 \mathrm{E}+00$ & $2.86 \mathrm{E}-06$ & $2.79 \mathrm{E}-06$ & $1.78 \mathrm{E}-02$ & $5.71 \mathrm{E}-03$ & $6.47 \mathrm{E}-03$ \\
\hline PO4-3 & $9.77 \mathrm{E}-02$ & $5.68 \mathrm{E}-02$ & $8.05 \mathrm{E}-06$ & $7.84 \mathrm{E}-06$ & $5.01 \mathrm{E}-02$ & $5.49 \mathrm{E}-02$ & $5.46 \mathrm{E}-02$ \\
\hline$A m+4$ & $5.94 \mathrm{E}-08$ & $3.45 \mathrm{E}-08$ & $1.25 \mathrm{E}-11$ & $1.22 \mathrm{E}-11$ & $7.77 \mathrm{E}-08$ & $8.55 \mathrm{E}-08$ & $8.50 \mathrm{E}-08$ \\
\hline $\mathrm{Br}-$ & $2.47 \mathrm{E}-07$ & $1.43 \mathrm{E}-07$ & $1.81 \mathrm{E}-11$ & $1.76 \mathrm{E}-11$ & $1.06 \mathrm{E}-07$ & $1.16 \mathrm{E}-07$ & 1.16E-07 \\
\hline $\mathrm{Ce}+4$ & $3.60 \mathrm{E}-05$ & $2.09 \mathrm{E}-05$ & $4.37 \mathrm{E}-09$ & $4.26 \mathrm{E}-09$ & $2.72 \mathrm{E}-05$ & $2.99 \mathrm{E}-05$ & $2.97 \mathrm{E}-05$ \\
\hline $\mathrm{Eu}+3$ & $4.10 \mathrm{E}-07$ & $2.38 \mathrm{E}-07$ & $5.40 \mathrm{E}-11$ & $5.25 \mathrm{E}-11$ & $3.35 \mathrm{E}-07$ & $3.69 \mathrm{E}-07$ & 3.67E-07 \\
\hline $\mathrm{Gd}+3$ & $1.23 \mathrm{E}-04$ & $7.15 \mathrm{E}-05$ & $1.68 \mathrm{E}-08$ & $1.63 \mathrm{E}-08$ & $1.04 \mathrm{E}-04$ & $1.15 \mathrm{E}-04$ & $1.14 \mathrm{E}-04$ \\
\hline $\mathrm{Ge}+4$ & $7.11 \mathrm{E}-09$ & $4.13 \mathrm{E}-09$ & $4.47 \mathrm{E}-13$ & $4.36 \mathrm{E}-13$ & $2.78 \mathrm{E}-09$ & $3.06 \mathrm{E}-09$ & 3.04E-09 \\
\hline $\ln +3$ & $1.14 \mathrm{E}-06$ & $6.63 \mathrm{E}-07$ & $1.14 \mathrm{E}-10$ & $1.11 \mathrm{E}-10$ & $7.06 \mathrm{E}-07$ & $7.76 \mathrm{E}-07$ & $7.72 \mathrm{E}-07$ \\
\hline $1-$ & $2.74 \mathrm{E}-06$ & $1.59 \mathrm{E}-06$ & $3.19 \mathrm{E}-10$ & $3.10 \mathrm{E}-10$ & $1.86 \mathrm{E}-06$ & $2.06 \mathrm{E}-06$ & $2.04 \mathrm{E}-06$ \\
\hline $\mathrm{La}+3$ & $7.43 \mathrm{E}-06$ & $4.32 \mathrm{E}-06$ & $8.95 \mathrm{E}-10$ & $8.71 \mathrm{E}-10$ & $5.56 \mathrm{E}-06$ & $6.12 \mathrm{E}-06$ & $6.08 \mathrm{E}-06$ \\
\hline
\end{tabular}


Table A-22. Mass balance, Tank WM-189 waste (continued).

\begin{tabular}{|c|c|c|c|c|c|c|c|}
\hline Stream \# & 101 & 102 & 103 & 104 & 106 & 107 & 108 \\
\hline Chemical Composition & Mol/liter & Mol/liter & $\mathrm{lb} / \mathrm{wscf}$ & $\mathrm{lb} / \mathrm{wscf}$ & Wt frac & Wt frac & Wt frac \\
\hline $\mathrm{Li}+$ & $4.93 \mathrm{E}-04$ & $2.86 \mathrm{E}-04$ & $2.83 \mathrm{E}-09$ & $2.76 \mathrm{E}-09$ & $1.76 \mathrm{E}-05$ & $2.03 E-05$ & $2.01 \mathrm{E}-05$ \\
\hline $\mathrm{Mg}+2$ & $1.76 \mathrm{E}-02$ & $1.02 \mathrm{E}-02$ & $3.36 \mathrm{E}-07$ & $3.28 \mathrm{E}-07$ & $2.09 \mathrm{E}-03$ & $2.55 \mathrm{E}-03$ & $2.52 \mathrm{E}-03$ \\
\hline $\mathrm{Mo}+6$ & $5.31 \mathrm{E}-02$ & $3.09 \mathrm{E}-02$ & $4.42 \mathrm{E}-06$ & $4.30 \mathrm{E}-06$ & $2.75 \mathrm{E}-02$ & $3.02 \mathrm{E}-02$ & $3.00 \mathrm{E}-02$ \\
\hline $\mathrm{Nd}+3$ & $2.40 \mathrm{E}-05$ & $1.39 \mathrm{E}-05$ & $3.00 \mathrm{E}-09$ & $2.92 \mathrm{E}-09$ & $1.86 \mathrm{E}-05$ & $2.05 \mathrm{E}-05$ & $2.04 \mathrm{E}-05$ \\
\hline $\mathrm{Np}+4$ & $2.49 \mathrm{E}-06$ & $1.45 \mathrm{E}-06$ & $5.12 \mathrm{E}-10$ & $4.98 \mathrm{E}-10$ & $3.18 \mathrm{E}-06$ & $3.50 \mathrm{E}-06$ & $3.48 \mathrm{E}-06$ \\
\hline $\mathrm{Nb}+5$ & $1.04 \mathrm{E}-03$ & $6.03 \mathrm{E}-04$ & $8.35 \mathrm{E}-08$ & $8.13 \mathrm{E}-08$ & $5.19 \mathrm{E}-04$ & $5.71 \mathrm{E}-04$ & $5.67 \mathrm{E}-04$ \\
\hline$\overline{P d+4}$ & $1.54 \mathrm{E}-03$ & $8.93 \mathrm{E}-04$ & $1.29 \mathrm{E}-07$ & $1.25 \mathrm{E}-07$ & $7.99 \mathrm{E}-04$ & $9.73 \mathrm{E}-04$ & $9.62 \mathrm{E}-04$ \\
\hline $\mathrm{Pu}+4$ & $6.03 \mathrm{E}-06$ & $3.51 \mathrm{E}-06$ & $1.28 \mathrm{E}-09$ & $1.24 \mathrm{E}-09$ & $7.93 \mathrm{E}-06$ & $8.72 \mathrm{E}-06$ & $8.67 \mathrm{E}-06$ \\
\hline $\mathrm{Pr}+4$ & $6.76 \mathrm{E}-06$ & $3.93 \mathrm{E}-06$ & $8.26 \mathrm{E}-10$ & $8.04 \mathrm{E}-10$ & $5.13 \mathrm{E}-06$ & $5.64 \mathrm{E}-06$ & $5.61 \mathrm{E}-06$ \\
\hline $\mathrm{Pm}+3$ & $1.36 \mathrm{E}-09$ & $7.89 \mathrm{E}-10$ & $1.71 \mathrm{E}-13$ & $1.66 \mathrm{E}-13$ & $1.06 \mathrm{E}-09$ & $1.17 \mathrm{E}-09$ & $1.16 \mathrm{E}-09$ \\
\hline $\mathrm{Rh}+4$ & $2.91 \mathrm{E}-06$ & 1.69E-06 & $2.60 \mathrm{E}-10$ & $2.53 \mathrm{E}-10$ & $1.62 \mathrm{E}-06$ & $1.78 \mathrm{E}-06$ & $1.77 \mathrm{E}-06$ \\
\hline $\mathrm{Rb}+$ & 4.49E-06 & $2.61 \mathrm{E}-06$ & $3.18 \mathrm{E}-10$ & $3.09 \mathrm{E}-10$ & $1.97 \mathrm{E}-06$ & $2.28 \mathrm{E}-06$ & $2.26 \mathrm{E}-06$ \\
\hline $\mathrm{Ru}+3$ & $8.05 \mathrm{E}-04$ & $4.68 \mathrm{E}-04$ & $7.05 \mathrm{E}-08$ & $6.87 \mathrm{E}-08$ & $4.38 \mathrm{E}-04$ & $4.82 \mathrm{E}-04$ & $4.79 \mathrm{E}-04$ \\
\hline $\mathrm{Sm}+3$ & $4.47 \mathrm{E}-06$ & $2.60 \mathrm{E}-06$ & $5.82 \mathrm{E}-10$ & $5.67 \mathrm{E}-10$ & $3.62 \mathrm{E}-06$ & $3.98 \mathrm{E}-06$ & $3.96 \mathrm{E}-06$ \\
\hline$\overline{S i+4}$ & $8.95 \mathrm{E}-02$ & $5.20 \mathrm{E}-02$ & $2.18 \mathrm{E}-06$ & $2.12 \mathrm{E}-06$ & $1.35 \mathrm{E}-02$ & $1.49 \mathrm{E}-02$ & $1.48 \mathrm{E}-02$ \\
\hline $\mathrm{Sr}+2$ & $1.08 \mathrm{E}-04$ & $6.29 \mathrm{E}-05$ & $7.45 \mathrm{E}-09$ & $7.26 \mathrm{E}-09$ & $4.63 \mathrm{E}-05$ & $5.64 \mathrm{E}-05$ & $5.58 \mathrm{E}-05$ \\
\hline $\mathrm{Tc}+7$ & $5.33 \mathrm{E}-06$ & $3.10 \mathrm{E}-06$ & $4.13 \mathrm{E}-09$ & $4.02 \mathrm{E}-09$ & $2.56 \mathrm{E}-05$ & $1.56 \mathrm{E}-06$ & $3.08 \mathrm{E}-06$ \\
\hline $\mathrm{Te}+4$ & $5.22 \mathrm{E}-06$ & $3.03 \mathrm{E}-06$ & $5.77 \mathrm{E}-10$ & $5.62 \mathrm{E}-10$ & $3.59 \mathrm{E}-06$ & $3.94 \mathrm{E}-06$ & $3.92 \mathrm{E}-06$ \\
\hline $\mathrm{Tb}+4$ & $1.71 \mathrm{E}-09$ & $9.95 \mathrm{E}-10$ & $2.36 \mathrm{E}-13$ & $2.29 \mathrm{E}-13$ & $1.47 \mathrm{E}-09$ & $1.61 \mathrm{E}-09$ & $1.60 \mathrm{E}-09$ \\
\hline$T h+4$ & $2.59 \mathrm{E}-05$ & $1.51 \mathrm{E}-05$ & $5.21 \mathrm{E}-09$ & $5.07 \mathrm{E}-09$ & $3.24 \mathrm{E}-05$ & $3.56 \mathrm{E}-05$ & $3.54 \mathrm{E}-05$ \\
\hline$S n+4$ & $1.75 \mathrm{E}-03$ & $1.02 \mathrm{E}-03$ & $1.80 \mathrm{E}-07$ & $1.75 \mathrm{E}-07$ & $1.12 \mathrm{E}-03$ & $1.23 \mathrm{E}-03$ & $1.22 \mathrm{E}-03$ \\
\hline $\mathrm{Ti+4}$ & $9.03 \mathrm{E}-04$ & $5.24 \mathrm{E}-04$ & $3.74 \mathrm{E}-08$ & $3.65 \mathrm{E}-08$ & $2.33 \mathrm{E}-04$ & $2.56 \mathrm{E}-04$ & $2.54 \mathrm{E}-04$ \\
\hline $\mathrm{Y}+3$ & $5.54 \mathrm{E}-06$ & $3.22 \mathrm{E}-06$ & $4.27 \mathrm{E}-10$ & $4.16 \mathrm{E}-10$ & $2.65 \mathrm{E}-06$ & $2.92 \mathrm{E}-06$ & $2.90 \mathrm{E}-06$ \\
\hline$\overline{\mathrm{OH}-}$ & & $4.11 \mathrm{E}-08$ & $3.26 \mathrm{E}-10$ & $3.18 \mathrm{E}-10$ & $2.03 \mathrm{E}-06$ & $1.47 \mathrm{E}-06$ & $1.50 \mathrm{E}-06$ \\
\hline $\mathrm{H} 2 \mathrm{O}$ & $4.43 \mathrm{E}+01$ & $2.74 \mathrm{E}+01$ & $1.45 \mathrm{E}-02$ & $1.41 \mathrm{E}-02$ & 1.33E-02 & $4.91 \mathrm{E}-03$ & $5.44 \mathrm{E}-03$ \\
\hline$\overline{\mathrm{SO} 2}$ & & $4.33 \mathrm{E}-08$ & $2.82 \mathrm{E}-12$ & $2.74 \mathrm{E}-12$ & & & \\
\hline $\mathrm{H} 2 \mathrm{~S}$ & & & $3.93 \mathrm{E}-05$ & $3.83 \mathrm{E}-05$ & & & \\
\hline $\mathrm{CO}$ & & $9.04 \mathrm{E}-11$ & $3.53 \mathrm{E}-04$ & $3.43 \mathrm{E}-04$ & & & \\
\hline $\mathrm{CO} 2$ & & $6.51 \mathrm{E}-06$ & $6.13 \mathrm{E}-02$ & $5.97 \mathrm{E}-02$ & & & \\
\hline $\mathrm{H} 2$ & & 1.16E-09 & $5.66 \mathrm{E}-04$ & $5.51 \mathrm{E}-04$ & & & \\
\hline N2 & & $2.13 \mathrm{E}-10$ & 1.26E-03 & $3.16 \mathrm{E}-03$ & & & \\
\hline $\mathrm{NO}$ & & $2.67 \mathrm{E}-09$ & $1.41 \mathrm{E}-04$ & $1.37 \mathrm{E}-04$ & & & \\
\hline NO2 & & $4.14 \mathrm{E}-07$ & $2.06 \mathrm{E}-06$ & $2.01 \mathrm{E}-06$ & & & \\
\hline $\mathrm{O} 2$ & & $5.69 \mathrm{E}-10$ & & & & & \\
\hline S (other) & & $6.48 \mathrm{E}-10$ & $1.54 \mathrm{E}-07$ & $1.50 \mathrm{E}-07$ & $9.59 \mathrm{E}-04$ & $1.11 \mathrm{E}-03$ & $1.10 \mathrm{E}-03$ \\
\hline $\mathrm{CO} 3$ & $2.77 \mathrm{E}-05$ & $1.61 \mathrm{E}-05$ & $4.79 \mathrm{E}-05$ & $4.66 \mathrm{E}-05$ & $2.98 \mathrm{E}-01$ & $3.26 \mathrm{E}-01$ & $3.24 \mathrm{E}-01$ \\
\hline \multicolumn{8}{|l|}{ C (reductant) } \\
\hline $\mathrm{O}$ (oxides) & $4.70 \mathrm{E}-01$ & $2.73 \mathrm{E}-01$ & $1.84 \mathrm{E}-05$ & $1.79 \mathrm{E}-05$ & $11.43 \%$ & $12.23 \%$ & $12.18 \%$ \\
\hline C (organic) & $1.10 \mathrm{E}-02$ & $2.52 \mathrm{E}+01$ & $5.53 \mathrm{E}-03$ & $5.38 \mathrm{E}-03$ & $5.52 \mathrm{E}-02$ & $8.56 \mathrm{E}-04$ & $4.28 \mathrm{E}-03$ \\
\hline $\mathrm{H}$ (organic) & $2.93 \mathrm{E}-02$ & $6.73 \mathrm{E}+01$ & $1.04 \mathrm{E}-03$ & $1.01 \mathrm{E}-03$ & $9.84 \mathrm{E}-03$ & $1.53 \mathrm{E}-04$ & $7.63 \mathrm{E}-04$ \\
\hline $\mathrm{O}$ (organic) & $3.66 \mathrm{E}-03$ & $8.41 \mathrm{E}+00$ & & & & & \\
\hline \\
\hline Mass Flow $(\mathrm{kg} / \mathrm{hr})$ : & $4.72 \mathrm{E}+02$ & $8.13 \mathrm{E}+02$ & $2.08 \mathrm{E}+03$ & $2.13 \mathrm{E}+03$ & $3.94 \mathrm{E}+00$ & $5.86 \mathrm{E}+01$ & $6.26 \mathrm{E}+01$ \\
\hline Canister Rate, canisters/day & & & & & & & 1.32 \\
\hline \multirow[t]{2}{*}{ Total Canisters Generated } & & & & & & & 179 \\
\hline & $\mathrm{w} / \mathrm{m} 3$ & $\mathrm{w} / \mathrm{m} 3$ & $\mathrm{w} / \mathrm{m} 3$ & $\mathrm{w} / \mathrm{m} 3$ & $\mathrm{w} / \mathrm{m} 3$ & $\mathrm{w} / \mathrm{m} 3$ & $\mathrm{w} / \mathrm{m} 3$ \\
\hline Heat Generation & $4.65 \mathrm{E}-01$ & $2.70 \mathrm{E}-01$ & & & $3.08 \mathrm{E}+00$ & $3.88 \mathrm{E}+00$ & $3.88 \mathrm{E}+00$ \\
\hline
\end{tabular}


Table A-22. Mass balance, Tank WM-189 waste (continued).

\begin{tabular}{|c|c|c|c|c|c|c|c|}
\hline Stream \# & 101 & 102 & 103 & 104 & 106 & 107 & 108 \\
\hline Radiological Composition & $\mathrm{Ci} / \mathrm{L}$ & $\mathrm{Ci} / \mathrm{L}$ & Ci/wscm & Ci/wscm & $\mathrm{Ci} / \mathrm{kg}$ & $\mathrm{Ci} / \mathrm{kg}$ & $\mathrm{Ci} / \mathrm{kg}$ \\
\hline $\mathrm{H}-3$ & $8.12 \mathrm{E}-06$ & $4.72 \mathrm{E}-06$ & $1.96 \mathrm{E}-06$ & $1.90 \mathrm{E}-06$ & & & \\
\hline C-14 & $1.31 \mathrm{E}-10$ & $7.60 \mathrm{E}-11$ & $3.13 \mathrm{E}-11$ & $3.04 \mathrm{E}-11$ & $9.99 \mathrm{E}-12$ & $5.76 \mathrm{E}-12$ & $6.02 \mathrm{E}-12$ \\
\hline Co-60 & $2.65 \mathrm{E}-05$ & $1.54 \mathrm{E}-05$ & $3.69 \mathrm{E}-07$ & $3.59 \mathrm{E}-07$ & $1.43 \mathrm{E}-04$ & $1.57 \mathrm{E}-04$ & $1.56 \mathrm{E}-04$ \\
\hline $\mathrm{Ni}-59$ & $1.11 \mathrm{E}-06$ & $6.47 \mathrm{E}-07$ & $1.40 \mathrm{E}-08$ & $1.37 \mathrm{E}-08$ & $5.44 \mathrm{E}-06$ & $6.63 \mathrm{E}-06$ & $6.56 \mathrm{E}-06$ \\
\hline $\mathrm{Ni}-63$ & $3.47 \mathrm{E}-05$ & $2.02 \mathrm{E}-05$ & $4.37 \mathrm{E}-07$ & $4.26 \mathrm{E}-07$ & $1.70 \mathrm{E}-04$ & $2.07 \mathrm{E}-04$ & $2.04 \mathrm{E}-04$ \\
\hline Se-79 & $4.75 \mathrm{E}-07$ & $2.76 \mathrm{E}-07$ & $6.59 \mathrm{E}-09$ & $6.41 \mathrm{E}-09$ & $2.56 \mathrm{E}-06$ & $2.81 \mathrm{E}-06$ & $2.79 \mathrm{E}-06$ \\
\hline Sr-90 & $3.15 \mathrm{E}-02$ & $1.83 \mathrm{E}-02$ & $3.96 \mathrm{E}-04$ & $3.86 \mathrm{E}-04$ & $1.54 \mathrm{E}-01$ & $1.87 \mathrm{E}-01$ & $1.85 \mathrm{E}-01$ \\
\hline $\mathrm{Y}-90$ & $3.15 \mathrm{E}-02$ & $1.83 \mathrm{E}-02$ & $4.37 \mathrm{E}-04$ & $4.26 \mathrm{E}-04$ & $1.70 \mathrm{E}-01$ & $1.87 \mathrm{E}-01$ & $1.85 \mathrm{E}-01$ \\
\hline Zr-93 & $1.76 \mathrm{E}-06$ & $1.02 \mathrm{E}-06$ & $2.45 \mathrm{E}-08$ & $2.38 \mathrm{E}-08$ & $9.49 \mathrm{E}-06$ & $1.04 \mathrm{E}-05$ & $1.04 \mathrm{E}-05$ \\
\hline $\mathrm{Nb}-93 \mathrm{~m}$ & $1.36 \mathrm{E}-06$ & $7.89 \mathrm{E}-07$ & $1.89 \mathrm{E}-08$ & $1.84 \mathrm{E}-08$ & $7.32 \mathrm{E}-06$ & $8.04 \mathrm{E}-06$ & $8.00 \mathrm{E}-06$ \\
\hline Nb-94 & $1.23 \mathrm{E}-06$ & $7.17 \mathrm{E}-07$ & $1.71 \mathrm{E}-08$ & $1.67 \mathrm{E}-08$ & $6.65 \mathrm{E}-06$ & $7.31 \mathrm{E}-06$ & $7.27 \mathrm{E}-06$ \\
\hline Tc-99 & $1.25 \mathrm{E}-05$ & $7.28 \mathrm{E}-06$ & $1.59 \mathrm{E}-06$ & $1.54 \mathrm{E}-06$ & $6.14 \mathrm{E}-04$ & $3.74 \mathrm{E}-05$ & $7.37 \mathrm{E}-05$ \\
\hline Ru-106 & $1.01 \mathrm{E}-06$ & $5.90 \mathrm{E}-07$ & $1.41 \mathrm{E}-08$ & $1.37 \mathrm{E}-08$ & $5.47 \mathrm{E}-06$ & $6.01 \mathrm{E}-06$ & $5.98 \mathrm{E}-06$ \\
\hline Rh-102 & $6.86 \mathrm{E}-10$ & $3.99 \mathrm{E}-10$ & $9.53 \mathrm{E}-12$ & $9.28 \mathrm{E}-12$ & 3.70E-09 & 4.06E-09 & 4.04E-09 \\
\hline Rh-106 & $1.01 \mathrm{E}-06$ & $5.90 \mathrm{E}-07$ & $1.41 \mathrm{E}-08$ & $1.37 \mathrm{E}-08$ & $5.47 \mathrm{E}-06$ & $6.01 \mathrm{E}-06$ & $5.98 \mathrm{E}-06$ \\
\hline $\mathrm{Pd}-107$ & $1.31 \mathrm{E}-08$ & $7.64 \mathrm{E}-09$ & $1.66 \mathrm{E}-10$ & $1.61 \mathrm{E}-10$ & $6.42 \mathrm{E}-08$ & $7.83 \mathrm{E}-08$ & $7.74 \mathrm{E}-08$ \\
\hline Cd-113m & $2.64 \mathrm{E}-06$ & $1.54 \mathrm{E}-06$ & $1.49 \mathrm{E}-07$ & $1.45 \mathrm{E}-07$ & $5.77 \mathrm{E}-05$ & $1.27 \mathrm{E}-05$ & $1.56 \mathrm{E}-05$ \\
\hline Sn-121m & $5.32 \mathrm{E}-08$ & $3.09 \mathrm{E}-08$ & $7.39 \mathrm{E}-10$ & $7.19 \mathrm{E}-10$ & $2.87 \mathrm{E}-07$ & $3.15 \mathrm{E}-07$ & $3.13 \mathrm{E}-07$ \\
\hline Sn-126 & $4.47 \mathrm{E}-07$ & $2.60 \mathrm{E}-07$ & $6.20 \mathrm{E}-09$ & $6.04 \mathrm{E}-09$ & $2.41 \mathrm{E}-06$ & $2.65 \mathrm{E}-06$ & $2.63 \mathrm{E}-06$ \\
\hline Sb-125 & $3.73 \mathrm{E}-04$ & $2.17 \mathrm{E}-04$ & $5.18 \mathrm{E}-06$ & $5.04 \mathrm{E}-06$ & $2.01 \mathrm{E}-03$ & $2.21 \mathrm{E}-03$ & $2.20 \mathrm{E}-03$ \\
\hline Sb-126 & $4.58 \mathrm{E}-08$ & $2.66 \mathrm{E}-08$ & $6.36 \mathrm{E}-10$ & $6.19 \mathrm{E}-10$ & $2.47 \mathrm{E}-07$ & $2.71 \mathrm{E}-07$ & $2.70 \mathrm{E}-07$ \\
\hline Te-125m & $2.51 \mathrm{E}-06$ & $1.46 \mathrm{E}-06$ & $3.48 \mathrm{E}-08$ & $3.39 \mathrm{E}-08$ & $1.35 \mathrm{E}-05$ & $1.48 \mathrm{E}-05$ & $1.48 \mathrm{E}-05$ \\
\hline $\mathrm{I}-129$ & $5.80 \mathrm{E}-08$ & $3.37 \mathrm{E}-08$ & $8.51 \mathrm{E}-10$ & $8.28 \mathrm{E}-10$ & $3.10 \mathrm{E}-07$ & $3.43 \mathrm{E}-07$ & $3.41 \mathrm{E}-07$ \\
\hline Cs-134 & $5.76 \mathrm{E}-05$ & $3.35 \mathrm{E}-05$ & 5.39E-06 & $5.25 \mathrm{E}-06$ & $2.09 \mathrm{E}-03$ & $2.21 \mathrm{E}-04$ & $3.39 \mathrm{E}-04$ \\
\hline Cs-135 & $9.08 \mathrm{E}-07$ & $5.28 \mathrm{E}-07$ & $8.51 \mathrm{E}-08$ & $8.28 \mathrm{E}-08$ & $3.30 \mathrm{E}-05$ & $3.49 \mathrm{E}-06$ & $5.35 \mathrm{E}-06$ \\
\hline Cs-137 & $4.70 \mathrm{E}-02$ & $2.73 \mathrm{E}-02$ & $4.41 \mathrm{E}-03$ & $4.29 \mathrm{E}-03$ & $1.71 \mathrm{E}+00$ & $1.81 \mathrm{E}-01$ & $2.77 \mathrm{E}-01$ \\
\hline Ba-137m & $4.45 \mathrm{E}-02$ & $2.58 \mathrm{E}-02$ & $5.60 \mathrm{E}-04$ & $5.45 \mathrm{E}-04$ & $2.17 \mathrm{E}-01$ & $2.65 \mathrm{E}-01$ & $2.62 \mathrm{E}-01$ \\
\hline Ce-144 & $6.85 \mathrm{E}-07$ & $3.98 \mathrm{E}-07$ & $9.52 \mathrm{E}-09$ & $9.26 \mathrm{E}-09$ & $3.69 \mathrm{E}-06$ & $4.06 \mathrm{E}-06$ & $4.04 \mathrm{E}-06$ \\
\hline Pr-144 & $6.85 \mathrm{E}-07$ & $3.98 \mathrm{E}-07$ & $9.52 \mathrm{E}-09$ & $9.26 \mathrm{E}-09$ & $3.69 \mathrm{E}-06$ & $4.06 \mathrm{E}-06$ & $4.04 \mathrm{E}-06$ \\
\hline $\mathrm{Pm}-146$ & $4.05 \mathrm{E}-08$ & $2.35 \mathrm{E}-08$ & $5.63 \mathrm{E}-10$ & $5.48 \mathrm{E}-10$ & $2.18 \mathrm{E}-07$ & $2.40 \mathrm{E}-07$ & $2.39 \mathrm{E}-07$ \\
\hline $\mathrm{Pm}-147$ & $1.85 \mathrm{E}-04$ & $1.08 \mathrm{E}-04$ & $2.57 \mathrm{E}-06$ & $2.50 \mathrm{E}-06$ & $9.96 \mathrm{E}-04$ & $1.10 \mathrm{E}-03$ & $1.09 \mathrm{E}-03$ \\
\hline Sm-151 & $3.66 \mathrm{E}-04$ & $2.13 \mathrm{E}-04$ & $5.08 \mathrm{E}-06$ & $4.94 \mathrm{E}-06$ & $1.97 \mathrm{E}-03$ & $2.17 \mathrm{E}-03$ & $2.15 \mathrm{E}-03$ \\
\hline Eu-152 & $2.35 \mathrm{E}-06$ & $1.36 \mathrm{E}-06$ & $3.26 \mathrm{E}-08$ & $3.17 \mathrm{E}-08$ & $1.26 \mathrm{E}-05$ & 1.39E-05 & 1.38E-05 \\
\hline Eu-154 & $1.40 \mathrm{E}-04$ & $8.14 \mathrm{E}-05$ & $1.95 \mathrm{E}-06$ & $1.89 \mathrm{E}-06$ & $7.55 \mathrm{E}-04$ & $8.30 \mathrm{E}-04$ & $8.25 \mathrm{E}-04$ \\
\hline Eu-155 & $1.32 \mathrm{E}-04$ & $7.67 \mathrm{E}-05$ & $1.83 \mathrm{E}-06$ & $1.78 \mathrm{E}-06$ & $7.10 \mathrm{E}-04$ & $7.81 \mathrm{E}-04$ & $7.77 \mathrm{E}-04$ \\
\hline Th-230 & $8.63 \mathrm{E}-10$ & $5.01 \mathrm{E}-10$ & $1.20 \mathrm{E}-11$ & $1.17 \mathrm{E}-11$ & $4.65 \mathrm{E}-09$ & 5.11E-09 & $5.08 \mathrm{E}-09$ \\
\hline Pa-233 & $2.33 \mathrm{E}-06$ & $1.36 \mathrm{E}-06$ & $3.24 \mathrm{E}-08$ & $3.15 \mathrm{E}-08$ & $1.26 \mathrm{E}-05$ & $1.38 \mathrm{E}-05$ & $1.37 \mathrm{E}-05$ \\
\hline $\mathrm{U}-232$ & $2.02 \mathrm{E}-09$ & $1.17 \mathrm{E}-09$ & $2.80 \mathrm{E}-11$ & $2.73 \mathrm{E}-11$ & $1.09 \mathrm{E}-08$ & $1.20 \mathrm{E}-08$ & $1.19 \mathrm{E}-08$ \\
\hline $\mathrm{U}-233$ & $7.12 \mathrm{E}-11$ & $4.13 \mathrm{E}-11$ & $9.88 \mathrm{E}-13$ & $9.61 \mathrm{E}-13$ & $3.83 \mathrm{E}-10$ & $4.21 \mathrm{E}-10$ & $4.19 \mathrm{E}-10$ \\
\hline $\mathrm{U}-234$ & $1.40 \mathrm{E}-06$ & $8.11 \mathrm{E}-07$ & $1.94 \mathrm{E}-08$ & $1.89 \mathrm{E}-08$ & $7.52 \mathrm{E}-06$ & $8.27 \mathrm{E}-06$ & $8.22 \mathrm{E}-06$ \\
\hline $\mathrm{U}-235$ & $5.63 \mathrm{E}-08$ & $3.27 \mathrm{E}-08$ & $7.82 \mathrm{E}-10$ & $7.61 \mathrm{E}-10$ & $3.03 \mathrm{E}-07$ & $3.33 \mathrm{E}-07$ & $3.32 \mathrm{E}-07$ \\
\hline $\mathrm{U}-236$ & $6.53 \mathrm{E}-08$ & $3.79 \mathrm{E}-08$ & $9.07 \mathrm{E}-10$ & $8.82 \mathrm{E}-10$ & $3.52 \mathrm{E}-07$ & $3.87 \mathrm{E}-07$ & $3.84 \mathrm{E}-07$ \\
\hline $\mathrm{U}-237$ & $5.12 \mathrm{E}-09$ & $2.98 \mathrm{E}-09$ & $7.11 \mathrm{E}-11$ & $6.92 \mathrm{E}-11$ & $2.76 \mathrm{E}-08$ & $3.03 \mathrm{E}-08$ & $3.01 \mathrm{E}-08$ \\
\hline $\mathrm{U}-238$ & $3.10 \mathrm{E}-08$ & $1.80 \mathrm{E}-08$ & $4.30 \mathrm{E}-10$ & $4.19 \mathrm{E}-10$ & $1.67 \mathrm{E}-07$ & $1.83 \mathrm{E}-07$ & $1.82 \mathrm{E}-07$ \\
\hline $\mathrm{Np}-237$ & $4.16 \mathrm{E}-07$ & $2.42 \mathrm{E}-07$ & 5.77E-09 & $5.62 \mathrm{E}-09$ & $2.24 \mathrm{E}-06$ & $2.46 \mathrm{E}-06$ & $2.45 \mathrm{E}-06$ \\
\hline Pu-236 & $3.29 \mathrm{E}-09$ & $1.91 \mathrm{E}-09$ & $4.57 \mathrm{E}-11$ & $4.45 \mathrm{E}-11$ & $1.77 \mathrm{E}-08$ & $1.95 \mathrm{E}-08$ & $1.94 \mathrm{E}-08$ \\
\hline Pu-238 & $7.60 \mathrm{E}-04$ & $4.42 \mathrm{E}-04$ & $1.05 \mathrm{E}-05$ & $1.03 \mathrm{E}-05$ & $4.09 \mathrm{E}-03$ & $4.50 \mathrm{E}-03$ & $4.47 \mathrm{E}-03$ \\
\hline Pu-239 & $8.30 \mathrm{E}-05$ & $4.82 \mathrm{E}-05$ & $1.15 \mathrm{E}-06$ & $1.12 \mathrm{E}-06$ & $4.47 \mathrm{E}-04$ & $4.91 \mathrm{E}-04$ & $4.89 \mathrm{E}-04$ \\
\hline Pu-240 & $1.23 \mathrm{E}-05$ & $7.13 \mathrm{E}-06$ & $1.70 \mathrm{E}-07$ & $1.66 \mathrm{E}-07$ & $6.61 \mathrm{E}-05$ & $7.27 \mathrm{E}-05$ & $7.22 \mathrm{E}-05$ \\
\hline Pu-241 & $6.65 \mathrm{E}-04$ & $3.86 \mathrm{E}-04$ & $9.23 \mathrm{E}-06$ & $8.98 \mathrm{E}-06$ & $3.58 \mathrm{E}-03$ & $3.94 \mathrm{E}-03$ & $3.91 \mathrm{E}-03$ \\
\hline Pu-242 & $9.61 \mathrm{E}-09$ & $5.58 \mathrm{E}-09$ & $1.33 \mathrm{E}-10$ & $1.30 \mathrm{E}-10$ & $5.17 \mathrm{E}-08$ & $5.69 \mathrm{E}-08$ & $5.66 \mathrm{E}-08$ \\
\hline Pu-244 & $2.86 \mathrm{E}-16$ & $1.66 \mathrm{E}-16$ & $3.98 \mathrm{E}-18$ & $3.87 \mathrm{E}-18$ & $1.54 \mathrm{E}-15$ & $1.70 \mathrm{E}-15$ & $1.69 \mathrm{E}-15$ \\
\hline Am-241 & $6.48 \mathrm{E}-05$ & 3.77E-05 & $9.00 \mathrm{E}-07$ & $8.76 \mathrm{E}-07$ & $3.49 \mathrm{E}-04$ & 3.84E-04 & $3.82 \mathrm{E}-04$ \\
\hline Am-242m & $1.22 \mathrm{E}-08$ & $7.07 \mathrm{E}-09$ & $1.69 \mathrm{E}-10$ & $1.64 \mathrm{E}-10$ & $6.55 \mathrm{E}-08$ & $7.21 \mathrm{E}-08$ & $7.16 \mathrm{E}-08$ \\
\hline
\end{tabular}


Table A-22. Mass balance, Tank WM-189 waste (continued).

\begin{tabular}{|c|c|c|c|c|c|c|c|}
\hline Stream \# & 101 & 102 & 103 & 104 & 106 & 107 & 108 \\
\hline Radiological Composition & $\mathrm{Ci} / \mathrm{L}$ & $\mathrm{Ci} / \mathrm{L}$ & $\mathrm{Ci} / \mathrm{wscm}$ & Ci/wscm & $\mathrm{Ci} / \mathrm{kg}$ & $\mathrm{Ci} / \mathrm{kg}$ & $\mathrm{Ci} / \mathrm{kg}$ \\
\hline $\mathrm{Am}-243$ & $2.07 E-08$ & $1.21 \mathrm{E}-08$ & $2.88 \mathrm{E}-10$ & $2.80 \mathrm{E}-10$ & $1.12 \mathrm{E}-07$ & $1.23 \mathrm{E}-07$ & $1.22 \mathrm{E}-07$ \\
\hline $\mathrm{Cm}-242$ & $2.43 \mathrm{E}-08$ & $1.41 \mathrm{E}-08$ & $3.37 \mathrm{E}-10$ & $3.28 \mathrm{E}-10$ & $1.31 \mathrm{E}-07$ & $1.44 \mathrm{E}-07$ & $1.43 \mathrm{E}-07$ \\
\hline $\mathrm{Cm}-243$ & 2.99E-08 & $1.74 \mathrm{E}-08$ & $4.15 \mathrm{E}-10$ & $4.04 \mathrm{E}-10$ & $1.61 \mathrm{E}-07$ & $1.77 \mathrm{E}-07$ & $1.76 \mathrm{E}-07$ \\
\hline $\mathrm{Cm}-244$ & $1.27 \mathrm{E}-06$ & 7.35E-07 & $1.76 \mathrm{E}-08$ & $1.71 \mathrm{E}-08$ & $6.82 \mathrm{E}-06$ & $7.49 \mathrm{E}-06$ & 7.45E-06 \\
\hline $\mathrm{Cm}-245$ & $3.15 \mathrm{E}-10$ & $1.83 \mathrm{E}-10$ & $4.37 \mathrm{E}-12$ & $4.25 \mathrm{E}-12$ & 1.69E-09 & 1.86E-09 & 1.85E-09 \\
\hline $\mathrm{Cm}-246$ & $2.06 \mathrm{E}-11$ & $1.20 \mathrm{E}-11$ & $2.87 \mathrm{E}-13$ & $2.79 \mathrm{E}-13$ & $1.11 \mathrm{E}-10$ & $1.22 \mathrm{E}-10$ & $1.22 \mathrm{E}-10$ \\
\hline TRU & 9.41E-04 & $5.47 \mathrm{E}-04$ & $1.31 \mathrm{E}-05$ & 1.27E-05 & 5.07E-03 & $5.57 \mathrm{E}-03$ & $5.54 \mathrm{E}-03$ \\
\hline & & & & & & & \\
\hline & & & & & & & \\
\hline $\begin{array}{l}\text { Gas Stream Bulk } \\
\text { Composition (Wet Basis) }\end{array}$ & $\begin{array}{l}\text { mol\% or } \\
\text { ppmv }\end{array}$ & $\begin{array}{l}\text { mol\% or } \\
\text { ppmv }\end{array}$ & $\begin{array}{l}\text { mol\% or } \\
\text { ppmv }\end{array}$ & $\begin{array}{l}\text { mol\% or } \\
\text { ppmv }\end{array}$ & $\begin{array}{l}\text { mol\% or } \\
\text { ppmv }\end{array}$ & $\begin{array}{l}\text { mol\% or } \\
\text { ppmv }\end{array}$ & $\begin{array}{l}\text { mol\% or } \\
\text { ppmv }\end{array}$ \\
\hline $\mathrm{H} 2 \mathrm{O}, \mathrm{mol} \%$ & & & $30.94 \%$ & $30.12 \%$ & & & \\
\hline $\mathrm{O} 2, \mathrm{~mol} \%$ & & & & & & & \\
\hline $\mathrm{N} 2, \mathrm{~mol} \%$ & & & $1.74 \%$ & $4.35 \%$ & & & \\
\hline $\mathrm{H} 2, \mathrm{~mol} \%$ & & & $10.82 \%$ & $10.54 \%$ & & & \\
\hline $\mathrm{CO} 2, \mathrm{~mol} \%$ & & & $53.64 \%$ & $52.21 \%$ & & & \\
\hline COgas, ppmv & & & $4.85 \mathrm{E}+03$ & $4.72 \mathrm{E}+03$ & & & \\
\hline $\mathrm{NO}, \mathrm{ppmv}$ & & & $1.81 \mathrm{E}+03$ & $1.76 \mathrm{E}+03$ & & & \\
\hline NO2, ppmv & & & $1.73 \mathrm{E}+01$ & $1.68 \mathrm{E}+01$ & & & \\
\hline SO2, ppmv & & & 1.69E-05 & $1.65 \mathrm{E}-05$ & & & \\
\hline $\mathrm{Cl}, \mathrm{ppmv}$ & & & $6.57 \mathrm{E}+00$ & $6.40 \mathrm{E}+00$ & & & \\
\hline $\mathrm{F}, \mathrm{ppmv}$ & & & $2.02 \mathrm{E}+01$ & $1.97 \mathrm{E}+01$ & & & \\
\hline C (organic), ppmv & & & $1.77 \mathrm{E}+05$ & $1.73 \mathrm{E}+05$ & & & \\
\hline $\mathrm{H}$ (organic), ppmv & & & $3.97 \mathrm{E}+05$ & $3.86 \mathrm{E}+05$ & & & \\
\hline & & & & & & & \\
\hline $\mathrm{Hg}, \mathrm{ug} / \mathrm{wscm}$ & & & $4.44 \mathrm{E}+05$ & $4.33 \mathrm{E}+05$ & & & \\
\hline $\mathrm{PM}, \mathrm{mg} / \mathrm{wscm}$ & & & $1.08 \mathrm{E}+05$ & $1.05 \mathrm{E}+05$ & & & \\
\hline SVM, ug/wscm & & & $2.94 \mathrm{E}+04$ & $2.86 \mathrm{E}+04$ & & & \\
\hline LVM, ug/wscm & & & $4.76 \mathrm{E}+03$ & $4.64 \mathrm{E}+03$ & & & \\
\hline & & & & & & & \\
\hline $\begin{array}{l}\text { Gas Stream Bulk } \\
\text { Composition (Dry Basis) }\end{array}$ & $\mathrm{mol} \%$ & $\mathrm{~mol} \%$ & $\mathrm{~mol} \%$ & $\mathrm{~mol} \%$ & $\mathrm{~mol} \%$ & $\mathrm{~mol} \%$ & $\mathrm{~mol} \%$ \\
\hline $\mathrm{O} 2, \mathrm{~mol} \%$, dry basis & & & & & & & \\
\hline $\mathrm{N} 2, \mathrm{~mol} \%$, dry basis & & & $2.51 \%$ & $6.23 \%$ & & & \\
\hline $\mathrm{H} 2, \mathrm{~mol} \%$, dry basis & & & $15.67 \%$ & $15.08 \%$ & & & \\
\hline $\mathrm{CO} 2, \mathrm{~mol} \%$, dry basis & & & $77.67 \%$ & $74.71 \%$ & & & \\
\hline $\begin{array}{l}\text { Gas Stream Bulk } \\
\text { Composition (Dry Basis, } \\
\text { Corrected to } 7 \% \text { O2 with } \\
100 \% \text { O2 Combustion Air) }\end{array}$ & $\begin{array}{l}\mathrm{ppmv}, \text { or } \\
\mathrm{ug} / \mathrm{dscm} \\
\text { or } \\
\mathrm{mg} / \mathrm{dscm}\end{array}$ & $\begin{array}{l}\text { ppmv, or } \\
\text { ug/dscm } \\
\text { or } \\
\text { mg/dscm }\end{array}$ & $\begin{array}{l}\mathrm{ppmv}, \text { or } \\
\text { ug/dscm } \\
\text { or } \\
\mathrm{mg} / \mathrm{dscm}\end{array}$ & $\begin{array}{l}\mathrm{ppmv}, \text { or } \\
\text { ug/dscm } \\
\text { or } \\
\mathrm{mg} / \mathrm{dscm}\end{array}$ & $\begin{array}{l}\text { ppmv, or } \\
\text { ug/dscm or } \\
\text { mg/dscm }\end{array}$ & $\begin{array}{l}\text { ppmv, or } \\
\text { ug/dscm or } \\
\text { mg/dscm }\end{array}$ & $\begin{array}{l}\text { ppmv, or } \\
\text { ug/dscm or } \\
\text { mg/dscm }\end{array}$ \\
\hline COgas, ppmv, dry basis & & & $9.83 \mathrm{E}+02$ & $9.46 \mathrm{E}+02$ & & & \\
\hline $\mathrm{NO}$, ppmv, dry basis & & & $3.67 \mathrm{E}+02$ & $3.53 \mathrm{E}+02$ & & & \\
\hline NO2, ppmv, dry basis & & & $3.50 \mathrm{E}+00$ & $3.37 \mathrm{E}+00$ & & & \\
\hline SO2, ppmv, dry basis & & & $3.43 E-06$ & $3.30 \mathrm{E}-06$ & & & \\
\hline $\mathrm{Cl}, \mathrm{ppmv}$, dry basis & & & $1.33 \mathrm{E}+00$ & $1.28 \mathrm{E}+00$ & & & \\
\hline F, ppmv, dry basis & & & $4.10 \mathrm{E}+00$ & $3.94 \mathrm{E}+00$ & & & \\
\hline C (organic), ppmv, dry basis & & & $3.59 \mathrm{E}+04$ & $3.46 \mathrm{E}+04$ & & & \\
\hline $\mathrm{H}$ (organic), ppmv, dry basis & & & $8.04 \mathrm{E}+04$ & $7.73 \mathrm{E}+04$ & & & \\
\hline $\mathrm{Hg}, \mathrm{ug} / \mathrm{dscm}$ & & & $9.01 \mathrm{E}+04$ & $8.66 \mathrm{E}+04$ & & & \\
\hline $\mathrm{PM}, \mathrm{mg} / \mathrm{dscm}$ & & & $2.19 \mathrm{E}+04$ & $2.10 \mathrm{E}+04$ & & & \\
\hline SVM, ug/dscm & & & $4.25 \mathrm{E}+04$ & $4.14 \mathrm{E}+04$ & & & \\
\hline LVM, ug/dscm & & & $6.90 \mathrm{E}+03$ & $6.71 \mathrm{E}+03$ & & & \\
\hline
\end{tabular}


Table A-22. Mass balance, Tank WM-189 waste (continued).

\begin{tabular}{|c|c|c|c|c|c|c|c|c|}
\hline \begin{tabular}{|l|} 
PFD Number \\
\end{tabular} & PFD-2 & PFD-2 & PFD-3 & PFD-3 & PFD-3 & PFD-3 & PFD-3 & PFD-3 \\
\hline Stream Number & 108 & 109 & 110 & 111 & 112 & $113 \mathrm{~A}$ & $113 \mathrm{~B}$ & 114 \\
\hline Stream Name & $\begin{array}{l}\text { Product } \\
\text { Shipping } \\
\text { Canisters }\end{array}$ & $\begin{array}{c}\text { Off-Gas } \\
\text { from Filter } \\
\text { to Oxidizer }\end{array}$ & $\begin{array}{l}\text { Outlet of } \\
\text { Oxidizer }\end{array}$ & $\begin{array}{c}\text { Quenched } \\
\text { Oxidizer } \\
\text { Off-gas }\end{array}$ & Scrub & $\begin{array}{l}\text { Packed } \\
\text { Scrubber } \\
\text { Drain }\end{array}$ & $\begin{array}{l}\text { Demister } \\
\text { Drain }\end{array}$ & $\begin{array}{l}\text { Off-Gas to } \\
\text { Demister }\end{array}$ \\
\hline Rate or Volume & $1.32 \mathrm{E}+00$ & $1.99 \mathrm{E}+05$ & $6.53 \mathrm{E}+05$ & $3.61 \mathrm{E}+05$ & $9.89 \mathrm{E}+03$ & $9.91 \mathrm{E}+03$ & 0 & $3.63 \mathrm{E}+05$ \\
\hline Volume Flow (standard, wet) & & $5.61 \mathrm{E}+04$ & $9.61 \mathrm{E}+04$ & $1.79 \mathrm{E}+05$ & & & & $1.77 \mathrm{E}+05$ \\
\hline Volume Flow (standard, dry) & & $3.94 \mathrm{E}+04$ & $5.26 \mathrm{E}+04$ & $5.26 \mathrm{E}+04$ & & & & $5.24 \mathrm{E}+04$ \\
\hline Rate Units & cont/day & $\mathrm{ft} 3 / \mathrm{hr}$ & $\mathrm{ft} 3 / \mathrm{hr}$ & $\mathrm{ft} 3 / \mathrm{hr}$ & $\mathrm{gal} / \mathrm{hr}$ & $\mathrm{gal} / \mathrm{hr}$ & $\mathrm{gal} / \mathrm{hr}$ & $\mathrm{ft} 3 / \mathrm{hr}$ \\
\hline Rate or Volume, metric & $6.26 \mathrm{E}+01$ & $1.59 \mathrm{E}+03$ & $2.72 \mathrm{E}+03$ & $5.06 \mathrm{E}+03$ & $3.75 \mathrm{E}+04$ & $3.75 \mathrm{E}+04$ & 1 & $5.02 \mathrm{E}+03$ \\
\hline Rate Units & $\mathrm{kg} / \mathrm{hr}$ & wscm/hr & wscm/hr & wscm/hr & $\mathrm{L} / \mathrm{hr}$ & $\mathrm{L} / \mathrm{hr}$ & $\mathrm{L} / \mathrm{hr}$ & wscm/hr \\
\hline Temperature, ${ }^{\circ} \mathrm{C}$ & 56 & 397 & 1000 & 100 & 77 & 79 & 339 & 78 \\
\hline Temperature, ${ }^{\circ} \mathrm{F}$ & 133 & 747 & 1832 & 212 & 171 & 174 & 171 & 172 \\
\hline Pressure, psia & 9.50 & 9.50 & 9.39 & 9.28 & 37.52 & 8.96 & 8.42 & 8.60 \\
\hline Specific Gravity & 1.42 & $3.81 \mathrm{E}-04$ & $1.92 \mathrm{E}-04$ & $5.19 \mathrm{E}-04$ & 1.03 & 1.03 & 1.03 & $5.15 \mathrm{E}-04$ \\
\hline Chemical Composition & Wt frac & $\mathrm{lb} / \mathrm{wscf}$ & $\mathrm{lb} / \mathrm{wscf}$ & $\mathrm{lb} / \mathrm{wscf}$ & Mol/liter & Mol/liter & Mol/liter & Ib/wscf \\
\hline $\mathrm{H}+$ & $5.57 \mathrm{E}-05$ & $2.27 \mathrm{E}-06$ & $1.69 \mathrm{E}-08$ & $9.10 \mathrm{E}-09$ & $9.36 \mathrm{E}-01$ & $9.34 \mathrm{E}-01$ & & $1.20 \mathrm{E}-07$ \\
\hline $\mathrm{Al}+3$ & $9.31 \mathrm{E}-02$ & & & & $9.19 \mathrm{E}-03$ & $9.17 \mathrm{E}-03$ & & \\
\hline $\mathrm{Sb}+5$ & $1.18 \mathrm{E}-05$ & & & & $2.58 \mathrm{E}-07$ & $2.57 \mathrm{E}-07$ & & \\
\hline$A s+3$ & $3.62 \mathrm{E}-05$ & & & & $1.29 \mathrm{E}-06$ & $1.28 \mathrm{E}-06$ & & \\
\hline $\mathrm{Ba}+2$ & $5.04 \mathrm{E}-05$ & & & & & & & \\
\hline $\mathrm{Be}+2$ & $1.08 \mathrm{E}-06$ & & & & & & & \\
\hline $\mathrm{B}+3$ & $1.06 \mathrm{E}-03$ & & & & $2.61 \mathrm{E}-04$ & $2.60 \mathrm{E}-04$ & & \\
\hline $\mathrm{Cd}+2$ & $1.96 \mathrm{E}-03$ & & & & & & & \\
\hline $\mathrm{Ca}+2$ & $1.32 \mathrm{E}-02$ & & & & & & & \\
\hline $\mathrm{Cr}+3$ & $1.43 \mathrm{E}-03$ & & & & & & & \\
\hline $\mathrm{Co}+2$ & $1.35 \mathrm{E}-05$ & & & & $6.10 \mathrm{E}-07$ & $6.09 \mathrm{E}-07$ & & \\
\hline Cs+ & $4.34 \mathrm{E}-05$ & & & & & & & \\
\hline $\mathrm{Cu}+2$ & $2.99 \mathrm{E}-04$ & & & & & & & \\
\hline $\mathrm{Fe}+3$ & $8.87 \mathrm{E}-03$ & $5.79 \mathrm{E}-09$ & $3.38 \mathrm{E}-09$ & $1.82 \mathrm{E}-09$ & $1.10 \mathrm{E}-04$ & $1.10 \mathrm{E}-04$ & & \\
\hline $\mathrm{Pb}+2$ & $1.10 \mathrm{E}-03$ & & & & & & & \\
\hline $\mathrm{Hg}+2$ & $2.17 \mathrm{E}-05$ & $2.67 \mathrm{E}-05$ & $1.56 \mathrm{E}-05$ & $8.37 \mathrm{E}-06$ & $7.92 \mathrm{E}-02$ & $7.91 \mathrm{E}-02$ & & $4.33 \mathrm{E}-06$ \\
\hline $\mathrm{Mn+4}$ & $4.85 \mathrm{E}-03$ & & & & $2.35 \mathrm{E}-04$ & $2.35 \mathrm{E}-04$ & & \\
\hline $\mathrm{Ni}+2$ & $6.55 \mathrm{E}-04$ & & & & & & & \\
\hline $\mathrm{K}+$ & $4.23 \mathrm{E}-02$ & & & & & & & \\
\hline $\mathrm{Se}+4$ & $1.09 \mathrm{E}-04$ & & & & & & & \\
\hline $\mathrm{Ag}+$ & $2.34 \mathrm{E}-04$ & & & & & & & \\
\hline $\mathrm{Na}+$ & $2.10 \mathrm{E}-01$ & & & & & & & \\
\hline $\mathrm{Tl}+3$ & $5.21 \mathrm{E}-06$ & & & & $6.79 \mathrm{E}-08$ & $6.78 \mathrm{E}-08$ & & \\
\hline $\mathrm{U}+4$ & $7.17 \mathrm{E}-04$ & & & & & & & \\
\hline $\mathrm{V}+3$ & $8.62 \mathrm{E}-06$ & & & & & & & \\
\hline$Z n+2$ & $3.30 \mathrm{E}-04$ & & & & & & & \\
\hline $\mathrm{Zr}+4$ & $1.90 \mathrm{E}-02$ & & & & & & & \\
\hline $\mathrm{Cl}-$ & $3.88 \mathrm{E}-03$ & $3.49 \mathrm{E}-08$ & $2.04 \mathrm{E}-08$ & $1.10 \mathrm{E}-08$ & $4.00 \mathrm{E}-04$ & $4.00 \mathrm{E}-04$ & & $6.72 \mathrm{E}-09$ \\
\hline F- & $3.19 \mathrm{E}-03$ & $5.28 \mathrm{E}-07$ & $3.08 \mathrm{E}-07$ & $1.66 \mathrm{E}-07$ & $4.86 \mathrm{E}-03$ & $4.86 \mathrm{E}-03$ & & $1.46 \mathrm{E}-07$ \\
\hline SO4-2 & $1.53 \mathrm{E}-08$ & & $2.54 \mathrm{E}-10$ & $1.37 \mathrm{E}-10$ & $4.51 \mathrm{E}-06$ & $4.50 \mathrm{E}-06$ & & \\
\hline NO3- & $6.47 \mathrm{E}-03$ & $1.06 \mathrm{E}-11$ & $6.21 \mathrm{E}-12$ & $3.34 \mathrm{E}-12$ & $8.98 \mathrm{E}-01$ & $8.96 \mathrm{E}-01$ & & $6.87 \mathrm{E}-06$ \\
\hline PO4-3 & $5.46 \mathrm{E}-02$ & & & & & & & \\
\hline$A m+4$ & $8.50 \mathrm{E}-08$ & & & & $9.31 \mathrm{E}-10$ & $9.30 \mathrm{E}-10$ & & \\
\hline $\mathrm{Br}-\mathrm{-}$ & $1.16 \mathrm{E}-07$ & $1.04 \mathrm{E}-12$ & $6.09 \mathrm{E}-13$ & $3.27 \mathrm{E}-13$ & $5.30 \mathrm{E}-09$ & $5.30 \mathrm{E}-09$ & & $2.01 \mathrm{E}-13$ \\
\hline $\mathrm{Ce}+4$ & $2.97 \mathrm{E}-05$ & & & & $5.65 \mathrm{E}-07$ & $5.64 \mathrm{E}-07$ & & \\
\hline $\mathrm{Eu}+3$ & $3.67 \mathrm{E}-07$ & & & & $6.43 \mathrm{E}-09$ & $6.42 \mathrm{E}-09$ & & \\
\hline $\mathrm{Gd}+3$ & $1.14 \mathrm{E}-04$ & & & & $1.93 \mathrm{E}-06$ & $1.93 \mathrm{E}-06$ & & \\
\hline $\mathrm{Ge}+4$ & $3.04 \mathrm{E}-09$ & & & & & & & \\
\hline $\ln +3$ & $7.72 \mathrm{E}-07$ & & & & $1.79 \mathrm{E}-08$ & $1.79 \mathrm{E}-08$ & & \\
\hline $\mid-$ & $2.04 \mathrm{E}-06$ & $1.84 \mathrm{E}-11$ & $1.07 \mathrm{E}-11$ & $5.77 \mathrm{E}-12$ & $5.89 \mathrm{E}-08$ & $5.89 \mathrm{E}-08$ & & $3.54 \mathrm{E}-12$ \\
\hline $\mathrm{La}+3$ & $6.08 \mathrm{E}-06$ & & & & 1.17E-07 & $1.16 \mathrm{E}-07$ & & \\
\hline
\end{tabular}


Table A-22. Mass balance, Tank WM-189 waste (continued).

\begin{tabular}{|c|c|c|c|c|c|c|c|c|}
\hline Stream \# & 108 & 109 & 110 & 111 & 112 & $113 \mathrm{~A}$ & $113 \mathrm{~B}$ & 114 \\
\hline Chemical Composition & Wt frac & $\mathrm{lb} / \mathrm{wscf}$ & $\mathrm{lb} / \mathrm{wscf}$ & Ib/wscf & Mol/liter & Mol/liter & Mol/liter & $\mathrm{lb} / \mathrm{wscf}$ \\
\hline $\mathrm{Li}+$ & $2.01 \mathrm{E}-05$ & & & & & & & \\
\hline $\mathrm{Mg}+2$ & $2.52 \mathrm{E}-03$ & & & & & & & \\
\hline $\mathrm{Mo}+6$ & $3.00 \mathrm{E}-02$ & & & & & & & \\
\hline $\mathrm{Nd}+3$ & $2.04 \mathrm{E}-05$ & & & & $3.76 \mathrm{E}-07$ & $3.75 \mathrm{E}-07$ & & \\
\hline $\mathrm{Np}+4$ & $3.48 \mathrm{E}-06$ & & & & $3.90 \mathrm{E}-08$ & $3.90 \mathrm{E}-08$ & & \\
\hline $\mathrm{Nb}+5$ & $5.67 \mathrm{E}-04$ & & & & & & & \\
\hline $\mathrm{Pd}+4$ & $9.62 \mathrm{E}-04$ & & & & & & & \\
\hline $\mathrm{Pu}+4$ & $8.67 \mathrm{E}-06$ & & & & & & & \\
\hline $\mathrm{Pr}+4$ & $5.61 \mathrm{E}-06$ & & & & $1.06 \mathrm{E}-07$ & $1.06 \mathrm{E}-07$ & & \\
\hline $\mathrm{Pm}+3$ & $1.16 \mathrm{E}-09$ & & & & $2.13 \mathrm{E}-11$ & $2.13 \mathrm{E}-11$ & & \\
\hline $\mathrm{Rh}+4$ & $1.77 \mathrm{E}-06$ & & & & $4.57 \mathrm{E}-08$ & $4.57 \mathrm{E}-08$ & & \\
\hline $\mathrm{Rb}+$ & $2.26 \mathrm{E}-06$ & & & & & & & \\
\hline $\mathrm{Ru}+3$ & $4.79 \mathrm{E}-04$ & & & & $1.26 \mathrm{E}-05$ & $1.26 \mathrm{E}-05$ & & \\
\hline $\mathrm{Sm}+3$ & $3.96 \mathrm{E}-06$ & & & & $7.01 \mathrm{E}-08$ & $7.00 \mathrm{E}-08$ & & \\
\hline$S i+4$ & $1.48 \mathrm{E}-02$ & & & & & & & \\
\hline $\mathrm{Sr}+2$ & $5.58 \mathrm{E}-05$ & & & & & & & \\
\hline $\mathrm{Tc}+7$ & $3.08 \mathrm{E}-06$ & $8.35 \mathrm{E}-12$ & $4.87 \mathrm{E}-12$ & $2.62 \mathrm{E}-12$ & $9.15 \mathrm{E}-08$ & $9.14 \mathrm{E}-08$ & & \\
\hline $\mathrm{Te}+4$ & $3.92 \mathrm{E}-06$ & & & & & & & \\
\hline $\mathrm{Tb}+4$ & $1.60 \mathrm{E}-09$ & & & & $2.68 \mathrm{E}-11$ & $2.68 \mathrm{E}-11$ & & \\
\hline $\mathrm{Th}+4$ & $3.54 \mathrm{E}-05$ & & & & & & & \\
\hline$S n+4$ & $1.22 \mathrm{E}-03$ & & & & & & & \\
\hline $\mathrm{Ti}+4$ & $2.54 \mathrm{E}-04$ & & & & & & & \\
\hline $\mathrm{Y}+3$ & $2.90 \mathrm{E}-06$ & & & & $8.69 \mathrm{E}-08$ & $8.68 \mathrm{E}-08$ & & \\
\hline $\mathrm{OH}-$ & $1.50 \mathrm{E}-06$ & & $1.79 \mathrm{E}-20$ & $3.69 \mathrm{E}-11$ & $1.32 \mathrm{E}-06$ & $1.31 \mathrm{E}-06$ & & \\
\hline $\mathrm{H} 2 \mathrm{O}$ & $5.44 \mathrm{E}-03$ & $1.39 \mathrm{E}-02$ & $2.12 \mathrm{E}-02$ & $3.30 \mathrm{E}-02$ & $5.30 \mathrm{E}+01$ & $5.29 \mathrm{E}+01$ & & $3.31 \mathrm{E}-02$ \\
\hline & & & & & & & & \\
\hline $\mathrm{SO} 2$ & & $2.71 \mathrm{E}-12$ & $4.15 \mathrm{E}-05$ & $2.23 \mathrm{E}-05$ & $1.39 \mathrm{E}-06$ & $1.46 \mathrm{E}-06$ & & $2.25 \mathrm{E}-05$ \\
\hline $\mathrm{H} 2 \mathrm{~S}$ & & $3.78 \mathrm{E}-05$ & $1.54 \mathrm{E}-20$ & $8.28 \mathrm{E}-21$ & & & & \\
\hline $\mathrm{CO}$ & & $3.39 \mathrm{E}-04$ & $2.29 \mathrm{E}-08$ & $1.23 \mathrm{E}-08$ & $2.90 \mathrm{E}-09$ & $2.99 \mathrm{E}-09$ & & $1.24 \mathrm{E}-08$ \\
\hline $\mathrm{CO} 2$ & & $5.90 \mathrm{E}-02$ & $5.53 \mathrm{E}-02$ & $2.97 \mathrm{E}-02$ & $2.08 \mathrm{E}-04$ & $3.14 \mathrm{E}-04$ & & $2.99 \mathrm{E}-02$ \\
\hline $\mathrm{H} 2$ & & $5.45 \mathrm{E}-04$ & $8.82 \mathrm{E}-10$ & $4.74 \mathrm{E}-10$ & $3.71 \mathrm{E}-08$ & $4.17 \mathrm{E}-08$ & & $3.75 \mathrm{E}-08$ \\
\hline N2 & & $3.97 \mathrm{E}-03$ & $2.36 \mathrm{E}-03$ & $1.27 \mathrm{E}-03$ & $6.83 \mathrm{E}-09$ & $2.72 \mathrm{E}-07$ & & $1.28 \mathrm{E}-03$ \\
\hline $\mathrm{NO}$ & & $1.36 \mathrm{E}-04$ & $1.94 \mathrm{E}-05$ & $1.04 \mathrm{E}-05$ & $8.55 \mathrm{E}-08$ & $1.37 \mathrm{E}-07$ & & $1.05 \mathrm{E}-05$ \\
\hline NO2 & & $1.98 \mathrm{E}-06$ & $1.16 \mathrm{E}-06$ & $6.23 \mathrm{E}-07$ & $1.33 \mathrm{E}-05$ & $1.33 \mathrm{E}-05$ & & $6.28 \mathrm{E}-07$ \\
\hline $\mathrm{O} 2$ & & & $2.50 \mathrm{E}-03$ & $1.34 \mathrm{E}-03$ & $1.82 \mathrm{E}-08$ & $4.34 \mathrm{E}-07$ & & $1.35 \mathrm{E}-03$ \\
\hline$S$ (other) & $1.10 \mathrm{E}-03$ & & & & $2.08 \mathrm{E}-08$ & $2.14 \mathrm{E}-08$ & & \\
\hline $\mathrm{CO} 3$ & $3.24 \mathrm{E}-01$ & & & & $1.23 \mathrm{E}-10$ & $1.85 \mathrm{E}-10$ & & \\
\hline $\mathrm{C}$ (reductant) & & & $2.57 \mathrm{E}-17$ & $1.38 \mathrm{E}-17$ & & & & \\
\hline $\mathrm{O}$ (oxides) & $12.18 \%$ & $2.49 \mathrm{E}-09$ & $1.46 \mathrm{E}-09$ & $7.82 \mathrm{E}-10$ & $1.65 \mathrm{E}-04$ & $1.65 \mathrm{E}-04$ & & \\
\hline C (organic) & & $5.31 \mathrm{E}-03$ & $4.92 \mathrm{E}-33$ & $2.64 \mathrm{E}-33$ & & & & \\
\hline $\mathrm{H}$ (organic) & & $9.97 \mathrm{E}-04$ & $1.65 \mathrm{E}-33$ & $8.88 \mathrm{E}-34$ & & & & \\
\hline \multicolumn{9}{|l|}{ O (organic) } \\
\hline & & & & & & & & \\
\hline Mass Flow $(\mathrm{kg} / \mathrm{hr}):$ & $6.26 \mathrm{E}+01$ & $2.15 \mathrm{E}+03$ & $3.55 E+03$ & $5.30 \mathrm{E}+03$ & $3.85 \mathrm{E}+04$ & $3.85 E+04$ & & $5.29 \mathrm{E}+03$ \\
\hline \multirow{2}{*}{\multicolumn{9}{|c|}{$\begin{array}{l}\text { Canister Rate, canisters/day } \\
\text { Total Canisters Generated }\end{array}$}} \\
\hline & & & & & & & & \\
\hline & & & & & & & & \\
\hline & w/Canister & $\mathrm{w} / \mathrm{m} 3$ & $\mathrm{w} / \mathrm{m} 3$ & $\mathrm{w} / \mathrm{m} 3$ & $\mathrm{w} / \mathrm{m} 3$ & $\mathrm{w} / \mathrm{m} 3$ & $\mathrm{w} / \mathrm{m} 3$ & $\mathrm{w} / \mathrm{m} 3$ \\
\hline Heat Generation & $3.10 \mathrm{E}+00$ & & & & $2.78 \mathrm{E}-03$ & $2.77 \mathrm{E}-03$ & & \\
\hline
\end{tabular}


Table A-22. Mass balance, Tank WM-189 waste (continued).

\begin{tabular}{|c|c|c|c|c|c|c|c|c|}
\hline Stream \# & 108 & 109 & 110 & 111 & 112 & $113 \mathrm{~A}$ & $113 \mathrm{~B}$ & 114 \\
\hline Radiological Composition & Ci/Canister & Ci/wscm & $\mathrm{Ci} / \mathrm{wscm}$ & Ci/wscm & $\mathrm{Ci} / \mathrm{L}$ & $\mathrm{Ci} / \mathrm{L}$ & $\mathrm{Ci} / \mathrm{L}$ & Ci/wscm \\
\hline $\mathrm{H}-3$ & & $1.88 \mathrm{E}-06$ & 1.10E-06 & 5.91E-07 & 1.02E-06 & 1.02E-06 & & 5.92E-07 \\
\hline $\mathrm{C}-14$ & $6.83 \mathrm{E}-09$ & $3.01 \mathrm{E}-11$ & $1.76 \mathrm{E}-11$ & $9.45 \mathrm{E}-12$ & $1.82 \mathrm{E}-16$ & $2.75 \mathrm{E}-16$ & & $9.52 \mathrm{E}-12$ \\
\hline Co-60 & $1.77 \mathrm{E}-01$ & & & & $4.16 \mathrm{E}-07$ & 4.16E-07 & & \\
\hline $\mathrm{Ni}-59$ & $7.44 \mathrm{E}-03$ & & & & & & & \\
\hline $\mathrm{Ni}-63$ & $2.32 \mathrm{E}-01$ & & & & & & & \\
\hline Se-79 & $3.17 \mathrm{E}-03$ & & & & & & & \\
\hline Sr-90 & $2.10 \mathrm{E}+02$ & & & & & & & \\
\hline $\mathrm{Y}-90$ & $2.10 \mathrm{E}+02$ & & & & $4.94 \mathrm{E}-04$ & $4.93 \mathrm{E}-04$ & & \\
\hline Zr-93 & $1.18 \mathrm{E}-02$ & & & & & & & \\
\hline $\mathrm{Nb}-93 \mathrm{~m}$ & 9.07E-03 & & & & & & & \\
\hline $\mathrm{Nb}-94$ & $8.24 \mathrm{E}-03$ & & & & & & & \\
\hline Tc-99 & $8.36 \mathrm{E}-02$ & $3.20 \mathrm{E}-09$ & 1.87E-09 & 1.01E-09 & $2.15 \mathrm{E}-07$ & $2.15 \mathrm{E}-07$ & & \\
\hline Ru-106 & $6.78 \mathrm{E}-03$ & & & & $1.59 \mathrm{E}-08$ & 1.59E-08 & & \\
\hline Rh-102 & $4.58 \mathrm{E}-06$ & & & & $1.08 \mathrm{E}-11$ & $1.07 \mathrm{E}-11$ & & \\
\hline Rh-106 & $6.78 \mathrm{E}-03$ & & & & $1.59 \mathrm{E}-08$ & $1.59 \mathrm{E}-08$ & & \\
\hline $\mathrm{Pd}-107$ & 8.78E-05 & & & & & & & \\
\hline $\mathrm{Cd}-113 \mathrm{~m}$ & $1.76 \mathrm{E}-02$ & & & & & & & \\
\hline Sn-121m & $3.55 \mathrm{E}-04$ & & & & & & & \\
\hline Sn-126 & $2.98 \mathrm{E}-03$ & & & & & & & \\
\hline Sb-125 & $2.49 \mathrm{E}+00$ & & & & 5.85E-06 & 5.84E-06 & & \\
\hline Sb-126 & 3.06E-04 & & & & $7.18 \mathrm{E}-10$ & $7.17 \mathrm{E}-10$ & & \\
\hline Te-125m & 1.67E-02 & & & & & & & \\
\hline I-129 & 3.86E-04 & $4.91 \mathrm{E}-11$ & $2.87 \mathrm{E}-11$ & $1.54 \mathrm{E}-11$ & $1.25 \mathrm{E}-09$ & 1.24E-09 & & $9.45 \mathrm{E}-12$ \\
\hline Cs-134 & $3.85 \mathrm{E}-01$ & & & & & & & \\
\hline Cs-135 & $6.06 \mathrm{E}-03$ & & & & & & & \\
\hline Cs-137 & $3.14 \mathrm{E}+02$ & & & & & & & \\
\hline Ba-137m & $2.97 \mathrm{E}+02$ & & & & & & & \\
\hline $\mathrm{Ce}-144$ & $4.58 \mathrm{E}-03$ & & & & $1.08 \mathrm{E}-08$ & 1.07E-08 & & \\
\hline Pr-144 & $4.58 \mathrm{E}-03$ & & & & $1.08 \mathrm{E}-08$ & 1.07E-08 & & \\
\hline $\mathrm{Pm}-146$ & $2.71 \mathrm{E}-04$ & & & & $6.35 \mathrm{E}-10$ & $6.34 \mathrm{E}-10$ & & \\
\hline $\mathrm{Pm}-147$ & $1.24 \mathrm{E}+00$ & & & & $2.90 \mathrm{E}-06$ & $2.90 \mathrm{E}-06$ & & \\
\hline Sm-151 & $2.44 \mathrm{E}+00$ & & & & $5.74 \mathrm{E}-06$ & $5.73 \mathrm{E}-06$ & & \\
\hline Eu-152 & 1.57E-02 & & & & $3.68 \mathrm{E}-08$ & $3.68 \mathrm{E}-08$ & & \\
\hline Eu-154 & $9.36 \mathrm{E}-01$ & & & & $2.20 \mathrm{E}-06$ & $2.19 \mathrm{E}-06$ & & \\
\hline Eu-155 & $8.81 \mathrm{E}-01$ & & & & 2.07E-06 & $2.07 \mathrm{E}-06$ & & \\
\hline Th-230 & $5.76 \mathrm{E}-06$ & & & & & & & \\
\hline $\mathrm{Pa}-233$ & $1.56 \mathrm{E}-02$ & & & & & & & \\
\hline U-232 & 1.35E-05 & & & & & & & \\
\hline U-233 & $4.75 \mathrm{E}-07$ & & & & & & & \\
\hline U-234 & 9.32E-03 & & & & & & & \\
\hline U-235 & $3.76 \mathrm{E}-04$ & & & & & & & \\
\hline U-236 & 4.36E-04 & & & & & & & \\
\hline U-237 & $3.42 \mathrm{E}-05$ & & & & & & & \\
\hline U-238 & 2.07E-04 & & & & & & & \\
\hline Np-237 & $2.78 \mathrm{E}-03$ & & & & $6.52 \mathrm{E}-09$ & $6.51 \mathrm{E}-09$ & & \\
\hline Pu-236 & $2.20 \mathrm{E}-05$ & & & & & & & \\
\hline Pu-238 & $5.07 \mathrm{E}+00$ & & & & & & & \\
\hline Pu-239 & $5.54 \mathrm{E}-01$ & & & & & & & \\
\hline Pu-240 & $8.19 \mathrm{E}-02$ & & & & & & & \\
\hline Pu-241 & $4.44 \mathrm{E}+00$ & & & & & & & \\
\hline Pu-242 & 6.42E-05 & & & & & & & \\
\hline Pu-244 & $1.91 \mathrm{E}-12$ & & & & & & & \\
\hline Am-241 & $4.33 \mathrm{E}-01$ & & & & $1.02 \mathrm{E}-06$ & $1.02 \mathrm{E}-06$ & & \\
\hline Am-242m & $8.13 E-05$ & & & & $1.91 \mathrm{E}-10$ & $1.90 \mathrm{E}-10$ & & \\
\hline
\end{tabular}


Table A-22. Mass balance, Tank WM-189 waste (continued).

\begin{tabular}{|c|c|c|c|c|c|c|c|c|}
\hline Stream \# & 108 & 109 & 110 & 111 & 112 & $113 \mathrm{~A}$ & $113 \mathrm{~B}$ & 114 \\
\hline Radiological Composition & Ci/Canister & $\mathrm{Ci} / \mathrm{wscm}$ & Ci/wscm & $\mathrm{Ci} / \mathrm{wscm}$ & $\mathrm{Ci} / \mathrm{L}$ & $\mathrm{Ci} / \mathrm{L}$ & $\mathrm{Ci} / \mathrm{L}$ & Ci/wscm \\
\hline Am-243 & 1.39E-04 & & & & $3.25 \mathrm{E}-10$ & $3.25 \mathrm{E}-10$ & & \\
\hline $\mathrm{Cm}-242$ & $1.62 \mathrm{E}-04$ & & & & & & & \\
\hline $\mathrm{Cm}-243$ & $2.00 \mathrm{E}-04$ & & & & & & & \\
\hline $\mathrm{Cm}-244$ & $8.45 \mathrm{E}-03$ & & & & & & & \\
\hline $\mathrm{Cm}-245$ & $2.10 \mathrm{E}-06$ & & & & & & & \\
\hline $\mathrm{Cm}-246$ & $1.38 \mathrm{E}-07$ & & & & & & & \\
\hline TRU & $6.28 \mathrm{E}+00$ & & & & $1.02 \mathrm{E}-06$ & $1.02 \mathrm{E}-06$ & & \\
\hline & & & & & & & & \\
\hline & & & & & & & & \\
\hline $\begin{array}{l}\text { Gas Stream Bulk } \\
\text { Composition (Wet Basis) }\end{array}$ & $\begin{array}{c}\text { mol\% or } \\
\text { ppmv }\end{array}$ & $\begin{array}{l}\text { mol\% or } \\
\text { ppmv }\end{array}$ & $\begin{array}{l}\text { mol\% or } \\
\text { ppmv }\end{array}$ & $\begin{array}{l}\text { mol\% or } \\
\text { ppmv }\end{array}$ & $\begin{array}{l}\text { mol\% or } \\
\text { ppmv }\end{array}$ & $\begin{array}{c}\text { mol\% or } \\
\text { ppmv }\end{array}$ & $\begin{array}{c}\text { mol\% or } \\
\text { ppmv }\end{array}$ & $\begin{array}{c}\text { mol\% or } \\
\text { ppmv }\end{array}$ \\
\hline $\mathrm{H} 2 \mathrm{O}, \mathrm{mol} \%$ & & $29.78 \%$ & $45.30 \%$ & $70.61 \%$ & & & & $70.49 \%$ \\
\hline $\mathrm{O} 2, \mathrm{~mol} \%$ & & & $3.0108 \%$ & $1.6176 \%$ & & & & $1.6244 \%$ \\
\hline $\mathrm{N} 2, \mathrm{~mol} \%$ & & $5.46 \%$ & $3.25 \%$ & $1.75 \%$ & & & & $1.75 \%$ \\
\hline $\mathrm{H} 2, \mathrm{~mol} \%$ & & $10.42 \%$ & $0.00002 \%$ & $0.00001 \%$ & & & & $0.0007 \%$ \\
\hline $\mathrm{CO} 2, \mathrm{~mol} \%$ & & $51.63 \%$ & $48.38 \%$ & $25.99 \%$ & & & & $26.10 \%$ \\
\hline COgas, ppmv & & $4.67 \mathrm{E}+03$ & $3.16 \mathrm{E}-01$ & $1.70 \mathrm{E}-01$ & & & & 1.70E-01 \\
\hline $\mathrm{NO}, \mathrm{ppmv}$ & & $1.74 \mathrm{E}+03$ & $2.49 \mathrm{E}+02$ & $1.34 \mathrm{E}+02$ & & & & $1.35 \mathrm{E}+02$ \\
\hline NO2, ppmv & & $1.66 \mathrm{E}+01$ & $9.71 \mathrm{E}+00$ & $5.22 \mathrm{E}+00$ & & & & $5.24 \mathrm{E}+00$ \\
\hline SO2, ppmv & & 1.63E-05 & $2.50 \mathrm{E}+02$ & $1.34 \mathrm{E}+02$ & & & & $1.35 \mathrm{E}+02$ \\
\hline $\mathrm{Cl}, \mathrm{ppmv}$ & & $3.79 \mathrm{E}-01$ & $2.22 \mathrm{E}-01$ & 1.19E-01 & & & & $7.27 \mathrm{E}-02$ \\
\hline $\mathrm{F}, \mathrm{ppmv}$ & & $1.07 \mathrm{E}+01$ & $6.25 \mathrm{E}+00$ & $3.36 \mathrm{E}+00$ & & & & $2.94 \mathrm{E}+00$ \\
\hline C (organic), ppmv & & $1.70 \mathrm{E}+05$ & & & & & & \\
\hline $\mathrm{H}$ (organic), ppmv & & $3.81 \mathrm{E}+05$ & & & & & & \\
\hline $\mathrm{Hg}, \mathrm{ug} / \mathrm{wscm}$ & & $4.27 E+05$ & $2.50 \mathrm{E}+05$ & $1.34 \mathrm{E}+05$ & & & & \\
\hline $\mathrm{PM}, \mathrm{mg} / \mathrm{wscm}$ & & $1.01 \mathrm{E}+05$ & $2.55 \mathrm{E}+02$ & $1.43 \mathrm{E}+02$ & & & & $8.43 E+00$ \\
\hline SVM, ug/wscm & & & & & & & & \\
\hline LVM, ug/wscm & & & & & & & & \\
\hline & & & & & & & & \\
\hline $\begin{array}{l}\text { Gas Stream Bulk } \\
\text { Composition (Dry Basis) }\end{array}$ & $\mathrm{mol} \%$ & $\mathrm{~mol} \%$ & $\mathrm{~mol} \%$ & $\mathrm{~mol} \%$ & $\mathrm{~mol} \%$ & $\mathrm{~mol} \%$ & $\mathrm{~mol} \%$ & $\mathrm{~mol} \%$ \\
\hline $\mathrm{O} 2, \mathrm{~mol} \%$, dry basis & & & $5.50 \%$ & $5.50 \%$ & & & & $5.50 \%$ \\
\hline $\mathrm{N} 2, \mathrm{~mol} \%$, dry basis & & $7.78 \%$ & $5.95 \%$ & $5.95 \%$ & & & & $5.95 \%$ \\
\hline $\mathrm{H} 2, \mathrm{~mol} \%$, dry basis & & $14.84 \%$ & $0.00003 \%$ & $0.00003 \%$ & & & & $0.002 \%$ \\
\hline $\mathrm{CO} 2, \mathrm{~mol} \%$, dry basis & & $73.53 \%$ & $88.45 \%$ & $88.45 \%$ & & & & $88.44 \%$ \\
\hline $\begin{array}{l}\text { Gas Stream Bulk } \\
\text { Composition (Dry Basis, } \\
\text { Corrected to } 7 \% \text { O2 with } \\
100 \% \text { O2 Combustion Air) } \\
\end{array}$ & $\begin{array}{l}\text { ppmv, or } \\
\text { ug/dscm or } \\
\mathrm{mg} / \mathrm{dscm}\end{array}$ & $\begin{array}{l}\text { ppmv, or } \\
\text { ug/dscm } \\
\text { or } \\
\mathrm{mg} / \mathrm{dscm}\end{array}$ & $\begin{array}{l}\text { ppmv, or } \\
\text { ug/dscm } \\
\text { or } \\
\mathrm{mg} / \mathrm{dscm}\end{array}$ & $\begin{array}{l}\text { ppmv, or } \\
\text { ug/dscm } \\
\text { or } \\
\mathrm{mg} / \mathrm{dscm}\end{array}$ & $\begin{array}{l}\mathrm{ppmv}, \text { or } \\
\mathrm{ug} / \mathrm{dscm} \\
\text { or } \\
\mathrm{mg} / \mathrm{dscm}\end{array}$ & $\begin{array}{l}\text { ppmv, or } \\
\text { ug/dscm } \\
\text { or } \\
\mathrm{mg} / \mathrm{dscm}\end{array}$ & $\begin{array}{l}\mathrm{ppmv}, \text { or } \\
\mathrm{ug} / \mathrm{dscm} \\
\text { or } \\
\mathrm{mg} / \mathrm{dscm}\end{array}$ & $\begin{array}{l}\text { ppmv, or } \\
\text { ug/dscm } \\
\text { or } \\
\mathrm{mg} / \mathrm{dscm}\end{array}$ \\
\hline COgas, ppmv, dry basis & & $9.31 \mathrm{E}+02$ & $8.08 \mathrm{E}-02$ & $8.08 \mathrm{E}-02$ & & & & $8.08 \mathrm{E}-02$ \\
\hline $\mathrm{NO}, \mathrm{ppmv}$, dry basis & & $3.48 \mathrm{E}+02$ & $6.39 \mathrm{E}+01$ & $6.39 \mathrm{E}+01$ & & & & $6.39 \mathrm{E}+01$ \\
\hline NO2, ppmv, dry basis & & $3.31 \mathrm{E}+00$ & $2.49 \mathrm{E}+00$ & $2.49 \mathrm{E}+00$ & & & & $2.49 \mathrm{E}+00$ \\
\hline SO2, ppmv, dry basis & & $3.25 \mathrm{E}-06$ & $6.40 \mathrm{E}+01$ & $6.40 \mathrm{E}+01$ & & & & $6.40 \mathrm{E}+01$ \\
\hline $\mathrm{Cl}, \mathrm{ppmv}$, dry basis & & $7.56 \mathrm{E}-02$ & 5.67E-02 & $5.67 \mathrm{E}-02$ & & & & $3.45 \mathrm{E}-02$ \\
\hline $\mathrm{F}, \mathrm{ppmv}$, dry basis & & $2.13 \mathrm{E}+00$ & $1.60 \mathrm{E}+00$ & $1.60 \mathrm{E}+00$ & & & & $1.40 \mathrm{E}+00$ \\
\hline $\mathrm{C}$ (organic), ppmv, dry basis & & $3.40 \mathrm{E}+04$ & 4.04E-26 & 4.04E-26 & & & & \\
\hline $\mathrm{H}$ (organic), ppmv, dry basis & & $7.60 \mathrm{E}+04$ & $1.62 \mathrm{E}-25$ & $1.62 \mathrm{E}-25$ & & & & \\
\hline $\mathrm{Hg}, \mathrm{ug} / \mathrm{dscm}$ & & $8.52 \mathrm{E}+04$ & $6.39 \mathrm{E}+04$ & $6.39 \mathrm{E}+04$ & & & & \\
\hline $\mathrm{PM}, \mathrm{mg} / \mathrm{dscm}$ & & $2.02 \mathrm{E}+04$ & $6.54 \mathrm{E}+01$ & $6.80 \mathrm{E}+01$ & & & & $4.00 \mathrm{E}+00$ \\
\hline SVM, ug/dscm & & & & & & & & \\
\hline LVM, ug/dscm & & & & & & & & \\
\hline
\end{tabular}


Table A-22. Mass balance, Tank WM-189 waste (continued).

\begin{tabular}{|c|c|c|c|c|c|c|}
\hline PFD Number & PFD-3 & PFD-3 & PFD-3 & PFD-3 & PFD-3 & PFD-3 \\
\hline Stream Number & 115 & 116 & 117 & 118 & 119 & 120 \\
\hline Stream Name & $\begin{array}{l}\text { Scrubbed } \\
\text { Off-Gas to } \\
\text { Preheater }\end{array}$ & $\begin{array}{c}\text { Preheated } \\
\text { Off-gas to } \\
\text { GAC }\end{array}$ & $\begin{array}{c}\text { Pressure } \\
\text { Control } \\
\text { Bleed Air }\end{array}$ & $\begin{array}{c}\text { Final HEPA } \\
\text { Off-Gas } \\
\text { Outlet }\end{array}$ & $\begin{array}{l}\text { Off-gas to } \\
\text { Exhaust } \\
\text { Blower }\end{array}$ & Spent GAC \\
\hline Rate or Volume & $3.97 \mathrm{E}+05$ & $4.90 \mathrm{E}+05$ & $1.58 \mathrm{E}+04$ & $5.96 \mathrm{E}+05$ & $6.37 \mathrm{E}+05$ & $5.14 \mathrm{E}+00$ \\
\hline Volume Flow (standard, wet) ${ }^{*}$ & $1.78 \mathrm{E}+05$ & $1.78 \mathrm{E}+05$ & $1.56 \mathrm{E}+04$ & $1.78 \mathrm{E}+05$ & $1.93 \mathrm{E}+05$ & \\
\hline Volume Flow (standard, dry) & $5.26 \mathrm{E}+04$ & $5.26 \mathrm{E}+04$ & $1.56 \mathrm{E}+04$ & $5.26 \mathrm{E}+04$ & $6.80 \mathrm{E}+04$ & \\
\hline Rate Units & $\mathrm{ft} 3 / \mathrm{hr}$ & $\mathrm{ft} 3 / \mathrm{hr}$ & $\mathrm{ft} 3 / \mathrm{hr}$ & $\mathrm{ft} 3 / \mathrm{hr}$ & $\mathrm{ft} 3 / \mathrm{hr}$ & $\mathrm{lb} / \mathrm{hr}$ \\
\hline Rate or Volume, metric & $5.04 \mathrm{E}+03$ & $5.04 \mathrm{E}+03$ & $4.41 \mathrm{E}+02$ & $5.04 \mathrm{E}+03$ & $5.47 \mathrm{E}+03$ & $2.33 \mathrm{E}+00$ \\
\hline Rate Units & wscm $/ \mathrm{hr}$ & wscm $/ \mathrm{hr}$ & wscm/hr & wscm $/ \mathrm{hr}$ & wscm $/ \mathrm{hr}$ & $\mathrm{kg} / \mathrm{hr}$ \\
\hline Temperature, ${ }^{\circ} \mathrm{C}$ & 77 & 120 & 25 & 120 & 114 & 27 \\
\hline Temperature, ${ }^{\circ} \mathrm{F}$ & 171 & 248 & 77 & 248 & 237 & 81 \\
\hline Pressure, psia & 7.88 & 7.16 & 14.70 & 5.89 & 5.89 & 14.70 \\
\hline Specific Gravity & $4.70 \mathrm{E}-04$ & $3.81 \mathrm{E}-04$ & $1.18 \mathrm{E}-03$ & $3.13 \mathrm{E}-04$ & $3.22 \mathrm{E}-04$ & $5.86 \mathrm{E}-01$ \\
\hline Chemical Composition & $\mathrm{lb} /$ wscf & $\mathrm{lb} / \mathrm{wscf}$ & $\mathrm{lb} /$ wscf & $\mathrm{lb} / \mathrm{wscf}$ & $\mathrm{lb} / \mathrm{wscf}$ & wt frac \\
\hline $\mathrm{H}+$ & $1.19 \mathrm{E}-07$ & $1.19 \mathrm{E}-07$ & & $1.19 \mathrm{E}-07$ & $1.10 \mathrm{E}-07$ & $4.88 \mathrm{E}-06$ \\
\hline \multicolumn{7}{|l|}{$\mathrm{Al}+3$} \\
\hline \multicolumn{7}{|l|}{$\mathrm{Sb}+5$} \\
\hline \multirow{2}{*}{\multicolumn{7}{|c|}{$\mathrm{As}+3$}} \\
\hline \multirow{2}{*}{\multicolumn{7}{|c|}{$\frac{\mathrm{Ba}+2}{\mathrm{Be}+2}$}} \\
\hline & & & & & & \\
\hline \multicolumn{7}{|l|}{$\frac{\mathrm{Be}+2}{\mathrm{~B}+3}$} \\
\hline \multicolumn{7}{|l|}{$\mathrm{Cd}+2$} \\
\hline \multicolumn{7}{|l|}{$\mathrm{Ca}+2$} \\
\hline \multicolumn{7}{|l|}{$\mathrm{Cr}+3$} \\
\hline \multirow{2}{*}{\multicolumn{7}{|c|}{$\mathrm{Co}+2$}} \\
\hline \multirow{2}{*}{\multicolumn{7}{|c|}{$\frac{\mathrm{Cs}+}{\mathrm{Cu}+2}$}} \\
\hline & & & & & & \\
\hline \multicolumn{7}{|l|}{$\begin{array}{l}\mathrm{Cu}+2 \\
\mathrm{Fe}+3\end{array}$} \\
\hline \multicolumn{3}{|l|}{$\frac{\mathrm{Fe}+3}{\mathrm{~Pb}+2}$} & & & & \\
\hline $\mathrm{Hg}+2$ & $4.31 \mathrm{E}-06$ & $4.31 \mathrm{E}-06$ & & $2.29 \mathrm{E}-09$ & $2.11 \mathrm{E}-09$ & $1.49 \mathrm{E}-01$ \\
\hline \multicolumn{7}{|l|}{$\mathrm{Mn}+4$} \\
\hline \multicolumn{7}{|l|}{$\mathrm{Ni}+2$} \\
\hline \multirow{2}{*}{\multicolumn{7}{|c|}{$\frac{\mathrm{K}+}{\mathrm{Se}+4}$}} \\
\hline \multirow{2}{*}{\multicolumn{7}{|c|}{$\frac{\mathrm{Se}+4}{\mathrm{Ag}+}$}} \\
\hline & & & & & & \\
\hline \multicolumn{7}{|l|}{$\frac{\mathrm{Na}}{\mathrm{Na}}$} \\
\hline \multicolumn{7}{|l|}{ 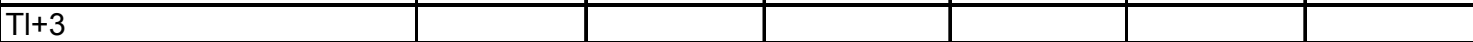 } \\
\hline $\mathrm{U}+4$ & & & & & & \\
\hline$\overline{V+3}$ & & & & & & \\
\hline$Z n+2$ & & & & & & \\
\hline $\mathrm{Zr}+4$ & & & & & & \\
\hline Cl- & $6.69 \mathrm{E}-09$ & $6.69 \mathrm{E}-09$ & & $1.74 \mathrm{E}-09$ & 1.61E-09 & $1.71 \mathrm{E}-04$ \\
\hline F- & $1.45 \mathrm{E}-07$ & $1.45 \mathrm{E}-07$ & & $1.45 \mathrm{E}-07$ & $1.34 \mathrm{E}-07$ & \\
\hline SO4-2 & & & & & & \\
\hline NO3- & $6.84 \mathrm{E}-06$ & $6.84 \mathrm{E}-06$ & & $6.84 \mathrm{E}-06$ & $6.30 \mathrm{E}-06$ & \\
\hline $\mathrm{PO} 4-3$ & & & & & & \\
\hline$\overline{A m+4}$ & & & & & & \\
\hline $\mathrm{Br}-$ & $2.00 \mathrm{E}-13$ & $2.00 \mathrm{E}-13$ & & $5.21 \mathrm{E}-14$ & $4.80 \mathrm{E}-14$ & $5.12 \mathrm{E}-09$ \\
\hline $\mathrm{Ce}+4$ & & & & & & \\
\hline $\mathrm{Eu+3}$ & & & & & & \\
\hline $\mathrm{Gd}+3$ & & & & & & \\
\hline $\mathrm{Ge}+4$ & & & & & & \\
\hline $\ln +3$ & & & & & & \\
\hline $1-$ & $3.53 \mathrm{E}-12$ & $3.53 \mathrm{E}-12$ & & $9.19 \mathrm{E}-13$ & $8.47 \mathrm{E}-13$ & 9.04E-08 \\
\hline$L a+3$ & & & & & & \\
\hline
\end{tabular}


Table A-22. Mass balance, Tank WM-189 waste (continued).

\begin{tabular}{|c|c|c|c|c|c|c|}
\hline Stream \# & 115 & 116 & 117 & 118 & 119 & 120 \\
\hline Chemical Composition & $\mathrm{lb} / \mathrm{wscf}$ & $\mathrm{lb} / \mathrm{wscf}$ & $\mathrm{lb} / \mathrm{wscf}$ & $\mathrm{lb} / \mathrm{wscf}$ & $\mathrm{lb} / \mathrm{wscf}$ & wt frac \\
\hline \multicolumn{7}{|l|}{$\mathrm{Li}+$} \\
\hline \multicolumn{7}{|l|}{$M g+2$} \\
\hline \multicolumn{7}{|l|}{ Mo+6 } \\
\hline \multicolumn{7}{|l|}{$\mathrm{Nd}+3$} \\
\hline \multicolumn{7}{|l|}{$\mathrm{Np}+4$} \\
\hline \multicolumn{7}{|l|}{$\mathrm{Nb}+5$} \\
\hline \multicolumn{7}{|l|}{$\mathrm{Pd}+4$} \\
\hline \multicolumn{7}{|l|}{$\mathrm{Pu}+4$} \\
\hline \multicolumn{7}{|l|}{$\mathrm{Pr}+4$} \\
\hline \multicolumn{7}{|l|}{$\mathrm{Pm}+3$} \\
\hline \multicolumn{7}{|l|}{$\mathrm{Rh}+4$} \\
\hline \multicolumn{7}{|l|}{$\mathrm{Rb}+$} \\
\hline \multicolumn{7}{|l|}{$\mathrm{Ru}+3$} \\
\hline \multicolumn{7}{|l|}{$\mathrm{Sm}+3$} \\
\hline \multicolumn{7}{|l|}{$\mathrm{Si}+4$} \\
\hline \multicolumn{7}{|l|}{$\mathrm{Sr}+2$} \\
\hline \multicolumn{7}{|l|}{$\mathrm{Tc}+7$} \\
\hline \multicolumn{7}{|l|}{$\mathrm{Te}+4$} \\
\hline \multicolumn{7}{|l|}{$\mathrm{Tb}+4$} \\
\hline \multicolumn{7}{|l|}{$\mathrm{Th}+4$} \\
\hline \multicolumn{7}{|l|}{$\mathrm{Sn+4}$} \\
\hline $\mathrm{Ti}+4$ & & & & & & \\
\hline $\mathrm{Y}+3$ & & & & & & \\
\hline $\mathrm{OH}-$ & & & & & & \\
\hline $\mathrm{H} 2 \mathrm{O}$ & $3.30 \mathrm{E}-02$ & $3.30 \mathrm{E}-02$ & 4.68E-06 & 3.30E-02 & 3.04E-02 & \\
\hline $\mathrm{SO} 2$ & 2.24E-05 & 2.24E-05 & & 2.24E-05 & 2.07E-05 & \\
\hline $\mathrm{H} 2 \mathrm{~S}$ & & & & & & \\
\hline $\mathrm{CO}$ & $1.24 \mathrm{E}-08$ & $1.24 \mathrm{E}-08$ & & $1.24 \mathrm{E}-08$ & 1.14E-08 & \\
\hline $\mathrm{CO} 2$ & $2.98 \mathrm{E}-02$ & $2.98 \mathrm{E}-02$ & & $2.98 \mathrm{E}-02$ & $2.75 \mathrm{E}-02$ & \\
\hline $\mathrm{H} 2$ & & & & & & \\
\hline N2 & $1.28 \mathrm{E}-03$ & $1.28 \mathrm{E}-03$ & $5.75 \mathrm{E}-02$ & $1.28 \mathrm{E}-03$ & $5.80 \mathrm{E}-03$ & \\
\hline NO & $1.05 \mathrm{E}-05$ & $1.05 \mathrm{E}-05$ & & $1.05 \mathrm{E}-05$ & $9.66 \mathrm{E}-06$ & \\
\hline $\mathrm{NO} 2$ & $6.26 \mathrm{E}-07$ & $6.26 \mathrm{E}-07$ & & $6.26 \mathrm{E}-07$ & $5.77 \mathrm{E}-07$ & \\
\hline $\mathrm{O} 2$ & $1.35 \mathrm{E}-03$ & 1.35E-03 & 1.74E-02 & $1.35 \mathrm{E}-03$ & $2.65 \mathrm{E}-03$ & \\
\hline S (other) & & & & & & \\
\hline $\mathrm{CO} 3$ & & & & & & \\
\hline C (reductant) & & & & & & 8.50E-01 \\
\hline $\mathrm{O}$ (oxides) & & & & & & \\
\hline C (organic) & & & & & & \\
\hline $\mathrm{H}$ (organic) & & & & & & \\
\hline $\mathrm{O}$ (organic) & & & & & & \\
\hline & & & & & & \\
\hline Mass Flow $(\mathrm{kg} / \mathrm{hr})$ : & $5.29 E+03$ & $5.29 E+03$ & $5.29 E+02$ & $5.29 E+03$ & $5.81 \mathrm{E}+03$ & $2.33 E+00$ \\
\hline Canister Rate, canister & & & & & & \\
\hline Total Canisters Genera & & & & & & \\
\hline & $\mathrm{w} / \mathrm{m} 3$ & $\mathrm{w} 3$ & $1 m^{3}$ & $\mathrm{w} / \mathrm{m}^{3}$ & $1 \mathrm{~m}^{3}$ & $5 / m 3$ \\
\hline Heat Generation & & & & & & $4.58 \mathrm{E}-14$ \\
\hline
\end{tabular}


Table A-22. Mass balance, Tank WM-189 waste (continued).

\begin{tabular}{|c|c|c|c|c|c|c|}
\hline Stream \# & 115 & 116 & 117 & 118 & 119 & 120 \\
\hline Radiological Composition & Ci/wscm & Ci/wscm & $\mathrm{Ci} / \mathrm{wscm}$ & Ci/wscm & Ci/wscm & $\mathrm{Ci} / \mathrm{kg}$ \\
\hline $\mathrm{H}-3$ & $5.89 \mathrm{E}-07$ & $5.89 \mathrm{E}-07$ & & $5.89 \mathrm{E}-07$ & $5.43 \mathrm{E}-07$ & \\
\hline $\mathrm{C}-14$ & $9.49 \mathrm{E}-12$ & $9.49 \mathrm{E}-12$ & & $9.49 \mathrm{E}-12$ & $8.74 \mathrm{E}-12$ & $6.70 \mathrm{E}-11$ \\
\hline \multicolumn{7}{|l|}{\begin{tabular}{|l|} 
Co-60 \\
Ni-59
\end{tabular}} \\
\hline $\mathrm{Ni}-59$ & & & & & & \\
\hline \multicolumn{7}{|l|}{$\mathrm{Ni}-63$} \\
\hline \multicolumn{7}{|l|}{ Se-79 } \\
\hline \multicolumn{7}{|l|}{ Sr-90 } \\
\hline \multicolumn{7}{|l|}{ Y-90 } \\
\hline \multicolumn{7}{|l|}{ Zr-93 } \\
\hline \multirow{2}{*}{\multicolumn{7}{|c|}{\begin{tabular}{|l|}
$\mathrm{Nb}-93 \mathrm{~m}$ \\
$\mathrm{Nb}-94$
\end{tabular}}} \\
\hline & & & & & & \\
\hline \multicolumn{7}{|l|}{ Tc-99 } \\
\hline \multicolumn{7}{|l|}{ Ru-106 } \\
\hline \multicolumn{7}{|l|}{\begin{tabular}{l|} 
Rh-102 \\
Rh-106
\end{tabular}} \\
\hline \multirow{2}{*}{\multicolumn{7}{|c|}{\begin{tabular}{|l|} 
Rh-106 \\
Pd-107
\end{tabular}}} \\
\hline & & & & & & \\
\hline \multicolumn{7}{|l|}{ Cd-113m } \\
\hline \multicolumn{7}{|l|}{ Sn-121m } \\
\hline \multirow{2}{*}{\multicolumn{7}{|c|}{\begin{tabular}{|l|} 
Sn-126 \\
Sb-125
\end{tabular}}} \\
\hline \multirow{2}{*}{\multicolumn{7}{|c|}{\begin{tabular}{|l|} 
Sb-125 \\
Sb-126
\end{tabular}}} \\
\hline & & & & & & \\
\hline \multicolumn{7}{|l|}{ Te-125m } \\
\hline $\mathrm{I}-129$ & $9.42 \mathrm{E}-12$ & $9.42 \mathrm{E}-12$ & & $2.45 \mathrm{E}-12$ & $2.26 \mathrm{E}-12$ & $1.51 \mathrm{E}-08$ \\
\hline \multicolumn{7}{|l|}{ Cs-134 } \\
\hline \multicolumn{7}{|l|}{ Cs-135 } \\
\hline Cs-137 & & & & & & \\
\hline Ba-137m & & & & & & \\
\hline Ce-144 & & & & & & \\
\hline Pr-144 & & & & & & \\
\hline$P m-146$ & & & & & & \\
\hline$P m-147$ & & & & & & \\
\hline Sm-151 & & & & & & \\
\hline Eu-152 & & & & & & \\
\hline Eu-154 & & & & & & \\
\hline Eu-155 & & & & & & \\
\hline Th-230 & & & & & & \\
\hline $\mathrm{Pa}-233$ & & & & & & \\
\hline $\mathrm{U}-232$ & & & & & & \\
\hline $\mathrm{U}-233$ & & & & & & \\
\hline $\mathrm{U}-234$ & & & & & & \\
\hline $\mathrm{U}-235$ & & & & & & \\
\hline $\mathrm{U}-236$ & & & & & & \\
\hline $\mathrm{U}-237$ & & & & & & \\
\hline $\mathrm{U}-238$ & & & & & & \\
\hline $\mathrm{Np}-237$ & & & & & & \\
\hline $\mathrm{Pu}-236$ & & & & & & \\
\hline Pu-238 & & & & & & \\
\hline Pu-239 & & & & & & \\
\hline Pu-240 & & & & & & \\
\hline Pu-241 & & & & & & \\
\hline Pu-242 & & & & & & \\
\hline Pu-244 & & & & & & \\
\hline Am-241 & & & & & & \\
\hline $\mathrm{Am}-242 \mathrm{~m}$ & & & & & & \\
\hline
\end{tabular}


Table A-22. Mass balance, Tank WM-189 waste (continued).

\begin{tabular}{|c|c|c|c|c|c|c|}
\hline Stream \# & 115 & 116 & 117 & 118 & 119 & 120 \\
\hline Radiological Composition & Ci/wscm & Ci/wscm & Ci/wscm & Ci/wscm & Ci/wscm & $\mathrm{Ci} / \mathrm{kg}$ \\
\hline \multicolumn{7}{|l|}{$\mathrm{Am}-243$} \\
\hline \multicolumn{7}{|l|}{$\mathrm{Cm}-242$} \\
\hline \multicolumn{7}{|l|}{$\mathrm{Cm}-243$} \\
\hline \multicolumn{7}{|l|}{$\mathrm{Cm}-244$} \\
\hline \multicolumn{7}{|l|}{$\mathrm{Cm}-245$} \\
\hline \multicolumn{7}{|l|}{ Cm-246 } \\
\hline \multicolumn{7}{|l|}{ TRU } \\
\hline & & & & & & \\
\hline & & & & & & \\
\hline $\begin{array}{l}\text { Gas Stream Bulk } \\
\text { Composition (Wet Basis) }\end{array}$ & $\begin{array}{c}\mathrm{mol} \% \text { or } \\
\mathrm{ppmv}\end{array}$ & $\begin{array}{c}\mathrm{mol} \% \text { or } \\
\mathrm{ppmv}\end{array}$ & $\begin{array}{c}\mathrm{mol} \% \text { or } \\
\text { ppmv }\end{array}$ & $\begin{array}{c}\mathrm{mol} \% \text { or } \\
\mathrm{ppmv}\end{array}$ & $\begin{array}{c}\mathrm{mol} \% \text { or } \\
\text { ppmv }\end{array}$ & $\begin{array}{c}\text { mol\% or } \\
\text { ppmv }\end{array}$ \\
\hline $\mathrm{H} 2 \mathrm{O}, \mathrm{mol} \%$ & $70.48 \%$ & $70.48 \%$ & $0.01 \%$ & $70.48 \%$ & $64.82 \%$ & \\
\hline $\mathrm{O} 2, \mathrm{~mol} \%$ & $1.62 \%$ & $1.62 \%$ & $20.99 \%$ & $1.62 \%$ & $3.18 \%$ & \\
\hline $\mathrm{N} 2, \mathrm{~mol} \%$ & $1.76 \%$ & $1.76 \%$ & $79.00 \%$ & $1.76 \%$ & $7.96 \%$ & \\
\hline \multicolumn{7}{|l|}{$\mathrm{H} 2, \mathrm{~mol} \%$} \\
\hline $\mathrm{CO} 2, \mathrm{~mol} \%$ & $26.11 \%$ & $26.11 \%$ & & $26.11 \%$ & $24.01 \%$ & \\
\hline COgas, ppmv & $1.70 \mathrm{E}-01$ & $1.70 \mathrm{E}-01$ & & $1.70 \mathrm{E}-01$ & $1.57 \mathrm{E}-01$ & \\
\hline NO, ppmv & $1.35 \mathrm{E}+02$ & $1.35 \mathrm{E}+02$ & & $1.35 \mathrm{E}+02$ & $1.24 \mathrm{E}+02$ & \\
\hline NO2, ppmv & $5.24 \mathrm{E}+00$ & $5.24 \mathrm{E}+00$ & & $5.24 \mathrm{E}+00$ & $4.82 \mathrm{E}+00$ & \\
\hline SO2, ppmv & $1.35 \mathrm{E}+02$ & $1.35 \mathrm{E}+02$ & & $1.35 \mathrm{E}+02$ & $1.24 \mathrm{E}+02$ & \\
\hline $\mathrm{Cl}, \mathrm{ppmv}$ & $7.27 \mathrm{E}-02$ & $7.27 \mathrm{E}-02$ & & $1.90 \mathrm{E}-02$ & $1.74 \mathrm{E}-02$ & \\
\hline $\mathrm{F}, \mathrm{ppmv}$ & $2.94 \mathrm{E}+00$ & $2.94 \mathrm{E}+00$ & & $2.94 \mathrm{E}+00$ & $2.71 \mathrm{E}+00$ & \\
\hline \multicolumn{7}{|l|}{ C (organic), ppmv } \\
\hline \multirow{2}{*}{\multicolumn{7}{|c|}{$\mathrm{H}$ (organic), ppmv }} \\
\hline & & & & & & \\
\hline $\mathrm{Hg}, \mathrm{ug} / \mathrm{wscm}$ & $3.67 \mathrm{E}+04$ & $3.67 \mathrm{E}+04$ & & $3.67 \mathrm{E}+01$ & $3.38 \mathrm{E}+01$ & \\
\hline $\mathrm{PM}, \mathrm{mg} / \mathrm{wscm}$ & $4.53 \mathrm{E}+01$ & $4.53 \mathrm{E}+01$ & $1.58 \mathrm{E}+01$ & $8.69 \mathrm{E}+00$ & $9.29 \mathrm{E}+00$ & \\
\hline \multicolumn{7}{|l|}{ SVM, ug/wscm } \\
\hline \multicolumn{7}{|l|}{ LVM, ug/wscm } \\
\hline Gas Stream Bulk & & & & & & \\
\hline Composition (Dry Basis) & $\mathrm{mol} \%$ & $\mathrm{~mol} \%$ & $\mathrm{~mol} \%$ & $\mathrm{~mol} \%$ & $\mathrm{~mol} \%$ & $\mathrm{~mol} \%$ \\
\hline $\mathrm{O} 2, \mathrm{~mol} \%$, dry basis & $5.50 \%$ & $5.50 \%$ & $20.99 \%$ & $0.77 \%$ & $9.04 \%$ & \\
\hline $\mathrm{N} 2, \mathrm{~mol} \%$, dry basis & $5.95 \%$ & $5.95 \%$ & $79.01 \%$ & $5.95 \%$ & $22.64 \%$ & \\
\hline \multicolumn{7}{|l|}{$\mathrm{H} 2, \mathrm{~mol} \%$, dry basis } \\
\hline $\mathrm{CO} 2, \mathrm{~mol} \%$, dry basis & $88.44 \%$ & $88.44 \%$ & & $88.44 \%$ & $68.24 \%$ & \\
\hline $\begin{array}{l}\text { Gas Stream Bulk } \\
\text { Composition (Dry Basis, } \\
\text { Corrected to } 7 \% \text { O2 with } \\
100 \% \text { O2 Combustion Air) }\end{array}$ & $\begin{array}{l}\mathrm{ppmv}, \text { or } \\
\text { ug/dscm or } \\
\mathrm{mg} / \mathrm{dscm}\end{array}$ & $\begin{array}{l}\text { ppmv, or } \\
\text { ug/dscm or } \\
\mathrm{mg} / \mathrm{dscm}\end{array}$ & $\begin{array}{l}\text { ppmv, or } \\
\text { ug/dscm or } \\
\mathrm{mg} / \mathrm{dscm}\end{array}$ & $\begin{array}{l}\text { ppmv, or } \\
\text { ug/dscm or } \\
\mathrm{mg} / \mathrm{dscm}\end{array}$ & $\begin{array}{c}\text { ppmv, or } \\
\text { ug/dscm or } \\
\mathrm{mg} / \mathrm{dscm}\end{array}$ & $\begin{array}{l}\mathrm{ppmv} \text {, or } \\
\mathrm{ug} / \mathrm{dscm} \text { or } \\
\mathrm{mg} / \mathrm{dscm}\end{array}$ \\
\hline COgas, ppmv, dry basis & $8.08 \mathrm{E}-02$ & $8.08 \mathrm{E}-02$ & & $8.08 \mathrm{E}-02$ & $6.24 \mathrm{E}-02$ & \\
\hline NO, ppmv, dry basis & $6.39 \mathrm{E}+01$ & $6.39 \mathrm{E}+01$ & & $6.39 \mathrm{E}+01$ & $4.93 \mathrm{E}+01$ & \\
\hline NO2, ppmv, dry basis & $2.49 \mathrm{E}+00$ & $2.49 \mathrm{E}+00$ & & $2.49 \mathrm{E}+00$ & $1.92 \mathrm{E}+00$ & \\
\hline SO2, ppmv, dry basis & $6.40 \mathrm{E}+01$ & $6.40 \mathrm{E}+01$ & & $6.40 \mathrm{E}+01$ & $4.94 \mathrm{E}+01$ & \\
\hline Cl, ppmv, dry basis & $3.45 \mathrm{E}-02$ & $3.45 \mathrm{E}-02$ & & $9.00 \mathrm{E}-03$ & $6.94 \mathrm{E}-03$ & \\
\hline F, ppmv, dry basis & $1.40 \mathrm{E}+00$ & $1.40 \mathrm{E}+00$ & & $1.40 \mathrm{E}+00$ & $1.08 \mathrm{E}+00$ & \\
\hline \multicolumn{7}{|l|}{$\mathrm{C}$ (organic), ppmv, dry basis } \\
\hline \multicolumn{7}{|l|}{$\mathrm{H}$ (organic), ppmv, dry basis } \\
\hline $\mathrm{Hg}, \mathrm{ug} / \mathrm{dscm}$ & $1.74 \mathrm{E}+04$ & $1.74 \mathrm{E}+04$ & & $1.74 \mathrm{E}+01$ & $1.35 \mathrm{E}+01$ & \\
\hline $\mathrm{PM}, \mathrm{mg} / \mathrm{dscm}$ & $2.15 \mathrm{E}+01$ & $2.15 \mathrm{E}+01$ & $2.22 \mathrm{E}+00$ & $4.12 \mathrm{E}+00$ & $3.70 \mathrm{E}+00$ & \\
\hline \multicolumn{7}{|l|}{ SVM, ug/dscm } \\
\hline LVM, ug/dscm & & & & & & \\
\hline
\end{tabular}


Table A-22. Mass balance, Tank WM-189 waste (continued).

\begin{tabular}{|c|c|c|c|c|c|c|c|}
\hline PFD Number & PFD-3 & PFD-3 & PFD-3 & PFD-3 & PFD-3 & PFD-3 & PFD-2 \\
\hline Stream Number & 203 & 204 & 205 & 206 & 207 & 301 & 303 \\
\hline Stream Name & $\begin{array}{l}\text { Fluidizing } \\
\text { Gas to } \\
\text { Reformer }\end{array}$ & $\begin{array}{c}\text { Propane to } \\
\text { Oxidizer }\end{array}$ & $\begin{array}{l}\text { Water to } \\
\text { Spray } \\
\text { Quench }\end{array}$ & $\begin{array}{l}\text { ANN to } \\
\text { Scrub for } F \\
\text { Adjust }\end{array}$ & $\begin{array}{l}\text { HNO3 to } \\
\text { Scrub for } \\
\mathrm{H}+\text { Adjust }\end{array}$ & Isopropanol & Bed Media \\
\hline Rate or Volume & $1.11 \mathrm{E}+04$ & $9.05 \mathrm{E}+02$ & $4.67 \mathrm{E}+02$ & $3.12 \mathrm{E}-02$ & $1.13 \mathrm{E}+00$ & $8.21 \mathrm{E}+01$ & $1.38 \mathrm{E}+00$ \\
\hline Volume Flow (standard, wet) ${ }^{*}$ & $1.09 \mathrm{E}+04$ & $2.56 \mathrm{E}+03$ & & & & & \\
\hline Volume Flow (standard, dry) ${ }^{*}$ & $1.09 \mathrm{E}+04$ & $2.56 \mathrm{E}+03$ & & & & & \\
\hline Rate Units & $\mathrm{ft} 3 / \mathrm{hr}$ & $\mathrm{ft} 3 / \mathrm{hr}$ & $\mathrm{gal} / \mathrm{hr}$ & $\mathrm{gal} / \mathrm{hr}$ & gal $/ \mathrm{hr}$ & gal/hr & $\mathrm{lb} / \mathrm{hr}$ \\
\hline Rate or Volume, metric & $3.10 \mathrm{E}+02$ & $7.26 \mathrm{E}+01$ & $1.77 \mathrm{E}+03$ & $1.18 \mathrm{E}-01$ & $4.29 \mathrm{E}+00$ & $3.11 \mathrm{E}+02$ & $6.26 \mathrm{E}-01$ \\
\hline Rate Units & wscm $/ \mathrm{hr}$ & wscm/hr & $\mathrm{L} / \mathrm{hr}$ & $\mathrm{L} / \mathrm{hr}$ & $\mathrm{L} / \mathrm{hr}$ & $\mathrm{L} / \mathrm{hr}$ & $\mathrm{kg} / \mathrm{hr}$ \\
\hline Temperature, ${ }^{\circ} \mathrm{C}$ & 580 & 25 & 25 & 25 & 25 & 15 & 15 \\
\hline Temperature, ${ }^{\circ} \mathrm{F}$ & 1076 & 77 & 77 & 77 & 77 & 59 & 59 \\
\hline Pressure, psia & 42.30 & 42.30 & 112.30 & 14.70 & 42.30 & 14.70 & 14.70 \\
\hline Specific Gravity & $1.81 \mathrm{E}-03$ & $5.19 \mathrm{E}-03$ & $9.93 \mathrm{E}-01$ & $1.80 \mathrm{E}+00$ & $1.23 \mathrm{E}+00$ & $1.03 \mathrm{E}+00$ & $1.58 \mathrm{E}+00$ \\
\hline Chemical Composition & $\mathrm{Mol} \%$ & Ib/wscf & Mol/liter & Mol/liter & Mol/liter & Mol/liter & Wt frac \\
\hline $\mathrm{H}+$ & & & $9.95 \mathrm{E}-08$ & & $1.33 \mathrm{E}+01$ & & \\
\hline $\mathrm{Al}+3$ & & & & $2.20 \mathrm{E}+00$ & & & 0.529 \\
\hline \multirow{2}{*}{\multicolumn{8}{|c|}{$\frac{S b+5}{A s+3}$}} \\
\hline & & & & & & & \\
\hline \multicolumn{8}{|l|}{$\mathrm{Ba}+2$} \\
\hline \multicolumn{8}{|l|}{$\mathrm{Be}+2$} \\
\hline \multirow{2}{*}{\multicolumn{8}{|c|}{$\frac{B+3}{C d+2}$}} \\
\hline & & & & & & & \\
\hline \multicolumn{8}{|l|}{$\mathrm{Ca}+2$} \\
\hline \multicolumn{8}{|l|}{$\mathrm{Cr}+3$} \\
\hline \multicolumn{8}{|l|}{$\mathrm{Co}+2$} \\
\hline \multirow{2}{*}{\multicolumn{8}{|c|}{$\frac{\mathrm{Cs}+}{\mathrm{Cu}+2}$}} \\
\hline & & & & & & & \\
\hline \multicolumn{8}{|l|}{$\mathrm{Fe}+3$} \\
\hline \multicolumn{8}{|l|}{$\mathrm{Pb}+2$} \\
\hline \multirow{2}{*}{\multicolumn{8}{|c|}{$\frac{\mathrm{Hg}+2}{\mathrm{Mn}+4}$}} \\
\hline & & & & & & & \\
\hline \multicolumn{8}{|l|}{$\begin{array}{l}\mathrm{Mn}+4 \\
\mathrm{Ni}+2 \\
\end{array}$} \\
\hline \multicolumn{8}{|l|}{$\mathrm{K}+$} \\
\hline \multicolumn{8}{|l|}{$\mathrm{Se}+4$} \\
\hline \multirow{2}{*}{\multicolumn{8}{|c|}{$\frac{\mathrm{Ag}+}{\mathrm{Na}+}$}} \\
\hline \multirow{2}{*}{\multicolumn{8}{|c|}{$\frac{\mathrm{Na}+}{\mathrm{Tl}+3}$}} \\
\hline & & & & & & & \\
\hline \multicolumn{8}{|l|}{$\frac{T I+3}{U+4}$} \\
\hline \multicolumn{8}{|l|}{$\mathrm{V}+3$} \\
\hline $\mathrm{Zn}+2$ & & & & & & & \\
\hline $\mathrm{Zr}+4$ & & & & & & & \\
\hline $\mathrm{Cl}-$ & & & & & & & \\
\hline $\mathrm{F}-$ & & & & & & & \\
\hline SO4-2 & & & & & & & \\
\hline NO3- & & & & $6.60 \mathrm{E}+00$ & $1.33 \mathrm{E}+01$ & & \\
\hline & & & & & & & \\
\hline $\mathrm{CO} 2$ & 100 & & & & & & \\
\hline$\overline{\mathrm{OH}-}$ & & & $9.95 \mathrm{E}-08$ & $7.31 \mathrm{E}-08$ & & & \\
\hline $\mathrm{H} 2 \mathrm{O}$ & & & $5.51 \mathrm{E}+01$ & $7.41 \mathrm{E}+01$ & $2.17 \mathrm{E}+01$ & & \\
\hline $\mathrm{O}$ (oxides) & & & & & & & 0.471 \\
\hline C (organic) & & 9.35E-02 & & & & $5.15 \mathrm{E}+01$ & \\
\hline $\mathrm{H}$ (organic) & & $2.09 \mathrm{E}-02$ & & & & $1.37 \mathrm{E}+02$ & \\
\hline O (organic) & & & & & & $1.72 \mathrm{E}+01$ & \\
\hline Mass Flow $(\mathrm{kg} / \mathrm{hr})$ : & $5.66 \mathrm{E}+02$ & $1.33 E+02$ & $1.76 \mathrm{E}+03$ & $2.13 \mathrm{E}-01$ & $5.28 \mathrm{E}+00$ & $3.20 \mathrm{E}+02$ & $6.26 \mathrm{E}-01$ \\
\hline
\end{tabular}


Table A-22. Mass balance, Tank WM-189 waste (continued).

\begin{tabular}{|c|c|c|c|c|c|c|c|}
\hline PFD Number & PFD-3 & PFD-3 & PFD-3 & PFD-3 & PFD-2 & PFD-2 & PFD-3 \\
\hline Stream Number & 305 & 401 & 402 & 404 & 404 & 503 & 505 \\
\hline Stream Name & $\begin{array}{l}\text { Grout Mix } \\
\text { for Scrub } \\
\text { Blowdown }\end{array}$ & $\begin{array}{l}\text { Scrub } \\
\text { Recycled } \\
\text { to Feed }\end{array}$ & $\begin{array}{c}\text { Scrub } \\
\text { Blowdown } \\
\text { to Grout } \\
\text { Mixer }\end{array}$ & $\begin{array}{l}\text { MLLW } \\
\text { Grout from } \\
\text { Scrub }\end{array}$ & $\begin{array}{l}\text { MLLW } \\
\text { Grout } \\
\text { Drums }\end{array}$ & $\begin{array}{c}\text { Feed } \\
\text { Atomizing } \\
\text { Gas }\end{array}$ & $\begin{array}{c}\text { Oxygen to } \\
\text { Oxidizer }\end{array}$ \\
\hline Rate or Volume & $6.61 \mathrm{E}+00$ & $5.23 \mathrm{E}+00$ & $2.57 \mathrm{E}-01$ & $8.82 \mathrm{E}+00$ & $2.29 \mathrm{E}-01$ & $5.09 \mathrm{E}+03$ & $5.45 \mathrm{E}+03$ \\
\hline $\bar{V}_{\text {Volume Flow (stand, wet }}^{*}$ & & & & & & $1.46 \mathrm{E}+04$ & $3.37 \mathrm{E}+04$ \\
\hline Volume Flow (standard, dry) $)^{*}$ & & & & & & $1.46 \mathrm{E}+04$ & $3.37 \mathrm{E}+04$ \\
\hline Rate Units & $\mathrm{lb} / \mathrm{hr}$ & $\mathrm{gal} / \mathrm{hr}$ & gal/hr & $\mathrm{lb} / \mathrm{hr}$ & Drums/day & $\mathrm{ft} 3 / \mathrm{hr}$ & $\mathrm{ft} 3 / \mathrm{hr}$ \\
\hline Rate or Volume, metric & $3.00 \mathrm{E}+00$ & $1.98 \mathrm{E}+01$ & $9.74 \mathrm{E}-01$ & $4.00 \mathrm{E}+00$ & $4.00 \mathrm{E}+00$ & $4.13 \mathrm{E}+02$ & $9.53 \mathrm{E}+02$ \\
\hline Rate Units & $\mathrm{kg} / \mathrm{hr}$ & $\mathrm{L} / \mathrm{hr}$ & $\mathrm{L} / \mathrm{hr}$ & $\mathrm{kg} / \mathrm{hr}$ & $\mathrm{kg} / \mathrm{hr}$ & wscm $/ \mathrm{hr}$ & wscm $/ \mathrm{hr}$ \\
\hline Temperature, ${ }^{\circ} \mathrm{C}$ & 25 & 70 & 70 & 54 & 54 & 21 & 25 \\
\hline Temperature, ${ }^{\circ} \mathrm{F}$ & 77 & 158 & 158 & 129 & 129 & 70 & 77 \\
\hline Pressure, psia & 14.70 & 29.98 & 29.98 & 14.70 & 14.70 & 42.30 & 92.30 \\
\hline Specific Gravity & $2.01 \mathrm{E}-01$ & $1.03 \mathrm{E}+00$ & $1.03 \mathrm{E}+00$ & $2.10 \mathrm{E}+00$ & $2.10 \mathrm{E}+00$ & $5.25 \mathrm{E}-03$ & $8.21 \mathrm{E}-03$ \\
\hline Chemical Composition & Wt frac & Mol/liter & Mol/liter & Wt frac & Wt frac & $\mathrm{lb} / \mathrm{wscf}$ & $\mathrm{lb} / \mathrm{wscf}$ \\
\hline $\mathrm{H}+$ & & $9.36 \mathrm{E}-01$ & $9.36 \mathrm{E}-01$ & $2.30 \mathrm{E}-04$ & $2.30 \mathrm{E}-04$ & & \\
\hline $\mathrm{Al}+3$ & & $9.19 \mathrm{E}-03$ & $9.19 \mathrm{E}-03$ & $0.01 \%$ & $0.01 \%$ & & \\
\hline$S b+5$ & & $2.58 \mathrm{E}-07$ & $2.58 \mathrm{E}-07$ & $7.64 \mathrm{E}-09$ & $7.64 \mathrm{E}-09$ & & \\
\hline $\mathrm{As}+3$ & & $1.29 \mathrm{E}-06$ & $1.29 \mathrm{E}-06$ & $2.35 \mathrm{E}-08$ & $2.35 \mathrm{E}-08$ & & \\
\hline \multicolumn{8}{|l|}{$\mathrm{Ba}+2$} \\
\hline \multicolumn{8}{|l|}{$\mathrm{Be}+2$} \\
\hline $\mathrm{B}+3$ & & $2.61 \mathrm{E}-04$ & $2.61 \mathrm{E}-04$ & $6.87 \mathrm{E}-07$ & $6.87 \mathrm{E}-07$ & & \\
\hline \multicolumn{8}{|l|}{$\mathrm{Cd}+2$} \\
\hline \multicolumn{8}{|l|}{$\mathrm{Ca}+2$} \\
\hline \multicolumn{8}{|l|}{$\mathrm{Cr}+3$} \\
\hline $\mathrm{Co}+2$ & & $6.10 \mathrm{E}-07$ & $6.10 \mathrm{E}-07$ & $8.75 \mathrm{E}-09$ & $8.75 \mathrm{E}-09$ & & \\
\hline \multicolumn{8}{|l|}{$\mathrm{Cs}+$} \\
\hline \multicolumn{8}{|l|}{$\mathrm{Cu}+2$} \\
\hline $\mathrm{Fe}+3$ & & $1.10 \mathrm{E}-04$ & $1.10 \mathrm{E}-04$ & $1.49 \mathrm{E}-06$ & $1.49 \mathrm{E}-06$ & & \\
\hline \multicolumn{8}{|l|}{$\mathrm{Pb}+2$} \\
\hline $\mathrm{Hg}+2$ & & $7.92 \mathrm{E}-02$ & $7.92 \mathrm{E}-02$ & $3.87 \mathrm{E}-03$ & $3.87 \mathrm{E}-03$ & & \\
\hline $\mathrm{Mn}+4$ & & $2.35 \mathrm{E}-04$ & $2.35 \mathrm{E}-04$ & $3.14 \mathrm{E}-06$ & $3.14 \mathrm{E}-06$ & & \\
\hline \multicolumn{8}{|l|}{$\mathrm{Ni+2}$} \\
\hline \multirow{2}{*}{\multicolumn{8}{|c|}{$\begin{array}{ll}\mathrm{K}+ \\
\mathrm{Se}+4\end{array}$}} \\
\hline & & & & & & & $\mathrm{Se}+4$ \\
\hline \multirow{2}{*}{\multicolumn{8}{|c|}{$\frac{\mathrm{Ag}+}{\mathrm{Na}+}$}} \\
\hline & & & & & & & \\
\hline $\mathrm{T} \mathrm{T}+3$ & & $6.79 \mathrm{E}-08$ & $6.79 \mathrm{E}-08$ & $3.38 \mathrm{E}-09$ & $3.38 \mathrm{E}-09$ & & \\
\hline \multicolumn{8}{|l|}{$\mathrm{U}+4$} \\
\hline \multirow{2}{*}{\multicolumn{8}{|c|}{$\begin{array}{l}\mathrm{V}+3 \\
\mathrm{n}+2\end{array}$}} \\
\hline \multirow{2}{*}{\multicolumn{8}{|c|}{$\frac{Z n+2}{Z r+4}$}} \\
\hline & & & & & & & \\
\hline$\overline{\mathrm{Cl}-}$ & & $4.00 \mathrm{E}-04$ & $4.00 \mathrm{E}-04$ & $3.45 \mathrm{E}-06$ & $3.45 \mathrm{E}-06$ & & \\
\hline F- & & $4.86 \mathrm{E}-03$ & $4.86 \mathrm{E}-03$ & $2.25 \mathrm{E}-05$ & $2.25 \mathrm{E}-05$ & & \\
\hline SO4-2 & & $4.51 \mathrm{E}-06$ & $4.51 \mathrm{E}-06$ & $1.05 \mathrm{E}-07$ & $1.05 \mathrm{E}-07$ & & \\
\hline NO3- & & $8.98 \mathrm{E}-01$ & $8.98 \mathrm{E}-01$ & $1.35 \mathrm{E}-02$ & $1.35 \mathrm{E}-02$ & & \\
\hline \multicolumn{8}{|l|}{$\mathrm{PO} 4-3$} \\
\hline $\mathrm{Am}+4$ & & $9.31 \mathrm{E}-10$ & $9.31 \mathrm{E}-10$ & $5.51 \mathrm{E}-11$ & $5.51 \mathrm{E}-11$ & & \\
\hline $\mathrm{Br}-$ & & $5.30 \mathrm{E}-09$ & $5.30 \mathrm{E}-09$ & $1.03 \mathrm{E}-10$ & $1.03 \mathrm{E}-10$ & & \\
\hline $\mathrm{Ce}+4$ & & $5.65 \mathrm{E}-07$ & $5.65 \mathrm{E}-07$ & $1.93 \mathrm{E}-08$ & $1.93 \mathrm{E}-08$ & & \\
\hline $\mathrm{Eu}+3$ & & $6.43 \mathrm{E}-09$ & $6.43 \mathrm{E}-09$ & $2.38 \mathrm{E}-10$ & $2.38 \mathrm{E}-10$ & & \\
\hline $\mathrm{Gd}+3$ & & $1.93 \mathrm{E}-06$ & $1.93 \mathrm{E}-06$ & $7.39 \mathrm{E}-08$ & $7.39 \mathrm{E}-08$ & & \\
\hline $\mathrm{Ge}+4$ & & & & & & & \\
\hline $\ln +3$ & & $1.79 \mathrm{E}-08$ & $1.79 \mathrm{E}-08$ & $5.00 \mathrm{E}-10$ & $5.00 \mathrm{E}-10$ & & \\
\hline I- & & $5.89 \mathrm{E}-08$ & $5.89 \mathrm{E}-08$ & 1.82E-09 & $1.82 \mathrm{E}-09$ & & \\
\hline$L a+3$ & & $1.17 \mathrm{E}-07$ & $1.17 \mathrm{E}-07$ & $3.94 \mathrm{E}-09$ & $3.94 \mathrm{E}-09$ & & \\
\hline
\end{tabular}


Table A-22. Mass balance, Tank WM-189 waste (continued).

\begin{tabular}{|c|c|c|c|c|c|c|c|}
\hline Stream \# & 305 & 401 & 402 & 404 & 404 & 503 & 505 \\
\hline Chemical Composition & Wt frac & Mol/liter & Mol/liter & Wt frac & Wt frac & $\mathrm{lb} / \mathrm{wscf}$ & $\mathrm{lb} / \mathrm{wscf}$ \\
\hline \multicolumn{8}{|l|}{$\mathrm{Li}+\mathrm{Cl}$} \\
\hline \multicolumn{8}{|l|}{$M g+2$} \\
\hline \multicolumn{8}{|l|}{$\mathrm{Mo}+6$} \\
\hline $\mathrm{Nd}+3$ & & $3.76 \mathrm{E}-07$ & $3.76 \mathrm{E}-07$ & $1.32 \mathrm{E}-08$ & $1.32 \mathrm{E}-08$ & & \\
\hline $\mathrm{Np}+4$ & & $3.90 \mathrm{E}-08$ & $3.90 \mathrm{E}-08$ & $2.25 \mathrm{E}-09$ & $2.25 \mathrm{E}-09$ & & \\
\hline \multicolumn{8}{|l|}{$\mathrm{Nb}+5$} \\
\hline \multicolumn{8}{|l|}{$\mathrm{Pd}+4$} \\
\hline \multicolumn{8}{|l|}{$\mathrm{Pu}+4$} \\
\hline $\mathrm{Pr}+4$ & & $1.06 \mathrm{E}-07$ & $1.06 \mathrm{E}-07$ & $3.64 \mathrm{E}-09$ & $3.64 \mathrm{E}-09$ & & \\
\hline $\mathrm{Pm}+3$ & & $2.13 \mathrm{E}-11$ & $2.13 \mathrm{E}-11$ & $7.52 \mathrm{E}-13$ & $7.52 \mathrm{E}-13$ & & \\
\hline $\mathrm{Rh}+4$ & & $4.57 \mathrm{E}-08$ & $4.57 \mathrm{E}-08$ & $1.15 \mathrm{E}-09$ & $1.15 \mathrm{E}-09$ & & \\
\hline \multicolumn{8}{|l|}{$\mathrm{Rb}+$} \\
\hline $\mathrm{Ru}+3$ & & $1.26 \mathrm{E}-05$ & $1.26 \mathrm{E}-05$ & $3.11 \mathrm{E}-07$ & $3.11 \mathrm{E}-07$ & & \\
\hline $\mathrm{Sm}+3$ & & $7.01 \mathrm{E}-08$ & $7.01 \mathrm{E}-08$ & $2.57 \mathrm{E}-09$ & $2.57 \mathrm{E}-09$ & & \\
\hline $\mathrm{Si+4}$ & $4.67 \mathrm{E}-01$ & & & $3.51 \mathrm{E}-01$ & $3.51 \mathrm{E}-01$ & & \\
\hline \multicolumn{8}{|l|}{$\mathrm{Sr}+2$} \\
\hline $\mathrm{Tc}+7$ & & $9.15 \mathrm{E}-08$ & $9.15 \mathrm{E}-08$ & $2.18 \mathrm{E}-09$ & $2.18 \mathrm{E}-09$ & & \\
\hline \multicolumn{8}{|l|}{$\mathrm{Te}+4$} \\
\hline $\mathrm{Tb}+4$ & & $2.68 \mathrm{E}-11$ & $2.68 \mathrm{E}-11$ & $1.04 \mathrm{E}-12$ & $1.04 \mathrm{E}-12$ & & \\
\hline \multicolumn{8}{|l|}{$T h+4$} \\
\hline \multicolumn{8}{|l|}{$S n+4$} \\
\hline \multicolumn{8}{|l|}{$\mathrm{Ti}+4$} \\
\hline $\mathrm{Y}+3$ & & $8.69 \mathrm{E}-08$ & $8.69 \mathrm{E}-08$ & $1.88 \mathrm{E}-09$ & $1.88 \mathrm{E}-09$ & & \\
\hline $\mathrm{OH}-$ & & $1.32 \mathrm{E}-06$ & $1.32 \mathrm{E}-06$ & $5.45 \mathrm{E}-09$ & $5.45 \mathrm{E}-09$ & & \\
\hline \multirow[t]{2}{*}{$\mathrm{H} 2 \mathrm{O}$} & & $5.30 \mathrm{E}+01$ & $5.30 \mathrm{E}+01$ & $2.32 \mathrm{E}-01$ & 2.32E-01 & & \\
\hline & & & & & & & \\
\hline $\mathrm{SO} 2$ & & $1.39 \mathrm{E}-06$ & $1.39 \mathrm{E}-06$ & $2.16 \mathrm{E}-08$ & $2.16 \mathrm{E}-08$ & & \\
\hline \multicolumn{8}{|l|}{$\mathrm{H} 2 \mathrm{~S}$} \\
\hline $\mathrm{CO}$ & & $2.90 \mathrm{E}-09$ & $2.90 \mathrm{E}-09$ & $1.97 \mathrm{E}-11$ & $1.97 \mathrm{E}-11$ & & \\
\hline $\mathrm{CO} 2$ & & $2.08 \mathrm{E}-04$ & $2.08 \mathrm{E}-04$ & $2.23 \mathrm{E}-06$ & $2.23 \mathrm{E}-06$ & $1.14 \mathrm{E}-01$ & \\
\hline $\mathrm{H} 2$ & & $3.71 \mathrm{E}-08$ & $3.71 \mathrm{E}-08$ & $1.82 \mathrm{E}-11$ & $1.82 \mathrm{E}-11$ & & \\
\hline N2 & & 6.83E-09 & 6.83E-09 & $4.66 \mathrm{E}-11$ & $4.66 \mathrm{E}-11$ & & \\
\hline $\mathrm{NO}$ & & $8.55 \mathrm{E}-08$ & $8.55 \mathrm{E}-08$ & $6.24 \mathrm{E}-10$ & $6.24 \mathrm{E}-10$ & & \\
\hline NO2 & & $1.33 \mathrm{E}-05$ & $1.33 \mathrm{E}-05$ & $1.49 \mathrm{E}-07$ & $1.49 \mathrm{E}-07$ & & \\
\hline \begin{tabular}{|l}
$\mathrm{O} 2$ \\
\end{tabular} & & 1.82E-08 & 1.82E-08 & $1.42 \mathrm{E}-10$ & $1.42 \mathrm{E}-10$ & & $8.30 \mathrm{E}-02$ \\
\hline S (other) & & $2.08 \mathrm{E}-08$ & $2.08 \mathrm{E}-08$ & $1.62 \mathrm{E}-10$ & 1.62E-10 & & \\
\hline \multicolumn{8}{|l|}{$\mathrm{CO} 3$} \\
\hline \multicolumn{8}{|l|}{ C (reductant) } \\
\hline $\mathrm{O}$ (oxides) & $53.26 \%$ & $1.65 \mathrm{E}-04$ & $1.65 \mathrm{E}-04$ & $39.94 \%$ & $39.94 \%$ & & \\
\hline \multicolumn{8}{|l|}{ C (organic) } \\
\hline \multicolumn{8}{|l|}{$\mathrm{H}$ (organic) } \\
\hline \multirow{2}{*}{\multicolumn{8}{|c|}{$\mathrm{O}$ (organic) }} \\
\hline & & & & & & & \\
\hline Mass Flow (kg/hr): & $3.00 \mathrm{E}+00$ & $2.03 \mathrm{E}+01$ & $1.00 \mathrm{E}+00$ & $4.00 \mathrm{E}+00$ & $4.00 \mathrm{E}+00$ & $7.56 \mathrm{E}+02$ & $1.27 \mathrm{E}+03$ \\
\hline Canister Rate, canisters/day & & & & & 0.23 & & \\
\hline Total Canisters Generated & & & & & 31 & & \\
\hline Heat Generation & $\mathrm{w} / \mathrm{m} 3$ & $\mathrm{w} / \mathrm{m} 3$ & $\mathrm{w} / \mathrm{m} 3$ & $\mathrm{w} / \mathrm{m} 3$ & w/Drum & $\mathrm{w} / \mathrm{m} 3$ & $\mathrm{w} / \mathrm{m} 3$ \\
\hline Heat Generation & & $2.78 \mathrm{E}-03$ & $2.78 \mathrm{E}-03$ & $1.42 \mathrm{E}-03$ & $2.84 \mathrm{E}-04$ & & \\
\hline
\end{tabular}


Table A-22. Mass balance, Tank WM-189 waste (continued).

\begin{tabular}{|c|c|c|c|c|c|c|c|}
\hline Stream \# & 305 & 401 & 402 & 404 & 404 & 503 & 505 \\
\hline Radiological Composition & $\mathrm{Ci} / \mathrm{kg}$ & $\mathrm{Ci} / \mathrm{L}$ & $\mathrm{Ci} / \mathrm{L}$ & $\mathrm{Ci} / \mathrm{kg}$ & Ci/Drum & Ci/wscm & $\mathrm{Ci} / \mathrm{wscm}$ \\
\hline \multicolumn{8}{|l|}{$\mathrm{H}-3$} \\
\hline $\mathrm{C}-14$ & & $1.82 \mathrm{E}-16$ & $1.82 \mathrm{E}-16$ & $4.44 \mathrm{E}-17$ & $1.86 \mathrm{E}-14$ & & \\
\hline Co-60 & & $4.16 \mathrm{E}-07$ & $4.16 \mathrm{E}-07$ & 1.01E-07 & $4.26 \mathrm{E}-05$ & & \\
\hline \multicolumn{8}{|l|}{ Ni-59 } \\
\hline \multicolumn{8}{|l|}{$\mathrm{Ni}-63$} \\
\hline \multicolumn{8}{|l|}{ Se-79 } \\
\hline \multicolumn{8}{|l|}{ Sr-90 } \\
\hline Y-90 & & $4.94 \mathrm{E}-04$ & $4.94 \mathrm{E}-04$ & $1.20 \mathrm{E}-04$ & $5.05 \mathrm{E}-02$ & & \\
\hline \multicolumn{8}{|l|}{ Zr-93 } \\
\hline \multirow{2}{*}{\multicolumn{8}{|c|}{\begin{tabular}{l|}
$\mathrm{Nb}-93 \mathrm{~m}$ \\
$\mathrm{Nb}-94$
\end{tabular}}} \\
\hline & & & & & & & \\
\hline Tc-99 & & $2.15 \mathrm{E}-07$ & $2.15 \mathrm{E}-07$ & $5.23 \mathrm{E}-08$ & $2.20 \mathrm{E}-05$ & & \\
\hline Ru-106 & & $1.59 \mathrm{E}-08$ & $1.59 \mathrm{E}-08$ & $3.88 \mathrm{E}-09$ & $1.63 \mathrm{E}-06$ & & \\
\hline Rh-102 & & $1.08 \mathrm{E}-11$ & $1.08 \mathrm{E}-11$ & $2.62 \mathrm{E}-12$ & $1.10 \mathrm{E}-09$ & & \\
\hline Rh-106 & & $1.59 \mathrm{E}-08$ & $1.59 \mathrm{E}-08$ & $3.88 \mathrm{E}-09$ & $1.63 \mathrm{E}-06$ & & \\
\hline \multicolumn{8}{|l|}{ Pd-107 } \\
\hline \multicolumn{8}{|l|}{ Cd-113m } \\
\hline \multicolumn{8}{|l|}{ Sn-121m } \\
\hline \multicolumn{8}{|l|}{ Sn-126 } \\
\hline Sb-125 & & $5.85 \mathrm{E}-06$ & $5.85 \mathrm{E}-06$ & $1.42 \mathrm{E}-06$ & $5.98 \mathrm{E}-04$ & & \\
\hline Sb-126 & & $7.18 \mathrm{E}-10$ & $7.18 \mathrm{E}-10$ & $1.75 \mathrm{E}-10$ & $7.34 \mathrm{E}-08$ & & \\
\hline \multicolumn{8}{|l|}{ Te-125m } \\
\hline \begin{tabular}{|l}
$1-129$ \\
\end{tabular} & & $1.25 \mathrm{E}-09$ & $1.25 \mathrm{E}-09$ & $3.03 \mathrm{E}-10$ & $1.27 \mathrm{E}-07$ & & \\
\hline \multicolumn{8}{|l|}{ Cs-134 } \\
\hline \multicolumn{8}{|l|}{ Cs-135 } \\
\hline \multirow{2}{*}{\multicolumn{8}{|c|}{\begin{tabular}{|l|} 
Cs-137 \\
Ba-137m \\
\end{tabular}}} \\
\hline & & & & & \multicolumn{3}{|c|}{ Ba-137m } \\
\hline Ce-144 & & $1.08 \mathrm{E}-08$ & $1.08 \mathrm{E}-08$ & $2.62 \mathrm{E}-09$ & $1.10 \mathrm{E}-06$ & & \\
\hline Pr-144 & & $1.08 \mathrm{E}-08$ & $1.08 \mathrm{E}-08$ & $2.62 \mathrm{E}-09$ & $1.10 \mathrm{E}-06$ & & \\
\hline Pm-146 & & $6.35 \mathrm{E}-10$ & $6.35 \mathrm{E}-10$ & $1.55 \mathrm{E}-10$ & $6.50 \mathrm{E}-08$ & & \\
\hline Pm-147 & & $2.90 \mathrm{E}-06$ & $2.90 \mathrm{E}-06$ & $7.06 \mathrm{E}-07$ & $2.97 \mathrm{E}-04$ & & \\
\hline Sm-151 & & $5.74 \mathrm{E}-06$ & $5.74 \mathrm{E}-06$ & $1.40 \mathrm{E}-06$ & $5.87 \mathrm{E}-04$ & & \\
\hline Eu-152 & & $3.68 \mathrm{E}-08$ & $3.68 \mathrm{E}-08$ & $8.96 \mathrm{E}-09$ & $3.76 \mathrm{E}-06$ & & \\
\hline Eu-154 & & $2.20 \mathrm{E}-06$ & $2.20 \mathrm{E}-06$ & $5.35 \mathrm{E}-07$ & $2.25 \mathrm{E}-04$ & & \\
\hline Eu-155 & & $2.07 \mathrm{E}-06$ & $2.07 \mathrm{E}-06$ & $5.04 \mathrm{E}-07$ & $2.11 \mathrm{E}-04$ & & \\
\hline Th-230 & & & & 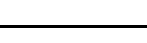 & & & \\
\hline Pa-233 & & & & & & & \\
\hline \begin{tabular}{|l|l|}
$\mathrm{a}-232$ \\
\end{tabular} & & & 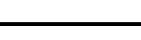 & 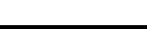 & 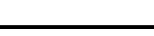 & & \\
\hline $\mathrm{U}-233$ & & & & & & & \\
\hline & & & & & & & \\
\hline U-234 & & & & & & & \\
\hline U-235 & & & & & & & \\
\hline $\mathrm{U}-236$ & & & & & & & \\
\hline U-237 & & & & & & & \\
\hline U-238 & & & & & & & \\
\hline Np-237 & & $6.52 \mathrm{E}-09$ & $6.52 \mathrm{E}-09$ & $1.59 \mathrm{E}-09$ & $6.67 \mathrm{E}-07$ & & \\
\hline Pu-236 & & & & & & & \\
\hline Pu-238 & & & & & & & \\
\hline \begin{tabular}{|l|} 
Pu-239 \\
\end{tabular} & & & & & & & \\
\hline Pu-240 & & & & & & & \\
\hline Pu-241 & & & & & & & \\
\hline Pu-242 & & & & & & & \\
\hline Pu-244 & & & & & & & \\
\hline \begin{tabular}{|l|}
$\mathrm{Am}-241$ \\
\end{tabular} & & $1.02 \mathrm{E}-06$ & $1.02 \mathrm{E}-06$ & $2.48 \mathrm{E}-07$ & $1.04 \mathrm{E}-04$ & & \\
\hline Am-242m & & $1.91 \mathrm{E}-10$ & $1.91 \mathrm{E}-10$ & $4.64 \mathrm{E}-11$ & $1.95 \mathrm{E}-08$ & & \\
\hline
\end{tabular}


Table A-22. Mass balance, Tank WM-189 waste (continued).

\begin{tabular}{|c|c|c|c|c|c|c|c|}
\hline Stream \# & 305 & 401 & 402 & 404 & 404 & 503 & 505 \\
\hline Radiological Composition & $\mathrm{Ci} / \mathrm{kg}$ & $\mathrm{Ci} / \mathrm{L}$ & $\mathrm{Ci} / \mathrm{L}$ & $\mathrm{Ci} / \mathrm{kg}$ & Ci/Drum & $\mathrm{Ci} / \mathrm{wscm}$ & Ci/wscm \\
\hline Am-243 & & $3.25 \mathrm{E}-10$ & $3.25 \mathrm{E}-10$ & $7.92 \mathrm{E}-11$ & $3.33 \mathrm{E}-08$ & & \\
\hline \multicolumn{8}{|l|}{\begin{tabular}{|l|l|}
$\mathrm{Cm}-242$ \\
$\mathrm{Cm}-243$
\end{tabular}} \\
\hline $\mathrm{Cm}-243$ & & & & & & & \\
\hline \multicolumn{8}{|l|}{$\mathrm{Cm}-244$} \\
\hline \multicolumn{8}{|l|}{$\mathrm{Cm}-245$} \\
\hline \multicolumn{8}{|l|}{$\mathrm{Cm}-246$} \\
\hline TRU & & $1.02 \mathrm{E}-06$ & $1.02 \mathrm{E}-06$ & $2.49 \mathrm{E}-07$ & $1.05 \mathrm{E}-04$ & & \\
\hline & & & & & & & \\
\hline & & & & & & & \\
\hline $\begin{array}{l}\text { Gas Stream Bulk Composition } \\
\text { (Wet Basis) }\end{array}$ & $\begin{array}{c}\text { mol\% or } \\
\text { ppmv }\end{array}$ & $\begin{array}{c}\text { mol\% or } \\
\text { ppmv }\end{array}$ & $\begin{array}{c}\text { mol\% or } \\
\text { ppmv }\end{array}$ & $\begin{array}{c}\mathrm{mol} \% \text { or } \\
\text { ppmv }\end{array}$ & $\begin{array}{c}\text { mol\% or } \\
\text { ppmv }\end{array}$ & $\begin{array}{c}\text { mol\% or } \\
\text { ppmv }\end{array}$ & $\begin{array}{c}\text { mol\% or } \\
\text { ppmv }\end{array}$ \\
\hline \multicolumn{8}{|l|}{$\mathrm{H} 2 \mathrm{O}, \mathrm{mol} \%$} \\
\hline $\mathrm{O} 2, \mathrm{~mol} \%$ & & & & & & & $100 \%$ \\
\hline \multicolumn{8}{|l|}{$\mathrm{N} 2, \mathrm{~mol} \%$} \\
\hline \multicolumn{8}{|l|}{$\mathrm{H} 2, \mathrm{~mol} \%$} \\
\hline $\mathrm{CO} 2, \mathrm{~mol} \%$ & & & & & & $100 \%$ & \\
\hline \multicolumn{8}{|l|}{ COgas, ppmv } \\
\hline \multirow{2}{*}{\multicolumn{8}{|c|}{$\frac{\mathrm{NO}, \mathrm{ppmv}}{\mathrm{NO} 2, \mathrm{ppmv}}$}} \\
\hline & & & & & & & \\
\hline \multicolumn{8}{|l|}{ SO2, ppmv } \\
\hline \multicolumn{8}{|l|}{$\mathrm{Cl}, \mathrm{ppmv}$} \\
\hline \multicolumn{8}{|l|}{ F, ppmv } \\
\hline \multirow{2}{*}{\multicolumn{8}{|c|}{$\frac{\mathrm{C} \text { (organic), ppmv }}{\mathrm{H} \text { (organic), ppmv }}$}} \\
\hline & & & & & & & \\
\hline \multirow{2}{*}{\multicolumn{8}{|c|}{$\mathrm{Hg}, \mathrm{ug} / \mathrm{wscm}$}} \\
\hline & & & & & & & \\
\hline $\mathrm{PM}, \mathrm{mg} / \mathrm{wscm}$ & & & & & & & $9.46 \mathrm{E}-02$ \\
\hline \multicolumn{8}{|l|}{ SVM, ug/wscm } \\
\hline \multicolumn{8}{|l|}{ LVM, ug/wscm } \\
\hline $\begin{array}{l}\text { Gas Stream Bulk Composition } \\
\text { (Dry Basis) }\end{array}$ & $\mathrm{mol} \%$ & $\mathrm{~mol} \%$ & $\mathrm{~mol} \%$ & $\mathrm{~mol} \%$ & $\mathrm{~mol} \%$ & $\mathrm{~mol} \%$ & $\mathrm{~mol} \%$ \\
\hline \multicolumn{8}{|l|}{$\mathrm{O} 2, \mathrm{~mol} \%$, dry basis } \\
\hline \multirow{2}{*}{\multicolumn{8}{|c|}{\begin{tabular}{|l|}
$\mathrm{N} 2, \mathrm{~mol} \%$, dry basis \\
$\mathrm{H} 2, \mathrm{~mol} \%$, dry basis
\end{tabular}}} \\
\hline & & & & & & & \\
\hline \multicolumn{8}{|l|}{$\begin{array}{l}\mathrm{H} 2, \mathrm{~mol} \% \text {, ary basis } \\
\mathrm{CO} 2, \mathrm{~mol} \% \text {, dry basis }\end{array}$} \\
\hline $\begin{array}{l}\text { Gas Stream Bulk Composition } \\
\text { (Dry Basis, Corrected to } 7 \% \\
\text { O2 with } 100 \% \text { O2 Combustion } \\
\text { Air) }\end{array}$ & $\begin{array}{l}\text { ppmv, or } \\
\text { ug/dscm or } \\
\mathrm{mg} / \mathrm{dscm}\end{array}$ & $\begin{array}{l}\mathrm{ppmv}, \text { or } \\
\mathrm{ug} / \mathrm{dscm} \text { or } \\
\mathrm{mg} / \mathrm{dscm}\end{array}$ & $\begin{array}{c}\mathrm{ppmv}, \text { or } \\
\mathrm{ug} / \mathrm{dscm} \text { or } \\
\mathrm{mg} / \mathrm{dscm}\end{array}$ & $\begin{array}{l}\text { ppmv, or } \\
\text { ug/dscm or } \\
\mathrm{mg} / \mathrm{dscm}\end{array}$ & $\begin{array}{l}\text { ppmv, or } \\
\text { ug/dscm or } \\
\mathrm{mg} / \mathrm{dscm}\end{array}$ & $\begin{array}{l}\text { ppmv, or } \\
\text { ug/dscm or } \\
\mathrm{mg} / \mathrm{dscm}\end{array}$ & $\begin{array}{l}\mathrm{ppmv} \text {, or } \\
\mathrm{ug} / \mathrm{dscm} \\
\text { or } \\
\mathrm{mg} / \mathrm{dscm}\end{array}$ \\
\hline \multicolumn{8}{|l|}{ COgas, ppmv, dry basis } \\
\hline NO, ppmv, dry basis & & & & & & & \\
\hline NO2, ppmv, dry basis & & & & & & & \\
\hline SO2, ppmv, dry basis & & & & & & & \\
\hline $\mathrm{Cl}, \mathrm{ppmv}$, dry basis & & & & & & & \\
\hline F, ppmv, dry basis & & & & & & & \\
\hline C (organic), ppmv, dry basis & & & & & & & \\
\hline $\mathrm{H}$ (organic), ppmv, dry basis & & & & & & & \\
\hline $\mathrm{Hg}, \mathrm{ug} / \mathrm{dscm}$ & & & & & & & \\
\hline $\mathrm{PM}, \mathrm{mg} / \mathrm{dscm}$ & & & & & & & \\
\hline SVM, ug/dscm & & & & & & & \\
\hline LVM, ug/dscm & & & & & & & \\
\hline
\end{tabular}


Table A-22. Mass balance, Tank WM-189 waste (continued).

\begin{tabular}{|c|c|c|c|c|c|c|c|c|}
\hline PFD Number & PFD-3 & PFD-3 & PFD-3 & PFD-3 & PFD-3 & PFD-3 & PFD-3 & PFD-3 \\
\hline Stream Number & 510 & 511 & 512 & 513 & 514 & 515 & 516 & 517 \\
\hline Stream Name & $\begin{array}{l}\text { Gas to } \\
\text { Cool } \\
\text { Product }\end{array}$ & $\begin{array}{l}\text { Hot Gas } \\
\text { after } \\
\text { Product } \\
\text { Cooling }\end{array}$ & $\begin{array}{c}\text { Backpulse } \\
\text { Gas for } \\
\text { Candle } \\
\text { Filters }\end{array}$ & $\begin{array}{c}\text { Bed/ Solid } \\
\text { Reductant } \\
\text { Transport } \\
\text { Gas } \\
\end{array}$ & \begin{tabular}{|c|} 
TF \\
Transfer \& \\
Tank \\
Sparge Air \\
\end{tabular} & $\begin{array}{c}\text { Ventilation } \\
\text { Air }\end{array}$ & New GAC & $\begin{array}{l}\text { Off-gas } \\
\text { Preaheater } \\
\text { Steam }\end{array}$ \\
\hline Rate or Volume & $2.19 \mathrm{E}+02$ & $6.85 \mathrm{E}+02$ & $2.22 \mathrm{E}+02$ & $6.48 \mathrm{E}-01$ & $1.52 \mathrm{E}+01$ & $2.89 \mathrm{E}+01$ & $4.73 \mathrm{E}+00$ & $1.31 \mathrm{E}+03$ \\
\hline Volume Flow (standard, wet) ${ }^{*}$ & $1.48 \mathrm{E}+03$ & $1.48 \mathrm{E}+03$ & $6.51 \mathrm{E}+02$ & $1.90 \mathrm{E}+00$ & $4.45 \mathrm{E}+01$ & $2.94 \mathrm{E}+01$ & & $6.52 \mathrm{E}+03$ \\
\hline Volume Flow (standard, dry) ${ }^{*}$ & $1.48 \mathrm{E}+03$ & $1.48 \mathrm{E}+03$ & $6.51 \mathrm{E}+02$ & $1.90 \mathrm{E}+00$ & $4.45 \mathrm{E}+01$ & $2.94 \mathrm{E}+01$ & & $0.00 \mathrm{E}+00$ \\
\hline Rate Units & $\mathrm{ft} 3 / \mathrm{hr}$ & $\mathrm{ft} 3 / \mathrm{hr}$ & $\mathrm{ft} 3 / \mathrm{hr}$ & $\mathrm{ft} 3 / \mathrm{hr}$ & $\mathrm{ft} 3 / \mathrm{hr}$ & $\mathrm{ft} 3 / \mathrm{hr}$ & $\mathrm{lb} / \mathrm{hr}$ & $\mathrm{ft} 3 / \mathrm{hr}$ \\
\hline Rate or Volume, metric & $4.18 \mathrm{E}+01$ & $4.18 \mathrm{E}+01$ & $1.84 \mathrm{E}+01$ & $5.37 \mathrm{E}-02$ & $1.26 \mathrm{E}+00$ & 8.34E-01 & $2.15 \mathrm{E}+00$ & $1.84 \mathrm{E}+02$ \\
\hline Rate Units & wscm/hr & wscm/hr & wscm/hr & wscm/hr & wscm/hr & wscm/hr & $\mathrm{kg} / \mathrm{hr}$ & wscm/hr \\
\hline Temperature, ${ }^{\circ} \mathrm{C}$ & 0 & 580 & 15 & 15 & 15 & 15 & 25 & 177 \\
\hline Temperature, ${ }^{\circ} \mathrm{F}$ & 32 & 1077 & 59 & 59 & 59 & 59 & 77 & 350 \\
\hline Pressure, psia & 92.30 & 92.30 & 42.30 & 42.30 & 42.30 & 14.70 & 14.70 & 112.30 \\
\hline Specific Gravity & $7.85 \mathrm{E}-03$ & $2.51 \mathrm{E}-03$ & $3.41 \mathrm{E}-03$ & $3.41 \mathrm{E}-03$ & $3.51 \mathrm{E}-03$ & $1.22 \mathrm{E}-03$ & $4.81 \mathrm{E}-01$ & $3.73 \mathrm{E}-03$ \\
\hline Chemical Composition & $\mathrm{lb} / \mathrm{wscf}$ & $\mathrm{lb} / \mathrm{wscf}$ & $\mathrm{lb} / \mathrm{wscf}$ & $\mathrm{lb} / \mathrm{wscf}$ & $\mathrm{lb} / \mathrm{wscf}$ & $\mathrm{lb} / \mathrm{wscf}$ & Wt. $\%$ & $\mathrm{lb} / \mathrm{wscf}$ \\
\hline C (reductant) & & & & & & & $100 \%$ & \\
\hline & & & & & & & & \\
\hline Gas Composition, wet basis & $\mathrm{mol} \mathrm{\%}$ & $\mathrm{mol} \%$ & $\mathrm{~mol} \mathrm{\%}$ & $\mathrm{mol} \%$ & $\mathrm{~mol} \%$ & $\mathrm{~mol} \%$ & $\mathrm{~mol} \%$ & $\mathrm{~mol} \%$ \\
\hline $\mathrm{H} 2 \mathrm{O}, \mathrm{mol} \%$ & & & & & $0.01 \%$ & $0.01 \%$ & & $100 \%$ \\
\hline $\mathrm{O} 2, \mathrm{~mol} \%$ & & & & & $21 \%$ & $21 \%$ & & \\
\hline $\mathrm{N} 2, \mathrm{~mol} \%$ & $100 \%$ & $100 \%$ & $100 \%$ & $100 \%$ & $79 \%$ & $79 \%$ & & \\
\hline $\mathrm{PM}, \mathrm{mg} / \mathrm{wscm}$ & $1.99 \mathrm{E}+01$ & 1.99E+01 & $1.98 \mathrm{E}+01$ & $1.95 \mathrm{E}+01$ & $1.55 \mathrm{E}+01$ & $1.57 \mathrm{E}+01$ & & $1.20 \mathrm{E}+01$ \\
\hline & & & & & & & & \\
\hline $\mathrm{kg} / \mathrm{hr}$ & $4.87 \mathrm{E}+01$ & $4.87 \mathrm{E}+01$ & $2.15 \mathrm{E}+01$ & $6.26 \mathrm{E}-02$ & $1.51 \mathrm{E}+00$ & $1.00 \mathrm{E}+00$ & $2.15 \mathrm{E}+00$ & $1.38 \mathrm{E}+02$ \\
\hline
\end{tabular}




\section{A-7. UTILITIES SUMMARY}

Utilities directly supporting the TTT reforming process are listed in Table A-23 along with an estimate of the consumption rate (electrical) or total consumption (steam use, cooling water, and cooling air). It is assumed that low pressure steam (35 psig) will be available to supply heat to the nitrogen and oxygen vaporizers (HE-300 and HE-301) as well as the off-gas heater (HE-304) upstream of the carbon bed. The primary user of cooling water is the scrub cooler (HE-303), while cooling air is used for the offgas cooler (HE-305) upstream of the sintered metal sinter. The largest electrical loads are drawn by the off-gas compressor and auxiliary heating of the steam reforming vessel.

Table A-23. Summary of the anticipated major utility uses for the TTT process.

\begin{tabular}{lccccc}
\hline & WM-180 & WM-187 & WM-188 & WM-189 & Total \\
\hline Low pressure steam $^{\mathrm{a}}$ (MMBtu) & 1910 & 1200 & 1960 & 2020 & 7090 \\
Low Pressure steam $^{\mathrm{a}}(\mathrm{lbs})$ & $2.06 \times 10^{6}$ & $1.30 \times 10^{6}$ & $2.11 \times 10^{6}$ & $2.19 \times 10^{6}$ & $7.66 \times 10^{6}$ \\
Cooling water (gal) & $1.16 \times 10^{6}$ & $.58 \times 10^{6}$ & $.61 \times 10^{6}$ & $1.24 \times 10^{6}$ & $3.6 \times 10^{6}$ \\
Cooling air (scf) & $1.08 \times 10^{9}$ & $.68 \times 10^{9}$ & $1.12 \times 10^{9}$ & $1.15 \times 10^{9}$ & $4.0 \times 10^{9}$ \\
Electrical: & & & & & \\
Compressor (kW) & 230 & 227 & 233 & 247 & \\
Reformer $(\mathrm{kW})$ & 472 & 425 & 443 & 425 & \\
\hline
\end{tabular}

a - low pressure steam assumed available at 35 psig.

Utilities directly supporting the TWR reforming process are listed in Table A-24 along with an estimate of the consumption rate (electrical) or total consumption (steam use, cooling water, and cooling air). It is assumed that low pressure steam (47.5 psig) will be available to supply heat to the carbon dioxide and oxygen vaporizers (HE-300 and HE-301), as well as the off-gas heater (HE-304). The primary user of cooling water is the scrub cooler (HE-303), while cooling air is used for the off-gas cooler (HE-305). The largest electrical loads are drawn by the off-gas compressor, the heating of the fluidization gas (HE-302), and auxiliary heating of the steam reforming vessel.

Table A-24. Summary of the anticipated major utility uses for the TRW process.

\begin{tabular}{lccccc}
\hline & WM-180 & WM-187 & WM-188 & WM-189 & Total \\
\hline Low pressure steam $^{\mathrm{a}}(\mathrm{MMBtu})$ & 3,130 & 2,000 & 3,150 & 3,060 & $1.13 \times 10^{4}$ \\
Low pressure steam $^{\mathrm{a}}(\mathrm{lbs})$ & $3.38 \times 10^{6}$ & $2.16 \times 10^{6}$ & $3.40 \times 10^{6}$ & $3.30 \times 10^{6}$ & $12.2 \times 10^{6}$ \\
Cooling water (gal) & $1.31 \times 10^{6}$ & $0.84 \times 10^{6}$ & $1.35 \times 10^{6}$ & $1.30 \times 10^{6}$ & $4.81 \times 10^{6}$ \\
Cooling air (scf) & $1.087 \times 10^{9}$ & $0.67 \times 10^{9}$ & $1.10 \times 10^{9}$ & $1.07 \times 10^{9}$ & $3.92 \times 10^{9}$ \\
Electrical: & & & & & \\
Compressor (kW) & 226 & 223 & 224 & 228 & \\
Reformer (kW) & 510 & 443 & 457 & 431 & \\
Fluid. gas heater (kW) & 147 & 147 & 147 & 147 & \\
\hline
\end{tabular}

a. Low pressure steam assumed available at $47.5 \mathrm{psig}$. 


\section{A-8. EQUIPMENT LISTS AND PLANT SCALE}

A preliminary equipment list for the TTT process is given in Table A-25. Equipment sizing was based on ASPEN simulation results and knowledge of the performance of existing equipment at the NWCF.

Most process operations in the Steam Reforming Treatment Facility are performed on the lower floor. Changing the scale of the process, i.e., designing for a shorter treatment schedule, would have the most effect on this plan. Two areas of the facility in particular - the process hot cells and the lag storage area for full waste canisters - would be most affected.

The major equipment in the process hot cells includes the SBW feed tanks, the reformer, product cooling bins, the oxidizer, and other off-gas treatment equipment. Keeping all other factors equal, decreasing the processing schedule would increase the floor space required for most of this equipment by the inverse ratio of the decrease to the two-thirds power. For example if the schedule was decreased from 2.5 years to 1 year, the floor space for tanks would increase by $(2.5 / 1)^{0.667}=1.84$ or an $84 \%$ increase. A more detailed evaluation would be needed to determine if multiple trains would be needed for any equipment or process systems. If so, the floor space increase would be greater than $84 \%$.

The rate at which RH canisters of reformer product are produced is inversely proportional to the processing schedule. For the 2.5 -year schedule, on average, one canister is filled in about 14 hours. A single fill station could likely accommodate shorter processing schedules. Even a one-year schedule would allow an average of about 5.5 hours to fill a canister. Thus it is likely that the effect of a shortened treatment schedule on floor space to package the reformer product is small or negligible.

Finally, the interim storage for RH waste is dependent upon the initial lag period between producing the first container of waste and having approval to ship that container to WIPP and the rate at which WIPP can accept waste. Based on discussions with WIPP personnel, it is expected that the disposal facility can receive at least $6 \mathrm{RH}$ canisters per week from the INEEL, or about 300 per year. For a 2.5-year operating schedule and assuming six months operation prior to waste shipment, storage for 176 canisters would be needed for the initial production. Storage for another 128 would be needed for the excess between the number produced and the number shipped in the later 2 years of operation. For a 1year schedule, these storage requirements increase to 439 and 289 canisters respectively. Thus the lag storage area would need to increase by the factor $(439+289) /(176+128)$ or $239 \%$.

In terms of floor space, for a 1-year schedule the lower floor hot cell space would increase to about $11,000 \mathrm{ft}^{2}$ from $7,000 \mathrm{ft}^{2}$ (for the 2.5-yr schedule) and the required storage area for canisters to about $4,400 \mathrm{ft}^{2}$ from $2,000 \mathrm{ft}^{2}$.

The preceding discussion pertains to the TWR process without change. A preliminary equipment list with estimated sizing is given below in Table A-26 for TWR. Similar unit operations are common between the TTT and TWR flowsheets. The TWR process modeled in this study uses a single liquid reductant in the feed (isopropyl alcohol) and also a single gas (carbon dioxide) for flows within the system. This results in an overall simplification of the equipment in the flow sheet. 


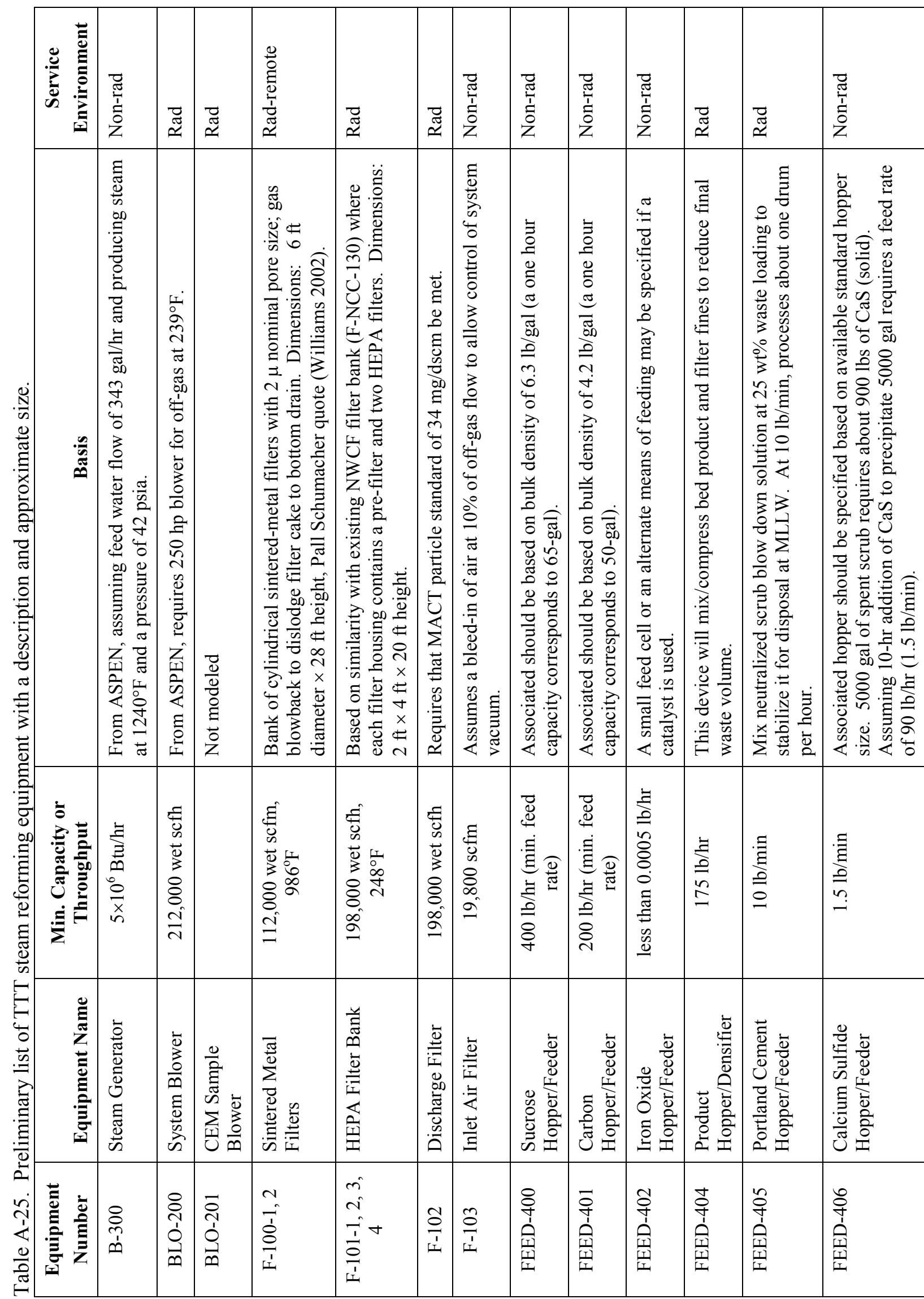




\begin{tabular}{|c|c|c|c|c|c|c|c|c|c|c|c|c|c|}
\hline 旅 & 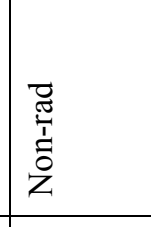 & 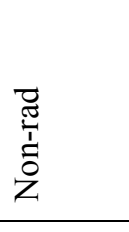 & 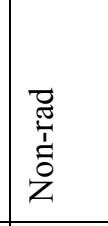 & 胥 & 丞 & 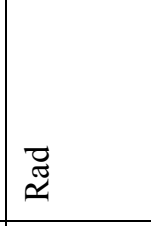 & 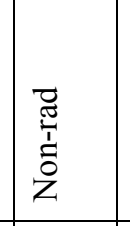 & 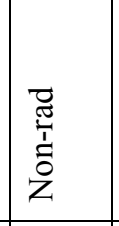 & 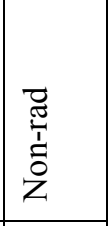 & 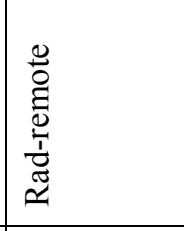 & 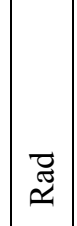 & & 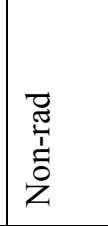 \\
\hline 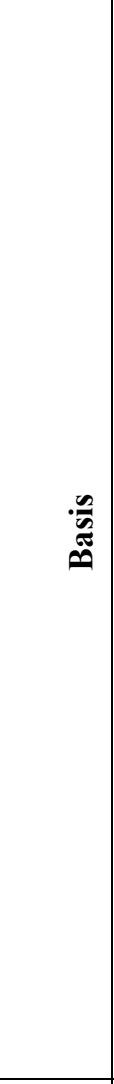 & 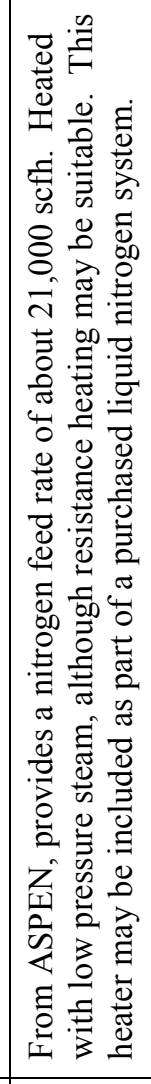 & 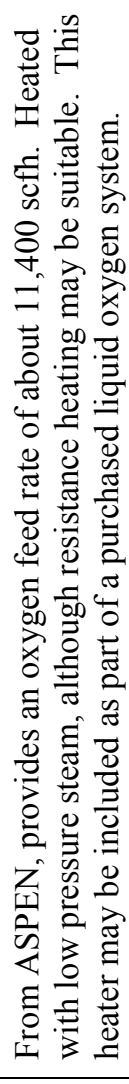 & 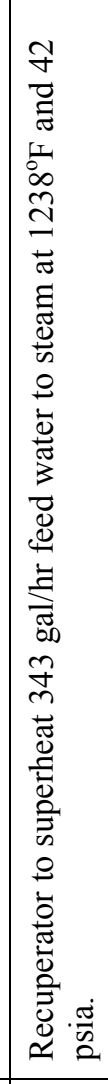 & 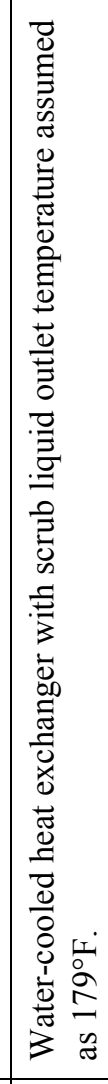 & 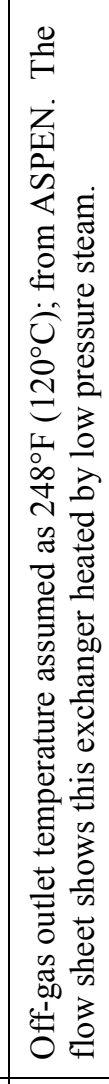 & 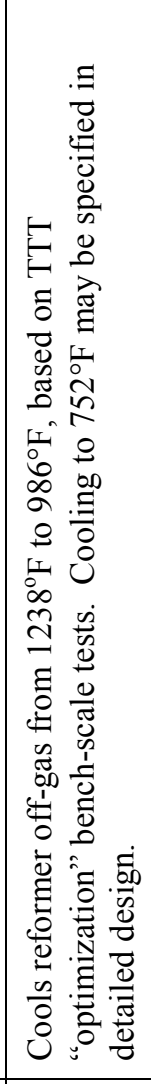 & 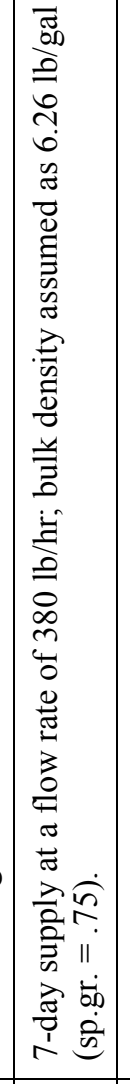 & 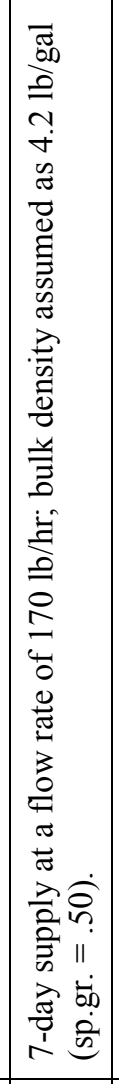 & 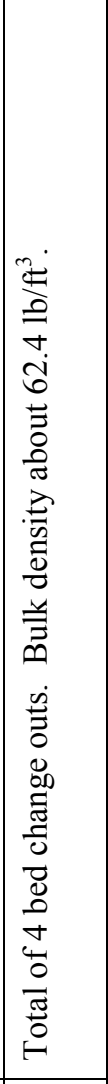 & 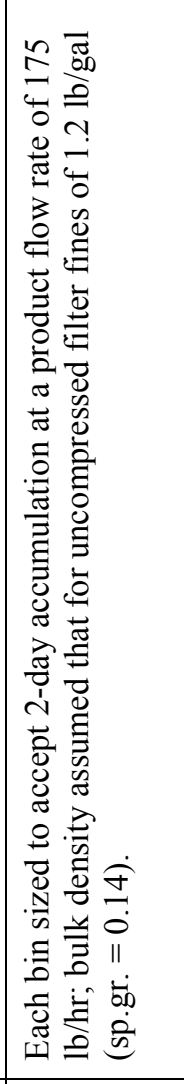 & 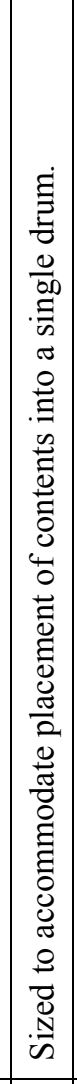 & 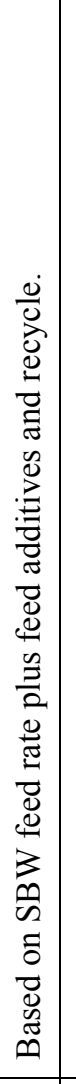 & 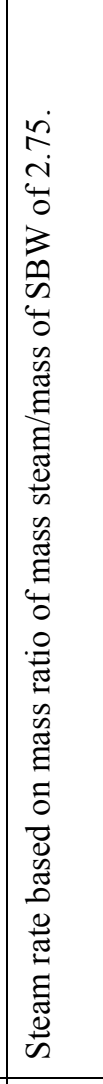 \\
\hline 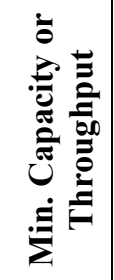 & 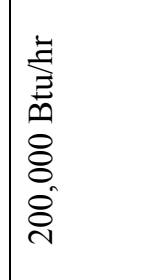 & 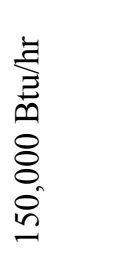 & 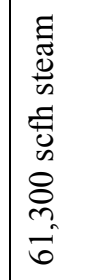 & 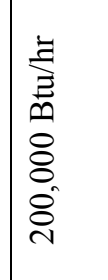 & 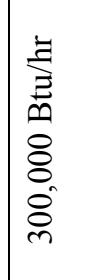 & 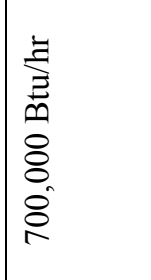 & 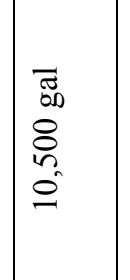 & 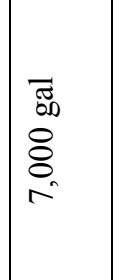 & 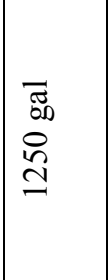 & 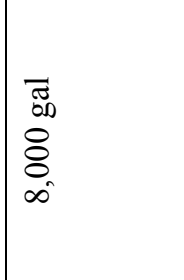 & 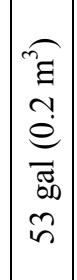 & & 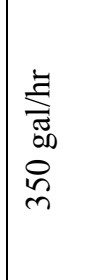 \\
\hline 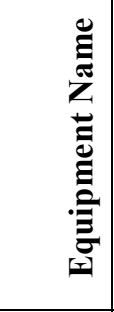 & 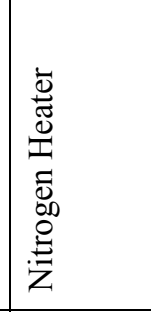 & 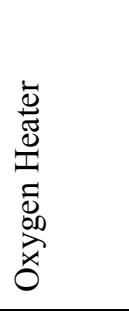 & 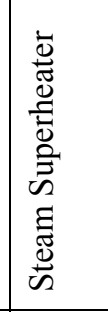 & 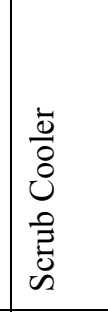 & 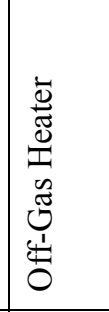 & 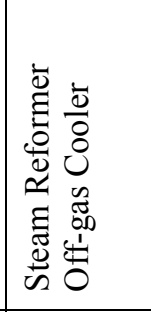 & 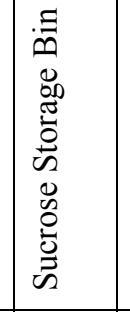 & 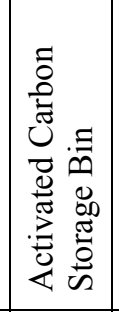 & 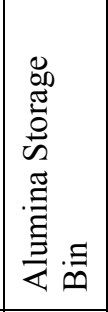 & 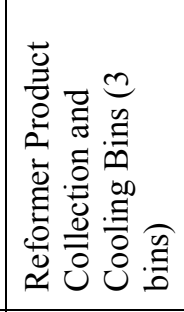 & 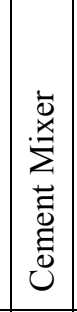 & 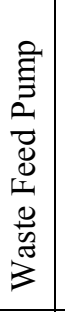 & 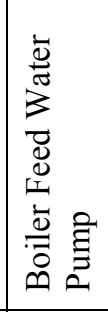 \\
\hline 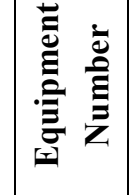 & 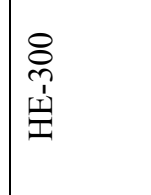 & $\begin{array}{l}\overrightarrow{0} \\
\stackrel{\oplus}{ \pm}\end{array}$ & 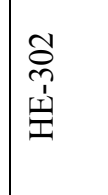 & 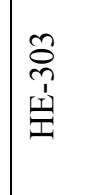 & $\begin{array}{l}\text { 总 } \\
\text { 思 }\end{array}$ & 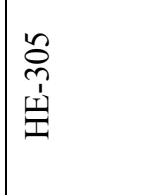 & 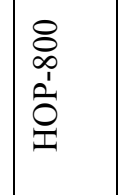 & 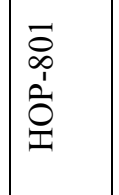 & 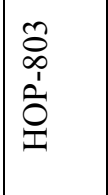 & 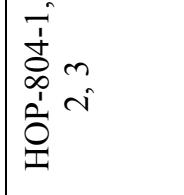 & $\begin{array}{l}\frac{8}{\dot{x}} \\
\frac{\dot{x}}{2}\end{array}$ & & ì \\
\hline
\end{tabular}




\begin{tabular}{|c|c|c|c|c|c|c|c|c|c|c|c|}
\hline 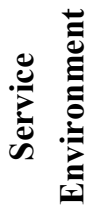 & $\underset{\mathscr{Z}}{\mathscr{Z}}$ & ゙ָ̈ & \begin{tabular}{l}
$\vec{Z}$ \\
\multirow{2}{*}{} \\
$\tilde{0}$ \\
$Z$
\end{tabular} & $\begin{array}{l}\overrightarrow{\widetilde{T}} \\
\stackrel{\pi}{1} \\
\tilde{0} \\
z\end{array}$ & 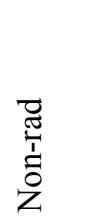 & $\underset{\mathscr{Z}}{\mathscr{Z}}$ & 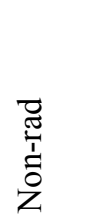 & 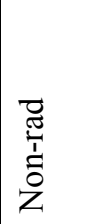 & $\underset{\mathscr{Z}}{\mathscr{I}}$ & $\underset{\mathscr{Z}}{\widetilde{Z}}$ & $\underset{\mathscr{I}}{\mathscr{Z}}$ \\
\hline $\begin{array}{l}\mathscr{n} \\
\mathscr{n} \\
\mathscr{n}\end{array}$ & 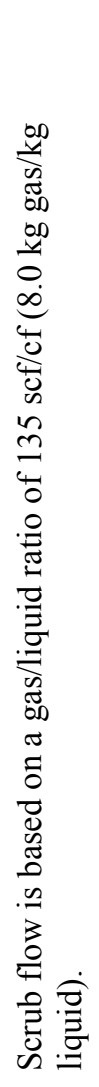 & 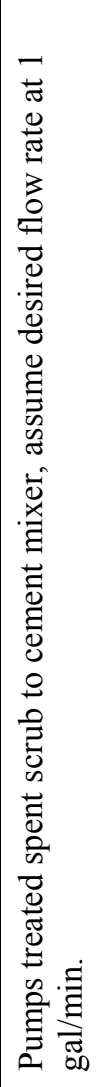 & 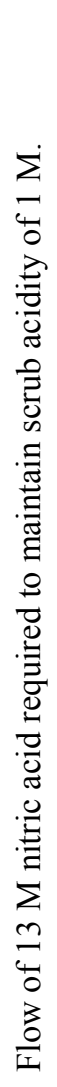 & 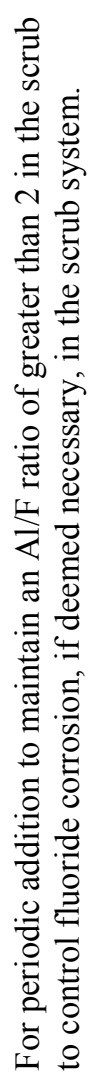 & 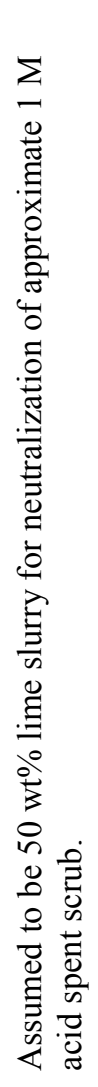 & 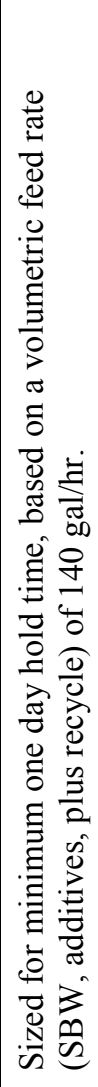 & 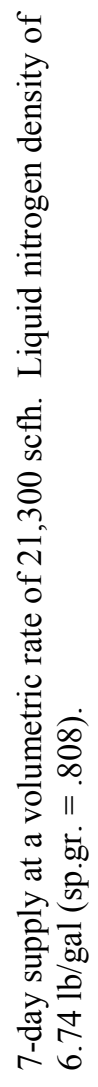 & 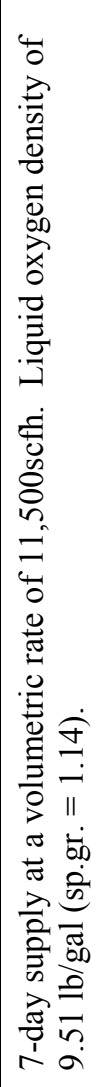 & 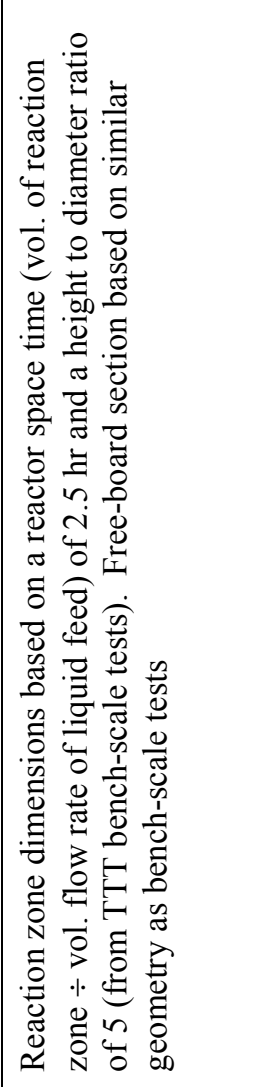 & 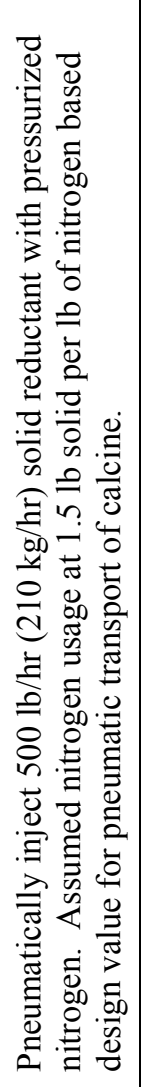 & 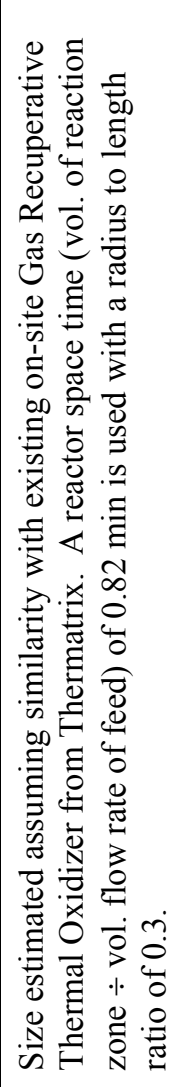 \\
\hline 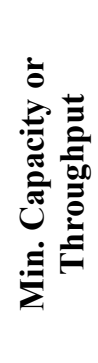 & $\begin{array}{l}\text { ż } \\
\text { 品 } \\
8 \\
8 \\
0 \\
=\end{array}$ & 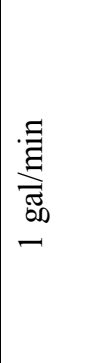 & 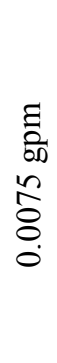 & 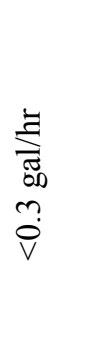 & $\begin{array}{l}\frac{z}{2} \\
\stackrel{0}{n} \\
n \\
0\end{array}$ & $\begin{array}{l}\overline{7} \\
0 \\
8 \\
8 \\
i\end{array}$ & 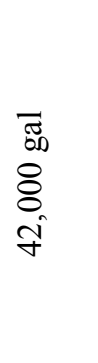 & $\begin{array}{l}\overrightarrow{\widetilde{D}} \\
8 \\
8 \\
8 \\
\stackrel{i}{0}\end{array}$ & 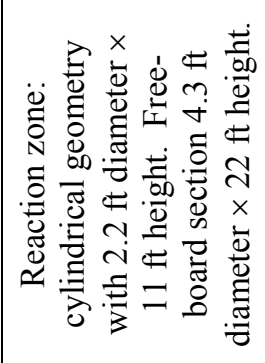 & 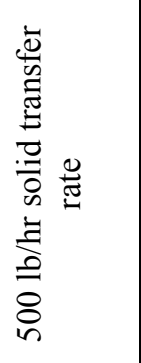 & 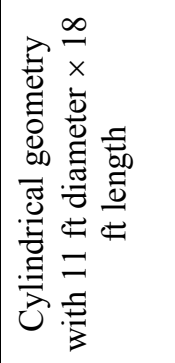 \\
\hline 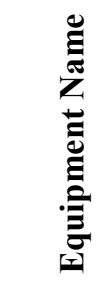 & 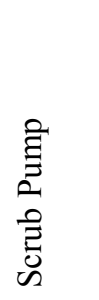 & 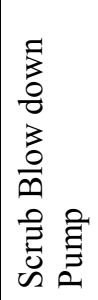 & 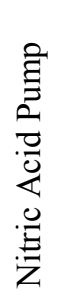 & 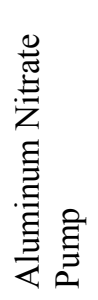 & 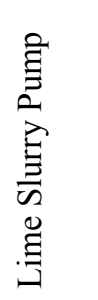 & 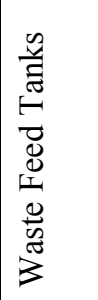 & 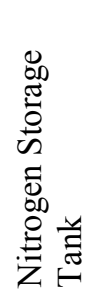 & 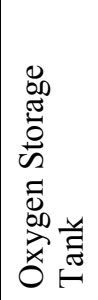 & 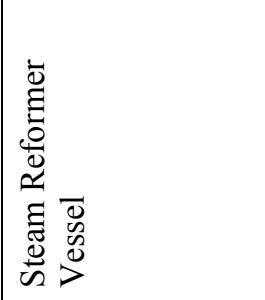 & 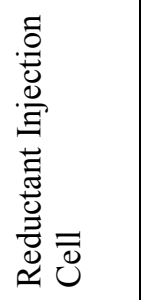 & : \\
\hline 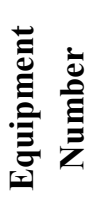 & $\begin{array}{l}\text { ָे } \\
\text { ปิ }\end{array}$ & $\begin{array}{l}\text { 令 } \\
\text { L }\end{array}$ & $\begin{array}{c}\stackrel{J}{0} \\
\stackrel{1}{a}\end{array}$ & 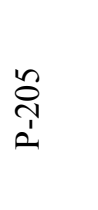 & 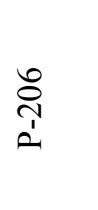 & 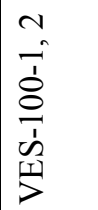 & \begin{tabular}{l}
$\overrightarrow{0}$ \\
$\dot{1}$ \\
11 \\
\multicolumn{1}{c}{}
\end{tabular} & 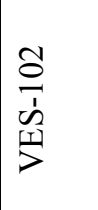 & $\begin{array}{l}0 \\
0 \\
\dot{1} \\
\dot{1} \\
>\end{array}$ & 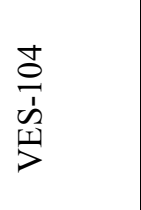 & $\begin{array}{l}n \\
0 \\
\dot{1} \\
n \\
>1 \\
>\end{array}$ \\
\hline
\end{tabular}




\begin{tabular}{|c|c|c|c|c|c|c|c|c|c|c|}
\hline 这 & ت્ّ & تृ & ت゙્ & ت্ّ & $\stackrel{\widetilde{Z}}{\widetilde{\simeq}}$ & ت゙્ & $\begin{array}{l}\frac{\pi}{\pi} \\
\frac{\pi}{1} \\
z \\
z\end{array}$ & ت゙્ & $\begin{array}{l}\frac{Z}{\pi} \\
\frac{\pi}{10} \\
z \\
z\end{array}$ & $\begin{array}{l}\bar{\pi} \\
\frac{\pi}{1} \\
z \\
z\end{array}$ \\
\hline 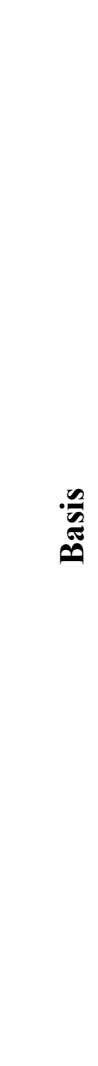 & 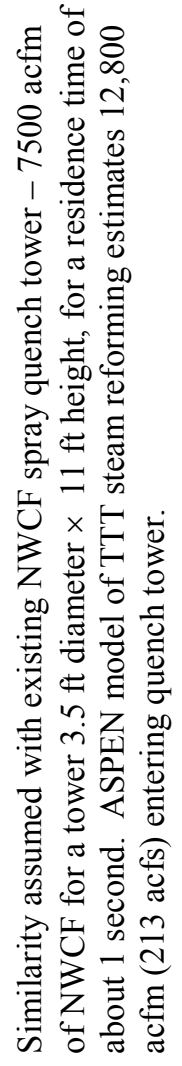 & 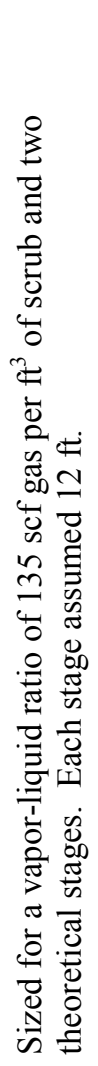 & 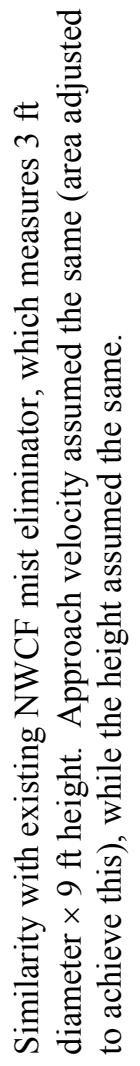 & 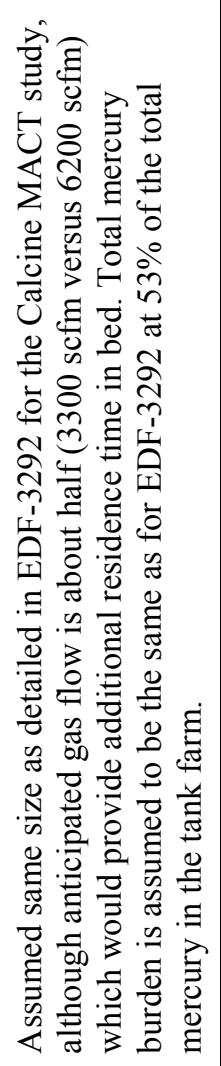 & 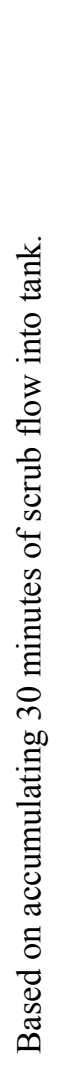 & 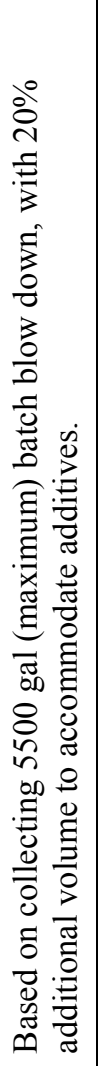 & 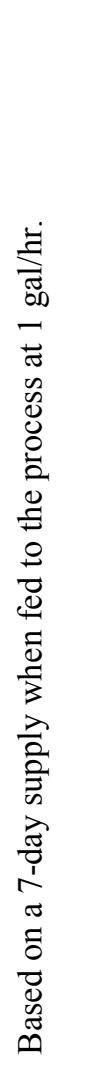 & 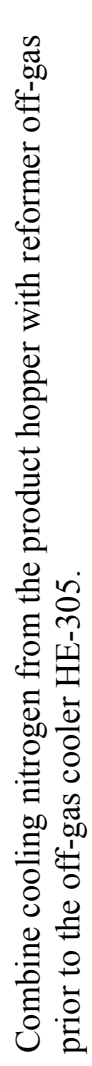 & 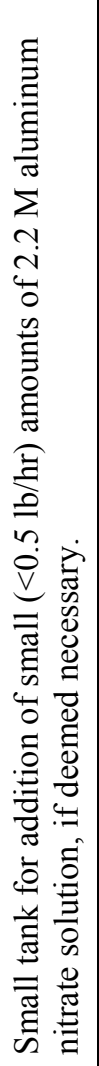 & 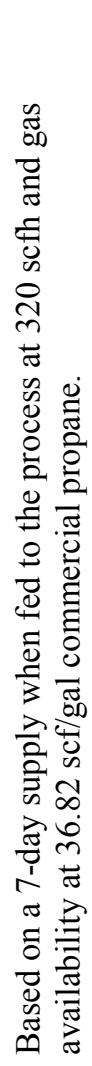 \\
\hline 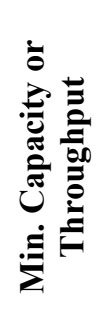 & 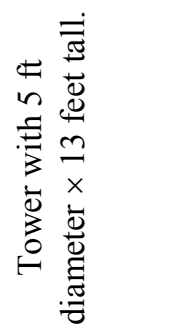 & 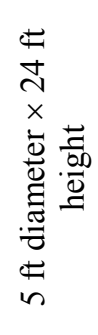 & 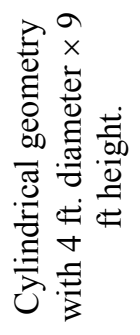 & 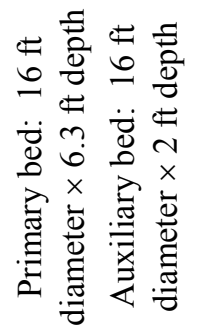 & 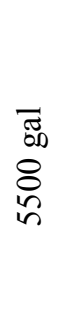 & $\begin{array}{l}\overline{5} \\
\text { o } \\
8 \\
8\end{array}$ & $\begin{array}{l}\overline{\widetilde{J}} \\
\stackrel{0}{ } \\
\stackrel{d}{N}\end{array}$ & & $\begin{array}{l}\overline{\mathscr{J}} \\
\text { D } \\
\text { D }\end{array}$ & $\begin{array}{l}\bar{I} \\
\text { on } \\
8 \\
\stackrel{0}{n}\end{array}$ \\
\hline 范 & 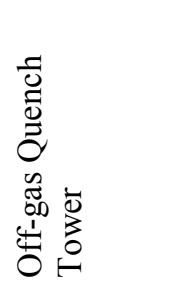 & 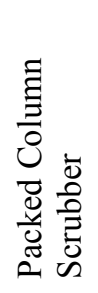 & 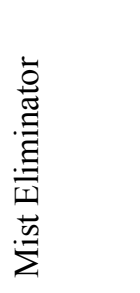 & 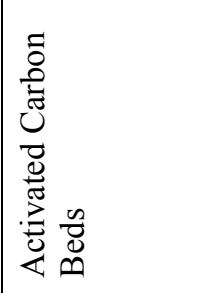 & 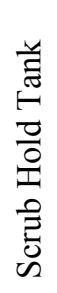 & 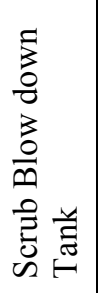 & 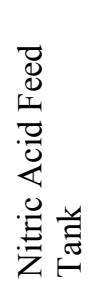 & 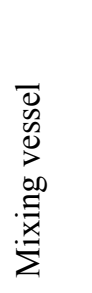 & 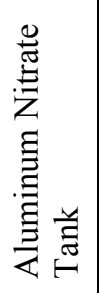 & 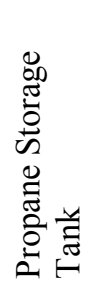 \\
\hline 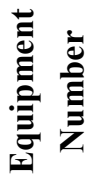 & \begin{tabular}{l}
0 \\
$\circ$ \\
\\
\multicolumn{2}{c}{} \\
$\stackrel{1}{>}$
\end{tabular} & $\begin{array}{l}\hat{0} \\
\dot{0} \\
\text { ஸे } \\
\stackrel{1}{>}\end{array}$ & $\begin{array}{l}\infty \\
0 \\
\dot{1} \\
\text { ஸे } \\
\dot{1} \\
>\end{array}$ & $\begin{array}{l}8 \\
\frac{1}{1} \\
\dot{1} \\
\frac{1}{>}\end{array}$ & $\begin{array}{l}\stackrel{0}{\Xi} \\
\stackrel{1}{2} \\
\stackrel{1}{>}\end{array}$ & $\begin{array}{l}\exists \\
\exists \\
\dot{1} \\
\dot{1} \\
>\end{array}$ & $\begin{array}{l}\stackrel{N}{J} \\
\dot{1} \\
\stackrel{1}{>}\end{array}$ & 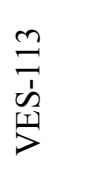 & $\begin{array}{l}\Xi \\
\doteq \\
\dot{1} \\
\stackrel{1}{>}\end{array}$ & 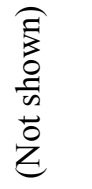 \\
\hline
\end{tabular}




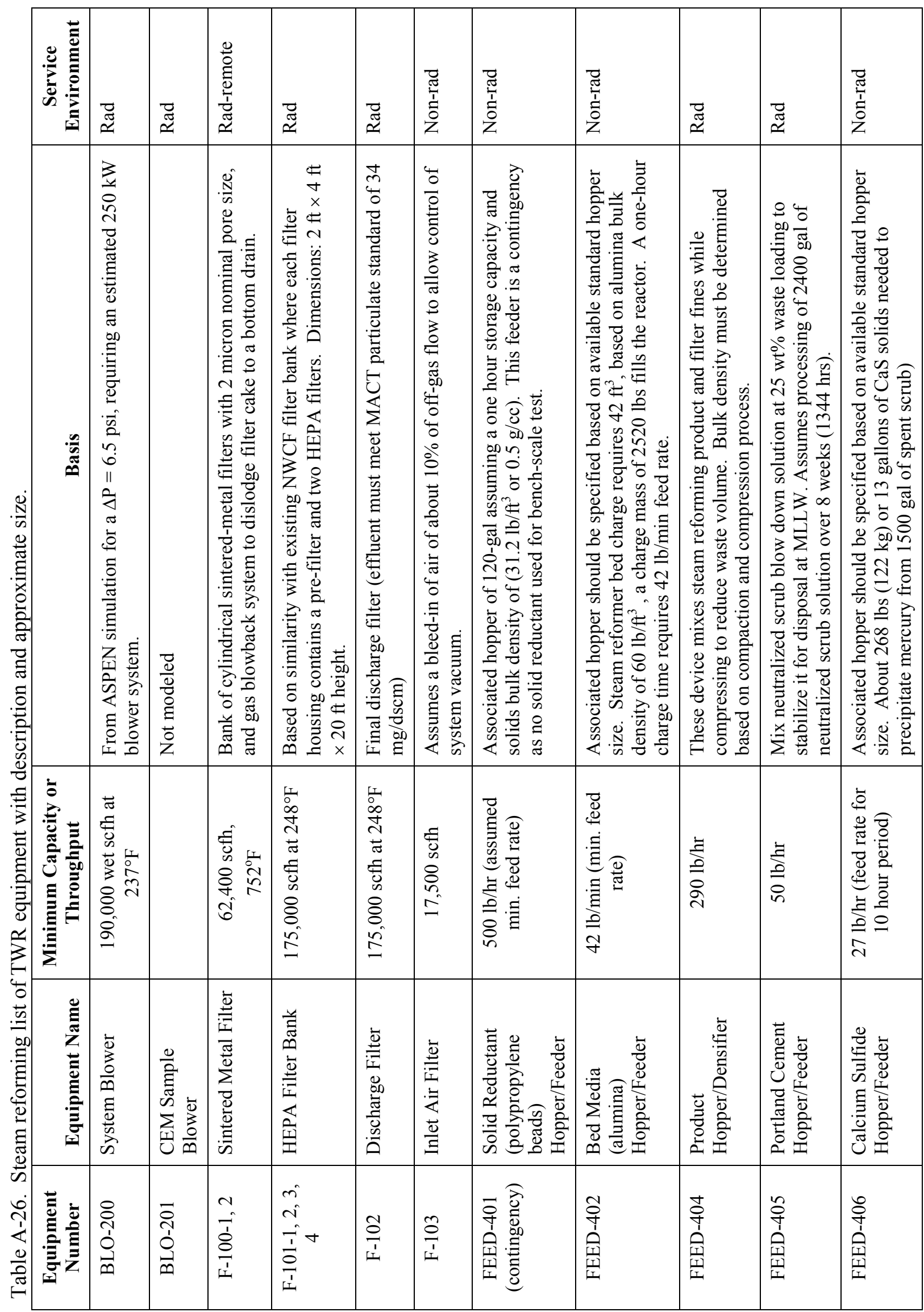




\begin{tabular}{|c|c|c|c|c|c|c|c|c|c|c|c|}
\hline $\begin{array}{l}\vec{\pi} \\
\frac{\pi}{1} \\
0 \\
z\end{array}$ & 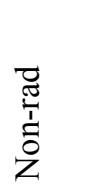 & 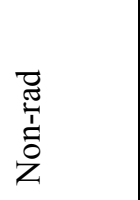 & ゙ָ̈ & $\underset{\mathscr{Z}}{\widetilde{Z}}$ & $\underset{\mathscr{I}}{\mathscr{\simeq}}$ & $\begin{array}{l}\overrightarrow{\widetilde{T}} \\
\stackrel{\pi}{0} \\
\tilde{0} \\
z\end{array}$ & 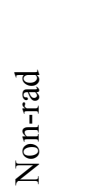 & 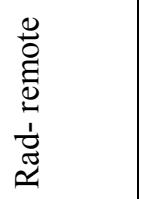 & تี & 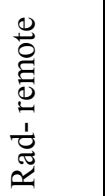 & $\widetilde{\widetilde{Z}}$ \\
\hline 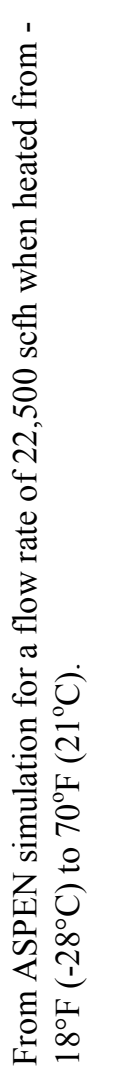 & 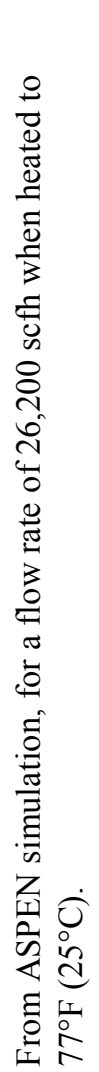 & 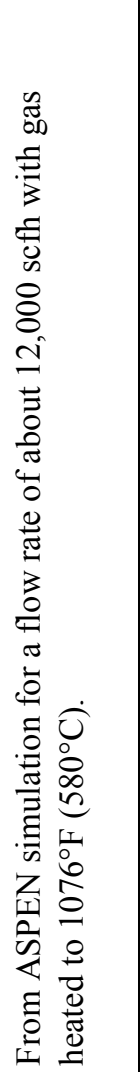 & 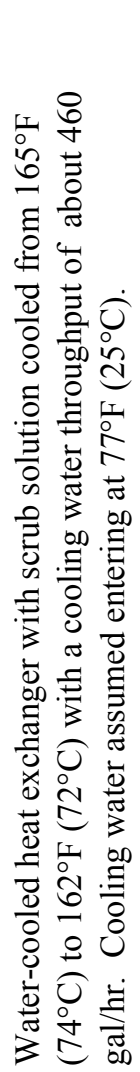 & 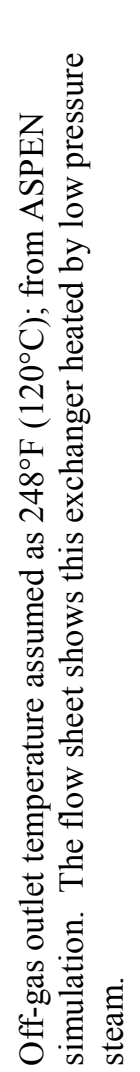 & 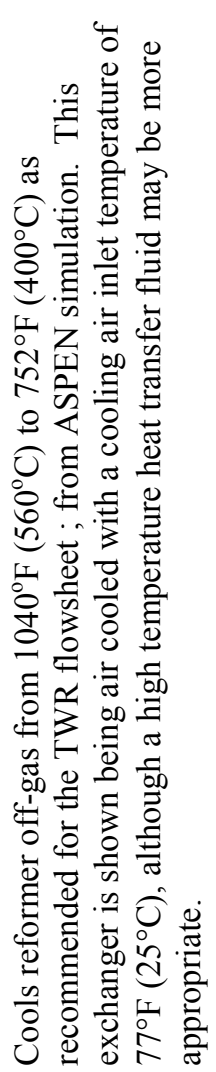 & 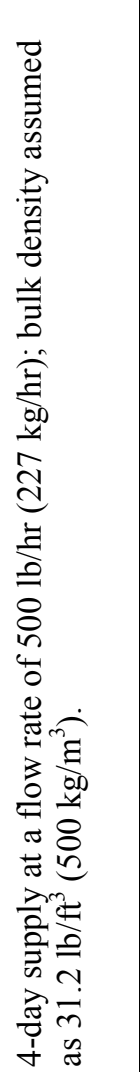 & 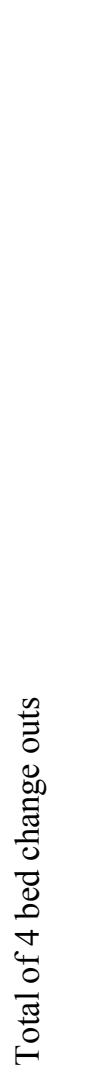 & 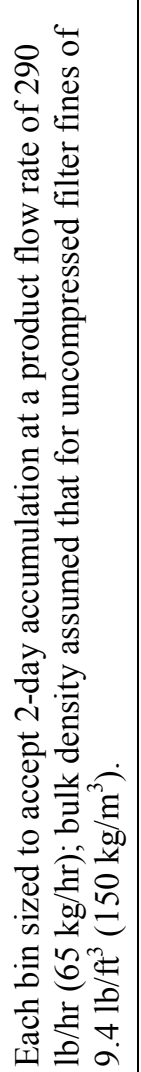 & 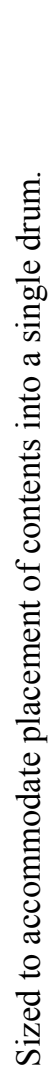 & 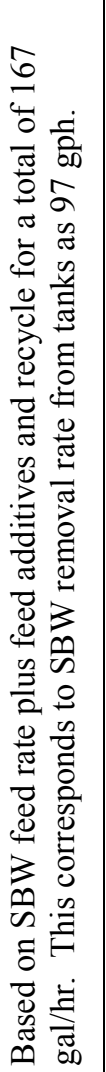 & 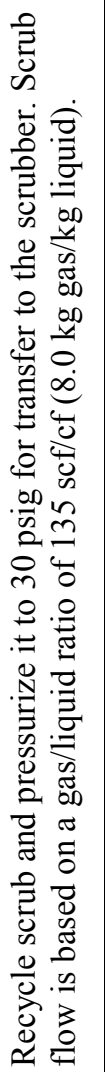 \\
\hline 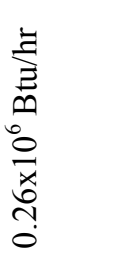 & 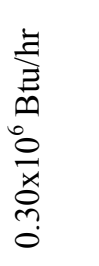 & 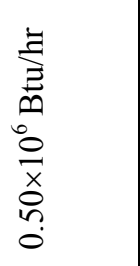 & 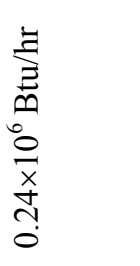 & 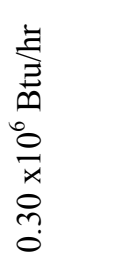 & 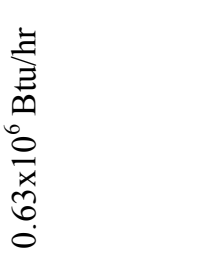 & 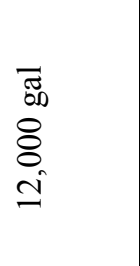 & $\begin{array}{l}\vec{F} \\
\infty \\
8 \\
\infty \\
0\end{array}$ & 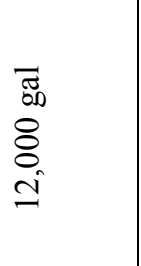 & 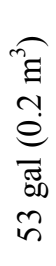 & 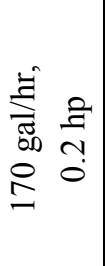 & 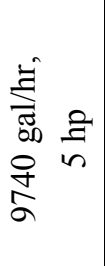 \\
\hline 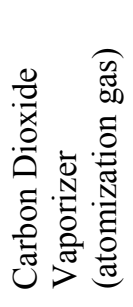 & 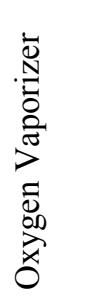 & 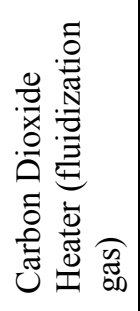 & $\begin{array}{l}\frac{\dot{0}}{0} \\
\dot{0} \\
0 \\
\overrightarrow{0} \\
\dot{0}\end{array}$ & 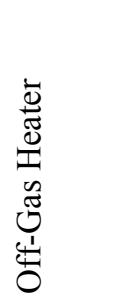 & 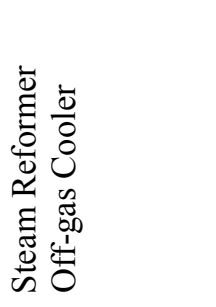 & 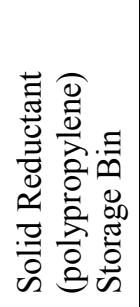 & 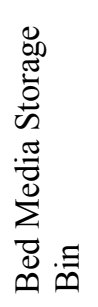 & 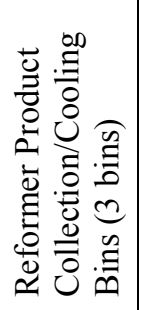 & 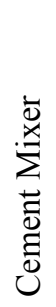 & 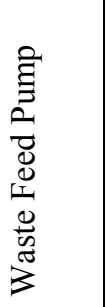 & 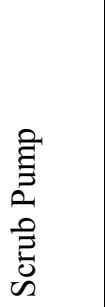 \\
\hline $\begin{array}{l}8 \\
\text { : } \\
1 \\
11\end{array}$ & 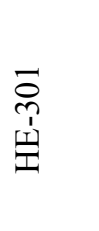 & 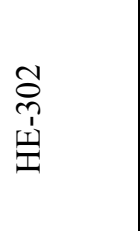 & $\begin{array}{l}\text { ô } \\
\text { n} \\
\text { 壵 }\end{array}$ & 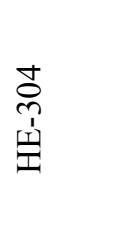 & 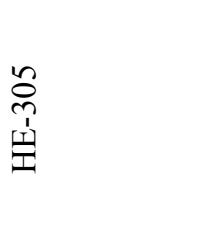 & 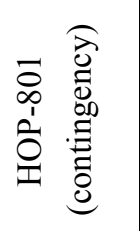 & 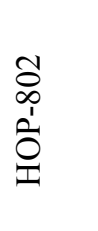 & 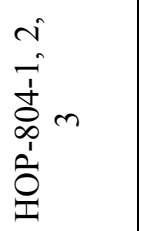 & $\frac{8}{\frac{8}{x}}$ & $\begin{array}{l}\stackrel{\leftrightarrow}{\Lambda} \\
\text { İ }\end{array}$ & $\begin{array}{c}\text { ָे } \\
\text { İ } \\
\text { I }\end{array}$ \\
\hline
\end{tabular}




\begin{tabular}{|c|c|c|c|c|c|c|c|c|c|c|c|}
\hline $\overrightarrow{\widetilde{Z}}$ & \begin{tabular}{l}
$\widetilde{T}$ \\
\multirow{2}{*}{} \\
$\tilde{0}$ \\
$Z$
\end{tabular} & 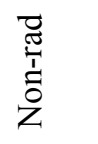 & 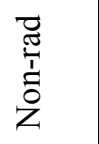 & ્ָટ & 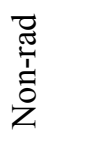 & $\begin{array}{l}\frac{\pi}{\pi} \\
\frac{\pi}{10} \\
z \\
z\end{array}$ & 苂 & & ت゙્ & $\overrightarrow{\widetilde{Z}}$ & 芯 \\
\hline 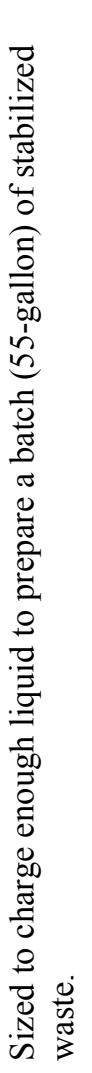 & 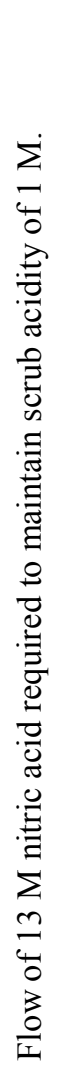 & 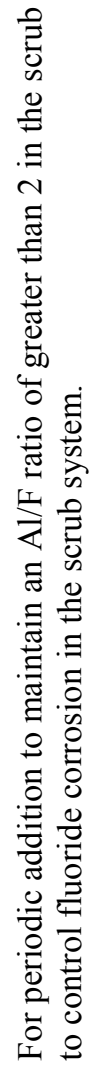 & 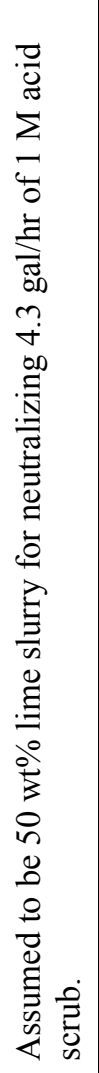 & 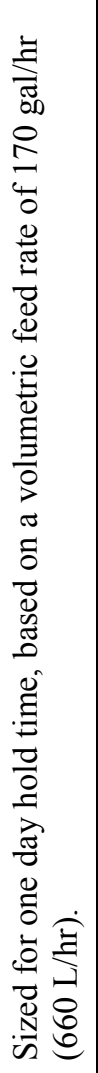 & 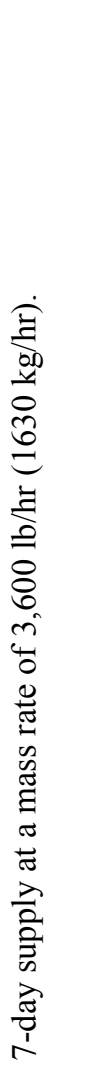 & 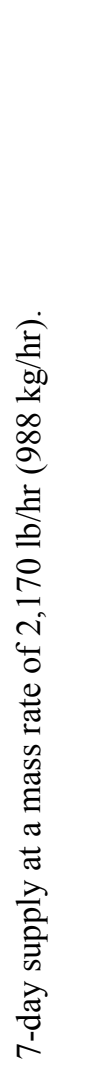 & 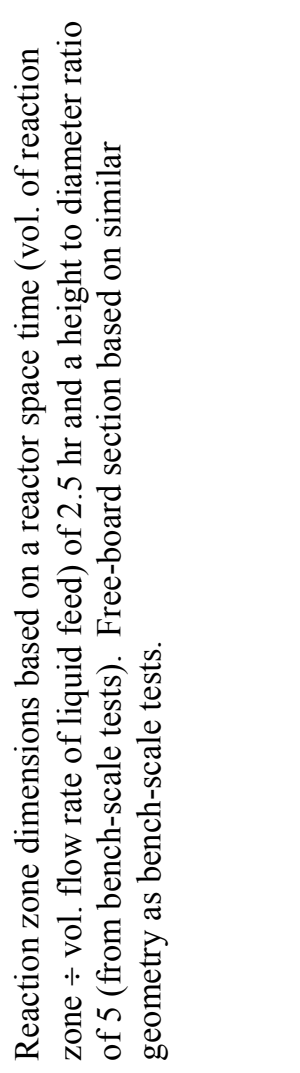 & 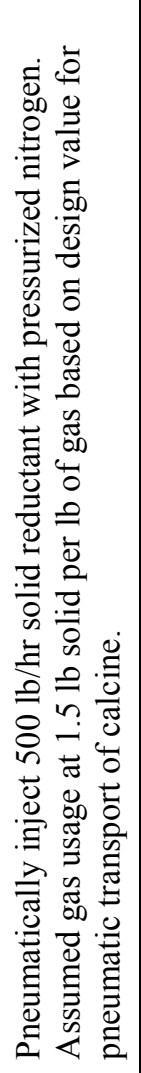 & 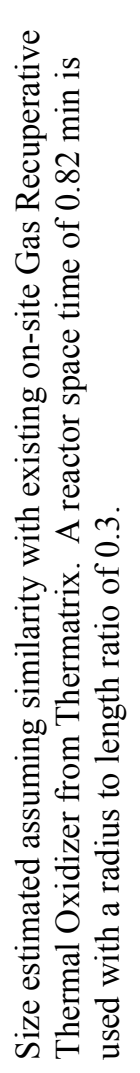 & 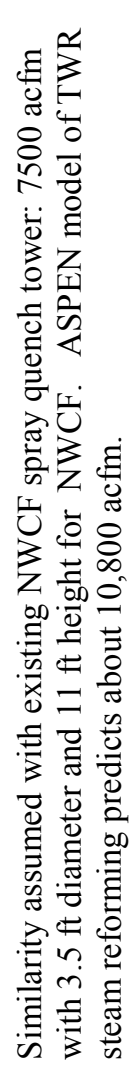 & 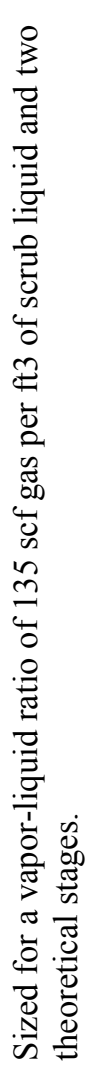 \\
\hline 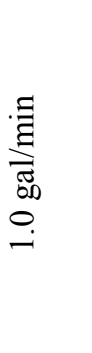 & 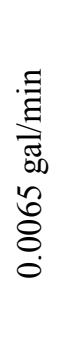 & 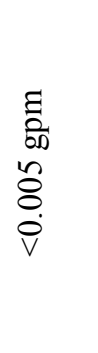 & $\begin{array}{l}\stackrel{\jmath}{ٍ} \\
\stackrel{m}{-}\end{array}$ & $\begin{array}{l}\bar{F} \\
\text { on } \\
8 \\
0 \\
n \\
+\end{array}$ & $\begin{array}{l}\bar{F} \\
\text { 品 } \\
8 \\
0 \\
\infty \\
\infty\end{array}$ & 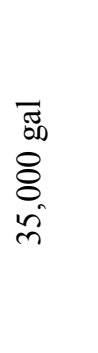 & 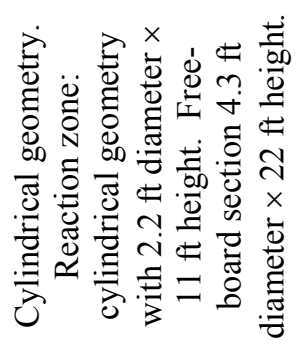 & 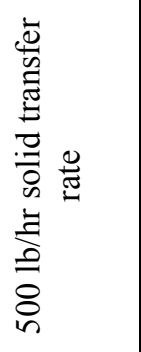 & 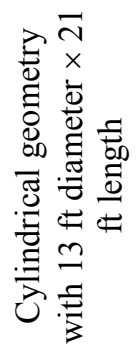 & 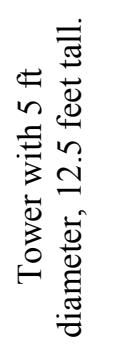 & 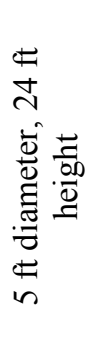 \\
\hline 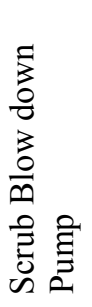 & 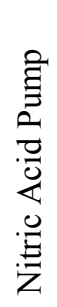 & 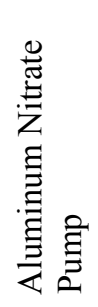 & 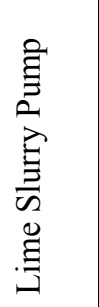 & 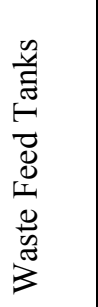 & 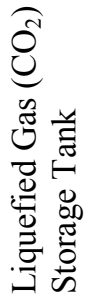 & 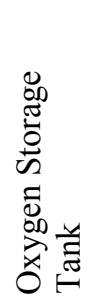 & 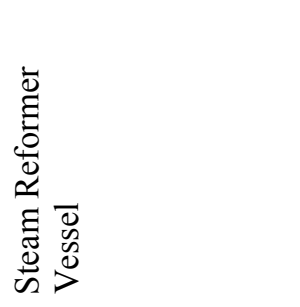 & 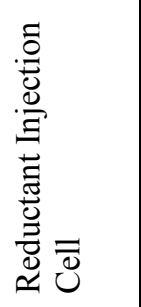 & : & 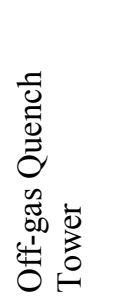 & 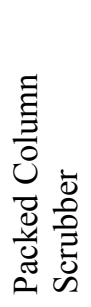 \\
\hline $\begin{array}{c}\stackrel{\text { }}{\Lambda} \\
\stackrel{1}{\sim}\end{array}$ & 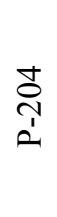 & 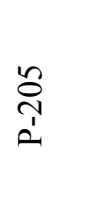 & 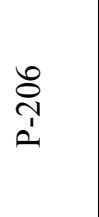 & 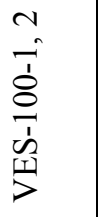 & $\begin{array}{l}\overrightarrow{0} \\
\overline{1} \\
\text { ñy } \\
\frac{1}{>}\end{array}$ & 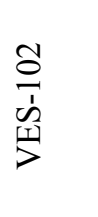 & $\begin{array}{l}m \\
0 \\
1 \\
\dot{n} \\
\dot{1} \\
>\end{array}$ & 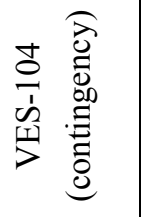 & $\begin{array}{l}n \\
0 \\
\dot{1} \\
\stackrel{1}{>}\end{array}$ & 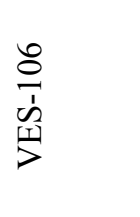 & $\begin{array}{l}\hat{s} \\
= \\
\text { s } \\
\text { İ } \\
>\end{array}$ \\
\hline
\end{tabular}




\begin{tabular}{|c|c|c|c|c|c|c|c|c|}
\hline 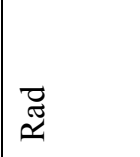 & 亗 & $\begin{array}{l}\overrightarrow{\mathbb{E}} \\
\tilde{\approx}\end{array}$ & 莺 & 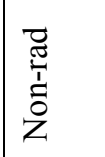 & $\begin{array}{l}\ddot{\mathscr{E}} \\
\simeq\end{array}$ & 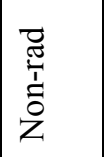 & 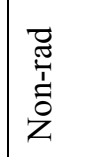 & 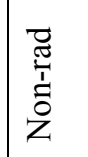 \\
\hline 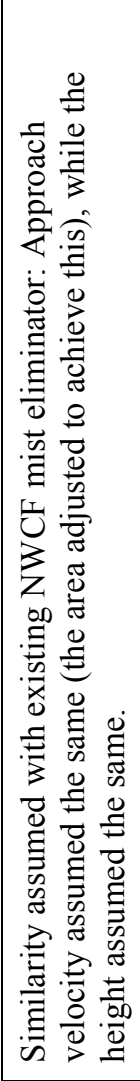 & 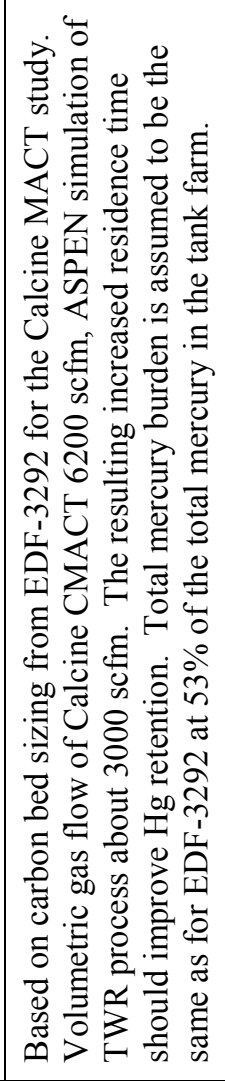 & 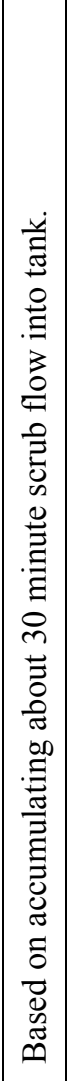 & 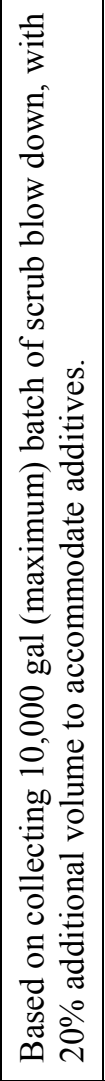 & 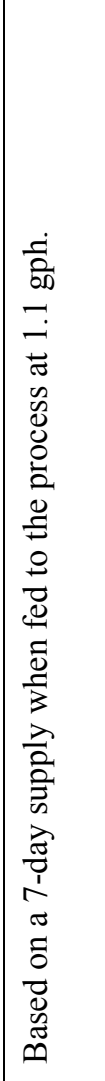 & 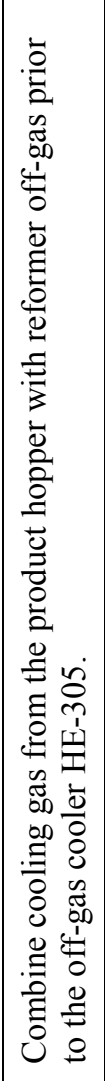 & 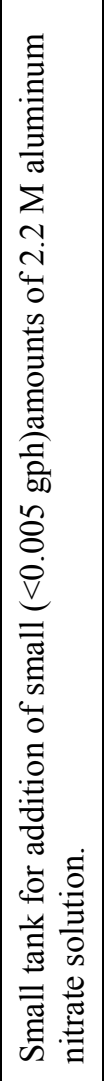 & 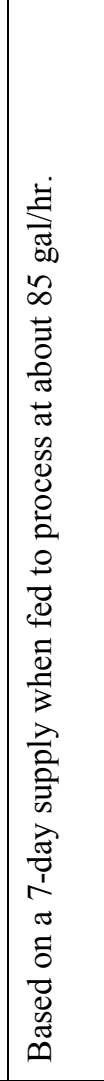 & 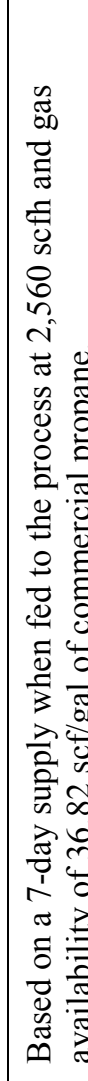 \\
\hline 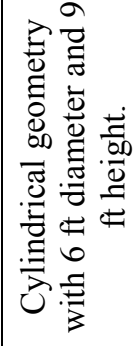 & 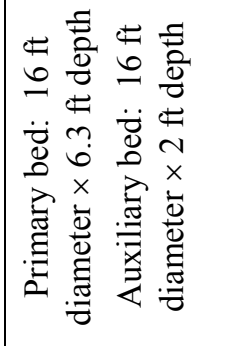 & $\begin{array}{l}\overrightarrow{\widetilde{D}} \\
8 \\
8 \\
i \\
i\end{array}$ & $\begin{array}{l}\overline{\widetilde{J}} \\
\overline{0} \\
8 \\
8 \\
0 \\
0\end{array}$ & $\begin{array}{l}\overrightarrow{\widetilde{5}} \\
\stackrel{్}{0} \\
\stackrel{్}{2}\end{array}$ & & 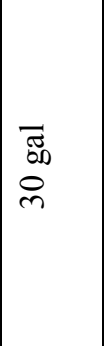 & 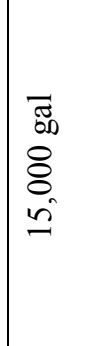 & 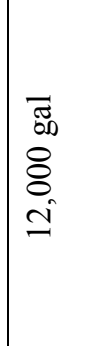 \\
\hline 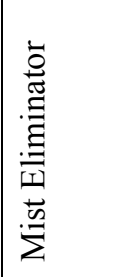 & 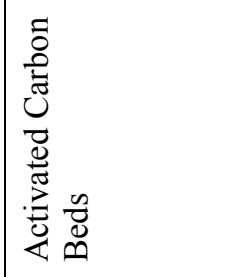 & 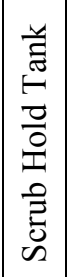 & 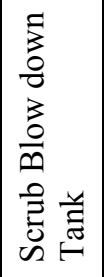 & 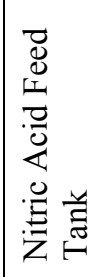 & 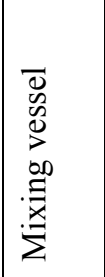 & 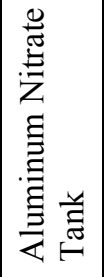 & 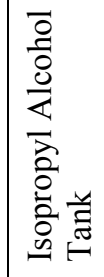 & 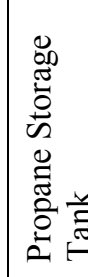 \\
\hline 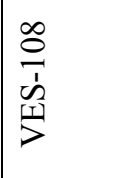 & 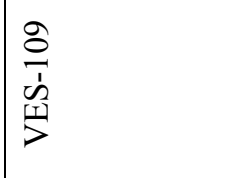 & $\begin{array}{l}0 \\
\overline{1} \\
\dot{\omega} \\
\underbrace{\prime}\end{array}$ & 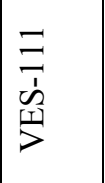 & 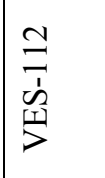 & $\begin{array}{l}\stackrel{m}{=} \\
\dot{\omega} \\
\stackrel{1}{>}\end{array}$ & $\begin{array}{l}\stackrel{ \pm}{=} \\
\dot{\omega} \\
\stackrel{y}{>}\end{array}$ & 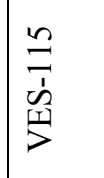 & 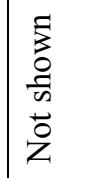 \\
\hline
\end{tabular}




\section{A-9. LAYOUT DRAWINGS}

Layout drawings for each floor of the Steam Reforming Treatment Facility are shown on the following pages. The primary purpose of these drawings is to estimate floor space requirements needed in the facility. Equipment and room arrangements have not been optimized, nor has sufficient engineering design been performed to provide a basis for all of the areas of the facility. The layout drawings were based on those prepared for the Steam Reforming Feasibility Study (Williams, 2002). Per the 2002 study, the layout drawings show a separate warehouse for empty waste canisters. Adequate storage space is provided on the lower hot cell floor for 280 filled canisters, sufficient for an initial sixmonth period plus storage of the difference between the production rate and the shipping rate. 


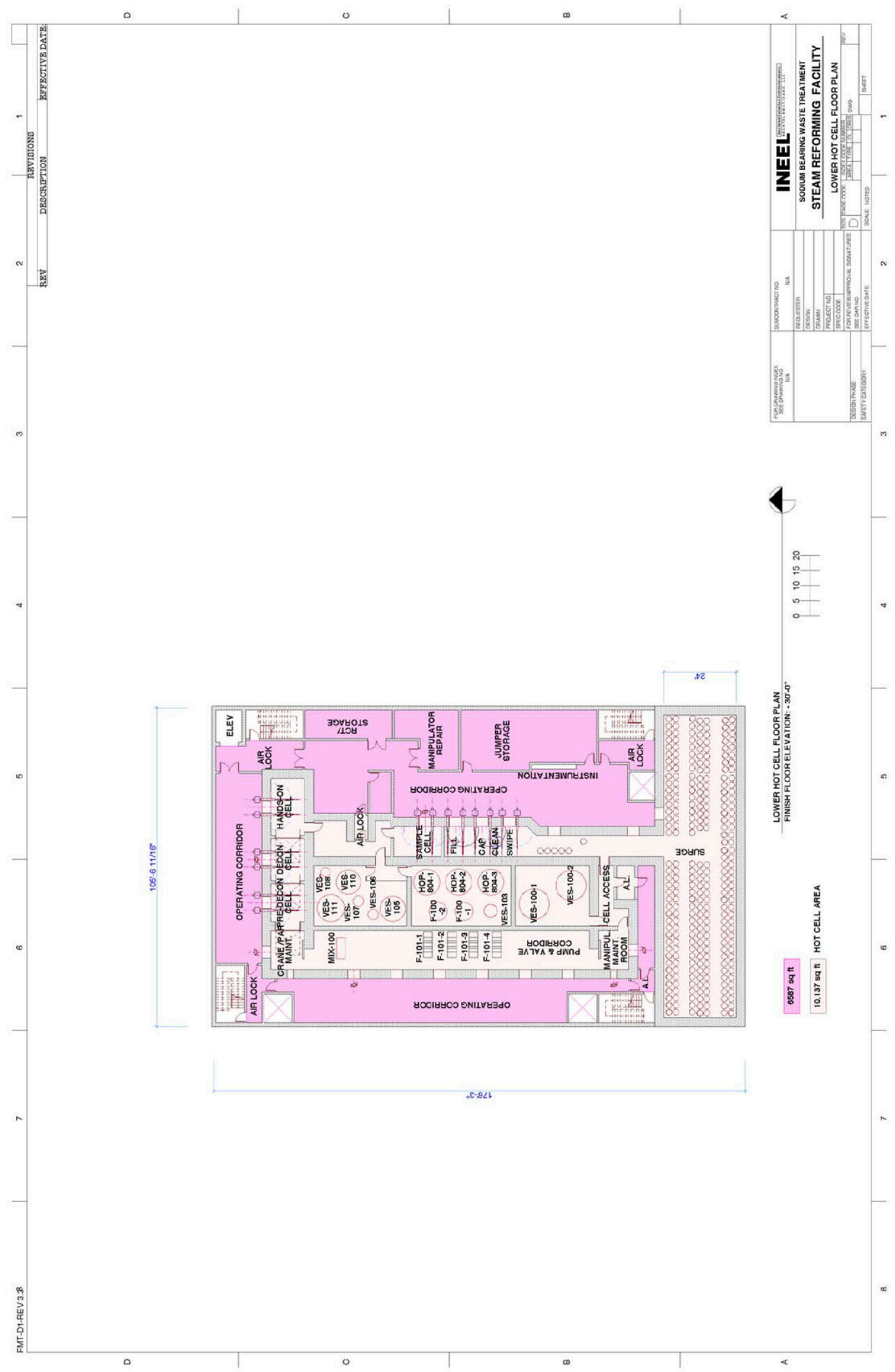




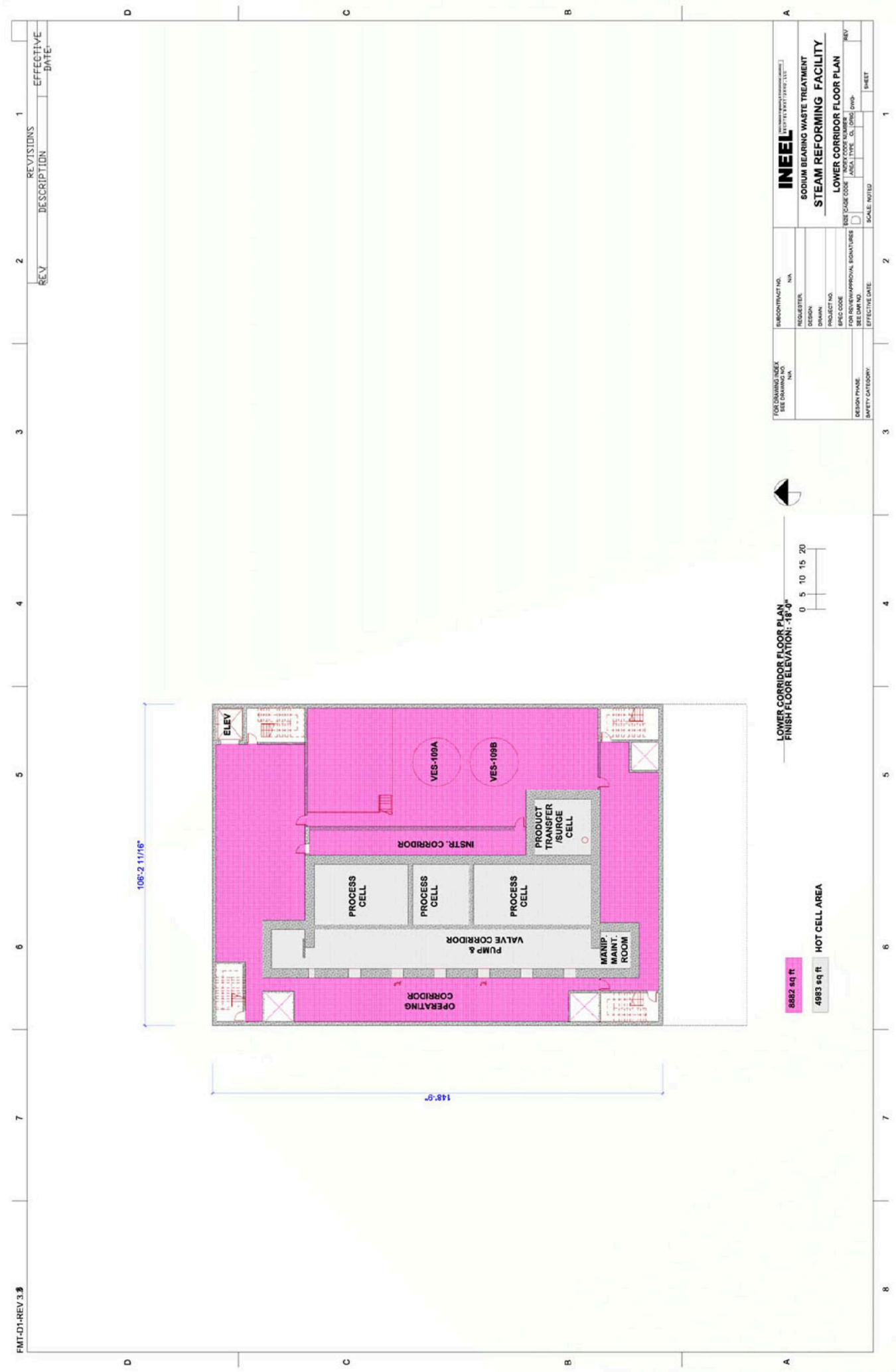




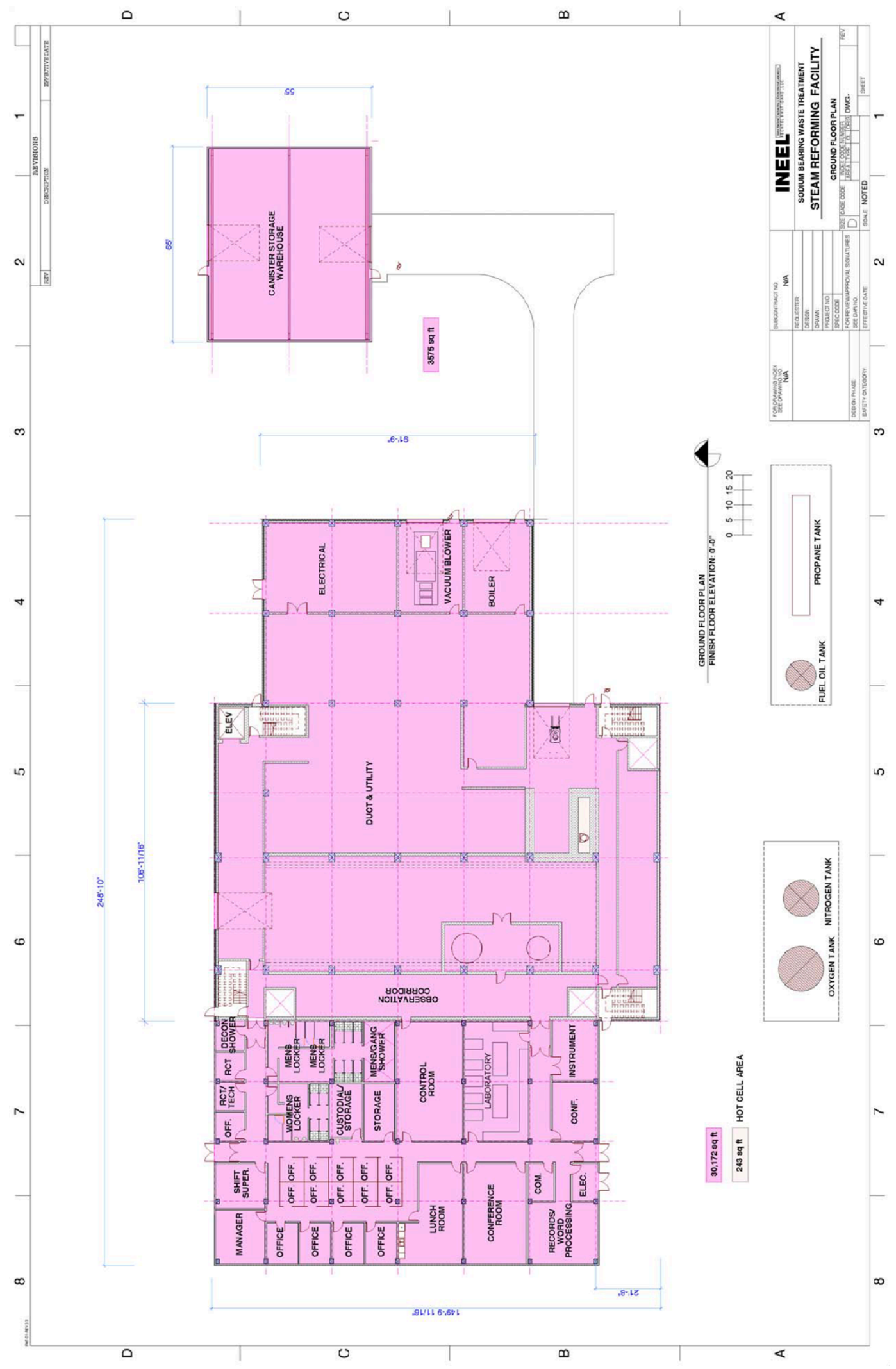

तิ 


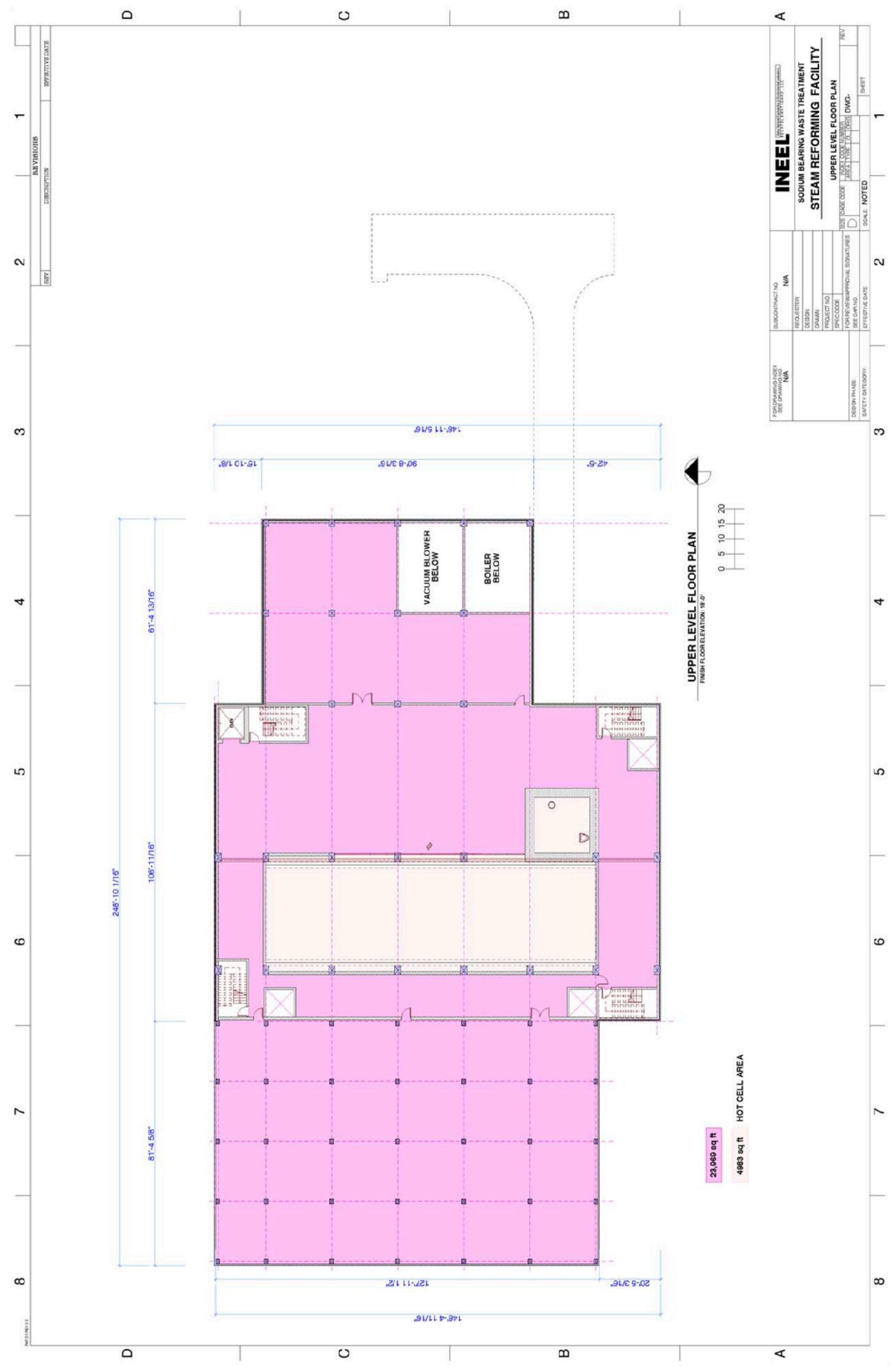

ָ̊ 


\section{A-10. REFERENCES}

Ashworth, S. and D. D. Siemer, (2002), Wet Scrubbing of Mercury, unpublished white paper sent as an attachment to S. Ashworth Interoffice Memorandum, April 15, 2002.

ASPEN Plus, (2002), Version 11.1 for Windows 2000, Aspen Technology, Inc., Cambridge, Massachusetts

Barnes, C. M, and C. B. Millet, (2002), "INTEC Tank Farm Facility Management Plan," PLN-1112, September 30, 2002.

Barnes, C. M., R. A. Wood, and B. H. O’Brien, (2003a), "Calcination with MACT Upgrade Process Design,” Engineering Design File EDF-3387, Rev. 0.

Barnes, C. M., S. K. Janikowski, and C. B. Millet, (2003b), Feed Composition for the Sodium-Bearing Waste Process, INEEL/EXT-2000-01378, Rev. 3.

Beitel, G. A., (2003), Technical and Functional Requirements for the Steam Reformer SBW Treatment Alternative (Draft), TFR-216, Rev. a.

Childs, K. F., R. I. Donovan, and M. C. Swenson, (1982), The Ninth Processing Campaign in the Waste Calcining Facility, ENICO-1100, April 1982.

Clark, M. L., (2003), "Study 18 - Calcine Packaging Facility Layout - Idaho Tank Farm Project Calcination with MACT Upgrade SBW Treatment Alternative," Engineering Design File EDF3281, April 30, 2003.

Del Debbio, J. A., T. L. Watson, and J. B. Heintzelman, (2003), Long-Term Performance of SulfurImpregnated, Granulated Activated Carbon (GAC) for Mercury Removal from NWCF Off-Gas, INEEL/EXT-03-01102.

Federal Energy Technology Center (FETC), (1997), Steam Reforming of Low-Level Mixed Waste, Technology Development Data Sheet from the Office of Science and Technology, Contract No.: DE-AR21-95MC32091.

Freeman, H. M., (1998), Standard Handbook of Hazardous Waste Treatment and Disposal, 2nd edition, McGraw-Hill.

Gentilucci, J. E, J. E. Miller, R. L. Treat, and W. W. Schulz, (2001), Technical Review of the Applicability of the Studsvik Inc. THOR ${ }^{\text {sm }}$ Process to INEEL SBW, July, 2001.

Haefner, D., B. O’Brien, and S. O. Bates, (2003), "Steam Reforming Process Design," Engineering Design File EDF-3827, September 30, 2003.

Herbst, A. K., J. A. DelDebbio, R. J. Kirkam, B. A. Scholes, and T. L. Watson, (2002), Idaho Nuclear Technology and Engineering Center Sodium-Bearing Waste Treatment Research and Development FY-2002 Status Report, INEEL/EXT-02-00985, September, 2002.

ICF Kaiser, (1995), Tank Farm Heel Removal Project Conceptual Design Report, RPT-034, February 3, 1995. 
INEEL, (2002), Risk Management Plan for the SBW Treatment Facility Project, PLN-1073, Rev. 0. The database is available at http://setest.inel.gov/sbwrisk/index.cfm.

Jantzen, C. M., (2002), Engineering Study of the Hanford Low Activity Waste (LAW) Steam Reforming Process (U), WSRC-TR-2002-00317, Rev.0. The report is available at http://www.osti.gov/bridge.

Jantzen, C. M., (2003), Characterization and Performance of Fluidized Bed Steam Reforming (FBSR) Product as a Final Waste Form, WSRC-MS-2003-00595, Rev. 0.

Kirk-Othmer, (1997), Kirk-Othmer Encyclopedia of Chemical Technology, Wiley Interscience, accessed through the INEEL technical library homepage on October 16, 2003 at http://www.mrw.interscience.wiley.com/kirk/articles/steaball.

Kohl, A. L., and F. C. Riesenfeld, (1979), Gas Purification, 3rd Edition, Gulf Publishing.

Marshall, D. W., (2003c), document review comment for Steam Reforming Process Design, accessible through SBW project server at Fserob1/Projects/Idaho Tank Farm Project/SBWT Facility Project/Supporting Information/Process Design/Steam Reforming.

Marshall, D. W., and N. R. Soelberg, (2003a), TWR Bench-Scale Steam Reforming Demonstration, INEEL/EXT-03-00436.

Marshall, D. W., and N. R. Soelberg, (2003c) TWR Bench-scale Steam Reforming DemonstrationProprietary Addendum, INEEL/EXT-03-00436a, May 2003.

Marshall, D. W., N. R. Soelberg, and K. M. Shaber, (2003b), THOR ${ }^{\mathrm{sm}}$ Bench-Scale Steam Reforming Demonstration, INEEL/EXT-03-00437.

Marshall, D., and Jenn-Hai Pao, (2000), Scoping Tests for Steam Reformation of Simulated Low-Activity Waste, INEEL/EXT-2000-01318.

Miller, J. E., and P. B. Kuehne, (1995), Steam Reforming of DOE Waste Simulants, SAND 95-0436C.

Morrell, D. K., and D. T. Clark, (2003), "Mixing Pumps for Homogenizing TFF SBW Liquids and Solids - Calcination with MACT Upgrade SBW Treatment Alternative," Engineering Design File EDF3307, April 24, 2003.

MSE Technology Applications, (2000), Controlled Emissions Demonstration Project Final Report Noxidizer Demonstration Testing, PTP-72, September, 2000.

MSE Technology Applications, (2001), John Zink Noxidizer Propane-Fired Performance Assessment and Mercury Emission Speciation, PTP-81, February, 2001.

Nichols, T. T., and D. D. Taylor, (2003), Thermodynamic Phase and Chemical Equilibrium at 0-110oC for the $\mathrm{H}+-\mathrm{K}+-\mathrm{Na}+-\mathrm{Cl}--\mathrm{H}_{2} \mathrm{O}$ System up to 16 Molar and the $\mathrm{HNO}_{3}-\mathrm{H}_{2} \mathrm{O}$ System up to 20 Molar Using an Association-Based Pitzer Model Compatible With ASPEN-Plus, INEEL/EXT-03-01167, Rev. 0, September, 2003

Perry, R. H., and C. H. Hilton, (1973), Chemical Engineers' Handbook, 5th edition, McGraw-Hill.

Shreve, R. N., (1967), Chemical Process Industries, 3rd edition, McGraw-Hill. 
Siemer, D. D., D. R. Haefner, and C. M. Barnes, (2003), "Preliminary Evaluation of Glycolic Acid Enhanced Evaporation to Process Sodium Bearing Waste," Engineering Design File EDF-4029, October 20, 2003.

Soelberg, N. R., (2003), "Off-gas Mercury Control for the Idaho Tank Farm Project - Calcination with MACT Upgrade SBW Treatment Alternative,” Engineering Design File EDF-3292, Rev. 0.

Soelberg, N. R., D. W. Marshall, S. O. Bates, and D. D. Taylor, (2004a), Phase 2 THOR $^{\text {sm }}$ Steam Reforming Tests for Sodium-Bearing Waste Treatment, INEEL/EXT-04-01493, January 30, 2004.

Soelberg, N. R., D. W. Marshall, S. O. Bates, and D. D. Taylor, (2004b), Phase 2 TWR Steam Reforming Tests for Sodium-Bearing Waste Treatment, INEEL/EXT-04-01494, January 30, 2004.

Stegen, G. E. and C. N. Wilson, (1996), Vectra GSI, Inc. Low-Level Waste Melter Testing Phase I Test Report, Document number: WHC-SD-WM-VI-031, Westinghouse Hanford Company, Richland WA.

Taylor, D. D., and T. T. Nichols, (2003), Revised Computer Program to Regress Parameters for Pitzer's Model, INEEL/EXT-03-01146, Rev. 0, September, 2003

ThermoChem, Inc., (June 1998), Steam Reforming of Low-Level Mixed Waste, Final Report, DOE/MC/32091-3.

THOR, (2003), THOR ${ }^{\text {sm }}$ Steam Reforming Process for Hazardous and Radioactive Wastes, TR-SR02-1, Rev. 1. Report available at http://www.thortt.com/library.

Treybal, R. E., (1968), Mass-Transfer Operations, 2nd Edition, McGraw-Hill.

U. S. Nuclear Regulatory Commission, 2002, Packaging and Transportation of Radioactive Material, 10 CFR 71.

Voelker, G. E., W. G. Steedman, and R. R. Chandran, (1996), Steam Reforming of Low-Level Mixed Waste, Federal Energy Technology Center Publications - 1996 Conference Proceedings, Available at http://www.netl.doe.gov/publications.

Williams, C. L., R. A. Wood, B. L. Blakely, and W. H. Landman, (2002), Feasibility Study Report for the Treatment of Sodium-Bearing Waste by Steam Reforming (Draft), INEEL/EXT-02-01211.

WIPP, (2003), TRUPACT-II Safety Analysis Report, NRC-Docket-71-9218/Rev. 19c, canister information given in Appendix 1.3.4, "Specification for RH-TRU Waste Containers," available at http://www.wipp.carlsbad.nm.us/library/caolib.htm.

Wood, R. A., and D. K. Morrell, (2003), "Heel Solids Processing Evaluation for the SBW Treatment Project - Calcination with MACT Upgrade Alternative," Engineering Design File EDF-3049, Rev. 0, December 4, 2002.

Wood, R. A., and D. R. Marshall, (2000), Modeling of a Multi-Stage Combustor (Noxidizer ${ }^{\mathrm{TM}}$ ) for Treatment of NWCF Off-gas, INEEL/INT-2000-01317, September, 2000 
Wood, R. A., D. Tyson, B. Bonnema, C. Olsen, A. P. Pinto, D. Wendt, S. Reese, and B. Raivo, (2001), Feasibility Study for the Idaho Waste Vitrification Facilities Off-gas Treatment for Sodium-Bearing Waste, INEEL/EXT-01-00995, September 2001.

Zenz, F. A., and D. F. Othmer, (1960), Fluidization and Fluid-Particle Systems, Reinhold Publishing. 
INEEL/EXT-04-01692

Appendix B

\section{Appendix B CsIX/TRU Grout Process}

Charles M. Barnes

Sam C. Ashworth

\section{February 2004}


This page intentionally left blank. 


\section{CONTENTS}

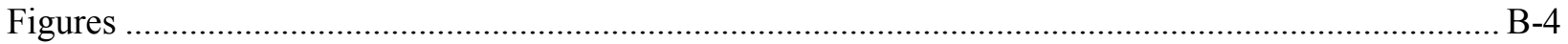

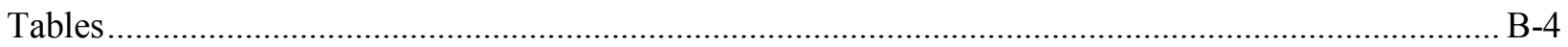

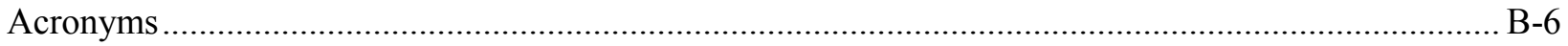

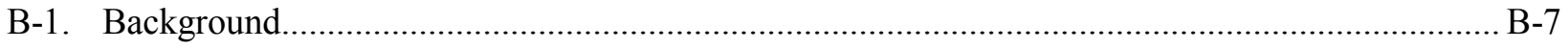

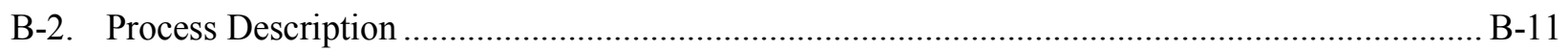

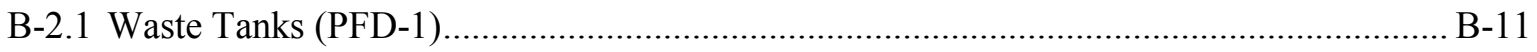

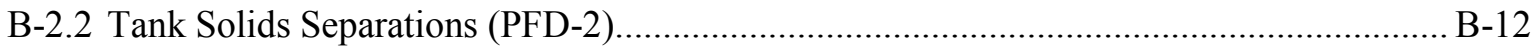

B-2.3 SBW Filtration \& Cesium Removal (PFD-3) ............................................................... B-12

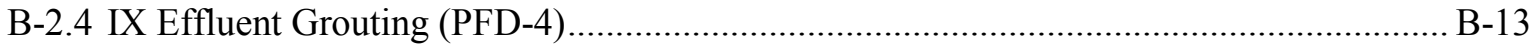

B-2.5 ETS, LET\&D Fractionator, \& PEWE (PFD- 5, -6 \& -7) ............................................ B-14

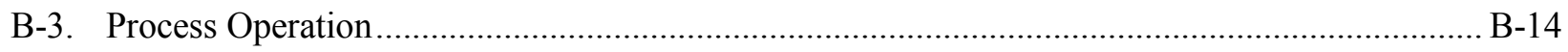

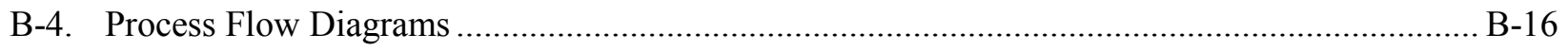

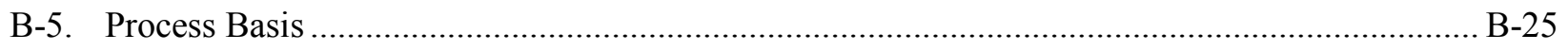

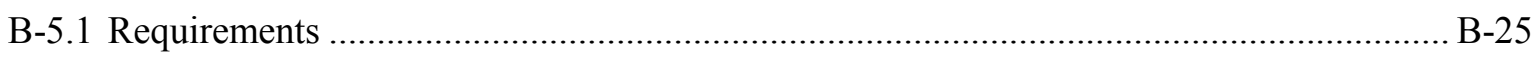

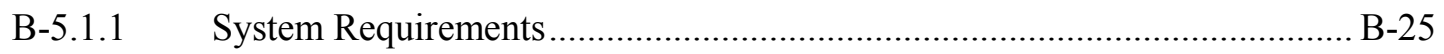

B-5.1.2 Systems, Subsystem, and Major Components....................................... B-29

B-5.1.3 Boundaries and Interfaces .................................................................... B-30

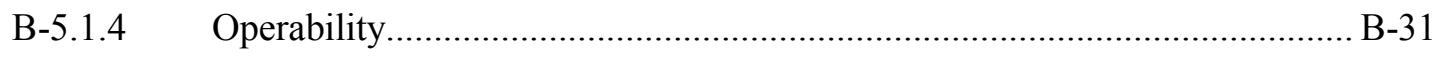

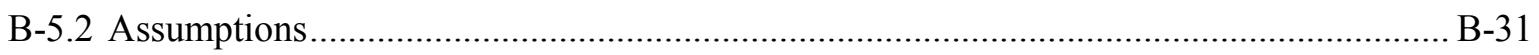

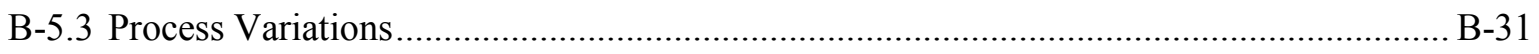

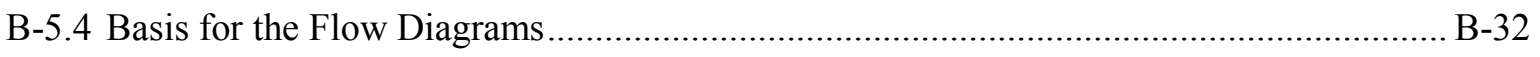

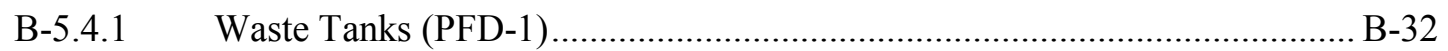

B-5.4.2 Tank Solids Separation (PFD-2) ....................................................... B-32

B-5.4.3 SBW Filtration and Cesium Removal (PFD-3) ...................................... B-33

B-5.4.4 IX Effluent Grouting (PFD-4) ................................................................ B-34

B-5.4.5 ETS, PEWE and LET\&D Fractionator (PFD-5, PFD-6, and PFD-7) ........... B-34

B-5.5 Basis for the Mass Balances......................................................................................... B-34

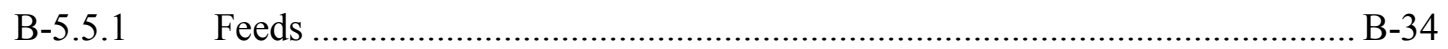

B-5.5.2 Stream Factor, Operating Schedule and Feed Rate................................... B-36

B-5.5.3 Feed Transfers from Tank Farm.............................................................. B-36 


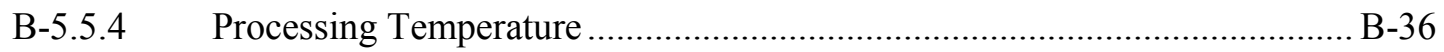

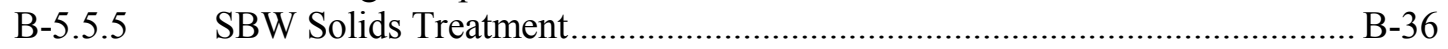

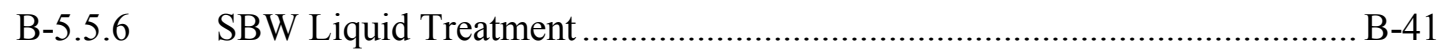

B-5.5.7 Waste Water Treatment..................................................................... B-45

B-5.5.8 Utilities ................................................................................... B-46

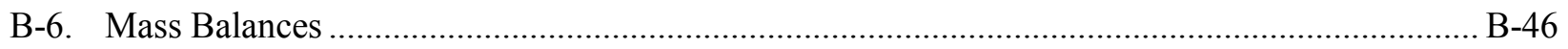

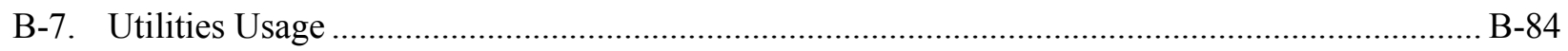

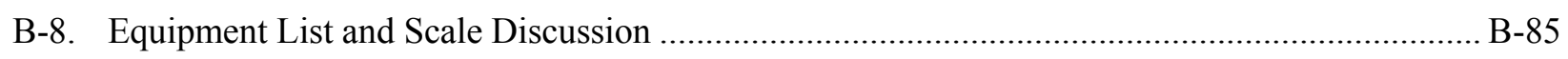

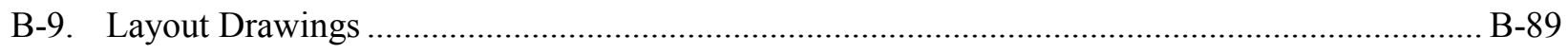

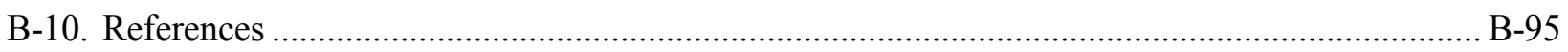

FIGURES

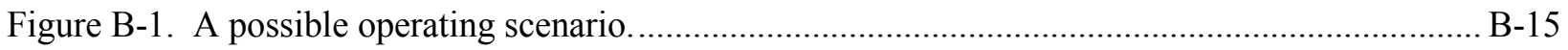

Figure B-2. Comparison of actual $500 \mathrm{~cm}^{3}$ column data with the $\mathrm{K}_{\mathrm{d}}$ values for the 2 and $500 \mathrm{~cm}^{3}$ column equilibrium models.................................................................. B-43

Figure B-3. Typical breakthrough curves for a series of three columns of CST removing Cs............. B-43

\section{TABLES}

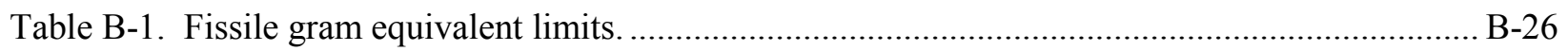

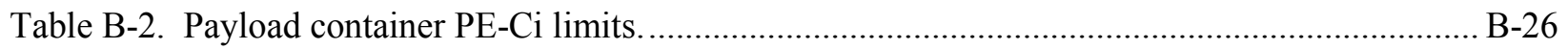

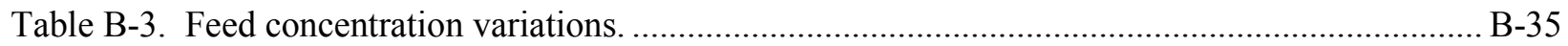

Table B-4. PEW WAC limits compared to Tank Farm liquid compositions before dilution with flush

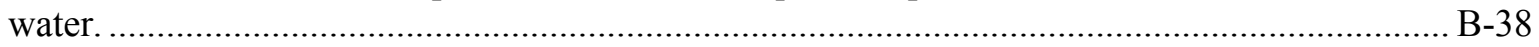

Table B-5. Limiting case volumes for processing supernate in PEWE. ............................................ B-38

Table B-6. Calculated liquid waste volumes after ETS/PEWE/LET\&D processing of tank flush

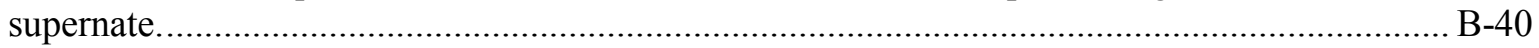

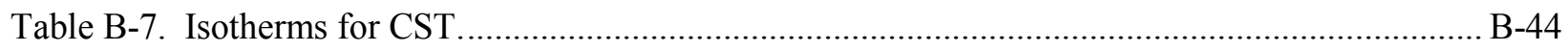

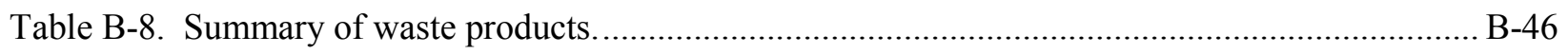

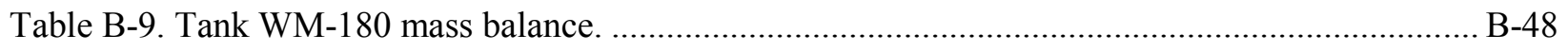




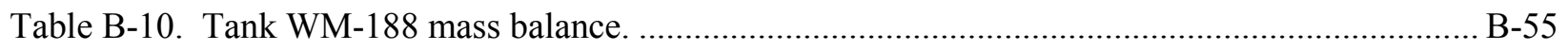

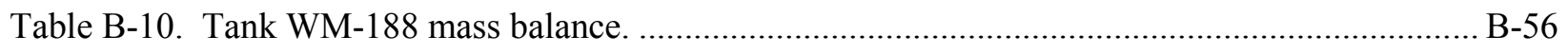

Table B-11. Tank WM-189 mass balance. ..................................................................................... B-63

Table B-11. Tank WM-189 mass balance. …................................................................................. B-64

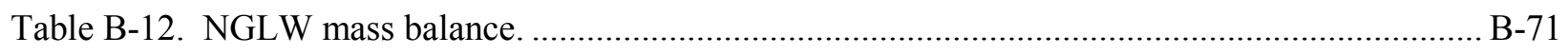

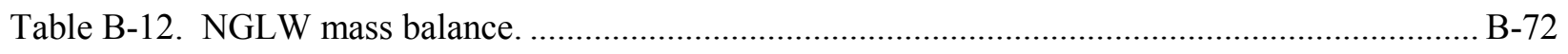

Table B-13. Grouted Waste Properties ……................................................................................. B-81

Table B-14. Spent Ion Exchange Media Properties....................................................................... B-82

Table B-15. Dried Tank Solids Properties.................................................................................. B-83

Table B-16. Chemicals and materials consumption...................................................................... B-84

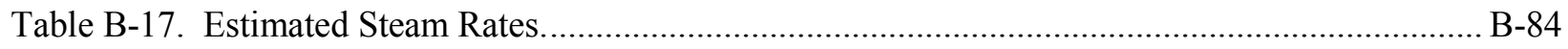

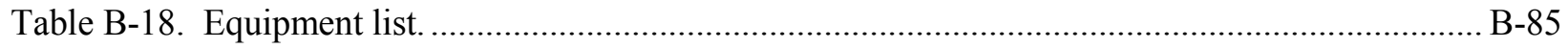

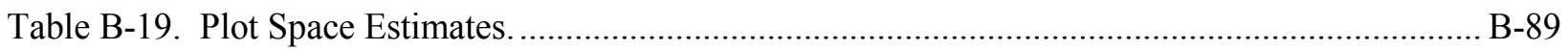




\section{ACRONYMS}

AMP

ASTM

BFS

CERCLA

$\mathrm{CH}$

CST

DBE

DOE

ETS

FGE

FY

HEPA

HLLWE

HVAC

INEEL

INTEC

LDR

LET\&D

NGLW

NWCF

PAN

PEWE

PFC

RCRA

$\mathrm{RH}$

SBW

T\&FR

TCLP

TEC

TFF

TPC

TRU

TSS

VOC

WAC

WIPP ammonium molydophosphate

American Society for Testing and Materials

blast furnace slag

Comprehensive Environmental Response, Compensation and Liability Act contact handled

crystalline silicotitanate

design basis element

Department of Energy

Evaporative Tank System

fissile gram equivalent

fiscal year

high-efficiency particulate air (filter)

High Level Liquid Waste Evaporator

heating, ventilation and air conditioning (equipment or systems)

Idaho National Engineering and Environmental Laboratory

Idaho Nuclear Technology and Engineering Center

land disposal restrictions (in RCRA)

Liquid Effluent Treatment and Disposal Facility

newly generated liquid waste

New Waste Calcining Facility

polyacrylonitrile

Process Equipment Waste Evaporator

process flow diagram

Resource Conservation and Recovery Act

remote-handled

sodium bearing waste

Technical and Functional Requirements

Toxicity Characteristic Leaching Procedure

total estimated cost

Tank Farm Facility

total project cost

transuranic (waste)

total suspended solids

volatile organic compounds

waste acceptance criteria

Waste Isolation Pilot Plant 


\section{Appendix B \\ Details of the CsIX TRU Grout Process}

\section{B-1. BACKGROUND}

The concept of the CsIX/Transuranic (TRU) Grout process for treatment of sodium-bearing waste (SBW) developed out of engineering studies and research and development (R\&D) testing of separations processes performed in the mid-1990s. These studies were aimed at developing treatment processes for both dissolved calcine and SBW which separated certain radionuclides from the initial Idaho Nuclear Technology and Engineering Center (INTEC) waste streams in order to produce both a relatively small volume of vitrified high activity waste and a larger volume of low-level waste grout.

Raytheon (Raytheon Engineers \& Constructors, 1994) performed a feasibility study in 1994 that included separation of actinides and ${ }^{90} \mathrm{Sr}$ by solvent extraction, separation of cesium by ion exchange, vitrification of the separated radionuclides and grouting the low-level waste streams. During the period 1994-1997, researchers at the Idaho National Engineering and Environmental Laboratory (INEEL) tested many of the unit operations of this separation process, including cesium removal by ion exchange and grouting of low-level waste (Miller, 1995; Herbst, 1996; Herbst, 1997; Todd, 2001). A later study by Fluor Daniel (Fluor Daniel, 1997) updated the design of the process based on these development studies, process studies and the evolving project assumptions.

In late 1997, G. E. Stegen proposed a much simplified separations process based on the assumption that SBW would be classified as TRU waste rather than high level waste (Stegen, 1997). Stegen's proposed process included cesium removal by adsorption on a non-regenerable ion exchange resin followed by grouting the cesium free liquid and grouting the spent resin. Stegen calculated that removal of $99.9 \%$ of the cesium from SBW would result in a surface dose rate from product drums of less than $100 \mathrm{mR} / \mathrm{hr}$.

Stegen amplified his treatment concept in two additional memorandums issued in March 1998 (Stegen, 1998a, 1998b). The objective of the proposed treatment process, according to the first of these memorandums, was to eliminate accumulation and storage of radioactive liquid waste by treating SBW, tank heels, and any "ongoing radioactive liquid waste" (now referred to as newly generated liquid waste [NGLW]) to produce solidified waste products and to accomplish this at a relatively low cost compared to other options being considered. An accompanying flow diagram showed (1) filtration of SBW to remove solids, (2) ion exchange columns to remove cesium from the filtrate, (3) grouting of the ion exchange effluent, and (4) grouting of the separated solids. Stegen discussed several options for disposal of the spent ion exchange media and also various options for use of existing facilities for all or part of the treatment process (Stegen, 1998a). In a separate memorandum, Stegen discussed the successful use of crystalline silicotitanate (CST) to remove cesium from a Melton Valley waste (Stegen, 1998b).

The first mass balances for the CsIX/TRU Grout process treating SBW and NGLW were prepared in early 1998 to supply information for the Idaho High Level Waste and Facilities Disposition Environmental Impact Statement (Barnes, 1998). A more detailed feasibility study was performed later that year (Losinski, 1998). The treatment process defined in this study included removal of undissolved solids by filtration, removal of cesium by ion exchange using CST, and cement-based grouting. Filtered undissolved solids and spend ion exchange media were dried and packaged for interim storage and disposal.

The Losinski CsIX/TRU Grout facility feasibility study included an investigation of alternative absorbents such as a low-density silica based absorbent, a clay based absorbent and a polymer based 
absorbent. However, based on a set of evaluation criteria, the cement-based grout was recommended. The treatment process feed included SBW and NGLW, but not tank heels. The study included preparing functional and operational requirements, developing a process flow diagram and mass balances, defining facility concepts and drawings, defining process and project uncertainties and estimating the project and life cycle costs and the project schedule. The mass balance showed that $7,500 \mathrm{~m}^{3}$ of grout, $12 \mathrm{~m}^{3}$ of spent ion exchange media and $10 \mathrm{~m}^{3}$ of undissolved solids waste would be produced. The schedule showed performing the conceptual design over three years from 2000-2002, treating the waste in five years (20092013), and completing shipments to the Waste Isolation Pilot Plant (WIPP) by the end of 2022. The total estimate cost (TEC) for a new facility was estimated to be \$102 million, and total project cost (TPC) \$134 million, or $\$ 92$ million if discounted.

Additional engineering studies of the CsIX/TRU Grout process were performed in 1999 (Valles, 1999). These studies sought to further simplify the process, reduce costs and final waste volume by evaluating each of the major unit operations. Recommended changes to the 1998 feasibility study design included (a) changing from vertical leaf pressure filters to a crossflow filter for UDS removal, (b) air drying the spent ion exchange media in place, and (c) using a higher waste loading grout formulation. The higher waste loading resulted a grout waste volume of about $4,400 \mathrm{~m}^{3}$. Recommended changes were estimated to reduce the total estimated cost (TEC) of the 1998 study by about $\$ 7$ million.

Raytheon performed a feasibility study of the CsIX/TRU Grout process in 2000 (Raytheon, 2000) for treatment of SBW. The Raytheon feasibility study report includes:

(a) Descriptions of process equipment and systems

(b) Descriptions of site, facility and utility systems

(c) A proposed approach to project execution

(d) Project cost and schedule estimates

(e) Technical evaluations and assessments of shielding, criticality, environmental and permitting considerations, waste analysis, sampling and waste characterization requirements, quality assurance, technology development needs

(f) A technical and functional requirements document

(g) Process flow diagrams and mass balances

(h) Drawings of equipment arrangement, HVAC diagrams, electrical single line diagrams, hot cell, remote operations and pump and valve corridor details

(i) Facility floor plans

(j) Details of interim storage areas

(k) A process design basis document

(1) A preliminary hazards analysis

(m) Evaluations and test results of equipment and systems selected to optimize the process. 
The treatment process defined in the Raytheon study included the following unit operations:

- $\mathrm{SBW} /$ suspended undissolved solids receiving and storage

- Solids/liquid separation to remove UDS from the feed

- Collection and transfer of separated UDS to the Tank Farm Facility (TFF)

- Cesium removal by ion exchange using ammonium molybdophosphate (AMP) supported on a polyacrylonitrile (PAN) substrate

- Neutralization of the ion exchange effluent

- Sampling and waste characterization

- Grouting of the neutralized ion exchange effluent

- Packaging, examination and interim storage of grouted SBW

- Waste certification and shipping

- Treatment and disposal of secondary waste (spent sorbent).

The Raytheon mass balances were based on about 1.1 million gallons of SBW, but did not include tank heels. The mass balance showed that about $4,700 \mathrm{~m}^{3}$ of grouted contact-handled $(\mathrm{CH})$ waste would be produced. Spent ion exchange media was processed by dissolving the AMP with caustic, grouting the dissolved AMP, dewatering the separated PAN and loading the PAN into high integrity containers for disposal. About $43 \mathrm{~m}^{3}$ of grouted AMP would be produced and $0.35 \mathrm{~m}^{3}$ of PAN waste.

In the Raytheon study, the process included a cartridge type (Fundabac) filter to separate undissolved solids, and the separated solids were returned to the INTEC Tank Farm. Ion exchange effluent was neutralized with dry calcium oxide to a $\mathrm{pH}$ of about seven prior to grouting. Neutralized ion exchange effluent was fed into 55-gal drums, dry grouting ingredients added and mixing performed in the drum with a paddle that was left in place in the grouted waste. The grouting facility included three grouting lines.

Raytheon's TEC estimate was $\$ 285$ million and TPC estimate $\$ 322$ million. These estimates included the CsIX facility, the grouting facility, an interim storage facility and site development. The project schedule assumed approval of critical decision (CD) 0 in June 2001, start of operations in January 2010 and completion of treatment three years later at the end of December 2012.

Development of ion exchange as a method to remove cesium from acidic reprocessing aqueous wastes began at the INEEL in 1961 (Wilding, 1961). These early tests used ammonium phosphomolybdate as the ion exchange media. Testing of AMP for removal of cesium from dissolved calcine was performed in 1994 (Miller, 1994), and AMP-PAN was tested for cesium removal from SBW and dissolved calcine in 1995 (Miller, 1995). More recent development of AMP as a cesium ion exchange media for treatment of INTEC tank waste has focused on the development of an inorganic support material (Todd, 2003; Tranter, 2003a).

The Department of Energy (DOE) has funded the development and commercialization of CST as an ion exchange media applicable to the treatment of wastes at multiple DOE sites (Miller, 1997). After 
development studies that demonstrated the effectiveness of CST to remove cesium from various aqueous solutions, UOP was selected as a technology transfer partner to commercialize CST (Miller, 1997). Starting in 1994, UOP has supplied CST for both testing and use at various DOE sites, including Savannah River, Oak Ridge National Laboratory, Los Alamos National Laboratory, the Pacific Northwest Laboratory and the INEEL (Miller, 1997).

Testing of CST to remove cesium from SBW began in 1997 (Todd, 2001). Initial tests were performed in a $1-\mathrm{cm}^{3}$ column with actual waste from Tank WM-183 and from a simulant representing an average SBW composition. Additional tests with CST, also known as IONSIV IE-911, were performed in 1999 (Mann, 1999), 2000 (Mann, 2000), and 2003 (Tranter, 2003a).

Conclusions drawn from the 2000 test results included:

- Batch equilibrium experiments performed with IONSIV IE-911 and an SBW composite simulant showed that the sorbent displayed a high affinity for ${ }^{137} \mathrm{Cs}$. Moreover, cesium loading was higher for batch experiments performed at $25^{\circ} \mathrm{C}$ compared to experiments performed at $50^{\circ} \mathrm{C}$. IONSIV IE-911 failed to load ${ }^{241} \mathrm{Am},{ }^{203} \mathrm{Hg}$, and ${ }^{238} \mathrm{Pu}$ under similar conditions.

- Batch equilibrium experiments performed with IONSIV IE-911 and solutions containing 15 $\mu \mathrm{g} / \mathrm{mL} \mathrm{Cs}, 10 \mu \mathrm{g} / \mathrm{mL} \mathrm{Sr}$ and various combinations and concentrations of $\mathrm{HNO}_{3}, \mathrm{NaNO}_{3}$ and $\mathrm{KNO}_{3}$ displayed a decrease in $\mathrm{K}_{\mathrm{d}}$ when increasing $\mathrm{HNO}_{3}$ from $1 \underline{\mathrm{M}}$ to $2 \underline{\mathrm{M}}$. Further, it appeared that $\mathrm{HNO}_{3}$ had a larger effect on cesium loading than $\mathrm{NaNO}_{3}$. A further decrease in cesium loading was observed with the addition of $\mathrm{KNO}_{3}$.

- Small-scale dynamic column experiments performed with IONSIV IE-911 and the composite tank waste simulant showed that the sorbent displayed a high affinity for ${ }^{137} \mathrm{Cs}$. Am-241 and ${ }^{203} \mathrm{Hg}$ in addition to various competing metals ( $\mathrm{Na}, \mathrm{K}, \mathrm{Cd}, \mathrm{Ca}, \mathrm{Cr}, \mathrm{Eu}, \mathrm{Fe}, \mathrm{Pb}, \mathrm{Mn}, \mathrm{Mo}, \mathrm{Ni}$, $\mathrm{Zr}$, and $\mathrm{Ba}$ ) were not sorbed by IONSIV IE-911.

Ion exchange tests performed in 2003 focused on (a) determining the stability of both AMP on an inorganic support and CST and in both SBW simulants and partially neutralized simulants, (b) determining the TRU content of spend ion exchange media to ensure proper disposal and (c) generating breakthrough curves using bench-scale columns (Tranter, 2003a; Tranter, 2003b). Results of these tests are discussed in later sections of this report.

Initial formulations for grouting SBW simulants were developed in 1997 (Herbst, 1997), and typically had waste loadings of $25-40 \%$. With the advent of the concept of disposal of the grouted waste product at WIPP, the constraint of grout compressive strength was removed, and new formulations were developed with higher waste loadings (McCray, 1999; Herbst, 1999; Herbst, 2000; Raman, 2003). Conclusions from 1999 tests included:

- Approximately $80 \mathrm{wt} \%$ waste loading is the maximum achievable for either CsIX or NGLW waste.

- Land Disposal Restriction (LDR) Toxicity Characteristic Leaching Procedure (TCLP) limits can likely also be met at these high waste loadings.

- Considerably better grout products, both chemically and physically, can be attained by lowering the waste loading to $70-75 \mathrm{wt} \%$. 
- Heat generation from waste acid neutralization and cement powders hydration is significant. On a large-scale process, a mix system equipped with cooling coils or other means of cooling may be required, regardless of the grout formulation chosen.

Optimization of the formulation for CsIX grout continued in FY 2000 (Herbst, 2000). FY 2000 testing was performed to determine grout viscosities during mixing, power requirements for mixing, the change in viscosity after cooling, water loss during curing, and the potential for formation of liquid water in grout drums during thermal cycling. These tests resulted in improvements to the grout formulation to improve viscosity, verification of acceptability of grouts with a $70 \%$ waste loading, alleviation of concerns of free liquid formation due to thermal cycling, and a recommendation of a revised formulation to reduce mass loss via evaporation during grout mixing and cooling.

Separate tests were performed in 2000 of a continuous mixer (Readco) at the vendor's test facility using two grout formulations (Herbst, 2000). These demonstration tests showed that a continuous mixer was capable of blending the SBW stimulant and dry powder additives to produce a homogenous grout mix. An expanded matrix of grout formulations was tested with a different continuous mixer in FY 2003 (Scholes, 2003). The optimum waste loading determined in these recent tests for WM-180 simulant was $73 \%$; for WM-189 simulant, a lower waste loading of $64 \%$ was required.

FY 2003 grout development focused on obtaining a better scientific, as opposed to simply empirical, understanding of the grouting process in order to be better prepared for scale up of the process (Raman, 2003).

The third major unit operation in the CsIX/TRU grout process, besides ion exchange and grouting, is solids filtration. Two types of filters have been tested for removal of solids from the CsIX feeds. Tests in 1997-1998 demonstrated the removal of undissolved calcine solids and undissolved solids in SBW using a cross-flow filter (Tripp, 1997; Mann, 1998). As part of the FY 2000 feasibility study of the CsIX process (Raytheon, 2000, Appendix F), initial tests were performed to demonstrate a Fundabac filter for removal of solids from SBW. The Fundabac filter demonstration used a $0.13 \mathrm{ft}^{2}$ filter and test feeds of $\mathrm{TiO}_{2}$ and $\mathrm{TiO}_{2}-\mathrm{SiO}_{2}-\mathrm{ZrO}_{2}$ mixtures.

Based on additional SBW solids characterization in late FY 2003, an improved SBW solids simulate has being developed (Barnes, 2003). Reviews of both crossflow and Fundabac filtration have been preformed in light of this characterization (Pao, 2003).

\section{B-2. PROCESS DESCRIPTION}

\section{B-2.1 Waste Tanks (PFD-1)}

Process flow diagram (PFD)-1 shows the existing seven storage tanks that will hold the feed to the CsIX TRU grout process. Tanks VES-WM-180 and VES-WM-189 presently contain SBW at near their administrative capacity limits. Tank VES-WM-188 is presently approximately $80 \%$ full of SBW, and will be filled to capacity as new wastes are generated and concentrated by the Evaporative Tank System (ETS, formerly the High Level Liquid Waste Evaporator [HLLWE]). Over the past few years solids that were in five tanks have been flushed to Tank VES-WM-187, which is being used as a collection tank for flush water and solids from operations to clean and close other TFF tanks. Upon flushing one more tank, VES-WM-181, the collection of solids in WM-187 will be complete.

After 2005, NGLW will be stored in the three tanks VES-WM-101, VES-WM-101, and VES-WM-102. 
Existing steam jets in each of the tanks plus an existing airlift in VES-WM-189 will be used to transfer the SBW liquid/solids mixture from the TFF to the CsIX TRU Grout Facility SBW Receiving Tanks. Waste will be transferred to the treatment facility intermittently in batches at a rate of about 50 gpm. A transfer of approximately 4,000 gallons of liquid would provide a 2-day supply of feed to the treatment process. All equipment shown on PFD-1 presently exists.

\section{B-2.2 Tank Solids Separations (PFD-2)}

PFD-2 shows the Solids Settling/Decant Tanks, the Solids Filters and the Waste Water Tank. Waste from Tank VES-WM-187 would be transferred in batches of approximately 4000 gallons to one of two Solids Tanks. One tank would receive and decant waste while the other was feeding waste to treatment. Initial transfers from VES-WM-187 are expected to contain a high concentration of solids and require no decanting. The Solids Tanks are equipped with mixers and pump recycle to keep the solids in suspension while the waste is being fed to treatment.

A small amount of $50 \%$ caustic would be added to waste received in one of the Solids Tank. The caustic promotes flocculation of particles that in turn increases the particle-settling rate. After settling, supernate would overflow through side withdrawal ports into a surge tank and from there be transferred by steam jet to the Waste Water Tank. Upon reaching a solids concentration of $20 \mathrm{wt} \%$, the tank contents would be mixed and sampled and are then ready to feed the Solids Filter.

The Solids Filter is a Fundabac filter and has six perforated tubes around a central tube. On the outside of these tubes is a filter cloth. The feed slurry would enter the filter vessel and liquid flow through the cloth into one of the six tubes. Liquid in these tubes would flow down and then enter the central tube and flow up to the discharge. Solids would be collected on the filter cloth. At the beginning of a filter cycle, a filter aid could be pumped through the filter to lay down an initial coating of solids to aid filtration of SBW solids. Alternatively, the filter discharge liquid could be recycled to the Settling Tank until adequate solids removal was achieved.

During filtration, solids would build up in a cake on the filter cloth. Upon reaching a set pressure drop across the filter, feed would be stopped and the filter drained. A steam jet would be used to transfer the filter liquid back to the Solids Tank. The cake would then be washed by flowing water through the filter to reduce the acidity of the solids waste. Discharge water would be collected in the Waste Water Tank. The cake would then be dried by passing low-pressure steam through the cake. Steam effluent from cake drying would be condensed and collected in the Waste Water Tank.

When the water content of the solids cake has been reduced to about $25 \mathrm{wt} \%$, the steam flow would be stopped and pulses of compressed air used to cause the filter cloth to billow, in turn causing the filter cake to crack, drop off the cloth and fall by gravity into a waste canister.

The Waste Water Tank collects decant water from the Decant Tank, solids wash water effluent, filter cake drying steam condensate, vent gas condensate, and ion exchange media flush water. The Tank is sized to hold 4,000 gallons, equivalent to about a 3-day volume of waste water. Upon reaching capacity, the contents are steam jetted to the ETS for concentration.

\section{B-2.3 SBW Filtration \& Cesium Removal (PFD-3)}

SBW from Tanks VES-WM-180, VES-WM-188 and VES-WM-189 would be received from the INTEC Tank Farm by the SBW Receiving Tanks, shown on PFD-3. One tank would receive, neutralize and hold waste while the other tank feeds liquid SBW treatment. The acidity of the SBW varies from about $1 \mathrm{M}$ to $3 \mathrm{M}$. To minimize degradation of the ion exchange media, the acidity of the waste would be 
reduced to $0.5 \mathrm{M}$ by the addition of $50 \mathrm{wt} \%$ caustic to the Receiving Tank. At this $\mathrm{pH}$, ion exchange media stability is acceptable and precipitation from neutralization is avoided. During neutralization, mixing will be achieved by a recirculation line with a mixing jet on the discharge pump. Each Receiving Tank is sized for 2 days of waste.

Undissolved solids in the neutralized SBW are then removed by filtration using a crossflow filter. Crossflow filtration operates by recirculating the feed flow parallel to the filter membrane. A high fluidcirculation rate tangential to the membrane sweeps away particles on the membrane, thereby limiting the thickness of the filter cake. The recirculation rate is usually determined experimentally. For this design a nominal rate of $130 \mathrm{gpm}$ was assumed, the mid-range value for a MOTT crossflow filter.

The permeate from the filter is collected in the Filtrate Receiver Tank and then pumped through three ion exchange columns in series to the Ion Exchange Effluent Tank. Three columns are used to achieve $99.9 \%$ removal of cesium from the SBW while also obtaining a reasonable cesium loading of the media. A fourth column is off-line. When breakthrough of cesium is detected in the effluent of the first column, it is taken offline for preparation for disposal. The fourth column is placed in service, with the second column becoming the first in the series and the newly on-line column the third in the series.

Each of the columns contains 60 gallons of CST sorbent. CST has been shown to be highly selective for removal of cesium from SBW and is commercially available.

Loading of an ion exchange column with fresh CST could take place outside of the hot cell. Sand would first be added to the column followed by fresh ion exchange media. The sand acts as a filter for loaded sorbent particles that would otherwise contaminate the effluent. After loading CST into the column, the bed would be backwashed with water to remove fine particles and to better distribute the bed. The water lines would then be disconnected and the bed transferred into position in the hot cell. Alternatively, the columns could be purchased pre-loaded from a vendor. In this case, backwashing would still be performed prior to moving a column into the hot cell.

The equipment required for disposal of spent ion exchange media has not been fully defined, but the processing steps are described here. After a column is taken off line from SBW flow, the bed would be rinsed with water and drained. The bed of CST and sand would then be dried using hot air until the moisture level falls below $25 \mathrm{wt} \%$. Once dry, the bed column would be remotely disconnected from feed and product lines and transferred to a loading area. The entire column would be loaded into a remotehandled (RH)-TRU canister for interim storage and shipment to WIPP.

\section{B-2.4 IX Effluent Grouting (PFD-4)}

PFD-4 shows equipment to grout ion exchange effluent and treat process vent gas. Grout bulk materials, including Portland cement, calcium hydroxide and blast furnace slag, are received from suppliers into storage hoppers. Metering hoppers are used to control the rate at which these dry ingredients are fed to a mixer and then the Grout Mixer, to be mixed with ion exchange effluent. The Grout Mixer discharges into 55-gal drums. A drum would be filled every 0.5 hours, and 48 drums (average) would be produced per day. After filling with waste, a drum would be moved to a curing area and held for 24 hours. The drum surface would then be smeared to check for surface contamination. If found, the drum would be decontaminated. The head-space of the drum would then be sampled for organic compounds and the drum inspected for free liquid. Passing inspection, the drum would either be sent to interim storage or loaded into a Halfpact and shipped to WIPP.

Ion exchange effluent is used to flush the grout mixer, although alternatively water could be used. Effluent flush is collected in the Flush Water Receiver/Feed Tank and then can be reused or grouted. 
Vent gas from process vessels is collected in a header and sent to a demister. Demister condensate flows by gravity to the Waste Water Tank. Vapor and gases from the demister are superheated and flow through a high efficiency particulate air (HEPA) filter prior to being sent to the INTEC stack via a blower.

\section{B-2.5 ETS, LET\&D Fractionator, \& PEWE (PFD- 5, -6 \& -7)}

Wastewater collected in the Wastewater Tank (PFD-2) is treated in existing INTEC equipment. The wastewater is sent to VES-NCC-101, the Blend Tank used as the ETS feed tank. The waste is air lifted from the Blend Tank to the ETS Feed Head Tank and flows by gravity to the ETS evaporator, also called the "Flash Column". Evaporator bottoms is collected in VES-NCC-119 and transferred by steam jet to VES-WM-100, VES-WM-101 or VES-WM-102. This waste will be treated by the CsIX TRU Grout liquid processing equipment. The evaporator overhead is condensed and collected in VES-NCC122. From this tank, the condensate is transferred to the Process Equipment Waste Evaporation (PEWE) for further treatment.

The condensate is processed through the PEWE to achieve additional decontamination. The ETS condensate is received into a feed collection tank, pumped to a feed head tank, and flows by gravity into one of two duplicate evaporators. Concentrate from the evaporator would be transferred by steam jet to WM-100, WM-101 or WM-102. Condensate from the PEWE evaporator is collected in storage tanks and periodically transferred to the Liquid Effluent Treatment and Disposal (LET\&D) facility.

The LET\&D Fractionator concentrates the condensate, thereby reducing the amount of waste that would require treatment and disposal. The condensate is fed by gravity from the LET\&D Feed Tank to one of two identical fractionators, each containing 15 sieve trays. Each fractionator typically recovers at least $99 \%$ of the nitric acid from the feed into the bottoms product. The bottoms product is usually 12 molar nitric acid and contains a high fraction of the other impurities in the feed condensate (HCl, fluoride, $\mathrm{HgCl}_{2}$ ). The fractionator overhead is steam with trace impurities. A partial condenser provides reflux to the column. The effluent from the condenser goes to a vapor/liquid separator, with the vapor from the separator superheated, filtered and discharged via a blower to the INTEC-708 stack. When the desired density of the fractionator bottoms is reached, a portion of the solution is drained out of the column and stored in the Bottoms Tank. This recovered acid is cooled by heat exchange with cooling water prior to entering the Bottoms Tank.

Except for the feed and bottoms tank, all acid fractionation equipment (fractionator with reboiler and condenser, separator, superheater, filter and blower) is duplicated. Recovered acid would be available for reuse in other INTEC activities. A 22,500-gal acid recycle tank, not shown of PFD-6, is available to collect and store LET\&D bottoms for use in the New Waste Calcining Facility (NWCF). However, the projected volume needed for expected INTEC operations is much less than the amount that would be generated by the SBW treatment process, and hence the acid will be processed with SBW in the CsIX TRU Grout Treatment Facility.

\section{B-3. PROCESS OPERATION}

This section describes a possible operation scenario.

By 2005, flushing TFF heels to WM-187 tanks will be complete except for solids in the full SBW tanks that will not be transferred until the treatment facility is on-line. Tank WM-188 will be full. Subsequent to 2005, Tanks WM-100, WM-101, and WM-102 will be used to collect and store NGLW. 
After startup, SBW liquid and tank solids would be processed simultaneously. Treatment is shown pictorially in Figure B-1.

Waste from WM-189 would be fed to the liquid processing section of the facility while solids in WM-187 would be carried in flush water to the solids treatment equipment. Tank WM-189, thought to contain the most heel solids of the three liquid SBW tanks, would be processed first, so that the heel solids could be flushed early to WM-187. As discussed in more detail in Section B-5.5.5, an estimated 414,000 gallons of water will be needed to flush WM-187 solids. At the completion of treatment of WM189 liquid, about half of total volume of WM-187 flush water will have been sent to treatment.

When Tank WM-189 is empty (at heel level) of liquid, heel would immediately be flushed to WM187. This flush, estimated to be about 87,000 gallons, will help flush solids remaining in WM-187, as will flushes from WM-180. While liquid from the last TFF tank (WM-188 according to Figure B-1) is being processed, the last flush from WM-187 will be performed, leaving WM-187 ready to close. The heel from WM-189 will then be flushed, either to WM-187 or preferably directly to treatment. The final solids are processed in a short period ( $\sim 1.4$ months as shown on Figure B-1) while NGLW is being processed in the liquid treatment portion of the facility.

4000-gal transfers of liquid SBW to the treatment facility will be made approximately every 1.3 hours. Waste is received and neutralized in one receiving tank, while being fed to treatment from a second tank. Liquid processing is continuous from the feed tank to the crossflow filter to the ion exchange columns to the grouting operation.

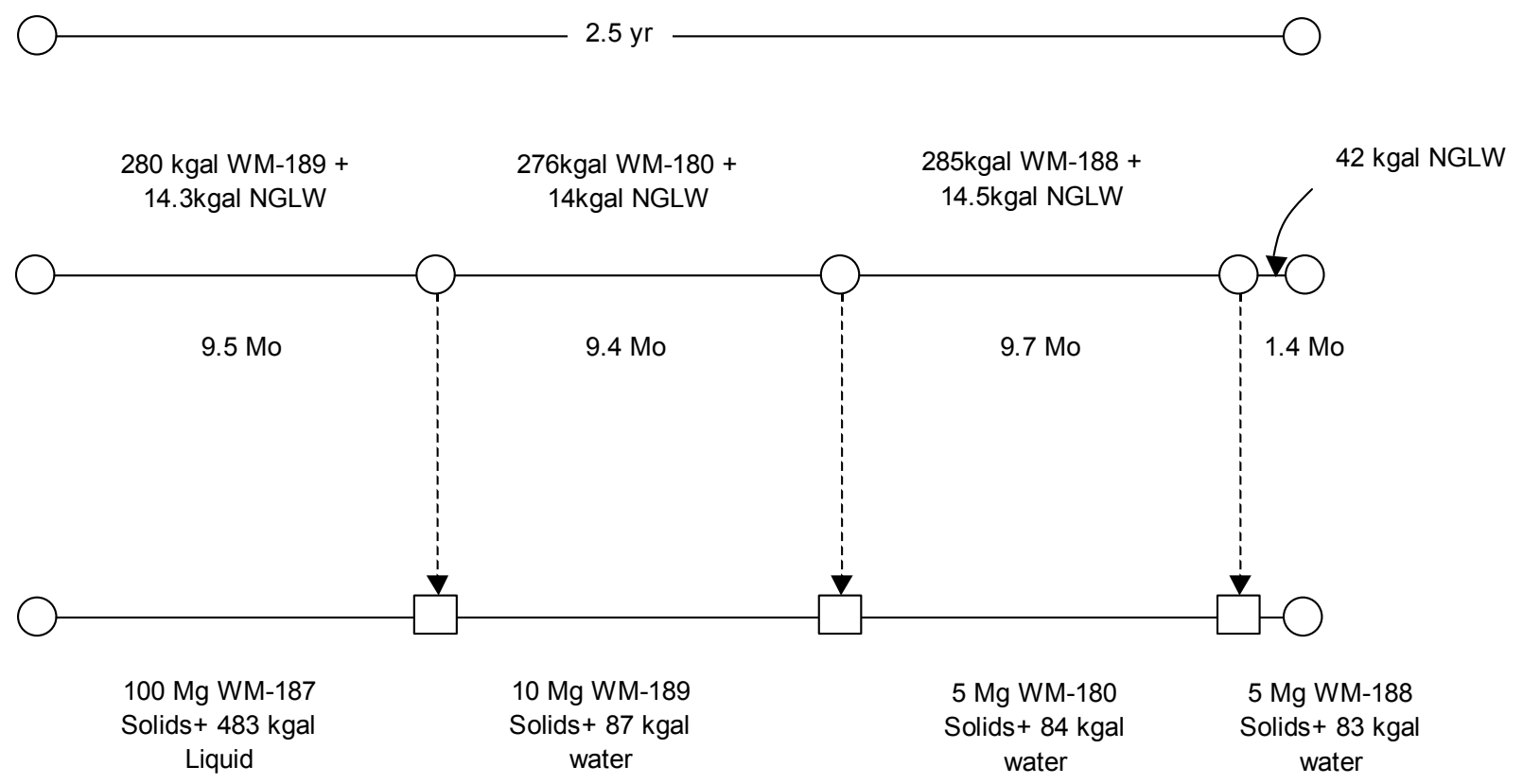

Figure B-1. A possible operating scenario.

When breakthrough is achieved in the leading ion exchange column, the flow is valved to the next column in series, with a new column coming on line in the third position. The spent column is drained, the media flushed and dried while still in the column, and then the column is packaged in a canister for disposal. 
The grout mixer has an effluent recycle line so that during the time when a full drum of grout is disconnected from the mixer effluent line and replaced with an empty one, grout exiting the mixer would be recycled to the mixer inlet. Ion exchange effluent would be used to flush the grout mixer if needed. This flush liquid would be bled into the grout feed line for grouting and disposal.

A purge of concentrated solids from the crossflow filter would be jet-pumped continuously from the Crossflow Filter Feed Tank to one of two Solids Tanks. The solids in TFF Tank WM-187 would be flushed to the Solids Tank periodically using existing jets at $50 \mathrm{gpm}$. 50\% sodium hydroxide would then be mixed with the Solids Tank content to bring the $\mathrm{pH}$ to approximately 5 . There would be several periods or time cycles for each batch. The first one is rapid mixing to form microfloc done by high-speed agitation using the mixer and recirculation. Two hours was allowed for this although it is expected to require less time. Flocculation can be accomplished via $\mathrm{pH}$ adjustment only if there is enough aluminum present to form the insoluble hydroxide. The small, slow settling particles will agglomerate to form larger, faster settling particles, thus reducing settling time. The resulting time cycle is based on an assumed settling of $95 \%$ of the solids in the top $5 \mathrm{ft}$ of the tank at a cut diameter of $5 \mu \mathrm{m}$ where this cut diameter is increased in size via flocculation from the received diameter of $2 \mu \mathrm{m}$. The settling takes 30 hours. The sampling time cycle can be accomplished prior to flocculation or prior to filter feed but it is assumed prior to filter feed when the batch at a $20 \mathrm{wt} \%$ maximum solids is pumped through the Fundabac ${ }^{\circledR}$ filter.

Recirculation after settling is done to evenly suspend solids and has a time cycle associated with sampling of two hours. Filtration is done by pumping at a nominal flow of 10 gpm until 100 liters of cake is collected. This would take six minutes. A short cycle is defined as filtration (6 minutes), draining (12 minutes), and steam drying (20 minutes). There are $8-9$ short cycles per batch. Since it takes 26 minutes to receive a batch using the $50 \mathrm{gpm}$ jet, the total batch cycle time is 40 hours. There are two Solids Tanks so that one can receive waste while the other is doing the other operations. The above describes the system based on the design and the overall volume to be treated using WM-187 suspended solids.

However, because of the uncertainties in flush volumes, the tank sizes were increased by a factor of three. This will also reduce the number of Tank Farm transfers and provide more flexibility in clarifier equipment operations.

\section{B-4. PROCESS FLOW DIAGRAMS}

Process flow diagrams are shown on the following pages:

Page

$\begin{array}{llll}\text { B-19 } & \text { PFD-1 } & \text { Waste Tanks } & \text { (Existing TFF) } \\ \text { B-20 } & \text { PFD-2 } & \text { Tank Solids Separations } & \text { (New solids processing equipment) } \\ \text { B-21 } & \text { PFD-3 } & \text { SBW Filtration and Cesium Removal } & \text { (New liquid processing equipment) } \\ \text { B-22 } & \text { PFD-4 } & \text { IX Effluent Grouting } & \text { (New equipment) } \\ \text { B-23 } & \text { PFD-5 } & \text { Evaporator Tank System } & \text { (Existing ETS) } \\ \text { B-24 } & \text { PFD-6 } & \text { LET\&D Fractionator } & \text { (Existing LET\&D) } \\ \text { B-25 } & \text { PFD-7 } & \text { PEWE Evaporator } & \text { (Existing PEWE) }\end{array}$




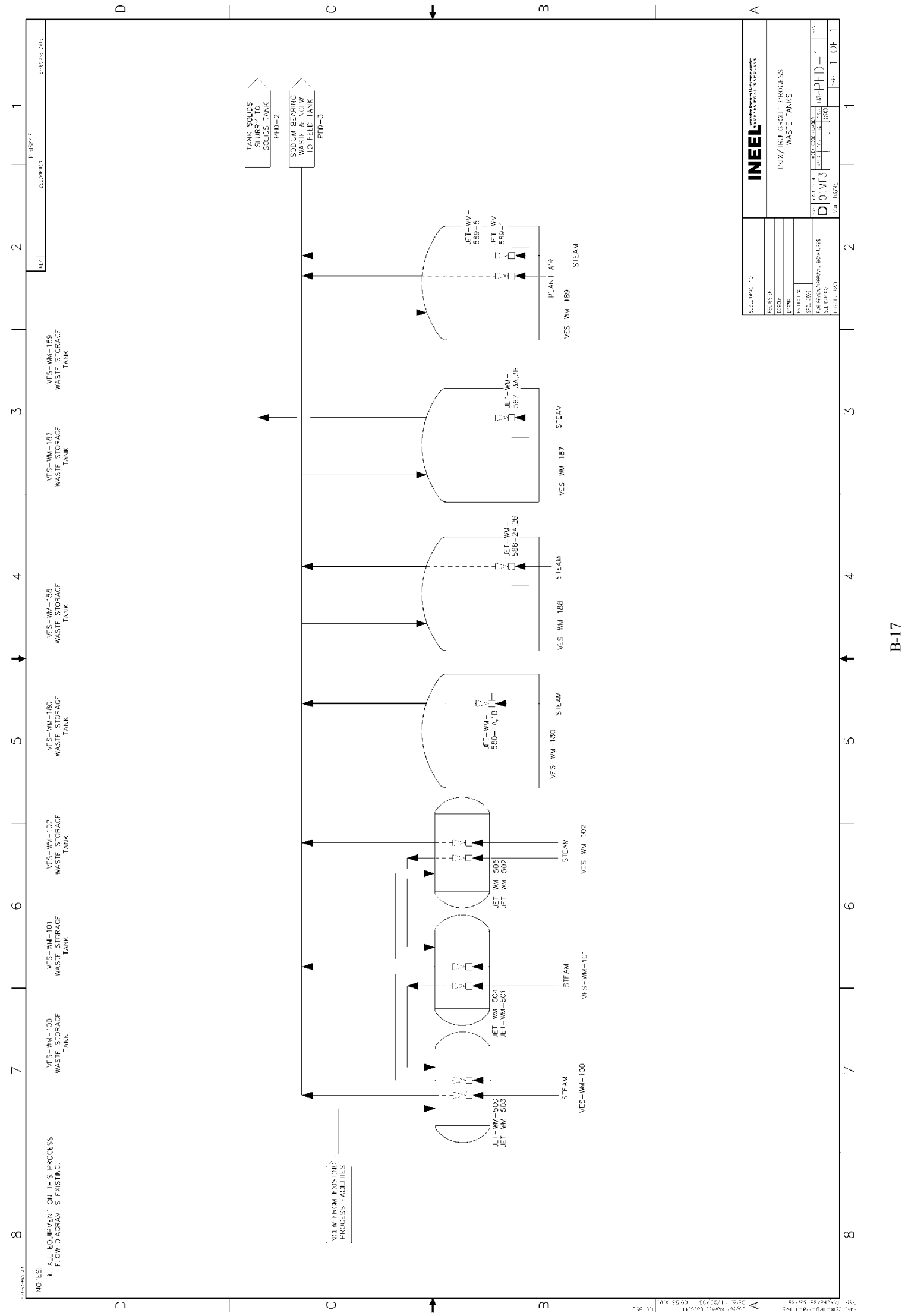




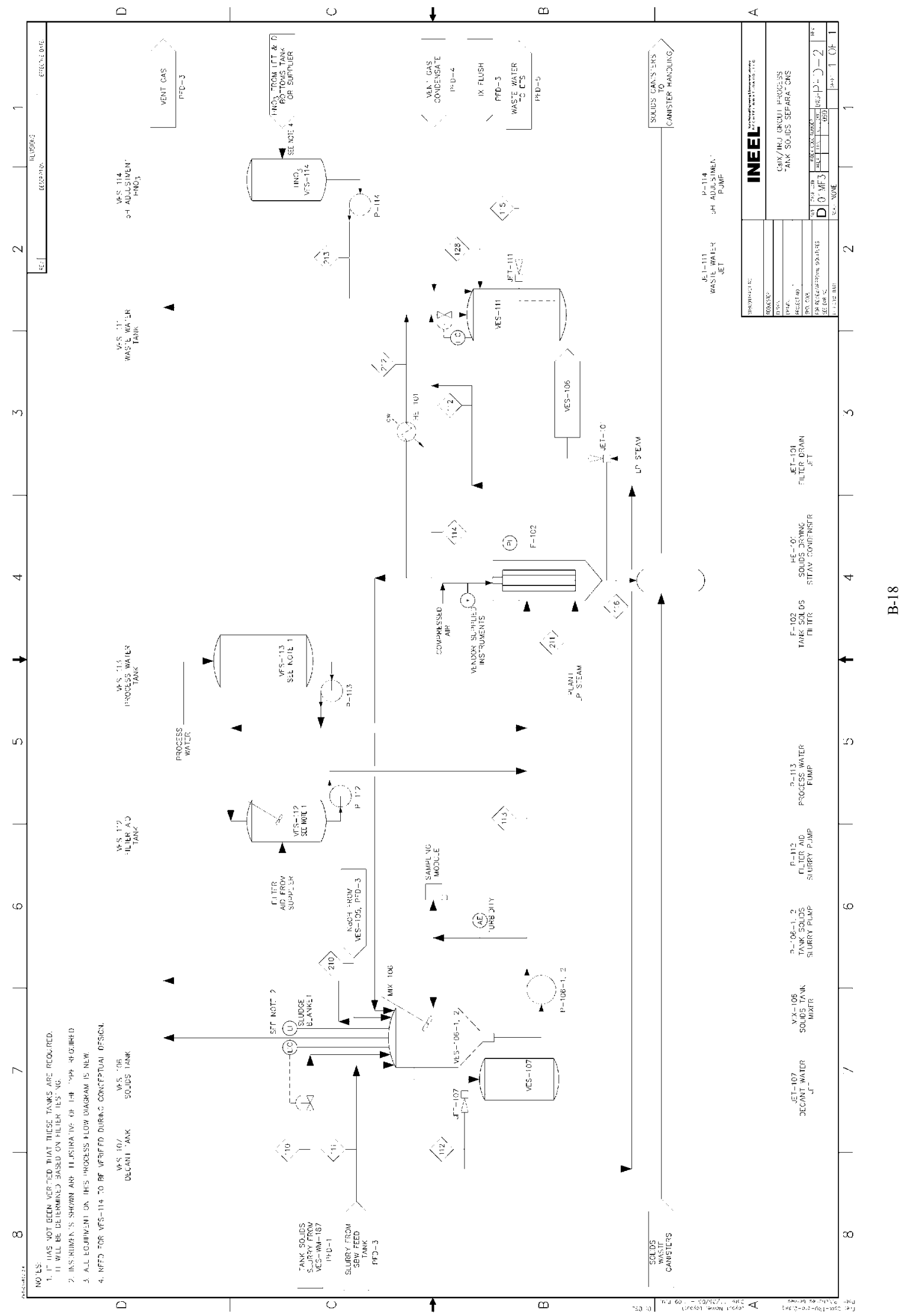




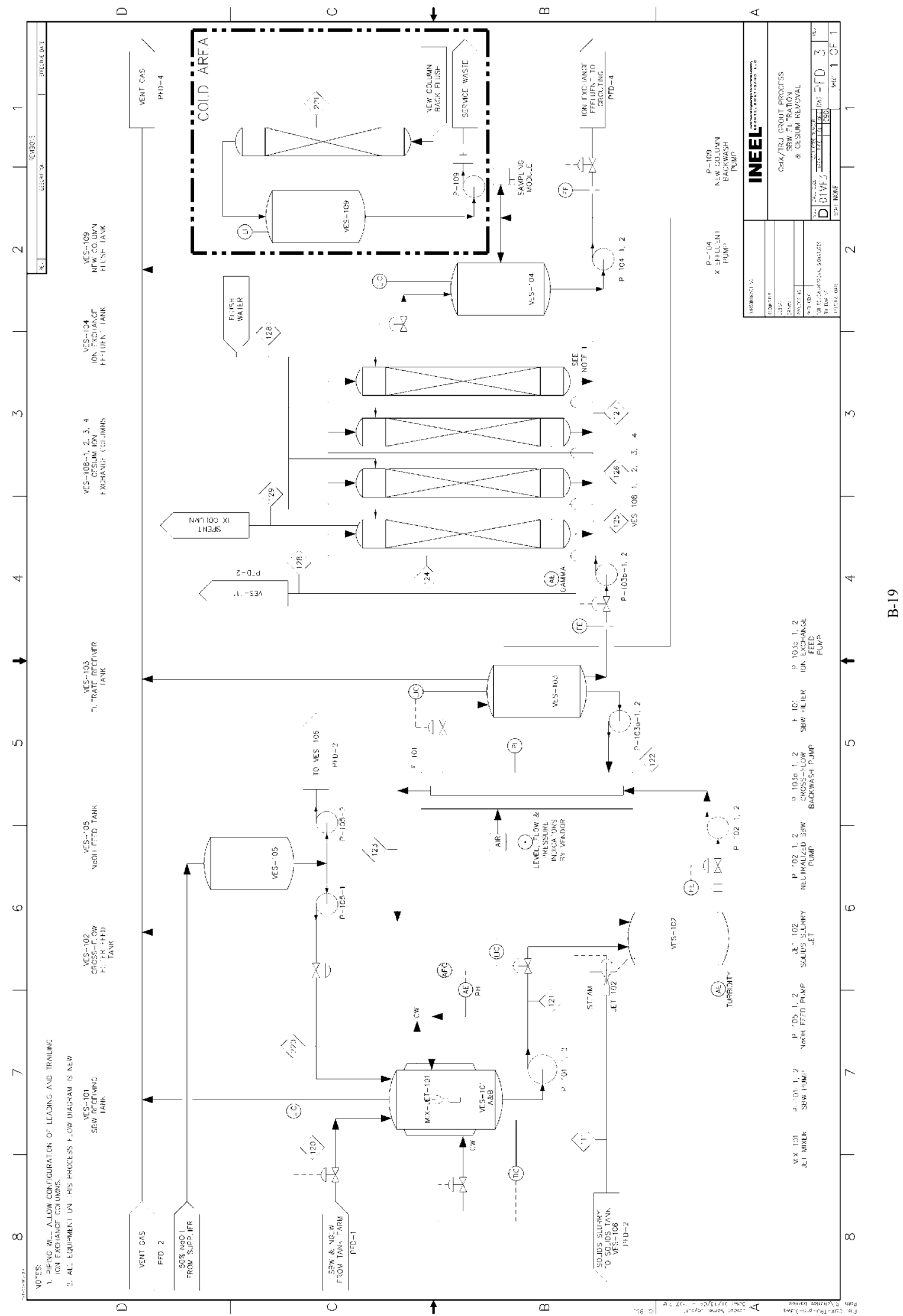




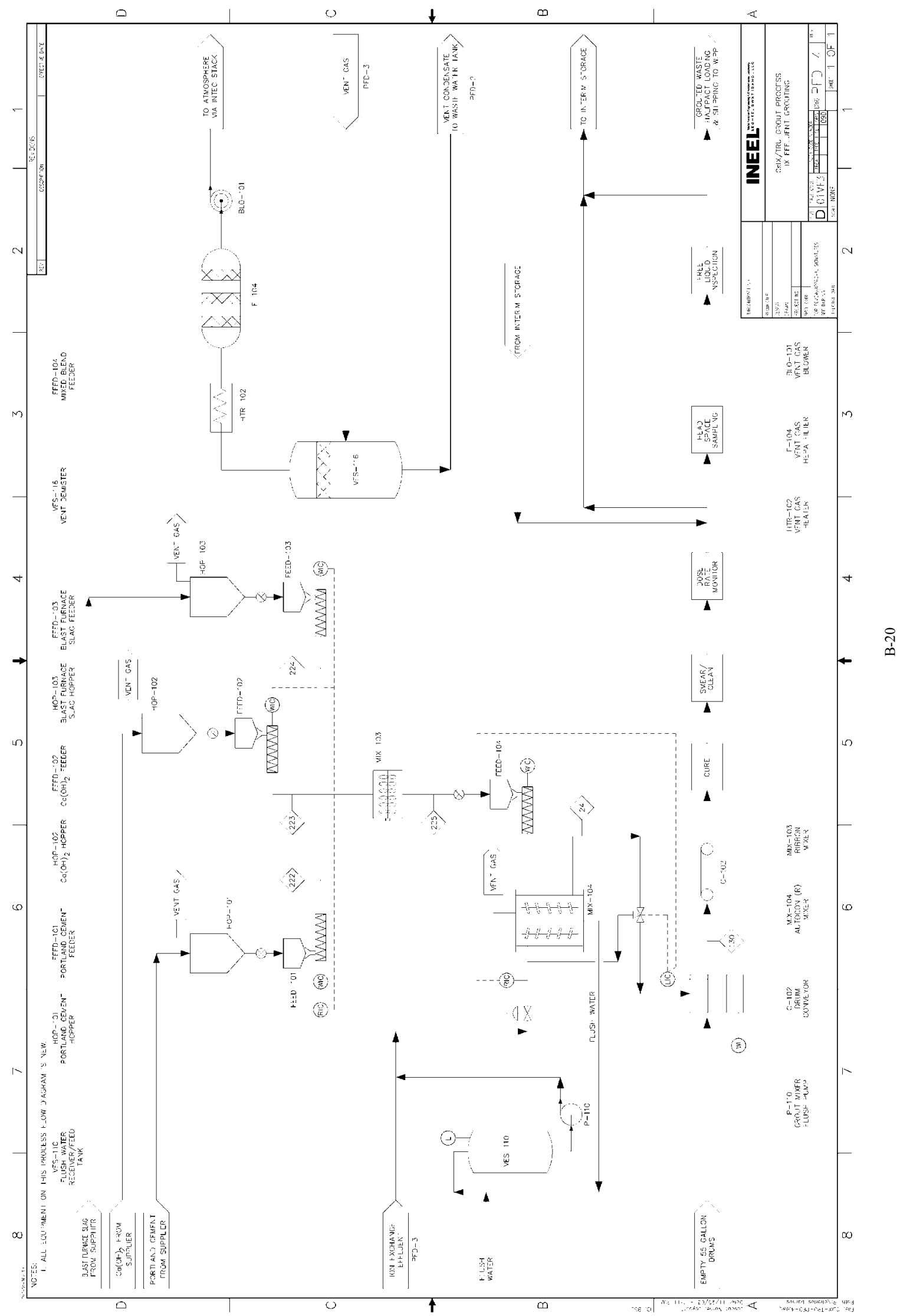




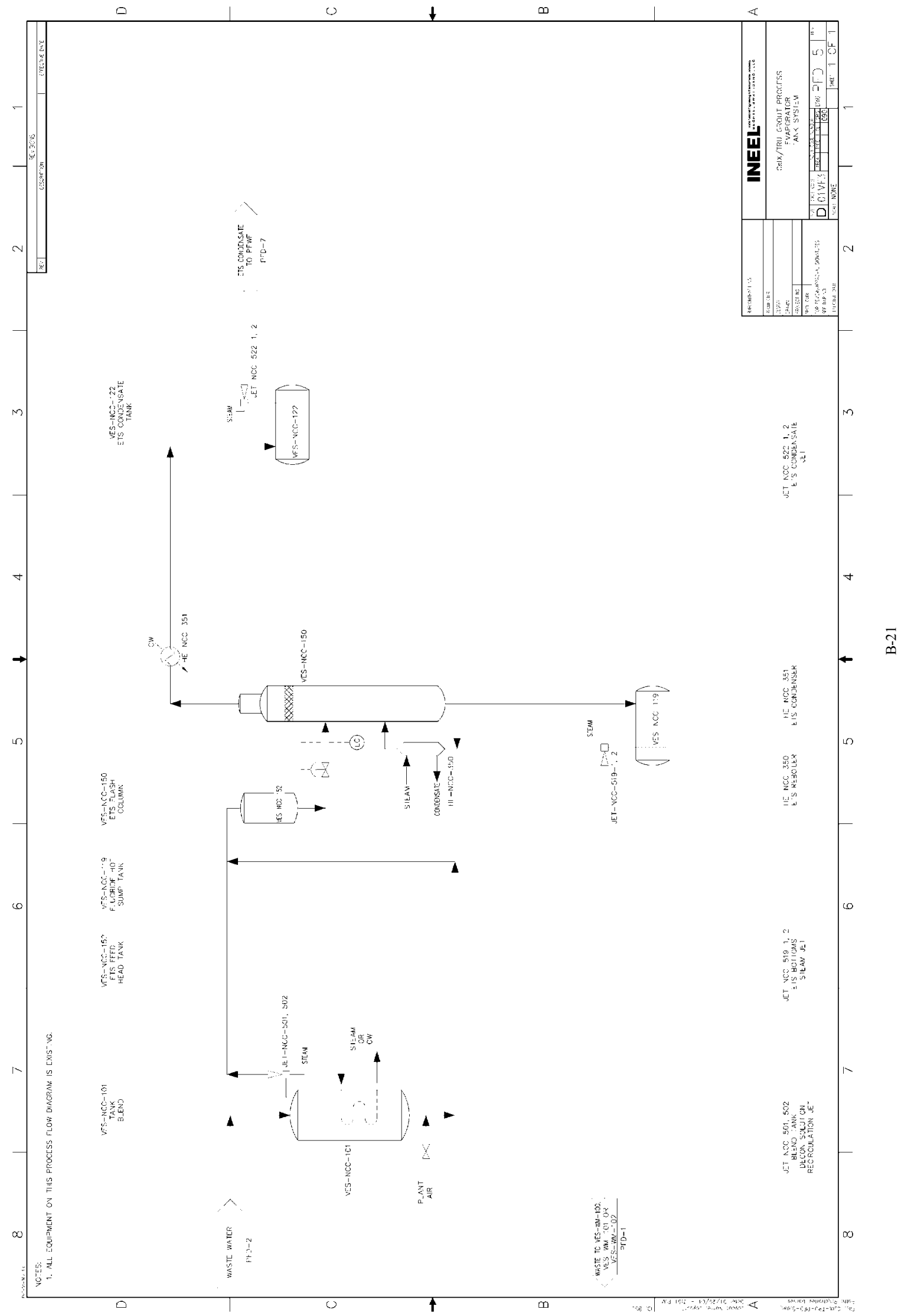




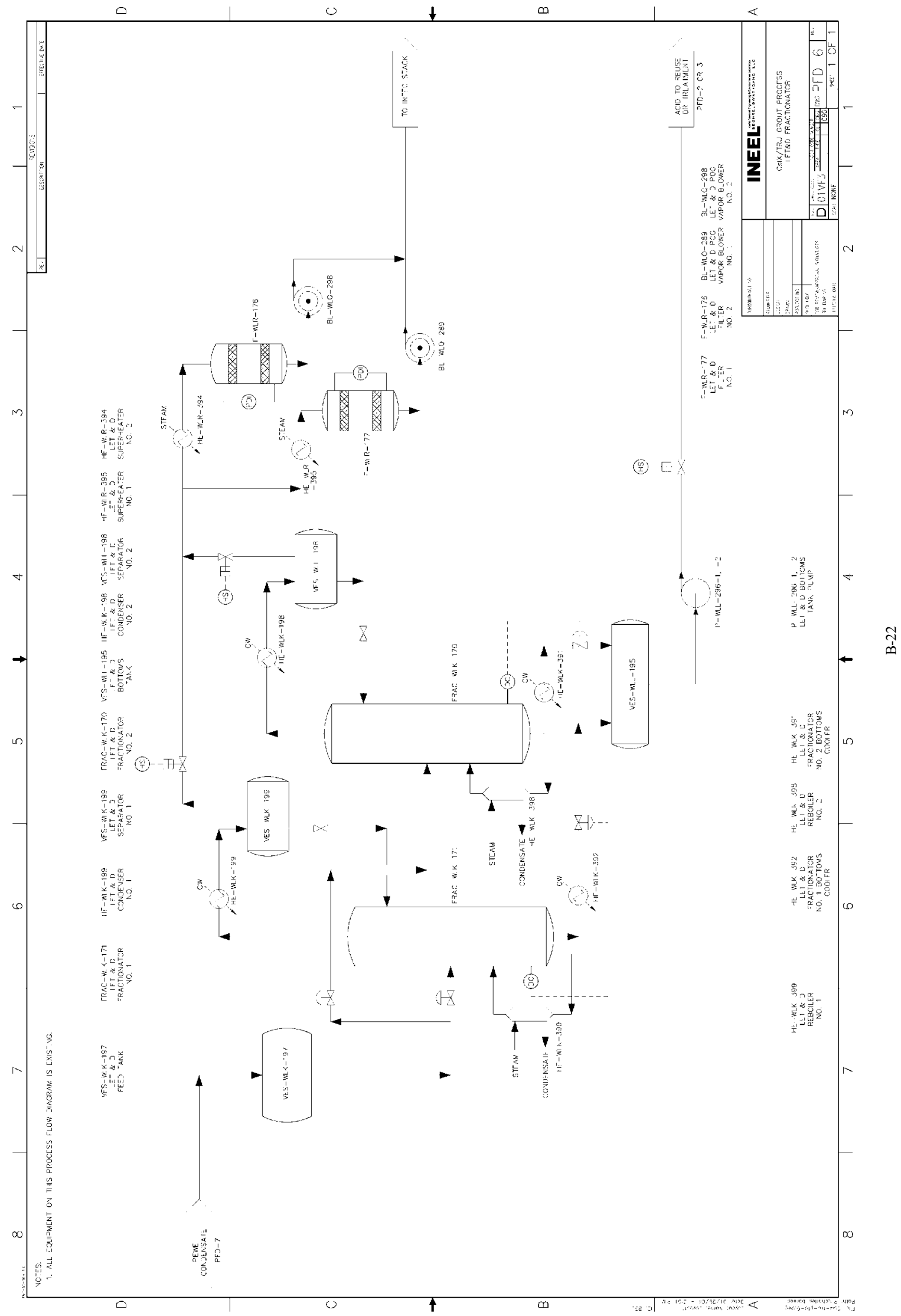




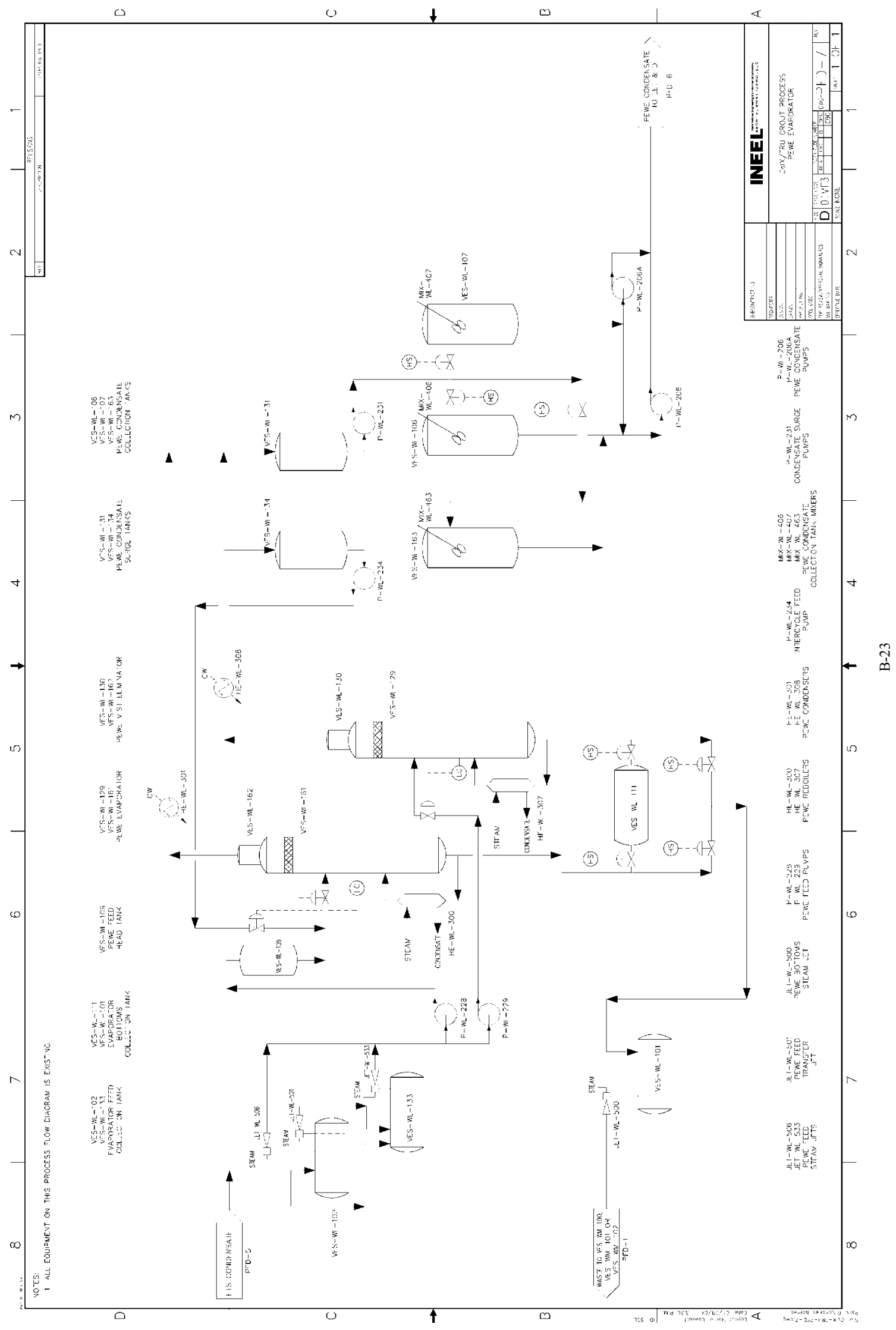




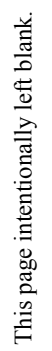




\section{B-5. PROCESS BASIS}

This section includes a summary of technical and functional requirements, process assumptions, a discussion of variations of the CsIX/TRU Grout process, the basis for the process shown on the process flow diagrams, and the basis for the mass balances.

\section{B-5.1 Requirements}

A draft technical and functional requirements document (T\&FR) was prepared for the CsIX/TRU Grout process (Barden, 2003). Most functional and performance requirements identified in the T\&FR document are summarized below. For other requirements, including special requirements, design requirements, testing and maintenance requirements and codes, standards and regulations refer to the T\&FR document. The T\&FR document also provides the basis or reference for each requirement.

\section{B-5.1.1 System Requirements}

B-5.1.1.1 Retrieve Waste. The system shall have the capability to retrieve SBW from WM-180, WM-187, WM-188, and WM-189 and NGLW from WM-100, WM-101 and WM-102.

B-5.1.1.2 Treat Waste - Feed Volume. The system shall have the capability to treat 1.2 million gallons of waste. Current estimates of waste volumes are 926,000 gallons of liquid waste, and 88,000 gallons of waste in Tank WM-187 containing 100,000 kg of solids. The maximum volume of liquid volume is $20 \%$ greater than the expected and the maximum amount of solids is $60 \%$ greater that the present estimate.

B-5.1.1.3 Treat Waste - Throughput. The facility design throughput shall provide the capability of processing all SBW, both liquids and solids, and NGLW generated through 2011 within 3.0 years. This timeframe assumes six months of hot start-up, leaving 2.5 years for processing SBW and NGLW volumes.

B-5.1.1.4 Treat Waste - Location. The waste shall be treated and packaged at the INEEL, within the INTEC security enclosure.

B-5.1.1.5 Treat Waste - Remove Solids from Ion Exchange Column Feed. Solids shall be removed from liquid feeds to the extent that particulate does not contribute more than $10 \%$ to the grouted waste dose rate and solids not removed are not detrimental to ion exchange column performance.

B-5.1.1.6 Treat Waste - Remove Cesium from Liquid Waste. To assure the grouted SBW is a contact handled waste, the ion exchange effluent can contain no more than $0.02 \mu \mathrm{Ci} / \mathrm{mL}$ of Cs- 137 .

B-5.1.1.7 Treat Waste - Solidify and Package. All waste streams shall be treated to comply with either RH or CH-TRU waste acceptance criteria (WAC) for WIPP. The effluent from the ion exchange process shall be solidified into a hydraulic cement grout matrix. The SBW solids shall be treated and packaged to meet the proposed WIPP RH-TRU WAC. This treatment will include removing liquid such that there is no free liquid and that the gas generation is below the limits established in the applicable TRUCON content code. The spent ion exchange media shall be characterized, treated, and packaged to meet the proposed WIPP RH-WAC. This treatment will include removing liquid such that there is no free liquid and that gas generation is below the limits established in the applicable TRUCON content code. Packaged solidified ion exchange media shall be stored for a minimum of 4 days for $\mathrm{CH}-$ TRU Waste and 15 days for RH-TRU waste to allow concentrations of flammable gases in the headspace to reach equilibrium. 
Portland cement used in grouting must meet American Society for Testing and Materials (ASTM) C150-02, "Standard Specification for Portland Cement." Blast furnace slag must meet ASTM C989-99, "Standard Specification for Ground Granulated Blast-Furnace Slag for Use in Concrete and Mortars." Blast furnace slag (BFS) Grade 120 must be further specified to designate the fineness of the slag powder.

\section{$\underline{\mathrm{CH} T R U}$ Waste Requirements}

1. TRU waste payload containers shall contain more than $100 \mathrm{nCi} / \mathrm{g}$ of alpha-emitting TRU isotopes with half-lives greater than 20 years.

2. The activity and mass of each of the following radionuclides shall be established on a payload container basis: Am-241, Pu-238, Pu-239, Pu-240, Pu-242, U-233, U-234, U-238, Sr-90, and Cs-137.

3. If the radionuclides listed in (b) do not account for at least $95 \%$ of the total waste product radioactivity, the mass and activity of additional radionuclides shall be determined until $95 \%$ of the waste product activity is accounted for.

4. Any payload container intended to be shipped as $\mathrm{CH}-\mathrm{TRU}$ shall have a dose rate at the external surface of the container less than $200 \mathrm{mrem} / \mathrm{h}$.

5. The ${ }^{239} \mathrm{Pu}$ FGE plus two times the associated total measurement uncertainty expressed in terms of one standard deviation shall be as shown in Table B-1.

Table B-1. Fissile gram equivalent limits.

\begin{tabular}{lc}
\hline \multicolumn{1}{c}{ Container Type } & ${ }^{239}$ Pu FGE Limit \\
\hline 55 gallon drum & $\leq 200$ \\
Standard Waste Box (SWB) & $\leq 325$ \\
HalfPACT & TBD \\
TRUPACT II containing fourteen 55 gallon drums or 1 TDOP & $\leq 325$ \\
\hline
\end{tabular}

6. The concentration of alpha-emitting TRU isotopes with half-lives greater than 20 years in the waste, exclusive of the packaging, shall be greater than $100 \mathrm{nCi} / \mathrm{g}$.

7. The ${ }^{239} \mathrm{Pu}$ equivalent curie limits in a payload container shall be less than the values shown in Table B-2.

Table B-2. Payload container PE-Ci limits.

\begin{tabular}{lcc}
\multicolumn{1}{c}{ Waste Container } & Packing Configuration & ${ }^{239}$ Pu FGE Limit \\
\hline $\begin{array}{l}55 \text { gallon drum in good } \\
\text { condition }\end{array}$ & direct load, all approved waste forms & $\leq 80$ \\
$\begin{array}{l}55 \text { gallon drum in good } \\
\text { condition }\end{array}$ & direct load, solidified/vitrified waste only & $\leq 1,800$ \\
\hline
\end{tabular}

8. The sum of the decay heat for each payload container plus its TMU shall be less than or equal to the limits for the assigned shipping category. 
9. The final payload container shall contain no detectable liquid.

10. PCB concentrations in wastes destined for WIPP shall not exceed $50 \mathrm{ppm}$.

11. CH-TRU grout shall be sampled to determine the hazardous waste code assignment by acceptable knowledge.

12. Flammable volatile organic compound (VOC) concentrations shall be less than $500 \mathrm{ppm}$ in the payload container headspace.

13. The physical waste form for newly generated CH-TRU mixed homogenous solids waste shall be characterized by documentation and verification.

14. CH-TRU waste shall be packaged in DOT type 7A steel drums.

15. The gross weight of 55-gallon drums shall be less than $1000 \mathrm{lbs}$.

16. Surface contamination on loaded payload containers, payload assemblies, and packages shall not exceed $20-\mathrm{dpm} / 100 \mathrm{~cm} 2$ alpha and $200-\mathrm{dpm} / 100 \mathrm{~cm} 2$ beta-gamma.

17. Unvented payload containers shall be aspirated for a length of time as described in the TRAMPACT to assure equilibration of any gases that may have accumulated in the closed payload container.

18. Each 55-gallon drum payload container shall have one filter vent. Each SWB shall have two filter vents.

\section{$\underline{R H}$ TRU Waste Requirements}

1. The activity and mass of each of the following radionuclides shall be established on a payload container Basis: Am-241, Pu-238, Pu-239, Pu-240, Pu-242, U-233, U-234, U-238, Sr-90, and Cs-137.

2. If the radionuclides listed in (a) do not account for at least $95 \%$ of the total waste product radioactivity, the mass and activity of additional radionuclides shall be determined until $95 \%$ of the waste product activity is accounted for.

3. Any RH-TRU waste product payload container shall have a dose rate at the surface of the container greater than $200 \mathrm{mrem} / \mathrm{h}$ and less than $1000 \mathrm{rem} / \mathrm{h}$.

4. The ${ }^{239} \mathrm{Pu}$ FGE in each payload container, plus its associated uncertainty expressed in terms of one standard deviation, shall be less than $325{ }^{239} \mathrm{Pu}$ FGE.

5. The TRU radionuclide alpha activity concentration, exclusive of the packaging, shall be greater than $100 \mathrm{nCi} / \mathrm{g}$.

6. The ${ }^{239} \mathrm{Pu}$ equivalent curie (PE-Ci) quantities in a payload container shall not exceed $80 \mathrm{PE}-$ $\mathrm{Ci}$ for direct loaded RH-TRU waste canisters and shall not exceed 240 PE-Ci for RH-TRU canisters loaded with three 30 -gallon or 55 -gallon drums.

7. The sum of the decay heat for each RH-TRU payload container plus its TMU shall be less than or equal to 50 watts per RH-TRU 72-B cask. 
8. The loaded payload container shall contain no detectable liquid.

9. The hydrogen gas generation rate within each payload container shall not exceed the limit specified in the applicable content code. In any case, the hydrogen gas concentration within each payload container shall not exceed $5 \%$ by volume.

10. PCB concentrations in wastes destined for WIPP shall not exceed $50 \mathrm{ppm}$.

11. Concentrations of flammable VOCs in the payload container headspace shall not exceed 500 ppm. If calculations cannot show that the VOC concentration in the headspace is less than $500 \mathrm{ppm}$ if all potentially flammable VOCs vaporized into the headspace, sampling of the containers shall be required.

12. The RH-TRU waste shall be placed in a WIPP approved RH-TRU waste canister.

13. The gross weight of a direct loaded RH-TRU canister shall not exceed $5250 \mathrm{lb}$.

14. Surface contamination on loaded RH-TRU payload containers, payload assemblies, and packages shall not exceed $20 \mathrm{dpm} / 100 \mathrm{~cm} 2$ alpha and $200 \mathrm{dpm} / 100 \mathrm{~cm} 2$ beta-gamma.

15. Each payload container shall have one or more filter vents. Any sealed secondary or internal container overpacked in the RH-TRU canister shall have one or more filter vents. Use of filter vents with metal internal containers greater than 4 liters in size that contain only solid inorganic waste is optional.

\section{B-5.1.1.8 Transfer waste to WIPP.}

1. CH-TRU waste shall be shipped over-the-road in a TRUPACT-II or in a HalfPACT package.

2. The maximum number of the 55-gallon drums in a TRUPACT-II is 14 . The maximum weight of a TRUPACT II payload assembly of 14 55-gallon drums, including pallet, guide tubes, slip sheets (optional), reinforcing plates, and banding material is 7,265 pounds.

3. The payload container assemblies to be shipped in the TRUPACT-II must meet the center of gravity requirements specified in the $\mathrm{CH}$-TRAMPAC. The external dose rate at the surface of the TRUPACT II shall be $\leq 200 \mathrm{mrem} / \mathrm{h}$ at the surface and $\leq 10 \mathrm{mrem} / \mathrm{h}$ at $2 \mathrm{~m}$.

4. The maximum number of the 55-gallons drums in a HalfPACT is 7. The maximum weight of the HalfPACT payload assembly weight is $7600 \mathrm{lbs}$. The external dose rate at the surface of the HalfPACT shall be $\leq 200 \mathrm{mrem} / \mathrm{h}$ at the surface and $\leq 10 \mathrm{mrem} / \mathrm{h}$ at $2 \mathrm{~m}$.

5. RH-TRU waste shall be shipped in a RH-TRU 72-B cask.

6. The maximum number of payload containers in a RH-TRU 72-B cask is one. The maximum weight of a loaded $72-\mathrm{B}$ cask is 45,000 pounds.

7. A minimum of $4+$ TBD days of storage of packaged solidified CsIX CH-TRU waste shall be provided to accommodate $\mathrm{CH}-\mathrm{TRU}$ lag storage. A minimum of $15+\mathrm{TBD}$ days of storage of packaged solidified CsIX RH-TRU waste shall be provided to accommodate RH-TRU lag storage. 
8. A cask loading station compatible with loading WIPP supplied TRUPACT II and HalfPACT casks delivered on WIPP supplied transport vehicles shall be provided. The facility shall provide the capability to receive the cask transporters in an enclosed area, raise the casks for loading, load the waste payload assemblies and close the cask on the transporters.

9. A cask loading station compatible with loading WIPP supplied RH-TRU 72-B casks delivered on WIPP supplied transport vehicles shall be provided. The facility shall provide the capability to receive RH-TRU 72-B cask transporters in an enclosed area, raise the cask for loading, load the waste payload assemblies into RH-TRU 72-B cask, and close and lower the RH-TRU 72-B cask onto the transporters.

\section{B-5.1.1.9 Close Facility.}

1. The CsIX TRU Grout Facility shall be placed in standby after treating SBW and NGLW waste.

2. Prior to December 31, 2012, the CsIX TRU Grout Facility shall have all hazardous waste and hazardous waste residues removed and placed in safe shutdown for future decommissioning.

3. The hazardous waste and hazardous waste residues shall be solidified and prepared for acceptable disposal prior to December 31, 2012.

\section{B-5.1.2 Systems, Subsystem, and Major Components} include:

This system shall utilize major support systems and utilities that are available at the INEEL. These

1. Analytical services.

2. Fire protection. This includes a manned fire station, central alarm system, and fire water lines near the waste treatment facility; it does not include fire protection within the physical structures designed and built or used by this project.

3. Electrical power. Both normal and standby electrical power are available for use near the INTEC Tank Farm from the INTEC electrical distribution system (EDS).

4. Steam

5. Telephone

6. Optical cable

7. Security

8. Medical

9. Radiation records

10. Central Facilities maintenance

11. Document control 
12. Configuration management

13. Office buildings. The project shall provide adequate office space only for any operations staff, engineers, technicians, supervisors, and managers whose presence is required in the facility full-time during operations, plus limited turn-around office space.

14. Roads

15. Bus transportation.

\section{B-5.1.3 Boundaries and Interfaces}

B-5.1.3.1 Liquid Waste Retrieval Feed System. The liquid waste retrieval system interfaces with Tank Farm Operations. All operations within the tank farm will be the responsibility of Tank Farm Operations. Prior to operation of the SBW treatment facility, all tank waste shall be delivered to Tanks WM-180, WM-187, WM-188, and WM-189. Any changes necessary to retrieve the SBW liquids and solids from these tanks will be the responsibility of this project.

\section{B-5.1.3.2 Utility Interfaces.}

1. Electric power will be obtained from existing sources at INTEC. The project shall provide the necessary equipment for distribution of power within the facility. The use of existing standby, emergency, or uninterruptible power supplies at INTEC shall be evaluated as the first choice during conceptual design.

2. The project shall tie in to the existing INTEC steam distribution piping at an appropriate location adjacent to the facility.

3. The project shall tie in to the existing INTEC water systems piping (de-mineralized, deionized, distilled, fire water, etc.) at an appropriate location adjacent to the facility.

B-5.1.3.3 Sample Handling. Radioactive sample handling shall be compatible with the existing pneumatic sample transport system.

B-5.1.3.4 Tank Farm Closure Project. The system shall interface with the Tank Farm Closure Project. The Tank Farm Closure Project will sequentially remove all liquids and waste solids from the eleven Tank Farm tanks WM-180 through 190. The TFC Project will also clean the tanks to performance parameters, grout the tanks, piping and vaults, and close the tanks by December 2012 in accordance with the INTEC TFF Management Plan. The SBW Treatment project retrieves the wastes from tanks with the support of the TFC Project and treats it in the Direct Evaporation Facility.

B-5.1.3.5 Tank Farm Soils Project. The system shall interface with the Tank Farm Soils Project, which is responsible to remediate, as required, and close the Tank Farm under the Comprehensive Environmental Response, Compensation and Liability Act (CERCLA) program. The Tank Closure Project shall coordinate all work scope with the INTEC CERCLA Environmental Restoration Program (WAG 3) to ensure no conflicts.

B-5.1.3.6 ETS, PEWE and LET\&D Facilities. The treatment process will interface with the ETS located in the NWCF. Per current operations, it is expected that condensate from the ETS evaporator will be sent to the PEWE and PEWE condensate to the LET\&D facility, located in CPP-1618. Any modifications required to ETS, PEWE or LET\&D treatment equipment, or associated piping and 
instrumentation, will be the responsibility of this project. Operation of the ETS and LET\&D facility and transfers of waste to and from the NWCF and/or LET\&D facilities will be coordinated with the SBW Treatment Project but the responsibility of INTEC Operations.

\section{B-5.1.4 Operability}

The operability requirements for the SBW facility shall support the completion of SBW treatment project by December 31, 2012.

B-5.1.4.1 Operational Lifetime. The facility shall have an operational lifetime adequate to process all SBW and tank heel solids without replacement of any process piping or major system components (tanks, hoppers, reaction vessels, etc).

B-5.1.4.2 Installed Spares. Installed spares shall be provided for all rotating or reciprocating machinery located in remotely operated areas of the facility.

B-5.1.4.3 Annual Operation. The facility shall be designed to operate continuously for at least 200 days per year for at least 3 sequential years. Note that three-year window is based on a 6-month hot startup of the facility and a two and a half year processing period for the entire SBW and NGLW.

\section{B-5.2 Assumptions}

Process designs considered for the CsIX TRU Grout process have been based on the following major assumptions. Additional assumptions used in the mass balance are identified in Section B-5.5.

1. The SBW, both liquids and solids, and NGLW to be treated are not high-level waste.

2. The solidified SBW waste can be qualified for disposal at WIPP.

3. The existing ETS, PEWE and LET\&D facility will be available for use if needed to process waste water.

4. Toxic Substance Control Act regulations do not apply.

5. The facility will not require NRC licensing.

\section{B-5.3 Process Variations}

Variations of the CsIX TRU grout process have been described in past studies (Raytheon, 2000; Valles, 1999; Losinski, 1998), and are briefly reviewed in Section 2.1. Variations in the CsIX TRU grout process could be categorized as differences in:

1. How tank solids are treated

2. How solids are filtered from liquid SBW

3. What sorbent is used to remove cesium

4. How the spent sorbent is treated

5. How the ion exchange effluent liquid is solidified

6. How the tank solids and spent sorbent wastes are disposed

7. What if any existing INTEC facilities are used. 
In all processes proposed to date in which tank solids were processed, they were first filtered and then either dried or grouted, followed by packaging for disposal. Various types of filters and other equipment have been proposed for filtering both the tank solids, and the undissolved solids in the liquid SBW. One study (Raytheon, 2000) reviews various types of cartridge filters, centrifuges, hydroclones, Spintek filters and crossflow filters for separation of undissolved solids from SBW liquid.

Several studies have evaluated cesium sorbents for use in the CsIX TRU grout process (Todd, 2003; Tranter, 2003a, Tranter, 2003c; Kimmitt, 2003a). While the most recent of these recommend CST, development has shown that other sorbents are feasible. The method of treatment of spent ion exchange media depends, in part, on the particular sorbent used and also on its planned disposal site. The current process is based on disposal of the spent sorbent at WIPP as a remote handled TRU waste.

Alternatives to a Portland cement-based solidified waste form for the ion exchange effluent are possible. Waste loadings have been determined for adsorption of the ion exchange effluent on silica gel (Raman, 2003; Herbst, 2003). Ashworth compared the use of silica gel as an adsorbent to Portland-based grout based on 10 criteria (Ashworth, 2003). Although six of the ten criteria favor silica, the remaining four have higher weighting factors, and result in the recommendation of the grout waste form. An alternatively use of silica gel in the CsIX process involves an evaporation step of the waste-loaded silica gel. A process design for this scheme is contained in Barnes (2002a) and has been demonstrated by Kirkham (Herbst, 2002). Other solidification agents that have been reviewed for use in the CsIX process include clay-based binders and chemically bonded phosphate ceramics (Raytheon, 2000). During the Raytheon feasibility study, bench-scale solidification tests were performed using Metalplex, a clay-based waste solidification agent (Raytheon, 2000, Appendix F).

Early studies of the CsIX process considered the use of existing INTEC facilities for housing part of the process. However, Losinski (1999) found that meeting the 2012 treatment deadline was not feasible for the scenario of locating the high-radiation equipment in the NWCF. The same study identified eight other issues with using the NWCF for the CsIX process. Use of the FPR facility for the CsIX process has also been suggested, but no evaluation has been performed.

\section{B-5.4 Basis for the Flow Diagrams}

\section{B-5.4.1 Waste Tanks (PFD-1)}

PFD-1 shows the SBW feed tanks VES-WM-180, VES-WM-187, VES-WM-188 and VES-WM189, and the NGLW tanks VES-WM-100, VES-WM-101 and VES-WM-102. These tanks currently exist and are in use. Tank WM-187 is being used to collect solids from flushing other Tank Farm tanks. According to current plans, solids from all but the SBW tanks will have been flushed to WM-187 by 2005. Thus, solids will settle in WM-187 over several years prior to the beginning of treatment. It is anticipated that flushing of WM-187 to treatment will be similar to flushing the heel of other tanks. Existing jets would be used to transfer waste out of the tank. A "wash ball" would be added to the tank to break up solids and entrain them into the flush water, which would be added as needed.

\section{B-5.4.2 Tank Solids Separation (PFD-2)}

In an evaluation of methods to remove undissolved solids from SBW liquid, Raytheon recommended the Fundabac filter (Raytheon, 2000, Appendix E). Their evaluation was based on six criteria that included the state of process development of the separation method, the process and mechanical complexity, the operability and dose rate, the life cycle cost and impacts to the schedule. In later development of the CsIX process (Barnes 2002a; Ashworth, 2003), the Fundabac filter was retained 
for removal of SBW solids because of the advantages identified by Raytheon, as well as the ability to dry the solids in place on the filter rather than needing a separate drying step.

Initial demonstration tests of a laboratory $\left(0.13 \mathrm{ft}^{2}\right)$ Fundabac filter were performed in 2000 (Raytheon, 2000, Appendix F). These tests used $0.1 \mathrm{wt} \%, 0.3 \mathrm{wt} \%$ or $5 \mathrm{wt} \%$ of $\mathrm{ZrO}_{2}, \mathrm{SiO}_{2}, \mathrm{TiO}_{2}$ and a mixture of these solids in $30 \mathrm{wt} \% \mathrm{NaNO}_{3}$ adjusted to $\mathrm{pH} 2$ with nitric acid as the SBW simulant. In eight runs of various concentrations and combinations of solids, a clear filtrate was obtained in 0.5 to 4 minutes.

Additional filtration tests pertaining to Fundabac filtration were performed in 2003 by Jenn-Hai Pao and Russel Lewis (Pao, 2003). The SBW simulant for these tests utilized a liquid composition based on Tank WM-180 analysis and solids prepared by a metathesis procedure. The filtration apparatus consisted of $600 \mathrm{ml}$ filtration cylinder, a filtrate collection vessel, a pressurized air supply, an air heater, a pressure regulator and a mass flow meter. Two types of filter cloths, polyvinylidene fluoride and polypropylene sulfide were used in the tests. Conclusions from the tests included:

- The removal of solid particles from the SBW slurry is feasible and will result in low moisture content dry cakes that separate from the filter cloth.

- Without filter-aid, the filter cake from SBW-solids simulant was found to have low-moderate compressibility and high resistance; higher filtration pressures lead to higher filtration rates.

- Without cake washing, the low moisture content relatively hard cakes from filtering the SBW-solids simulant were easily separated from the filter medium.

\section{B-5.4.3 SBW Filtration and Cesium Removal (PFD-3)}

PFD-3 includes equipment to remove solids and cesium from waste from Tanks WM-180, WM188 and WM-189. Solids are removed by a crossflow filter, cesium is removed by CST sorbent contained in a series of ion exchange columns.

Several types of filters could be used to remove the small amount of solids in the feed waste. Crossflow filtration was first recommended for the CsIX TRU grout process in 1999 (Valles, 1999) in a study that compared four filtration methods. Testing of a crossflow filter using actual Tank Farm waste was performed in 1997 (Tripp, 1997) and concluded that crossflow filtration was feasible.

As mentioned in Section B-5.3, several studies have evaluated cesium sorbents for use in the CsIX TRU grout process (Todd, 2003; Tranter, 2003a, Tranter, 2003c; Kimmitt, 2003a). While other sorbents are feasible, CST is the most common recommendation of these studies because of its demonstrated performance using SBW simulants and its commercial availability.

The basis for the number of ion exchange columns and the method of loading fresh media and processing spent media is given in Kimmitt (2003b). The number of columns is based on achieving the required cesium removal efficiency and producing an acceptable spent sorbent waste while minimizing the frequency of column changeout. Likewise, operational factors are of primary concern in determining the methods of column loading and spent column. Loading and preparing each column is done in a cold area rather than the alternative of sluicing and backwashing sorbent into an installed column in the hot cell. Likewise, packaging the entire column as waste involves fewer steps than sluicing spent sorbent into a different vessel for drying, following by then either disposing of that container or transferring the dried waste sorbent into a canister. 


\section{B-5.4.4 IX Effluent Grouting (PFD-4)}

The equipment to grout the ion exchange effluent is shown on PFD-4. Storage and transfer vessels are shown for grouting ingredients based on the optimum grout formulation developed at the INEEL for this waste (Herbst, 1999; Herbst, 2000; Herbst, 2002; Raman, 2003). This formulation includes Portland cement, calcium hydroxide and blast furnace slag. Equipment is shown to mix these ingredients prior to mixing them with the SBW ion exchange effluent.

The method of mixing the dry ingredients with the liquid ion exchange effluent has been the subject of several studies (Raytheon, 2000; Williams, 2003; Ashworth, 2003). Both an in-drum, lost paddle approach and out-of-drum mixers have been evaluated. Based on the most recent study (Ashworth, 2003), the PFD shows out-of-drum mixing. This and previous evaluations show that either method of mixing is feasible and neither have major advantages over the other. Out-of-drum mixing was selected for the flowsheet because of small advantages in waste volume/cost, process operability and plant layout. Demonstration tests of an out-of-drum (Readco) mixer were first performed in 2000 (Raytheon, 2000, Appendix F). Further tests using a 6-inch diameter Autocon mixer were performed in 2003 (Scholes, 2003).

\section{B-5.4.5 ETS, PEWE and LET\&D Fractionator (PFD-5, PFD-6, and PFD-7)}

The ETS, PEWE and LET\&D are existing INTEC processes that have operated successfully for many years. The ETS, rather than the PEWE, is used to concentrate waste water because the PEWE has feed acceptance criteria that would require high dilution ratios. This in turn would multiply the operating time and the energy requirements. However, the PEWE is then used to concentrate the ETS concentrate to provide the decontamination needed prior to fractionating the condensate in the LET\&D.

\section{B-5.5 Basis for the Mass Balances}

Mass balances were developed for the CsIX TRU grout process to provide a basis for sizing equipment, determining feed chemical requirements, determining utility requirements, estimating emissions and determining waste volumes, compositions and properties. The sections below document the basis for the mass balance. The basis is capsulated in succinct statements, called "design basis elements" (DBEs), shown in italics in the following paragraphs. The DBEs are amplified with background information, references, explanation, and, in some cases, a discussion of uncertainties.

\section{B-5.5.1 Feeds}

Feeds to the CsIX TRU grout process include NGLW and SBW from the INTEC TFF. According to present Tank Farm management plans (Barnes, 2002b), waste presently in the TFF tanks will be consolidated into four tanks, WM-180, WM-187, WM-188 and WM-189, by the end of 2005. Tanks WM-180, WM-188 and WM-189 will contain liquid SBW with relatively small quantities of undissolved solids, while WM-187 will be used as a collection tank for solids and dilute liquid wastes. Waste generated after 2005 will be collected in WM-100, WM-101 and WM-102.

Mass balances are shown in Section B-6 for four liquid feed cases and one solids feed case. The liquid feed cases correspond to present estimates of waste quantities and compositions in Tanks WM-180, WM-188, WM-189 and a combined NGLW feed. The mass balance shows these wastes processed through the liquid treatment equipment. Waste from Tank WM-187 is processed through the solids treatment equipment. 


\section{DBE \#1: Four waste compositions, corresponding to waste in Tanks WM-180, WM-188, WM-189 and a combined $N G L W$, are assumed to adequately envelope the liquid waste fed to the $S B W$ Treatment Facility for conceptual design purposes. Waste in Tank WM-187 is assumed representative of the solids waste.}

Table B-3 shows the variability of feed composition between the tanks for major species. Variation in feed composition in addition to what is shown in Table B-3 could be due to (a) uncertainties in sample analyses and (b) uncertainties in NGLW quantity and composition. However, the magnitude of these uncertainties is small compared to the range in concentration of a species from tank to tank. Feed composition uncertainties and expected composition ranges are discussed in more detail by Barnes (2003).

The estimated quantity of solids in Tank WM-187 is 100,000 kg (Barnes, 2003). The estimated quantity of solids present in all the liquid tanks, including NGLW, is about 22,000 kg (Barnes, 2003). Solids removed by the crossflow filter and solids in the heels of the liquid tanks will periodically be added to the Solids Decant Tank and fed to the solids treatment equipment. However, because the quantity added will be small relative to the amount of solids coming from WM-187, and analyses of solids from different tanks (see Barnes, 2003) shows the composition of solids from tank to tank is somewhat similar, the composition of solids in WM-187 should be adequate for the design of treatment equipment. It should be noted that the solids composition could vary from what is shown in the material balance not only because of the addition of solids from other tanks but also because of uncertainties in solids analysis and unrepresentative solids samples. However, the solids treatment equipment is very insensitive to solids composition, dependent more on physical properties.

Table B-3. Feed concentration variations.

\begin{tabular}{lccc}
\hline & $\begin{array}{c}\text { Minimum } \\
\text { mol/liter }\end{array}$ & $\begin{array}{c}\text { Maximum } \\
\text { mol/liter }\end{array}$ & Max/Min Ratio \\
\hline $\mathrm{H}+$ & 1.12 & 4.10 & 3.65 \\
$\mathrm{Al}+3$ & 0.14 & 0.71 & 4.96 \\
$\mathrm{Ca}+2$ & 0.014 & 0.07 & 5.13 \\
$\mathrm{Cs}+$ & $1.0 \mathrm{E}-05$ & $2.9 \mathrm{E}-05$ & 2.91 \\
$\mathrm{Cl}-$ & 0.011 & 0.030 & 2.81 \\
$\mathrm{~F}-$ & 0.014 & 0.042 & 3.09 \\
$\mathrm{Fe}+3$ & 0.006 & 0.027 & 4.33 \\
$\mathrm{Mg}+2$ & 0.011 & 0.022 & 1.95 \\
$\mathrm{Mn}+4$ & 0.013 & 0.022 & 1.67 \\
$\mathrm{NO}_{3}-$ & 7.16 & 7.52 & 1.46 \\
$\mathrm{PO}_{4}-3$ & 0.0004 & 0.013 & 32.45 \\
$\mathrm{~K}+$ & 0.18 & 0.73 & 3.95 \\
$\mathrm{Na}^{-}$ & 1.43 & 2.04 & 1.43 \\
$\mathrm{SO}_{4}-2$ & 0.015 & 0.11 & 7.25 \\
$\mathrm{U}+4$ & 0.0004 & 0.011 & 30.54 \\
\hline
\end{tabular}




\section{B-5.5.2 Stream Factor, Operating Schedule and Feed Rate}

The Technical and Functional Requirements (Barden, 2003) specifies a 3-year processing time that includes six months of hot start up. A stream factor of 200 days per year is assumed. The mass balance is based on a feed rate of 5\% in excess of that required for continuous processing to allow for down time at the end of processing liquid while tank heels would still be processed or for processing additional waste. The average feed rate is thus:

$$
972,300 \mathrm{gal} \times 1.05 /(2.5 \mathrm{yrs} * 200 \mathrm{~d} / \mathrm{yr} * 24 \mathrm{hr} / \text { day })=85 \mathrm{gal} / \mathrm{hr} \text { or } 1.4 \mathrm{gal} / \mathrm{min}
$$

The feed rate of slurry to the solids processing equipment is based on a total flush water volume of 654,000 gallons (414,000 for WM-187 and 80,000 each for three other tanks) (see Section B-5.5.5 for the basis for the flush water volume). The corresponding total volume of slurry to treatment is:

$$
753,100 \mathrm{gal} \times 1.05 /(2.5 \mathrm{yrs} * 200 \mathrm{~d} / \mathrm{yr} * 24 \mathrm{hr} / \mathrm{day})=65.9 \mathrm{gal} / \mathrm{hr} \text { or } 1.1 \mathrm{gal} / \mathrm{min}
$$

DBE \#2: The total liquid and solids waste inventory will be processed over 2.5 years, during 20024 hr operating days per year. The average liquid feed rate of $1.4 \mathrm{gpm}$. The average feed rate to solids treatment is $1.1 \mathrm{gpm}$.

\section{B-5.5.3 Feed Transfers from Tank Farm}

Transfers from the Tank Farm will be made using existing steam jets. Based on past operation, dilution of the feed with steam is equivalent to about $5 \%$.

\section{DBE \#3: A dilution of 5 vol \% is assumed for waste transferred from the Tank Farm due to steam condensate from jet transfer.}

\section{B-5.5.4 Processing Temperature}

All processing in the CsIX TRU Grout Facility except waste drying steps are expected to be done at or near ambient temperature. Heat generated during liquid feed neutralization will be removed by cooling water plus heat losses and heat generated during grout mixing and curing will be dissipated by heat loss over time. The maximum temperature reached during grout mixing is estimated to be approximately $48^{\circ} \mathrm{C}$ (Ashworth, 2003).

\section{DBE \#4: Processing in the CSIX TRU Grout Facility will occur at or near ambient temperature.}

\section{B-5.5.5 SBW Solids Treatment}

A brief evaluation was performed to determine the volume of slurry to the solids treatment equipment. The following are constraints on the design and operation of lines and equipment to transfer and treating the tank solids:

- Waste Transfers: The solids loading must be below about $100 \mathrm{~g} / \mathrm{L}$ (as total suspended solids [TSS] and UDS) to prevent line/nozzle plugging (Wood, 2002).

- $\quad$ Tank Cleaning: Enough flush water must be used to remove solids. Data obtained from mock up cleaning tests (Poloski, 2001) was used to estimate the amount of flush water to remove solids from WM-187 based on this criteria. Recent flushing experience for Tanks 
WM-182, WM-183 and WM-185 indicates that 80,000 gal per tank is adequate and this amount was assumed for the three SBW tanks.

- Pumping Slurry to Filter: Based on previous testing at the INEEL of separation methods for use for INTEC wastes (Tripp, 1997), the maximum amount of solids in the feed to the Fundabac ${ }^{\circledR}$ filter is $20 \mathrm{wt} \%$, as this is about the maximum solids loading that can be pumped.

- $\quad$ PEWE WAC: The solids/flush water from the Tank Farm will be received by a Solids (decant) Tank in the CsIX TRU Grout Facility. If the decanted liquid ("supernate") and filtrate from the Fundabac ${ }^{\circledR}$ filter is then fed to the PEWE:

- The waste water must meet the PEWE waste acceptance criteria (WAC) (PRD-166). Table B-4 provides non-RCRA limits for feed concentrations. The gross $\beta$ was determined by summing all components that have numerical $\beta$ energies.

- The feed rate to the PEWE must not exceed about 30,000 gal/week.

- $\quad$ ETS WAC: If the supernate and filtrate were to be processed in the ETS, the following constraints would need to be met:

- The maximum bottoms free HF at boiling temperature is $50 \mathrm{mg} / \mathrm{L}$ for corrosion control (bottoms Al/F ratio is greater than about 3) (PLN-17).

- The maximum ETS bottoms temperature is $117 \mathrm{oC}$ to prevent red oil formation (TSR 3.103.1-B)

- The maximum ETS bottoms density is $1.35 \mathrm{Sp}$. G. (based on the TFF design specification)

- The ETS feed rate is typically one 4,400 gal batch per day. The operating permit limits the feed input to 10,000 gal per day (TPR-7112).

A comparison of tank farm liquid compositions to the PEWE Waste Acceptance Criteria and are shown in Table B-4. Calculations of required dilution assume that the initial solids concentration in the heel is 16 vol\% (Barnes, 2003). The maximum ratio to the PEWE limits for each tank shown in bold italics. Table B-4 shows that $\mathrm{SO}_{4}^{-2}$ has the highest ratio to the limit for all of the tanks. These calculations indicate that dilution factors of 4.5 (for WM-187 heel) to 103 (for WM-189 heel) would be required for the supernate solutions to meet the PEWE WAC limits.

Table B-5 provides the volumes required to meet the worst case for processing supernate in the PEWE. All cases are based on diluting the sulfate to meet the PEW WAC except for tank WM-187, which has a maximum flush volume of 414,000 gal based on the mock up test results (Poloski, 2001). The total volume over the assumed 2.5-year treatment period does not exceed the planning rate of 30,000 gal/wk., i.e., the processing rate is $55,000 \mathrm{gal} / \mathrm{month}$. 
Table B-4. PEW WAC limits compared to Tank Farm liquid compositions before dilution with flush water.

\begin{tabular}{|c|c|c|c|c|c|c|c|c|c|}
\hline \multirow[t]{2}{*}{ Component } & \multirow{2}{*}{$\begin{array}{l}\text { Limit } \\
\mathrm{mg} / \mathrm{L}\end{array}$} & \multicolumn{2}{|c|}{ WM-180 } & \multicolumn{2}{|c|}{ WM-187 } & \multicolumn{2}{|c|}{ WM-188 } & \multicolumn{2}{|c|}{ WM-189 } \\
\hline & & $\mathrm{mg} / \mathrm{L}$ & Ratio & $\mathrm{mg} / \mathrm{L}$ & Ratio & $\mathrm{mg} / \mathrm{L}$ & Ratio & $\mathrm{mg} / \mathrm{L}$ & Ratio \\
\hline $\mathrm{Al}$ & 2600 & $1.63 \mathrm{E}+04$ & 6.28 & $9.66 \mathrm{E}+02$ & 0.37 & $1.51 \mathrm{E}+04$ & 5.80 & 19200.00 & 7.38 \\
\hline B & 75 & $1.16 \mathrm{E}+02$ & 1.55 & $2.23 \mathrm{E}+01$ & 0.30 & $1.89 \mathrm{E}+02$ & 2.52 & 229.00 & 3.05 \\
\hline $\mathrm{Ce}$ & 200 & $6.25 \mathrm{E}+00$ & 0.03 & $2.50 \mathrm{E}-01$ & 0.00 & $3.88 \mathrm{E}+00$ & 0.02 & 4.92 & 0.02 \\
\hline Cs & 200 & $1.33 \mathrm{E}+00$ & 0.01 & $2.58 \mathrm{E}-01$ & 0.00 & $3.85 \mathrm{E}+00$ & 0.02 & 3.56 & 0.02 \\
\hline $\mathrm{Cl}$ & 50 & $1.07 \mathrm{E}+03$ & 21.30 & $5.62 \mathrm{E}+01$ & 1.12 & $9.42 \mathrm{E}+02$ & 18.85 & 729.00 & 14.58 \\
\hline $\mathrm{Cu}$ & 150 & $4.18 \mathrm{E}+01$ & 0.28 & 7.03E-01 & 0.00 & $4.13 \mathrm{E}+01$ & 0.28 & 60.60 & 0.40 \\
\hline $\mathrm{F}$ & 200 & $8.06 \mathrm{E}+02$ & 4.03 & $2.11 \mathrm{E}+02$ & 1.06 & $3.33 \mathrm{E}+02$ & 1.67 & 261.00 & 1.31 \\
\hline $\mathrm{Mg}$ & 250 & $2.76 \mathrm{E}+02$ & 1.10 & $5.39 \mathrm{E}+01$ & 0.22 & $5.01 \mathrm{E}+02$ & 2.00 & 537.00 & 2.15 \\
\hline $\mathrm{Mn}$ & 150 & $7.31 \mathrm{E}+02$ & 4.87 & $8.52 \mathrm{E}+01$ & 0.57 & $8.51 \mathrm{E}+02$ & 5.68 & 1070.00 & 7.13 \\
\hline $\mathrm{Hg}$ & 80 & $2.70 \mathrm{E}+02$ & 3.38 & $2.45 \mathrm{E}+01$ & 0.31 & $1.21 \mathrm{E}+03$ & 15.14 & 1300.00 & 16.25 \\
\hline Mo & 150 & $1.74 \mathrm{E}+01$ & 0.12 & $7.75 \mathrm{E}-01$ & 0.01 & $2.14 \mathrm{E}+01$ & 0.14 & 26.90 & 0.18 \\
\hline $\mathrm{Ni}$ & 130 & $8.48 \mathrm{E}+01$ & 0.65 & $9.80 \mathrm{E}+00$ & 0.08 & $1.08 \mathrm{E}+02$ & 0.83 & 136.00 & 1.05 \\
\hline $\mathrm{PO}_{4}$ & 950 & $2.74 \mathrm{E}+02$ & 0.29 & & & & & & \\
\hline $\mathrm{Pu}$ & 5 & 4.84E-01 & 0.10 & $1.36 \mathrm{E}-01$ & 0.03 & $1.05 \mathrm{E}+00$ & 0.21 & 0.93 & 0.19 \\
\hline K & 39000 & $7.20 \mathrm{E}+03$ & 0.18 & $6.19 \mathrm{E}+02$ & 0.02 & $1.36 \mathrm{E}+04$ & 0.35 & 8800.00 & 0.23 \\
\hline $\mathrm{Si}$ & 20 & $8.48 \mathrm{E}-03$ & 0.00 & $4.55 \mathrm{E}+00$ & 0.23 & $1.67 \mathrm{E}+01$ & 0.84 & 8.64 & 0.43 \\
\hline $\mathrm{Na}$ & 23000 & $4.54 \mathrm{E}+04$ & 1.98 & $2.60 \mathrm{E}+03$ & 0.11 & $3.28 \mathrm{E}+04$ & 1.43 & 46900.00 & 2.04 \\
\hline $\mathrm{SO}_{4}$ & 100 & $4.96 \mathrm{E}+03$ & 49.7 & $4.49 \mathrm{E}+02$ & 4.49 & $2.68 \mathrm{E}+03$ & 26.8 & 10300.00 & 103. \\
\hline $\mathrm{Zn}$ & 100 & $6.48 \mathrm{E}+01$ & 0.65 & $7.17 \mathrm{E}-01$ & 0.01 & $5.27 \mathrm{E}+01$ & 0.53 & 69.90 & 0.70 \\
\hline \multirow[t]{2}{*}{ U-235 } & 20 & $1.83 \mathrm{E}+01$ & 0.91 & $8.25 \mathrm{E}-01$ & 0.04 & $4.39 \mathrm{E}+01$ & 2.20 & 27.80 & 1.39 \\
\hline & $\mathrm{mCi} / \mathrm{L}$ & $\mathrm{mCi} / \mathbf{L}$ & Ratio & $\mathrm{mCi} / \mathbf{L}$ & Ratio & $\mathbf{m C i} / \mathbf{L}$ & Ratio & $\mathbf{m C i} / \mathbf{L}$ & Ratio \\
\hline Gross beta & 4.50 & $4.71 \mathrm{E}+01$ & 10.45 & $1.03 \mathrm{E}+01$ & 2.28 & $5.58 \mathrm{E}+01$ & 12.39 & 90.31 & 20.05 \\
\hline
\end{tabular}

Alternatively, the supernate could be processed in the ETS. In this scenario, the ETS condensate would be processed in the PEWE, and the PEWE condensate processed through LET\&D Facility. Bottoms products from the two evaporators and the LET\&D fractionator would be collected in the NGLW tanks.

Most previous ETS operation used relatively concentrated feeds with boil-down ratios of at most 4 to 1; however, the ETS has recently been successfully evaporating dilute decanted tank flush solution from WM-187 at high boil-down ratios. Therefore, evaporation of dilute solutions such as the supernate in the ETS has been successfully demonstrated. 
Table B-5. Limiting case volumes for processing supernate in PEWE.

\begin{tabular}{|c|c|c|c|c|}
\hline & WM-180 & WM-187 & WM-188 & WM-189 \\
\hline Liquid Density, kg/L & 1.26 & 1.01 & 1.3 & 1.33 \\
\hline Floor Volume, gal & 4128 & 82563 & 4128 & 8256 \\
\hline Liquid Volume, gal & 3468 & 69353 & 3468 & 6935 \\
\hline Height on floor, in & 3.37 & 67.45 & 3.37 & 6.74 \\
\hline Expected mass (solids), $\mathrm{kg}$ & 5000 & 100000 & 5000 & 10000 \\
\hline Flush Volume@ @ 80kgal/tank, gal & 80000 & 80000 & 80000 & 80000 \\
\hline Flush Volume by EDF-15722-048, gal & 35000 & 414029 & 30201 & 50403 \\
\hline Flush Volume to meet PEW WAC, gal ${ }^{\mathrm{a}}$ & 168700 & 242108 & 89537 & 707398 \\
\hline Flush Volume to prevent plugging, gal & 11598 & 231962 & 11598 & 23196 \\
\hline \multicolumn{5}{|c|}{ Limiting/Worst Case Volumes for PEWE Processing } \\
\hline Total liquid volume/tank, gal & 172167 & 483381 & 93004 & 714333 \\
\hline Total volume/tank, gal & 172828 & 496592 & 93665 & 715654 \\
\hline PEWE Operating time, month & 3 & 9 & 2 & 13 \\
\hline TSS in feed to clarifier, $g / L$ by limiting case & 8 & 55 & 14 & 4 \\
\hline $\mathrm{Wt} \%$ by limiting case & $0.77 \%$ & $5.47 \%$ & $1.42 \%$ & $0.37 \%$ \\
\hline
\end{tabular}

a. PEW WAC for $\mathrm{SO}_{4}{ }^{=}$of $100 \mathrm{mg} / \mathrm{L}$

The tank flush solutions after solids removal for the three different flush volume cases and for all four tanks were evaluated in the same ASPEN ETS model (Nenni, 2003) used for evaluating ETS feeds for the HLLWE Operational Run Plan (PLN-17). This model also calculates flows and concentrations for processing the resulting ETS condensate through the PEWE and LET\&D. The resulting net waste volumes after processing the supernates through the ETS, PEWE, and LET\&D are summarized in Table B-6. Note that the ETS was evaluated for three cases - the flush water volume based on (a) 80,000 gallons per tank, (b) tank mock test results (Poloski, 2001), and (c) the criteria to prevent line plugging.

For all tanks but WM-187, none of the cases challenged the limits for bottoms from ETS or condensate to PEWE. The flush water volume impacted the final liquid waste volume for each tank by less than $13 \%$. In general, the final waste volumes were very close to the original tank farm heel liquid volumes, with WM-189 being the worst case, requiring a 25\% increase in liquid waste volume. For WM187 , the original heel liquid is already quite dilute even without flush water addition since it has received heel flushes from WM-182, 183, 185 and 186, but the boil-down in the ETS was limited by the $100 \mathrm{~g} / \mathrm{L}$ solids limit to prevent plugging in bottoms collection tank NCC-119 or in transfer lines. Net waste volumes for WM-187 might decrease if more than $95 \%$ of the heel solids are removed from the tank flush solution in the settler/clarifier.

Although past ETS condensates usually required dilution with other wastes to meet the PEW limit of $50 \mathrm{mg}$ chloride per liter, the ETS condensates from processing the supernates can all be sent directly to PEWE without dilution with other wastes.

Based on this review, the mass balances are based on 414,000 gallons of flush water to transfer solids from tank WM-187 and 80,000 gal for each of the other tanks. If actual volumes used prove less 
than these, the feed rate the Solids Tank will be less than shown in the mass balance and less waste water will be processed in the ETS, PEWE and LET\&D.

Table B-6. Calculated liquid waste volumes after ETS/PEWE/LET\&D processing of tank flush supernate.

\begin{tabular}{lcccc}
\hline Original Supernate Volumes: & WM-180 & WM-187 & WM-188 & WM-189 \\
\hline Flush + Tank Liquid Volume @ 80kgal per tank, gal & 83,468 & 149,353 & 83,468 & 86,935 \\
Flush + Tank Liquid Volume from EDF-15722-048, gal & 35,003 & 483,380 & 30,204 & 50,410 \\
Flush + Tank Liquid Volume to prevent plugging, gal & 16,598 & 331,962 & 16,598 & 33,196 \\
\hline Final Volume of ETS Bottoms, PEWE Bottoms, and LET\&D Bottoms: & & & \\
Volume from Flush Volume @ 80kgal per tank, gal & 3,574 & 15,507 & 3,768 & 8,541 \\
Bottoms Volume from Flush Volume by EDF-15722-048, gal & 3,278 & 18,143 & 3,533 & 8,663 \\
Bottoms Volume from Flush Volume to Prevent Plugging, gal & 3,161 & 16,672 & 3,485 & 8,480 \\
\hline
\end{tabular}

Note: The remainder of the liquid volume is water that is vaporized and discharged from LET\&D to the main INTEC stack.

B-5.5.5.1 Solids Content of Slurry Feed. The solids content of waste transferred from WM187 to the Solids Tank will vary. The estimated total amount of solids in the waste tanks is $120,000 \mathrm{~kg}$. Thus the average solids concentration of solids in the slurry feed is:

$$
120,000 \mathrm{~kg} \mathrm{x} 1000 \mathrm{~g} / \mathrm{kg} /(753,100 \text { gal x } 3.785 \text { liters/gal })=42 \mathrm{~g} / \mathrm{liter}
$$

\section{DBE \#5: Waste from Tank WM-187 will have an average undissolved solids content of 42 g/liter.}

B-5.5.5.2 Decant Tank. The decant tank is sized to allow settling of $95 \%$ of the solids fed to the tank. After settling and decanting the average solids concentration of the tank contents will be $20 \mathrm{wt} \%$. According to recent data for WM-187 solids, it takes approximately 16 days to settle $1 / 3-1 / 2$ of the solids. The settling velocity of a $2 \mu \mathrm{m}$ particle is $2 \times 10^{-6} \mathrm{ft} / \mathrm{s}$ based on the wet density and the Stokes Law region verifying a lengthy settling time. Based on this data flocculation is required to achieve reasonable settling times. A pH adjustment will result in flocculation due to aluminum hydroxide precipitation. Adding sufficient sodium hydroxide to bring the $\mathrm{pH}$ to about 5 is expected to increase a $2-\mu \mathrm{m}$ particle to $5 \mu \mathrm{m}$ and reduce the settling time (for $95 \%$ of the particles) to 30 hours.

\section{DBE \#6: The mass balance assumes settling of 95\% of the solids fed to it with the settled solids having an average concentration of $20 \mathrm{wt} \%$. Settling will require 30 hours.}

B-5.5.5.3 Solids Filtration. Filtration efficiency for the Fundabac filter will depend on several variables. A PVDF fabric can be specified as $1 \mu \mathrm{m}$ nominal. Since the particle distribution for WM-183 has $98.88 \%$ greater than this size, a reasonable assumption for the filtration efficiency is $98 \%$.

Based on FY 2003 filtration studies (Pao, 2003), the mass balance assumes no filter aid is added to the filter feed. The process maintains the capability to add filter aid until more complete testing can be performed.

\section{DBE \#7: The Fundabac filter is assumed to remove $98 \%$ of the solids fed to it, and will require no filter aid.}




\section{B-5.5.5.4 Solids Drying.}

DBE \#8: Solids are dried to a moisture content of $25 \mathrm{wt} \%$. It is assumed this water content will meet the WIPP WAC for no liquids.

B-5.5.5.5 Wet Filter Cake Volume and Dry Density. The solids filter is designed to collect 100 liters of solids. The dried filter cake is assumed to occupy the same volume and have a density of $0.68 \mathrm{~g} / \mathrm{cm}^{3}$. This density is based on a measured density of a sample of solids, washed and dried, taken from WM-187 in FY 2004. It is not known whether the dried filter cake density will differ from this value.

DBE \#9: The filter cycle is based on collecting 100 liters of solids. Removing water to 25 wt\% is expected to reduce the density to $0.68 \mathrm{~g} / \mathrm{cm}^{3}$.

B-5.5.5.6 Solids Packaging. The solids will be packaged in WIPP RH TRU canisters, which have a capacity of $0.89 \mathrm{~m}^{3}$. On average, the solids waste will fill $90 \%$ of the canister volume, or $0.8 \mathrm{~m}^{3}$. Approximately 2 inches of silica gel, equivalent to $0.015 \mathrm{~m}^{3}$ per canister will be added on top of the waste.

DBE \#10: The solids waste volume is assumed to average $0.8 \mathrm{~m}^{3}$ per canister.

\section{B-5.5.6 SBW Liquid Treatment}

B-5.5.6.1 Neutralization. Neutralization of the liquid feed is required to minimize degradation of CST by the acidic waste. Neutralization of the feed with caustic to a $\mathrm{pH}$ of 0.5 has been shown to result in acceptable CST performance (Tranter, 2003a; Tranter, 2003d).

DBE \#11: SBW liquid will be neutralized in the receiving tank to a pH of 0.5 using $50 \mathrm{wt} \% \mathrm{NaOH}$.

B-5.5.6.2 Filtration. The nominal filter pore diameter is $0.5 \mu \mathrm{m}$ and $99.94 \%$ of the particles are greater than this size based on the particle size distribution for WM-183. While the filtration solids removal efficiency is a function of several variables, a removal efficiency of $99 \%$ is reasonable based on present data.

DBE \#12: The crossflow filter will remove 99\% of the undissolved solids in the SBW and NGLW feeds.

The recirculation rate provides the high axial velocity required for the system. The vendor's range is $5-15 \mathrm{ft} / \mathrm{s}$. The midpoint of $10 \mathrm{ft} / \mathrm{s}$ corresponds to $130 \mathrm{gpm}$ and was assumed for the mass balance. Other crossflow design parameters were based on scale-up from test data (Mann, 1998), and scale-up based on shear stress would indicate a velocity of $6.25 \mathrm{ft} / \mathrm{s}$.

Permeate is based on testing of filtration of dissolved calcine (Mann, 1998). These tests, using a feed with $2.44 \mathrm{wt} \%$ solids, showed a filtrate flux of $0.04 \mathrm{gpm} / \mathrm{ft}^{2}$ at a pressure of about $33 \mathrm{psig}$. Based on this flux, 2 MOTT filter systems are required to obtain the required permeate flow of $315 \mathrm{~L} / \mathrm{hr}$.

These parameters of circulation rate, pressure, permeate flux and solids concentration will need to be optimized during start-up. Permeate flux increases with increasing pressure and decreasing solids concentration. In addition, it is not known if SBW solids will behave like undissolved calcine particles.

$D B E$ \#13: The crossflow filter circulation rate is based on a velocity of $10 \mathrm{ft} / \mathrm{s}$. The permeate rate is based on a flux of $0.04 \mathrm{gpm} / \mathrm{ft}^{2}$. 
B-5.5.6.3 Ion Exchange. The mass balance assumes a $90 \%$ cesium removal efficiency for each ion exchange column, thus the total cesium removal efficiency is $99.9 \%$. This removal efficiency is based on the requirement to produce a $\mathrm{CH}$ waste and on test results at both bench- and intermediate-scale. The most recent bench-scale dynamic tests used a $2-\mathrm{cm}^{3}$ column (Tranter, 2003a). Tranter (2003a) shows breakthrough curves - plots of effluent to feed cesium concentration ratios, $\mathrm{C} / \mathrm{Co}$, versus bed volumes for flow rates of 5 and 10 bed volumes per hour and using a simulant for Tank WM-189 waste. Similar breakthrough curves were obtained earlier for a different SBW stimulant (Mann, 2000).

A bench-scale test using a larger column $\left(500 \mathrm{~cm}^{3}\right)$ was recently completed and provided an additional breakthrough curve for Tank WM-189 simulant (Tranter 2003d). This test was conducted to support design efforts, specifically to compare the models developed from the $2 \mathrm{~cm}^{3}$ column with those based on the $500 \mathrm{~cm}^{3}$ column.

Two models have been developed to design the ion exchange column based on test data (Kimmitt, 2003c). One model solves material balance and mass transfer differential equations using a finite difference method. An iterative approach is applied to obtain agreement between the model output and experimental breakthrough curves. The second model uses the experimental breakthrough data to estimate an effective distribution coefficient, $\mathrm{K}_{\mathrm{d}}$. The ion exchange column is modeled as equilibrium stages in series using $\mathrm{K}_{\mathrm{d}}$, which is constant over the range of interest.

The modeling procedures have been applied to the 2 and $500 \mathrm{~cm}^{3}$ test results (breakthrough curves). The key parameters in both models are nearly the same for the two sets of tests (Kimmitt 2003d). The effective distribution coefficient, $K_{d}$, for the equilibrium model was 0.513 and 0.497 for the 2 and $500 \mathrm{~cm}^{3}$ tests, respectively. Figure B-2 shows the $500 \mathrm{~cm}^{3}$ column data plotted with the equilibrium model results for the different $\mathrm{K}_{\mathrm{d}}$ values. Very little difference is seen in predicted column performance for the differing $\mathrm{K}_{\mathrm{d}}$ values, particularly at low cesium effluent concentrations. For the mass transfer model, the adjusted mass transfer coefficient, $\mathrm{k}_{\mathrm{p}} \mathrm{a}$, was 0.0035 for both column sizes, therefore predicted curves for the mass transfer model would be exactly the same for either sized column. The significance of having the key parameters being very nearly the same for different column sizes is that this suggests the models can predict breakthrough curves independent of column size - in other words, one set of breakthrough curves can be used to infer column behavior when plotted as shown in Figure B-3 below. The inference is that the models can provide reasonable scale-up for larger systems, with the restriction that liquid flow rates be approximately $10 \mathrm{BV} / \mathrm{hr}$. The specific design information of interest would include column sizing, liquid throughput, and change-out schedule for operating to a predetermined cesium breakthrough value (Kimmitt 2003b). 


\section{Breakthrough $500 \mathrm{ml}$ Column Test}

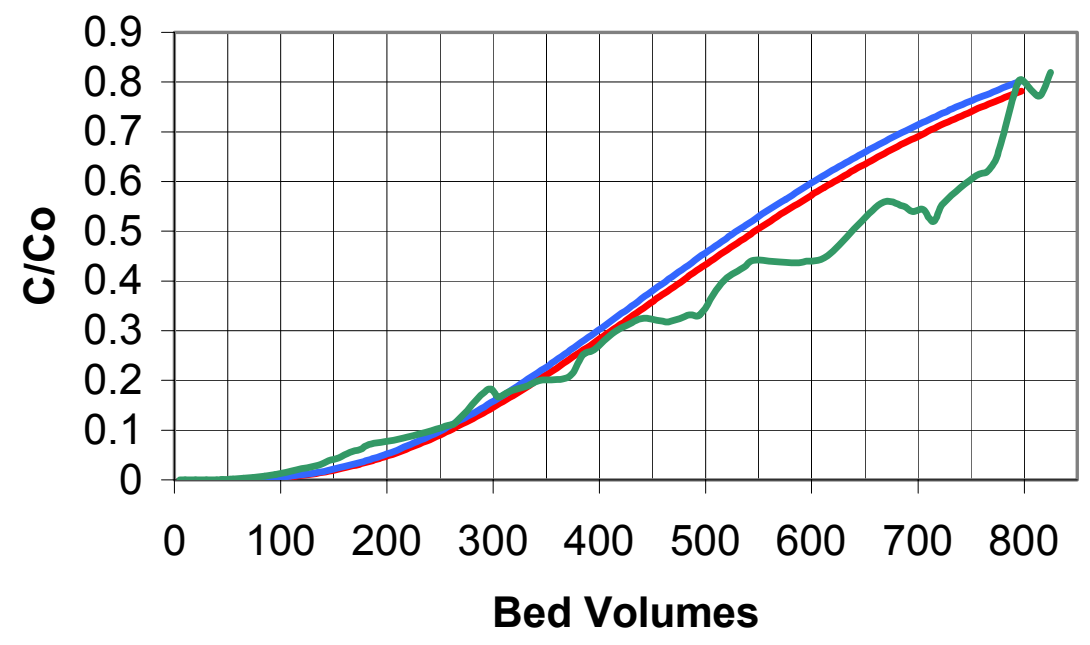
Kd $=513 \mathrm{ml} / \mathrm{gram}$
$\mathrm{Kd}=497 \mathrm{ml} / \mathrm{gram}$
Actual

Figure B-2. Comparison of actual $500 \mathrm{~cm}^{3}$ column data with the $\mathrm{K}_{\mathrm{d}}$ values for the 2 and $500 \mathrm{~cm}^{3}$ column equilibrium models (Kimmitt 2003e).

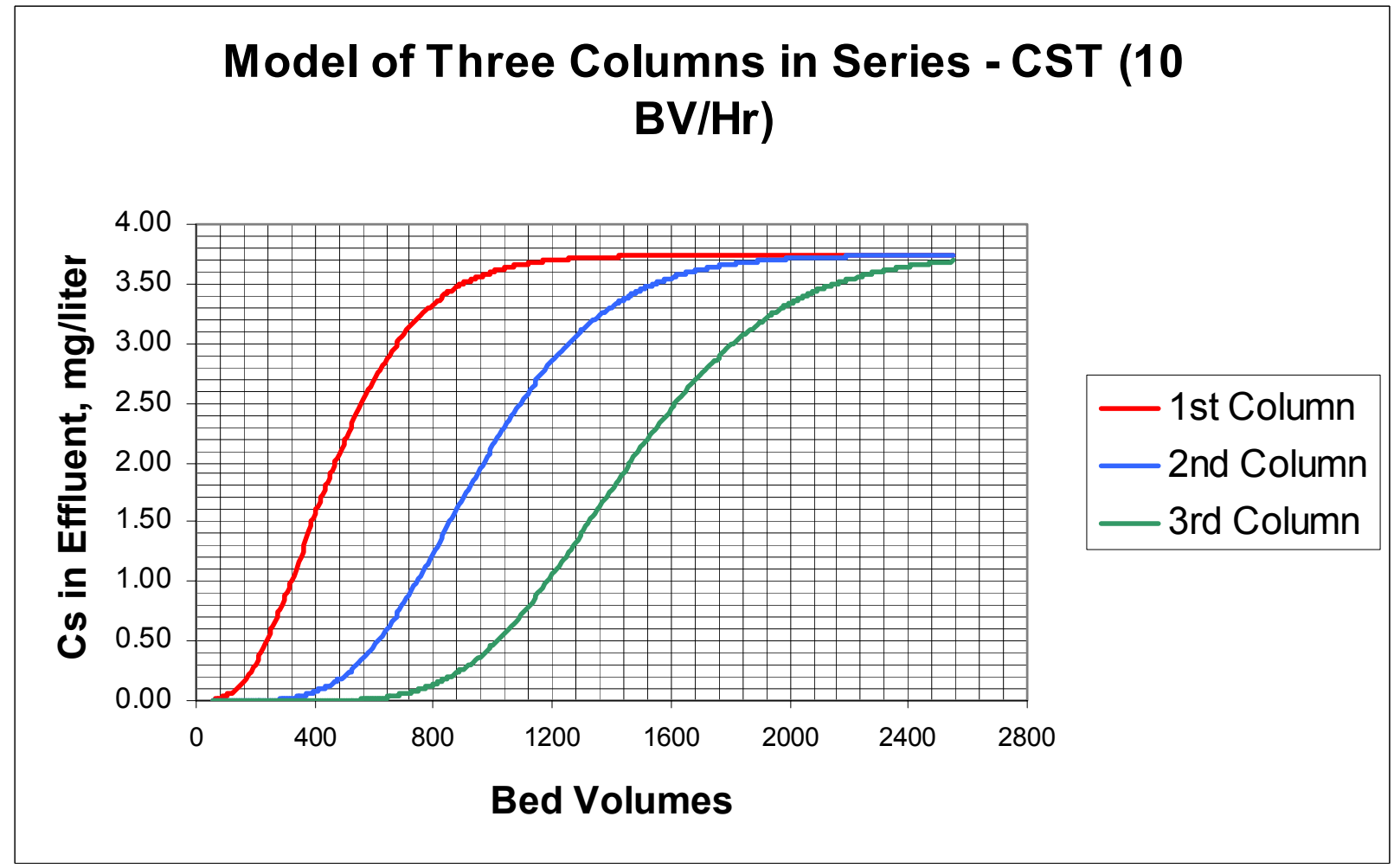

Figure B-3. Typical breakthrough curves for a series of three columns of CST removing Cs (Kimmit 2003b). 
Figure B-3 was generated from the equilibrium model and shows the behavior of a set of three columns of CST resin operated in series. Details are given in the referenced report, but generally the curves show that when fed with a solution containing cesium with about $3.5 \mathrm{mg} / \mathrm{L}$ at a rate of $10 \mathrm{BV} / \mathrm{hr}$, the effluent from the third column will be nearly free of cesium until about 500 bed volumes of liquid are treated (Kimmitt 2003b). When the cesium level in the effluent from the third column reaches a predetermined "breakpoint" value, the lead column is removed from service and a fresh column placed at the end of the series. Cascading columns in this manner allows the lead column to become nearly saturated with cesium, while maintaining low cesium levels in the process effluent.

Nick Mann obtained breakthrough curves not only for cesium, but also for ${ }^{241} \mathrm{Am}, \mathrm{Hg}, \mathrm{Ba}, \mathrm{Ca}, \mathrm{Cd}$, $\mathrm{Cr}, \mathrm{Eu}, \mathrm{Fe}, \mathrm{K}, \mathrm{Mn}, \mathrm{Mo}, \mathrm{Na}, \mathrm{Ni}, \mathrm{Pb}$, and $\mathrm{Zr}$ (Mann, 2000). These data show that sorption of all species except cesium is negligible and can be assumed to be zero.

$D B E$ \#14: The ion exchange columns will remove greater than $99.9 \%$ of the cesium in the feed $S B W$. The mass balance is based on this minimum removal efficiency. Removal of all other species by the columns is assumed to be zero.

Isotherms for CST have been determined by Tranter (2003d) and Mann (2000). These data, as shown in Table B-7, have been used to determine cesium capacities for CST.

Table B-7. Isotherms for CST.

\begin{tabular}{lcc}
\hline Waste & $\begin{array}{c}\text { Cs in waste } \\
\text { (mg/liter) }\end{array}$ & $\begin{array}{c}\text { CST capacity } \\
\text { (mg/g CST) }\end{array}$ \\
\hline WM-180 & 1.34 & 1.22 \\
WM-188 & 3.87 & 2.52 \\
WM-189 & 3.59 & 2.40 \\
NGLW & 2.94 & 2.09 \\
\hline
\end{tabular}

\section{DBE \#15: The mass balance assumes sorbent cesium capacities of 1.22 to $2.52 \mathrm{mg}$ Cs/g CST depending on the waste feed.}

The volume of sand at the bottom of the columns is $0.9 \mathrm{ft}^{3}$. The corresponding sand height of 6inches is assumed sufficient to capture fines from eroded media. The small amount of particles passing through the cross-flow filtration elements will be captured, likely due to Brownian motion mechanisms onto the media so that the effluent from the columns will be virtually particle free.

\section{DBE \#16: A sand volume of $0.9 \mathrm{ft}^{3}$ in the ion exchange column is assumed sufficient to capture all residual $S B W$ solids and eroded media particles.}

CST has an "as-shipped" bulk density of $60 \mathrm{lbs} / \mathrm{ft}^{3}$ (UOP). The particle density is $2.44 \mathrm{~g} / \mathrm{cm}^{3}$ (UOP).

DBE \#17: The bulk density of CST is $60 \mathrm{lb} / \mathrm{ft}$; the particle density is $2.44 \mathrm{~g} / \mathrm{cm}^{3}$.

Washing with ten bed volumes (600 gallons) is assumed sufficient to reduce the acidity of the spent ion exchange media to levels meeting WIPP WAC.

DBE \#18: The spent ion exchange media will be washed with 10 bed volumes of water. 
The spent ion exchange media requires drying to meet the WIPP criteria for "no free liquids" and to minimize radiolytic hydrogen generation in the waste package. While the moisture level to satisfy the second requirement cannot be determined until a waste code has been assigned to the waste, based on a review of other wastes disposed at WIPP, a moisture level of $25 \mathrm{wt} \%$ is expected to meet the requirement.

DBE \#19: The spent ion exchange column beds will be dried to a moisture content of 25 wt\% or less.

After drying the ion exchange column bed, the entire column will be loaded into a RH-TRU waste canister for interim storage and disposal.

\section{DBE \#20: Spent ion exchange columns will be disposed in $R H-T R U$ waste canisters, one column per canister.}

B-5.5.6.4 Grouting. Based on the most recent SBW grout testing (Raman, 2003), different waste loadings are recommended for the different tank wastes. For Tank WM-180 waste, a waste loading of 72 $\mathrm{wt} \%$ was recommended, while for Tank WM-189 waste, a waste loading of $68 \mathrm{wt} \%$ was recommended (Raman, 2003). However, when based on the waste after partial neutralization, the waste loading is the same for these two wastes. The recommended formulation for both WM-180 and WM-189 wastes includes $7 \mathrm{wt} \% \mathrm{Ca}(\mathrm{OH})_{2}, 6 \mathrm{wt} \%$ BFS and $12 \mathrm{wt} \%$ Portland cement. The product density using this formulation was 1.47 and $1.53 \mathrm{~g} / \mathrm{cm}^{3}$ respectively. The mass balance uses a grout density of $1.5 \mathrm{~g} / \mathrm{cm}^{3}$ for all tank wastes, the average of these two experimentally determined densities.

\section{DBE \#21: The grout formulation is 75 wt\% waste partially neutralized with $50 \% \mathrm{NaOH}, 7 \mathrm{wt} \%$ calcium hydroxide, $6 \mathrm{wt} \%$ blast furnace slag and $12 \mathrm{wt} \%$ Portland cement. (Note: the $\mathrm{NaOH}$ is added upstream of filtration and ion exchange.) This density of waste grouted with this formulation is $1.5 \mathrm{~kg} / \mathrm{m}^{3}$.}

The mass balance assumes bulk densities of calcium hydroxide, blast furnace slag and Portland cement of $480 \mathrm{~kg} / \mathrm{m}^{3}, 1200 \mathrm{~kg} / \mathrm{m}^{3}$ and $1200 \mathrm{~kg} / \mathrm{m}^{3}$ respectively. The density of calcium hydroxide is the average of densities for $\mathrm{Ca}(\mathrm{OH})_{2}$ from three suppliers. The density for blast furnace slag was also based on supplier literature and is a typical density for packed, air-cooled blast furnace slag. The density for Portland cement is consistent with manufacturers' literature for aerated ordinary Portland cement.

DBE \#22: The mass balance assumes bulk densities of $480 \mathrm{~kg} / \mathrm{m}^{3}, 1200 \mathrm{~kg} / \mathrm{m}^{3}$ and $1200 \mathrm{~kg} / \mathrm{m}^{3}$ for calcium hydroxide, blast furnace slag and Portland cement respectively.

Grout will be packaged in 55-gal drums. The mass balance assumes a fill volume of $0.2 \mathrm{~m}^{3}$, equivalent to approximately $94 \%$ of the capacity of standard 55-gal drums.

DBE \#23: The mass balance assumes a grouted waste volume of $0.2 \mathrm{~m}^{3}$ per drum.

\section{B-5.5.7 Waste Water Treatment}

B-5.5.7.1 ETS and PEWE Feedrate. Based on past operation, the ETS will process about 10,000 gallons of waste water per day, and the PEWE about 4,500 gallons per day.

B-5.5.7.2 ETS and PEWE Endpoint. Waste fed to the ETS or PEWE is concentrated to a bottoms specific gravity of 1.3. 
B-5.5.7.3 LET\&D Feedrate. The minimum L\&T\&D feed flow rate is $275 \mathrm{gal} / \mathrm{hr}$; the maximum is $550 \mathrm{gal} / \mathrm{hr}$; the nominal feed flow rate of $500 \mathrm{gal} / \mathrm{hr}$. The mass balance assumes that the LET\&D process the ETS condensate at the same rate as it is generated, about $400 \mathrm{gal} / \mathrm{hr}$.

B-5.5.7.4 LET\&D Removal Efficiency and Bottoms Composition. The LET\&D concentrates acidic feed streams to a bottoms concentration of 12 molar nitric acid. The LET\&D recovers $99 \%$ of the nitric acid in the feed. The mass balance assumes a decontamination factor (moles or curies in feed divided by moles or curies in vapor from the partial condenser) of 1000 for all nonvolatile species as well as volatile metals such as mercury. ${ }^{\mathrm{a}}$

\section{B-5.5.8 Utilities}

B-5.5.8.1 Cooling Water. Cooling water is used to remove heat of solution and reaction generated in the SBW Receiving Tank during neutralization of SBW feed. For purposes of the material balances, the supply temperature of the cooling water was assumed to be $20^{\circ} \mathrm{C}\left(68^{\circ} \mathrm{F}\right)$, and the return temperature $35^{\circ} \mathrm{C}\left(95^{\circ} \mathrm{F}\right)$.

B-5.5.8.2 Steam. Steam is used to dry the separated tank solids prior to packaging for disposal and for jet transfer of liquids. Steam is available at INTEC at 35 psig and 150 psig.

B-5.5.8.3 Air. Air is available from the INTEC plant air supply at 100 psig and 50 psig. This air will be filtered and regulated to 20 psig for instrument air. In addition, atmospheric air is assumed available for use in drying spent CST beds.

\section{B-6. MASS BALANCES}

A summary of waste products is shown in Table B-8. Tank solids and spent ion exchange media are packaged in nominally 2 - $\mathrm{ft}$ diameter by 10 -ft high $\mathrm{RH}$ canisters. One ion exchange column is packaged in a canister. Twenty-one spent columns are produced during treatment, and three additional columns will be packaged for disposal during decommissioning. The ion exchange effluent is grouted and packaged in 55-gal drums.

Table B-8. Summary of waste products.

\begin{tabular}{lccccccc}
\hline \multicolumn{1}{c}{ Waste Products } & WM-180 & WM-188 & WM-189 & NGLW & Decommissioning & Total & Total $\mathbf{~ m}^{\mathbf{3}}$ \\
\hline Tank Solids & & & & & & 279 & 223 \\
$\quad$ RH canisters & 87 & 90 & 89 & 13 & & & $10^{\mathrm{a}}$ \\
$\begin{array}{l}\text { Spent CST } \\
\quad \text { RH canisters }\end{array}$ & 5 & 8 & 7 & 1 & 3 & 24 & \\
CH Grout & & & & & & 24,000 & \\
$\quad$ CH drums & 6,820 & 8,030 & 7,950 & 1,200 & & 200 \\
\hline
\end{tabular}

${ }^{\mathrm{a}}$ Volume of spent CST based on volume of IX columns and includes sand filter

a This decontamination factor of 1000 is given in the LET\&D Safety Analysis, PSD 8.6A, for "nonvolatile radionuclides" and $\mathrm{Hg}, \mathrm{Cd}$ and $\mathrm{Pu}$. 
A mass balance for treating waste from WM-180 is shown in Table B-9. Similar mass balances for WM-188, WM-189 and NGLW are shown in Tables B-10, B-11, and B-12 respectively. Tables B-13 through B-15 show properties of the product wastes and Table B-16 shows consumption of feed chemicals and materials. 
Table B-9. Tank WM-180 mass balance.

\begin{tabular}{|c|c|c|c|c|c|c|c|c|}
\hline PFD Sheet \# & PFD-2 & PFD-2 & PFD-2 & PFD-2 & PFD-2 & PFD-2 & PFD-2 & PFD-3 \\
\hline Stream \# & 110 & 111 & 112 & 113 & 114 & 115 & 116 & 120 \\
\hline Stream Name & $\begin{array}{l}\text { Slurry from } \\
\text { TFF Tank } \\
\text { WM-187 }\end{array}$ & $\begin{array}{c}\text { Crossflow } \\
\text { Filter Recirc } \\
\text { Purge }\end{array}$ & $\begin{array}{l}\text { Decant } \\
\text { Liquid }\end{array}$ & $\begin{array}{c}\text { Solids } \\
\text { Treatment } \\
\text { Filter Feed }\end{array}$ & $\begin{array}{l}\text { Solids } \\
\text { Treatment } \\
\text { Filtrate }\end{array}$ & $\begin{array}{c}\text { Combined } \\
\text { Waste Water }\end{array}$ & $\begin{array}{l}\text { Dried Solids } \\
\text { Waste }\end{array}$ & $\begin{array}{c}\text { Liquid SBW } \\
\text { from Tank } \\
\text { WM-180 }\end{array}$ \\
\hline Rate, gal/hr & 65.9 & 1.1 & 55.0 & 14.7 & 8.3 & 67.0 & 6.3 & 85.2 \\
\hline Rate, $\mathrm{lb} / \mathrm{hr}$ & 571 & 12 & 467 & 137 & 70 & 569 & 36 & 886 \\
\hline Rate, peak, gpm & 50 & 0.019 & 50 & 10 & 0.14 & 190 & 4.32 & 50 \\
\hline Operation & Batch & Cont & Batch & Batch & Batch & Batch & Batch & Batch \\
\hline Temperature, ${ }^{\circ} \mathrm{F}$ & 70 & 83 & 70 & 70 & 70 & 70 & 70 & 70 \\
\hline Pressure, psia & 12.8 & 12.8 & 12.8 & 50.0 & 12.8 & 12.8 & 12.8 & 12.8 \\
\hline Specific Gravity & 1.04 & 1.25 & 1.02 & 1.12 & 1.02 & 1.02 & 0.68 & 1.25 \\
\hline Chemical Composition & Mol/liter & Mol/liter & Mol/liter & Mol/liter & Mol/liter & Mol/liter & Wt \% & Mol/liter \\
\hline $\mathrm{H}+$ & $3.13 \mathrm{E}-02$ & $4.76 \mathrm{E}-01$ & $3.90 \mathrm{E}-02$ & $3.90 \mathrm{E}-02$ & $3.90 \mathrm{E}-02$ & $3.59 \mathrm{E}-02$ & $9.63 \mathrm{E}-04$ & $1.21 \mathrm{E}+00$ \\
\hline$\overline{A l+3}$ & $3.04 \mathrm{E}-02$ & $5.24 \mathrm{E}-01$ & $1.51 \mathrm{E}-02$ & $1.24 \mathrm{E}-01$ & $1.72 \mathrm{E}-02$ & $2.71 \mathrm{E}-02$ & $1.00 \mathrm{E}+00$ & $5.56 \mathrm{E}-01$ \\
\hline$A m+4$ & $8.51 \mathrm{E}-10$ & $2.84 \mathrm{E}-08$ & $1.33 \mathrm{E}-09$ & $1.33 \mathrm{E}-09$ & $1.33 \mathrm{E}-09$ & $1.24 \mathrm{E}-09$ & 7.84E-09 & $3.09 \mathrm{E}-08$ \\
\hline $\mathrm{Sb}+5$ & $1.51 \mathrm{E}-05$ & $8.83 \mathrm{E}-06$ & $8.64 \mathrm{E}-07$ & $6.52 \mathrm{E}-05$ & $2.10 \mathrm{E}-06$ & $8.31 \mathrm{E}-06$ & $2.66 \mathrm{E}-03$ & $6.85 \mathrm{E}-07$ \\
\hline As+5 & $1.28 \mathrm{E}-04$ & $4.64 \mathrm{E}-04$ & $1.40 \mathrm{E}-05$ & 5.57E-04 & 2.44E-05 & $7.68 \mathrm{E}-05$ & $1.38 \mathrm{E}-02$ & $4.30 \mathrm{E}-04$ \\
\hline $\mathrm{Ba}+2$ & $2.76 \mathrm{E}-05$ & 5.89E-05 & 2.89E-06 & $1.18 \mathrm{E}-04$ & 5.09E-06 & 1.61E-05 & $5.35 \mathrm{E}-03$ & $4.82 \mathrm{E}-05$ \\
\hline $\mathrm{Be}+2$ & $6.21 \mathrm{E}-06$ & $9.62 \mathrm{E}-06$ & $4.65 \mathrm{E}-07$ & $2.68 \mathrm{E}-05$ & 9.71E-07 & $3.52 \mathrm{E}-06$ & 8.07E-05 & $6.81 \mathrm{E}-06$ \\
\hline$B+3$ & $7.79 \mathrm{E}-04$ & $9.41 \mathrm{E}-03$ & $4.63 \mathrm{E}-04$ & $2.58 \mathrm{E}-03$ & $5.04 \mathrm{E}-04$ & $6.80 \mathrm{E}-04$ & $7.88 \mathrm{E}-03$ & 9.95E-03 \\
\hline $\mathrm{Br}-$ & $4.45 \mathrm{E}-09$ & $1.48 \mathrm{E}-07$ & $6.94 \mathrm{E}-09$ & $6.94 \mathrm{E}-09$ & $6.94 \mathrm{E}-09$ & $6.49 \mathrm{E}-09$ & $1.36 \mathrm{E}-08$ & $1.61 \mathrm{E}-07$ \\
\hline $\mathrm{Cd}+2$ & $1.50 \mathrm{E}-04$ & $6.97 \mathrm{E}-04$ & $8.40 \mathrm{E}-05$ & $4.28 \mathrm{E}-04$ & $9.06 \mathrm{E}-05$ & 1.17E-04 & $1.34 \mathrm{E}-02$ & $7.10 \mathrm{E}-04$ \\
\hline $\mathrm{Ca}+2$ & $2.14 \mathrm{E}-03$ & $3.34 \mathrm{E}-02$ & $1.42 \mathrm{E}-03$ & $7.13 \mathrm{E}-03$ & $1.53 \mathrm{E}-03$ & $2.00 \mathrm{E}-03$ & $7.89 \mathrm{E}-02$ & $3.56 \mathrm{E}-02$ \\
\hline $\mathrm{Ce}+4$ & $1.39 \mathrm{E}-05$ & $4.62 \mathrm{E}-05$ & 1.67E-06 & 5.97E-05 & $2.79 \mathrm{E}-06$ & $8.37 \mathrm{E}-06$ & $2.76 \mathrm{E}-03$ & $4.22 \mathrm{E}-05$ \\
\hline Cs+ & $5.55 \mathrm{E}-05$ & $3.96 \mathrm{E}-05$ & $3.52 \mathrm{E}-06$ & $2.39 \mathrm{E}-04$ & $8.03 \mathrm{E}-06$ & $3.07 \mathrm{E}-05$ & $1.06 \mathrm{E}-02$ & $1.04 \mathrm{E}-05$ \\
\hline $\mathrm{Cl}-$ & $3.23 \mathrm{E}-03$ & $2.71 \mathrm{E}-02$ & $8.22 \mathrm{E}-04$ & $1.36 \mathrm{E}-02$ & $1.07 \mathrm{E}-03$ & $2.28 \mathrm{E}-03$ & $1.55 \mathrm{E}-01$ & $2.77 \mathrm{E}-02$ \\
\hline $\mathrm{Cr}+3$ & $5.85 \mathrm{E}-04$ & 3.37E-03 & $1.42 \mathrm{E}-04$ & $2.38 \mathrm{E}-03$ & $1.85 \mathrm{E}-04$ & $3.95 \mathrm{E}-04$ & 3.96E-02 & $3.36 \mathrm{E}-03$ \\
\hline $\mathrm{Co}+2$ & $4.86 \mathrm{E}-06$ & $1.97 \mathrm{E}-04$ & $3.74 \mathrm{E}-06$ & $2.39 \mathrm{E}-05$ & $4.13 \mathrm{E}-06$ & $6.05 \mathrm{E}-06$ & $4.09 \mathrm{E}-04$ & $2.11 \mathrm{E}-04$ \\
\hline $\mathrm{Cu}+2$ & $1.55 \mathrm{E}-04$ & $6.36 \mathrm{E}-04$ & 1.97E-05 & $6.75 \mathrm{E}-04$ & $3.22 \mathrm{E}-05$ & $9.53 \mathrm{E}-05$ & $1.41 \mathrm{E}-02$ & $6.01 \mathrm{E}-04$ \\
\hline $\mathrm{Eu}+3$ & $7.38 \mathrm{E}-09$ & $2.46 \mathrm{E}-07$ & $1.15 \mathrm{E}-08$ & $1.15 \mathrm{E}-08$ & $1.15 \mathrm{E}-08$ & $1.08 \mathrm{E}-08$ & $4.29 \mathrm{E}-08$ & $2.68 \mathrm{E}-07$ \\
\hline F- & $2.82 \mathrm{E}-02$ & $5.14 \mathrm{E}-02$ & $3.63 \mathrm{E}-03$ & 1.17E-01 & $5.80 \mathrm{E}-03$ & $1.66 \mathrm{E}-02$ & $7.32 \mathrm{E}-01$ & $4.03 \mathrm{E}-02$ \\
\hline $\mathrm{Gd}+3$ & $2.82 \mathrm{E}-05$ & $1.61 \mathrm{E}-04$ & $4.96 \mathrm{E}-06$ & $1.21 \mathrm{E}-04$ & $7.18 \mathrm{E}-06$ & $1.83 \mathrm{E}-05$ & $6.20 \mathrm{E}-03$ & $1.59 \mathrm{E}-04$ \\
\hline $\mathrm{Ge}+4$ & $1.28 \mathrm{E}-10$ & $4.28 \mathrm{E}-09$ & $2.00 \mathrm{E}-10$ & $2.00 \mathrm{E}-10$ & $2.00 \mathrm{E}-10$ & $1.87 \mathrm{E}-10$ & $3.56 \mathrm{E}-10$ & $4.66 \mathrm{E}-09$ \\
\hline $\ln +3$ & $2.02 \mathrm{E}-08$ & $6.77 \mathrm{E}-07$ & $3.16 \mathrm{E}-08$ & $3.16 \mathrm{E}-08$ & $3.16 \mathrm{E}-08$ & $2.95 \mathrm{E}-08$ & $8.88 \mathrm{E}-08$ & $7.36 \mathrm{E}-07$ \\
\hline I- & $3.70 \mathrm{E}-08$ & $1.23 \mathrm{E}-06$ & $5.76 \mathrm{E}-08$ & $5.76 \mathrm{E}-08$ & $5.76 \mathrm{E}-08$ & $5.39 \mathrm{E}-08$ & $1.79 \mathrm{E}-07$ & $1.34 \mathrm{E}-06$ \\
\hline $\mathrm{Fe}+3$ & 1.07E-02 & $2.16 \mathrm{E}-02$ & $1.11 \mathrm{E}-03$ & $4.56 \mathrm{E}-02$ & 1.97E-03 & $6.24 \mathrm{E}-03$ & $8.44 \mathrm{E}-01$ & $1.73 \mathrm{E}-02$ \\
\hline $\mathrm{La+3}$ & $1.34 \mathrm{E}-07$ & 4.47E-06 & $2.09 \mathrm{E}-07$ & $2.09 \mathrm{E}-07$ & $2.09 \mathrm{E}-07$ & $1.96 \mathrm{E}-07$ & $7.12 \mathrm{E}-07$ & 4.87E-06 \\
\hline $\mathrm{Pb}+2$ & $6.39 \mathrm{E}-05$ & $1.15 \mathrm{E}-03$ & $3.86 \mathrm{E}-05$ & $2.39 \mathrm{E}-04$ & $4.24 \mathrm{E}-05$ & $6.01 \mathrm{E}-05$ & $1.43 \mathrm{E}-02$ & $1.23 \mathrm{E}-03$ \\
\hline Li+ & $3.18 \mathrm{E}-04$ & $4.96 \mathrm{E}-04$ & $2.37 \mathrm{E}-05$ & $1.38 \mathrm{E}-03$ & $4.97 \mathrm{E}-05$ & $1.80 \mathrm{E}-04$ & $3.19 \mathrm{E}-03$ & $3.52 \mathrm{E}-04$ \\
\hline $\mathrm{Mg}+2$ & $1.23 \mathrm{E}-03$ & $1.07 \mathrm{E}-02$ & $5.27 \mathrm{E}-04$ & $4.49 \mathrm{E}-03$ & $6.03 \mathrm{E}-04$ & $9.55 \mathrm{E}-04$ & $3.30 \mathrm{E}-02$ & $1.11 \mathrm{E}-02$ \\
\hline $\mathrm{Mn}+4$ & $4.45 \mathrm{E}-04$ & $1.22 \mathrm{E}-02$ & $4.29 \mathrm{E}-04$ & $1.42 \mathrm{E}-03$ & $4.48 \mathrm{E}-04$ & $5.22 \mathrm{E}-04$ & $1.91 \mathrm{E}-02$ & $1.31 \mathrm{E}-02$ \\
\hline $\mathrm{Hg}+2$ & $3.11 \mathrm{E}-05$ & $1.22 \mathrm{E}-03$ & $3.81 \mathrm{E}-05$ & $9.92 \mathrm{E}-05$ & $3.93 \mathrm{E}-05$ & $4.35 \mathrm{E}-05$ & $4.34 \mathrm{E}-03$ & $1.32 \mathrm{E}-03$ \\
\hline $\mathrm{Mo}+6$ & $8.91 \mathrm{E}-04$ & $6.49 \mathrm{E}-04$ & $5.38 \mathrm{E}-05$ & $3.84 \mathrm{E}-03$ & $1.26 \mathrm{E}-04$ & $4.92 \mathrm{E}-04$ & $1.23 \mathrm{E}-01$ & $1.82 \mathrm{E}-04$ \\
\hline $\mathrm{Nd}+3$ & $4.32 \mathrm{E}-07$ & $1.44 \mathrm{E}-05$ & $6.74 \mathrm{E}-07$ & $6.74 \mathrm{E}-07$ & $6.74 \mathrm{E}-07$ & $6.31 \mathrm{E}-07$ & $2.38 \mathrm{E}-06$ & $1.57 \mathrm{E}-05$ \\
\hline $\mathrm{Np}+4$ & $2.47 \mathrm{E}-07$ & $7.78 \mathrm{E}-06$ & $3.77 \mathrm{E}-07$ & $3.77 \mathrm{E}-07$ & 3.77E-07 & $3.53 \mathrm{E}-07$ & $2.19 \mathrm{E}-06$ & 8.47E-06 \\
\hline $\mathrm{Ni}+2$ & $2.03 \mathrm{E}-04$ & $1.34 \mathrm{E}-03$ & $5.40 \mathrm{E}-05$ & $8.22 \mathrm{E}-04$ & $6.87 \mathrm{E}-05$ & $1.41 \mathrm{E}-04$ & $1.54 \mathrm{E}-02$ & $1.35 \mathrm{E}-03$ \\
\hline $\mathrm{Nb}+5$ & $1.47 \mathrm{E}-03$ & $8.10 \mathrm{E}-04$ & $8.28 \mathrm{E}-05$ & $6.36 \mathrm{E}-03$ & $2.03 E-04$ & $8.09 \mathrm{E}-04$ & $1.98 \mathrm{E}-01$ & $1.08 \mathrm{E}-05$ \\
\hline NO3- & $6.65 \mathrm{E}-02$ & $4.62 \mathrm{E}+00$ & $1.45 \mathrm{E}-01$ & 1.47E-01 & $1.45 \mathrm{E}-01$ & $1.38 \mathrm{E}-01$ & $2.61 \mathrm{E}-01$ & $5.03 \mathrm{E}+00$ \\
\hline $\mathrm{O}-2$ & $2.23 \mathrm{E}-01$ & $1.23 \mathrm{E}-01$ & $1.25 \mathrm{E}-02$ & $9.64 \mathrm{E}-01$ & $3.08 \mathrm{E}-02$ & $1.23 \mathrm{E}-01$ & $5.16 \mathrm{E}+00$ & $1.57 \mathrm{E}-03$ \\
\hline $\mathrm{Pd}+4$ & $2.45 \mathrm{E}-03$ & $1.35 \mathrm{E}-03$ & $1.38 \mathrm{E}-04$ & $1.06 \mathrm{E}-02$ & $3.38 \mathrm{E}-04$ & $1.35 \mathrm{E}-03$ & 3.77E-01 & $2.39 \mathrm{E}-05$ \\
\hline PO4-3 & 1.39E-01 & $8.68 \mathrm{E}-02$ & 8.04E-03 & 5.97E-01 & $1.93 \mathrm{E}-02$ & $7.63 \mathrm{E}-02$ & $1.90 \mathrm{E}+01$ & $1.28 \mathrm{E}-02$ \\
\hline $\mathrm{Pu}+4$ & $7.79 \mathrm{E}-08$ & $5.05 \mathrm{E}-06$ & $1.64 \mathrm{E}-07$ & $1.64 \mathrm{E}-07$ & $1.64 \mathrm{E}-07$ & $1.56 \mathrm{E}-07$ & $9.56 \mathrm{E}-07$ & $5.49 \mathrm{E}-06$ \\
\hline $\mathrm{K}+$ & $1.86 \mathrm{E}-02$ & $1.93 \mathrm{E}-01$ & $6.23 \mathrm{E}-03$ & $7.62 \mathrm{E}-02$ & $7.57 \mathrm{E}-03$ & $1.41 \mathrm{E}-02$ & $9.34 \mathrm{E}-01$ & $2.01 \mathrm{E}-01$ \\
\hline $\mathrm{Pr}+4$ & $1.22 \mathrm{E}-07$ & $4.07 \mathrm{E}-06$ & $1.90 \mathrm{E}-07$ & $1.90 \mathrm{E}-07$ & $1.90 \mathrm{E}-07$ & $1.78 \mathrm{E}-07$ & $6.57 \mathrm{E}-07$ & $4.42 \mathrm{E}-06$ \\
\hline $\mathrm{Rh}+4$ & $5.26 \mathrm{E}-08$ & $1.75 \mathrm{E}-06$ & $8.20 \mathrm{E}-08$ & $8.20 \mathrm{E}-08$ & $8.20 \mathrm{E}-08$ & $7.67 \mathrm{E}-08$ & 2.07E-07 & $1.91 \mathrm{E}-06$ \\
\hline
\end{tabular}


Table B-9 Tank WM-180 mass balance (continued).

\begin{tabular}{|c|c|c|c|c|c|c|c|c|}
\hline Stream \# & 110 & 111 & 112 & 113 & 114 & 115 & 116 & 120 \\
\hline Stream Name & $\begin{array}{l}\text { Slurry from } \\
\text { TFF Tank } \\
\text { WM-187 }\end{array}$ & $\begin{array}{c}\text { Crossflow } \\
\text { Filter Recirc } \\
\text { Purge }\end{array}$ & $\begin{array}{l}\text { Decant } \\
\text { Liquid }\end{array}$ & $\begin{array}{c}\text { Solids } \\
\text { Treatment } \\
\text { Filter Feed }\end{array}$ & $\begin{array}{l}\text { Solids } \\
\text { Treatment } \\
\text { Filtrate }\end{array}$ & $\begin{array}{c}\text { Combined } \\
\text { Waste Water }\end{array}$ & $\begin{array}{l}\text { Dried Solids } \\
\quad \text { Waste }\end{array}$ & $\begin{array}{l}\text { Liquid SBW } \\
\text { from TFF }\end{array}$ \\
\hline Chemical Composition & Mol/liter & Mol/liter & Mol/liter & Mol/liter & Mol/liter & Mol/liter & Wt \% & Mol/liter \\
\hline $\mathrm{Rb}+$ & $8.10 \mathrm{E}-08$ & $2.70 \mathrm{E}-06$ & $1.26 \mathrm{E}-07$ & $1.26 \mathrm{E}-07$ & $1.26 \mathrm{E}-07$ & $1.18 \mathrm{E}-07$ & 2.64E-07 & $2.94 \mathrm{E}-06$ \\
\hline $\mathrm{Ru}+3$ & $1.03 \mathrm{E}-03$ & $6.68 \mathrm{E}-04$ & $5.98 \mathrm{E}-05$ & 4.43E-03 & $1.44 \mathrm{E}-04$ & $5.66 \mathrm{E}-04$ & $1.50 \mathrm{E}-01$ & $1.20 \mathrm{E}-04$ \\
\hline $\mathrm{Sm+3}$ & $8.02 \mathrm{E}-08$ & $2.67 \mathrm{E}-06$ & 1.25E-07 & $1.25 \mathrm{E}-07$ & $1.25 \mathrm{E}-07$ & 1.17E-07 & $4.61 \mathrm{E}-07$ & $2.91 \mathrm{E}-06$ \\
\hline $\mathrm{Se}+4$ & $3.82 \mathrm{E}-04$ & $2.19 \mathrm{E}-04$ & $2.16 \mathrm{E}-05$ & $1.65 \mathrm{E}-03$ & $5.28 \mathrm{E}-05$ & $2.10 \mathrm{E}-04$ & $4.36 \mathrm{E}-02$ & $1.26 \mathrm{E}-05$ \\
\hline $\mathrm{Si+4}$ & $1.26 \mathrm{E}-01$ & $6.90 \mathrm{E}-02$ & $7.07 \mathrm{E}-03$ & $5.42 \mathrm{E}-01$ & $1.73 \mathrm{E}-02$ & $6.90 \mathrm{E}-02$ & $5.10 \mathrm{E}+00$ & $8.95 \mathrm{E}-04$ \\
\hline $\mathrm{Ag}+$ & $5.89 \mathrm{E}-04$ & $3.27 \mathrm{E}-04$ & 3.37E-05 & $2.54 \mathrm{E}-03$ & $8.17 \mathrm{E}-05$ & $3.23 \mathrm{E}-04$ & $9.16 \mathrm{E}-02$ & $8.31 \mathrm{E}-06$ \\
\hline $\mathrm{Na}+$ & $4.19 \mathrm{E}-02$ & $2.38 \mathrm{E}+00$ & $5.76 \mathrm{E}-02$ & $1.70 \mathrm{E}-01$ & $5.98 \mathrm{E}-02$ & $6.89 \mathrm{E}-02$ & $9.09 \mathrm{E}-01$ & $1.89 \mathrm{E}+00$ \\
\hline $\mathrm{Sr}+2$ & $8.53 \mathrm{E}-06$ & $1.03 \mathrm{E}-04$ & $2.37 \mathrm{E}-06$ & $3.78 \mathrm{E}-05$ & $3.05 \mathrm{E}-06$ & $6.45 \mathrm{E}-06$ & $1.06 \mathrm{E}-03$ & 1.07E-04 \\
\hline SO4-2 & $6.39 \mathrm{E}-04$ & $4.37 \mathrm{E}-02$ & $1.38 \mathrm{E}-03$ & $1.38 \mathrm{E}-03$ & $1.38 \mathrm{E}-03$ & $1.32 \mathrm{E}-03$ & $3.26 \mathrm{E}-03$ & $4.75 \mathrm{E}-02$ \\
\hline $\mathrm{Tc}+7$ & $9.40 \mathrm{E}-08$ & $5.01 \mathrm{E}-06$ & $1.79 \mathrm{E}-07$ & 1.79E-07 & 1.79E-07 & $1.69 \mathrm{E}-07$ & $4.25 \mathrm{E}-07$ & $5.45 \mathrm{E}-06$ \\
\hline $\mathrm{Te}+4$ & $4.21 \mathrm{E}-08$ & $1.47 \mathrm{E}-06$ & $6.68 \mathrm{E}-08$ & $6.68 \mathrm{E}-08$ & $6.68 \mathrm{E}-08$ & $6.26 \mathrm{E}-08$ & $2.09 \mathrm{E}-07$ & $1.60 \mathrm{E}-06$ \\
\hline $\mathrm{Tb}+4$ & $3.09 \mathrm{E}-11$ & $1.03 \mathrm{E}-09$ & $4.81 \mathrm{E}-11$ & $4.81 \mathrm{E}-11$ & $4.81 \mathrm{E}-11$ & $4.50 \mathrm{E}-11$ & $1.87 \mathrm{E}-10$ & $1.12 \mathrm{E}-09$ \\
\hline $\mathrm{Th}+4$ & $2.87 \mathrm{E}-12$ & $8.88 \mathrm{E}-07$ & $1.54 \mathrm{E}-08$ & $1.54 \mathrm{E}-08$ & $1.54 \mathrm{E}-08$ & $1.53 \mathrm{E}-08$ & $8.73 \mathrm{E}-08$ & $9.66 \mathrm{E}-07$ \\
\hline $\mathrm{Sn}+4$ & $2.63 \mathrm{E}-03$ & $1.44 \mathrm{E}-03$ & $1.48 \mathrm{E}-04$ & $1.13 \mathrm{E}-02$ & $3.62 \mathrm{E}-04$ & $1.44 \mathrm{E}-03$ & $4.51 \mathrm{E}-01$ & 1.97E-05 \\
\hline $\mathrm{Ti}+4$ & $1.29 \mathrm{E}-03$ & $7.55 \mathrm{E}-04$ & $7.42 \mathrm{E}-05$ & $5.56 \mathrm{E}-03$ & 1.79E-04 & 7.09E-04 & 8.91E-02 & $6.12 \mathrm{E}-05$ \\
\hline $\mathrm{U}+4$ & $3.83 \mathrm{E}-05$ & $7.84 \mathrm{E}-04$ & $2.09 \mathrm{E}-05$ & $1.59 \mathrm{E}-04$ & $2.35 \mathrm{E}-05$ & $3.62 \mathrm{E}-05$ & $1.12 \mathrm{E}-02$ & $8.34 \mathrm{E}-04$ \\
\hline $\mathrm{V}+5$ & $1.12 \mathrm{E}-05$ & $7.76 \mathrm{E}-04$ & $1.73 \mathrm{E}-05$ & 4.97E-05 & $1.79 \mathrm{E}-05$ & $2.06 \mathrm{E}-05$ & $5.81 \mathrm{E}-04$ & $8.40 \mathrm{E}-04$ \\
\hline $\mathrm{Y}+3$ & $1.00 \mathrm{E}-07$ & $3.33 \mathrm{E}-06$ & $1.56 \mathrm{E}-07$ & $1.56 \mathrm{E}-07$ & $1.56 \mathrm{E}-07$ & $1.46 \mathrm{E}-07$ & $3.40 \mathrm{E}-07$ & $3.63 \mathrm{E}-06$ \\
\hline$Z n+2$ & $8.90 \mathrm{E}-05$ & 8.92E-04 & $2.10 \mathrm{E}-05$ & 3.93E-04 & $2.81 \mathrm{E}-05$ & $6.39 \mathrm{E}-05$ & $8.29 \mathrm{E}-03$ & $9.18 \mathrm{E}-04$ \\
\hline $\mathrm{Zr}+4$ & $5.35 \mathrm{E}-02$ & 2.94E-02 & $3.10 \mathrm{E}-03$ & $2.30 \mathrm{E}-01$ & $7.46 \mathrm{E}-03$ & $2.94 \mathrm{E}-02$ & $7.04 \mathrm{E}+00$ & 4.91E-04 \\
\hline $\mathrm{H} 2 \mathrm{O}$ & $5.55 \mathrm{E}+01$ & $4.76 \mathrm{E}+01$ & $5.55 \mathrm{E}+01$ & $5.42 \mathrm{E}+01$ & $5.54 \mathrm{E}+01$ & $5.47 \mathrm{E}+01$ & $5.70 \mathrm{E}+01$ & $4.72 \mathrm{E}+01$ \\
\hline Canisters/day & & & & & & & 0.59 & \\
\hline Canisters total & & & & & & & 87 & \\
\hline Radiological Composition & Ci/liter & Ci/liter & Ci/liter & Ci/liter & Ci/liter & Ci/liter & $\mathrm{Ci} / \mathrm{kg}$ & Ci/liter \\
\hline Ra-226 & $1.15 \mathrm{E}-13$ & $3.87 \mathrm{E}-12$ & $1.80 \mathrm{E}-13$ & $1.80 \mathrm{E}-13$ & $1.80 \mathrm{E}-13$ & $1.69 \mathrm{E}-13$ & $4.42 \mathrm{E}-14$ & $4.21 \mathrm{E}-12$ \\
\hline Ac-227 & $5.43 \mathrm{E}-13$ & $1.82 \mathrm{E}-11$ & $8.49 \mathrm{E}-13$ & $8.49 \mathrm{E}-13$ & $8.49 \mathrm{E}-13$ & $7.94 \mathrm{E}-13$ & $2.08 \mathrm{E}-13$ & $1.98 \mathrm{E}-11$ \\
\hline Th-228 & $4.51 \mathrm{E}-11$ & $1.51 \mathrm{E}-09$ & $7.04 \mathrm{E}-11$ & $7.04 \mathrm{E}-11$ & $7.04 \mathrm{E}-11$ & $6.59 \mathrm{E}-11$ & $1.73 \mathrm{E}-11$ & $1.64 \mathrm{E}-09$ \\
\hline Th-230 & $3.37 \mathrm{E}-10$ & $5.67 \mathrm{E}-10$ & $3.64 \mathrm{E}-11$ & 1.42E-09 & $6.29 \mathrm{E}-11$ & $1.96 \mathrm{E}-10$ & $4.71 \mathrm{E}-09$ & $4.25 \mathrm{E}-10$ \\
\hline Th-232 & $9.98 \mathrm{E}-18$ & $3.34 \mathrm{E}-16$ & $1.56 \mathrm{E}-17$ & $1.56 \mathrm{E}-17$ & $1.56 \mathrm{E}-17$ & $1.46 \mathrm{E}-17$ & $3.82 \mathrm{E}-18$ & $3.64 \mathrm{E}-16$ \\
\hline Pa-231 & $1.26 \mathrm{E}-12$ & $4.22 \mathrm{E}-11$ & $1.97 \mathrm{E}-12$ & $1.97 \mathrm{E}-12$ & $1.97 \mathrm{E}-12$ & $1.84 \mathrm{E}-12$ & $4.82 \mathrm{E}-13$ & $4.59 \mathrm{E}-11$ \\
\hline Pa-233 & $4.13 \mathrm{E}-08$ & $1.38 \mathrm{E}-06$ & $6.45 \mathrm{E}-08$ & $6.45 \mathrm{E}-08$ & $6.45 \mathrm{E}-08$ & $6.04 \mathrm{E}-08$ & $1.58 \mathrm{E}-08$ & $1.51 \mathrm{E}-06$ \\
\hline U-232 & $6.99 \mathrm{E}-10$ & $1.31 \mathrm{E}-09$ & $8.14 \mathrm{E}-11$ & $2.94 \mathrm{E}-09$ & $1.36 \mathrm{E}-10$ & $4.09 \mathrm{E}-10$ & $9.70 \mathrm{E}-09$ & $1.03 \mathrm{E}-09$ \\
\hline U-233 & $1.27 \mathrm{E}-11$ & $4.41 \mathrm{E}-11$ & $2.41 \mathrm{E}-12$ & $5.16 \mathrm{E}-11$ & $3.35 \mathrm{E}-12$ & $8.00 \mathrm{E}-12$ & $1.68 \mathrm{E}-10$ & $4.11 \mathrm{E}-11$ \\
\hline U-234 & $2.08 \mathrm{E}-07$ & $1.05 \mathrm{E}-06$ & $3.85 \mathrm{E}-08$ & $8.77 \mathrm{E}-07$ & $5.46 \mathrm{E}-08$ & 1.34E-07 & $2.85 \mathrm{E}-06$ & $1.03 \mathrm{E}-06$ \\
\hline U-235 & $1.09 \mathrm{E}-08$ & $4.15 \mathrm{E}-08$ & $1.45 \mathrm{E}-09$ & $4.68 \mathrm{E}-08$ & $2.32 \mathrm{E}-09$ & 6.67E-09 & $1.54 \mathrm{E}-07$ & $3.88 \mathrm{E}-08$ \\
\hline U-236 & $1.43 \mathrm{E}-08$ & $5.88 \mathrm{E}-08$ & $2.12 \mathrm{E}-09$ & $6.11 \mathrm{E}-08$ & $3.25 \mathrm{E}-09$ & $8.90 \mathrm{E}-09$ & $2.01 \mathrm{E}-07$ & $5.58 \mathrm{E}-08$ \\
\hline U-237 & $9.07 \mathrm{E}-11$ & $3.04 \mathrm{E}-09$ & $1.42 \mathrm{E}-10$ & $1.42 \mathrm{E}-10$ & $1.42 \mathrm{E}-10$ & $1.33 \mathrm{E}-10$ & $3.47 \mathrm{E}-11$ & $3.30 \mathrm{E}-09$ \\
\hline U-238 & $1.23 \mathrm{E}-09$ & $2.11 \mathrm{E}-08$ & $8.25 \mathrm{E}-10$ & $4.22 \mathrm{E}-09$ & $8.90 \mathrm{E}-10$ & $1.17 \mathrm{E}-09$ & 1.17E-08 & $2.24 \mathrm{E}-08$ \\
\hline Np-236 & $6.44 \mathrm{E}-14$ & $1.78 \mathrm{E}-12$ & $9.40 \mathrm{E}-14$ & $9.40 \mathrm{E}-14$ & $9.40 \mathrm{E}-14$ & $8.76 \mathrm{E}-14$ & $2.30 \mathrm{E}-14$ & $1.93 \mathrm{E}-12$ \\
\hline $\mathrm{Np}-237$ & 1.02E-07 & 1.33E-06 & $6.65 \mathrm{E}-08$ & $3.27 \mathrm{E}-07$ & $7.15 \mathrm{E}-08$ & $9.25 \mathrm{E}-08$ & 8.98E-07 & $1.42 \mathrm{E}-06$ \\
\hline Pu-236 & $1.22 \mathrm{E}-09$ & 1.93E-09 & $1.54 \mathrm{E}-10$ & 5.07E-09 & $2.49 \mathrm{E}-10$ & $7.17 \mathrm{E}-10$ & 1.67E-08 & $1.42 \mathrm{E}-09$ \\
\hline Pu-238 & $6.41 \mathrm{E}-04$ & $8.39 \mathrm{E}-04$ & $6.25 \mathrm{E}-05$ & $2.71 \mathrm{E}-03$ & $1.13 \mathrm{E}-04$ & 3.67E-04 & $8.99 \mathrm{E}-03$ & $5.46 \mathrm{E}-04$ \\
\hline Pu-239 & $6.72 \mathrm{E}-05$ & $1.08 \mathrm{E}-04$ & 5.95E-06 & $2.88 \mathrm{E}-04$ & $1.13 \mathrm{E}-05$ & 3.84E-05 & $9.56 \mathrm{E}-04$ & $7.85 \mathrm{E}-05$ \\
\hline Pu-240 & $4.68 \mathrm{E}-06$ & $7.26 \mathrm{E}-06$ & $5.49 \mathrm{E}-07$ & 1.95E-05 & $9.13 \mathrm{E}-07$ & 2.72E-06 & $6.45 \mathrm{E}-05$ & $5.27 \mathrm{E}-06$ \\
\hline Pu-241 & 5.07E-04 & 3.96E-04 & 4.54E-05 & $2.13 \mathrm{E}-03$ & 8.54E-05 & 2.86E-04 & 7.09E-03 & $1.41 \mathrm{E}-04$ \\
\hline Pu-242 & $3.69 \mathrm{E}-09$ & $5.60 \mathrm{E}-09$ & $5.76 \mathrm{E}-10$ & $1.49 \mathrm{E}-08$ & $8.50 \mathrm{E}-10$ & $2.20 \mathrm{E}-09$ & $4.87 \mathrm{E}-08$ & $4.11 \mathrm{E}-09$ \\
\hline Pu-244 & $2.89 \mathrm{E}-16$ & $4.69 \mathrm{E}-16$ & $2.21 \mathrm{E}-17$ & 1.25E-15 & $4.56 \mathrm{E}-17$ & $1.64 \mathrm{E}-16$ & $4.16 \mathrm{E}-15$ & $3.40 \mathrm{E}-16$ \\
\hline Am-241 & $1.71 \mathrm{E}-05$ & $7.06 \mathrm{E}-05$ & $2.80 \mathrm{E}-06$ & $7.18 \mathrm{E}-05$ & $4.12 \mathrm{E}-06$ & $1.07 \mathrm{E}-05$ & $2.35 \mathrm{E}-04$ & $6.73 \mathrm{E}-05$ \\
\hline Am-242m & $2.14 \mathrm{E}-10$ & $7.19 \mathrm{E}-09$ & $3.35 \mathrm{E}-10$ & $3.35 \mathrm{E}-10$ & $3.35 \mathrm{E}-10$ & $3.13 \mathrm{E}-10$ & $8.21 \mathrm{E}-11$ & $7.82 \mathrm{E}-09$ \\
\hline Am-243 & 6.07E-09 & 1.33E-08 & $7.96 \mathrm{E}-10$ & $2.53 \mathrm{E}-08$ & 1.27E-09 & 3.61E-09 & 8.34E-08 & 1.11E-08 \\
\hline $\mathrm{Cm}-242$ & $2.00 \mathrm{E}-10$ & $6.57 \mathrm{E}-09$ & $2.88 \mathrm{E}-10$ & $3.87 \mathrm{E}-10$ & $2.90 \mathrm{E}-10$ & $2.82 \mathrm{E}-10$ & $4.05 \mathrm{E}-10$ & $7.14 \mathrm{E}-09$ \\
\hline
\end{tabular}


Table B-9. Tank WM-180 mass balance (continued).

\begin{tabular}{|c|c|c|c|c|c|c|c|}
\hline Stream \# & 110 & 111 & 112 & 113 & 114 & 115 & 116 \\
\hline Stream Name & $\begin{array}{l}\text { Slurry from } \\
\text { TFF Tank } \\
\text { WM-187 }\end{array}$ & $\begin{array}{c}\text { Crossflow } \\
\text { Filter Recirc } \\
\text { Purge }\end{array}$ & $\begin{array}{l}\text { Decant } \\
\text { Liquid }\end{array}$ & $\begin{array}{c}\text { Solids } \\
\text { Treatment } \\
\text { Filter Feed }\end{array}$ & $\begin{array}{l}\text { Solids } \\
\text { Treatment } \\
\text { Filtrate }\end{array}$ & $\begin{array}{c}\text { Combined } \\
\text { Waste Water }\end{array}$ & $\begin{array}{l}\text { Dried Solids } \\
\quad \text { Waste }\end{array}$ \\
\hline & Ci/liter & Ci/liter & Ci/liter & $\mathrm{Ci} /$ liter & $\mathrm{Ci} /$ liter & $\mathrm{Ci} /$ liter & $\mathrm{Ci} / \mathrm{kg}$ \\
\hline $\mathrm{Cm}-243$ & $1.23 \mathrm{E}-08$ & 1.99E-08 & 1.29E-09 & $5.18 \mathrm{E}-08$ & 2.26E-09 & 7.10E-09 & 1.72E-07 \\
\hline $\mathrm{Cm}-244$ & $7.68 \mathrm{E}-07$ & $1.21 \mathrm{E}-06$ & $8.00 \mathrm{E}-08$ & $3.24 \mathrm{E}-06$ & $1.41 \mathrm{E}-07$ & 4.44E-07 & $1.08 \mathrm{E}-05$ \\
\hline $\mathrm{Cm}-245$ & $1.30 \mathrm{E}-10$ & $2.10 \mathrm{E}-10$ & $1.36 \mathrm{E}-11$ & $5.49 \mathrm{E}-10$ & $2.39 \mathrm{E}-11$ & $7.52 \mathrm{E}-11$ & 1.82E-09 \\
\hline $\mathrm{Cm}-246$ & $8.44 \mathrm{E}-12$ & $1.38 \mathrm{E}-11$ & $8.91 \mathrm{E}-13$ & $3.56 \mathrm{E}-11$ & $1.56 \mathrm{E}-12$ & $4.88 \mathrm{E}-12$ & $1.18 \mathrm{E}-10$ \\
\hline $\mathrm{H}-3$ & $392 F_{-07}$ & $157 F_{-05}$ & 6 57F-07 & 6 57F-07 & $657 \mathrm{~F}-07$ & 6 17F-07 & \\
\hline $\mathrm{Be}-10$ & $4.23 \mathrm{E}-14$ & $1.42 \mathrm{E}-12$ & $6.60 \mathrm{E}-14$ & $6.60 \mathrm{E}-14$ & $6.60 \mathrm{E}-14$ & $6.18 \mathrm{E}-14$ & $1.62 \mathrm{E}-14$ \\
\hline C-14 & $5.49 \mathrm{E}-11$ & $8.58 \mathrm{E}-11$ & $5.62 \mathrm{E}-12$ & $2.32 \mathrm{E}-10$ & $9.96 \mathrm{E}-12$ & $3.16 \mathrm{E}-11$ & $7.68 \mathrm{E}-10$ \\
\hline Se-79 & $1.98 \mathrm{E}-07$ & $3.11 \mathrm{E}-07$ & $2.04 \mathrm{E}-08$ & $8.35 \mathrm{E}-07$ & $3.60 \mathrm{E}-08$ & $1.14 \mathrm{E}-07$ & $2.77 \mathrm{E}-06$ \\
\hline $\mathrm{Rb}-87$ & $4.13 \mathrm{E}-13$ & $1.38 \mathrm{E}-11$ & $6.45 \mathrm{E}-13$ & $6.45 \mathrm{E}-13$ & $6.45 \mathrm{E}-13$ & $6.03 E-13$ & $1.58 \mathrm{E}-13$ \\
\hline Sr-90 & 1.06E-03 & 1.86E-02 & 9.97E-04 & $2.66 \mathrm{E}-03$ & 1.03E-03 & $1.12 \mathrm{E}-03$ & $5.88 \mathrm{E}-03$ \\
\hline $\mathrm{Y}-90$ & 1.06E-03 & 1.86E-02 & 9.97E-04 & 2.66E-03 & 1.03E-03 & 1.12E-03 & $5.88 \mathrm{E}-03$ \\
\hline$Z r-93$ & $3.12 \mathrm{E}-08$ & $1.05 \mathrm{E}-06$ & $4.88 \mathrm{E}-08$ & $4.88 \mathrm{E}-08$ & $4.88 \mathrm{E}-08$ & $4.56 \mathrm{E}-08$ & $1.19 \mathrm{E}-08$ \\
\hline Nb-93m & $2.41 \mathrm{E}-08$ & 8.06E-07 & $3.76 \mathrm{E}-08$ & $3.76 \mathrm{E}-08$ & $3.76 \mathrm{E}-08$ & $3.51 \mathrm{E}-08$ & $9.21 \mathrm{E}-09$ \\
\hline $\mathrm{Nb}-94$ & $5.10 \mathrm{E}-07$ & 8.09E-07 & $5.28 \mathrm{E}-08$ & $2.15 \mathrm{E}-06$ & 9.31E-08 & $2.94 \mathrm{E}-07$ & $7.14 \mathrm{E}-06$ \\
\hline Tc-98 & $3.63 E-14$ & $1.22 \mathrm{E}-12$ & $5.68 \mathrm{E}-14$ & $5.68 \mathrm{E}-14$ & $5.68 \mathrm{E}-14$ & $5.31 \mathrm{E}-14$ & $1.39 \mathrm{E}-14$ \\
\hline Tc-99 & $4.43 E-06$ & 1.07E-05 & $5.40 \mathrm{E}-07$ & 1.87E-05 & 8.89E-07 & $2.63 E-06$ & $6.18 \mathrm{E}-05$ \\
\hline Ru-106 & 4.27E-07 & 6.66E-07 & $4.37 \mathrm{E}-08$ & $1.80 \mathrm{E}-06$ & $7.75 \mathrm{E}-08$ & $2.46 \mathrm{E}-07$ & $5.98 \mathrm{E}-06$ \\
\hline Rh-102 & $4.14 \mathrm{E}-07$ & $2.28 \mathrm{E}-07$ & $2.32 \mathrm{E}-08$ & $1.78 \mathrm{E}-06$ & $5.70 \mathrm{E}-08$ & $2.27 \mathrm{E}-07$ & $5.98 \mathrm{E}-06$ \\
\hline Pd-107 & $2.33 \mathrm{E}-10$ & $7.80 \mathrm{E}-09$ & $3.64 \mathrm{E}-10$ & $3.64 \mathrm{E}-10$ & $3.64 \mathrm{E}-10$ & $3.40 \mathrm{E}-10$ & $8.91 \mathrm{E}-11$ \\
\hline Cd-113m & 4.68E-08 & 1.57E-06 & 7.31E-08 & 7.31E-08 & 7.31E-08 & 6.84E-08 & 1.79E-08 \\
\hline $\ln -115$ & $1.42 \mathrm{E}-18$ & $4.75 \mathrm{E}-17$ & $2.22 \mathrm{E}-18$ & $2.22 \mathrm{E}-18$ & $2.22 \mathrm{E}-18$ & $2.07 \mathrm{E}-18$ & $5.43 \mathrm{E}-19$ \\
\hline Sn-121m & $9.42 \mathrm{E}-10$ & $3.16 \mathrm{E}-08$ & 1.47E-09 & 1.47E-09 & 1.47E-09 & 1.38E-09 & $3.61 \mathrm{E}-10$ \\
\hline Sn-126 & 1.87E-07 & 2.93E-07 & $1.92 \mathrm{E}-08$ & 7.89E-07 & 3.39E-08 & 1.08E-07 & $2.61 \mathrm{E}-06$ \\
\hline Sb-125 & 5.93E-04 & 3.31E-04 & $3.38 \mathrm{E}-05$ & $2.56 \mathrm{E}-03$ & $8.22 \mathrm{E}-05$ & $3.26 \mathrm{E}-04$ & $8.56 \mathrm{E}-03$ \\
\hline Sb-126 & $8.10 \mathrm{E}-10$ & $2.71 \mathrm{E}-08$ & 1.27E-09 & 1.27E-09 & 1.27E-09 & 1.18E-09 & $3.10 \mathrm{E}-10$ \\
\hline Te-123 & $5.40 \mathrm{E}-21$ & $1.81 \mathrm{E}-19$ & $8.43 \mathrm{E}-21$ & $8.43 \mathrm{E}-21$ & $8.43 \mathrm{E}-21$ & $7.89 \mathrm{E}-21$ & $2.07 \mathrm{E}-21$ \\
\hline Te-125m & 4.44E-08 & $1.49 \mathrm{E}-06$ & $6.93 \mathrm{E}-08$ & 6.93E-08 & 6.93E-08 & $6.48 \mathrm{E}-08$ & 1.70E-08 \\
\hline I-129 & $2.42 \mathrm{E}-08$ & $3.48 \mathrm{E}-08$ & $2.44 \mathrm{E}-09$ & 1.02E-07 & $4.35 \mathrm{E}-09$ & $1.39 \mathrm{E}-08$ & $3.38 \mathrm{E}-07$ \\
\hline Cs-134 & $4.92 \mathrm{E}-05$ & $3.17 \mathrm{E}-05$ & $3.64 \mathrm{E}-06$ & $2.09 E-04$ & $7.58 \mathrm{E}-06$ & $2.74 \mathrm{E}-05$ & $6.99 \mathrm{E}-04$ \\
\hline Cs-135 & $3.49 \mathrm{E}-07$ & 5.91E-07 & $3.78 \mathrm{E}-08$ & 1.47E-06 & $6.54 \mathrm{E}-08$ & $2.02 \mathrm{E}-07$ & $4.88 \mathrm{E}-06$ \\
\hline Cs-137 & 1.98E-02 & $3.35 \mathrm{E}-02$ & $2.17 \mathrm{E}-03$ & $8.36 \mathrm{E}-02$ & $3.73 \mathrm{E}-03$ & $1.15 \mathrm{E}-02$ & $2.77 \mathrm{E}-01$ \\
\hline Ba-137m & 1.88E-02 & $3.17 \mathrm{E}-02$ & $2.05 \mathrm{E}-03$ & 7.91E-02 & $3.53 E-03$ & $1.09 \mathrm{E}-02$ & $2.62 \mathrm{E}-01$ \\
\hline La-138 & $2.69 \mathrm{E}-18$ & $9.01 \mathrm{E}-17$ & $4.20 \mathrm{E}-18$ & $4.20 \mathrm{E}-18$ & $4.20 \mathrm{E}-18$ & $3.93 \mathrm{E}-18$ & $1.03 \mathrm{E}-18$ \\
\hline Ce-142 & $4.21 \mathrm{E}-13$ & $1.41 \mathrm{E}-11$ & $6.58 \mathrm{E}-13$ & $6.58 \mathrm{E}-13$ & $6.58 \mathrm{E}-13$ & $6.16 \mathrm{E}-13$ & $1.61 \mathrm{E}-13$ \\
\hline Ce-144 & $2.90 \mathrm{E}-07$ & $4.50 \mathrm{E}-07$ & 2.96E-08 & $1.22 \mathrm{E}-06$ & $5.25 \mathrm{E}-08$ & 1.67E-07 & 4.06E-06 \\
\hline Nd-144 & $2.27 \mathrm{E}-17$ & $7.59 \mathrm{E}-16$ & $3.54 \mathrm{E}-17$ & $3.54 \mathrm{E}-17$ & $3.54 \mathrm{E}-17$ & $3.31 \mathrm{E}-17$ & $8.67 \mathrm{E}-18$ \\
\hline Pm-146 & $7.17 \mathrm{E}-10$ & $2.40 \mathrm{E}-08$ & 1.12E-09 & 1.12E-09 & $1.12 \mathrm{E}-09$ & $1.05 \mathrm{E}-09$ & $2.74 \mathrm{E}-10$ \\
\hline Pm-147 & $7.68 \mathrm{E}-05$ & $1.21 \mathrm{E}-04$ & 7.92E-06 & $3.24 \mathrm{E}-04$ & 1.40E-05 & 4.43E-05 & $1.08 \mathrm{E}-03$ \\
\hline Sm-146 & $3.89 \mathrm{E}-15$ & $1.30 \mathrm{E}-13$ & $6.07 \mathrm{E}-15$ & 6.07E-15 & $6.07 \mathrm{E}-15$ & $5.68 \mathrm{E}-15$ & $1.49 \mathrm{E}-15$ \\
\hline Sm-147 & $1.04 \mathrm{E}-13$ & $3.48 \mathrm{E}-12$ & $1.62 \mathrm{E}-13$ & $1.62 \mathrm{E}-13$ & $1.62 \mathrm{E}-13$ & $1.52 \mathrm{E}-13$ & $3.97 \mathrm{E}-14$ \\
\hline Sm-148 & 5.33E-19 & $1.79 \mathrm{E}-17$ & $8.33 \mathrm{E}-19$ & 8.33E-19 & 8.33E-19 & $7.79 \mathrm{E}-19$ & $2.04 \mathrm{E}-19$ \\
\hline Sm-149 & 4.73E-20 & $1.59 \mathrm{E}-18$ & $7.39 \mathrm{E}-20$ & $7.39 E-20$ & $7.39 \mathrm{E}-20$ & $6.92 \mathrm{E}-20$ & $1.81 \mathrm{E}-20$ \\
\hline Sm-151 & 1.53E-04 & $2.40 \mathrm{E}-04$ & 1.57E-05 & $6.47 \mathrm{E}-04$ & $2.78 \mathrm{E}-05$ & $8.84 \mathrm{E}-05$ & $2.15 \mathrm{E}-03$ \\
\hline Eu-152 & 5.55E-07 & 1.47E-06 & $8.45 \mathrm{E}-08$ & $2.29 \mathrm{E}-06$ & 1.27E-07 & $3.37 \mathrm{E}-07$ & $7.51 \mathrm{E}-06$ \\
\hline Eu-154 & $2.44 \mathrm{E}-05$ & 5.36E-05 & $5.49 \mathrm{E}-06$ & 9.38E-05 & $7.19 \mathrm{E}-06$ & $1.54 \mathrm{E}-05$ & $3.01 \mathrm{E}-04$ \\
\hline Eu-155 & $3.50 \mathrm{E}-05$ & 9.32E-05 & $4.19 \mathrm{E}-06$ & 1.49E-04 & 6.96E-06 & $2.08 \mathrm{E}-05$ & 4.91E-04 \\
\hline Gd-152 & $2.00 \mathrm{E}-20$ & $6.71 \mathrm{E}-19$ & $3.13 \mathrm{E}-20$ & $3.13 \mathrm{E}-20$ & $3.13 \mathrm{E}-20$ & $2.93 \mathrm{E}-20$ & $7.67 \mathrm{E}-21$ \\
\hline Ho-166m & $6.49 \mathrm{E}-13$ & $2.17 \mathrm{E}-11$ & $1.01 \mathrm{E}-12$ & $1.01 \mathrm{E}-12$ & $1.01 \mathrm{E}-12$ & $9.48 \mathrm{E}-13$ & $2.48 \mathrm{E}-13$ \\
\hline Co-60 & $2.81 \mathrm{E}-06$ & $5.09 \mathrm{E}-06$ & $1.11 \mathrm{E}-06$ & $8.98 \mathrm{E}-06$ & $1.26 \mathrm{E}-06$ & $1.93 \mathrm{E}-06$ & $2.70 \mathrm{E}-05$ \\
\hline $\mathrm{Ni}-63$ & 1.48E-05 & 2.95E-05 & $2.09 \mathrm{E}-06$ & $6.10 \mathrm{E}-05$ & $3.22 \mathrm{E}-06$ & 8.81E-06 & $2.00 \mathrm{E}-04$ \\
\hline
\end{tabular}


Table B-9. Tank WM-180 mass balance (continued).

\begin{tabular}{|c|c|c|c|c|c|c|c|c|}
\hline PFD Sheet \# & PFD-3 & PFD-3 & PFD-3 & PFD-3 & PFD-3 & PFD-3 & PFD-3 & PFD-3 \\
\hline Stream \# & 121 & 122 & 123 & 124 & 125 & 126 & 127 & 128 \\
\hline Stream Name & $\begin{array}{c}\text { Neutralized } \\
\text { Feed }\end{array}$ & $\begin{array}{l}\text { SBW Liquid } \\
\text { Filter Feed }\end{array}$ & $\begin{array}{c}\text { Filter } \\
\text { Recirculation }\end{array}$ & $\begin{array}{l}\text { IX Column } \\
\text { Feed }\end{array}$ & $\begin{array}{c}\text { Column } 1 \\
\text { Effluent }\end{array}$ & $\begin{array}{c}\text { Column } 2 \\
\text { Effluent }\end{array}$ & $\begin{array}{c}\text { Column } 3 \\
\text { Effluent }\end{array}$ & $\begin{array}{l}\text { Spent IX } \\
\text { Rinse }\end{array}$ \\
\hline Rate, gal/hr & 88.2 & 7800 & 7712 & 88.2 & 88.2 & 88.2 & 88.2 & 0.5 \\
\hline Rate, lb/hr & 925 & 81796 & 80871 & 925 & 925 & 925 & 925 & 5 \\
\hline Rate, peak, gpm & 1.47 & 130 & 129 & 1.47 & 1.47 & 1.47 & 1.47 & 2.99 \\
\hline Operation & Cont & Cont & Cont & Cont & Cont & Cont & Cont & Batch \\
\hline Temperature, ${ }^{\circ} \mathrm{F}$ & 70 & 70 & 70 & 70 & 70 & 70 & 70 & 70 \\
\hline Pressure, psia & 12.8 & 35.0 & 35.0 & 24.8 & 20.8 & 16.8 & 12.8 & 12.8 \\
\hline Specific Gravity & 1.26 & 1.26 & 1.26 & 1.26 & 1.26 & 1.26 & 1.26 & 1.05 \\
\hline Chemical Composition & Mol/liter & Mol/liter & Mol/liter & Mol/liter & Mol/liter & Mol/liter & Mol/liter & Mol/liter \\
\hline $\mathrm{H}+$ & $5.00 \mathrm{E}-01$ & $5.00 \mathrm{E}-01$ & $5.00 \mathrm{E}-01$ & $5.00 \mathrm{E}-01$ & $5.00 \mathrm{E}-01$ & $5.00 \mathrm{E}-01$ & $5.00 \mathrm{E}-01$ & $1.01 \mathrm{E}-01$ \\
\hline $\mathrm{Al}+3$ & $5.36 \mathrm{E}-01$ & $5.51 \mathrm{E}-01$ & $5.51 \mathrm{E}-01$ & $5.36 \mathrm{E}-01$ & 5.36E-01 & $5.36 \mathrm{E}-01$ & 5.36E-01 & $1.08 \mathrm{E}-01$ \\
\hline $\mathrm{Am}+4$ & $2.98 \mathrm{E}-08$ & $2.98 \mathrm{E}-08$ & $2.98 \mathrm{E}-08$ & $2.98 \mathrm{E}-08$ & $2.98 \mathrm{E}-08$ & $2.98 \mathrm{E}-08$ & $2.98 \mathrm{E}-08$ & $6.02 \mathrm{E}-09$ \\
\hline $\mathrm{Sb}+5$ & $6.61 \mathrm{E}-07$ & $9.34 \mathrm{E}-06$ & $9.44 \mathrm{E}-06$ & 5.59E-07 & $5.59 \mathrm{E}-07$ & 5.59E-07 & 5.59E-07 & 1.13E-07 \\
\hline As+5 & $4.15 \mathrm{E}-04$ & $4.88 \mathrm{E}-04$ & $4.89 \mathrm{E}-04$ & $4.14 \mathrm{E}-04$ & $4.14 \mathrm{E}-04$ & $4.14 \mathrm{E}-04$ & $4.14 \mathrm{E}-04$ & $8.36 \mathrm{E}-05$ \\
\hline $\mathrm{Ba}+2$ & $4.65 \mathrm{E}-05$ & $6.20 \mathrm{E}-05$ & $6.21 \mathrm{E}-05$ & $4.63 \mathrm{E}-05$ & $4.63 \mathrm{E}-05$ & $4.63 \mathrm{E}-05$ & $4.63 \mathrm{E}-05$ & $9.36 \mathrm{E}-06$ \\
\hline $\mathrm{Be}+2$ & $6.57 \mathrm{E}-06$ & $1.01 \mathrm{E}-05$ & $1.02 \mathrm{E}-05$ & $6.53 \mathrm{E}-06$ & $6.53 \mathrm{E}-06$ & $6.53 \mathrm{E}-06$ & $6.53 \mathrm{E}-06$ & $1.32 \mathrm{E}-06$ \\
\hline$B+3$ & $9.60 \mathrm{E}-03$ & $9.88 \mathrm{E}-03$ & $9.89 \mathrm{E}-03$ & $9.60 \mathrm{E}-03$ & $9.60 \mathrm{E}-03$ & $9.60 \mathrm{E}-03$ & $9.60 \mathrm{E}-03$ & $1.94 \mathrm{E}-03$ \\
\hline $\mathrm{Br}-$ & $1.56 \mathrm{E}-07$ & $1.56 \mathrm{E}-07$ & 1.56E-07 & $1.56 \mathrm{E}-07$ & $1.56 \mathrm{E}-07$ & $1.56 \mathrm{E}-07$ & $1.56 \mathrm{E}-07$ & $3.15 \mathrm{E}-08$ \\
\hline $\mathrm{Cd}+2$ & $6.86 \mathrm{E}-04$ & $7.32 \mathrm{E}-04$ & 7.33E-04 & $6.85 \mathrm{E}-04$ & $6.85 \mathrm{E}-04$ & $6.85 \mathrm{E}-04$ & $6.85 \mathrm{E}-04$ & $1.38 \mathrm{E}-04$ \\
\hline $\mathrm{Ca}+2$ & $3.43 \mathrm{E}-02$ & $3.51 \mathrm{E}-02$ & $3.51 \mathrm{E}-02$ & $3.43 \mathrm{E}-02$ & $3.43 \mathrm{E}-02$ & $3.43 \mathrm{E}-02$ & $3.43 \mathrm{E}-02$ & $6.93 \mathrm{E}-03$ \\
\hline $\mathrm{Ce}+4$ & $4.07 \mathrm{E}-05$ & $4.85 \mathrm{E}-05$ & $4.86 \mathrm{E}-05$ & $4.06 \mathrm{E}-05$ & $4.06 \mathrm{E}-05$ & $4.06 \mathrm{E}-05$ & $4.06 \mathrm{E}-05$ & $8.21 \mathrm{E}-06$ \\
\hline Cs+ & $1.01 \mathrm{E}-05$ & $4.18 \mathrm{E}-05$ & $4.22 \mathrm{E}-05$ & $9.70 \mathrm{E}-06$ & $9.70 \mathrm{E}-07$ & $9.70 \mathrm{E}-08$ & 9.70E-09 & 1.96E-09 \\
\hline $\mathrm{Cl}-$ & $2.68 \mathrm{E}-02$ & $2.85 \mathrm{E}-02$ & $2.85 \mathrm{E}-02$ & 2.67E-02 & $2.67 \mathrm{E}-02$ & $2.67 \mathrm{E}-02$ & 2.67E-02 & $5.41 \mathrm{E}-03$ \\
\hline $\mathrm{Cr}+3$ & $3.24 \mathrm{E}-03$ & $3.55 \mathrm{E}-03$ & $3.55 \mathrm{E}-03$ & $3.24 \mathrm{E}-03$ & $3.24 \mathrm{E}-03$ & $3.24 \mathrm{E}-03$ & $3.24 \mathrm{E}-03$ & $6.55 \mathrm{E}-04$ \\
\hline $\mathrm{Co}+2$ & $2.04 \mathrm{E}-04$ & 2.07E-04 & $2.07 \mathrm{E}-04$ & $2.04 \mathrm{E}-04$ & $2.04 \mathrm{E}-04$ & $2.04 \mathrm{E}-04$ & $2.04 \mathrm{E}-04$ & $4.12 \mathrm{E}-05$ \\
\hline $\mathrm{Cu}+2$ & $5.80 \mathrm{E}-04$ & $6.69 \mathrm{E}-04$ & $6.70 \mathrm{E}-04$ & $5.79 \mathrm{E}-04$ & 5.79E-04 & $5.79 \mathrm{E}-04$ & 5.79E-04 & 1.17E-04 \\
\hline $\mathrm{Eu}+3$ & $2.58 \mathrm{E}-07$ & $2.58 \mathrm{E}-07$ & $2.58 \mathrm{E}-07$ & $2.58 \mathrm{E}-07$ & $2.58 \mathrm{E}-07$ & $2.58 \mathrm{E}-07$ & $2.58 \mathrm{E}-07$ & $5.22 \mathrm{E}-08$ \\
\hline F- & $3.88 \mathrm{E}-02$ & $5.41 \mathrm{E}-02$ & $5.43 \mathrm{E}-02$ & $3.87 \mathrm{E}-02$ & 3.87E-02 & 3.87E-02 & 3.87E-02 & $7.81 \mathrm{E}-03$ \\
\hline $\mathrm{Gd}+3$ & $1.53 \mathrm{E}-04$ & $1.69 \mathrm{E}-04$ & $1.69 \mathrm{E}-04$ & $1.53 \mathrm{E}-04$ & $1.53 \mathrm{E}-04$ & $1.53 \mathrm{E}-04$ & 1.53E-04 & $3.09 \mathrm{E}-05$ \\
\hline $\mathrm{Ge}+4$ & $4.49 \mathrm{E}-09$ & 4.49E-09 & 4.49E-09 & 4.49E-09 & 4.49E-09 & $4.49 \mathrm{E}-09$ & 4.49E-09 & $9.08 \mathrm{E}-10$ \\
\hline $\ln +3$ & $7.10 \mathrm{E}-07$ & $7.10 \mathrm{E}-07$ & $7.10 \mathrm{E}-07$ & $7.10 \mathrm{E}-07$ & $7.10 \mathrm{E}-07$ & $7.10 \mathrm{E}-07$ & $7.10 \mathrm{E}-07$ & $1.44 \mathrm{E}-07$ \\
\hline I- & $1.29 \mathrm{E}-06$ & $1.29 \mathrm{E}-06$ & $1.29 \mathrm{E}-06$ & $1.29 \mathrm{E}-06$ & $1.29 \mathrm{E}-06$ & $1.29 \mathrm{E}-06$ & $1.29 \mathrm{E}-06$ & $2.62 \mathrm{E}-07$ \\
\hline $\mathrm{Fe}+3$ & 1.67E-02 & $2.27 \mathrm{E}-02$ & $2.28 \mathrm{E}-02$ & 1.67E-02 & 1.67E-02 & 1.67E-02 & 1.67E-02 & $3.37 \mathrm{E}-03$ \\
\hline $\mathrm{La+3}$ & $4.69 \mathrm{E}-06$ & $4.69 \mathrm{E}-06$ & $4.69 \mathrm{E}-06$ & $4.69 \mathrm{E}-06$ & $4.69 \mathrm{E}-06$ & $4.69 \mathrm{E}-06$ & $4.69 \mathrm{E}-06$ & $9.49 \mathrm{E}-07$ \\
\hline $\mathrm{Pb}+2$ & $1.18 \mathrm{E}-03$ & $1.21 \mathrm{E}-03$ & $1.21 \mathrm{E}-03$ & $1.18 \mathrm{E}-03$ & $1.18 \mathrm{E}-03$ & $1.18 \mathrm{E}-03$ & $1.18 \mathrm{E}-03$ & $2.39 \mathrm{E}-04$ \\
\hline $\mathrm{Li+}$ & $3.40 \mathrm{E}-04$ & $5.22 \mathrm{E}-04$ & $5.24 \mathrm{E}-04$ & 3.37E-04 & 3.37E-04 & 3.37E-04 & 3.37E-04 & $6.82 \mathrm{E}-05$ \\
\hline$M g+2$ & $1.07 \mathrm{E}-02$ & $1.13 \mathrm{E}-02$ & $1.13 \mathrm{E}-02$ & 1.07E-02 & 1.07E-02 & 1.07E-02 & 1.07E-02 & $2.17 \mathrm{E}-03$ \\
\hline $\mathrm{Mn}+4$ & $1.26 \mathrm{E}-02$ & $1.28 \mathrm{E}-02$ & $1.28 \mathrm{E}-02$ & $1.26 \mathrm{E}-02$ & $1.26 \mathrm{E}-02$ & $1.26 \mathrm{E}-02$ & $1.26 \mathrm{E}-02$ & $2.55 \mathrm{E}-03$ \\
\hline $\mathrm{Hg}+2$ & $1.27 \mathrm{E}-03$ & $1.28 \mathrm{E}-03$ & $1.28 \mathrm{E}-03$ & $1.27 \mathrm{E}-03$ & $1.27 \mathrm{E}-03$ & $1.27 \mathrm{E}-03$ & $1.27 \mathrm{E}-03$ & $2.56 \mathrm{E}-04$ \\
\hline $\mathrm{Mo}+6$ & $1.75 \mathrm{E}-04$ & $6.86 \mathrm{E}-04$ & $6.92 \mathrm{E}-04$ & $1.69 \mathrm{E}-04$ & $1.69 \mathrm{E}-04$ & $1.69 \mathrm{E}-04$ & $1.69 \mathrm{E}-04$ & $3.42 \mathrm{E}-05$ \\
\hline $\mathrm{Nd}+3$ & $1.51 \mathrm{E}-05$ & $1.51 \mathrm{E}-05$ & $1.51 \mathrm{E}-05$ & $1.51 \mathrm{E}-05$ & $1.51 \mathrm{E}-05$ & $1.51 \mathrm{E}-05$ & $1.51 \mathrm{E}-05$ & $3.06 \mathrm{E}-06$ \\
\hline $\mathrm{Np}+4$ & $8.17 \mathrm{E}-06$ & 8.17E-06 & 8.17E-06 & $8.17 \mathrm{E}-06$ & $8.17 \mathrm{E}-06$ & 8.17E-06 & 8.17E-06 & 1.65E-06 \\
\hline $\mathrm{Ni}+2$ & $1.30 \mathrm{E}-03$ & $1.41 \mathrm{E}-03$ & $1.41 \mathrm{E}-03$ & $1.30 \mathrm{E}-03$ & $1.30 \mathrm{E}-03$ & $1.30 \mathrm{E}-03$ & $1.30 \mathrm{E}-03$ & $2.63 \mathrm{E}-04$ \\
\hline $\mathrm{Nb}+5$ & $1.04 \mathrm{E}-05$ & 8.57E-04 & $8.66 \mathrm{E}-04$ & $4.03 \mathrm{E}-07$ & $4.03 \mathrm{E}-07$ & $4.03 E-07$ & $4.03 \mathrm{E}-07$ & $8.14 \mathrm{E}-08$ \\
\hline NO3- & $4.85 \mathrm{E}+00$ & $4.85 \mathrm{E}+00$ & $4.85 \mathrm{E}+00$ & $4.85 \mathrm{E}+00$ & $4.85 \mathrm{E}+00$ & $4.85 \mathrm{E}+00$ & $4.85 \mathrm{E}+00$ & $9.80 \mathrm{E}-01$ \\
\hline $\mathrm{O}-2$ & $1.51 \mathrm{E}-03$ & $1.30 \mathrm{E}-01$ & $1.31 \mathrm{E}-01$ & & & & & \\
\hline$P d+4$ & $2.31 \mathrm{E}-05$ & $1.43 \mathrm{E}-03$ & $1.45 \mathrm{E}-03$ & $6.50 \mathrm{E}-06$ & $6.50 \mathrm{E}-06$ & $6.50 \mathrm{E}-06$ & $6.50 \mathrm{E}-06$ & $1.31 \mathrm{E}-06$ \\
\hline PO4-3 & $1.23 \mathrm{E}-02$ & $9.18 \mathrm{E}-02$ & $9.27 \mathrm{E}-02$ & $1.14 \mathrm{E}-02$ & $1.14 \mathrm{E}-02$ & $1.14 \mathrm{E}-02$ & $1.14 \mathrm{E}-02$ & $2.30 \mathrm{E}-03$ \\
\hline $\mathrm{Pu}+4$ & $5.30 \mathrm{E}-06$ & $5.30 \mathrm{E}-06$ & $5.30 \mathrm{E}-06$ & $5.30 \mathrm{E}-06$ & $5.30 \mathrm{E}-06$ & $5.30 \mathrm{E}-06$ & $5.30 \mathrm{E}-06$ & $1.07 \mathrm{E}-06$ \\
\hline $\mathrm{K}+$ & $1.94 \mathrm{E}-01$ & 2.03E-01 & $2.03 \mathrm{E}-01$ & 1.93E-01 & 1.93E-01 & 1.93E-01 & 1.93E-01 & 3.91E-02 \\
\hline $\mathrm{Pr}+4$ & $4.27 \mathrm{E}-06$ & $4.27 \mathrm{E}-06$ & $4.27 \mathrm{E}-06$ & $4.27 \mathrm{E}-06$ & $4.27 \mathrm{E}-06$ & $4.27 \mathrm{E}-06$ & $4.27 \mathrm{E}-06$ & $8.63 \mathrm{E}-07$ \\
\hline $\mathrm{Rh}+4$ & $1.84 \mathrm{E}-06$ & $1.84 \mathrm{E}-06$ & $1.84 \mathrm{E}-06$ & $1.84 \mathrm{E}-06$ & $1.84 \mathrm{E}-06$ & $1.84 \mathrm{E}-06$ & $1.84 \mathrm{E}-06$ & $3.72 \mathrm{E}-07$ \\
\hline
\end{tabular}


Table B-9. Tank WM-180 mass balance (continued).

\begin{tabular}{|c|c|c|c|c|c|c|c|c|}
\hline Stream \# & 121 & 122 & 123 & 124 & 125 & 126 & 127 & 128 \\
\hline Stream Name & $\begin{array}{l}\text { Neutralized } \\
\text { Feed }\end{array}$ & $\begin{array}{l}\text { SBW Liquid } \\
\text { Filter Feed }\end{array}$ & $\begin{array}{c}\text { Filter } \\
\text { Recirculation }\end{array}$ & $\begin{array}{l}\text { IX Column } \\
\text { Feed }\end{array}$ & $\begin{array}{c}\text { Column } 1 \\
\text { Effluent }\end{array}$ & $\begin{array}{c}\text { Column } 2 \\
\text { Effluent }\end{array}$ & $\begin{array}{c}\text { Column } 3 \\
\text { Effluent }\end{array}$ & $\begin{array}{l}\text { Spent IX } \\
\text { Rinse }\end{array}$ \\
\hline Chemical Composition & Mol/liter & Mol/liter & Mol/liter & Mol/liter & Mol/liter & Mol/liter & Mol/liter & Mol/liter \\
\hline $\mathrm{Rb}+$ & $2.83 \mathrm{E}-06$ & $2.83 \mathrm{E}-06$ & $2.83 \mathrm{E}-06$ & $2.83 \mathrm{E}-06$ & $2.83 \mathrm{E}-06$ & $2.83 \mathrm{E}-06$ & $2.83 \mathrm{E}-06$ & $5.73 \mathrm{E}-07$ \\
\hline$R u+3$ & 1.16E-04 & $7.06 \mathrm{E}-04$ & 7.13E-04 & $1.09 \mathrm{E}-04$ & 1.09E-04 & $1.09 \mathrm{E}-04$ & 1.09E-04 & $2.21 \mathrm{E}-05$ \\
\hline $\mathrm{Sm}+3$ & $2.81 \mathrm{E}-06$ & $2.81 \mathrm{E}-06$ & $2.81 \mathrm{E}-06$ & $2.81 \mathrm{E}-06$ & $2.81 \mathrm{E}-06$ & 2.81E-06 & $2.81 \mathrm{E}-06$ & 5.67E-07 \\
\hline $\mathrm{Se}+4$ & $1.21 \mathrm{E}-05$ & $2.31 \mathrm{E}-04$ & $2.34 \mathrm{E}-04$ & $9.56 \mathrm{E}-06$ & $9.56 \mathrm{E}-06$ & $9.56 \mathrm{E}-06$ & $9.56 \mathrm{E}-06$ & $1.93 \mathrm{E}-06$ \\
\hline $\mathrm{Si+4}$ & 8.63E-04 & $7.30 \mathrm{E}-02$ & $7.38 \mathrm{E}-02$ & 1.38E-05 & 1.38E-05 & 1.38E-05 & 1.38E-05 & $2.79 \mathrm{E}-06$ \\
\hline$\overline{\mathrm{Ag}+}$ & $8.02 \mathrm{E}-06$ & $3.46 \mathrm{E}-04$ & $3.50 \mathrm{E}-04$ & $4.04 \mathrm{E}-06$ & $4.04 \mathrm{E}-06$ & $4.04 \mathrm{E}-06$ & 4.04E-06 & $8.16 \mathrm{E}-07$ \\
\hline $\mathrm{Na}+$ & $2.49 \mathrm{E}+00$ & $2.50 \mathrm{E}+00$ & $2.50 \mathrm{E}+00$ & $2.49 \mathrm{E}+00$ & $2.49 \mathrm{E}+00$ & $2.49 \mathrm{E}+00$ & $2.49 \mathrm{E}+00$ & 5.02E-01 \\
\hline$\widehat{\mathrm{Sr}+2}$ & 1.03E-04 & $1.08 \mathrm{E}-04$ & $1.08 \mathrm{E}-04$ & 1.03E-04 & 1.03E-04 & 1.03E-04 & 1.03E-04 & $2.09 \mathrm{E}-05$ \\
\hline SO4-2 & 4.59E-02 & $4.59 \mathrm{E}-02$ & $4.59 \mathrm{E}-02$ & 4.59E-02 & 4.59E-02 & 4.59E-02 & 4.59E-02 & $9.27 \mathrm{E}-03$ \\
\hline$T c+7$ & $5.26 \mathrm{E}-06$ & $5.26 \mathrm{E}-06$ & $5.26 \mathrm{E}-06$ & $5.26 \mathrm{E}-06$ & $5.26 \mathrm{E}-06$ & $5.26 \mathrm{E}-06$ & $5.26 \mathrm{E}-06$ & $1.06 \mathrm{E}-06$ \\
\hline $\mathrm{Te}+4$ & $1.54 \mathrm{E}-06$ & $1.54 \mathrm{E}-06$ & $1.54 \mathrm{E}-06$ & $1.54 \mathrm{E}-06$ & $1.54 \mathrm{E}-06$ & $1.54 \mathrm{E}-06$ & $1.54 \mathrm{E}-06$ & $3.12 \mathrm{E}-07$ \\
\hline$T b+4$ & $1.08 \mathrm{E}-09$ & $1.08 \mathrm{E}-09$ & $1.08 \mathrm{E}-09$ & $1.08 \mathrm{E}-09$ & $1.08 \mathrm{E}-09$ & $1.08 \mathrm{E}-09$ & $1.08 \mathrm{E}-09$ & $2.18 \mathrm{E}-10$ \\
\hline $\mathrm{Th}+4$ & 9.33E-07 & $9.33 \mathrm{E}-07$ & $9.33 \mathrm{E}-07$ & $9.33 \mathrm{E}-07$ & $9.33 \mathrm{E}-07$ & $9.33 \mathrm{E}-07$ & $9.33 \mathrm{E}-07$ & $1.88 \mathrm{E}-07$ \\
\hline$S n+4$ & $1.90 \mathrm{E}-05$ & $1.53 \mathrm{E}-03$ & $1.55 \mathrm{E}-03$ & $1.26 \mathrm{E}-06$ & $1.26 \mathrm{E}-06$ & $1.26 \mathrm{E}-06$ & $1.26 \mathrm{E}-06$ & $2.55 \mathrm{E}-07$ \\
\hline $\mathrm{Ti}+4$ & $5.91 \mathrm{E}-05$ & $7.99 \mathrm{E}-04$ & 8.07E-04 & $5.04 \mathrm{E}-05$ & $5.04 \mathrm{E}-05$ & $5.04 \mathrm{E}-05$ & $5.04 \mathrm{E}-05$ & $1.02 \mathrm{E}-05$ \\
\hline $\mathrm{U}+4$ & $8.05 \mathrm{E}-04$ & $8.23 \mathrm{E}-04$ & $8.24 \mathrm{E}-04$ & $8.05 \mathrm{E}-04$ & $8.05 \mathrm{E}-04$ & $8.05 \mathrm{E}-04$ & $8.05 \mathrm{E}-04$ & $1.63 \mathrm{E}-04$ \\
\hline$V+5$ & $8.10 \mathrm{E}-04$ & $8.14 \mathrm{E}-04$ & $8.14 \mathrm{E}-04$ & $8.10 \mathrm{E}-04$ & $8.10 \mathrm{E}-04$ & $8.10 \mathrm{E}-04$ & $8.10 \mathrm{E}-04$ & $1.64 \mathrm{E}-04$ \\
\hline$Y+3$ & $3.50 \mathrm{E}-06$ & $3.50 \mathrm{E}-06$ & $3.50 \mathrm{E}-06$ & $3.50 \mathrm{E}-06$ & $3.50 \mathrm{E}-06$ & $3.50 \mathrm{E}-06$ & $3.50 \mathrm{E}-06$ & $7.07 \mathrm{E}-07$ \\
\hline$Z n+2$ & 8.86E-04 & $9.36 \mathrm{E}-04$ & 9.37E-04 & $8.86 \mathrm{E}-04$ & 8.86E-04 & $8.86 \mathrm{E}-04$ & 8.86E-04 & $1.79 \mathrm{E}-04$ \\
\hline$Z r+4$ & $4.74 \mathrm{E}-04$ & $3.11 \mathrm{E}-02$ & $3.15 \mathrm{E}-02$ & $1.13 \mathrm{E}-04$ & $1.13 \mathrm{E}-04$ & $1.13 \mathrm{E}-04$ & 1.13E-04 & $2.28 \mathrm{E}-05$ \\
\hline $\mathrm{H} 2 \mathrm{O}$ & $4.77 \mathrm{E}+01$ & $4.68 \mathrm{E}+01$ & $4.68 \mathrm{E}+01$ & $4.77 \mathrm{E}+01$ & $4.77 \mathrm{E}+01$ & $4.77 \mathrm{E}+01$ & $4.77 \mathrm{E}+01$ & $5.36 \mathrm{E}+01$ \\
\hline & & & & & & & & \\
\hline Radiological Composition & Ci/liter & Ci/liter & Ci/liter & Ci/liter & $\mathrm{Ci} / \mathrm{liter}$ & Ci/liter & $\mathrm{Ci} / \mathrm{liter}$ & Ci/liter \\
\hline Ra-226 & $4.06 \mathrm{E}-12$ & $4.06 \mathrm{E}-12$ & $4.06 \mathrm{E}-12$ & 4.06E-12 & $4.06 \mathrm{E}-12$ & $4.06 \mathrm{E}-12$ & $4.06 \mathrm{E}-12$ & $8.20 \mathrm{E}-13$ \\
\hline Ac-227 & $1.91 \mathrm{E}-11$ & $1.91 \mathrm{E}-11$ & $1.91 \mathrm{E}-11$ & $1.91 \mathrm{E}-11$ & $1.91 \mathrm{E}-11$ & $1.91 \mathrm{E}-11$ & $1.91 \mathrm{E}-11$ & $3.86 \mathrm{E}-12$ \\
\hline Th-228 & 1.59E-09 & $1.59 \mathrm{E}-09$ & $1.59 \mathrm{E}-09$ & $1.59 \mathrm{E}-09$ & $1.59 \mathrm{E}-09$ & $1.59 \mathrm{E}-09$ & $1.59 \mathrm{E}-09$ & $3.21 \mathrm{E}-10$ \\
\hline Th-230 & $4.10 \mathrm{E}-10$ & $5.96 \mathrm{E}-10$ & $5.99 \mathrm{E}-10$ & $4.07 \mathrm{E}-10$ & $4.07 \mathrm{E}-10$ & $4.07 \mathrm{E}-10$ & $4.07 \mathrm{E}-10$ & $8.24 \mathrm{E}-11$ \\
\hline Th-232 & $3.51 \mathrm{E}-16$ & $6.30 \mathrm{E}-11$ & $3.51 \mathrm{E}-16$ & $3.51 \mathrm{E}-16$ & $3.51 \mathrm{E}-16$ & $3.51 \mathrm{E}-16$ & $3.51 \mathrm{E}-16$ & $7.09 \mathrm{E}-17$ \\
\hline $\mathrm{Pa}-231$ & $4.43 \mathrm{E}-11$ & $4.43 \mathrm{E}-11$ & $4.43 \mathrm{E}-11$ & $4.43 \mathrm{E}-11$ & $4.43 \mathrm{E}-11$ & $4.43 \mathrm{E}-11$ & $4.43 \mathrm{E}-11$ & $8.95 \mathrm{E}-12$ \\
\hline $\mathrm{Pa}-233$ & $1.45 \mathrm{E}-06$ & $1.45 \mathrm{E}-06$ & $1.45 \mathrm{E}-06$ & $1.45 \mathrm{E}-06$ & $1.45 \mathrm{E}-06$ & $1.45 \mathrm{E}-06$ & $1.45 \mathrm{E}-06$ & 2.94E-07 \\
\hline U-232 & $9.89 \mathrm{E}-10$ & 1.37E-09 & $1.38 \mathrm{E}-09$ & $9.85 \mathrm{E}-10$ & $9.85 \mathrm{E}-10$ & $9.85 \mathrm{E}-10$ & $9.85 \mathrm{E}-10$ & $1.99 \mathrm{E}-10$ \\
\hline U-233 & $3.97 \mathrm{E}-11$ & $4.63 \mathrm{E}-11$ & $4.64 \mathrm{E}-11$ & $3.96 \mathrm{E}-11$ & $3.96 \mathrm{E}-11$ & $3.96 \mathrm{E}-11$ & $3.96 \mathrm{E}-11$ & $8.01 \mathrm{E}-12$ \\
\hline U-234 & 9.95E-07 & $1.11 \mathrm{E}-06$ & $1.11 \mathrm{E}-06$ & $9.94 \mathrm{E}-07$ & 9.94E-07 & 9.94E-07 & 9.94E-07 & $2.01 \mathrm{E}-07$ \\
\hline U-235 & $3.75 \mathrm{E}-08$ & $4.36 \mathrm{E}-08$ & $4.37 \mathrm{E}-08$ & $3.74 \mathrm{E}-08$ & $3.74 \mathrm{E}-08$ & $3.74 \mathrm{E}-08$ & $3.74 \mathrm{E}-08$ & $7.56 \mathrm{E}-09$ \\
\hline U-236 & $5.38 \mathrm{E}-08$ & $6.17 \mathrm{E}-08$ & $6.18 \mathrm{E}-08$ & $5.37 \mathrm{E}-08$ & $5.37 \mathrm{E}-08$ & $5.37 \mathrm{E}-08$ & $5.37 \mathrm{E}-08$ & $1.09 \mathrm{E}-08$ \\
\hline U-237 & $3.19 \mathrm{E}-09$ & $3.19 \mathrm{E}-09$ & $3.19 \mathrm{E}-09$ & $3.19 \mathrm{E}-09$ & $3.19 \mathrm{E}-09$ & $3.19 \mathrm{E}-09$ & $3.19 \mathrm{E}-09$ & $6.45 \mathrm{E}-10$ \\
\hline U-238 & $2.17 \mathrm{E}-08$ & $2.21 \mathrm{E}-08$ & $2.21 \mathrm{E}-08$ & $2.16 \mathrm{E}-08$ & $2.16 \mathrm{E}-08$ & $2.16 \mathrm{E}-08$ & $2.16 \mathrm{E}-08$ & $4.37 \mathrm{E}-09$ \\
\hline Np-236 & $1.87 \mathrm{E}-12$ & $1.87 \mathrm{E}-12$ & $1.87 \mathrm{E}-12$ & $1.87 \mathrm{E}-12$ & $1.87 \mathrm{E}-12$ & $1.87 \mathrm{E}-12$ & $1.87 \mathrm{E}-12$ & $3.77 \mathrm{E}-13$ \\
\hline $\mathrm{Np}-237$ & $1.37 \mathrm{E}-06$ & $1.40 \mathrm{E}-06$ & $1.40 \mathrm{E}-06$ & $1.37 \mathrm{E}-06$ & $1.37 \mathrm{E}-06$ & $1.37 \mathrm{E}-06$ & $1.37 \mathrm{E}-06$ & $2.76 \mathrm{E}-07$ \\
\hline Pu-236 & $1.37 \mathrm{E}-09$ & $2.04 \mathrm{E}-09$ & $2.04 \mathrm{E}-09$ & $1.36 \mathrm{E}-09$ & $1.36 \mathrm{E}-09$ & $1.36 \mathrm{E}-09$ & 1.36E-09 & $2.76 \mathrm{E}-10$ \\
\hline Pu-238 & $5.27 \mathrm{E}-04$ & 8.83E-04 & $8.88 \mathrm{E}-04$ & $5.22 \mathrm{E}-04$ & $5.22 \mathrm{E}-04$ & $5.22 \mathrm{E}-04$ & 5.22E-04 & $1.06 \mathrm{E}-04$ \\
\hline Pu-239 & $7.57 \mathrm{E}-05$ & $1.14 \mathrm{E}-04$ & $1.14 \mathrm{E}-04$ & $7.53 \mathrm{E}-05$ & $7.53 \mathrm{E}-05$ & $7.53 \mathrm{E}-05$ & $7.53 \mathrm{E}-05$ & $1.52 \mathrm{E}-05$ \\
\hline Pu-240 & $5.08 \mathrm{E}-06$ & $7.64 \mathrm{E}-06$ & 7.67E-06 & $5.05 \mathrm{E}-06$ & $5.05 \mathrm{E}-06$ & $5.05 \mathrm{E}-06$ & $5.05 \mathrm{E}-06$ & $1.02 \mathrm{E}-06$ \\
\hline Pu-241 & 1.37E-04 & $4.18 \mathrm{E}-04$ & $4.21 \mathrm{E}-04$ & $1.33 \mathrm{E}-04$ & $1.33 \mathrm{E}-04$ & $1.33 \mathrm{E}-04$ & 1.33E-04 & $2.69 \mathrm{E}-05$ \\
\hline Pu-242 & $3.96 \mathrm{E}-09$ & $5.89 \mathrm{E}-09$ & 5.92E-09 & $3.94 \mathrm{E}-09$ & $3.94 \mathrm{E}-09$ & $3.94 \mathrm{E}-09$ & $3.94 \mathrm{E}-09$ & $7.97 \mathrm{E}-10$ \\
\hline Pu-244 & $3.28 \mathrm{E}-16$ & $4.93 \mathrm{E}-16$ & $4.95 \mathrm{E}-16$ & $3.26 \mathrm{E}-16$ & $3.26 \mathrm{E}-16$ & $3.26 \mathrm{E}-16$ & $3.26 \mathrm{E}-16$ & $6.59 \mathrm{E}-17$ \\
\hline Am-241 & $6.49 \mathrm{E}-05$ & $7.42 \mathrm{E}-05$ & 7.43E-05 & $6.48 \mathrm{E}-05$ & $6.48 \mathrm{E}-05$ & $6.48 \mathrm{E}-05$ & $6.48 \mathrm{E}-05$ & $1.31 \mathrm{E}-05$ \\
\hline Am-242m & $7.55 \mathrm{E}-09$ & $7.55 \mathrm{E}-09$ & $7.55 \mathrm{E}-09$ & $7.55 \mathrm{E}-09$ & $7.55 \mathrm{E}-09$ & $7.55 \mathrm{E}-09$ & $7.55 \mathrm{E}-09$ & $1.52 \mathrm{E}-09$ \\
\hline Am-243 & $1.07 \mathrm{E}-08$ & $1.40 \mathrm{E}-08$ & $1.40 \mathrm{E}-08$ & $1.06 \mathrm{E}-08$ & $1.06 \mathrm{E}-08$ & $1.06 \mathrm{E}-08$ & $1.06 \mathrm{E}-08$ & $2.15 \mathrm{E}-09$ \\
\hline $\mathrm{Cm}-242$ & $6.89 \mathrm{E}-09$ & 6.90E-09 & 6.90E-09 & $6.89 \mathrm{E}-09$ & 6.89E-09 & 6.89E-09 & $6.89 \mathrm{E}-09$ & $1.39 \mathrm{E}-09$ \\
\hline
\end{tabular}


Table B-9. Tank WM-180 mass balance (continued).

\begin{tabular}{|c|c|c|c|c|c|c|c|c|}
\hline Stream \# & 121 & 122 & 123 & 124 & 125 & 126 & 127 & 128 \\
\hline Stream Name & $\begin{array}{l}\text { Neutralized } \\
\text { Feed }\end{array}$ & $\begin{array}{l}\text { SBW Liquid } \\
\text { Filter Feed }\end{array}$ & $\begin{array}{c}\text { Filter } \\
\text { Recirculation }\end{array}$ & $\begin{array}{l}\text { IX Column } \\
\text { Feed }\end{array}$ & $\begin{array}{c}\text { Column } 1 \\
\text { Effluent }\end{array}$ & $\begin{array}{c}\text { Column } 2 \\
\text { Effluent }\end{array}$ & $\begin{array}{c}\text { Column } 3 \\
\text { Effluent }\end{array}$ & $\begin{array}{l}\text { Spent IX } \\
\text { Rinse }\end{array}$ \\
\hline & Ci/liter & $\mathrm{Ci} / \mathrm{liter}$ & $\mathrm{Ci} /$ liter & $\mathrm{Ci} / \mathrm{liter}$ & $\mathrm{Ci} /$ liter & $\mathrm{Ci} / \mathrm{liter}$ & $\mathrm{Ci} /$ liter & $\mathrm{Ci} /$ liter \\
\hline \begin{tabular}{|l|}
$\mathrm{Cm}-243$ \\
\end{tabular} & 1.42E-08 & $2.10 \mathrm{E}-08$ & $2.11 \mathrm{E}-08$ & $1.41 \mathrm{E}-08$ & $1.41 \mathrm{E}-08$ & 1.41E-08 & $1.41 \mathrm{E}-08$ & $2.85 \mathrm{E}-09$ \\
\hline$\overline{\mathrm{Cm}-244}$ & 8.48E-07 & $1.28 \mathrm{E}-06$ & $1.28 \mathrm{E}-06$ & 8.43E-07 & 8.43E-07 & 8.43E-07 & $8.43 \mathrm{E}-07$ & $1.70 \mathrm{E}-07$ \\
\hline $\mathrm{Cm}-245$ & $1.49 \mathrm{E}-10$ & $2.21 \mathrm{E}-10$ & $2.22 \mathrm{E}-10$ & $1.48 \mathrm{E}-10$ & $1.48 \mathrm{E}-10$ & $1.48 \mathrm{E}-10$ & $1.48 \mathrm{E}-10$ & $2.99 \mathrm{E}-11$ \\
\hline $\mathrm{Cm}-246$ & $9.80 \mathrm{E}-12$ & $1.45 \mathrm{E}-11$ & $1.45 \mathrm{E}-11$ & $9.74 \mathrm{E}-12$ & $9.74 \mathrm{E}-12$ & $9.74 \mathrm{E}-12$ & $9.74 \mathrm{E}-12$ & $1.97 \mathrm{E}-12$ \\
\hline $\mathrm{H}-3$ & $1.65 \mathrm{E}-05$ & $1.65 \mathrm{E}-05$ & $1.65 \mathrm{E}-05$ & $1.65 \mathrm{E}-05$ & $1.65 \mathrm{E}-05$ & $1.65 \mathrm{E}-05$ & $1.65 \mathrm{E}-05$ & $3.34 \mathrm{E}-06$ \\
\hline Be-10 & $1.49 \mathrm{E}-12$ & $1.49 \mathrm{E}-12$ & $1.49 \mathrm{E}-12$ & $1.49 \mathrm{E}-12$ & $1.49 \mathrm{E}-12$ & $1.49 \mathrm{E}-12$ & $1.49 \mathrm{E}-12$ & $3.01 \mathrm{E}-13$ \\
\hline $\begin{array}{l}\mathrm{C}-14 \\
\end{array}$ & 5.98E-11 & $9.03 \mathrm{E}-11$ & $9.07 \mathrm{E}-11$ & 5.95E-11 & 5.95E-11 & $5.95 \mathrm{E}-11$ & $5.95 \mathrm{E}-11$ & $1.20 \mathrm{E}-11$ \\
\hline Se-79 & $2.18 \mathrm{E}-07$ & $3.28 \mathrm{E}-07$ & $3.29 \mathrm{E}-07$ & $2.16 \mathrm{E}-07$ & $2.16 \mathrm{E}-07$ & $2.16 \mathrm{E}-07$ & $2.16 \mathrm{E}-07$ & $4.37 \mathrm{E}-08$ \\
\hline Rb-87 & $1.45 \mathrm{E}-11$ & $1.45 \mathrm{E}-11$ & $1.45 \mathrm{E}-11$ & $1.45 \mathrm{E}-11$ & $1.45 \mathrm{E}-11$ & $1.45 \mathrm{E}-11$ & $1.45 \mathrm{E}-11$ & $2.93 \mathrm{E}-12$ \\
\hline Sr-90 & 1.93E-02 & 1.96E-02 & 1.96E-02 & 1.93E-02 & 1.93E-02 & 1.93E-02 & 1.93E-02 & $3.91 \mathrm{E}-03$ \\
\hline Y-90 & 1.93E-02 & 1.96E-02 & 1.96E-02 & 1.93E-02 & 1.93E-02 & 1.93E-02 & 1.93E-02 & $3.91 \mathrm{E}-03$ \\
\hline Zr-93 & $1.10 \mathrm{E}-06$ & $1.10 \mathrm{E}-06$ & $1.10 \mathrm{E}-06$ & $1.10 \mathrm{E}-06$ & $1.10 \mathrm{E}-06$ & $1.10 \mathrm{E}-06$ & $1.10 \mathrm{E}-06$ & $2.22 \mathrm{E}-07$ \\
\hline Nb-93m & $8.46 \mathrm{E}-07$ & 8.46E-07 & 8.46E-07 & $8.46 \mathrm{E}-07$ & 8.46E-07 & 8.46E-07 & $8.46 \mathrm{E}-07$ & $1.71 \mathrm{E}-07$ \\
\hline Nb-94 & 5.68E-07 & $8.51 \mathrm{E}-07$ & $8.54 \mathrm{E}-07$ & 5.65E-07 & 5.65E-07 & 5.65E-07 & 5.65E-07 & $1.14 \mathrm{E}-07$ \\
\hline Tc-98 & $1.28 \mathrm{E}-12$ & $1.28 \mathrm{E}-12$ & $1.28 \mathrm{E}-12$ & $1.28 \mathrm{E}-12$ & $1.28 \mathrm{E}-12$ & $1.28 \mathrm{E}-12$ & $1.28 \mathrm{E}-12$ & $2.58 \mathrm{E}-13$ \\
\hline Tc-99 & $8.85 \mathrm{E}-06$ & $1.13 \mathrm{E}-05$ & $1.13 \mathrm{E}-05$ & $8.82 \mathrm{E}-06$ & $8.82 \mathrm{E}-06$ & $8.82 \mathrm{E}-06$ & $8.82 \mathrm{E}-06$ & $1.78 \mathrm{E}-06$ \\
\hline Ru-106 & 4.64E-07 & $7.01 \mathrm{E}-07$ & $7.04 \mathrm{E}-07$ & $4.61 \mathrm{E}-07$ & $4.61 \mathrm{E}-07$ & $4.61 \mathrm{E}-07$ & $4.61 \mathrm{E}-07$ & $9.31 \mathrm{E}-08$ \\
\hline Rh-102 & $3.23 \mathrm{E}-09$ & $2.41 \mathrm{E}-07$ & $2.43 \mathrm{E}-07$ & $4.27 \mathrm{E}-10$ & $4.27 \mathrm{E}-10$ & $4.27 \mathrm{E}-10$ & $4.27 \mathrm{E}-10$ & $8.63 \mathrm{E}-11$ \\
\hline Pd-107 & $8.19 \mathrm{E}-09$ & 8.19E-09 & 8.19E-09 & $8.19 \mathrm{E}-09$ & $8.19 \mathrm{E}-09$ & $8.19 \mathrm{E}-09$ & $8.19 \mathrm{E}-09$ & $1.65 \mathrm{E}-09$ \\
\hline Cd-113m & $1.65 \mathrm{E}-06$ & $1.65 \mathrm{E}-06$ & $1.65 \mathrm{E}-06$ & $1.65 \mathrm{E}-06$ & $1.65 \mathrm{E}-06$ & 1.65E-06 & $1.65 \mathrm{E}-06$ & 3.33E-07 \\
\hline In-115 & $4.99 \mathrm{E}-17$ & $4.99 \mathrm{E}-17$ & $4.99 \mathrm{E}-17$ & $4.99 \mathrm{E}-17$ & $4.99 \mathrm{E}-17$ & $4.99 \mathrm{E}-17$ & $4.99 \mathrm{E}-17$ & $1.01 \mathrm{E}-17$ \\
\hline Sn-121m & $3.31 \mathrm{E}-08$ & $3.31 \mathrm{E}-08$ & $3.31 \mathrm{E}-08$ & $3.31 \mathrm{E}-08$ & $3.31 \mathrm{E}-08$ & $3.31 \mathrm{E}-08$ & $3.31 \mathrm{E}-08$ & $6.70 \mathrm{E}-09$ \\
\hline Sn-126 & $2.05 \mathrm{E}-07$ & $3.09 \mathrm{E}-07$ & $3.10 \mathrm{E}-07$ & $2.04 \mathrm{E}-07$ & 2.04E-07 & $2.04 \mathrm{E}-07$ & $2.04 \mathrm{E}-07$ & $4.11 \mathrm{E}-08$ \\
\hline Sb-125 & $1.04 \mathrm{E}-05$ & $3.50 \mathrm{E}-04$ & $3.54 \mathrm{E}-04$ & $6.40 \mathrm{E}-06$ & $6.40 \mathrm{E}-06$ & $6.40 \mathrm{E}-06$ & $6.40 \mathrm{E}-06$ & $1.29 \mathrm{E}-06$ \\
\hline Sb-126 & $2.85 \mathrm{E}-08$ & $2.85 \mathrm{E}-08$ & $2.85 \mathrm{E}-08$ & $2.85 \mathrm{E}-08$ & $2.85 \mathrm{E}-08$ & $2.85 \mathrm{E}-08$ & $2.85 \mathrm{E}-08$ & $5.76 \mathrm{E}-09$ \\
\hline Te-123 & $1.90 \mathrm{E}-19$ & $1.90 \mathrm{E}-19$ & $1.90 \mathrm{E}-19$ & $1.90 \mathrm{E}-19$ & $1.90 \mathrm{E}-19$ & $1.90 \mathrm{E}-19$ & $1.90 \mathrm{E}-19$ & $3.84 \mathrm{E}-20$ \\
\hline $\mathrm{Te}-125 \mathrm{~m}$ & $1.56 \mathrm{E}-06$ & $1.56 \mathrm{E}-06$ & $1.56 \mathrm{E}-06$ & $1.56 \mathrm{E}-06$ & $1.56 \mathrm{E}-06$ & $1.56 \mathrm{E}-06$ & $1.56 \mathrm{E}-06$ & $3.15 \mathrm{E}-07$ \\
\hline $1-129$ & $2.33 \mathrm{E}-08$ & $3.67 \mathrm{E}-08$ & $3.68 \mathrm{E}-08$ & $2.31 \mathrm{E}-08$ & $2.31 \mathrm{E}-08$ & $2.31 \mathrm{E}-08$ & $2.31 \mathrm{E}-08$ & $4.67 \mathrm{E}-09$ \\
\hline Cs-134 & $5.75 \mathrm{E}-06$ & $3.35 \mathrm{E}-05$ & $3.38 \mathrm{E}-05$ & $5.42 \mathrm{E}-06$ & 5.42E-07 & $5.42 \mathrm{E}-08$ & $5.42 \mathrm{E}-09$ & $1.10 \mathrm{E}-09$ \\
\hline Cs-135 & $4.28 \mathrm{E}-07$ & $6.22 \mathrm{E}-07$ & $6.24 \mathrm{E}-07$ & $4.26 \mathrm{E}-07$ & $4.26 \mathrm{E}-08$ & $4.26 \mathrm{E}-09$ & $4.26 \mathrm{E}-10$ & $8.61 \mathrm{E}-11$ \\
\hline Cs-137 & $2.42 \mathrm{E}-02$ & $3.52 \mathrm{E}-02$ & $3.53 \mathrm{E}-02$ & $2.41 \mathrm{E}-02$ & $2.41 \mathrm{E}-03$ & $2.41 \mathrm{E}-04$ & $2.41 \mathrm{E}-05$ & $4.87 \mathrm{E}-06$ \\
\hline Ba-137m & $2.29 \mathrm{E}-02$ & $3.33 \mathrm{E}-02$ & $3.34 \mathrm{E}-02$ & $2.28 \mathrm{E}-02$ & $2.28 \mathrm{E}-02$ & $2.28 \mathrm{E}-02$ & $2.28 \mathrm{E}-02$ & $4.61 \mathrm{E}-03$ \\
\hline La-138 & $9.46 \mathrm{E}-17$ & $9.46 \mathrm{E}-17$ & $9.46 \mathrm{E}-17$ & $9.46 \mathrm{E}-17$ & $9.46 \mathrm{E}-17$ & $9.46 \mathrm{E}-17$ & $9.46 \mathrm{E}-17$ & $1.91 \mathrm{E}-17$ \\
\hline Ce-142 & $1.48 \mathrm{E}-11$ & $1.48 \mathrm{E}-11$ & $1.48 \mathrm{E}-11$ & $1.48 \mathrm{E}-11$ & $1.48 \mathrm{E}-11$ & $1.48 \mathrm{E}-11$ & $1.48 \mathrm{E}-11$ & $2.99 \mathrm{E}-12$ \\
\hline Ce-144 & $3.12 \mathrm{E}-07$ & $4.74 \mathrm{E}-07$ & $4.75 \mathrm{E}-07$ & $3.11 \mathrm{E}-07$ & $3.11 \mathrm{E}-07$ & $3.11 \mathrm{E}-07$ & $3.11 \mathrm{E}-07$ & $6.28 \mathrm{E}-08$ \\
\hline Nd-144 & $7.97 \mathrm{E}-16$ & $7.97 \mathrm{E}-16$ & $7.97 \mathrm{E}-16$ & $7.97 \mathrm{E}-16$ & $7.97 \mathrm{E}-16$ & $7.97 \mathrm{E}-16$ & $7.97 \mathrm{E}-16$ & $1.61 \mathrm{E}-16$ \\
\hline $\mathrm{Pm}-146$ & $2.52 \mathrm{E}-08$ & $2.52 \mathrm{E}-08$ & $2.52 \mathrm{E}-08$ & $2.52 \mathrm{E}-08$ & $2.52 \mathrm{E}-08$ & $2.52 \mathrm{E}-08$ & $2.52 \mathrm{E}-08$ & $5.10 \mathrm{E}-09$ \\
\hline Pm-147 & $8.49 \mathrm{E}-05$ & $1.28 \mathrm{E}-04$ & $1.28 \mathrm{E}-04$ & $8.44 \mathrm{E}-05$ & $8.44 \mathrm{E}-05$ & $8.44 \mathrm{E}-05$ & $8.44 \mathrm{E}-05$ & $1.71 \mathrm{E}-05$ \\
\hline Sm-146 & $1.37 \mathrm{E}-13$ & $1.37 \mathrm{E}-13$ & $1.37 \mathrm{E}-13$ & $1.37 \mathrm{E}-13$ & $1.37 \mathrm{E}-13$ & $1.37 \mathrm{E}-13$ & $1.37 \mathrm{E}-13$ & $2.76 \mathrm{E}-14$ \\
\hline Sm-147 & $3.65 \mathrm{E}-12$ & $3.65 \mathrm{E}-12$ & $3.65 \mathrm{E}-12$ & $3.65 \mathrm{E}-12$ & $3.65 \mathrm{E}-12$ & $3.65 \mathrm{E}-12$ & $3.65 \mathrm{E}-12$ & $7.38 \mathrm{E}-13$ \\
\hline Sm-148 & $1.88 \mathrm{E}-17$ & $1.88 \mathrm{E}-17$ & $1.88 \mathrm{E}-17$ & $1.88 \mathrm{E}-17$ & $1.88 \mathrm{E}-17$ & $1.88 \mathrm{E}-17$ & $1.88 \mathrm{E}-17$ & $3.79 \mathrm{E}-18$ \\
\hline Sm-149 & $1.66 \mathrm{E}-18$ & $1.66 \mathrm{E}-18$ & $1.66 \mathrm{E}-18$ & $1.66 \mathrm{E}-18$ & $1.66 \mathrm{E}-18$ & $1.66 \mathrm{E}-18$ & $1.66 \mathrm{E}-18$ & $3.36 \mathrm{E}-19$ \\
\hline Sm-151 & 1.67E-04 & $2.52 \mathrm{E}-04$ & $2.53 \mathrm{E}-04$ & $1.66 \mathrm{E}-04$ & $1.66 \mathrm{E}-04$ & $1.66 \mathrm{E}-04$ & $1.66 \mathrm{E}-04$ & $3.36 \mathrm{E}-05$ \\
\hline Eu-152 & $1.25 \mathrm{E}-06$ & $1.55 \mathrm{E}-06$ & $1.55 \mathrm{E}-06$ & $1.25 \mathrm{E}-06$ & $1.25 \mathrm{E}-06$ & $1.25 \mathrm{E}-06$ & $1.25 \mathrm{E}-06$ & $2.52 \mathrm{E}-07$ \\
\hline Eu-154 & $4.45 \mathrm{E}-05$ & $5.64 \mathrm{E}-05$ & $5.65 \mathrm{E}-05$ & $4.43 \mathrm{E}-05$ & $4.43 \mathrm{E}-05$ & $4.43 \mathrm{E}-05$ & $4.43 \mathrm{E}-05$ & $8.96 \mathrm{E}-06$ \\
\hline Eu-155 & $7.85 \mathrm{E}-05$ & $9.80 \mathrm{E}-05$ & $9.82 \mathrm{E}-05$ & 7.83E-05 & 7.83E-05 & 7.83E-05 & $7.83 \mathrm{E}-05$ & $1.58 \mathrm{E}-05$ \\
\hline Gd-152 & $7.04 \mathrm{E}-19$ & $7.04 \mathrm{E}-19$ & $7.04 \mathrm{E}-19$ & $7.04 \mathrm{E}-19$ & $7.04 \mathrm{E}-19$ & $7.04 \mathrm{E}-19$ & $7.04 \mathrm{E}-19$ & $1.42 \mathrm{E}-19$ \\
\hline Ho-166m & $2.28 \mathrm{E}-11$ & $2.28 \mathrm{E}-11$ & $2.28 \mathrm{E}-11$ & $2.28 \mathrm{E}-11$ & $2.28 \mathrm{E}-11$ & $2.28 \mathrm{E}-11$ & $2.28 \mathrm{E}-11$ & $4.61 \mathrm{E}-12$ \\
\hline Co-60 & $4.29 \mathrm{E}-06$ & $5.35 \mathrm{E}-06$ & $5.37 \mathrm{E}-06$ & $4.28 \mathrm{E}-06$ & $4.28 \mathrm{E}-06$ & $4.28 \mathrm{E}-06$ & $4.28 \mathrm{E}-06$ & $8.65 \mathrm{E}-07$ \\
\hline Ni-63 & $2.31 \mathrm{E}-05$ & $3.10 \mathrm{E}-05$ & $3.11 \mathrm{E}-05$ & $2.30 \mathrm{E}-05$ & $2.30 \mathrm{E}-05$ & $2.30 \mathrm{E}-05$ & $2.30 \mathrm{E}-05$ & $4.64 \mathrm{E}-06$ \\
\hline
\end{tabular}


Table B-9. Tank WM-180 mass balance (continued).

\begin{tabular}{|c|c|c|c|c|c|c|c|c|}
\hline PFD Sheet \# & & PFD-4 & PFD Sheet \# & & PFD-4 & & & PFD-4 \\
\hline Stream \# & 129 & 130 & Stream \# & 129 & 130 & & 129 & 130 \\
\hline Stream Name & $\begin{array}{c}\text { Spent IX } \\
\text { Media }\end{array}$ & $\begin{array}{l}\text { Grouted } \\
\text { Waste }\end{array}$ & Stream Name & $\begin{array}{l}\text { Spent IX } \\
\text { Media }\end{array}$ & $\begin{array}{l}\text { Grouted } \\
\text { Waste }\end{array}$ & & $\begin{array}{c}\text { Spent IX } \\
\text { Media }\end{array}$ & $\begin{array}{l}\text { Grouted } \\
\text { Waste }\end{array}$ \\
\hline Rate, gal/hr & 0.08 & 99 & & Wt $\%$ & Wt $\%$ & & $\mathrm{Ci} / \mathrm{kg}$ & $\mathrm{Ci} / \mathrm{kg}$ \\
\hline Rate, Ib/hr & 0.93 & 1233 & $\mathrm{Rb}+$ & $1.22 \mathrm{E}-06$ & $1.44 \mathrm{E}-05$ & $\mathrm{Cm}-243$ & $2.85 \mathrm{E}-09$ & $8.39 \mathrm{E}-09$ \\
\hline Rate, total columns & 5 & $\mathrm{~N} / \mathrm{A}$ & $\mathrm{Ru}+3$ & $5.58 \mathrm{E}-05$ & $6.58 \mathrm{E}-04$ & $\mathrm{Cm}-244$ & $1.70 \mathrm{E}-07$ & 5.03E-07 \\
\hline Operation & Batch & Cont & $\mathrm{Sm}+3$ & $2.13 \mathrm{E}-06$ & $2.51 \mathrm{E}-05$ & $\mathrm{Cm}-245$ & $2.99 \mathrm{E}-11$ & $8.81 \mathrm{E}-11$ \\
\hline Temperature, ${ }^{\circ} \mathrm{F}$ & 70 & 70 & $\mathrm{Se}+4$ & $3.81 \mathrm{E}-06$ & $4.50 \mathrm{E}-05$ & $\mathrm{Cm}-246$ & & \\
\hline Pressure, psia & 12.8 & 12.8 & $\mathrm{Si+4}$ & 1.96E-06 & $2.31 \mathrm{E}-05$ & & 1.97E-12 & $5.81 \mathrm{E}-12$ \\
\hline Specific Gravity & 1.33 & 1.50 & $\mathrm{Ag}+^{+}$ & $2.20 \mathrm{E}-06$ & $2.60 \mathrm{E}-05$ & $\mathrm{H}-3$ & 3.34E-06 & $9.85 \mathrm{E}-06$ \\
\hline Chemical Composition & Wt \% & Wt \% & $\mathrm{Na}+$ & $2.89 \mathrm{E}-01$ & $3.41 \mathrm{E}+00$ & $\mathrm{Be}-10$ & $3.01 \mathrm{E}-13$ & $8.86 \mathrm{E}-13$ \\
\hline CST & $7.30 \mathrm{E}+01$ & $\mathrm{~N} / \mathrm{A}$ & $\mathrm{Sr}+2$ & $4.57 \mathrm{E}-05$ & $5.40 \mathrm{E}-04$ & C-14 & $1.20 \mathrm{E}-11$ & $3.54 \mathrm{E}-11$ \\
\hline $\mathrm{H}+$ & $2.55 \mathrm{E}-03$ & N/A & SO4-2 & $2.22 \mathrm{E}-02$ & $2.63 \mathrm{E}-01$ & Se-79 & $4.37 \mathrm{E}-08$ & $1.29 \mathrm{E}-07$ \\
\hline $\mathrm{Al}+3$ & $7.30 \mathrm{E}-02$ & $8.62 \mathrm{E}-01$ & $\mathrm{Tc}+7$ & $2.58 \mathrm{E}-06$ & $3.04 \mathrm{E}-05$ & Rb-87 & $2.93 \mathrm{E}-12$ & $8.65 \mathrm{E}-12$ \\
\hline$A m+4$ & $3.63 \mathrm{E}-08$ & $4.28 \mathrm{E}-07$ & $\mathrm{Te}+4$ & 9.96E-07 & 1.17E-05 & Sr-90 & $3.91 \mathrm{E}-03$ & 1.15E-02 \\
\hline $\mathrm{Sb}+5$ & $3.44 \mathrm{E}-07$ & $4.05 \mathrm{E}-06$ & $\mathrm{~Tb}+4$ & $8.67 \mathrm{E}-10$ & $1.02 \mathrm{E}-08$ & $Y-90$ & $3.91 \mathrm{E}-03$ & $1.15 \mathrm{E}-02$ \\
\hline$A s+5$ & 1.57E-04 & $1.85 \mathrm{E}-03$ & $\mathrm{Th}+4$ & $1.09 \mathrm{E}-06$ & $1.29 \mathrm{E}-05$ & Zr-93 & $2.22 \mathrm{E}-07$ & $6.54 \mathrm{E}-07$ \\
\hline $\mathrm{Ba}+2$ & $3.21 \mathrm{E}-05$ & $3.79 \mathrm{E}-04$ & $\mathrm{Sn}+4$ & 7.58E-07 & $8.94 \mathrm{E}-06$ & $\mathrm{Nb}-93 \mathrm{~m}$ & $1.71 \mathrm{E}-07$ & $5.04 \mathrm{E}-07$ \\
\hline $\mathrm{Be}+2$ & $2.97 \mathrm{E}-07$ & $3.51 \mathrm{E}-06$ & $\mathrm{Ti}+4$ & $1.22 \mathrm{E}-05$ & $1.44 \mathrm{E}-04$ & Nb-94 & $1.14 \mathrm{E}-07$ & $3.36 \mathrm{E}-07$ \\
\hline $\mathrm{B}+3$ & $5.24 \mathrm{E}-04$ & $6.18 \mathrm{E}-03$ & $\mathrm{U}+4$ & 9.67E-04 & $1.14 \mathrm{E}-02$ & Tc-98 & $2.58 \mathrm{E}-13$ & $7.62 \mathrm{E}-13$ \\
\hline $\mathrm{Br}-$ & $6.29 \mathrm{E}-08$ & 7.42E-07 & $\mathrm{V}+5$ & $2.08 \mathrm{E}-04$ & $2.46 \mathrm{E}-03$ & Tc-99 & $1.78 \mathrm{E}-06$ & $5.26 \mathrm{E}-06$ \\
\hline$C d+2$ & $3.89 \mathrm{E}-04$ & $4.59 \mathrm{E}-03$ & $\mathrm{Y}+3$ & 1.57E-06 & $1.85 \mathrm{E}-05$ & Ru-106 & 9.31E-08 & $2.75 \mathrm{E}-07$ \\
\hline $\mathrm{Ca}+2$ & $6.95 \mathrm{E}-03$ & $8.20 \mathrm{E}-02$ & $Z n+2$ & 2.93E-04 & $3.45 \mathrm{E}-03$ & Rh-102 & 8.63E-11 & $2.55 \mathrm{E}-10$ \\
\hline $\mathrm{Ce}+4$ & 2.87E-05 & 3.39E-04 & $\mathrm{Zr}+4$ & $5.20 \mathrm{E}-05$ & $6.14 \mathrm{E}-04$ & $\mathrm{Pd}-107$ & 1.65E-09 & 4.88E-09 \\
\hline Cs+ & $6.51 \mathrm{E}-09$ & 7.68E-08 & $\mathrm{H} 2 \mathrm{O}$ & $2.50 \mathrm{E}+01$ & & Cd-113m & $3.33 \mathrm{E}-07$ & 9.81E-07 \\
\hline $\mathrm{Cl}-$ & 4.79E-03 & 5.65E-02 & Canisters total & 5 & & $\ln -115$ & $1.01 \mathrm{E}-17$ & $2.97 \mathrm{E}-17$ \\
\hline $\mathrm{Cr}+3$ & $8.51 \mathrm{E}-04$ & $1.00 \mathrm{E}-02$ & \begin{tabular}{|l|} 
Drums/day \\
\end{tabular} & & 46 & Sn-121m & 6.70E-09 & 1.97E-08 \\
\hline $\mathrm{Co}+2$ & $6.07 \mathrm{E}-05$ & $7.16 \mathrm{E}-04$ & Radiological Comp. & $\mathrm{Ci} / \mathrm{kg}$ & $\mathrm{Ci} / \mathrm{kg}$ & \begin{tabular}{|l|} 
Sn-126 \\
\end{tabular} & $4.11 \mathrm{E}-08$ & $1.21 \mathrm{E}-07$ \\
\hline $\mathrm{Cu}+2$ & $1.86 \mathrm{E}-04$ & $2.19 \mathrm{E}-03$ & Ra-226 & $8.20 \mathrm{E}-13$ & $2.42 \mathrm{E}-12$ & Sb-125 & $1.29 \mathrm{E}-06$ & $3.81 \mathrm{E}-06$ \\
\hline $\mathrm{Eu}+3$ & $1.98 \mathrm{E}-07$ & $2.34 \mathrm{E}-06$ & Ac-227 & $3.86 \mathrm{E}-12$ & $1.14 \mathrm{E}-11$ & Sb-126 & 5.76E-09 & $1.70 \mathrm{E}-08$ \\
\hline F- & $3.71 \mathrm{E}-03$ & $4.38 \mathrm{E}-02$ & Th-228 & $3.21 \mathrm{E}-10$ & $9.45 \mathrm{E}-10$ & Te-123 & $3.84 \mathrm{E}-20$ & 1.13E-19 \\
\hline $\mathrm{Gd}+3$ & $1.22 \mathrm{E}-04$ & $1.43 \mathrm{E}-03$ & Th-230 & $8.24 \mathrm{E}-11$ & $2.43 \mathrm{E}-10$ & Te-125m & $3.15 \mathrm{E}-07$ & $9.30 \mathrm{E}-07$ \\
\hline $\mathrm{Ge}+4$ & $1.65 \mathrm{E}-09$ & $1.94 \mathrm{E}-08$ & Th-232 & $7.09 \mathrm{E}-17$ & $2.09 \mathrm{E}-16$ & $1-129$ & 4.67E-09 & $1.38 \mathrm{E}-08$ \\
\hline $\ln +3$ & $4.12 \mathrm{E}-07$ & $4.86 \mathrm{E}-06$ & $\mathrm{~Pa}-231$ & $8.95 \mathrm{E}-12$ & $2.64 \mathrm{E}-11$ & Cs-134 & $1.10 \mathrm{E}-09$ & $3.23 \mathrm{E}-09$ \\
\hline I- & $8.29 \mathrm{E}-07$ & $9.79 \mathrm{E}-06$ & $\mathrm{~Pa}-233$ & 2.94E-07 & $8.66 \mathrm{E}-07$ & Cs-135 & $8.61 \mathrm{E}-11$ & $2.54 \mathrm{E}-10$ \\
\hline $\mathrm{Fe}+3$ & $4.70 \mathrm{E}-03$ & $5.54 \mathrm{E}-02$ & $\mathrm{U}-232$ & $1.99 \mathrm{E}-10$ & $5.87 \mathrm{E}-10$ & Cs-137 & $4.87 \mathrm{E}-06$ & $1.44 \mathrm{E}-05$ \\
\hline $\mathrm{La}+3$ & $3.29 \mathrm{E}-06$ & $3.89 \mathrm{E}-05$ & $\mathrm{U}-233$ & $8.01 \mathrm{E}-12$ & $2.36 \mathrm{E}-11$ & Ba-137m & 4.61E-06 & $1.36 \mathrm{E}-05$ \\
\hline $\mathrm{Pb}+2$ & $1.24 \mathrm{E}-03$ & $1.46 \mathrm{E}-02$ & \begin{tabular}{|l|}
$U-234$ \\
\end{tabular} & $2.01 \mathrm{E}-07$ & $5.92 \mathrm{E}-07$ & \begin{tabular}{|l} 
La-138 \\
\end{tabular} & $1.91 \mathrm{E}-17$ & $5.64 \mathrm{E}-17$ \\
\hline $\mathrm{Li}+$ & $1.18 \mathrm{E}-05$ & $1.40 \mathrm{E}-04$ & $\mathrm{U}-235$ & 7.56E-09 & $2.23 \mathrm{E}-08$ & Ce-142 & $2.99 \mathrm{E}-12$ & $8.83 \mathrm{E}-12$ \\
\hline $\mathrm{Mg}+2$ & $1.32 \mathrm{E}-03$ & $1.55 \mathrm{E}-02$ & U-236 & $1.09 \mathrm{E}-08$ & $3.20 \mathrm{E}-08$ & $\mathrm{Ce}-144$ & $6.28 \mathrm{E}-08$ & 1.85E-07 \\
\hline $\mathrm{Mn}+4$ & $3.50 \mathrm{E}-03$ & $4.13 \mathrm{E}-02$ & \begin{tabular}{|l|}
$\mathrm{U}-237$ \\
\end{tabular} & $6.45 \mathrm{E}-10$ & $1.90 \mathrm{E}-09$ & Nd-144 & $1.61 \mathrm{E}-16$ & $4.75 \mathrm{E}-16$ \\
\hline $\mathrm{Hg}+2$ & $1.29 \mathrm{E}-03$ & $1.52 \mathrm{E}-02$ & U-238 & $4.37 \mathrm{E}-09$ & $1.29 \mathrm{E}-08$ & Pm-146 & $5.10 \mathrm{E}-09$ & $1.50 \mathrm{E}-08$ \\
\hline $\mathrm{Mo}+6$ & $8.20 \mathrm{E}-05$ & $9.67 \mathrm{E}-04$ & Np-236 & $3.77 \mathrm{E}-13$ & $1.11 \mathrm{E}-12$ & Pm-147 & $1.71 \mathrm{E}-05$ & $5.03 \mathrm{E}-05$ \\
\hline $\mathrm{Nd}+3$ & $1.10 \mathrm{E}-05$ & $1.30 \mathrm{E}-04$ & $\mathrm{~Np}-237$ & $2.76 \mathrm{E}-07$ & $8.14 \mathrm{E}-07$ & Sm-146 & $2.76 \mathrm{E}-14$ & $8.15 \mathrm{E}-14$ \\
\hline $\mathrm{Np}+4$ & $9.78 \mathrm{E}-06$ & $1.15 \mathrm{E}-04$ & Pu-236 & $2.76 \mathrm{E}-10$ & $8.13 \mathrm{E}-10$ & Sm-147 & $7.38 \mathrm{E}-13$ & $2.18 \mathrm{E}-12$ \\
\hline $\mathrm{Ni}+2$ & $3.86 \mathrm{E}-04$ & $4.55 \mathrm{E}-03$ & Pu-238 & 1.06E-04 & $3.11 \mathrm{E}-04$ & Sm-148 & $3.79 \mathrm{E}-18$ & $1.12 \mathrm{E}-17$ \\
\hline $\mathrm{Nb}+5$ & $1.89 \mathrm{E}-07$ & $2.23 \mathrm{E}-06$ & Pu-239 & $1.52 \mathrm{E}-05$ & $4.49 \mathrm{E}-05$ & Sm-149 & $3.36 \mathrm{E}-19$ & $9.92 \mathrm{E}-19$ \\
\hline NO3- & $1.52 \mathrm{E}+00$ & $1.79 \mathrm{E}+01$ & Pu-240 & $1.02 \mathrm{E}-06$ & $3.01 \mathrm{E}-06$ & Sm-151 & $3.36 \mathrm{E}-05$ & $9.91 \mathrm{E}-05$ \\
\hline $\mathrm{Pd}+4$ & $3.49 \mathrm{E}-06$ & $4.12 \mathrm{E}-05$ & Pu-241 & $2.69 \mathrm{E}-05$ & 7.94E-05 & Eu-152 & $2.52 \mathrm{E}-07$ & $7.43 \mathrm{E}-07$ \\
\hline PO4-3 & $5.46 \mathrm{E}-03$ & $6.44 \mathrm{E}-02$ & Pu-242 & $7.97 \mathrm{E}-10$ & $2.35 \mathrm{E}-09$ & Eu-154 & 8.96E-06 & $2.64 \mathrm{E}-05$ \\
\hline $\mathrm{Pu}+4$ & $6.40 \mathrm{E}-06$ & $7.52 \mathrm{E}-05$ & Pu-244 & $6.59 \mathrm{E}-17$ & $1.94 \mathrm{E}-16$ & Eu-155 & $1.58 \mathrm{E}-05$ & 4.67E-05 \\
\hline $\mathrm{K}+$ & $3.82 \mathrm{E}-02$ & $4.51 \mathrm{E}-01$ & Am-241 & $1.31 \mathrm{E}-05$ & $3.86 \mathrm{E}-05$ & Gd-152 & 1.42E-19 & $4.20 \mathrm{E}-19$ \\
\hline $\mathrm{Pr}+4$ & $3.04 \mathrm{E}-06$ & $3.58 \mathrm{E}-05$ & Am-242m & $1.52 \mathrm{E}-09$ & $4.50 \mathrm{E}-09$ & Ho-166m & $4.61 \mathrm{E}-12$ & $1.36 \mathrm{E}-11$ \\
\hline \multirow[t]{2}{*}{$\mathrm{Rh}+4$} & 9.57E-07 & $1.13 \mathrm{E}-05$ & Am-243 & $2.15 \mathrm{E}-09$ & $6.34 \mathrm{E}-09$ & Co-60 & 8.65E-07 & $2.55 \mathrm{E}-06$ \\
\hline & & & Cm-242 & $1.39 \mathrm{E}-09$ & $4.11 \mathrm{E}-09$ & $\mathrm{Ni}-63$ & 4.64E-06 & 1.37E-05 \\
\hline
\end{tabular}


Table B-9. Tank WM-180 mass balance (continued).

\begin{tabular}{|c|c|c|c|c|c|}
\hline & PFD-2 & PFD-2 & PFD-2 & PFD-3 & PFD-3 \\
\hline & 210 & 211 & 212 & 213 & 220 \\
\hline Stream Name & $\begin{array}{c}\text { Solids } \\
\text { Flocculent }\end{array}$ & $\begin{array}{c}\text { Steam to } \\
\text { Fundabac } \\
\text { Filter }\end{array}$ & Filter Condensate & Nitric Acid & $\begin{array}{c}50 \% \\
\text { Caustic }\end{array}$ \\
\hline Rate, gal/hr & 0.1 & 0.8 & 4.6 & TBD & 3.1 \\
\hline Rate, lb/hr & 1.7 & 6.4 & 38 & & 39 \\
\hline Rate, peak, gpm & Batch & 0.20 & 1.2 & & 10 \\
\hline Operation & Batch & Batch & Batch & & Batch \\
\hline Temperature, ${ }^{\circ} \mathrm{F}$ & 75 & 328 & 114 & & 70 \\
\hline Pressure, psia & 12.8 & 112.8 & 12.8 & 0.0 & 12.8 \\
\hline Specific Gravity & 1.51 & 1.00 & 1.00 & 100 & 1.51 \\
\hline Stream Composition & Wt \% & Wt \% & Wt \% & Wt \% & Wt \% \\
\hline $50 \% \mathrm{NaOH}$ & $100 \%$ & & & & $100 \%$ \\
\hline $\mathrm{HNO} 3$ & & & & $100 \%$ & \\
\hline Steam & & $100 \%$ & & & \\
\hline Water & & & $100 \%$ & & \\
\hline & & & & & \\
\hline \multicolumn{6}{|l|}{ CST } \\
\hline \multicolumn{6}{|l|}{ Portland cement } \\
\hline \multicolumn{6}{|l|}{$\mathrm{Ca}(\mathrm{OH}) 2$} \\
\hline Blast furnace slag & & & & & \\
\hline
\end{tabular}

\begin{tabular}{|c|c|c|c|c|c|}
\hline PFD Sheet \# & PFD-3 & $\begin{array}{l}\text { PFD-4 } \\
\end{array}$ & PFD-4 & PFD-4 & PFD-4 \\
\hline Stream \# & 221 & 222 & 223 & 224 & 225 \\
\hline Stream Name & $\begin{array}{c}\text { Fresh IX } \\
\text { Media }\end{array}$ & $\begin{array}{l}\text { Portland } \\
\text { Cement }\end{array}$ & Calcium Hydroxide & $\begin{array}{c}\text { Blast } \\
\text { Furnace } \\
\text { Slag }\end{array}$ & Solid Blend \\
\hline Rate, gal/hr & 0.08 & 14.81 & 21.59 & 7.40 & 36.40 \\
\hline Rate, $\mathrm{lb} / \mathrm{hr}$ & 0.67 & 148 & 86 & 74 & 308 \\
\hline Rate, peak, gpm & $\mathrm{NA}$ & TBD & TBD & TBD & 0.61 \\
\hline Operation & Batch & Cont & Cont & Cont & Cont \\
\hline Temperature, ${ }^{\circ} \mathrm{F}$ & 70 & 70 & 70 & 70 & 70 \\
\hline Pressure, psia & 12.8 & 12.8 & 12.8 & 12.8 & 12.8 \\
\hline \multirow[t]{2}{*}{ Specific Gravity } & 0.96 & 1.20 & 0.48 & 1.20 & 1.02 \\
\hline & Wt \% & Wt \% & Wt \% & Wt \% & Wt \% \\
\hline \multicolumn{6}{|l|}{ Chemical Composition } \\
\hline \multicolumn{6}{|l|}{$\mathrm{NaOH}$} \\
\hline \multicolumn{6}{|l|}{$\mathrm{H} 2 \mathrm{O}$} \\
\hline CST & $100 \%$ & & & & \\
\hline Portland cement & & $100 \%$ & & & \\
\hline $\mathrm{Ca}(\mathrm{OH}) 2$ & & & $100 \%$ & & \\
\hline Blast furnace slag & & & & $100 \%$ & \\
\hline No. Replacement col's & 5 & & & & \\
\hline & & & & & \\
\hline & & & & & \\
\hline & & & & & \\
\hline & & & & & \\
\hline & & & & & \\
\hline
\end{tabular}


Table B-10. Tank WM-188 mass balance.

\begin{tabular}{|c|c|c|c|c|c|c|c|c|}
\hline PFD Sheet \# & PFD-2 & PFD-2 & PFD-2 & PFD-2 & PFD-2 & PFD-2 & PFD-2 & PFD-3 \\
\hline Stream \# & 110 & 111 & 112 & 113 & 114 & 115 & 116 & 120 \\
\hline Stream Name & $\begin{array}{l}\text { Slurry from } \\
\text { TFF Tank } \\
\text { WM-187 }\end{array}$ & $\begin{array}{c}\text { Crossflow } \\
\text { Filter Recirc } \\
\text { Purge }\end{array}$ & $\begin{array}{l}\text { Decant } \\
\text { Liquid }\end{array}$ & $\begin{array}{c}\text { Solids } \\
\text { Treatment } \\
\text { Filter Feed }\end{array}$ & $\begin{array}{l}\text { Solids } \\
\text { Treatment } \\
\text { Filtrate }\end{array}$ & $\begin{array}{c}\text { Combined } \\
\text { Waste Water }\end{array}$ & $\begin{array}{l}\text { Dried Solids } \\
\text { Waste }\end{array}$ & $\begin{array}{c}\text { Liquid SBW } \\
\text { from Tank } \\
\text { WM-180 }\end{array}$ \\
\hline Rate, gal/hr & 65.9 & 1.8 & 55.9 & 14.7 & 8.3 & 67.9 & 6.3 & 85.2 \\
\hline Rate, Ib/hr & 571 & 19 & 475 & 138 & 71 & 577 & 36 & 930 \\
\hline Rate, peak, gpm & 50 & 0.030 & 50 & 10 & 0.14 & 190 & 4.32 & 50.00 \\
\hline Operation & Batch & Cont & Batch & Batch & Batch & Batch & Batch & Batch \\
\hline Temperature, ${ }^{\circ} \mathrm{F}$ & 70 & 83 & 70 & 70 & 70 & 70 & 70 & 70 \\
\hline Pressure, psia & 12.8 & 12.8 & 12.8 & 50.0 & 12.8 & 12.8 & 12.8 & 12.8 \\
\hline Specific Gravity & 1.04 & 1.32 & 1.02 & 1.12 & 1.02 & 1.02 & 0.68 & 1.31 \\
\hline Chemical Composition & Mol/liter & Mol/liter & Mol/liter & Mol/liter & Mol/liter & Mol/liter & Wt \% & Mol/liter \\
\hline $\mathrm{H}+$ & $3.13 \mathrm{E}-02$ & $4.76 \mathrm{E}-01$ & $4.31 \mathrm{E}-02$ & $4.31 \mathrm{E}-02$ & $4.31 \mathrm{E}-02$ & $3.96 \mathrm{E}-02$ & $1.06 \mathrm{E}-03$ & $2.76 \mathrm{E}+00$ \\
\hline$\overline{A l+3}$ & $3.04 \mathrm{E}-02$ & $4.52 \mathrm{E}-01$ & $1.78 \mathrm{E}-02$ & $1.27 \mathrm{E}-01$ & 1.99E-02 & $2.95 \mathrm{E}-02$ & $1.01 \mathrm{E}+00$ & $5.13 \mathrm{E}-01$ \\
\hline$A m+4$ & $8.51 \mathrm{E}-10$ & $5.48 \mathrm{E}-08$ & $2.28 \mathrm{E}-09$ & $2.28 \mathrm{E}-09$ & $2.28 \mathrm{E}-09$ & $2.14 \mathrm{E}-09$ & $1.35 \mathrm{E}-08$ & $6.42 \mathrm{E}-08$ \\
\hline $\mathrm{Sb}+5$ & $1.51 \mathrm{E}-05$ & $1.25 \mathrm{E}-05$ & $9.57 \mathrm{E}-07$ & $6.53 \mathrm{E}-05$ & $2.20 \mathrm{E}-06$ & $8.41 \mathrm{E}-06$ & $2.67 \mathrm{E}-03$ & $5.06 \mathrm{E}-06$ \\
\hline As+5 & $1.28 \mathrm{E}-04$ & $7.58 \mathrm{E}-05$ & $7.25 \mathrm{E}-06$ & $5.50 \mathrm{E}-04$ & 1.77E-05 & 7.01E-05 & $1.39 \mathrm{E}-02$ & $8.17 \mathrm{E}-06$ \\
\hline $\mathrm{Ba}+2$ & 2.76E-05 & $6.62 \mathrm{E}-05$ & $3.47 \mathrm{E}-06$ & $1.18 \mathrm{E}-04$ & 5.68E-06 & 1.67E-05 & 5.37E-03 & 6.05E-05 \\
\hline $\mathrm{Be}+2$ & $6.21 \mathrm{E}-06$ & $1.51 \mathrm{E}-05$ & $6.65 \mathrm{E}-07$ & $2.70 \mathrm{E}-05$ & 1.17E-06 & 3.71E-06 & $8.11 \mathrm{E}-05$ & $1.38 \mathrm{E}-05$ \\
\hline$B+3$ & $7.79 \mathrm{E}-04$ & $1.40 \mathrm{E}-02$ & $6.66 \mathrm{E}-04$ & $2.78 \mathrm{E}-03$ & $7.06 \mathrm{E}-04$ & $8.70 \mathrm{E}-04$ & $7.96 \mathrm{E}-03$ & $1.61 \mathrm{E}-02$ \\
\hline $\mathrm{Br}-$ & $4.45 \mathrm{E}-09$ & $2.86 \mathrm{E}-07$ & $1.19 \mathrm{E}-08$ & $1.19 \mathrm{E}-08$ & $1.19 \mathrm{E}-08$ & $1.12 \mathrm{E}-08$ & $2.34 \mathrm{E}-08$ & $3.35 \mathrm{E}-07$ \\
\hline $\mathrm{Cd}+2$ & $1.50 \mathrm{E}-04$ & $2.04 \mathrm{E}-03$ & $1.25 \mathrm{E}-04$ & $4.69 \mathrm{E}-04$ & $1.31 \mathrm{E}-04$ & $1.56 \mathrm{E}-04$ & $1.35 \mathrm{E}-02$ & $2.33 \mathrm{E}-03$ \\
\hline $\mathrm{Ca}+2$ & $2.14 \mathrm{E}-03$ & $4.28 \mathrm{E}-02$ & 1.97E-03 & 7.67E-03 & $2.08 \mathrm{E}-03$ & $2.51 \mathrm{E}-03$ & $7.98 \mathrm{E}-02$ & $4.93 \mathrm{E}-02$ \\
\hline $\mathrm{Ce}+4$ & $1.39 \mathrm{E}-05$ & $3.03 \mathrm{E}-05$ & $1.60 \mathrm{E}-06$ & $5.96 \mathrm{E}-05$ & $2.72 \mathrm{E}-06$ & $8.28 \mathrm{E}-06$ & $2.77 \mathrm{E}-03$ & $2.69 \mathrm{E}-05$ \\
\hline Cs+ & $5.55 \mathrm{E}-05$ & $5.36 \mathrm{E}-05$ & $3.95 \mathrm{E}-06$ & $2.39 \mathrm{E}-04$ & $8.49 \mathrm{E}-06$ & $3.11 \mathrm{E}-05$ & $1.07 \mathrm{E}-02$ & $2.79 \mathrm{E}-05$ \\
\hline $\mathrm{Cl}-$ & $3.23 \mathrm{E}-03$ & $2.26 \mathrm{E}-02$ & $9.34 \mathrm{E}-04$ & $1.38 \mathrm{E}-02$ & $1.18 \mathrm{E}-03$ & $2.38 \mathrm{E}-03$ & $1.56 \mathrm{E}-01$ & $2.46 \mathrm{E}-02$ \\
\hline $\mathrm{Cr}+3$ & $5.85 \mathrm{E}-04$ & $4.12 \mathrm{E}-03$ & $1.89 \mathrm{E}-04$ & $2.42 \mathrm{E}-03$ & $2.33 \mathrm{E}-04$ & $4.39 \mathrm{E}-04$ & $3.98 \mathrm{E}-02$ & $4.50 \mathrm{E}-03$ \\
\hline $\mathrm{Co}+2$ & $4.86 \mathrm{E}-06$ & $2.25 \mathrm{E}-04$ & $6.28 \mathrm{E}-06$ & $2.65 \mathrm{E}-05$ & $6.67 \mathrm{E}-06$ & $8.38 \mathrm{E}-06$ & $4.14 \mathrm{E}-04$ & $2.61 \mathrm{E}-04$ \\
\hline $\mathrm{Cu}+2$ & $1.55 \mathrm{E}-04$ & $5.91 \mathrm{E}-04$ & $2.34 \mathrm{E}-05$ & $6.79 \mathrm{E}-04$ & $3.61 \mathrm{E}-05$ & $9.87 \mathrm{E}-05$ & $1.42 \mathrm{E}-02$ & $5.95 \mathrm{E}-04$ \\
\hline $\mathrm{Eu}+3$ & $7.38 \mathrm{E}-09$ & $4.75 \mathrm{E}-07$ & $1.98 \mathrm{E}-08$ & $1.98 \mathrm{E}-08$ & $1.98 \mathrm{E}-08$ & $1.86 \mathrm{E}-08$ & $7.36 \mathrm{E}-08$ & $5.56 \mathrm{E}-07$ \\
\hline F- & $2.82 \mathrm{E}-02$ & $2.96 \mathrm{E}-02$ & $3.36 \mathrm{E}-03$ & 1.17E-01 & $5.55 \mathrm{E}-03$ & $1.63 \mathrm{E}-02$ & $7.35 \mathrm{E}-01$ & $1.78 \mathrm{E}-02$ \\
\hline $\mathrm{Gd}+3$ & $2.82 \mathrm{E}-05$ & $1.40 \mathrm{E}-04$ & $5.73 \mathrm{E}-06$ & $1.22 \mathrm{E}-04$ & 7.97E-06 & $1.89 \mathrm{E}-05$ & $6.23 \mathrm{E}-03$ & $1.47 \mathrm{E}-04$ \\
\hline $\mathrm{Ge}+4$ & $1.28 \mathrm{E}-10$ & $8.26 \mathrm{E}-09$ & $3.44 \mathrm{E}-10$ & $3.44 \mathrm{E}-10$ & $3.44 \mathrm{E}-10$ & $3.23 \mathrm{E}-10$ & $6.12 \mathrm{E}-10$ & $9.67 \mathrm{E}-09$ \\
\hline $\ln +3$ & $2.02 \mathrm{E}-08$ & $1.47 \mathrm{E}-06$ & $5.86 \mathrm{E}-08$ & $5.86 \mathrm{E}-08$ & $5.86 \mathrm{E}-08$ & $5.52 \mathrm{E}-08$ & $1.65 \mathrm{E}-07$ & $1.72 \mathrm{E}-06$ \\
\hline I- & $3.70 \mathrm{E}-08$ & $2.38 \mathrm{E}-06$ & $9.90 \mathrm{E}-08$ & $9.90 \mathrm{E}-08$ & $9.90 \mathrm{E}-08$ & $9.30 \mathrm{E}-08$ & $3.08 \mathrm{E}-07$ & $2.79 \mathrm{E}-06$ \\
\hline $\mathrm{Fe}+3$ & $1.07 \mathrm{E}-02$ & $2.23 \mathrm{E}-02$ & $1.27 \mathrm{E}-03$ & $4.58 \mathrm{E}-02$ & $2.13 \mathrm{E}-03$ & $6.39 \mathrm{E}-03$ & $8.48 \mathrm{E}-01$ & 1.95E-02 \\
\hline $\mathrm{La+3}$ & $1.34 \mathrm{E}-07$ & $8.63 \mathrm{E}-06$ & $3.59 \mathrm{E}-07$ & $3.59 \mathrm{E}-07$ & $3.59 \mathrm{E}-07$ & 3.37E-07 & $1.22 \mathrm{E}-06$ & $1.01 \mathrm{E}-05$ \\
\hline $\mathrm{Pb}+2$ & $6.39 \mathrm{E}-05$ & $8.10 \mathrm{E}-04$ & $3.97 \mathrm{E}-05$ & $2.41 \mathrm{E}-04$ & $4.36 \mathrm{E}-05$ & $6.05 \mathrm{E}-05$ & $1.44 \mathrm{E}-02$ & $9.19 \mathrm{E}-04$ \\
\hline Li+ & $3.18 \mathrm{E}-04$ & $4.14 \mathrm{E}-04$ & $2.43 \mathrm{E}-05$ & $1.38 \mathrm{E}-03$ & $5.05 \mathrm{E}-05$ & $1.81 \mathrm{E}-04$ & $3.20 \mathrm{E}-03$ & $2.84 \mathrm{E}-04$ \\
\hline $\mathrm{Mg}+2$ & $1.23 \mathrm{E}-03$ & 1.72E-02 & $7.88 \mathrm{E}-04$ & $4.75 \mathrm{E}-03$ & $8.64 \mathrm{E}-04$ & $1.20 \mathrm{E}-03$ & 3.33E-02 & 1.95E-02 \\
\hline $\mathrm{Mn}+4$ & $4.45 \mathrm{E}-04$ & $1.30 \mathrm{E}-02$ & $5.60 \mathrm{E}-04$ & $1.55 \mathrm{E}-03$ & $5.80 \mathrm{E}-04$ & $6.42 \mathrm{E}-04$ & $1.93 \mathrm{E}-02$ & $1.51 \mathrm{E}-02$ \\
\hline $\mathrm{Hg}+2$ & $3.11 \mathrm{E}-05$ & $4.76 \mathrm{E}-03$ & $1.43 \mathrm{E}-04$ & $2.04 \mathrm{E}-04$ & $1.44 \mathrm{E}-04$ & $1.44 \mathrm{E}-04$ & $4.87 \mathrm{E}-03$ & $5.57 \mathrm{E}-03$ \\
\hline $\mathrm{Mo}+6$ & $8.91 \mathrm{E}-04$ & $6.70 \mathrm{E}-04$ & $5.53 \mathrm{E}-05$ & $3.84 \mathrm{E}-03$ & $1.28 \mathrm{E}-04$ & $4.94 \mathrm{E}-04$ & $1.24 \mathrm{E}-01$ & $2.22 \mathrm{E}-04$ \\
\hline $\mathrm{Nd}+3$ & $4.32 \mathrm{E}-07$ & $2.78 \mathrm{E}-05$ & $1.16 \mathrm{E}-06$ & $1.16 \mathrm{E}-06$ & $1.16 \mathrm{E}-06$ & $1.09 \mathrm{E}-06$ & $4.10 \mathrm{E}-06$ & $3.26 \mathrm{E}-05$ \\
\hline $\mathrm{Np}+4$ & $2.47 \mathrm{E}-07$ & $2.23 \mathrm{E}-06$ & $3.00 \mathrm{E}-07$ & $3.00 \mathrm{E}-07$ & $3.00 \mathrm{E}-07$ & $2.74 \mathrm{E}-07$ & $1.74 \mathrm{E}-06$ & $2.61 \mathrm{E}-06$ \\
\hline $\mathrm{Ni}+2$ & $2.03 \mathrm{E}-04$ & $1.56 \mathrm{E}-03$ & 7.09E-05 & $8.39 \mathrm{E}-04$ & 8.57E-05 & $1.56 \mathrm{E}-04$ & $1.55 \mathrm{E}-02$ & $1.71 \mathrm{E}-03$ \\
\hline $\mathrm{Nb}+5$ & $1.47 \mathrm{E}-03$ & $8.29 \mathrm{E}-04$ & $8.25 \mathrm{E}-05$ & $6.36 \mathrm{E}-03$ & $2.04 \mathrm{E}-04$ & $8.10 \mathrm{E}-04$ & $1.99 \mathrm{E}-01$ & 3.87E-05 \\
\hline NO3- & $6.65 \mathrm{E}-02$ & $5.38 \mathrm{E}+00$ & $2.07 \mathrm{E}-01$ & $2.09 \mathrm{E}-01$ & $2.07 \mathrm{E}-01$ & $1.95 \mathrm{E}-01$ & $3.55 \mathrm{E}-01$ & $6.30 \mathrm{E}+00$ \\
\hline $\mathrm{O}-2$ & $2.23 \mathrm{E}-01$ & $1.23 \mathrm{E}-01$ & $1.24 \mathrm{E}-02$ & $9.64 \mathrm{E}-01$ & $3.08 \mathrm{E}-02$ & $1.23 \mathrm{E}-01$ & $5.19 \mathrm{E}+00$ & $2.42 \mathrm{E}-03$ \\
\hline $\mathrm{Pd}+4$ & $2.45 \mathrm{E}-03$ & $1.60 \mathrm{E}-03$ & $1.43 \mathrm{E}-04$ & $1.06 \mathrm{E}-02$ & $3.45 \mathrm{E}-04$ & $1.35 \mathrm{E}-03$ & 3.79E-01 & $3.17 \mathrm{E}-04$ \\
\hline PO4-3 & 1.39E-01 & 7.64E-02 & $7.78 \mathrm{E}-03$ & 5.97E-01 & $1.92 \mathrm{E}-02$ & $7.61 \mathrm{E}-02$ & $1.91 \mathrm{E}+01$ & $1.94 \mathrm{E}-03$ \\
\hline $\mathrm{Pu}+4$ & $7.79 \mathrm{E}-08$ & $3.79 \mathrm{E}-06$ & $1.76 \mathrm{E}-07$ & $1.76 \mathrm{E}-07$ & $1.76 \mathrm{E}-07$ & $1.65 \mathrm{E}-07$ & $1.03 \mathrm{E}-06$ & $4.44 \mathrm{E}-06$ \\
\hline $\mathrm{K}+$ & $1.86 \mathrm{E}-02$ & 3.07E-01 & $1.09 \mathrm{E}-02$ & $8.09 \mathrm{E}-02$ & $1.23 \mathrm{E}-02$ & $1.85 \mathrm{E}-02$ & $9.42 \mathrm{E}-01$ & $3.50 \mathrm{E}-01$ \\
\hline $\mathrm{Pr}+4$ & $1.22 \mathrm{E}-07$ & $7.84 \mathrm{E}-06$ & $3.27 \mathrm{E}-07$ & $3.27 \mathrm{E}-07$ & $3.27 \mathrm{E}-07$ & $3.07 \mathrm{E}-07$ & $1.13 \mathrm{E}-06$ & $9.19 \mathrm{E}-06$ \\
\hline $\mathrm{Rh}+4$ & $5.26 \mathrm{E}-08$ & $3.38 \mathrm{E}-06$ & $1.41 \mathrm{E}-07$ & $1.41 \mathrm{E}-07$ & $1.41 \mathrm{E}-07$ & $1.32 \mathrm{E}-07$ & $3.55 \mathrm{E}-07$ & $3.96 \mathrm{E}-06$ \\
\hline
\end{tabular}


Table B-10. Tank WM-188 mass balance (continued).

\begin{tabular}{|c|c|c|c|c|c|c|c|c|}
\hline Stream \# & 110 & 111 & 112 & 113 & 114 & 115 & 116 & 120 \\
\hline Stream Name & $\begin{array}{l}\text { Slurry from } \\
\text { TFF Tank } \\
\text { WM-187 }\end{array}$ & $\begin{array}{l}\text { Crossflow } \\
\text { Filter Recirc } \\
\text { Purge }\end{array}$ & $\begin{array}{l}\text { Decant } \\
\text { Liquid }\end{array}$ & $\begin{array}{c}\text { Solids } \\
\text { Treatment } \\
\text { Filter Feed }\end{array}$ & $\begin{array}{l}\text { Solids } \\
\text { Treatment } \\
\text { Filtrate }\end{array}$ & $\begin{array}{c}\text { Combined } \\
\text { Waste Water }\end{array}$ & $\begin{array}{c}\text { Dried Solids } \\
\text { Waste }\end{array}$ & $\begin{array}{c}\text { Liquid SBW } \\
\text { from TFF }\end{array}$ \\
\hline Chemical Composition & Mol/liter & Mol/liter & Mol/liter & Mol/liter & Mol/liter & Mol/liter & Wt \% & Mol/liter \\
\hline $\mathrm{Rb}+$ & $8.10 \mathrm{E}-08$ & $5.21 \mathrm{E}-06$ & $2.17 \mathrm{E}-07$ & $2.17 \mathrm{E}-07$ & $2.17 \mathrm{E}-07$ & $2.04 \mathrm{E}-07$ & $4.54 \mathrm{E}-07$ & $6.10 \mathrm{E}-06$ \\
\hline $\mathrm{Ru}+3$ & $1.03 \mathrm{E}-03$ & 6.79E-04 & $6.04 \mathrm{E}-05$ & 4.43E-03 & $1.45 \mathrm{E}-04$ & 5.67E-04 & $1.51 \mathrm{E}-01$ & $1.46 \mathrm{E}-04$ \\
\hline $\mathrm{Sm}+3$ & $8.02 \mathrm{E}-08$ & $5.16 \mathrm{E}-06$ & $2.15 \mathrm{E}-07$ & $2.15 \mathrm{E}-07$ & $2.15 \mathrm{E}-07$ & $2.02 \mathrm{E}-07$ & $7.91 \mathrm{E}-07$ & $6.04 \mathrm{E}-06$ \\
\hline $\mathrm{Se}+4$ & $3.82 \mathrm{E}-04$ & $2.13 \mathrm{E}-04$ & $2.13 \mathrm{E}-05$ & $1.65 \mathrm{E}-03$ & $5.27 \mathrm{E}-05$ & $2.10 \mathrm{E}-04$ & 4.37E-02 & 8.47E-06 \\
\hline $\mathrm{Si}+4$ & $1.26 \mathrm{E}-01$ & 6.94E-02 & $7.01 \mathrm{E}-03$ & 5.42E-01 & $1.73 \mathrm{E}-02$ & $6.90 \mathrm{E}-02$ & $5.12 \mathrm{E}+00$ & $1.92 \mathrm{E}-03$ \\
\hline $\mathrm{Ag}+$ & $5.89 \mathrm{E}-04$ & $3.26 \mathrm{E}-04$ & $3.33 \mathrm{E}-05$ & $2.54 \mathrm{E}-03$ & $8.17 \mathrm{E}-05$ & $3.23 \mathrm{E}-04$ & $9.20 \mathrm{E}-02$ & $9.67 \mathrm{E}-06$ \\
\hline $\mathrm{Na}+$ & $4.19 \mathrm{E}-02$ & $3.08 \mathrm{E}+00$ & $9.78 \mathrm{E}-02$ & $2.10 \mathrm{E}-01$ & 9.99E-02 & $1.06 \mathrm{E}-01$ & $9.35 \mathrm{E}-01$ & $1.39 \mathrm{E}+00$ \\
\hline $\mathrm{Sr}+2$ & $8.53 \mathrm{E}-06$ & $7.12 \mathrm{E}-05$ & $2.43 \mathrm{E}-06$ & 3.79E-05 & $3.12 \mathrm{E}-06$ & $6.45 \mathrm{E}-06$ & 1.06E-03 & $7.81 \mathrm{E}-05$ \\
\hline SO4-2 & $6.39 \mathrm{E}-04$ & $2.22 \mathrm{E}-02$ & $1.21 \mathrm{E}-03$ & $1.21 \mathrm{E}-03$ & $1.21 \mathrm{E}-03$ & $1.13 \mathrm{E}-03$ & $2.85 \mathrm{E}-03$ & $2.60 \mathrm{E}-02$ \\
\hline$\overline{T c+7}$ & $9.40 \mathrm{E}-08$ & 1.06E-05 & $3.74 \mathrm{E}-07$ & $3.74 \mathrm{E}-07$ & 3.74E-07 & $3.54 \mathrm{E}-07$ & $8.88 \mathrm{E}-07$ & $1.25 \mathrm{E}-05$ \\
\hline $\mathrm{Te}+4$ & $4.21 \mathrm{E}-08$ & $3.10 \mathrm{E}-06$ & $1.23 \mathrm{E}-07$ & 1.23E-07 & $1.23 \mathrm{E}-07$ & 1.16E-07 & $3.86 \mathrm{E}-07$ & $3.64 \mathrm{E}-06$ \\
\hline $\mathrm{Tb}+4$ & $3.09 \mathrm{E}-11$ & 1.99E-09 & $8.27 \mathrm{E}-11$ & $8.27 \mathrm{E}-11$ & $8.27 \mathrm{E}-11$ & $7.77 \mathrm{E}-11$ & $3.22 \mathrm{E}-10$ & $2.33 \mathrm{E}-09$ \\
\hline $\mathrm{Th}+4$ & $2.87 \mathrm{E}-12$ & $2.20 \mathrm{E}-05$ & $5.83 \mathrm{E}-07$ & $5.83 \mathrm{E}-07$ & $5.83 \mathrm{E}-07$ & $5.60 \mathrm{E}-07$ & $3.31 \mathrm{E}-06$ & $2.58 \mathrm{E}-05$ \\
\hline$S n+4$ & $2.63 \mathrm{E}-03$ & 1.47E-03 & $1.47 \mathrm{E}-04$ & $1.13 \mathrm{E}-02$ & $3.63 \mathrm{E}-04$ & $1.44 \mathrm{E}-03$ & $4.53 \mathrm{E}-01$ & $6.53 \mathrm{E}-05$ \\
\hline $\mathrm{Ti}+4$ & $1.29 \mathrm{E}-03$ & $7.50 \mathrm{E}-04$ & $7.38 \mathrm{E}-05$ & $5.56 \mathrm{E}-03$ & $1.80 \mathrm{E}-04$ & 7.09E-04 & 8.95E-02 & $6.42 \mathrm{E}-05$ \\
\hline$U+4$ & $3.83 \mathrm{E}-05$ & $7.10 \mathrm{E}-04$ & $2.59 \mathrm{E}-05$ & $1.64 \mathrm{E}-04$ & $2.86 \mathrm{E}-05$ & $4.06 \mathrm{E}-05$ & $1.13 \mathrm{E}-02$ & $8.12 \mathrm{E}-04$ \\
\hline $\mathrm{V}+5$ & $1.12 \mathrm{E}-05$ & $2.35 \mathrm{E}-05$ & $4.43 E-06$ & $3.68 \mathrm{E}-05$ & $5.05 \mathrm{E}-06$ & $7.82 \mathrm{E}-06$ & $5.67 \mathrm{E}-04$ & $2.27 \mathrm{E}-05$ \\
\hline $\mathrm{Y}+3$ & $1.00 \mathrm{E}-07$ & $6.43 \mathrm{E}-06$ & $2.68 \mathrm{E}-07$ & $2.68 \mathrm{E}-07$ & $2.68 \mathrm{E}-07$ & $2.52 \mathrm{E}-07$ & $5.84 \mathrm{E}-07$ & $7.53 \mathrm{E}-06$ \\
\hline$Z n+2$ & $8.90 \mathrm{E}-05$ & $6.89 \mathrm{E}-04$ & $2.33 \mathrm{E}-05$ & $3.96 \mathrm{E}-04$ & $3.05 \mathrm{E}-05$ & $6.57 \mathrm{E}-05$ & $8.33 \mathrm{E}-03$ & $7.52 \mathrm{E}-04$ \\
\hline $\mathrm{Zr}+4$ & $5.35 \mathrm{E}-02$ & $3.13 \mathrm{E}-02$ & $3.12 \mathrm{E}-03$ & $2.31 \mathrm{E}-01$ & $7.51 \mathrm{E}-03$ & $2.95 \mathrm{E}-02$ & $7.07 \mathrm{E}+00$ & $2.94 \mathrm{E}-03$ \\
\hline $\mathrm{H} 2 \mathrm{O}$ & $5.55 \mathrm{E}+01$ & $4.79 \mathrm{E}+01$ & $5.52 \mathrm{E}+01$ & $5.39 \mathrm{E}+01$ & $5.51 \mathrm{E}+01$ & $5.45 \mathrm{E}+01$ & $5.67 E+01$ & $4.66 \mathrm{E}+01$ \\
\hline Canisters/day & & & & & & & 0.59 & \\
\hline Canisters total & & & & & & & 90 & \\
\hline Radiological Composition & Ci/liter & Ci/liter & Ci/liter & Ci/liter & Ci/liter & Ci/liter & $\mathrm{Ci} / \mathrm{kg}$ & Ci/liter \\
\hline Ra-226 & 1.15E-13 & $8.39 \mathrm{E}-12$ & 3.35E-13 & $3.35 \mathrm{E}-13$ & $3.35 \mathrm{E}-13$ & $3.15 \mathrm{E}-13$ & $8.21 \mathrm{E}-14$ & $9.83 \mathrm{E}-12$ \\
\hline Ac-227 & $5.43 \mathrm{E}-13$ & $3.95 \mathrm{E}-11$ & $1.58 \mathrm{E}-12$ & $1.58 \mathrm{E}-12$ & $1.58 \mathrm{E}-12$ & $1.48 \mathrm{E}-12$ & $3.86 \mathrm{E}-13$ & $4.63 \mathrm{E}-11$ \\
\hline Th-228 & $4.51 \mathrm{E}-11$ & $3.28 \mathrm{E}-09$ & $1.31 \mathrm{E}-10$ & $1.31 \mathrm{E}-10$ & $1.31 \mathrm{E}-10$ & $1.23 \mathrm{E}-10$ & $3.21 \mathrm{E}-11$ & $3.84 \mathrm{E}-09$ \\
\hline Th-230 & $3.37 \mathrm{E}-10$ & 1.02E-09 & $5.17 \mathrm{E}-11$ & 1.44E-09 & $7.84 \mathrm{E}-11$ & $2.10 \mathrm{E}-10$ & 4.73E-09 & $9.91 \mathrm{E}-10$ \\
\hline Th-232 & $9.98 \mathrm{E}-18$ & $7.26 \mathrm{E}-16$ & $2.90 \mathrm{E}-17$ & $2.90 \mathrm{E}-17$ & $2.90 \mathrm{E}-17$ & $2.72 \mathrm{E}-17$ & $7.10 \mathrm{E}-18$ & $8.50 \mathrm{E}-16$ \\
\hline $\mathrm{Pa}-231$ & $1.26 \mathrm{E}-12$ & $9.15 \mathrm{E}-11$ & $3.65 \mathrm{E}-12$ & $3.65 \mathrm{E}-12$ & $3.65 \mathrm{E}-12$ & $3.44 \mathrm{E}-12$ & $8.95 \mathrm{E}-13$ & $1.07 \mathrm{E}-10$ \\
\hline $\mathrm{Pa}-233$ & 4.13E-08 & $3.00 \mathrm{E}-06$ & $1.20 \mathrm{E}-07$ & $1.20 \mathrm{E}-07$ & $1.20 \mathrm{E}-07$ & 1.13E-07 & $2.94 \mathrm{E}-08$ & $3.52 \mathrm{E}-06$ \\
\hline U-232 & $6.99 \mathrm{E}-10$ & $2.40 \mathrm{E}-09$ & $1.19 \mathrm{E}-10$ & $2.97 \mathrm{E}-09$ & $1.74 \mathrm{E}-10$ & $4.45 \mathrm{E}-10$ & $9.75 \mathrm{E}-09$ & $2.39 \mathrm{E}-09$ \\
\hline U-233 & $1.27 \mathrm{E}-11$ & $8.83 \mathrm{E}-11$ & $3.91 \mathrm{E}-12$ & $5.31 \mathrm{E}-11$ & $4.86 \mathrm{E}-12$ & $9.43 \mathrm{E}-12$ & 1.69E-10 & $9.61 \mathrm{E}-11$ \\
\hline $\mathrm{U}-234$ & $2.08 \mathrm{E}-07$ & $1.02 \mathrm{E}-06$ & $4.60 \mathrm{E}-08$ & $8.85 \mathrm{E}-07$ & $6.22 \mathrm{E}-08$ & $1.41 \mathrm{E}-07$ & 2.87E-06 & $1.07 \mathrm{E}-06$ \\
\hline $\mathrm{U}-235$ & $1.09 \mathrm{E}-08$ & $8.18 \mathrm{E}-08$ & 2.84E-09 & $4.82 \mathrm{E}-08$ & $3.72 \mathrm{E}-09$ & 8.00E-09 & $1.55 \mathrm{E}-07$ & $8.92 \mathrm{E}-08$ \\
\hline U-236 & 1.43E-08 & $4.29 \mathrm{E}-08$ & $2.16 \mathrm{E}-09$ & $6.11 \mathrm{E}-08$ & $3.30 \mathrm{E}-09$ & 8.92E-09 & $2.01 \mathrm{E}-07$ & $4.15 \mathrm{E}-08$ \\
\hline U-237 & $9.07 \mathrm{E}-11$ & $6.59 \mathrm{E}-09$ & $2.63 \mathrm{E}-10$ & $2.63 \mathrm{E}-10$ & $2.63 \mathrm{E}-10$ & $2.48 \mathrm{E}-10$ & $6.45 \mathrm{E}-11$ & $7.73 \mathrm{E}-09$ \\
\hline $\mathrm{U}-238$ & 1.23E-09 & $1.14 \mathrm{E}-08$ & $7.53 \mathrm{E}-10$ & 4.15E-09 & $8.19 \mathrm{E}-10$ & 1.09E-09 & 1.17E-08 & $1.28 \mathrm{E}-08$ \\
\hline Np-236 & $6.44 \mathrm{E}-14$ & $3.15 \mathrm{E}-12$ & $1.46 \mathrm{E}-13$ & $1.46 \mathrm{E}-13$ & $1.46 \mathrm{E}-13$ & $1.37 \mathrm{E}-13$ & $3.58 \mathrm{E}-14$ & $3.69 \mathrm{E}-12$ \\
\hline Np-237 & 1.02E-07 & 4.06E-07 & $5.35 \mathrm{E}-08$ & $3.14 \mathrm{E}-07$ & $5.85 \mathrm{E}-08$ & $7.93 \mathrm{E}-08$ & $8.99 \mathrm{E}-07$ & 4.37E-07 \\
\hline Pu-236 & 1.22E-09 & $3.45 \mathrm{E}-09$ & $2.05 \mathrm{E}-10$ & $5.13 E-09$ & $3.00 \mathrm{E}-10$ & $7.66 \mathrm{E}-10$ & $1.68 \mathrm{E}-08$ & $3.31 \mathrm{E}-09$ \\
\hline Pu-238 & $6.41 \mathrm{E}-04$ & $7.76 \mathrm{E}-04$ & $6.49 \mathrm{E}-05$ & $2.71 \mathrm{E}-03$ & 1.16E-04 & 3.69E-04 & 9.02E-03 & $5.16 \mathrm{E}-04$ \\
\hline Pu-239 & $6.72 \mathrm{E}-05$ & $8.80 \mathrm{E}-05$ & $6.03 E-06$ & $2.88 \mathrm{E}-04$ & 1.15E-05 & 3.85E-05 & $9.60 \mathrm{E}-04$ & $6.12 \mathrm{E}-05$ \\
\hline Pu-240 & $4.68 \mathrm{E}-06$ & $1.29 \mathrm{E}-05$ & 7.37E-07 & $1.97 \mathrm{E}-05$ & $1.10 \mathrm{E}-06$ & $2.91 \mathrm{E}-06$ & $6.48 \mathrm{E}-05$ & $1.22 \mathrm{E}-05$ \\
\hline Pu-241 & 5.07E-04 & $5.41 \mathrm{E}-04$ & $5.00 \mathrm{E}-05$ & $2.14 \mathrm{E}-03$ & 9.03E-05 & $2.90 \mathrm{E}-04$ & $7.12 \mathrm{E}-03$ & $3.24 \mathrm{E}-04$ \\
\hline Pu-242 & $3.69 \mathrm{E}-09$ & 9.97E-09 & $7.21 \mathrm{E}-10$ & $1.50 \mathrm{E}-08$ & $9.98 \mathrm{E}-10$ & $2.34 \mathrm{E}-09$ & $4.89 \mathrm{E}-08$ & $9.56 \mathrm{E}-09$ \\
\hline Pu-244 & $2.89 \mathrm{E}-16$ & $8.44 \mathrm{E}-16$ & $3.47 \mathrm{E}-17$ & $1.26 \mathrm{E}-15$ & $5.84 \mathrm{E}-17$ & $1.76 \mathrm{E}-16$ & $4.18 \mathrm{E}-15$ & $8.06 \mathrm{E}-16$ \\
\hline Am-241 & 1.71E-05 & $5.47 \mathrm{E}-05$ & $2.93 \mathrm{E}-06$ & 7.19E-05 & $4.26 \mathrm{E}-06$ & $1.08 \mathrm{E}-05$ & $2.36 \mathrm{E}-04$ & $5.38 \mathrm{E}-05$ \\
\hline Am-242m & $2.14 \mathrm{E}-10$ & $1.60 \mathrm{E}-08$ & $6.33 \mathrm{E}-10$ & $6.33 \mathrm{E}-10$ & $6.33 \mathrm{E}-10$ & $5.96 \mathrm{E}-10$ & $1.55 \mathrm{E}-10$ & $1.88 \mathrm{E}-08$ \\
\hline Am-243 & 6.07E-09 & $2.57 \mathrm{E}-08$ & $1.21 \mathrm{E}-09$ & $2.58 \mathrm{E}-08$ & 1.69E-09 & 4.01E-09 & $8.39 \mathrm{E}-08$ & $2.65 \mathrm{E}-08$ \\
\hline $\mathrm{Cm}-242$ & $2.00 \mathrm{E}-10$ & $3.51 \mathrm{E}-08$ & 1.10E-09 & $1.20 \mathrm{E}-09$ & $1.10 \mathrm{E}-09$ & 1.06E-09 & $6.06 \mathrm{E}-10$ & $4.11 \mathrm{E}-08$ \\
\hline
\end{tabular}


Table B-10. Tank WM-188 mass balance (continued).

\begin{tabular}{|c|c|c|c|c|c|c|c|c|}
\hline Stream \# & 110 & 111 & 112 & 113 & 114 & 115 & 116 & 120 \\
\hline Stream Name & $\begin{array}{l}\text { Slurry from } \\
\text { TFF Tank } \\
\text { WM-187 }\end{array}$ & $\begin{array}{c}\text { Crossflow } \\
\text { Filter Recirc } \\
\text { Purge }\end{array}$ & $\begin{array}{l}\text { Decant } \\
\text { Liquid }\end{array}$ & $\begin{array}{l}\text { Solids } \\
\text { Treatment } \\
\text { Filter Feed }\end{array}$ & $\begin{array}{l}\text { Solids } \\
\text { Treatment } \\
\text { Filtrate }\end{array}$ & $\begin{array}{c}\text { Combined } \\
\text { Waste Water }\end{array}$ & $\begin{array}{c}\text { Dried Solids } \\
\text { Waste }\end{array}$ & $\begin{array}{c}\text { Liquid SBW } \\
\text { from Tank } \\
\text { WM-180 }\end{array}$ \\
\hline & $\mathrm{Ci} /$ liter & Ci/liter & Ci/liter & $\mathrm{Ci} /$ liter & Ci/liter & Ci/liter & $\mathrm{Ci} / \mathrm{kg}$ & Ci/liter \\
\hline $\mathrm{Cm}-243$ & $1.23 \mathrm{E}-08$ & $3.56 \mathrm{E}-08$ & 1.82E-09 & $5.23 \mathrm{E}-08$ & $2.80 \mathrm{E}-09$ & 7.61E-09 & $1.72 \mathrm{E}-07$ & $3.42 \mathrm{E}-08$ \\
\hline Cm-244 & $7.68 \mathrm{E}-07$ & $1.20 \mathrm{E}-06$ & $8.64 \mathrm{E}-08$ & $3.25 \mathrm{E}-06$ & $1.48 \mathrm{E}-07$ & $4.50 \mathrm{E}-07$ & $1.08 \mathrm{E}-05$ & $9.34 \mathrm{E}-07$ \\
\hline $\mathrm{Cm}-245$ & $1.30 \mathrm{E}-10$ & $3.75 \mathrm{E}-10$ & $1.92 \mathrm{E}-11$ & $5.55 \mathrm{E}-10$ & $2.95 \mathrm{E}-11$ & $8.05 E-11$ & 1.83E-09 & $3.60 \mathrm{E}-10$ \\
\hline $\mathrm{Cm}-246$ & $8.44 \mathrm{E}-12$ & $2.46 \mathrm{E}-11$ & $1.26 \mathrm{E}-12$ & $3.60 \mathrm{E}-11$ & $1.93 \mathrm{E}-12$ & $5.24 \mathrm{E}-12$ & $1.19 \mathrm{E}-10$ & $2.37 \mathrm{E}-11$ \\
\hline $\mathrm{H}-3$ & $3.92 \mathrm{E}-07$ & $1.18 \mathrm{E}-05$ & $6.95 \mathrm{E}-07$ & $6.95 \mathrm{E}-07$ & $6.95 \mathrm{E}-07$ & $6.45 \mathrm{E}-07$ & $1.70 \mathrm{E}-07$ & 1.39E-05 \\
\hline $\mathrm{Be}-10$ & $4.23 E-14$ & $3.07 \mathrm{E}-12$ & $1.23 \mathrm{E}-13$ & $1.23 \mathrm{E}-13$ & $1.23 \mathrm{E}-13$ & $1.15 \mathrm{E}-13$ & $3.01 \mathrm{E}-14$ & $3.60 \mathrm{E}-12$ \\
\hline C-14 & $5.49 \mathrm{E}-11$ & $1.52 \mathrm{E}-10$ & $7.86 \mathrm{E}-12$ & $2.34 \mathrm{E}-10$ & $1.22 \mathrm{E}-11$ & $3.38 \mathrm{E}-11$ & $7.72 \mathrm{E}-10$ & $1.45 \mathrm{E}-10$ \\
\hline Se-79 & $1.98 \mathrm{E}-07$ & $5.53 \mathrm{E}-07$ & $2.85 \mathrm{E}-08$ & 8.43E-07 & $4.42 \mathrm{E}-08$ & $1.22 \mathrm{E}-07$ & $2.78 \mathrm{E}-06$ & $5.26 \mathrm{E}-07$ \\
\hline $\mathrm{Rb}-87$ & $4.13 \mathrm{E}-13$ & $3.00 \mathrm{E}-11$ & $1.20 \mathrm{E}-12$ & $1.20 \mathrm{E}-12$ & $1.20 \mathrm{E}-12$ & $1.13 \mathrm{E}-12$ & $2.94 \mathrm{E}-13$ & $3.52 \mathrm{E}-11$ \\
\hline Sr-90 & 1.06E-03 & $3.90 \mathrm{E}-02$ & $1.70 \mathrm{E}-03$ & $3.36 \mathrm{E}-03$ & $1.73 \mathrm{E}-03$ & 1.79E-03 & $6.07 \mathrm{E}-03$ & $4.54 \mathrm{E}-02$ \\
\hline $\begin{array}{l}\mathrm{Y}-90 \\
\end{array}$ & $1.06 \mathrm{E}-03$ & $3.90 \mathrm{E}-02$ & $1.70 \mathrm{E}-03$ & $3.36 \mathrm{E}-03$ & $1.73 \mathrm{E}-03$ & 1.79E-03 & $6.07 \mathrm{E}-03$ & $4.54 \mathrm{E}-02$ \\
\hline Zr-93 & $3.12 \mathrm{E}-08$ & $2.27 \mathrm{E}-06$ & $9.06 \mathrm{E}-08$ & $9.06 \mathrm{E}-08$ & $9.06 \mathrm{E}-08$ & $8.52 \mathrm{E}-08$ & $2.22 \mathrm{E}-08$ & $2.66 \mathrm{E}-06$ \\
\hline Nb-93m & $2.41 \mathrm{E}-08$ & $1.75 \mathrm{E}-06$ & $6.98 \mathrm{E}-08$ & $6.98 \mathrm{E}-08$ & $6.98 \mathrm{E}-08$ & $6.57 \mathrm{E}-08$ & $1.71 \mathrm{E}-08$ & $2.05 \mathrm{E}-06$ \\
\hline Nb-94 & $5.10 \mathrm{E}-07$ & $1.44 \mathrm{E}-06$ & $7.40 \mathrm{E}-08$ & $2.17 \mathrm{E}-06$ & $1.15 \mathrm{E}-07$ & $3.15 \mathrm{E}-07$ & $7.17 \mathrm{E}-06$ & $1.37 \mathrm{E}-06$ \\
\hline Tc-98 & $3.63 \mathrm{E}-14$ & $2.64 \mathrm{E}-12$ & $1.05 \mathrm{E}-13$ & $1.05 \mathrm{E}-13$ & $1.05 \mathrm{E}-13$ & $9.92 \mathrm{E}-14$ & $2.58 \mathrm{E}-14$ & $3.10 \mathrm{E}-12$ \\
\hline Tc-99 & $4.43 E-06$ & $2.02 E-05$ & 8.64E-07 & 1.90E-05 & $1.22 \mathrm{E}-06$ & $2.94 \mathrm{E}-06$ & $6.21 \mathrm{E}-05$ & $2.10 \mathrm{E}-05$ \\
\hline Ru-106 & $4.27 \mathrm{E}-07$ & $1.18 \mathrm{E}-06$ & $6.10 \mathrm{E}-08$ & 1.82E-06 & $9.50 \mathrm{E}-08$ & $2.63 \mathrm{E}-07$ & $6.01 \mathrm{E}-06$ & $1.12 \mathrm{E}-06$ \\
\hline Rh-102 & $4.14 \mathrm{E}-07$ & $2.28 \mathrm{E}-07$ & $2.30 \mathrm{E}-08$ & $1.78 \mathrm{E}-06$ & $5.70 \mathrm{E}-08$ & $2.27 \mathrm{E}-07$ & $6.00 \mathrm{E}-06$ & $5.52 \mathrm{E}-09$ \\
\hline Pd-107 & $2.33 \mathrm{E}-10$ & 1.69E-08 & $6.75 \mathrm{E}-10$ & $6.75 \mathrm{E}-10$ & $6.75 \mathrm{E}-10$ & $6.35 \mathrm{E}-10$ & $1.66 \mathrm{E}-10$ & $1.98 \mathrm{E}-08$ \\
\hline Cd-113m & $4.68 \mathrm{E}-08$ & $3.40 \mathrm{E}-06$ & 1.36E-07 & 1.36E-07 & $1.36 \mathrm{E}-07$ & $1.28 \mathrm{E}-07$ & $3.33 \mathrm{E}-08$ & $3.99 \mathrm{E}-06$ \\
\hline $\ln -115$ & $1.42 \mathrm{E}-18$ & $1.03 \mathrm{E}-16$ & $4.12 \mathrm{E}-18$ & $4.12 \mathrm{E}-18$ & $4.12 \mathrm{E}-18$ & $3.87 \mathrm{E}-18$ & $1.01 \mathrm{E}-18$ & $1.21 \mathrm{E}-16$ \\
\hline Sn-121m & $9.42 \mathrm{E}-10$ & $6.85 \mathrm{E}-08$ & $2.73 \mathrm{E}-09$ & $2.73 \mathrm{E}-09$ & $2.73 \mathrm{E}-09$ & $2.57 \mathrm{E}-09$ & $6.70 \mathrm{E}-10$ & $8.03 \mathrm{E}-08$ \\
\hline Sn-126 & $1.87 \mathrm{E}-07$ & $5.20 \mathrm{E}-07$ & $2.68 \mathrm{E}-08$ & $7.96 \mathrm{E}-07$ & $4.17 \mathrm{E}-08$ & $1.15 \mathrm{E}-07$ & $2.63 E-06$ & $4.95 \mathrm{E}-07$ \\
\hline Sb-125 & $5.93 \mathrm{E}-04$ & $3.38 \mathrm{E}-04$ & $3.37 \mathrm{E}-05$ & $2.56 \mathrm{E}-03$ & $8.24 \mathrm{E}-05$ & $3.26 \mathrm{E}-04$ & $8.59 \mathrm{E}-03$ & $2.19 \mathrm{E}-05$ \\
\hline Sb-126 & $8.10 \mathrm{E}-10$ & $5.89 \mathrm{E}-08$ & $2.35 \mathrm{E}-09$ & $2.35 \mathrm{E}-09$ & $2.35 \mathrm{E}-09$ & $2.21 \mathrm{E}-09$ & $5.76 \mathrm{E}-10$ & $6.90 \mathrm{E}-08$ \\
\hline Te-123 & $5.40 \mathrm{E}-21$ & $3.93 \mathrm{E}-19$ & $1.57 \mathrm{E}-20$ & $1.57 \mathrm{E}-20$ & $1.57 \mathrm{E}-20$ & $1.47 \mathrm{E}-20$ & $3.84 \mathrm{E}-21$ & $4.60 \mathrm{E}-19$ \\
\hline Te-125m & $4.44 \mathrm{E}-08$ & $3.23 \mathrm{E}-06$ & 1.29E-07 & 1.29E-07 & $1.29 \mathrm{E}-07$ & $1.21 \mathrm{E}-07$ & $3.16 \mathrm{E}-08$ & $3.78 \mathrm{E}-06$ \\
\hline $\mathrm{I}-129$ & $2.42 \mathrm{E}-08$ & $6.75 \mathrm{E}-08$ & $3.49 \mathrm{E}-09$ & 1.03E-07 & 5.42E-09 & $1.49 \mathrm{E}-08$ & $3.40 \mathrm{E}-07$ & $6.42 \mathrm{E}-08$ \\
\hline Cs-134 & $4.92 \mathrm{E}-05$ & $3.02 \mathrm{E}-05$ & $3.61 \mathrm{E}-06$ & $2.09 \mathrm{E}-04$ & $7.58 \mathrm{E}-06$ & $2.74 \mathrm{E}-05$ & $7.02 \mathrm{E}-04$ & $4.81 \mathrm{E}-06$ \\
\hline Cs-135 & $3.49 \mathrm{E}-07$ & $1.07 \mathrm{E}-06$ & $5.39 \mathrm{E}-08$ & $1.49 \mathrm{E}-06$ & $8.16 \mathrm{E}-08$ & $2.17 \mathrm{E}-07$ & $4.90 \mathrm{E}-06$ & $1.04 \mathrm{E}-06$ \\
\hline Cs-137 & $1.98 \mathrm{E}-02$ & $1.65 \mathrm{E}-02$ & $1.91 \mathrm{E}-03$ & $8.33 \mathrm{E}-02$ & $3.49 \mathrm{E}-03$ & $1.13 \mathrm{E}-02$ & $2.78 \mathrm{E}-01$ & $7.26 \mathrm{E}-03$ \\
\hline Ba-137m & $1.88 \mathrm{E}-02$ & 1.56E-02 & $1.81 \mathrm{E}-03$ & 7.88E-02 & $3.30 \mathrm{E}-03$ & 1.07E-02 & $2.63 \mathrm{E}-01$ & $6.87 \mathrm{E}-03$ \\
\hline La-138 & $2.69 \mathrm{E}-18$ & $1.96 \mathrm{E}-16$ & $7.80 \mathrm{E}-18$ & $7.80 \mathrm{E}-18$ & $7.80 \mathrm{E}-18$ & $7.34 \mathrm{E}-18$ & $1.91 \mathrm{E}-18$ & $2.29 \mathrm{E}-16$ \\
\hline Ce-142 & $4.21 \mathrm{E}-13$ & $3.06 \mathrm{E}-11$ & $1.22 \mathrm{E}-12$ & $1.22 \mathrm{E}-12$ & $1.22 \mathrm{E}-12$ & $1.15 \mathrm{E}-12$ & $3.00 \mathrm{E}-13$ & $3.59 \mathrm{E}-11$ \\
\hline Ce-144 & $2.90 \mathrm{E}-07$ & $7.96 \mathrm{E}-07$ & $4.12 \mathrm{E}-08$ & $1.24 \mathrm{E}-06$ & $6.43 \mathrm{E}-08$ & $1.78 \mathrm{E}-07$ & $4.08 \mathrm{E}-06$ & $7.55 \mathrm{E}-07$ \\
\hline Nd-144 & $2.27 \mathrm{E}-17$ & $1.65 \mathrm{E}-15$ & $6.57 \mathrm{E}-17$ & $6.57 \mathrm{E}-17$ & $6.57 \mathrm{E}-17$ & $6.18 \mathrm{E}-17$ & $1.61 \mathrm{E}-17$ & $1.93 \mathrm{E}-15$ \\
\hline Pm-146 & $7.17 \mathrm{E}-10$ & $5.21 \mathrm{E}-08$ & $2.08 \mathrm{E}-09$ & $2.08 \mathrm{E}-09$ & $2.08 \mathrm{E}-09$ & 1.96E-09 & $5.10 \mathrm{E}-10$ & $6.11 \mathrm{E}-08$ \\
\hline Pm-147 & $7.68 \mathrm{E}-05$ & $2.15 \mathrm{E}-04$ & $1.11 \mathrm{E}-05$ & $3.28 \mathrm{E}-04$ & $1.72 \mathrm{E}-05$ & $4.74 \mathrm{E}-05$ & $1.08 \mathrm{E}-03$ & $2.05 \mathrm{E}-04$ \\
\hline Sm-146 & $3.89 \mathrm{E}-15$ & $2.83 \mathrm{E}-13$ & 1.13E-14 & $1.13 \mathrm{E}-14$ & $1.13 \mathrm{E}-14$ & 1.06E-14 & $2.76 \mathrm{E}-15$ & $3.31 \mathrm{E}-13$ \\
\hline Sm-147 & $1.04 \mathrm{E}-13$ & $7.55 \mathrm{E}-12$ & $3.01 \mathrm{E}-13$ & $3.01 \mathrm{E}-13$ & $3.01 \mathrm{E}-13$ & $2.83 E-13$ & $7.38 \mathrm{E}-14$ & $8.84 \mathrm{E}-12$ \\
\hline Sm-148 & $5.33 \mathrm{E}-19$ & $3.88 \mathrm{E}-17$ & $1.55 \mathrm{E}-18$ & $1.55 \mathrm{E}-18$ & $1.55 \mathrm{E}-18$ & $1.46 \mathrm{E}-18$ & $3.79 \mathrm{E}-19$ & $4.54 \mathrm{E}-17$ \\
\hline Sm-149 & $4.73 \mathrm{E}-20$ & $3.44 \mathrm{E}-18$ & $1.37 \mathrm{E}-19$ & 1.37E-19 & $1.37 \mathrm{E}-19$ & $1.29 \mathrm{E}-19$ & $3.37 \mathrm{E}-20$ & $4.03 \mathrm{E}-18$ \\
\hline Sm-151 & $1.53 \mathrm{E}-04$ & $4.25 \mathrm{E}-04$ & $2.20 \mathrm{E}-05$ & $6.54 \mathrm{E}-04$ & $3.42 \mathrm{E}-05$ & $9.44 \mathrm{E}-05$ & $2.16 \mathrm{E}-03$ & 4.05E-04 \\
\hline Eu-152 & $5.55 \mathrm{E}-07$ & $2.86 \mathrm{E}-06$ & 1.32E-07 & $2.34 \mathrm{E}-06$ & $1.74 \mathrm{E}-07$ & $3.82 \mathrm{E}-07$ & $7.56 \mathrm{E}-06$ & $3.03 \mathrm{E}-06$ \\
\hline Eu-154 & $2.44 \mathrm{E}-05$ & $3.11 \mathrm{E}-05$ & $5.24 \mathrm{E}-06$ & $9.35 \mathrm{E}-05$ & $6.95 \mathrm{E}-06$ & $1.51 \mathrm{E}-05$ & $3.02 \mathrm{E}-04$ & $2.33 \mathrm{E}-05$ \\
\hline Eu-155 & $3.50 \mathrm{E}-05$ & $2.57 \mathrm{E}-05$ & $3.06 \mathrm{E}-06$ & $1.48 \mathrm{E}-04$ & $5.85 \mathrm{E}-06$ & 1.97E-05 & $4.92 \mathrm{E}-04$ & $8.68 \mathrm{E}-06$ \\
\hline Gd-152 & $2.00 \mathrm{E}-20$ & $1.46 \mathrm{E}-18$ & $5.81 \mathrm{E}-20$ & $5.81 \mathrm{E}-20$ & $5.81 \mathrm{E}-20$ & $5.47 \mathrm{E}-20$ & $1.42 \mathrm{E}-20$ & $1.71 \mathrm{E}-18$ \\
\hline Ho-166m & $6.49 \mathrm{E}-13$ & $4.72 \mathrm{E}-11$ & $1.88 \mathrm{E}-12$ & $1.88 \mathrm{E}-12$ & $1.88 \mathrm{E}-12$ & $1.77 \mathrm{E}-12$ & $4.61 \mathrm{E}-13$ & $5.53 \mathrm{E}-11$ \\
\hline Co-60 & $2.81 \mathrm{E}-06$ & $4.96 \mathrm{E}-06$ & $1.14 \mathrm{E}-06$ & $9.01 \mathrm{E}-06$ & $1.29 \mathrm{E}-06$ & 1.95E-06 & $2.71 \mathrm{E}-05$ & $4.64 \mathrm{E}-06$ \\
\hline $\mathrm{Ni}-63$ & $1.48 \mathrm{E}-05$ & $4.01 \mathrm{E}-05$ & $2.55 \mathrm{E}-06$ & $6.15 \mathrm{E}-05$ & $3.69 \mathrm{E}-06$ & $9.26 \mathrm{E}-06$ & $2.01 \mathrm{E}-04$ & $3.83 \mathrm{E}-05$ \\
\hline
\end{tabular}


Table B-10. Tank WM-188 mass balance (continued).

\begin{tabular}{|c|c|c|c|c|c|c|c|c|}
\hline PFD Sheet \# & PFD-3 & PFD-3 & PFD-3 & PFD-3 & PFD-3 & PFD-3 & PFD-3 & PFD-3 \\
\hline Stream \# & 121 & 122 & 123 & 124 & 125 & 126 & 127 & 128 \\
\hline Stream Name & $\begin{array}{l}\text { Neutralized } \\
\text { Feed }\end{array}$ & $\begin{array}{l}\text { SBW Liquid } \\
\text { Filter Feed }\end{array}$ & $\begin{array}{c}\text { Filter } \\
\text { Recirculation }\end{array}$ & $\begin{array}{l}\text { IX Column } \\
\text { Feed }\end{array}$ & $\begin{array}{c}\text { Column } 1 \\
\text { Effluent }\end{array}$ & $\begin{array}{c}\text { Column } 2 \\
\text { Effluent }\end{array}$ & $\begin{array}{c}\text { Column } 3 \\
\text { Effluent }\end{array}$ & $\begin{array}{l}\text { Spent IX } \\
\text { Rinse }\end{array}$ \\
\hline Rate, gal/hr & 95.0 & 7800 & 7705 & 95.0 & 95.0 & 95.0 & 95.0 & 0.5 \\
\hline Rate, Ib/hr & 1054 & 86569 & 85515 & 1054 & 1054 & 1054 & 1054 & 5 \\
\hline Rate, peak, gpm & 1.58 & 130 & 128 & 1.58 & 1.58 & 1.58 & 1.58 & 2.99 \\
\hline Operation & Cont & Cont & Cont & Cont & Cont & Cont & Cont & Batch \\
\hline Temperature, ${ }^{\circ} \mathrm{F}$ & 70 & 70 & 70 & 70 & 70 & 70 & 70 & 70 \\
\hline Pressure, psia & 12.8 & 35.0 & 35.0 & 24.8 & 20.8 & 16.8 & 12.8 & 12.8 \\
\hline Specific Gravity & 1.33 & 1.33 & 1.33 & 1.33 & 1.33 & 1.33 & 1.33 & 1.07 \\
\hline Chemical Composition & Mol/liter & Mol/liter & Mol/liter & Mol/liter & Mol/liter & Mol/liter & Mol/liter & Mol/liter \\
\hline $\mathrm{H}+$ & $5.00 \mathrm{E}-01$ & $5.00 \mathrm{E}-01$ & $5.00 \mathrm{E}-01$ & $5.00 \mathrm{E}-01$ & $5.00 \mathrm{E}-01$ & $5.00 \mathrm{E}-01$ & $5.00 \mathrm{E}-01$ & 1.01E-01 \\
\hline $\mathrm{Al}+3$ & $4.60 \mathrm{E}-01$ & $4.75 \mathrm{E}-01$ & $4.75 \mathrm{E}-01$ & $4.59 \mathrm{E}-01$ & $4.59 \mathrm{E}-01$ & $4.59 \mathrm{E}-01$ & $4.59 \mathrm{E}-01$ & $9.29 \mathrm{E}-02$ \\
\hline $\mathrm{Am}+4$ & $5.75 \mathrm{E}-08$ & $5.75 \mathrm{E}-08$ & $5.75 \mathrm{E}-08$ & $5.75 \mathrm{E}-08$ & $5.75 \mathrm{E}-08$ & $5.75 \mathrm{E}-08$ & $5.75 \mathrm{E}-08$ & $1.16 \mathrm{E}-08$ \\
\hline $\mathrm{Sb}+5$ & $4.53 \mathrm{E}-06$ & 1.37E-05 & $1.38 \mathrm{E}-05$ & $4.38 \mathrm{E}-06$ & $4.38 \mathrm{E}-06$ & $4.38 \mathrm{E}-06$ & $4.38 \mathrm{E}-06$ & 8.86E-07 \\
\hline As+5 & $7.32 \mathrm{E}-06$ & $8.44 \mathrm{E}-05$ & $8.54 \mathrm{E}-05$ & $6.08 \mathrm{E}-06$ & $6.08 \mathrm{E}-06$ & $6.08 \mathrm{E}-06$ & $6.08 \mathrm{E}-06$ & $1.23 \mathrm{E}-06$ \\
\hline $\mathrm{Ba}+2$ & $5.43 \mathrm{E}-05$ & $7.06 \mathrm{E}-05$ & $7.08 \mathrm{E}-05$ & $5.40 \mathrm{E}-05$ & $5.40 \mathrm{E}-05$ & $5.40 \mathrm{E}-05$ & $5.40 \mathrm{E}-05$ & $1.09 \mathrm{E}-05$ \\
\hline $\mathrm{Be}+2$ & $1.24 \mathrm{E}-05$ & $1.61 \mathrm{E}-05$ & $1.62 \mathrm{E}-05$ & $1.23 \mathrm{E}-05$ & $1.23 \mathrm{E}-05$ & $1.23 \mathrm{E}-05$ & $1.23 \mathrm{E}-05$ & $2.49 \mathrm{E}-06$ \\
\hline $\mathrm{B}+3$ & $1.44 \mathrm{E}-02$ & $1.47 \mathrm{E}-02$ & $1.47 \mathrm{E}-02$ & $1.44 \mathrm{E}-02$ & $1.44 \mathrm{E}-02$ & $1.44 \mathrm{E}-02$ & $1.44 \mathrm{E}-02$ & $2.91 \mathrm{E}-03$ \\
\hline $\mathrm{Br}-$ & $3.01 \mathrm{E}-07$ & $3.01 \mathrm{E}-07$ & $3.01 \mathrm{E}-07$ & $3.01 \mathrm{E}-07$ & $3.01 \mathrm{E}-07$ & $3.01 \mathrm{E}-07$ & $3.01 \mathrm{E}-07$ & $6.08 \mathrm{E}-08$ \\
\hline $\mathrm{Cd}+2$ & $2.09 \mathrm{E}-03$ & $2.14 \mathrm{E}-03$ & $2.14 \mathrm{E}-03$ & $2.09 \mathrm{E}-03$ & $2.09 \mathrm{E}-03$ & $2.09 \mathrm{E}-03$ & $2.09 \mathrm{E}-03$ & $4.22 \mathrm{E}-04$ \\
\hline $\mathrm{Ca}+2$ & $4.42 \mathrm{E}-02$ & $4.50 \mathrm{E}-02$ & $4.50 \mathrm{E}-02$ & $4.42 \mathrm{E}-02$ & $4.42 \mathrm{E}-02$ & $4.42 \mathrm{E}-02$ & $4.42 \mathrm{E}-02$ & 8.93E-03 \\
\hline $\mathrm{Ce}+4$ & $2.41 \mathrm{E}-05$ & $3.24 \mathrm{E}-05$ & $3.25 \mathrm{E}-05$ & $2.40 \mathrm{E}-05$ & $2.40 \mathrm{E}-05$ & $2.40 \mathrm{E}-05$ & $2.40 \mathrm{E}-05$ & $4.85 \mathrm{E}-06$ \\
\hline Cs+ & $2.50 \mathrm{E}-05$ & $5.84 \mathrm{E}-05$ & $5.88 \mathrm{E}-05$ & $2.44 \mathrm{E}-05$ & $2.44 \mathrm{E}-06$ & $2.44 \mathrm{E}-07$ & $2.44 \mathrm{E}-08$ & 4.94E-09 \\
\hline $\mathrm{Cl}-$ & $2.21 \mathrm{E}-02$ & $2.39 \mathrm{E}-02$ & $2.39 \mathrm{E}-02$ & $2.20 \mathrm{E}-02$ & $2.20 \mathrm{E}-02$ & $2.20 \mathrm{E}-02$ & $2.20 \mathrm{E}-02$ & $4.45 \mathrm{E}-03$ \\
\hline $\mathrm{Cr}+3$ & $4.03 \mathrm{E}-03$ & $4.35 \mathrm{E}-03$ & $4.35 \mathrm{E}-03$ & $4.03 \mathrm{E}-03$ & $4.03 \mathrm{E}-03$ & $4.03 \mathrm{E}-03$ & $4.03 \mathrm{E}-03$ & $8.14 \mathrm{E}-04$ \\
\hline $\mathrm{Co}+2$ & $2.34 \mathrm{E}-04$ & 2.37E-04 & $2.37 \mathrm{E}-04$ & $2.34 \mathrm{E}-04$ & $2.34 \mathrm{E}-04$ & $2.34 \mathrm{E}-04$ & $2.34 \mathrm{E}-04$ & 4.73E-05 \\
\hline $\mathrm{Cu}+2$ & 5.33E-04 & $6.26 \mathrm{E}-04$ & $6.27 \mathrm{E}-04$ & $5.31 \mathrm{E}-04$ & $5.31 \mathrm{E}-04$ & 5.31E-04 & $5.31 \mathrm{E}-04$ & 1.07E-04 \\
\hline $\mathrm{Eu}+3$ & $4.98 \mathrm{E}-07$ & $4.98 \mathrm{E}-07$ & $4.98 \mathrm{E}-07$ & $4.98 \mathrm{E}-07$ & $4.98 \mathrm{E}-07$ & $4.98 \mathrm{E}-07$ & $4.98 \mathrm{E}-07$ & $1.01 \mathrm{E}-07$ \\
\hline F- & $1.60 \mathrm{E}-02$ & $3.21 \mathrm{E}-02$ & $3.23 \mathrm{E}-02$ & 1.57E-02 & 1.57E-02 & 1.57E-02 & 1.57E-02 & $3.17 \mathrm{E}-03$ \\
\hline $\mathrm{Gd}+3$ & $1.31 \mathrm{E}-04$ & $1.48 \mathrm{E}-04$ & $1.48 \mathrm{E}-04$ & $1.31 \mathrm{E}-04$ & 1.31E-04 & 1.31E-04 & 1.31E-04 & $2.65 \mathrm{E}-05$ \\
\hline $\mathrm{Ge}+4$ & 8.67E-09 & 8.67E-09 & 8.67E-09 & 8.67E-09 & 8.67E-09 & 8.67E-09 & 8.67E-09 & 1.75E-09 \\
\hline $\ln +3$ & $1.54 \mathrm{E}-06$ & $1.54 \mathrm{E}-06$ & $1.54 \mathrm{E}-06$ & $1.54 \mathrm{E}-06$ & $1.54 \mathrm{E}-06$ & $1.54 \mathrm{E}-06$ & $1.54 \mathrm{E}-06$ & $3.12 \mathrm{E}-07$ \\
\hline I- & $2.50 \mathrm{E}-06$ & $2.50 \mathrm{E}-06$ & $2.50 \mathrm{E}-06$ & $2.50 \mathrm{E}-06$ & $2.50 \mathrm{E}-06$ & $2.50 \mathrm{E}-06$ & $2.50 \mathrm{E}-06$ & 5.04E-07 \\
\hline $\mathrm{Fe}+3$ & $1.75 \mathrm{E}-02$ & $2.38 \mathrm{E}-02$ & $2.39 \mathrm{E}-02$ & $1.74 \mathrm{E}-02$ & $1.74 \mathrm{E}-02$ & 1.74E-02 & 1.74E-02 & $3.52 \mathrm{E}-03$ \\
\hline $\mathrm{La+3}$ & $9.06 \mathrm{E}-06$ & 9.06E-06 & $9.06 \mathrm{E}-06$ & 9.06E-06 & 9.06E-06 & 9.06E-06 & 9.06E-06 & $1.83 \mathrm{E}-06$ \\
\hline $\mathrm{Pb}+2$ & $8.24 \mathrm{E}-04$ & $8.52 \mathrm{E}-04$ & $8.53 \mathrm{E}-04$ & $8.23 \mathrm{E}-04$ & $8.23 \mathrm{E}-04$ & $8.23 \mathrm{E}-04$ & $8.23 \mathrm{E}-04$ & $1.66 \mathrm{E}-04$ \\
\hline $\mathrm{Li+}$ & $2.54 \mathrm{E}-04$ & $4.46 \mathrm{E}-04$ & $4.49 \mathrm{E}-04$ & $2.51 \mathrm{E}-04$ & $2.51 \mathrm{E}-04$ & $2.51 \mathrm{E}-04$ & $2.51 \mathrm{E}-04$ & 5.07E-05 \\
\hline$M g+2$ & $1.75 \mathrm{E}-02$ & $1.80 \mathrm{E}-02$ & $1.81 \mathrm{E}-02$ & $1.75 \mathrm{E}-02$ & $1.75 \mathrm{E}-02$ & $1.75 \mathrm{E}-02$ & $1.75 \mathrm{E}-02$ & $3.53 \mathrm{E}-03$ \\
\hline $\mathrm{Mn}+4$ & $1.35 \mathrm{E}-02$ & 1.37E-02 & 1.37E-02 & $1.35 \mathrm{E}-02$ & $1.35 \mathrm{E}-02$ & $1.35 \mathrm{E}-02$ & $1.35 \mathrm{E}-02$ & $2.73 \mathrm{E}-03$ \\
\hline $\mathrm{Hg}+2$ & $4.99 \mathrm{E}-03$ & $5.00 \mathrm{E}-03$ & $5.00 \mathrm{E}-03$ & $4.99 \mathrm{E}-03$ & $4.99 \mathrm{E}-03$ & $4.99 \mathrm{E}-03$ & $4.99 \mathrm{E}-03$ & $1.01 \mathrm{E}-03$ \\
\hline $\mathrm{Mo}+6$ & $1.99 \mathrm{E}-04$ & 7.37E-04 & $7.44 \mathrm{E}-04$ & $1.90 \mathrm{E}-04$ & 1.90E-04 & $1.90 \mathrm{E}-04$ & $1.90 \mathrm{E}-04$ & 3.85E-05 \\
\hline $\mathrm{Nd}+3$ & $2.92 \mathrm{E}-05$ & $2.92 \mathrm{E}-05$ & $2.92 \mathrm{E}-05$ & $2.92 \mathrm{E}-05$ & 2.92E-05 & $2.92 \mathrm{E}-05$ & 2.92E-05 & $5.90 \mathrm{E}-06$ \\
\hline $\mathrm{Np}+4$ & $2.34 \mathrm{E}-06$ & $2.34 \mathrm{E}-06$ & $2.34 \mathrm{E}-06$ & $2.34 \mathrm{E}-06$ & $2.34 \mathrm{E}-06$ & $2.34 \mathrm{E}-06$ & $2.34 \mathrm{E}-06$ & $4.73 \mathrm{E}-07$ \\
\hline $\mathrm{Ni}+2$ & $1.53 \mathrm{E}-03$ & $1.64 \mathrm{E}-03$ & $1.64 \mathrm{E}-03$ & $1.53 \mathrm{E}-03$ & $1.53 \mathrm{E}-03$ & $1.53 \mathrm{E}-03$ & $1.53 \mathrm{E}-03$ & $3.09 \mathrm{E}-04$ \\
\hline $\mathrm{Nb}+5$ & $3.46 \mathrm{E}-05$ & $9.26 \mathrm{E}-04$ & $9.38 \mathrm{E}-04$ & $2.03 \mathrm{E}-05$ & $2.03 \mathrm{E}-05$ & $2.03 \mathrm{E}-05$ & $2.03 \mathrm{E}-05$ & $4.10 \mathrm{E}-06$ \\
\hline NO3- & $5.65 \mathrm{E}+00$ & $5.65 \mathrm{E}+00$ & $5.65 \mathrm{E}+00$ & $5.65 \mathrm{E}+00$ & $5.65 \mathrm{E}+00$ & $5.65 \mathrm{E}+00$ & $5.65 \mathrm{E}+00$ & $1.14 \mathrm{E}+00$ \\
\hline $\mathrm{O}-2$ & $2.17 \mathrm{E}-03$ & 1.37E-01 & $1.39 \mathrm{E}-01$ & & & & & \\
\hline$P d+4$ & $2.84 \mathrm{E}-04$ & $1.77 \mathrm{E}-03$ & $1.79 \mathrm{E}-03$ & $2.61 \mathrm{E}-04$ & $2.61 \mathrm{E}-04$ & $2.61 \mathrm{E}-04$ & $2.61 \mathrm{E}-04$ & $5.27 \mathrm{E}-05$ \\
\hline PO4-3 & $1.74 \mathrm{E}-03$ & $8.55 \mathrm{E}-02$ & $8.66 \mathrm{E}-02$ & $3.90 \mathrm{E}-04$ & $3.90 \mathrm{E}-04$ & $3.90 \mathrm{E}-04$ & $3.90 \mathrm{E}-04$ & $7.88 \mathrm{E}-05$ \\
\hline $\mathrm{Pu}+4$ & $3.98 \mathrm{E}-06$ & $3.98 \mathrm{E}-06$ & $3.98 \mathrm{E}-06$ & $3.98 \mathrm{E}-06$ & $3.98 \mathrm{E}-06$ & $3.98 \mathrm{E}-06$ & $3.98 \mathrm{E}-06$ & $8.05 \mathrm{E}-07$ \\
\hline $\mathrm{K}+$ & $3.14 \mathrm{E}-01$ & 3.23E-01 & $3.24 \mathrm{E}-01$ & 3.13E-01 & 3.13E-01 & 3.13E-01 & 3.13E-01 & $6.33 \mathrm{E}-02$ \\
\hline $\mathrm{Pr}+4$ & $8.23 \mathrm{E}-06$ & $8.23 \mathrm{E}-06$ & $8.23 \mathrm{E}-06$ & $8.23 \mathrm{E}-06$ & $8.23 \mathrm{E}-06$ & $8.23 \mathrm{E}-06$ & $8.23 \mathrm{E}-06$ & $1.66 \mathrm{E}-06$ \\
\hline $\mathrm{Rh}+4$ & $3.55 \mathrm{E}-06$ & $3.55 \mathrm{E}-06$ & $3.55 \mathrm{E}-06$ & $3.55 \mathrm{E}-06$ & $3.55 \mathrm{E}-06$ & $3.55 \mathrm{E}-06$ & $3.55 \mathrm{E}-06$ & $7.18 \mathrm{E}-07$ \\
\hline
\end{tabular}


Table B-10. Tank WM-188 mass balance (continued).

\begin{tabular}{|c|c|c|c|c|c|c|c|c|}
\hline Stream \# & 121 & 122 & 123 & 124 & 125 & 126 & 127 & 128 \\
\hline Stream Name & $\begin{array}{l}\text { Neutralized } \\
\text { Feed }\end{array}$ & $\begin{array}{l}\text { SBW Liquid } \\
\text { Filter Feed }\end{array}$ & $\begin{array}{c}\text { Filter } \\
\text { Recirculation }\end{array}$ & $\begin{array}{l}\text { IX Column } \\
\text { Feed }\end{array}$ & $\begin{array}{c}\text { Column } 1 \\
\text { Effluent }\end{array}$ & $\begin{array}{c}\text { Column } 2 \\
\text { Effluent }\end{array}$ & $\begin{array}{c}\text { Column } 3 \\
\text { Effluent }\end{array}$ & $\begin{array}{l}\text { Spent IX } \\
\text { Rinse }\end{array}$ \\
\hline Chemical Composition & Mol/liter & Mol/liter & Mol/liter & Mol/liter & Mol/liter & Mol/liter & Mol/liter & Mol/liter \\
\hline $\mathrm{Rb}+$ & $5.47 \mathrm{E}-06$ & $5.47 \mathrm{E}-06$ & $5.47 \mathrm{E}-06$ & $5.47 \mathrm{E}-06$ & $5.47 \mathrm{E}-06$ & 5.47E-06 & $5.47 \mathrm{E}-06$ & $1.11 \mathrm{E}-06$ \\
\hline $\mathrm{Ru}+3$ & $1.31 \mathrm{E}-04$ & $7.52 \mathrm{E}-04$ & $7.60 \mathrm{E}-04$ & $1.21 \mathrm{E}-04$ & $1.21 \mathrm{E}-04$ & $1.21 \mathrm{E}-04$ & $1.21 \mathrm{E}-04$ & 2.44E-05 \\
\hline $\mathrm{Sm+3}$ & $5.41 \mathrm{E}-06$ & $5.41 \mathrm{E}-06$ & $5.41 \mathrm{E}-06$ & 5.41E-06 & $5.41 \mathrm{E}-06$ & $5.41 \mathrm{E}-06$ & 5.41E-06 & 1.09E-06 \\
\hline $\mathrm{Se}+4$ & 7.59E-06 & $2.39 \mathrm{E}-04$ & $2.42 \mathrm{E}-04$ & $3.87 \mathrm{E}-06$ & $3.87 \mathrm{E}-06$ & $3.87 \mathrm{E}-06$ & $3.87 \mathrm{E}-06$ & $7.83 \mathrm{E}-07$ \\
\hline $\mathrm{Si+4}$ & 1.72E-03 & 7.77E-02 & 7.87E-02 & 4.96E-04 & 4.96E-04 & 4.96E-04 & 4.96E-04 & $1.00 \mathrm{E}-04$ \\
\hline $\mathrm{Ag}+$ & 8.67E-06 & $3.64 \mathrm{E}-04$ & $3.69 \mathrm{E}-04$ & $2.95 \mathrm{E}-06$ & $2.95 \mathrm{E}-06$ & $2.95 \mathrm{E}-06$ & $2.95 \mathrm{E}-06$ & 5.96E-07 \\
\hline $\mathrm{Na}+$ & $3.22 \mathrm{E}+00$ & $3.23 \mathrm{E}+00$ & $3.23 \mathrm{E}+00$ & $3.22 \mathrm{E}+00$ & $3.22 \mathrm{E}+00$ & $3.22 \mathrm{E}+00$ & $3.22 \mathrm{E}+00$ & $6.50 \mathrm{E}-01$ \\
\hline $\mathrm{Sr}+2$ & 7.00E-05 & $7.51 \mathrm{E}-05$ & $7.51 \mathrm{E}-05$ & 6.99E-05 & 6.99E-05 & $6.99 \mathrm{E}-05$ & 6.99E-05 & 1.41E-05 \\
\hline SO4-2 & $2.33 \mathrm{E}-02$ & $2.33 \mathrm{E}-02$ & $2.33 \mathrm{E}-02$ & $2.33 \mathrm{E}-02$ & $2.33 \mathrm{E}-02$ & $2.33 \mathrm{E}-02$ & $2.33 \mathrm{E}-02$ & $4.71 \mathrm{E}-03$ \\
\hline $\mathrm{Tc}+7$ & 1.12E-05 & $1.12 \mathrm{E}-05$ & 1.12E-05 & 1.12E-05 & $1.12 \mathrm{E}-05$ & 1.12E-05 & $1.12 \mathrm{E}-05$ & $2.26 \mathrm{E}-06$ \\
\hline $\mathrm{Te}+4$ & $3.26 \mathrm{E}-06$ & $3.26 \mathrm{E}-06$ & $3.26 \mathrm{E}-06$ & $3.26 \mathrm{E}-06$ & $3.26 \mathrm{E}-06$ & $3.26 \mathrm{E}-06$ & $3.26 \mathrm{E}-06$ & $6.59 \mathrm{E}-07$ \\
\hline $\mathrm{Tb}+4$ & $2.08 \mathrm{E}-09$ & $2.08 \mathrm{E}-09$ & $2.08 \mathrm{E}-09$ & 2.08E-09 & $2.08 \mathrm{E}-09$ & $2.08 \mathrm{E}-09$ & $2.08 \mathrm{E}-09$ & $4.21 \mathrm{E}-10$ \\
\hline $\mathrm{Th}+4$ & $2.31 \mathrm{E}-05$ & $2.31 \mathrm{E}-05$ & $2.31 \mathrm{E}-05$ & $2.31 \mathrm{E}-05$ & $2.31 \mathrm{E}-05$ & $2.31 \mathrm{E}-05$ & $2.31 \mathrm{E}-05$ & 4.67E-06 \\
\hline $\mathrm{Sn+4}$ & $5.86 \mathrm{E}-05$ & $1.65 \mathrm{E}-03$ & 1.67E-03 & $3.30 \mathrm{E}-05$ & $3.30 \mathrm{E}-05$ & $3.30 \mathrm{E}-05$ & $3.30 \mathrm{E}-05$ & 6.67E-06 \\
\hline $\mathrm{Ti+4}$ & $5.75 \mathrm{E}-05$ & 8.37E-04 & $8.47 \mathrm{E}-04$ & $4.50 \mathrm{E}-05$ & $4.50 \mathrm{E}-05$ & $4.50 \mathrm{E}-05$ & $4.50 \mathrm{E}-05$ & $9.09 \mathrm{E}-06$ \\
\hline $\mathrm{U}+4$ & $7.28 \mathrm{E}-04$ & $7.47 \mathrm{E}-04$ & 7.47E-04 & $7.27 \mathrm{E}-04$ & $7.27 \mathrm{E}-04$ & $7.27 \mathrm{E}-04$ & $7.27 \mathrm{E}-04$ & 1.47E-04 \\
\hline $\mathrm{V}+5$ & $2.03 \mathrm{E}-05$ & $2.49 \mathrm{E}-05$ & $2.50 \mathrm{E}-05$ & $2.03 E-05$ & $2.03 \mathrm{E}-05$ & $2.03 \mathrm{E}-05$ & $2.03 \mathrm{E}-05$ & $4.10 \mathrm{E}-06$ \\
\hline $\mathrm{Y}+3$ & $6.75 \mathrm{E}-06$ & $6.75 \mathrm{E}-06$ & $6.75 \mathrm{E}-06$ & $6.75 \mathrm{E}-06$ & $6.75 \mathrm{E}-06$ & $6.75 \mathrm{E}-06$ & $6.75 \mathrm{E}-06$ & 1.36E-06 \\
\hline$Z n+2$ & $6.74 \mathrm{E}-04$ & $7.27 \mathrm{E}-04$ & $7.28 \mathrm{E}-04$ & $6.73 \mathrm{E}-04$ & $6.73 \mathrm{E}-04$ & $6.73 \mathrm{E}-04$ & $6.73 \mathrm{E}-04$ & $1.36 \mathrm{E}-04$ \\
\hline $\mathrm{Zr}+4$ & $2.64 \mathrm{E}-03$ & $3.49 \mathrm{E}-02$ & $3.54 \mathrm{E}-02$ & $2.12 \mathrm{E}-03$ & $2.12 \mathrm{E}-03$ & $2.12 \mathrm{E}-03$ & $2.12 \mathrm{E}-03$ & $4.28 \mathrm{E}-04$ \\
\hline $\mathrm{H} 2 \mathrm{O}$ & $4.81 \mathrm{E}+01$ & $4.71 \mathrm{E}+01$ & $4.71 \mathrm{E}+01$ & $4.81 \mathrm{E}+01$ & $4.81 \mathrm{E}+01$ & $4.81 \mathrm{E}+01$ & $4.81 \mathrm{E}+01$ & $5.37 \mathrm{E}+01$ \\
\hline & & & & & & & & \\
\hline Radiological Composition & $\mathrm{Ci} / \mathrm{liter}$ & $\mathrm{Ci} /$ liter & $\mathrm{Ci} /$ liter & $\mathrm{Ci} /$ liter & $\mathrm{Ci} /$ liter & $\mathrm{Ci} /$ liter & $\mathrm{Ci} /$ liter & $\mathrm{Ci} / \mathrm{liter}$ \\
\hline Ra-226 & $8.81 \mathrm{E}-12$ & $8.81 \mathrm{E}-12$ & $8.81 \mathrm{E}-12$ & $8.81 \mathrm{E}-12$ & $8.81 \mathrm{E}-12$ & $8.81 \mathrm{E}-12$ & $8.81 \mathrm{E}-12$ & $1.78 \mathrm{E}-12$ \\
\hline Ac-227 & $4.15 \mathrm{E}-11$ & $4.15 \mathrm{E}-11$ & $4.15 \mathrm{E}-11$ & $4.15 \mathrm{E}-11$ & $4.15 \mathrm{E}-11$ & $4.15 \mathrm{E}-11$ & $4.15 \mathrm{E}-11$ & $8.39 \mathrm{E}-12$ \\
\hline Th-228 & $3.44 \mathrm{E}-09$ & $3.44 \mathrm{E}-09$ & $3.44 \mathrm{E}-09$ & $3.44 \mathrm{E}-09$ & 3.44E-09 & $3.44 \mathrm{E}-09$ & $3.44 \mathrm{E}-09$ & $6.96 \mathrm{E}-10$ \\
\hline Th-230 & $8.88 \mathrm{E}-10$ & $1.08 \mathrm{E}-09$ & 1.09E-09 & $8.85 \mathrm{E}-10$ & $8.85 \mathrm{E}-10$ & $8.85 \mathrm{E}-10$ & $8.85 \mathrm{E}-10$ & $1.79 \mathrm{E}-10$ \\
\hline Th-232 & $7.62 \mathrm{E}-16$ & $6.67 \mathrm{E}-11$ & $7.62 \mathrm{E}-16$ & $7.62 \mathrm{E}-16$ & $7.62 \mathrm{E}-16$ & $7.62 \mathrm{E}-16$ & $7.62 \mathrm{E}-16$ & $1.54 \mathrm{E}-16$ \\
\hline Pa-231 & $9.61 \mathrm{E}-11$ & $9.61 \mathrm{E}-11$ & $9.61 \mathrm{E}-11$ & $9.61 \mathrm{E}-11$ & $9.61 \mathrm{E}-11$ & $9.61 \mathrm{E}-11$ & $9.61 \mathrm{E}-11$ & $1.94 \mathrm{E}-11$ \\
\hline Pa-233 & $3.15 \mathrm{E}-06$ & $3.15 \mathrm{E}-06$ & $3.15 \mathrm{E}-06$ & $3.15 \mathrm{E}-06$ & $3.15 \mathrm{E}-06$ & $3.15 \mathrm{E}-06$ & $3.15 \mathrm{E}-06$ & $6.37 \mathrm{E}-07$ \\
\hline $\mathrm{U}-232$ & $2.14 \mathrm{E}-09$ & $2.55 \mathrm{E}-09$ & $2.56 \mathrm{E}-09$ & $2.14 \mathrm{E}-09$ & $2.14 \mathrm{E}-09$ & $2.14 \mathrm{E}-09$ & $2.14 \mathrm{E}-09$ & $4.32 \mathrm{E}-10$ \\
\hline $\mathrm{U}-233$ & $8.61 \mathrm{E}-11$ & $9.31 \mathrm{E}-11$ & $9.32 \mathrm{E}-11$ & $8.60 \mathrm{E}-11$ & $8.60 \mathrm{E}-11$ & $8.60 \mathrm{E}-11$ & $8.60 \mathrm{E}-11$ & $1.74 \mathrm{E}-11$ \\
\hline U-234 & $9.58 \mathrm{E}-07$ & $1.08 \mathrm{E}-06$ & $1.08 \mathrm{E}-06$ & $9.56 \mathrm{E}-07$ & $9.56 \mathrm{E}-07$ & $9.56 \mathrm{E}-07$ & $9.56 \mathrm{E}-07$ & $1.93 \mathrm{E}-07$ \\
\hline$U-235$ & $7.99 \mathrm{E}-08$ & $8.63 \mathrm{E}-08$ & $8.64 \mathrm{E}-08$ & $7.98 \mathrm{E}-08$ & $7.98 \mathrm{E}-08$ & $7.98 \mathrm{E}-08$ & $7.98 \mathrm{E}-08$ & $1.61 \mathrm{E}-08$ \\
\hline U-236 & $3.72 \mathrm{E}-08$ & $4.56 \mathrm{E}-08$ & $4.57 \mathrm{E}-08$ & $3.70 \mathrm{E}-08$ & $3.70 \mathrm{E}-08$ & $3.70 \mathrm{E}-08$ & $3.70 \mathrm{E}-08$ & $7.49 \mathrm{E}-09$ \\
\hline U-237 & $6.92 \mathrm{E}-09$ & $6.92 \mathrm{E}-09$ & $6.92 \mathrm{E}-09$ & $6.92 \mathrm{E}-09$ & $6.92 \mathrm{E}-09$ & $6.92 \mathrm{E}-09$ & $6.92 \mathrm{E}-09$ & $1.40 \mathrm{E}-09$ \\
\hline $\mathrm{U}-238$ & $1.15 \mathrm{E}-08$ & $1.20 \mathrm{E}-08$ & $1.20 \mathrm{E}-08$ & $1.15 \mathrm{E}-08$ & $1.15 \mathrm{E}-08$ & $1.15 \mathrm{E}-08$ & $1.15 \mathrm{E}-08$ & $2.32 \mathrm{E}-09$ \\
\hline Np-236 & $3.31 \mathrm{E}-12$ & $3.31 \mathrm{E}-12$ & $3.31 \mathrm{E}-12$ & $3.31 \mathrm{E}-12$ & $3.31 \mathrm{E}-12$ & $3.31 \mathrm{E}-12$ & $3.31 \mathrm{E}-12$ & $6.68 \mathrm{E}-13$ \\
\hline Np-237 & $3.92 \mathrm{E}-07$ & $4.29 \mathrm{E}-07$ & $4.29 \mathrm{E}-07$ & $3.91 \mathrm{E}-07$ & $3.91 \mathrm{E}-07$ & $3.91 \mathrm{E}-07$ & $3.91 \mathrm{E}-07$ & $7.90 \mathrm{E}-08$ \\
\hline Pu-236 & $2.96 \mathrm{E}-09$ & $3.66 \mathrm{E}-09$ & $3.67 \mathrm{E}-09$ & $2.95 \mathrm{E}-09$ & $2.95 \mathrm{E}-09$ & $2.95 \mathrm{E}-09$ & $2.95 \mathrm{E}-09$ & $5.97 \mathrm{E}-10$ \\
\hline Pu-238 & $4.63 \mathrm{E}-04$ & $8.39 \mathrm{E}-04$ & $8.44 \mathrm{E}-04$ & $4.57 \mathrm{E}-04$ & $4.57 \mathrm{E}-04$ & $4.57 \mathrm{E}-04$ & $4.57 \mathrm{E}-04$ & $9.23 \mathrm{E}-05$ \\
\hline Pu-239 & $5.49 \mathrm{E}-05$ & $9.49 \mathrm{E}-05$ & $9.54 \mathrm{E}-05$ & $5.42 \mathrm{E}-05$ & $5.42 \mathrm{E}-05$ & $5.42 \mathrm{E}-05$ & $5.42 \mathrm{E}-05$ & $1.10 \mathrm{E}-05$ \\
\hline Pu-240 & $1.10 \mathrm{E}-05$ & 1.37E-05 & $1.37 \mathrm{E}-05$ & 1.09E-05 & $1.09 \mathrm{E}-05$ & $1.09 \mathrm{E}-05$ & $1.09 \mathrm{E}-05$ & $2.21 \mathrm{E}-06$ \\
\hline Pu-241 & $2.90 \mathrm{E}-04$ & $5.87 \mathrm{E}-04$ & $5.91 \mathrm{E}-04$ & $2.86 \mathrm{E}-04$ & $2.86 \mathrm{E}-04$ & $2.86 \mathrm{E}-04$ & $2.86 \mathrm{E}-04$ & $5.77 \mathrm{E}-05$ \\
\hline Pu-242 & $8.57 \mathrm{E}-09$ & $1.06 \mathrm{E}-08$ & $1.06 \mathrm{E}-08$ & $8.53 \mathrm{E}-09$ & $8.53 \mathrm{E}-09$ & $8.53 \mathrm{E}-09$ & $8.53 \mathrm{E}-09$ & $1.72 \mathrm{E}-09$ \\
\hline Pu-244 & $7.22 \mathrm{E}-16$ & $8.97 \mathrm{E}-16$ & $8.99 \mathrm{E}-16$ & $7.20 \mathrm{E}-16$ & $7.20 \mathrm{E}-16$ & $7.20 \mathrm{E}-16$ & $7.20 \mathrm{E}-16$ & $1.45 \mathrm{E}-16$ \\
\hline Am-241 & $4.83 \mathrm{E}-05$ & $5.81 \mathrm{E}-05$ & $5.82 \mathrm{E}-05$ & $4.81 \mathrm{E}-05$ & $4.81 \mathrm{E}-05$ & $4.81 \mathrm{E}-05$ & $4.81 \mathrm{E}-05$ & $9.72 \mathrm{E}-06$ \\
\hline$A m-242 m$ & $1.68 \mathrm{E}-08$ & $1.68 \mathrm{E}-08$ & $1.68 \mathrm{E}-08$ & $1.68 \mathrm{E}-08$ & $1.68 \mathrm{E}-08$ & $1.68 \mathrm{E}-08$ & $1.68 \mathrm{E}-08$ & $3.40 \mathrm{E}-09$ \\
\hline Am-243 & $2.38 \mathrm{E}-08$ & $2.73 \mathrm{E}-08$ & $2.73 \mathrm{E}-08$ & $2.37 \mathrm{E}-08$ & $2.37 \mathrm{E}-08$ & $2.37 \mathrm{E}-08$ & $2.37 \mathrm{E}-08$ & $4.79 \mathrm{E}-09$ \\
\hline $\mathrm{Cm}-242$ & $3.68 \mathrm{E}-08$ & $3.68 \mathrm{E}-08$ & $3.68 \mathrm{E}-08$ & $3.68 \mathrm{E}-08$ & $3.68 \mathrm{E}-08$ & $3.68 \mathrm{E}-08$ & $3.68 \mathrm{E}-08$ & $7.44 \mathrm{E}-09$ \\
\hline
\end{tabular}


Table B-10. Tank WM-188 mass balance (continued).

\begin{tabular}{|c|c|c|c|c|c|c|c|c|}
\hline Stream \# & 121 & 122 & 123 & 124 & 125 & 126 & 127 & 128 \\
\hline Stream Name & $\begin{array}{l}\text { Neutralized } \\
\text { Feed }\end{array}$ & $\begin{array}{c}\text { SBW Liquid } \\
\text { Filter Feed }\end{array}$ & $\begin{array}{c}\text { Filter } \\
\text { Recirculation }\end{array}$ & $\begin{array}{l}\text { IX Column } \\
\text { Feed }\end{array}$ & $\begin{array}{c}\text { Column } 1 \\
\text { Effluent }\end{array}$ & $\begin{array}{c}\text { Column } 2 \\
\text { Effluent }\end{array}$ & $\begin{array}{c}\text { Column } 3 \\
\text { Effluent }\end{array}$ & $\begin{array}{l}\text { Spent IX } \\
\text { Rinse }\end{array}$ \\
\hline & Ci/liter & Ci/liter & Ci/liter & Ci/liter & Ci/liter & $\mathrm{Ci} /$ liter & Ci/liter & Ci/liter \\
\hline $\mathrm{Cm}-243$ & $3.07 \mathrm{E}-08$ & $3.79 \mathrm{E}-08$ & $3.80 \mathrm{E}-08$ & $3.06 \mathrm{E}-08$ & $3.06 \mathrm{E}-08$ & 3.06E-08 & $3.06 \mathrm{E}-08$ & $6.18 \mathrm{E}-09$ \\
\hline $\mathrm{Cm}-244$ & 8.37E-07 & 1.29E-06 & $1.29 \mathrm{E}-06$ & 8.30E-07 & 8.30E-07 & $8.30 \mathrm{E}-07$ & 8.30E-07 & $1.68 \mathrm{E}-07$ \\
\hline $\mathrm{Cm}-245$ & $3.22 \mathrm{E}-10$ & $3.98 \mathrm{E}-10$ & $3.99 \mathrm{E}-10$ & $3.21 \mathrm{E}-10$ & $3.21 \mathrm{E}-10$ & $3.21 \mathrm{E}-10$ & $3.21 \mathrm{E}-10$ & $6.49 \mathrm{E}-11$ \\
\hline $\mathrm{Cm}-246$ & $2.12 \mathrm{E}-11$ & $2.62 \mathrm{E}-11$ & $2.62 \mathrm{E}-11$ & $2.12 \mathrm{E}-11$ & $2.12 \mathrm{E}-11$ & $2.12 \mathrm{E}-11$ & $2.12 \mathrm{E}-11$ & $4.28 \mathrm{E}-12$ \\
\hline $\mathrm{H}-3$ & $1.24 \mathrm{E}-05$ & $1.24 \mathrm{E}-05$ & $1.24 \mathrm{E}-05$ & 1.24E-05 & 1.24E-05 & $1.24 \mathrm{E}-05$ & 1.24E-05 & $2.51 \mathrm{E}-06$ \\
\hline $\mathrm{Be}-10$ & $3.23 \mathrm{E}-12$ & $3.23 \mathrm{E}-12$ & $3.23 \mathrm{E}-12$ & $3.23 \mathrm{E}-12$ & $3.23 \mathrm{E}-12$ & $3.23 \mathrm{E}-12$ & $3.23 \mathrm{E}-12$ & $6.52 \mathrm{E}-13$ \\
\hline C-14 & $1.30 \mathrm{E}-10$ & 1.62E-10 & $1.62 \mathrm{E}-10$ & $1.29 \mathrm{E}-10$ & $1.29 \mathrm{E}-10$ & $1.29 \mathrm{E}-10$ & $1.29 \mathrm{E}-10$ & $2.61 \mathrm{E}-11$ \\
\hline Se-79 & $4.72 \mathrm{E}-07$ & 5.87E-07 & 5.89E-07 & 4.70E-07 & 4.70E-07 & 4.70E-07 & 4.70E-07 & $9.50 \mathrm{E}-08$ \\
\hline $\mathrm{Rb}-87$ & $3.15 \mathrm{E}-11$ & $3.15 \mathrm{E}-11$ & $3.15 \mathrm{E}-11$ & $3.15 \mathrm{E}-11$ & $3.15 \mathrm{E}-11$ & $3.15 \mathrm{E}-11$ & $3.15 \mathrm{E}-11$ & $6.37 \mathrm{E}-12$ \\
\hline Sr-90 & 4.07E-02 & 4.09E-02 & $4.09 \mathrm{E}-02$ & 4.07E-02 & 4.07E-02 & 4.07E-02 & 4.07E-02 & $8.22 \mathrm{E}-03$ \\
\hline Y-90 & 4.07E-02 & 4.09E-02 & 4.09E-02 & 4.07E-02 & 4.07E-02 & 4.07E-02 & 4.07E-02 & $8.22 \mathrm{E}-03$ \\
\hline Zr-93 & $2.38 \mathrm{E}-06$ & $2.38 \mathrm{E}-06$ & $2.38 \mathrm{E}-06$ & $2.38 \mathrm{E}-06$ & $2.38 \mathrm{E}-06$ & $2.38 \mathrm{E}-06$ & $2.38 \mathrm{E}-06$ & $4.82 \mathrm{E}-07$ \\
\hline $\mathrm{Nb}-93 \mathrm{~m}$ & 1.84E-06 & 1.84E-06 & 1.84E-06 & 1.84E-06 & 1.84E-06 & 1.84E-06 & $1.84 \mathrm{E}-06$ & 3.71E-07 \\
\hline $\mathrm{Nb}-94$ & $1.23 \mathrm{E}-06$ & 1.53E-06 & $1.53 \mathrm{E}-06$ & $1.23 \mathrm{E}-06$ & $1.23 \mathrm{E}-06$ & $1.23 \mathrm{E}-06$ & $1.23 \mathrm{E}-06$ & $2.48 \mathrm{E}-07$ \\
\hline Tc-98 & $2.77 \mathrm{E}-12$ & $2.77 \mathrm{E}-12$ & $2.77 \mathrm{E}-12$ & $2.77 \mathrm{E}-12$ & $2.77 \mathrm{E}-12$ & $2.77 \mathrm{E}-12$ & $2.77 \mathrm{E}-12$ & $5.61 \mathrm{E}-13$ \\
\hline Tc-99 & $1.88 \mathrm{E}-05$ & $2.14 \mathrm{E}-05$ & $2.14 \mathrm{E}-05$ & 1.87E-05 & 1.87E-05 & 1.87E-05 & 1.87E-05 & $3.79 \mathrm{E}-06$ \\
\hline Ru-106 & $1.00 \mathrm{E}-06$ & 1.25E-06 & $1.26 \mathrm{E}-06$ & 1.00E-06 & 1.00E-06 & 1.00E-06 & $1.00 \mathrm{E}-06$ & $2.02 \mathrm{E}-07$ \\
\hline Rh-102 & 4.95E-09 & $2.55 \mathrm{E}-07$ & $2.58 \mathrm{E}-07$ & $9.27 \mathrm{E}-10$ & $9.27 \mathrm{E}-10$ & $9.27 \mathrm{E}-10$ & $9.27 \mathrm{E}-10$ & $1.87 \mathrm{E}-10$ \\
\hline Pd-107 & $1.78 \mathrm{E}-08$ & $1.78 \mathrm{E}-08$ & $1.78 \mathrm{E}-08$ & $1.78 \mathrm{E}-08$ & $1.78 \mathrm{E}-08$ & $1.78 \mathrm{E}-08$ & $1.78 \mathrm{E}-08$ & 3.59E-09 \\
\hline Cd-113m & 3.57E-06 & 3.57E-06 & $3.57 \mathrm{E}-06$ & $3.57 \mathrm{E}-06$ & $3.57 \mathrm{E}-06$ & 3.57E-06 & $3.57 \mathrm{E}-06$ & $7.22 \mathrm{E}-07$ \\
\hline $\ln -115$ & $1.08 \mathrm{E}-16$ & $1.08 \mathrm{E}-16$ & $1.08 \mathrm{E}-16$ & $1.08 \mathrm{E}-16$ & $1.08 \mathrm{E}-16$ & $1.08 \mathrm{E}-16$ & $1.08 \mathrm{E}-16$ & $2.19 \mathrm{E}-17$ \\
\hline Sn-121m & 7.19E-08 & 7.19E-08 & $7.19 \mathrm{E}-08$ & $7.19 \mathrm{E}-08$ & $7.19 \mathrm{E}-08$ & 7.19E-08 & $7.19 \mathrm{E}-08$ & $1.45 \mathrm{E}-08$ \\
\hline Sn-126 & 4.44E-07 & 5.53E-07 & $5.54 \mathrm{E}-07$ & 4.42E-07 & $4.42 \mathrm{E}-07$ & 4.42E-07 & $4.42 \mathrm{E}-07$ & $8.93 \mathrm{E}-08$ \\
\hline Sb-125 & $1.97 \mathrm{E}-05$ & $3.78 \mathrm{E}-04$ & $3.83 E-04$ & $1.39 \mathrm{E}-05$ & $1.39 \mathrm{E}-05$ & $1.39 \mathrm{E}-05$ & $1.39 \mathrm{E}-05$ & $2.81 \mathrm{E}-06$ \\
\hline Sb-126 & $6.19 \mathrm{E}-08$ & $6.19 \mathrm{E}-08$ & $6.19 \mathrm{E}-08$ & $6.19 \mathrm{E}-08$ & $6.19 \mathrm{E}-08$ & $6.19 \mathrm{E}-08$ & $6.19 \mathrm{E}-08$ & $1.25 \mathrm{E}-08$ \\
\hline Te-123 & $4.12 \mathrm{E}-19$ & $4.12 \mathrm{E}-19$ & $4.12 \mathrm{E}-19$ & $4.12 \mathrm{E}-19$ & $4.12 \mathrm{E}-19$ & $4.12 \mathrm{E}-19$ & $4.12 \mathrm{E}-19$ & $8.33 \mathrm{E}-20$ \\
\hline Te-125m & $3.39 \mathrm{E}-06$ & 3.39E-06 & $3.39 \mathrm{E}-06$ & $3.39 \mathrm{E}-06$ & $3.39 \mathrm{E}-06$ & $3.39 \mathrm{E}-06$ & $3.39 \mathrm{E}-06$ & $6.85 \mathrm{E}-07$ \\
\hline I-129 & $5.76 \mathrm{E}-08$ & $7.17 \mathrm{E}-08$ & 7.19E-08 & $5.73 \mathrm{E}-08$ & $5.73 \mathrm{E}-08$ & $5.73 \mathrm{E}-08$ & 5.73E-08 & $1.16 \mathrm{E}-08$ \\
\hline Cs-134 & $4.31 \mathrm{E}-06$ & $3.36 \mathrm{E}-05$ & 3.39E-05 & $3.84 \mathrm{E}-06$ & $3.84 \mathrm{E}-07$ & 3.84E-08 & $3.84 \mathrm{E}-09$ & $7.76 \mathrm{E}-10$ \\
\hline Cs-135 & $9.28 \mathrm{E}-07$ & $1.13 \mathrm{E}-06$ & $1.13 \mathrm{E}-06$ & $9.25 \mathrm{E}-07$ & $9.25 \mathrm{E}-08$ & $9.25 \mathrm{E}-09$ & $9.25 \mathrm{E}-10$ & $1.87 \mathrm{E}-10$ \\
\hline Cs-137 & $6.51 \mathrm{E}-03$ & 1.81E-02 & $1.82 \mathrm{E}-02$ & $6.32 \mathrm{E}-03$ & $6.32 \mathrm{E}-04$ & $6.32 \mathrm{E}-05$ & $6.32 \mathrm{E}-06$ & $1.28 \mathrm{E}-06$ \\
\hline $\mathrm{Ba}-137 \mathrm{~m}$ & $6.16 \mathrm{E}-03$ & $1.71 \mathrm{E}-02$ & $1.72 \mathrm{E}-02$ & $5.98 \mathrm{E}-03$ & $5.98 \mathrm{E}-03$ & $5.98 \mathrm{E}-03$ & $5.98 \mathrm{E}-03$ & $1.21 \mathrm{E}-03$ \\
\hline La-138 & $2.05 \mathrm{E}-16$ & $2.05 \mathrm{E}-16$ & $2.05 \mathrm{E}-16$ & $2.05 \mathrm{E}-16$ & $2.05 \mathrm{E}-16$ & $2.05 \mathrm{E}-16$ & $2.05 \mathrm{E}-16$ & $4.15 \mathrm{E}-17$ \\
\hline Ce-142 & $3.22 \mathrm{E}-11$ & $3.22 \mathrm{E}-11$ & $3.22 \mathrm{E}-11$ & $3.22 \mathrm{E}-11$ & $3.22 \mathrm{E}-11$ & $3.22 \mathrm{E}-11$ & $3.22 \mathrm{E}-11$ & $6.50 \mathrm{E}-12$ \\
\hline Ce-144 & $6.77 \mathrm{E}-07$ & 8.47E-07 & $8.49 \mathrm{E}-07$ & $6.74 \mathrm{E}-07$ & $6.74 \mathrm{E}-07$ & $6.74 \mathrm{E}-07$ & $6.74 \mathrm{E}-07$ & $1.36 \mathrm{E}-07$ \\
\hline Nd-144 & $1.73 \mathrm{E}-15$ & $1.73 \mathrm{E}-15$ & $1.73 \mathrm{E}-15$ & $1.73 \mathrm{E}-15$ & $1.73 \mathrm{E}-15$ & $1.73 \mathrm{E}-15$ & $1.73 \mathrm{E}-15$ & $3.50 \mathrm{E}-16$ \\
\hline Pm-146 & $5.47 \mathrm{E}-08$ & $5.47 \mathrm{E}-08$ & $5.47 \mathrm{E}-08$ & $5.47 \mathrm{E}-08$ & $5.47 \mathrm{E}-08$ & $5.47 \mathrm{E}-08$ & $5.47 \mathrm{E}-08$ & $1.11 \mathrm{E}-08$ \\
\hline Pm-147 & $1.84 \mathrm{E}-04$ & $2.29 \mathrm{E}-04$ & $2.29 \mathrm{E}-04$ & $1.83 \mathrm{E}-04$ & $1.83 \mathrm{E}-04$ & 1.83E-04 & $1.83 \mathrm{E}-04$ & $3.70 \mathrm{E}-05$ \\
\hline Sm-146 & $2.97 \mathrm{E}-13$ & $2.97 \mathrm{E}-13$ & $2.97 \mathrm{E}-13$ & $2.97 \mathrm{E}-13$ & $2.97 \mathrm{E}-13$ & $2.97 \mathrm{E}-13$ & $2.97 \mathrm{E}-13$ & $6.00 \mathrm{E}-14$ \\
\hline Sm-147 & $7.92 \mathrm{E}-12$ & $7.92 \mathrm{E}-12$ & $7.92 \mathrm{E}-12$ & $7.92 \mathrm{E}-12$ & $7.92 \mathrm{E}-12$ & $7.92 \mathrm{E}-12$ & $7.92 \mathrm{E}-12$ & $1.60 \mathrm{E}-12$ \\
\hline Sm-148 & $4.07 \mathrm{E}-17$ & $4.07 \mathrm{E}-17$ & $4.07 \mathrm{E}-17$ & $4.07 \mathrm{E}-17$ & $4.07 \mathrm{E}-17$ & $4.07 \mathrm{E}-17$ & $4.07 \mathrm{E}-17$ & $8.23 \mathrm{E}-18$ \\
\hline Sm-149 & $3.61 \mathrm{E}-18$ & $3.61 \mathrm{E}-18$ & $3.61 \mathrm{E}-18$ & $3.61 \mathrm{E}-18$ & $3.61 \mathrm{E}-18$ & $3.61 \mathrm{E}-18$ & $3.61 \mathrm{E}-18$ & $7.30 \mathrm{E}-19$ \\
\hline Sm-151 & 3.63E-04 & 4.52E-04 & 4.53E-04 & 3.61E-04 & $3.61 \mathrm{E}-04$ & 3.61E-04 & $3.61 \mathrm{E}-04$ & $7.30 \mathrm{E}-05$ \\
\hline Eu-152 & $2.71 \mathrm{E}-06$ & 3.03E-06 & $3.03 \mathrm{E}-06$ & $2.71 \mathrm{E}-06$ & $2.71 \mathrm{E}-06$ & $2.71 \mathrm{E}-06$ & $2.71 \mathrm{E}-06$ & $5.47 \mathrm{E}-07$ \\
\hline Eu-154 & $2.09 \mathrm{E}-05$ & $3.35 \mathrm{E}-05$ & $3.36 \mathrm{E}-05$ & $2.07 E-05$ & $2.07 E-05$ & $2.07 \mathrm{E}-05$ & $2.07 E-05$ & 4.19E-06 \\
\hline Eu-155 & $7.78 \mathrm{E}-06$ & $2.83 \mathrm{E}-05$ & $2.86 \mathrm{E}-05$ & $7.45 \mathrm{E}-06$ & $7.45 \mathrm{E}-06$ & $7.45 \mathrm{E}-06$ & $7.45 \mathrm{E}-06$ & $1.51 \mathrm{E}-06$ \\
\hline Gd-152 & $1.53 \mathrm{E}-18$ & $1.53 \mathrm{E}-18$ & $1.53 \mathrm{E}-18$ & $1.53 \mathrm{E}-18$ & $1.53 \mathrm{E}-18$ & $1.53 \mathrm{E}-18$ & $1.53 \mathrm{E}-18$ & $3.09 \mathrm{E}-19$ \\
\hline Ho-166m & $4.95 \mathrm{E}-11$ & $4.95 \mathrm{E}-11$ & $4.95 \mathrm{E}-11$ & $4.95 \mathrm{E}-11$ & $4.95 \mathrm{E}-11$ & $4.95 \mathrm{E}-11$ & $4.95 \mathrm{E}-11$ & $1.00 \mathrm{E}-11$ \\
\hline Co-60 & $4.16 \mathrm{E}-06$ & $5.28 \mathrm{E}-06$ & $5.29 \mathrm{E}-06$ & $4.14 \mathrm{E}-06$ & $4.14 \mathrm{E}-06$ & $4.14 \mathrm{E}-06$ & $4.14 \mathrm{E}-06$ & 8.37E-07 \\
\hline Ni-63 & $3.43 \mathrm{E}-05$ & $4.27 \mathrm{E}-05$ & $4.28 \mathrm{E}-05$ & $3.42 \mathrm{E}-05$ & $3.42 \mathrm{E}-05$ & $3.42 \mathrm{E}-05$ & $3.42 \mathrm{E}-05$ & $6.91 \mathrm{E}-06$ \\
\hline
\end{tabular}


Table B-10. Tank WM-188 mass balance (continued).

\begin{tabular}{|c|c|c|c|c|c|c|c|c|}
\hline PFD Sheet \# & & PFD-4 & PFD Sheet \# & & PFD-4 & & & PFD-4 \\
\hline Stream \# & 129 & 130 & Stream \# & 129 & 130 & & 129 & 130 \\
\hline Stream Name & $\begin{array}{c}\text { Spent IX } \\
\text { Media }\end{array}$ & $\begin{array}{l}\text { Grouted } \\
\text { Waste }\end{array}$ & Stream Name & $\begin{array}{c}\text { Spent IX } \\
\text { Media }\end{array}$ & $\begin{array}{l}\text { Grouted } \\
\text { Waste }\end{array}$ & & $\begin{array}{c}\text { Spent IX } \\
\text { Media }\end{array}$ & $\begin{array}{l}\text { Grouted } \\
\text { Waste }\end{array}$ \\
\hline Rate, gal $/ \mathrm{hr}$ & 0.13 & 112 & & Wt \% & Wt $\%$ & & $\mathrm{Ci} / \mathrm{kg}$ & $\mathrm{Ci} / \mathrm{kg}$ \\
\hline Rate, Ib/hr & 1.45 & 1405 & $\mathrm{Rb}+$ & $2.36 \mathrm{E}-06$ & $2.63 \mathrm{E}-05$ & $\mathrm{Cm}-243$ & $6.18 \mathrm{E}-09$ & $1.72 \mathrm{E}-08$ \\
\hline Rate, total columns & 8 & N/A & $\mathrm{Ru}+3$ & $6.17 \mathrm{E}-05$ & $6.88 \mathrm{E}-04$ & $\mathrm{Cm}-244$ & $1.68 \mathrm{E}-07$ & 4.67E-07 \\
\hline Operation & Batch & Cont & $\mathrm{Sm}+3$ & $4.11 \mathrm{E}-06$ & $4.58 \mathrm{E}-05$ & $\mathrm{Cm}-245$ & $6.49 \mathrm{E}-11$ & $1.81 \mathrm{E}-10$ \\
\hline Temperature, ${ }^{\circ} \mathrm{F}$ & 70 & 70 & $\mathrm{Se}+4$ & $1.54 \mathrm{E}-06$ & $1.72 \mathrm{E}-05$ & $\mathrm{Cm}-246$ & & \\
\hline Pressure, psia & 12.8 & 12.8 & $\mathrm{Si}+4$ & $7.03 \mathrm{E}-05$ & $7.84 \mathrm{E}-04$ & & $4.28 \mathrm{E}-12$ & $1.19 \mathrm{E}-11$ \\
\hline Specific Gravity & 1.34 & 1.50 & $\mathrm{Ag}^{+}$ & 1.61E-06 & 1.79E-05 & $\mathrm{H}-3$ & $2.51 \mathrm{E}-06$ & $7.00 \mathrm{E}-06$ \\
\hline Chemical Composition & $\mathrm{Wt} \%$ & Wt \% & $\mathrm{Na}+$ & $3.73 \mathrm{E}-01$ & $4.16 \mathrm{E}+00$ & $\mathrm{Be}-10$ & $6.52 \mathrm{E}-13$ & $1.82 \mathrm{E}-12$ \\
\hline CST & $7.27 \mathrm{E}+01$ & $\mathrm{~N} / \mathrm{A}$ & $\mathrm{Sr}+2$ & $3.10 \mathrm{E}-05$ & $3.45 \mathrm{E}-04$ & C-14 & $2.61 \mathrm{E}-11$ & $7.27 \mathrm{E}-11$ \\
\hline $\mathrm{H}+$ & $2.55 \mathrm{E}-03$ & $\mathrm{~N} / \mathrm{A}$ & SO4-2 & $1.13 \mathrm{E}-02$ & $1.26 \mathrm{E}-01$ & Se-79 & $9.50 \mathrm{E}-08$ & $2.65 \mathrm{E}-07$ \\
\hline $\mathrm{Al}+3$ & $6.26 \mathrm{E}-02$ & $6.98 \mathrm{E}-01$ & $\mathrm{Tc}+7$ & $5.47 \mathrm{E}-06$ & $6.10 \mathrm{E}-05$ & $\mathrm{Rb}-87$ & 6.37E-12 & $1.78 \mathrm{E}-11$ \\
\hline $\mathrm{Am}+4$ & $7.00 \mathrm{E}-08$ & $7.80 \mathrm{E}-07$ & $\mathrm{Te}+4$ & $2.10 \mathrm{E}-06$ & $2.34 \mathrm{E}-05$ & Sr-90 & $8.22 \mathrm{E}-03$ & $2.29 \mathrm{E}-02$ \\
\hline $\mathrm{Sb}+5$ & $2.70 \mathrm{E}-06$ & $3.01 \mathrm{E}-05$ & $\mathrm{~Tb}+4$ & $1.67 \mathrm{E}-09$ & $1.87 \mathrm{E}-08$ & $Y-90$ & $8.22 \mathrm{E}-03$ & $2.29 \mathrm{E}-02$ \\
\hline As+5 & $2.30 \mathrm{E}-06$ & $2.57 \mathrm{E}-05$ & $\mathrm{Th}+4$ & $2.71 \mathrm{E}-05$ & $3.02 \mathrm{E}-04$ & Zr-93 & $4.82 \mathrm{E}-07$ & $1.34 \mathrm{E}-06$ \\
\hline $\mathrm{Ba}+2$ & $3.75 \mathrm{E}-05$ & $4.18 \mathrm{E}-04$ & $S n+4$ & $1.98 \mathrm{E}-05$ & $2.21 \mathrm{E}-04$ & Nb-93m & $3.71 \mathrm{E}-07$ & $1.03 \mathrm{E}-06$ \\
\hline $\mathrm{Be}+2$ & $5.61 \mathrm{E}-07$ & $6.26 \mathrm{E}-06$ & $\mathrm{Ti+4}$ & $1.09 \mathrm{E}-05$ & $1.21 \mathrm{E}-04$ & Nb-94 & $2.48 \mathrm{E}-07$ & $6.90 \mathrm{E}-07$ \\
\hline $\mathrm{B}+3$ & $7.86 \mathrm{E}-04$ & $8.76 \mathrm{E}-03$ & $\mathrm{U}+4$ & $8.74 \mathrm{E}-04$ & $9.75 \mathrm{E}-03$ & Tc-98 & $5.61 \mathrm{E}-13$ & $1.56 \mathrm{E}-12$ \\
\hline $\mathrm{Br}-$ & $1.21 \mathrm{E}-07$ & $1.35 \mathrm{E}-06$ & $\mathrm{~V}+5$ & $5.21 \mathrm{E}-06$ & $5.81 \mathrm{E}-05$ & Tc-99 & $3.79 \mathrm{E}-06$ & $1.06 \mathrm{E}-05$ \\
\hline $\mathrm{Cd}+2$ & $1.19 \mathrm{E}-03$ & $1.32 \mathrm{E}-02$ & $Y+3$ & $3.03 E-06$ & 3.38E-05 & Ru-106 & $2.02 \mathrm{E}-07$ & 5.63E-07 \\
\hline $\mathrm{Ca}+2$ & $8.95 \mathrm{E}-03$ & 9.97E-02 & $Z n+2$ & $2.22 \mathrm{E}-04$ & $2.48 \mathrm{E}-03$ & Rh-102 & $1.87 \mathrm{E}-10$ & $5.22 \mathrm{E}-10$ \\
\hline $\mathrm{Ce}+4$ & 1.70E-05 & $1.89 \mathrm{E}-04$ & $2 r+4$ & $9.75 \mathrm{E}-04$ & 1.09E-02 & $\mathrm{Pd}-107$ & 3.59E-09 & $1.00 \mathrm{E}-08$ \\
\hline Cs+ & $1.64 \mathrm{E}-08$ & $1.83 \mathrm{E}-07$ & $\mathrm{H} 2 \mathrm{O}$ & $2.50 \mathrm{E}+01$ & & Cd-113m & $7.22 \mathrm{E}-07$ & $2.01 \mathrm{E}-06$ \\
\hline $\mathrm{Cl}-$ & $3.94 \mathrm{E}-03$ & $4.40 \mathrm{E}-02$ & Canisters total & 8 & & $\ln -115$ & $2.19 \mathrm{E}-17$ & $6.10 \mathrm{E}-17$ \\
\hline $\mathrm{Cr}+3$ & $1.06 \mathrm{E}-03$ & $1.18 \mathrm{E}-02$ & Drums/day & & 52 & Sn-121m & 1.45E-08 & $4.05 \mathrm{E}-08$ \\
\hline $\mathrm{Co}+2$ & 6.96E-05 & $7.76 \mathrm{E}-04$ & Radiological Comp. & $\mathrm{Ci} / \mathrm{kg}$ & $\mathrm{Ci} / \mathrm{kg}$ & \begin{tabular}{|l|} 
Sn-126 \\
\end{tabular} & $8.93 \mathrm{E}-08$ & $2.49 \mathrm{E}-07$ \\
\hline $\mathrm{Cu}+2$ & $1.71 \mathrm{E}-04$ & $1.90 \mathrm{E}-03$ & Ra-226 & $1.78 \mathrm{E}-12$ & $4.96 \mathrm{E}-12$ & Sb-125 & $2.81 \mathrm{E}-06$ & $7.83 \mathrm{E}-06$ \\
\hline $\mathrm{Eu}+3$ & 3.83E-07 & $4.26 \mathrm{E}-06$ & Ac-227 & $8.39 \mathrm{E}-12$ & $2.34 \mathrm{E}-11$ & Sb-126 & $1.25 \mathrm{E}-08$ & $3.48 \mathrm{E}-08$ \\
\hline F- & $1.51 \mathrm{E}-03$ & $1.68 \mathrm{E}-02$ & Th-228 & $6.96 \mathrm{E}-10$ & 1.94E-09 & Te-123 & $8.33 E-20$ & $2.32 \mathrm{E}-19$ \\
\hline $\mathrm{Gd}+3$ & $1.04 \mathrm{E}-04$ & $1.16 \mathrm{E}-03$ & Th-230 & $1.79 \mathrm{E}-10$ & $4.98 \mathrm{E}-10$ & Te-125m & $6.85 \mathrm{E}-07$ & $1.91 \mathrm{E}-06$ \\
\hline $\mathrm{Ge}+4$ & $3.18 \mathrm{E}-09$ & $3.54 \mathrm{E}-08$ & Th-232 & $1.54 \mathrm{E}-16$ & $4.29 \mathrm{E}-16$ & $1-129$ & $1.16 \mathrm{E}-08$ & $3.23 E-08$ \\
\hline $\ln +3$ & $8.95 \mathrm{E}-07$ & 9.97E-06 & $\mathrm{Pa}-231$ & $1.94 \mathrm{E}-11$ & $5.41 \mathrm{E}-11$ & Cs-134 & $7.76 \mathrm{E}-10$ & $2.16 \mathrm{E}-09$ \\
\hline $1-$ & $1.60 \mathrm{E}-06$ & $1.78 \mathrm{E}-05$ & $\mathrm{~Pa}-233$ & $6.37 \mathrm{E}-07$ & $1.78 \mathrm{E}-06$ & Cs-135 & $1.87 \mathrm{E}-10$ & $5.21 \mathrm{E}-10$ \\
\hline $\mathrm{Fe}+3$ & $4.91 \mathrm{E}-03$ & $5.48 \mathrm{E}-02$ & U-232 & $4.32 \mathrm{E}-10$ & $1.20 \mathrm{E}-09$ & Cs-137 & $1.28 \mathrm{E}-06$ & $3.56 \mathrm{E}-06$ \\
\hline $\mathrm{La}+3$ & $6.35 \mathrm{E}-06$ & $7.08 \mathrm{E}-05$ & U-233 & $1.74 \mathrm{E}-11$ & $4.84 \mathrm{E}-11$ & Ba-137m & $1.21 \mathrm{E}-06$ & $3.37 \mathrm{E}-06$ \\
\hline $\mathrm{Pb}+2$ & $8.62 \mathrm{E}-04$ & $9.61 \mathrm{E}-03$ & U-234 & $1.93 \mathrm{E}-07$ & $5.38 \mathrm{E}-07$ & \begin{tabular}{|l} 
La-138 \\
\end{tabular} & $4.15 \mathrm{E}-17$ & $1.16 \mathrm{E}-16$ \\
\hline $\mathrm{Li+}$ & $8.80 \mathrm{E}-06$ & $9.81 \mathrm{E}-05$ & U-235 & $1.61 \mathrm{E}-08$ & $4.49 \mathrm{E}-08$ & Ce-142 & $6.50 \mathrm{E}-12$ & $1.81 \mathrm{E}-11$ \\
\hline $\mathrm{Mg}+2$ & $2.14 \mathrm{E}-03$ & $2.39 \mathrm{E}-02$ & $\mathrm{U}-236$ & $7.49 \mathrm{E}-09$ & $2.09 \mathrm{E}-08$ & Ce-144 & $1.36 \mathrm{E}-07$ & $3.80 \mathrm{E}-07$ \\
\hline$M n+4$ & $3.75 \mathrm{E}-03$ & $4.18 \mathrm{E}-02$ & U-237 & $1.40 \mathrm{E}-09$ & $3.90 \mathrm{E}-09$ & Nd-144 & $3.50 \mathrm{E}-16$ & $9.74 \mathrm{E}-16$ \\
\hline $\mathrm{Hg}+2$ & $5.05 \mathrm{E}-03$ & $5.63 \mathrm{E}-02$ & $\mathrm{U}-238$ & $2.32 \mathrm{E}-09$ & $6.46 \mathrm{E}-09$ & Pm-146 & $1.11 \mathrm{E}-08$ & $3.08 \mathrm{E}-08$ \\
\hline $\mathrm{Mo}+6$ & $9.22 \mathrm{E}-05$ & $1.03 \mathrm{E}-03$ & $\mathrm{~Np}-236$ & $6.68 \mathrm{E}-13$ & $1.86 \mathrm{E}-12$ & Pm-147 & $3.70 \mathrm{E}-05$ & 1.03E-04 \\
\hline $\mathrm{Nd}+3$ & $2.13 \mathrm{E}-05$ & $2.37 \mathrm{E}-04$ & Np-237 & $7.90 \mathrm{E}-08$ & $2.20 \mathrm{E}-07$ & Sm-146 & $6.00 \mathrm{E}-14$ & $1.67 \mathrm{E}-13$ \\
\hline $\mathrm{Np}+4$ & $2.80 \mathrm{E}-06$ & $3.12 \mathrm{E}-05$ & Pu-236 & $5.97 \mathrm{E}-10$ & $1.66 \mathrm{E}-09$ & Sm-147 & $1.60 \mathrm{E}-12$ & $4.46 \mathrm{E}-12$ \\
\hline $\mathrm{Ni}+2$ & $4.54 \mathrm{E}-04$ & $5.06 \mathrm{E}-03$ & $\mathrm{Pu}-238$ & $9.23 \mathrm{E}-05$ & $2.57 \mathrm{E}-04$ & Sm-148 & $8.23 \mathrm{E}-18$ & $2.29 \mathrm{E}-17$ \\
\hline $\mathrm{Nb}+5$ & $9.53 \mathrm{E}-06$ & $1.06 \mathrm{E}-04$ & Pu-239 & $1.10 \mathrm{E}-05$ & $3.05 \mathrm{E}-05$ & Sm-149 & $7.30 \mathrm{E}-19$ & $2.04 \mathrm{E}-18$ \\
\hline NO3- & $1.77 \mathrm{E}+00$ & $1.97 \mathrm{E}+01$ & Pu-240 & $2.21 \mathrm{E}-06$ & $6.15 \mathrm{E}-06$ & Sm-151 & $7.30 \mathrm{E}-05$ & $2.03 \mathrm{E}-04$ \\
\hline $\mathrm{Pd}+4$ & $1.40 \mathrm{E}-04$ & $1.56 \mathrm{E}-03$ & Pu-241 & $5.77 \mathrm{E}-05$ & $1.61 \mathrm{E}-04$ & Eu-152 & 5.47E-07 & 1.52E-06 \\
\hline PO4-3 & 1.87E-04 & $2.09 \mathrm{E}-03$ & Pu-242 & $1.72 \mathrm{E}-09$ & $4.80 \mathrm{E}-09$ & Eu-154 & $4.19 \mathrm{E}-06$ & 1.17E-05 \\
\hline $\mathrm{Pu}+4$ & $4.81 \mathrm{E}-06$ & 5.34E-05 & $\mathrm{Pu}-244$ & $1.45 \mathrm{E}-16$ & $4.05 \mathrm{E}-16$ & Eu-155 & $1.51 \mathrm{E}-06$ & $4.20 \mathrm{E}-06$ \\
\hline $\mathrm{K}+$ & $6.19 \mathrm{E}-02$ & $6.90 \mathrm{E}-01$ & Am-241 & $9.72 \mathrm{E}-06$ & $2.71 \mathrm{E}-05$ & Gd-152 & $3.09 \mathrm{E}-19$ & $8.61 \mathrm{E}-19$ \\
\hline $\mathrm{Pr}+4$ & $5.86 \mathrm{E}-06$ & $6.53 \mathrm{E}-05$ & $A m-242 m$ & $3.40 \mathrm{E}-09$ & $9.47 \mathrm{E}-09$ & Ho-166m & $1.00 \mathrm{E}-11$ & $2.79 \mathrm{E}-11$ \\
\hline \multirow[t]{2}{*}{$\mathrm{Rh}+4$} & $1.85 \mathrm{E}-06$ & $2.06 \mathrm{E}-05$ & $\mathrm{Am}-243$ & $4.79 \mathrm{E}-09$ & $1.34 \mathrm{E}-08$ & Co-60 & $8.37 \mathrm{E}-07$ & $2.33 \mathrm{E}-06$ \\
\hline & & & Cm-242 & $7.44 \mathrm{E}-09$ & $2.07 \mathrm{E}-08$ & $\mathrm{Ni}-63$ & 6.91E-06 & $1.92 \mathrm{E}-05$ \\
\hline
\end{tabular}


Table B-10. Tank WM-188 mass balance (continued).

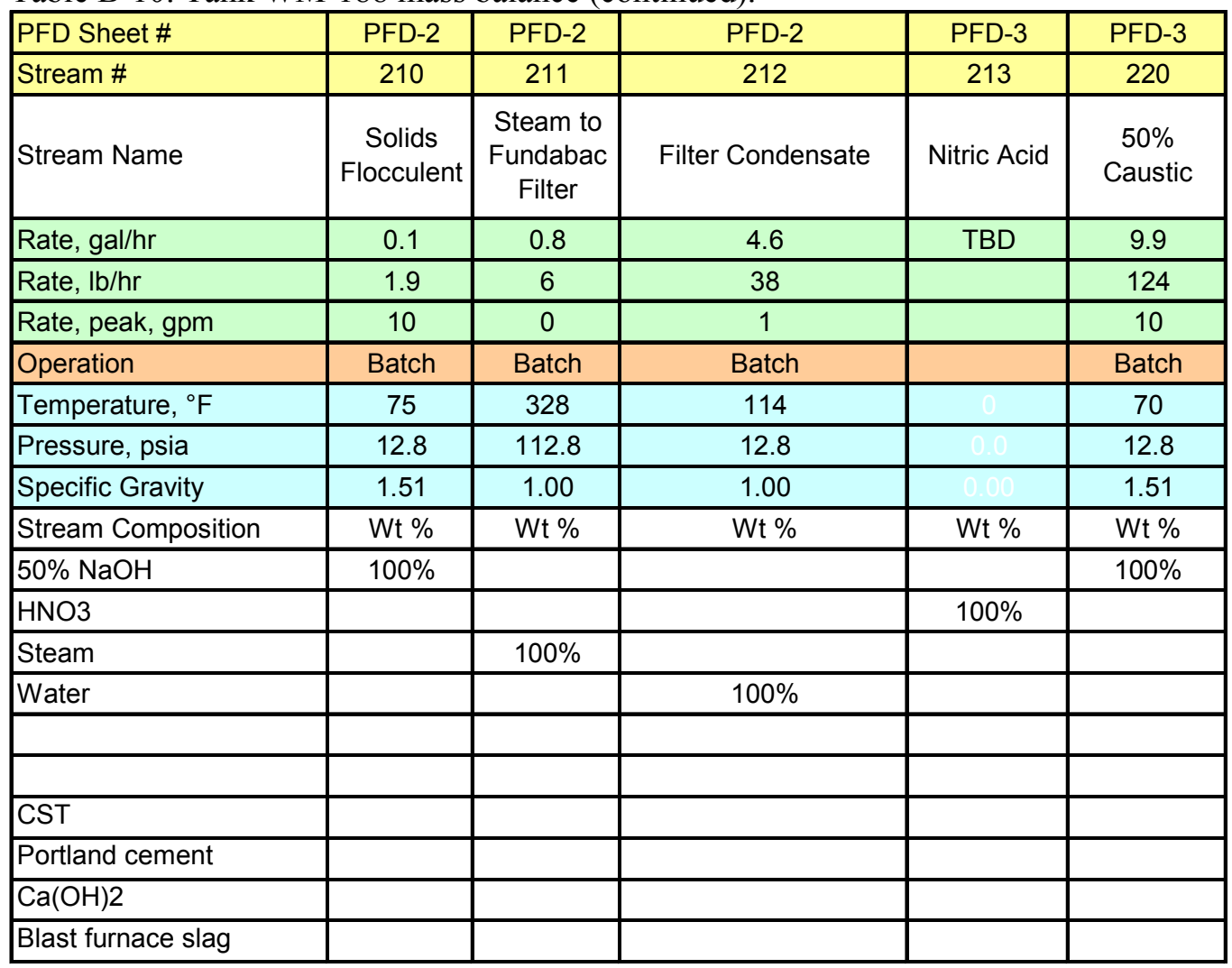

\begin{tabular}{|c|c|c|c|c|c|}
\hline PFD Sheet \# & PFD-3 & PFD-4 & PFD-4 & PFD-4 & PFD-4 \\
\hline Stream \# & 221 & 222 & 223 & 224 & 225 \\
\hline Stream Name & $\begin{array}{c}\text { Fresh IX } \\
\text { Media }\end{array}$ & $\begin{array}{l}\text { Portland } \\
\text { Cement }\end{array}$ & Calcium Hydroxide & $\begin{array}{c}\text { Blast } \\
\text { Furnace } \\
\text { Slag }\end{array}$ & Solid Blend \\
\hline Rate, gal/hr & 0.13 & 16.87 & 24.60 & 8.44 & 41.48 \\
\hline Rate, Ib/hr & 1.04 & 169 & 98 & 84 & 351 \\
\hline Rate, peak, gpm & NA & TBD & TBD & TBD & 0.69 \\
\hline Operation & Batch & Cont & Cont & Cont & Cont \\
\hline Temperature, ${ }^{\circ} \mathrm{F}$ & 70 & 70 & 70 & 70 & 70 \\
\hline Pressure, psia & 12.8 & 12.8 & 12.8 & 12.8 & 12.8 \\
\hline \multirow[t]{2}{*}{ Specific Gravity } & 0.96 & 1.20 & 0.48 & 1.20 & 1.02 \\
\hline & Wt \% & Wt \% & Wt \% & Wt \% & $\mathrm{Wt} \%$ \\
\hline \multicolumn{6}{|l|}{ Chemical Composition } \\
\hline \multicolumn{6}{|l|}{$\mathrm{NaOH}$} \\
\hline \multicolumn{6}{|l|}{$\mathrm{H} 2 \mathrm{O}$} \\
\hline CST & $100 \%$ & & & & \\
\hline Portland cement & & $100 \%$ & & & \\
\hline $\mathrm{Ca}(\mathrm{OH}) 2$ & & & $100 \%$ & & \\
\hline Blast furnace slag & & & & $100 \%$ & \\
\hline No. Replacement col's & 8 & & & & \\
\hline & & & & & \\
\hline & & & & & \\
\hline
\end{tabular}


Table B-11. Tank WM-189 mass balance.

\begin{tabular}{|c|c|c|c|c|c|c|c|c|}
\hline PFD Sheet \# & PFD-2 & PFD-2 & PFD-2 & PFD-2 & PFD-2 & PFD-2 & PFD-2 & PFD-3 \\
\hline Stream \# & 110 & 111 & 112 & 113 & 114 & 115 & 116 & 120 \\
\hline Stream Name & $\begin{array}{l}\text { Slurry from } \\
\text { TFF Tank } \\
\text { WM-187 }\end{array}$ & $\begin{array}{l}\text { Crossflow } \\
\text { Filter Recirc } \\
\text { Purge }\end{array}$ & $\begin{array}{l}\text { Decant } \\
\text { Liquid }\end{array}$ & $\begin{array}{c}\text { Solids } \\
\text { Treatment } \\
\text { Filter Feed }\end{array}$ & $\begin{array}{l}\text { Solids } \\
\text { Treatment } \\
\text { Filtrate }\end{array}$ & $\begin{array}{c}\text { Combined } \\
\text { Waste Water }\end{array}$ & $\begin{array}{l}\text { Dried Solids } \\
\text { Waste }\end{array}$ & $\begin{array}{c}\text { Liquid SBW } \\
\text { from Tank } \\
\text { WM-180 }\end{array}$ \\
\hline Rate, gal/hr & 65.9 & 0.8 & 54.7 & 14.6 & 8.3 & 66.6 & 6.3 & 85.2 \\
\hline Rate, $\mathrm{lb} / \mathrm{hr}$ & 571 & 9 & 464 & 137 & 70 & 566 & 36 & 935 \\
\hline Rate, peak, gpm & 50 & 0.013 & 50 & 10 & 0.14 & 190 & 4.32 & 50.00 \\
\hline Operation & Batch & Cont & Batch & Batch & Batch & Batch & Batch & Batch \\
\hline Temperature, ${ }^{\circ} \mathrm{F}$ & 70 & 83 & 70 & 70 & 70 & 70 & 70 & 70 \\
\hline Pressure, psia & 12.8 & 12.8 & 12.8 & 50.0 & 12.8 & 12.8 & 12.8 & 12.8 \\
\hline Specific Gravity & 1.04 & 1.32 & 1.02 & 1.12 & 1.02 & 1.02 & 0.68 & 1.32 \\
\hline Chemical Composition & Mol/liter & Mol/liter & Mol/liter & Mol/liter & Mol/liter & Mol/liter & Wt \% & Mol/liter \\
\hline $\mathrm{H}+$ & $3.13 \mathrm{E}-02$ & $4.76 \mathrm{E}-01$ & $3.65 \mathrm{E}-02$ & $3.65 \mathrm{E}-02$ & $3.65 \mathrm{E}-02$ & $3.36 \mathrm{E}-02$ & $9.01 \mathrm{E}-04$ & $2.82 \mathrm{E}+00$ \\
\hline $\mathrm{Al}+3$ & 3.04E-02 & $5.69 \mathrm{E}-01$ & $1.28 \mathrm{E}-02$ & $1.21 \mathrm{E}-01$ & 1.49E-02 & $2.51 \mathrm{E}-02$ & $1.00 \mathrm{E}+00$ & $6.52 \mathrm{E}-01$ \\
\hline $\mathrm{Am}+4$ & $8.51 \mathrm{E}-10$ & $4.84 \mathrm{E}-08$ & $1.41 \mathrm{E}-09$ & $1.41 \mathrm{E}-09$ & $1.41 \mathrm{E}-09$ & $1.35 \mathrm{E}-09$ & $8.32 \mathrm{E}-09$ & $5.69 \mathrm{E}-08$ \\
\hline $\mathrm{Sb}+5$ & $1.51 \mathrm{E}-05$ & $1.46 \mathrm{E}-05$ & $9.31 \mathrm{E}-07$ & $6.53 \mathrm{E}-05$ & $2.16 \mathrm{E}-06$ & $8.38 \mathrm{E}-06$ & $2.66 \mathrm{E}-03$ & $7.44 \mathrm{E}-06$ \\
\hline$A s+5$ & $1.28 \mathrm{E}-04$ & $7.14 \mathrm{E}-05$ & $7.21 \mathrm{E}-06$ & $5.50 \mathrm{E}-04$ & $1.76 \mathrm{E}-05$ & $7.00 \mathrm{E}-05$ & $1.38 \mathrm{E}-02$ & $2.31 \mathrm{E}-06$ \\
\hline $\mathrm{Ba}+2$ & $2.76 \mathrm{E}-05$ & 5.92E-05 & $2.65 \mathrm{E}-06$ & $1.17 \mathrm{E}-04$ & 4.85E-06 & $1.59 \mathrm{E}-05$ & $5.35 \mathrm{E}-03$ & $5.22 \mathrm{E}-05$ \\
\hline $\mathrm{Be}+2$ & $6.21 \mathrm{E}-06$ & $1.91 \mathrm{E}-05$ & 5.43E-07 & $2.69 \mathrm{E}-05$ & $1.05 \mathrm{E}-06$ & $3.60 \mathrm{E}-06$ & 8.07E-05 & $1.85 \mathrm{E}-05$ \\
\hline$B+3$ & $7.79 \mathrm{E}-04$ & $1.68 \mathrm{E}-02$ & $5.00 \mathrm{E}-04$ & $2.62 \mathrm{E}-03$ & $5.41 \mathrm{E}-04$ & $7.25 \mathrm{E}-04$ & $7.89 \mathrm{E}-03$ & 1.94E-02 \\
\hline $\mathrm{Br}-$ & $4.45 \mathrm{E}-09$ & $2.53 \mathrm{E}-07$ & 7.37E-09 & $7.37 \mathrm{E}-09$ & $7.37 \mathrm{E}-09$ & $7.05 \mathrm{E}-09$ & $1.44 \mathrm{E}-08$ & $2.97 \mathrm{E}-07$ \\
\hline$C d+2$ & $1.50 \mathrm{E}-04$ & $3.08 \mathrm{E}-03$ & $1.09 \mathrm{E}-04$ & $4.53 \mathrm{E}-04$ & $1.15 \mathrm{E}-04$ & $1.43 \mathrm{E}-04$ & 1.34E-02 & $3.57 \mathrm{E}-03$ \\
\hline $\mathrm{Ca}+2$ & $2.14 \mathrm{E}-03$ & 5.77E-02 & $1.53 \mathrm{E}-03$ & $7.23 \mathrm{E}-03$ & $1.64 \mathrm{E}-03$ & $2.14 \mathrm{E}-03$ & 7.90E-02 & $6.69 \mathrm{E}-02$ \\
\hline $\mathrm{Ce}+4$ & 1.39E-05 & $3.60 \mathrm{E}-05$ & 1.34E-06 & 5.94E-05 & $2.45 \mathrm{E}-06$ & $8.05 E-06$ & $2.76 \mathrm{E}-03$ & $3.35 \mathrm{E}-05$ \\
\hline Cs+ & $5.55 \mathrm{E}-05$ & $5.19 \mathrm{E}-05$ & $3.63 \mathrm{E}-06$ & $2.39 \mathrm{E}-04$ & $8.13 \mathrm{E}-06$ & $3.08 \mathrm{E}-05$ & $1.06 \mathrm{E}-02$ & $2.56 \mathrm{E}-05$ \\
\hline $\mathrm{Cl}-$ & $3.23 \mathrm{E}-03$ & $1.79 \mathrm{E}-02$ & $5.75 \mathrm{E}-04$ & $1.34 \mathrm{E}-02$ & $8.20 \mathrm{E}-04$ & $2.04 \mathrm{E}-03$ & $1.55 \mathrm{E}-01$ & $1.91 \mathrm{E}-02$ \\
\hline $\mathrm{Cr}+3$ & $5.85 \mathrm{E}-04$ & $4.80 \mathrm{E}-03$ & 1.42E-04 & $2.38 \mathrm{E}-03$ & $1.85 \mathrm{E}-04$ & $3.97 \mathrm{E}-04$ & 3.96E-02 & $5.30 \mathrm{E}-03$ \\
\hline $\mathrm{Co}+2$ & $4.86 \mathrm{E}-06$ & $2.04 \mathrm{E}-04$ & $2.75 \mathrm{E}-06$ & $2.29 \mathrm{E}-05$ & $3.13 \mathrm{E}-06$ & $5.17 \mathrm{E}-06$ & $4.08 \mathrm{E}-04$ & $2.37 \mathrm{E}-04$ \\
\hline $\mathrm{Cu}+2$ & 1.55E-04 & $8.24 \mathrm{E}-04$ & $1.88 \mathrm{E}-05$ & $6.74 \mathrm{E}-04$ & $3.14 \mathrm{E}-05$ & $9.49 \mathrm{E}-05$ & $1.41 \mathrm{E}-02$ & $8.69 \mathrm{E}-04$ \\
\hline $\mathrm{Eu}+3$ & $7.38 \mathrm{E}-09$ & $4.20 \mathrm{E}-07$ & $1.22 \mathrm{E}-08$ & $1.22 \mathrm{E}-08$ & $1.22 \mathrm{E}-08$ & $1.17 \mathrm{E}-08$ & $4.55 \mathrm{E}-08$ & 4.93E-07 \\
\hline F- & $2.82 \mathrm{E}-02$ & $2.66 \mathrm{E}-02$ & $3.14 \mathrm{E}-03$ & 1.17E-01 & $5.31 \mathrm{E}-03$ & $1.61 \mathrm{E}-02$ & 7.32E-01 & $1.42 \mathrm{E}-02$ \\
\hline $\mathrm{Gd}+3$ & $2.82 \mathrm{E}-05$ & $1.25 \mathrm{E}-04$ & $3.74 \mathrm{E}-06$ & $1.20 \mathrm{E}-04$ & $5.96 \mathrm{E}-06$ & $1.71 \mathrm{E}-05$ & $6.19 \mathrm{E}-03$ & $1.29 \mathrm{E}-04$ \\
\hline $\mathrm{Ge}+4$ & $1.28 \mathrm{E}-10$ & $7.30 \mathrm{E}-09$ & $2.12 \mathrm{E}-10$ & $2.12 \mathrm{E}-10$ & $2.12 \mathrm{E}-10$ & $2.03 \mathrm{E}-10$ & $3.78 \mathrm{E}-10$ & 8.57E-09 \\
\hline $\ln +3$ & $2.02 \mathrm{E}-08$ & $1.15 \mathrm{E}-06$ & $3.35 \mathrm{E}-08$ & $3.35 \mathrm{E}-08$ & $3.35 \mathrm{E}-08$ & $3.20 \mathrm{E}-08$ & $9.42 \mathrm{E}-08$ & $1.35 \mathrm{E}-06$ \\
\hline I- & $3.70 \mathrm{E}-08$ & $2.10 \mathrm{E}-06$ & $6.12 \mathrm{E}-08$ & $6.12 \mathrm{E}-08$ & $6.12 \mathrm{E}-08$ & $5.85 \mathrm{E}-08$ & $1.90 \mathrm{E}-07$ & $2.47 \mathrm{E}-06$ \\
\hline $\mathrm{Fe}+3$ & $1.07 \mathrm{E}-02$ & $2.67 \mathrm{E}-02$ & $1.09 \mathrm{E}-03$ & $4.56 \mathrm{E}-02$ & $1.94 \mathrm{E}-03$ & $6.22 \mathrm{E}-03$ & $8.44 \mathrm{E}-01$ & $2.47 \mathrm{E}-02$ \\
\hline $\mathrm{La}+3$ & 1.34E-07 & $7.62 \mathrm{E}-06$ & $2.22 \mathrm{E}-07$ & $2.22 \mathrm{E}-07$ & $2.22 \mathrm{E}-07$ & $2.12 \mathrm{E}-07$ & $7.56 \mathrm{E}-07$ & $8.96 \mathrm{E}-06$ \\
\hline $\mathrm{Pb}+2$ & $6.39 \mathrm{E}-05$ & $9.48 \mathrm{E}-04$ & $3.00 \mathrm{E}-05$ & $2.31 \mathrm{E}-04$ & $3.38 \mathrm{E}-05$ & $5.20 \mathrm{E}-05$ & $1.43 \mathrm{E}-02$ & $1.08 \mathrm{E}-03$ \\
\hline $\mathrm{Li+}$ & $3.18 \mathrm{E}-04$ & $4.84 \mathrm{E}-04$ & $2.19 \mathrm{E}-05$ & $1.38 \mathrm{E}-03$ & $4.78 \mathrm{E}-05$ & $1.79 \mathrm{E}-04$ & $3.19 \mathrm{E}-03$ & $3.65 \mathrm{E}-04$ \\
\hline $\mathrm{Mg}+2$ & $1.23 \mathrm{E}-03$ & $1.83 \mathrm{E}-02$ & $5.60 \mathrm{E}-04$ & $4.52 \mathrm{E}-03$ & $6.36 \mathrm{E}-04$ & $9.97 \mathrm{E}-04$ & $3.30 \mathrm{E}-02$ & $2.09 \mathrm{E}-02$ \\
\hline $\mathrm{Mn}+4$ & $4.45 \mathrm{E}-04$ & $1.60 \mathrm{E}-02$ & $4.09 \mathrm{E}-04$ & $1.40 \mathrm{E}-03$ & $4.28 \mathrm{E}-04$ & $5.11 \mathrm{E}-04$ & $1.90 \mathrm{E}-02$ & $1.87 \mathrm{E}-02$ \\
\hline $\mathrm{Hg}+2$ & $3.11 \mathrm{E}-05$ & $5.09 \mathrm{E}-03$ & $7.69 \mathrm{E}-05$ & $1.38 \mathrm{E}-04$ & $7.81 \mathrm{E}-05$ & $8.47 \mathrm{E}-05$ & $4.53 \mathrm{E}-03$ & $5.97 \mathrm{E}-03$ \\
\hline $\mathrm{Mo}+6$ & $8.91 \mathrm{E}-04$ & $7.14 \mathrm{E}-04$ & $5.38 \mathrm{E}-05$ & $3.84 \mathrm{E}-03$ & $1.26 \mathrm{E}-04$ & $4.92 \mathrm{E}-04$ & $1.23 \mathrm{E}-01$ & $2.69 \mathrm{E}-04$ \\
\hline $\mathrm{Nd}+3$ & $4.32 \mathrm{E}-07$ & $2.46 \mathrm{E}-05$ & 7.16E-07 & 7.16E-07 & $7.16 \mathrm{E}-07$ & $6.85 \mathrm{E}-07$ & $2.53 \mathrm{E}-06$ & $2.89 \mathrm{E}-05$ \\
\hline $\mathrm{Np}+4$ & $2.47 \mathrm{E}-07$ & $2.31 \mathrm{E}-06$ & $2.71 \mathrm{E}-07$ & $2.71 \mathrm{E}-07$ & $2.71 \mathrm{E}-07$ & $2.48 \mathrm{E}-07$ & $1.58 \mathrm{E}-06$ & $2.71 \mathrm{E}-06$ \\
\hline $\mathrm{Ni}+2$ & $2.03 \mathrm{E}-04$ & $1.92 \mathrm{E}-03$ & $5.41 \mathrm{E}-05$ & $8.22 \mathrm{E}-04$ & $6.88 \mathrm{E}-05$ & $1.42 \mathrm{E}-04$ & 1.54E-02 & $2.14 \mathrm{E}-03$ \\
\hline $\mathrm{Nb}+5$ & $1.47 \mathrm{E}-03$ & $8.10 \mathrm{E}-04$ & $8.31 \mathrm{E}-05$ & $6.36 \mathrm{E}-03$ & $2.03 E-04$ & $8.09 \mathrm{E}-04$ & $1.98 \mathrm{E}-01$ & $7.42 \mathrm{E}-06$ \\
\hline NO3- & 6.65E-02 & $6.09 \mathrm{E}+00$ & 1.37E-01 & $1.39 \mathrm{E}-01$ & 1.37E-01 & $1.33 \mathrm{E}-01$ & $2.49 \mathrm{E}-01$ & $7.16 \mathrm{E}+00$ \\
\hline $\mathrm{O}-2$ & $2.23 \mathrm{E}-01$ & $1.23 \mathrm{E}-01$ & $1.26 \mathrm{E}-02$ & 9.64E-01 & $3.08 \mathrm{E}-02$ & $1.23 \mathrm{E}-01$ & $5.16 \mathrm{E}+00$ & $1.06 \mathrm{E}-03$ \\
\hline $\mathrm{Pd}+4$ & $2.45 \mathrm{E}-03$ & $1.35 \mathrm{E}-03$ & $1.38 \mathrm{E}-04$ & 1.06E-02 & $3.38 \mathrm{E}-04$ & $1.35 \mathrm{E}-03$ & 3.77E-01 & 1.99E-05 \\
\hline PO4-3 & 1.39E-01 & 7.77E-02 & 7.90E-03 & 5.97E-01 & $1.92 \mathrm{E}-02$ & $7.61 \mathrm{E}-02$ & $1.90 \mathrm{E}+01$ & $2.60 \mathrm{E}-03$ \\
\hline $\mathrm{Pu}+4$ & $7.79 \mathrm{E}-08$ & $3.18 \mathrm{E}-06$ & 1.14E-07 & $1.14 \mathrm{E}-07$ & $1.14 \mathrm{E}-07$ & $1.08 \mathrm{E}-07$ & $6.67 \mathrm{E}-07$ & $3.73 \mathrm{E}-06$ \\
\hline $\mathrm{K}+$ & $1.86 \mathrm{E}-02$ & $2.11 \mathrm{E}-01$ & $5.43 \mathrm{E}-03$ & $7.54 \mathrm{E}-02$ & $6.77 \mathrm{E}-03$ & $1.34 \mathrm{E}-02$ & $9.33 \mathrm{E}-01$ & $2.38 \mathrm{E}-01$ \\
\hline $\mathrm{Pr}+4$ & $1.22 \mathrm{E}-07$ & $6.93 \mathrm{E}-06$ & $2.02 \mathrm{E}-07$ & $2.02 \mathrm{E}-07$ & $2.02 \mathrm{E}-07$ & 1.93E-07 & 6.97E-07 & $8.14 \mathrm{E}-06$ \\
\hline $\mathrm{Rh}+4$ & $5.26 \mathrm{E}-08$ & $2.99 \mathrm{E}-06$ & $8.71 \mathrm{E}-08$ & $8.71 \mathrm{E}-08$ & $8.71 \mathrm{E}-08$ & $8.33 \mathrm{E}-08$ & $2.20 \mathrm{E}-07$ & $3.51 \mathrm{E}-06$ \\
\hline
\end{tabular}


Table B-11. Tank WM-189 mass balance (continued).

\begin{tabular}{|c|c|c|c|c|c|c|c|c|}
\hline Stream \# & 110 & 111 & 112 & 113 & 114 & 115 & 116 & 120 \\
\hline Stream Name & $\begin{array}{l}\text { Slurry from } \\
\text { TFF Tank } \\
\text { WM-187 }\end{array}$ & $\begin{array}{c}\text { Crossflow } \\
\text { Filter Recirc } \\
\text { Purge }\end{array}$ & $\begin{array}{l}\text { Decant } \\
\text { Liquid }\end{array}$ & $\begin{array}{c}\text { Solids } \\
\text { Treatment } \\
\text { Filter Feed }\end{array}$ & $\begin{array}{l}\text { Solids } \\
\text { Treatment } \\
\text { Filtrate }\end{array}$ & $\begin{array}{c}\text { Combined } \\
\text { Waste Water }\end{array}$ & $\begin{array}{l}\text { Dried Solids } \\
\text { Waste }\end{array}$ & $\begin{array}{l}\text { Liquid SBW } \\
\text { from TFF }\end{array}$ \\
\hline Chemical Composition & Mol/liter & Mol/liter & Mol/liter & Mol/liter & Mol/liter & Mol/liter & Wt \% & Mol/liter \\
\hline $\mathrm{Rb}+$ & $8.10 \mathrm{E}-08$ & $4.60 \mathrm{E}-06$ & $1.34 \mathrm{E}-07$ & $1.34 \mathrm{E}-07$ & $1.34 \mathrm{E}-07$ & $1.28 \mathrm{E}-07$ & $2.81 \mathrm{E}-07$ & $5.41 \mathrm{E}-06$ \\
\hline $\mathrm{Ru}+3$ & $1.03 \mathrm{E}-03$ & $7.02 \mathrm{E}-04$ & $5.98 \mathrm{E}-05$ & $4.43 \mathrm{E}-03$ & $1.43 \mathrm{E}-04$ & $5.66 \mathrm{E}-04$ & $1.50 \mathrm{E}-01$ & $1.68 \mathrm{E}-04$ \\
\hline $\mathrm{Sm}+3$ & $8.02 \mathrm{E}-08$ & $4.56 \mathrm{E}-06$ & $1.33 \mathrm{E}-07$ & $1.33 \mathrm{E}-07$ & $1.33 \mathrm{E}-07$ & $1.27 \mathrm{E}-07$ & $4.89 \mathrm{E}-07$ & $5.35 \mathrm{E}-06$ \\
\hline $\mathrm{Se}+4$ & $3.82 \mathrm{E}-04$ & $2.11 \mathrm{E}-04$ & $2.15 \mathrm{E}-05$ & 1.65E-03 & $5.26 \mathrm{E}-05$ & $2.10 \mathrm{E}-04$ & 4.36E-02 & 3.05E-06 \\
\hline $\mathrm{Si}+4$ & $1.26 \mathrm{E}-01$ & $6.92 \mathrm{E}-02$ & $7.10 \mathrm{E}-03$ & $5.42 \mathrm{E}-01$ & $1.73 \mathrm{E}-02$ & $6.90 \mathrm{E}-02$ & $5.10 \mathrm{E}+00$ & 8.87E-04 \\
\hline $\mathrm{Ag}+$ & $5.89 \mathrm{E}-04$ & $3.23 \mathrm{E}-04$ & $3.37 \mathrm{E}-05$ & $2.54 \mathrm{E}-03$ & $8.16 \mathrm{E}-05$ & $3.23 \mathrm{E}-04$ & $9.16 \mathrm{E}-02$ & $3.01 \mathrm{E}-06$ \\
\hline $\mathrm{Na}+$ & $4.19 \mathrm{E}-02$ & $3.59 \mathrm{E}+00$ & $5.87 \mathrm{E}-02$ & $1.71 \mathrm{E}-01$ & $6.09 \mathrm{E}-02$ & 7.19E-02 & $9.09 \mathrm{E}-01$ & $1.94 \mathrm{E}+00$ \\
\hline $\mathrm{Sr}+2$ & $8.53 \mathrm{E}-06$ & $1.18 \mathrm{E}-04$ & $2.01 \mathrm{E}-06$ & $3.74 \mathrm{E}-05$ & $2.68 \mathrm{E}-06$ & $6.14 \mathrm{E}-06$ & $1.06 \mathrm{E}-03$ & $1.33 \mathrm{E}-04$ \\
\hline SO4-2 & $6.39 \mathrm{E}-04$ & 8.33E-02 & $1.61 \mathrm{E}-03$ & $1.61 \mathrm{E}-03$ & $1.61 \mathrm{E}-03$ & $1.58 \mathrm{E}-03$ & $3.79 \mathrm{E}-03$ & $9.78 \mathrm{E}-02$ \\
\hline $\mathrm{Tc}+7$ & $9.40 \mathrm{E}-08$ & $4.91 \mathrm{E}-06$ & $1.50 \mathrm{E}-07$ & $1.50 \mathrm{E}-07$ & $1.50 \mathrm{E}-07$ & $1.43 \mathrm{E}-07$ & $3.58 \mathrm{E}-07$ & $5.76 \mathrm{E}-06$ \\
\hline $\mathrm{Te}+4$ & $4.21 \mathrm{E}-08$ & $5.76 \mathrm{E}-06$ & $1.09 \mathrm{E}-07$ & $1.09 \mathrm{E}-07$ & 1.09E-07 & $1.08 \mathrm{E}-07$ & $3.42 \mathrm{E}-07$ & $6.77 \mathrm{E}-06$ \\
\hline $\mathrm{Tb}+4$ & $3.09 \mathrm{E}-11$ & $1.75 \mathrm{E}-09$ & $5.11 \mathrm{E}-11$ & $5.11 \mathrm{E}-11$ & $5.11 \mathrm{E}-11$ & $4.89 \mathrm{E}-11$ & $1.99 \mathrm{E}-10$ & $2.06 \mathrm{E}-09$ \\
\hline $\mathrm{Th}+4$ & $2.87 \mathrm{E}-12$ & $2.78 \mathrm{E}-05$ & $3.26 \mathrm{E}-07$ & $3.26 \mathrm{E}-07$ & $3.26 \mathrm{E}-07$ & $3.39 \mathrm{E}-07$ & $1.85 \mathrm{E}-06$ & $3.26 \mathrm{E}-05$ \\
\hline $\mathrm{Sn}+4$ & $2.63 \mathrm{E}-03$ & $1.48 \mathrm{E}-03$ & $1.48 \mathrm{E}-04$ & 1.13E-02 & 3.63E-04 & $1.44 \mathrm{E}-03$ & $4.51 \mathrm{E}-01$ & 5.12E-05 \\
\hline $\mathrm{Ti}+4$ & $1.29 \mathrm{E}-03$ & $7.66 \mathrm{E}-04$ & 7.43E-05 & $5.56 \mathrm{E}-03$ & $1.79 \mathrm{E}-04$ & $7.09 \mathrm{E}-04$ & 8.91E-02 & $7.49 \mathrm{E}-05$ \\
\hline $\mathrm{U}+4$ & $3.83 \mathrm{E}-05$ & $9.54 \mathrm{E}-04$ & $1.86 \mathrm{E}-05$ & $1.56 \mathrm{E}-04$ & $2.13 \mathrm{E}-05$ & $3.44 \mathrm{E}-05$ & $1.12 \mathrm{E}-02$ & $1.10 \mathrm{E}-03$ \\
\hline$V+5$ & $1.12 \mathrm{E}-05$ & $2.59 \mathrm{E}-05$ & $4.23 \mathrm{E}-06$ & $3.66 \mathrm{E}-05$ & $4.85 \mathrm{E}-06$ & $7.64 \mathrm{E}-06$ & $5.65 \mathrm{E}-04$ & $2.56 \mathrm{E}-05$ \\
\hline $\mathrm{Y}+3$ & $1.00 \mathrm{E}-07$ & $5.68 \mathrm{E}-06$ & $1.65 \mathrm{E}-07$ & $1.65 \mathrm{E}-07$ & $1.65 \mathrm{E}-07$ & $1.58 \mathrm{E}-07$ & $3.61 \mathrm{E}-07$ & $6.68 \mathrm{E}-06$ \\
\hline$Z n+2$ & $8.90 \mathrm{E}-05$ & $8.90 \mathrm{E}-04$ & $1.63 \mathrm{E}-05$ & $3.89 E-04$ & $2.34 \mathrm{E}-05$ & $5.96 \mathrm{E}-05$ & $8.29 \mathrm{E}-03$ & $9.90 \mathrm{E}-04$ \\
\hline $\mathrm{Zr}+4$ & $5.35 \mathrm{E}-02$ & $2.97 \mathrm{E}-02$ & $3.11 \mathrm{E}-03$ & $2.30 \mathrm{E}-01$ & $7.46 \mathrm{E}-03$ & $2.94 \mathrm{E}-02$ & $7.04 \mathrm{E}+00$ & $6.38 \mathrm{E}-04$ \\
\hline $\mathrm{H} 2 \mathrm{O}$ & $5.55 E+01$ & $4.48 \mathrm{E}+01$ & $5.55 E+01$ & $5.42 \mathrm{E}+01$ & $5.54 \mathrm{E}+01$ & $5.47 \mathrm{E}+01$ & $5.70 \mathrm{E}+01$ & $4.29 E+01$ \\
\hline Canisters/day & & & & & & & 0.59 & \\
\hline Canisters total & & & & & & & 89 & \\
\hline Radiological Composition & Ci/liter & Ci/liter & Ci/liter & Ci/liter & Ci/liter & Ci/liter & $\mathrm{Ci} / \mathrm{kg}$ & $\mathrm{Ci} /$ liter \\
\hline Ra-226 & $1.15 \mathrm{E}-13$ & $6.58 \mathrm{E}-12$ & $1.91 \mathrm{E}-13$ & $1.91 \mathrm{E}-13$ & $1.91 \mathrm{E}-13$ & $1.83 \mathrm{E}-13$ & $4.69 \mathrm{E}-14$ & $7.73 \mathrm{E}-12$ \\
\hline Ac-227 & $5.43 \mathrm{E}-13$ & $3.10 \mathrm{E}-11$ & $9.00 \mathrm{E}-13$ & $9.00 \mathrm{E}-13$ & $9.00 \mathrm{E}-13$ & $8.61 \mathrm{E}-13$ & $2.21 \mathrm{E}-13$ & $3.64 \mathrm{E}-11$ \\
\hline Th-228 & $4.51 \mathrm{E}-11$ & $2.57 \mathrm{E}-09$ & $7.47 \mathrm{E}-11$ & $7.47 \mathrm{E}-11$ & $7.47 \mathrm{E}-11$ & $7.15 \mathrm{E}-11$ & $1.83 \mathrm{E}-11$ & $3.02 \mathrm{E}-09$ \\
\hline Th-230 & $3.37 \mathrm{E}-10$ & $8.39 \mathrm{E}-10$ & $3.75 \mathrm{E}-11$ & 1.42E-09 & $6.40 \mathrm{E}-11$ & $1.97 \mathrm{E}-10$ & 4.71E-09 & $7.77 \mathrm{E}-10$ \\
\hline Th-232 & $9.98 \mathrm{E}-18$ & $5.69 \mathrm{E}-16$ & $1.65 \mathrm{E}-17$ & $1.65 \mathrm{E}-17$ & $1.65 \mathrm{E}-17$ & $1.58 \mathrm{E}-17$ & $4.05 \mathrm{E}-18$ & $6.68 \mathrm{E}-16$ \\
\hline $\mathrm{Pa}-231$ & $1.26 \mathrm{E}-12$ & $7.17 \mathrm{E}-11$ & $2.09 \mathrm{E}-12$ & $2.09 \mathrm{E}-12$ & $2.09 \mathrm{E}-12$ & $2.00 \mathrm{E}-12$ & $5.11 \mathrm{E}-13$ & $8.43 \mathrm{E}-11$ \\
\hline $\mathrm{Pa}-233$ & $4.13 \mathrm{E}-08$ & $2.35 \mathrm{E}-06$ & $6.84 \mathrm{E}-08$ & $6.84 \mathrm{E}-08$ & $6.84 \mathrm{E}-08$ & $6.55 \mathrm{E}-08$ & $1.68 \mathrm{E}-08$ & $2.77 \mathrm{E}-06$ \\
\hline U-232 & $6.99 \mathrm{E}-10$ & $1.96 \mathrm{E}-09$ & $8.42 \mathrm{E}-11$ & $2.94 \mathrm{E}-09$ & $1.39 \mathrm{E}-10$ & $4.13 \mathrm{E}-10$ & $9.70 \mathrm{E}-09$ & $1.88 \mathrm{E}-09$ \\
\hline $\mathrm{U}-233$ & 1.27E-11 & $7.06 \mathrm{E}-11$ & $2.52 \mathrm{E}-12$ & $5.17 \mathrm{E}-11$ & $3.46 \mathrm{E}-12$ & $8.14 \mathrm{E}-12$ & $1.68 \mathrm{E}-10$ & $7.55 \mathrm{E}-11$ \\
\hline U-234 & $2.08 \mathrm{E}-07$ & $1.50 \mathrm{E}-06$ & $3.85 \mathrm{E}-08$ & 8.77E-07 & $5.46 \mathrm{E}-08$ & 1.35E-07 & $2.85 \mathrm{E}-06$ & $1.64 \mathrm{E}-06$ \\
\hline U-235 & 1.09E-08 & $5.48 \mathrm{E}-08$ & 1.41E-09 & 4.67E-08 & $2.28 \mathrm{E}-09$ & 6.66E-09 & $1.54 \mathrm{E}-07$ & $5.75 \mathrm{E}-08$ \\
\hline U-236 & $1.43 \mathrm{E}-08$ & $7.02 \mathrm{E}-08$ & $1.98 \mathrm{E}-09$ & $6.10 \mathrm{E}-08$ & $3.10 \mathrm{E}-09$ & 8.79E-09 & $2.00 \mathrm{E}-07$ & $7.36 \mathrm{E}-08$ \\
\hline $\mathrm{U}-237$ & $9.07 \mathrm{E}-11$ & 5.17E-09 & $1.50 \mathrm{E}-10$ & $1.50 \mathrm{E}-10$ & $1.50 \mathrm{E}-10$ & $1.44 \mathrm{E}-10$ & $3.68 \mathrm{E}-11$ & 6.07E-09 \\
\hline U-238 & $1.23 \mathrm{E}-09$ & $3.50 \mathrm{E}-08$ & $8.76 \mathrm{E}-10$ & 4.27E-09 & $9.41 \mathrm{E}-10$ & $1.24 \mathrm{E}-09$ & $1.17 \mathrm{E}-08$ & $4.06 \mathrm{E}-08$ \\
\hline Np-236 & $6.44 \mathrm{E}-14$ & $2.47 \mathrm{E}-12$ & $9.26 \mathrm{E}-14$ & $9.26 \mathrm{E}-14$ & $9.26 \mathrm{E}-14$ & $8.75 \mathrm{E}-14$ & $2.27 \mathrm{E}-14$ & $2.90 \mathrm{E}-12$ \\
\hline \begin{tabular}{|l|}
$\mathrm{Np}-237$ \\
\end{tabular} & $1.02 \mathrm{E}-07$ & $4.19 \mathrm{E}-07$ & $4.88 \mathrm{E}-08$ & 3.09E-07 & $5.37 \mathrm{E}-08$ & $7.50 \mathrm{E}-08$ & $8.94 \mathrm{E}-07$ & $4.53 \mathrm{E}-07$ \\
\hline Pu-236 & 1.22E-09 & 3.26E-09 & $1.63 \mathrm{E}-10$ & 5.08E-09 & $2.57 \mathrm{E}-10$ & $7.27 \mathrm{E}-10$ & 1.67E-08 & $3.09 \mathrm{E}-09$ \\
\hline Pu-238 & $6.41 \mathrm{E}-04$ & $7.20 \mathrm{E}-04$ & $5.86 \mathrm{E}-05$ & $2.70 \mathrm{E}-03$ & $1.09 \mathrm{E}-04$ & $3.63 \mathrm{E}-04$ & 8.99E-03 & $4.48 \mathrm{E}-04$ \\
\hline Pu-239 & $6.72 \mathrm{E}-05$ & 7.92E-05 & $5.23 \mathrm{E}-06$ & $2.87 \mathrm{E}-04$ & $1.06 \mathrm{E}-05$ & $3.77 \mathrm{E}-05$ & $9.56 \mathrm{E}-04$ & $5.07 \mathrm{E}-05$ \\
\hline $\mathrm{Pu}-240$ & $4.68 \mathrm{E}-06$ & $1.22 \mathrm{E}-05$ & $5.82 \mathrm{E}-07$ & $1.96 \mathrm{E}-05$ & $9.45 \mathrm{E}-07$ & $2.76 \mathrm{E}-06$ & $6.45 \mathrm{E}-05$ & 1.14E-05 \\
\hline Pu-241 & 5.07E-04 & $6.50 \mathrm{E}-04$ & $4.78 \mathrm{E}-05$ & $2.14 \mathrm{E}-03$ & $8.78 \mathrm{E}-05$ & $2.88 \mathrm{E}-04$ & $7.09 \mathrm{E}-03$ & $4.50 \mathrm{E}-04$ \\
\hline Pu-242 & 3.69E-09 & 9.42E-09 & $6.02 \mathrm{E}-10$ & 1.49E-08 & $8.76 \mathrm{E}-10$ & 2.23E-09 & 4.87E-08 & $8.92 \mathrm{E}-09$ \\
\hline Pu-244 & $2.89 \mathrm{E}-16$ & $1.95 \mathrm{E}-16$ & $1.72 \mathrm{E}-17$ & $1.24 \mathrm{E}-15$ & $4.07 \mathrm{E}-17$ & $1.59 \mathrm{E}-16$ & $4.16 \mathrm{E}-15$ & $4.41 \mathrm{E}-17$ \\
\hline Am-241 & 1.71E-05 & $6.81 \mathrm{E}-05$ & 2.43E-06 & 7.14E-05 & 3.75E-06 & $1.04 \mathrm{E}-05$ & $2.35 \mathrm{E}-04$ & 6.97E-05 \\
\hline Am-242m & $2.14 \mathrm{E}-10$ & $1.22 \mathrm{E}-08$ & $3.55 \mathrm{E}-10$ & $3.55 \mathrm{E}-10$ & $3.55 \mathrm{E}-10$ & $3.40 \mathrm{E}-10$ & $8.70 \mathrm{E}-11$ & $1.44 \mathrm{E}-08$ \\
\hline Am-243 & 6.07E-09 & $2.04 \mathrm{E}-08$ & $8.25 \mathrm{E}-10$ & $2.54 \mathrm{E}-08$ & 1.29E-09 & 3.64E-09 & 8.34E-08 & $2.03 E-08$ \\
\hline $\mathrm{Cm}-242$ & $2.00 \mathrm{E}-10$ & $2.41 \mathrm{E}-08$ & $4.58 \mathrm{E}-10$ & $5.56 \mathrm{E}-10$ & $4.60 \mathrm{E}-10$ & $4.63 \mathrm{E}-10$ & $4.46 \mathrm{E}-10$ & 2.82E-08 \\
\hline
\end{tabular}


Table B-11. Tank WM-189 mass balance (continued).

\begin{tabular}{|c|c|c|c|c|c|c|c|c|}
\hline Stream \# & 110 & 111 & 112 & 113 & 114 & 115 & 116 & 120 \\
\hline Stream Name & $\begin{array}{l}\text { Slurry from } \\
\text { TFF Tank } \\
\text { WM-187 }\end{array}$ & $\begin{array}{c}\text { Crossflow } \\
\text { Filter Recirc } \\
\text { Purge }\end{array}$ & $\begin{array}{l}\text { Decant } \\
\text { Liquid }\end{array}$ & $\begin{array}{c}\text { Solids } \\
\text { Treatment } \\
\text { Filter Feed }\end{array}$ & $\begin{array}{l}\text { Solids } \\
\text { Treatment } \\
\text { Filtrate }\end{array}$ & $\begin{array}{c}\text { Combined } \\
\text { Waste Water }\end{array}$ & $\begin{array}{l}\text { Dried Solids } \\
\text { Waste }\end{array}$ & $\begin{array}{c}\text { Liquid SBW } \\
\text { from Tank } \\
\text { WM-180 }\end{array}$ \\
\hline & Ci/liter & Ci/liter & Ci/liter & $\mathrm{Ci} /$ liter & Ci/liter & $\mathrm{Ci} /$ liter & $\mathrm{Ci} / \mathrm{kg}$ & Ci/liter \\
\hline $\mathrm{Cm}-243$ & $1.23 \mathrm{E}-08$ & $2.93 \mathrm{E}-08$ & $1.33 \mathrm{E}-09$ & $5.18 \mathrm{E}-08$ & $2.30 \mathrm{E}-09$ & $7.15 \mathrm{E}-09$ & $1.72 \mathrm{E}-07$ & $2.69 \mathrm{E}-08$ \\
\hline $\mathrm{Cm}-244$ & $7.68 \mathrm{E}-07$ & $1.26 \mathrm{E}-06$ & $7.64 \mathrm{E}-08$ & $3.24 \mathrm{E}-06$ & 1.37E-07 & $4.41 \mathrm{E}-07$ & 1.07E-05 & $1.00 \mathrm{E}-06$ \\
\hline $\mathrm{Cm}-245$ & $1.30 \mathrm{E}-10$ & $3.09 \mathrm{E}-10$ & $1.41 \mathrm{E}-11$ & $5.50 \mathrm{E}-10$ & $2.43 \mathrm{E}-11$ & $7.57 \mathrm{E}-11$ & 1.82E-09 & $2.82 \mathrm{E}-10$ \\
\hline $\mathrm{Cm}-246$ & $8.44 \mathrm{E}-12$ & $2.03 \mathrm{E}-11$ & $9.19 \mathrm{E}-13$ & $3.56 \mathrm{E}-11$ & $1.58 \mathrm{E}-12$ & $4.92 \mathrm{E}-12$ & $1.18 \mathrm{E}-10$ & $1.86 \mathrm{E}-11$ \\
\hline $\mathrm{H}-3$ & 3.92E-07 & $8.01 \mathrm{E}-06$ & $4.81 \mathrm{E}-07$ & $4.81 \mathrm{E}-07$ & $4.81 \mathrm{E}-07$ & 4.46E-07 & $1.18 \mathrm{E}-07$ & $9.41 \mathrm{E}-06$ \\
\hline $\mathrm{Be}-10$ & $4.23 \mathrm{E}-14$ & $2.41 \mathrm{E}-12$ & $7.00 \mathrm{E}-14$ & $7.00 \mathrm{E}-14$ & $7.00 \mathrm{E}-14$ & $6.70 \mathrm{E}-14$ & $1.72 \mathrm{E}-14$ & $2.83 \mathrm{E}-12$ \\
\hline $\mathrm{C}-14$ & $5.49 \mathrm{E}-11$ & $1.26 \mathrm{E}-10$ & $5.80 \mathrm{E}-12$ & $2.32 \mathrm{E}-10$ & $1.01 \mathrm{E}-11$ & $3.19 \mathrm{E}-11$ & $7.69 \mathrm{E}-10$ & $1.13 \mathrm{E}-10$ \\
\hline Se-79 & 1.98E-07 & $4.56 \mathrm{E}-07$ & $2.10 \mathrm{E}-08$ & 8.36E-07 & 3.66E-08 & 1.15E-07 & 2.77E-06 & $4.13 \mathrm{E}-07$ \\
\hline Rb-87 & $4.13 \mathrm{E}-13$ & $2.35 \mathrm{E}-11$ & $6.84 \mathrm{E}-13$ & $6.84 \mathrm{E}-13$ & $6.84 \mathrm{E}-13$ & $6.54 \mathrm{E}-13$ & $1.68 \mathrm{E}-13$ & $2.76 \mathrm{E}-11$ \\
\hline Sr-90 & 1.06E-03 & $3.16 \mathrm{E}-02$ & $1.05 \mathrm{E}-03$ & $2.71 \mathrm{E}-03$ & $1.08 \mathrm{E}-03$ & 1.19E-03 & 5.89E-03 & $3.68 \mathrm{E}-02$ \\
\hline $\mathrm{Y}-90$ & 1.06E-03 & $3.16 \mathrm{E}-02$ & 1.05E-03 & $2.71 \mathrm{E}-03$ & $1.08 \mathrm{E}-03$ & $1.19 \mathrm{E}-03$ & $5.89 \mathrm{E}-03$ & $3.68 \mathrm{E}-02$ \\
\hline Zr-93 & $3.12 \mathrm{E}-08$ & $1.78 \mathrm{E}-06$ & $5.17 \mathrm{E}-08$ & $5.17 \mathrm{E}-08$ & $5.17 \mathrm{E}-08$ & $4.95 \mathrm{E}-08$ & $1.27 \mathrm{E}-08$ & $2.09 \mathrm{E}-06$ \\
\hline Nb-93m & $2.41 \mathrm{E}-08$ & $1.37 \mathrm{E}-06$ & $3.98 \mathrm{E}-08$ & $3.98 \mathrm{E}-08$ & $3.98 \mathrm{E}-08$ & $3.81 \mathrm{E}-08$ & 9.77E-09 & $1.61 \mathrm{E}-06$ \\
\hline $\mathrm{Nb}-94$ & $5.10 \mathrm{E}-07$ & $1.19 \mathrm{E}-06$ & $5.44 \mathrm{E}-08$ & $2.15 \mathrm{E}-06$ & $9.46 \mathrm{E}-08$ & $2.96 \mathrm{E}-07$ & $7.14 \mathrm{E}-06$ & $1.08 \mathrm{E}-06$ \\
\hline Tc-98 & $3.63 \mathrm{E}-14$ & $2.07 \mathrm{E}-12$ & $6.02 \mathrm{E}-14$ & $6.02 \mathrm{E}-14$ & $6.02 \mathrm{E}-14$ & $5.76 \mathrm{E}-14$ & $1.48 \mathrm{E}-14$ & $2.43 \mathrm{E}-12$ \\
\hline Tc-99 & 4.43E-06 & $1.06 \mathrm{E}-05$ & 4.93E-07 & 1.87E-05 & $8.41 \mathrm{E}-07$ & $2.59 \mathrm{E}-06$ & $6.18 \mathrm{E}-05$ & $9.69 \mathrm{E}-06$ \\
\hline Ru-106 & 4.27E-07 & $9.74 \mathrm{E}-07$ & $4.50 \mathrm{E}-08$ & $1.81 \mathrm{E}-06$ & $7.87 \mathrm{E}-08$ & $2.48 \mathrm{E}-07$ & 5.98E-06 & $8.79 \mathrm{E}-07$ \\
\hline Rh-102 & $4.14 \mathrm{E}-07$ & $2.28 \mathrm{E}-07$ & $2.33 \mathrm{E}-08$ & $1.78 \mathrm{E}-06$ & $5.70 \mathrm{E}-08$ & $2.27 \mathrm{E}-07$ & 5.98E-06 & $2.77 \mathrm{E}-09$ \\
\hline \begin{tabular}{|l|}
$\mathrm{Pd}-107$ \\
\end{tabular} & $2.33 \mathrm{E}-10$ & 1.33E-08 & $3.86 \mathrm{E}-10$ & $3.86 \mathrm{E}-10$ & $3.86 \mathrm{E}-10$ & $3.69 \mathrm{E}-10$ & $9.45 \mathrm{E}-11$ & $1.56 \mathrm{E}-08$ \\
\hline Cd-113m & $4.68 \mathrm{E}-08$ & $2.67 \mathrm{E}-06$ & $7.75 \mathrm{E}-08$ & $7.75 \mathrm{E}-08$ & $7.75 \mathrm{E}-08$ & $7.42 \mathrm{E}-08$ & $1.90 \mathrm{E}-08$ & $3.13 \mathrm{E}-06$ \\
\hline \begin{tabular}{|l|}
$\ln -115$ \\
\end{tabular} & $1.42 \mathrm{E}-18$ & $8.08 \mathrm{E}-17$ & $2.35 \mathrm{E}-18$ & $2.35 \mathrm{E}-18$ & $2.35 \mathrm{E}-18$ & $2.25 \mathrm{E}-18$ & $5.76 \mathrm{E}-19$ & $9.50 \mathrm{E}-17$ \\
\hline Sn-121m & $9.42 \mathrm{E}-10$ & $5.37 \mathrm{E}-08$ & 1.56E-09 & $1.56 \mathrm{E}-09$ & $1.56 \mathrm{E}-09$ & $1.49 \mathrm{E}-09$ & $3.83 \mathrm{E}-10$ & $6.31 \mathrm{E}-08$ \\
\hline Sn-126 & 1.87E-07 & $4.29 \mathrm{E}-07$ & $1.98 \mathrm{E}-08$ & $7.89 \mathrm{E}-07$ & $3.45 \mathrm{E}-08$ & $1.08 \mathrm{E}-07$ & $2.61 \mathrm{E}-06$ & $3.88 \mathrm{E}-07$ \\
\hline Sb-125 & $5.93 \mathrm{E}-04$ & 3.36E-04 & $3.40 \mathrm{E}-05$ & $2.56 \mathrm{E}-03$ & $8.22 \mathrm{E}-05$ & $3.26 \mathrm{E}-04$ & $8.56 \mathrm{E}-03$ & $1.50 \mathrm{E}-05$ \\
\hline Sb-126 & $8.10 \mathrm{E}-10$ & $4.62 \mathrm{E}-08$ & 1.34E-09 & $1.34 \mathrm{E}-09$ & $1.34 \mathrm{E}-09$ & $1.28 \mathrm{E}-09$ & $3.29 \mathrm{E}-10$ & $5.42 \mathrm{E}-08$ \\
\hline Te-123 & $5.40 \mathrm{E}-21$ & $3.08 \mathrm{E}-19$ & $8.95 \mathrm{E}-21$ & $8.95 \mathrm{E}-21$ & $8.95 \mathrm{E}-21$ & $8.56 \mathrm{E}-21$ & $2.19 \mathrm{E}-21$ & $3.61 \mathrm{E}-19$ \\
\hline Te-125m & 4.44E-08 & $2.53 \mathrm{E}-06$ & $7.35 \mathrm{E}-08$ & $7.35 \mathrm{E}-08$ & $7.35 \mathrm{E}-08$ & 7.03E-08 & $1.80 \mathrm{E}-08$ & $2.97 \mathrm{E}-06$ \\
\hline I-129 & $2.42 \mathrm{E}-08$ & $5.56 \mathrm{E}-08$ & $2.58 \mathrm{E}-09$ & 1.02E-07 & $4.48 \mathrm{E}-09$ & $1.40 \mathrm{E}-08$ & 3.38E-07 & $5.04 \mathrm{E}-08$ \\
\hline Cs-134 & 4.92E-05 & $5.83 \mathrm{E}-05$ & 3.93E-06 & $2.10 \mathrm{E}-04$ & $7.87 \mathrm{E}-06$ & $2.76 \mathrm{E}-05$ & $6.99 \mathrm{E}-04$ & $3.75 \mathrm{E}-05$ \\
\hline Cs-135 & $3.49 \mathrm{E}-07$ & $8.75 \mathrm{E}-07$ & $3.91 \mathrm{E}-08$ & 1.47E-06 & $6.65 \mathrm{E}-08$ & $2.03 \mathrm{E}-07$ & $4.88 \mathrm{E}-06$ & $8.12 \mathrm{E}-07$ \\
\hline Cs-137 & $1.98 \mathrm{E}-02$ & $5.02 \mathrm{E}-02$ & $2.25 \mathrm{E}-03$ & $8.36 \mathrm{E}-02$ & $3.80 \mathrm{E}-03$ & $1.16 \mathrm{E}-02$ & $2.77 \mathrm{E}-01$ & $4.68 \mathrm{E}-02$ \\
\hline Ba-137m & $1.88 \mathrm{E}-02$ & $4.75 \mathrm{E}-02$ & $2.12 \mathrm{E}-03$ & 7.91E-02 & $3.60 \mathrm{E}-03$ & $1.10 \mathrm{E}-02$ & $2.62 \mathrm{E}-01$ & $4.42 \mathrm{E}-02$ \\
\hline \begin{tabular}{|l|} 
La-138 \\
\end{tabular} & $2.69 \mathrm{E}-18$ & $1.53 \mathrm{E}-16$ & $4.46 \mathrm{E}-18$ & $4.46 \mathrm{E}-18$ & $4.46 \mathrm{E}-18$ & $4.26 \mathrm{E}-18$ & $1.09 \mathrm{E}-18$ & $1.80 \mathrm{E}-16$ \\
\hline Ce-142 & $4.21 \mathrm{E}-13$ & $2.40 \mathrm{E}-11$ & $6.98 \mathrm{E}-13$ & $6.98 \mathrm{E}-13$ & $6.98 \mathrm{E}-13$ & $6.68 \mathrm{E}-13$ & $1.71 \mathrm{E}-13$ & $2.82 \mathrm{E}-11$ \\
\hline Ce-144 & $2.90 \mathrm{E}-07$ & $6.57 \mathrm{E}-07$ & $3.04 \mathrm{E}-08$ & 1.23E-06 & $5.33 \mathrm{E}-08$ & $1.68 \mathrm{E}-07$ & 4.06E-06 & $5.92 \mathrm{E}-07$ \\
\hline Nd-144 & $2.27 \mathrm{E}-17$ & $1.29 \mathrm{E}-15$ & $3.75 \mathrm{E}-17$ & $3.75 \mathrm{E}-17$ & $3.75 \mathrm{E}-17$ & $3.59 \mathrm{E}-17$ & $9.20 \mathrm{E}-18$ & $1.52 \mathrm{E}-15$ \\
\hline Pm-146 & $7.17 \mathrm{E}-10$ & $4.09 \mathrm{E}-08$ & 1.19E-09 & 1.19E-09 & 1.19E-09 & 1.14E-09 & $2.91 \mathrm{E}-10$ & $4.80 \mathrm{E}-08$ \\
\hline Pm-147 & $7.68 \mathrm{E}-05$ & $1.78 \mathrm{E}-04$ & $8.16 \mathrm{E}-06$ & $3.25 \mathrm{E}-04$ & $1.42 \mathrm{E}-05$ & $4.46 \mathrm{E}-05$ & $1.08 \mathrm{E}-03$ & $1.61 \mathrm{E}-04$ \\
\hline Sm-146 & $3.89 \mathrm{E}-15$ & $2.21 \mathrm{E}-13$ & $6.44 \mathrm{E}-15$ & $6.44 \mathrm{E}-15$ & $6.44 \mathrm{E}-15$ & $6.16 \mathrm{E}-15$ & $1.58 \mathrm{E}-15$ & $2.60 \mathrm{E}-13$ \\
\hline Sm-147 & $1.04 \mathrm{E}-13$ & $5.91 \mathrm{E}-12$ & $1.72 \mathrm{E}-13$ & $1.72 \mathrm{E}-13$ & $1.72 \mathrm{E}-13$ & $1.65 \mathrm{E}-13$ & $4.21 \mathrm{E}-14$ & $6.95 \mathrm{E}-12$ \\
\hline Sm-148 & $5.33 \mathrm{E}-19$ & $3.04 \mathrm{E}-17$ & 8.84E-19 & $8.84 \mathrm{E}-19$ & $8.84 \mathrm{E}-19$ & $8.45 \mathrm{E}-19$ & $2.17 \mathrm{E}-19$ & $3.57 \mathrm{E}-17$ \\
\hline Sm-149 & $4.73 \mathrm{E}-20$ & $2.70 \mathrm{E}-18$ & $7.84 \mathrm{E}-20$ & $7.84 \mathrm{E}-20$ & 7.84E-20 & $7.50 \mathrm{E}-20$ & $1.92 \mathrm{E}-20$ & $3.17 \mathrm{E}-18$ \\
\hline Sm-151 & $1.53 \mathrm{E}-04$ & $3.51 \mathrm{E}-04$ & 1.62E-05 & $6.48 \mathrm{E}-04$ & $2.83 \mathrm{E}-05$ & $8.89 \mathrm{E}-05$ & $2.15 \mathrm{E}-03$ & 3.17E-04 \\
\hline Eu-152 & $5.55 \mathrm{E}-07$ & $2.31 \mathrm{E}-06$ & $8.80 \mathrm{E}-08$ & $2.30 \mathrm{E}-06$ & $1.30 \mathrm{E}-07$ & $3.41 \mathrm{E}-07$ & 7.52E-06 & $2.38 \mathrm{E}-06$ \\
\hline Eu-154 & 2.44E-05 & 1.57E-04 & $6.49 \mathrm{E}-06$ & $9.48 \mathrm{E}-05$ & $8.18 \mathrm{E}-06$ & 1.64E-05 & 3.01E-04 & $1.71 \mathrm{E}-04$ \\
\hline Eu-155 & $3.50 \mathrm{E}-05$ & $1.48 \mathrm{E}-04$ & 4.43E-06 & $1.49 \mathrm{E}-04$ & $7.19 \mathrm{E}-06$ & $2.11 \mathrm{E}-05$ & $4.91 \mathrm{E}-04$ & $1.52 \mathrm{E}-04$ \\
\hline \begin{tabular}{|l|} 
Gd-152 \\
\end{tabular} & $2.00 \mathrm{E}-20$ & $1.14 \mathrm{E}-18$ & $3.32 \mathrm{E}-20$ & $3.32 \mathrm{E}-20$ & $3.32 \mathrm{E}-20$ & $3.17 \mathrm{E}-20$ & $8.13 \mathrm{E}-21$ & $1.34 \mathrm{E}-18$ \\
\hline Ho-166m & $6.49 \mathrm{E}-13$ & $3.70 \mathrm{E}-11$ & $1.07 \mathrm{E}-12$ & $1.07 \mathrm{E}-12$ & $1.07 \mathrm{E}-12$ & $1.03 \mathrm{E}-12$ & $2.63 \mathrm{E}-13$ & $4.34 \mathrm{E}-11$ \\
\hline Co-60 & $2.81 \mathrm{E}-06$ & $2.95 \mathrm{E}-05$ & $1.38 \mathrm{E}-06$ & $9.25 \mathrm{E}-06$ & $1.53 \mathrm{E}-06$ & $2.21 \mathrm{E}-06$ & $2.70 \mathrm{E}-05$ & 3.35E-05 \\
\hline $\mathrm{Ni}-63$ & 1.48E-05 & 3.31E-05 & $2.02 \mathrm{E}-06$ & $6.09 \mathrm{E}-05$ & $3.14 \mathrm{E}-06$ & $8.75 \mathrm{E}-06$ & $2.00 \mathrm{E}-04$ & $3.00 \mathrm{E}-05$ \\
\hline
\end{tabular}


Table B-11. Tank WM-189 mass balance (continued).

\begin{tabular}{|c|c|c|c|c|c|c|c|c|}
\hline PFD Sheet \# & PFD-3 & PFD-3 & PFD-3 & PFD-3 & PFD-3 & PFD-3 & PFD-3 & PFD-3 \\
\hline Stream \# & 121 & 122 & 123 & 124 & 125 & 126 & 127 & 128 \\
\hline Stream Name & $\begin{array}{l}\text { Neutralized } \\
\text { Feed }\end{array}$ & $\begin{array}{l}\text { SBW Liquid } \\
\text { Filter Feed }\end{array}$ & $\begin{array}{c}\text { Filter } \\
\text { Recirculation }\end{array}$ & $\begin{array}{l}\text { IX Column } \\
\text { Feed }\end{array}$ & $\begin{array}{c}\text { Column } 1 \\
\text { Effluent }\end{array}$ & $\begin{array}{c}\text { Column } 2 \\
\text { Effluent }\end{array}$ & $\begin{array}{c}\text { Column } 3 \\
\text { Effluent }\end{array}$ & $\begin{array}{l}\text { Spent IX } \\
\text { Rinse }\end{array}$ \\
\hline Rate, gal/hr & 95.3 & 7800 & 7705 & 95.3 & 95.3 & 95.3 & 95.3 & 0.5 \\
\hline Rate, Ib/hr & 1062 & 86975 & 85913 & 1062 & 1062 & 1062 & 1062 & 5 \\
\hline Rate, peak, gpm & 1.59 & 130 & 128 & 1.59 & 1.59 & 1.59 & 1.59 & 2.99 \\
\hline Operation & Cont & Cont & Cont & Cont & Cont & Cont & Cont & Batch \\
\hline Temperature, ${ }^{\circ} \mathrm{F}$ & 70 & 70 & 70 & 70 & 70 & 70 & 70 & 70 \\
\hline Pressure, psia & 12.8 & 35.0 & 35.0 & 24.8 & 20.8 & 16.8 & 12.8 & 12.8 \\
\hline Specific Gravity & 1.34 & 1.34 & 1.34 & 1.34 & 1.34 & 1.34 & 1.34 & 1.07 \\
\hline Chemical Composition & Mol/liter & Mol/liter & Mol/liter & Mol/liter & Mol/liter & Mol/liter & Mol/liter & Mol/liter \\
\hline $\mathrm{H}+$ & $5.00 \mathrm{E}-01$ & $5.00 \mathrm{E}-01$ & $5.00 \mathrm{E}-01$ & $5.00 \mathrm{E}-01$ & $5.00 \mathrm{E}-01$ & $5.00 \mathrm{E}-01$ & $5.00 \mathrm{E}-01$ & $1.01 \mathrm{E}-01$ \\
\hline $\mathrm{Al}+3$ & $5.82 \mathrm{E}-01$ & $5.98 \mathrm{E}-01$ & $5.98 \mathrm{E}-01$ & $5.82 \mathrm{E}-01$ & 5.82E-01 & 5.82E-01 & 5.82E-01 & $1.18 \mathrm{E}-01$ \\
\hline $\mathrm{Am}+4$ & $5.08 \mathrm{E}-08$ & $5.08 \mathrm{E}-08$ & $5.08 \mathrm{E}-08$ & $5.08 \mathrm{E}-08$ & $5.08 \mathrm{E}-08$ & $5.08 \mathrm{E}-08$ & $5.08 \mathrm{E}-08$ & $1.03 \mathrm{E}-08$ \\
\hline $\mathrm{Sb}+5$ & $6.65 \mathrm{E}-06$ & $1.59 \mathrm{E}-05$ & $1.60 \mathrm{E}-05$ & $6.58 \mathrm{E}-06$ & $6.58 \mathrm{E}-06$ & $6.58 \mathrm{E}-06$ & $6.58 \mathrm{E}-06$ & 1.33E-06 \\
\hline As+5 & $2.06 \mathrm{E}-06$ & $8.02 \mathrm{E}-05$ & $8.12 \mathrm{E}-05$ & $1.52 \mathrm{E}-06$ & $1.52 \mathrm{E}-06$ & $1.52 \mathrm{E}-06$ & $1.52 \mathrm{E}-06$ & $3.08 \mathrm{E}-07$ \\
\hline $\mathrm{Ba}+2$ & $4.67 \mathrm{E}-05$ & $6.32 \mathrm{E}-05$ & $6.34 \mathrm{E}-05$ & $4.66 \mathrm{E}-05$ & $4.66 \mathrm{E}-05$ & $4.66 \mathrm{E}-05$ & $4.66 \mathrm{E}-05$ & $9.41 \mathrm{E}-06$ \\
\hline $\mathrm{Be}+2$ & $1.65 \mathrm{E}-05$ & 2.03E-05 & 2.03E-05 & $1.65 \mathrm{E}-05$ & $1.65 \mathrm{E}-05$ & $1.65 \mathrm{E}-05$ & $1.65 \mathrm{E}-05$ & 3.33E-06 \\
\hline$B+3$ & $1.73 \mathrm{E}-02$ & 1.76E-02 & 1.76E-02 & $1.73 \mathrm{E}-02$ & $1.73 \mathrm{E}-02$ & 1.73E-02 & 1.73E-02 & $3.50 \mathrm{E}-03$ \\
\hline $\mathrm{Br}-$ & $2.66 \mathrm{E}-07$ & $2.66 \mathrm{E}-07$ & $2.66 \mathrm{E}-07$ & $2.66 \mathrm{E}-07$ & $2.66 \mathrm{E}-07$ & $2.66 \mathrm{E}-07$ & $2.66 \mathrm{E}-07$ & 5.37E-08 \\
\hline $\mathrm{Cd}+2$ & $3.19 \mathrm{E}-03$ & $3.24 \mathrm{E}-03$ & $3.24 \mathrm{E}-03$ & $3.19 \mathrm{E}-03$ & $3.19 \mathrm{E}-03$ & $3.19 \mathrm{E}-03$ & $3.19 \mathrm{E}-03$ & $6.45 \mathrm{E}-04$ \\
\hline $\mathrm{Ca}+2$ & $5.98 \mathrm{E}-02$ & $6.06 \mathrm{E}-02$ & $6.06 \mathrm{E}-02$ & $5.98 \mathrm{E}-02$ & $5.98 \mathrm{E}-02$ & $5.98 \mathrm{E}-02$ & $5.98 \mathrm{E}-02$ & $1.21 \mathrm{E}-02$ \\
\hline $\mathrm{Ce}+4$ & $3.00 \mathrm{E}-05$ & $3.83 \mathrm{E}-05$ & $3.84 \mathrm{E}-05$ & $2.99 \mathrm{E}-05$ & $2.99 \mathrm{E}-05$ & $2.99 \mathrm{E}-05$ & $2.99 \mathrm{E}-05$ & $6.05 \mathrm{E}-06$ \\
\hline Cs+ & $2.28 \mathrm{E}-05$ & 5.67E-05 & $5.72 \mathrm{E}-05$ & $2.26 \mathrm{E}-05$ & $2.26 \mathrm{E}-06$ & $2.26 \mathrm{E}-07$ & $2.26 \mathrm{E}-08$ & 4.57E-09 \\
\hline $\mathrm{Cl}-$ & $1.71 \mathrm{E}-02$ & 1.90E-02 & $1.90 \mathrm{E}-02$ & $1.71 \mathrm{E}-02$ & $1.71 \mathrm{E}-02$ & $1.71 \mathrm{E}-02$ & $1.71 \mathrm{E}-02$ & $3.46 \mathrm{E}-03$ \\
\hline $\mathrm{Cr}+3$ & $4.74 \mathrm{E}-03$ & $5.06 \mathrm{E}-03$ & 5.07E-03 & $4.74 \mathrm{E}-03$ & $4.74 \mathrm{E}-03$ & $4.74 \mathrm{E}-03$ & $4.74 \mathrm{E}-03$ & $9.58 \mathrm{E}-04$ \\
\hline $\mathrm{Co}+2$ & $2.12 \mathrm{E}-04$ & $2.15 \mathrm{E}-04$ & $2.15 \mathrm{E}-04$ & $2.12 \mathrm{E}-04$ & $2.12 \mathrm{E}-04$ & $2.12 \mathrm{E}-04$ & $2.12 \mathrm{E}-04$ & $4.28 \mathrm{E}-05$ \\
\hline $\mathrm{Cu}+2$ & 7.77E-04 & $8.71 \mathrm{E}-04$ & $8.73 \mathrm{E}-04$ & 7.76E-04 & 7.76E-04 & 7.76E-04 & 7.76E-04 & 1.57E-04 \\
\hline $\mathrm{Eu}+3$ & $4.41 \mathrm{E}-07$ & $4.41 \mathrm{E}-07$ & $4.41 \mathrm{E}-07$ & $4.41 \mathrm{E}-07$ & $4.41 \mathrm{E}-07$ & $4.41 \mathrm{E}-07$ & $4.41 \mathrm{E}-07$ & $8.90 \mathrm{E}-08$ \\
\hline F- & $1.27 \mathrm{E}-02$ & $2.90 \mathrm{E}-02$ & $2.92 \mathrm{E}-02$ & $1.26 \mathrm{E}-02$ & $1.26 \mathrm{E}-02$ & $1.26 \mathrm{E}-02$ & $1.26 \mathrm{E}-02$ & $2.54 \mathrm{E}-03$ \\
\hline $\mathrm{Gd}+3$ & $1.16 \mathrm{E}-04$ & 1.32E-04 & 1.32E-04 & $1.15 \mathrm{E}-04$ & $1.15 \mathrm{E}-04$ & $1.15 \mathrm{E}-04$ & 1.15E-04 & $2.33 \mathrm{E}-05$ \\
\hline $\mathrm{Ge}+4$ & 7.66E-09 & 7.66E-09 & $7.66 \mathrm{E}-09$ & 7.66E-09 & 7.66E-09 & 7.66E-09 & 7.66E-09 & 1.55E-09 \\
\hline $\ln +3$ & $1.21 \mathrm{E}-06$ & $1.21 \mathrm{E}-06$ & $1.21 \mathrm{E}-06$ & $1.21 \mathrm{E}-06$ & $1.21 \mathrm{E}-06$ & $1.21 \mathrm{E}-06$ & $1.21 \mathrm{E}-06$ & $2.44 \mathrm{E}-07$ \\
\hline I- & $2.21 \mathrm{E}-06$ & $2.21 \mathrm{E}-06$ & $2.21 \mathrm{E}-06$ & $2.21 \mathrm{E}-06$ & $2.21 \mathrm{E}-06$ & $2.21 \mathrm{E}-06$ & $2.21 \mathrm{E}-06$ & $4.46 \mathrm{E}-07$ \\
\hline $\mathrm{Fe}+3$ & $2.21 \mathrm{E}-02$ & $2.85 \mathrm{E}-02$ & $2.85 \mathrm{E}-02$ & $2.20 \mathrm{E}-02$ & $2.20 \mathrm{E}-02$ & $2.20 \mathrm{E}-02$ & $2.20 \mathrm{E}-02$ & $4.45 \mathrm{E}-03$ \\
\hline $\mathrm{La}+3$ & $8.00 \mathrm{E}-06$ & $8.00 \mathrm{E}-06$ & $8.00 \mathrm{E}-06$ & $8.00 \mathrm{E}-06$ & $8.00 \mathrm{E}-06$ & $8.00 \mathrm{E}-06$ & $8.00 \mathrm{E}-06$ & $1.62 \mathrm{E}-06$ \\
\hline $\mathrm{Pb}+2$ & $9.69 \mathrm{E}-04$ & $9.98 \mathrm{E}-04$ & $9.98 \mathrm{E}-04$ & $9.68 \mathrm{E}-04$ & $9.68 \mathrm{E}-04$ & $9.68 \mathrm{E}-04$ & $9.68 \mathrm{E}-04$ & 1.96E-04 \\
\hline $\mathrm{Li+}$ & $3.26 \mathrm{E}-04$ & $5.21 \mathrm{E}-04$ & $5.24 \mathrm{E}-04$ & $3.25 \mathrm{E}-04$ & $3.25 \mathrm{E}-04$ & $3.25 \mathrm{E}-04$ & $3.25 \mathrm{E}-04$ & $6.56 \mathrm{E}-05$ \\
\hline$M g+2$ & $1.86 \mathrm{E}-02$ & 1.92E-02 & $1.92 \mathrm{E}-02$ & $1.86 \mathrm{E}-02$ & $1.86 \mathrm{E}-02$ & 1.86E-02 & 1.86E-02 & $3.77 \mathrm{E}-03$ \\
\hline $\mathrm{Mn}+4$ & 1.67E-02 & $1.68 \mathrm{E}-02$ & $1.68 \mathrm{E}-02$ & 1.67E-02 & 1.67E-02 & 1.67E-02 & 1.67E-02 & 3.37E-03 \\
\hline $\mathrm{Hg}+2$ & $5.33 \mathrm{E}-03$ & $5.34 \mathrm{E}-03$ & $5.34 \mathrm{E}-03$ & $5.33 \mathrm{E}-03$ & $5.33 \mathrm{E}-03$ & $5.33 \mathrm{E}-03$ & $5.33 \mathrm{E}-03$ & $1.08 \mathrm{E}-03$ \\
\hline $\mathrm{Mo}+6$ & $2.40 \mathrm{E}-04$ & $7.86 \mathrm{E}-04$ & $7.93 \mathrm{E}-04$ & 2.37E-04 & 2.37E-04 & 2.37E-04 & 2.37E-04 & $4.78 \mathrm{E}-05$ \\
\hline $\mathrm{Nd}+3$ & $2.58 \mathrm{E}-05$ & $2.58 \mathrm{E}-05$ & $2.58 \mathrm{E}-05$ & $2.58 \mathrm{E}-05$ & $2.58 \mathrm{E}-05$ & $2.58 \mathrm{E}-05$ & $2.58 \mathrm{E}-05$ & $5.22 \mathrm{E}-06$ \\
\hline $\mathrm{Np}+4$ & $2.42 \mathrm{E}-06$ & $2.42 \mathrm{E}-06$ & $2.42 \mathrm{E}-06$ & $2.42 \mathrm{E}-06$ & $2.42 \mathrm{E}-06$ & $2.42 \mathrm{E}-06$ & $2.42 \mathrm{E}-06$ & 4.90E-07 \\
\hline $\mathrm{Ni}+2$ & $1.91 \mathrm{E}-03$ & $2.02 \mathrm{E}-03$ & $2.02 \mathrm{E}-03$ & 1.91E-03 & $1.91 \mathrm{E}-03$ & $1.91 \mathrm{E}-03$ & 1.91E-03 & $3.86 \mathrm{E}-04$ \\
\hline $\mathrm{Nb}+5$ & $6.63 \mathrm{E}-06$ & $9.11 \mathrm{E}-04$ & $9.22 \mathrm{E}-04$ & $3.98 \mathrm{E}-07$ & $3.98 \mathrm{E}-07$ & $3.98 \mathrm{E}-07$ & $3.98 \mathrm{E}-07$ & $8.05 \mathrm{E}-08$ \\
\hline NO3- & $6.40 \mathrm{E}+00$ & $6.40 \mathrm{E}+00$ & $6.40 \mathrm{E}+00$ & $6.40 \mathrm{E}+00$ & $6.40 \mathrm{E}+00$ & $6.40 \mathrm{E}+00$ & $6.40 \mathrm{E}+00$ & $1.29 \mathrm{E}+00$ \\
\hline $\mathrm{O}-2$ & 9.44E-04 & $1.38 \mathrm{E}-01$ & $1.40 \mathrm{E}-01$ & & & & & \\
\hline$P d+4$ & $1.78 \mathrm{E}-05$ & $1.52 \mathrm{E}-03$ & $1.54 \mathrm{E}-03$ & $7.42 \mathrm{E}-06$ & $7.42 \mathrm{E}-06$ & $7.42 \mathrm{E}-06$ & $7.42 \mathrm{E}-06$ & $1.50 \mathrm{E}-06$ \\
\hline PO4-3 & $2.32 \mathrm{E}-03$ & $8.72 \mathrm{E}-02$ & $8.83 \mathrm{E}-02$ & $1.74 \mathrm{E}-03$ & $1.74 \mathrm{E}-03$ & $1.74 \mathrm{E}-03$ & $1.74 \mathrm{E}-03$ & 3.52E-04 \\
\hline $\mathrm{Pu}+4$ & $3.34 \mathrm{E}-06$ & $3.34 \mathrm{E}-06$ & $3.34 \mathrm{E}-06$ & $3.34 \mathrm{E}-06$ & $3.34 \mathrm{E}-06$ & $3.34 \mathrm{E}-06$ & $3.34 \mathrm{E}-06$ & $6.75 \mathrm{E}-07$ \\
\hline $\mathrm{K}+$ & $2.12 \mathrm{E}-01$ & $2.23 \mathrm{E}-01$ & $2.23 \mathrm{E}-01$ & $2.12 \mathrm{E}-01$ & $2.12 \mathrm{E}-01$ & $2.12 \mathrm{E}-01$ & $2.12 \mathrm{E}-01$ & $4.29 \mathrm{E}-02$ \\
\hline $\mathrm{Pr}+4$ & $7.28 \mathrm{E}-06$ & $7.28 \mathrm{E}-06$ & $7.28 \mathrm{E}-06$ & $7.28 \mathrm{E}-06$ & $7.28 \mathrm{E}-06$ & $7.28 \mathrm{E}-06$ & $7.28 \mathrm{E}-06$ & $1.47 \mathrm{E}-06$ \\
\hline $\mathrm{Rh}+4$ & $3.14 \mathrm{E}-06$ & $3.14 \mathrm{E}-06$ & $3.14 \mathrm{E}-06$ & $3.14 \mathrm{E}-06$ & $3.14 \mathrm{E}-06$ & $3.14 \mathrm{E}-06$ & $3.14 \mathrm{E}-06$ & $6.35 \mathrm{E}-07$ \\
\hline
\end{tabular}


Table B-11. Tank WM-189 mass balance (continued).

\begin{tabular}{|c|c|c|c|c|c|c|c|c|}
\hline Stream \# & 121 & 122 & 123 & 124 & 125 & 126 & 127 & 128 \\
\hline Stream Name & $\begin{array}{l}\text { Neutralized } \\
\text { Feed }\end{array}$ & $\begin{array}{l}\text { SBW Liquid } \\
\text { Filter Feed }\end{array}$ & $\begin{array}{c}\text { Filter } \\
\text { Recirculation }\end{array}$ & $\begin{array}{l}\text { IX Column } \\
\text { Feed }\end{array}$ & $\begin{array}{l}\text { Column } 1 \\
\text { Effluent }\end{array}$ & $\begin{array}{c}\text { Column } 2 \\
\text { Effluent }\end{array}$ & $\begin{array}{l}\text { Column } 3 \\
\text { Effluent }\end{array}$ & $\begin{array}{l}\text { Spent IX } \\
\text { Rinse }\end{array}$ \\
\hline Chemical Composition & Mol/liter & Mol/liter & Mol/liter & Mol/liter & Mol/liter & Mol/liter & Mol/liter & Mol/liter \\
\hline $\mathrm{Rb}+$ & 4.83E-06 & $4.83 \mathrm{E}-06$ & $4.83 \mathrm{E}-06$ & $4.83 \mathrm{E}-06$ & $4.83 \mathrm{E}-06$ & 4.83E-06 & $4.83 \mathrm{E}-06$ & 9.77E-07 \\
\hline $\mathrm{Ru}+3$ & $1.50 \mathrm{E}-04$ & $7.80 \mathrm{E}-04$ & 7.87E-04 & $1.45 \mathrm{E}-04$ & $1.45 \mathrm{E}-04$ & $1.45 \mathrm{E}-04$ & $1.45 \mathrm{E}-04$ & 2.94E-05 \\
\hline $\mathrm{Sm}+3$ & $4.79 \mathrm{E}-06$ & $4.79 \mathrm{E}-06$ & 4.79E-06 & 4.79E-06 & 4.79E-06 & $4.79 \mathrm{E}-06$ & 4.79E-06 & 9.67E-07 \\
\hline $\mathrm{Se}+4$ & $2.72 \mathrm{E}-06$ & $2.37 \mathrm{E}-04$ & $2.40 \mathrm{E}-04$ & $1.11 \mathrm{E}-06$ & 1.11E-06 & $1.11 \mathrm{E}-06$ & $1.11 \mathrm{E}-06$ & $2.24 \mathrm{E}-07$ \\
\hline $\mathrm{Si}+4$ & 7.93E-04 & $7.78 \mathrm{E}-02$ & $7.88 \mathrm{E}-02$ & $2.62 \mathrm{E}-04$ & $2.62 \mathrm{E}-04$ & $2.62 \mathrm{E}-04$ & $2.62 \mathrm{E}-04$ & 5.29E-05 \\
\hline $\mathrm{Ag}^{+}$ & $2.69 \mathrm{E}-06$ & $3.63 \mathrm{E}-04$ & $3.68 \mathrm{E}-04$ & 2.06E-07 & $2.06 \mathrm{E}-07$ & $2.06 \mathrm{E}-07$ & $2.06 \mathrm{E}-07$ & $4.16 \mathrm{E}-08$ \\
\hline $\mathrm{Na}+$ & $3.75 \mathrm{E}+00$ & $3.77 \mathrm{E}+00$ & $3.77 \mathrm{E}+00$ & $3.75 \mathrm{E}+00$ & $3.75 \mathrm{E}+00$ & $3.75 \mathrm{E}+00$ & $3.75 \mathrm{E}+00$ & $7.59 \mathrm{E}-01$ \\
\hline $\mathrm{Sr}+2$ & 1.19E-04 & $1.24 \mathrm{E}-04$ & $1.24 \mathrm{E}-04$ & $1.19 \mathrm{E}-04$ & 1.19E-04 & 1.19E-04 & 1.19E-04 & $2.41 \mathrm{E}-05$ \\
\hline SO4-2 & $8.75 \mathrm{E}-02$ & $8.75 \mathrm{E}-02$ & $8.75 \mathrm{E}-02$ & $8.75 \mathrm{E}-02$ & $8.75 \mathrm{E}-02$ & $8.75 \mathrm{E}-02$ & $8.75 \mathrm{E}-02$ & 1.77E-02 \\
\hline $\mathrm{Tc}+7$ & $5.15 \mathrm{E}-06$ & $5.15 \mathrm{E}-06$ & $5.15 \mathrm{E}-06$ & $5.15 \mathrm{E}-06$ & $5.15 \mathrm{E}-06$ & $5.15 \mathrm{E}-06$ & $5.15 \mathrm{E}-06$ & $1.04 \mathrm{E}-06$ \\
\hline $\mathrm{Te}+4$ & $6.05 \mathrm{E}-06$ & $6.05 \mathrm{E}-06$ & $6.05 \mathrm{E}-06$ & $6.05 \mathrm{E}-06$ & $6.05 \mathrm{E}-06$ & $6.05 \mathrm{E}-06$ & $6.05 \mathrm{E}-06$ & $1.22 \mathrm{E}-06$ \\
\hline $\mathrm{Tb}+4$ & 1.84E-09 & $1.84 \mathrm{E}-09$ & 1.84E-09 & 1.84E-09 & 1.84E-09 & 1.84E-09 & 1.84E-09 & $3.72 \mathrm{E}-10$ \\
\hline $\mathrm{Th}+4$ & $2.92 \mathrm{E}-05$ & $2.92 \mathrm{E}-05$ & $2.92 \mathrm{E}-05$ & $2.92 \mathrm{E}-05$ & $2.92 \mathrm{E}-05$ & $2.92 \mathrm{E}-05$ & $2.92 \mathrm{E}-05$ & $5.90 \mathrm{E}-06$ \\
\hline $\mathrm{Sn}+4$ & $4.57 \mathrm{E}-05$ & $1.66 \mathrm{E}-03$ & $1.68 \mathrm{E}-03$ & $3.46 \mathrm{E}-05$ & $3.46 \mathrm{E}-05$ & $3.46 \mathrm{E}-05$ & $3.46 \mathrm{E}-05$ & $7.00 \mathrm{E}-06$ \\
\hline $\mathrm{Ti}+4$ & $6.70 \mathrm{E}-05$ & 8.57E-04 & $8.67 \mathrm{E}-04$ & $6.15 \mathrm{E}-05$ & $6.15 \mathrm{E}-05$ & $6.15 \mathrm{E}-05$ & $6.15 \mathrm{E}-05$ & $1.24 \mathrm{E}-05$ \\
\hline $\mathrm{U}+4$ & $9.83 \mathrm{E}-04$ & $1.00 \mathrm{E}-03$ & $1.00 \mathrm{E}-03$ & $9.83 \mathrm{E}-04$ & $9.83 \mathrm{E}-04$ & $9.83 \mathrm{E}-04$ & $9.83 \mathrm{E}-04$ & $1.99 \mathrm{E}-04$ \\
\hline $\mathrm{V}+5$ & $2.29 \mathrm{E}-05$ & $2.76 \mathrm{E}-05$ & $2.76 \mathrm{E}-05$ & $2.29 \mathrm{E}-05$ & $2.29 \mathrm{E}-05$ & $2.29 \mathrm{E}-05$ & $2.29 \mathrm{E}-05$ & $4.62 \mathrm{E}-06$ \\
\hline $\mathrm{Y}+3$ & $5.97 \mathrm{E}-06$ & 5.97E-06 & $5.97 \mathrm{E}-06$ & 5.97E-06 & 5.97E-06 & 5.97E-06 & $5.97 \mathrm{E}-06$ & $1.21 \mathrm{E}-06$ \\
\hline$Z n+2$ & $8.84 \mathrm{E}-04$ & $9.38 \mathrm{E}-04$ & $9.39 \mathrm{E}-04$ & $8.84 \mathrm{E}-04$ & $8.84 \mathrm{E}-04$ & $8.84 \mathrm{E}-04$ & $8.84 \mathrm{E}-04$ & $1.79 \mathrm{E}-04$ \\
\hline $\mathrm{Zr}+4$ & $5.70 \mathrm{E}-04$ & 3.33E-02 & $3.37 \mathrm{E}-02$ & $3.45 \mathrm{E}-04$ & $3.45 \mathrm{E}-04$ & $3.45 \mathrm{E}-04$ & $3.45 \mathrm{E}-04$ & $6.97 \mathrm{E}-05$ \\
\hline $\mathrm{H} 2 \mathrm{O}$ & $4.48 \mathrm{E}+01$ & $4.38 E+01$ & $4.38 \mathrm{E}+01$ & $4.48 \mathrm{E}+01$ & $4.48 \mathrm{E}+01$ & $4.48 \mathrm{E}+01$ & $4.48 \mathrm{E}+01$ & $5.30 \mathrm{E}+01$ \\
\hline & & & & & & & & \\
\hline Radiological Composition & Ci/liter & Ci/liter & Ci/liter & $\mathrm{Ci} /$ liter & Ci/liter & Ci/liter & $\mathrm{Ci} / \mathrm{liter}$ & $\mathrm{Ci} /$ liter \\
\hline Ra-226 & $6.91 \mathrm{E}-12$ & $6.91 \mathrm{E}-12$ & $6.91 \mathrm{E}-12$ & $6.91 \mathrm{E}-12$ & $6.91 \mathrm{E}-12$ & $6.91 \mathrm{E}-12$ & $6.91 \mathrm{E}-12$ & $1.40 \mathrm{E}-12$ \\
\hline Ac-227 & $3.25 \mathrm{E}-11$ & $3.25 \mathrm{E}-11$ & $3.25 \mathrm{E}-11$ & $3.25 \mathrm{E}-11$ & $3.25 \mathrm{E}-11$ & $3.25 \mathrm{E}-11$ & $3.25 \mathrm{E}-11$ & $6.57 \mathrm{E}-12$ \\
\hline Th-228 & $2.70 \mathrm{E}-09$ & $2.70 \mathrm{E}-09$ & $2.70 \mathrm{E}-09$ & $2.70 \mathrm{E}-09$ & $2.70 \mathrm{E}-09$ & $2.70 \mathrm{E}-09$ & $2.70 \mathrm{E}-09$ & $5.45 \mathrm{E}-10$ \\
\hline Th-230 & $6.95 \mathrm{E}-10$ & $8.94 \mathrm{E}-10$ & $8.97 E-10$ & $6.93 \mathrm{E}-10$ & $6.93 \mathrm{E}-10$ & $6.93 \mathrm{E}-10$ & $6.93 \mathrm{E}-10$ & $1.40 \mathrm{E}-10$ \\
\hline Th-232 & $5.97 \mathrm{E}-16$ & $6.70 \mathrm{E}-11$ & $5.97 \mathrm{E}-16$ & $5.97 \mathrm{E}-16$ & $5.97 \mathrm{E}-16$ & $5.97 \mathrm{E}-16$ & $5.97 \mathrm{E}-16$ & $1.21 \mathrm{E}-16$ \\
\hline Pa-231 & $7.53 \mathrm{E}-11$ & $7.53 \mathrm{E}-11$ & $7.53 \mathrm{E}-11$ & $7.53 \mathrm{E}-11$ & $7.53 \mathrm{E}-11$ & $7.53 \mathrm{E}-11$ & $7.53 \mathrm{E}-11$ & $1.52 \mathrm{E}-11$ \\
\hline $\mathrm{Pa}-233$ & $2.47 \mathrm{E}-06$ & $2.47 \mathrm{E}-06$ & $2.47 \mathrm{E}-06$ & $2.47 \mathrm{E}-06$ & $2.47 \mathrm{E}-06$ & 2.47E-06 & $2.47 \mathrm{E}-06$ & 4.99E-07 \\
\hline $\mathrm{U}-232$ & $1.68 \mathrm{E}-09$ & $2.09 \mathrm{E}-09$ & $2.10 \mathrm{E}-09$ & $1.68 \mathrm{E}-09$ & $1.68 \mathrm{E}-09$ & $1.68 \mathrm{E}-09$ & $1.68 \mathrm{E}-09$ & $3.39 \mathrm{E}-10$ \\
\hline $\mathrm{U}-233$ & $6.75 \mathrm{E}-11$ & $7.46 \mathrm{E}-11$ & $7.47 \mathrm{E}-11$ & $6.74 \mathrm{E}-11$ & $6.74 \mathrm{E}-11$ & $6.74 \mathrm{E}-11$ & $6.74 \mathrm{E}-11$ & $1.36 \mathrm{E}-11$ \\
\hline U-234 & $1.46 \mathrm{E}-06$ & $1.58 \mathrm{E}-06$ & $1.59 \mathrm{E}-06$ & $1.46 \mathrm{E}-06$ & $1.46 \mathrm{E}-06$ & $1.46 \mathrm{E}-06$ & $1.46 \mathrm{E}-06$ & 2.96E-07 \\
\hline U-235 & $5.14 \mathrm{E}-08$ & $5.79 \mathrm{E}-08$ & $5.80 \mathrm{E}-08$ & $5.14 \mathrm{E}-08$ & $5.14 \mathrm{E}-08$ & $5.14 \mathrm{E}-08$ & $5.14 \mathrm{E}-08$ & $1.04 \mathrm{E}-08$ \\
\hline $\mathrm{U}-236$ & $6.58 \mathrm{E}-08$ & $7.43 \mathrm{E}-08$ & $7.44 \mathrm{E}-08$ & $6.57 \mathrm{E}-08$ & $6.57 \mathrm{E}-08$ & $6.57 \mathrm{E}-08$ & $6.57 \mathrm{E}-08$ & $1.33 \mathrm{E}-08$ \\
\hline $\mathrm{U}-237$ & $5.43 \mathrm{E}-09$ & $5.43 \mathrm{E}-09$ & 5.43E-09 & $5.43 \mathrm{E}-09$ & $5.43 \mathrm{E}-09$ & 5.43E-09 & 5.43E-09 & $1.10 \mathrm{E}-09$ \\
\hline U-238 & $3.63 \mathrm{E}-08$ & $3.68 \mathrm{E}-08$ & $3.68 \mathrm{E}-08$ & $3.63 \mathrm{E}-08$ & $3.63 \mathrm{E}-08$ & $3.63 \mathrm{E}-08$ & $3.63 \mathrm{E}-08$ & $7.34 \mathrm{E}-09$ \\
\hline Np-236 & $2.59 \mathrm{E}-12$ & $2.59 \mathrm{E}-12$ & $2.59 \mathrm{E}-12$ & $2.59 \mathrm{E}-12$ & $2.59 \mathrm{E}-12$ & $2.59 \mathrm{E}-12$ & $2.59 \mathrm{E}-12$ & $5.24 \mathrm{E}-13$ \\
\hline Np-237 & $4.05 \mathrm{E}-07$ & $4.43 \mathrm{E}-07$ & $4.43 \mathrm{E}-07$ & $4.05 \mathrm{E}-07$ & 4.05E-07 & $4.05 \mathrm{E}-07$ & $4.05 \mathrm{E}-07$ & $8.18 \mathrm{E}-08$ \\
\hline Pu-236 & $2.76 \mathrm{E}-09$ & 3.47E-09 & $3.48 \mathrm{E}-09$ & $2.76 \mathrm{E}-09$ & $2.76 \mathrm{E}-09$ & $2.76 \mathrm{E}-09$ & $2.76 \mathrm{E}-09$ & $5.57 \mathrm{E}-10$ \\
\hline Pu-238 & $4.00 \mathrm{E}-04$ & $7.81 \mathrm{E}-04$ & $7.86 \mathrm{E}-04$ & $3.98 \mathrm{E}-04$ & $3.98 \mathrm{E}-04$ & $3.98 \mathrm{E}-04$ & $3.98 \mathrm{E}-04$ & 8.04E-05 \\
\hline Pu-239 & 4.53E-05 & $8.58 \mathrm{E}-05$ & $8.64 \mathrm{E}-05$ & $4.50 \mathrm{E}-05$ & $4.50 \mathrm{E}-05$ & $4.50 \mathrm{E}-05$ & $4.50 \mathrm{E}-05$ & $9.09 \mathrm{E}-06$ \\
\hline Pu-240 & $1.02 \mathrm{E}-05$ & $1.30 \mathrm{E}-05$ & $1.30 \mathrm{E}-05$ & $1.02 \mathrm{E}-05$ & $1.02 \mathrm{E}-05$ & $1.02 \mathrm{E}-05$ & $1.02 \mathrm{E}-05$ & $2.06 \mathrm{E}-06$ \\
\hline Pu-241 & $4.02 \mathrm{E}-04$ & $7.03 \mathrm{E}-04$ & 7.07E-04 & $4.00 \mathrm{E}-04$ & $4.00 \mathrm{E}-04$ & $4.00 \mathrm{E}-04$ & $4.00 \mathrm{E}-04$ & $8.08 \mathrm{E}-05$ \\
\hline Pu-242 & 7.97E-09 & $1.00 \mathrm{E}-08$ & $1.01 \mathrm{E}-08$ & $7.96 \mathrm{E}-09$ & 7.96E-09 & 7.96E-09 & 7.96E-09 & 1.61E-09 \\
\hline Pu-244 & $3.94 \mathrm{E}-17$ & $2.16 \mathrm{E}-16$ & $2.18 \mathrm{E}-16$ & $3.82 \mathrm{E}-17$ & $3.82 \mathrm{E}-17$ & $3.82 \mathrm{E}-17$ & $3.82 \mathrm{E}-17$ & $7.71 \mathrm{E}-18$ \\
\hline Am-241 & $6.23 \mathrm{E}-05$ & $7.22 \mathrm{E}-05$ & $7.23 \mathrm{E}-05$ & $6.22 \mathrm{E}-05$ & $6.22 \mathrm{E}-05$ & $6.22 \mathrm{E}-05$ & $6.22 \mathrm{E}-05$ & $1.26 \mathrm{E}-05$ \\
\hline$A m-242 m$ & $1.28 \mathrm{E}-08$ & $1.28 \mathrm{E}-08$ & $1.28 \mathrm{E}-08$ & $1.28 \mathrm{E}-08$ & $1.28 \mathrm{E}-08$ & $1.28 \mathrm{E}-08$ & $1.28 \mathrm{E}-08$ & $2.59 \mathrm{E}-09$ \\
\hline Am-243 & $1.81 \mathrm{E}-08$ & $2.16 \mathrm{E}-08$ & $2.17 \mathrm{E}-08$ & $1.81 \mathrm{E}-08$ & $1.81 \mathrm{E}-08$ & $1.81 \mathrm{E}-08$ & $1.81 \mathrm{E}-08$ & 3.66E-09 \\
\hline $\mathrm{Cm}-242$ & $2.52 \mathrm{E}-08$ & $2.53 \mathrm{E}-08$ & $2.53 \mathrm{E}-08$ & $2.52 \mathrm{E}-08$ & $2.52 \mathrm{E}-08$ & $2.52 \mathrm{E}-08$ & $2.52 \mathrm{E}-08$ & $5.10 \mathrm{E}-09$ \\
\hline
\end{tabular}


Table B-11. Tank WM-189 mass balance (continued).

\begin{tabular}{|c|c|c|c|c|c|c|c|c|}
\hline Stream \# & 121 & 122 & 123 & 124 & 125 & 126 & 127 & 128 \\
\hline Stream Name & $\begin{array}{l}\text { Neutralized } \\
\text { Feed }\end{array}$ & $\begin{array}{l}\text { SBW Liquid } \\
\text { Filter Feed }\end{array}$ & $\begin{array}{c}\text { Filter } \\
\text { Recirculation }\end{array}$ & $\begin{array}{l}\text { IX Column } \\
\text { Feed }\end{array}$ & $\begin{array}{c}\text { Column } 1 \\
\text { Effluent }\end{array}$ & $\begin{array}{c}\text { Column } 2 \\
\text { Effluent }\end{array}$ & $\begin{array}{c}\text { Column } 3 \\
\text { Effluent }\end{array}$ & $\begin{array}{l}\text { Spent IX } \\
\text { Rinse }\end{array}$ \\
\hline & Ci/liter & Ci/liter & Ci/liter & Ci/liter & Ci/liter & Ci/liter & Ci/liter & Ci/liter \\
\hline $\mathrm{Cm}-243$ & $2.40 \mathrm{E}-08$ & $3.13 \mathrm{E}-08$ & $3.14 \mathrm{E}-08$ & $2.40 \mathrm{E}-08$ & $2.40 \mathrm{E}-08$ & $2.40 \mathrm{E}-08$ & $2.40 \mathrm{E}-08$ & $4.84 \mathrm{E}-09$ \\
\hline $\mathrm{Cm}-244$ & 8.94E-07 & 1.35E-06 & 1.36E-06 & 8.91E-07 & 8.91E-07 & 8.91E-07 & 8.91E-07 & $1.80 \mathrm{E}-07$ \\
\hline $\mathrm{Cm}-245$ & $2.52 \mathrm{E}-10$ & $3.29 \mathrm{E}-10$ & $3.30 \mathrm{E}-10$ & $2.52 \mathrm{E}-10$ & $2.52 \mathrm{E}-10$ & $2.52 \mathrm{E}-10$ & $2.52 \mathrm{E}-10$ & $5.09 \mathrm{E}-11$ \\
\hline $\mathrm{Cm}-246$ & $1.66 \mathrm{E}-11$ & $2.16 \mathrm{E}-11$ & $2.17 \mathrm{E}-11$ & $1.66 \mathrm{E}-11$ & $1.66 \mathrm{E}-11$ & $1.66 \mathrm{E}-11$ & $1.66 \mathrm{E}-11$ & $3.35 \mathrm{E}-12$ \\
\hline $\mathrm{H}-3$ & $8.41 \mathrm{E}-06$ & $8.41 \mathrm{E}-06$ & $8.41 \mathrm{E}-06$ & $8.41 \mathrm{E}-06$ & $8.41 \mathrm{E}-06$ & $8.41 \mathrm{E}-06$ & $8.41 \mathrm{E}-06$ & $1.70 \mathrm{E}-06$ \\
\hline $\mathrm{Be}-10$ & $2.53 \mathrm{E}-12$ & $2.53 \mathrm{E}-12$ & $2.53 \mathrm{E}-12$ & $2.53 \mathrm{E}-12$ & $2.53 \mathrm{E}-12$ & $2.53 \mathrm{E}-12$ & $2.53 \mathrm{E}-12$ & $5.11 \mathrm{E}-13$ \\
\hline $\mathrm{C}-14$ & $1.01 \mathrm{E}-10$ & $1.34 \mathrm{E}-10$ & $1.34 \mathrm{E}-10$ & $1.01 \mathrm{E}-10$ & $1.01 \mathrm{E}-10$ & $1.01 \mathrm{E}-10$ & $1.01 \mathrm{E}-10$ & $2.04 \mathrm{E}-11$ \\
\hline Se-79 & 3.69E-07 & $4.86 \mathrm{E}-07$ & $4.88 \mathrm{E}-07$ & $3.68 \mathrm{E}-07$ & 3.68E-07 & $3.68 \mathrm{E}-07$ & $3.68 \mathrm{E}-07$ & 7.44E-08 \\
\hline $\mathrm{Rb}-87$ & $2.47 \mathrm{E}-11$ & $2.47 \mathrm{E}-11$ & $2.47 \mathrm{E}-11$ & $2.47 \mathrm{E}-11$ & $2.47 \mathrm{E}-11$ & $2.47 \mathrm{E}-11$ & $2.47 \mathrm{E}-11$ & $4.99 \mathrm{E}-12$ \\
\hline Sr-90 & $3.29 \mathrm{E}-02$ & $3.31 \mathrm{E}-02$ & $3.32 \mathrm{E}-02$ & $3.29 \mathrm{E}-02$ & $3.29 \mathrm{E}-02$ & $3.29 \mathrm{E}-02$ & $3.29 \mathrm{E}-02$ & $6.65 \mathrm{E}-03$ \\
\hline Y-90 & $3.29 \mathrm{E}-02$ & 3.31E-02 & $3.32 \mathrm{E}-02$ & $3.29 \mathrm{E}-02$ & $3.29 \mathrm{E}-02$ & $3.29 \mathrm{E}-02$ & $3.29 \mathrm{E}-02$ & $6.65 \mathrm{E}-03$ \\
\hline Zr-93 & 1.87E-06 & 1.87E-06 & 1.87E-06 & 1.87E-06 & 1.87E-06 & 1.87E-06 & 1.87E-06 & $3.78 \mathrm{E}-07$ \\
\hline Nb-93m & $1.44 \mathrm{E}-06$ & $1.44 \mathrm{E}-06$ & $1.44 \mathrm{E}-06$ & $1.44 \mathrm{E}-06$ & $1.44 \mathrm{E}-06$ & $1.44 \mathrm{E}-06$ & $1.44 \mathrm{E}-06$ & $2.91 \mathrm{E}-07$ \\
\hline Nb-94 & 9.63E-07 & 1.27E-06 & $1.27 \mathrm{E}-06$ & 9.61E-07 & 9.61E-07 & 9.61E-07 & 9.61E-07 & 1.94E-07 \\
\hline Tc-98 & $2.17 \mathrm{E}-12$ & $2.17 \mathrm{E}-12$ & $2.17 \mathrm{E}-12$ & $2.17 \mathrm{E}-12$ & $2.17 \mathrm{E}-12$ & $2.17 \mathrm{E}-12$ & $2.17 \mathrm{E}-12$ & $4.40 \mathrm{E}-13$ \\
\hline Tc-99 & $8.66 \mathrm{E}-06$ & 1.13E-05 & 1.13E-05 & $8.64 \mathrm{E}-06$ & 8.64E-06 & $8.64 \mathrm{E}-06$ & $8.64 \mathrm{E}-06$ & 1.75E-06 \\
\hline Ru-106 & 7.86E-07 & 1.04E-06 & 1.04E-06 & 7.84E-07 & 7.84E-07 & 7.84E-07 & 7.84E-07 & $1.58 \mathrm{E}-07$ \\
\hline Rh-102 & $2.48 \mathrm{E}-09$ & $2.56 \mathrm{E}-07$ & $2.59 \mathrm{E}-07$ & $7.27 \mathrm{E}-10$ & 7.27E-10 & $7.27 \mathrm{E}-10$ & $7.27 \mathrm{E}-10$ & $1.47 \mathrm{E}-10$ \\
\hline Pd-107 & $1.39 \mathrm{E}-08$ & $1.39 \mathrm{E}-08$ & $1.39 \mathrm{E}-08$ & 1.39E-08 & 1.39E-08 & 1.39E-08 & $1.39 \mathrm{E}-08$ & 2.82E-09 \\
\hline Cd-113m & $2.80 \mathrm{E}-06$ & $2.80 \mathrm{E}-06$ & $2.80 \mathrm{E}-06$ & $2.80 \mathrm{E}-06$ & $2.80 \mathrm{E}-06$ & $2.80 \mathrm{E}-06$ & $2.80 \mathrm{E}-06$ & $5.66 \mathrm{E}-07$ \\
\hline $\ln -115$ & $8.49 \mathrm{E}-17$ & $8.49 \mathrm{E}-17$ & $8.49 \mathrm{E}-17$ & $8.49 \mathrm{E}-17$ & $8.49 \mathrm{E}-17$ & $8.49 \mathrm{E}-17$ & $8.49 \mathrm{E}-17$ & $1.72 \mathrm{E}-17$ \\
\hline Sn-121m & $5.64 \mathrm{E}-08$ & $5.64 \mathrm{E}-08$ & $5.64 \mathrm{E}-08$ & $5.64 \mathrm{E}-08$ & $5.64 \mathrm{E}-08$ & $5.64 \mathrm{E}-08$ & 5.64E-08 & $1.14 \mathrm{E}-08$ \\
\hline Sn-126 & 3.47E-07 & $4.58 \mathrm{E}-07$ & 4.59E-07 & 3.46E-07 & 3.46E-07 & $3.46 \mathrm{E}-07$ & $3.46 \mathrm{E}-07$ & $7.00 \mathrm{E}-08$ \\
\hline Sb-125 & $1.34 \mathrm{E}-05$ & $3.77 \mathrm{E}-04$ & $3.81 \mathrm{E}-04$ & $1.09 \mathrm{E}-05$ & $1.09 \mathrm{E}-05$ & $1.09 \mathrm{E}-05$ & $1.09 \mathrm{E}-05$ & $2.20 \mathrm{E}-06$ \\
\hline Sb-126 & $4.85 \mathrm{E}-08$ & $4.85 \mathrm{E}-08$ & $4.85 \mathrm{E}-08$ & $4.85 \mathrm{E}-08$ & $4.85 \mathrm{E}-08$ & $4.85 \mathrm{E}-08$ & $4.85 \mathrm{E}-08$ & $9.80 \mathrm{E}-09$ \\
\hline Te-123 & $3.23 E-19$ & $3.23 \mathrm{E}-19$ & $3.23 E-19$ & $3.23 \mathrm{E}-19$ & $3.23 \mathrm{E}-19$ & $3.23 \mathrm{E}-19$ & $3.23 \mathrm{E}-19$ & $6.53 \mathrm{E}-20$ \\
\hline Te-125m & $2.66 \mathrm{E}-06$ & $2.66 \mathrm{E}-06$ & $2.66 \mathrm{E}-06$ & $2.66 \mathrm{E}-06$ & $2.66 \mathrm{E}-06$ & $2.66 \mathrm{E}-06$ & $2.66 \mathrm{E}-06$ & $5.37 \mathrm{E}-07$ \\
\hline \begin{tabular}{|l}
$1-129$ \\
\end{tabular} & $4.50 \mathrm{E}-08$ & $5.94 \mathrm{E}-08$ & $5.95 \mathrm{E}-08$ & $4.49 \mathrm{E}-08$ & $4.49 \mathrm{E}-08$ & $4.49 \mathrm{E}-08$ & $4.49 \mathrm{E}-08$ & $9.08 \mathrm{E}-09$ \\
\hline Cs-134 & $3.35 \mathrm{E}-05$ & $6.32 \mathrm{E}-05$ & $6.36 \mathrm{E}-05$ & $3.33 \mathrm{E}-05$ & $3.33 \mathrm{E}-06$ & $3.33 \mathrm{E}-07$ & $3.33 \mathrm{E}-08$ & $6.73 \mathrm{E}-09$ \\
\hline Cs-135 & $7.26 \mathrm{E}-07$ & $9.33 \mathrm{E}-07$ & $9.36 \mathrm{E}-07$ & $7.25 \mathrm{E}-07$ & $7.25 \mathrm{E}-08$ & $7.25 \mathrm{E}-09$ & $7.25 \mathrm{E}-10$ & $1.46 \mathrm{E}-10$ \\
\hline Cs-137 & $4.18 \mathrm{E}-02$ & $5.35 \mathrm{E}-02$ & $5.37 \mathrm{E}-02$ & $4.17 \mathrm{E}-02$ & 4.17E-03 & $4.17 \mathrm{E}-04$ & 4.17E-05 & $8.43 \mathrm{E}-06$ \\
\hline Ba-137m & $3.95 \mathrm{E}-02$ & $5.06 \mathrm{E}-02$ & $5.08 \mathrm{E}-02$ & $3.95 \mathrm{E}-02$ & $3.95 \mathrm{E}-02$ & $3.95 \mathrm{E}-02$ & $3.95 \mathrm{E}-02$ & $7.97 \mathrm{E}-03$ \\
\hline La-138 & $1.61 \mathrm{E}-16$ & $1.61 \mathrm{E}-16$ & $1.61 \mathrm{E}-16$ & $1.61 \mathrm{E}-16$ & $1.61 \mathrm{E}-16$ & $1.61 \mathrm{E}-16$ & $1.61 \mathrm{E}-16$ & $3.25 \mathrm{E}-17$ \\
\hline Ce-142 & $2.52 \mathrm{E}-11$ & $2.52 \mathrm{E}-11$ & $2.52 \mathrm{E}-11$ & $2.52 \mathrm{E}-11$ & $2.52 \mathrm{E}-11$ & $2.52 \mathrm{E}-11$ & $2.52 \mathrm{E}-11$ & $5.09 \mathrm{E}-12$ \\
\hline Ce-144 & $5.30 \mathrm{E}-07$ & 7.02E-07 & $7.04 \mathrm{E}-07$ & $5.28 \mathrm{E}-07$ & $5.28 \mathrm{E}-07$ & $5.28 \mathrm{E}-07$ & $5.28 \mathrm{E}-07$ & 1.07E-07 \\
\hline Nd-144 & $1.36 \mathrm{E}-15$ & $1.36 \mathrm{E}-15$ & $1.36 \mathrm{E}-15$ & $1.36 \mathrm{E}-15$ & $1.36 \mathrm{E}-15$ & $1.36 \mathrm{E}-15$ & $1.36 \mathrm{E}-15$ & $2.74 \mathrm{E}-16$ \\
\hline $\mathrm{Pm}-146$ & $4.29 \mathrm{E}-08$ & $4.29 \mathrm{E}-08$ & $4.29 \mathrm{E}-08$ & $4.29 \mathrm{E}-08$ & $4.29 \mathrm{E}-08$ & $4.29 \mathrm{E}-08$ & $4.29 \mathrm{E}-08$ & 8.67E-09 \\
\hline Pm-147 & $1.44 \mathrm{E}-04$ & $1.89 \mathrm{E}-04$ & $1.90 \mathrm{E}-04$ & $1.44 \mathrm{E}-04$ & $1.44 \mathrm{E}-04$ & $1.44 \mathrm{E}-04$ & $1.44 \mathrm{E}-04$ & $2.90 \mathrm{E}-05$ \\
\hline Sm-146 & $2.33 \mathrm{E}-13$ & $2.33 \mathrm{E}-13$ & $2.33 \mathrm{E}-13$ & $2.33 \mathrm{E}-13$ & $2.33 \mathrm{E}-13$ & $2.33 \mathrm{E}-13$ & $2.33 \mathrm{E}-13$ & $4.70 \mathrm{E}-14$ \\
\hline Sm-147 & $6.21 \mathrm{E}-12$ & $6.21 \mathrm{E}-12$ & $6.21 \mathrm{E}-12$ & $6.21 \mathrm{E}-12$ & $6.21 \mathrm{E}-12$ & $6.21 \mathrm{E}-12$ & $6.21 \mathrm{E}-12$ & $1.26 \mathrm{E}-12$ \\
\hline Sm-148 & $3.19 \mathrm{E}-17$ & 3.19E-17 & $3.19 \mathrm{E}-17$ & $3.19 \mathrm{E}-17$ & 3.19E-17 & $3.19 \mathrm{E}-17$ & $3.19 \mathrm{E}-17$ & $6.45 \mathrm{E}-18$ \\
\hline Sm-149 & $2.83 \mathrm{E}-18$ & $2.83 \mathrm{E}-18$ & $2.83 \mathrm{E}-18$ & $2.83 \mathrm{E}-18$ & $2.83 \mathrm{E}-18$ & $2.83 \mathrm{E}-18$ & $2.83 \mathrm{E}-18$ & $5.73 \mathrm{E}-19$ \\
\hline Sm-151 & $2.84 \mathrm{E}-04$ & $3.75 \mathrm{E}-04$ & $3.76 \mathrm{E}-04$ & $2.83 \mathrm{E}-04$ & $2.83 \mathrm{E}-04$ & $2.83 \mathrm{E}-04$ & $2.83 \mathrm{E}-04$ & $5.72 \mathrm{E}-05$ \\
\hline Eu-152 & $2.12 \mathrm{E}-06$ & $2.44 \mathrm{E}-06$ & $2.45 \mathrm{E}-06$ & $2.12 \mathrm{E}-06$ & $2.12 \mathrm{E}-06$ & $2.12 \mathrm{E}-06$ & $2.12 \mathrm{E}-06$ & $4.29 \mathrm{E}-07$ \\
\hline Eu-154 & $1.53 \mathrm{E}-04$ & 1.65E-04 & $1.65 \mathrm{E}-04$ & $1.52 \mathrm{E}-04$ & $1.52 \mathrm{E}-04$ & $1.52 \mathrm{E}-04$ & $1.52 \mathrm{E}-04$ & $3.08 \mathrm{E}-05$ \\
\hline Eu-155 & $1.36 \mathrm{E}-04$ & $1.57 \mathrm{E}-04$ & $1.57 \mathrm{E}-04$ & $1.36 \mathrm{E}-04$ & $1.36 \mathrm{E}-04$ & $1.36 \mathrm{E}-04$ & $1.36 \mathrm{E}-04$ & $2.74 \mathrm{E}-05$ \\
\hline Gd-152 & $1.20 \mathrm{E}-18$ & $1.20 \mathrm{E}-18$ & $1.20 \mathrm{E}-18$ & $1.20 \mathrm{E}-18$ & $1.20 \mathrm{E}-18$ & $1.20 \mathrm{E}-18$ & $1.20 \mathrm{E}-18$ & $2.42 \mathrm{E}-19$ \\
\hline Ho-166m & $3.88 \mathrm{E}-11$ & $3.88 \mathrm{E}-11$ & $3.88 \mathrm{E}-11$ & $3.88 \mathrm{E}-11$ & $3.88 \mathrm{E}-11$ & $3.88 \mathrm{E}-11$ & $3.88 \mathrm{E}-11$ & $7.84 \mathrm{E}-12$ \\
\hline Co-60 & $2.99 \mathrm{E}-05$ & $3.11 \mathrm{E}-05$ & $3.11 \mathrm{E}-05$ & $2.99 \mathrm{E}-05$ & $2.99 \mathrm{E}-05$ & $2.99 \mathrm{E}-05$ & $2.99 \mathrm{E}-05$ & $6.05 \mathrm{E}-06$ \\
\hline $\mathrm{Ni}-63$ & $2.69 \mathrm{E}-05$ & $3.53 \mathrm{E}-05$ & $3.54 \mathrm{E}-05$ & $2.68 \mathrm{E}-05$ & $2.68 \mathrm{E}-05$ & $2.68 \mathrm{E}-05$ & $2.68 \mathrm{E}-05$ & $5.42 \mathrm{E}-06$ \\
\hline
\end{tabular}


Table B-11. Tank WM-189 mass balance (continued).

\begin{tabular}{|c|c|c|c|c|c|c|c|c|}
\hline PFD Sheet \# & & PFD-4 & PFD Sheet \# & & PFD-4 & & & PFD-4 \\
\hline Stream \# & 129 & 130 & Stream \# & 129 & 130 & & 129 & 130 \\
\hline Stream Name & $\begin{array}{c}\text { Spent IX } \\
\text { Media }\end{array}$ & $\begin{array}{l}\text { Grouted } \\
\text { Waste }\end{array}$ & Stream Name & $\begin{array}{c}\text { Spent IX } \\
\text { Media }\end{array}$ & $\begin{array}{l}\text { Grouted } \\
\text { Waste }\end{array}$ & & $\begin{array}{c}\text { Spent IX } \\
\text { Media }\end{array}$ & $\begin{array}{l}\text { Grouted } \\
\text { Waste }\end{array}$ \\
\hline Rate, gal $/ \mathrm{hr}$ & 0.12 & 113 & & Wt \% & Wt \% & & $\mathrm{Ci} / \mathrm{kg}$ & $\mathrm{Ci} / \mathrm{kg}$ \\
\hline Rate, Ib/hr & 1.29 & 1416 & $\mathrm{Rb}+$ & $2.09 \mathrm{E}-06$ & $2.32 \mathrm{E}-05$ & $\mathrm{Cm}-243$ & $4.84 \mathrm{E}-09$ & $1.34 \mathrm{E}-08$ \\
\hline Rate, total columns & 7 & $\mathrm{~N} / \mathrm{A}$ & $\mathrm{Ru}+3$ & $7.42 \mathrm{E}-05$ & $8.23 \mathrm{E}-04$ & $\mathrm{Cm}-244$ & $1.80 \mathrm{E}-07$ & 4.99E-07 \\
\hline Operation & Batch & Cont & $\mathrm{Sm}+3$ & $3.63 \mathrm{E}-06$ & 4.03E-05 & $\mathrm{Cm}-245$ & $5.09 \mathrm{E}-11$ & $1.41 \mathrm{E}-10$ \\
\hline Temperature, ${ }^{\circ} \mathrm{F}$ & 70 & 70 & $\mathrm{Se}+4$ & 4.42E-07 & $4.90 \mathrm{E}-06$ & $\mathrm{Cm}-246$ & & \\
\hline Pressure, psia & 12.8 & 12.8 & $\mathrm{Si}+4$ & $3.71 \mathrm{E}-05$ & $4.12 \mathrm{E}-04$ & & 3.35E-12 & $9.29 \mathrm{E}-12$ \\
\hline Specific Gravity & 1.34 & 1.50 & $\mathrm{Ag}^{+}$ & 1.12E-07 & $1.24 \mathrm{E}-06$ & $\mathrm{H}-3$ & $1.70 \mathrm{E}-06$ & $4.71 \mathrm{E}-06$ \\
\hline Chemical Composition & $\mathrm{Wt} \%$ & Wt \% & $\mathrm{Na}+$ & $4.36 \mathrm{E}-01$ & $4.84 \mathrm{E}+00$ & $\mathrm{Be}-10$ & $5.11 \mathrm{E}-13$ & $1.42 \mathrm{E}-12$ \\
\hline CST & $7.23 E+01$ & $\mathrm{~N} / \mathrm{A}$ & $\mathrm{Sr}+2$ & $5.28 \mathrm{E}-05$ & $5.85 \mathrm{E}-04$ & C-14 & $2.04 \mathrm{E}-11$ & $5.67 \mathrm{E}-11$ \\
\hline $\mathrm{H}+$ & $2.55 \mathrm{E}-03$ & $\mathrm{~N} / \mathrm{A}$ & SO4-2 & $4.24 \mathrm{E}-02$ & $4.71 \mathrm{E}-01$ & Se-79 & 7.44E-08 & 2.06E-07 \\
\hline $\mathrm{Al}+3$ & 7.94E-02 & $8.80 \mathrm{E}-01$ & $\mathrm{Tc}+7$ & $2.52 \mathrm{E}-06$ & $2.80 \mathrm{E}-05$ & $\mathrm{Rb}-87$ & 4.99E-12 & $1.38 \mathrm{E}-11$ \\
\hline$A m+4$ & $6.19 \mathrm{E}-08$ & $6.86 \mathrm{E}-07$ & $\mathrm{Te}+4$ & $3.90 \mathrm{E}-06$ & $4.33 \mathrm{E}-05$ & Sr-90 & $6.65 \mathrm{E}-03$ & 1.84E-02 \\
\hline $\mathrm{Sb}+5$ & $4.05 \mathrm{E}-06$ & $4.49 \mathrm{E}-05$ & $\mathrm{~Tb}+4$ & $1.48 \mathrm{E}-09$ & $1.64 \mathrm{E}-08$ & $Y-90$ & $6.65 \mathrm{E}-03$ & $1.84 \mathrm{E}-02$ \\
\hline As+5 & 5.76E-07 & $6.39 \mathrm{E}-06$ & $\mathrm{Th}+4$ & $3.42 \mathrm{E}-05$ & $3.79 \mathrm{E}-04$ & Zr-93 & $3.78 \mathrm{E}-07$ & $1.05 \mathrm{E}-06$ \\
\hline $\mathrm{Ba}+2$ & $3.23 \mathrm{E}-05$ & $3.59 \mathrm{E}-04$ & $S n+4$ & $2.08 \mathrm{E}-05$ & $2.30 \mathrm{E}-04$ & Nb-93m & $2.91 \mathrm{E}-07$ & 8.07E-07 \\
\hline $\mathrm{Be}+2$ & $7.50 \mathrm{E}-07$ & $8.32 \mathrm{E}-06$ & $\mathrm{Ti+4}$ & $1.49 \mathrm{E}-05$ & $1.65 \mathrm{E}-04$ & Nb-94 & 1.94E-07 & $5.38 \mathrm{E}-07$ \\
\hline $\mathrm{B}+3$ & $9.46 \mathrm{E}-04$ & $1.05 \mathrm{E}-02$ & $\mathrm{U}+4$ & $1.18 \mathrm{E}-03$ & $1.31 \mathrm{E}-02$ & Tc-98 & $4.40 \mathrm{E}-13$ & $1.22 \mathrm{E}-12$ \\
\hline $\mathrm{Br}-$ & 1.07E-07 & $1.19 \mathrm{E}-06$ & $\mathrm{~V}+5$ & $5.88 \mathrm{E}-06$ & $6.53 \mathrm{E}-05$ & Tc-99 & $1.75 \mathrm{E}-06$ & $4.84 \mathrm{E}-06$ \\
\hline $\mathrm{Cd}+2$ & $1.81 \mathrm{E}-03$ & $2.01 \mathrm{E}-02$ & $Y+3$ & $2.68 \mathrm{E}-06$ & 2.97E-05 & Ru-106 & $1.58 \mathrm{E}-07$ & 4.39E-07 \\
\hline $\mathrm{Ca}+2$ & $1.21 \mathrm{E}-02$ & $1.34 \mathrm{E}-01$ & $Z n+2$ & $2.92 \mathrm{E}-04$ & $3.24 \mathrm{E}-03$ & Rh-102 & $1.47 \mathrm{E}-10$ & $4.07 \mathrm{E}-10$ \\
\hline $\mathrm{Ce}+4$ & $2.12 \mathrm{E}-05$ & $2.35 \mathrm{E}-04$ & $2 r+4$ & $1.59 \mathrm{E}-04$ & $1.76 \mathrm{E}-03$ & $\mathrm{Pd}-107$ & 2.82E-09 & 7.81E-09 \\
\hline Cs+ & $1.52 \mathrm{E}-08$ & $1.68 \mathrm{E}-07$ & $\mathrm{H} 2 \mathrm{O}$ & $2.50 \mathrm{E}+01$ & & Cd-113m & $5.66 \mathrm{E}-07$ & $1.57 \mathrm{E}-06$ \\
\hline $\mathrm{Cl}-$ & $3.06 \mathrm{E}-03$ & $3.40 \mathrm{E}-02$ & Canisters total & 7 & & $\ln -115$ & $1.72 \mathrm{E}-17$ & $4.76 \mathrm{E}-17$ \\
\hline $\mathrm{Cr}+3$ & $1.24 \mathrm{E}-03$ & $1.38 \mathrm{E}-02$ & Drums/day & & 53 & Sn-121m & 1.14E-08 & $3.16 \mathrm{E}-08$ \\
\hline $\mathrm{Co}+2$ & $6.30 \mathrm{E}-05$ & $6.99 \mathrm{E}-04$ & Radiological Comp. & $\mathrm{Ci} / \mathrm{kg}$ & $\mathrm{Ci} / \mathrm{kg}$ & \begin{tabular}{|l|} 
Sn-126 \\
\end{tabular} & $7.00 \mathrm{E}-08$ & 1.94E-07 \\
\hline $\mathrm{Cu}+2$ & $2.49 \mathrm{E}-04$ & $2.76 \mathrm{E}-03$ & Ra-226 & $1.40 \mathrm{E}-12$ & $3.87 \mathrm{E}-12$ & Sb-125 & $2.20 \mathrm{E}-06$ & $6.10 \mathrm{E}-06$ \\
\hline $\mathrm{Eu}+3$ & $3.38 \mathrm{E}-07$ & $3.75 \mathrm{E}-06$ & Ac-227 & $6.57 \mathrm{E}-12$ & $1.82 \mathrm{E}-11$ & Sb-126 & $9.80 \mathrm{E}-09$ & $2.72 \mathrm{E}-08$ \\
\hline F- & $1.21 \mathrm{E}-03$ & $1.34 \mathrm{E}-02$ & Th-228 & $5.45 \mathrm{E}-10$ & $1.51 \mathrm{E}-09$ & Te-123 & $6.53 \mathrm{E}-20$ & $1.81 \mathrm{E}-19$ \\
\hline $\mathrm{Gd}+3$ & $9.17 \mathrm{E}-05$ & $1.02 \mathrm{E}-03$ & Th-230 & $1.40 \mathrm{E}-10$ & $3.89 \mathrm{E}-10$ & Te-125m & $5.37 \mathrm{E}-07$ & $1.49 \mathrm{E}-06$ \\
\hline $\mathrm{Ge}+4$ & $2.81 \mathrm{E}-09$ & $3.12 \mathrm{E}-08$ & Th-232 & $1.21 \mathrm{E}-16$ & $3.35 \mathrm{E}-16$ & I-129 & $9.08 \mathrm{E}-09$ & $2.52 \mathrm{E}-08$ \\
\hline $\ln +3$ & $7.01 \mathrm{E}-07$ & $7.78 \mathrm{E}-06$ & $\mathrm{~Pa}-231$ & $1.52 \mathrm{E}-11$ & $4.22 \mathrm{E}-11$ & Cs-134 & $6.73 \mathrm{E}-09$ & $1.87 \mathrm{E}-08$ \\
\hline $1-$ & $1.41 \mathrm{E}-06$ & $1.57 \mathrm{E}-05$ & $\mathrm{~Pa}-233$ & $4.99 \mathrm{E}-07$ & $1.39 \mathrm{E}-06$ & Cs-135 & $1.46 \mathrm{E}-10$ & $4.06 \mathrm{E}-10$ \\
\hline $\mathrm{Fe}+3$ & $6.21 \mathrm{E}-03$ & $6.89 \mathrm{E}-02$ & U-232 & $3.39 \mathrm{E}-10$ & $9.39 \mathrm{E}-10$ & Cs-137 & $8.43 \mathrm{E}-06$ & $2.34 \mathrm{E}-05$ \\
\hline $\mathrm{La}+3$ & $5.62 \mathrm{E}-06$ & $6.23 \mathrm{E}-05$ & $\mathrm{U}-233$ & $1.36 \mathrm{E}-11$ & $3.78 \mathrm{E}-11$ & Ba-137m & 7.97E-06 & $2.21 \mathrm{E}-05$ \\
\hline $\mathrm{Pb}+2$ & $1.01 \mathrm{E}-03$ & $1.12 \mathrm{E}-02$ & U-234 & $2.96 \mathrm{E}-07$ & $8.20 \mathrm{E}-07$ & \begin{tabular}{|l} 
La-138 \\
\end{tabular} & $3.25 \mathrm{E}-17$ & $9.02 \mathrm{E}-17$ \\
\hline $\mathrm{Li+}$ & $1.14 \mathrm{E}-05$ & $1.26 \mathrm{E}-04$ & U-235 & $1.04 \mathrm{E}-08$ & $2.88 \mathrm{E}-08$ & Ce-142 & $5.09 \mathrm{E}-12$ & $1.41 \mathrm{E}-11$ \\
\hline $\mathrm{Mg}+2$ & $2.29 \mathrm{E}-03$ & $2.54 \mathrm{E}-02$ & $\mathrm{U}-236$ & $1.33 \mathrm{E}-08$ & $3.68 \mathrm{E}-08$ & Ce-144 & $1.07 \mathrm{E}-07$ & $2.96 \mathrm{E}-07$ \\
\hline$M n+4$ & $4.63 \mathrm{E}-03$ & $5.14 \mathrm{E}-02$ & U-237 & $1.10 \mathrm{E}-09$ & 3.04E-09 & Nd-144 & $2.74 \mathrm{E}-16$ & $7.60 \mathrm{E}-16$ \\
\hline $\mathrm{Hg}+2$ & $5.40 \mathrm{E}-03$ & $6.00 \mathrm{E}-02$ & $\mathrm{U}-238$ & $7.34 \mathrm{E}-09$ & $2.04 \mathrm{E}-08$ & Pm-146 & $8.67 \mathrm{E}-09$ & $2.40 \mathrm{E}-08$ \\
\hline $\mathrm{Mo}+6$ & $1.15 \mathrm{E}-04$ & $1.27 \mathrm{E}-03$ & $\mathrm{~Np}-236$ & $5.24 \mathrm{E}-13$ & $1.45 \mathrm{E}-12$ & Pm-147 & $2.90 \mathrm{E}-05$ & $8.05 \mathrm{E}-05$ \\
\hline $\mathrm{Nd}+3$ & $1.88 \mathrm{E}-05$ & $2.09 \mathrm{E}-04$ & Np-237 & $8.18 \mathrm{E}-08$ & $2.27 \mathrm{E}-07$ & Sm-146 & $4.70 \mathrm{E}-14$ & $1.30 \mathrm{E}-13$ \\
\hline $\mathrm{Np}+4$ & $2.90 \mathrm{E}-06$ & $3.22 \mathrm{E}-05$ & Pu-236 & $5.57 \mathrm{E}-10$ & $1.54 \mathrm{E}-09$ & Sm-147 & $1.26 \mathrm{E}-12$ & $3.48 \mathrm{E}-12$ \\
\hline $\mathrm{Ni}+2$ & 5.67E-04 & $6.29 \mathrm{E}-03$ & $\mathrm{Pu}-238$ & $8.04 \mathrm{E}-05$ & $2.23 \mathrm{E}-04$ & Sm-148 & $6.45 \mathrm{E}-18$ & $1.79 \mathrm{E}-17$ \\
\hline $\mathrm{Nb}+5$ & $1.87 \mathrm{E}-07$ & $2.07 \mathrm{E}-06$ & Pu-239 & $9.09 \mathrm{E}-06$ & $2.52 \mathrm{E}-05$ & Sm-149 & $5.73 \mathrm{E}-19$ & $1.59 \mathrm{E}-18$ \\
\hline NO3- & $2.00 \mathrm{E}+00$ & $2.22 \mathrm{E}+01$ & Pu-240 & $2.06 \mathrm{E}-06$ & $5.72 \mathrm{E}-06$ & Sm-151 & $5.72 \mathrm{E}-05$ & $1.59 \mathrm{E}-04$ \\
\hline $\mathrm{Pd}+4$ & $3.99 \mathrm{E}-06$ & $4.42 \mathrm{E}-05$ & Pu-241 & $8.08 \mathrm{E}-05$ & $2.24 \mathrm{E}-04$ & Eu-152 & 4.29E-07 & 1.19E-06 \\
\hline PO4-3 & $8.34 \mathrm{E}-04$ & $9.26 \mathrm{E}-03$ & Pu-242 & $1.61 \mathrm{E}-09$ & $4.46 \mathrm{E}-09$ & Eu-154 & $3.08 \mathrm{E}-05$ & $8.55 \mathrm{E}-05$ \\
\hline $\mathrm{Pu}+4$ & $4.03 \mathrm{E}-06$ & $4.45 \mathrm{E}-05$ & $\mathrm{Pu}-244$ & $7.71 \mathrm{E}-18$ & $2.14 \mathrm{E}-17$ & Eu-155 & $2.74 \mathrm{E}-05$ & $7.60 \mathrm{E}-05$ \\
\hline $\mathrm{K}+$ & $4.19 \mathrm{E}-02$ & $4.65 \mathrm{E}-01$ & Am-241 & $1.26 \mathrm{E}-05$ & $3.49 \mathrm{E}-05$ & Gd-152 & $2.42 \mathrm{E}-19$ & $6.72 \mathrm{E}-19$ \\
\hline $\mathrm{Pr}+4$ & $5.18 \mathrm{E}-06$ & $5.75 \mathrm{E}-05$ & $A m-242 m$ & $2.59 \mathrm{E}-09$ & $7.19 \mathrm{E}-09$ & Ho-166m & $7.84 \mathrm{E}-12$ & $2.18 \mathrm{E}-11$ \\
\hline \multirow[t]{2}{*}{$\mathrm{Rh}+4$} & $1.63 \mathrm{E}-06$ & $1.81 \mathrm{E}-05$ & $\mathrm{Am}-243$ & $3.66 \mathrm{E}-09$ & $1.01 \mathrm{E}-08$ & Co-60 & $6.05 \mathrm{E}-06$ & $1.68 \mathrm{E}-05$ \\
\hline & & & Cm-242 & $5.10 \mathrm{E}-09$ & $1.42 \mathrm{E}-08$ & $\mathrm{Ni}-63$ & $5.42 \mathrm{E}-06$ & $1.50 \mathrm{E}-05$ \\
\hline
\end{tabular}


Table B-11. Tank WM-189 mass balance (continued).

\begin{tabular}{|c|c|c|c|c|c|}
\hline PFD Sheet \# & PFD-2 & PFD-2 & PFD-2 & PFD-3 & PFD-3 \\
\hline Stream \# & 210 & 211 & 212 & 213 & 220 \\
\hline Stream Name & $\begin{array}{c}\text { Solids } \\
\text { Flocculent }\end{array}$ & $\begin{array}{c}\text { Steam to } \\
\text { Fundabac } \\
\text { Filter }\end{array}$ & Filter Condensate & Nitric Acid & $\begin{array}{c}50 \% \\
\text { Caustic }\end{array}$ \\
\hline Rate, gal/hr & 0.1 & 0.8 & 4.6 & TBD & 10.1 \\
\hline Rate, $\mathrm{lb} / \mathrm{hr}$ & 2 & 6 & 38 & & 127 \\
\hline Rate, peak, gpm & 10 & 0 & 1 & & 10 \\
\hline Operation & Batch & Batch & Batch & & Batch \\
\hline Temperature, ${ }^{\circ} \mathrm{F}$ & 75 & 328 & 114 & 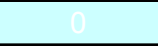 & 70 \\
\hline Pressure, psia & 12.8 & 112.8 & 12.8 & 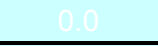 & 12.8 \\
\hline Specific Gravity & 1.51 & 1.00 & 1.00 & $x_{0}$ & 1.51 \\
\hline Stream Composition & Wt \% & Wt \% & Wt \% & Wt \% & Wt \% \\
\hline $50 \% \mathrm{NaOH}$ & $100 \%$ & & & & $100 \%$ \\
\hline HNO3 & & & & $100 \%$ & \\
\hline Steam & & $100 \%$ & & & \\
\hline Water & & & $100 \%$ & & \\
\hline & & & & & \\
\hline \multicolumn{6}{|l|}{ CST } \\
\hline \multicolumn{6}{|l|}{ Portland cement } \\
\hline \multicolumn{6}{|l|}{$\mathrm{Ca}(\mathrm{OH}) 2$} \\
\hline Blast furnace slag & & & & & \\
\hline
\end{tabular}

\begin{tabular}{|c|c|c|c|c|c|}
\hline PFD Sheet \# & PFD-3 & PFD-4 & PFD-4 & PFD-4 & PFD-4 \\
\hline Stream \# & 221 & 222 & 223 & 224 & 225 \\
\hline Stream Name & $\begin{array}{c}\text { Fresh IX } \\
\text { Media }\end{array}$ & $\begin{array}{l}\text { Portland } \\
\text { Cement }\end{array}$ & Calcium Hydroxide & $\begin{array}{c}\text { Blast } \\
\text { Furnace } \\
\text { Slag }\end{array}$ & Solid Blend \\
\hline Rate, gal/hr & 0.12 & 17.00 & 24.79 & 8.50 & 41.79 \\
\hline Rate, lb/hr & 0.93 & 170 & 99 & 85 & 354 \\
\hline Rate, peak, gpm & NA & TBD & TBD & TBD & 0.70 \\
\hline Operation & Batch & Cont & Cont & Cont & Cont \\
\hline Temperature, ${ }^{\circ} \mathrm{F}$ & 70 & 70 & 70 & 70 & 70 \\
\hline Pressure, psia & 12.8 & 12.8 & 12.8 & 12.8 & 12.8 \\
\hline \multirow[t]{2}{*}{ Specific Gravity } & 0.96 & 1.20 & 0.48 & 1.20 & 1.02 \\
\hline & Wt \% & Wt \% & $\mathrm{Wt} \%$ & Wt \% & $\mathrm{Wt} \%$ \\
\hline \multicolumn{6}{|l|}{ Chemical Composition } \\
\hline \multicolumn{6}{|l|}{$\mathrm{NaOH}$} \\
\hline \multicolumn{6}{|l|}{$\mathrm{H} 2 \mathrm{O}$} \\
\hline CST & $100 \%$ & & & & \\
\hline Portland cement & & $100 \%$ & & & \\
\hline $\mathrm{Ca}(\mathrm{OH}) 2$ & & & $100 \%$ & & \\
\hline Blast furnace slag & & & & $100 \%$ & \\
\hline No. Replacement col's & 7 & & & & \\
\hline & & & & & \\
\hline & & & & & \\
\hline
\end{tabular}


Table B-12. NGLW mass balance.

\begin{tabular}{|c|c|c|c|c|c|c|c|c|}
\hline PFD Sheet \# & PFD-2 & PFD-2 & PFD-2 & PFD-2 & PFD-2 & PFD-2 & PFD-2 & PFD-3 \\
\hline Stream \# & 110 & 111 & 112 & 113 & 114 & 115 & 116 & 120 \\
\hline Stream Name & $\begin{array}{l}\text { Slurry from } \\
\text { TFF Tank } \\
\text { WM-187 }\end{array}$ & $\begin{array}{c}\text { Crossflow } \\
\text { Filter Recirc } \\
\text { Purge }\end{array}$ & $\begin{array}{l}\text { Decant } \\
\text { Liquid }\end{array}$ & $\begin{array}{c}\text { Solids } \\
\text { Treatment } \\
\text { Filter Feed }\end{array}$ & $\begin{array}{l}\text { Solids } \\
\text { Treatment } \\
\text { Filtrate }\end{array}$ & $\begin{array}{c}\text { Combined } \\
\text { Waste Water }\end{array}$ & $\begin{array}{l}\text { Dried Solids } \\
\text { Waste }\end{array}$ & $\begin{array}{c}\text { Liquid SBW } \\
\text { from Tank } \\
\text { WM-180 }\end{array}$ \\
\hline Rate, gal/hr & 65.9 & 9.9 & 65.9 & 15.7 & 8.9 & 79.0 & 6.8 & 85.2 \\
\hline Rate, Ib/hr & 571 & 109 & 559 & 147 & 75 & 671 & 38 & 935 \\
\hline Rate, peak, gpm & 50 & 0.165 & 50 & 10 & 0.15 & 190 & 4.32 & 50.00 \\
\hline Operation & Batch & Cont & Batch & Batch & Batch & Batch & Batch & Batch \\
\hline Temperature, ${ }^{\circ} \mathrm{F}$ & 70 & 83 & 70 & 70 & 70 & 70 & 70 & 70 \\
\hline Pressure, psia & 12.8 & 12.8 & 12.8 & 50.0 & 12.8 & 12.8 & 12.8 & 12.8 \\
\hline Specific Gravity & 1.04 & 1.33 & 1.02 & 1.12 & 1.02 & 1.02 & 0.68 & 1.32 \\
\hline Chemical Composition & Mol/liter & Mol/liter & Mol/liter & Mol/liter & Mol/liter & Mol/liter & Wt \% & Mol/liter \\
\hline $\mathrm{H}+$ & 3.13E-02 & $4.76 \mathrm{E}-01$ & 8.98E-02 & $8.98 \mathrm{E}-02$ & 8.98E-02 & $8.17 \mathrm{E}-02$ & $2.22 \mathrm{E}-03$ & $3.91 \mathrm{E}+00$ \\
\hline $\mathrm{Al}+3$ & $3.04 \mathrm{E}-02$ & $1.25 \mathrm{E}-01$ & $2.01 \mathrm{E}-02$ & $1.29 \mathrm{E}-01$ & $2.24 \mathrm{E}-02$ & $3.12 \mathrm{E}-02$ & $1.01 \mathrm{E}+00$ & $1.38 \mathrm{E}-01$ \\
\hline$A m+4$ & $8.51 \mathrm{E}-10$ & $4.12 \mathrm{E}-08$ & $6.16 \mathrm{E}-09$ & $6.16 \mathrm{E}-09$ & $6.16 \mathrm{E}-09$ & $5.61 \mathrm{E}-09$ & $3.64 \mathrm{E}-08$ & $5.08 \mathrm{E}-08$ \\
\hline $\mathrm{Sb}+5$ & $1.51 \mathrm{E}-05$ & $1.74 \mathrm{E}-05$ & 1.97E-06 & 6.64E-05 & $3.29 \mathrm{E}-06$ & $9.40 \mathrm{E}-06$ & $2.66 \mathrm{E}-03$ & $1.22 \mathrm{E}-05$ \\
\hline As+5 & $1.28 \mathrm{E}-04$ & $9.84 \mathrm{E}-05$ & $1.02 \mathrm{E}-05$ & $5.54 \mathrm{E}-04$ & $2.13 \mathrm{E}-05$ & 7.34E-05 & $1.38 \mathrm{E}-02$ & $4.28 \mathrm{E}-05$ \\
\hline $\mathrm{Ba}+2$ & $2.76 \mathrm{E}-05$ & $3.45 \mathrm{E}-05$ & 4.49E-06 & 1.19E-04 & $6.84 \mathrm{E}-06$ & 1.76E-05 & $5.36 \mathrm{E}-03$ & $2.59 \mathrm{E}-05$ \\
\hline $\mathrm{Be}+2$ & $6.21 \mathrm{E}-06$ & $5.52 \mathrm{E}-06$ & 5.99E-07 & $2.70 \mathrm{E}-05$ & $1.14 \mathrm{E}-06$ & $3.66 \mathrm{E}-06$ & 8.07E-05 & 2.99E-06 \\
\hline $\mathrm{B}+3$ & $7.79 \mathrm{E}-04$ & $3.44 \mathrm{E}-03$ & $6.86 \mathrm{E}-04$ & $2.80 \mathrm{E}-03$ & $7.30 \mathrm{E}-04$ & $8.74 \mathrm{E}-04$ & $7.94 \mathrm{E}-03$ & $3.93 \mathrm{E}-03$ \\
\hline $\mathrm{Br}-$ & $4.45 \mathrm{E}-09$ & $2.15 \mathrm{E}-07$ & $3.22 \mathrm{E}-08$ & $3.22 \mathrm{E}-08$ & $3.22 \mathrm{E}-08$ & $2.94 \mathrm{E}-08$ & $6.31 \mathrm{E}-08$ & $2.66 \mathrm{E}-07$ \\
\hline $\mathrm{Cd}+2$ & $1.50 \mathrm{E}-04$ & $5.72 \mathrm{E}-04$ & $1.34 \mathrm{E}-04$ & $4.79 \mathrm{E}-04$ & $1.41 \mathrm{E}-04$ & $1.62 \mathrm{E}-04$ & $1.35 \mathrm{E}-02$ & $6.55 \mathrm{E}-04$ \\
\hline $\mathrm{Ca}+2$ & $2.14 \mathrm{E}-03$ & $1.17 \mathrm{E}-02$ & $2.21 \mathrm{E}-03$ & 7.92E-03 & $2.33 \mathrm{E}-03$ & $2.68 \mathrm{E}-03$ & 7.97E-02 & $1.37 \mathrm{E}-02$ \\
\hline $\mathrm{Ce}+4$ & $1.39 \mathrm{E}-05$ & $3.51 \mathrm{E}-05$ & $4.53 \mathrm{E}-06$ & $6.26 \mathrm{E}-05$ & $5.72 \mathrm{E}-06$ & $1.10 \mathrm{E}-05$ & $2.77 \mathrm{E}-03$ & $3.48 \mathrm{E}-05$ \\
\hline Cs+ & $5.55 \mathrm{E}-05$ & $4.73 \mathrm{E}-05$ & $5.25 \mathrm{E}-06$ & $2.41 \mathrm{E}-04$ & $1.01 \mathrm{E}-05$ & $3.26 \mathrm{E}-05$ & $1.06 \mathrm{E}-02$ & $2.43 \mathrm{E}-05$ \\
\hline $\mathrm{Cl}-$ & $3.23 \mathrm{E}-03$ & $9.90 \mathrm{E}-03$ & $1.43 \mathrm{E}-03$ & $1.43 \mathrm{E}-02$ & $1.69 \mathrm{E}-03$ & $2.81 \mathrm{E}-03$ & $1.55 \mathrm{E}-01$ & $1.04 \mathrm{E}-02$ \\
\hline $\mathrm{Cr}+3$ & $5.85 \mathrm{E}-04$ & $3.54 \mathrm{E}-03$ & 5.07E-04 & $2.74 \mathrm{E}-03$ & $5.53 \mathrm{E}-04$ & $7.26 \mathrm{E}-04$ & $4.00 \mathrm{E}-02$ & $4.05 E-03$ \\
\hline $\mathrm{Co}+2$ & $4.86 \mathrm{E}-06$ & $3.26 \mathrm{E}-03$ & $4.29 \mathrm{E}-04$ & $4.49 \mathrm{E}-04$ & $4.29 \mathrm{E}-04$ & $3.94 \mathrm{E}-04$ & $1.02 \mathrm{E}-03$ & $4.02 \mathrm{E}-03$ \\
\hline $\mathrm{Cu}+2$ & $1.55 \mathrm{E}-04$ & $1.59 \mathrm{E}-04$ & $1.89 \mathrm{E}-05$ & $6.75 \mathrm{E}-04$ & $3.23 \mathrm{E}-05$ & $9.47 \mathrm{E}-05$ & $1.41 \mathrm{E}-02$ & $1.01 \mathrm{E}-04$ \\
\hline $\mathrm{Eu}+3$ & $7.38 \mathrm{E}-09$ & $3.57 \mathrm{E}-07$ & $5.34 \mathrm{E}-08$ & $5.34 \mathrm{E}-08$ & $5.34 \mathrm{E}-08$ & $4.87 \mathrm{E}-08$ & $1.99 \mathrm{E}-07$ & $4.40 \mathrm{E}-07$ \\
\hline F- & $2.82 \mathrm{E}-02$ & $4.18 \mathrm{E}-02$ & $6.24 \mathrm{E}-03$ & $1.20 \mathrm{E}-01$ & $8.57 \mathrm{E}-03$ & $1.91 \mathrm{E}-02$ & $7.33 \mathrm{E}-01$ & $3.51 \mathrm{E}-02$ \\
\hline $\mathrm{Gd}+3$ & $2.82 \mathrm{E}-05$ & $1.32 \mathrm{E}-04$ & $1.76 \mathrm{E}-05$ & $1.34 \mathrm{E}-04$ & $2.00 \mathrm{E}-05$ & $2.98 \mathrm{E}-05$ & $6.25 \mathrm{E}-03$ & $1.46 \mathrm{E}-04$ \\
\hline $\mathrm{Ge}+4$ & $1.28 \mathrm{E}-10$ & $6.21 \mathrm{E}-09$ & $9.29 \mathrm{E}-10$ & $9.29 \mathrm{E}-10$ & $9.29 \mathrm{E}-10$ & $8.46 \mathrm{E}-10$ & $1.65 \mathrm{E}-09$ & $7.66 \mathrm{E}-09$ \\
\hline $\ln +3$ & $2.02 \mathrm{E}-08$ & $1.03 \mathrm{E}-06$ & $1.54 \mathrm{E}-07$ & $1.54 \mathrm{E}-07$ & $1.54 \mathrm{E}-07$ & $1.40 \mathrm{E}-07$ & $4.32 \mathrm{E}-07$ & $1.28 \mathrm{E}-06$ \\
\hline I- & $3.70 \mathrm{E}-08$ & $1.79 \mathrm{E}-06$ & 2.67E-07 & 2.67E-07 & $2.67 \mathrm{E}-07$ & $2.44 \mathrm{E}-07$ & 8.32E-07 & $2.21 \mathrm{E}-06$ \\
\hline $\mathrm{Fe}+3$ & $1.07 \mathrm{E}-02$ & $1.05 \mathrm{E}-02$ & $1.38 \mathrm{E}-03$ & $4.59 \mathrm{E}-02$ & $2.30 \mathrm{E}-03$ & $6.52 \mathrm{E}-03$ & $8.44 \mathrm{E}-01$ & $6.55 \mathrm{E}-03$ \\
\hline $\mathrm{La}+3$ & $1.34 \mathrm{E}-07$ & $6.49 \mathrm{E}-06$ & $9.71 \mathrm{E}-07$ & $9.71 \mathrm{E}-07$ & 9.71E-07 & $8.84 \mathrm{E}-07$ & $3.30 \mathrm{E}-06$ & $8.00 \mathrm{E}-06$ \\
\hline $\mathrm{Pb}+2$ & $6.39 \mathrm{E}-05$ & $5.20 \mathrm{E}-04$ & $8.19 \mathrm{E}-05$ & $2.83 \mathrm{E}-04$ & $8.61 \mathrm{E}-05$ & 9.83E-05 & $1.45 \mathrm{E}-02$ & $6.12 \mathrm{E}-04$ \\
\hline $\mathrm{Li}+$ & $3.18 \mathrm{E}-04$ & $4.43 \mathrm{E}-04$ & $5.16 \mathrm{E}-05$ & $1.41 \mathrm{E}-03$ & $7.94 \mathrm{E}-05$ & 2.07E-04 & $3.19 \mathrm{E}-03$ & $3.50 \mathrm{E}-04$ \\
\hline $\mathrm{Mg}+2$ & $1.23 \mathrm{E}-03$ & 1.45E-02 & $2.15 \mathrm{E}-03$ & $6.11 \mathrm{E}-03$ & $2.23 \mathrm{E}-03$ & $2.42 \mathrm{E}-03$ & 3.39E-02 & 1.73E-02 \\
\hline$M n+4$ & $4.45 \mathrm{E}-04$ & $1.73 \mathrm{E}-02$ & $2.46 \mathrm{E}-03$ & $3.45 \mathrm{E}-03$ & $2.48 \mathrm{E}-03$ & $2.36 \mathrm{E}-03$ & $2.18 \mathrm{E}-02$ & $2.12 \mathrm{E}-02$ \\
\hline $\mathrm{Hg}+2$ & $3.11 \mathrm{E}-05$ & $1.59 \mathrm{E}-03$ & $2.24 \mathrm{E}-04$ & $2.85 \mathrm{E}-04$ & $2.25 \mathrm{E}-04$ & $2.11 \mathrm{E}-04$ & $5.25 \mathrm{E}-03$ & $1.95 \mathrm{E}-03$ \\
\hline $\mathrm{Mo}+6$ & $8.91 \mathrm{E}-04$ & $6.65 \mathrm{E}-04$ & $6.89 \mathrm{E}-05$ & $3.86 \mathrm{E}-03$ & 1.47E-04 & $5.10 \mathrm{E}-04$ & $1.23 \mathrm{E}-01$ & $2.71 \mathrm{E}-04$ \\
\hline $\mathrm{Nd}+3$ & $4.32 \mathrm{E}-07$ & $2.09 \mathrm{E}-05$ & $3.13 \mathrm{E}-06$ & $3.13 \mathrm{E}-06$ & $3.13 \mathrm{E}-06$ & $2.85 \mathrm{E}-06$ & $1.11 \mathrm{E}-05$ & $2.58 \mathrm{E}-05$ \\
\hline $\mathrm{Np}+4$ & 2.47E-07 & $3.70 \mathrm{E}-06$ & $7.02 \mathrm{E}-07$ & $7.02 \mathrm{E}-07$ & $7.02 \mathrm{E}-07$ & $6.38 \mathrm{E}-07$ & $4.08 \mathrm{E}-06$ & 4.57E-06 \\
\hline $\mathrm{Ni+2}$ & $2.03 \mathrm{E}-04$ & $7.50 \mathrm{E}-04$ & $1.15 \mathrm{E}-04$ & $8.84 \mathrm{E}-04$ & $1.30 \mathrm{E}-04$ & $1.95 \mathrm{E}-04$ & $1.55 \mathrm{E}-02$ & $8.14 \mathrm{E}-04$ \\
\hline $\mathrm{Nb}+5$ & $1.47 \mathrm{E}-03$ & $8.16 \mathrm{E}-04$ & $7.51 \mathrm{E}-05$ & $6.36 \mathrm{E}-03$ & $2.04 \mathrm{E}-04$ & $8.10 \mathrm{E}-04$ & $1.98 \mathrm{E}-01$ & 9.71E-05 \\
\hline NO3- & $6.65 \mathrm{E}-02$ & $5.78 \mathrm{E}+00$ & $8.18 \mathrm{E}-01$ & $8.20 \mathrm{E}-01$ & $8.18 \mathrm{E}-01$ & $7.46 \mathrm{E}-01$ & $1.28 \mathrm{E}+00$ & $7.13 \mathrm{E}+00$ \\
\hline $\mathrm{O}-2$ & $2.23 \mathrm{E}-01$ & $1.23 \mathrm{E}-01$ & $1.12 \mathrm{E}-02$ & $9.64 \mathrm{E}-01$ & $3.08 \mathrm{E}-02$ & $1.23 \mathrm{E}-01$ & $5.16 \mathrm{E}+00$ & $1.35 \mathrm{E}-02$ \\
\hline $\mathrm{Pd}+4$ & $2.45 \mathrm{E}-03$ & $1.43 \mathrm{E}-03$ & $1.35 \mathrm{E}-04$ & $1.06 \mathrm{E}-02$ & $3.49 \mathrm{E}-04$ & $1.36 \mathrm{E}-03$ & $3.77 \mathrm{E}-01$ & $2.52 \mathrm{E}-04$ \\
\hline PO4-3 & $1.39 \mathrm{E}-01$ & $7.72 \mathrm{E}-02$ & $7.20 \mathrm{E}-03$ & 5.97E-01 & $1.93 \mathrm{E}-02$ & $7.62 \mathrm{E}-02$ & $1.90 \mathrm{E}+01$ & $9.90 \mathrm{E}-03$ \\
\hline $\mathrm{Pu}+4$ & $7.79 \mathrm{E}-08$ & $3.69 \mathrm{E}-06$ & $5.53 \mathrm{E}-07$ & $5.53 \mathrm{E}-07$ & $5.53 \mathrm{E}-07$ & $5.04 \mathrm{E}-07$ & $3.23 \mathrm{E}-06$ & $4.55 \mathrm{E}-06$ \\
\hline $\mathrm{K}+$ & $1.86 \mathrm{E}-02$ & $5.71 \mathrm{E}-01$ & 7.67E-02 & $1.47 \mathrm{E}-01$ & $7.81 \mathrm{E}-02$ & $7.82 \mathrm{E}-02$ & $1.00 \mathrm{E}+00$ & $6.94 \mathrm{E}-01$ \\
\hline $\mathrm{Pr}+4$ & $1.22 \mathrm{E}-07$ & $5.90 \mathrm{E}-06$ & $8.82 \mathrm{E}-07$ & 8.82E-07 & $8.82 \mathrm{E}-07$ & $8.04 \mathrm{E}-07$ & $3.05 \mathrm{E}-06$ & $7.28 \mathrm{E}-06$ \\
\hline $\mathrm{Rh}+4$ & $5.26 \mathrm{E}-08$ & $2.54 \mathrm{E}-06$ & 3.81E-07 & $3.81 \mathrm{E}-07$ & $3.81 \mathrm{E}-07$ & $3.47 \mathrm{E}-07$ & $9.60 \mathrm{E}-07$ & 3.14E-06 \\
\hline
\end{tabular}


Table B-12. NGLW mass balance (continued).

\begin{tabular}{|c|c|c|c|c|c|c|c|c|}
\hline Stream \# & 110 & 111 & 112 & 113 & 114 & 115 & 116 & 120 \\
\hline Stream Name & $\begin{array}{c}\text { Slurry from } \\
\text { TFF Tank } \\
\text { WM-187 }\end{array}$ & $\begin{array}{l}\text { Crossflow } \\
\text { Filter Recirc } \\
\text { Purge }\end{array}$ & $\begin{array}{l}\text { Decant } \\
\text { Liquid }\end{array}$ & $\begin{array}{c}\text { Solids } \\
\text { Treatment } \\
\text { Filter Feed }\end{array}$ & $\begin{array}{l}\text { Solids } \\
\text { Treatment } \\
\text { Filtrate }\end{array}$ & $\begin{array}{c}\text { Combined } \\
\text { Waste Water }\end{array}$ & $\begin{array}{l}\text { Dried Solids } \\
\text { Waste }\end{array}$ & $\begin{array}{l}\text { Liquid SBW } \\
\text { from TFF }\end{array}$ \\
\hline Chemical Composition & Mol/liter & Mol/liter & Mol/liter & Mol/liter & Mol/liter & Mol/liter & Wt \% & Mol/liter \\
\hline $\mathrm{Rb}+$ & $8.10 \mathrm{E}-08$ & $3.92 \mathrm{E}-06$ & $5.86 \mathrm{E}-07$ & $5.86 \mathrm{E}-07$ & $5.86 \mathrm{E}-07$ & $5.34 \mathrm{E}-07$ & $1.23 \mathrm{E}-06$ & $4.83 \mathrm{E}-06$ \\
\hline $\mathrm{Ru}+3$ & $1.03 \mathrm{E}-03$ & $6.75 \mathrm{E}-04$ & $6.66 \mathrm{E}-05$ & $4.44 \mathrm{E}-03$ & $1.56 \mathrm{E}-04$ & $5.77 \mathrm{E}-04$ & $1.50 \mathrm{E}-01$ & 1.99E-04 \\
\hline $\mathrm{Sm}+3$ & $8.02 \mathrm{E}-08$ & $3.88 \mathrm{E}-06$ & $5.80 \mathrm{E}-07$ & $5.80 \mathrm{E}-07$ & $5.80 \mathrm{E}-07$ & $5.29 \mathrm{E}-07$ & $2.14 \mathrm{E}-06$ & $4.78 \mathrm{E}-06$ \\
\hline $\mathrm{Se}+4$ & $3.82 \mathrm{E}-04$ & $2.18 \mathrm{E}-04$ & $2.03 \mathrm{E}-05$ & $1.65 \mathrm{E}-03$ & 5.37E-05 & $2.11 \mathrm{E}-04$ & $4.36 \mathrm{E}-02$ & $3.32 \mathrm{E}-05$ \\
\hline $\mathrm{Si}+4$ & $1.26 \mathrm{E}-01$ & $6.92 \mathrm{E}-02$ & $6.37 \mathrm{E}-03$ & $5.42 \mathrm{E}-01$ & $1.74 \mathrm{E}-02$ & $6.90 \mathrm{E}-02$ & $5.10 \mathrm{E}+00$ & $7.89 \mathrm{E}-03$ \\
\hline $\mathrm{Ag}^{+}$ & $5.89 \mathrm{E}-04$ & $3.26 \mathrm{E}-04$ & $3.05 \mathrm{E}-05$ & $2.54 \mathrm{E}-03$ & $8.19 \mathrm{E}-05$ & $3.24 \mathrm{E}-04$ & 9.16E-02 & 3.91E-05 \\
\hline $\mathrm{Na}+$ & $4.19 \mathrm{E}-02$ & $4.28 \mathrm{E}+00$ & $5.76 \mathrm{E}-01$ & $6.89 \mathrm{E}-01$ & $5.78 \mathrm{E}-01$ & $5.39 \mathrm{E}-01$ & $1.20 \mathrm{E}+00$ & $1.94 \mathrm{E}+00$ \\
\hline $\mathrm{Sr}+2$ & $8.53 \mathrm{E}-06$ & 9.05E-05 & 1.19E-05 & $4.74 \mathrm{E}-05$ & 1.26E-05 & 1.51E-05 & $1.08 \mathrm{E}-03$ & 1.06E-04 \\
\hline SO4-2 & $6.39 \mathrm{E}-04$ & $1.14 \mathrm{E}-02$ & $2.06 \mathrm{E}-03$ & $2.06 \mathrm{E}-03$ & $2.06 \mathrm{E}-03$ & $1.87 \mathrm{E}-03$ & $4.85 \mathrm{E}-03$ & $1.41 \mathrm{E}-02$ \\
\hline $\mathrm{Tc}+7$ & $9.40 \mathrm{E}-08$ & $6.43 \mathrm{E}-06$ & $9.28 \mathrm{E}-07$ & $9.28 \mathrm{E}-07$ & $9.28 \mathrm{E}-07$ & $8.46 \mathrm{E}-07$ & $2.21 \mathrm{E}-06$ & $7.93 \mathrm{E}-06$ \\
\hline $\mathrm{Te}+4$ & $4.21 \mathrm{E}-08$ & $3.25 \mathrm{E}-06$ & $4.65 \mathrm{E}-07$ & $4.65 \mathrm{E}-07$ & $4.65 \mathrm{E}-07$ & 4.24E-07 & $1.45 \mathrm{E}-06$ & $4.01 \mathrm{E}-06$ \\
\hline $\mathrm{Tb}+4$ & $3.09 \mathrm{E}-11$ & $1.49 \mathrm{E}-09$ & $2.23 \mathrm{E}-10$ & $2.23 \mathrm{E}-10$ & $2.23 \mathrm{E}-10$ & $2.04 \mathrm{E}-10$ & $8.70 \mathrm{E}-10$ & $1.84 \mathrm{E}-09$ \\
\hline $\mathrm{Th}+4$ & $2.87 \mathrm{E}-12$ & $1.61 \mathrm{E}-05$ & $2.13 \mathrm{E}-06$ & $2.13 \mathrm{E}-06$ & $2.13 \mathrm{E}-06$ & 1.94E-06 & $1.21 \mathrm{E}-05$ & 1.99E-05 \\
\hline $\mathrm{Sn}+4$ & $2.63 \mathrm{E}-03$ & $1.46 \mathrm{E}-03$ & $1.35 \mathrm{E}-04$ & $1.13 \mathrm{E}-02$ & $3.65 \mathrm{E}-04$ & $1.45 \mathrm{E}-03$ & $4.51 \mathrm{E}-01$ & $1.85 \mathrm{E}-04$ \\
\hline $\mathrm{Ti}+4$ & $1.29 \mathrm{E}-03$ & $7.54 \mathrm{E}-04$ & $7.18 \mathrm{E}-05$ & $5.56 \mathrm{E}-03$ & $1.85 \mathrm{E}-04$ & $7.14 \mathrm{E}-04$ & 8.91E-02 & $1.35 \mathrm{E}-04$ \\
\hline $\mathrm{U}+4$ & 3.83E-05 & $8.27 \mathrm{E}-03$ & $1.09 \mathrm{E}-03$ & $1.23 \mathrm{E}-03$ & $1.10 \mathrm{E}-03$ & $1.01 \mathrm{E}-03$ & $1.75 \mathrm{E}-02$ & $1.02 \mathrm{E}-02$ \\
\hline $\mathrm{V}+5$ & $1.12 \mathrm{E}-05$ & $5.10 \mathrm{E}-05$ & $9.66 \mathrm{E}-06$ & $4.21 \mathrm{E}-05$ & $1.03 \mathrm{E}-05$ & $1.26 \mathrm{E}-05$ & $5.72 \mathrm{E}-04$ & $5.82 \mathrm{E}-05$ \\
\hline $\mathrm{Y}+3$ & $1.00 \mathrm{E}-07$ & $4.84 \mathrm{E}-06$ & $7.23 \mathrm{E}-07$ & $7.23 \mathrm{E}-07$ & 7.23E-07 & 6.59E-07 & $1.58 \mathrm{E}-06$ & 5.97E-06 \\
\hline$Z n+2$ & $8.90 \mathrm{E}-05$ & $3.90 \mathrm{E}-04$ & 5.07E-05 & $4.24 \mathrm{E}-04$ & $5.83 \mathrm{E}-05$ & 9.02E-05 & $8.34 \mathrm{E}-03$ & $4.26 \mathrm{E}-04$ \\
\hline $\mathrm{Zr}+4$ & $5.35 \mathrm{E}-02$ & 3.04E-02 & $2.91 \mathrm{E}-03$ & 2.31E-01 & $7.58 \mathrm{E}-03$ & $2.95 \mathrm{E}-02$ & $7.04 \mathrm{E}+00$ & 4.53E-03 \\
\hline $\mathrm{H} 2 \mathrm{O}$ & $5.55 \mathrm{E}+01$ & $4.59 \mathrm{E}+01$ & $5.23 E+01$ & $5.10 \mathrm{E}+01$ & $5.22 \mathrm{E}+01$ & $5.19 \mathrm{E}+01$ & $5.56 \mathrm{E}+01$ & $4.33 \mathrm{E}+01$ \\
\hline Canisters/day & & & & & & & 0.59 & \\
\hline \multicolumn{9}{|l|}{ Canisters total } \\
\hline Radiological Composition & Ci/liter & Ci/liter & Ci/liter & Ci/liter & Ci/liter & Ci/liter & $\mathrm{Ci} / \mathrm{kg}$ & Ci/liter \\
\hline Ra-226 & $1.15 \mathrm{E}-13$ & $5.91 \mathrm{E}-12$ & $8.78 \mathrm{E}-13$ & $8.78 \mathrm{E}-13$ & $8.78 \mathrm{E}-13$ & $8.00 \mathrm{E}-13$ & $2.15 \mathrm{E}-13$ & $7.29 \mathrm{E}-12$ \\
\hline Ac-227 & $5.43 \mathrm{E}-13$ & $2.78 \mathrm{E}-11$ & $4.13 \mathrm{E}-12$ & $4.13 \mathrm{E}-12$ & $4.13 \mathrm{E}-12$ & $3.77 \mathrm{E}-12$ & $1.01 \mathrm{E}-12$ & $3.43 \mathrm{E}-11$ \\
\hline Th-228 & $4.51 \mathrm{E}-11$ & $2.31 \mathrm{E}-09$ & $3.43 \mathrm{E}-10$ & $3.43 \mathrm{E}-10$ & $3.43 \mathrm{E}-10$ & $3.13 \mathrm{E}-10$ & $8.41 \mathrm{E}-11$ & $2.85 \mathrm{E}-09$ \\
\hline Th-230 & $3.37 \mathrm{E}-10$ & $7.72 \mathrm{E}-10$ & $1.04 \mathrm{E}-10$ & $1.49 \mathrm{E}-09$ & $1.33 \mathrm{E}-10$ & $2.59 \mathrm{E}-10$ & 4.72E-09 & $7.51 \mathrm{E}-10$ \\
\hline Th-232 & $9.98 \mathrm{E}-18$ & $5.11 \mathrm{E}-16$ & $7.59 \mathrm{E}-17$ & $7.59 \mathrm{E}-17$ & $7.59 \mathrm{E}-17$ & $6.92 \mathrm{E}-17$ & $1.86 \mathrm{E}-17$ & $6.30 \mathrm{E}-16$ \\
\hline $\mathrm{Pa}-231$ & $1.26 \mathrm{E}-12$ & $6.44 \mathrm{E}-11$ & $9.57 \mathrm{E}-12$ & $9.57 \mathrm{E}-12$ & $9.57 \mathrm{E}-12$ & $8.72 \mathrm{E}-12$ & $2.35 \mathrm{E}-12$ & $7.95 \mathrm{E}-11$ \\
\hline $\mathrm{Pa}-233$ & $4.13 \mathrm{E}-08$ & $2.11 \mathrm{E}-06$ & $3.14 \mathrm{E}-07$ & $3.14 \mathrm{E}-07$ & $3.14 \mathrm{E}-07$ & $2.86 \mathrm{E}-07$ & $7.70 \mathrm{E}-08$ & $2.61 \mathrm{E}-06$ \\
\hline $\mathrm{U}-232$ & $6.99 \mathrm{E}-10$ & $1.80 \mathrm{E}-09$ & $2.47 \mathrm{E}-10$ & $3.11 \mathrm{E}-09$ & $3.05 \mathrm{E}-10$ & $5.62 \mathrm{E}-10$ & $9.75 \mathrm{E}-09$ & $1.81 \mathrm{E}-09$ \\
\hline $\mathrm{U}-233$ & $1.27 \mathrm{E}-11$ & $6.40 \mathrm{E}-11$ & $9.15 \mathrm{E}-12$ & $5.84 \mathrm{E}-11$ & $1.02 \mathrm{E}-11$ & $1.42 \mathrm{E}-11$ & $1.69 \mathrm{E}-10$ & $7.18 \mathrm{E}-11$ \\
\hline U-234 & $2.08 \mathrm{E}-07$ & $1.12 \mathrm{E}-06$ & $1.53 \mathrm{E}-07$ & $9.92 \mathrm{E}-07$ & $1.70 \mathrm{E}-07$ & $2.38 \mathrm{E}-07$ & $2.88 \mathrm{E}-06$ & $1.26 \mathrm{E}-06$ \\
\hline U-235 & $1.09 \mathrm{E}-08$ & $5.61 \mathrm{E}-08$ & 7.37E-09 & $5.28 \mathrm{E}-08$ & $8.30 \mathrm{E}-09$ & $1.21 \mathrm{E}-08$ & $1.56 \mathrm{E}-07$ & $6.27 \mathrm{E}-08$ \\
\hline U-236 & $1.43 \mathrm{E}-08$ & $5.36 \mathrm{E}-08$ & $7.16 \mathrm{E}-09$ & $6.62 \mathrm{E}-08$ & 8.37E-09 & $1.35 \mathrm{E}-08$ & $2.02 \mathrm{E}-07$ & $5.76 \mathrm{E}-08$ \\
\hline U-237 & $9.07 \mathrm{E}-11$ & 4.64E-09 & $6.90 \mathrm{E}-10$ & $6.90 \mathrm{E}-10$ & $6.90 \mathrm{E}-10$ & $6.28 \mathrm{E}-10$ & $1.69 \mathrm{E}-10$ & $5.72 \mathrm{E}-09$ \\
\hline U-238 & 1.23E-09 & $2.09 \mathrm{E}-08$ & $3.11 \mathrm{E}-09$ & $6.51 \mathrm{E}-09$ & $3.18 \mathrm{E}-09$ & 3.23E-09 & $1.23 \mathrm{E}-08$ & $2.53 \mathrm{E}-08$ \\
\hline Np-236 & $6.44 \mathrm{E}-14$ & $2.31 \mathrm{E}-12$ & $3.60 \mathrm{E}-13$ & $3.60 \mathrm{E}-13$ & $3.60 \mathrm{E}-13$ & $3.28 \mathrm{E}-13$ & $8.83 \mathrm{E}-14$ & $2.85 \mathrm{E}-12$ \\
\hline Np-237 & 1.02E-07 & $6.52 \mathrm{E}-07$ & $1.20 \mathrm{E}-07$ & $3.81 \mathrm{E}-07$ & $1.26 \mathrm{E}-07$ & $1.40 \mathrm{E}-07$ & $9.12 \mathrm{E}-07$ & 7.67E-07 \\
\hline Pu-236 & $1.22 \mathrm{E}-09$ & 2.75E-09 & $3.96 \mathrm{E}-10$ & $5.32 \mathrm{E}-09$ & $4.97 \mathrm{E}-10$ & $9.42 \mathrm{E}-10$ & $1.68 \mathrm{E}-08$ & $2.68 \mathrm{E}-09$ \\
\hline Pu-238 & $6.41 \mathrm{E}-04$ & $7.45 \mathrm{E}-04$ & $1.01 \mathrm{E}-04$ & $2.75 \mathrm{E}-03$ & $1.56 \mathrm{E}-04$ & $4.05 \mathrm{E}-04$ & $9.00 \mathrm{E}-03$ & $5.36 \mathrm{E}-04$ \\
\hline Pu-239 & $6.72 \mathrm{E}-05$ & $8.73 \mathrm{E}-05$ & 1.09E-05 & $2.93 \mathrm{E}-04$ & $1.67 \mathrm{E}-05$ & $4.32 \mathrm{E}-05$ & $9.58 \mathrm{E}-04$ & $6.69 \mathrm{E}-05$ \\
\hline Pu-240 & $4.68 \mathrm{E}-06$ & $1.03 \mathrm{E}-05$ & $1.44 \mathrm{E}-06$ & $2.04 \mathrm{E}-05$ & $1.83 \mathrm{E}-06$ & $3.56 \mathrm{E}-06$ & $6.47 \mathrm{E}-05$ & $9.92 \mathrm{E}-06$ \\
\hline Pu-241 & $5.07 \mathrm{E}-04$ & $5.14 \mathrm{E}-04$ & $7.08 \mathrm{E}-05$ & $2.16 \mathrm{E}-03$ & $1.14 \mathrm{E}-04$ & $3.11 \mathrm{E}-04$ & $7.10 \mathrm{E}-03$ & 3.32E-04 \\
\hline Pu-242 & $3.69 \mathrm{E}-09$ & 7.95E-09 & $1.26 \mathrm{E}-09$ & $1.56 \mathrm{E}-08$ & $1.55 \mathrm{E}-09$ & $2.84 \mathrm{E}-09$ & $4.88 \mathrm{E}-08$ & 7.74E-09 \\
\hline Pu-244 & $2.89 \mathrm{E}-16$ & $4.80 \mathrm{E}-16$ & $5.73 \mathrm{E}-17$ & $1.29 \mathrm{E}-15$ & $8.25 \mathrm{E}-17$ & $1.97 \mathrm{E}-16$ & $4.17 \mathrm{E}-15$ & $4.15 \mathrm{E}-16$ \\
\hline Am-241 & 1.71E-05 & $6.03 E-05$ & 8.31E-06 & 7.74E-05 & $9.72 \mathrm{E}-06$ & 1.57E-05 & $2.36 \mathrm{E}-04$ & $6.44 \mathrm{E}-05$ \\
\hline Am-242m & $2.14 \mathrm{E}-10$ & $1.11 \mathrm{E}-08$ & $1.65 \mathrm{E}-09$ & $1.65 \mathrm{E}-09$ & $1.65 \mathrm{E}-09$ & $1.50 \mathrm{E}-09$ & $4.04 \mathrm{E}-10$ & $1.37 \mathrm{E}-08$ \\
\hline Am-243 & 6.07E-09 & $1.88 \mathrm{E}-08$ & $2.61 \mathrm{E}-09$ & $2.72 \mathrm{E}-08$ & $3.12 \mathrm{E}-09$ & $5.28 \mathrm{E}-09$ & $8.39 \mathrm{E}-08$ & $1.97 \mathrm{E}-08$ \\
\hline Cm-242 & $2.00 \mathrm{E}-10$ & $2.08 \mathrm{E}-08$ & $2.89 \mathrm{E}-09$ & $2.99 \mathrm{E}-09$ & $2.90 \mathrm{E}-09$ & $2.65 \mathrm{E}-09$ & $1.04 \mathrm{E}-09$ & $2.57 \mathrm{E}-08$ \\
\hline
\end{tabular}


Table B-12. NGLW mass balance (continued).

\begin{tabular}{|c|c|c|c|c|c|c|c|}
\hline Stream \# & 110 & 111 & 112 & 113 & 114 & 115 & 116 \\
\hline Stream Name & $\begin{array}{l}\text { Slurry from } \\
\text { TFF Tank } \\
\text { WM-187 }\end{array}$ & $\begin{array}{c}\text { Crossflow } \\
\text { Filter Recirc } \\
\text { Purge }\end{array}$ & $\begin{array}{l}\text { Decant } \\
\text { Liquid }\end{array}$ & $\begin{array}{c}\text { Solids } \\
\text { Treatment } \\
\text { Filter Feed }\end{array}$ & $\begin{array}{l}\text { Solids } \\
\text { Treatment } \\
\text { Filtrate }\end{array}$ & $\begin{array}{c}\text { Combined } \\
\text { Waste Water }\end{array}$ & $\begin{array}{l}\text { Dried Solids } \\
\text { Waste }\end{array}$ \\
\hline & Ci/liter & Ci/liter & Ci/liter & Ci/liter & $\mathrm{Ci} /$ liter & $\mathrm{Ci} /$ liter & $\mathrm{Ci} / \mathrm{kg}$ \\
\hline $\mathrm{Cm}-243$ & $1.23 \mathrm{E}-08$ & $2.70 \mathrm{E}-08$ & 3.64E-09 & $5.42 \mathrm{E}-08$ & $4.68 \mathrm{E}-09$ & $9.29 \mathrm{E}-09$ & $1.72 \mathrm{E}-07$ \\
\hline $\mathrm{Cm}-244$ & $7.68 \mathrm{E}-07$ & $1.16 \mathrm{E}-06$ & $1.58 \mathrm{E}-07$ & 3.33E-06 & $2.23 E-07$ & 5.18E-07 & $1.08 \mathrm{E}-05$ \\
\hline $\mathrm{Cm}-245$ & $1.30 \mathrm{E}-10$ & $2.84 \mathrm{E}-10$ & $3.83 \mathrm{E}-11$ & $5.75 \mathrm{E}-10$ & $4.93 \mathrm{E}-11$ & $9.82 \mathrm{E}-11$ & $1.83 \mathrm{E}-09$ \\
\hline $\mathrm{Cm}-246$ & $8.44 \mathrm{E}-12$ & $1.87 \mathrm{E}-11$ & $2.52 \mathrm{E}-12$ & $3.73 \mathrm{E}-11$ & $3.23 E-12$ & $6.40 \mathrm{E}-12$ & $1.18 \mathrm{E}-10$ \\
\hline $\mathrm{H}-3$ & 3.92E-07 & 1.09E-05 & $1.78 \mathrm{E}-06$ & 1.78E-06 & $1.78 \mathrm{E}-06$ & $1.62 \mathrm{E}-06$ & 4.35E-07 \\
\hline $\mathrm{Be}-10$ & $4.23 \mathrm{E}-14$ & $2.16 \mathrm{E}-12$ & $3.22 \mathrm{E}-13$ & $3.22 \mathrm{E}-13$ & $3.22 \mathrm{E}-13$ & $2.93 \mathrm{E}-13$ & $7.88 \mathrm{E}-14$ \\
\hline C-14 & $5.49 \mathrm{E}-11$ & $1.16 \mathrm{E}-10$ & $1.55 \mathrm{E}-11$ & $2.42 \mathrm{E}-10$ & $2.02 \mathrm{E}-11$ & $4.09 \mathrm{E}-11$ & $7.71 \mathrm{E}-10$ \\
\hline Se-79 & $1.98 \mathrm{E}-07$ & $4.20 \mathrm{E}-07$ & $5.64 \mathrm{E}-08$ & $8.72 \mathrm{E}-07$ & $7.32 \mathrm{E}-08$ & $1.48 \mathrm{E}-07$ & $2.78 \mathrm{E}-06$ \\
\hline Rb-87 & $4.13 \mathrm{E}-13$ & $2.11 \mathrm{E}-11$ & $3.14 \mathrm{E}-12$ & $3.14 \mathrm{E}-12$ & $3.14 \mathrm{E}-12$ & $2.86 \mathrm{E}-12$ & $7.70 \mathrm{E}-13$ \\
\hline Sr-90 & 1.06E-03 & $2.80 \mathrm{E}-02$ & $4.25 \mathrm{E}-03$ & $5.91 \mathrm{E}-03$ & $4.29 \mathrm{E}-03$ & $4.07 \mathrm{E}-03$ & $6.67 \mathrm{E}-03$ \\
\hline$Y-90$ & $1.06 \mathrm{E}-03$ & $2.80 \mathrm{E}-02$ & $4.25 \mathrm{E}-03$ & $5.91 \mathrm{E}-03$ & $4.29 \mathrm{E}-03$ & $4.07 \mathrm{E}-03$ & $6.67 \mathrm{E}-03$ \\
\hline Zr-93 & $3.12 \mathrm{E}-08$ & $1.60 \mathrm{E}-06$ & $2.37 \mathrm{E}-07$ & $2.37 \mathrm{E}-07$ & $2.37 \mathrm{E}-07$ & $2.16 \mathrm{E}-07$ & $5.82 \mathrm{E}-08$ \\
\hline $\mathrm{Nb}-93 \mathrm{~m}$ & $2.41 \mathrm{E}-08$ & $1.23 \mathrm{E}-06$ & $1.83 \mathrm{E}-07$ & $1.83 \mathrm{E}-07$ & 1.83E-07 & 1.67E-07 & $4.48 \mathrm{E}-08$ \\
\hline $\mathrm{Nb}-94$ & $5.10 \mathrm{E}-07$ & $1.09 \mathrm{E}-06$ & $1.47 \mathrm{E}-07$ & $2.25 \mathrm{E}-06$ & $1.90 \mathrm{E}-07$ & $3.82 \mathrm{E}-07$ & $7.16 \mathrm{E}-06$ \\
\hline Tc-98 & 3.63E-14 & $1.86 \mathrm{E}-12$ & $2.76 \mathrm{E}-13$ & $2.76 \mathrm{E}-13$ & $2.76 \mathrm{E}-13$ & $2.52 \mathrm{E}-13$ & $6.77 \mathrm{E}-14$ \\
\hline Tc-99 & $4.43 \mathrm{E}-06$ & $1.31 \mathrm{E}-05$ & $1.77 \mathrm{E}-06$ & $2.00 \mathrm{E}-05$ & $2.15 \mathrm{E}-06$ & $3.76 \mathrm{E}-06$ & $6.21 \mathrm{E}-05$ \\
\hline Ru-106 & $4.27 \mathrm{E}-07$ & $8.98 \mathrm{E}-07$ & $1.20 \mathrm{E}-07$ & $1.88 \mathrm{E}-06$ & 1.57E-07 & $3.18 \mathrm{E}-07$ & $6.00 \mathrm{E}-06$ \\
\hline Rh-102 & $4.14 \mathrm{E}-07$ & $2.28 \mathrm{E}-07$ & $2.09 \mathrm{E}-08$ & $1.78 \mathrm{E}-06$ & $5.71 \mathrm{E}-08$ & $2.27 \mathrm{E}-07$ & $5.98 \mathrm{E}-06$ \\
\hline $\mathrm{Pd}-107$ & $2.33 \mathrm{E}-10$ & $1.19 \mathrm{E}-08$ & 1.77E-09 & 1.77E-09 & 1.77E-09 & $1.61 \mathrm{E}-09$ & $4.34 \mathrm{E}-10$ \\
\hline Cd-113m & $4.68 \mathrm{E}-08$ & $2.39 \mathrm{E}-06$ & $3.56 \mathrm{E}-07$ & 3.56E-07 & $3.56 \mathrm{E}-07$ & $3.24 \mathrm{E}-07$ & $8.72 \mathrm{E}-08$ \\
\hline $\ln -115$ & $1.42 \mathrm{E}-18$ & $7.26 \mathrm{E}-17$ & $1.08 \mathrm{E}-17$ & $1.08 \mathrm{E}-17$ & $1.08 \mathrm{E}-17$ & $9.83 \mathrm{E}-18$ & $2.64 \mathrm{E}-18$ \\
\hline Sn-121m & $9.42 \mathrm{E}-10$ & $4.82 \mathrm{E}-08$ & 7.16E-09 & $7.16 \mathrm{E}-09$ & 7.16E-09 & $6.53 \mathrm{E}-09$ & 1.76E-09 \\
\hline Sn-126 & 1.87E-07 & $3.95 \mathrm{E}-07$ & $5.31 \mathrm{E}-08$ & $8.24 \mathrm{E}-07$ & $6.89 \mathrm{E}-08$ & $1.39 \mathrm{E}-07$ & 2.62E-06 \\
\hline Sb-125 & $5.93 \mathrm{E}-04$ & $3.34 \mathrm{E}-04$ & $3.14 \mathrm{E}-05$ & $2.56 \mathrm{E}-03$ & $8.32 \mathrm{E}-05$ & $3.27 \mathrm{E}-04$ & $8.56 \mathrm{E}-03$ \\
\hline Sb-126 & $8.10 \mathrm{E}-10$ & $4.15 \mathrm{E}-08$ & $6.16 \mathrm{E}-09$ & $6.16 \mathrm{E}-09$ & $6.16 \mathrm{E}-09$ & $5.62 \mathrm{E}-09$ & $1.51 \mathrm{E}-09$ \\
\hline Te-123 & $5.40 \mathrm{E}-21$ & $2.76 \mathrm{E}-19$ & $4.11 \mathrm{E}-20$ & $4.11 \mathrm{E}-20$ & $4.11 \mathrm{E}-20$ & $3.74 \mathrm{E}-20$ & $1.01 \mathrm{E}-20$ \\
\hline Te-125m & $4.44 \mathrm{E}-08$ & $2.27 \mathrm{E}-06$ & 3.37E-07 & 3.37E-07 & 3.37E-07 & $3.08 \mathrm{E}-07$ & $8.27 \mathrm{E}-08$ \\
\hline \begin{tabular}{|l}
$1-129$ \\
\end{tabular} & $2.42 \mathrm{E}-08$ & $5.04 \mathrm{E}-08$ & $6.78 \mathrm{E}-09$ & 1.06E-07 & $8.83 \mathrm{E}-09$ & 1.79E-08 & 3.39E-07 \\
\hline Cs-134 & 4.92E-05 & 3.93E-05 & $4.85 \mathrm{E}-06$ & $2.11 \mathrm{E}-04$ & $9.07 \mathrm{E}-06$ & $2.87 \mathrm{E}-05$ & $6.99 \mathrm{E}-04$ \\
\hline Cs-135 & $3.49 \mathrm{E}-07$ & $8.05 \mathrm{E}-07$ & $1.09 \mathrm{E}-07$ & $1.55 \mathrm{E}-06$ & 1.39E-07 & $2.68 \mathrm{E}-07$ & $4.90 \mathrm{E}-06$ \\
\hline Cs-137 & $1.98 \mathrm{E}-02$ & $3.17 \mathrm{E}-02$ & $4.37 \mathrm{E}-03$ & $8.59 \mathrm{E}-02$ & $6.04 \mathrm{E}-03$ & $1.36 \mathrm{E}-02$ & $2.77 \mathrm{E}-01$ \\
\hline Ba-137m & $1.88 \mathrm{E}-02$ & $3.00 \mathrm{E}-02$ & $4.13 \mathrm{E}-03$ & $8.12 \mathrm{E}-02$ & $5.71 \mathrm{E}-03$ & $1.29 \mathrm{E}-02$ & $2.62 \mathrm{E}-01$ \\
\hline \begin{tabular}{|l|} 
La-138 \\
\end{tabular} & $2.69 \mathrm{E}-18$ & $1.38 \mathrm{E}-16$ & $2.05 \mathrm{E}-17$ & $2.05 \mathrm{E}-17$ & $2.05 \mathrm{E}-17$ & $1.86 \mathrm{E}-17$ & $5.01 \mathrm{E}-18$ \\
\hline Ce-142 & $4.21 \mathrm{E}-13$ & $2.16 \mathrm{E}-11$ & $3.20 \mathrm{E}-12$ & $3.20 \mathrm{E}-12$ & $3.20 \mathrm{E}-12$ & $2.92 \mathrm{E}-12$ & $7.85 \mathrm{E}-13$ \\
\hline Ce-144 & $2.90 \mathrm{E}-07$ & $6.06 \mathrm{E}-07$ & $8.13 \mathrm{E}-08$ & $1.28 \mathrm{E}-06$ & 1.06E-07 & $2.15 \mathrm{E}-07$ & 4.07E-06 \\
\hline Nd-144 & $2.27 \mathrm{E}-17$ & $1.16 \mathrm{E}-15$ & $1.72 \mathrm{E}-16$ & $1.72 \mathrm{E}-16$ & $1.72 \mathrm{E}-16$ & $1.57 \mathrm{E}-16$ & $4.22 \mathrm{E}-17$ \\
\hline Pm-146 & $7.17 \mathrm{E}-10$ & $3.67 \mathrm{E}-08$ & $5.45 \mathrm{E}-09$ & $5.45 \mathrm{E}-09$ & $5.45 \mathrm{E}-09$ & 4.97E-09 & 1.34E-09 \\
\hline Pm-147 & $7.68 \mathrm{E}-05$ & $1.64 \mathrm{E}-04$ & $2.20 \mathrm{E}-05$ & $3.39 \mathrm{E}-04$ & $2.85 \mathrm{E}-05$ & 5.74E-05 & $1.08 \mathrm{E}-03$ \\
\hline Sm-146 & $3.89 \mathrm{E}-15$ & $1.99 \mathrm{E}-13$ & $2.96 \mathrm{E}-14$ & $2.96 \mathrm{E}-14$ & $2.96 \mathrm{E}-14$ & $2.69 \mathrm{E}-14$ & $7.24 \mathrm{E}-15$ \\
\hline Sm-147 & $1.04 \mathrm{E}-13$ & $5.31 \mathrm{E}-12$ & $7.89 \mathrm{E}-13$ & $7.89 \mathrm{E}-13$ & $7.89 \mathrm{E}-13$ & $7.19 \mathrm{E}-13$ & $1.93 \mathrm{E}-13$ \\
\hline Sm-148 & $5.33 \mathrm{E}-19$ & $2.73 \mathrm{E}-17$ & $4.06 \mathrm{E}-18$ & $4.06 \mathrm{E}-18$ & $4.06 \mathrm{E}-18$ & $3.70 \mathrm{E}-18$ & $9.94 \mathrm{E}-19$ \\
\hline Sm-149 & $4.73 \mathrm{E}-20$ & $2.42 \mathrm{E}-18$ & $3.60 \mathrm{E}-19$ & $3.60 \mathrm{E}-19$ & $3.60 \mathrm{E}-19$ & $3.28 \mathrm{E}-19$ & $8.82 \mathrm{E}-20$ \\
\hline Sm-151 & $1.53 \mathrm{E}-04$ & $3.23 \mathrm{E}-04$ & $4.34 \mathrm{E}-05$ & $6.76 \mathrm{E}-04$ & $5.64 \mathrm{E}-05$ & 1.14E-04 & $2.15 \mathrm{E}-03$ \\
\hline Eu-152 & $5.55 \mathrm{E}-07$ & $2.10 \mathrm{E}-06$ & $2.96 \mathrm{E}-07$ & $2.51 \mathrm{E}-06$ & $3.41 \mathrm{E}-07$ & 5.31E-07 & 7.57E-06 \\
\hline Eu-154 & $2.44 \mathrm{E}-05$ & $7.60 \mathrm{E}-05$ & $1.27 \mathrm{E}-05$ & $1.01 \mathrm{E}-04$ & $1.45 \mathrm{E}-05$ & $2.20 \mathrm{E}-05$ & 3.03E-04 \\
\hline Eu-155 & $3.50 \mathrm{E}-05$ & $8.35 \mathrm{E}-05$ & $1.11 \mathrm{E}-05$ & $1.56 \mathrm{E}-04$ & $1.41 \mathrm{E}-05$ & $2.72 \mathrm{E}-05$ & $4.92 \mathrm{E}-04$ \\
\hline Gd-152 & $2.00 \mathrm{E}-20$ & $1.03 \mathrm{E}-18$ & $1.52 \mathrm{E}-19$ & $1.52 \mathrm{E}-19$ & $1.52 \mathrm{E}-19$ & 1.39E-19 & $3.73 \mathrm{E}-20$ \\
\hline Ho-166m & $6.49 \mathrm{E}-13$ & $3.32 \mathrm{E}-11$ & $4.93 \mathrm{E}-12$ & $4.93 \mathrm{E}-12$ & $4.93 \mathrm{E}-12$ & $4.49 \mathrm{E}-12$ & $1.21 \mathrm{E}-12$ \\
\hline Co-60 & $2.81 \mathrm{E}-06$ & $1.25 \mathrm{E}-05$ & $2.44 \mathrm{E}-06$ & 1.03E-05 & $2.60 \mathrm{E}-06$ & $3.14 \mathrm{E}-06$ & $2.73 \mathrm{E}-05$ \\
\hline $\mathrm{Ni}-63$ & $1.48 \mathrm{E}-05$ & $3.25 \mathrm{E}-05$ & $4.80 \mathrm{E}-06$ & $6.38 \mathrm{E}-05$ & $6.01 \mathrm{E}-06$ & $1.13 \mathrm{E}-05$ & $2.01 \mathrm{E}-04$ \\
\hline
\end{tabular}


Table B-12. NGLW mass balance (continued).

\begin{tabular}{|c|c|c|c|c|c|c|c|c|}
\hline PFD Sheet \# & PFD-3 & PFD-3 & PFD-3 & PFD-3 & PFD-3 & PFD-3 & PFD-3 & PFD-3 \\
\hline Stream \# & 121 & 122 & 123 & 124 & 125 & 126 & 127 & 128 \\
\hline Stream Name & $\begin{array}{l}\text { Neutralized } \\
\text { Feed }\end{array}$ & $\begin{array}{l}\text { SBW Liquid } \\
\text { Filter Feed }\end{array}$ & $\begin{array}{c}\text { Filter } \\
\text { Recirculation }\end{array}$ & $\begin{array}{l}\text { IX Column } \\
\text { Feed }\end{array}$ & $\begin{array}{c}\text { Column } 1 \\
\text { Effluent }\end{array}$ & $\begin{array}{c}\text { Column } 2 \\
\text { Effluent }\end{array}$ & $\begin{array}{c}\text { Column } 3 \\
\text { Effluent }\end{array}$ & $\begin{array}{l}\text { Spent IX } \\
\text { Rinse }\end{array}$ \\
\hline Rate, gal/hr & 99.9 & 7800 & 7700 & 99.9 & 99.9 & 99.9 & 99.9 & 0.5 \\
\hline Rate, Ib/hr & 1121 & 87554 & 86433 & 1121 & 1121 & 1121 & 1121 & 5 \\
\hline Rate, peak, gpm & 1.66 & 130 & 128 & 1.66 & 1.66 & 1.66 & 1.66 & 2.99 \\
\hline Operation & Cont & Cont & Cont & Cont & Cont & Cont & Cont & Batch \\
\hline Temperature, ${ }^{\circ} \mathrm{F}$ & 70 & 70 & 70 & 70 & 70 & 70 & 70 & 70 \\
\hline Pressure, psia & 12.8 & 35.0 & 35.0 & 24.8 & 20.8 & 16.8 & 12.8 & 12.8 \\
\hline Specific Gravity & 1.35 & 1.35 & 1.35 & 1.35 & 1.35 & 1.35 & 1.35 & 1.07 \\
\hline Chemical Composition & Mol/liter & Mol/liter & Mol/liter & Mol/liter & Mol/liter & Mol/liter & Mol/liter & Mol/liter \\
\hline $\mathrm{H}+$ & $5.00 \mathrm{E}-01$ & $5.00 \mathrm{E}-01$ & $5.00 \mathrm{E}-01$ & $5.00 \mathrm{E}-01$ & $5.00 \mathrm{E}-01$ & $5.00 \mathrm{E}-01$ & $5.00 \mathrm{E}-01$ & $1.01 \mathrm{E}-01$ \\
\hline$\overline{\mathrm{Al}+3}$ & $1.18 \mathrm{E}-01$ & $1.32 \mathrm{E}-01$ & 1.32E-01 & $1.16 \mathrm{E}-01$ & 1.16E-01 & 1.16E-01 & 1.16E-01 & $2.35 \mathrm{E}-02$ \\
\hline$\overline{A m+4}$ & 4.33E-08 & $4.33 \mathrm{E}-08$ & 4.33E-08 & 4.33E-08 & 4.33E-08 & 4.33E-08 & 4.33E-08 & 8.74E-09 \\
\hline $\mathrm{Sb}+5$ & 1.04E-05 & 1.90E-05 & 1.91E-05 & $9.58 \mathrm{E}-06$ & $9.58 \mathrm{E}-06$ & $9.58 \mathrm{E}-06$ & $9.58 \mathrm{E}-06$ & 1.94E-06 \\
\hline As+5 & $3.64 \mathrm{E}-05$ & 1.09E-04 & $1.10 \mathrm{E}-04$ & $2.98 \mathrm{E}-05$ & $2.98 \mathrm{E}-05$ & $2.98 \mathrm{E}-05$ & $2.98 \mathrm{E}-05$ & $6.03 E-06$ \\
\hline $\mathrm{Ba}+2$ & $2.20 \mathrm{E}-05$ & $3.74 \mathrm{E}-05$ & $3.76 \mathrm{E}-05$ & 2.06E-05 & 2.06E-05 & 2.06E-05 & $2.06 \mathrm{E}-05$ & 4.17E-06 \\
\hline $\mathrm{Be}+2$ & $2.55 \mathrm{E}-06$ & $6.08 \mathrm{E}-06$ & $6.13 \mathrm{E}-06$ & $2.23 \mathrm{E}-06$ & $2.23 \mathrm{E}-06$ & $2.23 \mathrm{E}-06$ & $2.23 \mathrm{E}-06$ & $4.50 \mathrm{E}-07$ \\
\hline$B+3$ & $3.35 \mathrm{E}-03$ & $3.63 E-03$ & $3.63 E-03$ & $3.32 \mathrm{E}-03$ & 3.32E-03 & $3.32 \mathrm{E}-03$ & 3.32E-03 & $6.71 \mathrm{E}-04$ \\
\hline $\mathrm{Br}-$ & $2.26 \mathrm{E}-07$ & $2.26 \mathrm{E}-07$ & $2.26 \mathrm{E}-07$ & $2.26 \mathrm{E}-07$ & $2.26 \mathrm{E}-07$ & $2.26 \mathrm{E}-07$ & $2.26 \mathrm{E}-07$ & $4.57 \mathrm{E}-08$ \\
\hline $\mathrm{Cd}+2$ & $5.58 \mathrm{E}-04$ & $6.04 \mathrm{E}-04$ & $6.04 \mathrm{E}-04$ & $5.53 \mathrm{E}-04$ & $5.53 \mathrm{E}-04$ & $5.53 \mathrm{E}-04$ & $5.53 \mathrm{E}-04$ & $1.12 \mathrm{E}-04$ \\
\hline $\mathrm{Ca}+2$ & $1.16 \mathrm{E}-02$ & $1.24 \mathrm{E}-02$ & $1.24 \mathrm{E}-02$ & $1.16 \mathrm{E}-02$ & $1.16 \mathrm{E}-02$ & $1.16 \mathrm{E}-02$ & 1.16E-02 & $2.34 \mathrm{E}-03$ \\
\hline $\mathrm{Ce}+4$ & $2.97 \mathrm{E}-05$ & $3.74 \mathrm{E}-05$ & $3.76 \mathrm{E}-05$ & $2.90 \mathrm{E}-05$ & $2.90 \mathrm{E}-05$ & $2.90 \mathrm{E}-05$ & $2.90 \mathrm{E}-05$ & $5.85 \mathrm{E}-06$ \\
\hline $\mathrm{Cs}+$ & $2.07 E-05$ & $5.22 \mathrm{E}-05$ & $5.26 \mathrm{E}-05$ & $1.78 \mathrm{E}-05$ & $1.78 \mathrm{E}-06$ & $1.78 \mathrm{E}-07$ & $1.78 \mathrm{E}-08$ & $3.61 \mathrm{E}-09$ \\
\hline $\mathrm{Cl}$ & 8.82E-03 & $1.05 \mathrm{E}-02$ & $1.06 \mathrm{E}-02$ & $8.66 \mathrm{E}-03$ & $8.66 \mathrm{E}-03$ & $8.66 \mathrm{E}-03$ & 8.66E-03 & $1.75 \mathrm{E}-03$ \\
\hline $\mathrm{Cr}+3$ & $3.45 \mathrm{E}-03$ & $3.75 \mathrm{E}-03$ & $3.75 \mathrm{E}-03$ & $3.42 \mathrm{E}-03$ & $3.42 \mathrm{E}-03$ & $3.42 \mathrm{E}-03$ & 3.42E-03 & $6.91 \mathrm{E}-04$ \\
\hline $\mathrm{Co}+2$ & $3.42 \mathrm{E}-03$ & $3.42 \mathrm{E}-03$ & $3.42 \mathrm{E}-03$ & $3.42 \mathrm{E}-03$ & $3.42 \mathrm{E}-03$ & $3.42 \mathrm{E}-03$ & $3.42 \mathrm{E}-03$ & $6.91 \mathrm{E}-04$ \\
\hline $\mathrm{Cu}+2$ & $8.63 \mathrm{E}-05$ & $1.74 \mathrm{E}-04$ & $1.75 \mathrm{E}-04$ & 7.84E-05 & 7.84E-05 & 7.84E-05 & $7.84 \mathrm{E}-05$ & $1.58 \mathrm{E}-05$ \\
\hline $\mathrm{Eu+3}$ & $3.75 \mathrm{E}-07$ & $3.75 \mathrm{E}-07$ & $3.75 \mathrm{E}-07$ & $3.75 \mathrm{E}-07$ & $3.75 \mathrm{E}-07$ & $3.75 \mathrm{E}-07$ & $3.75 \mathrm{E}-07$ & $7.58 \mathrm{E}-08$ \\
\hline F- & $2.99 \mathrm{E}-02$ & $4.51 \mathrm{E}-02$ & 4.53E-02 & $2.85 \mathrm{E}-02$ & $2.85 \mathrm{E}-02$ & $2.85 \mathrm{E}-02$ & $2.85 \mathrm{E}-02$ & 5.77E-03 \\
\hline $\mathrm{Gd}+3$ & $1.25 \mathrm{E}-04$ & $1.40 \mathrm{E}-04$ & $1.40 \mathrm{E}-04$ & $1.23 \mathrm{E}-04$ & $1.23 \mathrm{E}-04$ & $1.23 \mathrm{E}-04$ & $1.23 \mathrm{E}-04$ & 2.49E-05 \\
\hline $\mathrm{Ge}+4$ & $6.52 \mathrm{E}-09$ & $6.52 \mathrm{E}-09$ & $6.52 \mathrm{E}-09$ & $6.52 \mathrm{E}-09$ & $6.52 \mathrm{E}-09$ & $6.52 \mathrm{E}-09$ & $6.52 \mathrm{E}-09$ & 1.32E-09 \\
\hline $\ln +3$ & $1.09 \mathrm{E}-06$ & $1.09 \mathrm{E}-06$ & $1.09 \mathrm{E}-06$ & 1.09E-06 & 1.09E-06 & 1.09E-06 & 1.09E-06 & $2.19 \mathrm{E}-07$ \\
\hline I- & $1.88 \mathrm{E}-06$ & $1.88 \mathrm{E}-06$ & $1.88 \mathrm{E}-06$ & $1.88 \mathrm{E}-06$ & $1.88 \mathrm{E}-06$ & $1.88 \mathrm{E}-06$ & $1.88 \mathrm{E}-06$ & $3.80 \mathrm{E}-07$ \\
\hline $\mathrm{Fe}+3$ & $5.57 \mathrm{E}-03$ & $1.15 \mathrm{E}-02$ & $1.16 \mathrm{E}-02$ & $5.03 \mathrm{E}-03$ & $5.03 \mathrm{E}-03$ & $5.03 E-03$ & $5.03 E-03$ & $1.02 \mathrm{E}-03$ \\
\hline $\mathrm{La+3}$ & $6.81 E-06$ & $6.81 \mathrm{E}-06$ & $6.81 \mathrm{E}-06$ & $6.81 \mathrm{E}-06$ & $6.81 \mathrm{E}-06$ & $6.81 \mathrm{E}-06$ & $6.81 \mathrm{E}-06$ & $1.38 \mathrm{E}-06$ \\
\hline $\mathrm{Pb}+2$ & $5.21 \mathrm{E}-04$ & $5.48 \mathrm{E}-04$ & $5.49 \mathrm{E}-04$ & $5.19 \mathrm{E}-04$ & $5.19 \mathrm{E}-04$ & $5.19 \mathrm{E}-04$ & $5.19 \mathrm{E}-04$ & $1.05 \mathrm{E}-04$ \\
\hline Li+ & $2.98 \mathrm{E}-04$ & $4.79 \mathrm{E}-04$ & 4.82E-04 & $2.81 \mathrm{E}-04$ & $2.81 \mathrm{E}-04$ & $2.81 \mathrm{E}-04$ & $2.81 \mathrm{E}-04$ & $5.69 \mathrm{E}-05$ \\
\hline $\mathrm{Mg}+2$ & $1.47 \mathrm{E}-02$ & $1.52 \mathrm{E}-02$ & $1.52 \mathrm{E}-02$ & $1.46 \mathrm{E}-02$ & $1.46 \mathrm{E}-02$ & $1.46 \mathrm{E}-02$ & $1.46 \mathrm{E}-02$ & $2.96 \mathrm{E}-03$ \\
\hline$M n+4$ & $1.81 \mathrm{E}-02$ & $1.82 \mathrm{E}-02$ & $1.82 \mathrm{E}-02$ & $1.81 \mathrm{E}-02$ & $1.81 \mathrm{E}-02$ & $1.81 \mathrm{E}-02$ & 1.81E-02 & $3.65 \mathrm{E}-03$ \\
\hline $\mathrm{Hg}+2$ & $1.66 \mathrm{E}-03$ & 1.67E-03 & $1.67 \mathrm{E}-03$ & $1.66 \mathrm{E}-03$ & $1.66 \mathrm{E}-03$ & $1.66 \mathrm{E}-03$ & $1.66 \mathrm{E}-03$ & $3.36 \mathrm{E}-04$ \\
\hline $\mathrm{Mo}+6$ & $2.31 \mathrm{E}-04$ & $7.38 \mathrm{E}-04$ & $7.46 \mathrm{E}-04$ & $1.85 \mathrm{E}-04$ & $1.85 \mathrm{E}-04$ & $1.85 \mathrm{E}-04$ & $1.85 \mathrm{E}-04$ & $3.74 \mathrm{E}-05$ \\
\hline $\mathrm{Nd}+3$ & $2.20 \mathrm{E}-05$ & $2.20 \mathrm{E}-05$ & $2.20 \mathrm{E}-05$ & $2.20 \mathrm{E}-05$ & $2.20 \mathrm{E}-05$ & $2.20 \mathrm{E}-05$ & $2.20 \mathrm{E}-05$ & 4.44E-06 \\
\hline $\mathrm{Np}+4$ & $3.89 \mathrm{E}-06$ & $3.89 \mathrm{E}-06$ & $3.89 \mathrm{E}-06$ & $3.89 \mathrm{E}-06$ & $3.89 \mathrm{E}-06$ & 3.89E-06 & $3.89 \mathrm{E}-06$ & $7.86 \mathrm{E}-07$ \\
\hline $\mathrm{Ni}+2$ & $6.93 \mathrm{E}-04$ & $7.96 \mathrm{E}-04$ & $7.97 \mathrm{E}-04$ & $6.84 \mathrm{E}-04$ & $6.84 \mathrm{E}-04$ & $6.84 \mathrm{E}-04$ & $6.84 \mathrm{E}-04$ & $1.38 \mathrm{E}-04$ \\
\hline $\mathrm{Nb}+5$ & $8.27 \mathrm{E}-05$ & $9.23 \mathrm{E}-04$ & $9.35 \mathrm{E}-04$ & $6.78 \mathrm{E}-06$ & $6.78 \mathrm{E}-06$ & $6.78 \mathrm{E}-06$ & $6.78 \mathrm{E}-06$ & 1.37E-06 \\
\hline NO3- & $6.07 \mathrm{E}+00$ & $6.07 \mathrm{E}+00$ & $6.07 \mathrm{E}+00$ & $6.07 \mathrm{E}+00$ & $6.07 \mathrm{E}+00$ & $6.07 \mathrm{E}+00$ & $6.07 \mathrm{E}+00$ & $1.23 \mathrm{E}+00$ \\
\hline $\mathrm{O}-2$ & $1.15 \mathrm{E}-02$ & 1.39E-01 & $1.41 \mathrm{E}-01$ & & & & & \\
\hline $\mathrm{Pd}+4$ & $2.14 \mathrm{E}-04$ & $1.61 \mathrm{E}-03$ & 1.63E-03 & $8.81 \mathrm{E}-05$ & $8.81 \mathrm{E}-05$ & $8.81 \mathrm{E}-05$ & $8.81 \mathrm{E}-05$ & $1.78 \mathrm{E}-05$ \\
\hline PO4-3 & $8.43 \mathrm{E}-03$ & $8.74 \mathrm{E}-02$ & 8.85E-02 & $1.30 \mathrm{E}-03$ & $1.30 \mathrm{E}-03$ & $1.30 \mathrm{E}-03$ & $1.30 \mathrm{E}-03$ & $2.62 \mathrm{E}-04$ \\
\hline $\mathrm{Pu}+4$ & $3.87 \mathrm{E}-06$ & $3.87 \mathrm{E}-06$ & $3.87 \mathrm{E}-06$ & $3.87 \mathrm{E}-06$ & $3.87 \mathrm{E}-06$ & $3.87 \mathrm{E}-06$ & $3.87 \mathrm{E}-06$ & $7.83 \mathrm{E}-07$ \\
\hline $\mathrm{K}+$ & 5.91E-01 & $6.00 \mathrm{E}-01$ & $6.00 \mathrm{E}-01$ & $5.90 \mathrm{E}-01$ & 5.90E-01 & $5.90 \mathrm{E}-01$ & $5.90 \mathrm{E}-01$ & $1.19 \mathrm{E}-01$ \\
\hline $\mathrm{Pr}+4$ & $6.19 \mathrm{E}-06$ & $6.19 \mathrm{E}-06$ & $6.19 \mathrm{E}-06$ & $6.19 \mathrm{E}-06$ & $6.19 \mathrm{E}-06$ & $6.19 \mathrm{E}-06$ & $6.19 \mathrm{E}-06$ & $1.25 \mathrm{E}-06$ \\
\hline $\mathrm{Rh}+4$ & 2.67E-06 & $2.67 \mathrm{E}-06$ & $2.67 \mathrm{E}-06$ & $2.67 \mathrm{E}-06$ & 2.67E-06 & $2.67 \mathrm{E}-06$ & 2.67E-06 & $5.40 \mathrm{E}-07$ \\
\hline
\end{tabular}


Table B-12. NGLW mass balance (continued).

\begin{tabular}{|c|c|c|c|c|c|c|c|c|}
\hline Stream \# & 121 & 122 & 123 & 124 & 125 & 126 & 127 & 128 \\
\hline Stream Name & $\begin{array}{l}\text { Neutralized } \\
\text { Feed }\end{array}$ & $\begin{array}{l}\text { SBW Liquid } \\
\text { Filter Feed }\end{array}$ & $\begin{array}{c}\text { Filter } \\
\text { Recirculation }\end{array}$ & $\begin{array}{l}\text { IX Column } \\
\text { Feed }\end{array}$ & $\begin{array}{c}\text { Column } 1 \\
\text { Effluent }\end{array}$ & $\begin{array}{c}\text { Column } 2 \\
\text { Effluent }\end{array}$ & $\begin{array}{c}\text { Column } 3 \\
\text { Effluent }\end{array}$ & $\begin{array}{l}\text { Spent IX } \\
\text { Rinse }\end{array}$ \\
\hline Chemical Composition & Mol/liter & Mol/liter & Mol/liter & Mol/liter & Mol/liter & Mol/liter & Mol/liter & Mol/liter \\
\hline $\mathrm{Rb}+$ & $4.11 \mathrm{E}-06$ & $4.11 \mathrm{E}-06$ & $4.11 \mathrm{E}-06$ & $4.11 \mathrm{E}-06$ & $4.11 \mathrm{E}-06$ & $4.11 \mathrm{E}-06$ & $4.11 \mathrm{E}-06$ & $8.31 \mathrm{E}-07$ \\
\hline $\mathrm{Ru}+3$ & $1.70 \mathrm{E}-04$ & $7.55 \mathrm{E}-04$ & $7.63 \mathrm{E}-04$ & 1.17E-04 & 1.17E-04 & 1.17E-04 & 1.17E-04 & $2.36 \mathrm{E}-05$ \\
\hline $\mathrm{Sm+3}$ & 4.07E-06 & 4.07E-06 & $4.07 \mathrm{E}-06$ & $4.07 \mathrm{E}-06$ & 4.07E-06 & $4.07 \mathrm{E}-06$ & $4.07 \mathrm{E}-06$ & $8.23 \mathrm{E}-07$ \\
\hline $\mathrm{Se}+4$ & $2.83 \mathrm{E}-05$ & $2.46 \mathrm{E}-04$ & $2.49 \mathrm{E}-04$ & 8.64E-06 & $8.64 \mathrm{E}-06$ & $8.64 \mathrm{E}-06$ & $8.64 \mathrm{E}-06$ & $1.75 \mathrm{E}-06$ \\
\hline $\mathrm{Si+4}$ & $6.71 \mathrm{E}-03$ & $7.83 \mathrm{E}-02$ & 7.94E-02 & $2.47 \mathrm{E}-04$ & $2.47 \mathrm{E}-04$ & $2.47 \mathrm{E}-04$ & $2.47 \mathrm{E}-04$ & $4.98 \mathrm{E}-05$ \\
\hline $\mathrm{Ag}+^{+}$ & $3.33 \mathrm{E}-05$ & $3.69 \mathrm{E}-04$ & 3.73E-04 & $3.00 \mathrm{E}-06$ & $3.00 \mathrm{E}-06$ & $3.00 \mathrm{E}-06$ & $3.00 \mathrm{E}-06$ & $6.06 \mathrm{E}-07$ \\
\hline $\mathrm{Na}+$ & $4.48 \mathrm{E}+00$ & $4.49 \mathrm{E}+00$ & $4.49 \mathrm{E}+00$ & $4.48 \mathrm{E}+00$ & $4.48 \mathrm{E}+00$ & $4.48 \mathrm{E}+00$ & $4.48 \mathrm{E}+00$ & $9.05 \mathrm{E}-01$ \\
\hline $\mathrm{Sr}+2$ & $9.07 \mathrm{E}-05$ & $9.54 \mathrm{E}-05$ & $9.55 \mathrm{E}-05$ & $9.02 \mathrm{E}-05$ & $9.02 \mathrm{E}-05$ & $9.02 \mathrm{E}-05$ & $9.02 \mathrm{E}-05$ & $1.82 \mathrm{E}-05$ \\
\hline SO4-2 & $1.20 \mathrm{E}-02$ & $1.20 \mathrm{E}-02$ & $1.20 \mathrm{E}-02$ & $1.20 \mathrm{E}-02$ & $1.20 \mathrm{E}-02$ & $1.20 \mathrm{E}-02$ & $1.20 \mathrm{E}-02$ & $2.42 \mathrm{E}-03$ \\
\hline$T c+7$ & $6.75 \mathrm{E}-06$ & $6.75 \mathrm{E}-06$ & $6.75 \mathrm{E}-06$ & $6.75 \mathrm{E}-06$ & $6.75 \mathrm{E}-06$ & $6.75 \mathrm{E}-06$ & $6.75 \mathrm{E}-06$ & $1.36 \mathrm{E}-06$ \\
\hline $\mathrm{Te}+4$ & $3.42 \mathrm{E}-06$ & $3.42 \mathrm{E}-06$ & $3.42 \mathrm{E}-06$ & $3.42 \mathrm{E}-06$ & $3.42 \mathrm{E}-06$ & $3.42 \mathrm{E}-06$ & $3.42 \mathrm{E}-06$ & $6.90 \mathrm{E}-07$ \\
\hline$T b+4$ & $1.57 \mathrm{E}-09$ & 1.57E-09 & 1.57E-09 & 1.57E-09 & $1.57 \mathrm{E}-09$ & 1.57E-09 & $1.57 \mathrm{E}-09$ & $3.17 \mathrm{E}-10$ \\
\hline $\mathrm{Th}+4$ & $1.70 \mathrm{E}-05$ & $1.70 \mathrm{E}-05$ & $1.70 \mathrm{E}-05$ & $1.70 \mathrm{E}-05$ & $1.70 \mathrm{E}-05$ & $1.70 \mathrm{E}-05$ & $1.70 \mathrm{E}-05$ & $3.43 \mathrm{E}-06$ \\
\hline$S n+4$ & 1.57E-04 & $1.66 \mathrm{E}-03$ & $1.68 \mathrm{E}-03$ & $2.20 \mathrm{E}-05$ & $2.20 \mathrm{E}-05$ & $2.20 \mathrm{E}-05$ & $2.20 \mathrm{E}-05$ & $4.44 \mathrm{E}-06$ \\
\hline$T i+4$ & $1.15 \mathrm{E}-04$ & $8.50 \mathrm{E}-04$ & $8.60 \mathrm{E}-04$ & $4.86 \mathrm{E}-05$ & $4.86 \mathrm{E}-05$ & $4.86 \mathrm{E}-05$ & $4.86 \mathrm{E}-05$ & $9.81 \mathrm{E}-06$ \\
\hline $\mathrm{U}+4$ & $8.67 \mathrm{E}-03$ & $8.69 \mathrm{E}-03$ & $8.69 \mathrm{E}-03$ & $8.67 \mathrm{E}-03$ & $8.67 \mathrm{E}-03$ & 8.67E-03 & $8.67 \mathrm{E}-03$ & $1.75 \mathrm{E}-03$ \\
\hline$V+5$ & $4.96 \mathrm{E}-05$ & $5.39 \mathrm{E}-05$ & $5.40 \mathrm{E}-05$ & $4.92 \mathrm{E}-05$ & $4.92 \mathrm{E}-05$ & $4.92 \mathrm{E}-05$ & $4.92 \mathrm{E}-05$ & $9.94 \mathrm{E}-06$ \\
\hline$Y+3$ & $5.08 \mathrm{E}-06$ & $5.08 \mathrm{E}-06$ & $5.08 \mathrm{E}-06$ & $5.08 \mathrm{E}-06$ & $5.08 \mathrm{E}-06$ & $5.08 \mathrm{E}-06$ & $5.08 \mathrm{E}-06$ & $1.03 \mathrm{E}-06$ \\
\hline$Z n+2$ & 3.63E-04 & $4.13 \mathrm{E}-04$ & $4.14 \mathrm{E}-04$ & $3.59 \mathrm{E}-04$ & $3.59 \mathrm{E}-04$ & $3.59 \mathrm{E}-04$ & $3.59 \mathrm{E}-04$ & $7.25 \mathrm{E}-05$ \\
\hline $\mathrm{Zr}+4$ & $3.85 \mathrm{E}-03$ & $3.43 \mathrm{E}-02$ & $3.47 \mathrm{E}-02$ & $1.10 \mathrm{E}-03$ & $1.10 \mathrm{E}-03$ & $1.10 \mathrm{E}-03$ & $1.10 \mathrm{E}-03$ & $2.23 \mathrm{E}-04$ \\
\hline $\mathrm{H} 2 \mathrm{O}$ & $4.58 \mathrm{E}+01$ & $4.49 E+01$ & $4.49 \mathrm{E}+01$ & $4.59 \mathrm{E}+01$ & $4.59 \mathrm{E}+01$ & $4.59 \mathrm{E}+01$ & $4.59 \mathrm{E}+01$ & $5.33 E+01$ \\
\hline & & & & & & & & \\
\hline Radiological Composition & $\mathrm{Ci} / \mathrm{liter}$ & $\mathrm{Ci} /$ liter & $\mathrm{Ci} / \mathrm{liter}$ & $\mathrm{Ci} / \mathrm{liter}$ & Ci/liter & $\mathrm{Ci} /$ liter & $\mathrm{Ci} /$ liter & $\mathrm{Ci} /$ liter \\
\hline $\mathrm{Ra}-226$ & $6.20 \mathrm{E}-12$ & $6.20 \mathrm{E}-12$ & $6.20 \mathrm{E}-12$ & $6.20 \mathrm{E}-12$ & $6.20 \mathrm{E}-12$ & $6.20 \mathrm{E}-12$ & $6.20 \mathrm{E}-12$ & $1.25 \mathrm{E}-12$ \\
\hline Ac-227 & $2.92 \mathrm{E}-11$ & $2.92 \mathrm{E}-11$ & $2.92 \mathrm{E}-11$ & $2.92 \mathrm{E}-11$ & $2.92 \mathrm{E}-11$ & $2.92 \mathrm{E}-11$ & $2.92 \mathrm{E}-11$ & $5.90 \mathrm{E}-12$ \\
\hline Th-228 & 2.42E-09 & $2.42 \mathrm{E}-09$ & $2.42 \mathrm{E}-09$ & $2.42 \mathrm{E}-09$ & $2.42 \mathrm{E}-09$ & $2.42 \mathrm{E}-09$ & $2.42 \mathrm{E}-09$ & $4.90 \mathrm{E}-10$ \\
\hline Th-230 & $6.39 \mathrm{E}-10$ & $8.25 \mathrm{E}-10$ & $8.28 \mathrm{E}-10$ & $6.23 \mathrm{E}-10$ & $6.23 \mathrm{E}-10$ & $6.23 \mathrm{E}-10$ & $6.23 \mathrm{E}-10$ & $1.26 \mathrm{E}-10$ \\
\hline Th-232 & $5.36 \mathrm{E}-16$ & $6.74 \mathrm{E}-11$ & $5.36 \mathrm{E}-16$ & $5.36 \mathrm{E}-16$ & $5.36 \mathrm{E}-16$ & $5.36 \mathrm{E}-16$ & $5.36 \mathrm{E}-16$ & $1.08 \mathrm{E}-16$ \\
\hline $\mathrm{Pa}-231$ & 6.76E-11 & $6.76 \mathrm{E}-11$ & $6.76 \mathrm{E}-11$ & $6.76 \mathrm{E}-11$ & $6.76 \mathrm{E}-11$ & $6.76 \mathrm{E}-11$ & $6.76 \mathrm{E}-11$ & $1.37 \mathrm{E}-11$ \\
\hline $\mathrm{Pa}-233$ & $2.22 \mathrm{E}-06$ & $2.22 \mathrm{E}-06$ & $2.22 \mathrm{E}-06$ & $2.22 \mathrm{E}-06$ & $2.22 \mathrm{E}-06$ & $2.22 \mathrm{E}-06$ & $2.22 \mathrm{E}-06$ & $4.49 \mathrm{E}-07$ \\
\hline U-232 & $1.54 \mathrm{E}-09$ & 1.92E-09 & 1.93E-09 & $1.50 \mathrm{E}-09$ & $1.50 \mathrm{E}-09$ & $1.50 \mathrm{E}-09$ & $1.50 \mathrm{E}-09$ & $3.04 \mathrm{E}-10$ \\
\hline U-233 & $6.11 \mathrm{E}-11$ & $6.77 \mathrm{E}-11$ & $6.78 \mathrm{E}-11$ & $6.05 \mathrm{E}-11$ & $6.05 \mathrm{E}-11$ & $6.05 \mathrm{E}-11$ & $6.05 \mathrm{E}-11$ & $1.22 \mathrm{E}-11$ \\
\hline U-234 & $1.07 \mathrm{E}-06$ & $1.18 \mathrm{E}-06$ & $1.18 \mathrm{E}-06$ & $1.06 \mathrm{E}-06$ & $1.06 \mathrm{E}-06$ & $1.06 \mathrm{E}-06$ & $1.06 \mathrm{E}-06$ & $2.14 \mathrm{E}-07$ \\
\hline U-235 & $5.34 \mathrm{E}-08$ & $5.94 \mathrm{E}-08$ & $5.95 \mathrm{E}-08$ & $5.28 \mathrm{E}-08$ & $5.28 \mathrm{E}-08$ & $5.28 \mathrm{E}-08$ & $5.28 \mathrm{E}-08$ & $1.07 \mathrm{E}-08$ \\
\hline U-236 & $4.90 \mathrm{E}-08$ & $5.69 \mathrm{E}-08$ & $5.70 \mathrm{E}-08$ & $4.83 \mathrm{E}-08$ & $4.83 \mathrm{E}-08$ & $4.83 \mathrm{E}-08$ & $4.83 \mathrm{E}-08$ & 9.76E-09 \\
\hline U-237 & 4.87E-09 & 4.87E-09 & 4.87E-09 & $4.87 \mathrm{E}-09$ & $4.87 \mathrm{E}-09$ & $4.87 \mathrm{E}-09$ & $4.87 \mathrm{E}-09$ & $9.85 \mathrm{E}-10$ \\
\hline U-238 & $2.15 \mathrm{E}-08$ & $2.20 \mathrm{E}-08$ & $2.20 \mathrm{E}-08$ & $2.15 \mathrm{E}-08$ & $2.15 \mathrm{E}-08$ & $2.15 \mathrm{E}-08$ & $2.15 \mathrm{E}-08$ & $4.34 \mathrm{E}-09$ \\
\hline $\mathrm{Np}-236$ & $2.43 \mathrm{E}-12$ & $2.43 \mathrm{E}-12$ & $2.43 \mathrm{E}-12$ & $2.43 \mathrm{E}-12$ & $2.43 \mathrm{E}-12$ & $2.43 \mathrm{E}-12$ & $2.43 \mathrm{E}-12$ & $4.90 \mathrm{E}-13$ \\
\hline Np-237 & $6.53 \mathrm{E}-07$ & $6.88 \mathrm{E}-07$ & $6.88 \mathrm{E}-07$ & $6.50 \mathrm{E}-07$ & $6.50 \mathrm{E}-07$ & $6.50 \mathrm{E}-07$ & $6.50 \mathrm{E}-07$ & $1.31 \mathrm{E}-07$ \\
\hline Pu-236 & $2.28 \mathrm{E}-09$ & $2.94 \mathrm{E}-09$ & $2.95 \mathrm{E}-09$ & $2.22 \mathrm{E}-09$ & $2.22 \mathrm{E}-09$ & $2.22 \mathrm{E}-09$ & $2.22 \mathrm{E}-09$ & $4.49 \mathrm{E}-10$ \\
\hline Pu-238 & $4.56 \mathrm{E}-04$ & $8.11 \mathrm{E}-04$ & $8.16 \mathrm{E}-04$ & $4.24 \mathrm{E}-04$ & $4.24 \mathrm{E}-04$ & $4.24 \mathrm{E}-04$ & $4.24 \mathrm{E}-04$ & $8.58 \mathrm{E}-05$ \\
\hline Pu-239 & $5.69 \mathrm{E}-05$ & $9.46 \mathrm{E}-05$ & $9.52 \mathrm{E}-05$ & $5.35 \mathrm{E}-05$ & $5.35 \mathrm{E}-05$ & $5.35 \mathrm{E}-05$ & $5.35 \mathrm{E}-05$ & $1.08 \mathrm{E}-05$ \\
\hline Pu-240 & $8.44 \mathrm{E}-06$ & $1.10 \mathrm{E}-05$ & $1.10 \mathrm{E}-05$ & $8.21 \mathrm{E}-06$ & $8.21 \mathrm{E}-06$ & $8.21 \mathrm{E}-06$ & $8.21 \mathrm{E}-06$ & $1.66 \mathrm{E}-06$ \\
\hline Pu-241 & $2.83 \mathrm{E}-04$ & 5.62E-04 & $5.66 \mathrm{E}-04$ & $2.57 \mathrm{E}-04$ & $2.57 \mathrm{E}-04$ & $2.57 \mathrm{E}-04$ & $2.57 \mathrm{E}-04$ & $5.20 \mathrm{E}-05$ \\
\hline $\mathrm{Pu}-242$ & $6.58 \mathrm{E}-09$ & $8.50 \mathrm{E}-09$ & $8.53 \mathrm{E}-09$ & $6.41 \mathrm{E}-09$ & $6.41 \mathrm{E}-09$ & $6.41 \mathrm{E}-09$ & $6.41 \mathrm{E}-09$ & $1.30 \mathrm{E}-09$ \\
\hline Pu-244 & $3.53 \mathrm{E}-16$ & $5.17 \mathrm{E}-16$ & $5.20 \mathrm{E}-16$ & $3.38 \mathrm{E}-16$ & $3.38 \mathrm{E}-16$ & $3.38 \mathrm{E}-16$ & $3.38 \mathrm{E}-16$ & $6.83 \mathrm{E}-17$ \\
\hline Am-241 & $5.48 \mathrm{E}-05$ & $6.40 \mathrm{E}-05$ & $6.42 \mathrm{E}-05$ & $5.40 \mathrm{E}-05$ & $5.40 \mathrm{E}-05$ & $5.40 \mathrm{E}-05$ & $5.40 \mathrm{E}-05$ & $1.09 \mathrm{E}-05$ \\
\hline Am-242m & $1.17 \mathrm{E}-08$ & 1.17E-08 & 1.17E-08 & $1.17 \mathrm{E}-08$ & $1.17 \mathrm{E}-08$ & $1.17 \mathrm{E}-08$ & 1.17E-08 & $2.36 \mathrm{E}-09$ \\
\hline Am-243 & $1.67 \mathrm{E}-08$ & $2.00 \mathrm{E}-08$ & $2.01 \mathrm{E}-08$ & $1.64 \mathrm{E}-08$ & $1.64 \mathrm{E}-08$ & $1.64 \mathrm{E}-08$ & $1.64 \mathrm{E}-08$ & $3.32 \mathrm{E}-09$ \\
\hline $\mathrm{Cm}-242$ & $2.19 \mathrm{E}-08$ & $2.19 \mathrm{E}-08$ & $2.19 \mathrm{E}-08$ & $2.19 \mathrm{E}-08$ & $2.19 \mathrm{E}-08$ & $2.19 \mathrm{E}-08$ & $2.19 \mathrm{E}-08$ & $4.42 \mathrm{E}-09$ \\
\hline
\end{tabular}


Table B-12. NGLW mass balance (continued).

\begin{tabular}{|c|c|c|c|c|c|c|c|c|}
\hline Stream \# & 121 & 122 & 123 & 124 & 125 & 126 & 127 & 128 \\
\hline Stream Name & $\begin{array}{l}\text { Neutralized } \\
\text { Feed }\end{array}$ & $\begin{array}{l}\text { SBW Liquid } \\
\text { Filter Feed }\end{array}$ & $\begin{array}{c}\text { Filter } \\
\text { Recirculation }\end{array}$ & $\begin{array}{l}\text { IX Column } \\
\text { Feed }\end{array}$ & $\begin{array}{c}\text { Column } 1 \\
\text { Effluent }\end{array}$ & $\begin{array}{c}\text { Column } 2 \\
\text { Effluent }\end{array}$ & $\begin{array}{c}\text { Column } 3 \\
\text { Effluent }\end{array}$ & $\begin{array}{l}\text { Spent IX } \\
\text { Rinse }\end{array}$ \\
\hline & Ci/liter & $\mathrm{Ci} /$ liter & $\mathrm{Ci} /$ liter & $\mathrm{Ci} / \mathrm{liter}$ & $\mathrm{Ci} /$ liter & $\mathrm{Ci} / \mathrm{liter}$ & $\mathrm{Ci} /$ liter & Ci/liter \\
\hline \begin{tabular}{|l|}
$\mathrm{Cm}-243$ \\
\end{tabular} & $2.21 \mathrm{E}-08$ & $2.89 \mathrm{E}-08$ & $2.90 \mathrm{E}-08$ & $2.15 \mathrm{E}-08$ & $2.15 \mathrm{E}-08$ & $2.15 \mathrm{E}-08$ & $2.15 \mathrm{E}-08$ & $4.35 \mathrm{E}-09$ \\
\hline $\mathrm{Cm}-244$ & 8.32E-07 & $1.26 \mathrm{E}-06$ & $1.26 \mathrm{E}-06$ & 7.94E-07 & 7.94E-07 & 7.94E-07 & 7.94E-07 & $1.60 \mathrm{E}-07$ \\
\hline $\mathrm{Cm}-245$ & $2.32 \mathrm{E}-10$ & $3.04 \mathrm{E}-10$ & $3.05 \mathrm{E}-10$ & $2.26 \mathrm{E}-10$ & $2.26 \mathrm{E}-10$ & $2.26 \mathrm{E}-10$ & $2.26 \mathrm{E}-10$ & $4.57 \mathrm{E}-11$ \\
\hline $\mathrm{Cm}-246$ & $1.53 \mathrm{E}-11$ & $2.00 \mathrm{E}-11$ & $2.00 \mathrm{E}-11$ & $1.49 \mathrm{E}-11$ & $1.49 \mathrm{E}-11$ & $1.49 \mathrm{E}-11$ & $1.49 \mathrm{E}-11$ & $3.01 \mathrm{E}-12$ \\
\hline $\mathrm{H}-3$ & $1.14 \mathrm{E}-05$ & $1.14 \mathrm{E}-05$ & $1.14 \mathrm{E}-05$ & $1.14 \mathrm{E}-05$ & $1.14 \mathrm{E}-05$ & $1.14 \mathrm{E}-05$ & $1.14 \mathrm{E}-05$ & $2.31 \mathrm{E}-06$ \\
\hline $\mathrm{Be}-10$ & $2.27 \mathrm{E}-12$ & $2.27 \mathrm{E}-12$ & $2.27 \mathrm{E}-12$ & $2.27 \mathrm{E}-12$ & $2.27 \mathrm{E}-12$ & $2.27 \mathrm{E}-12$ & $2.27 \mathrm{E}-12$ & $4.59 \mathrm{E}-13$ \\
\hline $\begin{array}{l}\mathrm{C}-14 \\
\end{array}$ & $9.36 \mathrm{E}-11$ & $1.24 \mathrm{E}-10$ & $1.24 \mathrm{E}-10$ & $9.09 \mathrm{E}-11$ & $9.09 \mathrm{E}-11$ & $9.09 \mathrm{E}-11$ & $9.09 \mathrm{E}-11$ & $1.84 \mathrm{E}-11$ \\
\hline Se-79 & $3.41 \mathrm{E}-07$ & $4.50 \mathrm{E}-07$ & $4.51 \mathrm{E}-07$ & $3.31 \mathrm{E}-07$ & $3.31 \mathrm{E}-07$ & $3.31 \mathrm{E}-07$ & $3.31 \mathrm{E}-07$ & $6.68 \mathrm{E}-08$ \\
\hline Rb-87 & $2.22 \mathrm{E}-11$ & $2.22 \mathrm{E}-11$ & $2.22 \mathrm{E}-11$ & $2.22 \mathrm{E}-11$ & $2.22 \mathrm{E}-11$ & $2.22 \mathrm{E}-11$ & $2.22 \mathrm{E}-11$ & $4.48 \mathrm{E}-12$ \\
\hline Sr-90 & $2.92 \mathrm{E}-02$ & $2.94 \mathrm{E}-02$ & $2.94 \mathrm{E}-02$ & $2.91 \mathrm{E}-02$ & 2.91E-02 & $2.91 \mathrm{E}-02$ & 2.91E-02 & $5.89 \mathrm{E}-03$ \\
\hline Y-90 & 2.92E-02 & $2.94 \mathrm{E}-02$ & $2.94 \mathrm{E}-02$ & $2.91 \mathrm{E}-02$ & $2.91 \mathrm{E}-02$ & $2.91 \mathrm{E}-02$ & 2.91E-02 & $5.89 \mathrm{E}-03$ \\
\hline Zr-93 & $1.68 \mathrm{E}-06$ & $1.68 \mathrm{E}-06$ & $1.68 \mathrm{E}-06$ & $1.68 \mathrm{E}-06$ & $1.68 \mathrm{E}-06$ & $1.68 \mathrm{E}-06$ & $1.68 \mathrm{E}-06$ & $3.39 \mathrm{E}-07$ \\
\hline Nb-93m & $1.29 \mathrm{E}-06$ & $1.29 \mathrm{E}-06$ & $1.29 \mathrm{E}-06$ & $1.29 \mathrm{E}-06$ & $1.29 \mathrm{E}-06$ & $1.29 \mathrm{E}-06$ & $1.29 \mathrm{E}-06$ & $2.61 \mathrm{E}-07$ \\
\hline $\mathrm{Nb}-94$ & 8.88E-07 & 1.17E-06 & 1.17E-06 & 8.63E-07 & 8.63E-07 & 8.63E-07 & 8.63E-07 & $1.74 \mathrm{E}-07$ \\
\hline Tc-98 & $1.95 \mathrm{E}-12$ & $1.95 \mathrm{E}-12$ & $1.95 \mathrm{E}-12$ & $1.95 \mathrm{E}-12$ & $1.95 \mathrm{E}-12$ & $1.95 \mathrm{E}-12$ & $1.95 \mathrm{E}-12$ & $3.95 \mathrm{E}-13$ \\
\hline Tc-99 & $1.15 \mathrm{E}-05$ & $1.40 \mathrm{E}-05$ & $1.40 \mathrm{E}-05$ & $1.13 \mathrm{E}-05$ & $1.13 \mathrm{E}-05$ & $1.13 \mathrm{E}-05$ & $1.13 \mathrm{E}-05$ & $2.29 \mathrm{E}-06$ \\
\hline Ru-106 & $7.25 \mathrm{E}-07$ & $9.61 \mathrm{E}-07$ & $9.65 \mathrm{E}-07$ & 7.04E-07 & $7.04 \mathrm{E}-07$ & $7.04 \mathrm{E}-07$ & $7.04 \mathrm{E}-07$ & $1.42 \mathrm{E}-07$ \\
\hline Rh-102 & $2.20 \mathrm{E}-08$ & $2.58 \mathrm{E}-07$ & $2.61 \mathrm{E}-07$ & $6.53 \mathrm{E}-10$ & $6.53 \mathrm{E}-10$ & $6.53 \mathrm{E}-10$ & $6.53 \mathrm{E}-10$ & $1.32 \mathrm{E}-10$ \\
\hline Pd-107 & $1.25 \mathrm{E}-08$ & $1.25 \mathrm{E}-08$ & $1.25 \mathrm{E}-08$ & $1.25 \mathrm{E}-08$ & $1.25 \mathrm{E}-08$ & $1.25 \mathrm{E}-08$ & $1.25 \mathrm{E}-08$ & $2.53 \mathrm{E}-09$ \\
\hline Cd-113m & $2.51 \mathrm{E}-06$ & $2.51 \mathrm{E}-06$ & $2.51 \mathrm{E}-06$ & $2.51 \mathrm{E}-06$ & $2.51 \mathrm{E}-06$ & $2.51 \mathrm{E}-06$ & $2.51 \mathrm{E}-06$ & $5.08 \mathrm{E}-07$ \\
\hline $\ln -115$ & $7.62 \mathrm{E}-17$ & $7.62 \mathrm{E}-17$ & $7.62 \mathrm{E}-17$ & $7.62 \mathrm{E}-17$ & $7.62 \mathrm{E}-17$ & $7.62 \mathrm{E}-17$ & $7.62 \mathrm{E}-17$ & $1.54 \mathrm{E}-17$ \\
\hline Sn-121m & $5.06 \mathrm{E}-08$ & $5.06 \mathrm{E}-08$ & $5.06 \mathrm{E}-08$ & $5.06 \mathrm{E}-08$ & $5.06 \mathrm{E}-08$ & $5.06 \mathrm{E}-08$ & $5.06 \mathrm{E}-08$ & $1.02 \mathrm{E}-08$ \\
\hline Sn-126 & $3.20 \mathrm{E}-07$ & $4.23 \mathrm{E}-07$ & $4.25 \mathrm{E}-07$ & $3.11 \mathrm{E}-07$ & $3.11 \mathrm{E}-07$ & $3.11 \mathrm{E}-07$ & $3.11 \mathrm{E}-07$ & $6.29 \mathrm{E}-08$ \\
\hline Sb-125 & $4.03 \mathrm{E}-05$ & $3.78 \mathrm{E}-04$ & 3.83E-04 & $9.78 \mathrm{E}-06$ & $9.78 \mathrm{E}-06$ & $9.78 \mathrm{E}-06$ & $9.78 \mathrm{E}-06$ & $1.98 \mathrm{E}-06$ \\
\hline Sb-126 & $4.35 \mathrm{E}-08$ & $4.35 \mathrm{E}-08$ & $4.35 \mathrm{E}-08$ & $4.35 \mathrm{E}-08$ & $4.35 \mathrm{E}-08$ & $4.35 \mathrm{E}-08$ & $4.35 \mathrm{E}-08$ & $8.80 \mathrm{E}-09$ \\
\hline Te-123 & $2.90 \mathrm{E}-19$ & $2.90 \mathrm{E}-19$ & $2.90 \mathrm{E}-19$ & $2.90 \mathrm{E}-19$ & $2.90 \mathrm{E}-19$ & $2.90 \mathrm{E}-19$ & $2.90 \mathrm{E}-19$ & $5.86 \mathrm{E}-20$ \\
\hline $\mathrm{Te}-125 \mathrm{~m}$ & $2.38 \mathrm{E}-06$ & $2.38 \mathrm{E}-06$ & $2.38 \mathrm{E}-06$ & $2.38 \mathrm{E}-06$ & $2.38 \mathrm{E}-06$ & $2.38 \mathrm{E}-06$ & $2.38 \mathrm{E}-06$ & $4.82 \mathrm{E}-07$ \\
\hline I-129 & $4.06 \mathrm{E}-08$ & $5.39 \mathrm{E}-08$ & $5.41 \mathrm{E}-08$ & $3.94 \mathrm{E}-08$ & $3.94 \mathrm{E}-08$ & $3.94 \mathrm{E}-08$ & $3.94 \mathrm{E}-08$ & $7.96 \mathrm{E}-09$ \\
\hline Cs-134 & $1.59 \mathrm{E}-05$ & $4.34 \mathrm{E}-05$ & $4.38 \mathrm{E}-05$ & $1.34 \mathrm{E}-05$ & $1.34 \mathrm{E}-06$ & $1.34 \mathrm{E}-07$ & $1.34 \mathrm{E}-08$ & $2.70 \mathrm{E}-09$ \\
\hline Cs-135 & $6.68 \mathrm{E}-07$ & $8.60 \mathrm{E}-07$ & $8.63 \mathrm{E}-07$ & $6.51 \mathrm{E}-07$ & $6.51 \mathrm{E}-08$ & $6.51 \mathrm{E}-09$ & $6.51 \mathrm{E}-10$ & $1.32 \mathrm{E}-10$ \\
\hline Cs-137 & $2.32 \mathrm{E}-02$ & $3.41 \mathrm{E}-02$ & $3.43 \mathrm{E}-02$ & $2.22 \mathrm{E}-02$ & $2.22 \mathrm{E}-03$ & $2.22 \mathrm{E}-04$ & $2.22 \mathrm{E}-05$ & $4.50 \mathrm{E}-06$ \\
\hline Ba-137m & $2.20 \mathrm{E}-02$ & $3.23 \mathrm{E}-02$ & $3.24 \mathrm{E}-02$ & $2.10 \mathrm{E}-02$ & $2.10 \mathrm{E}-02$ & $2.10 \mathrm{E}-02$ & $2.10 \mathrm{E}-02$ & $4.25 \mathrm{E}-03$ \\
\hline La-138 & $1.45 \mathrm{E}-16$ & $1.45 \mathrm{E}-16$ & $1.45 \mathrm{E}-16$ & $1.45 \mathrm{E}-16$ & $1.45 \mathrm{E}-16$ & $1.45 \mathrm{E}-16$ & $1.45 \mathrm{E}-16$ & $2.92 \mathrm{E}-17$ \\
\hline Ce-142 & $2.26 \mathrm{E}-11$ & $2.26 \mathrm{E}-11$ & $2.26 \mathrm{E}-11$ & $2.26 \mathrm{E}-11$ & $2.26 \mathrm{E}-11$ & $2.26 \mathrm{E}-11$ & $2.26 \mathrm{E}-11$ & $4.57 \mathrm{E}-12$ \\
\hline Ce-144 & $4.89 \mathrm{E}-07$ & $6.49 \mathrm{E}-07$ & $6.51 \mathrm{E}-07$ & $4.74 \mathrm{E}-07$ & 4.74E-07 & $4.74 \mathrm{E}-07$ & 4.74E-07 & $9.59 \mathrm{E}-08$ \\
\hline $\mathrm{Nd}-144$ & $1.22 \mathrm{E}-15$ & $1.22 \mathrm{E}-15$ & $1.22 \mathrm{E}-15$ & $1.22 \mathrm{E}-15$ & $1.22 \mathrm{E}-15$ & $1.22 \mathrm{E}-15$ & $1.22 \mathrm{E}-15$ & $2.46 \mathrm{E}-16$ \\
\hline $\mathrm{Pm}-146$ & $3.85 \mathrm{E}-08$ & $3.85 \mathrm{E}-08$ & $3.85 \mathrm{E}-08$ & $3.85 \mathrm{E}-08$ & $3.85 \mathrm{E}-08$ & $3.85 \mathrm{E}-08$ & $3.85 \mathrm{E}-08$ & $7.79 \mathrm{E}-09$ \\
\hline Pm-147 & 1.33E-04 & $1.75 \mathrm{E}-04$ & 1.76E-04 & $1.29 \mathrm{E}-04$ & $1.29 \mathrm{E}-04$ & $1.29 \mathrm{E}-04$ & $1.29 \mathrm{E}-04$ & $2.61 \mathrm{E}-05$ \\
\hline Sm-146 & $2.09 \mathrm{E}-13$ & $2.09 \mathrm{E}-13$ & $2.09 \mathrm{E}-13$ & $2.09 \mathrm{E}-13$ & $2.09 \mathrm{E}-13$ & $2.09 \mathrm{E}-13$ & $2.09 \mathrm{E}-13$ & $4.22 \mathrm{E}-14$ \\
\hline Sm-147 & $5.58 \mathrm{E}-12$ & $5.58 \mathrm{E}-12$ & $5.58 \mathrm{E}-12$ & $5.58 \mathrm{E}-12$ & $5.58 \mathrm{E}-12$ & $5.58 \mathrm{E}-12$ & $5.58 \mathrm{E}-12$ & $1.13 \mathrm{E}-12$ \\
\hline Sm-148 & $2.87 \mathrm{E}-17$ & $2.87 \mathrm{E}-17$ & $2.87 \mathrm{E}-17$ & $2.87 \mathrm{E}-17$ & $2.87 \mathrm{E}-17$ & $2.87 \mathrm{E}-17$ & $2.87 \mathrm{E}-17$ & $5.79 \mathrm{E}-18$ \\
\hline Sm-149 & $2.54 \mathrm{E}-18$ & $2.54 \mathrm{E}-18$ & $2.54 \mathrm{E}-18$ & $2.54 \mathrm{E}-18$ & $2.54 \mathrm{E}-18$ & $2.54 \mathrm{E}-18$ & $2.54 \mathrm{E}-18$ & $5.14 \mathrm{E}-19$ \\
\hline Sm-151 & $2.62 \mathrm{E}-04$ & $3.46 \mathrm{E}-04$ & $3.48 \mathrm{E}-04$ & $2.54 \mathrm{E}-04$ & $2.54 \mathrm{E}-04$ & $2.54 \mathrm{E}-04$ & $2.54 \mathrm{E}-04$ & $5.14 \mathrm{E}-05$ \\
\hline Eu-152 & $1.93 \mathrm{E}-06$ & $2.23 \mathrm{E}-06$ & $2.23 \mathrm{E}-06$ & $1.91 \mathrm{E}-06$ & $1.91 \mathrm{E}-06$ & $1.91 \mathrm{E}-06$ & $1.91 \mathrm{E}-06$ & $3.85 \mathrm{E}-07$ \\
\hline Eu-154 & $6.89 \mathrm{E}-05$ & $8.08 \mathrm{E}-05$ & $8.09 \mathrm{E}-05$ & $6.79 \mathrm{E}-05$ & $6.79 \mathrm{E}-05$ & $6.79 \mathrm{E}-05$ & $6.79 \mathrm{E}-05$ & 1.37E-05 \\
\hline Eu-155 & $6.98 \mathrm{E}-05$ & $8.92 \mathrm{E}-05$ & $8.94 \mathrm{E}-05$ & $6.81 \mathrm{E}-05$ & $6.81 \mathrm{E}-05$ & $6.81 \mathrm{E}-05$ & 6.81E-05 & $1.38 \mathrm{E}-05$ \\
\hline Gd-152 & $1.08 \mathrm{E}-18$ & $1.08 \mathrm{E}-18$ & $1.08 \mathrm{E}-18$ & $1.08 \mathrm{E}-18$ & $1.08 \mathrm{E}-18$ & $1.08 \mathrm{E}-18$ & $1.08 \mathrm{E}-18$ & $2.18 \mathrm{E}-19$ \\
\hline Ho-166m & $3.49 \mathrm{E}-11$ & $3.49 \mathrm{E}-11$ & $3.49 \mathrm{E}-11$ & $3.49 \mathrm{E}-11$ & $3.49 \mathrm{E}-11$ & $3.49 \mathrm{E}-11$ & $3.49 \mathrm{E}-11$ & $7.04 \mathrm{E}-12$ \\
\hline Co-60 & $1.22 \mathrm{E}-05$ & 1.32E-05 & 1.32E-05 & $1.21 \mathrm{E}-05$ & $1.21 \mathrm{E}-05$ & $1.21 \mathrm{E}-05$ & $1.21 \mathrm{E}-05$ & $2.44 \mathrm{E}-06$ \\
\hline Ni-63 & $2.69 \mathrm{E}-05$ & $3.48 \mathrm{E}-05$ & $3.49 \mathrm{E}-05$ & $2.61 \mathrm{E}-05$ & $2.61 \mathrm{E}-05$ & $2.61 \mathrm{E}-05$ & $2.61 \mathrm{E}-05$ & $5.28 \mathrm{E}-06$ \\
\hline
\end{tabular}


Table B-12. NGLW mass balance (continued).

\begin{tabular}{|c|c|c|c|c|c|c|c|c|}
\hline PFD Sheet \# & & PFD-4 & PFD Sheet \# & & PFD-4 & & & PFD-4 \\
\hline Stream \# & 129 & 130 & Stream \# & 129 & 130 & & 129 & 130 \\
\hline Stream Name & $\begin{array}{c}\text { Spent IX } \\
\text { Media }\end{array}$ & $\begin{array}{l}\text { Grouted } \\
\text { Waste }\end{array}$ & Stream Name & $\begin{array}{c}\text { Spent IX } \\
\text { Media }\end{array}$ & $\begin{array}{l}\text { Grouted } \\
\text { Waste }\end{array}$ & & $\begin{array}{c}\text { Spent IX } \\
\text { Media }\end{array}$ & $\begin{array}{l}\text { Grouted } \\
\text { Waste }\end{array}$ \\
\hline Rate, gal/hr & 0.12 & 120 & & Wt \% & Wt \% & & $\mathrm{Ci} / \mathrm{kg}$ & $\mathrm{Ci} / \mathrm{kg}$ \\
\hline Rate, Ib/hr & 1.29 & 1494 & $\mathrm{Rb}+$ & $1.78 \mathrm{E}-06$ & $1.96 \mathrm{E}-05$ & $\mathrm{Cm}-243$ & $4.35 \mathrm{E}-09$ & $1.20 \mathrm{E}-08$ \\
\hline Rate, total columns & 1 & N/A & $\mathrm{Ru}+3$ & $5.95 \mathrm{E}-05$ & $6.56 \mathrm{E}-04$ & $\mathrm{Cm}-244$ & 1.60E-07 & $4.42 \mathrm{E}-07$ \\
\hline Operation & Batch & Cont & $\mathrm{Sm}+3$ & $3.09 \mathrm{E}-06$ & $3.41 \mathrm{E}-05$ & $\mathrm{Cm}-245$ & $4.57 \mathrm{E}-11$ & $1.26 \mathrm{E}-10$ \\
\hline Temperature, ${ }^{\circ} \mathrm{F}$ & 70 & 70 & $\mathrm{Se}+4$ & $3.45 \mathrm{E}-06$ & 3.80E-05 & $\mathrm{Cm}-246$ & & \\
\hline Pressure, psia & 12.8 & 12.8 & $\mathrm{Si}+4$ & $3.50 \mathrm{E}-05$ & $3.86 \mathrm{E}-04$ & & $3.01 \mathrm{E}-12$ & $8.29 \mathrm{E}-12$ \\
\hline Specific Gravity & 1.34 & 1.50 & $\mathrm{Ag}^{+}$ & $1.63 \mathrm{E}-06$ & $1.80 \mathrm{E}-05$ & $\mathrm{H}-3$ & $2.31 \mathrm{E}-06$ & 6.37E-06 \\
\hline Chemical Composition & $\mathrm{Wt} \%$ & Wt \% & $\mathrm{Na}+$ & $5.20 \mathrm{E}-01$ & $5.73 \mathrm{E}+00$ & $\mathrm{Be}-10$ & $4.59 \mathrm{E}-13$ & $1.26 \mathrm{E}-12$ \\
\hline CST & $7.24 \mathrm{E}+01$ & $\mathrm{~N} / \mathrm{A}$ & $\mathrm{Sr}+2$ & $3.99 \mathrm{E}-05$ & $4.40 \mathrm{E}-04$ & C-14 & $1.84 \mathrm{E}-11$ & $5.06 \mathrm{E}-11$ \\
\hline $\mathrm{H}+$ & $2.55 \mathrm{E}-03$ & $\mathrm{~N} / \mathrm{A}$ & SO4-2 & $5.82 \mathrm{E}-03$ & $6.42 \mathrm{E}-02$ & Se-79 & $6.68 \mathrm{E}-08$ & 1.84E-07 \\
\hline $\mathrm{Al}+3$ & $1.59 \mathrm{E}-02$ & $1.75 \mathrm{E}-01$ & $\mathrm{Tc}+7$ & $3.31 \mathrm{E}-06$ & $3.65 \mathrm{E}-05$ & $\mathrm{Rb}-87$ & $4.48 \mathrm{E}-12$ & $1.24 \mathrm{E}-11$ \\
\hline $\mathrm{Am}+4$ & $5.26 \mathrm{E}-08$ & $5.80 \mathrm{E}-07$ & $\mathrm{Te}+4$ & $2.20 \mathrm{E}-06$ & $2.43 \mathrm{E}-05$ & Sr-90 & $5.89 \mathrm{E}-03$ & 1.62E-02 \\
\hline $\mathrm{Sb}+5$ & $5.89 \mathrm{E}-06$ & $6.50 \mathrm{E}-05$ & $\mathrm{~Tb}+4$ & $1.26 \mathrm{E}-09$ & $1.39 \mathrm{E}-08$ & $Y-90$ & $5.89 \mathrm{E}-03$ & $1.62 \mathrm{E}-02$ \\
\hline As+5 & $1.13 \mathrm{E}-05$ & $1.24 \mathrm{E}-04$ & $\mathrm{Th}+4$ & $1.99 \mathrm{E}-05$ & $2.19 \mathrm{E}-04$ & Zr-93 & $3.39 \mathrm{E}-07$ & 9.34E-07 \\
\hline $\mathrm{Ba}+2$ & $1.43 \mathrm{E}-05$ & $1.58 \mathrm{E}-04$ & $S n+4$ & $1.32 \mathrm{E}-05$ & $1.45 \mathrm{E}-04$ & Nb-93m & $2.61 \mathrm{E}-07$ & $7.20 \mathrm{E}-07$ \\
\hline $\mathrm{Be}+2$ & $1.01 \mathrm{E}-07$ & $1.12 \mathrm{E}-06$ & $\mathrm{Ti+4}$ & $1.17 \mathrm{E}-05$ & $1.29 \mathrm{E}-04$ & Nb-94 & $1.74 \mathrm{E}-07$ & $4.80 \mathrm{E}-07$ \\
\hline $\mathrm{B}+3$ & $1.81 \mathrm{E}-04$ & $2.00 \mathrm{E}-03$ & $\mathrm{U}+4$ & $1.04 \mathrm{E}-02$ & $1.15 \mathrm{E}-01$ & Tc-98 & $3.95 \mathrm{E}-13$ & 1.09E-12 \\
\hline $\mathrm{Br}-$ & $9.13 \mathrm{E}-08$ & $1.01 \mathrm{E}-06$ & $\mathrm{~V}+5$ & $1.27 \mathrm{E}-05$ & 1.39E-04 & Tc-99 & $2.29 \mathrm{E}-06$ & $6.31 \mathrm{E}-06$ \\
\hline $\mathrm{Cd}+2$ & $3.14 \mathrm{E}-04$ & $3.46 \mathrm{E}-03$ & $\mathrm{Y}+3$ & $2.28 \mathrm{E}-06$ & $2.51 \mathrm{E}-05$ & Ru-106 & $1.42 \mathrm{E}-07$ & 3.92E-07 \\
\hline $\mathrm{Ca}+2$ & $2.34 \mathrm{E}-03$ & $2.58 \mathrm{E}-02$ & $Z n+2$ & $1.18 \mathrm{E}-04$ & $1.31 \mathrm{E}-03$ & Rh-102 & $1.32 \mathrm{E}-10$ & $3.63 \mathrm{E}-10$ \\
\hline $\mathrm{Ce}+4$ & $2.05 E-05$ & $2.26 \mathrm{E}-04$ & $2 r+4$ & $5.08 \mathrm{E}-04$ & $5.60 \mathrm{E}-03$ & $\mathrm{Pd}-107$ & 2.53E-09 & 6.96E-09 \\
\hline Cs+ & $1.20 \mathrm{E}-08$ & 1.32E-07 & $\mathrm{H} 2 \mathrm{O}$ & $2.50 \mathrm{E}+01$ & & Cd-113m & $5.08 \mathrm{E}-07$ & $1.40 \mathrm{E}-06$ \\
\hline $\mathrm{Cl}-$ & $1.55 \mathrm{E}-03$ & $1.71 \mathrm{E}-02$ & Canisters total & 1 & & $\ln -115$ & $1.54 \mathrm{E}-17$ & $4.24 \mathrm{E}-17$ \\
\hline $\mathrm{Cr}+3$ & $8.98 \mathrm{E}-04$ & $9.90 \mathrm{E}-03$ & Drums/day & & 56 & Sn-121m & $1.02 \mathrm{E}-08$ & $2.82 \mathrm{E}-08$ \\
\hline $\mathrm{Co}+2$ & $1.02 \mathrm{E}-03$ & $1.12 \mathrm{E}-02$ & Radiological Comp. & $\mathrm{Ci} / \mathrm{kg}$ & $\mathrm{Ci} / \mathrm{kg}$ & \begin{tabular}{|l|} 
Sn-126 \\
\end{tabular} & $6.29 \mathrm{E}-08$ & $1.73 \mathrm{E}-07$ \\
\hline $\mathrm{Cu}+2$ & $2.52 \mathrm{E}-05$ & $2.77 \mathrm{E}-04$ & Ra-226 & $1.25 \mathrm{E}-12$ & $3.45 \mathrm{E}-12$ & Sb-125 & $1.98 \mathrm{E}-06$ & $5.44 \mathrm{E}-06$ \\
\hline $\mathrm{Eu}+3$ & $2.88 \mathrm{E}-07$ & 3.17E-06 & Ac-227 & $5.90 \mathrm{E}-12$ & $1.63 \mathrm{E}-11$ & Sb-126 & $8.80 \mathrm{E}-09$ & $2.42 \mathrm{E}-08$ \\
\hline F- & $2.74 \mathrm{E}-03$ & $3.02 \mathrm{E}-02$ & Th-228 & $4.90 \mathrm{E}-10$ & 1.35E-09 & Te-123 & $5.86 \mathrm{E}-20$ & $1.62 \mathrm{E}-19$ \\
\hline $\mathrm{Gd}+3$ & $9.78 \mathrm{E}-05$ & $1.08 \mathrm{E}-03$ & Th-230 & $1.26 \mathrm{E}-10$ & $3.47 \mathrm{E}-10$ & Te-125m & $4.82 \mathrm{E}-07$ & $1.33 \mathrm{E}-06$ \\
\hline $\mathrm{Ge}+4$ & $2.39 \mathrm{E}-09$ & $2.64 \mathrm{E}-08$ & Th-232 & $1.08 \mathrm{E}-16$ & $2.99 \mathrm{E}-16$ & I-129 & 7.96E-09 & $2.19 \mathrm{E}-08$ \\
\hline $\ln +3$ & $6.30 \mathrm{E}-07$ & $6.94 \mathrm{E}-06$ & $\mathrm{~Pa}-231$ & $1.37 \mathrm{E}-11$ & $3.77 \mathrm{E}-11$ & Cs-134 & $2.70 \mathrm{E}-09$ & $7.45 \mathrm{E}-09$ \\
\hline $1-$ & $1.20 \mathrm{E}-06$ & $1.33 \mathrm{E}-05$ & $\mathrm{~Pa}-233$ & $4.49 \mathrm{E}-07$ & $1.24 \mathrm{E}-06$ & Cs-135 & $1.32 \mathrm{E}-10$ & $3.62 \mathrm{E}-10$ \\
\hline $\mathrm{Fe}+3$ & $1.42 \mathrm{E}-03$ & $1.57 \mathrm{E}-02$ & U-232 & $3.04 \mathrm{E}-10$ & $8.38 \mathrm{E}-10$ & Cs-137 & $4.50 \mathrm{E}-06$ & $1.24 \mathrm{E}-05$ \\
\hline $\mathrm{La}+3$ & $4.78 \mathrm{E}-06$ & 5.27E-05 & U-233 & $1.22 \mathrm{E}-11$ & $3.37 \mathrm{E}-11$ & Ba-137m & $4.25 \mathrm{E}-06$ & 1.17E-05 \\
\hline $\mathrm{Pb}+2$ & $5.43 \mathrm{E}-04$ & $5.98 \mathrm{E}-03$ & U-234 & $2.14 \mathrm{E}-07$ & $5.90 \mathrm{E}-07$ & \begin{tabular}{|l} 
La-138 \\
\end{tabular} & $2.92 \mathrm{E}-17$ & $8.05 \mathrm{E}-17$ \\
\hline $\mathrm{Li}+$ & 9.87E-06 & $1.09 \mathrm{E}-04$ & U-235 & $1.07 \mathrm{E}-08$ & $2.94 \mathrm{E}-08$ & Ce-142 & $4.57 \mathrm{E}-12$ & $1.26 \mathrm{E}-11$ \\
\hline $\mathrm{Mg}+2$ & $1.80 \mathrm{E}-03$ & $1.98 \mathrm{E}-02$ & $\mathrm{U}-236$ & $9.76 \mathrm{E}-09$ & $2.69 \mathrm{E}-08$ & Ce-144 & $9.59 \mathrm{E}-08$ & $2.64 \mathrm{E}-07$ \\
\hline $\mathrm{Mn}+4$ & $5.01 \mathrm{E}-03$ & $5.52 \mathrm{E}-02$ & U-237 & $9.85 \mathrm{E}-10$ & $2.71 \mathrm{E}-09$ & Nd-144 & $2.46 \mathrm{E}-16$ & $6.78 \mathrm{E}-16$ \\
\hline $\mathrm{Hg}+2$ & $1.68 \mathrm{E}-03$ & $1.86 \mathrm{E}-02$ & U-238 & $4.34 \mathrm{E}-09$ & $1.20 \mathrm{E}-08$ & Pm-146 & 7.79E-09 & $2.14 \mathrm{E}-08$ \\
\hline $\mathrm{Mo}+6$ & $8.97 \mathrm{E}-05$ & $9.89 \mathrm{E}-04$ & Np-236 & $4.90 \mathrm{E}-13$ & $1.35 \mathrm{E}-12$ & Pm-147 & $2.61 \mathrm{E}-05$ & $7.18 \mathrm{E}-05$ \\
\hline $\mathrm{Nd}+3$ & $1.60 \mathrm{E}-05$ & $1.76 \mathrm{E}-04$ & Np-237 & $1.31 \mathrm{E}-07$ & $3.62 \mathrm{E}-07$ & Sm-146 & $4.22 \mathrm{E}-14$ & $1.16 \mathrm{E}-13$ \\
\hline $\mathrm{Np}+4$ & $4.65 \mathrm{E}-06$ & $5.13 \mathrm{E}-05$ & Pu-236 & $4.49 \mathrm{E}-10$ & $1.24 \mathrm{E}-09$ & Sm-147 & $1.13 \mathrm{E}-12$ & $3.10 \mathrm{E}-12$ \\
\hline $\mathrm{Ni}+2$ & $2.03 \mathrm{E}-04$ & $2.23 \mathrm{E}-03$ & $\mathrm{Pu}-238$ & $8.58 \mathrm{E}-05$ & $2.36 \mathrm{E}-04$ & Sm-148 & $5.79 \mathrm{E}-18$ & $1.60 \mathrm{E}-17$ \\
\hline $\mathrm{Nb}+5$ & $3.18 \mathrm{E}-06$ & $3.51 \mathrm{E}-05$ & Pu-239 & $1.08 \mathrm{E}-05$ & $2.98 \mathrm{E}-05$ & Sm-149 & $5.14 \mathrm{E}-19$ & $1.42 \mathrm{E}-18$ \\
\hline NO3- & $1.90 \mathrm{E}+00$ & $2.10 \mathrm{E}+01$ & Pu-240 & $1.66 \mathrm{E}-06$ & $4.57 \mathrm{E}-06$ & Sm-151 & $5.14 \mathrm{E}-05$ & $1.41 \mathrm{E}-04$ \\
\hline $\mathrm{Pd}+4$ & $4.73 \mathrm{E}-05$ & $5.22 \mathrm{E}-04$ & Pu-241 & $5.20 \mathrm{E}-05$ & $1.43 \mathrm{E}-04$ & Eu-152 & $3.85 \mathrm{E}-07$ & 1.06E-06 \\
\hline PO4-3 & $6.22 \mathrm{E}-04$ & $6.86 \mathrm{E}-03$ & Pu-242 & $1.30 \mathrm{E}-09$ & $3.57 \mathrm{E}-09$ & Eu-154 & 1.37E-05 & $3.78 \mathrm{E}-05$ \\
\hline $\mathrm{Pu}+4$ & $4.68 \mathrm{E}-06$ & $5.13 \mathrm{E}-05$ & $\mathrm{Pu}-244$ & $6.83 \mathrm{E}-17$ & $1.88 \mathrm{E}-16$ & Eu-155 & $1.38 \mathrm{E}-05$ & $3.79 \mathrm{E}-05$ \\
\hline $\mathrm{K}+$ & 1.17E-01 & $1.28 \mathrm{E}+00$ & Am-241 & $1.09 \mathrm{E}-05$ & $3.00 \mathrm{E}-05$ & Gd-152 & $2.18 \mathrm{E}-19$ & $5.99 \mathrm{E}-19$ \\
\hline $\mathrm{Pr}+4$ & $4.41 \mathrm{E}-06$ & $4.86 \mathrm{E}-05$ & $A m-242 m$ & $2.36 \mathrm{E}-09$ & $6.50 \mathrm{E}-09$ & Ho-166m & $7.04 \mathrm{E}-12$ & $1.94 \mathrm{E}-11$ \\
\hline \multirow[t]{2}{*}{$\mathrm{Rh}+4$} & $1.39 \mathrm{E}-06$ & $1.53 \mathrm{E}-05$ & $\mathrm{Am}-243$ & $3.32 \mathrm{E}-09$ & $9.16 \mathrm{E}-09$ & Co-60 & $2.44 \mathrm{E}-06$ & $6.72 \mathrm{E}-06$ \\
\hline & & & Cm-242 & $4.42 \mathrm{E}-09$ & $1.22 \mathrm{E}-08$ & $\mathrm{Ni}-63$ & $5.28 \mathrm{E}-06$ & 1.46E-05 \\
\hline
\end{tabular}


Table B-12. NGLW mass balance (continued).

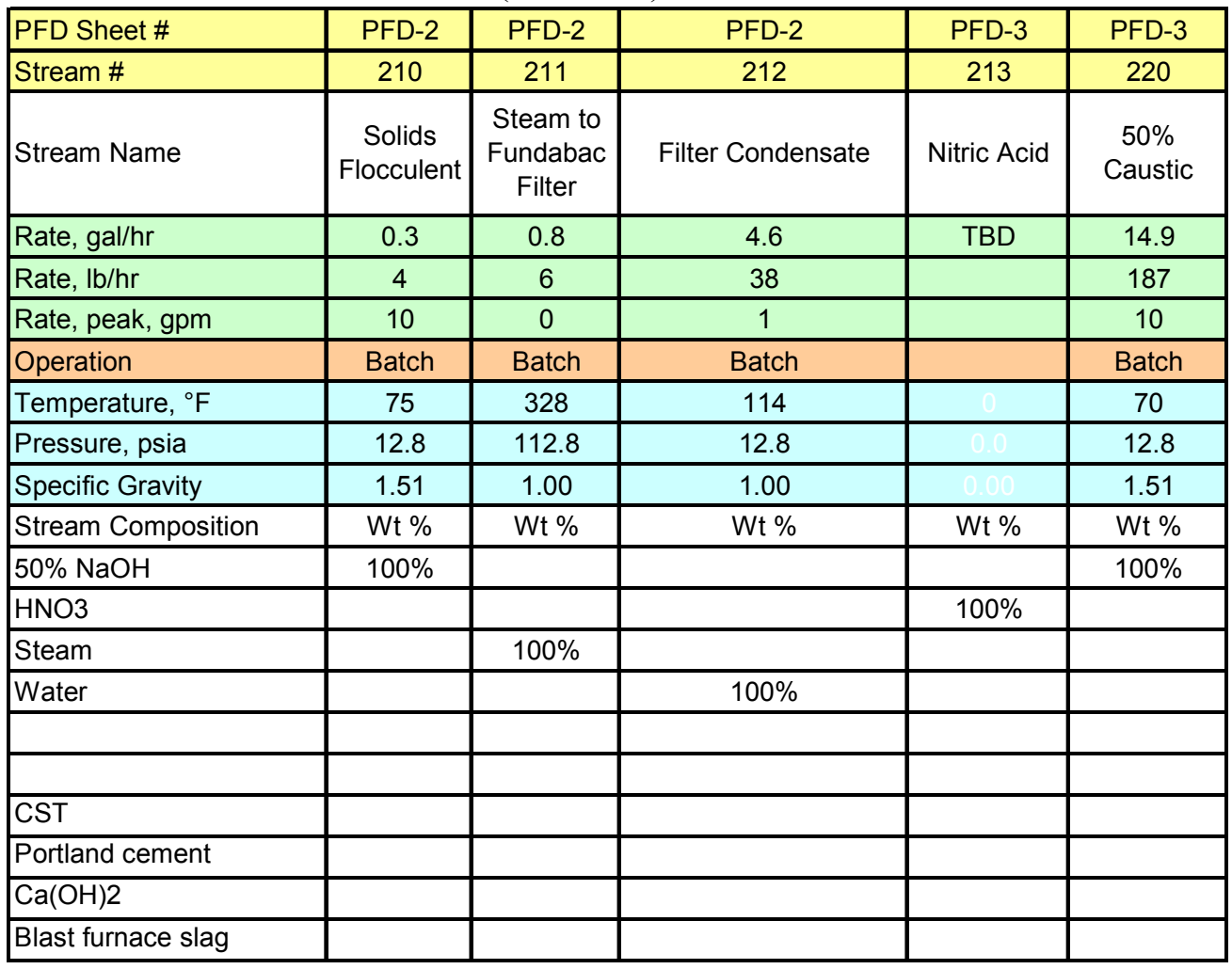

\begin{tabular}{|c|c|c|c|c|c|}
\hline PFD Sheet \# & PFD-3 & PFD-4 & PFD-4 & PFD-4 & PFD-4 \\
\hline Stream \# & 221 & 222 & 223 & 224 & 225 \\
\hline Stream Name & $\begin{array}{c}\text { Fresh IX } \\
\text { Media }\end{array}$ & $\begin{array}{l}\text { Portland } \\
\text { Cement }\end{array}$ & Calcium Hydroxide & $\begin{array}{c}\text { Blast } \\
\text { Furnace } \\
\text { Slag }\end{array}$ & Solid Blend \\
\hline Rate, gal/hr & 0.12 & 17.94 & 26.17 & 8.97 & 44.11 \\
\hline Rate, $\mathrm{Ib} / \mathrm{hr}$ & 0.93 & 179 & 105 & 90 & 374 \\
\hline Rate, peak, gpm & NA & TBD & TBD & TBD & 0.74 \\
\hline Operation & Batch & Cont & Cont & Cont & Cont \\
\hline Temperature, ${ }^{\circ} \mathrm{F}$ & 70 & 70 & 70 & 70 & 70 \\
\hline Pressure, psia & 12.8 & 12.8 & 12.8 & 12.8 & 12.8 \\
\hline \multirow[t]{2}{*}{ Specific Gravity } & 0.96 & 1.20 & 0.48 & 1.20 & 1.02 \\
\hline & Wt \% & Wt \% & Wt \% & Wt \% & Wt \% \\
\hline \multicolumn{6}{|l|}{ Chemical Composition } \\
\hline \multicolumn{6}{|l|}{$\mathrm{NaOH}$} \\
\hline \multicolumn{6}{|l|}{$\mathrm{H} 2 \mathrm{O}$} \\
\hline CST & $100 \%$ & & & & \\
\hline Portland cement & & $100 \%$ & & & \\
\hline $\mathrm{Ca}(\mathrm{OH}) 2$ & & & $100 \%$ & & \\
\hline Blast furnace slag & & & & $100 \%$ & \\
\hline No. Replacement col's & 1 & & & & \\
\hline & & & & & \\
\hline & & & & & \\
\hline
\end{tabular}


Dose rates and heat generation rates have not been calculated for waste compositions shown in the present mass balances. However, the grout dose rate is expected to be very similar to that calculated in 2003 (Durante, 2003). Based on a previous mass balance, Durante and Kimmitt estimated surface dose rates of 11-21 mR/hr for grout from WM-180 waste and 74-84 mR/hr for grout from WM-188 waste, based on decay to 2010. Durante concluded that one-third of the waste drums from the CsIX process would have a surface dose rate less than $50 \mathrm{mR} / \mathrm{hr}$ and two-thirds between 50 and $100 \mathrm{MR} / \mathrm{hr}$ if shipped in the 2010-2012 time period (Durante, 2003). The estimated heat generation rate for the grouted waste is 0.04 Watts (Bohn, 2002). Table B-13 shows properties for the $\mathrm{CH}$ grout product, including activities and masses of major radionuclides per drum, the fissile gram equivalent, ${ }^{239} \mathrm{Pu}$ equivalent activity, and TRU content.

Shielding calculations for the spent ion exchange media waste were performed in 2003 (Kimmitt, 2003b). The canister surface with 0.5 -inch of steel shielding would have a surface dose rate of $758 \mathrm{R} / \mathrm{hr}$, and a normal cask with 0.5 -inch of steel shielding would have a 1-meter dose of $5.8 \mathrm{mR} / \mathrm{hr}$ (Kimmitt, 2003b). Properties of the spent ion exchange media are shown in Table B-14.

The estimated average surface dose rate for the waste solids canisters in 2010 is $86 \mathrm{R} / \mathrm{hr}$ and heat generation rate 3.2 Watts/canister (Bohn, 2002). Table B-15 shows properties for the tank solids waste product. Properties are very similar for the different TFF tanks because a high percentage of the solids are from a single tank, WM-187, and the solids are assumed homogeneous in this tank.

Table B-16 shows the estimated chemicals and materials consumption. 
Table B-13. Grouted Waste Properties

\begin{tabular}{|c|c|c|c|c|}
\hline Major Radionuclides & $\begin{array}{c}\text { WM-180 } \\
\text { (Ci/drum) }\end{array}$ & $\begin{array}{l}\text { WM-188 } \\
\text { (Ci/drum) }\end{array}$ & $\begin{array}{l}\text { WM-189 } \\
\text { (Ci/drum) }\end{array}$ & $\begin{array}{c}\text { NGLW } \\
\text { (Ci/drum) }\end{array}$ \\
\hline Am-241 & $1.16 \mathrm{E}-02$ & $8.12 \mathrm{E}-03$ & $1.05 \mathrm{E}-02$ & $9.01 \mathrm{E}-03$ \\
\hline $\mathrm{Pu}-238$ & $9.34 \mathrm{E}-02$ & $7.72 \mathrm{E}-02$ & $6.69 \mathrm{E}-02$ & 7.09E-02 \\
\hline $\mathrm{Pu}-239$ & $1.35 \mathrm{E}-02$ & $9.16 \mathrm{E}-03$ & 7.57E-03 & 8.94E-03 \\
\hline $\mathrm{Pu}-240$ & $9.03 \mathrm{E}-04$ & $1.85 \mathrm{E}-03$ & $1.71 \mathrm{E}-03$ & $1.37 \mathrm{E}-03$ \\
\hline $\mathrm{Pu}-242$ & $7.05 \mathrm{E}-07$ & $1.44 \mathrm{E}-06$ & $1.34 \mathrm{E}-06$ & $1.07 \mathrm{E}-06$ \\
\hline $\mathrm{U}-233$ & $2.84 \mathrm{E}-07$ & $5.82 \mathrm{E}-07$ & $4.54 \mathrm{E}-07$ & 4.05E-07 \\
\hline U-234 & 7.29E-08 & $1.49 \mathrm{E}-07$ & $1.17 \mathrm{E}-07$ & $1.04 \mathrm{E}-07$ \\
\hline U-238 & $3.87 \mathrm{E}-06$ & $1.94 \mathrm{E}-06$ & $6.11 \mathrm{E}-06$ & $3.59 \mathrm{E}-06$ \\
\hline Sr-90 & $3.46 \mathrm{E}+00$ & $6.87 \mathrm{E}+00$ & $5.53 \mathrm{E}+00$ & $4.87 \mathrm{E}+00$ \\
\hline Cs-137 & $4.31 \mathrm{E}-03$ & $1.07 \mathrm{E}-03$ & $7.01 \mathrm{E}-03$ & $3.72 \mathrm{E}-03$ \\
\hline Y-90 & $3.46 \mathrm{E}+00$ & $6.87 \mathrm{E}+00$ & $5.53 \mathrm{E}+00$ & $4.87 \mathrm{E}+00$ \\
\hline Ba-137 & $4.08 \mathrm{E}-03$ & $1.01 \mathrm{E}-03$ & $6.63 \mathrm{E}-03$ & $3.51 \mathrm{E}-03$ \\
\hline Total of above 12 & $7.04 \mathrm{E}+00$ & $1.38 \mathrm{E}+01$ & $1.12 \mathrm{E}+01$ & $9.83 \mathrm{E}+00$ \\
\hline Total of all nuclides & $7.15 \mathrm{E}+00$ & $1.40 \mathrm{E}+01$ & $1.14 \mathrm{E}+01$ & $9.97 \mathrm{E}+00$ \\
\hline \multirow[t]{2}{*}{ Fraction } & $9.86 \mathrm{E}-01$ & $9.88 \mathrm{E}-01$ & $9.82 \mathrm{E}-01$ & $9.86 \mathrm{E}-01$ \\
\hline & g/drum & g/drum & g/drum & g/drum \\
\hline Am-241 & $3.38 \mathrm{E}-03$ & $2.37 \mathrm{E}-03$ & $3.05 \mathrm{E}-03$ & $2.63 \mathrm{E}-03$ \\
\hline $\mathrm{Pu}-238$ & $5.46 \mathrm{E}-03$ & $4.51 \mathrm{E}-03$ & $3.91 \mathrm{E}-03$ & $4.14 \mathrm{E}-03$ \\
\hline Pu-239 & $2.17 \mathrm{E}-01$ & $1.47 \mathrm{E}-01$ & $1.22 \mathrm{E}-01$ & $1.44 \mathrm{E}-01$ \\
\hline $\mathrm{Pu}-240$ & $3.96 \mathrm{E}-03$ & $8.10 \mathrm{E}-03$ & $7.52 \mathrm{E}-03$ & $6.02 \mathrm{E}-03$ \\
\hline $\mathrm{Pu}-242$ & $1.85 \mathrm{E}-04$ & $3.78 \mathrm{E}-04$ & $3.50 \mathrm{E}-04$ & $2.80 \mathrm{E}-04$ \\
\hline $\mathrm{U}-233$ & $2.93 \mathrm{E}-05$ & $6.01 \mathrm{E}-05$ & 4.69E-05 & 4.18E-05 \\
\hline U-234 & $1.17 \mathrm{E}-05$ & $2.39 \mathrm{E}-05$ & $1.87 \mathrm{E}-05$ & $1.66 \mathrm{E}-05$ \\
\hline U-238 & $1.15 \mathrm{E}+01$ & $5.76 \mathrm{E}+00$ & $1.82 \mathrm{E}+01$ & $1.07 \mathrm{E}+01$ \\
\hline Sr-90 & $2.54 \mathrm{E}-02$ & $5.04 \mathrm{E}-02$ & $4.06 \mathrm{E}-02$ & $3.57 \mathrm{E}-02$ \\
\hline Cs-137 & $4.96 \mathrm{E}-05$ & $1.23 \mathrm{E}-05$ & $8.06 \mathrm{E}-05$ & 4.27E-05 \\
\hline Y-90 & $6.36 \mathrm{E}-06$ & $1.26 \mathrm{E}-05$ & $1.02 \mathrm{E}-05$ & 8.94E-06 \\
\hline Ba-137 & $7.58 \mathrm{E}-12$ & $1.88 \mathrm{E}-12$ & $1.23 \mathrm{E}-11$ & $6.53 \mathrm{E}-12$ \\
\hline \multicolumn{5}{|c|}{ Fissile Gram Equivalent (FGE), g/drum } \\
\hline Total FGE & 3.3 & 6.4 & 4.1 & 4.2 \\
\hline \multicolumn{5}{|c|}{ Pu-239 Equivalent Curies (PE-Ci), Ci/drum } \\
\hline $\mathrm{PE} \mathrm{Ci}$ & $1.1 \mathrm{E}-01$ & $9.0 \mathrm{E}-02$ & $8.2 \mathrm{E}-02$ & $8.5 \mathrm{E}-02$ \\
\hline TRU nCi/g & 399 & 321 & 289 & 301 \\
\hline
\end{tabular}


Table B-14. Spent Ion Exchange Media Properties

\begin{tabular}{|c|c|c|c|c|}
\hline Major Radionuclides & $\begin{array}{c}\text { WM-180 } \\
\text { (Ci/column) }\end{array}$ & $\begin{array}{c}\text { WM-188 } \\
\text { (Ci/column) }\end{array}$ & $\begin{array}{c}\text { WM-189 } \\
\text { (Ci/column) }\end{array}$ & $\begin{array}{c}\text { NGLW } \\
\text { (Ci/column) }\end{array}$ \\
\hline Am-241 & $3.95 \mathrm{E}-03$ & $2.95 \mathrm{E}-03$ & $3.83 \mathrm{E}-03$ & $3.32 \mathrm{E}-03$ \\
\hline $\mathrm{Pu}-238$ & $3.19 \mathrm{E}-02$ & $2.80 \mathrm{E}-02$ & $2.45 \mathrm{E}-02$ & $2.61 \mathrm{E}-02$ \\
\hline Pu-239 & $4.59 \mathrm{E}-03$ & $3.32 \mathrm{E}-03$ & $2.77 \mathrm{E}-03$ & $3.29 \mathrm{E}-03$ \\
\hline $\mathrm{Pu}-240$ & $3.08 \mathrm{E}-04$ & $6.70 \mathrm{E}-04$ & $6.28 \mathrm{E}-04$ & $5.05 \mathrm{E}-04$ \\
\hline $\mathrm{Pu}-242$ & $2.40 \mathrm{E}-07$ & $5.23 \mathrm{E}-07$ & $4.90 \mathrm{E}-07$ & $3.95 \mathrm{E}-07$ \\
\hline U-233 & $9.68 \mathrm{E}-08$ & $2.11 \mathrm{E}-07$ & $1.66 \mathrm{E}-07$ & $1.49 \mathrm{E}-07$ \\
\hline U-234 & $2.49 \mathrm{E}-08$ & $5.42 \mathrm{E}-08$ & 4.27E-08 & $3.83 \mathrm{E}-08$ \\
\hline U-238 & $1.32 \mathrm{E}-06$ & 7.03E-07 & $2.24 \mathrm{E}-06$ & $1.32 \mathrm{E}-06$ \\
\hline Sr-90 & $1.18 \mathrm{E}+00$ & $2.49 \mathrm{E}+00$ & $2.03 \mathrm{E}+00$ & $1.79 \mathrm{E}+00$ \\
\hline Cs-137 & $6.10 \mathrm{E}+03$ & $5.24 \mathrm{E}+02$ & $4.43 \mathrm{E}+03$ & $6.24 \mathrm{E}+03$ \\
\hline Y-90 & $1.18 \mathrm{E}+00$ & $2.49 \mathrm{E}+00$ & $2.03 \mathrm{E}+00$ & $1.79 \mathrm{E}+00$ \\
\hline Ва-137 & $5.77 \mathrm{E}+03$ & $4.96 \mathrm{E}+02$ & $4.19 \mathrm{E}+03$ & $5.91 \mathrm{E}+03$ \\
\hline Total of above 12 & $1.19 \mathrm{E}+04$ & $1.02 \mathrm{E}+03$ & $8.62 \mathrm{E}+03$ & $1.22 \mathrm{E}+04$ \\
\hline Total of all nuclides & $1.19 \mathrm{E}+04$ & $1.03 \mathrm{E}+03$ & $8.62 \mathrm{E}+03$ & $1.22 \mathrm{E}+04$ \\
\hline \multirow[t]{2}{*}{ Fraction } & 1.000 & 1.000 & 1.000 & 1.000 \\
\hline & g/column & g/column & g/column & g/column \\
\hline Am-241 & $1.15 \mathrm{E}-03$ & 8.59E-04 & $1.12 \mathrm{E}-03$ & $9.68 \mathrm{E}-04$ \\
\hline $\mathrm{Pu}-238$ & $1.86 \mathrm{E}-03$ & $1.64 \mathrm{E}-03$ & $1.43 \mathrm{E}-03$ & $1.53 \mathrm{E}-03$ \\
\hline Pu-239 & 7.39E-02 & $5.35 \mathrm{E}-02$ & $4.46 \mathrm{E}-02$ & $5.30 \mathrm{E}-02$ \\
\hline $\mathrm{Pu}-240$ & $1.35 \mathrm{E}-03$ & $2.94 \mathrm{E}-03$ & $2.76 \mathrm{E}-03$ & $2.22 \mathrm{E}-03$ \\
\hline $\mathrm{Pu}-242$ & $6.30 \mathrm{E}-05$ & $1.37 \mathrm{E}-04$ & $1.28 \mathrm{E}-04$ & $1.03 \mathrm{E}-04$ \\
\hline U-233 & $1.00 \mathrm{E}-05$ & $2.18 \mathrm{E}-05$ & $1.72 \mathrm{E}-05$ & $1.54 \mathrm{E}-05$ \\
\hline U-234 & $3.98 \mathrm{E}-06$ & $8.68 \mathrm{E}-06$ & $6.83 \mathrm{E}-06$ & $6.13 \mathrm{E}-06$ \\
\hline $\mathrm{U}-238$ & $3.93 \mathrm{E}+00$ & $2.09 \mathrm{E}+00$ & $6.65 \mathrm{E}+00$ & $3.93 \mathrm{E}+00$ \\
\hline Sr-90 & $8.65 \mathrm{E}-03$ & $1.83 \mathrm{E}-02$ & $1.49 \mathrm{E}-02$ & $1.31 \mathrm{E}-02$ \\
\hline Cs-137 & $7.01 \mathrm{E}+01$ & $6.03 \mathrm{E}+00$ & $5.09 \mathrm{E}+01$ & $7.18 \mathrm{E}+01$ \\
\hline Y-90 & $2.17 \mathrm{E}-06$ & $4.58 \mathrm{E}-06$ & $3.72 \mathrm{E}-06$ & $3.29 \mathrm{E}-06$ \\
\hline Ва-137 & $1.07 \mathrm{E}-05$ & $9.22 \mathrm{E}-07$ & 7.79E-06 & $1.10 \mathrm{E}-05$ \\
\hline \multicolumn{5}{|c|}{ Fissile Gram Equivalent (FGE), g/column } \\
\hline Total FGE & 1.1 & 2.3 & 1.5 & 1.6 \\
\hline \multicolumn{5}{|c|}{ Pu-239 Equivalent Curies (PE-Ci), Ci/column } \\
\hline $\mathrm{PE} \mathrm{Ci}$ & $3.8 \mathrm{E}-02$ & $3.3 \mathrm{E}-02$ & $3.0 \mathrm{E}-02$ & $3.1 \mathrm{E}-02$ \\
\hline TRU content, $\mathbf{n C i} / \mathbf{g}$ & 135 & 115 & 104 & 109 \\
\hline
\end{tabular}


Table B-15. Dried Tank Solids Properties.

\begin{tabular}{|c|c|c|c|c|}
\hline Major Radionuclides & $\begin{array}{c}\text { WM-180 } \\
\text { (Ci/column) }\end{array}$ & $\begin{array}{c}\text { WM-188 } \\
\text { (Ci/column) }\end{array}$ & $\begin{array}{c}\text { WM-189 } \\
\text { (Ci/column) }\end{array}$ & $\begin{array}{c}\text { NGLW } \\
\text { (Ci/column) } \\
\end{array}$ \\
\hline Am-241 & $1.28 \mathrm{E}-01$ & $1.28 \mathrm{E}-01$ & $1.28 \mathrm{E}-01$ & $1.28 \mathrm{E}-01$ \\
\hline $\mathrm{Pu}-238$ & $4.89 \mathrm{E}+00$ & $4.91 \mathrm{E}+00$ & $4.89 \mathrm{E}+00$ & $4.89 \mathrm{E}+00$ \\
\hline $\mathrm{Pu}-239$ & $5.20 \mathrm{E}-01$ & $5.22 \mathrm{E}-01$ & $5.20 \mathrm{E}-01$ & $5.21 \mathrm{E}-01$ \\
\hline $\mathrm{Pu}-240$ & $3.51 \mathrm{E}-02$ & $3.52 \mathrm{E}-02$ & $3.51 \mathrm{E}-02$ & $3.52 \mathrm{E}-02$ \\
\hline $\mathrm{Pu}-242$ & $2.65 \mathrm{E}-05$ & $2.66 \mathrm{E}-05$ & $2.65 \mathrm{E}-05$ & $2.66 \mathrm{E}-05$ \\
\hline $\mathrm{U}-233$ & $9.11 \mathrm{E}-08$ & $9.17 \mathrm{E}-08$ & $9.11 \mathrm{E}-08$ & $9.20 \mathrm{E}-08$ \\
\hline U-234 & $1.55 \mathrm{E}-03$ & $1.56 \mathrm{E}-03$ & $1.55 \mathrm{E}-03$ & $1.57 \mathrm{E}-03$ \\
\hline U-238 & $6.37 \mathrm{E}-06$ & $6.39 \mathrm{E}-06$ & $6.38 \mathrm{E}-06$ & $6.67 \mathrm{E}-06$ \\
\hline Sr-90 & $3.20 \mathrm{E}+00$ & $3.30 \mathrm{E}+00$ & $3.20 \mathrm{E}+00$ & $3.63 \mathrm{E}+00$ \\
\hline Cs-137 & $1.50 \mathrm{E}+02$ & $1.51 \mathrm{E}+02$ & $1.50 \mathrm{E}+02$ & $1.51 \mathrm{E}+02$ \\
\hline Y-90 & $3.20 \mathrm{E}+00$ & $3.30 \mathrm{E}+00$ & $3.20 \mathrm{E}+00$ & $3.63 \mathrm{E}+00$ \\
\hline Ba-137 & $1.42 \mathrm{E}+02$ & $1.43 \mathrm{E}+02$ & $1.42 \mathrm{E}+02$ & $1.43 \mathrm{E}+02$ \\
\hline Total of above 12 & $3.05 \mathrm{E}+02$ & $3.06 \mathrm{E}+02$ & $3.05 \mathrm{E}+02$ & $3.06 \mathrm{E}+02$ \\
\hline Total of all nuclides & $3.16 \mathrm{E}+02$ & $3.17 \mathrm{E}+02$ & $3.16 \mathrm{E}+02$ & $3.17 \mathrm{E}+02$ \\
\hline \multirow[t]{2}{*}{ Fraction } & 0.964 & 0.964 & 0.964 & 0.964 \\
\hline & g/column & g/column & g/column & g/column \\
\hline Am-241 & $3.72 \mathrm{E}-02$ & $3.74 \mathrm{E}-02$ & $3.72 \mathrm{E}-02$ & $3.74 \mathrm{E}-02$ \\
\hline $\mathrm{Pu}-238$ & $2.86 \mathrm{E}-01$ & $2.87 \mathrm{E}-01$ & $2.86 \mathrm{E}-01$ & $2.86 \mathrm{E}-01$ \\
\hline $\mathrm{Pu}-239$ & $8.37 \mathrm{E}+00$ & $8.40 \mathrm{E}+00$ & $8.37 \mathrm{E}+00$ & $8.38 \mathrm{E}+00$ \\
\hline $\mathrm{Pu}-240$ & $1.54 \mathrm{E}-01$ & $1.55 \mathrm{E}-01$ & $1.54 \mathrm{E}-01$ & $1.54 \mathrm{E}-01$ \\
\hline $\mathrm{Pu}-242$ & $6.93 \mathrm{E}-03$ & $6.97 \mathrm{E}-03$ & $6.93 \mathrm{E}-03$ & $6.96 \mathrm{E}-03$ \\
\hline $\mathrm{U}-233$ & $9.41 \mathrm{E}-06$ & $9.47 \mathrm{E}-06$ & $9.42 \mathrm{E}-06$ & $9.51 \mathrm{E}-06$ \\
\hline U-234 & $2.48 \mathrm{E}-01$ & $2.50 \mathrm{E}-01$ & $2.48 \mathrm{E}-01$ & $2.51 \mathrm{E}-01$ \\
\hline $\mathrm{U}-238$ & $1.89 \mathrm{E}+01$ & $1.90 \mathrm{E}+01$ & $1.90 \mathrm{E}+01$ & $1.99 \mathrm{E}+01$ \\
\hline $\operatorname{Sr}-90$ & $2.34 \mathrm{E}-02$ & $2.42 \mathrm{E}-02$ & $2.35 \mathrm{E}-02$ & $2.66 \mathrm{E}-02$ \\
\hline Cs-137 & $1.73 \mathrm{E}+00$ & $1.74 \mathrm{E}+00$ & $1.73 \mathrm{E}+00$ & $1.73 \mathrm{E}+00$ \\
\hline Y-90 & 5.87E-06 & $6.07 \mathrm{E}-06$ & 5.89E-06 & $6.67 \mathrm{E}-06$ \\
\hline Ba-137 & $2.65 \mathrm{E}-07$ & $2.66 \mathrm{E}-07$ & $2.65 \mathrm{E}-07$ & $2.65 \mathrm{E}-07$ \\
\hline \multicolumn{5}{|c|}{ Fissile Gram Equivalent (FGE), g/column } \\
\hline Total FGE & 47.3 & 47.6 & 47.3 & 47.7 \\
\hline \multicolumn{5}{|c|}{ Pu-239 Equivalent Curies (PE-Ci), Ci/column } \\
\hline $\mathrm{PE} \mathrm{Ci}$ & $5.2 \mathrm{E}+00$ & $5.2 \mathrm{E}+00$ & $5.2 \mathrm{E}+00$ & $5.2 \mathrm{E}+00$ \\
\hline TRU content, $\mathrm{nCi} / \mathrm{g}$ & $1.02 \mathrm{E}+04$ & $1.03 \mathrm{E}+04$ & $1.02 \mathrm{E}+04$ & $1.03 \mathrm{E}+04$ \\
\hline
\end{tabular}


Table B-16. Chemicals and materials consumption.

\begin{tabular}{lccccc}
\hline & WM-180 & WM-188 & WM-189 & NGLW & Total \\
\hline 50\% NaOH, gallons & 12,900 & 38,600 & 38,500 & 22,400 & 112,400 \\
CST, gallons & 420 & 720 & 720 & 180 & 2,040 \\
Portland cement, lb & 530,000 & 624,000 & 618,000 & 93,000 & $1,865,000$ \\
BFS, lb & 265,000 & 312,000 & 309,000 & 47,000 & 933,000 \\
Calcium Hydroxide, lb & 309,000 & 364,000 & 360,000 & 54,000 & $1,087,000$ \\
\hline
\end{tabular}

\section{B-7. UTILITIES USAGE}

The CsIX TRU grout process requires steam, cooling water and electricity. Process equipment that uses electricity includes various pumps, the vent gas heater and the vent gas blower. All of these services are small. In the CsIX TRU Grout treatment facility, the only cooling water services are two intermittent users, the SBW Feed Tank cooling jacket ${ }^{\mathrm{b}}$ and the Solids Drying Steam Condenser. The SBW Feed Tank cooling jacket (or coil) will use cooling water at a rate of $50 \mathrm{gpm}$ and have a duty of 590,000 Btu/hr. The Solids Drying Steam Condenser will use about cooling water at a rate of $27 \mathrm{gpm}$ and have a duty of $370,000 \mathrm{Bu} / \mathrm{hr}$.

Steam is also used only intermittently in the new treatment facility. An estimated rate of $400 \mathrm{lb} / \mathrm{hr}$ of low-pressure steam will be required to dry the solids filter cake on the Fundabac filters prior to discharge into waste canisters. Steam would also be used to jet waste water to the ETS, at an estimated peak rate of $200 \mathrm{lb} / \mathrm{hr}$.

Other steam uses are existing equipment at INTEC, including the jets would that transfer SBW to the CsIX TRU grout facility and reboilers for the EVS, PEWE and LET\&D fractionator. All of these services are intermittent. Estimated steam rates for these services when operated are shown in Table B17.

Table B-17. Estimated Steam Rates.

\begin{tabular}{lc}
\hline \multicolumn{1}{c}{ Steam Services } & $\mathbf{L b} / \mathbf{h r}$ \\
\hline Steam to jet liquid SBW to treatment facility: & 35 \\
Steam to jet tank heel slurry to treatment facility & 27 \\
ETS reboiler steam & 2900 \\
PEWE reboiler steam & 1400 \\
LET\&D reboiler steam & 6000. \\
\hline
\end{tabular}

Condenser duties for the ETS, PEWE and LET\&D reboiler are estimated to be 3.7 million, 1.7 million and 2.6 million Btu/hr respectively, corresponding to cooling water rates of 270, 120 and 190 gpm.

b Coiling coils could be used rather than a cooling jacket to provide more heat transfer area 


\title{
B-8. EQUIPMENT LIST AND SCALE DISCUSSION
}

\author{
The equipment list is shown in Table B-18, based on a 2.5 -yr operating schedule.
}

Table B-18. Equipment list.

\begin{tabular}{|c|c|c|c|c|c|}
\hline \multicolumn{6}{|c|}{ Tanks } \\
\hline Tank & $\begin{array}{l}\text { Size } \\
\text { (gal) }\end{array}$ & $\begin{array}{l}\text { Diameter } \\
\text { (ft) }\end{array}$ & $\begin{array}{l}\text { Height } \\
\text { (ft) } \\
\text { Note } 1\end{array}$ & Material & Function/Features \\
\hline $\begin{array}{l}\text { VES-101 } 1 \& 2-\text { SBW } \\
\text { Receiving Tank }\end{array}$ & 4000 & 8.4 & 14.5 & $304 \mathrm{~L}$ & $\begin{array}{l}\text { Receiver Tank (2). Receive liquid waste from TFF tank and adjust } \\
\text { acid with NaOH. Dish top and bottom, jet mixer on pump } \\
\text { discharge, instrument wells, vent outlet, bottom pump suction, } \\
\text { ports. Water-cooling coils or jacket required for removing heat of } \\
\text { neutralization. Located on ground level. }\end{array}$ \\
\hline $\begin{array}{l}\text { VES-102 - Crossflow } \\
\text { Filter Feed Tank }\end{array}$ & 500 & 4.2 & 8.3 & $304 \mathrm{~L}$ & $\begin{array}{l}\text { Cross-Flow Filter Feed/Surge Tank. Dish bottom, instrument wells, } \\
\text { bottom feed outlet, vent, cooling water jacket, jet well. Inlets for } \\
\text { feed and recirculation. Located on ground level. }\end{array}$ \\
\hline $\begin{array}{l}\text { VES-103 - Filtrate } \\
\text { Receiver Tank }\end{array}$ & 4000 & 8.4 & 14.5 & $304 \mathrm{~L}$ & $\begin{array}{l}\text { Surge for crossflow permeate. Dish top and bottom, instrument } \\
\text { wells, vent outlet, bottom drain/pump suction. Located on ground } \\
\text { level. }\end{array}$ \\
\hline $\begin{array}{l}\text { VES-104 - IX Effluent } \\
\text { Tank }\end{array}$ & 4000 & 8.4 & 14.5 & $304 \mathrm{~L}$ & $\begin{array}{l}\text { Surge for ion exchange effluent. Dish top and bottom, instrument } \\
\text { wells, vent outlet, bottom drain/pump suction. Located on ground } \\
\text { level. }\end{array}$ \\
\hline $\begin{array}{l}\text { VES-105- NaOH Feed } \\
\text { Tank }\end{array}$ & 1780 & 6.4 & 11.6 & $304 \mathrm{~L}$ & $\begin{array}{l}\text { Caustic feed for VES-101. Instrument wells, inlet port, bottom } \\
\text { outlet, vent. Located on } 2 \text { nd floor }\end{array}$ \\
\hline $\begin{array}{l}\text { VES-106 1\&2 - Solids } \\
\text { Tank }\end{array}$ & 4000 & 8.5 & 15.1 & $304 \mathrm{~L}$ & $\begin{array}{l}\text { Batch Settling Tank (2). } 30^{\circ} \text { cone bottom, sludge level detector, } \\
\text { instrument wells, cone bottom pump outlet, side penetrations for } \\
\text { decantate withdraw. Located on ground level. }\end{array}$ \\
\hline $\begin{array}{l}\text { VES-107 - Decant Surge } \\
\text { Tank }\end{array}$ & 250 & 3.3 & 7.0 & $304 \mathrm{~L}$ & $\begin{array}{l}\text { VES-106 decant surge tank. Instrument wells, inlet port, jet port top } \\
\text { outlet, vent. Located on ground level }\end{array}$ \\
\hline $\begin{array}{l}\text { VES-109 - New Column } \\
\text { Flush Tank }\end{array}$ & 200 & 3.1 & 6.6 & $\mathrm{CS}$ & $\begin{array}{l}\text { Collect flush water from new IX column flushing. Dome } \\
\text { bottom/top, instrument wells, bottom pump outlet, vent. Located on } \\
2 \text { nd floor. }\end{array}$ \\
\hline $\begin{array}{l}\text { VES-110 - Flush Water } \\
\text { Receiver/Feed Tank }\end{array}$ & 250 & 3.3 & 7.0 & $304 \mathrm{~L}$ & $\begin{array}{l}\text { Grout Mixer Flush Receiver. Receives flush liquid from flushing } \\
\text { mixer M-101 with liquid from VES-104 and feeds back into mixer. } \\
\text { Located on ground level in grout area. }\end{array}$ \\
\hline $\begin{array}{l}\text { VES-111 - Waste Water } \\
\text { Tank }\end{array}$ & 4000 & 8.4 & 14.5 & $304 \mathrm{~L}$ & $\begin{array}{l}\text { Receive Fundabac filtrate and clarifier decantate. Dish bottom, } \\
\text { instrument wells, vent, bottom drain/pump suction. Located on } \\
\text { ground level. }\end{array}$ \\
\hline $\begin{array}{l}\text { VES-112 - Filter Aid } \\
\text { Tank }\end{array}$ & 200 & 3 & 7 & $\mathrm{CS}$ & $\begin{array}{l}\text { Tank for adding chemicals and operations for flocculent or filter } \\
\text { aides, Fundabac filter. May not be required. Located on } 2 \text { nd floor. }\end{array}$ \\
\hline $\begin{array}{l}\text { VES-113 - Process Water } \\
\text { Tank }\end{array}$ & 200 & 3 & 7 & $\mathrm{CS}$ & $\begin{array}{l}\text { Tank for adding chemicals and operations for flocculent or filter } \\
\text { aides, Fundabac filter. May not be required. Located on } 2 \text { nd floor. }\end{array}$ \\
\hline $\begin{array}{l}\text { VES-114 - Nitric Acid } \\
\text { Tank }\end{array}$ & 200 & 3 & 7 & $304 \mathrm{~L}$ & $\begin{array}{l}\text { Tank for adding nitric acid to adjust } \mathrm{pH} \text { for the ETS Evaporator. } \\
\text { May not be required. Located on } 2 \text { nd floor. }\end{array}$ \\
\hline VES-116 - Vent Demister & $\sim 5$ & 1 & 2 & $304 \mathrm{~L}$ & $\begin{array}{l}\text { Pre-HEPA mist eliminator. Standard industrial demister, } 0.7 \mathrm{ft}^{2} \text { flow } \\
\text { area minimum. }\end{array}$ \\
\hline $\begin{array}{l}\text { HOP-101 - Portland } \\
\text { Cement Hopper }\end{array}$ & 3000 & 7.8 & 13.9 & $\mathrm{CS}$ & $\begin{array}{l}\text { Portland Cement Feed Hopper. Cone bottom, } 30^{\circ} \text {, instrument wells, } \\
\text { vent, bottom feed system. Locate on } 2 \text { nd floor above grout mixer } \\
\text { MIX-103/104. Has Acrison 105z supply hopper and } 407-105 \text { weigh } \\
\text { feeder. }\end{array}$ \\
\hline $\begin{array}{l}\mathrm{HOP}-102-\mathrm{Ca}(\mathrm{OH})_{2} \\
\text { Hopper }\end{array}$ & 4500 & 8.9 & 15.7 & $\mathrm{CS}$ & $\begin{array}{l}\text { Lime Hopper. Cone bottom, } 30^{\circ} \text {, instrument wells, vent, bottom } \\
\text { feed system. Locate on } 2 \text { nd floor above grout mixer MIX-103/104. } \\
\text { Has Acrison } 105 \mathrm{z} \text { supply hopper and } 407-105 \text { weigh feeder. }\end{array}$ \\
\hline $\begin{array}{l}\text { HOP-103 - Blast Furnace } \\
\text { Slag Hopper }\end{array}$ & 1500 & 6.2 & 11.5 & CS & $\begin{array}{l}\text { Blast Furnace Slag. Cone bottom, } 30^{\circ} \text {, instrument wells, vent, } \\
\text { bottom feed screw auger system. Locate on } 2 \text { nd floor above grout } \\
\text { mixer MIX-103/104. Has Acrison } 101-0 \text { supply hopper and } 407- \\
101-0 \text { weigh feeder. }\end{array}$ \\
\hline
\end{tabular}

Note 1: Height is overall, including legs, barrel, and top 


\section{Other Equipment}

\section{Filters}

\begin{tabular}{|c|c|c|c|c|}
\hline Part & $\begin{array}{l}\text { Filter Area } \\
\text { (ft2) }\end{array}$ & $\begin{array}{c}\text { Skid Height, } \\
\mathbf{f t}\end{array}$ & $\begin{array}{l}\text { Skid } \\
\text { Footprint, ft } \\
\quad \times \text { ft }\end{array}$ & Function/Features \\
\hline $\begin{array}{l}\text { F-101-1\&2-SBW } \\
\text { Filter }\end{array}$ & 21 & TBD & $2 \times 12$ & $\begin{array}{l}\text { Cross-flow Filtration (2). Remove particles from feed to ion } \\
\text { exchange. } 6 \text {-in. diameter x } 120 \text {-in. Located on ground level. }\end{array}$ \\
\hline $\begin{array}{l}\text { F-102-1\&2 - Solids } \\
\text { Filter }\end{array}$ & 32 & 8 & $5 \times 10$ & $\begin{array}{l}\text { Fundabac Type Filter (2). Removes particles from WM-187 } \\
\text { settled solids from the underflow of VES-106. Located on } \\
\text { ground level above canister loading. }\end{array}$ \\
\hline $\begin{array}{l}\text { F-104-1\&2 - HEPA } \\
\text { Filter }\end{array}$ & \multicolumn{3}{|c|}{50 SCFM, 5.5 in by 5.5 in or larger } & Two 3-filter standard banks in parallel \\
\hline
\end{tabular}

Ion Exchange Columns

\begin{tabular}{|c|c|c|c|c|}
\hline Part & Size (ft3) & Diameter, $\mathrm{ft}$ & Height (ft) & Function/Features \\
\hline $\begin{array}{l}\text { VES-108-x - Cs Ion } \\
\text { Exchange Columns }\end{array}$ & 15.4 & 1.5 & 8.7 & $\begin{array}{l}\text { Cs ion exchange (Note 2). Top inlet, bottom outlet. CST } \\
\text { sorbent. Wells on top and bottom for air and water. Instrument } \\
\text { wells. Located on ground level }\end{array}$ \\
\hline \multicolumn{5}{|c|}{ Pumps } \\
\hline Part & Flow, gpm & Sp.G & Wt \% Solids & Function/Features \\
\hline $\begin{array}{l}\text { P-101-1\&2-SBW } \\
\text { Pump }\end{array}$ & 15 & 1.3 & 0.5 & $\begin{array}{l}\text { Pump acid-adjusted feed to cross-flow filter feed tank (2). } \\
\text { Ground level. }\end{array}$ \\
\hline $\begin{array}{l}\text { P-102-1\&2- } \\
\text { Neutralized SBW } \\
\text { Pump }\end{array}$ & 130 & 1.3 & 2.15 & Crossflow filter feed (2). Ground level. \\
\hline $\begin{array}{l}\text { P-103a } 1 \& 2- \\
\text { Crossflow Backwash } \\
\text { Pump }\end{array}$ & TBD & 1.3 & 0 & Crossflow back wash (2). Ground level. \\
\hline $\begin{array}{l}\text { P-103b } 1 \& 2-\text { Ion } \\
\text { Exchange Feed Pump }\end{array}$ & 1.58 & 1.3 & 0 & Ion Exchange Feed (2) Ground level. \\
\hline $\begin{array}{l}\text { P-104 1\&2 - IX } \\
\text { Effluent Pump }\end{array}$ & 1.58 & 1.3 & 0 & Grout process feed (2) Ground level. \\
\hline $\begin{array}{l}\text { P-105-1\&2- NaOH } \\
\text { Feed Pump }\end{array}$ & 50 & 1.5 & 0 & $\mathrm{NaOH}$ liquid feed pump (2). 2nd Floor. \\
\hline $\begin{array}{l}\text { P-106-1\&2 - Tank } \\
\text { Solids Slurry Pump }\end{array}$ & 10 & 1.13 & 20 & Fundabac slurry feed (2) Ground level. \\
\hline $\begin{array}{l}\text { P-109 - IX Media } \\
\text { Wash Effluent Pump }\end{array}$ & 10 & 1.0 & 0.01 & IX Flush collection to service waste header. 2nd Floor. \\
\hline $\begin{array}{l}\text { P-110-1\&2- Grout } \\
\text { Mixer Flush Pump }\end{array}$ & 10 & 1.3 & 0 & MIX-104 flush pump (2) Ground level. \\
\hline $\begin{array}{l}\text { P-112 - Filter Aid } \\
\text { Slurry Pump }\end{array}$ & TBD & TBD & TBD & Pumps for chemical additive, filters. 2nd Floor. \\
\hline $\begin{array}{l}\text { P-113 - Process Water } \\
\text { Pump }\end{array}$ & TBD & TBD & TBD & Pumps for chemical additive, filters. 2nd Floor. \\
\hline $\begin{array}{l}\mathrm{P}-114-\mathrm{pH} \\
\text { Adjustment Pump }\end{array}$ & TBD & TBD & TBD & Pumps for $\mathrm{pH}$ adjustment. 2nd Floor. \\
\hline
\end{tabular}

Note 2: 4 columns installed at any one time. 24 total required 
Table B-18. Equipment list (continued)

\begin{tabular}{|c|c|c|c|c|}
\hline \multicolumn{5}{|r|}{ Mixers } \\
\hline Part & $\begin{array}{l}\text { Width } \\
\text { (in) }\end{array}$ & $\begin{array}{l}\text { Length } \\
\text { (in) }\end{array}$ & $\begin{array}{l}\text { Height } \\
\text { (in) }\end{array}$ & Function/Features \\
\hline $\begin{array}{l}\text { MIX-103 - Ribbon } \\
\text { Mixer }\end{array}$ & 22 & 58 & 18 & $\begin{array}{l}\text { Mixer. Blends } 3 \text { grout solid ingredients. Located on } 2 \text { nd floor below } \\
\text { HOP- } 102 \text {. Acrison M } 350 \text {. Has Acrison } 105 \text { z supply hopper and } 407- \\
105 \text { weigh feeder. }\end{array}$ \\
\hline $\begin{array}{l}\text { MIX-104 - Autocon } \\
\text { Mixer }\end{array}$ & 20 & 48 & 48 & $\begin{array}{l}\text { Mixer. Mixes solid grout formulation with liquid effluent and feeds to } \\
\text { drums. Located on ground level below HOP-104. Autocon specified, } \\
\text { no substitutions. 2nd floor. }\end{array}$ \\
\hline \multicolumn{5}{|r|}{ Feeders } \\
\hline $\begin{array}{l}\text { Feed-101 - Portland } \\
\text { Cement Feeder }\end{array}$ & 31 & 50 & 32 & Volumetric feeder, Acrison 105z. Feeds Portland cement. 2nd Floor. \\
\hline $\begin{array}{l}\text { Feed-102-Lime } \\
\text { Feeder }\end{array}$ & 31 & 50 & 32 & Volumetric feeder, Acrison 105z. Feeds lime. 2nd Floor. \\
\hline $\begin{array}{l}\text { Feed-103 - Blast } \\
\text { Furnace Slag Feeder }\end{array}$ & 24 & 32 & 30 & Volumetric feeder, Acrison 101-0. Feeds blast furnace slag. 2nd Floor. \\
\hline $\begin{array}{l}\text { Feed-104 - Mixed } \\
\text { Blend Feeder }\end{array}$ & 24 & 32 & 30 & Volumetric feeder, Acrison 105z. Feeds grout blend. 2nd Floor. \\
\hline $\begin{array}{l}\text { Weigh Feeders for } \\
\text { Feed-101,-102,-104 }\end{array}$ & 23 & 43 & 34.5 & $\begin{array}{l}\text { Weigh feeders ( } 3 \text { ), Acrison 407-105, goes with 105z. Feeds cement, } \\
\text { lime, and blend. 2nd Floor. }\end{array}$ \\
\hline $\begin{array}{l}\text { Weigh Feeders for } \\
\text { Feed-103 }\end{array}$ & 25 & 40 & 48 & $\begin{array}{l}\text { Weigh feeder, Acrison 407-101-0. Goes with 101-0. Feeds slag. 2nd } \\
\text { Floor. }\end{array}$ \\
\hline \multicolumn{5}{|r|}{ Jets } \\
\hline JET & $\begin{array}{l}\text { Flow } \\
(\text { gpm) }\end{array}$ & $\begin{array}{l}\text { Lift } \\
\text { (ft) }\end{array}$ & $\begin{array}{l}\text { Pressure } \\
\quad(p s i)\end{array}$ & Function/Features \\
\hline $\begin{array}{l}\text { JET-101 - Filter } \\
\text { Drain Jet }\end{array}$ & $<1$ & N/A & 25 & $\begin{array}{l}\text { Provides motive force for draining Fundabac filter prior to solids } \\
\text { drying. }\end{array}$ \\
\hline $\begin{array}{l}\text { JET-MIX-101 - Jet } \\
\text { Mixer }\end{array}$ & 12 & $\mathrm{~N} / \mathrm{A}$ & $\mathrm{N} / \mathrm{A}$ & $\begin{array}{l}\text { Jet mixer on discharge of P-101. Decreases mix time in neutralization } \\
\text { of VES-101. }\end{array}$ \\
\hline $\begin{array}{l}\text { JET-102 - Solids } \\
\text { Slurry Jet }\end{array}$ & 5 & 10 & 100 & $\begin{array}{l}\text { Jet pump for removing slurry from VES-102. } 11 / 2 \text { inch with control } \\
\text { orifice to control flow and keep solids concentration in VES-102 } \\
\text { relatively constant. }\end{array}$ \\
\hline $\begin{array}{l}\text { JET-107 - Decant } \\
\text { Water Jet }\end{array}$ & 50 & 5 & 100 & $11 / 2$ inch Jet pump for transferring decant from VES-106 to VES-111. \\
\hline $\begin{array}{l}\text { JET-111 - Waste } \\
\text { Water Jet }\end{array}$ & 50 & 15 & 100 & $\begin{array}{l}21 / 2 \text { inch Jet pump for transferring VES-111 to high level waste } \\
\text { evaporator. }\end{array}$ \\
\hline \multicolumn{5}{|r|}{ Other Equipment } \\
\hline $\begin{array}{l}\text { BLO-101-1\&2 - } \\
\text { Vent Gas Blower }\end{array}$ & $50 \mathrm{scfm}$ & \multicolumn{2}{|c|}{$\begin{array}{l}12 \text { in. water } \\
\text { vacuum }\end{array}$} & Vessel ventilation blower (2) \\
\hline $\begin{array}{l}\text { HE-101 - Solids } \\
\text { Drying Steam } \\
\text { Condenser }\end{array}$ & $18 \mathrm{~kg} / \mathrm{hr}$ & \multicolumn{2}{|c|}{$50,000 \mathrm{Btu} / \mathrm{hr}$} & $\begin{array}{l}\text { Water cooled shell and tube heat exchanger, condensing steam in } \\
\text { tubes. Peak steam rate } 10 \mathrm{~kg} / \mathrm{hr}\end{array}$ \\
\hline HTR-102 & $50 \mathrm{scfm}$ & \multicolumn{2}{|c|}{$0.3 \mathrm{~kW}$} & HEPA filter pre-heater. Electric. \\
\hline
\end{tabular}


The CsIX TRU Grout Facility includes waste loading and canister handling operations in the lower level; hot cell process equipment for both liquid and solids processing on the ground floor and cold activities on the second floor. The control room, offices, a decontamination room, a manipulator repair room and other support activities are also located on the ground floor. Changing the scale of the process, i.e., designing for a shorter treatment schedule, would primarily affect two areas of the facility - the process hot cell and the grouting operation floor space. Minimal effects are expected in the basement with waste packaging and canister handling and canister storage, although this conclusion will need to be confirmed in conceptual design.

Equipment in the process hot cell includes the ion exchange columns, seven tanks and both the crossflow and Fundabac filters. A maximum of about a $40 \%$ increase in capacity of the ion exchange columns could be achieved without exceeding the column dimension that would fit a RH $2-\mathrm{ft}$ by $10-\mathrm{ft}$ canister. Thus reducing the operating schedule up to about 1.8 years would have no effect on the ion exchange columns, reducing the schedule further would mean more frequent change-out of columns.

Keeping all other factors equal, decreasing the processing schedule would increase the floor space required for process tanks by the ratio of the decrease to the two-thirds power. For example if the schedule was decreased from 2.5 years to 1 year, the floor space would increase by $(2.5 / 1)^{0.667}=1.84$ or an $84 \%$ increase. Approximately the same increase in space requirements could be expected for the filters, although a trade off study would be needed to better evaluate whether to increase the filter size or install multiple smaller filters.

For the 2.5-year operating schedule, 24,000 drums of grout are produced, equivalent to an average of two drums per hour. If in-drum mixing were used, multiple grouting lines would be required. The FY 2000 feasibility study for the CsIX TRU grout process (Raytheon, 2000) included three grouting lines. However, by using an out-of-drum mixer, a single line could meet the production rate in 2.5 years or a shorter schedule as well. The area required for grouting equipment would increase by the ratio of the schedule decrease to the two-thirds power. However, the area required for storage of grouted waste drums depends only on the lag storage requirement. The lag storage in turn depends of the rate at which WIPP can receive shipments and the initial lag between start of production and sending the first shipment. The expected shipping rate of $\mathrm{CH}$ grout to WIPP is 17 shipments (357 drums) per week for 71 weeks for the 2.5-year operating schedule (DOE-ID, 2003). If the treatment schedule is shortened, it is likely WIPP could receive additional shipments per week. Thus, additional storage is not requiring because of shipping rate limitations. Also, the initial lag time for sending shipments to WIPP could likely be shortened for a shorter schedule, resulting in no dependency of drum storage area on processing schedule.

Insufficient design work has been performed on the RH packaging systems for the CsIX process to evaluate the effect of reducing schedule on basement floor space. The number of ion exchange columns would decrease for a shorter schedule because each would hold more ion media, hence it is unlikely there would be any significant change in floor space for spent ion exchange column packaging. For the same 1-year processing schedule, the rate of production of solids canisters would increase from one per 43 hours to one per 17 hours. Whether this higher rate would require multiple packaging lines has yet to be determined.

Finally, the interim storage for packaged waste is dependent on the initial lag period between producing the first container of waste and having approval to ship that container to WIPP, and also on the rate at which WIPP can accept waste. While the process will generate, for a 2.5 year schedule, an average of 800 drums of grout and 9 canisters of solids per month, it will take about 3-months to generate the second spent IX column. Perhaps this period could be shortened by starting up on waste with the highest cesium content. However, it is not certain how long a production WIPP would require before qualifying 
the spent ion exchange media waste canisters. The area shown below is based on a production of two spent ion exchange columns.

Based on discussion with WIPP, it is expected that the disposal facility can receive at least $6 \mathrm{RH}$ canisters per week from the INEEL, or about 300 per year. The total number of RH canisters generated for a 2.5-year production schedule is 302 , while for a shorter schedule would be less than 302 . HENCE, WIPP could receive waste without any increase in storage at the INEEL for any treatment schedule of a year or longer. A summary of plot space estimates is given in Table B-19.

Table B-19. Plot Space Estimates.

\begin{tabular}{lcc}
\hline & $\begin{array}{c}\text { 2.5-year } \\
\text { treatment schedule }\end{array}$ & $\begin{array}{c}\text { 1-year } \\
\text { treatment schedule }\end{array}$ \\
\hline $\begin{array}{l}\text { Process equipment } \\
\text { (Process cell and pump and valve corridor, no } \\
\text { change assumed in P\&V corridor) }\end{array}$ & $2,000 \mathrm{ft}^{2}$ & $3,400 \mathrm{ft}^{2}$ \\
$\begin{array}{l}\text { Grouting equipment and drum storage } \\
\begin{array}{l}\text { Waste packaging \& canister handling } \\
\text { (assumes no change, to be verified in } \\
\text { conceptual design) }\end{array}\end{array}$ & $13,000 \mathrm{ft}^{2}$ & $21,000 \mathrm{ft}^{2}$ \\
$\begin{array}{l}\text { Lag storage for RH canisters } \\
\text { (to be verified in conceptual design) }\end{array}$ & $5,800 \mathrm{ft}^{2}$ & $5,800 \mathrm{ft}^{2}$ \\
\hline
\end{tabular}

\section{B-9. LAYOUT DRAWINGS}

Layout drawings for each floor of the CsIX TRU Grout Treatment Facility are shown on the following pages. The primary purpose of these drawings is to estimate floor space requirements needed in the facility. Equipment and room arrangements have not been optimized, nor has sufficient engineering design been performed to provide a basis for all of the areas of the facility. The layout drawings were based on those prepared for the Direct Evaporation Feasibility Study (Kimmitt, 2003f) and the Raytheon CsIX TRU Grout feasibility study (Raytheon, 2000).

The basement shows significantly more space for canister storage than is estimated to be required for lag storage. The storage space shown is based on provided structural integrity, that is, the storage area walls are a lower extension of the ground floor hot cell walls. 
This page intentionally left blank. 


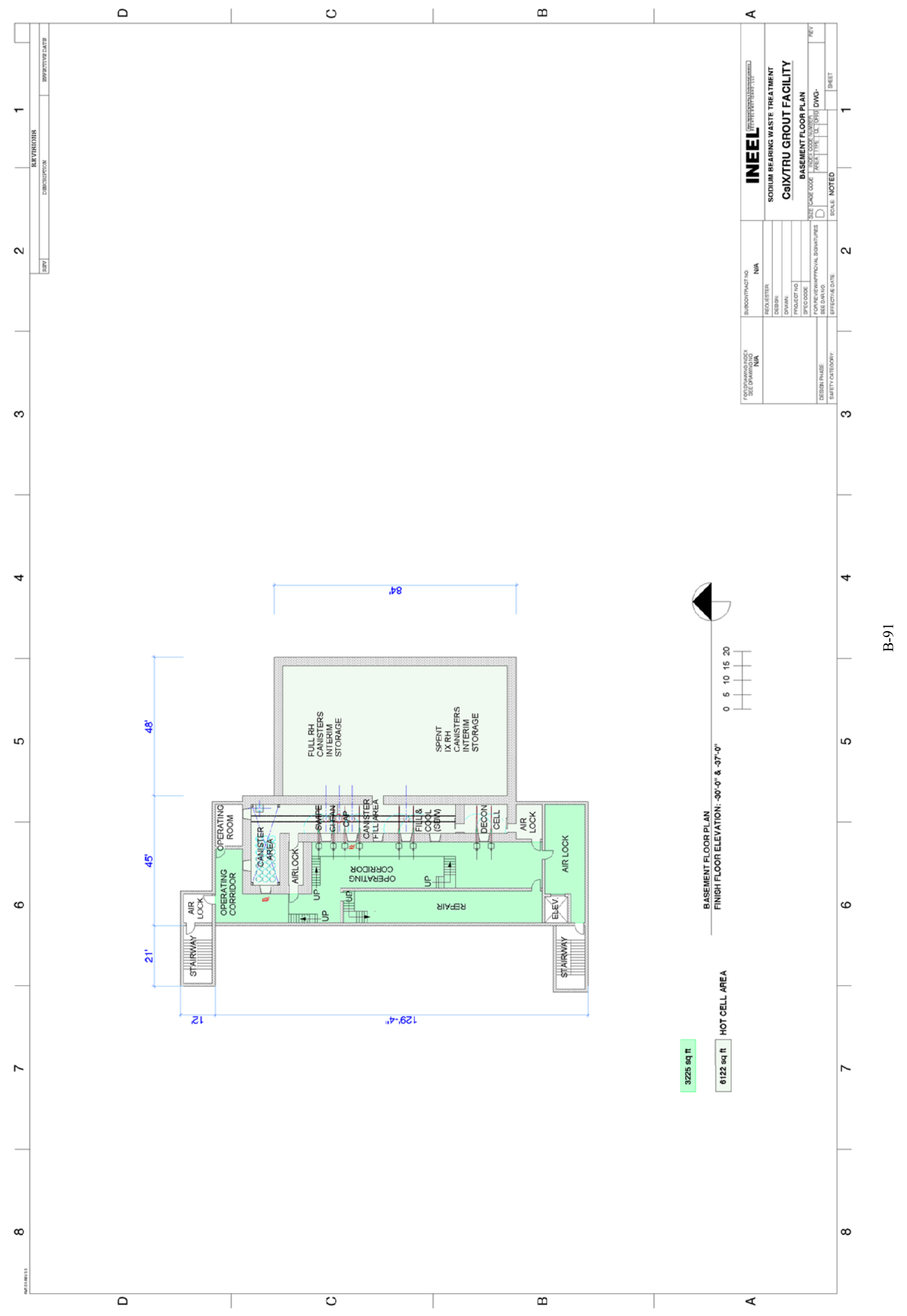




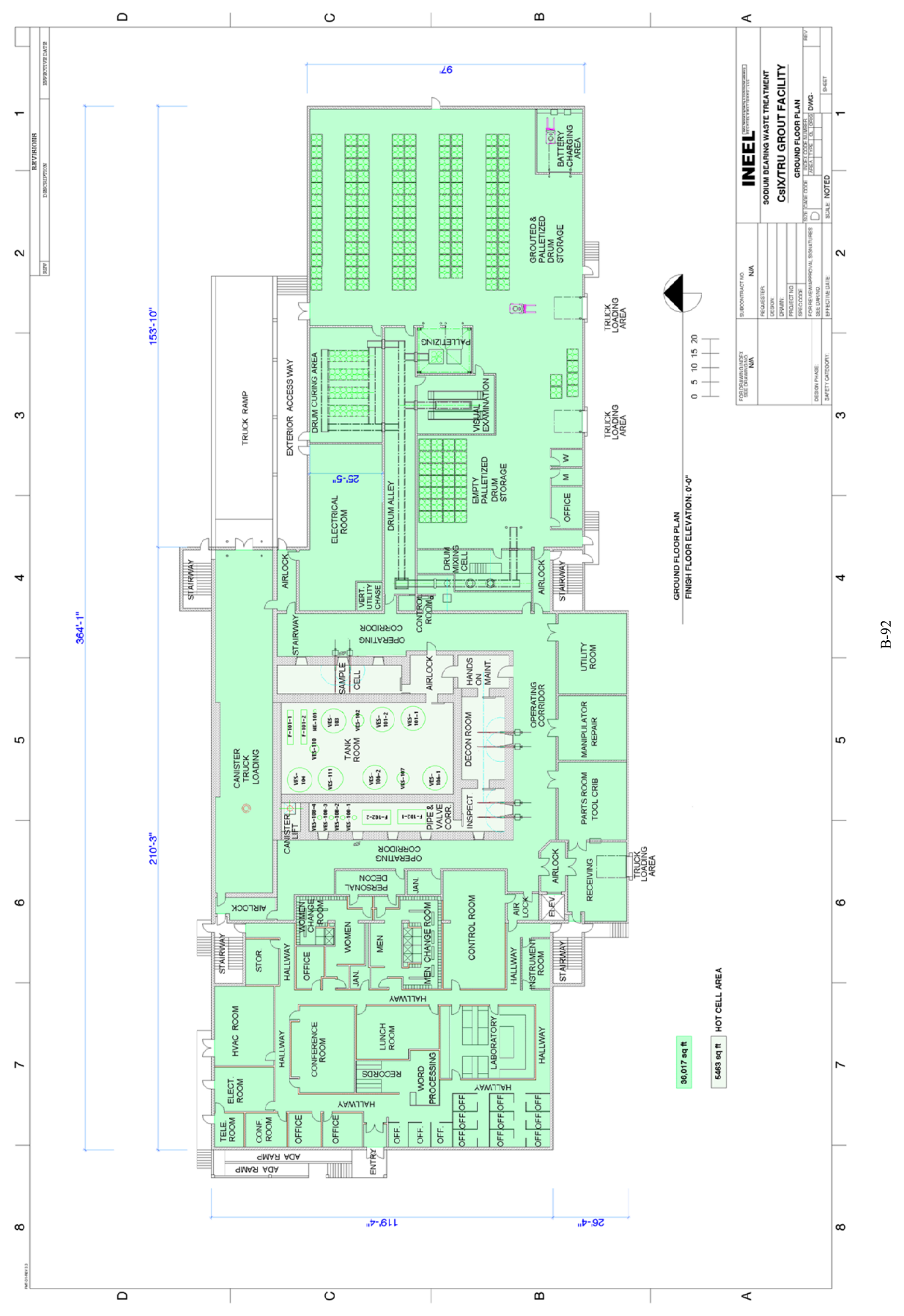




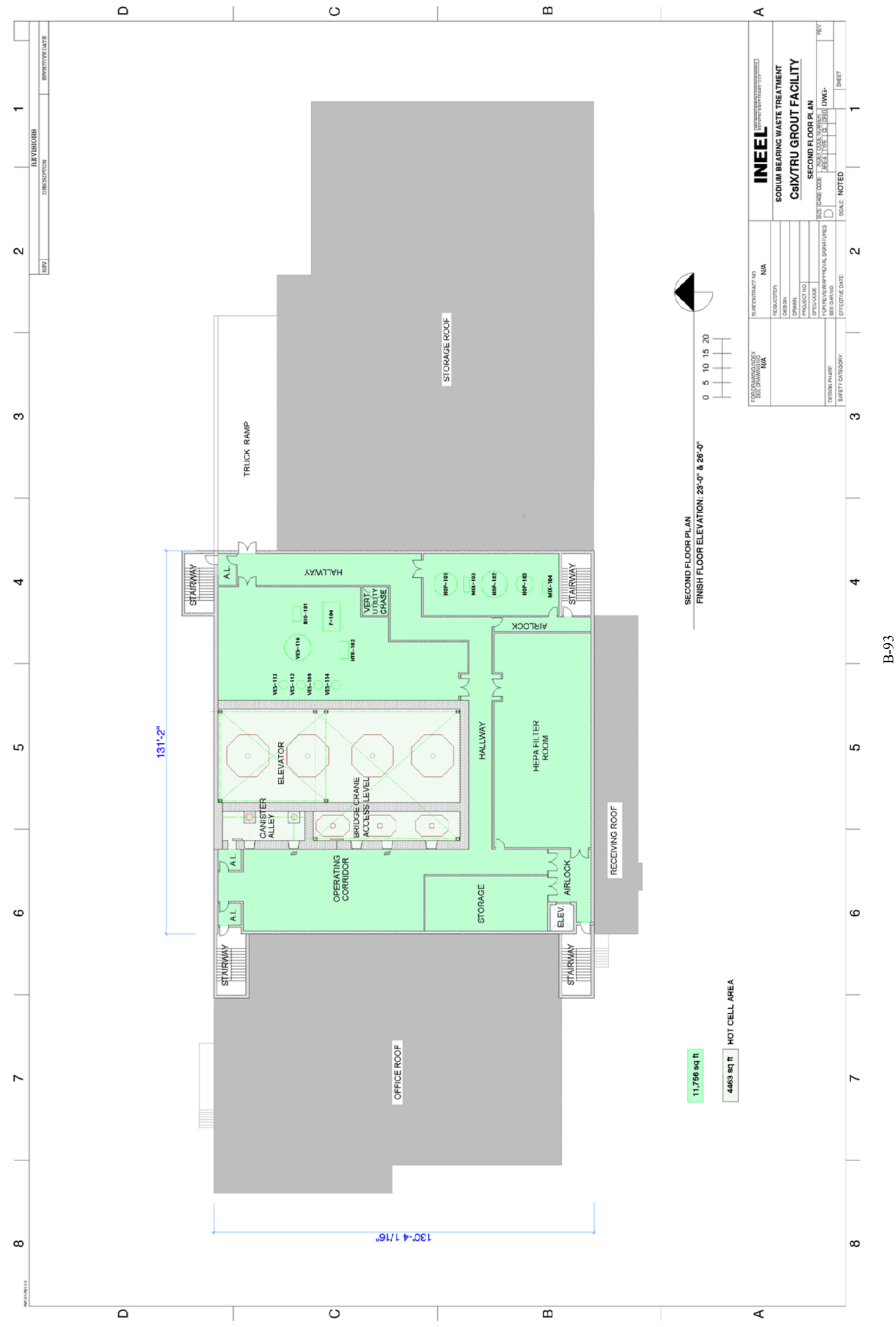




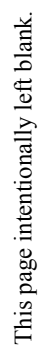




\section{B-10. REFERENCES}

Ashworth, S. C., (2003), "Sodium Bearing Waste Cesium Ion Exchange Evaluation of Solidification and Mixing," Engineering Design File EDF-4020, September 26, 2003.

Barden, R. R., (2003), “Technical and Functional Requirements for the CsIX TRU Grout SBW Treatment Alternative,” TFR-180, draft, April 2003.

Barnes, C. M., (1998), "Process Assumptions, Description, Diagrams and Calculations for P110," HLW EIS Project Data Sheet Studies, Engineering Design File EDF-PDS-D-008, 1998.

Barnes, C. M., (2002a), "Process Design of SBW Treatment Alternatives," Engineering Design File 2373, September 10, 2002.

Barnes, C. M., and C. B. Millet, (2002b), "INTEC Tank Farm Facility Management Plan," PLN-1112, September 30, 2002.

Barnes, C. M., S. K. Janikowski, and C. B. Millet, (2003), Feed Composition for the Sodium-Bearing Waste Treatment Process, INEEL/EXT-2000-01378, Revision 3, September 2003.

Bohn, T. S., (2002), Radiological Shielding Calculations for Screening of Sodium Bearing Waste (SBW) Treatment Alternatives, Rev. 1, INEEL Interoffice Memorandum TSB-20, August 26, 2002.

Bostick, D. T., S. M. DePaoli, and B. Guo, (2001), "Treatment of Low-Level Radioactive Wastewaters with IONSIV ${ }^{\mathrm{TM}} \mathrm{IE}-911$ and Charbazite Zeolite," Separation Science and Technology 36, pp. 975998, 2001.

Durante, R. P., and R. R. Kimmitt, (2003), "Estimated Exposure Rates for Grout Drums from the CsIX Process,” INEEL Interoffice Memorandum RPD-02-03/RRK-03-03, September 22, 2003.

Fluor Daniel, (1997), Idaho Chemical Processing Plant Waste Treatment Facilities Feasibility Study Report, DOE/ID/13206, December 1997.

Herbst, A. K., to J. H. Valentine, (1996), FY-96 Status Report on the Low-Level Waste Grout Stabilization Development Program, Lockheed Martin Idaho Technologies Company Interdepartmental Communication AKH-07-96, September 18, 1996.

Herbst, A. K., to J. H. Valentine, (1997), FY-97 Status Report on the Low-Level Waste Grout Stabilization Development Program, Lockheed Martin Idaho Technologies Company Interdepartmental Communication AKH-02-97, October 20, 1997.

Herbst, A. K., J. A. McCray, R. J. Kirkham, J. Pao, and S. H. Hinckley, (1999), Idaho Nuclear Technology and Engineering Center Low-Activity Waste Process Technology Program FY-99 Status Report, INEEL/EXT-99-00973, September 1999.

Herbst, A. K., J. A. McCray, R. J. Kirkham, J. Pao, M. D. Argyle, L. Lauerhass, C. L. Bendixsen, and S. H. Hinckley, (2000), Idaho Nuclear Technology and Engineering Center Low-Activity Waste Process Technology Program FY-2000 Status Report, INEEL/EXT-2000-01167, October, 2000. 
Herbst, A. K., J. A. DelDebbio, R. J. Kirkham, B. A. Scholes, and T. L. Watson, (2002), Idaho Nuclear Technology and Engineering Center Low-Activity Waste Process Technology Program FY-2002 Status Report, INEEL/EXT-02-00985, September, 2002.

Herbst, A. K., (2003), "Preliminary Results for Solidification of Simulated Liquid Effluents from Sodium-Bearing Waste Treatment Options," Engineering Design File EDF-3788, May 15, 2003.

Kimmitt, R. R., (2003a), Review of Cesium-Selective Ion Exchange Materials for Use in the Sodium Bearing Waste Treatment Facility, Engineering Design File EDF-3601, September 15, 2003.

Kimmitt, R. R., and W. St. Michel, (2003b), Ion Exchange Operations in the Cesium Ion Exchange TRU Grout Alternative for Treating SBW, Engineering Design File EDF-3846, September 17, 2003.

Kimmitt, R. R., T. Tranter, and R. Nims, (2003c), "Two Computer Models for Design of CesiumSelective Ion Exchange System,” Engineering Design File EDF-4071, September 30, 2003.

Kimmitt, R. R., T. Tranter, and R. Nims, (2003d), "Development of Two Computer Models to Support Design of Selective Ion Exchange Systems," to be presented at Waste Management 04 Conference, February, Tuscon, AZ.

Kimmitt, R. R., (2003e), Personal communication on December 1, 2003.

Kimmitt, R. R., (2003f), "Feasibility Study for Direct Evaporation of Sodium Bearing Waste," Engineering Design File EDF-2524, Rev. 0, May 20, 2003.

Losinski, S. J., C. M. Barnes, and B. K. Grover, (1998), CsIX/TRU Grout Feasibility Study, INEEL/EXT99-00034, November 1998.

Mann, N. R., and T. A. Todd, (1998), Evaluation and Testing of the Cells Unit Crossflow Filter on INEEL Dissolved Calcine Slurries, INEEL/EXT-98-00749, August 1998.

Mann, N. R., T. A. Todd, K. N. Brewer, D. J. Wood, T. J. Tranter, and P. A. Tullock, (1999), Evaluation and Testing of IONSIV IE-911 for the Removal of Cesium-137 from INEEL Tank Waste and Dissolved Calcine, INEEL/EXT-99-00332, April 1999.

Mann, N. R., and T. A. Todd, (2000), Removal of Cesium-137 from INEEL Tank Waste Using IONSIV IE-911 Sorbent, INEEL/EXT-2000-01570 (draft), September 2000.

McCray, J. A., to A. K. Herbst, (1999), Status Report for CsIX and NGLW Demonstration Grout Testing, Lockheed Martin Idaho Technologies Company Interdepartmental Communication JAM-03-99, September 30, 1999.

Miller, C. J., T. L. Watson, and T. A. Todd, (1994), An Evaluation of Extraction Chromatography Resins for Actinide and Strontium Removal and Ammonium Molybdophosphate for the Removal of Cesium from Dissolved Pilot Plant Calcine Solutions, WIN-374, July, 1994.

Miller, C. J., A. L. Olson, and C. K. Johnson, (1995), Cesium Absorption from Acidic Solutions Using Ammonium Molybdophosphate on a Polyacrylonitrile Support (AMP-PAN), INEL-95/00516, November 17, 1995. 
Miller, J. E., (1997), Development and Properties of Crystalline Silicotitanate (CST) Ion Exchangers for Radioactive Waste Applications, SAND97-0771, April 1997.

Nenni, J. A., (2003), "Process Data In Support of Aspen Plus Validation,” Engineering Design File EDF3936, August 14, 2003.

Pao, Jenn-Hai, and R. Lewis, (2003), Experimental Study of Filtration and Solids' Post Treatment from Simulated Sodium-Bearing Waste Derived Slurry, INEEL/EXT-03-01106, September 2003.

PLN-17, “High Level Liquid Waste Evaporator (HLLWE) Operational Run Plan,” January 30, 2003

Poloski, A. P., (2001), FY-2000 Tank Farm Closure Mock-Up Test Report, Engineering Design File EDF-15722-048, April 26, 2001.

PRD-166, “INTEC PEW Chemical Acceptance Criteria,” Rev. 4, October 21, 1999.

Raman, S. V., A. K. Herbst, B. A. Scholes, S. H. Hinckley, and R. D. Colby, (2003), Solidification of Simulated Liquid Effluents Originating form Sodium-Bearing Waste at the Idaho Nuclear Technology and Engineering Center, FY-03 Report, INEEL/EXT-03-01096, September 2003.

Raytheon Engineers and Constructors, (1994), Idaho Chemical Processing Plant Waste Immobilization Facility Feasibility Study Report, October 1994.

Raytheon Engineers and Constructors, (2000), Idaho National Technology \& Engineering Center Sodium Bearing Waste (SBW) Treatment Facility Project CsIX/TRU Grout Process Feasibility Study Report, Volumes 1-4, September 2000.

Scholes, B. A., A. K. Herbst, S. V. Raman, and S. H. Hinckley, (2003), Out-of-Drum Grout Mixer Testing with Simulated Liquid Effluents Originating form Sodium-Bearing Waste at the Idaho Nuclear Technology and Engineering Center, INEEL/EXT-03-01095, September 2003.

Stegen, G. E., to W. B. Palmer, (1997),“Additional SBW Separation and Backup Options,” DSI97-GES051, November 11, 1997.

Stegen, G. E., to V. Jacobson, (1998a), “Scoping Evaluation of Waste Treatment Concepts," DSI98-GES007, March 5, 1998.

Stegen, G. E., to V. Jacobson, (1998b), “Additional Information on Waste Treatment,” DSI98-GES-011, March 19, 1998.

Todd, T. A., K. N. Brewer, D. J. Wood, P. A. Tullock, N. R. Mann, and L. G. Olson, (2001), "Evaluation and Testing of Inorganic Ion Exchange Sorbents for the Removal of Cesium-137 from Actual Idaho Nuclear Technology and Engineering Center Acidic Tank Waste, Separation Science and Technology 36, pp. 999-1016.

Todd, T. A., (2003), A Review of Cesium Ion Exchange Technologies Applicable to the Treatment of INEEL Tank Waste, Engineering Design File EDF-2558, April 10, 2003.

TPR-7112, “Operate the HLLWE without the Batch Program, September 19, 2003. 
Tranter, T. J., M. D. Argyle, and R. D. Tillotson, (2003a), Evaluation of IONSIV IE911 and AMPCenosphere Composites as Cesium Removal Options for INEEL Acidic Tank Waste - Phase 1, INEEL/EXT-03-00757, July 2003.

Tranter, T. J., (2003b), “AMP-CST Static Stability Testing,” Engineering Design File EDF-3655, August $13,2003$.

Tranter, T. J., (2003c), "Evaluation of IONSIV IE911 and AMP-Cenosphere Composites as Cesium Removal Options for INEEL Acidic Tank Waste," Engineering Design File EDF-3602, May 23, 2003.

Tranter, T. J., R.D. Tillotson, M.D. Argyle, and T.A. Batcheller, (2004a), "Evaluation of IONSIV ${ }^{\mathrm{TM}}$ IE911 as a Cesium Removal Option for INEEL Acidic Tank Waste: Phase II Semi-Scale Column Tests," Engineering Design File EDF-4536, January 30, 2004.

Tranter, T. J., (2004b), "Dynamic Stability Testing of IONSIV® IE911 for Cesium Removal from INEEL Acidic Tank Waste,” Engineering Design File EDF-4612, February 24, 2004.

Tripp, J. L., and E. L. Wade, (1997), FY-97 Experimental Results of the Cells Unit Cross-Flow Filter Tests at the INEEL, INEEL/EXT-97-01232, November, 1997.

TSR-103, “Technical Safety Requirements, New Waste Calciner Facility,” April 18, 2001.

U. S. Department of Energy, Idaho Operations Office, (2003), WIPP Waste Certification Strategy for the Sodium-Bearing Waste Treatment Project, DOE/ID-11057, Rev. 1, August, 2003.

UOP, IONSIV ${ }^{\mathrm{TM}}$ IE-910 Ion Exchanger Series Product Information

Valles, N. O., C. M. Barnes, C. R. Edinborough, K. H. Liebelt, B. D. Raivo, and D. S. Wendt, (1999), Sodium-Bearing Waste to WIPP Waste (SBW ${ }^{3}$ ) Optimization Study, INEEL/EXT-99-00975, November, 1999.

Wilding, M. W., (1961), Cesium Removal from Acidic Radioactive Waste Solutions, IDO-14544, April $14,1961$.

Williams, C. L., (2003), Grouting Methods and Equipment for the CsIX-SBW Treatment Alternative, Engineering Design File EDF-3415, April 17, 2003 (draft).

Wood, R. A., and D. K. Morrell, (2002), Heel Solids Processing Evaluation for the SBW Treatment Project - Calcination with MACT Upgrade Alternative, Engineering Design File EDF-3049, December 4, 2002. 
INEEL/EXT-04-01692

Appendix C

\section{Appendix C \\ Direct Evaporation Process}

Charles M. Barnes

Barry H. O'Brien

February 2004 
This page intentionally left blank. 


\section{CONTENTS}

Contents C-3

Tables. C-4

Acronyms C-6

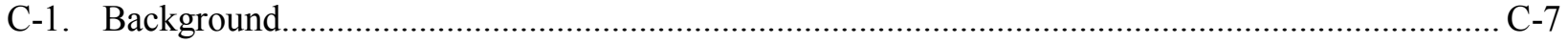

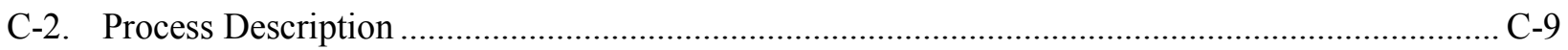

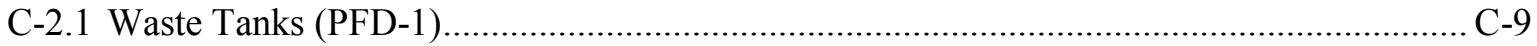

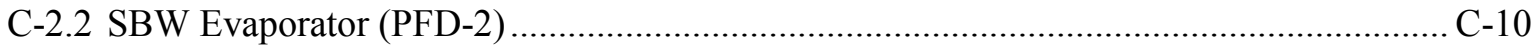

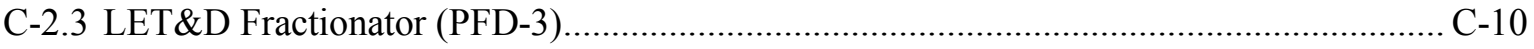

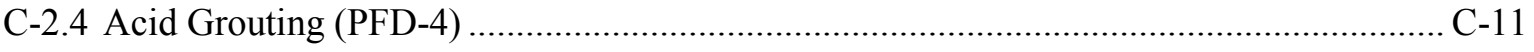

C-2.5 Canister Loading and Handling (PFD-5) ..................................................................... C-12

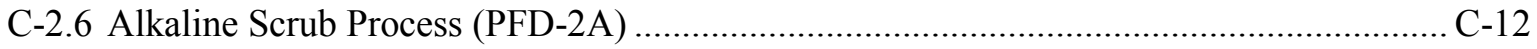

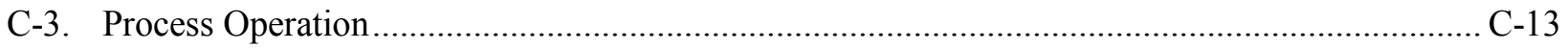

C-4. Process Flow Diagrams ........................................................................................................

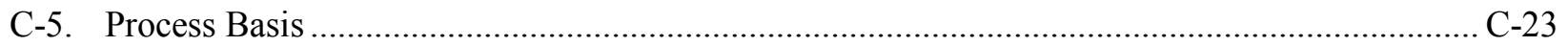

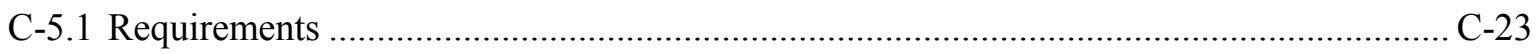

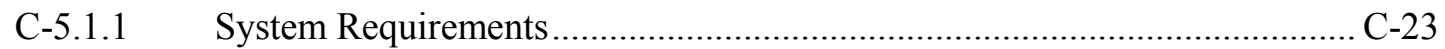

C-5.1.2 Systems, subsystem, and major components.......................................... C-25

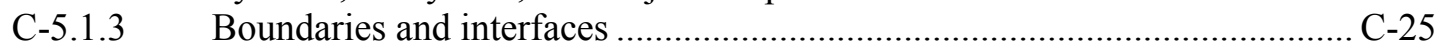

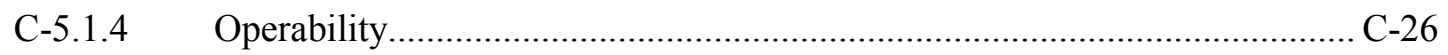

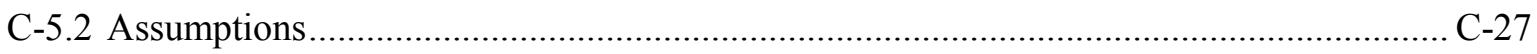

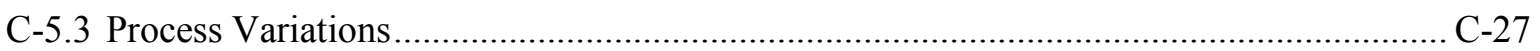

C-5.3.1 Initial and Revised Baseline Process.................................................... C-27

C-5.3.2 Alternative Evaporator Types ................................................................. C-29

C-5.3.3 Alternative Condensate Treatment Schemes.......................................... C-29

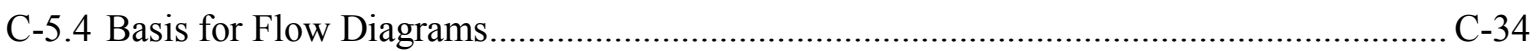

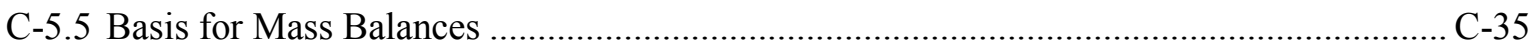

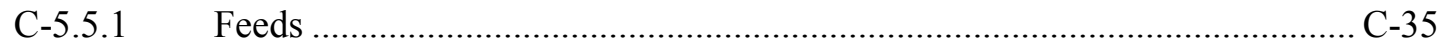

C-5.5.2 Stream Factor, Operating Schedule and Feed Rate....................................... C-37

C-5.5.3 Tank Mixing and Feed Transfer from Tank Farm ...................................... C-37 
C-5.5.4 Evaporation and Disengagement......................................................... -38

C-5.5.5 Condensate Treatment - Revised Baseline Scheme.................................... C-40

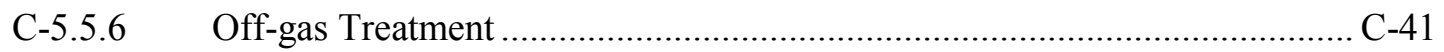

C-5.5.7 Evaporator Product Packaging ............................................................. C-42

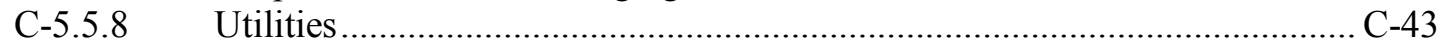

C-5.5.9 Alkaline Scrub Scheme............................................................... C-4

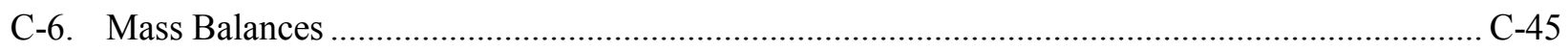

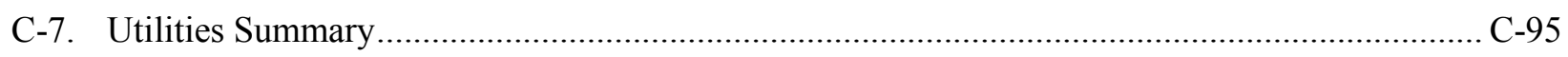

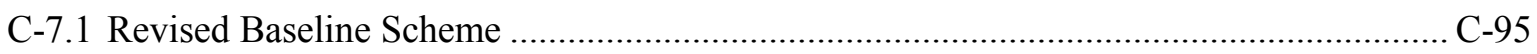

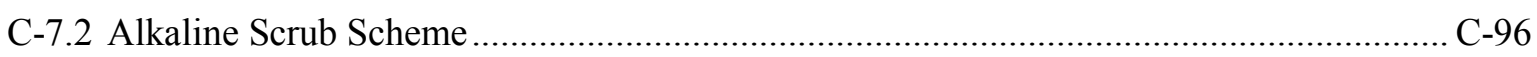

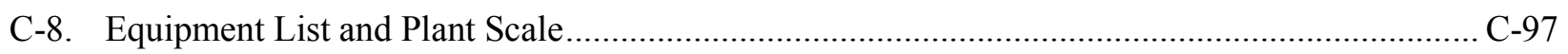

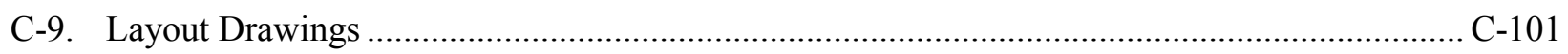

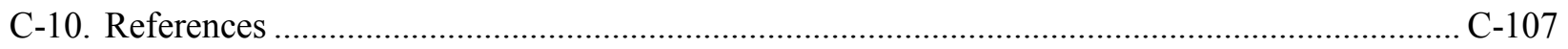

\section{TABLES}

Table C-1. Comparison of alkaline scrubbing process to the baseline process. ${ }^{\mathrm{a}}$................................. C-32

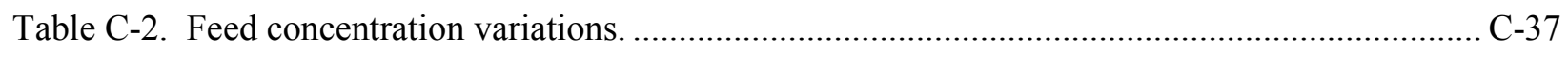

Table C-3. Products from and consumable feeds for the direct evaporation process. ......................... C-45

Table C-4. Revised baseline mass balance, Tank WM-180 waste........................................................

Table C-5. Revised baseline mass balance, Tank WM-187 waste........................................................ C-54

Table C-6. Revised baseline mass balance, Tank WM-188 waste...................................................... C-62

Table C-7. Revised baseline mass balance, Tank WM-189 waste....................................................... C-70

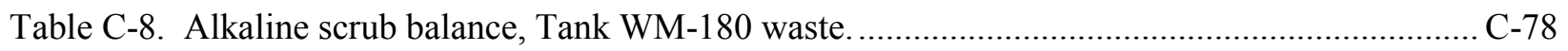

Table C-9. Alkaline scrub balance, Tank WM-187 waste.............................................................. C-81

Table C-9. Alkaline scrub balance, Tank WM-187 waste................................................................ C-82

Table C-10. Alkaline scrub balance, Tank WM-188 waste. ............................................................. C-85

Table C-10. Alkaline scrub balance, Tank WM-188 waste. ............................................................ C-86

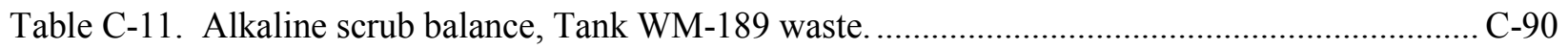

Table C-12. Revised baseline scheme evaporator product waste properties. ..................................... C-94 
Table C-13. Grouted waste properties compared to Hanford and Envirocare WAC. C-95

Table C-14. Expected utilities for revised baseline scheme ......................................................... C-96

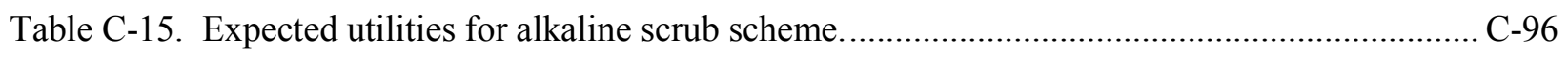

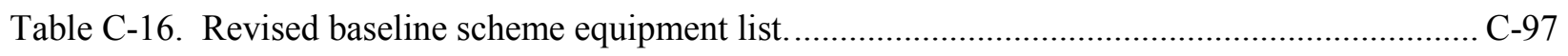

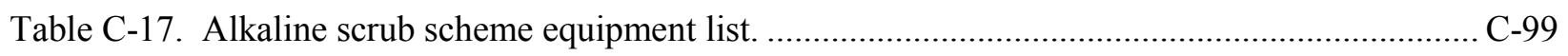

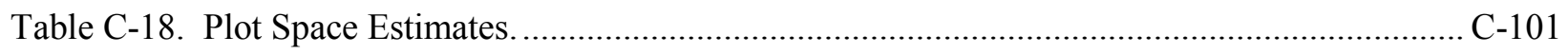




\section{ACRONYMS}

BFS blast furnace slag

CERCLA Comprehensive Environmental Response, Compensation and Liability Act

DBE design basis element

DF decontamination factor

DOE Department of Energy

DWPF Defense Waste Processing Facility

ETS Evaporative Tank System

FGE fissile gram equivalent

GAC granulated activated carbon

HEME high-efficiency mist eliminator

HEPA high-efficiency particulate air (filter)

INEEL Idaho National Engineering and Environmental Laboratory

INTEC Idaho Nuclear Technology and Engineering Center

LET\&D Liquid Effluent Treatment and Disposal Facility

NGLW newly generated liquid waste

OPC ordinary Portland cement

PEWE Process Effluent Waste Evaporator

PFD Process Flow Diagram

POG process off-gas

RCRA Resource Conservation and Recovery Act

SBW

sodium bearing waste

SCR

selective catalytic reduction (process or reactor)

T\&FR technical and functional requirements

TCLP Toxicity Characteristic Leaching Procedure

THC

TFF

total hydrocarbons

Tank Farm Facility

VOG vessel off-gas

WAC waste acceptance criteria

WESP wet electrostatic precipitator

WIPP Waste Isolation Pilot Plant 


\section{Appendix C \\ Details of the Direct Evaporation process}

\section{C-1. BACKGROUND}

Evaporation is a common practice at U.S. Department of Energy (DOE) facilities in the treatment of radioactive waste. The Idaho National Engineering and Environmental Laboratory (INEEL) currently operates two evaporators to concentrate dilute aqueous, acidic wastes, the Process Effluent Waste Evaporator (PEWE) and the Evaporative Tank System (ETS) (former called the High Level Liquid Waste Evaporator). The PEWE has been in operation since 1984 and the ETS since 1987. Unlike these current evaporators, the concept behind the direct evaporation process is to continue the evaporation of the feed to the point that upon cooling, the concentrated waste will solidify into a form acceptable for disposal.

The predecessors of the direct evaporation concept for treatment of sodium bearing waste (SBW) were the freeze crystallization and evaporation/precipitation concepts proposed and tested by John McCray in the mid-1990s (McCray, 1994; McCray, 1995), and a concept of SBW evaporation followed by vitrification with spent high efficiency particulate air (HEPA) filters proposed by Robert Kirkham in 1992 (Kirkham, 2003). In June 1994, small-scale evaporation tests at reduced pressures were performed on SBW simulants followed by cooling to promote precipitation (McCray, 1994). The evaporation/precipitation process was proposed as a means to reduce the volume of stored liquid waste by $70 \%$ by evaporation. The process was designed to produce a sodium nitrate low-level waste product (McCray, 1995). Sodium nitrate crystals would have been separated from the evaporator bottoms, and the liquid treated by additional processing such as calcination.

In September 1997, Robert Kirkham performed initial tests to determine whether it was possible to evaporate SBW to the extent that it would solidify upon cooling (Kirkham, 1998). The intent at the time was to then ship the solidified waste to another site such as Hanford, where it would be re-liquefied and blended with a much larger quantity of similar waste in a treatment facility that would produce a final waste form. The tests showed that evaporation to a solidified waste form was feasible, with the initial 500 $\mathrm{ml}$ sample being concentrated to a liquid-free solid of about $180 \mathrm{ml}$ (Kirkham, 1998).

Development of the direct evaporation process for treatment of SBW was resumed in 2002 (McCray, 2002). Scoping tests were performed by heating $750 \mathrm{ml}$ samples of starting waste simulant in a 1-liter flask to determine evaporator temperature, pressure and extent of evaporation. Two other tests were performed, one to represent an "in-can" approach where the waste would be evaporated in its shipping container, and another in external equipment at vacuum. Conclusions from these tests included the following:

- WM-180 simulant solidification could be accomplished by means of in-container evaporation or by a separate evaporation vessel. The expected mass reduction is approximately $65-72 \%$ and corresponding volume reduction $74-81 \%$.

- At ambient pressure, a temperature greater than $130^{\circ} \mathrm{C}$ is required to reach sufficient concentration. If the pressure is reduced to $550 \mathrm{~mm} \mathrm{Hg}$, the evaporation can be carried out below $100^{\circ} \mathrm{C}$.

- The solidified waste product is hygroscopic and deliquescent. A thin layer of silica gel on top of the solidified waste was shown to absorb moisture accumulated on the solid waste surface. 
- The acidic evaporator condensate contained mercury above the Resource Conservation and Recovery Act (RCRA) wastewater limit, but $\mathrm{Pb}, \mathrm{Cr}$ and $\mathrm{Cd}$ below regulatory concerns. Entrainment carryover fractions of all species measured did not exceed $0.02 \%$.

- Acid, mercury and most anion concentrations in the condensate rapidly increased toward the end of the evaporation.

- There was no evidence of expansion or contraction of the concentrated waste as it cooled and solidified.

An engineering feasibility study of the direct evaporation process was also performed in 2002 (Kimmitt, 2003). The feasibility study was based on a process that included separate treatment of solids, processing the evaporator condensate through the PEWE evaporator and Liquid Effluent Treatment and Disposal (LET\&D) fractionator, and grouting the LET\&D bottoms. In addition to defining the process by preparation of process flow diagrams, mass balances and an equipment list, architectural drawings were prepared, the facility was described and project cost and schedule estimated. The total project cost was estimated to be $\$ 210$ million at an $85 \%$ confidence level. The schedule assumed start of conceptual design in November 2002 and operation between October 2008 and November 2011.

In the spring of 2003, a series of sixteen direct evaporation small-scale test runs using WM-189 SBW simulant was conducted (Kirkham, 2003). A known quantity of feed was boiled down to a concentration endpoint suitable to produce a solid bottoms product upon cooling. The operating temperatures and pressures were varied for each run. From the results of these studies, the preferred operating pressure for SBW-simulant evaporation was determined to be $380 \mathrm{~mm} \mathrm{Hg}$. At this absolute pressure and a temperature below $125^{\circ} \mathrm{C}$, a "hard" bottoms product was produced at a mass reduction ratio of $60 \%$.

In April and June of 2003, two series of tests at LCI Corporation test facilities in South Carolina provided performance data using a $1.4 \mathrm{ft}^{2}$ agitated thin-film evaporator. The first test used simulated SBW without solids; the second test used a feed of simulated SBW blended with tank heel solids (Griffith, 2003a; Griffith, 2003b). The first series included four runs with WM-189 simulant and two with WM-180 simulant; the second series included four runs of WM-189 simulant without undissolved solids and fourteen runs with several different undissolved solids. All of the bottoms samples solidified after cooling, demonstrating successful operation over a reasonably wide range of operating conditions. Test results showed that bottoms temperature could be used to control the process. Acceptable evaporator operation was demonstrated for all feeds for bottoms temperatures ranging from 110 to $118^{\circ} \mathrm{C}$. No operational problems were experienced in the runs with solids, demonstrating that the SBW solids can be processed through the evaporator.

Development work performed in FY 2002 determined that the LET\&D bottoms could be grouted with a waste loading of $35 \mathrm{wt} \%$ (Herbst, 2002). Continued testing of this formulation in 2003 demonstrated that the concentrations of hazardous metals in the leachate from Toxicity Characteristic Leaching Procedure (TCLP) tests of the grout were less than the Land Disposal Restrictions Universal Treatment Standards, even if the mercury level in the LET\&D bottoms was more than 3 times the expected level (Raman, 2003).

An engineering study was performed in 2003 to evaluate alterative schemes for processing the evaporator overhead or condensate (Barnes, 2003a). Five schemes were evaluated:

1. A revised baseline process which replaced the PEWE with new equipment and processed tank solids through the SBW evaporator 
2. A scheme in which the SBW evaporator feed was neutralized prior to evaporation

3. A scheme in which the nitric acid in the evaporator overhead is reduced in an SCR reactor

4. A scheme that includes alkaline scrubbing of evaporator vapor with the resulting salt waste grouted

5. A scheme that includes alkaline scrubbing of evaporator vapor with the resulting salt recycled to the SBW evaporator.

Although it produces a higher volume of RH waste, the alkaline scrub scheme with recycled salt showed significant advantages over the other schemes. Testing of this scheme was recommended, and initial tests performed in November 2003 at the LCI facility. The results from these tests showed that $\mathrm{MgO}$ or aluminum hydroxide added to the feed to simulate either scrubbing with $\mathrm{MgO}$ or neutralization of condensate with either reagent resulted in acceptable evaporator bottoms products.

Process information for two direct evaporation schemes is provided in this Appendix. One scheme, referred to as the "revised baseline" includes evaporation of a mixed SBW liquid/undissolved solids feed, condensing the evaporator overhead, processing the condensate through the existing LET\&D facility and grouting the LET\&D bottoms. Of all direct evaporation alternatives, this scheme has the least risk because all major operations have been demonstrated. The second process discussed in this Appendix is the alkaline scrub with recycle to the evaporator. Testing of this scheme has been performed with a feed simulating the expected recycle, but no tests have been performed yet of the scrubber or with actual recycle.

Section C--2 describes the process as shown on the Process Flow Diagrams (PFDs), which are given in Section C-4. PFDs 1-5 show the revised baseline process. PFDs 1,2A and 5 show the alkaline scrub process. Section C-5 discusses the basis for the process design, including major requirements, assumptions and variations or options that have been considered for process equipment and configurations. Section C-5 also discusses the basis for the mass balance, including feed compositions and rates, operating conditions, and assumed or expected equipment performance.

\section{C-2. PROCESS DESCRIPTION \\ C-2.1 Waste Tanks (PFD-1)}

PFD-1 shows the seven existing storage tanks that will hold the feed to the direct evaporation process. Tanks VES-WM-180 and VES-WM-189 presently contain SBW near their administrative capacity limits. Tank VES-WM-188 is presently approximately $80 \%$ full of SBW, and will be filled to capacity as new wastes are generated and concentrated by the ETS. Over the past few years solids that were in five tanks have been flushed to Tank VES-WM-187, which is being used as a collection tank for flush water and solids from operations to clean and close Tank Farm Facility (TFF) tanks. Upon flushing one more tank, VES-WM-181, the collection of solids in WM-187 will be complete. Then prior to SBW treatment new mixing pumps will be installed in VES-WM-187, VES-WM-188 and VES-WM-189 and waste transfers will be made back and forth between these three tanks to distribute the solids somewhat evenly between them.

After 2005, newly generated liquid waste (NGLW) will be stored in the three tanks, VES-WM-100, VES-WM-101, and VES-WM-102. The NGLW can be blended with SBW during the solids blending transfers or can be held in the three NGLW tanks and transferred directly to the treatment process feed tank. 
Existing steam jets in each of the tanks plus an existing airlift in VES-WM-189 will be used to transfer the SBW liquid/solids mixture from the TFF to the Direct Evaporation SBW Feed Tank. Waste will be transferred to the Direct Evaporation facility intermittently in batches at a rate of about 50 gpm. Transfers of approximately 8,500 gallons would provide a four-day supply of feed to the treatment process.

All equipment shown on PFD-1 is existing except for the mixing pumps (P-287, P-288 and P-289), which would be installed in tanks WM-187, WM-188 and WM-189 to keep solids in suspension.

\section{C-2.2 SBW Evaporator (PFD-2)}

SBW will be received from the TFF by the SBW Feed Tank, equipped with an air sparge (or other means of mixing the tank contents) and ability to mix fluid in the tank by use of the discharge pump. The air sparge and pump discharge recycle will keep the solids homogeneously suspended in the tank liquid. The SBW liquid/solids mixture will be continuously pumped at a rate of $1.5 \mathrm{gpm}$ to the SBW Evaporator, a thin film type evaporator that is expected to operate at about $120^{\circ} \mathrm{C}$ and about 8.6 -psia pressure. The evaporator is operated at a vacuum to keep the temperature below that at which "red oil" could form. Red oil is an explosive nitrated organic which can form above $130^{\circ} \mathrm{C}$.

Evaporator concentrate will fall directly from the bottom of the evaporator through a short transfer pipe into its final waste container. Evaporator vapor will exit the top of the evaporator passing through an internal mist eliminator and then an external demister. The demister provides separation of entrained liquid and solids from the evaporator vapor. Liquid collected in the demister is periodically pumped to the SBW Feed Tank. The mesh pads in the demister can also be washed with condensate. Vapor from the demister is condensed by heat exchange with cooling water. Condensed liquid drains from the Condensate Demister to the Condensate Tank. Tests to date have shown the evaporator vapor to be totally condensable, but if any noncondensible gases were present in the condenser effluent, they would be superheated and HEPA-filtered before being boosted in pressure and released to the atmosphere through the Idaho Nuclear Technology and Engineering Center (INTEC) stack.

The evaporator is heated with 150 psig steam that is generated within the new facility.

The SBW Feed Tank and Neutralization Tank are vented to a vessel off-gas (VOG) collection header. VOG passes through a demister, followed by a superheater, HEPA filter, and blower, and is then discharged through the INTEC stack. Liquid collected in the VOG demister would be pumped to the SBW Feed Tank.

Potential corrosion in the Condensate Tank by hydrofluoric acid is controlled by the addition of aluminum nitrate solution, which will react with hydrofluoric acid and form soluble aluminum fluoride complexes. Aluminum nitrate solution is received from a supplier into the Aluminum Nitrate Tank and pumped as needed to the Condensate Tank. Condensate collected in the Condensate Tank is pumped to the LET\&D, an existing fractionator at INTEC. A separate pump can transfer condensate to the evaporator vapor demister to provide washing of mesh pads.

All equipment shown on PFD-2 is new equipment.

\section{C-2.3 LET\&D Fractionator (PFD-3)}

The existing LET\&D Fractionator would be used to concentrate the condensate, thereby reducing the amount of waste that would require disposal. The condensate is fed by gravity from the LET\&D Feed Tank to one of two identical fractionators, each containing15 sieve trays. A reboiler supplies heat to the 
liquid in the lower section of the column. Vapors from the boiling liquid rise through sieve trays. Fractionation occurs as the vapor contacts the liquid flowing across the trays and down the column.

Because the feed would be much more concentrated in nitric acid than typical PEWE condensate, the fractionators may need to be modified to allow feeding at a lower tray than is presently used. The fractionator typically recovers at least $99 \%$ of the nitric acid from the feed into the bottoms product. The bottoms product is usually 12 molar nitric acid and contains a high fraction of the other impurities in the feed condensate including $\mathrm{HCl}$, fluorides, and mercury compounds such as $\mathrm{HgCl}_{2}$.

The fractionator overhead is steam with trace impurities. A partial condenser provides reflux to the column. The effluent from the condenser goes to a vapor/liquid separator, with the vapor from the separator superheated, filtered and discharged via a blower to the INTEC-708 stack. When the desired density of the fractionator bottoms is reached, a portion of the solution is drained out of the column and stored in the Bottoms Tank. This recovered acid is cooled by heat exchange with cooling water prior to entering the Bottoms Tank.

Except for the feed and bottoms tank, all acid fractionation equipment (fractionator with reboiler and condenser, separator, superheater, filter and blower) is duplicated. A new tie-in would be required to the Bottoms Tank Pump discharge line to route the recovered acid to a new acid grouting facility. The recovered acid would be available for reuse in other INTEC activities. A 22,500-gal acid recycle tank, VES-NCR-171, is available to collect and store LET\&D bottoms for use in the NWCF. However, the projected volume needed for expected INTEC operations is much less than the amount that would be generated by the direct evaporation process.

\section{C-2.4 Acid Grouting (PFD-4)}

LET\&D bottoms would be transferred in batches to a Neutralization Tank. Once a batch was received, solid calcium hydroxide would be added to the tank to neutralize the recovered nitric acid. The Neutralization Tank is equipped with a mixer to ensure adequate mixing of the solid calcium hydroxide with the acid waste, and with a cooling jacket or cooling coils to remove heat generated by the neutralization reaction.

After the addition and mixing of calcium hydroxide is complete, the neutralized acid would be pumped to a hold tank where it is continuously fed to a grout mixer. Blended Portland cement and blast furnace slag (BFS) in a weight ratio of 3:1 cement:slag would be mixed with the neutralized waste in the grout mixer, which would discharge directly into 55-gal drums, the final waste container. Filled drums would be conveyed to a swipe/decontamination area where the drum exterior surface would be swiped to test for radioactive contamination. If contamination was found the drum would be decontaminated and re-swiped. Drums that pass the surface swipe would be transferred to a curing area and held for 24-72 hours to complete curing.

Following curing, the drum would be visually inspected for free water. If water were found, silica gel would be added. Following inspection, the drum would move by conveyor to the pallet loading area. A gantry crane would place four drums on a pallet, which when full would be moved to temporary storage until a full load of drums (approximately 60) is available for shipment to the disposal site. An average of 14 drums would be produced per day, although, depending on the feed, as few as seven or as many as 17 drums would be produced in a day. 


\section{C-2.5 Canister Loading and Handling (PFD-5)}

PFD-5 summarizes pictorially the steps in loading waste into canisters and loading canisters onto a transport truck. Concentrate from the evaporator will be discharged into RH-TRU canisters. During filling, the canister would be kept under vacuum by a pathway through the fill line and evaporator. When the waste in a canister reached a set fill level, the steam to the evaporator would be shut off and then the feed to the evaporator shut off. This procedure flushes the evaporator. The full canister would be moved out of the fill position to the cooling area. An empty canister would be moved into the fill position. Feed to the evaporator would be restarted and then steam.

The full canister would be moved to the cooling area. A vacuum line would be attached to maintain a slight negative pressure in the canister, a clamp-on cooler would be attached and cooling water lines connected to the cooler. The waste temperature and off-gas moisture level would be monitored during cooling. Upon reaching a set temperature and moisture level, a plug would be inserted into the pintle fill hole and the canister moved to lag storage.

Empty canisters with pre-welded lids would be lowered into the Treatment Facility using an overhead crane and placed in a staging area until needed. Each empty canister would be weighed. When needed, a canister would be moved into an airlock, then out of the airlock, and into position to be attached to the evaporator discharge. After filling and cooling, the canister would be visually inspected. After attaching the pintle plug, the canister would be moved into the airlock and after closing the airlock door a surface swipe is performed. If analysis shows no contamination, the canister would be weighed, the dose rate of the canister measured, and the canister moved out of the airlock. If surface contamination were found on the swipe, the canister would be decontaminated, dried, and re-swiped.

In separate operations, a cask would be loaded onto a transporter and the transporter would be positioned over the canister load-out port. The filled canister would be lifted out of the treatment facility and into the cask. An inner lid would be attached to the cask. The transporter would then be repositioned and the outer lid attached to the cask. The cask would then be rotated into the transport position; the transporter inspected and then would depart from the facility.

Through a combination of sampling the feed material, acceptable knowledge, and measurements made during packaging, the waste would be certified to meet the Waste Isolation Pilot Plant (WIPP) RHTRU Waste Acceptance Criteria (WAC).

Equipment shown on PFD-5 is new equipment.

\section{C-2.6 Alkaline Scrub Process (PFD-2A)}

The alkaline scrub process is an alternative direct evaporation scheme that offers the potential for reducing the number of equipment items required, reducing the plot space and reducing the cost of the treatment facility. PFDs 1 and 5 as described above would be included, but PFD-3 and PFD-4 are eliminated. Changes to PFD-2, shown on PFD-2A, are described below.

As in the revised baseline process, overhead from the SBW evaporator would pass through both an internal mist eliminator and then an external demister vessel. However, the vapor from the demister would then enter a packed bed scrubber. The scrubber would contain magnesium oxide pellets that would react with nitric and hydrochloric acid vapors in the evaporator vapor. An aqueous scrub solution would be sprayed into the top of the column. As the liquid flows down through the bed, it would dissolve the products of the $\mathrm{MgO}$ /acid gas reactions. The scrub solution exiting the bed would be recycled to the top of the column, with a purge sent to the evaporator and makeup water added to maintain the liquid flow 
rate. The scrubber temperature would be controlled by a cooler on the scrub circulation stream. The scrub solution would be purged to the evaporator.

$\mathrm{MgO}$ would be continually added to the bed to replace that removed by reaction and dissolution. The $\mathrm{MgO}$ would be carried into the bed as a slurry with make-up water. Other reactions would occur in the scrubber, such as the formation of magnesium hydroxide by reaction of $\mathrm{MgO}$ with steam. The scrubber may also remove some of the mercury from the evaporator vapor. However, the concentration of mercury in the vapor leaving the scrubber is determined by vapor-liquid equilibrium of the recirculated liquid and the exiting gas. As mercury builds up in the scrub solution over time, the concentration in the vapor will increase proportionately.

The scrubber effluent gas is superheated and then mercury is removed in a bed of sulfurimpregnated (S-) granulated activated carbon (GAC). The bed contains sufficient carbon to process the entire inventory of SBW and NGLW. Vapors exiting the GAC bed are HEPA-filtered and boosted in pressure to be exhausted through the INTEC stack.

\section{C-3. PROCESS OPERATION}

This section describes the operation of the direct evaporation process.

Prior to processing, mixing pumps would have been installed in Tanks WM-187, WM-188 and WM-189, and the solids initially in Tank WM-187 would be distributed between these three tanks by a series of waste transfers between these tanks. After these transfers, all concentrated NGLW in tanks WM-100, WM-101 and WM-102 would be transferred to WM-187. This NGLW transfer is needed to be able to continue using these smaller tanks to receive and collect NGLW. The four waste tanks - WM180, WM-187, WM-188 and WM-189 - would then be sampled and the analytical results used to support qualification of the wastes from the treatment process.

After plant start-up activities are complete, waste from Tank WM-187 would be processed. Processing this tank first would free it up to receive flush water from the other tanks after they are emptied. Following the completion of processing Tank WM-187 waste, waste from one of the other tanks (WM-180, WM-188 or WM-189) would be processed. When waste has been withdrawn to heel level, estimated to be about 3000 gallons, processing of waste from a second of these three tanks would begin. Simultaneously, heel from the emptied tank would be flushed with water to Tank WM-187. After this sequence is repeated for another tank, the treatment process will be processing waste from the last tank, and heel from two tanks will have been collected in WM-187.

The contents of Tank WM-187, mostly flush water, would then be evaporated (using presently existing INTEC evaporators), with the concentrate sent to WM-100, WM-101 or WM-102 to be held for treatment. After completing treatment of waste in the final waste tank, its heel would be flushed to WM187, which initially contains a dilute heel. The final evaporation of WM-187 would produce about 30004000 gallons of concentrate, which would either be treated in the SBW treatment facility or dispositioned with other NGLW collected during 2012. The heel in WM-187, dilute to begin with, would be flushed using multiple washes to the NGLW tanks. This waste, if concentrated separately from other wastes, would produce only an estimated 300 gallons of concentrate. Thus, flush from WM- 187 would more likely be evaporated with other NGLW collected in late 2012, and the concentrate dispositioned with this NGLW.

Transfers of about 10,000 gallons from the Tank Farm to the Direct Evaporation Facility would be made once every 5 days. At a rate of $50 \mathrm{gpm}$, the transfer would take about 3.3 hours. Waste qualification would be based on analysis of samples taken from the TFF tanks, hence no holdup is 
designed into the treatment facility feed tank for sample turnaround. The treatment facility would thus have a single tank to receive SBW from the TFF and feed it to a single operating evaporator. The evaporator is fed continuously while a canister is being filled. Feed is introduced into the evaporator and then steam to the evaporator steam jacket. Bottoms product falls directly into a waste canister. When the canister is full, the steam to the evaporator is shut off, followed by the SBW feed. The time interval required to replace the full canister with an empty one is estimated to be on average about 2.5 hours, longer when the facility starts up and then decreasing. When a new canister is in place, the feed is restarted to the evaporator and then the steam. The evaporator downtime fraction (ratio of downtime to total time) due to canister switch operations is expected to be about 0.2 for the revised baseline scheme or 0.3 for the alkaline scrubbing scheme.

A spare evaporator and evaporator overhead demister would be installed to reduce the risk of shutdown due to mechanical or other problems related to evaporator operation.

Vapors from the demister are condensed and condensate collected in a single condensate tank. Condensate would be collected for approximately six days and then sent to the LET\&D at a rate of 500 $\mathrm{gal} / \mathrm{hr}$. The contents of the condensate tank would be processed in the LET\&D in 20 hours.

The LET\&D bottoms tank has a capacity of 200 gallons. The tank will be filled and transferred to the direct evaporation facility for grouting every 1.4-3.4 hours, depending on the feed case. The transfer rate would exceed the generation rate by about a factor of two. A 200-gallon batch of acid would be received in the neutralization tank and as acid is received, $\mathrm{CaO}$ would be added, neutralizing the waste. Mixing the contents of the neutralization would continue for about 15 minutes after feed had stopped. Periodically the neutralized acid would then be transferred to the Grout Feed Tank.

The Grout Feed Tank and Neutralization Tank need to have a combined capacity to supply a continuous feed to the grout mixer during the downtime for the LET\&D. This requires a combined volume of about 3000 gallons. Assuming equal capacity for each of these two tanks, the Neutralization Tank would feed the Grout Feed Tank until it reached its capacity of 1500 gallons. Then transfers would be stopped until the Grout Feed Tank reached a low-level set point. Then the contents of the Neutralization Tank would again be transferred to the Grout Feed Tank. At some point, the Neutralization Tank would reach an "empty" level and remain so until operation of the LET\&D resumed. However, waste in the Grout Feed Tank would continuously supply feed to the grout mixer during this

time. A single grout mixer would be in operation at all times; a spare would be available but not installed.

\section{C-4. PROCESS FLOW DIAGRAMS}

Process flow diagrams are shown on the following pages:

$\underline{\text { Page }}$

$\begin{array}{llll}25 & \text { T-1 } & \text { Title Sheet and Legend } & \\ 26 & \text { PFD-1 } & \text { Waste Tanks } & \text { (Existing TFF) } \\ 27 & \text { PFD-2 } & \text { SBW Evaporator } & \text { (New equipment, baseline scheme) } \\ 28 & \text { PFD-2a } & \text { SBW Evaporator, Alkaline Scrub } & \text { (New equipment, alkaline scrub scheme) } \\ 29 & \text { PFD-3 } & \text { LET\&D Fractionator } & \text { (Existing equipment, baseline scheme) } \\ 30 & \text { PFD-4 } & \text { Acid Grouting } & \text { (New equipment, baseline scheme) } \\ 31 & \text { PFD-5 } & \text { Canister Loading and Handling } & \text { (New equipment) }\end{array}$




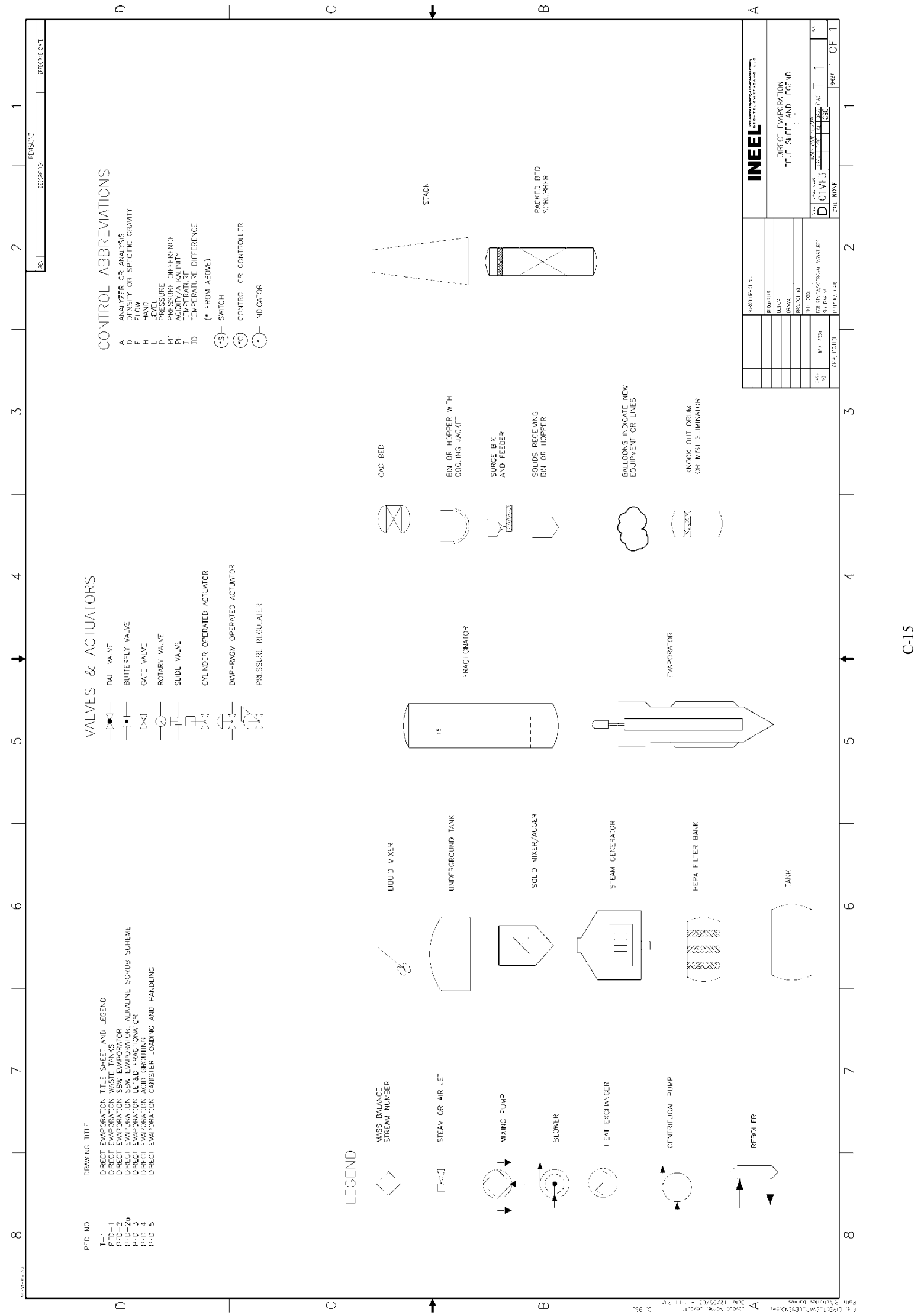




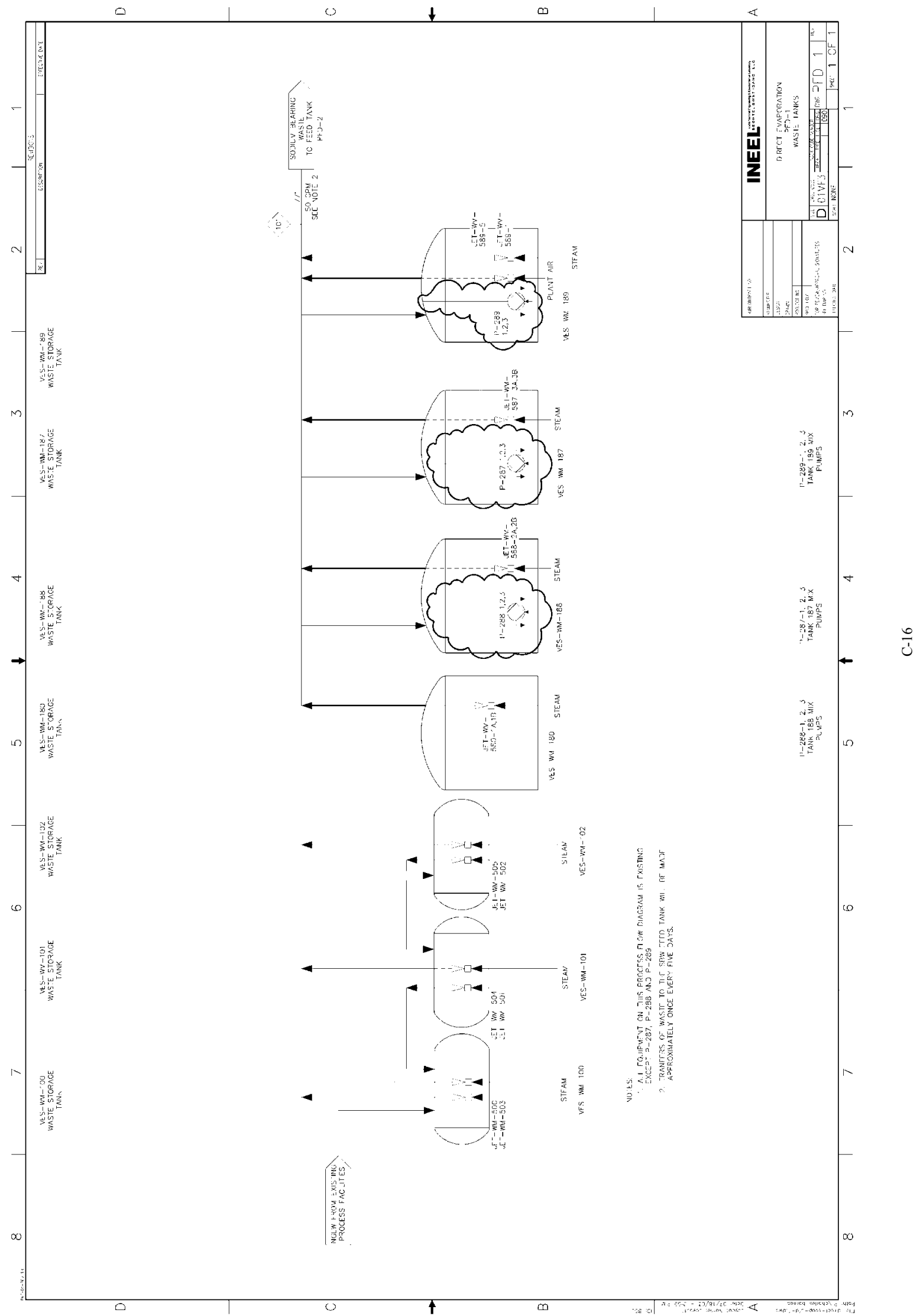




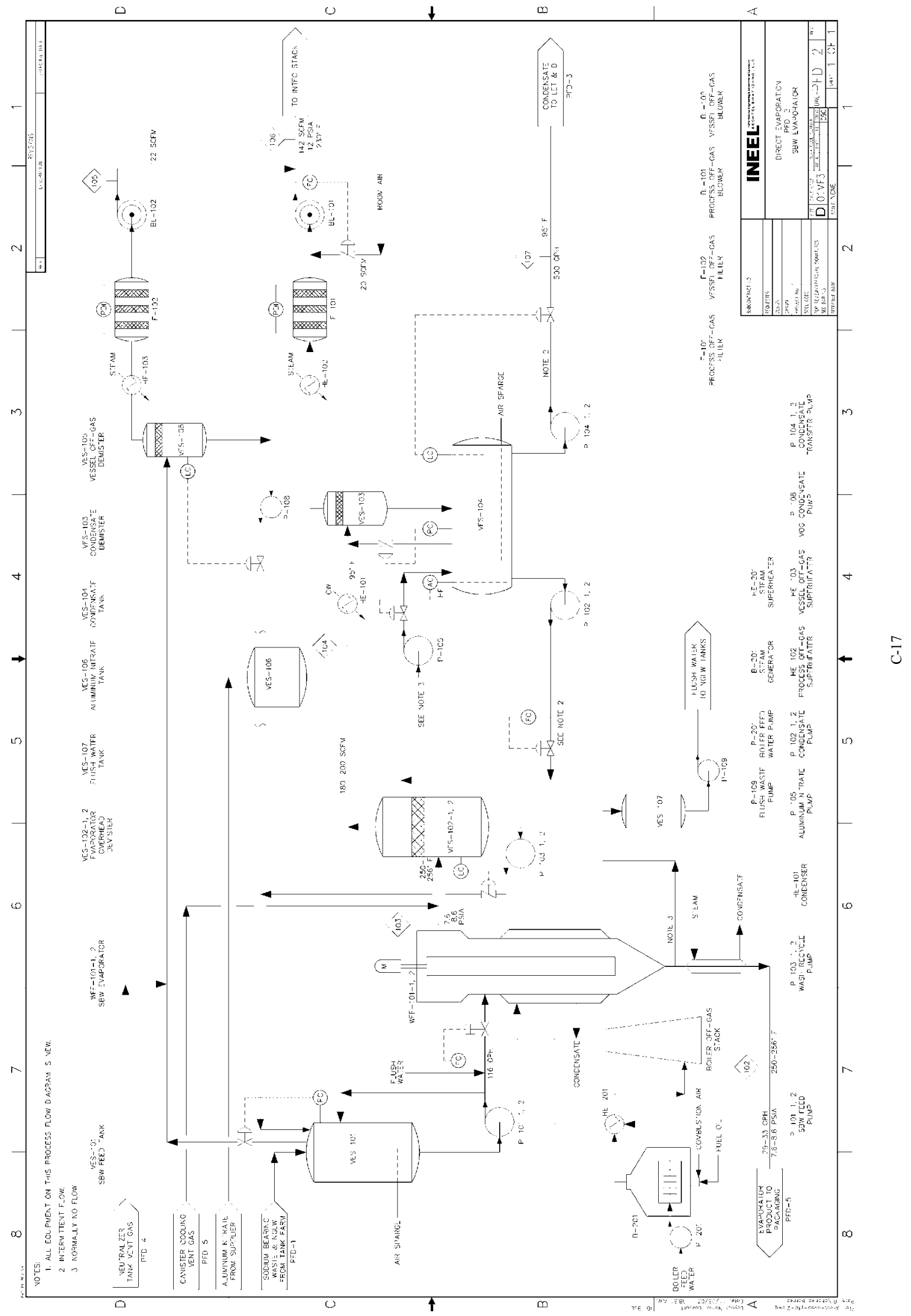




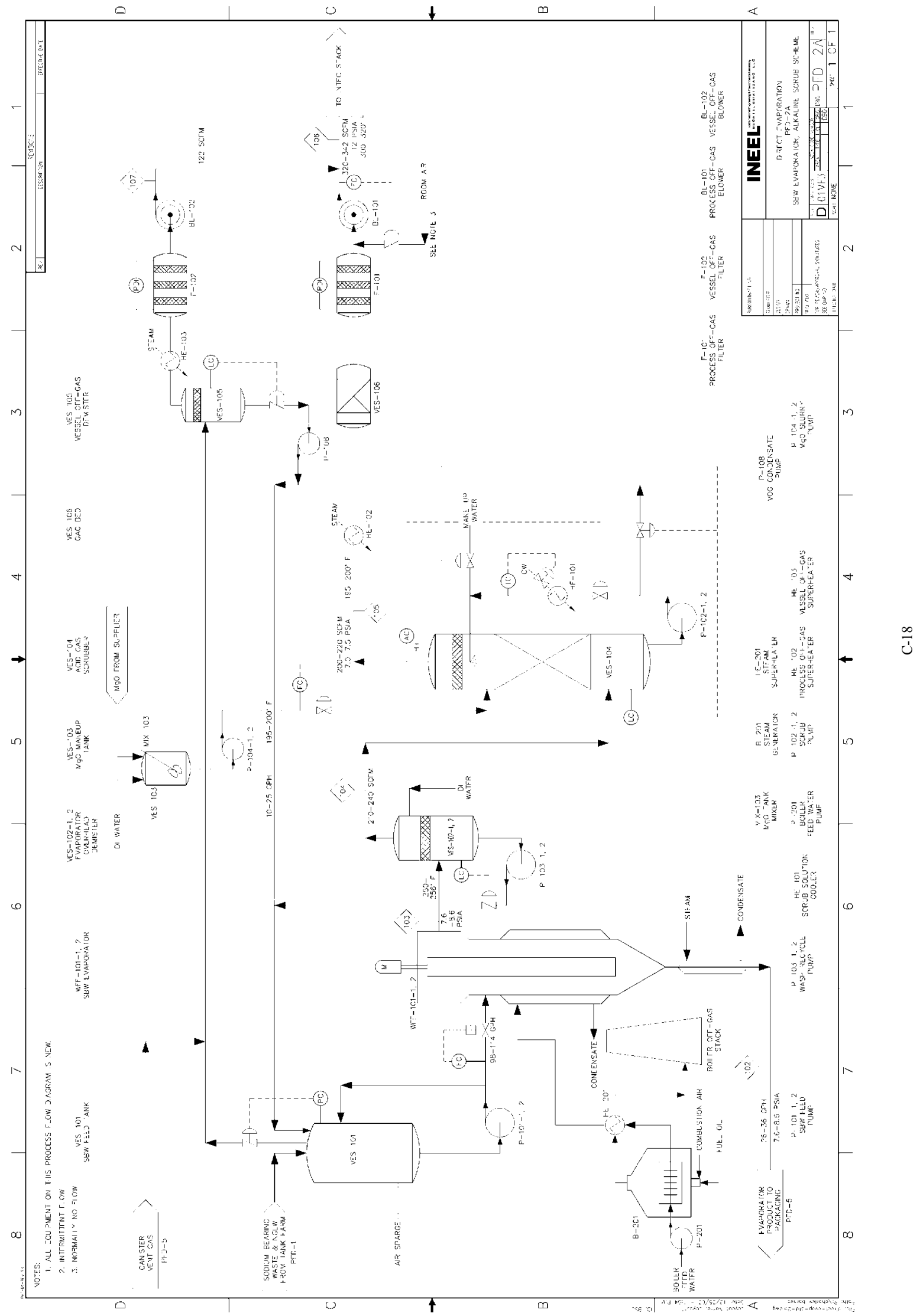




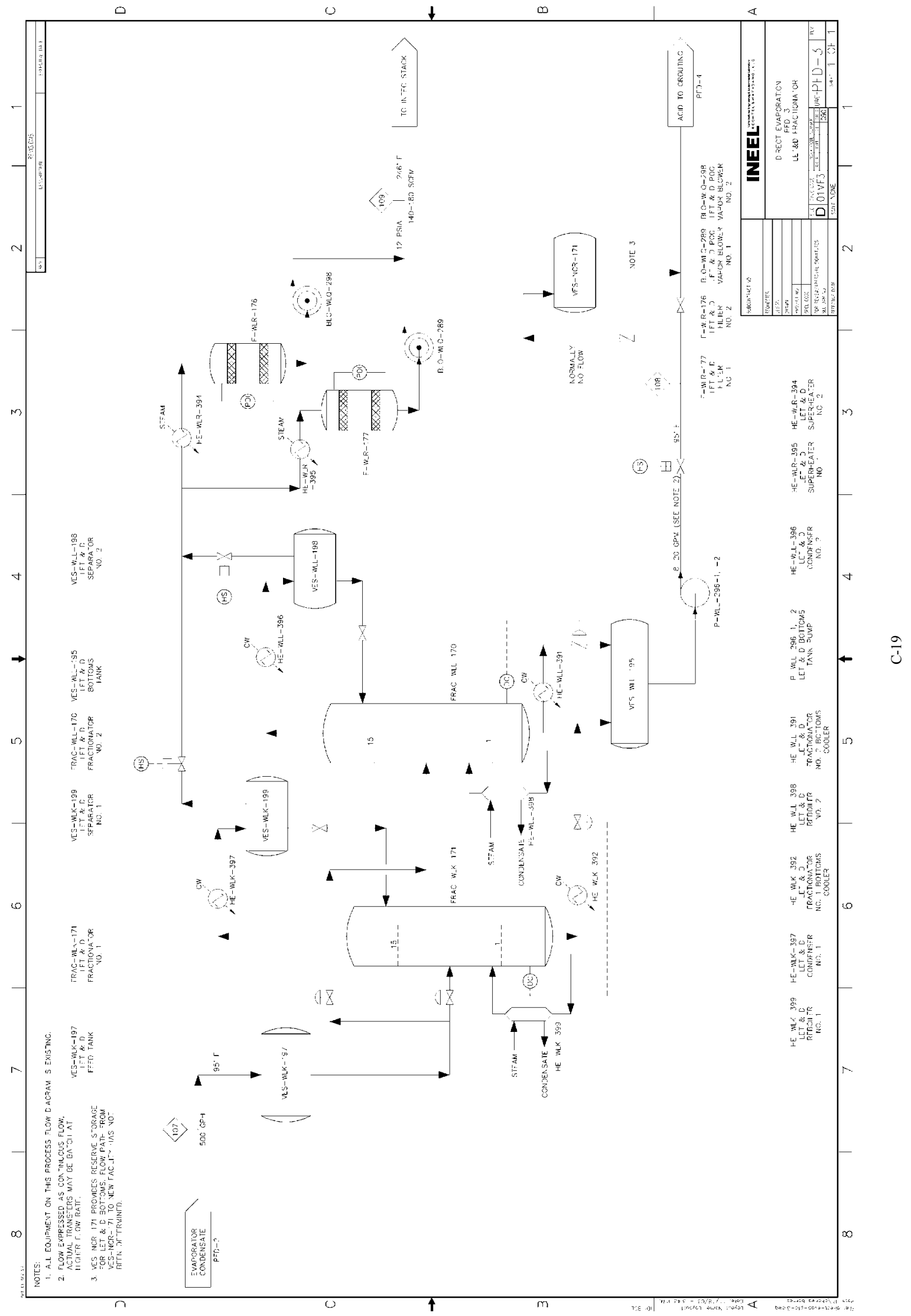




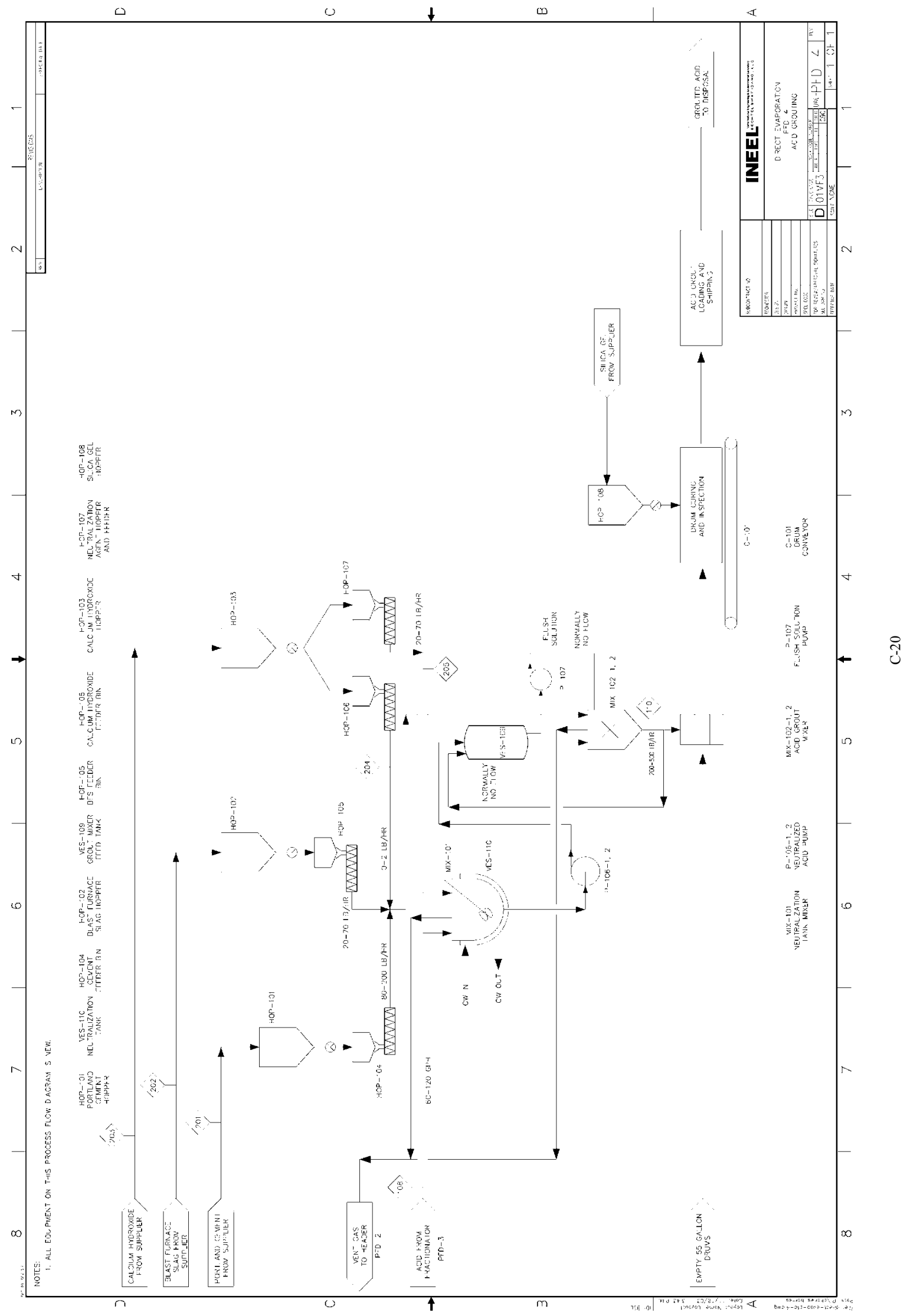




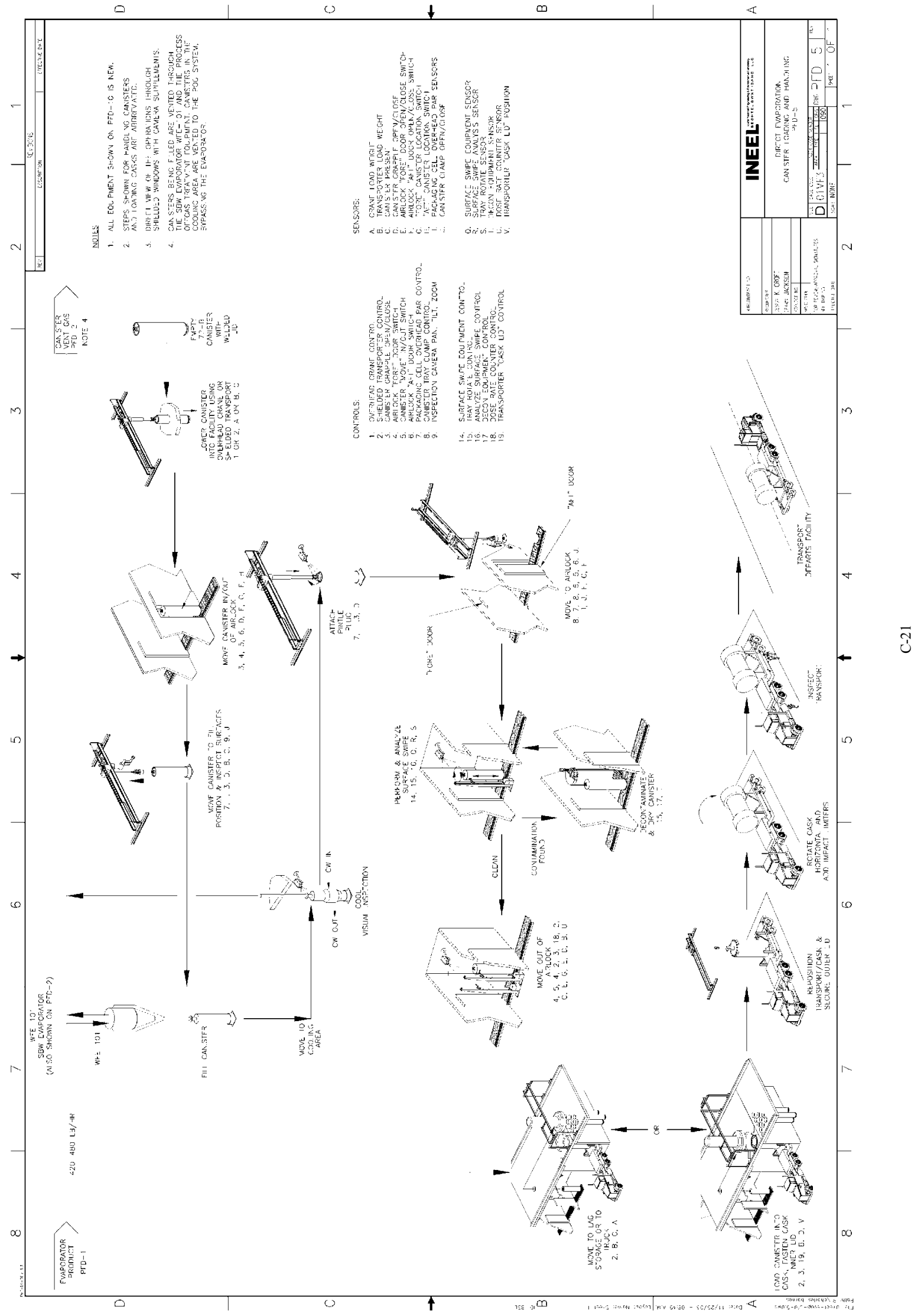




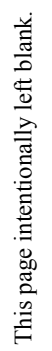




\section{C-5. PROCESS BASIS}

This section includes a summary of technical and functional requirements, process assumptions, a discussion of variations of the direct evaporation process, the basis for the process shown on the process flow diagrams, and the basis for the mass balances.

\section{C-5.1 Requirements}

A draft technical and functional requirements document (T\&FR) was prepared for the direct evaporation process (Barden, 2003). Most functional and performance requirements identified in the T\&FR document are summarized below. For other requirements, including special requirements, design requirements, testing and maintenance requirements and codes, standards and regulations refer to the T\&FR document. The T\&FR document also provides the basis or reference for each requirement.

\section{C-5.1.1 System Requirements}

C-5.1.1.1 Retrieve Waste. The system shall have the capability to retrieve SBW from WM-180, WM-187, WM-188, and WM-189 and NGLW from WM-100, WM-101 and WM-102.

C-5.1.1.2 Treat Waste - Volume. The facility shall be capable of processing 1.2 million gallons of waste.

C-5.1.1.3 Treat Waste - Throughput. The facility design throughput shall provide the capability of processing all SBW including liquids and tank solids and NGLW collected through 2011 within 3.0 years. This time-period includes six months of hot start-up.

C-5.1.1.4 Treat Waste - Location. The waste shall be treated and packaged at the INEEL, within the INTEC security enclosure, or outside of the INTEC security enclosure with its own security system.

C-5.1.1.5 Treat Waste - Meet WIPP RH-TRU WAC for the Evaporator Bottoms. The activity and mass of each of the following radionuclides shall be established on a payload container basis: Am-241, Pu-238, Pu-239, Pu-240, Pu-242, U-233, U-234, U-238, Sr-90, and Cs-137. If these radionuclides do not account for at least $95 \%$ of the total waste product radioactivity, the mass and activity of additional radionuclides shall be determined until $95 \%$ of the waste product activity is accounted for.

1. Any RH-TRU waste product payload container shall have a dose rate at the surface of the container greater than $200 \mathrm{mrem} / \mathrm{h}$ and less than $1000 \mathrm{rem} / \mathrm{h}$.

2. The ${ }^{239} \mathrm{Pu}$ fissile gram equivalent (FGE) in each payload container, plus its associated uncertainty expressed in terms of one standard deviation, shall be less than $325{ }^{239} \mathrm{Pu}$ FGE.

3. The TRU radionuclide alpha activity concentration, exclusive of the packaging, shall be greater than $100 \mathrm{nCi} / \mathrm{g}$.

4. The ${ }^{239} \mathrm{Pu}$ equivalent curie (PE-Ci) quantities in a payload container shall not exceed $80 \mathrm{PE}-$ $\mathrm{Ci}$ for direct loaded RH-TRU waste canisters and shall not exceed 240 PE-Ci for RH-TRU canisters loaded with three 30 gallon or 55 gallon drums.

5. The loaded payload container shall contain no detectable liquid. 
6. The hydrogen gas generation rate within each payload container shall not exceed the limit specified in the applicable content code.

7. PCB concentrations in wastes destined for WIPP shall not exceed $50 \mathrm{ppm}$.

8. Concentrations of flammable volatile organic compounds in the payload container headspace shall not exceed $500 \mathrm{ppm}$. If calculations cannot show that the VOC concentration in the headspace is less than $500 \mathrm{ppm}$ if all potentially flammable VOCs vaporized into the headspace, sampling of the containers shall be required.

9. The RH-TRU waste shall be placed in a WIPP approved RH-TRU waste canister.

10. The gross weight of a direct loaded RH-TRU canister shall not exceed $5250 \mathrm{lb}$.

11. Surface contamination on loaded RH-TRU payload containers, payload assemblies, and packages shall not exceed $20 \mathrm{dpm} / 100 \mathrm{~cm}^{2}$ alpha and $200 \mathrm{dpm} / 100 \mathrm{~cm}^{2}$ beta-gamma.

\section{C-5.1.1.6 Treat Waste - Meet Mixed Low Level WAC for the Grouted Acid Waste.}

1. SBW acid grout shall contain less than $10 \mathrm{nCi} / \mathrm{g}$ TRU alpha contamination so it may be sent to a qualified mixed low-level waste disposal facility.

2. SBW acid grout shall meet the Land Disposal Restrictions of a permitted near surface disposal unit.

C-5.1.1.7 Transfer Waste to WIPP. All transuranic wastes will be sent to WIPP for disposal. Facilities will be provided to transfer certified payload containers to WIPP-supplied transport vehicles. The SBW Treatment Project will be responsible to load the waste into approved transport casks and prepare those casks for shipment. WIPP will be responsible for providing the casks, trucks, drivers, and receipt of the waste at WIPP in Carlsbad, Mew Mexico.

1. RH-TRU waste shall be shipped in a RH-TRU 72-B cask.

2. The maximum number of payload containers in a RH-TRU 72-B cask is one.

3. The maximum weight of a loaded $72-\mathrm{B}$ cask is 45,000 pounds.

4. All RH-TRU wastes intended for shipment to WIPP shall meet an approved content code listed in the RH-TRUCON.

5. Sufficient lag storage of RH-TRU waste shall be provided in order to meet WIPP requirements for waste qualification and shipping/receiving rates.

6. A cask loading station compatible with loading WIPP supplied RH-TRU 72-B casks delivered on WIPP supplied transport vehicles shall be provided. The facility shall provide the capability to receive RH-TRU 72-B cask transporters in an enclosed area, raise the cask for loading, load the waste payload assemblies into RH-TRU 72-B cask, and close and lower the RH-TRU 72-B cask onto the transporters.

7. One of the cask loading facilities shall have the capability to wash road grime from the transporters and shipping casks prior to moving into the loading area. 


\section{C-5.1.1.8 Close Facility.}

1. The Direct Evaporation Facility shall be placed in standby after treating the volumes of waste identified above.

2. Prior to December 31, 2012, the Direct Evaporation Facility shall have all hazardous waste and hazardous waste residues removed and placed in safe shutdown until decommissioning.

\section{C-5.1.2 Systems, subsystem, and major components}

The direct evaporation facility shall utilize major support systems and utilities that are available at the INEEL. These include:

1. Analytical services.

2. Fire protection. This includes a manned fire station, central alarm system, and fire water lines near the waste treatment facility; it does not include fire protection within the physical structures designed and built or used by this project.

3. Electrical power. Both normal and standby electrical power are available for use near the INTEC Tank Farm from the INTEC electrical distribution system.

4. Steam

5. Telephone

6. Optical cable

7. Security

8. Medical

9. Radiation records

10. Central Facilities maintenance

11. Document control

12. Configuration management

13. Office buildings. The project shall provide adequate office space only for any operations staff, engineers, technicians, supervisors, and managers whose presence is required in the facility full-time during operations, plus limited turn-around office space.

14. Roads

15. Bus transportation.

\section{C-5.1.3 Boundaries and interfaces}

C-5.1.3.1 Liquid Waste Retrieval Feed System. The liquid waste retrieval system interfaces with Tank Farm Operations. All operations within the tank farm will be the responsibility of Tank Farm 
Operations. Prior to operation of the SBW Treatment Facility, all SBW shall be contained in Tanks WM180, WM-187, WM-188, and WM-189, and NGLW in WM-100, WM-101 and WM-102. Any modifications necessary to retrieve the SBW liquids and solids from these tanks will be the responsibility of this project.

\section{C-5.1.3.2 Utility Interfaces.}

1. Electric power will be obtained from existing sources at INTEC. The project shall provide the necessary equipment for distribution of power within the facility. The use of existing standby, emergency, or uninterruptible power supplies at INTEC shall be evaluated as the first choice during conceptual design.

2. The project shall tie in to the existing INTEC steam distribution piping at an appropriate location adjacent to the facility. If supplemental steam is required, electrical steam generation is preferred to avoid air permitting issues related to direct fired steam boilers.

3. The project shall tie in to the existing INTEC water systems piping (de-mineralized, deionized, distilled, fire water, etc.) at an appropriate location adjacent to the facility.

C-5.1.3.3 Sample Handling. Radioactive sample handling shall be compatible with the existing pneumatic sample transport system.

C-5.1.3.4 Tank Farm Closure Project. The system shall interface with the Tank Farm Closure Project. The Tank Farm Closure Project will sequentially remove all liquids and waste solids from the eleven Tank Farm tanks WM-180 through 190. The Tank Farm Closure Project will also clean the tanks to performance parameters, grout the tanks, piping and vaults, and close the tanks by December 2012 in accordance with the INTEC TFF Management Plan. The SBW Treatment project retrieves the wastes from tanks with the support of the TFC Project and treats it in the Direct Evaporation Facility.

C-5.1.3.5 Tank Farm Soils Project. The system shall interface with the Tank Farm Soils Project, which is responsible to remediate, as required, and close the Tank Farm under the Comprehensive Environmental Response, Compensation and Liability Act (CERCLA) program. The Tank Closure Project shall coordinate all work scope with the INTEC CERCLA Environmental Restoration Program (WAG 3) to ensure no conflicts.

C-5.1.3.6 LET\&D Facility and Nitric Acid Recycle Hold Tank. The treatment process may interface with the LET\&D facility, located in CPP-1618, and the Nitric Acid Recycle Hold Tank, located in CPP-659. Any modifications required to the LET\&D treatment equipment, Nitric Acid Recycle Hold Tank, or associated piping and instrumentation will be the responsibility of the SBW Treatment Project. Operation of the LET\&D facility and transfers of waste to and from the LET\&D facility and the Nitric Acid Recycle Hold Tank will be coordinated with the SBW Treatment Project but the responsibility of INTEC Operations.

\section{C-5.1.4 Operability}

The operability requirements for the SBW facility shall support the completion of SBW treatment by December 31, 2011.

C-5.1.4.1 Operational Lifetime. The facility shall have an operational lifetime adequate to process all SBW and tank solids without replacement of any process piping or major system components (tanks, hoppers, reaction vessels, etc). 
C-5.1.4.2 Installed Spares. Installed spares shall be provided for all rotating or reciprocating machinery located in remotely operated areas of the facility.

C-5.1.4.3 Annual Operation. The facility shall be designed to operate continuously for at least 200 days per year for at least three sequential years. Note that three-year window is based on a 6-month hot startup of the facility and a two and one-half year processing period for the entire inventory of SBW and NGLW.

\section{C-5.2 Assumptions}

Process designs considered for the direct evaporation process have been based on the following major assumptions. Additional assumptions used in the mass balance are identified in Section 2.5.4.

1. The SBW, both liquids and solids, and NGLW to be treated are not high-level waste.

2. The solidified SBW evaporator waste can be qualified for disposal at the WIPP.

3. No neutralization of waste prior to evaporation is required in order to satisfy WIPP's requirements for waste carrying the U-134 hazardous waste code.

4. The existing Evaporator Tank System (ETS) and LET\&D acid fractionator will be available for use if needed to process SBW evaporator condensate.

5. The grouted acid waste can be disposed of at a mixed low-level waste disposal site, such as Hanford or Envirocare, provided that the waste meets the site's WAC.

6. Toxic Substance Control Act regulations do not apply.

7. The facility will not require Nuclear Regulatory Commission licensing.

\section{C-5.3 Process Variations}

Many variations of the direct evaporation process are possible. Some have been tested and evaluated while others have not. In general, variations in the process could be categorized as differences in (a) how tank solids are treated, (b) the type of evaporator used, (c) details of how the evaporator concentrate is handled and packaged, (d) how the evaporator overhead vapors are processed, and (e) what existing INTEC facilities or equipment are used.

\section{C-5.3.1 Initial and Revised Baseline Process}

The FY 2002 direct evaporation feasibility study (Kimmitt, 2003) defined a process that included separate solids processing, dilution of evaporator condensate to process it through the PEWE and LET\&D, and grouting the LET\&D bottoms after dilution. The process defined in the FY 2002 feasibility study was synthesized prior to most development work and trade-off studies. It was intended that the cost estimate developed as part of the study be conservative, i.e., there would be low risk of exceeding the estimate due to later changes in the process.

Later evaluations and results of testing led to several improvements to this process scheme, including:

1. Direct grouting of 12 molar LET\&D bottoms rather than dilution to 3 molar prior to grouting, 
2. Coprocessing solids through the SBW evaporator rather than separate processing, and

3. Using an additional mist eliminator rather than processing the condensate through the PEWE.

Initial attempts to develop a grout formulation for the LET\&D acid obtained from processing the evaporator condensate indicated that because of highly exothermic reactions, dilution of the acid to about 3 molar would be needed prior to grouting. Grout tests determined that an acceptable grout could be made with this diluted acid and a $47.5 \%$ waste loading (Herbst, 2002). This formulation was used in the initial baseline process. However, subsequent tests showed that 12 molar could be successfully grouted without dilution using a waste loading of $35 \%$ (Herbst, 2002). This improved formulation resulting in a reduction in the grout volume by a factor of 3.1 .

The initial baseline process assumed that heel solids would be processed separately from the SBW. This configuration was selected due to concerns regarding the ability of evaporator systems to accommodate the high solids loading of an SBW/heel solids mixture. While coprocessing heel solids with SBW is not trivial, the ability to coprocess would provide significant benefits both in terms of cost and overall reduced system complexity. Coprocessing of these two waste streams would remove several major components including the heel solids feed tank and associated pumps, the heel solids canister loading station, and the FUNDABAC $®$ filter with its associated pumps, valves, filter cake drying system and monitors. An evaluation of different evaporator types was completed using the assumption of solids coprocessing (Packer, 2003) and found that several evaporator types will likely be able to process the mixed liquid/solids feed. Tests of a simulant containing undissolved solids were performed in June 2003, and were successful (Griffith, 2003b). As a result of the above benefits and based on the results from tests of solids-containing feeds, solids coprocessing was selected over separate processing for the present flowsheet.

The initial baseline process condensed the evaporator vapor, and then utilizing existing treatment equipment, re-evaporated the condensate in the PEWE. The PEWE bottoms was returned to the SBW evaporator feed tank, while the PEWE overhead was fractionated in the existing LET\&D, releasing water vapor to the atmosphere and producing a concentrated acid that was grouted. Because the PEWE has an acceptance criteria limit of $50 \mathrm{mg} / \mathrm{L}$ chloride (PRD-166, 1999), the SBW evaporator condensate would need to be diluted with deionized water by a factor of about 8 . The primary function of the PEWE is to concentrate dilute aqueous wastes, allowing the overhead to be processed by the downstream, contacthandled LET\&D and reducing the volume of the original waste. Since the PEWE equipment already exists, the costs to process the SBW evaporator condensate are operating rather than capital. The major operating cost is to re-vaporize not only the condensate but also about eight times the condensate volume due to required dilution, and also to then condense this dilute acid and re-vaporize it again in the LET\&D reboilers.

An alternative to processing the condensate through the PEWE is a new demister. At the INEEL, evaporators with various mist eliminator designs have been used to consistently achieve decontamination factors (DFs) in the range needed by the direct evaporation process. These DFs have been achieved using a combination of mist eliminator units. The PEWE consistently achieves a DF of $10^{6}$. Its mist eliminator system consists of a baffle, followed by 8 -inches of 931 York style (or equivalent) knitted mesh, 6-inches of York style 326 knitted mesh, and 6-inches of York style 421 knitted mesh. The EVS typically achieves a DF on the order of $10^{5}$ with a cyclone followed by 6 inches of York style 421 knitted mesh. Based on this information, it is reasonable to assume that a multiple effect demister can be designed for the direct evaporation process that will result in a DF of $10^{6}$ or greater.

Incorporating the above changes - grouting of LET\&D bottoms without dilution, coprocessing solids in the evaporator, and replacing the PEWE with a new demister - into the process synthesized in 
FY 2002 results in the present process flow diagrams and is called the "revised baseline" direct evaporation process.

\section{C-5.3.2 Alternative Evaporator Types}

An initial evaluation of evaporators for the direct evaporation process (Packer, 2003) reviewed the capabilities of thermosiphon evaporators, kettle evaporators, falling film evaporators, rising film evaporators, forced circulation evaporators, thin film evaporators, in-canister evaporation and evaporator combinations. The evaluation concluded that several evaporator types might be applicable to SBW evaporation; the types with the most promise being forced circulation evaporators and the agitated thin film evaporators (Packer, 2003). As of the end of CY 2003, three successful pilot tests had been performed with an agitated thin film evaporator (Griffith, 2003a; Griffith 2003b, Griffith, 2004). Testing of a forced circulation evaporator was planned for FY 2004, but due to the success of the agitated thin film evaporator, was cancelled.

\section{C-5.3.3 Alternative Condensate Treatment Schemes}

Vapor from the evaporator contains water, nitric acid, and small concentrations of $\mathrm{HCl}, \mathrm{HF}$, mercury, ${ }^{3} \mathrm{H}$, and carryover nonvolatile species. The concentration of nonvolatiles, including both radionuclides and RCRA hazardous metals, will depend on the evaporator design and whether additional de-entrainment equipment is added as part of the evaporator or subsequent to it. Concentrations of volatile species will depend primarily on the extent of evaporation.

Treatment of the evaporator vapor is required to render the vapor stream into forms that can either be released to the atmosphere or disposed at a waste repository or both. If processed into a waste, the evaporator vapor would first be condensed, then possibly concentrated, and the concentrate solidified while the water vapor would be released to the atmosphere. Alternatively, various technologies could be used to decompose or react nitric acid to form nitrogen that could be released to the atmosphere. A third type of treatment could be used to remove the contaminants, including nitric acid, from the vapor without condensing the water vapor. Each of these options for treating the condensate is summarized in the sections below. Additional information is available in the Condensate Treatment Alternatives Evaluation (Barnes, 2003a).

C-5.3.3.1 Alternatives to Processing Condensate through the PEWE. Because the condensate would require a high dilution with water in order to process it in the PEWE, alternatives have been considered. A new demister, as discussed above, was selected for the revised baseline process. Other alternatives are reviewed in this section.

Use of the EVS is a possible option for the removal of radionuclides from the SBW evaporator overheads. Bottoms product from the EVS would be recycled back to the SBW feed tank. Chlorine feed limits (if any) to the EVS for a given waste stream have in the past been established in Run Plans based on the calculated amount of chlorine expected in the EVS overheads and subsequently sent to the PEWE. Because the PEWE would be bypassed in this option, chloride feed limits to the EVS would likely be based upon those ultimately established for the LET\&D. The EVS option, while feasible, is presently not recommended for the direct evaporation process. The cost associated with re-evaporating the evaporator overheads and the possible EVS modification expenses would likely make this option more costly than incorporating a new mist eliminator system.

HEPA filtration of the evaporator's overheads would be expected to provide decontamination sufficient to allow downstream equipment to be contact-handled. The use of a HEPA filter would require as a minimum the installation of a reheater to raise the temperature of the evaporator overheads $20-30^{\circ} \mathrm{F}$ 
above its dew point temperature. A stand-alone mist eliminator upstream of the HEPA would also be considered desirable to prevent any liquid that may bypass the evaporator's internal mist eliminator from wetting the HEPA filters. Before HEPA filtration can be considered as a viable option for this application, however, validation tests would need to be carried out to determine if an appropriate sealant is available to accommodate the acidic environment of the evaporator overheads.

Wet Electrostatic Precipitators (WESPs) are used in industry to remove particles smaller than 1 micron. Depending upon the vapor velocities through the WESP, removal efficiencies have been reported as high as $99.99 \%$ for particle sizes between 0.4 and 1.0 micron $^{\mathrm{a}}$. These units come in a variety of materials of construction to accommodate acidic and basic gas streams. While DFs would be expected to be very high, a WESP is not considered an economically attractive option due to its high capital cost and the large footprint required to house the unit.

C-5.3.3.2 Alternatives to Grouting Acid in Condensate. An alternative to grouting the acid recovered from the condensate is to chemically react the acid to form nitrogen that can then be released to the atmosphere. Selective catalytic reduction (SCR) is one of several methods that could be used to reduce the nitric acid in the evaporator vapor to diatomic nitrogen gas. SCR was selected to represent these options because of its widespread use in industry. Vapor from the evaporator would be routed through a secondary mist eliminator or other solids separator, and then reheated and HEPA filtered to remove the majority of the radioactive contamination. The off-gas stream at this point in the process would consist primarily of nitric acid and water vapor, with small amounts of tritium, iodine, and mercury. This off-gas stream would be preheated to approximately $400^{\circ} \mathrm{C}$ prior to entering the SCR reactor. The SCR reactor would be a packed bed of hydrogen mordenite or similar zeolite catalyst. ${ }^{b}$ Ammonia would also be injected into the reactor as the reducing agent. In the reactor, nitric acid and $\mathrm{NO}_{\mathrm{x}}$ (formed during reheating) would be reduced to nitrogen and oxygen. Significant cost savings associated with this scenario could be realized in these areas:

- $\quad$ Operating Costs - by eliminating or reducing the need to operate the PEWE and LET\&D, operating expenditures would be reduced

- Waste Disposal Costs - by eliminating a secondary waste stream (grout), costs associated with characterization and disposal could be reduced

- $\quad$ Capital Costs - by eliminating the acid waste stream, significant money could be saved by eliminating the need for a new grout facility ${ }^{\mathrm{c}}$.

a AirPol Inc., 2002, personal communication to Bart Packer

b A hydrogen mordenite or zeolite catalyst is preferred over a precious metal or vanadium/tungsten catalyst due to the presence of mercury in the off-gas. It is believed that mercury would rapidly poison these types of catalysts.

c The savings from elimination of the grout plant need to be compared against the capital cost to install the SCR and associated off-gas monitoring and treatment equipment. However, before a capital estimate can be made for the SCR equipment, a regulatory analysis should be performed to determine how the facility would be permitted (i.e., if the facility will require permitting to MACT standards, additional off-gas cleanup unit operations and monitoring instrumentation will also be required). 
However, there are also several drawbacks to implementing the SCR technology. The most significant concerns include:

- $\quad$ Safety Concerns - The transportation, storage, and use of anhydrous ammonia presents a personnel safety hazard. Alternatives to anhydrous ammonia could be considered, but these alternatives introduce additional process complexities (i.e., the use of aqueous ammonia would require a vaporizer and a larger storage facility; the use of urea would require a decomposition reactor to convert it to ammonia).

- $\quad$ Permitting Concerns - Evaporation is a relatively low-temperature process (i.e., $<130^{\circ} \mathrm{C}$ ). It is conceivable that inclusion of the SCR (which operates at $400-550^{\circ} \mathrm{C}$ ) could result in classification of the process as a miscellaneous thermal treatment unit by the EPA and/or State regulators. Such a designation would likely require a more involved permitting process, and could include requirements such as a trial burn and adherence to MACT air emissions standards. If MACT compliance were imposed, it would also be necessary to add a thermal or catalytic oxidizer for control of total hydrocarbons (THC) and CO, and a carbon bed for control of mercury emissions. It is likely that these additional permitting requirements would negatively impact both project cost and schedule.

- Based on a process simulation of this scheme (see Barnes, 2003a), five reactor stages would be required to destroy the $\mathrm{HNO}_{3}$ in the off-gas from the SBW evaporator. This is not typical for an SCR installation and would add cost, plot space, and complexity to the process.

Due to feasibility issues discussed above, and due to potential safety, permitting, and schedule issues associated with this scenario, it is not recommended.

C-5.3.3.3 Alkaline Scrub Process. This scheme involves scrubbing the evaporator vapor using an alkaline scrubbing agent to both remove contaminants and convert acids to salts that can be returned to the SBW evaporator. The alkaline scrubber would neutralize and remove nitric acid from the vapor, neutralize and remove other trace acids such as $\mathrm{HCl}$ and $\mathrm{HF}$, remove oxidized forms of mercury and remove carry-over particulate. A purge from the scrubber would be recycled to the evaporator. The scrubbed vapor could then be released to the atmosphere after superheating and HEPA filtration ${ }^{\mathrm{d}}$. The volume of primary waste would increase due to the recycled salts, but no secondary waste grout is produced, eliminating all grouting and grout packaging equipment and reducing the plot space and facility size needed relative to the revised baseline process.

The scrub purge that is returned to the evaporator would have a neutral $\mathrm{pH}$ and, thus, would be expected to have a minimal effect on feed and evaporation chemistry. Magnesium oxide is recommended as the scrubbing agent because of its ability to hydrate in the concentrated waste as it cools. The scrub purge would add about $12-28 \%$ to the feed rate. Other comparisons are shown in Table C-1. For reference Table C-1 also show a comparison to the initial baseline process, which is described in the FY 2002 feasibility study (Kimmitt, 2003). The higher evaporator bottoms volume of the revised baseline compared to the initial baseline process is due to a $30 \mathrm{wt} \%$ increase in feed mass due to the addition of solids, an updated feed volume estimate and a small change in the assumed degree of evaporation.

The caustic scrubbing process would have capital cost savings over the revised baseline process, due to the elimination of the grouting facility. Based on unit costs of $\$ 13,352 / \mathrm{m}^{3}$ for disposal or $\mathrm{RH}$

d. Additional test data for this process is needed to confirm that no other removal steps would be required. 
wastes at WIPP and $\$ 1,854 / \mathrm{m}^{3}$ for disposal of $\mathrm{CH}$ low-level mixed waste, the caustic scrub process would have a disposal cost of $\$ 2.6$ million greater than the revised baseline cost. The savings in capital cost is \$20 million (see Barnes, 2003a).

Table C-1. Comparison of alkaline scrubbing process to the baseline process. ${ }^{\mathrm{a}}$

\begin{tabular}{lccc}
\hline \multicolumn{1}{c}{ Ratios } & $\begin{array}{c}\text { Initial Baseline } \\
\text { Process }\end{array}$ & $\begin{array}{c}\text { Revised Baseline } \\
\text { Process }\end{array}$ & $\begin{array}{c}\text { Alkaline Scrub } \\
\text { Process }\end{array}$ \\
\hline Relative Evaporator Feed Ratio & 1.00 & 1.00 & 1.23 \\
Relative Evaporator Bottoms Volume & 0.71 & 1.00 & 1.30 \\
Relative Evaporator Reboiler Duty & 1.00 & 1.00 & 1.07 \\
Relative Condenser Duty & 1.00 & 1.00 & 0.21 \\
Relative Grout Volume & 2.56 & 1.00 & 0 \\
\hline \multicolumn{1}{c}{ Volumes } & $\mathbf{m}^{\mathbf{3}}$ & $\mathbf{m}^{\mathbf{3}}$ & $\mathbf{m}^{\mathbf{3}}$ \\
\hline Evaporator Bottoms & 727 & 1021 & 1387 \\
Tank Solids & 106 & 0 & 0 \\
Grouted Acid & 3550 & 1387 & 0 \\
\hline
\end{tabular}

a Waste volumes shown in this table differ slightly from those shown in later sections of this report because the two sets of estimates were based on different revisions of the Feed Composition Report (Barnes, 2003b).

The present alkaline scrub process (see PFD-2A) shows a packed bed MgO scrubber. Magnesium oxide and magnesium hydroxide are commonly used in water treatment and commercial forms are available that could be used in a packed bed. $\mathrm{MgO}$ scrubbers have been used for $\mathrm{SO}_{2}$ removal from offgas of coal-fired boilers and other industrial processes. $\mathrm{MgO}$ scrubbers in these facilities are often venturi-type scrubbers or other slurry-based systems. However, to minimize maintenance of the scrubber, a packed bed may be preferable. Nitric acid vapor in the scrubber feed would react with bed particles forming magnesium nitrate, which would be dissolved by the down-flowing aqueous stream. A portion of the scrubber effluent liquid would be purged to the evaporator, and the remainder recycled to the top of the bed. The liquid stream would be nearly solids-free. Bed material would be continually added to the top of the bed; however, it's expected that the rate of addition would only be a small fraction, about $1 \%$, of the volume of the bed per hour.

Other scrubber types, such as moving bed, fluidized bed, or slurry systems designs, could also be considered.

C-5.3.3.4 Condensation/Fractionation/Neutralization Option. Another possible flow scheme would condense the evaporator vapor, concentrate the acid using the LET\&D, and neutralize the concentrated acid with either $\mathrm{MgO}$ or aluminum hydroxide. The resultant salt solution would be recycled to the evaporator. This scheme is similar to the alkaline scrub process, except that the existing LET\&D is used, and the scrubber is replaced by a neutralization tank. While this scheme retains the use of the LET\&D and hence incurs its operating expenses, it does not require a scrubber nor is it likely that it would require a GAC bed, and would thus have no spent carbon waste. The scheme would require a neutralization tank, either a new tank or possible modifications to an existing tank. Because no testing has yet been performed of a scrubber in an SBW evaporation process, neutralization of the LET\&D bottoms presents less technical risk than scrubbing. A cost comparison of this scheme versus the alkaline scrub scheme has not been performed. 
C-5.3.3.5 Neutralization of SBW Feed. An indirect method to eliminate condensate treatment is to neutralize the SBW feed to the evaporator. Partial neutralization of the feed would convert a large fraction of the nitric acid in the feed to nitrate salts, greatly reducing the quantity of nitric acid in the condensate that would need to be treated and disposed as a secondary waste. Complete neutralization of the feed has the potential for elimination of the secondary waste completely.

However, this scheme has several disadvantages. As SBW is neutralized, a point is reached at which dissolved aluminum in the waste will precipitate in a gelatinous form. Other species such as iron may also precipitate. The precipitated solids may present handling problems transferring the waste to the evaporator and scaling problems in the evaporator. The solids loading in the waste may be high, resulting in extremely high viscosities, as the waste is concentrated in the evaporator, so that evaporation becomes infeasible or requires high maintenance equipment. In addition, neutralization could cause chemistry changes that would prevent the concentrated product from complete solidification upon cooling. For these reasons, and because the alkaline scrub and condensation/fractionation/neutralization options retains the benefits of this process without some of the disadvantages, neutralization of the feed has not be given further consideration.

C-5.3.3.6 Removal of Mercury and/or Chloride from Noncondensable Gases. Small concentrations of mercury, as volatilized chloride or other forms, and hydrochloride acid vapor will be present in the evaporator overhead. Rates of mercury released to the atmosphere are expected to be well under regulatory or risk-based limits. For the revised baseline scheme, or other schemes in which the evaporator vapor is totally condensed, the emissions of mercury should be undetectable. However, for the alkaline scrub process or others in which the evaporator vapor is not totally condensed, mercury emissions would likely exceed MACT standards. While it is not known whether regulators would apply MACT requirements to this process, reduction in mercury emissions could be required. Mercury could be removed in the gas phase with a carbon bed. Alternatively, the vapor could be condensed and mercury removed from the condensate by either ion exchange or sulfide precipitation. The treated condensate would then need to be vaporized.

Present mass balances show mercury concentrations of approximately $100 \mathrm{mg} / \mathrm{kg}$ in the grouted acid. This concentration is well below the Land Disposal Restriction limits for mercury of $260 \mathrm{mg} / \mathrm{kg}$, and thus mercury removal from the condensate is not required for schemes in which the condensate is grouted.

The need for chloride removal is based on WAC for the LET\&D and will need to be reviewed for the revised baseline case after LET\&D criteria are established. Testing is needed to confirm that an alkaline scrubber can remove $\mathrm{HCl}$ to levels meeting emission requirements.

Additional information about methods to remove mercury and chloride are contained in Barnes (2003a), Soelberg (2003) and O’Brien (2003).

C-5.3.3.7 Acid Fractionation Options. The LET\&D is an existing INTEC fractionator that currently processes condensate from the Process Equipment Waste Evaporator. To utilize the LET\&D in the direct evaporation process, a new line would be needed from the SBW facility to the LET\&D in CPP1618. Based on the location of the Direct Evaporation Facility defined in the Feasibility Study (Kimmet, 2003), just north of the TFF, a line of approximately $1000 \mathrm{ft}$ would be needed to carry condensate to the LET\&D. Also, because the evaporator condensate would be about 10-15 times higher in acid concentration than PEWE condensate, column modifications would be required to introduce the feed on a lower tray. Modifications in piping and/or fluid transfer equipment may also be needed to use the Nitric Acid Recycle Hold Tank. 
Alternatively, a new fractionator could be designed and built to perform concentration of the SBW evaporator overhead.

\section{C-5.4 Basis for Flow Diagrams}

C-5.4.1.1 Waste Tanks (PFD-1). PFD-1 shows the SBW feed tanks VES-WM-180, VES-WM187, VES-WM-188 and VES-WM-189, and the NGLW tanks VES-WM-100, VES-WM-101 and VESWM-102. These tanks are all existing tanks and currently in use. New mixing pumps would be installed in VES-WM-187, VES-WM-188 and VES-WM-189 to distribute solids evenly between these three tanks. The basis for the number of pumps ( 3 per tank) and the blending scenario has been documented by Wood (2002).

A follow-on study (Morrell, 2003) evaluated different mixing pumps and recommended submersible pumps over long shaft pump technology. Both Wood and Morrell recommend mockup testing of the mixing pumps. Mockup testing is needed to confirm and further define the basis for the mixing pumps.

Based on present estimates of total tank solids, it is anticipated that new pumps will be required in three tanks. If future tank samples indicate the total solids are significantly less than current estimates, pumps would only be installed in two tanks.

C-5.4.1.2 SBW Evaporator (PFD-2). PFD-2 shows the SBW Evaporator, evaporator overhead demister, condenser, and other demisters, superheaters and HEPA filters for both process and vent gases.

An evaluation of different evaporator types (Packer, 2003) concluded that several types were likely feasible, including agitated thin film, in-can, thermosiphon, and semi-batch kettle evaporator. Three series of tests have been performed with an agitated thin film evaporator and have been very successful (Griffith, 2003a; Griffith, 2003b, Griffith, 2004). Based on the results of these tests, an agitated thin film evaporator is shown on PFD-2.

Based on DFs achieved by INEEL existing evaporators, sufficient decontamination can be achieved by the use of multiple mesh pads. The evaporator will have an internal demister pad, and then the vapor will pass through an external demister. Entrained liquid captured by the mesh pads will be pumped to the SBW Feed Tank. To maintain a high removal efficiency and minimal pressure drop, condensate will be pumped intermittently to the pads to wash off solids that have accumulated.

The condenser removes sufficient heat from the evaporator overhead to effect total condensation, preventing release of nitric acid to the atmosphere. At the expected operating pressure, ASPEN Plus predicts a bubble point temperature for the overhead of $83^{\circ} \mathrm{C}$, hence total condensation should be achievable using cooling water as the coolant. The amount of nitric acid, $\mathrm{HCl}$, and $\mathrm{HF}$ carried into the off-gas will be dependent on the efficiency of the demister.

Off-gas from the demister is superheated to avoid condensation in the downstream HEPA filters. The HEPA filters serve as the final protection against release of nonvolatile hazardous and radioactive species.

C-5.4.1.3 LET\&D (PFD-3). Equipment contained in the existing LET\&D is shown on PFD-3. The equipment includes a feed tank, a bottoms tank and spared 15-tray acid fractionators with reboilers, partial condensers, vapor liquid separators, superheaters, off-gas filters and vapor blowers. 
Each of the two fractionators was designed to process 550 gallons per hour of feed (INTEC RCRA Interim Status Document ISD-1, 2002). Typically, $99 \%$ of the acid in the feed is recovered in the bottoms product while $1 \%$ is lost in the vapor from the overhead separators exhausted to the stack (Interim Status Document ISD-1, 2002).

A new line from the SBW Direct Evaporation Facility to CPP-604 would be required. In addition, modifications to the fractionators would be required to allow feed to enter on a lower tray.

C-5.4.1.4 Acid Grouting (PFD-4). PFD-4 shows equipment to grout and package the recovered nitric acid from the LET\&D. Neutralization of this 12 molar acid with calcium hydroxide followed by grouting with a combination of BFS and Portland cement is based on demonstration tests in 2002 and 2003 (Herbst, 2002; Raman, 2003).

C-5.4.1.5 Canister Loading and Handling (PFD-5). An initial concept for packaging the concentrated SBW waste was identified and briefly described in the Direct Evaporation Feasibility Study (Kimmitt, 2003). Additional details for a related packaging system (for calcine waste) were developed in a separate study (Clark, 2003) and are summarized on PFD-5. Several packaging requirements are inherent in SBW evaporator waste that were not present for the calcine packaging system, i.e., filling the canister with hot waste, cooling of waste in the canister, and possible addition of absorbent on top of the waste. Translating these additional requirements into a mechanical design has not yet been done.

C-5.4.1.6 Alkaline Scrub (PFD-2A). The basis for the alkaline scrub process is an evaluation (Barnes, 2003a) that showed that this process had advantages - reduced footprint, less equipment, a single waste form - than the revised baseline scheme. The scrubbing agent assumed in Barnes' evaluation was caustic. Later tests showed that the use of caustic would result in an unsatisfactory evaporator product. Tests performed in FY 2004 have shown that a simulated waste that includes neutralization of overhead with $\mathrm{MgO}$ produces a good waste product. Based on the results of these tests, $\mathrm{MgO}$ was selected as the scrubbing agent.

\section{C-5.5 Basis for Mass Balances}

Mass balances were developed for the Direct Evaporation alternative to provide a basis for sizing equipment, determining feed chemical requirements, determining utility requirements, estimating emissions and determining waste volumes, compositions and properties. The sections below document the basis for the mass balance. The basis is capsulated in succinct statements, called "design basis elements" (DBEs), shown in italics in the following paragraphs. The DBEs are amplified with background information, references, explanation, and, in some cases, a discussion of uncertainties.

\section{C-5.5.1 Feeds}

Feeds to the Direct Evaporation process include NGLW and SBW from the INTEC TFF.

C-5.5.1.1 Tank Farm Waste Feeds. According to present Tank Farm management plans (Barnes, 2002b), waste presently in the TFF tanks will be consolidated into four tanks, WM-180, WM187, WM-188 and WM-189 by the end of 2005. Tanks WM-180, WM-188 and WM-189 will contain liquid SBW with relatively small quantities of undissolved solids, while WM-187 will be used as a collection tank for solids and dilute liquid wastes. Waste generated after 2005 will be collected in WM100, WM-101 and WM-102. Shortly before treatment, transfers will be made between tanks to distribute solids that have been collected in WM-187 between the three tanks WM-187, WM-188 and WM-189. 
DBE \#1: Solids that have been collected in Tank WM-187 will be distributed between Tanks WM188, WM-189 and WM-187 by waste transfers between these tanks prior to feeding to the treatment process.

Pumps will be installed in these three tanks to homogeneously mix the solids with the tank liquid. Approximately equal amounts of waste from WM-188 and WM-189 will first be transferred to Tank WM-187, and then similar volumes of the mixed solids/liquid slurry in WM-187 will be returned to Tanks WM-188 and WM-189 to restore these tanks to their full capacity. The tank-blending scenario is described in more detail by Wood (2002) and Barnes (2003b).

Mass balances are shown in Section C-6 for four feed cases. The feed cases correspond to present estimates of waste quantities and compositions that would be transferred from Tanks WM-180, WM-187, WM-188 and WM-189 after waste in these tanks is blended. The WM-187 mass balance case represents a blend of the waste in WM-187, after transfers to Tanks WM-188 and WM-189, with newly generated liquid waste (NGLW). Whether the NGLW is added to WM-187 or sent directly from WM-100, WM101 or WM-102 to the SBW Treatment Facility feed tanks is of no consequence to the mass balance.

\section{DBE \#2: Four waste compositions, corresponding to waste in Tanks WM-180, WM-188, WM-189 and a combined residual in WM-187 plus $N G L W$, are assumed to adequately envelope the waste fed to the $S B W$ Treatment Facility for conceptual design purposes.}

Table C-2 shows the variability of feed composition between the tanks for major species. Additional variation in feed composition is possible due to (a) uncertainties in sample analyses (b) uncertainties in the amount of waste solids in the feed (c) uncertainties in solids composition (d) uncertainties in NGLW quantity and composition and (e) potential changes in the tank mixing or Tank Farm Management scenario. The magnitude of some of these uncertainties is known or can be estimated, and is generally within the range shown in Table C-2. Feed composition uncertainties and expected composition ranges are discussed in more detail by Barnes (2003a and 2003b). 
Table C-2. Feed concentration variations.

\begin{tabular}{lccc}
\hline & Minimum & Maximum & Max/Min \\
\hline $\mathrm{H}+$ & $1.07 \mathrm{E}+00$ & $2.43 \mathrm{E}+00$ & 2.28 \\
$\mathrm{Al}+3$ & $3.61 \mathrm{E}-01$ & $6.08 \mathrm{E}-01$ & 1.68 \\
$\mathrm{~B}+3$ & $1.04 \mathrm{E}-02$ & $1.83 \mathrm{E}-02$ & 1.76 \\
$\mathrm{Ca}+2$ & $3.47 \mathrm{E}-02$ & $6.13 \mathrm{E}-02$ & 1.77 \\
$\mathrm{Cs}+$ & $8.42 \mathrm{E}-05$ & 3.27 \\
$\mathrm{Cl}-$ & $2.86 \mathrm{E}-02$ & 1.59 \\
$\mathrm{Cr}+3$ & $1.80 \mathrm{E}-02$ & $5.13 \mathrm{E}-03$ & 1.53 \\
$\mathrm{~F}-$ & $3.36 \mathrm{E}-03$ & $5.61 \mathrm{E}-02$ & 1.69 \\
$\mathrm{Fe}+3$ & $3.32 \mathrm{E}-02$ & $2.97 \mathrm{E}-02$ & 1.54 \\
$\mathrm{Mg}+2$ & $1.92 \mathrm{E}-02$ & $1.94 \mathrm{E}-02$ & 1.76 \\
$\mathrm{Hg}+2$ & $1.10 \mathrm{E}-02$ & $5.44 \mathrm{E}-03$ & 3.73 \\
$\mathrm{NO}_{3}-$ & $1.46 \mathrm{E}-03$ & $6.63 \mathrm{E}+00$ & 1.34 \\
$\mathrm{PO}_{4}-3$ & $4.93 \mathrm{E}+00$ & $1.75 \mathrm{E}-01$ & 8.95 \\
$\mathrm{~K}+$ & $1.95 \mathrm{E}-02$ & $3.13 \mathrm{E}-01$ & 1.78 \\
$\mathrm{Na}+$ & $1.76 \mathrm{E}-01$ & $1.89 \mathrm{E}+00$ & 1.40 \\
$\mathrm{SO}_{4}-2$ & $1.35 \mathrm{E}+00$ & $9.08 \mathrm{E}-02$ & 2.24 \\
$\mathrm{Zr}^{-}+4$ & $4.05 \mathrm{E}-02$ & $6.66 \mathrm{E}-02$ & 50.33 \\
\hline
\end{tabular}

\section{C-5.5.2 Stream Factor, Operating Schedule and Feed Rate}

The Technical and Functional Requirements (Barden, 2003) specifies a 3-year processing time that includes six months of hot start up. A stream factor of 200 days per year is assumed. The average feed rate is thus:

$$
1,029,450 \mathrm{gal} /(2.5 \mathrm{yrs} * 200 \mathrm{~d} / \mathrm{yr} * 24 \mathrm{hr} / \mathrm{day})=85.8 \mathrm{gal} / \mathrm{hr} \text { or } 1.43 \mathrm{gal} / \mathrm{min}
$$

Because of downtime for canister change-out, feed to the evaporator is intermittent, and the actual feed rate is expected to be $1.8 \mathrm{gpm}$ for the revised baseline scheme and $2 \mathrm{gpm}$ for the alkaline scrubber scheme.

$D B E$ \#3: The total SBW waste inventory will be processed over 2.5 years, during 200 24-hr operating days per year and an average feed rate of $1.4 \mathrm{gpm}$.

\section{C-5.5.3 Tank Mixing and Feed Transfer from Tank Farm}

In an evaluation of options for processing solids in the calciner, R. Wood (2003) and D. Morrell (2003) recommend mixing the solids in the TFF tanks. The concept of mix pumps in the tanks was taken from an earlier conceptual design (ICF Kaiser, 1995) for tank heel removal. While mock-up tests are recommended to validate the effectiveness of this mix scheme, the studies by Wood and Morrell included an assessment of mixing pump performance and issues (see Section 3 of Wood). 
Transfers of waste to the treatment facility will be made using existing steam jets. The mass balance assumed that the volume of waste increases by $5 \%$ due to steam used to in the jets. This increase is based on past transfers of waste to the NWCF.

DBE \#4: Mix pumps installed in TFF tanks are assumed to keep solids uniformly distributed throughout the tank waste. Waste will be transferred to the treatment facility using existing steam jets, diluting the waste by $5 \%$.

\section{C-5.5.4 Evaporation and Disengagement}

This section documents the basis for and assumptions used in the mass balance of the SBW evaporator and demister.

C-5.5.4.1 Evaporation Temperature and Pressure. Lab and pilot-scale testing of the direct evaporation process indicate that the optimal operating pressure is around $390 \mathrm{~mm} \mathrm{Hg}$ absolute (7.5 psia) (Kirkham, 2003). Lowering the pressure beyond this value results in film boiling, while increasing the pressure results in a higher endpoint temperature (i.e., $>130^{\circ} \mathrm{C}$ ) and visible $\mathrm{NO}_{2}$ generation. Hence, for the material balances, the minimum evaporator operating vacuum pressure was selected in order to achieve a predicted endpoint temperature between $120^{\circ} \mathrm{C}$ and $126^{\circ} \mathrm{C}$.

DBE \#5: An evaporator pressure of 8.6 psia was assumed for the mass balances. At this pressure, the APEN model predicted temperatures of $117-124^{\circ} \mathrm{C}$ for the revised baseline scheme and 120$126^{\circ} \mathrm{C}$ for alkaline scrub scheme.

C-5.5.4.2 Extent of Evaporation. In lab scale testing for WM-189 simulant, it was observed that the product would set up "rock hard" when concentrated above $59.8 \mathrm{wt} \%$ (Kirkham, 2003). The maximum concentration achieved was $64.7 \mathrm{wt} \%$. Hence, for the revised baseline material balance for WM-189, the extent of evaporation was specified near the middle of this range at $62 \mathrm{wt} \%$. However, for other tank compositions, mass balance modeling showed that the extent of evaporation needed to be increased in order to tie up all water in the bottoms product as $\mathrm{Al}\left(\mathrm{NO}_{3}\right)_{3} \bullet 9 \mathrm{H}_{2} \mathrm{O}, \mathrm{Ca}\left(\mathrm{NO}_{3}\right)_{2} \bullet 4 \mathrm{H}_{2} \mathrm{O}$, and $\mathrm{Na}_{2}\left(\mathrm{SO}_{4}\right) \cdot 10 \mathrm{H}_{2} \mathrm{O}$. Hence, for the other revised baseline cases, the extent of evaporation was adjusted in the mass balance until all free water was tied up.

For the alkaline scrubber cases, the extent of evaporation was adjusted in the mass balance model to maintain a bottoms temperature of less than $125^{\circ} \mathrm{C}$. Water in the bottoms will be tied up as hydrated nitrates of aluminum, iron, magnesium and other metals. Because of the use of magnesium as the scrubbing agent, which forms both $\mathrm{Mg}\left(\mathrm{NO}_{3}\right) \cdot 2 \mathrm{H}_{2} \mathrm{O}$ and $\mathrm{Mg}\left(\mathrm{NO}_{3}\right) \cdot 6 \mathrm{H}_{2} \mathrm{O}$, an acceptable product can be produced over a range of water concentrations. The mass balances assume magnesium nitrate solidifies as dihydrate unless this results in an endpoint evaporator temperature greater than $125^{\circ} \mathrm{C}$, in which case, the amount of water is increased to maintain a temperature less than $125^{\circ} \mathrm{C}$.

DBE \#6: Extent of evaporation was set at 62 wt\% for the WM-189 revised baseline material balance to match laboratory results. Extent of evaporation for the WM-180, WM-187, and WM-188 revised baseline material balances was adjusted to tie up all free water as $\mathrm{Al}\left(\mathrm{NO}_{3}\right)_{3} \bullet 9 \mathrm{H}_{2} \mathrm{O}$, $\mathrm{Ca}\left(\mathrm{NO}_{3}\right)_{2} \bullet 4 \mathrm{H}_{2} \mathrm{O}$, and $\mathrm{Na}_{2}\left(\mathrm{SO}_{4}\right) \bullet 10 \mathrm{H}_{2} \mathrm{O}$. For all alkaline scrubber material balances, the extent of evaporation was adjusted for formation of a combination of $\mathrm{Mg}\left(\mathrm{NO}_{3}\right)_{2} \cdot 2 \mathrm{H}_{2} \mathrm{O}$ and $\mathrm{Mg}\left(\mathrm{NO}_{3}\right)_{2}{ }^{\circ} \mathrm{HH}_{2} \mathrm{O}$ that maintained the evaporator temperature equal to or below $126^{\circ} \mathrm{C}$.

\section{C-5.5.4.3 Evaporation and Disengagement Decontamination Factors/Entrainment.}

The DF for the evaporator was specified as 10,000 based on PEWE performance, and consistent with that determined for most feed components achieved in wiped film evaporator tests conducted at Oak Ridge 
(Boring, 1994). Also, discussions with LCI personnel indicated that entrainment for a wiped film evaporator should be essentially zero for the gas flow rates recently tested during SBW trials at LCI (see Griffith, 2003a). According to LCI, the gas velocity would have to double before any entrainment would be expected to occur. This is also consistent with the observation of zero liquid collected by the mist eliminator during the LCI tests.

The DF for the demister was specified as 100. Perry's Chemical Engineers' Handbook states that wire mesh mist eliminators can achieve a collection efficiency of $99.8 \%$ (i.e., DF $=500$ ). However, this may be overly optimistic based on testing within the DOE complex. Testing of a high-efficiency mist eliminator (HEME) at Hanford only achieved a DF of 400, and Defense Waste Processing Facility (DWPF) uses a DF of 50 for their HEME (see Wood, 2001). A conventional mist eliminator (i.e., not a HEME) cannot reasonably be expected to perform even this well. However, by sequencing correspondingly finer meshes, it should be possible to achieve a DF of 100 using standard demister designs.

The DFs assumed for the evaporator and demister must be verified in pilot-scale tests to eliminate the uncertainty associated with these assumptions.

\section{DBE \#7: The DF for the evaporator was specified as 10,000 and the DF for the mist eliminator was specified as 100, yielding a combined DF of 106.}

C-5.5.4.4 Partitioning of Volatile Species. Partitioning of volatile chemical species such as chloride, fluoride, and iodine was estimated using Aspen vapor liquid equilibrium (VLE) models. Property data for this model has been tuned to match prior sampling results from the existing EVS and PEWE evaporators.

Partitioning of mercury was based on lab and pilot-scale test results (Kirkham, 2003, Griffith, 2003a) which indicate that only 3-10\% of the mercury is partitioned to the evaporator condensate, while the remainder is partitioned to the bottoms product. This was accomplished in the Aspen model by diverting $90 \%$ of the mercury predicted in the condensate to the bottoms product. For the alkaline scrubber material balances, ASPEN predicted that over $99.9 \%$ of the mercury remained in the bottoms. This may have been due to an interaction with the magnesium and related species, or due to dilution of the feed by the scrub recycle. The FY 2004 direct evaporation tests with MgO (Griffith, 2004) did not include mercury in the simulant, so this improved retention has not been experimentally verified.

Tritium was assumed to partition the same as water in the model, which was predicted using Aspen VLE models. Radioactive iodine was assumed to partition the same as chloride in the model. Ruthenium and cesium were treated as nonvolatile species in the model (i.e., a DF of $10^{6}$ was applied over the evaporator and demister combination). The DFs assumed for radioactive species must be verified in pilot-scale tests (likely using surrogates) to eliminate the uncertainty associated with these assumptions.

DBE \#8: Aspen VLE models were used for partitioning of halides. Aspen VLE models were also used for partitioning mercury, but a correction was accomplished by diverting $90 \%$ of the mercury in the condensate to the bottoms product in order to better match experimental data. Ruthenium and cesium were treated as nonvolatile species in the material balance. For all alkaline scrubber material balances, no correction was needed. 


\section{C-5.5.5 Condensate Treatment - Revised Baseline Scheme}

The basis for and assumptions used in the mass balance of the condenser, condensate tank, and grout facility are documented in this section for the revised baseline case. The basis for the evaporator vapor treatment for the alkaline scrubber scheme is given in Section C-5.5.9.

C-5.5.5.1 Condenser Temperature and Pressure Drop. The condenser temperature was specified at $35^{\circ} \mathrm{C}$, which represents a reasonable value based on typical cooling water supply temperatures. The pressure drop through the condenser and demister combination was assigned a value of 15 inches $\mathrm{H}_{2} \mathrm{O}$. These process parameters are not perceived to significantly impact process feasibility, but will influence the size of the condenser and process off-gas (POG) blower. Hence, a more rigorous effort should be made to quantify these parameters as part of detailed design.

DBE \#9: The condenser temperature was specified at $35^{\circ} \mathrm{C}$, and the pressure drop across the condenser and demister combination was assigned a value of 15 inches $\mathrm{H}_{2} \mathrm{O}$.

C-5.5.5.2 Demister Efficiency. Particulate and droplet carryover through the condenser and demister was assigned a DF of 10,000. This value has been used commonly in existing NWCF process models. However, pilot-scale testing should be performed to validate this value, as overestimation of this DF could adversely impact downstream HEPA filter change-out frequency.

DBE \#10: The DF for the condenser/demister combination was specified as 10,000.

C-5.5.5.3 Aluminum Nitrate Addition. Addition of aluminum nitrate may be required in order to complex fluoride in the condensate prior to processing in the LET\&D. However, the amount is expected to be very small ( $<0.2$ liter/day of $2.2 \mathrm{M}$ aluminum nitrate), and hence was not included in this mass balance. Based on model predictions of HF in the condensate a total of 20 gallons of 2.2 molar aluminum nitrate will be sufficient to process all the waste.

\section{DBE \#11: Aluminum nitrate addition to complex fluoride in the feed to LET\&D will be less than 0.2 liters per day.}

C-5.5.5.4 LET\&D Acid Recovery Efficiency. The LET\&D was modeled in Aspen as a 16-tray distillation column with a molar reflux ratio of 0.82 . The distillate to feed ratio was varied to achieve a 12 molar acid product. Acid recovery was estimated by Aspen based on vapor-liquid equilibrium. For all four waste feeds, acid recovery is estimated to exceed $99 \%$. This is consistent with past LET\&D performance.

\section{DBE \#12: Acid recovery in the LET\&D is predicted to exceed 99\% for all four waste feeds based on Aspen VLE calculations.}

C-5.5.5.5 Partitioning of Volatile Species in LET\&D. Partitioning of volatile chemical species such as chloride, fluoride, and iodine was estimated using Aspen VLE models. Property data for this model has been tuned to match prior sampling results from the existing EVS and PEWE evaporators.

Tritium was assumed to partition the same as water in the model, which was predicted using Aspen VLE models. Radioactive iodine was assumed to partition the same as chloride in the model. Ruthenium and cesium were treated as nonvolatile species in the model (i.e., a DF of $10^{6}$ was applied over the evaporator and demister combination). 
DBE \#13: Aspen VLE models were used for partitioning of halides in the LET\&D. Ruthenium and cesium were treated as nonvolatile species in the material balance for the LET\&D.

C-5.5.5.6 Acid Grout Formulation. The acid grout formulation and grout density used in the material balance were taken from Herbst (2002, Table 3) and are based on lab-scale testing. The formulation on a weight basis is as follows:

- $\quad 35.0 \% 12$ molar acid (LET\&D bottoms)

- $12.5 \% \mathrm{Ca}(\mathrm{OH})_{2}$

- $13.1 \%$ BFS

- $39.4 \%$ ordinary Portland cement (OPC).

Grout density was assumed to be $2.06 \mathrm{~g} / \mathrm{cm}^{3}$ as reported in the same tests (Herbst, 2003).

$D B E$ \#14: Grout density was assumed to be $2.06 \mathrm{~g} / \mathrm{cm}^{3}$. The following grout formulation was used in the mass balance: 35.0 wt.\% $12 \mathrm{M}$ nitric acid, $12.5 \mathrm{wt. \%} \mathrm{Ca}(\mathrm{OH})_{2}, 13.1 \mathrm{wt} \% \mathrm{BFS}$, and 39.4 $w t . \%$ OPC.

C-5.5.5.7 Acid Grout Packaging. The mass balance assumes that of the acid grout will be packaged in 55-gal drums. A capacity of $200 \mathrm{~L}$ (52.8 gal) of grout per drum was assumed.

DBE \#15: Acid grout will be packaged in 55-gal drums. A drum capacity of 200 L (52.8 gal) of waste was assumed.

\section{C-5.5.6 Off-gas Treatment}

The basis for and assumptions used in the mass balance of the process and VOG systems are documented in this section.

C-5.5.6.1 Process Off-gas and Vessel Off-gas Superheater Outlet Temperature. The POG and VOG superheater outlet temperatures were specified at $100^{\circ} \mathrm{C}$ in order to reduce the potential for condensation in the HEPA filters. This temperature is significantly above the dew point for these offgas streams, but was over-specified in order to effectively tolerate process upsets. For the alkaline scrubber material balances, the process off superheater outlet temperature was increased to $120^{\circ} \mathrm{C}$, which is the optimal operating temperature for the S-GAC bed based on laboratory testing.

DBE \#16: POG and VOG superheater outlet temperatures were specified at $100^{\circ} \mathrm{C}$ in order to reduce the potential for condensation in the downstream HEPA filters. For the alkaline scrubber material balances, the POG superheater outlet temperature was specified at $120^{\circ} \mathrm{C}$ for optimal performance of the mercury removal S-GAC beds.

C-5.5.6.2 Off-gas Mercury Removal. For the revised baseline scheme, or other schemes in which the evaporator vapor is totally condensed, the emissions of mercury should be undetectable. However, for the alkaline scrub process or others in which the evaporator vapor is not totally condensed, mercury emissions would likely exceed MACT standards. While it is not known whether regulators would apply MACT requirements to this process, reduction in mercury emissions could be required. Mercury could be removed in the gas phase with a fixed bed of sulfur impregnated granular activated carbon (S-GAC). The size of this bed is not expected to be large, since the model predicts that $>99.9 \%$ of the mercury would be retained in the evaporator bottoms. 
DBE \#16a: For the alkaline scrubber process, a bed of S-GAC downstream of the POG superheater is assumed to remove $99.9 \%$ of the mercury from the POG.

C-5.5.6.3 HEPA Filter Efficiency. Each HEPA housing was assumed to contain three filters (either three HEPAs, or a prefilter and two HEPAs). A DF of 1000 (efficiency $=99.9 \%$ ) was assumed for the first HEPA, a DF of 500 (efficiency $=99.8 \%$ ) was assumed for the second HEPA, and a DF of 1 (efficiency $=0.0 \%$ ) was assumed for the third HEPA. These DFs are in line with the recommendations of DOE AE Standard 1554-4 4.2(b)1. Hence, the overall DF for a HEPA bank is 500,000.

DBE \#17: A HEPA filter bank DF of 500,000 was assumed.

C-5.5.6.4 Process Off-gas and Vessel Off-gas Rates. Off-gas flow rates in the material balances were calculated by the Aspen model, and are a result of numerous process parameters.

However, some assumptions were made that impact the off-gas flow rate, including the following:

- The airlift flow transferring waste from WM-189 was assumed to be $23 \mathrm{~L}$ air/L SBW. Although only one TFF tank has an airlift, this air rate was used in the mass balance for all four tank wastes for both the revised baseline and alkaline scrub schemes.

- The sparge air flow to the SBW feed tank flow was assumed constant at $143 \mathrm{ft}^{3} / \mathrm{min}$ for all four tank wastes for both the revised baseline and alkaline scrub schemes.

- The vacuum control air flow was arbitrarily assumed to be constant at $22.5 \mathrm{ft}^{3} / \mathrm{min}$ for all four waste tanks for the revised baseline scenario only.

These values are engineering estimates only, and will need to be refined as part of final design. This is especially true for the vacuum control airflow rate - this flow could be significantly different from the assumed value.

DBE \#18: Off-gas flow rates were calculated by Aspen based on numerous other model inputs. However, the following input assumptions were made that will impact off-gas flow rate: (1) airlift flow to the TFF tank was assumed to be $23 \mathrm{~L}$ air / L SBW, (2) sparge air flow to the SBW feed tank was assumed to be constant at $143 \mathrm{ft}^{3} / \mathrm{min}$, and (3) vacuum control air flow was assumed to be $22.5 \mathrm{ft}^{3} / \mathrm{min}$ (revised baseline scenario only).

C-5.5.6.5 Process Off-gas and Vessel Off-gas Blower Outlet Conditions. Off-gas blowers were modeled as single-stage isentropic compressors in Aspen with an isentropic efficiency of $72 \%$. Outlet pressures were specified as 12.3 psia based on atmospheric pressure at INTEC. Based on these specifications, the model calculated the outlet temperature for the blowers (range $=119^{\circ} \mathrm{C}$ to $180^{\circ} \mathrm{C}$ ). The higher temperature of $180^{\circ} \mathrm{C}$ is at the upper limit for many blowers; hence, it may be necessary to implement a two-stage blower scenario with intercooling. This should be investigated as part of detailed design.

DBE \#19: For purposes of the mass balance, single stage blowers were assumed. An outlet pressure of 12.3 psia was specified.

\section{C-5.5.7 Evaporator Product Packaging}

The basis for and assumptions used in the mass balance of the evaporator product packaging system is documented in this section. 
C-5.5.7.1 Canister Volume. Canisters would be used for packaging of the evaporator product. A capacity of $800 \mathrm{~L}$ of waste per canister was assumed.

DBE \#20: Evaporator product will be packaged in canisters. An average canister waste fill volume of $800 \mathrm{~L}$ was assumed.

C-5.5.7.2 Cooling Time. The material balance does not assume a specific cooling time for RH canisters. This parameter will, however, impact interim storage requirements, canister loading system design and operational procedures. Unaided cooling of 5-gallons of product in a bucket required approximately 18 hours to reach $40^{\circ} \mathrm{C}$ (Griffith, 2003a). Cooling profiles were recorded for the waste in 15-gal drums generated in the November 2003 tests (Griffith, 2004). At 50 hours the centerline temperature for waste from a $62 \%$ mass reduction run with magnesium recycle was about $38^{\circ} \mathrm{C}$ (see Griffith, 2004, Figure 25). Data for cooling of waste in canisters is needed. In lieu of this data, it was assumed that with cooling jackets, three days would be sufficient cooling time per canister, achieving the maximum required production rate of three canisters per day with one canister packaging line.

\section{C-5.5.8 Utilities}

The basis for and assumptions used in the mass balances relative to utility availability and usage is documented in this section.

C-5.5.8.1 Steam. Based on pilot-scale tests (Griffith, 2003a), the temperature of the steam should be approximately $50-60^{\circ} \mathrm{C}$ above the operating temperature of the evaporator. Hence, 150 psig steam $\left(\mathrm{t}_{\mathrm{sat}}=185^{\circ} \mathrm{C}\right)$ was assumed for the material balances. It is also assumed that a new boiler and superheater at INTEC would be required to support the direct evaporation process. Aspen was used to calculate the steam usage, based on $150 \mathrm{psig}$ saturated steam as an input, and 147 psig condensate returning from the evaporator.

DBE \#21: 150 psig saturated steam was assumed as the heat source for the evaporator. A 3 psig pressure drop was assumed in the evaporator jacket, resulting in 147 psig condensate returning from the evaporator.

C-5.5.8.2 Cooling Water. Cooling water is used in several locations throughout the process. It was assumed in the revised baseline that cooling water would be sufficient to condense the evaporator vapor (i.e., a chiller will not be required). For purposes of the material balances, the supply temperature of the cooling water was assumed to be $20^{\circ} \mathrm{C}\left(68^{\circ} \mathrm{F}\right)$. The maximum allowable return temperature was assumed to be $50^{\circ} \mathrm{C}\left(122^{\circ} \mathrm{F}\right)$.

DBE \#22: For the material balances, cooling water was assumed to be available with a supply temperature of $20^{\circ} \mathrm{C}\left(68^{\circ} \mathrm{F}\right)$. The maximum allowable return temperature was assumed to be $50^{\circ} \mathrm{C}\left(122^{\circ} \mathrm{F}\right)$.

C-5.5.8.3 Air. The material balances assume that pressurized air at $50 \mathrm{psig}$ will be available to airlift waste to the SBW feed tank. In addition, atmospheric air is assumed available for use in various process locations, such as for vacuum control on the POG system.

DBE \#23: 50 psig and atmospheric air are assumed to be available for process use. 


\section{C-5.5.9 Alkaline Scrub Scheme.}

Assumptions used in the mass balance for the unique sections of the alkaline scrub process are provided in this section. Sections C-5.5.1, C-5.5.2, C-5.5.3, C-5.5.4, C-5.5.6, C-5.5.7 and C-5.5.8 also apply to the alkaline scrub process.

C-5.5.9.1 Liquid to Vapor Ratio. Lab or pilot-scale testing is needed to evaluate the optimal L/V for the caustic scrubber. Perry's Chemical Engineer's Handbook (5th edition) cites that scrub flows of 4.3 to $11 \mathrm{gpm} / 1000 \mathrm{cfm}$ have been used for sulfuric acid and phosphoric acid scrubbers (Perry, 1973). These corresponded to $\mathrm{L} / \mathrm{V}$ molar ratios of 1 to 2 . A conservative value of slightly over 2 was assumed for the liquid to vapor molar ratio of the scrubber.

DBE \#24: A liquid to vapor molar rate of about 2.0 was assumed for the $\mathrm{MgO}$ scrubber.

C-5.5.9.2 Scrub Cooler Duty. In the material balances, the duty of the scrub cooler was varied to achieve the desired L/V in the scrubber. Hence, the scrub cooler outlet temperature varies for each case depending on the flow rate, acid content, and composition of the evaporator vapor inlet stream. For the four SBW waste tanks to be processed, the temperature varies from a low of $38^{\circ} \mathrm{C}$ for WM- 188 to a high of $65^{\circ} \mathrm{C}$ for WM-180. Correspondingly, the estimated cooler duty varies between $20 \mathrm{~kW}$ for WM180 to $42 \mathrm{~kW}$ for WM-188.

DBE \#25: Scrub cooler duty was varied in the material balances to achieve the desired scrubber $L / V$ ratio.

C-5.5.9.3 Scrub Purge Rate. The scrub purge rate was varied for each case in order to keep the concentration of salts in the scrub low enough to minimize plugging the off-gas HEPA filters from salts in entrained mist. Hence, the purge rate was adjusted to limit the concentration of magnesium nitrate in the scrub system to about 3 molar. The scrub salts concentration must also be kept below magnesium nitrate solubility of about 6 molar to avoid precipitation.

$D B E$ \#26: The scrub purge rate was set to limit $\mathrm{Mg}\left(\mathrm{NO}_{3}\right)_{2}$ concentration in the scrub solution to about 3 molar.

C-5.5.9.4 Make-up MgO Concentration and Addition Rate. The MgO make-up concentration was assumed to be a slurry at $20 \mathrm{wt} \%$. The make-up rate was set to match the $\mathrm{HNO}_{3}$ input from the evaporator off-gas while maintaining the scrub slightly acidic.

DBE \#27: MgO was slurried with water to a concentration of $20 \mathrm{wt} \%$ and added to the scrubber to replace bed material removed by reaction with nitric acid vapors and dissolution in the scrub liquid. 


\section{C-6. MASS BALANCES}

A summary of waste products generated materials consumed in the direct evaporation process is shown in Table C-3. Mass balances with stream compositions are shown in Tables 4-7 for the revised baseline scheme and in Tables C-8 through C-11 for the alkaline scrub scheme. Tables C-12 and C-13 show additional properties of the waste products.

Table C-3. Products from and consumable feeds for the direct evaporation process.

\section{Total}

WM-180 WM-187 WM-188 WM-189 containers Total m3

Products

Revised Baseline Scheme

Canisters of SBW evaporator waste

$\begin{array}{llllll}340 & 182 & 354 & 382 & 1,259 & 1,007\end{array}$

Drums of grouted LET\&D bottoms

971

$712 \quad 1,997$

2,161

$5,841 \quad 1,168$

\section{Alkaline Scrub Scheme}

Canisters of SBW evaporator waste

425

224

501

529

1,678

1,342

Drums of spent GAC

2

0.3

\section{Consumables}

Revised Baseline Scheme

Portland cement

Blast furnace slag

Calcium hydroxide

\begin{tabular}{ccccc}
$\mathbf{l b}$ & $\mathbf{l b}$ & $\mathbf{l b}$ & $\mathbf{l b}$ & Total lb \\
\hline $2.91 \mathrm{E}+05$ & $2.13 \mathrm{E}+05$ & $5.98 \mathrm{E}+05$ & $2.87 \mathrm{E}+05$ & $1.39 \mathrm{E}+06$ \\
$9.69 \mathrm{E}+04$ & $7.10 \mathrm{E}+04$ & $1.99 \mathrm{E}+05$ & $9.58 \mathrm{E}+04$ & $4.63 \mathrm{E}+05$ \\
$9.23 \mathrm{E}+04$ & $6.77 \mathrm{E}+04$ & $1.90 \mathrm{E}+05$ & $9.12 \mathrm{E}+04$ & $4.41 \mathrm{E}+05$
\end{tabular}

Alkaline Scrub Scheme

lb

lb

lb

lb

Total lb

Granulated activated carbon

290

Magnesium oxide

$\begin{array}{lllll}4.9 \mathrm{E}+04 & 3.6 \mathrm{E}+04 & 1.0 \mathrm{E}+05 & 1.1 \mathrm{E}+05 & 3.0 \mathrm{E}+05\end{array}$


Table C-4. Revised baseline mass balance, Tank WM-180 waste.

\begin{tabular}{|c|c|c|c|c|c|c|c|c|}
\hline PFD Sheet \# & PFD-1 & PFD-2 & PFD-2 & PFD-2 & PFD-2 & PFD-2 & PFD-2 & PFD-3 \\
\hline Stream \# & 101 & 102 & 103 & 104 & 105 & 106 & 107 & 108 \\
\hline Stream Name & $\begin{array}{l}\text { SBW } \\
\text { Feed }\end{array}$ & $\begin{array}{c}\text { Evap. } \\
\text { Bottoms }\end{array}$ & $\begin{array}{c}\text { Evap. } \\
\text { Overheads } \\
\text { Vapor }\end{array}$ & $\begin{array}{c}\text { Mist } \\
\text { Eliminator } \\
\text { Vapor }\end{array}$ & $\begin{array}{l}\text { VOG } \\
\text { Offgas } \\
\text { Vapor }\end{array}$ & \begin{tabular}{|c|} 
POG + VOG \\
Offgas \\
Vapor
\end{tabular} & $\begin{array}{l}\text { LET\&D } \\
\text { Feed } \\
\text { Liquid }\end{array}$ & $\begin{array}{l}\text { LET\&D } \\
\text { Acid } \\
\text { Liquid }\end{array}$ \\
\hline Rate or Volume Flow (actual) & 85.8 & 21.3 & $2.68 \mathrm{E}+04$ & $2.73 \mathrm{E}+04$ & $1.17 \mathrm{E}+04$ & $1.37 \mathrm{E}+04$ & 65.7 & 7.87 \\
\hline Volume Flow (standard, wet) & & & $1.15 \mathrm{E}+04$ & $1.15 \mathrm{E}+04$ & $7.34 \mathrm{E}+03$ & $8.57 \mathrm{E}+03$ & & \\
\hline Volume Flow (standard, dry) & & & $2.76 \mathrm{E}+02$ & $2.76 \mathrm{E}+02$ & $7.13 E+03$ & $8.36 \mathrm{E}+03$ & & \\
\hline Rate Units & $\mathrm{gal} / \mathrm{hr}$ & $\mathrm{gal} / \mathrm{hr}$ & $\mathrm{ft} 3 / \mathrm{hr}$ & $\mathrm{ft} 3 / \mathrm{hr}$ & $\mathrm{ft} 3 / \mathrm{hr}$ & $\mathrm{ft} 3 / \mathrm{hr}$ & $\mathrm{gal} / \mathrm{hr}$ & $\mathrm{gal} / \mathrm{hr}$ \\
\hline Temperature, ${ }^{\circ} \mathrm{C}$ & 25 & 124 & 124 & 122 & 108 & 109 & 35 & 35 \\
\hline Temperature, ${ }^{\circ} \mathrm{F}$ & 77 & 255 & 255 & 252 & 226 & 229 & 95 & 95 \\
\hline Pressure, psia & 12.3 & 8.6 & 8.6 & 8.4 & 12.0 & 12.0 & 12.0 & 12.2 \\
\hline Specific Gravity & 1.24 & 1.75 & $3.43 \mathrm{E}-04$ & $3.36 \mathrm{E}-04$ & 7.46E-04 & 7.44E-04 & 1.04 & 1.16 \\
\hline Chemical Composition & Mol/Liter & Mol/Liter & $\mathrm{lb} /$ wscf & $\mathrm{lb} /$ wscf & $\mathrm{lb} /$ wscf & $\mathrm{lb} /$ wscf & Mol/Liter & Mol/Liter \\
\hline $\mathrm{H}+$ & $1.07 \mathrm{E}+00$ & $2.47 \mathrm{E}-01$ & $6.29 \mathrm{E}-05$ & 6.29E-05 & $3.83 \mathrm{E}-11$ & 8.49E-09 & $1.31 \mathrm{E}+00$ & $1.09 \mathrm{E}+01$ \\
\hline \multicolumn{9}{|l|}{$\mathrm{OH}-$} \\
\hline $\mathrm{Al}+3$ & $5.83 \mathrm{E}-01$ & $2.35 \mathrm{E}+00$ & $9.77 \mathrm{E}-08$ & $9.77 \mathrm{E}-10$ & & & $7.61 \mathrm{E}-07$ & $6.35 \mathrm{E}-06$ \\
\hline $\mathrm{Sb+5}$ & $1.39 \mathrm{E}-06$ & $5.60 \mathrm{E}-06$ & $1.05 \mathrm{E}-12$ & $1.05 E-14$ & & & $1.82 \mathrm{E}-12$ & $1.52 \mathrm{E}-11$ \\
\hline As+5 & 4.47E-04 & $1.80 \mathrm{E}-03$ & $2.08 \mathrm{E}-10$ & $2.08 \mathrm{E}-12$ & & & $5.84 \mathrm{E}-10$ & 4.87E-09 \\
\hline $\mathrm{Ba}+2$ & $5.00 \mathrm{E}-05$ & $2.01 \mathrm{E}-04$ & $4.27 \mathrm{E}-11$ & $4.27 \mathrm{E}-13$ & & & $6.53 \mathrm{E}-11$ & $5.45 \mathrm{E}-10$ \\
\hline $\mathrm{Be}+2$ & $7.82 \mathrm{E}-06$ & $3.15 E-05$ & $4.38 \mathrm{E}-13$ & $4.38 \mathrm{E}-15$ & & & $1.02 \mathrm{E}-11$ & $8.52 \mathrm{E}-11$ \\
\hline $\mathrm{B}+3$ & $1.04 \mathrm{E}-02$ & $4.19 \mathrm{E}-02$ & $6.99 \mathrm{E}-10$ & $6.99 \mathrm{E}-12$ & & & $1.36 \mathrm{E}-08$ & $1.13 \mathrm{E}-07$ \\
\hline $\mathrm{Cd}+2$ & $7.16 \mathrm{E}-04$ & $2.88 \mathrm{E}-03$ & $5.00 \mathrm{E}-10$ & $5.00 \mathrm{E}-12$ & & & $9.35 \mathrm{E}-10$ & 7.81E-09 \\
\hline $\mathrm{Ca}+2$ & $3.69 \mathrm{E}-02$ & $1.49 \mathrm{E}-01$ & $9.20 \mathrm{E}-09$ & $9.20 \mathrm{E}-11$ & & & $4.82 \mathrm{E}-08$ & 4.03E-07 \\
\hline $\mathrm{Cr}+3$ & $3.36 \mathrm{E}-03$ & $1.35 \mathrm{E}-02$ & $1.09 \mathrm{E}-09$ & $1.09 \mathrm{E}-11$ & & & $4.39 E-09$ & $3.67 \mathrm{E}-08$ \\
\hline $\mathrm{Co}+2$ & $1.83 \mathrm{E}-05$ & 7.37E-05 & $6.70 \mathrm{E}-12$ & $6.70 \mathrm{E}-14$ & & & $2.39 \mathrm{E}-11$ & $1.99 \mathrm{E}-10$ \\
\hline Cs+ & $2.57 \mathrm{E}-05$ & $1.04 \mathrm{E}-04$ & $2.13 \mathrm{E}-11$ & $2.13 \mathrm{E}-13$ & & & $3.36 \mathrm{E}-11$ & $2.81 \mathrm{E}-10$ \\
\hline $\mathrm{Cu}+2$ & $6.32 \mathrm{E}-04$ & $2.55 \mathrm{E}-03$ & $2.50 \mathrm{E}-10$ & $2.50 \mathrm{E}-12$ & & & $8.25 \mathrm{E}-10$ & 6.89E-09 \\
\hline $\mathrm{Fe}+3$ & 1.92E-02 & $7.75 \mathrm{E}-02$ & 6.68E-09 & $6.68 \mathrm{E}-11$ & & & $2.51 \mathrm{E}-08$ & $2.10 \mathrm{E}-07$ \\
\hline $\mathrm{Pb}+2$ & 1.26E-03 & $5.08 \mathrm{E}-03$ & 1.62E-09 & $1.62 \mathrm{E}-11$ & & & 1.65E-09 & $1.38 \mathrm{E}-08$ \\
\hline $\mathrm{Hg}+2$ & $1.46 \mathrm{E}-03$ & $5.71 \mathrm{E}-03$ & $5.16 \mathrm{E}-07$ & $5.15 \mathrm{E}-07$ & $2.97 \mathrm{E}-11$ & $2.54 \mathrm{E}-11$ & $2.39 \mathrm{E}-10$ & $2.00 \mathrm{E}-09$ \\
\hline $\mathrm{Mn}+4$ & 1.27E-02 & $5.13 E-02$ & 4.34E-09 & 4.34E-11 & & & 1.66E-08 & 1.39E-07 \\
\hline $\mathrm{Ni}+2$ & 1.39E-03 & 5.59E-03 & $5.06 \mathrm{E}-10$ & $5.06 \mathrm{E}-12$ & & & 1.81E-09 & $1.51 \mathrm{E}-08$ \\
\hline $\mathrm{K}+$ & $1.76 \mathrm{E}-01$ & 7.09E-01 & $4.28 \mathrm{E}-08$ & $4.28 \mathrm{E}-10$ & & & $2.30 \mathrm{E}-07$ & 1.92E-06 \\
\hline $\mathrm{Se}+4$ & 7.67E-05 & 3.09E-04 & $3.77 \mathrm{E}-11$ & $3.77 \mathrm{E}-13$ & & & $1.00 \mathrm{E}-10$ & 8.37E-10 \\
\hline $\mathrm{Ag}+$ & $6.08 \mathrm{E}-06$ & $2.45 \mathrm{E}-05$ & $4.07 \mathrm{E}-12$ & $4.07 E-14$ & & & $7.93 \mathrm{E}-12$ & $6.62 \mathrm{E}-11$ \\
\hline $\mathrm{Na}+$ & $1.89 \mathrm{E}+00$ & $7.60 \mathrm{E}+00$ & $2.70 \mathrm{E}-07$ & $2.70 \mathrm{E}-09$ & & & $2.46 \mathrm{E}-06$ & $2.06 \mathrm{E}-05$ \\
\hline $\mathrm{TI}+3$ & $2.75 \mathrm{E}-05$ & $1.11 \mathrm{E}-04$ & $3.49 \mathrm{E}-11$ & $3.49 \mathrm{E}-13$ & & & $3.59 \mathrm{E}-11$ & $2.99 \mathrm{E}-10$ \\
\hline $\mathrm{U}+4$ & $3.61 \mathrm{E}-04$ & $1.46 \mathrm{E}-03$ & $5.34 \mathrm{E}-10$ & $5.34 \mathrm{E}-12$ & & & $4.72 \mathrm{E}-10$ & 3.94E-09 \\
\hline$V+3$ & 8.76E-04 & $3.53 \mathrm{E}-03$ & $2.77 \mathrm{E}-10$ & $2.77 \mathrm{E}-12$ & & & $1.14 \mathrm{E}-09$ & $9.55 \mathrm{E}-09$ \\
\hline$Z n+2$ & $9.51 \mathrm{E}-04$ & 3.83E-03 & $3.86 \mathrm{E}-10$ & $3.86 \mathrm{E}-12$ & & & 1.24E-09 & 1.04E-08 \\
\hline$Z r+4$ & $1.32 \mathrm{E}-03$ & $5.33 \mathrm{E}-03$ & $7.50 \mathrm{E}-10$ & $7.50 \mathrm{E}-12$ & & & $1.73 \mathrm{E}-09$ & $1.44 \mathrm{E}-08$ \\
\hline $\mathrm{Cl}-$ & $2.86 \mathrm{E}-02$ & 9.04E-02 & 1.35E-05 & $1.35 \mathrm{E}-05$ & $1.26 \mathrm{E}-11$ & 1.80E-09 & $7.88 \mathrm{E}-03$ & 1.15E-09 \\
\hline F- & $4.02 \mathrm{E}-02$ & $1.62 \mathrm{E}-01$ & $3.71 \mathrm{E}-08$ & $3.22 \mathrm{E}-08$ & $3.82 \mathrm{E}-13$ & $4.65 \mathrm{E}-12$ & 3.57E-05 & $2.98 \mathrm{E}-04$ \\
\hline SO4-2 & 4.96E-02 & $2.00 \mathrm{E}-01$ & $2.96 \mathrm{E}-08$ & $2.96 \mathrm{E}-10$ & & & $6.48 \mathrm{E}-08$ & 5.41E-07 \\
\hline NO3- & $4.92 \mathrm{E}+00$ & $1.58 \mathrm{E}+01$ & $3.85 \mathrm{E}-03$ & $3.85 \mathrm{E}-03$ & $2.35 \mathrm{E}-09$ & $5.19 \mathrm{E}-07$ & $1.30 \mathrm{E}+00$ & $1.09 \mathrm{E}+01$ \\
\hline PO4-3 & 1.95E-02 & $7.86 \mathrm{E}-02$ & 1.15E-08 & $1.15 \mathrm{E}-10$ & & & $2.55 \mathrm{E}-08$ & $2.13 \mathrm{E}-07$ \\
\hline$A m+4$ & $3.16 \mathrm{E}-08$ & $1.27 \mathrm{E}-07$ & $4.77 \mathrm{E}-14$ & 4.77E-16 & & & $4.12 \mathrm{E}-14$ & $3.44 \mathrm{E}-13$ \\
\hline $\mathrm{Br}-$ & $1.55 \mathrm{E}-07$ & $4.91 \mathrm{E}-07$ & $1.66 \mathrm{E}-10$ & $1.66 \mathrm{E}-10$ & $1.55 \mathrm{E}-16$ & $2.21 \mathrm{E}-14$ & $4.30 \mathrm{E}-08$ & \\
\hline $\mathrm{Ce}+4$ & 4.36E-05 & $1.76 \mathrm{E}-04$ & $3.79 \mathrm{E}-11$ & $3.79 \mathrm{E}-13$ & & & $5.69 \mathrm{E}-11$ & $4.75 \mathrm{E}-10$ \\
\hline $\mathrm{Eu}+3$ & $2.58 \mathrm{E}-07$ & 1.04E-06 & $2.43 \mathrm{E}-13$ & $2.43 \mathrm{E}-15$ & & & $3.36 \mathrm{E}-13$ & $2.81 \mathrm{E}-12$ \\
\hline$G d+3$ & 1.61E-04 & $6.47 \mathrm{E}-04$ & $1.57 \mathrm{E}-10$ & $1.57 \mathrm{E}-12$ & & & $2.10 \mathrm{E}-10$ & 1.75E-09 \\
\hline $\mathrm{Ge}+4$ & 4.48E-09 & $1.81 \mathrm{E}-08$ & $2.02 \mathrm{E}-15$ & $2.02 \mathrm{E}-17$ & & & $5.85 \mathrm{E}-15$ & $4.89 \mathrm{E}-14$ \\
\hline $\ln +3$ & 7.05E-07 & $2.84 \mathrm{E}-06$ & $5.03 E-13$ & 5.03E-15 & & & $9.21 \mathrm{E}-13$ & $7.69 \mathrm{E}-12$ \\
\hline I- & $1.35 \mathrm{E}-06$ & $4.31 \mathrm{E}-06$ & $2.19 E-09$ & 2.19E-09 & $2.05 \mathrm{E}-15$ & $2.92 \mathrm{E}-13$ & 3.57E-07 & $6.20 \mathrm{E}-13$ \\
\hline $\mathrm{La}+3$ & $4.68 \mathrm{E}-06$ & 1.89E-05 & $4.04 \mathrm{E}-12$ & 4.04E-14 & & & $6.11 \mathrm{E}-12$ & $5.10 \mathrm{E}-11$ \\
\hline $\mathrm{Li}+$ & 4.44E-04 & 1.79E-03 & $1.92 \mathrm{E}-11$ & $1.92 \mathrm{E}-13$ & & & $5.80 \mathrm{E}-10$ & 4.84E-09 \\
\hline$M g+2$ & $1.10 \mathrm{E}-02$ & 4.43E-02 & 1.66E-09 & $1.66 \mathrm{E}-11$ & & & $1.43 \mathrm{E}-08$ & $1.20 \mathrm{E}-07$ \\
\hline $\mathrm{Mo}+6$ & $1.88 \mathrm{E}-04$ & $7.56 \mathrm{E}-04$ & $1.12 \mathrm{E}-10$ & $1.12 \mathrm{E}-12$ & & & $2.45 \mathrm{E}-10$ & 2.05E-09 \\
\hline
\end{tabular}


Table C-4. Revised baseline mass balance, Tank WM-180 waste (Continued).

\begin{tabular}{|c|c|c|c|c|c|c|c|c|}
\hline Stream \# & 101 & 102 & 103 & 104 & 105 & 106 & 107 & 108 \\
\hline Stream Name & $\begin{array}{l}\text { SBW } \\
\text { Feed }\end{array}$ & $\begin{array}{l}\text { Evap. } \\
\text { Bottoms }\end{array}$ & $\begin{array}{l}\text { Evap. } \\
\text { Overheads } \\
\text { Vapor }\end{array}$ & $\begin{array}{c}\text { Mist } \\
\text { Eliminator } \\
\text { Vapor }\end{array}$ & $\begin{array}{l}\text { VOG } \\
\text { Offgas } \\
\text { Vapor }\end{array}$ & $\begin{array}{c}\text { POG + VOG } \\
\text { Offgas } \\
\text { Vapor }\end{array}$ & $\begin{array}{l}\text { LET\&D } \\
\text { Feed } \\
\text { Liquid }\end{array}$ & $\begin{array}{l}\text { LET\&D } \\
\text { Acid } \\
\text { Liquid }\end{array}$ \\
\hline Chemical Composition & Mol/Liter & Mol/Liter & $\mathrm{lb} / \mathrm{wscf}$ & lb/wscf & $\mathrm{lb} / \mathrm{wscf}$ & $\mathrm{Ib} / \mathrm{wscf}$ & Mol/Liter & Mol/Liter \\
\hline $\mathrm{Nd}+3$ & 1.51E-05 & $6.08 \mathrm{E}-05$ & $1.35 \mathrm{E}-11$ & $1.35 \mathrm{E}-13$ & & & 1.97E-11 & $1.65 \mathrm{E}-10$ \\
\hline $\mathrm{Np}+4$ & $8.73 \mathrm{E}-06$ & $3.52 \mathrm{E}-05$ & $1.29 \mathrm{E}-11$ & $1.29 \mathrm{E}-13$ & & & $1.14 \mathrm{E}-11$ & $9.52 \mathrm{E}-11$ \\
\hline $\mathrm{Nb}+5$ & 4.46E-04 & $1.80 \mathrm{E}-03$ & $2.58 \mathrm{E}-10$ & $2.58 \mathrm{E}-12$ & & & $5.83 \mathrm{E}-10$ & 4.87E-09 \\
\hline $\mathrm{Pd}+4$ & $3.13 \mathrm{E}-05$ & $1.26 \mathrm{E}-04$ & $2.07 \mathrm{E}-11$ & $2.07 \mathrm{E}-13$ & & & $4.08 \mathrm{E}-11$ & $3.41 \mathrm{E}-10$ \\
\hline $\mathrm{Pu}+4$ & $9.98 \mathrm{E}-06$ & $4.02 \mathrm{E}-05$ & $1.51 \mathrm{E}-11$ & $1.51 \mathrm{E}-13$ & & & $1.30 \mathrm{E}-11$ & $1.09 \mathrm{E}-10$ \\
\hline $\mathrm{Pr}+4$ & $4.26 \mathrm{E}-06$ & $1.71 \mathrm{E}-05$ & $3.73 \mathrm{E}-12$ & $3.73 \mathrm{E}-14$ & & & $5.56 \mathrm{E}-12$ & $4.64 \mathrm{E}-11$ \\
\hline $\mathrm{Rh}+4$ & 1.84E-06 & $7.40 \mathrm{E}-06$ & $1.17 \mathrm{E}-12$ & $1.17 \mathrm{E}-14$ & & & $2.40 \mathrm{E}-12$ & $2.00 \mathrm{E}-11$ \\
\hline $\mathrm{Rb}+$ & $2.83 \mathrm{E}-06$ & 1.14E-05 & $1.50 \mathrm{E}-12$ & $1.50 \mathrm{E}-14$ & & & $3.69 \mathrm{E}-12$ & $3.08 \mathrm{E}-11$ \\
\hline $\mathrm{Ru}+3$ & 1.26E-04 & $5.08 \mathrm{E}-04$ & $7.93 \mathrm{E}-11$ & $7.93 \mathrm{E}-13$ & & & $1.65 \mathrm{E}-10$ & 1.38E-09 \\
\hline $\mathrm{Sm}+3$ & $2.80 \mathrm{E}-06$ & $1.13 \mathrm{E}-05$ & $2.62 \mathrm{E}-12$ & $2.62 \mathrm{E}-14$ & & & $3.66 \mathrm{E}-12$ & 3.05E-11 \\
\hline $\mathrm{Si+4}$ & $3.08 \mathrm{E}-03$ & $1.24 \mathrm{E}-02$ & $5.37 \mathrm{E}-10$ & $5.37 \mathrm{E}-12$ & & & 4.02E-09 & $3.35 \mathrm{E}-08$ \\
\hline $\mathrm{Sr}+2$ & $1.08 \mathrm{E}-04$ & $4.34 \mathrm{E}-04$ & $5.86 \mathrm{E}-11$ & $5.86 \mathrm{E}-13$ & & & $1.41 \mathrm{E}-10$ & $1.17 \mathrm{E}-09$ \\
\hline $\mathrm{Tc}+7$ & $5.30 \mathrm{E}-06$ & $2.13 \mathrm{E}-05$ & $3.19 \mathrm{E}-12$ & $3.19 \mathrm{E}-14$ & & & $6.91 \mathrm{E}-12$ & $5.77 \mathrm{E}-11$ \\
\hline $\mathrm{Te}+4$ & 1.47E-06 & 5.93E-06 & 1.17E-12 & 1.17E-14 & & & 1.92E-12 & $1.60 \mathrm{E}-11$ \\
\hline $\mathrm{Tb}+4$ & 1.08E-09 & 4.34E-09 & $1.06 \mathrm{E}-15$ & $1.06 \mathrm{E}-17$ & & & $1.41 \mathrm{E}-15$ & 1.17E-14 \\
\hline $\mathrm{Th}+4$ & $1.05 \mathrm{E}-10$ & $4.23 \mathrm{E}-10$ & $1.51 \mathrm{E}-16$ & $1.51 \mathrm{E}-18$ & & & $1.37 \mathrm{E}-16$ & $1.15 \mathrm{E}-15$ \\
\hline $\mathrm{Sn}+4$ & 7.38E-05 & 2.97E-04 & $5.45 \mathrm{E}-11$ & $5.45 \mathrm{E}-13$ & & & 9.64E-11 & $8.05 \mathrm{E}-10$ \\
\hline $\mathrm{Ti}+4$ & 1.34E-04 & $5.41 \mathrm{E}-04$ & $4.00 \mathrm{E}-11$ & $4.00 \mathrm{E}-13$ & & & $1.75 \mathrm{E}-10$ & 1.47E-09 \\
\hline$\overline{Y+3}$ & $3.49 \mathrm{E}-06$ & $1.41 \mathrm{E}-05$ & $1.93 \mathrm{E}-12$ & $1.93 \mathrm{E}-14$ & & & $4.56 \mathrm{E}-12$ & $3.81 \mathrm{E}-11$ \\
\hline O-2 (oxides) & 1.33E-02 & 5.34E-02 & 1.32E-09 & $1.32 \mathrm{E}-11$ & & & $1.73 \mathrm{E}-08$ & $1.45 \mathrm{E}-07$ \\
\hline $\mathrm{H} 2 \mathrm{O}$ & $4.76 \mathrm{E}+01$ & $2.48 \mathrm{E}+01$ & $4.58 \mathrm{E}-02$ & $4.58 \mathrm{E}-02$ & $1.35 \mathrm{E}-03$ & 1.16E-03 & $5.34 \mathrm{E}+01$ & $2.66 \mathrm{E}+01$ \\
\hline $\mathrm{O} 2$ & & $1.86 \mathrm{E}-10$ & $1.65 \mathrm{E}-07$ & $1.65 \mathrm{E}-07$ & 1.69E-02 & $1.70 \mathrm{E}-02$ & 8.54E-05 & \\
\hline$\overline{\mathrm{N} 2}$ & & $2.27 \mathrm{E}-10$ & $2.76 \mathrm{E}-07$ & $2.76 \mathrm{E}-07$ & $5.58 \mathrm{E}-02$ & $5.60 \mathrm{E}-02$ & 1.36E-04 & \\
\hline C (organic) & $1.78 \mathrm{E}-02$ & 7.06E-02 & 1.92E-07 & 1.91E-07 & $2.42 \mathrm{E}-13$ & $2.58 \mathrm{E}-11$ & 3.34E-04 & $2.79 \mathrm{E}-03$ \\
\hline $\mathrm{H}$ (organic) & $1.78 \mathrm{E}-02$ & 7.06E-02 & $1.61 \mathrm{E}-08$ & $1.60 \mathrm{E}-08$ & $2.03 \mathrm{E}-14$ & $2.17 \mathrm{E}-12$ & 3.34E-04 & $2.79 \mathrm{E}-03$ \\
\hline \multicolumn{9}{|l|}{ SiO2, BFS, or OPC Additives } \\
\hline $\begin{array}{l}\text { Gas Stream Bulk Composition } \\
\text { (wet basis) }\end{array}$ & $\begin{array}{l}\text { mol. } \% \\
\text { or ppmv }\end{array}$ & $\begin{array}{l}\text { mol. } \% \\
\text { or ppmv }\end{array}$ & $\begin{array}{c}\text { mol.\% } \\
\text { or ppmv }\end{array}$ & $\begin{array}{c}\text { mol. \% or } \\
\text { ppmv }\end{array}$ & $\begin{array}{c}\text { mol. } \% \\
\text { or ppmv }\end{array}$ & $\begin{array}{l}\text { mol.\% } \\
\text { or ppmv }\end{array}$ & $\begin{array}{c}\text { mol. } \% \\
\text { or ppmv }\end{array}$ & $\begin{array}{c}\text { mol. } \% \\
\text { or ppmv }\end{array}$ \\
\hline $\mathrm{H} 2 \mathrm{O}, \mathrm{mol} . \%$ & & & 97.60 & 97.60 & 2.89 & 2.49 & & \\
\hline $\mathrm{O} 2, \mathrm{~mol} . \%$ & & & 0.0002 & 0.0002 & 20.39 & 20.48 & & \\
\hline $\mathrm{N} 2, \mathrm{~mol} . \%$ & & & 0.0004 & 0.0004 & 76.72 & 77.04 & & \\
\hline \multicolumn{9}{|l|}{$\mathrm{CO} 2, \mathrm{~mol} . \%$} \\
\hline HNO3, mol. \% & & & 2.40 & 2.40 & 0.000001 & 0.0003 & & \\
\hline \multicolumn{9}{|l|}{$\mathrm{CO}, \mathrm{ppmv}$} \\
\hline \multicolumn{9}{|l|}{ NO, ppmv } \\
\hline \multicolumn{9}{|l|}{ NO2, ppmv } \\
\hline $\mathrm{C}$ (organic), ppmv & & & 6 & 6 & 0.00001 & 0.0008 & & \\
\hline $\mathrm{H}$ (organic), ppmv & & & 6 & 6 & 0.00001 & 0.0008 & & \\
\hline PM (mg/wscm) & & & 1 & 0.01 & & & & \\
\hline $\begin{array}{l}\text { Gas Stream Bulk Composition } \\
\text { (dry basis) }\end{array}$ & $\begin{array}{c}\text { mol.\% } \\
\text { or ppmv }\end{array}$ & $\begin{array}{c}\text { mol. } \% \\
\text { or ppmv }\end{array}$ & $\begin{array}{c}\text { mol.\% } \\
\text { or ppmv }\end{array}$ & $\begin{array}{c}\text { mol. } \% \\
\text { or ppmv }\end{array}$ & $\begin{array}{c}\text { mol. } \% \\
\text { or ppmv }\end{array}$ & $\begin{array}{c}\text { mol.\% } \\
\text { or ppmv }\end{array}$ & $\begin{array}{c}\text { mol. } \% \\
\text { or ppmv }\end{array}$ & $\begin{array}{c}\text { mol. } \% \\
\text { or ppmv }\end{array}$ \\
\hline $\mathrm{O} 2, \mathrm{~mol} . \%$ & & & 0.01 & 0.01 & 21.00 & 21.00 & & \\
\hline $\mathrm{N} 2, \mathrm{~mol} . \%$ & & & 0.02 & 0.02 & 79.00 & 79.00 & & \\
\hline \multicolumn{9}{|l|}{$\mathrm{CO} 2, \mathrm{~mol} . \%$} \\
\hline HNO3, mol. \% & & & 99.90 & 99.96 & 0.000002 & 0.0003 & & \\
\hline \multicolumn{9}{|l|}{$\mathrm{CO}, \mathrm{ppmv}$} \\
\hline \multicolumn{9}{|l|}{$\mathrm{NO}, \mathrm{ppmv}$} \\
\hline \multicolumn{9}{|l|}{ NO2, ppmv } \\
\hline $\mathrm{C}$ (organic), ppmv & & & 256 & 254 & 0.00001 & 0.0009 & & \\
\hline $\mathrm{H}$ (organic), ppmv & & & 256 & 254 & 0.00001 & 0.0009 & & \\
\hline $\mathrm{PM}(\mathrm{mg} / \mathrm{dscm})$ & & & 59 & 1 & & & & \\
\hline & & & & & & & & \\
\hline
\end{tabular}


Table C-4. Revised baseline mass balance, Tank WM-180 waste (Continued).

\begin{tabular}{|c|c|c|c|c|c|c|c|c|}
\hline Stream \# & 101 & 102 & 103 & 104 & 105 & 106 & 107 & 108 \\
\hline Stream Name & $\begin{array}{l}\text { SBW } \\
\text { Feed }\end{array}$ & $\begin{array}{l}\text { Evap. } \\
\text { Bottoms }\end{array}$ & $\begin{array}{c}\text { Evap. } \\
\text { Overheads } \\
\text { Vapor }\end{array}$ & $\begin{array}{c}\text { Mist } \\
\text { Eliminator } \\
\text { Vapor }\end{array}$ & $\begin{array}{l}\text { VOG } \\
\text { Offgas } \\
\text { Vapor }\end{array}$ & $\begin{array}{c}\text { POG + VOG } \\
\text { Offgas } \\
\text { Vapor }\end{array}$ & $\begin{array}{l}\text { LET\&D } \\
\text { Feed } \\
\text { Liquid }\end{array}$ & $\begin{array}{l}\text { LET\&D } \\
\text { Acid } \\
\text { Liquid }\end{array}$ \\
\hline Canister Rate, canisters/day & & 2.42 & & & & & & \\
\hline Total Canisters Generated & & 340 & & & & & & \\
\hline \multicolumn{9}{|l|}{ Drum Rate, drums/day } \\
\hline \multicolumn{9}{|l|}{ Total Drums Generated } \\
\hline Radiological Composition & $\mathrm{Ci} / \mathrm{L}$ & $\mathrm{Ci} / \mathrm{L}$ & $\mathrm{Ci} / \mathrm{wscm}$ & $\mathrm{Ci} / \mathrm{wscm}$ & $\mathrm{Ci} / \mathrm{wscm}$ & $\mathrm{Ci} / \mathrm{wscm}$ & $\mathrm{Ci} / \mathrm{L}$ & $\mathrm{Ci} / \mathrm{L}$ \\
\hline Ra-226 & $4.04 \mathrm{E}-12$ & $1.63 \mathrm{E}-11$ & $4.02 \mathrm{E}-16$ & $4.02 \mathrm{E}-18$ & $1.26 \mathrm{E}-25$ & $1.09 \mathrm{E}-25$ & $5.27 \mathrm{E}-18$ & $4.40 \mathrm{E}-17$ \\
\hline Ac-227 & $1.90 \mathrm{E}-11$ & $7.66 \mathrm{E}-11$ & $1.89 \mathrm{E}-15$ & $1.89 \mathrm{E}-17$ & $5.94 \mathrm{E}-25$ & $5.14 \mathrm{E}-25$ & $2.48 \mathrm{E}-17$ & $2.07 \mathrm{E}-16$ \\
\hline Th-228 & $1.58 \mathrm{E}-09$ & 6.36E-09 & $1.57 \mathrm{E}-13$ & $1.57 \mathrm{E}-15$ & 4.93E-23 & $4.27 \mathrm{E}-23$ & $2.06 \mathrm{E}-15$ & $1.72 \mathrm{E}-14$ \\
\hline Th-230 & $4.30 \mathrm{E}-10$ & 1.73E-09 & $4.28 \mathrm{E}-14$ & $4.28 \mathrm{E}-16$ & $1.34 \mathrm{E}-23$ & $1.16 \mathrm{E}-23$ & $5.62 \mathrm{E}-16$ & $4.69 \mathrm{E}-15$ \\
\hline Th-232 & $3.49 \mathrm{E}-16$ & $1.41 \mathrm{E}-15$ & $3.48 \mathrm{E}-20$ & $3.48 \mathrm{E}-22$ & $1.09 \mathrm{E}-29$ & $9.44 \mathrm{E}-30$ & $4.56 \mathrm{E}-22$ & $3.81 \mathrm{E}-21$ \\
\hline $\mathrm{Pa}-231$ & $4.41 \mathrm{E}-11$ & $1.78 \mathrm{E}-10$ & $4.39 \mathrm{E}-15$ & $4.39 \mathrm{E}-17$ & $1.38 \mathrm{E}-24$ & 1.19E-24 & $5.75 \mathrm{E}-17$ & $4.80 \mathrm{E}-16$ \\
\hline $\mathrm{Pa}-233$ & $1.45 \mathrm{E}-06$ & $5.82 \mathrm{E}-06$ & $1.44 \mathrm{E}-10$ & $1.44 \mathrm{E}-12$ & $4.52 \mathrm{E}-20$ & $3.91 \mathrm{E}-20$ & $1.89 \mathrm{E}-12$ & $1.58 \mathrm{E}-11$ \\
\hline $\mathrm{U}-232$ & 1.04E-09 & 4.18E-09 & $1.03 E-13$ & $1.03 E-15$ & $3.24 \mathrm{E}-23$ & $2.80 \mathrm{E}-23$ & $1.35 \mathrm{E}-15$ & $1.13 \mathrm{E}-14$ \\
\hline U-233 & $4.04 \mathrm{E}-11$ & $1.63 \mathrm{E}-10$ & $4.02 \mathrm{E}-15$ & $4.02 \mathrm{E}-17$ & $1.26 \mathrm{E}-24$ & 1.09E-24 & $5.28 \mathrm{E}-17$ & $4.41 \mathrm{E}-16$ \\
\hline U-234 & $1.04 \mathrm{E}-06$ & $4.18 \mathrm{E}-06$ & $1.03 \mathrm{E}-10$ & $1.03 \mathrm{E}-12$ & $3.24 \mathrm{E}-20$ & $2.81 \mathrm{E}-20$ & $1.36 \mathrm{E}-12$ & $1.13 \mathrm{E}-11$ \\
\hline U-235 & 3.79E-08 & $1.53 \mathrm{E}-07$ & $3.78 \mathrm{E}-12$ & $3.78 \mathrm{E}-14$ & 1.19E-21 & 1.03E-21 & $4.95 \mathrm{E}-14$ & $4.14 \mathrm{E}-13$ \\
\hline U-236 & 5.63E-08 & $2.27 \mathrm{E}-07$ & $5.60 \mathrm{E}-12$ & $5.60 \mathrm{E}-14$ & $1.76 \mathrm{E}-21$ & $1.52 \mathrm{E}-21$ & $7.35 \mathrm{E}-14$ & $6.14 \mathrm{E}-13$ \\
\hline $\mathrm{U}-237$ & $3.17 \mathrm{E}-09$ & $1.28 \mathrm{E}-08$ & $3.16 \mathrm{E}-13$ & $3.16 \mathrm{E}-15$ & $9.91 \mathrm{E}-23$ & $8.58 \mathrm{E}-23$ & $4.14 \mathrm{E}-15$ & $3.46 \mathrm{E}-14$ \\
\hline U-238 & $2.24 \mathrm{E}-08$ & 9.04E-08 & $2.23 \mathrm{E}-12$ & $2.23 E-14$ & $7.01 \mathrm{E}-22$ & $6.06 \mathrm{E}-22$ & $2.93 \mathrm{E}-14$ & $2.45 \mathrm{E}-13$ \\
\hline Np-236 & $1.88 \mathrm{E}-12$ & $7.58 \mathrm{E}-12$ & $1.87 \mathrm{E}-16$ & $1.87 \mathrm{E}-18$ & $5.88 \mathrm{E}-26$ & 5.09E-26 & $2.46 \mathrm{E}-18$ & $2.05 \mathrm{E}-17$ \\
\hline $\mathrm{Np}-237$ & $1.46 \mathrm{E}-06$ & 5.89E-06 & $1.46 \mathrm{E}-10$ & $1.46 \mathrm{E}-12$ & 4.57E-20 & $3.95 E-20$ & $1.91 \mathrm{E}-12$ & $1.59 \mathrm{E}-11$ \\
\hline Pu-236 & $2.08 \mathrm{E}-09$ & 8.38E-09 & $2.07 \mathrm{E}-13$ & $2.07 \mathrm{E}-15$ & $6.49 \mathrm{E}-23$ & $5.62 E-23$ & $2.71 \mathrm{E}-15$ & $2.27 \mathrm{E}-14$ \\
\hline Pu-238 & $9.48 \mathrm{E}-04$ & $3.82 \mathrm{E}-03$ & 9.44E-08 & $9.44 \mathrm{E}-10$ & $2.96 \mathrm{E}-17$ & $2.56 \mathrm{E}-17$ & 1.24E-09 & $1.03 \mathrm{E}-08$ \\
\hline Pu-239 & 1.44E-04 & $5.80 \mathrm{E}-04$ & 1.43E-08 & $1.43 \mathrm{E}-10$ & $4.49 \mathrm{E}-18$ & $3.89 \mathrm{E}-18$ & $1.88 \mathrm{E}-10$ & 1.57E-09 \\
\hline Pu-240 & $7.81 \mathrm{E}-06$ & $3.15 E-05$ & $7.77 \mathrm{E}-10$ & $7.77 \mathrm{E}-12$ & $2.44 \mathrm{E}-19$ & $2.11 \mathrm{E}-19$ & $1.02 \mathrm{E}-11$ & $8.51 \mathrm{E}-11$ \\
\hline Pu-241 & $5.71 \mathrm{E}-04$ & $2.30 \mathrm{E}-03$ & $5.68 \mathrm{E}-08$ & $5.68 \mathrm{E}-10$ & $1.78 \mathrm{E}-17$ & $1.54 \mathrm{E}-17$ & $7.45 \mathrm{E}-10$ & $6.22 \mathrm{E}-09$ \\
\hline Pu-242 & 6.02E-09 & $2.43 \mathrm{E}-08$ & $5.99 \mathrm{E}-13$ & $5.99 \mathrm{E}-15$ & $1.88 \mathrm{E}-22$ & $1.63 \mathrm{E}-22$ & $7.86 \mathrm{E}-15$ & $6.56 \mathrm{E}-14$ \\
\hline Pu-244 & $5.16 \mathrm{E}-16$ & $2.08 \mathrm{E}-15$ & $5.13 E-20$ & $5.13 E-22$ & 1.61E-29 & 1.39E-29 & $6.73 E-22$ & $5.62 \mathrm{E}-21$ \\
\hline Am-241 & 6.87E-05 & $2.77 \mathrm{E}-04$ & 6.84E-09 & $6.84 \mathrm{E}-11$ & $2.15 \mathrm{E}-18$ & $1.86 \mathrm{E}-18$ & 8.97E-11 & $7.49 \mathrm{E}-10$ \\
\hline Am-242m & 7.50E-09 & $3.02 \mathrm{E}-08$ & $7.47 \mathrm{E}-13$ & $7.47 \mathrm{E}-15$ & $2.34 \mathrm{E}-22$ & $2.03 E-22$ & $9.79 \mathrm{E}-15$ & $8.18 \mathrm{E}-14$ \\
\hline $\mathrm{Am}-243$ & $1.09 \mathrm{E}-08$ & $4.39 \mathrm{E}-08$ & $1.08 \mathrm{E}-12$ & $1.08 \mathrm{E}-14$ & $3.40 \mathrm{E}-22$ & $2.94 \mathrm{E}-22$ & $1.42 \mathrm{E}-14$ & $1.19 \mathrm{E}-13$ \\
\hline $\mathrm{Cm}-242$ & 6.19E-09 & $2.49 \mathrm{E}-08$ & $6.16 \mathrm{E}-13$ & $6.16 \mathrm{E}-15$ & 1.93E-22 & 1.67E-22 & $8.08 \mathrm{E}-15$ & $6.75 \mathrm{E}-14$ \\
\hline $\mathrm{Cm}-243$ & $1.78 \mathrm{E}-08$ & 7.17E-08 & $1.77 \mathrm{E}-12$ & $1.77 \mathrm{E}-14$ & $5.56 \mathrm{E}-22$ & $4.81 \mathrm{E}-22$ & $2.32 \mathrm{E}-14$ & $1.94 \mathrm{E}-13$ \\
\hline $\mathrm{Cm}-244$ & $1.11 \mathrm{E}-06$ & $4.45 \mathrm{E}-06$ & $1.10 \mathrm{E}-10$ & $1.10 \mathrm{E}-12$ & $3.45 \mathrm{E}-20$ & $2.99 \mathrm{E}-20$ & $1.44 \mathrm{E}-12$ & $1.20 \mathrm{E}-11$ \\
\hline $\mathrm{Cm}-245$ & $1.87 \mathrm{E}-10$ & $7.54 \mathrm{E}-10$ & $1.86 \mathrm{E}-14$ & $1.86 \mathrm{E}-16$ & $5.85 \mathrm{E}-24$ & 5.06E-24 & $2.44 \mathrm{E}-16$ & $2.04 \mathrm{E}-15$ \\
\hline $\mathrm{Cm}-246$ & $1.23 \mathrm{E}-11$ & 4.95E-11 & $1.22 \mathrm{E}-15$ & $1.22 \mathrm{E}-17$ & $3.84 \mathrm{E}-25$ & 3.32E-25 & $1.60 \mathrm{E}-17$ & $1.34 \mathrm{E}-16$ \\
\hline $\mathrm{Cm}-247$ & $2.92 \mathrm{E}-18$ & $1.18 \mathrm{E}-17$ & $2.91 \mathrm{E}-22$ & $2.91 \mathrm{E}-24$ & & & $3.81 \mathrm{E}-24$ & $3.18 \mathrm{E}-23$ \\
\hline $\mathrm{H}-3$ & 1.73E-05 & $9.02 E-06$ & $1.48 \mathrm{E}-05$ & 1.48E-05 & $2.98 \mathrm{E}-07$ & $2.57 \mathrm{E}-07$ & 1.94E-05 & $9.64 \mathrm{E}-06$ \\
\hline $\mathrm{Be}-10$ & $1.48 \mathrm{E}-12$ & $5.96 \mathrm{E}-12$ & $1.47 \mathrm{E}-16$ & 1.47E-18 & $4.62 \mathrm{E}-26$ & $4.00 \mathrm{E}-26$ & 1.93E-18 & $1.61 \mathrm{E}-17$ \\
\hline C-14 & $6.21 \mathrm{E}-11$ & $2.50 \mathrm{E}-10$ & $6.18 \mathrm{E}-15$ & $6.18 \mathrm{E}-17$ & 1.94E-24 & $1.68 \mathrm{E}-24$ & $8.10 \mathrm{E}-17$ & $6.77 \mathrm{E}-16$ \\
\hline Co-60 & $3.98 \mathrm{E}-06$ & $1.60 \mathrm{E}-05$ & $3.96 \mathrm{E}-10$ & $3.96 \mathrm{E}-12$ & $1.24 \mathrm{E}-19$ & $1.08 \mathrm{E}-19$ & $5.19 \mathrm{E}-12$ & $4.34 \mathrm{E}-11$ \\
\hline \begin{tabular}{|l|}
$\mathrm{Ni}-59$ \\
\end{tabular} & $9.14 \mathrm{E}-08$ & $3.68 \mathrm{E}-07$ & $9.09 \mathrm{E}-12$ & $9.09 \mathrm{E}-14$ & $2.85 \mathrm{E}-21$ & $2.47 \mathrm{E}-21$ & 1.19E-13 & $9.96 \mathrm{E}-13$ \\
\hline $\mathrm{Ni}-63$ & $2.42 E-05$ & 9.73E-05 & $2.40 \mathrm{E}-09$ & $2.40 \mathrm{E}-11$ & $7.54 \mathrm{E}-19$ & $6.53 \mathrm{E}-19$ & $3.15 \mathrm{E}-11$ & $2.63 \mathrm{E}-10$ \\
\hline Se-79 & $2.26 \mathrm{E}-07$ & $9.10 \mathrm{E}-07$ & $2.25 \mathrm{E}-11$ & $2.25 \mathrm{E}-13$ & $7.05 E-21$ & $6.10 \mathrm{E}-21$ & $2.95 \mathrm{E}-13$ & $2.46 \mathrm{E}-12$ \\
\hline Rb-87 & $1.45 \mathrm{E}-11$ & $5.82 \mathrm{E}-11$ & $1.44 \mathrm{E}-15$ & $1.44 \mathrm{E}-17$ & $4.51 \mathrm{E}-25$ & $3.91 \mathrm{E}-25$ & $1.89 \mathrm{E}-17$ & $1.58 \mathrm{E}-16$ \\
\hline Sr-90 & 1.95E-02 & $7.86 \mathrm{E}-02$ & $1.94 \mathrm{E}-06$ & 1.94E-08 & $6.10 \mathrm{E}-16$ & $5.27 \mathrm{E}-16$ & $2.55 \mathrm{E}-08$ & $2.13 \mathrm{E}-07$ \\
\hline Y-90 & 1.95E-02 & 7.86E-02 & 1.94E-06 & 1.94E-08 & $6.10 \mathrm{E}-16$ & $5.27 \mathrm{E}-16$ & $2.55 \mathrm{E}-08$ & $2.13 \mathrm{E}-07$ \\
\hline Zr-93 & 1.09E-06 & $4.40 \mathrm{E}-06$ & $1.09 \mathrm{E}-10$ & 1.09E-12 & $3.41 \mathrm{E}-20$ & $2.95 E-20$ & $1.43 \mathrm{E}-12$ & $1.19 \mathrm{E}-11$ \\
\hline $\mathrm{Nb}-93 \mathrm{~m}$ & $8.42 \mathrm{E}-07$ & 3.39E-06 & $8.38 \mathrm{E}-11$ & $8.38 \mathrm{E}-13$ & $2.63 \mathrm{E}-20$ & $2.28 \mathrm{E}-20$ & $1.10 \mathrm{E}-12$ & $9.18 \mathrm{E}-12$ \\
\hline $\mathrm{Nb}-94$ & 5.89E-07 & $2.37 \mathrm{E}-06$ & $5.86 \mathrm{E}-11$ & $5.86 \mathrm{E}-13$ & $1.84 \mathrm{E}-20$ & $1.59 \mathrm{E}-20$ & $7.69 \mathrm{E}-13$ & $6.42 \mathrm{E}-12$ \\
\hline Tc-98 & $1.27 \mathrm{E}-12$ & $5.12 \mathrm{E}-12$ & $1.27 \mathrm{E}-16$ & $1.27 \mathrm{E}-18$ & $3.97 \mathrm{E}-26$ & $3.44 \mathrm{E}-26$ & $1.66 \mathrm{E}-18$ & $1.39 \mathrm{E}-17$ \\
\hline Tc-99 & $9.03 E-06$ & 3.64E-05 & $8.99 \mathrm{E}-10$ & $8.99 \mathrm{E}-12$ & $2.82 \mathrm{E}-19$ & $2.44 \mathrm{E}-19$ & $1.18 \mathrm{E}-11$ & $9.84 \mathrm{E}-11$ \\
\hline
\end{tabular}


Table C-4. Revised baseline mass balance, Tank WM-180 waste (Continued).

\begin{tabular}{|c|c|c|c|c|c|c|c|c|}
\hline & 101 & 102 & 103 & 104 & 105 & 106 & 107 & 108 \\
\hline Stream Name & $\begin{array}{l}\text { SBW } \\
\text { Feed }\end{array}$ & $\begin{array}{c}\text { Evap. } \\
\text { Bottoms }\end{array}$ & $\begin{array}{c}\text { Evap. } \\
\text { Overheads } \\
\text { Vapor }\end{array}$ & $\begin{array}{c}\text { Mist } \\
\text { Eliminator } \\
\text { Vapor }\end{array}$ & $\begin{array}{l}\text { VOG } \\
\text { Offgas } \\
\text { Vapor }\end{array}$ & $\begin{array}{c}\text { POG + VOG } \\
\text { Offgas } \\
\text { Vapor }\end{array}$ & $\begin{array}{l}\text { LET\&D } \\
\text { Feed } \\
\text { Liquid }\end{array}$ & $\begin{array}{l}\text { LET\&D } \\
\text { Acid } \\
\text { Liquid }\end{array}$ \\
\hline Radiological Composition & $\mathrm{Ci} / \mathrm{L}$ & $\mathrm{Ci} / \mathrm{L}$ & $\mathrm{Ci} / \mathrm{wscm}$ & $\mathrm{Ci} / \mathrm{wscm}$ & $\mathrm{Ci} / \mathrm{wscm}$ & $\mathrm{Ci} / \mathrm{wscm}$ & $\mathrm{Ci} / \mathrm{L}$ & $\mathrm{Ci} / \mathrm{L}$ \\
\hline Ru-106 & $4.81 \mathrm{E}-07$ & 1.94E-06 & $4.79 \mathrm{E}-11$ & $4.79 \mathrm{E}-13$ & $1.50 \mathrm{E}-20$ & $1.30 \mathrm{E}-20$ & $6.28 \mathrm{E}-13$ & $5.25 \mathrm{E}-12$ \\
\hline Rh-102 & $4.25 \mathrm{E}-10$ & 1.71E-09 & $4.23 E-14$ & $4.23 E-16$ & $1.33 E-23$ & $1.15 \mathrm{E}-23$ & $5.55 \mathrm{E}-16$ & $4.63 E-15$ \\
\hline Rh-106 & $4.81 \mathrm{E}-07$ & 1.94E-06 & $4.79 \mathrm{E}-11$ & $4.79 \mathrm{E}-13$ & $1.50 \mathrm{E}-20$ & $1.30 \mathrm{E}-20$ & $6.28 \mathrm{E}-13$ & $5.25 \mathrm{E}-12$ \\
\hline $\mathrm{Pd}-107$ & $8.15 \mathrm{E}-09$ & $3.28 \mathrm{E}-08$ & $8.11 \mathrm{E}-13$ & $8.11 \mathrm{E}-15$ & $2.54 \mathrm{E}-22$ & $2.20 \mathrm{E}-22$ & $1.06 \mathrm{E}-14$ & $8.88 \mathrm{E}-14$ \\
\hline Cd-113m & $1.64 \mathrm{E}-06$ & $6.60 \mathrm{E}-06$ & $1.63 \mathrm{E}-10$ & $1.63 \mathrm{E}-12$ & $5.12 \mathrm{E}-20$ & $4.43 E-20$ & $2.14 \mathrm{E}-12$ & $1.79 \mathrm{E}-11$ \\
\hline $\ln -115$ & $4.96 \mathrm{E}-17$ & $2.00 \mathrm{E}-16$ & $4.94 \mathrm{E}-21$ & $4.94 \mathrm{E}-23$ & $1.55 \mathrm{E}-30$ & $1.34 \mathrm{E}-30$ & $6.48 \mathrm{E}-23$ & $5.41 \mathrm{E}-22$ \\
\hline Sn-121m & $3.30 \mathrm{E}-08$ & $1.33 \mathrm{E}-07$ & $3.28 \mathrm{E}-12$ & $3.28 \mathrm{E}-14$ & $1.03 E-21$ & $8.91 \mathrm{E}-22$ & $4.30 \mathrm{E}-14$ & $3.59 \mathrm{E}-13$ \\
\hline Sn-126 & $2.12 \mathrm{E}-07$ & $8.56 \mathrm{E}-07$ & $2.11 \mathrm{E}-11$ & $2.11 \mathrm{E}-13$ & $6.64 \mathrm{E}-21$ & $5.74 \mathrm{E}-21$ & $2.77 \mathrm{E}-13$ & $2.32 \mathrm{E}-12$ \\
\hline Sb-125 & $7.91 \mathrm{E}-06$ & 3.19E-05 & $7.87 \mathrm{E}-10$ & $7.87 \mathrm{E}-12$ & $2.47 \mathrm{E}-19$ & $2.14 \mathrm{E}-19$ & $1.03 \mathrm{E}-11$ & $8.62 \mathrm{E}-11$ \\
\hline Sb-126 & $2.84 \mathrm{E}-08$ & 1.14E-07 & $2.82 \mathrm{E}-12$ & $2.82 \mathrm{E}-14$ & $8.86 \mathrm{E}-22$ & $7.67 \mathrm{E}-22$ & $3.70 \mathrm{E}-14$ & $3.09 E-13$ \\
\hline Te-123 & $1.89 \mathrm{E}-19$ & $7.61 \mathrm{E}-19$ & $1.88 \mathrm{E}-23$ & $1.88 \mathrm{E}-25$ & & & $2.47 \mathrm{E}-25$ & $2.06 \mathrm{E}-24$ \\
\hline Te-125m & $1.55 \mathrm{E}-06$ & $6.26 \mathrm{E}-06$ & $1.55 \mathrm{E}-10$ & $1.55 \mathrm{E}-12$ & $4.85 \mathrm{E}-20$ & $4.20 \mathrm{E}-20$ & $2.03 \mathrm{E}-12$ & $1.69 \mathrm{E}-11$ \\
\hline I-129 & $2.40 \mathrm{E}-08$ & $7.60 \mathrm{E}-08$ & $5.13 \mathrm{E}-09$ & 5.12E-09 & $4.80 \mathrm{E}-15$ & $6.83 \mathrm{E}-13$ & $6.72 \mathrm{E}-09$ & $9.84 \mathrm{E}-16$ \\
\hline Cs-134 & $5.14 \mathrm{E}-06$ & $2.07 \mathrm{E}-05$ & $5.11 \mathrm{E}-10$ & $5.11 \mathrm{E}-12$ & $1.60 \mathrm{E}-19$ & $1.39 \mathrm{E}-19$ & $6.70 \mathrm{E}-12$ & $5.60 \mathrm{E}-11$ \\
\hline Cs-135 & 4.42E-07 & $1.78 \mathrm{E}-06$ & $4.40 \mathrm{E}-11$ & $4.40 \mathrm{E}-13$ & $1.38 \mathrm{E}-20$ & $1.20 \mathrm{E}-20$ & $5.77 \mathrm{E}-13$ & $4.82 \mathrm{E}-12$ \\
\hline Cs-137 & $2.59 \mathrm{E}-02$ & 1.04E-01 & $2.58 \mathrm{E}-06$ & $2.58 \mathrm{E}-08$ & $8.09 \mathrm{E}-16$ & $7.00 \mathrm{E}-16$ & $3.38 \mathrm{E}-08$ & $2.83 \mathrm{E}-07$ \\
\hline Ba-137m & $2.45 \mathrm{E}-02$ & 9.88E-02 & $2.44 \mathrm{E}-06$ & $2.44 \mathrm{E}-08$ & $7.66 \mathrm{E}-16$ & $6.63 \mathrm{E}-16$ & $3.20 \mathrm{E}-08$ & $2.67 E-07$ \\
\hline La-138 & $9.42 \mathrm{E}-17$ & $3.79 \mathrm{E}-16$ & $9.37 \mathrm{E}-21$ & 9.37E-23 & $2.94 \mathrm{E}-30$ & $2.54 \mathrm{E}-30$ & $1.23 \mathrm{E}-22$ & 1.03E-21 \\
\hline Ce-142 & $1.47 \mathrm{E}-11$ & $5.94 \mathrm{E}-11$ & $1.47 \mathrm{E}-15$ & $1.47 \mathrm{E}-17$ & $4.61 \mathrm{E}-25$ & $3.98 \mathrm{E}-25$ & $1.92 \mathrm{E}-17$ & $1.61 \mathrm{E}-16$ \\
\hline Ce-144 & $3.24 \mathrm{E}-07$ & $1.31 \mathrm{E}-06$ & $3.23 \mathrm{E}-11$ & $3.23 \mathrm{E}-13$ & $1.01 \mathrm{E}-20$ & $8.77 \mathrm{E}-21$ & $4.23 \mathrm{E}-13$ & $3.54 \mathrm{E}-12$ \\
\hline Pr-144 & $3.24 \mathrm{E}-07$ & $1.31 \mathrm{E}-06$ & $3.23 \mathrm{E}-11$ & $3.23 \mathrm{E}-13$ & $1.01 \mathrm{E}-20$ & $8.77 \mathrm{E}-21$ & $4.23 \mathrm{E}-13$ & $3.54 \mathrm{E}-12$ \\
\hline Nd-144 & $7.93 \mathrm{E}-16$ & $3.19 \mathrm{E}-15$ & $7.89 \mathrm{E}-20$ & $7.89 E-22$ & $2.48 \mathrm{E}-29$ & $2.14 \mathrm{E}-29$ & $1.03 \mathrm{E}-21$ & $8.64 \mathrm{E}-21$ \\
\hline Pm-146 & $2.51 \mathrm{E}-08$ & $1.01 \mathrm{E}-07$ & $2.50 \mathrm{E}-12$ & $2.50 \mathrm{E}-14$ & $7.84 \mathrm{E}-22$ & $6.78 \mathrm{E}-22$ & $3.28 \mathrm{E}-14$ & $2.74 \mathrm{E}-13$ \\
\hline Pm-147 & $8.80 \mathrm{E}-05$ & $3.55 \mathrm{E}-04$ & 8.76E-09 & $8.76 \mathrm{E}-11$ & $2.75 \mathrm{E}-18$ & $2.38 \mathrm{E}-18$ & $1.15 \mathrm{E}-10$ & $9.60 \mathrm{E}-10$ \\
\hline Sm-146 & $1.36 \mathrm{E}-13$ & $5.48 \mathrm{E}-13$ & $1.35 \mathrm{E}-17$ & $1.35 \mathrm{E}-19$ & $4.25 \mathrm{E}-27$ & $3.68 \mathrm{E}-27$ & $1.78 \mathrm{E}-19$ & $1.48 \mathrm{E}-18$ \\
\hline Sm-147 & $3.63 \mathrm{E}-12$ & $1.46 \mathrm{E}-11$ & $3.62 \mathrm{E}-16$ & $3.62 \mathrm{E}-18$ & $1.13 \mathrm{E}-25$ & $9.82 \mathrm{E}-26$ & $4.74 \mathrm{E}-18$ & $3.96 \mathrm{E}-17$ \\
\hline Sm-148 & $1.87 \mathrm{E}-17$ & $7.52 \mathrm{E}-17$ & $1.86 \mathrm{E}-21$ & $1.86 \mathrm{E}-23$ & & & $2.44 \mathrm{E}-23$ & $2.04 \mathrm{E}-22$ \\
\hline Sm-149 & $1.66 \mathrm{E}-18$ & $6.67 \mathrm{E}-18$ & $1.65 \mathrm{E}-22$ & $1.65 \mathrm{E}-24$ & & & $2.16 \mathrm{E}-24$ & $1.81 \mathrm{E}-23$ \\
\hline Sm-151 & $1.74 \mathrm{E}-04$ & 6.99E-04 & 1.73E-08 & $1.73 \mathrm{E}-10$ & $5.42 \mathrm{E}-18$ & $4.69 \mathrm{E}-18$ & $2.27 \mathrm{E}-10$ & 1.89E-09 \\
\hline Eu-152 & $1.26 \mathrm{E}-06$ & $5.06 \mathrm{E}-06$ & $1.25 \mathrm{E}-10$ & $1.25 \mathrm{E}-12$ & $3.93 E-20$ & $3.40 \mathrm{E}-20$ & $1.64 \mathrm{E}-12$ & $1.37 \mathrm{E}-11$ \\
\hline Eu-154 & $4.51 \mathrm{E}-05$ & $1.82 \mathrm{E}-04$ & $4.49 \mathrm{E}-09$ & $4.49 \mathrm{E}-11$ & $1.41 \mathrm{E}-18$ & $1.22 \mathrm{E}-18$ & $5.89 \mathrm{E}-11$ & $4.92 \mathrm{E}-10$ \\
\hline Eu-155 & $8.20 \mathrm{E}-05$ & $3.30 \mathrm{E}-04$ & $8.16 \mathrm{E}-09$ & $8.16 \mathrm{E}-11$ & $2.56 \mathrm{E}-18$ & $2.22 \mathrm{E}-18$ & $1.07 \mathrm{E}-10$ & $8.94 \mathrm{E}-10$ \\
\hline Gd-152 & $7.01 \mathrm{E}-19$ & $2.82 \mathrm{E}-18$ & $6.98 \mathrm{E}-23$ & $6.98 \mathrm{E}-25$ & & & $9.15 \mathrm{E}-25$ & $7.64 \mathrm{E}-24$ \\
\hline Ho-166m & $2.27 \mathrm{E}-11$ & $9.15 \mathrm{E}-11$ & $2.26 \mathrm{E}-15$ & $2.26 \mathrm{E}-17$ & $7.09 \mathrm{E}-25$ & $6.14 \mathrm{E}-25$ & $2.96 \mathrm{E}-17$ & $2.48 \mathrm{E}-16$ \\
\hline \multicolumn{9}{|l|}{ Other Stream Data: } \\
\hline TRU, nCi/gm & $9.57 \mathrm{E}+02$ & $2.73 \mathrm{E}+03$ & & & & & $1.48 \mathrm{E}-03$ & 1.11E-02 \\
\hline Heat Generation, W/m3 & $2.92 \mathrm{E}-01$ & $1.18 \mathrm{E}+00$ & & & & & $1.04 \mathrm{E}-06$ & $3.51 \mathrm{E}-06$ \\
\hline Mass Flow, kg/hr & $4.04 \mathrm{E}+02$ & $1.41 \mathrm{E}+02$ & $2.60 \mathrm{E}+02$ & $2.60 \mathrm{E}+02$ & $2.47 \mathrm{E}+02$ & $2.88 \mathrm{E}+02$ & $2.60 \mathrm{E}+02$ & $3.47 \mathrm{E}+01$ \\
\hline
\end{tabular}


Table C-4. Revised baseline mass balance, Tank WM-180 waste (Continued).

\begin{tabular}{|c|c|c|c|c|c|c|c|}
\hline PFD Sheet \# & PFD-3 & PFD-4 & PFD-4 & PFD-4 & PFD-4 & PFD-4 & PFD-4 \\
\hline Stream \# & 109 & 110 & 201 & 202 & 203 & 204 & 205 \\
\hline Stream Name & $\begin{array}{l}\text { LET\&D } \\
\text { Offgas } \\
\text { Vapor }\end{array}$ & $\begin{array}{l}\text { Acid } \\
\text { Grout } \\
\text { Solid }\end{array}$ & $\begin{array}{c}\text { OPC } \\
\text { Grout } \\
\text { Additive }\end{array}$ & $\begin{array}{c}\text { BFS } \\
\text { Grout } \\
\text { Additive }\end{array}$ & $\begin{array}{c}\mathrm{Ca}(\mathrm{OH}) 2 \\
\text { Total } \\
\text { Additive }\end{array}$ & $\begin{array}{c}\mathrm{Ca}(\mathrm{OH}) 2 \\
\text { Grout } \\
\text { Additive }\end{array}$ & $\begin{array}{c}\mathrm{Ca}(\mathrm{OH}) 2 \\
\text { Neutraliz. } \\
\text { Additive }\end{array}$ \\
\hline Rate or Volume Flow (actual) & $1.69 \mathrm{E}+04$ & 219 & 86.0 & 28.7 & 27.3 & 0.87 & 26.4 \\
\hline Volume Flow (standard, wet) ${ }^{*}$ & $1.06 \mathrm{E}+04$ & & & & & & \\
\hline Volume Flow (standard, dry) ${ }^{*}$ & $1.70 \mathrm{E}+00$ & & & & & & \\
\hline Rate Units & $\mathrm{ft} 3 / \mathrm{hr}$ & $\mathrm{Ib} / \mathrm{hr}$ & $\mathrm{lb} / \mathrm{hr}$ & $\mathrm{Ib} / \mathrm{hr}$ & $\mathrm{Ib} / \mathrm{hr}$ & $\mathrm{Ib} / \mathrm{hr}$ & $\mathrm{lb} / \mathrm{hr}$ \\
\hline Temperature, ${ }^{\circ} \mathrm{C}$ & 119 & 64 & 25 & 25 & 25 & 25 & 25 \\
\hline Temperature, ${ }^{\circ} \mathrm{F}$ & 246 & 148 & 77 & 77 & 77 & 77 & 77 \\
\hline Pressure, psia & 12.3 & 12.2 & 12.3 & 12.3 & 12.3 & 12.3 & 12.3 \\
\hline Specific Gravity & $4.71 \mathrm{E}-04$ & 2.06 & 1.2 & 1.2 & 0.48 & 0.48 & 0.48 \\
\hline Chemical Composition & $\mathrm{lb} /$ wscf & Wt. \% & Wt. \% & Wt. \% & Wt. \% & Wt. \% & Wt. \% \\
\hline $\mathrm{H}+$ & $4.13 E-07$ & $8.88 \mathrm{E}-06$ & & & & & \\
\hline $\mathrm{OH}-$ & & 1.77E-01 & & & $4.59 \mathrm{E}+01$ & $4.59 \mathrm{E}+01$ & $4.59 \mathrm{E}+01$ \\
\hline $\mathrm{Al}+3$ & & $5.15 \mathrm{E}-06$ & & & & & \\
\hline $\mathrm{Sb}+5$ & & $5.55 \mathrm{E}-11$ & & & & & \\
\hline$A s+5$ & & $1.10 \mathrm{E}-08$ & & & & & \\
\hline $\mathrm{Ba}+2$ & & $2.25 \mathrm{E}-09$ & & & & & \\
\hline $\mathrm{Be}+2$ & & $2.31 \mathrm{E}-11$ & & & & & \\
\hline $\mathrm{B}+3$ & & $3.68 \mathrm{E}-08$ & & & & & \\
\hline $\mathrm{Cd}+2$ & & $2.64 \mathrm{E}-08$ & & & & & \\
\hline $\mathrm{Ca}+2$ & & $6.76 \mathrm{E}+00$ & & & $5.41 \mathrm{E}+01$ & $5.41 \mathrm{E}+01$ & $5.41 \mathrm{E}+01$ \\
\hline $\mathrm{Cr}+3$ & & $5.73 \mathrm{E}-08$ & & & & & \\
\hline $\mathrm{Co}+2$ & & $3.53 \mathrm{E}-10$ & & & & & \\
\hline $\mathrm{Cs}+$ & & 1.12E-09 & & & & & \\
\hline $\mathrm{Cu}+2$ & & $1.32 \mathrm{E}-08$ & & & & & \\
\hline $\mathrm{Fe}+3$ & & $3.52 \mathrm{E}-07$ & & & & & \\
\hline $\mathrm{Pb}+2$ & & $8.57 \mathrm{E}-08$ & & & & & \\
\hline $\mathrm{Hg}+2$ & & $1.20 \mathrm{E}-08$ & & & & & \\
\hline $\mathrm{Mn}+4$ & & $2.29 \mathrm{E}-07$ & & & & & \\
\hline $\mathrm{Ni}+2$ & & $2.67 \mathrm{E}-08$ & & & & & \\
\hline $\mathrm{K}+$ & & $2.25 \mathrm{E}-06$ & & & & & \\
\hline $\mathrm{Se}+4$ & & 1.99E-09 & & & & & \\
\hline $\mathrm{Ag}+$ & & $2.15 \mathrm{E}-10$ & & & & & \\
\hline $\mathrm{Na}+$ & & 1.42E-05 & & & & & \\
\hline $\mathrm{TI}+3$ & & 1.84E-09 & & & & & \\
\hline $\mathrm{U}+4$ & & $2.82 \mathrm{E}-08$ & & & & & \\
\hline $\mathrm{V}+3$ & & $1.46 \mathrm{E}-08$ & & & & & \\
\hline$Z n+2$ & & $2.04 \mathrm{E}-08$ & & & & & \\
\hline $\mathrm{Zr}+4$ & & $3.95 \mathrm{E}-08$ & & & & & \\
\hline $\mathrm{Cl}-$ & $1.45 \mathrm{E}-05$ & 1.23E-09 & & & & & \\
\hline F- & & 1.70E-04 & & & & & \\
\hline SO4-2 & & $1.56 \mathrm{E}-06$ & & & & & \\
\hline NO3- & 3.14E-09 & $2.03 \mathrm{E}+01$ & & & & & \\
\hline PO4-3 & & $6.07 \mathrm{E}-07$ & & & & & \\
\hline $\mathrm{Am}+4$ & & $2.51 \mathrm{E}-12$ & & & & & \\
\hline $\mathrm{Br}-$ & $1.79 \mathrm{E}-10$ & & & & & & \\
\hline $\mathrm{Ce}+4$ & & $2.00 \mathrm{E}-09$ & & & & & \\
\hline $\mathrm{Eu}+3$ & & $1.28 \mathrm{E}-11$ & & & & & \\
\hline $\mathrm{Gd}+3$ & & 8.27E-09 & & & & & \\
\hline $\mathrm{Ge}+4$ & & 1.07E-13 & & & & & \\
\hline $\ln +3$ & & $2.65 \mathrm{E}-11$ & & & & & \\
\hline I- & $2.36 \mathrm{E}-09$ & $2.36 \mathrm{E}-12$ & & & & & \\
\hline $\mathrm{La+3}$ & & $2.13 \mathrm{E}-10$ & & & & & \\
\hline $\mathrm{Li}+$ & & 1.01E-09 & & & & & \\
\hline $\mathrm{Mg}+2$ & & $8.75 \mathrm{E}-08$ & & & & & \\
\hline $\mathrm{Mo}+6$ & & 5.90E-09 & & & & & \\
\hline
\end{tabular}


Table C-4. Revised baseline mass balance, Tank WM-180 waste (Continued).

\begin{tabular}{|c|c|c|c|c|c|c|c|}
\hline Stream \# & 109 & 110 & 201 & 202 & 203 & 204 & 205 \\
\hline Stream Name & $\begin{array}{l}\text { LET\&D } \\
\text { Offgas } \\
\text { Vapor }\end{array}$ & $\begin{array}{l}\text { Acid } \\
\text { Grout } \\
\text { Solid }\end{array}$ & $\begin{array}{c}\text { OPC } \\
\text { Grout } \\
\text { Additive }\end{array}$ & $\begin{array}{c}\text { BFS } \\
\text { Grout } \\
\text { Additive }\end{array}$ & $\begin{array}{c}\mathrm{Ca}(\mathrm{OH}) 2 \\
\text { Total } \\
\text { Additive }\end{array}$ & $\begin{array}{c}\mathrm{Ca}(\mathrm{OH}) 2 \\
\text { Grout } \\
\text { Additive }\end{array}$ & $\begin{array}{c}\mathrm{Ca}(\mathrm{OH}) 2 \\
\text { Neutraliz. } \\
\text { Additive }\end{array}$ \\
\hline Chemical Composition & $\mathrm{lb} /$ wscf & Wt. \% & Wt. \% & Wt. \% & Wt. \% & Wt. \% & Wt. \% \\
\hline $\mathrm{Nd}+3$ & & $7.14 \mathrm{E}-10$ & & & & & \\
\hline $\mathrm{Np}+4$ & & $6.78 \mathrm{E}-10$ & & & & & \\
\hline $\mathrm{Nb}+5$ & & 1.36E-08 & & & & & \\
\hline $\mathrm{Pd}+4$ & & 1.09E-09 & & & & & \\
\hline $\mathrm{Pu}+4$ & & $7.98 \mathrm{E}-10$ & & & & & \\
\hline $\mathrm{Pr}+4$ & & $1.97 \mathrm{E}-10$ & & & & & \\
\hline $\mathrm{Rh}+4$ & & $6.19 \mathrm{E}-11$ & & & & & \\
\hline $\mathrm{Rb}+$ & & $7.91 \mathrm{E}-11$ & & & & & \\
\hline Ru+3 & & 4.18E-09 & & & & & \\
\hline $\mathrm{Sm}+3$ & & $1.38 \mathrm{E}-10$ & & & & & \\
\hline $\mathrm{Si}+4$ & & $2.83 \mathrm{E}-08$ & & & & & \\
\hline $\mathrm{Sr}+2$ & & 3.09E-09 & & & & & \\
\hline $\mathrm{Tc}+7$ & & $1.68 \mathrm{E}-10$ & & & & & \\
\hline $\mathrm{Te}+4$ & & $6.15 \mathrm{E}-11$ & & & & & \\
\hline $\mathrm{Tb}+4$ & & $5.61 \mathrm{E}-14$ & & & & & \\
\hline $\mathrm{Th}+4$ & & $7.99 \mathrm{E}-15$ & & & & & \\
\hline $\mathrm{Sn}+4$ & & 2.87E-09 & & & & & \\
\hline $\mathrm{Ti}+4$ & & $2.11 \mathrm{E}-09$ & & & & & \\
\hline $\mathrm{Y}+3$ & & $1.02 \mathrm{E}-10$ & & & & & \\
\hline O-2 (oxides) & & $6.95 \mathrm{E}-08$ & & & & & \\
\hline $\mathrm{H} 2 \mathrm{O}$ & 4.70E-02 & $2.03 \mathrm{E}+01$ & & & & & \\
\hline $\mathrm{O} 2$ & $1.42 \mathrm{E}-07$ & & & & & & \\
\hline $\mathrm{N} 2$ & $1.98 \mathrm{E}-07$ & & & & & & \\
\hline C (organic) & & $1.01 \mathrm{E}-03$ & & & & & \\
\hline $\mathrm{H}$ (organic) & & $8.44 \mathrm{E}-05$ & & & & & \\
\hline SiO2, BFS, or OPC Additives & & $5.25 \mathrm{E}+01$ & $1.00 \mathrm{E}+02$ & $1.00 \mathrm{E}+02$ & & & \\
\hline $\begin{array}{l}\text { Gas Stream Bulk Composition } \\
\text { (wet basis) }\end{array}$ & $\begin{array}{l}\text { mol. \% } \\
\text { or ppmv }\end{array}$ & $\begin{array}{l}\text { mol. \% } \\
\text { or ppmv }\end{array}$ & $\begin{array}{l}\text { mol. \% } \\
\text { or ppmv }\end{array}$ & $\begin{array}{l}\text { mol.\% } \\
\text { or ppmv }\end{array}$ & $\begin{array}{l}\text { mol. } \% \\
\text { or ppmv }\end{array}$ & $\begin{array}{l}\text { mol. } \% \\
\text { or ppmv }\end{array}$ & $\begin{array}{l}\text { mol. \% } \\
\text { or ppmv }\end{array}$ \\
\hline $\mathrm{H} 2 \mathrm{O}, \mathrm{mol} . \%$ & 99.98 & & & & & & \\
\hline $\mathrm{O} 2, \mathrm{~mol} . \%$ & 0.0002 & & & & & & \\
\hline $\mathrm{N} 2, \mathrm{~mol} . \%$ & 0.0003 & & & & & & \\
\hline \multicolumn{8}{|l|}{$\mathrm{CO} 2, \mathrm{~mol} . \%$} \\
\hline HNO3, mol. \% & 0.02 & & & & & & \\
\hline \multicolumn{8}{|l|}{$\mathrm{CO}, \mathrm{ppmv}$} \\
\hline \multicolumn{8}{|l|}{$\mathrm{NO}, \mathrm{ppmv}$} \\
\hline \multicolumn{8}{|l|}{ NO2, ppmv } \\
\hline \multicolumn{8}{|l|}{ C (organic), ppmv } \\
\hline \multicolumn{8}{|l|}{$\mathrm{H}$ (organic), ppmv } \\
\hline \multicolumn{8}{|l|}{$\mathrm{PM}$ (mg/wscm) } \\
\hline & & & & & & & \\
\hline $\begin{array}{l}\text { Gas Stream Bulk Composition } \\
\text { (dry basis) }\end{array}$ & $\begin{array}{l}\text { mol. \% } \\
\text { or ppmv }\end{array}$ & $\begin{array}{l}\text { mol. } \% \\
\text { or ppmv }\end{array}$ & $\begin{array}{l}\text { mol. \% } \\
\text { or ppmv }\end{array}$ & $\begin{array}{l}\text { mol.\% } \\
\text { or ppmv }\end{array}$ & $\begin{array}{l}\text { mol.\% } \\
\text { or ppmv }\end{array}$ & $\begin{array}{l}\text { mol. \% } \\
\text { or ppmv }\end{array}$ & $\begin{array}{l}\text { mol. } \% \\
\text { or ppmv }\end{array}$ \\
\hline $\mathrm{O} 2, \mathrm{~mol} . \%$ & 1.05 & & & & & & \\
\hline $\mathrm{N} 2, \mathrm{~mol} . \%$ & 1.67 & & & & & & \\
\hline \multicolumn{8}{|l|}{$\mathrm{CO} 2, \mathrm{~mol} . \%$} \\
\hline $\mathrm{HNO} 3, \mathrm{~mol} . \%$ & 97.25 & & & & & & \\
\hline \multicolumn{8}{|l|}{$\mathrm{CO}, \mathrm{ppmv}$} \\
\hline \multicolumn{8}{|l|}{$\mathrm{NO}, \mathrm{ppmv}$} \\
\hline \multicolumn{8}{|l|}{ NO2, ppmv } \\
\hline \multicolumn{8}{|l|}{ C (organic), ppmv } \\
\hline \multicolumn{8}{|l|}{$\mathrm{H}$ (organic), ppmv } \\
\hline $\mathrm{PM}(\mathrm{mg} / \mathrm{dscm})$ & & & & & & & \\
\hline & & & & & & & \\
\hline
\end{tabular}


Table C-4. Revised baseline mass balance, Tank WM-180 waste (Continued).

\begin{tabular}{|c|c|c|c|c|c|c|c|}
\hline Stream \# & 109 & 110 & 201 & 202 & 203 & 204 & 205 \\
\hline Stream Name & $\begin{array}{l}\text { LET\&D } \\
\text { Offgas } \\
\text { Vapor }\end{array}$ & $\begin{array}{l}\text { Acid } \\
\text { Grout } \\
\text { Solid }\end{array}$ & $\begin{array}{c}\text { OPC } \\
\text { Grout } \\
\text { Additive }\end{array}$ & $\begin{array}{c}\text { BFS } \\
\text { Grout } \\
\text { Additive }\end{array}$ & $\begin{array}{l}\mathrm{Ca}(\mathrm{OH}) 2 \\
\text { Total } \\
\text { Additive }\end{array}$ & $\begin{array}{c}\mathrm{Ca}(\mathrm{OH}) 2 \\
\text { Grout } \\
\text { Additive }\end{array}$ & $\begin{array}{l}\mathrm{Ca}(\mathrm{OH}) 2 \\
\text { Neutraliz. } \\
\text { Additive }\end{array}$ \\
\hline \multicolumn{8}{|l|}{ Canister Rate, canisters/day } \\
\hline \multicolumn{8}{|l|}{ Total Canisters Generated } \\
\hline Drum Rate, drums/day & & 6.90 & & & & & \\
\hline \multicolumn{8}{|l|}{ Total Drums Generated } \\
\hline Radiological Composition & $\mathrm{Ci} / \mathrm{wscm}$ & $\mathrm{Ci} / \mathrm{kg}$ & $\mathrm{Ci} / \mathrm{kg}$ & $\mathrm{Ci} / \mathrm{kg}$ & $\mathrm{Ci} / \mathrm{kg}$ & $\mathrm{Ci} / \mathrm{kg}$ & $\mathrm{Ci} / \mathrm{kg}$ \\
\hline Ra-226 & $8.78 \mathrm{E}-30$ & $1.32 \mathrm{E}-17$ & & & & & \\
\hline Ac-227 & $4.13 \mathrm{E}-29$ & $6.23 \mathrm{E}-17$ & & & & & \\
\hline Th-228 & $3.43 \mathrm{E}-27$ & $5.17 \mathrm{E}-15$ & & & & & \\
\hline Th-230 & $9.35 \mathrm{E}-28$ & $1.41 \mathrm{E}-15$ & & & & & \\
\hline Th-232 & & $1.14 \mathrm{E}-21$ & & & & & \\
\hline Pa-231 & $9.57 \mathrm{E}-29$ & $1.44 \mathrm{E}-16$ & & & & & \\
\hline Pa-233 & $3.14 \mathrm{E}-24$ & $4.74 \mathrm{E}-12$ & & & & & \\
\hline $\mathrm{U}-232$ & $2.25 \mathrm{E}-27$ & $3.40 \mathrm{E}-15$ & & & & & \\
\hline $\mathrm{U}-233$ & $8.78 \mathrm{E}-29$ & $1.32 \mathrm{E}-16$ & & & & & \\
\hline $\mathrm{U}-234$ & $2.26 \mathrm{E}-24$ & $3.40 \mathrm{E}-12$ & & & & & \\
\hline $\mathrm{U}-235$ & $8.24 \mathrm{E}-26$ & $1.24 \mathrm{E}-13$ & & & & & \\
\hline $\mathrm{U}-236$ & $1.22 \mathrm{E}-25$ & $1.84 \mathrm{E}-13$ & & & & & \\
\hline $\mathrm{U}-237$ & $6.89 \mathrm{E}-27$ & $1.04 \mathrm{E}-14$ & & & & & \\
\hline $\mathrm{U}-238$ & $4.87 \mathrm{E}-26$ & $7.35 \mathrm{E}-14$ & & & & & \\
\hline Np-236 & $4.09 \mathrm{E}-30$ & $6.17 \mathrm{E}-18$ & & & & & \\
\hline Np-237 & $3.18 \mathrm{E}-24$ & $4.79 \mathrm{E}-12$ & & & & & \\
\hline Pu-236 & $4.52 \mathrm{E}-27$ & $6.81 \mathrm{E}-15$ & & & & & \\
\hline Pu-238 & $2.06 \mathrm{E}-21$ & $3.11 \mathrm{E}-09$ & & & & & \\
\hline Pu-239 & $3.13 \mathrm{E}-22$ & $4.71 \mathrm{E}-10$ & & & & & \\
\hline Pu-240 & $1.70 \mathrm{E}-23$ & $2.56 \mathrm{E}-11$ & & & & & \\
\hline Pu-241 & $1.24 \mathrm{E}-21$ & $1.87 \mathrm{E}-09$ & & & & & \\
\hline Pu-242 & $1.31 \mathrm{E}-26$ & $1.97 \mathrm{E}-14$ & & & & & \\
\hline Pu-244 & & $1.69 \mathrm{E}-21$ & & & & & \\
\hline Am-241 & $1.49 \mathrm{E}-22$ & $2.25 \mathrm{E}-10$ & & & & & \\
\hline Am-242m & $1.63 \mathrm{E}-26$ & $2.46 \mathrm{E}-14$ & & & & & \\
\hline $\mathrm{Am}-243$ & $2.37 \mathrm{E}-26$ & $3.57 \mathrm{E}-14$ & & & & & \\
\hline $\mathrm{Cm}-242$ & $1.34 \mathrm{E}-26$ & $2.03 \mathrm{E}-14$ & & & & & \\
\hline $\mathrm{Cm}-243$ & $3.86 \mathrm{E}-26$ & $5.83 \mathrm{E}-14$ & & & & & \\
\hline $\mathrm{Cm}-244$ & $2.40 \mathrm{E}-24$ & $3.62 \mathrm{E}-12$ & & & & & \\
\hline $\mathrm{Cm}-245$ & $4.07 \mathrm{E}-28$ & $6.13 \mathrm{E}-16$ & & & & & \\
\hline $\mathrm{Cm}-246$ & $2.67 \mathrm{E}-29$ & $4.03 \mathrm{E}-17$ & & & & & \\
\hline $\mathrm{Cm}-247$ & & $9.56 \mathrm{E}-24$ & & & & & \\
\hline $\mathrm{H}-3$ & $1.52 \mathrm{E}-05$ & $2.90 \mathrm{E}-06$ & & & & & \\
\hline $\mathrm{Be}-10$ & $3.21 \mathrm{E}-30$ & $4.85 \mathrm{E}-18$ & & & & & \\
\hline C-14 & $1.35 \mathrm{E}-28$ & $2.03 \mathrm{E}-16$ & & & & & \\
\hline Co-60 & $8.64 \mathrm{E}-24$ & $1.30 \mathrm{E}-11$ & & & & & \\
\hline Ni-59 & $1.98 \mathrm{E}-25$ & $2.99 \mathrm{E}-13$ & & & & & \\
\hline $\mathrm{Ni}-63$ & $5.25 \mathrm{E}-23$ & $7.91 \mathrm{E}-11$ & & & & & \\
\hline Se-79 & $4.91 \mathrm{E}-25$ & $7.40 \mathrm{E}-13$ & & & & & \\
\hline Rb-87 & $3.14 \mathrm{E}-29$ & $4.74 \mathrm{E}-17$ & & & & & \\
\hline Sr-90 & $4.24 \mathrm{E}-20$ & $6.39 \mathrm{E}-08$ & & & & & \\
\hline$Y-90$ & $4.24 \mathrm{E}-20$ & $6.39 \mathrm{E}-08$ & & & & & \\
\hline Zr-93 & $2.37 \mathrm{E}-24$ & $3.58 \mathrm{E}-12$ & & & & & \\
\hline $\mathrm{Nb}-93 \mathrm{~m}$ & 1.83E-24 & $2.76 \mathrm{E}-12$ & & & & & \\
\hline Nb-94 & $1.28 \mathrm{E}-24$ & $1.93 \mathrm{E}-12$ & & & & & \\
\hline Tc-98 & $2.76 \mathrm{E}-30$ & $4.17 \mathrm{E}-18$ & & & & & \\
\hline Tc-99 & $1.96 \mathrm{E}-23$ & $2.96 \mathrm{E}-11$ & & & & & \\
\hline & & & & & & & \\
\hline
\end{tabular}


Table C-4. Revised baseline mass balance, Tank WM-180 waste (Continued).

\begin{tabular}{|c|c|c|c|c|c|c|c|}
\hline Stream \# & 109 & 110 & 201 & 202 & 203 & 204 & 205 \\
\hline Stream Name & $\begin{array}{l}\text { LET\&D } \\
\text { Offgas } \\
\text { Vapor }\end{array}$ & $\begin{array}{l}\text { Acid } \\
\text { Grout } \\
\text { Solid }\end{array}$ & $\begin{array}{c}\text { OPC } \\
\text { Grout } \\
\text { Additive }\end{array}$ & $\begin{array}{c}\text { BFS } \\
\text { Grout } \\
\text { Additive }\end{array}$ & $\begin{array}{c}\mathrm{Ca}(\mathrm{OH}) 2 \\
\text { Total } \\
\text { Additive }\end{array}$ & $\begin{array}{c}\mathrm{Ca}(\mathrm{OH}) 2 \\
\text { Grout } \\
\text { Additive }\end{array}$ & $\begin{array}{c}\mathrm{Ca}(\mathrm{OH}) 2 \\
\text { Neutraliz. } \\
\text { Additive }\end{array}$ \\
\hline Radiological Composition & $\mathrm{Ci} / \mathrm{wscm}$ & $\mathrm{Ci} / \mathrm{kg}$ & $\mathrm{Ci} / \mathrm{kg}$ & $\mathrm{Ci} / \mathrm{kg}$ & $\mathrm{Ci} / \mathrm{kg}$ & $\mathrm{Ci} / \mathrm{kg}$ & $\mathrm{Ci} / \mathrm{kg}$ \\
\hline Ru-106 & 1.05E-24 & $1.58 \mathrm{E}-12$ & & & & & \\
\hline Rh-102 & $9.23 \mathrm{E}-28$ & 1.39E-15 & & & & & \\
\hline Rh-106 & $1.05 \mathrm{E}-24$ & $1.58 \mathrm{E}-12$ & & & & & \\
\hline $\mathrm{Pd}-107$ & $1.77 \mathrm{E}-26$ & $2.67 E-14$ & & & & & \\
\hline Cd-113m & $3.56 \mathrm{E}-24$ & $5.37 \mathrm{E}-12$ & & & & & \\
\hline $\ln -115$ & & 1.63E-22 & & & & & \\
\hline Sn-121m & $7.16 \mathrm{E}-26$ & $1.08 \mathrm{E}-13$ & & & & & \\
\hline Sn-126 & $4.61 \mathrm{E}-25$ & $6.96 \mathrm{E}-13$ & & & & & \\
\hline Sb-125 & $1.72 \mathrm{E}-23$ & $2.59 \mathrm{E}-11$ & & & & & \\
\hline Sb-126 & $6.16 \mathrm{E}-26$ & $9.29 \mathrm{E}-14$ & & & & & \\
\hline Te-123 & & $6.19 \mathrm{E}-25$ & & & & & \\
\hline Te-125m & $3.37 E-24$ & $5.09 \mathrm{E}-12$ & & & & & \\
\hline I-129 & 5.59E-09 & $2.96 \mathrm{E}-16$ & & & & & \\
\hline Cs-134 & $1.12 \mathrm{E}-23$ & $1.68 \mathrm{E}-11$ & & & & & \\
\hline Cs-135 & $9.61 \mathrm{E}-25$ & $1.45 \mathrm{E}-12$ & & & & & \\
\hline Cs-137 & $5.63 \mathrm{E}-20$ & $8.49 \mathrm{E}-08$ & & & & & \\
\hline Ba-137m & $5.33 \mathrm{E}-20$ & 8.03E-08 & & & & & \\
\hline La-138 & & $3.08 \mathrm{E}-22$ & & & & & \\
\hline Ce-142 & $3.20 \mathrm{E}-29$ & $4.83 \mathrm{E}-17$ & & & & & \\
\hline Ce-144 & $7.05 E-25$ & $1.06 \mathrm{E}-12$ & & & & & \\
\hline Pr-144 & $7.05 E-25$ & $1.06 \mathrm{E}-12$ & & & & & \\
\hline Nd-144 & & $2.60 \mathrm{E}-21$ & & & & & \\
\hline $\mathrm{Pm}-146$ & $5.45 \mathrm{E}-26$ & $8.22 \mathrm{E}-14$ & & & & & \\
\hline $\mathrm{Pm}-147$ & 1.91E-22 & $2.88 \mathrm{E}-10$ & & & & & \\
\hline Sm-146 & & $4.46 \mathrm{E}-19$ & & & & & \\
\hline Sm-147 & $7.89 E-30$ & $1.19 \mathrm{E}-17$ & & & & & \\
\hline Sm-148 & & $6.12 \mathrm{E}-23$ & & & & & \\
\hline Sm-149 & & $5.43 E-24$ & & & & & \\
\hline Sm-151 & $3.77 \mathrm{E}-22$ & $5.69 \mathrm{E}-10$ & & & & & \\
\hline Eu-152 & $2.73 \mathrm{E}-24$ & $4.12 \mathrm{E}-12$ & & & & & \\
\hline Eu-154 & $9.79 \mathrm{E}-23$ & $1.48 \mathrm{E}-10$ & & & & & \\
\hline Eu-155 & $1.78 \mathrm{E}-22$ & $2.69 \mathrm{E}-10$ & & & & & \\
\hline Gd-152 & & $2.30 \mathrm{E}-24$ & & & & & \\
\hline Ho-166m & 4.93E-29 & $7.44 \mathrm{E}-17$ & & & & & \\
\hline \multicolumn{8}{|l|}{ Other Stream Data: } \\
\hline TRU, nCi/gm & & 3.89E-03 & & & & & \\
\hline Heat Generation, W/m3 & & $1.82 \mathrm{E}-06$ & & & & & \\
\hline Mass Flow, kg/hr & $2.25 \mathrm{E}+02$ & $9.91 \mathrm{E}+01$ & $3.90 \mathrm{E}+01$ & $1.30 \mathrm{E}+01$ & $1.24 \mathrm{E}+01$ & 3.93E-01 & $1.20 \mathrm{E}+01$ \\
\hline
\end{tabular}

* Standard conditions $=68^{\circ} \mathrm{F}$ and 1 atmosphere 
Table C-5. Revised baseline mass balance, Tank WM-187 waste.

\begin{tabular}{|c|c|c|c|c|c|c|c|c|}
\hline PFD Sheet \# & PFD-1 & PFD-2 & PFD-2 & PFD-2 & PFD-2 & PFD-2 & PFD-2 & PFD-3 \\
\hline Stream \# & 101 & 102 & 103 & 104 & 105 & 106 & 107 & 108 \\
\hline Stream Name & $\begin{array}{l}\text { SBW } \\
\text { Feed }\end{array}$ & $\begin{array}{c}\text { Evaporator } \\
\text { Bottoms }\end{array}$ & $\begin{array}{c}\text { Evaporator } \\
\text { Overheads } \\
\text { Vapor }\end{array}$ & $\begin{array}{c}\text { Mist } \\
\text { Eliminator } \\
\text { Vapor }\end{array}$ & $\begin{array}{l}\text { VOG } \\
\text { Offgas } \\
\text { Vapor }\end{array}$ & $\begin{array}{c}\text { POG + VOG } \\
\text { Offgas } \\
\text { Vapor }\end{array}$ & $\begin{array}{c}\text { LET\&D } \\
\text { Feed } \\
\text { Liquid }\end{array}$ & $\begin{array}{l}\text { LET\&D } \\
\text { Acid } \\
\text { Liquid }\end{array}$ \\
\hline Rate or Volume Flow (actual) & 85.8 & 21.5 & $2.68 \mathrm{E}+04$ & $2.75 \mathrm{E}+04$ & $1.17 \mathrm{E}+04$ & $1.37 \mathrm{E}+04$ & 66.7 & 10.9 \\
\hline Volume Flow (standard, wet) ${ }^{*}$ & & & $1.16 \mathrm{E}+04$ & $1.16 \mathrm{E}+04$ & $7.34 \mathrm{E}+03$ & $8.57 \mathrm{E}+03$ & & \\
\hline Volume Flow (standard, dry) ${ }^{*}$ & & & $3.80 \mathrm{E}+02$ & $3.80 \mathrm{E}+02$ & $7.13 E+03$ & $8.35 E+03$ & & \\
\hline Rate Units & gal/hr & $\mathrm{gal} / \mathrm{hr}$ & $\mathrm{ft} 3 / \mathrm{hr}$ & $\mathrm{ft} 3 / \mathrm{hr}$ & $\mathrm{ft} 3 / \mathrm{hr}$ & $\mathrm{ft} 3 / \mathrm{hr}$ & $\mathrm{gal} / \mathrm{hr}$ & $\mathrm{gal} / \mathrm{hr}$ \\
\hline Temperature, ${ }^{\circ} \mathrm{C}$ & 25.0 & 120.2 & 120.2 & 120.0 & 107.5 & 109.4 & 35.0 & 35.0 \\
\hline Temperature, ${ }^{\circ} \mathrm{F}$ & 77.0 & 248.4 & 248.4 & 248.0 & 225.6 & 228.8 & 95.0 & 95.0 \\
\hline Pressure, psia & 12.3 & 8.6 & 8.6 & 8.4 & 12.0 & 12.0 & 12.0 & 12.2 \\
\hline Specific Gravity & 1.27 & 1.75 & 0.00035 & 0.00034 & 0.00075 & 0.00074 & 1.06 & 1.17 \\
\hline Chemical Composition & Mol/Liter & Mol/Liter & $\mathrm{lb} / \mathrm{wscf}$ & Ib/wscf & Ib/wscf & Ib/wscf & Mol/Liter & Mol/Liter \\
\hline $\mathrm{H}+$ & $1.47 \mathrm{E}+00$ & 3.66E-01 & $8.55 \mathrm{E}-05$ & $8.55 \mathrm{E}-05$ & $3.93 \mathrm{E}-12$ & 1.16E-08 & $1.77 \mathrm{E}+00$ & $1.09 \mathrm{E}+01$ \\
\hline $\mathrm{OH}-$ & & 7.36E-09 & & & & & & \\
\hline $\mathrm{Al}+3$ & $3.61 \mathrm{E}-01$ & $1.44 \mathrm{E}+00$ & $5.99 \mathrm{E}-08$ & $5.99 \mathrm{E}-10$ & & & $4.64 \mathrm{E}-07$ & $2.85 \mathrm{E}-06$ \\
\hline $\mathrm{Sb}+5$ & $2.78 \mathrm{E}-05$ & $1.11 \mathrm{E}-04$ & $2.08 \mathrm{E}-11$ & $2.08 \mathrm{E}-13$ & & & $3.58 \mathrm{E}-11$ & $2.20 \mathrm{E}-10$ \\
\hline As+5 & 1.77E-04 & $7.06 \mathrm{E}-04$ & $8.14 \mathrm{E}-11$ & $8.14 \mathrm{E}-13$ & & & $2.27 \mathrm{E}-10$ & 1.40E-09 \\
\hline $\mathrm{Ba}+2$ & $7.12 \mathrm{E}-05$ & $2.84 \mathrm{E}-04$ & $6.01 \mathrm{E}-11$ & $6.01 \mathrm{E}-13$ & & & $9.15 \mathrm{E}-11$ & $5.62 \mathrm{E}-10$ \\
\hline $\mathrm{Be}+2$ & 1.67E-05 & $6.66 \mathrm{E}-05$ & $9.24 \mathrm{E}-13$ & $9.24 \mathrm{E}-15$ & & & $2.14 \mathrm{E}-11$ & $1.32 \mathrm{E}-10$ \\
\hline $\mathrm{B}+3$ & $1.10 \mathrm{E}-02$ & $4.40 \mathrm{E}-02$ & $7.33 \mathrm{E}-10$ & $7.33 \mathrm{E}-12$ & & & $1.42 \mathrm{E}-08$ & $8.71 \mathrm{E}-08$ \\
\hline $\mathrm{Cd}+2$ & 1.87E-03 & 7.47E-03 & 1.29E-09 & $1.29 E-11$ & & & $2.41 \mathrm{E}-09$ & $1.48 \mathrm{E}-08$ \\
\hline $\mathrm{Ca}+2$ & $3.47 E-02$ & $1.39 \mathrm{E}-01$ & $8.56 \mathrm{E}-09$ & $8.56 \mathrm{E}-11$ & & & $4.47 \mathrm{E}-08$ & $2.74 \mathrm{E}-07$ \\
\hline $\mathrm{Cr}+3$ & $4.71 \mathrm{E}-03$ & $1.88 \mathrm{E}-02$ & 1.51E-09 & $1.51 \mathrm{E}-11$ & & & 6.06E-09 & 3.72E-08 \\
\hline $\mathrm{Co}+2$ & 1.94E-03 & $7.74 \mathrm{E}-03$ & $7.03 \mathrm{E}-10$ & $7.03 \mathrm{E}-12$ & & & 2.49E-09 & $1.53 \mathrm{E}-08$ \\
\hline Cs+ & 8.42E-05 & 3.36E-04 & $6.88 \mathrm{E}-11$ & $6.88 \mathrm{E}-13$ & & & $1.08 \mathrm{E}-10$ & $6.65 \mathrm{E}-10$ \\
\hline $\mathrm{Cu}+2$ & $5.12 \mathrm{E}-04$ & $2.04 \mathrm{E}-03$ & $2.00 \mathrm{E}-10$ & $2.00 \mathrm{E}-12$ & & & $6.59 \mathrm{E}-10$ & 4.05E-09 \\
\hline $\mathrm{Fe}+3$ & $2.53 E-02$ & $1.01 \mathrm{E}-01$ & 8.69E-09 & $8.69 \mathrm{E}-11$ & & & $3.25 \mathrm{E}-08$ & $2.00 \mathrm{E}-07$ \\
\hline $\mathrm{Pb}+2$ & $6.73 \mathrm{E}-04$ & $2.69 \mathrm{E}-03$ & $8.58 \mathrm{E}-10$ & $8.58 \mathrm{E}-12$ & & & $8.66 \mathrm{E}-10$ & $5.32 \mathrm{E}-09$ \\
\hline $\mathrm{Hg}+2$ & $3.40 \mathrm{E}-03$ & $1.26 \mathrm{E}-02$ & 3.03E-06 & 3.03E-06 & $1.14 \mathrm{E}-11$ & $4.21 \mathrm{E}-10$ & $3.16 \mathrm{E}-04$ & 1.93E-03 \\
\hline $\mathrm{Mn}+4$ & 1.87E-02 & 7.45E-02 & 6.30E-09 & $6.30 \mathrm{E}-11$ & & & $2.40 \mathrm{E}-08$ & 1.47E-07 \\
\hline $\mathrm{Ni}+2$ & $1.41 \mathrm{E}-03$ & 5.63E-03 & $5.09 \mathrm{E}-10$ & 5.09E-12 & & & 1.81E-09 & $1.11 \mathrm{E}-08$ \\
\hline $\mathrm{K}+$ & $3.13 E-01$ & $1.25 \mathrm{E}+00$ & $7.53 \mathrm{E}-08$ & $7.53 \mathrm{E}-10$ & & & 4.03E-07 & $2.47 \mathrm{E}-06$ \\
\hline $\mathrm{Se}+4$ & $4.61 \mathrm{E}-04$ & $1.84 \mathrm{E}-03$ & $2.24 \mathrm{E}-10$ & $2.24 \mathrm{E}-12$ & & & $5.93 \mathrm{E}-10$ & 3.64E-09 \\
\hline $\mathrm{Ag}+$ & $7.08 \mathrm{E}-04$ & $2.82 \mathrm{E}-03$ & $4.69 \mathrm{E}-10$ & $4.69 \mathrm{E}-12$ & & & $9.10 \mathrm{E}-10$ & 5.59E-09 \\
\hline $\mathrm{Na}+$ & $1.76 \mathrm{E}+00$ & $7.04 \mathrm{E}+00$ & $2.49 \mathrm{E}-07$ & 2.49E-09 & & & $2.27 \mathrm{E}-06$ & 1.39E-05 \\
\hline $\mathrm{TI}+3$ & 6.31E-06 & $2.52 \mathrm{E}-05$ & $7.93 \mathrm{E}-12$ & $7.93 \mathrm{E}-14$ & & & $8.12 \mathrm{E}-12$ & $4.99 \mathrm{E}-11$ \\
\hline $\mathrm{U}+4$ & $2.90 \mathrm{E}-04$ & 1.16E-03 & $4.25 \mathrm{E}-10$ & $4.25 \mathrm{E}-12$ & & & $3.73 \mathrm{E}-10$ & 2.29E-09 \\
\hline $\mathrm{V}+3$ & $6.14 \mathrm{E}-05$ & $2.45 \mathrm{E}-04$ & 1.92E-11 & $1.92 \mathrm{E}-13$ & & & $7.90 \mathrm{E}-11$ & $4.85 \mathrm{E}-10$ \\
\hline$Z n+2$ & $7.21 \mathrm{E}-04$ & $2.88 \mathrm{E}-03$ & $2.90 \mathrm{E}-10$ & $2.90 \mathrm{E}-12$ & & & $9.27 \mathrm{E}-10$ & 5.70E-09 \\
\hline $\mathrm{Zr}+4$ & 6.66E-02 & $2.66 \mathrm{E}-01$ & $3.73 \mathrm{E}-08$ & $3.73 \mathrm{E}-10$ & & & $8.56 \mathrm{E}-08$ & 5.26E-07 \\
\hline $\mathrm{Cl}-$ & 1.80E-02 & $6.02 \mathrm{E}-02$ & $6.39 \mathrm{E}-06$ & $6.38 \mathrm{E}-06$ & $4.04 \mathrm{E}-12$ & $8.71 \mathrm{E}-10$ & 3.77E-03 & $3.85 \mathrm{E}-03$ \\
\hline F- & 5.61E-02 & $2.24 \mathrm{E}-01$ & $4.71 \mathrm{E}-08$ & $4.06 \mathrm{E}-08$ & & 5.52E-12 & 4.47E-05 & $2.74 \mathrm{E}-04$ \\
\hline SO4-2 & 4.99E-02 & $1.99 \mathrm{E}-01$ & $2.95 \mathrm{E}-08$ & $2.95 \mathrm{E}-10$ & & & 6.42E-08 & 3.94E-07 \\
\hline \begin{tabular}{|l|} 
NO3- \\
\end{tabular} & $4.62 \mathrm{E}+00$ & $1.29 \mathrm{E}+01$ & $5.25 \mathrm{E}-03$ & $5.25 \mathrm{E}-03$ & $2.42 \mathrm{E}-10$ & $7.14 \mathrm{E}-07$ & $1.77 \mathrm{E}+00$ & $1.09 \mathrm{E}+01$ \\
\hline PO4-3 & $1.75 \mathrm{E}-01$ & 6.96E-01 & 1.02E-07 & 1.02E-09 & & & $2.24 \mathrm{E}-07$ & $1.38 \mathrm{E}-06$ \\
\hline$A m+4$ & 6.56E-08 & $2.62 \mathrm{E}-07$ & $9.80 \mathrm{E}-14$ & $9.80 \mathrm{E}-16$ & & & $8.43 \mathrm{E}-14$ & $5.18 \mathrm{E}-13$ \\
\hline $\mathrm{Br}-$ & $2.21 \mathrm{E}-07$ & 7.01E-07 & $2.23 E-10$ & $2.23 \mathrm{E}-10$ & $1.41 \mathrm{E}-16$ & $3.04 \mathrm{E}-14$ & 5.84E-08 & 5.97E-08 \\
\hline $\mathrm{Ce}+4$ & 4.07E-05 & 1.62E-04 & $3.50 \mathrm{E}-11$ & $3.50 \mathrm{E}-13$ & & & $5.23 \mathrm{E}-11$ & $3.21 \mathrm{E}-10$ \\
\hline $\mathrm{Eu}+3$ & 3.68E-07 & 1.47E-06 & $3.44 \mathrm{E}-13$ & $3.44 \mathrm{E}-15$ & & & $4.73 \mathrm{E}-13$ & $2.90 \mathrm{E}-12$ \\
\hline$G d+3$ & 1.37E-04 & $5.46 \mathrm{E}-04$ & $1.32 \mathrm{E}-10$ & $1.32 \mathrm{E}-12$ & & & $1.76 \mathrm{E}-10$ & 1.08E-09 \\
\hline $\mathrm{Ge}+4$ & 6.37E-09 & $2.54 \mathrm{E}-08$ & $2.85 \mathrm{E}-15$ & $2.85 \mathrm{E}-17$ & & & $8.19 \mathrm{E}-15$ & $5.03 \mathrm{E}-14$ \\
\hline $\ln +3$ & 1.07E-06 & $4.26 \mathrm{E}-06$ & $7.54 \mathrm{E}-13$ & $7.54 \mathrm{E}-15$ & & & $1.37 \mathrm{E}-12$ & $8.43 \mathrm{E}-12$ \\
\hline I- & $3.12 \mathrm{E}-06$ & 1.09E-05 & 2.94E-09 & 2.94E-09 & $1.86 \mathrm{E}-15$ & $4.01 \mathrm{E}-13$ & 4.84E-07 & 4.96E-07 \\
\hline $\mathrm{La}+3$ & 6.66E-06 & 2.66E-05 & $5.69 \mathrm{E}-12$ & 5.69E-14 & & & $8.56 \mathrm{E}-12$ & $5.26 \mathrm{E}-11$ \\
\hline Li+ & $6.26 \mathrm{E}-04$ & $2.50 \mathrm{E}-03$ & $2.67 \mathrm{E}-11$ & $2.67 \mathrm{E}-13$ & & & $8.05 \mathrm{E}-10$ & 4.94E-09 \\
\hline $\mathrm{Mg}+2$ & 1.54E-02 & $6.14 \mathrm{E}-02$ & $2.30 \mathrm{E}-09$ & $2.30 \mathrm{E}-11$ & & & $1.98 \mathrm{E}-08$ & $1.22 \mathrm{E}-07$ \\
\hline $\mathrm{Mo}+6$ & $1.23 \mathrm{E}-03$ & $4.91 \mathrm{E}-03$ & $7.26 \mathrm{E}-10$ & $7.26 \mathrm{E}-12$ & & & 1.58E-09 & $9.73 \mathrm{E}-09$ \\
\hline
\end{tabular}


Table C-5. Revised baseline mass balance, Tank WM-187 waste (Continued).

\begin{tabular}{|c|c|c|c|c|c|c|c|c|}
\hline Stream \# & 101 & 102 & 103 & 104 & 105 & 106 & 107 & 108 \\
\hline Stream Name & $\begin{array}{l}\text { SBW } \\
\text { Feed }\end{array}$ & $\begin{array}{l}\text { Evaporator } \\
\text { Bottoms }\end{array}$ & $\begin{array}{c}\text { Evaporator } \\
\text { Overheads } \\
\text { Vapor } \\
\end{array}$ & $\begin{array}{c}\text { Mist } \\
\text { Eliminator } \\
\text { Vapor }\end{array}$ & $\begin{array}{l}\text { VOG } \\
\text { Offgas } \\
\text { Vapor }\end{array}$ & $\begin{array}{c}\text { POG + VOG } \\
\text { Offgas } \\
\text { Vapor } \\
\end{array}$ & $\begin{array}{l}\text { LET\&D } \\
\text { Feed } \\
\text { Liquid }\end{array}$ & $\begin{array}{l}\text { LET\&D } \\
\text { Acid } \\
\text { Liquid } \\
\end{array}$ \\
\hline Chemical Composition & Mol/Liter & Mol/Liter & $\mathrm{lb} / \mathrm{wscf}$ & $\mathrm{lb} / \mathrm{wscf}$ & $\mathrm{lb} / \mathrm{wscf}$ & $\mathrm{lb} / \mathrm{wscf}$ & Mol/Liter & Mol/Liter \\
\hline $\mathrm{Nd}+3$ & $2.15 \mathrm{E}-05$ & 8.57E-05 & $1.90 \mathrm{E}-11$ & $1.90 \mathrm{E}-13$ & & & $2.76 \mathrm{E}-11$ & $1.70 \mathrm{E}-10$ \\
\hline $\mathrm{Np}+4$ & $3.28 \mathrm{E}-06$ & 1.31E-05 & $4.78 \mathrm{E}-12$ & $4.78 \mathrm{E}-14$ & & & $4.22 \mathrm{E}-12$ & $2.59 \mathrm{E}-11$ \\
\hline $\mathrm{Nb}+5$ & 1.86E-03 & $7.41 \mathrm{E}-03$ & 1.06E-09 & $1.06 \mathrm{E}-11$ & & & $2.39 \mathrm{E}-09$ & 1.47E-08 \\
\hline $\mathrm{Pd}+4$ & $3.01 \mathrm{E}-03$ & $1.20 \mathrm{E}-02$ & 1.97E-09 & 1.97E-11 & & & 3.87E-09 & $2.38 \mathrm{E}-08$ \\
\hline $\mathrm{Pu}+4$ & $8.75 \mathrm{E}-06$ & $3.49 \mathrm{E}-05$ & $1.31 \mathrm{E}-11$ & $1.31 \mathrm{E}-13$ & & & $1.13 \mathrm{E}-11$ & $6.91 \mathrm{E}-11$ \\
\hline $\mathrm{Pr}+4$ & $6.05 \mathrm{E}-06$ & $2.42 \mathrm{E}-05$ & $5.25 \mathrm{E}-12$ & $5.24 \mathrm{E}-14$ & & & $7.78 \mathrm{E}-12$ & $4.78 \mathrm{E}-11$ \\
\hline $\mathrm{Rh}+4$ & $2.61 \mathrm{E}-06$ & 1.04E-05 & $1.65 \mathrm{E}-12$ & $1.65 \mathrm{E}-14$ & & & $3.36 \mathrm{E}-12$ & $2.06 \mathrm{E}-11$ \\
\hline $\mathrm{Rb}+$ & 4.02E-06 & 1.60E-05 & $2.11 \mathrm{E}-12$ & $2.11 \mathrm{E}-14$ & & & $5.17 \mathrm{E}-12$ & $3.18 \mathrm{E}-11$ \\
\hline $\mathrm{Ru}+3$ & 1.36E-03 & 5.43E-03 & $8.46 \mathrm{E}-10$ & $8.46 \mathrm{E}-12$ & & & 1.75E-09 & $1.08 \mathrm{E}-08$ \\
\hline $\mathrm{Sm}+3$ & 4.03E-06 & 1.61E-05 & $3.72 \mathrm{E}-12$ & $3.72 \mathrm{E}-14$ & & & $5.18 \mathrm{E}-12$ & $3.18 \mathrm{E}-11$ \\
\hline $\mathrm{Si+4}$ & 1.59E-01 & $6.33 \mathrm{E}-01$ & $2.74 \mathrm{E}-08$ & $2.74 \mathrm{E}-10$ & & & $2.04 \mathrm{E}-07$ & 1.25E-06 \\
\hline $\mathrm{Sr}+2$ & $8.80 \mathrm{E}-05$ & 3.51E-04 & $4.74 \mathrm{E}-11$ & $4.74 \mathrm{E}-13$ & & & $1.13 \mathrm{E}-10$ & $6.95 \mathrm{E}-10$ \\
\hline $\mathrm{Tc}+7$ & $6.42 \mathrm{E}-06$ & $2.56 \mathrm{E}-05$ & $3.83 \mathrm{E}-12$ & $3.83 \mathrm{E}-14$ & & & $8.25 \mathrm{E}-12$ & $5.07 \mathrm{E}-11$ \\
\hline $\mathrm{Te}+4$ & $3.48 \mathrm{E}-06$ & $1.39 \mathrm{E}-05$ & $2.73 \mathrm{E}-12$ & $2.73 \mathrm{E}-14$ & & & $4.48 \mathrm{E}-12$ & $2.75 \mathrm{E}-11$ \\
\hline $\mathrm{Tb}+4$ & 1.53E-09 & $6.12 \mathrm{E}-09$ & $1.50 \mathrm{E}-15$ & $1.50 \mathrm{E}-17$ & & & 1.97E-15 & $1.21 \mathrm{E}-14$ \\
\hline $\mathrm{Th}+4$ & $1.85 \mathrm{E}-05$ & $7.37 \mathrm{E}-05$ & $2.63 \mathrm{E}-11$ & $2.63 \mathrm{E}-13$ & & & $2.37 \mathrm{E}-11$ & $1.46 \mathrm{E}-10$ \\
\hline $\mathrm{Sn+4}$ & 3.23E-03 & 1.29E-02 & 2.36E-09 & $2.36 \mathrm{E}-11$ & & & 4.15E-09 & $2.55 \mathrm{E}-08$ \\
\hline$\overline{T i+4}$ & $1.62 \mathrm{E}-03$ & $6.46 \mathrm{E}-03$ & $4.77 \mathrm{E}-10$ & $4.77 \mathrm{E}-12$ & & & $2.08 \mathrm{E}-09$ & $1.28 \mathrm{E}-08$ \\
\hline $\mathrm{Y}+3$ & $4.96 \mathrm{E}-06$ & 1.98E-05 & $2.71 \mathrm{E}-12$ & $2.71 \mathrm{E}-14$ & & & $6.38 \mathrm{E}-12$ & $3.92 \mathrm{E}-11$ \\
\hline O-2 (oxides) & $2.70 \mathrm{E}-01$ & $1.08 \mathrm{E}+00$ & $2.66 \mathrm{E}-08$ & $2.66 \mathrm{E}-10$ & & & $3.47 \mathrm{E}-07$ & $2.13 \mathrm{E}-06$ \\
\hline $\mathrm{H} 2 \mathrm{O}$ & $4.71 \mathrm{E}+01$ & $2.68 \mathrm{E}+01$ & 4.54E-02 & 4.54E-02 & $1.36 \mathrm{E}-03$ & $1.17 \mathrm{E}-03$ & $5.27 \mathrm{E}+01$ & $2.66 \mathrm{E}+01$ \\
\hline $\mathrm{O} 2$ & & $1.78 \mathrm{E}-10$ & 1.69E-07 & 1.69E-07 & 1.69E-02 & 1.70E-02 & 7.84E-05 & \\
\hline N2 & & $2.16 \mathrm{E}-10$ & $2.81 \mathrm{E}-07$ & $2.81 \mathrm{E}-07$ & $5.58 \mathrm{E}-02$ & $5.60 \mathrm{E}-02$ & $1.18 \mathrm{E}-04$ & \\
\hline C (organic) & $1.96 \mathrm{E}-01$ & 7.72E-01 & 1.77E-06 & $1.76 \mathrm{E}-06$ & $2.34 \mathrm{E}-12$ & $2.41 \mathrm{E}-10$ & $3.06 \mathrm{E}-03$ & $1.88 \mathrm{E}-02$ \\
\hline $\mathrm{H}$ (organic) & 1.96E-01 & 7.72E-01 & 1.49E-07 & $1.48 \mathrm{E}-07$ & $1.96 \mathrm{E}-13$ & $2.02 \mathrm{E}-11$ & 3.06E-03 & $1.88 \mathrm{E}-02$ \\
\hline \multicolumn{9}{|l|}{ SiO2, BFS, or OPC Additives } \\
\hline $\begin{array}{l}\text { Gas Stream Bulk Composition } \\
\text { (wet basis) }\end{array}$ & $\begin{array}{l}\text { mol. } \% \\
\text { or ppmv }\end{array}$ & $\begin{array}{l}\text { mol. } \% \\
\text { or ppmv }\end{array}$ & $\begin{array}{l}\text { mol. } \% \\
\text { or ppmv }\end{array}$ & $\begin{array}{l}\text { mol. } \% \\
\text { or ppmv }\end{array}$ & $\begin{array}{l}\text { mol. } \% \\
\text { or ppmv }\end{array}$ & $\begin{array}{l}\text { mol. } \% \\
\text { or ppmv }\end{array}$ & $\begin{array}{l}\text { mol. } \% \\
\text { or ppmv }\end{array}$ & $\begin{array}{l}\text { mol. } \% \\
\text { or ppmv }\end{array}$ \\
\hline $\mathrm{H} 2 \mathrm{O}, \mathrm{mol} . \%$ & & & 96.74 & 96.74 & 2.91 & 2.50 & & \\
\hline $\mathrm{O} 2, \mathrm{~mol} . \%$ & & & 0.0002 & 0.0002 & 20.39 & 20.47 & & \\
\hline $\mathrm{N} 2, \mathrm{~mol} . \%$ & & & 0.0004 & 0.0004 & 76.71 & 77.02 & & \\
\hline \multicolumn{9}{|l|}{$\mathrm{CO} 2, \mathrm{~mol} . \%$} \\
\hline HNO3, mol. \% & & & 3.26 & 3.26 & $1.5 \mathrm{E}-07$ & 4.4E-04 & & \\
\hline \multicolumn{9}{|l|}{$\mathrm{CO}, \mathrm{ppmv}$} \\
\hline \multicolumn{9}{|l|}{$\mathrm{NO}, \mathrm{ppmv}$} \\
\hline \multicolumn{9}{|l|}{ NO2, ppmv } \\
\hline C (organic), ppmv & & & 57 & 56 & $7.5 \mathrm{E}-05$ & $7.7 \mathrm{E}-03$ & & \\
\hline $\mathrm{H}$ (organic), ppmv & & & 57 & 56 & $7.5 \mathrm{E}-05$ & $7.7 \mathrm{E}-03$ & & \\
\hline $\mathrm{PM}$ (mg/wscm) & & & 7 & 0.07 & & & & \\
\hline $\begin{array}{l}\text { Gas Stream Bulk Composition } \\
\text { (dry basis) }\end{array}$ & $\begin{array}{c}\text { mol. } \% \\
\text { or ppmv }\end{array}$ & $\begin{array}{l}\text { mol.\% } \\
\text { or ppmv }\end{array}$ & $\begin{array}{c}\text { mol. } \% \\
\text { or ppmv }\end{array}$ & $\begin{array}{l}\text { mol. } \% \\
\text { or ppmv }\end{array}$ & $\begin{array}{l}\text { mol.\% } \\
\text { or ppmv }\end{array}$ & $\begin{array}{l}\text { mol. } \% \\
\text { or ppmv }\end{array}$ & $\begin{array}{l}\text { mol. } \% \\
\text { or ppmv }\end{array}$ & $\begin{array}{l}\text { mol. } \% \\
\text { or ppmv }\end{array}$ \\
\hline $\mathrm{O} 2, \mathrm{~mol} . \%$ & & & 0.01 & 0.01 & 21.00 & 21.00 & & \\
\hline $\mathrm{N} 2, \mathrm{~mol} . \%$ & & & 0.01 & 0.01 & 79.00 & 79.00 & & \\
\hline \multicolumn{9}{|l|}{$\mathrm{CO} 2, \mathrm{~mol} . \%$} \\
\hline $\mathrm{HNO} 3, \mathrm{~mol} . \%$ & & & 99.84 & 99.88 & $1.5 \mathrm{E}-07$ & 4.6E-04 & & \\
\hline \multicolumn{9}{|l|}{$\mathrm{CO}, \mathrm{ppmv}$} \\
\hline \multicolumn{9}{|l|}{ NO, ppmv } \\
\hline \multicolumn{9}{|l|}{ NO2, ppmv } \\
\hline C (organic), ppmv & & & 1,737 & 1,724 & 7.7E-05 & 7.9E-03 & & \\
\hline $\mathrm{H}$ (organic), ppmv & & & 1,737 & 1,724 & $7.7 \mathrm{E}-05$ & $7.9 \mathrm{E}-03$ & & \\
\hline $\mathrm{PM}(\mathrm{mg} / \mathrm{dscm})$ & & & 212 & 2 & & & & \\
\hline & & & & & & & & \\
\hline
\end{tabular}


Table C-5. Revised baseline mass balance, Tank WM-187 waste (Continued).

\begin{tabular}{|c|c|c|c|c|c|c|c|c|}
\hline Stream \# & 101 & 102 & 103 & 104 & 105 & 106 & 107 & 108 \\
\hline Stream Name & $\begin{array}{l}\text { SBW } \\
\text { Feed }\end{array}$ & $\begin{array}{c}\text { Evaporator } \\
\text { Bottoms }\end{array}$ & $\begin{array}{c}\text { Evaporator } \\
\text { Overheads } \\
\text { Vapor }\end{array}$ & $\begin{array}{c}\text { Mist } \\
\text { Eliminator } \\
\text { Vapor }\end{array}$ & $\begin{array}{l}\text { VOG } \\
\text { Offgas } \\
\text { Vapor }\end{array}$ & $\begin{array}{c}\text { POG + VOG } \\
\text { Offgas } \\
\text { Vapor }\end{array}$ & $\begin{array}{l}\text { LET\&D } \\
\text { Feed } \\
\text { Liquid }\end{array}$ & $\begin{array}{l}\text { LET\&D } \\
\text { Acid } \\
\text { Liquid }\end{array}$ \\
\hline Canister Rate, canisters/day & & 2.44 & & & & & & \\
\hline Total Canisters Generated & & 182 & & & & & & \\
\hline \multicolumn{9}{|l|}{ Drum Rate, drums/day } \\
\hline \multicolumn{9}{|l|}{ Total Drums Generated } \\
\hline Radiological Composition & $\mathrm{Ci} / \mathrm{L}$ & $\mathrm{Ci} / \mathrm{L}$ & $\mathrm{Ci} / \mathrm{wscm}$ & $\mathrm{Ci} / \mathrm{wscm}$ & $\mathrm{Ci} / \mathrm{wscm}$ & $\mathrm{Ci} / \mathrm{wscm}$ & $\mathrm{Ci} / \mathrm{L}$ & $\mathrm{Ci} / \mathrm{L}$ \\
\hline Ra-226 & $6.30 \mathrm{E}-12$ & $2.52 \mathrm{E}-11$ & $6.21 \mathrm{E}-16$ & $6.21 \mathrm{E}-18$ & 1.97E-25 & $1.70 \mathrm{E}-25$ & $8.10 \mathrm{E}-18$ & $4.98 \mathrm{E}-17$ \\
\hline Ac-227 & $2.97 \mathrm{E}-11$ & $1.18 \mathrm{E}-10$ & $2.92 \mathrm{E}-15$ & $2.92 \mathrm{E}-17$ & $9.28 \mathrm{E}-25$ & $8.03 \mathrm{E}-25$ & $3.82 \mathrm{E}-17$ & $2.34 \mathrm{E}-16$ \\
\hline Th-228 & $2.46 \mathrm{E}-09$ & 9.83E-09 & $2.43 \mathrm{E}-13$ & $2.43 \mathrm{E}-15$ & $7.70 \mathrm{E}-23$ & $6.66 \mathrm{E}-23$ & $3.17 \mathrm{E}-15$ & $1.95 \mathrm{E}-14$ \\
\hline Th-230 & 1.03E-09 & 4.10E-09 & $1.01 \mathrm{E}-13$ & $1.01 \mathrm{E}-15$ & $3.21 \mathrm{E}-23$ & $2.78 \mathrm{E}-23$ & $1.32 \mathrm{E}-15$ & $8.11 \mathrm{E}-15$ \\
\hline Th-232 & $5.45 \mathrm{E}-16$ & $2.18 \mathrm{E}-15$ & 5.37E-20 & $5.37 \mathrm{E}-22$ & $1.70 \mathrm{E}-29$ & 1.47E-29 & $7.01 \mathrm{E}-22$ & $4.31 \mathrm{E}-21$ \\
\hline $\mathrm{Pa}-231$ & $6.88 \mathrm{E}-11$ & $2.74 \mathrm{E}-10$ & $6.77 \mathrm{E}-15$ & $6.77 \mathrm{E}-17$ & $2.15 \mathrm{E}-24$ & 1.86E-24 & $8.84 \mathrm{E}-17$ & $5.43 \mathrm{E}-16$ \\
\hline $\mathrm{Pa}-233$ & $2.26 \mathrm{E}-06$ & $9.00 \mathrm{E}-06$ & $2.22 \mathrm{E}-10$ & $2.22 \mathrm{E}-12$ & 7.05E-20 & $6.10 \mathrm{E}-20$ & $2.90 \mathrm{E}-12$ & $1.78 \mathrm{E}-11$ \\
\hline U-232 & $2.35 \mathrm{E}-09$ & $9.36 \mathrm{E}-09$ & $2.31 \mathrm{E}-13$ & $2.31 \mathrm{E}-15$ & $7.33 \mathrm{E}-23$ & $6.34 \mathrm{E}-23$ & $3.02 \mathrm{E}-15$ & $1.85 \mathrm{E}-14$ \\
\hline $\mathrm{U}-233$ & $7.56 \mathrm{E}-11$ & $3.02 \mathrm{E}-10$ & $7.45 \mathrm{E}-15$ & $7.45 \mathrm{E}-17$ & $2.36 \mathrm{E}-24$ & $2.04 \mathrm{E}-24$ & $9.72 \mathrm{E}-17$ & $5.97 \mathrm{E}-16$ \\
\hline U-234 & $1.24 \mathrm{E}-06$ & 4.93E-06 & $1.22 \mathrm{E}-10$ & $1.22 \mathrm{E}-12$ & $3.86 \mathrm{E}-20$ & $3.34 \mathrm{E}-20$ & $1.59 \mathrm{E}-12$ & $9.76 \mathrm{E}-12$ \\
\hline U-235 & $6.49 \mathrm{E}-08$ & $2.59 \mathrm{E}-07$ & $6.39 \mathrm{E}-12$ & $6.39 \mathrm{E}-14$ & $2.03 \mathrm{E}-21$ & 1.76E-21 & $8.35 \mathrm{E}-14$ & $5.13 \mathrm{E}-13$ \\
\hline U-236 & $6.04 \mathrm{E}-08$ & $2.41 \mathrm{E}-07$ & $5.95 \mathrm{E}-12$ & $5.95 \mathrm{E}-14$ & 1.89E-21 & 1.63E-21 & $7.77 \mathrm{E}-14$ & $4.77 \mathrm{E}-13$ \\
\hline U-237 & 4.95E-09 & $1.98 \mathrm{E}-08$ & $4.88 \mathrm{E}-13$ & $4.88 \mathrm{E}-15$ & $1.55 \mathrm{E}-22$ & $1.34 \mathrm{E}-22$ & $6.37 \mathrm{E}-15$ & $3.91 \mathrm{E}-14$ \\
\hline U-238 & $2.12 \mathrm{E}-08$ & $8.46 \mathrm{E}-08$ & $2.09 \mathrm{E}-12$ & $2.09 \mathrm{E}-14$ & $6.63 \mathrm{E}-22$ & $5.73 \mathrm{E}-22$ & $2.73 \mathrm{E}-14$ & $1.68 \mathrm{E}-13$ \\
\hline Np-236 & $2.43 \mathrm{E}-12$ & $9.70 \mathrm{E}-12$ & $2.39 \mathrm{E}-16$ & $2.39 \mathrm{E}-18$ & $7.60 \mathrm{E}-26$ & $6.57 \mathrm{E}-26$ & $3.12 \mathrm{E}-18$ & $1.92 \mathrm{E}-17$ \\
\hline Np-237 & $5.64 \mathrm{E}-07$ & $2.25 \mathrm{E}-06$ & $5.56 \mathrm{E}-11$ & $5.56 \mathrm{E}-13$ & 1.76E-20 & $1.53 \mathrm{E}-20$ & $7.25 \mathrm{E}-13$ & $4.46 \mathrm{E}-12$ \\
\hline Pu-236 & 3.70E-09 & $1.48 \mathrm{E}-08$ & $3.65 \mathrm{E}-13$ & $3.65 \mathrm{E}-15$ & $1.16 \mathrm{E}-22$ & $1.00 \mathrm{E}-22$ & $4.76 \mathrm{E}-15$ & $2.93 \mathrm{E}-14$ \\
\hline $\mathrm{Pu}-238$ & $1.13 \mathrm{E}-03$ & 4.53E-03 & 1.12E-07 & 1.12E-09 & $3.55 \mathrm{E}-17$ & $3.07 \mathrm{E}-17$ & 1.46E-09 & 8.96E-09 \\
\hline Pu-239 & 1.25E-04 & 4.98E-04 & 1.23E-08 & $1.23 \mathrm{E}-10$ & $3.90 \mathrm{E}-18$ & $3.37 \mathrm{E}-18$ & $1.60 \mathrm{E}-10$ & $9.85 \mathrm{E}-10$ \\
\hline $\mathrm{Pu}-240$ & $1.39 \mathrm{E}-05$ & 5.54E-05 & 1.37E-09 & $1.37 \mathrm{E}-11$ & 4.34E-19 & $3.75 \mathrm{E}-19$ & $1.78 \mathrm{E}-11$ & $1.10 \mathrm{E}-10$ \\
\hline Pu-241 & $8.71 \mathrm{E}-04$ & $3.47 \mathrm{E}-03$ & $8.57 \mathrm{E}-08$ & $8.57 \mathrm{E}-10$ & $2.72 \mathrm{E}-17$ & $2.35 \mathrm{E}-17$ & $1.12 \mathrm{E}-09$ & $6.88 \mathrm{E}-09$ \\
\hline Pu-242 & $1.09 \mathrm{E}-08$ & 4.35E-08 & $1.07 \mathrm{E}-12$ & $1.07 \mathrm{E}-14$ & $3.41 \mathrm{E}-22$ & $2.95 \mathrm{E}-22$ & $1.40 \mathrm{E}-14$ & $8.61 \mathrm{E}-14$ \\
\hline Pu-244 & $6.59 \mathrm{E}-16$ & $2.63 \mathrm{E}-15$ & $6.49 \mathrm{E}-20$ & $6.49 \mathrm{E}-22$ & $2.06 \mathrm{E}-29$ & $1.78 \mathrm{E}-29$ & $8.48 \mathrm{E}-22$ & $5.21 \mathrm{E}-21$ \\
\hline Am-241 & $6.74 \mathrm{E}-05$ & $2.69 \mathrm{E}-04$ & 6.64E-09 & $6.64 \mathrm{E}-11$ & $2.11 \mathrm{E}-18$ & $1.82 \mathrm{E}-18$ & $8.66 \mathrm{E}-11$ & $5.32 \mathrm{E}-10$ \\
\hline Am-242m & $1.19 \mathrm{E}-08$ & $4.74 \mathrm{E}-08$ & $1.17 \mathrm{E}-12$ & $1.17 \mathrm{E}-14$ & $3.71 \mathrm{E}-22$ & $3.21 \mathrm{E}-22$ & $1.53 \mathrm{E}-14$ & $9.38 \mathrm{E}-14$ \\
\hline Am-243 & $2.36 \mathrm{E}-08$ & 9.43E-08 & $2.33 \mathrm{E}-12$ & $2.33 \mathrm{E}-14$ & $7.39 \mathrm{E}-22$ & $6.39 E-22$ & $3.04 \mathrm{E}-14$ & $1.87 \mathrm{E}-13$ \\
\hline $\mathrm{Cm}-242$ & $2.36 \mathrm{E}-08$ & 9.41E-08 & $2.32 \mathrm{E}-12$ & $2.32 \mathrm{E}-14$ & $7.37 \mathrm{E}-22$ & $6.38 \mathrm{E}-22$ & $3.03 \mathrm{E}-14$ & $1.86 \mathrm{E}-13$ \\
\hline $\mathrm{Cm}-243$ & $3.60 \mathrm{E}-08$ & $1.44 \mathrm{E}-07$ & $3.54 \mathrm{E}-12$ & $3.54 \mathrm{E}-14$ & $1.12 \mathrm{E}-21$ & $9.73 \mathrm{E}-22$ & $4.63 \mathrm{E}-14$ & $2.84 \mathrm{E}-13$ \\
\hline Cm-244 & $1.63 \mathrm{E}-06$ & $6.51 \mathrm{E}-06$ & $1.61 \mathrm{E}-10$ & $1.61 \mathrm{E}-12$ & $5.10 \mathrm{E}-20$ & $4.41 \mathrm{E}-20$ & $2.10 \mathrm{E}-12$ & $1.29 \mathrm{E}-11$ \\
\hline $\mathrm{Cm}-245$ & $3.79 \mathrm{E}-10$ & $1.51 \mathrm{E}-09$ & $3.74 \mathrm{E}-14$ & $3.74 \mathrm{E}-16$ & 1.19E-23 & 1.03E-23 & $4.88 \mathrm{E}-16$ & $3.00 \mathrm{E}-15$ \\
\hline $\mathrm{Cm}-246$ & $2.48 \mathrm{E}-11$ & $9.91 \mathrm{E}-11$ & $2.45 \mathrm{E}-15$ & $2.45 \mathrm{E}-17$ & $7.76 \mathrm{E}-25$ & $6.72 \mathrm{E}-25$ & $3.19 \mathrm{E}-17$ & $1.96 \mathrm{E}-16$ \\
\hline $\mathrm{Cm}-247$ & $1.09 \mathrm{E}-17$ & $4.36 \mathrm{E}-17$ & $1.08 \mathrm{E}-21$ & $1.08 \mathrm{E}-23$ & & & 1.40E-23 & $8.63 \mathrm{E}-23$ \\
\hline $\mathrm{H}-3$ & $9.86 \mathrm{E}-06$ & $5.51 \mathrm{E}-06$ & $8.30 \mathrm{E}-06$ & $8.30 \mathrm{E}-06$ & $7.80 \mathrm{E}-08$ & $6.79 \mathrm{E}-08$ & $1.08 \mathrm{E}-05$ & $5.46 \mathrm{E}-06$ \\
\hline $\mathrm{Be}-10$ & $2.31 \mathrm{E}-12$ & $9.21 \mathrm{E}-12$ & $2.27 \mathrm{E}-16$ & $2.27 \mathrm{E}-18$ & $7.22 \mathrm{E}-26$ & $6.24 \mathrm{E}-26$ & $2.97 \mathrm{E}-18$ & $1.82 \mathrm{E}-17$ \\
\hline $\mathrm{C}-14$ & $1.58 \mathrm{E}-10$ & $6.29 \mathrm{E}-10$ & $1.55 \mathrm{E}-14$ & $1.55 \mathrm{E}-16$ & $4.92 \mathrm{E}-24$ & 4.26E-24 & $2.03 \mathrm{E}-16$ & $1.24 \mathrm{E}-15$ \\
\hline Co-60 & 1.65E-05 & $6.59 \mathrm{E}-05$ & 1.63E-09 & $1.63 \mathrm{E}-11$ & $5.16 \mathrm{E}-19$ & $4.46 \mathrm{E}-19$ & $2.12 \mathrm{E}-11$ & $1.30 \mathrm{E}-10$ \\
\hline \begin{tabular}{|l|}
$\mathrm{Ni}-59$ \\
\end{tabular} & $2.06 \mathrm{E}-06$ & $8.22 \mathrm{E}-06$ & $2.03 \mathrm{E}-10$ & $2.03 \mathrm{E}-12$ & $6.43 \mathrm{E}-20$ & 5.57E-20 & $2.65 \mathrm{E}-12$ & $1.63 \mathrm{E}-11$ \\
\hline $\mathrm{Ni}-63$ & $4.29 \mathrm{E}-05$ & $1.71 \mathrm{E}-04$ & $4.22 \mathrm{E}-09$ & $4.22 \mathrm{E}-11$ & $1.34 \mathrm{E}-18$ & $1.16 \mathrm{E}-18$ & $5.51 \mathrm{E}-11$ & $3.39 \mathrm{E}-10$ \\
\hline Se-79 & $5.71 \mathrm{E}-07$ & $2.28 \mathrm{E}-06$ & $5.62 \mathrm{E}-11$ & $5.62 \mathrm{E}-13$ & $1.78 \mathrm{E}-20$ & $1.54 \mathrm{E}-20$ & $7.34 \mathrm{E}-13$ & $4.51 \mathrm{E}-12$ \\
\hline $\mathrm{Rb}-87$ & $2.26 \mathrm{E}-11$ & $9.00 \mathrm{E}-11$ & $2.22 \mathrm{E}-15$ & $2.22 \mathrm{E}-17$ & $7.05 \mathrm{E}-25$ & $6.10 \mathrm{E}-25$ & $2.90 \mathrm{E}-17$ & $1.78 \mathrm{E}-16$ \\
\hline Sr-90 & 3.04E-02 & $1.21 \mathrm{E}-01$ & $2.99 \mathrm{E}-06$ & $2.99 \mathrm{E}-08$ & $9.49 \mathrm{E}-16$ & $8.21 \mathrm{E}-16$ & $3.90 \mathrm{E}-08$ & $2.40 \mathrm{E}-07$ \\
\hline Y-90 & 3.04E-02 & $1.21 \mathrm{E}-01$ & $2.99 \mathrm{E}-06$ & $2.99 \mathrm{E}-08$ & $9.49 \mathrm{E}-16$ & $8.21 \mathrm{E}-16$ & $3.90 \mathrm{E}-08$ & $2.40 \mathrm{E}-07$ \\
\hline Zr-93 & 1.70E-06 & $6.80 \mathrm{E}-06$ & $1.68 \mathrm{E}-10$ & $1.68 \mathrm{E}-12$ & $5.33 \mathrm{E}-20$ & $4.61 \mathrm{E}-20$ & $2.19 \mathrm{E}-12$ & $1.35 \mathrm{E}-11$ \\
\hline $\mathrm{Nb}-93 \mathrm{~m}$ & $1.31 \mathrm{E}-06$ & $5.24 \mathrm{E}-06$ & $1.29 \mathrm{E}-10$ & $1.29 \mathrm{E}-12$ & $4.10 \mathrm{E}-20$ & $3.55 \mathrm{E}-20$ & $1.69 \mathrm{E}-12$ & $1.04 \mathrm{E}-11$ \\
\hline $\mathrm{Nb}-94$ & $1.48 \mathrm{E}-06$ & 5.92E-06 & $1.46 \mathrm{E}-10$ & $1.46 \mathrm{E}-12$ & $4.63 \mathrm{E}-20$ & $4.01 \mathrm{E}-20$ & $1.91 \mathrm{E}-12$ & $1.17 \mathrm{E}-11$ \\
\hline Tc-98 & $1.99 \mathrm{E}-12$ & $7.92 \mathrm{E}-12$ & $1.95 \mathrm{E}-16$ & $1.95 \mathrm{E}-18$ & $6.20 \mathrm{E}-26$ & 5.37E-26 & $2.55 \mathrm{E}-18$ & $1.57 \mathrm{E}-17$ \\
\hline Tc-99 & 1.69E-05 & $6.75 \mathrm{E}-05$ & 1.67E-09 & 1.67E-11 & $5.28 \mathrm{E}-19$ & $4.57 \mathrm{E}-19$ & $2.17 \mathrm{E}-11$ & $1.34 \mathrm{E}-10$ \\
\hline
\end{tabular}


Table C-5. Revised baseline mass balance, Tank WM-187 waste (Continued).

\begin{tabular}{|c|c|c|c|c|c|c|c|c|}
\hline Stream \# & 101 & 102 & 103 & 104 & 105 & 106 & 107 & 108 \\
\hline Stream Name & $\begin{array}{l}\text { SBW } \\
\text { Feed }\end{array}$ & $\begin{array}{c}\text { Evaporator } \\
\text { Bottoms }\end{array}$ & $\begin{array}{c}\text { Evaporator } \\
\text { Overheads } \\
\text { Vapor }\end{array}$ & $\begin{array}{c}\text { Mist } \\
\text { Eliminator } \\
\text { Vapor }\end{array}$ & $\begin{array}{l}\text { VOG } \\
\text { Offgas } \\
\text { Vapor }\end{array}$ & $\begin{array}{c}\text { POG + VOG } \\
\text { Offgas } \\
\text { Vapor }\end{array}$ & $\begin{array}{l}\text { LET\&D } \\
\text { Feed } \\
\text { Liquid }\end{array}$ & $\begin{array}{l}\text { LET\&D } \\
\text { Acid } \\
\text { Liquid }\end{array}$ \\
\hline Radiological Composition & $\mathrm{Ci} / \mathrm{L}$ & $\mathrm{Ci} / \mathrm{L}$ & $\mathrm{Ci} / \mathrm{wscm}$ & $\mathrm{Ci} / \mathrm{wscm}$ & $\mathrm{Ci} / \mathrm{wscm}$ & $\mathrm{Ci} / \mathrm{wscm}$ & $\mathrm{Ci} / \mathrm{L}$ & $\mathrm{Ci} / \mathrm{L}$ \\
\hline Ru-106 & $1.22 \mathrm{E}-06$ & $4.88 \mathrm{E}-06$ & $1.20 \mathrm{E}-10$ & $1.20 \mathrm{E}-12$ & $3.82 \mathrm{E}-20$ & $3.31 \mathrm{E}-20$ & $1.57 \mathrm{E}-12$ & $9.66 \mathrm{E}-12$ \\
\hline Rh-102 & $6.63 \mathrm{E}-10$ & $2.65 E-09$ & $6.53 E-14$ & $6.53 \mathrm{E}-16$ & $2.07 E-23$ & 1.79E-23 & $8.53 \mathrm{E}-16$ & $5.24 \mathrm{E}-15$ \\
\hline Rh-106 & $1.22 \mathrm{E}-06$ & $4.88 \mathrm{E}-06$ & $1.20 \mathrm{E}-10$ & $1.20 \mathrm{E}-12$ & $3.82 \mathrm{E}-20$ & $3.31 \mathrm{E}-20$ & $1.57 \mathrm{E}-12$ & $9.66 \mathrm{E}-12$ \\
\hline $\mathrm{Pd}-107$ & $1.27 \mathrm{E}-08$ & 5.07E-08 & $1.25 \mathrm{E}-12$ & $1.25 \mathrm{E}-14$ & $3.97 \mathrm{E}-22$ & $3.44 \mathrm{E}-22$ & $1.63 \mathrm{E}-14$ & $1.00 \mathrm{E}-13$ \\
\hline $\mathrm{Cd}-113 \mathrm{~m}$ & $2.56 \mathrm{E}-06$ & $1.02 \mathrm{E}-05$ & $2.52 \mathrm{E}-10$ & $2.52 \mathrm{E}-12$ & $7.99 \mathrm{E}-20$ & $6.91 \mathrm{E}-20$ & $3.29 \mathrm{E}-12$ & $2.02 \mathrm{E}-11$ \\
\hline In-115 & $7.75 \mathrm{E}-17$ & $3.09 \mathrm{E}-16$ & $7.63 E-21$ & $7.63 E-23$ & $2.42 \mathrm{E}-30$ & $2.09 \mathrm{E}-30$ & $9.96 \mathrm{E}-23$ & $6.12 \mathrm{E}-22$ \\
\hline Sn-121m & $5.15 \mathrm{E}-08$ & $2.05 \mathrm{E}-07$ & $5.07 \mathrm{E}-12$ & $5.07 \mathrm{E}-14$ & $1.61 \mathrm{E}-21$ & $1.39 \mathrm{E}-21$ & $6.61 \mathrm{E}-14$ & $4.06 \mathrm{E}-13$ \\
\hline Sn-126 & $5.38 \mathrm{E}-07$ & $2.15 \mathrm{E}-06$ & $5.30 \mathrm{E}-11$ & $5.30 \mathrm{E}-13$ & $1.68 \mathrm{E}-20$ & $1.45 \mathrm{E}-20$ & $6.92 \mathrm{E}-13$ & $4.25 \mathrm{E}-12$ \\
\hline Sb-125 & $7.14 \mathrm{E}-04$ & $2.85 \mathrm{E}-03$ & 7.04E-08 & $7.03 E-10$ & $2.23 \mathrm{E}-17$ & $1.93 \mathrm{E}-17$ & $9.18 \mathrm{E}-10$ & 5.64E-09 \\
\hline Sb-126 & $4.43 E-08$ & 1.77E-07 & $4.36 \mathrm{E}-12$ & $4.36 \mathrm{E}-14$ & $1.38 \mathrm{E}-21$ & $1.20 \mathrm{E}-21$ & $5.69 \mathrm{E}-14$ & $3.50 \mathrm{E}-13$ \\
\hline Te-123 & $2.95 \mathrm{E}-19$ & $1.18 \mathrm{E}-18$ & $2.90 \mathrm{E}-23$ & $2.90 \mathrm{E}-25$ & & & $3.79 \mathrm{E}-25$ & $2.33 \mathrm{E}-24$ \\
\hline Te-125m & $2.42 \mathrm{E}-06$ & 9.67E-06 & $2.39 \mathrm{E}-10$ & $2.39 \mathrm{E}-12$ & $7.57 \mathrm{E}-20$ & $6.55 \mathrm{E}-20$ & $3.12 \mathrm{E}-12$ & $1.91 \mathrm{E}-11$ \\
\hline I-129 & 6.94E-08 & $2.32 \mathrm{E}-07$ & 1.11E-08 & 1.11E-08 & $7.04 \mathrm{E}-15$ & $1.51 \mathrm{E}-12$ & $1.45 \mathrm{E}-08$ & $1.49 \mathrm{E}-08$ \\
\hline Cs-134 & 7.29E-05 & $2.91 \mathrm{E}-04$ & $7.18 \mathrm{E}-09$ & $7.18 \mathrm{E}-11$ & $2.28 \mathrm{E}-18$ & $1.97 \mathrm{E}-18$ & $9.37 \mathrm{E}-11$ & $5.76 \mathrm{E}-10$ \\
\hline Cs-135 & $1.08 \mathrm{E}-06$ & $4.29 \mathrm{E}-06$ & $1.06 \mathrm{E}-10$ & $1.06 \mathrm{E}-12$ & $3.36 \mathrm{E}-20$ & $2.91 \mathrm{E}-20$ & $1.38 \mathrm{E}-12$ & $8.49 \mathrm{E}-12$ \\
\hline Cs-137 & 4.44E-02 & 1.77E-01 & 4.37E-06 & 4.37E-08 & $1.39 \mathrm{E}-15$ & $1.20 \mathrm{E}-15$ & $5.70 \mathrm{E}-08$ & $3.50 \mathrm{E}-07$ \\
\hline Ba-137m & $4.20 \mathrm{E}-02$ & 1.67E-01 & $4.13 \mathrm{E}-06$ & $4.13 E-08$ & $1.31 \mathrm{E}-15$ & $1.13 \mathrm{E}-15$ & $5.40 \mathrm{E}-08$ & 3.31E-07 \\
\hline La-138 & $1.47 \mathrm{E}-16$ & $5.86 \mathrm{E}-16$ & $1.45 \mathrm{E}-20$ & $1.45 \mathrm{E}-22$ & $4.59 E-30$ & $3.97 \mathrm{E}-30$ & 1.89E-22 & 1.16E-21 \\
\hline $\mathrm{Ce}-142$ & $2.30 \mathrm{E}-11$ & $9.18 \mathrm{E}-11$ & $2.27 \mathrm{E}-15$ & $2.27 \mathrm{E}-17$ & $7.19 \mathrm{E}-25$ & $6.22 \mathrm{E}-25$ & $2.96 \mathrm{E}-17$ & $1.82 \mathrm{E}-16$ \\
\hline Ce-144 & $8.27 \mathrm{E}-07$ & $3.30 \mathrm{E}-06$ & $8.14 \mathrm{E}-11$ & $8.14 \mathrm{E}-13$ & $2.58 \mathrm{E}-20$ & $2.24 \mathrm{E}-20$ & $1.06 \mathrm{E}-12$ & $6.53 \mathrm{E}-12$ \\
\hline Pr-144 & 8.27E-07 & 3.30E-06 & $8.14 \mathrm{E}-11$ & $8.14 \mathrm{E}-13$ & $2.58 \mathrm{E}-20$ & $2.24 \mathrm{E}-20$ & $1.06 \mathrm{E}-12$ & $6.53 \mathrm{E}-12$ \\
\hline Nd-144 & $1.24 \mathrm{E}-15$ & $4.94 \mathrm{E}-15$ & $1.22 \mathrm{E}-19$ & $1.22 \mathrm{E}-21$ & $3.87 \mathrm{E}-29$ & $3.34 \mathrm{E}-29$ & $1.59 \mathrm{E}-21$ & $9.77 \mathrm{E}-21$ \\
\hline $\mathrm{Pm}-146$ & $4.00 \mathrm{E}-08$ & $1.60 \mathrm{E}-07$ & $3.94 \mathrm{E}-12$ & $3.94 \mathrm{E}-14$ & $1.25 \mathrm{E}-21$ & $1.08 \mathrm{E}-21$ & $5.14 \mathrm{E}-14$ & $3.16 \mathrm{E}-13$ \\
\hline $\mathrm{Pm}-147$ & $2.22 \mathrm{E}-04$ & 8.87E-04 & $2.19 \mathrm{E}-08$ & $2.19 \mathrm{E}-10$ & $6.95 E-18$ & $6.01 \mathrm{E}-18$ & $2.86 \mathrm{E}-10$ & 1.76E-09 \\
\hline Sm-146 & $2.12 \mathrm{E}-13$ & $8.47 \mathrm{E}-13$ & $2.09 \mathrm{E}-17$ & $2.09 \mathrm{E}-19$ & $6.63 \mathrm{E}-27$ & $5.74 \mathrm{E}-27$ & $2.73 E-19$ & $1.68 \mathrm{E}-18$ \\
\hline Sm-147 & $5.67 \mathrm{E}-12$ & $2.26 \mathrm{E}-11$ & $5.58 \mathrm{E}-16$ & $5.58 \mathrm{E}-18$ & 1.77E-25 & $1.53 \mathrm{E}-25$ & $7.29 \mathrm{E}-18$ & $4.48 \mathrm{E}-17$ \\
\hline Sm-148 & $2.91 \mathrm{E}-17$ & $1.16 \mathrm{E}-16$ & $2.87 \mathrm{E}-21$ & $2.87 E-23$ & & & $3.74 \mathrm{E}-23$ & $2.30 \mathrm{E}-22$ \\
\hline Sm-149 & $2.59 \mathrm{E}-18$ & $1.03 \mathrm{E}-17$ & $2.55 \mathrm{E}-22$ & $2.55 E-24$ & & & $3.32 \mathrm{E}-24$ & $2.04 \mathrm{E}-23$ \\
\hline Sm-151 & $4.40 \mathrm{E}-04$ & $1.76 \mathrm{E}-03$ & $4.34 \mathrm{E}-08$ & $4.34 \mathrm{E}-10$ & $1.38 \mathrm{E}-17$ & $1.19 \mathrm{E}-17$ & $5.66 \mathrm{E}-10$ & $3.48 \mathrm{E}-09$ \\
\hline Eu-152 & $2.57 \mathrm{E}-06$ & 1.03E-05 & $2.53 \mathrm{E}-10$ & $2.53 \mathrm{E}-12$ & $8.04 \mathrm{E}-20$ & $6.96 \mathrm{E}-20$ & $3.31 \mathrm{E}-12$ & $2.03 E-11$ \\
\hline Eu-154 & $9.75 \mathrm{E}-05$ & 3.89E-04 & $9.60 \mathrm{E}-09$ & $9.60 \mathrm{E}-11$ & $3.05 E-18$ & $2.64 \mathrm{E}-18$ & $1.25 \mathrm{E}-10$ & $7.70 \mathrm{E}-10$ \\
\hline Eu-155 & 1.03E-04 & $4.11 \mathrm{E}-04$ & $1.01 \mathrm{E}-08$ & $1.01 \mathrm{E}-10$ & $3.22 \mathrm{E}-18$ & $2.78 \mathrm{E}-18$ & $1.32 \mathrm{E}-10$ & $8.13 E-10$ \\
\hline Gd-152 & $1.09 \mathrm{E}-18$ & $4.37 \mathrm{E}-18$ & $1.08 \mathrm{E}-22$ & $1.08 \mathrm{E}-24$ & & & $1.41 \mathrm{E}-24$ & $8.64 \mathrm{E}-24$ \\
\hline Ho-166m & $3.54 \mathrm{E}-11$ & $1.41 \mathrm{E}-10$ & $3.49 \mathrm{E}-15$ & $3.49 \mathrm{E}-17$ & $1.11 \mathrm{E}-24$ & $9.58 \mathrm{E}-25$ & $4.55 \mathrm{E}-17$ & $2.80 \mathrm{E}-16$ \\
\hline \multicolumn{9}{|l|}{ Other Stream Data: } \\
\hline TRU, nCi/gm & $1.08 \mathrm{E}+03$ & $3.12 \mathrm{E}+03$ & & & & & 1.66E-03 & 9.27E-03 \\
\hline Heat Generation, W/m3 & $4.62 \mathrm{E}-01$ & $1.84 \mathrm{E}+00$ & & & & & $9.66 \mathrm{E}-07$ & $3.84 \mathrm{E}-06$ \\
\hline Mass Flow, kg/hr & $4.07 \mathrm{E}+02$ & $1.42 \mathrm{E}+02$ & $2.68 \mathrm{E}+02$ & $2.68 \mathrm{E}+02$ & $2.47 \mathrm{E}+02$ & $2.88 \mathrm{E}+02$ & $2.68 \mathrm{E}+02$ & $4.79 \mathrm{E}+01$ \\
\hline
\end{tabular}


Table C-5. Revised baseline mass balance, Tank WM-187 waste (Continued).

\begin{tabular}{|c|c|c|c|c|c|c|c|}
\hline PFD Sheet \# & PFD-3 & PFD-4 & PFD-4 & PFD-4 & PFD-4 & PFD-4 & PFD-4 \\
\hline Stream \# & 109 & 110 & 201 & 202 & 203 & 204 & 205 \\
\hline Stream Name & $\begin{array}{c}\text { LET\&D } \\
\text { Offgas } \\
\text { Vapor }\end{array}$ & $\begin{array}{l}\text { Acid } \\
\text { Grout } \\
\text { Solid }\end{array}$ & $\begin{array}{c}\text { OPC } \\
\text { Grout } \\
\text { Additive }\end{array}$ & $\begin{array}{c}\text { BFS } \\
\text { Grout } \\
\text { Additive }\end{array}$ & $\begin{array}{c}\mathrm{Ca}(\mathrm{OH}) 2 \\
\text { Total } \\
\text { Additive }\end{array}$ & $\begin{array}{c}\mathrm{Ca}(\mathrm{OH}) 2 \\
\text { Grout } \\
\text { Additive }\end{array}$ & $\begin{array}{c}\mathrm{Ca}(\mathrm{OH}) 2 \\
\text { Neutraliz. } \\
\text { Additive }\end{array}$ \\
\hline Rate or Volume Flow (actual) & $1.65 \mathrm{E}+04$ & 301.8 & 119 & 39.6 & 37.7 & 1.20 & 36.5 \\
\hline Volume Flow (standard, wet) & $1.03 E+04$ & & & & & & \\
\hline Volume Flow (standard, dry) ${ }^{*}$ & $7.11 \mathrm{E}-01$ & & & & & & \\
\hline Rate Units & $\mathrm{ft} 3 / \mathrm{hr}$ & $\mathrm{lb} / \mathrm{hr}$ & $\mathrm{lb} / \mathrm{hr}$ & $\mathrm{lb} / \mathrm{hr}$ & $\mathrm{lb} / \mathrm{hr}$ & $\mathrm{lb} / \mathrm{hr}$ & $\mathrm{lb} / \mathrm{hr}$ \\
\hline Temperature, ${ }^{\circ} \mathrm{C}$ & 119.1 & 64.3 & 25.0 & 25.0 & 25.0 & 25.0 & 25.0 \\
\hline Temperature, ${ }^{\circ} \mathrm{F}$ & 246.3 & 147.8 & 77.0 & 77.0 & 77.0 & 77.0 & 77.0 \\
\hline Pressure, psia & 12.3 & 12.2 & 12.3 & 12.3 & 12.3 & 12.3 & 12.3 \\
\hline Specific Gravity & 0.00047 & 2.06 & 1.2 & 1.2 & 0.48 & 2.24 & 0.48 \\
\hline Chemical Composition & Ib/wscf & Wt. \% & Wt. \% & Wt. \% & Wt. \% & Wt. \% & Wt. \% \\
\hline $\mathrm{H}+$ & $1.70 \mathrm{E}-07$ & $8.23 \mathrm{E}-06$ & & & & & \\
\hline $\mathrm{OH}-$ & & $1.81 \mathrm{E}-01$ & & & $4.59 \mathrm{E}+01$ & $4.59 \mathrm{E}+01$ & $4.59 \mathrm{E}+01$ \\
\hline $\mathrm{Al}+3$ & & $2.31 \mathrm{E}-06$ & & & & & \\
\hline $\mathrm{Sb}+5$ & & $8.04 \mathrm{E}-10$ & & & & & \\
\hline$A s+5$ & & $3.14 \mathrm{E}-09$ & & & & & \\
\hline $\mathrm{Ba}+2$ & & $2.32 \mathrm{E}-09$ & & & & & \\
\hline $\mathrm{Be}+2$ & & $3.57 \mathrm{E}-11$ & & & & & \\
\hline $\mathrm{B}+3$ & & $2.83 \mathrm{E}-08$ & & & & & \\
\hline $\mathrm{Cd}+2$ & & 4.99E-08 & & & & & \\
\hline $\mathrm{Ca}+2$ & & $6.76 \mathrm{E}+00$ & & & $5.41 \mathrm{E}+01$ & $5.41 \mathrm{E}+01$ & $5.41 \mathrm{E}+01$ \\
\hline $\mathrm{Cr}+3$ & & $5.81 \mathrm{E}-08$ & & & & & \\
\hline $\mathrm{Co}+2$ & & $2.71 \mathrm{E}-08$ & & & & & \\
\hline Cs+ & & 2.65E-09 & & & & & \\
\hline $\mathrm{Cu}+2$ & & 7.73E-09 & & & & & \\
\hline $\mathrm{Fe}+3$ & & $3.35 \mathrm{E}-07$ & & & & & \\
\hline $\mathrm{Pb}+2$ & & $3.31 \mathrm{E}-08$ & & & & & \\
\hline $\mathrm{Hg}+2$ & & $1.16 \mathrm{E}-02$ & & & & & \\
\hline $\mathrm{Mn}+4$ & & $2.43 \mathrm{E}-07$ & & & & & \\
\hline $\mathrm{Ni}+2$ & & $1.96 \mathrm{E}-08$ & & & & & \\
\hline $\mathrm{K}+$ & & $2.90 \mathrm{E}-06$ & & & & & \\
\hline $\mathrm{Se}+4$ & & $8.64 \mathrm{E}-09$ & & & & & \\
\hline $\mathrm{Ag}+$ & & $1.81 \mathrm{E}-08$ & & & & & \\
\hline $\mathrm{Na}+$ & & $9.62 \mathrm{E}-06$ & & & & & \\
\hline $\mathrm{TI}+3$ & & $3.06 \mathrm{E}-10$ & & & & & \\
\hline$U+4$ & & $1.64 \mathrm{E}-08$ & & & & & \\
\hline$V+3$ & & $7.42 \mathrm{E}-10$ & & & & & \\
\hline$Z n+2$ & & 1.12E-08 & & & & & \\
\hline $\mathrm{Zr}+4$ & & $1.44 \mathrm{E}-06$ & & & & & \\
\hline $\mathrm{Cl}-$ & 5.99E-06 & $4.10 \mathrm{E}-03$ & & & & & \\
\hline F- & & $1.57 \mathrm{E}-04$ & & & & & \\
\hline SO4-2 & & 1.14E-06 & & & & & \\
\hline NO3- & 4.59E-09 & $2.03 E+01$ & & & & & \\
\hline PO4-3 & & $3.93 \mathrm{E}-06$ & & & & & \\
\hline $\mathrm{Am}+4$ & & $3.78 \mathrm{E}-12$ & & & & & \\
\hline $\mathrm{Br}-$ & $2.09 \mathrm{E}-10$ & 1.43E-07 & & & & & \\
\hline $\mathrm{Ce}+4$ & & 1.35E-09 & & & & & \\
\hline $\mathrm{Eu}+3$ & & 1.33E-11 & & & & & \\
\hline$G d+3$ & & $5.10 \mathrm{E}-09$ & & & & & \\
\hline $\mathrm{Ge}+4$ & & $1.10 \mathrm{E}-13$ & & & & & \\
\hline $\ln +3$ & & $2.91 \mathrm{E}-11$ & & & & & \\
\hline I- & $2.76 \mathrm{E}-09$ & 1.89E-06 & & & & & \\
\hline $\mathrm{La}+3$ & & $2.19 \mathrm{E}-10$ & & & & & \\
\hline $\mathrm{Li}+$ & & 1.03E-09 & & & & & \\
\hline $\mathrm{Mg}+2$ & & $8.88 \mathrm{E}-08$ & & & & & \\
\hline $\mathrm{Mo}+6$ & & $2.80 \mathrm{E}-08$ & & & & & \\
\hline
\end{tabular}


Table C-5. Revised baseline mass balance, Tank WM-187 waste (Continued).

\begin{tabular}{|c|c|c|c|c|c|c|c|}
\hline Stream \# & 109 & 110 & 201 & 202 & 203 & 204 & 205 \\
\hline Stream Name & $\begin{array}{l}\text { LET\&D } \\
\text { Offgas } \\
\text { Vapor }\end{array}$ & $\begin{array}{l}\text { Acid } \\
\text { Grout } \\
\text { Solid }\end{array}$ & $\begin{array}{c}\text { OPC } \\
\text { Grout } \\
\text { Additive }\end{array}$ & $\begin{array}{c}\text { BFS } \\
\text { Grout } \\
\text { Additive }\end{array}$ & $\begin{array}{c}\mathrm{Ca}(\mathrm{OH}) 2 \\
\text { Total } \\
\text { Additive }\end{array}$ & $\begin{array}{c}\mathrm{Ca}(\mathrm{OH}) 2 \\
\text { Grout } \\
\text { Additive }\end{array}$ & $\begin{array}{c}\mathrm{Ca}(\mathrm{OH}) 2 \\
\text { Neutraliz. } \\
\text { Additive }\end{array}$ \\
\hline Chemical Composition & $\mathrm{lb} / \mathrm{wscf}$ & Wt. \% & Wt. \% & Wt. \% & Wt. \% & Wt. \% & Wt. \% \\
\hline $\mathrm{Nd}+3$ & & $7.35 \mathrm{E}-10$ & & & & & \\
\hline $\mathrm{Np}+4$ & & $1.85 \mathrm{E}-10$ & & & & & \\
\hline $\mathrm{Nb}+5$ & & 4.09E-08 & & & & & \\
\hline $\mathrm{Pd}+4$ & & 7.61E-08 & & & & & \\
\hline $\mathrm{Pu}+4$ & & $5.07 \mathrm{E}-10$ & & & & & \\
\hline $\mathrm{Pr}+4$ & & $2.02 \mathrm{E}-10$ & & & & & \\
\hline $\mathrm{Rh}+4$ & & $6.38 \mathrm{E}-11$ & & & & & \\
\hline $\mathrm{Rb}+$ & & $8.15 \mathrm{E}-11$ & & & & & \\
\hline Ru+3 & & $3.26 \mathrm{E}-08$ & & & & & \\
\hline $\mathrm{Sm}+3$ & & $1.44 \mathrm{E}-10$ & & & & & \\
\hline $\mathrm{Si+4}$ & & 1.06E-06 & & & & & \\
\hline $\mathrm{Sr}+2$ & & 1.83E-09 & & & & & \\
\hline $\mathrm{Tc}+7$ & & $1.48 \mathrm{E}-10$ & & & & & \\
\hline $\mathrm{Te}+4$ & & $1.05 \mathrm{E}-10$ & & & & & \\
\hline $\mathrm{Tb}+4$ & & $5.78 \mathrm{E}-14$ & & & & & \\
\hline $\mathrm{Th}+4$ & & 1.02E-09 & & & & & \\
\hline $\mathrm{Sn}+4$ & & 9.09E-08 & & & & & \\
\hline $\mathrm{Ti}+4$ & & 1.84E-08 & & & & & \\
\hline $\mathrm{Y}+3$ & & $1.05 \mathrm{E}-10$ & & & & & \\
\hline O-2 (oxides) & & $1.03 E-06$ & & & & & \\
\hline $\mathrm{H} 2 \mathrm{O}$ & 4.70E-02 & $2.03 \mathrm{E}+01$ & & & & & \\
\hline $\mathrm{O} 2$ & 1.35E-07 & & & & & & \\
\hline $\mathrm{N} 2$ & $1.78 \mathrm{E}-07$ & & & & & & \\
\hline C (organic) & & 6.79E-03 & & & & & \\
\hline $\mathrm{H}$ (organic) & & $5.70 \mathrm{E}-04$ & & & & & \\
\hline SiO2, BFS, or OPC Additives & & $5.25 \mathrm{E}+01$ & $1.00 \mathrm{E}+02$ & $1.00 \mathrm{E}+02$ & & & \\
\hline $\begin{array}{l}\text { Gas Stream Bulk Composition } \\
\text { (wet basis) }\end{array}$ & $\begin{array}{l}\text { mol. } \% \\
\text { or ppmv }\end{array}$ & $\begin{array}{l}\text { mol. \% } \\
\text { or ppmv }\end{array}$ & $\begin{array}{l}\text { mol. } \% \\
\text { or ppmv }\end{array}$ & $\begin{array}{l}\text { mol. } \% \\
\text { or ppmv }\end{array}$ & $\begin{array}{l}\text { mol.\% } \\
\text { or ppmv }\end{array}$ & $\begin{array}{l}\text { mol. \% } \\
\text { or ppmv }\end{array}$ & $\begin{array}{l}\text { mol. \% } \\
\text { or ppmv }\end{array}$ \\
\hline $\mathrm{H} 2 \mathrm{O}, \mathrm{mol} . \%$ & 99.99 & & & & & & \\
\hline $\mathrm{O} 2, \mathrm{~mol} . \%$ & 0.00 & & & & & & \\
\hline $\mathrm{N} 2, \mathrm{~mol} . \%$ & 0.00 & & & & & & \\
\hline \multicolumn{8}{|l|}{$\mathrm{CO} 2, \mathrm{~mol} . \%$} \\
\hline HNO3, mol. \% & 0.01 & & & & & & \\
\hline \multicolumn{8}{|l|}{$\mathrm{CO}, \mathrm{ppmv}$} \\
\hline \multicolumn{8}{|l|}{$\mathrm{NO}, \mathrm{ppmv}$} \\
\hline \multicolumn{8}{|l|}{$\mathrm{NO} 2, \mathrm{ppmv}$} \\
\hline \multicolumn{8}{|l|}{ C (organic), ppmv } \\
\hline \multicolumn{8}{|l|}{$\mathrm{H}$ (organic), ppmv } \\
\hline \multicolumn{8}{|l|}{ PM (mg/wscm) } \\
\hline $\begin{array}{l}\text { Gas Stream Bulk Composition } \\
\text { (dry basis) } \\
\end{array}$ & $\begin{array}{c}\text { mol. } \% \\
\text { or ppmv }\end{array}$ & $\begin{array}{c}\text { mol. } \% \\
\text { or ppmv }\end{array}$ & $\begin{array}{l}\text { mol. } \% \\
\text { or ppmv }\end{array}$ & $\begin{array}{c}\text { mol. } \% \\
\text { or ppmv }\end{array}$ & $\begin{array}{c}\text { mol. } \% \\
\text { or ppmv }\end{array}$ & $\begin{array}{l}\text { mol. } \% \\
\text { or ppmv }\end{array}$ & $\begin{array}{c}\text { mol. } \% \\
\text { or ppmv }\end{array}$ \\
\hline $\mathrm{O} 2, \mathrm{~mol} . \%$ & 2.35 & & & & & & \\
\hline $\mathrm{N} 2, \mathrm{~mol} . \%$ & 3.54 & & & & & & \\
\hline \multicolumn{8}{|l|}{$\mathrm{CO} 2, \mathrm{~mol} . \%$} \\
\hline HNO3, mol. \% & 94.12 & & & & & & \\
\hline \multicolumn{8}{|l|}{$\mathrm{CO}, \mathrm{ppmv}$} \\
\hline \multicolumn{8}{|l|}{$\mathrm{NO}, \mathrm{ppmv}$} \\
\hline \multicolumn{8}{|l|}{ NO2, ppmv } \\
\hline \multicolumn{8}{|l|}{ C (organic), ppmv } \\
\hline \multicolumn{8}{|l|}{$\mathrm{H}$ (organic), ppmv } \\
\hline $\mathrm{PM}(\mathrm{mg} / \mathrm{dscm})$ & & & & & & & \\
\hline
\end{tabular}


Table C-5. Revised baseline mass balance, Tank WM-187 waste (Continued).

\begin{tabular}{|c|c|c|c|c|c|c|c|}
\hline Stream \# & 109 & 110 & 201 & 202 & 203 & 204 & 205 \\
\hline Stream Name & $\begin{array}{l}\text { LET\&D } \\
\text { Offgas } \\
\text { Vapor }\end{array}$ & $\begin{array}{l}\text { Acid } \\
\text { Grout } \\
\text { Solid }\end{array}$ & $\begin{array}{c}\text { OPC } \\
\text { Grout } \\
\text { Additive }\end{array}$ & $\begin{array}{c}\text { BFS } \\
\text { Grout } \\
\text { Additive }\end{array}$ & $\begin{array}{c}\mathrm{Ca}(\mathrm{OH}) 2 \\
\text { Total } \\
\text { Additive }\end{array}$ & $\begin{array}{c}\mathrm{Ca}(\mathrm{OH}) 2 \\
\text { Grout } \\
\text { Additive }\end{array}$ & $\begin{array}{c}\mathrm{Ca}(\mathrm{OH}) 2 \\
\text { Neutraliz. } \\
\text { Additive }\end{array}$ \\
\hline Chemical Composition & $\mathrm{lb} / \mathrm{wscf}$ & Wt. \% & Wt. \% & Wt. \% & Wt. \% & Wt. \% & Wt. \% \\
\hline $\mathrm{Nd}+3$ & & $7.35 \mathrm{E}-10$ & & & & & \\
\hline $\mathrm{Np}+4$ & & $1.85 \mathrm{E}-10$ & & & & & \\
\hline $\mathrm{Nb}+5$ & & 4.09E-08 & & & & & \\
\hline $\mathrm{Pd}+4$ & & 7.61E-08 & & & & & \\
\hline $\mathrm{Pu}+4$ & & $5.07 \mathrm{E}-10$ & & & & & \\
\hline $\mathrm{Pr}+4$ & & $2.02 \mathrm{E}-10$ & & & & & \\
\hline $\mathrm{Rh}+4$ & & $6.38 \mathrm{E}-11$ & & & & & \\
\hline $\mathrm{Rb}+$ & & $8.15 \mathrm{E}-11$ & & & & & \\
\hline Ru+3 & & $3.26 \mathrm{E}-08$ & & & & & \\
\hline $\mathrm{Sm}+3$ & & $1.44 \mathrm{E}-10$ & & & & & \\
\hline $\mathrm{Si+4}$ & & 1.06E-06 & & & & & \\
\hline $\mathrm{Sr}+2$ & & 1.83E-09 & & & & & \\
\hline $\mathrm{Tc}+7$ & & $1.48 \mathrm{E}-10$ & & & & & \\
\hline $\mathrm{Te}+4$ & & $1.05 \mathrm{E}-10$ & & & & & \\
\hline $\mathrm{Tb}+4$ & & $5.78 \mathrm{E}-14$ & & & & & \\
\hline $\mathrm{Th}+4$ & & 1.02E-09 & & & & & \\
\hline $\mathrm{Sn}+4$ & & 9.09E-08 & & & & & \\
\hline $\mathrm{Ti}+4$ & & 1.84E-08 & & & & & \\
\hline $\mathrm{Y}+3$ & & $1.05 \mathrm{E}-10$ & & & & & \\
\hline O-2 (oxides) & & $1.03 E-06$ & & & & & \\
\hline $\mathrm{H} 2 \mathrm{O}$ & 4.70E-02 & $2.03 \mathrm{E}+01$ & & & & & \\
\hline $\mathrm{O} 2$ & 1.35E-07 & & & & & & \\
\hline $\mathrm{N} 2$ & $1.78 \mathrm{E}-07$ & & & & & & \\
\hline C (organic) & & 6.79E-03 & & & & & \\
\hline $\mathrm{H}$ (organic) & & $5.70 \mathrm{E}-04$ & & & & & \\
\hline SiO2, BFS, or OPC Additives & & $5.25 \mathrm{E}+01$ & $1.00 \mathrm{E}+02$ & $1.00 \mathrm{E}+02$ & & & \\
\hline $\begin{array}{l}\text { Gas Stream Bulk Composition } \\
\text { (wet basis) }\end{array}$ & $\begin{array}{l}\text { mol. } \% \\
\text { or ppmv }\end{array}$ & $\begin{array}{l}\text { mol. \% } \\
\text { or ppmv }\end{array}$ & $\begin{array}{l}\text { mol. } \% \\
\text { or ppmv }\end{array}$ & $\begin{array}{l}\text { mol. } \% \\
\text { or ppmv }\end{array}$ & $\begin{array}{l}\text { mol.\% } \\
\text { or ppmv }\end{array}$ & $\begin{array}{l}\text { mol. \% } \\
\text { or ppmv }\end{array}$ & $\begin{array}{l}\text { mol. \% } \\
\text { or ppmv }\end{array}$ \\
\hline $\mathrm{H} 2 \mathrm{O}, \mathrm{mol} . \%$ & 99.99 & & & & & & \\
\hline $\mathrm{O} 2, \mathrm{~mol} . \%$ & 0.00 & & & & & & \\
\hline $\mathrm{N} 2, \mathrm{~mol} . \%$ & 0.00 & & & & & & \\
\hline \multicolumn{8}{|l|}{$\mathrm{CO} 2, \mathrm{~mol} . \%$} \\
\hline HNO3, mol. \% & 0.01 & & & & & & \\
\hline \multicolumn{8}{|l|}{$\mathrm{CO}, \mathrm{ppmv}$} \\
\hline \multicolumn{8}{|l|}{$\mathrm{NO}, \mathrm{ppmv}$} \\
\hline \multicolumn{8}{|l|}{$\mathrm{NO} 2, \mathrm{ppmv}$} \\
\hline \multicolumn{8}{|l|}{ C (organic), ppmv } \\
\hline \multicolumn{8}{|l|}{$\mathrm{H}$ (organic), ppmv } \\
\hline \multicolumn{8}{|l|}{ PM (mg/wscm) } \\
\hline $\begin{array}{l}\text { Gas Stream Bulk Composition } \\
\text { (dry basis) } \\
\end{array}$ & $\begin{array}{c}\text { mol. } \% \\
\text { or ppmv }\end{array}$ & $\begin{array}{c}\text { mol. } \% \\
\text { or ppmv }\end{array}$ & $\begin{array}{l}\text { mol. } \% \\
\text { or ppmv }\end{array}$ & $\begin{array}{c}\text { mol. } \% \\
\text { or ppmv }\end{array}$ & $\begin{array}{c}\text { mol. } \% \\
\text { or ppmv }\end{array}$ & $\begin{array}{l}\text { mol. } \% \\
\text { or ppmv }\end{array}$ & $\begin{array}{c}\text { mol. } \% \\
\text { or ppmv }\end{array}$ \\
\hline $\mathrm{O} 2, \mathrm{~mol} . \%$ & 2.35 & & & & & & \\
\hline $\mathrm{N} 2, \mathrm{~mol} . \%$ & 3.54 & & & & & & \\
\hline \multicolumn{8}{|l|}{$\mathrm{CO} 2, \mathrm{~mol} . \%$} \\
\hline HNO3, mol. \% & 94.12 & & & & & & \\
\hline \multicolumn{8}{|l|}{$\mathrm{CO}, \mathrm{ppmv}$} \\
\hline \multicolumn{8}{|l|}{$\mathrm{NO}, \mathrm{ppmv}$} \\
\hline \multicolumn{8}{|l|}{ NO2, ppmv } \\
\hline \multicolumn{8}{|l|}{ C (organic), ppmv } \\
\hline \multicolumn{8}{|l|}{$\mathrm{H}$ (organic), ppmv } \\
\hline $\mathrm{PM}(\mathrm{mg} / \mathrm{dscm})$ & & & & & & & \\
\hline
\end{tabular}


Table C-5. Revised baseline mass balance, Tank WM-187 waste (Continued).

\begin{tabular}{|c|c|c|c|c|c|c|c|}
\hline Stream \# & 109 & 110 & 201 & 202 & 203 & 204 & 205 \\
\hline Stream Name & $\begin{array}{l}\text { LET\&D } \\
\text { Offgas } \\
\text { Vapor }\end{array}$ & $\begin{array}{l}\text { Acid } \\
\text { Grout } \\
\text { Solid }\end{array}$ & $\begin{array}{c}\text { OPC } \\
\text { Grout } \\
\text { Additive }\end{array}$ & $\begin{array}{c}\text { BFS } \\
\text { Grout } \\
\text { Additive }\end{array}$ & $\begin{array}{c}\mathrm{Ca}(\mathrm{OH}) 2 \\
\text { Total } \\
\text { Additive }\end{array}$ & $\begin{array}{c}\mathrm{Ca}(\mathrm{OH}) 2 \\
\text { Grout } \\
\text { Additive }\end{array}$ & $\begin{array}{c}\mathrm{Ca}(\mathrm{OH}) 2 \\
\text { Neutraliz. } \\
\text { Additive }\end{array}$ \\
\hline Radiological Composition & $\mathrm{Ci} / \mathrm{wscm}$ & $\mathrm{Ci} / \mathrm{kg}$ & $\mathrm{Ci} / \mathrm{kg}$ & $\mathrm{Ci} / \mathrm{kg}$ & $\mathrm{Ci} / \mathrm{kg}$ & $\mathrm{Ci} / \mathrm{kg}$ & $\mathrm{Ci} / \mathrm{kg}$ \\
\hline Ru-106 & $2.72 \mathrm{E}-24$ & $2.90 \mathrm{E}-12$ & & & & & \\
\hline Rh-102 & $1.47 \mathrm{E}-27$ & $1.57 \mathrm{E}-15$ & & & & & \\
\hline Rh-106 & $2.72 \mathrm{E}-24$ & $2.90 \mathrm{E}-12$ & & & & & \\
\hline Pd-107 & $2.82 E-26$ & $3.02 \mathrm{E}-14$ & & & & & \\
\hline Cd-113m & $5.68 \mathrm{E}-24$ & $6.06 \mathrm{E}-12$ & & & & & \\
\hline $\ln -115$ & & $1.84 \mathrm{E}-22$ & & & & & \\
\hline Sn-121m & $1.14 \mathrm{E}-25$ & $1.22 \mathrm{E}-13$ & & & & & \\
\hline Sn-126 & $1.20 \mathrm{E}-24$ & $1.28 \mathrm{E}-12$ & & & & & \\
\hline Sb-125 & 1.59E-21 & 1.69E-09 & & & & & \\
\hline Sb-126 & $9.83 E-26$ & $1.05 \mathrm{E}-13$ & & & & & \\
\hline \begin{tabular}{|l|} 
Te-123 \\
\end{tabular} & & $7.00 \mathrm{E}-25$ & & & & & \\
\hline Te-125m & $5.38 \mathrm{E}-24$ & $5.75 \mathrm{E}-12$ & & & & & \\
\hline \begin{tabular}{|l|}
$I-129$ \\
\end{tabular} & 1.04E-08 & 4.48E-09 & & & & & \\
\hline Cs-134 & $1.62 \mathrm{E}-22$ & $1.73 \mathrm{E}-10$ & & & & & \\
\hline Cs-135 & $2.39 \mathrm{E}-24$ & $2.55 \mathrm{E}-12$ & & & & & \\
\hline Cs-137 & $9.86 \mathrm{E}-20$ & $1.05 \mathrm{E}-07$ & & & & & \\
\hline Ba-137m & 9.32E-20 & 9.96E-08 & & & & & \\
\hline \begin{tabular}{|l} 
La-138 \\
\end{tabular} & & $3.49 \mathrm{E}-22$ & & & & & \\
\hline Ce-142 & 5.11E-29 & $5.46 \mathrm{E}-17$ & & & & & \\
\hline Ce-144 & $1.84 \mathrm{E}-24$ & $1.96 \mathrm{E}-12$ & & & & & \\
\hline Pr-144 & $1.84 \mathrm{E}-24$ & $1.96 \mathrm{E}-12$ & & & & & \\
\hline Nd-144 & & $2.93 \mathrm{E}-21$ & & & & & \\
\hline $\mathrm{Pm}-146$ & $8.88 \mathrm{E}-26$ & $9.48 \mathrm{E}-14$ & & & & & \\
\hline $\mathrm{Pm}-147$ & $4.94 \mathrm{E}-22$ & $5.27 \mathrm{E}-10$ & & & & & \\
\hline Sm-146 & & $5.04 \mathrm{E}-19$ & & & & & \\
\hline Sm-147 & $1.26 \mathrm{E}-29$ & $1.34 \mathrm{E}-17$ & & & & & \\
\hline Sm-148 & & $6.91 \mathrm{E}-23$ & & & & & \\
\hline Sm-149 & & $6.13 E-24$ & & & & & \\
\hline Sm-151 & $9.78 \mathrm{E}-22$ & 1.04E-09 & & & & & \\
\hline Eu-152 & $5.72 \mathrm{E}-24$ & $6.10 \mathrm{E}-12$ & & & & & \\
\hline Eu-154 & $2.17 \mathrm{E}-22$ & $2.31 \mathrm{E}-10$ & & & & & \\
\hline Eu-155 & $2.29 \mathrm{E}-22$ & $2.44 \mathrm{E}-10$ & & & & & \\
\hline Gd-152 & & $2.60 \mathrm{E}-24$ & & & & & \\
\hline Ho-166m & $7.87 E-29$ & $8.40 \mathrm{E}-17$ & & & & & \\
\hline \multicolumn{8}{|l|}{ Other Stream Data: } \\
\hline TRU, nCi/gm & & $3.25 E-03$ & & & & & \\
\hline Heat Generation, W/m3 & & 1.99E-06 & & & & & \\
\hline Mass Flow, kg/hr & $2.20 \mathrm{E}+02$ & $1.37 \mathrm{E}+02$ & $5.39 \mathrm{E}+01$ & $1.80 \mathrm{E}+01$ & $1.71 \mathrm{E}+01$ & $5.42 \mathrm{E}-01$ & $1.66 \mathrm{E}+01$ \\
\hline
\end{tabular}

* Standard conditions $=68 \mathrm{oF}$ and 1 atmosphere 
Table C-6. Revised baseline mass balance, Tank WM-188 waste.

\begin{tabular}{|c|c|c|c|c|c|c|c|}
\hline PFD Sheet \# & PFD-1 & PFD-2 & PFD-2 & PFD-2 & PFD-2 & PFD-2 & PFD-2 \\
\hline Stream \# & 101 & 102 & 103 & 104 & 105 & 106 & 107 \\
\hline Stream Name & $\begin{array}{l}\text { SBW } \\
\text { Feed }\end{array}$ & $\begin{array}{c}\text { Evaporator } \\
\text { Bottoms }\end{array}$ & $\begin{array}{c}\text { Evaporator } \\
\text { Overheads } \\
\text { Vapor }\end{array}$ & $\begin{array}{c}\text { Mist } \\
\text { Eliminator } \\
\text { Vapor }\end{array}$ & $\begin{array}{l}\text { VOG } \\
\text { Offgas } \\
\text { Vapor }\end{array}$ & $\begin{array}{c}\text { POG + VOG } \\
\text { Offgas } \\
\text { Vapor }\end{array}$ & $\begin{array}{l}\text { LET\&D } \\
\text { Feed } \\
\text { Liquid }\end{array}$ \\
\hline Rate or Volume Flow (actual) & 85.8 & 21.4 & $2.59 \mathrm{E}+04$ & $2.68 \mathrm{E}+04$ & $1.17 \mathrm{E}+04$ & $1.37 \mathrm{E}+04$ & 65.7 \\
\hline Volume Flow (standard, wet) ${ }^{*}$ & & & $1.14 \mathrm{E}+04$ & $1.14 \mathrm{E}+04$ & $7.34 \mathrm{E}+03$ & $8.57 \mathrm{E}+03$ & \\
\hline Volume Flow (standard, dry) & & & $5.48 \mathrm{E}+02$ & $5.48 \mathrm{E}+02$ & $7.13 \mathrm{E}+03$ & $8.36 \mathrm{E}+03$ & \\
\hline Rate Units & $\mathrm{gal} / \mathrm{hr}$ & gal/hr & $\mathrm{ft} 3 / \mathrm{hr}$ & $\mathrm{ft} 3 / \mathrm{hr}$ & $\mathrm{ft} 3 / \mathrm{hr}$ & $\mathrm{ft} 3 / \mathrm{hr}$ & gal/hr \\
\hline Temperature, ${ }^{\circ} \mathrm{C}$ & 25.0 & 116.7 & 116.7 & 120.0 & 107.5 & 109.4 & 35.0 \\
\hline Temperature, ${ }^{\circ} \mathrm{F}$ & 77.0 & 242.0 & 242.0 & 248.0 & 225.6 & 228.8 & 95.0 \\
\hline Pressure, psia & 12.3 & 8.6 & 8.6 & 8.4 & 12.0 & 12.0 & 12.0 \\
\hline Specific Gravity & 1.26 & 1.75 & 0.00037 & 0.00036 & 0.00075 & 0.00074 & 1.09 \\
\hline Chemical Composition & Mol/Liter & Mol/Liter & $\mathrm{lb} / \mathrm{wscf}$ & $\mathrm{lb} / \mathrm{wscf}$ & $\mathrm{lb} / \mathrm{wscf}$ & $\mathrm{lb} / \mathrm{wscf}$ & Mol/Liter \\
\hline $\mathrm{H}+$ & $2.18 \mathrm{E}+00$ & 7.40E-01 & $1.26 \mathrm{E}-04$ & 1.26E-04 & $5.81 \mathrm{E}-12$ & $1.68 \mathrm{E}-08$ & $2.60 \mathrm{E}+00$ \\
\hline \multicolumn{8}{|l|}{$\mathrm{OH}-$} \\
\hline $\mathrm{Al}+3$ & $5.07 \mathrm{E}-01$ & $2.03 E+00$ & $8.61 \mathrm{E}-08$ & $8.61 \mathrm{E}-10$ & & & 6.61E-07 \\
\hline $\mathrm{Sb}+5$ & $1.58 \mathrm{E}-05$ & $6.34 \mathrm{E}-05$ & $1.22 \mathrm{E}-11$ & $1.22 \mathrm{E}-13$ & & & $2.07 \mathrm{E}-11$ \\
\hline As+5 & $8.88 \mathrm{E}-05$ & $3.55 \mathrm{E}-04$ & $4.19 \mathrm{E}-11$ & $4.19 \mathrm{E}-13$ & & & $1.16 \mathrm{E}-10$ \\
\hline $\mathrm{Ba}+2$ & $7.24 \mathrm{E}-05$ & $2.90 \mathrm{E}-04$ & $6.26 \mathrm{E}-11$ & $6.26 \mathrm{E}-13$ & & & $9.45 \mathrm{E}-11$ \\
\hline $\mathrm{Be}+2$ & $1.83 \mathrm{E}-05$ & $7.31 \mathrm{E}-05$ & $1.04 \mathrm{E}-12$ & $1.04 \mathrm{E}-14$ & & & $2.38 \mathrm{E}-11$ \\
\hline $\mathrm{B}+3$ & $1.59 \mathrm{E}-02$ & $6.35 \mathrm{E}-02$ & $1.08 \mathrm{E}-09$ & $1.08 \mathrm{E}-11$ & & & $2.07 \mathrm{E}-08$ \\
\hline $\mathrm{Cd}+2$ & $2.42 \mathrm{E}-03$ & $9.70 \mathrm{E}-03$ & 1.72E-09 & $1.72 \mathrm{E}-11$ & & & 3.16E-09 \\
\hline $\mathrm{Ca}+2$ & $4.83 \mathrm{E}-02$ & $1.93 \mathrm{E}-01$ & $1.22 \mathrm{E}-08$ & $1.22 \mathrm{E}-10$ & & & $6.30 \mathrm{E}-08$ \\
\hline $\mathrm{Cr}+3$ & $4.48 \mathrm{E}-03$ & 1.79E-02 & 1.47E-09 & $1.47 \mathrm{E}-11$ & & & $5.85 \mathrm{E}-09$ \\
\hline $\mathrm{Co}+2$ & 3.97E-05 & 1.59E-04 & $1.47 \mathrm{E}-11$ & $1.47 \mathrm{E}-13$ & & & $5.18 \mathrm{E}-11$ \\
\hline Cs+ & $5.75 \mathrm{E}-05$ & $2.30 \mathrm{E}-04$ & $4.82 \mathrm{E}-11$ & $4.82 \mathrm{E}-13$ & & & $7.51 \mathrm{E}-11$ \\
\hline $\mathrm{Cu}+2$ & $6.78 \mathrm{E}-04$ & $2.71 \mathrm{E}-03$ & $2.71 \mathrm{E}-10$ & $2.71 \mathrm{E}-12$ & & & $8.85 \mathrm{E}-10$ \\
\hline $\mathrm{Fe}+3$ & $2.49 \mathrm{E}-02$ & 9.98E-02 & 8.77E-09 & $8.77 \mathrm{E}-11$ & & & $3.25 \mathrm{E}-08$ \\
\hline $\mathrm{Pb}+2$ & $7.98 \mathrm{E}-04$ & $3.19 \mathrm{E}-03$ & 1.04E-09 & $1.04 \mathrm{E}-11$ & & & 1.04E-09 \\
\hline $\mathrm{Hg}+2$ & $5.11 \mathrm{E}-03$ & 1.93E-02 & 3.70E-06 & 3.70E-06 & $1.31 \mathrm{E}-11$ & $5.02 \mathrm{E}-10$ & 3.82E-04 \\
\hline$M n+4$ & $1.43 \mathrm{E}-02$ & $5.73 \mathrm{E}-02$ & 4.96E-09 & $4.96 \mathrm{E}-11$ & & & $1.87 \mathrm{E}-08$ \\
\hline $\mathrm{Ni}+2$ & $1.78 \mathrm{E}-03$ & $7.11 \mathrm{E}-03$ & $6.57 \mathrm{E}-10$ & $6.57 \mathrm{E}-12$ & & & $2.32 \mathrm{E}-09$ \\
\hline $\mathrm{K}+$ & 1.82E-01 & $7.29 \mathrm{E}-01$ & 4.49E-08 & $4.49 \mathrm{E}-10$ & & & $2.38 \mathrm{E}-07$ \\
\hline $\mathrm{Se}+4$ & $2.34 \mathrm{E}-04$ & 9.36E-04 & $1.16 \mathrm{E}-10$ & $1.16 \mathrm{E}-12$ & & & $3.05 \mathrm{E}-10$ \\
\hline $\mathrm{Ag}+$ & 3.64E-04 & $1.46 \mathrm{E}-03$ & $2.47 \mathrm{E}-10$ & $2.47 \mathrm{E}-12$ & & & $4.75 \mathrm{E}-10$ \\
\hline $\mathrm{Na}+$ & $1.35 \mathrm{E}+00$ & $5.41 \mathrm{E}+00$ & 1.96E-07 & 1.96E-09 & & & 1.76E-06 \\
\hline $\mathrm{TI}+3$ & $4.42 \mathrm{E}-06$ & 1.77E-05 & $5.70 \mathrm{E}-12$ & $5.70 \mathrm{E}-14$ & & & $5.77 \mathrm{E}-12$ \\
\hline $\mathrm{U}+4$ & $3.50 \mathrm{E}-04$ & $1.40 \mathrm{E}-03$ & $5.25 \mathrm{E}-10$ & $5.25 \mathrm{E}-12$ & & & $4.57 \mathrm{E}-10$ \\
\hline $\mathrm{V}+3$ & 3.62E-05 & $1.45 \mathrm{E}-04$ & $1.16 \mathrm{E}-11$ & $1.16 \mathrm{E}-13$ & & & $4.72 \mathrm{E}-11$ \\
\hline$Z n+2$ & $7.85 \mathrm{E}-04$ & $3.14 \mathrm{E}-03$ & $3.23 \mathrm{E}-10$ & $3.23 \mathrm{E}-12$ & & & 1.02E-09 \\
\hline$Z \mathrm{Zr}+4$ & $3.65 E-02$ & $1.46 \mathrm{E}-01$ & $2.09 \mathrm{E}-08$ & $2.09 \mathrm{E}-10$ & & & $4.76 \mathrm{E}-08$ \\
\hline $\mathrm{Cl}-$ & $2.39 \mathrm{E}-02$ & 7.56E-02 & 1.11E-05 & 1.11E-05 & $4.63 \mathrm{E}-12$ & $1.48 \mathrm{E}-09$ & $6.49 \mathrm{E}-03$ \\
\hline F- & $3.45 \mathrm{E}-02$ & $1.38 \mathrm{E}-01$ & $2.80 \mathrm{E}-08$ & $2.39 \mathrm{E}-08$ & & $3.17 \mathrm{E}-12$ & $2.61 \mathrm{E}-05$ \\
\hline SO4-2 & $4.05 \mathrm{E}-02$ & 1.62E-01 & $2.45 \mathrm{E}-08$ & $2.45 \mathrm{E}-10$ & & & 5.29E-08 \\
\hline NO3- & $5.35 \mathrm{E}+00$ & $1.34 \mathrm{E}+01$ & $7.77 \mathrm{E}-03$ & $7.76 \mathrm{E}-03$ & $3.57 \mathrm{E}-10$ & $1.03 E-06$ & $2.59 \mathrm{E}+00$ \\
\hline PO4-3 & $9.61 \mathrm{E}-02$ & $3.84 \mathrm{E}-01$ & $5.75 \mathrm{E}-08$ & $5.75 \mathrm{E}-10$ & & & $1.25 \mathrm{E}-07$ \\
\hline $\mathrm{Am}+4$ & $6.68 \mathrm{E}-08$ & 2.67E-07 & $1.02 \mathrm{E}-13$ & $1.02 \mathrm{E}-15$ & & & $8.72 \mathrm{E}-14$ \\
\hline $\mathrm{Br}-$ & $2.87 \mathrm{E}-07$ & 8.88E-07 & $3.30 \mathrm{E}-10$ & $3.30 \mathrm{E}-10$ & $1.38 \mathrm{E}-16$ & $4.39 \mathrm{E}-14$ & $8.55 \mathrm{E}-08$ \\
\hline $\mathrm{Ce}+4$ & 3.31E-05 & 1.32E-04 & $2.92 \mathrm{E}-11$ & $2.92 \mathrm{E}-13$ & & & $4.32 \mathrm{E}-11$ \\
\hline Eu+3 & 4.77E-07 & 1.91E-06 & $4.57 \mathrm{E}-13$ & $4.57 \mathrm{E}-15$ & & & $6.23 \mathrm{E}-13$ \\
\hline $\mathrm{Gd}+3$ & $1.41 \mathrm{E}-04$ & 5.63E-04 & $1.39 \mathrm{E}-10$ & $1.39 \mathrm{E}-12$ & & & $1.84 \mathrm{E}-10$ \\
\hline $\mathrm{Ge}+4$ & 8.29E-09 & 3.32E-08 & 3.79E-15 & $3.79 \mathrm{E}-17$ & & & $1.08 \mathrm{E}-14$ \\
\hline $\ln +3$ & $1.46 \mathrm{E}-06$ & 5.83E-06 & $1.05 \mathrm{E}-12$ & $1.05 \mathrm{E}-14$ & & & $1.90 \mathrm{E}-12$ \\
\hline I- & 3.07E-06 & $1.01 \mathrm{E}-05$ & 4.35E-09 & 4.35E-09 & $1.82 \mathrm{E}-15$ & $5.78 \mathrm{E}-13$ & $7.10 \mathrm{E}-07$ \\
\hline $\mathrm{La+3}$ & $8.66 \mathrm{E}-06$ & $3.47 \mathrm{E}-05$ & $7.58 \mathrm{E}-12$ & $7.58 \mathrm{E}-14$ & & & $1.13 \mathrm{E}-11$ \\
\hline Li+ & $4.52 \mathrm{E}-04$ & $1.81 \mathrm{E}-03$ & $1.98 \mathrm{E}-11$ & $1.98 \mathrm{E}-13$ & & & $5.90 \mathrm{E}-10$ \\
\hline $\mathrm{Mg}+2$ & $1.78 \mathrm{E}-02$ & $7.10 \mathrm{E}-02$ & $2.72 \mathrm{E}-09$ & $2.72 \mathrm{E}-11$ & & & $2.32 \mathrm{E}-08$ \\
\hline $\mathrm{Mo}+6$ & $7.29 \mathrm{E}-04$ & $2.92 \mathrm{E}-03$ & $4.40 \mathrm{E}-10$ & $4.40 \mathrm{E}-12$ & & & $9.51 \mathrm{E}-10$ \\
\hline
\end{tabular}


Table C-6. Revised baseline mass balance, Tank WM-188 waste (Continued).

\begin{tabular}{|c|c|c|c|c|c|c|c|}
\hline Stream \# & 101 & 102 & 103 & 104 & 105 & 106 & 107 \\
\hline Stream Name & $\begin{array}{l}\text { SBW } \\
\text { Feed }\end{array}$ & $\begin{array}{c}\text { Evaporator } \\
\text { Bottoms }\end{array}$ & $\begin{array}{c}\text { Evaporator } \\
\text { Overheads } \\
\text { Vapor }\end{array}$ & $\begin{array}{c}\text { Mist } \\
\text { Eliminator } \\
\text { Vapor }\end{array}$ & $\begin{array}{l}\text { VOG } \\
\text { Offgas } \\
\text { Vapor }\end{array}$ & $\begin{array}{c}\text { POG + VOG } \\
\text { Offgas } \\
\text { Vapor }\end{array}$ & $\begin{array}{l}\text { LET\&D } \\
\text { Feed } \\
\text { Liquid }\end{array}$ \\
\hline Chemical Composition & Mol/Liter & Mol/Liter & $\mathrm{lb} / \mathrm{wscf}$ & $\mathrm{lb} /$ wscf & $\mathrm{Ib} / \mathrm{wscf}$ & $\mathrm{lb} /$ wscf & Mol/Liter \\
\hline $\mathrm{Nd}+3$ & $2.79 \mathrm{E}-05$ & 1.12E-04 & $2.54 \mathrm{E}-11$ & $2.54 \mathrm{E}-13$ & & & $3.64 \mathrm{E}-11$ \\
\hline $\mathrm{Np}+4$ & $2.55 \mathrm{E}-06$ & 1.02E-05 & $3.81 \mathrm{E}-12$ & $3.81 \mathrm{E}-14$ & & & $3.33 \mathrm{E}-12$ \\
\hline $\mathrm{Nb}+5$ & 1.05E-03 & 4.19E-03 & $6.12 \mathrm{E}-10$ & $6.12 \mathrm{E}-12$ & & & 1.37E-09 \\
\hline $\mathrm{Pd}+4$ & $1.70 \mathrm{E}-03$ & $6.79 \mathrm{E}-03$ & 1.14E-09 & $1.14 \mathrm{E}-11$ & & & $2.22 \mathrm{E}-09$ \\
\hline $\mathrm{Pu}+4$ & $6.59 \mathrm{E}-06$ & $2.64 \mathrm{E}-05$ & $1.01 \mathrm{E}-11$ & $1.01 \mathrm{E}-13$ & & & $8.60 \mathrm{E}-12$ \\
\hline $\mathrm{Pr}+4$ & 7.87E-06 & $3.15 \mathrm{E}-05$ & $6.99 \mathrm{E}-12$ & $6.99 \mathrm{E}-14$ & & & $1.03 \mathrm{E}-11$ \\
\hline $\mathrm{Rh}+4$ & $3.40 \mathrm{E}-06$ & 1.36E-05 & $2.20 \mathrm{E}-12$ & $2.20 \mathrm{E}-14$ & & & $4.43 \mathrm{E}-12$ \\
\hline $\mathrm{Rb}+$ & $5.23 \mathrm{E}-06$ & 2.09E-05 & $2.81 \mathrm{E}-12$ & $2.81 \mathrm{E}-14$ & & & $6.82 \mathrm{E}-12$ \\
\hline $\mathrm{Ru}+3$ & $7.86 \mathrm{E}-04$ & $3.14 \mathrm{E}-03$ & $5.00 \mathrm{E}-10$ & $5.00 \mathrm{E}-12$ & & & 1.03E-09 \\
\hline $\mathrm{Sm+3}$ & $5.20 \mathrm{E}-06$ & $2.08 \mathrm{E}-05$ & $4.93 \mathrm{E}-12$ & $4.93 \mathrm{E}-14$ & & & $6.79 \mathrm{E}-12$ \\
\hline $\mathrm{Si+4}$ & 8.92E-02 & 3.57E-01 & $1.58 \mathrm{E}-08$ & $1.58 \mathrm{E}-10$ & & & 1.16E-07 \\
\hline $\mathrm{Sr}+2$ & 7.77E-05 & $3.11 \mathrm{E}-04$ & $4.29 \mathrm{E}-11$ & $4.29 \mathrm{E}-13$ & & & $1.01 \mathrm{E}-10$ \\
\hline $\mathrm{Tc}+7$ & 1.01E-05 & 4.05E-05 & $6.19 \mathrm{E}-12$ & $6.19 \mathrm{E}-14$ & & & $1.32 \mathrm{E}-11$ \\
\hline $\mathrm{Te}+4$ & $3.46 \mathrm{E}-06$ & $1.38 \mathrm{E}-05$ & $2.78 \mathrm{E}-12$ & $2.78 \mathrm{E}-14$ & & & $4.51 \mathrm{E}-12$ \\
\hline $\mathrm{Tb}+4$ & 1.99E-09 & 7.98E-09 & $2.00 \mathrm{E}-15$ & $2.00 \mathrm{E}-17$ & & & $2.60 \mathrm{E}-15$ \\
\hline $\mathrm{Th}+4$ & $2.29 \mathrm{E}-05$ & $9.18 \mathrm{E}-05$ & $3.35 \mathrm{E}-11$ & $3.35 \mathrm{E}-13$ & & & $2.99 \mathrm{E}-11$ \\
\hline $\mathrm{Sn}+4$ & 1.73E-03 & $6.91 \mathrm{E}-03$ & 1.29E-09 & $1.29 \mathrm{E}-11$ & & & $2.25 \mathrm{E}-09$ \\
\hline $\mathrm{Ti}+4$ & 8.83E-04 & $3.53 \mathrm{E}-03$ & $2.66 \mathrm{E}-10$ & $2.66 \mathrm{E}-12$ & & & 1.15E-09 \\
\hline $\mathrm{Y}+3$ & $6.46 \mathrm{E}-06$ & $2.58 \mathrm{E}-05$ & $3.62 \mathrm{E}-12$ & $3.62 \mathrm{E}-14$ & & & $8.43 \mathrm{E}-12$ \\
\hline O-2 (oxides) & 1.41E-01 & $5.63 \mathrm{E}-01$ & 1.42E-08 & $1.42 \mathrm{E}-10$ & & & $1.84 \mathrm{E}-07$ \\
\hline $\mathrm{H} 2 \mathrm{O}$ & $4.63 \mathrm{E}+01$ & $3.20 \mathrm{E}+01$ & 4.47E-02 & $4.47 \mathrm{E}-02$ & 1.36E-03 & 1.17E-03 & $5.14 \mathrm{E}+01$ \\
\hline $\mathrm{O} 2$ & & $1.62 \mathrm{E}-10$ & 1.54E-07 & 1.54E-07 & 1.69E-02 & 1.70E-02 & $6.56 \mathrm{E}-05$ \\
\hline N2 & & $1.95 \mathrm{E}-10$ & $2.57 \mathrm{E}-07$ & $2.57 \mathrm{E}-07$ & $5.58 \mathrm{E}-02$ & $5.60 \mathrm{E}-02$ & 9.51E-05 \\
\hline C (organic) & 6.89E-02 & $2.73 \mathrm{E}-01$ & 5.03E-07 & 4.99E-07 & $8.19 \mathrm{E}-13$ & $6.69 \mathrm{E}-11$ & 8.62E-04 \\
\hline $\mathrm{H}$ (organic) & 6.89E-02 & $2.73 \mathrm{E}-01$ & $4.22 \mathrm{E}-08$ & 4.19E-08 & $6.88 \mathrm{E}-14$ & $5.62 \mathrm{E}-12$ & $8.62 \mathrm{E}-04$ \\
\hline \multicolumn{8}{|l|}{ SiO2, BFS, or OPC Additives } \\
\hline $\begin{array}{l}\text { Gas Stream Bulk Composition } \\
\text { (wet basis) }\end{array}$ & $\begin{array}{c}\text { mol.\% } \\
\text { or ppmv }\end{array}$ & $\begin{array}{l}\text { mol. } \% \\
\text { or ppmv }\end{array}$ & $\begin{array}{l}\text { mol. } \% \\
\text { or ppmv }\end{array}$ & $\begin{array}{l}\text { mol. } \% \\
\text { or ppmv }\end{array}$ & $\begin{array}{l}\text { mol. } \% \\
\text { or ppmv }\end{array}$ & $\begin{array}{l}\text { mol. } \% \\
\text { or ppmv }\end{array}$ & $\begin{array}{l}\text { mol. } \% \\
\text { or ppmv }\end{array}$ \\
\hline $\mathrm{H} 2 \mathrm{O}, \mathrm{mol} . \%$ & & & 95.18 & 95.18 & 2.91 & 2.50 & \\
\hline $\mathrm{O} 2, \mathrm{~mol} . \%$ & & & 0.0002 & 0.0002 & 20.39 & 20.47 & \\
\hline $\mathrm{N} 2, \mathrm{~mol} . \%$ & & & 0.0004 & 0.0004 & 76.70 & 77.02 & \\
\hline \multicolumn{8}{|l|}{$\mathrm{CO} 2, \mathrm{~mol} . \%$} \\
\hline HNO3, mol. \% & & & 4.82 & 4.82 & $2.2 \mathrm{E}-07$ & $6.4 \mathrm{E}-04$ & \\
\hline \multicolumn{8}{|l|}{$\mathrm{CO}, \mathrm{ppmv}$} \\
\hline \multicolumn{8}{|l|}{$\mathrm{NO}, \mathrm{ppmv}$} \\
\hline \multicolumn{8}{|l|}{ NO2, ppmv } \\
\hline C (organic), ppmv & & & 16 & 16 & $2.6 \mathrm{E}-05$ & $2.1 \mathrm{E}-03$ & \\
\hline $\mathrm{H}$ (organic), ppmv & & & 16 & 16 & $2.6 \mathrm{E}-05$ & $2.1 \mathrm{E}-03$ & \\
\hline $\mathrm{PM}$ (mg/wscm) & & & 4 & 0.04 & & & \\
\hline $\begin{array}{l}\text { Gas Stream Bulk Composition } \\
\text { (dry basis) }\end{array}$ & $\begin{array}{c}\text { mol.\% } \\
\text { or ppmv }\end{array}$ & $\begin{array}{c}\text { mol.\% } \\
\text { or ppmv }\end{array}$ & $\begin{array}{c}\text { mol.\% } \\
\text { or ppmv }\end{array}$ & $\begin{array}{c}\text { mol.\% } \\
\text { or ppmv }\end{array}$ & $\begin{array}{c}\text { mol.\% } \\
\text { or ppmv }\end{array}$ & $\begin{array}{c}\text { mol. } \% \\
\text { or ppmv }\end{array}$ & $\begin{array}{l}\text { mol. } \% \\
\text { or ppmv }\end{array}$ \\
\hline $\mathrm{O} 2, \mathrm{~mol} . \%$ & & & 0.00 & 0.00 & 21.00 & 21.00 & \\
\hline $\mathrm{N} 2, \mathrm{~mol} . \%$ & & & 0.01 & 0.01 & 79.00 & 79.00 & \\
\hline \multicolumn{8}{|l|}{$\mathrm{CO} 2, \mathrm{~mol} . \%$} \\
\hline HNO3, mol. \% & & & 99.93 & 99.96 & $2.3 \mathrm{E}-07$ & $6.6 \mathrm{E}-04$ & \\
\hline \multicolumn{8}{|l|}{$\mathrm{CO}, \mathrm{ppmv}$} \\
\hline \multicolumn{8}{|l|}{$\mathrm{NO}, \mathrm{ppmv}$} \\
\hline \multicolumn{8}{|l|}{ NO2, ppmv } \\
\hline C (organic), ppmv & & & 334 & 331 & 2.7E-05 & $2.2 \mathrm{E}-03$ & \\
\hline $\mathrm{H}$ (organic), ppmv & & & 334 & 331 & 2.7E-05 & $2.2 \mathrm{E}-03$ & \\
\hline $\mathrm{PM}(\mathrm{mg} / \mathrm{dscm})$ & & & 84 & 1 & & & \\
\hline
\end{tabular}


Table C-6. Revised baseline mass balance, Tank WM-188 waste (Continued).

\begin{tabular}{|c|c|c|c|c|c|c|c|}
\hline Stream \# & 101 & 102 & 103 & 104 & 105 & 106 & 107 \\
\hline Stream Name & $\begin{array}{l}\text { SBW } \\
\text { Feed }\end{array}$ & $\begin{array}{c}\text { Evaporator } \\
\text { Bottoms }\end{array}$ & $\begin{array}{c}\text { Evaporator } \\
\text { Overheads } \\
\text { Vapor }\end{array}$ & $\begin{array}{c}\text { Mist } \\
\text { Eliminator } \\
\text { Vapor }\end{array}$ & $\begin{array}{l}\text { VOG } \\
\text { Offgas } \\
\text { Vapor }\end{array}$ & $\begin{array}{c}\text { POG + VOG } \\
\text { Offgas } \\
\text { Vapor }\end{array}$ & $\begin{array}{l}\text { LET\&D } \\
\text { Feed } \\
\text { Liquid }\end{array}$ \\
\hline Canister Rate, canisters/day & & 2.43 & & & & & \\
\hline Total Canisters Generated & & 354 & & & & & \\
\hline \multicolumn{8}{|l|}{ Drum Rate, drums/day } \\
\hline \multicolumn{8}{|l|}{ Total Drums Generated } \\
\hline Radiological Composition & $\mathrm{Ci} / \mathrm{L}$ & $\mathrm{Ci} / \mathrm{L}$ & $\mathrm{Ci} / \mathrm{wscm}$ & $\mathrm{Ci} / \mathrm{wscm}$ & $\mathrm{Ci} / \mathrm{wscm}$ & $\mathrm{Ci} / \mathrm{wscm}$ & $\mathrm{Ci} / \mathrm{L}$ \\
\hline Ra-226 & $8.48 \mathrm{E}-12$ & $3.39 \mathrm{E}-11$ & $8.56 \mathrm{E}-16$ & $8.55 \mathrm{E}-18$ & $2.65 \mathrm{E}-25$ & $2.29 \mathrm{E}-25$ & $1.11 \mathrm{E}-17$ \\
\hline Ac-227 & $3.99 \mathrm{E}-11$ & $1.60 \mathrm{E}-10$ & $4.03 E-15$ & $4.03 \mathrm{E}-17$ & $1.25 \mathrm{E}-24$ & $1.08 \mathrm{E}-24$ & $5.21 \mathrm{E}-17$ \\
\hline Th-228 & 3.31E-09 & $1.33 \mathrm{E}-08$ & $3.34 \mathrm{E}-13$ & $3.34 \mathrm{E}-15$ & $1.04 \mathrm{E}-22$ & $8.96 \mathrm{E}-23$ & $4.32 \mathrm{E}-15$ \\
\hline Th-230 & 1.06E-09 & 4.23E-09 & $1.07 \mathrm{E}-13$ & $1.07 \mathrm{E}-15$ & $3.30 \mathrm{E}-23$ & $2.86 \mathrm{E}-23$ & $1.38 \mathrm{E}-15$ \\
\hline Th-232 & $7.33 \mathrm{E}-16$ & $2.93 E-15$ & $7.40 \mathrm{E}-20$ & $7.40 \mathrm{E}-22$ & $2.29 \mathrm{E}-29$ & $1.98 \mathrm{E}-29$ & $9.57 \mathrm{E}-22$ \\
\hline Pa-231 & $9.25 \mathrm{E}-11$ & $3.70 \mathrm{E}-10$ & $9.33 \mathrm{E}-15$ & $9.33 \mathrm{E}-17$ & $2.89 \mathrm{E}-24$ & $2.50 \mathrm{E}-24$ & $1.21 \mathrm{E}-16$ \\
\hline Pa-233 & 3.03E-06 & 1.21E-05 & $3.06 \mathrm{E}-10$ & $3.06 \mathrm{E}-12$ & $9.48 \mathrm{E}-20$ & $8.20 \mathrm{E}-20$ & $3.96 \mathrm{E}-12$ \\
\hline $\mathrm{U}-232$ & 2.49E-09 & 9.96E-09 & $2.51 \mathrm{E}-13$ & $2.51 \mathrm{E}-15$ & $7.77 \mathrm{E}-23$ & $6.73 \mathrm{E}-23$ & $3.25 \mathrm{E}-15$ \\
\hline U-233 & 9.02E-11 & $3.61 \mathrm{E}-10$ & $9.10 \mathrm{E}-15$ & $9.10 \mathrm{E}-17$ & $2.82 \mathrm{E}-24$ & $2.44 \mathrm{E}-24$ & $1.18 \mathrm{E}-16$ \\
\hline U-234 & 1.11E-06 & 4.45E-06 & $1.12 \mathrm{E}-10$ & $1.12 \mathrm{E}-12$ & $3.47 \mathrm{E}-20$ & $3.00 \mathrm{E}-20$ & $1.45 \mathrm{E}-12$ \\
\hline U-235 & $8.17 \mathrm{E}-08$ & 3.27E-07 & $8.24 \mathrm{E}-12$ & $8.24 \mathrm{E}-14$ & $2.55 \mathrm{E}-21$ & $2.21 \mathrm{E}-21$ & 1.07E-13 \\
\hline U-236 & 4.79E-08 & 1.92E-07 & $4.84 \mathrm{E}-12$ & $4.84 \mathrm{E}-14$ & $1.50 \mathrm{E}-21$ & 1.30E-21 & $6.26 \mathrm{E}-14$ \\
\hline U-237 & 6.66E-09 & $2.67 \mathrm{E}-08$ & $6.72 \mathrm{E}-13$ & $6.72 \mathrm{E}-15$ & $2.08 \mathrm{E}-22$ & $1.80 \mathrm{E}-22$ & 8.69E-15 \\
\hline $\mathrm{U}-238$ & $1.46 \mathrm{E}-08$ & 5.83E-08 & $1.47 \mathrm{E}-12$ & $1.47 \mathrm{E}-14$ & $4.55 \mathrm{E}-22$ & 3.94E-22 & $1.90 \mathrm{E}-14$ \\
\hline Np-236 & $3.19 \mathrm{E}-12$ & $1.28 \mathrm{E}-11$ & $3.22 \mathrm{E}-16$ & $3.22 \mathrm{E}-18$ & 9.97E-26 & $8.63 \mathrm{E}-26$ & $4.16 \mathrm{E}-18$ \\
\hline Np-237 & 4.33E-07 & 1.73E-06 & $4.37 \mathrm{E}-11$ & $4.37 \mathrm{E}-13$ & $1.35 \mathrm{E}-20$ & 1.17E-20 & $5.66 \mathrm{E}-13$ \\
\hline Pu-236 & 3.63E-09 & $1.45 \mathrm{E}-08$ & $3.66 \mathrm{E}-13$ & $3.66 \mathrm{E}-15$ & $1.13 \mathrm{E}-22$ & 9.82E-23 & $4.74 \mathrm{E}-15$ \\
\hline Pu-238 & 8.27E-04 & 3.31E-03 & 8.35E-08 & $8.34 \mathrm{E}-10$ & $2.58 \mathrm{E}-17$ & $2.24 \mathrm{E}-17$ & 1.08E-09 \\
\hline \begin{tabular}{|l} 
Pu-239 \\
\end{tabular} & $9.26 \mathrm{E}-05$ & 3.70E-04 & 9.34E-09 & $9.34 \mathrm{E}-11$ & $2.89 \mathrm{E}-18$ & $2.50 \mathrm{E}-18$ & $1.21 \mathrm{E}-10$ \\
\hline Pu-240 & 1.35E-05 & 5.41E-05 & 1.36E-09 & $1.36 \mathrm{E}-11$ & $4.23 \mathrm{E}-19$ & $3.66 \mathrm{E}-19$ & $1.77 \mathrm{E}-11$ \\
\hline \begin{tabular}{|l} 
Pu-241 \\
\end{tabular} & $6.01 \mathrm{E}-04$ & $2.41 \mathrm{E}-03$ & 6.07E-08 & $6.07 \mathrm{E}-10$ & $1.88 \mathrm{E}-17$ & $1.63 \mathrm{E}-17$ & $7.85 \mathrm{E}-10$ \\
\hline Pu-242 & 1.06E-08 & 4.24E-08 & $1.07 \mathrm{E}-12$ & $1.07 \mathrm{E}-14$ & $3.31 \mathrm{E}-22$ & $2.86 \mathrm{E}-22$ & $1.38 \mathrm{E}-14$ \\
\hline Pu-244 & $8.08 \mathrm{E}-16$ & $3.23 E-15$ & $8.16 \mathrm{E}-20$ & $8.15 E-22$ & $2.53 \mathrm{E}-29$ & $2.18 E-29$ & $1.05 \mathrm{E}-21$ \\
\hline Am-241 & 5.83E-05 & $2.33 \mathrm{E}-04$ & 5.88E-09 & $5.88 \mathrm{E}-11$ & $1.82 \mathrm{E}-18$ & $1.58 \mathrm{E}-18$ & $7.61 \mathrm{E}-11$ \\
\hline Am-242m & $1.61 \mathrm{E}-08$ & $6.46 \mathrm{E}-08$ & $1.63 \mathrm{E}-12$ & $1.63 \mathrm{E}-14$ & $5.05 \mathrm{E}-22$ & 4.37E-22 & $2.11 \mathrm{E}-14$ \\
\hline Am-243 & $2.63 \mathrm{E}-08$ & $1.05 \mathrm{E}-07$ & $2.65 \mathrm{E}-12$ & $2.65 \mathrm{E}-14$ & $8.21 \mathrm{E}-22$ & $7.11 \mathrm{E}-22$ & $3.43 \mathrm{E}-14$ \\
\hline $\mathrm{Cm}-242$ & $3.50 \mathrm{E}-08$ & 1.40E-07 & $3.53 \mathrm{E}-12$ & $3.53 \mathrm{E}-14$ & $1.09 \mathrm{E}-21$ & $9.46 \mathrm{E}-22$ & $4.56 \mathrm{E}-14$ \\
\hline $\mathrm{Cm}-243$ & $3.65 \mathrm{E}-08$ & $1.46 \mathrm{E}-07$ & $3.69 \mathrm{E}-12$ & $3.69 \mathrm{E}-14$ & $1.14 \mathrm{E}-21$ & $9.88 \mathrm{E}-22$ & $4.77 \mathrm{E}-14$ \\
\hline $\mathrm{Cm}-244$ & 1.27E-06 & $5.10 \mathrm{E}-06$ & $1.29 \mathrm{E}-10$ & $1.29 \mathrm{E}-12$ & $3.98 \mathrm{E}-20$ & $3.44 \mathrm{E}-20$ & $1.66 \mathrm{E}-12$ \\
\hline Cm-245 & $3.85 \mathrm{E}-10$ & 1.54E-09 & $3.88 \mathrm{E}-14$ & $3.88 \mathrm{E}-16$ & $1.20 \mathrm{E}-23$ & 1.04E-23 & $5.02 \mathrm{E}-16$ \\
\hline Cm-246 & $2.53 \mathrm{E}-11$ & $1.01 \mathrm{E}-10$ & $2.55 \mathrm{E}-15$ & $2.55 \mathrm{E}-17$ & $7.89 \mathrm{E}-25$ & $6.83 \mathrm{E}-25$ & 3.30E-17 \\
\hline $\mathrm{Cm}-247$ & $5.52 \mathrm{E}-18$ & $2.21 \mathrm{E}-17$ & $5.57 \mathrm{E}-22$ & $5.57 \mathrm{E}-24$ & & & $7.20 \mathrm{E}-24$ \\
\hline $\mathrm{H}-3$ & 1.18E-05 & 7.94E-06 & 9.86E-06 & 9.86E-06 & $7.68 \mathrm{E}-08$ & $6.71 \mathrm{E}-08$ & $1.28 \mathrm{E}-05$ \\
\hline Be-10 & $3.11 \mathrm{E}-12$ & $1.24 \mathrm{E}-11$ & $3.13 \mathrm{E}-16$ & $3.13 \mathrm{E}-18$ & $9.70 \mathrm{E}-26$ & $8.40 \mathrm{E}-26$ & $4.05 \mathrm{E}-18$ \\
\hline C-14 & $1.59 \mathrm{E}-10$ & $6.37 \mathrm{E}-10$ & $1.61 \mathrm{E}-14$ & $1.61 \mathrm{E}-16$ & 4.97E-24 & $4.30 \mathrm{E}-24$ & $2.08 \mathrm{E}-16$ \\
\hline Co-60 & 8.97E-06 & 3.59E-05 & $9.05 \mathrm{E}-10$ & $9.05 \mathrm{E}-12$ & $2.80 \mathrm{E}-19$ & $2.43 \mathrm{E}-19$ & 1.17E-11 \\
\hline $\mathrm{Ni}-59$ & 1.10E-06 & 4.41E-06 & $1.11 \mathrm{E}-10$ & $1.11 \mathrm{E}-12$ & $3.44 \mathrm{E}-20$ & $2.98 \mathrm{E}-20$ & $1.44 \mathrm{E}-12$ \\
\hline $\mathrm{Ni}-63$ & $4.22 \mathrm{E}-05$ & 1.69E-04 & $4.26 \mathrm{E}-09$ & $4.26 \mathrm{E}-11$ & $1.32 \mathrm{E}-18$ & $1.14 \mathrm{E}-18$ & $5.51 \mathrm{E}-11$ \\
\hline Se-79 & $5.78 \mathrm{E}-07$ & $2.31 \mathrm{E}-06$ & $5.83 \mathrm{E}-11$ & $5.83 \mathrm{E}-13$ & $1.81 \mathrm{E}-20$ & $1.56 \mathrm{E}-20$ & $7.54 \mathrm{E}-13$ \\
\hline Rb-87 & $3.03 \mathrm{E}-11$ & $1.21 \mathrm{E}-10$ & $3.06 \mathrm{E}-15$ & $3.06 \mathrm{E}-17$ & $9.48 \mathrm{E}-25$ & $8.20 \mathrm{E}-25$ & $3.96 \mathrm{E}-17$ \\
\hline Sr-90 & $3.98 \mathrm{E}-02$ & $1.59 \mathrm{E}-01$ & $4.02 \mathrm{E}-06$ & $4.02 E-08$ & $1.24 \mathrm{E}-15$ & $1.08 \mathrm{E}-15$ & $5.20 \mathrm{E}-08$ \\
\hline $\mathrm{Y}-90$ & 3.98E-02 & 1.59E-01 & $4.02 E-06$ & $4.02 \mathrm{E}-08$ & $1.24 \mathrm{E}-15$ & $1.08 \mathrm{E}-15$ & $5.20 \mathrm{E}-08$ \\
\hline Zr-93 & $2.29 \mathrm{E}-06$ & $9.18 \mathrm{E}-06$ & $2.31 \mathrm{E}-10$ & $2.31 \mathrm{E}-12$ & $7.17 \mathrm{E}-20$ & $6.20 \mathrm{E}-20$ & $2.99 \mathrm{E}-12$ \\
\hline $\mathrm{Nb}-93 \mathrm{~m}$ & 1.77E-06 & 7.07E-06 & $1.78 \mathrm{E}-10$ & $1.78 \mathrm{E}-12$ & $5.52 \mathrm{E}-20$ & $4.78 \mathrm{E}-20$ & $2.31 \mathrm{E}-12$ \\
\hline $\mathrm{Nb}-94$ & $1.50 \mathrm{E}-06$ & $6.01 \mathrm{E}-06$ & $1.52 \mathrm{E}-10$ & $1.52 \mathrm{E}-12$ & $4.70 \mathrm{E}-20$ & $4.06 \mathrm{E}-20$ & $1.96 \mathrm{E}-12$ \\
\hline Tc-98 & $2.67 \mathrm{E}-12$ & $1.07 \mathrm{E}-11$ & $2.69 \mathrm{E}-16$ & $2.69 \mathrm{E}-18$ & $8.34 \mathrm{E}-26$ & $7.22 \mathrm{E}-26$ & $3.48 \mathrm{E}-18$ \\
\hline Tc-99 & $2.09 \mathrm{E}-05$ & 8.37E-05 & $2.11 \mathrm{E}-09$ & $2.11 \mathrm{E}-11$ & $6.53 \mathrm{E}-19$ & $5.65 \mathrm{E}-19$ & $2.73 \mathrm{E}-11$ \\
\hline
\end{tabular}


Table C-6. Revised baseline mass balance, Tank WM-188 waste (Continued).

\begin{tabular}{|c|c|c|c|c|c|c|c|}
\hline Stream \# & 101 & 102 & 103 & 104 & 105 & 106 & 107 \\
\hline Stream Name & $\begin{array}{l}\text { SBW } \\
\text { Feed }\end{array}$ & $\begin{array}{c}\text { Evaporator } \\
\text { Bottoms }\end{array}$ & $\begin{array}{c}\text { Evaporator } \\
\text { Overheads } \\
\text { Vapor }\end{array}$ & $\begin{array}{c}\text { Mist } \\
\text { Eliminator } \\
\text { Vapor }\end{array}$ & $\begin{array}{l}\text { VOG } \\
\text { Offgas } \\
\text { Vapor }\end{array}$ & $\begin{array}{c}\text { POG + VOG } \\
\text { Offgas } \\
\text { Vapor }\end{array}$ & $\begin{array}{l}\text { LET\&D } \\
\text { Feed } \\
\text { Liquid }\end{array}$ \\
\hline Radiological Composition & $\mathrm{Ci} / \mathrm{L}$ & $\mathrm{Ci} / \mathrm{L}$ & $\mathrm{Ci} / \mathrm{wscm}$ & $\mathrm{Ci} / \mathrm{wscm}$ & $\mathrm{Ci} / \mathrm{wscm}$ & $\mathrm{Ci} / \mathrm{wscm}$ & $\mathrm{Ci} / \mathrm{L}$ \\
\hline Ru-106 & 1.23E-06 & 4.94E-06 & $1.25 \mathrm{E}-10$ & $1.25 \mathrm{E}-12$ & $3.86 \mathrm{E}-20$ & $3.34 \mathrm{E}-20$ & $1.61 \mathrm{E}-12$ \\
\hline Rh-102 & $8.92 \mathrm{E}-10$ & 3.57E-09 & $9.00 \mathrm{E}-14$ & $9.00 \mathrm{E}-16$ & $2.79 \mathrm{E}-23$ & $2.41 \mathrm{E}-23$ & $1.16 \mathrm{E}-15$ \\
\hline Rh-106 & $1.23 \mathrm{E}-06$ & 4.94E-06 & $1.25 \mathrm{E}-10$ & $1.25 \mathrm{E}-12$ & $3.86 \mathrm{E}-20$ & $3.34 \mathrm{E}-20$ & $1.61 \mathrm{E}-12$ \\
\hline $\mathrm{Pd}-107$ & $1.71 \mathrm{E}-08$ & $6.84 \mathrm{E}-08$ & $1.73 \mathrm{E}-12$ & $1.73 \mathrm{E}-14$ & $5.34 \mathrm{E}-22$ & $4.62 \mathrm{E}-22$ & $2.23 \mathrm{E}-14$ \\
\hline Cd-113m & $3.44 \mathrm{E}-06$ & 1.38E-05 & $3.47 \mathrm{E}-10$ & $3.47 \mathrm{E}-12$ & 1.07E-19 & $9.29 \mathrm{E}-20$ & $4.49 \mathrm{E}-12$ \\
\hline $\ln -115$ & $1.04 \mathrm{E}-16$ & $4.17 \mathrm{E}-16$ & $1.05 \mathrm{E}-20$ & $1.05 \mathrm{E}-22$ & $3.26 \mathrm{E}-30$ & $2.82 \mathrm{E}-30$ & $1.36 \mathrm{E}-22$ \\
\hline Sn-121m & $6.92 \mathrm{E}-08$ & $2.77 \mathrm{E}-07$ & $6.98 \mathrm{E}-12$ & $6.98 \mathrm{E}-14$ & $2.16 \mathrm{E}-21$ & 1.87E-21 & $9.03 \mathrm{E}-14$ \\
\hline Sn-126 & 5.44E-07 & $2.18 \mathrm{E}-06$ & $5.49 \mathrm{E}-11$ & $5.49 \mathrm{E}-13$ & $1.70 \mathrm{E}-20$ & 1.47E-20 & $7.10 \mathrm{E}-13$ \\
\hline Sb-125 & $3.69 \mathrm{E}-04$ & $1.48 \mathrm{E}-03$ & $3.72 \mathrm{E}-08$ & $3.72 \mathrm{E}-10$ & $1.15 \mathrm{E}-17$ & $9.97 \mathrm{E}-18$ & $4.81 \mathrm{E}-10$ \\
\hline Sb-126 & $5.95 \mathrm{E}-08$ & $2.38 \mathrm{E}-07$ & $6.01 \mathrm{E}-12$ & $6.01 \mathrm{E}-14$ & $1.86 \mathrm{E}-21$ & $1.61 \mathrm{E}-21$ & $7.77 \mathrm{E}-14$ \\
\hline Te-123 & $3.97 \mathrm{E}-19$ & $1.59 \mathrm{E}-18$ & $4.00 \mathrm{E}-23$ & $4.00 \mathrm{E}-25$ & & & $5.18 \mathrm{E}-25$ \\
\hline $\mathrm{Te}-125 \mathrm{~m}$ & $3.26 \mathrm{E}-06$ & 1.30E-05 & $3.29 \mathrm{E}-10$ & $3.29 \mathrm{E}-12$ & $1.02 \mathrm{E}-19$ & $8.81 \mathrm{E}-20$ & $4.25 \mathrm{E}-12$ \\
\hline $\mathrm{I}-129$ & $7.06 \mathrm{E}-08$ & $2.24 \mathrm{E}-07$ & $1.48 \mathrm{E}-08$ & $1.48 \mathrm{E}-08$ & $6.19 \mathrm{E}-15$ & $1.97 \mathrm{E}-12$ & $1.92 \mathrm{E}-08$ \\
\hline Cs-134 & $3.69 \mathrm{E}-05$ & $1.48 \mathrm{E}-04$ & $3.72 \mathrm{E}-09$ & $3.72 \mathrm{E}-11$ & $1.15 \mathrm{E}-18$ & $9.97 \mathrm{E}-19$ & $4.81 \mathrm{E}-11$ \\
\hline Cs-135 & $1.11 \mathrm{E}-06$ & $4.45 E-06$ & $1.12 \mathrm{E}-10$ & $1.12 \mathrm{E}-12$ & $3.47 \mathrm{E}-20$ & $3.00 \mathrm{E}-20$ & $1.45 \mathrm{E}-12$ \\
\hline Cs-137 & $2.28 \mathrm{E}-02$ & $9.12 \mathrm{E}-02$ & $2.30 \mathrm{E}-06$ & $2.30 \mathrm{E}-08$ & $7.12 \mathrm{E}-16$ & $6.16 \mathrm{E}-16$ & $2.97 \mathrm{E}-08$ \\
\hline Ba-137m & $2.16 \mathrm{E}-02$ & $8.63 \mathrm{E}-02$ & $2.18 \mathrm{E}-06$ & $2.18 \mathrm{E}-08$ & $6.74 \mathrm{E}-16$ & $5.83 \mathrm{E}-16$ & $2.81 \mathrm{E}-08$ \\
\hline La-138 & $1.98 \mathrm{E}-16$ & $7.91 \mathrm{E}-16$ & 1.99E-20 & $1.99 \mathrm{E}-22$ & $6.17 \mathrm{E}-30$ & $5.34 \mathrm{E}-30$ & $2.58 \mathrm{E}-22$ \\
\hline Ce-142 & $3.09 \mathrm{E}-11$ & $1.24 \mathrm{E}-10$ & $3.12 \mathrm{E}-15$ & $3.12 \mathrm{E}-17$ & 9.67E-25 & 8.37E-25 & $4.04 \mathrm{E}-17$ \\
\hline $\mathrm{Ce}-144$ & 8.33E-07 & 3.33E-06 & $8.41 \mathrm{E}-11$ & $8.40 \mathrm{E}-13$ & $2.60 \mathrm{E}-20$ & $2.25 \mathrm{E}-20$ & $1.09 \mathrm{E}-12$ \\
\hline \multicolumn{8}{|l|}{ Pr-144m } \\
\hline Pr-144 & $8.33 \mathrm{E}-07$ & $3.33 \mathrm{E}-06$ & $8.41 \mathrm{E}-11$ & $8.40 \mathrm{E}-13$ & $2.60 \mathrm{E}-20$ & $2.25 \mathrm{E}-20$ & $1.09 \mathrm{E}-12$ \\
\hline $\mathrm{Nd}-144$ & $1.66 \mathrm{E}-15$ & $6.66 \mathrm{E}-15$ & $1.68 \mathrm{E}-19$ & $1.68 \mathrm{E}-21$ & $5.20 \mathrm{E}-29$ & $4.50 \mathrm{E}-29$ & $2.17 \mathrm{E}-21$ \\
\hline $\mathrm{Pm}-146$ & $5.27 \mathrm{E}-08$ & $2.11 \mathrm{E}-07$ & $5.32 \mathrm{E}-12$ & $5.31 \mathrm{E}-14$ & $1.65 \mathrm{E}-21$ & 1.42E-21 & $6.87 \mathrm{E}-14$ \\
\hline $\mathrm{Pm}-147$ & $2.25 \mathrm{E}-04$ & $9.01 \mathrm{E}-04$ & $2.27 \mathrm{E}-08$ & $2.27 \mathrm{E}-10$ & $7.03 \mathrm{E}-18$ & $6.08 \mathrm{E}-18$ & $2.94 \mathrm{E}-10$ \\
\hline Sm-146 & $2.85 \mathrm{E}-13$ & $1.14 \mathrm{E}-12$ & $2.88 \mathrm{E}-17$ & $2.88 \mathrm{E}-19$ & $8.92 \mathrm{E}-27$ & $7.72 \mathrm{E}-27$ & $3.73 \mathrm{E}-19$ \\
\hline Sm-147 & $7.62 \mathrm{E}-12$ & $3.05 \mathrm{E}-11$ & $7.69 \mathrm{E}-16$ & $7.69 \mathrm{E}-18$ & $2.38 \mathrm{E}-25$ & $2.06 \mathrm{E}-25$ & $9.95 \mathrm{E}-18$ \\
\hline Sm-148 & $3.92 \mathrm{E}-17$ & $1.57 \mathrm{E}-16$ & $3.95 \mathrm{E}-21$ & $3.95 \mathrm{E}-23$ & $1.22 \mathrm{E}-30$ & $1.06 \mathrm{E}-30$ & $5.11 \mathrm{E}-23$ \\
\hline Sm-149 & $3.48 \mathrm{E}-18$ & $1.39 \mathrm{E}-17$ & $3.51 \mathrm{E}-22$ & $3.51 \mathrm{E}-24$ & & & $4.54 \mathrm{E}-24$ \\
\hline Sm-151 & $4.45 \mathrm{E}-04$ & $1.78 \mathrm{E}-03$ & 4.49E-08 & $4.49 \mathrm{E}-10$ & $1.39 \mathrm{E}-17$ & $1.20 \mathrm{E}-17$ & $5.81 \mathrm{E}-10$ \\
\hline \multicolumn{8}{|l|}{ Eu-150 } \\
\hline Eu-152 & $2.94 \mathrm{E}-06$ & 1.18E-05 & $2.97 \mathrm{E}-10$ & $2.97 \mathrm{E}-12$ & $9.20 \mathrm{E}-20$ & $7.96 \mathrm{E}-20$ & $3.84 \mathrm{E}-12$ \\
\hline Eu-154 & $5.00 \mathrm{E}-05$ & $2.00 \mathrm{E}-04$ & $5.05 \mathrm{E}-09$ & $5.04 \mathrm{E}-11$ & $1.56 \mathrm{E}-18$ & $1.35 \mathrm{E}-18$ & $6.53 \mathrm{E}-11$ \\
\hline Eu-155 & $4.31 \mathrm{E}-05$ & 1.73E-04 & $4.35 \mathrm{E}-09$ & $4.35 \mathrm{E}-11$ & $1.35 \mathrm{E}-18$ & $1.17 \mathrm{E}-18$ & $5.63 \mathrm{E}-11$ \\
\hline Gd-152 & $1.47 \mathrm{E}-18$ & $5.89 \mathrm{E}-18$ & $1.48 \mathrm{E}-22$ & $1.48 \mathrm{E}-24$ & & & 1.92E-24 \\
\hline Ho-166m & $4.76 \mathrm{E}-11$ & $1.91 \mathrm{E}-10$ & $4.81 \mathrm{E}-15$ & $4.81 \mathrm{E}-17$ & 1.49E-24 & $1.29 \mathrm{E}-24$ & $6.22 \mathrm{E}-17$ \\
\hline \multicolumn{8}{|l|}{ Other Stream Data: } \\
\hline TRU, nCi/gm & $8.00 \mathrm{E}+02$ & $2.31 \mathrm{E}+03$ & & & & & $1.21 \mathrm{E}-03$ \\
\hline Heat Generation, W/m3 & $4.10 \mathrm{E}-01$ & $1.64 \mathrm{E}+00$ & & & & & $9.74 \mathrm{E}-07$ \\
\hline Mass Flow, kg/hr & $4.07 \mathrm{E}+02$ & $1.42 \mathrm{E}+02$ & $2.71 \mathrm{E}+02$ & $2.71 \mathrm{E}+02$ & $2.47 \mathrm{E}+02$ & $2.88 \mathrm{E}+02$ & $2.71 \mathrm{E}+02$ \\
\hline
\end{tabular}


Table C-6. Revised baseline mass balance, Tank WM-188 waste (Continued).

\begin{tabular}{|c|c|c|c|c|c|c|c|c|}
\hline PFD Sheet \# & PFD-3 & PFD-3 & PFD-4 & PFD-4 & PFD-4 & PFD-4 & PFD-4 & PFD-4 \\
\hline Stream \# & 108 & 109 & 110 & 201 & 202 & 203 & 204 & 205 \\
\hline Stream Name & $\begin{array}{l}\text { LET\&D } \\
\text { Acid } \\
\text { Liquid }\end{array}$ & $\begin{array}{l}\text { LET\&D } \\
\text { Offgas } \\
\text { Vapor }\end{array}$ & $\begin{array}{l}\text { Acid } \\
\text { Grout } \\
\text { Solid }\end{array}$ & $\begin{array}{c}\text { OPC } \\
\text { Grout } \\
\text { Additive }\end{array}$ & $\begin{array}{c}\text { BFS } \\
\text { Grout } \\
\text { Additive }\end{array}$ & $\begin{array}{c}\mathrm{Ca}(\mathrm{OH}) 2 \\
\text { Total } \\
\text { Additive }\end{array}$ & $\begin{array}{c}\mathrm{Ca}(\mathrm{OH}) 2 \\
\text { Grout } \\
\text { Additive }\end{array}$ & $\begin{array}{c}\mathrm{Ca}(\mathrm{OH}) 2 \\
\text { Neutraliz. } \\
\text { Additive }\end{array}$ \\
\hline Rate or Volume Flow (actual) & 15.7 & $1.51 \mathrm{E}+04$ & 435 & 171 & 57.1 & 54.4 & 1.71 & 52.7 \\
\hline Volume Flow (standard, wet) ${ }^{*}$ & & $9.47 \mathrm{E}+03$ & & & & & & \\
\hline Volume Flow (standard, dry) ${ }^{*}$ & & $1.24 \mathrm{E}+00$ & & & & & & \\
\hline Rate Units & gal/hr & $\mathrm{ft} 3 / \mathrm{hr}$ & $\mathrm{lb} / \mathrm{hr}$ & $\mathrm{lb} / \mathrm{hr}$ & $\mathrm{lb} / \mathrm{hr}$ & $\mathrm{lb} / \mathrm{hr}$ & $\mathrm{lb} / \mathrm{hr}$ & $\mathrm{lb} / \mathrm{hr}$ \\
\hline Temperature, ${ }^{\circ} \mathrm{C}$ & 35.0 & 119.1 & 64.2 & 25.0 & 25.0 & 25.0 & 25.0 & 25.0 \\
\hline Temperature, ${ }^{\circ} \mathrm{F}$ & 95.0 & 246.3 & 147.5 & 77.0 & 77.0 & 77.0 & 77.0 & 77.0 \\
\hline Pressure, psia & 12.2 & 12.3 & 12.2 & 12.3 & 12.3 & 12.3 & 12.3 & 12.3 \\
\hline Specific Gravity & 1.16 & 0.00047 & 2.06 & 1.2 & 1.2 & 0.48 & 0.48 & 0.48 \\
\hline Chemical Composition & Mol/Liter & $\mathrm{lb} / \mathrm{wscf}$ & Wt. \% & Wt. \% & Wt. \% & Wt. \% & Wt. \% & Wt. \% \\
\hline $\mathrm{H}+$ & $1.09 \mathrm{E}+01$ & $3.35 \mathrm{E}-07$ & $3.24 \mathrm{E}-06$ & & & & & \\
\hline $\mathrm{OH}-$ & & & $1.78 \mathrm{E}-01$ & & & $4.59 \mathrm{E}+01$ & $4.59 \mathrm{E}+01$ & $4.59 \mathrm{E}+01$ \\
\hline $\mathrm{Al}+3$ & $2.77 \mathrm{E}-06$ & & $2.25 \mathrm{E}-06$ & & & & & \\
\hline $\mathrm{Sb}+5$ & $8.67 \mathrm{E}-11$ & & $3.17 \mathrm{E}-10$ & & & & & \\
\hline$A s+5$ & $4.86 \mathrm{E}-10$ & & 1.09E-09 & & & & & \\
\hline $\mathrm{Ba}+2$ & $3.96 \mathrm{E}-10$ & & 1.63E-09 & & & & & \\
\hline $\mathrm{Be}+2$ & $1.00 \mathrm{E}-10$ & & $2.71 \mathrm{E}-11$ & & & & & \\
\hline $\mathrm{B}+3$ & 8.69E-08 & & $2.82 \mathrm{E}-08$ & & & & & \\
\hline $\mathrm{Cd}+2$ & 1.33E-08 & & 4.48E-08 & & & & & \\
\hline $\mathrm{Ca}+2$ & $2.64 \mathrm{E}-07$ & & $6.76 \mathrm{E}+00$ & & & $5.41 \mathrm{E}+01$ & $5.41 \mathrm{E}+01$ & $5.41 \mathrm{E}+01$ \\
\hline $\mathrm{Cr}+3$ & $2.45 \mathrm{E}-08$ & & 3.83E-08 & & & & & \\
\hline $\mathrm{Co}+2$ & $2.17 \mathrm{E}-10$ & & $3.84 \mathrm{E}-10$ & & & & & \\
\hline $\mathrm{Cs}+$ & $3.15 \mathrm{E}-10$ & & 1.26E-09 & & & & & \\
\hline $\mathrm{Cu}+2$ & 3.71E-09 & & $7.08 \mathrm{E}-09$ & & & & & \\
\hline $\mathrm{Fe}+3$ & $1.36 \mathrm{E}-07$ & & $2.29 \mathrm{E}-07$ & & & & & \\
\hline $\mathrm{Pb}+2$ & 4.37E-09 & & $2.72 \mathrm{E}-08$ & & & & & \\
\hline $\mathrm{Hg}+2$ & 1.57E-03 & & $9.66 \mathrm{E}-03$ & & & & & \\
\hline $\mathrm{Mn}+4$ & $7.84 \mathrm{E}-08$ & & 1.29E-07 & & & & & \\
\hline $\mathrm{Ni+2}$ & 9.73E-09 & & $1.71 \mathrm{E}-08$ & & & & & \\
\hline $\mathrm{K}+$ & 9.97E-07 & & 1.17E-06 & & & & & \\
\hline $\mathrm{Se}+4$ & $1.28 \mathrm{E}-09$ & & 3.04E-09 & & & & & \\
\hline$\overline{\mathrm{Ag}+}$ & 1.99E-09 & & $6.46 \mathrm{E}-09$ & & & & & \\
\hline $\mathrm{Na}+$ & $7.40 \mathrm{E}-06$ & & $5.11 \mathrm{E}-06$ & & & & & \\
\hline$\overline{\mathrm{TI}+3}$ & $2.42 \mathrm{E}-11$ & & $1.49 \mathrm{E}-10$ & & & & & \\
\hline $\mathrm{U}+4$ & 1.92E-09 & & 1.37E-08 & & & & & \\
\hline $\mathrm{V}+3$ & $1.98 \mathrm{E}-10$ & & $3.03 \mathrm{E}-10$ & & & & & \\
\hline$Z n+2$ & 4.30E-09 & & 8.44E-09 & & & & & \\
\hline $\mathrm{Zr}+4$ & $2.00 \mathrm{E}-07$ & & $5.47 \mathrm{E}-07$ & & & & & \\
\hline $\mathrm{Cl}-$ & $3.14 \mathrm{E}-03$ & 1.18E-05 & $2.90 \mathrm{E}-02$ & & & & & \\
\hline F- & 1.09E-04 & & $6.24 \mathrm{E}-05$ & & & & & \\
\hline SO4-2 & $2.22 \mathrm{E}-07$ & & $6.40 \mathrm{E}-07$ & & & & & \\
\hline NO3- & $1.09 \mathrm{E}+01$ & 1.17E-08 & $2.03 \mathrm{E}+01$ & & & & & \\
\hline PO4-3 & $5.26 \mathrm{E}-07$ & & $1.50 \mathrm{E}-06$ & & & & & \\
\hline$A m+4$ & $3.66 \mathrm{E}-13$ & & $2.67 \mathrm{E}-12$ & & & & & \\
\hline $\mathrm{Br}-$ & $4.15 \mathrm{E}-08$ & $3.50 \mathrm{E}-10$ & 8.61E-07 & & & & & \\
\hline $\mathrm{Ce}+4$ & $1.81 \mathrm{E}-10$ & & $7.63 \mathrm{E}-10$ & & & & & \\
\hline $\mathrm{Eu}+3$ & $2.61 \mathrm{E}-12$ & & 1.19E-11 & & & & & \\
\hline $\mathrm{Gd}+3$ & $7.71 \mathrm{E}-10$ & & 3.64E-09 & & & & & \\
\hline $\mathrm{Ge}+4$ & $4.54 \mathrm{E}-14$ & & $9.90 \mathrm{E}-14$ & & & & & \\
\hline $\ln +3$ & $7.98 \mathrm{E}-12$ & & $2.75 \mathrm{E}-11$ & & & & & \\
\hline I- & 3.44E-07 & 4.61E-09 & 1.14E-05 & & & & & \\
\hline $\mathrm{La+3}$ & $4.74 \mathrm{E}-11$ & & $1.98 \mathrm{E}-10$ & & & & & \\
\hline Li+ & $2.48 \mathrm{E}-09$ & & $5.16 \mathrm{E}-10$ & & & & & \\
\hline $\mathrm{Mg}+2$ & $9.72 \mathrm{E}-08$ & & $7.10 \mathrm{E}-08$ & & & & & \\
\hline $\mathrm{Mo}+6$ & 3.99E-09 & & $1.15 \mathrm{E}-08$ & & & & & \\
\hline
\end{tabular}


Table C-6. Revised baseline mass balance, Tank WM-188 waste (Continued).

\begin{tabular}{|c|c|c|c|c|c|c|c|c|}
\hline Stream \# & 108 & 109 & 110 & 201 & 202 & 203 & 204 & 205 \\
\hline Stream Name & $\begin{array}{c}\text { LET\&D } \\
\text { Acid } \\
\text { Liquid }\end{array}$ & $\begin{array}{c}\text { LET\&D } \\
\text { Offgas } \\
\text { Vapor }\end{array}$ & $\begin{array}{l}\text { Acid } \\
\text { Grout } \\
\text { Solid }\end{array}$ & $\begin{array}{c}\text { OPC } \\
\text { Grout } \\
\text { Additive }\end{array}$ & $\begin{array}{c}\text { BFS } \\
\text { Grout } \\
\text { Additive }\end{array}$ & $\begin{array}{c}\mathrm{Ca}(\mathrm{OH}) 2 \\
\text { Total } \\
\text { Additive }\end{array}$ & $\begin{array}{c}\mathrm{Ca}(\mathrm{OH}) 2 \\
\text { Grout } \\
\text { Additive }\end{array}$ & $\begin{array}{c}\mathrm{Ca}(\mathrm{OH}) 2 \\
\text { Neutraliz. } \\
\text { Additive }\end{array}$ \\
\hline Chemical Composition & Mol/Liter & $\mathrm{Ib} / \mathrm{wscf}$ & Wt. \% & Wt. \% & Wt. \% & Wt. \% & Wt. \% & Wt. \% \\
\hline $\mathrm{Nd}+3$ & $1.53 \mathrm{E}-10$ & & $6.62 \mathrm{E}-10$ & & & & & \\
\hline $\mathrm{Np}+4$ & $1.40 \mathrm{E}-11$ & & $9.95 \mathrm{E}-11$ & & & & & \\
\hline $\mathrm{Nb}+5$ & 5.73E-09 & & 1.60E-08 & & & & & \\
\hline $\mathrm{Pd}+4$ & 9.29E-09 & & $2.97 \mathrm{E}-08$ & & & & & \\
\hline $\mathrm{Pu}+4$ & $3.61 \mathrm{E}-11$ & & $2.64 \mathrm{E}-10$ & & & & & \\
\hline $\mathrm{Pr}+4$ & $4.31 \mathrm{E}-11$ & & $1.82 \mathrm{E}-10$ & & & & & \\
\hline $\mathrm{Rh}+4$ & $1.86 \mathrm{E}-11$ & & $5.75 \mathrm{E}-11$ & & & & & \\
\hline $\mathrm{Rb}+$ & $2.86 \mathrm{E}-11$ & & $7.35 \mathrm{E}-11$ & & & & & \\
\hline Ru+3 & 4.30E-09 & & $1.31 \mathrm{E}-08$ & & & & & \\
\hline $\mathrm{Sm}+3$ & $2.85 \mathrm{E}-11$ & & $1.29 \mathrm{E}-10$ & & & & & \\
\hline $\mathrm{Si}+4$ & $4.88 \mathrm{E}-07$ & & $4.12 \mathrm{E}-07$ & & & & & \\
\hline $\mathrm{Sr}+2$ & $4.25 \mathrm{E}-10$ & & $1.12 \mathrm{E}-09$ & & & & & \\
\hline $\mathrm{Tc}+7$ & $5.55 \mathrm{E}-11$ & & $1.62 \mathrm{E}-10$ & & & & & \\
\hline $\mathrm{Te}+4$ & 1.89E-11 & & $7.25 \mathrm{E}-11$ & & & & & \\
\hline $\mathrm{Tb}+4$ & $1.09 \mathrm{E}-14$ & & $5.21 \mathrm{E}-14$ & & & & & \\
\hline$T \mathrm{Th}+4$ & $1.26 \mathrm{E}-10$ & & $8.76 \mathrm{E}-10$ & & & & & \\
\hline $\mathrm{Sn+4}$ & $9.45 E-09$ & & 3.37E-08 & & & & & \\
\hline $\mathrm{Ti}+4$ & 4.83E-09 & & $6.95 \mathrm{E}-09$ & & & & & \\
\hline $\mathrm{Y}+3$ & $3.53 \mathrm{E}-11$ & & $9.44 \mathrm{E}-11$ & & & & & \\
\hline O-2 (oxides) & $7.70 \mathrm{E}-07$ & & 3.70E-07 & & & & & \\
\hline $\mathrm{H} 2 \mathrm{O}$ & $2.66 \mathrm{E}+01$ & $4.70 \mathrm{E}-02$ & $2.03 \mathrm{E}+01$ & & & & & \\
\hline $\mathrm{O} 2$ & & $1.22 \mathrm{E}-07$ & & & & & & \\
\hline N2 & & $1.54 \mathrm{E}-07$ & & & & & & \\
\hline C (organic) & $3.61 \mathrm{E}-03$ & & 1.30E-03 & & & & & \\
\hline $\mathrm{H}$ (organic) & $3.61 \mathrm{E}-03$ & & 1.09E-04 & & & & & \\
\hline SiO2, BFS, or OPC Additives & & & $5.25 \mathrm{E}+01$ & $1.00 \mathrm{E}+02$ & $1.00 \mathrm{E}+02$ & & & \\
\hline $\begin{array}{l}\text { Gas Stream Bulk Composition } \\
\text { (wet basis) }\end{array}$ & $\begin{array}{l}\text { mol. } \% \\
\text { or ppmv }\end{array}$ & $\begin{array}{l}\text { mol. } \% \\
\text { or ppmv }\end{array}$ & $\begin{array}{l}\text { mol.\% } \\
\text { or ppmv }\end{array}$ & $\begin{array}{l}\text { mol.\% } \\
\text { or ppmv }\end{array}$ & $\begin{array}{l}\text { mol.\% } \\
\text { or ppmv }\end{array}$ & $\begin{array}{l}\text { mol. \% } \\
\text { or ppmv }\end{array}$ & $\begin{array}{l}\text { mol. } \% \\
\text { or ppmv }\end{array}$ & $\begin{array}{l}\text { mol. } \% \\
\text { or ppmv }\end{array}$ \\
\hline $\mathrm{H} 2 \mathrm{O}, \mathrm{mol} . \%$ & & 99.99 & & & & & & \\
\hline $\mathrm{O} 2, \mathrm{~mol} . \%$ & & $1.5 \mathrm{E}-04$ & & & & & & \\
\hline $\mathrm{N} 2, \mathrm{~mol} . \%$ & & $2.1 \mathrm{E}-04$ & & & & & & \\
\hline \multicolumn{9}{|l|}{$\mathrm{CO} 2, \mathrm{~mol} . \%$} \\
\hline HNO3, mol. \% & & 0.01 & & & & & & \\
\hline \multicolumn{9}{|l|}{ CO, ppmv } \\
\hline \multicolumn{9}{|l|}{$\mathrm{NO}, \mathrm{ppmv}$} \\
\hline \multicolumn{9}{|l|}{ NO2, ppmv } \\
\hline \multicolumn{9}{|l|}{ C (organic), ppmv } \\
\hline \multicolumn{9}{|l|}{$\mathrm{H}$ (organic), ppmv } \\
\hline \multicolumn{9}{|l|}{$\mathrm{PM}$ (mg/wscm) } \\
\hline $\begin{array}{l}\text { Gas Stream Bulk Composition } \\
\text { (dry basis) }\end{array}$ & $\begin{array}{l}\text { mol. \% } \\
\text { or ppmv }\end{array}$ & $\begin{array}{l}\text { mol. } \% \\
\text { or ppmv }\end{array}$ & $\begin{array}{l}\text { mol. } \% \\
\text { or ppmv }\end{array}$ & $\begin{array}{l}\text { mol. } \% \\
\text { or ppmv }\end{array}$ & $\begin{array}{l}\text { mol. } \% \\
\text { or ppmv }\end{array}$ & $\begin{array}{l}\text { mol. } \% \\
\text { or ppmv }\end{array}$ & $\begin{array}{l}\text { mol. } \% \\
\text { or ppmv }\end{array}$ & $\begin{array}{l}\text { mol. } \% \\
\text { or ppmv }\end{array}$ \\
\hline O2, mol.\% & & 1.11 & & & & & & \\
\hline $\mathrm{N} 2, \mathrm{~mol} . \%$ & & 1.61 & & & & & & \\
\hline \multicolumn{9}{|l|}{$\mathrm{CO} 2, \mathrm{~mol} . \%$} \\
\hline HNO3, mol. \% & & 97.28 & & & & & & \\
\hline \multicolumn{9}{|l|}{$\mathrm{CO}, \mathrm{ppmv}$} \\
\hline \multicolumn{9}{|l|}{ NO, ppmv } \\
\hline \multicolumn{9}{|l|}{ NO2, ppmv } \\
\hline \multicolumn{9}{|l|}{ C (organic), ppmv } \\
\hline \multicolumn{9}{|l|}{$\mathrm{H}$ (organic), ppmv } \\
\hline PM (mg/dscm) & & & & & & & & \\
\hline
\end{tabular}


Table C-6. Revised baseline mass balance, Tank WM-188 waste (Continued).

\begin{tabular}{|c|c|c|c|c|c|c|c|c|}
\hline Stream \# & 108 & 109 & 110 & 201 & 202 & 203 & 204 & 205 \\
\hline Stream Name & $\begin{array}{l}\text { LET\&D } \\
\text { Acid } \\
\text { Liquid }\end{array}$ & $\begin{array}{l}\text { LET\&D } \\
\text { Offgas } \\
\text { Vapor }\end{array}$ & $\begin{array}{l}\text { Acid } \\
\text { Grout } \\
\text { Solid }\end{array}$ & $\begin{array}{c}\text { OPC } \\
\text { Grout } \\
\text { Additive }\end{array}$ & $\begin{array}{c}\text { BFS } \\
\text { Grout } \\
\text { Additive }\end{array}$ & $\begin{array}{c}\mathrm{Ca}(\mathrm{OH}) 2 \\
\text { Total } \\
\text { Additive }\end{array}$ & $\begin{array}{c}\mathrm{Ca}(\mathrm{OH}) 2 \\
\text { Grout } \\
\text { Additive }\end{array}$ & $\begin{array}{c}\mathrm{Ca}(\mathrm{OH}) 2 \\
\text { Neutraliz. } \\
\text { Additive }\end{array}$ \\
\hline \multicolumn{9}{|l|}{ Canister Rate, canisters/day } \\
\hline \multicolumn{9}{|l|}{ Total Canisters Generated } \\
\hline Drum Rate, drums/day & & & 13.74 & & & & & \\
\hline Total Drums Generated & & & 1,997 & & & & & \\
\hline Radiological Composition & $\mathrm{Ci} / \mathrm{L}$ & Ci/wscm & $\mathrm{Ci} / \mathrm{kg}$ & $\mathrm{Ci} / \mathrm{kg}$ & $\mathrm{Ci} / \mathrm{kg}$ & $\mathrm{Ci} / \mathrm{kg}$ & $\mathrm{Ci} / \mathrm{kg}$ & $\mathrm{Ci} / \mathrm{kg}$ \\
\hline Ra-226 & $4.64 \mathrm{E}-17$ & $2.05 \mathrm{E}-29$ & $1.39 \mathrm{E}-17$ & & & & & \\
\hline Ac-227 & $2.19 \mathrm{E}-16$ & 9.67E-29 & $6.57 \mathrm{E}-17$ & & & & & \\
\hline Th-228 & $1.81 \mathrm{E}-14$ & $8.02 \mathrm{E}-27$ & $5.45 \mathrm{E}-15$ & & & & & \\
\hline Th-230 & $5.78 \mathrm{E}-15$ & $2.56 \mathrm{E}-27$ & $1.74 \mathrm{E}-15$ & & & & & \\
\hline Th-232 & $4.01 \mathrm{E}-21$ & & $1.21 \mathrm{E}-21$ & & & & & \\
\hline Pa-231 & $5.06 \mathrm{E}-16$ & $2.24 \mathrm{E}-28$ & $1.52 \mathrm{E}-16$ & & & & & \\
\hline $\mathrm{Pa}-233$ & $1.66 \mathrm{E}-11$ & $7.35 \mathrm{E}-24$ & $4.99 \mathrm{E}-12$ & & & & & \\
\hline $\mathrm{U}-232$ & $1.36 \mathrm{E}-14$ & $6.03 E-27$ & $4.09 \mathrm{E}-15$ & & & & & \\
\hline U-233 & 4.94E-16 & $2.18 \mathrm{E}-28$ & $1.48 \mathrm{E}-16$ & & & & & \\
\hline U-234 & $6.08 \mathrm{E}-12$ & $2.69 \mathrm{E}-24$ & $1.83 \mathrm{E}-12$ & & & & & \\
\hline U-235 & $4.47 \mathrm{E}-13$ & $1.98 \mathrm{E}-25$ & $1.34 \mathrm{E}-13$ & & & & & \\
\hline U-236 & $2.62 \mathrm{E}-13$ & $1.16 \mathrm{E}-25$ & $7.88 \mathrm{E}-14$ & & & & & \\
\hline U-237 & $3.65 \mathrm{E}-14$ & 1.61E-26 & $1.10 \mathrm{E}-14$ & & & & & \\
\hline $\mathrm{U}-238$ & $7.97 \mathrm{E}-14$ & $3.53 \mathrm{E}-26$ & $2.39 \mathrm{E}-14$ & & & & & \\
\hline $\mathrm{Np}-236$ & $1.75 \mathrm{E}-17$ & $7.73 E-30$ & $5.25 \mathrm{E}-18$ & & & & & \\
\hline $\mathrm{Np}-237$ & $2.37 \mathrm{E}-12$ & $1.05 \mathrm{E}-24$ & $7.13 \mathrm{E}-13$ & & & & & \\
\hline Pu-236 & $1.99 \mathrm{E}-14$ & $8.79 \mathrm{E}-27$ & 5.97E-15 & & & & & \\
\hline Pu-238 & 4.53E-09 & $2.00 \mathrm{E}-21$ & 1.36E-09 & & & & & \\
\hline Pu-239 & $5.07 \mathrm{E}-10$ & $2.24 \mathrm{E}-22$ & $1.52 \mathrm{E}-10$ & & & & & \\
\hline Pu-240 & $7.40 \mathrm{E}-11$ & $3.28 \mathrm{E}-23$ & $2.22 \mathrm{E}-11$ & & & & & \\
\hline Pu-241 & 3.29E-09 & 1.46E-21 & $9.89 \mathrm{E}-10$ & & & & & \\
\hline Pu-242 & $5.80 \mathrm{E}-14$ & $2.57 \mathrm{E}-26$ & $1.74 \mathrm{E}-14$ & & & & & \\
\hline Pu-244 & $4.42 \mathrm{E}-21$ & & 1.33E-21 & & & & & \\
\hline Am-241 & $3.19 \mathrm{E}-10$ & $1.41 \mathrm{E}-22$ & $9.59 \mathrm{E}-11$ & & & & & \\
\hline Am-242m & $8.84 \mathrm{E}-14$ & $3.91 \mathrm{E}-26$ & $2.66 \mathrm{E}-14$ & & & & & \\
\hline Am-243 & $1.44 \mathrm{E}-13$ & $6.37 \mathrm{E}-26$ & $4.32 \mathrm{E}-14$ & & & & & \\
\hline $\mathrm{Cm}-242$ & $1.91 \mathrm{E}-13$ & $8.47 \mathrm{E}-26$ & $5.75 \mathrm{E}-14$ & & & & & \\
\hline $\mathrm{Cm}-243$ & $2.00 \mathrm{E}-13$ & $8.85 E-26$ & $6.01 \mathrm{E}-14$ & & & & & \\
\hline $\mathrm{Cm}-244$ & $6.97 \mathrm{E}-12$ & $3.09 E-24$ & $2.10 \mathrm{E}-12$ & & & & & \\
\hline $\mathrm{Cm}-245$ & $2.10 \mathrm{E}-15$ & $9.31 \mathrm{E}-28$ & $6.32 \mathrm{E}-16$ & & & & & \\
\hline $\mathrm{Cm}-246$ & $1.38 \mathrm{E}-16$ & $6.12 \mathrm{E}-29$ & $4.15 \mathrm{E}-17$ & & & & & \\
\hline $\mathrm{Cm}-247$ & $3.02 \mathrm{E}-23$ & & $9.08 \mathrm{E}-24$ & & & & & \\
\hline $\mathrm{H}-3$ & $6.60 \mathrm{E}-06$ & 1.04E-05 & $1.98 \mathrm{E}-06$ & & & & & \\
\hline $\mathrm{Be}-10$ & $1.70 \mathrm{E}-17$ & $7.52 \mathrm{E}-30$ & $5.11 \mathrm{E}-18$ & & & & & \\
\hline $\mathrm{C}-14$ & $8.71 \mathrm{E}-16$ & $3.85 E-28$ & $2.62 \mathrm{E}-16$ & & & & & \\
\hline Co-60 & $4.91 \mathrm{E}-11$ & $2.17 \mathrm{E}-23$ & $1.48 \mathrm{E}-11$ & & & & & \\
\hline Ni-59 & $6.03 \mathrm{E}-12$ & $2.67 \mathrm{E}-24$ & $1.81 \mathrm{E}-12$ & & & & & \\
\hline Ni-63 & $2.31 \mathrm{E}-10$ & 1.02E-22 & $6.94 \mathrm{E}-11$ & & & & & \\
\hline Se-79 & $3.16 \mathrm{E}-12$ & 1.40E-24 & $9.50 \mathrm{E}-13$ & & & & & \\
\hline Rb-87 & $1.66 \mathrm{E}-16$ & $7.35 \mathrm{E}-29$ & $4.99 \mathrm{E}-17$ & & & & & \\
\hline Sr-90 & $2.18 \mathrm{E}-07$ & 9.65E-20 & $6.55 \mathrm{E}-08$ & & & & & \\
\hline $\mathrm{Y}-90$ & $2.18 \mathrm{E}-07$ & $9.65 \mathrm{E}-20$ & $6.55 \mathrm{E}-08$ & & & & & \\
\hline Zr-93 & $1.26 \mathrm{E}-11$ & $5.55 \mathrm{E}-24$ & $3.77 \mathrm{E}-12$ & & & & & \\
\hline $\begin{array}{ll}\mathrm{Nb}-93 \mathrm{~m} \\
\end{array}$ & $9.67 \mathrm{E}-12$ & $4.28 \mathrm{E}-24$ & $2.91 \mathrm{E}-12$ & & & & & \\
\hline \begin{tabular}{|l|}
$\mathrm{Nb}-94$ \\
\end{tabular} & $8.23 \mathrm{E}-12$ & $3.64 \mathrm{E}-24$ & $2.47 \mathrm{E}-12$ & & & & & \\
\hline Tc-98 & $1.46 \mathrm{E}-17$ & $6.47 \mathrm{E}-30$ & 4.39E-18 & & & & & \\
\hline Tc-99 & $1.14 \mathrm{E}-10$ & $5.06 \mathrm{E}-23$ & $3.44 \mathrm{E}-11$ & & & & & \\
\hline
\end{tabular}


Table C-6. Revised baseline mass balance, Tank WM-188 waste (Continued).

\begin{tabular}{|c|c|c|c|c|c|c|c|c|}
\hline Stream \# & 108 & 109 & 110 & 201 & 202 & 203 & 204 & 205 \\
\hline Stream Name & $\begin{array}{l}\text { LET\&D } \\
\text { Acid } \\
\text { Liquid }\end{array}$ & $\begin{array}{l}\text { LET\&D } \\
\text { Offgas } \\
\text { Vapor }\end{array}$ & $\begin{array}{l}\text { Acid } \\
\text { Grout } \\
\text { Solid }\end{array}$ & $\begin{array}{c}\text { OPC } \\
\text { Grout } \\
\text { Additive }\end{array}$ & $\begin{array}{c}\text { BFS } \\
\text { Grout } \\
\text { Additive }\end{array}$ & $\begin{array}{c}\mathrm{Ca}(\mathrm{OH}) 2 \\
\text { Total } \\
\text { Additive }\end{array}$ & $\begin{array}{c}\mathrm{Ca}(\mathrm{OH}) 2 \\
\text { Grout } \\
\text { Additive }\end{array}$ & $\begin{array}{c}\mathrm{Ca}(\mathrm{OH}) 2 \\
\text { Neutraliz. } \\
\text { Additive }\end{array}$ \\
\hline Radiological Composition & $\mathrm{Ci} / \mathrm{L}$ & Ci/wscm & $\mathrm{Ci} / \mathrm{kg}$ & $\mathrm{Ci} / \mathrm{kg}$ & $\mathrm{Ci} / \mathrm{kg}$ & $\mathrm{Ci} / \mathrm{kg}$ & $\mathrm{Ci} / \mathrm{kg}$ & $\mathrm{Ci} / \mathrm{kg}$ \\
\hline Ru-106 & $6.76 \mathrm{E}-12$ & $2.99 \mathrm{E}-24$ & $2.03 \mathrm{E}-12$ & & & & & \\
\hline Rh-102 & $4.88 \mathrm{E}-15$ & $2.16 \mathrm{E}-27$ & $1.47 \mathrm{E}-15$ & & & & & \\
\hline Rh-106 & $6.76 \mathrm{E}-12$ & $2.99 \mathrm{E}-24$ & $2.03 \mathrm{E}-12$ & & & & & \\
\hline $\mathrm{Pd}-107$ & $9.36 \mathrm{E}-14$ & $4.14 \mathrm{E}-26$ & $2.81 \mathrm{E}-14$ & & & & & \\
\hline Cd-113m & $1.88 \mathrm{E}-11$ & $8.33 \mathrm{E}-24$ & $5.65 \mathrm{E}-12$ & & & & & \\
\hline $\ln -115$ & $5.70 \mathrm{E}-22$ & & $1.71 \mathrm{E}-22$ & & & & & \\
\hline Sn-121m & $3.79 \mathrm{E}-13$ & $1.68 \mathrm{E}-25$ & $1.14 \mathrm{E}-13$ & & & & & \\
\hline Sn-126 & $2.98 \mathrm{E}-12$ & $1.32 \mathrm{E}-24$ & $8.94 \mathrm{E}-13$ & & & & & \\
\hline Sb-125 & 2.02E-09 & 8.93E-22 & $6.06 \mathrm{E}-10$ & & & & & \\
\hline Sb-126 & $3.26 \mathrm{E}-13$ & $1.44 \mathrm{E}-25$ & $9.79 \mathrm{E}-14$ & & & & & \\
\hline Te-123 & $2.17 \mathrm{E}-24$ & & $6.52 \mathrm{E}-25$ & & & & & \\
\hline Te-125m & $1.78 \mathrm{E}-11$ & $7.90 \mathrm{E}-24$ & $5.36 \mathrm{E}-12$ & & & & & \\
\hline $\mathrm{I}-129$ & 9.39E-09 & $1.57 \mathrm{E}-08$ & $2.82 \mathrm{E}-09$ & & & & & \\
\hline Cs-134 & $2.02 \mathrm{E}-10$ & $8.93 \mathrm{E}-23$ & $6.06 \mathrm{E}-11$ & & & & & \\
\hline Cs-135 & $6.08 \mathrm{E}-12$ & $2.69 \mathrm{E}-24$ & $1.83 \mathrm{E}-12$ & & & & & \\
\hline Cs-137 & $1.25 \mathrm{E}-07$ & $5.52 \mathrm{E}-20$ & $3.75 \mathrm{E}-08$ & & & & & \\
\hline Ba-137m & 1.18E-07 & $5.22 \mathrm{E}-20$ & $3.55 \mathrm{E}-08$ & & & & & \\
\hline La-138 & $1.08 \mathrm{E}-21$ & & $3.25 \mathrm{E}-22$ & & & & & \\
\hline Ce-142 & $1.69 \mathrm{E}-16$ & $7.49 \mathrm{E}-29$ & $5.09 \mathrm{E}-17$ & & & & & \\
\hline Ce-144 & $4.56 \mathrm{E}-12$ & $2.02 \mathrm{E}-24$ & $1.37 \mathrm{E}-12$ & & & & & \\
\hline \multicolumn{9}{|l|}{ Pr-144m } \\
\hline $\mathrm{Pr}-144$ & $4.56 \mathrm{E}-12$ & $2.02 \mathrm{E}-24$ & $1.37 \mathrm{E}-12$ & & & & & \\
\hline $\mathrm{Nd}-144$ & $9.11 \mathrm{E}-21$ & & $2.74 \mathrm{E}-21$ & & & & & \\
\hline $\mathrm{Pm}-146$ & $2.88 \mathrm{E}-13$ & $1.28 \mathrm{E}-25$ & $8.66 \mathrm{E}-14$ & & & & & \\
\hline $\mathrm{Pm}-147$ & 1.23E-09 & $5.45 \mathrm{E}-22$ & $3.70 \mathrm{E}-10$ & & & & & \\
\hline Sm-146 & $1.56 \mathrm{E}-18$ & & $4.70 \mathrm{E}-19$ & & & & & \\
\hline Sm-147 & $4.17 \mathrm{E}-17$ & 1.85E-29 & $1.25 \mathrm{E}-17$ & & & & & \\
\hline Sm-148 & $2.14 \mathrm{E}-22$ & & $6.44 \mathrm{E}-23$ & & & & & \\
\hline Sm-149 & $1.90 \mathrm{E}-23$ & & $5.72 \mathrm{E}-24$ & & & & & \\
\hline Sm-151 & 2.43E-09 & $1.08 \mathrm{E}-21$ & $7.32 \mathrm{E}-10$ & & & & & \\
\hline \multicolumn{9}{|l|}{ Eu-150 } \\
\hline Eu-152 & $1.61 \mathrm{E}-11$ & 7.13E-24 & $4.84 \mathrm{E}-12$ & & & & & \\
\hline Eu-154 & $2.74 \mathrm{E}-10$ & $1.21 \mathrm{E}-22$ & $8.22 \mathrm{E}-11$ & & & & & \\
\hline Eu-155 & $2.36 \mathrm{E}-10$ & 1.04E-22 & $7.10 \mathrm{E}-11$ & & & & & \\
\hline Gd-152 & $8.05 \mathrm{E}-24$ & & $2.42 \mathrm{E}-24$ & & & & & \\
\hline Ho-166m & $2.61 \mathrm{E}-16$ & 1.15E-28 & $7.84 \mathrm{E}-17$ & & & & & \\
\hline \multicolumn{9}{|l|}{ Other Stream Data: } \\
\hline TRU, nCi/gm & $4.75 \mathrm{E}-03$ & & $1.66 \mathrm{E}-03$ & & & & & \\
\hline Heat Generation, W/m3 & $2.47 \mathrm{E}-06$ & & $1.28 \mathrm{E}-06$ & & & & & \\
\hline Mass Flow, kg/hr & $6.91 \mathrm{E}+01$ & $2.02 \mathrm{E}+02$ & $1.97 \mathrm{E}+02$ & $7.77 \mathrm{E}+01$ & $2.59 \mathrm{E}+01$ & $2.47 \mathrm{E}+01$ & 7.77E-01 & $2.39 \mathrm{E}+01$ \\
\hline
\end{tabular}

$*$ Standard conditions $=68 \mathrm{oF}$ and 1 atmosphere 
Table C-7. Revised baseline mass balance, Tank WM-189 waste.

\begin{tabular}{|c|c|c|c|c|c|c|c|c|}
\hline PFD Sheet \# & PFD-1 & PFD-2 & PFD-2 & PFD-2 & PFD-2 & PFD-2 & PFD-2 & PFD-3 \\
\hline Stream \# & 101 & 102 & 103 & 104 & 105 & 106 & 107 & 108 \\
\hline Stream Name & $\begin{array}{l}\text { SBW } \\
\text { Feed }\end{array}$ & $\begin{array}{c}\text { Evaporator } \\
\text { Bottoms }\end{array}$ & $\begin{array}{c}\text { Evaporator } \\
\text { Overheads } \\
\text { Vapor }\end{array}$ & $\begin{array}{c}\text { Mist } \\
\text { Eliminator } \\
\text { Vapor }\end{array}$ & $\begin{array}{l}\text { VOG } \\
\text { Offgas } \\
\text { Vapor }\end{array}$ & $\begin{array}{c}\text { POG + VOG } \\
\text { Offgas } \\
\text { Vapor }\end{array}$ & $\begin{array}{l}\text { LET\&D } \\
\text { Feed } \\
\text { Liquid }\end{array}$ & $\begin{array}{l}\text { LET\&D } \\
\text { Acid } \\
\text { Liquid }\end{array}$ \\
\hline Rate or Volume Flow (actual) & 85.8 & 24.2 & $2.44 \mathrm{E}+04$ & $2.49 \mathrm{E}+04$ & $1.17 \mathrm{E}+04$ & $1.37 \mathrm{E}+04$ & 61.5 & 17.7 \\
\hline Volume Flow (standard, wet) & & & $1.06 \mathrm{E}+04$ & $1.06 \mathrm{E}+04$ & $7.33 E+03$ & $8.56 \mathrm{E}+03$ & & \\
\hline Volume Flow (standard, dry) & & & $6.19 \mathrm{E}+02$ & $6.18 \mathrm{E}+02$ & $7.13 E+03$ & $8.36 \mathrm{E}+03$ & & \\
\hline Rate Units & $\mathrm{gal} / \mathrm{hr}$ & $\mathrm{gal} / \mathrm{hr}$ & $\mathrm{ft} 3 / \mathrm{hr}$ & $\mathrm{ft} 3 / \mathrm{hr}$ & $\mathrm{ft} 3 / \mathrm{hr}$ & $\mathrm{ft} 3 / \mathrm{hr}$ & $\mathrm{gal} / \mathrm{hr}$ & $\mathrm{gal} / \mathrm{hr}$ \\
\hline Temperature, ${ }^{\circ} \mathrm{C}$ & 25.0 & 120.9 & 121.0 & 120.0 & 107.5 & 109.4 & 35.0 & 35.0 \\
\hline Temperature, ${ }^{\circ} \mathrm{F}$ & 77.0 & 249.7 & 249.7 & 248.0 & 225.6 & 228.8 & 95.0 & 95.0 \\
\hline Pressure, psia & 12.3 & 8.6 & 8.6 & 8.4 & 12.0 & 12.0 & 12.0 & 12.2 \\
\hline Specific Gravity & 1.30 & 1.75 & 0.00037 & 0.00037 & 0.00075 & 0.00074 & 1.11 & 1.16 \\
\hline Chemical Composition & Mol/Liter & Mol/Liter & $\mathrm{lb} /$ wscf & $\mathrm{lb} /$ wscf & $\mathrm{lb} / \mathrm{wscf}$ & $\mathrm{lb} /$ wscf & Mol/Liter & Mol/Liter \\
\hline $\mathrm{H}+$ & $2.43 \mathrm{E}+00$ & $6.27 E-01$ & $1.54 \mathrm{E}-04$ & $1.54 \mathrm{E}-04$ & 2.86E-09 & $2.14 \mathrm{E}-08$ & $3.14 \mathrm{E}+00$ & $1.09 \mathrm{E}+01$ \\
\hline $\mathrm{OH}-$ & & 9.52E-09 & & & & & & \\
\hline $\mathrm{Al}+3$ & $6.08 \mathrm{E}-01$ & $2.16 \mathrm{E}+00$ & $1.11 \mathrm{E}-07$ & $1.11 \mathrm{E}-09$ & & & $8.47 \mathrm{E}-07$ & $2.94 \mathrm{E}-06$ \\
\hline $\mathrm{Sb}+5$ & $1.81 \mathrm{E}-05$ & $6.41 \mathrm{E}-05$ & $1.49 \mathrm{E}-11$ & $1.49 \mathrm{E}-13$ & & & $2.52 \mathrm{E}-11$ & $8.75 \mathrm{E}-11$ \\
\hline$A s+5$ & $9.02 \mathrm{E}-05$ & $3.20 \mathrm{E}-04$ & $4.58 \mathrm{E}-11$ & $4.58 \mathrm{E}-13$ & & & $1.26 \mathrm{E}-10$ & $4.37 \mathrm{E}-10$ \\
\hline $\mathrm{Ba}+2$ & $6.85 E-05$ & $2.43 E-04$ & $6.38 \mathrm{E}-11$ & $6.38 \mathrm{E}-13$ & & & $9.56 \mathrm{E}-11$ & $3.32 \mathrm{E}-10$ \\
\hline $\mathrm{Be}+2$ & $2.23 E-05$ & $7.90 \mathrm{E}-05$ & $1.36 \mathrm{E}-12$ & $1.36 \mathrm{E}-14$ & & & $3.11 \mathrm{E}-11$ & $1.08 \mathrm{E}-10$ \\
\hline $\mathrm{B}+3$ & $1.83 \mathrm{E}-02$ & $6.49 \mathrm{E}-02$ & 1.34E-09 & $1.34 \mathrm{E}-11$ & & & $2.55 \mathrm{E}-08$ & $8.86 \mathrm{E}-08$ \\
\hline $\mathrm{Cd}+2$ & $3.27 E-03$ & $1.16 \mathrm{E}-02$ & 2.49E-09 & $2.49 \mathrm{E}-11$ & & & $4.55 \mathrm{E}-09$ & $1.58 \mathrm{E}-08$ \\
\hline $\mathrm{Ca}+2$ & $6.13 E-02$ & $2.17 \mathrm{E}-01$ & $1.67 \mathrm{E}-08$ & $1.67 \mathrm{E}-10$ & & & $8.55 \mathrm{E}-08$ & 2.97E-07 \\
\hline $\mathrm{Cr}+3$ & $5.13 \mathrm{E}-03$ & $1.82 \mathrm{E}-02$ & $1.81 \mathrm{E}-09$ & $1.81 \mathrm{E}-11$ & & & $7.16 \mathrm{E}-09$ & $2.49 \mathrm{E}-08$ \\
\hline $\mathrm{Co}+2$ & $4.28 \mathrm{E}-05$ & $1.52 \mathrm{E}-04$ & $1.71 \mathrm{E}-11$ & $1.71 \mathrm{E}-13$ & & & $5.97 \mathrm{E}-11$ & $2.07 \mathrm{E}-10$ \\
\hline Cs+ & $6.10 \mathrm{E}-05$ & $2.16 \mathrm{E}-04$ & $5.50 \mathrm{E}-11$ & $5.50 \mathrm{E}-13$ & & & $8.51 \mathrm{E}-11$ & $2.96 \mathrm{E}-10$ \\
\hline $\mathrm{Cu}+2$ & 8.80E-04 & $3.12 \mathrm{E}-03$ & $3.79 \mathrm{E}-10$ & $3.79 \mathrm{E}-12$ & & & 1.23E-09 & 4.26E-09 \\
\hline $\mathrm{Fe}+3$ & 2.97E-02 & $1.05 \mathrm{E}-01$ & $1.12 \mathrm{E}-08$ & $1.12 \mathrm{E}-10$ & & & $4.14 \mathrm{E}-08$ & $1.44 \mathrm{E}-07$ \\
\hline $\mathrm{Pb}+2$ & 9.97E-04 & $3.54 \mathrm{E}-03$ & 1.40E-09 & $1.40 \mathrm{E}-11$ & & & 1.39E-09 & 4.83E-09 \\
\hline $\mathrm{Hg}+2$ & 5.44E-03 & $1.80 \mathrm{E}-02$ & 5.09E-06 & $5.09 \mathrm{E}-06$ & $2.68 \mathrm{E}-10$ & $8.58 \mathrm{E}-10$ & $5.23 \mathrm{E}-04$ & 1.79E-03 \\
\hline $\mathrm{Mn}+4$ & 1.65E-02 & $5.84 \mathrm{E}-02$ & $6.13 E-09$ & $6.13 \mathrm{E}-11$ & & & $2.29 \mathrm{E}-08$ & 7.97E-08 \\
\hline $\mathrm{Ni}+2$ & $2.09 E-03$ & $7.40 \mathrm{E}-03$ & $8.30 \mathrm{E}-10$ & $8.30 \mathrm{E}-12$ & & & 2.91E-09 & $1.01 \mathrm{E}-08$ \\
\hline $\mathrm{K}+$ & $2.02 \mathrm{E}-01$ & $7.15 \mathrm{E}-01$ & 5.34E-08 & $5.34 \mathrm{E}-10$ & & & $2.81 \mathrm{E}-07$ & 9.77E-07 \\
\hline $\mathrm{Se}+4$ & $2.59 \mathrm{E}-04$ & $9.18 \mathrm{E}-04$ & $1.38 \mathrm{E}-10$ & $1.38 \mathrm{E}-12$ & & & $3.61 \mathrm{E}-10$ & 1.25E-09 \\
\hline $\mathrm{Ag}+$ & $4.05 \mathrm{E}-04$ & $1.44 \mathrm{E}-03$ & $2.96 \mathrm{E}-10$ & $2.96 \mathrm{E}-12$ & & & $5.64 \mathrm{E}-10$ & $1.96 \mathrm{E}-09$ \\
\hline $\mathrm{Na}+$ & $1.70 \mathrm{E}+00$ & $6.03 \mathrm{E}+00$ & $2.65 \mathrm{E}-07$ & $2.65 E-09$ & & & $2.37 \mathrm{E}-06$ & $8.23 E-06$ \\
\hline $\mathrm{TI}+3$ & $4.76 \mathrm{E}-06$ & 1.69E-05 & $6.59 \mathrm{E}-12$ & $6.59 \mathrm{E}-14$ & & & $6.64 \mathrm{E}-12$ & $2.31 \mathrm{E}-11$ \\
\hline $\mathrm{U}+4$ & 5.63E-04 & $2.00 \mathrm{E}-03$ & $9.08 \mathrm{E}-10$ & $9.07 \mathrm{E}-12$ & & & $7.85 \mathrm{E}-10$ & 2.73E-09 \\
\hline$V+3$ & 3.17E-05 & 1.13E-04 & $1.10 \mathrm{E}-11$ & $1.09 \mathrm{E}-13$ & & & $4.42 \mathrm{E}-11$ & $1.54 \mathrm{E}-10$ \\
\hline$Z n+2$ & 9.43E-04 & $3.34 \mathrm{E}-03$ & $4.18 \mathrm{E}-10$ & $4.18 \mathrm{E}-12$ & & & 1.31E-09 & 4.57E-09 \\
\hline $\mathrm{Zr}+4$ & $3.89 \mathrm{E}-02$ & $1.38 \mathrm{E}-01$ & $2.40 \mathrm{E}-08$ & $2.40 \mathrm{E}-10$ & & & $5.42 \mathrm{E}-08$ & $1.88 \mathrm{E}-07$ \\
\hline $\mathrm{Cl}-$ & $2.05 \mathrm{E}-02$ & 5.94E-02 & $8.93 \mathrm{E}-06$ & $8.93 \mathrm{E}-06$ & $1.72 \mathrm{E}-10$ & 1.25E-09 & $5.18 \mathrm{E}-03$ & $3.58 \mathrm{E}-03$ \\
\hline F- & $3.32 \mathrm{E}-02$ & $1.18 \mathrm{E}-01$ & $2.27 \mathrm{E}-08$ & 1.85E-08 & $1.55 \mathrm{E}-12$ & $3.61 \mathrm{E}-12$ & $2.00 \mathrm{E}-05$ & $6.94 \mathrm{E}-05$ \\
\hline SO4-2 & $9.08 \mathrm{E}-02$ & $3.22 \mathrm{E}-01$ & 5.91E-08 & $5.91 \mathrm{E}-10$ & & & $1.27 \mathrm{E}-07$ & $4.40 \mathrm{E}-07$ \\
\hline NO3- & $6.22 \mathrm{E}+00$ & $1.41 \mathrm{E}+01$ & $9.44 \mathrm{E}-03$ & $9.44 \mathrm{E}-03$ & $1.76 \mathrm{E}-07$ & 1.32E-06 & $3.13 \mathrm{E}+00$ & $1.09 \mathrm{E}+01$ \\
\hline PO4-3 & 1.07E-01 & 3.81E-01 & 6.91E-08 & $6.91 \mathrm{E}-10$ & & & $1.50 \mathrm{E}-07$ & $5.20 \mathrm{E}-07$ \\
\hline $\mathrm{Am}+4$ & $6.52 \mathrm{E}-08$ & $2.31 \mathrm{E}-07$ & $1.07 \mathrm{E}-13$ & $1.07 \mathrm{E}-15$ & & & $9.09 \mathrm{E}-14$ & $3.16 \mathrm{E}-13$ \\
\hline $\mathrm{Br}-$ & $2.71 \mathrm{E}-07$ & $7.65 \mathrm{E}-07$ & $3.00 \mathrm{E}-10$ & $3.00 \mathrm{E}-10$ & $5.78 \mathrm{E}-15$ & $4.20 \mathrm{E}-14$ & $7.73 \mathrm{E}-08$ & $5.34 \mathrm{E}-08$ \\
\hline $\mathrm{Ce}+4$ & 3.96E-05 & $1.40 \mathrm{E}-04$ & $3.76 \mathrm{E}-11$ & $3.76 \mathrm{E}-13$ & & & $5.52 \mathrm{E}-11$ & $1.92 \mathrm{E}-10$ \\
\hline $\mathrm{Eu+3}$ & 4.51E-07 & $1.60 \mathrm{E}-06$ & $4.64 \mathrm{E}-13$ & $4.64 \mathrm{E}-15$ & & & $6.28 \mathrm{E}-13$ & $2.18 \mathrm{E}-12$ \\
\hline $\mathrm{Gd}+3$ & 1.35E-04 & $4.80 \mathrm{E}-04$ & $1.44 \mathrm{E}-10$ & $1.44 \mathrm{E}-12$ & & & $1.89 \mathrm{E}-10$ & $6.55 \mathrm{E}-10$ \\
\hline $\mathrm{Ge}+4$ & 7.82E-09 & $2.77 \mathrm{E}-08$ & $3.85 \mathrm{E}-15$ & $3.85 \mathrm{E}-17$ & & & $1.09 \mathrm{E}-14$ & $3.79 \mathrm{E}-14$ \\
\hline $\ln +3$ & 1.26E-06 & $4.45 \mathrm{E}-06$ & $9.76 \mathrm{E}-13$ & $9.76 \mathrm{E}-15$ & & & $1.75 \mathrm{E}-12$ & $6.08 \mathrm{E}-12$ \\
\hline I- & $3.02 \mathrm{E}-06$ & $9.06 \mathrm{E}-06$ & 3.96E-09 & 3.96E-09 & $7.63 \mathrm{E}-14$ & $5.54 \mathrm{E}-13$ & $6.42 \mathrm{E}-07$ & $4.43 \mathrm{E}-07$ \\
\hline $\mathrm{La}+3$ & $8.17 \mathrm{E}-06$ & $2.90 \mathrm{E}-05$ & $7.69 \mathrm{E}-12$ & $7.69 \mathrm{E}-14$ & & & $1.14 \mathrm{E}-11$ & $3.96 \mathrm{E}-11$ \\
\hline Li+ & 5.42E-04 & 1.92E-03 & $2.55 \mathrm{E}-11$ & $2.55 \mathrm{E}-13$ & & & $7.55 \mathrm{E}-10$ & 2.62E-09 \\
\hline $\mathrm{Mg}+2$ & 1.94E-02 & $6.87 \mathrm{E}-02$ & 3.19E-09 & $3.19 \mathrm{E}-11$ & & & $2.70 \mathrm{E}-08$ & $9.38 \mathrm{E}-08$ \\
\hline $\mathrm{Mo}+6$ & 8.37E-04 & $2.97 \mathrm{E}-03$ & $5.44 \mathrm{E}-10$ & $5.44 \mathrm{E}-12$ & & & 1.17E-09 & $4.05 E-09$ \\
\hline
\end{tabular}


Table C-7. Revised baseline mass balance, Tank WM-189 waste (Continued).

\begin{tabular}{|c|c|c|c|c|c|c|c|c|}
\hline Stream \# & 101 & 102 & 103 & 104 & 105 & 106 & 107 & 108 \\
\hline Stream Name & $\begin{array}{l}\text { SBW } \\
\text { Feed }\end{array}$ & $\begin{array}{c}\text { Evaporator } \\
\text { Bottoms }\end{array}$ & $\begin{array}{c}\text { Evaporator } \\
\text { Overheads } \\
\text { Vapor }\end{array}$ & $\begin{array}{c}\text { Mist } \\
\text { Eliminator } \\
\text { Vapor }\end{array}$ & $\begin{array}{l}\text { VOG } \\
\text { Offgas } \\
\text { Vapor }\end{array}$ & $\begin{array}{c}\text { POG + VOG } \\
\text { Offgas } \\
\text { Vapor }\end{array}$ & $\begin{array}{l}\text { LET\&D } \\
\text { Feed } \\
\text { Liquid }\end{array}$ & $\begin{array}{l}\text { LET\&D } \\
\text { Acid } \\
\text { Liquid }\end{array}$ \\
\hline Chemical Composition & Mol/Liter & Mol/Liter & $\mathrm{lb} / \mathrm{wscf}$ & $\mathrm{lb} / \mathrm{wscf}$ & $\mathrm{lb} / \mathrm{wscf}$ & $\mathrm{Ib} / \mathrm{wscf}$ & Mol/Liter & Mol/Liter \\
\hline $\mathrm{Nd}+3$ & $2.64 \mathrm{E}-05$ & 9.35E-05 & $2.58 \mathrm{E}-11$ & $2.58 \mathrm{E}-13$ & & & $3.68 \mathrm{E}-11$ & $1.28 \mathrm{E}-10$ \\
\hline $\mathrm{Np}+4$ & $2.74 \mathrm{E}-06$ & 9.71E-06 & $4.40 \mathrm{E}-12$ & $4.40 \mathrm{E}-14$ & & & $3.82 \mathrm{E}-12$ & $1.33 \mathrm{E}-11$ \\
\hline $\mathrm{Nb}+5$ & $1.14 \mathrm{E}-03$ & 4.05E-03 & $7.18 \mathrm{E}-10$ & $7.18 \mathrm{E}-12$ & & & 1.59E-09 & $5.53 \mathrm{E}-09$ \\
\hline $\mathrm{Pd}+4$ & 1.69E-03 & 5.99E-03 & $1.22 \mathrm{E}-09$ & $1.22 \mathrm{E}-11$ & & & 2.36E-09 & $8.18 \mathrm{E}-09$ \\
\hline $\mathrm{Pu}+4$ & $6.57 \mathrm{E}-06$ & $2.33 \mathrm{E}-05$ & $1.09 \mathrm{E}-11$ & $1.09 \mathrm{E}-13$ & & & $9.16 \mathrm{E}-12$ & $3.18 \mathrm{E}-11$ \\
\hline $\mathrm{Pr}+4$ & $7.43 E-06$ & $2.64 \mathrm{E}-05$ & $7.09 \mathrm{E}-12$ & $7.09 \mathrm{E}-14$ & & & $1.04 \mathrm{E}-11$ & $3.60 \mathrm{E}-11$ \\
\hline $\mathrm{Rh}+4$ & $3.21 \mathrm{E}-06$ & $1.14 \mathrm{E}-05$ & $2.24 \mathrm{E}-12$ & $2.24 \mathrm{E}-14$ & & & $4.47 \mathrm{E}-12$ & $1.55 \mathrm{E}-11$ \\
\hline $\mathrm{Rb}+$ & 4.93E-06 & $1.75 \mathrm{E}-05$ & $2.86 \mathrm{E}-12$ & $2.86 \mathrm{E}-14$ & & & $6.88 \mathrm{E}-12$ & $2.39 \mathrm{E}-11$ \\
\hline Ru+3 & 8.85E-04 & $3.14 \mathrm{E}-03$ & $6.06 \mathrm{E}-10$ & $6.06 \mathrm{E}-12$ & & & 1.23E-09 & 4.29E-09 \\
\hline $\mathrm{Sm}+3$ & 4.91E-06 & $1.74 \mathrm{E}-05$ & $5.01 \mathrm{E}-12$ & $5.01 \mathrm{E}-14$ & & & $6.85 \mathrm{E}-12$ & $2.38 \mathrm{E}-11$ \\
\hline $\mathrm{Si}+4$ & 9.84E-02 & $3.49 E-01$ & $1.87 \mathrm{E}-08$ & $1.87 \mathrm{E}-10$ & & & $1.37 \mathrm{E}-07$ & 4.77E-07 \\
\hline $\mathrm{Sr}+2$ & 1.19E-04 & $4.22 \mathrm{E}-04$ & $7.06 \mathrm{E}-11$ & $7.06 \mathrm{E}-13$ & & & $1.66 \mathrm{E}-10$ & $5.76 \mathrm{E}-10$ \\
\hline $\mathrm{Tc}+7$ & $5.86 \mathrm{E}-06$ & 2.08E-05 & 3.85E-12 & $3.85 \mathrm{E}-14$ & & & 8.18E-12 & $2.84 \mathrm{E}-11$ \\
\hline $\mathrm{Te}+4$ & $5.74 \mathrm{E}-06$ & $2.04 \mathrm{E}-05$ & $4.96 \mathrm{E}-12$ & $4.96 \mathrm{E}-14$ & & & $8.01 \mathrm{E}-12$ & $2.78 \mathrm{E}-11$ \\
\hline $\mathrm{Tb}+4$ & $1.88 \mathrm{E}-09$ & 6.67E-09 & $2.03 E-15$ & $2.03 \mathrm{E}-17$ & & & $2.62 \mathrm{E}-15$ & $9.11 \mathrm{E}-15$ \\
\hline $\mathrm{Th}+4$ & $2.85 E-05$ & 1.01E-04 & $4.48 \mathrm{E}-11$ & $4.48 \mathrm{E}-13$ & & & 3.97E-11 & $1.38 \mathrm{E}-10$ \\
\hline $\mathrm{Sn}+4$ & 1.92E-03 & $6.82 \mathrm{E}-03$ & 1.55E-09 & $1.55 \mathrm{E}-11$ & & & $2.68 \mathrm{E}-09$ & $9.31 \mathrm{E}-09$ \\
\hline $\mathrm{Ti}+4$ & 9.93E-04 & $3.52 \mathrm{E}-03$ & $3.22 \mathrm{E}-10$ & $3.22 \mathrm{E}-12$ & & & 1.38E-09 & $4.81 \mathrm{E}-09$ \\
\hline $\mathrm{Y}+3$ & 6.09E-06 & $2.16 \mathrm{E}-05$ & $3.67 \mathrm{E}-12$ & $3.67 \mathrm{E}-14$ & & & $8.50 \mathrm{E}-12$ & $2.95 \mathrm{E}-11$ \\
\hline O-2 (oxides) & 1.57E-01 & 5.57E-01 & $1.70 \mathrm{E}-08$ & $1.70 \mathrm{E}-10$ & & & 2.19E-07 & $7.61 \mathrm{E}-07$ \\
\hline $\mathrm{H} 2 \mathrm{O}$ & $4.47 \mathrm{E}+01$ & $2.83 \mathrm{E}+01$ & $4.42 \mathrm{E}-02$ & 4.42E-02 & $1.25 \mathrm{E}-03$ & $1.08 \mathrm{E}-03$ & $5.05 \mathrm{E}+01$ & $2.66 \mathrm{E}+01$ \\
\hline $\mathrm{O} 2$ & & $1.42 \mathrm{E}-10$ & $1.42 \mathrm{E}-07$ & $1.42 \mathrm{E}-07$ & 1.70E-02 & $1.70 \mathrm{E}-02$ & 5.87E-05 & \\
\hline $\mathrm{N} 2$ & & $1.72 \mathrm{E}-10$ & 2.36E-07 & 2.36E-07 & $5.59 \mathrm{E}-02$ & $5.61 \mathrm{E}-02$ & $8.43 \mathrm{E}-05$ & \\
\hline C (organic) & 4.79E-02 & $1.68 \mathrm{E}-01$ & $4.26 \mathrm{E}-07$ & 4.22E-07 & $1.52 \mathrm{E}-12$ & $5.34 \mathrm{E}-11$ & $7.23 \mathrm{E}-04$ & $2.51 \mathrm{E}-03$ \\
\hline $\mathrm{H}$ (organic) & $4.79 \mathrm{E}-02$ & $1.68 \mathrm{E}-01$ & $3.57 \mathrm{E}-08$ & $3.54 \mathrm{E}-08$ & $1.27 \mathrm{E}-13$ & $4.48 \mathrm{E}-12$ & $7.23 \mathrm{E}-04$ & $2.51 \mathrm{E}-03$ \\
\hline \multicolumn{9}{|l|}{ SiO2, BFS, or OPC Additives } \\
\hline $\begin{array}{c}\text { Gas Stream Bulk Composition } \\
\text { (wet basis) }\end{array}$ & $\begin{array}{l}\text { mol. } \% \\
\text { or ppmv }\end{array}$ & $\begin{array}{l}\mathrm{mol} . \% \\
\text { or ppmv }\end{array}$ & $\begin{array}{l}\text { mol. } \% \\
\text { or ppmv }\end{array}$ & $\begin{array}{l}\text { mol. } \% \\
\text { or ppmv }\end{array}$ & $\begin{array}{l}\text { mol.\% } \\
\text { or ppmv }\end{array}$ & $\begin{array}{l}\text { mol. } \% \\
\text { or ppmv }\end{array}$ & $\begin{array}{l}\text { mol. } \% \\
\text { or ppmv }\end{array}$ & $\begin{array}{l}\text { mol. } \% \\
\text { or ppmv }\end{array}$ \\
\hline $\mathrm{H} 2 \mathrm{O}, \mathrm{mol} . \%$ & & & 94.15 & 94.15 & 2.68 & 2.31 & & \\
\hline O2, mol.\% & & & 0.0002 & 0.0002 & 20.44 & 20.51 & & \\
\hline $\mathrm{N} 2, \mathrm{~mol} . \%$ & & & 0.0003 & 0.0003 & 76.88 & 77.17 & & \\
\hline \multicolumn{9}{|l|}{$\mathrm{CO} 2, \mathrm{~mol} . \%$} \\
\hline HNO3, mol. \% & & & 5.85 & 5.85 & 1.1E-04 & 8.2E-04 & & \\
\hline \multicolumn{9}{|l|}{$\mathrm{CO}, \mathrm{ppmv}$} \\
\hline \multicolumn{9}{|l|}{$\mathrm{NO}, \mathrm{ppmv}$} \\
\hline \multicolumn{9}{|l|}{ NO2, ppmv } \\
\hline C (organic), ppmv & & & 14 & 13 & 4.9E-05 & 1.7E-03 & & \\
\hline $\mathrm{H}$ (organic), ppmv & & & 14 & 13 & 4.9E-05 & 1.7E-03 & & \\
\hline PM (mg/wscm) & & & 6 & 0.1 & & & & \\
\hline $\begin{array}{c}\text { Gas Stream Bulk Composition } \\
\text { (dry basis) }\end{array}$ & $\begin{array}{l}\text { mol.\% } \\
\text { or ppmv }\end{array}$ & $\begin{array}{l}\text { mol. } \% \\
\text { or ppmv }\end{array}$ & $\begin{array}{l}\text { mol. } \% \\
\text { or ppmv }\end{array}$ & $\begin{array}{l}\text { mol. } \% \\
\text { or ppmv }\end{array}$ & $\begin{array}{l}\text { mol. } \% \\
\text { or ppmv }\end{array}$ & $\begin{array}{l}\text { mol. } \% \\
\text { or ppmv }\end{array}$ & $\begin{array}{l}\text { mol. } \% \\
\text { or ppmv }\end{array}$ & $\begin{array}{l}\text { mol. } \% \\
\text { or ppmv }\end{array}$ \\
\hline $\mathrm{O} 2, \mathrm{~mol} . \%$ & & & 0.003 & 0.003 & 21.00 & 21.00 & & \\
\hline N2, mol.\% & & & 0.01 & 0.01 & 79.00 & 79.00 & & \\
\hline \multicolumn{9}{|l|}{$\mathrm{CO} 2, \mathrm{~mol} . \%$} \\
\hline HNO3, mol. \% & & & 99.94 & 99.96 & $1.1 \mathrm{E}-04$ & 8.4E-04 & & \\
\hline \multicolumn{9}{|l|}{$\mathrm{CO}, \mathrm{ppmv}$} \\
\hline \multicolumn{9}{|l|}{$\mathrm{NO}, \mathrm{ppmv}$} \\
\hline \multicolumn{9}{|l|}{ NO2, ppmv } \\
\hline C (organic), ppmv & & & 232 & 230 & $5.0 \mathrm{E}-05$ & $1.8 \mathrm{E}-03$ & & \\
\hline $\mathrm{H}$ (organic), ppmv & & & 232 & 230 & $5.0 \mathrm{E}-05$ & $1.8 \mathrm{E}-03$ & & \\
\hline $\mathrm{PM}(\mathrm{mg} / \mathrm{dscm})$ & & & 95 & 1 & & & & \\
\hline
\end{tabular}


Table C-7. Revised baseline mass balance, Tank WM-189 waste (Continued).

\begin{tabular}{|c|c|c|c|c|c|c|c|c|}
\hline Stream \# & 101 & 102 & 103 & 104 & 105 & 106 & 107 & 108 \\
\hline Stream Name & $\begin{array}{l}\text { SBW } \\
\text { Feed }\end{array}$ & $\begin{array}{c}\text { Evaporator } \\
\text { Bottoms }\end{array}$ & $\begin{array}{c}\text { Evaporator } \\
\text { Overheads } \\
\text { Vapor } \\
\end{array}$ & $\begin{array}{c}\text { Mist } \\
\text { Eliminator } \\
\text { Vapor }\end{array}$ & $\begin{array}{l}\text { VOG } \\
\text { Offgas } \\
\text { Vapor } \\
\end{array}$ & $\begin{array}{c}\text { POG + VOG } \\
\text { Offgas } \\
\text { Vapor }\end{array}$ & $\begin{array}{l}\text { LET\&D } \\
\text { Feed } \\
\text { Liquid } \\
\end{array}$ & $\begin{array}{l}\text { LET\&D } \\
\text { Acid } \\
\text { Liquid } \\
\end{array}$ \\
\hline Canister Rate, canisters/day & & 2.75 & & & & & & \\
\hline Total Canisters Generated & & 382 & & & & & & \\
\hline \multicolumn{9}{|l|}{ Drum Rate, drums/day } \\
\hline \multicolumn{9}{|l|}{ Total Drums Generated } \\
\hline Radiological Composition & $\mathrm{Ci} / \mathrm{L}$ & $\mathrm{Ci} / \mathrm{L}$ & $\mathrm{Ci} / \mathrm{wscm}$ & $\mathrm{Ci} / \mathrm{wscm}$ & $\mathrm{Ci} / \mathrm{wscm}$ & $\mathrm{Ci} /$ wscm & $\mathrm{Ci} / \mathrm{L}$ & $\mathrm{Ci} / \mathrm{L}$ \\
\hline Ra-226 & $7.31 \mathrm{E}-12$ & $2.59 \mathrm{E}-11$ & $7.94 \mathrm{E}-16$ & $7.94 \mathrm{E}-18$ & $2.29 \mathrm{E}-25$ & $1.98 \mathrm{E}-25$ & $1.02 \mathrm{E}-17$ & $3.54 \mathrm{E}-17$ \\
\hline Ac-227 & $3.44 \mathrm{E}-11$ & $1.22 \mathrm{E}-10$ & $3.74 \mathrm{E}-15$ & $3.74 \mathrm{E}-17$ & $1.08 \mathrm{E}-24$ & $9.32 \mathrm{E}-25$ & $4.80 \mathrm{E}-17$ & $1.67 \mathrm{E}-16$ \\
\hline Th-228 & $2.86 \mathrm{E}-09$ & $1.01 \mathrm{E}-08$ & $3.10 \mathrm{E}-13$ & $3.10 \mathrm{E}-15$ & $8.94 \mathrm{E}-23$ & $7.74 \mathrm{E}-23$ & $3.98 \mathrm{E}-15$ & $1.38 \mathrm{E}-14$ \\
\hline Th-230 & $9.63 \mathrm{E}-10$ & 3.42E-09 & $1.05 \mathrm{E}-13$ & $1.05 \mathrm{E}-15$ & $3.01 \mathrm{E}-23$ & $2.61 \mathrm{E}-23$ & $1.34 \mathrm{E}-15$ & 4.67E-15 \\
\hline Th-232 & $6.32 \mathrm{E}-16$ & $2.24 \mathrm{E}-15$ & $6.86 \mathrm{E}-20$ & $6.86 \mathrm{E}-22$ & $1.98 \mathrm{E}-29$ & $1.71 \mathrm{E}-29$ & $8.82 \mathrm{E}-22$ & $3.06 \mathrm{E}-21$ \\
\hline $\mathrm{Pa}-231$ & $7.98 \mathrm{E}-11$ & $2.83 \mathrm{E}-10$ & $8.66 \mathrm{E}-15$ & $8.66 \mathrm{E}-17$ & $2.50 \mathrm{E}-24$ & $2.16 \mathrm{E}-24$ & $1.11 \mathrm{E}-16$ & $3.86 \mathrm{E}-16$ \\
\hline $\mathrm{Pa}-233$ & $2.62 \mathrm{E}-06$ & $9.28 \mathrm{E}-06$ & $2.84 \mathrm{E}-10$ & $2.84 \mathrm{E}-12$ & $8.19 \mathrm{E}-20$ & 7.09E-20 & $3.65 \mathrm{E}-12$ & $1.27 \mathrm{E}-11$ \\
\hline U-232 & 2.26E-09 & 8.00E-09 & $2.45 \mathrm{E}-13$ & $2.45 \mathrm{E}-15$ & $7.06 \mathrm{E}-23$ & $6.11 \mathrm{E}-23$ & $3.14 \mathrm{E}-15$ & $1.09 \mathrm{E}-14$ \\
\hline U-233 & $7.97 \mathrm{E}-11$ & $2.83 \mathrm{E}-10$ & $8.65 E-15$ & $8.65 \mathrm{E}-17$ & $2.49 \mathrm{E}-24$ & $2.16 \mathrm{E}-24$ & $1.11 \mathrm{E}-16$ & $3.86 \mathrm{E}-16$ \\
\hline U-234 & $1.56 \mathrm{E}-06$ & $5.55 \mathrm{E}-06$ & $1.70 \mathrm{E}-10$ & $1.70 \mathrm{E}-12$ & 4.89E-20 & $4.23 \mathrm{E}-20$ & $2.18 \mathrm{E}-12$ & $7.57 \mathrm{E}-12$ \\
\hline U-235 & $6.30 \mathrm{E}-08$ & $2.24 \mathrm{E}-07$ & $6.84 \mathrm{E}-12$ & $6.84 \mathrm{E}-14$ & 1.97E-21 & 1.71E-21 & $8.79 \mathrm{E}-14$ & $3.05 \mathrm{E}-13$ \\
\hline U-236 & 7.31E-08 & 2.59E-07 & $7.93 \mathrm{E}-12$ & 7.93E-14 & $2.29 \mathrm{E}-21$ & $1.98 \mathrm{E}-21$ & $1.02 \mathrm{E}-13$ & $3.54 \mathrm{E}-13$ \\
\hline U-237 & $5.75 \mathrm{E}-09$ & $2.04 \mathrm{E}-08$ & $6.24 \mathrm{E}-13$ & $6.24 \mathrm{E}-15$ & $1.80 \mathrm{E}-22$ & $1.56 \mathrm{E}-22$ & $8.01 \mathrm{E}-15$ & $2.78 \mathrm{E}-14$ \\
\hline U-238 & $3.48 \mathrm{E}-08$ & 1.23E-07 & $3.77 \mathrm{E}-12$ & $3.77 \mathrm{E}-14$ & 1.09E-21 & $9.41 \mathrm{E}-22$ & $4.85 \mathrm{E}-14$ & $1.68 \mathrm{E}-13$ \\
\hline Np-236 & $2.76 \mathrm{E}-12$ & $9.77 \mathrm{E}-12$ & $2.99 \mathrm{E}-16$ & $2.99 \mathrm{E}-18$ & $8.62 E-26$ & $7.46 \mathrm{E}-26$ & $3.84 \mathrm{E}-18$ & $1.33 \mathrm{E}-17$ \\
\hline Np-237 & 4.66E-07 & 1.65E-06 & $5.05 \mathrm{E}-11$ & $5.05 \mathrm{E}-13$ & $1.46 \mathrm{E}-20$ & $1.26 \mathrm{E}-20$ & $6.49 \mathrm{E}-13$ & $2.25 \mathrm{E}-12$ \\
\hline Pu-236 & $3.68 \mathrm{E}-09$ & $1.30 \mathrm{E}-08$ & $3.99 \mathrm{E}-13$ & $3.99 \mathrm{E}-15$ & $1.15 \mathrm{E}-22$ & $9.95 \mathrm{E}-23$ & $5.13 \mathrm{E}-15$ & $1.78 \mathrm{E}-14$ \\
\hline Pu-238 & 8.44E-04 & $2.99 \mathrm{E}-03$ & $9.16 \mathrm{E}-08$ & $9.16 \mathrm{E}-10$ & $2.64 \mathrm{E}-17$ & $2.29 \mathrm{E}-17$ & 1.18E-09 & $4.09 \mathrm{E}-09$ \\
\hline Pu-239 & $9.22 \mathrm{E}-05$ & $3.27 \mathrm{E}-04$ & 1.00E-08 & $1.00 \mathrm{E}-10$ & $2.89 \mathrm{E}-18$ & $2.50 \mathrm{E}-18$ & $1.29 \mathrm{E}-10$ & $4.47 \mathrm{E}-10$ \\
\hline Pu-240 & $1.37 \mathrm{E}-05$ & 4.86E-05 & 1.49E-09 & 1.49E-11 & $4.29 \mathrm{E}-19$ & $3.71 \mathrm{E}-19$ & $1.91 \mathrm{E}-11$ & $6.64 \mathrm{E}-11$ \\
\hline Pu-241 & 7.39E-04 & $2.62 \mathrm{E}-03$ & $8.02 \mathrm{E}-08$ & $8.02 \mathrm{E}-10$ & $2.31 \mathrm{E}-17$ & $2.00 \mathrm{E}-17$ & 1.03E-09 & $3.58 \mathrm{E}-09$ \\
\hline Pu-242 & 1.07E-08 & 3.81E-08 & $1.16 \mathrm{E}-12$ & $1.16 \mathrm{E}-14$ & $3.36 \mathrm{E}-22$ & $2.91 \mathrm{E}-22$ & $1.50 \mathrm{E}-14$ & $5.20 \mathrm{E}-14$ \\
\hline \begin{tabular}{|l|}
$\mathrm{Pu}-244$ \\
\end{tabular} & $3.17 \mathrm{E}-16$ & 1.13E-15 & $3.44 \mathrm{E}-20$ & $3.44 \mathrm{E}-22$ & $9.93 \mathrm{E}-30$ & $8.59 \mathrm{E}-30$ & 4.43E-22 & $1.54 \mathrm{E}-21$ \\
\hline Am-241 & $7.25 \mathrm{E}-05$ & $2.57 \mathrm{E}-04$ & 7.87E-09 & $7.87 \mathrm{E}-11$ & $2.27 \mathrm{E}-18$ & $1.96 \mathrm{E}-18$ & $1.01 \mathrm{E}-10$ & $3.51 \mathrm{E}-10$ \\
\hline Am-242m & $1.36 \mathrm{E}-08$ & 4.84E-08 & $1.48 \mathrm{E}-12$ & $1.48 \mathrm{E}-14$ & $4.27 \mathrm{E}-22$ & $3.69 \mathrm{E}-22$ & $1.90 \mathrm{E}-14$ & $6.61 \mathrm{E}-14$ \\
\hline Am-243 & $2.32 \mathrm{E}-08$ & $8.22 \mathrm{E}-08$ & $2.52 \mathrm{E}-12$ & $2.52 \mathrm{E}-14$ & $7.25 \mathrm{E}-22$ & $6.28 \mathrm{E}-22$ & $3.23 \mathrm{E}-14$ & $1.12 \mathrm{E}-13$ \\
\hline Cm-242 & $2.72 \mathrm{E}-08$ & 9.66E-08 & $2.96 \mathrm{E}-12$ & $2.96 \mathrm{E}-14$ & $8.53 \mathrm{E}-22$ & $7.38 \mathrm{E}-22$ & $3.80 \mathrm{E}-14$ & $1.32 \mathrm{E}-13$ \\
\hline $\mathrm{Cm}-243$ & $3.34 \mathrm{E}-08$ & $1.18 \mathrm{E}-07$ & $3.62 \mathrm{E}-12$ & $3.62 \mathrm{E}-14$ & $1.04 \mathrm{E}-21$ & $9.03 \mathrm{E}-22$ & $4.65 \mathrm{E}-14$ & $1.62 \mathrm{E}-13$ \\
\hline $\mathrm{Cm}-244$ & $1.41 \mathrm{E}-06$ & $5.00 \mathrm{E}-06$ & $1.53 \mathrm{E}-10$ & $1.53 \mathrm{E}-12$ & $4.41 \mathrm{E}-20$ & $3.82 \mathrm{E}-20$ & $1.97 \mathrm{E}-12$ & $6.83 \mathrm{E}-12$ \\
\hline $\mathrm{Cm}-245$ & $3.51 \mathrm{E}-10$ & $1.25 \mathrm{E}-09$ & $3.81 \mathrm{E}-14$ & $3.81 \mathrm{E}-16$ & $1.10 \mathrm{E}-23$ & $9.51 \mathrm{E}-24$ & $4.90 \mathrm{E}-16$ & $1.70 \mathrm{E}-15$ \\
\hline $\mathrm{Cm}-246$ & $2.31 \mathrm{E}-11$ & $8.18 \mathrm{E}-11$ & $2.50 \mathrm{E}-15$ & $2.50 \mathrm{E}-17$ & $7.22 \mathrm{E}-25$ & $6.24 \mathrm{E}-25$ & $3.21 \mathrm{E}-17$ & $1.12 \mathrm{E}-16$ \\
\hline Cm-247 & $6.19 \mathrm{E}-18$ & $2.20 \mathrm{E}-17$ & $6.72 \mathrm{E}-22$ & $6.72 \mathrm{E}-24$ & & & $8.63 E-24$ & $3.00 \mathrm{E}-23$ \\
\hline $\mathrm{H}-3$ & $9.12 \mathrm{E}-06$ & $5.76 \mathrm{E}-06$ & $8.00 \mathrm{E}-06$ & $8.00 \mathrm{E}-06$ & 1.83E-07 & $1.58 \mathrm{E}-07$ & $1.03 \mathrm{E}-05$ & $5.41 \mathrm{E}-06$ \\
\hline $\mathrm{Be}-10$ & $2.68 \mathrm{E}-12$ & $9.50 \mathrm{E}-12$ & $2.91 \mathrm{E}-16$ & $2.91 \mathrm{E}-18$ & $8.38 \mathrm{E}-26$ & $7.25 \mathrm{E}-26$ & $3.74 \mathrm{E}-18$ & $1.30 \mathrm{E}-17$ \\
\hline $\mathrm{C}-14$ & $1.46 \mathrm{E}-10$ & $5.18 \mathrm{E}-10$ & $1.58 \mathrm{E}-14$ & $1.58 \mathrm{E}-16$ & $4.57 \mathrm{E}-24$ & $3.95 \mathrm{E}-24$ & $2.04 \mathrm{E}-16$ & $7.07 \mathrm{E}-16$ \\
\hline Co-60 & 2.97E-05 & 1.05E-04 & 3.23E-09 & $3.23 E-11$ & $9.31 \mathrm{E}-19$ & $8.05 E-19$ & $4.15 \mathrm{E}-11$ & $1.44 \mathrm{E}-10$ \\
\hline $\mathrm{Ni}-59$ & $1.23 \mathrm{E}-06$ & $4.35 \mathrm{E}-06$ & $1.33 \mathrm{E}-10$ & $1.33 \mathrm{E}-12$ & $3.83 E-20$ & $3.32 \mathrm{E}-20$ & $1.71 \mathrm{E}-12$ & $5.93 \mathrm{E}-12$ \\
\hline $\mathrm{Ni}-63$ & 3.87E-05 & 1.37E-04 & $4.20 \mathrm{E}-09$ & $4.20 \mathrm{E}-11$ & $1.21 \mathrm{E}-18$ & $1.05 \mathrm{E}-18$ & $5.40 \mathrm{E}-11$ & $1.88 \mathrm{E}-10$ \\
\hline Se-79 & 5.30E-07 & $1.88 \mathrm{E}-06$ & $5.75 \mathrm{E}-11$ & $5.75 \mathrm{E}-13$ & $1.66 \mathrm{E}-20$ & $1.43 \mathrm{E}-20$ & $7.39 \mathrm{E}-13$ & $2.57 \mathrm{E}-12$ \\
\hline Rb-87 & $2.62 \mathrm{E}-11$ & $9.28 \mathrm{E}-11$ & $2.84 \mathrm{E}-15$ & $2.84 \mathrm{E}-17$ & $8.19 \mathrm{E}-25$ & $7.08 \mathrm{E}-25$ & $3.65 \mathrm{E}-17$ & $1.27 \mathrm{E}-16$ \\
\hline Sr-90 & 3.53E-02 & $1.25 \mathrm{E}-01$ & 3.83E-06 & 3.83E-08 & $1.11 \mathrm{E}-15$ & $9.56 \mathrm{E}-16$ & 4.92E-08 & $1.71 \mathrm{E}-07$ \\
\hline Y-90 & $3.53 E-02$ & $1.25 \mathrm{E}-01$ & $3.83 \mathrm{E}-06$ & $3.83 \mathrm{E}-08$ & $1.11 \mathrm{E}-15$ & $9.56 \mathrm{E}-16$ & $4.92 \mathrm{E}-08$ & $1.71 \mathrm{E}-07$ \\
\hline Zr-93 & $1.98 \mathrm{E}-06$ & $7.02 \mathrm{E}-06$ & $2.15 \mathrm{E}-10$ & $2.15 \mathrm{E}-12$ & $6.19 \mathrm{E}-20$ & $5.36 \mathrm{E}-20$ & $2.76 \mathrm{E}-12$ & $9.58 \mathrm{E}-12$ \\
\hline $\mathrm{Nb}-93 \mathrm{~m}$ & $1.52 \mathrm{E}-06$ & 5.41E-06 & $1.65 \mathrm{E}-10$ & $1.65 \mathrm{E}-12$ & $4.77 \mathrm{E}-20$ & $4.13 \mathrm{E}-20$ & $2.12 \mathrm{E}-12$ & $7.38 \mathrm{E}-12$ \\
\hline Nb-94 & $1.38 \mathrm{E}-06$ & 4.89E-06 & $1.50 \mathrm{E}-10$ & $1.50 \mathrm{E}-12$ & $4.31 \mathrm{E}-20$ & $3.73 \mathrm{E}-20$ & $1.92 \mathrm{E}-12$ & $6.67 \mathrm{E}-12$ \\
\hline Tc-98 & $2.30 \mathrm{E}-12$ & $8.17 \mathrm{E}-12$ & $2.50 \mathrm{E}-16$ & $2.50 \mathrm{E}-18$ & $7.21 \mathrm{E}-26$ & $6.24 \mathrm{E}-26$ & $3.21 \mathrm{E}-18$ & $1.12 \mathrm{E}-17$ \\
\hline Tc-99 & $1.40 \mathrm{E}-05$ & 4.95E-05 & 1.52E-09 & $1.52 \mathrm{E}-11$ & $4.37 \mathrm{E}-19$ & $3.78 \mathrm{E}-19$ & $1.95 \mathrm{E}-11$ & $6.76 \mathrm{E}-11$ \\
\hline
\end{tabular}


Table C-7. Revised baseline mass balance, Tank WM-189 waste (Continued).

\begin{tabular}{|c|c|c|c|c|c|c|c|c|}
\hline Stream \# & 101 & 102 & 103 & 104 & 105 & 106 & 107 & 108 \\
\hline Stream Name & $\begin{array}{l}\text { SBW } \\
\text { Feed }\end{array}$ & $\begin{array}{c}\text { Evaporator } \\
\text { Bottoms }\end{array}$ & $\begin{array}{c}\text { Evaporator } \\
\text { Overheads } \\
\text { Vapor }\end{array}$ & $\begin{array}{c}\text { Mist } \\
\text { Eliminator } \\
\text { Vapor }\end{array}$ & $\begin{array}{l}\text { VOG } \\
\text { Offgas } \\
\text { Vapor }\end{array}$ & $\begin{array}{c}\text { POG + VOG } \\
\text { Offgas } \\
\text { Vapor }\end{array}$ & $\begin{array}{l}\text { LET\&D } \\
\text { Feed } \\
\text { Liquid }\end{array}$ & $\begin{array}{l}\text { LET\&D } \\
\text { Acid } \\
\text { Liquid }\end{array}$ \\
\hline Radiological Composition & $\mathrm{Ci} / \mathrm{L}$ & $\mathrm{Ci} / \mathrm{L}$ & Ci/wscm & $\mathrm{Ci} / \mathrm{wscm}$ & Ci/wscm & $\mathrm{Ci} / \mathrm{wscm}$ & $\mathrm{Ci} / \mathrm{L}$ & $\mathrm{Ci} / \mathrm{L}$ \\
\hline Ru-106 & $1.13 \mathrm{E}-06$ & 4.02E-06 & $1.23 \mathrm{E}-10$ & $1.23 \mathrm{E}-12$ & $3.54 \mathrm{E}-20$ & 3.07E-20 & $1.58 \mathrm{E}-12$ & $5.48 \mathrm{E}-12$ \\
\hline Rh-102 & $7.70 \mathrm{E}-10$ & $2.73 E-09$ & $8.35 E-14$ & $8.35 E-16$ & $2.41 \mathrm{E}-23$ & $2.08 \mathrm{E}-23$ & $1.07 \mathrm{E}-15$ & $3.73 \mathrm{E}-15$ \\
\hline Rh-106 & $1.13 \mathrm{E}-06$ & $4.02 \mathrm{E}-06$ & $1.23 \mathrm{E}-10$ & $1.23 \mathrm{E}-12$ & $3.54 \mathrm{E}-20$ & 3.07E-20 & $1.58 \mathrm{E}-12$ & $5.48 \mathrm{E}-12$ \\
\hline $\mathrm{Pd}-107$ & $1.47 \mathrm{E}-08$ & $5.23 E-08$ & $1.60 \mathrm{E}-12$ & $1.60 \mathrm{E}-14$ & $4.62 \mathrm{E}-22$ & $3.99 \mathrm{E}-22$ & $2.06 \mathrm{E}-14$ & $7.14 \mathrm{E}-14$ \\
\hline Cd-113m & $2.97 \mathrm{E}-06$ & 1.05E-05 & $3.22 \mathrm{E}-10$ & $3.22 \mathrm{E}-12$ & $9.28 \mathrm{E}-20$ & $8.03 E-20$ & $4.13 \mathrm{E}-12$ & $1.44 \mathrm{E}-11$ \\
\hline In-115 & $8.99 \mathrm{E}-17$ & $3.19 \mathrm{E}-16$ & $9.76 \mathrm{E}-21$ & $9.75 \mathrm{E}-23$ & $2.81 \mathrm{E}-30$ & $2.43 \mathrm{E}-30$ & $1.25 \mathrm{E}-22$ & $4.35 \mathrm{E}-22$ \\
\hline Sn-121m & 5.97E-08 & $2.12 \mathrm{E}-07$ & $6.48 \mathrm{E}-12$ & $6.48 \mathrm{E}-14$ & 1.87E-21 & $1.62 \mathrm{E}-21$ & $8.32 \mathrm{E}-14$ & $2.89 \mathrm{E}-13$ \\
\hline Sn-126 & 4.99E-07 & $1.77 \mathrm{E}-06$ & $5.41 \mathrm{E}-11$ & $5.41 \mathrm{E}-13$ & $1.56 \mathrm{E}-20$ & $1.35 \mathrm{E}-20$ & $6.95 \mathrm{E}-13$ & $2.42 \mathrm{E}-12$ \\
\hline Sb-125 & 4.10E-04 & $1.45 \mathrm{E}-03$ & $4.45 \mathrm{E}-08$ & $4.45 \mathrm{E}-10$ & $1.28 \mathrm{E}-17$ & $1.11 \mathrm{E}-17$ & $5.72 \mathrm{E}-10$ & 1.99E-09 \\
\hline Sb-126 & $5.13 \mathrm{E}-08$ & $1.82 \mathrm{E}-07$ & $5.57 \mathrm{E}-12$ & $5.57 \mathrm{E}-14$ & 1.61E-21 & 1.39E-21 & $7.16 \mathrm{E}-14$ & $2.49 \mathrm{E}-13$ \\
\hline Te-123 & $3.42 \mathrm{E}-19$ & $1.21 \mathrm{E}-18$ & $3.71 \mathrm{E}-23$ & $3.71 \mathrm{E}-25$ & & & 4.77E-25 & $1.66 \mathrm{E}-24$ \\
\hline Te-125m & $2.81 \mathrm{E}-06$ & 9.97E-06 & $3.05 E-10$ & $3.05 E-12$ & $8.80 \mathrm{E}-20$ & $7.61 \mathrm{E}-20$ & $3.92 \mathrm{E}-12$ & $1.36 \mathrm{E}-11$ \\
\hline I-129 & $6.47 \mathrm{E}-08$ & $1.88 \mathrm{E}-07$ & $1.28 \mathrm{E}-08$ & 1.27E-08 & $2.46 \mathrm{E}-13$ & $1.78 \mathrm{E}-12$ & $1.64 \mathrm{E}-08$ & $1.14 \mathrm{E}-08$ \\
\hline Cs-134 & 6.39E-05 & $2.27 \mathrm{E}-04$ & 6.94E-09 & $6.94 \mathrm{E}-11$ & $2.00 \mathrm{E}-18$ & $1.73 \mathrm{E}-18$ & $8.92 \mathrm{E}-11$ & $3.10 \mathrm{E}-10$ \\
\hline Cs-135 & $1.01 \mathrm{E}-06$ & $3.60 \mathrm{E}-06$ & $1.10 \mathrm{E}-10$ & $1.10 \mathrm{E}-12$ & $3.17 \mathrm{E}-20$ & $2.74 \mathrm{E}-20$ & $1.41 \mathrm{E}-12$ & $4.91 \mathrm{E}-12$ \\
\hline Cs-137 & $5.25 \mathrm{E}-02$ & $1.86 \mathrm{E}-01$ & $5.70 \mathrm{E}-06$ & $5.70 \mathrm{E}-08$ & $1.64 \mathrm{E}-15$ & $1.42 \mathrm{E}-15$ & 7.32E-08 & $2.54 \mathrm{E}-07$ \\
\hline Ba-137m & 4.97E-02 & 1.76E-01 & 5.39E-06 & $5.39 \mathrm{E}-08$ & $1.55 \mathrm{E}-15$ & 1.34E-15 & $6.92 \mathrm{E}-08$ & $2.40 \mathrm{E}-07$ \\
\hline La-138 & $1.70 \mathrm{E}-16$ & $6.05 \mathrm{E}-16$ & $1.85 \mathrm{E}-20$ & 1.85E-22 & $5.33 \mathrm{E}-30$ & $4.61 \mathrm{E}-30$ & $2.38 \mathrm{E}-22$ & $8.26 \mathrm{E}-22$ \\
\hline Ce-142 & $2.67 \mathrm{E}-11$ & $9.47 \mathrm{E}-11$ & $2.90 \mathrm{E}-15$ & $2.90 \mathrm{E}-17$ & $8.35 \mathrm{E}-25$ & $7.23 E-25$ & $3.72 \mathrm{E}-17$ & $1.29 \mathrm{E}-16$ \\
\hline Ce-144 & $7.65 \mathrm{E}-07$ & $2.71 \mathrm{E}-06$ & $8.30 \mathrm{E}-11$ & $8.30 \mathrm{E}-13$ & $2.39 \mathrm{E}-20$ & $2.07 \mathrm{E}-20$ & $1.07 \mathrm{E}-12$ & $3.70 \mathrm{E}-12$ \\
\hline Pr-144 & $7.65 \mathrm{E}-07$ & $2.71 \mathrm{E}-06$ & $8.30 \mathrm{E}-11$ & $8.30 \mathrm{E}-13$ & $2.39 \mathrm{E}-20$ & $2.07 \mathrm{E}-20$ & $1.07 \mathrm{E}-12$ & $3.70 \mathrm{E}-12$ \\
\hline $\mathrm{Nd}-144$ & $1.44 \mathrm{E}-15$ & $5.09 \mathrm{E}-15$ & $1.56 \mathrm{E}-19$ & $1.56 \mathrm{E}-21$ & 4.49E-29 & 3.89E-29 & $2.00 \mathrm{E}-21$ & $6.95 \mathrm{E}-21$ \\
\hline \begin{tabular}{|l|l}
$\mathrm{Pm}-146$ \\
\end{tabular} & 4.54E-08 & $1.61 \mathrm{E}-07$ & $4.93 \mathrm{E}-12$ & $4.93 \mathrm{E}-14$ & 1.42E-21 & $1.23 \mathrm{E}-21$ & $6.33 \mathrm{E}-14$ & $2.20 \mathrm{E}-13$ \\
\hline Pm-147 & $2.06 \mathrm{E}-04$ & 7.32E-04 & $2.24 \mathrm{E}-08$ & $2.24 \mathrm{E}-10$ & $6.46 \mathrm{E}-18$ & $5.59 \mathrm{E}-18$ & $2.88 \mathrm{E}-10$ & $9.99 \mathrm{E}-10$ \\
\hline Sm-146 & $2.46 \mathrm{E}-13$ & $8.73 E-13$ & $2.67 \mathrm{E}-17$ & $2.67 E-19$ & $7.71 \mathrm{E}-27$ & $6.67 \mathrm{E}-27$ & $3.43 E-19$ & $1.19 \mathrm{E}-18$ \\
\hline Sm-147 & $6.58 \mathrm{E}-12$ & $2.33 \mathrm{E}-11$ & $7.14 \mathrm{E}-16$ & $7.14 \mathrm{E}-18$ & $2.06 \mathrm{E}-25$ & $1.78 \mathrm{E}-25$ & $9.17 \mathrm{E}-18$ & $3.18 \mathrm{E}-17$ \\
\hline Sm-148 & $3.38 \mathrm{E}-17$ & $1.20 \mathrm{E}-16$ & $3.67 \mathrm{E}-21$ & $3.67 \mathrm{E}-23$ & $1.06 \mathrm{E}-30$ & & $4.71 \mathrm{E}-23$ & 1.64E-22 \\
\hline Sm-149 & $3.00 \mathrm{E}-18$ & $1.06 \mathrm{E}-17$ & $3.26 \mathrm{E}-22$ & $3.26 \mathrm{E}-24$ & & & $4.18 \mathrm{E}-24$ & $1.45 \mathrm{E}-23$ \\
\hline Sm-151 & $4.08 \mathrm{E}-04$ & $1.45 \mathrm{E}-03$ & $4.43 \mathrm{E}-08$ & $4.43 \mathrm{E}-10$ & $1.28 \mathrm{E}-17$ & $1.10 \mathrm{E}-17$ & $5.69 \mathrm{E}-10$ & $1.98 \mathrm{E}-09$ \\
\hline Eu-152 & $2.62 \mathrm{E}-06$ & 9.31E-06 & $2.85 \mathrm{E}-10$ & $2.85 \mathrm{E}-12$ & $8.22 E-20$ & $7.11 \mathrm{E}-20$ & $3.66 \mathrm{E}-12$ & 1.27E-11 \\
\hline Eu-154 & 1.57E-04 & $5.56 \mathrm{E}-04$ & $1.70 \mathrm{E}-08$ & $1.70 \mathrm{E}-10$ & $4.91 \mathrm{E}-18$ & $4.25 \mathrm{E}-18$ & $2.19 \mathrm{E}-10$ & $7.59 \mathrm{E}-10$ \\
\hline Eu-155 & $1.47 \mathrm{E}-04$ & $5.23 E-04$ & $1.60 \mathrm{E}-08$ & $1.60 \mathrm{E}-10$ & $4.61 \mathrm{E}-18$ & $3.99 \mathrm{E}-18$ & $2.06 \mathrm{E}-10$ & $7.14 \mathrm{E}-10$ \\
\hline Gd-152 & $1.27 \mathrm{E}-18$ & $4.50 \mathrm{E}-18$ & $1.38 \mathrm{E}-22$ & $1.38 \mathrm{E}-24$ & 3.97E-32 & $3.44 \mathrm{E}-32$ & 1.77E-24 & $6.15 \mathrm{E}-24$ \\
\hline Ho-166m & $4.11 \mathrm{E}-11$ & $1.46 \mathrm{E}-10$ & $4.46 \mathrm{E}-15$ & $4.46 \mathrm{E}-17$ & $1.29 \mathrm{E}-24$ & $1.11 \mathrm{E}-24$ & $5.73 \mathrm{E}-17$ & $1.99 \mathrm{E}-16$ \\
\hline \multicolumn{9}{|l|}{ Other Stream Data: } \\
\hline TRU, nCi/gm & $8.01 \mathrm{E}+02$ & $2.12 \mathrm{E}+03$ & & & & & $1.32 \mathrm{E}-03$ & $4.35 \mathrm{E}-03$ \\
\hline Heat Generation, W/m3 & $5.23 \mathrm{E}-01$ & $1.85 \mathrm{E}+00$ & & & & & $1.08 \mathrm{E}-06$ & $2.72 \mathrm{E}-06$ \\
\hline Mass Flow, kg/hr & $4.21 \mathrm{E}+02$ & $1.60 \mathrm{E}+02$ & $2.58 \mathrm{E}+02$ & $2.58 \mathrm{E}+02$ & $2.46 \mathrm{E}+02$ & $2.88 \mathrm{E}+02$ & $2.58 \mathrm{E}+02$ & $7.81 \mathrm{E}+01$ \\
\hline
\end{tabular}


Table C-7. Revised baseline mass balance, Tank WM-189 waste (Continued).

\begin{tabular}{|c|c|c|c|c|c|c|c|}
\hline PFD Sheet \# & PFD-3 & PFD-4 & PFD-4 & PFD-4 & PFD-4 & PFD-4 & PFD-4 \\
\hline Stream \# & 109 & 110 & 201 & 202 & 203 & 204 & 205 \\
\hline Stream Name & $\begin{array}{l}\text { LET\&D } \\
\text { Offgas } \\
\text { Vapor }\end{array}$ & $\begin{array}{l}\text { Acid } \\
\text { Grout } \\
\text { Solid }\end{array}$ & $\begin{array}{c}\text { OPC } \\
\text { Grout } \\
\text { Additive }\end{array}$ & $\begin{array}{c}\text { BFS } \\
\text { Grout } \\
\text { Additive }\end{array}$ & $\begin{array}{c}\mathrm{Ca}(\mathrm{OH}) 2 \\
\text { Total } \\
\text { Additive }\end{array}$ & $\begin{array}{c}\mathrm{Ca}(\mathrm{OH}) 2 \\
\text { Grout } \\
\text { Additive }\end{array}$ & $\begin{array}{c}\mathrm{Ca}(\mathrm{OH}) 2 \\
\text { Neutraliz. } \\
\text { Additive }\end{array}$ \\
\hline Rate or Volume Flow (actual) & $1.35 \mathrm{E}+04$ & 492.0 & 193.7 & 64.6 & 61.5 & 1.94 & 59.6 \\
\hline Volume Flow (standard, wet) ${ }^{*}$ & $8.43 \mathrm{E}+03$ & & & & & & \\
\hline Volume Flow (standard, dry) ${ }^{*}$ & $8.45 \mathrm{E}-01$ & & & & & & \\
\hline Rate Units & $\mathrm{ft} 3 / \mathrm{hr}$ & $\mathrm{lb} / \mathrm{hr}$ & $\mathrm{lb} / \mathrm{hr}$ & $\mathrm{lb} / \mathrm{hr}$ & $\mathrm{lb} / \mathrm{hr}$ & $\mathrm{lb} / \mathrm{hr}$ & $\mathrm{lb} / \mathrm{hr}$ \\
\hline Temperature, ${ }^{\circ} \mathrm{C}$ & 119.1 & 64.2 & 25.0 & 25.0 & 25.0 & 25.0 & 25.0 \\
\hline Temperature, ${ }^{\circ} \mathrm{F}$ & 246.3 & 147.5 & 77.0 & 77.0 & 77.0 & 77.0 & 77.0 \\
\hline Pressure, psia & 12.3 & 12.2 & 12.3 & 12.3 & 12.3 & 12.3 & 12.3 \\
\hline Specific Gravity & 0.00047 & 2.06 & 1.2 & 1.2 & 0.48 & 0.48 & 0.48 \\
\hline Chemical Composition & $\mathrm{lb} / \mathrm{wscf}$ & Wt. \% & Wt. \% & Wt. \% & Wt. \% & Wt. \% & Wt. \% \\
\hline $\mathrm{H}+$ & $2.55 \mathrm{E}-07$ & $2.02 \mathrm{E}-06$ & & & & & \\
\hline $\mathrm{OH}-$ & & 1.79E-01 & & & $4.59 E+01$ & $4.59 \mathrm{E}+01$ & $4.59 \mathrm{E}+01$ \\
\hline $\mathrm{Al}+3$ & & $2.39 \mathrm{E}-06$ & & & & & \\
\hline $\mathrm{Sb}+5$ & & $3.20 \mathrm{E}-10$ & & & & & \\
\hline$A s+5$ & & $9.84 \mathrm{E}-10$ & & & & & \\
\hline $\mathrm{Ba}+2$ & & 1.37E-09 & & & & & \\
\hline $\mathrm{Be}+2$ & & $2.92 \mathrm{E}-11$ & & & & & \\
\hline$B+3$ & & $2.88 \mathrm{E}-08$ & & & & & \\
\hline $\mathrm{Cd}+2$ & & $5.34 \mathrm{E}-08$ & & & & & \\
\hline $\mathrm{Ca}+2$ & & $3.58 \mathrm{E}-07$ & & & $5.41 \mathrm{E}+01$ & $5.41 \mathrm{E}+01$ & $5.41 \mathrm{E}+01$ \\
\hline $\mathrm{Cr}+3$ & & $3.88 \mathrm{E}-08$ & & & & & \\
\hline $\mathrm{Co}+2$ & & 3.67E-10 & & & & & \\
\hline Cs+ & & 1.18E-09 & & & & & \\
\hline $\mathrm{Cu}+2$ & & $8.14 \mathrm{E}-09$ & & & & & \\
\hline $\mathrm{Fe}+3$ & & $2.41 \mathrm{E}-07$ & & & & & \\
\hline $\mathrm{Pb}+2$ & & $3.01 \mathrm{E}-08$ & & & & & \\
\hline $\mathrm{Hg}+2$ & & 1.09E-02 & & & & & \\
\hline $\mathrm{Mn}+4$ & & $1.32 \mathrm{E}-07$ & & & & & \\
\hline $\mathrm{Ni}+2$ & & $1.78 \mathrm{E}-08$ & & & & & \\
\hline $\mathrm{K}+$ & & $1.15 \mathrm{E}-06$ & & & & & \\
\hline $\mathrm{Se}+4$ & & 2.97E-09 & & & & & \\
\hline $\mathrm{Ag}+$ & & $6.35 \mathrm{E}-09$ & & & & & \\
\hline $\mathrm{Na}+$ & & 5.69E-06 & & & & & \\
\hline $\mathrm{Tl}+3$ & & $1.42 \mathrm{E}-10$ & & & & & \\
\hline$U+4$ & & 1.95E-08 & & & & & \\
\hline $\mathrm{V}+3$ & & $2.35 \mathrm{E}-10$ & & & & & \\
\hline$Z n+2$ & & $8.97 \mathrm{E}-09$ & & & & & \\
\hline $\mathrm{Zr}+4$ & & $5.16 \mathrm{E}-07$ & & & & & \\
\hline $\mathrm{Cl}-$ & 8.95E-06 & 1.92E-02 & & & & & \\
\hline F- & & $3.96 \mathrm{E}-05$ & & & & & \\
\hline SO4-2 & & 1.27E-06 & & & & & \\
\hline NO3- & $1.85 \mathrm{E}-08$ & $2.03 E+01$ & & & & & \\
\hline PO4-3 & & $1.48 \mathrm{E}-06$ & & & & & \\
\hline $\mathrm{Am}+4$ & & $2.30 \mathrm{E}-12$ & & & & & \\
\hline $\mathrm{Br}-$ & $3.01 \mathrm{E}-10$ & $6.45 \mathrm{E}-07$ & & & & & \\
\hline $\mathrm{Ce}+4$ & & $8.07 \mathrm{E}-10$ & & & & & \\
\hline $\mathrm{Eu}+3$ & & $9.96 \mathrm{E}-12$ & & & & & \\
\hline $\mathrm{Gd}+3$ & & 3.09E-09 & & & & & \\
\hline $\mathrm{Ge}+4$ & & $8.26 \mathrm{E}-14$ & & & & & \\
\hline $\ln +3$ & & $2.10 \mathrm{E}-11$ & & & & & \\
\hline I- & 3.97E-09 & $8.50 \mathrm{E}-06$ & & & & & \\
\hline $\mathrm{La}+3$ & & $1.65 \mathrm{E}-10$ & & & & & \\
\hline $\mathrm{Li+}$ & & $5.47 \mathrm{E}-10$ & & & & & \\
\hline $\mathrm{Mg}+2$ & & $6.85 \mathrm{E}-08$ & & & & & \\
\hline $\mathrm{Mo}+6$ & & $1.17 \mathrm{E}-08$ & & & & & \\
\hline
\end{tabular}


Table C-7. Revised baseline mass balance, Tank WM-189 waste (Continued).

\begin{tabular}{|c|c|c|c|c|c|c|c|}
\hline Stream \# & 109 & 110 & 201 & 202 & 203 & 204 & 205 \\
\hline Stream Name & $\begin{array}{c}\text { LET\&D } \\
\text { Offgas } \\
\text { Vapor }\end{array}$ & $\begin{array}{l}\text { Acid } \\
\text { Grout } \\
\text { Solid }\end{array}$ & $\begin{array}{c}\text { OPC } \\
\text { Grout } \\
\text { Additive }\end{array}$ & $\begin{array}{c}\text { BFS } \\
\text { Grout } \\
\text { Additive }\end{array}$ & $\begin{array}{c}\mathrm{Ca}(\mathrm{OH}) 2 \\
\text { Total } \\
\text { Additive }\end{array}$ & $\begin{array}{c}\mathrm{Ca}(\mathrm{OH}) 2 \\
\text { Grout } \\
\text { Additive }\end{array}$ & $\begin{array}{c}\mathrm{Ca}(\mathrm{OH}) 2 \\
\text { Neutraliz. } \\
\text { Additive }\end{array}$ \\
\hline Chemical Composition & $\mathrm{lb} / \mathrm{wscf}$ & Wt. \% & Wt. \% & Wt. \% & Wt. \% & Wt. \% & Wt. \% \\
\hline $\mathrm{Nd}+3$ & & $5.53 \mathrm{E}-10$ & & & & & \\
\hline $\mathrm{Np}+4$ & & $9.44 \mathrm{E}-11$ & & & & & \\
\hline $\mathrm{Nb}+5$ & & $1.54 \mathrm{E}-08$ & & & & & \\
\hline $\mathrm{Pd}+4$ & & $2.62 \mathrm{E}-08$ & & & & & \\
\hline $\mathrm{Pu}+4$ & & $2.33 \mathrm{E}-10$ & & & & & \\
\hline $\mathrm{Pr}+4$ & & $1.52 \mathrm{E}-10$ & & & & & \\
\hline $\mathrm{Rh}+4$ & & $4.80 \mathrm{E}-11$ & & & & & \\
\hline $\mathrm{Rb}+$ & & $6.14 \mathrm{E}-11$ & & & & & \\
\hline Ru+3 & & $1.30 \mathrm{E}-08$ & & & & & \\
\hline $\mathrm{Sm}+3$ & & 1.07E-10 & & & & & \\
\hline $\mathrm{Si}+4$ & & 4.02E-07 & & & & & \\
\hline $\mathrm{Sr}+2$ & & 1.52E-09 & & & & & \\
\hline $\mathrm{Tc}+7$ & & $8.28 \mathrm{E}-11$ & & & & & \\
\hline $\mathrm{Te}+4$ & & $1.07 \mathrm{E}-10$ & & & & & \\
\hline $\mathrm{Tb}+4$ & & $4.35 \mathrm{E}-14$ & & & & & \\
\hline $\mathrm{Th}+4$ & & $9.62 \mathrm{E}-10$ & & & & & \\
\hline $\mathrm{Sn}+4$ & & $3.32 \mathrm{E}-08$ & & & & & \\
\hline $\mathrm{Ti}+4$ & & 6.92E-09 & & & & & \\
\hline $\mathrm{Y}+3$ & & $7.88 \mathrm{E}-11$ & & & & & \\
\hline O-2 (oxides) & & $3.66 \mathrm{E}-07$ & & & & & \\
\hline $\mathrm{H} 2 \mathrm{O}$ & $4.70 \mathrm{E}-02$ & $2.03 \mathrm{E}+01$ & & & & & \\
\hline $\mathrm{O} 2$ & $1.14 \mathrm{E}-07$ & & & & & & \\
\hline N2 & $1.44 \mathrm{E}-07$ & & & & & & \\
\hline C (organic) & & $9.06 \mathrm{E}-04$ & & & & & \\
\hline $\mathrm{H}$ (organic) & & $7.60 \mathrm{E}-05$ & & & & & \\
\hline SiO2, BFS, or OPC Additives & & $5.25 \mathrm{E}+01$ & $1.33 \mathrm{E}+02$ & $4.00 \mathrm{E}+02$ & & & \\
\hline $\begin{array}{c}\text { Gas Stream Bulk Composition } \\
\text { (wet basis) }\end{array}$ & $\begin{array}{l}\text { mol.\% } \\
\text { or ppmv }\end{array}$ & $\begin{array}{l}\text { mol. \% } \\
\text { or ppmv }\end{array}$ & $\begin{array}{l}\text { mol.\% } \\
\text { or ppmv }\end{array}$ & $\begin{array}{l}\text { mol. } \% \\
\text { or ppmv }\end{array}$ & $\begin{array}{l}\text { mol. } \% \\
\text { or ppmv }\end{array}$ & $\begin{array}{l}\text { mol. } \% \\
\text { or ppmv }\end{array}$ & $\begin{array}{l}\text { mol.\% } \\
\text { or ppmv }\end{array}$ \\
\hline $\mathrm{H} 2 \mathrm{O}, \mathrm{mol} . \%$ & 99.99 & & & & & & \\
\hline $\mathrm{O} 2, \mathrm{~mol} . \%$ & $1.4 \mathrm{E}-04$ & & & & & & \\
\hline $\mathrm{N} 2, \mathrm{~mol} . \%$ & $2.0 \mathrm{E}-04$ & & & & & & \\
\hline \multicolumn{8}{|l|}{$\mathrm{CO} 2, \mathrm{~mol} . \%$} \\
\hline HNO3, mol. \% & 0.01 & & & & & & \\
\hline \multicolumn{8}{|l|}{$\mathrm{CO}, \mathrm{ppmv}$} \\
\hline \multicolumn{8}{|l|}{ NO, ppmv } \\
\hline \multicolumn{8}{|l|}{ NO2, ppmv } \\
\hline \multicolumn{8}{|l|}{ C (organic), ppmv } \\
\hline \multicolumn{8}{|l|}{$\mathrm{H}$ (organic), ppmv } \\
\hline \multicolumn{8}{|l|}{$\mathrm{PM}(\mathrm{mg} / \mathrm{wscm})$} \\
\hline $\begin{array}{c}\text { Gas Stream Bulk Composition } \\
\text { (dry basis) }\end{array}$ & $\begin{array}{l}\text { mol. } \% \\
\text { or ppmv }\end{array}$ & $\begin{array}{c}\text { mol. } \% \\
\text { or ppmv }\end{array}$ & $\begin{array}{l}\text { mol. } \% \\
\text { or ppmv }\end{array}$ & $\begin{array}{c}\text { mol. } \% \\
\text { or ppmv }\end{array}$ & $\begin{array}{c}\text { mol. } \% \\
\text { or ppmv }\end{array}$ & $\begin{array}{c}\text { mol. } \% \\
\text { or ppmv }\end{array}$ & $\begin{array}{l}\text { mol. } \% \\
\text { or ppmv }\end{array}$ \\
\hline $\mathrm{O} 2, \mathrm{~mol} . \%$ & 1.37 & & & & & & \\
\hline $\mathrm{N} 2, \mathrm{~mol} . \%$ & 1.96 & & & & & & \\
\hline \multicolumn{8}{|l|}{$\mathrm{CO} 2, \mathrm{~mol} . \%$} \\
\hline HNO3, mol. \% & 96.66 & & & & & & \\
\hline \multicolumn{8}{|l|}{$\mathrm{CO}, \mathrm{ppmv}$} \\
\hline \multicolumn{8}{|l|}{$\mathrm{NO}, \mathrm{ppmv}$} \\
\hline \multicolumn{8}{|l|}{$\mathrm{NO} 2, \mathrm{ppmv}$} \\
\hline \multicolumn{8}{|l|}{$\mathrm{C}$ (organic), ppmv } \\
\hline \multicolumn{8}{|l|}{$\mathrm{H}$ (organic), ppmv } \\
\hline $\mathrm{PM}(\mathrm{mg} / \mathrm{dscm})$ & & & & & & & \\
\hline
\end{tabular}


Table C-7. Revised baseline mass balance, Tank WM-189 waste (Continued).

\begin{tabular}{|c|c|c|c|c|c|c|c|}
\hline Stream \# & 109 & 110 & 201 & 202 & 203 & 204 & 205 \\
\hline Stream Name & $\begin{array}{l}\text { LET\&D } \\
\text { Offgas } \\
\text { Vapor }\end{array}$ & $\begin{array}{l}\text { Acid } \\
\text { Grout } \\
\text { Solid }\end{array}$ & $\begin{array}{c}\text { OPC } \\
\text { Grout } \\
\text { Additive }\end{array}$ & $\begin{array}{c}\text { BFS } \\
\text { Grout } \\
\text { Additive }\end{array}$ & $\begin{array}{c}\mathrm{Ca}(\mathrm{OH}) 2 \\
\text { Total } \\
\text { Additive }\end{array}$ & $\begin{array}{c}\mathrm{Ca}(\mathrm{OH}) 2 \\
\text { Grout } \\
\text { Additive }\end{array}$ & $\begin{array}{c}\mathrm{Ca}(\mathrm{OH}) 2 \\
\text { Neutraliz. } \\
\text { Additive }\end{array}$ \\
\hline \multicolumn{8}{|l|}{ Canister Rate, canisters/day } \\
\hline \multicolumn{8}{|l|}{ Total Canisters Generated } \\
\hline Drum Rate, drums/day & & 15.53 & & & & & \\
\hline Total Drums Generated & & 2,161 & & & & & \\
\hline Radiological Composition & $\mathrm{Ci} / \mathrm{wscm}$ & $\mathrm{Ci} / \mathrm{kg}$ & $\mathrm{Ci} / \mathrm{kg}$ & $\mathrm{Ci} / \mathrm{kg}$ & $\mathrm{Ci} / \mathrm{kg}$ & $\mathrm{Ci} / \mathrm{kg}$ & $\mathrm{Ci} / \mathrm{kg}$ \\
\hline Ra-226 & $1.99 \mathrm{E}-29$ & $1.06 \mathrm{E}-17$ & & & & & \\
\hline Ac-227 & $9.37 \mathrm{E}-29$ & $5.01 \mathrm{E}-17$ & & & & & \\
\hline Th-228 & $7.78 \mathrm{E}-27$ & $4.16 \mathrm{E}-15$ & & & & & \\
\hline Th-230 & $2.62 \mathrm{E}-27$ & $1.40 \mathrm{E}-15$ & & & & & \\
\hline Th-232 & & $9.20 \mathrm{E}-22$ & & & & & \\
\hline $\mathrm{Pa}-231$ & $2.17 \mathrm{E}-28$ & $1.16 \mathrm{E}-16$ & & & & & \\
\hline $\mathrm{Pa}-233$ & $7.12 \mathrm{E}-24$ & $3.81 \mathrm{E}-12$ & & & & & \\
\hline $\mathrm{U}-232$ & $6.14 \mathrm{E}-27$ & $3.28 \mathrm{E}-15$ & & & & & \\
\hline $\mathrm{U}-233$ & $2.17 \mathrm{E}-28$ & $1.16 \mathrm{E}-16$ & & & & & \\
\hline $\mathrm{U}-234$ & $4.26 \mathrm{E}-24$ & $2.28 \mathrm{E}-12$ & & & & & \\
\hline $\mathrm{U}-235$ & $1.72 \mathrm{E}-25$ & $9.17 \mathrm{E}-14$ & & & & & \\
\hline $\mathrm{U}-236$ & $1.99 \mathrm{E}-25$ & $1.06 \mathrm{E}-13$ & & & & & \\
\hline $\mathrm{U}-237$ & $1.56 \mathrm{E}-26$ & $8.36 \mathrm{E}-15$ & & & & & \\
\hline $\mathrm{U}-238$ & $9.46 \mathrm{E}-26$ & $5.06 \mathrm{E}-14$ & & & & & \\
\hline Np-236 & $7.50 \mathrm{E}-30$ & $4.01 \mathrm{E}-18$ & & & & & \\
\hline Np-237 & $1.27 \mathrm{E}-24$ & $6.77 \mathrm{E}-13$ & & & & & \\
\hline Pu-236 & $1.00 \mathrm{E}-26$ & $5.35 \mathrm{E}-15$ & & & & & \\
\hline Pu-238 & $2.30 \mathrm{E}-21$ & $1.23 \mathrm{E}-09$ & & & & & \\
\hline Pu-239 & $2.51 \mathrm{E}-22$ & $1.34 \mathrm{E}-10$ & & & & & \\
\hline $\mathrm{Pu}-240$ & $3.73 \mathrm{E}-23$ & $1.99 \mathrm{E}-11$ & & & & & \\
\hline $\mathrm{Pu}-241$ & $2.01 \mathrm{E}-21$ & $1.08 \mathrm{E}-09$ & & & & & \\
\hline Pu-242 & $2.92 \mathrm{E}-26$ & $1.56 \mathrm{E}-14$ & & & & & \\
\hline $\mathrm{Pu}-244$ & & $4.62 \mathrm{E}-22$ & & & & & \\
\hline $\mathrm{Am}-241$ & 1.97E-22 & $1.06 \mathrm{E}-10$ & & & & & \\
\hline Am-242m & $3.71 \mathrm{E}-26$ & $1.99 \mathrm{E}-14$ & & & & & \\
\hline Am-243 & $6.31 \mathrm{E}-26$ & $3.37 \mathrm{E}-14$ & & & & & \\
\hline $\mathrm{Cm}-242$ & $7.41 \mathrm{E}-26$ & $3.96 \mathrm{E}-14$ & & & & & \\
\hline $\mathrm{Cm}-243$ & $9.08 \mathrm{E}-26$ & $4.86 \mathrm{E}-14$ & & & & & \\
\hline $\mathrm{Cm}-244$ & $3.84 \mathrm{E}-24$ & $2.05 \mathrm{E}-12$ & & & & & \\
\hline $\mathrm{Cm}-245$ & $9.56 \mathrm{E}-28$ & $5.11 \mathrm{E}-16$ & & & & & \\
\hline $\mathrm{Cm}-246$ & $6.27 \mathrm{E}-29$ & $3.36 \mathrm{E}-17$ & & & & & \\
\hline $\mathrm{Cm}-247$ & & $9.01 \mathrm{E}-24$ & & & & & \\
\hline $\mathrm{H}-3$ & $8.51 \mathrm{E}-06$ & $1.63 \mathrm{E}-06$ & & & & & \\
\hline $\mathrm{Be}-10$ & $7.29 \mathrm{E}-30$ & $3.90 \mathrm{E}-18$ & & & & & \\
\hline C-14 & $3.97 \mathrm{E}-28$ & $2.12 \mathrm{E}-16$ & & & & & \\
\hline Co-60 & $8.09 \mathrm{E}-23$ & $4.33 \mathrm{E}-11$ & & & & & \\
\hline Ni-59 & $3.33 \mathrm{E}-24$ & $1.78 \mathrm{E}-12$ & & & & & \\
\hline Ni-63 & $1.05 \mathrm{E}-22$ & $5.64 \mathrm{E}-11$ & & & & & \\
\hline Se-79 & $1.44 \mathrm{E}-24$ & $7.71 \mathrm{E}-13$ & & & & & \\
\hline $\mathrm{Rb}-87$ & $7.12 \mathrm{E}-29$ & $3.81 \mathrm{E}-17$ & & & & & \\
\hline Sr-90 & $9.61 \mathrm{E}-20$ & $5.14 \mathrm{E}-08$ & & & & & \\
\hline Y-90 & $9.61 \mathrm{E}-20$ & $5.14 \mathrm{E}-08$ & & & & & \\
\hline Zr-93 & $5.38 \mathrm{E}-24$ & $2.88 \mathrm{E}-12$ & & & & & \\
\hline $\mathrm{Nb}-93 \mathrm{~m}$ & $4.15 \mathrm{E}-24$ & $2.22 \mathrm{E}-12$ & & & & & \\
\hline $\mathrm{Nb}-94$ & $3.75 \mathrm{E}-24$ & $2.00 \mathrm{E}-12$ & & & & & \\
\hline Tc-98 & $6.27 \mathrm{E}-30$ & 3.35E-18 & & & & & \\
\hline Tc-99 & $3.80 \mathrm{E}-23$ & $2.03 \mathrm{E}-11$ & & & & & \\
\hline
\end{tabular}


Table C-7. Revised baseline mass balance, Tank WM-189 waste (Continued).

\begin{tabular}{|c|c|c|c|c|c|c|c|}
\hline Stream \# & 109 & 110 & 201 & 202 & 203 & 204 & 205 \\
\hline Stream Name & $\begin{array}{l}\text { LET\&D } \\
\text { Offgas } \\
\text { Vapor }\end{array}$ & $\begin{array}{l}\text { Acid } \\
\text { Grout } \\
\text { Solid }\end{array}$ & $\begin{array}{c}\text { OPC } \\
\text { Grout } \\
\text { Additive }\end{array}$ & $\begin{array}{c}\text { BFS } \\
\text { Grout } \\
\text { Additive }\end{array}$ & $\begin{array}{c}\mathrm{Ca}(\mathrm{OH}) 2 \\
\text { Total } \\
\text { Additive }\end{array}$ & $\begin{array}{c}\mathrm{Ca}(\mathrm{OH}) 2 \\
\text { Grout } \\
\text { Additive }\end{array}$ & $\begin{array}{l}\mathrm{Ca}(\mathrm{OH}) 2 \\
\text { Neutraliz. } \\
\text { Additive }\end{array}$ \\
\hline Radiological Composition & $\mathrm{Ci} / \mathrm{wscm}$ & $\mathrm{Ci} / \mathrm{kg}$ & $\mathrm{Ci} / \mathrm{kg}$ & $\mathrm{Ci} / \mathrm{kg}$ & $\mathrm{Ci} / \mathrm{kg}$ & $\mathrm{Ci} / \mathrm{kg}$ & $\mathrm{Ci} / \mathrm{kg}$ \\
\hline Ru-106 & $3.08 \mathrm{E}-24$ & $1.65 \mathrm{E}-12$ & & & & & \\
\hline Rh-102 & $2.09 \mathrm{E}-27$ & $1.12 \mathrm{E}-15$ & & & & & \\
\hline Rh-106 & $3.08 \mathrm{E}-24$ & $1.65 \mathrm{E}-12$ & & & & & \\
\hline Pd-107 & $4.01 \mathrm{E}-26$ & $2.15 \mathrm{E}-14$ & & & & & \\
\hline Cd-113m & $8.07 \mathrm{E}-24$ & $4.31 \mathrm{E}-12$ & & & & & \\
\hline $\ln -115$ & & $1.31 \mathrm{E}-22$ & & & & & \\
\hline$S n-121 m$ & $1.62 \mathrm{E}-25$ & $8.69 \mathrm{E}-14$ & & & & & \\
\hline Sn-126 & 1.36E-24 & $7.26 \mathrm{E}-13$ & & & & & \\
\hline Sb-125 & $1.12 \mathrm{E}-21$ & $5.97 \mathrm{E}-10$ & & & & & \\
\hline Sb-126 & $1.40 \mathrm{E}-25$ & $7.47 \mathrm{E}-14$ & & & & & \\
\hline Te-123 & & $4.98 \mathrm{E}-25$ & & & & & \\
\hline Te-125m & $7.65 \mathrm{E}-24$ & $4.09 \mathrm{E}-12$ & & & & & \\
\hline $1-129$ & $1.28 \mathrm{E}-08$ & $3.42 \mathrm{E}-09$ & & & & & \\
\hline Cs-134 & $1.74 \mathrm{E}-22$ & $9.30 \mathrm{E}-11$ & & & & & \\
\hline Cs-135 & $2.76 \mathrm{E}-24$ & $1.48 \mathrm{E}-12$ & & & & & \\
\hline Cs-137 & $1.43 \mathrm{E}-19$ & $7.64 \mathrm{E}-08$ & & & & & \\
\hline Ba-137m & $1.35 \mathrm{E}-19$ & $7.23 \mathrm{E}-08$ & & & & & \\
\hline La-138 & & $2.48 \mathrm{E}-22$ & & & & & \\
\hline Ce-142 & $7.26 \mathrm{E}-29$ & $3.88 \mathrm{E}-17$ & & & & & \\
\hline Ce-144 & $2.08 \mathrm{E}-24$ & $1.11 \mathrm{E}-12$ & & & & & \\
\hline Pr-144 & $2.08 \mathrm{E}-24$ & $1.11 \mathrm{E}-12$ & & & & & \\
\hline Nd-144 & & $2.09 \mathrm{E}-21$ & & & & & \\
\hline Pm-146 & $1.24 \mathrm{E}-25$ & $6.61 \mathrm{E}-14$ & & & & & \\
\hline $\mathrm{Pm}-147$ & $5.62 \mathrm{E}-22$ & $3.00 \mathrm{E}-10$ & & & & & \\
\hline Sm-146 & & $3.58 \mathrm{E}-19$ & & & & & \\
\hline Sm-147 & 1.79E-29 & $9.57 \mathrm{E}-18$ & & & & & \\
\hline Sm-148 & & $4.92 \mathrm{E}-23$ & & & & & \\
\hline $\mathrm{Sm}-149$ & & $4.37 \mathrm{E}-24$ & & & & & \\
\hline $\mathrm{Sm}-151$ & $1.11 \mathrm{E}-21$ & $5.94 \mathrm{E}-10$ & & & & & \\
\hline Eu-152 & $7.14 \mathrm{E}-24$ & $3.82 \mathrm{E}-12$ & & & & & \\
\hline Eu-154 & $4.27 \mathrm{E}-22$ & $2.28 \mathrm{E}-10$ & & & & & \\
\hline Eu-155 & $4.01 \mathrm{E}-22$ & $2.14 \mathrm{E}-10$ & & & & & \\
\hline Gd-152 & $3.45 \mathrm{E}-36$ & $1.85 \mathrm{E}-24$ & & & & & \\
\hline Ho-166m & $1.12 \mathrm{E}-28$ & $5.98 \mathrm{E}-17$ & & & & & \\
\hline \multicolumn{8}{|l|}{ Other Stream Data: } \\
\hline TRU, nCi/gm & & $1.52 \mathrm{E}-03$ & & & & & \\
\hline Heat Generation, W/m3 & & $1.41 \mathrm{E}-06$ & & & & & \\
\hline Mass Flow, kg/hr & $1.80 \mathrm{E}+02$ & $2.23 \mathrm{E}+02$ & $8.79 \mathrm{E}+01$ & $2.93 \mathrm{E}+01$ & $2.79 \mathrm{E}+01$ & 8.79E-01 & $2.70 \mathrm{E}+01$ \\
\hline
\end{tabular}

* Standard conditions $=68 \mathrm{oF}$ and 1 atmosphere 
Table C-8. Alkaline scrub balance, Tank WM-180 waste.

\begin{tabular}{|c|c|c|c|c|c|c|c|c|c|}
\hline $\begin{array}{|ll|}\text { WM-180 } & \text { PFD Sheet \# }\end{array}$ & PFD-2A & PFD-2A & PFD-2A & PFD-2A & PFD-2A & PFD-2A & PFD-2A & PFD-2A & PFD-2A \\
\hline Stream \# & 101 & 102 & 103 & 104 & 105 & 106 & 107 & 108 & 201 \\
\hline Stream Name & $\begin{array}{l}\text { SBW } \\
\text { Feed }\end{array}$ & $\begin{array}{c}\text { Evaporator } \\
\text { Bottoms }\end{array}$ & $\begin{array}{c}\text { Evaporator } \\
\text { Overheads } \\
\text { Vapor }\end{array}$ & $\begin{array}{c}\text { Mist } \\
\text { Eliminator } \\
\text { Vapor }\end{array}$ & $\begin{array}{l}\text { Alkaline } \\
\text { Scrubber } \\
\text { Vapor }\end{array}$ & $\begin{array}{c}\text { POG + VOG } \\
\text { Offgas } \\
\text { Vapor }\end{array}$ & $\begin{array}{l}\text { VOG } \\
\text { Offgas } \\
\text { Vapor }\end{array}$ & $\begin{array}{l}\text { Scrubber } \\
\text { Recycle to } \\
\text { Feed }\end{array}$ & $\begin{array}{c}20 \mathrm{wt} . \% \\
\mathrm{MgO} \\
\text { Additive }\end{array}$ \\
\hline Rate or Volume Flow (actual) & 85.8 & 26.6 & 36,397 & 36,842 & 34,297 & 38,784 & 11,747 & 14.8 & 72.2 \\
\hline Volume Flow (standard, wet) ${ }^{*}$ & & & 14,010 & 14,010 & 13,293 & 20,694 & 7,387 & & \\
\hline Volume Flow (standard, dry) & & & 275.4 & 275.2 & 0.53 & 7,124 & 7,137 & & \\
\hline Rate Units & $\mathrm{gal} / \mathrm{hr}$ & $\mathrm{gal} / \mathrm{hr}$ & $\mathrm{ft} 3 / \mathrm{hr}$ & $\mathrm{ft} 3 / \mathrm{hr}$ & $\mathrm{ft} 3 / \mathrm{hr}$ & $\mathrm{ft} 3 / \mathrm{hr}$ & $\mathrm{ft} 3 / \mathrm{hr}$ & $\mathrm{gal} / \mathrm{hr}$ & $\mathrm{Ib} / \mathrm{hr}$ \\
\hline Temperature, ${ }^{\circ} \mathrm{C}$ & 25.0 & 120 & 120 & 114 & 88 & 175 & 108 & 88.1 & 25.0 \\
\hline Temperature, ${ }^{\circ} \mathrm{F}$ & 77.0 & 247 & 247 & 237 & 190 & 348 & 226 & 191 & 77.0 \\
\hline Pressure, psia & 12.3 & 7.6 & 7.6 & 7.4 & 7.0 & 12.0 & 12.0 & 30.0 & 12.3 \\
\hline Specific Gravity & 1.23 & 1.75 & 0.0003 & 0.0003 & 0.00029 & 0.00048 & 0.00074 & 1.28 & 1.20 \\
\hline Chemical Composition & Mol/Liter & Mol/Liter & $\mathrm{Ib} / \mathrm{wscf}$ & $\mathrm{Ib} / \mathrm{wscf}$ & $\mathrm{Ib} / \mathrm{wscf}$ & Ib/wscf & $\mathrm{Ib} / \mathrm{wscf}$ & Mol/Liter & Wt. \% \\
\hline $\mathrm{H}+$ & $1.07 \mathrm{E}+00$ & $2.11 \mathrm{E}-01$ & $5.15 \mathrm{E}-05$ & $5.15 \mathrm{E}-05$ & $1.09 \mathrm{E}-09$ & 1.56E-09 & 2.40E-09 & 1.31E-04 & $2.64 \mathrm{E}-12$ \\
\hline $\mathrm{OH}-$ & & & $1.00 \mathrm{E}-14$ & & $1.06 \mathrm{E}-11$ & $1.30 \mathrm{E}-13$ & & $6.25 \mathrm{E}-05$ & $1.69 E+01$ \\
\hline$A \mathrm{Al}+3$ & $5.83 \mathrm{E}-01$ & $1.88 \mathrm{E}+00$ & $8.04 \mathrm{E}-08$ & $1.23 \mathrm{E}-11$ & $1.44 \mathrm{E}-14$ & & & $5.25 \mathrm{E}-08$ & \\
\hline $\mathrm{Sb}+5$ & $1.39 \mathrm{E}-06$ & $4.49 \mathrm{E}-06$ & $8.65 \mathrm{E}-13$ & $8.65 \mathrm{E}-15$ & $1.01 \mathrm{E}-17$ & & & $8.17 \mathrm{E}-12$ & \\
\hline $\mathrm{As+3}$ & $4.47 \mathrm{E}-04$ & $1.44 \mathrm{E}-03$ & $1.71 \mathrm{E}-10$ & $1.71 \mathrm{E}-12$ & $2.00 \mathrm{E}-15$ & & & $2.62 \mathrm{E}-09$ & \\
\hline $\mathrm{Ba}+2$ & $5.00 \mathrm{E}-05$ & $1.61 \mathrm{E}-04$ & $3.51 \mathrm{E}-11$ & $7.18 \mathrm{E}-15$ & $8.38 \mathrm{E}-18$ & & & $6.00 \mathrm{E}-12$ & \\
\hline $\mathrm{Be}+2$ & $7.82 \mathrm{E}-06$ & $2.52 \mathrm{E}-05$ & $3.60 \mathrm{E}-13$ & $4.01 \mathrm{E}-16$ & $4.68 \mathrm{E}-19$ & & & $5.11 \mathrm{E}-12$ & \\
\hline$B+3$ & $1.04 \mathrm{E}-02$ & $3.36 \mathrm{E}-02$ & $5.74 \mathrm{E}-10$ & $1.08 \mathrm{E}-13$ & $1.26 \mathrm{E}-16$ & & & 1.15E-09 & \\
\hline$C d+2$ & $7.16 \mathrm{E}-04$ & $2.31 \mathrm{E}-03$ & $4.11 \mathrm{E}-10$ & $3.74 \mathrm{E}-14$ & $4.36 \mathrm{E}-17$ & & & $3.82 \mathrm{E}-11$ & \\
\hline $\mathrm{Ca}+2$ & $3.69 \mathrm{E}-02$ & $1.19 \mathrm{E}-01$ & $7.57 \mathrm{E}-09$ & $9.08 \mathrm{E}-13$ & $1.06 \mathrm{E}-15$ & & & $2.60 \mathrm{E}-09$ & \\
\hline $\mathrm{Cr}+3$ & $3.36 \mathrm{E}-03$ & $1.09 \mathrm{E}-02$ & $8.93 \mathrm{E}-10$ & $1.44 \mathrm{E}-13$ & $1.68 \mathrm{E}-16$ & & & $3.18 \mathrm{E}-10$ & \\
\hline $\mathrm{Co}+2$ & $1.83 \mathrm{E}-05$ & $5.91 \mathrm{E}-05$ & $5.51 \mathrm{E}-12$ & $3.17 \mathrm{E}-15$ & $3.70 \mathrm{E}-18$ & & & $6.17 \mathrm{E}-12$ & \\
\hline Cs + & $2.61 \mathrm{E}-05$ & $8.43 \mathrm{E}-05$ & $1.51 \mathrm{E}-11$ & $1.15 \mathrm{E}-13$ & $1.34 \mathrm{E}-16$ & & & $9.95 \mathrm{E}-11$ & \\
\hline $\mathrm{Cu}+2$ & $6.32 \mathrm{E}-04$ & $2.04 \mathrm{E}-03$ & $2.05 \mathrm{E}-10$ & $2.87 \mathrm{E}-14$ & $3.35 \mathrm{E}-17$ & & & $5.19 \mathrm{E}-11$ & \\
\hline $\mathrm{Fe}+3$ & $1.92 \mathrm{E}-02$ & $6.21 \mathrm{E}-02$ & 5.49E-09 & $4.25 \mathrm{E}-12$ & $4.96 \mathrm{E}-15$ & & & $8.74 \mathrm{E}-09$ & \\
\hline $\mathrm{Pb}+2$ & $1.26 \mathrm{E}-03$ & $4.08 \mathrm{E}-03$ & $1.34 \mathrm{E}-09$ & $1.11 \mathrm{E}-13$ & $1.29 \mathrm{E}-16$ & & & $6.13 \mathrm{E}-11$ & \\
\hline $\mathrm{Hg}+2$ & $1.46 \mathrm{E}-03$ & $4.72 \mathrm{E}-03$ & $9.49 \mathrm{E}-07$ & $9.48 \mathrm{E}-07$ & $1.22 \mathrm{E}-08$ & $3.44 \mathrm{E}-11$ & $7.43 \mathrm{E}-11$ & $5.53 \mathrm{E}-04$ & \\
\hline $\mathrm{Mn}+4$ & $1.27 \mathrm{E}-02$ & $4.11 \mathrm{E}-02$ & 3.57E-09 & $3.57 \mathrm{E}-11$ & $4.17 \mathrm{E}-14$ & & & $7.47 \mathrm{E}-08$ & \\
\hline $\mathrm{Ni}+2$ & $1.39 \mathrm{E}-03$ & $4.48 \mathrm{E}-03$ & $4.16 \mathrm{E}-10$ & $5.83 \mathrm{E}-14$ & $6.80 \mathrm{E}-17$ & & & $1.14 \mathrm{E}-10$ & \\
\hline $\mathrm{K}+$ & $1.76 \mathrm{E}-01$ & $5.69 \mathrm{E}-01$ & $3.52 \mathrm{E}-08$ & $3.10 \mathrm{E}-12$ & $3.62 \mathrm{E}-15$ & & & $9.12 \mathrm{E}-09$ & \\
\hline $\mathrm{Se}+4$ & $7.67 \mathrm{E}-05$ & $2.48 \mathrm{E}-04$ & $3.10 \mathrm{E}-11$ & $3.10 \mathrm{E}-13$ & $3.62 \mathrm{E}-16$ & & & $4.51 \mathrm{E}-10$ & \\
\hline$\overline{\mathrm{Ag}^{+}}$ & $6.24 \mathrm{E}-06$ & $2.02 \mathrm{E}-05$ & $2.50 \mathrm{E}-12$ & $1.20 \mathrm{E}-14$ & $1.40 \mathrm{E}-17$ & & & $1.28 \mathrm{E}-11$ & \\
\hline $\mathrm{Na}+$ & $1.96 \mathrm{E}+00$ & $6.33 \mathrm{E}+00$ & $1.41 \mathrm{E}-07$ & $1.72 \mathrm{E}-10$ & $2.01 \mathrm{E}-13$ & & & $8.61 \mathrm{E}-07$ & \\
\hline$T \mid+3$ & $2.75 \mathrm{E}-05$ & $8.87 \mathrm{E}-05$ & $2.87 \mathrm{E}-11$ & $2.87 \mathrm{E}-13$ & $3.35 \mathrm{E}-16$ & & & $1.61 \mathrm{E}-10$ & \\
\hline $\mathrm{U}+4$ & $3.61 \mathrm{E}-04$ & $1.17 \mathrm{E}-03$ & $4.39 \mathrm{E}-10$ & $4.39 \mathrm{E}-12$ & $5.16 \mathrm{E}-15$ & & & $2.13 \mathrm{E}-09$ & \\
\hline$V+3$ & $8.76 \mathrm{E}-04$ & $2.83 \mathrm{E}-03$ & $2.28 \mathrm{E}-10$ & $2.28 \mathrm{E}-12$ & $2.66 \mathrm{E}-15$ & & & $5.14 \mathrm{E}-09$ & \\
\hline$Z n+2$ & $9.51 \mathrm{E}-04$ & $3.07 \mathrm{E}-03$ & $3.18 \mathrm{E}-10$ & $4.14 \mathrm{E}-14$ & $4.83 \mathrm{E}-17$ & & & $7.27 \mathrm{E}-11$ & \\
\hline $\mathrm{Zr}+4$ & $1.32 \mathrm{E}-03$ & $4.27 \mathrm{E}-03$ & $6.17 \mathrm{E}-10$ & $6.17 \mathrm{E}-12$ & $7.20 \mathrm{E}-15$ & & & 7.77E-09 & \\
\hline $\mathrm{Cl}-$ & $2.86 \mathrm{E}-02$ & $9.26 \mathrm{E}-02$ & $1.55 \mathrm{E}-05$ & $1.55 \mathrm{E}-05$ & $2.22 \mathrm{E}-08$ & $2.43 \mathrm{E}-10$ & $1.37 \mathrm{E}-10$ & $5.01 \mathrm{E}-02$ & \\
\hline F- & $4.02 \mathrm{E}-02$ & $1.30 \mathrm{E}-01$ & $1.72 \mathrm{E}-08$ & $1.72 \mathrm{E}-08$ & $1.02 \mathrm{E}-09$ & $6.55 \mathrm{E}-10$ & $8.16 \mathrm{E}-12$ & $9.85 \mathrm{E}-05$ & \\
\hline SO4-2 & $4.96 \mathrm{E}-02$ & $1.60 \mathrm{E}-01$ & $2.44 \mathrm{E}-08$ & $2.44 \mathrm{E}-10$ & $2.84 \mathrm{E}-13$ & & & $2.91 \mathrm{E}-07$ & \\
\hline NO3- & $4.94 \mathrm{E}+00$ & $1.59 \mathrm{E}+01$ & $3.14 \mathrm{E}-03$ & $3.14 \mathrm{E}-03$ & $3.73 \mathrm{E}-06$ & $9.32 \mathrm{E}-08$ & $1.48 \mathrm{E}-07$ & $5.82 \mathrm{E}+00$ & \\
\hline PO4-3 & $1.95 \mathrm{E}-02$ & $6.30 \mathrm{E}-02$ & 9.46E-09 & $9.46 \mathrm{E}-11$ & $1.10 \mathrm{E}-13$ & & & $1.14 \mathrm{E}-07$ & \\
\hline$A m+4$ & $3.16 \mathrm{E}-08$ & $1.02 \mathrm{E}-07$ & $3.89 \mathrm{E}-14$ & $3.89 \mathrm{E}-16$ & $4.57 \mathrm{E}-19$ & & & $1.86 \mathrm{E}-13$ & \\
\hline $\mathrm{Br}-$ & $1.55 \mathrm{E}-07$ & $5.04 \mathrm{E}-07$ & $1.90 \mathrm{E}-10$ & $1.90 \mathrm{E}-10$ & $2.74 \mathrm{E}-13$ & $2.99 \mathrm{E}-15$ & $1.68 \mathrm{E}-15$ & $2.74 \mathrm{E}-07$ & \\
\hline $\mathrm{Ce}+4$ & $4.36 \mathrm{E}-05$ & $1.41 \mathrm{E}-04$ & $3.12 \mathrm{E}-11$ & $3.12 \mathrm{E}-13$ & $3.67 \mathrm{E}-16$ & & & $2.56 \mathrm{E}-10$ & \\
\hline $\mathrm{Eu}+3$ & $2.58 \mathrm{E}-07$ & $8.32 \mathrm{E}-07$ & $2.00 \mathrm{E}-13$ & $3.04 \mathrm{E}-19$ & $3.54 \mathrm{E}-22$ & & & $2.30 \mathrm{E}-16$ & \\
\hline $\mathrm{Gd}+3$ & $1.61 \mathrm{E}-04$ & $5.19 \mathrm{E}-04$ & $1.29 \mathrm{E}-10$ & $1.71 \mathrm{E}-14$ & $2.00 \mathrm{E}-17$ & & & $1.25 \mathrm{E}-11$ & \\
\hline $\mathrm{Ge}+4$ & $4.48 \mathrm{E}-09$ & 1.45E-08 & $1.66 \mathrm{E}-15$ & $1.66 \mathrm{E}-17$ & $1.95 \mathrm{E}-20$ & & & $2.64 \mathrm{E}-14$ & \\
\hline $\ln +3$ & $7.05 \mathrm{E}-07$ & $2.28 \mathrm{E}-06$ & $4.14 \mathrm{E}-13$ & & & & & & \\
\hline $1-$ & $1.35 \mathrm{E}-06$ & $4.37 \mathrm{E}-06$ & $2.51 \mathrm{E}-09$ & $2.51 \mathrm{E}-09$ & $3.61 \mathrm{E}-12$ & $3.94 \mathrm{E}-14$ & $2.22 \mathrm{E}-14$ & $2.27 \mathrm{E}-06$ & \\
\hline $\mathrm{La}+3$ & $4.68 \mathrm{E}-06$ & $1.51 \mathrm{E}-05$ & $3.32 \mathrm{E}-12$ & & & & & & \\
\hline Li+ & $4.58 \mathrm{E}-04$ & $1.48 \mathrm{E}-03$ & $1.12 \mathrm{E}-11$ & $4.25 \mathrm{E}-14$ & $4.96 \mathrm{E}-17$ & & & $7.04 \mathrm{E}-10$ & \\
\hline$M g+2$ & $1.08 \mathrm{E}-02$ & $1.67 \mathrm{E}+00$ & $6.42 \mathrm{E}-08$ & & $7.25 \mathrm{E}-07$ & $9.32 \mathrm{E}-14$ & & $2.94 \mathrm{E}+00$ & $1.21 \mathrm{E}+01$ \\
\hline$\overline{\mathrm{Mo}+6}$ & $1.88 \mathrm{E}-04$ & $6.06 \mathrm{E}-04$ & $9.20 \mathrm{E}-11$ & $9.20 \mathrm{E}-13$ & $1.07 \mathrm{E}-15$ & & & $1.10 \mathrm{E}-09$ & \\
\hline
\end{tabular}


Table C-8. Alkaline scrub balance, Tank WM-180 waste. (Continued).

\begin{tabular}{|c|c|c|c|c|c|c|c|c|c|}
\hline Stream \# & 101 & 102 & 103 & 104 & 105 & 106 & 107 & 108 & 201 \\
\hline Chemical Composition & Mol/Liter & Mol/Liter & $\mathrm{lb} / \mathrm{wscf}$ & $\mathrm{lb} / \mathrm{wscf}$ & $\mathrm{lb} /$ wscf & $\mathrm{lb} /$ wscf & $\mathrm{Ib} / \mathrm{wscf}$ & Mol/Liter & Wt. \% \\
\hline $\mathrm{Nd}+3$ & $1.51 \mathrm{E}-05$ & $4.88 \mathrm{E}-05$ & $1.11 \mathrm{E}-11$ & & & & & & \\
\hline $\mathrm{Np}+4$ & $8.73 \mathrm{E}-06$ & $2.82 \mathrm{E}-05$ & $1.06 \mathrm{E}-11$ & 1.06E-13 & $1.24 \mathrm{E}-16$ & & & $5.13 \mathrm{E}-11$ & \\
\hline $\mathrm{Nb}+5$ & $4.46 \mathrm{E}-04$ & $1.44 \mathrm{E}-03$ & $2.12 \mathrm{E}-10$ & $2.12 \mathrm{E}-12$ & $2.47 \mathrm{E}-15$ & & & $2.62 \mathrm{E}-09$ & \\
\hline $\mathrm{Pd}+4$ & $3.13 \mathrm{E}-05$ & $1.01 \mathrm{E}-04$ & $1.70 \mathrm{E}-11$ & 1.70E-13 & $1.99 \mathrm{E}-16$ & & & $1.84 \mathrm{E}-10$ & \\
\hline $\mathrm{Pu}+4$ & $1.01 \mathrm{E}-05$ & $3.25 \mathrm{E}-05$ & $1.23 \mathrm{E}-11$ & $1.23 \mathrm{E}-13$ & $1.44 \mathrm{E}-16$ & & & $5.92 \mathrm{E}-11$ & \\
\hline $\mathrm{Pr}+4$ & $4.26 \mathrm{E}-06$ & $1.37 \mathrm{E}-05$ & $3.07 \mathrm{E}-12$ & $3.07 \mathrm{E}-14$ & $3.60 \mathrm{E}-17$ & & & $2.50 \mathrm{E}-11$ & \\
\hline $\mathrm{Rh}+4$ & $1.84 \mathrm{E}-06$ & $5.93 \mathrm{E}-06$ & $9.66 \mathrm{E}-13$ & $9.66 \mathrm{E}-15$ & $1.14 \mathrm{E}-17$ & & & $1.08 \mathrm{E}-11$ & \\
\hline $\mathrm{Rb}+$ & $2.94 \mathrm{E}-06$ & $9.49 \mathrm{E}-06$ & $7.84 \mathrm{E}-13$ & $8.74 \mathrm{E}-16$ & $1.02 \mathrm{E}-18$ & & & $1.17 \mathrm{E}-12$ & \\
\hline $\mathrm{Ru}+3$ & $1.26 \mathrm{E}-04$ & $4.08 \mathrm{E}-04$ & $6.52 \mathrm{E}-11$ & $7.58 \mathrm{E}-14$ & $8.84 \mathrm{E}-17$ & & & $8.61 \mathrm{E}-11$ & \\
\hline $\mathrm{Sm+3}$ & $2.80 \mathrm{E}-06$ & $9.05 \mathrm{E}-06$ & $2.15 \mathrm{E}-12$ & $1.57 \mathrm{E}-17$ & $1.83 \mathrm{E}-20$ & & & $1.20 \mathrm{E}-14$ & \\
\hline $\mathrm{Si+4}$ & $3.08 \mathrm{E}-03$ & $9.94 \mathrm{E}-03$ & $4.42 \mathrm{E}-10$ & $4.42 \mathrm{E}-12$ & $5.15 \mathrm{E}-15$ & & & $1.81 \mathrm{E}-08$ & \\
\hline $\mathrm{Sr}+2$ & $1.08 \mathrm{E}-04$ & $3.48 \mathrm{E}-04$ & $4.82 \mathrm{E}-11$ & $4.64 \mathrm{E}-15$ & $5.42 \mathrm{E}-18$ & & & $6.09 \mathrm{E}-12$ & \\
\hline $\mathrm{Tc}+7$ & $5.30 \mathrm{E}-06$ & $1.71 \mathrm{E}-05$ & $2.63 \mathrm{E}-12$ & & & & & & \\
\hline $\mathrm{Te}+4$ & 1.47E-06 & $4.75 \mathrm{E}-06$ & $9.59 \mathrm{E}-13$ & $9.59 \mathrm{E}-15$ & $1.13 \mathrm{E}-17$ & & & $8.65 \mathrm{E}-12$ & \\
\hline $\mathrm{Tb}+4$ & $1.08 \mathrm{E}-09$ & $3.48 \mathrm{E}-09$ & $8.75 \mathrm{E}-16$ & $8.75 \mathrm{E}-18$ & $1.03 \mathrm{E}-20$ & & & $6.34 \mathrm{E}-15$ & \\
\hline $\mathrm{Th}+4$ & $1.05 \mathrm{E}-10$ & $3.39 \mathrm{E}-10$ & $1.25 \mathrm{E}-16$ & $1.25 \mathrm{E}-18$ & 1.46E-21 & & & $6.18 \mathrm{E}-16$ & \\
\hline $\mathrm{Sn}+4$ & $7.38 \mathrm{E}-05$ & $2.38 \mathrm{E}-04$ & $4.48 \mathrm{E}-11$ & $4.48 \mathrm{E}-13$ & $5.23 \mathrm{E}-16$ & & & 4.33E-10 & \\
\hline$T i+4$ & $1.34 \mathrm{E}-04$ & $4.34 \mathrm{E}-04$ & $3.29 \mathrm{E}-11$ & $3.29 \mathrm{E}-13$ & $3.85 \mathrm{E}-16$ & & & $7.90 \mathrm{E}-10$ & \\
\hline$Y+3$ & $3.49 \mathrm{E}-06$ & $1.13 \mathrm{E}-05$ & $1.59 \mathrm{E}-12$ & $2.28 \mathrm{E}-20$ & $2.66 \mathrm{E}-23$ & & & $2.94 \mathrm{E}-17$ & \\
\hline $\mathrm{O}-2$ (oxides) & $5.11 \mathrm{E}-02$ & $1.65 \mathrm{E}-01$ & $4.18 \mathrm{E}-09$ & $4.18 \mathrm{E}-11$ & $4.88 \mathrm{E}-14$ & & & $3.00 \mathrm{E}-07$ & \\
\hline $\mathrm{H} 2 \mathrm{O}$ & $5.05 \mathrm{E}+01$ & $2.50 \mathrm{E}+01$ & $4.60 \mathrm{E}-02$ & 4.60E-02 & $4.69 \mathrm{E}-02$ & 3.07E-02 & $1.58 \mathrm{E}-03$ & $4.70 \mathrm{E}+01$ & $7.11 \mathrm{E}+01$ \\
\hline $\mathrm{O} 2$ & & & $1.42 \mathrm{E}-07$ & $1.42 \mathrm{E}-07$ & $1.50 \mathrm{E}-07$ & $6.01 \mathrm{E}-03$ & $1.69 \mathrm{E}-02$ & $1.94 \mathrm{E}-10$ & \\
\hline N2 & & & $2.40 \mathrm{E}-07$ & $2.40 \mathrm{E}-07$ & $2.53 \mathrm{E}-07$ & $1.98 \mathrm{E}-02$ & $5.55 \mathrm{E}-02$ & $2.22 \mathrm{E}-10$ & \\
\hline C (organic) & $1.78 \mathrm{E}-02$ & $5.74 \mathrm{E}-02$ & $1.64 \mathrm{E}-07$ & 1.64E-07 & $5.01 \mathrm{E}-10$ & $3.22 \mathrm{E}-10$ & $7.15 \mathrm{E}-13$ & $1.54 \mathrm{E}-03$ & \\
\hline $\mathrm{H}$ (organic) & $1.78 \mathrm{E}-02$ & $5.74 \mathrm{E}-02$ & $1.37 \mathrm{E}-08$ & 1.37E-08 & $4.21 \mathrm{E}-11$ & $2.70 \mathrm{E}-11$ & $6.00 \mathrm{E}-14$ & $1.54 \mathrm{E}-03$ & \\
\hline $\begin{array}{l}\text { Gas Stream Bulk Composition } \\
\text { (wet basis) }\end{array}$ & $\begin{array}{l}\text { mol.\% } \\
\text { or ppmv } \\
\end{array}$ & $\begin{array}{l}\mathrm{mol} . \% \\
\text { or ppmv }\end{array}$ & $\begin{array}{l}\text { mol. \% } \\
\text { or ppmv } \\
\end{array}$ & $\begin{array}{l}\text { mol.\% } \\
\text { or ppmv }\end{array}$ & $\begin{array}{l}\text { mol.\% } \\
\text { or ppmv }\end{array}$ & $\begin{array}{l}\text { mol.\% } \\
\text { or ppmv }\end{array}$ & $\begin{array}{l}\text { mol.\% } \\
\text { or ppmv }\end{array}$ & $\begin{array}{l}\text { mol.\% } \\
\text { or ppmv } \\
\end{array}$ & $\begin{array}{l}\text { mol.\% } \\
\text { or ppmv } \\
\end{array}$ \\
\hline $\mathrm{H} 2 \mathrm{O}, \mathrm{mol} . \%$ & & & 98.03 & 98.04 & 100.00 & 65.57 & 3.39 & & \\
\hline $\mathrm{O} 2, \mathrm{~mol} . \%$ & & & 0.0002 & 0.0002 & 0.0002 & 7.23 & 20.29 & & \\
\hline $\mathrm{N} 2, \mathrm{~mol} . \%$ & & & 0.0003 & 0.0003 & 0.0003 & 27.20 & 76.32 & & \\
\hline \multicolumn{10}{|l|}{$\mathrm{CO} 2, \mathrm{~mol} . \%$} \\
\hline HNO3, mol. \% & & & 1.96 & 1.96 & 0.00004 & 0.00006 & 0.00009 & & \\
\hline \multicolumn{10}{|l|}{ CO, ppmv } \\
\hline $\mathrm{HCl}, \mathrm{ppmv}$ & & & 167 & 167 & 0.24 & 0.003 & 0.001 & & \\
\hline C (organic), ppmv & & & 5 & 5 & 0.016 & 0.010 & 0.00002 & & \\
\hline $\mathrm{H}$ (organic), ppmv & & & 5 & 5 & 0.016 & 0.010 & 0.00002 & & \\
\hline $\mathrm{PM}(\mathrm{mg} / \mathrm{wscm})$ & & & 1 & 0.01 & 0.000015 & & & & \\
\hline $\mathrm{Hg}$ (ug/wscm) & & & $1.5 \mathrm{E}+04$ & $1.5 \mathrm{E}+04$ & 196 & 1 & 1 & & \\
\hline $\begin{array}{l}\text { Gas Stream Bulk Composition } \\
\text { (dry basis) }\end{array}$ & $\begin{array}{l}\mathrm{mol} . \% \\
\text { or ppmv }\end{array}$ & $\begin{array}{l}\mathrm{mol} . \% \\
\text { or ppmv }\end{array}$ & $\begin{array}{l}\text { mol.\% } \\
\text { or ppmv }\end{array}$ & $\begin{array}{l}\text { mol.\% } \\
\text { or ppmv }\end{array}$ & $\begin{array}{l}\text { mol.\% } \\
\text { or ppmv }\end{array}$ & $\begin{array}{l}\text { mol.\% } \\
\text { or ppmv }\end{array}$ & $\begin{array}{l}\mathrm{mol} . \% \\
\text { or ppmv }\end{array}$ & $\begin{array}{l}\mathrm{mol} . \% \\
\text { or ppmv }\end{array}$ & $\begin{array}{l}\text { mol.\% } \\
\text { or ppmv }\end{array}$ \\
\hline $\mathrm{O} 2, \mathrm{~mol} . \%$ & & & 0.009 & 0.009 & 4.48 & 21.00 & 21.00 & & \\
\hline $\mathrm{N} 2, \mathrm{~mol} . \%$ & & & 0.017 & 0.017 & 8.64 & 79.00 & 79.00 & & \\
\hline \multicolumn{10}{|l|}{$\mathrm{CO} 2, \mathrm{~mol} . \%$} \\
\hline HNO3, mol. \% & & & 99.89 & 99.95 & 1.03 & 0.0002 & 0.0001 & & \\
\hline \multicolumn{10}{|l|}{$\mathrm{CO}, \mathrm{ppmv}$} \\
\hline $\mathrm{HCl}, \mathrm{ppmv}$ & & & $8.5 \mathrm{E}+03$ & $8.5 \mathrm{E}+03$ & $6.0 \mathrm{E}+03$ & 0.008 & 0.002 & & \\
\hline C (organic), ppmv & & & $2.7 \mathrm{E}+02$ & $2.7 \mathrm{E}+02$ & $4.0 \mathrm{E}+02$ & 0.03 & 0.00002 & & \\
\hline $\mathrm{H}$ (organic), ppmv & & & 266 & 267 & 400 & 0.03 & 0.00002 & & \\
\hline $\mathrm{PM}(\mathrm{mg} / \mathrm{dscm})$ & & & 66 & 1 & 0.38 & & & & \\
\hline $\mathrm{Hg}$ (ug/wscm) & & & $2.0 \mathrm{E}+06$ & $2.0 \mathrm{E}+06$ & $1.3 \mathrm{E}+07$ & 3 & 2 & & \\
\hline Total Mass Flow, kg/hr & $4.22 \mathrm{E}+02$ & $1.76 \mathrm{E}+02$ & $3.13 \mathrm{E}+02$ & $3.13 \mathrm{E}+02$ & $2.83 \mathrm{E}+02$ & $5.31 \mathrm{E}+02$ & $2.48 \mathrm{E}+02$ & $7.17 \mathrm{E}+01$ & $3.27 \mathrm{E}+01$ \\
\hline Canister Rate, canisters/day & & 3.02 & & & & & & & \\
\hline Total Canisters Generated & & 425 & & & & & & & \\
\hline
\end{tabular}


Table C-8. Alkaline scrub balance, Tank WM-180 waste. (Continued).

\begin{tabular}{|c|c|c|c|c|c|c|c|c|c|}
\hline Stream \# & 101 & 102 & 103 & 104 & 105 & 106 & 107 & 108 & 201 \\
\hline Radiological Composition & $\mathrm{Ci} / \mathrm{L}$ & $\mathrm{Ci} / \mathrm{L}$ & $\mathrm{Ci} / \mathrm{wscm}$ & $\mathrm{Ci} / \mathrm{wscm}$ & $\mathrm{Ci} / \mathrm{wscm}$ & $\mathrm{Ci} / \mathrm{wscm}$ & $\mathrm{Ci} / \mathrm{wscm}$ & $\mathrm{Ci} / \mathrm{L}$ & \\
\hline Ra-226 & $4.03 \mathrm{E}-12$ & $1.30 \mathrm{E}-11$ & $3.30 \mathrm{E}-16$ & $3.30 \mathrm{E}-18$ & $3.79 \mathrm{E}-21$ & $4.95 \mathrm{E}-26$ & $1.25 \mathrm{E}-25$ & $2.33 \mathrm{E}-17$ & \\
\hline Ac-227 & $1.90 \mathrm{E}-11$ & $6.13 \mathrm{E}-11$ & $1.55 \mathrm{E}-15$ & $1.55 \mathrm{E}-17$ & $1.79 \mathrm{E}-20$ & $2.33 \mathrm{E}-25$ & $5.89 \mathrm{E}-25$ & 1.10E-16 & \\
\hline Th-228 & $1.57 \mathrm{E}-09$ & $5.08 \mathrm{E}-09$ & $1.29 \mathrm{E}-13$ & $1.29 \mathrm{E}-15$ & $1.48 \mathrm{E}-18$ & $1.94 \mathrm{E}-23$ & $4.89 \mathrm{E}-23$ & $9.12 \mathrm{E}-15$ & \\
\hline Th-230 & $4.29 \mathrm{E}-10$ & 1.39E-09 & $3.51 \mathrm{E}-14$ & $3.51 \mathrm{E}-16$ & $4.04 \mathrm{E}-19$ & $5.28 \mathrm{E}-24$ & $1.33 \mathrm{E}-23$ & $2.49 \mathrm{E}-15$ & \\
\hline Th-232 & $3.48 \mathrm{E}-16$ & $1.13 \mathrm{E}-15$ & $2.85 \mathrm{E}-20$ & $2.85 \mathrm{E}-22$ & $3.28 \mathrm{E}-25$ & $4.28 \mathrm{E}-30$ & $1.08 \mathrm{E}-29$ & $2.02 \mathrm{E}-21$ & \\
\hline Pa-231 & 4.40E-11 & $1.42 \mathrm{E}-10$ & $3.60 \mathrm{E}-15$ & 3.60E-17 & $4.14 \mathrm{E}-20$ & $5.40 \mathrm{E}-25$ & $1.37 \mathrm{E}-24$ & $2.55 \mathrm{E}-16$ & \\
\hline Pa-233 & 1.44E-06 & $4.66 \mathrm{E}-06$ & $1.18 \mathrm{E}-10$ & $1.18 \mathrm{E}-12$ & $1.36 \mathrm{E}-15$ & $1.77 \mathrm{E}-20$ & $4.48 \mathrm{E}-20$ & $8.36 \mathrm{E}-12$ & \\
\hline U-232 & 1.03E-09 & 3.34E-09 & $8.47 \mathrm{E}-14$ & $8.47 \mathrm{E}-16$ & $9.73 \mathrm{E}-19$ & $1.27 \mathrm{E}-23$ & $3.21 \mathrm{E}-23$ & $5.99 \mathrm{E}-15$ & \\
\hline $\mathrm{U}-233$ & $4.03 \mathrm{E}-11$ & $1.30 \mathrm{E}-10$ & $3.30 \mathrm{E}-15$ & 3.30E-17 & $3.79 \mathrm{E}-20$ & $4.96 \mathrm{E}-25$ & 1.25E-24 & $2.34 \mathrm{E}-16$ & \\
\hline $\mathrm{U}-234$ & 1.04E-06 & $3.35 \mathrm{E}-06$ & $8.48 \mathrm{E}-11$ & $8.48 \mathrm{E}-13$ & $9.75 \mathrm{E}-16$ & $1.27 \mathrm{E}-20$ & $3.22 \mathrm{E}-20$ & $6.00 \mathrm{E}-12$ & \\
\hline U-235 & $3.78 \mathrm{E}-08$ & $1.22 \mathrm{E}-07$ & $3.10 \mathrm{E}-12$ & $3.10 \mathrm{E}-14$ & $3.56 \mathrm{E}-17$ & $4.65 \mathrm{E}-22$ & $1.18 \mathrm{E}-21$ & $2.19 \mathrm{E}-13$ & \\
\hline U-236 & $5.62 \mathrm{E}-08$ & $1.81 \mathrm{E}-07$ & $4.60 \mathrm{E}-12$ & 4.60E-14 & $5.29 \mathrm{E}-17$ & $6.91 \mathrm{E}-22$ & $1.74 \mathrm{E}-21$ & $3.25 \mathrm{E}-13$ & \\
\hline $\mathrm{U}-237$ & 3.17E-09 & 1.02E-08 & $2.59 \mathrm{E}-13$ & $2.59 \mathrm{E}-15$ & $2.98 \mathrm{E}-18$ & $3.89 \mathrm{E}-23$ & 9.83E-23 & 1.83E-14 & \\
\hline $\mathrm{U}-238$ & $2.24 \mathrm{E}-08$ & $7.22 \mathrm{E}-08$ & 1.83E-12 & 1.83E-14 & $2.11 \mathrm{E}-17$ & $2.75 \mathrm{E}-22$ & $6.95 \mathrm{E}-22$ & $1.30 \mathrm{E}-13$ & \\
\hline Np-236 & $1.88 \mathrm{E}-12$ & $6.06 \mathrm{E}-12$ & $1.54 \mathrm{E}-16$ & $1.54 \mathrm{E}-18$ & $1.77 \mathrm{E}-21$ & $2.31 \mathrm{E}-26$ & $5.83 \mathrm{E}-26$ & $1.09 \mathrm{E}-17$ & \\
\hline Np-237 & $1.46 \mathrm{E}-06$ & $4.71 \mathrm{E}-06$ & $1.19 \mathrm{E}-10$ & $1.19 \mathrm{E}-12$ & $1.37 \mathrm{E}-15$ & $1.79 \mathrm{E}-20$ & $4.53 \mathrm{E}-20$ & $8.45 \mathrm{E}-12$ & \\
\hline Pu-236 & $2.08 \mathrm{E}-09$ & $6.70 \mathrm{E}-09$ & 1.70E-13 & 1.70E-15 & 1.95E-18 & $2.55 \mathrm{E}-23$ & $6.45 \mathrm{E}-23$ & 1.20E-14 & \\
\hline Pu-238 & $9.47 \mathrm{E}-04$ & 3.06E-03 & $7.75 \mathrm{E}-08$ & $7.75 \mathrm{E}-10$ & $8.91 \mathrm{E}-13$ & $1.16 \mathrm{E}-17$ & $2.94 \mathrm{E}-17$ & 5.49E-09 & \\
\hline Pu-239 & 1.44E-04 & $4.64 \mathrm{E}-04$ & 1.18E-08 & $1.18 \mathrm{E}-10$ & $1.35 \mathrm{E}-13$ & $1.77 \mathrm{E}-18$ & $4.46 \mathrm{E}-18$ & $8.32 \mathrm{E}-10$ & \\
\hline $\mathrm{Pu}-240$ & $7.80 \mathrm{E}-06$ & $2.52 \mathrm{E}-05$ & $6.38 \mathrm{E}-10$ & $6.38 \mathrm{E}-12$ & $7.34 \mathrm{E}-15$ & $9.58 \mathrm{E}-20$ & $2.42 \mathrm{E}-19$ & $4.52 \mathrm{E}-11$ & \\
\hline Pu-241 & $5.70 \mathrm{E}-04$ & $1.84 \mathrm{E}-03$ & 4.67E-08 & 4.67E-10 & $5.37 \mathrm{E}-13$ & $7.01 \mathrm{E}-18$ & 1.77E-17 & 3.30E-09 & \\
\hline Pu-242 & $6.01 \mathrm{E}-09$ & $1.94 \mathrm{E}-08$ & $4.92 \mathrm{E}-13$ & $4.92 \mathrm{E}-15$ & $5.66 \mathrm{E}-18$ & $7.39 \mathrm{E}-23$ & $1.87 \mathrm{E}-22$ & $3.48 \mathrm{E}-14$ & \\
\hline Pu-244 & $5.15 \mathrm{E}-16$ & $1.66 \mathrm{E}-15$ & $4.21 \mathrm{E}-20$ & $4.22 \mathrm{E}-22$ & $4.85 \mathrm{E}-25$ & $6.33 \mathrm{E}-30$ & $1.60 \mathrm{E}-29$ & $2.98 \mathrm{E}-21$ & \\
\hline Am-241 & $6.86 \mathrm{E}-05$ & $2.21 \mathrm{E}-04$ & 5.61E-09 & $5.61 \mathrm{E}-11$ & $6.45 \mathrm{E}-14$ & $8.43 \mathrm{E}-19$ & $2.13 \mathrm{E}-18$ & $3.97 \mathrm{E}-10$ & \\
\hline$\overline{A m-242 m}$ & $7.48 \mathrm{E}-09$ & $2.42 \mathrm{E}-08$ & $6.13 \mathrm{E}-13$ & $6.13 \mathrm{E}-15$ & $7.04 \mathrm{E}-18$ & $9.20 \mathrm{E}-23$ & $2.32 \mathrm{E}-22$ & $4.34 \mathrm{E}-14$ & \\
\hline Am-243 & $1.09 \mathrm{E}-08$ & $3.51 \mathrm{E}-08$ & $8.89 \mathrm{E}-13$ & $8.89 \mathrm{E}-15$ & $1.02 \mathrm{E}-17$ & $1.34 \mathrm{E}-22$ & 3.37E-22 & $6.29 \mathrm{E}-14$ & \\
\hline $\mathrm{Cm}-242$ & $6.17 \mathrm{E}-09$ & $1.99 \mathrm{E}-08$ & $5.05 \mathrm{E}-13$ & $5.05 \mathrm{E}-15$ & $5.81 \mathrm{E}-18$ & $7.59 \mathrm{E}-23$ & $1.92 \mathrm{E}-22$ & $3.58 \mathrm{E}-14$ & \\
\hline $\mathrm{Cm}-243$ & $1.78 \mathrm{E}-08$ & $5.73 \mathrm{E}-08$ & $1.45 \mathrm{E}-12$ & $1.45 \mathrm{E}-14$ & $1.67 \mathrm{E}-17$ & $2.18 \mathrm{E}-22$ & $5.51 \mathrm{E}-22$ & $1.03 \mathrm{E}-13$ & \\
\hline $\mathrm{Cm}-244$ & 1.10E-06 & $3.56 \mathrm{E}-06$ & $9.03 \mathrm{E}-11$ & $9.03 \mathrm{E}-13$ & $1.04 \mathrm{E}-15$ & $1.36 \mathrm{E}-20$ & $3.43 \mathrm{E}-20$ & $6.39 \mathrm{E}-12$ & \\
\hline $\mathrm{Cm}-245$ & $1.87 \mathrm{E}-10$ & $6.03 \mathrm{E}-10$ & $1.53 \mathrm{E}-14$ & $1.53 \mathrm{E}-16$ & $1.76 \mathrm{E}-19$ & $2.30 \mathrm{E}-24$ & $5.80 \mathrm{E}-24$ & $1.08 \mathrm{E}-15$ & \\
\hline $\mathrm{Cm}-246$ & 1.23E-11 & $3.96 \mathrm{E}-11$ & $1.00 \mathrm{E}-15$ & 1.00E-17 & 1.15E-20 & $1.51 \mathrm{E}-25$ & $3.81 \mathrm{E}-25$ & 7.11E-17 & \\
\hline $\mathrm{H}-3$ & $1.72 \mathrm{E}-05$ & $8.38 \mathrm{E}-06$ & 1.37E-05 & 1.37E-05 & 1.24E-05 & $8.10 \mathrm{E}-06$ & 4.71E-07 & 1.38E-05 & \\
\hline $\mathrm{Be}-10$ & $1.48 \mathrm{E}-12$ & $4.77 \mathrm{E}-12$ & $1.21 \mathrm{E}-16$ & $1.21 \mathrm{E}-18$ & 1.39E-21 & $1.81 \mathrm{E}-26$ & $4.58 \mathrm{E}-26$ & $8.55 \mathrm{E}-18$ & \\
\hline C-14 & $6.19 \mathrm{E}-11$ & $2.00 \mathrm{E}-10$ & $5.07 \mathrm{E}-15$ & $5.07 \mathrm{E}-17$ & $5.83 \mathrm{E}-20$ & $7.61 \mathrm{E}-25$ & $1.92 \mathrm{E}-24$ & $3.59 \mathrm{E}-16$ & \\
\hline Co-60 & 3.97E-06 & $1.28 \mathrm{E}-05$ & $3.25 \mathrm{E}-10$ & $3.25 \mathrm{E}-12$ & $3.73 \mathrm{E}-15$ & $4.88 \mathrm{E}-20$ & 1.23E-19 & $2.30 \mathrm{E}-11$ & \\
\hline Ni-59 & $9.14 \mathrm{E}-08$ & $2.95 \mathrm{E}-07$ & $7.48 \mathrm{E}-12$ & $7.48 \mathrm{E}-14$ & $8.60 \mathrm{E}-17$ & $1.12 \mathrm{E}-21$ & $2.84 \mathrm{E}-21$ & $5.29 \mathrm{E}-13$ & \\
\hline Ni-63 & $2.41 \mathrm{E}-05$ & $7.78 \mathrm{E}-05$ & 1.97E-09 & 1.97E-11 & $2.27 \mathrm{E}-14$ & $2.96 \mathrm{E}-19$ & $7.48 \mathrm{E}-19$ & 1.40E-10 & \\
\hline Se-79 & $2.25 \mathrm{E}-07$ & $7.27 \mathrm{E}-07$ & $1.84 \mathrm{E}-11$ & $1.84 \mathrm{E}-13$ & $2.12 \mathrm{E}-16$ & $2.77 \mathrm{E}-21$ & $7.00 \mathrm{E}-21$ & $1.31 \mathrm{E}-12$ & \\
\hline Rb-87 & 1.44E-11 & $4.66 \mathrm{E}-11$ & $1.18 \mathrm{E}-15$ & $1.18 \mathrm{E}-17$ & $1.36 \mathrm{E}-20$ & $1.77 \mathrm{E}-25$ & $4.48 \mathrm{E}-25$ & $8.35 \mathrm{E}-17$ & \\
\hline Sr-90 & 1.95E-02 & $6.29 \mathrm{E}-02$ & 1.59E-06 & $1.59 \mathrm{E}-08$ & $1.83 \mathrm{E}-11$ & $2.39 \mathrm{E}-16$ & $6.05 \mathrm{E}-16$ & 1.13E-07 & \\
\hline $\begin{array}{l}\mathrm{Y}-90 \\
\end{array}$ & $1.95 \mathrm{E}-02$ & $6.29 \mathrm{E}-02$ & $1.59 \mathrm{E}-06$ & $1.59 \mathrm{E}-08$ & $1.83 \mathrm{E}-11$ & $2.39 \mathrm{E}-16$ & $6.05 \mathrm{E}-16$ & $1.13 \mathrm{E}-07$ & \\
\hline $\begin{array}{l}\mathrm{Zr}-93 \\
\end{array}$ & $1.09 \mathrm{E}-06$ & $3.52 \mathrm{E}-06$ & $8.92 \mathrm{E}-11$ & $8.92 \mathrm{E}-13$ & $1.03 \mathrm{E}-15$ & $1.34 \mathrm{E}-20$ & $3.38 \mathrm{E}-20$ & $6.32 \mathrm{E}-12$ & \\
\hline Nb-93m & $8.40 \mathrm{E}-07$ & $2.71 \mathrm{E}-06$ & $6.87 \mathrm{E}-11$ & $6.87 \mathrm{E}-13$ & $7.90 \mathrm{E}-16$ & $1.03 E-20$ & $2.61 \mathrm{E}-20$ & $4.87 \mathrm{E}-12$ & \\
\hline Nb-94 & $5.87 \mathrm{E}-07$ & $1.90 \mathrm{E}-06$ & $4.81 \mathrm{E}-11$ & $4.81 \mathrm{E}-13$ & $5.53 \mathrm{E}-16$ & $7.22 \mathrm{E}-21$ & $1.82 \mathrm{E}-20$ & $3.40 \mathrm{E}-12$ & \\
\hline Tc-98 & $1.27 \mathrm{E}-12$ & 4.10E-12 & $1.04 \mathrm{E}-16$ & 1.04E-18 & 1.19E-21 & $1.56 \mathrm{E}-26$ & $3.94 \mathrm{E}-26$ & 7.35E-18 & \\
\hline Tc-99 & $9.01 \mathrm{E}-06$ & $2.91 \mathrm{E}-05$ & $7.37 \mathrm{E}-10$ & $7.37 \mathrm{E}-12$ & $8.48 \mathrm{E}-15$ & $1.11 \mathrm{E}-19$ & $2.80 \mathrm{E}-19$ & $5.22 \mathrm{E}-11$ & \\
\hline Ru-106 & $4.80 \mathrm{E}-07$ & $1.55 \mathrm{E}-06$ & $3.93 \mathrm{E}-11$ & $3.93 \mathrm{E}-13$ & $4.52 \mathrm{E}-16$ & $5.90 \mathrm{E}-21$ & $1.49 \mathrm{E}-20$ & $2.78 \mathrm{E}-12$ & \\
\hline Rh-102 & $4.24 \mathrm{E}-10$ & 1.37E-09 & $3.47 \mathrm{E}-14$ & $3.47 \mathrm{E}-16$ & $3.99 \mathrm{E}-19$ & $5.21 \mathrm{E}-24$ & 1.32E-23 & $2.46 \mathrm{E}-15$ & \\
\hline Rh-106 & $4.80 \mathrm{E}-07$ & $1.55 \mathrm{E}-06$ & $3.93 \mathrm{E}-11$ & 3.93E-13 & $4.52 \mathrm{E}-16$ & $5.90 \mathrm{E}-21$ & $1.49 \mathrm{E}-20$ & $2.78 \mathrm{E}-12$ & \\
\hline Pd-107 & 8.13E-09 & $2.62 \mathrm{E}-08$ & $6.65 \mathrm{E}-13$ & $6.65 \mathrm{E}-15$ & $7.65 \mathrm{E}-18$ & $9.99 \mathrm{E}-23$ & $2.52 \mathrm{E}-22$ & 4.71E-14 & \\
\hline Cd-113m & $1.63 \mathrm{E}-06$ & $5.28 \mathrm{E}-06$ & $1.34 \mathrm{E}-10$ & $1.34 \mathrm{E}-12$ & $1.54 \mathrm{E}-15$ & $2.01 \mathrm{E}-20$ & $5.07 \mathrm{E}-20$ & $9.47 \mathrm{E}-12$ & \\
\hline$\overline{S n-121 m}$ & $3.29 \mathrm{E}-08$ & $1.06 \mathrm{E}-07$ & $2.69 \mathrm{E}-12$ & $2.69 \mathrm{E}-14$ & $3.10 \mathrm{E}-17$ & $4.04 \mathrm{E}-22$ & $1.02 \mathrm{E}-21$ & $1.91 \mathrm{E}-13$ & \\
\hline Sn-126 & 2.12E-07 & $6.84 \mathrm{E}-07$ & 1.73E-11 & 1.73E-13 & 1.99E-16 & $2.61 \mathrm{E}-21$ & $6.58 \mathrm{E}-21$ & 1.23E-12 & \\
\hline Sb-125 & $7.89 \mathrm{E}-06$ & $2.55 \mathrm{E}-05$ & $6.46 \mathrm{E}-10$ & $6.46 \mathrm{E}-12$ & $7.43 \mathrm{E}-15$ & $9.71 \mathrm{E}-20$ & $2.45 \mathrm{E}-19$ & $4.57 \mathrm{E}-11$ & \\
\hline Sb-126 & $2.83 \mathrm{E}-08$ & $9.14 \mathrm{E}-08$ & $2.32 \mathrm{E}-12$ & $2.32 \mathrm{E}-14$ & $2.66 \mathrm{E}-17$ & $3.48 \mathrm{E}-22$ & $8.79 \mathrm{E}-22$ & 1.64E-13 & \\
\hline
\end{tabular}


Table C-8. Alkaline scrub balance, Tank WM-180 waste. (Continued).

\begin{tabular}{|c|c|c|c|c|c|c|c|c|c|}
\hline Stream \# & 101 & 102 & 103 & 104 & 105 & 106 & 107 & 108 & 201 \\
\hline Radiological Composition & $\mathrm{Ci} / \mathrm{L}$ & $\mathrm{Ci} / \mathrm{L}$ & $\mathrm{Ci} / \mathrm{wscm}$ & $\mathrm{Ci} / \mathrm{wscm}$ & $\mathrm{Ci} / \mathrm{wscm}$ & $\mathrm{Ci} / \mathrm{wscm}$ & $\mathrm{Ci} / \mathrm{wscm}$ & $\mathrm{Ci} / \mathrm{L}$ & \\
\hline Te-125m & $1.55 \mathrm{E}-06$ & $5.00 \mathrm{E}-06$ & $1.27 \mathrm{E}-10$ & $1.27 \mathrm{E}-12$ & $1.46 \mathrm{E}-15$ & $1.90 \mathrm{E}-20$ & $4.81 \mathrm{E}-20$ & $8.98 \mathrm{E}-12$ & \\
\hline $\mid-129$ & $2.40 \mathrm{E}-08$ & $7.82 \mathrm{E}-08$ & $5.90 \mathrm{E}-09$ & $5.90 \mathrm{E}-09$ & $8.65 \mathrm{E}-12$ & $9.42 \mathrm{E}-14$ & $5.22 \mathrm{E}-14$ & $4.32 \mathrm{E}-08$ & \\
\hline Cs-134 & $5.12 \mathrm{E}-06$ & $1.65 \mathrm{E}-05$ & $4.19 \mathrm{E}-10$ & $4.19 \mathrm{E}-12$ & $4.82 \mathrm{E}-15$ & $6.30 \mathrm{E}-20$ & $1.59 \mathrm{E}-19$ & $2.97 \mathrm{E}-11$ & \\
\hline Cs-135 & $4.41 \mathrm{E}-07$ & 1.42E-06 & $3.61 \mathrm{E}-11$ & $3.61 \mathrm{E}-13$ & $4.15 \mathrm{E}-16$ & $5.42 \mathrm{E}-21$ & 1.37E-20 & $2.56 \mathrm{E}-12$ & \\
\hline Cs-137 & $2.59 \mathrm{E}-02$ & 8.35E-02 & $2.12 \mathrm{E}-06$ & $2.12 \mathrm{E}-08$ & $2.43 \mathrm{E}-11$ & $3.18 \mathrm{E}-16$ & $8.03 \mathrm{E}-16$ & $1.50 \mathrm{E}-07$ & \\
\hline Ba-137m & $2.45 \mathrm{E}-02$ & $7.90 \mathrm{E}-02$ & $2.00 \mathrm{E}-06$ & $2.00 \mathrm{E}-08$ & $2.30 \mathrm{E}-11$ & $3.01 \mathrm{E}-16$ & $7.59 \mathrm{E}-16$ & 1.42E-07 & \\
\hline La-138 & $9.39 \mathrm{E}-17$ & $3.03 \mathrm{E}-16$ & $7.69 \mathrm{E}-21$ & $7.69 \mathrm{E}-23$ & $8.84 \mathrm{E}-26$ & $1.15 \mathrm{E}-30$ & $2.92 \mathrm{E}-30$ & $5.44 \mathrm{E}-22$ & \\
\hline Ce-142 & 1.47E-11 & 4.75E-11 & $1.20 \mathrm{E}-15$ & $1.20 \mathrm{E}-17$ & $1.38 \mathrm{E}-20$ & $1.81 \mathrm{E}-25$ & 4.57E-25 & $8.52 \mathrm{E}-17$ & \\
\hline Ce-144 & $3.24 \mathrm{E}-07$ & 1.04E-06 & $2.65 \mathrm{E}-11$ & $2.65 \mathrm{E}-13$ & $3.04 \mathrm{E}-16$ & $3.98 \mathrm{E}-21$ & 1.00E-20 & $1.87 \mathrm{E}-12$ & \\
\hline Pr-144 & $3.24 \mathrm{E}-07$ & 1.04E-06 & $2.65 \mathrm{E}-11$ & $2.65 \mathrm{E}-13$ & $3.04 \mathrm{E}-16$ & $3.98 \mathrm{E}-21$ & $1.00 \mathrm{E}-20$ & $1.87 \mathrm{E}-12$ & \\
\hline Nd-144 & $7.91 \mathrm{E}-16$ & $2.55 \mathrm{E}-15$ & $6.47 \mathrm{E}-20$ & $6.47 \mathrm{E}-22$ & $7.44 \mathrm{E}-25$ & $9.72 \mathrm{E}-30$ & $2.46 \mathrm{E}-29$ & $4.58 \mathrm{E}-21$ & \\
\hline Pm-146 & $2.50 \mathrm{E}-08$ & $8.08 \mathrm{E}-08$ & $2.05 \mathrm{E}-12$ & $2.05 \mathrm{E}-14$ & $2.36 \mathrm{E}-17$ & $3.08 \mathrm{E}-22$ & $7.77 \mathrm{E}-22$ & $1.45 \mathrm{E}-13$ & \\
\hline Pm-147 & $8.78 \mathrm{E}-05$ & $2.84 \mathrm{E}-04$ & 7.19E-09 & $7.19 \mathrm{E}-11$ & $8.26 \mathrm{E}-14$ & $1.08 \mathrm{E}-18$ & $2.73 \mathrm{E}-18$ & $5.09 \mathrm{E}-10$ & \\
\hline Sm-146 & 1.36E-13 & $4.38 \mathrm{E}-13$ & $1.11 \mathrm{E}-17$ & $1.11 \mathrm{E}-19$ & $1.28 \mathrm{E}-22$ & $1.67 \mathrm{E}-27$ & $4.21 \mathrm{E}-27$ & $7.86 \mathrm{E}-19$ & \\
\hline Sm-147 & $3.62 \mathrm{E}-12$ & 1.17E-11 & $2.97 \mathrm{E}-16$ & $2.97 \mathrm{E}-18$ & $3.41 \mathrm{E}-21$ & $4.45 \mathrm{E}-26$ & 1.13E-25 & $2.10 \mathrm{E}-17$ & \\
\hline Sm-151 & 1.73E-04 & $5.59 \mathrm{E}-04$ & 1.42E-08 & $1.42 \mathrm{E}-10$ & $1.63 \mathrm{E}-13$ & $2.13 \mathrm{E}-18$ & $5.38 \mathrm{E}-18$ & 1.00E-09 & \\
\hline Eu-152 & 1.25E-06 & 4.05E-06 & 1.03E-10 & 1.03E-12 & $1.18 \mathrm{E}-15$ & $1.54 \mathrm{E}-20$ & $3.89 \mathrm{E}-20$ & $7.26 \mathrm{E}-12$ & \\
\hline Eu-154 & $4.50 \mathrm{E}-05$ & 1.45E-04 & 3.68E-09 & $3.68 \mathrm{E}-11$ & $4.23 \mathrm{E}-14$ & $5.53 \mathrm{E}-19$ & 1.40E-18 & $2.61 \mathrm{E}-10$ & \\
\hline Eu-155 & $8.18 \mathrm{E}-05$ & $2.64 \mathrm{E}-04$ & $6.70 \mathrm{E}-09$ & $6.70 \mathrm{E}-11$ & $7.70 \mathrm{E}-14$ & $1.01 \mathrm{E}-18$ & $2.54 \mathrm{E}-18$ & $4.74 \mathrm{E}-10$ & \\
\hline Ho-166m & $2.26 \mathrm{E}-11$ & $7.31 \mathrm{E}-11$ & $1.85 \mathrm{E}-15$ & $1.85 \mathrm{E}-17$ & $2.13 \mathrm{E}-20$ & $2.78 \mathrm{E}-25$ & $7.03 \mathrm{E}-25$ & $1.31 \mathrm{E}-16$ & \\
\hline
\end{tabular}


Table C-9. Alkaline scrub balance, Tank WM-187 waste.

\begin{tabular}{|c|c|c|c|c|c|c|c|c|c|}
\hline $\begin{array}{|ll|}\text { WM-187 } & \text { PFD Sheet \# }\end{array}$ & PFD-2A & PFD-2A & PFD-2A & PFD-2A & PFD-2A & PFD-2A & PFD-2A & PFD-2A & PFD-2A \\
\hline Stream \# & 101 & 102 & 103 & 104 & 105 & 106 & 107 & 108 & 201 \\
\hline Stream Name & $\begin{array}{l}\text { SBW } \\
\text { Feed }\end{array}$ & $\begin{array}{c}\text { Evaporator } \\
\text { Bottoms }\end{array}$ & $\begin{array}{c}\text { Evaporator } \\
\text { Overheads } \\
\text { Vapor }\end{array}$ & $\begin{array}{c}\text { Mist } \\
\text { Eliminator } \\
\text { Vapor }\end{array}$ & $\begin{array}{l}\text { Alkaline } \\
\text { Scrubber } \\
\text { Vapor }\end{array}$ & $\begin{array}{c}P O G+V O G \\
\text { Offgas } \\
\text { Vapor }\end{array}$ & $\begin{array}{l}\text { VOG } \\
\text { Offgas } \\
\text { Vapor }\end{array}$ & $\begin{array}{l}\text { Scrubber } \\
\text { Recycle to } \\
\text { Feed }\end{array}$ & $\begin{array}{l}20 \text { wt. } \% \\
\text { MgO } \\
\text { Additive }\end{array}$ \\
\hline Rate or Volume Flow (actual) & 85.8 & 26.4 & 35,835 & 36,269 & 31,503 & 36,574 & 11,770 & 19.5 & 101.3 \\
\hline Volume Flow (standard, wet) ${ }^{\star}$ & & & 13,574 & 13,574 & 12,188 & 19,601 & 7,401 & & \\
\hline Volume Flow (standard, dry) ${ }^{\star}$ & & & 386.9 & 386.8 & 0.55 & 7,113 & 7,125 & & \\
\hline Rate Units & gal/hr & $\mathrm{gal} / \mathrm{hr}$ & $\mathrm{ft} 3 / \mathrm{hr}$ & $\mathrm{ft} 3 / \mathrm{hr}$ & $\mathrm{ft} 3 / \mathrm{hr}$ & $\mathrm{ft} 3 / \mathrm{hr}$ & $\mathrm{ft} 3 / \mathrm{hr}$ & $\mathrm{gal} / \mathrm{hr}$ & $\mathrm{lb} / \mathrm{hr}$ \\
\hline Temperature, ${ }^{\circ} \mathrm{C}$ & 25.0 & 126 & 126 & 120.2 & 88.7 & 173 & 108 & 88.8 & 25.0 \\
\hline Temperature, ${ }^{\circ} \mathrm{F}$ & 77.0 & 259 & 259 & 248 & 192 & 344 & 226 & 192 & 77.0 \\
\hline Pressure, psia & 12.3 & 7.6 & 7.6 & 7.4 & 7.0 & 12.0 & 12.0 & 30.0 & 12.3 \\
\hline Specific Gravity & 1.26 & 1.75 & 0.00030 & 0.00030 & 0.00029 & 0.00049 & 0.00074 & 1.30 & 1.20 \\
\hline Chemical Composition & Mol/Liter & Mol/Liter & $\mathrm{Ib} / \mathrm{wscf}$ & $\mathrm{lb} / \mathrm{wscf}$ & $\mathrm{Ib} / \mathrm{wscf}$ & $\mathrm{lb} / \mathrm{wscf}$ & $\mathrm{lb} / \mathrm{wscf}$ & Mol/Liter & Wt. \% \\
\hline $\mathrm{H}+$ & $1.47 \mathrm{E}+00$ & $2.18 \mathrm{E}-01$ & $7.46 \mathrm{E}-05$ & $7.46 \mathrm{E}-05$ & $1.28 \mathrm{E}-09$ & $1.80 \mathrm{E}-08$ & $4.56 \mathrm{E}-08$ & $1.40 \mathrm{E}-04$ & $2.64 \mathrm{E}-12$ \\
\hline $\mathrm{OH}-$ & & & $2.70 \mathrm{E}-14$ & & $1.35 \mathrm{E}-11$ & 1.45E-13 & & $7.13 \mathrm{E}-05$ & $1.69 \mathrm{E}+01$ \\
\hline $\mathrm{Al}+3$ & $3.61 \mathrm{E}-01$ & $1.18 \mathrm{E}+00$ & $5.14 \mathrm{E}-08$ & $4.70 \mathrm{E}-11$ & $4.36 \mathrm{E}-14$ & & & $1.45 \mathrm{E}-07$ & \\
\hline $\mathrm{Sb}+5$ & $2.78 \mathrm{E}-05$ & $9.06 \mathrm{E}-05$ & $1.79 \mathrm{E}-11$ & $1.79 \mathrm{E}-13$ & $1.66 \mathrm{E}-16$ & & & $1.23 \mathrm{E}-10$ & \\
\hline $\mathrm{As}+3$ & $1.77 \mathrm{E}-04$ & $5.76 \mathrm{E}-04$ & $6.99 \mathrm{E}-11$ & $6.99 \mathrm{E}-13$ & $6.49 \mathrm{E}-16$ & & & $7.78 \mathrm{E}-10$ & \\
\hline$\overline{\mathrm{Ba}+2}$ & $7.12 \mathrm{E}-05$ & $2.32 \mathrm{E}-04$ & $5.16 \mathrm{E}-11$ & $2.39 \mathrm{E}-13$ & $2.21 \mathrm{E}-16$ & & & $1.45 \mathrm{E}-10$ & \\
\hline $\mathrm{Be}+2$ & $1.67 \mathrm{E}-05$ & $5.43 \mathrm{E}-05$ & $7.93 \mathrm{E}-13$ & $4.04 \mathrm{E}-15$ & $3.75 \mathrm{E}-18$ & & & $3.74 \mathrm{E}-11$ & \\
\hline $\mathrm{B}+3$ & $1.10 \mathrm{E}-02$ & $3.59 \mathrm{E}-02$ & $6.29 \mathrm{E}-10$ & $4.51 \mathrm{E}-13$ & $4.18 \mathrm{E}-16$ & & & $3.48 \mathrm{E}-09$ & \\
\hline $\mathrm{Cd}+2$ & 1.87E-03 & $6.09 \mathrm{E}-03$ & $1.11 \mathrm{E}-09$ & $6.28 \mathrm{E}-13$ & $5.82 \mathrm{E}-16$ & & & $4.66 \mathrm{E}-10$ & \\
\hline $\mathrm{Ca}+2$ & 3.47E-02 & 1.13E-01 & 7.34E-09 & $4.02 \mathrm{E}-12$ & $3.73 \mathrm{E}-15$ & & & $8.36 \mathrm{E}-09$ & \\
\hline $\mathrm{Cr}+3$ & $4.71 \mathrm{E}-03$ & $1.53 \mathrm{E}-02$ & $1.29 \mathrm{E}-09$ & $1.88 \mathrm{E}-12$ & $1.74 \mathrm{E}-15$ & & & $3.01 \mathrm{E}-09$ & \\
\hline $\mathrm{Co}+2$ & $1.94 \mathrm{E}-03$ & $6.32 \mathrm{E}-03$ & $6.03 \mathrm{E}-10$ & $1.94 \mathrm{E}-14$ & $1.80 \mathrm{E}-17$ & & & $2.75 \mathrm{E}-11$ & \\
\hline Cs+ & $8.43 \mathrm{E}-05$ & $2.74 \mathrm{E}-04$ & $5.29 \mathrm{E}-11$ & $4.70 \mathrm{E}-13$ & $4.35 \mathrm{E}-16$ & & & $2.95 \mathrm{E}-10$ & \\
\hline $\mathrm{Cu}+2$ & $5.12 \mathrm{E}-04$ & 1.67E-03 & $1.72 \mathrm{E}-10$ & $6.25 \mathrm{E}-13$ & $5.80 \mathrm{E}-16$ & & & $8.20 \mathrm{E}-10$ & \\
\hline $\mathrm{Fe}+3$ & $2.53 \mathrm{E}-02$ & $8.24 \mathrm{E}-02$ & $7.46 \mathrm{E}-09$ & $3.76 \mathrm{E}-11$ & $3.49 \mathrm{E}-14$ & & & $5.62 \mathrm{E}-08$ & \\
\hline $\mathrm{Pb}+2$ & $6.73 \mathrm{E}-04$ & $2.19 \mathrm{E}-03$ & $7.36 \mathrm{E}-10$ & $6.58 \mathrm{E}-13$ & $6.11 \mathrm{E}-16$ & & & $2.65 \mathrm{E}-10$ & \\
\hline $\mathrm{Hg}+2$ & $3.40 \mathrm{E}-03$ & $1.11 \mathrm{E}-02$ & $1.11 \mathrm{E}-06$ & $1.11 \mathrm{E}-06$ & $1.11 \mathrm{E}-08$ & $1.73 \mathrm{E}-10$ & $3.66 \mathrm{E}-10$ & $4.58 \mathrm{E}-04$ & \\
\hline $\mathrm{Mn}+4$ & $1.87 \mathrm{E}-02$ & $6.08 \mathrm{E}-02$ & $5.41 \mathrm{E}-09$ & $5.41 \mathrm{E}-11$ & $5.02 \mathrm{E}-14$ & & & $8.21 \mathrm{E}-08$ & \\
\hline $\mathrm{Ni}+2$ & $1.41 \mathrm{E}-03$ & $4.59 \mathrm{E}-03$ & $4.37 \mathrm{E}-10$ & $7.60 \mathrm{E}-13$ & $7.05 \mathrm{E}-16$ & & & $1.08 \mathrm{E}-09$ & \\
\hline $\mathrm{K}+$ & 3.13E-01 & $1.02 \mathrm{E}+00$ & $6.46 \mathrm{E}-08$ & $4.35 \mathrm{E}-11$ & $4.04 \mathrm{E}-14$ & & & $9.28 \mathrm{E}-08$ & \\
\hline $\mathrm{Se}+4$ & 4.61E-04 & $1.50 \mathrm{E}-03$ & $1.92 \mathrm{E}-10$ & $1.92 \mathrm{E}-12$ & $1.78 \mathrm{E}-15$ & & & 2.03E-09 & \\
\hline $\mathrm{Ag}^{+}$ & $7.08 \mathrm{E}-04$ & $2.30 \mathrm{E}-03$ & $4.02 \mathrm{E}-10$ & $4.01 \mathrm{E}-12$ & $3.72 \mathrm{E}-15$ & & & $3.10 \mathrm{E}-09$ & \\
\hline $\mathrm{Na}+$ & $1.77 \mathrm{E}+00$ & $5.77 \mathrm{E}+00$ & $1.12 \mathrm{E}-07$ & $1.27 \mathrm{E}-10$ & $1.18 \mathrm{E}-13$ & & & $4.60 \mathrm{E}-07$ & \\
\hline $\mathrm{TI}+3$ & $6.31 \mathrm{E}-06$ & $2.06 \mathrm{E}-05$ & $6.81 \mathrm{E}-12$ & $6.81 \mathrm{E}-14$ & $6.31 \mathrm{E}-17$ & & & $2.78 \mathrm{E}-11$ & \\
\hline $\mathrm{U}+4$ & $2.90 \mathrm{E}-04$ & $9.45 \mathrm{E}-04$ & $3.64 \mathrm{E}-10$ & $3.64 \mathrm{E}-12$ & $3.38 \mathrm{E}-15$ & & & $1.28 \mathrm{E}-09$ & \\
\hline $\mathrm{V+3}$ & $6.14 \mathrm{E}-05$ & $2.00 \mathrm{E}-04$ & $1.65 \mathrm{E}-11$ & $1.65 \mathrm{E}-13$ & $1.54 \mathrm{E}-16$ & & & $2.72 \mathrm{E}-10$ & \\
\hline$Z n+2$ & $7.21 \mathrm{E}-04$ & $2.35 \mathrm{E}-03$ & $2.49 \mathrm{E}-10$ & $3.77 \mathrm{E}-13$ & $3.50 \mathrm{E}-16$ & & & $4.81 \mathrm{E}-10$ & \\
\hline $\mathrm{Zr}+4$ & $6.66 \mathrm{E}-02$ & $2.17 \mathrm{E}-01$ & $3.20 \mathrm{E}-08$ & $3.20 \mathrm{E}-10$ & $2.97 \mathrm{E}-13$ & & & 2.93E-07 & \\
\hline $\mathrm{Cl}-$ & $1.80 \mathrm{E}-02$ & $5.87 \mathrm{E}-02$ & $1.15 \mathrm{E}-05$ & 1.15E-05 & 1.14E-08 & $5.41 \mathrm{E}-10$ & 1.10E-09 & $2.69 \mathrm{E}-02$ & \\
\hline F- & $5.61 \mathrm{E}-02$ & 1.83E-01 & $2.19 \mathrm{E}-08$ & $1.86 \mathrm{E}-08$ & $9.87 \mathrm{E}-10$ & $6.30 \mathrm{E}-10$ & $4.42 \mathrm{E}-11$ & $7.77 \mathrm{E}-05$ & \\
\hline SO4-2 & $4.99 \mathrm{E}-02$ & $1.62 \mathrm{E}-01$ & $2.53 \mathrm{E}-08$ & $2.53 \mathrm{E}-10$ & $2.35 \mathrm{E}-13$ & & & $2.19 \mathrm{E}-07$ & \\
\hline NO3- & $4.57 \mathrm{E}+00$ & $1.44 \mathrm{E}+01$ & $4.57 \mathrm{E}-03$ & $4.57 \mathrm{E}-03$ & $4.31 \mathrm{E}-06$ & $1.10 \mathrm{E}-06$ & $2.80 \mathrm{E}-06$ & $6.15 \mathrm{E}+00$ & \\
\hline PO4-3 & $1.75 \mathrm{E}-01$ & $5.68 \mathrm{E}-01$ & $8.74 \mathrm{E}-08$ & $8.74 \mathrm{E}-10$ & $8.11 \mathrm{E}-13$ & & & $\begin{array}{l}7.67 \mathrm{E}-07 \\
\end{array}$ & \\
\hline $\mathrm{Am}+4$ & $6.58 \mathrm{E}-08$ & $2.14 \mathrm{E}-07$ & 8.37E-14 & 8.37E-16 & 7.76E-19 & & & $2.89 \mathrm{E}-13$ & \\
\hline $\mathrm{Br}-$ & $2.21 \mathrm{E}-07$ & $7.19 \mathrm{E}-07$ & $4.00 \mathrm{E}-10$ & $4.00 \mathrm{E}-10$ & $3.99 \mathrm{E}-13$ & $1.89 \mathrm{E}-14$ & $3.85 \mathrm{E}-14$ & 4.17E-07 & \\
\hline $\mathrm{Ce}+4$ & 4.07E-05 & $1.32 \mathrm{E}-04$ & $3.01 \mathrm{E}-11$ & $3.01 \mathrm{E}-13$ & $2.79 \mathrm{E}-16$ & & & $1.79 \mathrm{E}-10$ & \\
\hline $\mathrm{Eu}+3$ & $3.68 \mathrm{E}-07$ & $1.20 \mathrm{E}-06$ & $2.95 \mathrm{E}-13$ & $9.84 \mathrm{E}-18$ & $9.13 \mathrm{E}-21$ & & & $5.40 \mathrm{E}-15$ & \\
\hline $\mathrm{Gd}+3$ & $1.37 \mathrm{E}-04$ & $4.45 \mathrm{E}-04$ & $1.13 \mathrm{E}-10$ & $2.74 \mathrm{E}-13$ & $2.54 \mathrm{E}-16$ & & & $1.45 \mathrm{E}-10$ & \\
\hline $\mathrm{Ge}+4$ & $6.37 \mathrm{E}-09$ & $2.07 \mathrm{E}-08$ & $2.44 \mathrm{E}-15$ & $2.44 \mathrm{E}-17$ & $2.26 \mathrm{E}-20$ & & & $2.80 \mathrm{E}-14$ & \\
\hline $\mathrm{In}+3$ & 1.07E-06 & $3.48 \mathrm{E}-06$ & $6.47 \mathrm{E}-13$ & & & & & & \\
\hline I- & $3.12 \mathrm{E}-06$ & $1.01 \mathrm{E}-05$ & $5.28 \mathrm{E}-09$ & $5.27 \mathrm{E}-09$ & $5.26 \mathrm{E}-12$ & $2.49 \mathrm{E}-13$ & $5.07 \mathrm{E}-13$ & $3.47 \mathrm{E}-06$ & \\
\hline $\mathrm{La}+3$ & $6.66 \mathrm{E}-06$ & 2.17E-05 & $4.88 \mathrm{E}-12$ & & & & & & \\
\hline Li+ & $6.27 \mathrm{E}-04$ & $2.04 \mathrm{E}-03$ & $1.87 \mathrm{E}-11$ & $1.46 \mathrm{E}-13$ & $1.35 \mathrm{E}-16$ & & & 1.75E-09 & \\
\hline $\mathrm{Mg}+2$ & $1.42 \mathrm{E}-02$ & $2.33 \mathrm{E}+00$ & $9.17 \mathrm{E}-08$ & & $8.34 \mathrm{E}-07$ & $1.04 \mathrm{E}-13$ & & $3.09 \mathrm{E}+00$ & $1.21 \mathrm{E}+01$ \\
\hline $\mathrm{Mo}+6$ & $1.23 \mathrm{E}-03$ & $4.01 \mathrm{E}-03$ & $6.23 \mathrm{E}-10$ & $6.23 \mathrm{E}-12$ & $5.78 \mathrm{E}-15$ & & & $5.42 \mathrm{E}-09$ & \\
\hline
\end{tabular}


Table C-9. Alkaline scrub balance, Tank WM-187 waste. (Continued).

\begin{tabular}{|c|c|c|c|c|c|c|c|c|c|}
\hline Stream \# & 101 & 102 & 103 & 104 & 105 & 106 & 107 & 108 & 201 \\
\hline Chemical Composition & Mol/Liter & Mol/Liter & $\mathrm{Ib} / \mathrm{wscf}$ & $\mathrm{Ib} / \mathrm{wscf}$ & $\mathrm{Ib} / \mathrm{wscf}$ & $\mathrm{Ib} / \mathrm{wscf}$ & $\mathrm{Ib} / \mathrm{wscf}$ & Mol/Liter & Wt. \% \\
\hline $\mathrm{Nd}+3$ & $2.15 \mathrm{E}-05$ & $6.99 \mathrm{E}-05$ & $1.63 \mathrm{E}-11$ & & & & & & \\
\hline$N p+4$ & $3.28 \mathrm{E}-06$ & $1.07 \mathrm{E}-05$ & $4.10 \mathrm{E}-12$ & $4.10 \mathrm{E}-14$ & $3.81 \mathrm{E}-17$ & & & $1.44 \mathrm{E}-11$ & \\
\hline $\mathrm{Nb}+5$ & $1.86 \mathrm{E}-03$ & $6.05 \mathrm{E}-03$ & $9.10 \mathrm{E}-10$ & $9.10 \mathrm{E}-12$ & $8.44 \mathrm{E}-15$ & & & $8.17 \mathrm{E}-09$ & \\
\hline $\mathrm{Pd}+4$ & $3.01 \mathrm{E}-03$ & $9.81 \mathrm{E}-03$ & $1.69 \mathrm{E}-09$ & $1.69 \mathrm{E}-11$ & $1.57 \mathrm{E}-14$ & & & $1.33 \mathrm{E}-08$ & \\
\hline $\mathrm{Pu}+4$ & $8.87 \mathrm{E}-06$ & $2.89 \mathrm{E}-05$ & $1.12 \mathrm{E}-11$ & $1.12 \mathrm{E}-13$ & $1.04 \mathrm{E}-16$ & & & $3.90 \mathrm{E}-11$ & \\
\hline $\mathrm{Pr}+4$ & $6.06 \mathrm{E}-06$ & $1.97 \mathrm{E}-05$ & $4.50 \mathrm{E}-12$ & $4.50 \mathrm{E}-14$ & $4.17 \mathrm{E}-17$ & & & $2.66 \mathrm{E}-11$ & \\
\hline $\mathrm{Rh}+4$ & $2.61 \mathrm{E}-06$ & $8.50 \mathrm{E}-06$ & $1.42 \mathrm{E}-12$ & $1.42 \mathrm{E}-14$ & $1.31 \mathrm{E}-17$ & & & 1.15E-11 & \\
\hline $\mathrm{Rb}+$ & $4.04 \mathrm{E}-06$ & $1.32 \mathrm{E}-05$ & $9.28 \mathrm{E}-13$ & $6.85 \mathrm{E}-16$ & $6.35 \mathrm{E}-19$ & & & $6.68 \mathrm{E}-13$ & \\
\hline $\mathrm{Ru}+3$ & $1.36 \mathrm{E}-03$ & $4.43 \mathrm{E}-03$ & $7.26 \mathrm{E}-10$ & $6.70 \mathrm{E}-12$ & $6.21 \mathrm{E}-15$ & & & $5.52 \mathrm{E}-09$ & \\
\hline$S m+3$ & $4.03 \mathrm{E}-06$ & $1.31 \mathrm{E}-05$ & $3.19 \mathrm{E}-12$ & $3.65 \mathrm{E}-16$ & $3.39 \mathrm{E}-19$ & & & $2.02 \mathrm{E}-13$ & \\
\hline$S i+4$ & $1.59 \mathrm{E}-01$ & $5.17 \mathrm{E}-01$ & $2.35 \mathrm{E}-08$ & $2.35 \mathrm{E}-10$ & $2.18 \mathrm{E}-13$ & & & $6.98 \mathrm{E}-07$ & \\
\hline $\mathrm{Sr+2}$ & $8.80 \mathrm{E}-05$ & $2.86 \mathrm{E}-04$ & $4.07 \mathrm{E}-11$ & $4.94 \mathrm{E}-14$ & $4.58 \mathrm{E}-17$ & & & $4.70 \mathrm{E}-11$ & \\
\hline $\mathrm{Tc}+7$ & $6.42 \mathrm{E}-06$ & $2.09 \mathrm{E}-05$ & $3.28 \mathrm{E}-12$ & & & & & & \\
\hline $\mathrm{Te}+4$ & $3.48 \mathrm{E}-06$ & $1.13 \mathrm{E}-05$ & $2.34 \mathrm{E}-12$ & $2.34 \mathrm{E}-14$ & $2.17 \mathrm{E}-17$ & & & $1.53 \mathrm{E}-11$ & \\
\hline$T \mathrm{~Tb}+4$ & $1.53 \mathrm{E}-09$ & $4.99 \mathrm{E}-09$ & $1.29 \mathrm{E}-15$ & $1.29 \mathrm{E}-17$ & $1.19 \mathrm{E}-20$ & & & $6.74 \mathrm{E}-15$ & \\
\hline$T h+4$ & $1.85 \mathrm{E}-05$ & $6.01 \mathrm{E}-05$ & $2.26 \mathrm{E}-11$ & $2.26 \mathrm{E}-13$ & $2.10 \mathrm{E}-16$ & & & $8.12 \mathrm{E}-11$ & \\
\hline$S n+4$ & $3.23 \mathrm{E}-03$ & $1.05 \mathrm{E}-02$ & $2.02 \mathrm{E}-09$ & $2.02 \mathrm{E}-11$ & $1.87 \mathrm{E}-14$ & & & $1.42 \mathrm{E}-08$ & \\
\hline$T i+4$ & $1.62 \mathrm{E}-03$ & $5.27 \mathrm{E}-03$ & $4.09 \mathrm{E}-10$ & $4.09 \mathrm{E}-12$ & $3.79 \mathrm{E}-15$ & & & $7.12 \mathrm{E}-09$ & \\
\hline $\mathrm{Y}+3$ & $4.96 \mathrm{E}-06$ & $1.62 \mathrm{E}-05$ & $2.33 \mathrm{E}-12$ & $6.24 \mathrm{E}-20$ & $5.78 \mathrm{E}-23$ & & & $5.85 \mathrm{E}-17$ & \\
\hline $\mathrm{O}-2$ (oxides) & $3.25 \mathrm{E}-01$ & $1.06 \mathrm{E}+00$ & $2.75 \mathrm{E}-08$ & $2.75 \mathrm{E}-10$ & $2.55 \mathrm{E}-13$ & & & $1.43 \mathrm{E}-06$ & \\
\hline $\mathrm{H} 2 \mathrm{O}$ & $4.50 \mathrm{E}+01$ & $2.16 \mathrm{E}+01$ & $4.56 \mathrm{E}-02$ & $4.56 \mathrm{E}-02$ & $4.69 \mathrm{E}-02$ & $2.98 \mathrm{E}-02$ & $1.74 \mathrm{E}-03$ & $4.66 \mathrm{E}+01$ & $7.11 \mathrm{E}+01$ \\
\hline $\mathrm{O} 2$ & & & $1.29 \mathrm{E}-07$ & $1.29 \mathrm{E}-07$ & $1.44 \mathrm{E}-07$ & $6.34 \mathrm{E}-03$ & $1.68 \mathrm{E}-02$ & $1.81 \mathrm{E}-10$ & \\
\hline N2 & & & $2.19 \mathrm{E}-07$ & $2.19 \mathrm{E}-07$ & $2.44 \mathrm{E}-07$ & $2.09 \mathrm{E}-02$ & $5.53 \mathrm{E}-02$ & $2.08 \mathrm{E}-10$ & \\
\hline C (organic) & $1.96 \mathrm{E}-01$ & $6.38 \mathrm{E}-01$ & $3.15 \mathrm{E}-06$ & $3.15 \mathrm{E}-06$ & $7.67 \mathrm{E}-09$ & $4.79 \mathrm{E}-09$ & $6.37 \mathrm{E}-11$ & $2.19 \mathrm{E}-02$ & \\
\hline $\mathrm{H}$ (organic) & $1.96 \mathrm{E}-01$ & $6.38 \mathrm{E}-01$ & $2.64 \mathrm{E}-07$ & $2.64 \mathrm{E}-07$ & $6.44 \mathrm{E}-10$ & $4.02 \mathrm{E}-10$ & $5.34 \mathrm{E}-12$ & $2.19 \mathrm{E}-02$ & \\
\hline $\begin{array}{l}\text { Gas Stream Bulk Composition } \\
\text { (wet basis) }\end{array}$ & $\begin{array}{l}\text { mol. } \% \\
\text { or ppmv }\end{array}$ & $\begin{array}{l}\text { mol.\% } \\
\text { or ppmv }\end{array}$ & $\begin{array}{l}\text { mol.\% } \\
\text { or ppmv }\end{array}$ & $\begin{array}{l}\text { mol.\% } \\
\text { or ppmv }\end{array}$ & $\begin{array}{l}\text { mol.\% } \\
\text { or ppmv }\end{array}$ & $\begin{array}{l}\text { mol. } \% \\
\text { or ppmv }\end{array}$ & $\begin{array}{l}\text { mol.\% } \\
\text { or ppmv }\end{array}$ & $\begin{array}{l}\text { mol.\% } \\
\text { or ppmv }\end{array}$ & $\begin{array}{l}\text { mol. } \% \\
\text { or ppmv }\end{array}$ \\
\hline $\mathrm{H} 2 \mathrm{O}, \mathrm{mol} . \%$ & & & 97.15 & 97.15 & 100.00 & 63.71 & 3.72 & & \\
\hline $\mathrm{O} 2, \mathrm{~mol} . \%$ & & & 0.0002 & 0.0002 & 0.0002 & 7.62 & 20.22 & & \\
\hline $\mathrm{N} 2, \mathrm{~mol} . \%$ & & & 0.0003 & 0.0003 & 0.0003 & 28.67 & 76.06 & & \\
\hline \multicolumn{10}{|l|}{$\mathrm{CO} 2, \mathrm{~mol} . \%$} \\
\hline HNO3, mol. \% & & & 2.84 & 2.84 & 0.00005 & 0.0007 & 0.002 & & \\
\hline \multicolumn{10}{|l|}{$\mathrm{CO}, \mathrm{ppmv}$} \\
\hline $\mathrm{HCl}, \mathrm{ppmv}$ & & & 124 & 124 & 0.12 & 0.006 & 0.012 & & \\
\hline C (organic), ppmv & & & 101 & 101 & 0.25 & 0.15 & 0.002 & & \\
\hline $\mathrm{H}$ (organic), ppmv & & & 101 & 101 & 0.25 & 0.15 & 0.002 & & \\
\hline $\mathrm{PM}(\mathrm{mg} / \mathrm{wscm})$ & & & 6 & 0.06 & 0.0001 & & & & \\
\hline $\mathrm{Hg}(\mathrm{ug} / \mathrm{wscm})$ & & & $3.1 \mathrm{E}+03$ & $3.1 \mathrm{E}+03$ & 32 & 2 & 6 & & \\
\hline $\begin{array}{l}\text { Gas Stream Bulk Composition } \\
\text { (dry basis) }\end{array}$ & $\begin{array}{l}\text { mol.\% } \\
\text { or ppmv }\end{array}$ & $\begin{array}{l}\text { mol.\% } \\
\text { or ppmv }\end{array}$ & $\begin{array}{l}\text { mol.\% } \\
\text { or ppmv }\end{array}$ & $\begin{array}{l}\text { mol.\% } \\
\text { or ppmv }\end{array}$ & $\begin{array}{l}\text { mol.\% } \\
\text { or ppmv }\end{array}$ & $\begin{array}{l}\text { mol.\% } \\
\text { or ppmv }\end{array}$ & $\begin{array}{l}\text { mol. } \% \\
\text { or ppmv }\end{array}$ & $\begin{array}{l}\text { mol.\% } \\
\text { or ppmv }\end{array}$ & $\begin{array}{l}\text { mol.\% } \\
\text { or ppmv }\end{array}$ \\
\hline $\mathrm{O} 2, \mathrm{~mol} . \%$ & & & 0.005 & 0.005 & 3.82 & 21.00 & 21.00 & & \\
\hline $\mathrm{N} 2, \mathrm{~mol} . \%$ & & & 0.011 & 0.011 & 7.40 & 79.00 & 79.00 & & \\
\hline \multicolumn{10}{|l|}{$\mathrm{CO} 2, \mathrm{~mol} . \%$} \\
\hline HNO3, mol. \% & & & 99.77 & 99.81 & 1.08 & 0.002 & 0.002 & & \\
\hline \multicolumn{10}{|l|}{$\mathrm{CO}, \mathrm{ppmv}$} \\
\hline $\mathrm{HCl}, \mathrm{ppmv}$ & & & $4.4 \mathrm{E}+03$ & $4.4 \mathrm{E}+03$ & $2.7 \mathrm{E}+03$ & 0.02 & 0.01 & & \\
\hline $\mathrm{C}$ (organic), ppmv & & & $3.5 \mathrm{E}+03$ & $3.5 \mathrm{E}+03$ & $5.4 \mathrm{E}+03$ & 0.42 & 0.002 & & \\
\hline $\mathrm{H}$ (organic), ppmv & & & 3,535 & 3,536 & 5,433 & 0.42 & 0.002 & & \\
\hline $\mathrm{PM}(\mathrm{mg} / \mathrm{dscm})$ & & & 216 & 2 & 1 & & & & \\
\hline \multirow[t]{2}{*}{$\mathrm{Hg}$ (ug/wscm) } & & & $2.9 \mathrm{E}+05$ & $2.9 \mathrm{E}+05$ & $1.8 \mathrm{E}+06$ & 11 & 10 & & \\
\hline & $394 \mathrm{~F}+02$ & $175 \mathrm{~F}+02$ & $309 \mathrm{E}+02$ & $309 \mathrm{~F}+02$ & $260 \mathrm{E}+02$ & $507 \mathrm{~F}+02$ & $248 \mathrm{E}+02$ & $957 \mathrm{~F}+01$ & $459 \mathrm{E}+01$ \\
\hline Canister Rate, canisters/day & & 2.99 & & & & & & & \\
\hline Total Canisters Generated & & 224 & & & & & & & \\
\hline & & & & & & & & & \\
\hline
\end{tabular}


Table C-9. Alkaline scrub balance, Tank WM-187 waste. (Continued).

\begin{tabular}{|c|c|c|c|c|c|c|c|c|c|}
\hline Stream \# & 101 & 102 & 103 & 104 & 105 & 106 & 107 & 108 & 201 \\
\hline Radiological Composition & $\mathrm{Ci} / \mathrm{L}$ & $\mathrm{Ci} / \mathrm{L}$ & Ci/wscm & $\mathrm{Ci} / \mathrm{wscm}$ & $\mathrm{Ci} / \mathrm{wscm}$ & $\mathrm{Ci} / \mathrm{wscm}$ & $\mathrm{Ci} / \mathrm{wscm}$ & $\mathrm{Ci} / \mathrm{L}$ & \\
\hline Ra-226 & $6.10 \mathrm{E}-12$ & $1.99 \mathrm{E}-11$ & $5.15 \mathrm{E}-16$ & $5.15 \mathrm{E}-18$ & $4.78 \mathrm{E}-21$ & $7.73 \mathrm{E}-26$ & $1.89 \mathrm{E}-25$ & $2.68 \mathrm{E}-17$ & \\
\hline Ac-227 & $2.87 \mathrm{E}-11$ & $9.35 \mathrm{E}-11$ & $2.43 \mathrm{E}-15$ & $2.43 \mathrm{E}-17$ & $2.25 \mathrm{E}-20$ & $3.64 \mathrm{E}-25$ & $8.90 \mathrm{E}-25$ & $1.26 \mathrm{E}-16$ & \\
\hline Th-228 & $2.38 \mathrm{E}-09$ & $7.76 \mathrm{E}-09$ & $2.01 \mathrm{E}-13$ & $2.01 \mathrm{E}-15$ & $1.87 \mathrm{E}-18$ & $3.02 \mathrm{E}-23$ & $7.39 \mathrm{E}-23$ & $1.05 \mathrm{E}-14$ & \\
\hline Th-230 & $1.01 \mathrm{E}-09$ & $3.27 \mathrm{E}-09$ & $8.50 \mathrm{E}-14$ & $8.50 \mathrm{E}-16$ & $7.88 \mathrm{E}-19$ & $1.28 \mathrm{E}-23$ & $3.12 \mathrm{E}-23$ & $4.42 \mathrm{E}-15$ & \\
\hline Th-232 & $5.27 \mathrm{E}-16$ & $1.72 \mathrm{E}-15$ & $4.46 \mathrm{E}-20$ & $4.46 \mathrm{E}-22$ & $4.13 \mathrm{E}-25$ & $6.69 \mathrm{E}-30$ & $1.63 \mathrm{E}-29$ & $2.32 \mathrm{E}-21$ & \\
\hline Pa-231 & $6.65 \mathrm{E}-11$ & $2.17 \mathrm{E}-10$ & $5.62 \mathrm{E}-15$ & $5.62 \mathrm{E}-17$ & $5.21 \mathrm{E}-20$ & $8.43 \mathrm{E}-25$ & $2.06 \mathrm{E}-24$ & $2.93 \mathrm{E}-16$ & \\
\hline $\mathrm{Pa}-233$ & $2.18 \mathrm{E}-06$ & $7.10 \mathrm{E}-06$ & $1.84 \mathrm{E}-10$ & $1.84 \mathrm{E}-12$ & $1.71 \mathrm{E}-15$ & $2.77 \mathrm{E}-20$ & $6.77 \mathrm{E}-20$ & $9.60 \mathrm{E}-12$ & \\
\hline $\mathrm{U}-232$ & $2.30 \mathrm{E}-09$ & $7.48 \mathrm{E}-09$ & $1.94 \mathrm{E}-13$ & $1.94 \mathrm{E}-15$ & $1.80 \mathrm{E}-18$ & $2.91 \mathrm{E}-23$ & $7.12 \mathrm{E}-23$ & $1.01 \mathrm{E}-14$ & \\
\hline $\mathrm{U}-233$ & $7.36 \mathrm{E}-11$ & $2.40 \mathrm{E}-10$ & $6.22 \mathrm{E}-15$ & $6.22 \mathrm{E}-17$ & $5.77 \mathrm{E}-20$ & $9.33 \mathrm{E}-25$ & $2.28 \mathrm{E}-24$ & $3.24 \mathrm{E}-16$ & \\
\hline $\mathrm{U}-234$ & $1.20 \mathrm{E}-06$ & $3.92 \mathrm{E}-06$ & $1.02 \mathrm{E}-10$ & $1.02 \mathrm{E}-12$ & $9.43 \mathrm{E}-16$ & $1.53 \mathrm{E}-20$ & $3.73 \mathrm{E}-20$ & $5.29 \mathrm{E}-12$ & \\
\hline U-235 & $6.32 \mathrm{E}-08$ & $2.06 \mathrm{E}-07$ & $5.34 \mathrm{E}-12$ & $5.34 \mathrm{E}-14$ & $4.95 \mathrm{E}-17$ & $8.02 \mathrm{E}-22$ & $1.96 \mathrm{E}-21$ & $2.78 \mathrm{E}-13$ & \\
\hline $\mathrm{U}-236$ & $5.90 \mathrm{E}-08$ & $1.92 \mathrm{E}-07$ & $4.99 \mathrm{E}-12$ & $4.99 \mathrm{E}-14$ & $4.63 \mathrm{E}-17$ & $7.48 \mathrm{E}-22$ & $1.83 \mathrm{E}-21$ & $2.60 \mathrm{E}-13$ & \\
\hline $\mathrm{U}-237$ & $4.79 \mathrm{E}-09$ & $1.56 \mathrm{E}-08$ & $4.05 \mathrm{E}-13$ & $4.05 \mathrm{E}-15$ & $3.76 \mathrm{E}-18$ & $6.08 \mathrm{E}-23$ & $1.49 \mathrm{E}-22$ & $2.11 \mathrm{E}-14$ & \\
\hline $\mathrm{U}-238$ & $2.06 \mathrm{E}-08$ & $6.69 \mathrm{E}-08$ & $1.74 \mathrm{E}-12$ & $1.74 \mathrm{E}-14$ & $1.61 \mathrm{E}-17$ & $2.61 \mathrm{E}-22$ & $6.37 \mathrm{E}-22$ & $9.04 \mathrm{E}-14$ & \\
\hline Np-236 & $2.35 \mathrm{E}-12$ & $7.65 \mathrm{E}-12$ & $1.99 \mathrm{E}-16$ & $1.99 \mathrm{E}-18$ & $1.84 \mathrm{E}-21$ & $2.98 \mathrm{E}-26$ & $7.29 \mathrm{E}-26$ & $1.03 \mathrm{E}-17$ & \\
\hline Np-237 & $5.48 \mathrm{E}-07$ & $1.78 \mathrm{E}-06$ & $4.63 \mathrm{E}-11$ & $4.63 \mathrm{E}-13$ & $4.30 \mathrm{E}-16$ & $6.95 \mathrm{E}-21$ & $1.70 \mathrm{E}-20$ & $2.41 \mathrm{E}-12$ & \\
\hline Pu-236 & 3.63E-09 & $1.18 \mathrm{E}-08$ & 3.07E-13 & $3.07 \mathrm{E}-15$ & $2.84 \mathrm{E}-18$ & $4.60 \mathrm{E}-23$ & $1.13 \mathrm{E}-22$ & $1.60 \mathrm{E}-14$ & \\
\hline Pu-238 & $1.12 \mathrm{E}-03$ & $3.65 \mathrm{E}-03$ & $9.48 \mathrm{E}-08$ & $9.48 \mathrm{E}-10$ & $8.79 \mathrm{E}-13$ & $1.42 \mathrm{E}-17$ & $3.48 \mathrm{E}-17$ & $4.94 \mathrm{E}-09$ & \\
\hline Pu-239 & $1.23 \mathrm{E}-04$ & $4.01 \mathrm{E}-04$ & $1.04 \mathrm{E}-08$ & $1.04 \mathrm{E}-10$ & $9.66 \mathrm{E}-14$ & $1.56 \mathrm{E}-18$ & $3.82 \mathrm{E}-18$ & $5.42 \mathrm{E}-10$ & \\
\hline $\mathrm{Pu}-240$ & $1.36 \mathrm{E}-05$ & $4.43 \mathrm{E}-05$ & $1.15 \mathrm{E}-09$ & $1.15 \mathrm{E}-11$ & $1.07 \mathrm{E}-14$ & $1.72 \mathrm{E}-19$ & $4.22 \mathrm{E}-19$ & $5.98 \mathrm{E}-11$ & \\
\hline Pu-241 & $8.61 \mathrm{E}-04$ & $2.80 \mathrm{E}-03$ & $7.28 \mathrm{E}-08$ & $7.28 \mathrm{E}-10$ & $6.75 \mathrm{E}-13$ & $1.09 \mathrm{E}-17$ & $2.67 \mathrm{E}-17$ & $3.79 \mathrm{E}-09$ & \\
\hline Pu-242 & $1.07 \mathrm{E}-08$ & $3.48 \mathrm{E}-08$ & $9.03 \mathrm{E}-13$ & $9.03 \mathrm{E}-15$ & $8.37 \mathrm{E}-18$ & $1.35 \mathrm{E}-22$ & $3.31 \mathrm{E}-22$ & $4.70 \mathrm{E}-14$ & \\
\hline Pu-244 & $6.49 \mathrm{E}-16$ & $2.11 \mathrm{E}-15$ & $5.49 \mathrm{E}-20$ & $5.49 \mathrm{E}-22$ & $5.09 \mathrm{E}-25$ & $8.23 \mathrm{E}-30$ & $2.01 \mathrm{E}-29$ & $2.86 \mathrm{E}-21$ & \\
\hline Am-241 & $6.58 \mathrm{E}-05$ & $2.14 \mathrm{E}-04$ & $5.56 \mathrm{E}-09$ & $5.56 \mathrm{E}-11$ & $5.16 \mathrm{E}-14$ & $8.35 \mathrm{E}-19$ & $2.04 \mathrm{E}-18$ & $2.90 \mathrm{E}-10$ & \\
\hline Am-242m & $1.15 \mathrm{E}-08$ & $3.74 \mathrm{E}-08$ & $9.71 \mathrm{E}-13$ & $9.71 \mathrm{E}-15$ & $9.01 \mathrm{E}-18$ & $1.46 \mathrm{E}-22$ & $3.56 \mathrm{E}-22$ & $5.05 E-14$ & \\
\hline Am-243 & $2.31 \mathrm{E}-08$ & $7.52 \mathrm{E}-08$ & $1.95 \mathrm{E}-12$ & 1.95E-14 & $1.81 \mathrm{E}-17$ & $2.93 \mathrm{E}-22$ & $7.16 \mathrm{E}-22$ & $1.02 \mathrm{E}-13$ & \\
\hline Cm-242 & $2.28 \mathrm{E}-08$ & $7.43 \mathrm{E}-08$ & $1.93 \mathrm{E}-12$ & $1.93 \mathrm{E}-14$ & $1.79 \mathrm{E}-17$ & $2.89 \mathrm{E}-22$ & $7.07 \mathrm{E}-22$ & $1.00 \mathrm{E}-13$ & \\
\hline $\mathrm{Cm}-243$ & $3.53 \mathrm{E}-08$ & $1.15 \mathrm{E}-07$ & $2.98 \mathrm{E}-12$ & $2.98 \mathrm{E}-14$ & $2.76 \mathrm{E}-17$ & $4.47 \mathrm{E}-22$ & $1.09 \mathrm{E}-21$ & $1.55 \mathrm{E}-13$ & \\
\hline $\mathrm{Cm}-244$ & $1.61 \mathrm{E}-06$ & $5.24 \mathrm{E}-06$ & $1.36 \mathrm{E}-10$ & $1.36 \mathrm{E}-12$ & $1.26 \mathrm{E}-15$ & $2.04 \mathrm{E}-20$ & $4.99 \mathrm{E}-20$ & 7.07E-12 & \\
\hline Cm-245 & $3.72 \mathrm{E}-10$ & $1.21 \mathrm{E}-09$ & $3.14 \mathrm{E}-14$ & $3.14 \mathrm{E}-16$ & $2.91 \mathrm{E}-19$ & $4.71 \mathrm{E}-24$ & $1.15 \mathrm{E}-23$ & $1.64 \mathrm{E}-15$ & \\
\hline $\mathrm{Cm}-246$ & $2.43 \mathrm{E}-11$ & $7.93 \mathrm{E}-11$ & $2.06 \mathrm{E}-15$ & $2.06 \mathrm{E}-17$ & $1.91 \mathrm{E}-20$ & $3.09 \mathrm{E}-25$ & $7.55 \mathrm{E}-25$ & $1.07 \mathrm{E}-16$ & \\
\hline $\mathrm{H}-3$ & $9.54 \mathrm{E}-06$ & $4.44 \mathrm{E}-06$ & $8.34 \mathrm{E}-06$ & $8.34 \mathrm{E}-06$ & $7.59 \mathrm{E}-06$ & 4.84E-06 & $3.18 \mathrm{E}-07$ & $8.34 \mathrm{E}-06$ & \\
\hline Be-10 & $2.23 \mathrm{E}-12$ & $7.27 \mathrm{E}-12$ & $1.89 \mathrm{E}-16$ & $1.89 \mathrm{E}-18$ & $1.75 \mathrm{E}-21$ & $2.83 \mathrm{E}-26$ & $6.93 \mathrm{E}-26$ & $9.83 \mathrm{E}-18$ & \\
\hline C-14 & $1.55 \mathrm{E}-10$ & $5.03 \mathrm{E}-10$ & $1.31 \mathrm{E}-14$ & $1.31 \mathrm{E}-16$ & $1.21 \mathrm{E}-19$ & $1.96 \mathrm{E}-24$ & $4.79 \mathrm{E}-24$ & $6.80 \mathrm{E}-16$ & \\
\hline Co-60 & $1.61 \mathrm{E}-05$ & $5.23 \mathrm{E}-05$ & $1.36 \mathrm{E}-09$ & 1.36E-11 & $1.26 \mathrm{E}-14$ & $2.04 \mathrm{E}-19$ & $4.98 \mathrm{E}-19$ & $7.06 \mathrm{E}-11$ & \\
\hline Ni-59 & $2.06 \mathrm{E}-06$ & $6.70 \mathrm{E}-06$ & $1.74 \mathrm{E}-10$ & $1.74 \mathrm{E}-12$ & $1.61 \mathrm{E}-15$ & $2.61 \mathrm{E}-20$ & $6.38 \mathrm{E}-20$ & $9.06 \mathrm{E}-12$ & \\
\hline $\mathrm{Ni}-63$ & $4.20 \mathrm{E}-05$ & $1.37 \mathrm{E}-04$ & $3.55 \mathrm{E}-09$ & $3.55 \mathrm{E}-11$ & $3.29 \mathrm{E}-14$ & $5.33 \mathrm{E}-19$ & $1.30 \mathrm{E}-18$ & $1.85 \mathrm{E}-10$ & \\
\hline Se-79 & $5.60 \mathrm{E}-07$ & $1.82 \mathrm{E}-06$ & $4.73 \mathrm{E}-11$ & $4.73 \mathrm{E}-13$ & $4.39 \mathrm{E}-16$ & $7.10 \mathrm{E}-21$ & $1.74 \mathrm{E}-20$ & $2.46 \mathrm{E}-12$ & \\
\hline Rb-87 & $2.18 \mathrm{E}-11$ & $7.10 \mathrm{E}-11$ & $1.84 \mathrm{E}-15$ & $1.84 \mathrm{E}-17$ & $1.71 \mathrm{E}-20$ & $2.77 \mathrm{E}-25$ & $6.76 \mathrm{E}-25$ & $9.60 \mathrm{E}-17$ & \\
\hline Sr-90 & $2.94 \mathrm{E}-02$ & $9.57 \mathrm{E}-02$ & $2.48 \mathrm{E}-06$ & $2.48 \mathrm{E}-08$ & $2.30 \mathrm{E}-11$ & $3.73 \mathrm{E}-16$ & $9.11 \mathrm{E}-16$ & $1.29 \mathrm{E}-07$ & \\
\hline Y-90 & $2.94 \mathrm{E}-02$ & $9.57 \mathrm{E}-02$ & $2.48 \mathrm{E}-06$ & $2.48 \mathrm{E}-08$ & $2.30 \mathrm{E}-11$ & $3.73 \mathrm{E}-16$ & $9.11 \mathrm{E}-16$ & $1.29 \mathrm{E}-07$ & \\
\hline Zr-93 & $1.65 \mathrm{E}-06$ & $5.37 \mathrm{E}-06$ & $1.39 \mathrm{E}-10$ & $1.39 \mathrm{E}-12$ & $1.29 \mathrm{E}-15$ & $2.09 \mathrm{E}-20$ & $5.11 \mathrm{E}-20$ & $7.26 \mathrm{E}-12$ & \\
\hline Nb-93m & $1.27 \mathrm{E}-06$ & $4.14 \mathrm{E}-06$ & $1.07 \mathrm{E}-10$ & $1.07 \mathrm{E}-12$ & $9.96 \mathrm{E}-16$ & $1.61 \mathrm{E}-20$ & $3.94 \mathrm{E}-20$ & $5.59 \mathrm{E}-12$ & \\
\hline Nb-94 & $1.45 \mathrm{E}-06$ & $4.73 \mathrm{E}-06$ & $1.23 \mathrm{E}-10$ & $1.23 \mathrm{E}-12$ & $1.14 \mathrm{E}-15$ & $1.84 \mathrm{E}-20$ & $4.51 \mathrm{E}-20$ & $6.39 \mathrm{E}-12$ & \\
\hline Tc-98 & $1.92 \mathrm{E}-12$ & $6.25 \mathrm{E}-12$ & 1.62E-16 & $1.62 \mathrm{E}-18$ & $1.51 \mathrm{E}-21$ & $2.44 \mathrm{E}-26$ & $5.95 \mathrm{E}-26$ & $8.45 \mathrm{E}-18$ & \\
\hline Tc-99 & $1.66 \mathrm{E}-05$ & $5.39 \mathrm{E}-05$ & 1.40E-09 & 1.40E-11 & $1.30 \mathrm{E}-14$ & $2.10 \mathrm{E}-19$ & $5.13 \mathrm{E}-19$ & $7.28 \mathrm{E}-11$ & \\
\hline Ru-106 & $1.20 \mathrm{E}-06$ & $3.91 \mathrm{E}-06$ & $1.01 \mathrm{E}-10$ & $1.01 \mathrm{E}-12$ & $9.40 \mathrm{E}-16$ & $1.52 \mathrm{E}-20$ & $3.72 \mathrm{E}-20$ & $5.28 \mathrm{E}-12$ & \\
\hline Rh-102 & $6.42 \mathrm{E}-10$ & $2.09 \mathrm{E}-09$ & $5.42 \mathrm{E}-14$ & $5.42 \mathrm{E}-16$ & $5.03 \mathrm{E}-19$ & $8.14 \mathrm{E}-24$ & $1.99 \mathrm{E}-23$ & $2.82 \mathrm{E}-15$ & \\
\hline Rh-106 & $1.20 \mathrm{E}-06$ & $3.91 \mathrm{E}-06$ & $1.01 \mathrm{E}-10$ & 1.01E-12 & $9.40 \mathrm{E}-16$ & $1.52 \mathrm{E}-20$ & $3.72 \mathrm{E}-20$ & $5.28 \mathrm{E}-12$ & \\
\hline Pd-107 & $1.23 \mathrm{E}-08$ & $4.00 \mathrm{E}-08$ & $1.04 \mathrm{E}-12$ & $1.04 \mathrm{E}-14$ & $9.64 \mathrm{E}-18$ & $1.56 \mathrm{E}-22$ & $3.81 \mathrm{E}-22$ & $5.41 \mathrm{E}-14$ & \\
\hline Cd-113m & $2.47 \mathrm{E}-06$ & $8.05 \mathrm{E}-06$ & $2.09 \mathrm{E}-10$ & $2.09 \mathrm{E}-12$ & $1.94 \mathrm{E}-15$ & $3.13 \mathrm{E}-20$ & $7.66 \mathrm{E}-20$ & $1.09 \mathrm{E}-11$ & \\
\hline Sn-121m & $4.98 \mathrm{E}-08$ & $1.62 \mathrm{E}-07$ & $4.21 \mathrm{E}-12$ & $4.21 \mathrm{E}-14$ & $3.90 \mathrm{E}-17$ & $6.31 \mathrm{E}-22$ & $1.54 \mathrm{E}-21$ & $2.19 \mathrm{E}-13$ & \\
\hline Sn-126 & $5.28 \mathrm{E}-07$ & $1.72 \mathrm{E}-06$ & $4.46 \mathrm{E}-11$ & $4.46 \mathrm{E}-13$ & $4.14 \mathrm{E}-16$ & $6.69 \mathrm{E}-21$ & $1.64 \mathrm{E}-20$ & $2.32 \mathrm{E}-12$ & \\
\hline Sb-125 & $7.14 \mathrm{E}-04$ & $2.32 \mathrm{E}-03$ & $6.03 \mathrm{E}-08$ & $6.03 \mathrm{E}-10$ & $5.59 \mathrm{E}-13$ & $9.05 \mathrm{E}-18$ & $2.21 \mathrm{E}-17$ & $3.14 \mathrm{E}-09$ & \\
\hline$\overline{S b-126}$ & $4.28 \mathrm{E}-08$ & $1.39 \mathrm{E}-07$ & $3.62 \mathrm{E}-12$ & $3.62 \mathrm{E}-14$ & $3.36 \mathrm{E}-17$ & $5.43 \mathrm{E}-22$ & $1.33 \mathrm{E}-21$ & $1.88 \mathrm{E}-13$ & \\
\hline
\end{tabular}


Table C-9. Alkaline scrub balance, Tank WM-187 waste. (Continued).

\begin{tabular}{|c|c|c|c|c|c|c|c|c|c|}
\hline Stream \# & 101 & 102 & 103 & 104 & 105 & 106 & 107 & 108 & 201 \\
\hline Radiological Composition & $\mathrm{Ci} / \mathrm{L}$ & $\mathrm{Ci} / \mathrm{L}$ & Ci/wscm & $\mathrm{Ci} / \mathrm{wscm}$ & $\mathrm{Ci} / \mathrm{wscm}$ & $\mathrm{Ci} / \mathrm{wscm}$ & $\mathrm{Ci} / \mathrm{wscm}$ & $\mathrm{Ci} / \mathrm{L}$ & \\
\hline Te-125m & $2.35 \mathrm{E}-06$ & $7.63 \mathrm{E}-06$ & $1.98 \mathrm{E}-10$ & $1.98 \mathrm{E}-12$ & $1.84 \mathrm{E}-15$ & $2.97 \mathrm{E}-20$ & $7.27 \mathrm{E}-20$ & $1.03 \mathrm{E}-11$ & \\
\hline $\mid-129$ & $6.81 \mathrm{E}-08$ & $2.18 \mathrm{E}-07$ & $1.92 \mathrm{E}-08$ & $1.92 \mathrm{E}-08$ & $1.83 \mathrm{E}-11$ & $8.98 \mathrm{E}-13$ & $1.85 \mathrm{E}-12$ & $9.54 \mathrm{E}-08$ & \\
\hline Cs-134 & $7.24 \mathrm{E}-05$ & $2.36 \mathrm{E}-04$ & $6.12 \mathrm{E}-09$ & $6.12 \mathrm{E}-11$ & $5.67 \mathrm{E}-14$ & $9.18 \mathrm{E}-19$ & $2.24 \mathrm{E}-18$ & $3.18 \mathrm{E}-10$ & \\
\hline Cs-135 & $1.05 \mathrm{E}-06$ & $3.43 \mathrm{E}-06$ & $8.90 \mathrm{E}-11$ & $8.91 \mathrm{E}-13$ & $8.26 \mathrm{E}-16$ & $1.34 \mathrm{E}-20$ & $3.27 \mathrm{E}-20$ & $4.63 \mathrm{E}-12$ & \\
\hline Cs-137 & $4.37 \mathrm{E}-02$ & $1.42 \mathrm{E}-01$ & $3.69 \mathrm{E}-06$ & $3.69 \mathrm{E}-08$ & $3.42 \mathrm{E}-11$ & $5.54 \mathrm{E}-16$ & $1.35 \mathrm{E}-15$ & $1.92 \mathrm{E}-07$ & \\
\hline Ba-137m & $4.13 \mathrm{E}-02$ & $1.35 \mathrm{E}-01$ & $3.49 \mathrm{E}-06$ & $3.49 \mathrm{E}-08$ & $3.24 \mathrm{E}-11$ & $5.24 \mathrm{E}-16$ & $1.28 \mathrm{E}-15$ & $1.82 \mathrm{E}-07$ & \\
\hline La-138 & $1.42 \mathrm{E}-16$ & $4.63 \mathrm{E}-16$ & $1.20 \mathrm{E}-20$ & $1.20 \mathrm{E}-22$ & $1.11 \mathrm{E}-25$ & $1.80 \mathrm{E}-30$ & $4.41 \mathrm{E}-30$ & $6.25 \mathrm{E}-22$ & \\
\hline Ce-142 & $2.23 \mathrm{E}-11$ & $7.25 \mathrm{E}-11$ & $1.88 \mathrm{E}-15$ & $1.88 \mathrm{E}-17$ & $1.74 \mathrm{E}-20$ & $2.82 \mathrm{E}-25$ & $6.90 \mathrm{E}-25$ & $9.79 \mathrm{E}-17$ & \\
\hline Ce-144 & $8.11 \mathrm{E}-07$ & $2.64 \mathrm{E}-06$ & $6.85 \mathrm{E}-11$ & $6.85 \mathrm{E}-13$ & $6.36 \mathrm{E}-16$ & $1.03 \mathrm{E}-20$ & $2.51 \mathrm{E}-20$ & $3.57 \mathrm{E}-12$ & \\
\hline Pr-144 & $8.11 \mathrm{E}-07$ & $2.64 \mathrm{E}-06$ & $6.85 \mathrm{E}-11$ & $6.85 \mathrm{E}-13$ & $6.36 \mathrm{E}-16$ & $1.03 \mathrm{E}-20$ & $2.51 \mathrm{E}-20$ & $3.57 \mathrm{E}-12$ & \\
\hline Nd-144 & $1.20 \mathrm{E}-15$ & $3.90 \mathrm{E}-15$ & $1.01 \mathrm{E}-19$ & $1.01 \mathrm{E}-21$ & $9.38 \mathrm{E}-25$ & $1.52 \mathrm{E}-29$ & $3.71 \mathrm{E}-29$ & $5.26 \mathrm{E}-21$ & \\
\hline Pm-146 & $3.87 \mathrm{E}-08$ & $1.26 \mathrm{E}-07$ & $3.27 \mathrm{E}-12$ & $3.27 \mathrm{E}-14$ & $3.03 \mathrm{E}-17$ & $4.90 \mathrm{E}-22$ & $1.20 \mathrm{E}-21$ & $1.70 \mathrm{E}-13$ & \\
\hline Pm-147 & $2.18 \mathrm{E}-04$ & $7.10 \mathrm{E}-04$ & $1.84 \mathrm{E}-08$ & $1.84 \mathrm{E}-10$ & 1.71E-13 & $2.76 \mathrm{E}-18$ & $6.76 \mathrm{E}-18$ & $9.59 \mathrm{E}-10$ & \\
\hline Sm-146 & $2.05 \mathrm{E}-13$ & $6.69 \mathrm{E}-13$ & $1.74 \mathrm{E}-17$ & $1.74 \mathrm{E}-19$ & $1.61 \mathrm{E}-22$ & $2.60 \mathrm{E}-27$ & $6.37 \mathrm{E}-27$ & $9.03 \mathrm{E}-19$ & \\
\hline Sm-147 & $5.48 \mathrm{E}-12$ & $1.79 \mathrm{E}-11$ & $4.63 \mathrm{E}-16$ & $4.63 \mathrm{E}-18$ & $4.30 \mathrm{E}-21$ & $6.95 \mathrm{E}-26$ & $1.70 \mathrm{E}-25$ & $2.41 \mathrm{E}-17$ & \\
\hline \begin{tabular}{|l|}
$S m-151$ \\
\end{tabular} & $4.32 \mathrm{E}-04$ & $1.41 \mathrm{E}-03$ & $3.65 \mathrm{E}-08$ & $3.65 \mathrm{E}-10$ & $3.39 \mathrm{E}-13$ & $5.48 \mathrm{E}-18$ & $1.34 \mathrm{E}-17$ & $1.90 \mathrm{E}-09$ & \\
\hline Eu-152 & $2.51 \mathrm{E}-06$ & $8.17 \mathrm{E}-06$ & $2.12 \mathrm{E}-10$ & $2.12 \mathrm{E}-12$ & 1.97E-15 & $3.18 \mathrm{E}-20$ & $7.78 \mathrm{E}-20$ & $1.10 \mathrm{E}-11$ & \\
\hline Eu-154 & $9.52 \mathrm{E}-05$ & $3.10 \mathrm{E}-04$ & $8.04 \mathrm{E}-09$ & $8.04 \mathrm{E}-11$ & $7.46 \mathrm{E}-14$ & $1.21 \mathrm{E}-18$ & $2.95 \mathrm{E}-18$ & $4.19 \mathrm{E}-10$ & \\
\hline Eu-155 & $1.01 \mathrm{E}-04$ & $3.29 \mathrm{E}-04$ & $8.53 \mathrm{E}-09$ & $8.53 \mathrm{E}-11$ & $7.91 \mathrm{E}-14$ & $1.28 \mathrm{E}-18$ & $3.13 \mathrm{E}-18$ & $4.44 \mathrm{E}-10$ & \\
\hline Ho-166m & $3.43 \mathrm{E}-11$ & $1.12 \mathrm{E}-10$ & $2.90 \mathrm{E}-15$ & $2.90 \mathrm{E}-17$ & $2.69 \mathrm{E}-20$ & $4.35 \mathrm{E}-25$ & $1.06 \mathrm{E}-24$ & $1.51 \mathrm{E}-16$ & \\
\hline
\end{tabular}


Table C-10. Alkaline scrub balance, Tank WM-188 waste.

\begin{tabular}{|c|c|c|c|c|c|c|c|c|c|}
\hline \begin{tabular}{|ll} 
WM-188 & PFD Sheet \#
\end{tabular} & PFD-2A & PFD-2A & PFD-2A & PFD-2A & PFD-2A & PFD-2A & PFD-2A & PFD-2A & PFD-2A \\
\hline Stream \# & 101 & 102 & 103 & 104 & 105 & 106 & 107 & 108 & 201 \\
\hline Stream Name & $\begin{array}{l}\text { SBW } \\
\text { Feed }\end{array}$ & $\begin{array}{c}\text { Evaporator } \\
\text { Bottoms }\end{array}$ & $\begin{array}{c}\text { Evaporator } \\
\text { Overheads } \\
\text { Vapor }\end{array}$ & $\begin{array}{c}\text { Mist } \\
\text { Eliminator } \\
\text { Vapor }\end{array}$ & $\begin{array}{l}\text { Alkaline } \\
\text { Scrubber } \\
\text { Vapor }\end{array}$ & $\begin{array}{c}\text { POG + VOG } \\
\text { Offgas } \\
\text { Vapor }\end{array}$ & $\begin{array}{l}\text { VOG } \\
\text { Offgas } \\
\text { Vapor }\end{array}$ & $\begin{array}{l}\text { Scrubber } \\
\text { Recycle to } \\
\text { Feed }\end{array}$ & $\begin{array}{l}20 \text { wt.\% } \\
\text { MgO } \\
\text { Additive }\end{array}$ \\
\hline Rate or Volume Flow (actual) & 85.8 & 30.2 & 39,564 & 40,100 & 33,918 & 38,489 & 11,768 & 28.7 & 144.8 \\
\hline Volume Flow (standard, wet) ${ }^{*}$ & & & 15,224 & 15,224 & 13,137 & 20,550 & 7,400 & & \\
\hline Volume Flow (standard, dry) ${ }^{\star}$ & & & 552.5 & 552.3 & 0.66 & 7,118 & 7,130 & & \\
\hline Rate Units & $\mathrm{gal} / \mathrm{hr}$ & gal/hr & $\mathrm{ft} 3 / \mathrm{hr}$ & $\mathrm{ft} 3 / \mathrm{hr}$ & $\mathrm{ft} 3 / \mathrm{hr}$ & $\mathrm{ft} 3 / \mathrm{hr}$ & $\mathrm{ft} 3 / \mathrm{hr}$ & $\mathrm{gal} / \mathrm{hr}$ & $\mathrm{lb} / \mathrm{hr}$ \\
\hline Temperature, ${ }^{\circ} \mathrm{C}$ & 25.0 & 120 & 120 & 114.6 & 88.3 & 175 & 108 & 88.4 & 25.0 \\
\hline Temperature, ${ }^{\circ} \mathrm{F}$ & 77.0 & 248 & 248 & 238 & 191 & 347 & 226 & 191 & 77.0 \\
\hline Pressure, psia & 12.3 & 7.6 & 7.6 & 7.4 & 7.0 & 12.0 & 12.0 & 30.0 & 12.3 \\
\hline Specific Gravity & 1.24 & 1.75 & 0.0003 & 0.0003 & 0.00029 & 0.00048 & 0.00074 & 1.29 & 1.20 \\
\hline Chemical Composition & Mol/Liter & Mol/Liter & $\mathrm{lb} / \mathrm{wscf}$ & $\mathrm{Ib} / \mathrm{wscf}$ & $\mathrm{Ib} / \mathrm{wscf}$ & $\mathrm{Ib} / \mathrm{wscf}$ & $\mathrm{lb} / \mathrm{wscf}$ & Mol/Liter & Wt. \% \\
\hline $\mathrm{H}+$ & $2.18 \mathrm{E}+00$ & $4.81 \mathrm{E}-01$ & $9.52 \mathrm{E}-05$ & $9.52 \mathrm{E}-05$ & $5.52 \mathrm{E}-09$ & $3.66 \mathrm{E}-09$ & $3.55 \mathrm{E}-10$ & $6.70 \mathrm{E}-04$ & $2.64 \mathrm{E}-12$ \\
\hline $\mathrm{OH}-$ & & & $1.31 \mathrm{E}-14$ & & $2.91 \mathrm{E}-12$ & 1.63E-13 & & 1.34E-05 & $1.69 \mathrm{E}+01$ \\
\hline$A \mathrm{Al}+3$ & $5.07 \mathrm{E}-01$ & $1.44 \mathrm{E}+00$ & $6.43 \mathrm{E}-08$ & $2.45 \mathrm{E}-11$ & $1.94 \mathrm{E}-14$ & & & $5.76 \mathrm{E}-08$ & \\
\hline $\mathrm{Sb}+5$ & $1.58 \mathrm{E}-05$ & $4.50 \mathrm{E}-05$ & $9.07 \mathrm{E}-12$ & $9.07 \mathrm{E}-14$ & $7.20 \mathrm{E}-17$ & & & $4.73 \mathrm{E}-11$ & \\
\hline$A s+3$ & $8.88 \mathrm{E}-05$ & $2.52 \mathrm{E}-04$ & $3.13 \mathrm{E}-11$ & $3.13 \mathrm{E}-13$ & $2.48 \mathrm{E}-16$ & & & $2.65 \mathrm{E}-10$ & \\
\hline $\mathrm{Ba}+2$ & $7.24 \mathrm{E}-05$ & $2.05 \mathrm{E}-04$ & $4.67 \mathrm{E}-11$ & 1.13E-13 & $8.99 \mathrm{E}-17$ & & & $5.24 \mathrm{E}-11$ & \\
\hline $\mathrm{Be}+2$ & $1.83 \mathrm{E}-05$ & $5.19 \mathrm{E}-05$ & $7.74 \mathrm{E}-13$ & $2.29 \mathrm{E}-15$ & $1.82 \mathrm{E}-18$ & & & $1.61 \mathrm{E}-11$ & \\
\hline$B+3$ & $1.59 \mathrm{E}-02$ & $4.50 \mathrm{E}-02$ & $8.07 \mathrm{E}-10$ & $3.01 \mathrm{E}-13$ & $2.39 \mathrm{E}-16$ & & & $1.77 \mathrm{E}-09$ & \\
\hline$C d+2$ & $2.42 \mathrm{E}-03$ & $6.88 \mathrm{E}-03$ & $1.28 \mathrm{E}-09$ & $3.32 \mathrm{E}-13$ & $2.63 \mathrm{E}-16$ & & & $1.88 \mathrm{E}-10$ & \\
\hline $\mathrm{Ca}+2$ & $4.83 \mathrm{E}-02$ & 1.37E-01 & $9.10 \mathrm{E}-09$ & $2.37 \mathrm{E}-12$ & $1.88 \mathrm{E}-15$ & & & 3.76E-09 & \\
\hline $\mathrm{Cr}+3$ & $4.48 \mathrm{E}-03$ & $1.27 \mathrm{E}-02$ & 1.10E-09 & $9.86 \mathrm{E}-13$ & $7.82 \mathrm{E}-16$ & & & $1.20 \mathrm{E}-09$ & \\
\hline $\mathrm{Co}+2$ & 3.97E-05 & $1.13 \mathrm{E}-04$ & $1.10 \mathrm{E}-11$ & $1.03 \mathrm{E}-14$ & $8.21 \mathrm{E}-18$ & & & $1.11 \mathrm{E}-11$ & \\
\hline Cs+ & $5.76 \mathrm{E}-05$ & $1.64 \mathrm{E}-04$ & $3.29 \mathrm{E}-11$ & $2.19 \mathrm{E}-13$ & $1.74 \mathrm{E}-16$ & & & $1.05 \mathrm{E}-10$ & \\
\hline $\mathrm{Cu}+2$ & $6.78 \mathrm{E}-04$ & 1.92E-03 & $2.03 \mathrm{E}-10$ & $2.92 \mathrm{E}-13$ & $2.31 \mathrm{E}-16$ & & & $2.91 \mathrm{E}-10$ & \\
\hline $\mathrm{Fe}+3$ & $2.49 \mathrm{E}-02$ & $7.08 \mathrm{E}-02$ & $6.55 \mathrm{E}-09$ & $1.78 \mathrm{E}-11$ & $1.41 \mathrm{E}-14$ & & & $2.02 \mathrm{E}-08$ & \\
\hline $\mathrm{Pb}+2$ & $7.98 \mathrm{E}-04$ & $2.27 \mathrm{E}-03$ & $7.77 \mathrm{E}-10$ & $3.35 \mathrm{E}-13$ & $2.66 \mathrm{E}-16$ & & & 1.03E-10 & \\
\hline $\mathrm{Hg}+2$ & $5.11 \mathrm{E}-03$ & $1.45 \mathrm{E}-02$ & 1.04E-06 & 1.04E-06 & 7.97E-09 & $4.18 \mathrm{E}-11$ & $1.02 \mathrm{E}-10$ & $3.24 \mathrm{E}-04$ & \\
\hline$M n+4$ & $1.43 \mathrm{E}-02$ & $4.07 \mathrm{E}-02$ & $3.70 \mathrm{E}-09$ & $3.70 \mathrm{E}-11$ & $2.94 \mathrm{E}-14$ & & & $4.28 \mathrm{E}-08$ & \\
\hline $\mathrm{Ni}+2$ & $1.78 \mathrm{E}-03$ & $5.04 \mathrm{E}-03$ & $4.90 \mathrm{E}-10$ & $4.25 \mathrm{E}-13$ & $3.37 \mathrm{E}-16$ & & & $4.60 \mathrm{E}-10$ & \\
\hline $\mathrm{K}+$ & $1.82 \mathrm{E}-01$ & $5.17 \mathrm{E}-01$ & $3.35 \mathrm{E}-08$ & $2.24 \mathrm{E}-11$ & $1.77 \mathrm{E}-14$ & & & $3.63 \mathrm{E}-08$ & \\
\hline $\mathrm{Se}+4$ & 2.34E-04 & $6.64 \mathrm{E}-04$ & $8.69 \mathrm{E}-11$ & $8.69 \mathrm{E}-13$ & $6.89 \mathrm{E}-16$ & & & $6.99 \mathrm{E}-10$ & \\
\hline $\mathrm{Ag}^{+}$ & $3.64 \mathrm{E}-04$ & $1.03 \mathrm{E}-03$ & $1.84 \mathrm{E}-10$ & $1.83 \mathrm{E}-12$ & 1.45E-15 & & & $1.08 \mathrm{E}-09$ & \\
\hline $\mathrm{Na}+$ & $1.36 \mathrm{E}+00$ & $3.85 \mathrm{E}+00$ & 1.16E-07 & $8.75 \mathrm{E}-11$ & $6.94 \mathrm{E}-14$ & & & $2.42 \mathrm{E}-07$ & \\
\hline $\mathrm{TI}+3$ & $4.42 \mathrm{E}-06$ & 1.26E-05 & $4.25 \mathrm{E}-12$ & $4.25 \mathrm{E}-14$ & 3.37E-17 & & & $1.32 \mathrm{E}-11$ & \\
\hline $\mathrm{U}+4$ & $3.50 \mathrm{E}-04$ & $9.93 \mathrm{E}-04$ & $3.92 \mathrm{E}-10$ & $3.92 \mathrm{E}-12$ & $3.11 \mathrm{E}-15$ & & & $1.05 \mathrm{E}-09$ & \\
\hline $\mathrm{V}+3$ & $3.62 \mathrm{E}-05$ & $1.03 \mathrm{E}-04$ & $8.66 \mathrm{E}-12$ & $8.66 \mathrm{E}-14$ & $6.87 \mathrm{E}-17$ & & & $1.08 \mathrm{E}-10$ & \\
\hline$Z n+2$ & $7.85 \mathrm{E}-04$ & $2.23 \mathrm{E}-03$ & $2.41 \mathrm{E}-10$ & $1.86 \mathrm{E}-13$ & $1.47 \mathrm{E}-16$ & & & $1.80 \mathrm{E}-10$ & \\
\hline $\mathrm{Zr}+4$ & $3.65 \mathrm{E}-02$ & 1.03E-01 & 1.56E-08 & $1.56 \mathrm{E}-10$ & $1.24 \mathrm{E}-13$ & & & $1.09 \mathrm{E}-07$ & \\
\hline $\mathrm{Cl}-$ & $2.39 \mathrm{E}-02$ & $6.78 \mathrm{E}-02$ & 1.54E-05 & 1.54E-05 & $1.55 \mathrm{E}-08$ & $5.99 \mathrm{E}-10$ & $4.54 \mathrm{E}-11$ & $2.77 \mathrm{E}-02$ & \\
\hline$\overline{F-}$ & $3.45 \mathrm{E}-02$ & $9.80 \mathrm{E}-02$ & $1.12 \mathrm{E}-08$ & $9.59 \mathrm{E}-09$ & $4.77 \mathrm{E}-09$ & $3.05 \mathrm{E}-09$ & $1.02 \mathrm{E}-12$ & $2.67 \mathrm{E}-05$ & \\
\hline SO4-2 & $4.05 \mathrm{E}-02$ & 1.15E-01 & 1.83E-08 & 1.83E-10 & $1.45 \mathrm{E}-13$ & & & $1.21 \mathrm{E}-07$ & \\
\hline NO3- & $5.31 \mathrm{E}+00$ & $1.51 \mathrm{E}+01$ & $5.83 \mathrm{E}-03$ & $5.83 \mathrm{E}-03$ & 4.94E-06 & $2.14 \mathrm{E}-07$ & $2.18 \mathrm{E}-08$ & $5.96 \mathrm{E}+00$ & \\
\hline PO4-3 & $9.61 \mathrm{E}-02$ & $2.73 \mathrm{E}-01$ & $4.29 \mathrm{E}-08$ & $4.29 \mathrm{E}-10$ & $3.40 \mathrm{E}-13$ & & & $2.87 \mathrm{E}-07$ & \\
\hline$A m+4$ & $6.69 \mathrm{E}-08$ & $1.90 \mathrm{E}-07$ & $7.59 \mathrm{E}-14$ & $7.59 \mathrm{E}-16$ & $6.02 \mathrm{E}-19$ & & & $2.00 \mathrm{E}-13$ & \\
\hline $\mathrm{Br}-$ & $2.87 \mathrm{E}-07$ & $8.16 \mathrm{E}-07$ & $4.58 \mathrm{E}-10$ & $4.58 \mathrm{E}-10$ & $4.62 \mathrm{E}-13$ & $1.78 \mathrm{E}-14$ & $1.35 \mathrm{E}-15$ & $3.65 \mathrm{E}-07$ & \\
\hline $\mathrm{Ce}+4$ & $3.31 \mathrm{E}-05$ & $9.40 \mathrm{E}-05$ & $2.18 \mathrm{E}-11$ & $2.18 \mathrm{E}-13$ & $1.73 \mathrm{E}-16$ & & & $9.89 \mathrm{E}-11$ & \\
\hline$E u+3$ & $4.77 \mathrm{E}-07$ & $1.35 \mathrm{E}-06$ & $3.41 \mathrm{E}-13$ & $4.65 \mathrm{E}-18$ & $3.69 \mathrm{E}-21$ & & & $1.94 \mathrm{E}-15$ & \\
\hline $\mathrm{Gd}+3$ & $1.41 \mathrm{E}-04$ & $4.00 \mathrm{E}-04$ & $1.04 \mathrm{E}-10$ & $1.28 \mathrm{E}-13$ & $1.02 \mathrm{E}-16$ & & & $5.17 \mathrm{E}-11$ & \\
\hline $\mathrm{Ge}+4$ & $8.29 \mathrm{E}-09$ & $2.35 \mathrm{E}-08$ & $2.83 \mathrm{E}-15$ & $2.83 \mathrm{E}-17$ & $2.25 \mathrm{E}-20$ & & & $2.48 \mathrm{E}-14$ & \\
\hline $\ln +3$ & $1.46 \mathrm{E}-06$ & $4.14 \mathrm{E}-06$ & $7.87 \mathrm{E}-13$ & & & & & & \\
\hline $\mid-$ & $3.07 \mathrm{E}-06$ & $8.73 \mathrm{E}-06$ & $6.04 \mathrm{E}-09$ & $6.04 \mathrm{E}-09$ & $6.09 \mathrm{E}-12$ & $2.35 \mathrm{E}-13$ & $1.78 \mathrm{E}-14$ & $3.03 \mathrm{E}-06$ & \\
\hline $\mathrm{La}+3$ & $8.66 \mathrm{E}-06$ & $2.46 \mathrm{E}-05$ & $5.66 \mathrm{E}-12$ & & & & & & \\
\hline Li+ & $4.53 \mathrm{E}-04$ & $1.29 \mathrm{E}-03$ & $1.31 \mathrm{E}-11$ & $7.06 \mathrm{E}-14$ & $5.60 \mathrm{E}-17$ & & & $6.46 \mathrm{E}-10$ & \\
\hline$M g+2$ & $1.71 \mathrm{E}-02$ & $2.89 \mathrm{E}+00$ & $1.17 \mathrm{E}-07$ & & $9.10 \mathrm{E}-07$ & $1.16 \mathrm{E}-13$ & & $3.00 \mathrm{E}+00$ & $1.21 \mathrm{E}+01$ \\
\hline $\mathrm{Mo}+6$ & $7.29 \mathrm{E}-04$ & $2.07 \mathrm{E}-03$ & $3.29 \mathrm{E}-10$ & $3.29 \mathrm{E}-12$ & $2.61 \mathrm{E}-15$ & & & $2.18 \mathrm{E}-09$ & \\
\hline
\end{tabular}


Table C-10. Alkaline scrub balance, Tank WM-188 waste. (Continued).

\begin{tabular}{|c|c|c|c|c|c|c|c|c|c|}
\hline Stream \# & 101 & 102 & 103 & 104 & 105 & 106 & 107 & 108 & 201 \\
\hline Chemical Composition & Mol/Liter & Mol/Liter & Ib/wscf & $\mathrm{Ib} / \mathrm{wscf}$ & $\mathrm{Ib} / \mathrm{wscf}$ & $\mathrm{Ib} / \mathrm{wscf}$ & $\mathrm{Ib} / \mathrm{wscf}$ & Mol/Liter & Wt. \% \\
\hline $\mathrm{Nd}+3$ & $2.79 \mathrm{E}-05$ & $7.93 E-05$ & $1.89 \mathrm{E}-11$ & & & & & & \\
\hline $\mathrm{Np}+4$ & $2.55 \mathrm{E}-06$ & $7.25 \mathrm{E}-06$ & $2.85 \mathrm{E}-12$ & $2.85 \mathrm{E}-14$ & $2.26 \mathrm{E}-17$ & & & $7.62 \mathrm{E}-12$ & \\
\hline $\mathrm{Nb}+5$ & $1.05 \mathrm{E}-03$ & $2.97 \mathrm{E}-03$ & $4.57 \mathrm{E}-10$ & $4.57 \mathrm{E}-12$ & $3.63 \mathrm{E}-15$ & & & $3.12 \mathrm{E}-09$ & \\
\hline $\mathrm{Pd}+4$ & $1.70 \mathrm{E}-03$ & $4.82 \mathrm{E}-03$ & $8.50 \mathrm{E}-10$ & $8.50 \mathrm{E}-12$ & $6.74 \mathrm{E}-15$ & & & $5.07 E-09$ & \\
\hline $\mathrm{Pu}+4$ & $6.65 \mathrm{E}-06$ & $1.89 \mathrm{E}-05$ & $7.47 \mathrm{E}-12$ & $7.47 \mathrm{E}-14$ & $5.93 \mathrm{E}-17$ & & & $1.99 \mathrm{E}-11$ & \\
\hline $\mathrm{Pr}+4$ & $7.87 \mathrm{E}-06$ & $2.24 \mathrm{E}-05$ & $5.22 \mathrm{E}-12$ & $5.22 \mathrm{E}-14$ & $4.14 \mathrm{E}-17$ & & & $2.35 \mathrm{E}-11$ & \\
\hline $\mathrm{Rh}+4$ & $3.40 \mathrm{E}-06$ & $9.64 \mathrm{E}-06$ & $1.64 \mathrm{E}-12$ & $1.64 \mathrm{E}-14$ & $1.30 \mathrm{E}-17$ & & & $1.01 \mathrm{E}-11$ & \\
\hline $\mathrm{Rb}+$ & $5.24 \mathrm{E}-06$ & $1.49 \mathrm{E}-05$ & $1.66 \mathrm{E}-12$ & $8.47 \mathrm{E}-16$ & $6.72 \mathrm{E}-19$ & & & $6.29 \mathrm{E}-13$ & \\
\hline $\mathrm{Ru}+3$ & $7.86 \mathrm{E}-04$ & $2.23 \mathrm{E}-03$ & $3.74 \mathrm{E}-10$ & $3.17 \mathrm{E}-12$ & $2.52 \mathrm{E}-15$ & & & $1.99 \mathrm{E}-09$ & \\
\hline $\mathrm{Sm}+3$ & $5.20 \mathrm{E}-06$ & $1.48 \mathrm{E}-05$ & $3.68 \mathrm{E}-12$ & $1.74 \mathrm{E}-16$ & $1.38 \mathrm{E}-19$ & & & $7.35 \mathrm{E}-14$ & \\
\hline$S i+4$ & $8.92 \mathrm{E}-02$ & $2.53 \mathrm{E}-01$ & $1.18 \mathrm{E}-08$ & $1.18 \mathrm{E}-10$ & $9.35 \mathrm{E}-14$ & & & $2.67 \mathrm{E}-07$ & \\
\hline $\mathrm{Sr}+2$ & $7.77 \mathrm{E}-05$ & $2.21 \mathrm{E}-04$ & $3.20 \mathrm{E}-11$ & $2.54 \mathrm{E}-14$ & $2.02 \mathrm{E}-17$ & & & $1.84 \mathrm{E}-11$ & \\
\hline $\mathrm{Tc}+7$ & $1.01 \mathrm{E}-05$ & $2.88 \mathrm{E}-05$ & $4.62 \mathrm{E}-12$ & & & & & & \\
\hline $\mathrm{Te}+4$ & $3.46 \mathrm{E}-06$ & $9.82 \mathrm{E}-06$ & $2.07 \mathrm{E}-12$ & $2.07 \mathrm{E}-14$ & $1.65 \mathrm{E}-17$ & & & $1.03 \mathrm{E}-11$ & \\
\hline$T \mathrm{~Tb}+4$ & $1.99 \mathrm{E}-09$ & $5.66 \mathrm{E}-09$ & $1.49 \mathrm{E}-15$ & $1.49 \mathrm{E}-17$ & $1.18 \mathrm{E}-20$ & & & $5.95 \mathrm{E}-15$ & \\
\hline $\mathrm{Th}+4$ & $2.29 \mathrm{E}-05$ & $6.51 \mathrm{E}-05$ & $2.50 \mathrm{E}-11$ & $2.50 \mathrm{E}-13$ & $1.99 \mathrm{E}-16$ & & & $6.85 \mathrm{E}-11$ & \\
\hline$S n+4$ & $1.73 \mathrm{E}-03$ & $4.90 \mathrm{E}-03$ & $9.64 \mathrm{E}-10$ & $9.64 \mathrm{E}-12$ & $7.64 \mathrm{E}-15$ & & & $5.16 \mathrm{E}-09$ & \\
\hline$T i+4$ & $8.83 \mathrm{E}-04$ & $2.51 \mathrm{E}-03$ & $1.99 \mathrm{E}-10$ & $1.99 \mathrm{E}-12$ & $1.58 \mathrm{E}-15$ & & & $2.64 \mathrm{E}-09$ & \\
\hline $\mathrm{Y}+3$ & $6.46 \mathrm{E}-06$ & $1.83 \mathrm{E}-05$ & $2.70 \mathrm{E}-12$ & $4.31 \mathrm{E}-20$ & $3.42 \mathrm{E}-23$ & & & $3.08 \mathrm{E}-17$ & \\
\hline $\mathrm{O}-2$ (oxides) & $1.83 \mathrm{E}-01$ & $5.20 \mathrm{E}-01$ & $1.38 \mathrm{E}-08$ & $1.38 \mathrm{E}-10$ & $1.09 \mathrm{E}-13$ & & & $5.47 \mathrm{E}-07$ & \\
\hline $\mathrm{H} 2 \mathrm{O}$ & $4.85 \mathrm{E}+01$ & $2.79 \mathrm{E}+01$ & $4.52 \mathrm{E}-02$ & $4.52 \mathrm{E}-02$ & $4.69 \mathrm{E}-02$ & $3.06 \mathrm{E}-02$ & $1.70 \mathrm{E}-03$ & $4.68 \mathrm{E}+01$ & $7.11 \mathrm{E}+01$ \\
\hline $\mathrm{O} 2$ & & & $1.21 \mathrm{E}-07$ & $1.21 \mathrm{E}-07$ & $1.40 \mathrm{E}-07$ & $6.05 \mathrm{E}-03$ & $1.68 \mathrm{E}-02$ & $1.79 \mathrm{E}-10$ & \\
\hline N2 & & & $2.05 E-07$ & $2.05 \mathrm{E}-07$ & $2.38 \mathrm{E}-07$ & $1.99 \mathrm{E}-02$ & $5.53 \mathrm{E}-02$ & $2.07 \mathrm{E}-10$ & \\
\hline C (organic) & $6.88 \mathrm{E}-02$ & $1.95 \mathrm{E}-01$ & $5.40 \mathrm{E}-07$ & $5.40 \mathrm{E}-07$ & $1.00 \mathrm{E}-09$ & $6.42 \mathrm{E}-10$ & $1.12 \mathrm{E}-12$ & $2.81 \mathrm{E}-03$ & \\
\hline $\mathrm{H}$ (organic) & $6.88 \mathrm{E}-02$ & $1.95 \mathrm{E}-01$ & $4.53 \mathrm{E}-08$ & $4.53 \mathrm{E}-08$ & $8.42 \mathrm{E}-11$ & $5.38 \mathrm{E}-11$ & $9.44 \mathrm{E}-14$ & $2.81 \mathrm{E}-03$ & \\
\hline $\begin{array}{l}\begin{array}{l}\text { Gas Stream Bulk Composition } \\
\text { (wet basis) }\end{array} \\
\end{array}$ & $\begin{array}{l}\text { mol. \% } \\
\text { or ppmv }\end{array}$ & $\begin{array}{l}\text { mol. \% } \\
\text { or ppmv }\end{array}$ & $\begin{array}{l}\text { mol.\% } \\
\text { or ppmv }\end{array}$ & $\begin{array}{l}\text { mol.\% } \\
\text { or ppmv }\end{array}$ & $\begin{array}{c}\mathrm{mol} . \% \\
\text { or ppmv }\end{array}$ & $\begin{array}{l}\text { mol.\% } \\
\text { or ppmv }\end{array}$ & $\begin{array}{l}\mathrm{mol} . \% \\
\text { or ppmv }\end{array}$ & $\begin{array}{c}\mathrm{mol} . \% \\
\text { or ppmv }\end{array}$ & $\begin{array}{c}\text { mol.\% } \\
\text { or ppmv }\end{array}$ \\
\hline $\mathrm{H} 2 \mathrm{O}, \mathrm{mol} . \%$ & & & 96.37 & 96.37 & 100.00 & 65.36 & 3.64 & & \\
\hline $\mathrm{O} 2, \mathrm{~mol} . \%$ & & & 0.0001 & 0.0001 & 0.0002 & 7.27 & 20.23 & & \\
\hline N2, mol.\% & & & 0.0003 & 0.0003 & 0.0003 & 27.36 & 76.12 & & \\
\hline \multicolumn{10}{|l|}{$\mathrm{CO} 2, \mathrm{~mol} . \%$} \\
\hline HNO3, mol. \% & & & 3.63 & 3.63 & 0.0002 & 0.0001 & 0.00001 & & \\
\hline \multicolumn{10}{|l|}{$\mathrm{CO}, \mathrm{ppmv}$} \\
\hline $\mathrm{HCl}, \mathrm{ppmv}$ & & & 167 & 167 & 0.17 & 0.006 & 0.0005 & & \\
\hline C (organic), ppmv & & & 17 & 17 & 0.032 & 0.021 & 0.00004 & & \\
\hline $\mathrm{H}$ (organic), ppmv & & & 17 & 17 & 0.032 & 0.021 & 0.00004 & & \\
\hline $\mathrm{PM}(\mathrm{mg} / \mathrm{wscm})$ & & & 3 & 0.03 & 0.00003 & & & & \\
\hline $\mathrm{Hg}(\mathrm{ug} / \mathrm{wscm})$ & & & $1.7 \mathrm{E}+04$ & $1.7 \mathrm{E}+04$ & 128 & 0.7 & 1.6 & & \\
\hline $\begin{array}{l}\text { Gas Stream Bulk Composition } \\
\text { (dry basis) }\end{array}$ & $\begin{array}{l}\text { mol. } \% \\
\text { or ppmv }\end{array}$ & $\begin{array}{l}\text { mol. } \% \\
\text { or ppmv }\end{array}$ & $\begin{array}{l}\text { mol. } \% \\
\text { or ppmv }\end{array}$ & $\begin{array}{l}\text { mol.\% } \\
\text { or ppmv }\end{array}$ & $\begin{array}{l}\text { mol. } \% \\
\text { or ppmv }\end{array}$ & $\begin{array}{l}\text { mol. } \% \\
\text { or ppmv }\end{array}$ & $\begin{array}{l}\text { mol. } \% \\
\text { or ppmv }\end{array}$ & $\begin{array}{l}\text { mol. } \% \\
\text { or ppmv }\end{array}$ & $\begin{array}{l}\text { mol. } \% \\
\text { or ppmv }\end{array}$ \\
\hline $\mathrm{O} 2, \mathrm{~mol} . \%$ & & & 0.004 & 0.004 & 3.36 & 21.00 & 21.00 & & \\
\hline $\mathrm{N} 2, \mathrm{~mol} . \%$ & & & 0.008 & 0.008 & 6.53 & 79.00 & 79.00 & & \\
\hline \multicolumn{10}{|l|}{$\mathrm{CO} 2, \mathrm{~mol} . \%$} \\
\hline HNO3, mol. \% & & & 99.92 & 99.96 & 4.21 & 0.0004 & 0.00001 & & \\
\hline \multicolumn{10}{|l|}{ CO, ppmv } \\
\hline $\mathrm{HCl}, \mathrm{ppmv}$ & & & $4.6 \mathrm{E}+03$ & $4.6 \mathrm{E}+03$ & $3.4 \mathrm{E}+03$ & 0.02 & 0.0005 & & \\
\hline C (organic), ppmv & & & $4.8 \mathrm{E}+02$ & $4.8 \mathrm{E}+02$ & $6.4 \mathrm{E}+02$ & 0.06 & 0.00004 & & \\
\hline $\mathrm{H}$ (organic), ppmv & & & 476 & 476 & 642 & 0.06 & 0.00004 & & \\
\hline $\mathrm{PM}(\mathrm{mg} / \mathrm{dscm})$ & & & 88 & 0.88 & 0.51 & & & & \\
\hline $\mathrm{Hg}$ (ug/wscm) & & & $1.2 \mathrm{E}+06$ & $1.2 \mathrm{E}+06$ & $6.6 \mathrm{E}+06$ & 3.6 & 2.7 & & \\
\hline Total Mass Flow, kg/hr & $4.19 \mathrm{E}+02$ & $2.00 \mathrm{E}+02$ & $3.53 E+02$ & $3.53 E+02$ & $2.80 \mathrm{E}+02$ & $5.28 \mathrm{E}+02$ & $2.48 \mathrm{E}+02$ & $1.40 \mathrm{E}+02$ & $6.57 \mathrm{E}+01$ \\
\hline Canister Rate, canisters/day & & 3.43 & & & & & & & \\
\hline Total Canisters Generated & & 499 & & & & & & & \\
\hline
\end{tabular}


Table C-10. Alkaline scrub balance, Tank WM-188 waste. (Continued).

\begin{tabular}{|c|c|c|c|c|c|c|c|c|c|}
\hline Stream \# & 101 & 102 & 103 & 104 & 105 & 106 & 107 & 108 & 201 \\
\hline Radiological Composition & $\mathrm{Ci} / \mathrm{L}$ & $\mathrm{Ci} / \mathrm{L}$ & Ci/wscm & $\mathrm{Ci} / \mathrm{wscm}$ & $\mathrm{Ci} / \mathrm{wscm}$ & $\mathrm{Ci} / \mathrm{wscm}$ & $\mathrm{Ci} / \mathrm{wscm}$ & $\mathrm{Ci} / \mathrm{L}$ & \\
\hline Ra-226 & $8.33 \mathrm{E}-12$ & $2.36 \mathrm{E}-11$ & $6.27 \mathrm{E}-16$ & $6.27 \mathrm{E}-18$ & $4.83 \mathrm{E}-21$ & $9.91 \mathrm{E}-26$ & $2.58 \mathrm{E}-25$ & $2.40 \mathrm{E}-17$ & \\
\hline Ac-227 & $3.92 \mathrm{E}-11$ & $1.11 \mathrm{E}-10$ & $2.95 \mathrm{E}-15$ & $2.95 \mathrm{E}-17$ & $2.27 \mathrm{E}-20$ & $4.67 \mathrm{E}-25$ & $1.22 \mathrm{E}-24$ & $1.13 \mathrm{E}-16$ & \\
\hline Th-228 & $3.25 \mathrm{E}-09$ & $9.24 \mathrm{E}-09$ & $2.45 \mathrm{E}-13$ & $2.45 \mathrm{E}-15$ & $1.89 \mathrm{E}-18$ & $3.87 \mathrm{E}-23$ & $1.01 \mathrm{E}-22$ & $9.38 \mathrm{E}-15$ & \\
\hline Th-230 & $1.04 \mathrm{E}-09$ & $2.96 \mathrm{E}-09$ & $7.84 \mathrm{E}-14$ & $7.84 \mathrm{E}-16$ & $6.04 \mathrm{E}-19$ & $1.24 \mathrm{E}-23$ & $3.23 \mathrm{E}-23$ & $3.00 \mathrm{E}-15$ & \\
\hline Th-232 & $7.20 \mathrm{E}-16$ & $2.04 \mathrm{E}-15$ & $5.42 \mathrm{E}-20$ & $5.42 \mathrm{E}-22$ & $4.18 \mathrm{E}-25$ & $8.57 \mathrm{E}-30$ & $2.23 \mathrm{E}-29$ & $2.08 \mathrm{E}-21$ & \\
\hline $\mathrm{Pa}-231$ & $9.08 \mathrm{E}-11$ & $2.58 \mathrm{E}-10$ & $6.84 \mathrm{E}-15$ & $6.84 \mathrm{E}-17$ & $5.27 \mathrm{E}-20$ & $1.08 \mathrm{E}-24$ & $2.82 \mathrm{E}-24$ & $2.62 \mathrm{E}-16$ & \\
\hline $\mathrm{Pa}-233$ & $2.98 \mathrm{E}-06$ & $8.46 \mathrm{E}-06$ & $2.24 \mathrm{E}-10$ & $2.24 \mathrm{E}-12$ & $1.73 E-15$ & $3.55 \mathrm{E}-20$ & $9.24 \mathrm{E}-20$ & $8.59 \mathrm{E}-12$ & \\
\hline $\mathrm{U}-232$ & $2.45 \mathrm{E}-09$ & $6.96 \mathrm{E}-09$ & $1.85 \mathrm{E}-13$ & $1.85 \mathrm{E}-15$ & $1.42 \mathrm{E}-18$ & $2.92 \mathrm{E}-23$ & $7.60 \mathrm{E}-23$ & $7.07 \mathrm{E}-15$ & \\
\hline U-233 & $8.87 \mathrm{E}-11$ & $2.52 \mathrm{E}-10$ & $6.68 \mathrm{E}-15$ & $6.68 \mathrm{E}-17$ & $5.15 \mathrm{E}-20$ & $1.06 \mathrm{E}-24$ & $2.75 \mathrm{E}-24$ & $2.56 \mathrm{E}-16$ & \\
\hline U-234 & $1.09 \mathrm{E}-06$ & $3.10 \mathrm{E}-06$ & $8.24 \mathrm{E}-11$ & $8.24 \mathrm{E}-13$ & $6.34 \mathrm{E}-16$ & $1.30 \mathrm{E}-20$ & $3.39 \mathrm{E}-20$ & $3.15 \mathrm{E}-12$ & \\
\hline $\mathrm{U}-235$ & $8.04 \mathrm{E}-08$ & $2.28 \mathrm{E}-07$ & $6.05 \mathrm{E}-12$ & $6.05 \mathrm{E}-14$ & $4.66 \mathrm{E}-17$ & $9.57 \mathrm{E}-22$ & $2.49 \mathrm{E}-21$ & $2.32 \mathrm{E}-13$ & \\
\hline U-236 & $4.72 \mathrm{E}-08$ & $1.34 \mathrm{E}-07$ & $3.56 \mathrm{E}-12$ & $3.56 \mathrm{E}-14$ & $2.74 \mathrm{E}-17$ & $5.62 \mathrm{E}-22$ & $1.46 \mathrm{E}-21$ & $1.36 \mathrm{E}-13$ & \\
\hline U-237 & $6.54 \mathrm{E}-09$ & $1.86 \mathrm{E}-08$ & $4.93 \mathrm{E}-13$ & $4.93 \mathrm{E}-15$ & $3.79 \mathrm{E}-18$ & $7.79 \mathrm{E}-23$ & $2.03 \mathrm{E}-22$ & $1.89 \mathrm{E}-14$ & \\
\hline U-238 & $1.43 \mathrm{E}-08$ & $4.06 \mathrm{E}-08$ & $1.08 \mathrm{E}-12$ & $1.08 \mathrm{E}-14$ & $8.30 \mathrm{E}-18$ & $1.70 \mathrm{E}-22$ & $4.44 \mathrm{E}-22$ & $4.13 \mathrm{E}-14$ & \\
\hline Np-236 & $3.13 \mathrm{E}-12$ & $8.90 \mathrm{E}-12$ & $2.36 \mathrm{E}-16$ & $2.36 \mathrm{E}-18$ & $1.82 \mathrm{E}-21$ & $3.73 \mathrm{E}-26$ & $9.72 \mathrm{E}-26$ & $9.04 \mathrm{E}-18$ & \\
\hline Np-237 & $4.26 \mathrm{E}-07$ & $1.21 \mathrm{E}-06$ & $3.21 \mathrm{E}-11$ & $3.21 \mathrm{E}-13$ & $2.47 \mathrm{E}-16$ & $5.08 \mathrm{E}-21$ & $1.32 \mathrm{E}-20$ & $1.23 \mathrm{E}-12$ & \\
\hline Pu-236 & $3.58 \mathrm{E}-09$ & $1.02 \mathrm{E}-08$ & $2.70 \mathrm{E}-13$ & $2.70 \mathrm{E}-15$ & $2.08 \mathrm{E}-18$ & $4.26 \mathrm{E}-23$ & $1.11 \mathrm{E}-22$ & $1.03 E-14$ & \\
\hline $\mathrm{Pu}-238$ & $8.19 \mathrm{E}-04$ & $2.33 \mathrm{E}-03$ & $6.17 \mathrm{E}-08$ & $6.17 \mathrm{E}-10$ & $4.75 \mathrm{E}-13$ & $9.75 \mathrm{E}-18$ & $2.54 \mathrm{E}-17$ & $2.36 \mathrm{E}-09$ & \\
\hline Pu-239 & $9.16 \mathrm{E}-05$ & $2.60 \mathrm{E}-04$ & $6.90 \mathrm{E}-09$ & $6.90 \mathrm{E}-11$ & $5.31 \mathrm{E}-14$ & $1.09 \mathrm{E}-18$ & $2.84 \mathrm{E}-18$ & $2.64 \mathrm{E}-10$ & \\
\hline Pu-240 & $1.33 \mathrm{E}-05$ & $3.79 \mathrm{E}-05$ & $1.00 \mathrm{E}-09$ & $1.00 \mathrm{E}-11$ & $7.73 \mathrm{E}-15$ & $1.59 \mathrm{E}-19$ & $4.13 \mathrm{E}-19$ & $3.84 \mathrm{E}-11$ & \\
\hline Pu-241 & $5.96 \mathrm{E}-04$ & $1.69 \mathrm{E}-03$ & $4.49 \mathrm{E}-08$ & $4.49 \mathrm{E}-10$ & $3.46 \mathrm{E}-13$ & $7.09 \mathrm{E}-18$ & $1.85 \mathrm{E}-17$ & $1.72 \mathrm{E}-09$ & \\
\hline Pu-242 & $1.04 \mathrm{E}-08$ & $2.97 \mathrm{E}-08$ & $7.87 \mathrm{E}-13$ & $7.87 \mathrm{E}-15$ & $6.06 \mathrm{E}-18$ & $1.24 \mathrm{E}-22$ & $3.24 \mathrm{E}-22$ & $3.01 \mathrm{E}-14$ & \\
\hline Pu-244 & $7.97 \mathrm{E}-16$ & $2.26 \mathrm{E}-15$ & $6.00 \mathrm{E}-20$ & $6.00 \mathrm{E}-22$ & $4.62 \mathrm{E}-25$ & $9.49 \mathrm{E}-30$ & $2.47 \mathrm{E}-29$ & $2.30 \mathrm{E}-21$ & \\
\hline Am-241 & $5.74 \mathrm{E}-05$ & $1.63 \mathrm{E}-04$ & $4.33 \mathrm{E}-09$ & $4.33 \mathrm{E}-11$ & $3.33 \mathrm{E}-14$ & $6.84 \mathrm{E}-19$ & $1.78 \mathrm{E}-18$ & $1.66 \mathrm{E}-10$ & \\
\hline Am-242m & $1.59 \mathrm{E}-08$ & $4.50 \mathrm{E}-08$ & $1.19 \mathrm{E}-12$ & $1.19 \mathrm{E}-14$ & $9.20 \mathrm{E}-18$ & $1.89 \mathrm{E}-22$ & $4.92 \mathrm{E}-22$ & $4.57 \mathrm{E}-14$ & \\
\hline Am-243 & $2.59 \mathrm{E}-08$ & $7.35 \mathrm{E}-08$ & $1.95 \mathrm{E}-12$ & $1.95 \mathrm{E}-14$ & $1.50 \mathrm{E}-17$ & $3.08 \mathrm{E}-22$ & $8.02 \mathrm{E}-22$ & $7.46 \mathrm{E}-14$ & \\
\hline $\mathrm{Cm}-242$ & $3.44 \mathrm{E}-08$ & $9.75 \mathrm{E}-08$ & $2.59 \mathrm{E}-12$ & $2.59 \mathrm{E}-14$ & $1.99 \mathrm{E}-17$ & $4.09 \mathrm{E}-22$ & $1.06 \mathrm{E}-21$ & $9.91 \mathrm{E}-14$ & \\
\hline Cm-243 & $3.60 \mathrm{E}-08$ & $1.02 \mathrm{E}-07$ & $2.71 \mathrm{E}-12$ & $2.71 \mathrm{E}-14$ & $2.09 \mathrm{E}-17$ & $4.29 \mathrm{E}-22$ & $1.12 \mathrm{E}-21$ & $1.04 \mathrm{E}-13$ & \\
\hline $\mathrm{Cm}-244$ & $1.26 \mathrm{E}-06$ & $3.58 \mathrm{E}-06$ & $9.49 \mathrm{E}-11$ & $9.49 \mathrm{E}-13$ & $7.31 \mathrm{E}-16$ & $1.50 \mathrm{E}-20$ & $3.90 \mathrm{E}-20$ & $3.63 \mathrm{E}-12$ & \\
\hline Cm-245 & $3.79 \mathrm{E}-10$ & $1.08 \mathrm{E}-09$ & $2.86 \mathrm{E}-14$ & $2.86 \mathrm{E}-16$ & $2.20 \mathrm{E}-19$ & $4.51 \mathrm{E}-24$ & $1.18 \mathrm{E}-23$ & $1.09 \mathrm{E}-15$ & \\
\hline \begin{tabular}{|l|}
$\mathrm{Cm}-246$ \\
\end{tabular} & $2.49 \mathrm{E}-11$ & $7.07 \mathrm{E}-11$ & $1.88 \mathrm{E}-15$ & $1.88 \mathrm{E}-17$ & $1.44 \mathrm{E}-20$ & $2.96 \mathrm{E}-25$ & $7.72 \mathrm{E}-25$ & $7.18 \mathrm{E}-17$ & \\
\hline $\mathrm{H}-3$ & $116 E-05$ & $6.36 \mathrm{E}-06$ & $9.17 \mathrm{E}-06$ & $917 E-06$ & $798 \mathrm{E}-06$ & $522 \mathrm{E}-06$ & $3,46 \mathrm{E}-07$ & $874 \mathrm{E}-06$ & \\
\hline $\mathrm{Be}-10$ & $3.05 \mathrm{E}-12$ & $8.66 \mathrm{E}-12$ & $2.30 \mathrm{E}-16$ & $2.30 \mathrm{E}-18$ & $1.77 \mathrm{E}-21$ & $3.63 \mathrm{E}-26$ & $9.46 \mathrm{E}-26$ & $8.80 \mathrm{E}-18$ & \\
\hline C-14 & $1.57 \mathrm{E}-10$ & $4.45 \mathrm{E}-10$ & $1.18 \mathrm{E}-14$ & $1.18 \mathrm{E}-16$ & $9.10 \mathrm{E}-20$ & $1.87 \mathrm{E}-24$ & $4.86 \mathrm{E}-24$ & $4.52 \mathrm{E}-16$ & \\
\hline Co-60 & $8.84 \mathrm{E}-06$ & $2.51 \mathrm{E}-05$ & $6.66 \mathrm{E}-10$ & $6.66 \mathrm{E}-12$ & $5.13 \mathrm{E}-15$ & $1.05 \mathrm{E}-19$ & $2.74 \mathrm{E}-19$ & $2.55 \mathrm{E}-11$ & \\
\hline Ni-59 & $1.10 \mathrm{E}-06$ & $3.13 \mathrm{E}-06$ & $8.30 \mathrm{E}-11$ & $8.30 \mathrm{E}-13$ & $6.39 \mathrm{E}-16$ & $1.31 \mathrm{E}-20$ & $3.41 \mathrm{E}-20$ & $3.18 \mathrm{E}-12$ & \\
\hline Ni-63 & $4.16 \mathrm{E}-05$ & $1.18 \mathrm{E}-04$ & 3.13E-09 & $3.14 \mathrm{E}-11$ & $2.41 \mathrm{E}-14$ & $4.95 \mathrm{E}-19$ & $1.29 \mathrm{E}-18$ & $1.20 \mathrm{E}-10$ & \\
\hline Se-79 & $5.70 \mathrm{E}-07$ & $1.62 \mathrm{E}-06$ & $4.29 \mathrm{E}-11$ & $4.29 \mathrm{E}-13$ & 3.30E-16 & $6.78 \mathrm{E}-21$ & $1.77 \mathrm{E}-20$ & $1.64 \mathrm{E}-12$ & \\
\hline Rb-87 & $2.98 \mathrm{E}-11$ & $8.46 \mathrm{E}-11$ & $2.24 \mathrm{E}-15$ & $2.24 \mathrm{E}-17$ & $1.73 \mathrm{E}-20$ & $3.55 \mathrm{E}-25$ & $9.24 \mathrm{E}-25$ & $8.59 \mathrm{E}-17$ & \\
\hline Sr-90 & $3.91 \mathrm{E}-02$ & $1.11 \mathrm{E}-01$ & $2.95 \mathrm{E}-06$ & $2.95 \mathrm{E}-08$ & $2.27 \mathrm{E}-11$ & $4.66 \mathrm{E}-16$ & $1.21 \mathrm{E}-15$ & $1.13 \mathrm{E}-07$ & \\
\hline$Y-90$ & $3.91 \mathrm{E}-02$ & $1.11 \mathrm{E}-01$ & $2.95 \mathrm{E}-06$ & $2.95 \mathrm{E}-08$ & $2.27 \mathrm{E}-11$ & $4.66 \mathrm{E}-16$ & $1.21 \mathrm{E}-15$ & $1.13 \mathrm{E}-07$ & \\
\hline Zr-93 & $2.25 \mathrm{E}-06$ & $6.39 \mathrm{E}-06$ & $1.70 \mathrm{E}-10$ & $1.70 \mathrm{E}-12$ & $1.31 \mathrm{E}-15$ & $2.68 \mathrm{E}-20$ & $6.98 \mathrm{E}-20$ & $6.50 \mathrm{E}-12$ & \\
\hline Nb-93m & $1.74 \mathrm{E}-06$ & $4.93 \mathrm{E}-06$ & $1.31 \mathrm{E}-10$ & $1.31 \mathrm{E}-12$ & $1.01 \mathrm{E}-15$ & $2.07 \mathrm{E}-20$ & $5.38 \mathrm{E}-20$ & $5.00 \mathrm{E}-12$ & \\
\hline Nb-94 & $1.48 \mathrm{E}-06$ & $4.21 \mathrm{E}-06$ & $1.12 \mathrm{E}-10$ & $1.12 \mathrm{E}-12$ & $8.60 \mathrm{E}-16$ & $1.76 \mathrm{E}-20$ & $4.60 \mathrm{E}-20$ & $4.27 \mathrm{E}-12$ & \\
\hline Tc-98 & $2.62 \mathrm{E}-12$ & $7.44 \mathrm{E}-12$ & $1.98 \mathrm{E}-16$ & $1.98 \mathrm{E}-18$ & $1.52 \mathrm{E}-21$ & $3.12 \mathrm{E}-26$ & $8.13 \mathrm{E}-26$ & $7.56 \mathrm{E}-18$ & \\
\hline Tc-99 & $2.06 \mathrm{E}-05$ & $5.85 \mathrm{E}-05$ & $1.55 \mathrm{E}-09$ & $1.55 \mathrm{E}-11$ & $1.20 \mathrm{E}-14$ & $2.45 \mathrm{E}-19$ & $6.39 \mathrm{E}-19$ & $5.94 \mathrm{E}-11$ & \\
\hline Ru-106 & $1.22 \mathrm{E}-06$ & $3.45 \mathrm{E}-06$ & $9.17 \mathrm{E}-11$ & $9.17 \mathrm{E}-13$ & $7.06 \mathrm{E}-16$ & 1.45E-20 & $3.77 \mathrm{E}-20$ & $3.51 \mathrm{E}-12$ & \\
\hline Rh-102 & $8.76 \mathrm{E}-10$ & $2.49 \mathrm{E}-09$ & $6.60 \mathrm{E}-14$ & $6.60 \mathrm{E}-16$ & $5.08 \mathrm{E}-19$ & $1.04 \mathrm{E}-23$ & $2.72 \mathrm{E}-23$ & $2.53 \mathrm{E}-15$ & \\
\hline Rh-106 & $1.22 \mathrm{E}-06$ & $3.45 \mathrm{E}-06$ & $9.17 \mathrm{E}-11$ & $9.17 \mathrm{E}-13$ & $7.06 \mathrm{E}-16$ & $1.45 \mathrm{E}-20$ & $3.77 \mathrm{E}-20$ & $3.51 \mathrm{E}-12$ & \\
\hline Pd-107 & $1.68 \mathrm{E}-08$ & 4.77E-08 & $1.27 \mathrm{E}-12$ & $1.27 \mathrm{E}-14$ & $9.74 \mathrm{E}-18$ & $2.00 \mathrm{E}-22$ & $5.21 \mathrm{E}-22$ & $4.84 \mathrm{E}-14$ & \\
\hline \begin{tabular}{|l|}
$C d-113 m$ \\
\end{tabular} & $3.38 \mathrm{E}-06$ & $9.59 \mathrm{E}-06$ & $2.54 \mathrm{E}-10$ & $2.54 \mathrm{E}-12$ & $1.96 \mathrm{E}-15$ & $4.02 \mathrm{E}-20$ & 1.05E-19 & $9.74 \mathrm{E}-12$ & \\
\hline Sn-121m & $6.80 \mathrm{E}-08$ & $1.93 \mathrm{E}-07$ & $5.12 \mathrm{E}-12$ & $5.12 \mathrm{E}-14$ & $3.94 \mathrm{E}-17$ & $8.09 \mathrm{E}-22$ & $2.11 \mathrm{E}-21$ & $1.96 \mathrm{E}-13$ & \\
\hline Sn-126 & $5.36 \mathrm{E}-07$ & $1.52 \mathrm{E}-06$ & $4.04 \mathrm{E}-11$ & $4.04 \mathrm{E}-13$ & $3.11 \mathrm{E}-16$ & $6.38 \mathrm{E}-21$ & $1.66 \mathrm{E}-20$ & $1.55 \mathrm{E}-12$ & \\
\hline Sb-125 & $3.68 \mathrm{E}-04$ & $1.05 \mathrm{E}-03$ & $2.78 \mathrm{E}-08$ & $2.78 \mathrm{E}-10$ & $2.14 \mathrm{E}-13$ & $4.39 \mathrm{E}-18$ & $1.14 \mathrm{E}-17$ & $1.06 \mathrm{E}-09$ & \\
\hline Sb-126 & $5.85 \mathrm{E}-08$ & $1.66 \mathrm{E}-07$ & $4.40 \mathrm{E}-12$ & $4.40 \mathrm{E}-14$ & $3.39 \mathrm{E}-17$ & $6.96 \mathrm{E}-22$ & $1.81 \mathrm{E}-21$ & $1.69 \mathrm{E}-13$ & \\
\hline
\end{tabular}


Table C-10. Alkaline scrub balance, Tank WM-188 waste. (Continued).

\begin{tabular}{|c|c|c|c|c|c|c|c|c|c|}
\hline Stream \# & 101 & 102 & 103 & 104 & 105 & 106 & 107 & 108 & 201 \\
\hline Radiological Composition & $\mathrm{Ci} / \mathrm{L}$ & $\mathrm{Ci} / \mathrm{L}$ & $\mathrm{Ci} / \mathrm{wscm}$ & $\mathrm{Ci} / \mathrm{wscm}$ & $\mathrm{Ci} / \mathrm{wscm}$ & $\mathrm{Ci} / \mathrm{wscm}$ & $\mathrm{Ci} / \mathrm{wscm}$ & $\mathrm{Ci} / \mathrm{L}$ & \\
\hline Te-125m & $3.20 \mathrm{E}-06$ & 9.09E-06 & $2.41 \mathrm{E}-10$ & $2.41 \mathrm{E}-12$ & $1.86 \mathrm{E}-15$ & $3.81 \mathrm{E}-20$ & $9.93 \mathrm{E}-20$ & $9.23 \mathrm{E}-12$ & \\
\hline $\mid-129$ & $6.96 \mathrm{E}-08$ & 1.97E-07 & $2.02 \mathrm{E}-08$ & $2.02 \mathrm{E}-08$ & $1.96 \mathrm{E}-11$ & $7.56 \mathrm{E}-13$ & $5.96 \mathrm{E}-14$ & $7.68 \mathrm{E}-08$ & \\
\hline Cs-134 & $3.67 \mathrm{E}-05$ & $1.04 \mathrm{E}-04$ & $2.77 \mathrm{E}-09$ & $2.77 \mathrm{E}-11$ & $2.13 \mathrm{E}-14$ & $4.37 \mathrm{E}-19$ & $1.14 \mathrm{E}-18$ & $1.06 \mathrm{E}-10$ & \\
\hline Cs-135 & $1.10 \mathrm{E}-06$ & $3.11 \mathrm{E}-06$ & $8.25 \mathrm{E}-11$ & $8.25 \mathrm{E}-13$ & $6.35 \mathrm{E}-16$ & $1.30 \mathrm{E}-20$ & $3.40 \mathrm{E}-20$ & $3.16 \mathrm{E}-12$ & \\
\hline Cs-137 & $2.26 \mathrm{E}-02$ & $6.42 \mathrm{E}-02$ & $1.70 \mathrm{E}-06$ & $1.70 \mathrm{E}-08$ & $1.31 \mathrm{E}-11$ & $2.69 \mathrm{E}-16$ & $7.01 \mathrm{E}-16$ & $6.52 \mathrm{E}-08$ & \\
\hline Ba-137m & $2.14 \mathrm{E}-02$ & $6.07 \mathrm{E}-02$ & $1.61 \mathrm{E}-06$ & $1.61 \mathrm{E}-08$ & $1.24 \mathrm{E}-11$ & $2.55 \mathrm{E}-16$ & $6.63 \mathrm{E}-16$ & $6.17 \mathrm{E}-08$ & \\
\hline La-138 & $1.94 \mathrm{E}-16$ & $5.51 \mathrm{E}-16$ & 1.46E-20 & $1.46 \mathrm{E}-22$ & $1.13 \mathrm{E}-25$ & $2.31 \mathrm{E}-30$ & $6.02 \mathrm{E}-30$ & $5.60 \mathrm{E}-22$ & \\
\hline Ce-142 & $3.04 \mathrm{E}-11$ & $8.63 \mathrm{E}-11$ & $2.29 \mathrm{E}-15$ & $2.29 \mathrm{E}-17$ & $1.76 \mathrm{E}-20$ & $3.62 \mathrm{E}-25$ & $9.42 \mathrm{E}-25$ & $8.76 \mathrm{E}-17$ & \\
\hline Ce-144 & $8.21 \mathrm{E}-07$ & 2.33E-06 & $6.19 \mathrm{E}-11$ & $6.19 \mathrm{E}-13$ & $4.76 \mathrm{E}-16$ & $9.78 \mathrm{E}-21$ & $2.55 \mathrm{E}-20$ & $2.37 \mathrm{E}-12$ & \\
\hline Pr-144 & $8.21 \mathrm{E}-07$ & $2.33 \mathrm{E}-06$ & $6.19 \mathrm{E}-11$ & $6.19 \mathrm{E}-13$ & $4.76 \mathrm{E}-16$ & $9.78 \mathrm{E}-21$ & $2.55 \mathrm{E}-20$ & $2.37 \mathrm{E}-12$ & \\
\hline $\mathrm{Nd}-144$ & $1.63 \mathrm{E}-15$ & $4.64 \mathrm{E}-15$ & $1.23 \mathrm{E}-19$ & $1.23 \mathrm{E}-21$ & $9.48 \mathrm{E}-25$ & $1.95 \mathrm{E}-29$ & $5.07 \mathrm{E}-29$ & $4.71 \mathrm{E}-21$ & \\
\hline Pm-146 & $5.17 \mathrm{E}-08$ & 1.47E-07 & $3.90 \mathrm{E}-12$ & $3.90 \mathrm{E}-14$ & $3.00 \mathrm{E}-17$ & $6.16 \mathrm{E}-22$ & $1.60 \mathrm{E}-21$ & 1.49E-13 & \\
\hline Pm-147 & $2.22 \mathrm{E}-04$ & $6.30 \mathrm{E}-04$ & $1.67 \mathrm{E}-08$ & $1.67 \mathrm{E}-10$ & $1.29 \mathrm{E}-13$ & $2.64 \mathrm{E}-18$ & $6.88 \mathrm{E}-18$ & $6.40 \mathrm{E}-10$ & \\
\hline Sm-146 & $2.80 \mathrm{E}-13$ & $7.96 \mathrm{E}-13$ & $2.11 \mathrm{E}-17$ & $2.11 \mathrm{E}-19$ & $1.63 \mathrm{E}-22$ & $3.34 \mathrm{E}-27$ & $8.69 \mathrm{E}-27$ & $8.09 \mathrm{E}-19$ & \\
\hline Sm-147 & $7.49 \mathrm{E}-12$ & $2.13 \mathrm{E}-11$ & $5.64 \mathrm{E}-16$ & $5.64 \mathrm{E}-18$ & $4.34 \mathrm{E}-21$ & $8.91 \mathrm{E}-26$ & $2.32 \mathrm{E}-25$ & $2.16 \mathrm{E}-17$ & \\
\hline Sm-151 & $4.39 \mathrm{E}-04$ & $1.25 \mathrm{E}-03$ & $3.30 \mathrm{E}-08$ & $3.30 \mathrm{E}-10$ & $2.54 \mathrm{E}-13$ & $5.22 \mathrm{E}-18$ & $1.36 \mathrm{E}-17$ & $1.26 \mathrm{E}-09$ & \\
\hline Eu-152 & $2.90 \mathrm{E}-06$ & $8.23 \mathrm{E}-06$ & $2.18 \mathrm{E}-10$ & $2.18 \mathrm{E}-12$ & $1.68 \mathrm{E}-15$ & $3.45 \mathrm{E}-20$ & $8.98 \mathrm{E}-20$ & $8.36 \mathrm{E}-12$ & \\
\hline Eu-154 & 4.93E-05 & $1.40 \mathrm{E}-04$ & $3.72 \mathrm{E}-09$ & $3.72 \mathrm{E}-11$ & $2.86 \mathrm{E}-14$ & $5.87 \mathrm{E}-19$ & $1.53 \mathrm{E}-18$ & 1.42E-10 & \\
\hline Eu-155 & $4.28 \mathrm{E}-05$ & $1.21 \mathrm{E}-04$ & $3.22 \mathrm{E}-09$ & $3.22 \mathrm{E}-11$ & $2.48 \mathrm{E}-14$ & $5.09 \mathrm{E}-19$ & $1.33 \mathrm{E}-18$ & $1.23 \mathrm{E}-10$ & \\
\hline Ho- $166 \mathrm{~m}$ & $4.68 \mathrm{E}-11$ & 1.33E-10 & $3.53 \mathrm{E}-15$ & 3.53E-17 & 2.71E-20 & $5.57 \mathrm{E}-25$ & $1.45 \mathrm{E}-24$ & $1.35 \mathrm{E}-16$ & \\
\hline
\end{tabular}


Table C-11. Alkaline scrub balance, Tank WM-189 waste.

\begin{tabular}{|c|c|c|c|c|c|c|c|c|c|}
\hline WM-189 PFD Sheet \# & PFD-2A & PFD-2A & PFD-2A & PFD-2A & PFD-2A & PFD-2A & PFD-2A & PFD-2A & PFD-2A \\
\hline Stream \# & 101 & 102 & 103 & 104 & 105 & 106 & 107 & 108 & 201 \\
\hline Stream Name & $\begin{array}{l}\text { SBW } \\
\text { Feed }\end{array}$ & $\begin{array}{c}\text { Evaporator } \\
\text { Bottoms }\end{array}$ & $\begin{array}{c}\text { Evaporator } \\
\text { Overheads } \\
\text { Vapor }\end{array}$ & $\begin{array}{c}\text { Mist } \\
\text { Eliminator } \\
\text { Vapor }\end{array}$ & $\begin{array}{l}\text { Alkaline } \\
\text { Scrubber } \\
\text { Vapor }\end{array}$ & $\begin{array}{c}\text { POG + VOG } \\
\text { Offgas } \\
\text { Vapor }\end{array}$ & $\begin{array}{l}\text { VOG } \\
\text { Offgas } \\
\text { Vapor }\end{array}$ & $\begin{array}{l}\text { Scrubber } \\
\text { Recycle to } \\
\text { Feed }\end{array}$ & $\begin{array}{c}20 \mathrm{wt} \% \\
\mathrm{MgO} \\
\text { Additive }\end{array}$ \\
\hline Rate or Volume Flow (actual) & 85.8 & 35.3 & 38,498 & 39,007 & 33,434 & 38,034 & 11,770 & 29.2 & 159.4 \\
\hline Volume Flow (standard, wet) & & & 14,821 & 14,821 & 12,911 & 20,325 & 7,401 & & \\
\hline Volume Flow (standard, dry)* & & & 607.8 & 607.7 & 0.68 & 7,124 & 7,136 & & \\
\hline Rate Units & $\mathrm{gal} / \mathrm{hr}$ & $\mathrm{gal} / \mathrm{hr}$ & $\mathrm{ft} 3 / \mathrm{hr}$ & $\mathrm{ft} 3 / \mathrm{hr}$ & $\mathrm{ft} 3 / \mathrm{hr}$ & $\mathrm{ft} 3 / \mathrm{hr}$ & $\mathrm{ft} 3 / \mathrm{hr}$ & $\mathrm{gal} / \mathrm{hr}$ & $\mathrm{lb} / \mathrm{hr}$ \\
\hline Temperature, ${ }^{\circ} \mathrm{C}$ & 25.0 & 120 & 120 & 114.3 & 89.4 & 175 & 108 & 89.5 & 25.0 \\
\hline Temperature, ${ }^{\circ} \mathrm{F}$ & 77.0 & 247 & 247 & 238 & 193 & 347 & 226 & 193 & 77.0 \\
\hline Pressure, psia & 12.3 & 7.6 & 7.6 & 7.4 & 7.0 & 12.0 & 12.0 & 30.0 & 12.3 \\
\hline Specific Gravity & 1.27 & 1.75 & 0.0003 & 0.0003 & 0.00029 & 0.00049 & 0.00074 & 1.31 & 1.20 \\
\hline Chemical Composition & Mol/Liter & Mol/Liter & $\mathrm{lb} / \mathrm{wscf}$ & $\mathrm{lb} / \mathrm{wscf}$ & $\mathrm{Ib} / \mathrm{wscf}$ & $\mathrm{lb} / \mathrm{wscf}$ & $\mathrm{lb} / \mathrm{wscf}$ & Mol/Liter & Wt. \% \\
\hline $\mathrm{H}+$ & $2.43 \mathrm{E}+00$ & $5.28 \mathrm{E}-01$ & $1.08 \mathrm{E}-04$ & $1.08 \mathrm{E}-04$ & $1.66 \mathrm{E}-09$ & 1.32E-09 & $7.15 \mathrm{E}-10$ & $1.06 \mathrm{E}-04$ & $2.64 \mathrm{E}-12$ \\
\hline $\mathrm{OH}-$ & & & 1.26E-14 & & $1.60 \mathrm{E}-11$ & 1.76E-13 & & 1.14E-04 & $1.69 \mathrm{E}+01$ \\
\hline$A \mathrm{Al}+3$ & $6.08 \mathrm{E}-01$ & $1.48 \mathrm{E}+00$ & $7.92 \mathrm{E}-08$ & $2.77 \mathrm{E}-11$ & $2.12 \mathrm{E}-14$ & & & $6.24 \mathrm{E}-08$ & \\
\hline $\mathrm{Sb}+5$ & $1.81 \mathrm{E}-05$ & $4.40 \mathrm{E}-05$ & $1.06 \mathrm{E}-11$ & $1.06 \mathrm{E}-13$ & $8.17 \mathrm{E}-17$ & & & $5.34 \mathrm{E}-11$ & \\
\hline $\mathrm{As}+3$ & 9.02E-05 & $2.20 \mathrm{E}-04$ & $3.27 \mathrm{E}-11$ & $3.27 \mathrm{E}-13$ & $2.50 \mathrm{E}-16$ & & & $2.65 \mathrm{E}-10$ & \\
\hline $\mathrm{Ba}+2$ & $6.85 \mathrm{E}-05$ & 1.67E-04 & $4.55 \mathrm{E}-11$ & $1.30 \mathrm{E}-13$ & $9.92 \mathrm{E}-17$ & & & $5.75 \mathrm{E}-11$ & \\
\hline $\mathrm{Be}+2$ & 2.23E-05 & $5.42 \mathrm{E}-05$ & $9.70 \mathrm{E}-13$ & $2.56 \mathrm{E}-15$ & 1.96E-18 & & & $1.73 \mathrm{E}-11$ & \\
\hline$B+3$ & 1.83E-02 & 4.45E-02 & $9.55 \mathrm{E}-10$ & 3.31E-13 & $2.53 \mathrm{E}-16$ & & & 1.86E-09 & \\
\hline$C d+2$ & $3.27 \mathrm{E}-03$ & 7.95E-03 & 1.77E-09 & $3.74 \mathrm{E}-13$ & $2.86 \mathrm{E}-16$ & & & $2.03 \mathrm{E}-10$ & \\
\hline $\mathrm{Ca}+2$ & $6.13 \mathrm{E}-02$ & 1.49E-01 & $1.19 \mathrm{E}-08$ & $2.64 \mathrm{E}-12$ & $2.02 \mathrm{E}-15$ & & & $4.01 \mathrm{E}-09$ & \\
\hline $\mathrm{Cr}+3$ & $5.13 E-03$ & 1.25E-02 & 1.29E-09 & $1.11 \mathrm{E}-12$ & $8.51 \mathrm{E}-16$ & & & 1.30E-09 & \\
\hline $\mathrm{Co}+2$ & $4.28 \mathrm{E}-05$ & 1.04E-04 & $1.22 \mathrm{E}-11$ & $1.16 \mathrm{E}-14$ & $8.91 \mathrm{E}-18$ & & & $1.20 \mathrm{E}-11$ & \\
\hline $\mathrm{Cs}^{+}$ & $6.12 \mathrm{E}-05$ & 1.49E-04 & $3.60 \mathrm{E}-11$ & $2.59 \mathrm{E}-13$ & $1.98 \mathrm{E}-16$ & & & $1.19 \mathrm{E}-10$ & \\
\hline $\mathrm{Cu}+2$ & $8.80 \mathrm{E}-04$ & $2.14 \mathrm{E}-03$ & $2.70 \mathrm{E}-10$ & $3.34 \mathrm{E}-13$ & $2.56 \mathrm{E}-16$ & & & $3.20 \mathrm{E}-10$ & \\
\hline $\mathrm{Fe}+3$ & 2.97E-02 & $7.22 \mathrm{E}-02$ & 8.01E-09 & $2.04 \mathrm{E}-11$ & $1.56 \mathrm{E}-14$ & & & $2.22 \mathrm{E}-08$ & \\
\hline $\mathrm{Pb}+2$ & 9.97E-04 & $2.43 \mathrm{E}-03$ & $9.98 \mathrm{E}-10$ & $3.80 \mathrm{E}-13$ & $2.91 \mathrm{E}-16$ & & & 1.12E-10 & \\
\hline $\mathrm{Hg}+2$ & $5.44 \mathrm{E}-03$ & $1.32 \mathrm{E}-02$ & $1.20 \mathrm{E}-06$ & $1.20 \mathrm{E}-06$ & $9.34 \mathrm{E}-09$ & $3.95 \mathrm{E}-11$ & $9.23 \mathrm{E}-11$ & $3.65 \mathrm{E}-04$ & \\
\hline $\mathrm{Mn}+4$ & 1.65E-02 & $4.00 \mathrm{E}-02$ & 4.37E-09 & $4.37 \mathrm{E}-11$ & $3.34 \mathrm{E}-14$ & & & 4.84E-08 & \\
\hline $\mathrm{Ni}+2$ & $2.09 \mathrm{E}-03$ & $5.08 \mathrm{E}-03$ & $5.92 \mathrm{E}-10$ & $4.76 \mathrm{E}-13$ & $3.64 \mathrm{E}-16$ & & & $4.93 \mathrm{E}-10$ & \\
\hline $\mathrm{K}+$ & $2.02 \mathrm{E}-01$ & 4.91E-01 & $3.81 \mathrm{E}-08$ & $2.53 \mathrm{E}-11$ & $1.94 \mathrm{E}-14$ & & & $3.94 \mathrm{E}-08$ & \\
\hline $\mathrm{Se}+4$ & $2.59 \mathrm{E}-04$ & $6.29 \mathrm{E}-04$ & $9.87 \mathrm{E}-11$ & $9.87 \mathrm{E}-13$ & 7.55E-16 & & & $7.61 \mathrm{E}-10$ & \\
\hline $\mathrm{Ag}+$ & 4.05E-04 & $9.85 \mathrm{E}-04$ & $2.11 \mathrm{E}-10$ & $2.10 \mathrm{E}-12$ & $1.61 \mathrm{E}-15$ & & & 1.19E-09 & \\
\hline $\mathrm{Na}+$ & $1.71 \mathrm{E}+00$ & $4.16 \mathrm{E}+00$ & $1.48 \mathrm{E}-07$ & $2.13 \mathrm{E}-10$ & 1.63E-13 & & & $5.64 \mathrm{E}-07$ & \\
\hline $\mathrm{TI}+3$ & $4.76 \mathrm{E}-06$ & $1.16 \mathrm{E}-05$ & $4.70 \mathrm{E}-12$ & $4.70 \mathrm{E}-14$ & $3.60 \mathrm{E}-17$ & & & 1.40E-11 & \\
\hline$U+4$ & $5.63 \mathrm{E}-04$ & $1.37 \mathrm{E}-03$ & $6.47 \mathrm{E}-10$ & $6.47 \mathrm{E}-12$ & $4.95 \mathrm{E}-15$ & & & 1.66E-09 & \\
\hline $\mathrm{V}+3$ & $3.17 \mathrm{E}-05$ & $7.72 \mathrm{E}-05$ & $7.81 \mathrm{E}-12$ & $7.81 \mathrm{E}-14$ & $6.04 \mathrm{E}-17$ & & & $9.44 \mathrm{E}-11$ & \\
\hline$Z n+2$ & $9.43 \mathrm{E}-04$ & $2.29 \mathrm{E}-03$ & $2.98 \mathrm{E}-10$ & $2.11 \mathrm{E}-13$ & $1.62 \mathrm{E}-16$ & & & $1.97 \mathrm{E}-10$ & \\
\hline $\mathrm{Zr}+4$ & 3.89E-02 & 9.45E-02 & $1.71 \mathrm{E}-08$ & $1.71 \mathrm{E}-10$ & 1.31E-13 & & & 1.14E-07 & \\
\hline Cl- & 2.05E-02 & 4.98E-02 & 9.90E-06 & $9.90 \mathrm{E}-06$ & 1.07E-08 & 1.24E-10 & 4.32E-11 & 1.69E-02 & \\
\hline F- & $3.32 \mathrm{E}-02$ & $8.08 \mathrm{E}-02$ & $9.10 \mathrm{E}-09$ & 7.30E-09 & 1.07E-09 & $6.78 \mathrm{E}-10$ & 1.03E-12 & $2.10 \mathrm{E}-05$ & \\
\hline SO4-2 & $9.08 \mathrm{E}-02$ & $2.21 \mathrm{E}-01$ & $4.21 \mathrm{E}-08$ & $4.21 \mathrm{E}-10$ & $3.22 \mathrm{E}-13$ & & & $2.67 \mathrm{E}-07$ & \\
\hline NO3- & $6.18 \mathrm{E}+00$ & $1.48 \mathrm{E}+01$ & $6.60 \mathrm{E}-03$ & $6.60 \mathrm{E}-03$ & $5.15 \mathrm{E}-06$ & $7.86 \mathrm{E}-08$ & 4.40E-08 & $6.48 \mathrm{E}+00$ & \\
\hline PO4-3 & 1.07E-01 & $2.61 \mathrm{E}-01$ & 4.93E-08 & 4.93E-10 & $3.77 \mathrm{E}-13$ & & & $3.16 \mathrm{E}-07$ & \\
\hline$A m+4$ & $6.53 \mathrm{E}-08$ & $1.59 \mathrm{E}-07$ & $7.60 \mathrm{E}-14$ & $7.60 \mathrm{E}-16$ & $5.81 \mathrm{E}-19$ & & & $1.92 \mathrm{E}-13$ & \\
\hline $\mathrm{Br}-$ & $2.71 \mathrm{E}-07$ & $6.59 \mathrm{E}-07$ & 3.33E-10 & 3.33E-10 & $3.59 \mathrm{E}-13$ & 4.16E-15 & 1.45E-15 & $2.53 \mathrm{E}-07$ & \\
\hline $\mathrm{Ce}+4$ & 3.96E-05 & 9.63E-05 & $2.68 \mathrm{E}-11$ & $2.68 \mathrm{E}-13$ & $2.05 \mathrm{E}-16$ & & & $1.16 \mathrm{E}-10$ & \\
\hline $\mathrm{Eu}+3$ & $4.51 \mathrm{E}-07$ & 1.10E-06 & $3.31 \mathrm{E}-13$ & $5.32 \mathrm{E}-18$ & 4.07E-21 & & & $2.13 \mathrm{E}-15$ & \\
\hline $\mathrm{Gd}+3$ & $1.35 \mathrm{E}-04$ & $3.29 \mathrm{E}-04$ & $1.03 \mathrm{E}-10$ & $1.47 \mathrm{E}-13$ & $1.12 \mathrm{E}-16$ & & & $5.68 \mathrm{E}-11$ & \\
\hline $\mathrm{Ge}+4$ & $7.82 \mathrm{E}-09$ & $1.90 \mathrm{E}-08$ & $2.74 \mathrm{E}-15$ & $2.74 \mathrm{E}-17$ & $2.10 \mathrm{E}-20$ & & & $2.30 \mathrm{E}-14$ & \\
\hline $\ln +3$ & $1.25 \mathrm{E}-06$ & $3.05 \mathrm{E}-06$ & $6.96 \mathrm{E}-13$ & & & & & & \\
\hline I- & $3.02 \mathrm{E}-06$ & 7.33E-06 & 4.39E-09 & 4.39E-09 & $4.73 \mathrm{E}-12$ & $5.49 \mathrm{E}-14$ & $1.92 \mathrm{E}-14$ & $2.10 \mathrm{E}-06$ & \\
\hline $\mathrm{La}+3$ & 8.17E-06 & 1.99E-05 & $5.48 \mathrm{E}-12$ & & & & & & \\
\hline $\mathrm{Li+}$ & $5.44 \mathrm{E}-04$ & 1.32E-03 & $1.59 \mathrm{E}-11$ & $8.72 \mathrm{E}-14$ & $6.67 \mathrm{E}-17$ & & & $7.65 \mathrm{E}-10$ & \\
\hline $\mathrm{Mg}+2$ & $1.86 \mathrm{E}-02$ & $2.73 \mathrm{E}+00$ & 1.32E-07 & & 9.92E-07 & $1.26 \mathrm{E}-13$ & & $3.25 \mathrm{E}+00$ & $1.21 \mathrm{E}+01$ \\
\hline $\mathrm{Mo}+6$ & $8.36 \mathrm{E}-04$ & $2.04 \mathrm{E}-03$ & $3.88 \mathrm{E}-10$ & $3.88 \mathrm{E}-12$ & $2.97 \mathrm{E}-15$ & & & $2.46 \mathrm{E}-09$ & \\
\hline
\end{tabular}


Table C-11. Alkaline scrub balance, Tank WM-189 waste. (Continued).

\begin{tabular}{|c|c|c|c|c|c|c|c|c|c|}
\hline Stream \# & 101 & 102 & 103 & 104 & 105 & 106 & 107 & 108 & 201 \\
\hline Chemical Composition & Mol/Liter & Mol/Liter & $\mathrm{lb} / \mathrm{wscf}$ & $\mathrm{lb} / \mathrm{wscf}$ & $\mathrm{lb} / \mathrm{wscf}$ & $\mathrm{lb} / \mathrm{wscf}$ & $\mathrm{lb} / \mathrm{wscf}$ & Mol/Liter & Wt. \% \\
\hline $\mathrm{Nd}+3$ & $2.64 \mathrm{E}-05$ & $6.41 \mathrm{E}-05$ & $1.84 \mathrm{E}-11$ & & & & & & \\
\hline$N p+4$ & $2.74 \mathrm{E}-06$ & $6.66 \mathrm{E}-06$ & $3.13 \mathrm{E}-12$ & $3.13 \mathrm{E}-14$ & $2.40 \mathrm{E}-17$ & & & 8.05E-12 & \\
\hline $\mathrm{Nb}+5$ & 1.14E-03 & $2.78 \mathrm{E}-03$ & $5.12 \mathrm{E}-10$ & $5.12 \mathrm{E}-12$ & $3.92 \mathrm{E}-15$ & & & 3.36E-09 & \\
\hline$P d+4$ & 1.69E-03 & $4.11 \mathrm{E}-03$ & $8.69 \mathrm{E}-10$ & $8.69 \mathrm{E}-12$ & $6.65 \mathrm{E}-15$ & & & 4.97E-09 & \\
\hline $\mathrm{Pu}+4$ & $6.64 \mathrm{E}-06$ & $1.61 \mathrm{E}-05$ & $7.66 \mathrm{E}-12$ & $7.66 \mathrm{E}-14$ & $5.86 \mathrm{E}-17$ & & & $1.95 \mathrm{E}-11$ & \\
\hline $\mathrm{Pr}+4$ & 7.43E-06 & $1.81 \mathrm{E}-05$ & $5.06 \mathrm{E}-12$ & $5.06 \mathrm{E}-14$ & $3.87 \mathrm{E}-17$ & & & $2.19 \mathrm{E}-11$ & \\
\hline $\mathrm{Rh}+4$ & $3.21 \mathrm{E}-06$ & $7.80 \mathrm{E}-06$ & $1.59 \mathrm{E}-12$ & 1.59E-14 & $1.22 \mathrm{E}-17$ & & & $9.43 \mathrm{E}-12$ & \\
\hline $\mathrm{Rb}+$ & $4.96 \mathrm{E}-06$ & $1.21 \mathrm{E}-05$ & $1.59 \mathrm{E}-12$ & $1.97 \mathrm{E}-15$ & $1.51 \mathrm{E}-18$ & & & $1.41 \mathrm{E}-12$ & \\
\hline $\mathrm{Ru}+3$ & $8.85 \mathrm{E}-04$ & $2.15 \mathrm{E}-03$ & $4.32 \mathrm{E}-10$ & $3.63 \mathrm{E}-12$ & $2.78 \mathrm{E}-15$ & & & $2.19 \mathrm{E}-09$ & \\
\hline $\mathrm{Sm}+3$ & 4.91E-06 & $1.20 \mathrm{E}-05$ & $3.57 \mathrm{E}-12$ & $1.99 \mathrm{E}-16$ & $1.52 \mathrm{E}-19$ & & & $8.06 \mathrm{E}-14$ & \\
\hline$S i+4$ & $9.84 \mathrm{E}-02$ & $2.39 \mathrm{E}-01$ & $1.34 \mathrm{E}-08$ & $1.34 \mathrm{E}-10$ & $1.02 \mathrm{E}-13$ & & & $2.90 \mathrm{E}-07$ & \\
\hline $\mathrm{Sr}+2$ & 1.19E-04 & $2.90 \mathrm{E}-04$ & $5.04 \mathrm{E}-11$ & $2.87 \mathrm{E}-14$ & $2.20 \mathrm{E}-17$ & & & $2.00 \mathrm{E}-11$ & \\
\hline $\mathrm{Tc}+7$ & $5.86 \mathrm{E}-06$ & $1.43 \mathrm{E}-05$ & $2.75 \mathrm{E}-12$ & & & & & & \\
\hline $\mathrm{Te}+4$ & $5.74 \mathrm{E}-06$ & $1.40 \mathrm{E}-05$ & $3.54 \mathrm{E}-12$ & $3.54 \mathrm{E}-14$ & $2.71 \mathrm{E}-17$ & & & 1.69E-11 & \\
\hline $\mathrm{Tb}+4$ & $1.88 \mathrm{E}-09$ & $4.58 \mathrm{E}-09$ & $1.44 \mathrm{E}-15$ & $1.44 \mathrm{E}-17$ & 1.10E-20 & & & $5.53 \mathrm{E}-15$ & \\
\hline $\mathrm{Th}+4$ & 2.85E-05 & 6.93E-05 & $3.19 \mathrm{E}-11$ & $3.19 \mathrm{E}-13$ & $2.44 \mathrm{E}-16$ & & & $8.38 \mathrm{E}-11$ & \\
\hline$S n+4$ & $1.92 \mathrm{E}-03$ & $4.68 \mathrm{E}-03$ & 1.10E-09 & 1.10E-11 & $8.43 E-15$ & & & 5.65E-09 & \\
\hline $\mathrm{Ti+4}$ & $9.93 \mathrm{E}-04$ & $2.42 \mathrm{E}-03$ & $2.30 \mathrm{E}-10$ & $2.30 \mathrm{E}-12$ & 1.76E-15 & & & $2.92 \mathrm{E}-09$ & \\
\hline $\mathrm{Y}+3$ & $6.09 \mathrm{E}-06$ & $1.48 \mathrm{E}-05$ & $2.62 \mathrm{E}-12$ & $4.71 \mathrm{E}-20$ & $3.60 \mathrm{E}-23$ & & & $3.23 \mathrm{E}-17$ & \\
\hline O-2 (oxides) & $2.06 \mathrm{E}-01$ & $5.01 \mathrm{E}-01$ & 1.59E-08 & $1.59 \mathrm{E}-10$ & $1.22 \mathrm{E}-13$ & & & $6.05 \mathrm{E}-07$ & \\
\hline $\mathrm{H} 2 \mathrm{O}$ & $4.83 \mathrm{E}+01$ & $2.76 \mathrm{E}+01$ & $4.50 \mathrm{E}-02$ & $4.50 \mathrm{E}-02$ & $4.69 \mathrm{E}-02$ & $3.04 \mathrm{E}-02$ & $1.67 \mathrm{E}-03$ & $4.62 \mathrm{E}+01$ & $7.11 \mathrm{E}+01$ \\
\hline $\mathrm{O} 2$ & & & 1.17E-07 & 1.17E-07 & 1.34E-07 & $6.12 \mathrm{E}-03$ & 1.68E-02 & 1.64E-10 & \\
\hline N2 & & & 1.99E-07 & 1.99E-07 & $2.29 \mathrm{E}-07$ & $2.02 \mathrm{E}-02$ & $5.54 \mathrm{E}-02$ & $1.90 \mathrm{E}-10$ & \\
\hline C (organic) & 4.79E-02 & 1.17E-01 & $3.24 \mathrm{E}-07$ & $3.24 \mathrm{E}-07$ & $6.37 \mathrm{E}-10$ & $4.05 \mathrm{E}-10$ & $8.20 \mathrm{E}-13$ & $1.65 \mathrm{E}-03$ & \\
\hline $\mathrm{H}$ (organic) & 4.79E-02 & 1.17E-01 & $2.72 \mathrm{E}-08$ & $2.72 \mathrm{E}-08$ & $5.35 \mathrm{E}-11$ & $3.40 \mathrm{E}-11$ & $6.88 \mathrm{E}-14$ & 1.65E-03 & \\
\hline $\begin{array}{l}\text { Gas Stream Bulk Composition } \\
\text { (wet basis) }\end{array}$ & $\begin{array}{c}\text { mol.\% } \\
\text { or ppmv }\end{array}$ & $\begin{array}{c}\text { mol.\% } \\
\text { or ppmv }\end{array}$ & $\begin{array}{l}\mathrm{mol} . \% \\
\text { or ppmv }\end{array}$ & $\begin{array}{c}\mathrm{mol} . \% \\
\text { or ppmv } \\
\end{array}$ & $\begin{array}{c}\mathrm{mol} . \% \\
\text { or ppmv } \\
\end{array}$ & $\begin{array}{c}\mathrm{mol} . \% \\
\text { or ppmv } \\
\end{array}$ & $\begin{array}{l}\mathrm{mol} . \% \\
\text { or ppmv }\end{array}$ & $\begin{array}{c}\text { mol. } \% \\
\text { or ppmv } \\
\end{array}$ & $\begin{array}{l}\mathrm{mol} . \% \\
\text { or ppmv } \\
\end{array}$ \\
\hline $\mathrm{H} 2 \mathrm{O}, \mathrm{mol} . \%$ & & & 95.90 & 95.90 & 99.99 & 64.95 & 3.58 & & \\
\hline O2, mol.\% & & & 0.0001 & 0.0001 & 0.0002 & 7.36 & 20.25 & & \\
\hline $\mathrm{N} 2, \mathrm{~mol} . \%$ & & & 0.0003 & 0.0003 & 0.0003 & 27.69 & 76.17 & & \\
\hline \multicolumn{10}{|l|}{$\mathrm{CO} 2, \mathrm{~mol} . \%$} \\
\hline HNO3, mol. \% & & & 4.10 & 4.10 & 0.00006 & 0.0001 & 0.00003 & & \\
\hline \multicolumn{10}{|l|}{ CO, ppmv } \\
\hline $\mathrm{HCl}, \mathrm{ppmv}$ & & & 107 & 107 & 0.12 & 0.001 & 0.0005 & & \\
\hline C (organic), ppmv & & & 10 & 10 & 0.020 & 0.013 & 0.00003 & & \\
\hline $\mathrm{H}$ (organic), ppmv & & & 10 & 10 & 0.020 & 0.013 & 0.00003 & & \\
\hline $\mathrm{PM}(\mathrm{mg} / \mathrm{wscm})$ & & & 4 & 0.04 & 0.00003 & & & & \\
\hline $\mathrm{Hg}$ (ug/wscm) & & & $1.9 \mathrm{E}+04$ & $1.9 \mathrm{E}+04$ & 150 & 0.6 & 1.5 & & \\
\hline $\begin{array}{l}\text { Gas Stream Bulk Composition } \\
\text { (dry basis) }\end{array}$ & $\begin{array}{l}\mathrm{mol} . \% \\
\text { or ppmv }\end{array}$ & $\begin{array}{c}\mathrm{mol} . \% \\
\text { or ppmv } \\
\end{array}$ & $\begin{array}{l}\mathrm{mol} . \% \\
\text { or ppmv }\end{array}$ & $\begin{array}{c}\mathrm{mol} . \% \\
\text { or ppmv } \\
\end{array}$ & $\begin{array}{c}\mathrm{mol} . \% \\
\text { or ppmv } \\
\end{array}$ & $\begin{array}{l}\mathrm{mol} . \% \\
\text { or ppmv }\end{array}$ & $\begin{array}{c}\mathrm{mol} . \% \\
\text { or ppmv }\end{array}$ & $\begin{array}{c}\text { mol. } \% \\
\text { or ppmv } \\
\end{array}$ & $\begin{array}{c}\mathrm{mol} . \% \\
\text { or ppmv } \\
\end{array}$ \\
\hline $\mathrm{O} 2, \mathrm{~mol} . \%$ & & & 0.003 & 0.003 & 3.07 & 21.00 & 21.00 & & \\
\hline $\mathrm{N} 2, \mathrm{~mol} . \%$ & & & 0.007 & 0.007 & 5.98 & 79.00 & 79.00 & & \\
\hline \multicolumn{10}{|l|}{$\mathrm{CO} 2, \mathrm{~mol} . \%$} \\
\hline HNO3, mol. \% & & & 99.93 & 99.97 & 1.21 & 0.0001 & 0.00003 & & \\
\hline \multicolumn{10}{|l|}{ CO, ppmv } \\
\hline $\mathrm{HCl}, \mathrm{ppmv}$ & & & $2.6 \mathrm{E}+03$ & $2.6 \mathrm{E}+03$ & $2.2 \mathrm{E}+03$ & 0.004 & 0.0005 & & \\
\hline C (organic), ppmv & & & $2.5 \mathrm{E}+02$ & $2.5 \mathrm{E}+02$ & $3.9 \mathrm{E}+02$ & 0.04 & 0.00003 & & \\
\hline $\mathrm{H}$ (organic), ppmv & & & 253 & 253 & 388 & 0.04 & 0.00003 & & \\
\hline $\mathrm{PM}(\mathrm{mg} / \mathrm{dscm})$ & & & 101 & 1.0 & 0.6 & & & & \\
\hline $\mathrm{Hg}$ (ug/wscm) & & & $1.2 \mathrm{E}+06$ & $1.2 \mathrm{E}+06$ & $7.4 \mathrm{E}+06$ & 3.4 & 2.4 & & \\
\hline Total Mass Flow, kg/hr & $4.42 \mathrm{E}+02$ & $2.34 \mathrm{E}+02$ & $3.48 \mathrm{E}+02$ & $3.48 \mathrm{E}+02$ & $2.75 \mathrm{E}+02$ & $5.23 \mathrm{E}+02$ & $2.48 \mathrm{E}+02$ & $1.45 \mathrm{E}+02$ & $7.23 \mathrm{E}+01$ \\
\hline Canister Rate, canisters/day & & 4.00 & & & & & & & \\
\hline Total Canisters Generated & & 557 & & & & & & & \\
\hline
\end{tabular}


Table C-11. Alkaline scrub balance, Tank WM-189 waste. (Continued).

\begin{tabular}{|c|c|c|c|c|c|c|c|c|c|}
\hline Stream \# & 101 & 102 & 103 & 104 & 105 & 106 & 107 & 108 & 201 \\
\hline Radiological Composition & $\mathrm{Ci} / \mathrm{L}$ & $\mathrm{Ci} / \mathrm{L}$ & $\mathrm{Ci} / \mathrm{wscm}$ & $\mathrm{Ci} / \mathrm{wscm}$ & Ci/wscm & $\mathrm{Ci} / \mathrm{wscm}$ & $\mathrm{Ci} / \mathrm{wscm}$ & $\mathrm{Ci} / \mathrm{L}$ & \\
\hline Ra-226 & $7.17 \mathrm{E}-12$ & $1.74 \mathrm{E}-11$ & $5.55 \mathrm{E}-16$ & $5.55 \mathrm{E}-18$ & $4.81 \mathrm{E}-21$ & $8.70 \mathrm{E}-26$ & $2.22 \mathrm{E}-25$ & $2.39 \mathrm{E}-17$ & \\
\hline Ac-227 & $3.38 \mathrm{E}-11$ & $8.21 \mathrm{E}-11$ & $2.61 \mathrm{E}-15$ & $2.61 \mathrm{E}-17$ & $2.26 \mathrm{E}-20$ & $4.10 \mathrm{E}-25$ & $1.05 \mathrm{E}-24$ & $1.13 \mathrm{E}-16$ & \\
\hline Th-228 & $2.80 \mathrm{E}-09$ & $6.82 \mathrm{E}-09$ & $2.17 \mathrm{E}-13$ & $2.17 \mathrm{E}-15$ & $1.88 \mathrm{E}-18$ & $3.40 \mathrm{E}-23$ & $8.68 \mathrm{E}-23$ & $9.34 \mathrm{E}-15$ & \\
\hline Th-230 & $9.49 \mathrm{E}-10$ & $2.31 \mathrm{E}-09$ & $7.34 \mathrm{E}-14$ & $7.34 \mathrm{E}-16$ & $6.06 \mathrm{E}-19$ & $1.15 \mathrm{E}-23$ & $2.94 \mathrm{E}-23$ & $3.01 \mathrm{E}-15$ & \\
\hline Th-232 & $6.20 \mathrm{E}-16$ & $1.51 \mathrm{E}-15$ & $4.80 \mathrm{E}-20$ & $4.80 \mathrm{E}-22$ & $4.16 \mathrm{E}-25$ & $7.53 \mathrm{E}-30$ & $1.92 \mathrm{E}-29$ & $2.07 \mathrm{E}-21$ & \\
\hline $\mathrm{Pa}-231$ & $7.82 \mathrm{E}-11$ & $1.90 \mathrm{E}-10$ & $6.05 \mathrm{E}-15$ & $6.05 \mathrm{E}-17$ & $5.24 \mathrm{E}-20$ & $9.49 \mathrm{E}-25$ & $2.42 \mathrm{E}-24$ & $2.61 \mathrm{E}-16$ & \\
\hline Pa-233 & $2.57 \mathrm{E}-06$ & $6.24 \mathrm{E}-06$ & $1.99 \mathrm{E}-10$ & $1.99 \mathrm{E}-12$ & $1.72 \mathrm{E}-15$ & $3.11 \mathrm{E}-20$ & $7.95 \mathrm{E}-20$ & $8.55 \mathrm{E}-12$ & \\
\hline U-232 & 2.22E-09 & $5.40 \mathrm{E}-09$ & $1.72 \mathrm{E}-13$ & $1.72 \mathrm{E}-15$ & $1.43 \mathrm{E}-18$ & $2.69 \mathrm{E}-23$ & $6.88 \mathrm{E}-23$ & $7.08 \mathrm{E}-15$ & \\
\hline U-233 & $7.83 \mathrm{E}-11$ & $1.90 \mathrm{E}-10$ & $6.06 \mathrm{E}-15$ & $6.06 \mathrm{E}-17$ & $5.14 \mathrm{E}-20$ & $9.49 \mathrm{E}-25$ & $2.43 \mathrm{E}-24$ & $2.55 \mathrm{E}-16$ & \\
\hline U-234 & $1.54 \mathrm{E}-06$ & $3.74 \mathrm{E}-06$ & $1.19 \mathrm{E}-10$ & $1.19 \mathrm{E}-12$ & $6.84 \mathrm{E}-16$ & $1.82 \mathrm{E}-20$ & $4.76 \mathrm{E}-20$ & $3.40 \mathrm{E}-12$ & \\
\hline U-235 & $6.19 \mathrm{E}-08$ & $1.51 \mathrm{E}-07$ & $4.79 \mathrm{E}-12$ & $4.79 \mathrm{E}-14$ & $4.58 \mathrm{E}-17$ & $7.57 \mathrm{E}-22$ & $1.92 \mathrm{E}-21$ & $2.27 \mathrm{E}-13$ & \\
\hline U-236 & $7.18 \mathrm{E}-08$ & 1.75E-07 & $5.56 \mathrm{E}-12$ & $5.56 \mathrm{E}-14$ & $3.00 \mathrm{E}-17$ & $8.49 \mathrm{E}-22$ & $2.23 \mathrm{E}-21$ & 1.49E-13 & \\
\hline U-237 & 5.63E-09 & 1.37E-08 & $4.36 \mathrm{E}-13$ & $4.36 \mathrm{E}-15$ & $3.78 \mathrm{E}-18$ & $6.84 \mathrm{E}-23$ & $1.75 \mathrm{E}-22$ & $1.88 \mathrm{E}-14$ & \\
\hline U-238 & $3.41 \mathrm{E}-08$ & $8.29 \mathrm{E}-08$ & $2.64 \mathrm{E}-12$ & $2.64 \mathrm{E}-14$ & $1.02 \mathrm{E}-17$ & $3.98 \mathrm{E}-22$ & $1.06 \mathrm{E}-21$ & $5.07 \mathrm{E}-14$ & \\
\hline Np-236 & $2.70 \mathrm{E}-12$ & $6.57 \mathrm{E}-12$ & $2.09 \mathrm{E}-16$ & $2.09 \mathrm{E}-18$ & $1.81 \mathrm{E}-21$ & $3.28 \mathrm{E}-26$ & $8.37 \mathrm{E}-26$ & $8.99 \mathrm{E}-18$ & \\
\hline $\mathrm{Np}-237$ & 4.57E-07 & 1.11E-06 & $3.54 \mathrm{E}-11$ & $3.54 \mathrm{E}-13$ & $2.54 \mathrm{E}-16$ & $5.48 \mathrm{E}-21$ & $1.42 \mathrm{E}-20$ & $1.26 \mathrm{E}-12$ & \\
\hline Pu-236 & 3.62E-09 & $8.81 \mathrm{E}-09$ & $2.80 \mathrm{E}-13$ & $2.80 \mathrm{E}-15$ & $2.11 \mathrm{E}-18$ & $4.35 \mathrm{E}-23$ & $1.12 \mathrm{E}-22$ & 1.05E-14 & \\
\hline Pu-238 & 8.36E-04 & 2.03E-03 & $6.47 \mathrm{E}-08$ & $6.47 \mathrm{E}-10$ & $4.85 \mathrm{E}-13$ & $1.00 \mathrm{E}-17$ & $2.59 \mathrm{E}-17$ & $2.41 \mathrm{E}-09$ & \\
\hline Pu-239 & $9.13 \mathrm{E}-05$ & $2.22 \mathrm{E}-04$ & 7.06E-09 & $7.06 \mathrm{E}-11$ & $5.40 \mathrm{E}-14$ & $1.10 \mathrm{E}-18$ & $2.83 \mathrm{E}-18$ & $2.68 \mathrm{E}-10$ & \\
\hline Pu-240 & $1.35 \mathrm{E}-05$ & $3.28 \mathrm{E}-05$ & $1.04 \mathrm{E}-09$ & $1.04 \mathrm{E}-11$ & $7.88 \mathrm{E}-15$ & $1.62 \mathrm{E}-19$ & $4.18 \mathrm{E}-19$ & $3.91 \mathrm{E}-11$ & \\
\hline Pu-241 & 7.31E-04 & $1.78 \mathrm{E}-03$ & $5.66 \mathrm{E}-08$ & $5.66 \mathrm{E}-10$ & 3.63E-13 & $8.72 \mathrm{E}-18$ & 2.27E-17 & $1.81 \mathrm{E}-09$ & \\
\hline Pu-242 & 1.06E-08 & $2.57 \mathrm{E}-08$ & $8.18 \mathrm{E}-13$ & $8.18 \mathrm{E}-15$ & $6.17 \mathrm{E}-18$ & $1.27 \mathrm{E}-22$ & $3.28 \mathrm{E}-22$ & 3.07E-14 & \\
\hline Pu-244 & $3.15 \mathrm{E}-16$ & $7.67 \mathrm{E}-16$ & $2.44 \mathrm{E}-20$ & $2.44 \mathrm{E}-22$ & $4.27 \mathrm{E}-25$ & $4.10 \mathrm{E}-30$ & $9.77 \mathrm{E}-30$ & $2.12 \mathrm{E}-21$ & \\
\hline Am-241 & $7.13 \mathrm{E}-05$ & 1.73E-04 & $5.52 \mathrm{E}-09$ & $5.52 \mathrm{E}-11$ & $3.51 \mathrm{E}-14$ & $8.49 \mathrm{E}-19$ & $2.21 \mathrm{E}-18$ & $1.74 \mathrm{E}-10$ & \\
\hline Am-242m & $1.34 \mathrm{E}-08$ & $3.25 \mathrm{E}-08$ & $1.03 \mathrm{E}-12$ & $1.03 \mathrm{E}-14$ & $9.13 \mathrm{E}-18$ & 1.63E-22 & $4.15 \mathrm{E}-22$ & $4.54 \mathrm{E}-14$ & \\
\hline Am-243 & $2.28 \mathrm{E}-08$ & $5.55 \mathrm{E}-08$ & 1.76E-12 & 1.76E-14 & $1.50 \mathrm{E}-17$ & $2.76 \mathrm{E}-22$ & 7.07E-22 & 7.45E-14 & \\
\hline $\mathrm{Cm}-242$ & $2.67 \mathrm{E}-08$ & $6.50 \mathrm{E}-08$ & $2.07 \mathrm{E}-12$ & $2.07 \mathrm{E}-14$ & $1.96 \mathrm{E}-17$ & $3.26 \mathrm{E}-22$ & $8.28 \mathrm{E}-22$ & $9.73 \mathrm{E}-14$ & \\
\hline $\mathrm{Cm}-243$ & $3.29 \mathrm{E}-08$ & $8.00 \mathrm{E}-08$ & $2.54 \mathrm{E}-12$ & $2.54 \mathrm{E}-14$ & $2.10 \mathrm{E}-17$ & $3.98 \mathrm{E}-22$ & $1.02 \mathrm{E}-21$ & $1.04 \mathrm{E}-13$ & \\
\hline $\mathrm{Cm}-244$ & 1.39E-06 & $3.39 \mathrm{E}-06$ & $1.08 \mathrm{E}-10$ & $1.08 \mathrm{E}-12$ & $7.55 \mathrm{E}-16$ & 1.67E-20 & $4.31 \mathrm{E}-20$ & $3.75 \mathrm{E}-12$ & \\
\hline $\mathrm{Cm}-245$ & $3.46 \mathrm{E}-10$ & $8.42 \mathrm{E}-10$ & $2.68 \mathrm{E}-14$ & $2.68 \mathrm{E}-16$ & $2.21 \mathrm{E}-19$ & $4.19 \mathrm{E}-24$ & 1.07E-23 & $1.10 \mathrm{E}-15$ & \\
\hline $\mathrm{Cm}-246$ & 2.27E-11 & $5.53 \mathrm{E}-11$ & $1.76 \mathrm{E}-15$ & 1.76E-17 & 1.45E-20 & $2.75 \mathrm{E}-25$ & 7.04E-25 & 7.20E-17 & \\
\hline $\mathrm{H}-3$ & $8.94 \mathrm{E}-06$ & $5.17 \mathrm{E}-06$ & $7.49 \mathrm{E}-06$ & $7.49 \mathrm{E}-06$ & $7.48 \mathrm{E}-06$ & 4.85E-06 & $2.79 \mathrm{E}-07$ & $8.10 \mathrm{E}-06$ & \\
\hline Be-10 & $2.63 \mathrm{E}-12$ & $6.39 \mathrm{E}-12$ & $2.03 \mathrm{E}-16$ & $2.03 \mathrm{E}-18$ & 1.76E-21 & $3.19 \mathrm{E}-26$ & $8.14 \mathrm{E}-26$ & $8.75 \mathrm{E}-18$ & \\
\hline C-14 & $1.44 \mathrm{E}-10$ & $3.50 \mathrm{E}-10$ & $1.11 \mathrm{E}-14$ & $1.11 \mathrm{E}-16$ & $9.14 \mathrm{E}-20$ & $1.74 \mathrm{E}-24$ & $4.46 \mathrm{E}-24$ & $4.54 \mathrm{E}-16$ & \\
\hline Co-60 & 2.92E-05 & $7.10 \mathrm{E}-05$ & 2.26E-09 & 2.26E-11 & $7.02 \mathrm{E}-15$ & 3.38E-19 & $9.04 \mathrm{E}-19$ & 3.49E-11 & \\
\hline Ni-59 & 1.23E-06 & $2.98 \mathrm{E}-06$ & $9.48 \mathrm{E}-11$ & $9.48 \mathrm{E}-13$ & $6.61 \mathrm{E}-16$ & 1.47E-20 & $3.80 \mathrm{E}-20$ & $3.28 \mathrm{E}-12$ & \\
\hline $\mathrm{Ni}-63$ & $3.82 \mathrm{E}-05$ & $9.29 \mathrm{E}-05$ & $2.95 \mathrm{E}-09$ & $2.95 \mathrm{E}-11$ & $2.42 \mathrm{E}-14$ & $4.62 \mathrm{E}-19$ & $1.18 \mathrm{E}-18$ & $1.20 \mathrm{E}-10$ & \\
\hline Se-79 & $5.22 \mathrm{E}-07$ & $1.27 \mathrm{E}-06$ & $4.04 \mathrm{E}-11$ & $4.04 \mathrm{E}-13$ & $3.32 \mathrm{E}-16$ & $6.31 \mathrm{E}-21$ & 1.62E-20 & 1.65E-12 & \\
\hline Rb-87 & $2.56 \mathrm{E}-11$ & $6.24 \mathrm{E}-11$ & $1.98 \mathrm{E}-15$ & $1.98 \mathrm{E}-17$ & $1.72 \mathrm{E}-20$ & $3.11 \mathrm{E}-25$ & $7.95 \mathrm{E}-25$ & $8.55 \mathrm{E}-17$ & \\
\hline Sr-90 & 3.46E-02 & $8.42 \mathrm{E}-02$ & $2.68 \mathrm{E}-06$ & $2.68 \mathrm{E}-08$ & 2.27E-11 & 4.20E-16 & 1.07E-15 & $1.13 \mathrm{E}-07$ & \\
\hline Y-90 & $3.46 \mathrm{E}-02$ & $8.42 \mathrm{E}-02$ & $2.68 \mathrm{E}-06$ & $2.68 \mathrm{E}-08$ & $2.27 \mathrm{E}-11$ & $4.20 \mathrm{E}-16$ & $1.07 \mathrm{E}-15$ & $1.13 \mathrm{E}-07$ & \\
\hline Zr-93 & 1.94E-06 & $4.72 \mathrm{E}-06$ & $1.50 \mathrm{E}-10$ & $1.50 \mathrm{E}-12$ & $1.30 \mathrm{E}-15$ & $2.35 \mathrm{E}-20$ & $6.01 \mathrm{E}-20$ & $6.46 \mathrm{E}-12$ & \\
\hline $\mathrm{Nb}-93 \mathrm{~m}$ & 1.49E-06 & $3.64 \mathrm{E}-06$ & $1.16 \mathrm{E}-10$ & 1.16E-12 & $1.00 \mathrm{E}-15$ & $1.81 \mathrm{E}-20$ & 4.63E-20 & $4.98 \mathrm{E}-12$ & \\
\hline Nb-94 & 1.36E-06 & $3.30 \mathrm{E}-06$ & $1.05 \mathrm{E}-10$ & $1.05 \mathrm{E}-12$ & $8.63 \mathrm{E}-16$ & $1.64 \mathrm{E}-20$ & $4.21 \mathrm{E}-20$ & $4.29 \mathrm{E}-12$ & \\
\hline Tc-98 & $2.26 \mathrm{E}-12$ & $5.49 \mathrm{E}-12$ & $1.75 \mathrm{E}-16$ & $1.75 \mathrm{E}-18$ & $1.51 \mathrm{E}-21$ & $2.74 \mathrm{E}-26$ & $7.00 \mathrm{E}-26$ & $7.52 \mathrm{E}-18$ & \\
\hline Tc-99 & $1.38 \mathrm{E}-05$ & 3.35E-05 & 1.07E-09 & $1.07 \mathrm{E}-11$ & $1.15 \mathrm{E}-14$ & $1.70 \mathrm{E}-19$ & $4.27 \mathrm{E}-19$ & $5.74 \mathrm{E}-11$ & \\
\hline Ru-106 & $1.12 \mathrm{E}-06$ & $2.72 \mathrm{E}-06$ & $8.64 \mathrm{E}-11$ & $8.64 \mathrm{E}-13$ & $7.09 \mathrm{E}-16$ & $1.35 \mathrm{E}-20$ & $3.46 \mathrm{E}-20$ & $3.52 \mathrm{E}-12$ & \\
\hline Rh-102 & $7.54 \mathrm{E}-10$ & 1.84E-09 & $5.84 \mathrm{E}-14$ & $5.84 \mathrm{E}-16$ & $5.06 \mathrm{E}-19$ & $9.16 \mathrm{E}-24$ & $2.34 \mathrm{E}-23$ & $2.51 \mathrm{E}-15$ & \\
\hline Rh-106 & 1.12E-06 & $2.72 \mathrm{E}-06$ & $8.64 \mathrm{E}-11$ & $8.64 \mathrm{E}-13$ & $7.09 \mathrm{E}-16$ & 1.35E-20 & $3.46 \mathrm{E}-20$ & $3.52 \mathrm{E}-12$ & \\
\hline Pd-107 & $1.45 \mathrm{E}-08$ & $3.52 \mathrm{E}-08$ & $1.12 \mathrm{E}-12$ & $1.12 \mathrm{E}-14$ & $9.70 \mathrm{E}-18$ & $1.76 \mathrm{E}-22$ & $4.48 \mathrm{E}-22$ & $4.82 \mathrm{E}-14$ & \\
\hline Cd-113m & $2.91 \mathrm{E}-06$ & $7.07 \mathrm{E}-06$ & $2.25 \mathrm{E}-10$ & $2.25 \mathrm{E}-12$ & $1.95 \mathrm{E}-15$ & $3.53 \mathrm{E}-20$ & $9.01 \mathrm{E}-20$ & $9.69 \mathrm{E}-12$ & \\
\hline Sn-121m & $5.85 \mathrm{E}-08$ & $1.42 \mathrm{E}-07$ & $4.53 \mathrm{E}-12$ & $4.53 \mathrm{E}-14$ & $3.92 \mathrm{E}-17$ & $7.10 \mathrm{E}-22$ & $1.81 \mathrm{E}-21$ & $1.95 \mathrm{E}-13$ & \\
\hline Sn-126 & 4.92E-07 & $1.20 \mathrm{E}-06$ & $3.80 \mathrm{E}-11$ & $3.80 \mathrm{E}-13$ & $3.12 \mathrm{E}-16$ & $5.94 \mathrm{E}-21$ & $1.52 \mathrm{E}-20$ & $1.55 \mathrm{E}-12$ & \\
\hline Sb-125 & $4.10 \mathrm{E}-04$ & 9.97E-04 & $3.17 \mathrm{E}-08$ & $3.17 \mathrm{E}-10$ & $2.21 \mathrm{E}-13$ & $4.91 \mathrm{E}-18$ & $1.27 \mathrm{E}-17$ & 1.10E-09 & \\
\hline Sb-126 & $5.03 \mathrm{E}-08$ & $1.22 \mathrm{E}-07$ & $3.90 \mathrm{E}-12$ & $3.90 \mathrm{E}-14$ & $3.38 \mathrm{E}-17$ & $6.11 \mathrm{E}-22$ & $1.56 \mathrm{E}-21$ & $1.68 \mathrm{E}-13$ & \\
\hline
\end{tabular}


Table C-11. Alkaline scrub balance, Tank WM-189 waste. (Continued).

\begin{tabular}{|c|c|c|c|c|c|c|c|c|c|}
\hline Stream \# & 101 & 102 & 103 & 104 & 105 & 106 & 107 & 108 & 201 \\
\hline Radiological Composition & $\mathrm{Ci} / \mathrm{L}$ & $\mathrm{Ci} / \mathrm{L}$ & $\mathrm{Ci} / \mathrm{wscm}$ & $\mathrm{Ci} / \mathrm{wscm}$ & $\mathrm{Ci} / \mathrm{wscm}$ & $\mathrm{Ci} / \mathrm{wscm}$ & $\mathrm{Ci} / \mathrm{wscm}$ & $\mathrm{Ci} / \mathrm{L}$ & \\
\hline Te-125m & $2.76 \mathrm{E}-06$ & $6.71 \mathrm{E}-06$ & $2.13 \mathrm{E}-10$ & $2.13 \mathrm{E}-12$ & $1.85 \mathrm{E}-15$ & $3.35 \mathrm{E}-20$ & $8.54 \mathrm{E}-20$ & $9.19 \mathrm{E}-12$ & \\
\hline $\mid-129$ & $6.38 \mathrm{E}-08$ & $1.71 \mathrm{E}-07$ & $1.54 \mathrm{E}-08$ & $1.54 \mathrm{E}-08$ & $2.13 \mathrm{E}-11$ & $2.40 \mathrm{E}-13$ & $6.72 \mathrm{E}-14$ & $7.49 \mathrm{E}-08$ & \\
\hline Cs-134 & $6.33 \mathrm{E}-05$ & $1.54 \mathrm{E}-04$ & $4.90 \mathrm{E}-09$ & $4.90 \mathrm{E}-11$ & $2.40 \mathrm{E}-14$ & $7.45 \mathrm{E}-19$ & $1.96 \mathrm{E}-18$ & 1.19E-10 & \\
\hline Cs-135 & $9.99 \mathrm{E}-07$ & $2.43 \mathrm{E}-06$ & $7.73 \mathrm{E}-11$ & $7.73 E-13$ & $6.37 \mathrm{E}-16$ & $1.21 \mathrm{E}-20$ & $3.10 \mathrm{E}-20$ & $3.17 \mathrm{E}-12$ & \\
\hline Cs-137 & $5.17 \mathrm{E}-02$ & $1.26 \mathrm{E}-01$ & $4.00 \mathrm{E}-06$ & $4.00 \mathrm{E}-08$ & $1.59 \mathrm{E}-11$ & $6.04 \mathrm{E}-16$ & $1.60 \mathrm{E}-15$ & $7.91 \mathrm{E}-08$ & \\
\hline Ba-137m & $4.89 \mathrm{E}-02$ & 1.19E-01 & $3.79 \mathrm{E}-06$ & $3.79 \mathrm{E}-08$ & $1.51 \mathrm{E}-11$ & $5.71 \mathrm{E}-16$ & $1.52 \mathrm{E}-15$ & $7.48 \mathrm{E}-08$ & \\
\hline La-138 & $1.67 \mathrm{E}-16$ & $4.07 \mathrm{E}-16$ & $1.29 \mathrm{E}-20$ & $1.29 \mathrm{E}-22$ & $1.12 \mathrm{E}-25$ & $2.03 \mathrm{E}-30$ & $5.18 \mathrm{E}-30$ & $5.57 \mathrm{E}-22$ & \\
\hline Ce-142 & $2.62 \mathrm{E}-11$ & $6.37 \mathrm{E}-11$ & $2.02 \mathrm{E}-15$ & $2.02 \mathrm{E}-17$ & $1.75 \mathrm{E}-20$ & $3.18 \mathrm{E}-25$ & $8.11 \mathrm{E}-25$ & $8.72 \mathrm{E}-17$ & \\
\hline Ce-144 & $7.54 \mathrm{E}-07$ & $1.83 \mathrm{E}-06$ & $5.83 \mathrm{E}-11$ & $5.83 \mathrm{E}-13$ & $4.78 \mathrm{E}-16$ & $9.11 \mathrm{E}-21$ & $2.34 \mathrm{E}-20$ & $2.38 \mathrm{E}-12$ & \\
\hline 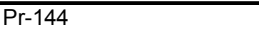 & $7.54 \mathrm{E}-07$ & $1.83 \mathrm{E}-06$ & $5.83 \mathrm{E}-11$ & $5.83 \mathrm{E}-13$ & $4.78 \mathrm{E}-16$ & $9.11 \mathrm{E}-21$ & $2.34 \mathrm{E}-20$ & $2.38 \mathrm{E}-12$ & \\
\hline Nd-144 & $1.41 \mathrm{E}-15$ & $3.42 \mathrm{E}-15$ & $1.09 \mathrm{E}-19$ & $1.09 \mathrm{E}-21$ & $9.44 \mathrm{E}-25$ & $1.71 \mathrm{E}-29$ & $4.36 \mathrm{E}-29$ & $4.69 \mathrm{E}-21$ & \\
\hline $\mathrm{Pm}-146$ & $4.45 \mathrm{E}-08$ & $1.08 \mathrm{E}-07$ & $3.45 \mathrm{E}-12$ & $3.45 \mathrm{E}-14$ & $2.99 \mathrm{E}-17$ & $5.41 \mathrm{E}-22$ & $1.38 \mathrm{E}-21$ & $1.48 \mathrm{E}-13$ & \\
\hline $\mathrm{Pm}-147$ & $2.03 \mathrm{E}-04$ & $4.95 \mathrm{E}-04$ & $1.57 \mathrm{E}-08$ & $1.57 \mathrm{E}-10$ & $1.29 \mathrm{E}-13$ & $2.46 \mathrm{E}-18$ & $6.30 \mathrm{E}-18$ & $6.42 \mathrm{E}-10$ & \\
\hline Sm-146 & $2.41 \mathrm{E}-13$ & $5.87 \mathrm{E}-13$ & $1.87 \mathrm{E}-17$ & $1.87 \mathrm{E}-19$ & $1.62 \mathrm{E}-22$ & $2.93 \mathrm{E}-27$ & $7.48 \mathrm{E}-27$ & $8.05 \mathrm{E}-19$ & \\
\hline Sm-147 & $6.45 \mathrm{E}-12$ & $1.57 \mathrm{E}-11$ & $4.99 \mathrm{E}-16$ & $4.99 \mathrm{E}-18$ & $4.32 \mathrm{E}-21$ & $7.83 \mathrm{E}-26$ & $2.00 \mathrm{E}-25$ & $2.15 \mathrm{E}-17$ & \\
\hline $\mathrm{Sm}-151$ & $4.02 \mathrm{E}-04$ & $9.78 \mathrm{E}-04$ & $3.11 \mathrm{E}-08$ & $3.11 \mathrm{E}-10$ & $2.55 \mathrm{E}-13$ & $4.86 \mathrm{E}-18$ & $1.25 \mathrm{E}-17$ & $1.27 \mathrm{E}-09$ & \\
\hline Eu-152 & $2.58 \mathrm{E}-06$ & $6.28 \mathrm{E}-06$ & $2.00 \mathrm{E}-10$ & $2.00 \mathrm{E}-12$ & $1.68 \mathrm{E}-15$ & $3.13 \mathrm{E}-20$ & $8.00 \mathrm{E}-20$ & $8.35 \mathrm{E}-12$ & \\
\hline Eu-154 & $1.54 \mathrm{E}-04$ & $3.75 \mathrm{E}-04$ & $1.19 \mathrm{E}-08$ & $1.19 \mathrm{E}-10$ & $3.84 \mathrm{E}-14$ & $1.79 \mathrm{E}-18$ & $4.77 \mathrm{E}-18$ & $1.91 \mathrm{E}-10$ & \\
\hline Eu-155 & $1.45 \mathrm{E}-04$ & $3.53 \mathrm{E}-04$ & $1.12 \mathrm{E}-08$ & $1.12 \mathrm{E}-10$ & $3.43 \mathrm{E}-14$ & $1.68 \mathrm{E}-18$ & $4.49 \mathrm{E}-18$ & $1.70 \mathrm{E}-10$ & \\
\hline Ho-166m & $4.03 \mathrm{E}-11$ & $9.80 \mathrm{E}-11$ & $3.12 \mathrm{E}-15$ & $3.12 \mathrm{E}-17$ & $2.70 \mathrm{E}-20$ & $4.89 \mathrm{E}-25$ & $1.25 \mathrm{E}-24$ & $1.34 \mathrm{E}-16$ & \\
\hline
\end{tabular}


Table C-12 shows the expected properties of the evaporator bottoms product.

Table C-12. Revised baseline scheme evaporator product waste properties.

\begin{tabular}{|c|c|c|c|c|}
\hline Major Radionuclides & $\begin{array}{c}\text { WM-180 } \\
\text { Ci/canister }\end{array}$ & $\begin{array}{l}\text { WM-187 } \\
\text { Ci/canister }\end{array}$ & $\begin{array}{c}\text { WM-188 } \\
\text { Ci/canister }\end{array}$ & $\begin{array}{c}\text { WM-189 } \\
\text { Ci/canister }\end{array}$ \\
\hline Am-241 & $2.22 \mathrm{E}-01$ & $2.15 \mathrm{E}-01$ & $1.87 \mathrm{E}-01$ & $2.06 \mathrm{E}-01$ \\
\hline $\mathrm{Pu}-238$ & $3.06 \mathrm{E}+00$ & $3.62 \mathrm{E}+00$ & $2.65 \mathrm{E}+00$ & $2.39 \mathrm{E}+00$ \\
\hline Pu-239 & 4.64E-01 & $3.98 \mathrm{E}-01$ & $2.96 \mathrm{E}-01$ & 2.62E-01 \\
\hline $\mathrm{Pu}-240$ & $2.52 \mathrm{E}-02$ & $4.43 \mathrm{E}-02$ & 4.33E-02 & $3.89 \mathrm{E}-02$ \\
\hline $\mathrm{Pu}-242$ & $1.94 \mathrm{E}-05$ & $3.48 \mathrm{E}-05$ & 3.39E-05 & $3.04 \mathrm{E}-05$ \\
\hline U-233 & $1.30 \mathrm{E}-07$ & $2.41 \mathrm{E}-07$ & 2.89E-07 & $2.26 \mathrm{E}-07$ \\
\hline $\mathrm{U}-234$ & $3.35 \mathrm{E}-03$ & $3.95 \mathrm{E}-03$ & $3.56 \mathrm{E}-03$ & 4.44E-03 \\
\hline $\mathrm{U}-238$ & 7.23E-05 & $6.77 \mathrm{E}-05$ & $4.66 \mathrm{E}-05$ & $9.86 \mathrm{E}-05$ \\
\hline Sr-90 & $6.29 \mathrm{E}+01$ & $9.69 \mathrm{E}+01$ & $1.28 \mathrm{E}+02$ & $1.00 \mathrm{E}+02$ \\
\hline Cs-137 & $8.35 \mathrm{E}+01$ & $1.42 \mathrm{E}+02$ & $7.30 \mathrm{E}+01$ & $1.49 \mathrm{E}+02$ \\
\hline Y-90 & $6.29 \mathrm{E}+01$ & $9.69 \mathrm{E}+01$ & $1.28 \mathrm{E}+02$ & $1.00 \mathrm{E}+02$ \\
\hline Ba-137 & $7.90 \mathrm{E}+01$ & $1.34 \mathrm{E}+02$ & $6.90 \mathrm{E}+01$ & $1.41 \mathrm{E}+02$ \\
\hline Total of above 12 nuclides & 292 & 474 & 400 & 493 \\
\hline Total of all nuclides & 295 & 482 & 406 & 499 \\
\hline \multirow[t]{2}{*}{ Fraction of the 12 to the total } & 0.989 & 0.983 & 0.985 & 0.987 \\
\hline & g/canister & g/canister & g/canister & g/canister \\
\hline Am-241 & $6.45 \mathrm{E}-02$ & $6.27 \mathrm{E}-02$ & $5.44 \mathrm{E}-02$ & 5.99E-02 \\
\hline $\mathrm{Pu}-238$ & $1.78 \mathrm{E}-01$ & 2.12E-01 & $1.55 \mathrm{E}-01$ & $1.40 \mathrm{E}-01$ \\
\hline Pu-239 & $7.46 \mathrm{E}+00$ & $6.41 \mathrm{E}+00$ & $4.77 \mathrm{E}+00$ & $4.21 \mathrm{E}+00$ \\
\hline $\mathrm{Pu}-240$ & $1.10 \mathrm{E}-01$ & $1.94 \mathrm{E}-01$ & $1.90 \mathrm{E}-01$ & $1.71 \mathrm{E}-01$ \\
\hline $\mathrm{Pu}-242$ & $5.08 \mathrm{E}-03$ & $9.12 \mathrm{E}-03$ & $8.88 \mathrm{E}-03$ & 7.98E-03 \\
\hline U-233 & $1.35 \mathrm{E}-05$ & $2.49 \mathrm{E}-05$ & $2.98 \mathrm{E}-05$ & $2.34 \mathrm{E}-05$ \\
\hline $\mathrm{U}-234$ & $5.36 \mathrm{E}-01$ & $6.31 \mathrm{E}-01$ & $5.69 \mathrm{E}-01$ & 7.10E-01 \\
\hline $\mathrm{U}-238$ & $2.15 \mathrm{E}+02$ & $2.01 \mathrm{E}+02$ & $1.39 \mathrm{E}+02$ & $2.93 \mathrm{E}+02$ \\
\hline Sr-90 & $4.61 \mathrm{E}-01$ & $7.11 \mathrm{E}-01$ & $9.35 \mathrm{E}-01$ & 7.34E-01 \\
\hline Cs-137 & $9.60 \mathrm{E}-01$ & $1.63 \mathrm{E}+00$ & 8.39E-01 & $1.71 \mathrm{E}+00$ \\
\hline Y-90 & $1.16 \mathrm{E}-04$ & $1.78 \mathrm{E}-04$ & $2.34 \mathrm{E}-04$ & $1.84 \mathrm{E}-04$ \\
\hline Ba-137 & $1.47 \mathrm{E}-07$ & $2.49 \mathrm{E}-07$ & $1.28 \mathrm{E}-07$ & $2.62 \mathrm{E}-07$ \\
\hline Fissile Gram Equivalents, g/canister & 64 & 102 & 126 & 87 \\
\hline Pu Equivalent Curies & 3.5 & 4.0 & 3.0 & 2.7 \\
\hline TRU content, $\mathrm{nCi} / \mathrm{g}$ & $2.7 \mathrm{E}+03$ & $3.1 \mathrm{E}+03$ & $2.3 \mathrm{E}+03$ & $2.1 \mathrm{E}+03$ \\
\hline
\end{tabular}


Dose rates and heat generation rates have not been calculated for waste compositions shown in the present mass balances. However, the evaporator waste dose rate is expected to be very similar to that calculated for previous material balances. Based on a date of 2010, the evaporator product canisters are expected to have an average dose rate of $28 \mathrm{R} / \mathrm{hr}$ and generate 1.4 Watts of heat per canister (Bohn, 2002). The drums of grouted acid are estimated to have a dose rate of $0.015 \mathrm{mR} / \mathrm{hr}$ and a heat generation rate of $1.3 \times 10^{-7}$ Watts per drum (Bohn, 2002).

Table C-13 shows additional properties of the grouted waste product. Radionuclides in the grouted waste sum to $2-5 \%$ of Hanford limit for Category 1 waste, or less than $1 \%$ of the Envirocare limits.

Table C-13. Grouted waste properties compared to Hanford and Envirocare WAC.

\begin{tabular}{lcccc}
\hline & $\begin{array}{c}\text { Hanford Cat } \mathbf{1} \text { WAC } \\
\mathbf{C i} / \mathbf{m} 3\end{array}$ & $\begin{array}{c}\text { Envirocare WAC } \\
\mathbf{p C i} / \mathbf{g}\end{array}$ \\
\hline $\mathrm{Sr}-90$ & $1.6 \mathrm{E}-02$ & $2.5 \mathrm{E}+04$ & \\
$\mathrm{Tc}-99$ & $2.3 \mathrm{E}-02$ & $1.9 \mathrm{E}+05$ & \\
$\mathrm{I}-129$ & $8.5 \mathrm{E}-03$ & $3.1 \mathrm{E}+03$ & \\
$\mathrm{Cs}-137$ & $5.5 \mathrm{E}-03$ & $6.0 \mathrm{E}+04$ & & \\
Pu-241 & $6.1 \mathrm{E}-02$ & $3.5 \mathrm{E}+05$ & & \\
Pu-238 & $4.7 \mathrm{E}-03$ & $1.0 \mathrm{E}+04$ & & \\
Pu-239 & $1.9 \mathrm{E}-03$ & $1.0 \mathrm{E}+04$ & & \\
Pu-240 & $1.9 \mathrm{E}-03$ & $1.0 \mathrm{E}+04$ & & \\
Am-241 & $2.1 \mathrm{E}-03$ & $1.0 \mathrm{E}+04$ & & \\
\hline & Sum of fractions & & Total Ci/g \\
\hline WM-180 & 0.04 & 0.004 & $3.9 \mathrm{E}-03$ & $6.4 \mathrm{E}-04$ \\
WM-187 & 0.05 & 0.006 & $3.2 \mathrm{E}-03$ & $4.0 \mathrm{E}-04$ \\
WM-188 & 0.02 & 0.004 & $1.7 \mathrm{E}-03$ & $4.4 \mathrm{E}-04$ \\
WM-189 & 0.04 & 0.005 & $1.5 \mathrm{E}-03$ & $3.8 \mathrm{E}-04$ \\
\hline
\end{tabular}

\section{C-7. UTILITIES SUMMARY}

\section{C-7.1 Revised Baseline Scheme}

The direct evaporation process requires steam, fuel oil and cooling water. Fuel oil is required for the boiler that generates 150-psig steam for the evaporator. The LET\&D reboiler uses 100-psig steam. Low-pressure steam is used in POG, VOG and LET\&D off-gas superheaters. Steam is also used in the jets that transfer waste from the tank farm. Typically, the steam requirement for waste transfer is $5 \%$ of the waste volume.

Cooling water is used in as the cooling medium for the evaporator condenser, canister cooler, LET\&D condenser, LET\&D bottoms cooler and neutralization tank cooler. Expected utilities usages for the revised baseline scheme are shown in Table C-14. 
Table C-14. Expected utilities for revised baseline scheme

\begin{tabular}{lcccc}
\hline & & Duty, MM Btu/hr & Ave Usage & Total usage \\
\hline Fuel oil & 0.92 & $10 \mathrm{gph}$ & 115,000 gallons \\
Steam & & & \\
$\quad$ (evap) & $150 \mathrm{psig*}$ & 0.69 & $573 \mathrm{lb} / \mathrm{hr}$ & $6.9 \mathrm{E}+06 \mathrm{lb}$ \\
$\quad($ LET\&D) & $100 \mathrm{psig}$ & 6.7 & $5,660 \mathrm{lb} / \mathrm{hr}$ & $9.0 \mathrm{E}+06 \mathrm{lb}$ \\
$\quad$ (Stm jet) & $100 \mathrm{psig}$ & 1.5 & $1,250 \mathrm{lb} / \mathrm{hr}$ & $4.3 \mathrm{E}+05 \mathrm{lb}$ \\
& $35 \mathrm{psig}$ & 0.022 & $19 \mathrm{lb} / \mathrm{hr}$ & $2.3 \mathrm{E}+05 \mathrm{lb}$ \\
Cooling Water & & 1.3 & $60 \mathrm{gpm}$ & $4.2 \mathrm{E}+07$ gallons \\
\hline
\end{tabular}

* 150 psig steam is generated in the treatment facility

\section{C-7.2 Alkaline Scrub Scheme}

Expected utilities for the alkaline scrub scheme are shown in Table C-15.

Table C-15. Expected utilities for alkaline scrub scheme.

\begin{tabular}{lcccc}
\hline & & Duty, MM Btu/hr & Ave Usage & Total usage \\
\hline Fuel oil & 1.5 & $16 \mathrm{gph}$ & 188,000 gallons \\
Steam & & & \\
\multicolumn{1}{c}{$($ evap) } & $150 \mathrm{psig}^{*}$ & 0.9 & $710 \mathrm{lb} / \mathrm{hr}$ & $8.5 \mathrm{E}+06 \mathrm{lb}$ \\
$\quad$ Stm jet) & $100 \mathrm{psig}$ & 1.5 & $1,250 \mathrm{lb} / \mathrm{hr}$ & $4.3 \mathrm{E}+05 \mathrm{lb}$ \\
& $35 \mathrm{psig}$ & 0.034 & $29 \mathrm{lb} / \mathrm{hr}$ & $3.5 \mathrm{E}+05 \mathrm{lb}$ \\
Cooling Water & & 0.19 & $9 \mathrm{gpm}$ & $6.2 \mathrm{E}+06$ gallons \\
\hline
\end{tabular}

* 150 psig steam is generated in the treatment facility 


\section{C-8. EQUIPMENT LIST AND PLANT SCALE}

The equipment list for the revised baseline scheme is shown in Table C-16, and for the alkaline scrub scheme in Table C-17. These tables present equipment sizes based on a 2.5 -yr operating schedule. Following the tables is a discussion of how a shorter operating schedule would affect facility size.

Table C-16. Revised baseline scheme equipment list.

\begin{tabular}{|c|c|c|c|c|c|c|c|c|}
\hline ID & Component Item & $\begin{array}{l}\text { Number } \\
\text { Required }\end{array}$ & $\begin{array}{l}\text { Size or } \\
\text { Capacity }\end{array}$ & Units & $\begin{array}{l}\text { Diameter } \\
\text { (ft.) }\end{array}$ & $\begin{array}{c}\text { Height } \\
\text { (ft.) }\end{array}$ & Notes & $\begin{array}{l}\text { Materials of } \\
\text { Construction }\end{array}$ \\
\hline \multicolumn{9}{|c|}{ Tanks } \\
\hline VES-101 & SBW Feed Tank & 1 & 12,400 & gal & 12.0 & 13.5 & $\mathrm{a}$ & SS \\
\hline VES-102 & SBW Mist Eliminator Zone A & 1 & 13 & $\mathrm{ft}^{3}$ & 2.0 & 4.0 & $\mathrm{~b}$ & Alloy \\
\hline VES-102 & SBW Mist Eliminator Zone B & 1 & 6 & $\mathrm{ft}^{3}$ & 2.0 & 2.0 & $\mathrm{~b}$ & Alloy \\
\hline VES-103 & Condensate Demister & 1 & 16 & $\mathrm{ft}^{3}$ & 2.0 & 5.0 & $\mathrm{c}$ & Alloy \\
\hline VES-104 & Condensate Tank & 1 & 12,400 & gal & 12.0 & 13.5 & & SS \\
\hline VES-105 & VOG Demister & 1 & 9 & $\mathrm{ft}^{3}$ & 2.0 & 3.0 & $\mathrm{c}$ & SS \\
\hline VES-106 & Aluminum Nitrate Tank & 1 & 30 & gal & & & & SS \\
\hline VES-107 & Flush Water Tank & 1 & 70 & gal & & & & SS \\
\hline VES-109 & Grout Mixer Feed Tank & 1 & 3,300 & gal & 8.0 & 9.0 & & SS \\
\hline VES-110 & Neutralization Tank & 1 & 3,300 & gal & 8.0 & 9.0 & & SS \\
\hline \multicolumn{9}{|c|}{ Hoppers } \\
\hline HOP-101 & Portland Cement Hopper & 1 & 1,860 & $\mathrm{ft}^{3}$ & 10.5 & 22 & & $\mathrm{CS}$ \\
\hline HOP-102 & BFS Hopper & 1 & 620 & $\mathrm{ft}^{3}$ & 7.5 & 14 & & CS \\
\hline HOP-103 & Calcium Hydroxide Hopper & 1 & 1,480 & $\mathrm{ft}^{3}$ & 10.5 & 17 & & CS \\
\hline HOP-104 & Cement Feeder Bin & 1 & 300 & gal & & & & $\mathrm{CS}$ \\
\hline HOP-105 & BFS Feeder Bin & 1 & 100 & gal & & & & $\mathrm{CS}$ \\
\hline HOP-106 & $\mathrm{Ca}(\mathrm{OH}) 2$ Feeder Bin & 1 & 230 & gal & & & & $\mathrm{CS}$ \\
\hline HOP-107 & $\begin{array}{l}\text { Neut. Agent Hopper \& } \\
\text { Feeder }\end{array}$ & 1 & 230 & gal & & & & $\mathrm{CS}$ \\
\hline HOP-108 & Silica Gel Hopper & 1 & 30 & gal & & & & $\mathrm{CS}$ \\
\hline \multicolumn{9}{|c|}{ Evaporator } \\
\hline WFE-101 & Wiped Film Evaporator & 2 & 1,100 & $\mathrm{lb} / \mathrm{hr}$ & 1.3 & 10.67 & $\mathrm{~d}$ & Alloy \\
\hline \multicolumn{9}{|c|}{ Heat Exchangers } \\
\hline HE-101 & Condenser & 1 & 60 & $\mathrm{ft} 2$ & & & & Alloy \\
\hline HE-102 & POG Superheater & 1 & 2 & $\mathrm{ft} 2$ & & & & Alloy \\
\hline HE-103 & VOG Superheater & 1 & 2 & $\mathrm{ft} 2$ & & & & Alloy \\
\hline
\end{tabular}


Table C-16. Revised baseline scheme equipment list (continued).

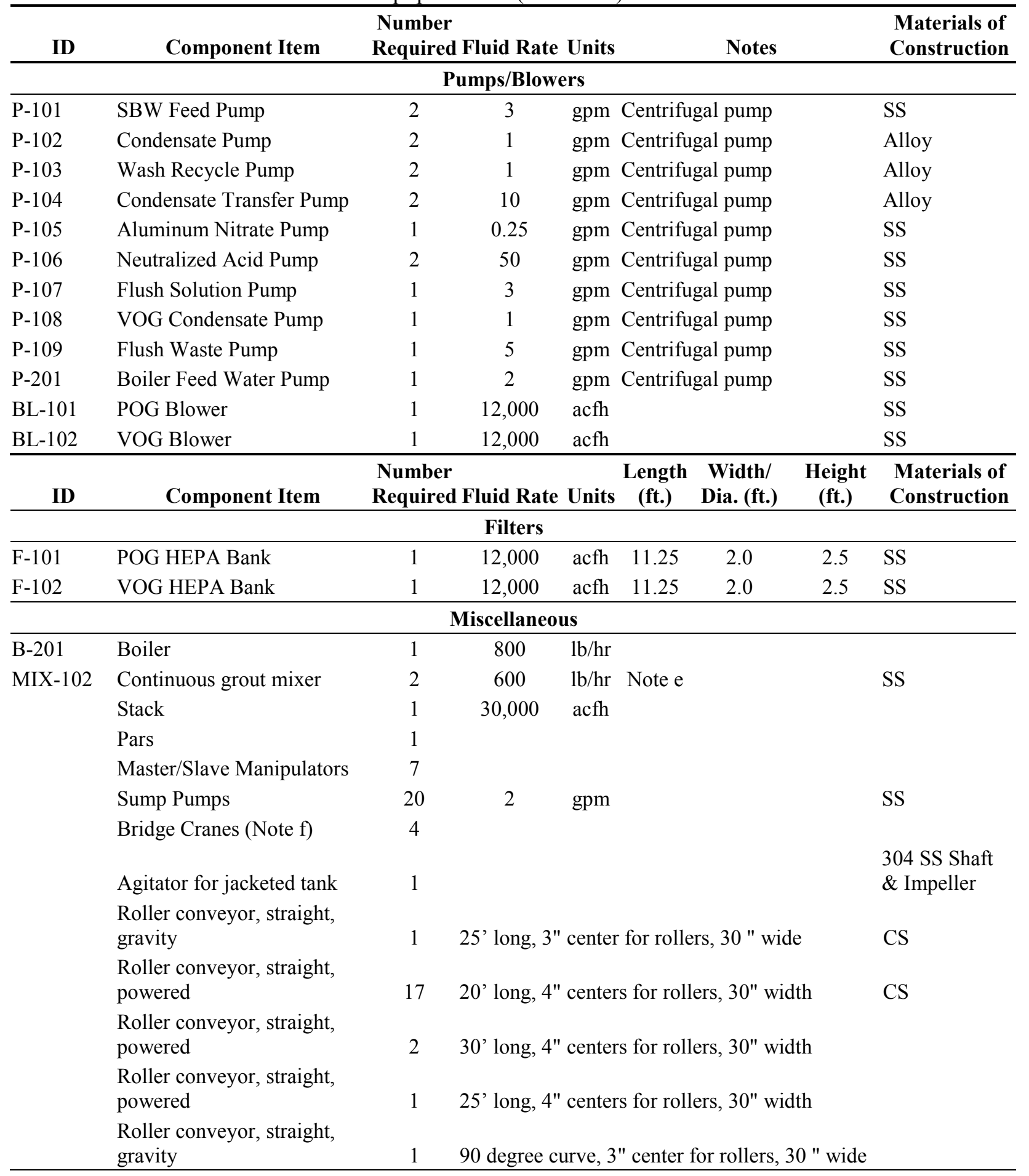

Notes

a. Include sparge

b. Include 12" mesh pad

c. Include 8" pad of York style 931

d. Provide an additional $11 \mathrm{ft}$ of vertical clearance to remove shaft

e. One installed, the other a spare to be installed when needed

f. $5 \mathrm{~T}$ with multiple remote operating stations 
Table C-17. Alkaline scrub scheme equipment list.

\begin{tabular}{llllllll}
\hline \multicolumn{1}{c}{ ID } & \multicolumn{1}{c}{$\begin{array}{c}\text { Number } \\
\text { Required }\end{array}$} & $\begin{array}{c}\text { Size or } \\
\text { Capacity }\end{array}$ & $\begin{array}{c}\text { Diameter } \\
\text { Units }\end{array}$ & $\begin{array}{c}\text { Height } \\
\text { (ft.) }\end{array}$ & $\begin{array}{c}\text { Materials of } \\
\text { (ft.) }\end{array}$ & Notes & $\begin{array}{c}\text { Construction } \\
\text { Tanks }\end{array}$ \\
\hline VES-101 SBW Feed Tank & 1 & 12,400 & gal & 12.0 & 13.5 & $\mathrm{a}$ & $\mathrm{SS}$ \\
VES-102 SBW Mist Eliminator Zone A 1 & 13 & $\mathrm{ft}^{3}$ & 2.25 & 4.0 & $\mathrm{~b}$ & Alloy \\
VES-102 SBW Mist Eliminator Zone B 1 & 6 & $\mathrm{ft}^{3}$ & 2.25 & 2.0 & $\mathrm{~b}$ & Alloy \\
VES-103 MgO Makeup Tank & 1 & 32 & $\mathrm{ft}^{3}$ & 3.0 & 4.5 & $\mathrm{c}$ & $\mathrm{CS}$ \\
VES-104 Alkaline Scrubber & 1 & 42,000 & $\mathrm{SCFH} 1.5$ & 11.0 & & Alloy \\
VES-105 VOG Demister & 1 & 9 & $\mathrm{ft}^{3}$ & 2.0 & 3.0 & $\mathrm{c}$ & $\mathrm{SS}$ \\
VES-106 GAC Bed & 1 & 38 & $\mathrm{ft}^{3}$ & 3.5 & 4.0 & & $\mathrm{SS}$ \\
\hline
\end{tabular}

Evaporator

\begin{tabular}{|c|c|c|c|c|c|c|c|c|}
\hline $\begin{array}{l}\text { WFE- } \\
101\end{array}$ & Wiped Film Evaporator & 2 & 1,250 & $\mathrm{lb} / \mathrm{hr}$ & 1.3 & 10.67 & $\mathrm{~d}$ & Alloy \\
\hline ID & Component Item & $\begin{array}{c}\text { Number } \\
\text { Required }\end{array}$ & \multicolumn{2}{|c|}{$\begin{array}{c}\text { Heat Transfer } \\
\text { Area }\end{array}$} & Units & & & $\begin{array}{l}\text { Materials of } \\
\text { Construction }\end{array}$ \\
\hline \multicolumn{9}{|c|}{ Heat Exchangers } \\
\hline HE-101 & Scrub Solution Cooler & 1 & 50 & \multicolumn{2}{|r|}{$\mathrm{ft}^{2}$} & & \multicolumn{2}{|r|}{ Alloy } \\
\hline HE-102 & POG Superheater & 1 & 2 & \multicolumn{2}{|r|}{$\mathrm{ft}^{2}$} & & \multicolumn{2}{|r|}{ Alloy } \\
\hline HE-103 & VOG Superheater & 1 & 2 & \multicolumn{2}{|r|}{$\mathrm{ft}^{2}$} & & \multicolumn{2}{|r|}{ Alloy } \\
\hline ID & Component Item & $\begin{array}{l}\text { Number } \\
\text { Required }\end{array}$ & $\begin{array}{l}\text { Fluid } \\
\text { Rate }\end{array}$ & Units & Notes & & & $\begin{array}{l}\text { Materials of } \\
\text { Construction }\end{array}$ \\
\hline \multicolumn{9}{|c|}{ Pumps/Blowers } \\
\hline P-101 & SBW Feed Pump & 2 & 4 & gpm & \multicolumn{2}{|c|}{ Centrifugal pump } & \multicolumn{2}{|r|}{ SS } \\
\hline P-102 & Scrub Pump & 2 & 6 & gpm & \multicolumn{2}{|c|}{ Centrifugal pump } & \multicolumn{2}{|r|}{ Alloy } \\
\hline P-103 & Wash Recycle Pump & 2 & 1 & gpm & \multicolumn{2}{|c|}{ Centrifugal pump } & \multicolumn{2}{|r|}{ Alloy } \\
\hline P-104 & MgO Slurry Pump & 2 & 0.5 & gpm & \multicolumn{2}{|c|}{ Centrifugal pump } & \multicolumn{2}{|r|}{ CS } \\
\hline P-108 & VOG Condensate Pump & 1 & 1 & gpm & \multicolumn{2}{|c|}{ Centrifugal pump } & \multicolumn{2}{|r|}{ SS } \\
\hline P-201 & Boiler Feed Water Pump & 1 & 2 & gpm & \multicolumn{2}{|c|}{ Centrifugal pump } & \multicolumn{2}{|r|}{ SS } \\
\hline BL-101 & POG Blower & 1 & 34,000 & acfh & & & \multicolumn{2}{|r|}{ SS } \\
\hline BL-102 & VOG Blower & 1 & 12,000 & acfh & & & & SS \\
\hline ID & Component Item & $\begin{array}{l}\text { Number } \\
\text { Required }\end{array}$ & $\begin{array}{l}\text { Fluid } \\
\text { Rate }\end{array}$ & Units & $\begin{array}{c}\text { Length } \\
\text { (ft.) }\end{array}$ & $\begin{array}{l}\text { Width or } \\
\text { Dia. (ft.) }\end{array}$ & $\begin{array}{l}\text { Height } \\
\text { (ft.) }\end{array}$ & $\begin{array}{c}\text { Materials of } \\
\text { Construction }\end{array}$ \\
\hline & & & Filters & & & & & \\
\hline F-101 & POG HEPA Bank & 1 & 34,000 & acfh & 11.25 & 2.0 & 2.5 & SS \\
\hline F-102 & VOG HEPA Bank & 1 & 12,000 & acfh & 11.25 & 2.0 & 2.5 & SS \\
\hline & & & Miscellane & ous & & & & \\
\hline B-201 & Boiler & 1 & 1,000 & $\mathrm{lb} / \mathrm{hr}$ & & & & \\
\hline
\end{tabular}

Notes
a. Include sparge
b. Include 12" mesh pad
c. Include 8" pad of York style 931
d. Provide an additional $11 \mathrm{ft}$ of vertical clearance to remove shaft
e. Stack, pars, manipulators, sump, cranes and conveyors same as Table C-14 
The Direct Evaporation Treatment Facility includes waste loading and canister handling operations in the lower level; hot cell process equipment on the ground floor and cold (nonradioactive) activities plus process and vent gas HEPA filtration on the second floor. The control room, offices, a decontamination room, a manipulator repair room and other support activities are located on the ground floor. Changing the scale of the process, i.e., designing for a shorter treatment schedule, would primarily affect four areas of the facility - the process hot cells, the grouting operation floor space, waste packaging and canister handling in the basement and lag storage for waste canisters.

The major equipment in the process hot cell includes the evaporator and four tanks. Other equipment is small and effects of scale on floor space for this equipment is minimal. The evaporator as sized for 2.5 years operation has an outside diameter of 191/4 inches and a length of 10-ft 8-in, and heat transfer area of $21.6 \mathrm{ft}^{2}$. For a shorter schedule, the heat transfer area would need to be increased by the schedule reduction ratio. Thus for a schedule of 1-year, the heat transfer area would be $21.6 \times 2.5=54$ $\mathrm{ft}^{2}$. This area could be achieved in an evaporator 1.5-ft ID by about 17 feet or 2-ft ID by 14-ft. The effect on floor space due would be small, but the hot cell and building height would need to increase to accommodate clearance for evaporator shaft and rotor removal. Alternatively, two evaporators could be used.

Keeping all other factors equal, decreasing the processing schedule would increase the floor space required for process tanks by the inverse ratio of the decrease to the two-thirds power. For example if the schedule was decreased from 2.5 years to 1 year, the floor space for tanks would increase by $(2.5 / 1)^{0.667}=$ 1.84 or an $84 \%$ increase.

For the 2.5-year operating schedule, 5,840 drums of grout are produced, equivalent to an average of 12 drums per day. At this production rate, a single grouting line is adequate and would be adequate for shorter treatment schedules as well. Additional space would likely be included in a facility designed for a shorter schedule for empty 55-gal drums fed to the grouting line, to maintain the same schedule for receiving drums.

The area required for storage of grouted waste drums depends only on the lag storage requirement. Lag storage space in turn depends of the rate at which the disposal site can receive shipments and the initial lag between start of production and sending the first shipment. The intended disposal site for the grouted waste from the direct evaporator process has been Hanford or Envirocare. It is expected that either site could receive this waste at a shipment rate equal to the rate generated for either a $2.5-\mathrm{yr}$ or a shorter schedule. However, this assumption has not been confirmed.

The alkaline scrub variation of the direct evaporation process, as well as the variation that neutralizes LET\&D bottoms, does not produce a grouted waste product. Besides eliminating the grouting equipment and floor space, these schemes eliminate two of the four major tanks of the process.

The rate at which $\mathrm{RH}$ canisters of evaporator product are produced is directly proportional to the inverse of the processing schedule. For the 2.5-year schedule, on average, one canister is filled in about 10 hours. A single fill station could accommodate shorter processing schedules, but more floor space would be required in the cooling area. The cooling time has yet to be adequately determined, but is expected to be several days. Assuming three days for cooling, space for eight canisters would be needed for a 2.5-year schedule or twenty for a 1-year schedule.

Finally, the interim storage for RH waste is dependent upon the initial lag period between producing the first container of waste and having approval to ship that container to WIPP and the rate at which WIPP can accept waste. Based on discussions with WIPP personnel, it is expected that the disposal facility can receive at least 6 RH canisters per week from the INEEL, or about 300 per year. 
Assuming an initial continuous 40-day operating period prior to waste shipment, storage for 80 canisters would be needed. However, since 1260 total RH canisters of waste are produced and WIPP can receive only about 300 per year, storage for 590 canisters would be required for a 2.5-year schedule, or 1,040 for a 1 -year schedule. This equates into a $76 \%$ increase in storage space. A summary of plot space estimates is given in Table $\mathrm{C}-18$ for the revised baseline scheme:

Table C-18. Plot Space Estimates.

\begin{tabular}{lcc}
\hline & $\begin{array}{c}\mathbf{2 . 5} \text {-year } \\
\text { treatment schedule }\end{array}$ & $\begin{array}{c}\text { 1-year } \\
\text { treatment schedule }\end{array}$ \\
\hline $\begin{array}{l}\text { Process equipment (hot cell space, ground } \\
\text { floor) }\end{array}$ & $5,200 \mathrm{ft}^{2}$ & $6,200 \mathrm{ft}^{2}$ \\
$\begin{array}{l}\text { Grouting equipment and drum storage } \\
\begin{array}{l}\text { Waste packaging \& canister handling } \\
\text { (to be verified in conceptual design) }\end{array}\end{array}$ & $18,000 \mathrm{ft}^{2}$ & $20,000 \mathrm{ft}^{2}$ \\
Lag storage for RH canisters & $1,200 \mathrm{ft}^{2}$ & $1,300 \mathrm{ft}^{2}$ \\
(to be verified in conceptual design) & $3,300 \mathrm{ft}^{2}$ & $5,800 \mathrm{ft}^{2}$ \\
\hline
\end{tabular}

\section{C-9. LAYOUT DRAWINGS}

Layout drawings for each floor of the Direct Evaporation Treatment Facility are shown on the following pages. The primary purpose of these drawings is to estimate floor space requirements needed in the facility. Equipment and room arrangements have not been optimized, nor has sufficient engineering design been performed to provide a basis for all of the areas of the facility. The layout drawings are similar to those prepared for the Direct Evaporation Feasibility Study (Kimmitt, 2003).

Storage is provided in the basement for 590 full waste canisters. This quantity is required for (a) lag storage prior to the initial shipment assumed to be equivalent to 40 production days plus (b) storage for the difference between the production rate and the shipping rate over the 2.5 -year operating phase of the treatment process. 
This page intentionally left blank. 


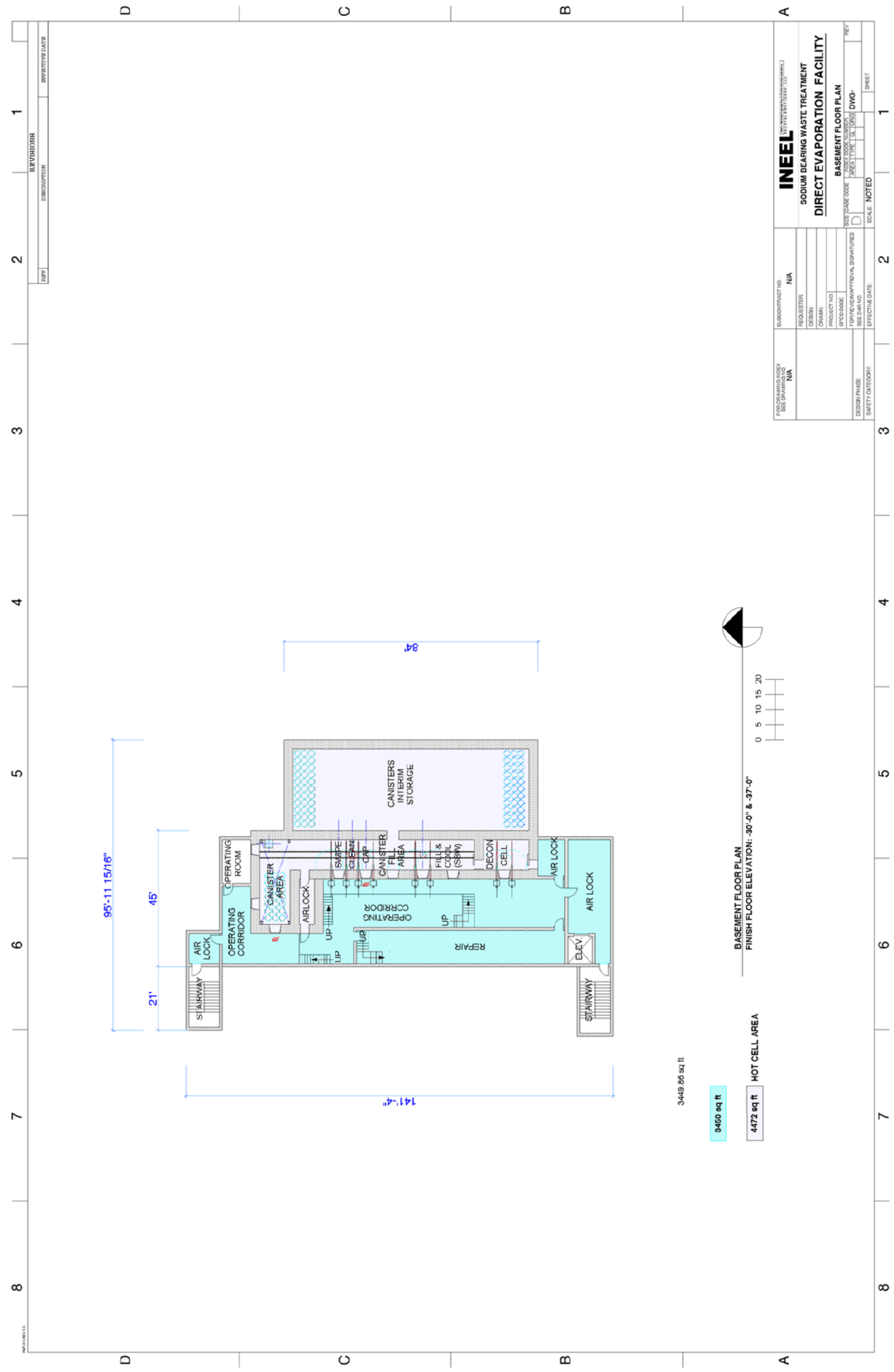




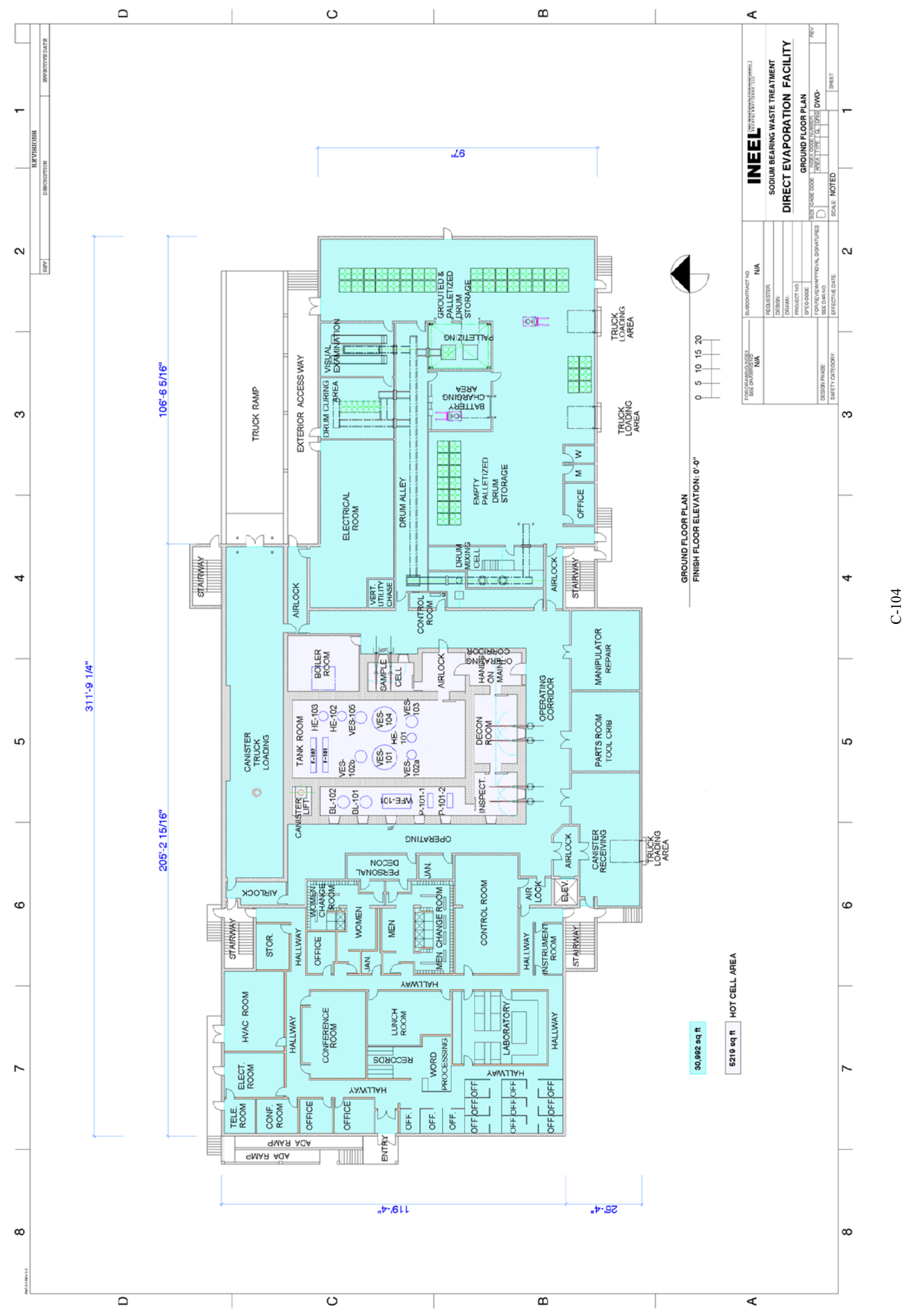




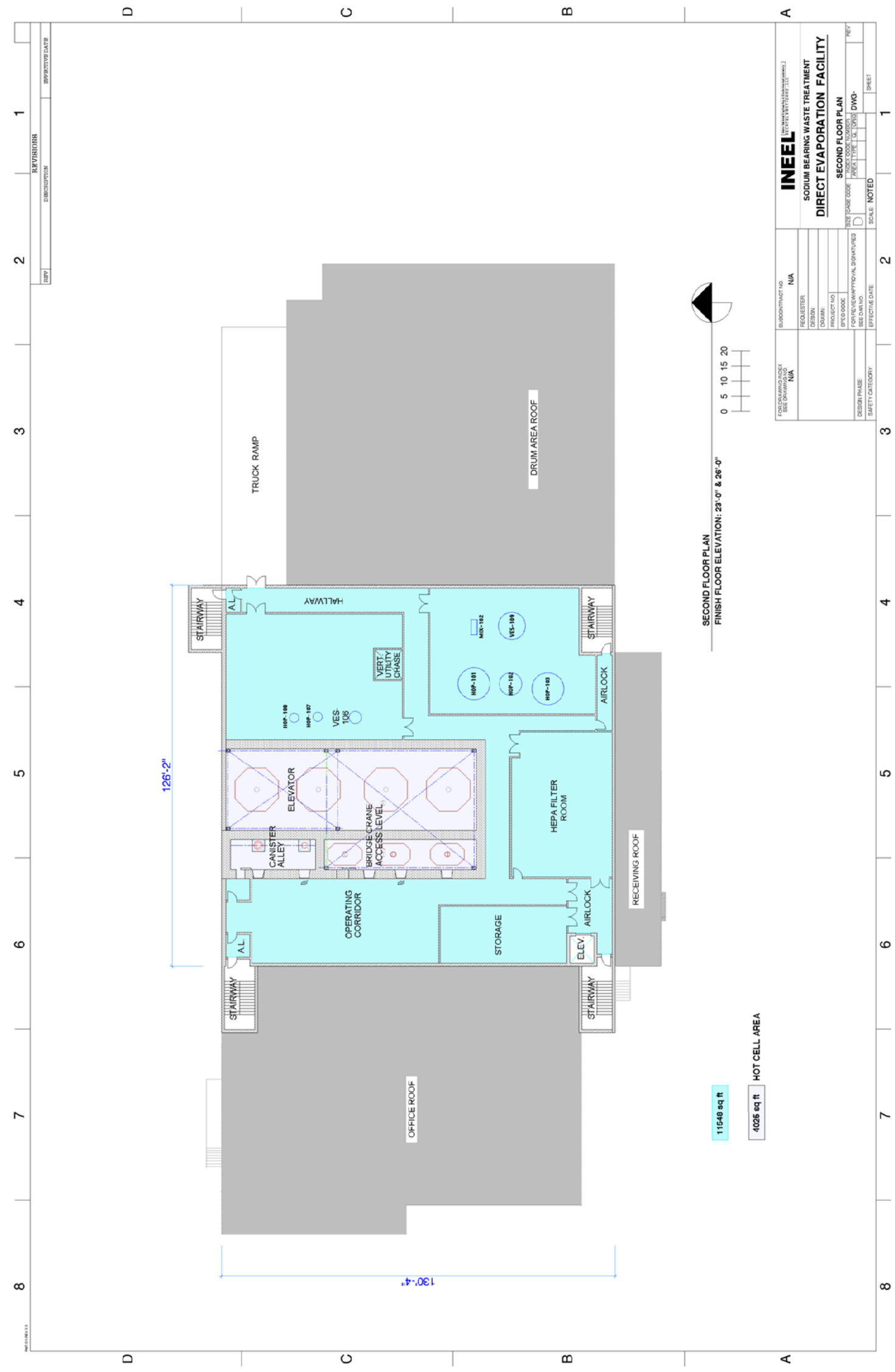




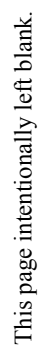




\section{C-10. REFERENCES}

Barden, R. R. (2003), "Technical and Functional Requirements for the Direct Evaporation SBW Treatment Alternative,” TFR-186, draft, May 2003.

Barnes, C. M., and C. B. Millet, (2002b), "INTEC Tank Farm Facility Management Plan," PLN-1112, September 30, 2002.

Barnes, C. M., R. A. Wood, and B. H. O'Brien, (2002a) "Process Design of SBW Treatment Alternatives," Engineering Design File EDF-2373, September 10, 2002.

Barnes, C. M., R. A. Wood, E. B. Packer, and N. A. Chipman, (2003a) "Direct Evaporator Process Condensate Treatment Alternatives Evaluation," Engineering Design File EDF-3356, July 22, 2003.

Barnes, C. M., S. K. Janikowski, and C. B. Millet, (2003b), Feed Composition for the Sodium-Bearing Waste Treatment Process," INEEL/EXT-2000-01378, Rev. 3, September 2003.

Bohn, T. S. (2002), Radiological Shielding Calculations for Screening of Sodium Bearing Waste (SBW) Treatment Alternatives, Rev. 1, INEEL Interoffice Memorandum TSB-20, August 26, 2002.

Boring, M. D., L. L. Fair, V. L. Fowler, and J. D. Hewitt, (1994), Concentration of Melton Valley Storage Tank Surrogates with a Wiped Film Evaporator, ORNL/TM-12768, August 1994.

Clark, M. L., (2003), "Study 18 - Calcine Packaging Facility Layout - Idaho Tank Farm Project Calcination with MACT Upgrade SBW Treatment Alternative," Engineering Design File EDF3281, April 30, 2003.

Griffith, D. L. (2003b), Testing an Agitated Thin Film Evaporator for Solidifying a Mixture of Simulated Sodium-Bearing Waste and Undissolved Solids (Test Series 2), INEEL/EXT-03-01189, November 2003.

Griffith, D. L., R. J. Kirkham, L. G. Olson, W. D. St. Michel, and S. J. Losinski, (2004), Converting Simulated Sodium-Bearing Waste into a Single Solid Waste Form By Evaporation: Laboratory-and Pilot-Scale Test Results on Recycling Evaporator Overheads, INEEL/EXT-04-00172, January, 2004.

Griffith, D. L., S. J. Losinski, B. E. Olaveson, and L. G. Olson, (2003a), Testing an Agitated Thin Film Evaporator for Solidifying Simulated Sodium-Bearing Waste (Test Series 1), INEEL/EXT-0300680, June 2003.

Herbst, A. K., J. A. DelDebbio, R. J. Kirkham, B. A. Scholes, and T. L. Watson, (2002), Idaho Nuclear Technology and Engineering Center Sodium-Bearing Waste Treatment Research and Development FY-2002 Status Report, INEEL/EXT-02-00985, September, 2002.

ICF Kaiser, (1995), Tank Farm Heel Removal Project Conceptual Design Report, RPT-034, February 3, 1995.

INTEC RCRA Interim Status Document - Liquid Effluent Treatment and Disposal Facility: Section A General Description, Interim Status Document ISD-1, January 31, 2002. 
Kimmitt, R. R. (2003), “Feasibility Study for Direct Evaporation of Sodium Bearing Waste,” Engineering Design File EDF-2524, Rev. 0, May 20, 2003.

Kirkham, R. J., S. J. Losinski, B. E. Olaveson, and L. G. Olson, (2003), Direct Evaporation of SodiumBearing Waste: Flask-Scale Testing of WM-189 Simulant, INEEL/EXT-03-0657, July 2003.

Kirkham, R. J., to W. H. Landman, (1998) "Sodium Bearing Waste Solidification by Evaporation", Lockheed Martin Idaho Technologies Company Internal Correspondence RJK-6-98, September 29, 1998.

McCray, J. A., L. G. Olson, and S. J. Losinski, (2002), Investigation of Direct Evaporation and Fractional Crystallization Technologies for Sodium-Bearing Waste Treatment, INEEL/EXT-0201043, September 2002.

McCray, J. A., to D. V. Croson, (1994), "Report on Freeze Crystallization and Evaporation/ Precipitation Testing for Sodium-Bearing Waste Treatment," Idaho National Engineering Laboratory Correspondence JAM-11-94, September 30, 1994.

McCray, J. A., to D. V. Croson, (1995), “Analysis of Sodium-Bearing Waste Treatment Using Evaporation/ Precipitation Technology Concept," Lockheed Idaho Technologies Company Interdepartmental Communications JAM-01-95, February 23, 1995.

Morrell, D. K., and D. T. Clark, (2003), "Mixing Pumps for Homogenizing TFF SBW Liquids and Solids - Calcination with MACT Upgrade SBW Treatment Alternative," Engineering Design File EDF3307, April 24, 2003.

O’Brien, B. H. (2003), "Off-gas Chloride Control for the Calciner MACT Compliance Facility," Engineering Design File EDF-2205, April 3, 2003.

Packer, E. Bart "'Preliminary Assessment of Evaporator Technologies for Direct Evaporation of Sodium Bearing Waste," Engineering Design File EDF-3443, April 8, 2003.

Perry, R. H., and C. H. Chilton, Chemical Engineer's Handbook, $5^{\text {th }}$ ed., 1973, New York:McGraw-Hill.

PRD-166, (1999) “INTEC PEW Chemical Acceptance Criteria Program Requirements Document," Rev. 4, October 1999.

Raman, S. V., A. K. Herbst, B. A. Scholes, S. H. Hinckley, and R. D. Colby, (2003), Solidification of Simulated Liquid Effluents Originating form Sodium-Bearing Waste at the Idaho Nuclear Technology and Engineering Center, FY-03 Report, INEEL/EXT-03-01096, September 2003.

Soelberg, N. R. (2003), "Offgas Mercury Control for the Idaho Tank Farm Project - Calcination with MACT Upgrade SBW Treatment Alternative," Engineering Design File EDF-3292, February 28, 2003.

Wood, R. ,D. Tyson, B. Bonnema, C. Olsen, A. P. Pinto, D. Wendt, S. Reese, and B. Raivo, (2001), Feasibility Study for the Idaho Waste Vitrification Facilities Off-gas Treatment for Sodium-Bearing Waste, INEEL/EXT-01-00995, September, 2001. 
Wood, R., and D. K. Morrell, (2002) "Heel Solids Processing Evaluation for the SBW Treatment Project - Calcination with MACT Upgrade Alternative," Engineering Design File EDF-3049, December 4, 2002. 